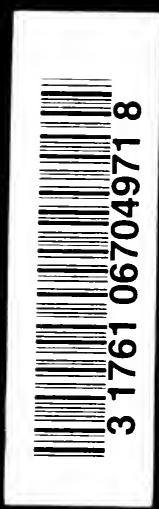

LWNVG:

tonanig

Laskat 





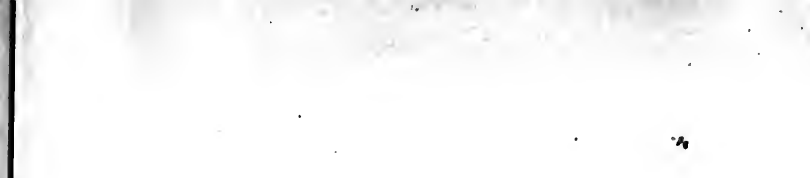

$$
\cdots
$$

. 

E

\section{NEOPLASTIC DISEASES}

A TREATISE ON TUMORS

By

JAMES EWING, A. M., M. D., Sc. D.

Professor of Pathology at Cornell University Medical College, New York City;

Pathologist to the Memorial Hospital

SECOND EDITION, REVISED AND ENLARGED

WITH 514 ILLUSTRATIONS 
Copyright, 1919, by W. B. Saunders Company. Revised, entirely reset, reprinted, and recopyrighted January, 1922

Copyright, 1922, by WW. B. Saunders Company
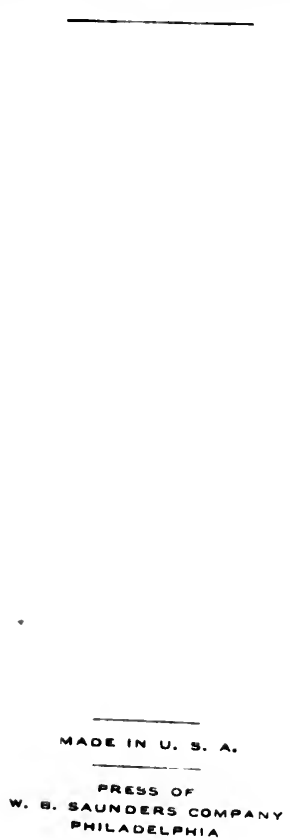
To the memory of

JAMES DOUGLAS

A man of ideas, ideals, and affairs

In token of the deep respect of the author

This work is dedicated 



\section{PREFACE TO THE SECOND EDITION}

THE early call for a reprinting of this work has given a welcome opportunity to revise and enlarge certain chapters and to add many new references and data that have been contributed or collected during the past two years.

Few portions of the work have passed without minor addenda, while the chapters on tumors of bone and on neurofibromatosis have been extensively recast. No reason has appeared for distrusting the reliance on photographs for illustrations, of which thirty-three have been added.

The writer wishes to acknowledge with thanks the great assistance he has received from many sources, through reviews, correspondence, case records, and material, all of which have been gladly received and freely employed.

January, 1922. 



\section{PREFACE}

IT is the object of this work to present within reasonable space and in accessible form the main features of the origin, structure, and natural history of tumors.

Up to a very recent time it has been the prevailing impression that tumors fall into a limited number of grand classes in which the forms occurring in the several organs are so nearly related as to be virtually identical. Hence the practical physician or surgeon has been content to regard all fibromas, sarcomas, or cancers as equivalent conditions without regard to the organ involved, and on this theory to treat the members of each class alike. Upon this theory also it was legitimate to conceive of a universal causative agent of malignant tumors and thus to subordinate many very obvious differences which clinical experience has established in the origin and behavior of different related tumors.

I believe that this point of view has greatly retarded the progress of the knowledge of tumors, and it has been the writer's effort to combat such a conception, so far as present knowledge permits. He has endeavored to analyze the numerous etiologic factors which meet in such diverse fashions in the inception of tumors, to emphasize the general dependence of clinical course upon histologic structure, to trace the histogenesis to the last degree, impressing its essential importance when known, and to enumerate and contrast the more striking clinical features which are often highly characteristic of different tumors.

No one would think of confusing lobar pneumonia with pneumonic plague, although both are examples of acute exudative pneumonitis, but it is quite the rule to identify for statistical studies several equally different forms of mammary cancer. The former diseases are related only as forms of inflammation, the latter only as types of neoplasia. From this point of view it may safely be said that there are more distinct clinical and pathologic entities within the groups of neoplasms than exist outside of them.

While a great volume of information regarding the clinical phenomena of the main forms of tumors is available in special works on medicine, surgery, and the specialties, the task of unraveling their separate varieties, tracing their mode of origin and growth, and establishing the nature of the less common forms, falls to the lot of the pathologist. For the final classification of tumors must depend chiefly on histogenesis and structure. Present-day oncology is chiefly concerned with these topics and the space devoted to them can safely be reduced only when our knowledge is much further advanced.

In spite of several laborious years spent in the task the writer acknowledges disappointment with the results attained in many departments, but can only claim that the effort to present tumors as specific diseases is in the right direction. He first undertook to write a book on the general principles of oncology, but soon found that the significant facts about tumors are not of general application, but are best revealed in the study of special tumor groups or even of special cases.

In the compilation of the work the writer has endeavored to consult with 
due respect all the standard authorities, and as far as possible the original contributions in the literature. The rather extensive bibliographic lists seem necessary for the guidance of the reader who desires complete information and to whom the work is chiefly addressed. The recent rapid increase in original contributions from the United States has made it impossible to do full justice to American literature.

It is a pleasure to acknowledge the valuable assistance received from many friends and colleagues here and abroad. The author acknowledges especially his indebtedness to Messrs. W. B. Saunders Company for undertaking the publication of a highly specialized work. Through the painstaking assistance of Mr. William Dunn it has been possible to rely almost entirely upon photographs for illustrations.

While confessing a deep interest in the theoretic problems which render oncology the most complex and fascinating field in pathology, the chief object and hope of the author have been that by rendering more accessible to English readers the knowledge of tumors he may contribute something toward the reduction of the mortality from cancer.

James Ewing. 


\section{CON'TEN'TS}

\section{GENERAL ONCOLOGY}

CHAPTER I PAGE

Historical. . . . . . . . . . . 17

\section{CHAPTER II}

Definition. Classification. General Pathology................ 25

CHAPTER III

Malignancy and Its Effects On the Organism................ 62

CHAPTER IV

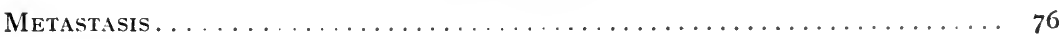

CHAPTER $V$

CHAPTER VI

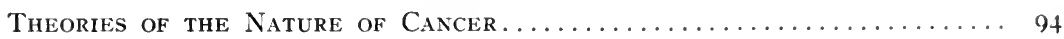

CHAPTER VII

The Special Etiology of Tumors; Trauma.................... 109

CHAPTER VIII

The Parasitic Theory............................... I 3

CHAPTER IX

Experimental Cancer Research....................... 126

\section{SPECIAL ONCOLOGY}

CHAPTER $\times$

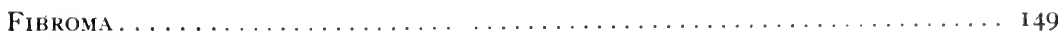


Myxoma

CHAPTER XII

LIPOMA

CHAPTER XIII

Chondroma 185

CHAPTER XIV

OSTEOMA 196

CHAPTER XV

Mroms. 203

CHAPTER XVI

ANgIOMA 223

CHAPTER XVII

SARCOM. 240

CHAPTER XVIH

Clinical Types of Sarcoma 249

CHAPTER XIX

SARCOMAS OF BONE AND BONE-MaRROW. 269

CHAPTER XX

ENdothelioma 299

CHAPTER XXI

LMMPHOMA AND LYMPHOSARCOMA. 348

CHAPTER XXII

TUMORS OF THE BraIN 393

CHAPTER XXIII

Tumors of Nerve Trunks. 427

CHAPTER XXIV

Tumors of Spinal Cord and Membranes. 436

\section{CHAPTER XXV}

Gexeral Pathology of Epithelial Tumors. 
CHAPTER XXVI

Epithelial and Other Tumors of the Breast.

\section{CHAPTER XXVII}

Cancer of Uterus, Vulva, Vagina.

\section{CHAPTER XXVIII}

CHORIOMA (CHORIONEPITHELIOMA) 566

CHAPTER XXIX

Cysts and Epithelial Tumors of the Ovary................. 582

CHAPTER XXX

The Ovarian Teratoma.............................. $61_{3}$

CHAPTER XXXI

Carcinoma of Stomach. . . . . . . . . . . . . . . . . . . . . . 625

CHAPTER XXXII

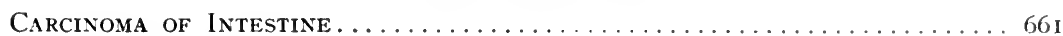

CHAPTER XXXIII

Epithelial Hyperpiasta and Tumors of Liver.................... 675

CHAPTER XXXIV

Tumors of Pancreas............................... 702

CHAPTER XXXV

Maxillary Tumors of Dental Origin...................... 707

CHAPTER XXXVI

Epithelial Tumors of the Salivary Glands............... 725

CHAPTER XXXVII

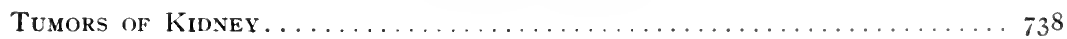

CHAPTER XXXVIII

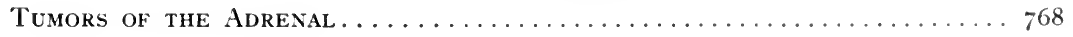

\section{CHAPTER XXXIX}

Tumors of Prostate.

CHAPTER XL

Tumors OF TEstis. 
Tumors of Luxg.

CH $\mathrm{PTER}$ XI.II

Epidermoid Carcinoma.

\section{CHAPTER XLIII}

Epidermoid Cincisoma (Continued)

\section{CHAPTER XLIV}

Melanoma . . . . . . . . . . . . . . . . . 87 I

\section{CHAPTER XLV}

TUMORS OF THE THYroID.

\section{CHAPTER XLVI}

The Thymes aNd Its Tumors 912

\section{CHAPTER XLVII}

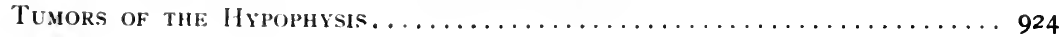

CHAPTER XLVIII

The Pinfal Giand and Its Tumors 948

\section{CHAPTER XLIX}

Teratology

BibliogR.I PHY 987

INDEX. 


\title{
NEOPLASTIC DISEASES
}

\section{GENERAL ONCOLOGY}

\author{
CHAPTER I
}

\section{HISTORICAL}

The Ancients knew cancer well. They treated it by excision and by a variety of escharotics, including the Egyptian arsenical ointment. Cancer is mentioned in the Papyrus Ebers (B. C. I 500) and in the oldest remnants of the literature of India and Persia.

Hippocrates (B. C. 460 to 375 ) received from earlier days a considerable body of descriptive facts regarding cancer of the skin, breast, uterus, and internal organs, and he first employed the terms $x \alpha \rho \% \gamma_{j} \omega s$ for all indolent

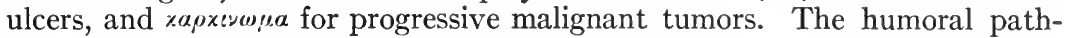
ology then disseminated conceptions of the origin of cancer. Deficiency or excess of blood, mucus, or bile formed the basis of all disease. Herodotus mentions that Democedes (B. C. 520) cured Atossa, the daughter of Darius Hystaspis, of breast cancer, and Hippocrates burnt out a carcinoma of the neck, the earliest record of diathermia.

Celsus (B. C. 30 to A. D. 38 ) distinguished several gross varieties of cancer, and he excised breast cancer, advising against removal of the pectoralis major. Treatment by charcoal was employed by Cato, and a variety of crude internal remedies are mentioned by Pliny.

Galen (A. D. I3I to 203), the founder of experimental physiology and pathology, failed to make any significant advance in the conception of cancer, but the presentation of the humoral doctrine of atra bilis in his writings formed a scripture which dominated medical thought for more than a thousand years. Swellings were secundam naturam (gravid uterus), supra naturam (callous formations), or preter naturam (true tumors). The pneuma, composed of solid parts and four fluids, blood, mucus, yellow and black bile, ruled the processes of the body. Cancer developed from the concentration of black bile.

Suppression of menses and hemorrhoids, preventing the discharge of black bile, were chiefly responsible for cancer, which appeared where the bile gravitated in face, lips, breast, etc. No modern writer has been able to reflect the thought of that period or to explain the firm entrenchment of the crude humoral theories. Since capable logic could not have been lacking, one must suppose that religious and esthetic tendencies in the race unfitted the human mind for natural thought regarding the structure and functions of the human body. For internal cancer, of which little was known, a diet chiefly vegetable was recommended. Walnuts were specifically forbidden.

Diagnosis rested chiefly on the cause of the disease, while treatment by excision, ligation of vessels, and cautery was comparatively successful. 
Leonides of Alexandria (A. D. I80) broke away from Hippocrates' conservatism, dissected out breast cancer extensively, cutting through healthy tissue with knife and cautery, and approached closely to the modern technics of this operation.

In the Byzantine period (475 to I 500) considerable progress was made in the description of various tumors. Paulus of Ægina (625 to 690) separated chronic metritis from uterine cancer. In Arabia, Avicenna (980 to I037) introduced the internal use of arsenic, and Avenzoar (1070 to 1 r62) employed the esophageal sound and the nutrient enema. The $3_{3}$ th to $5_{5}$ th centuries were the Dark Ages for medicine, and especially for surgery. At the Council of Tours (II62), and later, at the Fourth Lateran Conference, the ban of the Church was laid upon the free pursuit of surgery. The fact that surgery could be studied only in private and practised only by nomads was possibly both cause and effect of these ecclesiastic orders. At the Universities of Salerno and Montpellier (II50) the Church designated what books should be used. The Monks jealously dominated medical speculation and concerned themselves mainly with translations of the classics. Walsh would defend the influence of the Popes of this period on science, and refers to publications on anatomy and surgery from Catholic schools where these branches of learning were undoubtedly cultivated. Lanfranchi, of Lyons, an exile from Mailand, established scientific surgery in France (I 290) with two notable works. He strongly urged the radical operation for cancer with free bleeding of the affected part. Henri de Mondeville and Guy de Chaulliac (I300 to I368), physician to Clemens VII, wrote text-books on surgery, excised cancer with the knife, and developed to a high degree extirpation by caustic arsenic. Yet the Medicine of the Monks must be rated as unfruitful and as incompatible with the progress achieved shortly thereafter in the Renaissance.

The Renaissance ( $I_{500}$ to I 700), bringing the discovery of the printing press and the circulation of the blood (Harvey, I6 28), greatly facilitated the spread of knowledge and aided the more accurate diagnosis and better treatment of cancer, but threw little light on etiology. Andreas Vesal (I 5I4 to I564) began the attack on many of the concepts of Galen, identifying deepseated with ulcerating cancer. Fabricius (I537 to r6I9) separated many inflammatory swellings from cancer, warned rigidly against incomplete removal, extirpated the uterus, and experimented with internal remedies.

Marcus Aurelius Severinus ( 1580 to I $_{56} 6$ ) described myxosarcoma, separated cancer from benign tumors of the breast, and extirpated the axillary nodes.

In Prag, Sennert ( 1572 to 1637 ), and in Lisbon, Lusitanus (1642) first claimed that cancer was contagious, a doctrine which prevailed extensively and with little dissent. Paracelsus (I4I3 to $\mathrm{I}_{54 \mathrm{I}}$ ) stands out as the first successful opponent of Galen's theory of atra bilis as the cause of cancer. He claimed that the disease was due to mineral salts in the blood. He seemed to think that cancer developed where various animal salts became concentrated and sought an outlet. At this period it is interesting to note that the decline of Galen's authority and distrust of even his crude theories of etiology led to complete demoralization in the treatment of cancer, encouraged great abuse of arsenic and other internal and external remedies, permitted the faith cure career of Queen Elizabeth $\left(\mathrm{I}_{602}\right)$, and developed many fantastic theories regarding the nature of cancer.

The Lymph Theory.-In the I 7 th century Galen's doctrine was completely demolished by the discovery of the circulation by Harvey (I628), of the lymph-vessels by Olens $\left(\mathrm{I}_{52}\right)$, and of the red blood-cells by Malpighi
(I66I). 
Malpighi used the microscope which had been in existence since I 592 . The black bile was nowhere to be found, but everywhere was blood and lymph. Lymph coagulated and foamed on boiling, hence cancer was composed of lymph varying in density, alkalescence, or acidity, and in malignant tumors fermenting and degenerating. Louis ( $\mathrm{I}_{72}$ to $\mathrm{I} 7 \mathrm{9}^{2}$ ) distinguished gelatinous lymph -(goiter) and albuminous lymph (scirrhus). LeDran (I685 to I770) studied cancer by many autopsies, emphasized the local nature of cancer of the skin, and the internal origin of breast cancer. He conceived that if a drop of cancer lymph passed the adjacent nodes it contaminated the entire system.

Astruc (I684 to I766) separated cysts from true tumors, showed that scirrhus and soft cancer were of the same nature, pointed out important differences in prognosis of different types of carcinoma, and by incineration proved that cancer and muscle tissue contained the same salts. Yet he likened the growth of cancer from lymph to the heating and swelling of gypsum in water.

Morgagni ( $\mathrm{I} 682$ to $\mathrm{I} 772$ ) established the importance of the pathological anatomy of cancer, describing many internal tumors studied at autopsy, and separating gumma, struma, exostosis, and lipoma from cancer. He distrusted, without replacing, the lymph theory.

A notable event in the history of cancer research was the essay of Peyrilhe (I735 to I804) submitted to the Academy of Lyons in answer to the question, $Q u$ ' est ce que le cancer? This was the first systematic investigation of the whole subject and dealt with the cancer toxin, the nature of the disease, the manner of growth, and the treatment.

He spoke of the local origin, the production of a specific virus from degeneration of the tumor, and the development of cachexia from this source.

$\mathrm{He}$ endeavored to demonstrate the virus by injecting tumor emulsion beneath the skin of a dog, but an abscess resulted and his servant drowned the animal. He treated ulceration effectively with the newly discovered carbonic acid. His imagination was satisfied by the Cartesian lymph theory of the times.

In Germany the $I 7$ th century was occupied by the exploitation of many personal views of the origin of cancer and other diseases.

Chemical conceptions held sway, and cancer was attributed usually to excess of acid, to be treated by alkali (Helmont, Ettmüller).

Stahl (I660 to I734) supposed that stasis and thickening of the blood were the essential factors. Hoffmann held that life and health depended on normal movements of the tissues, cancer and other diseases resulting from atony, stasis, and abnormal fermentation of blood and lymph. He constructed his anodyne as a panacea to restore tissue tone.

In England, John Hunter's (I 728 to I 793) conception of the lymph theory represented a distinct advance toward the cellular pathology. He held that tumors grew from the coagulable lymph constantly thrown out of the blood, that tumor tissue like normal tissue was nourished by the organism, and developed according to the same biological laws. In I802 The Society for Investigating the Nature and Cause of Cancer was formed in London, and formulated the problems of the disease as they stand today. The society dissolved in 1806 .

The pathological anatomy of malignant tumors chiefly interested the English observers, and Hey (I736 to I8I9) described in detail the structure of certain vascular tumors which he found to be composed of organized bloodfluid and a large proportion of lymph, and for which he employed the term "fungus hematodes." Wardrop, I809, described in great detail miscella- 
neous tumors of this gross type, attempting to separate them from cancer. Many of his cases occurred in children, some involved the eye, and one arose from a wart.

Abernethy, I 804 , attempted to define the old term "sarcoma" by applying it to various soft tumors, and he called Hey's fungus hematodes medullary sarcoma. Maunoir, 1820 , showed the beneficent result of anatomical study by announcing that each tumor is the result of a morbid change of the fluid or tissue from which it arises, and retains the original characters of this tissue. He employed many crude chemical and physical tests to support this claim.

The close attention then being given to clinical data led Pott ( 1775 ) to recognize and describe chimney-sweeps' cancer and to point out its etiology. The English contributions of this period were completed by Home, who, using the microscope, described and depicted rounded bodies which he regarded as lymph corpuscles.

In France, Bichat's studies of tissue structure opened a new era with the appearance of his Anatomie generale in ISOI. He first distinguished the stroma from the parenchyma of tumors, deriving the former from the originating tissue and the latter by proliferation from the stroma. Without reliance upon the microscope he regarded the stroma as cellular and conceived of its growth by proliferation.

Following Bichat, Laennec made a systematic study of the gross features of cancer in thin slices, and introduced the term "encephaloid" for the soft parts. Dupuytren attempted to prove the infectiousness of the disease by ingestion and intravenous inoculation of cancer tissue. Bayle and Cayol argued in favor of the constitutional nature of the disease and fully pointed out the difference between chronic mastitis and cancer.

Broussais' doctrine that cancer was the sequel of recurrent inflammation had made some influence on the thought of this period. Based on chemical observation, his claim, that cancer never arises in normal tissues but only after inflammatory alterations, found many adherents and is of interest in connection with later theories.

Lobstein used the term "plastic lymph" and formulated the view that the tumor-forming lymph was not under control of the biological laws of the organism. Recamier studied especially the infiltration of cancer, observed the destruction of veins, and applied the term "metastases" to nodules in the brain in cases of mammary cancer. He recognized the importance of supernumerary organs and nevi as sources of cancer. Andral reflected the confusion of ideas then prevailing by offering his theory that products of secretion became organized into cancer. Cruveilhier regarded cancer as a malignant degeneration which, like inflammation, affected all the organs. Its chief pathognomonic feature was the cancer juice exuded on pressure. Andral thought tumor masses floating in the veins were derived from the fibrin, but Velpeau, in a case of intravenous tumor, searched the blood in vain for cancerous elements. Such questions as the identity of encephaloid or soft cancer with brain tissue were hardly settled. Tubercle was commonly confused with cancer and gummata imperfectly distinguished from it. Cancers were classified upon crude gross characters, as in Jager's Handworterbuch, Leipsic, $18_{37}$, where they were divided into four types, hard, soft, pigmented, and blood-cancer.

Thus, during the sway of the lymph theory, English and French students while adding important contributions to the descriptive history and gross pathology of malignant tumors, failed to pass beyond the limits of the prevailing theoretical conceptions of the time. It was the great period of individual surmises which in some instances, notably with Hunter, Home, Lobstein 
and Recamier, approached closely toward and prepared the way for modern conceptions.

In Germany, Richter, Walther, and others engaged in the current discussions without adding essentially new data.

It was the general conception that the elements of cancer were fluid and traveled in the veins.

Great significance was attached to the observation of tumor masses in the vessels. A special variety of "blood-cancer" supposed to come from irritating elements was described by Langstaff, I8I 7 , and Carswell, I834.

Histological Period.-With the construction of the achromatic microscope in Paris, I824, a new era opened in cancer research. Wolf finds that the first fruitful studies of the structure of vegetable and animal tissues by this instrument were made by Raspail in 1826 , who showed that the growth of tissues resulted from the multiplication of cells. He clearly stated the doctrine of the cell, finding that tissues were composed of microscopic vesicles. The structure and growth of fat tissue he described in detail. Collard, 1828 , also described rather clearly the stages in the embryonal development of tissues, assuming, however, that the cells originated from plastic lymph. Schwann, I838, established this doctrine of cellular structure as a universal principle and discovered the nucleus and nucleolus of the cell.

In the same year J. Müller published his classical study of malignant tumors. He found them all to be composed of groups of cells, each containing nuclei and nucleoli. The various current types of cancer he found to be distinguished only by different proportions and groupings of cell masses and stroma. Certain elongated or racquet-shaped cells, "geschwanzten Korperchen," he regarded as on the way to fiber formation, but not as specific cancer elements, which he was unable anywhere to detect. Hence his diagnosis of cancer rested on clinical signs and the anatomical grouping of the cells. He held the interesting view that cancer developed not from normal tissue, but from germ cells which as a "seminium morbi" lay scattered between the tissue elements.

In regard to the origin of the cells it was held that most of them developed from the plastic lymph or blastema by a process of budding. Others resulted from division of nuclei and cell body, or by the transformation of intracellular blastema lying in spaces within the cell body (Virchow's Brutraume). The originating tumor-cells were not derivatives of the normal tissue cells, but came from the seminium morbi, hence there were urgent demand and search for specific characters in the tumor-cells. Lebert especially described such specific cells and designated as pseudocancer all tumors, as rodent ulcer, which failed to contain them. Following this principle, Hannover carefully described the group of tumors arising from stratified squamous epithelium and separated them from cancer under the term "epithelioma."

Hannover believed that cancer-cells circulated in the blood and produced metastases as pus-cells produced pyemia.

The doctrine of the specific cancer-cell now became the chief topic of discussion and was supported by many writers. It was opposed by Bruch, who added many details to Müller's work; by Virchow, who found the tailed corpuscles in the normal bladder epithelium; and by Velpeau, who, with Hannover, established the microscopical diagnosis of tumors. Endogenous cell formation then took the place denied the "tailed corpuscle" as the pathognomonic sign of cancer, Bruch, Virchow, Remak and many others accepting the importance of this supposed type of cell growth. The significance of tumor stroma also attracted new attention, especially from Rokitansky, who explained many of the gross features from variations in this element. He also studied 
the secondary degenerations of cancer tissue, including inflammation, necrosis, and saponification, and considered the possibility of a spontaneous cure by these processes.

In spite of their very careful histological studies of tumor tissue in the fresh condition all the authoritative writers of this period including Virchow were led to believe in the origin of cancer from a fluid blastema. Cancer was defined as an organized exudate from the blood with overnutrition and overgrowth. Much controversy arose concerning the various types of blastemas supposed to exist and the changes in the blood from which they were all necessarily derived. Vogel thought there were as many blastemas as tissues, different tumors arising in different tissues according to the type or analogy of the tissue involved (law of analogous blastemas). These views led directly to the conception of cancer as a constitutional dyscrasia (Rokitansky), a belief that gained wide acceptance and seemed to be supported by the anemia of the disease. The help of the chemists was sought to separate from the blood the different blastemas, and Führer was able to distinguish albuminous, chondrinous, and glutinous varieties, each of which gave a suitable color reaction with nitric acid, and which were offered as sources of the corresponding tumors. The idea that exuded elements of the blood could become organized into cancer was vigorously opposed by Cruveilhier, who urged that extravasated blood never became organized, but had lost all claim to vitality. Cancer could therefore develop only in the vessels. This reasoning led Langenbeck to study cancerous masses in the veins which he found to be composed of fibrin, pus-cells, and cancer-cells. He therefore drew the important conclusion that cancer-cells possess a remarkable capacity for independent existence and that they were carried through the veins, producing secondary tumors or metastases. After Peyrilhe, Alibert, I806, and Dupuytren, I8I7, produced only suppuration by intravenous injections of tumor emulsion, but Langenbeck, Follin and Velpeau claimed to have observed tumor nodules in the lungs of dogs receiving intravenous injections. These results, although failing to receive confirmation, seemed then to prove not only the origin of metastases, but the contagiousness of cancer. Yet Bruch at once interpreted them as transportation of cancer-cells and not as infection. He held the lymph-vessels to be the chief channels of transport and with Meckel described backward transport in lymphatics.

While still burdened by the blastema theory of origin the study of cancer had succeeded up to 1860 in rather accurately describing and classifying the main classes of tumors chiefly according to microscopical structure. The description of the benign tumors, the existence of various types of carcinoma, the malignancy of epithelioma and the separate position of sarcomas, were generally accepted facts. Correct conceptions of the histogenesis, however, were impossible until Virchow founded the cellular pathology upon the doctrine of Omnis cellula e cellula. There had been several opponents of the idea that cells could be formed from exuded lymph, notably Cruveilhier, while Remak at the same time with Virchow claimed that cells grew exclusively from other cells by endogenous reproduction; but Virchow applied the new principle rigidly to all departments and especially to the origin and growth of tumors. Coincidently with this memorable service he fell into two grave errors. He failed to correctly interpret the deceptive evidence of endogenous cell formation and he was led to believe that cancer-cells originated from connective-tissue cells. The latter error he never fully relinquished and possibly on this account his monumental work "Die krankhaften Geschwülste" was never extended over the field of cancer.

Remak immediately attacked the connective tissue theory which had 
become very popular, but which conflicted with the significant principle of the immutability of the three separate germ layers which he with His had already established. Remak insisted that epithelial cells could arise only from epithelium. The appearance of such cells in abnormal places he referred to the misplacement of embryonal cell groups.

Virchow's authority, however, prevented the general acceptance of Remak's views for many years, until the appearance of Thiersch's famous monograph on epithelial cancer.

Although many authors opposed the theory of the connective origin of cancer, and Meckel in I857 had traced the origin of a buccal tumor directly from the lining epithelium, it remained for Thiersch to present convincing evidence of the invariable derivation of epithelioma from lining epithelium. $\mathrm{By}$ improvements in hardening, cutting and staining the tissues in serial sections he traced the growth of several epitheliomas from the Malpighian layer or glandular structures of the skin and demonstrated the principle, omnis cellula e cellula ejusdem generis. He also placed great importance upon previous changes in the epithelial connective tissue as precipitating the downward growth of epithelium.

By a process of involution associated with diminished nutrition, functional capacity, and mechanical resistance the histogenetic equilibrium between stroma and epithelium became disturbed and overgrowth of epithelium resulted. This was the first competent physiological conception of the pathogenesis of cancer and with it Thiersch introduced the modern era of our knowledge of the nature of the disease. Waldeyer extended Thiersch's observations to the internal organs and traced the origin of cancer of stomach, liver, and kidney to the epithelial cells of these organs. He first described the isolation of these cells by indurated connective tissue and held that tumors developed from these isolated cells. The formation of secondary tumors he demonstrated to be the result of continuous growth through blood- and lymph-vessels as well as of cell emboli.

The acceptance of the epithelial theory was briefly delayed by the studies of Recklinghausen and Koster, who brought forward evidence to show that many cancers arose from the endothelium of lymph spaces. The outcome of this controversy was the establishment of the group of endothelioma. That true cancer has no connection with lymphatic endothelium was shown by Carmalt, who in Waldeyer's laboratory shook thin sections of fresh cancer in silver solution, thereby removing the cancer-cells from the lymph spaces and leaving the silvered endothelium intact. Yet Virchow's influence still predominated and led to the assumption that cancer developed from almost any mesoblastic cell, as from sarcolemma (Popper), perimysium (R. Volkmann), cartilage (Weber), vessel wall (Gussenbauer), and leukocytes (Classen, Rollett).

Through the untiring industry of himself and his pupils Waldeyer slowly forced the acceptance of his views of the origin of cancer. Billroth slowly abandoned much of his early allegiance to the connective tissue doctrine. It was of great assistance when the regeneration of epithelium over wounds was shown to advance from the edges of intact skin. By the minute study of a large number of cancers of many varieties Waldeyer eventually established the principle of the exclusive epithelial origin of cancer and of its growth from its own resources, and demolished the former belief that normal tissues could become transformed into cancer.

The classification of tumors upon the histogenetic basis was accomplished with the data furnished by the studies of this period. The theory of a cancerous dyscrasia necessarily lost ground with the demonstration that the 
disease has a local origin and that secondary tumors arise from transported cells. Likewise it became evident that the parasitic theory which had enjoyed a general acceptation must be set aside as an inadequate explanation of the new facts of tumor origin and growth.

Further than this the cellular pathology seemed incapable of carrying progress in the etiological explanation of cancer. Irritation, trauma, and infection seemed to be connected with the origin of the disease, but the obscure nature of the relation of any of these factors was fully recognized.

The last decades of the nineteenth century were thus occupied with the detailed study of the morphology of tumors, the separation of varieties of the disease, the elucidation of histogenesis, and the writing of the natural history of malignant diseases. The twentieth century opens as the experimental era with the systematic study of tumors throughout the animal kingdom, and it seems likely to become noteworthy as the period of specific etiological investigation which promises to widely separate many neoplastic diseases formerly held to be closely related. It may thereby prove to be the era of successful therapeutics and prophylaxis. 
CHAPTER II

\section{DEFINITION. CLASSIFICATION. GENERAL PATHOLOGY}

Definition.-A tumor is an autonomous new growth of tissue.

Among most authors there is virtual agreement regarding the essential parts of the definition of tumors. v. Rindfleisch says briefly: "A tumor is a localized degenerative excess of growth." Ziegler defines a tumor as a new growth of tissue which apparently originates and grows spontaneously, possesses an atypical structure, does not subserve the uses of the organism, and reaches no definite termination of its growth. According to BirchHirschfeld, tumors are progressive new growths of tissue arising spontaneously from cells of its own, and separated from normal tissue both in morphology and in function. Ribbert states that tumors are new growths of tissue, self-centered, largely or wholly uncontrolled by the organism which supplies only their nutrition, with a structure never entirely normal, and reaching no definite termination of growth.

Lubarsch includes among tumors all spontaneously arising tissue growths which, while typical in form, differ in histology from the originating tissue, and in spite of organic connection with the body pursue an autonomous course rarely to the benefit of the whole body. Borst emphasizes in the tumor process the unknown etiology, the local overgrowth, the peculiar autonomy, the purposeless and endless course, and the atypical morphology and biology of the tumor product. Adami accepts White's descriptive definition: "A tumor proper is a mass of cells, tissues, or organs, resembling those normally present but arranged atypically. It grows at the expense of the organism without at the same time subserving any useful function."

I believe with Prudden that beyond the autonomy of tumor growth it is difficult to add any element to our definition which may apply to all true blastomas. The more descriptive definitions are doubtless more readily applied but with increasing knowledge the unknown etiology, progressive growth, and even the atypical morphology, may become less significant, while the physiological conception of autonomy grows more substantial. Certain obvious features of tumor growth emphasize the importance of their autonomy.

The nature of tumor growth is different from that of the normal tissues and from inflammatory hyperplasia, and is something new and foreign to the organism in which it occurs.

In most tumors growth is progressive, and no natural termination is realized, the tumor growing most rapidly at the death of the patient. Yet the progressive quality is not invariable, as some tumors spontaneously regress; others, as scirrhous cancer, long remain practically quiescent, and others degenerate or necrose or become extruded, while fibrosis overtakes a few.

The growth of tumors is new to the organism in that it is usually expansive, emanating from an isolated group of cells and pushing contiguous tissue before it. Such tumors are more or less encapsulated, appearing as isolated or parasitic organoid structures. In some tumors the growth is infiltrative, single cells or cell groups pushing their way through and destroying adjacent tissues. In many tumors growth is extensive, beginning in a small focus and gradually precipitating the contiguous normal cells into the tumor process. 
Tumor-cells acquire new and greatly increased powers of growth often exceeding those possessed by any parallel normal tissue.

Ribbert claims that tumor-cells do not reveal any abnormal capacity for growth since these are determined in the ovum, but merely exhibit their innate capacities because the restraints to growth have been lost. It is true that the organized regenerative properties of tissue cells are determined in the fertilized ovum, but the power of multiplication without organization is subject to great variations from the environment, and observation and experiment show that tumor-cells have acquired in this sense abnormal capacity for proliferation. The growth of tumor-cells is peculiar in that it proceeds under abnormal conditions when the cells have been separated from their natural connections, and even when they are transferred to other animals of the same species. In transplanted tumors the cells often grow with increased activity, when normal cells rapidly necrose or undergo slow regression, or adjust themselves to the organization. Thus in many particulars the growth of tumors differs from the organized regeneration of normal tissues.

These physiological peculiarities will later be considered in detail.

\section{CLASSIFICATION AND NOMENCLATURE}

There are three obvious and useful plans for the classification of tumors: histological, regional, and etiological.

I. A thorough etiological classification of tumors cannot with our present knowledge be accomplished with sufficient detail for most practical purposes. Yet many useful terms, which have more or less etiological significance, are employed to designate tumors. Most tumors are post-natal in origin; many are congenital, as the multiple chondromas of the spinal column, sarcoma of the kidney, and angioma of the skin.

There are some notable tumors which show a striking hereditary character, as glioma of the retina. A large class of tumors of many varieties are apparently of traumatic origin. Partly etiological is the grouping of tumors into adult and embryonal, arising from adult or embryonal cells and reproducing adult or embryonal cells and structures.

The list of known specific causes of tumors is small but enlarging, including the cancers of pipe-smokers, chimney-sweepers, and workers in paraffin, $x$-ray cancers, Kangri cancer and that of chewers of betelnut among Africans. Some tumors probably result from exaggerated response to functional stimuli, as the thyroid tumors of fish; others from prolonged mechanical, chemical or thermal irritation; some are closely related to tuberculous, syphilitic and other infectious processes. As knowledge advances it seems probable that a much wider extension of etiological classification along these and other lines will be accomplished.

2. The regional classification of tumors is of great practical utility, but as a rule it only deals with superficial characters of neoplasms and usually resolves itself into a grouping of distinct histological varieties occurring in different organs. Yet there are exceptions to the rule. Hypernephroma is a regional term referring to tumors chiefly of the head of the kidney, but including several histological and structural types. Multiple myeloma varies in structure, but is practically the sole primary tumor of bone-marrow. Teratoma of the testis and adamantinoma of the maxillæ are almost the only tumors arising in these regions and both cover such a wide variety of structures that it is convenient to employ regional terms to designate such tumors. Moreover, the marked peculiarities in the origin and course of similar histological tumors in different organs often render it advisable to emphasize 
the organ involved rather than the structure of the tumors. The morphologist speaks of cancer of mamma, larynx, uterus, etc., as one and the same disease, while the clinician properly conceives of mammary cancer, laryngeal cancer, uterine cancer, etc., as quite distinct clinical entities. There is much practical value in emphasizing the regional point of view, and as knowledge advances it may well prove that the strictly histological grouping of many tumors is based on structural resemblances which are less significant than are now supposed.

3. The histological structure of tumors offers the simplest means of classification.

A tumor receives a name and position according to the tissue which it most resembles. Yet this plan meets difficulties at once and applies readily only to simple and benign tumors. Many malignant tumors do not resemble any normal tissue and terms drawn from gross pathology, sarcoma and carcinoma, have been drafted to designate the microscopical structure of the two main classes of tumors.

Moreover, to name a tumor according to its microscopical structure often conveys very little information about it.

Tumor nomenclature aims to be more specific and there is a constant tendency to replace histological with histogenetic terms wherever possible. The main classes of tumors are therefore named from their histology, while the specific forms are designated, if possible, by their histogenesis, as neurofibroma, adenoma sebaceum, etc.

Where structure varies, histogenesis dominates the nomenclature, as with adamantinoma, hypernephroma, etc.

\section{Histological Classification}

GROUP I.-TYPE: CONNECTIVE TISSUE

(a) Fibroma, composed of connective tissue.

(b) Chondroma, composed of cartilage.

(c) Chordoma, composed of tissue of chorda dorsalis.

(d) Osteoma, composed of bone.

(e) Myxoma, composed of mucous tissue.

(f) Lipoma, composed of fat tissue.

(g) Angioma, composed of blood-vessels.

(h) Lymphoma, composed of lymphatic tissue.

(i) Sarcoma, a cellular tumor composed of anaplastic tissue of any of the above types.

GROUP II.-TYPE: MUSCLE TISSUE. MYOMA AND MYOSARCOMA

(a) Leiomyoma, composed of smooth muscle tissue.

(b) Rhabdomyoma, composed of striated muscle.

GROUP III.-TYPE: THE ELEMENTS OF THE NERVOUS SYSTEM

(a) Neuroma, composed of nerve-fibers.

(b) Neuroma ganglionare, composed of nerve-fibers and ganglion cells.

(c) Glioma, composed of glia tissue.

(d) Neuro-epithelioma, composed of neuro-epithelium. 


\section{GROUP V.-TYPE: EPITHELIUM, PAVEMENT OR GLANDULAR}

(a) Papilloma, a tumor of pavement epithelium, with supporting tissues in normal arrangement.

(b) Adenoma, a benign tumor of glandular epithelium, with supporting tissue, in normal arrangement.

(c) Epithelioma, or epidermoid carcinoma, epithelium in atypical arrangement.

(d) Carcinoma, a tumor of glandular epithelium in atypical arrangement.

\section{GROUP VI.-TYPE: COMPLEX TISSUES}

(a) Simple mixed tumors, composed of more than one type of neoplastic tissue - named according to composition: as chondro-epithelioma, adenosarcoma.

(b) Teratoma, composed of tissues and organs of one, two, or three germinal layers, as monodermal, bidermal, or tridermal types.

(c) Embryoma, composed of tissues from three germinal layers in more or less orderly imitation of a fetus.

The variable structure of many simple tumors requires complex terms for the accurate designation of structure. A fibroma with many'neoplastic blood-vessels is called an angiofibroma, while a tumor chiefly composed of blood-bessels with much new connective tissue is called a fibro-angioma.

A sarcomatous quality is indicated as fibro-, chondro-, or osteosarcoma. So fibro-adenoma and adenofibroma indicate predominance of one or another structure. Histioid tumors are composed of a simple tissue, organoid growths reproduce the structure of organs. Homologous tumors resemble the tissue in which they arise, as adenoma mammæ; heterologous tumors present a structure foreign to the tissue in which they grow, as chondroma mammæ.

A strictly embryologic classification of tumors presents many advantages over others, but for good reasons has never gained general adoption.

The early segnenting embryo first consists of a mass (morula, blastula) of large undifferentiated cells (blastomeres). Soon these cells form two distinct layers, epiblast and hypoblast, both of which have the characters of lining membranes, the pavement quality being more marked in the former. Next the hypoblast gives rise to a group of cells forming the mesoblast and lying intermediate between the other two layers. The mesoblast cells lose their pavement qualities and appear in an undifferentiated cell mass, but, later, certain of its cells grow out between the epiblast and hypoblast in modified pavement form and enclose the body cavity (cœlom). Thus the mesoblast comes to possess a lining portion, mesothelium, and a pulp portion, mesenchyme.

The cœlom with its mesothelial lining eventually becomes the pleura, pericardium and peritoneum. From a portion of the mesothelium is also developed the parent tissue of the striated muscles, and later modified mesoblastic cells form the lining cells of the blood and lymphatic vessels. Thus the lining cells of the great serous cavities, and the vessels, and the striated muscles, are derived from mesoblastic cells which have at one time lost their pavement quality while merged in the mesoblast.

At the same period with these changes, the epiblast proliferates in the region of the primitive groove and forces inward a mass of cells, which become isolated and modified, going to form the central nervous system. Coincidently, a portion of hypoblast retaining some of its pavement qualities arises to form the notochord.

Adami points out that these early embryological processes lead to the development of two main classes of tissue which permanently retain their early characters, viz.: lining membrane tissues and pulp tissues, and which he proposes to call lepidic ( $\lambda \epsilon \pi$ is, membrane) and hylic ( $\dot{v} \lambda \eta$. crude matter). Lepidic tissues are composed of groups of specific rells which are not penetrated by blood-vessels and possess no intercellular stroma. The epiblast and hypoblast are primary, the mesothelium and endothelium are secondary lepidic tissues. Hylic or pulp tissues of which the mesenchyme is the type, are composed of cells separated by a homogeneous fibrillar stroma which may or nay not be penetrated by vessels.

'pon this basis Adami has divided the blastomas into two main groups: Lepidoma and IIyloma. 


\section{LEPIDOMATA}

A. Primary. (I) Epilepidomata, typical (papilloma and adenoma), and atypical (carcinoma), all from epiblast. (2) Hypolepidomata, typical (adenoma) and atypical, (carcinoma) all from hypoblast.

B. Secondary. (3) Mesolepidomata, typical (adenoma of kidney, etc.) and atypical (carcinoma of kidney, etc.), all from mesothelium. (4) Endothelial lepidomata.

\section{Hylomata, or Pulp Tumors}

I. Epihylomata, typical (neuroma, glioma) and atypical (gliosarcoma).

2. Hypohylomata, chordoma.

3. Mesohylomata, typical (fibroma, lipoma, etc.) and atypical (sarcoma) from mesenchymal tissues. Mesothelial hylomata or rhabdomyonata.

There is no doubt that in the above scheme Adami has accomplished a successful classification and legitimate nomenclature for tissues and tumors, based upon accredited data. In several directions this classification is of value in the interpretation of many peculiarities of tumor growth. Thus the essential distinctions between lepidic or pavement tissues and hylic or pulp tissues established in the early embryo have a strong influence upon tumors derived from these tissues. The degrees and types of the pavement quality observed in embryonal epiblast, hypoblast, and mesothelium reappear in much of the history of tumors derived from such tissues, especially the mesenchymal relations of endothelium. To a less extent, I think, the reversionary tendencies of tumorcells show the influence of embryological relations.

Yet there appear to me to be two main objections to the adoption of this most successful embryological classification and nomenclature:

First, the behavior of tumor-cells is very much more influenced by the acquired characters of the cells of origin than by their embryological derivation.

Second, the neoplastic process does not consist in retracing the steps of embryological development, but probably in a progressive loss of original cell potencies, so that the cells become a prey to all manner of new external conditions which control their nutrition, greatly influence their form and render them significant chiefly as pathological parasitic cells and tissues.

Embryology has been of great value in clearing up many of the disturbances of development, which lead to the growth of heterologous tumors and teratomas, it aids in the interpretation of many phenomena of tumor growth, especially of teratomas, and will likely be of further aid in this field, but with the main element in the neoplastic process, anaplasia, it has nothing to do.

Oncology is not a department of embryology, but a separate chapter in the biology of the cell. Hence strictly embryological classification and nomenclature have never gained great favor, because they do not serve the largest needs of the student of tumors. The generally accepted plan of classification and terminology which is based on histology, modified as much as possible by histogenesis, is a natural product which has become very firmly established and probably deserves to prevail against the varying prominence of embryology, chemistry, and etiology.

\section{GENERAL MORPHOLOGY OF TUMORS}

The external appearances of tumors present extremely wide variations far exceeding those of normal structures. Yet these gross features are as a rule distinctive, so that the recognition of a tumor may usually be made by inspection and palpation of the tissue.

The terms "sarcoma" and "carcinoma" refer to the exuberant fleshy character of connective tissue growths and the rough similarity of cancer to the crab.

The local circumscription of many tumors is a sufficient gross sign of many blastomas, but fails to separate miliary or nodular granulomas from many forms of multiple tumors. There are some cases of diffuse carcinoma, as of the breast, or carcinosis, as of the meninges, in which no visible tumor is produced, and only a minute attention to color, consistence, and distribution saves the gross diagnostician from error.

The color of tumor tissue is often distinctive.

The normal color of tissues is almost invariably altered, becoming opaque, lighter and often with a tinge of yellow or white, where groups of cancer-cells 
are growing. Virchow used to emphasize three zones in a cancer nodule, a central light or yellowish area of degeneration or cicatrization, a middle, firm, opaque, cellular zone, and a peripheral reddish area of congestive reaction. The congestion which marks the advance of many cancers in the skin may simulate a bacterial process.

Blood content and hemorrhage chiefly determine the color of most tumors as of other tissues. Most cancers are comparatively bloodless while sarcomas are usually more vascular and often contain extravasated blood. Angiomas and angiosarcomas can usually be recognized in the gross from the congeries of blood-vessels composing them. Some sarcomas of bone are traversed by wide blood sinuses surrounded by little tumor tissue, and deserve the name of bone aneurism. Multiple hemorrhagic sarcoma of the skin (Kaposi) is marked by frequent hemorrhages and pigmentation. Certain types of carcinoma, as chorioma, and teratoid cancer of the testis usually produce notably hemorrhagic tumors.

The fungus hematodes of older writers is a cauliflower-like growth rich in blood sinuses. Hemorrhagic infarction may overtake many tumors causing them to assume a very dark red or rusty color. Secondary pigmentation of large portions of tumors may result from altered extravasated blood. Carcinoma of the breast in dogs is nearly always pigmented from this source.

Melanoma of the skin and choroid is a brown or black tumor containing autochthonous pigment, but in rapidly growing metastases it may progressively lose its pigment. The greenish yellow color of chloroma is a distinctive feature possibly caused by lipochromes.

A pearl gray color is seen in chondroma, myxoma, and some scirrhous cancers. Hypernephroma, except in its highly embryonal types and in rapidly growing metastases, has an ochre yellow tint.

The consistence yields significant indications of the nature of many tumors. Osteoma and chondroma reveal themselves at once by stony hardness, but hard fibroma may simulate chondroma.

The peculiar density of many carcinomatous nodules in the breast and of the bases and borders of carcinomatous ulcers is their chief diagnostic feature. There are few organs in which the density of carcinoma is equaled by any other process, but gumma of the testis is usually firmer than the embryonal cancer of this organ.

Many tumors are softened by edema, hemorrhage, necrosis, mucous degeneration and cyst formation, and some become very hard from fibrosis or calcification. In each organ carcinoma is usually firmer than adenoma and sarcoma, which have less fibrous tissue and more vessels. Rapidly growing and embryonal cancers fail to show the density of older types, and from this and other characters are difficult to distinguish in the gross from sarcoma. The soft elasticity of lipoma and especially of myxoma and colloid tumors is somewhat characteristic. Glioma is usually softer and more vascular than adjacent brain tissue.

Cystic tumors are tense, but yielding or fluctuating. Angiomas may be compressible. Lymphosarcoma is rather less firm than Hodgkin's granuloma, leukemia, or slow tuberculosis of lymph-nodes.

Mucous carcinoma of peritoneum produces jelly-like infiltration or huge intraperitoneal masses, and endothelioma of pleura may yield peculiar dry crumbly material. The "sand tumors" of the dura (endothelioma) and ovary (adenoma) are easily recognized on palpation, and the waxy plates of cholesteatoma and sebaceous contents of dermoids are equally characteristic.

The texture and markings revealed by inspection of the surface and section of tumors often disclose their true nature. 
The firm translucent surface of a fibroma, the glistening homogeneous luster of a chondroma, the intertwined fascicles of muscle fibers in a myoma, and the branching twigs of a papillary tumor, are readily recognizable nakedeye features. Likewise the jelly-like material in myxoma and colloid cancer, the whorls of many endotheliomas of the dura, the vascular twigs of angiosarcoma, and the lobules of fat tissue in lipoma furnish a ready basis for gross diagnosis.

In dealing with cellular tumors there are many criteria which lead one to a correct conclusion.

Very cellular tumors without much stroma are invariably soft and fragile or pultaceous (encephaloid). Soft but coherent tumor masses usually show abundant stroma. Cellular tumors whether sarcoma or carcinoma are usually lighter colored and more opaque than the surrounding normal tissues. As a rule, sarcomas are more uniform in texture than carcinomas. The former may produce wide areas of new tissue unbroken by bands of connective or areas of necrosis although frequently interrupted by hemorrhages. The latter exhibit the tendency of carcinoma to excite the growth of connective tissue and to undergo focal fatty degeneration which produce points and masses of yellowish or whitish material. The necrosis of sarcoma is usually more massive.

Vertical section through an acanthoma shows compact columnar masses or whorls of cells, of pearly white luster which even when infiltrating are well banked against the surrounding tissue. Early ulceration is also characteristic of this common tumor, but it rarely produces bulky masses, either primary or secondary.

There are peculiar forms of carcinoma, as in the pylorus and rectum, marked by mucous degeneration and fibrosis, which are sometimes more safely identified in the gross than by the microscope. They produce annular constrictions, or wide infiltrations of the submucosa, but most of the cells may be lost by mucous degeneration.

By naked eye inspection one can frequently recognize the main characters of tumor structure many of which, from too exclusive reliance on the microscope, have come to possess chiefly a histological significance.

The growth of a tumor may be mainly central so that it displaces the surrounding tissue by lateral pressure, as in myoma uteri.

Central growth is well shown in some angiosarcomas in which actively growing blood-vessels sheathed with tumor-cells compose the central portions, while in the periphery the growth is solid and grades into a firm fibrous capsule. Or the growth may be peripheral, in which case the central portions are apt to be fibrosed or degenerated and depressed.

Infiltrative growth is revealed by lack of capsule or other demarcation and by opaque cords of tumor-cells stretching out into the lymph spaces or vessels. Extensive intravascular growth characterizes hypernephroma, adenoma of liver, and chorioma. Polypoid, papillary and dendritic, and cystic architectures reveal themselves best to the naked eye, while more minute structures designated as fascicular, plexiform, and alveolar can usually be detected by careful inspection.

Soft cellular tumors are often called medullary, or encephaloid, from resemblance to brain tissue, to which the counterpart is the hard scirrhus.

Thrombosis of blood-vessels, edema, fatty, hyaline, mucoid and colloid degeneration, calcification and ossification, are all encountered in extreme degrees in the gross anatomy of tumors.

Cysts of varying origin, form, and content are notable gross features of many tumors. 
Retention cysts filled with retained secretion develop in many adenomas especially of the ovary, some of which are the largest tumors ever observed. Originating in a closed group of alveoli whose secreting function is active dilatation of alveoli is accompanied by complex systems of papillary ingrowths which constantly add their quota of secretion. Eventually inflammatory exudate and hemorrhage are added with rapid increase in volume. The contents of such cysts are therefore serous fluid with much nucleo-albumin and mucus, or with pus and disintegrated chocolate colored blood. The walls are lined with varying quantities of papillary tumor growth.

Liquefaction cysts with smooth walls form in fibromyomas, sarcomas, etc., from local edema, necrosis, and autolysis of solid elements. Their contents

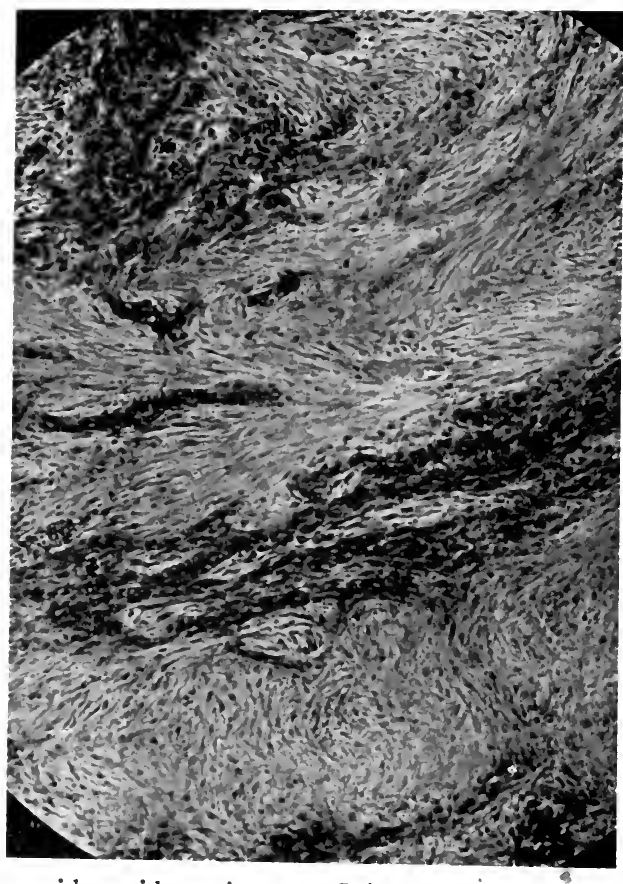

FIG. I.-Fibrosing epidermoid carcinoma. Spindle-shaped tumor-cells. Illustrating infiltrative growth.

are thin and serous or mingled with granular or fatty detritus, blood or pus; or the fluids are removed by absorption. Extravasation cysts form after hemorrhages and exhibit all stages of alteration and absorption of blood. Dilated lymph- or blood-vessels contribute to the structure of some cystic sarcomas of bone and other tissues. Cystosarcoma phylloides is an old term sometimes applied to tumors showing a branching system of more or less parallel cysts recalling the veins of a leaf.

It is often difficult to distinguish between true cystic blastomas and simple cysts with inflammatory overgrowth of lining cells.

In most simple retention cysts there is a tendency for the epithelium to grow up into papillary projections, as in chronic mastitis, and with several
forms of encysted animal parasites. Such inflammatory overgrowth may 
reach a considerable grade, but usually lacks the distinctive morphology of a tumor, and regresses when the cyst is evacuated. Yet some of them may go on to produce genuine neoplasms.

Ulceration overtakes most tumors of the skin and mucous membranes. The inadequacy and fragility of blood-vessels, degeneration and autolysis of tumor-cells, trauma, mechanical pressure and tension, contact with irritants causing inflammation, and bacterial infection, are the chief factors in its origin.

In ulcerating acanthoma the base is formed of dense eroded tumor tissue; the secretion is scanty or abundant pus, serum and opaque particles of tumorcells; the edges are raised, very hard, in mucous membranes often undermined, rounded, irregular, or serpiginous; and the vicinity is indurated and inflamed according to the extent of infiltration and infection. Erosion of bone and cartilage with pain, and of blóod-vessels with hemorrhage may occur, and perforation into adjoining hollow viscera. Considerable masses

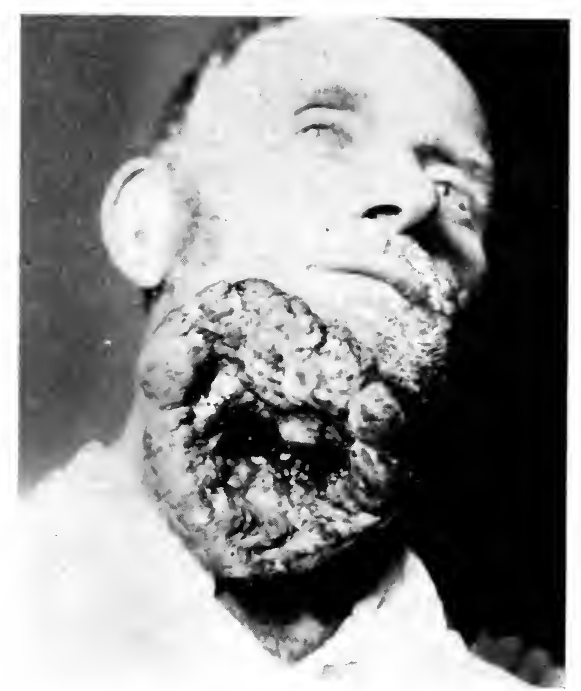

FIG. 2.-Fungating carcinoma of neck. A recurrence from the lip.

may slough, and new skin or mucous membrane may replace portions of the ulcer. Extensive tracts of suppuration may follow invasion of cellular tissues.

In adenocarcinoma of mucous membranes thrombosis and infection commonly leads to the formation of large sloughing excoriated ulcers with much hemorrhage. Flat, indurated, annular ulcers with extensive infiltration of the vicinity and construction of the lumen form in carcinoma of the rectum. Necrosis of large portions of the tumor together with the distended overlying skin in carcinoma of the breast and other cancers produces extensive ulcers in which the sloughing tumor may protrude or suffer deep excavation. In sarcoma, ulceration is usually marked by massive necrosis and hemorrhage.

The onset of ulceration frequently transforms a comparatively harmless tumor into a rapidly fatal process through local or general infection, suppuration, absorption of toxic products and hemorrhage, to which chiefly must be attributed the cachexia of cancer.

In general it may be said that the outward morphology of tumors con- 
stitutes an important and very practical chapter in their study since it not only reveals the general architecture of neoplasms, but allows their separation in most cases from other processes, and usually permits an accurate diagnosis of their histological strurture.

\section{HISTOPATHOLOGY}

The study of tumor growth must begin with its earliest stages.

In one large group this study leads to the scrutiny of misplaced and chiefly embryonal cells, or even to the consideration of single cells, giving rise to the large group of tumors to which Cohnheim's theory applies. In another equally important class the initial changes affect cells which so far

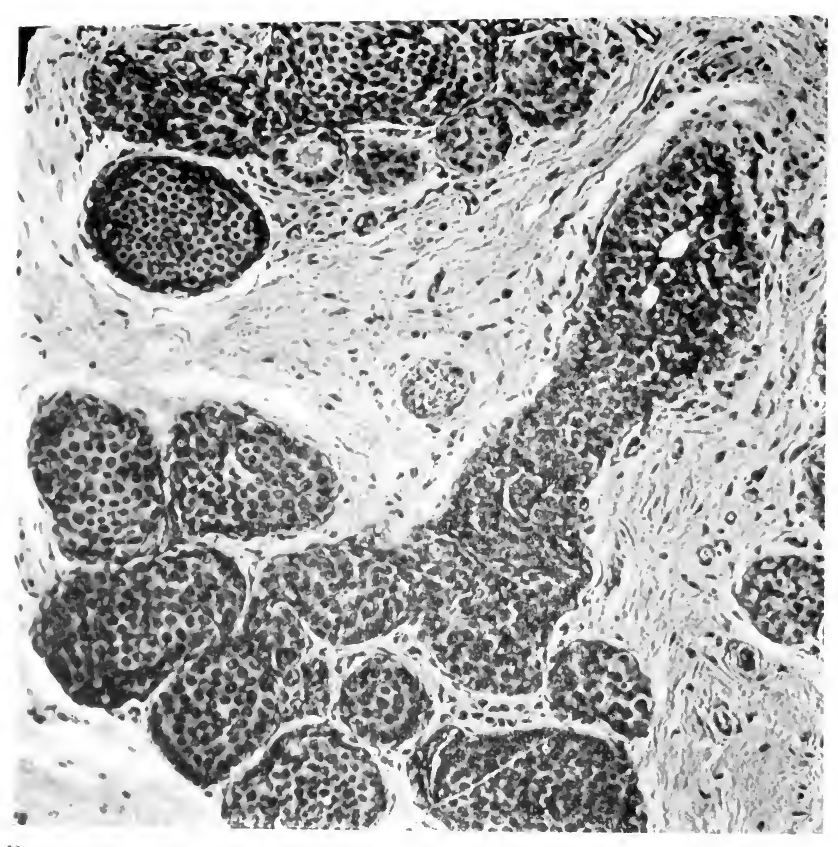

Fili. 3.-Precancerous changes in breast. Atypical epithelial proliferation in ducts and acini found in a small portion of the breast.

as we know are normal and here a series of preliminary changes occurs, belonging to the so-called precancerous stage, which is of importance in the origin and growth of tumors.

Precancerous Stage of Tumor Growth.-Processes occasionally followed by neoplastic growth are observed in syphilitic leukoplakia, tuberculosis, Hodgkin's granuloma, chronic eczema, chronic mastitis, ulcer of the stomach, cirrhosis of liver, $x$-ray dermatitis, and the chronic processes associated with mechanical, chemical and thermal irritation.

It is impossible to analyze all the factors acting in such processes. Upon their interpretation have been built many theories of the nature of tumor growth. Yet it is possible to enumerate several very constant morphological changes which in certain combinations seem to be most effective in exciting
the subsequent autonomous growth. 
In a study of precancerous changes in several situations Bonney finds constant loss of elastic tissue, and usually hyaline changes in the collagen, lymphocytic exudate, and fraying of the edges of the epithelium.

In multiple epithelioma of the skin Janeway has observed that the tumors are often preceded by a period of local erythema which may slowly disappear or be followed by cancer. Sections of the skin show focal dilatation of the blood capillaries adjacent to the basal epithelium about which the epithelium is hypertrophied, hyperplastic, and with hyperchromatic nuclei.

In leukoplakia, hyperkeratosis, thickening of the Malpighian layer, hypertrophy of the epithelium, round-cell infiltration, increased vascularity and edema long precede epithelioma of the tongue.

In ulcer of the stomach disordered gland alveoli are drawn into new celluar connective tissue, detached from their normal relations, pass through

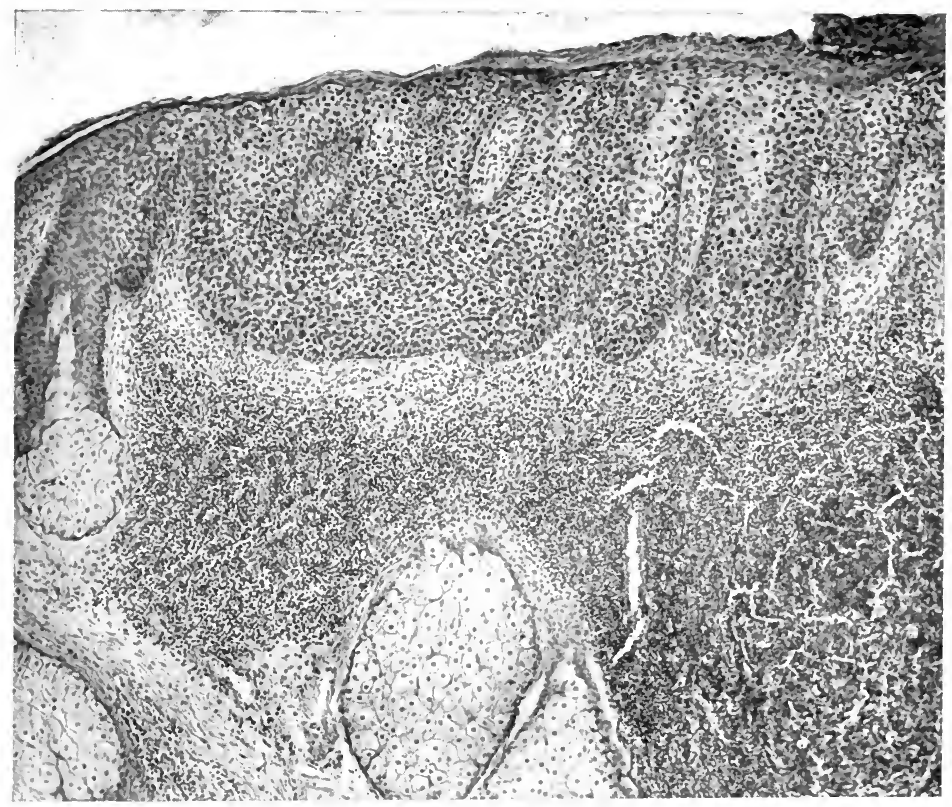

FIG. 4.-Atypical epithelial hyperplasia on edge of a beginnnig carcinoma of skin.

stages of proliferation, hypertrophy of cells and nuclei, and gradually or suddenly assume neoplastic characters. Here, as in cancer following cirrhosis of the liver, it is sometimes difficult to determine just when a true neoplastic process exists.

In chronic mastitis single small cancer nodules may appear and I believe their preliminary stages may be traced in other portions of the breast, chiefly in the ducts. Here one finds hyperplasia of lining cells with hypertrophy and increased eosin stain, cyst formation and papillary outgrowths into them, in which condition they seem usually to be diverted from further dangerous tendencies. Yet other cells show more active proliferation, increase in size and in nuclear chromatin; their polarity is lost by growth of round-cells and connective tissue, and it is possible to trace such cells into types closely approaching those seen in early carcinoma. 
It may be true, as Ribbert asserts, that no one has ever seen the beginning of cancer of the breast, but the changes just enumerated seem to form a common preliminary to the onset of true cancer, which on such a basis develops rapidly and is accompanied as a rule with fatty degeneration in the affected cells.

In rare cases of Hodgkin's granuloma the proliferation of large mononuclear cells and of giant-cells, originally of inflammatory origin, takes on more autonomous and sarcomatoid qualities. The cells become more numerous, the stroma less, the nuclei larger, mitoses more abundant, metatases composed of large round-cells without stroma occur, and the process has many of the qualities of a blastoma. Yet the true neoplastic properties of these cases must be doubted, although they exhibit a certain progress toward a genuine autonomous new growth.

The abnormal physiology associated with the precancerous state will be discussed under General Etiology.

Beginning thus either in isolated embryonal cell groups or in tissues long subjected to the influences of the precancerous stage, tumors grow, always chiefly, sometimes exclusively, from their own resources, $i$. e., from the original cells involved in the process.

Modes of Origin.--Four modes of the origin and extension of tumors may be discerned:

I. Growth is unicentric and exclusively from the original cell group involved in the tumor process. In the large class of blastomas arising from embryonal cell groups, the originating cells are isolated from the first, and the tumor-cells remain isolated during their entire history. Thus hypernephroma may begin in a small group of cells misplaced in the renal cortex, produce a large tumor of the kidney, invade the renal vein and produce metastases in many organs, the cells in all situations being the direct descendants of those composing the original cell complex.

2. Multicentric growth is observed in many tumors. In multiple epithelioma of the skin, $x$-ray cancers, cancers of the gastro-intestinal tract following multiple polypi, fibrosarcoma of nerve trunks, and others, several centers originating tumor-cells appear simultaneously or at intervals. These centers may gradually coalesce, producing one large tumor area, or they may long remain discrete.

Petersen by reconstruction in wax has shown that certain cancers of the breast arise from several discrete centers of proliferation, and a similar multiple origin in an adrenal tumor is described by Woolley; and for carcinomatosis of the liver, by Von Heukelom and Oertel.

A remarkable case of multiple angiosarcoma or endothelioma, involving nearly the entire skeleton, is described by Marckwald.

Of such cases some must be referred to the wide diffusion of the inciting factors, as in $x$-ray cancers; others to multiple foci of embryonal displaced or superfluous cells as Hauser would explain the multiple cancers of the intestine; while the conditions in a few cases suggest some complex influence of an existing tumor upon neighboring cell groups predisposed to tumor growth.

Multiple tumors of the same type in paired organs is a special case of multicentric growth. Carcinoma arises in both breasts, teratoma in both ovaries, lymphosarcoma in both testes. In each such case different influences seem to be concerned, but in all the influence both of local and of constitutional factors is suggested.

The occurrence of multiple tumors of different types in the same organ or in different organs of the same subject is also observed.

In some instances a second tumor may result from conditions brought 
about by the presence of the first, as when a submucous fibromyoma causes ulceration followed by cancer of the uterine mucosa.

Tumors have been observed simultaneously in the uterus, oraries, and breast, a combination suggesting the influence of the functional relation existing between these organs. The rather common occurrence of two or more tumors in different organs of the same subject suggests nothing more than the accidental coincidence in several organs of the general etiological factors in the genesis of tumors.

Many cases illustrating several groups of multiple tumors have been collected by Walter and Woolley. Among rooo autopsies on tumor cases Hansemann found 5 with multiple primary growths, and in 1225 cases collected by Redlich I4 showed more than, one primary tumor. When a minute search is carefully conducted and all forms of tumor growth included, the proportion of multiple tumors is much greater.

Symmers in 2 I 5 autopsies found tumors in 55 , in 22 of which more than one type existed. Gotting observed 3 cancers of different organs, several adenomas of bile-ducts, and an angioma of liver, in a man of 58 years.

3. The question whether a tumor process may gradually extend from an originating focus over the surrounding normal cells cannot at present be determined. Such a possibility is commonly denied. Borst argues that it is impossible to conceive how such an influence can be transferred from one cell to another. Ribbert, after strongly opposing the idea of transference of the tumor tendency from a tumor-cell to normal cells, admits that there may be wide-spread multiple foci of origin which exhibit neoplastic growth one after the other, thus simulating the lateral diffusion of the tumor process. Unless one assumes, as White actually does, that tumors develop from single cells, it is necessary to assume some such gradual implication of the several cells in the originating group, so that Ribbert has practically admitted the gradual extension of a tumor by progressive involvement of normal cells, not by transfer of influence from one cell to another, yet by the effects of underlying factors upon one cell after another. It is not necessary to attribute this influence to the mere contact of tumor-cells or to suppose that it involves a parasite.

Increased vascularity, mechanical displacement, disordered function, chemical stimuli, and toxic agents may all be included among the factors resulting from the presence of tumor-cells. There is also a certain complex tropism observed in the growth of tissues in lower animals, whereby processes at work in one tissue induce similar tendencies in contiguous cells. There appears to be adequate theoretical basis for the extension of a tumor process over normal cells, and observation indicates that in some cases such extension actually occurs.

First, in the neighborhood of many epithelial tumors the contiguous cells show a peculiar increase in number, size, and chromatin content.

This collateral hypertrophy is more common about primary tumors, but may be seen in metastases. Usually it does not reach a neoplastic grade, but it is sometimes difficult to distinguish from an advance of the tumor.

In adenoma of the sigmoid such transformations may occur, the outlying cells first increasing in size, then becoming opaque and granular, while the nuclei become hyperchromatic. Some extensive superficial adenomas of the sigmoid appear to have developed in this manner.

Hauser depicts definite stages in the transformation of normal glands into hypertrophic forms which he believes precede the development of cancer. Haaland has described a neoplastic transformation of the pulmonary cells in metastatic carcinoma in the mouse, an observation which needs to be 
confirmed, but the sarcomatous transformation of the stroma of transplanted cancers of the mouse, if genuine, is probably a sufficiently attested instance of the tropism exerted by tumor epithelium upon adult connective tissue.

In adenocarcinoma of the human ovary is sometimes seen a sarcomatoid proliferation of the stroma.

Yet these instances of lateral extension of tumor processes, if they eventually stand the test of criticism, are rare, and it should be emphasized that the great majority of tumor-cells are isolated in origin and throughout their history.

4. The systemic round-cell sarcomas present a difficult problem in the analysis of their mode of origin and extension. Multiple myeloma arises simultaneously at numerous widely distant foci in the bone-marrow and may early show a diffuse tumor process throughout many bones. Hence one must assume the existence of so many foci of origin as to suggest the simultaneous involvement of the entire bone-marrow system.

Multiple metastases cannot satisfactorily explain the course of these tumors, and it would seem probable that there occurs a lateral extension of the tumor process over adjacent normal areas as well as infiltrative growth from the multiple foci. In the group of lymphocytomas there are examples very strongly indicating simultaneous tumor growth originating in a large portion of the lymphatic system. In some intestinal cases (Wells, Symmers) there is a diffuse lymphadenomatous hyperplasia involving the entire lymphoid apparatus, from the cardiac orifice to the anus, the superficial, the thoracic and the abdominal nodes, and the bone-marrow. Leucocytosis is absent and the spleen is little affected. A rapid lateral extension from very many foci or a primary involvement of the entire system seems to be the only reasonable explanation of such cases. There are systemic highly malignant large-cell sarcomas of equal extent. Chloroma, with or without leukemia, occurs as a multiple or diffuse tumor process affecting wide areas of marrow and lymphatic system for which a lateral extension from many foci or a primary diffuse origin seems a necessary assumption. In thyroid tumors of fish the hyperplasia which seems to result from exaggerated response to functional stimulus is felt in all the widely disseminated alveoli of the gland. Some of these hyperplastic glands may regress (Marine), but beyond a certain stage they become autonomous.

Cell Division in Tumors.-Normal and many abnormal types of cell division occur in tumors, including mitosis, direct nuclear division, and direct and indirect fragmentation.

This subject has been very fully investigated by Stroebe, Vit. Müller, Cornil, Arnold, Pianese, Galeotti, Hansemann, and many others, who agree that the chief mode of cell division in tumors is by mitosis. In the benign tumors in which the growth is comparatively slow the scanty mitotic figures usually appear normal, but degeneration, inflammation, and very rapid growth are marked by increasing irregularity in the mitotic process.

Pianese classifies the irregularities occurring in cancer as follows:

A. Relatively typical mitosis, which may be bipolar and symmetrical, hyper- or hypochromatic, and giant.

B. Atypical mitosis, either asymmetrical or multipolar.

C. Abortive mitoses, marked by suppressed or atypical polarization of chromosomes, by aberration of chromosomes, by loss of polar bodies, and by
degeneration of cytoplasm.

As a rule he found the first two types in comparatively intact cells, while abortive mitoses occurred chiefly in degenerating cells. Irregularities in 
all the phases and elements of the mitotic process occur in malignant tumors and bear a certain relation to the morphological and physiological variation of the tissue from the normal (Hansemann). The number and size of the chromosomes, the structure of the spindles, the coördination of the division phases, the position of the elements in the cell, contribute numerous abnormalities in the process. Hyperchromatic cells may arise from asymmetric mitosis. Hypochromatism results from asymmetric mitosis and loss of chromosomes.

Asymmetric mitosis has been referred to secondary subdivision of centrosomes, one of which may divide into as many as four parts, each forming attraction spheres. Under these conditions the migration of chromosomes is often delayed and unequal (Galeotti).

The results of abortive mitosis give rise to a great variety of structures which have been elaborately illustrated by Pianese. In this class of structures have been found many of the pseudoparasites of cancer. Thus abnormalities in the mitotic process have been observed rather more abundantly in carcinoma than in sarcoma, but many of them have been seen in inflammatory and regenerative hyperplasia (Stroebe).

Striking peculiarities in such a fundamental process as cell division may well suggest that here is disclosed the essential nature of tumor growth. Hansemann has interpreted the studies in this field in such a manner, holding that asymmetrical, multipolar and abortive mitoses mean unequal distribution of cell potencies, loss of cell differentiation, freedom from normal restraints to growth, and exaggeration of growth over functional capacities. These physiological properties he includes in the conception of anaplasia, the morphological side of which is illustrated in resting and dividing tumorcells. To a considerable extent this interpretation may be valid, and yet the majority of tumor-cells divide by normal mitosis (Pfitzner, Hauser), and the minute study of the conditions favoring abnormal mitoses indicates that these changes are secondary results of tumor growth and not primary and essential.

Abnormal, asymmetric, and multipolar mitoses are readily produced experimentally by heat and by many chemicals (Galeotti, O. Hertwig).

Gametoid Mitosis.-In the maturation of sex cells the mitotic nucleus exhibits only one-half the number of chromosomes of the somatic or general body cells, and these chromosomes instead of assuming a $\mathbf{V}$ shape and radial arrangement and splitting lengthwise in the monaster, are ring-like or loop shaped, or composed of coarse granules arranged in the long axis of the spindle. This type of division with reduction of chromosomes is called gametogenous or heterotype.

Farmer, Moore and Walker have found that this type of division, here called gametoid, is common in the growing edges of epithelioma and other tumors. On this ground they at first assumed that tumor-cells possess the physiological significance of sexual cells, but this view has been abandoned since gametoid mitosis is by no means constant in tumors, and the peculiar mitoses may not signify the properties of sex cells. Yet I think the observation remains suggestive, since tumor-cells may show three properties of sex cells, gametoid mitosis, increased growth capacities, and remarkable altruistic relations.

Amitosis is frequently observed in rapidly growing tumors and in some cases it may be the chief mode of cell division. Accordingly to Nedjelsky it begins with swelling of the nucleolus followed by elongation, budding or cleavage of the nucleus. It results in the formation of giant-cells with pale nuclei which may later undergo budding or mitotic division, or by division 
of the cell body it often leads to rapid regeneration of viable tumor-cells (V. Müller).

Flemming and Trambusti hold that amitosis always results from degenerative changes in the cell and may yield giant-cells, but not fully viable tumorcells. Marchand and most observers believe that mitosis contributes, as does amitosis, to the rapid regeneration of tumor-cells, but fails to convey to the progeny all the properties of the parent cells. Pianese in his elaborate study of cancer-cells could not find that amitosis was ever followed by cell division, but was always abortive, leading to the formation of multinucleated giant-cells.

Howard has endeavored to trace in the nuclear changes of tumor-cells many processes parallel to those observed in the protozoön nucleus.

The impression that tumor-cells must owe their exaggerated growth capacities to some form of fertilization has led to an interpretation of certain nuclear changes suggested by this point of view, but now abandoned. Klebs once thought that the nuclei of englobed leukocytes fused with the nuclei of tumor-cells. Recklinghausen saw appearances suggesting the fusion of the nuclei of endothelium and fibroblast.

Auerbach and Bashford pictured the fusion of the nuclei of adjoining tumor-cells, and Schleich thought that endogenous cell formation constituted a form of cell infection which accounted for overgrowth.

Neoplastic vs. Inflammatory Hyperplasia.-Neoplastic hyperplasia is usually more rapid than the multiplication of cells in inflammation, or regenerative growth. Lymphosarcoma of a few months' growth may reach a bulk constituting a large portion of the body weight. Carcinoma of the liver may largely replace the tissue of this organ in a brief period. In the terminal stages of many malignant tumors growth is much accelerated so as to divert much nutriment from the other tissues. This rapid growth is associated with a great abundance of mitotic nuclei in the tumor-cells which often far exceeds that seen in any inflammatory process.

Yet rapidity of growth is not an essential distinguishing feature of tumor processes. Few tumors grow as rapidly as the fetus in utero.

Leucocytes multiply with great rapidity in pneumonia; and epithelium covers denuded skin far more actively than the growth of such cells in many epitheliomas.

A more important distinction of tumor growth is found in its progressive tendencies. Tumors commonly grow to a bulk far exceeding the limits of any inflammatory or physiological process affecting the same area, and they continue to grow to whatever extent nutrition is provided. Physiological regeneration observes a certain adaptation to the function of the organ; inflammatory overgrowth is measured by the intensity and duration of certain irritants and ceases when these irritants are removed; tumor growth ignores all these limitations and ceases only with the death of the patient or the curtailment of blood-supply. Yet there are cyclic variations in the growth of tumors, periods of activity being followed by standstill or regression.

Indeed, there appears to be a natural limit to the growth of some tumors in some subjects. Progressive fibrosis brings some cancers of the breast to comparative quiescence in an innocuous scirrhus form, and the menopause marks the acme of growth of some uterine myomas, and has been followed by the spontaneous regression even of cancer of the breast (WatsonCherne).

The frequency of tumor regression in lower animals, the increasing number of spontaneous regressions in human tumors (Czerny), and observations of the unhindered growth of tumors over many years in old persons, indicate 
that limitless growth is not a power possessed by all malignant tumors. The universal employment of surgical measures renders this fact less obvious than it might be.

The morphology of neoplastic hyperplasia differs from inflammatory overgrowth and physiological regeneration. A productive inflammation is usually a diffuse process involving a whole organ or tissue area and it grades off insensibly into normal tissue (chronic mastitis), while a neoplasm is usually circumscribed on account of its origin from an isolated group of cells (fibroma mammax). Productive inflammation involves all the elements of a tissue; tumor processes usually reside in a single-cell type. Inflammatory neoplasia is confined by narrow limits, which in tumors are yery wide.

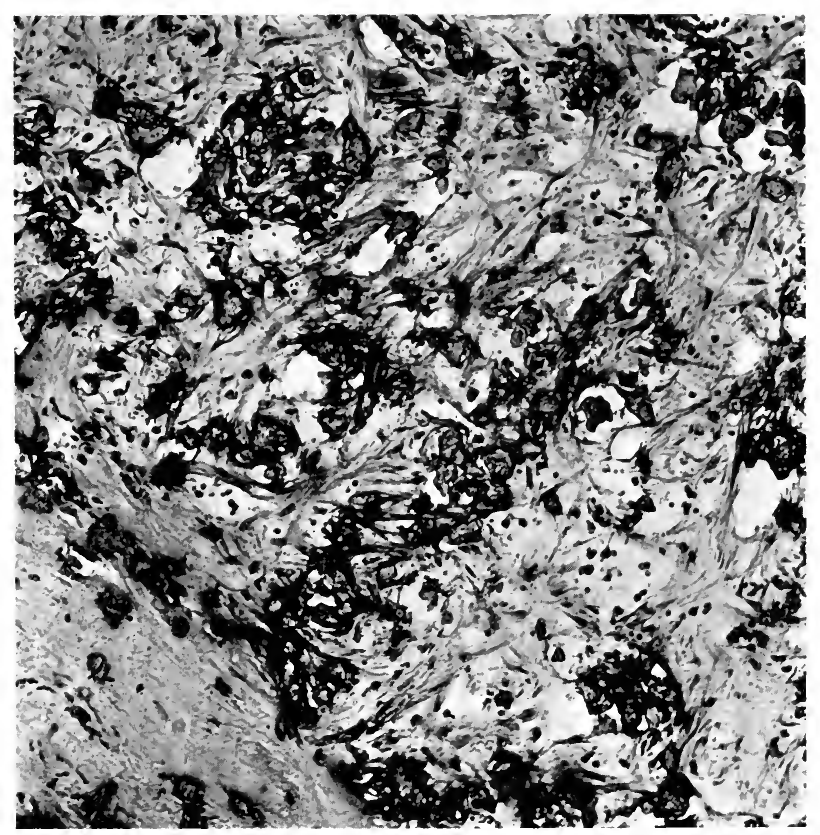

FIG. 5.-Mammary cancer. Spontaneous local regression, with formation of fibrous tissue, calcific granules, and fatty cry'stals.

Tumor-cells show the features of anaplasia, loss of specific form, and of polarity, variation in size, nuclear hyperchromatism, multiformed nuclei and atypical mitoses, all of which are absent or less marked in inflammatory hyperplasia.

Yet inflammatory overgrowth passes by insensible gradations into neoplastic hyperplasia. This significant principle of pathology has an important and yet a limited application. As a rule tumor growth differs sharply from any inflammatory hyperplasia affecting the same tissue, but there are exceptions to this rule.

(a) There are inflammatory hyperplasias of the bone-marrow cells, leukemic processes with some of the features of a neoplasm, and chloromas which are true tumors of marrow-cells. Most cases fall clearly into one of these categories, but others are intermediate in type and it is difficult to determine whether they are strictly inflammatory or truly neoplastic. Similar 
intermediate grades of hyperplasia, difficult to classify, are occasionally encountered with nearly every tissue cell in the body.

(b) A process beginning as a simple inflammatory hyperplasia may in the same individual gradually assume neoplastic properties.

In the thyroid gland of goiter, especially in fish, in the prostate gland of hypertrophic prostatitis, in the uterine mucosa of a glandular endometritis, in the mammary gland of chronic mastitis, and in the lymph-nodes of Hodgkin's granuloma, are occasionally seen transformations of a functional or inflammatory hyperplasia into a more or less typical neoplasm. Here it would appear that a hyperplastic process incited by external irritants may continue and increase long after the removal of the irritant. As with certain chemical reactions so with pathological processes the importance of momentum must be recognized.

It may be objected that when a neoplasm appears in the course of inflammatory hyperplasia something new is added.

This objection may be valid for certain cases where the neoplasm appears suddenly and in widely different form from that exhibited by the preëxisting hyperplasia, but not for other cases in which observation shows the change to be gradual. Since with every increase in quantity there is a change in quality it is reasonable to expect that gradually accumulating tendencies in the life of the cell may express themselves at times in quite sudden and radical changes in morphology and behavior. All these considerations strengthen the view that the "precancerous condition" is of wide occurrence and of much theoretical and practical importance in oncology. In this condition one finds tissues and cells in a state of overgrowth intermediate between inflammatory and neoplastic hyperplasia, exhibiting certain tumor characters which must be judged from different standards for each tissue, and which experience shows are often followed by genuine and usually malignant tumors.

The theoretical distinctions which a general survey establishes between neoplastic and inflammatory hyperplasia are sharp and fundamental, but these distinctions fail us when we have to search for them in processes of doubtful nature. Here we assume them to exist from our general knowledge, but we cannot prove their presence.

Thus, as Borst assumes, inflammatory overgrowth results from exaggerated response to external irritants while neoplastic growth arises from loss of normal restraints to growth. The one is purposeful, organized, self-limiting, typical, accelerated hyperplasia, while the other is baneful, lawless, progressive, atypical degenerative growth excess. But this is a composite picture and the practical problem is to decide how much it is reflected in individual doubtful cases. Virchow recognized this difficulty and some observers, finding it insurmountable, have concluded that there is only a difference in degree between healing of wounds, inflammatory hyperplasia, and tumor formation (Brosch, Fabian).

This view cannot be accepted. Inflammatory hyperplastic processes pass by insensible gradation into neoplastic growth according to our present analysis. Syphilitic leukoplakia becomes epithelioma, and Hodgkin's granuloma may take on a sarcomatoid character. Yet these are complex processes and although we cannot detect when growth from external stimulation is succeeded by growth from loss of growth restraints, yet theoretical considerations require us to assume that at some point such a change actually occurs. I have elsewhere suggested that some hyperplastic processes, owing their inception to the influence of parasites, become at length established and progress without the stimulus of the parasite. Here one may suppose that the declining stimulus is replaced by progressive loss of growth restraints, 
but the reasoning would still show that inflammatory hyperplasia passes into neoplastic growth.

On the other hand, I do not believe that growth restraints are fully maintained in some inflammatory hyperplasias. In proud flesh mechanical restraints seem obviously defective and in hypertrophic endometritis several signs of lowered tissue tension are visible. External stimulus and loss of growth restraints are here combined, and it cannot be said that neoplastic hyperplasia is wholly different in nature from inflammatory overgrowth.

Thus, however closely the processes are analyzed, the conclusion remains that inflammatory hyperplasia passes into neoplastic.

Regressive Changes in Tumor-cells.-Tumor-cells in general suffer the same degenerative changes as occur in other tissues, but some special interest attaches to this field on account of the frequency and extent of these changes in tumors, and also from the very minute analysis which has been required because of the former interpretation of many intracellular structures as parasites. The presence of degenerative changes in tumors is the direct result of the activity of growth, the accelerated metabolism, and the uncertainty of blood-supply.

Fatty degeneration is nearly constant in cancer, less common in sarcoma. It contributes a light opaque color to tumor cords which is one of the earliest gross diagnostic signs of many cancers. It seldom reaches an extreme degree, the fat being commonly limited to fine droplets which first appear about the nucleus.

It is most prominent in tumors of glands which normally secrete fatty substances as in adenoma sebaceum, adrenal tumors, and cancer of the breast. In rapidly growing, well-nourished tumors the active metabolism is unfavorable to the deposit of visible fat, which appears chiefly in areas of necrosis.

The chemical nature of the lipoids of tumors is no better known than is that of the fats in other conditions. In a series of cancers Pianese could find no fat blackening with osmic acid.

Stains by Sudan III and Scharlach $R$ commonly reveal many fine fat droplets in such tumors. To what extent the fatty degeneration of tumors belongs in the class of lipoid or myelinic degenerations has not been determined, but Kaiserling and Orgler found myelin droplets in the cells of many cancers and sarcomas.

White has described several varieties of fatty crystals occurring in frozen sections in and about the cells of carcinoma, sarcoma, and adenoma. Some of these were large plates or needles which did not melt readily and which he regarded as cholesterin.

Smaller needles which melt and solidify into globules on cooling he identified as fats. Other small needles and anisotropic crystals were classed as mixtures of cholesterin with lecithin and as myelin forms. The crystals seem to be associated with cell-growth rather than with degeneration, and White suggests that they are in some way concerned in the regulation of cell growth.

Hydropic degeneration occurs in the edematous portions of both benign and malignant tumors, and affects both nuclei and cell bodies.

It is seen in marked degree in the lining cells of cystic adenoma and in the cells of any tumor that is constantly bathed in fluids as in papillary epithelioma of the bladder. Exfoliated cells lying in tissue spaces are commonly ballooned out with imbibed fluids. Virchow's "physaliden" were in part hydropic vacuoles in nuclei or cytoplasm which in the fresh condition appeared hyaline, but on hardening yielded a central granular or homogeneous mass. 
Glycogenic degeneration of tumors has been carefully studied by several observers. According to Gierke it appears in tumors under the same conditions as in other cells. These are mainly as a primary physiological constituent of actively growing or embryonal cells and as a secondary result of disturbed nutrition and degeneration. In the former class it would naturally characterize malignant, rapidly growing and embryonal tumors, and in the latter it would be associated with and have much the same significance as fatty changes. Observation has shown that glycogen is commonly found in most rapidly growing tumors (Best), but in very variable quantities, and some malignant growths, as breast cancer, are usually free from it. In endothelioma it is very constant and abundant (Gierke). In I 544 tumors Lubarsch found glycogen in 477, 29 per cent. Fibroma, osteoma, glioma, and hemangioma were constantly glycogen free; lipoma and lymphangioma and adenoma nearly always so. The more certain the embryonal nature of the tumor, the more constant was the presence of glycogen. Thus in teratomas, hypernephromas, and choriomas it was present in all cases and usually abundant. It was about equally common in sarcomas ( 50.7 per cent.) and carcinomas (43.6 per cent.); in squamous cell epithelioma 70 per cent.

Four factors seemed to Lubarsch to chiefly determine its occurrence.

I. An origin of the tumor from embryonal cells.

2. An origin from cells normally containing glycogen.

3. The absence of mucous and colloid degeneration, and frequent presence of fatty changes.

4. The presence of numerous delicate blood-ressels in intimate relation to the parenchyma.

Secondary glycogen deposits appear in necrotic areas and about the inflamed edges of tumors which are elsewhere free from it (Best). Brault thought that glycogenic degeneration sometimes preceded mucous changes in mucoid carcinoma.

Müller concluded that actively growing, well-nourished cells contain no glycogen, which appears in cells with feeble metabolism. The relation of glycogen content to the prognosis of tumors was considered by Brault, who states that in sarcomas there is no parallel between glycogen content and malignancy, while with carcinoma the glycogen-holding tumors are regularly the more malignant. Best and Gierke, however, did not find this rule to hold.

Mucous degeneration is a wide-spread and important regressive change in many tumors. It may be a primary and essential feature of the neoplasms, arising from exaggerated development of the mucous constituents or from increased secretion, or it may overtake tumors whose cells have no original tendency toward its production.

It represents the matrix of primary myxoma, the secretion of many adenomas, and a degenerative product in chondroma, sarcoma, and carcinoma. It is readily recognizable by the homogeneous luster and viscid quality which it imparts to the tissue. True mucin is found chiefly in connectivetissue tumors, pseudomucin in epithelial growths. Certain tumors are characterized by a remarkable production of mucus. Primary myxoma of the skin consists of spindle- and star-shaped cells lying in a matrix of homogeneous finely fibrillated material which infiltrates the surrounding tissues and doubtless contributes to the local recurrence of these growths. Some gelatinous cancers of the colon early invade the peritoneum, spread through the cavity, and produce immense quantities of firm gelatinous material which distends the abdomen. In such cases it may be difficult to discover any remnants of living tumor-cells, and some such tumors seem to reach a standstill through 
excessive production of mucus. Cancer of the stomach may yield a localized growth or diffuse infiltration of the stomach wall in which are found scanty groups of tumor-cells widely distended by mucus.

Adenoma of the ovary may yield cystic tumors of very large dimensions composed of cysts distended by fluid containing large quantities of pseudomucin. Mixed chondroma of the parotid commonly shows areas of cartilage verging into myxoma, and recurrences of the tumor may eventually assume the type of pure myxoma. Actively growing lipomas are occasionally overtaken by diffuse mucoid degeneration.

Intracellular mucous globules are observed in a wide variety of tumors in which the process fails to make headway. As a rule, when mucous degeneration overtakes a tumor, while a rapid increase in size may follow, the tumor has reached the acme of its cellular activity and its malignancy is reduced. Since the nuclear constituents of the cell are chiefly concerned in mucus production, the appearance of intracellular mucous degeneration usually indicates a process intimately affecting the vitality of the cell.

Hyaline changes in cells, vessels, and stroma are the most common forms of regressive process occurring in tumors. Firm, homogeneous, acidophile material arises from the connective-tissue elements and vessels of the stroma, from the tumor-cell cytoplasm, and from exudates and secretions. Pronounced grades of the first type are seen in keloids, fibroma, fibrocarcinoma, endothelioma, and angiosarcoma.

It may strongly influence the course of the neoplasm, usually appearing in slowly growing or regressing tumors, to which qualities it contributes. Atrophy of cells usually keeps pace with the progress of hyaline changes, so that large tumors may be converted into nearly stationary growths composed largely of hyaline masses.

In some angiosarcomas the walls of vessels are converted into hyaline rings sheathed by ill-nourished tumor-cells. Calcification of these hyaline vessels produces the sand grains of psammoma.

Intracellular hyalinosis produces a wide variety of intracellular structures which have been studied and elaborately depicted by Pianese. A somewhat special variety is the keratinization of squamous-cell epitheliomas, in which group the appearance of hyaline globules, rings, and vacuolated bodies, within or about the nucleus, in the cytoplasm, or involving the cell borders, produces bizarre structures, many of which were once interpreted as parasites. Exudates and secretions becoming inspissated contribute a large portion of the hyaline material in some tumors. "Mulberry cells" containing small nuclei and many large acidophile globules resembling red blood-cells occur in certain tumors and in other conditions. Their appearance suggests an origin from englobed red cells or from intracellular hyaline degeneration. Weber depicts them in a case of myeloma, and I have found them very abundant in cancer of the stomach. True amyloidosis has been described by many authors in a variety of tumors, as carcinoma of esophagus by Wagner, tumor of the bladder (Billroth), carcinoma and sarcoma of larynx (Burow), carcinoma of lung (Langhans), chondroma of lung (Siegert), carcinoma of breast (Aoyama), and endosteal sarcoma (Hildebrandt). Some fibromas or sarcomas of the larynx are characterized by the development of large masses of amyloid. Usually the amyloid masses have a concentric or radial striation.

Colloid Degeneration.-Homogeneous, semisolid, acidophile material occurs in many tumors in large intercellular masses and in small intracellular globules. The largest collections are seen in ovarian adenomas, in which the ordinary mucin may present the qualities of firm colloid. Thyroid tera- 
tomas containing little else than thyroid tissue with distended alveoli are frequent in the ovary, and some ovarian adenomas, not of thyroidal nature, may show considerable masses of colloid substance. Carcinomas in various situations may present cylindrical intra-alveolar masses of colloid approaching the appearance of thyroid tissue. Colloid appears in the cells of many tumors in the form of globules of rather dense, strongly acidophile material. These structures are one of the chief sources of Russell's fuchsin bodies. Colloid droplets may appear in well-nourished mitotic cells or in cells showing advanced stages of other forms of degeneration.

Miscellaneous Peculiarities of Tumor-cells.-Besides the well-defined types of degeneration tumor-cells exhibit a wide variety of alterations which have been subjected to close scrutiny, but which it is difficult to interpret and classify.

These changes affect both nucleus and cytoplasm.

In the highly developed tumor-cell nucleus may be distinguished five elements:

I. Nuclein, a nucleoproteid, composing the chromatin which appears in densely basic staining clumps, normally arranged along the periphery of the nucleus or as an intranuclear network.

2. Paranuclein, an acidophile substance composing the nucleoli.

3. Linin, or plastin, an achromatic substance forming an intranuclear network.

4. Amphipyrenin, which some authors describe as constituting the nuclear membrane. It is distinguishable from linin. According to Albrecht the nuclear membrane is a lipoid substance.

5. Nuclear fluid, filling the meshes of the nuclear network.

As compared with normal cells all of these nuclear structures may be much more abundant in tumor-cells. In giant-cells, especially those of myogenous origin, and in various sarcomas and epitheliomas, the nuclei reach astonishing proportions from hypertrophy, chiefly of nuclein. In epitheliomas great excess and multiplicity of paranuclein bodies is somewhat characteristic, while in endotheliomas the nucleoli are relatively small. Shrinkage and pyknosis of nuclei occurs in degenerating and necrosing areas of many tumors. Wide distention from imbibition of fluids may accompany hydropic degeneration of the cytoplasm. Rarefaction of nuclein masses may result in a pale diffuse stain of the thickened chromatin network. Or the chromatin may appear in very thin strands, eventually disappearing in complete karyolysis. A common appearance in carcinomas is the presence of several discrete blocks of chromatin lying irregularly in a nucleus devoid of chromatic membrane. The various stages of karyorrhexis may be followed in degenerating or necrosing cells. The fragments of chromatin thus resulting may be scattered in the cytoplasm and remain pyknotic or become vacuolated or surrounded by cytoplasmic vacuoles or become dissolved. Extrusion of chromatin into the cytoplasm may result in the appearance of many basophile granules in the cell. The chromatin or linin may break up into many fine rings within the nucleus and these be discharged into the cytoplasm, as observed by Schuller and interpreted by him as the forms of an intranuclear parasite.

Under the term "pseudo-adipose and cystic degeneration of nucleus" Pianese described the appearance of homogeneous spheroidal, brownish, achromatic globules in the nucleoplasm, occurring especially in tumors of the liver. This author also describes a hyalinosis of the nucleoplasm which results in a transformation of part or the whole nucleus into a hyaline mass.

Fragmentation of the nucleus with formation of pseudonuclei occurs 
in many giant-cell tumors. In melanoma the nuclei often contain large masses of homogeneous material in which appear all stages of the formation of granular pigment.

In the cytoplasm of tumor-cells the early stages of degeneration, hydropic, mucous, lipoid, fatty, glycogenic, colloid, hyaline, and calcific, yield a great variety of distortions of the cell structure and abnormal granules, rings, globules and complex masses, most of which are identified by appropriate technics. The complex morphology of these products is illustrated in the extensive literature on the pseudoparasites of cancer, especially in the work by Pianese. Many of the bodies, especially those of epithelioma, I have found duplicated by diphtheria toxin necrosis of the corneal epithelium.

Phagocytosis by tumor-cells yields many intracellular bodies, as from red blood-cells, leukocytes, other tumor-cells, bacteria, pigment, and foreign bodies, all in stages of degeneration. Meser found throughout a deep cancer of the breast lycopodium seeds which had penetrated the tumor from the dressing and had been englobed in the cells.

Multiplication of centrosomes with their surrounding archoplasm may yield a number of cytoplasmic bodies containing a central chromatic point,

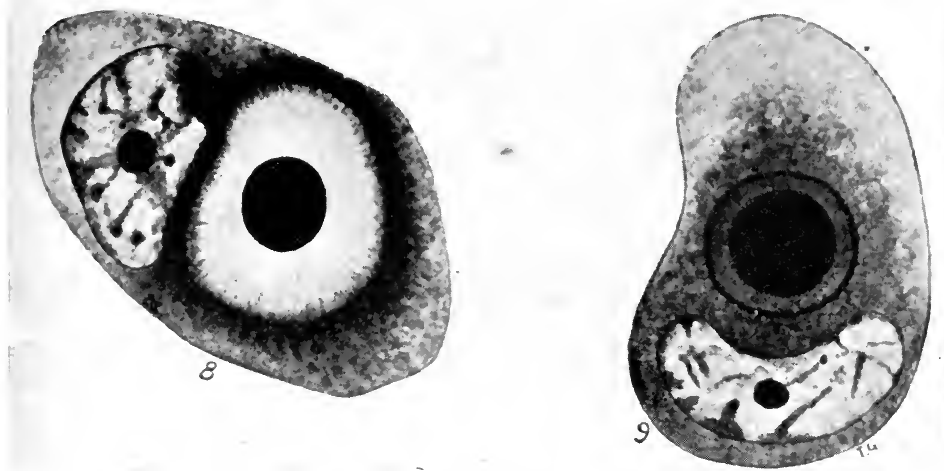

FIG. 6.-Bird's-eye inclusions in cancer-cells. (After Le Count.)

and a rim of condensed protoplasm more or less separated from the remaining cytoplasm. Hansemann found as many as twenty centrosomes in one cancercell with multipolar mitosis. In normal cells the occurrence of multiple centrosomes has been abundantly shown by many histologists, Heidenhain counting as many as one hundred in a giant-cell of the rabbit's marrow.

Comparing the centrosomes of the guinea-pig's spermatocytes with structures in cancer-cells, Borrel has shown that multiple centrosomes are a prolific source of the peculiar bird's-eye inclusion of Leyden which he and many others have regarded as a parasite. He finds that the centrosomes of cancer-cells may appear as chains or congeries of chromatin granules without special protoplasmic mantle or as multiple chromatic granules with well segregated and somewhat differentiated cytoplasm, or as single swollen chromatin bodies with large cytoplasmic masses lying in cell vacuoles.

LeCount's observations support Borrel's interpretation of a large class of intracellular bodies in cancer. Benda in dissenting from this view was able to show the presence of normal centrosomes in cells containing the bird'seye inclusion, possibly from some other source than centrosomes. 
Accumulation of secretory products greatly modifies the appearance of many tumor-cells and produces some structures which have been interpreted as parasites. Since the secretory activities of glandular epithelium are closely associated with that portion of the cell containing the centrosome, the presence of chromatin in many cancer bodies is perhaps not an argument against the origin of these bodies from secretory processes. That such an origin accounts for many of the peculiar structures in cancer-cells has been maintained by many observers. Pianese thus explained the majority of the bodies of the Thoma-Sjobing type which were small perinuclear bodies with relatively large chromatin granules. According to Greenough the characteristic bird's-eye inclusion of cancer-cells is practically limited to glandular carcinomas and is chiefly the result of secretory processes.

$\mathrm{He}$ found them nearly constantly in breast cancers, but absent in sarcomas and epitheliomas.

Somewhat similar conclusions have been reached by Nosske, Honda, and Klimenko. As a rule these authors found the typical inclusions in slowly growing cancers in which secretory function may be assumed to persist. In position they usually lie between the nucleus and the alveolar lumen (Greenough). They vary in size from minute globules to masses distending the cell. Stains show a central basophile portion which may be of large size or may be absent, and a rim of protoplasm which may be granular, or radially striated, or surrounded by a condensation capsule. While Plimmer stated that these bodies occur in enormous numbers in rapidly growing cancers, chiefly in the growing edges, later observers have not confirmed this statement, but have found them in moderate numbers, chiefly in slowly growing tumors, and well within the edges. Apolant and Embden noted very few inclusions in mitotic cells, an observation which accords equally well with the secretory origin as with the centrosome theory.

Thus the specific bird's-eye inclusions of cancer-cells, first described by Virchow, suggested by Foa as a possible parasite, appropriated and variously described by Thoma, Soudakewitsch, Leyden, Plimmer, Ruffer and Walker, and finally defended by Feinberg, must be ascribed to two main sources, multiplication of centrosomes and secretory processes in which centrosomes are probably concerned. The chromatin element of these bodies favors their origin from centrosomes, while the location in the cell and the morphology and occurrence chiefly in glandular carcinoma support the theory of retained secretion.

\section{STRUCTURE AND GROWTH}

The composition of tumors and the characteristics of their growth present many variations from the normal standards.

Some tumors, called histioid, reproduce simple tissues and are composed of a single and uniform parenchyma.

Others, called organoid, are of more complex architecture consisting of tumor-cells supported by normal stroma and blood-vessels, and reproduce the structure and preserve more or less the function of organs. The extremes of these types are well defined, as lymphosarcoma versus adrenal adenoma, but the great majority of tumors reveal some organoid characters.

Homotypic or homologous tumors reproduce the structure of the tissue in which they arise (adenoma of breast); heterotypic or heterologous growths differ in structure from the tissue in which they grow (chondroma of breast). Tumors may reproduce the structures of adult organs, and tumor cells may preserve the characters of adult cells, as in epithelioma of the lip, and there may be termed adult tumors. Others arise from groups of embryonal 
cells whose adult characters they never reproduce and these are conveniently termed embryonal. Examples are the so-called fetal adenoma of thyroid, and many teratomas of testicle and kidney. Such embryonal tumors are to be distinguished from those which, because of extreme metaplasia, differ widely from the originating structure. The organoid homotypic and adult characters tend to disappear as growth proceeds more rapidly and in metastases and recurrences, or in different parts of the same tumor. In ordinary cancer of the breast there may be wide variations in these qualities.

- Adamantinoma in successive recurrences may change from an adult acanthoma through an adenomatoid growth to a highly malignant tumor composed of closely packed, indifferent round and polyhedral cells.

Chondro-epithelioma of the parotid of comparatively adult type may recur as pure atypical myxoma. Recurrences after operation may differ widely from the original structure. I have observed typical perithelioma

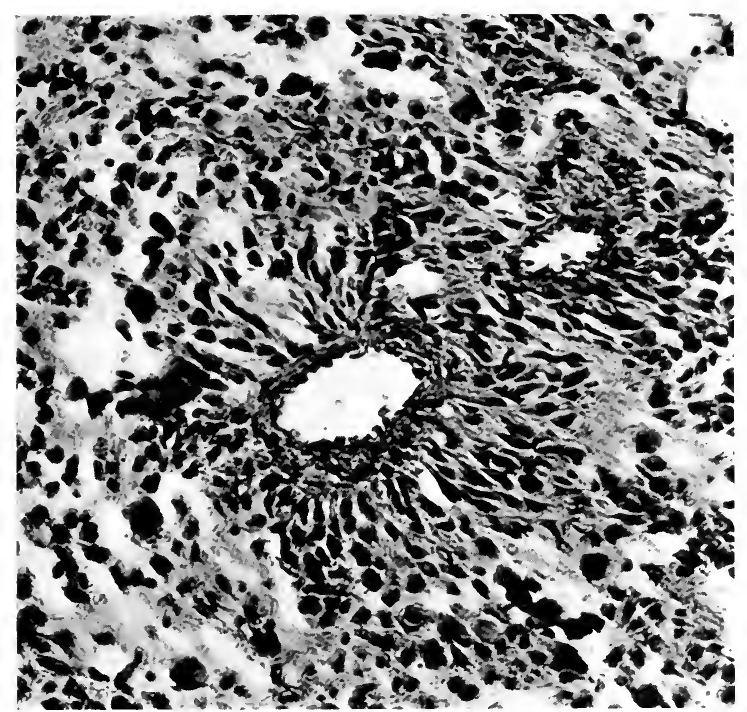

FIG. 7.-Peritheliomatous structure assumed by adenocarcinoma of uterus following curettage.

after the removal of adenocarcinoma of testis, and in a uterus removed shortly after curettage for typical adenoma malignum I have found a spindle-cell tumor of perithelial type.

Wholly spontaneous changes are illustrated in adenocarcinoma of adrenal which may invade the liver under the form of diffusely growing large cell carcinoma.

In normal growth cells maintain a certain quantitative relation to the other elements of the tissue of origin, and they have an orderly arrangement and a polarity toward each other and to contiguous structures, but in tumors there is a progressive loss of these relations. Even in benign tumors, as in chondroma, the cells are in excess over stroma and their relation and position are altered.

Some adenomas, as of thyroid and stomach, show a remarkable preservation of normal structure even while proving malignant, but in most pro- 
nounced blastomas cells increase enormously over stroma, eventually relations between individual cells and between cells and stroma are lost, and the cells grow diffusely. Several notable characters of tumors are dependent upon the gradual development of these tendencies. Benign tumors retain much of the tissue organization, grow from their own resources, provide themselves with their own stroma and vessels, and push surrounding tissues before them and remain encapsulated.

In malignant tumors the cells increase in number and size, become displaced from natural connections, lose their polarity, and invade and clestroy surrounding tissues.

Lining cells grow outward into polypoid projections, drawing bloodvessels with them, or downward, forming compressed pearls or convoluted cords; gland alveoli elongate and widen, or sacculated projections from sides or fundi produce simple tubules, or cystic or complex adenomas; diffuse masses of cancer or sarcoma cells disorganize the normal structure and force their way into neighboring spaces, alveoli, and vessels. The demands for nourishment lead to formation of elongated vessels sheathed with tumorcells producing the arrangement of perithelioma, which may appear in many varieties of tumors where the cells are pressed for food.

Necroses arise where nutrition fails. Finally, the disordered cells penetrating lymph- and blood-vessels, become loosened and pass into the circulation, lodging into adjacent lymph-nodes or distant organs, giving rise to secondary tumors.

Many of these characters of tumor growth must be referred to mechanical crowding of cells, and the demands for nutrition, influenced by whatever remnant of tissue organization the tumor may retain.

The local extension of malignant tumors gives rise to many changes in the invaded tissue and reveals many specific qualities of tumor-cells.

Local extension involves the passage of tumor-cells first into tissue spaces and lymph radicles. Whether tissue spaces directly communicate with lymph radicles (Recklinghausen), or lymph radicles form a series of closed anastomosing channels (Ranvier), there is no doubt that inflammatory and legenerative processes soon open the lymphatic vessels to the tumor-cells.

From this point the ameboid properties of the cells (Klebs, Waldeyer), mechanical pressure, and the natural course of fluids facilitate the progress of the tumor toward the larger lymph-vessels and nodes. Invasion of the terminal vessels occurs through the communication between the lymph and blood capillary plexuses (Sabine).

Through gland ducts, renal tubules, bile ducts, bronchioles, and tubular structures also, as well as through vessels, local extension may occur.

In muscle, extension of tumor-cells is affected by the muscular contractions, and especially in pyloric cancer the extension may be wide-spread before any local tumor develops. Blumenthal attributes the invasive properties of tumor-cells to their proteolytic enzymes which he conceived as dissolving the tissue barriers, but neither the histology of the process nor the known capacities of tumor ferments support such a view.

Inflammatory reaction frequently meets the invasion of tumor-cells. This is usually of a low grade, causing the appearance of lymphocytes, large mononuclear leukocytes, plasma-cells, and in certain cases polynuclear leukocytes. It is a highly significant feature of malignant tumor growth and must be regarded as a defensive process. Well-marked reaction signifies a pronounced capacity to limit the tumor growth, but not that the effort will be successful. Yet about many epitheliomas which are making little progress one finds a thick barrier of lymphocytes. In carcinoma of the breast one 
may find islands of tumor-cells inclosed in masses of lymphocytes and presenting clear signs of degeneration.

It is probable, as Orth concludes, that some degeneration of tumor-cells always precedes the tissue reaction.

Very actively growing malignant tumors and their late metastatic deposits often fail to show any inflammatory reaction, but the majority of infiltrating growths elicit considerable cellular response from invaded tissues. Degeneration and calcification of tumor-cells, formation of macrophages from fibroblasts, endothelium, and leukocytes, and appearance of giant tumor-cells, accompany the process.

Besides the general inflammatory reaction, which fails only in very rapid invasion, the different tissue cells exhibit interesting reactions against tumorcells.

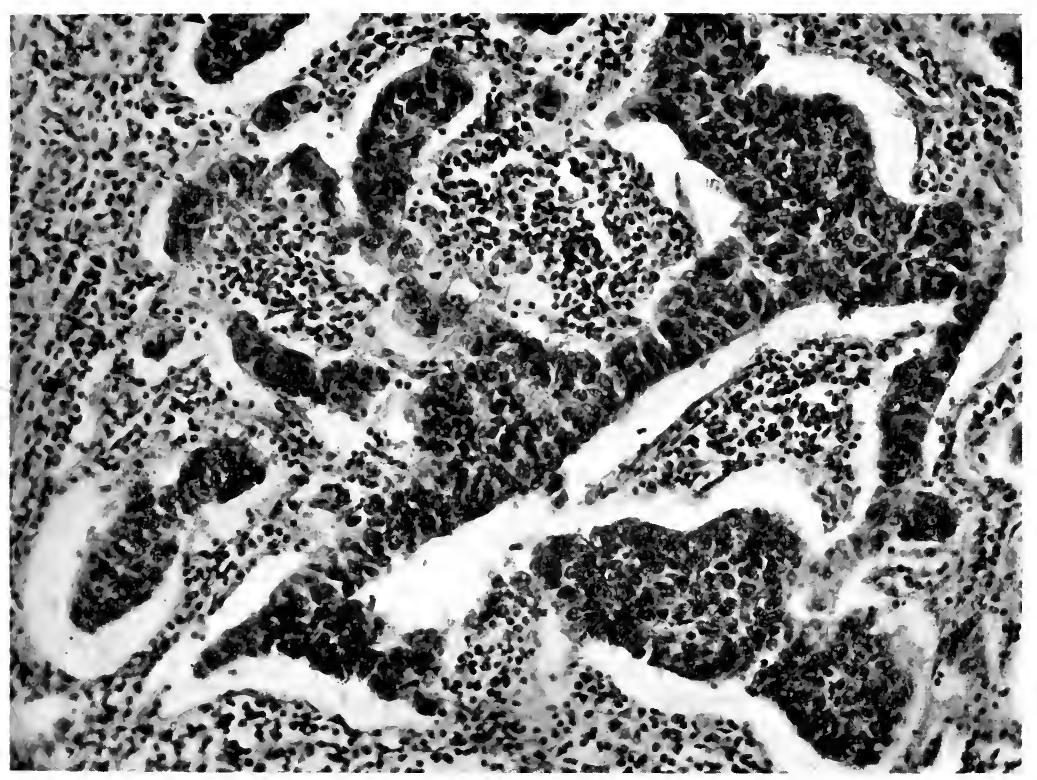

Fig. 8.-Diffuse lymphocytic infiltration in a rapidly growing carcinoma of breast.

Striated muscle-cells exhibit a relative immunity to many cancers which infiltrate the perimysium while long sparing the muscle-fibers. Eventually the sarcolemma is penetrated and the tumor-cells pass rapidly along the sheaths, absorbing the muscle substance, hollowing out central portions of fibers, causing fatty and hyaline degeneration, fragmentation, swelling of ends of fibers with formation of giant-cells, and exciting proliferation of nuclei of sarcolemma and muscle-cell (Fujinami).

Smooth muscle-cells also are rather resistant, but are frequently destroyed chiefly with simple atrophy.

Specialized gland epithelium is usually passive before the attack of invading tumors. The cells degenerate and atrophy, the membrana propria often persisting to inclose pseudo-alveoli of tumor-cells. Gland ducts are often filled or distended with tumor-cells which replace the lining cells or stimulate them to feeble multiplication. 
Squamous epithelium tends to hypertrophy when invaded by alien cells, with thickening of the Malpighian layer, formation of new papillæ and hyperkeratosis. With melanoma the swollen hyperchromatic and pigmented epithelium is often difficult to distinguish from the tumor-cells.

Endothelium usually degenerates, exfoliates and disappears, or forms macrophages. In blood-vessels it may proliferate and assist in the formation of stroma.

In connective tissues where the changes are not obscured by inflammation the invasion leads to degeneration, compression, and atrophy of fibroblasts, swelling, edema, fragmentation and solution of fibrils and matrix, and destruction of elastic fibers.

In many organs preëxisting stroma, vessels and trabeculæ, may long persist, as in the lung, where the air vesicles may be passively filled with invading tumor-cells. Strongly encapsulated organs, as testis, kidney, lymphnodes, spleen, may long resist invasion and rupture by tumor-cells.

Reactive growth of invaded connective tissue plays an important part in the course of desmoplastic tumors. In scirrhous and metastatic carcinoma it may far exceed the bulk of tumor-cells and may eventually lead to complete scarring of large quescent areas once the seat of active proliferation.

In fat tissue cells penetrate readily, replace the fat in the tissue cell envelops, incite mucous degeneration, form foreign body giant-cells about free fat, and sometimes induce proliferation of fat-cells.

Periosteum and perichondrium may successfully resist invasion from without, but when the element of pressure is added they yield to the invading cells. From within the attack on bone is more rapid. The matrix is absorbed, the tumor-cells forming lacunæ like osteoclasts, dissolving cancellous tissue, and in many cases of myeloma, sarcoma and metastatic hypernephroma and carcinoma, perforating the shaft. New blood-vessels accompany these inroads and new fibroblasts act as osteoclasts, facilitating the absorption. Formative osteitis accompanies many tumor processes in bone. Located in periosteum it may lead to bony encapsulation on the periphery keeping pace with bone absorption within. Epithelial tumor-cells invading bone are usually free from new bone formation, but in a group of carcinomas, especially of the prostate, bone-cells are excited to extensive proliferation possibly even to the extent of a secondary tumor growth. Such cases are wholly different from the tumors originating in bone-cells and yielding the extensive group of bone sarcomas. In certain very vascular tumors the bone is passively absorbed and replaced by large sinuses lined by giant-cells.

Nerve-trunks owing to their rich lymphatic sheathing are extensively invaded, especially by epithelial tumor-cells. The medullary sheaths atrophy from pressure, the axis-cylinders being the last element to disappear; while the nuclei may feebly multiply.

In a few instances the nerve-trunks seem to lead the way in local extensions, especially of epidermoid carcinoma. Brain tissue is quite passive to the invasion of tumors, suffers pressure atrophy with degeneration of all the specific elements, while necrosis often occurs from occlusion of vessels.

In the local extension of malignant tumors the inherent momentum of cell growth is doubtless the chief factor. As this property constitutes the great distinction between normal regeneration and malignant neoplasia, its analysis is of first importance, and will be discussed under general etiology. Here it may only be mentioned that the histological study of invaded tissues leads to the conclusions above stated, and offers little basis for the theories of attraxins drawing the cells deeply into the tissues, or of special toxic agents 
preparing the tissues for invasion, or for the belief that inflammatory changes greatly influence the invasive properties of the cells of an established tumor.

Functional activity in tumors may be retained in part or exhibited in exaggerated form. Geotropism, the quality of living cells to cover and grow along surfaces, is shown in epithelioma, endothelioma, and adenoma. Phagocytosis of bacteria, leukocytes, red cells, fragments of organ cells, other tumorcells, even in mitotic division, bile, fatty material and foreign bodies, is exhibited in great variety. Many tumor-cells may exhibit slow ameboid properties, as first observed by Virchow in chondroma.

The abundant matrix in many osteo- and chondrosarcomas, the mucus of myoma, fat-cells in liposarcoma, and the rigid alveolar structure of adenoma destruens even in metastases, are familiar indications of functional activity.

Clear examples of overfunction of tumor tissue are seen in mucous carcinoma of the colon and in melanoma of the skin. Some tumors of the hypophysis seem to be followed by overactivity of the influence of this gland on growth.

While tumors destroying the adrenal, pancreas, or thyroid may not be followed by Addison's disease, diabetes, or myxedema, yet the functional capacities of such tumor tissue have not been demonstrated by chemical and physiological tests, and the absence of specific disease with these tumors may be referable to the wide limit of safety of the organs involved or the presence of aberrant glands. Hansemann reports complete destruction of the pancreas by carcinoma without diabetes, although diabetes is common with cancer of the pancreas. The early bilateral adenomas of the adrenal may very well fail to give Addison's disease, but I have seen complete destruction of both adrenals by diffuse hypernephroma with bulky metastases, but no signs of Addison's disease. The disease was rapid and no search was made for aberrant adrenals.

Tetany followed extirpation of one-half of the thyroid for cancerous goiter by v. Eiselsberg, but disappeared on the development of a metastatic focus. When this focus was removed the symptoms of tetany recurred, in spite of new metastatic growths, these, Hansemann suggests, being more anaplastic. Since a large portion of the thyroid was not removed and since the symptoms suggest involvement of the parathyroid rather than the thyroid I think the interpretation of this case is very uncertain. Many thyroid adenocarcinomas contain colloid and functionate, but it is difficult to find any histological traces of functional activity in true cancer of the thyroid.

Adenoma and primary carcinoma of the liver usually fail to show traces of bile formation, yet Heller observed bile in the cells of a pulmonary metastasis from liver cancer. Hansemann found bile in the adenomatous portion, but none in the carcinomatous areas of an adenocarcinoma of the liver.

Chemical studies in general support the view that many tumors retain some of the function of their parent cells, as shown by the high content and qualities of lipoids in hypernephroma (Wells) and the considerable content of iodin in thyroid cancer (Beebe, Ewald). As a rule, functional activities diminish with increasing anaplasia, and original overactivity may be succeeded by complete failure, as in the pigment-free metastases of melanoma.

Stroma.-Benign or encapsulated tumors provide themselves with a new stroma, while infiltrating growths appropriate and alter the preëxisting stroma of the invaded tissue. In sarcoma it is usually much less abundant than in carcinoma and may consist entirely of blood-vessels, while in cancer it is more abundant and fails only in very anaplastic growths. In sarcoma a fine intercellular stroma is in some degree constant, but may be difficult 
to demonstrate. In carcinoma the cells are in immediate apposition without intervening fibrils. Endothelioma reveals its close embryological relation to sarcoma by fine fibrils which imperfectly penetrate the cell masses (Woolley).

In benign organoid tumors the neoplastic process is usually limited to one type of cell, in adenoma to epithelial cells, and the new stroma results from a formative influence on the cells of the connective tissue and vessels associated with cells originating the tumor.

Mechanical influences may be prominent in the behavior of this stroma or the cells may show active mitosis, and even participate in the tumor growth. Thus stroma may have adult characters, or as in fibro-adenoma it may be difficult to determine whether the epithelium or the stroma cells dominate the neoplastic process. In certain ovarian adenomas the periacinar stroma may be extremely cellular, as in sarcoma.

It seems necessary to sharply distinguish between those tumors whose stroma is clearly not neoplastic and those in which it constitutes a part of the tumor and which must, therefore, be regarded as mixed tumors. In chondro- and osteosarcoma the stroma is a combined product, consisting of intercellular substance derived from the tumor-cells and new vessels derived from the tumor or from peripheral tissues. In teratomatous cancer of the testis the stroma may be richly infiltrated by lymphocytes and even contain lymph-follicles, yielding a type of stroma not seen in any other carcinoma. In veins the stroma of an invading tumor may consist of capillaries arising from the vessel wall.

Elastic fibers are seldom reproduced in the stroma of tumors and if present belong chiefly to the preëxisting tissue (Williams).

According to Bonney they disappear in all areas of connective-tissue proliferation and cellular exudation, but in isolated areas in breast cancer he found bulky periacinar masses.

The stroma of an original growth may fail to be reproduced in metastases with increasing anaplasia, but often reappears in typical form, as in scirrhous carcinoma, or in the overdeveloped vessels of fungous hematodes.

The stroma of surrounding tissues may be (I) passive during the increase of tumor-cells, or it may be incited to (2) reactive growth, and there is considerable evidence to show that this reaction may reach almost if not quite to a (3) neoplastic grade.

Most highly organized tissues are passive during tumor invasion, as the liver in cancer. Very active growth of tumor-cells usually fails to excite any reaction even from simple tissues, as in lymphosarcoma in the skin.

Apart from collateral hypertrophy as previously discussed, epithelial tumor-cells exert a notable formative influence on connective tissue; they are desmoplastic.

So marked is this influence that many carcinomas are composed of much new connective tissue and relatively few cells both in primary tumors and in metastases. Originally this action is the result of a formative stimulus of epithelium upon connective tissue, but eventually it proves protective and some cancerous areas undergo complete regression from overgrowth of fibrous tissue.

An osteoplastic influence is exerted on bone tissue by some cancers, and active budlding and proliferation of muscle-cells is occasionally seen with rare myoplastic tumors.

That the presence of a tumor may excite its stroma or surrounding tissue to neoplastic growth has never been demonstrated. While improbable, such an action is not impossible, and has been suggested by several appearances encountered with spontaneous and transplanted tumors. Langhans con- 
sidered the possibility that teratoma testis might incite the spermatoblasts to tumor growth in certain complex tumors of this organ.

In certain lymphocytomas it is difficult to distinguish between the infiltrative growth, multicentric origin, and the contact influence of tumor-cells. Haaland thought he observed the transformation of pulmonary cells into tumor-cells about metastatic carcinoma in the mouse. Ehrlich, Loeb, and others have observed the sudden appearance of sarcoma in the course of transplantations of carcinoma in mice and rats, and Murray has strongly substantiated the belief that this sarcoma arises from the stroma and not from the altered cancer-cells.

The influence of transplantations renders these observations inapplicable to human tumors, and several uncertainties still surround the interpretation of this remarkable change of structure. No such stroma reaction has been observed in spontaneous tumors, although the reactive overgrowth of invaded tissues may at times exhibit some of the qualities of a neoplasm.

Regressive changes occur in tumor stroma very similar to those observed in the parenchyma. In some cases these changes greatly influence the character and course of the tumor. In progressive keloids the hyaline stroma is the chief portion of the tumor.

Cylindroma, or syphonoma, are terms applied to a tumor structure in which the stroma appears in the form of elongated, twisted, thickened cords of hyaline material. This structure is most often seen in basal-cell carcinomas. In old regressing carcinoma large areas of tissue may show very few tumor-cells in a mass of quiescent hyaline stroma.

Edema, mucous degeneration, and calcification of stroma are frequent factors influencing the course of tumors.

In cancers, fatty degeneration often affects not only the parenchyma but also the stroma. Calcification may begin in the stroma and gradually involve the entire substance of myomas, or it may form a hard shell about this or other growths. Solid or lamellated concretions of hyaline material of uncertain nature are occasionally seen in the stroma as in the parenchyma.

Experimental Analysis of Stroma Reactions.-Much light has been thrown on the meaning of the cellular reaction to invading tumors by experimental studies on transplantable tumors.

The inoculation of susceptible animals with a tumor graft is usually followed by a brief local polynuclear leukocytosis incited by diffusible products of tumor-cells, red cell detritus, and traumatism.

An excessive degree of this reaction may prevent the graft from surviving. With successful grafts there is later a gathering of lymphocytes in moderate number and these may form rich foci which interfere with the growth of the tumor-cells. The graft then becomes successfully implanted by inciting the growth of fibroblasts and capillaries about which the cells adhere. Often the vessels of the graft are appropriated and reopened. From this point the growth proceeds by multiplication of the transferred cells about the new stroma derived from the host's tissues. In its further progress the tumor may become encapsulated and thus be shielded from cellular attack, or it may early show infiltrative tendencies unopposed by any cellular reaction.

In some conditions animals become immunized by the temporary growth followed by spontaneous regression of the tumor (Gaylord, Clowes). In such cases one of the earliest changes observed is a focal degeneration of the tumor-cells accompanied by an accumulation, probably from chemotactic influences, of ameboid large and small lymphocytes, mononuclear leukocytes, and often many plasma cells. Minute hemorrhages may occur in the tumor about which eosinophile cells gather. These cells surround the tumor and penetrate within it, sometimes richly infiltrating the stroma.

Then follows contraction of the tumor mass, multiplication of spindle-cells, growth of new vessels, and encapsulation of the regressing tumor. During the absorption process the endothelial macrophages appear and giant-cells result from fusion of tumor elements. Even to a late stage mitosis may persist in such encapsulated regressing tumors and section of the capsule may excite renewed growth. 
In immune animals the implantation of cancer excites a more active emigration of polynuclear cells, the degeneration of parenchyma cells appears earlier and is more pronounced, and the lymphocytic reaction is less marked, plasma-cells being absent (De Fano).

With a sarcoma of the hare transplanted into rabbits v. Dungern and Coca obtained in the rabbit hypersensitization followed by complete immunity by successive implantations of the tumor. 'The second or third graft excited a rich production of endothelial macrophages which promptly surrounded and englobed the degenerating tumor-cells, and formed macrophage thrombi in the small vessels. Leukocytes were not prominent in this reaction. In the adjoining lymph-nodes were many similar endothelial macrophages.

With Jensen's mouse cancer Russell found that grafts in resistant mice suffered ex. tensive degeneration and the peripheral rim of surviving cells failed to excite a stroma reaction.

Vorking with Japanese mice Burgess observed that in both susceptible and nonsusceptible animals tumor grafts established themselves by exciting a stroma reaction, after which in the immunized mice the nutrition of the tumor was cut off by overgrowth of fibroblasts with much intercellular substance.

In the interpretation of these results one may conclude that in highly immunized and hypersensitized animals the reaction is pronounced, immediate, and exudative, preventing the growth of new stroma or by thrombosis (v. Dungern-Coca) shutting off blood-supply. In spontaneously immune or in alien animals the reaction is less pronounced, new stroma forms, but later degeneration of the graft leads to strangulation by connective-tissue overgrowth.

Analyzed in the light of experimental studies, the processes connected with the growth of tumors signify as follows:

Infiltrative growth without stroma or cellular reaction indicates absence of protective forces in the body.

The development of vascular stroma from the host's tissues, up to a certain degree, is favorable to the growth of the tumor.

Overgrowth of stroma with much intercellular substances is a menace to the tumor.

Polynuclear leukocytes gather about the tumor as the result of necrosis, trauma, bacterial processes and actively chemotactic tumor products, and signifies a sharp response against the tumor.

Lymphocytes and large mononuclear leukocytes signify a reaction of immunity, limiting the growth of cells, establishing local tissue immunity, and conveying immune forces to a distance. Plasma-cells are a later factor in the same process.

Eosinophile cells gather about hemorrhages.

Endothelial proliferation, if slight, figures as a part of the stroma reaction, but if excessive, macrophages form and oppose cell growth.

Degeneration of tumor areas, fusion giant-cells, and necrosis may result chiefly from withdrawal of nutriment.

It is obvious that most of these conclusions have been warranted by previous study of human material, but it must be claimed that the experimental studies have rendered them more precise.

Blood-vessels. - The relation of blood-vessels to tumor growth presents two highly important aspects, first in connection with the beginning of cancer, and second in the channels of nutrition in the progressive growth of tumors.

Franz Boll from a study of early epithelioma and of the growing edges of such tumors concluded that changes in the blood-vessels determined the proliferation of epithelium. He found the walls of capillaries thickened, opaque, and varicose. The adventitia of veins and arteries was invaded by cells, and the vessels lost their sharp demarcation from the surrounding tissue which took on an embryonal character.

These changes were found throughout the tumor and preceded the extension of the growth. An extensive disease of the vascular system of the whole 
area involved was in Boll's opinion an essential part of the cancerous process. The later study of the vascular system of malignant tumors, while not fully demonstrating the prime influence of vascular lesions, has yet emphasized the importance of the blood-supply in the origin and growth of many neoplasms.

Theilhaber, among recent writers, has emphasized the importance of arteriosclerosis in the origin of many cancers in elderly persons. He concludes that the local predisposition of aging tissues to cancer results from a diminution of the cellular elements and a contraction of the blood-vessels in the connective tissue. On the other hand, Warner reports a study of the blood-vessels of 206 cancerous organs, finding that I05 (5 I per cent.) showed obstructive arterial changes, II8 ( 57 per cent.) fibrosis, and 85 (42 per cent.) lymphocytic infiltration. Since normal vessels were present in almost half the cases endarteritis could not be considered a necessary factor in the production of the disease.

Possibly a very close analysis by various methods of the circulatory conditions about beginning carcinomas would show a more constant relation between this factor and carcinoma in elderly persons. On the contrary side, increased vascularity has been demonstrated in the beginnings of many carcinomas, and has been assumed to account for the awakening of many cell rests. Evidently the exact relation between blood-supply and the inception of carcinoma has not yet been elucidated.

Since the demands for nutrition in tumors exceed those of normal tissues the significance of the vascular system is correspondingly increased. In the older literature S. von der Kolk stated, as a result of injection experiments, that only arteries formed anew in tumors, the veins being those which became inclosed in the tumor.

Extensive lesions of the blood-vessels have been described by many authors as a significant feature of tumor growth (Eberth, Belapolsky). Virchow pointed out that the circulation in many tumors is to some degree independent of and different from the general circulation, a fact according with the parasitic nature of tumor growth. The difficulty of passing injection material from arteries through veins he attributed to compression, invasion, and thrombosis of the veins and to an indirect connection of veins with arteries.

The "saber-sheath" flat veins on the surface of many tumors result chiefly from compression. The turgescence of enlarged veins leading out from malignant tumors was one of the gross features giving rise to the term "cancer." Compared with the affected organ the vessels of actively growing tumors are more numerous, dichotomous branching of the arterial system is absent or irregular, and the main vessels are often tortuous and spirally wound.

The vascular system may be studied by corrosion preparations, by $x$-ray photographs of specimens injected with bismuth and oil, and by Hill's method of injection with India ink with subsequent clearing of tissues by alkali and glycerin.

From results obtained by the two latter methods Goldman observed the marked increase in vascularity in the periphery of growing tumors, while in quiescent cases the central portions were nearly devoid of vessels and those of the periphery reduced. The new vessels were usually of small size, tortuous, irregularly distributed, and the arteries rapidly broke up into capillaries.

In the growth of blood-vessels in tumors one may distinguish two factors, direct irritation by tumor products and a formative influence exerted by tumor-cells. Along the growing edges of advancing carcinoma there is often a zone of hyperemia, and section shows many new capillaries and roundcells, producing at times a structure which is equivalent to granulation tissue. Such changes, which soon regress, may be referred to inflammatory reaction. 
In the artificial implantation of tumor grafts, in the organization of intravascular tumor thrombi, in the natural growth of angiomas, and in the recurrence of many varieties of cellular tumors, new vessels develop apparently as a result of a formative influence centered on the vessels of surrounding tissues.

In many endotheliomas and some angiomas the vessels constitute the unit of the tumor; elsewhere they are devoid of neoplastic qualities. The structure known as perithelioma may develop solely as an expression of a formative influence of tumor-cells upon blood-vessels, and has been observed in angiosarcoma, fibroblastic sarcoma, hypernephroma, epithelioma, and teratoma.

The structure of the blood-ressels varies greatly.

In some benign growths the large arteries exhibit all three coats in proportions approaching the normal. In most malignant tumors muscle-tissue is deficient in walls of arteries, and the main type of vessel is composed of adult fibroblastic tissue lined by endothelium. In many endotheliomas the vessels are chiefly spaces lined by tumor-cells. In adenoma of thyroid and some primary carcinomas of the liver widely dilated capillaries lined by endothelium produce highly vascular growths. In telangiectatic sarcoma the capacious sinuses may be lined by endothelium backed by connective tissue. In bone-sarcoma widely dilated sinuses are lined by tumor giantcells, as in the pulsating sarcomas of tibia and humerus. Finally a feeble circulation may be maintained in intercellular tissue spaces without definite lining cells.

Lesions of blood-vessels are frequent and important for both the tumor and the organism. Functional inadequacy or compression of vessels is chiefly responsible for the degeneration and necrosis of the parenchyma of tumors.

Rupture from degeneration or trauma gives rise to hemorrhage and infiltration with blood. Strangulation of pedunculated ovarian cystomas or uterine myomas, cutaneous sarcomas, and even breast cancer may lead to complete hemorrhagic infarction, necrosis, and sloughing.

Hyaline degeneration of vessel walls yields a characteristic structure in certain sarcomas, as in the ovary.

The vessels of invaded tissues are passively compressed or react with periarteritis and round-cell infiltration or the walls are invaded by tumorcells by way of the vasa vasorum, and the perivascular lymphatics form a channel directing the course of invading cells. Arterial trunks long resist the attack of most tumors, but fall a ready prey to squamous epithelioma whose cells penetrate the adventitia, split up the muscularis, occlude the lumen, and thus cause necrosis of the invaded tissues. In such vessels the tumor-cells may largely disappear with fibrous organization. In the veins tumor-cells readily penetrate to the intima, producing nodular swellings, endophlebitis carcinomatosa, and eventually rupturing into the vein.

It has often been noted that very vascular tumors may long fail to develop metastases, yet the invasion of tissue veins is the chief source of metastatic growths in sarcoma and with many carcinomas. An intravascular position characterizes the growth of certain tumors throughout most of their history. Chorioma of the uterus and testis is remarkable for its limitation to pelvic, vaginal, abdominal, and pulmonary veins. According to Marchand and Risel other rare tumors growing in or invading the blood-vessels may imitate the hydatidiform appearance of chorioma. Hypernephroma often passes up the renal vein, and distends the vena cava and the hepatic veins before generalizing. An adenocarcinoma of the liver regularly appears as a circumscribed growth distending the large branches of the portal vein in this organ. 
Course and Rate of Growth.-Many variations are observed in the course and rate of growth of tumors.

I. Rapid and progressive multiplication of tumor-cells without notable intermission or hindrance is characteristic of most highly malignant neoplasms.

Lymphosarcoma in adult or young subjects may run its course in a few weeks with extensive metastases in many organs. Cancer of the liver in elderly subjects may terminate with steadily progressive symptoms in three months. Some melanotic tumors after becoming established run a very rapid course. Children are especially susceptible to such forms of fulminant blastomatosis.

Pregnancy usually accelerates tumor growth. I have observed cancer of the breast arising after gestation prove fatal, with wide-spread metastases, before term. With experimental tumors pregnancy sometimes accelerates, but at other times retards growth (Moreau, Loeb).

2. Intermittent periods of relative or complete quiescence occur in the course of many malignant tumors. It is probable that such quiescence is often more apparent than genuine. The progress of the edges of carcinomas and the involvement of lymph-nodes often appear to be sudden and the growth reaches certain dimensions in a few weeks after which progress is much slower. Exhaustion of growth capacities may partly account for such cases, but usually it is referable to pressure on a distended capsule or hindrance to the increased blood-supply. The onset of edema, hemorrhage, retention of secretions, inflammatory complications, or central necrosis, fibrosis, contraction, and evacuation of fluids, simulate changes in the rate of growth. Or a primary tumor may be at a standstill while internal metastases are active.

The general nutrition of the body has considerable influence on the rate and extent of growth of many tumors. This influence is most clearly seen when the tumor is encapsulated and secondary complications are absent. Any factor which influences the nutrition of the body as a whole is apt to affect the size of a tumor. Infectious diseases, starvation diet, alimentary obstruction, etc., by reducing the general nutrition often reduce the size of tumors, and may thus give a false impression of a favorable therapeutic influence. On the other hand, some lipomas have been observed to grow to a bulky size in emaciating patients.

Transplantable tumors of lower animals have been found to follow the normal laws of growth in many particulars.

Yet apart from these conditions there appears to be a frequent tendency in certain tumors to grow steadily to a certain bulk and then to remain stationary until trauma or other external factors initiate a new period of growth. A definite form of intermittent growth is observed in cancer of the stomach and epithelioma of mucous membranes which remain localized ulcers for a time, but suddenly extend more rapidly upon the invasion of liver or lymphnodes. Malignant change in a benign tumor is marked by sudden acceleration of growth, as when myosarcoma develops on a uterine myoma.

3. A natural limit exists to the course of some tumors, and the rule of endless increase is by no means invariable. Myomas of uterus may cease to grow at the climacteric. Warts and lipomas may be self-limiting. The relative bulk is in part an indication of the general momentum of growth of tumors. Thus squamous epithelioma does not as a rule produce a bulky growth, and seldom yields bulky metastases. Epithelioma of skin or even of esophagus may produce ulcers which after steadily growing to certain dimensions long remain practically quiescent, and on section one finds a 
firm basis of connective tissue beneath the ulcer and few or no mitoses in the tumor-cells. Martland has reported a mixed tumor of a salivary gland of 20 years' duration and long stationary. The same numerous and complex factors which interrupt growth may succeed in establishing a permanent limit. It is also beginning to appear that a local or general immunity may be slowly established and effectually prevent growth.

4. Spontaneous.cure is observed with certain tumors under peculiar conditions.

There is a considerable series of polypoid tumors of skin and mucous membranes where nutrition is cut off by constriction of the pedicle and which are either completely extruded or regress without detachment. Fibromas and chondromas may accidentally compress their nutrient vessels with regression or sloughing.

Within the tumor hyaline degeneration of vessels and mucous degeneration of stroma and parenchyma usually retard growth and may completely exhaust the capacity for growth. Calcification of parenchyma and capsule may incarcerate the tumor.

Inflammation about the borders and in the body of the tumor seems to cause the occasional extrusion of certain sarcoid growths of the skin (Randolph, Watson). Accidental infection by erysipelas has been followed by spontaneous regression of sarcomas.

For somewhat uncertain reasons benign tumors have been known to regress, as exostoses (Nasse, Starch) and papilloma of the bladder (Nitze). A local or general immunity seems to be the only explanation of an increasing list of spontaneous cures of highly malignant tumors.

Most of these cures are only partial. Among them are sarcomas, squamous epitheliomas, and glandular carcinomas (Czerny).

Tripier records a case of multiple round- and spindle-cell sarcomas of wide distribution with pulmonary hemorrhages which disappeared after the condition seemed hopeless. The exact nature of this case is uncertain. Kaposi has recorded the disappearance of lymphosarcoma of the upper jaw while internal metastases were in progress, and Reichel, of a spindle-cell sarcoma of forehead.

Some of the cures have followed persistent recurrence after operation (Shepherd, Watson). In dermatological literature there is a considerable list of reported spontaneous cures of cutaneous sarcomas. While the true nature of many of these processes is uncertain, I do not believe that they can properly be rejected, as Williams would, from the field of neoplasms.

Spontaneous recovery from chorioma belongs in a special category owing to the peculiar relations of this tumor. In I5 cases this process has been known to regress after the occurrence of pulmonary or vaginal metastases, in 3 of which neither uterus nor vaginal nodules were removed. In 7 other cases recovery followed partial removal (Ewing).

Recovery after partial removal of ovarian adenocarcinoma is not infrequent. Rotter has described a case of complete cure of an original malignant adenoma of the rectum, although after 3 years a metastasis of the iliac bone was found at autopsy. Here a complex form of local tissue immunity seems to have existed.

With cancer of the breast there is a group of cases in which the disease becomes locally extensive and internal metastases form, the patient becoming greatly debilitated, but at this stage the picture changes and very marked improvement sets in attended with regression or even disappearance of many tumors. Although these patients usually die of the disease, they may enjoy good health for many years. General, debility cannot account for such a 
course, and there seems to be no other explanation than the development of a relative general immunity. In Mackay's case improvement was coincident with absorption of a pleural exudate, and I have followed several very resistant cases which were marked by continuous ascites, sometimes chyliform (Hodenpyl).

Somewhat similar are those cases in which primary tumors disappear and the patient long enjoys fair health, during the very slow progress of internal metastases (Bryant, Gould, Broca, Hutchinson).

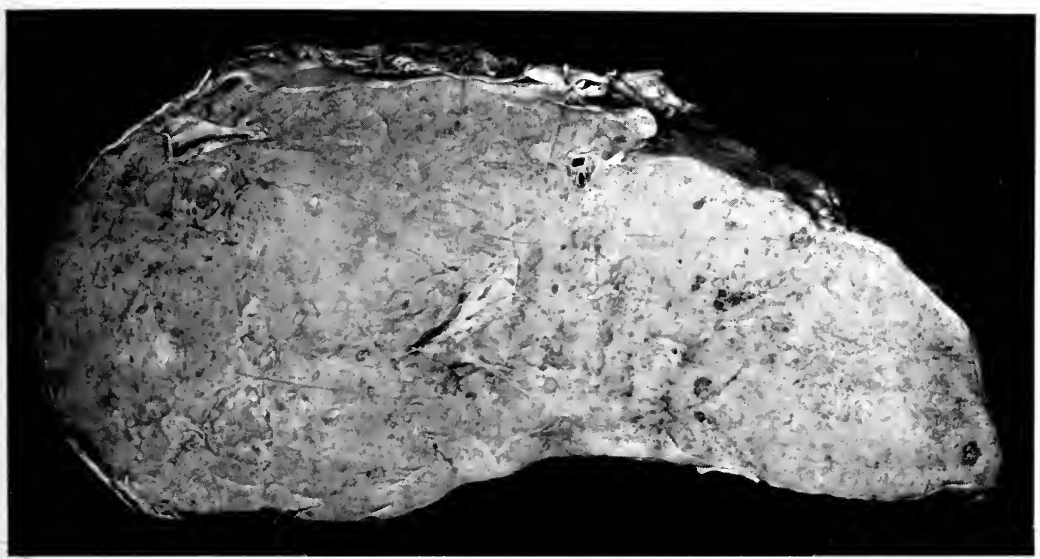

FIG. 9.-Almost complete replacement of liver by metastatic fibrosing carcinoma of breast. The external tumors in this patient had notably regressed.

The self-limiting tendencies of certain epitheliomas seem to have resulted in spontaneous cure in cases reported by Sanger. Many interesting cases of this general type are collected by Williams.

The morphology of spontaneous regression presents special characters with each type of tumor, among which are the calcification and giant-cell formation in epithelioma (Petersen), giant-cell degeneration in chorioma (Teacher) (Ewing), extreme hyalinosis in cancer of the liver, and simple atrophy of parenchyma in the large cell tumor of the testis. 


\section{CHAPTER III}

\section{MALIGNANCY AND ITS EFFECT ON THE ORGANISM}

The Significance of Malignancy.-The distinctions between benign and malignant tumors involve questions of great interest from both the theoretical and the practical sides. If malignancy were a purely clinical conception it would be impossible to draw any rigid distinctions between benign and malignant tumors, since nearly all tumors may occasionally prove fatal. Yet the tendency is to restrict the term to tumors which exhibit certain features which are essentially deleterious to the host. The most important of these features are infiltrative growth, local destructive properties, recurrence after removal, formation of metastases, local interference with function and general toxic action of absorbed tumor products. These elements involve both anatomical and clinical effects.

Infiltrative growth is the most important of the anatomical factors in malignancy This property facilitates local and general extension, renders removal difficult, is responsible for many recurrences, and is a constant preliminary to the local destruction of tissue. The controlling influence of encapsulation is seen in the harmless course of many very cellular tumors which may readily be enucleated while circumscribed, but which on rupture of the capsule take on infiltrative and malignant properties. It is also demonstrated in transplantable tumors which upon incision of the capsule may be transformed from regressing into malignant infiltrative growths (Loeb).

During experimental increase of growth Bashford and others have shown that originally circumscribed mouse tumors take on infiltrative qualities. Yet Borst notes that early fibroma of the kidney showing infiltrative growth later becomes encapsulated and the same observation applies to myomas.

Rapidity of growth is usually associated with infiltrative qualities and deleterious effects and if carefully separated from simple increase in size from other causes is a nearly constant sign of a dangerous tumor. Local destruction of tissue is a variable feature prominent only in certain types of tumors, appearing early in squamous epithelioma, and constituting the sole malignant quality of rodent ulcer. In benign tumors destruction of tissue is a secondary effect of pressure. Hemorrhage from destruction of vessels may be the chief instrument in the cachexia which marks the course of many ulcerating tumors as in the stomach and uterus. Pain from involvement of nerves is often the first and even the last clinical problem in the course of a lethal neoplasm.

Formation of metastases may be held to constitute any tumor malignant. Being usually the result of infiltrative growth, invasion of vessels, and cellular character, and signifying dissemination and collapse of defensive powers, it is the most impressive external sign of malignancy. It is, however, by no means constant in lethal tumor processes, as in glioma, rodent ulcer, etc., and certain tumors otherwise benign may occasionally yield distant secondary growths, as chondroma and myoma.

Local interference with function is the chief dangerous feature of many gliomas, epitheliomas of larynx, esophagus, cardia, and cancer of the pylorus and rectum, and many benign tumors become serious chiefly from effects of pressure. 
The general intoxication resulting from tumor growth is a complex subject which has long been recognized as one of the most obscure and important problems in the natural history of malignant tumors. Hemorrhage, mechanical interference with nutrition, pain, the psychical condition, abnormal secretions, destruction of important tissues, the toxic action of products absorbed from degenerating and ulcerating areas, and bacterial infection combine to produce the cachexia of tumors and require consideration in a separate chapter. When the clinical signs of malignancy declare themselves the conditions exciting them often belong to the past history of the disease and are often irremediable.

Diagnosis of Malignancy.-The prediction of the course that a given tumor will take is based upon two sources of information, anatomical and microscopical diagnosis and accumulated experience regarding the usual behavior of tumors of known histological structure. Fortunately a parallel exists to a very marked degree between the histological structure and the usual clinical course.

The main subdivisions of tumors into benign and malignant is accomplished at once and, as a rule, only by microscopical structure. Under some circumstances the microscopical structure may stand alone and override all other considerations. Far more usual is it to find the interpretation of structure to be greatly influenced by clinical information regarding the exact location of the tumor, its attachments, the presence of a capsule, and the age and condition of the patient.

Pathologists and surgeons alike commonly ignore these essential conditions of accurate diagnosis, a delinquency which is responsible for the wide and firm differences of opinion regarding the relation of microscopical structure and prognosis. It must also be urged that tumor diagnosis is commonly assigned to the relatively inexperienced, whereas the correct interpretation of structure in the light of clinical data requires a very wide experience not only with the general tendencies of specific structures, but with the observed clinical courses of different tumors.

A somewhat general estimate of the malignancy of tumors may be based on the distinctions between adult and embryonal or anaplastic growth. It has long been recognized that the greater the variation in type between a tumor and its originating tissue, the more malignant the tumor, and Hansemann has effectively emphasized this principle, pointing out that the morphological evidences of anaplasia have a physiological significance indicating the degree to which the process is freed from growth restraints and the control of the organization. Yet here is encountered the difficulty of distinguishing between original embryonal qualities and signs of acquired anaplasia. One group of tumors arises from embryonal cells which have lagged behind in development and such tumors bear an embryonal stamp. The histological signs of this embryonal character are often difficult to distinguish from the signs of anaplasia, and if they are wrongly interpreted an erroneous impression may be drawn of the malignancy of the tumor. The great theoretical value of the distinction between embryonal and anaplastic cells is not, however, equalled in practical importance, since both types of tumors, especially the latter, are usually quite malignant. The histological signs of anaplasia are a cellular character, marked variations in size in either direction from the originating cells, increase of chromatic nuclear substance, abundance and abnormality of mitoses, and loss of polarity and diffuse infiltrative growth of cells. In many instances lack of reaction of the tissues against the infiltration of tumor-cells is a significant feature. Equally important are the general signs of exalted nutrition and vitality of the cells. Upon these fea- 
tures one may safely base the estimate of growth capacity and potential malignancy of tumors.

The clinical course does not always accord with the signs of growth capacity. Many factors besides the surgeon's knife influence the progress of growth. One must distinguish between the potential malignancy and the clinical course of tumors. The histological signs of malignancy measure the potential malignancy of a tumor, but the clinical course is subject to wide variations from the position of the tumor, hemorrhage, trauma, changes in rate of growth, bacterial infection, etc., any one of which influences may greatly alter the course. With these important limitations it may be asserted that there is a close parallel between histological structure and the malignancy of a tumor.

In practice the most frequent source of discrepancy between histological signs of malignancy and clinical course arises from the confusion of embryonal with anaplastic qualities. Some of the most malignant looking tumors, as the congenital sarcoma of the kidney, teratoma testis, sarcoma of skin, adenoma of thyroid, gastro-intestinal tract and other organs, and large cell epithelioma of the skin, long remain localized and spare the lymph-nodes. These tumors are of embryonal origin, and the growth capacities of their abundant atypical undifferentiated cells are much below those of other tumors whose cells show true anaplasia and increasing loss of growth restraints. Recurrent adult and highly malignant squamous epithelioma yields cells with little trace of pavement character, which closely resemble those of a benign embryonal epithelioma. Here and in many other fields it is necessary to know the history and to divine the origin of the tumor in order to construct the prognosis.

Different standards apply to different tissues in estimating the significance of cell growth. The thyroid, liver, uterine mucosa, and lymph-nodes respond readily to various stimuli, and the gross and microscopical criteria applicable to those organs cannot be employed for the breast, kidney, or stomach.

The nature and origin of many round-cell growths are obscure and difficult to recognize, while their natural history is imperfectly known, so that in this group there are many apparent discrepancies between structure and prognosis. Finally, there is a natural limit to the onward growth of many malignant tumors and it should occasion no surprise if in advanced stages certain malignant tumors should advance more slowly, become stationary or even regress. Further knowledge may possibly show that under some conditions the presence of tumor tissue is a safeguard rather than a menace to the organism.

The transformation of a benign into a malignant tumor occurs in rare instances. To be separated from this category are those cases in which one element of teratoma gains the upper hand and eventually outstrips the relatively benign portions. Apparently this event may occur at any stage of the growth of teratomas. In a case which I have reported a teratoma of the testicle containing adult thyroid, epidermoid cysts and diffuse carcinoma, the malignant process was delayed for years. In an ovarian dermoid which had broken into the colon, I have seen epithelioma developing from the irritated epithelial surface. In another group of cases a pigmented mole or adrenal rest in the kidney after growing slowly for years may suddenly, perhaps after trauma, take on malignant qualities. Here the neoplastic nature of the originating cell group may be doubted.

When an encapsulated nearly stationary but potentially malignant tumor breaks through its capsule and grows rapidly there is no ground for assuming a change in the quality of the tumor. Different portions of many tumors 
differ widely in structure, and many apparent changes in type of tumors rest on the mistaken conclusions of a biopsy.

The impression that the tissue of a well-established benign tumor may after a long period take on diffuse malignant characters is based chiefly upon observations of uterine myomas. About 3 per cent. of such tumors are said to become sarcomatous, but the exact significance of this change has always been subject to doubt and discussion. In the great majority of such cases the impression that a malignant change has occurred is based on the discovery of a myosarcoma by operation on a tumor of which the previous history has been imperfectly traced. In a smaller proportion a very slowly growing fibroid has been known to exist for years when it suddenly begins to grow rapidly and on removal one portion of the tumor is found sarcomatous. This portion is often connected with an ulcerated surface of the endometrium, an observation which justifies the conclusion that the ulceration and the sarcomatous change are in some way related. In other cases the myoma has been cystic or angiomatous. Sarcomatous change of this sort has occurred in several authentic cases (Piquand). The true interpretation of this change remains uncertain. It may be that the sarcoma results from a new tumor process arising in the stroma; or a sarcomatous process becomes established in the stroma of the endometrium subsequently invading the myoma ( $\mathrm{W}$. Williams); or the more rapid growth and the cell changes represent the natural course of the original tumor which has not thereby acquired any new powers of growth (Borst). The enumeration of these possibilities suffices to reveal some of the complications of this subject.

Carcinoma has been known to develop from the epithelial elements present in many uterine fibroids, as in cases recorded by Klob, Rolly, and others, More often uterine fibroids are invaded by malignant tumors of adjacent or distant organs, as by cancer of the endometrium, lung (Schafer), or breast (Bender).

In the breast, cancer and sarcoma have been found in connection with fibro-adenoma, and it appears that the malignant tumor has arisen from the tissues of the benign. In the case of ovarian cystomas there is no evidence to show that varying grades of malignancy exhibited by these usually benign tumors constitute a definite change in type.

While therefore the transformation of certain benign tumors into malignant forms has been shown to exist, this event is among the rare occurrences in the natural history of tumors. In the fields where it is supposed to be most frequent $R$. Williams has shown that benign tumors are much less liable to change of type than are the normal tissues to develop cancer. This conclusion accords with the rule that a tumor process becomes established at a certain momentum which it tends to maintain throughout its entire course.

\section{EFFECTS ON THE BODY}

The emaciation accompanying tumor growth has long been recognized as one of the most significant and obscure of its many features. It may be said to be a constant effect of both very malignant and relatively benign tumors. It occurs early or late, with or without anemia, though probably always with diminution in the total volume of blood, sometimes with preservation of the fat deposits, and is preceded by distinct muscular weakness. It affects chiefly the muscular system but also all cellular organs and tissues. Inanition is undoubtedly the chief factor in the loss of weight. Mental depression leading to distaste of food, the lowering of digestive capacity, and mechanical obstruction to the alimentary passages, reduce the amount of food absorbed. 
In 6 cases of uterine cancer v. Noorden calculated the voluntary ingesta at 300 to 1200 calories. The importance of starvation is strikingly emphasized by the rapid increase in weight following relief of esophageal and pyloric stenosis.

Abnormal loss of proteins figures in many cases, by hemorrhage, exudation, albuminuria, and diarrhea. A febrile destruction of proteins occurs very often and is a somewhat neglected factor especially in low forms of infection, and the difficulty of securing bacteria-free tumor tissue suggests that bacterial poisons may often be at work when not suspected. The influence of actively regenerating tissues upon the general organism has been generally overlooked in discussions of the tissue atrophy of cancer. The exalted capacity of tumor-cells to absorb nutriment may long be successfully met by physiological processes, but there is a point, as mentioned by Cramer, when the general body tissues begin to yield their food supply to the tumor. That this process may actually lead to atrophy of normal tissues has been shown experimentally by Stockard who observed marked diminution of the remaining arms of Medusa during the normal regeneration of amputated arms. The restored individuals were sometimes only half as large as the original.

The conception of a toxic destruction of protein tissues emanated from the observations of F. Müller, I889, upon 7 febrile cancer patients. In 2 of these, pancreatic and mammary, a toxic destruction was not apparent, but in 4 gastric cases the nitrogen loss was excessive, and in a case of cancer of penis with metastases, an intake of $2 \mathrm{I} \mathrm{gm}$. N., 3064 calories, failed to balance the loss. These observations support the general belief that the products of degenerating necrosing and infected tumors cause toxic destruction of tissues in cancer as in other diseases.

Yet the belief in a specific toxic destruction of protein tissues in cancer has never been fully accepted. It is worth noting that Wilks in 1868 from the study of 2000 autopsies at Guy's hospital concluded that cancer as such does not cause cachexia. This conclusion has steadily gained support.

Many observers have failed to detect the toxic nitrogen loss described by Müller and verified by Klemperer. Many cases with entirely normal protein metabolism have been observed by Widal, Setti, Braunstein, Clowes, and Lewin, and even a nitrogen retention has been found in cases studied by Schopp and Moraczewski. It is possible that a correct interpretation of these cases must consider such factors as masking of a nitrogen loss by inadequate excretion by the kidneys, or by the sparing action of carbohydrate diet. Schmidt points out many resemblances between the supposed toxic destruction of cancer and that of fever, as the sparing of fat, and the high oxygen consumption, which is yet not in proportion to the protein catabolism. Yet these objections merely emphasize the difficulty of securing a suitable subject for investigation, among which gastric, hepatic, intestinal, and ulcerating or infected cases must not be included. In a case of mammary cancer which seemed to meet all obvious requirements Benedict and Gorslin at New York Hospital readily maintained nitrogen equilibrium and found no abnormality in the partition except marked increase of creatin over a terminal period of Io days. Our present knowledge derived from careful clinical study, from observation at autopsy, and finally from chemical investigation, seems to warrant the conclusion that a peculiar toxin secreted by cancer-cells and leading to cachexia does not exist.

This conclusion does not seem to need revision in the light of various chemical analyses of cancer tissue (Petry, Wolf, Beebe, Schaffer), since no new and abnormal toxic substances have been shown to exist in tumor tissue, although quantitative variations in their split products may occur. 
Tumor Ferments.- It is everywhere agreed that cellular tumors are very prone to degenerate and that their products may cause intoxication. This subject appears in a new phase in the study of tumor ferments. Petry emphasized the well-known rapid autolysis of tumor tissue which he referred to autolytic ferments. Buxton found quantitative but no qualitative differences between tumor and normal tissue ferments.

A heterolytic action of tumor ferments has been actively claimed by Neuberg, Jacoby, and Blumenthal and Wolff, but I think on insufficient grounds. None of these authors has considered the influence of bacterial or leukocytic ferments, factors which seem to me to influence their results and to explain their inconstancy, and warn against the hasty acceptance of the comprehensive deductions drawn from them. Kepinow has pointed out the obvious defects of this work and with Müller has shown that when free from leukocytes and bacteria cancer tissues do not accelerate autolysis in other organs. Petry also was unable to find evidence of specific ferment activities in the aseptic blood of cancer. Abderhalden and his associates have endeavored to show that the splitting of optically active polypeptids in the presence of tumor juice follows a different course than with extracts of normal tissue, but their results were inconstant.

The study of experimental tumors in lower animals has given additional support to the belief in the innocency of well-nourished tumor tissues. Especially in mice it is observed that tumors nearly as large as the animal itself may be well borne until the moment when ulceration, infection, or necrosis occur.

The Blood in Cancer.-Changes in the blood are of interest to the student of tumors chiefly in their relation to cachexia and to the problems of immunity.

Benign tumors exert little or no influence upon the blood, and the very slight change of the early stages of malignant tumors may be cited in support of the view that in the beginning cancer is a local disease. Likewise in the established stages of many malignant tumors the blood remains practically normal in quality, and even in advanced stages there are many records showing normal cells and hemoglobin. Cabot collected I9 cases of gastric cancer with over $5,000,000$ red cells. Laache noted the same immunity of the blood against the effects of a bleeding uterine carcinoma and concluded that there is an individual insusceptibility to the effects of malignant tumors. In some gastric cancers there may be a concentration of the blood, probably from diminished fluid ingesta which maintains the quality. Leichtenstern, Patrigeon, and the writer have observed cases of gastric cancer with increase of $\mathrm{Hb}$. to roo per cent., shortly before death. Even when marked emaciation occurs the blood may fail to deteriorate in quality, so that Hampeln, Neubert, and others speak of a marantic as opposed to the usual anemic type of malignant disease.

Yet the great majority of malignant tumors are associated throughout most of their course with progressive deterioration in the quality and quantity of the blood. Usually this anemia takes the form of a secondary chlorotic process, with loss of $\mathrm{Hb}$. exceeding the reduction of cells, low $\mathrm{Hb}$. index, and slight leukocytosis. In a notable group of cancers of the stomach the anemia dominates the clinical picture, and takes a secondary pernicious form. The sources of the anemia of malignant tumors are numerous and complex and being variously combined must be estimated separately for each case.

Hemorrhage is the most obvious source of deterioration of the blood, but occasional escape from its natural effects has been recorded as above. It is quite as capable of causing emaciation as anemia. Interference with ingestion of food leads to anemia and emaciation in cancer of esophagus and 
stomach. The absorption of hemolytic agents from ulcerating and infected surfaces and from necrosing areas of closed tumors is a chief factor in producing anemia. Maragliano, Kullman, Bard, Polk, and many others have demonstrated the presence of hemolytic properties in the blood of cancer. Elsberg found that normal red cells injected beneath the skin of cancerous subjects were soon hemolyzed, yielding a characteristic discoloration of the skin, and he proposed this method as a diagnostic test for carcinoma. In extracts of degenerating and necrosing tumors Weil demonstrated thermolabile and thermostabile hemolysins not differing from those obtained from necrosing normal organs. Clinical effects of the action of such hemolysins were observed by Bard who pointed out that in cancerous pleuritic exudates the red cells are often hemolyzed, while in other bloody pleural fluids the red cells are intact. The presence of these circulating hemolysins may render transfusion of cancer patients with normal blood a dangerous procedure. Against their own serum the red cells are resistant and since the specific gravity of the blood is usually low the red cells are resistant to hypotonic solutions. Since hemolytic agents and variations in the resistance of red cells are commonly present in other diseases these properties have not been found reliable in the diagnosis of cancer.

The specific gravity of the blood in most cases does not differ from that of other forms of secondary anemia, but in well-established cachexia the gravity has often been found remarkably low. Dieballa, Peiper, and Moraczewski found lower specific gravity, IOI 2 to I032, in cancer of stomach, than in pernicious anemia of equal grade. The albumins of both plasma and serum are distinctly low in such cases (Grawitz). In a case of gastric cancer Wendelstadt and Bleibtreu found $0.79 \mathrm{gm}$. instead of the normal 2 to $2.25 \mathrm{gm}$. in I $00 \mathrm{gm}$. blood-serum.

Rieder believed that the lymphocytosis of cancer resulted from accelerated flow of lymph and Grawitz attributed the dilution of the blood to the same factor, finding that watery extracts of cancer tissue when injected into rabbits produced a marked increase of lymph flow.

Leukocytosis was early recognized as a very frequent condition in cancer, having been described postmortem by Andral in 1823 , and in the circulating blood by Lucke and Virchow about i 867 . The leukocytes were long regarded as derivatives of the tumor. Virchow referred their presence to increased flow of lymph.

Although some disturbance of the leukocytes is very frequently observed, the uncomplicated tumor process seems to have no capacity to attract leukocytes to the circulation. This conclusion accords with the fact that tumors excite no uniform local reaction. The cause of leukocytosis must therefore be sought in some other factor than the presence of growing tumor tissue.

Ulceration or other inflammatory complications are the most definite causes of leukocytosis in cancer, and the resulting hemorrhage adds to the increase of white cells seen in bleeding and necrosing tumors. Bacterial infection, local and general, may intensify the effects of absorption of tissue toxins and loss of blood.

Degeneration and necrosis of tissue and foci of cells must account for the leukocytosis of many large cellular and rapidly growing tumors. The inflamed lymph-nodes in the vicinity of malignant tumors indicate absorption or irritants, but these nodes seem to furnish few new cells to the blood since the leukocytosis is usually polynuclear.

As a reaction of immunity may be considered the lymphocytosis associated with certain tumors about whose edges round-cells gather in masses, but there seems to be no ground in the human subject for assuming the same 
interpretation of polynuclear leukocytosis. With the onset of cachexia the way is open for the action of many causes of leukocytosis which are as numerous as the elements in the cachexia itself.

With these theoretical principles the results of leukocyte counting in tumor patients offer only a partial parallel. The temptation is often strong to assume some specific chemotactic influence residing in the tumor process to account for pronounced leukocytosis observed in certain cases. Hayem observed marked leukocytosis with scirrhus of the breast which subsided after operation only to return with recurrence of the growth. In many rapidly growing but apparently uncomplicated tumors, especially in children, pronounced leukocytosis has been observed without satisfactory explanation. In the extensive series of blood reports of Alexander, Price-Jones, Cabot, and others, it is not always possible to refer the leukocytosis to definite factors apart from the tumor process. In fulminant carcinosis of breast and other organs the disease may be actively febrile and associated with marked polynuclear or mixed leukocytosis. Renal carcinoma is very frequently marked by intermittent fever and leukocytosis. Satisfactory explanation of these peculiarities is wanting.

Certain specific events in the course of malignant tumors may be marked by definite changes in the leukocytes. Severe cachexia with anemia is usually attended with the presence of myelocytes, and myelocytes with nucleated red cells are often seen with marrow metastases. Kurpjuweit collected I 3 cases in which considerable numbers of myelocytes were associated with bone metastases. Eosinophilia is regarded by Neusser as diagnostic of bonesarcoma. In general, sarcoma affects the blood and leukocytes earlier and more than carcinoma, and in many cases of sarcoma the increase has reached the higher limits of inflammatory leukocytosis (Martin, Matthewson, Alexander). In certain cases of systemic lymphosarcoma, myeloma, and in leukemic chloroma it is probable that numbers of tumor-cells reach the blood and may be found in stained specimens. In 2 cases of localized sarcoma Loeper and Loeste claim to have found specific tumor-cells in the centrifuged sediment of the blood. The many details of differential diagnosis by the blood may be left to the special treatises upon this subject.

The total quantity of blood in cases of malignant tumor varies greatly, but, as Louis showed in 1846 , it is usually much diminished. The distinction between the anemic and the marantic cases is here quite pronounced, the vessels in the former type showing at autopsy a considerable volume of watery blood, while the others may give a much reduced quantity of concentrated blood of fair quality. The cases with arterial hypotension observed by Janeway must usually fall in the former class.

The regeneration of the blood in cancer has been found by Bierfreund to require much longer than in other surgical conditions, and in progressive cases the $\mathrm{Hb}$. seldom reaches the ration existing before operation. Yet it is impossible to establish any general rule in this matter. In a woman who in four years underwent six operations for recurrent adamantinoma of the superior maxilla, I found the highest Hb. ( 85 per cent.) and good general health, although the tumor had again recurred.

The resistance of the red cells to various destructive agents has been found about normal in early stages of cancer, but in advanced cases it is much increased, probably as a result of reaction to hemolytic factors (Schmidlechner). So marked may the increased resistance become that Conte regarded this property as of diagnostic significance.

Alkalescence of Blood.-Determinations of the alkalescence of the blood have yielded results which differ with the method employed and the particular 
factors brought into consideration. Klemperer found a reduced amount of $\mathrm{C}_{0} 2$ in the blood of advanced cancer. Peiper, titrating the whole blood, found very low grades of alkalescence in advanced cachexia, and Rumpf, Limbeck, and others, dealing with the whole blood, obtained uniform diminution of alkali in advanced cases. It is clear that the chief factor in these results was anemia, although, as claimed by v. Noorden, liberation of acids from destruction of proteins and acidosis may occasionally be present (Herter). Yet H. Strauss, titrating laked blood, found marked variations, some cases showing normal and some increased alkalescence.

Methods dealing with the serum yield directly opposite results. In I906 Moore and Wilson found that the serum alkalescence to dimethylamidoazobenzol showed a striking increase in cancer as compared with healthy subjects and other hospital patients, while the basic capacity of the inorganic salts after removal of proteins by incineration showed a small but distinct increase. Gamble, Royle and Watson secured similar results by the same methods in both carcinoma and sarcoma, but in very advanced cachexia the alkalinity was barely above normal. Neither extirpation nor $x$-ray treatment produced any change. Sturroch improved the di-methyl technic and found an average alkalinity of the serum of $0.190 \mathrm{~N}$. and often more than $0.200 \mathrm{~N}$. in cancer, while non-malignant cases gave an average of $0.173 \mathrm{~N}$.

IIyperglycemia with a percentage of glucose reaching 0.33 was regularly found in the blood of cancer by Freund and again in many cases by Trinkler. Yet Matrai could not find that the anemia of cancer varied in this respect from other forms. Lewis and Benedict found quite variable relations between sugar content of the blood and the progress of malignant tumors, but usually an increase in the later stages.

A retention of chlorin and phosphorus, especially the former, was observed by Moraczewski to be earlier and more pronounced in the anemia of cancer than in other forms.

Theis and Stone found the non-protein nitrogen and urea nitrogen generally low in the blood of cancer patients, especially in malignant cases. In 2 cases of melanoma the uric acid was increased. Blood-sugar was somewhat above normal in 26 per cent., and below normal in 13 per cent., of their cases.

Influence of Cancer on Digestion.-Gastric Digestion.- The absence of free $\mathrm{HCl}$ in the gastric contents of cancer of the stomach, discovered by Van der Velden in 1879 , and thoroughly investigated by many later writers, stands as a prime factor in the anemia and malnutrition of this disease. In all but Io to I3 per cent. of such cases free hydrochloric acid is missing (Richter). When cancer is grafted on simple ulcer the early stages of the process may be marked by the excess of free $\mathrm{HCl}$ belonging to the former condition, but as a rule this excess declines and eventually disappears, sometimes suddenly as the disease progresses (Rosenheim, Schneider). In most cases the loss occurs early in the disease (Riegel).

Several factors appear to be combined in determining the loss of free $\mathrm{HCl}$. In most cases of gastric cancer one finds a general chronic catarrhal inflammation, in some cases there is atrophy of the mucosa, and in one group a diffuse infiltration of mucosa and muscularis is observed. There is every reason to attribute to these anatomical lesions a loss of functional capacity partly accounting for the loss of $\mathrm{HCl}$. Yet the failure of free acid occurs in many early cases, and is relieved after excision of the cancer (Rosenheim). In other cancers not involving the stomach a similar deficiency of $\mathrm{HCl}$ occurs, so that it seems necessary to assume that malignant disease exerts some general influence which affects gastric secretion, even in the absence of severe anemia and cachexia. 
Reissner showed that in gastric cancer the total chlorids secreted are not below normal, that decrease of free acid is nearly always associated with increase of fixed chlorids, and that the acid is promptly combined in the stomach with alkalis of the blood and serum exuded from the tumor. Since an increased supply of $\mathrm{NaCl}$ from the tumor juices can probably be eliminated, it would appear that the loss of free acid in early cases results from its neutralization by alkalis and not from any functional deficiency of an apparently normal mucosa. This explanation can hardly apply to late and cachectic cases and to other forms of cancer in which the total chlorides are diminished. That the tumor yields such free alkalis is further indicated by Stahelin's observation that free $\mathrm{HCl}$ introduced into the cancerous stomach is actively neutralized; by the neutralization of $\mathrm{HCl}$ by cancer tissue in artificial digestive mixtures (Rosenberger); and by the increased quantity of protein found in the stomach juice after previous washing (Salomon).

Moore and Palmer, from the study of a series of cases of carcinoma, sarcoma, and non-malignant tumors, found in nearly all a diminution of $\mathrm{HCl}$, which they attribute to reduction of hydrogen ions of the blood. In many of these cases it is evident that they have to deal with the effects of cancerous cachexia, but in others which were not cachectic Parker suggests that the general unhygienic hospital surroundings were at fault. Copeman and Hake with Cramer secured the interesting result from observations on 500 mice that the implantation of cancer in these animals is followed by decided increase in the physiologically active $\mathrm{HCl}$.

There are, therefore, a specific influence of tumor juices neutralizing secreted $\mathrm{HCl}$ of the gastric juice, a diminished functional capacity of an organ damaged by an extensive tumor, and an effect of cachexia in reducing the hydrogen ions of the blood and impairing the secretion of acid, all to be considered in the relation of cancer to the acid of the gastric juice.

The gastric ferments persist long after the disappearance of free $\mathrm{HCl}$ (Oppler). According to Glassner both rennet and pepsin are diminished with tumors of the fundus in which there is considerable destruction of the mucosa, whereas pepsin alone is diminished with cancers of the pylorus in which region it is principally secreted. Emerson found that one hour after a test breakfast $\mathrm{i} 6.9$ per cent. of the proteins in the normal stomach has been hydrolyzed to the stage in which they are not precipitable by phosphotungstic acid, while in cancer of the stomach 27.6 per cent. reached this stage. In a digestion mixture containing a piece of fresh cancer tissue he found proteolytic digestion to proceed further than in mixtures of pepsin- $\mathrm{HCl}$. These experiments indicate that cancer tissue contains a ferment similar to that found in other autolyzing tissues. Whether this ferment is specific of cancer tissue; to what extent the advanced proteolysis observed in cancerous stomachs is the effect of this ferment; and the part played by leukocytes and bacteria, are matters yet to be elucidated. Rosenberg has verified these results and the method has been found of value in the diagnosis of gastric cancer.

Neubauer and $\mathrm{H}$. Fischer have found in the stomach washings of gastric cancer and in the expressed juice of carcinoma and sarcoma a ferment which splits glycyl-tryptophan and this test has been extensively employed in diagnosis with satisfactory results. Lately it appears to have been shown that this ferment is ptyalin from saliva. Fischer points out that amino-acids appearing in the abnormal digestion of cancer of stomach unite with free $\mathrm{HCl}$ through the amino-group, while the total acidity may remain normal on account of the acid reaction of the free carboxyl group.

Disturbance of motility of the stomach dominates the clinical signs in cancer of the pylorts, leading in many cases to dilatation of the stomach and 
stagnation with abnormal fermentations of the contents. Of the influence of cancer on absorption by the stomach little is definitely known. With diffuse infiltration of the wall and contraction of the cavity, evacuation of the organ may be accelerated and absorption must be greatly reduced.

As a result of diminished digestive capacity, disturbance of motility, and retarded absorption, abnormal fermentative processes become established and various bacterial species find a favorable soil. The products of fermentation include lactic, butyric, and acetic acids, alcohol, and protein decomposition products. Boas at one time claimed that the presence of lactic acid in the gastric contents was specific of carcinoma and appeared at an early stage when stagnation and loss of free $\mathrm{HCl}$ did not exist, but it has since been shown that the presence of lactic acid is dependent upon impaired motility and deficiency of $\mathrm{HCl}$ (Strauss, Wagner, Seelig). Since these conditions are present very early in cancer and since, as Sick has shown, formation of lactic acid is favored by decomposition products of cancer tissue, the test for lactic acid is of considerable diagnostic value.

Intestinal digestion suffers in many ways from malignant tumors of the gastro-intestinal tract and its accessory glands. With gastric cancer the discharge of abnormal digestive putrefactive and bacterial products into the intestine tends to incite disorder throughout the entire course of digestion, and Wasbutski, Kast, and others have shown that intestinal decomposition occurs especially in cases with loss of free $\mathrm{HCl}$ and active fermentation in the stomach. Rarely there is severe diarrhea in the terminal stages of such cases. The main effects of tumors of the intestinal tract are the result of stenosis and ulceration, and either of these conditions in pronounced form has a prompt influence on general nutrition. Detailed studies in this field are meager. Excessive indicanuria tells of absorption of putrefactive products in stenosis, and formation of fistulous tracts may complicate and terminate the course without much influence on the extent of this absorption.

Blood in the stools offers a means of diagnosis in ulcerating tumors, and constitutes a serious source of anemia. Acholic stools result from cancerous stenosis of the common duct, and impaired digestion of fats follows destruction of the pancreas and occlusion of its duct.

Tumors of the liver, bile passages, pancreas, cecum, and rectum produce various important clinical types of disease the full discussion of which may be left to works on special pathology. Here it may only be said that all of these conditions tend to produce cachexia through the combination of very numerous factors which must be analyzed for each case and the variety of which reveals the very complex nature of tumor cachexia.

Changes in the Urine.--Specific alterations in the urine of cancer have not been demonstrated, but its composition varies greatly according to general rules. Specific substances appear in the urine with melanoma and myeloma. The entire subject invites further study by improved methods.

The total nitrogen varies with the diet throughout the main course of the disease, while at the termination may be observed the increased nitrogen output which Müller interpreted as a sign of toxic destruction of proteins, or the diminished excretion of starvation. The distribution of the urinary nitrogen fails to show any specific characters. The percentage of urea is influenced mainly by starvation, falling to a very low figure in extreme cases.

Uric acid may be increased without relation to leukocytosis (Cario). The course of this substance in cases of various cellular tumors has not been determined. Brandenburg and Blumenthal found the nitrogen of alloxur bodies relatively high in most of their cases, while Setti failed to find any uniform increase. 
Ammonia nitrogen has not been found to show any variations other than those dependent on digestion and nutrition. Herter found high ammonia in the coma of gastric cancer.

Creatinin excretion has been found low in cachectic cases by Benedict and Gorslin, an observation which accords with Shaffer's view that the creatinin coefficient is a measure of muscular efficiency. Creatin appears in moderate amounts in cachexia.

Rest nitrogen, chiefly amino-acids, is higher than in some other abnormal states and increases with the progress of the disease (Setti). Salkowski has drawn attention to an increase in the colloidal nitrogen precipitable by alcohol, in cancer. This fraction corresponds partly to the amino-acids (Wolf). Later Salkowski estimated the nitrogen precipitable by lead acetate after removal of phosphates by alkaline barium chlorid, finding that this nitrogen fraction is greatly increased in cancer. The nitrogen estimated by Salkowski probably includes the oxyproteic acids and polypeptids.

An increase in unoxidized sulphur has also been observed by M. Weiss and others, and Salomon and Saxl have demonstrated such a notable and uniform increase in oxidizable neutral sulphur as to indicate its value in diagnosis. Lehman points out that the results of this test depend largely on the diet without the control of which the examination is useless. From observations in this field Saxl concludes that various analytic methods demonstrate the occurrence in notably increased amounts of protein derivatives, chiefly oxyproteic acids, which escape the normal transformation into urea. In general he finds a somewhat specific disturbance of metabolism in cancer marked by a nearly constant excess in total metabolism, relative reduction in urea formation, increased ammonia excretion and increase of protein derivatives which fail of oxidation into urea. In no other disease except certain special intoxications is the internal oxidation of protein so much disturbed as in cancer. Saxl goes on to show that the disturbance of oxidation is probably due to accumulation in the system of rhodan, an oxidation product of hydrocyanic acid, since this substance is increased in the urine in cancer and when administered to man reproduces the typical metabolic disturbance of cancer. The basis of this theory remains to be verified.

The urinary chlorids show no specific relation to cancer. Although Beneke supposed that the cancerous constitution was marked by deficiency of alkaline chlorids, observation shows that these urinary principles depend, as in other diseases, chiefly on the diet, the presence of disintegrating protein tissues, and the activity of the kidneys. In severe inanition the chlorides fall very low (Müller, Braunstein), but an increased excretion has often been observed (Schopp). Chlorid retention may occur with cardiac or renal insufficiency and may be associated with anemia and edema (Laudenheimer). Chlorin loss may result from active destruction of tumor or other tissues. Marked disproportion between urinary nitrogen and chlorine in favor of the latter occurs in states of inanition with little protein intake and much destruction of tissue, as in cancerous stenosis of esophagus. Schopp concluded that $\mathrm{NaCl}$ might be retained in the building up of tumor-tissue in cancer of the liver, but Laudenheimer found the same proportion of $\mathrm{NaCl}$ in liver tumor and normal liver tissue. Royle finds retention of both $\mathrm{Na}$ and $\mathrm{Cl}$ in cancer, that of $\mathrm{Na}$ being greater than $\mathrm{Cl}$. He concludes that extra $\mathrm{Na}$ combines with phosphoric acid and that the retention of chlorine seems to counterbalance the alkaline phosphates of the blood and maintain the proper relation of salts in the plasma. In ro cases of carcinoma Braunstein found no changes in the urine which could be regarded as specific. In 3 cases the excretion of $\mathrm{Na}$ and $\mathrm{Cl}$ was practically normal and the loss of $\mathrm{P}$ ran parallel with that 
of N. Phosphoric acid excretion is increased in most cases both absolutely and in proportion to the nitrogen with which in general it runs parallel. The $\mathrm{P}: \mathrm{N}$ ratio runs $\mathrm{I}: 4$ or $\mathrm{I}: 5: 6$ instead of the normal I $: 7$ (Braunstein). Its excess suggests a source in tissues rich in $\mathrm{P}$, as the bones, glandular organs, or the tumor tissue itself. Sulphuric acid excretion is parallel with that of nitrogen, but Cario observed somewhat increased proportions in 2 cases of cancer of esophagus. The partition of the sulphur has not been determined, but high percentages of ethereal sulphates may be expected, with ulcerating tumors and intestinal disturbance.

Urobilin.-Considerable urobilin was frequently observed and regarded as somewhat characteristic of cancer, especially of cachectic and hepatic cases, by F. Müller and Gerhardt. Yet Hoppe-Seyler had previously found little urobilin in several cases, and Blumenthal, who observed an excess in gastrointestinal and ulcerating cases, often found it absent in others. Braunstein from the study of 22 cases concludes that urobilin is missing in cancer so long as fever and complications are absent, but appears with metastases especially in the liver; with necrosis and decomposition of the tumor, and with complicating pneumonia. In cancer of the liver when the bile-ducts are closed he found that bilirubin replaced the urobilin.

Products of putrefaction of proteins are found in increased quantities in the urine of advanced cancer, but the exact source of these substances has not been fully determined. Indican, phenol, aromatic oxyacids, and ethereal sulphates have been estimated by several observers who attribute their presence to intestinal putrefaction, to decomposition of secretions, and to destruction of tumor-tissue. That intestinal putrefaction is not the sole source is indicated by the considerable quantities of such substances observed with decomposing tumors of breast and uterus (Brieger, Haberlin, Hennige). Lewin finds excess of aromatic acids in patients with $\mathrm{N}$ loss, but not with those showing $\mathrm{N}$ retention, and concludes that these substances are derived from the toxic destruction of tumor and tissue proteins and not wholly from intestinal putrefaction. Yet it still remains uncertain whether aromatic oxyacids, indol and phenol, may arise in the course of nitrogenous metabolism, and it is clear that patients laying on nitrogen are not likely to be suffering from excessive intestinal disturbance.

Acidosis of Cancer.- The acetone bodies in cancer follow the same rules as in other conditions. Their presence in the urine bears no relation to the growth of cancer as such, and these substances are absent in the early stages of the disease and when the patient is well fed (Waldvogel). With the advent of protein loss and cachexia acetone and diacetic acid commonly appear in the urine and beta-oxybutyric acid is added in severe inanition. Hirschfeld relieved the acidosis by giving sugar.

In cases chiefly of esophageal, gastric, and hepatic cancer v. Jaksch, Riess and Senator described a form of cancer coma in which the dyspnea, acidosis, and diminished alkalescence of the blood were present as in diabetes. In 2 such cases Klemperer observed a daily $\mathrm{N}$ loss of 5 to $9 \mathrm{gm}$. which he interpreted as signifying toxic destruction of tissues. The coma he referred not to the acidosis but to the general toxic process. v. Jaksch also stated that these patients were not always suffering from starvation, but that the appearance of acetone bodies ushered in a severe type of general cachexia. It is not entirely clear that cancer coma is purely an acid intoxication, and it seems probable that the rapid burning of tissue fats and proteins of which acidosis is only one result may be responsible for some of the sudden terminations of malignant tumors.

Von Noorden's observation of considerable quantities of lactic acid in the 
urine of 2 cases suggests that the acidosis of cancer may involve hepatic disturbance as well as pure acidemia.

Demineralization occurs in cancer cachexia as in other conditions and is associated with nitrogen loss, with which it maintains a parallel. Of II cases, Lewin observed loss of mineral salts, I. 36 to 8.9 gm., and of nitrogen, in 7 , and slight retention of both in 4 . One important source of these mineral salts is probably the bones, which become increasingly fragile in many wasting diseases. The shafts become thinner, the cancellous spaces and Haversian canals larger, and Campbell has shown that the breaking strain is greatly reduced. In some cases of cancer the calcium depots become greatly disturbed and extensive deposits of calcium salts are found frequently in the tumor, occasionally in the lung.

Albuminuria occurs in most cases of cancerous cachexia, but is absent in the early stages of the disease. According to Müller it occurs in 35 to 72 per cent. of all cases of carcinoma. In cancer of the intestinal tract albumoses are very often present (Ury, Lilienthal), either by direct absorption of digestive products through the ulcerated surface (Maixner) or from the disintegration of tumor tissues (Pacanowski). In many cases of myeloma a peculiar protein, Bence-Jones body, appears in the urine. 


\section{CHAPTER IV}

\section{METASTASIS}

The formation of secondary tumors is a cardinal property of malignant tumor growth. The appearance of a secondary tumor at some distance from the primary growth may represent one of several events. It may mean a multiple origin, and a new tumor; or a continuous extension from the primary growth; or a true metastatic growth arising from tumor-cell emboli. Only a critical estimate of the known tendencies of particular tumors can determine which of these events has occurred. Melanoma and multiple fibroma both produce very numerous tumors of the skin, the one from cell emboli, the other by multiple growth of new tumors.

Multiple origin of new tumors is observed with a considerable list of neoplasms, already considered, including fibroma molluscum, myeloma, epithelioma of skin, sarcoid growths of skin, adenoma and adenocarcinoma of mucous membranes, chondroma of spine, myoma, lipoma, etc.

Lymphatic Permeation.-By continuous extension of primary infiltrating tumors through the lymphatics, apparently discrete tumors often appear at points widely distant from the original growth. In multiple carcinoma of the skin continuous chains of cells may extend through convenient lymphatics until the cells reach a locality favorable for growth and there multiply, producing discrete tumors connected with the primary growth only by a thin strand of cells. It is conceivable that accidental breaks may occur in this chain of cells completely separating the secondary from the primary growth. There are many tumors in which this process leads to formation of secondary growths. At what remarkable distances secondary tumors may arise by this method has only recently been demonstrated.

In cancer of the breast Handley has shown that there may be continuous extension of tumor-cells through the lymphatics of the deep pectoral fascia to the axillary and supraclavicular nodes; thence to overlying skin and to humerus; through the deep lymphatics to the ribs, pleura, lung, and spine; across the chest wall to the opposite breast; downward through the abdominal wall to the epigastric region, and thence by the falciform ligaments to the liver; and further to the inguinal region with involvement of lymph-nodes, skin, and femur. Extension through superficial lymphatics is much less wide. Invasion of the humerus occurs at the deltoid insertion and of femur at the trochanter, where these bones are closest to the skin, through the deep lymphatics of which the bone invasion follows. The leg and arm bones escape infection, which is inconsistent with a free embolic origin through the bloodvessels. Passing through the deep lymphatics of chest or abdominal wall the cells enter the pleura and peritoneum and become implanted on the serous surfaces and produce superficial infiltrations of the lung, liver, intestines, and ovary. Or the viscera may be invaded through their main lymphatic vessels giving central tumors. The liver is involved through the lymphatics of the falciform and round ligaments, or by transperitoneal implantation, or by way of portal nodes; the lung by transpleural implantation or through bronchial nodes and hilus. Abdominal invasion by the epigastric route is earlier and more frequent than the thoracic, occurring without thoracic lesions in 12 per cent. of all cases. The diaphragm is invaded by the epigastric peri- 
toneal route, and through the descending lymphatics of its crura the retroperitoneal nodes and kidneys are attacked. This process of lymphatic permeation Handley believes is the master process of general dissemination in cancer. Lymphatic embolism he would reduce to a very secondary factor. Handley bases his conclusions on the study of long slices of thoracic and abdominal parietes in advanced cases of mammary cancer. In such material he finds the lymphatics continuously filled by tumor-cells. The only breaks occur as the result of perilymphatic fibrosis, an inflammatory reaction excited by the tumor process and resulting in complete atrophy of tumor-cell cords at many points.

While recognizing the value of Handley's studies it must be considered that the condition at autopsy may not clearly indicate the manner in which the lymphatics have become filled. It is conceivable that cell emboli lodging at different points in a lymphatic chain may grow in both directions and after a time fill the lymphatic completely. Many observers believe that lymphatic embolism is commonly associated with continuous permeation in most mammary and in many other cancers. However, the result of these processes is practically the same, ending, in prolonged cases, in very widespread and nearly continuous invasion of lymphatics.

The theory of continuous permeation is of fundamental importance in surgical procedure. To what extent this process prevails in different forms of cancer can be determined only by careful study in each case. Such studies are not yet available and are essential before this theory can safely be adopted. In epithelioma of esophagus Borrmann has found that the metastatic tumors in the stomach wall, which have long been regarded as implantations through the esophagus, are connected with the primary tumor by an unbroken chain of cancerous lymphatics. Bloodgood believes that successful extirpation of cancer of the tongue and infected lymph-nodes may be accomplished only by removal of all lymphatics joining ulcer and lymph-nodes.

Yet the majority of observers fail to find in early cases such uniform and continuous connection between primary cancers and infected regional lymphnodes and are agreed that such infection is usually by means of cell emboli. I have repeatedly failed to find any trace of invaded lymphatics connecting carcinomas in the breast with infected axillary nodes, and have been equally unsuccessful with cancer of tongue and penis. Metastases from lingual cancer often appear first in rather distant nodes, sometimes on the opposite side of the neck. The metastases from melanoma of foot and other regions are usually incompatible with the theory of continuous permeation. In general it appears probable that the rapidly growing epidermoid and glandular carcinomas disseminate chiefly by lymphatic embolism, while slowly growing and recurrent tumors, especially in the skin, often extend by continuous permeation. This belief is strengthened by such observations as those of Gussenbauer who found cancer-cells in the cervical lymph-nodes in 29 of 32 cases, and of Kuster, who found the axillary nodes free from cancer-cells in only 2 of II 7 operative cases of breast cancer. Since definite growing tumors of the lymph-nodes are much less common it is clear that embolic cancercells are frequently present in lymph-nodes and that they are probably destroyed there under some circumstances. Yet it is extremely rare to find in lymph-nodes any appearance suggesting the destruction of small groups of embolic tumor-cells. On the other hand, evidences of retardation of growth by fibrosis and encapsulation are relatively common.

The frequency of lymph-node metastases varies with different tumors, with different varieties of the same tumor, and with different positions of the tumor in the same organ, and must be separately considered for each case. 
Cancer of breast probably stands high on the list, very few fully malignant cases of this disease failing to involve the nodes if allowed to remain more than a few months. Williams found the nodes involved in 86 ( 73 per cent.) of I 8 cases at first examination, and in 40 (90 per cent.) of 44 autopsies. The variety of the cancer is here of paramount importance, and is a factor often underestimated.

Epithelioma of the tongue or tonsil very early and nearly constantly invades the lymph-nodes, but here again the type of the tumor is the determining factor. Williams recorded 86 ( 83 per cent.) of ${ }_{3} 04$ lingual cases infected at time of first examination, and $5^{6}$ of 57 cases coming to autopsy. Epithelioma of the lower lip invades the lymph-nodes more slowly, but eventually in almost all cases. In I86 cases of cancer of stomach examined at autopsy by Moore, Cuneo, and Colwell, lymph-nodes were involved in I 49.

The lymph-nodes escape in many cases of uterine cancer, especially with tumors of the fundus. Epithelioma of the skin is slow in reaching the lymphnodes, and rodent ulcer and adenoid cystic epithelioma are remarkable for the long or permanent immunity of adjacent lymph-nodes. Of 34 malignant tumors of the testis Butlin found the lymph-nodes free in only 3 , but in a series of I9 cases I found several which had not attacked the inguinal nodes. The tumor usually first affects the epigastric nodes.

The value of available statistics, however, is very limited, since most authors fail to distinguish between the different types of cancer, although it is well known that some are very prone to invade the lymphatics while others long remain circumscribed. It is little help to know that 75 per cent. of the cancers of the breast involve the lymph-nodes within a year of their onset, when the surgeon might learn that his case is an adenocarcinoma which long spares the nodes.

For the same reasons available statistics regarding the period at which lymph-node invasion occurs appear to be of uncertain value. Thus Fink observed it in breast cancer as early as the sixth and always after the thirteenth months, yet I have examined a mucoid breast cancer of ro years' duration, service of Dr. Hartley at Roosevelt Hospital, without invasion of the nodes. Similar variations are observed with many other tumors, while it should be noted that some cancers show general dissemination without palpable enlargement of regional nodes.

Changes in lymph-nodes draining malignant tumors show that the implantation of metastases is preceded by a period of preparation of the soil. For weeks or months before actual tumor invasion the regional lymph-nodes may be moderately swollen. During this period many new lymph-nodes may develop in the course of the vessels. On section the swollen nodes show diffuse hyperplasia with catarrhal exfoliation of sinus endothelium, or multiplication of follicles. It is not uncommon also to find the nodes atrophic and fibrous, or extensively invaded by fat tissue, conditions which reduce their effectiveness as filters. Owing to a variety of causes old changes of this latter class may permit the passage of cancer-cells through or around a given group only to lodge in a more central area. The former class of recent alterations must be referred to the absorption of toxic products from the tumor, autolytic and bacterial. There are no specific histological features of the precancerous condition of the lymph-nodes, but there are reasons to believe that regressing tumor-cells may be found in the sinuses before definite implantation has occurred. As previously noted, Kuster found that in only 2 of II 7 cases were the axillary nodes removed with breast cancer free from invasion. Yet the proportion of lymph-node recurrences when the nodes are not removed is very much less. Nevertheless it is extremely rare to find 
any metastatic focus in a lymph-node which does not appear to be safely implanted and capable of growth.

The earliest nodules appear in the sinuses, from which they invade and compress the pulp with complete atrophy of lymphoid tissue. From this point the cells may invade the capsule and the peripheral lymphatics, blood-vessels, fats, or fibrous tissue.

When the metastatic period is established lymph-node invasion may follow rapidly. In a case of cancer of breast a swollen axillary node examined in March showed only inflammatory hyperplasia with no demonstrable tumor in the breast, but in June the entire breast was infiltrated by a flat diffuse growth and some axillary nodes were completely replaced by cancer. Since many tumor metastases long remain confined to the regional lymph-nodes and fail to make any headway through the blood-stream it may be assumed that there is a regional and a general immunity against implantation. Yet in not a few cases cancer skips the regional nodes and yields distant metastases. According to Gross this occurred in I out of 7 cases of breast cancer. With some rapidly growing tumors both local and general susceptibility to metastasis exists at a very early period, and in most cases one must conclude that the growth capacities of the cells are of chief importance in determining the fate of cell emboli.

Invasion of the Thoracic Duct.-In not a few cases of advanced cancer the thoracic duct is invaded, an event which commonly leads to wide-spread dissemination by lymph and blood-stream.

One of the striking results of invasion of the thoracic duct is the appearance of enlarged nodes in the supraclavicular region. Troisier in I886 first emphasized the diagnostic importance of this symptom in cases of abdominal and especially of gastric and uterine cancer, and similar cases have since been reported by many observers (Lit. in Williams, p. 425).

It has also been observed with cancer of pancreas (Raw), testis (Poncet), prostate (Hurlemont), adrenal (Troisier). Nevertheless it is a late and, with improving diagnosis, a rare symptom of diminishing importance, and must be distinguished from other affections of the nodes, and according to Williams, from Verneuil's "pseudolipome sus-claviculare." According to Rousseau, in 37 cases presenting Troisier's symptom, the left nodes were involved in 29, the right in 4 , both sides in 4 .

Inguinal lymph-node invasion is also associated with and occurs without involvement of the thoracic duct, with abdominal cancers, especially of the uterine fundus, prostate (Viannay), bladder, rectum, chorion, and occasionally with many other primary tumors. Among other symptoms resulting from occlusion of the thoracic duct are chylous ascites (Leydbecker), chylothorax, and lymph varices which may lead to cysts of considerable size. The results of invasion of the blood-stream from the duct with multiple tumors of the lung are frequently observed.

Winkler has given a full description of 27 cases ( 12 original) of cancer and I of round-cell sarcoma of the thoracic duct. The invasion followed most frequently cancer of the stomach, then of uterus, while other cases arose from the gall-bladder, testis, colon, and kidney. In all these cases the abdominal nodes were enlarged and adherent to the duct, offering abundant opportunity for the invasion by this means. The duct presented one of three conditions: (I) Free masses of tumor-cells lying in a coagulum, with thrombosis or occlusion. (2) Papillary masses of tumor growth adherent to the wall and organized from it, usually at the valves, with numerous varices often resembling a string of pearls. (3) Complete thrombosis by tumor masses invading the wall and mingled with fibrin. 
Invasion of Serous Cavities.-The large serous cavities are penetrated by many pathways and in the course of a considerable number of tumors chiefly those of the contained organs. The most frequent source of free peritoneal growths is the ovarian adenocarcinoma which at any period may rupture its covering and be disseminated through the cavity. Its cells readily become implanted on the peritoneum, producing many miliary or large solid papillary or cystic tumors with a tendency to ascites. One of the most remarkable forms of peritoneal cancer arises from small adenocarcinomas of colon or appendix, which early perforate the peritoneum, spread rapidly throughout the greater sac and, retaining the large alveolar structure, produce enormous quantities of mucus greatly distending the abdomen. In very large tumors of this sort, several liters of gelatinous material may be evacuated and it may be extremely difficult to discover any trace of living tumor-cells. I have seen gelatinous cancer of the rectum, following an extraperitoneal route, fill the pelvis inclosing rectum and bladder, pass up the abdominal parietes in front and retroperitoneal tissues behind, separating liver from diaphragm, inclosing the entire abdominal cavity in a rigid shell 3 to $5 \mathrm{~cm}$. in thickness, and eventually invading the mesentery and subserous intestinal lymphatics, but without any trace of intraperitoneal growth. This condition may be designated as abdominal cancer en cuirasse.

Peritoneal invasion is a very common late complication of breast cancer, and occurs, as Handley has shown, often by way of the epigastric lymphatics. From the subperitoneal lymphatics and along the falciform and round ligaments of the liver he has found cancer nodules opening into the peritoneum. Loose cells often become implanted first in Douglas' sac or on the ovary. More frequently there is a wide-spread permeation of the subperitoneal lymphatics, which may become nearly universal. It is associated with active growth of hard fibrous tissue, producing nodules and adhesions. Eventually the entire peritoneum becomes thickened, leathery and opaque, and the omentum shrinks to a firm globular infiltrated mass. Such patients show marked resistance to cachexia and may suffer for months or years from increasing ascites which gradually drains their strength or suddenly terminates life by asphyxia.

The peritoneum seems to have very little capacity to destroy cancercells, so that the invasion once accomplished is usually progressive. Gravity and the muscular contraction of stomach, intestine, diaphragm, and parietes assure a general dissemination of cells. These movements also may cause the separation of tumor masses which have been found free in the peritoneal and pleural cavities, while the aspirated fluid nearly always contains exfoliated cancer-cells.

Intrapleural and intrapericardial cancerous invasion occurs chiefly by direct invasion from cancer of breast, bronchi, and lung, with results very similar to those produced by breast cancer in the peritoneum.

Penetration of the cerebrospinal membranes occurs in rare cases of carcinoma, chiefly of the breast, and may lead to a universal cerebrospinal carcinomatosis resembling meningitis. In a breast case observed at New York Hospital, service of Dr. Conner, isolated cells, small groups of cells and thin plates were found from the vertex to the cauda equina, infiltrating the pia, and penetrating many sulci, with little cellular or serous exudate.

Lymphatic Invasion by Sarcoma.- It is a somewhat important distinction that lymphatic invasion by carcinoma is frequent, by sarcoma rare. Not a few epithelial tumors violate this rule and travel through the bloodressels, but sarcomas that tend to invade lymphatics are much less numerous. The lymphosarcomas are the only sarcomas that frequently travel by the 
lymphatics, and the origin of these tumors within the lymph-nodes fully accounts for this tendency. The blood-vessels also serve as channels for the dissemination of lymphosarcoma, so that certain forms of this tumor yield the most abundant and widely disseminated metastases seen with any type of neoplasm.

Most statistical reports of lymph-node invasion in sarcoma are of doubtful value owing to the uncertainty connected with the diagnosis of sarcoma. Most of the sarcomas reported of the testis and thyroid are probably epithelial tumors, and commonly invade the lymph-nodes. Melanoma declares its specific nature by frequently (4I.7 per cent. Williams) affecting lymph-nodes. Of the bone-sarcomas the spindle-cell and giant-cell types rarely, the roundcell or lymphoid-cell types frequently involve the adjacent nodes (Gross). The frank spindle-cell sarcomas of the parietes and its contained structures, and those of the internal organs affect lymph-nodes in 5 to 5 per cent. of cases. As a rule the blood-vessels as well as the lymphatics may be the route of entry.

The comparative immunity of lymphatics against invasion of sarcoma is probably to be explained chiefly by the greater local fixation of sarcomacells as compared with the mobile and sometimes ameboid cancer-cell. It is in accordance with this view that the lymphosarcomas behave much like carcinomas. At the same time sarcomas are usually more vascular than carcinomas so that cells rapidly break into the blood-vessels whose walls are thin or deficient. The growth of most sarcomas is more expansive than with carcinomas and more often encapsulated, so that lymphatic channels are closed and only the nutrient blood-vessels remain pervious. I do not think that the original relations between lymphatics and epithelium or connectivetissue cells have an influence on the character of tumor metastases, since at the period when dissemination begins these relations are greatly altered.

Dissemination by Blood-vessels. - The blood-vessels are the chief channel of the extension of sarcomas, they are frequently invaded by carcinomas, and several epithelial tumors show a remarkable capacity to flourish in and travel through the veins. The abundance of thin-walled blood-vessels, the presence of naked sinuses, and the tendency toward necrosis and hemorrhage rather than fibrosis, may largely explain the predominance of blood-vessel invasion by sarcoma as compared to carcinoma. In many cases, as in fascial myxosarcoma, the tumor-cells appear to be mechanically forced into the veins by the presence of rapidly growing and swollen tumor tissue.

The entry into the veins is accomplished through the defective vessels within the tumor, or by invasion and rupture of adjoining vessels, or by infiltration along the vasa vasorum and intima, with gradual erosion of the wall. Carcinoma often penetrates the wall via the perivascular lymphatics.

The result of such invasion is at first a thrombosis complete or partial, at the point of entry. The tumor-cells may then be destroyed by organization and fibrosis of the thrombus with subsequent canalization (Schmidt), or the cells continue to grow within the vein, they become attached to the wall, produce flat or polypoid outgrowths, many cells or cell masses become detached from the wall or from the original tumor and are carried to the first zone of capillary vessels, usually the lungs, where they lodge as emboli. Here again they are exposed to destructive agencies and may perish, but more often develop secondary tumors.

If the detached masses are large they regularly lodge in the lungs, or become arrested in the right heart. Direct transport of emboli or continuous growth in the veins is by far the most important mode of dissemination in blood-vessels, and several well known tumors offer striking illustrations. 
Chorioma originates in the uterine sinuses and maintains throughout its intravascular and hemorrhagic tendencies, producing retrograde vaginal and renal metastases, honeycombing the uterine wall, and yielding forward metastases in the lungs. Portions of the stroma of villi as well as tumor-cells have been found in the pulmonary emboli of chorioma. Hypernephroma regularly perforates the renal vein and may extend in a solid column of tumor-tissue into the heart. Adenoma of the liver is commonly found distending the portal veins. The intravascular hydatid growths of teratoma testis are among the oldest observed forms of intravenous extension. When a sarcoma or carcinoma ruptures into a pulmonary vein wide-spread dissemination results and very numerous tumors appear rapidly in different tissues and organs.

The form assumed by tumors growing within vessels is sometimes influenced by the existing mechanical and nutritional conditions. Freedom from pressure allows the cells to reveal their natural tendencies, and the intravascular tumor may be more "typical" than the original. Or a more rapid and diffuse growth is assumed. In the axillary vein the cells of breast cancer may become larger and more anaplastic, and I have seen evidence of multiplication of loose cells in this situation and in the thoracic duct. The organization of a tumor thrombus with fibrosis and reopening of the lumen occurs rarely. The advancing mass of hypernephroma in the renal vein is usually very edematous and the cells hydropic. Fuhrmann and Risel believe that some tumors, especially myxoma, tend to assume an hydatidiform growth when invading blood-vessels, and they would attribute the chorion-like form of certain testicular teratomas to the influence of the intravascular position and not to any intrinsic chorionic character.

Are vagrant tumor-cells destroyed in the blood or lymph stream or their associated organs, and if so by what mechanism? 'To this fundamental question only a very incomplete answer can be given.

The scope of the conditions to which the question applies is very wide, including many different forms of tumors and cells, involving the natural life span of the cells and the facility with which they may reach the circulation. It must be assumed at once that in many systemic sarcomas and other rapidly growing neoplasms, cells are constantly reaching the circulation and perishing there at least by natural autolytic processes. It is a very prevalent belief, however, that cells of most carcinomas from an early period of their growth frequently reach the blood and lymph and are actively dissolved by antagonistic substances in the blood. Many general considerations favor this view. The active infiltration of capillaries and lymphatics by rather loose tumor-cells suggests an easy and frequent passage into the moving current. The list of observed emboli even of normal cells is quite large. The experimental cultivation of tumor-cells by Burrows shows that they are regularly capable of growth in the isolated state, and in many human tumors, as in thyroid adenoma, it has been shown that blood-clot may be penetrated by loose tumor-cells much as in an artificial culture. In several well-known tumors cited elsewhere, as chorioma, hepatic carcinoma, and mammary carcinoma, tumor masses and loose tumor-cells are observed within the vessel lumen.

The belief that such loose tumor-cells are destroyed in the blood- or lymph-stream is based on rather scanty evidence. Usually wide dissemination is observed to follow the invasion of blood-vessels. M. Schmidt, however, has shown that tumor-cell emboli are much more frequent in the lungs than are secondary tumors of these organs. In 28 cases he found emboli in the pulmonary arterioles in all, but definite metastases in only 13 . From the study of the lungs in these cases Schmidt concludes that in carcinoma of the 
abdominal organs there is frequently and repeatedly a discharge of cancercells which lodge in the small arteries of the lungs. Only a small portion of these cell emboli produce metastatic tumors or grow into the perivascular lymphatics. Most of them are inclosed in fibrin, organized, encapsulated, and in spite of retained vitality, rendered harmless. Some of them grow through their capsule into the capillaries and small veins whence they may form the source of late general metastases. All of these processes may occur without macroscopical changes in the lungs. The chief source of these emboli he believes to be through the pervious thoracic duct. The endophlebitis carcinomatosa which Goldmann finds to be nearly constant in generalized carcinoma, he regards as a sequel of pulmonary emboli. In chorio-adenoma it has been shown that pulmonary emboli occur in cases which recover after hysterectomy, but the mechanism of destruction of the embolic cells, if they exist, has not been followed. In many hepatomas the growth is from the first into the veins, but pulmonary or other metastases are almost unknown with this tumor.

In the lymph-nodes while it has been shown that microscopic invasion is more frequent than growing metastases, actual observation of late regressing cell emboli in the nodes is not satisfactorily reported. The lymph-nodes seem to retain and retard the growth rather than destroy the cells.

Experimental research has suggested the existence of a premetastatic period during which the organism is incapable of developing metastatic tumors. Gay found that he could not successfully reinoculate tumor-bearing animals until a certain period had elapsed after the first implantation. Yet these results are not uniform. Tyzzer by manipulating his grafted tumors was able to cause numerous metastases, apparently without regard to the period of growth.

Serological studies have in general failed to demonstrate in the blood serum of natural or immune animals any substances capable of destroying tumor-cells. The most direct evidence favoring the existence of cytolytic powers of the blood is contributed by Freund and Caminer. These observers claim to have shown that normal blood dissolves cancer-cells in vitro, while the blood of cancer patients has lost this property. With many others I have been unable to verify these observations, and believe that Freund was dealing with erratic autolytic and bacterial effects and not with true cytolytic agents. In any case Freund's studies would not encourage the view that tumor-cells are destroyed in the circulating blood of cancer patients.

Retrograde transport was first emphasized by v. Recklinghausen as an important process in the dissemination of tumors through both blood- and lymph-vessels. The basis of his conclusions had been laid in many previous observations on the fate of insoluble particles injected into the veins. Retrograde transport occurs chiefly in those organs in which there is a normal venous pulse, lungs, liver, kidneys, heart, and brain, and in which violent expiration or increase of intrathoracic pressure from any cause may transform the weak forward into a backward current. When the main lymphatic or vein is occluded the flow is entirely disordered and backward transport is facilitated. Occlusion of the trunk vessel is probably essential for backward metastasis in lymphatics, but not in veins. In either case the backward progress is probably slow and intermittent, although wide distances seem at times to be traversed (Arnold, Ernst). In the organs retrograde metastases arise from tumors originating in these organs and from distant tumors. Thus a large tumor thrombus lying in an arched vein of the kidney in primary sarcoma of tibia could originate only by backward passage through the renai vein of an embolus traveling from below. Emboli in a cerebral sinus with cancer of breast (Arnold), in an hepatic vein from intestinal or thyroid cancer 
(Heller, Bonome), and in cerebral, cardiac, pulmonary, and hepatic veins with hypernephroma (Ernst), are not infrequent examples. Backward passage through obstructed lymphatics may be assumed in cases of advanced cancer of bronchial nodes with subpleural lymphatic invasion, but without involvement of pulmonary parenchyma. Cases of gastric cancer with involvement of portal, lumbar, and inguinal nodes v. Recklinghausen referred to retrograde passage of obstructed lymphatics. Metastases in supraclavicular nodes from tumors of abdomen and testis, with invasion and occlusion of thoracic duct, have been explained by several observers as retrograde lymphatic growth (Troisier, Poncet, Most). A cancer of gall-bladder with retrograde metastases in renal lymphatics, the veins being free, has been described by Vogel. With cancer of pancreas is sometimes found continuous lymphatic invasion down the aorta to the pelvic nodes and discrete nodules along the mesentery. In many of these cases it may be difficult to eliminate backward permeation, as Handley has shown, and some writers, especially Ribbert, doubt or deny the occurrence of retrograde lymphatic embolism. Y reason for holding that such embolism frequently occurs especially when a lymphatic trunk is occluded.

That tissue- and tumor-cells pass through the pulmonary capillaries has frequently been demonstrated, and in certain tumors, especially the lymphosarcomas, general metastases develop in this way. Passing the lungs the embolic cells tend to lodge in organs with feeble circulation, as liver, bonemarrow, and subcutaneous tissue. Or being arrested in the lung the embolic cells may grow through the vessels and break into the pulmonary veins (secondary embolism). Zahn speaks even of a tertiary embolism, metastatic tumors of the liver producing pulmonary emboli which grow through the lung.

Abnormal communications between the cardiac chambers may permit the passage of larger emboli into the aorta. In a case of sarcoma of the seminal vesicles and in one of thyroid cancer, metastases were found in the kidneys, but not in the lungs, from which Zahn concluded that emboli had passed through a patent foramen ovale, a process which he termed paradoxic embolism. Finally the blood-vessels are reached by way of the thoracic duct.

Thus invasion of the blood-stream may occur by: (I) Direct invasion of vessels in primary or secondary tumors, (2) secondary embolism, (3) tertiary embolism, (4) retrograde transport, (5) paradoxic embolism, (6) passage of single cells through pulmonary venules, $(7)$ through the thoracic duct.

Metastasis of a benign tumor was first claimed by Cohnheim for thyroid adenoma, and similar observations have since been reported by many writers. The great vascularity of this gland, the immediate contact of epithelium with capillary endothelium, and the extreme hyperplasia which may follow functional stimuli, offer unusual conditions in the thyroid favoring the dislodgment of adenomatous alveoli and even of simple hyperplastic cells. The occurrence of such metastases has been accepted by the majority of observers (Borst), but Williams and many others believe that in all such cases a small malignant tumor exists in the thyroid which gives origin to the metastases. The question is further complicated by the great difficulty of estimating from their morphology the growth capacities of thyroid cells. It is a fact, however, that thyroid cells have unusual powers of proliferation and growth, and it would seem necessary to admit that under rare conditions thyroid adenoma and hyperplastic thyroid cells may give rise to distant benign tumors. Definite proof of such an event requires minute search of the main and any accessory thyroids, which has been lacking in my own and many other reports.

Similarly for the benign hydatid mole and even for the normal placenta there is a very suggestive group of facts which has led Marchand and others 
to believe in the occurrence of malignant metastases of normal chorionic epithelium.

Pure chondroma not infrequently enters veins and its ameboid cells produce distant metastases. In the notable case of Ernst's a chondroma of the vertebral column advanced through the lumbar, adrenal, spermatic, azygos, and cava inferior veins into heart and lungs. Discontinuous metastases usually in the lungs have often been observed from chondroma of pelvis, scapula, fibula, etc. (Lit. Ernst). The metaplasia of undifferentiated tumor-cells derived from teratomas or sarcomas must account for some chondromatous metastases from tumors of testis and probably from other organs.

Metastases have been observed with leiomyoma of the uterus (Klebs, Langerhans, Beetson, Schlagenhaufer); of the stomach (Hansemann, Moser, Borrmann); and jejunum (Ribbert). In some cases the structure of the tumor has not varied from the usual benign myoma, but in others the cells were larger and nuclei large and oval. Some were cystic and vascular. BirchHirschfeld speaks of the invasion of uterine lymph-vessels by myoma. Williams denies that the simple uterine myoma ever produces metastases.

Contact Infection.-Contact of a cancerous surface with an opposed, abraded or inflamed mucous surface is a rare source of transfer of tumors. The best known example is the infectious lymphosarcoma of dogs which is commonly transferred by coitus. This and other tumors of lower animals may sometimes be transferred by rubbing a granulating surface with a portion of tumor.

The cases of cancer a deux in man have figured prominently at times, but in none has the actual transfer by coitus been proved. Demarquay collected I34 cases of cancer of penis in only I of which transfer by coitus appeared possible. Of 27 such cases collected by Gueillott none seems to me beyond question. Yet the genuineness of some of these cases cannot be denied.

Transfer of epithelioma of lower lip to upper is difficult to establish, but seems to have occurred in a few cases (v. Bergmann). In a case reported by Hartmann and Lecene a cylindrical cell cancer of the cervix seems to have become implanted on the squamous epithelial surface of the vagina.

Implantation of epithelioma of esophagus in the stomach has been widely accepted as a fact (Borst), but Borrmann has found continuous permeation of the lymphatics connecting such tumors. Similar evidence has not been adduced against implantation of renal tumor fragments in the ureter (Butlin). Likewise, the contact transfer of carcinoma has been reported from one point to another in the urinary bladder, vagina, vulva (Thorn, Walter), cheek (Lucke), thighs (Williams), stomach (Klebs), vocal cords (Semon), and from hand to conjunctiva (Kaufmann). In most of these cases it is difficult to eliminate lymphatic extension and primary multiple tumors, so that the doctrine of contact infection has always excited a legitimate skepticism. Bucher, Kaufmann, and others have fully stated the many grounds for this skepticism and it is obvious that the evidence must be very complete before any case can be accepted. The transferred tumor should be of different structure from that arising in the implanted surface and the lymphatics should be free from invasion.

"Genius loci," or the particular susceptibility of a tissue to develop secondary tumors, is an interesting phase of the study of metastases. Familiar examples are the predominance of pulmonary metastases in chorioma, which is simply explained by the intravascular position of this tumor; the bonemarrow metastases of hypernephroma and cancer of prostate, and the multiple cutaneous tumors secondary to lymphosarcoma testis. The mechanism of the circulation will doubtless explain most of these peculiarities, for there 
is as yet no evidence that any one parenchymatous organ is more adapted than others to the growth of embolic tumor-cells. The spleen, however, seems to escape with peculiar frequency.

Virchow observed that organs frequently originating cancers are rarely the seat of metastatic cancer. Yet Zahn collected 16 metastatic cancers of the stomach, and 26 invasions of the ovary in 366 cases of mammary cancer.

The preservation of structural type in metastatic tumors is often remarkable, yet, as a rule, there is increased anaplasia and more rapid growth in metastatic tumors and especially in recurrences after operation.

Adenocarcinoma of the alimentary tract invades the lymph-nodes and the peritoneum, and produces distant metastases while rigidly maintaining its large alveolar type. Adenocarcinoma of the stomach has been recognized in a metastasis over the scapula. The metastases of benign tumors are always typical in structure. The bone metastases of hypernephroma are usually recognizable. Metastatic melanoma is usually but not always pigmented. On the other hand, the general metastases of chorioma tend to be overgrown by Langhans cells with loss of syncytium. Squamous epithelioma may yield spindle-cell tumors in the liver.

In general, there is a gradual loss of structure in metastatic tumors. The liver metastases of mammary cancer, hypernephroma, and chorioma may be indistinguishable. The more anaplastic the original tumor, the less is the room for change in its metastases. Mixed tumors tend therefore to become simple, and certain elements may be eliminated in metastases and recurrences. This rule is best illustrated in the great variety of simple metastatic growths arising from teratoma testis. A peculiar form of elimination of epithelium is seen in the regional metastases of mixed tumors of the parotid which are often purely myxomatous or chondromatous.

Tridermal metastases which rarely arise from teratoma of testis or ovary indicate either that all three tissues are contained in the cell embolus, or that undifferentiated cells unfold their potencies after lodging in distant organs.

The osteoplastic carcinoma of the prostate offers a remarkable change of type and tendency in its pulmonary metastases. Here osteosarcoma appears to accompany the carcinomatous elements. A satisfactory explanation of this process cannot be given, but Schmorl supposes that the original bone metastases incite osteosarcomatous growth from which embolic cells yield osteoid tumors in the lung.

It is characteristic of certain cases of several tumors that a very small original focus gives rise to very bulky secondary growths. This course is followed by some cancers of the breast with large tumors in the axilla, by melanoma of the skin or cancer of gall-bladder invading the liver, by mucoid cancer of colon involving the peritoneum. On the other hand, squamous epithelioma rarely produces bulky metastases.

Recurrence.- After apparently complete removal of malignant and of some otherwise benign tumors the growth may recur. The recurrence is usually in the operation wound or its immediate neighborhood (local recurrence), or in adjacent lymph-nodes (regional recurrence), or it may appear in distant organs from metastatic emboli. Sarcoma often recurs at a distance from the original growth, while local and lymphatic recurrence is the rule with carcinoma.

Recurrence by implantation from abdominal tumors in the laparotomy wound or in the peritoneum is a well-recognized hazard of operations. The return of many malignant tumors is prompt and local, but many years may elapse between the removal of choroidal melanoma and its reappearance in widely distant regions. 
The very wide variations in the periods of recurrences are the result of many complex factors so that statistical reports in this field are of limited significance. Yet Williams has gathered a very interesting collection of such records. Among the most striking of these reports are the long intervals sometimes elapsing between operation and recurrence, in cancer of breast, 30 years (Heurteaux, Verneuil); rectum, 21 years (English); of tongue, I8 years (Guinard); choroidal sarcoma, I4 years (Fischer and Box); uterine cancer, I5 years (Pomard). The records of repeated recurrence are also of interest as encouraging the surgeon. Thus Gross performed 22 operations in 54 recurrent tumors in a case of sarcoma of the breast during four years, the patient finally recovering.

Recurrent tumors may reproduce exactly the structure of the original or follow the rules already stated for metastatic growths. Local recurrences usually show increasing malignancy and anaplasia, and at times the recurring tumor shows an astonishing variation from the original type. Clinical observation is here supported by the results obtained with transplantable tumors which by repeated passage may be considerably heightened in activity. Squamous epithelioma is comparatively stable in type, as is also adenoma destruens. Most alveolar carcinomas tend to lose the alveolar form and recur diffusely. Myxoma of the skin recurs repeatedly with the same structure, while melanoma may retain or lose its pigment. Recurring mixed tumors tend rapidly to become simple. One sees pure chondroma in the returns of parotid tumors. In the second to fourth recurrences of a complex tridermal teratoma testis I found pure angiosarcoma. In a case of adamantinoma originating as squamous epithelioma, L'Esperance, in this laboratory, has recorded progressive changes through plexiform epithelioma, glandular carcinoma, diffuse small spindle-cell structures, alveolar round-cell sarcoma, and finally in the sixth operation, diffuse round-cell "sarcoma."

There appear to be no indications that a recurrent tumor may be less anaplastic than the original, but Apolant claims to have reduced an alveolar carcinoma to an adenoma by transplantations in immunized mice.

Recurrent tumors in the great majority of cases arise from cells or portions of the tumor which have escaped removal. Microscopic section of the edges of specimens removed at operation often reveals that the knife has passed through strands of tumor tissue or vessels containing tumor-cells. There can be little doubt that the rough manipulation of cellular tumors in the preparation of the patient and in the excision of the growth widens the field of infection by forcing cells through vessels and tissue spaces. Or, viable cells are scattered through the wound by piece-meal removal from inaccessible regions. The practice of removing a portion of the tumor for diagnosis may add to the dangers of local dissemination. With myxoma, a tumor which is characterized by persistent local recurrence, the semifluid tissue may very readily be forced into the surrounding fat tissue.

Arising from remnants of the original growth recurrence is usually prompt, multiple, in the line of incision or near by, and of the same type as the original growth or more malignant. Yet there are many records of cases in which recurrence in the line of incision has been delayed for years. Such cases require the conclusion that displaced cells may long remain dormant. The many instances in which partial removal of malignant tumors has been followed by regression of the remnant, as in ovarian carcinoma and in chorioma, indicate that the mechanical disturbance of nutrition, or removal of local sources of growth stimulus, or the general relief of the organism from toxic products, turn the balance in favor of the patient, and lead to absorption of tumor-cells. Thus tumors in rats sometimes regress after hemorrhagic 
infiltration following crushing trauma. The same factors may be assumed to retard the growth in more malignant tumors which spring up at varying periods after incomplete removal.

As a second source of recurrent tumors it has been suggested by v. Rindfleisch, Beneke, and others that tumors exert a pervasive formative influence leaving the surrounding tissues in a state of excitation and with a momentum toward neoplastic growth, so that after the removal of the tumor the adjacent tissues give origin to a new tumor. This somewhat vague conception is vigorously rejected by Ribbert and is quite irreconcilable with the theory of origin from isolated cell groups. Yet there are several considerations which render it inadvisable to discard such a theory of recurrences. This question is closely related to that of the influence of tumor growth upon surrounding tissues and to that of the multiple origin of tumors in the same organ.

Attention has already been called to the fact that some tumors advance by gradual lateral extension over previously normal cells, so that removal at one stage of this extension may leave other cells which later reveal their momentum toward tumor growth. The local recurrences of some lingual and laryngeal cancers seem to accord with this view. Further, the local predisposition to tumor growth which figures distinctly in many phases may, as Borst points out, extend over a considerable area, in all of which the tumor growth does not reveal itself at once, but develops in separate stages or foci. Such conditions seem rather clearly revealed in neurofibromas, and, according to Hauser, with various carcinomas, especially those of the gastro-intestinal tract.

Ovarian cystomas recur in the opposite ovary or in fragments of the first ovary or in misplaced ovarian structures in the neighborhood, showing the influence of local predisposition (Velits). Many authors have pointed out the great frequency of cancer after ovariotomy and have endeavored to show, without, I think, complete success, that loss of the ovaries greatly increases the local disposition of many tissues to cancer (Wells, Kratzenstein, Neugebauer, Williams).

A third source of recurrence is found in the multiple origin of many tumors. The removal of a small cancerous nodule from the breast with chronic mastitis may readily be followed by the appearance of a new tumor in an adjacent portion of the gland or in a supernumerary gland. One seldom hears of recurring uterine myomas, since these are so clearly multiple tumors, but multiplicity while less obvious is perhaps equally common with epithelioma and sarcoma of the skin where the new tumor is often regarded as a recurrence.

New tumors following operation differ from the true recurrences in not appearing in the scar, developing usually after an interval, reproducing the original growth without great change in malignancy, and often being solitary. 


\section{CHAPTER V \\ CHEMISTRY OF TUMORS ${ }^{1}$; SEROLOGY}

Constitution of Tumor Proteins.-The conception that tumor proteins must differ in essential respects from those of normal tissues has not been demonstrated by chemical methods. The nature of the problem involved appears to have presented itself in different forms in the minds of investigators who have attacked the problem.

A different distribution of normal proteins from that of normal tissues has been demonstrated in tumor tissue by Petry, Wolff, and Beebe, who found a higher content in nucleoprotein, more uncoagulable protein, and less globulin and albumin. It is probable that these results depend on the overgrowth of cell nuclei, degenerative and autolytic processes, and edema. Nucleohiston is present only in lymph-nodes among normal tissues and its presence in lymphatic metastases of tumors originally free from this protein indicates that metastatic tumors receive chemical impress from the tissue in which they are growing. Although nucleohiston is absent in primary carcinoma of breast and in the testis, Beebe found this substance in lymph-node metastases of mammary cancer and Bang in the lymphatic metastases of testicular carcinoma. Nucleohiston gives a precipitate when $\mathrm{CaCl}_{2}$ is added to a watery solution of the tumor.

Direct chemical analysis of the split products of cancer proteins by Wolff yielded a high proportion (35 per cent.) of glutaminic acid, while Bergell and Dorpinghaus found excess of alanin, phenylalanin, asparaginic acid, and diamino-acids. Yet these results conflicted with those of Petry, Neuberg, and Beebe.

Resistance to peptic and susceptibility to tryptic digestion was said by Blumenthal and Wolff to distinguish tumor from normal tissues. Yet their results were not uniform and were probably determined by the increased amount of nucleoproteid in some of the tumors.

Excess of potassium and deficiency in calcium in rapidly growing tumors free from necrosis, and the opposite relations in slowly growing or old and necrotic tumors have been demonstrated by Beebe and by Clowes.

Pentose was greatly increased in a fibrocarcinoma of the breast in comparison with the amount in the normal breast in cases studied by Beebe and Shaffer. These authors also found that the pentose content in different tumors varied and bore no relation to the nucleoprotein, or to the presence of degeneration. In hepatic carcinoma and in hepatic metastases of gastric carcinoma Neuberg found 70 to 80 per cent. more pentose than in normal liver.

Lactic acid appears in tumors, according to Fulci, in considerable quantities. It is more abundant in epithelial than in connective-tissue growths and increases in the more malignant actively growing tumors. Its formation is dependent on metabolic activity of the tumor-cells, its source the blood carbohydrates and possibly the proteins. It appears to have no relation to cachexia.

${ }^{1}$ For a fuller discussion of this subject with literature consult Wells, "Chemical Pathology." 
Of the total phosphorus of the normal liver B. Wolter found 24.68 per cent. as phosphatid phosphorus, in the tumor-free portions of a liver with primary carcinoma 22.04 per cent., and in the tumor nodules 16.28 per cent., while the protein phosphorus in the same materials ran 20 per cent., 25.5 per cent., and 26.70 per cent. In $0.0634 \mathrm{gm}$. of dried tumor substance he found $\mathrm{I} .40$ per cent. of cholesterin.

Tryptophan was markedly increased in an epidermoid carcinoma of skin, and in an hepatic carcinoma, over the proportions found in normal skin and liver in cases studied by Fasal, but this substance was absent in a mammary fibrocarcinoma.

Fats. - The chemistry of tumor fats has been studied extensively in renal and adrenal tumors (q. v.). In general it appears from Bossart's work that actively growing tumors free from degeneration contain little fat and much lecithin, while with degeneration and necrosis free fats replace lecithin.

Purin bodies were found by Wells and Long in about the same form and amount as in normal tissue and less abundantly than the nuclear content would suggest. The purin enzymes were also identical with those of normal tissues, guanase being constantly present and adenase absent.

The delicate methods of immunological studies indicate that there are biological differences between certain tumor-tissues which are probably based on chemical distinctions, but the results obtained in this field are not decisive. Following Michaelis' failure to produce specific immune bodies against mouse tumor cells, Beebe working with purified nucleoproteins of a leukemic spleen produced a serum which agglutinated the emulsified cells of this spleen and those of a lymphosarcoma, but acted feebly and only in strong concentration on cells and nucleoproteins of normal spleen and other tissues, as well as of cancer and spindle-cell sarcoma.

Other data bearing on this question are considered in the discussion of experimental immunization.

Tumor Ferments.- The study of special ferments in tumors by Buxton and Shaffer demonstrated no distinct differences in quantity or quality from equivalent normal tissues. Weil using the viscosimeter found more proteolytic activity in certain cellular tumors than in certain normal tissues, but was not prepared to say that the difference did not depend on leukocytes. Abderhalden, however, concluded that extracts of tumor-tissue and those of normal tissue split certain polypeptids in a different manner. Extracts of normal mouse liver cleave certain polypeptids slowly, while those of mouse tumor act much more quickly. In comparing the extracts of mouse and human tumors with those of normal tissues he found differences in the split products of the proteins after the action of these ferments. Comparing the peptolytic action of normal and tumor-tissue from lower animals on peptone, Abderhalden and Medigreceanu found occasional but no constant or striking differences.

Autolysis is often observed to proceed more rapidly in certain tumor tissues than in normal tissues, but it is probable that all such differences depend on the more cellular character, and presence of degenerating tissue, edema, leukocytes, and bacteria. It is extremely difficult to obtain normal tissue which may safely be compared with tumor-tissue in this respect.

The increased activity of autolysis is well illustrated in Yoshimoto's experiments in which an hepatic carcinoma yielded $7.2 \mathrm{gm} . \mathrm{N}$ in the split products as compared to $4.8 \mathrm{gr}$. in equivalent units of normal liver. With a mammary carcinoma the difference was even greater. In the tumor autolysate purin $\mathrm{N}$ was reduced, while that of diamino-acids, peptone, and ammonia was increased in proportion. 
It is commonly believed, on the basis of Jacoby's experiments, that autolytic ferments have a high degree of specificity for the proteins of the organs in which the ferments are found. Blumenthal and Wolff have reported that when measured amounts of tumor-tissue and of normal tissue are autolyzed separately in one series, and conjointly in another the autolysis is always greater in the conjoined series. They conclude that the tumor ferments attack the normal tissues and exhibit a heterolytic property. On this basis rests the claim that infiltrative growth and cachexia depend on the heterolytic activities of the tumor ferments. Baer and Ettinger demonstrated a proteolytic activity in cancerous ascitic fluid which failed to appear with ascitic fluid from other sources, but Kepinow and Hess and Saxl were unable to verify any of these observations. The possible presence of bacteria, necrosis, or postmortem decomposition has not apparently been considered, although Neuberg has subsequently denied that bacteria or leukocytes have any influence on the results.

Ascitic fluid of carcinomatous origin yielded little euglobulin (I 2.44 per cent.), increased albumin $(64.92$ per cent.), and the usual proportion of total globulin, 35.07 per cent. (Joachim, Wolff). Similar proportions of proteins were determined in edema fluid and expressed cancer juice by Wolff.

The milky character of carcinomatous ascitic fluid is ascribed by Wolff to the presence of cholesterin-acid-ester combined with euglobulin. In a series of cancerous and other ascitic fluids Weil demonstrated occasional but inconstant hemolytic and hemagglutinative properties, abundance of complement in two cancer cases, and some antitryptic action, but no constant distinction between the cancerous and non-cancerous fluids. Signs of active autolysis in a peritoneal exudate accompanying ovarian carcinoma are reported by Umber, who found increase in non-coagulable N, albumose, leucin, and tyrosin. Eppinger reports similar findings. In a bloody cancerous exudate K. Wiener demonstrated an ereptic ferment, but any tryptic ferment present was masked by the blood. In this fluid were traces of histidin and arginin.

J. W. Vaughan has observed a striking relation between anaphylactic sensibility of guinea-pigs inoculated with tumor residue and the lymphocytosis excited by the inoculation. Animals receiving the water-soluble residue of cancer-cells after their extraction with alkalized alcohol, or a vaccine of tumor-cell emulsion after extraction with alcohol, showed in many cases very high lymphocytosis, and in such sensitized animals anaphylactic death was readily produced. The sensitization was quite transitory, lasting only during the 4- to ro-hour period of lymphocytosis, and the author interprets the result as depending on the action of lymphocytic ferments on the tumorcells.

Wassermann Reaction in Cancer.- The results obtained with the Wassermann reaction in cancer patients have varied very widely. Caan secured positive reactions in $4 \mathrm{I}$ per cent. of 85 cases; in 6 of 7 lip carcinomas; in 9 per cent. of breast tumors; and in 17 per cent. of gastro-enteric tumors. All of these cases were free from clinical signs of lues. A. Foerster secured 36 negative reactions in 37 cases, and Noguchi 38 in 39, while F. J. Fox reports 5 positive reactions in 210 cases of cancer. Positive reactions in patients with cerebral tumors free from syphilis appear to be not uncommon (Cohn).

Complement Deviation with Autogenous Tumor Antigen.--In over roo cases of carcinoma and I 6 cases of sarcoma the complement deviation test of the blood-serum has been made, using tumor antigens of the same or similar tumors. In the majority of cases the test has been positive, but in a large number the results have been negative. Recently W. Barratt, using the 
patient's own tumor as antigen, got negative results in 6 cases. De Marchis often obtained negative results in both early and advanced cases. A positive result he regarded as of considerable diagnostic value, although it occasionally occurred in syphilis and other diseases. With an antigen prepared from human carcinoma v. Dungern claims to have obtained complement deviation with the serum of both carcinoma and many other tumors, such as lymphosarcoma, glioma, and even myoma. These paradoxical results failed of verification in the hands of Edzard, Schenk, and Coca.

It is evident that the subject of complement deviation in tumors is without substantial foundation.

Complement deviation by antigen of Micrococcus neoformans tested with serum of cancer patients, in all but 7 of I44 cases, was secured by Yamanouchi and Lytchkowsky. The reaction was also positive in 44 cases of lues, but not in benign tumors. F. Green obtained positive reactions in cancer with the antigen of Micrococcus neoformans and also with those of staphylococci and streptococci.

Antitryptic Power of the Blood.-Blood-serum has long been known to inhibit the action of certain ferments. Brieger and Trebing first found that the serum of cancer patients inhibits very markedly the action of trypsin. Using various technical methods, these authors, followed by Bergman and Meyer, Herzfeld, Roche, and others, showed that in about 90 per cent. of cancers there was marked increase in the antitryptic power of the blood-serum, but that considerable increase occurred also in many other diseases, especially in those attended with leukocytosis (Wiens, Schlecht). Weil by an exact quantitative method determined that the "antitrypsin" is increased in some cancer cases beyond that observed in any other diseases, while in other cases it failed to fall as low as the ratio observed in other diseases. All authors agree that the failure of any increase is rather strong evidence against the existence of established malignant disease. Brieger regarded the phenomenon as a sign of cachexia and an immunity reaction against excessive amounts of proteolytic ferments derived from tumor-cells. Wiens and Schlecht trace a close relation with leukocytosis. Weil points out that the exact nature of the inhibiting substance is undetermined and that no specific relation to trypsin need necessarily exist, since blood-serum also inhibits saponin. Among 57 cases of cancer and 3 of sarcoma S. M. Lewin found much increased antitryptic power in 57. He could not refer the antiferment action to leukocytes, but attributed it to a reaction to proteolytic ferments discharged into the blood from degenerating tumor-tissue.

Meiostagmin Reaction.- This reaction is based upon a change in the surface tension of blood-serum. When to a dilute solution of an alcoholether extract of cancer tissue is added a small portion of blood-serum from a carcinoma patient, a change in the surface tension is produced which may be estimated by the reduced number of drops of the fluid passing in a given time through a specially designed pipet or stalagmometer. This physiochemical alteration was employed by Ascoli in the study of cancer serum and was considered by him to result from a somewhat specific interaction of cancer antigen and cancer antibody developed in the serum. The specific character and immunological nature of the reaction was soon set aside when it was discovered that extracts from many organs and even synthetic substances, as linoleic and ricinic acids, were equally effective as antigens (Ascoli, Izar). It was also shown that the sera of a great variety of diseases and of pregnancy reacted in the same manner with a variety of antigens, thus establishing that the production of the reacting factors was the result of a constitutional process of wide-spread occurrence. 
To carry out the test one may best employ a solution of $0.2 \mathrm{gm}$. ricinic acid plus $0.5 \mathrm{gm}$. of linoleic acid in Io c.c. absolute alcohol. To I c.c. of serum is added, by dropping carefully from a pipet, 0.01 to 0.02 c.c. of the antigen solution and then 9 c.c. of 0.85 per cent. $\mathrm{NaCl}$ with thorough mixing. As a control I c.c. of serum is added to 9 c.c. of salt solution. Both tubes are kept I hour on a water-bath at $50^{\circ} \mathrm{C}$. and after 2 to 6 hours the drops are counted by the stalagmometer. The count increases in the sera receiving antigen, an increase of $\mathrm{I} .3$ or less being regarded as negative, I. $7+$ positive, intermediates uncertain.

Various other antigens may be employed according to the choice of the investigator. Burmeister prepared several antigens by different methods, all with practically identical results. The results obtained vary considerably. Ascoli and Izar secured nearly constant figures with tumor sera. N. Blumenthal (I9I 5) finds that 78 per cent. of gastro-intestinal carcinomas react positively, while in other forms the positive results are much less, and in cutaneous and hepatic carcinoma and sarcomas very few. He concludes that the test is of diagnostic value only in gastro-intestinal carcinoma, and in pregnancy, while it is always necessary to exclude diabetes, uremia, infectious fever, tuberculosis, lues, chronic joint diseases, and cirrhosis. The diagnostic importance is therefore practically nil.

Various hypotheses have been suggested in explanation of the reaction.

I. The presence in the serum of abnormal lipoids resulting from fermentative processes in the cancer-cells. This view is favored by the heat-resistance of the serum; by the ready removal of the active factors by extracting the serum with alcohol, ether, or chloroform (Izar, Michaeli, Cottoretti); by the discovery of synthetic antigens, as linoleic acid (Kohler, Luger); and by the demonstrated increase of lipoids in reacting sera (Michaeli, Cottoretti). The reaction may also depend on a diminution in blood cholesterin (Izar).

2. A diminution in blood albumins, and presence of peptone. This condition is observed in most diseases giving the reaction.

-3. Changes in the alkalescence of the blood. Heating the serum increases the reaction and drives off $\mathrm{CO}_{2}$ from the bicarbonates.

The epiphanin reaction of Weichardt depends upon a change in the alkalescence of the serum. This reaction has been found positive in $8 \mathrm{I}$ per cent. of a series of cases studied by Jozca and Tokioka. By this means E. Rosenthal finds it possible to demonstrate antibodies in the blood of guinea-pigs immunized by human or mouse tumor and in the serum of tumor-bearing mice.

Stammler's Reaction.-Most tumor extracts exhibit a slight or distinct opalescence. Stammler observed that the addition of cancer serum clears up this opalescence with the formation of a slight precipitate, while most normal sera fail to act in this manner.

Ransohoff's Test.-Ransohoff observed that the injection of 3 to 5 c.c. of blood-serum from cancer patients in guinea-pigs, which had previously been sensitized by I c.c. of such sera, caused none or very weak anaphylactic reaction, while normal human serum produced the usual violent reaction. Since the same immunity followed sensitization by tumor extract, he concluded that the immunizing substance of the serum was derived from the tumor. In a series of 30 cancer cases 92 per cent. gave a positive reaction, while the test was always negative in other conditions. F. Green was unable to verify these results. 


\section{CHAPTER VI}

\section{THEORIES OF THE NATURE OF CANCER}

The Embryonal Theory.-For many years before the appearance of Cohnheim's work observations had been accumulating to show that tumors were in some way related to the embryonal growth of tissue. Lobstein, I829, likened the growth of a tumor to that of embryonal tissues and conceived that neoplastic growth had lost the control of the organism. Recamier, I829, noted that cancer developed from irritated moles and pointed out that supernumerary organs, "tous les tissues extraordinaires accidentels," readily degenerated into cancer. Rokitansky regarded certain myxomas, in his class of collonema, as derived from embryonal connective tissue. Houel, I864, designated certain sarcomas as embryonal, because they seemed to represent an abnormal growth of the same elements which in the embryo formed normal organs and tissues. In the subsequent decade there were several observations of tumors arising in connection with abnormalities of development, and the embryonal theory was believed to apply to the group of complex teratomas (Lucke). A further basis of the embryonal theory existed in the belief maintained by Remak, I854, that cancer arose from misplaced islands of epithelial cells in tissue not normally containing epithelium. Paget in 1853 remarked that invisible defects in the formation of organs might render them or portions of them peculiarly apt for the seats of malignant disease.

In I874 Durante clearly stated the doctrine that all tumors arise from embryonal groups of cells. He observed sarcoma twice developing from pigmented moles, examined many nevi, and described the embryonal character of the cells composing them. Hence he concluded that similar circumstances must surround the origin of all tumors, especially the malignant growths.

The modern embryonal theory was placed on a comprehensive basis by Cohnheim, whose original views were ably supported by his own observations, and have been steadily strengthened by very numerous contributions from many sources during the past forty years. While this is not a theory of universal application, yet that embryonal cells possess more than any others the essential factors of tumor growth is, perhaps, the most important single fact in our knowledge of tumor genesis.

Cohnheim believed that tumors develop from masses of simple or complex tissue misplaced during embryonal development. Or they arise from small groups of superfluous cells which have retained their embryonal characters, but are not necessarily misplaced. The idea of the embryonal character of the cells appeared to him essential. Most of the embryonal cells result from overproduction after the formation of the germ layers and before the appearance of definite rudiments of the organs, and he held that these superfluous cells were either distributed throughout the viscera or were gathered at certain points, as the mucocutaneous junctions. The sudden development of the cells he referred chiefly to changes in the blood-supply.

The entire group of mixed tumors and the simple heterologous growths were at once included in the scope of the embryonal theory. Indeed, when one explores the group of teratomas there seem to be all intermediate forms 
from the complex sacral tumors up to parasitic implantations containing parts of organs or limbs and even up to such phenomena as identical twins. Likewise when one passes downward through the complex heterologous tumors of the parotid to the simple heterologous growths which arise from small groups of cells misplaced or remaining embryonal it would seem that tumor genesis were chiefly a question of the mechanics of development. The present support of Cohnheim's theory is extensive. Congenital tumors of many varieties, chiefly mesoblastic, but also epithelial, exist at birth or develop shortly after, and the evidence is clear that embryological disturbances are concerned in their origin. Such tumors include fibroma, myxoma, lipoma, chondroma, angioma, glioma, myoma, mixed tumors of kidney, dermoids, and teratomas.

Congenital cancers affect the kidneys (Leibert), adrenal (Reiman), testis (Phillip), vagina, ovary (Ahlfeld), pylorus (Cullingworth), pancreas (Bohn), skin (Braun), shoulder (Selbērg), neck (Kronlein), pleura (Muus), etc.

The embryonal tumors of adults which yield a type of tissue closely simulating the embryonal counterparts and resemble the congenital tumors of the same regions form a well-recognized group for which an identical etiology seems highly probable (Wilms). Among such tumors are myxomas, rhabdomyoma of heart, amyelinic neuroma, tubular neuro-epithelioma, and a great variety of very cellular malignant tumors whose histogenesis has not been fully traced. Rudimentary organs are specially susceptible to tumor growth, as undescended testes, Luschka's gland (Defosses), the paroöphoron (Ricker), the neurenteric canal (Kraske).

Supernumerary organs are frequent seats of tumors. The misplaced adrenal rests have a wide distribution and give origin to many heterotopic growths in and about the kidney, along the genito-urinary tract, in testis and ovary, throughout the pelvis, and elsewhere (Chiari, Askanazy, Lubarsch). Supernumerary breasts are occasional sources of mammary cancer. Aberrant portions of thyroid, thymus, pancreas, uterus, and ovary give rise to many tumors in the regions of these organs. Some authors assume the presence of straggling sex cells all the way from the cephalic to the caudal extremities of the embryo to account for complex teratomas in neck, thorax, and abdomen.

The association of tumors with local abnormalities of development strongly supports the embryonal theory. Prominent in this category are the melanomas arising from pigmented moles and pigment spots in sclera and choroid; the gliomas associated with spinal hydromyelia and various abnormalities of cord and brain; sarcoma in cystic kidneys; adenomyoma of uterus; gliomas, dermoids, and mixed tumors, with spina bifida; adenoma with cysts of liver.

Persistent remnants of embryonal structures clearly originate the branchiogenic cysts, epitheliomas, and chondromas. The thyroglossal duct gives cystic or solid tumors sometimes lined by ciliated epithelium at base of tongue, in floor of mouth, or deep in the neck. Similar cystic tumors with ciliated epithelium occur also in esophagus, peritoneum, and liver. Certain embryonal tumors of uterus and adnexa and bladder are probably derived from remnants of Wolffian body or Gartner's duct. The pars postanalis intestini yields epithelial tumors of the lumbar region, and the omphalomesenteric duct and urachus give rise to abdominal tumors.

The extensive lists of heterologous tumors are most readily explained as growths from aberrant cell groups. These include chondromas of tonsil, breast, parotid, bladder, uterus, thyroid; epithelial tumors in connective tissues and organs not normally containing such cells; the mixed tumors of uterus, bladder, breast; lipoma of pia and brain, and myolipoma of sper- 
matic cord (Sazarin). While the heterologous tumors and teratomas of the sex glands are probably derived from sex cells, the origin of teratoid tumors in abdomen, thorax, pharynx, and other organs may still be referred in part to aberrant cell groups of another type.

The predilection of tumors for mucocutaneous junctions, ostia at the points of fusion of embryonal structures, and fissures formed by incomplete union of such structures, impressed Cohnheim and all later observers as strongly favoring the theory of origin from superfluous cells.

Here must be mentioned the frequent cancers of lip, nares, anus, rectum, cardia, pylorus, portio vaginalis uteri, rete testis, and chose at the tracheal and esophageal junction where squamous and ciliated epithelial tumors have been found transposed across normal boundaries. Misplacements at embryonal fissures must be connected with the cholesteatomas and epidermoids of skin, breast, abdominal and cranial cavities, spinal canal, and middle ear. Many chondromas of the skeleton, as multiple chondroma of spine and spinal canal, trachea, etc., are referred to irregularities in formation and separation of islands of cartilage. The chordomas of spine, base of skull, and nares reproduce the embryonal chorda dorsalis. Dentigerous cysts, odontomas, and the frequent adamantinoma of alveolar borders arise from superfluous or isolated remnants of the teeth. Symmetrical tumors sometimes strongly suggest embryonal irregularities, examples of which are symmetrical nevi, xanthomas (Rayer, Ehrmann), lipomas (Grosch, Kottnitz), angiomas, myomas (Brigidi, Marcacci), sarcoma of epiphyses.

There are recorded many notable examples of symmetrical tumors in paired organs, as fibrolipoma of kidneys, adenoma and carcinoma of kidneys, ovaries, tubes, breast, adrenals, and lymphosarcoma of testis. Multiple systemic tumors of the nerve-trunks, neurofibroma, suggest an imperfect differentiation of endoneurium. From this point one is led into the extensive field of multiple primary tumors of the same or different organs. Studies in this field have usually led to the conclusion that developmental eccentricities were involved.

Later followers of Cohnheim's suggestions, especially Ribbert and Wilms, have urged that in extra-uterine life cell groups may become isolated and superfluous by various mechanisms, and form the basis of tumors. The history of phylogenetic and ontogenetic development suggests that certain tumors signify spasmodic reversions to the anatomic conditions of prehistoric man or of other closely related animal species. Thus the fusion of multiple renculi, the coalescence of multiple uteri and breasts, the reduction in length of stomach, intestine, appendix, and colon, loss of lymphoid tissue in the relatively narrow human cecum, and the elimination of hair and sebaceous follicles in the face, suggest a source of superfluous cells and lay a significant foundation for the atavistic growth of tumors in many organs. Such influences, however, probably form only the feeblest of the predisposing causes of tumors.

The studies of R. Williams, Lubarsch, Ribbert, Borrmann, Meyer, and many others have shown that embryonal rests are far more frequent than was at first imagined. Wiesel, for example, finds that adrenal rests are almost constantly present along the spermatic cord and in the pelvic tissues of both sexes. It is possible to argue with fair success, as $\mathrm{R}$. Williams has done, that all known tumors of the uterus arise from aberrant, or superfluous, or embryonal cell groups. From a study of the various tumors of the testis I have been led to the conclusion that practically all of them are derived from sex cells. For tumors of the ovary Ribbert reached a similar conclusion. Thus the more careful analysis of the origin of tumors has extended rather than 
restricted the number of tumors that are known to arise from cells that have lagged behind in development.

Limitations of Cohnheim's Theory.-The pursuit of the embryonal theory has added extensively to our knowledge of aberrant cell groups and at the same time revealed many new difficulties in the way of the embryonal theory.

It has been shown that neither the isolation nor misplacement nor abnormal persistence of cell groups are necessarily followed by tumor growth. The fate of tissue rests varies: (I) they may remain stationary; (2) they may pass through normal stages of development and eventually atrophy; (3) they may experience only a limited growth; (4) cysts may form from them, or (5) tumor growth may appear.

Various hypotheses have been suggested to account for neoplastic growth from rests. The period of isolation of the cells is an important factor. The earlier its occurrence, the less is the differentiation and the greater the capacity for growth. Early embryonal rests when starting to grow meet conditions which do not favor normal development.

Since only certain rests produce tumors it may be that the embryogenic disturbance which produces the rest stamps the cells with abnormal qualities (Aschoff). This disturbance may perhaps be associated with failure of the cells to experience their proper idioplastic development and thus leave them in a condition unusually favorable to growth when such potencies are in excess. Or the relation to the surrounding tissue may influence the growth of the rest. Separation from the normal nervous control of the tissue is here to be considered. Organized cell groups such as those composed of epithelium with supporting connective tissue, according to Ribbert, tend to develop in an orderly manner, while epithelium alone tends to develop a malignant tumor. Considering the great number of organized adrenal and thyroid rests, tumors from these sources are rare. The attempts to produce malignant tumors experimentally by transplantation of tissues, adult and embryonal, have not succeeded. On the contrary, the very numerous studies in this field have served to show that something more than the separation of cell groups from their natural environment is necessary for progressive growth (cf. section on Experimental Research).

Thus it becomes evident that Cohnheim's theory, while it explains the structure and occurrence of many tumors, wholly fails to reveal why the embryonal cells begin to grow and when growing produce malignant tumors instead of normal structures.

The limited growth of many benign tumors has been approached by the structures produced by transplanted tissues, but the complete emancipation of malignant neoplasms from the normal laws of growth remains the obscure and essential element. In the attempt to solve this difficulty the theory of cell autonomy has grown up.

Theory of Cell Autonomy.-The defects of Cohnheim's theory were brought to light by studies undertaken in its defense. They showed that the mere presence of embryonal cells was not sufficient to account for their growth in tumors, and that tumors grow where probably no embryonal cells exist. It is necessary to consider how tumors arise from cells which are neither originally misplaced nor essentially embryonal. In this field the theory of cell autonomy has developed.

The germ of the theory of cell autonomy appeared in the studies of Remak and Thiersch, who traced the antagonistic relation of epithelium and connective tissue throughout embryonal development. Epithelium seemed everywhere to be the dominating embryonal tissue and to cease growing when it met sufficient resistance from the connective tissues. Thiersch found 
in the weakening of the stroma of the involuting breast a relief of tension capable of releasing the formative tendencies of gland-cells with their longer span of vital activity. The decay of connective tissues he regarded as senile atrophy and the growth of cells as degenerative proliferation. The idea of cell autonomy appears also in Cohnheim's theory, but here it was overshadowed by the embryonal element in the originating cells.

To account for the limitless growth of cells, embryonal or adult, the theory of cell autonomy introduces the conception of tissue tension.

Ribbert endeavored to explain the growth of tumors solely through the removal of certain cells from the influence of a tissue tension by which their growth is normally restrained. He abandoned the theory that the disturbed cells must be embryonal, claiming that the regenerative capacities of adult cells are quite sufficient to account for all the characters of malignant tumors.

Conception of Tissue Tension.-The theory of tissue tension assumes that cells are capable of growing indefinitely unless restrained by a complex group of forces. This assumption is perhaps not yet proved, but there are many facts in its favor. The astonishing regenerative powers of injured tissues in plants, lower animals, and of many organs and tissues of vertebrates, the remarkable rate of growth of the vertebrate embryo, the hypertrophy of the gravid uterus, the indefinite reparative capacity of injured or transplanted epidermis, all suggest that normal cells, although eventually restrained by organized forces, yet in proper environment are capable of growing without limit. Ribbert insists that no unusual power of proliferation exists in cancercells, that these cells freed from the restraints of tissue tension are merely exhibiting the powers of growth with which they are endowed from the ovum. Weigert and Roux have also asserted that the regenerative capacities of cells are determined from the moment of their derivation from the ovum and can never be increased by any external stimulus.

Many observers have not accepted the Weigert-Roux hypothesis. Lubarsch finds it incompatible with the extensive growth of metastatic tumors and claims that external stimuli may induce an enormous increase in the regenerative capacities of cells. I believe it is here necessary to distinguish between the organized regenerative properties of tissue cells, which are truly determined in the ovum, and the mere power of multiplication without organization which is subject to immense variations from the influence of the environment. Tumor-cells seem to have more than their ancestral power of growth as when without pressure they erode bone. In any case the facts of normal and tumor growth seem to place the doctrine of growth restraints and tissue tension in an unassailable position.

What, then, are the restraints to growth which control the multiplication of cells and maintain the tissue in a physiological condition while permitting normal repair? At least four such factors are known:

I. Mechanical pressure of cells on each other.

2. The distribution of nutriment.

3. The influence of specialized functions.

4. Organization.

Under each of these heads belong important contributions to the theory of cell autonomy.

r. The influence of mechanical pressure may be traced both in the inception and in the course of tumor growth. In the former case it is somewhat difficult to visualize the action of pressure. Thiersch thought that relief of mechanical pressure followed the decay of the stroma about epithelial cell groups and permitted the expansion of the cell mass, but this element is inadequate to explain the inception of any tumor. The rapid lateral growth 
of regenerative epithelium in skin-grafts may be referred in part to the absence of lateral pressure. Loeb has made an effort to analyze this element experimentally. Given a certain grade of epithelial overgrowth, as in the tense ducts of chronic mastitis, trauma would relieve tension and facilitate expansion of the cell mass. Acting upon blood-vessels pressure is a prominent factor in controlling the progress of tumors. Fibrous encapsulation causes some tumors to regress, and relief of pressure by incision of capsule releases active growth. These events are frequently seen in both early and late stages of tumor growth.

2. Distribution of Nutriment.-Many clinical and experimental data have established the importance of blood-supply both for the physiological proliferation of cells and the inception and progress of tumor growth. The normal development of organs is regulated partly through the plan of the arterial system, and partial agenesia follows arterial defects. Cohnheim believed that cell rests begin to grow chiefly because of increased blood-supply. The minute study of many early tumors indicates that increased vascularity precedes the hypertrophy and multiplication of cells. Yet relative anemia is perhaps equally common.

While the blood-supply is often increased the abnormal capacity of tumorcells to absorb nutriment is a more obscure and significant feature of the disturbed nutrition of neoplastic cells. Its explanation would throw much light on the nature of the tumor process. The increased demands for function determine increased vascularity in certain tumors (thyroid cancer). In the case of chorioma an increased supply of lutein secretion, according to Pick and others, is associated with abnormal growth of the chorion over whose normal growth this secretion presides. It is possible that other hormones may by unusual activity favor the growth of tissues with which they are concerned, especially in the field of multiple tumors of paired or functionally related organs. Thus there is extreme fibrosis of the ovaries in many cases of mammary cancer. The theory that a specific nutritive substance, not itself the nutrient molecule, presides over the growth of tumors has been applied by Ehrlich to explain the results of his zig-zag transplantation of mouse tumors, from mouse to rat and back. While this theory seems out of accord with certain observations concerning these transplantable tumors and seems to have little bearing upon the problem of tumor growth in man, yet it presents a new point of view for the study of the origin of tumors in general.

3. The Infuence of Specialized Functions.-The energies of cells are normally divided between proliferation and specialized function, between work and growth. Normal function diverting the energies of the cells must be a constant restraint or regulator of growth. In most organs certain groups of cells are set apart for growth and from these are derived the more specialized functionating cells. The germ center cells of lymph-nodes, the cells at the bases of intestinal villi, and the basal cells of the epidermis are the ones that respond to demands for growth, and from them tumors arise. Overresponse to demands for growth and loss of functional requirements both seem to precede the development of cancer, as in the adenomas of cirrhotic livers, and in the atrophying breast. Adami has presented the general importance of this principle designating the tumor process as "the cumulative habit of growth, replacing the habit of work."

Thiersch and Beneke have interpreted the tumor process as a degenerative overgrowth of cells. That there is overfunction in some degenerating cells, as in the paralytic hypersecretion of glands, has been pointed out by Oertel who urges that overgrowth may also characterize degenerative proc- 
esses. In protozoa the germinative and vegetative functions reside in separate nuclear structures, the macro- and micronucleus, and it seems possible that the overgrowth of tumor-cells may be accompanied by excess of germinative nuclear material. This conception of idioplastic variations in the cells has been pursued by Rulf.

Active growth is usually associated with active function. In thyroid cancer of fish there is apparently an example of a certain type of malignant tumor developing as a result of overresponse to functional stimulus. There are many examples of adenomatoid hyperplasia from excessive functional stimuli and it seems probable that this element may be concerned in the inception of certain tumors. Proliferating chromatophores and mucous goblet cells exhibit excessive function. Thus both overfunction and loss of function seem to be related to tumor growth.

4. Organization.-The limitless growth of tumors early suggested that the cancerous tissue had lost the control of the organism. Recamier in I829 definitely formulated this idea and the conception has steadily enlarged with the progress of biology.

The fertilized ovum reproduces its kind because of very complex factors which are best interpreted as the influence of environment on hereditary characters. Yet after the last analysis there is always an unexplained residuum about regeneration which justifies the teleological concept. The forms of regeneration among the lower animals show many processes that seem to be purposeful. The regeneration of limbs at the breaking joints of crustaceans; the variable regeneration of one or two heads in flat worms according to line of incision; the regeneration of the lens in Triton indifferently from capsule, iris, choroid, or retina; the polarity of the hydroid Antennularia, which transforms twigs into roots according to its accidental needs, illustrate the extreme adaptability of regeneration to the uses of the organism. The mechanism by which these results are brought about, whether purposeful or not, is called organization. Some biologists, notably Morgan, claim that this influence can be defined only in terms of purpose, and cannot be wholly resolved into any known physical forces. However that may be, it is a fact that a tumor can be defined only in terms of purpose. All the known features of a malignant neoplasm are exhibited by the normal chorion, even the destructive invasion and cachexia, but the process is not a tumor because of its purpose, which shows that it is under the control of the organization.

Embryology and cellular biology have thrown much light on the nature of the forces concerned in organization.

First, it has been shown that at all stages the organization has an equilibrium which is nuaintained by the altruism of the tissues. The dividing embryo in some animals up to the sixteen-cell stage yields cells any one of which if separated from the others is capable of producing an entire embryo (Wilson, Zoja). Beyond this stage and usually before, the cells become differentiated, different cell groups going to produce different specific tissues. Accorling to Weigert and Roux the differentiation results from an unequal division among the cells of the properties of the ovum (the mosaic theory). According to others (Spencer, Hertwig) the differentiation is accompanied not by loss but by suppression of certain properties of the ovum. Owing to the unequal distribution or activity of qualities or potencies, some cells come to possess more of one type and others come to possess more of another, while the sum total of all the potencies is necessary to maintain the equilibrium of the organization. Hence the cells, tissues, and organs stand in a relation of antagonism or correlation which maintains an equilibrium, any change in one affecting more or less all the others. This interdependence has been called by Hansemann the altruism of the cells. Removal of one kidney is followed by compensatory hypertrophy of the other, through the physical factors of increased urea in the blood, increased blood-supply and nervous stimulus, all acting harmoniously under the influence of the organization. There is altruistic hypertrophy of bones with tumors of the hypophysis, and altruistic atrophy of the adrenals in anencephalic 
monsters. A definite chemical substance conveys this altruistic influence in corpus luteum secretion which controls the growth of the chorion.

Many recent observations and experiments have emphasized the importance of the current of nutrition in determining the growth of normal and neoplastic tissues. Murphy finds that the presence of a small fragment of growing splenic tissue prevents the growth of tumor-tissue implanted in the chicken egg, a result probably signifying that the growing splenic tissue diverts and absorbs the available nutriment. This principle has received experimental confirmation in an interesting way by J. Loeb in observations on plants. Stockard shows how the rate of regeneration of the amputated arms of the starfish depends on the number amputated. There are many clinical observations indicating that the growth and even the inception of tumors may depend chiefly on the stream nutrition to the part, and also upon the character of the nutrition.

Second, the study of cell division reveals a mechanism by which the altruism of the cells and the control of the organization may be disturbed. As long as the cells multiply

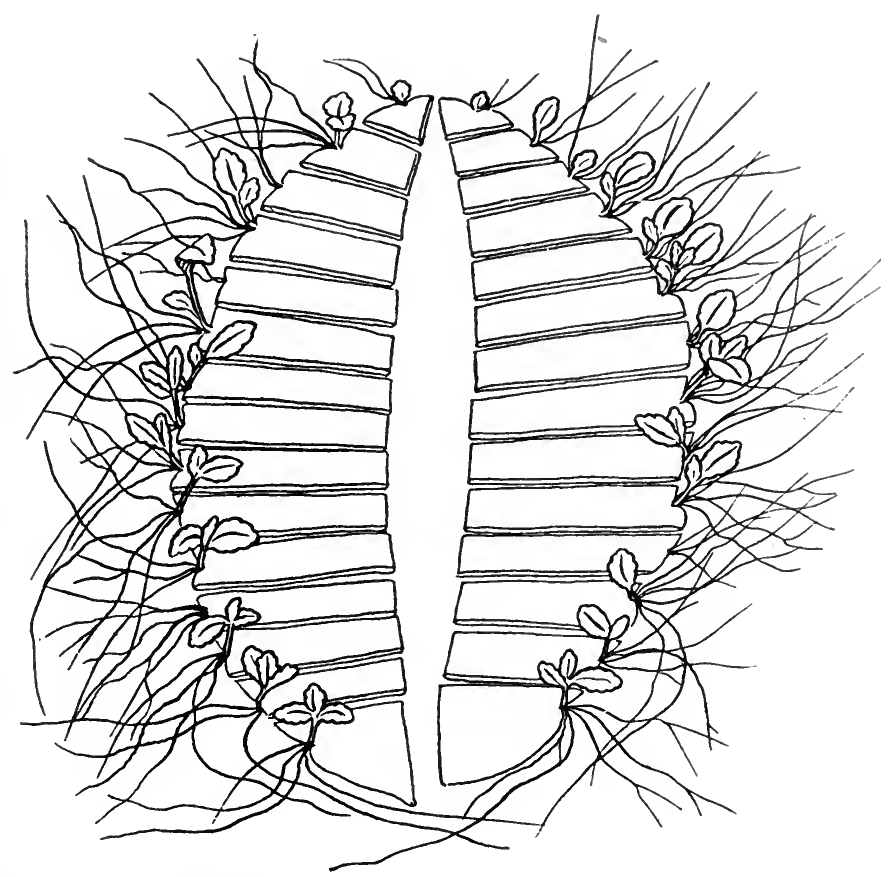

Fig. I0.-J. Loeb's experiment with leaf of Bryophyllum calicynum, illustrating growth restraints. The leaf, notched as above, fails to develop shoots while attached to stem, but does so when severed from stem. The influence is thought to depend on the flow of nutriment, which ceases to pass from leaf when it is severed.

by normal mitosis and each stage of differentiation in one group is accompanied by equivalent antagonistic qualities in others, equilibrium is maintained. But if inflammation or chenical or mechanical factors derange the mitotic process single chromosomes may be destroyed and the resulting cells will not have the proper antagonists to balance it. By repetition of this process a new type of cell may arise which is more and more removed from the normal and fails to receive any restraining influence from the organization.

The study of tumor-cells shows that irregular, asymmetric and multipolar mitoses and destruction of chromosomes are frequent in malignant tumors. Hansemann applies the term anaplasia to the condition of such cells, which signifies loss of normal differentiation, specific function, and organization. These physiological properties which characterize malignant tumor-cells are usually but not always associated with pronounced changes in morphology. Anaplastic cells are not embryonal cells, but a new 
type which have lost their place in the organization. More or less anaplastic cells occur in inflammation, but there are many degrees of anaplasia, and its occurrence in inflammation accords with the fact that inflammatory hyperplasia may pass into neoplastic.

The abnormal capacity for growth has long suggested that tumor-cells have some relation to sex cells. In some animals the entire series of sex cells from the fertilized ovum up to the new egg cell has been traced as a distinct series apart from the somatic cells. In this series the mitotic nucleus exhibits only one-half the usual number of chromosomes, and these instead of assuming a $\mathbf{V}$ shape and radial arrangement are ring or loop shaped or composed of coarse granules. This gametogenous mitosis has also been observed in the growing edges of tumors (Farmer, Moore, Walker) and it may be produced by chemical irritants. Its occurrence does not signify that tumor-cells are equivalent to sex cells, and yet in connection with the invasive properties and striking altruistic relations of both classes it shows that tumor-cells and sex cells have some interesting points of resemblance. Spencer, Hertwig, and others claim that the sex potencies of somatic cells are not lost, but only suppressed. On this basis Williams has built an elaborate and ingenious argument to show that tumor growth signifies the reawakening of the reproductive functions in the somatic cells. According to this theory tumor growth is a form of agamogenesis comparable to the budding of plants.

Many have supposed that tumor-cells are fertilized cells, through conjugation with leukocytes (Klebs), by parthenogenesis (Waldeyer), by conjugation of endothelium and fibroblasts (Recklinghausen), by nuclear conjugation (Auerbach, Bashford), or by endogenous cell infection.

None of these hypotheses has survived criticism and the theory of cell autonomy remains content in the position that tumor-cells emancipated from growth restraints are merely exhibiting their natural capacity for growth.

The theory of cell autonomy appropriates all the observations regarding the mechanisms of the isolation of cell groups. It also accepts the principle that adult cells which have not lagged behind in development and are not embryonal or superfluous may be isolated and freed from growth restraints.

Ribbert lays great stress on the mechanical separation of epithelial cells by new connective tissue. In early carcinoma of stomach, epithelioma of lip, and psoriasis linguæ he finds strands of new connective tissue growing between the epithelium and snaring off cell groups. Or round-cell infiltration of inflammatory origin beneath the epithelium creates an abnormal environment, and nullifies the restraints to grow. Central enchondromas and many exostoses he refers to groups of cells isolated by irregular ossification as in rickets. Malignant tumors he thinks may develop from groups of cells snared off in the course of benign tumors or from implantations during removal, as of ovarian cystomas. So carcinoma develops from isolated cell groups in cirrhosis of liver or contracted kidneys.

Further, it must be assumed that trauma isolates predisposed cell groups as in many bone-sarcomas. The evidence that malignant chorioma may develop from emboli of normal chorionic cells is practically conclusive (Marchand, Pick).

In $x$-ray carcinoma there is chronic edema, round-cell infiltration, and fibrosis altering the relation of the epithelium long before the downward growth of these cells. In chronic mastitis multiple cancer nodules appear at minute points where the fibrosis is unusually dense and the disturbance of normal relations most marked.

There is little doubt that the minute study of the conditions surrounding the early stages of cancer, as instituted by Ribbert, forms a strong support to 
the theory of cell autonomy, while its bearing on the early diagnosis and prevention of cancer is obvious. Yet these observations fail to show why the isolated cells so often regress and only rarely succeed in producing tumors, and it is clear that the mechanical isolation of cells is, like the embryonal character, only a predisposing factor in tumor genesis. To cover this defect various hypotheses have been maintained.

I. The isolated cells have been altered and their growth tendencies increased by previous irritation. 2. There is a local predisposition to tumor growth. 3. There is a general predisposition to tumor growth.

I. Billroth's dictum, "Without previous chronic inflammation cancer does not exist," while subject to exceptions, is yet so generally true as to establish the great significance of chronic irritation as a factor in tumor genesis. Interpreted according to the principles of the theory of cell autonomy it is clear that successive generations of cells subjected to chronic irritation will suffer increasing loss of growth restraints with disturbance of their normal rate of growth, the size of their nuclei and cytoplasm, their blood-supply, and their relations to neighboring cells. All of these changes are observable in the preliminary stages of lingual and other cancers. In this condition it seems but a short step to downward growth of such cells when the cumulative process reaches a sufficient stage or when more abrupt mechanical isolation of cells occurs. Whether these cells in their continued progress are merely exhibiting normal powers of growth or have acquired new powers constituting them an entirely new biological series it is difficult to decide. Here it must be urged that the continued growth of cancer is an entirely different problem from its inception. Many new factors intervene to facilitate extension and metastases. It is by no means clear that malignant tumor-cells are more viable than normal cells. They are more readily autolyzed and in early cases tumor emboli enjoy less resistance to destructive forces than normal cells.

For the inception of many tumor processes chronic irritation acting upon normal cells seems to adequately explain the observed phenomena. Thus the cumulative effects of chronic irritation alter the nutrition and growth activities of cells so that when they become isolated they are not normal cells, but are in a state of disturbed equilibrium with a tendency toward exaggerated growth. Such conditions are commonly observed in the precancerous lesion.

2. A local predisposition to tumor growth must be assumed to exist to account for the capricious development of many neoplasms. The factors which lead to the inception of tumors in one locality or individual often seem incapable of bringing this result under other circumstances. Many of the factors constituting this local predisposition form the basis of Cohnheim's theory, including, (I) Isolation without misplacement of embryonal cell groups. (2) Persistence of cell groups and organs which normally regress after embryonal life. (3) Formation of superfluous cell groups. (4) Gross abnormalities in development of regions, organs, and systems. (5) Disturbance in the idioplastic develepment of cell groups without visible changes in morphology.

In addition, Rindfleisch assumed that imperfect nervous control is one of the factors predisposing to exaggerated growth of tissues and tumor formation. While the effectiveness of such a factor may readily be granted, there seem to be no observations in favor of its existence except the absence of intrinsic nerves in tumor-tissue. Likewise imperfect idioplastic development may be conceded as certainly favoring tumor growth, but in the absence of definite criteria of such a condition this factor remains largely in the field of speculation and as a problem for future studies. A reasonable speculation 
also is that of Israel, who supposes that tissue cells, like bacteria, in the course of repeated or abnormal generations tend to vary and in some tissues they vary in the direction of overgrowth. Yet as normal cells do not vary in this way Israel is assuming an original local predisposition to overgrowth. Thus the well-established elements in the doctrine of local tissue predisposition belong under the embryonal theory, while the others still remain seductive hypotheses.

3. A constitutional predisposition toward tumor growth has long been a favorite field of discussion. There is a vast amount of literature concerning the relation or antagonism between cancer and tuberculosis, syphilis, malaria, diabetes, insanity, arthritism, obesity, gall-stones, dermatoses, heart disease, mental disturbance, etc. The many interesting observations in this field seem to have wholly failed to throw any light on the etiology of malignant tumors, nor have they established any definite connection between cancer and the diseases mentioned.

It has long been recognized that cancer is prone to develop in plethoric individuals. Williams sums up an elaborate argument with the conclusion that cancer occurs chiefly in the well nourished, the well-to-do, and the wellprotected against infectious disease, that it is especially prevalent in peoples with whom the consumption of meat is high, and that it is a tax on civilization. Yet all of these factors may exhaust themselves in increased longevity, by which more individuals of weaker type reach the cancer age, and none of them seems to establish any definite predisposition to the disease. It is conceivable that overnutrition should facilitate overgrowth, but it is not so clear why overnourished tissues should tend toward neoplastic instead of normal growth.

The immense variation in the nervous and physical conformation of individuals suggests to Borst that there may be equal divergences for or against neoplastic growth of tissue cells. The quality of the body proteins, the general organization of the body, the relation of tissues and organs to the vascular and nervous systems may give rise to individual peculiarities which are of importance in the origin or failure of tumor growth, but there are few facts to support this speculation. At best it can only mean that the capacity for tumor growth is far more general than the occurrence of tumors. The existence of multiple tumors is more properly referred to definite multiple disturbances of development or to the existence of several recognizable adequate factors than to any general predisposition.

The individual of cancerous type is described by Benecke as revealing a large heart and arteries, small lungs and pulmonary arteries, long and capacious intestine, and well-developed osseous, muscular, and adipose tissues. Yet these are just the subjects most likely to reach the cancer zone, where the age factor becomes far more prominent than anything we know about predisposition.

Imperfect balance between the sexual and other organs, the status of the thyroid gland, and the chromaffin system have been suggested as forming the basis of a cancerous predisposition, but the observations in these fields are as yet quite inadequate to form the ground of an effective argument. Many of the above considerations may be adduced in favor of the hereditary theory of cancer.

According to Williams the growth of malignant tumors is favored by an altered metabolism of the body consequent on the decline of the sexual functions. He refers to the increased frequency of cancer after removal or destruction of the ovaries (Wells, Kratzenstein), and in cases of congenital absence or defect of the sexual organs (Neugebauer). Stickler also found 
that 50 per cent. of a series of cattle and horses with malignant tumors were castrates.

The thyroid gland exhibits in most adult cancer patients that tendency toward involution which belongs to advancing years, but that this involution is more marked in cancer patients has not been shown; nor has any relation been established between cancer and the various members of the chromaffine system. A small proportion of adrenal tumors is associated with malignant tumors in other organs (Williams, Adams, Guthrie). 'The usual integrity of the pancreas does not favor the theory of Beard that cancer depends on defective pancreatic secretion.

Thus although the facts of tumor growth seem to demand the assumption of a constitutional predisposition, the basis of this doctrine is as yet imperfectly laid. Clinical observations, studies in histogenesis and gross anatomy, and chemical researches fail to reveal why the same factors succeed in one but fail in another subject to produce tumors. The most suggestive hypotheses seem to be those concerned with overnutrition, the decline of the sexual functions, and congenital disturbance in the idioplastic development of the cells. The constitutional element seems to be too subtle for ordinary methods of study, and to show itself most clearly in the relation of heredity to tumor growth, where, however, with certain exceptions, its practical importance diminishes almost to the vanishing point.

Heredity.- Nothing about cancer is more generally accepted than its hereditary nature, and nothing is less satisfactorily proved. The evidence favoring this doctrine consists chiefly in records, some very notable, of cancer families, and in statistical studies of the incidence of the disease in the relatives of numerous cancer patients. Recently experimental evidence has been adduced in its favor.

Cancer Families.-In I 837 Warren reported a family history in which the grandfather had cancer of the lip, while the son, his daughter, two sisters, and the daughter of one of them all died of cancer of the breast. The most famous cancer family is that reported by Broca, I866, of Madame Z, the details of which were furnished by a medical member of the family. Of 26 members, mother, children, and grandchildren, reaching the age of thirty, I6 died of cancer of breast, liver, or uterus. Napoleon I, his father, one brother and two sisters, are said to have died of cancer of the stomach. Sibley observed cancer of the left breast in a mother and her 5 daughters. Korteweg saw cancer of the breast, and Paget cancer of the uterus in mother, daughter, and granddaughter.

Retinal glioma occurred in ro of 16 children of healthy parents in a family encountered by Newton, and in 8 children of a family seen by Wilson. These, however, are instances of congenital, not hereditary origin. Williams has collected many other references to cancer families.

Levin analyzes the conditions under which the clinical study of heredity in cancer may be conducted and points out that the occurrence of cancer in a family member may not necessarily mean an hereditary influence. An extended investigation of the families of 5 cancer patients failed to show any unusual incidence of the disease. In fact, he seems to have encountered unusually resistant families in which cancer was of occasional but rare occurrence. Warthin, on the other hand, records several very striking histories of families in which cancer was unusually frequent. Thus in one family, I 7 of 48 descendants of a cancerous grandfather developed cancer, chiefly of uterus or stomach. In another, 8 descendants of a cancerous great-grandfather all died of the disease. Warthin adds that these families became extinct, but clearly not from cancer. These observations emphasize the occasional 
occurrence of cancer families, and suggest that members of such families may properly take definite precautions against the disease.

Statistical studies began with Paget, who traced cancer or other tumors in the relatives of 23.6 per cent. of 254 cancer patients, but in only I 8.3 per cent. of 147 non-cancerous tumor cases. Baker found cancer in the relatives of 24.2 per cent. of 322 cancer cases. Velpeau thought that at least one-third of his cancer patients had a family history of cancer. Leichtenstein collected histories of II 37 cancer cases in which heredity appeared in 17 per cent.

The proportion of women with cancer of breast in whose relatives there was a history of cancer has been estimated by Butlin at 37 per cent., by Nunn at 29.3 per cent., by Williams at 24.2 per cent., and by Leaf at 23 per cent. Yet Campiche and Lazarus-Barlow place the proportion in roo recent records of the Middlesex Hospital at I 5.6 per cent., Williams found a history of heredity in cancer of uterus in 19.7 per cent., of breast 24.2 per cent., of other female cases 23.9 per cent., and in men suffering from cancer ( 209 cases) II per cent.

On the other hand, Lebert placed the percentage of inheritance at 8.5 per cent.; Sibley in 305 cases at 8.5; Winniwarter in Billroth's clinic at 8.8 per cent. Billroth found one parent affected in 5.8 per cent. of I 70 cases. Tillmann quotes reports showing 8.5 per cent. and ro per cent. of hereditary cases of cancer of stomach. Ziel collected 200 cases with I I per cent. hereditary. H. Cripps excluded distant relatives, as cousins and aunts, and in I69 cases only 6.4 per cent. had a cancerous parent. He found that in England and Wales (I86I to I870) I in 29.I deaths occurring after 20 years of age was from cancer. Comparing this ratio with that of cancerous parents of cancer cases he concluded that cancer in one parent does not increase the liability to the disease. Recently Hillier and Tritsch made an elaborate study of family history in 3000 cancer cases in the Middlesex hospital. Of these, I3.I per cent. had a history of cancer in relatives, while of $4 \mathrm{I} 7$ non-cancerous cases I4 per cent. gave cancerous antecedents. Pearson, who interpreted these data, hesitated to accept the necessary conclusion that a family history. of cancer confers a very slight immunity to the disease, yet unless the collection of the data was very much biased it is clear that statistics do not support the doctrine of the general hereditary nature of cancer.

Bashford points out that in England of those living at 35 years of age I man in II, and I woman in 8 , die of cancer. This ratio signifies that a history of cancer will occur in I out of every 2 families of the general population. In the families of 669 cancer patients he obtained a history of cancer in 3 I I or 50 per cent. In Stuttgart, Weinberg, examining the cancer records from I 872 to I902, found no evidence that heredity plays a dominant part in the etiology of cancer. His data show that the deaths from cancer in each 100 relatives of cancer patients were, of the patients' parents, 6.6, brothers and sisters, 3.9; of their husbands and wives' parents, 5.9; brothers and sisters, 3.r.

There are numerous sources of error in the conclusions drawn from statistical studies. It is quite unlikely that any hospital population can give reliable information regarding the existence of cancer in their parents, much less in their other relatives. There are numerous errors both of omission and commission in the diagnosis of cancer. The nature of any hereditary element in cancer is wholly obscure so that it is impossible to decide whether cancer in cousins and aunts, or only in parents should be considered, whether the predisposition may skip generations, whether the influence affects the same organ or all the organs, or whether the influence of a similar environment, climate, food, and habits, may not submerge any form of heredity. Consideration of the age factor completely altered the conclusions suggested by 
Guillot's statistics. In $35^{2}$ patients he found a cancer history in ro per cent. of the non-cancerous and in 17.4 per cent. of the cancerous. But the average age of the living relatives of the non-cancerous was 37 years, that of the others $5^{2}$ years. So that the expectation that cancer would later develop in the relatives of the non-cancerous before their average age reached 52 changed the true percentage of cancerous relatives of the non-cancerous to 16 , or r.4 per cent. less than for the cancerous group. In view of all these considerations it must be confessed that the statistics of cancer heredity in the present state of our knowledge are inconclusive or even worthless.

The evidence presented by the many notable cancer families seems to have a different value. It seems impossible to attribute to coincidence, or environment, or erroneous observation the remarkable prevalence of cancer in the families mentioned by Warren, Broca, Manichon, Le Tulle, and others. These cases seem to show that in rare instances a pronounced hereditary predisposition to the disease exists. It is, however, important to emphasize that the history of these families indicates that when an hereditary predisposition exists it displays itself in unmistakable form, and it in no way justifies the assumption that hereditary influences prevail for cancer in general, on which point the evidence is wholly negative. These family histories show how very uncommon an effective hereditary influence really is. From the clinical and statistical evidence therefore one must, I think, subscribe to the conclusions of Le Doux-Le Bard. (I) Nothing authorizes us to affirm that cancer is hereditary. (2) In the interests of the public this doctrine ought to be combated.

The influence of heredity upon tumor incidence has been submitted to experimental test. Bashford and Murray were able to increase the incidence of mammary cancer in 340 mice of recent cancerous ancestry to 18.2 per cent., as compared with an incidence of 8.6 per cent. in 223 mice of remote cancerous ancestry.

Tyzzer has conducted breeding experiments with the offspring of a female mouse, suffering from a cystadenoma of the lung. Of roo offspring of this one cancerous parent 65 survived six months and 3 more than two and a half years. Of these, 20 ( 32 per cent.) developed one or more tumors, I7 being pulmonary tumors. Among 68 of these mice born of cancer-free parents 9 (I3 per cent.) developed tumors, while among 29 with one cancerous parent II (39 per cent.) developed tumors. The increasing frequency of tumors in the old mice ( 2 years) of this family indicated that cancer was almost inevitable for animals which survived infectious diseases. While no satisfactory controls were secured these interesting results strongly support the hereditary theory, at least for this family of mice.

Maud Slye has conducted elaborate experimental studies on the influence of heredity on the incidence of mouse tumors and has proved that inbreeding of tumor-bearing animals greatly increases the incidence of tumors. The material used in these studies is a pedigreed stock of mice, in her hands I 5 years. The tumors under study were all spontaneous, arising without manipulation of any sort except that of selective breeding. The work has now yielded over 30,000 autopsies and over 4000 primary spontaneous tumors. The results led her to the following conclusions:

(x) The inheritance behavior of neoplasms is that of a Mendelian recessive. (2) Double cancerous parentage yields roo per cent. tumor strains, except where some individuals die of infections before they reach the cancer age. (3) Single cancerous parentage yields heterozygotes (transmitting but not themselves developing cancer) in the first hybrid generation. These, whether inbred or hybridized with other heterozygotes, yield in the next 
hybrid generation non-cancerous, heterozygous and cancerous progeny, approximately in the proportion of $I: 2: I$. (4) The mating of a cancerous with a heterozygous individual gives approximately 50 per cent. cancerous and 50 per cent. heterozygous offspring. (5) Double non-cancerous parentage yields roo per cent. non-cancerous strains. (6) The tendency to cancer, therefore, is inheritable, as no character except one which is hereditary can behave in this manner. This segregating out of a character is the test of heredity. (7) The tendency to tumors of specific organs and of specific types is also inheritable. For example, this stock has yielded strains of roo per cent. lung tumor, or soo per cent. alveolar carcinoma of the mammary gland, or 50 per cent. liver adenoma, or 37 per cent. kidney tumor.

Other geneticists, however, do not accept all of Slye's conclusions from her published data. They are inclined to believe that there is, no doubt, a susceptibility to cancer in various families and strains of animals. The method of inheritance, they believe, does not follow a simple Mendelian procedure, but is probably understandable on a Mendelian hypothesis involving multiple factors.

While there are certain sources of error in the experimental studies which suggest themselves to the general pathologist, it may be assumed that these studies have demonstrated the prime influence of heredity under the conditions described. Nevertheless, the writer holds that it is still necessary to go out into the field of human tumors and determine by observation just how far any hereditary influence proves effective in the causation of tumors. This test seems reasonable, since there is abundant clinical evidence of the transmissibility of such diseases as hemophilia, glioma retinæ, and neurofibromatosis. Clinical and statistical studies, however, do not support the view that heredity plays an important part in the incidence of the main human tumors, and until these studies bear out the experimental results, the conclusions of the latter must be limited to the experimental conditions and not applied to the more complex field of human disease. Clinical studies indicate that there is among human beings a general susceptibility to tumors, which in rare instances has become pronounced and effective, but that, as a rule, this susceptibility is negligible and the disease does not develop until other exciting factors, which are the real effective causes of the disease, are brought into play.

It should be noted also that the cancerous predisposition may result from factors acting in each case in utero, that is, that the predisposition may be congenital without being hereditary. 


\section{CHAPTER VII}

\section{THE SPECIAL ETIOLOGY OF TUMORS; TRAUMA}

While one general and essential principle, best portrayed in the theory of cell autonomy, seems to underlie the inception of all tumors, yet it is becoming more and more apparent that the etiological factors are variously combined for nearly all neoplasms. In this sense it may almost be said that the etiology of each form of tumor is specific and that a practical knowledge of tumor etiology requires a minute analysis of all the factors concerned in each case.

Thus the conditions surrounding the inception of cancer of the breast are in many respects quite different from those concerned with cancer of the tongue. This distinctive quality is even more obvious in the clinical course and termination of different malignant tumors, so that there is theoretical basis for and practical value in considering each tumor as a distinct clinical entity. Well-known examples of specific etiology are seen in various tumors connected with occupations, as $x$-ray cancer, multiple epithelioma of the skin of workers in paraffin, and anilin dyes, scrotal cancer of chimney-sweeps, and the rare cancer of the lung in metal workers, weavers, and cigar makers.

The cumulative effects of the habits of the individual are seen in smokers' cancer of the lip, pharynx, and larynx, Kangri and Betel-nut cancer of Indian natives, and cancer of the skin in arsenic eaters.

Particular forms of chronic irritation and inflammation precede many well-known clinical forms of cancer, as epithelioma of tongue from jagged teeth, cancer of skin from chronic eczema, indolent ulcers, burns, or along chronic sinuses from necrosing bone, and with Paget's disease of the nipple.

Specific chronic granulomas occasionally lead to cancer, as syphilitic psoriasis linguæ, and lupus of the skin. Sarcoma also develops after lupus and syphilis of bones. Chronic inflammation of mucous membranes precedes cancer, as in uterus, stomach, urinary passages, nares, and with cholelithiasis. Chronic inflammation of glandular organs frequently forms the essential basis of cancer, as in the cirrhotic liver, contracted kidney, and hypertrophic prostate. Chronic hyperplastic and benign neoplastic processes rather rarely become transformed into malignant tumors, as warts, polyps, myoma uteri, and simple epithelial cysts of many organs. Abnormal involution designates the conditions leading to many cancers of the breast, appendix, prostate, and thyroid. Trauma seems to be the sole tangible factor in originating many tumors of bone, glia tissue, testicle, and many other organs.

The wide differences in the nature of the embryogenic disturbances which lead to isolation of embryonal cell groups and tissues set apart a very large class of tumors whose etiological factor is distinctive for each member as well as for the entire class. Thus branchiogenic cancer, Wilms' renal embryoma, and the teratoid testicular tumors which are probably derived from sex cells, deserve to be considered as enjoying quite a different etiology.

If, as seems probable, Hodgkin's granuloma becomes transformed into a nearly autonomous lymphosarcoma, there is very little resemblance between its etiology and that of bilateral lymphosarcoma of the testis of a child. If abnormalities of the lutein secretion enable emboli of normal chorionic cells to develop malignant tumors there is little wisdom in discussing its etiology with that of smokers' cancer of the larynx. The two diseases are as 
different in etiology as gout and erysipelas, both of which produce exudative inflammations of the skin.

Thus the special etiology of tumors presents extremely numerous and complex problems which have immediate practical bearing on diagnosis, treatment, and prevention, and which are probably much nearer solution than is that of the nature of the neoplastic process. It seems important to emphasize the separate nature of these departments of the subject of etiology and to point out that in many respects our knowledge of the etiology of tumors is very clear and precise. Yet the action of all these special etiological factors, as irritation, trauma, and inflammation, is mainly secondary and indirect, and without combination with other predisposing conditions they are incapable of inducing tumor growth. It is possible that heredity may at times furnish one of these predisposing factors. In many cases the above special factors exist, but no tumor develops.

Therefore the special etiology of tumors can deal only with the conditions under which tumors arise, but not with the intrinsic properties of cells which find their expression in the tumor process.

Trauma.-Mechanical trauma is an important factor in the causation of tumors. From the scope of trauma in this relation should be excluded the various forms of chronic irritation, inflammation, surgical wounds, cicatrization, and chemical escharotics. By trauma is here understood a single or repeated more or less contusing, crushing, or lacerating mechanical injury. This much discussed subject would be greatly simplified if the statistical tendency were replaced by clearer ideas of the results of trauma in different tissues and of the mechanism by which such lesions lead to tumor growth. Studies in this field are as meager as the statistical contributions are superabundant.

Important effects of trauma here are: (I) Solution of continuity, minute and gross; (2) separation of cell groups and tissue masses, as of skin, glands, bone; (3) necrosis of tissue; (4) confined hemorrhage requiring absorption or encapsulation; (5) accelerated regenerative processes with hyperemia, and new growth of specific cells, blood-vessels, and supporting tissue; (6) cicatrization.

All of these conditions are well-proved elements entering into the causation of tumors, and the failure of attempts to produce tumors by experimental trauma in given cases does not reduce their importance when associated with other necessary predisposing factors. When one or several of them meet with the relatively rare predisposing conditions, we have a somewhat satisfactory explanation of the facts observed regarding the relation of trauma to tumors.

The predisposing factors take many forms: (I) There may be a benign or a minute malignant tumor in the tissue before the injury. Many patients with cancer of the breast attribute their disease to some form of injury. Probably very few of these tumors are the direct sequel of the trauma, but a slowly growing cancerous nodule in chronic mastitis may be accelerated by a blow where the injury alone seems to be the immediate cause of the tumor. In any organ a preëxisting lesion renders the effects of ordinary injury more severe and more noticeable.

2. The precancerous condition may be precipitated into a malignant process by injury. Examples are wounds of a psoriatic tongue by the teeth, injuries of the breast altered by chronic mastitis, and incomplete surgical removal of indolent ulcers, mucous polyps, fistulous tracts, and benign tumors. Leukoplakia of the tongue is said never to develop cancer until it becomes complicated by cracks and fissures.

3. Misplaced and undeveloped organs are predisposed not only to tumor growth but also to trauma. The results of this unusual combination are seen in cancers of undescended testes and supernumerary breasts. 
4. Aberrant quiescent cell groups may be included in the damaged tissue. The best known example is the malignant melanoma arising from an injured mole. Definite injury frequently precedes the appearance of the various forms of teratoma testis, and since trauma is an effective method of producing artificial parthenogenesis, there is good reason to believe that the relation of the injury to the tumor is in this case direct, for these tumors develop from immature aberrant sex cells. Throughout the entire series of embryonal tumors there is a sound basis for ascribing more than ordinary significance to a history of severe or mild and repeated injury. Ribbert thinks that many adrenal rests are incited to growth by trauma.

5. Normal cells under the conditions established by trauma develop benign or malignant tumors. There seems to be no sufficient reason for denying the relation between trauma and many chondromas, osteomas, lipomas, fibromas, and fibromyxomas, with which the history of a blow is rather common, and there is little ground for assuming that the trauma acts on any but normal cells. Also with the two most striking forms of traumatic tumors, gliosarcoma and osteosarcoma, where a definite history of injury is frequent, there is no evidence that any but normal cells are involved.

The character of the injury followed by tumor growth varies widely. Traumatic cancer usually appears after repeated mild injuries often amounting to chronic irritation, while sarcoma commonly develops after a single blow. Yet many cancers have appeared after single injuries (Ziegler, Lowenthal, Jordan, Segond). Only the severer injuries are capable of reaching the deeper tissues from which sarcoma develops. Cancer has been clearly traced to lacerations by rough instruments, rusty nails and pins, thorn pricks, insect bites, surgical wounds, and to blows without visible destruction of tissue.

Fractures, lacerations of deep tissues, contusions and concussion, all of which are apt to cause hemorrhage, represent the usual type of injury preceding sarcoma and benign mesoblastic tumors.

The mechanism by which injury induces tumor growth is generally obscure. The traumatic dermoid clearly originates from implanted epidermis and fair success has attended its experimental production. Yet it has been abundantly proved that simple misplacement of other normal tissue cells is quite inadequate to explain tumor growth. Hyperemia may be the remaining factor necessary to excite the proliferation of aberrant rests, and precancerous foci. Blood extravasation requiring absorption or organization creates conditions especially favorable to mesoblastic overgrowth. Certain tissues are predisposed to excessive overgrowth in the presence of hemorrhage. The periosteum, the tissues of the scrotum and cord, the omentum, and the pia-mater frequently exhibit very active overgrowth of tissue following hemorrhage, yielding bulky products, which have the gross and many of the microscopical appearances of tumors. Such pseudosarcomas are especially frequent in the scrotum. Cornil and Berard have noted the very active proliferation about traumatic hematomas, and locate the early sarcomatous process in the cells about new blood-vessels. Some early sarcomas of the brain present appearances implicating the new vessels in the sarcomatous process.

The common superfluous callus about fractures reveals the great proliferative capacity of periosteum, and Benecke believes that the regenerative process in periosteum may run directly into sarcoma. In traumatic myositis the new tissue is often suggestive of sarcoma. Yet the full explanation of traumatic sarcoma seems to require the assumption of special local or general predisposing factors which have never been traced in microscopical structure. The chemical composition, blood-supply, and lymph supply of brain tissue are peculiar, but give no clue to the frequency of traumatic gliosarcoma. The 
presence of necrotic tissue should receive attention as a possible specific factor in the development of traumatic tumors. It is possible that products of autolysis or decomposition of protein tissue may exert peculiar influence on regenerative processes, as in the brain. Kottnitz cites many cases of lipoma in which a traumatic factor appears acting directly or through the nervous system.

Ribbert admits that we have not the slightest knowledge why trauma, which affects many cell types in large areas, excites tumors of one cell type in one focus. He thinks that the results of experimental separation of cell groups have often approached the condition of benign tumors some of which may regress. The most extensive proliferation is obtained by partial separation of tissues, as along three sides of a square, of the rabbit's ear. Epithelium completely separated dies, but regenerating connective tissue is much more resistant.

The frequency of the traumatic origin of tumors varies with different statisticians, according to their conception of trauma and their critical standards. Composite statistics vary from 2.5 per cent. by Kempf to 44.7 per cent. by Lowenthal. Of 6780 tumors of all varieties reported in the literature with reference to trauma 494 or 7 per cent. were regarded as of traumatic origin. Of 264I cancers, I07, 5 per cent., and of 938 sarcomas I86, or about I9 per cent., were classed as traumatic. In a large proportion of these cases the relation of the trauma was extremely uncertain. The figures represent the present attitude of most clinical writers which, as Lubarsch and Schimmelbusch have said, is often extremely uncritical. Only the statistics relating to single tumors are of practical value. Of 55 melanomas Werner-Rowe found I9 with a history of injury of a mole.

Of glioma of the brain Adler collected ro86 cases of which 8.8 per cent. were preceded by rather definite traumatic history. In this class of cases the relation between the trauma is often clear, the symptoms of trauma merging into those of tumor and occasionally a scar has been found in bone or dura directly connected with the tumor.

There are important medicolegal aspects of the relation of trauma to tumors. To establish the relation of trauma several classes of evidence are necessary, as is recognized especially by the French statutes.

I. The authenticity and sufficient importance of the trauma. (2) Previous integrity of the wounded part. (3) A reasonable time relation, three weeks to three years or more in certain cases. (4) Continuity of pathological changes or symptoms in the wounded part and the appearance of the tumor. Microscopical proof of the existence of a tumor.

It is necessary to distinguish also between complete and partial liability. When a healthy adult receives a blow on the head, remains unconscious for days, never recovers full mentality, shortly develops signs of brain tumor, and dies in a few months of gliosarcoma of suitable size and location, the liability is generally accepted as complete. When a patient with symptoms of abdominal disease receives a blow in the epigastrium and rapidly succumbs to carcinoma of an abdominal organ it is reasonable to assume that the trauma hastened the progress of an existing tumor. As Segond states, trauma reveals, aggravates, or serves as a pretext for the origin of tumors; so that in each case very careful consideration of all circumstances are required to establish the relation of the injury.

In the German courts definite liability has been assumed for cases in which the connection between trauma and tumor is very uncertain and incapable of proof, as with carcinoma of colon or stomach appearing several years after a blow on the abdomen. 


\section{CHAPTER VIII}

\section{THE PARASITIC THEORY}

The parasitic theory is the oldest hypothesis of the origin of cancer. It appealed to the ancients, was tacitly accepted throughout the Middle Ages, was definitely argued by modern observers, and reached the height of its popularity as a scientific theory about I895, but during the last fifteen years it has rapidly lost ground, and today few competent observers consider it as a possible explanation of the unknown element in blastomatosis.

The data concerning the parasitic theory fall into four main classes: (I) Studies of the incidence of cancer; (2) the search for specific parasites in tumors; (3) the experimental production of tumors; (4) theoretical consideration of the nature of cancer.

The Incidence of Cancer.-In I 809 Arnaudet reported that in certain rural districts in Normandy during a period of eight years, the cancer mortality reached $\mathbf{I} 4.88$ per cent. He noted the occurrence of cancer in several members of the same household, and he concluded that the neighborhood of a cancer patient was contaminated through the water.

Many similar reports of cancer districts and cancer houses soon appeared from Guelliot, Webb, Fiessinger, D'Arcy Power, Bosc, and others. Behla found the cancer mortality of the low-lying portions of Luckau to be four times that of the central portion. He noted the frequency of cancer in the dogs of the town, the large quantities of home-grown vegetables consumed by the cancer families, and concluded that cancer is an infectious disease carried to its victims through contamination of the soil.

In I892 Haviland, from an elaborate analysis of the English census, concluded that cancer is a disease of low-lying districts and of seasonably flooded riparial lands where subsoil is constantly moist, while in high dry localities it is rare.

Very minute studies of cancer localities have been made in England and other countries by numerous observers all tending to show a relation between cancer and all manner of local peculiarities; as living in "old and cancer houses" (Park), "infected streets" (Mason), houses with defective plumbing, leaky sewers (Nason), proximity to trees, especially large ones (Lloyd-Jones), collections of decaying vegetable matter, stagnant water (Poppelman), abundance of certain insects (Bosc), etc. The uncritical character of these observations hardly needs comment. Many of the fallacies involved in them have been pointed out by Sticker and by Prinzing.

At the height of their popularity Symons showed that there were no cancer houses in Bath where cancer mortality was the highest in England. Out of this fanciful discussion Williams draws the sane conclusion that cancer is a disease of civilization, choosing its victims from the well-to-do, the wellnourished and the well-protected against infectious diseases, and that it flourishes in just the opposite conditions from those which favor the spread of tuberculosis. At one time there was a tendency to report small epidemics of cancer among men (Webb, Fiessinger), and to point out its epidemic character. Later, epidemics of cancer among cattle were described (Loeb), or among rats and mice used for breeding purposes (Gaylord, Borrel, Loeb), for which old age and inbreeding are sufficient explanations. Bashford, in a 
very wide experience with captive rats and mice, found no evidence of any "cage infection."

Epidemics of thyroid cancer among artificially bred trout have menaced the fish industry in several countries. Pick and Plehne concluded that it arises from overcrowding, overfeeding, and inbreeding. Gaylord dernonstrated the true cancerous nature of a certain proportion of these cases, but Gudernatsch pointed out the remarkably wide distribution of the thyroid lobules in many species of fish and rendered doubtful the neoplastic character of the great majority of the "tumors." Marine and Lenhardt have shown that many of the hyperplastic thyroids regress under proper hygiene.

Statistics elaborately presented by Williams and by Hoffman show a remarkable increase in the recorded deaths from cancer during the last 50 years. In England in 1840 the cancer deaths were $I$ in $I 29$ of the total; in I905, I in I7. Very similar indications are found in the records of most civilized countries (Hoffman, Lit.). Williams calculates that in England, I900, I in $I_{5}$ of all men, and $I$ in 9 of all women, living at 35 years of age are destined to die of cancer. The increase seems to be affecting men more than women, although in England cancer is now a more fatal disease for women than is tuberculosis. The increase of cancer is concomitant with a decrease of tuberculosis.

Of the apparent increase in cancer deaths a considerable proportion must be referred to improved diagnosis. Riechelman shows for the Berlin hospitals that there was still, I902, room for a 20 per cent. increase in the recorded deaths from cancer through improved diagnosis alone. The remaining portion must be largely referred to the longer tenure of life through immunity to infectious diseases and improved hygiene, which permit a larger proportion of persons to reach the cancer age. In Prussia vital statistics show that the average person lives 25 years longer than in $\mathrm{r} 860$. Willcox in a critical analysis of this subject shows that the sources of the recorded increase are quite complex, including improved diagnosis, changes in age composition of population, elimination of old age as a cause of death, improved registration, and widening of the definition of cancer.

He shows that the increase is chiefly of inaccessible cancers, among the lower classes of society, in males rather than in females, in negroes and the foreign born, and in the country more than in the cities.

The impression that cancer is actually increasing to a slight extent which cannot wholly be explained by the above factors and is more often appearing at earlier ages, is probably correct, but such a fact cannot stand as an argument for the parasitic theory.

The contagiousness of cancer was at one time supposed to be proved by clinical observation. Lusitanus (I557) claimed to have observed infection of 3 children by a cancerous mother. Tulpius reported the direct transfer of the disease between human beings. In the $\mathrm{I} 7 \mathrm{th}$ and $\mathrm{I} 8$ th centuries cancer was regarded as quite as infectious as phthisis, with which it was often confounded. Lebert and Friedreich believed they had observed cancer infection of the fetuses of cancerous mothers.

The numerous observations on contact infection were supposed to point to a parasitic factor, but relatively few of these cases seem to have been genuine, and in these it must be held that the infecting agent is the cancer-cell. Of the many cases of cancer a deux reported by Budd and Guelliot none appears to be properly attested, while Demarquay found that in only I of I34 cases of cancer of the penis did the wife have cancer of the uterus, and Bossi reported that of I80 husbands of women with uterine cancer, none contracted the disease. The attempts of Alibert, Wickham, and Senn to inorulate them- 
selves with cancer, failed. Finally, it is the universal experience of surgeons that the disease is not contracted by the treatment and care of cancer patients.

The conclusion may thus be drawn, that nothing in the incidence of cancer points to its infectious nature.

Microörganisms in Cancer.-With the advent of microbiology each new class of microörganisms in turn was isolated from cancer tissue, many were grown in pure culture, and some were claimed to reproduce the disease upon inoculation.

(a) Bacteria.-Rappin, Schill, Francke, Lampiasi, and others made unwarranted claims for the data regarding the bacteria isolated by them and they were soon forgotten. In I 887 Scheurlen obtained pure cultures of a bacillus from mammary cancer and with it claimed to have produced tumors of the breast in dogs. Baumgarten showed this bacterium to be the potato bacillus. In I 890 Koubassoff offered a complete claim of evidence for a thick motile bacillus from gastric cancer. In I9Io Doyen announced his Micrococcus neoformans as the cause and cure of cancer. It was soon shown that this microörganism had nothing to do with cancer, and the Societe de Chirurgie (1905) reported it to be valueless in the amelioration of the disease. These studies led to certain results of positive value. Shattuck and Ballance showed that non-ulcerated tumor-tissue could easily be kept sterile. Verneuil in necrotic foci and Zahn in some metastatic growths at autopsy found many bacteria. Richet found pyogenic bacteria common in cutaneous cancers. Maragliano often found staphylococci in the blood of patients with ulcerating tumors, even without fever, but never with non-ulcerating growths.

(b) Coccidia, differing in many details, were described by several observers.

Darier's psorosperm of Paget's disease secured much support from Wickham, and the histological evidence alone sufficed to impress many with the belief that this structure was the true cause of this and other cancers. It was repudiated by its discoverer in 1904 .

Thoma (I888) and Sjöbring (I890) described much the same intracellular and intranuclear structure as a parasitic coccidium.

Metchnikoff supported Sudakiewitsch's intracellular coccidium.

Adamkiewicz asserted that all cancer-cells are parasitic coccidia (Coc. sarcolytum), giving origin to a specific toxin (cancroin) and much this same view was held by L. Pfeiffer and J. Clarke.

(c) Miscellaneous Protozoa.-Other observers could not regard all the cancer-cells as alien parasites, but certain of the tumor-cells they identified as parasitic amebæ because of their bizarre forms and long pseudopodia which stretched between adjacent cells. These were the Rhopalocephalus carcinomatosus of Korotneff, and Cancriamaba macroglossia of Eisen. L. Pfeiffer described and depicted intracellular structures which resembled the microsporidia of muscle-tissue.

Podwyssoski and Sawtchenko described as sporozoa a variety of free and encapsulated intracellular structures many of which resembled Sudakiewitsch's parasites. Ruffer and Walker improved the technical methods of demonstrating the cancer bodies and endeavored to distinguish between true and spurious parasites. Kahane thought he detected a minute protozoan in the circulating blood of cancer patients. In cancerous ascitic fluid Schaudinn observed a large ameboid cell which he named Leydenia gemmipara. Schüller traced the complete cycle of a minute intranuclear protozoön in cancer-cells which differed from nearly all other cancer parasites.

Bird's-eye Inclusion.-From the beginning of the search for the cancer parasite special interest always centered in a certain intracellular body called the "bird's-eye inclusion." 
This body appeared in the cycle of many of the parasites described by various authors. It was first cautiously suggested by Foa as the probable cancer parasite. In I902 Feinberg made a final effort to establish specific features in this body, double contour, metachromatism and nucleus surrounded by a clear zone, but these features were promptly and authoritatively discarded by Hertwig.

(d) Mycetozoa.-L. Pfeiffer early pointed out the resemblance of many cancer bodies to forms of mycetozoa, especially Plasmodiophora brassicæ. This organism was first described by Woronin as the cause of club root, a common disease of plants, and its complex cycle was worked out by Nawaschin. Some of its forms closely resemble the bird's-eye inclusion of cancer, while others are extremely minute and difficult of detection in the infected cells. Behla found the disease common in the gardens of his cancer houses in Luckau. Elaborate attempts to demonstrate that this or a similar organism is the cause of cancer have been made by Behla, Podwyssoski, Feinberg, Gaylord, and Robertson and Wade.

Several observers claimed to have secured cultures of these protozoa and to have reproduced tumors by inoculation of the cultures in animals. Sjöbring used a medium containing ascitic fluid, peptone, glucose, and soap made from human fat, and claimed to have isolated his rhizopod from human tumors. Cultures produced tumor-like growths in mice. Yet the German pathologists denied that the growths were neoplasms and Israel asserted that the rhizopods were fat droplets. By the inoculation of material from club root Podwyssoski and Gaylord produced granulomatous swellings in animal tissues, the cells of which contained the englobed parasites, which closely resembled some of the intranuclear bodies seen in cancer. Yet v. Tubeuf and others have inoculated many animals with club root and failed to produce any lesions resembling neoplasms. According to v. Tubeuf the histology of club root is not that of a neoplasm, the tumor resulting from distention and degeneration of cells surrounded by an area of inflammatory overgrowth. The picture of infected cells in club root distended with enormous numbers of very definite parasites is wholly different from anything seen in cancer.

Smith has shown on an elaborate scale that Bacillus tumefaciens, isolated from crown gall, is capable of producing a variety of tissue overgrowths in plants which he regards as true tumors, both simple and teratomatous. Yet this observer fails to distinguish between chronic productive inflammation in plants and neoplasia; he does not consider that even in animals inflammatory hyperplasia may pass into neoplastic, while his infectious "embryomas" in plants cannot have any relation to the embryogenic disturbances known to give rise to teratomas in the animal body.

Schüller claims to have cultivated his protozoön and to have produced by it both carcinoma and sarcoma. Yet no one else has been able to find this organism in cancer, and Schüller's technic fell under suspicion when Volcker pointed out that the very characteristic large forms of this protozoön exactly resemble cork cells.

(e) Spirocheta.-In 1905 Borrel reported the occurrence of spirochetæ in 2 mouse tumors without attributing to them etiological significance. Wenyon has shown that mice are susceptible to blood infection by these organisms. Gaylord and Calkins, finding a single type of spirochetæ in ro mouse cancers and in I 6 transplants therefrom, suggested that the spirochet $x$ were the cause of the tumors. Yet Gaylord and Clowes succeeded in freeing their tumors from spirochetæ by treatment with $\mathrm{KCN}$, and Tyzzer found many tumors in mice not infected with spirochetæ. In 35 human tumors and 25 in the 
dog I found spiral organisms only on ulcerated surfaces or in necrotic areas. Similar results were obtained by Mulzer and by Lowenthal.

(f) Blastomycetes.-In I 890 Russell fully described certain small and large round bodies in cancer-cells which stained intensely with fuchsin and which he recognized as parasitic budding fungi or yeasts. Although these bodies had long before been noted by Fox, and Klein and Lubarsch soon showed that they were found in many normal and diseased tissues, the yeast theory of cancer was taken up systematically by San Felice and the Italian school. From 1895 up to the present time San Felice has devoted much labor to the support of this theory. He experimented first with a culture obtained from fruits, Saccharomyces neoformans, and one from the lymphnodes of an ox dying with carcinoma of the liver, Saccharomyces litogeres. Later he obtained cultures from human tumors. With these cultures he produced infection of many animals, chiefly dogs.

With Saccharomyces neoformans he thought he produced a sarcoma in the breast of a bitch and in many other animals, but most of the lesions were clearly inflammatory. Saccharomyces litogenes produced very similar lesions.

Repeated passages increased the virulence of the strains and their neoplastic properties. Cultures from human tumors were non-pathogenic for animals. It was not possible to recover the organism from the tumors, a result which San Felice attributed to the death of the yeasts which then assumed the form of fuchsin bodies. In his latest studies San Felice combines the soluble toxins of yeasts with the living cultures, finding that the toxins rather than the bodies of the organism cause proliferation of cells. Nevertheless the lesions which he pictures seem to have the features of infectious granulomas and not those of tumors.

Many other observers claim to have isolated blastomyces from tumors and produced other tumors by inoculation, as Plimmer, Roncali, Corselli and Frisco, Curtis, Monsarrat, Leopold, Wlaeff, Klein, etc. Against their conclusions stand an equal number of very competent studies which show that blastomyces are rarely present in tumors and that they are incapable of producing neoplastic lesions. Foulerton and Richardson examined several hundred human tumors without securing a single yeast. Their rare presence in tumors is attributed by most bacteriologists to secondary infection, and the high proportion of successful cultures secured by many observers is clearly the result of air contamination. Meser found lycopodium seeds from the dressings deep within the tissue of carcinoma. The pathogenic action of a number of yeasts have been elaborately studied by many competent observers, including Mafucci and Sirleo, Foulerton, Rabinowitsch, Petersen and Exner, and Nichols, none of whom found any indication that these organisms can produce tumors.

The various blastomycetes known to be pathogenic for man produce characteristic granulomas, but not tumors. It is true that in certain chronic blastomycotic lesions, especially of the skin, considerable hyperplasia of epithelium resembling cancer has occasionally been seen, but there is no indication that this hyperplasia exceeds that observed with syphilis and tuberculosis. Genuine cancer sometimes follows such lesions and yet no one supposes that the tubercle bacillus is the cancer parasite. The identity of yeasts, dead or alive, with Russell's bodies has been subjected to careful criticism by many observers, especially by Sternberg and Nichols, who conclude that there is no adequate proof that blastomyces produce any considerable proportion of the fuchsin bodies.

Otto Schmidt claims to have isolated from human carcinoma and sarcoma an organism which he calls Mucor racemosus and which he believes falls in 
the class of mycetozoa. With this organism he has produced Io sarcomas in rats and mice, some of which he has transplanted through several generations, the tumor strain finally reaching roo per cent. of successful implantations. He is able to immunize rats against the organism and against its tumorproducing properties, and rats with tumors he is able to cure by inoculation with killed cultures of the mucor. Six out of I9 cancer cases at the Heidelberg Institute gave what he regards as anaphylactic reactions against the mucor. Baisch has succeeded in verifying some of Schmidt's results. On investigation it does not appear how often Schmidt secures cultures from human tumors nor with what precautions the successful cultures were obtained. The mucor cannot be identified in sections of the tumors. Some of the growing forms of the parasite Schuberg regards as fat droplets. From the experimental tumors the parasite is not recoverable. Ten tumors were produced, but hundreds of animals were used and it does not appear that the proportion of successful results greatly exceeds that of spontaneous tumors in rats subjected to other traumatic influences. The cure of the rat tumors is accomplished after very severe intoxication by the injected cultures and the death of many animals. Schmidt claims to have cured human cancer by his toxins, but others have not succeeded. There seem to be no grounds for accepting the conclusion that human patients show anaphylaxis to the cultures of mucor. In fact, Schmidt has not adduced satisfactory evidence that Mucor racemosus has anything to do with human cancer. What he seems to have accomplished is to add to the list of toxic agents that are known to excite hyperplastic inflammation which, especially in rats, may exhibit metaplastic changes and possibly pass over into tumors. He has not apparently controlled his work by equally energetic efforts to produce similar changes by other agents. Yet his observations seem to fall in line with those of other observers who by various means succeeded in causing notable hyperplasia and metaplasia of animal tissues, as Fischer with Sudan III, Jores with Scharlach R in paraffin, Stohr and Stoeber with naphthalamin, paratoluidin, and amidoazotoluol, and Stoeber and Wacker with indol and skatol. With the expressed juice or the nucleoproteids of yeasts Gaelotti and Pentimalli have also produced a considerable variety of tumor-like hyperplasia in dogs and rats, but they hesitate to assume that these processes are identical with progressive neoplasms. It is even more doubtful if the agents they used actually occur in spontaneous tumors.

The Experimental Study of Tumors.-The inoculability of carcinoma was once supposed to demonstrate its parasitic nature. Long after Peyrilhe's failure several experimenters claimed to have succeeded in transferring cancer to lower animals by intravenous inoculations of fresh emulsions (Langenbeck, Follin, Velpeau). These results were later met with many more negative reports by Virchow, Weigert, and Billroth. The more recent claims of successful implantations of human cancer into lower animals by Boinet, I894 (epithelioma into dogs); Juergens, I896 (melanoma into rabbits); Dagonet, I904 (epithelioma into a rat); and Werner, I907 (carcinoma into a dog), leave open the question how far human cancer may survive in these animals. Extensive melanosis of the organs as well as local tumors followed the inoculations of human melanoma by Goujon, Lang and Bosc, and Vedel, while Pfeiffer thought he was able to carry his melanoma into one of a second series of mice. Yet Fischer repeatedly failed to transfer melanoma to rats and Roux and Metchnikoff were unsuccessful with the chimpanzee. In none of the successful cases does it appear that the resulting process was anything more than a local or general reaction to the cells and pigment introduced. While it is possible that the reactions observed in some of these animals must be 
classed with the tumor-like processes produced by chemical agents acting upon tissue cells there is no proof that the injected human cells multiplied during the considerable periods over which the animals were observed.

Firket, Boinet, Gaylord, Lewin, Bosc and Vedel, and others claim to have produced cancerous nodules by inoculation into animals of various human cancers. While it is impossible to detect definite fallacies in all of these cases, especially in that of Dagonet, there are many difficulties in the way of their acceptance. A much greater number of negative results are recorded by many observers, as Shattuck and Ballance, Fischel, Sticker, Hemmeter, and many others. Herzog found that the nodules first forming invariably disappeared if the animal survived long enough, and this has been the common experience of many pathologists working in this field. The experience of the last decade with transplantable tumors, showing how narrowly balanced is the nutrition of tumor-cells, renders it extremely unlikely that human tumorcells can proliferate extensively in distantly related animals. The frequency with which spontaneous tumors occur in laboratory animals has been greatly underestimated by most observers, but has been clearly pointed out by Tyzzer.

The apparent impossibility of inoculating tumors from one lower animal to another of the same species long stood as evidence against their parasitic nature. Since the transfer of tumors among lower animals has become extensively practised it has become evident that this inoculability does not favor the parasitic theory, but rather stands strongly against it, since it has been shown that the tumor-cells are transplanted and no evidence of a parasitic agent has been obtained. It is, of course, conceivable that a parasite is transferred with the cells and maintains their tendency to growth, but no evidence of such a parasite has thus far been secured, and there is no indication of the existence of any such form of parasitism anywhere in the animal or vegetable kingdom (Lubarsch).

Therefore the results of the study of transplantable tumors are everywhere regarded as a new and serious obstacle in the way of the parasitic theory.

The development of epithelioma following $x$-ray burns is a phenomenon which upon analysis seems to prove that this tumor develops entirely apart from any parasite and arises through slow disturbance of the nutrition and mechanical relations of the epithelial cells.

Clunet has succeeded in producing a malignant sarcoma in a rat by repeated exposures to $x$-ray and, in a case reported by Senger, round-cell sarcoma seems to have developed after a lupus cancer treated with $x$-rays.

Chemical agents of great variety have been employed by many observers to produce tumors and not without a certain success.

Emanating from a different point of view, but falling in the same general class, are the results of San Felice, Schmidt and Galeotti, and Pentemalli, previously mentioned, who by means of extracts of yeasts seem to have succeeded in rare instances in producing genuine malignant tumors in lower animals. Since the great majority of such attempts are failures, the occasional success may be accepted without in any way involving the conclusion that such agents are commonly at work in producing spontaneous tumors in man or animals. They seem merely to illustrate the indirect action of irritants on predisposed tissues, which is a principle of tumor genesis long since established by clinical and pathological studies in man.

Likewise the striking observations of Rous that filtered extracts of a transplanted and very virulent sarcoma of chickens causes the growth of the tumor in a considerable proportion of cases, is probably to be classed as an instance of chemical stimulation of cells.

This observation has the additional interest that the chemical deriva- 
tives of this tumor possess an unusual capacity to excite the neoplastic hyperplasia and recalls the well-known observations frequently made in man, that normal cells are drawn into the tumor process by the gradual diffusion of some influence from the tumor-cells. As an alternate hypothesis one may assume that an invisible microöorganism passed through the filter, but here again the evidence of the existence of such a parasite is defective.

There are several other observations that chemical agents arising in the course of tumor growth possess a notable capacity to excite tumor-like growths of tissue cells, and it seems probable that in some instances genuine neoplasms have been produced by such agents.

By repeated subcutaneous injections of extracts of human cancer filtered through porcelain Mayet claims to have produced cancerous lesions in white rats, and Francotte and DeRechter report similar results with cancer juice in white mice. Hemmeter by local injections of filtered extract of cancer of the stomach from the dog has produced cancerous-like proliferation in the walls of experimental gastric ulcers in the dog.

Theoretical Objections to the Parasitic Theory.-It is often assumed that the establishment of a parasitic cause of cancer is surrounded by no more difficulties than those which beset the search for the causes of syphilis or other infectious diseases. It should be pointed out, however, that there are many theoretical objections which would render unusually difficult the establishment of any cancer parasite even though supported by strong objective data.

The known infectious diseases display features of incidence, course, and anatomical character which sharply separate them from malignant tumors. The study of the age and sex incidence of cancer presents a body of data which reveals a fundamental difference in the etiology of cancer and all known infectious diseases. The distribution of cancer is as wide-spread as inflammation in general, indicating that blastomatosis is not a subdepartment of the reaction of tissues to injury, but a distinct and separate phenomenon in the life of the cell. Over against degeneration and inflammation stand regeneration and neoplasia. The anatomical and physiological characters of malignant tumors differ essentially from those of known infectious processes. The isolation of the cells of origin of tumors is wholly different from anything recognized in parasitic diseases. The abnormal size of nucleus and cell body revealing overnutrition is contrary to the rule in infectious processes. The types and degrees of metaplasia observed in tumors find no parallels in inflammation. The progressive growth of malignant tumors reaches a degree which constitutes it a different pathological entity from the regenerative process in the healing of wounds and the reaction to irritants. Parasitic diseases cause regressive processes in the infected tissues and general deterioration of the system by toxic agents, but tumors exert no deleterious action on the system or on the organ involved or even on the cells affected except through secondary agencies. The metastases of tumors reveal conditions wholly different from any phenomena observed with infectious diseases. There is no more impressive illustration of the difference between tumors and infectious granulomas, which they most nearly resemble, than the comparison of the fate of tumor-cell emboli and of emboli from a tuberculous focus. In the former case the tumor-cells grow where they lodge, receiving only nutriment from the blood of the part; in the latter case the embolic cells die and the transported bacilli excite an inflammatory process in the adjacent tissues. Similarly, transplantable tumors survive in the progeny of the transferred cells.

It is conceivable that an invisible parasite lives with the tumor-cell 
stimulating its growth and nutrition, but there is no evidence of any such form of parasitism anywhere in the animal kingdom. Parasites occur in mitotic cells, especially in club root, but this process is not a tumor (Tubeuf). Where parasitism is known. it is at the expense, usually, of the life of the cells, while degenerative processes in tumors are secondary.

Weigert and Roux assume that the growth capacities of the cells are determined in the ovum and can never be increased except by fertilization, and Ribbert concludes that a tumor-producing parasite is therefore inconceivable. Yet it must be admitted that the growth of cells is greatly influenced by the environment and that peculiar external irritants of parasitic origin may greatly stimulate the nutrition and growth of cells. It has also been assumed that tumors result from release of the restraints of growth, but our knowledge of the nature of growth restraints seems hardly sufficient to warrant the conclusion that parasites may not be concerned in abolishing these restraints. Biology cannot argue the cancer parasite out of existence, but it can demand objective data in its support.

The general facts of the genesis of tumors are strongly against the possibility of a parasitic origin.

Tumors arise in some instances from a single cell (teratoma testis), in most cases from a narrowly circumscribed group of cells, and grow chiefly or exclusively from their own resources. The gradual inclusion of neighboring cells in the process is as well explained by the diffusion of chemical or other influences as by the transfer of a parasite, while there is much evidence directly in favor of the former hypothesis. The embryonic nature and isolation of the cells of origin of a large proportion of tumors, as embodied in Cohnheim's theory, are incompatible with a parasitic origin of such tumors, and yet they possess all the qualities of malignant neoplasms which are not known to be derived from embryonic cells. The occurrence of highly malignant congenital tumors in several members of the same family, as glioma of retina, reveals an embryogenic and hereditary disturbance as the essential factor. Clinical experience strongly impresses the importance of chemical and mechanical irritants and various disturbances of nutrition as the exciting causes of tumors. Here stand the numerous list of occupational cancers, from paraffin, anilin, chimney-soot, the predisposing influence of arsenic (Dubreuilh, Hutchinson) and tobacco, $x$-ray cancers, the influence of chronic mechanical irritation, and severe trauma. Likewise clinical observation and anatomical study reveal the fact that cancers which are not of embryogenic origin do not arise except after long continuous previous change in the tissue. These changes do not favor the establishment of any known form of parasitism, but rather suggest disturbances in the nutrition and function of the cells. Thus Billroth expressed the conviction that without previous changes in the originating tissue cancer does not exist, and his report of an epithelioma arising after many years over most of the area of an extensive scar from a burn, well illustrates the basis for his belief.

Few writers have ventured to suggest that benign tumors can be of parasitic origin, and yet occasionally tumors which are otherwise indistinguishable from benign growths, as adenoma of the thyroid and leiomyoma, may exhibit all the characters of malignancy.

The developmental history of many tumors exhibits the natural unfolding of embryonic potencies. However extensive may be the scope of metaplasia in tumors, it cannot cover the facts observed in the field of malignant teratomas. The teratomas arising from sex cells yield many pure forms of benign and malignant tumors, on the one hand, and highly complex structures approaching a parasitic fetus. The metastatic cells from such tumors again 
go on to develop specific structures in lymph-nodes and distant organs. Thus in a metastasis of an ovarian teratoma Lubarsch observed brain tissue and ependyma in orderly arrangement. Metastases of many tumors are distinctly organoid in character, maintaining polarity in arrangement and functionating as organs. If the above phenomena are of parasitic origin then, as Wilms points out, the whole history of embryonic development must be conceived as within the scope of parasitism. Hopeless dilemmas arise when one attempts to conceive of the necessary properties of the cancer parasite. It must pick out minute aberrant groups of embryonic cells in protected situations even in the fetus of a healthy mother. Misplaced and embryonal tissue invaded by metastases escapes infection by the parasite. Shaffer and Lubarsch have described misplaced islands of gastric mucosa in the esophagus invaded by epithelioma of the esophagus, but showing no hyperplasia. Berent observed a misplaced adrenal rest in the kidney unaffected by a metastatic nodule from epithelioma of the jaw. With the most actively growing metastases adult normal tissues are not infected, but as a rule behave passively. In chorioma the parasite invades the fetal but spares maternal tissues. In the metastases of melanoma the parasite produces extremely rapid proliferation of cells in one case, or it remains dormant for many years.

Contrary to all known forms of parasitism the cancer parasite stimulates growth and nutrition of cells and renders them viable after transplantation in the same or other animals. It stimulates the absorption of nitrogen by the host (Cramer) and fails, except through secondary influences, to exert any toxic or deleterious action on the body.

In view of the considerations thus briefly reviewed it is impossible to regard as a valid hypothesis the conception of a specific group of parasites living in symbiosis with the cancer-cell and stimulating its growth and nutrition. All the facts are reasonably explained by regarding the cancer parasite as the cancer-cell. The temporary popularity of the search for a specific parasite must be attributed to the undue influence of the germ theory of disease which can be effectually combated only by further knowledge of the biology of the cell.

The Scope of the Relation of Parasites to Cancer.-Although the conception of a specific cancer parasite living in symbiosis with the cell and stimulating its growth is without definite foundation and is incompatible with the nature of many tumors, there remains a certain field for parasites as etiological factors in tumors.

In the familiar coccidiosis of the rabbit's liver parasitic ova excite a tumorlike proliferation of the bile-ducts. The process is strictly dependent upon the presence of the ova and regresses when these are removed, and hence it is not a true tumor, but it has some of the qualities of a neoplasm and these are dependent on a parasitic irritant.

In Bilharzia infection cancer of the bladder follows in a certain proportion of cases. Here the disease is true cancer, it is less intimately connected with the presence of the parasite, but it is nevertheless rather obviously a sequel of the infection and reveals a peculiar capacity of this form of irritation to produce cancer.

Lowenstein has described papilloma of the bladder in rats infested with Trichodes. Primary carcinoma of the liver in cows suffering from Distomiasis has been referred to the irritation of this parasite (Haaland). In a papillomatous gastric tumor of pigeons Wasielewski has found a species of Dispharagus. Brumpt describes an adenoma of the stomach in monkeys as referable to infection by Physaloptera. Febiger has fully demonstrated the relation of Spiroptera to a gastric carcinoma in rats. Askanazy attributes 
to a trematode, Apistorchis felineus, an etiological relation to certain hepatic carcinomas in man, and similar relations have been claimed for Distomum spatulatum and Japonicum by Katsurada and others.

Many observers have considered the possible relation of trichinosis to carcinoma, and several have stated that carcinoma is peculiarly frequent in chronic trichinosis (Langenbeck, Babes). I rather frequently find trichinæ in cancerous tongues.

Borrel has observed several cases of sarcoma and adenocarcinoma of the rat in which a nematode worm was found in the center of the tumor. Regaud and Saul have reported similar cases and Bridrè found several sarcomas in rats growing about cysticerci. Borrel finds acari in the early spontaneous lesions of the lymphosarcoma of dogs. In 12 early epitheliomas of the face in man he found numerous acari inclosed in the tumor-cell nests. In the nipple of certain cases of Paget's disease and cancer of the breast he finds acari or another animal parasite, Demodex folliculorum. Borrel admits that these same parasites occur not infrequently in the healthy skin or nipple where they usually produce hypertrophy of the sebaceous glands. These ectoparasites he regards merely as the carriers of the true cancer virus.

Saul has also drawn attention to the occurrence of worms in mouse tumors, and in two human ovarian tumors he found a parasitic mite, Tarsonemus. It has been suggested that the Tarsonemus gained access to the material after its removal from the body.

All of these observations indicate that animal parasites or their derivatives have a peculiar capacity to excite hyperplasia in the tissues they infect. Yet it has not been proved that they are present in any but superficial tumors, or that their presence signifies anything more than a secondary invasion. Nor is it known that the tumor-like processes in which they are usually found have any other significance than the hyperplasia excited by sudan III, which regresses upon the removal of the irritant. In lower animals especially animal parasites of the skin are very common, while cutaneous tumors are rare.

Certain manifestations of syphilis have a close though indirect relation to tumors. The lingual cancer following leukoplakia may be regarded as the result of the disturbed nutrition and relations of the epithelial cells long established in the disease, and yet the frequency of this form of cancer reveals a certain unusual capacity of syphilis to excite neoplastic growth. Likewise the syphilitic sarcoma described by Hansemann shows this same peculiar influence exerted on mesoblastic cells, the full scope of which is possibly not yet recognized.

Tuberculosis in several of its phases has an indirect relation to certain tumors. In a small proportion of cases of lupus epithelioma develops through long disturbance of the nutrition and relation of the epithelial cells of the affected area. That there is something specific in the tuberculous process is suggested by the greater frequency of epithelioma after lupus than with simple chronic eczema.

In some forms of tuberculous lymphadenitis tumors of endothelial cells develop under conditions strongly suggesting that the neoplasm is a sequel of the tuberculous infection. Tuberculosis may cause a wide-spread hyperplasia of the lymphatic tissues of the body with miliary lymphomas in many organs. The frequent association of lymphatic leukemia with tuberculosis has suggested to many that this disease, which has several characters of a neoplasm, may be dependent on tuberculous infection or intoxication. Hodgkin's granuloma, which is either tuberculous or caused by a related organism, has been shown in several cases to pass over into a sarcomatous process. 
Hence the sequels of both syphilis and tuberculosis have a definite relation to tumors, which reveals the microörganisms as indirect cancer parasites. In both cases the resulting tumor process seems not to be dependent on the presence of the parasite, but to arise from the natural momentum of the disturbance originally excited by the parasite.

In the lower animals, especially in rats, the momentum of inflammatory processes which may lead to tumor growth seems distinctly less dependent on the presence of the parasites than in man.

In plants the observations on crown-gall, especially those of Smith, point to the conclusion that in the vegetable kingdom progressive hyperplasias originally excited by parasites may be still less dependent upon their con-

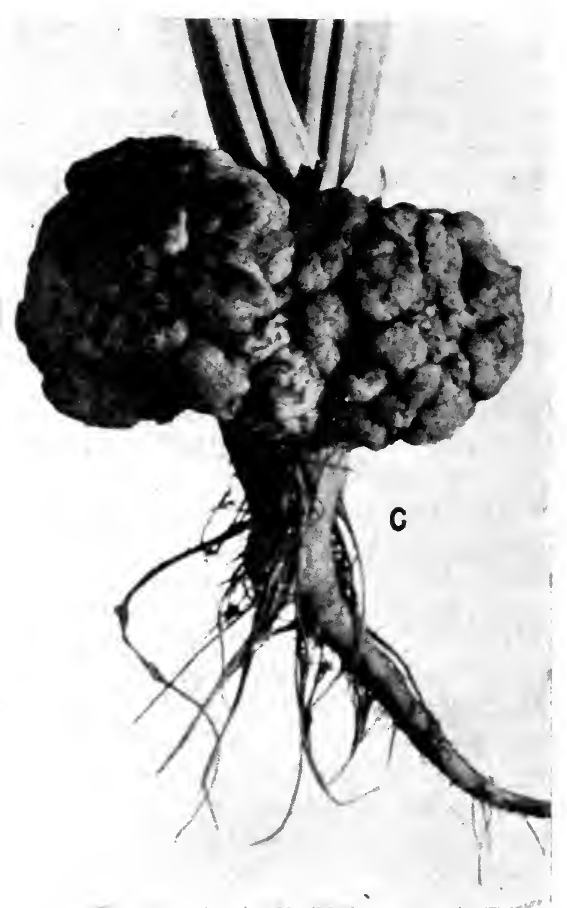

FIG. 11.-Growth resulting in $3 \mathrm{I}$ days from inoculation in sugar beet of Bacillus tumefaciens obtained from poplar gall. (After Smith.)

tinued presence than in animals. Levin and Levine find that the initial process excited by Bacillus tumefaciens in crown-gall is inflammatory, while the transformation of this inflammatory reaction into a malignant tumorlike process is due to some mechanism within the host, independent of the microörganisms.

All these considerations encourage the search for new microörganisms which may have a special capacity to excite inflammatory processes which tend to go on to tumor growth, but they offer no support to the theory of a specific cancer parasite living in symbiosis with the cancer-cell and constantly stimulating its growth.

The results in this field show that parasites may be concerned only with 
the inception of certain tumors, but that their influence cannot explain the continued autonomous growth of malignant neoplasms, wherein lies the real mystery of the cancer process.

The study of the parasitic relations of cancer suggests the following classification of tumors with regard to their possible connection with a parasitic origin:

I. Tumors of embryonal origin and their derivatives to which Conheim's theory applies, and to which parasites have no relation whatever.

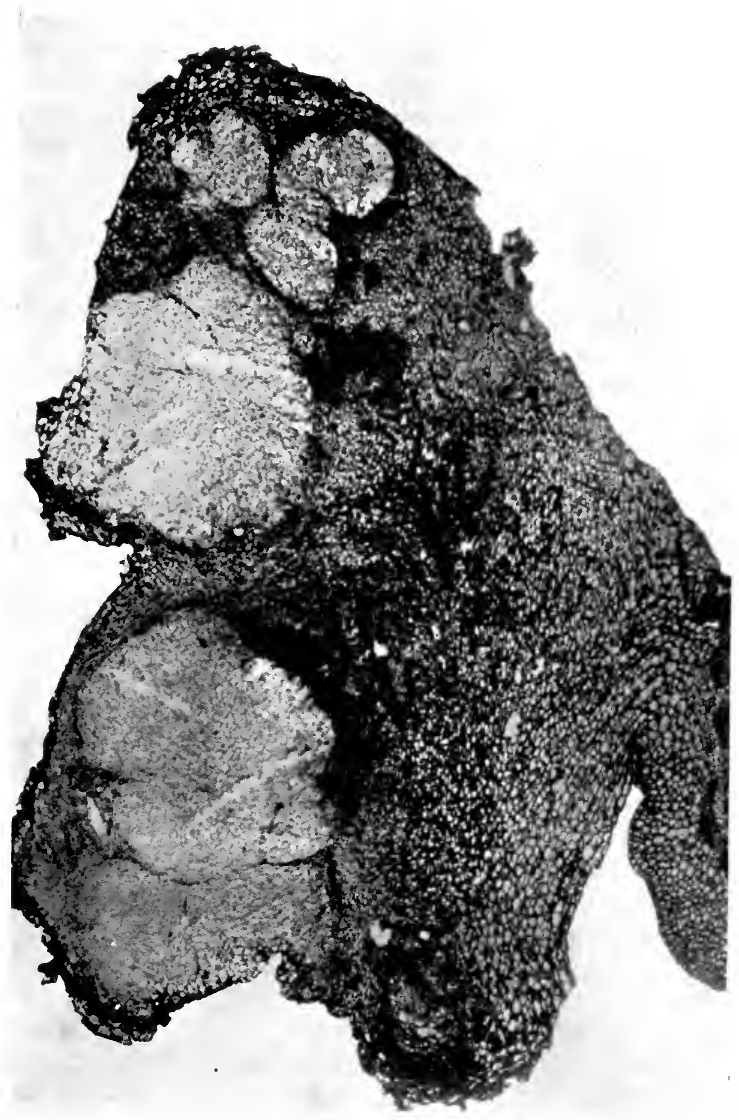

FIG. I2.-Centers of cell proliferation in daisy stem inoculated with Bacillus tumefaciens. (After Smith.)

2. Malignant tumors of exposed surfaces or internal organs with which parasites may figure as occasional indirect or direct exciting causes.

3. Sarcomas, especially those of lymphoid type, which are known to be the sequel of infectious diseases or on account of their imperfect neoplastic qualities may probably be regarded as more or less dependent upon the presence of parasites.

4. Tumors of lower animals in which the momentum of a parasitic process tends, more than in man, to assume autonomous qualities. 


\section{CHAPTER IX}

\section{EXPERIMENTAL CANCER RESEARCH}

\section{EXPERIMENTAL PRODUCTION OF TUMORS}

Among the various fields of the experimental study of cancer the oldest and probably the least fruitful is the attempted experimental production of tumors. Today it may be said that no one has succeeded in producing cancer under conditions that are strictly experimental, although cancer and sarcoma have been observed to follow $x$-ray inflammation in man and animals, and Febiger has observed the development of cancer on chronic gastritis produced by feeding rats with cockroaches infested by a nematode worm. Febiger's work may probably be accepted as presenting the controllable conditions of an experiment, but the proportion of cancers produced was small.

Efforts to produce tumors have passed through several phases suggested by theoretical views of the nature of tumors.

I. Transplantation of adult tissues or cells was early found by Zahn and Leopold to result in eventual and usually prompt absorption. Squamous epithelium from the combs of fowls Kaufmann found to persist for an unusual period, producing small cysts, and thus duplicating the observed mode of origin of traumatic epidermoids.

Lengemann followed the fate of misplaced cells of liver, kidney, and cartilage. He failed to observe any features of tumor growth, and concluded that misplacement alone, hyperemia, or partial degeneration of the cell mass were insufficient to excite tumor growth, while the weakened state of the animal had no influence on the fate of the cells. In his hands salivary gland and thyroid tissue exhibited the greatest proliferation.

Stilling chose the spleen as the most suitable soil and after transplanting fragments of uterine muscle and mucosa in rabbits found in some cases a growth of muscle-cells far exceeding their normal limits and resembling fibromyoma, and in other cases he observed the development of large cysts lined by proliferating epithelium.

Nichols in an extensive series of tests found definite proliferative powers of adult transplanted cells only in epidermis, uterine epithelium, cartilage, and placenta. I. Levin used cells from healing wounds without result. Passing interest was excited by Lack's report that multiple carcinoma developed in a rabbit's abdomen 14 months after scraping and leaving loose germinal epithelium from the ovary. Yet spontaneous carcinomas arise in this region in the rabbit (Boycott), and Fraenkel failed in several attempts to duplicate Lack's results.

Lubarsch appears to have encountered a similar coincidence in finding a large renal adenocarcinoma in the kidney of a rabbit into whose kidney he had some months previously transplanted a fragment of salivary gland.

Ribbert implanted portions of many organs in the peritoneum, skin, anterior chamber of eye, and lymph-nodes, always with eventual atrophy. He concluded that fully organized tissue masses might become implanted in these new positions and go on to functionate. The transplantation of whole organs, or large portions of ovary, thyroid, testis, mamma has been accom- 
plished by many observers. The transplanted thyroid may relieve myxedema (Eiselsberg); the transplanted ovary is said to have led to pregnancy (Krauer); the testis atrophies without necrosis; and transplanted mammary tissue has secreted milk in gestation (Ribbert).

2. Transplantation of embryonal tissues has been pursued to a formidable extent with the general result of showing that embryonal tissues exhibit much greater proliferative capacity than adult, but still lack an essential factor in tumor growth. Loeb, however, found certain adult tissues to grow better than the embryonal.

Leopold concluded that the younger the embryo, the better was the growth, while Tisenhausen specified 5 days as the optimum age of chick embryos. Zahn correlated the growth with the extent of vascularization of the transplant. Cartilage is said to have increased twenty times in bulk in Nichols' animals, and Leopold thought he obtained true chondromas. Intermittent heating of the organ seemed to increase the growth in the rabbit's liver of fragments of periosteum injected by Birch-Hirschfeld and Garten. Pregnancy was found to create a more favorable soil in the work of Askanazy and Jentzer in rats, but Shattock found it less favorable for cartilage growing in rabbits, while Rous found the most favorable period in mice to be after delivery.

Successive transfers of transplanted tissues at 8-day intervals seemed to increase the growth in Wilms' experiments with chicks, but an opposite result is recorded by Fichera. In most circumstances transplanted embryonal cells exhibit a certain amount of differentiation as well as of growth, but in the direction of normal tendencies rather than toward tumor growth.

Transplantation of complex tissues or portions of embryos, intact or comminuted, usually results in abortive teratoid growths. Freund secured such growths in 74 per cent. of rats inoculated intraperitoneally with emulsified rat embryos. Such emulsified cells grew for 6 weeks in rabbits (v. Hippel), or 8 weeks in chicks (v. Tiesenhausen), but gradually regressed after 6 to I 2 months. Fichera estimated the growth in rats at twenty times the original bulk, but saw no resemblance to true tumors. Cartilage and epiblastic tissues are usually the most active.

Rous mixed embryo with tumor-tissue, finding that both components grew in association, but the tumor-cells soon outgrew the normal cells, or both failed together, but growing tumor-cells did not stimulate growth in the others.

Traina implanted whole limbs and complex masses of embryonal tissue in the ovaries of guinea-pigs and secured striking degrees of proliferation, but no tumors.

The negative results of this extensive department of experimental research become easily explicable when one compares the obvious crudity of the experiments with the minute analysis of pathological data bearing on the conditions under which misplaced cells are observed to give origin to tumors. Thus among essential conditions for the neoplastic growth of misplaced cells are the following: ( $\mathrm{I}$ ) The cell group must not possess the intrinsic growth restraints of an organ. Otherwise it will grow or functionate as an organ. Evidently there is a nice adjustment between the demands for function and the capacity for growth in misplaced cell groups that develop tumors. (2) The misplaced tissue should not be completely divorced from its normal environment, but only partially so. Otherwise it will fail to receive sufficient blood-supply for active growth. (3) A considerable period is usually necessary for the misplaced cells to adjust themselves to the abnormal environment. Exceptions to this condition appear probably in some traumatic 
sarcomas. (4) An unusual source of hyperemia is usually necessary for tumor growth, for this factor facilitates nutrition, and lessens restraints to growth. (5) The misplaced cells must possess some special capacity for growth either by virtue of an embryonal character or from the retention of proliferative tendencies of more than ordinary degree. Thus tumors do not arise indifferently from all portions of an organ, but from the cells of ducts or from the germ centers of lymph-nodes. (6) The experimental studies ignore the importance of the preliminary changes which are observed to precede the development of tumors from normal or misplaced cells. These precancerous stages, the "indifferent vorstadium" of Ribbert, are not provided in the experimental transplantation of tissue cells.

Some of these objections have been met in the efforts to alter the character and accelerate the growth of cells before transplantation. G. Lewin injected emulsion of a human ovarian carcinoma into the peritoneum of a dog. After several weeks the omentum was found studded with miliary nodules which seemed to be of inflammatory character. Those were again emulsified and injected and the process repeated through 5 series of dogs, the resulting nodules presenting eventually a structure which Lewin regarded as sarcomatous. These observations have not yet been verified.

In somewhat the same manner Stieve produced granulomatous inflammation about injections of infusorial earth, transplanted this granulomatous tissue, and saw more and more atypical proliferation approaching that of sarcoma.

Influence of Lipoid Solvents on Epithelial Proliferation.-A series of experimental studies undertaken from various viewpoints have indicated that cells treated or influenced by lipoid solvents tend to exhibit increased and atypical proliferation.

Reinke, noting atypical epithelial growth after the injection of 4 per cent. ether into the eye of a salamander, transplanted the proliferating epithelium into the peritoneum of other salamanders, where it continued to grow more atypically so as to resemble carcinoma. Askanazy mixed emulsion of rat embryos with ether before inoculation and secured larger teratoid tumors than by means of untreated embryos, but Freund could not duplicate these results.

B. Fischer introduced an interesting field of study by inoculating under pressure a saturated solution of scharlach $\mathrm{R}$ in olive oil under the skin of rabbits' ears. The basal epithelium of skin and hair follicles after a few days began to proliferate and continued to grow downward inclosing oil globules and producing a picture resembling epidermoid carcinoma, but the growth ceased when the oil had been absorbed. In the mammary gland of rabbits a saturated ethereal solution of the dye produced a squamous epithelial growth replacing gland lobules. Since no such effect followed injection of simple oils and fats Fischer assumed that the dye contained a specific chemotactic substance (attraxin) stimulating epithelial growth. Many experimenters verified these observations, offering various explanations of the result. The wide variety of materials used in this connection and the general results obtained have been conveniently tabulated by Haga.

Meyer found that when the arteries of the ear were ligated olive oil alone produced the results, which he was inclined to refer to chronic inflammation. Stoeber and Wacker found certain components of scharlach $\mathrm{R}$ and sudan III equally effective, and by using 5 per cent. solutions of indol or skatol in rabbit fat they produced very active growth more closely resembling epidermoid carcinoma. White produced the usual changes with oleic acid, and Benthin used a great variety of materials, finding that the best were sudan 
III and scharlach R. These dyes have shown a variable influence upon transplantable tumors of animals.

In the mammary glands of rats (I. Levin, White), in the kiclnevs of rabbits (Meyer), and in the prostate, seminal vesicles, liver, and lung of rats (Bullock and Rohdenburg) injections of scharlach $\mathrm{R}$ have produced considerable atypical proliferation of inflammatory character, but much less

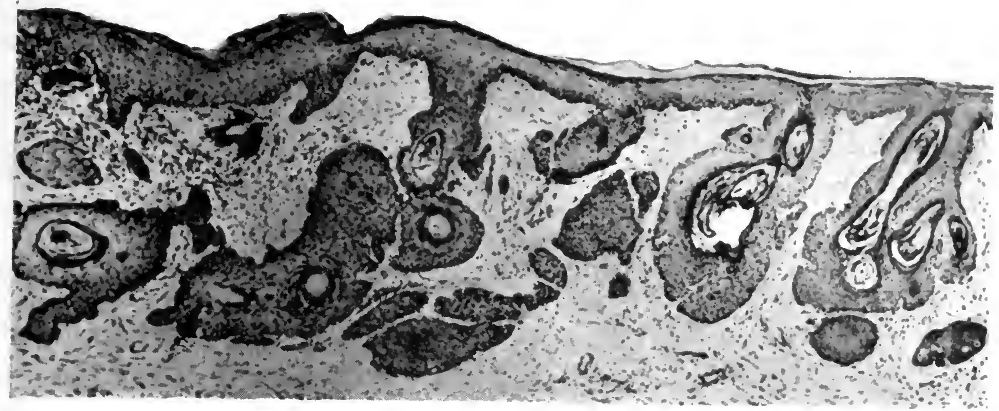

FIG. 13.-Atypical epithelial proliferation of epithelium caused by injection of scharlach R. (Bullock, Rohdenburg.)

marked than in the skin of the ear. Yet Haga succeeded in producing markedly atypical but self-limiting proliferation in the tongue, stomach, breast, and liver of rabbits, which in some cases was difficult to distinguish from malignant growth.

Reviewing these results, in general it appears that the property of certain lipoid solvents to excite epithelial proliferation has been established, but no true tumors have been produced (Wacker, Schmincke, Lit.).

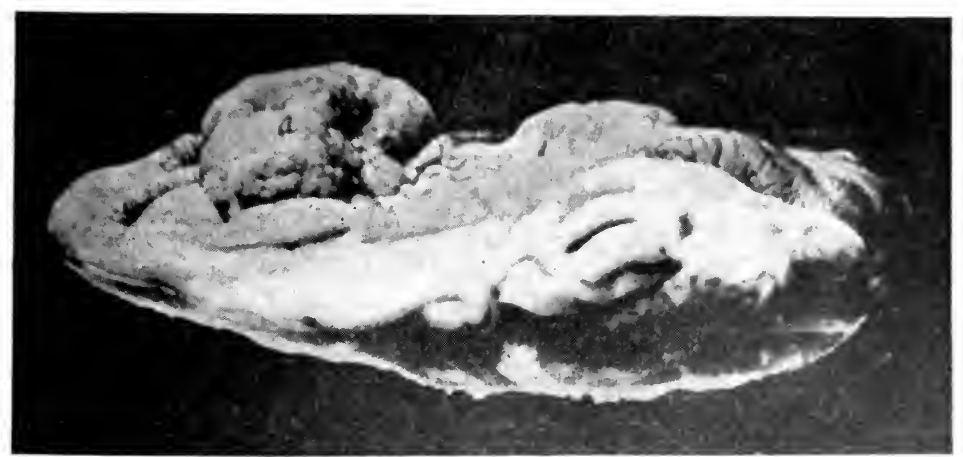

Fig. I 4.-Adenomatous growth of the rabbit's stomach, produced by feeding lanolin. (After IIaga.)

That the lipoid solvent property is an essential factor in the result, although suggested by much collateral evidence, appears uncertain. J. Loel) concluded that it was the lipoid solvent properties of the substance employed that produced artificial parthenogenesis. The theory of solution of a lipoid cell membrane has been pursued by Clowes as the key to atypical cell proliferation. Bullock and Rohdenburg reject this theory on the ground that 
all lipoid solvents do not act as does scharlach R. They secured considerable effect from injections of pine tar, indigo, and calomel, in oil, and they refer all the effects to cell death and regeneration. Nevertheless it is clear that certain fat stains exceed all other irritants so far tested in their ability to excite epithelial proliferation. That they possess somewhat specific properties in this respect is also indicated by their effective use to stimulate epithelial growth of ulcerating surfaces.

In later experiments Bullock and Rohdenberg by injections of scharlach $\mathrm{R}$ and by various mechanical irritants succeeded in producing the morphological picture of carcinoma in the stomach and other tissues of rats. Not observing metastases, and finding that continued growth was apparently

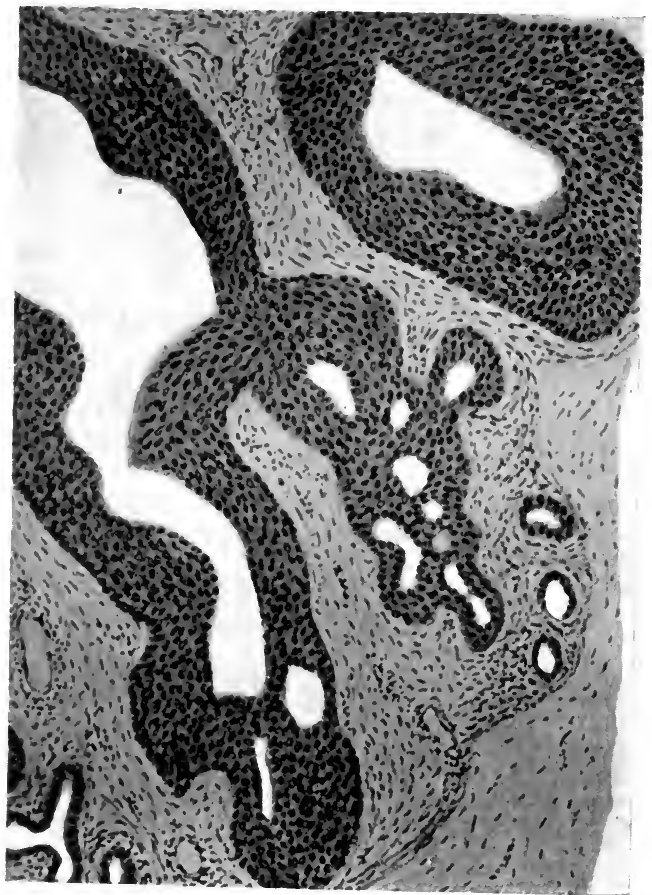

FIG. 15. - Epithelial proliferation of lining cells in ligated duct of salivary gland. (After Ribbert.)

dependent upon continuous irritation, they hesitate to accept the condition produced as equivalent to true experimental carcinoma.

Recently Yamagiwa and Ichikawa have succeeded in producing, first, precancerous changes and in 7 cases complete carcinomas, 2 with metastases, by applications of coal-tar over periods of 55 to 360 days in rabbits' ears.

Reviewing the results obtained by many observers with scharlach $\mathrm{R}$ and coal-tar, it seems to the writer that the processes excited must be regarded as essentially identical with certain carcinomas occurring in man, and as pointing to the probable mechanism of origin of such tumors in man. To demand that these experimental processes must continue after the irritant is removed and that metastases must always be produced is to ask more 
evidence of fully developed carcinoma than is often obtained in lesions which are regularly observed to progress in man to a fatal issue. The lower animals are not very susceptible to carcinoma, especially to epidermoid carcinoma and metastases of epidermoid carcinoma may not be so readily established in their tissues as in the human species. Thus the general object of the experimental studies, which is to prove that carcinoma may be induced in previously normal cells by continued irritation, seems to have been accomplished.

After exciting proliferation by means of sudan III, Lamezan and Haga have each attempted to transplant the growing cells to other tissues of the same animal or to other animals of the same species, but without success.

Chronic Inflammation in the Experimental Production of Tumors. Many experimenters have employed various forms of chronic inflammation in the endeavor to produce tumors.

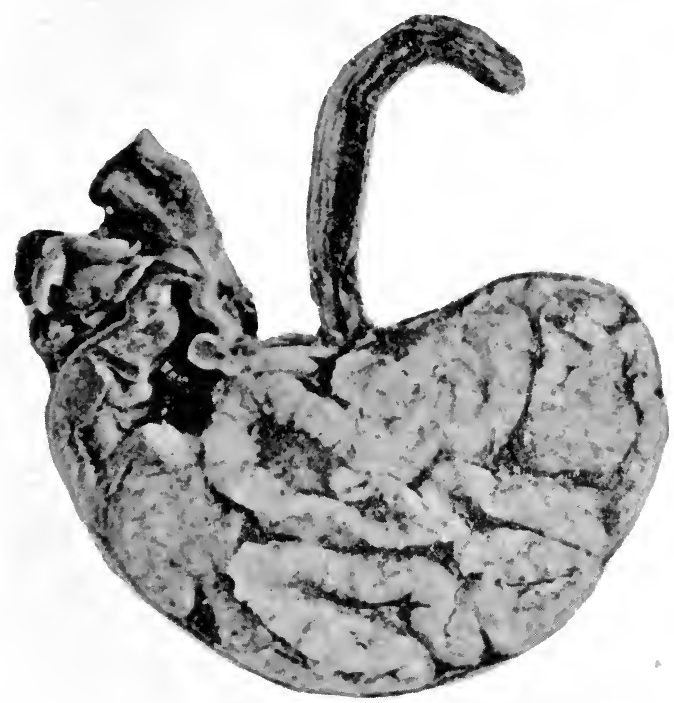

FIG. I6.-Gastric carcinoma in the rat, caused by infection with Spiroptera. (After Febiger.)

Ribbert produced small but typical fibro-epithelial papillomatous tumors of the rabbit's lip by repeatedly scraping the regenerating epithelium. In many organs he saw atypical growth of mechanically displaced epithelium, but in no instance was a true tumor observed. One of his most notable results was obtained by ligating the ducts of salivary glands in rabbits, which was followed by extensive atypical overgrowth of duct epithelium approaching the appearance of duct carcinoma.

Brosch assumes that preëxisting chronic productive inflammation is an essential condition in the experimental production of tumors by means of chronic irritants. After cauterizing and crushing a portion of the skin of the back in white rats he rubbed melted paraffin into the inflamed area over a period of 8 to 12 weeks and secured atypical epithelial proliferation closely resembling beginning carcinoma. 
Cazin employed a variety of traumatic influences in the unsuccessful effort to produce cancer from normal epithelium. Over a period of 5 months he rubbed soot into the inflamed skin of a dog without producing any atypical proliferation.

The particular chemical agents believed to be responsible for many of the forms of cancer observed in the trades, as in workers in paraffin, coaltar, soot, tobacco, etc., have been rather extensively employed in the study of experimental atypical epithelial proliferation, but in no case has a progressive tumor resulted (Haga, Lit.). Special interest attaches to the rather notable influence of tobacco extract in exciting epithelial proliferation.

Effect of Extract of Human Tumors. - Wacker and Schminke injected into the rabbit's ear fat extracts from mammary carcinoma, chondroma, and gastric carcinoma, and secured moderate epithelial proliferation. I have employed the alcoholic extract of a comedo-carcinoma of the breast in intra-

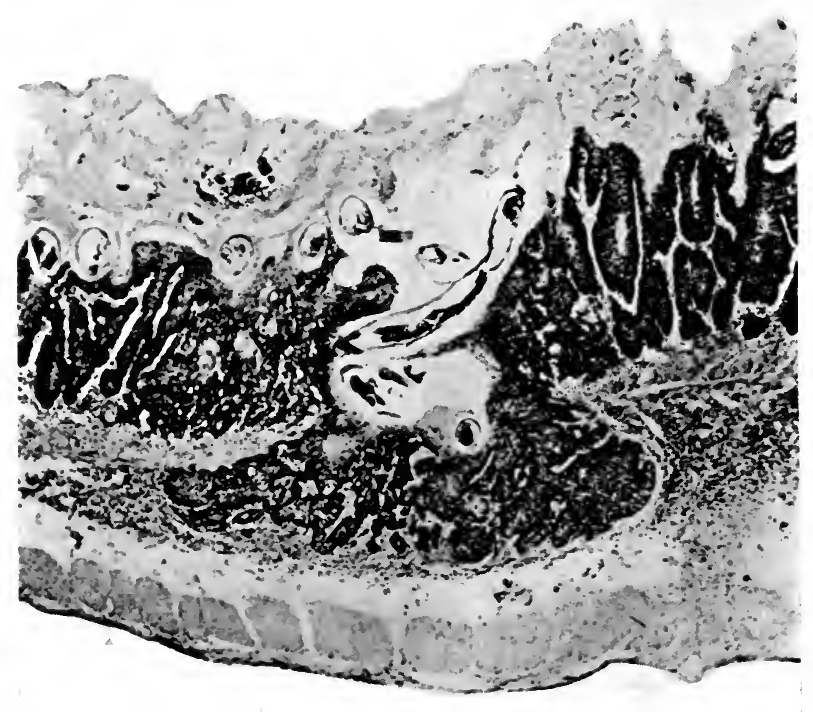

FIfi. 17.--Position of spiroptera in center of carcinoma of stomach in rat. (After Febiger.)

mammary injections in the rabbit, securing only an inflammatory reaction. These experiments were suggested by the impression that the extension of comedo-carcinoma in the breast keeps pace with the diffusion of fatty material in the obstructed ducts.

By mixing the expressed juice of carcinoma tissue with sudan III and injecting into the stomach wall of rabbits Haga secured well-marked tumefactions of the mucosa with considerable ingrowth of glandular epithelium presenting several histological characters of carcinoma.

Experimental Production of Tumors by the $x$-Ray.- While the frequency of development of carcinoma from $x$-ray dermatitis places these observations virtually in the experimental class, deliberate employment of the $x$-ray for the production of tumors has been reported by Marie, Clunet, and RaulotLa Pointe. These authors describe the exact dosage and the intervals of treatment followed in a successful effort to produce a malignant tumor in 
the rat. The growth was a spindle-cell sarcoma and proved transplantable in other rats.

Febiger's Experimental Gastric Carcinoma in the Rat.-Having discovered the remains of a nematode worm in a spontaneous gastric carcinoma in a rat, Febiger identified this parasite as one occurring in cockroaches (Periplaneta Americana and $\mathrm{P}$. orientalis). He designates this parasite as Spiroptera neoplastica. It was later found to infect other hosts. He next discovered a source of infested cockroaches in a Danish sugar factory and by feeding a series of rats with the bodies of these insects observed the development of chronic inflammation, papillomatosis, and carcinoma of the stomach. Of 62 rats which survived the feeding more than 60 days, I 2 developed carcinoma. The interval required was as brief as 64 days, and in one case metastases were observed after ro4 days.

The inflammation and papillomatosis affected a large portion of the gastric mucosa and reached such an extensive grade as to fill or even occlude

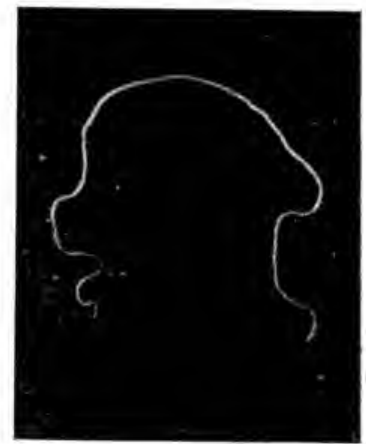

FIG. I 8.-Spiroptera neoplastica. (After Febiger.) the stomach. Structurally the process showed extensive papillary overgrowth of epithelium with marked keratosis, while the supporting tissue was richly infiltrated with eosinophile leukocytes. From this lesion there was progres-

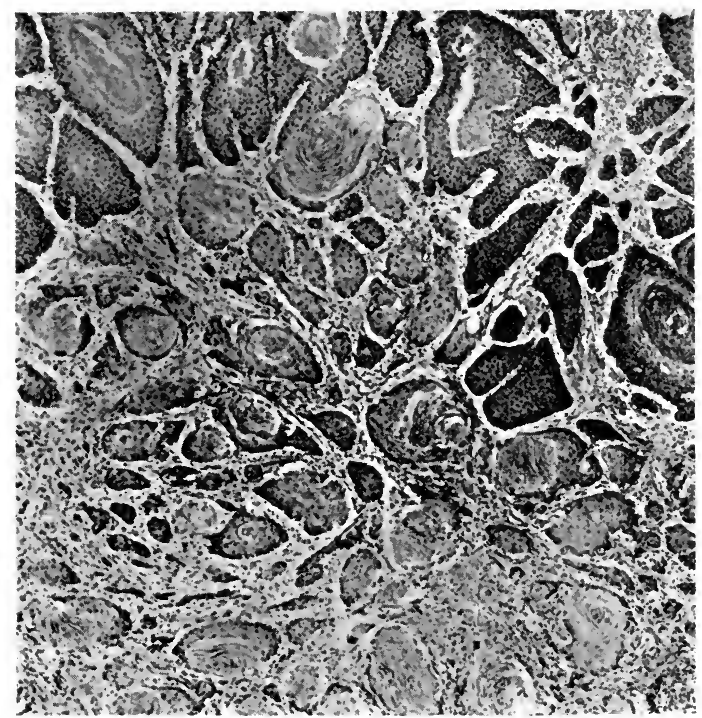

FIG. 19.- Structure of experimental carcinoma of stomach of rat, caused by Spiroptera. (After Febiger.)

sive invasion of the mucosa and stomach wall by proliferating epithelial masses producing the structure of carcinoma. In two animals pulmonary metastases were observed in which tumor-cells but no worms or ova were 
found. In the gastric tumor numerous spiropteræ with ova were commonly present within the proliferating masses.

This brilliant study presents an experimental production of a well-recognized form of gastric carcinoma in the rat, demonstrates the dependence of the malignant tumor upon the presence of a nematode worm, and reveals the gradual assumption by the epithelium of the power of independent growth apart from the irritant originally exciting its proliferation.

There appears to be no reason for introducing the idea of a constitutional dyscrasia in the particular animals yielding the carcinomas, nor for assuming the existence of a microörganism carried along with the nematode.

M. B. Stark has studied what appears to be an hereditary pigmented tumor of the fruit fly, Drosophila. This condition was observed by Bridges, who found that certain black-spotted male larvæ invariably died, and that the lethal factor which produced the tumor is sex-linked in inheritance, being located in the X-chromosome. Stark found that the black spots were composed of polyhedral and spindle-cells containing pigment which gathered at the periphery of the mass. She noted that the tumors caused death in about I 5 hours, that the removal of the spots prolonged the life of the larvæ, while the injection of tumor emulsion caused the death of normal larvæ apparently from toxic action.

\section{TRANSPLANTATION OF TUMORS}

Between Animals of Different Species.-The early period of attempts to transfer cancer from human beings to lower animals, from the time when Peyrilhe's servant mercifully terminated the sufferings of the dog into which he had injected cancer emulsion, down to the present date, has practically demonstrated the impossibility of this mode of transfer. The numerous failures recorded in the literature are doubtless far exceeded by the number of unreported experiments. Even using anthropoid apes Metchnikoff and Roux and Jobling had negative results, but it is perhaps not to be assumed that in these animals whose blood relationship to man is comparatively close, a successful transfer may not prove possible. The significance of the few alleged but paradoxical successes has already been considered.

Between Man and Man.-Cornil reported that an unnamed surgeon grafted a fragment of spindle-cell sarcoma of the breast into the other breast. At the end of two months the tumor reached the size of an almond and proved on section to resemble the original growth. With an adenocarcinoma a similar result was obtained, but the resulting nodule was not sectioned. Fortunately no other clinical record of successful deliberate transplantation seems available (cf. Milner). Hahn successfully transplanted a portion of skin containing a carcinomatous nodule.

From the period when vaccine treatment was very widely employed I have learned of 4 cases in which tumors grew from injected unkilled cells in the vaccine, some of which were mentioned by Coca. The study of spontaneous tumor metastases removes the necessity of experimental evidence in this field.

Between Related Species of Lower Animals.- The early observations on transplantable tumors of lower animals indicated that successful grafts could be made only on animals of the same species and even then only under favorable conditions, but later studies have shown that the suitability of the soil is of somewhat wider scope and extends with some tumors to closely connected or even distantly related species. Thus Sticker transferred the lymphosarcoma of dogs to foxes; v. Dungern carried his hare sarcoma into rabbits; Funk claims to have secured successive transplants of rat sarcoma 
in rabbits long fed on emulsified rat sarcoma. Murphy was able to cultivate mouse sarcoma in rats subjected to repeated $x$-raying of the spleen, but as soon as the lymphocytic function of the animals was restored the tumors regressed.

Strauch reports an anomalous result of transplanting Ehrlich's mouse carcinoma in rabbit. Three weeks after inoculation he secured in the rabbit a well-defined tumor which recurred after extirpation. The extirpated tumor then failed to grow in the mouse, but produced an increasingly malignant tumor in other rabbits, even giving metastases. Happe succeeded in transplanting rat sarcoma into the lens of a rabbit where it grew out into choroid and retina.

Thus it appears that the viability of the cells of certain tumors of lower animals is somewhat greater than once seemed likely, but is nevertheless sharply restricted to species enjoying a definite blood relationship. There seems to be no theoretical difficulty in the way of still further adaptation of the cells of one animal to growth in related species.

Between Lower Animals of the Same Species.-It is highly suggestive of the factors determining the results of transplantation of tumors that the first success was with the most easily transferable growth, the lymphosarcoma of dogs. Yet Novinsky in 1876 had only 2 positive results in 42 attempts. Hanau's transplantation (I889) of epidermoid carcinoma of the vulva in a rat was rather more impressive, since he dealt with a better known malignant tumor and secured extensive metastases. Yet Wehr at the same time interpreted the lymphosarcoma of dogs as a carcinoma, obtained metastases in lymph-nodes and spleen in inoculated dogs, and noted spontaneous regressions. In $\mathrm{I} 890 \mathrm{v}$. Eiselsberg succeeded in transferring to the mesenteric tissues a spindle-cell periosteal sarcoma from a rat.

The first systematic study of a transplanted tumor and one which revealed the possibilities of this method of study was that of Moreau (I89I). A cylindrical cell carcinoma of a mouse he carried through several generations, the tumor gradually losing viability. In the offspring of tumor mice as well as in pregnant animals it grew more rapidly. Traumatism produced metastases which were otherwise usually missing. Other animals were insusceptible. The tumor-tissue when not ulcerated was free from microörganisms. Firket carried a spindle-cell sarcoma through 3 generations of rats, and Velich lost his periosteal spindle-cell sarcoma only after it had passed 8 generations, when it failed to survive in a new breed of rats.

In I9OI and 1902 the general revival of interest in the cancer problem gave to the studies of Loeb in America and Jensen in Denmark a special interest. Loeb (I900) carried a cystic sarcoma of the rat's thyroid through 40 generations without observing change of structure or metastases, and reported observations on other transplantable rat tumors. Jensen ( 1902 to I903) reported the transmission of a mouse carcinoma through I9 generations again without metastases. Both these authors convinced themselves that the tumor grew from the transplanted cells, but their data on this point were not decisive and Loeb assumed the existence of an agent apart from the cells. Jensen found the tumor-cells very susceptible to heat, light, drying, and antiseptics, but was able to preserve their viability for $\mathrm{I} 8$ days at $-\mathrm{I}^{\circ} \mathrm{C}$. He further announced that he had seen the regression of large tumors in mice receiving injections of antiserum prepared in the rabbit. Borrel strengthened the evidence in favor of the carcinomatous nature of the mouse tumors by observing numerous metastases in lungs and lymph-nodes. With these and other studies shortly following the modern field of experimental cancer research definitely opened. 
Of the permanent results of this new era of experimental research it is too early to speak with certainty. In many respects the recent work has deepened our insight into the conditions of growth of tumors, especially the narrow range of their viability and at the same time their apparently unlimited capacity for growth in suitable environment. Particularly fortunate are the conditions provided for the detection of any immunity factors that may be concerned, and yet progress in this field has perhaps been more limited than in others. It is apparent that many phenomena encountered in the study of transplanted tumors and hastily interpreted as new observations had long before been fully recognized in spontaneous human tumors by those familiar with this field. Many observations on change of structure, the apparent discrepancy between histological appearance and biological behavior, the mechanism of tumor regression, the conditions governing metastasis, the elements contributing to cachexia, have proved little more than welcome reappearance, under experimental conditions, of facts and principles previously established in the human subject. The study of transplantable tumors has shed no light on the histogenesis or mode of origin of neoplasms. In the field of practical therapeutics it has opened up many new trails which unfortunately have led mostly astray. Chief among the less important results is the further evidence supplied by the extension of the work to various lower animals that different tumors are often quite different clinical and pathological entities, and that observations on one may not safely be applied to another.

The problems arising in the study of transplanted tumors may now be considered in brief detail. For a full discussion of this subject cf. Woglom.

Problems of Transplantable Tumors.-Nature and Origin of the Tumors.Many doubts were first expressed regarding the malignancy and even of the neoplastic nature of the transplantable tumors. Yet it has been abundantly shown that the chief tumors in mice often exhibit infiltrative growth and metastases. Bashford, Murray, and Cramer described in detail local infiltration and metastases in the Jensen tumor, and Apolant showed that this type of growth developed when the tumor met dense resisting tissues.

Murray found pulmonary metastases in about 50 per cent. of his spontaneous tumors, but they were often microscopic. The significance of the morphology of these tumors must also be regarded as unequivocal. When the whole list of mouse tumors now described is considered, it appears that this animal is subject to a considerable variety of tumors comparable to those of man, and a standard of tumor morphology for this animal has been established.

The origin of the chief mouse tumor has been shown by Bashford and Murray and Apolant to be from widely scattered mammary tissue. Many other mouse tumors have been described and traced to their probable origin, as epidermoid carcinoma of the mouth (Borrel, Haaland), adenocarcinoma of intestine, carcinoma of stomach, adenoma of liver, spindle-cell sarcoma of renal region (Bashford, Murray, and Cramer), chondroma (Ehrlich), adenocarcinoma of kidney, adenocarcinoma of ovary, melanoma, myoma uteri, adenocarcinoma of sebaceous glands, and many others (Haaland, Tyzzer). Apolant thought that 95 per cent. of mouse tumors arise in the mamma, but in Tyzzer's series of 70 tumors of mice 74 per cent. arose in the lung.

In the rat the series of observed tumors is almost equally extensive. Loeb's tumor was a spindle-cell growth apparently sarcomatous arising in the thyroid. Other sarcomas are described by Herzog and by Jensen. Stohr reports a fibro-epithelioma of the tongue, Flexner and Jobling an adenocarcinoma of the seminal vesicle; and Lewin and Michaelis a mammary 
carcinoma; Gaylord, a spindle-cell sarcoma. Many of these tumors have been extensively transplanted.

In the dog, mammary carcinoma is a common tumor which presents many peculiarities but all the essential features of malignancy. It has been transplanted in the Loomis Laboratory by Teague into animals weakened by distemper. The infectious lymphosarcoma of dogs has been variously interpreted. It is a large round or rather polyhedral cell-growth probably arising from the reticulum cells of the lymph-node. Beebe and the writer have shown in bacteria-free transplants that it arises from the transplanted cells. Whatever may be the conditions surrounding the inception of this tumor, when fully developed it presents all essential features of a malignant neoplasm. Many efforts to cultivate bacteria have failed, and spirochetæ occur only on ulcerating surfaces. The conditions permitting transplantation are similar to those observed with other tumors.

In the fowl lymphocytomas with and without leukemia are comparatively common. In Wernicke's summary of the recorded tumors of fowl it is seen

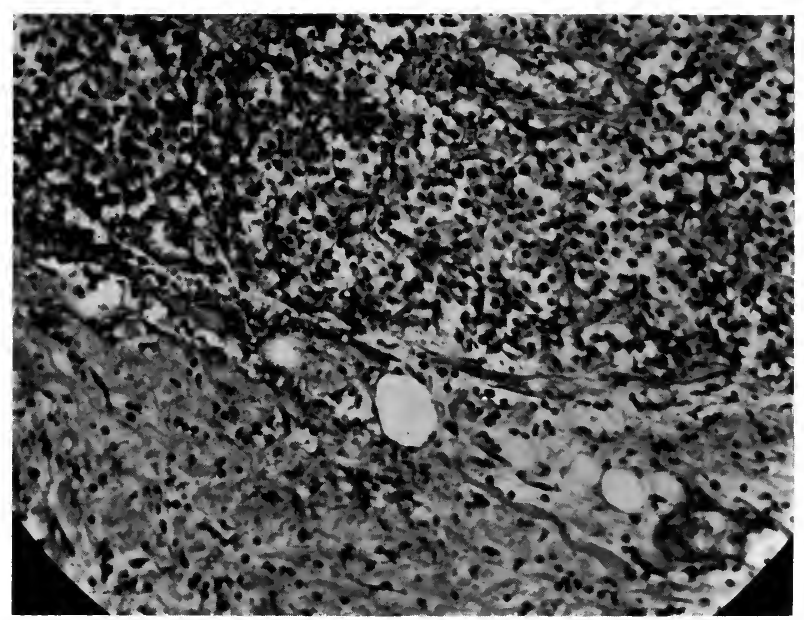

FIG. 20.- Structure of nodule of lymphosarcoma of dog 3 days after transplantation. Note line of separation between tumor-cells and host's tissues.

that a considerable variety of new growths has been observed. Ehrenreich found 7 tumors of carcinomatous or sarcomatous type among rooo old chickens. An attempt to transplant one of the carcinomas failed. Pick observed an epidermoid carcinoma of the mouth; Tyzzer reports myxosarcoma of the thigh and leiomyoma of the mesentery. Fujinami and Inamoto have described a myxosarcoma which was readily transplantable and produced numerous metastases in the inoculated fowl.

Rous has described 3 chicken sarcomas, spindle-cell and osteochondromatous, all of which are transplantable. The juice from the spindle-cell sarcoma strained through a Berkefeld filter (No. 5, medium) as well as the dried and powdered tumor-tissue reproduced the growth. The effective agent remained in the dried tissue for 7 months, but was destroyed by a temperature of $55^{\circ} \mathrm{C}$., and would not pass a Chamberland bougie (F. I). It was very susceptible to antiseptics. Quite remarkable was the increase in viability of this tumor, for while it was at first transferred with great diffi- 
culty, it eventually gave roo per cent. of successes in Plymouth Rocks. It could not be transplanted to other fowl.

In animals inoculated with the juice secondary tumors very often developed at points of trauma, but always much more slowly than in animals inoculated with cells. Filtrates from the spindle-cell tumor and from the chondroma reproduced only the original tumor. It thus appears that in Rous' sarcoma a tumor-producing agent can be separated from the tumorcells by coarse filtration. The nature of this agent has not been determined.

It seems probable that the action of this agent may be compared to that of substances diffusing from certain human tumors into surrounding tissues and causing peculiar collateral hyperplasia and even the gradual transformation of normal into tumor-cells. It may also be pointed out that various chemical agents have been shown to be specially adapted to the excitation

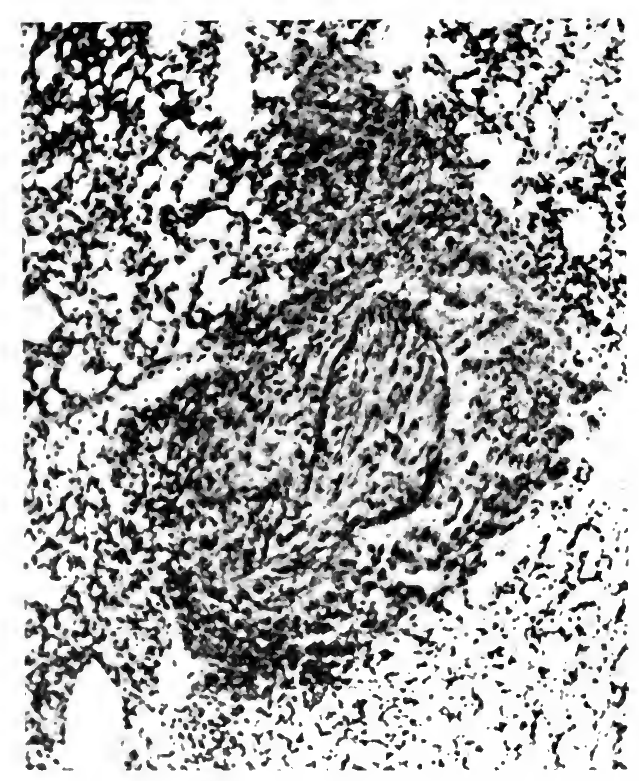

FIG. 21.- Section of a pulmonary metastasis of Rous' chicken sarcoma.

of atypical overgrowth of cells. Another factor of undetermined value may be a peculiar quality in the reaction of chicken tissues to irritants. The principles of avian pathology are not as yet fully expounded. It is of little moment to inquire whether or not the Rous sarcoma is a true tumor. It is obviously not a simple inflammation, but enjoys several essential features of a neoplasm. At present it must be regarded as a disease sui generis without exact parallel in other animal species.

In rabbits a considerable number of tumors have been described, but none appears to have been extensively transplanted. In the hare v. Dungern and Coca discovered a slowly growing spindle-cell sarcoma in the skin of the ear. It contained spindle-cells mingled with large polyhedral eosinophile cells and somewhat resembled a granuloma. It proved readily transplantable in rabbits, and from the serum reactions of the inoculated animals the authors concluded that the tumor-cells even in late generations retained the properties 
of hare protein. The significance of these serum reactions is, however, open to doubt.

Fate of the Transplanted Tumor.-The demonstration of the survival of the tumor-cells in the transplanted tumor has been a highly important result of experimental studies, since it establishes an essential distinction between neoplasms and infectious processes. The principle had, of course, been previously demonstrated by observations on metastatic processes in human subjects by the experimental evidence has been more decisive. Observations at intervals upon the implanted tumor grafts have shown for the tumors of Jensen, Loeb, Ehrlich, the lymphosarcoma of dogs, and Rous' chicken sarcoma, that while the central portions of the mass suffer simple necrosis, the peripheral cells stimulate the formation of new blood-vessels and the multiplication of fibroblasts, and with this support they multiply and produce the new tumor. This reaction has been attributed to somewhat specific formative powers acting on fibroblasts or angioblasts and producing chiefly connective-tissue or granulation tissue about the tumor graft (Ehrlich, Gierke). A leukocytic or a lymphocytic reaction is also observed, especially when there is much necrosis in the graft, or when it is infected. Much exudative inflammation often interferes with implantation. V. Dungern and Coca describe a phagocytic absorption of tumor-cells in the hare by means of large mononuclear leukocytes, which interfere with implantation. The stroma of the transplanted tissue usually undergoes hyaline transformation and absorption, but in some cases it may be preserved for a certain period. Thus Beebe and the writer, in the lymphosarcoma of dogs, found on the third day arterioles in the graft filled with circulating blood. Progressive proliferation of the transplanted stroma has not been satisfactorily traced.

The time required for the appearance of proliferation varies greatly with different tumors. It may be visible in 24 to 48 hours. A palpable tumor seldom appears before Io days, usually it may be detected in 2 or 3 weeks, but occasionally is delayed for months (Bridre, Stohr).

Adaptation.-Great variation in the proportions of successful transplants has nearly constantly appeared. Nearly all observers found difficulty in securing the first graft, while subsequent generations have usually followed much more readily, so that some tumors, as the Buffalo rat sarcoma, have eventually yielded Ioo per cent. of "takes." Careful analysis of the conditions of successful transplantation has been necessary in order to determine whether the differences were due to an increase in the growth capacity of the cells or to some peculiar adaptation to the experience of transplantation. The former possibility led to the employment of Ehrlich of the conception of increased virulence.

It was soon shown that the chief factors resided in the transplanted cells, since within the range of animals commonly employed the increase in number of successful grafts and in their rate of growth was invariable. Preliminary heating to $39^{\circ}$ to $4 \mathrm{I}^{\circ} \mathrm{C}$. or exposure to mercuric chloride or iodide, yielded a larger percentage of takes in the experience of Clowes and Baeslack. Heat beyond $45^{\circ} \mathrm{C}$. or a few minutes' exposure to $20^{\circ} \mathrm{C}$. kills most tumor-cells (Jensen, Loeb). Yet Ehrlich transplanted a carcinoma kept for 2 years at $-10^{\circ} \mathrm{C}$., although the tumor soon ceased to grow. Liquid air at a temperature of $-\mathrm{I} 95^{\circ} \mathrm{C}$. failed to kill all cells in the experiments of Moore and Walker, while Gaylord and Haaland eliminated the carcinomatous elements from a mixed carcinosarcoma by heating to $44^{\circ} \mathrm{C}$. for 30 minutes. With the exception of Rous' chicken tumors it has always been found necessary to transplant intact cells, while extensive comminution or drying abolished growth. Most tumor-cells are comparatively resistant to ordinary antiseptics. 
Spontaneous fluctuation in growth energy of the implanted tumor has been assumed in order to account for variations in the results of implantation. As stated by Bashford, Murray, and Cramer, "considerable variations in success attend the inoculations of one and the same tumor at different times. Thus one series of inoculations may give a small percentage of slowgrowing tumors which at a subsequent period may begin to grow rapidly or, on transplantation, while still growing slowly, give a high percentage of quickly growing tumors." From a large series of inoculation experiments taking into account the percentage of "takes" and the rate of growth, these authors constructed curves showing rhythmic variations in growth energy.

It is thus interesting to note that in this experimental field conclusions may be drawn which support clinical observations frequently made in the human subject, that the rate of growth of many tumors varies at different periods of their course. Whether the two sets of observations are exactly parallel may be doubted. It may further be concluded from clinical and pathological study that the rate of growth of different portions of the same tumor varies enormously, the active portions being usually peripheral, that one tumor may be active while another neighboring mass is quiescent, that superficial tumors may regress when internal metastases are active, and that in general the rate of growth of tumors is subject to fluctuations from a great variety of factors which are chiefly dependent on the uncertain nutrition of both tumor and host. Under these complex conditions it is extremely difficult to determine whether anything properly designated as increased virulence or adaptation of tumor-cells has so far been demonstrated in the experimental field. On the contrary, it would appear that successive transplantation involves a process of sifting out the more viable cells and providing for them a highly favorable soil.

Uniformity in technic of transplantation is an obvious essential in a comparison of results. Emulsions of cells in salt solution, or thin slices of tissue, are chiefly employed, and in either case it has been found that an optimum quantity exists, viz., I to $2 \mathrm{~cm}$. of emulsion and I or 2 cubic millimeters of tissue. Larger amounts may yield initially larger tumors, but in many cases subsequent standstill or regression are observed.

With large doses it has been assumed that partial immunity resulted from absorption of much of the tumor-tissue.

The site chosen for inoculation is ordinarily the subcutaneous tissue, into which the tumor material is injected or implanted. The internal organs, especially the spleen, have proved less suitable (Goldmann). Uhlenhuth secured grafts by rubbing an incised wound with the Jensen rat sarcoma, and Beebe and the writer transferred the dog sarcoma in this same manner, which is apparently the natural mode of transfer of this disease, during coitus.

Influence of Variations in the Soil.-That slight variations in the tissues of the host greatly influenced the results of transplantation was very early noted. Not only was the same species of animal required, but the results were more successful in animals of exactly the same color and antecedents. Brown and wild strains differed in susceptibility from white and domesticated families. Rous found it impossible to transfer his chicken sarcoma at first to any but Plymouth Rocks, preferably of the same brood. Haaland found it impossible to propagate on Danish mice fed on grain a Berlin tumor coming from mice fed on milk. Many others have had difficulty in transferring propagable tumors from the animals of one country to those of another.

The influence of diet has since been shown to be highly important. Beebe and Van Alstyne were able to render rats highly refractory to the Bufialo sarcoma by a previous course of carbohydrate-free diet. They have also 
shown that the course of this tumor is distinctly retarded by carbohydratefree diet, and accelerated by butyrates among the fats. Sweet also has observed increased resistance to implantation in animals on a carbohydrate-free diet. That these rules may not hold for all tumors is highly probable and specifically shown by Woglom's negative results in carcinoma. Benedict brought about the complete regression of large sarcomas in rats rendered diabetic by phloridzin.

Simple restriction of food has been shown by Moreschi to retard the growth of implanted tumors. With the Flexner-Jobling rat carcinoma Rous, however, failed to influence the growth by restricted diet.

By substituting lime substances for the usual diet Sweet, Corson, and Saxon rendered rats markedly insusceptible to implanted sarcoma, and when a graft succeeded in such animals its growth was accelerated by return to normal diet. Castration also favored growth. Centanni reports that a diet of wheat bread and corn but without green food renders mice highly insusceptible to tumor implantation. Oser and others find that tumors grow better in splenectomized animals.

Cholesterol administered subcutaneously in doses of $40 \mathrm{mgm}$. at intervals of 2 to 3 days was found by Robertson and Burnett to decidedly accelerate the growth of carcinoma in rats. These results were confirmed by Sweet, Corson, White, and Saxon. Tethelin, the active principle of the anterior lobe of the pituitary gland, was found to have a similar effect, and since both these substances contain a hydroxy-benzol group, Robertson and Burnett concluded that the accelerating influence resided in the hydroxy-benzol radical. An increased cholesterol content of the blood has been found in certain cases of cancer by Luden.

Spontaneous absorption of implanted tumors was first recorded by Loeb and its comparative frequency and the increased resistance to reinoculation were emphasized especially by Clowes and Baeslack. About 23 per cent. of tumors derived by Gaylord and Clowes from the Jensen tumor were found to regress, and Bashford reported as high as 50 per cent. Most of the regressing tumors have never reached a large size, but some very large growths appear to reach a critical point beyond which nutrition fails and complete absorption follows. It is a notable fact that during absorption the animals do not suffer from intoxication and that recovery may be complete. The histological changes in regressing tumors vary widely. A simple necrosis is often the primary event followed by absorption and fibrosis, or a progressive fibrosis may overtake the tissue and lead to gradual atrophy of tumor-cells. Phagocytic processes by mononuclear cells are frequently observed (Gaylord, Clowes, Bashford).

Structural Changes in Propagated Tumors.-While many tumors maintain a uniform structure through many generations, some carcinomas have exhibited notable changes in structure which are difficult to interpret. As a rule these changes duplicate those observed in the human subject, and on the whole they appear to be less violent than are frequently observed in human material. Thus Bashford's study of 85 propagable tumors, 35 of which had been in cultivation for 3 years, revealed the usual series of minor changes in the size and structure of alveoli, appearance, and disappearance of various forms of epithelial metaplasia, formation of bone and cartilage in sarcomas and variations in vascularity. Metastases were not always more atypical than the original growth. Apolant was disposed to attribute the reversion of a solid carcinoma to an adenoma to increased resistance in partly immunized animals, a conclusion from which Murray dissents.

Considering the variation observed in propagable tumors in comparison 
with the structural changes in recurrent human tumors the entire scope appears very much more restricted in the lower animals than in man.

The most important change credited to the propagable tumors is the sudden assumption of malignant neoplastic properties by the previously normal stroma. This change was first observed by Ehrlich and Apolant in the tenth generation of an adenocarcinoma of the mouse. A reverse transformation, viz., the disappearance of spindle-cells from an adenocarcinoma, is also recorded by Apolant, but in this instance it was concluded that the spindle-cells were merely altered epithelium.

Many later observations of the development of sarcoma in carcinoma have been recorded in both rats and mice (Loeb, Lewin, Bashford, Russell).

The change appears to occur rather suddenly after the 8th to Ioth or later generations. Russell fixed the period at which the change usually appears at the 55th to 6oth day of propagation and states that it is independent of the number of transfers. When once established it is usually progressive and in certain cases the spindle-cell tissue has eliminated the other element, or both structures persist together, or separate strains of sarcoma and carcinoma are obtained. The rate of growth of the mixed tumors appears increased rather than diminished. In metastases either sarcoma or carcinoma were encountered, but the sarcomatous element when present was more prominent in metastases (Haaland, Clunet).

Several theories have been advanced in explanation of the sarcomatous transformation of mouse carcinoma.

It was at first considered possible that the growths were originally mixed, but this theory was abandoned for lack of evidence.

Most observers have concluded that the sarcoma represents a neoplastic transformation of the stroma of the host caused by a stimulation of stromacells by the epithelium. The exact nature of this stimulus is not clearly defined, but it has been conceived as a sort of growth stimulus possibly carried by chemical derivatives of the epithelium.

Repeated transplantations are not necessary. Both Haaland and Russell describe in detail the appearance of foci of overcellular stroma located in the center of carcinoma nodules, the increase of mitotic figures in the stromacells, the survival of these altered stroma-cells in grafts and their rapid increase until they overgrow the epithelial elements. Haaland describes peculiar halos of large cells surrounding epithelial groups, and intermediate between epithelium and stroma-cells. Others have failed to trace the spindle-cells to either stroma or epithelium.

The third possibility, which seems to the writer to have been too hastily dismissed, is that the spindle-cells are altered epithelium. Such transformations are relatively common in the early or advanced stages of many human tumors and especially in recurrences after operation, and this fact establishes a probability that a similar change in mouse tumors has a similar significance. On the other hand, there is no parallel in human pathology for the development of sarcoma in stroma supporting carcinoma, so that observations in mice would introduce a new principle into oncology. In man spindlecell transformation usually goes with increased growth and malignancy, and such is the case in mice. It is difficult to conceive how an original carcinoma can be made to yield all its growth energy to normal stroma-cells and completely retire from the field, yet this anomalous result appears to be reached in the pure sarcomas developing from mouse carcinoma. The crucial evidence is that presented by Haaland and Russell of gradual transformation of stroma into sarcoma. Yet the interpretation of transitional pictures is notoriously hazardous, and few observers have been able to convince themselves that 
it actually occurs. Orth thought that Lewin's sarcoma-cells represented granulation tissue. I have examined, through the kindness of Woglom, several cases purporting to show the sarcomatous transformation of stromacells, but have been forced to draw from these sections the opposite conclusion, viz., that the spindle-cells were derivatives of epithelium. Since such an interpretation is at least admissible, it may be urged that further evidence is required before the sarcomatous transformation of mouse carcinoma can be accepted as proved.

Resistance.-In the elucidation of the factors concerned in natural and acquired resistance to tumor implantation the experimental studies have opened up a new and highly fruitful field. The results apply strictly to the artificial conditions in normal animals receiving tumor grafts, but not themselves developing spontaneous tumors. It has repeatedly been shown that animals resisting implantation may often develop spontaneous tumors (Bashford, Thorel, Clunet). Likewise very young animals which do not develop cancer may prove very favorable soil for implantation. This principle re-

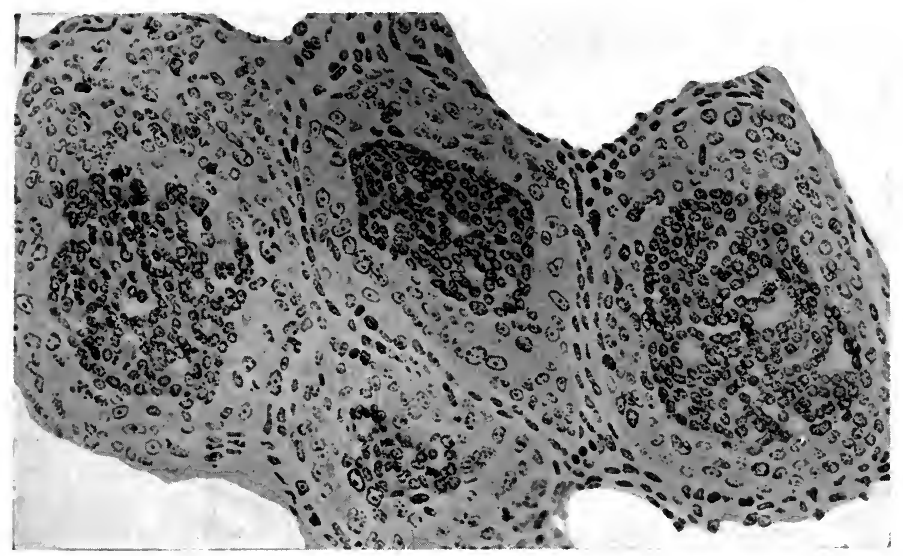

FIG. 22.- Halos of pale cells surrounding epithelial cell groups, in the sarcomatous transformation of carcinoma in the rat. (After Haaland.)

appears in the rapid growth of many carcinomas occurring in human subjects of youthful age. The problem of the continued growth of a tumor is therefore quite different from that of its inception. It follows also that an animal may be susceptible to one tumor while insusceptible to another, and it is equally clear that the body tissues of animals in general are very favorable soil for the growth of lawless cells which must therefore be restrained by purely local factors.

Natural Resistance.-Age has been shown to have no definite influence on the susceptibility to certain tumor grafts, since both young and old animals have proved suitable for experimental studies. Most observers have preferred stock from 4 to 8 weeks of age (Haaland, Albrecht, Hecht).

Racial differences were believed to account for the capricious behavior of many tumors when transferred from animals of one country to those of another. Yet it has appeared that many indications of resistance seemingly referable to racial differences were the result of altered diet, and Gierke strongly supported this view. In many instances strange animals at first 
resistant soon became susceptible when housed under the same conditions as the animals furnishing the graft (Stohr, Cuenot, Mercier). For absolutely comparable results the use of animals of one litter or parentage is probably advisable.

Good health appears to favor the growth of tumor grafts and poor condition to retard it. Yet Teague succeeded in transplanting mammary carcinoma of dogs only in animals weakened by distemper. Observations on the effect of pregnancy are conflicting, Moreau and Herzog finding acceleration, while Haaland, Bridre, and Cuenot saw retardation of growth in gestation.

Slye, comparing mammary tumor growth in two series each of 30 mice, found that without exception the amount of tumor grown by a female while reproductive was strikingly less than during her non-reproductive period. Similar results were observed during gestation. Reproductive females bearing small tumors of slow growth were often observed to succumb rapidly to their tumors after the birth of the last litter.

Acquired Resistance.-The first observations concerning acquired resistance were made by Gaylord, Clowes, and Baeslack, who noted frequent spontaneous regression of the Jensen rat tumor and also the insusceptibility of these recovered animals to reinoculation. Similar results were soon reported by many observers with various tumors and the question arose whether the diminishing proportion of successful takes in reinoculated animals was due to a gradual sifting of naturally immune animals or to active immunity caused by repeated absorption of the tumor grafts. The latter theory was strongly supported by Bridre, who found that by inoculating very large doses his resisting animals rapidly became quite immune. Michaelis, Borrel, and Ehrlich verified these results. It next became apparent that the resistance thus established while most pronounced against the tumor employed extended also but to a lesser degree to other tumors. Ehrlich was inclined to believe that the resistance was practically universal (pan-immunity).

That the actual temporary growth of tumor-cells was necessary to excite the resistance was strongly suggested by its failure to appear in animals treated by tumor-cells killed by heat or mercuric chlorid (Clowes); or killed by chloroform (Michaelis); or treated with the filtrate of tumor emulsion (Bridre): or by cells injured by grinding and freezing (Haaland). Whether the principle has been fully established is perhaps doubtful.

Normal tissue cells are almost equally effective in exciting resistance, as first shown by Bashford using red corpuscles, and later by Schone, using liver, testis, or emulsion of mouse embryo; by Borrel and Bridre, with spleen, liver, or testis; by Higuchi with blood-free placenta. With lactating mouse breast Moreschi secured variable results. Both Russell and Woglom produced the resistant state by emulsions of embryo skin and Woglom ascertained that the immunity reached a maximum ro days after inoculation, remained high until the twenty-fourth day, and vanished about the seventy-fifth day.

Devitalization of the normal tissue cells generally robbed them of their immunizing power, while Woglom showed that injection of the animal's own tissues were ineffective.

When these experiments were transferred to tumor-bearing animals the results became less definite, although Russell considerably reduced the proportion of successful secondary inoculations when the reimplantation was preceded by an injection of tumor or embryo-emulsion. The practical test of these methods in animals with spontaneous tumors proved entirely fruitless in the hands of Haaland who failed by this form of vaccination to influence the growth, prevent post-operative recurrence, or diminish metastases from spontaneous tumors. Likewise in the human subject, vaccination therapy 
employed on a vast scale, while attended by some paradoxical successes, has failed to establish its usefulness (Coca).

Passive Resistance.-Attempts to convey actively acquired resistance to other animals has not been distinctly successful. The use of the serum of resistant animals has usually failed to render new animals passively resistant to inoculation (Michaelis, Ehrlich, Haaland). Repeated reinoculations of immune rats by Uhlenhuth failed to render their serum effective in preventing successful inoculation in fresh animals receiving first serum and then tumor, or serum and tumor together. Bridre prepared in sheep and fowl an antiserum against mouse cancer which did not retard and even appeared to accelerate tumor growth. This method has been extensively employed in human therapy using as antigen either the whole tumor or various protein fractions, with uncertain results (Vidal, Beebe, Berkeley).

Nature of the Factors in Resistance.-The blood-serum having failed to yield proof of possession of the immunity factors, it became evident that resistance to tumor implantation must be of more complex nature than that excited by bacteria. In this emergency Ehrlich pronounced his theory of athrepsia to account for the failure of tumor grafts to grow in resistant animals. Having found that mouse carcinoma after surviving in rat tissues for a week may then be successfully restored to active growth by returning the graft to the mouse, he succeeded in conducting a series of zig-zag transfers from mouse to rat to mouse, etc., without permanent injury to the tumor. From this data he argued (I) that there were no cytolytic agents in the rat, otherwise the tumor-cells would have been injured, and (2) that the mouse graft failed to grow in the rat after a few days sojourn because it soon exhausted a specific nutritive substance, which was not the nutritive molecule itself, but acted as a stimulus to nutrition. Replacements in the mouse provided new supplies of the $x$-substance. This theory abandoned the idea of antagonistic substances in the resistant animal and substituted the conception of an inert lack of nutriment as the factor in resistance. The theory was possibly a correct interpretation of the experiments cited, but failed to meet the conditions in animals of the same species. Here two valid objections were soon raised by many authors (Uhlenhuth, Borrel, Hertwig and Poll, Bashford). It has been shown (I) that a tumor-bearing animal may usually be reinoculated, both tumors advancing or regressing together, the one not interfering with the nutrition of the other; and (2) that when a second inoculation fails it is usually due to an immunity excited by the partial absorption of the original graft.

While thus failing to account for actively produced resistance Ehrlich's athreptic theory has served to emphasize the probable importance of the nutritive qualities of the soil as opposed to antagonistic substances in the blood of resistant animals.

A further illustration of this principle may be found in the experiment of Beebe and Crile who regularly caused the disappearance of the lymphosarcoma of dogs by exsanguinating the animals and immediately transfusing them with the blood of resistant dogs. Here it may be assumed that an antagonistic substance was carried over in the blood, but it is simpler and equally reasonable to suppose that the transfused blood was better adapted to the normal than to the tumor-tissue, and that its presence turned the balance of nutrition in favor of the normal tissues and against the tumor, which then suffered slow atrophy and absorption. Rather specific evidence against the purely athreptic nature of tumor immunity has appeared in the successful cultivation of rat and mouse tumor-cells in various alien plasmas (Lambert, Hanes). 
Cultivation of tumor-cells in vitro. The conditions requisite for the prolonged survival or actual multiplication of tissue and tumor-cells in vitro seem to be a fluid medium permitting of nutrition and diffusion, fixed points of attachment for the cells, and a suitable temperature. Nutrient fluids exactly homogeneous with those to which the

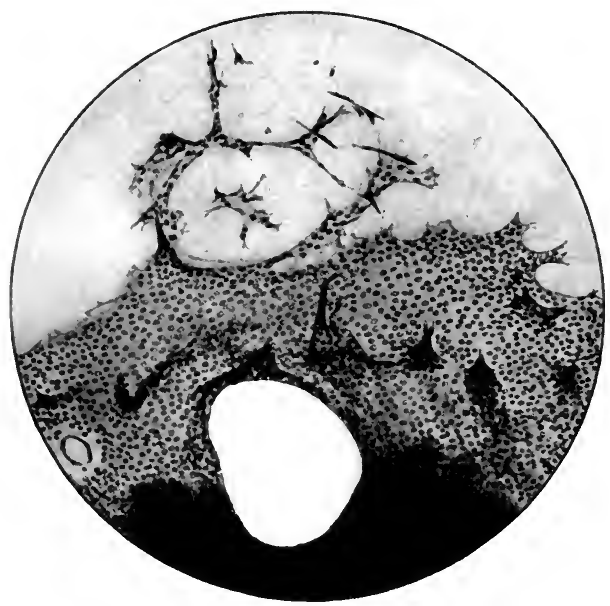

FIG. 23.-Cultivation of mouse carcinoma-cells in blood-plasma. (Lambert and Hanes.)

cell has been adapted are not necessary. These conditions were partially provided by Loeb who saw slight spreading of epithelial cells over the surface and into clefts of an agar medium.

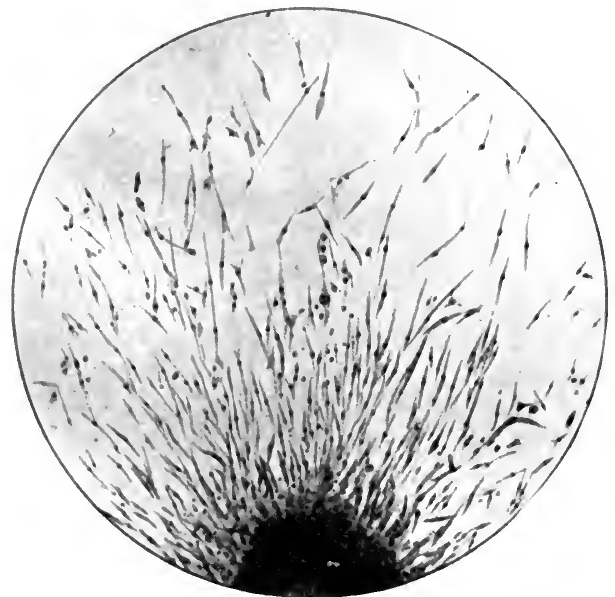

FIG. 24.-Rat sarcoma, growing in pigeon plasma. (Lambert, IIanes, Crocker Reports, Vol. 2.)

The fixed points of support were missing in the circulating whole blood used by Beebe and the writer, and we secured only prolonged survival of dog tumor-cells. An adaptation of Harrison's technic by Burrowes proved successful in his hands and Carrel's in permittng definite multiplcation of tissue cells in the fibrin meshes of coagulated plasma, and this method had been extensively used. Epithelial cells grow 
out in coherent sheets from the parent mass, while mesoblastic cells tend to grow out in long sprouts or become widely scattered. The importance of the results of experimental cultivation of tissue and tumor-cells in vitro has not been as great as might have been anticipated, but it has been shown that these cells are capable of living in much more varied media than once seemed likely, and that the natural immune substances supposed to exist in the serum of refractory animals has little influence on the growth. Tumor-cells as a rule seem to be less capable of survival or growth than corresponding normal tissue cells, and only after some difficulty has Burrowes succeeded in cultivating certain human tumor-cells.

Hypersusceptibility.-Various observers have reported phenomena suggesting hypersusceptibility to tumor growth in animals receiving grafts, emulsions, or saline extracts of tumors, but the data are insufficient to establish the existence of a specific hypersensitization (Woglom, Lit.).

The mechanism by which the resistant animal aborts the tumor graft offers suggestive evidence regarding the nature of the immunity factors. In resistant animals the unsuccessful graft fails to excite the specific stroma reaction and remains unprovided with the necessary channels of nutriment. Russell examining grafts in refractory animals at intervals found that the surviving cells continued to proliferate from 7 to Io days, but instead of exciting an active growth of fibroblasts they became sharply separated from the host's tissues. He concluded that there must be present in resisting animals something which inhibits the chemotactic influence of the cancer-cell upon the connective tissues of the host. Inflammatory reaction has also been shown to be responsible for the failure of grafting in resistant animals. v. Dungern and Coca, studying the inoculation site in immune animals, and in regressing sarcoma in the hare, observed that the necrosing graft gathered large numbers of macrophages in vessels and tissue spaces, as well as many lymphocytes and plasma-cells, which attacked and absorbed the tumorcells. Since this reaction failed in susceptible animals they interpreted it as a phenomenon of hypersusceptibility or allergie, provided by the previous immunizing dose. Burgess examining grafts in non-susceptible Japanese mice observed at first the formation of the usual vascular stroma, but after a week an inflammatory reaction ensued yielding polynuclears, eosinophils, lymphocytes, and plasma-cells, which shut off the blood-supply and induced fibrosis. Da Fano also noted a rich exudate of lymphocytes and plasmacells about the inoculated tumor in immunizing animals. In splenectomized animals Apolant finds that very slight immunity to tumors can be excited, and Baeslack observed marked fall in the circulating lymphocytes in susceptible animals with growing tumors, and a steady increase in resisting subjects with regressing tumors. Murphy finds that the chicken embryo possesses no ineans of preventing growth of implanted rat tumor until the chick hatches and develops spleen and lymphocytes, but if the embryo receives a fragment of adult chicken spleen or bone-marrow along with the tumor graft the latter fails to grow. After subjecting the rat spleen to the $x$-ray he found that the animal supported the growth of alien tumor-tissue for a considerable period and until the spleen recovered its lymphocytogenic function.

All these observations lend important support to the view, long since established in human pathology, that the lymphocyte is an important agent of defense of the organism against tumor growth.

Some light but no complete elucidation of the nature of tumor immunity has been furnished by collateral studies on tissue immunity.

Antibody production has been demonstrated against various tissue cells injected into foreign species, as tracheal epithelium (v. Dungern), or spermatozoa (Metalnikoff). These observations may justify the use of 
antisera prepared in lower animals against human carcinoma tissue or its derivatives.

Antibody production has been demonstrated against an animal's own tissues, injected either into the same individual or into other animals of the same species. Thus Adler prepared an autospermatoxin in guinea-pigs and Halpern showed, by complement deviation, the development of antibodies against an animal's own kidney, pancreas, and spleen. These observations further support the view that a human tumor-bearing subject may to some extent be immunized by injections of his own tumor-tissue.

A certain relationship has been found between the same organs in different species of animals, as with the testis (v. Dungern and Hirschfeld Schenk), and with the sexual cells of fish (Dunbar).

Nevertheless in the sera of animals rendered immune to transplantable tumors there has been no satisfactory demonstration of specific cytolysins, agglutinins, or precipitins. Nor have complement deviation tests in such sera been clearly successful.

Lambert and Hanes were able to cultivate rat sarcoma cells in the plasma of rats immunized against this sarcoma, although the sera of immunized alien animals inhibited the growth while the normal alien sera did not.

The serological evidence therefore points to some other form of immunity and this is apparently to be found in the local and general hypersensitization produced in the immunized animals elicited by absorption of regressing or inoculated tumor-tissue. v. Dungern and Coca with the hare sarcoma, and Weil with the Flexner-Jobling rat carcinoma demonstrated anaphylactic reactions in the inoculated animals and a local inflammatory allergetic reaction to inoculation. This conception of tumor immunity is in accord with the fact that complete resistance to implantation of a virulent sarcoma may exist in animals bearing an actively growing tumor of the same sort. 


\title{
SPECIAL ONCOLOGY
}

\author{
CHAPTER $\mathrm{X}$
}

\section{FIBROMA}

Fibroma is a term applied to tumors composed chiefly of connective tissue.

Many of these tumors are composed exclusively of connective tissue and are designated as pure fibromas. More of them are covered by hypertrophic lining epithelium, as in papillary fibroma, or contain glandular structures, adenofibroma. In the nerve-trunks they may show atrophic nerve-fibers for which, and on account of their origin, they are called neurofibroma.

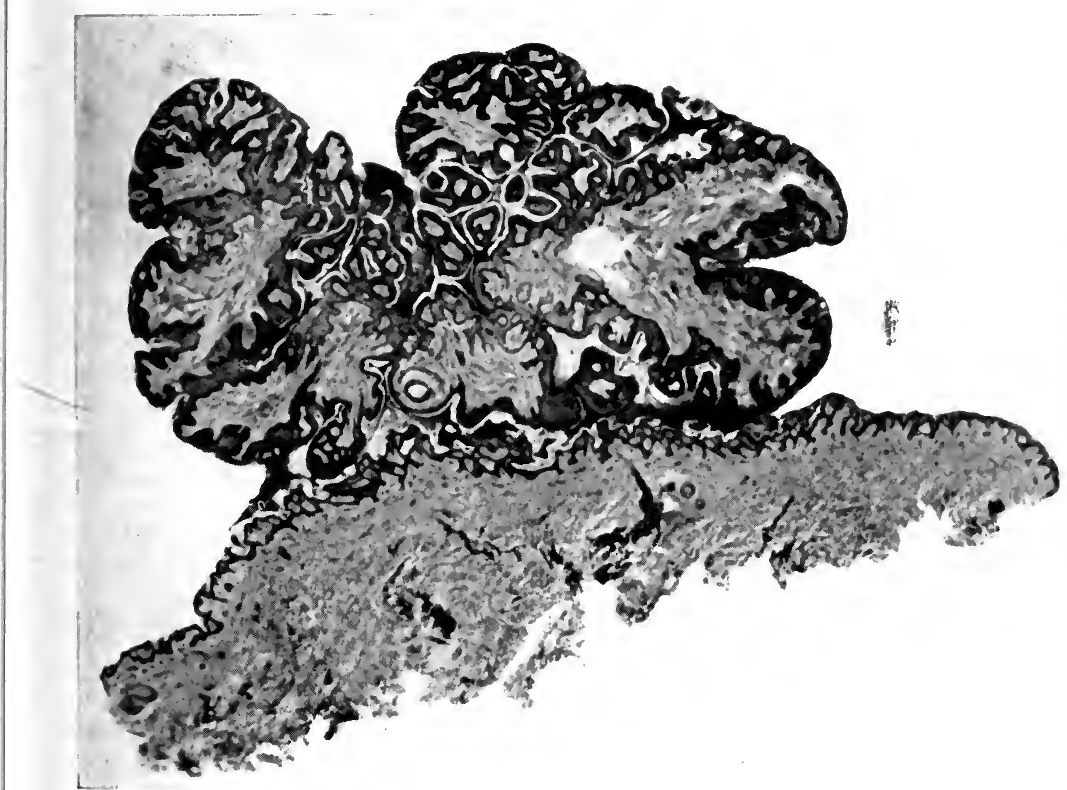

FIG. 25.-Benign pigmented fibro-epithelial wart. (Photo by B. H. Buxton.)

Blood-vessels may be very abundant or the lymph-vessels may be in excess, in angiofibroma and lymphangiofibroma. Neoplastic connective tissue is also observed in various other benign and some malignant tumors, as in fibrolipoma, fibrochondroma, etc.

The gross appearance of true fibromas is usually characteristic. They are circumscribed, encapsulated, usually lobulated, firm or soft tumors, and on section exhibit a grayish translucent appearance. 'The blood content varies, but is usually slight. Secondary changes yield corresponding alterations in texture. Multiplicity is a striking feature of neurofibromas both in the skin (fibroma molluscum) and in the organs. From this characteristic 
appearance there are all gradations merging toward the products of chronic inflammation. In some very early fibromas of breast and kidney the isolation of the tumor nodule is imperfect, although the later stages of such growths are well encapsulated. There is no sharp dividing line between chronic productive inflammation and fibroma, although characteristic examples of each are widely separate. The inflammatory nodule merges insensibly with the surrounding tissue, its histology lacks the definite features of a neoplasm and it occurs under clinical conditions which supply the inflammatory agent, as in chronic mastitis, and chronic rhinitis. Yet, as Ribbert remarks, the longer an inflammatory nodule persists, the more in some cases it may approach the quality of a neoplasm. It must be admitted that chronic productive inflammation may pass insensibly into fibroma, a fact most frequently illustrated with some forms of local elephantiasis.

The structure of fibromas varies greatly, but it commonly presents fibroblasts, fibrils, blood- and lymph-vessels.

Soft fibromas contain a considerable proportion of cells lying in a soft matrix which is often edematous. Much vascularity also reduces the consistence. Hard fibromas contain fewer cells and firmer, more abundant matrix which is often hyaline. In very dense fibromas arising from periosteum and tendons very few cells may be present and wide areas of hyaline matrix are observed. The consistence is also affected by calcification, mucoid degeneration, and the formation of cysts.

The cells of genuine fibromas are usually larger and more numerous than in adult connective tissue. In actively growing fibromas the chromatin content of the nuclei is also increased and the tissue has a distinctly neoplastic character. Even in some hard fibromas, as in keloid, the cells may be of large size throughout, but in many cases the active cells may be limited to the periphery or to isolated foci chiefly about vessels, while the main portions of the tumor are acellular. The arrangement of the cells is uniform and is determined by pressure or by the presence of originating structures as bloodvessels or nerve-trunks, or gland alveoli.

With an increasing proportion of cells, diminution of matrix, and irregularity in size and shape of the cells, the tumors show more active growth and a tendency to recurrence, and to these tumors the term fibrosarcoma is given. In distinguishing fibroma from sarcoma Borst relies chiefly on irregularity in size and shape of the cells as well as overgrowth of nucleus and absence of definite stroma. When the bulk of cells greatly exceeds the matrix the tumors are usually of active growth and require careful removal to prevent recurrence.

The matrix of fibromas varies greatly. In many cases it has the characters of embryonal connective tissue; in keloid it is hyaline; in dense tumors it may be very firm but fibrillar; and it is often edematous and infiltrated with leukocytes.

The fibroglia fibrils of Mallory are regularly present and may be very long and quite abundant. They are best demonstrated in material promptly fixed in Zenker's fluid and stained by a mixture of anilin blue, 0.5 ; orange G, 2.0; oxalic acid, 2.0; water, Io०. The bundles of wavy collagen fibrils are abundant chiefly in hard fibromas. Ribbert regards the matrix of fibromas as a product of cell activity, but not as a derivative of cell processes. The fibrils he considered to be the result of cell activity upon a secretory product which has many of the qualities of fibrin, but with an altered staining reaction. This origin would explain the great length of many fibrils, the great bulk of the matrix, and the presence of many fibrils and much matrix with very few cells. Elastic fibrils are usually scanty. 
Secondary changes occur in the matrix, including hyaline transformation, calcification, fatty and mucoid degeneration, edema, and necrosis, and in connection with bone, ossification. Also the remnants of invaded structures may be included in fibromas, as fat-cells, which may be incited to proliferation, gland alveoli, muscle-cells, and nerve fibrils. By metaplasia portions of fibromas may be converted into cartilage or bone.

Blood-vessels occur in normal form and number, or as irregular thin-walled spaces, or as sinuses lined by endothelium alone. In angiofibroma the vessels are very abundant. They appear as irregular spaces and canals lying in the connective tissue, they are lined by a moderate number of flat or cubical endothelium, but the connective tissue is the predominant element. Some authors include among fibromas the majority of angiomas. It seems better, however, to separate from fibromas all very vascular tumors in which bloodvessels form a predominant organoid unit. Fibroma is a histioid, not an organoid, tumor.

Lymph-vessels are commonly present in fibromas. In keloid the lymph spaces are so prominent as to suggest for this tumor the term lymphangiofibroma. In local elephantiasis which is closely related to fibroma the dilated lymph-vessels are numerous. Edema and lymph-cysts form from obstruction to the lymph flow.

The natural history of fibroma is that of a slowly growing tumor progressing steadily or intermittently over many years. In many instances, as in neurofibroma, there is a natural tendency for the tumor to reach a certain size and then to remain stationary, probably from mechanical interference with blood-supply. In very large fibromas, as of breast, skin and ovary, hyaline, calcific and necrotic areas bear witness to the regressive tendency that overtakes this type of tumor. A spontaneous cure may thus be accomplished. In a large fibroma of the quadriceps tendon I have seen almost complete destruction and permanent arrest of growth by central softening. Many mammary fibromas suffer hyaline change and arrest of growth after I $_{5}$ to 20 years. Kosinski has reported complete spontaneous atrophy of neurofibromata. Sarcomatous transformation is very rare, but occurs with neurofibroma. In the mixed adenofibroma of breast cancerous changes may develop in the epithelium. Recurrence after operation is characteristic of keloid, but usually consists of an extension of the tumor process over formerly intact areas.

Origin of Fibromas. - The exact point of origin of most fibromas is still undetermined. That many of them arise from misplaced islands of tissue, according to Cohnheim's theory, is very probable. Chiari observed a congenital polypoid fibroma at the perineal raphé, and Ribbert has seen a fibrolipoma in the same situation. Arnold described a congenital chondrofibroma of the skull which he referred to a disturbance in the development of the cranium. In several reported fibromas of the testis there were elements suggesting that the tumor represented a one-sided development of a teratoma. Certain fibromas in the adult appear in the region of embryonal clefts where superfluous cells may be supposed to exist. In the breast Williams found evidence that I4 per cent. of the adenofibromas of this organ arise from embryonal rests, and the frequent association of chondroma and osteoma with fibroma, especially in the dog's breast, favors this view.

For other fibromas one must apply the theory of local irritation and disturbance of nutrition, as in keloid, which commonly follows scarring; in papillary fibromas and adenofibromas of mucous membranes, which are associated with catarrhal inflammation; and in fibromas of periosteum and joint areas, which may follow repeated trauma. 
In a third group the clinical features point to a congenital or acquired local predisposition, of which multiple neurofibroma is the best example. Elephantiasis seems to exhibit a general predisposition often combined with the effect of recurring inflammation.

It is especially in the second and third etiological classes that one encounters the less definite tumor-like processes which it is sometimes difficult to. classify, and in which one must recognize the cumulative influence of inflammation and chronic disturbance of nutrition and the passage of inflammatory into self-perpetuating neoplastic processes.

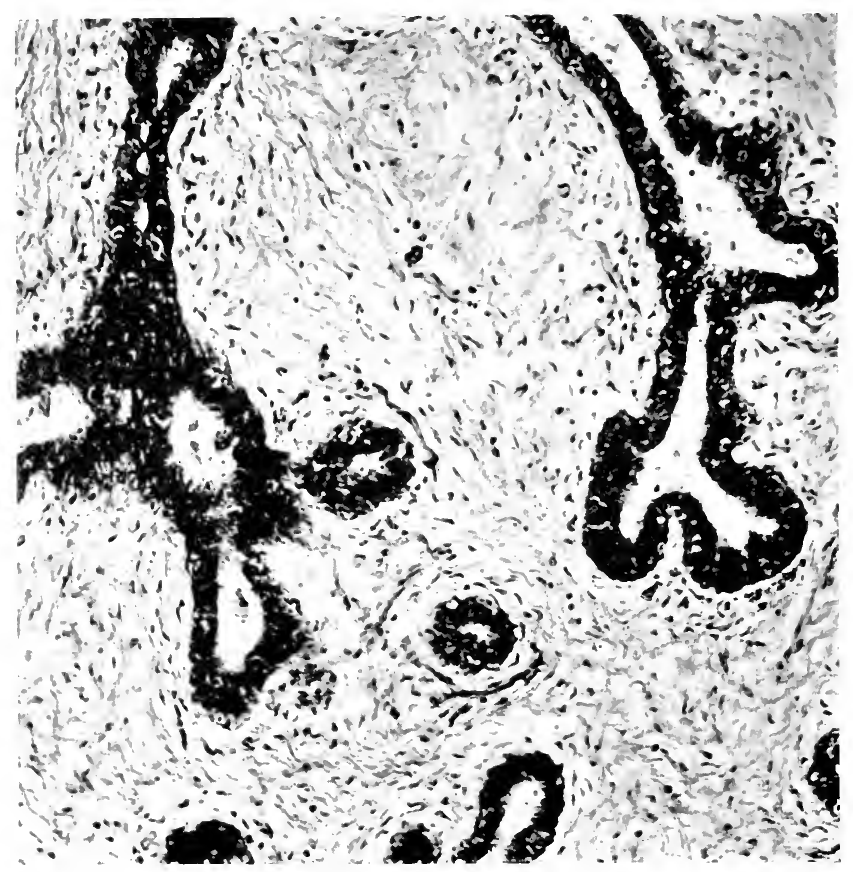

Fig. 26.-Fetal fibro-adenoma of breast in a girl of I 8 years. The tumor grew rapidly, measured $9 \mathrm{~cm}$., was soft, bluish, resembling sarcoma.

Clinical Types of Fibroma.-The clinical types of fibroma are numerous and some of them form characteristic clinical entities which not only illustrate the peculiarities of this group of neoplasms, but throw light on the nature of tumors in general.

\section{NEUROFIBROMATOSIS}

Tumors of the peripheral nervous system form a very important and complex group of neoplasms of frequent occurrence and varied clinical course.

A satisfactory classification of these tumors is difficult, owing to the complex structure of the nerve trunks, and the uncertainty of the source of the supporting cells which accompany nerve-fibers, such as the cells of the sheath of Schwann, the neurilemma, the endoneurium, and the lymph sheaths of nerve trunks, all of which participate in tumor growth.

In one general group the growth affects the connective-tissue structures 
of the nerve, epineurium, perineurium, and endoneurium, the specialized nerve elements undergo atrophy, the tumors are fibrous in character, and they are commonly classed with the fibromas.

In another group there is extensive overgrowth of specialized nerve structures, axis-cylinders, and myelin sheaths, and these tumors are designated as neuromas. Virchow recognized these main classes as false and true neuromas.

In many cases, especially the more actively progressive, there is overgrowth of both nervous and supporting elements, which are difficult to trace in the tumor, so that a complex histogenesis must be recognized, and these tumors do not readily fall in either of the above groups. Moreover, there may be one type of tumor in the skin, mainly fibrous, and others, mainly nervous, in the deeper regions (Genersich, Herxheimer).

All these considerations indicate that neurofibromatosis in all its forms is essentially one and the same disease, and that the underlying factor is a disturbance in the relations between the fetal ectoderm and the tissues which it innervates.

In most of the cases it is the motor and sensory nerves which are involved, in some, to what extent is not yet clear, the sympathetic system is concerned.

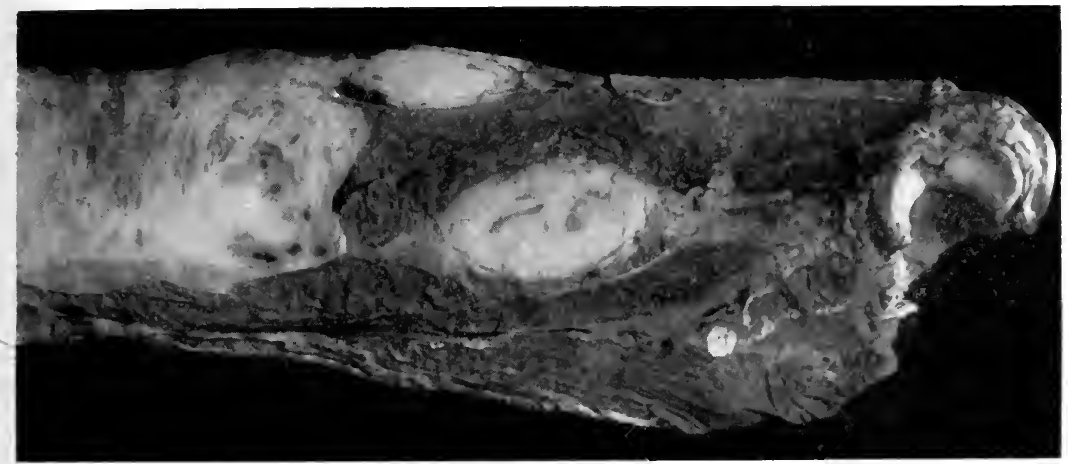

FIG. 27.-Recurrent neurogenic sarcoma of forearm. Diffuse invasion of skin and muscle. A new tumor arising in upper segment of nerve trunk.

It is probable that the great majority of fibromas and fibrosarcomas of the skin and deeper tissues of limbs and trunk are of neurogenic origin.

While theoretical considerations favor the classification of all tumors of nerve trunks and filaments in one group, there are practical advantages in separating the more fibrous growths from those in which specialized nerve structures are prominent.

Adopting, for the present, this latter point of view, the neurofibromas will be described as a type of fibroma, while the others will be considered in a separate chapter in connection with tumors of brain and spinal cord.

Fibrous tumors of the nerve trunks and filaments appear under two main forms:

I. Cutaneous neurofibroma. Single. Multiple (Fibroma molluscum). Recklinghausen's disease.

2. Neurofibroma of the subcutaneous and deeper nerve trunks. Plexiform neurofibroma. Visceral neurofibroma. Neurofibrosarcoma.

Cutaneous Neurofibroma. Fibroma Molluscum.-This most frequent tumor of the skin occurs as a single nodule, more often as very numerous 
projecting nodules, sometimes associated with slight local or general pigmentation. Recklinghausen showed that the tumors arise from the cutaneous nerve filaments, a conclusion which had been suggested by some previous writers and has been fully verified by many later studies.

The peculiar clinical characters of the disease were fully described by Kolliker, I860; Hitchcock, I862; Virchow, I863; Murray, I873; Atkinson, I875; Balzer, I879; and Winiwarter, I876; but Recklinghausen in I882 traced degenerating nerve-fibers in several cases and stated that all these tumors arise from nerve trunks or filaments. An extensive literature has accumulated on this subject and has been reviewed more recently by Bruns, Thomson, Adrian, Sarazanes, Verocay, and Herxheimer and Roth.

In typical fibroma molluscum the tumors are multiple and they may be so numerous as to nearly cover the surface of the body. Very often a single nodule attracts attention, but others may be found on careful search. They may be as small as a pin-head, more often the size of a pea, occasionally they

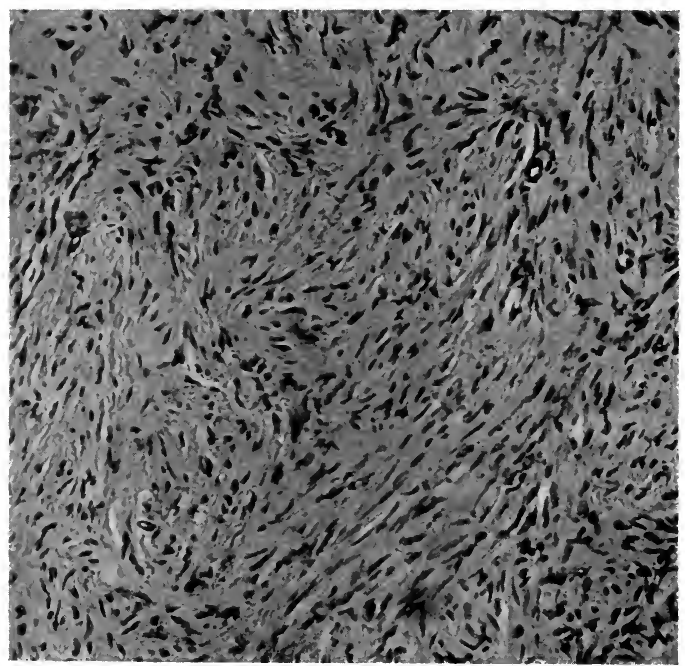

FIG. 28.- Structure of a recurrent neurofibroma of skin.

form broader flat elevations. Developing in the subcutaneous tissue they may reach several centimeters in diameter, and rarely they attain larger dimensions. A few become pendulous, and complete extrusion has been observed. All portions of the integument may be affected from the scalp to the soles of the feet, while in some cases the mucous and serous membranes and internal organs are involved.

In the skin they lie in the derma or just beneath it, where they form hard, movable and often painful nodules, of irregular distribution. Pigmentation of the overlying skin or other regions is often marked, so that the disease is designated as pigmented neurofibromatosis.

Typical fibroma molluscum may be complicated by other forms of neurofibroma or neurosarcoma, as in the cases of Genersich and Herxheimer.

The structure presents interlacing hyaline fibers between which lie few or many compressed tumor cells. The interlacing structure is usually very characteristic. The proportion of cells to intercellular fibers varies widely, 
the older tumors being almost completely fibrous. Nerve-fibers in stages of degeneration and atrophy may usually be demonstrated. The larger tumors frequently involve other structures of the skin, as the sebaceous and sweat glands. They may be quite vascular, and many authors trace a connection between neurofibroma and angiofibromas and nevi. They are sometimes associated with xanthoma (Delore, Poncet) and with multiple lipoma (Vallas, Mouchet). Some early lesions suggest an origin from the connective tissue about sweat glands, but even here the process has been traced to nerve filaments probably belonging to the sympathetic.

The exact nature of the cells involved in neurofibromas is not easily determined and probably not uniform. Herxheimer and Roth conclude that the small dermal tumors are chiefly fibromas. In the larger growths they find that the cells of the sheath of Schwann and of the endoneurium multiply. Concentric groups of cells which are so characteristic of this tumor they interpret as endothelium of the nerve sheaths. In the ganglionic lesions the endothelium of the capsules of the ganglion cells multiples actively. In fact, all the elements of the nerve filament or nerve trunk except the ganglion cells and nerve fibrils participate in the tumor process.

According to Verocay's conception the tissue of neurofibroma is not connective, but consists of nerve cells or their embryonal equivalents which have not been properly employed in the development of the nervous system. He therefore suggests the term "neurinoma." The validity of this view depends on the nature of the cells of the sheath of Schwann. If these are specialized nerve or glia cells Verocay's interpretation would seem acceptable, but it seems probable that this author has chiefly studied tumors which belong with the true neuromas and their derivatives.

Clinical Course.- The single nodules in the skin are of slow growth, may undergo fibrosis, and are usually excised without recurrence. When the nodules are very numerous they cannot well be excised, and the patient often presents other general stigmata of Recklinghausen's disease. Bruns calculates that about 8 per cent. of the cases of multiple neurofibroma are complicated by other phases of the disease and eventually die from extensions of the tumors. These cases belong to a group in which the larger nerve trunks are involved and they deserve separate consideration.

The typical molluscum persists for many years with slow increase in the size and number of tumors, but without notable change in character.

Neurofibroma of the Deeper Nerve Trunks. Visceral Neurofibroma. Neurofibrosarcoma.- In a numerous group of cases the subcutaneous and deeper nerve trunks, and especially the peripheral portions of the nerve trunks, are the seat of single or multiple tumors which are usually more cellular than the cutaneous fibromas, and carry a much more serious prognosis.

In the simplest form the tumor is a firm movable mass lying in the subcutaneous tissue, well circumscribed, of moderate dimensions, slow growth, and often permitting successful excision.

Very often, however, the single subcutaneous tumor is associated with other growths which appear later in the course of the same nerve trunk. Or the single superficial tumor is followed after excision by another growth at or near the original site. The subcutaneous and intermuscular regions of the arm, leg, popliteal space, neck, or back are favorite seats of such multiple tumors with recurrence after operation.

In certain cases the external tumors are associated with multiple small or single large tumors of the deep nerve trunks, which project into the pleural or peritoneal cavities, or externally, and give the impression of being metastatic growths. 
The plexiform or cirsoid neurofibroma occurs in the subcutaneous tissues of face, scalp, buttock, or other regions, and produces a bulky ill-defined swelling in which the thickened nerve trunks may be detected by palpation. On dissection many of the coiled and thickened nerve trunks may be unraveled. The blood-vessels may be abundant and dilated. On microscopic section one finds diffuse and nodular swelling of the nerve trunks due to increase of the cells within the epineurium and to mucous degeneration. The fibrous tissue about the nerves and vessels and throughout the fat tissues may be increased and infiltrated with mucus. Local recurrence is very frequent and sarcomatous changes have occurred in several cases, so that simple

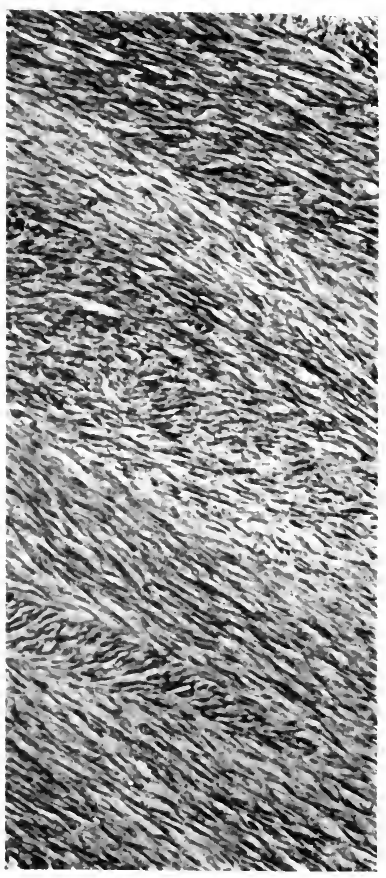

Fig. 29.-Hard recurrent fibrosarcoma of popliteal space. Probably neurogenic. surgical interference is generally contraindicated. Many other peculiarities are illustrated in the cases collected by Recklinghausen and later writers.

There is considerable variety in the structure of the larger tumors of nerve trunks. Some exhibit overgrowth of nerve elements and medullated nerve-fibers, and belong in the class of true neuromas. Most of them are rather cellular but fibrous, and show no reproduction of nerve-fibers, but they lack encapsulation, they recur locally with great persistence, so that the patient eventually loses the limb after repeated excision, or his life from metastatic growths. They are therefore properly designated as neurosarcoma. Local recurrence is observed with some rather fibrous tumors of this class. Or the cells are much more numerous than the fibers and appear as elongated spindlecells arranged in intertwining fascicles, and the tendency to recurrence is more marked. Very cellular growths present larger polyhedral or multipolar cells with little stroma. Giant-cells may be prominent. Myxomatous changes are frequently observed, so that the suspicion arises that most of the recurring myxomas of the skin and fasciæ may originate from nerve trunks. In actively growing neurosarcoma the cells are very numerous, small, spindle or rounded in form, and devoid of definite stroma.

The clinical course of neurofibrosarcoma of the deeper nerve trunks is generally unfavorable. The small tumors may begin to grow slowly and some appear to reach a natural limit. Many of them progress by successive involvement of more central portions of the condemned nerve trunk. A high proportion recur after each operation, with increasing extensions, more active proliferation of cells of more atypical quality, and eventually calling for amputation of the limb. Even wide excision may fail to remove the danger of local return, while new tumors continue to develop higher up the nerve trunk.

The usual postoperative recurrence presents an indurated scar, firmly adherent to an underlying mass which extends widely in and between the muscles, along the fascix, and even to the bone, which restricts movement, is accompanied by much pain, and is quite impossible of excision. 
In such conditions it is difficult to distinguish between local recurrence and the development of new tumors. The cellular growths invade the subcutaneous and deep veins, and produce pulmonary metastases which often reach large dimensions. Such metastases may exist when the local tumor is not regarded seriously or when amputation is advised.

The serious nature of the deeper neurofibromas is very imperfectly realized by most surgeons, so that the number of victims of this disease is much larger than is generally believed. The primary attack on these tumors must be undertaken with great caution. Little aid in their control can at present be expected from the ordinary methods of employing $x$-ray or radium as an adjuvant to surgery. Probably the best plan of treatment is to apply radium or $x$-ray to the limit of skin tolerance, followed by excision of the tumor and the use of radium in the fresh wound. The persistent use of the $x$-ray may effect a cure.

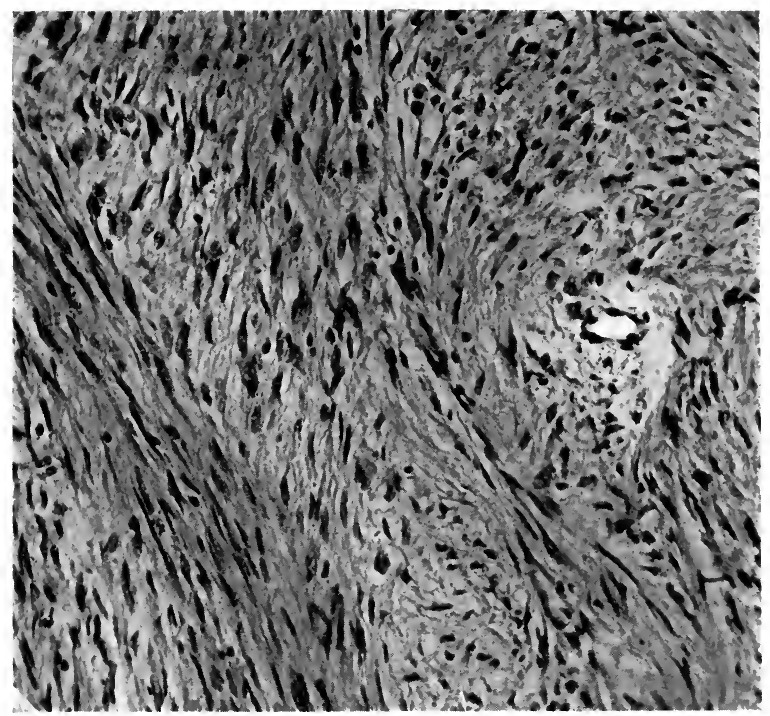

FIG. 30.-Markedly fasciculated structure of a neurosarcoma. Neurofibroma of skin.

Visceral Neurofibromatosis. - The internal nerve-trunks and filaments may be the seat of large plexiform or small multiple fibromas. In the intestinal form the tumors may occur at any point from the lips to the anus. Certain cases of macroglossia have been found to be diffuse neurofibromatosis and related to elephantiasis especially of the lymphangiectatic type (Virchow). Sarazanes has collected cases in which the tumors occurred in the tongue, stomach, jejunum, ileum, colon, vagina, and bladder. In Robin's case there was a large tumor of the solar plexus. Lotzbeck has described several large tumors pressing upon the lumbar spine and arising from the lumbar plexus, and Czerny observed a case involving both lumbar and sacral plexuses. Pomorski describes a remarkable case in which multiple tumors projected into the pleural cavity arising from the intercostal nerves. Herxheimer and Roth describe a remarkable case presenting multiple fibromas of skin of upper half of trunk; fibromas of intercostal and lumbar nerves; small sarcoma of lumbar plexus passing into a large pelvic tumor; tumor-like thickenings of 
terminal filaments of the sympathetic and lumbar intervertebral ganglia; tumor mass with chromaffin cells in adrenal medulla; multiple subserous nodules in jejunum; miliary endotheliomas of spinal dura.

In a well-defined group of cases the sympathetic system is the chief or exclusive seat of the lesions (Czerny, Adrian). In the case of Simon and Hoche the mesentery was much thickened and beset with many fine nodules.

Several cases with adrenal tumors and pigmentation serve to explain the long-suspected relation to Addison's disease (Chauffard, Suzuki, Hoffmann, Kawashima, Vignolo-Lutati). The tumors of the adrenal are composed of medullary chromaffin cells. Gliomas of the central nervous system have been observed in a few cases (Verocay).

Elephantiasis.-It has long been recognized that there exists a close connection between multiple tumors of nerve-trunks and some forms of ele-

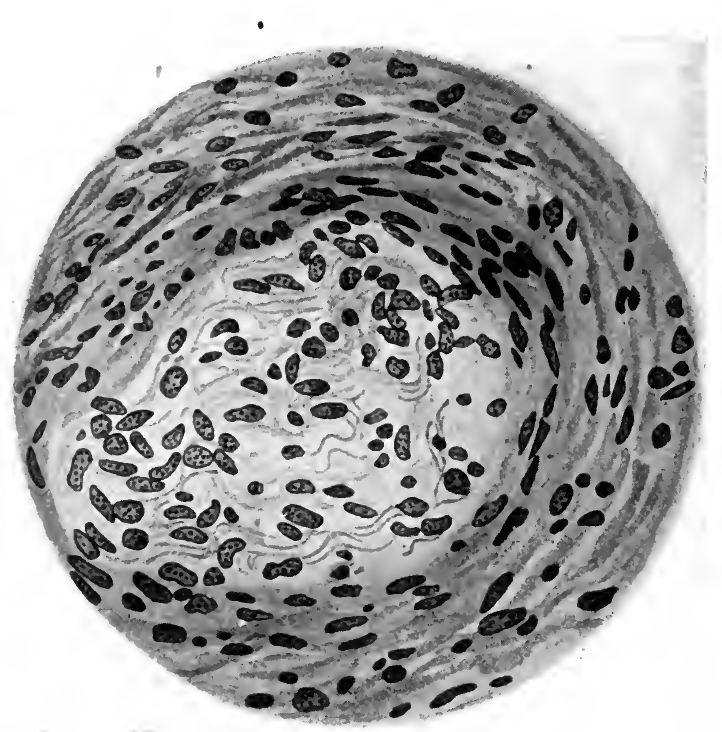

FIG. 31.-Neurofibromatosis. Detail of process in nerve-filaments. In the center, proliferation of sheath-cells; in intermediate zone, endothelial cells; in peripheral area, fibroblasts. (After IIerxheimer.)

phantiasis. The nature of this relation remains ill-defined, but it is strongly suggested by the occurrence of multiple fibromas in many cases of elephantiasis, by the hereditary and congenital character of both conditions, by the occasional presence in both of pigmentary changes in the skin, and by the partial histological resemblance of the structure of the affected tissues. The result of recurrent inflammation and lymph stasis are the two main factors which separate non-parasitic elephantiasis from fibromatosis.

Elephantiasis or diffuse fibromatosis affects the skin over large areas, as of an entire limb, or occurs in more circumscribed form when its neoplastic characters are more distinct. It is acquired and sporadic or congenital and endemic. Beginning early it produces a soft mucinous thickening or in later periods the tissue is firmer or sclerosed (elephantiasis molle and durum). The lymph-vessels are commonly increased, enlarged, or may be cystic (elephantiasis lymphangiectatica). 
Hygroma cysticum (Wernher) is a circumscribed form of congenital lymphangiectatic elephantiasis appearing in the neck, sacral and perineal regions of fetuses, infants, and children. The blood-vessels are sometimes much increased (elephantiasis telangiectatica, elephantiasis angiomatosa). In some remarkable cases the skin of the entire limb was greatly swollen, dark blue, and composed of many thickened varicose veins often containing phleboliths (Rokitansky, Pitha, Leisrink, Unna). All structures of the skin may be involved and there is increase of connective tissue diffusely and about nerves, vessels, and glands. The sweat-glands may be hypertrophic. The epidermis may be thickened, pigmented, and corrugated, and in the remarkable form of congenital ichthyosis it is hypertrophic and extensively hornified. In cases reported by Naegeli and by Morgan elephantiasis was associated with tumor-like thickening of the nerve-trunks supplying the area. In several cases reported by $P$. Bruns the elephantiasis was associated with definite neurofibromatosis. In certain cases usually congenital the thickened skin falls in overlapping folds like drapery (Mott) and for these cases the terms pachydermia or pachydermatocele are employed. Jordan distinguished two types of congenital elephantiasis, one of which is chiefly a diffuse neurofibromatosis, the other involving nerves, other cutaneous structures, and the muscle-tissue. In each case the blood-ressels gave origin to the new tissue.

In most cases of acquired elephantiasis the nerve-trunks play a subordinate part and the process involves all the cutaneous structures, yet in many cases of long-standing neurofibromatosis similar extension of the hyperplastic process is observed. The clinical history indicates that this extension is chiefly owing to recurrent inflammation and lymph stasis. Thus the disease commonly progresses with repeated attacks of erythema or erysipeloid, and it may develop in the course of chronic suppuration. These observations early led to the assumption that elephantiasis was of infective origin and confusion arose between elephantiasis arabum (diffuse fibromatosis) and elephantiasis græcorum, which was true leprosy. Carter has pointed out many points of resemblance between the two types, especially in the nerve lesions, and shown that true elephantiasis may complicate leprosy. Today both leprosy and filariasis may be readily separated from the other causes of elephantiasis.

These clinical relations of elephantiasis as well as the histological structure demonstrate that the process is not a true neoplasm, but belongs in the class of fibromatoid reactions of predisposed subjects to chronic disturbance of nutrition.

Neuropathic papilloma (Gerhardt), Nevus unius lateris (Barensprung), Nevus linearis (Unna), Nerve n€vus (Simon).-This lesion occurs as multiple flat or bean-shaped, warty outgrowths of hypertrophic dermal papillæ. They follow the distribution of definite cutaneous nerves like herpes zoster and may be more numerous along Voight's lines bounding the distribution of the nerves (Philipson). They may occur on any portion of the body and even in the buccal mucosa (Pott). In some cases they are extensively pigmented (Schönberg).

The condition is hereditary (Haegele) and often congenital, but may appear as late as the 27 th year. The onset may be rather acute, as in Beigel's case, but the cuurse is chronic, reaching a standstill or spontaneously regressing (Neumann). Pain may be pronounced, and paresis and paralysis may develop.

The histology shows an acute stage followed by chronic connective overgrowth of dermal papillæ, but the structure varies greatly and there are no 
reports to indicate that the process is a true tumor. In Elliot's case there was cystic distention of the cutaneous glands. The overlying epithelium may be hypertrophic. Recklinghausen states that the growth affects the terminal filaments of the nerves, that it follows neuritis and is related to ichthyosis with which it may be associated. Unna doubts this relation. Although the condition is vaguely defined it appears to represent most clearly the influence of nervous disorder upon fibromatosis.

General Etiology.- In the origin of the foregoing types of cutaneous fibromas many complex factors seem to be concerned, most of which center in the nervous system. Together they constitute a somewhat definite clinical entity or dyscrasia to which the term neurofibromatosis may be applied. The congenital and slightly hereditary character is prominent in all the forms of fibromatosis, suggesting the existence of abnormal qualities in the connective tissues of these subjects. The unfolding of the dyscrasia often proceeds in a somewhat orderly manner. Thus Debove observed pigmented plaques at birth, small tumors of the skin at 5 years of age, and tumors of the nerves at 8 . Yet in most cases the pigmentation and the tumors appear simultaneously at a later age, io to 30 years (Marie). French authors, especially Sarazanes, emphasize the nervous symptoms observed in many cases, and point to the pain, anesthesia, defects of special senses, neuroses, epilepsy, mental disturbances, idiocy, fragility of the bones, and infantilism and malformations, as evidence of general and especially of nervous deficiency in the subjects of neurofibromatosis. Various digestive disturbances, jaundice, urobilinuria, and alterations of nitrogenous metabolism have been cited in support of the theory of general dyscrasia. Feindel and Oppenheim describe marked forms of neurofibromatosis, in which the general symptoms are present with punctate or lenticular pigmented spots in the skin, while the tumors are absent, scanty, or appear later.

For general fibromatosis various theories of origin have been assumed, as the theory of recurrent or chronic infection as suggested by the recurrent inflammatory processes in elephantiasis and its resemblance to leprosy. The theory of auto-intoxication is suggested by many constitutional symptoms and especially by certain cases of neurofibroma which clinically resemble Addison's disease (Chauffard, Bernard). Feindel points out that the pigmentation and tumors may date from pregnancy, and alcoholism and overuse of arsenic have been reported as exciting causes. Brickner has described in pregnancy a peculiar punctate pigmentation of the skin which seems to fall with neurofibromatosis. It is generally assumed that the victims of fibromatosis are the subjects of a congenital malformation of the ectoderm which under a great variety of exciting causes may slowly or rapidly develop one or more of the manifestations of the disease. The great mass of data that has accumulated in this field seems to call for a general assent to this hypothesis, but there is danger that the conception of the scope of this dyscrasia may be extended over too many pathological phenomena.

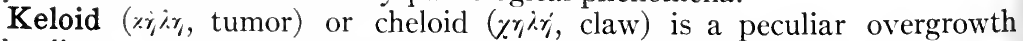
of hyaline connective tissue developing in the skin of predisposed subjects after trauma or scarring. The traumatic origin is usually obvious, as in the extensive scarring of burns. More localized keloids occur in which it is difficult to trace the probable traumatic origin, and these are called spontaneous.

The subjects of keloid may exhibit a special predisposition to overgrowth of connective tissue after injury and in the negro this predisposition is racial. The character of the trauma is diverse. Extensive burns by heat or escharotics are specially prone to develop keloid, but needle punctures (Gumbel), 
scratches, vaccination, repeated pressure of heavy weights, and surgical wounds have preceded the condition.

The typical keloid has a characteristic structure and consists of anastomosing strands of thick, hyaline, collagenous matrix between which lie many well-nourished fibroblasts. Small well-developed blood-vessels are present, and lymph spaces may be so prominent as to lead to the use of the term lymphangiofibroma for some cases of keloid. Elastic fibers are missing. Glandular structures of the skin are pushed aside and this fact leads Goldman to conclude that the growth of the tumor is chiefly central. The periphery is not well defined, but is often more cellular, with thinner fibers than the central portions. Yet this feature is not so pronounced as to deprive the growth

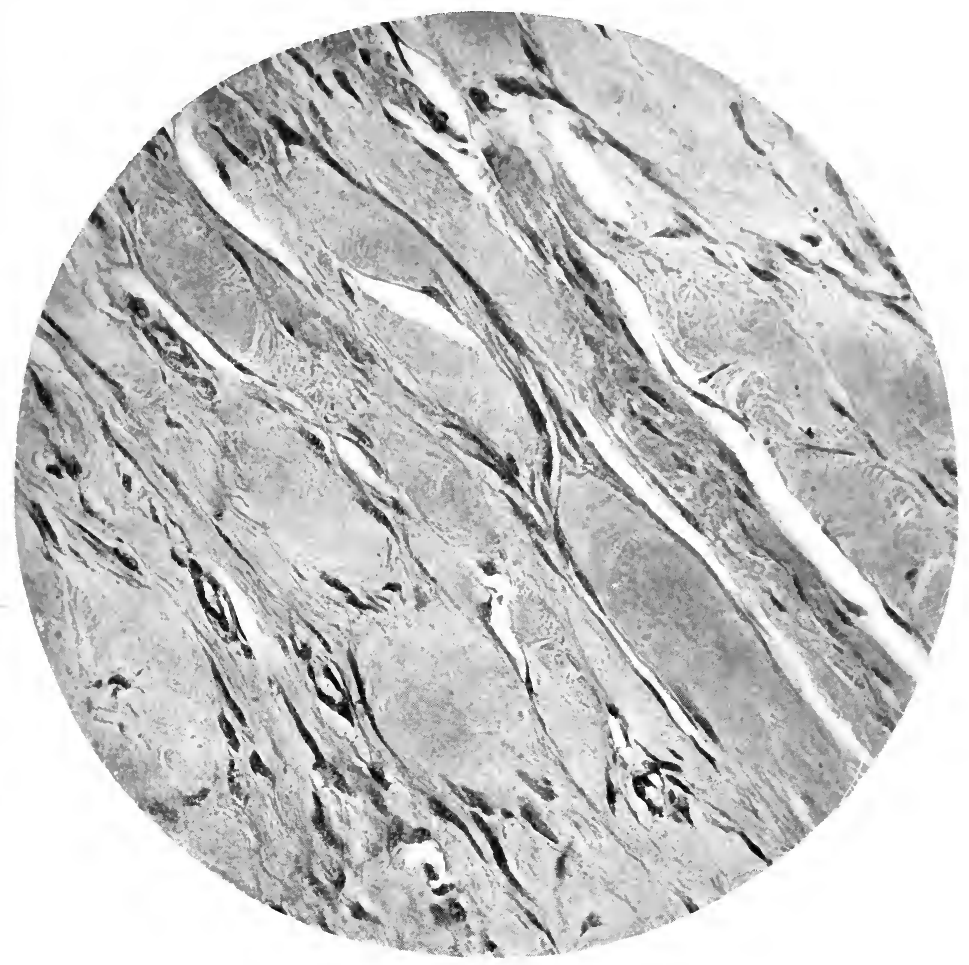

FIG. 32.--Structure of a keloid.

of its circumscribed character or to invalidate the statement of Ribbert that keloid grows largely from its own resources. Secondary changes are chiefly those of increasing hyalinosis.

Keloid occurs in the form of a broad flat elevated firm thickening of scar tissue which gradually extends over a considerable area, or as more circumscribed projecting or even polypoid outgrowths. The overlying epidermis is usually shiny, atrophic, and separated from the tumor by a thin layer of cutis. The natural history is of a slowly progressive growth which tends to involve the whole area of affected scar tissue, or remains limited to the seat of a trauma. After extirpation it often develops anew in predisposed tissues, but never produces metastases. It may be made to disappear under pressure. 
The neoplastic properties in keloid are not pronounced. Especially at its inception it belongs in the group of fibrous overgrowths which depend upon chronic disturbance of nutrition. Yet it sometimes possesses considerable momentum of growth, its cells exhibit nuclear hypertrophy, and especially in the so-called spontaneous circumscribed forms, it may sometimes be regarded as an imperfect neoplasm. The remarkable case of Porter's, in which a very bulky keloid of face and arm developed in ten months after a burn, suggests both the neoplastic nature of the process and a predisposition to the disease.

Xanthoma, or xanthelasma, as named by Wilson and Smith, is a common brownish-yellow tumor of the skin appearing most frequently in the eyelids (92 per cent., Poensgen), but occurring in multiple form in other regions (xanthoma multiplex, generalized xanthoma). It may appear first in the eyelid, later in other localities (Korach) and like multiple fibroma often follows the course of cutaneous nerves, especially along Voight's lines (Kobner), and symmetrically along folds of the skin. Apparently similar growths have been described in the respiratory and pharyngeal mucosa, the serous membranes, endocardium, tendon sheaths, on the cornea, and in the pancreas

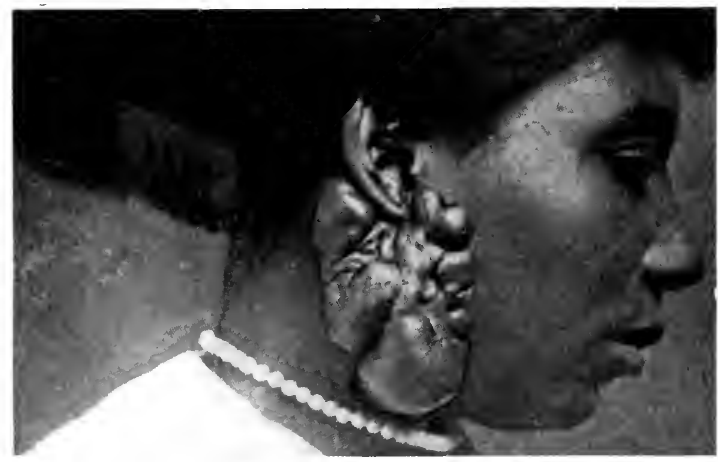

FIG. 33.-Tumor-like keloid following needle puncture. Remained stationary after a few months of rapid growth. (After Brenizer, A. S., 6I.)

in diabetes. According to Unna generalized xanthoma is sharply separated anatomically from xanthoma palpebrarum.

In the skin the growth is flat (xanthoma planum) or bean shaped (xanthoma tuberosum) or polypoid and pendulous. Usually of small size, they occasionally reach considerable dimensions (Poensgen, Ehrmann). They are often congenital, but rather more frequent in adults and old persons. It was shown by Chambard that general xanthoma may develop in the course of icterus or with other diseases of the liver and with diabetes (xanthoma diabeticorum). Not a few subjects of early xanthoma later develop diabetes, yet Johnston and Torok separate the skin affection in diabetes from the common xanthoma vulgare. The diabetic lesion may be transitory, while the other is permanent.

In some cases xanthoma tuberosum is associated with nevi, vascular and pigmented, illustrating a congenital predisposition, and this fact may support Borst's conclusion that the two conditions are anatomically related. Xanthoma may also be associated with multiple lipomas (Ehrmann). Virchow regarded xanthoma tissue as intermediate between connective and fat tissue and employed the term fibroma lipomatodes, believing that the cells were 
derived from fibroblasts. The histological and clinical features point to the existence of a peculiar formation of lipoids in the cells of a fibroma under the influence of a local nervous and a general metabolic disorder. In xanthoma palpebrarum the deposit of lipoids precedes the tumor growth and first appears as yellow streaks along the blood- and lymph-vessels and musclefibers.

The structure of xanthoma is characterized chiefly by the great abundance of lipoid globules in large swollen polyhedral and smaller spindle-shaped cells. This material is an orange-yellow lipochrome; more of it is stained by sudan III than by osmic acid, and Stoerk, with Pinkus and Pick, have showed that the globules are doubly refractive and of myelin nature or probably a cholesterin fatty acid ester. The central cells may become greatly distended with this material, while the peripheral cells may show the spindle shape of growing fibroblasts. Much fatty detritus may accumulate in these tumors and giant-cells similar to those in epulis and containing or surrounding fatty crystals or masses may form (Touton). In such cases the tumor may resemble a spindle- and giant-cell sarcoma. I have seen such a tumor from the peroneal tendon sheath of a diabetic subject who also showed xanthoma palpebrarum.

Waldeyer early showed that the first changes in xanthoma palpebrarum consist in the appearance of fatty globules in certain perivascular groups of star-shaped fibroblasts. Many authors, including Borst, trace the xanthoma cells to the lymph endothelium and he also finds a close structural resemblance between some xanthomas and pigmented nevi. Hallopeau and Torok consider the cells as equivalent to embryonal fat-cells which they may strongly resemble. Waldeyer found many plasma-cells about the vessels of the eyelid and believes that these become infiltrated with fat. Hebra, Wilson, Geyer, and others have observed a proliferation of the sebaceous glands, and Knauss of the hair follicles, sweat-glands, and blood-vessels. Pollitzer in cases of xanthoma palpebrarum traces the fatty cells to degenerating fibers of the orbicularis muscle, and he would separate this lesion from xanthoma multiplex, which he regards as a fibroma or keloid undergoing fatty degeneration. Unna also traces a close connection between the fatty cells of early xanthoma palpebrarum and the muscle-fibers of the orbicularis which lie close to the skin, but he considers the fat to be a deposit from the lymph-vessels.

It thus appears that many different anatomical elements may be concerned in the clinical lesions of xanthoma, and that not all of the clinical forms have a relation to tumors. Some of them, however, are certainly neoplastic and fibromatous. Since the fatty change seems to be identical in nature wherever it occurs there would seem to be room for the term xanthomatosis as applying to this process wherever it occurs and without limitation to a specific anatomical lesion.

\section{FIBROMA OF BONES, PERIOSTEUM, AND FASCIF}

Tumors of this class are comparatively rare, but of very general distribution. From the maxillæ, especially the superior, fibromas arise and reach moderate dimensions, projecting into the mouth with ulceration of the mucosa, or beneath the skin. In the interior of the maxillæ fibromas may develop from disturbances in the formation of the teeth (Blauel). The long bones are occasionally the seat of hard fibroma for which a history of trauma is common. In the fasciæ especially about the joints are encountered hard fibromas which may reach a considerable size. The osteal and periosteal fibromas frequently exhibit areas of calcification, chondrification, and true bone formation, in 
which processes characteristic osteoblasts engage. Bone also forms in fibromas apart from any connection with periosteum. In the large fascial fibromas hyalinosis, calcification, and central softening may occur leading to arrest of growth.

Desmoid Tumors.--This term was given by J. Muller and the older writers to the general class of hard fibromas. It is now used chiefly to designate a group of tumors arising from the rectus abdominis muscle. This condition is of rather frequent occurrence, Ledderhose having analyzed roo cases, and Bodenstein, $2 \mathrm{I}$.

The tumors occur chiefly in women after gestation, between the 2oth and $35^{\text {th }}$ years. Kramer and Greig have described congenital cases. They appear at any point in the abdominal wall between costal border and pubes, and grow slowly until they reach variable but often considerable dimensions, Bodenstein's tumor measuring $24 \times 27 \mathrm{~cm}$. Bouffleur's tumor filled groin

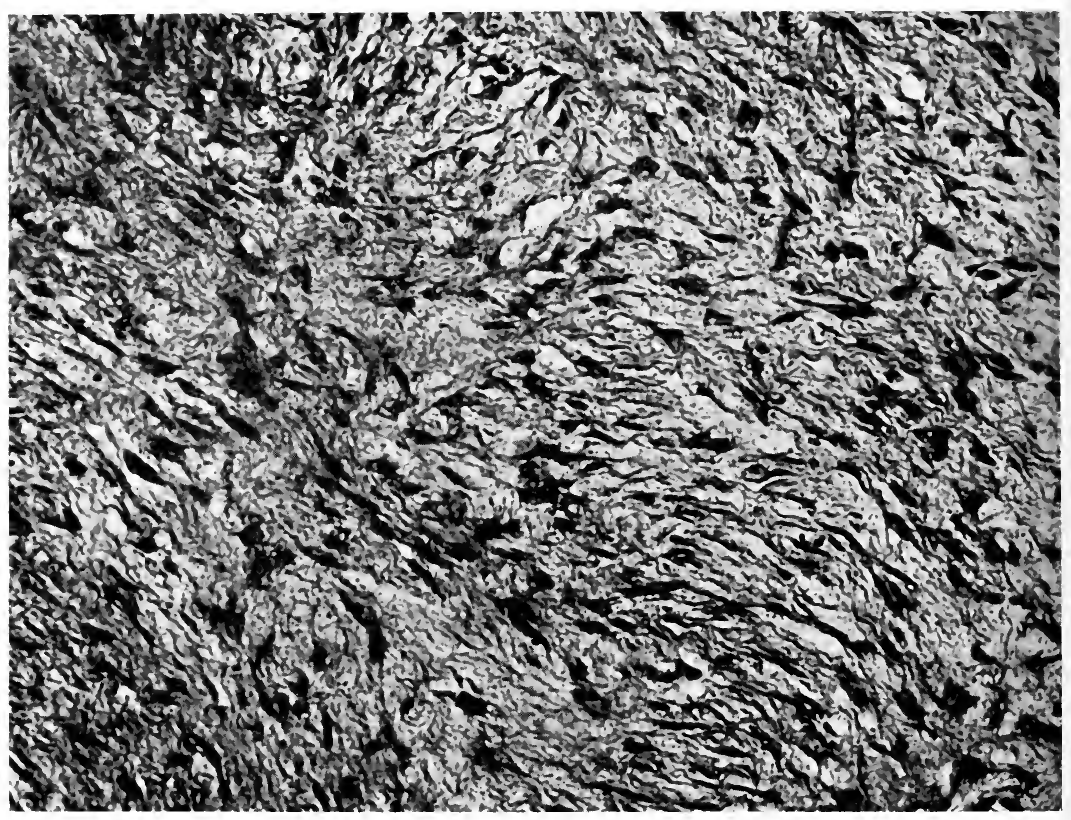

FIG. 34.- Structure of a recurrent bulky desmoid tumor of abdominal wall.

and pelvis and was attached to iliac spine. The growth usually springs from the transverse or vertical fibers on the posterior surface of the muscle, to which it is attached by a broad base or narrow pedicle. It frequently involves a considerable area of peritoneum, the excision of which forms the main difficulty in operation.

The structure varies from a hard acellular fibroma to a rather cellular fibrosarcoma, the latter type exhibiting somewhat active growth. The prognosis is good.

The origin of the tumors in adults, especially after gestation, may probably be referred to trauma of the muscle and to interference with the healing process by constant traction. Yet the congenital cases and those following laparotomy suggest that there is some anatomical predisposition to tumor 
growth in this region. The possible relation to neurofibroma is to be considered.

\section{FIBROMA OF GLANDULAR ORGANS}

Breast.-The fibromas of the breast are usually complex fibro-adenomas and will be considered under epithelial tumors.

Kidney.-Small fibromas of the kidney are of frequent occurrence. They are single or multiple, often bilateral, hard, opaque, homogeneous nodules which project under the capsule, or appear in the cortex or more frequently in the medulla at the bases of the pyramids. Many of them contain smooth muscle-fibers and in the cortex they usually inclose compressed or cystic renal tubules. These small tumors arise from disturbances of development of the kidney. According to Genewein they are not true neoplasms, but tumor-like nodules resulting from superfluous tissue (Albrecht's hamartoma).

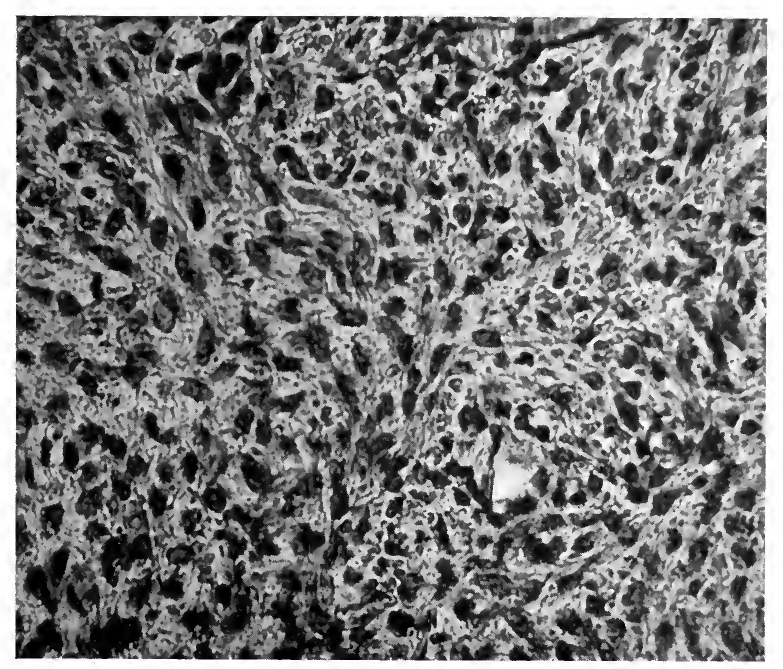

FIG. 35.-Fibroma of ovary.

Large fibromas occasionally grow from the capsule or in the hilus of the kidney, but they are generally associated with fat and smooth muscle-tissue, and exhibit myxomatous changes. Under the term myxoma fibromatosum Tillmann described a large tumor weighing so pounds which he extirpated from a woman of 28 years and which soon recurred. Claus, Wilks, and Busse and Reinach have reported large tumors of capsule, cortex, or hilus of the kidney which were nearly pure fibroma or mixed with lipoma, myxoma, or myoma. In a large tumor of this class I found lobules of nearly pure fibroma, with others of fat and myxomatous tissue. These growths must owe their origin to some developmental disturbances of the kidney, the nature of which is undetermined. Small fibromyomas or nearly pure multiple leiomyomas are not infrequently seen and have been described by Lartigau and Larkin.

Testis.-Pure fibroma of the testis is very rare (Langhans), but I find that tumors chiefly fibromatous have been described as occurring in the tunica albuginea, testis, epididymis, or cord. On close examination most of these tumors disclose evidence of complex composition, as in Chevassu's case. 
They have sometimes reached a large size. Their origin has been referred to the interstitial tissue of the affected locality, but that some of them are related to teratoma is very probable.

Fibroma in the ovary presents several features of interest. (a) The diffuse tumors arise chiefly at the lateral pole and involve much or all of the organ. They are capable of reaching enormous dimensions. A frequent accident is strangulation by torsion of the pedicle. The structure varies greatly in richness in cells, or vessels; from admixture with myoma; from secondary changes toward myxoma, osteoma, chondroma; and from necrosis, and cystic softening. Adler describes an ovarian intracanalicular adenofibroma. Ovarian hematoma may be connected with their origin (Brothers), and a special type develops about corpora lutea (Rokitansky). Others may be of teratomatous nature.

(b) Papillary fibroma appears as multiple warty outgrowths of one or both organs.

(c) Ovarium gyratum is a form of superficial fibrosis of both ovaries which obliterates the cortical structures and transforms the organ into an enlarged, hard, very opaque mass, with wart-like surface. The condition was so named by Adler and is not infrequent.

\section{FIBROMA OF THE NARES. NASAL POLYP}

Although there has been much debate over the relation of nasal polyps to true neoplasms, here as elsewhere it is impossible to draw a sharp line between the products of chronic inflammation and tumor processes, so that there is some justification in the current usage by rhinologists of the term fibroma for these common growths (Bosworth, Lit.). Nevertheless, it is quite clear that in the nares more than in any other mucous membrane the polypoid outgrowths of chronic inflammation lack the histological features of an autonomous new growth. In fact, as Chiari claimed in I887, many of them consist of nothing more than localized edematous areas of mucous membrane rendered protuberant by mechanical means, but without other changes. Once established, however, these masses are subject to various grades of hyperplasia of their elements which render them not only persistent, but often progressive, and in such cases there may be considerable change in the appearance and proportions of various cells. Since this change is seldom pronounced, the groups of nasal polyps must stand among the purest examples of pseudoneoplasms of inflammatory origin.

Nasal polyps are probably always preceded by chronic rhinitis and Tissier traces an unbroken series of cases from simple chronic rhinitis through hyperplastic rhinitis to polypoid inflammatory outgrowths. 'The tumors appear chiefly in young subjects and infants, rarely after 30 years, generally at the ostia of the mucous sinuses opening into the nares. Empyema of these sinuses is a common antecedent. They are commonly multiple, sometimes very numerous, and they occasionally reach a large size, pressing on adjacent structures, and widening the bridge of the nose, with obstruction of passages and various anatomical and clinical sequelæ. Heymann pictures a very large nasal polyp which filled the nasopharynx. They are soft in the early stages, firmer if old, gorged with blood, or gray and translucent from edema, with smooth warty or papillary surface. They may begin as numerous low elevations of the mucosa, but soon tend to become pedunculated.

Histologically three somewhat different varieties are described by Zarniko, fibroma edematodes simplex, adenofibroma edematodes, and fibroma edematodes cysticum. 
Fibroma Edematodes Simplex.-This tumor is composed of loose connective tissue with few or many spindle- or star-shaped fibroblasts. The stroma contains loose collagen and some elastic fibers. The grade of edema varies, but is usually pronounced, sometimes leaving wide spaces, or if slight, as in old cases, yielding rather firm cellular areas. Lymphocytes may be abundant, beneath the epithelium, about the vessels or in small foci. Eosinophile cells, Charcot-Leyden crystals, and even extensive pigmentation may form the sequel of hemorrhages. Nerve-fibers losing themselves in the stroma have been demonstrated by Billroth and by Kalischer. Blood-vessels are abundant, especially in the pedicle, but tend to disappear. In some old cases they are very numerous and cellular, suggesting angioma. Dilated venous sinuses add to the bulk of many cases. Lymph sinuses are less numerous. Areas of calcification and islands of true bone suggest an influence of the neighboring periosteum. The lining epithelium is hypertrophic, ciliated or transitional, or squamous from attrition. The edematous fluid is an albuminous transudate, but may contain considerable traces of mucin. Hyaline globules may also be present.

Adenofibroma Edematodes.-In some polyps new gland formation is prominent and much of the tissue is composed of various slightly distended alveoli lined by several layers of hypertrophic epithelium. Goblet cells may be very numerous. In some cases the epithelial hyperplasia is pronounced, but it rarely suggests a true neoplasm.

Fibroma Edematodes Cysticum.-Most of the large and some smaller polyps owe their bulk to the formation of cysts which arise from distended alveoli lined with overgrowing cells, or if ducts are occluded, with flat cells. The contents are serous fluid, mucus, pus, degenerating epithelium, fatty detritus, or hyaline or calcific deposits.

The derivation of all these features and many other details in the structure of nasal polyps have been fully traced by Heymann, Zuckerkandl, Wright, Zarniko, and many others.

The natural history of nasal polyps is that of a persistent and slowly progressive pseudoneoplasm. They seldom spontaneously disappear, except through accidental expulsion. They are frequently found as a complication of malignant tumors, but malignant transformation of a benign polyp is extremely rare, Heymann finding only 3 somewhat doubtful cases. They do not recur after complete removal, which is sometimes difficult, but new tumors often succeed those removed. In the etiology a special predisposition may possibly exist, but the growths are adequately explained as the result of chronic inflammation and the irritation of retained secretion.

Amyloid Tumors.-In an interesting and rare group of cases extensive amyloid degeneration appears to overtake fibromas, chondromas, occasionally sarcomas, and some chronic productive inflammatory processes, so that the cellular structure is almost completely replaced by bulky masses of amyloid. This condition is observed most frequently in the larynx and nares, but also in the conjunctiva, bladder, and intestine (Herxheimer, Lit.). The process is usually of slow and benign course, but the formation of amyloid may be rather persistent in otherwise harmless conditions.

Choanal Polyp.-Arising from the posterior nasal orifice very cellular firm tumors occur which lack the edema of the intranasal growths, but yet attain large dimensions, reaching into the pharynx or, as in Storck's case, to the larynx. Zaufal removed such a tumor weighing $122 \mathrm{gm}$. Zarniko observed extensive bone formation in one case with marked hyperplasia and metaplasia of the epithelium. Some of these growths show more definite neoplastic characters than the intranasal growths. The choanal polyp often 
originates from the mucosa of the accessory sinuses, maxillary and antral, ethmoidal, or sphenoidal. These tumors are characterized by a tendency to reach a large size, by the presence of a long pedicle, and by recurrence after removal of the tumor without the pedicle. After repeated recurrence it is usually found necessary to open the sinus and attack the growth at the base, and many rhinologists employ the radical method at once in dealing with these polyps (Moore, Syme).

Juvenile Nasopharyngeal Fibroma.- This rare and peculiar tumor occurs chiefly in males and almost invariably between the ages of 10 and 25 years. It is a very firm almost cartilaginous tumor which appears in the vault of the pharynx and grows in several definite directions. According to Bensch it produces an intrapharyngeal tumor when arising from the basilar fibro-

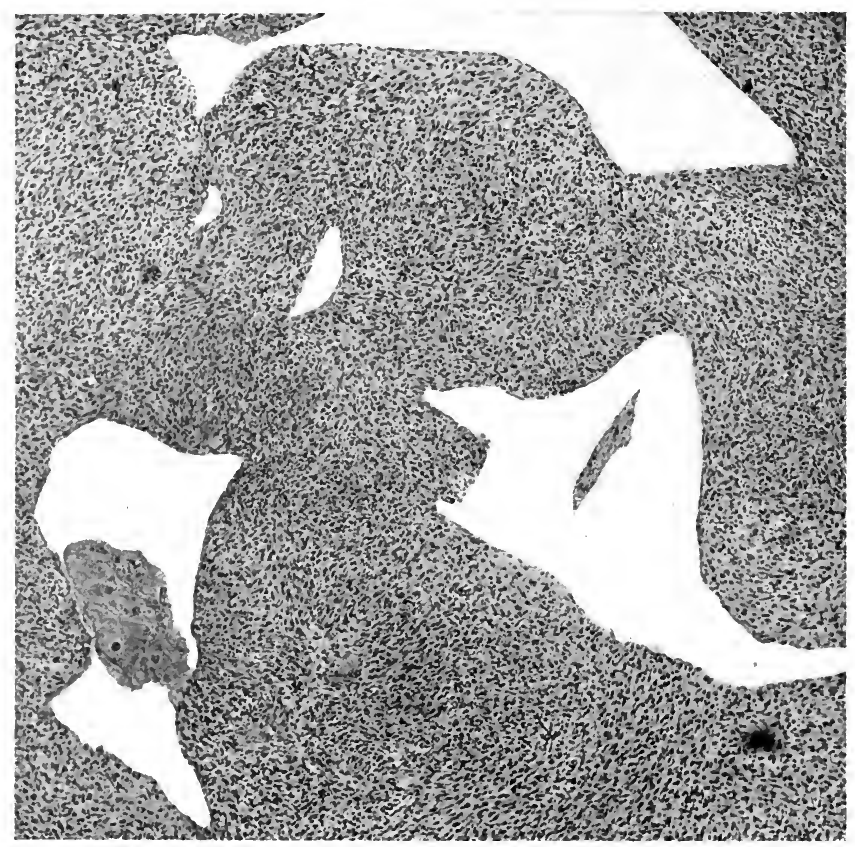

FIG. 36.-Malignant angiomyxoma growing from superior nares in a child.

cartilages, the upper cervical vertebræ, or the internal lamina of the pterygoid process; or an extrapharyngeal growth when arising from the cartilage of the foramen lac. ant., or the sphenopalatine fossa. The intrapharyngeal tumor extends forward into the nares and the adjacent sinuses, causing atrophy of the bony structures. From the sphenoidal origin the growth extends down between the masseter muscle and the mucosa; or it pushes between the pterygoid and styloid muscles into the temporal fossa and forward into the malar region; or through the inferior orbital fissure it extends into the orbit, or by way of the superior orbital fissure or lamina cribrosa it reaches the cranial cavity. From these points the courses from the two seats of origin overlap.

In structure the tumor is composed of dense fibrous and elastic tissue which rarely shows calcification, cartilage, or bone. The cells are round, spindle- or star-shaped fibroblasts which are rather scanty except in certain 
foci of young connective tissue, where they may be so numerous as to suggest fibrosarcoma. Mast cells and plasma-cells may be present. The vessels are numerous (pharyngeal angiofibroma) and sometimes cavernous. Involution changes follow thickening and hyalinosis of vessel walls, the stroma becoming hyaline and the tumor undergoing necrosis or fatty degeneration (Ballo).

The course is of an actively growing tumor which disturbs various functions by pressure, leads to anemia from hemorrhage, suffers ulceration and local infection, and may prove fatal in this way, or from cerebral disturbance. Metastases are not observed. A remarkable feature of this tumor attested by many observers is its complete spontaneous regression after partial removal (Bensch, Grünwald, König, Bruns, Zarniko). This event seems to occur chiefly toward the end of the period when the tumor may develop, $i$. e., the $25^{\text {th }}$ year. There is thus illustrated a form of natural immunity, which may be referred to natural anatomical changes at the point of origin from which alone the nutrition of the growth is maintained. According to Bensch the development of the male and female face and skull at puberty explains the predominance of the tumor in males, while the completion of the cranial development at the $25^{\text {th }}$ year determines the spontaneous disappearance of the tumor. It is not clear that this tumor is always of fibromatous structure. Naab observes that chondroma and sarcoma may appear under much the same conditions. I have seen a tumor corresponding in many particulars to the above description, but showing the structure of a sarcoma with indistinct chondromatous qualities. Confusion with chondroma seems possible. Many of the tumors are myxomatous or myxosarcomatous and these recur persistently and are commonly fatal. 


\section{CHAPTER XI}

\section{MYXOMA}

Myxoma is a tumor composed of mucous tissue.

Primary myxoma, a tumor which probably arises from embryonal mucous tissue, is rare, but mesoblastic tumors, such as fibroma, lipoma, and chondroma, which contain myxomatous portions are not infrequent. It is often difficult to determine whether the myxomatous portion of a complex tumor is originally of this type or represents a degeneration of the more adult tissue. In the former case one employs the terms myxofibroma or myxolipoma, in the latter case the myomatous degeneration is indicated by the suffix myxomatodes, as chondroma myxomatodes.

When mucous tissue is present in a neoplasm three possibilities as to its significance must be considered. The mucous tissue may be developed directly from embryonal mucous tissue and the tumor may be a primary myxoma. Or the tissue may represent a metaplastic product of other tissues and the tumor is a secondary myxoma. Or the tissue may result from chronic edema of other structures and represent a form of spurious mucous tissue, such as is seen in edematous nasal polyps and in atrophic fat tissue. These theoretical deductions cannot always be established in the practical examination of tumors.

Since mucous tissue does not exist in the adult body, a primary myxoma, according to the above definition, must be rare and always embryonal. Fully developed mucous tissue exists in the embryo only in the umbilical cord, and although a few myxomas of the navel have been described which probably arose from cord tissue the chief source of myxomas must be sought elsewhere. Such a source may be found in the early undifferentiated connective tissue of the embryo which has a mucous quality. Such mucous tissue is widely distributed in the embryo, especially in the subcutaneous areas. It is genetically related to fat tissue into which it is extensively transformed during normal growth. It may be assumed that many primary myxomas arise from islands of such embryonal tissue. Since such islands of mucous tissue may readily be associated with cartilage and fibrous and fat tissues, which are normally developed from the embryonal mesoblast, it is reasonable to explain the occurrence of many myxolipomas, fibromas, and chondromas by assuming their origin from islands of tissue which are partly differentiated, or which become so during the progress of the growth. It does not appear that pure myxomas ever tend to differentiate into fibroma or lipoma. It is not so easy to decide to what extent other mesoblastic tumors become transformed into true myxoma. Fibroma, lipoma, and chondroma undoubtedly become transformed by degeneration or imperfect growth into tumors containing areas of typical mucous tissue. The group of secondary myxoma develops in this way.

Virchow considered myxoma to be very closely related to lipoma and as occasionally arising from embryonal fat tissue. Certain myxomas of bone he believed might arise from cartilage. Ribbert, however, regards such secondary changes as spurious myxomas, on the ground that already differentiated tissues cannot become transformed into true mucous tissue. This objection seems to me adequate to separate the primary from secondary 
myxomas, but not to eliminate the latter class from the general category of myxoma.

In the sense that primary myxomas are foreign to the type of tissue in which they arise they may be regarded as always heterologous. Yet this character is not equivalent to that of many other heterologous tumors which arise from tissues widely displaced and genetically unrelated to the tissue in which the tumor occurs.

Myxoma is to be sharply distinguished from epithelial tumors undergoing mucous degeneration. Here the mucus is a form of hypersecretion, while in myxoma it is an integral part of the living tissue.

Anatomical Characters.-Myxomas are soft, lobulated, polypoid, or papillary tumors, which on section are smooth, translucent, and gelatinous. The loculation is determined by the growth about blood-vessels which $\mathrm{v}$. Rindfleisch finds on injection are of relatively large caliber with few capillaries. The encapsulation of myxomas is seldom complete, the mucinous material tending to infiltrate surrounding tissues. On this account complete extirpation is sometimes difficult and recurrence follows from persistence of widely disseminated mucous material and cells. In some cases the conditions suggest a transformation of normal tissues into myxoma under the influence of the mucinous material. Virchow observed the gradual extension of the myxomatous tendency from a recurrent tumor of the ulnar nerve over the greater part of the brachial plexus.

On extraction with weak alkali myxomas yield a solution of mucus which is precipitable by acetic acid. Alcohol precipitates mucus in membranous or reticulated form resembling fibrin.

The structure of myxoma presents typical features of spindle- and starshaped cells with processes anastomosing or disappearing in the matrix, and cytoplasm usually containing fatty and watery droplets. Hydropic degeneration may be extreme in edematous tumors.

The cells may be grouped chiefly about blood-vessels which when abundant may call for the designation "telangiectatic" or "cavernous." Or they may be more numerous in the capsule in cases showing peripheral growth. Various admixtures of connective tissue, fat, cartilage, or bone occur and may deserve suitable recognition in the terminology. Cystic myxoma results from local edema, or hemorrhage, and in one definite group of cases dilated lymph spaces or vessels yield cysts. Very cellular myxomas, pure or combined with other tissues, and primary or secondary, constitute the important group of myxosarcomas.

The matrix of myxomas varies greatly in bulk and in structure. It may be very abundant, widely separating the cells, and appearing as nearly homogeneous or faintly fibrillated or granular, but always basophilic material. In rapidly growing tumors and in cellular areas it may be very scanty. The bulk of the matrix lepends much on the capacity of the mucinous material to absorb water. In the secondary myxomas the gradual transformation of fibrous tissue, fat, cartilage, and bone may often be traced, the matrix of these tissues becoming transformed into mucous tissue and the cells assuming spindle and star shapes. Similar changes may sometimes be observed on the edges of infiltrating myxomas, but the invaded tissue is usually passively displaced by the tumor.

Epithelial and endothelial cells may become incorporated in the advancing myxoma and rendered indistinguishable from the tumor-cells. In some endotheliomas myxomatous changes may be so complete as to greatly obscure the true nature of the growth. The myxosarcomas exhibit many more cells, usually more vessels, and less matrix than the simple myxomas. As a rule 
the mucous tissue is associated with areas of fine spindle-cells often grouped about blood-ressels. In a bulky myxoma of the fascia of the thigh I found that all the spindle-cells could be traced to the walls of very numerous capillaries. This tumor was strictly a myxo-endothelioma. Myxomatous tissue is often combined with fat tissue, cartilage, bone, or other neoplastic elements. When the formation of mucous tissue in such a tumor is the predominating feature the growth may be designated as myxosarcoma, but when mucous degeneration affects only small areas the term sarcoma myxomatodes is employed. The former group constitutes a numerous class of tumors, among which the lipomatous and chondromatous varieties are most numerous. The malignancy of myxosarcoma depends chiefly on its local effects, metastases

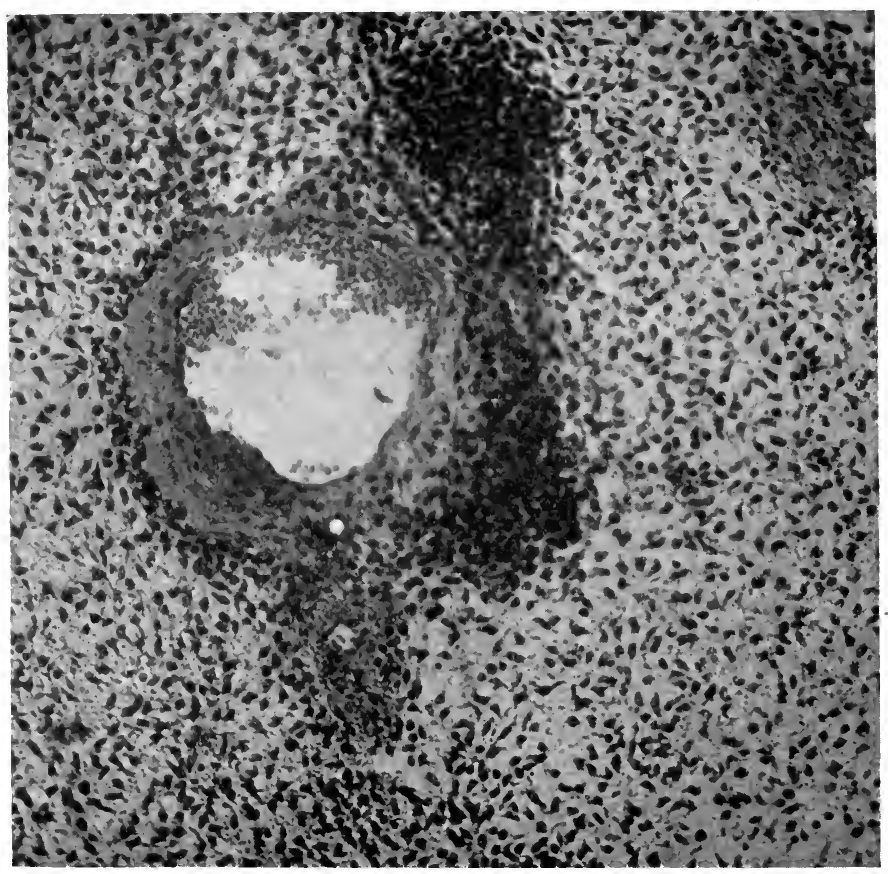

FIG. 37--Recurrent myxosarcoma of nares in a child.

not being very common. According to Malherbe, myxoma of striated muscle, fascix, and of certain viscera is usually malignant and equivalent to sarcoma, while in the nares, breast, bone, and nervous system it is almost always benign.

The clinical course of myxoma is of a slowly growing tumor which produces no symptoms except local swelling and pressure. After complete extirpation they do not recur, but thorough removal is not always readily accomplished and local recurrence of this benign tumor is not uncommon. In the skin and nerve-trunks they are frequently multiple and new tumors develop after extirpation of the old. Yet Virchow refers to 2 cases of myxoma of cheek, and of labium, which recurred after operation and eventually produced many myxomatous metastases. As a rule, malignant myxomas fall readily in the class of myxosarcoma. 
Simple myxoma rarely attains a large size and the onset of myxomatous changes usually marks a partial limitation of the growth capacities of a tumor. Yet the myxolipomas grow to very large size and for some of the diffuse forms of this tumor Virchow suggested a relation to elephantiasis. Such a case affecting the leg and thigh is reported by Barling. In certain situations the pressure symptoms of myxomas become serious (nares, brain, cord).

The location of myxomas is chiefly the subcutaneous and intermuscular tissue of localities where the structure is loose and much fat is commonly present. The regions most affected are the thigh, neck, cheek, leg, and peritoneum, and less frequently, the bones, meninges, nerve-trunks, mucous and serous membranes, and hilus of the kidney. I have seen a myxoma as large as an orange involving three lobes of the placenta.

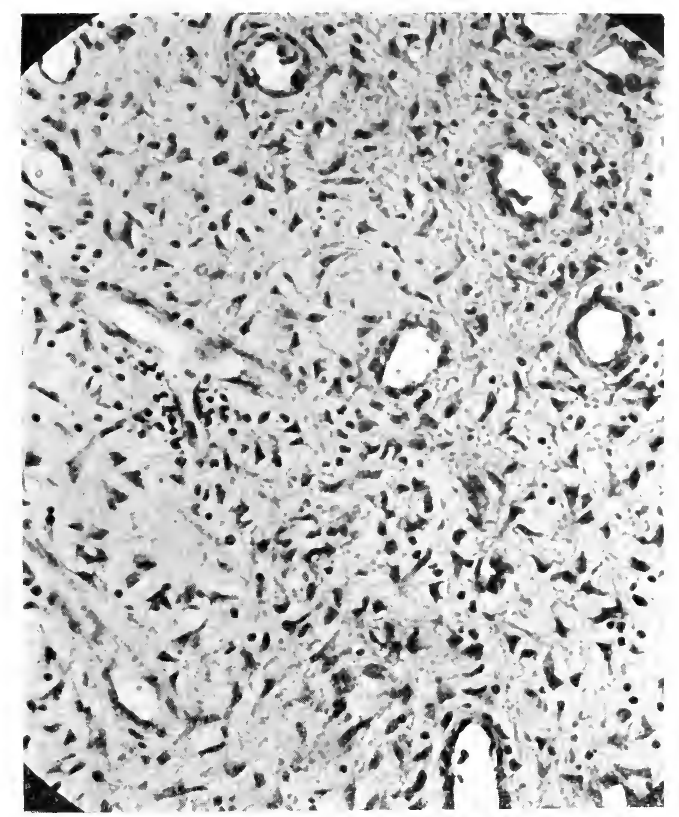

FIG. 38.-A vascular myxoma of superior nares.

Certain clinical forms of myxoma are rather well defined. Myxoma of the nervous system affects the peripheral nerves, meninges, and brain tissue. In the nerve-trunks single and multiple primary myxoma occurs under much the same conditions as multiple fibroma and probably represents a more embryonal form of this tumor. Some of the cutaneous myxomas are probably of neural origin. They arise in the endoneurium and perineurium probably from foci of embryonal tissue, and produce rounded or elongated, soft, but often painful tumors. After extirpation new tumors may develop. Wilms observed a large cystic myxoma of the ulnar nerve.

The optic nerve is especially susceptible to myxoma, which here tends to pursue a characteristic clinical course (Sattler, Parsons, Salzmann). The tumor occurs chiefly in young subjects, produces a fusiform swelling of the whole nerve-trunk, blindness results, but the eyeball is usually not invaded, and extirpation of the tumor is usually successful. In a case studied in this 
laboratory with Knapp the central portions of the tumor were softened and cystic, the nerve-fibers were nearly all destroyed, while the capsule presented the structure of an angiosarcoma. In the meninges several soft myxomas have been described, especially by the older authors, of which Virchow collected a series. In the brain tissue Virchow described as myxoma certain tumors which were probably gelatinous gliomas.

In the mucous membranes polypoid outgrowths in many regions present some of the features of myxoma. They are chiefly edematous fibro-adenomas or inflammatory hyperplasias and contain little or no mucin.

In the bones Virchow described both primary and secondary myxoma. The primary myxoma he believed might arise from mucous connective tissue in the marrow, but he was not sure that it did not develop from some trace

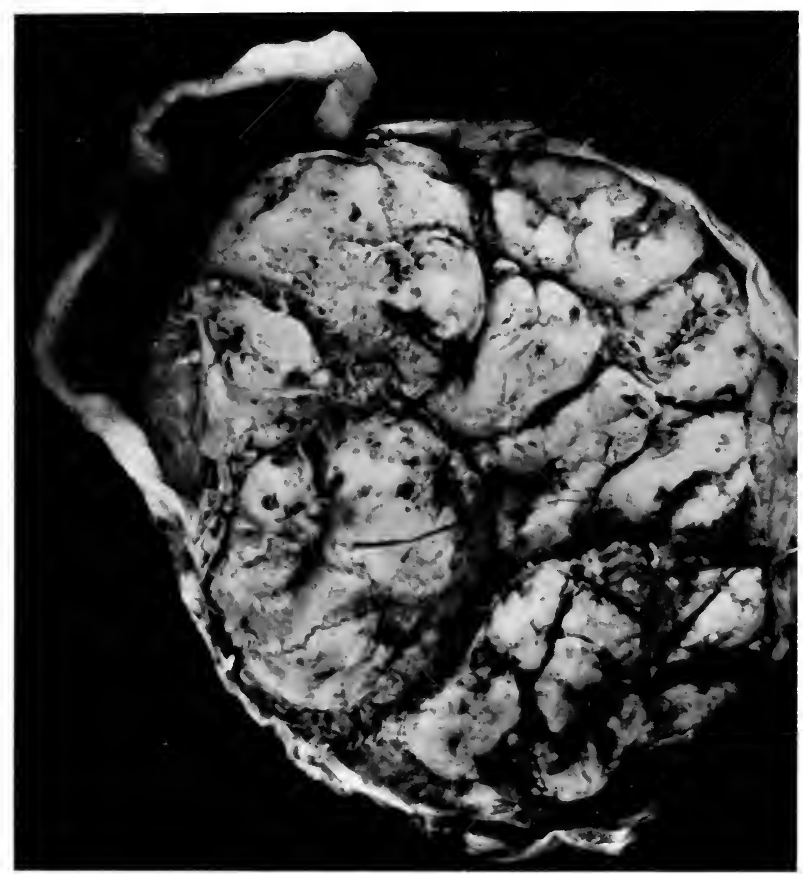

FIG. 39.-Myxoma of portion of placenta.

of cartilage. This tumor absorbs the shaft while the periosteum lays down an enlarging shell of bone, but it may perforate the shaft and periosteum and invade the soft tissues.

The misplaced islands of cartilage found in the marrow cavity in rickets and other cliseases of bone form a ready source of secondary myxoma, and fragments of cartilage or bone have often been found in central myxomas. Portions of benign giant-cell tumors of bone-marrow may present a myxomatous structure, but it is associated with other features of the benign process.

The majority if not all of the extramedullary myxomas of bone must be interpreted as degenerating chondromas or osteomas.

Whatever the origin, a myxomatous structure indicates a tendency to local recurrence. These tumors behave much as do recurring chondromas. 
Without much increase in cellular content they may produce local or distant metastases, especially after operation. Pseudomyxomatous changes in bone-marrow are not infrequent and should not be confused with true myxoma. Virchow warns against including spongy osteomas with mucoid marrow tissue in the category of myxoma.

Myxoma of bone is of rare occurrence, but rather wide distribution. A common seat is in the phalanges of the fingers, in which locality Bloodgood has collected a series of cases. Other locations are the tibia, femur, shoulder, and spine. The rather unsatisfactory results of surgical treatment, and the uncertainties of diagnosis, suggest that they should first be subjected to treatment by physical agents.

Congenital myxoma or myxosarcoma of the navel is described by Kaufmann with the report of a case and review of the literature. I have observed a cystic myxoma of the umbilical cord and navel measuring $10 \mathrm{~cm}$. in diameter, and associated with congenital malformation of the kidneys.

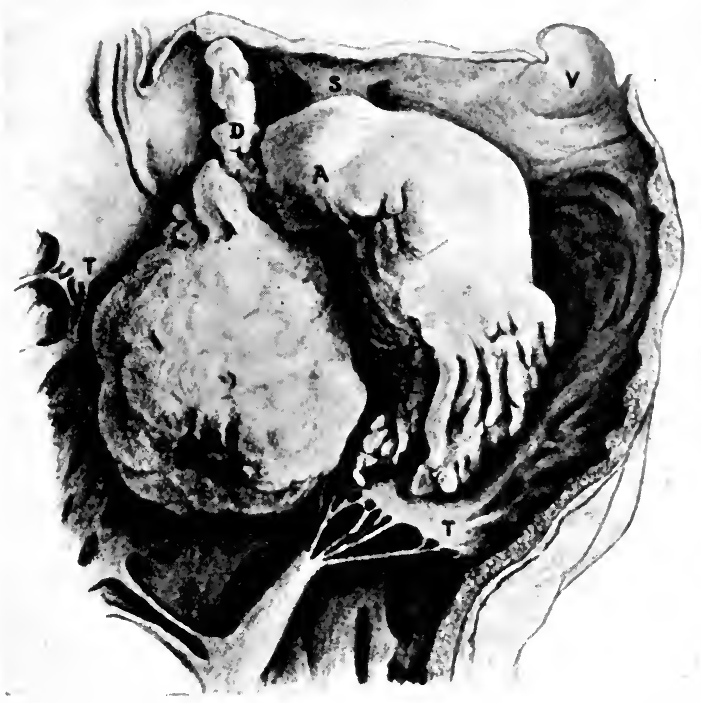

FIG. 40.-Lobulated myxoma of right auricle: $A$ and $B$, Main lobes of tumor; $D$, elongated portion; $V$, vena cava superior; $T$, tricuspid. (After Ribbert.)

Myxoma of the Heart.-The endocardium is the seat of a series of tumors which include small fibromas, larger soft myxomas, and more cellular myxosarcomas. The interpretation of any one of these tumors should be influenced by our knowledge of the entire group.

Brenner collected 33 reports of cardiac myxomas, of which 20 were located in the left auricle, chiefly on the septum, Io on the heart valves, 2 in the right ventricle, and $\mathrm{I}$ on the apex epicardium. The smaller tumors offer little difficulty of recognition, since they usually take the form of typical fibromyxoma. With increasing growth they assume a lobate, papillary, polypoid, or even villous form and the structure is greatly altered by edema, hemorrhage, and progressive thrombosis of blood with organization on the surface. In this form they are difficult to distinguish from organized thrombi. In fact, Czapek, Thorel, and others regard most of the reported cardiac myxomas 
as organized thrombi. The tumors are as small as a pea or as large as a hen's egg, and while they usually produce no symptoms, some seriously obstruct the circulation. They are usually covered with blood-clot, organized portions of which are fused with the tumor. Fibrin emboli may be detached, and Marchand found a growing tumor embolus in a cerebral vessel.

The structure of the tumors is rather uniform, but varies as the original myxomatous tissue is altered or replaced by edema, hemorrhage, perivascular infiltration with round cells, and organization of secondary thrombi. The framework consists of a system of blood-vessels radiating from the pedicle which support the overabundant mucinous material composing the bulk of the tumor. The vessels are usually thin arterioles or venules or capillaries surrounded by lymphocytes or large mononuclear cells. Muscle-fibers may be drawn into the pedicle from the myocardium. An abundance of elastic fibers is a common element which Brenner attributes to the mechanical influence of cardiac contraction upon the tumor stroma. In one of Czapek's cases the stroma was nearly cartilaginous. The separation from organized thrombi may be based on the highly mucinous character of the stroma, on the presence of orderly radiating blood-vessels and elastic fibers, and on the absence of masses of blood detritus undergoing organization. While many of the larger tumors may be difficult to identify (as in Oppenheimer's case) it seems unlikely that a simple organizing blood-clot can reproduce the positive features of the true myxoma. Hence the great majority of the reported cases of cardiac myxoma are probably genuine.

The origin of the tumors has been referred to superfluous embryonal tissue in the region of the foramen ovale where many of the growths are attached. Curtis expressed the view that the process is not a genuine neoplasm, but a result of chronic inflammation of rheumatic nature producing nodular outgrowths of the endocardium which continued to grow because of the mechanical influences to which they are exposed.

Primary sarcomas of the heart are probably connected in origin with the myxomas. Binder collected I $_{5}$ cases, of which 8 were located in the right auricle. They reach large dimensions, grow into the veins, occlude the heart, but rarely give metastases. The composition is chiefly of spindle-cells, but giant-cell and round-cell tumors also occur.

Apparently pure myxomas have been observed in the mesentery (Borst) and in the thymus (Winogradow).

Chondromyxoma may make up the bulk of the mixed tumor of the parotid, and in the fourth recurrence of an original cartilaginous tumor of the parotid I have found pure myxoma. Certain of the nasopharyngeal polyps which are of cartilaginous origin may appear, especially in recurrences, as pure myxosarcoma. In other organs also, as testis, mammæ, and bones, it seems probable that certain myxomas represent imperfect growth of cartilage in embryonal chondromas.

In several notable cases the intramuscular metastases of cartilaginous or choriomatous teratoma testis have appeared in the form of myxoma. In fact, there is reason to believe that the condition of nutrition of intravascular tumors strongly favors the myxomatous type of growth in many tumors.

The combination of myxoma and lipoma occurs in certain tumors of clinical importance and many of these growths reach a large size and prove malignant. In the retroperitoneal region, in the hilus of the left kidney, and in the spermatic cord are encountered locally malignant myxolipomas. In the eleventh recurrence of an original lipoma of the spermatic cord which I was able to follow over a period of I 5 years some lobules were pure myxoma. Robertson, in an analysis of $5^{\mathrm{I}}$ cases of lipoma myxomatodes, found that 
43 per cent. developed in the muscles of the lower extremities, 33 per cent. in the retroperitoneal region; I reached a weight of 65 pounds, and 33 per cent. were malignant.

In the etiology of myxomas special factors must be considered for different groups and an etiological classification becomes of interest.

A congenital origin has been established for myxomas of the navel which probably arise from foci of umbilical cord tissue, and for the mesenteric tumor described by Borst. The general source of myxomas must be sought in superfluous or aberrant foci of embryonal connective tissue, fat, and cartilage. Virchow regarded myxoma as arising indifferently from several embryonal mesoblastic tissues and from glia-tissue. Ribbert would limit their origin rather strictly to embryonal connective tissue. The conditions which give rise to secondary myxoma may apparently represent an embryonal reversion of connective tissue, fat, or cartilage, or it may result from chronic edema and a true mucous degeneration.

Special embryonic disturbances do not seem to be connected with myxomas, although Marchand suggested that abnormalities in the construction of the cardiac septa might be responsible for myxoma of the endocardium. As already stated, an intravascular position favors the appearance of myxomatous changes in a tumor. Chronic inflammation and edema lead to the growth of myxomatoid polyps of mucous membranes. Finally, in the testis, spermatic cord, and possibly in the kidney myxomas are probably of teratoid origin, and in some other regions, as parotid, breast, they represent mixed tumors.

Sanarelli and Splendore have studied a filterable virus which on inoculation produces multiple tumors resembling myxoma in the skin and organs of rabbits. Rous' tumor of chickens which he was able to reproduce by the inoculation of filtered extracts has a myxomatous character. 


\section{CHAPTER XII}

\section{LIPOMA}

A lipoma is a tumor composed of fat tissue.

The gross appearance of most of these tumors is characteristic. They form firm, elastic, rounded, usually multilobulated growths, which, without encapsulation, are sharply circumscribed from the surrounding tissue. Their size varies from that of a pea to masses weighing many pounds which produce serious results from weight and pressure. The consistence is usually that of normal fat tissue, lipoma molle, but this density may be reduced by



FIG. 4I.-Lipoma of forearm in adult, containing fetal fat tissue.

secondary changes, or, more often increased, by admixture with fibrous tissue or by forms of metaplasia. In many situations they become pendulous and by constriction of the pedicle spontaneous atrophy may result. The color is usually that of normal fat tissue, but xanthomatous changes may yield an orange yellow tint, or various secondary processes may give corresponding alterations. Very cellular areas with imperfect fat formation are recognizable by a lighter opaque color, while the fully developed tumor tissue is yellow and translucent. 
Lipomas exhibit a striking connection with the nutrient blood-vessels, each of the multiple lobules growing about a branch of the main vessel. Lateral anastomoses of these vessels are scanty, so that the tumor grows expansively and is readily shelled out of its position. With pure lipomas appositional growth is not observed, but this rule is less rigid for mixed lipoma and liposarcoma.

The microscopical structure resembles normal fat tissue, but the lobules vary greatly in size and the supporting stroma is irregular in distribution. The cells may be overdistended with fat, or may produce the smaller type of embryonal fat tissue, and often there are areas of polyhedral cells in which the fatty deposits are incomplete. It is from such cells lying in isolated foci or along the vessels that the growth of lipomas chiefly occurs. In some of

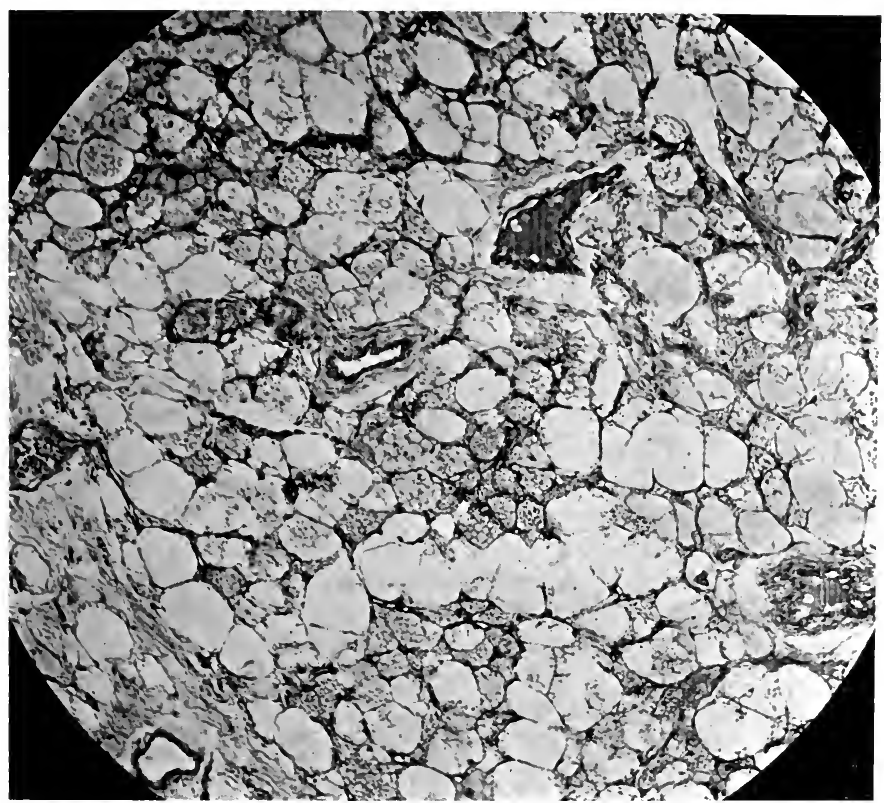

FIG. 42.--Subcutaneous lipoma. Gross texture of orange tint. Lipoid material appears in granular form as in xanthoma.

these foci an alveolar structure may be produced by polyhedral cells with incomplete fat deposits. Occasionally the young cells contain granular lipoid pigment and strongly resemble xanthoma cells, and this character may be diffuse throughout rather large lipomas, especially about the kidney. The blood-vessels are usually overabundant and many cellular arterioles are found in the stroma running out into the lobules and dividing into capillaries. Extensive overgrowth of blood-vessels leads to the formation of vascular fatty tumors, lipoma telangiectaticum or cavernosum, so that it is sometimes difficult to distinguish between vascular lipoma and angioma. Lymphvessels also may be overdeveloped, and Borst observed the transformation of a lipoma into a fibrous lymphangioma.

Secondary changes in lipomas are common in advanced stages of growth. One of the more frequent is a mucinous degeneration which occurs in atro- 
phic or edematous areas and may reach extensive proportions without constituting a true myxolipoma. Diffuse mucinous degeneration produces a peculiar tumor-tissue designated by Müller as collonema. Mucinous changes are more common in liposarcomas, where they usually represent a true myxolipoma. Borst has observed the transformation of the fat in portions of lipomas into a waxy material. Beriel and Delachanal followed the myxomatous and sarcomatous transformation of a lipoma of the sciatic sheath which eventually produced lipomatous and sarcomatous metastases in lymphnodes and lung. Calcification either of isolated foci or of the entire capsule may occur and is rarely followed by ossification (Lipoma petrificum ossificans, Virchow).

Cysts form in lipomas from the fluidification of the central portions of large lobules producing areas of fluid fat (oil cysts) which are said to result chiefly from trauma. In mucinous and edematous lipomas irregular cystic areas may form containing mucous or serous fluid, fatty crystals and calcific deposits. Lymph-cysts with clear fluid are occasionally observed.

Lipomas may exhibit an excess of fibrous tissue resulting from atrophy of fat and fibrous replacement or from admixture with true fibroma (fibrolipoma). More frequently true myxoma is combined with lipoma, and occasionally chondrolipomas are observed. In a considerable class of tumors all the cells are abundant and one has to deal with various types of liposarcoma. In the abdominal cavity of a dog I have observed a large tumor composed of adult fat, embryonal fat, myxoma, chondroma, and large areas of osteoma.

Many interesting features are occasionally observed in the clinical course of lipomas. Some internal growths, as in the thorax, abdomen, or cranium, reach such a size and exert such pressure as to cause serious symptoms or even death, with symptoms referable to the affected organs. Very large lipomas seem to be capable of diverting the nutrition of the body and inducing emaciation. This feature is prominent in many of the retroperitoneal tumors collected by Voeckler. The limit of growth of simple lipoma is often considerable, but in many of the very large tumors portions of the growth become more cellular and exhibit sarcomatous structure. The occurrence of multiple lipomas, even hundreds (Virchow), is evidence of a peculiar dyscrasia of the fat tissues of these subjects, in whom the tumors may appear in the lungs and liver where fat is normally absent. A remarkable case is that of Broca, in which, in a man of $3 \mathrm{r}$ years, after extirpation of a large lipoma of thigh, hundreds of small tumors appeared over the body and persisted for 40 years. The patient finally suffered from dysphagia, with regression of most of the tumors, but at autopsy a large fatty tumor surrounded and compressed most of the esophagus. Persistent local recurrence of lipomas is rare and usually associated with a cellular and vascular or sarcomatous structure. Yet in a case of recurrent lipoma of the spermatic cord extirpated many times in the course of I $_{5}$ years, the structure was never very cellular and varied little throughout the course.

The capacity of the organism to appropriate the fat of lipomas has been investigated by Wells, who found no peculiarity in the chemical composition of the fat of lipomas, no deficiency of lipase, and no ground for the common assumption that the fat of these tumors is beyond the reach of the fat-mobilizing factors of the body. Since many lipomas have continued to grow while the body was emaciating, and not a few remain unaffected during the loss of body fat in phthisis (Madelung) or gastritis (Kuster), it is necessary to assume that local conditions, possibly the character of the circulation, prevented the absorption of the lipoma.

Into the etiology of lipoma many factors seem to enter. An hereditary 
influence was recognized in a family observed by Murchison in which the father and 3 daughters had multiple symmetrical lipomas while 9 sons were free. In Blaschko's case only the male members were affected and the tumors appeared at puberty. Other hereditary cases are recorded by Meerbeck and Petren.

The occurrence of multiple symmetrical lipomas has suggested to many a connection with the peripheral nerves (Payer, Kottnitz). In a group of cases the tumors have been painful and associated with other lesions of the nervous system. Alsberg found several neurofibromas with many lipomas in the same case and he traced nerve-fibers into certain lipomas. Both xanthoma and multiple lipoma have also been observed with multiple neurofibroma. Yet the occurrence of lipomas with multiple neurofibroma is extremely rare and it has not been possible to establish for lipoma such a relation to the peripheral nerves as exists with fibroma. Grosch and others have argued that multiple lipomas may originate in connection with the sebaceous glands, as the result of a trophoneurosis, but later observers have been unable to find support for this theory (Lit. Goebel).

Virchow described as capsular lipoma certain limited but sometimes bulky growths occurring in atrophying organs as the kidney and breast. A similar growth of fat tissue replaces the atrophic thymus and bone-marrow and is very often seen in the atrophic lymph-nodes of cachectic or emaciated subjects and with chronic mastitis. Askanazy was able to show that in several cases of small multiple lipomas the tumors were located in atrophic lymphnodes, and he suggested that this was a common origin of lipomas. The term "replacement lipomatosis" seems more applicable to this process, which may produce fatty growths of moderate dimensions, but not true lipomas.

Multiple lipomas have been observed in many cases of disease or atrophy of the thyroid gland in cases of obesity and in alcoholism, but it does not appear that these constitutional conditions have any direct bearing on the ctiology of true lipoma (Curling, Madelung, Kottnitz). They seem more clearly connected with the forms of diffuse or regional overgrowth of fat tissue which bear the same relation to lipoma as diffuse fibromatosis holds to fibroma. In such conditions disturbances of the thyroid and pituitary glands seem to be an important factor.

A congenital tissue predisposition seems to be an essential factor in the origin of most lipomas. This predisposition may take the form of a disturbance of development of the fat tissue. It has been shown by Kolliker and Toldt that the fat lobules have a certain independence in development which constitute them a sort of primitive organ. The comparative isolation of the blood-supply of these lobules reappearing in lipomas suggests that the anomaly predisposing to lipoma is connected with the distribution of bloodvessels in the fatty tissues.

A more definite embryonic disturbance is probably responsible for the origin of many lipomas of the internal organs, as in the kidney, where misplaced islands of capsular fat are held to give rise to tumors (Selter, Lubarsch, Manasse, Müller).

More complex embryogenic anomalies are concerned with those lipomas which arise in the cranial cavity with cholesteatoma, where complex epidermal tissues are involved (Bostroem); with spina bifida (v. Recklinghausen, Arnold); with a rudimentary cervical rib (Völcker); in the uterus (Merckel).

Certain lipomas result from predominance of fat tissues in mixed tumors. Such is probably the nature of the recurrent lipoma of the spermatic cord. (Sazarin, Ehrendorfer, Porges).

Trauma of many types has acted as an exciting factor with many soli- 
tary superficial lipomas in subjects in which a local or general predisposition must be assumed to exist.

In the general etiological classification of lipoma and lipomatoid processes one must recognize many different forms of hypertrophy of fat tissue as follows: (I) Obesity; (2) localized overgrowth of fat tissue, lipoma anmulare colli; (3) replacement lipomatosis, as in atrophic organs, marrow, kidney, capsular lipoma, lymph-nodes; (4) homologous lipoma, a group including the majority of solitary subcutaneous lipomas; (5) heterologous lipoma from misplaced groups of embryonal tissue cells; (6) overgrowth of lipoma in mixed tumors and teratomas.

Clinical Types of Lipoma.-Subcutaneous.-The subcutaneous tissue is the commonest seat of lipoma, and the back, neck, shoulders, axilla, and abdominal wall are most frequently affected. According to Grosch, who has plated a large number of subcutaneous lipomas, these tumors occur in inverse proportion to the number of glandular structures in the skin. The cutaneous lipomas are single or multiple, unilateral or bilateral and symmetrical, small or large, and polypoid, or pendulated. They arise in the derma, beneath the superficial fascia, and in the deeper fascias.

Small congenital lipomas occur beneath the skin on the volar side of the fingers and hands. They arise from the deep fascia and may be connected with the tendon sheaths.

Lipoma annulare colli is a diffuse form of lipomatosis occurring in the neck, producing great enlargement of this region, and involving the fat tissues of the skin and subcutaneous and intramuscular structures (Madelung). It is not a true lipoma, but a localized overgrowth of the abundant fat tissues of this region. Somewhat similar overgrowths of fat tissue may occur in the hips and thighs (Shattock). More general hypertrophy of fat tissues is seen in adiposis dolorosa (Dercum). Subcutaneous lipomas often reaching considerable size occur in the scrotum and labia.

Synovial.-Lipoma and lipomatous processes affect the joints. Solitary lipoma may arise within the joint cavity through rupture of the synovial membrane and protrusion of the subcapsular fat which then goes on to continuous hyperplasia (König). Solitary lipomas may also arise in the joint from overgrowth of fat in synovial fringes (Otterbeck, Filter). Extra-articular lipomas arising from the subcapsular fat occur about the knee and hip. Lipoma arborescens is a characteristic extensive papillary outgrowth from the fat tissues of the synovial membrane and joint capsule of the knee which fills the joint spaces and interferes with function. It occurs with simple chronic and with tuberculous arthritis in which case it is probably of inflammatory origin with overgrowth of synovial fat (Kaufmann), but it occurs apart from any inflammatory processes as a true tumor of pure fat tissue in which the characteristic relation of lobules to branching subcapsular blood-ressels is observed (Borst, Ribbert).

Intermuscular.- In the cheek a congenital lipoma arises from a mass of fat in the canine fossa, on inner surface of masseter muscle, long known as corpus adiposum mala (Bichat), pushes its way beneath the skin and appears as a subcutaneous tumor of moderate dimensions. Several tumors of this origin have been collected by Bruns and Ransohoff. They may readily be mistaken for tumors of the parotid with which they are in intimate connection.

Beneath the pectoralis lipomas arise probably from extensions of the axillary fat which cause protrusion of the breast (Billroth). Deep lipomas are also observed in the orbit, at the base of the tongue, and in the larynx. Grosch has collected many lipomas occurring about the cranium.

Cranial.-In the cranial cavity lipomas have been observed in various 
situations in most of which small collections of fat tissue are normally present. The chief locations include the surface of the corpus callosum, the base of the cerebrum, the brain stem and cerebellum, the ventricles, and the roots of the cranial nerves (v. Sury, Lit.). They invariably arise from the pia, although many appear to be embedded in the brain tissue. Taubner argued that they may arise from the glia-tissue.

Virchow mentions 6 lipomas of the pia, I of the raphé of the corpus callosum, and 4 at the base. In the choroid plexus and nourished by its vessels they have been observed by Virchow, Wallmann, and Hackel. Rindfleisch saw small multiple lipomas of dura and ventricular ependyma. Fere and Francillon report symmetrical lipomas. Garnier's lipoma was attached to the crus cerebri. Small lipomas of the corpora quadrigemina are reported by Bernhard and Taubner. The most characteristic type is the lipoma which is nourished by the vessels of the corpus callosum, grows along this structure, and largely replaces the nerve tissue (Virchow, Benjamin, Coats). Perineural lipomas inclose the roots of the cranial nerves or olfactory bulbs (Shouppe). A central nucleus of bone has been found by Benjamin, Chiari, Ernst, and v. Sury.

Many of the tumors are small and fail to produce symptoms, while not a few reach sufficient bulk to cause atrophy of adjacent nerve tissue. Weil removed at operation a lipoma, ro $\times$ ro $\times 4 \mathrm{~cm}$. from the temporal fossa. Wurth's lipoma of the basal pia extended into the left hemisphere and caused epilepsy and hemiplegia. From the studies of Bostroem, who collected 28 cases, it appears probable that most intracranial lipomas originate from epidermal inclusions, although no dermal elements have been found in them. Nippe found a lipoma of the parietal lobe surrounded by gliosarcoma which he attributed to trauma.

Lipomas may develop in connection with defective development of the spinal canal and meninges. A series of tumors, some of which were pure lipoma, others composed of fat, fibrous tissue or cartilage, have been observed in connection with spina bifida and with scantily developed meningocele (v. Recklinghausen). Arnold's hairy fibrolipoma of the scalp appears to have originated from imperfect closure of a cranial suture.

Renal.-In the kidney small circumscribed multiple lipomas occur beneath the capsule or replacing a portion of cortex, or reaching to the medulla. The structure of these tumors varies. Some of them are composed of pure fat tissue; others contain considerable fibromatous tissue, so that Virchow and others have spoken of them as fibrolipoma. In either case the light yellow translucent color seems to distinguish them from the more common struma suprarenalis of Grawitz, which is opaque and of an orange tint. In not a few cases smooth muscle-tissue has been found in such tumors and Selter, Lubarsch, and Manasse have described them as myofibroma; while in other cases sarcomatous features are observed as in the case of Muiller's (myoliposarcoma). I have observed a very large liposarcoma of this type, in one lobe of which xanthomatous changes were pronounced. Borst and Selter describe symmetrical lipomas of the kidneys chiefly of the upper half of the organ. In Borst's case double myolipoma was associated with fibromuscular tumors of the same portions of both kidneys. All these features point to a congenital and embryonal origin of these tumors but the exact nature of the embryogenic disturbance is undetermined. That they may be connected in some way with Grawitz's struma suprarenalis is indicated by the presence of adrenal tissue in Müller's case, and by the occurrence of curious, complex, chiefly liposarcomatous tumors of the kidney, in one of which I have observed areas of perithelioma mingled with liposarcomatous 
tissue. Somewhat similar myolipomatous growths occur along the spermatic cord.

Partial or complete replacement lipomatosis of the kidney has been observed in a series of cases reported by Lacrampe-Loustan. Rayer and Epstein have described such cases in which no trace of renal tissue remained, while the intact capsule of the organ inclosed pure fat tissue. Selter called attention to the fact that in most of these cases a single large calculus occupies the renal pelvis. The proliferation of fat begins in the pelvic adipose tissue, and follows atrophy of the renal parenchyma.

True pelvic lipomas probably develop from such capsular tumors which early project into the cavity. Warthin described a very large pedunculated intrapelvic growth over which the mucosa and atrophic kidney tissue was widely stretched, while a tongue-like mass extended several inches into the ureter.

Perirenal lipomas containing connective tissue, mucoid areas, and often sarcomatous areas occur in infants and adults and may reach very large dimensions. In advanced stages they pass as retroperitoneal lipomas. Lauwers describes a tumor arising at birth and reaching a weight of 6 pounds at 7 years. Windle's liposarcoma weighed 50 pounds. Adami described 2 very large tumors composed of adult fat tissue with cellular stroma. The growth is usually slow, but with sarcomatous structure the progress may be rapid and recurrence is frequent.

Gastro-intestinal.-In the gastro-intestinal tract lipomas are of rare occurrence. They arise from the submucosa and from the appendices epiploicæ of the colon. Dewis collected 44 cases in 9 of which the tumor was expelled spontaneously, while intussusception occurred in $2 \mathrm{I}$. The subserous lipomas may be worked loose in the peritoneum. Ehrlich has collected 52 cases of intestinal lipomas. In the gastric submucosa small fatty tumors may occur. I have observed a large fatty tumor $4 \times$ ro $\mathrm{cm}$. surrounding the appendix which was the seat of chronic suppuration. Very large retroperitoneal lipomas have been observed. (Madelung, Waldeyer, Adami).

Mediastinal.-In the mediastinum localized overgrowth of fat tissue is not unknown, especially in alcoholic and obese subjects. True lipomas of the mediastinum may reach large dimensions and cause pressure symptoms from the thoracic organs. Arising from many different points in the subpleural fat, mediastinal lipomas may project into the pleural cavity or along the intercostal spaces (Rokitansky). Or the growth may perforate the chest wall and appear externally in the back (Czerny), beneath the breast (Gussenbauer) or on the front of the chest (Cruveilhier, Conner). In the writer's case the tumor encircled nearly all the structures in the thorax. In Fitz's case there was congenital hypoplasia of the left lung. Carless observed a large lipoma of the thyroid region of which the pedicle was traced into the mediastinum and which was associated with other multiple symmetrical lipomas. I have seen one mediastinal liposarcoma with tumor fragments in sputum and metastasis in deltoid muscle.

Cardiac.- In the heart lipomas have been described by Ribbert, Orth, Petrocchi, Spalty, Hagedorn, and others. They may originate in misplaced islands of epicardial fat tissue. Dittrich's tumor was congenital.

Uterine.- In the uterus Orth has observed a polypoid lipoma and Werkel 2 intramural lipomas, while lipomatous areas of mixed tumors of the uterus are described by Gebhart. Pollak thought his intrauterine lipoma originated from a portion of omentum protruding into a wound of the uterus.

Myelogenous.-A true lipoma of the marrow of the fibula in a young girl has been recorded by Wehrsig. 


\section{CHAPTER XIII}

\section{CHONDROMA}

Localized overgrowth of cartilage occurs in several forms between which it is sometimes difficult to draw sharp distinctions. Limited outgrowths of preëxisting cartilage occur on the ribs, in the larynx, and about joints, which exhibit the characters of a simple hyperplastic process, and are called ecchondroses. True progressive neoplasms composed of cartilage appear in the same situations and also in tissues not normally containing cartilage, and these are called chondromas or enchrondromas. That many ecchondroses possess some of the properties of tumors is indicated by the occasional occur.rence of large chondromas as a sequel of ecchondrosis. Thus chondromas as large as an apple occur at the chondrosternal junction. In a case of Weber's there was striking symmetry in the location of multiple chondromas and ecchondroses.

Virchow classed as ecchondroses the smooth diffuse outgrowths of permanent cartilage, and as enchondromas the large circumscribed lobulated tumors of transitory cartilage. Between these main classes are many intermediate forms which display more or less clearly the neoplastic qualities.

The ecchondroses are usually small, multiple, smooth and nodular, or diffuse outgrowths of preëexisting cartilage. After reaching a certain stage their growth tends to culminate, often with the natural growth of cartilage in the body, after which they may remain quiescent or undergo secondary changes, chiefly calcification or ossification. Amyloid deposits may also occur.

In structure the ecchondroses copy normal hyaline and fibrocartilage according to their points of origin, and show few of the atypical features of true chondroma. They are usually surrounded by normal perichondrium, from a portion of which, according to $\mathrm{v}$. Rindfleisch, they originate.

Ecchondroses are not extremely common, but rather widely distributed. The most familiar examples occur at the costochondral junctions, where they appear as smooth multiple nodular swellings which tend to calcify or ossify. Virchow and others believed that these and most other ecchondroses were a sequel of rickets and this origin probably applies to the ecchondroses arising in early life and soon tending to calcify.

A well-known form of ecchondrosis appears on the inner surface of the symphysis pubis, and may reach such dimensions as to obstruct labor (pelvis spinosa). About the epiphyses of any of the long bones small nodular outgrowths of cartilage may appear.

Localized cartilaginous outgrowths of the cricoid and thyroid cartilages have been described by Virchow and many later writers. They project inward with deformation of the larynx, but with rare exceptions do not obstruct breathing. The entire cricoid cartilage may be diffusely thickened by multiple ecchondroses. From the upper and lower surfaces of the tracheal rings multiple cartilaginous nodules may develop. They are connected with the rings by strands of fibrous tissue of perichondrium, and they may become so numerous as to cause fusion of many adjacent rings (Virchow, Recklinghausen, Mischaikoff). Ecchondroses usually of small size develop from the intervertebral discs and project outward or into the spinal canal. 
In the joint cavities multiple ecchondroses are a frequent result of chronic arthritis with osteochondritis. The modes of origin of these growths and the manner in which they become detached, forming joint mice, have been exhaustively discussed by Virchow.

Enchondroma.- The true chondroma is a rather common tumor, the varied features of which, perhaps more than any other benign tumor, illustrate the peculiarities of neoplasms. This result is perhaps dependent upon the facts that cartilage is essentially an embryonal and transitory tissue, and that cartilage cells although encased in a firm matrix have rather active proliferative powers, possess ameboid properties, and are readily subject to metaplastic changes. For the most striking display of these characters one must pass to the tumors of lower animals with which chondromas are more common than with man.

The chondroma produces a hard, rounded, lobulated tumor which often reaches large dimensions. The lobulated structure is referred to the expansive growth from multiple centers, as a result of which the surrounding tissues are pressed aside. In this way large tumors may form, composed of convoluted masses resembling the convolutions of the brain. Such growths are commonly encapsulated by perichondrium and are cartilaginous throughout at all stages. Other chondromas are imperfectly encapsulated, encroach upon surrounding tissues as a malignant tumor, and invade blood-vessels. In such cases one finds many stages of the formation of the perfected cartilage from more cellular tissue, the nature of which is not always clear. The nutrition of the solid chondromas is maintained by a system of lymph spaces from the periphery. Rindfleish and Borst have traced a rich system of lymphatics from the periphery of a submaxillary chondroma and communicating with the pericellular spaces. Through most of these growths there are irregular bands of connective tissue carrying blood-vessels and this feature becomes pronounced as the tumors tend to calcify or ossify. About many chondromas there is an excessive development of blood-vessels to which Virchow and v. Recklinghausen ascribe an important and primary influence in the development of the tumors. Such growths have been termed angiochondroma and a few of them have been deeply pigmented by diffusion of extravasated blood pigment (Siegert).

The capsule of subperiosteal chondromas may consist chiefly of the thickened periosteum which may go on to produce bone, and a bony capsule may surround chondromas developing in the marrow cavities.

While most chondromas are solid, many become softened by mucinous degeneration, and cysts filled with mucinous, serous, or fatty material may form. The skin over a chondroma may atrophy, and ulceration, excavation of portions of the tumor, and suppuration may be established. Although the chondromas are usually localized and benign they sometimes grow extensively in the blood-vessels, filling the lumen with nodular or solid masses and extending over wide areas. In a case reported by Ernst a chondroma of the lumbar spine invaded many of the abdominal and pelvic veins, traveled up the vena azygos and vena cava, filled the right ventricle and continued into the pulmonary artery. Discontinuous metastases also occur especially in the lungs, occasionally in the lymph-nodes.

The multiple character of certain chondromas has been a striking feature, and in some cases nearly every bone in the body has been affected. In the notable case reported by Recklinghausen the hands, feet, knee, elbow, and ribs were the seat of very numerous chondromas.

The location of skeletal chondromas is usually in the diaphyses of the bones near the epiphyses. They may project externally, pushing outward 
the periosteum, or growing in the medullary cavity they distend the cavity and are surrounded by the thin bony shaft.

The structure of chondromas reproduces that of the various normal types of cartilage, chiefly the hyaline variety. Ranvier classified chondromas, according to their structure, in four groups. (I) A single lobe of hyaline cartilage. (2) Several lobules of hyaline cartilage separated by fibrocartilage. (3) Fetal cartilage. (4) Cartilage with stellate cells. The cells may be more or less numerous than in normal cartilage. They vary greatly in size and usually lack the orderly arrangement into groups of cells with opposed surfaces flattened. Peculiar vacuoles are common in these cells and were described by Virchow under the term "physaliden." The cells are usually rounded and lie in distinct spaces, but with imperfect formation of chondrin matrix they become stellate with mucinous degeneration, fusiform as they approach the connective-tissue type, calcific granules may be deposited in them, and in some cases they show all transitions up to the bone cell in osteoid and osseous areas. The cells usually contain glycogen and fat granules and wellmarked fatty degeneration may occur.

The growing cells of a chondroma are not, as a rule, those inclosed in the matrix, but rather those on the periphery of the tumor. The matrix of the chondroma is usually hyaline, but may be fibrillated or may contain elastic fibrils. Ernst noted very active production of the elastic fibrils in a rapidly growing tumor. The matrix is not an integral part of the cells, but a product which is deposited under cellular influence (Ribbert). The chondrin is usually deficient and irregularly distributed and various types of secondary change are frequent.

Calcification with the deposit of phosphate and carbonate of lime affects first the matrix, producing an irregular network of densely basic staining material which is composed of granules compacted together and becoming homogeneous. The borders of the cell spaces may be first affected and from this point the deposit appears in the cells, so that in advanced stages both matrix and cells may become heavily incrusted. In such areas the cells assume a stellate form approaching the character of bone cells.

Ossification of chondromas occurs under several conditions. An imperfect formation of islands of bone may take place at different points throughout chondroma, this change being preceded by increased vascularity of the septa, and by calcification and final ossification.

More often the connective tissue in and about the chondroma having first laid down cartilage goes on to replace it by trabeculæ of well-formed bone surrounded by typical osteoblasts. In this process the normal events in the production of bone in cartilaginous matrix take place.

About central chondromas which invade the shaft of a bone the periosteum may be incited to the production of a bony capsule which is of inflammatory origin and not a part of the tumor.

In many chondromas the formation of cartilaginous matrix is imperfect and a simple hyaline or partly fibrillated or mucinous material takes its place. This imperfect chondrification is much more common than any form of softening of chondrified matrix. In the soft matrix the cells may attain large size and lie in very large round spaces, or, in myxochondroma they become stellate and the matrix fibrillated. Some of the soft chondromas are composed of a shell of perichondrium from which spring papillary fringes of poorly chondrified cartilage, while the central portions are filled with mucinous fluid (cystic, papillary chondroma).

Distinctly malignant cartilaginous tumors, chondrosarcomas, occur in several forms. Medullary chondromas may perforate the shafts of long 
bones and destroy the epiphyses, proving malignant from mechanical factors. Infiltrating chondromas in which very cellular peripheral portions invade surrounding tissues and produce nodules of cartilage are properly called chondrosarcoma. There is a considerable group of chondromas occurring in mixed tumors and teratomas, which are usually very cellular, containing other tissues besides cartilage, and which invade tissues, lymphnodes, and blood- and lymph-vessels. Invasion of veins is not confined to the embryonal or teratoid chondromas, for in Virchow's case of tumor of fibula and in Ernst's case of fibrochondroma of spine there were extensive intravascular growths, while the pelvic chondromas in several cases have invaded blood-vessels and lymphatics (Foerster, Weber, Biesiadecki). In this group the malignant properties are often out of proportion to the cel-

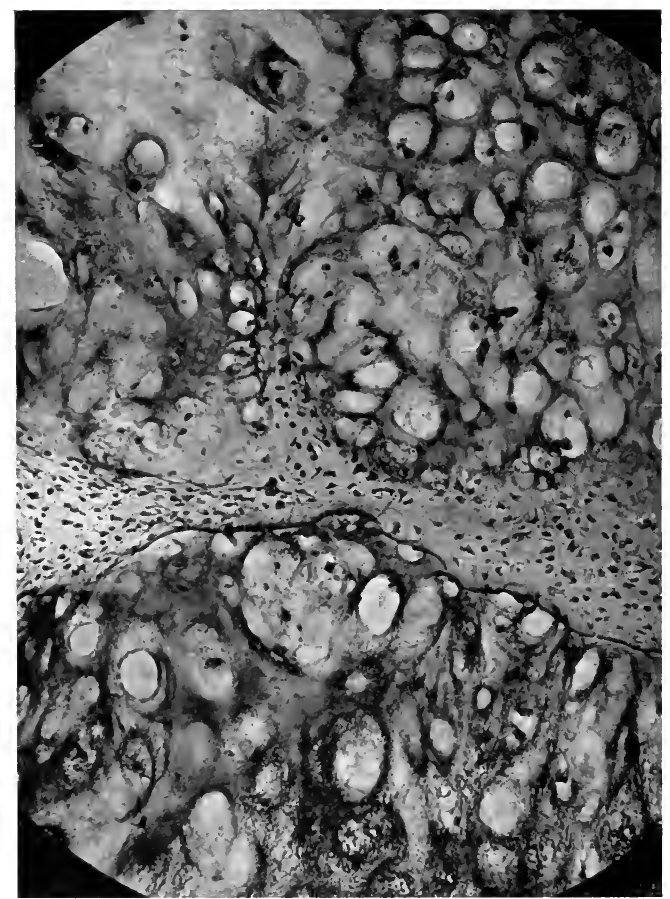

FIG. 43.-Structure of the wall of a cystic chondroma.

lular quality. In a group of sarcomas of long bones composed of cartilage, bone, and cellular tissue, the local destructive effects and recurrence after operation lead to their designation as osteochondrosarcoma.

While in general the more cellular and softer tumors, as myxochondroma, are the more malignant, both clinical and histological features should be regarded in applying the term "chondrosarcoma." The metastases of chondromas exhibit most of the characters observed with other malignant tumors and a special capacity to invade the large veins must be ascribed to them. Invasion of the lymphatics also occurs. The structure of these invasive chondromas does not always suggest malignancy. In Ernst's case the intravascular cartilage was provided with firm hyaline matrix, but this tumor did not produce embolic nodules. To account for the invasive properties 
one must assume that the active growth and infiltrative properties reside in the undifferentiated cells of the periphery of the cartilaginous nodules and in some cases extensive continuous growths in vessels and embolic metastases have consisted almost exclusively of such undifferentiated cells. By such changes a chondrosarcoma may lose much or possibly all of its chondromatous character. This possibility also suggests that certain tumors originating from the mother tissue of cartilage may never show pronounced chondromatous features, but appear as simple sarcoma or myxosarcoma. Certain nasopharyngeal sarcomas (polyps) are probably of this character.

Etiology.-The majority of chondromas occur in early life and about puberty. Some are congenital, and multiple chondroma of the spine is hereditary and congenital. Many of them are located at the growing ends of bones and in not a few there is a distinct tendency toward standstill and regression at the period when the development of the skeleton is complete. All of these facts point to an origin from disturbances in development of the cartilaginous elements in the formation of bones and joints. An inherited predisposition has been emphasized by Weber, who observed many chondromas and ecchondroses in several members and three generations of the

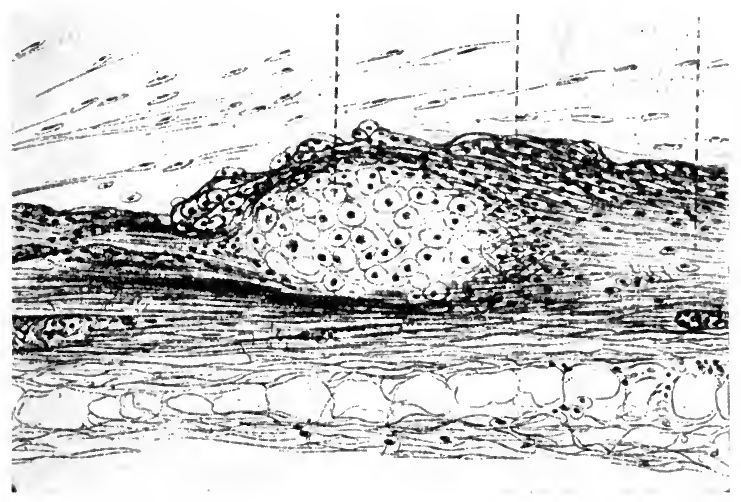

FIG. 44.-Island of superfluous cartilage in periosteum of rib. (E. Müller.)

same family. In the French family of Pellerin members of three generations were affected with multiple chondromas of tibia, ribs, and humerus (Ernst).

Virchow, Recklinghausen, and many others have shown that rickets is an important factor in the disturbance of the growth of bones that leads to chondroma. Borst has found widely misplaced islands of cartilage in rachitic bones and believes that these may give origin to chondroma of limited growth, but doubts the general applicability of the rachitic theory of skeletal chondroma. Ribbert also fails to find in this theory a sufficient explanation of any large group of chondromas and thinks the disturbance must result from some unknown fetal disorder of the bones. E. Müller has described islands of superfluous cartilage in the periosteum of the ribs and suggested that these structures may be the source of chondromas.

In the remarkable case of Recklinghausen's in which multiple chondromas of feet and hands were associated with angioma, the author assumed that congenital aplasia of the blood-vessels subsequently leading to passive dilatation and angioma, prevented the normal progress of replacement of cartilage by bone and resulted in overgrowth of cellular cartilage. The tumors ceased to grow at 22 years. 
For the large group of distinctly heterologous chondromas of breast, parotid, neck, uterus, etc., various embryonic disturbances must be assumed to exist, in most of which complex masses of tissue are involved. Chondroma of the sex glands results from the one-sided development of teratomas and it is probable that in other localities certain chondromas have a similar origin.

Trauma and inflammation are frequent causes of ecchondroses, and trauma is not infrequently reported as preceding chondroma and chondrosarcoma (50 per cent. Weber), especially of the long bones, in which cases it must be assumed that there is also a local or general predisposition.

Clinical Types of Chondroma.-The skeletal chondromas are the most frequent forms of this tumor. They occur most often as single or multiple tumors of the hands and feet, but may affect any of the bones (Nasse, Lit.). They arise from periosteum or in the medulla, usually near the diaphysis and regularly before puberty, tending to become stationary at that period. They deform the shafts of the bones and the joints. From the pelvic synchondroses they may grow to very large size, a case of Weber's having reached a diameter of 2 feet. These tumors as well as the pubic ecchondroses may obstruct labor. In a girl of I 5 years Pfeiffer observed a pelvic chondroma which measured I meter in circumference and weighed 27 pounds. Most of these large pelvic tumors have shown rapid growth (Ernst).

Pronounced cases of multiple osteochondroma represent a somewhat specific malady which is probably related to chondrodysplasia and other disturbances in the growth of bone (Ashurst, Lit.). It is responsible for a variety of skeletal deformities, as depicted by Hagen. The disease sometimes exhibits hereditary tendencies, being transmitted by affected males and females and by unaffected females. Honeij finds that subjects in the progressive stage of the disease, when placed on a diet poor in calcium, show excessive calcium elimination. The tumors may appear at birth, or be delayed until the 5oth year, and one tumor may be growing while another is regressing. There is generally a limit of growth about puberty. The growth of the long bones may be so inhibited that the subject is dwarfed. Growth of radius over that of ulna may produce a deformity resembling dislocation of the radius, and growth of tibia over fibula results in pes valgus. The $x$-ray shows osteoporosis of the ends of the bones, and often a cystic appearance, while the compact bone of the ends of shafts may be very deficient. At various points, usually about the joints, the multiple out growths appear. The structure shows a persistence and overgrowth of poorly ossified or calcified cartilage, in which the cells are irregular in size and form (Carman, Fisher). The ordinary epiphyseal line is irregular or obliterated. Late ossification leaves the bone deformed and covered with osteophytes. Lenormant collected a series of cases in which osteosarcoma appeared to develop on the basis of the early lesions.

Chondroma of the scapula arises from various portions of the bone, and exhibits the structure of the benign chondroma or that of chondrosarcoma, often myxomatous. The great variations in the structure and course of these tumors is fully presented by Deganello in a study of 39 cases. Osteoid chondroma is a peculiar form of skeletal chondroma arising beneath the periosteum of the long bones. As the growth enlarges the bone assumes a thickened spindle form. The structure is that of hyaline acidophilic osteoid tissue in which the cells approach the form of bone-cells, and true bone may form in them. Many of the osteosarcomas correspond to this description. Virchow describes an extensive case, largely ossified, arising in the soft intercostal tissues and producing metastases in the lung and pleura.

Branchiogenic chondromas occur in the neck from misplaced islands of 
cartilage derived from the branchial clefts. They are located in and about the sternomastoid ligament and muscle with branchiogenic cysts and fistulas, and near the parotid gland. Zahn has collected i 2 cases in which misplaced islands of cartilage were discovered in this region, and Bidder observed a congenital tumor arising from such an island. Recklinghausen's chondroma

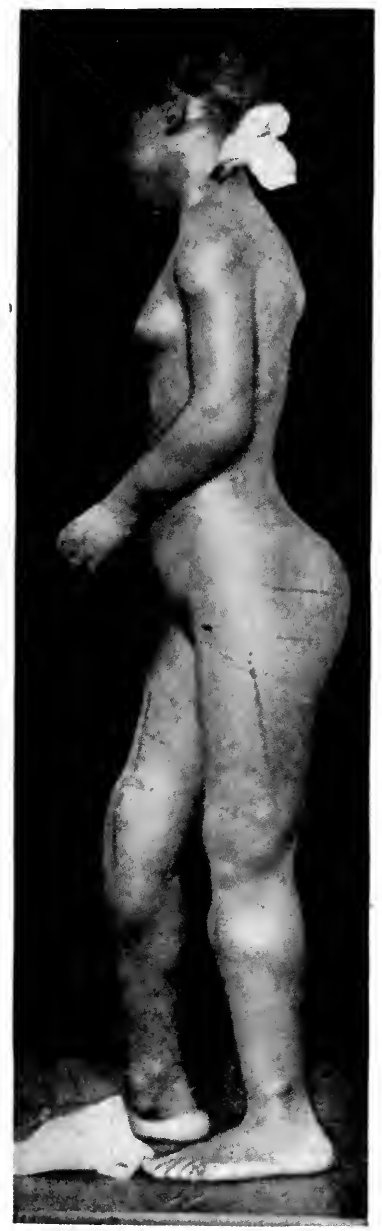

FIG. 45 . of the thyroid may have arisen from such a tissue rest. Symmetrical islands of cartilage and bone have been found in the tonsil, but do not seem to have given rise to tumors (Deichert).

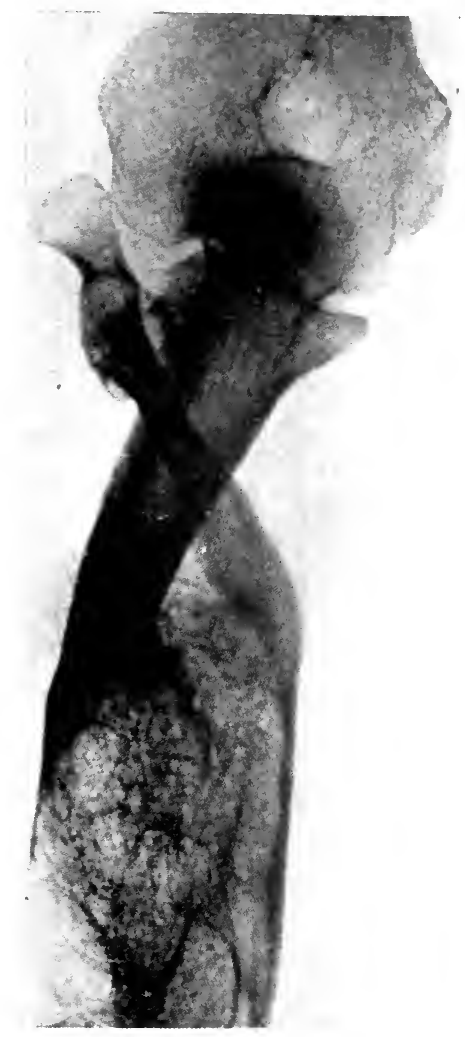

FIG. 46.

Fig. 45.-Multiple congenital ecchondroses in a negro girl of II years. (After Ashhurst, A.S., 63.)

FIG. 46.-Multiple congenital osteochondroma. $x$-Ray photograph of bones of forearm. (After Carman and Fisher, A. S., 6r.)

An auricular chondroma is described by Virchow as springing from a misplaced portion of the fibro-elastic cartilage of the ear.

The chondromas of the hyoid region described by Boeckel and Spisharny seem to have been connected entirely with abnormalities of the hyoid bone. 
The various embryological disturbances giving rise to cysts, rests, and tumors in this region will be discussed elsewhere.

Chondroma of the respiratory tract occurs in the trachea, bronchi, and lungs, apparently arising from the same structures that lead to ecchondroses. Virchow has described multiple chondromas of the lung, located at the root, in the parenchyma and on the pleura. While often purely cartilaginous, they may become calcified or ossified. In the lung rare tumors occur as multilobed growths of hyaline cartilage, subpleural or deep within the lobe, and their association with connective and fat tissue points to an origin for some of them from a complex mass of tissue. In Siegert's case the chondroma projected from the bronchial wall into a dilated bronchus. Chronic thickening of the pleura may yield flat plates resembling cartilage, but composed of hyaline or calcified connective tissue. Yet Kramer has described a lobulated chondroma of the pleural surface of the diaphragm.

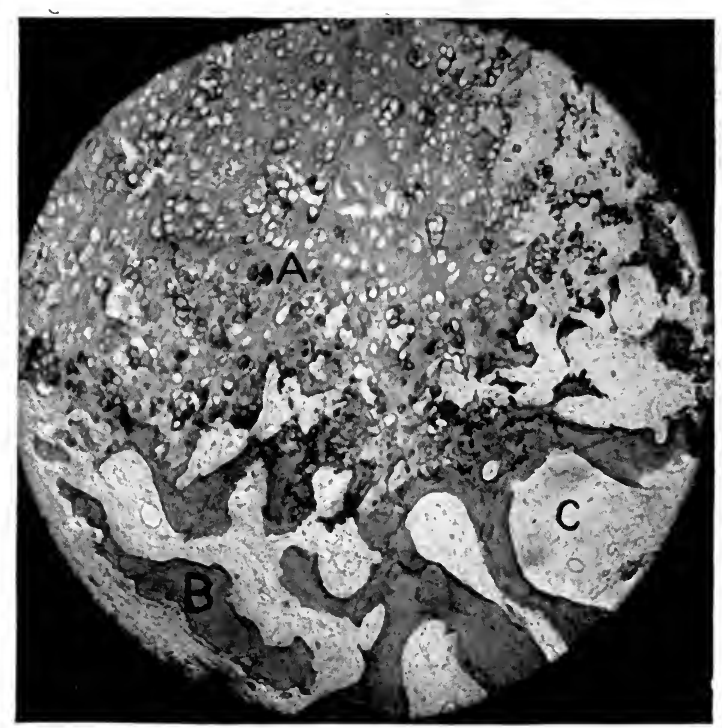

FIG. 47.--Structure of multiple osteochondroma. Section through outer bony shell: $A$, Hyaline cartilage with atypical cells and calcium deposit; $B$, bone trabeculæ; $C$, mucoid connective and fat tissue. (After Carman and Fisher.)

In the breast pure chondroma is occasionally observed, but more often the cartilaginous growth is a portion of a mixed tumor usually of epithelial type.

It is commonly assumed that in all instances these tumors arise from isolated masses of tissue which include a skeletal portion giving rise to the chondroma, but the correctness of this view is not demonstrated. It is possible that some of the cartilaginous tumors arise by metaplasia of other tissues. In the dog a large proportion of mammary cancers contain cartilage and the histological structure strongly suggests its formation by metaplasia from other tissues. Williams has collected many reports of cartilaginous and bony tumors of the breast and argues in favor of the origin from misplaced masses of complex tissue.

In the uterus, especially in the cervix, cartilaginous and bony tumors are occasionally observed (Williams, Lit.). It seems probable that they arise 
from misplaced islands containing skeletal tissue. Thus Meyer found a bony nodule in the cervix associated with a remnant of the Wolffian duct. Miller found an osseous tumor replacing the corpus in a subject in whom the vagina was absent. In several uterine myomas cartilage has been found (Williams).

In the mixed tumors of the salivary glands cartilaginous areas are very common, and especially in the submaxillary the tumor may be nearly pure chondroma. Here the cartilage is of imperfect hyaline variety and often of myxomatous type. In recurrences of these tumors there may be a tendency toward elimination of all but the cartilaginous elements. The various questions that concern these tumors will be discussed elsewhere.

Teratoid chondroma. A considerable group of chondromas, including many of those which invade the vessels and prove malignant, represent onesided development of the cartilaginous portions of teratomas. Most of these

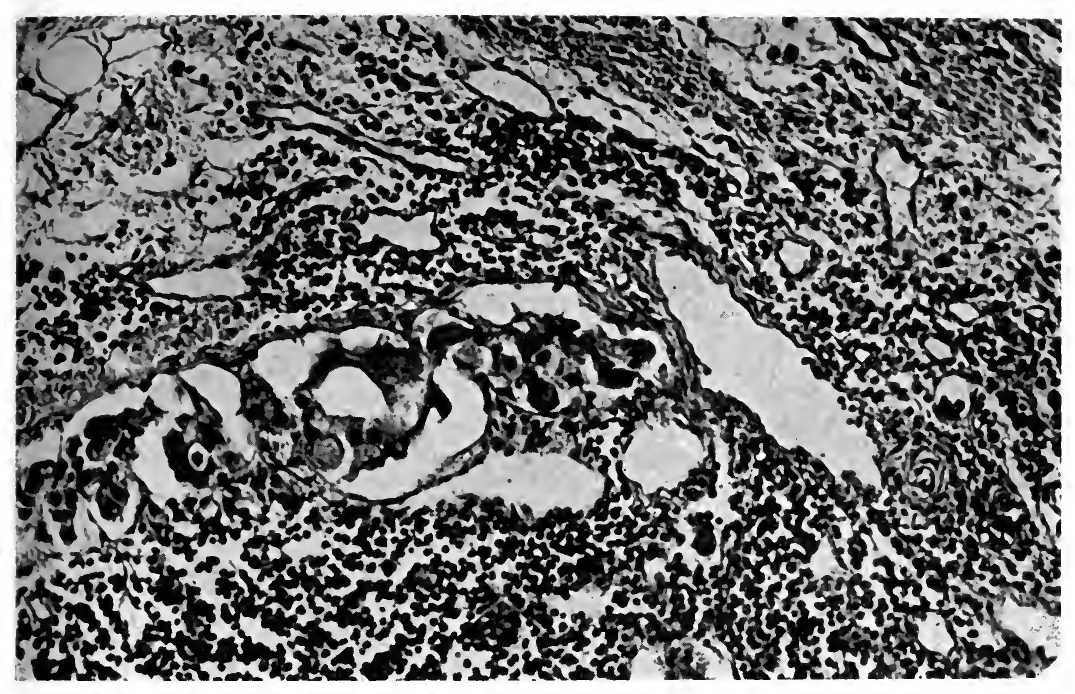

FIG. 48.-Metastasis from malignant chordoma in the cavernous sinus of a pelvic lymph-node. (Lewis' case.)

chondromas arise in the sex glands, ovary, and more especially the testis, but with the extensions of the limits of occurrence of teratomas it seems probable that chondromas of this origin may be more numerous than now appears. In the testis these tumors are solid or cystic, and other elements besides cartilage are usually present in abundance. The cartilage may be reduced to very minute traces which escape detection in many tumors or tridermal type (Ewing). In several notable cases extensive intravascular growths and metastases occurred (Paget, Ohkubo, Lit.).

Chordoma.-A remnant of the chorda dorsalis, a specific entodermal embryonal tissue about which the spinal column develops, regularly persists in infants in the centers of the intervertebral discs (Kolliker). It was shown by $H$. Müller that portions of chorda tissue commonly persist at the base of the skull and in the coccyx, and that this tissue probably gives origin to certain peculiar tumors which Virchow first described as ecchondrosis spheno- 
occipitalis. Ribbert found chorda remnants in the bony tissue and flat plates beneath the dura mater of the Clivus Blumenbachii, and held that they produced tumors only when misplaced and lying outside the bony encasement. He found the tumors in about 2 per cent. of a series of subjects, in the form of bean-shaped masses which perforate the dura and become adherent to the basilar artery with which they may be torn away in removing the brain. By careful sectioning he was able to show that the small intradural tumors are connected by a pedicle with the underlying bone in which their roots are embedded. They seldom reach a large size and being soft do not produce pressure or other symptoms. Yet Grahl observed a lobulated extradural chordoma of the sella turcica which produced fatal pressure symptoms. A large chordoma lying in front of the upper cervical vertebræ has been observed by Klebs. Hassner's tumor measured $5.5 \times 6.5 \mathrm{~cm}$. and had produced pressure symptoms for four years.

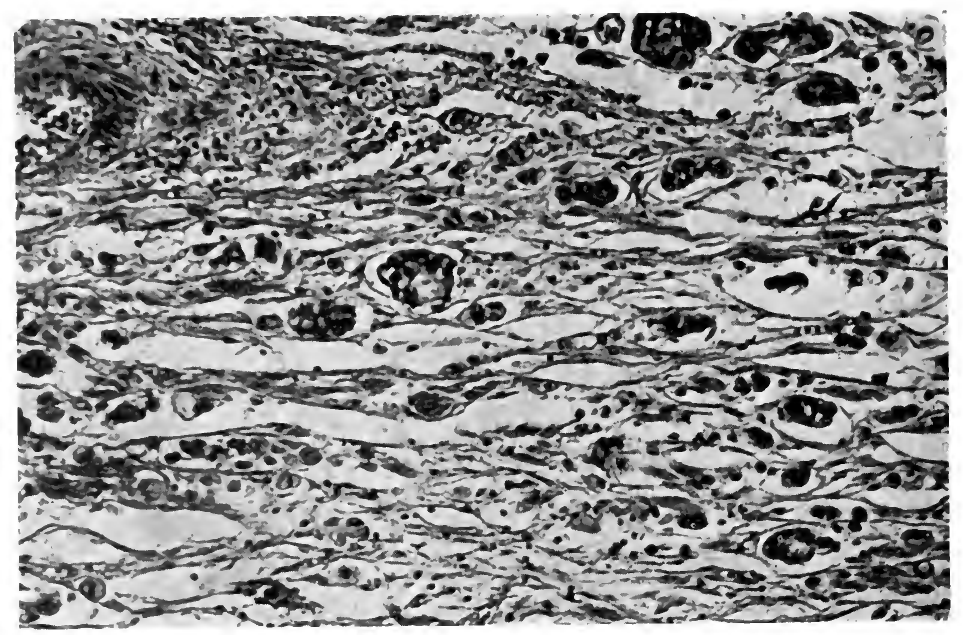

FIG. 49.-Malignant chordoma infiltrating the pelvic fat tissues. (From a case of Dr. N. D. C. Lewis.)

In structure chorda tissue is composed of groups of large vacuolated cells lying in soft homogeneous basic staining matrix, and the tumors accurately produce this structure. Virchow described the peculiar vacuolated cells of these growths under the term "ecchondrosis physalifora," and since somewhat similar vacuolation occurs in chondroma, he was inclined to regard all of them as chondromas. Although chorda tissue does not produce cartilage and there can be no transition forms between chondroma and chordoma, yet it may at times be difficult to distinguish between them.

Malignant Chordoma.-The exact limits of the occurrence and possible variations in structure and course of chordoma have not been determined. It would seem likely that a tissue as extensive as the chorda dorsalis might persist at various points along its normal location, and that it might give origin to structures varying from that of the simple chordoma.

Nebelthau has shown that remnants of chorda tissue may occur at several points along the base of the skull. Fischer has described a malignant chordoma arising beneath the dura of the clivus B., invading the spinal canal and 
compressing the cord. A small vein was invaded by polyhedral tumor-cells. The structure corresponded to that of chordoma, but some areas resembled cartilage.

Link obtained portions of a tumor in the pharyngeal vault, which affected the internal ear and compressed several cranial nerves and which he believed arose from the clivus B. Besides the usual chorda-like tissue it contained groups of polyhedral tumor-cells so that Linck recalls the entodermal origin of the chorda and discusses the question whether the malignant chordoma should be called sarcoma or carcinoma. Coccygeal chordomas are described by Feldmann and Vecchi, and in the latter case the growth recurred after operation. Albert describes a coccygeal chordoma which involved the rectal wall and recurred promptly after operation.

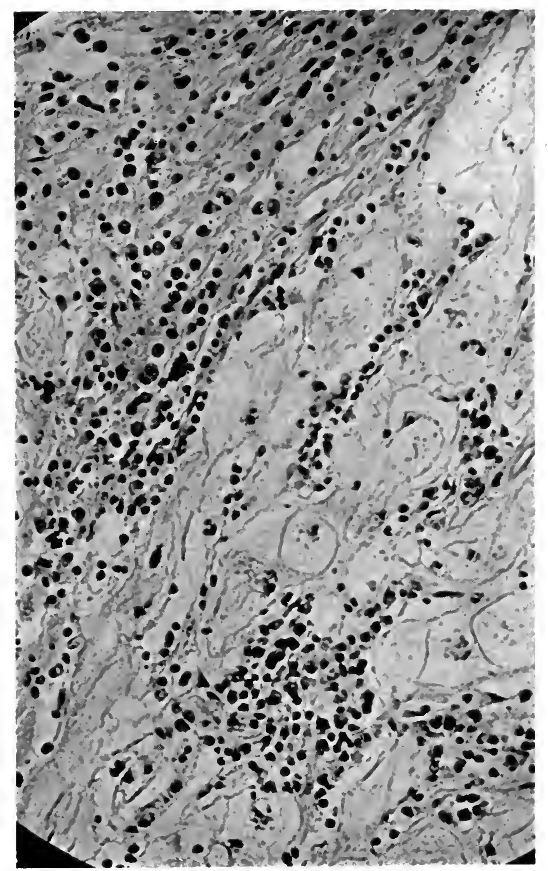

FIG. 50.-Sacral chordoma. (From a section received from Dr. Mallory.)

The positive identification of chordoma is not readily accomplished. Two common tumors closely simulate or even duplicate the structure attributed to chordoma-viz., myxochondroma and colloid carcinoma of the intestinal canal. The gross relations of the tumor should be given first consideration in the diagnosis. Especially in the sacral region chordoma is difficult to separate satisfactorily from chondroma or colloid carcinoma. Feldman analyzes in detail the features which distinguished his tumor which eroded the sacrum, from colloid carcinoma, but I have seen all these features, except the broad attachment to the sacrum, in colloid carcinoma of the rectum. In the pharyngeal region atypical chondromas are rather common and must be considered a more probable occurrence than chordoma. 


\section{CHAPTER XIV}

\section{OSTEOMA}

Circumscribed overgrowth of bone occurs under such a wide variety of conditions and the distinctions betwèen inflammatory and neoplastic hyperplasia of the tissue are so often obscure that it has never been possible to exactly define the limits of osteoma. True progressive neoplasms which adhere to Virchow's criteria and in which bone is the essential and not the secondary or accidental product, are not common, but chronic processes which result in bone formation from trauma, inflammation, and disturbances of nutrition are numerous. Volkmann concluded that all exostoses that arise from cartilage are true tumors, others not. Borst, who separates from the tumors all the bone-producing processes which are self-limiting or clearly associated with trauma and inflammation has little to say about true osteoma. Virchow and Ribbert largely agree in recognizing neoplastic qualities in many processes in which trauma and inflammation are originally concerned, and which, whether true tumors or not, illustrate the complex etiology of neoplasms.

Bone formation in necrotic tissue (brain, eve, kidney, aorta); in the floors of ulcers; in the course of syphilis, tuberculosis, and chronic suppuration in and about bones; after fractures, in chronic arthritis; at the insertion of the overused tendons; in bursæ; in the muscles of riders-are some of the processes in which neoplastic qualities are least prominent. Yet in all of them the size of the resulting bony mass, the long duration of the process, and its eventual independence of the original exciting factor occasionally reveal definite neoplastic characters. Hence in discussions of osteoma it is customary to include all forms of overgrowth of bone, thus securing an effective comparison of true and partial bony neoplasms.

Histological study fails as a rule to distinguish simple hyperplastic growth of bone from true osteomas. When originating in bone both processes show a participation of some or many osteoblasts which surround the edges of the new growth and add to its substance from one or many sides. Both processes yield dense lamellated bone with few Haversian canals or spongy bone with many vessels and abundant marrow spaces and cells. Bone forms in connective tissue without the appearance of many osteoblasts, the fixed cells being passively incorporated in osseous matrix, but this character does not always distinguish self-limiting from extensively progressive osteosis.

The gross and clinical features seem to form the best criteria by which to separate osteoma from simple hyperostosis.

Spontaneous or traumatic but non-inflammatory origin, progressive course, circumscribed form, active participation of osteoblasts, and derivation from cartilage, are features which are more prominent in true osteoma, while an inflammatory origin, self-limitation, multiplicity, diffuse form, reduced numbers or absence of osteoblasts, and origin from connective or other soft tissues, belong to the less definite or spurious bony neoplasms.

Terminology.- "Osteoma" is the term applied to tumors composed of bone. Strictly speaking, it should be limited to true neoplasms.

Exostoses are circumscribed masses which project above the bony surface.

Enostoses lie within compact or cancellous bone. 
Hyperostoses are more diffuse enlargements of bone. "Osteophyte" is the general term applied to inflammatory periosteal bony deposits.

The new tissue may be ivory-like with solid lamellæ with few or no Haversian canals (exostosis eburnea); or spongy with cancellous tissue (exostosis spongiosa); or it may contain wide marrow cavities or spaces (exostosis medullaris). The growth may affect bony structures or other tissues, and is thus hyperplastic or heteroplastic.

The osteophyte is an inflammatory cortical or supracortical deposit of bone from the periosteum. It appears as a flat, often extensive plate or more circumscribed mass deposited on preëxisting bone from which it may usually be detached. Microscopically it consists of many small islands and thin traveculæ of bone lying in the cellular and vascular connective tissue of the thickened periosteum and continuous with the preformed bone from which it springs. Many stages of the process of ossification are visible in early cases.

Any area of periosteum which is affected by chronic inflammation may produce osteophytes. They are most frequently observed on the shafts of long bones; in the neighborhood of chronic sinuses or ulcers; as a prominent feature in the course of chronic arthritis; on the maxillæ about inflamed teeth; and beneath the dura of the frontal or occipital bones in gestation.

The course of inflammatory peliosteal bony deposits varies with their etiology. Most cases reach a standstill as the inflammation subsides, leaving deformities and interference with function. Ankylosis of joints may result. In not a few cases osteophytic growth seems to have led to progressive overgrowth and the production of extensive tumor-like processes. In the case of Forcade's son, cited by Virchow, suppuration in the lacrimal region led to progressive enlargement of many bones of the face and skull continuing for 33 years, and resulting in extensive leontiasis ossea.

Hyperostosis.-Diffuse hypertrophy of large portions of bones, or whole bones, or several bones, occurs under many conditions. The most familiar form of hyperostosis results from osteomyelitis, periostitis, and arthritis. While the hypertrophies of this class may be extensive they are readily separated from neoplastic growths. In acromegaly there is diffuse overgrowth of bones of skull and extremities resulting from disturbance of function of the hypophysis (leontiasis ossea). The old and frequently cited case of Sancerotte of general leontiasis ossea is unique but of uncertain nature. The bones of the face and skull are specially prone to diffuse enlargement from local factors, trauma, inflammation, and rickets, and not all cases of craniosclerosis can be referred to acromegaly. Virchow collected many cases of diffuse enlargements of temporal, sphenoid, maxillary and malar bones, and head of femur, apparently resulting from varied causes, not distinctly inflammatory. He considered these overgrowths of bone as comparable to elephantiasis of the skin and lipomatosis.

Exostoses.-The circumscribed tumor-like masses projecting from the surface of bones and intimately connected with the shaft, present a varied form, course, and etiology. They are flat or tuberous, nodular, globular, or pointed or ragged growths, which appear in many striations, at epiphyseal junctions or on the shafts of long bones, and at the insertions of tendons and fascia. They are flat, smooth and ivory-like as is usually the case with exostoses of the skull (ex. clavata); or spongy with cancellous spaces communicating with those of the shaft. The porous exostoses may later become solid. They are usually multiple, often symmetrical, and some notable cases are recorded in which there was a prominent hereditary tendency transmitted through the males (Heymann, Reinecke). In a case of hereditary multiple 
exostoses E. Müller found very numerous islands of cartilage in the periosteum of long and flat bones.

In advanced forms the general development of the bones has been retarded and even dwarfism has occurred (Virchow). As a rule exostoses occur in young subjects before puberty during the period of active growth of the bones (exostoses de croissance).

Exostoses usually arise from overgrowth of subperiosteal bone and these must be attributed to an irritation affecting the periosteum. Yet the entire thickness of the shaft may be involved and new cancellous spaces of the exostosis communicate with the old. Or the new growth may lie within the bone shaft or marrow cavity (enostosis). In other cases the process is entirely superficial and periosteal and some of these may lie within the periosteum and apart from the shaft (parosteal exostosis). Trauma, with or without misplacement of a fragment of periosteum, or fracture, is often the exciting cause of such growths. About the ends of long bones portions of cartilage may be found in exostoses (ex. cartilaginea). An origin from a misplaced island of cartilage is probable, and may be referred to trauma, rickets, or preëxisting ecchondrosis. Microscopically exostoses usually begin with proliferation of the inner vascular layer of the periosteum and the formation of a network of osteoid tissue. This matrix may at once become eburnated, or vascular channels may persist and the tissue remain spongy. The underlying bone becomes sclerosed and fused with the new growth, or it becomes rarefied and the adjacent marrow spaces communicate with those of the exostosis. According to Virchow the former process may be called histioid, the latter organoid.

Clinical Groups of Exostoses. - The largest class of exostoses are those which form at the ends of long bones and are connected with disturbances of growth. They are multiple, tend to grow less rapidly as the subject reaches puberty and rarely they may spontaneously disappear (Hartmann). The long bones, the vertebræ, pelvis, scapula, and the skull are the chief sites affected. Partly cartilaginous exostoses occur about joints and may possess a synovial covering which is derived from and may communicate with the joint (exostoses bursata). Some of these are probably originally intra-articular and gradually become separated from the joint cavity (Bergmann, Volkmann).

Multiple exostoses occur on the bodies of the vertebræ and involving the intervertebral discs. In the cervical region they may produce palpable tumors.

Traumatic cxostoses constitute a well-defined class. They occur at the seat of fractures when excessive callus forms and after single or repeated trauma of the periosteum or bone and along the alveolar borders after extraction of teeth. Virchow cites 2 cases where broken splinters of bone hypertrophied, forming exostoses of moderate size. The great toe is the seat of a subungual exostosis which may occasionally develop into a true osteoma or osteochondroma. Continuous mild trauma is also responsible for the discontinuous exostoses which form in the deltoid in soldiers where the gun rests on the shoulder, and in the adductor muscles of riders.

In nearly all these situations occur larger growths with more distinct neoplastic characters, suggesting that the small exostoses may occasionally give origin to true osteomas.

In the trachea Ribbert and Mischaikoff have fully described the multiple, round, flat or ragged nodules and plates of bone which grow in the submucosa, and have found that most of them, like the tracheal ecchondroses, are connected with the perichondrium of the tracheal rings. In a very advanced case described by Heyman, with extensive ossification in the larynx, there were many bony masses with marrow spaces throughout the trachea. 


\section{TRUE OSTEOMA}

Osteoma of Facial Bones and Antra.- The bones of the face are the seat of a variety of bony overgrowths which are difficult to classify.

In the maxilla osteosclerosis and hyperplastic bone formation occurs in connection with dislocated and inflamed teeth, producing exostoses or enostoses, or hyperplastic masses some of which project externally or into the antrum of Highmore. Many of these must be regarded as inflammatory, while others are true osteomas.

In the orbit bony growths arise from any of the bones of this cavity, usually from the upper and inner segment.

They not infrequently appear as congenital tumors in young girls. Virchow concluded that they arose as enostoses of the orbital wall and grew

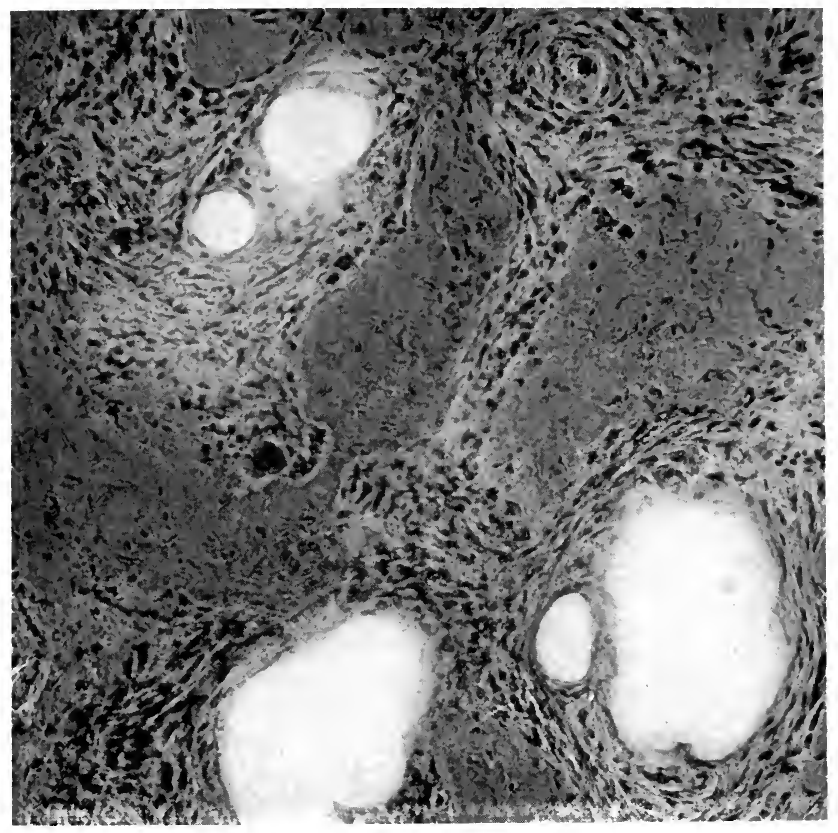

FIG. 5I.-Growing osteoma of antrum.

either into the orbit or nares or into the cranial cavity. He described 4 cases, in some of which cysts were found lined by ciliated epithelium. Many bony tumors have been described as arising from the ethmoid, frontal, and superior maxillary bones, projecting into the cranium, nares, frontal sinus, or antrum of Highmore.

LaGrange has collected 148 cases. Their origin appeared to be from the periosteum or, as Lesser states, from embryonal cartilaginous portions of the ethmoid. Projecting into the sinuses they present a covering of inflamed and often cystic mucosa (Panas). The chief site is the upper inner wall of the orbit. They may penetrate the skull, but seldom cause grave cerebral symptoms, while only 2 such cases were fatal. Knapp has reported 2 enucleations, in I of which the growth was broadly attached over the upper wall of the orbit. 
Osteoma of the frontal sinus is believed to arise from fragments of cartilage connected with the ethmoid. It produces a characteristic swelling over the orbit and progresses steadily until extirpated. Its structure is usually solid and lamellated. Most of the osteomas of the nares and antrum of Highmore also arise from the ethmoid (Bornhaupt). Tillmanns collected several cases in which these tumors were found loose in the nasal cavity or antrum (dead osteoma).

Secondary Osteoma.-Osteoma occurs as an element in many other tumors where it appears as a result of secondary ossification of the connective tissue. Here it may signify a terminal product in a series of transformations of the tissues of fibroma, lipoma, sarcoma (Borst). In periosteal fibroma bone is a natural end product of the growth (fibro-osteoma).

In certain cases it represents an integral part of a mixed tumor. In the common breast tumors of dogs, bone formation may be very active and exten-

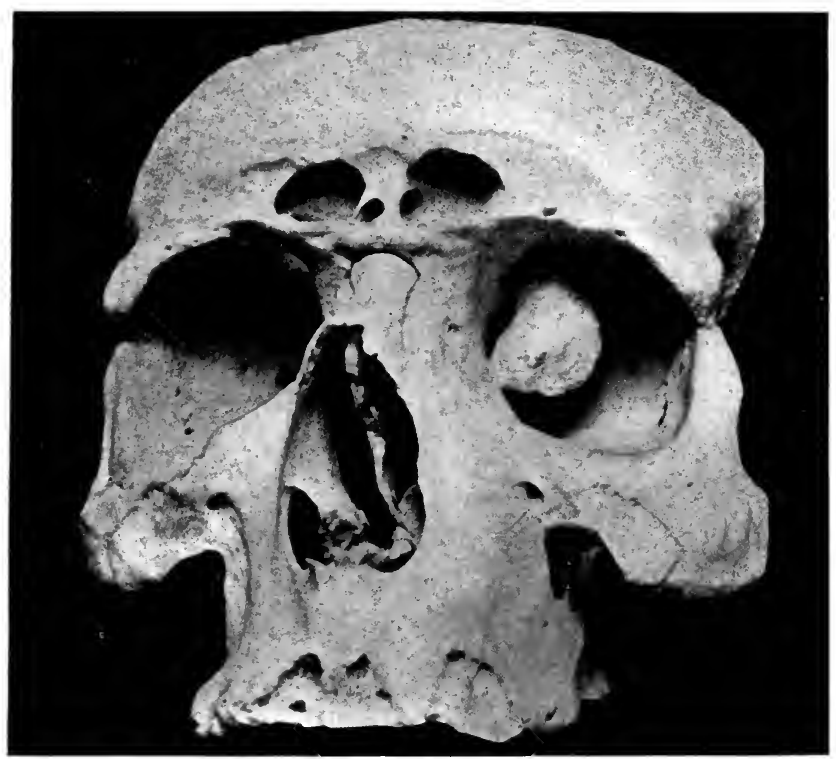

FIG. 52.-Osteoma of orbit. (After Knapp.)

sive, producing complex osteo-chondro-carcino-sarcomas. In the ovary osteoma occurs in several forms (Kroemer, Lit.). True bone with periosteum and marrow spaces is observed in connection with dermoids and teratomas. Ovarian stones composed of a thick shell of bone about a central cavity arise from ossification of cysts, some of which are from corpora lutea. They are usually preceded by active inflammation. Metaplastic bone formation occurs in ovarian fibromas and chondromas, either in small foci or throughout the tumor.

Osteoma in the Nervous System.--In a considerable portion of cachectic subjects, especially of the insane and epileptic, there are multiple nodules of bone in the cerebral or spinal arachnoid. Zanda finds that these nodules form about small vessels through degeneration, exfoliation, and sclerosis of endothelial cells and connective tissue, which form hyaline, then osteoid, and finally bone tissue. From these nodules or over large areas, especially in the 
falx cerebri and cerebral and spinal dura, larger masses of bone may form with cellular and fatty marrow spaces, yielding bony plates which are smooth and incorporated in the dura externally, and rough internally where growth progresses (Zanda, Ziegler, Borst).

Larger tumors compressing the brain or cord and lying within the brain tissue or arising from the membranes have been described by several authors (Virchow, Meschede, Ebstein, Bidder, DeVecchi).

The intracerebral growths were referred by Virchow to osteogenesis by the glia-tissue. Some are associated with encephalitis with softening. Others doubtless originate from misplaced islands of the bone-forming matrix of the dura.

Heteroplastic Bone Formation.-In heteroplastic bone formation the process begins either in a cartilaginous or a fibrous matrix. The cartilage becomes more vascular and ossification occurs about the small vessels through the activities of osteoblasts. In connective tissue the stroma becomes hyaline, calcification occurs under the influence of osteoblasts with the appearance of osteoid and finally osseous tissue. Here one must conclude that the process is metaplastic, the fibroblasts acquiring the function of osteoblasts.

Several factors may be regarded as tending to call forth osteoblastic properties in fibroblasts.

I. Proximity to Bone.- Some authors have assumed that bone formation always results from osteoblasts that have wandered out from periosteum (Busch). In myositis ossificans this theory finds a certain support as the process begins in the periosteum.

2. The presence of calcific deposits figures in many instances of ossification of necrotic tissue.

3. An active productive inflammation with organization of dead tissue and blood-clot is probably essential in the ossification of muscle-tissue after trauma (Busse, Berndt, Rapke, Lit.).

4. A special predisposition to calcification and ossification, possibly connected with disturbance of calcium metabolism, must be assumed to exist in certain cases, notably in those of reticulated osteoma of the lung.

In many cases heteroplastic bone formation occurs without the appearance of many osteoblasts, the fixed cells being passively incorporated in osteoid and osseous matrix.

Clinical Forms.-The lung is subject to an interesting and rare type of bone deposit through the progressive ossification of inflammatory connective tissue. This condition was first described by Luschka and may be termed reticulated osteoma.

The condition appears in three forms which are probably stages of the same process. It first produces multiple nodules or tuberosities in the connective tissue of one or several lobes.

In early cases in young subjects both lungs throughout presented about fifty pea- to bean-sized nodules with much new connective tissue (Wagner, Heschl). In later stages one or more lobes are the seat of extensive reticulated bony deposits radiating in stalactite form from the root of the lung (Forster, Bostrom, Picchini, Triboulet, Arnsperger). In a few cases one lobe is transformed into a nearly diffuse mass of bone while nodules appear in other lobes (Port, Cohn).

Bone masses in the wall of a cavity with nodules in other portions of the lung were observed by Rullier, Browning, and LeDiberder.

Microscopically the process begins as an interstitial growth of connective tissue, in the septa, walls of bronchi and alveoli, and about the large vessels, all of which are inclosed and eventually occluded by ossifying connective 
tissue. The vessels may show endarteritis obliterans (Picchini). The islands of bone are surrounded by numerous osteoblasts and marrow spaces filled with fat and marrow-cells may appear.

The majority of observers have concluded that the process, at least at its inception, is an ossifying interstitial pneumonia. In advanced stages it seems to acquire certain neoplastic characters, and Virchow regarded the diffuse form as a true osteoma. The reticulated osteoma is to be distinguished from ossifying chondroma of the lung, but in advanced cases this may be difficult to accomplish.

Simple calcification may occur in the walls of cavities (Krauss, Nusser) and the lung is a frequent seat of metastatic osteosarcoma (Gross).

In the penis of a subject 42 years old, Lenhossek has described the formation of bony and cartilaginous plates which probably resulted from the changes in inflammatory tissue in the fibrous septum and sheath. König refers to similar cases.

In the skin multiple nodules of compact bone appear in the epidermis or derma, sometimes about cartilaginous matrix (Virchow).

Coleman has described osseous plates in the sole of the foot.

In the eye, the sclera and choroid, are occasionally seen bone plates which result from metaplastic changes in inflammatory connective tissues.

Bone formation in necrotic tissue occurs in many situations, as in phthisis bulbi (Virchow), in the wall of the sclerotic aorta (Cohn, Klotz), in the walls of abscesses and in chronic pleurisy, and in advanced stages marrow spaces and cells may appear. After ligation of the main vessels of the kidney there may be extensive formation of bone and cellular marrow in the necrotic organ of the rabbit.

Myositis Ossificans.--Spongy bone may form as a result of trauma in the muscles of working people and has been observed in the biceps, digastric, vastus externus, adductor magnus, and diaphragm (Virchow). In these cases the ossification is self-limiting, but in not a few cases of traumatic origin there is a certain progressive tendency suggesting a relation to myositis ossificans.

The progressive ossification of one or many muscles, first fully described by Munchmayer and called progressive myositis ossificans, arises spontaneously, chiefly in young subjects and pursues a chronic intermittent course. It affects the shoulder, face, trunk and limbs, and may become quite extensive. In the early exacerbations there may be local congestion and pain.

Once established, it tends to progress until the affected muscle is completely ossified and immobile. Yet Elliott observed complete resolution of the preliminary myositis. According to Ribbert the ossification emanates from the periosteum, spreading along the fascia, tendons and the perimysium and endymysium, with atrophy of the muscle-fibers.

Lexer finds first, increase of connective tissue, then chondrification, and finally ossification, constituting three stages in the process.

Since the disease occurs at an early age in subjects with deformities of fingers and toes, is associated with multiple exostoses, and progresses independently of any originating trauma or other known factor, Ribbert regards the process as blastomatous. Since the intermuscular connective tissue may be regarded as a part of the skeletal system and intimately connected with the periosteum (Koester), it may be supposed that in these cases the former tissue retains more of the periosteal character than is normal, thus constituting a tissue predisposition toward bone formation (Mays). The occurrence of very similar lesions in tabes and syringomyelia suggests that a spinal trophic influence may be concerned in some cases (Kaufmann). 


\section{CHAPTER XV}

\section{MYOMA}

Myoma, a tumor of muscle-tissue, occurs in two types: leiomyoma, containing smooth muscle-tissue, and rhabdomyoma, composed of striated muscletissue. In both cases supporting connective tissue and blood-vessels usually accompany the muscle-cells and the structure is organoid. Yet pure leiomyoma occurs in the gastro-intestinal wall and the early stages of many myomas contain little or no connective tissue. According to the rule with

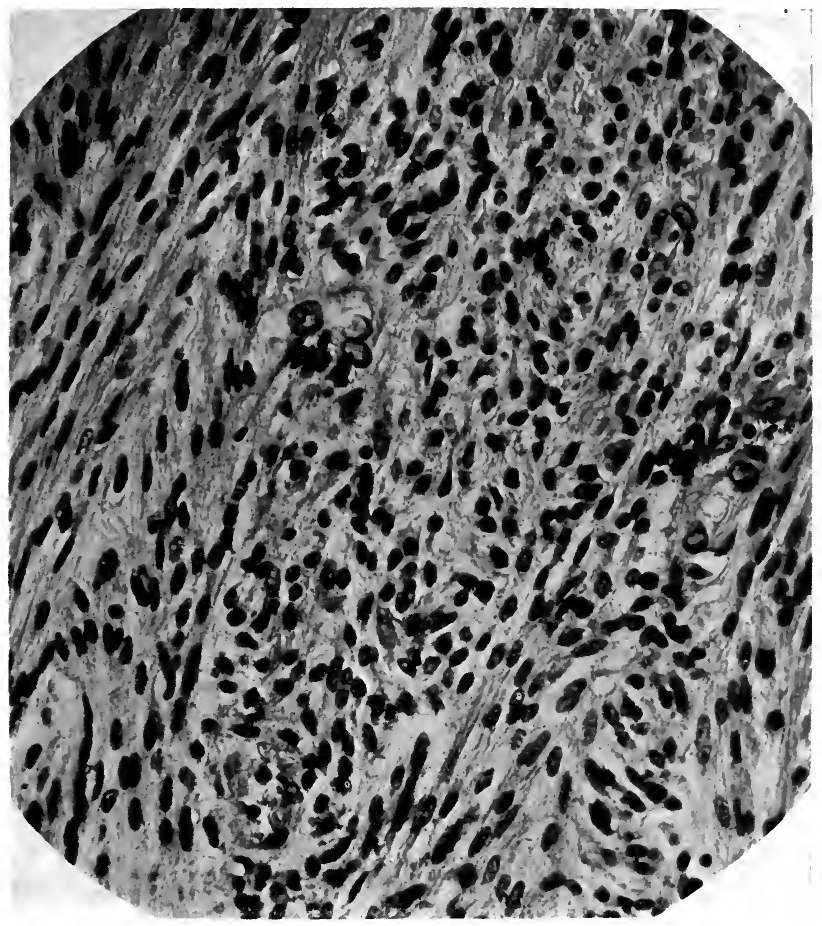

FIG. 53.-Structure of simple benign myoma uteri.

benign tumors, myomas are easily recognizable as such, and metaplasia and inflammatory factors play a very subordinate rôle in their history.

\section{LEIOMYOMA}

Leiomyoma occurs in the form of single or more often multiple, miliary or voluminous, firm and opaque, or cystic tumors chiefly in preëxisting smooth muscle-tissues. Some contain much connective tissue and are quite hard, leiomyoma durum; others are succulent and edematous, leiomyoma molle; rarely dilated blood-vessels are prominent, leiomyoma cavernosum; and occa- 
sionally cysts of dilated lymph-ressels, glandular structures, or softened edematous areas may form. Extreme congestion may render them dark and mottled, and after strangulation of vessels they become black. Fatty degeneration may produce yellowish soft areas, and calcification may transform entire tumors into stony masses.

On section leiomyoma presents characteristic striated or convoluted markings from intertwining bands of muscle-cells. The tumor is more opaque than normal muscle-tissue and is usually sharply circumscribed, often shelling readily out of its bed. The curved markings often center about blood-vessels

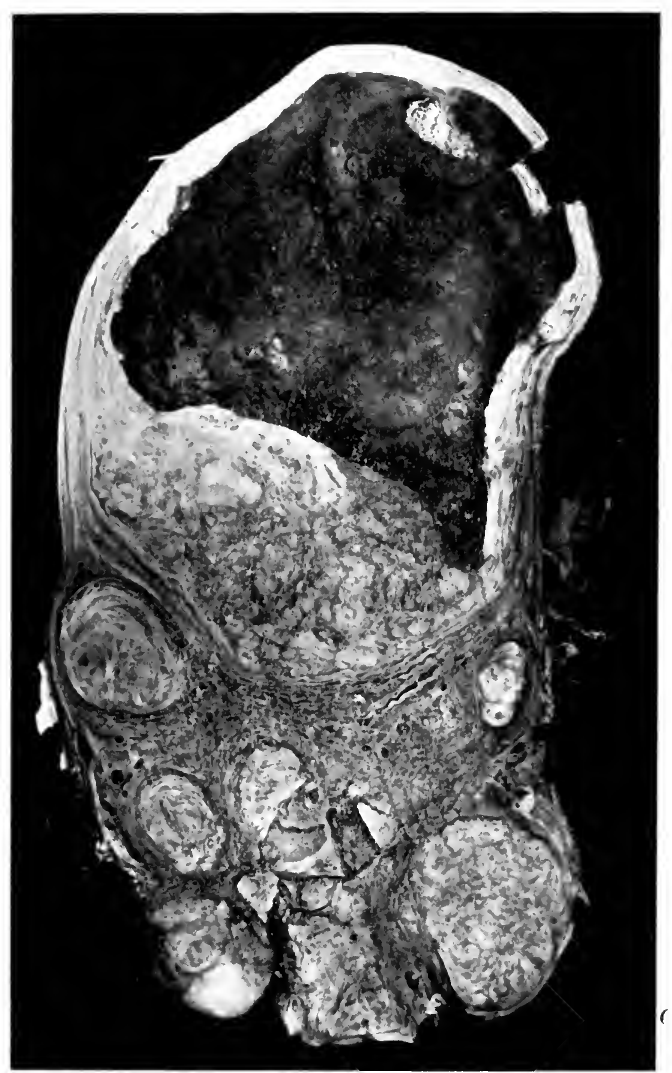

FIG. 54.-Multiple myomas of uterus, one cystic.

from which the growth appears to originate. In injected specimens Ribbert finds the blood-ressels small and scanty as compared with surrounding tissue, and the nutrition is maintained largely by lymph-vessels. Hence myomas grow slowly and are very subject to standstill and regression.

The structure of leiomyoma presents a system of intertwining bundles of muscle-cells supported by septa and fibrils of connective tissue. In some cases the muscle bundles are definitely related to small blood-vessels or glandular structures, but more often no such relation is apparent. The edges of the tumor are usually sharply defined, but may grade insensibly into the surround- 
ing tissues. The tumor-cells are thicker and shorter than normal smooth muscle-cells, the cytoplasm grows less acidophile, and the nuclei are larger and richer in chromatin. In actively growing tumors there is increase in the neoplastic characters of the cells, especially in their size, and in malignant leiomyomas very large spindle-cells appear, giant-cells form of enormous dimensions, and the connective-tissue stroma disappears.

The diagnosis between leiomyoma and spindle-cell sarcoma is often difficult and may be based on the clinical history, the acidophile character of muscle cytoplasm, the long oval nuclei, the arrangement of the cells and the presence of many adult collagenous fibrils running between the cells. Both cells and nuclei are more uniform in size and exhibit more evenly rounded ends than fibroblasts. Special stains facilitate the diagnosis. The appearance of crosssections of muscle bundles with nuclei embedded in acidophile fibers, alternating with longitudinal sections, is usually quite characteristic.

The connective-tissue stroma is acellular, often edematous or infiltrated with round-cells. Two types of blood-vessels are found in most myomas. In one the walls are normal, while the others participate in the neoplastic process, the walls are composed chiefly of hypertrophic muscle-cells and the adventitia is deficient or absent.

Regressive changes are frequently present. Fatty degeneration affects individual cells in moderate degree, and extreme fatty and hydropic degeneration or necrosis with edema produce softened areas. Hyaline degeneration may overtake small areas or an entire tumor. Calcification follows fatty degeneration or hyáline change and may become universal. Inflammatory changes in myomas are usually slight. Round-cell infiltration of the stroma is associated with edema, and about the included glandular structures of some myomas there is adenoid tissue, or lymph-follicles may develop. Mast cells may be very numerous (Reich, Gottschalk). Occasionally myomas become infected and infiltrated with polynuclear leukocytes, and cystic myomas may be found distended with pus.

The course of leiomyoma is slowly progressive over a period of years and the symptoms are mainly of mechanical origin, or referable to associated conditions. There is a natural tendency for many leiomyomas to reach a standstill after a long period of growth or to regress with atrophy of musclefibers, replacement fibrosis, hyaline degeneration, and calcification. In the uterus this tendency reveals itself especially at the menopause, but it is not constant. On the contrary, very rapid growth or sarcomatous transformation may begin at the menopause. Gibson observed the appearance of a uterine myoma and growth to the size of a child's head within 7 months after removal of both tubes and ovaries. Regressive changes may be hastened by pedunculation, by hyaline changes in stroma, by closure of vessels, by separation from attachments, and by inflammatory processes. Acceleration of growth may result from abnormal adhesions.

Myoma Malignum.-In a group of cases, now rather numerous, leiomyoma has proved malignant, breaking its natural boundaries, and producing metastases in liver, lungs, kidney, peritoneum, and lymph-nodes, and thereby acquiring the designation myoma malignum. From the uterus, stomach, esophagus, and intestine this usually benign tumor has developed extensive local and general secondary growths and proved fatal. This event has occurred in the later periods of the growth or after extirpation of tumors which had long remained benign, so that it would appear that a malignant change had taken place in the character of the tumor. The symptoms observed are a rapid increase in the size of a long quiescent growth, signs of secondary growths, ascites in the uterine cases, and cachexia. The main tumor has usually been 
adherent, with cavernous, softened, or cystic areas. In one of my cases the softening spread from a crater-like ulcer in the adherent endometrium. In Schlagenhaufer's case it was limited to an angiomatous area.

In most of the cases the structure of the soft pultaceous areas and of the metastatic growths which probably emanate from these areas, differed from that of ordinary leiomyoma. The cells were larger, the nuclei more massive and hyperchromatic, mitoses were present, the fusiform cells became shorter or rounded, giant-cells formed, intercellular connective tissue was scanty or absent, and the walls of vessels were defective (Krische, Langerhans, Williams, Beeston, Moser, Basso). Evans finds that the presence of many mitotic figures (estimated at 2200 to 12,000 per cubic millimeter of tissue) is a reliable sign of clinical malignancy in uterine myomas. With

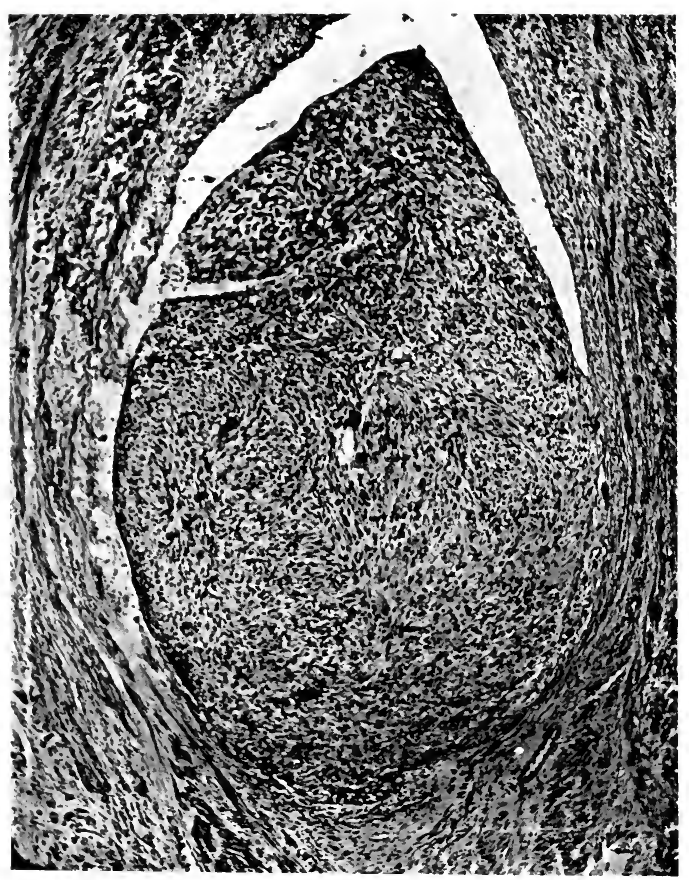

FIG. 55.-Miliary myoma in a uterine vein. The tumor is benign.

fewer mitotic figures or when they are absent the tumors do not recur. In Hansemann's malignant myoma of the stomach the cells were uniform, but short or rounded, and the nuclei very rich in chromatin. In Eising's esophageal myoma islands of adenocarcinoma were present and the muscle-cells much increased in size. In I of Evelt's cases the sarcoma was apparently melanotic. In 3 uterine cases I found the structural changes very marked. In I (346I) there was extensive mucoid degeneration. In another (2287) the spindlecells and giant-cells reached enormous dimensions. In all the derivation from muscle-cells was clear. So far as I have been able to learn no case has been fully studied in which definite variation from the usual structure of leiomyoma were wanting, although in several instances these variations have not been very pronounced. 
In one case of large uterine myoma I found the peritoneum of the uterus thickly beset with miliary myomas identical in structure with ordinary myoma, and in a case of recurrent multiple nodular myomas of the broad ligament the cells varied little from the usual type, although they were shorter and looser and the tissue was very vascular. In neither case were there distant metastases. Accordingly, it may be said that the structure of malignant myoma varies from that of the benign type.

It is not to be assumed without adequate proof that a malignant myoma represents the transformation of a previously benign tumor. Many and probably the majority of these tumors are malignant from the first. Malignant myoma usually occurs after the menopause, at an average age of about 50 years (Evans), but polypoid myomas of the cervix are usually malignant at all ages; adenomyomas are often cellular and adherent; while extra-uterine myomas, especially if multiple, frequently recur.

Ribbert has protested against the term "myosarcoma," employed by many for the malignant tumors, on the ground that sarcoma implies a tumor of connective tissues. Yet in a morphological sense this term seems admissible. Objection has also been made to the interpretation of the above changes as genuine sarcomatous degeneration of myomas. There has been a tendency on the part of gynecologists to multiply the cases of this sort, and to search for suspicious foci over wide areas of perfectly benign tumors. Some estimates place the frequency of the change at ro per cent. of all uterine myomas. From the reports of Winter, Henkel, Hofmeier, and Flautau, in I880 cases of fibroids 48 , or 2.5 per cent., were found to show sarcomatous changes. The manner in which these statistics were obtained is perhaps fairly indicated in the study of Winter. In his first series of 500 cases he examined only suspicious-looking areas and found I6 ( 3.2 per cent.) to contain sarcomatous foci. In 253 later cases he examined all parts of the tumor and succeeded in raising the number of sarcomas to II ( 4.3 per cent.). There were sometimes present general signs of malignancy, as rapid growth, polypoid masses on the main tumor, and changes in consistence, but local metastases occurred only once and cachexia resulted chiefly from hemorrhage. In the same manner Cullen has reported $\mathrm{I} 7$ cases of sarcomatous degeneration of uterine myomas.

These observations show that ordinary myomas vary in structure in different portions and probably at different periods, and they seem to justify the suspicion with which the gynecologist regards the entire group. But it must be considered that these suspicious changes may not always be progressive, but may signify merely a temporary or local acceleration of growth which may subside and even regress. They do not seem to justify their designation as sarcomatous transformations, for which much more extensively altered areas or even general metastases might well be demanded. Sarcomatous tendencies and precancerous changes do not constitute real sarcoma or cancer. I have encountered 3 malignant uterine myomas with general metastases and 2 with local recurrence in 20 years, and Winter found no case among 753 , so that malignant degeneration of myoma must be rare.

It has been claimed that certain myosarcomas arise through a malignant transformation of the connective tissue of leiomyoma. (Ricker, v. Franque, and Hauser). In Ricker's case the change occurred in numerous foci sharply separated from the muscle-tissue which was either infiltrated with the sarcomacells or underwent atrophy. Yet the descriptions of these authors are not entirely convincing that they were not dealing with the ordinary type of malignant change in muscle-cells.

Clinical Types of Leiomyoma.-Uterine Fibromyoma.-The uterus is the commonest seat of leiomyoma. The tumors are usually multiple, in 
fact, in several cases the entire uterus has been found to consist of a congeries of discrete myomatoid masses (Cruveilhier, Williams, Stone). The location is widely distributed, the posterior wall, anterior wall, sides, and cervix appearing in the order named. From 5 to 8 per cent. of these tumors are cervical (Fehling). Fxtra-uterine tumors of the same type and significance occur also in the broad ligaments, vagina, tubal junction, in the pelvic fasciæ, and in the round ligament as far down as the groin or labium majus (Cullen, Aschoff). The position of the uterine tumor may be subserous, interstitial, or submucous. Both subserous and submucous tumors tend to become pedunculated or even separated from the uterus, being rarely discharged per vaginam (Simpson), or lying free in the peritoneum (Turner), or becoming attached to surrounding organs. Submucous tumors may gradually separate from their

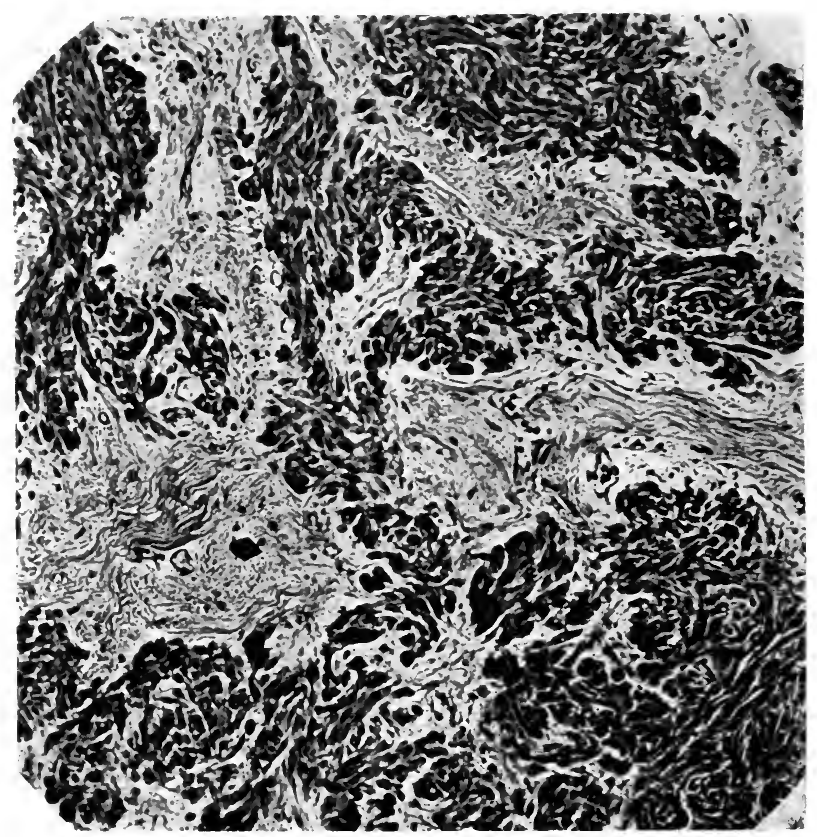

FIG. 56. - Structure of a multiple nodular recurrent myoma of broad ligament.

original site and become implanted on the opposed mucous surface (Leyden, Kustner). Diffuse myomatosis may cause thickening of a small focus or of a large area, or of almost the whole organ. Miliary myomas appear in the peritoneum during the course of larger tumors, while Hunter found at autopsy a single tumor weighing $\mathrm{I} 40$ pounds.

The form of uterine myoma is usually a rounded, sharply circumscribed solid and elastic mass which projects prominently from the cut surface. Many very early myomas and many adenomyomas are without definite demarcation, and the diffuse forms remain so, but with increasing age most tumors show more and more isolation and some become encapsulated. Cysts, multiple and superficial or large and central, form in many myomas from dilated gland alveoli, or from chronic edema and mucinous softening. Their contents are serous or mucinous fluid with cholesterin, or after infection they may become 
distended with pus. Some of the cystic myomas reach a very large size (Martin), that removed by Severanu of Bucharest being one of the largest tumors ever observed. They may contain pus or chocolate-brown or bloody fluid. The uterus, which is the seat of myoma, especially of the submucous or interstitial varieties, enlarges with the growth of the tumor and becomes extensively deformed. There is hypertrophy and hyperplasia of the muscle-cells as a result of contractions caused by irritation from the tumor (Bertelsman), but Tillaux saw the uterus greatly enlarged with a small tumor of the lower seg-

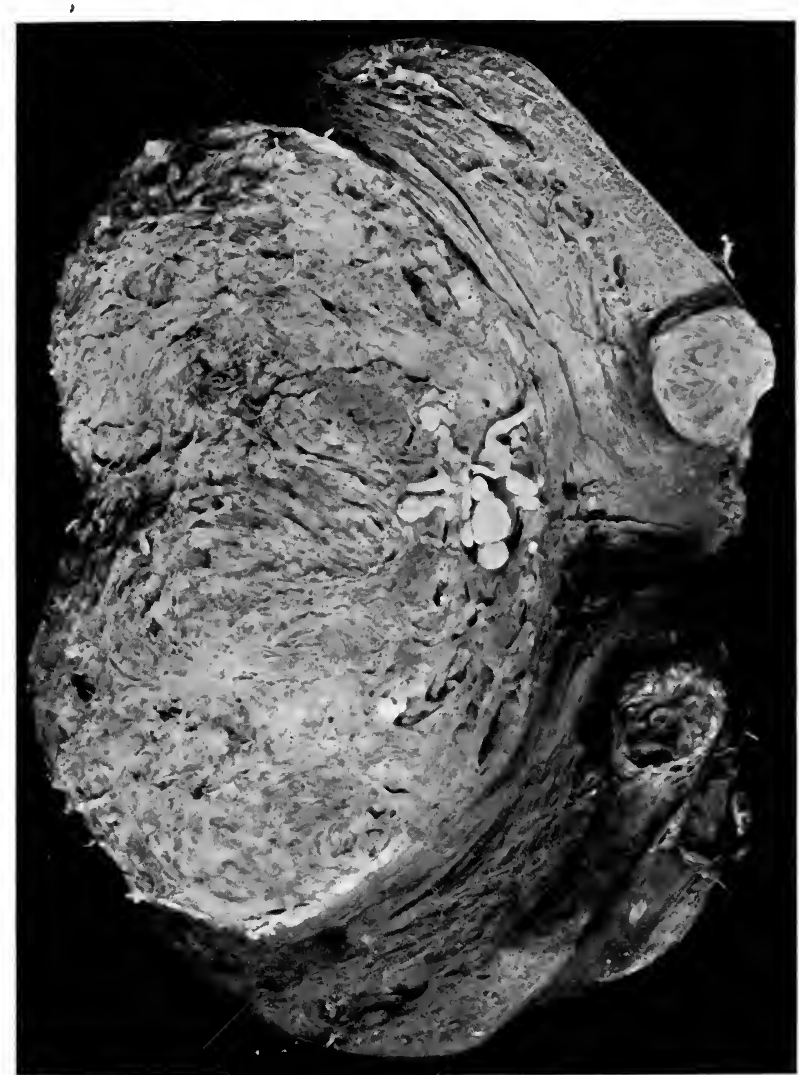

FIG. 57.-Myoma of uterus, confined under pressure with edema, small cysts, and polypoid growths into cysts.

ment. The vessels about the tumor are enlarged and the whole organ is hyperemic. Endometritis, interstitial or glandular, commonly results, especially with submucous varieties, and mucous and bloody discharge becomes a prominent symptom. Inflammatory hyperplasia may be limited to the mucosa overlying the tumor (Wyder), or it may extend also to the tubes (Fabricius) and ovaries (Bulius). Profuse hemorrhage between or prolonging the mentrual periods marks the course of many forms of myomas, and sometimes proves fatal. According to the position of the tumors the uterine cavity becomes elongated, irregular, or occluded. The stretching of the 
myometrium may produce peculiar rhombic spaces in the stroma and the stretching has even led to rupture of fundus from the cervix (Lennander, Hedren). Mural myomas may cause extensive torsion of the uterus, and submucous tumors may lead to prolapse or inversion (Kuster, Kotschau).

The gross section reveals the usual convoluted markings of myoma, or a congeries of multiple tumors. Islands of adenoid tissue and the sections of glands may be revealed by the hand glass. Multiple minute cysts appear when the alveoli are moderately dilated. The tumor vessels are usually scanty, while the surrounding tissue supports numerous dilated veins, but small areas (Cullen) or the entire tumor (Virchow, Martin) may be telangiectatic. Dilated lymph-vessels were prominent in two myosarcomas de-

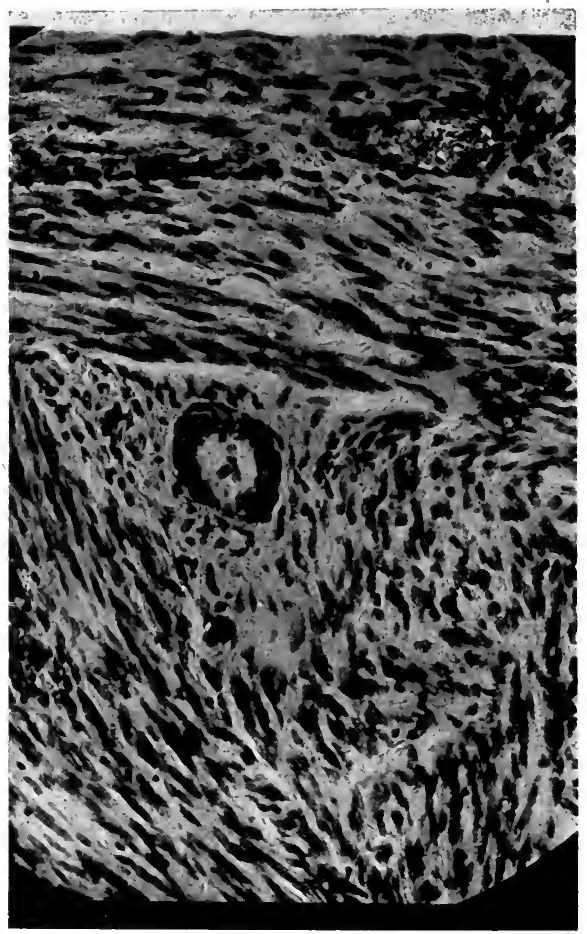

FIG. 58.- Structure of a locally infiltrating myoma uteri.

scribed by Menge. Submucous myomas are often traversed by glandular tracts, the openings of which may be seen on the mucous surface.

Extensive degenerative changes frequently alter the appearance of uterine myomas. Marked edema produces softening and cystic areas. Chronic edema leads to extensive mucinous degeneration with increase in the size of the tumor and formation of cysts. With increasing age there is a tendency toward atrophy and fibrosis which may greatly reduce the size of large tumors and render others very hard. Fatty degeneration of muscle-cells is associated with their atrophy and probably precedes calcification. Fatty changes may become very active during gestation, and Martin saw a large myoma almost wholly transformed into fatty semifluid material during ges- 
tation and the puerperium. Large tumors undergo fatty degeneration and may be reduced to small nodules or entirely disappear (Duncan, Hewitt, Gusserow). Hyaline degeneration may affect the blood-vessels, stroma, or muscle-tissue, in small foci or in considerable areas. According to Ribbert this change may precede calcification, the salts being deposited in the hyaline material. Stratz saw amyloid degeneration about the vessels of a polypoid myoma. Calcification overtakes fatty and atrophying myomas and completely transforms them into stony masses. Uterine stones of large dimensions are thus found in the cavity, wall, or on the peritoneal surface of the uterus (Henocque), and they have been discharged through the vagina or intestine, or into the bladder (Payr). Everett found the composition to be calcium carbonate 49 per cent., phosphate 29 per cent., sulphate I3 per cent., with traces of lithates and organic material. Turner and Wylie found a complete separation from the uterus of calcified subperitoneal myomas. Thrombosis of vessels is not an uncommon event, especially with pedunculated myomas, and leads to hemorrhagic infarction, necrosis, softening, and even to excavation and discharge of large portions of submucous tumors.

Sarcomatous changes, previously considered, occur in a small proportion of uterine myomas. Carcinoma is sometimes observed with uterine myoma and may result from several conditions. In adenomyoma, carcinoma may develop from the epithelial elements in the tumor. A submucous myoma may be complicated by carcinoma of the overlying and long inflamed mucosa, and the carcinoma may penetrate the myoma. Or the carcinoma may arise in a distant portion of the mucosa. Carcinoma of other organs has been known to produce metastases in uterine myomas (Liebmann, Schafer, Rolley).

The microscopical structure of uterine myomas reveals two forms of this tumor-fibromyoma and adenomyoma. The great majority of the tumors are of the former class and present the usual characteristic structure of fibromyoma. At their inception these tumors are nearly pure myomas and some remain so throughout most of their course, but with increasing age there is a tendency toward increasing development of connective tissue which in atrophying tumors may exceed the muscular tissue. The connective tissue is distributed in septa supporting vessels and as finer fibrils ramifying between the muscle-cells. Gebhard observed a large, purely fibrous nodule in the center of a solid myoma. The connective tissue does not participate in the tumor process, although some observers have claimed that it might give rise to sarcomatous changes in myomas. The tumor-cells are larger, thicker, and more granular than normal uterine muscle-cells, and the nuclei are larger, more rounded, and richer in chromatin. During gestation the cells of myomas increase in size. Their arrangement is in intertwining bundles running in all directions and without definite relation to any structural unit. Certain large tumors are composed of a congeries of somewhat discrete nodules in the centers of which are blood-vessels, and in some early myomas the growth centers chiefly about blood-vessels from the walls of which the tumor seems to spring. In such cases the adventitia of the vessel walls may be deficient (Rosger). It has been claimed that nerves have been demonstrated in uterine myoma (Hertz), but they are insensitive, and their existence has been denied.

In rare cases islands of bone have been found in uterine myoma (Lebert, Freund); a nodule of cartilage has been encountered by Bennet; and in one malignant case of adenomyosarcoma Kaufmann found both bone and cartilage. A fibrochondro-osteoma described by Kworostansky was possibly an atypical myoma suffering extensive metaplasia. 
Adenomyoma presents the usual structure of the muscle-tissue, but it contains glandular alveoli which appear in various forms. They usually form small groups of acini lined by cubical or cylindrical and sometimes ciliated epithelium supported by lymphoid stroma. These acini are identical in appearance with fragments of uterine mucosa with which they are often directly connected. Or the cells are more embryonal in type, with relatively large nuclei, with or without cilia, and the lymphoid stroma may be present or absent. Most observers have been unable to establish any constant distinctions between the epithelial structures in adenomyomas in different locations, but the development of the glands varies widely from a few scanty acini to large cyrstic tumors composed largely of epithelial lined cysts. Papillary projections may grow into the cysts, and Cullen saw adenocarcinoma developing from the papillæ. During menstruation there is congestion and hemorrhage into the glands (Cullen), and Amos has described decidual changes during gestation.

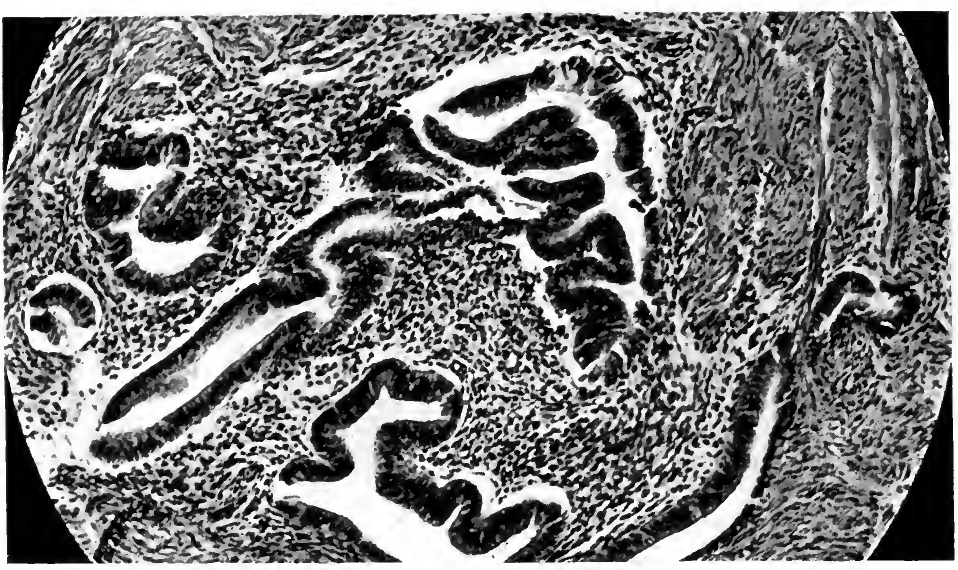

FIG. 59.-Structure of adenomyoma of broad ligament.

The occurrence of adenomyoma is relatively infrequent, but its distribution is wide-spread. They may be submucous, interstitial, or subperitoneal, and they have been found in the broad ligament, round ligament, and groin (Cullen), cervix, vaginal wall, Fallopian tube, and rectum.

Etiology.- - Uterine myoma is the most frequent of all tumors, its occurrence being estimated at 50 per cent. of all women over 50 years of age (Klob), and at 20 per cent. for those over 35 years. The incidence reaches its acme, 38.8 per cent., between the ages of 30 and 40 years (Gusserow). The negress is especially susceptible. Before puberty it is extremely rare, but Tillaux reported in a girl of ig a cervical myoma which had given symptoms for 6 years. Leopold's statement that the rudiments of myomas may be found in the uterus in children has not been verified. An hereditary influence seems to have existed in certain families in which several members suffered from myoma at an early age (Veit, Gusserow).

Sterility has long been held to be a factor in the etiology of myoma and Veit concludes that abnormal excitation and congestion of the organ without conception may excite tumor growth. It is highly probable that myoma favors sterility from the mechanical and inflammatory effects of the tumor. Both sterility and myoma are favored by the infantile type of uterus and by 
misplacements and other defects in the organ which go with each of these conditions. Virchow held that myoma is caused by local irritation. Many authors have pointed out sources of such irritation and shown that these act as secondary and exciting factors, which are not constant or essential.
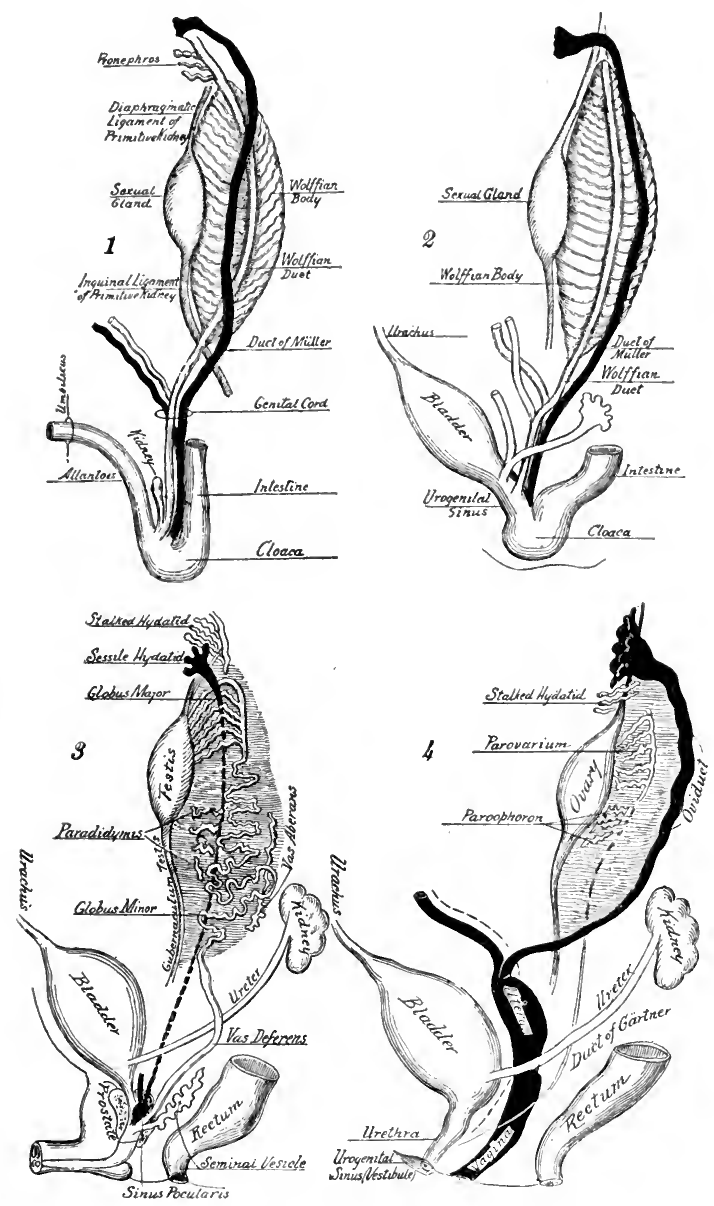

FIG. 60.-Diagrammatic representation of development of genito-urinary tract. (After Heisler.)

The Müllerian duct and derivatives are jet black. The Wolffian body and derivatives include all other structures except the sex glands, bladder, prostate, and rectum.

I, Indifferent type. 2, Indifferent type, later stage, the Wolffian and Müllerian ducts and primitive ureter now opening into the urogenital sinus. 3, Male type, lower ends of Müllerian ducts fused to form the sinus pocularis. 4, Female type.

The essential factor in the etiology of myoma is an embryogenic disturbance in the structure of the uterus. The remarkable degree of isolation of many myomas, their wide-spread occurrence apart from the uterine body, and the presence in many cases of heterotopic inclusions, epithelial, cartiaginous, osseous, fatty, and rhabdomyomatous, clearly point to an embry- 
onal origin. Moreover, as Williams has emphasized, uterine myomas are often associated with a large number of abnormalities in the genito-urinary system.

The nature of the embryogenic disturbance varies with different tumors.

(a) It is generally agreed that the common pure fibromyoma results from a disturbance in the formation of the tubes, uterus, and vagina from the Müllerian ducts, which split off from the Wolffian ducts at an early period and fuse to form the genital canal.

The relation of certain early myomas to the blood-vessels of the uterus has long impressed many observers and suggested that uterine myomas arise from disturbances in the growth of the blood-vessels from the walls of which the uterus and vagina originally receive their muscular tissue. Rosger, Kleinwachter, Sobotta, and others have traced the development of early myomas from the vessel walls and concluded that the blood-vessels control the origin and growth of uterine myomas. This view has much evidence to commend it, although it is seldom possible to demonstrate such a relation in advanced stages of the tumors. Yet in many pure, in adenomatous and in telangiectatic myomas, the blood-vessels are composed of neoplastic muscular tissue. In a case of multiple recurrent telangiectatic myoma of the broad ligament I found striking evidence of the origin of the tumor masses from blood-vessels.

The cases in which the whole uterus is composed of a congeries of myomas, or where a myoma replaces the cervix (Landau) or causes uterus didelphys (Pick), strongly suggest an origin from misfitting vascular units which go to make up the uterine muscle. Likewise single isolated myomas seem to represent overgrowth of muscle from single vascular units which have not fitted the general texture of the myometrium. Into many such tumors the invasion of glands has been traced from the mucosa. There are many who believe that the great majority of myomas of all types originate in this way.

(b) Regarding the origin of adenomyomas opinions are at variance and it is probable that no single mode of origin can apply to all these tumors.

I. A Müllerian origin accounts most satisfactorily for the majority of uterine adenomyomas, especially for the submucous, interstitial, and diffuse varieties. Duplication of the duct, fetal budding of the epithelium, misplacements of islands of fetal mucosa, postembryonal misplacement of gland tissue, and invasion of the myometrium by gland alveoli during inflammation have been observed or suggested as accounting for the origin of these tumors (Meyer, Pick, Hauser, Schroeder, Ruge).

In many submucous or deeper adenomyomas the epithelial structures have been traced to the uterine mucosa, on the surface of which they sometimes open by patent canals (Ribbert, Cullen). The invasion of a tubal myoma by glands of the tubal mucosa has been traced in serial sections by $v$. Franque. The structure of the glands in most adenomyomas is an exact duplicate of the uterine glands and they seem to functionate as does the uterine mucosa.

2. Mesonephric Adenomyoma.-The epithelial structures in certain adenomyomas were interpreted by Babes and by Recklinghausen as remnants of the Wolffian body or mesonephros, and many have supported this view (Ricker, Schroeder, Pick, Schickele, Ernst). Recklinghausen believed the glandular structures in certain cases presented the characteristics of the mesonephros which forms a closed system consisting of a chief canal or ampulla into which many parallel smaller canals open. The smaller canals, starting in wide ampullæ, advance in the form of convoluted secreting tubules lined by cubical epithelium and empty into the chief canal while lined by high or ciliated epithelium. Vascular polypoid projections of cellular tissue along the 
canals in certain tumors he interpreted as pseudoglomeruli. Occasional deposits of pigment he compared with the pigment of Giralde's organ (remnant of lower Wolffian tubules).

Recklinghausen and Pick have attempted to divide adenomyomas into submucous (central and ventral) and paroöphoral (dorsal and peripheral), the former deriving its glands from the endometrium, the latter from the paroöphoron, the remnant of the Wolffian body (lower tubules). Pick holds strongly to the dual origin of adenomyoma uteri, claiming that the tumors located in the broad ligament are of Wolffian origin and may be designated as parovarian and paroöphoral adenomyomas. Under the term "adenomyoma psammopapillare" he describes a remarkable multiple papillary tumor arising in the broad ligament which well illustrates Recklinghausen's voluminous paroophoral adenomyoma. As the chief histological distinction he states that lymphoid stroma usually but not always accompanies uterine glands in adenomyoma, while the alveoli of Wolffian origin lie in immediate contact with muscle tissue and lack the lymphoid stroma. Yet Cullen finds such lymphoid stroma in tumors of the round ligament.

While it is probable that certain extra-uterine adenomyomas originate from the parovarium or paroöphoron, the majority of observers have not been impressed with the mesonephric characters of these tumors. Neither the location of the tumors nor the arrangement of the ducts nor the character of the epithelium seem to be sufficient evidence to prove the mesonephric origin, since very similar structures are seen in submucous tumors and in others for which a Müllerian origin is extremely probable (Meyer, v. Franque). Cohen saw the characteristic arrangement of tubules in a submucous tumor of which the epithelium was clearly derived from the endometrium, and he found the pigment in adenomyoma to be ferruginous, while that of Giralde's organ is iron free. Aschoff found no uniformity in the location of the so-called paroöphoral type of adenomyoma, and Kossmann and Lockstadt found a general resemblance between uterine glandular tissue and the epithelial structures in all adenomyomas. Pick states that there are both adult and embryonal types of adenomyoma, and such a distinction appears to be not infrequent, but the distribution of these types does not accord with the other features which might separate two varieties of adenomyoma. The predominance of muscular tissue is more readily explained by a Müllerian origin, for muscle-cells are scanty in the Wolffian structures, and Pick regards this element as quite secondary and unessential in the anlage of mesonephric tumors.

3. Remnants of the Wolffian duct (Gärtner's duct) seem to give origin to certain extra-uterine adenomyomas. It has been shown by Recklinghausen, Ricker, Pick, and others that the whole region of the uterovaginal canal may contain isolated epithelial groups which are probably derived from the Wolffian duct. Borst found heterotopic Wolffian alveoli in the iliac lymphnodes. Klein traced a persistent Wolffian duct in two infants. The proximal portion traversed the broad ligament beneath the Fallopian tube to the uterus, and terminated in the uterine muscle at the os internum. The distal portion passed along the side of the uterus to the cervix, curved backward over the fornix, and passed downward in the wall of the vagina to the hymen. The canal was lined by cubical epithelium and supported by smooth muscle, and Klein concludes that this structure may give rise to various tumors along its course, as cysts, myoma, adenomyoma, and adenocarcinoma. Cervical and vaginal adenomyomas especially have been referred to this origin (Breus, Herpf, Pfannenstiel). Yet, except for their unusual position, the same difficulties exist in proving this origin as for the mesonephric type, since the structure of these tumors does not differ essentially from that of the Müllerian type (Cullen).

4. Besides the epithelial alveoli, subperitoneal myomas may contain acini lined by cubical endothelium derived from inclusions of the peritoneum (Aschoff, Meyer).

Summarizing the evidence, one may conclude that simple myoma uteri arises chiefly from a disturbance in the development of the tubes, uterus, and vagina from Müller's ducts, which often leads at the same time to gross deformities and infantile characters in these organs. 
Adenomyoma arises chiefly from the fetal or postembryonal inclusion of Müllerian epithelium in the tumor process. Extra-uterine adenomyomas in the broad and round ligaments and cervix may also arise from the mesonephric elements or from the Wolffian duct, but neither the position nor structure of these tumors permits their positive identification in every instance. The growth of many myomas is controlled by their relation to blood-vessels from which they are derived. The chief exciting factor is intermittent hyperemia connected with irregularities in the sexual functions.

Myoma in the Alimentary Tract.-The esophagus was the seat of small multiple leiomyomas containing also striated muscle-fibers and ganglioncells in a case described by Pickler. A larger single tumor was observed by Illig. Both were connected with the inner muscular coat. Eising's case was complicated with adenocarcinoma.

In the stomach Miodowski observed a bulky myoma which led to severe hemorrhage. He found 2 similar cases in the literature. Hansemann has reported a malignant gastric myoma with metastases in liver and pancreas. In a large tumor studied by Cohen there were islands of pancreas. Laboulbene saw 4 small myomas in the same stomach. Many gastric sarcomas are probably of myogenic origin (q.v.).

In the intestine leiomyoma is not uncommon. The tumors are single or multiple. Nazzari saw 40 small tumors in one case and 120 in another. Steiner found them either subperitoneal or submucous, the latter type causing stenosis or intussusception, the former producing diverticula. In Mercer's case fatal hemorrhage occurred. Cohen found islands of pancreas in an intestinal myoma which probably belonged with the adenomyomas of Trappe. Both gastric and intestinal myomas are apt to be associated with uterine myoma, and they appear to originate from isolated segments of the musculature, connected with blood-vessels or epithelial structures. According to Boetticher and Lode 3 types of intestinal myomas may be distinguished: (I) Small multiple nodular or polypoid tumors arise from local proliferation in the muscularis; the mucosa is free; $(2)$ broad thick tumor masses form in the muscle layers, while the mucosa becomes adherent to the tumor; (3) larger polypoid subserous myomas may project into the peritoneum.

Dermatomyoma.- A wide variety of myomas is observed in the skin. They occur on the buttock and extremities, at embryonal fissures (Babes), in the scrotum and labia (m. dartiques) (Besnier, Forster, Challard). They may be associated with keloid (Babes) or lymphangioma (Axel, Key), neuroma (Czerny), and xanthoma (Chambard, Gouilloud). Politzer has shown that certain xanthomas result from fatty degeneration of smooth muscle-fibers. In some cases the blood-vessels are very numerous and neoplastic, producing angiomyomas (Hess). The tumors are usually small and multiple, firm, movable, cutaneous or subcutaneous nodules or masses. They may be painful and tender. They usually occur in adults, but Hess observed early cases and Marc describes a congenital lymphangiectatic myoma. The origin has been traced to the arrectores pilorum (Judassohn, Walters) or to the cutaneous arterioles (Hess, Marc). Borst suggests that some of the dermatomyomas are really neurofibromas. Sobotka traced the process from simple hypertrophy of the arrectores up to tumor growth, followed by degeneration.

In the urinary bladder and passages myomas constitute a considerable proportion of the tumors occurring in those organs.

In the bladder they may reach large dimensions and their location is either subserous or submucous (Stein, Terrier, Hartmann). In an infant Kaufmann found a myoma of the trigonum containing cartilage. A large myocarcinoma is reported by Volkmann. In the ureter Buttner observed a 
large pure myoma. In the testis Rindfleisch described a myoma which contained nerve-fibers and ganglion-cells, and Becker reported I case of doubtful nature.

In chronic prostatitis there is often extensive hypertrophy of smooth muscle tissue which may approach the neoplastic grade. True myomas of this organ have not been clearly separated from this inflammatory overgrowth.

In the kidney small multiple myomas and myolipomas occur in capsule or cortex and appear to be derived from fragments of capsule tissue (Müller, Larkin). Jacobsens has collected several cases which were associated with cerebral sclerosis. In the breast leiomyoma has been observed by Abramow and Ribbert.

\section{RHABDOMYOMA}

Tumors of striated muscle are characterized by their rare occurrence, embryonal type, and common association with other constituents in mixed tumors. They occur in very wide distribution and, while the genito-urinary system furnishes the largest proportion, few muscular regions escape an occasional development of rhabdomyoma, and the homologous growths are fully equaled in number by heterotopic forms. They usually occur in early life, some are congenital, and a few examples have been first noted in advanced or old age (Wolfensberger, Fujinami).

In form they appear as single or multiple, nodular or voluminous, flat or rounded, circumscribed or diffuse, and even polypoid growths. They are usually soft and grayish on section, and markings produced by muscle bundles alternate with abundant connective-tissue stroma. Very cellular forms are more opaque, yellowish or reddish, soft, and often diffuse. The mixed rhabdomyomas present the varied appearance of teratomas. A cystic rhabdomyoma of the elbow has been described by Billroth.

The structure of rhabdomyoma presents chiefly a system of parallel bundles or intertwining strands of striped muscle-fibers, supported by adult or embryonal connective tissue. The fibers may encircle blood-vessels or other structures, but are more often diffuse. The cells of rhabdomyoma seldom exhibit adult characters. They are usually thin and much elongated and rarely they are branching (Zenker, Billroth). Both long and cross striation may be pronounced or the cross striation may be present in a part of the cell only or be entirely missing in embryonal and spindle-shaped cells which still retain longitudinal fibrillation. Or the cells may closely resemble smooth muscle-cells. In large vacuolated cells Ribbert describes concentric striation of the perinuclear cytoplasm. In a testicular tumor he observed long tubular fibers, the walls of which were of striated or hyaline muscle fibrils and the core of granular sarcoplasm with many nuclei in axial positions. The ends of the fibers may be swollen, rounded, and contain multiple nuclei. In the more embryonal types the cells are spindle-shaped, shorter, and even round, and they may lose their acidophile character and all resemblance to muscle-cells. Yet such marked anaplasia is seldom if ever universal, certain foci commonly retaining cells of definite myogenic character. Even the round-cells of lymph-node metastases may retain traces of striation (Wolfensberger, Eberth), or the metastases may consist of cells showing no features of muscle-cells (Benenati). In many myomas the cells contain an abundance of glycogen which is evidence of their embryonal character (Marchand).

The nuclei follow a type of large vesicular chromatic bodies, single or multiple, which lie in a central area of granular cytoplasm. Or they may project from the cell border like nuclei of sarcolemma. A definite sar- 
colemma is wanting, but traces of such a structure with its nuclei have been seen in rare cases (v. Franque, Marchand). The stroma is either loose adult connective, or embryonal, sarcomatous or myxosarcomatous, and in the teratomas bone, cartilage, and various other tissues may be present. Bloodvessels are usually abundant and sometimes, as in a large polypoid vaginal tumor described by Kaschewarowa, they are overdeveloped.

In parts of certain rhabdomyomas it is difficult to determine whether one has to deal with voluntary muscle-fibers which have lost their striation or with genuine smooth muscle tissue. The undifferentiated cells may closely resemble smooth muscle. Moreover, rhabdomyoma occurs especially where

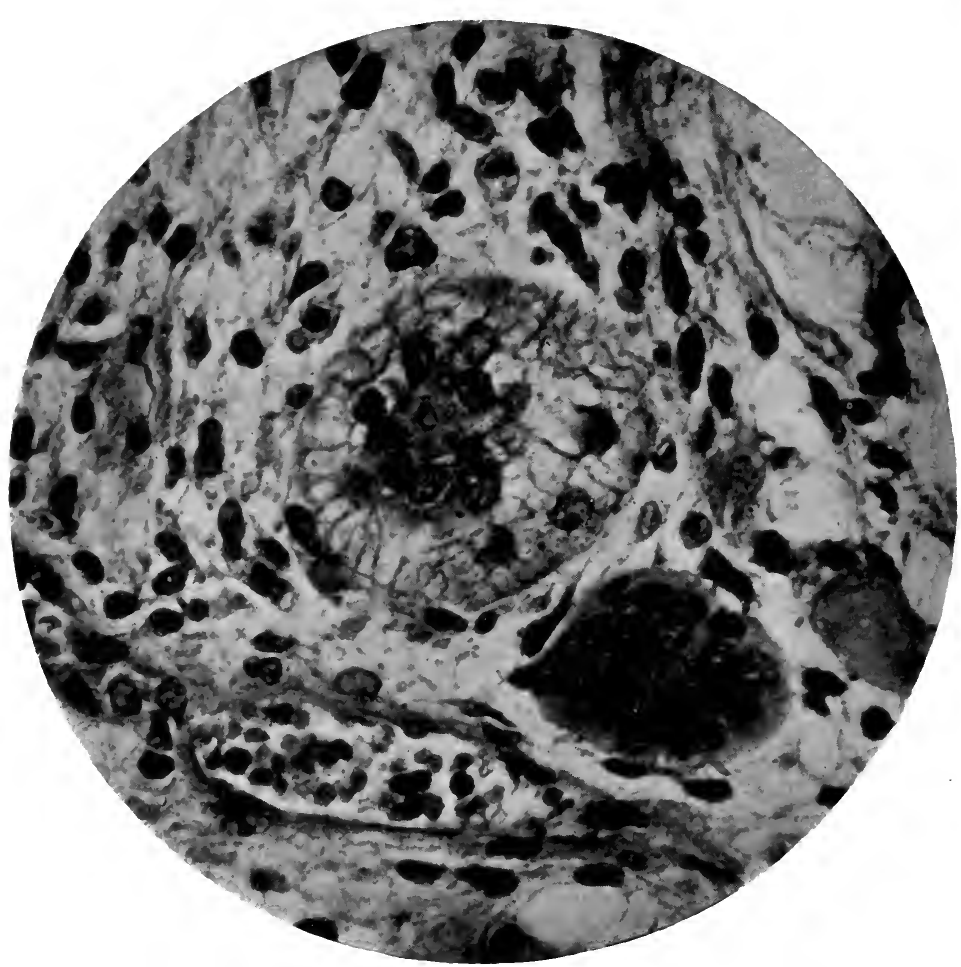

FIG. 6I.-Spider cell with multiple nuclei and centrosomes, believed to be derived from heart muscle, in rhabdomyosarcoma of ovary. (After Himwich.)

smooth muscle tissue exists (kidney, testis). To many observers it has appeared that smooth muscle tissue may give rise to striped muscle-fibers in tumors (Eberth, Rindfleisch, Arnold, Marchand). In a myosarcoma uteri v. Franque saw islands of striated muscle together with smooth muscle and spindle-cell sarcoma, and he concluded that the striped muscle arose by metaplasia of smooth muscle. Yet Girode and Nehrkorn both saw islands of striated muscle in the normal myometrium, and the embryology of this organ provides the possibility that portions of striped muscle tissue may be included in its structure. Arnold, in supporting a suspicion that metaplasia of smooth may produce striated muscle, derived a rhabdomyoma testis from the cremaster muscle, but the tumor lay within the albuginea, while this 
muscle lies without the organ. The embryology of muscle tissue, voluntary muscle being derived from mesothelial plates and involuntary muscle from the mesenchyme, does not favor the idea that one form may pass into the other. While many earlier observations suggested the change of smooth into striped muscle, most recent studies have not favored this view (Ribbert, Hauser, Kolisko).

Himwich has described from this laboratory a large rhabdomyosarcoma of the ovary of an infant, which recurred soon after extirpation. The structure presented many phases of the development and degeneration of striated muscle-cells. Large areas of the tumor had the appearance of a cellular myxoma, suggesting that pure myxoma may arise from this source. Other portions

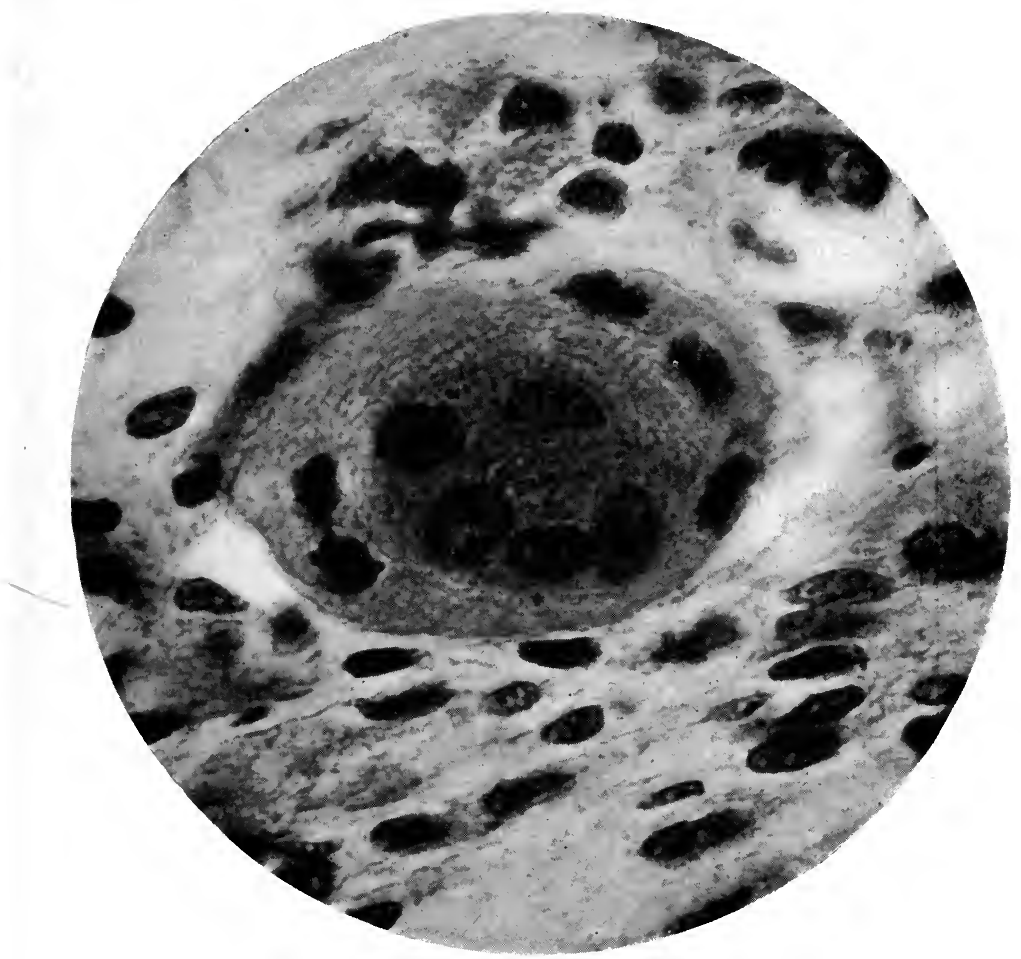

FIG. 62.- Striated giant-cell in rhabdomyosarcoma.

were composed of well-developed striated muscle-cells, and many large giantcells with concentric and cross striation were encountered. Most of the giantcells were extensively vacuolated, forming the so-called spider-cells which are found exclusively in rhabdomyoma of the heart. On this account the author concluded that the ovarian tumor represented was of teratoid origin, and derived from the heart muscle of a twin fetus.

The course of rhabdomyoma is usually progressive and in the sarcomatous and teratoid forms it is rapid. The more adult types may become encapsulated or assume the polypoid forms, but the embryonal tumors infiltrate surrounding tissue, multiplying muscle-cells being often preceded by a zone of proliferating connective tissue. 
Degenerative changes, glycogenic, hyaline, or amyloid, may occur without interfering with the growth. The malignant tumors of the kidney and testis reach large dimensions and produce metastases and cachexia, but the metastases are usually derived from other elements of the tumor.

\section{CLINICAL FORMS OF RHABDOMYOMA}

Adenomyosarcoma of Kidney.-The kidney is the commonest seat of tumors containing striated muscle, but since none of them has yet appeared in pure form they will be described under tumors of the kidney.

In the urinary bladder polypoid rhabdomyomas have been described by Cattani, Vincenzi, Stumpf, and Monckberg. They occurred in children or young adults, and formed movable growths about the urethral orifice or trigone.

Rhabdomyoma uteri appears almost exclusively as an element in the polypoid vaginal sarcoma of children and adults (Sarcoma botryoides, Pfan-

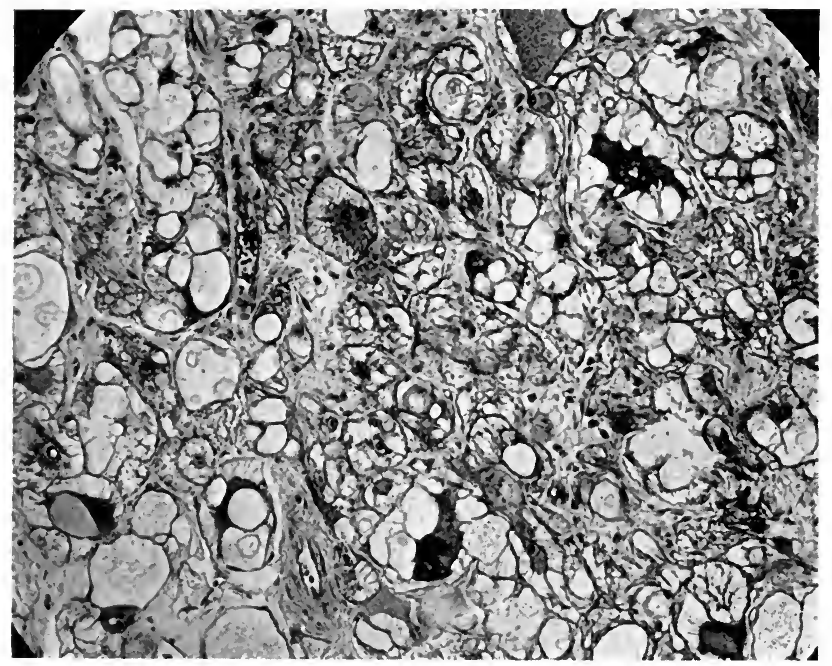

FIG. 63.-Structure of congenital rhabdomyoma of heart. (From a section of Wolbach's case.)

nenstiel). This process affects the vagina in children and chiefly the cervix in adults (Gow, Pick). It may exist at birth (Demme) and first appears as a rather broad thickening of the submucosa which soon becomes polypoid. v. Franque reported a somewhat similar tumor located on the fundus uteri in a multipara. The symptoms are hemorrhage, fetid discharge, and protrusion of a polypoid tumor from the vagina, with dysuria, pain, fever, and cachexia. The vagina is eventually filled with ulcerating masses and there are bulky extensions into the pelvis, and occasionally to regional lymphnodes, rarely to skin or lung (Kalustow, Rosthorn). In Demme's case a benign polyp after five years suddenly became malignant. The usual histology is that of a large spindle-cell sarcoma, with many blood-and lymph-vessels, myxomatous tendencies, and areas of striated muscle.

Pfannenstiel believed he could trace the development of muscle-cells from the spindle-cells. Kolisko found muscle tissue in all of 3 cases and 
believed it to be present in all cases. Islands of cartilage have been observed in the primary tumors (Rein, Pernici) or only in recurrences. The remarkable clinical characters of this tumor render it a very well-defined disease. The early appearance and complex histological structure of the tumors show that their origin must be referred to some embryogenic disturbance of the cervix and vagina, but the nature of this disturbance has never been clearly defined.

In the testis rhabdomyoma occurs in the form of nodular tumors as large as a walnut or a child's head. They may replace the entire organ or displace it to one side, or be found outside the tunica albuginea. In several cases the muscle tissue was associated with epithelial structures, and one of Ribbert's cases was combined with carcinoma and sarcoma. Neumann

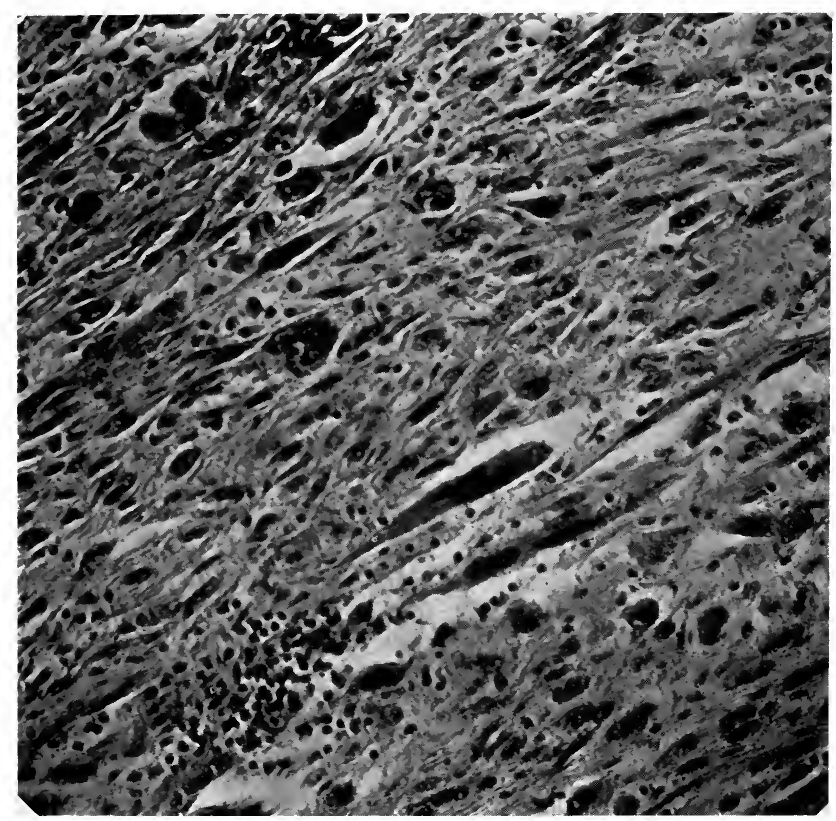

Fig. 64. - Rhabdomyosarcoma of voluntary muscle following fracture of femur. (After Miiller.)

and Ribbert found many spindle ${ }_{\tau}$ and round cells which were not readily identified as myomatous, and Neumann suggests that some spindle-cell sarcomas regarded as fibroblastic may really be of myogenous origin. Benenati describes a large rhabdomyoma of an undescended testis with round-cell metastases in the regional lymph-nodes. The tumor contained spindle-cell and round-cell areas as well as striped muscle. Stoerk observed a very malignant case with myomatous metastases in distant lymph-nodes. Wood found cartilage and epithelial cysts as well as muscle. All these observations lead one to accept the view that rhabdomyoma testis occurs only as a one-sided development of a teratoma. Rokitansky and Neumann derived their tumors from the gubernaculum of Hunter, but these growths were attached to the lower pole of the tunica and were not strictly tumors of the testis. 
Congenital rhabdomyoma of the heart is a very characteristic condition, of which 12 cases have been reported (Wolbach, Lit.). They occur as multiple sharply circumscribed areas or tumors, lying within the wall or projecting internally or externally. Ponfick pointed out that they are associated with diffuse sclerosis of the cerebral cortex and with disturbances of nutrition. Cesaris-Demel found also nodules in the kidney composed of embryogenic renal tissue, and Wolbach observed multiple neuroglia rests in the spinal meninges.

The structure of the tumor presents a peculiar spongy tissue in which it is difficult to make out the relation of certain large vacuoles to cells and fibrils. The vacuoles have been shown to lie in the very large cells of which the tumor is composed (Seifert, Wolbach). The cells are large rounded masses containing large nuclei and nucleoli, surrounded by partly striated cytoplasm which extends in many fibrils between the vacuoles to the cell border. They resemble early embryonal heart muscle-cells (Kolisko), and similar cells may be found in the fetal myocardium (Wolbach). The cross striations are formed of fuchsinophile granules grouped in the sarcous elements, and the long striations are made up of alternating sarcous elements and basophile fibrillary material. Either of these elements may be in excess. The resemblance of the cells to Purkinje's cells and those of the conducting bundles of the heart is mentioned by Knox and Shorer. That the tumors arise from an embryogenic disturbance in the structure of the heart is obvious. Kolisko mentions the early and extensive changes in the morphology of the heart as favoring errors in development. Bonome traces the pathogenesis to fetal malnutrition resulting in cerebral atrophy and sclerosis, and to fibrous overgrowth in the heart, which separates nests of embryonal cells from which the tumors develop.

Miscellaneous Rhabdomyomas.-Tumors containing striated muscle have been observed in isolated cases in many other organs and tissues; in the esophagus (Wolfensberger, Glinski); in the stomach (Brodowski), in the tongue (Pende), parotid gland (Prudden); in the breast (Billroth); in the prostate (E. Kaufmann); in place of the left lung (Helbing). Rhabdomyomas of the skeletal muscles in various parts of the body, orbit, neck, pelvis, buttocks, and different portions of the extremities have been collected by Fujinami from reports of various authors, and Benenati gives an extensive chronological list of cases. Among the regions affected are the spinal vertebræ and pectoralis major (Buhl), upper arm (Billroth), tibia (Lambl), thigh (Fujinami), and nose (Erdmann).

Of 16 cases of rhabdomyoma derived from adult voluntary striated muscle in which the cells were said to have retained cross striation, reported up to I9I3, Kuttner rejects 6 and regards the others as questionable. Tumors composed of more anaplastic cells in which-cross striation was lost and whose origin from muscle-cells was established on other grounds, are very rarely reported. Müller has reported from this laboratory one such case following repeated trauma of a long ununited fracture of the femur. The tumor was composed of large acidophile spindle-cells, some of which approached the dimensions of muscle-cells. A few cases of similar origin are reported. 


\section{CHAPTER XVI}

\section{ANGIOMA}

Angioma is a tumor composed of newly formed vessels. Both blood- and lymph-vessels are subject to neoplastic growth, giving the two classes of tumors-hemangioma and lymphangioma.

\section{HEMANGIOMA}

The determination of the scope of neoplastic processes affecting bloodvessels presents unusual difficulties. Vessels occupy an altruistic position in the physiology of organs, subordinating themselves to more specialized structures. Possessing less natural autonomy they may be expected to display pronounced neoplastic properties. Thus practically normal bloodvessels support highly malignant tumor tissue. The supply of vessels in a

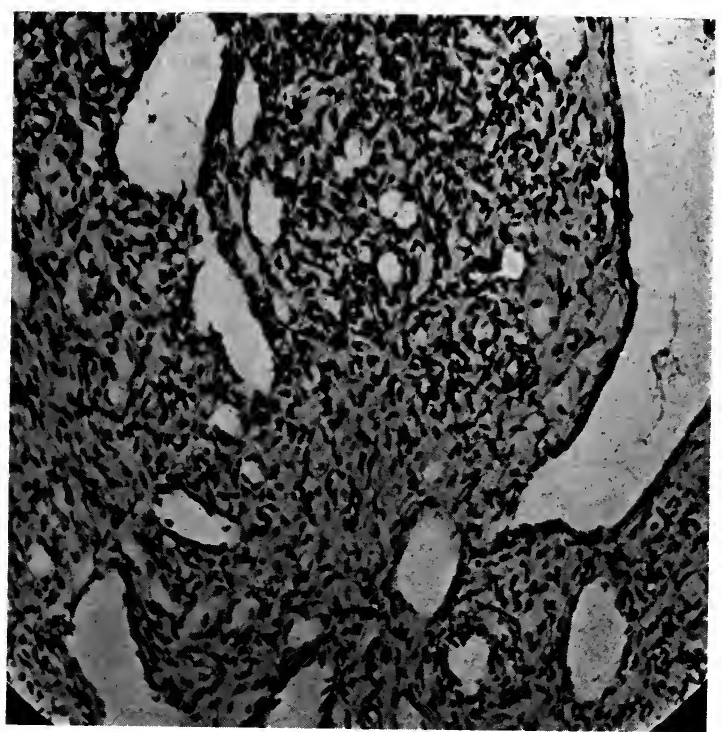

FIG. 65.-Plexiform angioma_of skin. Slowly growing and benign.

tissue or organ varies extremely in both normal and pathological conditions. Not being composed of a simple tissue, tumors of vessels must be organoid in character. The growth of vessels is markedly influenced by the element of mechanical pressure of the circulation, which is absent in other tumors. Finally, the nutrition of vessels, especially of those subject to tumor growth, is provided in a different manner from that existing in other tissues. Thus the physiology of vessels necessitates special standards in the interpretation of tumor processes, and this peculiarity has led to much difference of opinion as to what constitutes an angioma.

From the angiomas may at once be excluded several processes marked by overgrowth of vessels. 
Hypertrophic granulation tissue presents an extensive new growth of vessels in sinuses, abscess walls, suppurating cysts, and in closed tissues, with circumscribed enlargement of tissue. Yet it is self-limiting, regresses upon removal of the irritant, and the structure of the vessels shows absence of the histological signs of a neoplasm. In rare instances, however, without clinical data it may be difficult to separate this process from angioma, but a definite relation between chronic granulation tissue and any of the characteristic vascular tumors can rarely be established. On the other hand, there are many indications that certain sarcomas arise from granulation tissue.

Chronic varicosities are established in veins and arteries in several conditions which do not constitute tumors, as varicose veins in the limbs, spermatic cord, and broad ligament, and in diffuse aneurysms which follow trauma. In hemorrhoids the varicosities are the result of venous stasis and chronic inflammation. In inflammatory polyps extensive development of veins often results from venous stasis.

Excessive development of blood-vessels occurs in many tumors as a result of venous stasis or unusual demands for nutrition. Very often such vessels present a normal or nearly normal structure and do not exhibit neoplastic growth. Such tumors are not true angiomas, but their vascularity may be indicated by the term "angiomatosum," as fibroma angiomatosum. The demands for nutrition in rapidly growing cellular tumors may produce a type of growth which is composed chiefly of blood-vessels sheathed by masses of tumor-cells, and which is often called perithelioma, or telangiectatic sarcoma. This rather characteristic structure is not confined to any one class of tumors and it is not essentially a tumor of blood-vessels.

Over against these processes in which there is excessive development of more or less normal vessels stands a large class of tumors in which a neoplastic process affects the walls of vessels and usually also the supporting connective tissue, and these constitute the true angiomas.

In general, angiomas are of congenital or early development, slow growth and benign course, and they occur under rather characteristic clinical conditions.

Hemangioma Simplex. Vascular Nevus (Telangiectasis).-This type of angioma occurs in several forms.

Nevus vinosus, or the port-wine stain, consists of a circumscribed or diffuse dilatation and new growth of superficial capillaries and venules of the derma over which the epidermis is usually thin and delicate. The dilated capillaries lie immediately beneath the epidermis so that the color of the blood is imparted to the skin. They are usually congenital and the face is the chief locality affected. A similar process affects the subcutaneous tissue and fat, where it fails to yield a discoloration of the skin. According to Unna, nevus vinosus is to be separated from the true angiomas of the skin, since it consists of a simple telangiectasis of venous capillaries and does not progress after birth. Nevertheless it and the deeper congenital nevi have often been the source of extensive plexiform and cavernous angiomas. It may be classed with Albrecht's hamartomas.

Plexiform angioma consists of a new growth of dilated capillaries in which the length of the vessels is increased, but the number of new cells is not in great excess. Small veins and arterioles are also involved. This process causes a definite enlargement and tumor of the subcutaneous tissue, with flat or warty projections of the skin. Circumscribed tumors of this class occur in the skin of the face and especially in the eyelids of young children. In a few cases multiple and diffuse tumors with considerable new growth of connective tissue have occurred, chiefly in the arms and face of children 
and adults, and have been described under the terms "elephantiasis hemangiomatosa" or "nevus vasc. mollusciformis" (Seifert, Fox, Kaposi, Unna). In Jackson's case the process followed the course of the facial nerve. The plexiform angioma or deep nevus is located in the derma or subcutaneous tissue or in the fat tissue and commonly extends from one to the other. It may even invade the muscles and bones and eventually involve considerable areas and even a large portion of a limb. Occurring in embryonal fissures in face, cheek, lip, and neck, it has been called fissural angioma.

Angioma often begins in the vessels about the sweat-glands which may become atrophic and fibrosed or hypertrophic. An imperfect formation of lobules may be observed in relation to the cutaneous glands, or from natural

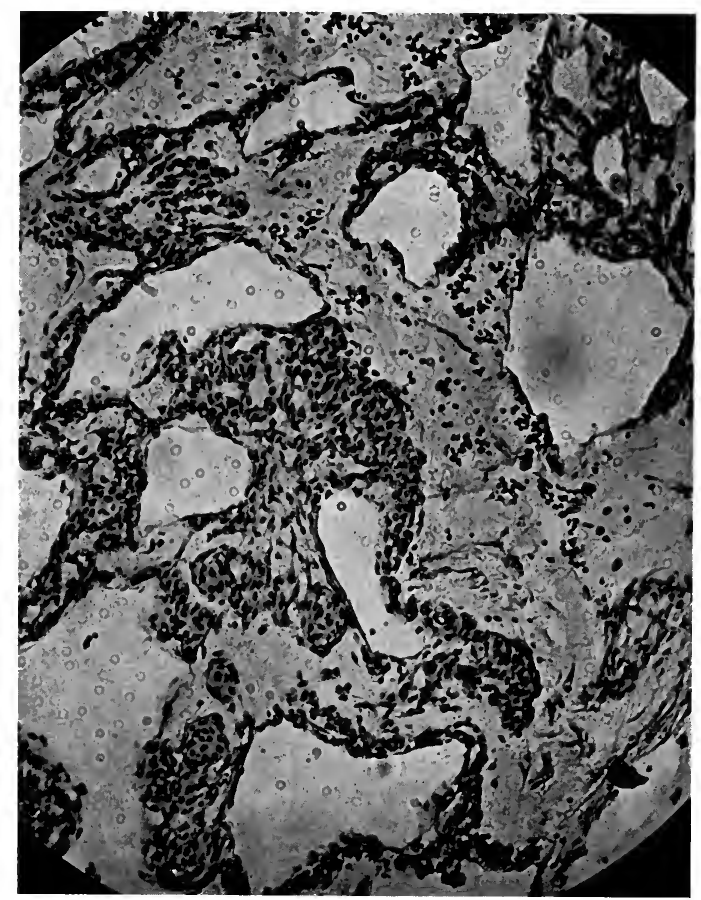

FIG. 66. - Simple cellular angioma, or angio-endothelioma.

anatomical septa in the fat tissue. The new vessels have the characters of arterioles rather than venules, but many sizes and types of vessels may occur in the same tumor. Cellular hyperplasia in the vessel walls is constant. The endothelium is much increased and appears in one or more layers. It is sometimes exfoliated or degenerated, and dilated vessels may be lined by giant-cells. Elastin is deficient or absent, and muscular tissue is usually deficient.

Angioma of the muscles occurs in subjects under 30 years of age, chiefly in the triceps femoris and forearm, occasionally in other regions. It produces moderate enlargement of the part with turgescence and impairment of function. Muscatello describes four histological varieties: (r) Capillary and progressive; (2) arterial, (3) venous, (4) cavernous. Pupovac observed multi- 
plication of lymph- as well as blood-vessels. In a series of 46 cases tabulated by Sutter the above histological varieties were variously combined. The process usually began with a proliferation of muscle-cells in the walls of venules and in several cases there were polypoid myomatous outgrowths into the lumen and fusion of contiguous vessel walls with subsequent dilatation of channels. The atrophic muscle-fibers were replaced by fat-cells. Angioma of muscles may extend to other tissues. I have examined a plexiform angioma involving the subcutaneous tissue, fat, and nearly all the musclés of the forearm of a young girl.

Hemangioma hypertrophicum (Ziegler) is a cellular form of capillary angioma occurring chiefly in the skin. Nauwerck described a very similar tumor occurring in the femur. It consists of a large number of small vessels lined by hypertrophic and neoplastic endothelium. The vessels usually maintain a scanty lumen, but the proliferation of endothelium may obliterate the lumen and yield compact groups of cells. In this form the tumor is virtually an endothelioma and in this and the transitional forms it may be designated as hemangio-endothelioma. Distention of the cellular vessels may also occur, giving a cavernous variety of the tumor. In many simple angiomas, especially in the growing edges and in fat lobules, the structure is that of hemangioma hypertrophicum. Pure tumors of this type are usually progressive and if very cellular may exhibit local malignancy.

Histogenesis. - Ribbert has analyzed in detail the origin and growth of simple angioma. By means of interstitial injection he finds that the vessels have few or no lateral anastomoses, while the injection mass passes freely into the efferent artery and afferent veins, but not into the tissue surrounding the tumor. This result indicates that the tumor process resides in an isolated segment of the vessel walls and produces elongation and varicosities in a more or less closed territory without gradual involvement of surrounding vessels. Where the tumor forms a new lobule or invades fat or other tissues it is not by extension of the tumor process to the healthy vessels of the new area but by the projection of new vessels which grow out from the tumor while the vessels of the invaded part are compressed and occluded. The microscopical structure shows that the process affects both the walls of the vessel and the supporting connective tissue. In the invaded fat lobules Ribbert finds isolated tumor vessels connected by long strands with the main tumor, while between the fat-cells parallel rows of endothelial cells appear which later become thickened and canalized. These new structures seem to have no connection with the normal vessels of the invaded tissue.

Thoma refers much of the growth of angioma to mechanical factors. Increase of blood-pressure and loss of support to vessel walls from changes in the surrounding tissue tend to excite new growth of vessels, while increased rapidity of flow favors elongation and dilatation of the wall. Many authors (Rokitansky, Borst) consider that many simple so-called angiomas represent simple hypertrophy of vascular segments without neoplastic overgrowth. These factors are doubtless important in determining the course of angiomas, but they cannot account for their origin, which must be referred to a developmental anomaly in the structure of certain vascular segments which do not fit into the circulatory system, and which retain embryonal characters. The congenital origin of the great majority of angiomas speaks strongly in favor of a tissue predisposition as a prominent factor in their genesis.

Virchow believed that angiomas result from the action of local irritation on imperfectly formed vessels, as those in embryonal fissures. Unna was led to believe that nevus vinosus especially occurs in areas which have been subjected to abnormal pressure during fetal life. The relation to nerve- 
trunks has suggested to many a neurotic theory of origin. In not a few cases a traumatic origin is clearly indicated (Lowenthal, Lit.).

Cavernous Angioma.- When the vascular channels are widely dilated and the connective tissue septa are thin, the angioma is designated as cavern-. ous. This process is of frequent occurrence in many situations and in nearly all tissues and organs. In the skin cavernous angiomas produce circumscribed or diffuse, flat or elevated lesions, involving the derma and subcutaneous tissue. If superficial, they are dark red in color. If covered by corrugated and thickened epidermis, they become warty. Changes in the circulation affect both the size and color of the tumors. Many of them are distinctly erectile and pulsating. Thrombosis and the formation of calcific

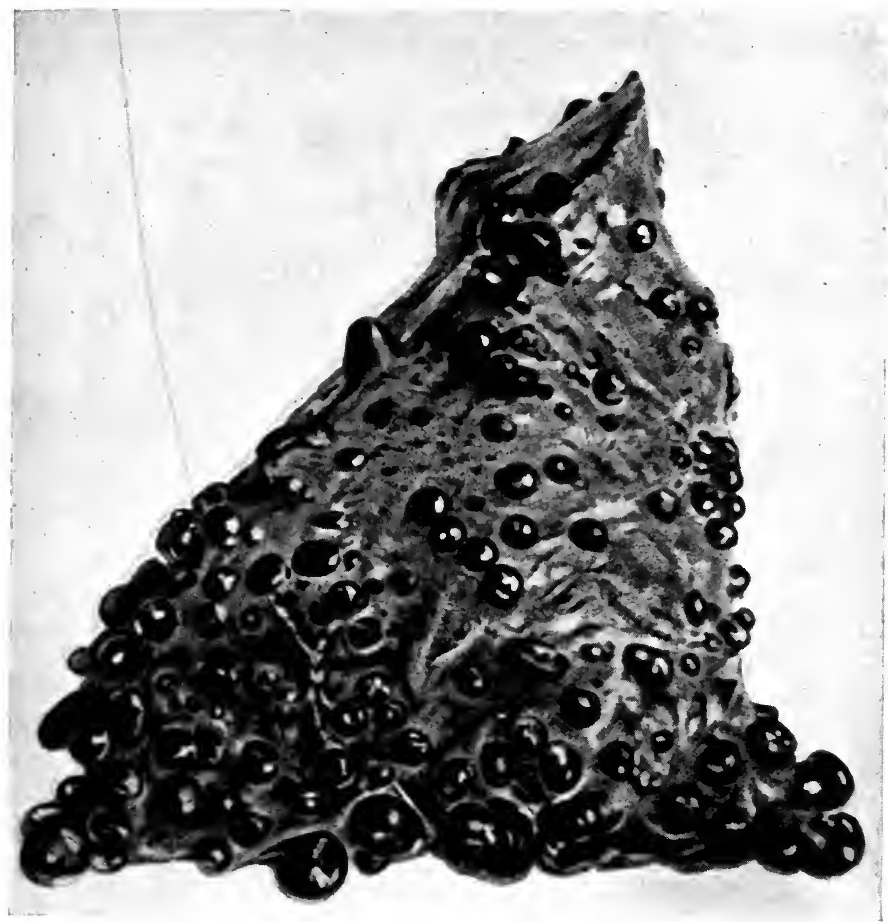

FIG. 67.-Metastasizing angioma, secondary nodules in lung. (Borrmann.)

phleboliths occur in dilated sinuses. The smaller tumors are usually encapsulated and stationary, but there are many cases of diffuse cavernous angioma in which a capsule is missing and the limits of the process gradually extend over many years.

The cavernous angioma first appears as a circumscribed tumor developing often on the basis of a congenital nevus and tends to enlarge steadily over a period of many years. They may be observed at birth or appear at any age. When allowed to progress they may attain very large dimensions, and successively invade neighboring tissues and organs. In some of the older cases, extraordinary results were produced. Gascoyen observed an angioma of the parotid which progressed for many years, produced a large polypoid 
tumor externally, while a pharyngeal portion eventually caused death by suffocation. Autopsy showed several nevi of the intestinal serosa and mucosa and one in the liver. Cruveilhier described a cavernous angioma involving the skin, muscles, tendons, synovial membranes, nerves, and periosteum of nearly the whole arm of a 65-year-old hemiplegic. Falkowski reports peculiar cavernomas of liver and spleen and angiomas of skin in an infant.

The subcutaneous angiomas may gradually involve the skin and the deep fascias, and some which first appear subcutaneously originate in much deeper tissues, or they establish wide communications with deep venous trunks.

Multiplicity of cavernous angioma is a prominent feature in some cases. The minute senile angiomas of the skin usually appear in considerable numbers. Large multiple cavernomas of widely separated regions occurred in a case of Hildebrand's. The smaller cavernous angiomas are often multiple,

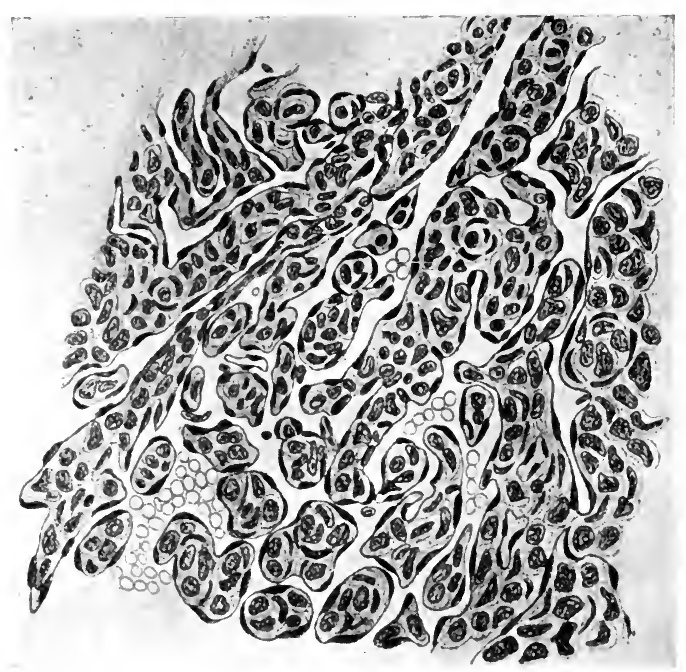

FIG. 68.-Structure of metastasizing angioma. (Borrmann.)

as many as 40 or even Ioo having been observed in one subject (Esmarch, Schuh).

Sharply contrasted with the multiple benign cavernous angiomas is a group of metastasizing cavernous or more cellular angiomas which exhibit certain peculiar features of malignancy, and are eventually fatal, chiefly through internal hemorrhage and anemia. Here belongs the remarkable case observed by Borrmann in which angioma of the skin of the breast in a subject of 23 years recurred repeatedly after operation and finally proved fatal with numerous secondary tumors in both lungs. The original tumor had the structure of simple angioma, but the secondary growths were more cellular.

In a case observed by the writer the breast was greatly enlarged by a bulky cavernous angioma, several tumors appeared on the skin and mucous membranes, and there were evidences of pulmonary involvement. The structure was very similar to that in Borrmann's case.

In Shennan's remarkable case, of 6 years' duration, marked by numerous 
hemoptyses, there were found at autopsy cavernous angiomas involving the whole of the spleen, much of the lungs, the thymus and mediastinal nodes, liver and bone-marrow, while many miliary angiomas occurred in other tissues. The structure was generally not malignant in appearance, but the small tumors and some of the larger were doubtless metastatic, and exhibited a local invasive tendency.

The course of cavernous angioma is usually slowly progressive. Beginning in congenital nevi or in deep tissues, at any period of life, they slowly enlarge by distention of the original vessels and by formation of new vessels. The growth is sometimes accelerated at the menstrual period and during

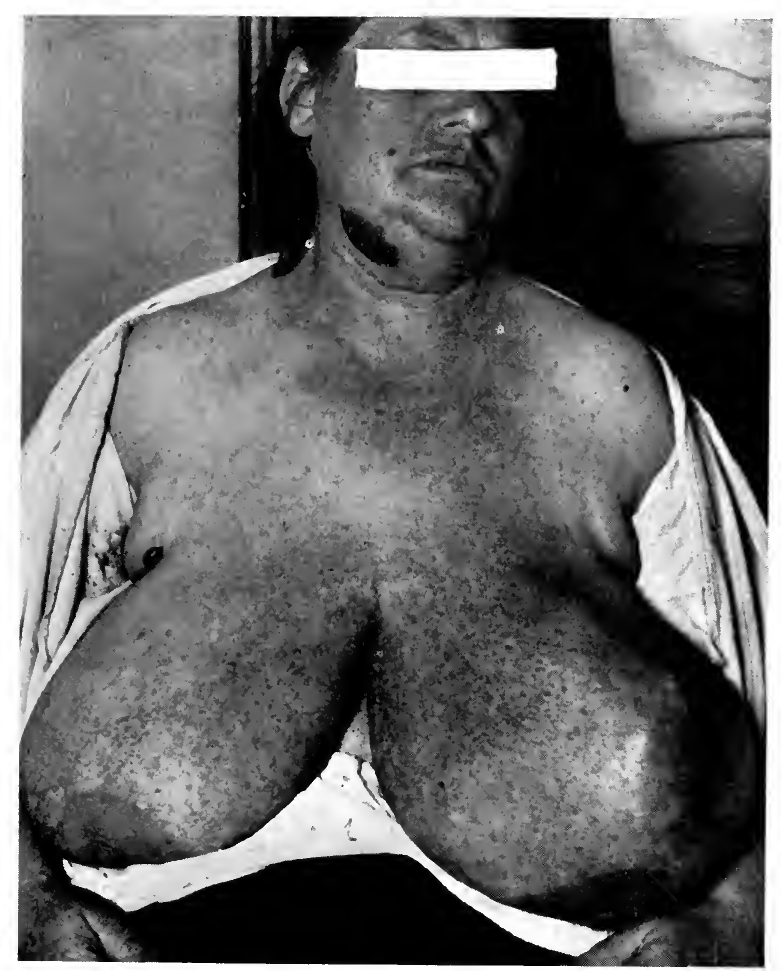

Fig. 69.-Metastasizing angioma of left breast. Note tumors of jaw, neck, under ear, and at right axilla. There were tumors also in pharynx and lungs.

gestation. Free anastomoses with large arteries or veins may become established and severe or fatal hemorrhage may occur. A capsule forms about many tumors which are then apt to remain stationary. Spontaneous regression may result from contraction of the capsule, thrombosis, inflammation, or ulceration, or a cure may be affected by continuous pressure, ligature of vessels, or excision. Very slight interference is sometimes sufficient. Intercurrent diseases or cachexia may initiate the regression. Recurrence after operation has often followed incomplete removal. A certain local malignancy is also exhibited by tumors which extend from one tissue to another, leading even to the erosion of bones, and occasionally pressure symptoms become serious. 
The structure of cavernous angioma presents chiefly a series of anastomosing vascular channels inclosed by thin septa. The appearance on section may be roughly compared to that of a sponge. Yet in less advanced cases it is possible to recognize simple spherical sacculations, and tubular dilatations, all connected with one main afferent and one efferent vessel. Large cystic dilatations may occur, filled with blood or serous fluid. The walls exhibit the structure of venules or less often of arterioles. Hyperplasia of cells may lead to nodular growths of new tissue projecting into the lumen. Many authors have described the budding of new vessels on the advancing edges of cavernous angioma. In progressing cases certain areas of the tumor may show the numerous cellular vessels of simple or plexiform angioma, so that it is not always possible to separate cavernous from simple angioma.

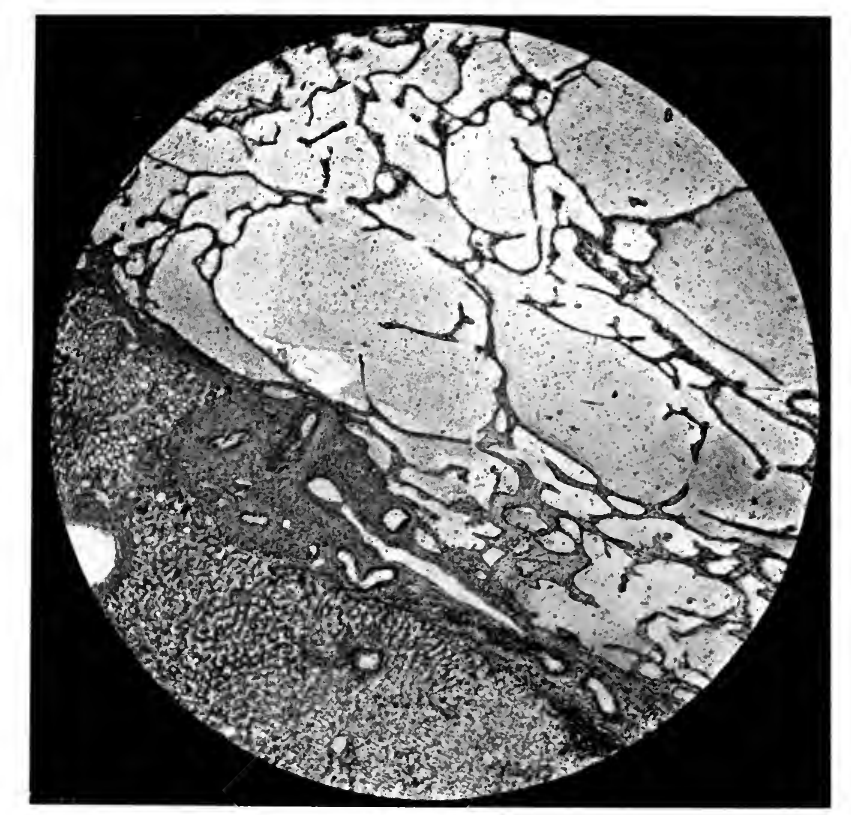

FIG. 70.-Cavernous angioma of liver.

v. Recklinghausen and others locate the earliest stages of the cavernous angioma in the walls of the veins, while many believe the capillaries are first affected (Bosrt). I have drawn the impression that either veins or capillaries may be involved in different cases.

Histogenesis.- In the development of cavernoma Rindfleisch and Borst attribute chief importance to a fibrocellular growth in and about the walls of the capillaries. The retraction of this new tissue tends to shorten the vessel, dilatation resulting from mechanical pressure. The same relation between connective-tissue growth and vascular dilatation is observed in cavernous fibromas, so that the cavernoma has been likened to a fibroma with excessive development of vessels. Loss of muscular and elastic tissue in the new or altered vessels must also greatly favor the dilatation. In regressing cases a fibrous capsule forms or fibrous areas appear in the tumor, the sinus walls thicken and contract, and eventually only scar tissue remains. 
The question of the neoplastic nature of cavernous angioma has been extensively discussed. When one examines a stationary cavernoma of the liver it is difficult to recognize any definite feature of a tumor and many have assumed that these and a considerable proportion of other localized dilatations of vessels should be separated from the angiomas and referred to mechanical factors. There is no doubt that simple varices may simulate the structure of cavernous angiomas and have often been classed with this tumor. The gross dissection of certain angiomas strongly suggests that simple saccular and tubular dilatations of preëxisting veins will explain their origin. Yet Virchow traced the earliest stages of cavernoma of liver to islands of proliferating connective tissue surrounding cellular capillaries, and Ribbert finds on the edges of cavernoma new vessels which communicate freely with those of the tumor, but imperfectly with those of the surrounding tissue. Local varices can usually be distinctly separated from angioma in the same tissue. The usual origin of cutaneous angioma is from congenital simple or plexiform angioma which is admittedly a neoplasm. It is thus apparent that in the growth of cavernous angioma there are essential factors other than mechanical dilatation of vessels, and it is most reasonable to regard these factors as partaking of the neoplastic order. Albrecht regards cavernoma of liver as an ill-fitted but practically normal segment of tissue which possess a limited capacity for aberrant growth. For this and many other benign tumors he introduced the term hamartoma.

The clinical features of cavernous angioma fall into several rather welldefined groups. The cutaneous tumors form the most numerous group. They are located in the derma or subcutaneous tissues, chiefly in regions where the skin is loose. While no region of the body escapes, the chief locations are the face, scalp, labia, scrotum, prepuce, extremities, and folds of the knees, axilla, and buttocks. The influence of embryonal fissures noted in the occurrence of simple angioma is observed also with the cavernous type. The wide variations in the origin, growth, size, number, and complications of these tumors is elaborately: set forth in the detailed reports of cases collected by Virchow.

Submucous cavernomas occur chiefly in the buccal region. The gum is the seat of a common cavernous tumor which may follow violent extraction of molar teeth. It usually remains of moderate dimensions, but may gradually extend until it involves a considerable portion of the alveolar tissues and establishes connections with large veins. At this stage its extirpation is difficult and has produced fatal hemorrhage.

In the tongue cavernous angioma arises usually in the tip of the organ and may extend until it produces an erectile tumor of large dimensions (Reiche). Both lymph- and blood-vessels participate in the process in certain cases of angiomatous macroglossia (Wegner). A form of angiomatous ranula has been described by Delbeau. The lips are frequent seats of congenital nevi and of cavernous angiomas.

Orbital angiomas affect either eyelid and may involve the conjunctiva. In the retroörbital fat a few diffuse and circumscribed cavernous angiomas have been described (Morton, Schuh, di Ricci). These tumors may extend along the optic nerve into the eyeball (Quackenboss).

Angioma of bone is rare, although overgrowth of vessels is a prominent feature of many malignant bone tumors, and bone-tissues are often eroded by extensions from angioma of adjacent tissues. The extensively cavernous tumors of the long bones, especially of humerus and tibia, must be classed histologically as sarcomas, although some of them may be successfully treated by curetage or ligation of the main vessels. True cavernous angioma may 
arise in the periosteum or in the marrow (Virchow, Kaufimann). They invade the outer layers or cause absorption of the shaft. Extensive angiomas of the skull have been observed by Kauffmann and by Schoene, and of the vertebræ by Gerhardt, Kauffmann, and Muthmann. I have studied one cavernous angioma of the head of the humerus which appeared beneath a thin shell of bone 2 years after trauma. In a case of slowly progressive paraplegia I found a cavernous angioma of a dorsal vertebra, which permitted collapse of the body and gave rise to a protruding tumor which compressed the cord.

In the glands angioma occurred in the parotid in the case of Gascoyen, and in several cases the breast has been extensively transformed into cavernous tissue by tumors arising in the fat (Virchow, Image, Borst, Hake).

In the internal organs cavernous angioma occurs chiefly in the liver, rarely in the spleen (Albrecht), kidney, and uterus (Virchow). Dowd has collected I 3 cases of angioma of the spleen, illustrating small tumors found at autopsy, very large growths removed at operation, malignant tumors producing metastases, multiple benign tumors in spleen, liver, omentum, and skin, and a progressive anemia accompanying the large growths.

The common cavernoma of the liver is of much theoretical interest, but rarely produces symptoms. They are single or multiple, as small as a pea or as large as a child's head (Ribbert), and usually lie just beneath or projecting from the surface. They are often associated with angioma of other organs. Their frequency increases with the age of the subjects, but congenital cases rarely occur. Veeder and Austin describe a remarkably extensive multiple congenital hemangioma of the liver. Virchow believed that they might spontaneously disappear, leaving scars. The structure shows central sinuses separated by thin fibrous or cellular septa which may contain islands of liver cells. On the edges are smaller vessels communicating with the sinuses. The tumor is sharply marked off from the parenchyma. Ribbert finds that interstitial injections of the tumor or of the surrounding parenchyma do not pass from one tissue to the other, the tumor vessels showing a marked independence of the normal vessels of the organ. Yet the tumor may be filled by injections through the portal or hepatic veins or hepatic artery (Virchow).

The origin and nature of the cavernoma of the liver has long been a subject of discussion, the later phases of which have been maintained by Ribbert and by Schmieden. On the whole, the argument seems to favor the contention of Ribbert, that these growths are partial neoplasms originating from embryogenic disturbances, through which a displaced segment of the organ comes to possess a limited power of aberrant growth. As already stated, Virchow traced their inception in areas of proliferating vascular connective tissue. The progressive dilatation of vessels naturally results from absence of muscular and elastic tissue in the walls.

In the ovary Gottschalk describes a diffuse bilateral cavernous transformation. Orth observed a remarkable case of multiple congenital hemangiomas in both ovaries, skin, and other organs of a child. In the placenta various tumors have been described as fibroma, myxoma, and angioma. They appear to be essentially overgrowths of blood-vessels with more or less admixture of fibrous or mucinous stroma. They are often multiple and may reach considerable dimensions, causing hemorrhage and interfering with the normal growth of the fetus, but none has proved malignant. (Albert, Lit.; Williams, Lit.)

Angioma of the brain occurs in two main forms, as (I) a more or less diffuse varicosity of the vessels of the meninges, and (2) a true angioma, simple or cavernous, of the brain tissue. La Villette has collected 18 cases of both 
types in various situations. Several cases have occurred in the region of the corpus striatum (Virchow, Luschka). They are probably of congenital origin, and in Hebold's case the intracranial was associated with cutaneous angiomas. They are of slow growth and usually produce only general symptoms. Wergman's cavernous angioma of the left cerebellar pedicle led to fatal hemorrhage. Orbison reported a racemose angioma of cerebral pia invading the brain tissue and giving a long history of epilepsy. Several cases of angioma in the substance of the pons are recorded by LaFora and by Enders. One of LaFora's cases terminated after severe general and focal symptoms lasting one month. The structure is that of simple or cavernous angioma.

Angioma arteriale racemosum consists of a dilatation and complex intertwining of many new formed and altered vessels of small caliber with subsequent involvement of normal vessels (Deetz). The condition occurs in infants or adults, and of 87 cases collected by Schuck 84 occurred on the head, a few on the extremities. They usually arise in close connection with the large carotid artery. The external appearance was likened by Virchow to a pulsating mass of earthworms. Arising externally they extend over the neck and scalp, erode the skull and penetrate the cranium, and in several cases they have involved the cerebral and meningeal vessels. The walls of the vessels show a variety of changes, as fatty degeneration and loss of muscle-tissue of the media (Heine), hypertrophy of media (Lablée) hypertrophy of interna (Kretchmann), or hypertrophy of all coats. Emanuel described leiomyomatous outgrowths of the media with degeneration, calcification, and formation of aneurysms. Deetz found uniform hypertrophy of small arterioles and new growth of cellular capillaries. The exact position of this process is difficult to determine, but most authors assume that it arises from a congenital tissue abnormality and that it belongs in the class of partial tumors.

\section{IYMPHANGIOMA}

Lymphangioma is a tumor composed of lymph-vessels.

It is therefore an organoid structure consisting of endothelial cells and supporting connective tissue, both of which are involved in the neoplastic process. Lymph-nodules or foci of round cells are often present to complete the parallel with a lymphatic structure. Lymphangioma has also been associated with minute islands of lipoma, proliferating smooth muscle-cells may be found in the septa, and Ritschl found an island of cartilage in an intermuscular lymphangioma, observations which suggest an embryogenic origin. New formed lymph-vessels are also present in many benign and malignant tumors, especially with endothelioma and sarcoma.

The clinical conditions which fall in the general class of lymphangioma include a variety of slowly growing, usually congenital, single or multiple tumors of the skin, subcutaneous tissues, deep areolar tissues and muscles, of the neck, trunk, lips, tongue, eye, and orbit, and mediastinal and retroperitoneal regions. In the pathogenesis of these conditions many factors are concerned and it is thus more difficult to distinguish between lymphangioma and lymphangiectasis than between true and spurious hemangioma.

Ribbert's definition of lymphangioma as an isolated group of vessels growing from their own resources and more or less disconnected from surrounding channels can seldom be directly applied. In some typical examples of lymphangioma the tumor has been found freely accessible to injections through afferent and efferent vessels (Langhans).

Proliferating buds of endothelium forming new lymph-vessels have been fully recognized in certain cases by Nasse, Schmidt, and Borst, but such 
structures are usually missing. Size is not a reliable criterion, since in some of the large cystic growths the signs of a neoplasm are least distinct. Moreover, simple occlusion of large lymph paths may, although rarely, be followed by extensive varicosities resembling lymphangioma. The appearance of the stroma sometimes suggests a neoplastic growth, but more often it is fibrous or comparatively acellular. The general clinical features seem to offer an important means of recognition of true lymphangioma, and many would include in the class only such processes as show a congenital origin and a progressive course, and absence of traumatic and inflammatory factors.

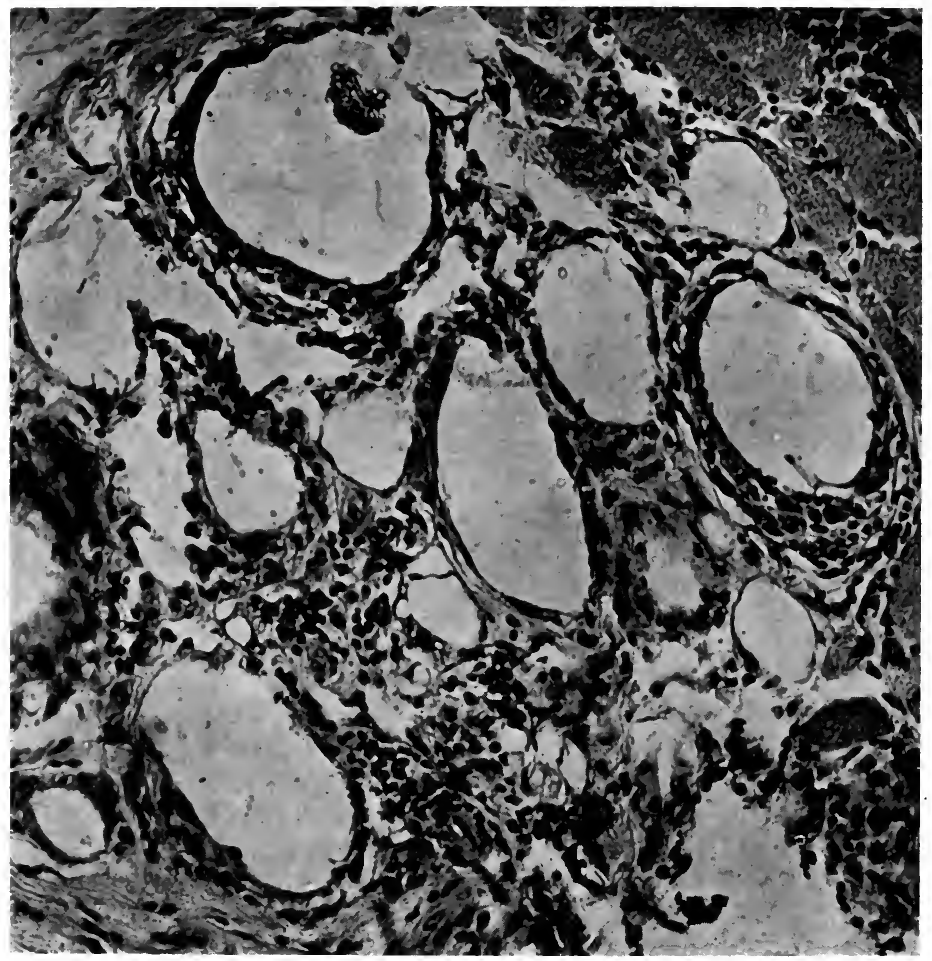

FIG. 7I.-I,ymphangioma of voluntary muscle; excavation of muscle-fibers by lymphvessels. Giant-cells.

The histological structure of lymphangioma was first fully described by Wegner, who recognized three groups of cases: I. Lymphangioma simplex consists of an anastomosing network of spaces and vessels of small and medium caliber. The septa are either reduced to thin strands of acellular connective tissue, or they are thicker and participate in the proliferation. The endothelium is flat or cubical and rarely it may appear in multiple layers or in the form of projecting buds (Freudweiler).

2. Lymphangioma cavernosum consists of a system of closed communicating lymph-spaces supported by thin walls or thicker septa, lined by flat endothelium and filled with fluid or coagulated lymph occasionally mixed with blood. 
3. Lymphangioma cystoides consists of a congeries of large and small cysts lined by flat endothelium and filled with lymph. Some of the cysts may be closed and the main lymphatic vessels are usually occluded.

The great majority of lymphangiomas fall readily among these classes, most of them being of the cavernous type. In not a few cases there is new growth of blood-vessels not communicating with the lymphatic system, and forming a mixed hemolymphangioma (Nasse, Sutter, Novack).

In the development of lymphangioma several factors seem to be concerned. Wegner believed that three modes of origin could be traced: (a) passive dilatation with inflammatory hyperplasia of preëxisting vessels, lymphangiectasis, $(b)$ neoplastic growth of vessels, and $(c)$ heteroplastic formation of lymph-vessels in granulation tissue.

(a) Lymph stasis doubtless influences the course of many lymphangiomas, especially the large cystic type with occluded vessels, to a less extent the saccular ectasiæ of the cavernous forms, but it has not been accepted as a sufficient explanation of any of the characteristic varieties of lymphangioma. Ribbert argues that any marked pressure would inhibit the growth of endothelium, and it is clear that with free afferent and efferent vessels the stasis must be slight. Ribbert assumes that lymphatic and other cystic tumors develop as a result of a process of growth located in the walls which steadily enlarges the circumference of the cysts and prevents the passive accumulation of fluids. Secondary dilatation of new-formed vessels must be regarded as an important factor in cavernous and cystic lymphangiomas. Intermittent attacks of inflammation influence the course and structure of many lymphangiomas (Kuttner).

(b) The evidence of new formation of vessels is conclusive, for in many cases have been observed hypertrophic and proliferating endothelium, multiple layers of endothelium, and sprouts of endothelium growing out into the connective tissue with subsequent canalization. Borst depicts the formation of new lymph-vessels in a small fibromatous tumor in fat tissue where lymph-vessels are normally scanty. In a type of cutaneous lymphangioma the predominance of endothelium has led many to designate the process as endothelioma and other types of endothelioma arise from lymphatic endothelium. The occasional occurrence of definite fibroma and lipoma in lymphangiomas illustrates the organoid character of these tumors and demonstrates the neoplastic growth of lymph-vessels.

(c) The heteroplastic formation of lymph-vessels in granulation tissue has not been satisfactorily demonstrated.

In the origin of lymphangioma it must be assumed that there exists a local predisposition resulting from an embryogenic disturbance similar to that assumed for hemangioma. Of the nature of this disturbance nothing is definitely known, but the congenital origin of most lymphangiomas is a striking feature in their etiology. A partial isolation of a segment of lymphvessels with imperfect development and retention of abnormal powers of growth may be supposed to exist. The occurrence of cartilage in one reported lymphangioma indicates that the isolation may be complete. I have seen a circumscribed lymphangioma $2 \mathrm{~cm}$. in diameter attached to the spermatic cord. The vessels were lined by large cuboidal endothelium and supported by embryonal connective tissue. In a congenital flat tumor of the subcutaneous tissue of the cheek I found many lymph-follicles and diffuse lymphoid tissue in the septa of a cavernous lymphangioma. The structure suggested an abortive attempt at the formation of a lymph-node.

The clinical forms of lymphangioma are numerous and difficult to classify. 
Simple lymphangioma, consisting of moderately dilated and slightly hyperplastic vessels in which a neoplastic element is either wanting or feebly developed, occurs in many situations, chiefly in the skin. In elephantiasis dilated lymph-vessels contribute largely to the increased bulk of the tissues. In the lymph-scrotum of filariasis the swelling is referable to lymphangiectasis and edema, and may be traced to occlusion of lymphatic trunks by the parent worms. The process commonly involves the bladder with chyluria and the presence of filaria embryos in the urine. The tissues show only inflammatory reaction and the process is not a tumor.

In the skin of face and neck congenital lymphangiectasis produces small or large flat or wart-like prominences which after trauma may exude serous fluid. They consist of dilated lymphatics of the derma. The endothelium

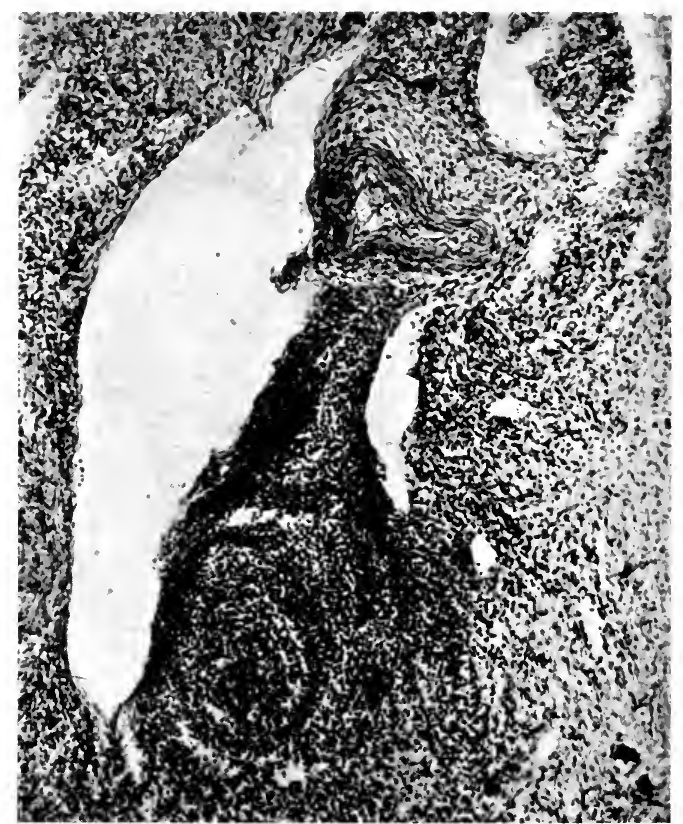

FIG. 72.- Structure of a congenital angiolymphoma occurring in skin of cheek of an infant.

is slightly hyperplastic, but their chief neoplastic character is the occasional relation to embryonal fissures and their congenital origin. In a case of Haug's the tongue was involved and there was a congenital auricular fistula.

In lymphangioma cutis circumscripta the skin of face, chest, or extremities is the seat of numerous small projecting translucent vesicopapules which on section prove to consist of many small cellular lymph-vessels. The endothelium is hypertrophic and proliferating, and the channels are small and filled with fluid or hyaline masses. There is extensive new formation of imperfect lymph-vessels and the process is a true neoplasm (Fox, Hutchinson, Noyes, Torok, Freudweiler, Waelsch).

Lymphangioma tuberosum multiplex was described by Kaposi as a small superficial multiple tumor of cellular lymph-vessels. Beneke's case falls in 
that histological group. The nature of other cases in dermatological literature is not clear (Crocker, Perry, Kromayer).

In the eye lymphangiectasiæ of congenital or inflammatory origin occur in the conjunctiva. They may reach considerable dimensions and occasionally there are evidences of considerable new formation of vessels (Steudener, Bull, Wintersteiner, Sachs).

Lymphangioma cavernosum occurs chiefly in the skin, but also in the intermuscular septa and in the mucous membranes. The dilated vessels are filled with coagulable lymph often mixed with blood, and the contents may be thrombosed, or hyaline, or extensively calcific. On analysis the aspirated

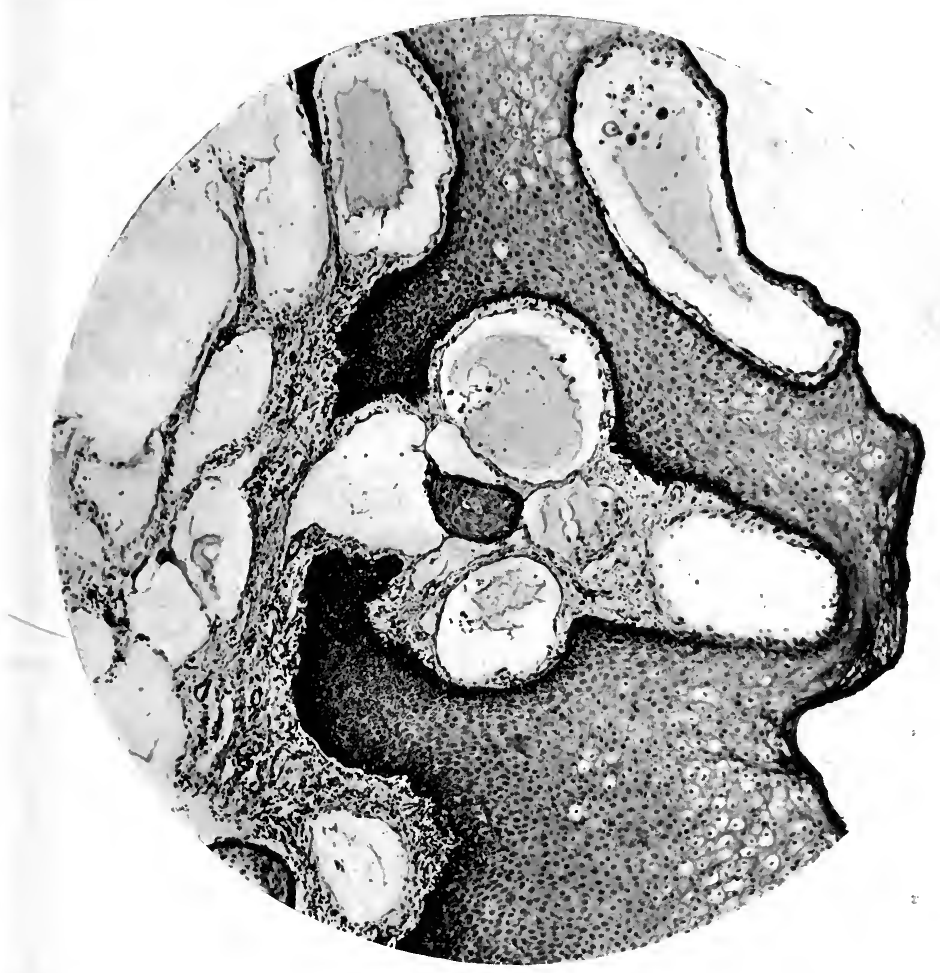

FIG. 73.-Superficial diffuse lymphangioma of entire tongue, existing since infancy in a boy of 19 years.

fluid shows a considerable content of blood-proteins and salts, many lymphocytes and exfoliated endothelial cells, and usually cholesterin crystals. It lacks the digestive ferments of pancreatic cysts (Sick, Bryck, Zeyneck). The septa are thin and acellular or thicker and composed of proliferating connective tissue, containing round-cells, lymph-nodules, and often much smooth muscletissue. New growth of small vessels is often associated with the larger ones. The tumors are small and circumscribed or extend diffusely over considerable areas.

In the skin elephantiasis lymphangiectatica may exhibit extensive dilatation of old and new formed lymph-vessels.

The most frequent forms of cavernous lymphangioma occur in the lip, 
cheek, and tongue, and are designated as macrocheilia, macromelia, and macroglossia. They are usually congenital and produce irregular and sometimes extensive enlargement of the tissues. The pharynx and larynx may also be involved (Suchstorff, Nasse).

Chylangioma of the mesentery is a cavernous lymphangioma containing milky fluid which arises from congenital or acquired obstruction to the lacteal vessels (Kruse, Schmidt, Ritter). Multiple dilatations of the submucous lymphatics of the intestine were observed by Krauss, and extensive grades of this condition have been reported by Blatteis, Thalheimer, and Takano.

Cavernous lymphangioma occasionally occurs in the conjunctiva, eyelids, and orbit (Michel, Steudener, Sachs). Klein described a case occurring in the vagina.

Extensive cavernous lymphangioma of the foot resembling elephantiasis, multiple lymphangioma of the finger, hands and pleura, and hyperplasia of many lymph-nodes, were associated in a remarkable case reported by Bryck.

Lymphangioma cysticum is a multilocular cystic tumor which occurs chiefly in the neck and sacral region. They are usually of congenital origin and exist at birth or shortly develop into tumors of considerable bulk and wide extent.

Hygroma cysticum colli is a lymphangioma which usually arises in the submaxillary region and ramifies upward toward the parotid and ear, inward toward the median line, and downward to the supraclavicular fossa and even into the pleura and mediastinum (Nasse, Suckstorff). One class of these tumors occurs deep in the neck, reaches below the sternal notch and seems to be connected with the thyroid (Otto). In one case which came to my notice the cyst lay close to the trachea and reached from the sternal notch to the inferior thyroid artery. Lymphangioma of the neck must be distinguished from hydrocele colli, which is a simple dilatation in a branchial cleft, lined by epithelium and walled by fibrous tissue containing lymphoid tissue (Frobenius). The axilla and thoracic wall are occasionally the seat of cystic lymphangioma (Nasse). Bilateral congenital cystic lymphangioma of the back of the neck has been described by Frobenius.

Retroperitoneal and mesenteric lymphangioma occurs in children and adults as a multilocular cavernous and cystic tumor, originating along the spinal column and ramifying into the pelvis behind the kidney or colon, upward to the liver, spleen and pancreas, and into the mesentery or omentum (Takano, Lit.). The receptaculum chyli and thoracic duct have been found unaffected by the process (Sick, Smoler, Schwarzenberger). Lion has reported a case of lymph-cyst of the broad ligament. In a case of the writer's the broad ligaments were chiefly involved with extensions throughout the pelvis and along the lumbar spine.

The very early stages and exact origin of these tumors have not been demonstrated, and as Hedinger points out, they require differential diagnosis from a variety of other abdominal cysts. Their neoplastic nature seems assured, since the walls contain cellular connective tissue, often much smooth muscle-tissue, and lymph-follicles. After partial removal the remaining portion may rapidly increase in bulk. Along the edges of the growths Sick found proliferating areas of cavernous lymphangioma. $\mathrm{He}$ assumes that they arise from misplaced and embryonal islands of connective tissue and lymph-vessels. In the omentum and mesentery of newborn cats and pigs Ranvier has demonstrated such misplaced islands of tissue.

In the wall of the stomach and gastrohepatic ligament Reimers and Tilger have found small lymph-cysts which they referred to obstruction of vessels following gastric ulcer. 
Sacral hygroma is a cystic tumor developing in the region of the sacrum and usually connected with defects in the spinal canal. Some of them are probably aberrant meningoceles. Borst found a large multilocular cystic tumor in front of a defective sacrum and connected by a strand of tissue with the spinal canal. Virchow described an external pedunculated hygroma attached to the sacrum of a negro infant and containing a lymph-cyst and portions of nervous tissue. Attached by a pedicle to the liver of a child of

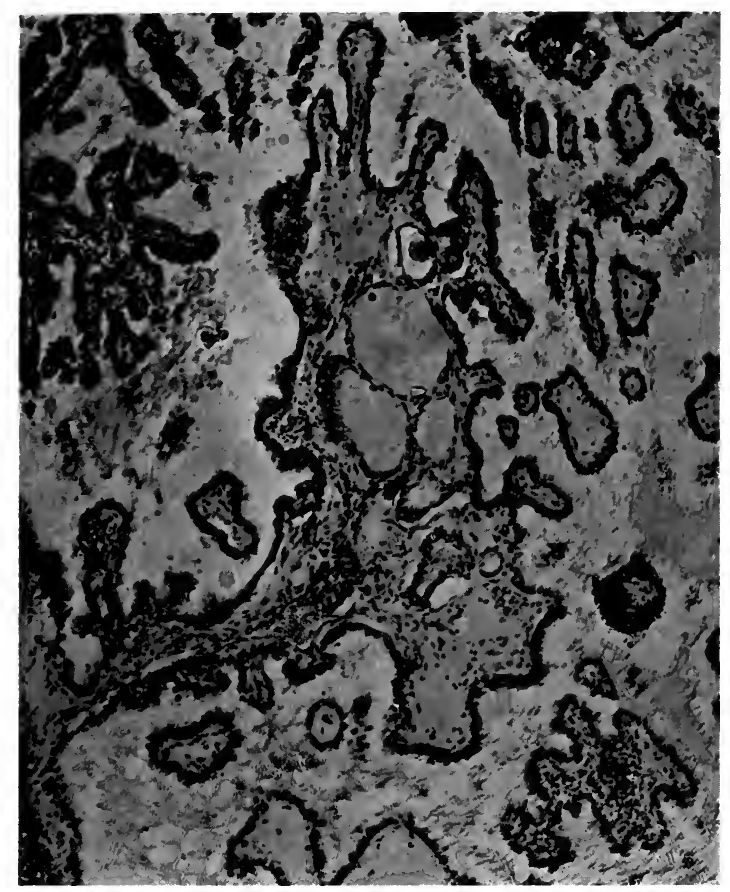

FIG. 74.-Papillary growth in a cervical lymph-node which was the seat of chronic lymph stasis. Papillary fibro-endothelioma.

5 years Maresch found a large cavernous and cystic lymphangioma. In the adrenal and in the uterine musculature lymph-cysts have been described by Sick.

In the ovary Kroemer finds lymphangioma of small dimensions not inrequent. Besides telangiectatic fibroma he describes two large cystic lymphingiomas, in one of which the endothelial cells were prominent, suggesting the designation fibro-endothelioma. 


\section{CHAPTER XVII}

\section{SARCOMA}

General Characters.-Sarcoma is a malignant tumor composed of cells of the connective-tissue type.

This definition is based on the morphology of the tumor-cells and on their histogenesis. It accomplishes the main object of separating a large group of malignant tumors from the carcinomas, but it overrides certain embryological considerations.

Thus it permits the inclusion of gliosarcomas which are of ectodermal origin, and it accepts as sarcoma certain tumors, chiefly of endothelial origin, while rejecting others. This portion seems justified by the history of the development of the conceptions of sarcoma, as ably sketched by Malherbe, by certain anatomical and clinical features of the group, and by the peculiar part played by the blood-vessels in the growth of many sarcomas. Nevertheless, it cannot be claimed that the effort to stretch the scope of the term "sarcoma" over an extensive field of neoplasms has been entirely successful.

The diseases included in this group, such as angiosarcoma, lymphosarcoma, and gliosarcoma, are of such varied origin and character that some writers have urged the elimination of the term sarcoma. This radical reform has not received approval, since there are many common features among sarcomas and the group as a whole is well-defined from carcinomas. Yet future investigation will doubtless reveal many new and more precise facts regarding the etiology, conditions of incidence, histogenesis, and clinical course which will warrant the recognition of many sarcomas as specific pathological entities.

The original conception of sarcoma as a tumor of fungating, soft or fleshy character reflected truly the gross character of most sarcomas, but the definition based on histogenesis is much more significant. Even this conception of sarcoma we believe is destined to be replaced by etiological criteria.

Since there is often much difficulty in determining the origin of cellular tumors the accepted scope of sarcomas has been subject to much revision. In recent years, especially, many tumors once interpreted as sarcomas have been proved to be of epithelial origin. Further inroad has resulted from the identification as endotheliomas, of many tumors once regarded as sarcoma, but it appears unlikely that the separation between the latter groups will be rendered complete.

In fact, the finer analysis of the origin and composition of many sarcomas reveals a prominent participation of endothelium in many tumors of distinct mesoblastic characters. In such cases the characters of the tumor-cells rather than their embryonal antecendents should determine the classification.

Even the spindle-cell sarcomas have not escaped suspicion, especially in epithelial organs, since it is a fact too often ignored that squamous and glandular epithelium may assume a fusiform shape during the vicissitudes of tumor growth.

Gross Anatomy.-The majority of sarcomas are bulky, soft, and vascular. In general, the bulky character results from the principle that sarcomas tend to grow expansively upon a scaffolding of new blood-vessels rather than to infiltrate preëxisting tissues as do carcinomas. The soft consistence reflects the cellular structure of a rapidly growing tumor. The vascularity reveals 
to the naked eye the prominence of blood-vessels as an essential part of the growth.

Some specific varieties of sarcoma fail to display the usual features. Fibrosarcoma is firm, resistant, and non-vascular; osteogenic sarcoma may be as hard as ivory. In fact, there are so many variations in the appearance of sarcoma that general statements are of little value and the gross characters are best detailed in connection with specific tumors. As a rule the gross appearance depends largely upon the tissue of origin, according to which one recognizes fibrosarcoma, chondrosarcoma, osteosarcoma, liposarcoma, myxosarcoma, angiosarcoma, myosarcoma, and lymphosarcoma. All adult mesoblastic derivatives are represented in the tissues of origin. Gliosarcoma occupies an especially peculiar position. While its cells of origin are derived from neural epithelium, the form and function of the adult glia-cells, and especially the characters of the tumors, do not encourage efforts to reform current nomenclature in this group.

Sarcomas are usually single, but multiple tumors arise in bone-marrow, nerve-trunks, and skin. Certain angiosarcomas are multiple or diffuse, and some forms represent systemic diseases, as lymphosarcoma and myeloma.

Characters of Growth. - The growth of sarcomas is usually rapid and locally destructive. The abundant blood-supply favors extremely active cell growth, so that the tumors soon reach a large size, and their bulk is often sharply increased by edema, hemorrhage, or mucoid degeneration. After reaching a certain limit the excessive circulation fails and large areas of necrosis form from infarction. The further growth of the tumor then depends on its capacity to infiltrate or produce metastases.

Hence some sarcomas regress after extensive central necrosis and not a few are remarkably susceptible to toxic agents or to absorbents such as $x$-ray or iodid of potash. Hemorrhage from the fragile vessels is prominent in many vascular tumors. Into the extravasated blood-mass sarcoma-cells are often observed to grow, replacing the clot.

Following necrosis, sarcomatous tissue is very susceptible to infection and many cases terminate with local suppuration and gangrene or with general sepsis. Accordingly, fever is often observed throughout the course of malignant sarcomas, but in some instances it appears to result from specific intoxication and not from infection.

Most sarcomas grow expansively, the central portions increasing with the peripheral. Hence they are rather sharply marked off from the surrounding tissue, although rarely presenting a capsule.

Infiltrative growth on the periphery fuses the tumor with the surrounding tissue so that it is difficult to extirpate and commonly recurs after operation. The more malignant growths infiltrate widely through fascial planes, along vessels and nerves and directly through the soft tissues. Penetration of the skin is followed by more rapid fungating growth with ulceration and hemorrhage. Cellular bone-sarcomas rapidly destroy bony tissue and perforate joint cavities. To the attack of other sarcomas bone is resistant. I have observed very wide infiltration of a spindle-cell periosteal sarcoma which produced a large tumor of the ilium and spread beneath the periosteum over the entire ilium, pubes, and upper third of the femur. The extensions of lymphosarcoma and of myeloma are partly by infiltration and largely by systemic development of new tumors.

Metastases are observed in the advanced stages of most sarcomas and it is characteristic of the disease that with rare exceptions the embolic cells travel through the blood-vessels. Except in lymphosarcoma the occurrence of metastases in lymph-nodes justifies the suspicion that the tumor is not a 
sarcoma. The strong tendency of sarcoma-cells to grow upon a framework of blood-vessels determines the early invasion of these vessels which occurs even in sclerosing osteogenic sarcoma, while the expansive growth, as opposed to the infiltration of preëxisting tissues in carcinoma, explains the usual failure to invade lymphatics. Sarcoma tissue itself is usually devoid of lymphatics.

Cell-emboli thus pass readily into the vessels and lodge first in the lungs which are the chief seat of sarcomatous metastases, and later they reach liver, kidney, spleen, and other organs and tissues.

General miliary sarcomatosis may result from discharge of very numerous loose cells, but it is much less frequent than general carcinomatosis. Mechanical pressure of actively growing sarcoma sometimes forces tumor-tissue into the blood-vessels and thus leads to metastases when the infiltrating power and capacity for independent growth of the cells appears to be limited. Thus myxosarcoma of the thigh may be forced into the saphenous and iliac veins and form a continuous mass filling vena cava, pulmonary veins, and even reaching the heart. The same extensions of sarcoma of the kidney have been observed.

Some sarcoma-cells seem comparatively resistant to any destructive influence that may be exerted by the blood. They have frequently been found in the peripheral blood. In the blood-vessels invaded by sarcoma thrombosis is not as common as in carcinoma. Borst states that they have a certain capacity to grow in the lumen without connection with the vessel wall. These considerations may tend to explain the frequency of metastases by blood-vessels. The metastases of sarcoma often occur much earlier than is generally conceived, and this fact adds to the gravity of the prognosis after surgical operation. In a considerable series of cases at the Memorial Hospital the $x$-ray photograph of the lungs has revealed pulmonary nodules in apparently operable cases, especially of bone sarcoma, and in not a few which had just recovered from operation.

Structure.-Sarcomas are chiefly histioid tumors. With the exception of mixed or teratoid growths, a definite arrangement of elements in organoid form is missing. Yet considerable differentiation of tissues occurs in the more mature types. Fibrosarcoma may present areas resembling cellular connective tissue; bony trabeculæ appear in many osteosarcomas; embryonal fat tissue occurs in liposarcoma; while angiosarcoma produces many functionating blood-vessels.

With increasing anaplasia the form and function of the originating cells are gradually lost.

The controlling factor in the structure of sarcomas is found in the natural tendencies of the cells of origin to reproduce the mother tissue. Many authors have traced in the growth of sarcoma a repetition of the embryonal development of the affected tissue.

In many cases the origin of the tumor is readily determined by the presence of cellular connective tissue, cartilage, bone, or fat.

In other cases the tumor is highly cellular and reveals few traces of the original form or function of its cells, and in the most anaplastic types the cells are round and completely undifferentiated.

According to cell form, sarcomas may be divided into spindle-cell, roundcell, and giant-cell groups, but these terms convey little information, and, unless the tissue or origin can be stated, no significant diagnosis has been reached. Since any one of the three above types of structure may arise in sarcomas from each mesoblastic tissue, it is desirable to avoid terms referring solely to the morphology of the cells.

According to histogenesis sarcomas may be classified as fibroblastic, 
angiosarcoma, chondrosarcoma, osteosarcoma, liposarcoma, myosarcoma, myxosarcoma, lymphosarcoma, and gliosarcoma. In typical cases the structure of each of these forms is specific.

In general, the structure of sarcomas presents an imperfect development of the tissue of origin. The cells are usually larger and always more numerous. Very large spindle-cells belong chiefly to malignant fibroblastic, angioblastic, and myxosarcomas. Quite small spindle-cells also appear in tumors of these same origins. Giant-cells of various types appear in many sarcomas, especially in the tumors of bone and smooth muscle. In muscle they result chiefly from hypertrophy of tumor-cells; in bone-tumors they have been interpreted as giant osteoclasts; in bone-marrow tumors they are likened to the myeloplaques; in many cases they evidently form by cell fusion in poorly nourished areas; in the epulis they represent fused endothelium surrounding foreign material; while amitotic nuclear division is responsible for many.

The origin and significance of the different forms of giant-cells is best considered in connection with specific tumors, since in many respects they

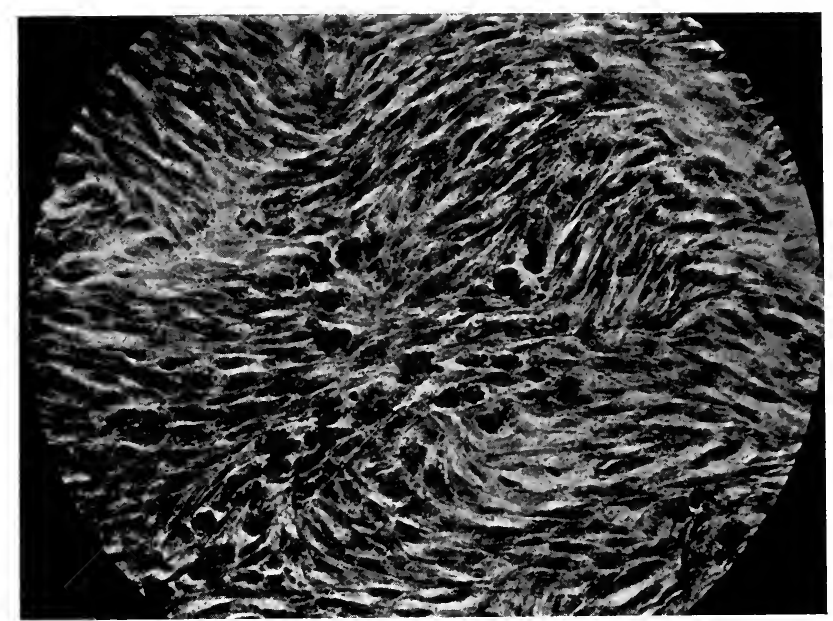

FIG. 75.-Large spindle-cell fibroblastic sarcoma.

are peculiar in each tumor and form an important means of diagnosis. Wakabayashi found the same groups of chromosomes and lack of mitoses in seven types of sarcoma as he observed in tuberculosis and syphilis. Howard, studying the morphology of many giant-cells, observed in the behavior of the nuclei many resemblances to protozoan nuclei.

Round-cells appear in lymphosarcoma, gliosarcoma, and myelosarcoma, but it is doubtful if true round-cell tumors arise from any other mesoblastic tissues. While very cellular and anaplastic growths composed of indifferent spheroidal cells arise from many tissues, perfectly fixed portions of these tumors regularly show the cells to be spindle or polyhedral, and the effort should be made to detect these features so as to exclude the tumor from the group of true round-cell growths.

Such distinctions are, however, rarely attempted and the term "round-cell sarcoma" is in very general use and enjoys recognition from practically all writers. Malherbe, however, separates sharply between round-cell sarcomas, which he regards as exclusively lymphoid, and true fibroblastic tumors. 
Virchow stated that a round-cell tumor could safely be identified as sarcoma only when a definite intercellular stroma was demonstrated. Borst describes the cells of small round-cell sarcomas as identical with the round-cells infiltrating granulation tissue, but the source of these cells he left undetermined. Highly malignant small round-cell tumors arise in the intermuscular septa, periosteum, submucous stroma, meninges, skin, kidney, testicle, and liver (Borst), and are said to represent the highest degree of sarcomatous degeneration (Rindfleisch). Yet it has never been shown and appears unlikely that any fixed connective-tissue cells in such numerous situations give origin to such tumors.

From my own observations I have been forced to conclude that with rare exceptions such round-cell growths are either lymphosarcomas or small cell carcinomas. They usually invade lymph-nodes.

It is chiefly among the sarcomas of bone that small round-cell tumors appear which suggest an origin from periosteal fibroblasts, but here the proximity of bone-marrow provides a possible origin from lymphoid cells. Many of the "round-cell" sarcomas of bone are diffuse endotheliomas.

Much the same uncertainties surround the origin of most of the so-called large round-cell sarcomas. These tumors may, as a rule, be divided among endotheliomas, tumors of lymphoid cells, carcinomas, and sarcomas of which the cells are not strictly round.

The round-cell tumors of the testis and thyroid are among the epithelial growths commonly misinterpreted as large round-cell and alveolar sarcoma.

The nuclei of sarcoma-cells exhibit the widest variations in size, form, chromatin content, nucleolar elements, and methods of division; all of which features reflect rapid and lawless growth and the accompanying degenerative processes. All the abnormalities observed in carcinoma cells are duplicated or even exaggerated in sarcoma. In pronounced grade they are specific of a malignant tumor process, but not of sarcoma. Favre and Regaud find the same mitochondria granules in sarcoma cells as in carcinoma.

Degenerative changes in the cells of sarcoma occur chiefly in areas of necrosis from infarction. In actively growing, well-nourished zones the cell cytoplasm is usually less subject to fatty and hydropic degeneration than in carcinoma. Yet under appropriate conditions the cells undergo the usual forms of cytoplasmic decay. The most common change is a mucoid transformation of cells and stroma which affects nearly all forms of sarcoma, especially those arising from connective tissue. Extensive deposits of bloodpigment may lead to confusion with melanoma. Calcific deposits are comparatively rare, except in osteogenic tumors. Glycogen is found in the cells of many rapidly growing sarcomas (Brault).

The arrangement of the cells reflects the structure of the mother tissue and often gives a clue to the origin, yet in cellular growths all traces of the originating tissue may be lost. Prominent in many sarcomas is the tendency of the cells to grow about blood-vessels simulating the type of angiosarcoma or perithelioma. The specific structure of these latter tumors may be all but duplicated by tumors which have no angioblastic relations whatever. On the growing edges of infiltrating sarcomas the cells are commonly gathered about the small blood-ressels. A true alveolar arrangement is rarely if ever assumed by sarcomas. While it cannot be asserted that the small spheroidal or polyhedral sarcoma-cells never appear in groups surrounded by stroma, the history of most alveolar sarcomas has terminated in their identification as carcinomas or endotheliomas.

The stroma of sarcomas is derived from remnants of preëxisting tissue, from blood-vessels appropriated for the nutrition of the tumor or new formed 
as an integral part of the neoplasm, and from the specific intercellular substance derived from the tumor-cells. In relatively adult growths an intercellular stroma is regularly laid down by the cells, and in tumors of bone, cartilage, mucoid tissue, and glia-tissue it may form an easily recognized element. Yet in very cellular tumors the attempt to demonstrate a stroma commonly fails, and it would appear that the diagnostic significance of this element in sarcomas has been overstated. W. C. White found a fine intercellular reticulum very constant in sarcomas, but absent in carcinomas. $\mathrm{He}$ identifies the reticulum of lymphosarcoma with white fibrous tissue. Seelig, in specimens digested by pancreatin, found a rather abundant fine reticulum in many sarcomas, but failed to note any constant differences in the reticulum of lymphosarcoma from that of other round-cell sarcomas. Bielschowsky's stain is particularly effective in demonstrating a reticulum in sarcoma as well as in carcinoma.

The blood-vessels form the most important part of the stroma. They are of various forms and sources. Following the analogy with granulation tissue the blood-vessels may represent elongations of those of the originating tissue about which the tumor-cells grow. Such vessels may be venous, arterial or capillary, and their walls are composed of adult normal cells. In the course of their growth they may become varicose or sinusoidal and the walls thin and fragile. Some of the well-formed vessels are probably appropriated directly from the invaded tissue. As the tumor develops, the adventitia of the vessels is lost in the stroma of the neoplasm. Eventually, nothing but a swollen endothelial cell separates blood-current from tumortissue. Malherbe describes the apparent sarcomatous transformation of the muscle-cells of vessel walls and points out the diagnostic importance of the swollen condition of the cells in the vessel walls of sarcoma. Many bloodspaces and channels appear to be formed by modified tumor-cells, but such structures are extremely fragile and leave the tumor-tissue subject to interstitial hemorrhage.

In a large group of angiosarcomas and of peritheliomas the tumor-cells exhibit true angioblastic properties and the tumors are composed essentially of a congeries of very cellular blood-vessels.

The continued growth of most sarcomas is closely dependent upon the capacity of the cells to excite the development of blood-vessels.

Where the vascular channels fail, growth ceases, and when they become occluded anemic necrosis promptly follows. Yet some very cellular actively growing small spindle-cell sarcomas contain few vessels.

Lymph circulation in sarcomas is very deficient and according to Ziegler lymph-vessels cannot be demonstrated. Yet in some varieties clefts and canals appear which probably represent lymph pockets or channels, and some cutaneous sarcomas have abundant lymph-vessels (Unna). Nerve-trunks invaded by sarcoma undergo pressure atrophy.

In neurosarcomas fragments of myelin sheaths and axis-cylinders may long persist. Definite new formation of nerve-fibers in sarcoma has not been demonstrated.

Etiology.-Of the specific etiology of sarcoma little is definitely known. These tumors demonstrate the very great proliferative capacity of mesoblastic cells released from the restraints to growth. This capacity may reasonably be estimated as even greater than with most epithelial tissues.

It is commonly assumed that normal adult cells are incapable of such great proliferation as occurs in sarcoma, and the further assumption is then necessitated that sarcomas as a rule grow from isolated, or superfluous, or embryonal cell groups. Thus when trauma is followed by an extremely malig- 
nant bone-sarcoma, the violent explosion of growth capacity seems to require some element which is not attributable to normal cells regenerating after contusion. The rarity of any definite observations of the beginnings of such sarcomas is a strong defense of this hypothesis. Moreover, there are many instances in which sarcomas are known to arise from isolated or embryonal cell groups, especially in the mixed tumors of teratoid type.

On the other hand, it may be urged that the regenerative capacities of mesoblastic cells are very great, especially in youth when most sarcomas occur. It must be admitted that the existence of isolated cell groups as the source of many sarcomas has not been proved.

Sarcoma has been observed to follow $x$-ray injury in rats, and in mice the continued growth of carcinoma is said to have excited sarcomatous proliferation of the tumor-stroma. Finally, increasing observations of very early sarcomas and of presarcomatous lesions of inflammatory origin favor the view that sarcomas often arise from previously normal adult cells.

It is clear that no general rule can be applied in this field and that the evidence must be worked out separately in each form of sarcoma.

Presarcomatous Lesions. - The occurrence of atypical productive inflammatory lesions leading to sarcoma must be regarded as fully in accord with established views regarding the nature of sarcoma. Many sarcomas show such marked histological resemblances to inflammatory processes that pathologists have long been inclined to accept in a certain sense the inflammatory or even the parasitic origin of certain sarcomas. The lymphosarcomas especially stand in this position, and Borst, among others, anticipates the ultimate identification of this group of neoplasms with the infectious granulomas. In such an event the sarcoma must be regarded as an indirect result of the parasite, much as some carcinomas are an indirect sequel of chronic irritation. Syphilis and tuberculosis are the most prominent infections which lead to sarcoma.

Relation of Tuberculosis and Syphilis to Lymphosarcoma.-Clinical observations have long indicated that tuberculous lymphadenitis may pass rapidly or after several recurrences into lymphosarcoma. Ricker in a case terminating after I4 years found typical tuberculosis associated with lymphosarcoma of neck, lungs, adrenals, and spinal cord. Müller saw general sarcomatosis from a primary tumor of the breast, miliary tubercles being nearly coextensive with wide-spread sarcomatous tumors. The rather frequent discovery of tubercle bacilli in lesions supposed to be pure lymphosarcoma suggests that a very slight difference separates some of such tumors from the immediate or distant presence of tubercle bacilli or their toxins. I have followed a case of cervical tumors originally tuberculous, recurring four times in 6 years and terminating with the picture of endothelioma of lymph-nodes. In this study it was shown that an infectious granuloma may cause proliferation of the reticulum cells, producing large-cell lymphosarcoma, or of the sinus endothelium producing endothelioma of lymph-nodes, and it was also shown that exactly similar tumors of lymph-nodes occur in the axilla without any histological evidence of granuloma. Various diphtheroid bacteria may be isolated from such tumors.

The transformation of Hodgkin's granuloma into sarcoma has been described by various authors, but I have found evidence that most of these tumors arise from the thymus where again they are implicated with granuloma.

The difficulty of distinguishing from sarcoma certain late syphilitic lesions in bone and muscle is mentioned by many authors, especially by v. Hansemann. The clinical diagnosis of syphilis is rendered difficult by the recurrence of the lesions after operation, by their multiplicity, and by the resistance to 
syphilitic treatment, while the structure presents very marked cellular overgrowth of round-cells or spindle-cells. Yet the syphilitic process tends toward necrosis and cicatrization rather than progressive growth. There are many indications that true sarcoma develops from such lesions, but satisfactory proof is lacking.

In the healing of fractures and of wounds of the periosteum the histological picture shows very active growth of bone, giant-cells and connectivetissue cells, and regenerating capillaries, and may distinctly resemble sarcoma. The actual transition into sarcoma has not been traced.

Traumatic myositis ossificans appears to occupy a position intermediate between inflammatory and neoplastic processes (Berndt). The clinical

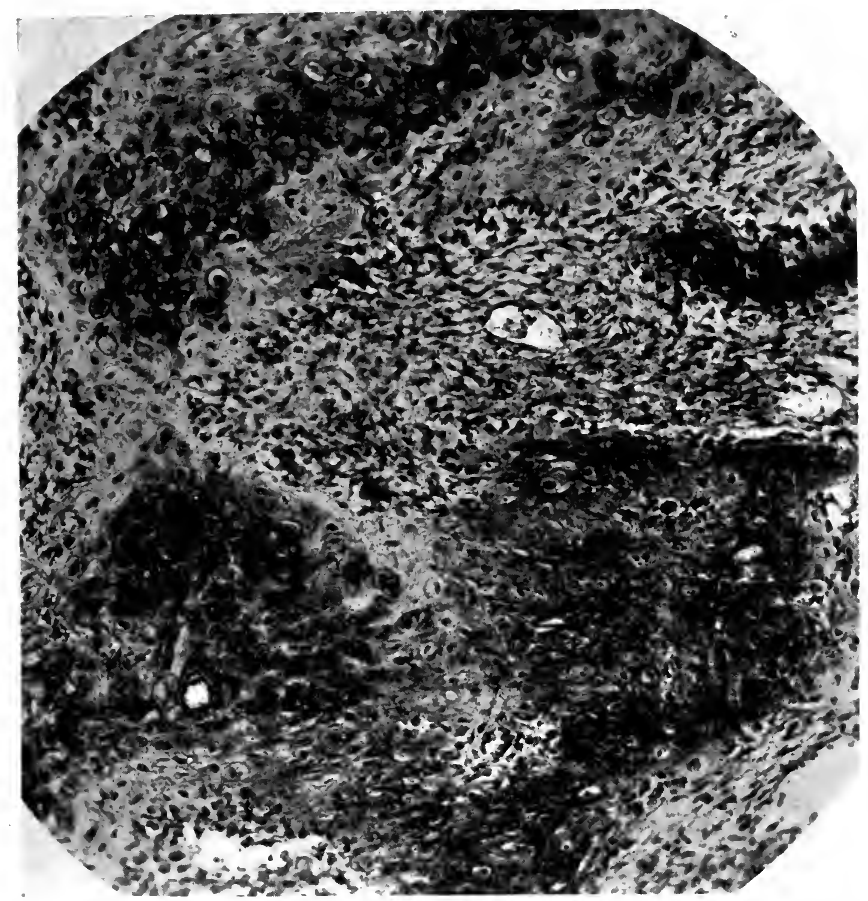

FIG. 76.-Structure of rapidly forming callus developing in muscle tissue 2 weeks after fracture of clavicle. Note resemblance to osteogenic sarcoma.

history of established cases is that of a self-limiting productive inflammation, but the histology of the early lesions may be difficult to distinguish from bone-sarcoma. It appears that the division of cases is accidental, some of the lesions progressing as myositis, others going on as traumatic sarcoma. I have observed the two conditions combined. Central giant-cell sarcoma of bone has often been interpreted as a form of productive inflammation, but its various phases show every gradation from the benign to a malignant process. Kolisko finds that osteitis fibrosa may be followed by progressive changes leading to giant-cell sarcoma of moderate malignancy.

Many clinical observations point to the development of sarcoma from granulation tissue, and the histological study of granulation tissue and of 
organizing blood-clots occasionally reveals pictures which closely approach the structure of sarcoma. Heukelom has traced in considerable detail the gradual transformation of the cells of granulation tissue into sarcoma-cells. Occasionally one finds small tumors following trauma and containing much blood-pigment, of which the structure is distinctly sarcomatous.

Against the hypothesis of the origin of sarcoma from previously normal cells stands the opinion of Birch-Hirschfeld, Borst, and others that sarcomas do not develop from previously normal cells, but from embryonal cell groups. This view must be regarded as an hypothesis without adequate proof. Ackermann was unable to find any evidence of the origin of a series of sarcomas from embryonal cells.

It seems highly probable that sarcomas, like carcinomas, arise through exaggerated inflammatory and regenerative overgrowth of tissue-cells. While in many cases the cells of origin are adult, theoretical considerations suggest that in some instances they are embryonal.

In the former group only would specific presarcomatous stages be expected.

The clinical transformation from benign into malignant mesoblastic tumors has repeatedly been observed, but the exact nature of the changes is somewhat uncertain. There is no doubt that in a small proportion of cases of fibroma, uterine myoma, neurofibroma, and lymphangioma, a long-standing and quiescent tumor eventually displays rapid growth and the structure proves highly cellular and malignant. In rare instances there is every indication that the slowly growing cells have taken on greatly increased powers of growth. In other cases it has been assumed that the malignant process arises de novo in some element of the tumors, as the blood-vessels. For the assumption that the sarcoma develops from embryonal cell groups included in the benign tumors there seems to be very insufficient basis. 


\section{CHAPTER XVIII}

\section{CLINICAL TYPES OF SARCOMA}

Spindle-cell Sarcoma; Fibroblastic Sarcoma.-This common tumor represents the purest form of fibroblastic neoplasm. It is of wide-spread occurrence, developing in nearly all situations where supporting connective tissue is found. The chief locations are in the subcutaneous and submucous tissues, the fasciæ and muscles, periosteum, and in the parenchymatous organs.

In the gross these tumors are usually single, but occasionally multiple. The form is rounded or lobulated and the borders are not sharply marked. The consistence is hard in the fibrous and small cell-growths, softer and more elastic with the large cell-tumors. The hard tumors are opaque and nonvascular, the soft ones are vascular, succulent, reddish, and variously altered by secondary changes. The minute markings are, as a rule, not distinctive. In size one encounters very small nodules in the earliest stages and bulky fungating and necrosing growths in the later periods. In the skin they form multiple, nodular, or globular growths which may become polypoid.

The rate of growth varying with the structure may be very slow or very rapid. Cessation of growth is rare, but cutaneous tumors may be spontaneously extruded. Extremely rapid and bulky tumors are observed especially after injuries. Being poorly circumscribed, local recurrence frequently follows removal even with the comparatively benign forms. Metastases in lungs, liver, and other organs frequently occur with large cell-growths, and rarely, as in Walter's case, one is surprised to find secondary growths from apparently benign small spindle-cell sarcoma.

The structure falls into two main classes which differ also in their clinical features: (I) small, and (2) large spindle-cell sarcoma.

The small cell-tumors are slowly growing, relatively benign neoplasms which approach the structure of fibrosarcoma on the one hand and on the other may approximate the type of large cell-tumors. Their rate of growth, density, and malignancy usually accord with these distinctions. The large cell-tumors show more active growth, are softer, more vascular, readily suffer from necrosis, ulceration and hemorrhage, and often recur locally and produce metastases.

The spindle-cells of either type resemble fusiform fibroblasts. They are much smaller than normal fibroblasts and more densely packed in the small cell-growths, but reach very large dimensions and are loosely arranged in the large cell type. The cytoplasm is granular, opaque, and acidophile, the nuclei vesicular and provided with one or more small nucleoli.

In the compact tumors cell borders are distinguished with difficulty and nuclei occupy most of the field, but when the texture is looser the cells exhibit elongated processes and an intercellular stroma becomes visible. This stroma when well developed is composed of fibroglia fibrils demonstrable by Van Gieson's or, better, by Mallory's stain. It may become abundant or be reduced to a trace or be altogether absent. Much of it radiates from the bloodvessels. Its diagnostic importance is not great. Besides the specific stroma of the tumor, remnants of invaded tissue often appear and these with the bloodvessels often determine the arrangement of the cells. 
The cells may run diffusely, but as a rule they lie in broad bundles or narrower columns following blood-vessels or inclosed in connective-tissue septa, or coursing in various directions so that longitudinal, cross, and oblique sections appear in the same field. Virchow and others have employed many terms to designate these structural details as sarcoma lamellosum, fasciculatum, trabeculare, radiare. As a rule these features do not give any clue to the histogenesis.

The spindle-cell sarcoma tends to infiltrate surrounding tissues, the cells pushing their way between fat-cells, vessels, or gland structures, all of which suffer atrophy. The walls of vessels may be infiltrated, penetrated and destroyed, and since the vessels are an easy prey metastases by these channels are common (Goldmann, Hedinger). Bone is penetrated by way of the Haversian canals, and readily absorbed.

The blood-vessels of spindle-cell sarcoma increase with the larger size of the cells and the more rapid growth. They present a variety of structural types. Scanty but well-formed vessels predominate in small cell-tumors, but in others the vessels may be very numerous and the walls are composed of single layers of endothelial cells supported by little or no adventitia. In several forms the vascular paths consist of a system of sinuses walled only by tumor-cells. Thus hemorrhage is common and metastases by way of blood-vessels is facilitated. While in many cases the vessels appear not to belong to the neoplastic process, as they become more abundant the cells usually appear hypertrophic and their nuclei hyperchromatic. The indications of lymph-paths are usually wanting, but Unna describes abundant dilated lymph-spaces in certain cutaneous sarcomas. In defective lymphpaths $v$. Heukelom saw a factor in the pathogenesis of sarcoma, but Lubarsch properly interprets the absence of lymph-channels when it exists as a result and not the cause of the sarcomatous process.

Secondary changes in spindle-cell sarcoma are not prominent. Fibrosis may appear in slowly growing small cell-tumors and an arrest of growth may rarely be established. Wide fibrous areas sometimes appear in large celltumors as a local result of occlusion of vessels. More often hemorrhage and necrosis follow rupture or thrombosis of vessels, and pseudomelanosis frequently recalls old extravasations of blood. Chronic edema affects old and large tumors in which venous stasis occurs, and a pseudomyxomatous change may overtake small foci or the whole tumor. Edema, fatty degeneration, and simple necrosis combine to produce cysts with serous or blood-stained contents.

In many soft vascular tumors the cells are polymorphous, small and large spindle, polyhedral and round-cells appearing in foci or throughout. Such growths are often called round-cell sarcoma, but a true round-cell sarcoma of fibroblastic origin probably does not occur. Likewise polynuclear cells may form and reach considerable dimensions, but the term "giant-cell sarcoma" is better limited to other classes of tumors.

Types of nuclear division are extensively illustrated, especially in the large cell-tumors. They occur in the elongated spindle-cells and especially in large round-cells mingled with them. Increasing numbers of round-cells result from multiplication of dividing cells and constitute a sign of malignancy. Many of the anomalies of cell division in tumors appear in the fibroblastic sarcomas (Stroebe).

The diagnosis of spindle-cell sarcoma seldom presents difficulty, but there are some notable exceptions to this rule and the proper terminology is still a matter of discussion. Typical structures occurring in ordinary situations, as skin, bone, etc., are readily identified. When cell-fibrils become prominent 
and are of adult type the tumor is relatively benign and deserves the designation fibrosarcoma. The numerous small cells and abundant fibrils serve to distinguish most fibrosarcomas from cellular fibromas which show more resemblance to large spindle-cell sarcoma. From myosarcoma the fibroblastic tumors may be distinguished by their location, the large vesicular nuclei, and lack of acidophile quality of the cytoplasm. Yet the myogenous origin of certain spindle-cell sarcomas has often been suggested and is well worthy of consideration (testis, kidney). Very vascular tumors may be called telangiectatic or angiosarcoma, but the latter term has repeatedly been condemned as wrongly inferring an origin from blood-vessels. The malignant (sarcomatous) angiomas have quite a different structure and origin. From typical perithelioma the large spindle-cell sarcoma can sometimes be distinguished with difficulty, but the perivascular units of radiating cells and the characteristic large giant-cells of the former growth are usually unmistakable.

Large spindle-cell sarcoma may be closely simulated by various tumors whose existence must be specially noted. Malignant epithelial tumors of the thyroid are very prone to assume the spindle-cell type, as do also the embryonal forms of the so-called hypernephroma. Metastatic malanoma may appear as a spindle-cell or large round-cell unpigmented tumor. Not a few epidermoid carcinomas present spindle-cells throughout most of their substance.

Diffuse endothelioma may exactly reproduce the structure of fibroblastic sarcoma, but the location of the former growths, the appearance of typical endothelial characters in small areas, the arrangement of the cells, and their minute structure usually but not always permit a positive separation of these closely allied types.

Histogenesis.- In the origin of spindle-cell sarcoma the adventitial cells of the blood-vessels appear to take a prominent part. The early stages of these tumors have many times been traced to foci of vascular proliferating connective tissue which has been identified on doubtful evidence with granulation tissue. Some of these tumors appear to follow trauma, and the processes which result from solution of continuity, extravasation, and absorption of blood often bear considerable resemblance to sarcoma. In organizing blood-clots there may be very active growth of vessels about and into the clot, and it may be supposed that an exaggerated growth of these structures leads in certain cases to sarcoma. Likewise in some established and spontaneous tumors the cells appear to be derived from proliferating adventitial fibroblasts. Such appearances are rather frequent and occasionally convincing, and Babes and Ackermann have expressed the opinion that fibroblastic sarcomas in general originate from blood-vessels. Lubarsch also described spindle-cell sarcomas in young subjects which on close analysis reveal themselves as obliterating cellular angiomas.

The part played by endothelial cells in the genesis of sarcoma has been variously estimated, but in the pure spindle-cell tumors they are not prominent. Many endotheliomas, however, closely resemble this type of sarcoma, and since it is desirable to adhere to the fibroblastic origin of spindle-cell sarcoma the endothelial tumors must be excluded from this group as long as endothelial characters are retained.

Smooth muscle tissue may also give origin to tumors of spindle-cells, as in myosarcoma uteri, but these tumors also must be separately classified. The intermuscular fasciæ and the peri- and endomysium give rise to fibroblastic sarcoma. Those occurring in the muscles of the thigh produce bulky tumors with a notable tendency toward mucoid degeneration. It appears 
probable that in certain situations (e. g., testis) spindle-cell sarcoma represents a one-sided development of teratoma, but to what extent this principle applies elsewhere remains to be determined.

Sarcoma of the Skin.- The skin is the primary seat of a variety of sarcomas or sarcomatoid processes, the exact nature and position of which have not been determined.

Some of these are of spontaneous origin, extend by displacement of normal tissues, grow progressively, produce metastases and cachexia, exhibit the definite histology of a malignant tumor usually composed of spindle-cells, and belong clearly in the group of sarcomas. Others, while producing single or multiple tumor-like growths, progress by infiltrating the tissues, may often regress or disappear, do not produce metastases although appearing in numerous foci, and they present in their earliest stages or throughout the histology of an inflammatory lesion which, however, may sometimes assume a more or less distinct neoplastic character. These conditions are often called sarcoids of the skin.

Of the latter group many types have been described (Pollard, Lit.), of which some have been finally disposed of among infectious granulomas. Such are: (I) mycosis fungoides, types of which are often mistaken for sarcoid (Paltauf); (2) multiple benign sarcoid of Boeck, which proves to be a form of tuberculosis; (3) various cases of benign sarcoid which consist of diffuse lymphocytic infiltrations, and which, while sometimes reaching considerable dimensions, show a distinct tendency toward regression (Spiegler, Joseph, Pollard). Numerous other peculiar cases have failed to receive a final interpretation.

The chief general significance of the study of these cases lies in the evidence which they offer that various inflammatory conditions may closely approach the true sarcomatous process. Spontaneous regression is perhaps not a sufficient ground, as Fano claims, for the exclusion of such processes from the group of tumors, since other true sarcomas may occasionally regress, but the entire clinical and histological picture justifies the current view that these processes are pseudoneoplasms. Not clearly separable from the regressing sarcoids, but distinguished from them by its capacity to develop genuine malignant tumor characters is the multiple hemorrhagic sarcoma of Kaposi.

Multiple Hemorrhagic Sarcoma (Kaposi).-This interesting malady, first fully described by Kaposi in 1872 , has attracted much interest from dermatologists. Recently the data have been fully analyzed by Mariani.

Observed chiefly in southern Europe, in elderly subjects, it exhibits a definite relation to certain predisposing factors, as general or local vascular lesions, sclerosis, ectasiæ, hemorrhagic diathesis, edema, and to trauma and alcoholism. A prodromal stage is marked by local cyanosis, edema and slight inflammation, or by definite vascular ectasiæ (De Amicis). In the stage of tumor growth multiple soft bluish nodules or flat infiltrations form in the derma of the outer surface of hands, feet, cheeks, nose, and elsewhere. They are often painful, inflamed, and may ulcerate, involving deeper tissues and even eroding bones. The nodules may be very numerous, Babes observing $45^{\circ}$ in one patient. Hemorrhage often occurs, leaving pigmentation prominent in some cases. The disease progresses from the extremities upward, some areas showing a tendency to heal, especially under arsenic. The lesions may be symmetrical. In the stage of cachexia the cutaneous lesions may become numerous or diffuse and in several fatal cases secondary nodules appeared in the pharynx, gastro-intestinal tract and liver, kidney, pancreas, lungs, and serous membranes (Phillipsohn, Mariani, Lit.). Most of the cases are eventually fatal from hemorrhage, diarrhea, fever, and cachexia. 
The histological structure exhibits in the early stages chiefly new and dilated lymph- and blood-vessels and infiltration by lymphocytes, plasmacells, mast-cells, and edema, and the lesion resembles that of an infectious granuloma. According to W. Pick the early lesion is indistinguishable from elephantiasis lymphangiectatica. Proliferation of endothelium and perivascular spindle- and round-cells may appear early and become so prominent as to lead to the diagnosis of angiosarcoma, etc. In the later stages spindlecells may predominate and the lesion is indistinguishable from spindle-cell fibroblastic sarcoma (Pick). In all stages vascular ectasiæ, hemorrhage, and pigmentation are constant or frequent.

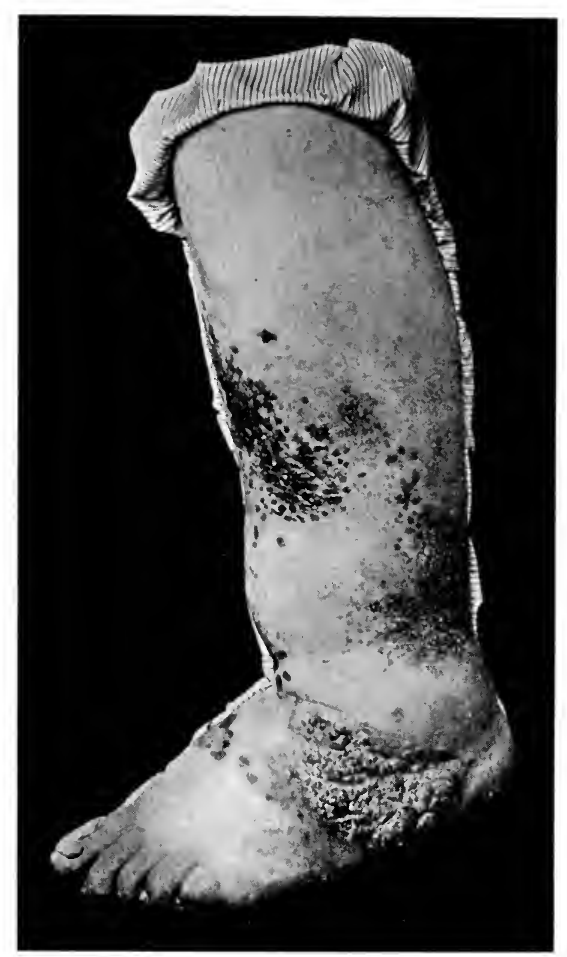

FIG. 77.-Multiple hemorrhagic sarcoma. Kaposi. (After W. Pick, A. D., 87.)

Regarding the nature of this disease opinions are naturally at variance, but the conclusion seems well founded that we have here to do with an infectious granuloma of unknown origin, which in its later stages in predisposed subjects and under suitable conditions may take on genuine neoplastic properties. Justus seems to have carried the agent through five generations of rabbits with the production of typical lesions. The secondary growths in the viscera are probably not metastases, but extensions to new originating foci.

Beginning in local lesions in the extremities in which an infectious agent is probably concerned and aided by the various predisposing factors, the entire vascular system seems to be overtaken by degeneration with over- 
growth of vasoformative cells leading in many instances to true tumor formation. In the histogenesis the numerous discussions have dealt with all the data regarding the interrelations of endothelium, perithelium, fibroblasts, plasma-cells, and polyblasts, but the final tumor product is a spindle-cell sarcoma, which on account of its early history and tendency toward regression has often been called sarcoid.

Spindle-cell Sarcoma of Skin.-These tumors appear as single large growths which tend to become pedunculated or as multiple small nodules widely distributed. Appearing in the derma or subcutaneous tissue they form circumscribed nodules or masses which vary widely in color, consistence, as well as in number and position. Some are hard, opaque, and fibrous,

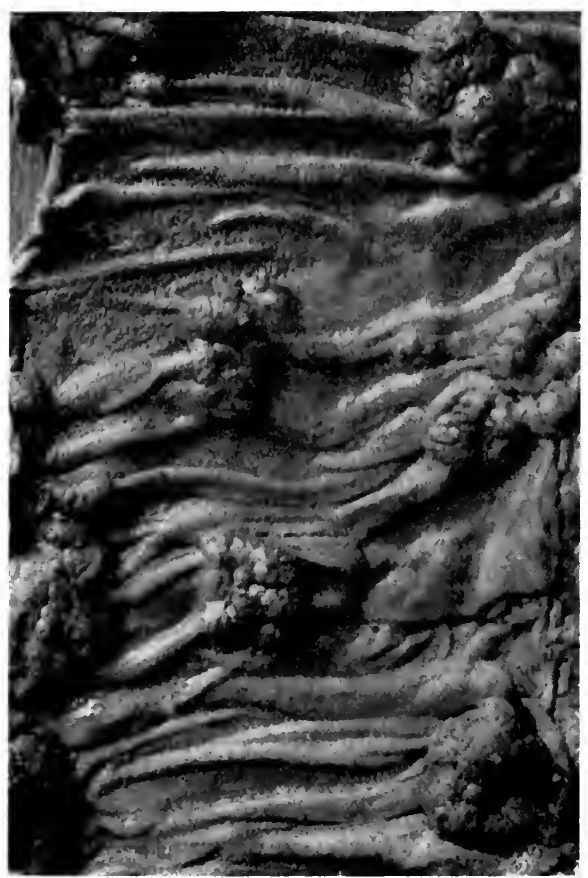

FIG. 78.-Multiple hemorrhagic sarcoma. Kaposi. Intestinal lesion. (After Mariana, A. D., 98.)

others soft, reddish, vascular, and edematous. Both the single and multiple tumors are usually of slow growth and relatively benign, but either type may develop greater malignancy and terminate with metastases and cachexia (Unna). As a rule these tumors show a limited capacity for growth, and while recurring locally are not directly fatal. Fibrosis (Linser), pedunculation, and spontaneous extrusion may occur. Ulceration and necrosis are rare. They appear at almost any age, and Maldovan and Pernet describe congenital cases.

The structure is in many instances that of the simplest form of small or large spindle-cell tumor, but many variations from this type are encountered. Blood-vessels may be numerous and the tumor-cells may appear to be 
derived from their adventitial cells. Varying in this direction spindle-cell sarcoma of the skin approaches angiosarcoma and perithelioma, from which types they are not always sharply separable. In one group endothelial proliferation appears to be prominent and yields many rounded and polyhedral cells (Johnston). Chronic edema may lead to secondary myxomatous characters. Unna describes a rich supply of lymph-spaces in some tumors.

The arrangement of cells also varies greatly. They may be closely packed, of small size, with little stroma, and quite diffusely arranged. An interfascicular form is yielded by cells whose axis is determined by the course of blood-vessels (Unna). The cells may form whorls or intertwining bundles which have suggested an origin from nerve-trunks. The cells penetrate sur-

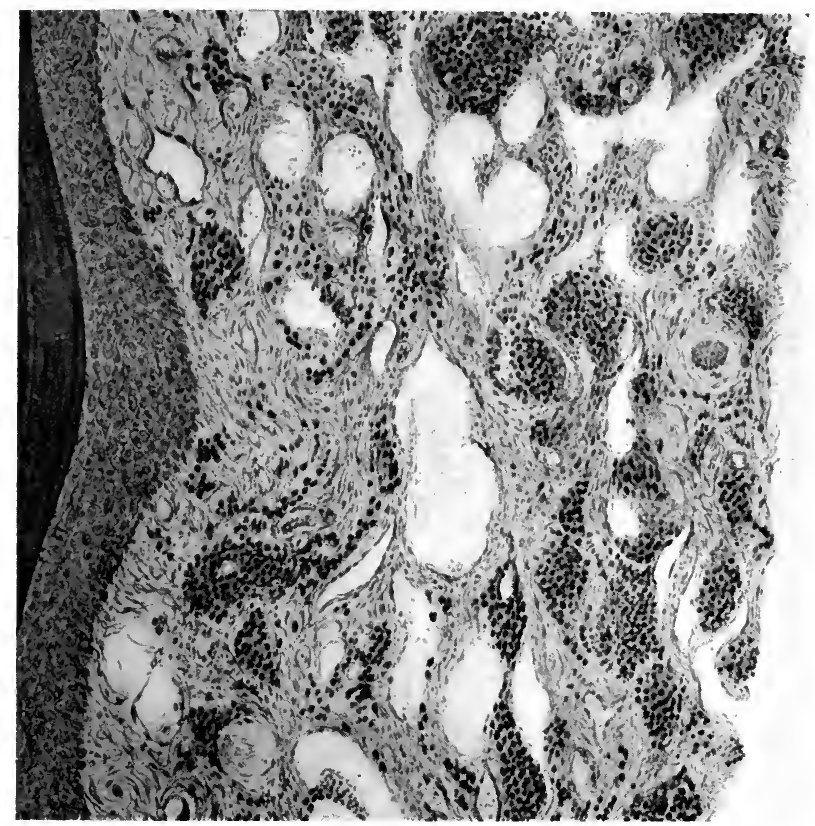

FIG. 79.-Structure of multiple hemorrhagic sarcoma. (After Mariani.)

rounding tissues and develop new outlying nodules, or they may remain well circumscribed. In the central portion of all true sarcomas of the skin Unna finds elastic fibrils missing.

The histogenesis of cutaneous spindle-cell sarcoma is a complex question, and it has not been possible to clearly define the subgroups probably existing in this class according to their exact origin. Yet the fibroblastic nature of the typical form seems reasonably certain. Many have concluded that the adventitial cells of blood-vessels and lymph-vessels are the chief seats of origin. In certain cases I have been impressed with the evident relation of the tumor-cells to numerous abortive blood-vessels which seemed to become lost in the tumor-tissue. Yet Unna believes that the importance of blood-vessels in the origin of these growths has been overestimated, especially by Babes. The connective tissue about cutaneous glands rarely seems 
to be specially concerned. That an endothelial element is present in some cases is highly probable, especially in tumors with rounded, polyhedral, or giant-cells. In cases of multiple symmetrical tumors with cells in intertwining bundles an origin from nerve-sheaths and an endothelial element are indicated. The occurrence of congenital cases indicates that misplaced or superfluous cell groups, and not normal tissues, are occasional or frequent points of origin. Finally it is clear that some tumors closely resembling fibroblastic sarcoma belong in the group of nevomelanomas, and that the highly malignant cutaneous sarcomas, as in Pini's case, must be regarded with suspicion from this standpoint.

In the etiology, various forms of trauma not infrequently precede the solitary sarcoma (Lowenstein). Virchow (II, 245) cites many cases of sarcoma arising in scar tissue which had been subjected to long irritation. For the multiple tumors a tissue predisposition and the various inflam-

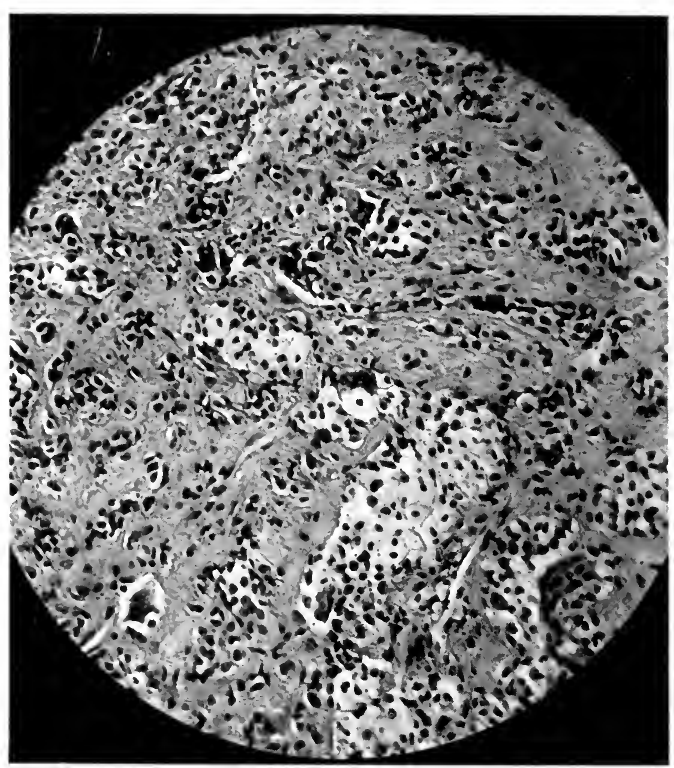

FIG. 80.-A giant-cell tumor of tendon sheath, probably of endothelial origin.

matory and nutritional changes illustrated in the sarcoids may be held responsible.

Giant-cell Sarcoma of Tendon Sheaths and Aponeuroses.--The specific structure and benign clinical course of a group of giant-cell sarcomas of tendon sheaths of the hand and feet have long been recognized. They have been fully described, especially by French observers, under the terms "myeloma" or "xanthosarcoma" (Gross, Paquet, Reverdin, Heurteaux, Spiess, Lit.).

The tumors arise chiefly in tendon sheaths and aponeuroses of hands and feet, and reach moderate dimensions after a period of $\mathrm{I}$ to 20 years. Sudden increase in size may rouse a suspicion of malignancy, but they seldom reach the size of an egg. They are well encapsulated, firm, opaque or yellowish and markedly lobulated, and are readily shelled out by operation. They never yield metastases and rarely recur after removal. 
The structure presents four main features: (r) Groups' of spindle- or polyhedral cells surrounded by dense connective tissue. Some of the tumors are exclusively of this type. Although the tumor may be quite cellular and free from giant-cells it still maintains its benign character. (2) Groups of large cells containing much doubly refractive lipoid material. These are the so-called xanthoma-cells. They may be so abundant as to duplicate the appearance of xanthoma of the skin, and their origin is referred to prolif-

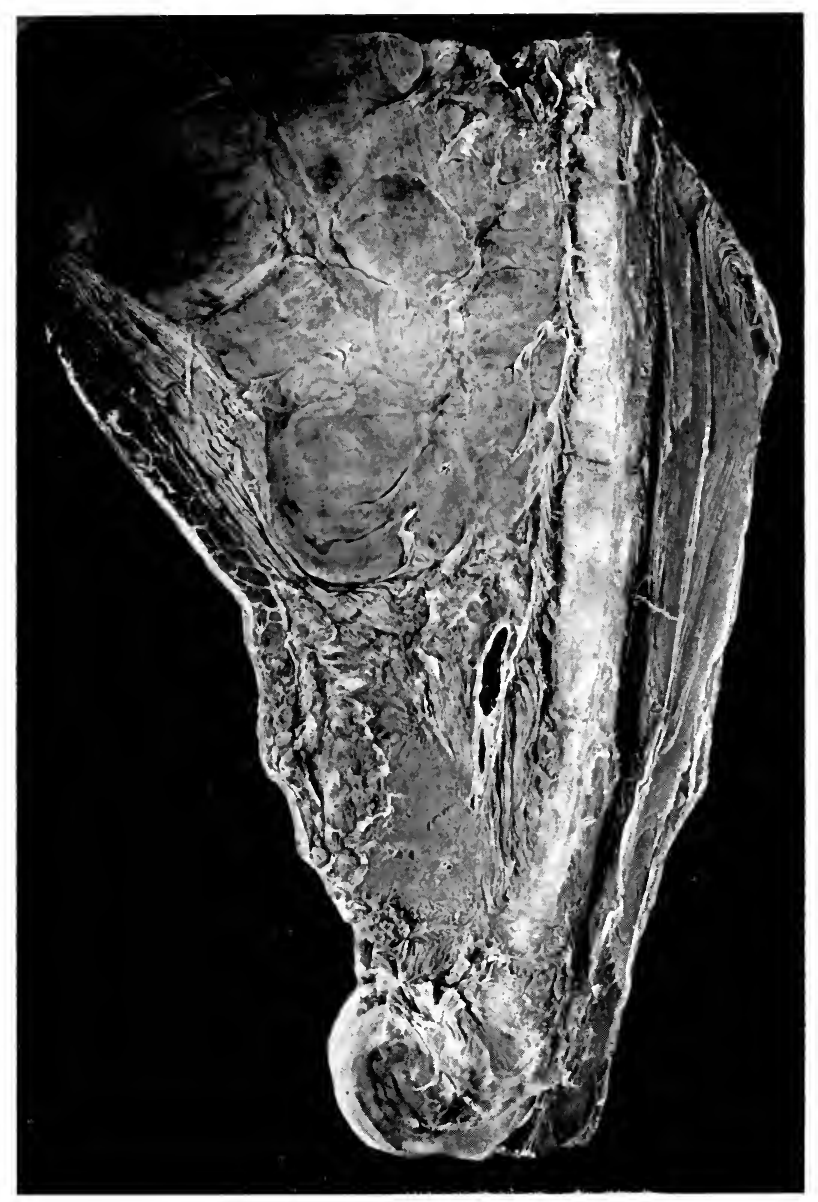

FIG. 8I.-Myxosarcoma of fascia of thigh.

erating fibroblasts or endothelium. (3) Giant-cells of very large size up to I25. $\mathrm{m}$. are commonly present in abundance, and present very numerous smail vesicular nuclei. The cytoplasm is granular, acidophile, and often contains much lipoid material. They belong to the class of foreign body giant-cells. (4) Pigment of the type of hemosiderin frequently collects in the tumor-cells.

Etiological factors are very indefinite, but the structure and benign char- 
acter of the process suggests that the tumors arise from a local inflammatory process connected with trauma and some peculiar disturbance of local lipoid metabolism. Flessig denies that they have any neoplastic quality and regards them as a form of granulation tissue, yet similar arguments apply almost equally well to many other sarcomas. They have often been classed with xanthoma.

Sarcoma of Muscles.-Primary sarcoma of the muscles arises from the connective tissue and vessels of the perimysium and endomysium and usually takes the form of fibrosarcoma or myxosarcoma with small or large spindlecells, and often with a marked prominence of capillary or larger blood-vessels. Occasionally they are described as round-cell sarcoma (Chambe, Pasteau, Kuttner).

They occur chiefly in young adults, the rapidity of growth varies with the structure, but is often rapid, they reach a large size if undisturbed, recur locally after operation, and the cellular forms produce metastases. Chambe has collected 39 cases illustrating many features. Many cases were fatal. Virchow observed that in the muscle fasciæ slowly growing fibrosarcoma or spindle-cell sarcoma predominate, while at tendon insertions many roundcell and malignant tumors appear. A characteristic fibrosarcoma arises from the sheath of the rectus abdominis (Bodenstein, Lit.).

The histogenesis has been traced in certain cases to the interstitial tissue (Birch-Hirschfeld), and the remarkable proliferative tendency of this tissue renders such an origin probable. The muscle-cells usually atrophy, but Sokalow, Guitton, and others reported the transformation of musclecells into sarcoma-cells. About many tumors of muscle there is a pronounced reactive myositis with various alterations in the form of the cells (W. Schaffer).

That sarcoma of muscle may be derived from groups of embryonal and undifferentiated muscle-cells has often been suggested (Borst). It seems probable that with myxosarcomas and malignant growths approaching the round-cell type the origin is from misplaced cell groups connected with bone formation. In a series of bulky sarcomas of the thigh I have observed gradations in structure from myxosarcoma to chondrosarcoma. In an early sarcoma of muscle I found very many small blood-vessels which seemed to be the source of the spindle-cells of the tumors. Peabody's study of Peters' cases suggested a vascular origin. Sokalow traced some tumors to the sarcolemma, but Cornil and Ranvier, Malherbe, and most authors assume that they arise from the interfibrillar connective tissue.

Periosteal sarcomas of many types commonly invade the muscles and some may appear largely intramuscular.

Sarcoma of the esophagus is a rare condition. Howard (I902) analyzed I 2 cases. Herxheimer, I9o8, refers to I 8 cases, 2 of which were combined carcinoma and sarcoma. Hacker, I909, gave a complete analysis of the subject with reference to $2 \mathrm{I}$ cases. They occurred usually in males, in the upper or lower segment, and in subjects from 4 to 70 years of age, and were rapidly fatal. They appear either as an ulcerative process or as a polypoid tumor. The former usually contain round or polyhedral cells, the latter, spindle-cells. Stephan describes a peculiar lymphosarcoma in an infant. In Eucken's case there were many giant-cells. With the round-cell growths metastases are early and wide-spread (Shaw, Stark, Rolleston). The spindle-cell tumors were usually free from metastases (Targett, Ogle, Livingood, Herxheimer).

It seems probable that two entirely different tumors have been included in this group. The round-cell growths strongly resemble embryonal carcinoma in gross appearance, and Stark suspected that his 2 cases might be 
carcinomatous. One case of this type with bulky metastases in the liver strongly suggested to me a carcinomatous origin (Norris). The spindlecell tumors are probably genuine sarcomas, but little effort has been made to determine their origin. Howard regarded his spindle-cell tumor as a myosarcoma. Wolfensberger and Glinsky have observed mixed tumors of the esophagus composed of striated muscle-, spindle-, and giant-cells. Herxheimer describes combined sarcoma and epithelioma arising apparently from the submucosa and the epithelial lining. Metastases were wanting. Baur describes a melanotic sarcoma of esophagus.

Sarcoma of Stomach.-The reported cases of sarcoma of the stomach constitute about I per cent. of all gastric tumors. On analysis the cases fall into three distinct groups: (I) Spindle-cell myosarcoma; (2) lymphosarcoma, and (3) miscellaneous round-cell or alveolar sarcomas, the nature of which is uncertain. Of all these, I50 cases have been collected by Ziesche and Davidsohn.

I. The spindle-cell myosarcomas constitute a well-defined group. They form solid or cystic growths, single or multiple, which project from the wall of the stomach into the peritoneum, or remain interstitial, or protrude into the cavity of the organ, where they suffer necrosis and ulceration. Very early small growths have been observed, while Brodowski's tumor grew to the size of a child's head between the mesenteric layers. Cantwell's case weighed I 2 pounds and Baldy's nearly filled the abdomen. Most of these tumors arise from the curvatures, rarely from the pylorus (Manges, Schlesinger).

The external pedunculated tumors may draw the stomach out into a funnel form; the interstitial tumors may cause dilatation; and Ewald found the organ reduced to a narrow canal surrounded by multiple or diffuse masses of fibrosarcoma. Ulceration and hemorrhage frequently occur. The large tumors are often cystic, and the bulky metastases which commonly form in the liver are often cystic and may transform this organ into a series of large cystic tumors (Hosch, Lit.). The course of gastric myosarcoma is relatively slow in the typical cases (3 $\frac{1}{2}$ years), but with greater anaplasia of the cells the growth is often quite rapid.

The structure varies between considerable limits. In some cases the type approaches that of a cellular myoma and the recognition of a myogenous origin is readily accomplished. Or the cells are of large spindle form and the designation as a myosarcoma depends on the attitude of the observer. Such a case is that of Hoesch-Kaufmann which appears to be identical in structure with others described as large spindle-cell sarcoma. There is little doubt also that some or many of the so-called large round-cell sarcomas are of muscular origin. In some cases careful examination has revealed typical myosarcoma in one portion of the tumor, indifferent spindle-cells in other portions, and elsewhere only round-cells (Moser). The arrangement of the cells in intertwining bundles is often a characteristic feature. The grouping of cells about blood-vessels has sometimes suggested that the tumor arose from the walls of the vessels as in myoma uteri (Kaufmann, Howard), or it has led to the diagnosis of angiosarcoma, or lymphangiosarcoma (Steudel). The gross appearance of many of these tumors of doubtful nature is very similar to that of definite myosarcoma.

In the etiology of myosarcoma a congenital anomaly must be sought for the stomach as for the uterus. Of the nature of this embryogenic disturbance there are scant indications. That isolated islands of muscular tissue occur in the stomach is shown by Alsleben's report of an adenomyoma in the pyloric mucosa, while the congenital occurrence of myosarcoma is probably demonstrated by Finlayson's case. 
2. Lymphomatous tumors of the stomach occur ( $\mathrm{r}$ ) as a part of the leukemic process; (2) in gastro-intestinal pseudoleukemia; (3) as a part of general lymphosarcoma, and (4) in the form of localized lymphosarcoma. They are considered in detail under these headings.

3. Miscellaneous Sarcomas of Stomach.-In not a few gastric sarcomas the structure has appeared not to fall into any of the common groups, and the tumor has been variously described as alveolar or mixed-cell, or angiosarcoma, or myxosarcoma. None of these histological features deserves special recognition as a separate variety of gastric tumor.

Dreyer described a pyloric tumor composed of spindle-cells and carcinoma occupying a large ulcerated area at the pylorus. The metastases were carcinomatous. Leube also observed mixed carcinoma and sarcoma. Such observations suggest the conclusion that carcinoma of the stomach may give histological appearances resembling sarcoma. Cammidge has mentioned 4 cases of this type and concludes that many so-called sarcomas of the stomach are really carcinomas (Wilson). This criticism appears to be justified and the writer would urge great caution in interpreting as sarcoma any tumor associated with an ulcer and of which the gross appearance strongly suggests carcinoma. That sarcoma of the stomach may follow ulcer appears possible (Moser, Kehr), but this relation has not been satisfactorily proved; I8 cases of metastatic sarcoma of the stomach, chiefly lymphoid and melanotic, have been collected by Ziesche and Davidsohn. They are not uncommon.

Sarcoma of Intestine.-The small and large intestines and the rectum are subject to the same types of sarcoma as the stomach. Baltzer called attention to the surgical aspects of this group of tumors in a report of 14 cases. Rademacher (1908) collected I40 cases. Of rectal sarcomas Key found 55 reports, to which he added 3. The majority of these cases were studied exclusively from the clinical standpoint, which emphasizes the symptoms of an abdominal tumor causing intestinal obstruction. The nature of the growths is seldom clearly stated, but the great majority appear to be lymphomatous, while a considerable proportion are fibroblastic.

I. Spindle-cell sarcoma arises chiefly from the muscular coat, and produces a large, solid or cystic growth lying external to an intact mucosa. A rather well-defined group is the myxosarcoma affecting chiefly the cecum and appendix and said to arise from the submucosa. It may contain large cysts. The subserous tissue is said to give origin to certain angiosarcomas. A congenital angiosarcoma of ileum is described by Stern, and a subserous angiosarcoma of the splenic flexure by Baltzer. I have examined a large spindle-cell vascular sarcoma of the omentum.

2. Miscellaneous sarcomas of intestine constitute a small group of illdefined and imperfectly described varieties. An interesting case is that of Lehmann, who designated as interfascicular endothelioma a diffuse thickening of the entire intestine with secondary growths in liver. Pigmented sarcomas occur in the rectum and ileum (Sandner, Treves), but the source of the pigment is not clear and intestinal metastases of melanoma of the skin are common. I have studied one slightly pigmented primary submucous melanoma arising around the opening of the appendix in a child (cf. Melanoma).

3. Lymphomatous tumors of the intestine are considered under that heading. They arise chiefly in the ileum, colon, and rectum. Rarely they develop the appendix (Warren, Carwardine).

Sarcoma of Ovary.- Primary sarcoma is a comparatively rare tumor constituting about 5 per cent. of all ovarian neoplasms (Kroemer). Yet in 
this small group many histological varieties are represented, concerning the histogenesis of which little is known. The process of organization of bloodclot in the corpus luteum and of regression of this structure offers conditions very favorable to the development of sarcoma, and it is probable that this source of myxosarcoma, perithelioma, etc., has not been given due attention. Other sarcomas, especially of the embryonal type, have been traced to a teratoid origin.

I. Spindle-cell sarcoma is a well-defined type which is closely related to fibroma and probably also to ovarian myoma. It appears as a diffuse process affecting the whole ovary and producing either a solid tumor of moderate dimensions or a large growth containing many cysts. The surface is nodular or lobulated and section reveals a smooth opaque texture, or many dilated vessels, or cysts which are more irregular in size and form than in cystic adenoma. Áreas of edema and mucoid degeneration may be present and hemorrhage or infarction may follow torsion.

The structure presents spindle-cells of moderate dimensions mingled usually with spheroidal cells. Trabeculæ of adult connective tissue separate the tumor into lobules, while between the cells there is little demonstrable stroma. The cells may grow diffusely, but more often they are definitely connected with small blood-vessels which are either thin walled and telangiectatic or compressed or occluded by hyaline degeneration. The main feature of some tumors is the abundance of hyaline vessels between which lie scanty spindle-cells. In the cystic tumors dilatation of lymph-vessels, edema, and liquefaction necrosis seem responsible for the irregular cavities which contain serous or bloody fluid and are lined by a ragged layer of sarcomatous tissue supported by connective tissue. Such growths may be interpreted as cystic lymphangiosarcomas. The spindle-cell tumors are of slow growth and relatively benign.

The origin of the spindle-cell tumors must be referred to the ovarian stroma, but to which elements is uncertain. The perivascular arrangement of the cells suggests an origin from the walls of vessels, but not an endothelial character. The perithelial tissue has been suggested as a possible source, but without definite basis, since the tumors are usually diffuse. Smooth musclecells have been observed in round-cell and spindle-cell sarcoma and it appears probable that some of these tumors are of myogenic origin (Basso, Gangolphe, Kroemer).

2. Round-cell sarcoma of the ovary is an ill-defined and rare form of malignant tumor which occurs chiefly in young subjects or children and proves rapidly fatal. The tumors are single or bilateral, reach a considerable size, are usually solid or contain softened or necrotic areas. They are soft, edematous, infiltrated with blood, and exhibit numerous necrotic foci. 'The structure is of small round-cells lying diffusely or grouped about thin vessels. In some cases the cells are of larger size and exhibit an alveolar arrangement (alveolar sarcoma). Or spindle-cells, small round-cells, large spheroidal cells, and giantcells may be variously combined.

In none of these cases does the structure offer any clue to the histogenesis and this group of tumors, therefore, invites subdivision according to the histogenetic principle. It seems probable that some of the tumors with indifferent round-cells are to be interpreted as one-sided developments of teratoma, but to which forms this view may apply it is difficult to decide. One form of large round-cell sarcoma of the ovary closely resembles a similar tumor of the testis and is probably of teratomatous nature. It is very prone to necrosis.

It also seems probable that some so-called alveolar sarcomas are really 
of epithelial origin arising from the cells of the granular layer of the follicles, since these cells in adenomatous tumors readily assume a small round form and diffuse arrangement. That the theca cells of the ovarian follicles give rise to certain sarcomas is claimed by Pinto, but this origin still lacks definite proof.

The relationship of cells to blood- and lymph-vessels has often led to the designation of such tumors as angiosarcoma and perithelioma. In favor of this view is the frequency of angiomas in the ovary and of sarcomas in which the blood-ressel is the prime unit, yet most of these tumors are composed of spindle-cells.

Against the origin from vessels is the well-known tendency of all tumorcells to thrive best in the proximity to blood-vessels, giving spurious angiosarcoma. Nevertheless, Kroemer recognizes a special group of ovarian angiosarcoma of blood and lymph-vascular origin. They are difficult to distinguish from the group of perithelioma $(q . v$.). In this field also one encounters evidences of the close relationship of endothelioma and sarcoma. The existence of a true ovarian endothelioma is elsewhere discussed.

A form of plexiform sarcoma is described by Kroemer. It consists of anastomosing columns of syncytial and giant-cells which he derives from the perivascular cells about lymph-vessels, a lymphangiosarcoma. The endothelial cells of the vessels proliferated slightly in places, but tended to disappear from the field. This nice histological analysis still leaves untouched more important questions of histogenesis.

Finally, among the polymorphous cell ovarian sarcomas there remains a rather well-defined type.

Myxosarcoma.-Areas of secondary myxomatous change are frequently seen in other forms of ovarian sarcoma. They occur about the blood-vessels and lymph-vessels, especially of fibrosarcoma and sometimes become so prominent as to call for the designation of myxosarcoma. Yet such cases are rare. Segalowitz found only one largely myxomatous tumor among 300 from the ovary, and Kroemer states that a pure myxosarcoma of the ovary does not exist. An interesting observation is that of Walker, who records 3 fatal myxosarcomas in sisters.

Myxomatous areas occur especially with chondrosarcoma, as in the case of Gibb, which was probably of teratoid origin, and myxomatous changes in the stroma of ovaries invaded by epithelial tumors is so common as to lead Glockner to regard it as a specific reaction of ovarian tissue. A specific variety of myxomatous tumor of the ovary has been described by Krunkenburg with Marchand's endorsement, under the term fibrosarcoma mucocellulare carcinomatodes (see section on Carcinoma of Ovary).

Chondrosarcoma occurs in the ovary as a one-sided development of a mixed tumor or of a teratoma, or as a metaplastic growth derived from the ovarian stroma.

The former class is illustrated by tumors in which islands of cartilage are associated with dermoids, or with other derivatives of the ectoderm or entoderm. The cartilaginous elements may predominate, and a characteristic case of this sort is that of Reis in which a large chondromatous tumor after extirpation was followed by a rapidly growing carcinoma. A similar origin probably applies to Gibb's myxochondrosarcoma occurring in a child of $2 \frac{1}{2}$ years. Donati observed very large giant-cells in a tumor containing cartilage and much round- and spindle-cell tissue. Here it seems impossible to decide whether the origin was teratomatous or metaplastic.

Some authors assume that ovarian and other chondromas of the female genital organs arise from inclusion of the Müllerian or Wolffian ducts (Wilms), 
and Kehrer states that such tumors contain areas of embryonal round-cell mesodermal tissue which differentiate into various tissues.

That cartilage arises by metaplasia from the ovarian stroma was strongly suggested by the old observation of Kiwisch, who found cartilaginous plates in the outer layers of a bilateral ovarian fibroma. Buet also described cartilaginous metaplasia in a fibrochondro-osteoma of the ovary. These processes were diffuse, involving the entire ovary. The most notable evidence of metaplastic development of cartilage in the ovary is furnished by the case of Jung.

In a multipara of 48 years with cervical endothelioma (epithelioma?) a myomatous uterus was removed with one-half of one ovary which was very slightly enlarged. This ovary was found to contain several islands of young and some ossifying cartilage embedded in edematous connective or in spindle-cell sarcomatoid tissue. There was a prompt recurrence of a pelvic tumor at the site of the ovary containing chiefly chondrosarcoma with areas of spindle-, round, and large polyhedral cells. Meyer interprets this case as a teratoma, Kehrer as a mixed growth of Wilms type, and Jung and Kroemer as a metaplastic chondrosarcoma. The diffuse distribution of multiple islands of cartilage in edematous connective tissue of the ovary seems to favor a metaplastic origin.

Sarcoma of the uterus occurs in two rather distinct forms which it is desirable to separate because of their anatomical position, histogenesis, and clinical features. These are: (I) mural sarcoma, and (2) sarcoma of the mucosa (Virchow).

It was early recognized that certain apparently benign uterine tumors recurred after operation, and these were described by English observers as recurrent fibroids (Hutchinson, Callender). In I864 Virchow described a group of uterine sarcomas and these tumors have since been fully discussed by Gusserow (1870), Tertillon (I890), Gessner (I899), Piquand (I905), and lately by Meyer (I908).

I. Mural Sarcoma.-This tumor occurs chiefly in the body, less often in the cervix (I 2 per cent. Gessner). It may appear as a primary sarcoma or develop secondarily in myoma. In either case the growths present many of the features of uterine myoma. Thus they are often encapsulated, more frequently diffuse, and their position is subserous, interstitial, or submucous. The cervical and submucous tumors are usually polypoid and the interstitial growths may early break into the mucosa and thus become indistinguishable from originally submucous tumors. A rare type encircles the tubal orifice (Meyer). All varieties may be single or multiple. Multiple sarcomatous fibromyomas have been described by v. Kahlden, Gessner, and Busse. The single tumors may reach a very large size, and Terrillon has recorded a weight of 20 kilos. The submucous tumors and especially the cervical growths may be extensively polypoid or even papillary.

On section, the uterine sarcomas are soft and opaque as compared with myoma and are subject to liquefaction necrosis and hemorrhage. Fatty degeneration, thrombosis of vessels and infarction, with extensive softening may produce large cysts in which suppuration may occur. While the mucosa is usually intact, many of the tumors which project into the cavity become ulcerated and extensively excavated. Overdevelopment of blood-vessels or lymph-vessels is observed, and Meyer describes a markedly lobulated tumor in which the lobules were separated by large lymph-sinuses. The more circumscribed tumors have the general outline of fibromyomas, but diffuse forms infiltrate the wall, the mucosa, and the neighboring structures.

Metastases appear late with encapsulated interstitial tumors, but when the parametrium is invaded secondary growths often appear in the regional 
areas and in the lungs as well as in many other tissues and organs (Lubman). Yet Gessner observed regional metastases with circumscribed tumors. The uterine veins and lymphatics have been found thrombosed by tumor masses without metastases. The ovaries may be extensively invaded through the vessels or by implantation.

The gross anatomic features suggest the following somewhat serviceable classification:

I. Circumscribed primary sarcoma, chiefly subserous or interstitial.

2. Diffuse sarcomas, chiefly submucous or infiltrating the wall and parametrium, with metastases, and subject to hemorrhage and necrosis.

3. Polypoid sarcomas of body or cervix.

4. Extra-uterine single or multiple myosarcoma.

5. Secondary sarcoma in myoma.

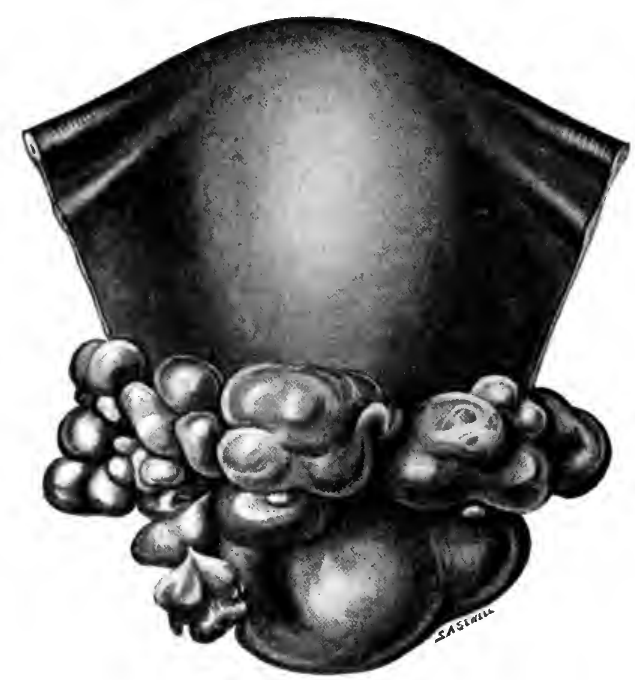

FIG. 82.-Polypoid sarcoma of cervix uteri in a subject of 52 years. (After McCann.)

Structure.-The structure of uterine sarcoma is extremely varied. Many authors have described them as myosarcoma, spindle-cell, round-cell, mixedcell, and giant-cell growths and other subvarieties. Yet there is strong reason to believe that the entire group, with rare exceptions, is of myogenic origin. It is well-known that the malignancy varies with the cell type, reaching its acme in round-cell and giant-cell structures, but it has repeatedly been shown that in the same tumor the structure of different portions may exhibit several cell types and that different tumors illustrate all transition phases of smooth muscle-cells to round-cells and giant-cells. Even in well-formed fibromyomas areas of round-cell sarcoma may occur (Meyer, Moraller, v. Franquè). In a case of large polypoid submucous sarcoma I found areas of adult myoma, very cellular lobules resembling the recurrent myoma, indifferent spindlecell sarcoma, numerous areas of round-cells (very short spindles), and in edematous portions alveolar sarcoma with many large polyhedral cells. Moraller observed a notable perivascular grouping of round-cells. Meyer concludes that the round-cell sarcoma of cervix is often regarded as endothelioma. 
In fact, it is evident that in this field too much importance has been attached to variations in cell forms and too little to histogenesis and gross anatomy.

From the prognostic standpoint, however, considerable significance attaches to the cell type and somewhat characteristic clinical varieties of uterine sarcoma are often associated with the predominance of cells showing different grades of anaplasia.

(a) Recurrent myoma is a term that may well designate a tumor occurring chiefly in the cervix, which recurs after a difficult extirpation and which contains rather long, loose spindle-cells and scanty imperfectly formed long giant-cells. In a case of this type the structure remained uniform after three recurrences, while the pelvis and vaginal wall were slowly infiltrated. A characteristic recurrent myoma of the broad ligament occurs in the form of multiple nodules composed of well-formed muscle-cells with marked vasoformative tendencies. The cells are short spindles with long nuclei and strongly acidophile cytoplasm. The arrangement of the cells is quite orderly, the vessels well formed, and giant-cells, hemorrhage, degeneration, necrosis, and metasstases are wanting.

(b) Large spindle-cells with granular cytoplasm, large vesicular nuclei, and occasional giant-cells compose the bulk of many tumors which invade parametrium, broad ligament and mucosa, with ulceration, necrosis, and cachexia. In some cases the giant-cells are

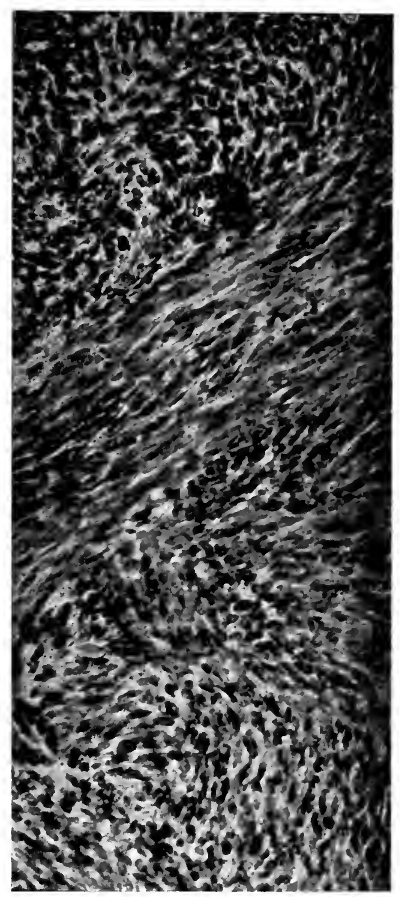

FIG. 83--Recurring myoma of cervix uteri. very numerous and of enormous dimensions.

(c) Predominance of round-cells usually marks rapidly growing tumors which are locally destructive, produce cachexia from necrosis and hemorrhage,

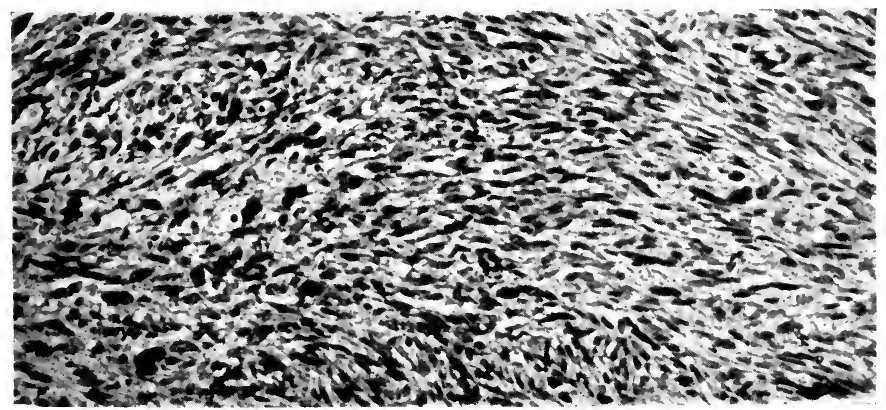

FIG. 84.- Section of a recurrent myosarcoma of vagina and cervix uteri.

and early yield local and distant metastases (Meyer). Yet many metastases contain chiefly spindle-cells. Heinrich found both spindle-cells and round 
cells in tubal metastases of spindle-cell tumors, and both original and metastatic tumors contained all transitions from muscle-cells to spindle-cells, giantcells, and round-cells in the cases of Busse and Masty.

Histogenesis and Etiology. - While many efforts have been made to inculpate the connective tissue of the uterus in the genesis of sarcoma there is no sufficient evidence to show that these tumors include any of fibroblastic origin.

On the other hand, the wide variation in form of neoplastic smooth musclecells demonstrated in these and other myosarcomas leaves little room for doubt that the entire group is essentially of myogenic origin. Yet there

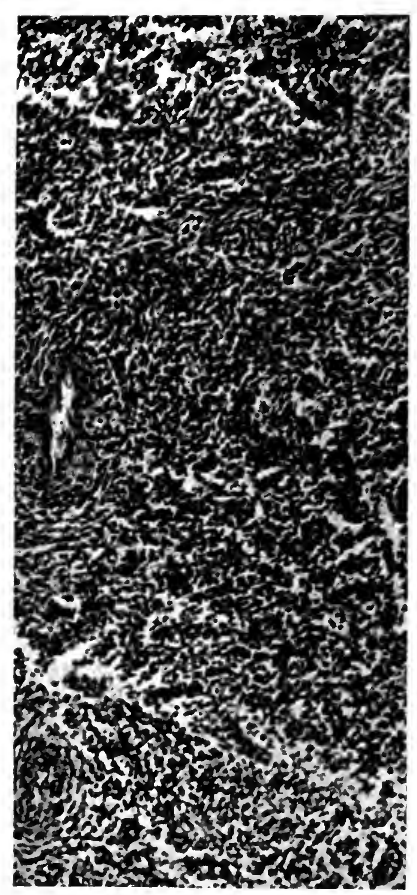

FIG. 85.-Myosarcoma of uterus. A rare type. The small cell groups surround minute vessels. remains the possibility that the group of cells giving rise to the tumor contains besides muscle-cells a portion of the specific stromacells of the uterine mucosa. Such isolated complex groups of cells have been shown to give rise to adenomyoma, but the adenosarcomas which doubtless originate from such cell groups have quite a different structure from myosarcoma and contain gland alveoli (v. Winckel, Amann).

In certain sarcomas areas of small roundcells traverse the main spindle-cell tumor in broad but sharply circumscribed bands, and some have derived these bands from the stroma of the mucosa. Yet the adult stroma is probably not connected with the origin of any of these tumors and similar round-cells are observed in encapsulated intramural growths.

Regarding the nature of the embryogenic disturbance giving origin to sarcoma, one must refer to the same data which serve for uterine myoma. (For the sarcomatous transformation of myoma see Myoma Uteri.)

2. Sarcoma of Uterine Mucosa.-Two main anatomic forms of this condition are commonly recognized, viz., (I) diffuse, and (2) polypoid. The latter represents a natural tendency of growth of the former, and there is no essential distinction between them.

Arising from fundus or cervix the diffuse forms are usually wide-spread, Piquand finding the entire mucosa involved in 33 of 54 reported cases, while a portion or even the whole of the tubal mucosa may be affected (Griffith, Simpson). In advanced cases polypoid outgrowths frequently form. From a broad base bulky tumors may develop over which the uterus is stretched with thinning or hypertrophy of the wall (Beckmann, Terillon). Occlusion of the uterine canal may lead to retention of serous, bloody, or purulent fluids. In one case Terillon withdrew I $_{5}$ liters of bloody fluid. A thin uterine wall may suffer inversion (Williams). The original mucosa may remain intact, but is gradually altered by the growth of the tumor, by interstitial endometritis, or by erosion and replacement by granulation tissue.

The tumor is at first sharply separated from the muscularis, but soon invasion of the wall occurs and extension follows to the parametrium, perito- 
neum and vagina. Metastases are observed in the regional nodes, lungs, and elsewhere (Gessner).

Secondary changes are common in both forms of sarcoma of the endometrium. The superficial portions early become eroded, infected, and necrotic. Hemorrhages, edema, myxomatous softening, wide areas of caseation, and fibrinous exudate combine to give a varied appearance.

The polypoid form is most pronounced in the cervix, where it is not readily distinguished from polypoid mural sarcoma and certain malignant teratoid growths. The teratoid tumors include the malignant myxosarcoma of Spiegelberg which is the most important cervical sarcoma and is to be sharply distinguished from the present group. Many reported polypoid sarcomas of the cervix refer to cellular polyps chiefly of inflammatory origin and it appears doubtful if the cervical mucosa is the seat of any true sarcomas apart from the two above types.

The structure of endometrial sarcoma is quite varied. Ruge describes 4 varieties: (I) Large round and spindle-cells; (2) decidua-like cells; (3) giant-cells with small round cells and spindle-cells, and (4) small round cells. It is probable that all these forms represent variations of one original cell type. Thus Meyer found round cells in the superficial layers, spindlecells in the deeper portions of a polypoid tumor. He interprets the decidual cells as transitory effects of the menstrual influence, and the giant-cells as regressive forms. In my own cases the chief features have been the diffuse arrangement of large and small rounded cells with hyperchromatic nuclei. The long spindles, parallel intertwining columns, and vasoformative tendencies of myosarcoma are wanting. The liability to necrosis is much more pronounced.

The diffuse distribution of many endometrial sarcomas is strong evidence that the tumor arises form the stroma-cells. Some have traced the earliest stages to the periacinar stroma. Wolgren describes as "fibromatosis uteri" a tumor-like thickening of the endometrium due to overgrowth of stromacells most marked about cystic glands, and Fellander places in an intermediate group between inflammatory and neoplastic processes his case of benign "elephantiasis fibrosarcomatosa" in which many giant-cells were mingled with short spindles. The occurrence of such intermediate forms of hyperplasia renders it probable that highly malignant tumors also arise from the stromacells. That some of the supposed sarcomas of the mucosa are advanced stages of mural myosarcoma is nevertheless probable. Ruge states that sarcoma follows periacinar interstitial endometritis and that the tumor process first affects groups of glands about which the stroma-cells increase in size and number.

For the endometrium a special factor in the genesis of sarcoma which should not be overlooked is the influence of gestation. Yet the varieties of sarcoma are well represented in children and virgins. Highly malignant lymphosarcomas are said to have existed in rare cases, but their exact nature is uncertain (Wilischamin, Wagner, Gow). Melanosarcomas result from imbibition of blood. Alveolar and angiosarcomas illustrate occasional secondary structural features.

Carcinosarcoma of Uterus.-In a series of cases carcinoma and sarcoma have been found in the same uterus, and the interpretation of these cases has given rise to much discussion (Gessner, Meyer, Herxheimer, H. Albrecht).

I. Simultaneous occurrence of two separate tumors has been observed in ro cases and constitutes the most frequent and least notable form of the combination. Usually a polypoid sarcoma is associated with diffuse adenocarcinoma of the body (Niebergall). 
2. Two tumors arise separately, but later one invades the other (Nebesky, Schaller).

3. At the point where a submucous or mural sarcoma meets the glandular layer carcinoma develops secondarily. This event seems to be not infrequent and is illustrated in Albrecht's case where carcinoma developed in the mucosa of a uterus inverted by a sarcomatous polyp. It may indicate a stimulating influence of sarcoma-cells upon epithelium or, more probably, the ordinary effects of the hyperemia and irritation to which the mucosa becomes exposed. I have seen it in connection with a benign submucous fibromyoma, and near a calcific myoma.

4. The glands included in sarcomatous polyps or in inflammatory polyps or in adenomyoma may become carcinomatous. In Gebhard's case the glands surrounded by round-cell sarcoma of the mucosa appeared to become carcinomatous. Amann's case has usually been interpreted as an adenosarcoma in which the glands became carcinomatous and appeared alone in the recurrences. Ballin assumed a simultaneous process in both stroma and glands.

5. The sarcomatous transformation of the stroma of a cervical carcinoma was assumed to have occurred in a case of Lindemann's, but the gross appearance of his specimen strongly suggests an ordinary cervical carcinoma with vesical fistula and with marked inflammatory cell changes.

6. Spurious cases are undoubtedly recorded in which atypical proliferation of glands was interpreted as carcinoma (Klein), or endothelial proliferation was so mistaken (Riederer), or in which carcinoma, becoming diffuse and its cells assuming a spindle form, bore a certain resemblance to sarcoma.

There is little doubt that many so-called carcinosarcomas result from the atypical growth of epithelial cells. Gessner refers all such tumors to this origin, pointing out that their metastases are usually carcinomatous. The exudation and inflammation accompanying tumors of the endometrium greatly favor atypical cell-growth, the scope of which is, I believe, underestimated. I have studied several tumors which appeared to exhibit sarcomatous and carcinomatous structure, but have regarded them as unfavorable material for accurate observations on histogenesis. R. Meyer holds that sarcomatous transformation of the stroma of a carcinoma has not yet been demonstrated. 


\section{CHAPTER XIX}

\section{SARCOMAS OF BONE AND BONE-MARROW}

The malignant tumors of bone constitute one of the most important departments of oncology. Their very frequent occurrence, local destructive tendencies resulting in loss of limbs, rapid extension with loss of life, and the difficulties in establishing the prognosis render them a complex surgical problem. Arising from cells whose natural function is to produce the most stable of tissues, these tumors rank with the most rapidly growing of neoplasms. Although often most clearly connected with a traumatic origin the mode of action of the trauma remains highly obscure, and while the neoplastic nature is usually most obvious, the general etiology of some of the growths reveals complex relations with normal growth of bone and physiological regeneration and with nutritional, inflammatory, and infectious processes.

Finally, the histological structure of this group of tumors while often highly characteristic is subject to wide variations, so that many problems of histogenesis remain unsolved.

Historical.-Although Astley Cooper recognized a distinction between periosteal and medullary tumors of bone, the older clinicians separated imperfectly between primary sarcoma, secondary carcinoma, and chronic inflammations of bone. Boyer first employed the term "osteosarcoma" and applied it to certain malignant bone-tumors. The microscopical distinctions between sarcoma and carcinoma of bone were established by Lebert in r845. Virchow very fully described the morphology of these tumors, but did not separate them sharply from cancer. In 1849 Robin described two types of marrow-cells which he believed gave origin to bone-tumors. One of these, the myeloplaque, was described as a large giant-cell with 6 to ro nuclei, each with nucleoli, and found chiefly in young mammals in the spongy tissue and between the marrow and the inner wall of the bone. These cells appeared abundantly in some tumors. The other type of cells, the medullocelles, were large round-cells with single nuclei and often made up the majority of marrowcells. They also were found in tumors. It is clear that Robin did not fully distinguish bone-forming from blood-forming cells in the marrow. Kolliker identified the myeloplaques with osteoclasts and the medullocelles with osteoblasts.

In 1853 Paget also recognized a variety of tumors containing large giantcells which he identified with the giant-cells of marrow, and Gray described these cells as forming a well-defined layer where the marrow comes in contact with the bone in subjects under 20 years of age. In I 860 Nólaton contributed an important monograph in which he emphasized the importance of Robin's cell groups and sharply distinguished between the malignant tumeur $\grave{a}$ medullocelles and the benign tumeur à myeloplacques. In 1879 Gross reviewed the history and morphology of giant-cell sarcoma, emphasizing, against Billroth's opinion, the benign prognostic importance of the giant-cell structure and showing in 4 fatal cases that this tumor might become malignant.

Gross's description of the origin, structure, clinical characters, and treatment of bone-sarcoma stands today as the classic contribution on this subject. 
Robin's derivation of the giant-cells from bone-forming cells of the marrow did not receive complete endorsement. Rindfleisch, Virchow, Wyss, Ziegler, and Hulke derived the giant-cells from hypertrophied bone-cells liberated during the absorption of bone (Gross). Wegner in Germany and Malassez and Monod in France and many later writers have claimed that these cells are vasoformative in nature and of endothelial origin. Many observers have shown that they are present in many forms of inflammation of bone (Rustizky, Fehr, Barrie).

In recent years studies in this field have dealt with the origin of the giant-cells, the relation of giant-cell sarcoma to inflammation, and the general etiology, prognosis, and treatment of these tumors.

Classification.-Tumors occurring primarily in bone include those arising from bone-cells or osteogenic tissue and those arising from bone-marrow. Bone-cells give origin to tumors which retain more or less of the function of bone production and are properly called osteogenic, while the marrowcells give origin to a very different class of tumors which are called myelomas. Malignant tumors of bone-cells, although differing markedly in many features, are essentially one and the same disease, which may be designated as osteogenic sarcoma. The anatomic varieties of osteogenic sarcoma result from exaggeration of bone production, as in sclerosing osteogenic sarcoma, or overgrowth of blood-vessels as in the telangiectatic type, or from the predominance of fibrocellular tissue as in the so-called periosteal sarcoma. Many osteogenic sarcomas combine all three of these features. The location of the growth is of minor importance, and yet periosteal tumors are usually fibrocellular, telangiectatic tumors either originate in the medullary portion of the shaft or soon involve the marrow cavity, and sclerosing sarcoma fills the marrow cavity with dense bone. In rare instances osteogenic sarcoma arises in two or more bones. The attempt to classify these tumors as periosteal or medullary is, therefore, unsatisfactory, since most of them involve marrow cavity, shaft, and periosteum.

The benign giant-cell sarcoma of epulis type is not osteogenic, but is essentially connected with the absorption of bone, and usually of cancellous bone. Its position is usually medullary. Its structure may arise as a secondary process in osteogenic sarcoma.

A peculiar and somewhat rare tumor of bone presents the structure of angio-endothelioma. It is not osteogenic, although it may arise from the blood-vessels of bone, or, more probably, from the vessels of the marrow. It is described as an endothelioma.

The myelomas, although destroying bone, have no relation to osteogenic sarcoma.

The entire group of primary sarcomatous tumors of bone and bone-marrow may be best classified as follows:

Primary sarcomatous tumors occurring in bone.

I. Osteogenic sarcoma:

a. Fibrocellular, chiefly periosteal.

b. Telangiectatic, involving marrow cavity, shaft, and periosteum.

c. Sclerosing, affecting marrow cavity, shaft, and periosteum.

2. Benign giant-cell sarcoma of epulis type.

3. Myeloma, arising from bone-marrow cells.

4. Endothelioma, or angio-endothelioma, arising from blood-vessels of bone or marrow. (Described under Endothelioma.)

Osteogenic Sarcoma.-(a) Periosteal Sarcoma.-The ends of the long bones, femur, tibia, and humerus are the chief seats, but the small bones of hands, feet and skull, and many other portions of the skeleton are involved. 
The site of origin of osteogenic sarcomas is nearly always at the ends of the shaft. A location in the middle third of the shaft is strong evidence against an osteogenic tumor. The main gross feature of the periosteal sarcoma is the production of a tumor arising in the inner layers of the periosteum, extending along and inclosing the shaft, with a sharply marked border, causing a relatively bulky rounded fusiform or lobulated tumor lying outside the shaft which long remains intact. This position favors a bulky growth so that periosteal

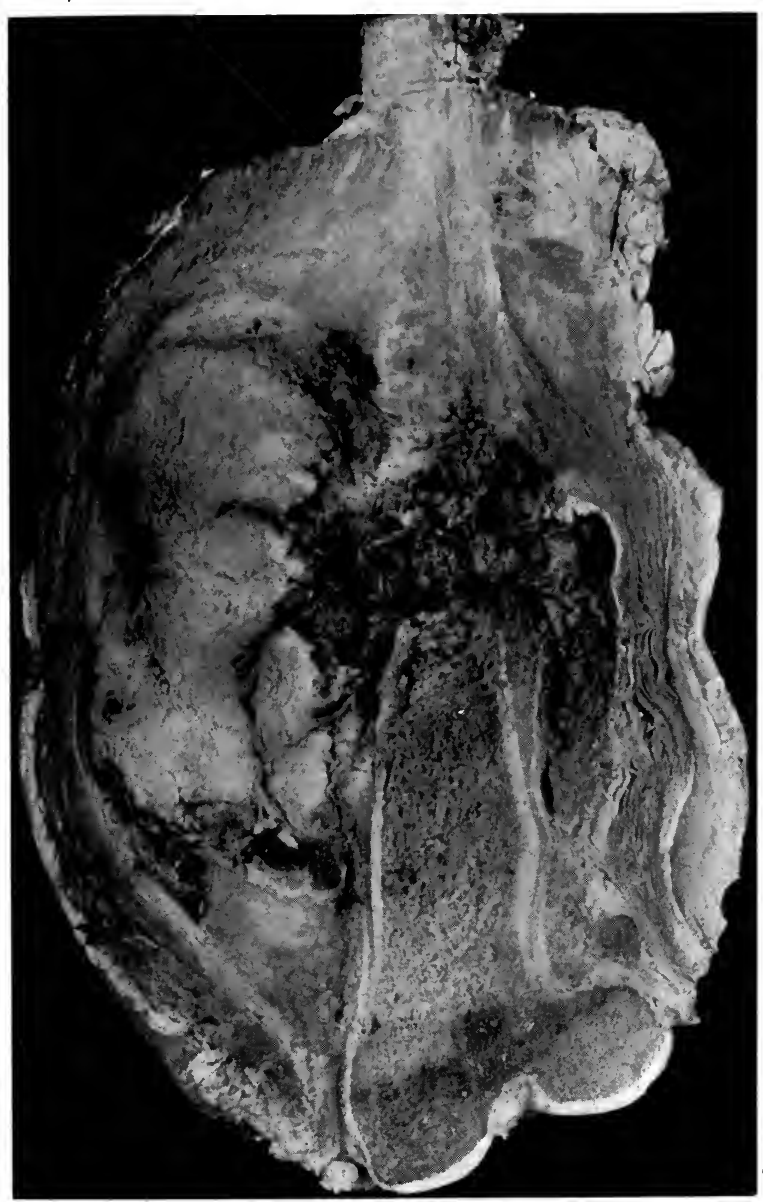

FIG. 86.-Periosteal sarcoma completely traversing shaft as a telangiectatic structure.

- sarcomas commonly reach a large size, some of them attaining very large dimensions after a long period. The integrity of the shaft is usual, but not constant, for the more cellular growths early penetrate and destroy the compact bone and lead to spontaneous fractures. Even thus the extramedullary tumor is usually more bulky than with corresponding central telangiectatic sarcoma.

According to the consistence periosteal sarcomas are: (I) soft, cellular, and highly malignant, or (2) firm and fibrous, or (3) irregularly ossified. En- 
capsulation by the periosteum is maintained for periods varying with the character of the growth, but the more malignant tumors invade not only the shaft but the surrounding muscles, fasciæ, vessels, and eventually the skin. Tendon insertions offer considerable resistance to progress of the growth. The general appearance of these tumors supports the view that the periosteum is not passively displaced by an underlying growth, but, as Marullaz finds, that the periosteum contributes to the growth and undergoes sarco-

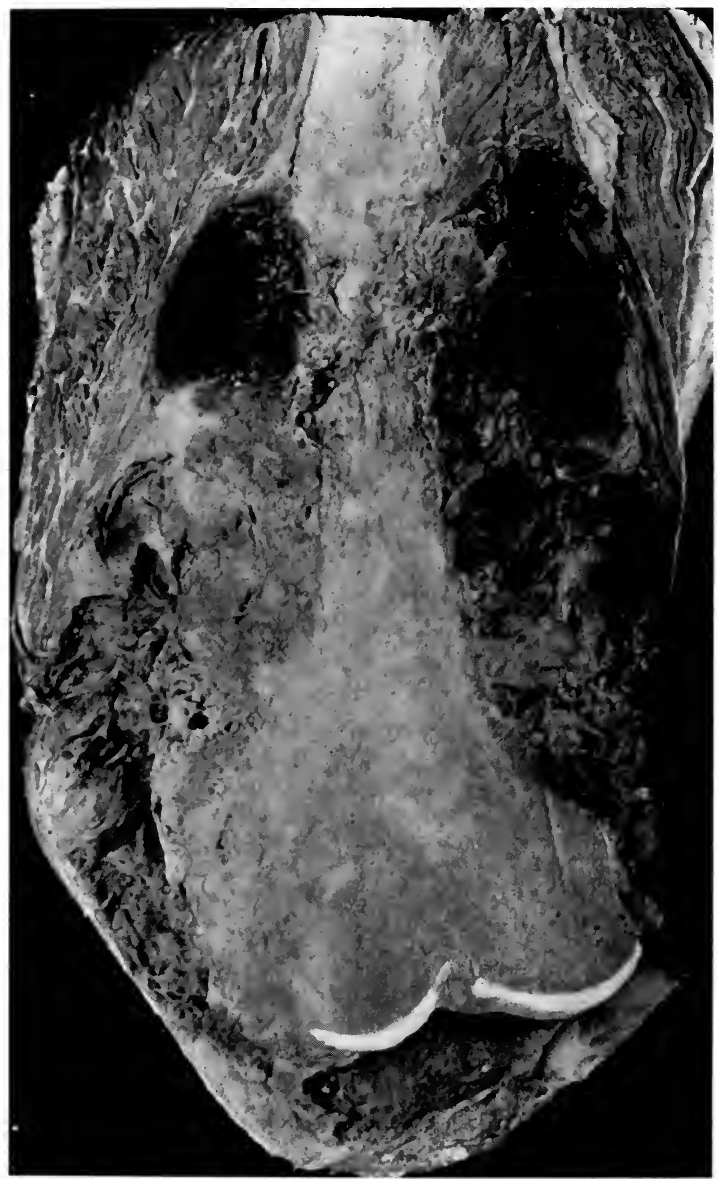

FIG. 87.-Periosteal sarcoma, combined with diffuse central sclerosing sarcoma.

matous transformation. A thin, bony capsule may be laid down by the periosteum.

Incision reveals a solid tumor mass in which the shaft is fused with ossifying tumors or eroded by cellular growths. An expansion of the shaft as noted with benign central giant-cell sarcoma is missing. Fatty and mucoid degenerations, hemorrhage, and necrosis may greatly alter the appearance. Maceration by removing the soft parts may leave bony trabeculæ radiating out from the shaft, or a globular mass resembling an exostosis, or isolated bony 
areas may be removed entirely. Many of the large tumors, especially of the spindle-cell variety, contain cysts filled with serous fluid, or semifluid detritus, or blood. Although sometimes quite vascular, periosteal sarcomas do not pulsate.

All the histological varieties of bone-sarcoma originate in the periosteum, but the spindle-cell type is most frequent. Giant-cells are absent in many cases, and always less numerous than in benign central sarcomas, being found usually in areas of dissolving bone. The tendency to calcification and ossification may be very prominent. In one group, in which the cells are usually large and polyhedral, the matrix is chiefly cartilaginous. Some of the most malignant bone-tumors are of this type, and at times the large polyhedral cells grow diffusely, while the cartilaginous matrix is missing or reduced to hyaline traces.

(b) Telangiectatic Bone-sarcoma. -A considerable proportion of bonesarcomas present an extensive development of blood-vessels supported by more or less cellular osteogenic tumor-tissue, they early destroy the shaft, penetrate the marrow cavity for a short distance, and rupturing the periosteum extend widely through fasciæ and muscle. The exact origin of these tumors is not satisfactorily located, but they seem to arise from the blood-vessels and bony tissue of the shafts of long bones. They are sometimes called central osteogenic sarcoma, but the majority of central tumors are not of this type. They are often confused with benign giant-cell sarcoma, which is a central medullary tumor, but is not osteogenic or malignant. The structure of this telangiectatic sarcoma is occasionally seen over an entire tumor, but more often it is associated with other structural types, so that it is best interpreted as an exaggeration of the bloodvessel element in osteogenic bone-

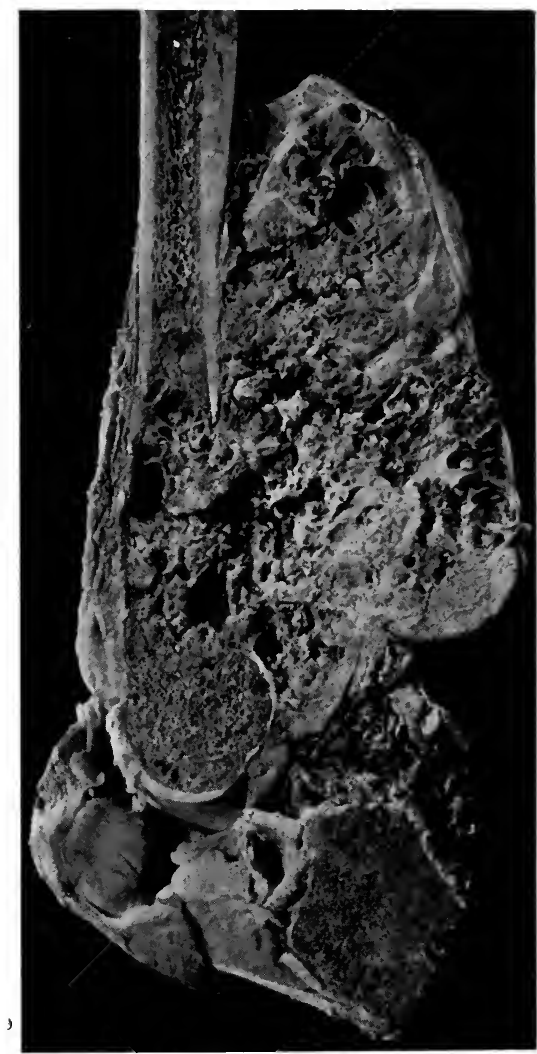

FIG. 88.-Telangiectatic, periosteal, spindle- and giant-cell sarcoma of femur. Perforation of shaft. sarcoma.

The absorption of bone occurs rapidly, and the line of absorption while ragged is rather sharp, suggesting that the growth has originated in the marrow, but the same type of absorption occurs in vascular portions of tumors which are chiefly periosteal. Spontaneous fracture quickly results in many cases and may be the first symptom of the disease to be detected.

Telangiectatic sarcomas of bone are osteogenic, but the production of bone and even of tumor-tissue may be slight. The more solid portions of such tumors usually present irregularly ossified or only calcified strands, 
plates, or spicules of imperfectly formed bone. In some cases the periosteum is displaced outward and succeeds in laying down a thin shell of bone which crepitates on pressure.

In the malignant bone aneurysm the vessels are widely dilated into irregular sinuses and the tumor-tissue is correspondingly reduced, or limited to an irregular layer disposed along the periphery. Formation of bony or osteoid tissue is also reduced and may be missing. The tumors may pulsate and yield a bruit.

The head of the humerus, upper end of tibia, and lower end of femur are the chief seats of malignant bone aneurysms. As a rule the tumor proves highly malignant and recurs locally or in the lungs after amputation.

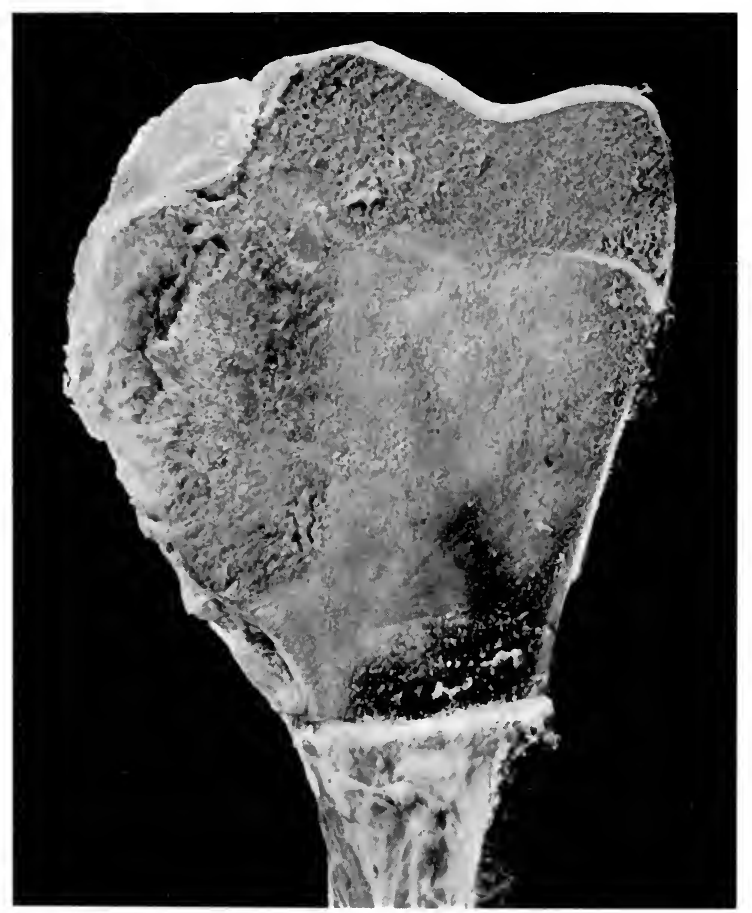

FIG. 89.-Malignant central sclerosing osteogenic sarcoma of lower end of femur. Note partial limitation at epiphyseal line.

The relation of the malignant bone aneurysm to cavernous angioma, benign giant-cell sarcoma, and simple blood-cysts of bone has been much discussed. The opinion expressed by Nélaton, Volkmann, Lucke, and Gross, that the great majority of bone aneurysms are forms of sarcoma, has been supported by recent observers (Gaylord, Nakayama, Bloodgood).

It is not always easy but yet highly important to distinguish between malignant bone aneurysm and very vascular forms of benign giant-cell sarcoma. The malignant disease is almost never found until it has destroyed much of the shaft of the bone, whereas the benign process long spares the shaft and expands so slowly that the periosteum is able to lay down a firm 
bone capsule about the expanding tumor. The great majority of simple tonecysts are not connected with tumors.

(c) Sclerosing Osteogenic Sarcoma.-The production of much dense solid bone occurs in portions of many bone-sarcomas, but occasionally it forms the bulk of the tumor or is exclusively present, and since it is associated with a prolonged clinical course, there are both anatomic and clinical grounds for recognizing it as a specific form of osteogenic sarcoma. These tumors were once the subject of much debate (cf. Gross), being regarded by many authors as bone cancers, until Volkmann and Virchow established their true nature and termed them "sclerosing osteoid sarcoma."

In typical cases a large portion of the end of a long bone is converted into a solid mass of dense almost ivory-like bone, which obliterates marrow cavity and shaft and produces a fusiform swelling of considerable dimensions. Bloodvessels are very scanty except about the periphery or over a small area of the tumor, yet metastases form in a high proportion of cases, so that this slowly advancing tumor has long been known to be fully as malignant as the others. They show comparatively little tendency to invade surrounding soft parts.

Areas of sclerosing sarcoma occur frequently with periosteal growths in which they show all grades of bone formation up to the very dense ivorylike type.

Capsular and Parosteal Sarcoma.-Osteogenic tumors not infrequently involve the capsules of joints or appear in the soft tissues about joints or along the shafts of bones. This group of cases is important not only for their clinical interest but also for their bearing on the origin of bony tumors and of certain neoplasms not always recognized as related to bone. The capsule of the knee-joint is the chief seat of capsular sarcoma, and here the tumors illustrate most of the clinical and histological features of bone-sarcoma (Rydygier, Lit.). They are distinguished chiefly by the failure to involve the joint-cavity or the bone, and their malignancy is distinctly less than that of corresponding tumors of periosteum or medulla. Yet malignant cases occur which are rapidly fatal after resection or amputation. Some produce a fusiform swelling of the joint, and being accompanied by fever the case resembles tuberculosis (Garre). Or a localized tumor appears at one point in the capsule (Marsh).

Pedunculated giant- and spindle-cell sarcomas have been described by Weir and by Dowd. The structural variations cover most of the scope of bone-sarcoma with corresponding prognosis.

Capsular sarcomas of the ankle exhibit much the same characters as in the knee (Moser, Lit.). The tendon-sheaths are a common seat of sarcoma which usually takes the form of a giant-cell tumor of slight malignancy. The deep fasciæ may be the seat of sarcomas of various osteogenic varieties which have no definite connection with the periosteum and the true nature of which may, therefore, be overlooked. They include giant-, spindle-, and round-cell types, and may belong in the group of chondrosarcoma or its derivative, myxochondrosarcoma.

Along the cranial sutures, base of skull, pharynx, nares, vertebræ, and pelvis occur many tumors of pronounced osteogenic character which belong in this group, and not a few in which the derivation from bone is only vaguely indicated by the structure.

The course of osteogenic sarcoma varies greatly and accords in general with the histological structure. As a rule they are malignant tumors appearing before the 3 oth year, recurring after incision or amputation and producing pain, fever, metastases, and cachexia. Coley reports 4 bone-sarcomas occurring in subjects aged 17 months to 9 years. Goebel has collected a series of con- 
genital sarcomas of bone and other tissues, and recorded a successful result with periosteal sarcoma by local extirpation and $x$-ray treatment. The spindlecell tumors grow rather slowly, but nevertheless tend to recur and cause death by local growth and internal metastasis. Jackson recorded an unusual case in which a small growth on the under side of the knee increased very slowly for 32 years, when after trauma it grew rapidly and produced a large medullary tumor. The highly osteoid tumors may also pursue a very chronic course, as with cases of Paget and Holmes, which proved fatal with metastases after $2 I$ and 25 years.

Metastases are frequent with osteogenic periosteal sarcoma, in the advanced stages of all types and very early with soft and cellular varieties. A period of months or years may intervene between the appearance of the primary and the secondary tumors or there may be almost simultaneous involvement of several bones (LeDentu, Zahn, Poncet). That these tumors are not myelomas is attested by Poncet's case. The lungs are chiefly involved by emboli through the blood-vessels and other widely distant organs may be reached by the same route. Neighboring or distant lymph-nodes are also occasionally invaded through lymphatics, but swollen lymph-nodes usually prove to be purely inflammatory. The occurrence of pulmonary tumors secondary to periosteal sarcomas was noted by Virchow, but the frequency and extent of such metastatic tumors has lately been emphasized by LeCount (Lit.). In malignant cellular and vascular tumors pulmonary metastases often occur quite early in the disease, so that operation in these cases should not be considered until the condition of the lungs is determined by $x$-ray photograph.

These tumors appear as multiple nodules, or as superficial pleural growths, or as bulky pleural or pulmonary tumors. Extensive diffuse pleural growths are reported by Virchow, Pitts, LeCount, and others, and pedunculated pleural tumors by Virchow and Cocks and Wilks. Massive intrapulmonary growths have been observed by Birch-Hirschfeld, Kuster, Allin, Meakin and others. In structure the tumors have been soft and cellular or cartilaginous and bony, or encased in a bony shell. In a remarkable case described by Bosch, the pulmonary metastases of an osteoid sarcoma of the femur exhibited lamellar structure as in the long bones, and enclosed groups of cells resembling lymphoid marrow. Pleural effusion may be absent and pulmonary symptoms may be slight, but bloody effusions, dyspnea, and hemoptysis frequently occur and suggest tuberculosis.

In a few cases extensive intravascular growths have been observed. Hektoen found bony masses from a tibial sarcoma, which nearly filled the right ventricle and produced secondary growths in the lungs. Feistmantel found cylindrical bone masses in one lung and large nodules in the opposite lung which probably resulted from metastases in the pulmonary artery from a femoral sarcoma. Pulmonary metastases appear to have occurred, while the primary tumors, of foot and wrist, were quite small in the cases of Pitts and of West. Usually they appear a few months after operation on the primary growth, while in Jenckel's case death from a pulmonary growth occurred I 5 years after amputation of the femur.

That metastases are often produced by the trauma of operation is indicated by McAuliffe's observation of tumor-cell emboli in many organs after death from resection of a sacral sarcoma.

Structure of Osteogenic Sarcoma.-The three main anatomical varieties of osteogenic sarcoma are marked by distinctive histological features, but the frequent occurrence of different structures in the same tumor indicates that the disease is essentially one and the same. 'This fact does not, however, 
assure that with the progress of knowledge it may not be possible to separate certain types of bone sarcoma with peculiar histological structure and which arise under peculiar conditions.

I. Spindle-cell tumors are the most frequent, and this is the usual form of periosteal growth. The cells have a short spindle form, or they appear larger and almost polyhedral, and in very anaplastic processes they are small and appear almost round, although never assuming lymphoid characters. Osteogenic sarcoma is never a round-cell tumor. All round-cell growths of bone arise from bone-marrow cells and should be sharply separated from tumors of bone-cells.

The spindle- or polyhedral or rounded cells of osteogenic sarcoma present many variations in size and chromatin content which are of importance in

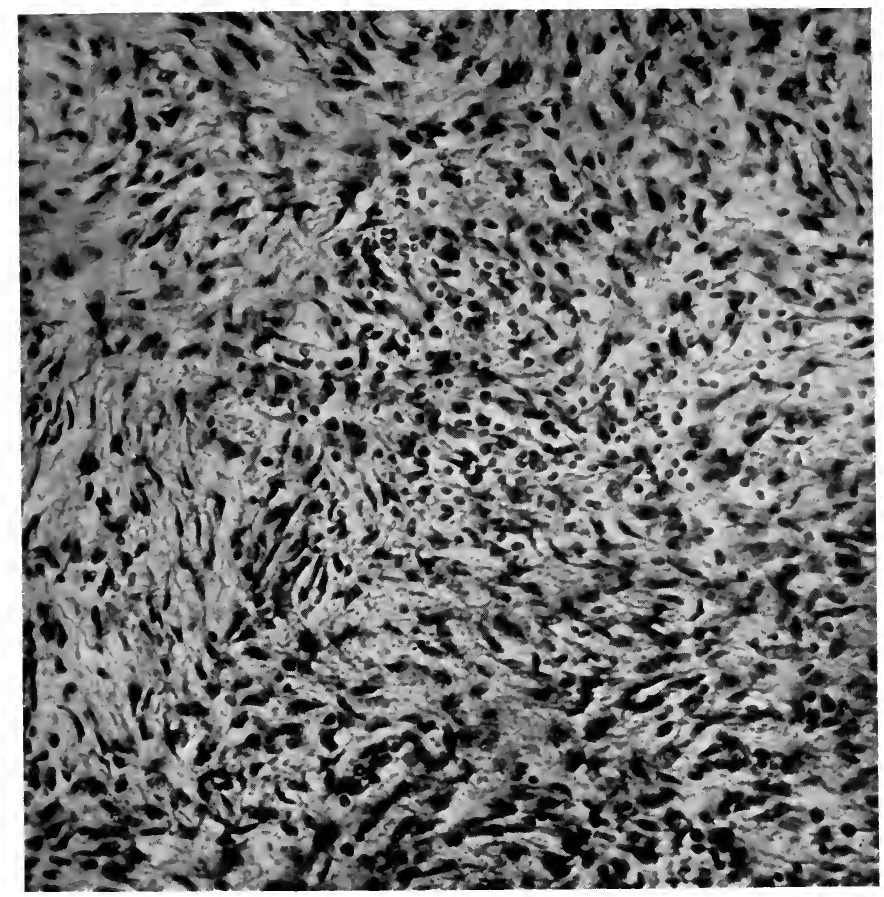

Fig. 90.-Myxosarcoma-like structure in benign central sarcoma.

prognosis. Intercellular substance may be considerable in the slowly growing fibrosarcomas, but it is reduced to a scant trace in more rapidly growing tumors, and may be entirely absent in the most malignant cases. It may appear in fibrillar, hyaline, or osteoid form, and when this tendency becomes marked the growth passes into the ossifying group. The more leisurely the growth, the more prominent, as a rule, is the production of bony stroma.

The slowly growing tumors are poor in vessels, but the malignant cellular forms may be quite vascular and yield early metastases. The walls of the larger vessels are often infiltrated with lymphocytes, occasionally they appear sarcomatous, and sometimes they are found distended with tumor tissue (Bristowe) (Fig. 90). In vascular tumors a close relation of the tumor-cells 
to the blood-vessels is common, and the structure of perithelioma may be simulated. A pseudo-alveolar structure is sometimes observed.

While usually well circumscribed, spindle-cell osteogenic sarcoma may extend widely along the periosteum, so that very extensive areas of the bone surface may be involved in a comparatively flat tumor. I have found the upper third of the femur and almost the entire surface of the pelvic bones invaaed by a rather flat growth of small spindle-cells. Similar tendencies may appear in the metastases of this tumor.

2. Telangiectatic sarcoma usually presents a complex histological picture. The dilated sinuses are lined by very cellular tumor-tissue in which many

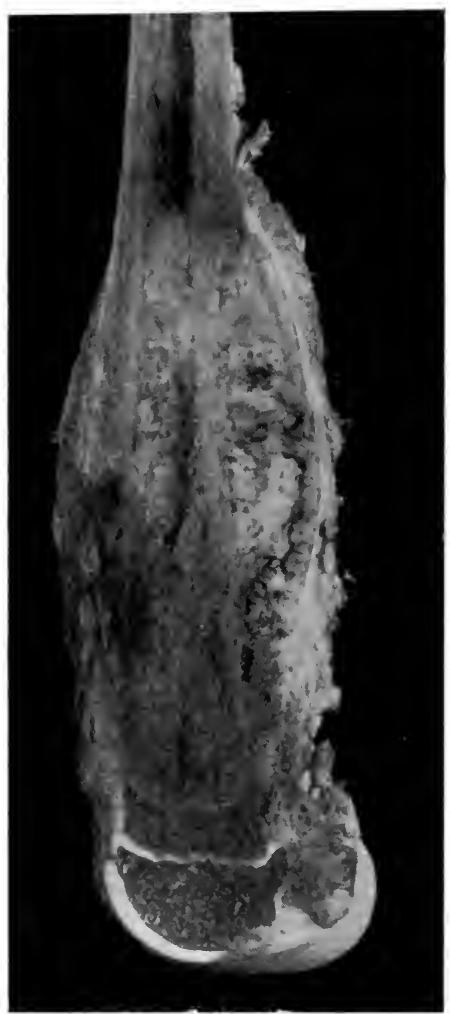

FIG. 91.-Osteogenic sarcoma, periosteal and central. foreign body giant-cells may appear and lead to an erroneous diagnosis of a benign process. Yet the intervening walls and septa are composed of large polyhedral or spindle-cells with hyperchromatic nuclei which fully attest the malignant character of the disease. Imperfect bone trabeculæ are often found in portions of these growths. In malignant bone aneurysm the tumor tissue may be greatly reduced by the distended sinuses. In solid portions which usually accompany the vascular tumors the cells are very abundant, the stroma scanty, and the vessels small. The surrounding muscles and fasciæ are invaded along the septa and by the bloodvessels. Rapidly advancing tumors of this type may cause separation of a large portion of the diaphysis, which lies loose in the center of the mass. In and about the tumor old or new bone in process of absorption may yield very complex pictures often marked by foreign body giantcells and irregular calcific deposits.

3. Sclerosing (Ossifying) Osteogenic Sarcoma.-The formation of dense bone tissue is a feature of the more slowly progressive bone tumors, in which the rate of proliferation of the cells is sufficiently restrained to permit them to exhibit their natural function. Cellular bone-free sarcomas, under $x$-ray treatment, may cease to grow and begin to lay down bone.

In periosteal tumors the bone usually appears in stalactite form radiating from the shaft and giving a characteristic appearance by the $x$-ray. The structure shows well-formed trabeculæ of variable size surrounded by and including many hyperchromatic osteblasts. In portions of such tumors much cartilage or osteoid tissue may appear and show gradual transformation into bone. Tumors composed chiefly of cartilage, osteochondrosarcoma, contain many large polyhedral cells, in compact groups or separated by hyaline stroma, and these are among the most malignant of osteogenic sarcomas.

In sclerosing. central sarcoma the very dense bone, filling the marrow 
cavity and distending the shaft, presents solid masses and irregular trabeculæ of bone between which lie compressed tumor-cells. More cellular foci often appear along the edges of the tumor or scattered through it. About many sclerosing sarcomas very cellular tumor tissue may infiltrate the muscle and invade the veins (Fig. 94).

In most ossifying sarcomas the production of bone is accompanied by its absorption in other portions of the growth, or the two processes may be found

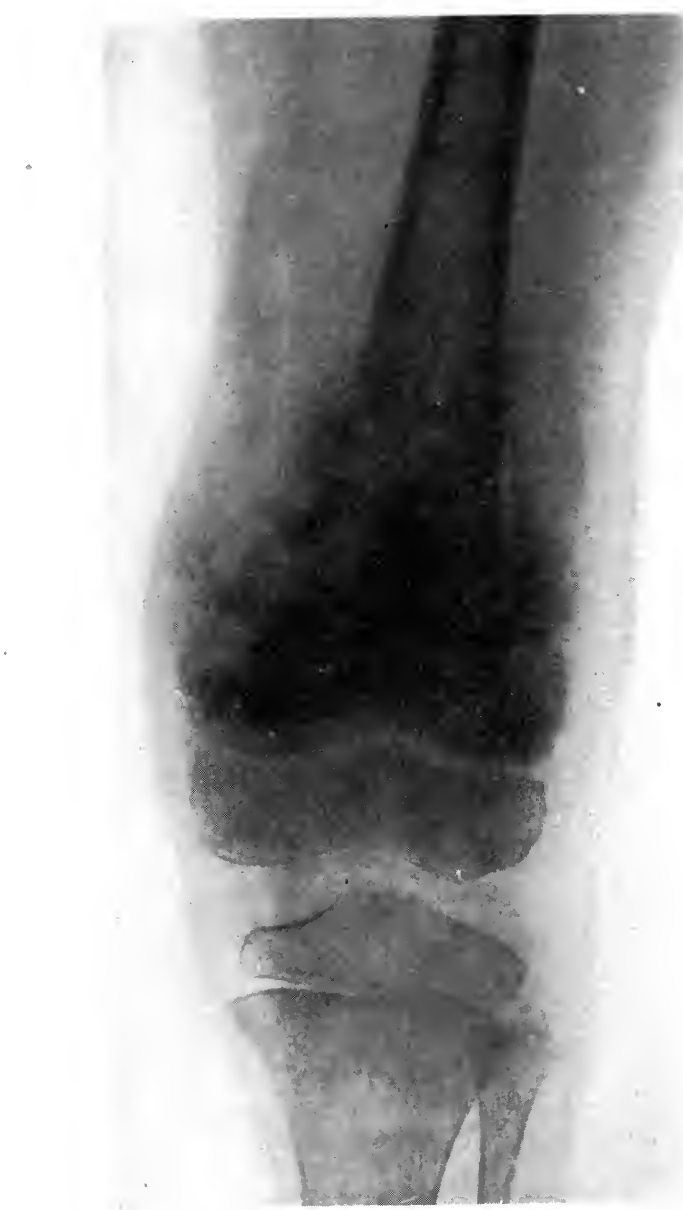

FIG. 92.-Malignant periosteal and central osteogenic sarcoma.

closely associated. When bone is absorbed the solid trabeculæ first exhibit interfibrillary clefts from decalcification, then canals are formed by advancing blood-vessels or groups of tumor-cells, or giant-cells form in lacunæ. In most cases where bone, normal or neoplastic, is being absorbed, the giant-cells tend to assume the foreign-body type of benign sarcoma.

When ossifying sarcoma invades the muscle the histological picture may closely resemble myositis ossificans. 
Benign Giant-cell Sarcoma (Epulis Type).-This peculiar condition was first fully described as a benign process in bone by Nélaton, who emphasized the reddish jelly-like appearance of the tumor-tissue, noted its capacity to absorb bone and widen the marrow cavity, and fully established its benign course and the wisdom of conservative treatment. All of these features have been fully recognized by most later observers, but not by all, so that many limbs have been needlessly sacrificed because of the failure to distinguish between this benign process and the malignant central telangiectatic sarcoma.

The disease appears at the ends of the long bones, but it very frequently arises in the maxillæ, leading to the extraction of teeth, so that it is often called

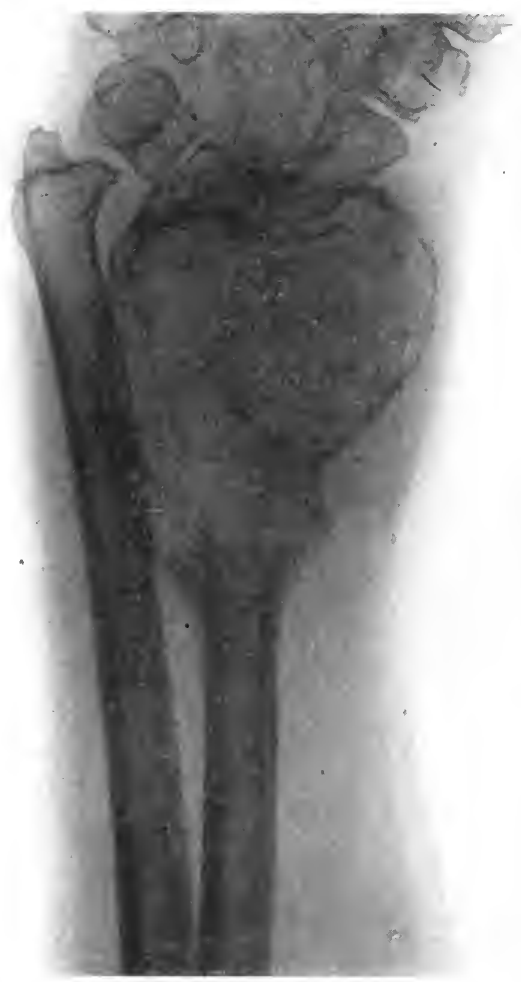

FIg. 93.-Benign central giant-cell sarcoma. Epulis type. Note limiting shell of bone and multicystic appearance.

the epulis type of sarcoma. Very often the first symptom noted is a spontaneous fracture at the point where the process has absorbed the shaft. It has frequently appeared as a multiple tumor, and one of the most remarkable of these cases is that of Martland in which tumors appeared in widely distant parts of the body over a long period without showing any evidence of local malignancy.

Very similar tumors occur in the capsules of the joint, and along the tendon sheaths and bursæ.

In the long bones the tumors produce reddish jelly-like tumor masses resembling granulation tissue and replacing the cancellous portions of the bone. They may also slowly absorb the shaft while the periosteum lays 
down an advancing shell of new bone. Eventually this shell may become thin and allow passage of the tumor-tissue, but there is seldom any tendency toward invasion of soft parts. Likewise the cartilaginous surfaces of the joint may be reached and absorbed and the joint surfaces may collapse, from simple absorption, but without infiltration (Gross, Stimson).

In more cellular tumors the jelly-like appearance is replaced by a more opaque and firmer texture, which occupies the periphery of the growth while the central portion is soft or cystic and infiltrated with blood.

Occasionally these tumors present a golden-yellow xanthomatous appearance, which is associated with the presence of many large cells distended with lipoid granules. Such tumors may be bulky, but they are not, as a rule, especially aggressive.

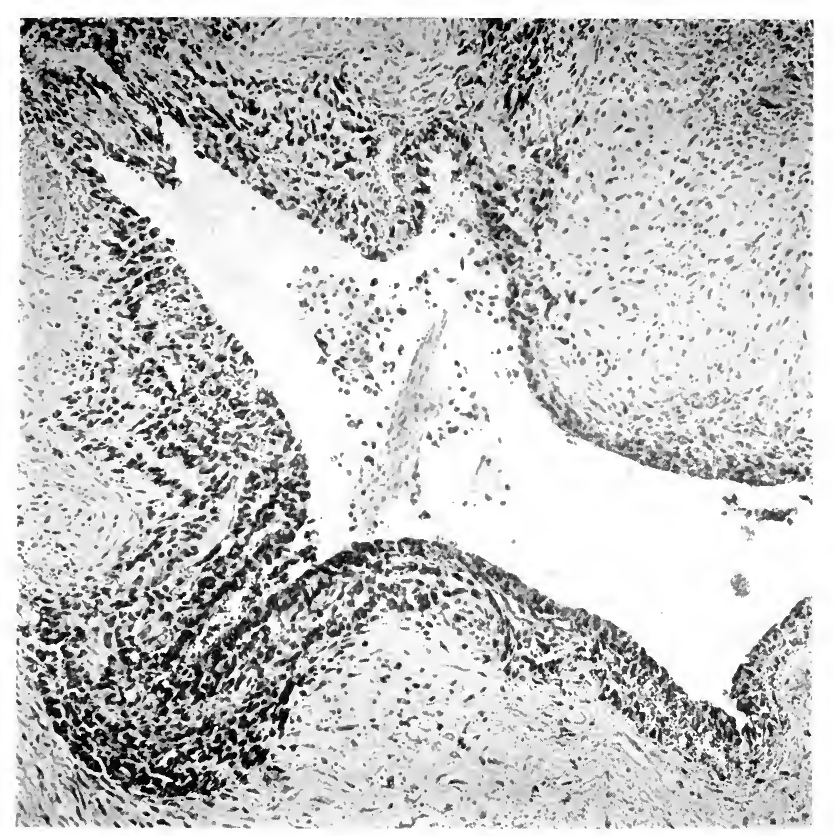

FIG. 94.-Periosteal sarcoma. Vein lined with tumor-cells. This structure explains the ready metastases of these tumors.

Finally, the tumor may exhibit the features of a myxoma or myxosarcoma, being more or less translucent and elastic. It seems probable that the socalled myxomas of the marrow cavity have mainly this origin. The characteristic giant-cells of epulis type may be found in many portions of the growth.

There is considerable variation in the rate of growth and bone absorption by benign giant-cell sarcoma. As the spindle-cells of the stroma become more active and abundant, the giant-cells diminish, the tumor shows less resemblance to granulation tissue, but becomes firmer and like spindle-cell sarcoma. Such growths recur locally and may destroy bone and infiltrate the soft tissues, but I have never known them to yield metastases. They regularly fail to produce bone.

The earliest stages of this disease have been traced to the walls of small cysts forming in osteitis fibrosa cystica. Here the process may be interpreted 
as a form of bone absorption or, as some prefer, rarefying osteitis or osteomyelitis, with the formation of cysts.

In some cases the process appears to subside, leaving cysts with smooth lining and serous contents. In most cases it is progressive and acquires the characters of a mild tumor process, and rarely it develops greater momentum, actively destroys bone, and exhibits certain clinically malignant tendencies.

While many of these tumors are successfully dealt with by curettage, there are several hazards in their surgical treatment. The growth may promptly recur after curettage and exhibit increased growth capacity, while the curetted bone is thinned and offers less resistance. The large cavity remaining after

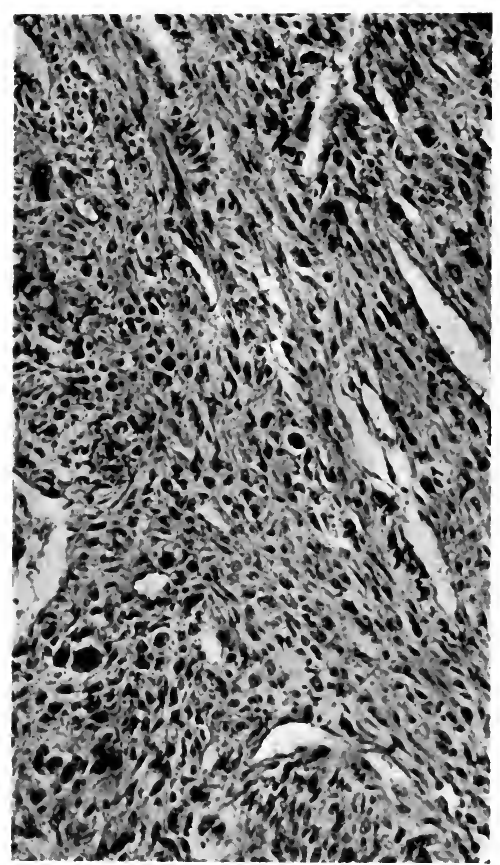

FIG. 95.-Malignant chondrosarcoma of femur.

curettage may fail to heal and a difficult surgical problem remains, which sometimes calls for amputation. Infection of various types is a sequel which may prove very difficult to control, since both the tumor-tissue and the exposed bone-marrow are particularly susceptible to bacterial invasion. All of these complications may greatly alter the natural gross appearance of giant-cell sarcoma.

Structure of Giant-cell Sarcoma.The typical benign giant-cell sarcoma presents a very characteristic picture which is chiefly marked by the abundance of large giant-cells containing many small separate oval nuclei. This peculiar structure indicates the presence of a peculiar disease, and it is, therefore, highly desirable to restrict the term "giant-cell sarcoma of bone" to this relatively benign process. Similar cells are sometimes seen in portions of malignant tumors. These cells are of large size, the cytoplasm is opaque and acidophile, and the nuclei are numerous, separate, oval, and usually grouped in the center of the cell. They often contain vacuoles, fatty detritus, homogeneous material, red blood-cells, and occasionally spicules of bone. They have the general characters of foreign body giant-cells (Fig. 96).

The bulk of these growths is composed of loosely packed spindle-cells surrounding many vessels, capillaries, and sinuses. There is much variation in the vascularity of these tumors and in the number, size, and nuclear chromatin of the spindle-cells, and there is a corresponding difference in their powers of growth.

Osteogenic sarcoma may contain giant-cells of the above type, especially where the tumor comes in contact with absorbing bone, but both periosteal and central sarcomas may exhibit giant-cells of distinctly different type and significance. These cells may be large or small, the cytoplasm stains poorly, the nuclei are large and vesicular and composed of many lobes which may become detached. As a rule these cells belong to malignant tumors. While the two types of giant-cells are usually distinct, it must be admitted that transitional 
forms seem to occur, especially when malignant tumors undergo regressive changes, erode bone, or become inflamed (Fig. 97).

The existence of different types of giant-cells in bone-sarcoma has been recognized by several observers. Poncet describes true and false myeloplaques. The former are as large as 3 to 4 ordinary tumor-cells and contain 5 to 6 nuclei; the latter are as large as 4 to 5 ordinary cells and contain 12 to 20 nuclei, and correspond to the common giantcell of the epulis. Borst states that the giant-cells of medullary sarcoma are more delicate than those of periosteal origin, in which the cytoplasm is more compact, often free from granules, and more sharply defined, and the cell processes are longer than in medullary tumors. Mallory distinguishes between true tumor giant-cells and the foreign body giant-cells of osteogenic and other sarcomas. The tumor giant-cells differ from other tumor-cells chiefly in size and number of nuclei which may multiply by mitosis. They produce specific fibrils. The foreign body giant-cells are of endothelial origin, and correspond in type to those of the epulis.

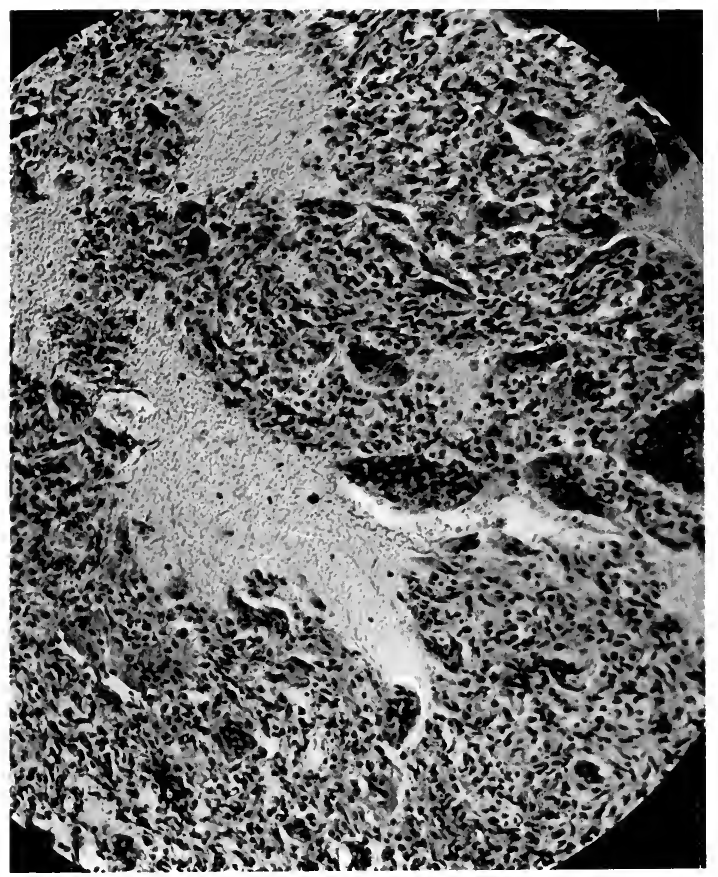

FIG. 96.-Benign giant-cell sarcoma of head of tibia. Epulis type of medullary sarcoma. Giant-cells forming about blood masses and lipoid material.

The origin of the giant-cells of the epulis type has been the subject of much discussion. Virchow and Rindfleisch believed that they were hypertrophied bone-cells set free by the absorption of the bone matrix and that they are identical with the osteoblasts of Kolliker. Röbin and Nélaton, in describing the giant-cell sarcoma of bone (tumeur a myeloplaxes), accepted the identity of the giant-cells with Kolliker's osteoblasts. This view is favored by the very frequent presence of giant-cells in many lesions of bone and has been widely accepted. The direct transformaton of bone-cells into giant-cells of sarcoma has been traced by Wyss, Ziegler, and others, but it is not clear that the giant-cells observed in the malignant tumors of those authors were identified with those of the epulis. Ziegler found that giant-cells are by no means constant in the absorption of bone and suspected that osteoclasts might have a variable origin.

Wegner, Malassez, and many later observers have been led to conclude that the giant-cells of the epulis are derived from modified endothelium or angioblastic cells, and 
Malassez classified this tumor with the angioblastic sarcomas. Very similar results were reached by Ritter, who traced the cells in Howship's lacunæ to proliferating endothelium, and he further concluded that these cells may take on the function of bone-cells. Borst recognizes the multiple origin of the giant-cells of sarcomas, but clings to the view that in the epulis they stand as evidence of the bony origin of the tumor matrix. Mallory holds that the giant-cells of epulis are transformed wandering endothelial leukocytes and do not belong to the tumor process.

Thus, both in morphology and in origin it is necessary to recognize two types of giant-cells in bone-sarcoma. One occurs chiefly in the benign epulis, is chiefly of endothelial origin, and belongs among the foreign body giant-cells, although participating in the tumor process. The other occurs chiefly in malignant periosteal tumors and is derived from tumor-cells.

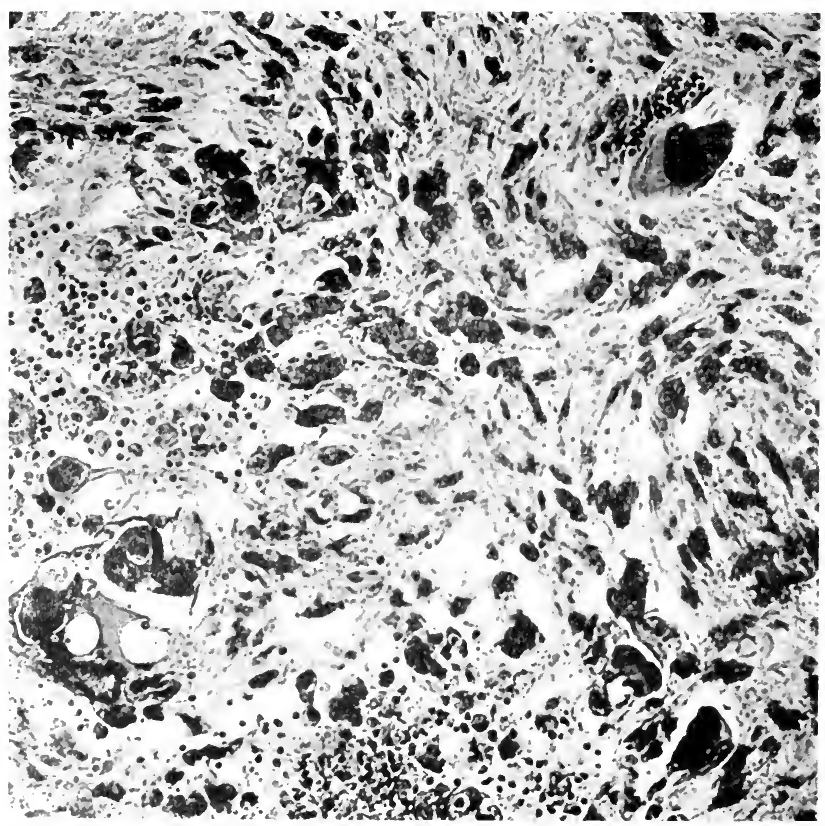

FIG. 97.-Periosteal sarcoma. Malignant giant-cell structure.

The giant-cells of bone sarcoma are, therefore, contributed both by the bone-cells and by proliferating angioblastic cells. Apparently the growing endothelium feels the influence of the mother tissue and may take on some of the form and function of bone-cells, giving the specific structure of the benign epulis which is rarely observed except in connection with bone.

On account of their chiefly angioblastic origin and benign course many have suggested that bone-tumors of the epulis type should be excluded from the class of sarcomas or even of true tumors. Yet these cases show many features of neoplasms, not a few are moderately malignant, and many transitional forms up to the malignant tumors are observed.

Prognosis of Bone Sarcoma.-The widest extremes are represented in the prognosis of different sarcomas of bone, and it becomes a matter of first importance to determine the prognosis in the light of all the data, clinical and pathological. 
The age of the patient, the duration of the disease, its rate of progress, the particular bone affected, the completeness of the operation, and, above all, the structure and essential malignancy of the tumor, must all be considered. Subjects under 20 years withstand the progress of malignant tumors poorly.

The duration of the disease must be considered from several standpoints. A tumor which grows very slowly is for that reason probably less malignant, yet there are numerous records of long existing tumors suddenly assuming active growth after trauma (Jackson). Certain slowly growing but malignant osteoid tumors appear not formidable in themselves, but early produce metastases (Le Count). The local damage to tissues occurring in the advanced stages of otherwise comparatively benign tumors is an important element in prognosis, chiefly because it complicates the operation, but also because it favors invasion of veins and metastasis. Kramer states that encapsulation is the most important factor in the prognosis of most bone sarcomas.

The rapidity of growth is a very reliable clinical sign of malignancy and should always control the deductions drawn from histological structure. Rapidly growing tumors are nearly always malignant and eventually fatal. A highly anaplastic, small spindle-cell, traumatic sarcoma of patella submitted to me by Dr. John Rogers proved fatal 6 weeks after the trauma, with very extensive local recurrence and metastases. Many would have described this growth as of round-cell structure.

Yet the essential capacity for growth cannot always be estimated clinically, encapsulation restrains growth, and it is possible for a tumor to change its rate of growth. A central tumor which long remains confined by the periosteum usually has a limited growth capacity, for very malignant tumors early perforate the shaft. Yet in one of Bloodgood's fatal cases symptoms had existed for 25 years.

It is an established rule that periosteal growths are much more malignant than central tumors, but this rule has little practical value. Gross found the average duration of life with round-cell, spindle-cell, and osteoid periosteal tumors with operation at $\mathrm{I} 8,20$, and 92.7 months respectively. While amputation in the early stages of spindle-cell or osteoid sarcoma of the extremities may be followed by complete recovery, the prognosis of any variety of periosteal sarcoma located in or near the trunk is very grave. The great majority of cases which seem to recover after operation eventually suffer from local recurrence or internal metastasis. Gross concluded that practically all cases of round-cell or spindle-cell periosteal sarcoma eventually die of the disease, while with osteoid sarcoma 65.62 per cent. of the cases prove fatal. More recent statistics show slightly better results and some dependence of the outcome upon the particular bone affected.

Kocher collected 48 cases of sarcoma of long bones which were regarded as cured. Of these, the radius was the seat of the growth in 4 , ulna I, humerus Io, femur 23, tibia 12. Coley collected 57 cases of apparent cures, 30 of the "myelogenous" type, I 5 periosteal, I 2 undetermined. Butlin collected I 8 cases of periosteal sarcoma of humerus which survived operation, but only one remained well after 3 years. Of 68 cases of periosteal sarcoma of femur only one doubtful case remained well 3 years. Yet McCosh had 3 recoveries of spindle-cell sarcoma of the femur among 7 cases. Bloodgood reports 26 medullary giant-cell sarcomas all living; 18 inoperable malignant sarcomas; 34 high amputations without a single cure; and 6 permanent cures (periosteal osteosarcoma, 3; myxochondrosarcoma, 2; fibrosarcoma, I).

Rheinhardt reported 7 permanent cures ( 8 to 1 2 years) out of 54 cases, including 4 round-cell tumors, of tibia 2, and humerus 2. Coley collected 
62 cases of sarcoma of clavicle, of which 6 recovered, but in many the result was not known. Nancrede could find no cases of recovery from sarcoma of the scapula. The interscapulothoracic amputation, while comparatively effective for benign tumors of the humerus, appears to have succeeded in curing only I advanced malignant tumor of the humerus (Berger's case) (Jeanbreau, Riche).

From a general survey it appears that recovery has occasionally followed operation for almost every variety of sarcoma of the long bones, but in none

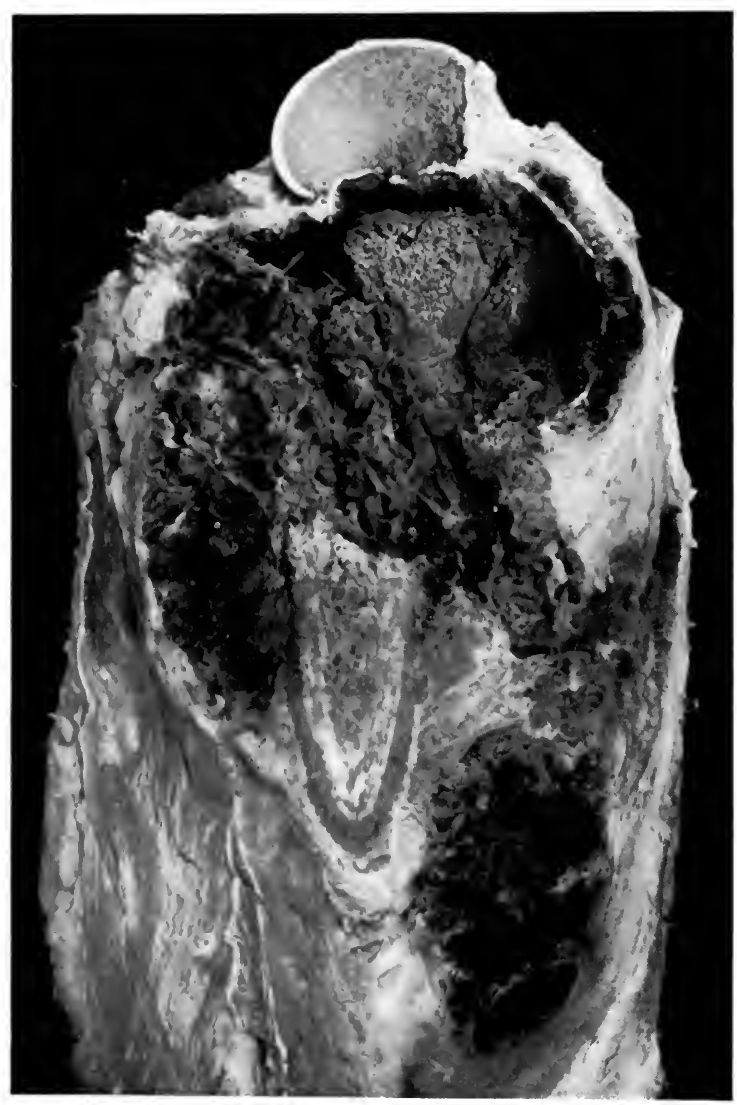

FIG. 98.-Rapidly growing telangiectatic osteogenic sarcoma of femur. A malignant bone aneurysm. Sequestration of end of diaphysis. Total duration 6 months.

of the statistical reports of Nasse, Reinhardt, Koeber, Butlin, Coley has adequate attention been given to the dependence of prognosis upon the location, origin, and structural type of the tumor.

Although Billroth asserted that the histological structure is of no aid in prognosis, this opinion cannot apply to the more malignant tumors, most of which prove fatal and among which recovery is determined by the location and stage of growth of the tumor. Contrary to the above opinion, histological studies have established the benign nature of the central giant-cell sar- 
coma, the greater malignancy of other types of giant-cell tumors, and the very unfavorable prognosis of the small spindle-round-cell growths. The chief source of difficulty and confusion in this field has arisen from the assumption that the tumors fall into a few sharply defined categories, as giant-, spindle-, and round-cell, whereas there are different grades of potential malignancy in each of these groups. When this factor is considered I believe it will be found in bone-sarcoma as with other groups of tumors that there is a definite relation between prognosis and structure.

I. The benign nature of central giant-cell sarcoma of the epulis type was early pointed out by Nélaton, later by Gross, Haberer, and others, and is now generally recognized. Bloodgood finds that practically all of these tumors respond to conservative operations, and that considerable invasion of the soft parts is no bar to conservative treatment. This treatment consists of curettage, or resection, followed by application to the wound of pure carbolic acid, and alcohol, or chlorid of zinc. Implantation of bone is employed when necessary. Among 26 cases curetted, Bloodgood records 5 recurrences, all of which were cured by a second curettage or by resection or by amputation. Kramer and Hinds report successful curettage when both condyles of the femur were involved. Most of these tumors respond well to radium.

It was early shown that certain giant-cell sarcomas of bone are very malignant (Ziegler, Wyss, Gross), and this fact has stood against the general recognition of the benign nature of the epulis type. Yet the giant-cells of the malignant tumor are quite different in appearance from those of the epulis and present single multilobed hyperchromatic nuclei, while the chief spindlecells of the tumor also show the features of malignancy, mitoses, and hyperchromatic nuclei. Gross found that the malignant giant-cell sarcomas exhibit calcific deposits and bony trabeculæ, but these features are wanting in the sarcomas of the epulis type.

2. The telangiectatic sarcoma and the bone aneurysm usually contain many giant-cells, but the prognosis of these tumors varies extremely, some proving highly malignant. Here I have several times observed that the character of the giant-cells is not a safe guide, but that in the malignant cases the chief or spindle-cells present unmistakable features of malignancy. My experience leads me to conclude that telangiectatic bone sarcoma tends to prove malignant when it occurs near the trunk, when round or spindle-cells with hyperchromatic nuclei predominate over giant-cells, and when perforation and destruction of the shaft occur early. In the malignant cases reported by Nakayama and Bloodgood the scanty tumor tissue was composed chiefly of round- and spindle-cell tissue.

3. A source of error arises when one portion of the tumor differs from another. When ulceration and necrosis occur the superficial portions of the tumor may lose their original structure and giant-cells assume the benign foreign-body type. I have made an erroneous prognosis of benign sarcoma from the fungating tissue about a sinus leading to a malignant, sclerosing, central and periosteal tumor.

4. With spindle-cell growths considerable differences in structure and malignancy exist which are constantly revealed by the minute characters of the cells. Comparatively adult types of spindle-cells with much fibrous or osteoid tissue belong to the slowly growing tumors which, however, recur locally and in advanced stages metastasize. Here the prognosis depends upon the location, extent, and complications of the tumor. At the other extreme stand the very malignant rapidly growing small spindle-cell tumors, often called round-cell sarcoma, for which a fatal prognosis must be given at all stages." Tumors of large polyhedral or rounded cells occur in bone 
which in general exhibit marked malignancy. The exact grade may be estimated by the minute characters of the cells, especially by the activity of cell division and by nuclear hyperchromatism. Fibrosarcoma and cellular chondromas are comparatively benign. Myxochondrosarcoma is also as a rule not very malignant, and if, as is often the case, such tumors are parosteal and encapsulated, the condition invites conservative treatment.

5. It should be emphasized that the histological structure indicates only the potential malignancy of a tumor, or its rate and capacity of growth. This factor is only one of many which determine the actual course of the tumor,

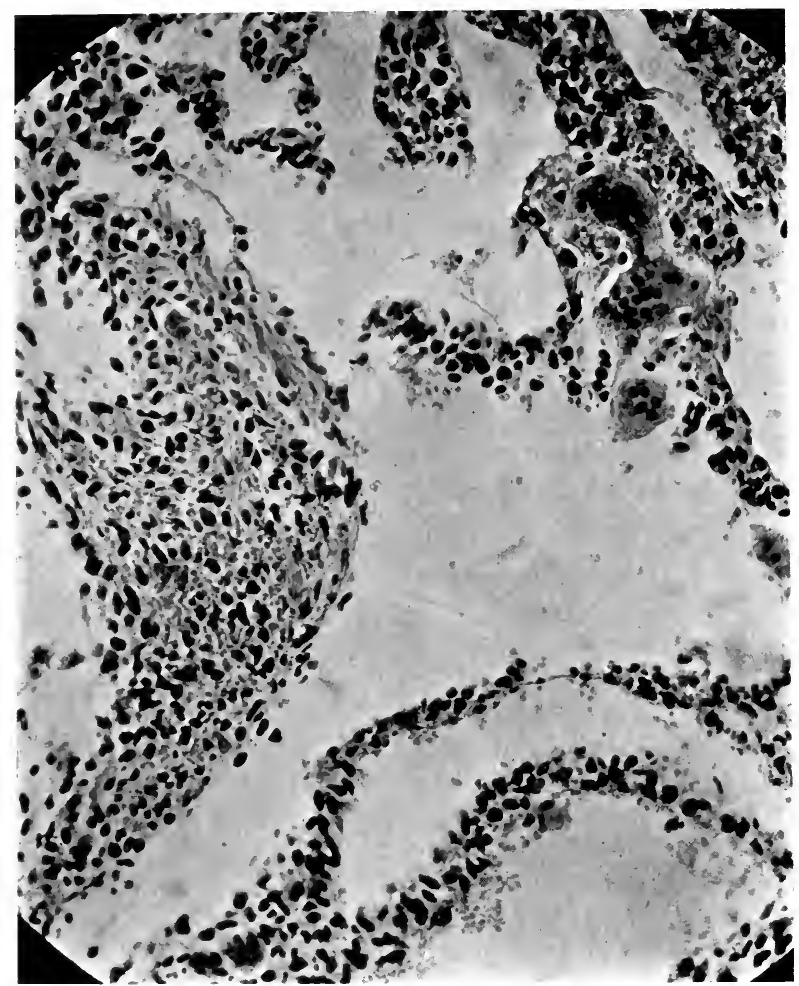

FIG. 99.-Malignant telangiectatic central sarcoma of head of humerus in a child. Malignant bone aneurysm. The giant-cells are of the epulis type, but the malignancy is indicated by the spindle-cells with very hyperchromatic nuclei.

which forms the clinical conception of malignancy. In the field of bone sarcoma when the clinical course does not accord with the structural characters the discrepancy should be attributed to lack of information regarding the gross and clinical features of the case.

Treatment.- The surgical treatment of bone tumors is highly unsatisfactory. Statistics show that nearly all osteogenic sarcomas eventually prove fatal, and while recovery seems to have occurred in rare cases in several locations, this fortunate outcome is the meager fruit of large numbers of useless amputations. Moreover, it must be recognized that the difficulty of separating the benign from the malignant conditions results in the needless sacrifice of 
many limbs, and raises a reasonable doubt about the nature of some of the reported surgical successes. Under these circumstances the demonstration that radium and $x$-rays, energetically employed, have a pronounced influence on the cellular tumors of bone and bone-marrow is a matter of first importance. Applied to the limit of skin tolerance, these agents, especially radium in large doses heavily filtered, seem to cause regression of the benign giant-cell sarcoma with gradual restoration of the shafts, produce rapid absorption of the cellular myelomas, and to have considerable restraining influence on certain malignant osteogenic sarcomas which in some cases has resulted in a clinical cure. If these results withstand the test of time, and some of those obtained at the Memorial Hospital are already of considerable duration, then the treatment of choice of all tumors of bone and bone-marrow is by means of

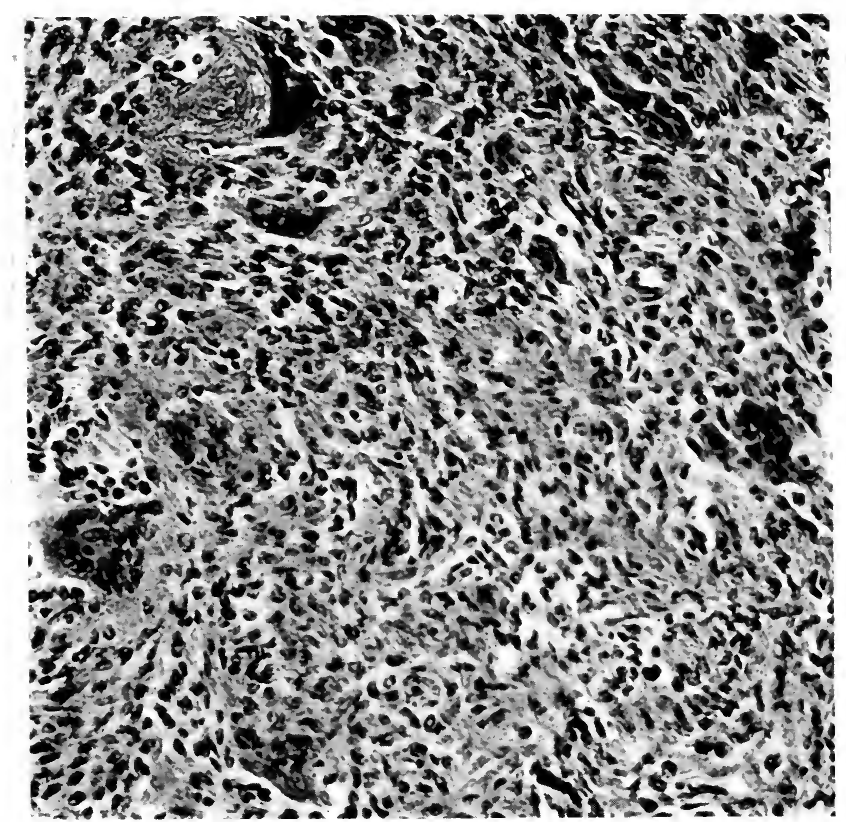

FIG. I00.-Central sarcoma of bone. Epulis type. This particular tumor is more cellular than usual, and is not entirely benign.

these physical agents. Not even incision for diagnosis can be recommended. Such incision may lead to infection, it often fails to yield material on which a positive diagnosis can be based, and it interferes with subsequent treatment. On the other hand, the clinical history, physical signs, $x$-ray findings, and results of treatment should permit a reasonably accurate estimate of the true nature of the case.

Etiology.- The etiology of bone sarcoma is highly obscure. While trauma must be accepted as a very common exciting factor, little is known about the growth or structure of bone which can explain the mode of action of the trauma. A study of the healing process in fractures is yet very suggestive of the mode of origin of traumatic sarcoma. In the average specimen of callus the proliferative activity of fibroblasts, osteoblasts, and endothelium is quite remarkable and often presents a picture which is difficult to separate 
from sarcoma. Especially in certain foci the cells may be quite as abundant as in sarcoma, but their arrangement is more orderly and the nuclei are not hyperchromatic (Fig. 66). The organization of considerable masses of blood provides conditions favorable for very free growth of atypical cells. Some organizing blood-clots after fracture I have found quite difficult to separate from sarcoma. Formation of progressive traumatic angiomas which gradually extend beneath the periosteum, often inclosed by a shell of new bone, may apparently precede the late development of traumatic sarcoma.

As a rule the trauma is moderately severe and may result in fracture or splintering, or in local hemorrhage, and the tumor seems to represent an exaggeration and perversion of the healing process. Klebs describes a very early medullary sarcoma in which the adventitial cells of the blood-vessels seemed to be chiefly concerned. There is some evidence that the presence of

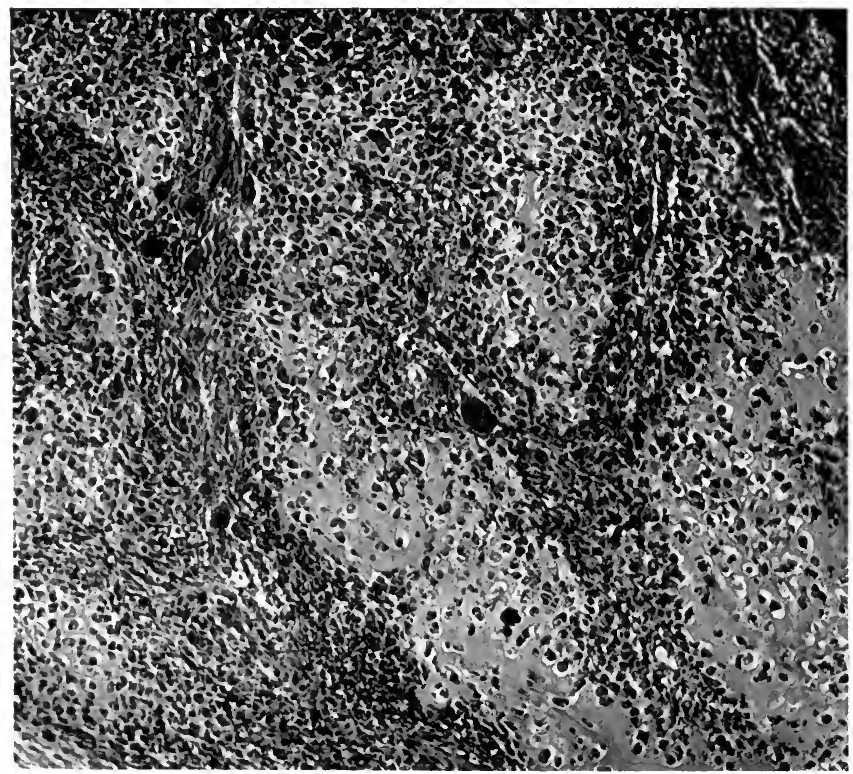

FIG. IOr.-Structure of malignant chondrosarcoma of bone.

blood exerts a peculiar stimulating influence on the regeneration of bone. Both these factors are present in the healing of fractures and other traumatic conditions.

All varieties of sarcoma have been attributed to trauma, but it is evident that the injury is only one of several essential factors, since many or most cases fail to give a history of trauma, many tumors arise in bones protected from injury, and occasionally bone-sarcomas are multiple. The traumatic origin applies chiefly to periosteal sarcoma. The fact that central sarcomas arise in the diaphysis, usually near the epiphyseal line, indicates that disorders in the growth of bone at this point are involved in their origin. Borst reports a remarkable case of periosteal ossifying sarcoma which contained islands of lymphoid cells and hematoblasts, and he suggests that the tumor arose in part from an aberrant portion of marrow tissue. For the common epulis of the alveolar border injury to the periosteum and bone from extraction of teeth 
may be responsible. Yet primary tumors of this type are common in the jaw bones, and they may precede and call for the extraction of teeth.

The origin of parosteal sarcoma must be referred to misplaced islands of bone-forming tissue. An imperfect differentiation of tissues neighboring to bone such as has been assumed to exist in myositis ossificans may also be included as an etiologic factor. The observed facts do not seem to require the theory of a metaplastic development of any recognized type of bonesarcoma.

Simple chronic inflammation of various types may be concerned with some of the capsular sarcomas. Syphilis and tuberculosis may be suggested as probable factors in certain of the round-cell and diffuse sarcomas, but the grounds for such an assumption are quite indefinite. The Wassermann reaction has been present in some of my cases of extensive bone-sarcoma.

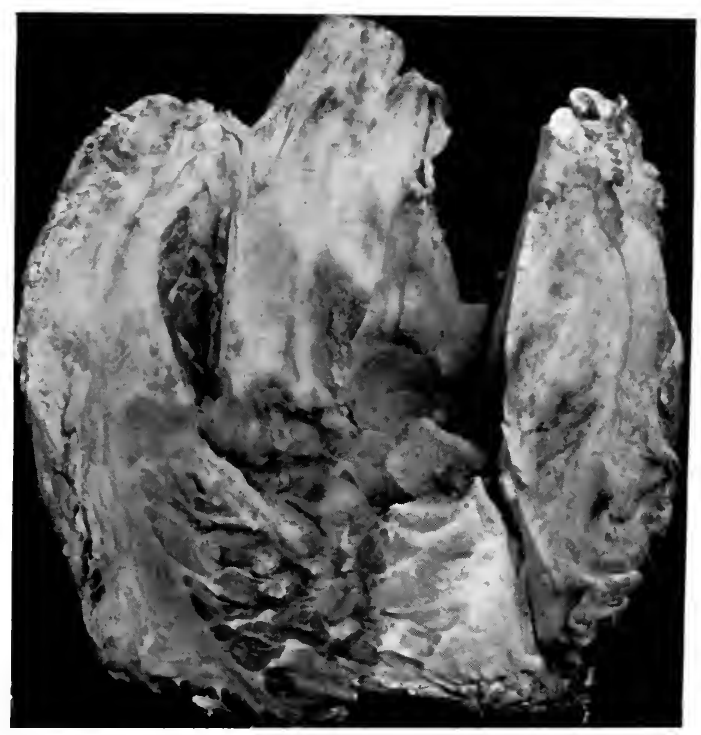

FIG. I02.-Excessive callus developing in 2 weeks about a comminuted fracture of inner end of clavicle. Clinically resembling sarcoma.

The idea that trauma or any other factor may lead to the development of sarcoma at the ends of long bones which are previously entirely normal is, I think, without satisfactory foundation. There is, however, abundant evidence that developmental disturbances, chiefly at the epiphyseal lines, form an essential factor. The routine examination of the ends of bones in infants shows not a few irregularities in growth resulting in the displacement of islands of cartilage in marrow cavity or periosteum. These irregularities I find especially frequent after rickets. Many osteogenic sarcomas are sharply separated from the marrow cavity by a septum of bony or osteoid tissue, indicating that the tissue of origin was also separated. After rickets definite masses of imperfectly ossified cartilage are often found in the marrow cavity. It is reasonable to assume that mild forms of bone dyscrasiæ exist which are not recognized clinically, but which are capable of causing abnormalities in the formation of the ends of the bones with a tissue predisposition to tumor growth. 
The elements of tissue predisposition and constitutional tendency become most apparent in connection with the disease osteitis fibrosa.

Osteitis Fibrosa.-This interesting malady, first fully described in I 891 , has important bearing on the etiology and significance of giant-cell sarcoma of bone of the epulis type (Haberer, Bloodgood, Lit.). It occurs chiefly in children and has been termed "osteodystrophia juvenilis" (Monckberg, Tietze, König). Barrie emphasizes the importance of abnormal bone formation and defective calcium metabolism (A. S., 7I, $58 \mathrm{I}$ ). It also occurs in elderly subjects where its relation to osteomalacia is apparent. It pursues a long course, but often ends fatally from cachexia or intercurrent disease. It is characterized by defective calcification of the bones which leads to thickening, weakening and deformity of one or many bones of the limbs, jaws, skull, or trunk. The bone tissue is replaced by cellular fibrous tissue which may invade the marrow cavity replacing the red marrow. From this stage the disease takes one of two courses, the cellular tissue softening and producing multiple bone cysts lined by fibrous tissue and filled with clear fluid, or in the walls of the cysts or with cyst formation giant-cell sarcoma develops. There has been much controversy regarding the relation of the different stages of this process, some holding that the cysts always follow the sarcomatous process, others finding that the sarcoma develops in the cysts. Both events seem to occur. A history of trauma and the presence of hemorrhage and pigmentation in the walls of cysts seem to show that the presence of blood coagulum influences the development both of cysts and of sarcoma. In some cases fibrous cysts are chiefly observed (Monckberg, Heinecker), in others cystic sarcomas (Haberer), or again solid sarcomas (Rehn). The long duration, 9 years (Rehn), and relatively benign quality of the sarcomatous process are notable features. Arising in the course of a nutritional disturbance of bone and being associated with inflammatory changes Rehn concludes that the entire process is inflammatory. Cystic softening of the tumors, transformation into fibrous tissue, and spontaneous disappearance have several times been observed. Lubarsch points out that very similar processes giving rise to a diagnosis of sarcoma occur at the points of fractures in cases of osteitis fibrosa and he inclines to the opinion that the process is not a true sarcoma (Gaugele).

A remarkable case reported by Martland of multiple giant-cell sarcomas involving many of the bones of the body probably belongs in this category and illustrates a universal tissue predisposition to the disease. Knaggs and Gruner describe an atypical case of fibrous osteitis affecting femur, tibia, and fibula, and ending in malignant sarcoma.

Kolisko identifies osteitis fibrosa with Paget's osteitis deformans and finds that in both the transformation of bone-marrow into fibrosa tissue may be followed by regressive changes leading to bone cysts or to progressive changes giving rise to giant-cell sarcoma of slight malignancy.

\section{MULTIPLE MYELOMA}

Multiple myeloma is a specific malignant tumor of the bone-marrow arising probably from a single cell type, and characterized chiefly by multiple foci of origin, a uniform and specific structure composed of plasma-cells or their derivatives, rare metastases, albumosuria, and a fatal termination. This orthodox definition of the classic picture of the disease is, however, subject to extensive modifications, as will appear in the context.

The disease was first described by McIntyre in I 850 under the term "mollities ossium," and has been variously interpreted by subsequent observers as senile osteomalacia (Marchand); myelogenous pseudoleukemia (Zahn); 
primary multiple sarcoma of bone-marrow (Buch). The general clinical characters were early recognized, McIntyre reporting the presence of BenceJones albumose in the urine, while the tendency of the disease to limit itself to the bone-marrow and the resemblance to an infectious granuloma have been repeatedly noted. Rusticky ( 1873 ) seems to have first recognized the disease as a specific affection of the bone-marrow and employed the term "myeloma." Kahler (I889) demonstrated the nearly constant occurrence of the albuminuria and the peculiar nature of the protein involved. The identity of the tumor-cells in many cases with plasma-cells was pointed out by Wright in r9oo. The etiology, pathogenesis, origin of the albumosuria, and the relation to other tumors and pathological processes in bone-marrow still remain obscure.

Gross Anatomy.- - The ribs and sternum are the chief original seats of the tumors, while the vertebra, skull, femur, pelvis, and humerus are less frequently involved, and in the order named. In 3 cases the ilium appears to have been the primary seat (Hoffmann). The tumors form small nodules, multiple and as large as a bean, or bulky growths as large as an orange, associated with many smaller tumors. Twenty-eight separate tumors have been observed in the skull (Winkler), and the diffusion of the process is often wide-spread, involving many bones and revealing a certain systemic quality which, however, is nearly always much less marked than that of leukemia. Yet the process occasionally appears in diffuse form and circumscribed tumors are missing (Winkler, Jochmann, Schumm, Abrickassoff, Hirschfeld, Lit.).

The tumors are soft or firm, translucent or opaque, and whitish, gray, or deep red according to vascularity. Hemorrhage, infarction, blood cysts, and necrotic areas may be present. The bone tissue suffers active absorption and the shafts become very thin, or multiple perforations result, or fractures occur. After passing the periosteum the surrounding tissues are diffusely invaded.

Many cases terminate without metastases in the organs, but distant secondary growths have been found in liver, spleen, kidney, lung, and ovary, while in certain very malignant cases that probably belong in this category nearly every organ in the body may be involved.

The clinical course of multiple myeloma is that of a progressive malignant tumor with gradual and often very extensive invasion of the bone-marrow, pronounced lesions in the other organs, a somewhat specific cachexia, and an invariably fatal termination. These same general clinical features are observed in all types of myeloma, in so-called myelosarcoma, and in endothelioma of bone, and were closely simulated by a case of chondrosarcoma reported by Seegelken.

The disease arises chiefly in males, in the $4^{\text {th }}$ and 5 th decades (24 to 69 years) and continues a few months or several years. The tumors are usually first detected from the pain they produce from erosion of periosteum, pressure on adjacent nerves, and from general toxemia, and continuous or paroxysmal pain usually persists. Recurring fever has been observed, but the temperature is usually subnormal. Fractures and extensive deformities of the skeleton mark the later stages. Prolonged cases may resemble osteomalacia (Marchand). Anemia may become very severe and exhibit pernicious features (Grawitz), but in many cases the blood shows no pronounced changes. Rarely there is an increase of myelocytes in the blood, in one case up to 2r.8 per cent. (Saltykow, Sternberg). In the blood of Aschoff's case Schridde found plasma-cells. Albumosuria has been noted first in the IIth week or $5^{\text {th }}$ year of the disease. Its occurrence may be continuous or periodic, and the quantity of albumose may be scanty or large, up to 6.9 per cent. 
It has been found in less than half the cases, and occurs in myxedema, leukemia, carcinoma of bone, etc.

Neither the exact nature, position, nor origin of the urinary protein are fully understood. It bears some relation to a severe, but non-specific nephritis which commonly marks the disease, and other proteins may occur in the urine. The peculiar qualities of this protein have been fully studied by Mag-

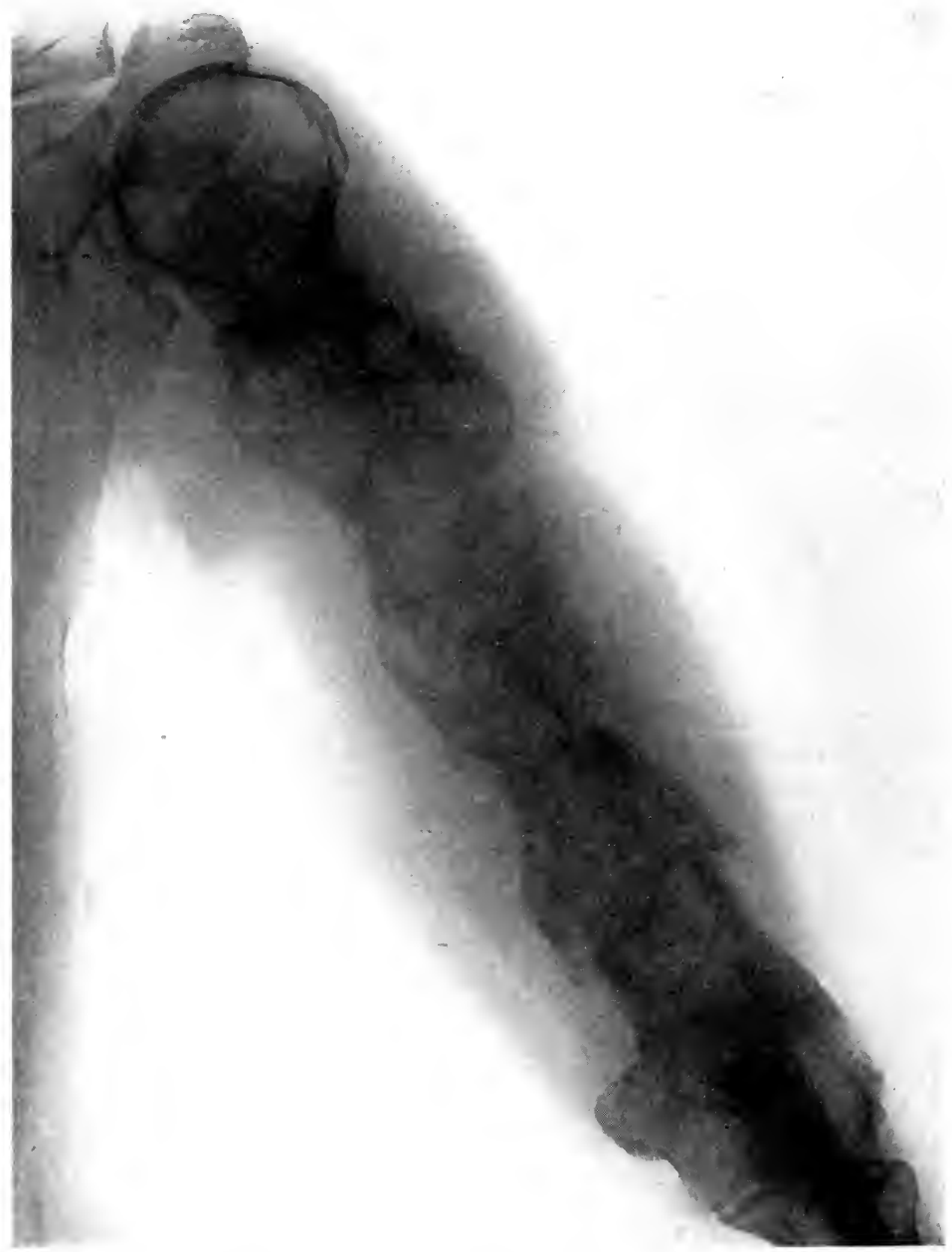

FIG. I03.-Diffuse myeloma of the humerus.

nus-Levy, Jochmann and Schumm, and others. It precipitates at $55^{\circ} \mathrm{C}$., the precipitate becomes most abundant at $65^{\circ} \mathrm{C}$, and gradually dissolves at $85^{\circ}$ C., and reappears on cooling. Williams finds that its physical properties may change from day to day, that it contains a carbohydrate radicle, no phosphorus, and falls in the group of mucins. According to Geis the albumose is derived from the elastin of bone tissue absorbed by the tumors. 
The spleen is often moderately enlarged, at times partly in compensation for the loss of bone-marrow, or from the general toxemia, and from metastases. The lymph-nodes are rarely hyperplastic (Weber, Scheele, Ewald). Extensive amyloid degeneration of the muscles of thorax and intestine was observed by Hueter. The termination of the disease is marked by anemia, emaciation, diarrhea, dyspnea, paralysis, and coma.

Structure.-While in some cases the cells of myeloma exhibit the features of plasma-cells, large or small, with single or multiple nuclei, yet in the entire scope of tumors which probably belong in this class the cells vary widely in size and character. In some cases the entire tumor is composed of loosely packed typical plasma-cells, 8 to I $2 \mu$ in diameter, round, oval, or polygonal, and with opaque, amphophile, non-granular cytoplasm. The single or multuple nuclei are relatively small, eccentric, or protruding from the cytoplasm,

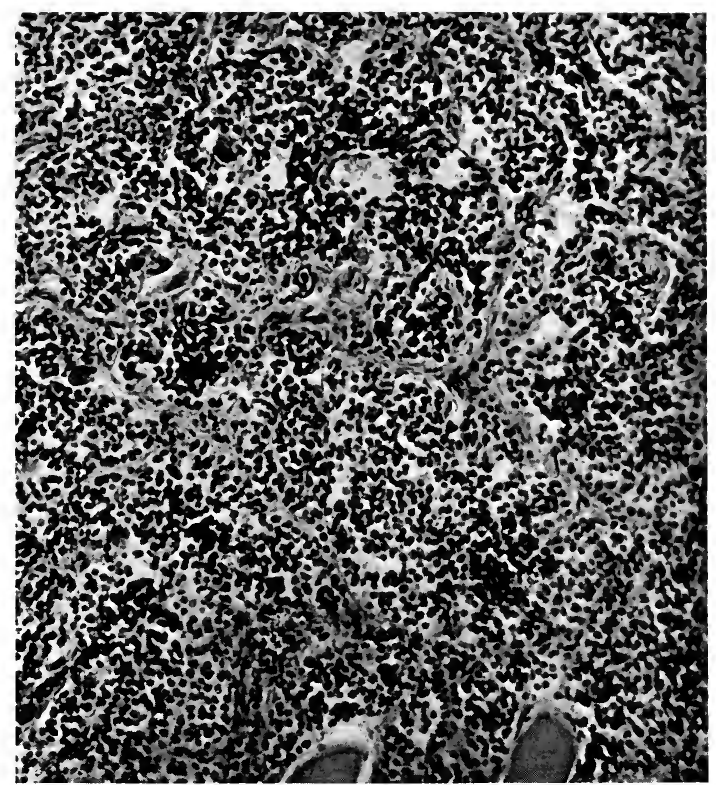

FIG. I04.-Structure of a diffuse lymphocytic myeloma involving whole shaft of humerus. (Same tumor as in Fig. .103)

surrounded by a clear zone, and presenting blocks of chromatin arranged along the nuclear membrane. Mitotic figures are observed in many cases, but are less numerous when the cells are typical plasma-cells. Certain cytoplasmic bodies have been interpreted as centrosomes (Christian). The clear zone about the nucleus has been described as a vacuolated secretory product. In rare cases the round cells are mingled with spindle-cells which probably result from pressure (Wieland).

In a second group of cases the cells are larger, giant-cells with multilobed nuclei appear, multiple and vesicular nuclei are more prominent, nucleoli are large and acidophile, the resemblance to plasma-cells is not striking, the tumors are more malignant and metastases occur. Here one finds a structure which recalls lymphosarcoma with large cells.

In this group are rare cases of very malignant large cell sarcoma of the 
bone-marrow with universal involvement of this tissue and very extensive growths in lymph-nodes, spleen and other organs, and in which the cells are uniformly round, large, granular, and with single or multiple vesicular and hyperchromatic nuclei. An extreme case of this group has been fully described by Norris. It seems probable that these tumors are a very malignant form of neoplasm derived from bone-marrow. The relation to myeloma is especially indicated by the fact that the tumors in certain regions may exhibit a structure closely resembling ordinary myeloma.

The arrangement of the cells in myeloma is diffuse, but there is often a scanty stroma separating groups of cells. Occasionally the cells are embedded in a hyaline acidophilic material, and in malignant forms the tumors are

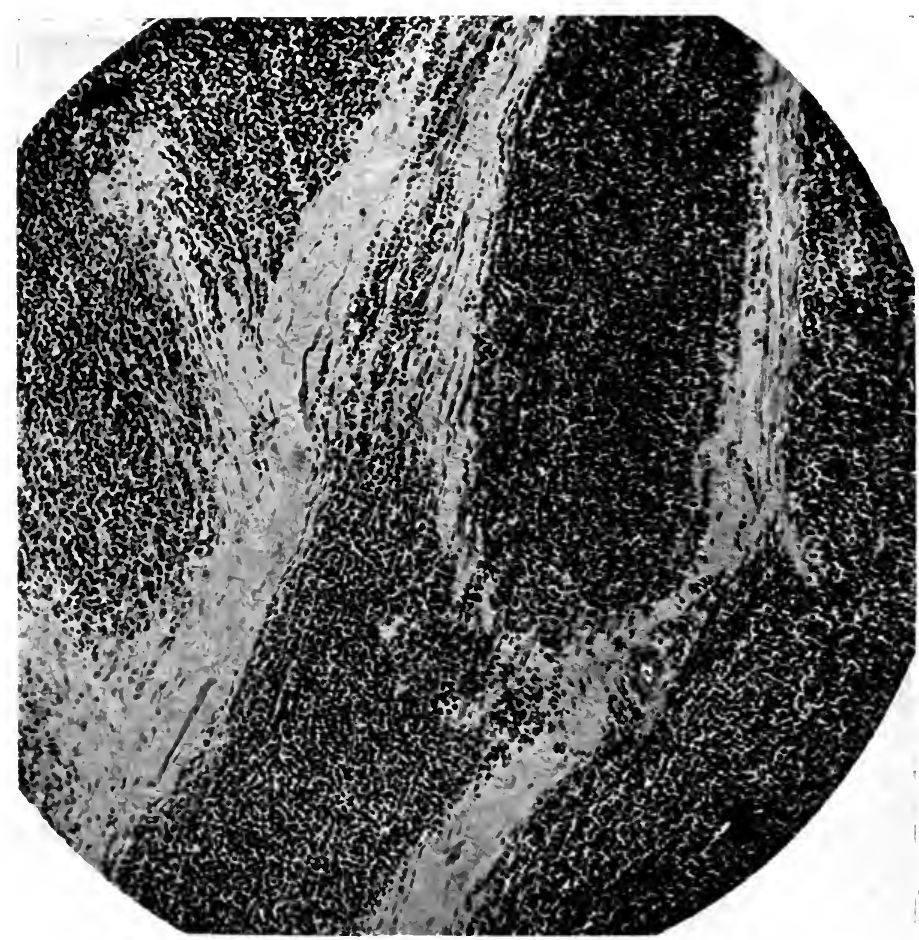

FIG. 105.-Structure of plasma-cell type of multiple myeloma.

very edematous. As the tumor progresses the other elements of the marrow disappear, but on the edges tumor and marrow-cells are intermingled. Bone trabecula undergo simple absorption or in the case of compact bone osteoblasts are found in lacunæ. The tumor never produces bone. An inflammatory reaction with exudate of lymphocytes and plasma-cells may mark the advance in bone-marrow, periosteum, or other tissues. Some of the tumors are oversupplied with small blood-vessels, and in Rusticky's case a frontal tumor pulsated. The larger vessels possess well-formed walls, but many small sinuses occur without definite walls, as in bone-marrow. Hemorrhage is frequent in the central growths and leads to softening, forms blood cysts, and leaves pigmentation. Extensive areas of necrosis occur and here 
the cells undergo the usual forms of degeneration. Similar degenerative changes overtake single cells in well-nourished tumors and have been used by Hoffmann as a basis of classification of cell types. Extensive fibrosis seems to be the natural termination of the process in many areas of typical myeloma.

Histogenesis.-The origin of the cells of multiple myeloma has been the subject of much discussion which necessarily invades the extensive fields of literature on the relations of bone-marrow cells and on the origin of plasmacells (Hirschfeld, Lit.). That the tumor-cells in one group of cases represent various forms of plasma-cells is strongly attested by the comparison made by Christian. In one group of cases the identity with plasma-cells is so striking as to suggest that those writers who doubt this identity have never seen such a case. Assuming that the plasma-cells are derived from adventitial cells of the vessels the myeloma must be derived from the supporting tissue of the blood-vessels of the marrow and not from blood-forming cells. Although the earliest stages of formation of myeloma from adventitial cells have not been traced, this theory of origin accords with certain important clinical features of the disease, such as its relatively benign character, and partial resemblance to a granuloma and serves to maintain the complete separation of the malady from leukemia. It also serves to explain in some degree the capacity of the tumor to destroy bone tissue. The origin from adventitial cells nearly related to endothelium is further supported by the occurrence of multiple perforating tumors of bone clinically resembling myeloma, but composed of cells with striking endothelial characters. Such cases have been recorded by Kahler and Marckwald, and observed once by the writer, and, in fact, if solitary marrow tumors are included they are not infrequent.

In another group of cases the cells lack close resemblance to plasma-cells and their form and arrangement strongly suggest an origin from the bloodforming cells of the marrow. Both the red-and the white-cell progenitors have been included in the scope of this origin. Sternberg, Lubarsch, Herz, and Charles were able to demonstrate a few neutrophile granules in the cells of their cases, and this evidence is believed by some to demonstrate an origin from the granular leukocyte series (leukocytoma). Since these granules are demonstrated with difficulty in tissues from leukemia and since they may well be absent in neoplastic cells, many, after careful study, admit the absence of granules, but still claim the identity of the tumor-cells with immature myelocytes, basing their conclusion on the other features of the cells (Menne, Lit.).

Against this view stands especially the fact that other tumor-like processes affecting myelocytes, as in leukemia, pursue a wholly different course from myeloma. The so-called myelocytomas being as a rule more active than the plasmomas, it may be urged that a greater degree of anaplasia may transform plasma-cells into cells resembling myelocytes.

Opinions regarding the scope of the disease, multiple myeloma, have been based not only on the histological structure of the tumors but also upon the clinical features. Many observers would adhere narrowly to certain general clinical characters which they assume to be essential and would eliminate from the group all cases which do not present these features. Thus Winkler insists upon the strict limitation of the process to the bone-marrow, the absence of metastases, the importance of albuminuria, and the perfectly uniform size and character of the cells. Yet it is clear, as Lubarsch pointed out, that the occurrence of metastases is more or less accidental, that albumosuria may be absent, and the size and form of the cells may vary considerably, in cases which in generaly strongly impress themselves as essentially the same disease. 
On structure chiefly Kaufmann separates from multiple myeloma a group of malignant lymphosarcomas of bone-marrow. In these the cells are larger than plasma-cells, giant-cells occur, a reticular matrix is present, and metastases occur. They correspond to the writer's group of more malignant myelomas. Yet the cases of Wieland to which Kaufmann refers present the main characteristics of malignant myeloma and it is clear that many authors have accepted such cases as true myeloma, so that the grounds for their separation seem inadequate. Both are multiple primary tumors of bonemarrow, perforating the compact tissue, sparing lymph-nodes, producing albumosuria, and following the same general clinical course, while variations in the cells seem to indicate only different grades of anaplasia.

A distinctly different condition from myeloma appears to have existed in the case described by Hammer in which severe recurrent fever, invasion of lymph-nodes, metastatic growths in the dura, and extensive thickening of many bones without albumosuria were associated with diffuse lymphoid hyperplasia without circumscribed tumors of the bone-marrow. Such a picture suggests a true pseudoleukemic process and differs essentially from multiple myeloma. Very similar cases of myelogenous pseudoleukemia have been described by Baumgarten and by Rubinstein. Hodgkin's granuloma affects the bone-marrow, but its structure is specific and quite different from that of multiple myeloma.

An erythroblastic origin of certain cases of myeloma is indicated by the observation of Ribbert, who found hemoglobin in the cells of his case. Norris has also encountered such a case. In a recent case of multiple perforating bone-tumors with enlarged regional lymph-nodes, I found most of the cells smaller than lymphocytes with pyknotic nuclei and strongly acidophile homogeneous cytoplasm.

Finally, in certain cases the tumor-cells seemed to the observers to lack the specific features of any of the adult marrow-cells and either to belong to the lymphocyte class or to represent an indifferent blood metrocyte. It is notable that certain of these cases have been very rapid and malignant forms of myeloma (Scheele, Herxheimer, Jellinek, Vignard, Norris).

Thus if one classifies myeloma according to the views of different observers concerning the origin of the tumor-cells the following groups appear: (I) Plasmocytoma, (2) erythroblastoma, (3) myelocytoma, adult and embryonal, (4) lymphocytoma. Whether such varied interests are actually represented in the scope of multiple myeloma or whether we have to deal with varying grades of anaplasia in a single cell or origin remains to be determined. At present the data seem to favor the former alternative.

Regarding the etiology of multiple myeloma little can be said. It is a disease of middle life, occurring chiefly in males ( 19 to 7 ) of the middle classes. Multiple tumors can hardly be closely connected with trauma, yet 3 cases were referred to injury (Marchand, Ewald, Winkler), and Winkler discusses at length its mode of action. Grawitz observed a case which followed typhoid fever. Stokvis found 2 cases in brothers. An.infectious origin has frequently been suggested from the clinical features, the general significance of plasma-cells, and the relatively slow course of many cases. As a late and indirect sequel of an infectious process acting in subjects with tissue predisposition the disease is perhaps well conceived. Yet several cases have indicated a dependence on nutritional disorders of the bone itself which in other subjects leads to osteitis or osteomalacia. 


\section{CHAPTER XX}

\section{ENDOTHELIOMA}

The term "endothelioma" is applied to an extensive group of tumors which are believed to be derived from the lining cells of blood-vessels, lymph-vessels and spaces, subdural spaces, and serous cavities.

The scope of endothelioma has suffered wide fluctuations because of the uncertainty surrounding the embryological relations of different classes of endothelial cells, the peculiar position occupied by these cells as intermediate between epithelium and fibroblasts, and the difficulty of determining the origin of many tumors supposed to belong in this class. Thus some authors find the group extremely numerous and complex, while others insisting upon more rigid data describe a very restricted class of tumors as endothelioma.

Origin and Functions of Endothelial Cells.-The embryological relations of the flat internal lining cells of the body deserve first consideration in the study of endothelioma both for the sake of accurate classification and for the bearing on many peculiarities of these tumors.

Although our knowledge in this field is still incomplete two main facts seem to be sufficiently attested: (I) The lining cells of the great body cavities, pleura, pericardium, and peritoneum, arise from cells lining the celomic cavity which forms early by the splitting of two layers of mesoderm; (2) the lining cells of the blood-and lymph-vessels, as well as the subdural membranes are derived from mesenchymal cells and are very closely related to connective tissue.

Through the researches of Huxley, Balfour, Lancaster, and the Hertwigs it has been shown that the mesoderm owes its origin in vertebrates to a separation of cells derived from both ectoderm and endoderm near the primitive groove. The definite layer thus produced splits into two lamellæ uniting with the ectoderm on the one hand to form the somatopleura, and with the endoderm to form the splanchnopleura, and inclosing the celomic cavity between them. In addition to the processes which gives rise to a definite middle germ layer, an entirely different process gives origin to the sustentative tissues of the body which spread out into the various ramifications of the main germ layers. O. and R. Hertwig (I88I) and others have shown that the sustentative tissues are derived from the middle germ layer by the splitting off of many ameboid cells which spread out between the epithelial germ layers and form the mesenchyme. From this embryonal tissue it is now generally agreed that the connective tissues and the lining cells of the vessels, blood-cells, lymphoid organs, the smooth involuntary muscles, heart muscle, and in some cases the striated muscles are derived. Concerning the exact mode of origin of the blood and vessels, opinions are still at variance, but the data are becoming more definite. According to Ziegler the fundaments of the blood- and lymphvessels are derived from the interstitial spaces of the primitive body cavity which persists as lacunæ and interstices in the advancing mesenchyme and become confluent in that tissue. A very similar process is described by others who assume that the first vessels arise from mesenchymal cells, which arrange themselves in rows and become canalized, forming the vessels. Finally, there are those who assume an entirely different origin for connective tissues and vascular endothelium, attributing the former to migrating cells from the mesoderm and the latter to cells of the endoderm which are constricted off as an independent tissue and give origin to the whole vascular system (Uskow, 
Gegenbauer, Ruckert). Thus, embryological data do not warrant fundamental distinctions between the various cells now commonly grouped as endothelium, since all are of mesodermal origin. Although the celomic lining cells which give origin to the serous endothelium maintain a lining character throughout, that fact does not warrant their identification with any forms of epithelium such as is derived from ectoderm or endoderm.

The vascular endothelium being of mesenchymal origin is closely related to the connective tissues, and this fact may serve to explain the resemblance of certain endotheliomas to connective-tissue tumors, while the difference in the embryological history of serous and vascular endothelium seems to find some expression in the morphology of tumors derived from these two types of cells. Tumors of the serous cells show certain cacrinomatous rather than sarcomatous tendencies. From the embryologic standpoint one might assume a certain relation between endothelioma and tumors of lymphoid or smooth muscle-tissue, but observation does not indicate that this relation is important. In the field of endothelioma as elsewhere the adult characters of the cells seem to be of more importance than their embryological history.

The physiology of endothelial cells establishes their position as intermediate between epithelium and connective tissue. They enjoy certain functions recalling those of epithelium, but less specialized. In the formation of lymph Heidenhain and Hamburger have shown that the lymphatic endothelium exerts a secretory action. The secretion of a mucinous lubricant is a constant property of serous endothelium. Thoma attributed to a secretory process the appearance of blood fluids in the closed cell layers of the area vasculosa of the chick. Metabolic functions are evidenced by the occurrence of fat droplets in the normal endothelium of the pleura (Thoma). Phagocytosis by vascular and serous endothelium is often extremely active, especially in the spleen and liver.

Considerable light has been thrown on the origin of endothelium and blood-cells by Stockard's study of fundulus embryos without a circulation. Here the serous endothelium secretes large amounts of fluid distending the pericardium until it deforms the embryo. Vascular endothelium forms locally throughout the mesenchyme from wandering mesenchymal cells. Similar wandering mesenchymal cells also produce chromatophores. The vascular endothelium never produces red or white blood-cells. The red blood-cells arise from a specialized portion of mesenchyme or intermediate cell mass, derived from the posterior region of the embryo. Some of these cells wander out on the yolk-sac, producing the blood islands of Pander. Leukocyte production is largely limited to mesenchymal cells in the anterior portion of the embryo, but later occurs throughout the mesenchyme, always from extravascular mesenchymal cells and not from endothelial cells.

According to these data vascular endothelium, leukocytes, and red bloodcells are separate cell series derived from different mesenchymal anlagen. Closely related to them in origin is a series of wandering chromatophores.

This study is in accord with others which indicate that lymphocytes are not derived from the reticulum cells of lymph-nodes. The results are probably not irreconcilable with the opinions of Councilman, Mallory, and others that lymphatic endothelium is a source of some large mononuclear cells of the blood.

The general pathology of endothelium most clearly illustrates the dual tendencies of these cells, revealing, on the one hand, the assumption of epithelial qualities and on the other the possession of certain potencies of connective-tissue cells. In the course of inflammation of serous surfaces endothelial cells multiply readily, and form giant-cells and concentric endothelial 
pearls. In granulation tissue they become ameboid and phagocytic. Pial tubercles may be composed exclusively of aggregations of proliferating endothelial cells. Birch-Hirschfeld found serous endothelium closely related to fibroblasts, observing that they participated largely in chronic thickenings of the peritoneum. In artificial adhesions of serous membranes Graser and Roloff traced the change of serous cells into fibroblasts, while fibroblasts in turn produced serous endothelium on new surfaces. Borst saw serous cells assume star and spindle shapes, and Lubarsch and Marchand have described the formation of intercellular substance and fibrils from these cells. Yet Ribbert, Ziegler, and others do not accept these observations as proving the transformation of serous cells into genuine fibroblasts, and it seems highly probable that the new tissues produced by proliferating endothelium have

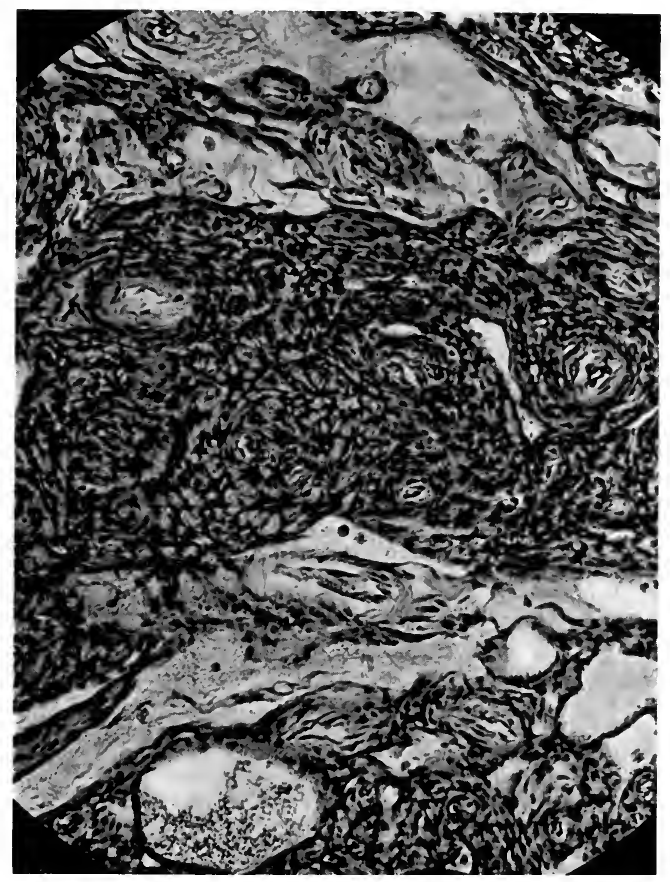

FIG. 106.-Angio-endothelioma arising in a thrombosed vein.

not the permanence of true connective tissue, but early undergo hyalinosis and atrophy.

Vascular endothelium also proliferates readily in inflammation and forms collections of cells which may resemble the groups of endothelioma or carcinoma. In endarteritis the endothelial cells may contribute largely to the new tissue, especially in syphilis. In tuberculous endarteritis very extensive new growth of endothelium is observed in the intima. In the organization of thrombi the endothelial cells are very active, wandering out into the bloodclot, acting as phagocytes, replacing the thrombus with strands of spindleor masses of flat cells, and conducting new capillaries. The subsequent cicatrization of the organized thrombus illustrates the usual fate of new tissues produced by proliferating endothelium. Many authors have described the 
alveolar structures produced by the reversion to the cubical type by endothelium of tissue spaces in chronic inflammation. In many cases of chronic inflammation of serous membranes groups of endothelial cells become incarcerated by adhesions and transformed into cubical or cylindrical cells producing structures resembling gland alveoli (Ribbert, Renngli, Meyer, Menetrier). In most tissues the endothelial cells are the chief source of the epithelioid elements in miliary tubercles.

In lymph-nodes some remarkable grades of endothelial proliferation are observed. In chronic lymphadenitis, probably of tuberculous origin, several types of large cell hyperplasia occur, some of which closely resemble or actually merge into neoplasms. In scrofulous lymphadenitis the entire node may be replaced by masses of poorly staining endothelium. In chronic congestion of the spleen the sinuses may appear lined by cubical endothelium. These and many other details, which have been extensively discussed by Borst, illustrate the behavior of endothelial cells under abnormal conditions, and they may serve as a standard of comparison in the interpretation of neoplastic processes in these cells.

History of Endothelioma.-The history of endothelioma has passed through many stages. Arising from a well-characterized cell type, these tumors are of such wide distribution and exhibit so many of the varied qualities and relations of the originating cells that much change and conflict of opinion have always existed regarding their scope and classification.

Koster first clearly maintained the opinion that the endothelium of lymph spaces and vessels gave origin to many tumors, which, however, he regarded as carcinomas. His demonstration that endothelium may give rise to tumors closely resembling carcinoma strengthened the old doctrine of Virchow that all cancers arise from the matrix of connective tissue. When Thiersch and Waldeyer established the epithelial origin of carcinoma and Carmalt showed that cancer-cells could be washed out of the invaded lymph-vessels, leaving the endothelial cells intact, the way was opened for the recognition of a special group of tumors derived from endothelium. Waldeyer's authority encouraged the interpretation of certain tumors in various organs as of endothelial origin.

In I 860 Golgi introduced the term "endothelioma," applying it especially to psammoma of the dura mater, which has always stood as a standard type of the growth. The use of this term became established slowly, many authors classifying endothelial tumors with sarcoma. Thus Waldeyer described the perivascular growths as angiosarcoma, and Kolaczek in an extensive study classified all the tumors of the endothelium of blood- and lymph-vessels as angiosarcoma. The mixed tumors of the salivary glands previously regarded as carcinomas were placed by Kaufmann among sarcomas until Wartmann in I879 first maintained that the polyhedral cells of these growths are derived from endothelium. Yet the endothelial origin of salivary gland tumors has never been universally accepted, and while Ribbert and many others have firmly maintained their epithelial nature, Kaufmann, Borst, and others accept in part the endothelial theory.

As early as I866 Lucke described a pulsating tumor following a fracture of the forearm, the adenoid structure of which he referred to a growth from indifferent cells of the connective tissue. Kocher (1868) and Billroth (1869) derived somewhat similar tumors of bone from the walls of blood-vessels. Tillmanns (1873) and Joppe trace these tumors to the endothelial or perithelial cells. Schweninger (I876) clearly traced the development of an adenoid bone tumor to the endothelial cells of blood- and lymph-vessels and lymph spaces. 
In the orbit Pagenstecher first described a tumor removed by Knapp, which by exclusion he was compelled to refer to proliferating endothelium of lymph spaces, and this view was endorsed by Knapp (1872) in the description of later cases. Yet it now appears probable that most of these tumors were derivatives of the lacrimal gland and that endothelioma of the orbit arises chiefly from the optic nerve sheath.

In I879 a group of ovarian endotheliomas was brought forward by Marchand, although Leopold had previously regarded certain large solid tumors of the ovary as of endothelial origin. In I870 Wagner recognized the endothelial origin of certain pleural tumors and his view was strongly supported by Neelsen's study. In I867 Massey and in I879 Hubl described endothelial tumors of the peritoneal serosa.

In the skin Kaposi described under the term lymphangioma tuberosum a tumor which Jarisch later grouped among the hemangio-endotheliomas, but it has long been known that this tumor is derived from the epithelial structures of the skin.

The lymph-nodes were drawn into the sources of endothelioma by Zahn in I874 under the title of sarcoma epithelioides, while in I88I Hoffmann and Schottelius described a similar tumor as endothelioma. Most of these tumors appear to have been classed as carcinomas, angiosarcomas, and alveolar sarcomas. The writer in I9I4 finds that tumors derived from the endothelium and reticulum cells of lymph-nodes form separate well-defined and rather frequent groups of tumors.

In the stomach Hansemann maintained that many of the so-called scirrhous cancers are derived from endothelial cells of the lymph spaces, a view which Borst accepts. Yet this interpretation is open to question and the existence of a true endothelioma of the gastro-intestinal tract remains unproved, if not improbable. Likewise the existence of an endothelioma of the cervix uteri is claimed by Amann, Hurdon, Kirschgessner, and denied by Sperber, R. Meyer, and others.

In the kidney a group of vascular tumors became generally recognized as probable endotheliomas. The common angiosarcoma of this organ was interpreted by de Paoli as a derivative of perithelial cells, but the exact nature of these tumors and their common origin has not yet been determined. Most of them are probably derived from adrenal rests or represent renal adenomas. In 1896 Hansemann claimed to have traced the origin of a vascular renal tumor to the lining endothelium of a small and originally angiomatous growth, but it has remained practically impossible to separate from the numerous renal tumors any which may safely be referred to this origin.

From time to time specific terms have been introduced to designate structural types of endothelioma or of tumors so interpreted. In 1856 Billroth described under the term cylindroma a tumor composed of glassy translucent cylinders of hyaline connective tissue between which ran a network of tumorcells. This structure, which is regarded by some authors as a form of interfascicular endothelioma, is still often designated as cylindroma.

v. Ohlen in 1893 pointed out that many authors had been recognizing a second form of cylindroma arising by degeneration of groups of cells in endothelial masses and the formation of cylinders of mucinous material. This structure likewise often received the designation of cylindroma, but it is doubtful if it is ever produced by endothelioma.

For many years the term "angiosarcoma" was loosely applied to certain endotheliomas. This term was first employed by Waldeyer and Kolaczek to designate all tumors originating from blood- and lymph-vessels, and thus it came to include those developing from endothelial cells lining the vessels. 
With the recognition that Eberth's perithelial cells, described originally for the cerebral vessels, exist also in many other organs, as testicle, adrenal, breast, and salivary glands (Arnold, Paltauf, Luschka, v. Brunn), and that tumors probably arise from these cells, a specific variety of angiosarcoma was admitted under the term "perithelioma." This term was especially recommended by Hildebrandt. It has remained firmly established in the nomenclature, but its relation to endothelioma has not yet been finally determined. According to Borrmann, perithelial cells are modified endothelial cells of adventitial lymph spaces. Many vessels possess not merely lymph spaces and perithelium, but also a definite plexus of lymph-vessels from which Borrmann believes tumors arise. He would, therefore, distinguish between perithelioma and peri-endothelioma, the cells of the former radiating from, those of the latter encircling the vessel. In any case perithelioma exhibits sarcomatous, not endotheliomatous characters, and in the writer's opinion this tumor should be grouped with the sarcomas (Barth, Hippel). Thus the old class of angiosarcomas is today resolved into perithelioma, periendothelioma, and the various forms of endotheliomas arising from bloodand lymph-vessels. The present tendency is to limit the term "angiosarcoma" to cellular angiomas in which the unit is the vessel and not the endothelial cell.

The scope of the group of endothelioma as commonly accepted, especially in Germany, reached its acme with the appearance of Volkmann's study in which he endeavored to establish the endothelial origin of the mixed tumors of the salivary glands. In 1897 Borst reviewed in great detail the behavior of endothelial cells under physiological and pathological conditions with a result favorable on the whole to the probable wide extent of endothelial tumors. In French the tendency to recognize endothelioma as of frequent occurrence was much less pronounced (Malassez, Berget, Collet). In recent years a rigid critique has emphasized the fact that comparatively few of the recognized groups are supported by satisfactory data. In I899 Hinsberg actively contested the endothelial origin of the salivary tumors and he has been supported by Ribbert, Marchand, and others, so that the fibro-epithelial nature of this group has become generally accepted. Lubarsch also has demolished the diagnosis of endothelioma in several types of tumors, including Mulert's multiple endothelioma of scalp, a so-called endothelioma of the orbit which he derived from the lacrimal gland, and a cylindromatous epithelioma of the nasal mucosa. Ribbert does not accept the endothelial origin of various cutaneous growths, cylindromas, and tumors of parotid. The so-called renal endothelioma he thinks is of epithelial origin, while the endothelial origin of bone-tumors, as in Sternberg's case, is not proved. No tumor of perithelial cells, he believes, has yet been demonstrated.

Thus while the existence of certain distinct classes and many well-defined forms of endothelioma was early demonstrated, the attempt to enlarge the scope to this group of tumors by assuming an endothelial origin for many growths of uncertain nature has met with only partial success. The studies of recent years have served chiefly to emphasize the difficulty of separating true endothelioma from many typical sarcomas, lymphosarcomas, carcinomas, and certain embryonal tumors.

In this dilemma one may follow Ribbert's plan of discriminating rigidly against the tendency to discover endothelial qualities in many tumors of uncertain origin. In that case the discussion of endothelioma becomes comparatively brief. Or one may accept Borst's conception that the scope of endothelioma is probably very wide, and one may thus give full expression to the views of many who favor the endothelial origin of a very wide variety of tumors whose exact nature has not been determined. While fully admit- 
ting the deficiencies of present knowledge, my own experience in this field inclines me to pursue the latter course.

Classification and Nomenclature.-Various plans of classification of endotheliomas have been suggested. Hansemann at one time recommended the elimination of the term "endothelioma" and the designation of these tumors as endothelial sarcoma, carcinoma, adenoma, etc. Ackermann classified the tumors according to their origin as intravascular, lymphangiomatous, and interfascicular (from lymph spaces). Many recognize perithelioma as a separate variety derived from blood-vessels.

Borrmann has constructed an ideal histogenetic scheme including hemangio-endothelioma, lymphangio-endothelioma, capillary endothelioma, perithelioma, and peri-endothelioma. Of capillary endothelioma, with proliferating capillaries filled with endothelial cells, he finds only 3 recorded cases (Langhans, Limacher (Case II), Borrmann). Peri-endothelioma refers to a growth of cells of perivascular lymph-vessels concentrically about the vessel wall. In a case described by Perthes he detects a combination of perithelioma and peri-endothelioma. However desirable such histogenetic distinctions may be, the practical difficulty of unraveling them has stood in the way of general adoption of this scheme. Borrmann's capillary endothelioma is generally, and I think, better designated as an angioma.

The writer agrees with Borst that the most serviceable grouping of endotheliomas is based on their morphology and he has long employed the following plan:

Histological Types of Endothelioma.-I. Interfascicular.-The cells grow in thin layers between strands of connective tissue. When these strands are swollen and hyaline the tumor may be designated as cylindroma.

2. Alveolar.-The cells grow in small or large groups, as in adenoma. When appearing in long sections the groups may be tubular. When mucinous degeneration occurs in these cell groups a variety of cylindroma is produced, but the use of this term for such structures leads to confusion and is inadvisable.

3. Plexiform.-The cells grow in convoluted columns often surrounding vascular paths. Papillary projections may arise from these columns suggesting the term "papillary endothelioma." This structure is represented by several tumors of doubtful origin.

4. Perivascular.-The cells surround definite vascular paths usually in concentric fashion, as in the dura mater. Psammoma, or sand tumor, applies to this growth when the units are calcified. Osteo-endothelioma forms rarely in the same manner (Perthes).

5. Diffuse.-The cells grow diffusely or without definite arrangement in uniform relations.

6. Miscellaneous changes in the stroma, progressive and regressive, are readily indicated by appropriate terms, as osteo-, myxo-, fibro-, cysto-, etc.

Perithelioma, whatever its origin, is a highly characteristic structure which exhibits the qualities of sarcoma or carcinoma, with which groups it should be classed.

Origin and Growth.-The exact origin of endotheliomas must be referred in general to the various endothelial cells, but the earliest stages of growth are rarely observed. While the absence of blood in any of the tumor spaces distinctly favors a lymphatic origin, its presence is of less significance, since abnormal communications form between the channels of tumors and the blood-vessels.

According to Borrmann, true hemendothelioma is extremely rare and he accepts only a few cases, some of which were traced to capillary endothelium. 
The endotheliomas of pleura and pericardium have been referred both to the lining cells and to the cells of superficial lymphatics. It seems probable that both elements give rise to tumors which differ somewhat in structure.

The rare and doubtful ovarian endotheliomas have been referred to the perivascular lymphatics. While Rosthorn traced capillary blood-vessels into the masses of tumor-cells, he was uncertain to what extent the lining cells of the vessels participated in the process. For the perivascular tumors an origin from the lymphatic endothelium lying outside of the lining cells must be assumed. Endothelioma of lymph-nodes arises from the lining cells of lymph and cavernous sinuses.

Thus in the great majority of cases it is the endothelium of small vessels or lymph spaces which gives origin to endothelioma. Many authors have claimed that they could trace tumors to more than one form of endothelial structure, as lymph spaces and lymph-vessels, or serous lining cells and endothelium of lymphatics (Volkmann), lymph spaces, and lining cells of bloodvessels (v. Ohlen, Franke). I do not find that the data presented by these authors is at all adequate for their conclusions, while some of them were probably not dealing with genuine endothelioma.

From this chief source the growth proceeds either by extension of the cells derived from the original territory or by the continuous transformation of adjoining normal cells into tumor-cells. Ribbert and Borrmann maintain that endotheliomas grow exclusively from their own resources. In various endotheliomas Borrmann finds that the proliferating cells of lymph capillaries and spaces distend and enlarge these structures and eventually break

r into other spaces, but they do not produce new capillaries and "contact infection" does not occur. On the other hand, Best and others describe the gradual involvement of normal endothelium in the extension of the tumor process. He finds this evidence chiefly along the growing edges of larger tumors, while in the very early stages of small growths the increase seems to be exclusively in the tumor-cells. The multiplicity of many endotheliomas renders the decision in this question unusually difficult. In certain tumors of the peritoneum Neelson and Glockner find a progressive involvement of neighboring lymphatics, the lining cells of which actively proliferate, producing a condition described as lymphangitis carcinomatosa (Schottelius) or prolifera (Schweninger). Lubarsch saw this process extend from the peritoneum into the liver. It has been held that secondary tumors may arise in this way without the transportation of any tumor-cells (Schulz, Neelson).

In many instances endothelioma appears to invade the tissues chiefly or exclusively through preëxisting channels, as lymph-vessels and spaces, and fails to split up the tissues as do carcinoma and epithelioma. This feature was especially notable in an endothelioma of the peritoneum studied by Wichern.

The growth of endotheliomas is usually slow but progressive, and in this respect they are comparatively benign. Especially in comparison with the forms of carcinoma and sarcoma which they resemble histologically, the course of endotheliomas is relatively favorable. When undisturbed by the knife the growth capacities of some of these tumors seem to be confined within certain limits which do not apply to carcinoma and sarcoma. In the dura very slow growth characterizes most of the perivascular tumors and pressure symptoms are often long delayed. On account probably of their infiltrating character thorough extirpation is often difficult, so that endotheliomas are notable for persistent local recurrence. Each recurrence is apt to show increasing capacity for growth and changes in structure. This fact 
encourages complete removal of the organ involved and not merely extirpation of the tumor.

Extension to the neighboring lymph-nodes is also late or entirely missing. Yet pleural endothelioma has invaded the lung and pulmonary nodes. As an exception to the rule certain endotheliomas of lymph-nodes described by Zahn, Volkmann, and the writer were rather malignant.

Hemangio-endothelioma.-Many authors have endeavored to emphasize and define a distinct group of tumors which they designate as above (Borrmann, Limacher, Borst).

Among the tumors commonly included in this group are certain growths of the type of angioma, but exhibiting an excess of endothelial cells, and certain well-known cases are specifically included in the group by the above authors (Borrmann, Lit.). Yet Nauwerck's case was designated by the authors as a simple hyperplastic telangiectasis. Langhans described a multiple

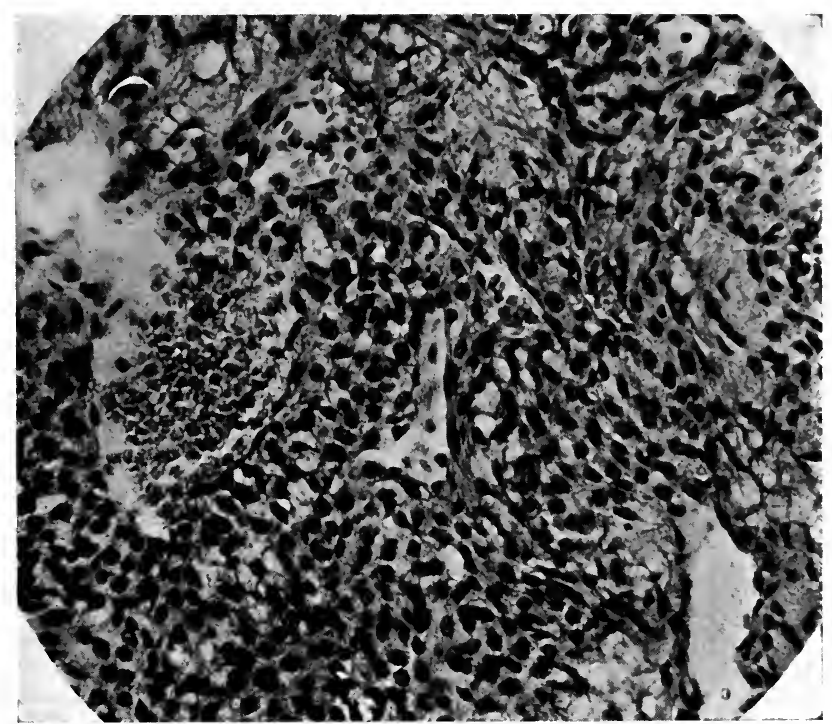

FIG. 107.-Angio-endothelioma about tendon sheath. A small, slowly growing tumor

pulsating cavernous angioma of the spleen. Borrmann's case seems to fall in the group of simple angiomas with moderate excess of cells, while Steudener describes a rapidly growing ulcerating tumor of the forehead which he thought resembled carcinoma. None of these tumors seems to deserve a special classification apart from angioma or perithelioma.

A second group includes the rare and somewhat obscure intravascular endothelioma of corpus cavernosum which cannot be classed with angioma. In several small encapsulated slowly growing subcutaneous tumors I have been able to trace the abundant cells to proliferating endothelium of dilated varicose veins. The circulation appeared to have been previously obstructed. The endothelium grows first in broad papillary masses which later become fused and enlarged, distending the vessel wall. The endothelial characters of the cells were maintained, but the nuclei became enlarged and hyperchromatic. Schlagenhaufer has described such tumors arising in hemorrhoidal veins. 
In the bones, while Narath's pulsating tumor of the tibia belongs with cellular angiomas, Marckwald's remarkable multiple tumor of the bones may well be designated as an endothelioma of vascular origin.

In the testis many authors have described as intravascular endothelioma tumors which in all probability are derivatives of teratomas and do not merit classification with any form of endothelioma. Of the old angiosarcomas most authors are agreed that none can be regarded as hemangio-endothelioma, and Borst would discard the term "angiosarcoma" as misleading.

Thus on analysis it appears that the various tumors which at one time or another have led various authors to employ the term "hemangio-endothelioma" fall readily into other groups and that this term has a very limited application and would include certain rare tumors as follows: (I) The doubtful endothelioma of corpus cavernosum; (2) Marckwald's multiple endo-

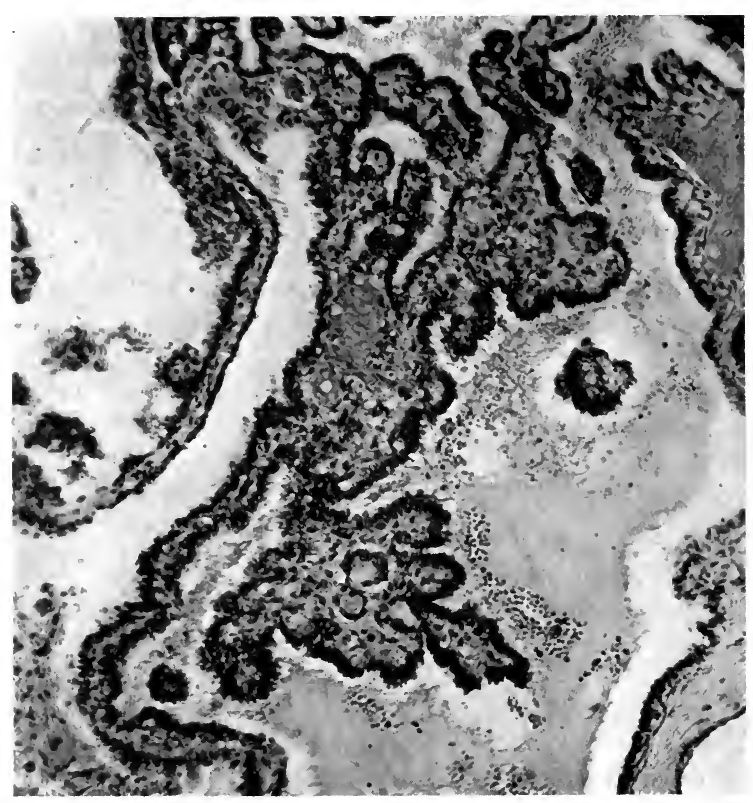

FIG. I08.-A type of papillary proliferation of endothelium arising in long distended lymphatics.

thelioma of bones; (3) a fibrocellular tumor of the thyroid described by Limacher in which the vessels were too scanty and the cells too numerous for angioma and in which many endothelial cells had become disseminated from the vessels (in the ovary somewhat similar endotheliomas may possibly occur); (4) a slowly growing tumor of the skin in which a moderate number of blood-ressels are lined by many layers of endothelium; (5) an intravascular endothelioma arising in hemorrhoidal and other dilated veins.

Gross Anatomy.- The gross appearances of endotheliomas are quite as varied as their other characters. Solid circumscribed slowly growing tumors occur regularly in the dura. In the serous membranes the growth begins as multiple flat or nodular outgrowths, which coalesce and extend gradually over a wide area of the membrane, producing thickenings and adhesions. In the pleura the entire surface of both sides may be diffusely 
thickened and the cavities obliterated. In the bones endothelioma tends to replace preëxisting tissues with little or no increase in the size of the bone, but with perforations. In the skin endothelioma is said to take the form of large single tumors. In the ovary cystic endothelioma is said to occur.

In the lymph-nodes endothelioma produces solid tumors which may reach considerable dimensions. They usually involve more than one node and some become systemic. They are often mistaken for tuberculous nodes at first, later for carcinoma. Rarely they are cystic.

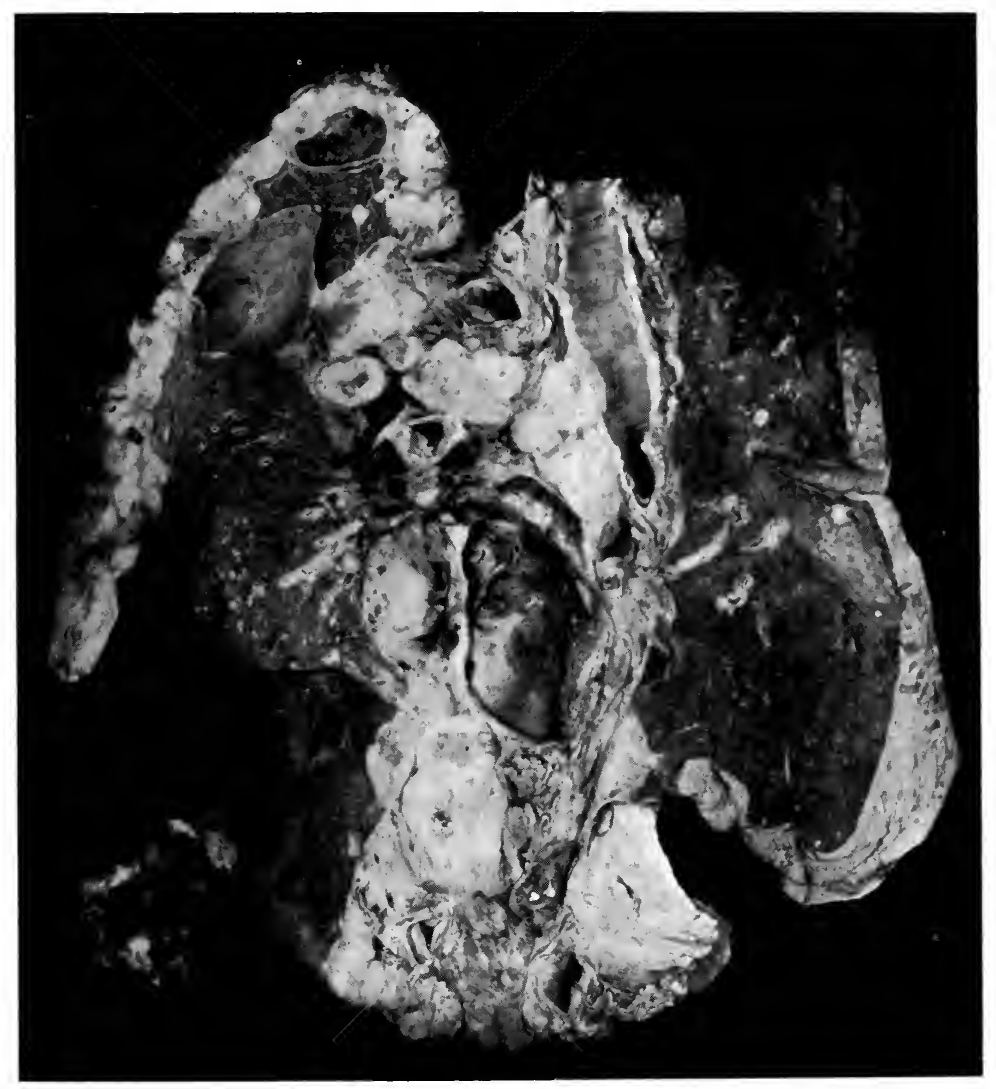

FIG. I09.-Extensive endothelioma of pleura with hydrothorax.

The various forms of secondary changes may chiefly determine the gross appearance of the tumor.

Multiplirity is one of the most constant characters of endothelioma. In the dura there may be a score of separate nodules. In the serous membranes the tumors begin as multiple coalescing nodules. In the spleen Weichselbaum observed a notable form of multiple endothelioma or cellular angioma. In the bones many have classed multiple myeloma with endothelioma (Zahn) and a remarkable case of multiple enclothelioma involving nearly all the bones is recorded by Marckwald. 
Structure of Endothelioma.-The endothelial cell in tumors usually retains some of its distinguishing features on which alone the recognition of the nature of the growth may often be based. The form is polyhedral, often pavement in type, and occasionally cylindrical. Under pressure it assumes a spindle form and in edematous tissues it swells to spheroidal form and considerable dimensions.

A well-defined cell-membrane, relatively clear cytoplasm, small pale vesicular nucleus with minute multiple nucleoli are features so frequently exhibited as to render them valuable diagnostic aids which are too often neglected. Especially in separating endothelioma from epithelioma these characters are of value, since the epithelial cell usually exhibits a granular opaque cytoplasm, prominent nuclei, and bulky acidophile nucleoli. Yet in some malignant tumors the endothelial characters of the cells are lost and embryonal epithelium resembles endothelium. In certain endotheliomas the cells tend to become flattened and spindle shaped and to form compact masses in which hyaline intercellular substance appears in increasing amount until finally the tumor is largely transformed into hyaline fibrous tissue. This process reveals the natural termination of old endotheliomas of the dura. It is sometimes assumed that we have to deal with the production of intercellular fibrils by endothelial cells and with the conversion of endothelial tissue into connective. Yet it appears more probable that the fibrils represent elongated and hyaline cell bodies and that the resulting hyaline mass represents the gradual conversion of the compressed cells into homo-

r geneous material. Definite formation of intercellular fibrils I have been unable to detect in endothelioma. In dural endothelioma their absence is conspicuous.

In various tissues normal endothelial cells show morphological differences which dominate the tumors arising in these tissues. Serous endothelium is notably flat, bulky, and pavement in type, and these features long persist in tumors of the peritoneum and pleura. Giant-cells of many forms have been encountered in various endotheliomas, but their occurrence is comparatively rare (Glockner, Lit.).

Syncytial masses with scattered nuclei were observed in cylindroma by Sattler. In 4 pleural or peritoneal endotheliomas Glockner found mononuclear and polynuclear giant-cells, in one case so numerous as to dominate the picture. In an abdominal endothelioma which he derived from the lymph-nodes Glockner found very large giant-cells measuring up to ${ }^{7} 6$ micra. In an endothelioma of the pleura Brosch describes giant-cells resembling the Langhans type in tubercle, and I have seen such cells in an endothelioma of the scalp. In dural tumors multinuclear giant-cells are not infrequent.

Retained secretion affects the appearance of endothelioma, and endothelial tumors of lymph-nodes may be cystic. Extensive pigmentation occurred in a peculiar tumor with numerous metastases which Rindfleisch interpreted as an endothelial myeloma. In an endothelial tumor of the sole of the foot attached to the bone I found considerable pigment evidently derived from extravasated blood. Secondary changes in the cells and stroma greatly alter or dominate the appearance of many of these tumors. Fatty degeneration is not prominent, but in a case of Ritter's it was quite pronounced. Glycogenic degeneration has been noted in many cases of actively growing endothelioma (Driessen, Volkmann). Accordingly to Best its abundance may distinguish endothelioma as a class from sarcoma.

Hyaline degeneration frequently affects the cells, stroma, and vessels of endothelioma. Involving the stroma of interfascicular growths it pro- 
duces the appearance of cylindroma. In psammoma it is associated with calcification of the concentric groups of cells and produces the sand grains. Other dural tumors may be rendered quiescent by nearly complete hyalinosis. In a slowly growing angio-endothelioma of dura I found the walls of all vessels thick and hyaline and many occluded by hyaline material, while the cells were intact.

In the true endothelioma mucinous degeneration is of rare occurrence, but is observed in certain tumors of serous membranes, and in the group of ovarian endothelioma, in both of which it may become extensive, with the formation of mucinous cysts.

Calcification overtakes many slowly growing endotheliomas, especially the perivascular and psammomatous tumors of the dura. The deposits appear in the cells, stroma, and walls of blood-vessels. In a subcutaneous tumor of the foot Perthes found extensive calcification leading eventually to ossification. In a remarkable form of endothelioma of the orbit I found very extensive deposits of calcific nodules producing a form of osteoid tissue.

The various arrangements of the cells in endotheliomas yield characteristic structures which form the basis of the plan of classification previously given. These classes by no means exhaust the architectural peculiarities and, as in carcinoma, combinations of several types may occur in the same tumor. Likewise, epithelioma may be simulated not only by the morphology of the cells, but by the formation of pearls with hyaline centers. Prickle cells and keratohyalin granules, however, are absent in the endothelial structures. The structure of sarcoma is simulated by many spindle-cell endotheliomas and in this group it becomes desirable to recognize as types of sarcoma certain tumors whose cells of origin may be ultimately of endothelial nature, although modified by location and function.

Vascular endothelioma tends to remain of smaller size and to assume cuboidal or cylindrical rather than pavement types. From the lymph spaces develop tumors of more embryonal type with cells lacking the above-mentioned adult endothelial characters. These cells are small with opaque granular cytoplasm and very intimate connections with the tissue stroma. From certain specialized derivatives of endothelial cells, as perithelium, and the germ center cells of lymph-nodes, the tumors are almost wholly lacking in endothelial qualities and constitute subvarieties of sarcoma.

An intimate relation to the supporting stroma is an important structural peculiarity which distinguishes many endotheliomas from epithelial tumors. The cells cling to the walls of spaces in spite of the shrinkage of hardening and the cell bodies may seem to pass insensibly into the substance of the stroma. Small fibroblasts and fine fibrils may ramify between the endothelial cells. Yet it has repeatedly been shown that these same peculiarities may be present in basal cell epithelioma and that they alone do not warrant the diagnosis of endothelioma.

Combination of Endothelioma and Sarcoma.-In some spindle-cell sarcomas there is evidence that endothelial cells are commingled with the main tumor element, but they lose their endothelial characters among the derivatives of fibroblasts. Borst mentions the occurrence of large spindlecell sarcomas in which are well-defined groups of neoplastic endothelial cells which may resemble carcinomatous alveoli except for the intimate relation with the sarcoma-cells. I have seen such appearances in teratomas. He also describes angiomatous spindle-cell sarcomas in which the numerous vessels are lined by proliferating cubical endothelium recalling gland alveoli. In lymphangio-endothelioma the stroma may be very cellular, or one part 
of an angioma may be very vascular and another part chiefly composed of cellular stroma.

Differential Diagnosis of Endothelioma.-Although there have been numerous attempts to establish differential diagnostic signs between endothelioma and other tumors, it must be admitted with Lubarsch that reliable criteria of general application are lacking. On this account the personal impressions of the individual observer have figured too prominently in the steady expansion of this group of tumors.

The most reliable sign of endothelioma is found in the minute characters of the cells as above described, but these characters are lost in many actively growing tumors and in recurrences. Yet a thorough search for the translucent polyhedral cells with pale nuclei devoid of acidophile nucleoli is often rewarded in tumors the bulk of which fails to show such cell types. Especially in edematous foci the pavement characters reassert themselves.

The concentric perivascular arrangement is a convincing appearance, but is limited chiefly to dural growths. All other arrangements of the cells exhibited by endothelioma are duplicated by other tumors.

The occurrence of endothelial pearls with hyaline or calcified centers, but without prickle cells and keratohyalin granules, as emphasized by many, stands in the opinion of Lubarsch and Ribbert a strong indication against endothelioma. Prickle cells and specific granules are often absent in epithelioma, and with the exception of psammoma I have never seen definite pearl formation in any undoubted endothelioma.

The relation of tumor-cells directly walling circulatory paths is a convincing feature of some endotheliomas, but it rarely exists unless the characters of the cells are even more indicative of endothelioma. The identification of lymph paths is usually difficult, while endothelioma of blood-vessels is rare. It must be recognized also that the presence of a column of blood in immediate contact with tumor-cells is not a certain criterion of endothelioma. Hemorrhage often fills the alveoli of carcinoma, and in epithelioma of the skin blood sinuses invade the epithelial cell groups, lose their endothelial lining, and the blood lies in immediate contact with cuboidal epithelial cells.

The intimate relation of the cells to the supporting stroma is regarded by Borst and many others as a most important criterion. In soft tissues the endothelial cells are said to grow out like young capillaries. Yet this sign led to error in the case of the spurious endotheliomas of the parotid.

The desmoplastic properties of many endotheliomas assist in their recognition, and a peculiar form of hyalinosis may sharpen this feature, as in cylindroma. On the edges of endothelioma the tumor-cells may fade in the advancing fibrosis in a manner quite foreign to carcinoma. Fine fibroblasts and even capillaries may grow out between the cells of alveoli both in endothelioma and carcinoma. The structure of cylindroma, especially that form resulting from mucous degeneration in cellular alveoli, is not distinctive of endothelioma, since hyaline and mucous degeneration of cell groups, stroma, and vessels often occurs in carcinomas (Lubarsch, Schmidt).

On the growing edges the tumor-cells are said to pass insensibly into the endothelium of lymph spaces (Volkmann, Hansemann), but this appearance occurs also in carcinoma (Ribbert, Borst). Proliferation of endothelium of vessels invaded by tumor-cells occurs both in endothelioma and carcinoma, but rather more in the former.

The location of the tumor may furnish important evidence, as when multiple endothelioma develops in lymph-nodes, bone-marrow, or in any tissue not containing epithelium. Yet unless the sources of primary tumors 
are carefully eliminated this evidence is worthless, and there remains the possibility of aberrant tissue rests.

Finally, it should be urged that the diagnosis of endothelioma should be accepted only when the evidence is clear and conclusive. Otherwise this group will continue to represent the resting place of miscellaneous tumors on which the data and study have been inadequate.

Etiology.- In the etiology of endothelioma the influence of chronic irritation or trauma and low grades of inflammation must be given a prominent place. In a few instances it has been possible clinically to establish the existence of such factors. Endothelioma of lymph-nodes appears to follow forms of chronic lymphadenitis. In the skin chronic trauma is abundantly provided, especially in the sole of the foot. In the serous membranes endothelioma in some cases appears to be connected with tuberculosis. As a rule, however, the tumor appears to develop spontaneously. On the other hand, it is necessary to assume a local predisposition to tumor growth, and in certain cases efforts have been made to establish a relation with definite embryogenic disturbances. It cannot be said that much success has attended these efforts. Many endotheliomas first appear in small encapsulated areas suggesting an embryonal isolation of the originating tissue. Eberth and Spude have described a small teratoid endothelioma of the dura mater. Ribbert suggests that endothelioma of the dura arises from groups of cells misplaced during the separation of the original membrane into pia and dura. Schmidt has described such collections of superfluous endothelial cells in the normal dura. I have observed a small perfectly isolated endothelioma embedded in the tissues of the spermatic cord. Yet the great majority of endotheliomas fail to give any tangible evidence of a relation to embryogenic disturbance.

The multiple origin of tumors appearing successively over many years (Marckwald), the genesis from minute lymph spaces and vessels, and the slow growth, all indicate that these tumors usually develop from adult endothelium under the influence of chronic irritation.

\section{CLINICAL TYPES OF ENDOTHELIOMA}

A. Tumor-like Hyperplasia of Endothelium.-Under certain conditions endothelium exhibits a grade of hyperplasia which is intermediate between inflammatory and neoplastic overgrowth.

I. In chronic lymphadenitis a peculiar form of endothelial hyperplasia may occur which exhibits some of the morphological and clinical features of a neoplasm. These cases occur in the group of chronic enlargements of the lymph-nodes of uncertain origin and they pursue the course of Hodgkin's disease, but specific Hodgkin's structure and specific signs of tuberculosis are missing.

Microscopically one finds irregular foci and broad sheets of large flat endothelial cells, obliterating the normal structure, grading insensibly into lymphoid tissues, and apparently originating in the sinus endothelium. The cells are slightly granular, clearly outlined, polyhedral or elongated, with large vesicular hyperchromatic nuclei. The appearance is quite different from that of the large cell hyperplasia of tuberculosis or of the common Hodgkin's structure and bears the stamp of a feeble neoplastic process.

2. Primary Splenomegaly (Type, Gaucher).-This remarkable condition consists of an extensive chronic overgrowth of endothelial cells of the spleen, lymph-nodes, and bone-marrow, with secondary foci in the portal canals of the liver, and is associated with anemia, hemorrhages, and pigmentation 
of skin and other organs. It begins in early life, chiefly in females, commonly affects more than one member of a single generation in the family, and progresses over many years (39 in Schlagenhaufer's case), proving fatal or terminated by complications. The spleen reaches enormous size ( 6250 gms. Bovaird) and the liver is greatly enlarged, while the lymph-nodes are much less prominent. The pigmentation affects skin, spleen, lymph-nodes, and smooth muscletissues.

Microscopically the splenic structure varies in different stages. It begins with the appearance of many large clear polyhedral cells lying loosely in the pulp tissue occluding and replacing sinuses, cords, and finally the Malphigian bodies. Scattered lymphocytes long persist, but eventually disappear. At the acme of the process, as in Bovaird's case, the tissue is

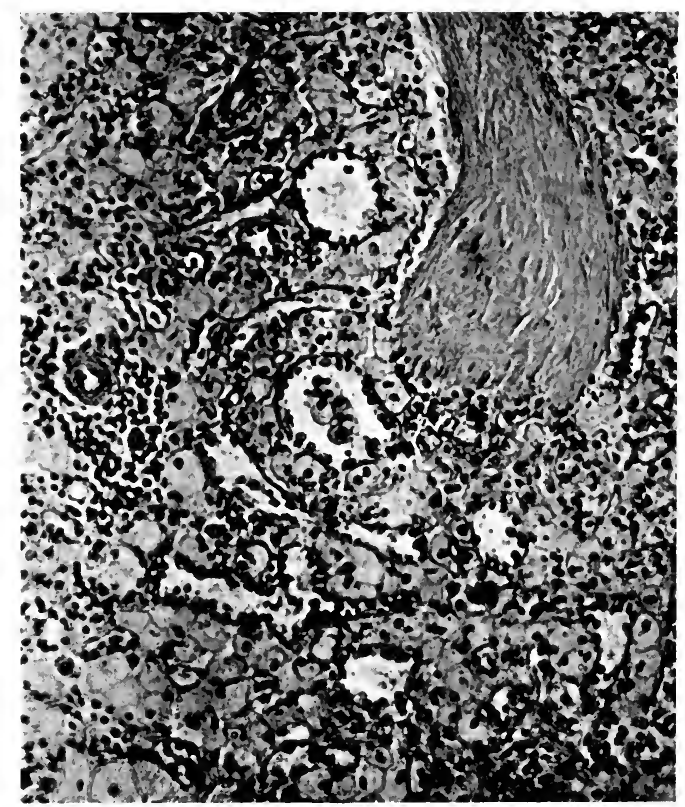

FIG. I I0.- Structure of splenic lesion in Gaucher's splenomegaly. Note that the large cells lie within the cords and do not line the splenic sinuses.

composed almost exclusively of closely packed, very large, clear polyhedral cells forming diffuse masses or large alveoli. Occasional mitoses may be observed and it is evident that the cells are not only well nourished, but possess considerable powers of growth. Yet the cells lack the hyperchromatic nuclei of tumor-cells and they carefully respect anatomical barriers. Necrosis is rare, but occurred in Schlagenhaufer's case. In the late stages a slow fibrosis overtakes the process and the hyperplastic cells fade into a form of hyaline or fibrillated tissue. In the lymph-nodes and marrow much the same process is observed, beginning in the sinuses. In the liver small foci of the large cells appear in the lymphatics of the portal canals.

From the study of 6 cases (Bovaird, Brill, 4 from New York Hospital) I am satisfied that the hyperplastic cells appear first within the pulp cords, that they are derived from the lining cells of the splenic sinuses is uncertain, 
that the process originates secondarily in the lymph-nodes and marrow, and that the hyperplasia falls below that of a true neoplasm. Whether the process in the liver arises in that organ or results from cell emboli appears uncertain.

In the etiology a chronic irritant affecting especially the lymphoid organs must be assumed to exist. Many features suggest an atypical tuberculosis. In 2 cases (Gaucher, Schlagenhaufer) tuberculous lesions coexisted. In Collier's case there was a caseous mass in the mediastinum, while in other cases tuberculosis was not eliminated. Lutz found an early stage of the process in diabetic lipemia.

It has recently been shown by Anitschkow that a very similar condition of spleen and bone-marrow may be produced experimentally in animals by feeding large amounts of cholesterin. The material in the cells does not give the staining reactions of myelin bodies, lipoids or fats, nor does it react to Weigert's myelin stain. Yet it is probably a peculiar lipoid '(Schlagenhaufer, Lutz).

B. Endothelioma.- - I. Endothelioma of Meninges.-This common tumor appears in two main forms: (I) As a perivascular endothelioma, and (2) as psammoma in which small perivascular units undergo hyalinosis and calcification. The tumors are single and as large as a hen's egg, or multiple and of miliary dimensions. They first appear as flat elevations on the inner side of the dura which slowly enlarge with corresponding displacement or atrophy of brain substance. They show little tendency to form adhesions, but may be surrounded by a vascular capsule. An infiltration between the thickened layers of the dura may occur. They are usually very firm in consistence, gray in color, with nodular surface or lobulated structure. Strands of connective tissue from the dura may be drawn into the growth separating lobules of endothelial tissue. The psammomas are extremely hard, usually of moderate dimensions, and the sand grains are visible in the gross. Occasionally the tumor becomes pedunculated. On gross inspection the concentric units may usually be discerned, but old hyaline forms exhibit a smooth, glassy luster.

The chief seats are the dura over the convexity, in the falx and tentorium, and along the basal vessels, and they may develop over the medulla, and down to the cauda. The cerebral tumors have been discussed by many authors, while the less known spinal tumors were described in detail by Eppinger. Schlesinger collected I8 spinal psammomas located chiefly in the central and lower segment. Hippel found a large cellular psammoma in the cerebellum and multiple calcified nodules throughout the spinal meninges. Small tumors are said to arise from the pia (Borst), and atypical forms have been observed in the ventricles arising from ependyma. The predisposition of the dura may be associated with the frequent occurrence of "sand grains" on its inner surface producing the condition called by Virchow meningitis arenosa. Virchow observed several cases in connection with exostoses. Henschen describes multiple endothelioma of the spinal dura associated with tuberculous pachymeningitis.

The structure is quite characteristic and the endothelial features are so obvious that this tumor was the first to receive the designation of endothelioma (Golgi, r869). The tissue is composed of systems of cells concentrically arranged about a central lumen, closed or patent, which represents a rudimentary vessel. These units may be very small, numerous and sharply defined, or large and ill defined. Considerable areas may be composed of diffuse cells without concentric arrangement, or the masses may be plexiform and convoluted. Borst in one case saw a uniform thickening of the 
meshes of subarachnoidal tissue by cells lying loosely within these strands. The cells are polyhedral or of elongated spindle form with the usual endothelial characters. In some very cellular forms the cell borders are less distinct and the nuclei hyperchromatic.

The origin of the cells has been referred chiefly to the endothelium of perivascular lymph spaces and to perithelium of proliferating vessels, while Engert regards them as derivatives of the lining cells of the dura. On the other hand, M. B. Schmidt has made it appear very probable that most or all of the endothelial tumors of the dura are derived from groups of endothelial cells which he finds in a large proportion of adults lying in the meshes of the pia, near small blood-vessels, and especially in connection with Pacchionian granulations.

In a notable case described by Lindner with multiple tumors of dura and similar growths in bladder and urethra, the cell masses inclosed spaces communicating with blood-vessels and containing groups of nucleated red blood-

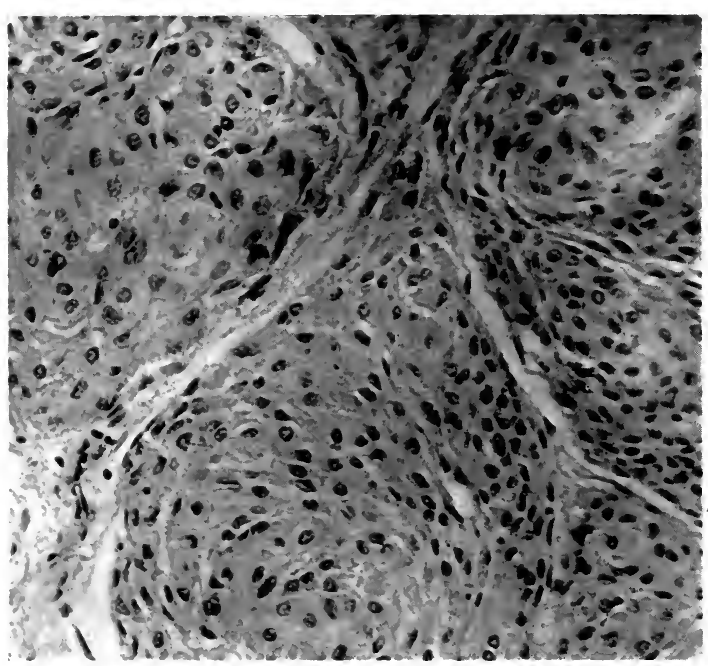

FIG. III.-Endothelioma of dura mater.

cells. According to Albrecht's bizarre interpretation of this case the tumor was derived from vasoformative cells which retained their capacity for red-cell formation.

The blood-ressels are usually scanty and are carried by the stroma derived from the dura.

Secondary changes are frequent. A form of fibrosis overtakes old tumors and may transform them into dense hyaline masses on the edges of which islands of growing cells may be preserved. Here the cell bodies become fused and hyaline without the intervention of any special intercellular substance. Chronic edema may occur in small or large areas in which the cells swell to large dimensions and polyhedral form.

Hyaline degeneration and calcification produce the characteristic structure of psammoma. These processes affect chiefly the central cells of the concentric units, hyalinosis preceding calcification. Entire cell groups may be calcified and almost the entire tumor may be composed of sand grains 
with traces of concentric or irregular striation. The walls of vessels may be extensively affected, hyaline changes altering the walls and the contents, and calcification appearing in or about the walls and in the contained material. According to Ernst hyaline material may first appear as an intercellular secretion.

Numerous variations from the typical perivascular structure occur in endothelioma of the meninges. Tumors that have undergone ionsiderable fibrosis are often called fibro-endothelioma. The appearance of alveolar endothelioma is sometimes produced by cells growing in a fine network of connective tissue derived from dura or pia. Very vascular tumers occur in which the vessels seem to belong to the tumor process and these may be designated as angio-endothelioma.

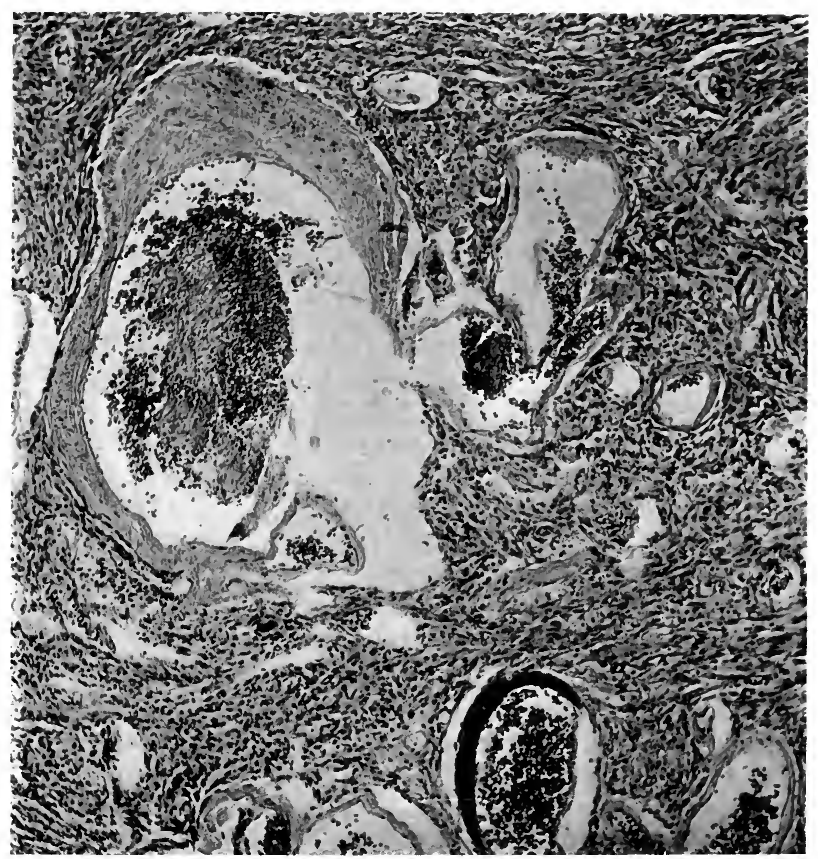

FIG. I 12.-Slowly growing angio-endothelioma of meninges of base of brain. Hyaline and calcific material forms the walls of many vessels.

In some of the vascular tumors a peculiar structure is produced by the growth of large cells in several layers covering or radiating from the vessels. They may involve the pia mater and extend down the sulci. Borst and others derive these cells from the perithelium of the blood-vessels and designate the tumors as perithelioma. Similar growths arise from the ependyma and choroid plexuses where they produce papillary structures. An endothelioma of the meninges of the dorsal cord, exhibiting the structure of perithelioma, has been reported from this laboratory by Schlapp. It was rather clearly referable to a severe trauma and while it grew rapidly, was completely eradicated by curetage. It is difficult to determine the exact position of these tumors, but in Schlapp's case the cylindrical and pavement character of the cells indicated true endothelial properties in the cells of origin. 
Very cellular growths are frequently described as sarcoma or endothelial sarcoma, but it was long ago pointed out by Newmann and by Bizzozero and Bozzolo, and lately by Zenoni, that these dural sarcomas are probably derived from the endothelial cells. Yet the careful analysis of Lens shows that true sarcoma of the meninges arises from the adventitial cells of the blood-vessels while an extensive proliferation of arachnoidal endothelium occurs as a secondary process which is not neoplastic. The true meningeal sarcomas usually take the form of angiosarcoma or large round-cell sarcoma. The clinical features of sarcoma are usually missing in the endotheliomas, which pursue a slow course without metastases or wide local extensions.

The course of dural endothelioma is slowly progressive. Pressure symptoms are usually late in appearance and many cases are first discovered at postmortem. Even the vascular growths may fail to alter this course, and I have observed an extremely vascular and very cellular diffuse endothelioma compressing the pons which progressed slowly over a period of four years.

On the other hand, pressure symptoms and recurrence after operation marked a case of Taylor's, and a small tumor between falx and convexity was found by Lunz in a case of cortical epilepsy. Henschen described a remarkable case of multiple endothelioma of the spinal dura associated with old tuberculous meningitis, while Dufour observed a somewhat similar case in the horse. Metastases are extremely rare, but Klebs found nodules in the lung in one case, and Lindner is said to have observed a metastasis in the bladder.

Psammoma of nerve-trunks has been described in a considerable group of cases; in the optic nerve (Knapp, Ernst, Tailer, Braunschweig, Lit.); or acoustic and facial (Fester); but the exact nature of some of these tumors is doubtful.

Acoustic neurofibromas form a characteristic group of intracranial tumors and are described under brain tumors.

Endothelioma of Nerve-trunks and Ganglia.-In many nerve-trunks and certain ganglia endotheliomas, usually of the perivascular type, have repeatedly been observed. In some of the common neurofibromas the tumor-cells present endothelial characters and are arranged in whorls as in plexiform endothelioma. It is difficult to distinguish such tumors from true endothelioma on the one hand and simple neurofibroma on the other.

In other tumors of nerve-trunks the structure is that of typical perivascular endothelioma as occurring in the dura mater, and psammomatous changes may appear in them.

In the orbit perivascular endothelioma arising from the sheath of the optic nerve is a well-defined condition. Of $\mathrm{I} 2$ extradural tumors of the optic nerve collected by Parsons 9 were this form of endothelioma. They are usually of slow growth, 6 to 8 years, but reach considerable size (Billroth), producing exophthalmos. They surround the nerve-trunk in conical form or are attached by a pedicle or appear disconnected from the optic nerve, in which case another origin may be assumed. The structure shows concentric masses of endothelial cells in which hyalinosis and calcification may be prominent.

Of 2 orbital tumors belonging to this group and not distinctly connected with the nerves, in I I found very extensive calcific deposits among concentric groups of cells showing very hyperchromatic nuclei and evidently malignant; in another, $\mathrm{I} \times 2 \mathrm{~cm}$., the cells in broad concentric groups were highly pavement in type. Both failed to recur.

In the extensive literature on tumors of the orbit it is clear that many 
other tumors, especially carcinoma of the lacrimal gland, have been interpreted as endothelioma. Thus in a full discussion of orbital tumors not connected with the optic nerve Parsons includes many growths of cylindromatous, peritheliomatous, and sarcomatous character, but none of clearly endotheliomatous type. Verhoef has shown that the common so-called endothelioma of the orbit is a carcinoma of the lacrimal gland. Yet it would be unwise to assume that all the suspected endotheliomas of this region are spurious. Thus Frank describes a congenital tumor attached to the periosteum of the inner wall, containing alveoli of cylindrical cells, diffuse cell masses, and vessels containing blood and lined by tumor-cells. Even here an embryonal carcinoma is difficult to eliminate. In the Gasserian ganglion Spiller describes a typical perivascular endothelioma with multiple miliary tumors of the adjacent dura.

Endothelioma of Serous Membranes.-Highly characteristic conditions are produced in the pleura and peritoneum by endothelioma of these structures and the gross and microscopical features were early recognized as the results of a specific process differing from ordinary carcinoma. There are such notable differences in the conditions produced in the pleura and in the peritoneum as to require their separate consideration.

(a) Pleural Endothelioma.-The process first appears in the form of multiple nodules or flat elevations widely distributed over one or both membranes. These nodules fuse, the layers of the pleura become thickened and adherent and converted into a diffuse firm opaque mass covering and compressing the entire lung. In Gutmann's case the tumor consisted of a limited number of nodules and distinctly papillary masses, and the pleura was only slightly thickened. The costal pleura only may be affected (Scagliosi). A serofibrinous or bloody exudate commonly accompanies the growth and the fluid gathers in small cysts or in larger collections. Invasion of the lung may be entirely missing, as in Wagner's and Benda's cases, or more often the tumor invades the septa and parenchyma producing irregular nodules and masses, and metastases appear in bronchial, axillary, cervical, and mediastinal nodes, and in the liver and spleen. Perls observed metastases in the dura and choroid, and Eberth found a second endothelioma primary in the pia. The process may extend to the peritoneum or to the pericardium (Bassoe), or from the peritoneum to the pleura (Rossier, Pollmann).

The structure in most cases is rather uniformly that of alveolar and tubular endothelioma. The cells are of moderate size, of polyhedral or flat form, with hyperchromatic vesicular nuclei and faint nucleoli. They lie in small alveoli or long single or multiple rows between cellular or hyaline connective tissue to which they are usually intimately adherent. Evidences of secretion are usually missing. Borst describes as an endothelioma of pleura a tumor containing very large cells closely resembling squamous epithelium and forming numerous pearls, but the massing of this tumor about the root of the lung and its perforation of the chest wall as well as its structure render the diagnosis uncertain.

The origin of the tumor has usually been referred to the cells of the subpleural lymph spaces (Wagner, Bostrom, Neelsen, Volkmann, Adler), which have been found affected in very early stages. Malassez, Rossier, Pollmann, and others were unable to trace the origin exactly, but were sure that the tumor did not arise from any epithelial structures. Benda and Guttmann were able to trace the tumor to the lining cells of the pleura, which several observers have found to show nodular thickenings due to proliferating endothelial plates. All agree that the process begins over a wide area. It is possible that two groups of serous endotheliomas should be recognized, one inva- 
sive, with metastases, and derived from the endothelium of lymph spaces, and another, superficial, nodular, or papillary, and originating from the lining cells.

The clinical course is that of chronic pleurisy with thickening and adhesions. Rossier especially emphasizes the inflammatory character of the process, claiming that it begins as a chronic pleurisy, and the wide extent of the growth suggests that it may signify a neoplastic process becoming established in predisposed subjects on the basis of inflammatory hyperplasia. Perls, Neelson, Glockner, and others even consider that an infectious agent is concerned in the disease and that it is not a true tumor, but a form of tumorlike lymphangitis. The multiple or diffuse origin and gradual extension over normal areas have often been observed and Neelson claims that the metastases are not caused by embolic cells, but arise de novo in the affected tissue. It may be noted that Brosch in a very early case of pleural endothelioma observed multiple nodules with caseous centers surrounded by proliferating vessels and many giant-cells of Langhans type. The resemblance to a hemorrhagic tuberculous pleurisy is mentioned by Kaufmann, and the difficulty of distinguishing the early nodules from miliary tubercles is noted by Wichern. Yet endothelioma of the pleura differs widely from the forms of hyperplastic lymphangitis and whatever its etiology the process is neoplastic. In the diagnosis Fraenkel and Kaufmann mention the importance of blood and tumor fragments in the fluid and retraction of the chest wall. The disease occurs chiefly in adults, but Hibler has observed it in a child of 5 years.

(b) Peritoneal Endothelioma.-Rokitansky (I 854) described primary cancers of the peritoneum composed of multiple translucent nodules lying under the serosa and covering many organs. Waldeyer found in primary peritoneal growths the structure of plexiform angiosarcoma and cylindroma. BirchHirschfeld (I895) distinguished clearly between primary jelly-like "angiosarcomas," which he derived from the lymphatic endothelium, and true colloid cancer, a bulky tumor which he referred to an aberrant portion of intestinal mucosa. A characteristic case of primary endothelioma with hundreds of small nodules universally distributed he pictures and describes as composed of small groups of endothelial cells which he traced to the endothelium of dilated lymph-vessels.

In I 897 Glockner collected 75 cases of endothelioma of serous membranes, pleura, peritoneum, or both, and since that time the condition has been generally recognized as the result of a specific process.

The structure of these tumors generally follows the description given by Birch-Hirschfeld and is fully presented in a recent case by Miller and Wynn. They found the nodules composed of a framework of connective tissue carrying numerous vessels and inclosing groups of large polyhedral cells with clear cytoplasm. The cell groups often contained mucinous material, and a large amount of mucilaginous ascitic fluid was present. Many small clear cells and many multinucleated giant-cells were present. The authors trace the tumor to the lining cells of the peritoneum, observing all stages of their proliferation up to the large nodules. They found intimate connections between the cells and the accompanying fine connective-tissue fibrils, and describe the fibrillation of cell processes leading to the formation of connective tissue. Hence they conclude that the tumor has sarcomatous qualities. The intestinal walls were invaded as far as the submucosa, the general peritoneum only in subserous tissue, and the lymph-nodes only in the capsular tissue.

The ascitic fluid was alkaline, specific gravity I $_{7}{ }_{7}$, when boiled solid still exuded a mucinous fluid, reduced Fehling's solution only after boiling with $\mathrm{HCl}$, and gave the reactions of albumins which precipitated on half-saturation with ammonium sulphate. 
A somewhat atypical case is described by Borst. The peritoneum was everywhere covered with nodules, plates, and papillary elevations, and numerous small and large cysts had resulted from inclosure of mucinous material not in the tumor-masses, but by adhesion of the inflamed peritoneum. The diaphragm was perforated and the pleura involved. The cells were flat, cubical, cylindrical, or syncytial. The structure was adenoid, interfascicular, papillary, and often peritheliomatous. The organs were not invaded, but the abdominal nodes were extensively infiltrated. The origin seemed to be from the subserous lymphatics, and Borst suspected that the ovarian region had first been involved.

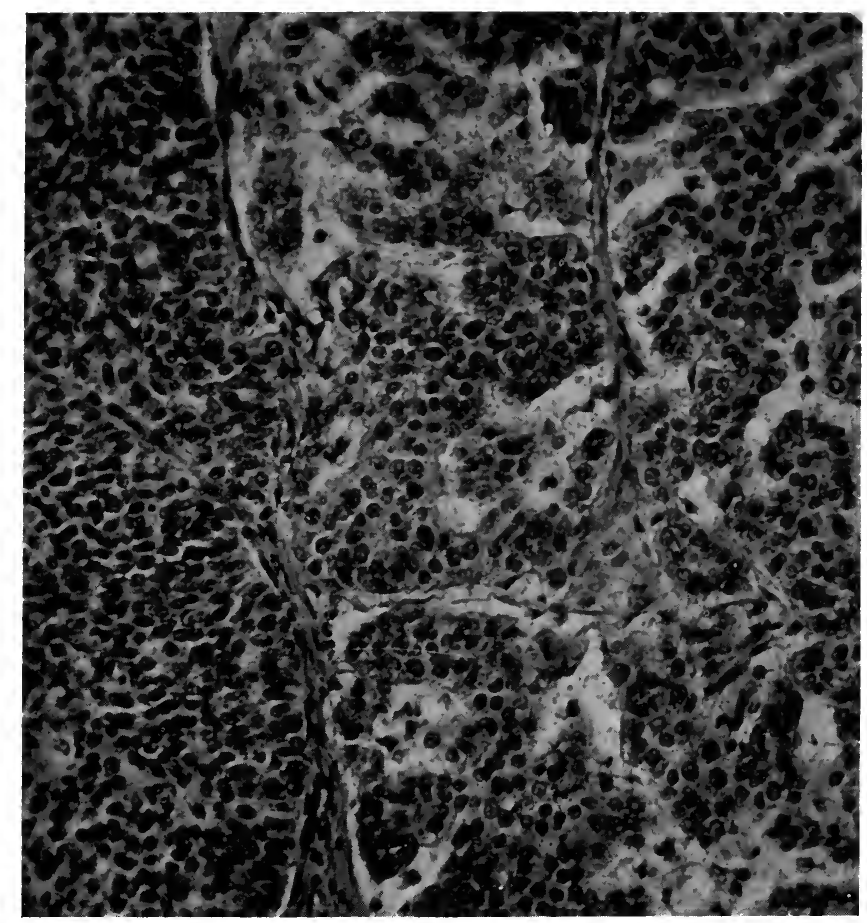

FIG. I I3.- Structure of a primary endothelioma of the peritoneum.

A still further variation from the usual type is described by Kaufmann (loc. cit., 542) who found multiple nodules, confluent plates, and flat masses of partly caseous material surrounded by strata of hyperplastic cells derived from the surface endothelium. In many alveoli there were cylindrical cells in palisade order. The diaphragm and omentum were chiefly affected, and a hemorrhagic exudate accompanied the process.

In cases reported by Nager and by Henke the tumor took the form of a multitude of miliary or larger cysts covering all the organs, causing universal adhesions, and marked thickening of the mesentery and omentum. Some of the reports of endothelioma of the peritoneum raise the suspicion that the authors are dealing with secondary carcinoma of intestinal origin in which the primary tumor has been overlooked. Especially when there is marked 
secretion of mucus the cases closely resemble the so-called pseudomyxoma peritonei which has been definitely traced to the rupture of comparatively benign ovarian cysts. This suspicion attaches to the cases of Nager and Hueter, in which the secretion of mucus was abundant, the cells cylindrical, and the structure adenocarcinomatous. Very similar conditions undoubtedly follow peritoneal dissemination of colloid carcinoma of the colon. Further studies in this field are desirable.

The proper classification of endothelioma of pleura and peritoneum still remains a somewhat fruitless matter of discussion. Ribbert insists that the lining cells of the large serous cavities are of celomic origin and epithelial nature and he describes these tumors as carcinoma. Kaufmann adheres strictly to principle in separating endothelial tumors derived from lymph spaces and carcinoma derived from lining cells, of both pleura and peritoneum. The majority of authors, the writer among them, are less impressed by embryological considerations than by the morphology and physiology of the cells and the general characters of the tumors derived from them, and they approve of the classification as endothelioma. The impression gained by Birch-Hirschfeld, Waldeyer, and Ziegler that all these tumors have sarcomatous qualities is supported by the recent observations of Miller and Wynn of connective-tissue formation by the tumor-cells. I cannot trace this process in the few cases at my disposal, but consider that other grounds fully support the grouping of these tumors among endotheliomas (cf. Monckberg).

Endothelioma of Bone.-The knowledge of endothelioma of bone has - progressed under many difficulties, so that the importance of the disease is very inadequately recognized. The chief difficulty has arisen from the fact that metastatic tumors of very variable structure frequently occur in bones, and since endothelioma is not always of highly specific appearance, it has been difficult to eliminate the possible origin from metastatic carcinoma. Carcinoma of stomach, thyroid, prostate, kidney, and adrenal may early produce bone metastasis, often as the first symptom of disease, and the structure of some of these tumors may closely resemble endothelioma.

It is further necessary to consider the possibility that embolic cells from normal organs may yield primary tumors of the bone-marrow. Gierke has collected much evidence to show that cell emboli from benign tumors of the thyroid and even from simple goiter may lodge in the bone-marrow and give rise to tumors. A certain number of tumors of bone described as endothelioma were probably of this nature (Gierke, Lit.). A similar mode of origin for certain bone endotheliomas which resemble papillary carcinoma of the kidney with clear cells has often been suggested. Pick argues that the close contact of normal adrenal cells with the capillary blood-stream, and the discovery by Stilling of buds of adrenal cells projecting into the veins in the regeneration which follows removal of the adrenal in animals, reveal abundant sources of emboli of normal adrenal cells. Yet it must be considered that such vagrant cell emboli probably soon perish.

Finally, it is possible that definite misplaced islands of thyroid or adrenal tissue may occur in the bone-marrow and give rise to tumors, since the rather wide distribution of accessory thyroid and adrenal tissue, mainly in their respective regions, is fully established.

All these considerations, however, seem contentious and have proved unavailing against the increasing evidence that endothelioma of bone is a rather well-defined turnor of bone occurring in several subvarieties, but usually submerged under the diagnosis of round-cell or alveolar sarcoma.

It is now sufficiently attested that endothelioma of bone is a specific neoplastic disease, marked by the appearance of single or multiple tumors arising 
from the endothelium of bone-marrow or bone, which are cellular and vascular, tending to absorb and perforate the bone, never producing bone, often growing to considerable dimensions, invading the soft parts, but usually slow in forming metastases, often accompanied by a peculiar anemia and cachexia, and tending to a fatal termination. One of their notable characteristics is marked susceptibility to $x$-ray and radium, which distinguishes them from osteogenic sarcoma.

A possible relation to some forms of multiple myeloma is suggested by the occasional occurrence of plasmacells, but this question remains to be determined. Hansemann, Herxheimer, and Schlagenhaufer expressed the feeling that some of these tumors may be derived from osteoblasts. They did not offer definite grounds for this impression, which I believe is incorrect.

Having found in a considerable series of cases all gradations in structure between solid diffuse cell masses at one extreme, up to tumors presenting very many capillary bloodor lymph-vessels lined by cells of the identical type at the other extreme, I am convinced that all these tumors belong in one general group, derived from the endothelium of blood- or lymph-vessels.

Three forms of endothelioma of bone seem to deserve recognition:

I. Solitary Bulky Cystic or Telangiectatic Angio-endothelioma. - This tumor develops in the ends or shafts of long bones. I have seen 3 cases at the lower end of the humerus. It grows steadily, perforates the shaft, invades the soft parts, and often produces pulmonary metastases, but less rapidly than does osteogenic sarcoma. The tumor may pulsate and yield a bruit and thus be classed with malignant bone aneurysm, which is usually a variety of telangiectatic

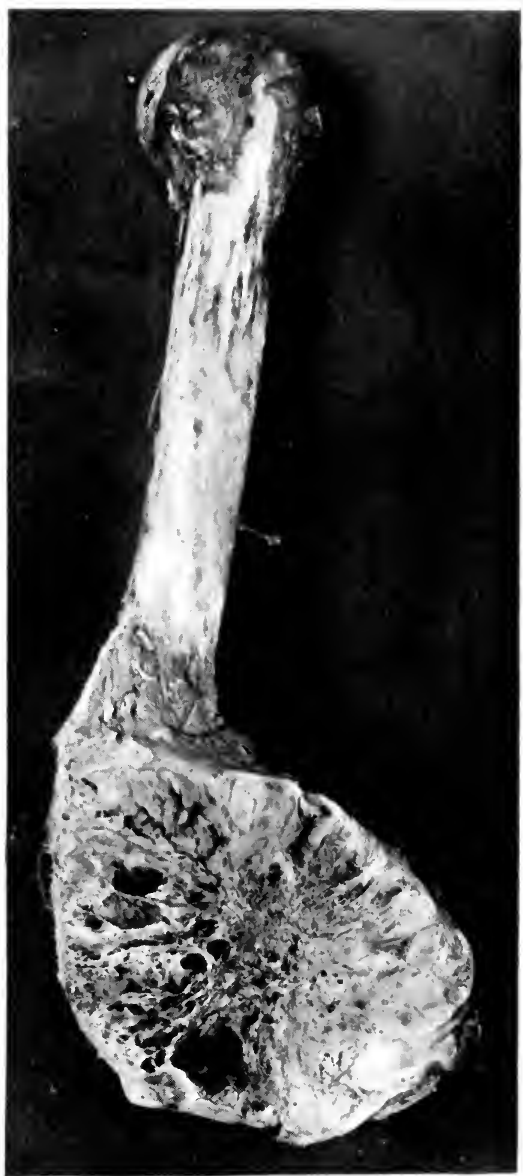

FIG. I 14.-Angio-endothelioma of humerus. osteogenic sarcoma. For this reason also notable fluctuations in size may be observed. The disease affects chiefly adults and elderly subjects (Figs. II 4 , II 5 ).

Volkman described a large multicystic tumor containing gelatinous fluid affecting the occiput in an aged woman. Most of these tumors appear to arise within the bone tissue or in the bone-marrow, but Hildebrand's tumor appears to have sprung from the periosteum. Periosteal endothelioma with calcification and bone formation has been described by Perthes, who finds 30 such cases in the literature. The growth is rather slow, occupying several 
months; it is accompanied by pain and disability and often results in spontaneous fracture, which may be the first prominent symptom.

The structure presents large cuboidal or cylindrical cells arranged in cords or columns or pseudo-alveoli, and inclosing freely circulating blood. The cells are large, with clear cytoplasm, sharp cell membrane, and small nucleus. Solid sheets of such cells may form, or alveoli lined by cylindrical cells, or wide cystic spaces filled with blood. Fragments of dissolving bone may appear on the periphery, but bone production is absent. A somewhat similar structure may be produced by metastatic carcinoma of the kidney or adrenal, which it is necessary to exclude before the diagnosis of endothelioma can be established.

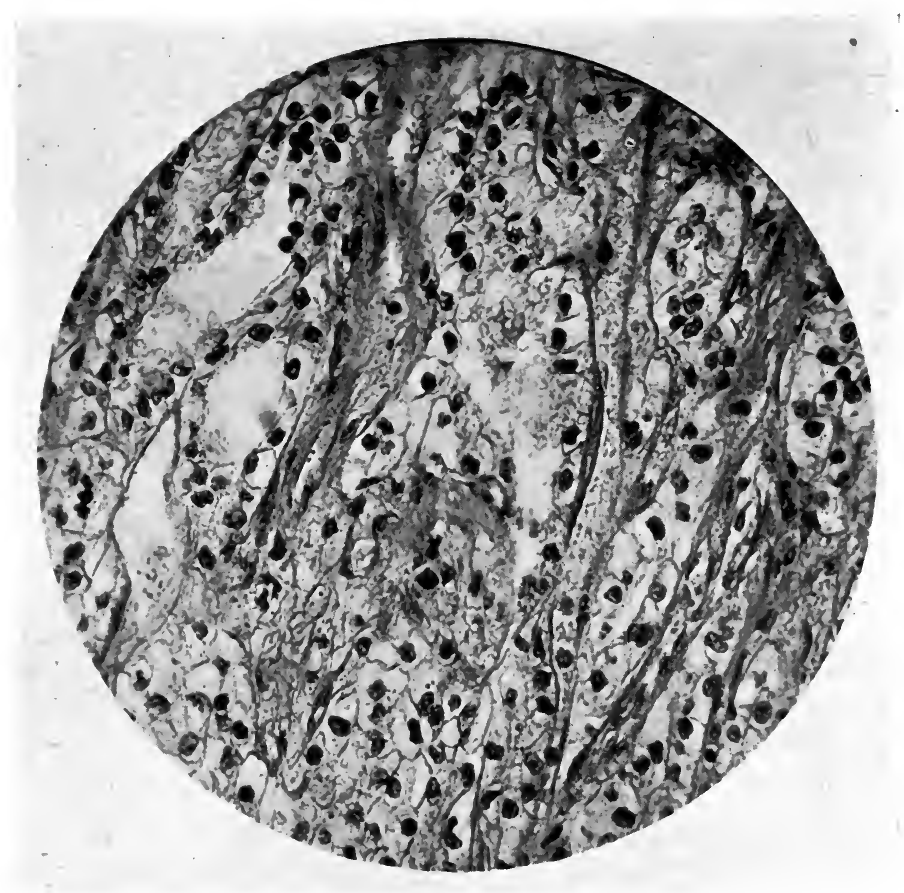

FiG. I I5.-Structure of angio-endothelioma of bone.

Structures of this type were recognized in solitary primary tumors of bone by Billroth (1869), by Kolaczek (1878), who included them under angiosarcoma, and by Hildebrand, who urged the designation endothelioma, and discussed their resemblance to benign bone aneurysm.

In a series of cases the tumor cells have not inclosed circulating blood, but formed small alveoli or small cysts containing mucinous material, the cells were more cylindrical, and the structure resembled carcinoma (Howard and Crile, Lit.). These authors generally assumed that such tumors had arisen from lymph-vessels. It is of these cases especially that the possibility of metastatic origin must be considered and eliminated.

2. Multiple Endothelioma of Bone.-Multiple small tumors of the bone marrow affecting nearly every bone in the body occurred in a remarkable 
case described by Marckwald, and stands as a type of genuine endothelioma of bone. The tumors were most numerous in the vertebræ and sternum, but many were found in skull and pelvis. Some had perforated the bone. All other organs were free. The patient was a male, aged 53 years, duration I4 months, with intermittent fever, pain, and advancing anemia and cachexia. The general condition resembled that of multiple myeloma, but the structure of the tumors was entirely different. The tumors were composed of a congeries of clear polyhedral cells in diffuse sheets, and often surrounding capillary channels containing intact blood. Neighboring tissue cells were pigmented. The diagnosis of intravascular endothelioma seems fully justified.

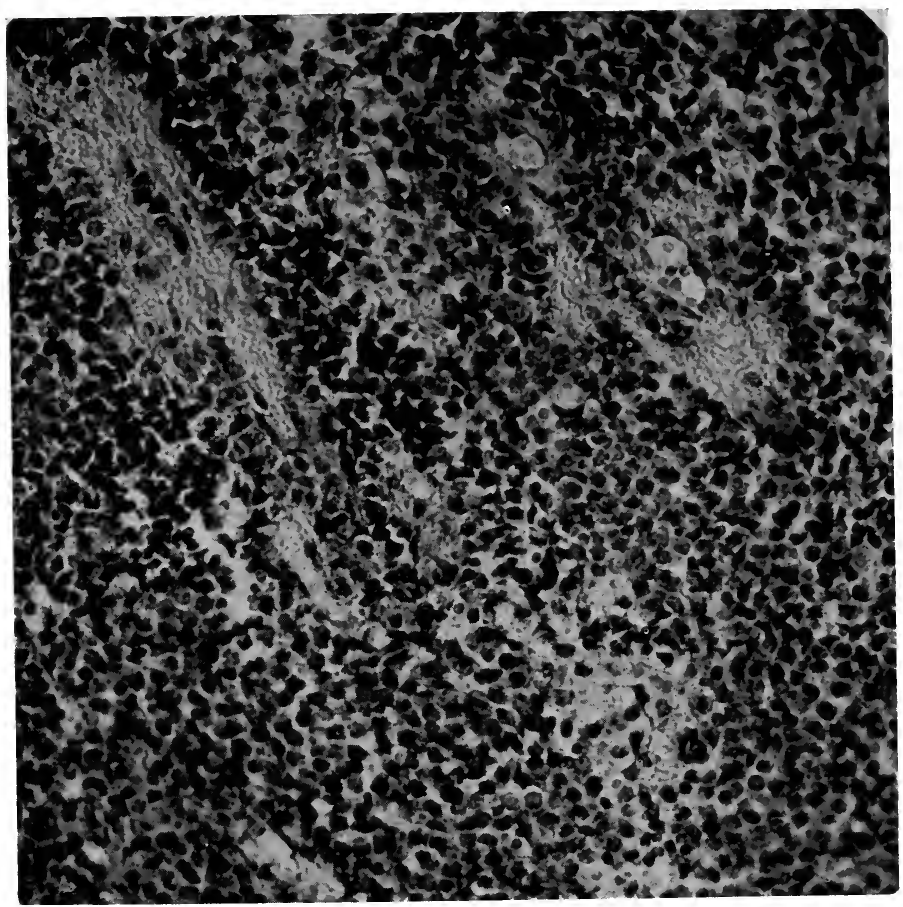

FIG. I I6.- Structure of diffuse endothelioma of bone.

Marckwald analyzed several cases from the literature, with the conclusion that similar tumors had been described by Rustizky, Zahn, Kahler, Wieland, and Klebs. Some of these, however, were probably multiple myeloma. Howard and Crile described multiple tumors of the bones which closely resemble Marckwald's case, and they offer an interpretation of the much discussed cases in the literature. They conclude that endothelioma arises from the endothelium of either blood- or lymph-vessels. In one of their cases there were primary tumors of the lumbar vertebre, others in the clavicle and ribs, and numerous metastases in liver, lungs, heart muscle, skin, and many lymphnodes. The cells were large, clear, and polyhedral. Giant-cells were numerous. The cells did not inclose blood, but formed small alveoli or solid cords. Their origin could apparently be traced to the endothelium of lymph-spaces in the marrow. 
The existence of this group of cases of multiple endothelioma of bonemarrow and their separation from metastatic carcinoma seems to be sufficiently attested. Their possible relation to multiple myeloma of plasmacell type remains for future studies to determine. It also appears uncertain whether the multiple tumors in the bones are metastases or multiple primary growths.

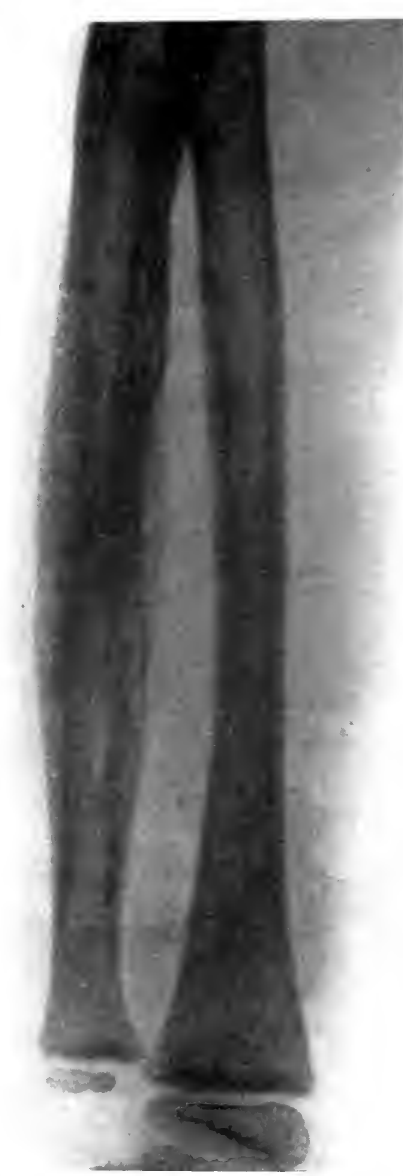

Fic. I17.-Diffuse endothelioma of ulna. Diffuse widening and absorption of whole shaft.
3. Solitary Diffuse Endothelioma.This relatively frequent and quite specific disease is usually unrecognized and passes under the clinical diagnosis of round-cell sarcoma of bone. It may be distinguished from osteogenic sarcoma by its somewhat peculiar clinical history, the location in a considerable portion of the shaft rather than at the end of the bone, by a characteristic radiographic picture, and by the marked susceptibility of the tumor tissue to radium and $x$-ray.

Most of the cases that I have seen have been in young subjects, 3 of them I4 years of age, the oldest in years. The disease begins without assignable cause with attacks of pain aggravated by exercise, but not very severe, so that the patient is able to go about for weeks or months. A spontaneous fracture may occur early. A diffuse swelling slowly forms over the affected bone, and the tumor tissue involves the soft parts. Fluctuations in the size of the tumor may then be noted. The growth is comparatively slow, several months elapsing before the external swelling demands attention. At this time the radiograph shows a smooth diffuse rarefaction of a large portion of the shaft of the long bone, the outlines of which are widened. The location in the shaft rather than at the end of the bone, and absence of bone production, distinguish the condition from solid osteogenic sarcoma, while the failure of early and sharply limited bone destruction is contrasted with the radiograph of telangiectatic bone sarcoma. Benign giant-cell tumors greatly expand the shaft over a sharply limited area and exhibit a bony capsule.

With this history and radiographic evidence it would seem to be unnecessary to incise these tumors for diagnosis, especially if they also respond promptly to radium or $x$-ray. The diagnosis of some form of periostitis has been strongly maintained in some of my cases, and has compelled the resort to a biopsy.

The later history of these cases it is difficult to determine. Although regressing rapidly under radium and $x$-ray, they are prone to recur unless the treatment is maintained, and the ultimate outcome of this method of treat- 
ment cannot at present be stated. Many tumors have been amputated, but this procedure is commonly followed by local recurrence and metastases in other bones, and in the lungs, lymph-nodes, and other organs. The tendency to involve the regional lymph-nodes is a further differential feature from osteogenic sarcoma.

Whether the tumors appearing in other bones are metastases or later primary manifestations of the disease it is impossible to determine, but this feature of the disease renders the prognosis always serious. Pulmonary

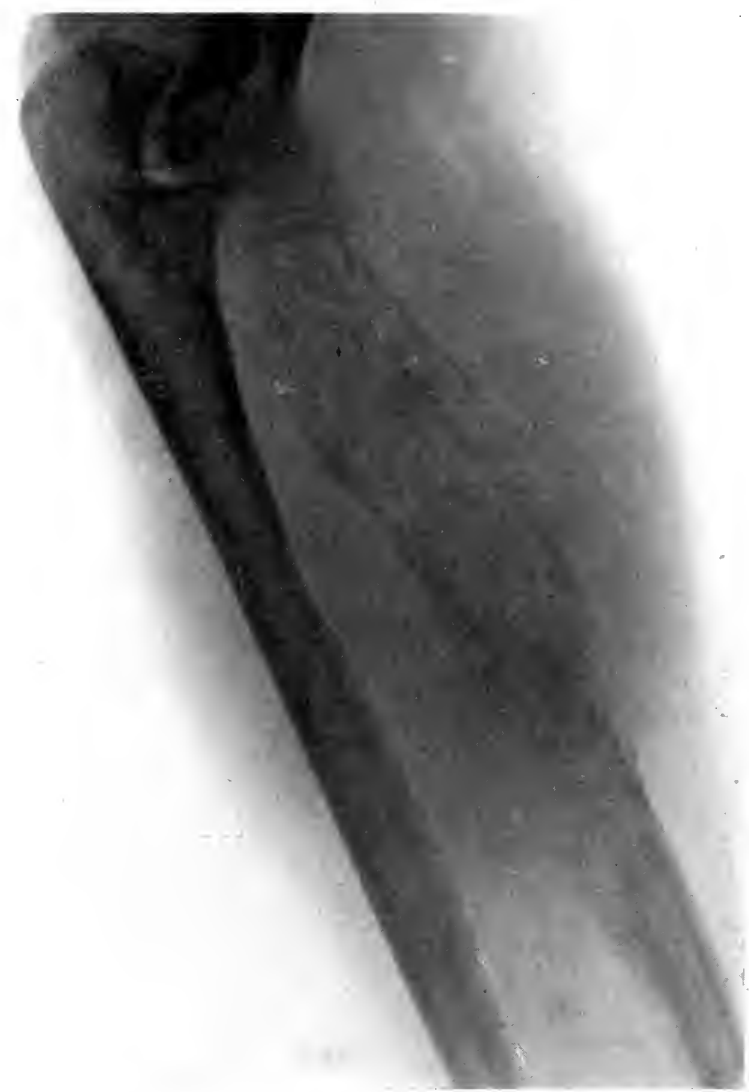

FIG. I I 8.-Diffuse endothelioma of radius. Diffuse absorption without destruction of shaft. Spontaneous fracture. Wide invasion of muscle. Duration I year.

metastases appear to form much later than with osteogenic sarcoma. The total duration in fatal cases has been about 2 years.

The structure presents solid broad sheets of small polyhedral cells, with clear cytoplasm, pronounced cell borders, small hyperchromatic nuclei, and absence of intercellular substance. If the tissue has been roughly handled or imperfectly fixed the resemblance to some form of lymphosarcoma may be considerable. Isolated foci of cells may show marked hydropic degeneration. Blood-vessels may be missing over large areas. Or the cells inclose very numerous blood channels of capillary size in which there is circulating blood. 
In the same tumor I have found both types of structure, but the solid growths without many capillaries are the more frequent. These distensible capillaries account for the fluctuations in the size of the tumors.

Rarely the cells approach the type of plasma cells, suggesting that there may be some relation between this disease and multiple myeloma.

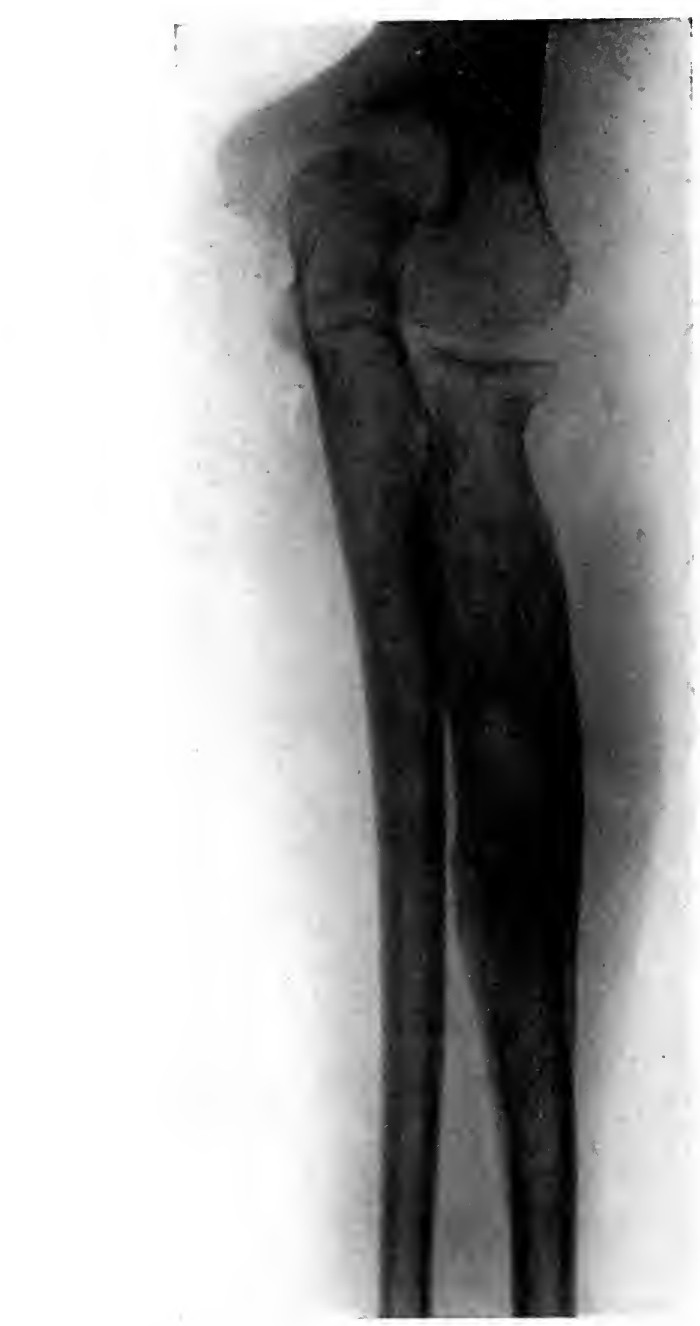

FIG. I 19.-Diffuse endothelioma. after radium Same case as Fig. I 8.

Fndothelioma of Skin.-In no department has the doctrine of endothelioma suffered such violent transformation as in the field of tumors of the skin. Here several quite distinct neoplasms have gradually acquired a wide or general acceptance as endothelioma only to lose their claims for recognition in this category through the more careful studies of critical observers. These tumors are the lymphangioma tuberosum multiplex of Kaposi, the endothe- 
liomas of the skin of Braun and others, and the soft nevus with its derivatives. With the elimination of these groups neither Darier nor Unna admit the existence of any genuine endothelioma of the skin.

Lymphangioma tuberosum multiplex was first described in 1868 by Kaposi as a tumor composed of lymph-vessels filled with proliferating endothelial cells. Although Jacquet and Darier in I 887 showed that these cell groups are derived from the epithelium of dilated sweat-glands, numerous writers continued to describe cases as endothelioma. Many authors have pointed out their true nature (Frick, Lit.). Stockman has reviewed the history of this tumor, fully supported its epithelial origin, and accepted its designation as hydrocystoma tuberosum multiplex.

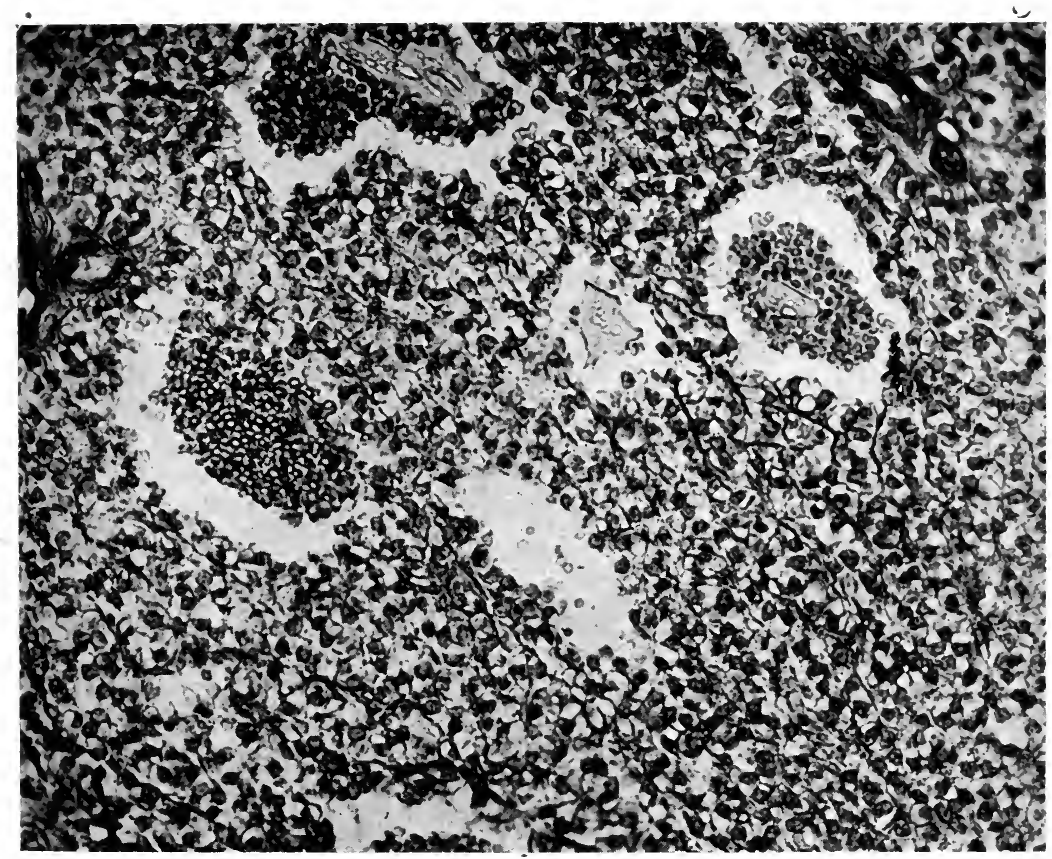

FIG. 120.-Diffuse endothelioma of bone. Blood lacunæ lined by tumor-cells.

A second group of tumors of the skin presenting the structure of cylindroma was long regarded as endothelioma. A typical case of this class and one much referred to is that of Braun. This tumor was clearly shown by Krompecher to be of epithelial origin and is now quite generally assigned to the class of epithelioma adenoides cysticum.

With another group of somewhat similar tumors it has been more difficult to reach a conclusion. In 1889 Spiegler described 3 cases of multiple tumors of the scalp which grew over a long period and eventually covered the head with numerous discrete tumors resembling small "billiard balls" or "tomatoes." The clinical condition was extremely characteristic. Similar tumors are also observed in other parts of the body, especially of face, chest, and abdomen. The clinical course, gross section, and microscopical structure show many resemblances to reticulated epithelioma, with which they are classed by Borrmann, Ribbert, and others. Mulert's case was disposed of by Lubarsch. 
Many others closely resemble some of the varieties of epithelioma extensively illustrated by Coenen. Juliusberger, who reviews this group at length, regards most of the cases as epithelioma, but considers Haslund's case and his own as true endothelioma. I do not find satisfactory evidence that the tumor described by Juliusberger was derived from endothelium, but in Haslund's case the clinical history and structure are rather distinct and it is difficult to eliminate an endothelial origin.

This patient suffered from multiple tumors of the scalp which grew rapidly, involved the regional lymph-nodes, and proved fatal in 18 months. Microscopically the polyhedral or spindle-cells, the formation of long intercellular fibrils, the absence of definite epithelial characters, the striking relation to

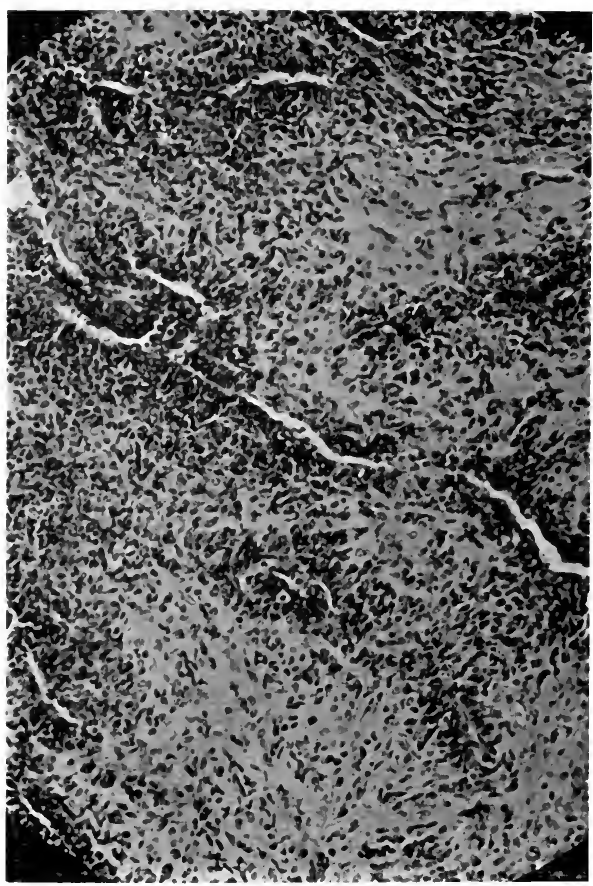

FIG. I21.-Fibro-angio-endothelioma. A circumscribed tumor beneath skin of thumb.

vascular spaces and channels, and the various stages of tumor growth which were traced in great detail from the lymphatic endothelium, all accord with an endothelial character. On the other hand, their appearance as multiple tumors of the scalp of the same general features as the characteristic epithelioma of this region raises the suspicion that Haslund had to deal with unusual histological features of an epithelioma. Nevertheless the minute histological study of this case brings to light so many endothelial characters as to leave in doubt the true nature of the growth, and with it the existence of a true endothelioma of the skin. It has already been pointed out that the presence of circulating blood in immediate contact with tumor-cells, which is the chief endothelial feature of Haslund's case, is not a reliable sign of endothelioma, but occurs also in epithelioma of the skin. 
The cellular nevus has been the subject of much discussion and difference of opinion. First regarded as an endothelioma derived from lymph-ressels (Recklinghausen, Demieville, Lowenbach), the opinion of Unna who interpreted the cells as misplaced embryonal epithelium has found very general acceptance by the majority of observers. Yet Kaufmann, Johnston, Schutz, and others believe that there are nevi of endothelial as well as those of epithelial nature. I do not find the distinctions between these groups sufficient to warrant any separation of the cases, which seem to me to fall in the class of melanoma ( $q . v$.).

There is a group of multiple small red or brown tumors of skin, in children or adults, which probably represent phases of regression, or rarely of slight or even active neoplastic growth of endothelial cells of capillary nevi. Wise describes them in 2 cases at length, but does not clearly connect them with capillary nevi, nor distinguish them from pigmented moles.

Endothelial psammoma of the skin is described by Winkler. This rare tumor occurs in the form of multiple hard nodules in the cutis or subcutis which follow the course of nerves and may be traced down to the periosteum. They are probably derived from the endothelium of nerve sheaths and traces of nerve-fibers may persist. They belong in the group of psammoma of nerve-trunks. They consist of concentric masses of endothelial cells which are often calcified. According to Winkler certain cases of calcified endothelioma of the skin (Perthes, Linser, Volkmann) have a similar origin. Psammoma of deeper nerve-trunks is more frequent.

Angio-endothelioma of the skin and subcutaneous tissue occurred in several cases which I have examined. They were slowly growing circumscribed tumors of the sole of the foot, finger, or face. They were composed of a moderate number of small blood channels traversing wide groups of endothelial cells. The preponderance of cells over vessels calls for the designation of endothelioma (cf. Hemangio-endothelioma).

Endothelioma of Corpus Cavernosum Penis.-This tumor appears in the form of multiple nodules or flat infiltrations of the corpora cavernosa behind the glands and along the dorsum of the penis. Four cases are recorded, in 2 of which the course was moderately slow, the growth circumscribed, and operation was followed by recovery (Alexander, Hildebrandt), while in the others the disease early involved the lymph-nodes and the patients died with general metastases (Maurer, Colmers). The structure consists of the cavernous spaces filled with large polyhedral or rounded, rather clear or granular cells with large nuclei and prominent nucleoli. The central portions of large cell groups are necrotic or occupied by circulating blood (Colmers). Invasion of the urethra, septa, skin, and especially of the nerve-sheaths is observed. Occasionally concentric groups of cells appear. The origin of this tumor from endothelium has not been fully determined, but primary tumors of the urethra were excluded. Yet Hildebrandt considers the possibility, and Borrmann assumes, an origin from urethral epithelium. In Alexander's case I must agree with Dunham that the structure was different from that of epithelioma and strongly suggestive of endothelioma.

Creite has described a peculiar urethral carcinoma of undetermined origin in a child of 2 years which may have some bearing on the present topic.

Endothelioma of Ovary.- Among the tumors of the ovary Leopold (I 874) described a very large growth which he derived from the endothelium of lymph-vessels, while numerous small cysts composing the bulk of the tumor he referred to dilated lymphatics. In I 879 Marchand described papillary, cystic, and tubular forms of ovarian tumor which he distinguished from sarcoma and considered as very probably derived from endothelium of lymph- 
or blood-vessels. Somewhat similar structures were next observed by Flaischlen in a cystic tumor combined with a dermoid cyst.

A definite relation of the tumor-cells to the endothelium of blood-vessels was claimed by Olshausen and Ackermann and by Eckardt, who described their tumors as endothelioma intravasculare. On the other hand, Pomorski and v. Velits traced cystic and myxomatous tumors to lymphatic endothelium, and Rosthorn referred a somewhat different structure, composed of large cell groups inclosing blood-masses, to proliferation of perithelial cells. All three structures seemed to Amann to deserve recognition as types of endothelioma. In the aisposition of the cells L. Pick recognized three types composed of (I) long rows and whorls of cells, (2) cell groups resembling adenoma or carcinoma, and (3) diffuse masses of cells resembling sarcoma. In 1896 a specific structure, designated as fibrosarcoma ovarii mucocellulare carcinomatodes, was described by Krunkenberg and referred in all probability to the endothelial cells of this organ (Krunkenberg's tumor).

Thus such a wide variety of structures of such uncertain origin were now included under endothelioma that suspicion arose regarding the validity of the rapidly growing scope of this ovarian tumor. Such obvious combinations as pyloric cancer and endothelioma of ovary were misinterpreted by Rosinski as multiple separate tumors, while Linck even saw in the pyloric cancer a metastasis from the ovarian growth. Likewise Krunkenberg found the typical structure of perivascular endothelioma in an ovarian tumor in a patient who 2 years later required hysterectomy for a similar tumor of the cervix. In Eymer's case also the cervix was involved.

The first inroad upon endothelioma of the ovary was accomplished by Schlagenhaufer, I902, who showed that the majority of bilateral tumors of the ovary are secondary to epithelial neoplasms of stomach, intestine, gall-bladder, or uterus, and the typical Krunkenberg tumor he and Glockner observed as a metastatic growth from pyloric cancer with mucous cells. Polano (I904) showed that ova-like structures and perithelioma could appear in adenocarcinoma, that metastatic gastric cancer in the ovary produced many of the structures of the so-called endothelioma, and that certain mucoid endotheliomas were really thyroid struma in this organ. Papaioannou also pointed out the close resemblance of metastatic ovarian carcinoma to endothelioma and fibrosarcoma. Stone has recently reviewed at length the subject of secondary ovarian carcinoma. Glockner denied the validity of Pick's histological criteria of endothelioma, especially the absence of an intact layer of endothelial cells in the invaded tissue spaces, and claimed that the diagnosis of ovarian endothelioma could not be based on histological structure alone, but required the elimination of other tumors, especially of the uterus, by autopsy. Thus grave doubts arose as to the existence of any genuine endothelioma of the ovary. R. Meyer was disposed to discard the entire group, and Ribbert states (I9I2) the extreme view that the endothelial origin of any ovarian tumor while possible is unproved.

During this long discussion lasting over 30 years it became apparent that unusual difficulties surround the diagnosis of endothelioma in the ovary. This organ does not meet one of the conditions laid down by Ribbert for the probable endothelial nature of any primary tumor, viz., that the organ should not contain epithelial structures. On the contrary, the remarkable physiology and changeable morphology of the germ epithelium, Pflüger's tubes, Graafian follicles, and corpus luteum, offer unusual sources of atypical epithelial tumors. The average quota of endothelial cells seems to be deficient in the ovary if one can accept Polano's observation that no lymph spaces lined by endothelial cells exist in this organ. Again it has transpired 
that the ovary is peculiarly liable to implantation metastases by its exposed position in the peritoneal cavity, and to deposits by embolism or permeation. Finally, the doctrine of overgrowth of one element in teratomas has offered a peculiar source of certain supposed endotheliomas of embryonal type or associated with dermoids (Flaischlen, Vogel) or teratomatous structures (Ribbert, Yamagiva).

One of the most conclusive features of certain endotheliomas is the relation of cell groups inclosing blood-masses as in angioblastic sarcoma. Yet this structure has been clearly traced in the testis to a teratomatous origin and an epithelial derivation (Wlassow), and while Sternberg and Monckberg have endeavored to establish the existence of a special variety of angioblastic sarcoma or endothelioma apart from teratoma and Pfannenstiel recognizes such tumors in the ovary, their teratomatous nature cannot be so readily discarded.

Thus critical research fully justifies the current skepticism regarding the endothelial nature of the present group of ovarian tumors and especially their origin from adult endothelial cells of any type.

There is nevertheless a restricted group of ovarian tumors for which very strong evidence points to an endothelial origin and their somewhat peculiar clinical course, gross appearance, and histological structure warrant their separate classification as probable endotheliomas.

These tumors are usually large, sometimes bilateral (22 per cent., Apelt), chiefly solid, yet often cystic. The solid growths show marked lobulation and the exterior is nodular, while the general form of the ovary is maintained. The cysts are small and numerous as from dilated lymph-vessels, or larger and exhibiting on minute inspection papillary outgrowths. A honeycomb appearance is noted by Pick. The consistence is firm, and the color varies greatly with the blood content. Hydropic areas and hyaline or colloid degeneration alter the appearance of the section, which in general resembles sarcoma.

The clinical course is of an actively growing tumor appearing at any period of life, but chiefly in adults. v. Velits observed a case continuing for 9 years, and Kubo I 2 years, while Glockner saw a fatal result in 6 months. Local adhesions with ascites and permeation through lymphatics are variable, but recurrence is frequent. Kubo found no case of recovery from bilateral tumors reported as endothelioma, but single tumors have been successfully eradicated by several operators (Pfannenstiel). It is generally agreed that the malignancy is less than that of carcinoma.

The structure falls into several classes (Pick, Pfannenstiel):

I. The cells are arranged in orderly rows or whorls between abundant fibrils, an appearance which has been likened to strings of pearls. An indefinite lumen between such single or multiple rows of small cuboidal cells has often been interpreted as a dilated lymph-channel, yet the origin of the cells from lymphatic endothelium has never been traced, and it is obvious that in a large tumor mass original lymph paths are wholly obliterated. On the other hand, the remarkable long and regular cell columns as pictured by Kubo may well signify the reassertion of the physiological tendencies of lymphatic endothelium in a desmoplastic tumor.

2. A definite lumen of small or large dimensions walled by one or more rows of tumor-cells and containing lymph or blood characterizes a second group of tumors designated as alveolar or intravascular endothelioma. The structural evidence of endothelial origin of this group is unusually striking, and there is little doubt that such cases as those of Marchand, Pfannenstiel, and Kubo are properly regarded as endothelioma. They show, 
moreover, numerous transitions up to structures which really deserve the name of angioma. Tumors consisting of one or more large cysts lined by layers of endothelial-like cells, described by Schurman and by Lange, are accepted by Monckberg as genuine endothelioma. If so they represent extreme examples of dilated lymph-vessels. Larger plexiform masses of cells often form in which small groups of cells surround marrow lumina (Kubo) or wide lumina filled with mucinous material appear. Ova-like structures formed by mucinous degeneration of cells occur in certain tumors of this type whose endothelial nature is doubtful. Similar bodies are found in undoubted adenocarcinomas. Hyaline globules in and between the cells may also become a prominent feature (Kworostansky). The lymph-channels may become dilated and papillary projections of tumor-cells may partially fill the small cysts (Eymer), or the channels may be filled with blood (Rosthorn).

3. Diffuse growth of small cuboidal cells forms the bulk of some endotheliomas and appears in portions of many others. Here indications of alveolar tendencies may be detected at many points and the tumors bear a resemblance to alveolar sarcoma. The true nature and origin of such tumors is, I think, open to question.

4. In a group of cases (Kubo, Lit.) the tumor-cells surround vascular channels which possess a well-defined wall of normal endothelial cells, giving the structure of perithelioma. There seems to be little reason to doubt that such tumors, $e . g$. ., Amann's case, are of endothelial nature, but the attempt to derive the cells from specialized perivascular endothelium has not been successful. Pfannenstiel interprets as angioplastic endothelioma ovarian tumors described by Michel and by himself in which numerous capillary blood channels without walls coursed throughout diffuse cell masses. This tumor is probably of teratomatous origin and choriomatous type, and while the cells may assume angioplastic qualities the tumor has a different significance from the present conception of endothelioma.

In all of these structural forms the features of the tumor-cells strongly indicate their endothelial nature. They are of very uniform small size, cuboidal or rounded form, pale staining cytoplasm, and regular oval nuclei with minute nucleoli.

In the stroma mucinous changes may be prominent or hyaline degeneration may yield the appearance of cylindroma.

In the effort to trace the origin of the tumors many have assumed that they could be subdivided into lymphangio-endothelioma and hemangio-endothelioma, intravascular and perivascular, and Kubo has arranged the reported cases according to this plan. Its validity, as well as the exact nature of many of the tumors included, remain to be established. Especially for the perivascular type the attempt to derive the growth from the hypothetical perithelial cells deserves little encouragement. Polano could not discover any traces of such cells in the ovarian vessels, and it is well know that this structure may be produced by many tumors.

Endothelioma of Uterus.- In the uterine mucosa and muscularis and in the cervix several tumors have been described as types of endothelioma, and derived by the authors from lining cells of blood- or lymph-vessels. From the descriptions of these cases I have not been convinced that a well-defined endothelioma of the uterus has been demonstrated and would agree with R. Meyer that, while such tumors may occur, it is more probable that the alleged cases should be distributed among epitheliomas and sarcomas. Meyer points out that a considerable grade of endothelial proliferation may occur in the cervix and uterus as a result of inflammation, that the remnants of Gartner's duct in the cervix have been overlooked as a possible source of 
atypical epithelial tumors, and that many of the structures of endothelioma uteri may be explained as atypical features of other tumors.

Neither in the clinical course nor in the gross or microscopical structure have the reported cases shown uniformity. Thus Pick finds endothelioma in a small portion of the mucosa connected with a myosarcoma. Hansen describes a large vascular tumor of the myometrium which showed many features of an angiosarcoma, and the structure in Silberberg's case falls in the same general class. Amann who recorded the first case was content to find no connections between the tumor-cells and the surface epithelium, but his case and that of Braetz strongly suggest embryonal epithelioma. Pohorecky, Kroemer, and Krischgessner describe structures often duplicated by epithelioma. Hurdon's case was clinically indistinguishable from cervical epithelioma with wide extensions, and while portions of the structure suggest endothelioma, other areas correspond to epithelioma. Rabb considered his case clinically epithelioma, and such is the impression clearly conveyed by Graefe's report.

Thus the recorded cases seem to fall into three groups, vascular tumors of the angiosarcomatous type, embryonal epithelioma, and atypical forms of ordinary epithelioma of the cervix. Further critical study would seem to be demanded before the existence of endothelioma uteri can be accepted.

Endothelioma of Stomach.-For many years a peculiar condition of cirrhosis of the stomach with pyloric stenosis has been recognized under various terms and interpretations. From the surgical side the history of the subject has been fully reviewed by Lyle, who employs Brinton's term linitis plastica for the general condition. It has long been known that some of these cases were atypical diffuse carcinoma. In a second group the process has been recognized as neoplastic and interpreted as endothelioma by Fick, Tilger, Jungmann, Cignozzi, Aldegarmann, and others, and Hansemann and Borst have lent some support to this view. Szobelew and others have found the condition both at the pylorus and in the colon. Yet in none of these cases has there been satisfactory evidence that the process is an endothelioma. The structure shows a diffuse and scanty infiltration of large cells with hyperchromatic nuclei, but of wholly indefinite origin. Many of the cells may show the mucous degeneration with signet-ring nuclei of gastric cancer, and some small foci of such cells are usually found on careful search. I have examined 5 cases of this type in all of which the characteristic mucous cells have been found, and in 3 prolonged search revealed alveoli of typical gastric cancer. Since the typical appearance of endothelioma may appear in undoubted gastric cancer there appears to be no ground for the assumption that endothelioma of this organ exists.

That the remaining cases of cirrhosis of the stomach and intestine with pyloric stenosis in the adult are the results of a chronic inflammatory process has been clearly shown by Krompecher, who attributes the changes to edema, fibrosis, muscular hypertrophy, and irritation by foreign bodies. Recently there has arisen a tendency on the part of clinicians to call many of these cases syphilis.

Endothelioma of Lymph-nodes.-Primary endothelioma of lymph-nodes was described by Chambard in I 880 under the term "primary carcinoma." He recognized a local form of the disease affecting one node or one chain, and a generalized form which was always fatal. The structure was alveolar. Zahn had previously interpreted an alveolar tumor of the cervical nodes as sarcoma alveolare epithelioides. In $\mathrm{x} 88 \mathrm{I}$ Hoffmann and Schottelius used the term "endothelioma" in their report of a case. The endothelial nature of these tumors became gradually accepted, so that Ziegler, Birch-Hirschfeld, and 
Kaufmann admitted their occurrence and referred to single cases of their own, and Recklinghausen pointed out certain distinctions between primary endothelioma and secondary carcinoma of lymph-nodes. More recently quite detailed reports of the gross anatomy, microscopic structure, and clinical course of these tumors have been furnished by Ravenna, Gallina, Parlavecchio, and the writer.

In the study of a series of cases collected during a period of several years I have drawn the conclusions that endothelioma of lymph-nodes is comparatively frequent in occurrence, that the tumors often arise on a basis of chronic granulomatous inflammation, that they develop from the endothelium of the lymph and cavernous sinuses and are then usually classed as secondary carcinoma, that somewhat similar tumors arise from the reticulum cells of the lymph-nodes, but usually take the form of large-cell lymphosarcoma, occasionally of carcinoma, and that the characteristic clinical course and microscopical structure of the tumors constitute them specific diseases.

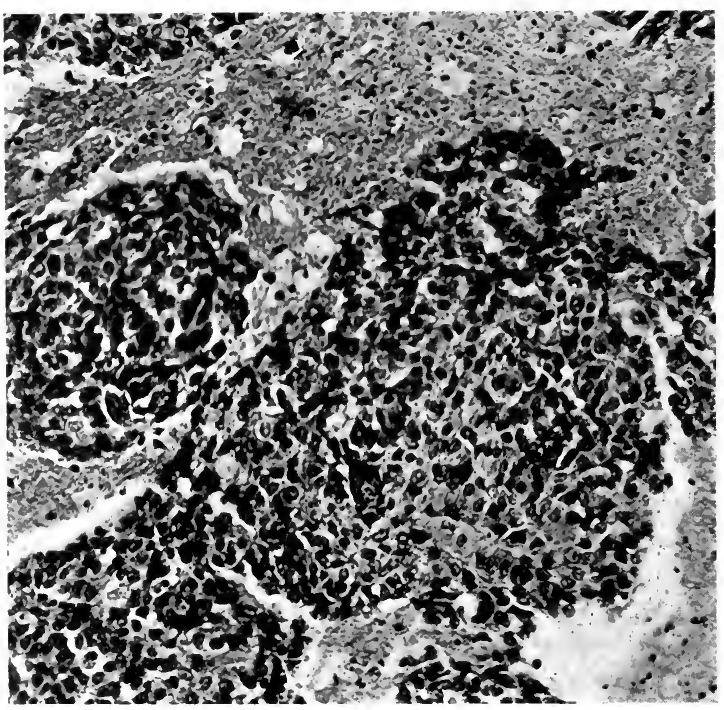

FIG. I22.- Perivascular endothelioma of lymph-nodes. Nearly all lymph-nodes were involved in this case. (Flournoy.)

Clinically the disease appears in the form of (I) a systemic involvement of many lymph-nodes with a progressive and fatal course lasting from one to several years, and (2) as single or multiple tumors of cervical, axiliary, or other lymphatic chains, often preceded by granulomatous infection, but eventually or from the first declaring itself as a locally aggressive recurring malignant tumor.

The systemic form of the disease usually suggests the diagnosis of pseudoleukemia, as emphasized by Parlevecchio. Most of my systemic cases were regarded as Hodgkin's granuloma and in some certain of the nodes presented the typical Sternberg-Reed histology. In one case of lymphatic leukemia I found alveolar endothelioma of the bronchial nodes. Flournoy's case ran a prolonged course of 4 years and developed cervical, thoracic, and very large abdominal tumors. Gallina's patient lived I 2 years.

The localized tumors develop chiefly in neck, axilla, and groin, and are 
commonly regarded as tuberculosis. In one case I followed an originally tuberculous infection of cervical nodes recurring repeatedly over a period of 5 years, and gradually assuming the structure of a malignant endothelioma with elimination of the tuberculous element. Usually the tumors are endotheliomatous from the first. In some cases I have been unable to separate the local, cervical, or axillary tumors from a systemic disease of the type of Hodgkin's granuloma. The local disease has a lethal tendency, but some patients enjoy long immunity after operation, although the ultimate outcome is usually unfavorable. Zahn and Volkmann describe rapidly fatal cases. Extensive tumors of the neck may early cause asphyxia and in others attempted removal proves fatal.

Gross Anatomy.- The systemic disease is marked by uniform enlargement of several chains of nodes, some of which reach large dimensions and give

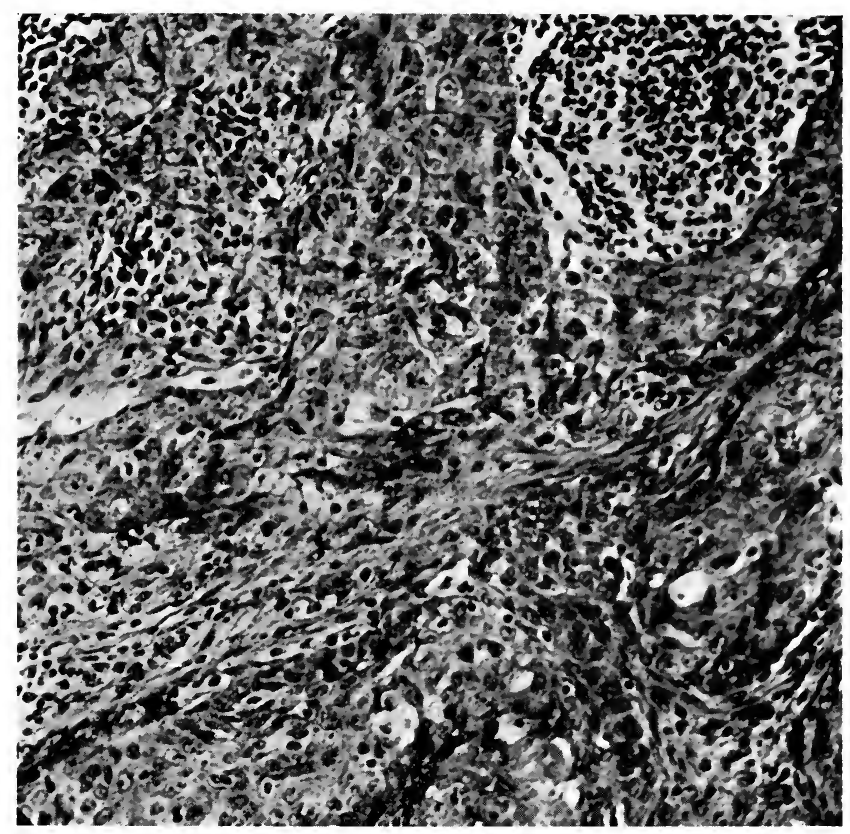

FIG. I23.-Diffuse endothelioma of cervical lymph-nodes.

pressure symptoms. The tumors are smooth, at first firm, later very hard, usually solid, opaque, and marked by areas of necrosis. Recklinghausen saw cystic distention of the abdominal chains, and involvement of many retroperitoneal nodes.

The local tumors are at first circumscribed and the capsules free, but later they become large, hard, adherent, and multiple. The infiltration of recurrent tumors involves the neighboring structures with compression or destruction, as in carcinoma, but the progress is usually distinctly slower than in epithelial growths. Very extensive fibrosis and large areas of retained desiccated lymph or necrotic tissue appear in late stages. Almost complete sclerosis may overtake some of the tumor masses.

Metastases are difficult to distinguish from systemic extensions, but the 
lesions tend to advance over wide areas, involving thoracic and abdominal nodes and spleen. There appear to be all gradations between the localized and the systemic form. Definite metastases were found by Gallina in the adrenal, and in the portal canals of the liver by Ravenna. Very extensive lymphatic lesions from which skin and muscles were invaded, with edema of chest wall, chylous hydrothorax, and metastases in pleura, stomach, and adrenals occurred in the case of Da Gradi and de Amicis. In Flournoy's case a very large tumor, I780 gm., surrounded the pancreas, nearly all lymphnodes were involved, and there were metastases in adrenal and thyroid.

Structure.-The structure encountered depends on the stage of the process and on the rapidity of its development. On the one hand, it may be difficult to separate the early stages from granulomatous inflammation, and on the

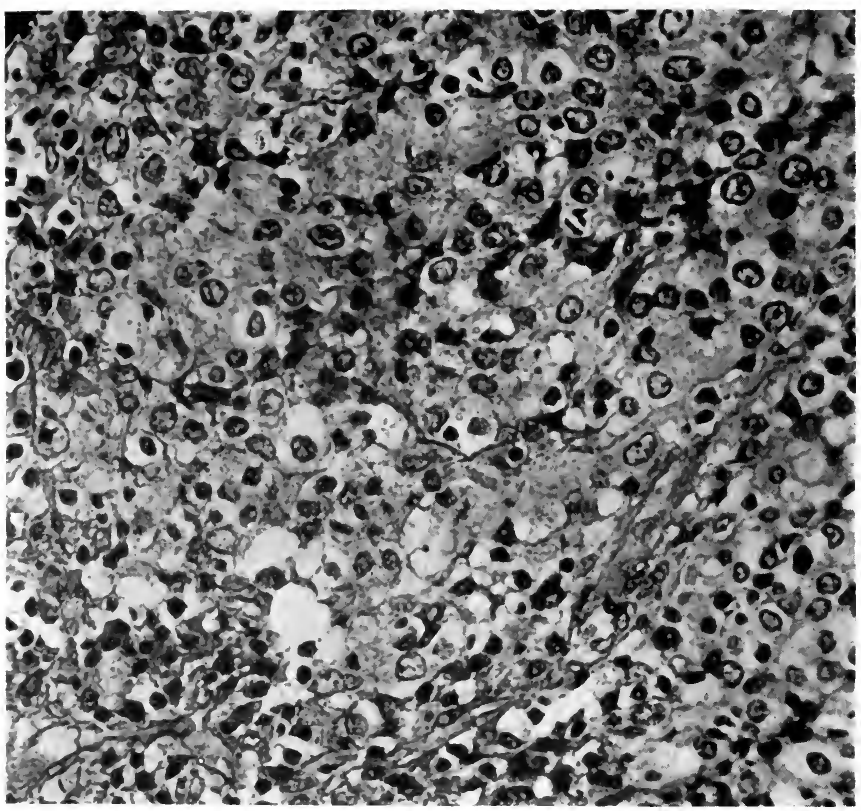

FIG. 124--Diffuse endothelioma of lymph-nodes. Coherent masses of large cells with clear cytoplasm.

other the late stages resemble carcinoma. The earliest stages of many cases appear as a granuloma in which groups or coherent syncytial sheets of sinus endothelium or reticulum cells stand out prominently by virtue of their size and nuclear hyperchromatism. The cells are large, elongated or polyhedral, with very large vesicular nuclei and diminutive nucleoli, and they spring in multiple foci from the lymphatic tissue. The isolation of invading tumorcells is conspicuously absent. The lymphocytes are passive and disappear. Ravenna emphasizes the imperfect outlines of the cells and their occurrence in broad sheets of cytoplasm interspersed with large vesicular nuclei.

In some cases fibrosis sets in early and then the tumor-cells become isolated and very prominent and the structure resembles alveolar carcinoma. Many patent lymph paths lined by tumor-cells may persist or cysts may form. Large convoluted masses of coherent endothelium may make up the bulk of 
large tumors in which irregular areas of necrotic tissue containing fatty crystals may appear. The structure may be designated as plexiform endothelioma.

In several cases I have found dilated lymph-channels lined by multiple layers of opaque, elongated, or polyhedral cells. Or the cells mingled with lymphocytes may encircle small arterioles as in perithelioma. These structures suggest the term perivascular endothelioma.

In some cases the whole node is replaced by a diffuse growth of large polyhedral or rounded cells which may cohere or fall apart. Giant-cells with multilobed nuclei may appear in these tumors, which may be called diffuse endothelioma. They may present the appearance of large-cell lymphosarcoma.

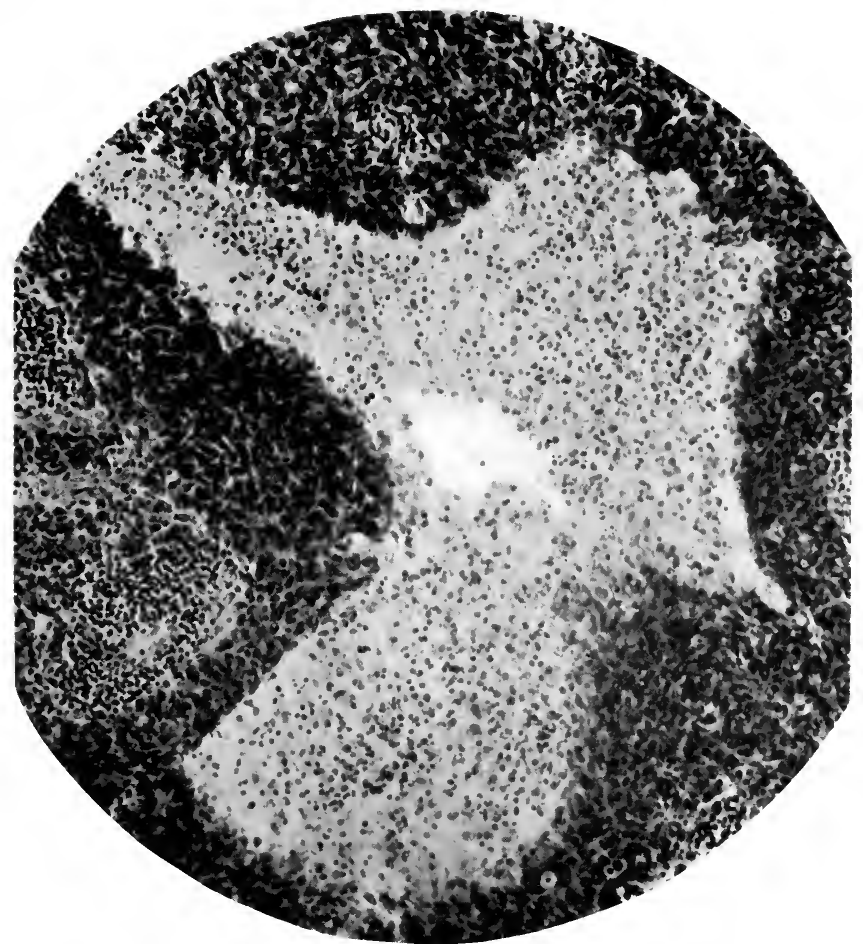

FIG. I25.-Perivascular endothelioma of cervical lymph-nodes.

Histogenesis.-Analysis of the origin of endothelioma of lymph-nodes involves at once the somewhat complicated questions of the structure of lymph-nodes. I have elsewhere discussed this subject in some detail with the following conclusions:

The reticulum cells of the lymph-nodes are derived from connective-tissue cells, and form a meshwork in which lymphocytes gather from without. 'The reticulum of the follicles and pulp does not produce lymphocytes, but these two cells constitute separate series (Saxer, Gulland). The reticulum of the nodes is not covered by separate endothelial cells, but is itself a cellular syncytial structure which incloses spaces continuous with the lymph paths of the node. In the cavernous and lymph sinuses the lining cells have pavement qualities of adult endothelium, while in the cords and follicles the 
reticulum cells retain more of their original mesoblastic tendencies. Hence tumors arising from sinus endothelium may be expected to show pavement or epithelial characters, while tumors of reticulum cells should exhibit some of the mesoblastic features of their parent cells.

To a large extent these expectations are realized in the study of the tumors. To the sinus endothelium has been traced the origin of several tumors presenting alveolar or perivascular structures composed of polyhedral cells (Ravenna, Ewing). The reticulum cells of the lymph cords are found multiplying in the early stages of many tumors, especially in the outlying nodes, and it is probable that changes in the form of these cells toward adult endothelium accounts for the preponderance of large polyhedral cells in the whole group of tumors. From the reticulum cells of the follicles I have repeatedly been able to trace the development of large round-cell tumors of the type of

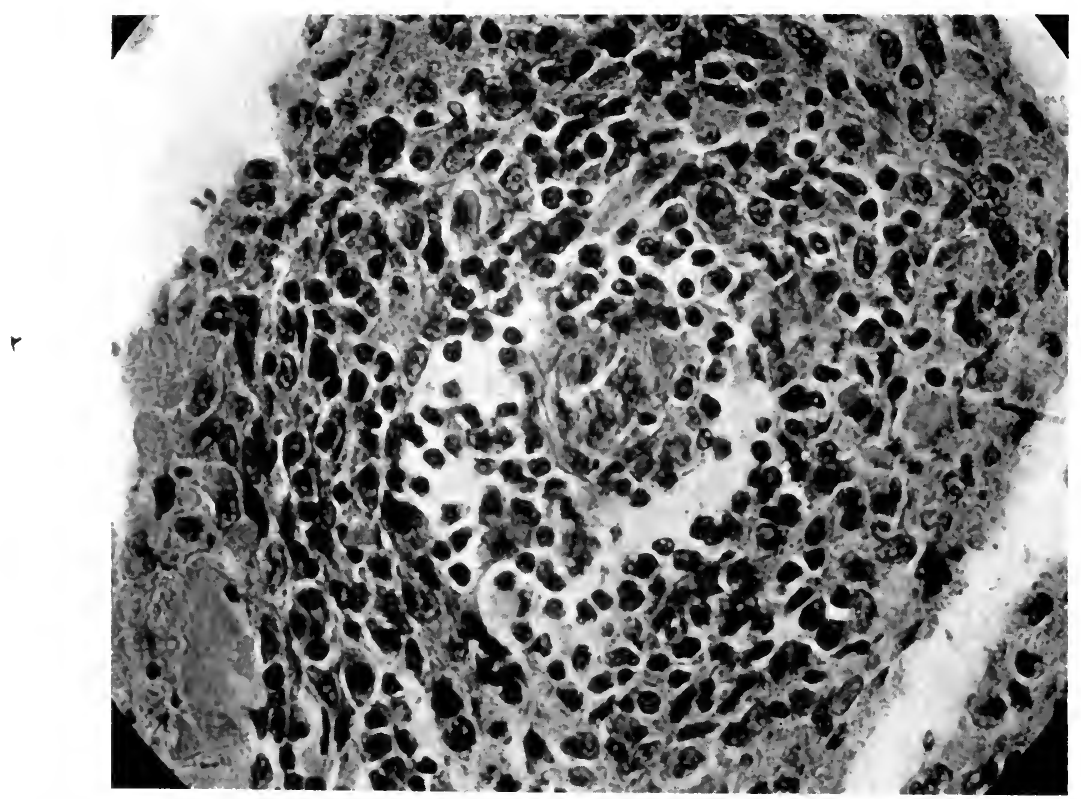

FIG. 126.- Unit structure in a tumor of lymph-nodes. Large cells growing in and about small blood-vessels.

large round- and giant-cell lymphosarcoma, which tumors must be separated from lymphocytoma.

That the tumors are not secondary carcinomas is sufficiently attested by the failure to find any primary tumor and by the very wide diffusion and slow course of the systemic disease. That they do not arise from aberrant epithelial rests within lymph-nodes has been proved by the detection of early stages throughout the whole node or several nodes of a chain (Parlavecchio).

With this complex morphology and histogenesis it is difficult to establish a legitimate nomenclature. The terms employed, endothelioma of various types, emphasize the form of the tumor-cells and their probable origin. The tumors of loose round-cells derived from the reticulum of the follicles differ markedly from the others and it seems unwise to urge any change in their usual designation as large-cell lymphosarcoma or reticulum-cell sarcoma. 
Etiology.-Many observers have been impressed by the apparent dependence of endothelioma of lymph-nodes upon lymphatic infection. The clinical features of the systemic cases closely resemble those of pseudoleukemia or of Hodgkin's granuloma. Parlavecchio stated that the histological structure revealed the transformation of an inflammatory into a neoplastic hyperplasia of endothelium and he clearly pictured these transitions. In the recurrences of one case I was able to trace an original tuberculous granuloma into the neoplasm with elimination of the tuberculous structure. I have reported several other cases in which various phases of remarkable endothelial overgrowth occurred in tuberculous or other granulomatous lesions. Many of the localized tumors present in outlying nodes the changes of frank or atypical tuberculosis. Most of the localized tumors and the advanced systemic lesions

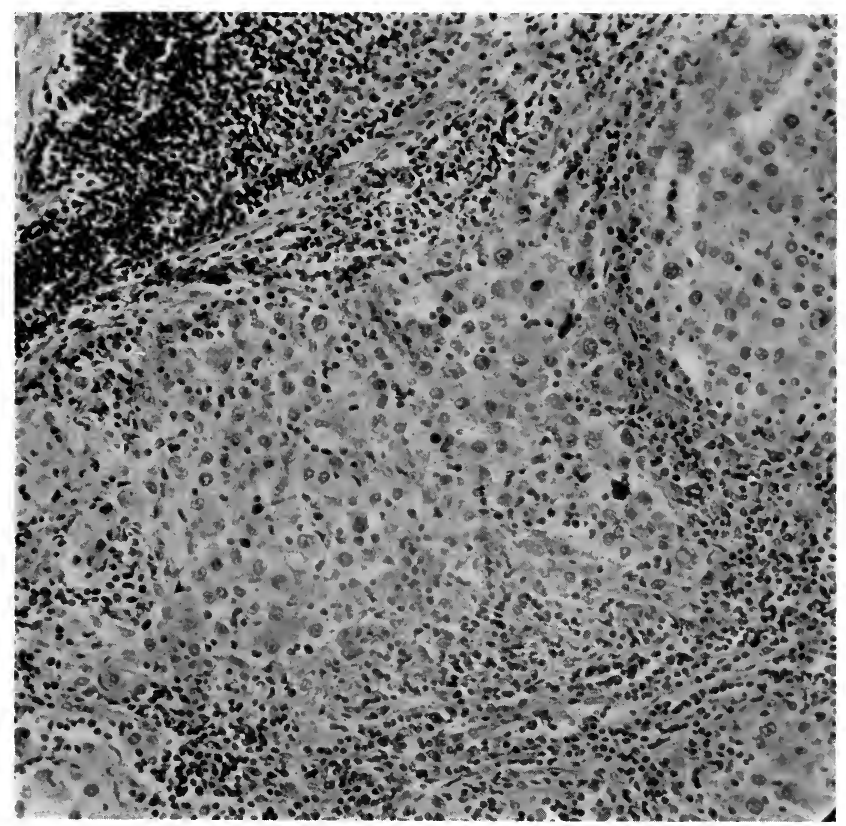

FIG. 127.-Early stage of diffuse endothelioma of lymph-nodes. These cells are derived from the reticulum and sinus endothelium.

fail to show any traces of granuloma, suggesting that the tumor soon overgrows the granuloma or arises immediately in response to the infection. Other infectious agencies besides tubercle probably figure in the disease, and of these Hodgkin's granuloma requires first consideration. Syphilis has not thus far been implicated.

\section{PERITHELIOMA}

That a definite group of tumors exists which deserves recognition under the term "perithelioma" must be admitted, but the exact origin of these tumors and their relation to other neoplasms are questions requiring more extensive data than we now possess.

The existence of a special type of cell about the blood-vessels of the pia mater was first shown by Eberth. In salt solution he was able to isolate 
a thin membrane composed of polygonal or multipolar cells surrounding these vessels. These cells were distinct from the true adventitial cells and formed an outer boundary between the vessel wall and the perivascular lymphatics. Although Eberth did not clearly separate these perithelial cells from the endothelium of perivascular lymphatics, and Kolliker (I896) describes only perivascular lymphatics about cerebral vessels, similar cells have been described about the blood-vessels of the hypophysis (Waldeyer), carotid gland (Paltauf), adrenal, breast, salivary glands (Eberth, Arnold, Sertoli, Luschka), and testis (Henle). Nevertheless the existence of such cells has not been accepted as a well-established fact in normal histology, and the derivation of an important group of tumors from them rests on an uncertain basis. The belief that certain tumors do arise from Eberth's perithelial cells is suggested chiefly by the peculiar arrangement of the tumorcells, radiating out from the supporting vessels, but it must be admitted again that all the tumors in this class do not exhibit this peculiar arrangement of cells. Indeed, one must agree with Ribbert that no tumor has yet been clearly traced to perithelial cells.

Notwithstanding these obscurities the group of perithelioma maintains its existence for the following reasons: ( $\mathrm{I}$ ) These tumors present as a structural unit the blood-ressel, and it is clear that they originate from some cells in the walls of the vessels. (2) Although originating from blood-vessels they differ in structure from angioma on the one hand and from endothelioma on the other. (3) Their gross and clinical characters differ from those of angioma and endothelioma.

From this point of view the term "perithelioma". cannot be regarded as fully applicable, and many other terms have been employed by different authors, as angiosarcoma (Kolaczek), plexiform angiosarcoma (Waldeyer), perivascular endothelioma, and peri-endothelioma (Borrmann). Yet many types of sarcoma are derived from blood-vessels and it seems better to limit the term "angiosarcoma" to the cellular and malignant forms of angioma. The term "endothelioma" is inadmissible since the perithelial tumors differ notably from recognized types of endothelioma and they have not been traced to an endothelial origin.

The position of perithelioma seems to be intermediate between sarcoma, especially angiosarcoma, and endothelioma, and atypical and diffuse forms tend to vary in either direction. Thus diffuse perithelioma may be difficult to distinguish from large spindle-cell sarcoma, while certain cases which it is difficult to eliminate from the peritheliomas (Paltauf, Hildebrand) resemble alveolar endothelioma.

An important source of confusion which has been prominent in the history of angiosarcoma and perithelioma arises from the fact that many other actively growing and malignant tumors assume the structure of perithelioma, either in the original tumor or in metastases or recurrences. Hence Roussy recognizes perithelioma only as a peculiar morphological structure without histogenetic significance. In primary carcinoma of liver and in the roundcell teratoid carcinoma of testis a peritheliomatous structure is frequently observed. In the third recurrence of a teratoma testis I found the entire tumor composed of blood-vessels sheathed with large radiating cells. For such structures the term secondary perithelioma may be suggested. Again, the tissue giving origin to a malignant epithelial tumor may be very vascular so that the tumor from the first assumes the form of spurious perithelioma. These conditions exist in kidney and adrenal, epithelial tumors of which have often been described as perithelioma. Indeed, it is open to question if a considerable number of supposed primary peritheliomas may not owe 
their structure to the excessive development of blood-vessels supporting rapidly growing embryonal epithelial tumors. Metastatic melanoma has a persistent tendency in this direction, so that one may well look for active pigmented moles in all cases of perithelioma of groin or axilla.

Gross Appearance.-Perithelioma usually appears as a well-circumscribed, very vascular, at first slowly, later rapidly growing tumor which may reach considerable dimensions. It is very subject to hemorrhage, necrosis, and ulceration. In early stages it may be freely movable in the tissues, exciting little local reaction, and may be firm, soft, or almost fluctuating or pulsating. On cross-section the tissue appears to be composed of thick-walled and variously twisted blood-vessels. This appearance strongly indicates that the predominant unit in the tumor is the blood-vessel, and since this unit is less pronounced in secondary and spurious perithelioma its presence is the best indication of the nature of the growth. Local extensions and metastases

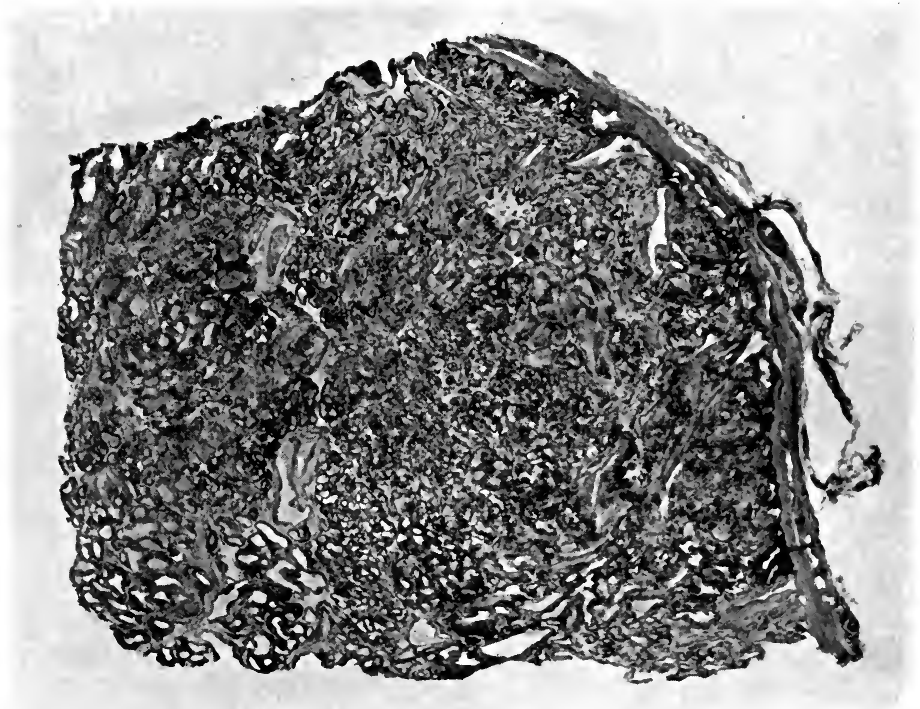

FIG. 128.-Low magnification of cross-section of perithelioma.

occur late, but after imperfect removal recurrence, rapid extension, metastases and cachexia supervene, so that the general prognosis is unfavorable.

Structure.-I. The typical perithelioma presents a very characteristic structure. It is composed of a congeries of small vessels of the type of arterioles sheathed with large polyhedral fusiform or rounded cells which radiate in numerous layers from the vessel wall. At a certain distance from the vessels nutrition often fails and the cells undergo anemic necrosis which serves to isolate and emphasize the vascular units. In many tumors the vessels disappear, the growth is of diffuse large spindle-cells, and the tumor resembles simple sarcoma. Giant-cells of small and large dimensions with multilobed nuclei are a frequent and characteristic feature, especially in the diffuse growths. Intercellular substance appears in many of these tumors and completes the resemblance to sarcoma.

2. An alveolar arrangement of distinctly polyhedral or cylindrical cells 
appears in another group of peritheliomas, especially in tumors of the carotid gland, bone, and skin (Paltauf, Hildebrand, Hanke). Since the cells remain polyhedral or rounded, and intercellular substance is scanty or absent, these tumors resemble endothelioma. At present their exact position must remain uncertain.

In both structural varieties of perithelioma, the walls of vessels and the stroma and tumor-cells may undergo hyaline and especially mucoid degeneration. Some tumors described as myxosarcoma are probably myxomatous forms of perithelioma. The hyaline degeneration may produce the structure of cylindroma.

Clinical Types of Perithelioma.-I. The subcutaneous tissue is one of the chief seats of typical perithelioma. The loose tissues of the anal region, orbit, axilla, popliteal space, and regions of embryonal fissures, but not the

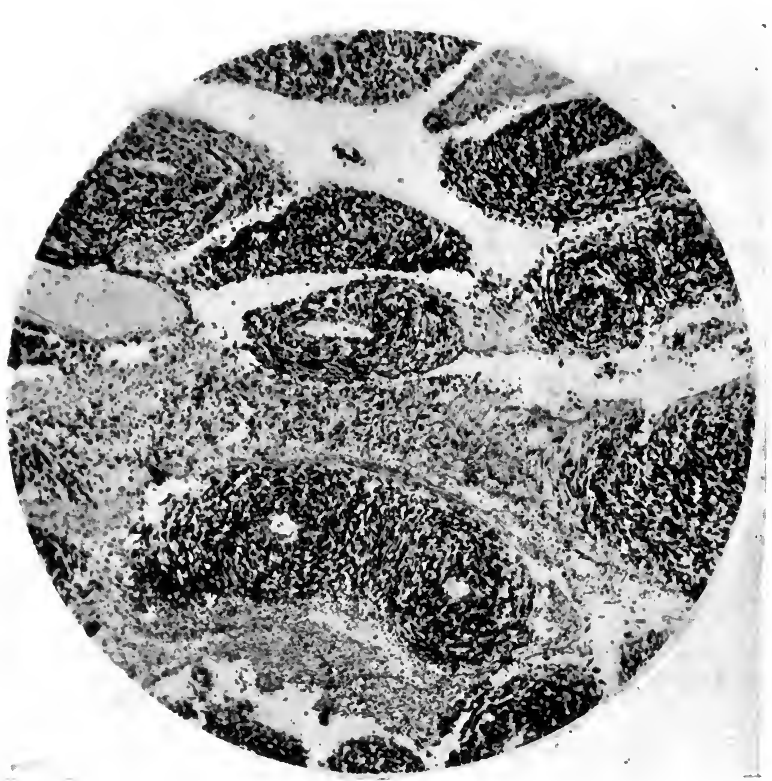

FIG. 129.-Topography of a true perithelioma.

more exposed regions, furnish the exact points of origin, and this distribution suggests a relation to embryonal disturbances of superfluous tissue.

The deep fascix and intermuscular septa are sometimes the point of attachment of peritheliomas which soon appear to be subcutaneous, as described by Kolaczek, Tillmanns, and others.

The buccal mucosa over the hard and soft palate is the seat of a welldefined clinical group of typical perithelioma which has been emphasized by Eisenmenger (Lit.).

2. The pia mater is the seat of a group of somewhat atypical peritheliomas. A well-circumscribed growth $5 \mathrm{~cm}$. in diameter, displacing the brain tissue, with cells concentrically arranged about numerous blood-vessels as in angioma, was early described by Arnold. A flat tumor of the dura mater of the base, involving roots of several cranial nerves and composed of many small vessels lying among loose round and polyhedral cells, was interpreted 
as a peri-endothelioma by Wells. It contained calcific areas and bone spicules. In the brain substance perithelioma has been described by Waldeyer and Billroth and many others, and Waldeyer showed that these tumors had previously been mistaken for primary carcinoma of the brain (Eberth, Arndt). Probably the majority of bulky sarcomas of the brain substance present this structure.

3. In the bones perithelioma appears as a soft vascular tumor, often pulsating, which early perforates the shaft, may invade the surrounding tissues and tends to produce distant metastases (Jaffe). Hildebrand has collected a series of cases and describes one in which the congeries of vessels was plainly evident in the gross. The origin he finds to be in the bone substance and the growth is often preceded by trauma or fracture (Kocher,

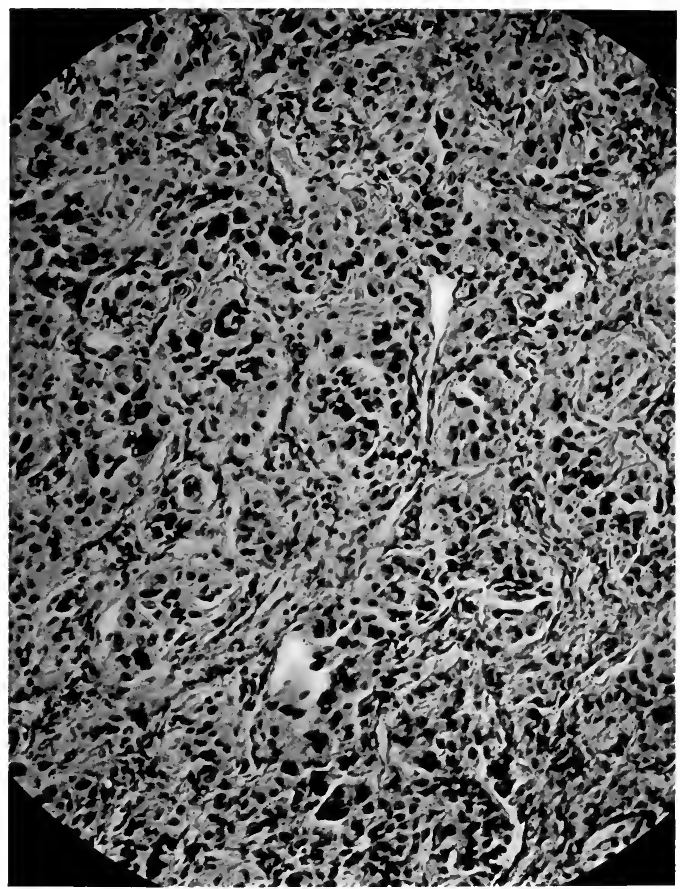

FIG. I30.-Structure of a carotid gland tumor.

Lucke). Howard and Crile interpret 9 cases in the literature and 3 of their own as arising from perivascular endothelium which they identify with perithelioma. The cells are epithelioid in form and their arrangement is either alveolar or tubular. It is not certain that all the tumors described under this term have been clearly separated from endothelioma or from epithelial tumors derived from aberrant cell groups. Many have been described as angiosarcoma, and before the studies of Billroth and Waldeyer they were commonly regarded as primary carcinoma of bone. They are probably identical with angio-endothelioma.

4. In the breast perithelioma is one of the common forms of vascular sarcoma. It occurs chiefly in adults after 50 years as a single circumscribed small or large tumor movable in the breast, but usually adherent to the skin. 
The density, retraction of skin, and involvement of lymph-nodes of carcinoma are absent. Growth is slow, but recurrence after operation is frequent (Schmidt).

In the ovary, among the many forms of so-called endothelioma there is one described by Amann which has certain features of perithelioma.

5. In the kidney, Paoli and others have described large vascular tumors which they interpreted as perithelioma, but it seems highly probable that these growths should be divided among adenocarcinoma of the kidney and hypernephroma. The cells exhibit epithelial characters. The cases of angiosarcoma of the liver described by Arnold exhibited the structure of this type, but whether they are primary or secondary remains to be shown. In cases of primary carcinomas of the liver, areas of typical perithelioma are very frequent ( $L$ 'Esperance).

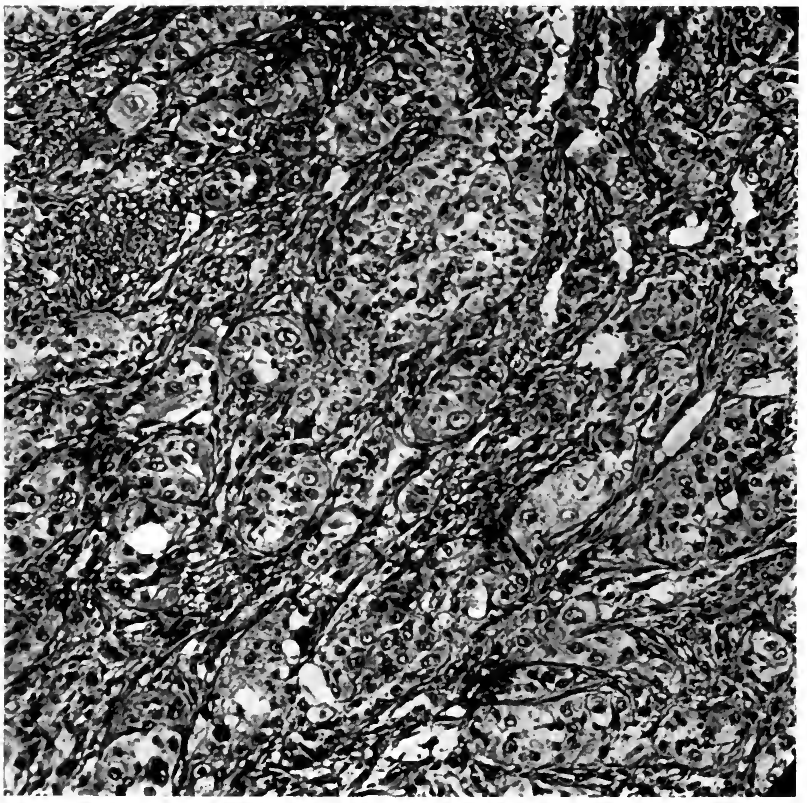

FIG. 13I.-Structure of a carotid gland tumor.

6. Perithelioma of Carotid Gland.-Since the appearance of the simultaneous studies of Marchand and Paltauf describing a characteristic tumor of the carotid gland, many other cases have been reported, minute analysis of the structure of these tumors has repeatedly been made, and the normal histology and embryology of the gland itself has been thoroughly investigated.

While Marchand suggested the origin from perithelial cells, and Paltauf fully accepted this view, later observers have been less confident regarding the exact nature of the growth. Kauffmann employed the term "alveolar tumor," and compared it to a hamartoma, in which all elements of the gland participated. The complex structure of the organ, as shown by Kahn, Stilling, and Monckberg, may serve to explain many peculiarities of the tumors and the difficulty of devising a satisfactory designation. While the tumor belongs in a class by itself, the term "perithelioma" recalls a prominent structural feature and has been very generally employed. 
These tumors arise usually about puberty and slowly reach sufficient dimensions to protrude from the side of the neck, producing a deformity which has been pictured in detail by Keen and Funke. Their location is first upon and behind the bifurcation of the carotid artery which is often inclosed, compressed, and even invaded by the growth. Owing to these attachments the tumors are movable laterally, but not vertically. They may bulge in the pharyngeal wall. Prolongations run in various directions, upward toward the skull, inward to pharynx (Reclus), and downward. Most of the tumors are rounded, lobulated, and encapsulated. Rupture of the capsule may occur (Monckberg, Leithoff). 'They are firm or soft, may yield a bruit, frequently pulsate, and Reclus was able to largely obliterate a tumor by compression. On section they are vascular, grayish red, or slightly brownish from chromaffin substance in the specific cells. The markings show vessels, plexiform cell masses, concentric structures, and hyaline areas.

The growth is slow, occupying years. Heinleth observed a very large tumor existing over 30 years. While numerous adhesions form and vessels may be infiltrated and penetrated, a skilful enucleation is usually successful in preventing recurrence, but Licini estimates the mortality from the operation at 35 per cent. In several cases recurrence has followed operation, and the cervical lymph-nodes have been found involved (Kaufmann, Kretschmar, Kopfstein). General metastases are not observed.

The structure is in general that of an alveolar perithelioma, but varies in many details. Postmortem changes occur rapidly and only prompt fixation of operative material in Zenker's or Orth's fluids preserves the true structural detail.

The chief cells are distinctly polyhedral, granular, and may contain chromaffin substance. They are arranged in compact groups without lumen, but surrounded by hyperplastic endothelium lining the numerous capillaries. Much larger cell groups are often observed, the central portions of which are prone to degenerate and form cavities in which blood may be found. Definite capillaries may also traverse the cell groups (Monckberg). Hyaline degeneration of the cells gives rise to intracellular globules, and affecting the stroma produces peculiar knobs projecting into the capillaries (Kaufmann). The numerous vessels may produce a cavernous tissue or they may be much reduced in hard tumors. They are lined by prominent endothelial cells which sometimes grow out into the vessels in syncytial buds, or mingle with the chief cells of the tumor, producing complex pictures. The stroma may be scanty and acellular or hyaline, or infiltrated with tumor-cells, and Leithoff describes a case in which he regarded the stroma as sarcomatous.

These and many other structural details which appear in many combinations in the reports of numerous authors show that the tumors of the carotid gland are sui generis. Both the specific cells and the endothelium participate in the tumor process, yielding organoid growths involving the entire gland. Although Monckberg thought he could identify ganglion-cells in one case, the numerous non-medullated nerve-fibers of the normal gland appear only in traces and do not participate in the tumor process. Since the tumors arise about the period when a physiological regression usually overtakes the gland, some disturbance in the course of this regression may be an etiological factor (Heinleth). Advanced osteomalacia occurred in a case of Oberndorfer's, and various authors mention other possible etiological factors. The occurrence of lemon-yellow pigmentation of the skin, disappearing after operation, in 2 cases reported by Reid indicates a disturbance of endocrine function. 


\section{CHAPTER XXI}

\section{LYMPHOMA AND LYMPHOSARCOMA}

Two important physiological properties influence the conception of tumors of lymphoid tissues.

First, lymphoid tissue responds to irritation with inflammatory hyperplasia far more actively than any other tissue. Second, lymphoid tissues are relatively mobile rather than fixed, and lymphocytes are not only ameboid, but are structurally placed in easy access to lymph and blood paths. Hence tumors and tumor-like processes in lymphoid tissues are frequent and as a rule tend to become widely diffused. These same factors render unusually difficult the distinction between simple and neoplastic hyperplasia of lymphoid tissue by introducing special standards, both clinical and anatomical, in the interpretation of lymphatic hyperplasia.

For these reasons the discussion of lymphoma in general necessarily includes the consideration of various processes, some of which are chiefly inflammatory, some neoplastic, and others intermediate in position. Lack of knowledge of etiological factors, the intricate relation of many forms of lymphoid hyperplasia, and the occasional transformation of one process to another, render this method of treatment practically advantageous and theoretically sound.

Extensive tumor-like hyperplasia of lymphoid tissues occurs in the following scheme of clinical conditions: (I) Simple lymphoma; (2) leukemia, lymphatic and myelocytic, in its various phases; (3) pseudoleukemia; (4) Hodgkin's disease; (5) lymphosarcoma.

The complexities of the subject of lymphoid tumors depend chiefly upon the lack of knowledge of etiology and partly upon the lack of accurate anatomical classification. It seems to the author that some advantage would result from the rigid application of simple anatomical principles in the classification of these processes, even at the risk of carrying the anatomical distinctions too far.

Anatomically there are three elements in lymphoid tissues capable of originating lymphoid tumors, viz.: (I) lymphocytes; (2) reticulum cells of follicles and pulp, and (3) endothelial cells of pulp and cavernous sinuses. Connective-tissue cells, endothelium of vessels, and cells of vessel walls do not appear to be concerned in the common tumors of lymph-nodes.

Classified according to present indications of histogenesis, tumors of lymphoid tissue appear as follows:

\section{Origin \\ Lymphocytes \\ Lymphocytoma}

Reticulum cells

Large round-cell hyperplasia or neoplasia

Endothelial cells Endothelial hyperplasia or neoplasia

\section{Clinical Type}

Simple lymphoma

Tuberculous lymphoma

Lymphatic leukemia

Pseudoleukemia

Malignant lymphocytoma

Granuloma malignum

Myeioid leukemia

Hodgkin's sarcoma

Large-cell lymphosarcoma

Endothelial hyperplasia

of tuberculosis, etc.

Endothelioma 
Several difficulties stand against the finality of this classification:

I. The relation between lymphocytes and reticulum cells is not fully determined. While the older anatomists, headed by Flemming, held that reticulum cells of the follicles become transformed into lymphocytes, practically all the recent work indicates that the two types of cells are entirely separate (Gulland).

It would greatly simplify the questions of histogenesis if separation of the two types of cells were established. Most pathologists, however, the writer among them, are unable to satisfy themselves that reticulum cells during the course of inflammatory and neoplastic changes may not assume the form of lymphocytes. Yet the separate nature of the two cell types is of such great

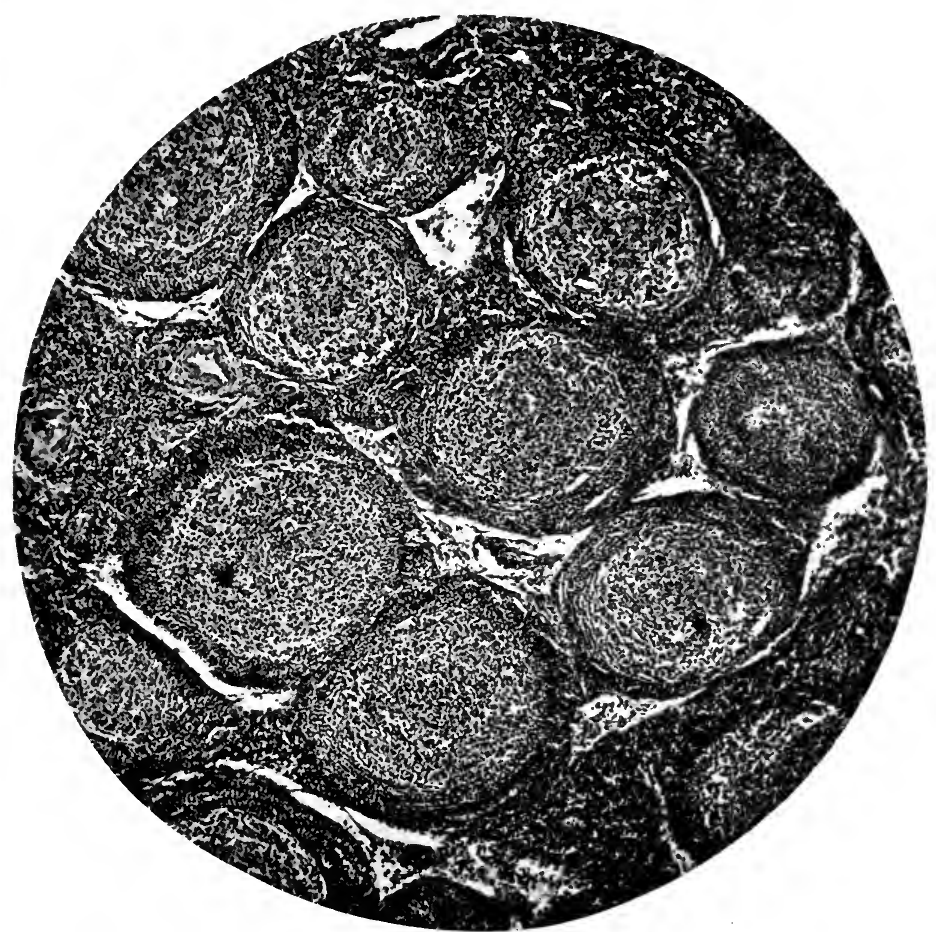

FIG. I32.-Multiplication of follicles in simple chronic lymphadenitis, in postpyloric nodes draining a chronic ulcer of stomach.

importance in the study of lymphoid tumors, that it seems wise to accept the dictum of embryology until it is proved incorrect. In most pathological processes the two cell types undoubtedly maintain their individuality.

2. The distinctions between reticulum cells and sinus endothelium are much less definite. In fact, in the development of lymph-nodes both arise from modified mesoblastic cells. Yet in a study of endothelioma of lymphnodes the writer has brought evidence to show that the reticulum cells give origin to the large-cell lymphosarcomas, while the sinus endothelium yields tumors of a different structure.

3. While the lymph-nodes in lymphatic leukemia contain an excess chiefly of lymphocytes, the origin of the larger round-cells in the nodes of myelocytic 
leukemia is not satisfactorily determined. Their classification as derivatives of reticulum cells must be regarded as tentative.

4. If a complete solution of the etiology of lymphoid tumors were provided it might destroy much of the value of an anatomical classification. The best known excitant of lymphoid enlargements, tuberculosis, produces overgrowth of lymphocytes, extensive or exclusive hyperplasia of reticulum cells and endothelium, and also introduces many blood-cells by exudation. Hodgkin's granuloma exhibits similar or identical tendencies.

Yet there is much value in classifying lymphatic tuberculosis from the anatomical standpoint, and similar value may remain for the anatomical subdivisions of other diseases when their etiology is known. For the definite tumors of lymph-nodes this objection loses much or all of its force, but it is difficult to determine when one is dealing with a granuloma or a tumor of this tissue.

\section{SIMPLE LYMPHOMA. LYMPHADENOMA. HYPERPLASTIC LYMPHADENITIS}

Local or regional, circumscribed, chronic enlargements of lymph-nodes which are self-limiting and do not lead to systemic invasion are rather com-

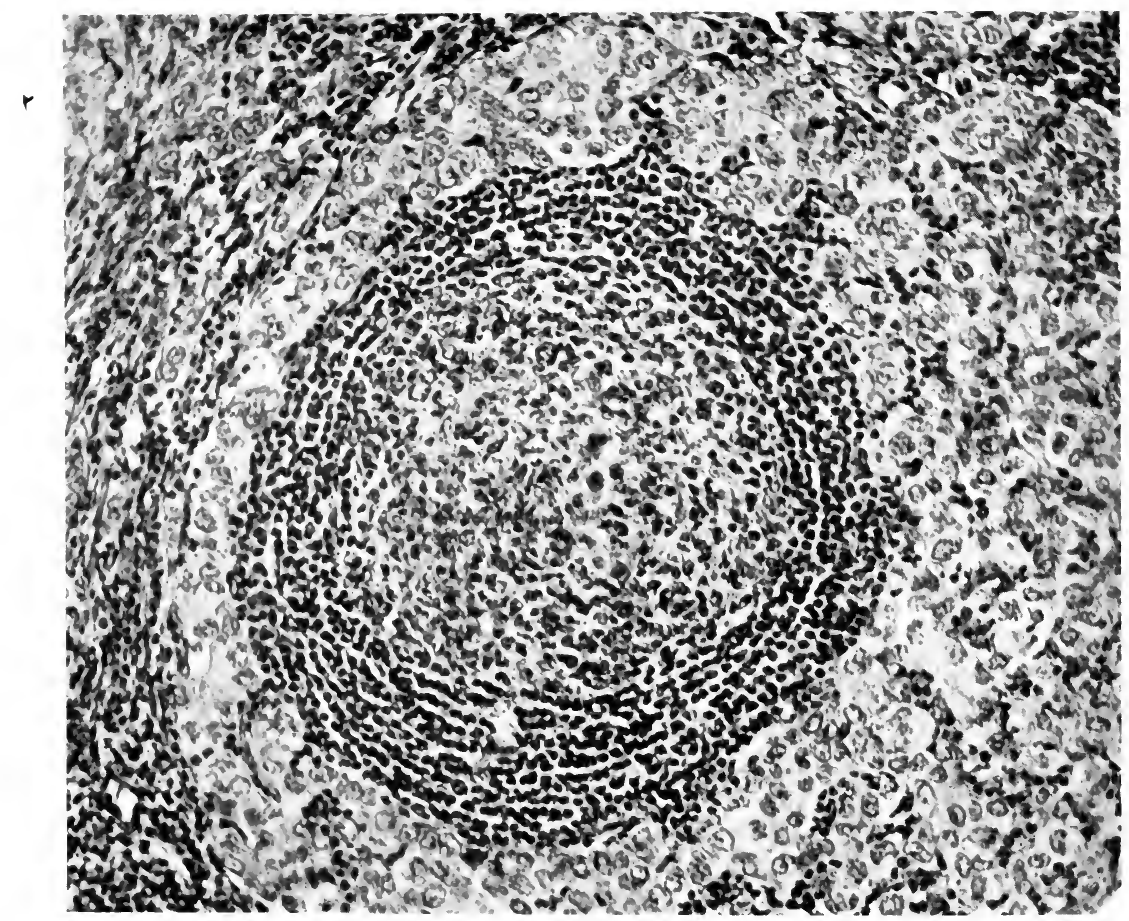

Fici. I33.- Inflammatory reticulum cell hyperplasia in a lymph-node.

mon pathological conditions (Le Count, Lit.). They appear in the neck, axilla, groin, subcutaneous tissue, submucous tissues, parotid and other 
glands, and occasionally in other regions. They form solid masses of tumorlike dimensions and firm consistence, and on section exhibit a smooth opaque uniform surface often presenting firm, light, more prominent areas, and revealing little or no tendency toward degeneration or necrosis. They may be composed of a single node or a congeries of nodes with fused capsules. After reaching a certain limit of growth they may remain stationary for months or years producing only local symptoms. The tendency toward regression is slight. I have observed a simple lymphoma of 4 years' duration occurring beneath the skin of the scapular region which failed to show signs of regression or fibrosis. The prolonged course is a feature of many cases, that of Le Count continuing for I 5 years, but still showing hyperplastic germ follicles. The tumor described by Vagner and Hewitt reached dimen-

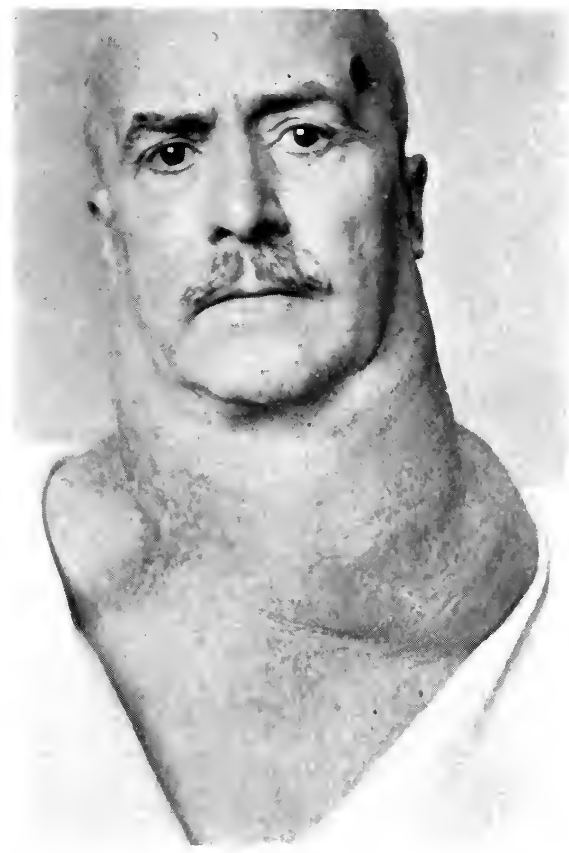

FIG. I34.-Malignant lymphadenoma.

sions of $7 \times 5 \mathrm{~cm}$. in 6 years. Internal growths of uncertain history may be found at autopsy.

The structure varies at different periods, but is dominated by diffuse overgrowth of typical small lymphocytes. In early stages lymph-follicles are increased in size and number, are readily visible in the gross and exhibit enlarged germ centers, the pulp tissue is composed of closely packed lymphocytes, the sinuses are indistinct or obliterated, the vessels compressed or packed with lymphocytes; or the normal landmarks are obliterated by a diffuse growth of lymphocytes and the distended capsule is infiltrated. Simple lymphoma may be separated from leukemia by the blood test, and from pseudoleukemia by its local character, but from neither on the histological structure alone. From lymphosarcoma the diagnosis may be difficult, and 
should be based on the absence of the usual signs of malignancy, as rapid growth, mitotic figures, active destruction of trabeculæ and capsule, and atypical quality of the cells.

However obvious may be the inflammatory origin of many cases of simple lymphoma, the excessive hyperplasia, prolonged course, and often the apparently idiopathic origin renders the condition of interest in the field of tumors and requires the recognition especially in chronic cases of certain features of true neoplasms.

I have observed one case, structurally requiring classification as lymphadenoma, persisting for I 8 years and eventually proving fatal with rapid local growth and asphyxia (Figs. I34-136).

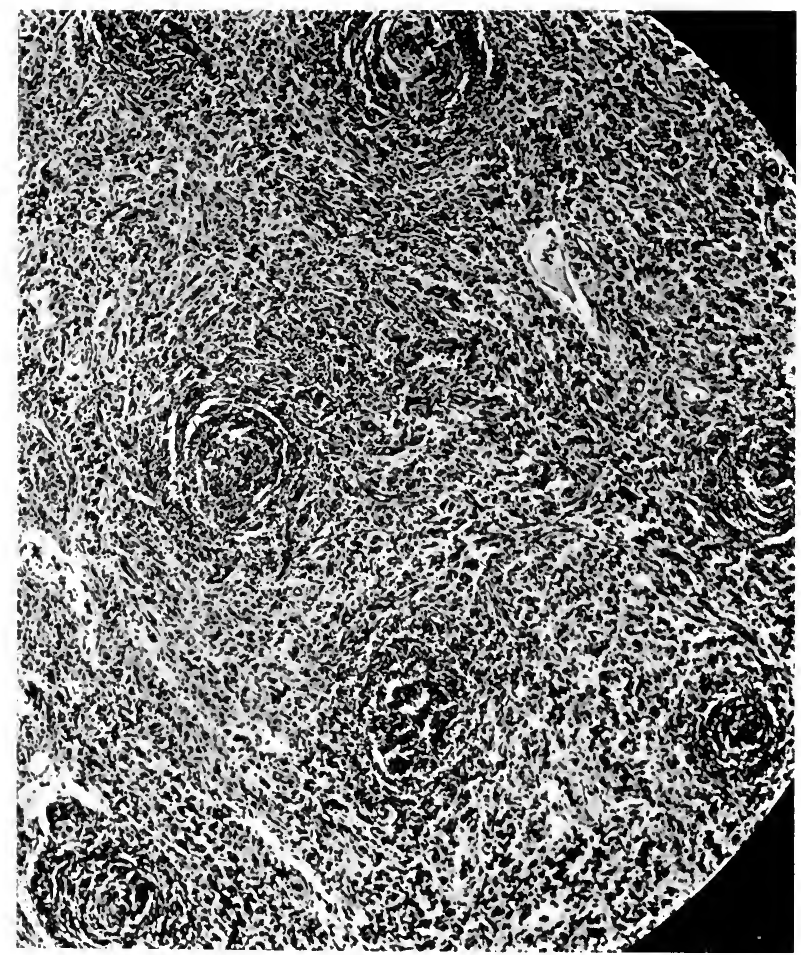

F1G. I35.-Malignant lymphadenoma. Same as Fig. I34.

In the etiology of simple lymphoma many forms of chronic bacterial infection appear to be concerned, especially tuberculosis. According to Paltauf the regeneration of lymphocytes following an acute infection may be excessive and prolonged, giving rise to a tumor-like overgrowth which persists long after the removal of bacteria and their products. In the case of tuberculosis, it has been shown by Bartels and others that the structure of simple lymphoma may be exhibited by lymph-nodes harboring viable but attenuated tubercle bacilli, and the frequency of simple lymphoma without tubercle bacilli in tuberculous subjects suggests that this lesion may be one of many which seem to be referable to atypical tuberculosis. Such 
cases may be designated as paratuberculous lymphadenitis (Adami). In certain cases it is impossible to establish any relation whatever to tuberculo-

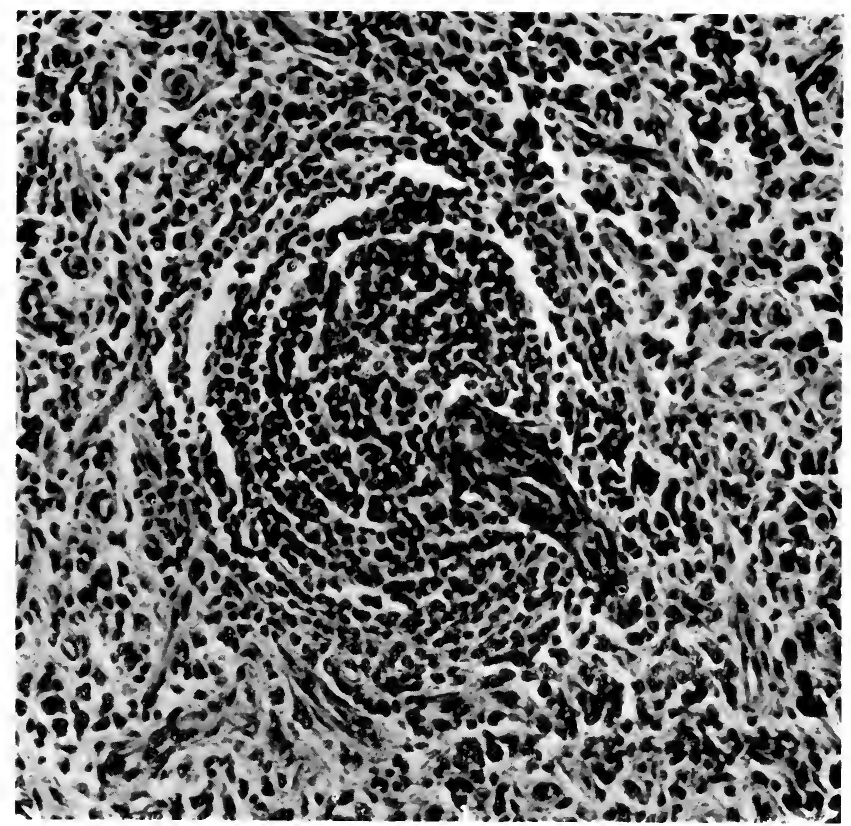

FIG. I36.-Malignant lymphadenoma of cervical nodes. Detail of Fig. I34.

sis, or any acute infection, or any chronic disturbance in neighboring mucous membranes or tissues.

\section{LEUKEMIC LYMPHOMA}

The leukemic process in its entire scope illustrates many phases of hyperplasia of lymphoid tissue. The disease may exhibit as its anatomical basis: (a) A transient leukemic blood-picture with slight hyperplasia of the cells of lymph-nodes or marrow; (b) acute leukemia associated with acute hyperplasia of lymphoid tissues; $(c)$ chronic leukemia, with leukemic lymphomas and hyperplasia of bone-marrow; $(d)$ chronic leukemia with partially neoplastic hyperplasia of lymphoid and myeloid tissues; $(e)$ chloroma.

While it is impossible to prove that leukemia is always caused by a single infectious agent or that it represents anything more than a clinical entity comparable to leukocytosis, these various lesions belonging to essentially the same condition illustrate every gradation from an acute inflammatory process up to a chronic and somewhat malignant neoplasm.

The lesions of leukemia, both lymphocytic and myelocytic, involve the bone-marrow, lymph-nodes, spleen, and the other preëxisting lymphoid tissues, while heterotopic growths of lymphoid tissue appear in the liver, kidney, lung, skin, serous membranes, and many other organs. The picture is that of a diffuse systemic involvement of blood-forming organs with secondary invasion of other tissues.

In the bone-marrow the process begins in multiple hyperplastic foci which 
enlarge, coalesce, and eventually extend the limits of lymphoid marrow throughout all portions of the skeleton. At the height of the process the new tissue is firm, light colored, opaque, or pyoid, or beset with foci of infarction, necrosis, hemorrhage, or mucoid softening. Fat cells, sinuses, and all other normal structures of the marrow are commonly obliterated by a diffuse growth of hyperplastic round-cells. Banti describes an initial hyperemia with widening of sinuses and atrophy of fat-cells. Islands of hyperplastic marrow-cells then appear in and about the sinuses and these are followed by pure lymphoid growths starting from the walls of arterioles and gradually invading the entire marrow. The spongy trabeculæ are often absorbed and even the shafts may be thinned, but a distinctly aggressive destruction of bone as in true tumors is missing. In rare cases of myelemia there is wide-spread osteosclerosis (Schmorl).

In the lymph-nodes the disease appears in one or more chains spreading to other chains and involving all the nodes of the chain simultaneously. Bulky tumors are thus produced in which all the nodes remain discrete or the capsules are fused by inflammatory reaction. The process in the lymph-nodes differs in the two main types of leukemia. In myelemia the pulp cords first show increase in size by the appearance of myelocytes which gradually distend the cords, obliterate the sinuses, cause passive atrophy of the lymph-follicles, and even infiltrate the capsule or the surrounding fat tissue. Giant-cells and nucleated red blood-cells may be present, but in the more pronounced lesions only large myelocytes are present.

In lymphemia the condition in the lymph-nodes varies. In some cases there is hypertrophy of lymph-follicles which become greatly enlarged and fusing with the pulp cords convert the entire node into diffuse lymphoid tissue. In such nodes the lesion is primary and these cases may be designated as lymphoplastic lymphemia (Pappenheim, Meyer, Heinicke, Hirschfeld). In other cases the lymph-follicles fail to enlarge, but seem passively compressed by new growth of lymphocytes beginning in the pulp cords. Opinions differ as to the relative frequency of the two types of lesions. According to Walz, Pappenheim, Grawitz, Kelly, Gulland, and Goodall lymphemia is always primarily myelogenic, while Ehrlich and Pincus maintain that the whole lymphoid system may be primarily involved. My own cases seem to illustrate both conditions, but I have not seen active growth of germ centers in lymph-nodes of leukemia. The extent of the affection of lymph-nodes varies greatly. Thus Walz found scanty changes in nodes, much in spleen and marrow; Pappenheim describes absence of changes in nodes, few in spleen, much in marrow; while Meyer and Heinicke record early and extensive lesions in nodes, later in spleen, and later and only focal hyperplasia in bone-marrow. Turk ventures to conclude from clinical signs that marrow lesions may be absent in early stages of lymphemia, and this view is substantiated by Rosenfeld and others, who believe that the marrow lesions may appear late in the disease.

The splenic lesions are very similar to those of the lymph-nodes. The organ exhibits a diffuse enlargement, a smooth opaque surface and section, areas of infarction and necrosis, but seldom any localized tumor growth. In myelemia there may be a simple myeloid transformation of pulp with atrophy of follicles. Here myelocytes, giant-cells, nucleated red blood-cells, and phagocytes are mingled, much as in the marrow. More often there is a diffuse infiltration with cells of the myelocyte class. In late stages and especially in very large spleens the cells are greatly reduced and fibrosis, hyaline degeneration, hemorrhage, and necrosis are prominent. In lymphemia the follicles may be intact and enlarged, but more often they are obliterated by a 
diffuse growth of lymphocytes which lie loosely in the disordered cords and sinuses.

In the submucous lymphoid tissues the process resembles that of the lymph-nodes.

The heteroplastic deposits of leukemia occur chiefly in the liver. Here there is a diffuse growth of round cells in the capillaries which has little tendency to destroy liver cords, but miliary or larger lymphomas occur in the portal canals. A certain grade of these lesions is practically constant in all forms of leukemia. In lymphemia the portal nodules are more extensive than in myelemia.

In the kidney leukemic infiltrations, focal or diffuse, frequently occur. The cutaneous manifestations of leukemia are varied and complex (Oertel, Lit.). Lymphemia has been observed with spindle-cell sarcoma of the skin, with multiple lymphomas, and with mycosis fungoides (Nekam, Pincus, Lit.). The characteristic lymphomas appear as multiple nodules chiefly on the face and neck or widely distributed as a papular eruption. They may

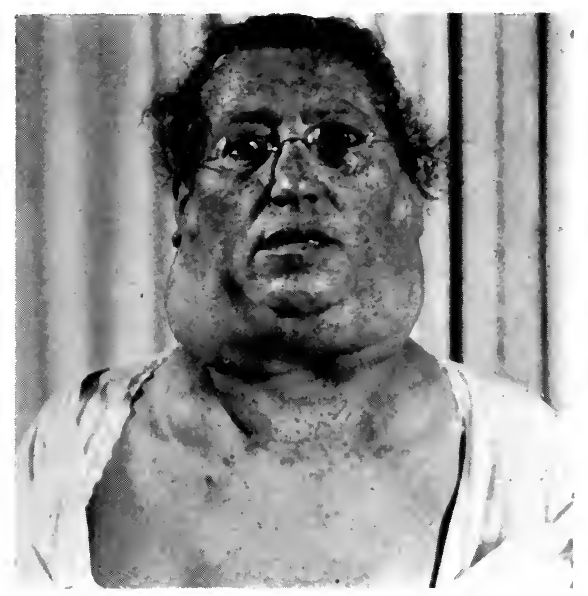

FIG. I37.-Advanced chronic myelocytic leukemia.

be preceded by or associated with other eruptions. The older lesions may ulcerate (Nicolau). They are composed of lymphocytes grouped about the vessels of the corium.

Cutaneous lesions occur with moderate frequency, but in great variety, and illustrate in their course many phases of a granulomatous inflammation unfolding into a tumor-like process. The lesions include petechiæ and hematomas, vesicles and bullæ, macules, papules and pustules, and localized or diffuse tumors. There may be marked pruritus and terminal bronzing (Hazen, Lit.). Very similar conditions occur without leukocytosis, as in Hodgkin's disease, pseudoleukemia, and mycosis fungoides. In rare cases the lesion is diffuse and universal (Arndt). Other organs and tissues are occasionally the seat of leukemic infiltrations.

There has been much discussion regarding the origin and nature of the heteroplastic deposits. Perhaps the majority of observers have concluded that in both forms of leukemia the hepatic deposits arise from hyperplasia of preëxisting lymphoid structures in this organ which undergo simple hyperplasia in lymphemia and myeloid changes in myelemia (Sternberg, Lit.). 
Some authors assume the existence of latent myeloid foci in liver, lung, etc., which serve as a source of the new tissue in myelemia (Dominici, Hirschfeld, Walz). From these foci may arise the myeloid changes so often seen in certain infectious diseases attended with anemia (Meyer, Heineke). Others accept the local origin of the heteroplastic lymphomas of lymphemia, but deny the existence of myeloid foci. Thus Sternberg distinguishes sharply between lymphemia and myelemia in the origin of their heteroplastic deposits, and Meyer and Heineke deny that any myeloid foci exist in normal nodes, spleen, or liver, although they find the source of lymphemic growths in slumbering lymphoid nodules. At the other extreme Banti holds that all heteroplastic leukemic nodules represent metastatic growths of embolic cells.

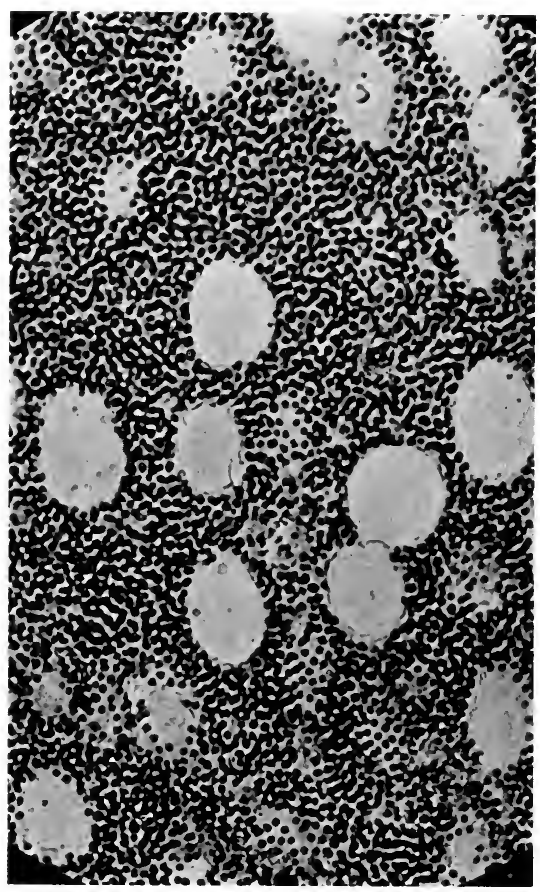

FIG. I38.-Bone-marrow in lymphatic leukemia. Focal hyperplasia of small lymphocytes in center of femur.

At present it seems impossible to offer proof of the entire validity of any of these theories. I believe, however, that too much importance may be placed upon the hypothetical lymphoid foci of such organs as the liver and kidney. In the normal adult human organs such foci are conspicuously wanting, but in lymphemia such organs rapidly develop lymphomas. For the more distant lymphomas of skin, brain, and serous membranes, especially those which reach a bulky growth, it is more difficult to accept an origin from preëxisting lymphoid foci, while the origin from embolic leukocytes seems much more probable.

Finally, in those cases in which the lesions exhibit more definite neoplastic properties, as in the leukosarcomatosis of Sternberg and in chloroma, it is 
highly probable that the laws of neoplasms control the development of the heteroplastic nodules. Hence it is necessary to assume that in mild forms

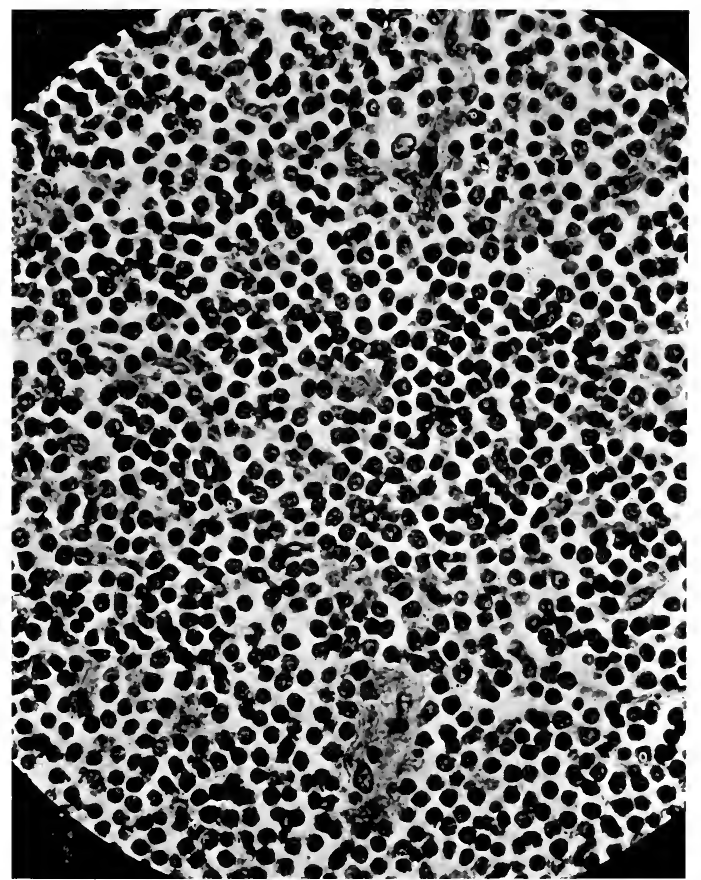

FIG. I39.-Lymph-node in chronic lymphatic leukemia. Note the very uniform size and distribution of the cells.

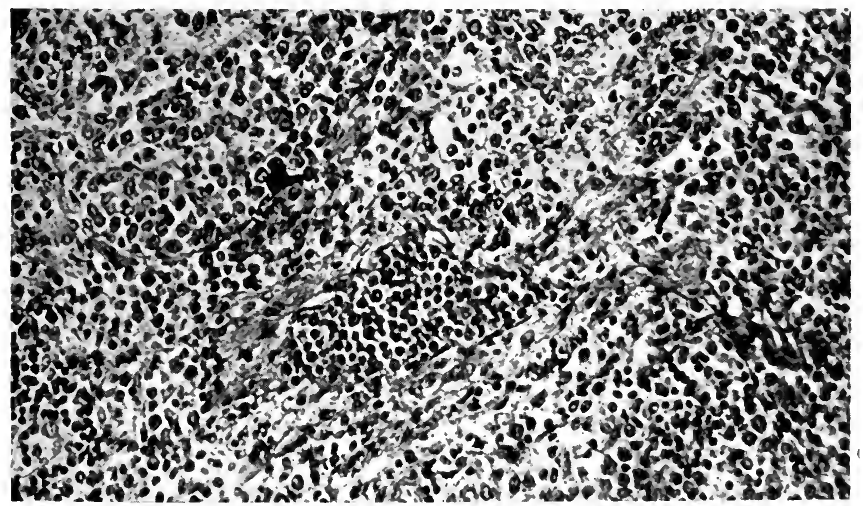

FIG. 140.-Chronic myelocytic leukemia. Section of lymph-node showing diffuse growth of cells of uniform size.

of lymphemia the heteroplastic nodules arise in loco, while in the more intense grades of leukemic hyperplasia they are chiefly embolic in origin or else one 
must accept their embolic origin throughout. The latter position seems to me the more logical and more in accordance with observed facts.

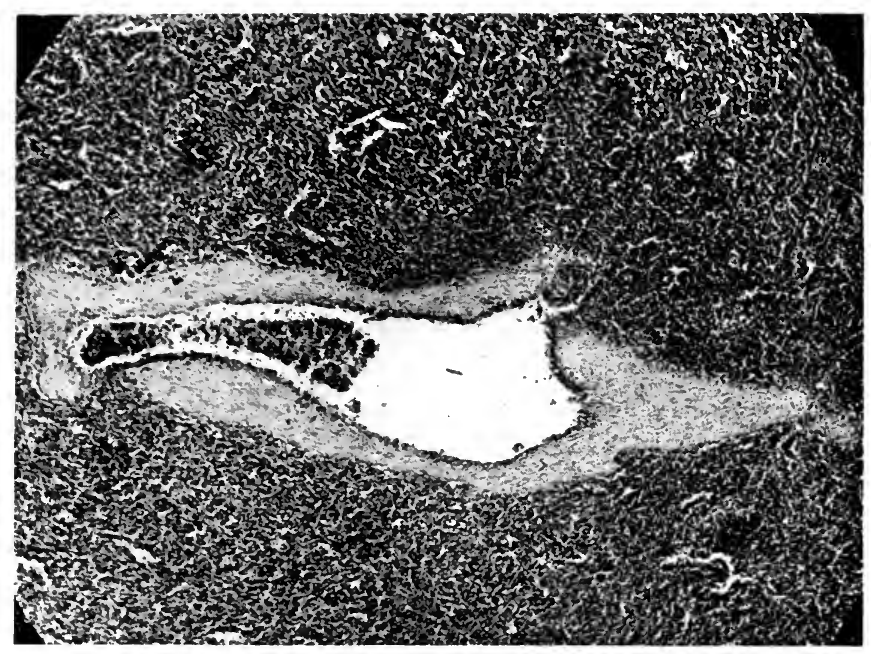

FIG. I4I.- Structure of spleen in a case of lymphatic leukemia. Focal and diffuse overgrowth of lymphocytes, which are found in cavernous sinuses, pulp sinuses, pulp cords, and in enlarged follicles.

In myelemia the evidence in favor of the embolic origin of heteroplastic foci is much more direct. Myeloid foci do not exist in normal adult liver or spleen, and while they develop there in infectious diseases and in anemia

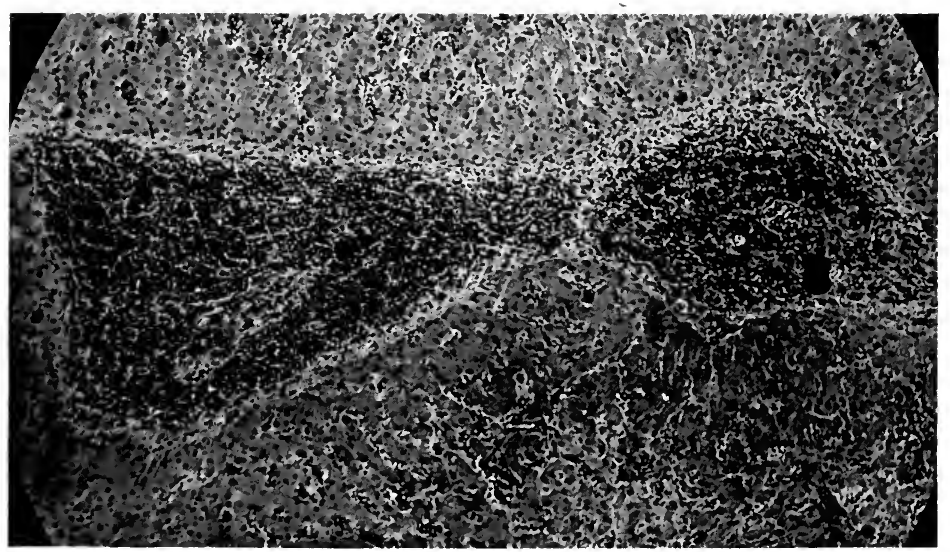

FIG. I 2.- Liver in lymphatic leukemia. Lymphocytic infiltration of portal canals and hepatic capillaries.

their constituent cells have not been satisfactorily traced to preëxisting cells in these organs. In leukemia the blood-ressels in and about myeloid areas often contain thrombi of myelocytes and giant-cells which offer the readiest 
explanation of heteroplastic nodules (Meyer, Heineke, Schwartz, Michaelis). Yet an embolic origin does not involve a neoplastic nature if, as Sternberg claims, leukemic cells are normal marrow-cells produced in large numbers, for sarcoma-cells are atypical and grow without normal restraint. Yet Ribbert and others find that the leukemic cells are undifferentiated and abnormal and exhibit in some degree the characters of tumor-cells. In ordinary myelemia the myeloid areas show little of these neoplastic properties, but in the atypical and rare forms bulky tumors arise, tissues are invaded, cells are atypical, and neoplastic characters are rather obvious.

Changes in Blood.-Closely dependent upon the organic lesions are the changes in the blood, the full discussion of which falls outside the scope of

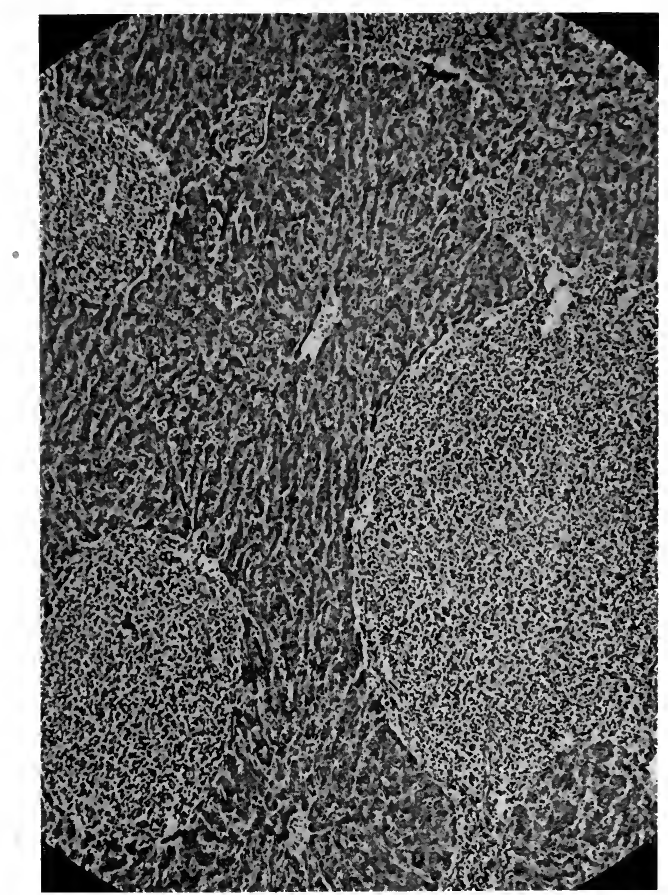

FIG. I43.-Miliary lymphocytomas in the liver of lymphatic leukemia.

this work. Briefly they consist in extensive increase of leukocytes and progressive anemia. In lymphemia the cells are small and medium-sized lymphocytes in chronic cases, and larger undifferentiated lymphocytes or mononuclear cells in acute cases. In myelemia the new cells are chiefly neutrophile myelocytes, many of which are of abnormally large size, atypical eosinophile myelocytes, excess of polynuclear neutrophile and eosinophile cells, mast cells, and various degenerative forms. While in typical cases the leukocytosis is the first demonstrable symptom, it varies greatly in degree, in its relation to the extent of the organic lesions, and in its permanence. While the leukocytes may outnumber the red cells, leukemia occurs with small numbers of atypical cells in the blood, and their numbers are subject to extensive variations from numerous factors. 
The cause of the access of large numbers of cells to the blood-stream still remains obscure. It is not dependent essentially on involvement of the bonemarrow, since apparently identical marrow lesions in pseudoleukemia fail to give leukocytosis. Multiplication in the blood-stream is a negligible factor. The theory of circulating chemotactic agents is unsatisfactory (Gulland, Goodall). In many cases it seems to be dependent upon the activity of the hyperplasia in the organs, but the more aggressive the local process in the neoplastic sense, the less is the leukocytosis. It may appear in the late stages of formerly aleukemic processes, and its inception has once been clearly traced to rupture of the tumor mass into a large vein. Essential conditions

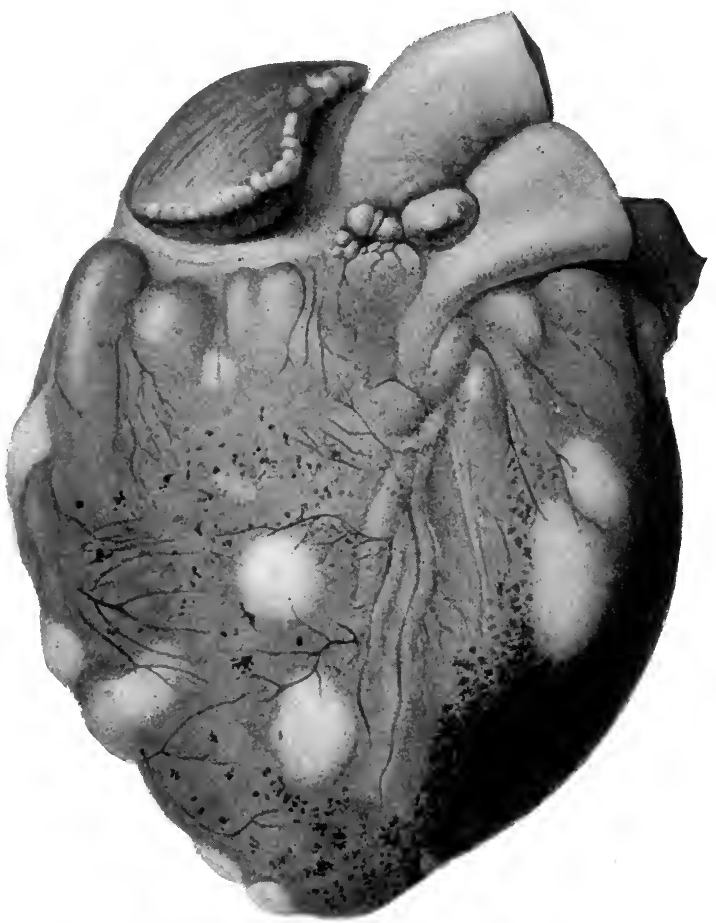

FIG. I44.-Leukemic deposits in the heart muscle. Similar tumors in spleen and kid. neys. Enlargement of thymus and many lymph-nodes. (After Seelig.)

seem to include the association of a certain grade of hyperplasia with integrity and patency of many small blood and lymph paths.

The course of leukemic lesions depends much on the rapidity of the process. Acute cases steadily progress until the fatal issue. In chronic cases there is a definite tendency for the cellular phases of the lesions to be followed by fibrosis and there is a distinct limit to growth. Likewise the leukemic lesions as well as the leukocytosis may be much altered by intercurrent infections, treatment by arsenic, $x$-ray, or without assignable cause.

Atypical Leukemia.-Certain atypical forms of leukemia vary widely from the common type and reveal the extensive scope of the anatomical processes associated with the condition.

Leukanemia.- Under this term Leube first described a condition of pro- 
gressive pernicious anemia with pronounced leukocytosis and cellular infiltrations in the organs resembling those of leukemia. It has long been known that in certain cases of pernicious anemia the marrow changes may be very similar to those of leukemia (myeloblastic degeneration of Naegeli).

In such cases the leukocytes are increased and myelocytes are present in considerable numbers. Meyer and Heineke report cases of pernicious anemia, leukanemia, and atypical leukemia in which the organic lesions are practically identical, and Preis and Nauwerck and Moritz describe cases in which the blood-picture at certain periods resembles that oi pernicious anemia. These observations seem to indicate that the leukemic process in a mild degree may be established in many states of anemia which call forth excessive proliferation and degeneration of blood-forming cells.

Mixed-cell Leukemia.- In rare cases of lymphemia myelocytes have been found in the blood and slight myeloid changes in the organs (Weber). While these cases suggest a mixed form of leukemia, Pappenheim and others interpret the myeloid changes as a secondary process of compensatory myelocytosis resulting from the anemia of the disease. More often a frank myeloid leukemia appears to change into a large-cell lymphemia (Seelig, v. der Wey, Wilkinson, Mellard, Turk). In these cases it appears probable that in the advanced stages of the disease degenerative processes affect the myelocytes so that they lose their granules without any genuine change in the type of the disease. Thus Wolff and Michaelis describe cases of leukemia in which they interpret the proliferating cell as the mother cell of both lymphocyte and myelocyte. They believe that the acute leukemias are chiefly of this nature. While Turk, Hirschfeld, and others argue strongly for the existence of mixed and transitional cases of leukemia the evidence in favor of their views is based chiefly on blood examinations, lacks the support of anatomical studies of the organs, and is unsatisfactory.

Aplastic leukemia is a term applied by Wolff to a condition marked by anemia, slight leukocytosis with 96 per cent. of large atypical lymphocytes, lymphomas in the liver and a deficiency of colorless cells in the marrow. The significance of such cases is not clear, but the condition appears more closely related to pseudoleukemia. Extensive fibrosis occurs in the bonemarrow in late myelemia so that this organ becomes virtually aplastic (Lehndorff, Zak).

Leukosarcomatosis (Sternberg).-While the neoplastic nature of the entire leukemic process has never received general endorsement it has long been recognized that in certain cases the anatomical condition varies from the standard type in the direction of a true neoplasm. The more conspicuous features of such atypical cases have been emphasized by Banti, who in view of this evidence has claimed that the leukemic process, at least the lymphatic, is always neoplastic. They consist of marked invasion of bone by the hyperplastic marrow, the penetration of the shaft and invasion of periosteum with miliary leukemic nodules, the fusion of leukemic lymph-nodes, the appearance of multiple nodules in serous membranes, the invasion of intestinal and cardiac muscle by lymphomas, and the atypical structure, large size, and indefinite character of the cells of leukemic infiltrations.

In a considerable series of cases a large sarcomatous tumor of the thymus with leukemic blood-picture has been the chief feature (Fabian, Lit.), so that leukemia with involvement of the thymus came to be recognized as an atypical and malignant variety. Orth speaks of these growths as malignant leukemic lymphoma. With thymic tumors the other lesions of leukemia have not always been fully developed (Heubner). Lymphemia is not infrequently associated with an infiltrating process in the tonsils (Askanazy). 
Other regions have also been the seat of lymphosarcomatous tumors with leukemic blood-picture. In a case of Warthin's the ileum was extensively involved in a large abdominal tumor which extended to the kidneys, stomach, and regional lymphatic system, but the marrow was normal. Of 90,000 leukocytes 86.6 per cent. were atypical mononuclears resembling those of the tumor. Moritz reports a somewhat similar case of tumor of the cecum in which spleen, nodes, and marrow were involved. Pernicious anemia with terminal leukocytosis suggesting leukemia may occur with extensive sarcomatous tumors of bones, lymph-nodes, and spleen (Grawitz, Lazarus).

At this point is encountered the possibility that tumors essentially different from leukemia may discharge many cells into the blood and simulate leukemia. The well-known case of Lucke's illustrates this event. Here a round- and spindle-cell sarcoma of the axilla ruptured into a subclavian vein and the patient died with extensive leukocytosis as in leukemia. That this case was not leukemia is shown by the absence of lesions in spleen and lymphnodes. The blood contained many large round and spindle cells. Martin and Matthewson report several cases of lymphosarcoma with leukocytosis in which they found it difficult to establish the diagnosis of leukemia. Where the usual systemic lesions are present there seems to be no other alternative than to class the condition with leukemia. In nearly all these atypical cases the blood-picture has varied from the usual types, and the abnormal cells, not always very numerous, have belonged in the class of large undifferentiated mononuclear leukocytes.

Out of this group Sternberg attempts to establish a special variety of leukemia, leukosarcomatosis, which he would sharply separate from the ordinary type, on the ground that it represents a true tumor process. The atypical characters of the cells he regards as the expression of a neoplastic quality, and he gathers from the literature a series of cases described chiefly as lymphatic, acute, and chronic, in which the presence of these cells and the infiltrating characters of the organic process indicate the existence of a neoplasm. Sternberg's views have been attacked by Turk and others, who have shown that many of the anatomical features of leukosarcomatosis are observed in otherwise typical leukemia. Nevertheless Sternberg's studies have been of service in contrasting different types of leukemia and in showing that there are different grades of hyperplasia associated with leukemia and that in some cases the process closely approaches a malignant tumor.

Lymphoid-cell leukemia is a term applied by Wolff to certain cases in which the blood contains many large indifferent mononuclear cells which he interprets as leukocytic metrocytes. The structure of the organic lesions in these cases shows many features of a neoplasm composed of proliferating atypical leukocytes (Babes). This condition does not differ essentially from Sternberg's leukosarcomatosis.

Chloroma.--The occurrence of greenish tumors of skull, vertebræ, ribs, and other organs had been recorded by many others before I 853 when King employed the term "chloroma" for these growths. In 1854 Aran with Lebert contributed an important study of the condition, but Recklinghausen in 1885 first recognized the lymphomatous nature of the process and its probable relation to leukemia, as a systemic disease of the lymphatic system. The association with a leukemic blood-picture seems to have been recognized first by Huber in 1878 , but observations on the blood remained imperfect until after Recklinghausen's study. In I 893 Dock fully reviewed the history of the disease, confirmed the relation to leukemia, and later with Warthin established the primary myelogenous origin of the tumors in their case. Detailed hematological studies by Turk, Klein and Steinhaus, Sternberg and 
Fabian have completed the parallel with leukemia by showing the existence of both lymphatic and myelocytic types of chloroma.

The disease occurs chiefly in young males, average age I 8.8 years, and runs an active course, terminating fatally within an average observed period of 5.5 months. More prolonged cases occur in older subjects. The tumors appear chiefly in the skull, affecting the orbit, dura, sinuses, auditory region, sphenoid and ethmoid sinuses, nares, antrum, pharynx, palate, and alveolar processes. The early symptoms are, therefore, often referable to the eye, ear, nose, and throat. Very often the tumors affect the sternum, vertebral column, ribs, pelvis, and occasionally the long bones. In such cases the distinctions from multiple myeloma are not clear. In the lymphatic system the cervical, axillary, mediastinal, or other deep chains are always involved in the lymphatic type, and have escaped in only one myelocytic case (Klein, Steinhaus). The bone-marrow is regularly involved, sometimes rather slightly or not at all, often very extensively (Sternberg). The spleen is much enlarged, and the gastro-intestinal lesions may be extensive. The heterotopic deposits while irregular are a very prominent feature, occur in almost every organ and tissue, and illustrate the widest scope of the leukemic process. The liver and kidney frequently show deposits, and the pancreas, thyroid, thymus, ovaries, mamma, and prostate have been found involved with tumor-like growths. A special tendency to invade the muscles and their tendinous insertions is observed, the infiltration passing out from the bone-marrow. The walls of vessels also suffer more than in typical leukemia. In several cases there have been infiltrations, eruptions, or tumors in the skin (Stevens, Hitschmann, Bramwell).

The most conspicuous gross feature is the color of the growths, which varies from a grass green to a faint greenish-yellow tinge, may be very pronounced throughout the marrow and all the tumors, or may be limited to a few areas. The heart clots and the sedimented leukocytes have been found greenish (Trevithick). A similar color may be observed in the marrow of typical acute leukemia. Its source is undetermined, but it has been referred to transformed blood pigment (Risel), or to fatty products of cell metabolism (Sternberg). Huber and Chiari attributed the color to greenish refractive lipoid granules which they found in the cells. Lang's surmise that pigmentproducing bacteria are present has been disproved by Lubarsch. Since the coloration is not constant and may be observed in typical leukemia, its presence is inadequate to separate the disease essentially from leukemia.

In form the tumors appear as broad flat masses I to $2 \mathrm{~cm}$. thick, firm or fibrous, surrounding the bones, invading muscles, tendons, and vessels, stripping up the periosteum, or yielding more circumscribed nodular growths. In the skull and spinal canal flat masses separate the dura from the bone or perforate the meninges and invade neighboring tissues. The lymph-nodes may reach large dimensions and become fused with each other. The lesion in the marrow is diffuse or focal. Diffuse infiltrations in the mucous membranes of eye, mouth, nose, throat, larynx, or gastro-intestinal tract produce deformities and ulceration.

The blood-picture in chloroma varies extremely. The cases fall into the two main groups, lymphemia and myelemia, while in certain instances the leukocytosis has been absent or transient. Hence one may assume that there are leukemic and aleukemic chloromas of both lymphocytic and myelocytic types. The lymphocytic type is much the more common. Here the circulating leukocytes have usually shown a large proportion of large indifferent mononuclear cells. Yet the cells have varied greatly in size, being small lymphocytes (Rosenblath, Stevens) or large lymphocytes (Dock 
et al.), while Trevithick commented on the extreme size of many cells. The numbers of leukocytes also vary greatly, Bramwell finding only 8000 , Stevens 491,000, while the increase appears early or late or in transient. Similar variations occur in the myelocytic variety. Dock and Warthin describe many peculiar cells, including eosinophile myelocytes, which were abundant in the tumor-tissue. Waldstein described a case of extensive chloroma in which the blood-picture of pernicious anemia suddenly became leukemic in the terminal weeks.

The structure of the tumors accords with that of lymphocytic and myelocytic leukemia. In the former class there is diffuse infiltration with large mononuclear non-granular cells displacing normal structures and exhibiting considerable power to invade capsules of nodes, blood-vessels, and other surrounding tissues. In the latter class the evidences of the myeloid character have been pronounced and neutrophile myelocytes have been abundant. In a case interpreted as lymphatic Warthin found a very large proportion of eosinophile cells.

The chief significance of chloroma lies in the fact that it represents a leukemic process of a pronounced neoplastic type. The bulky tumors, the destructive local infiltrations, the distant heterotopic metastases, the atypical character of the cells, and their very active proliferation provide all the essential features of a malignant neoplasm. 'The process belongs with the systemic sarcomas of blood-forming organs, affecting primarily either the lymphatic system proper or the bone-marrow. It appears to differ only in degree from the other more aggressive leukemic processes, so that Sternberg includes it in his class of leukosarcomatosis. While the clinical course may resemble acute leukemia the process is quite different from the ordinary acute febrile leukemia, in that the cells are much more atypical, the local process more aggressive, and the secondary growths are more clearly of embolic origin and of much wider distribution. The evidence furnished by chloroma, especially the myeloid type, tells strongly in favor of the metastatic origin of all heterotopic deposits in leukemia. Chloroma illustrates also the access to the blood-stream, permanent or transient, in small or large numbers, of specific tumor-cells.

There has been much discussion of the relation between chloroma and the lymphosarcomatosis of Kundrat, but the two conditions are anatomically distinct. Being clearly a tumor of lymphocytes and myelocytes the observations on chloroma indicate that it has no relation to myeloma, although albuminosuria has been detected in one case (Weinberger) and myeloma may yield many tumor-cells to the blood-stream. Regarding the etiology of chloroma nothing is known, but a combination with tuberculosis has been observed in several cases (Horing, Schmidt, Lubarsch, Risel).

Plasma-cell Leukemia.- Turk has described the frequent presence in the blood of a peculiar mononuclear cell with eccentric nucleus and amphophile cytoplasm, the "reizungsformen," and Hoffmann has identified them with plasma-cells. In a case of myeloma Schridde found in the blood typical plasma-cells in small numbers, but Gluzinski and Reichenstein report a case of myeloma in which a large proportion of 39,640 leukocytes were of the plasma-cell type, and they speak of plasma-cell leukemia. This isolated observation of an unusual number of tumor-cells reaching the blood is, however, quite inadequate to establish any relation between myeloma and any form of leukemia. Myeloma varies in other directions than toward leukemia, and it may be recalled that various tumor-cells even of cancer have appeared in the blood.

Nature of Leukemia.--The foregoing data relating to the anatomical 
process in leukemia seem to warrant certain conclusions regarding the nature and position of this disease. The ordinary picture of leukemia is so characteristic as to lead to the impression that it is a definite disease of uniform etiology, but when the wide scope of the clinical features and anatomical lesions of atypical leukemia are considered, the suspicion arises that this is not a definite disease, but merely a variable symptom complex of diverse etiology. From this point of view leukemia is comparable to leukocytosis. Both conditions represent a response of the blood-forming organs to irritation, but highly essential differences exist in the nature of this response. In leukocytosis there is a transitory multiplication of leukocytes of normal type which in its various phases may be regarded as covering the entire scope of simple hyperplasia. The organic changes may be considerable, but soon subside when the irritant is removed. In leukemia, on the other hand, the response is from the first of a different type. Although transient leukemic blood-pictures occur, yet with rare exceptions the appearance of signs of leukemia means the beginning of a progressive process which will terminate fatally. It is possible that some peculiar etiological agent will be found to explain this progressive quality, but it seems more probable that it depends on the nature of the process rather than on its cause. Two main anatomical features separate leukemia from leukocytosis, the abnormal types of cells produced and the permanence and extent of the organic lesions.

While there is much difference of opinion regarding the variations of leukemic cells from normal leukocytes and their progenitors, it is acknowledged that even in ordinary leukemia the new cells vary from the normal, and that new, possibly ancestral forms appear, while in atypical cases the cell changes may be quite pronounced. It is this variation in cell type even more than increased numbers that distinguishes the leukemic process, so that in recent times diagnoses are made of leukemia without leukocytosis. Nor is this variation in cell type degenerative, for it is not paralleled by any of the severe acute or chronic degenerative processes in leukocytosis. Moreover, it is accompanied by evidence of great proliferative capacity, such as mitotic figures in the circulating cells, increase in number beyond any limit of leukocytosis, and by permanent enlargements of the blood-forming tissues. That all these variations are steps in the direction of a neoplasm seems to the writer an inevitable conclusion. Leukemia represents more clearly than any other condition the phases of neoplastic transformation.

Yet it is not necessary to dismiss all leukemias as frank tumor processes. In the numerous discussions of the neoplastic theory of leukemia controversy has arisen not so much regarding the existence of neoplastic tendencies which have always been recognized, but on the hasty classification of leukemia with tumors:

Thus Banti, who emphasizes the tumor nature, especially of lymphemia (of myelemia he is less certain), seems to overlook important distinctions between acute febrile leukemia and prolonged chloroma. In the former, local aggressive properties are almost entirely wanting, the infiltrating cells show great respect for the invaded tissues, the lymph-node lesions may be very moderate, local lymphomas may be scanty, and any neoplastic quality present is confined to simple multiplication of atypical cells, while an acute toxic process is prominent. In chloroma, on the other hand, a very different grade of neoplastic properties is present, and the effects are so different that it seems unwarranted to exactly identify the two processes. Sternberg assumes a more defensible position in drawing the tumor line at the locally aggressive lesions of leukosarcomatosis. Below that line leukemias are for him peculiar forms of leukocytic overgrowth, above it they are neoplasms. 
Yet for the reasons mentioned there seems to be no ground for assuming that the specific quality in the leukemic process is anything other than neoplastic. Admitting that there are all grades of neoplastic hyperplasia, this theory accounts for the essential peculiarities of leukemia and admits of a uniform conception of the process in all its phases. The conception of leukemia as essentially neoplastic does not exclude the most complex etiology nor a close dependence of the process upon irritants or parasites. The general facts of the disease rather strongly suggest the continued presence of an irritant, of toxic or bacterial nature, as the exciting cause of the hyperplasia. If any one known bacterial agent is chiefly concerned it is the tubercle bacillus. The comparatively frequent association of some form of tuberculosis with lymphemia and the known tendency of tuberculosis to induce extensive lymphoid hyperplasia even without the demonstrable presence of the bacillus, creates a strong suspicion that this organism is a frequent excitant of leukemia. It seems possible that in tuberculosis may eventually be found a connecting link between lymphemia, pseudoleukemia, some cases of Hodgkin's disease, and some forms of lymphosarcoma. Among the other possible factors has been suggested the impulse toward lymphoid hyperplasia given by the typhoid process, a field in which specific data remain to be gathered.

For the etiology of myelemia there are even less definite indications, but here the numerous factors, which excite anemia, call forth the regeneration of granular leukocytes, and injure the bone-marrow, may well be kept in mind. That local and general predisposing factors exist can only be assumed from analogy. Leukemia is chiefly a disease of early years when the bloodforming organs are relatively active.

\section{PSEUDOLEUKEMIA}

In 1865 Cohnheim applied the term "pseudoleukemia" to a condition anatomically resembling leukemia, but with reduction of leukocytes in the blood. His patient in the course of 9 months developed a greatly enlarged spleen with prominent follicles, diffuse hyperplasia of cervical, inguinal, mediastinal and retroperitoneal lymph-nodes, miliary lymphomas of liver, and extensive diffuse lymphoid infiltration of the kidneys.

Since that time many authors have described as pseudoleukemia miscellaneous forms of systemic disease of the lymphatic system without leukocytosis, so that the term came to have a very wide scope. In recent years extensive inroads have been made upon the domain of pseudoleukemia, so that today it is somewhat doubtful in the minds of many if there is any condition to which this term may properly be applied.

The first definite condition to be separated from pseudoleukemia was lymphatic tuberculosis.

Lymphatic Tuberculosis. - That general tuberculous lymphadenitis cannot always be distinguished clinically from pseudoleukemia was early recognized and the scope of the disease was by some widened to include such cases. Many of the cases of intermittent fever with swelling of lymph-nodes described by Pel, Ebstein, Renvers, and others probably belong in this class (Combemale). In I887 Delafield showed that the course of pseudoleukemia could be exactly imitated by lesions of lymph-nodes which were typically tuberculous, with miliary tubercles, foci of necrosis, and stainable bacilli. Waetzoldt, however, found nodes which showed pure lymphoid hyperplasia, with scanty foci of hyaline material and yet many tubercle bacilli in sections. Finally Brentano and Tangl described a case in which with tuberculous ulcer of cecum and peritonitis the thoracic and retroperitoneal lymph-nodes showed 
only lymphoid hyperplasia, no bacilli in sections, but inoculation proved positive. In Sabrazes' case of "lymphadenoma" the firm nodes showed lymphoid hyperplasia without caseation, while in lungs, liver, spleen, and peritoneum were miliary lymphomas, microscopic criteria of tuberculosis were absent, but rabbits inoculated died with tuberculosis.

It thus appears that the clinical picture of pseudoleukemia may be associated with: (I) Ordinary miliary and caseous tuberculosis of many lymphnodes (Delafield); (2) Frank tuberculous lesions in one locality and pure lymphoid hyperplasia of many nodes which yield no stainable bacilli, but prove infective (Brentano, Tangl); (3) pure lymphoid hyperplasia without stainable bacilli, but giving positive results by inoculation (Waetzoldt, Sabrazes).

In all these cases in which a tuberculous infection has been demonstrated it appears that the extent of the lesion is rather less general and locally more destructive than in many examples of pseudoleukemia. Yet the purest forms of the disease exhibit these same characters as compared with leukemia. That the tuberculous infection in all these cases may be secondary or unrelated to the pseudoleukemic process appears to the writer quite unworthy of consideration. On the contrary, there can be no doubt that the clinical picture and anatomical lesions of pseudoleukemia are often produced by tuberculosis.

\section{HODGKIN'S GRANULOMA. LYMPHOGRANULOMA}

A considerable group of the cases formerly classed as pseudoleukemia present a specific histological structure which is now regarded as pathognomonic of Hodgkin's disease. The Vienna school of pathologists early recognized this characteristic structure and Sternberg clearly described it in a group of cases in most of which he claimed to find tubercle bacilli. This specific histology has been emphasized by Reed. The structure shows diffuse cellular hyperplasia with varying proportions, sometimes excessive, of proliferating endothelial cells, endothelial giant-cells, plasma-cells, and eosinophile leukocytes. While there are numerous variations in the proportions of these cells, especially of the endothelium, the typical picture of Hodgkin's granuloma with the confusion of so many cell forms is unmistakable, is very probably caused by a single agent, and forms a rational basis for classification of many obscure cases of clinical lymphomas.

Unfortunately, the clinical picture of Hodgkin's disease is not always associated with this particular histological structure. It is the common experience of pathologists, as stated by Christian and Warthin, to find an absence of the typical picture described by Sternberg, in nodes removed under the diagnosis of Hodgkin's disease, and in its place indefinite forms of lymphoid hyperplasia. Without the knowledge of the etiological factor it remains almost as difficult as before to determine the true scope of this infectious granuloma, for all the phases of the disease cannot be recognized with the same certainty. It is not known, for example, whether a pure lymphoid hyperplasia, or a pure plasmoma (myeloma), or pure endothelial hyperplasia belong in this group, and these questions cannot be settled until the problem of etiology is determined.

In this dilemma one may follow the plan adopted by H. Ziegler and include under Hodgkin's disease all conditions which seem to have any probable relation to the specific process. This plan means hardly less than to replace the term "pseudoleukemia" with Hodgkin's disease. Thus Ziegler describes Hodgkin's disease as (a) Acute, (b) Localized, (c) General, $(d)$ Mediastinal, (e) Larval, $(f)$ Splenic, $(g)$ Osteitic, $(h)$ Atypical (intestinal form, Mikulicz's disease), (i) Mycosis fungoides. 
In the description of these forms he includes many histological processes which differ considerably from Hodgkin's granuloma and whose relation to which is far from certain. Nevertheless there is a strong probability that the scope of this disease is very wide and that the above classification deserves adoption as a working hypothesis. The writer would prefer to exclude from Ziegler's scheme such diseases as multiple myeloma, mycosis fungoides, and any form of systemic pure lymphocytic hyperplasia.

Clinical Course.--The disease occasionally exhibits as prodromal symptoms an itching or eczematous eruption of the skin which may precede other symptoms by months or years, bears considerable relation to the subsequent course of lymph-node lesions, and may result in the definite and progressive cutaneous lesions of Hodgkin's disease. Gastro-intestinal disturbances may also be observed (Rolleston, Bramwell).

More often the initial symptom is enlargement of a chain of lymph-nodes, cervical (5o per cent.) axillary, inguinal, and the continuous or irregular extension to other chains. A splenic tumor develops in 60 to 70 per cent. of the cases, and may reach very large proportions. At the same time and with equal frequency the liver enlarges. With this generalization of the disease, fever, night-sweats, anemia, and cachexia appear. The fever occurs early or late, may be slight or absent, but is often- of sharply intermittent type, and may dominate the clinical picture.

The anemia is of the secondary chlorotic type and may become severe or pernicious. The leukocytes are usually under Io,০oo, often there is leukopenia, occasionally leukocytosis of 30,000 or more. Relative or absolute lymphocytosis usually prevails, but the neutrophile cells are much increased in exacerbations. Eosinophilia is so frequent and marked as to form an important diagnostic sign (Bunting). Mast cells may be increased. Evidences of a hemorrhagic tendency are frequently observed, as minute hemorrhages in skin, petechiæ, or purpura hæmorrhagica (Sabrazes, Hippel). The terminal cachexia is marked by numerous extensions or inflammatory complications in many organs, and in about 25 per cent. of the cases by miliary tuberculosis (Ziegler). Amyloidosis of spleen and other organs is recorded in 22 cases (Fabian). The duration varies from a few weeks in acute cases to many years, but is usually about i 8 months. The cutaneous lesions are more chronic. Clinical types are numerous.

Clinical Types.-I. Acute cases lasting 4 and 6 weeks, marked by fever, anemia, swelling of cervical and axillary nodes and spleen, or by general swelling of lymph-nodes, lymphomas in liver, and cutaneous ulcers are described by Hirschfeld and Isaak, and Beitzke. The structure of the lesions is characteristic of Hodgkin's granuloma. Focal necrosis in liver, nodes, and spleen are prominent in this group.

2. Chronic generalized lesions accompany the usual clinical course described above. In this group several chains of superficial nodes, usually the cervical, are extensively involved, and the lesion extends to thoracic and abdominal nodes, spleen, liver, and bone-marrow. All the lymphoid tissue of the body may be slightly hyperplastic. Secondary deposits in lungs and serous membranes are often observed.

3. Splenic Hodgkin's disease occurs as a primary lesion of this organ or as a part of the generalized infection. The peculiar color of the organ led Benda to apply the term "porphyry spleen" to this condition. The primary splenic lesion is extremely rare, some authors denying its existence. Yet in a case described by Symmers and studied in this laboratory the structure was practically identical with Hodgkin's granuloma. Primary splenic lesions extending to the lymph-nodes appear to have been observed also by Kummel who 
found tubercle bacilli in the tissues, while Doncaster has reported a case of primary splenic lesion which is probably genuine. The great majority of cases of primary splenomegaly which resemble Hodgkin's disease prove on examination to belong elsewhere, chiefly among splenic neoplasms. For the primary splenomegaly of Gaucher a relation to tuberculosis or Hodgkin's disease seems improbable.

The secondary invasion of the spleen in Hodgkin's disease is common, and not infrequently the splenomegaly becomes the dominant symptom. Here the primary lesion may appear in the peripheral nodes (Nowak) or in

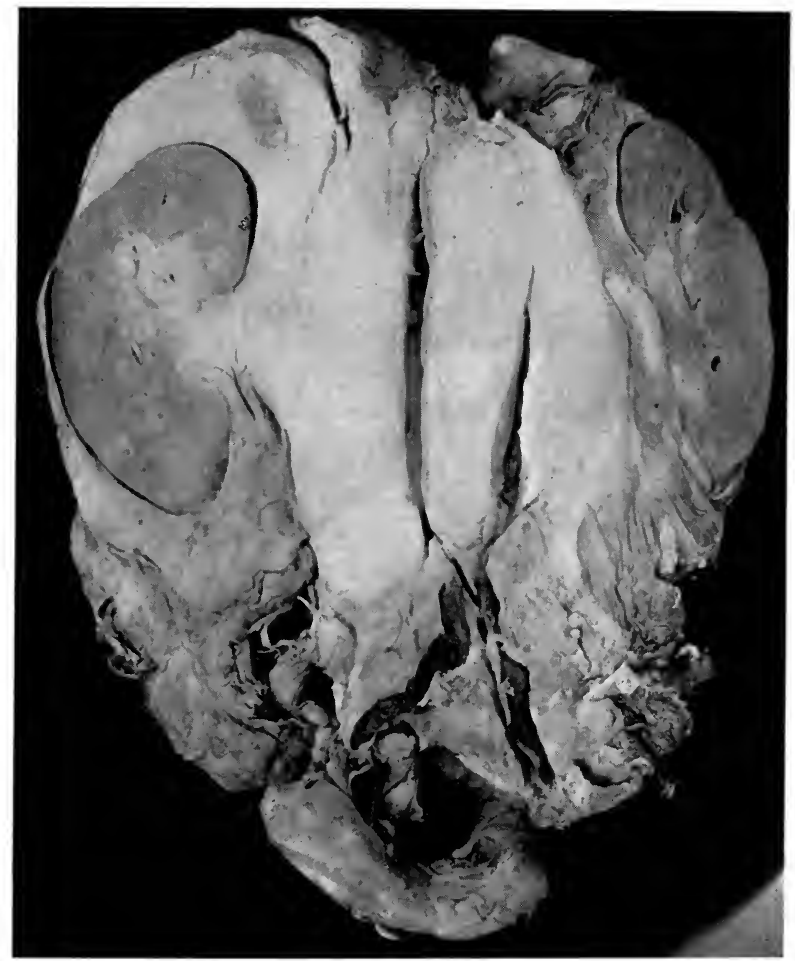

FIG. I 45.- Retroperitoneal region in a case of Hodgkin's disease. Lymph-nodes, mesentery, and perirenal tissues are fused into a mass of granulomatous tissue which occludes vena cava and constricts aorta.

deeper chiefly retroperitoneal chains. Remittent fever, anemia, and cachexia are leading clinical symptoms. In a case lasting I 6 months and marked by severe febrile attacks with intermittent swelling of cervical nodes, the spleen continued to enlarge and at autopsy was found to contain very numerous necrotic foci surrounded by typical Hodgkin's granuloma. The lymphnodes showed only hyaline areas. Tubercle bacilli could not be identified. Many of the cases of Ebstein's disease must be classed in this group.

4. Gastro-intestinal Hodgkin's gramuloma is an ill-defined condition difficult to separate from tuberculosis, on the one hand, and lymphosarcoma on the other. The group of diffuse lymphomas affecting a large portion of or the entire gastro-intestinal mucosa (Stoerk, Wells, Symmers) must, I believe, 
be given a separate position, since they differ widely in structure and distribution from typical Hodgkin's granuloma. There remains a considerable group of locally destructive hyperplastic lesions located in any portion of the gastro-intestinal tract, especially in stomach, ileum, and cecum, in which the structure is distinctly granulomatous and in which tubercle bacilli are missing. I have studied several cases of this type, with local ulceration and extensive swelling of regional nodes, in which the lesions resembled Hodgkin's granuloma. Coupland and La Roy have described typical cases, but reports in the literature are scanty, since most of the cases are interpreted as lymphosarcoma.

5. Mediastinal tumors may form the chief lesion in Hodgkin's disease, and their structure is usually typical of the granulomatous or of the sarcomatoid process arising in the thymus. With or without enlargement of palpable nodes it forms a bulky tumor of the mediastinum, compressing bronchi, vessels, and nerves with corresponding pressure symptoms. Extensions to pleura and pericardium, heart muscle, lung, liver, supraclavicular, and axillary nodes have been observed. (Palma, Lorrain, Schottelius, Jacquet, Brigidi, Piccoli). In the case of Weber and Ledingham a high grade of pulmonary osteo-arthropathy was observed. Although in this last-mentioned case the structure was typical and extensions were chiefly regional, yet in most cases the tumor is bulky, local invasive properties are pronounced, distant secondary deposits occur, and the tissue is composed chiefly of large round cells with few lymphocytes and no necrosis, so that many have regarded this condition as true lymphosarcoma. In a case studied in this laboratory by

- Symmers a bulky but encapsulated tumor occupied the mediastinum and had caused ulceration of the trachea. The nodes on both sides of the neck were difficult to separate from the tumor and the bronchial nodes were also included in the mass. There were no distant deposits. The structure approached the sarcomatous type, being composed of regularly placed bands of dense connective tissue inclosing areas of large round cells derived from the reticulum, a few lymphocytes, giant-cells, and eosinophile cells. Here some relation to Hodgkin's granuloma is probable, but neoplastic characters seemed to be present also. Leukemia occurs with somewhat similar processes in the thymus, and in a case reported by Coenen it is difficult to determine whether leukemia or granuloma should be recognized (cf. Tumors of Thymus).

6. Abdominal Hodgkin's granuloma often appears clinically as a larval form of the disease, since enlargement of palpable lymph-nodes is late or wanting. In this group intermittent or continuous fever is common, the course may be rapid, and a typhoidal condition is established. Local pain, gastrointestinal symptoms, jaundice, and a diazo-reaction of the urine strengthen the resemblance to typhoid fever.

The lesion usually begins in mesenteric or retroperitoneal nodes and extends along the spine into the thorax. It may remain in the abdominal nodes producing a bulky tumor (Symmers, longcope), or more often extends widely, involving spleen, liver, lungs, bone-marrow, and eventually superficial nodes. Or a primary affection of the cervical nodes may subside after extensive invasion of the thorax and abdomen (Gutig). The lesions are therefore the most extensive occurring in the disease.

The structure illustrates the typical granuloma and most of its variations. Focal or bulky necroses are common in severe febrile cases. Ziegler describes excess of plasma-cells in lesions in some of his cases. The gross appearance may strongly suggest a neoplasm and many cases have been described as lymphosarcoma. In Symmers' case the tumor was composed almost entirely of large, flat, endothelial cells. 
7. Mikulicz's disease is an infectious granuloma affecting the salivary and lacrimal glands, occasionally the lips and eyelids. It has sometimes been associated with leukemia; with systemic involvement of the lymphatic system interpreted as pseudoleukemia; and with tuberculosis (Brun, Fleischer). The cervical and axillary nodes, spleen, and liver are often involved in the later stages, and many of the symptoms resemble those of Hodgkin's disease. The structure varies, but in certain cases it has resembled Hodgkin's granuloma (Haeckel, Jacobeus).

8. Bone-marrow lesions of typical structure àre commonly observed in generalized Hodgkin's disease, but as a rule the bone tissue and periosteum remain intact. Ziegler, however, believes that many peculiar osteal and periosteal lesions attributed to primary tumors of bone and marrow or to obscure inflammatory processes belong in this group, and he has collected a series of such cases, including myeloma with plasma-cells, and lymphosarcoma, which he would class with Hodgkin's granuloma.

9. Dermal lesions occur in the course of many cases of Hodgkin's disease. The itchy eczematous or urticarial exanthem that marks the prodromal stages may be repeated throughout the later course and may lead to excoriation, scarring, and pigmentation so marked as to suggest a complicating affection of the adrenal. Or a bullous eruption may appear with extensive erythema and scaling. A second group of lesions, clinically and anatomically specific, have been designated as lymphogranulomatosis cutis. The specific nature of certain multiple, nodular, or ulcerated infiltrations of the skin, formerly classed as pseudoleukemia, was pointed out by Grosz in I906, who found in them the typical structure of Hodgkin's granuloma. Several cases of this character, with or without systemic lesions, have since been described (Arndt, Lit.). Pick emphasized the prominent eosinophile cells, and Arndt detected scanty acid-fast bacilli in one case. Extensive ulceration of the skin occurred in Beitzke's acute case. Ziegler would here include nearly all the cases of generalized lymphosarcomatosis of the skin, and it seems very probable that some of the sarcoid growths in dermatological literature may eventually be placed in this group. Ziegler finds many resemblances between mycosis fungoides and lymphogranulomatosis, but the structure of the two processes, as well as the clinical aspects, show notable differences, and Arndt from a careful comparison is not disposed to identify the diseases.

Io. Besides these more definite clinical varieties, lymphogranuloma has been observed in many other organs as thyroid, pancreas, adrenal, heart and voluntary muscles, esophagus, tonsils, breast, and ovary (Fabian, Lit.). Jessup has described a typical case in the uterus. In the lower animals the disease appears to be not infrequent (Ziegler, Lit.).

Structure.-Typical Hodgkin's granuloma presents a characteristic structure on which depends its recognition as a specific disease. It consists of a recticulum in which lie a few small lymphocytes, large lymphocytes, plasma cells, eosinophile cells, proliferating endothelium, and endothelial giant-cells. There are many variations in the proportions of these cells. In the early stages lymphocytes are abundant, but follicles and sinuses are soon obliterated. The nodes on the outskirts of invaded chains may show a simple hyperplastic lymphadenitis, but the specific process attacks the supporting tissue and endothelium and seems to cause little hyperplasia of lymphocytes. Later the lymphocytes largely disappear with the multiplication of other cells. Endothelial proliferation is prominent from the first, so that large, loose, rounded cells with clear cytoplasm and single or multilobed hyperchromatic nuclei are an important diagnostic feature. These cells may increase in size and number so that they may compose the bulk 
of the tissue. When in excess they may appear as large, flat, almost pavementlike cells, or as a collection of giant-cells, or as a diffuse growth resembling sarcoma. They long resist the advance of fibrosis.

Eosinophile cells are usually numerous, sometimes extremely abundant, occasionally absent. They are mononuclear or polynuclear, and they probably arise as a result of small extravasations of blood which mark the early lesions. A peculiar brownish-yellow color of the Hodgkin nodes on section may perhaps be connected with this tissue eosinophilia. Polynuclear neutrophile cells are not prominent. A few plasma-cells are regularly present and they may become so numerous as to lead to the designation of plasmoma. Necrosis is missing in the characteristic stages of the process, but in acute

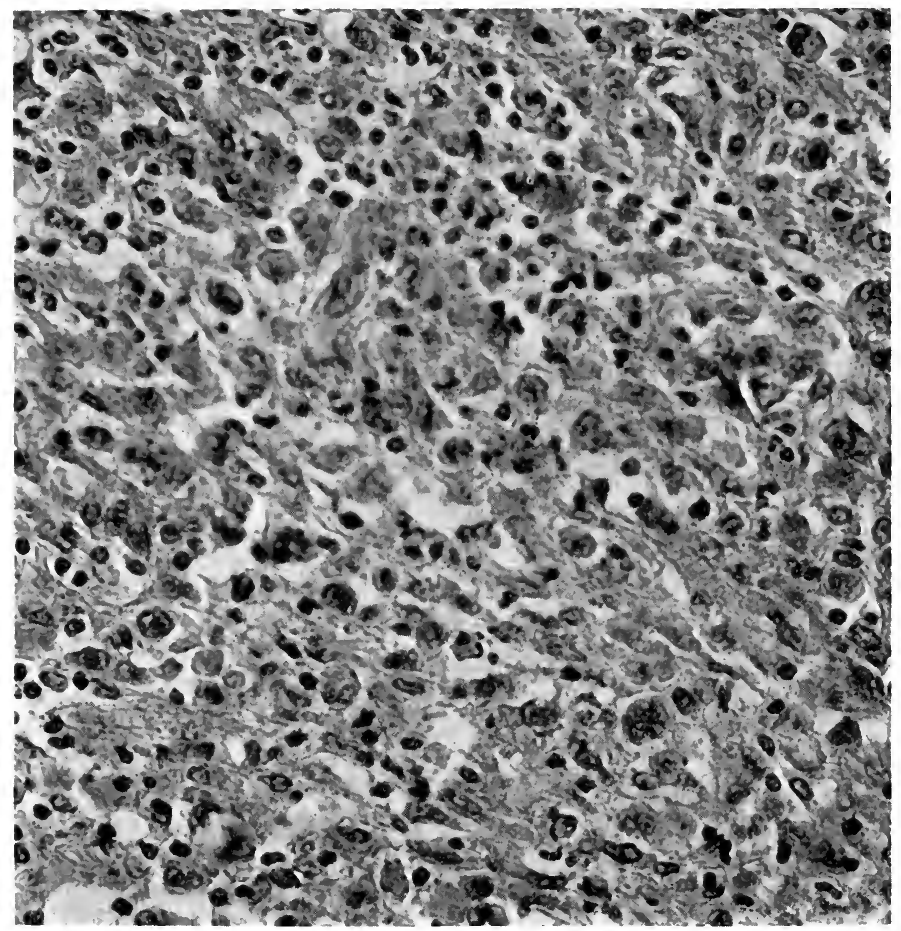

FIG. I 46.-Diffuse growth of reticulum cells in a case of Hodgkin's disease.

febrile cases and in old and bulky lesions there may be focal or extensive necrosis. Fibrosis becomes established in many advanced lesions and acellular connective tissue in bands and masses composes a considerable portion of certain bulky tumors.

Apart from the specific structure presented by typical stages of the process and the variations which are readily derived from it, the scope of this granuloma is difficult to determine. Very early lesions are often typical and the terminal stages are commonly recognizable. Besides the typical picture described by Sternberg and Reed, characteristic cases may be associated with pure lymphoid hyperplasia, various forms of proliferation of reticulum cells, as well as with subvarieties of tuberculosis (cf. Endothelioma of Lymphnodes). 
The locally invasive and destructive properties of lymphogranuloma constitute a notable feature. While the capsules of nodes long remain intact, yet from many primary foci the surrounding tissues may be invaded by a process which is typically granulomatous. The walls of blood-vessels may be extensively invaded, and the lumina may be occluded by the new cells. These appearances have often suggested that the process is sarcomatous. Yet similar lesions are observed in tuberculosis and so long as the new tissue maintains a typical structure it should not be classed with malignant tumors.

Hodgkin's Sarcoma.-The transformation of Hodgkin's granuloma into a sarcomatous process occurs in a certain proportion of cases, and since the new cells are of endothelial origin this tumor might be classed with endothe-

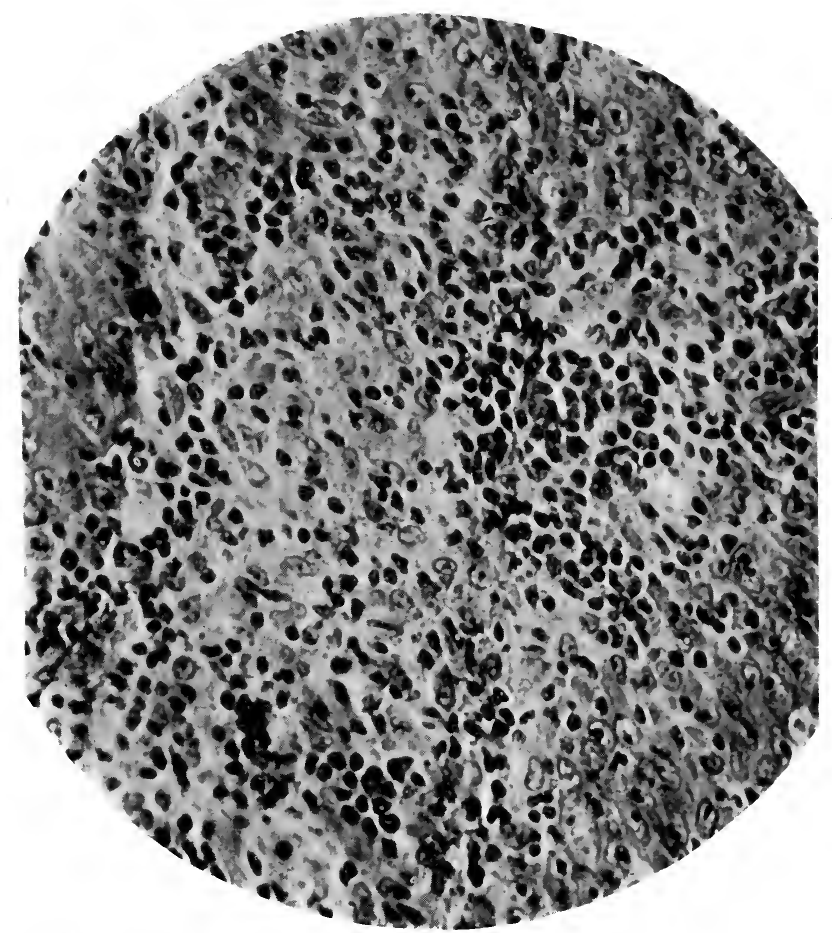

FIG. I 47.-Diffuse endothelial overgrowth in atypical Hodgkin's disease.

lioma. Yet the proliferating cells lose their endothelial characters and appear as large round cells, the tumors differ markedly in behavior from other endotheliomas, and they constitute a considerable group of the processes commonly called lymphosarcoma or pseudoleukemia, so that it seems admissible to consider them in this connection under the term "Hodgkin's sarcoma."

This process runs the clinical course of Hodgkin's disease, especially that form which terminates in invasive and destructive lesions resembling sarcoma. It also appears in single chains of nodes, chiefly the cervical, but recurring after operation it goes on to a fatal issue. These cases correspond to Billroth's malignant lymphoma. Mediastinal Hodgkin's disease furnishes a large proportion of the cases terminating in sarcoma. The spleen and bone- 
marrow are commonly involved, but the more malignant the growth, the less extensive is its distribution. Metastatic growths of considerable extent are found in the liver or lung. In Welch's case there was a metastatic tumor of the spinal dura causing paraplegia. On section the tumors are soft' and of peculiar brownish-yellow tint, or more often firmer, fibrous, and opaque.

The structure varies from a close counterpart of Hodgkin's granuloma to a tissue composed exclusively of large round cells with faintly staining granular cytoplasm and moderately chromatic vesicular nuclei. Large, round giant cells with multiple or multilobed nuclei may predominate. The proportion of lymphocytes may be considerable, as in Karsner's case, but in more definite neoplastic processes these cells are missing. The structure may not be uniform in all the lesions nor at all periods. The cells are not lymphocytes, but derivatives of the reticulum cells. That a true lymphosarcoma composed of neoplastic lymphocytes may develop in Hodgkin's disease has not been demonstrated. The original process does not tend to stimulate the growth of lymphocytes.

That the sarcomatous structure represents a terminal stage of Hodgkin's disease is strongly indicated by the observations of Yamasaki, Karsner, and others, and has been clearly shown in Welch's case. Here a cervical node removed 6 months before death showed Hodgkin's granuloma, while the tumors removed at autopsy from neck, dura mater, liver, and lung showed replacement of lymphocytes, chiefly by large round cells resembling sarcoma.

Cases of this type are by no means uncommon. Many cases described in the literature as lymphosarcoma are probably of this nature. Several have been observed in this laboratory, including Welch's. From a comparison of their very characteristic structure with that of certain malignant lymphomas I am led to believe that localized tumors of lymph-nodes of this same origin are relatively common.

The exact position of this form of sarcoma it is difficult to determine. Its clinical malignancy may depend on the dissemination of an infectious agent or its toxins, and the local recurrences are probably recrudescences in new lymphoid structures. Yet the metastatic growths in dura and lung and the changes in the type of the cells demonstrate that it is a malignant tumor.

The histological signs of malignancy are not very pronounced. The perforation of capsules of nodes is slow, and liver tissue seems to be passively displaced. A large mediastinal tumor I found still well encapsulated and an ulceration of a bronchus appeared to be mechanical. The nuclei of the chief round cells are uniform and only slightly hyperchromatic. The tendency to fibrosis is marked. Hence Hodgkin's sarcoma differs distinctly from true lymphosarcoma, in the type of cells, in its slight invasive power, and in its origin. Since Hodgkin's sarcoma is established by increasing proliferation of cells incited by an inflammatory agent there must be transitional stages of the change, and it becomes a difficult matter to decide when certain lesions should be classed with the granuloma and when with sarcoma. This difficulty has long disturbed the surgeon, and it appears throughout the case reports of Hodgkin's disease collected by Ziegler. I believe that clinical data are inadequate in this field and that the microscopical evidence of complete predominance of one atypical cell, and usually the presence of metastases, should be secured before the diagnosis of sarcoma is made. Hodgkin's sarcoma is therefore unique among tumor processes and a disease sui generis. It illustrates in the same patient and in its various gradations as a primary tumor, the transformation of an infectious granuloma into a true neoplasm. It demonstrates the relation of a tumor to the presence of a microörganism 
or its toxins. Its malignancy is apparently founded upon peculiar features, rather less upon cellular anaplasia than upon the association with a toxic agent which is readily disseminated and seems to prepare the soil for its subsequent changes.

Since the etiology of Hodgkin's disease is not determined, one is reduced to speculation regarding the mode of dependence of the tumor process upon the irritant. It seems reasonable to suggest that this condition is analogous to the epithelioma arising upon lupus or syphilis and to assume that the microörganism is present only in early stages, and that the tumor process, established indirectly, progresses of its own momentum and apart from the parasite.

In a recent study of thymus tumors I have collected evidence suggesting that many of the cases of Hodgkin's disease exhibiting sarcomatous qualities originate in the thymus, and that the peculiar characters of the infiltrating cells are referable to their origin from the epithelial reticulum cells of the thymus.

Etiology of Hodgkin's Granuloma.-The cause of Hodgkin's disease still remains imperfectly determined, the majority of writers holding that it is not tuberculous. The evidence supporting this position consists in the usual absence of tubercle bacilli in sections, the negative results of inoculation, and the specific structure. Karsner holds that the tuberculous lesion resembling Hodgkin's may be distinguished by somewhat minute characters of the giant-cells. Sternberg, who originally described the condition as a peculiar form of tuberculosis, has partly modified his position and is inclined to admit the existence of a non-tuberculous Hodgkin's granuloma.

The evidence in favor of the tuberculous origin is nevertheless somewhat formidable.

In the material as gathered in New York where the disease is very common tuberculosis follows Hodgkin's disease like a shadow. Tuberculous stigmata or tuberculous family history or tuberculous lesions in the body are the rule. In a case of phthisis I was much impressed by finding in three small adjoining bronchial nodes miliary tubercles in one, diffuse lymphocytes in another, and typical Hodgkin's granuloma in the third. The association of local or widespread tuberculous lesions with Hodgkin's granuloma is frequently observed. According to Ziegler about 20 per cent. of the cases show tuberculous lesions in the organs, and in ro to 2 per cent. tubercle bacilli have been demonstrated by inoculation. Acute miliary tuberculosis often terminates the disease.

The idea of a mixed infection coextensive with such wide-spread lesions is a strained hypothesis. An examination of the protocols of many inoculation tests shows that they were carried out in a perfunctory or incompetent manner. Some observers used rabbits. Others employed small quantities of single nodes in single guinea-pigs. In this laboratory 4 cases of Hodgkin's disease have infected guinea-pigs with tubercle after a period of 9 months to I year. Yet Longcope failed to infect monkeys, and I have had negative results in this animal with fresh emulsions and concentrated antifornin sediment. While the stainable bacilli are usually missing, yet Sternberg found them in many cases. I have never found them numerous, but after prolonged search have occasionally detected scanty acid-fast rods. It is clear that acid-fast tubercle bacilli are practically absent in these tissues. Yet they are regularly absent in many known tuberculous lymphomas. Much's claim that a granular form of the tubercle bacillus exists in many lesions demands attention in this field. Fraenkel and Much claim to find many of the granules in the antiformin sediment of Hodgkin's nodes and in sections of the bone-marrow stained by itensified Gram. O. Meyer found 
them in 8 of 9 cases, the remaining case giving tubercle bacilli. I have found these granules, but am not convinced of their tuberculous nature. Long ago Arrigo found slightly acid-fast tubercle bacilli and granules in old tuberculous nodes. Such granules are regularly present in Hodgkin's disease, but again their nature appears uncertain. Sticker states that tubercle bacilli, probably bovine, may be demonstrated in Hodgkin's granuloma by repeated passage of material through guinea-pigs.

All of these data seem to me to require further consideration of the tuberculous theory of Hodgkin's disease. Yet before this view can be accepted several deficiencies in the evidence must be supplied. Especially the absence of stainable bacilli, and the negative results of inoculation must be satisfactorily explained. Besides the tubercle bacillus, some other microörganisms, including the sporothrix of Beurmann, and the oildia seem to deserve consideration. Pick claims to have found Hodgkin's granuloma in the nodes of a case of prolonged staphylococcus infection, and Mosler found a very similar structure in leprosy. Several cases have occurred in active syphilitic subjects (Fabian, Lit.). Syphilitic lymphoma may clinically simulate Hodgkin's disease, but it regresses promptly under iodids and it has not been shown that syphilis can produce the characteristic structure of Hodgkin's granuloma.

Recent studies by Bunting and Yates have centered attention on the diphtheroid bacilli of Hodgkin's disease. These microörganisms are very frequently but not constantly to be isolated from Hodgkin's lymph-nodes, but they are present in many other diseases and their pathogenic powers in monkeys are unsatisfactory. Torrey reports the isolation of five different varieties, aërobic and anaërobic, of diphtheroids from Hodgkin's as well as some other diseased lymph-nodes. He describes the constant presence of a very minute anaërobic bacillus in the nodes, but its pathogenic properties are feeble. Bloomfield has also identified this same minute anaërobic bacillus.

\section{Systemic aleukemic lymphomatosis. True Pseudoleukemia}

The observation of Cohnheim that the anatomical picture of leukemia may occur without leukemic blood changes is constantly being verified, While it may very well be that the blood changes are of secondary importance and that their absence does not call for the separation of this form of pseudoleukemia from leukemia, nevertheless the enormous increase of leukocytes in leukemia forms a very notable morphological feature of this disease, and one which may prove to be of fundamental importance. Moreover, the pseudoleukemic counterparts of leukemia present some other peculiarities not observed with leukemia, so that, adhering to the morphological criteria on which we are chiefly dependent, there seem to be certain grounds for recognizing a somewhat separate, although closely related position of this group of cases.

The general features of aleukemic lymphomatosis have been clearly stated by Wunderlich: "There is a peculiar disease, pseudoleukemia, which is chiefly characterized by a gradual development of very numerous and sometimes very large swellings of superficial and internal lymph-nodes, and by peculiar deposits in the internal organs, especially in the spleen, also in liver, less often in kidneys, gastro-intestinal lymphatic system, lungs, pharynx, and elsewhere."

Histologically these wide-spread lesions have been found to consist in hyperplasia of lymphocytes, so that this form of pseudoleukemia is the counterpart of the lymphatic type of leukemia.

Uniform microscopical distinctions between the pseudoleukemic and the 
leukemic process have not been demonstrated. There is the same obliteration of sinuses and follicles, the respect for capsules, the wide diffusion of the process, the tendency to fibrosis, and the exclusive participation of lymphocytes, as in leukemia. Baumgarten found that the leukemic nodes permit the passage of injection fluids, while the pseudoleukemic do not.

The blood shows a relative lymphocytosis, and Ehrlich and Pincus find that a slight absolute increase of lymphocytes is characteristic of pseudoleukemia, but this condition is inconstant. The anemia is less severe than in lymphemia. The persistent lymphocytosis and low proportion of neutrophile and eosinophile cells distinguish pseudoleukemia from lymphogranuloma.

There are also certain clinical and anatomical features which frequently separate pseudoleukemia from leukemia. Acute forms of pseudoleukemia are very rare, most of the rapidly fatal lymphoid hyperplasias without leukocytosis proving sarcomatous. Moritz's cases of acute pseudoleukemia bear a suspicious resemblance to typhoid fever which may give very extensive lymphoid hyperplasia. As a rule the pseudoleukemic tumors of lymph-nodes are larger than the leukemic, and the invasions of organs while not destructive are more circumscribed and tend to reach a large size. The confluence of hyperplastic lymph-follicles in nodes and spleen I find more frequently than in lymphemia. The exclusive presence of typical normal lymphocytes is perhaps more uniform in pseudoleukemia. In the pseudoleukemic processes in the dog and fowl these distinctions are often quite pronounced. None of them seems to furnish any ground for assuming that the processes are essentially different.

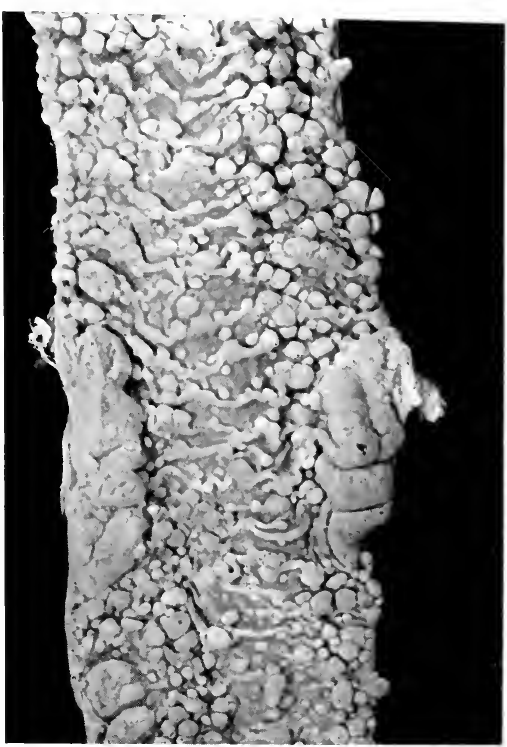

FIG. I48.-Ileum in diffuse gastrointestinal lymphomatosis, showing enlarged solitary follicles and Peyer's patches.

The necessity of recognizing clinical subvarieties of this form of pseudoleukemia is not urgent. A pure lymphoid splenic pseudoleukemia with diffuse hyperplasia of lymphocytes I do not find in the literature. Pappenheim, who recognizes splenic pseudoleukemia, states that the group is heterogeneous and imperfectly studied. Of the many forms of splenomegaly none shows lymphoid hyperplasia. French writers recognize a cutaneous pseudoleukemia (Jaccoud), but these cases seem to fall rather with lymphosarcoma. Medullary lesions of general pseudoleukemia are often pronounced and consist of focal or diffuse lymphoid hyperplasia. That a primary or predominant medullary pseudoleukemia exists remains doubtful. The custom once common of interpreting the marrow lesions of certain cases of pernicious anemia as medullary pseudoleukemia terminated with Litten's argument in 1877 . The excess of lymphocytes in the marrow of pernicious anemia, which may sometimes approach a leukemic picture, is probably a secondary condition and is not comparable to a primary pseudoleukemia (cf. Bloch and Hirschfeld). More recently a few cases of apparently pure and primary lymphoid 
hyperplasia of the bone-marrow have been described. In the cases of Baumgarten and Rubinstein this process was associated with extensive osteosclerosis and in one there were also lymphomas of liver and dura mater. Nothnagel's "lymphadenia ossium" probably belongs in this group. In a case of severe pernicious anemia of acute aplastic type Senator found diffuse lymphoid hyperplasia of the marrow. He interpreted the condition as medullary pseudoleukemia. A similar observation is recorded by Domarus.

The exact relation of these cases to other forms of pseudoleukemia is uncertain. Senator has collected a series of cases in which the marrow showed active lymphoid hyperplasia and which, he thinks, indicates a relation between pernicious anemia, leukanemia, lymphemia, and pseudoleukemia.

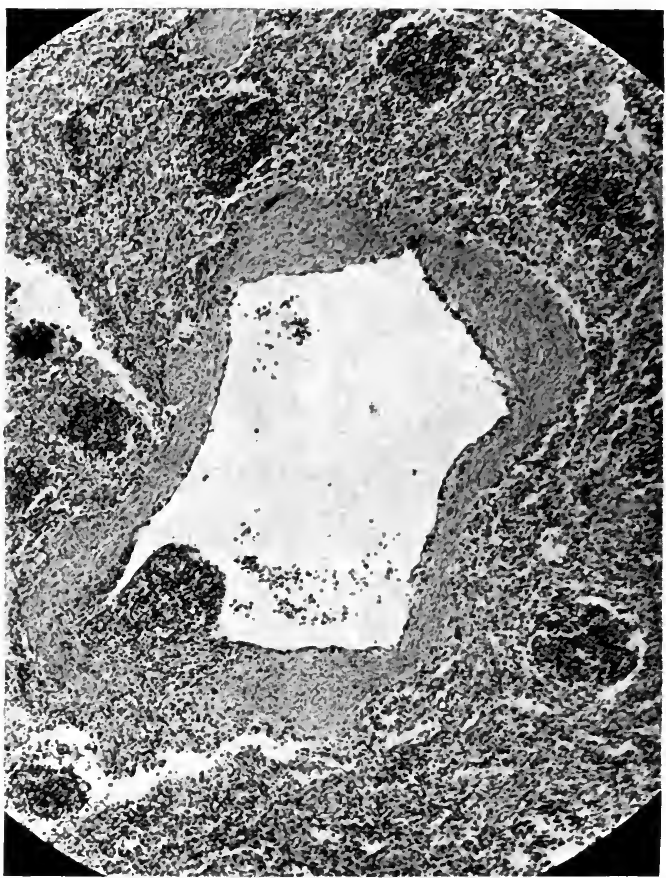

FIG. I49.-Pseudoleukemia. Anatomically, gastro-intestinal, mediastinal, and cervical lymphomatosis. Structurally, lymphadenoma. Section of spleen showing lymph-follicles in pulp and sinus.

The identity of this apparently secondary tissue lymphocytosis with the true pseudoleukemic process appears doubtful (cf. Hirschfeld).

Gastro-intestinal Pseudoleukemia.-The gastro-intestinal tract is the seat of a remarkable form of primary lymphoid hyperplasia which lacks the destructive character of lymphosarcoma and fails to give lymphocytosis in the blood. The process may be chiefly limited to a portion or involve the whole of the gastro-intestinal tract, or it may be associated with wide-spread lesions of most other lymphoid structures.

The disease was first mentioned by Briquet in 1835 , was fully described by Stoerk, and Symmers collected ro cases from the literature. The first symptoms may be enlargement of cervical nodes, or gastro-intestinal irritation with persistent diarrhea. 
In Symmer's case, studied in this laboratory, the extent of the lymphoid growth was enormous. The entire gastro-intestinal tract from cardia to anus was the seat of myriads of discrete pea-sized nodules projecting into the lumen. Peyer's patches were represented by flat polypoid tumors. In the stomach the lesion was diffuse and this organ was greatly thickened and enlarged. Cervical, mediastinal, retroperitoneal, and other chains of nodes were much enlarged or formed compact masses of nodes of which the outlines were still preserved. The spleen was much enlarged, and the bone-marrow diffusely affected. There was general peritonitis without demonstrable origin. The structure of the lesions was peculiar, consisting of extensive multiplication of lymphoid follicles which became greatly enlarged and eventually fused, a true lymphadenoma. Yet the cells in the centers of these aggregations of follicles were medium-sized lymphocytes. Mitoses were missing. The process showed no capacity to invade resisting structures. The changes were more localized in the gastro-intestinal tract and the pylorus was partly occluded in the case of Wells and Mayer.

Stoerk observed many superficial ulcers and peritonitis from perforation of a deep ulcer. He found considerable variations in the cell types, some foci resembling germ centers of follicles. In one of his cases the process penetrated the wall of the colon and invaded pelvic organs, thus exhibiting a transition to sarcoma. Ulceration of Peyer's patches may partly simulate the lesions of typhoid fever.

The etiology of this remarkable condition is quite obscure. The chief location of the process suggests the action of a diffusible irritant of intestinal origin, but the cervical nodes may be involved very early. Tuberculosis was present in one of Stoerk's cases, and in Glinski's case, limited chiefly to the colon, there was advanced pulmonary tuberculosis and foci of necrosis with giant-cells in the mesenteric nodes. The process illustrates the most extreme degree of orderly proliferation of lymphocytes, which seems to occupy the position of a relatively benign neoplasm. The occasional appearance of infiltrating properties illustrates the transition to and the sharp distinction from lymphosarcoma. Flexner describes 2 cases which seem to belong in this intermediate field. This form of pseudoleukemia is never followed by leukemia.

Myeloid Pseudoleukemia.--That the spleen, lymph-nodes, and marrow may be the seat of an extensive myeloidization, as the result of blood destruction and regeneration in many forms of infection, has recently been shown by numerous observers. The absence of leukocytosis places this condition in the light of a myeloid pseudoleukemia. Apart from these infectious diseases and their immediate sequels the existence of chronic myeloidization of the organs is imperfectly established. Sternberg, however, refers to cases of general myeloid transformation of the spleen and lymph-nodes without leukemic blood changes. In certain cases of splenomegaly with anemia the splenic changes approach the type of chronic myeloidization. It is possible to interpret the "anemia infantum" of v. Jaksch as a chronic myeloid pseudoleukemia.

Certain multiple myelomas, especially those composed of plasma-cells, have been compared with medullary pseudoleukemia, with the chief result of emphasizing their sharp morphological distinctions. The transformation of pseudoleukemia into lymphatic leukemia has been described in a considerable series of cases, thereby establishing a close relation between these processes.

Not all of these cases withstand critical analysis, as I have elsewhere noted, but some of them appear to be genuine. As a rule the leukemic blood changes appear a short period before the termination of the disease, and 
persist. Or the leukocytosis may diminish shortly before death. Turk observed intermittent leukemic changes in the blood of pseudoleukemia. On the part of leukemia approach to a pseudoleukemic condition is exhibited by those cases in which there is a temporary decline in the leukocytosis. That the histological structure of the lesions in the two conditions cannot be sharply separated has already been pointed out. Klein and Turk have especially emphasized the close parallel between leukemic and aleukemic lymphoma and lymphosarcoma. All of these features point to the conclusion that leukemia and pseudoleukemia are essentially the same disease (Turk, Klein).

On the other hand, it is clear that the great majority of cases of pseudoleukemia never show any tendency to develop leukemia. The transformations which are exclusively of the lymphatic type do not reproduce the typical picture of chronic lymphemia, but the leukocytosis is a terminal, or antemortem, or transitory condition. It does not reach the extreme degree of typical lymphemia, but recalls the leukocytosis of certain malignant tumors. There are some minor anatomical distinctions between leukemic and aleukemic lymphomas and some of the latter never run into leukemia. Hence there are many considerations which favor the view, emphasized especially by Pincus, that leukemia and pseudoleukemia are not different stages of the same disease. Until they are connected by an etiological factor there seems to the writer little advantage in merging the two conditions.

\section{LYMPHOSARCOMA}

A true malignant neoplasm arising in lymphatic tissue from proliferation of atypical lymphocytes occurs as a (I) localized or a (2) diffuse process. The former is more malignant than the latter, but its rapid course, as well as the occurrence of intermediate types of cases, indicate that there is no other important distinction between them.

Lymphosarcoma, formerly included with pseudoleukemia, secured an independent position chiefly through the studies of Kundrat and Paltauf, who recognized very notable gross and microscopical distinctions between this process and other aleukemic lymphomas. From the surgical side Billroth especially found it necessary to recognize under the term "malignant lymphoma" locally destructive and recurring tumors of lymph-nodes, but he could not separate the different types of such processes.

Kundrat described lymphosarcoma as a tumor arising only from groups of lymph-nodes, not from single nodes, and from adenoid tissue of mucous membranes, from which points it extended to neighboring nodes and the surrounding tissue, or along the submucous tissue. The systemic character of leukemia and pseudoleukemia was missing, and the spleen, marrow, and liver were rarely involved. Secondary tumors occurred almost exclusively in the intestinal mucosa, serous membranes, and lymph-nodes, and were joined by continuous growth through lymph-channels, two features distinguishing this process from true metastatic cancer. Robust males between the ages of 25 to 55 were chiefly attacked, and a tuberculous history was rare. Structurally the tumor was composed of lymphoid cells of variable size lying in an atypical reticular tissue. Cellular and fibrous stages of the process could be distinguished, but necrosis was rare.

Kundrat separated this process from the true neoplasms on the ground that it was not a spontaneous growth of multiple origin and with extensions by metastasis, but a regional disease of lymphoid tissues which propagated itself through the lymph paths. Yet it was one of the most malignant of 
diseases, resisting attempts to extirpation, and proving a veritable "nolime-tangere." It was more closely related to pseudoleukemia and "granuloma malignum," from both of which he had observed the development of lymphosarcoma.

The picture drawn by Kundrat is now generally accepted as depicting a specific group of cases which require separation from other lymphoid tumors. The extension of the process solely by permeation of lymphatics is probably not invariable, but in other details his criteria may be followed rigidly in diagnosis.

Anatomical and Clinical Features.-Lymphosarcoma is distinguished from other forms of lymphoma by its local destructive capacity and by the formation of true metastases in distant organs.

The tumors arise in a chain of lymph-nodes or in a localized lymphatic structure, and rapidly produce bulky growths which obliterate the outlines

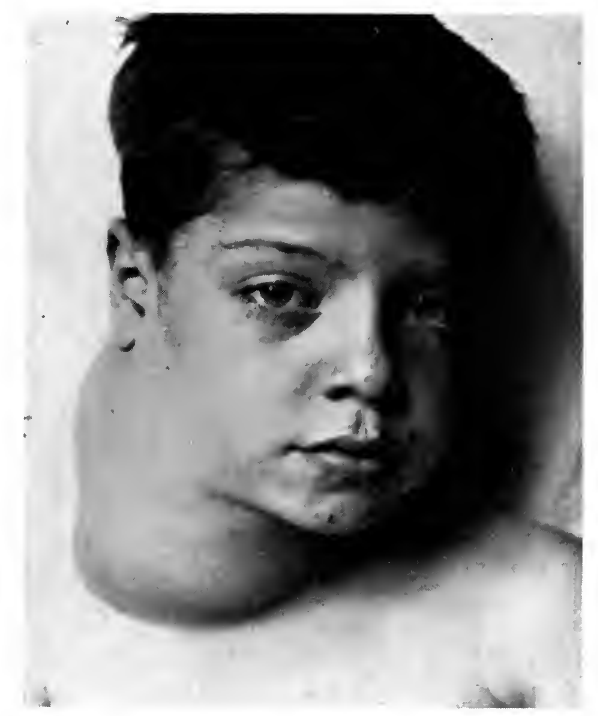

FIG. I 50.-Lymphosarcoma of cervical lymph-nodes.

of the separate nodes, infiltrate surrounding tissues, and tend to result in necrosis and ulceration of skin and mucous membrane. The more rapid cases are fatal while the growth is chiefly local, but wide-spread extensions and metastases are commonly observed. Fever is often a prominent symptom, but anemia and cachexia may not appear until toward the end of the disease. In comparing cases of lymphosarcoma with those suffering from Hodgkin's disease it may often be noted that the former do not appear to be very ill until shortly before death, while the typical Hodgkin's case is feeble, emaciated, and cachectic for a long period. Leukocytosis is usually present, and may be so marked as to suggest leukemia (Sadler). The excess is usually of polynuclear cells (Grawitz), occasionally of lymphocytes (Turk), rarely of eosinophile cells (Reinbach). Atypical cells found in the tumor may also appear in the blood and in such numbers as to suggest leukemia (Martin-Matthewson, Warthin). In fact, the distinctions between lymphosarcoma and leukemia cannot always be sharply drawn (cf. Leukosarcomatosis). 
Extension of the process in the earlier stages appears to be exclusively by way of the lymphatics, as stated by Kundrat, but in many advanced cases true metastatic growths form in the lungs, brain, kidneys, skin, and other organs, which are satisfactorily explained only by invasion of the blood-vessels.

The duration of the disease varies widely. Birch-Hirschfeld observed an intestinal case following typhoid fever which was fatal in 6 weeks. Kaufmann refers to a mediastinal tumor which caused suffocation after 3 months. Libmann's cases resembling appendicitis were very rapidly fatal. The usual course is progressive and fatal within a few months. Wide extensions are observed chiefly with more prolonged course. After local treatment, extirpation, internal use of arsenic, or application of $x$-ray, the disease has often appeared to be arrested only to recur after a brief period. Yet not a few cases have been reported as cured (Chiari, Lit.).' The true nature of some of these older cases is uncertain, but Ruff has collected a series of more recent reports illustrating the regression of lymphosarcoma after infectious diseases,

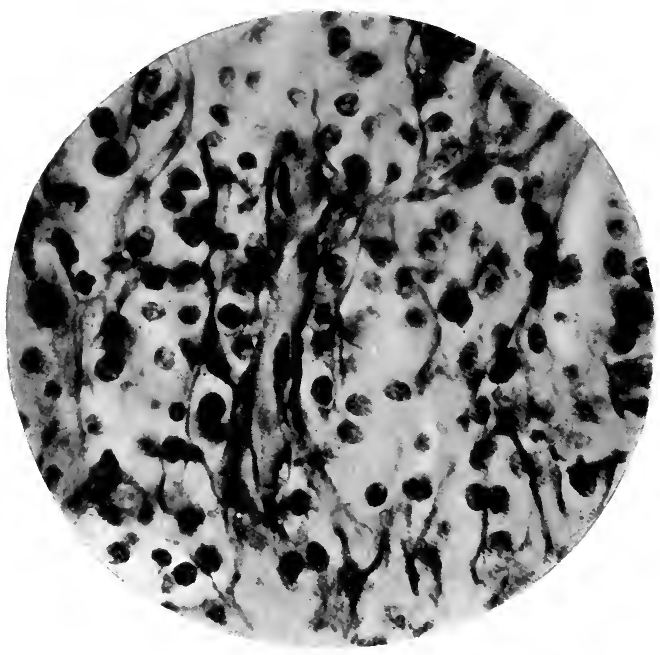

FIG. 151.-Reticulum in a lymphosarcoma. Tannin-silver stain. (After Hulisch.)

and irradiation. Koscher reports regression of a tunsillar growth after removal of a portion of tissue for diagnosis, followed by a recurrence in abdomen. Longcope reports the complete spontaneous disappearance of extensive lymphosarcomatous tumors followed by death of the patient from asthenia.

The structure of lymphosarcoma is rather specific. It presents a diffuse growth of lymphoid cells lying in reticular tissue. The structure of the affected node or follicle is obliterated. The cells I find to vary in size, being small, medium, or large. The nuclei are compact or vesicular, always hyperchromatic, and nucleoli are not prominent. Giant-cells do not belong to this process, but large multinuclear cells are occasionally seen.

In the origin of lymphosarcoma two specific cells participate, giving rise to two specific forms of the tumor. These cells are: (I) the reticulum cell of the germ centers of follicles and pulp cords, and (2) the lymphocyte. Thus arise two types of lymphosarcoma which may be designated as

I. Reticulum cell sarcoma, or large round-cell lymphosarcoma.

2. Malignant lymphocytoma. 
It may sometimes be possible to establish these two histological varieties as separate diseases. In the great majority of cases they retain permanently their separate identity, and they appear to arise under different clinical conditions and from different causative factors. But until the relation of the lymphocyte to the reticulum cell is fully established the two conditions may be discussed together.

The reticulum of the tumor is irregularly distributed, being deficient in places, and thickened in areas tending toward fibrosis. Kaufmann and Ribbert recommend washing out the cells for its demonstration.

The pure lymphoid type of structure is not always present. In certain cases there is an admixture of plasma-cells, eosinophile cells, and normal lymphocytes, which may signify a secondary inflammatory process (cf. Kanter). When these features of an infectious granuloma are pronounced the diagnosis of lymphosarcoma must remain uncertain.

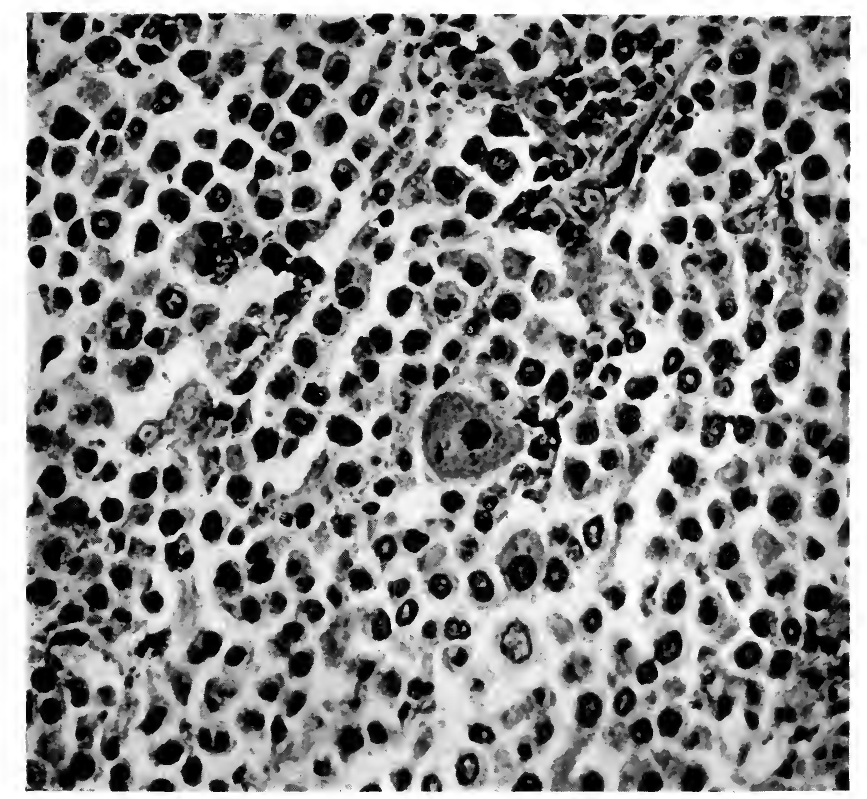

FIG. I 52.-Lymphosarcoma. Reticulum-cell sarcoma of lymph-nodes.

Regressive changes in lymphosarcoma are rare, but the occlusion of vessels in bulky growths may lead to extensive necrosis, while superficial ulceration is common.

The local aggressive quality of the process is a notable feature. The capsules of nodes, muscle tissue, and periosteum are readily penetrated and destroyed. The adventitia of large blood-vessels is invaded, but the media resists.

The primary lesions of lymphosarcoma in mucous membranes usually begin with swelling of many lymph-nodules, followed by ulceration and widening of the lumen, while secondary invasions, as of pharynx and intestine, are diffuse and may cause constriction.

The histolog ical characters of the process, together with its gross anatomy 
usually separate typical cases of lymphosarcoma from pseudoleukemia, leukemia, and Hodgkin's granuloma, and from other tumors of lymph-nodes. Yet since some of the above conditions may occasionally give rise to lymphosarcoma it is impossible always to distinguish between them without very full clinical data. Pseudoleukemia and lymphatic leukemia are lymphocytomas; in myelocytic leukemia the cells resemble those of lymphosarcoma, but are more evenly distributed, less atypical, and the process is less aggressive. Any group of lymph-nodes or any lymphoid structure may be the primary seat of lymphosarcoma, but the tumors of spleen and bone-marrow differ in many respects from those of lymph-nodes and are separately considered. In systemic lymphosarcoma the spleen is usually enlarged by a moderate overgrowth of follicles and pulp cells, and occasionally by metastases.

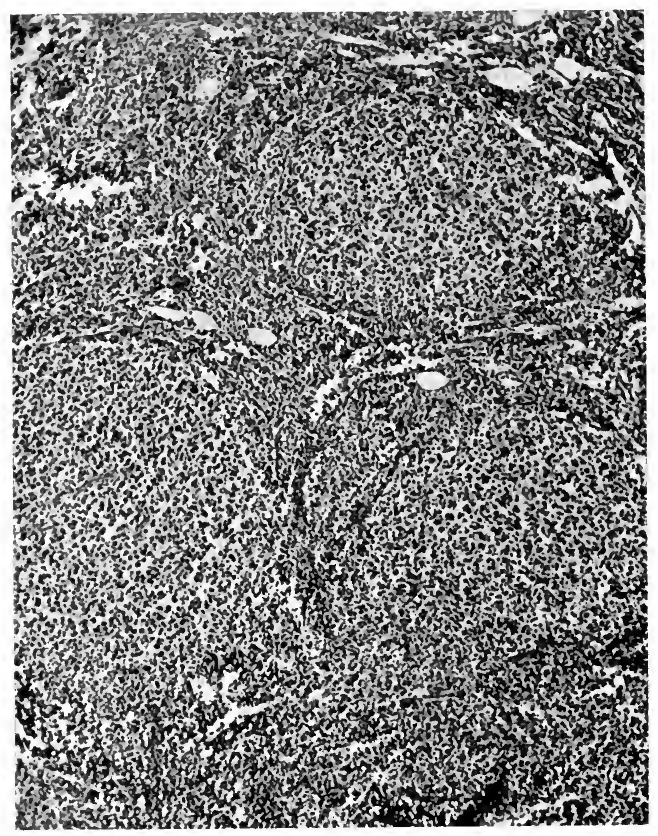

FIG. 153.-Beginning reticulum-cell sarcoma of lymph-nodes. Proliferation of reticulum cells in centers of three follicles.

Harbitz and Wieland describe primary myelogenous lymphosarcoma, but the exact nature of their cases is uncertain and their separation from myeloma appears impossible. In the discussion of myeloma the writer has pictured a case of diffuse lymphocytic myeloma of humerus which eventually proved fatal with systemic lesions (Figs. I03, ro4).

Of lymphatic lymphosarcoma several well-defined groups may be recognized.

Clinical Subvarieties.-The most frequent form of lymphosarcoma is a primary affection of a definite chain of lymph-nodes from which the disease extends to other chains of nodes, and often to the spleen and other organs. The cervical, mediastinal, retroperitoneal, and mesenteric nodes are commonly involved and in the order named, less often the inguinal and axillary. 
With the cervical nodes the lesion may invade the wall of the pharynx, tonsils, base of skull, or it may lead to extension into thorax and abdomen (Freudweiler). With the mediastinal type the bronchial nodes may be involved with extensions to pericardium, or pleura or lung, with marked pulmonary symptoms. Schlagenhaufer and Kundrat each report 2 cases of lymphosarcoma of bronchial nodes with extensive infiltration of esophagus. From the cervical, mediastinal, or peritoneal nodes very extensive invasion of the pleura and peritoneum may occur, in the form of diffuse infiltration, globular masses, or myriads of miliary nodules (Kundrat, Martin).

Most of the mediastinal lymphosarcomas arise from the thymus and are to be considered as varieties of thymoma ( $q$. v.).

Primary pharyngeal lymphosarcoma is a very common type, and the fact that with the cervical forms they constitute the majority of the cases, suggests that an infectious agent commonly enters through this area (Chiari, Lit.). The initial symptom is swelling of lymphoid tissue and diffuse infiltration of mucosa of tonsillar ring or pharyngeal wall, and the early appearances

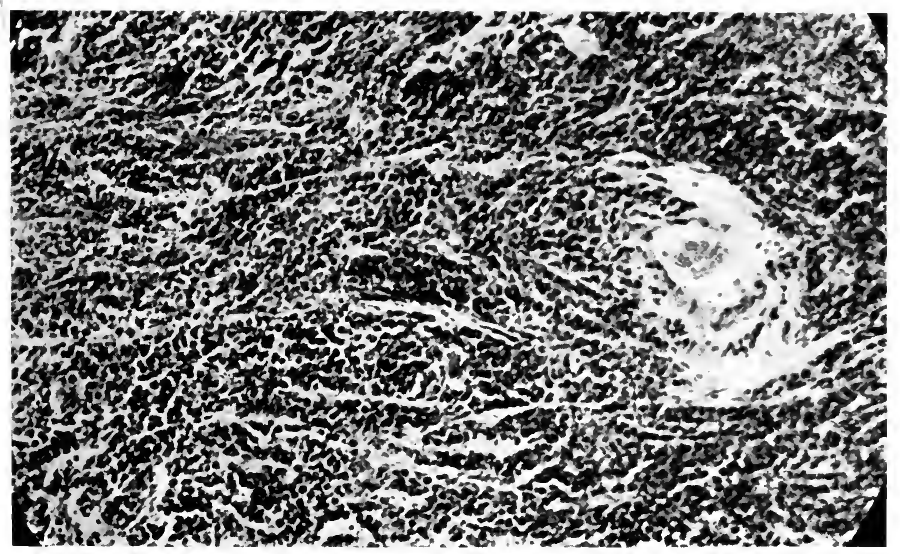

FIG. I54.-Malignant lymphocytoma of cervical lymph-nodes.

are characteristic (Kundrat). According to Eisenmenger the process regularly begins in the pharyngeal mucosa and not in the tonsils. Yet in several cases at the Memorial Hospital the disease was recognized shortly after removal of enlarged tonsils. In a fatal case with gastro-intestinal metastases I found the original ulcerative lesion of the sinus laryngis completely healed. Ulceration is soon established and remains a persistent or recurring complication. The process extends to the nares, cervical nodes, base of skull or cranial cavity, orbit, larynx, and thereafter in the usual manner to internal nodes, intestinal wall, and other organs. Local treatment, extirpation, and internal use of arsenic may cause marked or complete regression of the original lesion, but with rare exceptions the process recurs.

In the advanced stages the tissues of pharynx, face, and neck may become fused in a bulky infiltrating tumor which compresses the vessels without rupturing into them, and causes marked symptoms from involvement of vessels, nerves, trachea, and special sense organs. Many cases terminate without notable extension to other regions.

Lymphosarcoma of the gastro-intestinal tract is a common type. The seats of election are the stomach, lower ileum, and rectum. 
The literature on this subject presents a chaotic picture in which morphological details are obscure and the data regarding histogenesis and etiology are imperfectly presented. From the reported cases it is necessary to exclude, first, an infectious granuloma which is probably a form of Hodgkin's disease, but in which the structure is atypical, characteristic giant-cells being usually absent (cf. Haberer). Diffuse pseudoleukemia must also be excluded. Some of the so-called round-cell sarcomas of the stomach are probably of myogenic origin. Finally, it must be noted that diffuse carcinoma of the stomach may produce lesions which closely resemble lymphosarcoma in the gross, and a structure which can be identified as carcinomatous only when care and industry are used in the examination.

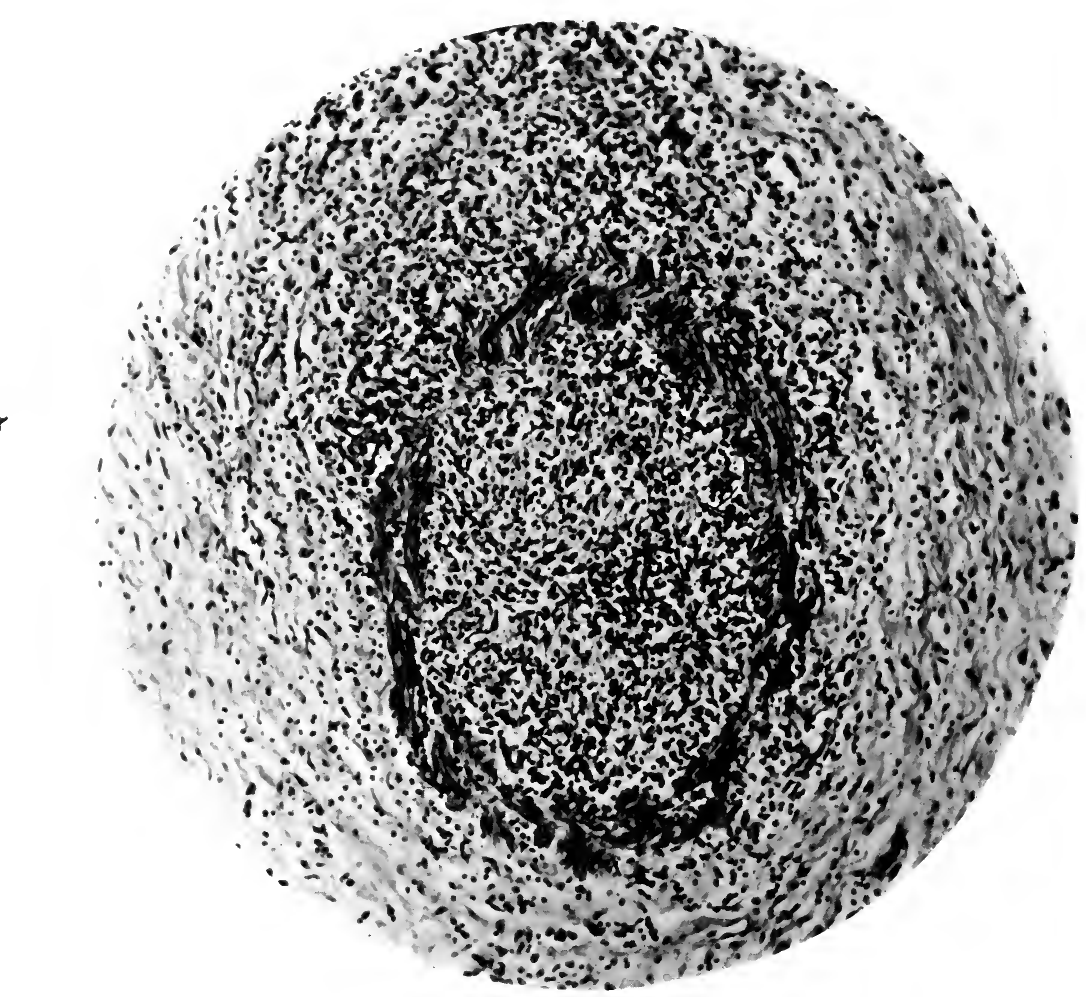

FIG. I 55.-Lymphosarcoma of stomach. Invasion of arteriole.

I. True lymphosarcoma of the stomach, which is always separated with difficulty from the granulomatous pseudosarcoma, occurs chiefly in young subjects and produces a bulky circumscribed or diffuse growth in the pylorus or in the curvatures. It is often difficult to determine whether the primary growth is in the wall of the organ or in adjacent lymph-nodes which are commonly involved. The majority of these tumors are diffuse (Kundrat, Cayley, Hammerschlag, Menge, Thursfield); others produce several projecting, submucous masses (Kaufmann); some are polypoid or pedunculated (Pitt); and a few become quite bulky (Stahelin, Howard). Ulceration and pyloric stenosis are frequent (Fleiner), and local or wide-spread extensions or metastases 
are common. Maas described a bulky subserous lymphosarcoma. The gastric tumor is often only a part of a very general lymphosarcomatosis, as illustrated by the case of Ziesche and Davidsohn. Here a very extensive tumor of the stomach was associated with lymphosarcoma of tonsils, mediastinal and retroperitoneal lymph-nodes, and the entire small intestine, while other growths occurred in the heart, kidney, and bone-marrow.

2. Lymphosarcoma of the intestine is an important type of the disease. While usually found in the lower ileum, it may appear at any point from the duodenum to the anus. The rectal tumors are less frequent, but equally characteristic (Glinski, Key, Lit.). Rarely the appendix is the primary site. The earliest stages appear as a localized thickening of the submucosa with or without ulceration. The process extends laterally and invades and destroys the muscular layers and appears as a subserous tumor which soon forms adhesions. Thereafter central ulceration excavates the tissue or produces aneurysmal dilatation which with peripheral growth yields a large tumor with roomy cavity. Large polypoid growths protruding into the widened lumen without ulceration are also observed (Baltzer). Rarely marked stenosis results and perforation may occur. Chronic peritonitis with chylous ascites is often observed.

Metastases appear early in regional nodes and they may extend to many of the organs (Libman). The disease often occurs in childhood as well as at other ages and usually runs a rapid course, terminating fatally in a few weeks or months. Acute cases resemble appendicitis (Libman, Jopson, White).

The structure of the growths is that of small or large round-cell lymphosarcoma. The reported cases are variously designated, but there is no satisfactory evidence that more than one type of tumor is represented in this group. The cells are large or medium mononuclears with liberal cytoplasm, lying irregularly in a meshwork of fine fibrils. Fragile blood-vessels and many small lymphocytes may accompany the tumor cells. It is especially in this group of cases that the process may resemble an infectious granuloma.

Retroperitoneal lymphosarcoma is a characteristic type of the disease. It produces a large abdominal tumor composed of fused lymph-nodes, while the extensions may be either very wide or rather limited. Such tumors may be associated with intestinal lesions so that it is difficult to determine the primary seat. Various complications arise in the course of these tumors, including peritonitis with chylous ascites, constriction of intestine, occlusion of bile-ducts with jaundice, and compression of vessels with edema.

Cutaneous lymphosarcoma occurs only as a metastatic process from other regions (Sedziak); mediastinum (Romberg, Packard); mesentery (Kutzner); antrum (Kaposi). The cutaneous tumors appear early or late in the disease and form multiple papules or subcutaneous nodules of varying size. They may regress spontaneously or after arsenic (Arning), and Kaposi saw a very large lymphoid tumor of the skin rapidly disappear. The exact nature of many of the cases in the literature is uncertain.

Lymphosarcoma of the tongue has been reported by several observers, but Schleinzer was able to collect only 4 cases which could be regarded as genuine, 2 of which recovered after operations. In I case the tumor distended the buccal cavity after i2 years' growth, and recurred after operation. In 3 cases the tumors early became ulcerated and invaded the cervical and once he abdominal nodes. Fripp and Iwan have collected cases of round-cell sarcoma of the tongue, the exact nature of which it is difficult to determine. Some of the difficulties of diagnosis in this field are illustrated in the case of pseudosarcoma described by Foote, in which the very chronic lesion con- 
sisted of a diffuse ilfiltration of large mononuclear cells somewhat resembling plasma cells. In this case there were also anal lesions.

Lymphosarcoma of the testis has been a common diagnosis, especially among French writers, but Chevassu has pointed out that many of these cases were probably embryonal carcinoma. Yet true lymphosarcoma has been described by Malassez, Ehrendorfer, and Debarnardi. I have studied 2 cases which seem to be genuine.

The disease occurs chiefly in young subjects, usually involves both testes, develops cutaneous and internal metastases, and runs a rapid course with fever. The exact origin of this tumor is uncertain. The testicle is sometimes the seat of metastatic lymphosarcoma.

The kidney is a favorite seat of metastatic lymphosarcoma in the form of minute foci, diffuse infiltrations, or bulky masses. In Turner's case both kidneys reached an enormous size from diffuse infiltration and much of the renal tissue was destroyed. The existence of primary lymphosarcoma of the kidney is uncertain. In a case of Dowd's I found the organ largely re-

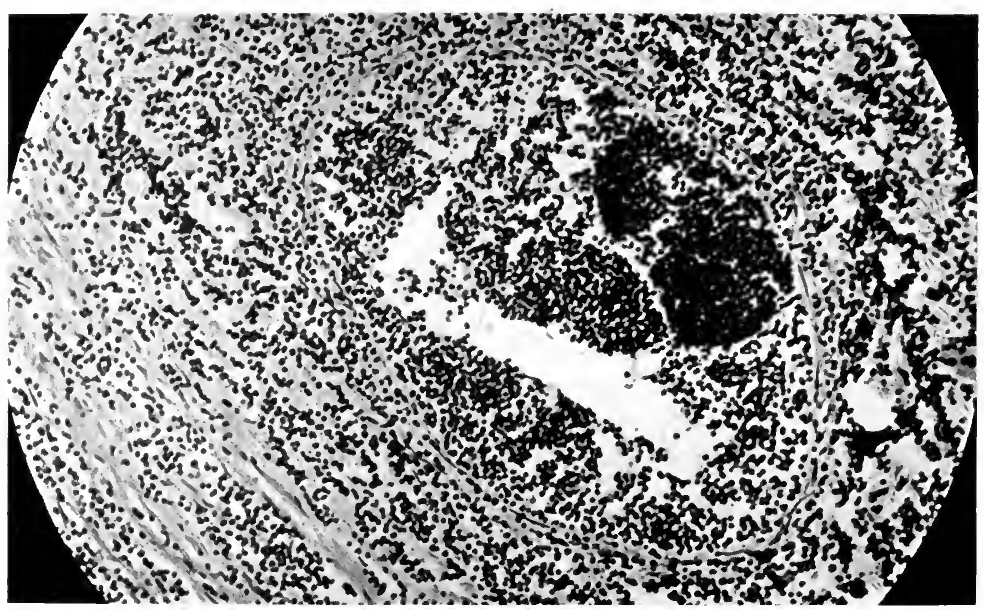

FIG. I56.-Malignant lymphocytoma. Invasion of venule in retroperitoneal fat.

placed and moderately enlarged by many round masses of tumor tissue composed of atypical lymphocytes with hyperchromatic nuclei.

Extramedullary Plasma-cell Tumors.-In a rather numerous group of tumors of mucous membranes and lymph-nodes the growth is composed exclusively of plasma-cells. Considering their comparative frequency and rather peculiar clinical course, they have received inadequate attention (Kusunoki, Frank, Lit.). The growths occur chiefly in the nasopharynx, alveolar borders, tongue, lips, and cervical lymph-nodes, but they have been observed in many other regions. The course is relatively slow, but they may recur after extirpation, and become associated with a chronic cachexia. They are commonly classed as lymphosarcoma, but being as a rule benign processes with very indistinct neoplastic properties, it is highly important that they should be separated from the malignant lymphosarcoma. The structure presents a diffuse growth of more or less typical plasma-cells.

Etiology.-Lymphosarcoma, representing a lawless proliferation of reticulum cells and their derivatives, may be attributed, $\dot{a}$ priori, to any 
irritant which influences these unstable cells over a considerable period or in a specific manner. There is no urgent necessity for assuming the existence of any unknown micro-organism or any other occult factors in the origin of this process, for when the above principle is applied in the study of cases a large proportion of lymphosarcomas are reasonably accounted for.

Thus Birch-Hirschfeld observed a rapidly fatal case which was practically a continuation of typhoid hyperplasia. The older observers were in the habit of attributing many of these cases to syphilis, but the grounds for this assumption have not been strengthened in recent years.

The more generalized forms of lymphosarcoma are not infrequently the sequel of pseudoleukemia or leukemia, for which an infectious origin is probable. The usual type of sarcoma following Hodgkin's granuloma has a specific structure which differs from that of lymphosarcoma and has been separately described.

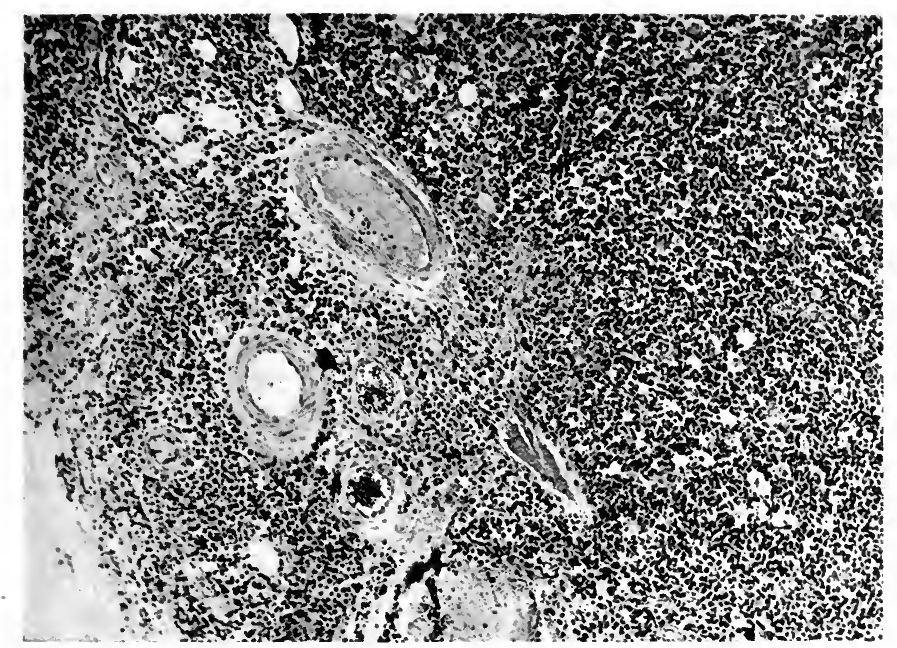

FIG. I 57.-Malignant lymphocytoma of groin, infiltrating fat tissue, walls of vessels, and lumina of small veins.

Tuberculosis occupies a prominent position as an excitant of lymphosarcoma. In fact, many observers, finding frank tuberculosis so intimately associated with lymphosarcoma, have been willing to identify the two conditions. Since the discovery of tubercle bacilli in pseudoleukemic tissue by Weigert, in pure lymphoid hyperplasia by Brentano and Tangl, and in generalized lymphomas of nodes, spleen, liver, and serious membranes by Sabrazes, only a slight difference has separated lymphosarcoma from the immediate or distant presence of tubercle bacilli or their toxins. Frank tuberculosis associated with lymphosarcoma is reported by Ricker in a case terminating after I4 years with sarcomatous infiltration of neck, lung, adrenals, spinal canal, and wide-spread caseous areas, with giant-cells and tubercle bacilli. Yet the structure of the sarcomatous tissue in this case suggests Hodgkin's granuloma. Müller, however, saw general sarcomatosis from a primary tumor of breast in which miliary tubercles were nearly coextensive with large sarcomatous masses. A significant case is that of Brandt's in which, with tuberculosis of left apex of lung, there was sarcomatous inva- 
sion of ileocecal region, many lymph-nodes, pharynx, Eustachian tube, parotid, middle ear, adrenal, and ovary. Inoculation of the pure sarcomatous tissue yielded only lymphoid hyperplasia of intestinal follicles in guinea-pigs, but inoculation of the tissue of these follicles gave frank tuberculosis in a second series of pigs.

Gastro-intestinal lymphosarcoma is especially difficult to separate from tuberculosis or some form of infectious granuloma. These lesions often exhibit lymphocytes, plasma-cells, eosinophile cells, and necrosis, and cases in which tubercle bacilli are missing may closely resemble hyperplastic tuberculosis in which acid-fast bacilli are present, but scanty. Tuberculous intestinal ulcers closely associated with wide-spread sarcomatous lesions in mesentery or retroperitoneal nodes are described by Nothnagel, Freudweiler, Munk, and others. Thus it seems highly probable that many cases of lymphosarcoma arise on a tuberculous basis and that the process thus established progresses of its own momentum and eventually apart from the presence of any bacilli or their toxins.

That tuberculosis is not always the, exciting factor must be granted. Kundrat stated that tuberculosis is not commonly implicated in lymphosarcomatosis. Lubarsch assumes that any infectious agent may serve as the exciting cause, and chiefly because of the close dependence of the process upon microörganisms and their products he would separate lymphosarcoma from other or true neoplasms. For the same reasons Borst anticipates the ultimate identification of lymphosarcoma with infectious granulomas. It may here be noted that the local aggressive quality which chiefly distinguishes lymphosarcoma from pseudoleukemia fails entirely to separate this process from the infectious granulomas, which also infiltrate and destroy tissues.

Not a few lymphosarcomas arise in infants and appear to have a congenital basis. The testicular cases are often of this character. The discovery of epithelial elements in the tumor masses indicates an origin from misplaced tissue masses (Finkler, Martland).

Ribbert assumes that all true lymphosarcomas arise from aberrant and undifferentiated cell groups, and that tuberculosis often serves as the necessary incitant to growth. Such a view seems reasonable for very malignant localized tumors, less so for the generalized forms resembling pseudoleukemia.

\section{SARCOMA OF SPLEEN}

While primary sarcoma of the spleen is rare, several varieties of this tumor have been reported (Jepson, Albert, Foix, Roemmele, Lit.). Three varieties of splenic sarcoma were briefly described by Weichselbaum in $\mathrm{r} 88 \mathrm{I}$, spindlecell sarcoma, endothelial sarcoma, and lymphosarcoma, and although it is impossible to determine the exact nature of many cases in the literature, most of them seem to fall in one of the above groups.

I. Spindle-cell sarcoma occurs as a small or bulky circumscribed tumor. The case of Jepson and Albert stands as a typical example of this rarest of types. The structure showed spindle- and round cells with trabeculæ and solid areas of fibrous tissue. This tumor is comparatively benign. A capsular sarcoma has been described by Heinricus, and angiosarcomas by Langhans, Theile, and Jores.

2. The endothelial sarcoma is the most frequent variety. As a rule it produces multiple nodules in a greatly enlarged organ, and many of these nodules may fuse into larger diffuse masses. Metastases are commonly present, having been observed by Bunting in liver, pancreas, and skin, and 
by Foix and Roemmele about the adrenal. This tumor is, therefore, quite malignant.

The structure consists of large cells with single or multiple vesicular nuclei and pale cytoplasm. They are round or elongated or polyhedral, and giantcells may form. The arrangement may be diffuse or alveolar. Bunting, in an alveolar tumor, concluded that the cells arose from the endothelium of the splenic tissue.

Foix and Roemmele in an elaborate analysis trace the origin to the reticulum cells of the splenic follicles. They liken the process to a neoplasm arising on an inflammatory hyperplasia of these follicles and are prepared to accept an infectious agent or an irritant of endogenous origin as the ultimate cause of the proliferation. Their designation of the tumor as "reticulosplenome

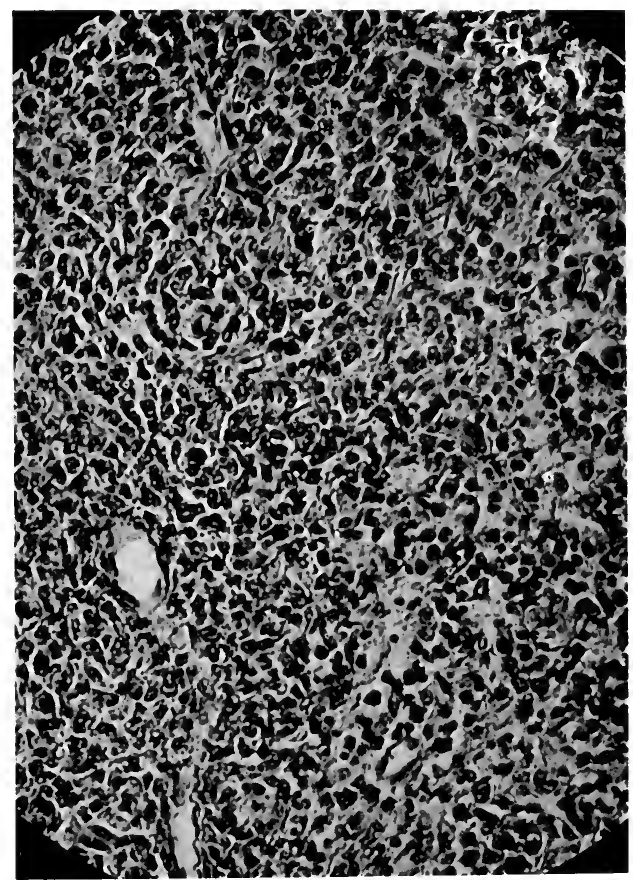

FIG. I58.-Structure of a primary diffuse sarcoma of the spleen.

nodulaire" is non-committal on the endothelial origin of the growth. Many of these cases resemble the endothelial sarcoma arising in Hodgkin's granuloma. A relation to "splenomegalie primitive, Gaucher" has not been indicated by any transitional cases so far described.

3. Primary lymphosarcoma of the spleen, derived from the lymphoid cells of pulp or follicles, has been described by several observers, but it is difficult to determine which of the recorded cases belong in this group. This tumor produces diffuse enlargement of the spleen, as in the cases of Kocher, Grohe, Clarke, and Kocher, or it may appear in the form of one or more large discrete masses (Warren, Herczel). Regional metastases are common, but not invariable. The normal structure of the spleen is largely destroyed by diffuse growth of small or large round cells of the type of lym- 
phocytes. Menetrier designates the tumors with small cells as lymphocytoma, and those with large cells as splenoma. I have studied one case of the latter type, reported by Janvrin, in which the entire organ, much enlarged but with capsule intact, was composed of somewhat peculiar, large round cells resembling the splenic lymphocytes. On account of the partial preservation of sinuses and follicles the truly neoplastic nature of the process appeared doubtful. Grohe's case, which appears to belong in this group, gave extensive metastases. 


\section{CHAPTER XXII}

\section{TUMORS OF THE BRAIN}

General Considerations.-Occurrence.-The frequency of occurrence of brain tumors cannot be accurately stated. Probably about I per cent. of deaths are from this cause. Bruns saw 2 Io cases among II,500 nervous patients, but Hirsch saw only I4 brain tumors among 8000 nervous cases. Tooth collected 500 cases in the service of the National Hospital, London, during Io years. Among I4,005 autopsies at Munich and Heidelberg, I35 brain tumors were recorded by Seydel and Beck. In Gurlt's tables covering I4,630 tumors, 2 I 8 of the brain are included.

Sex.-Including a small proportion of tubercle, males were affected in 744 cases, and females in 428 , in the series collected by Gowers, Bruns, and Tooth.

Age Incidence.-Of Tooth's 500 tumors, excluding tubercle, I39 occurred between I and 20 years (27.8 per cent.); 123 from 2I to 30 years ( 26 per cent.); I 37 from 3 I to 40 years (27.4 per cent.); 65 from 4 I to 50 years (I3 per cent.); 33 from $5 \mathrm{I}$ to 60 years (6.6 per cent.); and 3 after 6r years.

Starr shows that cerebellar tumors are relatively frequent in children. Of 45 cortical tumors, 3 occurred under 19 years, while of 29 cerebellar growths I I were found under I9 years. The gliomas, endotheliomas, and sarcomas show much the same age incidence. Gliomas, cholesteatomas, and dermoids are rarely congenital. Gowers found only the first 5 months immune.

Heredity was a negligible factor in Tooth's analysis, a cancerous family history being obtained in only 37 cases $(7.2$ per cent.). In no case was a history of brain tumor noted. There are, however, occasional exceptions to this rule.

Trauma.-The frequency of the traumatic origin of brain tumors has been variously estimated by different observers, but as a rule the estimates are notably high. Gerhardt accepted the traumatic origin of ro among 60 reported cases of glioma, and in 4 of I I cases of his own. Bruns holds that a direct traumatic origin is rare. Adler collected I 986 cases, accepting a relation to trauma in 8.8 per cent. He details I 8 cases of various types, illustrating many possible relations to trauma, some of them quite direct. Lowenthal found II gliomas which on somewhat uncertain evidence he referred to trauma. A traumatic origin of certain sarcomas resembling granulation tissue is more acceptable than with gliomas.

The trauma may have no influence whatever on the tumor. This relation must be assumed to exist in the cases in which the trauma is incapable of producing a lesion of the brain tissue, and may be assumed as probable when a long interval elapses between the injury and the appearance of symptoms.

The trauma may cause the outbreak of symptoms of a previously existing latent tumor. Such cases are observed when an unsuspected glioma causes epileptic attacks with head injury in falling (Bruns).

The trauma may accelerate the growth of a previously existing tumor. Burns refers to the rapid growth after trauma of mildly indicated gliomas, and supposes that the trauma produces hemorrhage in the vascular tumor. Oppenheim states that trauma may indirectly excite tumor growth by establishing 
a condition of epilepsy which eventually induces the neoplastic proliferation of glia-tissue. Such cases are reported also by P. Knapp and Osler.

Trauma may be the direct exciting cause of the tumor. In order to prove such a relation it is necessary to show that a sufficient injury has caused a lesion of the dura or pia with affection of the brain tissue. Evidences of old hemorrhage, with adhesions of brain to membranes beneath the point of injury, are the only satisfactory data that can be offered as proof. Such cases have been fully reported by Hitzig, Eppinger, Thomas and Bartlett, Keen, Annandale, Adler, Carara, and others. Both sarcoma and glioma are observed under such conditions. Somewhat less satisfactory and more numerous are the cases in which the tumor does not appear at the point of injury, but owing to the influence of contrecoup the brain lesion may be far removed from the point of the blow. Additional evidence may appear in the clinical history, when it is shown that the tumor symptoms were continuous with or appeared shortly after the injury. With increasing intervals between

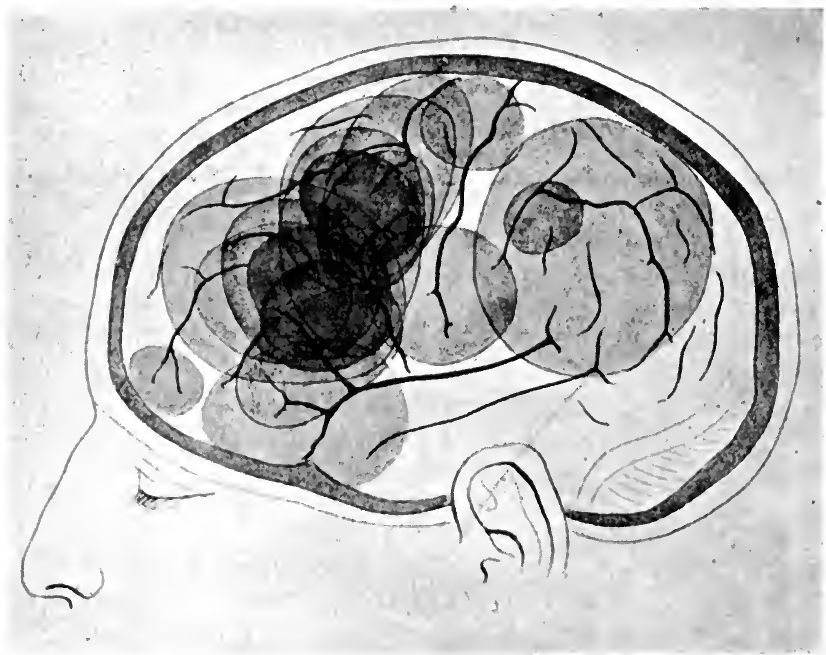

FIG. 1 59.-Location of a series of brain tumors. (v. Eiselsberg.)

trauma and tumor the relation must be regarded as less direct. Starr's assumption that a cerebral hemorrhage or contusion may pass directly into a tumor process is rejected by Oppenheim and Bruns, but such an interpretation is fully in accord with histological findings as well as clinical history. In the organization of a cerebral blood-clot the growth of capillaries and gliatissue may be very active.

The relative frequency of the different types of tumors may be judged by Tooth's analysis of $25^{8}$ cases verified at operation or postmortem as follows:

Glioma, I32 (55 per cent.); endothelioma, 37 (I4.3 per cent.); sarcoma, 2I (8. I per cent.); carcinoma, I5 (5.8 per cent.); tubercle, I4 (5.4 per cent.); fibroma, I3 (5 per cent.); cysts, 5 (I.9 per cent.); ventricular papilloma, 3 (I. I per cent.); cholesteatoma, 2 ( 0.7 per cent.); pituitary, 2 ( 0.7 per cent.); pineal, 4 (I.5 per cent.).

Fibrogliomas and fibromas belonged to the cerebellum. The endothe- 
liomas were confined to the anterior portion of the falx in 35 of the 37 cases. Of the carcinomas only one was certainly primary.

The general distribution of brain tumors is indicated in the following table, in which are included the series of cases of J. Collier and Tooth, together with those of Stern:

Frontal................. 129

Parietal............................. 73

Temporosphenoidal........... 63

Occipital.................. I 7

Corpus.callosum .............. 34

Mesencephalon.............. 57

Lateral ventricles.............. 3

Third and fourth ventricles.......
Optic thalamus............... 6

Pons..................... 65

Medulla.................. I

Base.................... 9

Pituitary................ I4

Pineal................... 4

Cerebellum................ I I 2

Extracerebellar.............. $\frac{40}{632}$

Including tubercle and gumma the following table of incidence results from combining the figures of Birch-Hirschfeld and Bernhardt:

\begin{tabular}{|c|c|c|c|c|c|c|}
\hline & Tubercle. & Gumma. & Sarcoma. & Glioma. & Osteoma. & $\begin{array}{c}\text { Cholestea- } \\
\text { toma. }\end{array}$ \\
\hline Cortex. & 50 & 15 & I6 & I 2 & o & o \\
\hline Lobes......... & 18 & 8 & 39 & 44 & o & 2 \\
\hline Basal ganglia ........ & 17 & 7 & I4 & I I & I & \\
\hline Corpora quadrigemina & 2 & 0 & 2 & 6 & & \\
\hline Pons $\ldots \ldots \ldots \ldots \ldots$ & 30 & 6 & 8 & 12 & I & \\
\hline Medulla........... & 9 & 2 & 5 & II & & \\
\hline Cerebellum.......... & 57 & 4 & 22 & 32 & 2 & \\
\hline Hypophysis . . . . . . . & o & 3 & 3 & o & o & \\
\hline Pineal. . . . . . . . . & 0 & 0 & 4 & o & o & \\
\hline & 183 & 45 & II 3 & I 28 & 4 & 2 \\
\hline
\end{tabular}

Multiple brain tumors are not infrequently observed. In the same case Hanel saw a cystic round-cell sarcoma, a ganglionic neuroma, a spindlecell sarcoma, several lymphangiomas, and a tumor-like thickening of the dura vessels.

Operability.-Conclusions regarding operability of brain tumors have not greatly changed in recent years, notwithstanding isolated reports of brilliant successes. In 1889 Starr concluded that of 300 brain tumors in children only i9 cases warranted operation, and only I6 could have been successfully removed, of which ro were tubercle.

In I914 Kuttner reports that in Ioo clinical cases 30 tumors were removed and 34 others were later demonstrated to exist, 45 died from the operation, I 4 died in 1 to 3 months, and 5 in 4 to 5 months. In 13 cases it was later shown that no tumor existed. Four blind cases recovered sight, and in cases remained well for 6 months to 6 years.

Henschen reports that of 205 operations, 48 patients died from the operation and 88 more in one month, while after 2 years all but $\mathrm{r}_{4}$ were dead. Bergmann and v. Eiselsberg both report operative mortalities of 38 per cent., while among 37 I cases Bergmann reports III not improved and 88 (23 per cent.) improved. Eichelberg concludes that 70 to 80 per cent. of brain tumors can be localized, and of these 5 per cent. are enucleable. Among 33 cases of brain tumor Cramer and Hippel found only 4 suitable for operation, 3 of which were cysts. Duret analyzed 400 operative cases, finding that 78 
(I9.5 per cent.) died immediately, 20 very soon from meningitis, and 47 within one month, so that a total of 145 ( 36 per cent.) received no benefit. Improvement in varying degree followed in 258 cases, among which there were $4 \mathrm{I}$ recurrences of the tumor. The surgical possibilities in favorable cases are well illustrated in the report of Alexander and Unger who located a calcific subpial endothelioma, $7 \times 7 \times 5 \mathrm{~cm}$., by means of the $x$-ray and removed it under cocain with complete cure, although the lateral ventricle had been opened.

Miscellaneous statistics are of practically no value in determining the probable outcome of any given case, whereas a very thorough clinical study of every feature of the condition, accurate localization, rate of growth of the tumor, age of the patient, a knowledge of the natural history of the different types of tumors, and of their pathological anatomy may together yield a comparatively reliable prognosis.

All of these subjects form a very extensive department of our knowledge of brain tumors and furnish the chief topic of several comprehensive works, such as those of Oppenheim, Bruns, Duret; Henschen, Stern, Starr, F. Krause, and many others.

The duration of brain tumors without operation was found by Tooth as follows: Glioma IO.I months for 2 I cases, extremes 9 years and 24 days. Endothelioma, 20.2 months for 6 cases unoperated. In $3^{I}$ cases operated upon the average duration was 4 years, extremes 20 years and 2 months. Sarcoma, 5 cases survived II.2 months, or, omitting one which lasted 4 years, the average was 5.I months. Of 7 cases diagnosed as pituitary the average survival period was 7.5 years. A notable fact was the long survival period of 6.5 years of $5 \mathrm{I}$ cases not localized.

Secondary Effects of Brain Tumors.-The continued growth of an intracranial tumor usually induces secondary changes in many intracranial structures, giving rise to complex and confusing cerebral symptoms and complicating the anatomic relations of the tumor. These effects are the result of many factors, including the pressure of the tumor mass, circulatory disturbances in the vicinity of the tumor and in the whole cranial cavity, interference with the paths of ventricular fluids, and local invasion and dissemination of the tumor.

(a) On the brain tissue. The tumor may act on the brain tissue by compression, by irritation, or by actual destruction of cells and fibers. Simple compression is best observed with tumors of the meninges, or encapsulated growths which displace the adjoining brain substance. Pressure atrophy gradually follows with loss of cells and myelin sheaths, but complete loss of functional capacity of the affected region is generally slow to appear. Extensive displacement of affected parts may result, especially in the cerebellum. Here bulky tumors have been known to force the medulla or even the cerebellum tightly into the foramen magnum or spinal canal. Compression effects are often observed at a distance, as when a tumor of one side compresses the structures of the opposite cerebral hemisphere with convulsions. Cerebral tumors may even compress the cerebellum, although the tentorium usually limits such effects in both directions.

Very extensive hernial protrusion of the brain may follow opening the skull. In some cases the relief of pressure accelerates the growth of an underlying or distant glioma or sarcoma which forces brain tissue or tumor tissue, or both, through the opening. Eichelberg describes a hernial protrusion almost as large as the brain itself. Compression of the cranial nerves vields some of the most reliable of localizing symptoms. These nerves may be directly compressed by the tumor, or displacement of portions of the brain 
may strangle the blood-vessels or the nerve-fibers or actually sever the nerve trunk (Wernicke).

The imperfectly formed blood-vessels of gliomas are especially susceptible to intermittent distention, producing intermittent pressure symptoms. Or, after rupture, extensive hemorrhage in or about the tumor may come from these vessels. Irritation of brain tissue usually precedes the pronounced stages of compression. The mechanism of the resulting spasms and paresthesias is not clear, but probably consists of local vascular disturbances with edema and exudation.

Destruction of brain tissue follows prolonged pressure, results from the gradual extension of glioma, and is rapidly effected by infiltrating sarcomas and especially by carcinomas. Inflammatory reaction about the tumor or extending over considerable portions of the brain is frequently observed and may greatly complicate accurate localization of the original growth. Collier

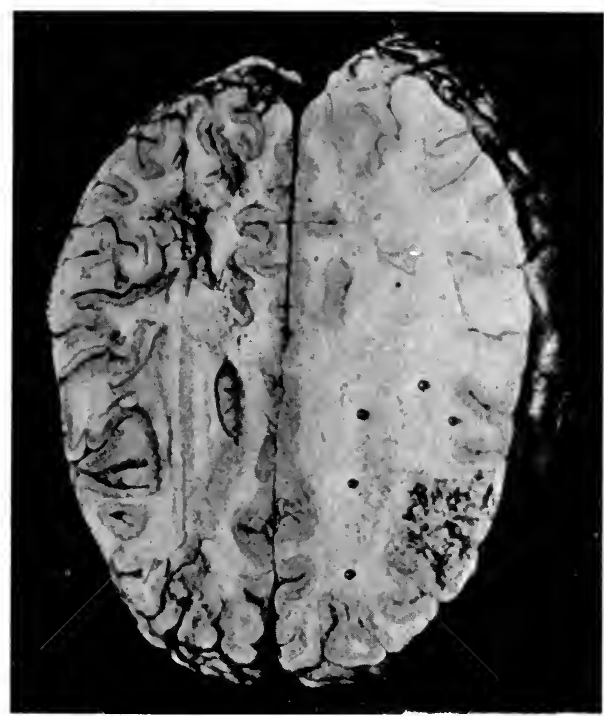

FIG. I60.-Acute edema of brain in region surrounding a hard, slowly growing tumor. (After J. Collier.)

analyzed false localizing signs occurring in 20 of 16 I cases, finding, as the source of the symptoms, extensive edema about slowly growing tumors, thrombosis or hemorrhage at a distance from the tumor, areas of softening, ventricular hydrops, and distortion of cranial nerves. These signs were encountered in I3 per cent. of supratentorial growths and only twice in cerebellar tumors. In two fibrosarcomas the false signs resulted from intracranial metastases.

General edema about small gliomas may involve an entire hemisphere and lead to rapidly deepening coma, convulsions, and paralysis. About a pial sarcoma invading the brain tissue Merzbacher observed very marked proliferation of glia-cells which he interpreted as a reactive glioma. In a series of I $_{2}$ cases he found no reaction of the glia-tissue against carcinoma, but considerable reaction in the presence of sarcoma.

The disappearance of localizing symptoms, which is a well-known feature 
of brain tumors, may result from a change in anatomical relations, with relief of pressure and subsidence of edema and exudate. Interesting cases of relief from symptoms of brain tumor are recorded by Hawthorne. In many brain tumors, especially gliomas, the entire brain tissue may be the seat of structural changes resulting probably from general disturbance of circulation. They consist in increase of glia nuclei, especially in the deep cell lavers, disturbed polarity of the cells, partial or complete tigrolysis, shrinkage or complete atrophy of cells, pyknosis, karyolysis, or homogenization of nuclei, and marked increase of pericellular nuclei (Stern).

(b) Disturbance of the circulation of blood, lymph, and cerebrospinal fluid becomes pronounced in the late stages of most brain tumors and leads to general cerebral compression, with symptoms of headache, increasing dulness, coma, slowing of pulse and respiration, and choked disk. On exposing the brain the convolutions may appear flattened and pulsation is absent. The essential feature of general cerebral compression appears to be capillary anemia. In nearly all cases there is ventricular hydrops, and this condition develops early in most tumors of the base, cerebellum, and fourth ventricle. It is generally indicated by deep coma and muscular spasticity. Secondary pressure effects of ventricular hydrops may be traced in several directions: as upon the plexus veins, vena Galeni, and the great sinuses; upon the surrounding brain tissue with atrophy; and upon the chiasm and cranial nerves with false localizing symptoms.

Choked disk may be referred chiefly to the mechanical effects of general cerebral compression. The fluid in the arachnoid spaces passes directly into the lymph sheath of the optic nerve, distends the sheath and the lymph vessels of the nerve, obstructs the blood flow of the central vein with varicosities in the retinal veins, and edema, hemorrhages, and eventual atrophy of the optic cusp. Secondary exudative and degenerative changes may follow and have led to the assumption that the changes are of toxic origin (Elschnig). The essential dependence of choked disk upon mechanical edema best explains the sudden changes which this condition may exhibit. A similar edema of the auditory labyrinth is also observed (Schwalbe).

(c) Spinal cord degeneration, affecting the posterior columns and ganglia, results from distention of the spinal arachnoid with stretching, tearing, and compression of nerve roots. Batten and Collier found such changes in 20 of 29 cases. The cervical cord was chiefly affected, the condition was especially common with cerebellar tumors, and the chief symptom was loss of kneejerks.

(d) Meningeal complications consist chiefly in local or diffuse invasion by the tumor process. While very pronounced conditions of this type belong to pial malanoma and rare forms of pial sarcoma, Verdun was able to collect 28 cases of more or less extensive invasion of the pia by various types of tumors. In one glioma of the pons he observed extensive pial thrombophlebitis.

(e) Changes in the skull. The bones of the skull may suffer absorption from local pressure or thinning from general cerebral pressure. Bony changes are most common with tumors of the base and in children, and by means of the $x$-ray they form an important class of localizing symptoms. Up to the age of puberty intracranial growths may separate the cranial sutures as does hydrocephalus. Perforation of the skull is observed with hypophyseal growths, which may discharge into the nares, and with sarcomas which penetrate the orbit or pharynx.

Anatomical Classification.-Of the numerous pathological conditions which fall in the clinical group of brain tumors several may for the present purpose be eliminated from the category of intracranial neoplasms. Such are the soli- 
tary tubercle, gummas, echinococcus cysts, and simple hydropic cysts. The remaining list of true tumors includes:

\section{Glioma $\left\{\begin{array}{l}\text { Astrocytoma, } \\ \text { Gliosarcoma, } \\ \text { Neuro-epithelioma. }\end{array}\right.$}

Sarcoma, derived from blood-vessels.

Endothelioma and psammoma, derived from endothelial structures of the meninges.

Papilloma and carcinoma of the ventricles and choroid plexus.

Neurofibroma, arising from cranial nerve-trunks.

Melanoma, derived from misplaced or wandering chromatophores.

Fibroma and myxoma, derived from the dural connective tissue or representing acellular forms of glioma, or endothelioma.

Osteoma.

Lipoma.

\section{Cholesteatoma.}

Finally, there are specific tumors of the pineal gland and the hypophysis.

The plan adopted for the discussion of these tumors recognizes the fact that some, especially the benign tumors, are of rare occurrence in the brain and are of more general interest. Fibroma, osteoma, lipoma, endothelioma, and melanoma are therefore included with similar growths in other situations.

Tumors of the pineal gland and hypophysis are treated as specific diseases of these organs. There remain, as true tumors belonging to the brain, glioma, sarcoma, and tumors of the ventricles. Glioma, the chief cerebral tumor, occurs in other situations and its various types and locations will be considered together.

\section{GLIOMA}

Glioma is the characteristic tumor of the central nervous system and consists of neoplastic neuro-epithelium or its adult derivative the glia-cell. Three structural forms of this tumor may be distinguished:

I. Astrocytoma, typical adult glioma.

2. Gliosarcoma, anaplastic, cellular.

3. Neuro-epithelioma, embryonal.

The typical glioma occurs in the brain, spinal cord, and roots of cranial nerves, while atypical forms arise both in these organs and in the eye and in teratomas. The adult form of glioma consists of well-developed glia-tissue, but in many embryonal tumors undifferentiated neural epithelium appears and the tumors are called neuro-epithelioma. There are considerable variations in the proportions of glia-cells and glia-fibers, giving rise to the terms glioma molle and durum, and the great predominance of round or spindle glia-cells deficient in fibrils produces cellular tumors called gliosarcoma. Some would limit the term "gliosarcoma" to mixed tumors derived from gliatissue and from mesoblastic elements in nervous tissue, but it is doubtful if such tumors exist, and in a morphological sense the term "gliosarcoma" is admissible for cellular and malignant gliomas. Scaffidi argues for the mesoblastic origin of a portion of the glia-cells. Glia tumor-cells often grow to large dimensicns and resemble ganglion-cells in tumors which may be called glioma ganglionare or gangliocellulare.

Gross Anatomy.-Gliomas appear as irregular or rounded segments of nervous tissue of greater or less density than the normal tissue, but often without definite enlargement of the affected part. They are usually more opaque than normal brain tissue and the blood content is increased so that the 
color is bluish. Hemorrhage or excessive development of blood-vessels, glioma telangiectaticum, may render them especially conspicuous. Yet the borders grade insensibly into the surrounding tissue so that it is often difficult to determine the limits or even the existence of a glioma from the naked-eye inspection. Gliomas obliterate the normal markings of the brain, some are distinctly nodular and quite firm; they may cause flat protrusions of the pia and a diffuse thickening is often produced. Ependymal gliomas occur in the form of projecting nodules or larger masses which may become pedunculated (Virchow, Linck). In Bielschowski's case and in Pfeiffer's the tumors were multiple and the ventricles dilated. In size gliomas vary from a small focus to a diffuse involvement of nearly a whole hemisphere, with distinct widening of the convolutions. They are seldom multiple, and local metastases

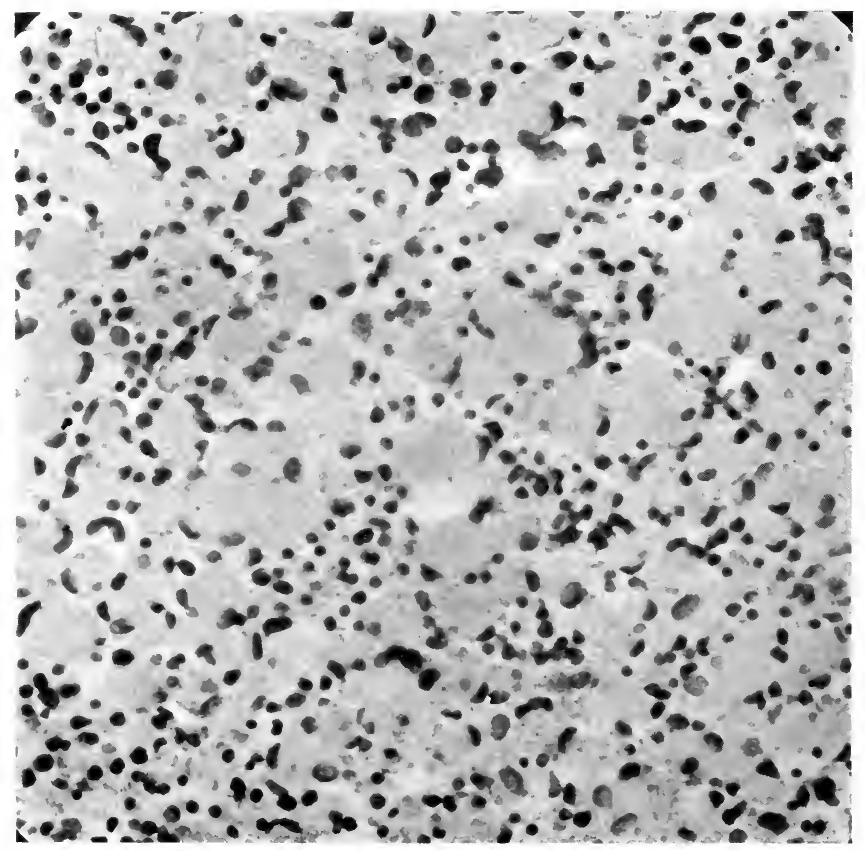

FIG. I6I.-Glioma cerebri.

are rare. Retinal gliomas differ from the cerebral in producing bulky fungating tumors. In the spinal cord the tumors may be localized, but usually they arise in the region of the central canal into which they extend in the form of long cords, destroying the gray matter, and sheathed by the white tissue. A dilated central canal may persist and the condition resembles syringomyelia (Miura, Saxer, Schlesinger). Neuro-epitheliomas frequently grow to large size and produce metastases.

Secondary degenerations in gliomas are relatively frequent. Hemorrhages, old, with formation of cysts and deposit of pigment, or recent and diffuse, belong to the natural course, especially of the cerebral tumors. Necrosis of large central areas is common. Hyaline degeneration may affect the vessels and calcification may appear in such vessels or in old necrotic areas. Globular myelin droplets may be very numerous and may become calcified. 
Soft gliomas may be extensively edematous, strands of tissue being widely separated by collections of fluid. Myxomatoid changes follow edema and have led to the use of the term "myxoglioma." Cysts form from absorbed necrotic areas, from extensive edema, areas of hemorrhage, and dilated canals.

The structure of glioma presents glia-cells and glia-fibers in their various forms and in varying proportions. In many cases the typical spider-cell (astrocyte) is predominant. This cell has a small compact nucleus and scanty cytoplasm, from which radiate very numerous comparatively short fibers. Golgi believed that the cells with short fibers characterize tumors

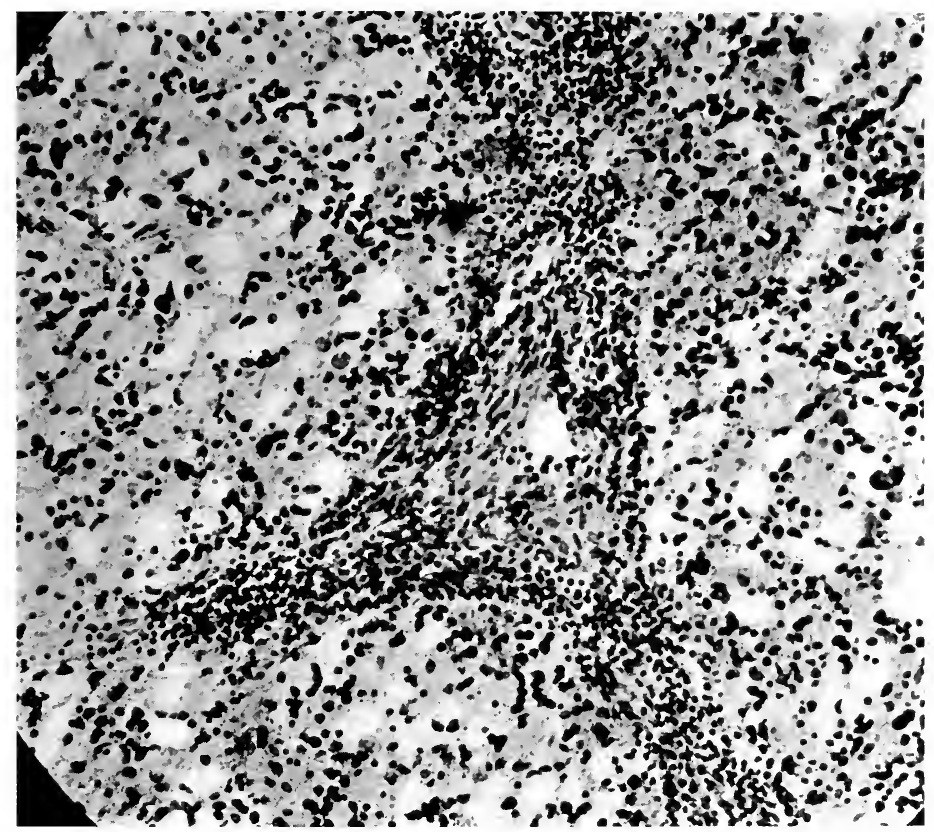

FIG. I62.-Edematous glioma of brain.

of the outer cortex (astromas), while cells with long fibers are found chiefly in the deeper or central gliomas, but this relation appears to be inconstant. In other cases the cells are larger and the fibers less numerous and much longer.

In certain tumors the large glia-cells possess cytoplasmic processes, large nuclei, with multiple nucleoli, and closely resemble ganglion-cells. Stroebe finds such cells most frequently about the edges of spaces lined by neuroepithelium, but they are not limited to such situations, and in some tumors all the cells may be of this type (Borst). Occasionally the cells contain multiple nuclei, so that Stroebe speaks of giant-cell glioma. The significance of these ganglionic glia-cells has generally been interpreted as an expression of the normal developmental tendency of the originating cells of the tumor, both glia- and ganglion-cells being derived from neural epithelium. Even in normal brain tissue Golgi and v. Kolliker find glia-cells approaching the form of ganglion-cells, and Renaut's view that glia-cells functionate as conducting nerve-cells is being regarded with increasing favor. Renaut described axis- 


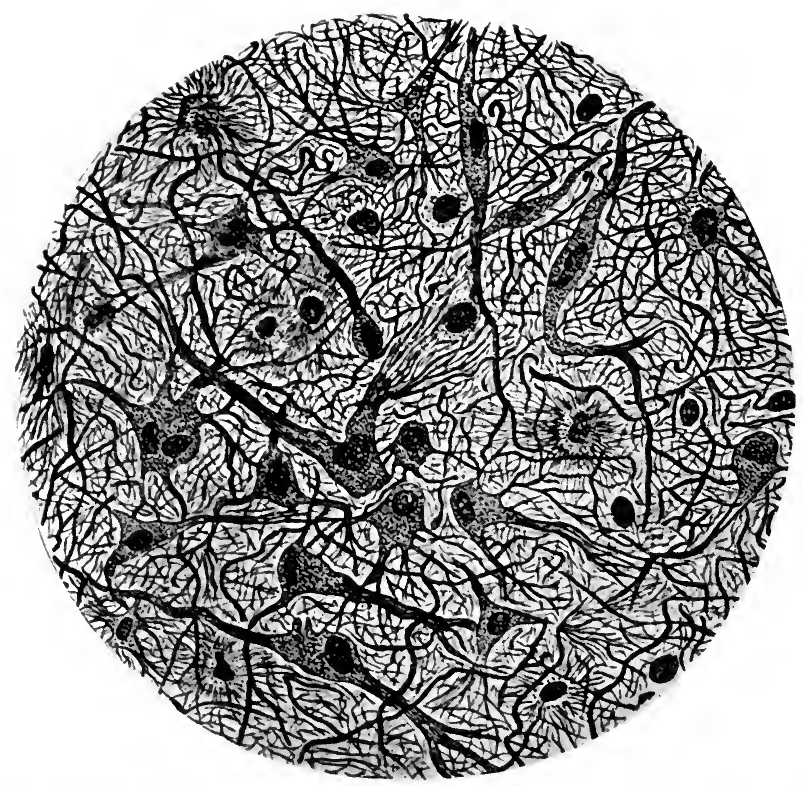

FIG. I63.-Detailed structure of glioma (astrocytoma). (After Stroebe.)

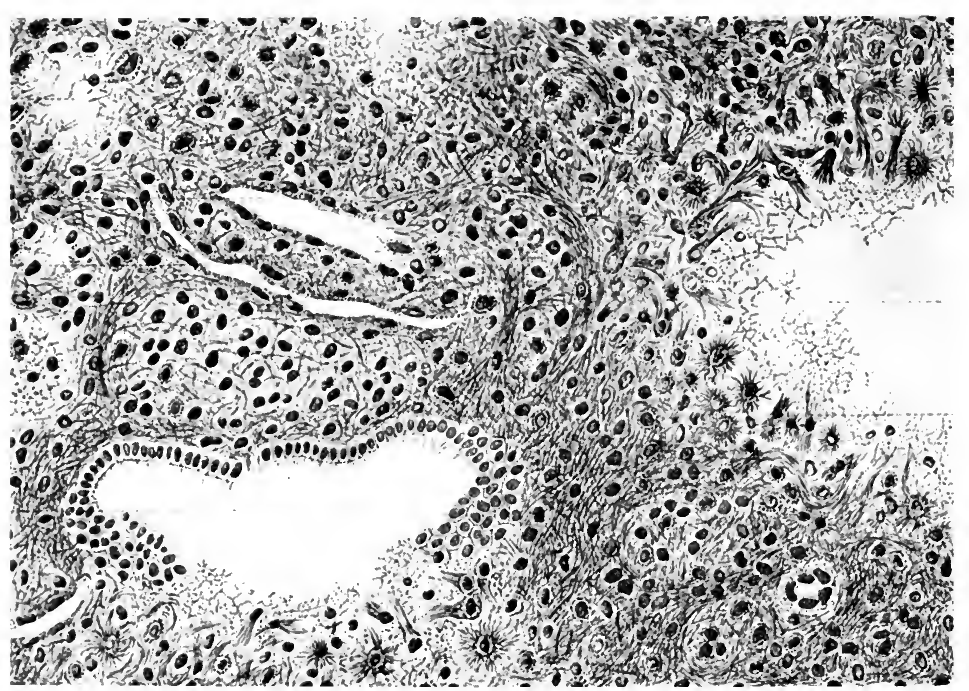

FIG. I64.-Details of structure of a glioma. Astrocytes, round cells, and cavities lined by neuro-epithelium. Müller's fluid. Mallory's hematoxylon. (After Stroebe.)

cylinder processes in the large cells of glioma, but Storch finds by means of Weigert's neuroglia stain that these processes have the brush-like ends of glia-fibers and often terminate in the adventitia of blood-vessels. Mallory 
finds that the cells in many gliomas contain from 2 to 30 of the glia granules described by Weigert in ependymal cells, and that these granules form a valuable diagnostic sign of glioma. Pigmented astrocytomas of optic thalamus and lateral ventricle with ependymitis granularis occurred in a case described by E. J. Kraus.

Finally, the cells in glioma may assume a spindle form, and when they are numerous the tumor resembles a spindle-cell sarcoma. From the poles of such spindle-cells the radiating fibers may usually be demonstrated, and portions of such tumors may show more typical glia structure.

The glia-fibers are the most characteristic element in glioma. Although Ranvier and Weigert claimed that the fibers are disconnected from the cells, Golgi, Kolliker, Lenhossek, and many later observers using specific staining

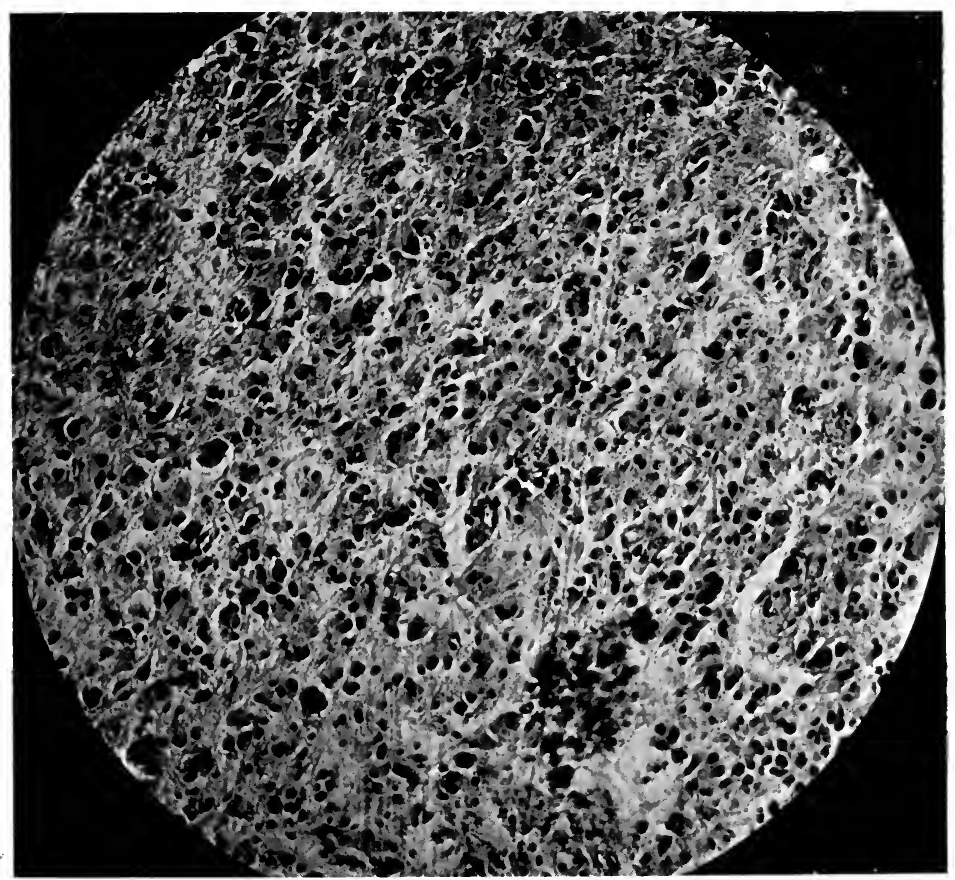

FIG. I65.- Large cell glioma of brain cortex.

methods have shown that the fibers emanate from the cells and under ordinary conditions retain their cellular connection. In normal tissue the great length which the fibers attain, reaching $1000 \mu$ (Kolliker) renders very difficult the determination of their origin, and in glioses where fibers increase and predominate over cells, solution of continuity may doubtless occur. The study of gliomas emphasizes the essential connection of fibers and the growing glia-cells into which they are readily traced (Stroebe, Storch, Borst). That occasional separation of fibers from cells may occur and that this condition is prominent in hard acellular gliomas can at the same time be admitted (Aschoff). Ribbert holds that the relation of glia-fibers and cells is much the same as that between fibroblastic fibers and cells.

The glia-fibers are very numerous, fine and short (astrocytes), or fewer, 
thicker, and longer, while from the large glioma-cells there may pass only a few coarse, irregular, protoplasmic processes which later assume the characters of glia-fibers and break up into a terminal brush. Anastomoses with other fibers or connections with other cells are not observed. Buchholz and Storch noted a striking accumulation of fibrils about the walls of bloodvessels, but this feature is not invariable. Mallory finds that glia fibrils may fuse together in a sort of membrane where they meet connective-tissue structures. Degenerating fibrils become varicose, fragmented, and granular. The proportion of fibers varies extremely, the hard gliomas exhibiting a predominance of them, while in certain round- and spindle-cell tumors they may be difficult to demonstrate. Stroebe claims that neuroglia fibers may always be demonstrated in gliomas, but this criterion cannot be demanded for retinal gliomas and it is supplied with difficulty for some gliosarcomas. Special stains are required for their complete demonstration, the best of which are Weigert's neuroglia stain and especially Mallory's phosphotungstic acid hematoxylon.

In a somewhat peculiar form of cerebral glioma the detritus of the invaded tissue becomes inclosed in large phagocytic tumor cells in the form of homogeneous globules and much of the affected area is composed of such cells. Between them lie many small cells with compact nuclei and scanty glia fibrils besides which are many large multinucleated cells of uncertain origin. On the edges of the tumor it seems possible to trace the ganglion-cells of the invaded cortex which become hypertrophied with hyperchromatic nuclei and are gradually lost among the numerous tumor cells. Their ultimate fate is uncertain, but they seem to participate in the very diffuse tumor process.

In many gliomas there are canals lined by small cuboidal cells which resemble ependymal epithelium. The lumen is minute or of considerable dimensions and the contents are watery fluid or granular detritus. The external borders of the cells merge insensibly into the surrounding tissue, and glia fibrils are scanty or missing. Canals of this character are to be distinguished from rosettes of embryonal and often cylindrical neuro-epithelium which inclose minute spaces and form large cysts in neuro-epitheliomas. The significance of the ependyma-like canals has been variously interpreted. Buchholz believed that glia-cells of the tumor became transformed into ependyma-like cells with which they are genetically related. Stroebe and many others have concluded that the canals arise from sprouts and misplaced groups of ependymal cells, and this view is suggested especially by the abundance of such structures in tumors of the basal ganglia and spinal cord. Misplaced islands of ependymal epithelium have been found by Arnold in a deformed hemicephalic brain, by Miura in syringomyelia, and by Aschoff in ependymitis granularis. A third origin has been suggested from spaces lined by endothelium. In my own cases it has been possible to trace the glia-cells of the tumor into cubical epithelium lining canals and spaces usually of considerable size, and in these cases the cubical cells are of neoplastic type. In other cases canals lined by cubical epithelium not of neoplastic type have appeared on the outer portions of the tumor and these I believe result from transformation of glia-cells of the invaded tissue. Similar changes may be seen in other pathological conditions. Borst describes them both in glioma and in multiple sclerosis. In central and spinal gliomas the inclusion of groups of ependymal epithelium probably accounts for some of the canals. The participation of endothelial cells in this process seems very doubtful, and the presence of the canals does not require an ependymal element in the tumor anlage, as urged by Stroebe, since any of the glia-cells may apparently produce the cubical cells in the formation of the canals. 
Neuro-epithelioma.- In a numerous group of cases the cells greatly exceed the fibrils, they assume a polyhedral or spindle form, rosettes of low cubical or high cylindrical cells surround central points or canals, and from its predominant cellular element the tumor is designated as neuro-epithelioma or neurocytoma. In this group one has to deal with a more embryonal form of glioma in which the cells develop but slightly from the original form of embryonal neural epithelium. The essentially gliomatous character of these tumors appears in the presence of very many well-formed neuroglia fibrils, which with specific stains are nearly always demonstrable and often form a very prominent structural feature. In edematous, degenerating, and spindlecell growths the fibrils, which are a highly important diagnostic feature, may be scanty and difficult to identify.

Neuro-epitheliomas are of wide distribution, occurring in the cortex, basal ganglia, arachnoid, eye, spinal cord, abdominal sympathetic, adrenal,

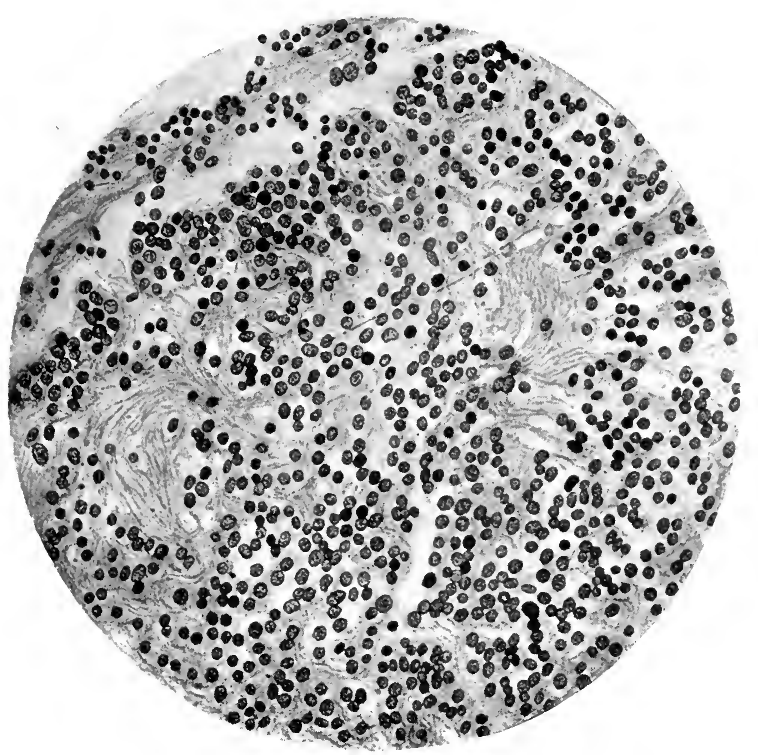

Fig. I66.-Structure of neurocytoma. (After Landau.)

and in teratomas in many situations. The retinal gliomas are often of this type. Many of them are very cellular and in this class fall most of the tumors to which the term "gliosarcoma" has been applied. It is probable that the gliomatous nature of many sarcomas connected with the nervous system has been overlooked.

The blood-vessels in gliomas are usually abundant. They appear as medium-sized arterioles with well-developed walls, or as capacious venules with thin walls, or as large sinuses. Areas of cavernous blood-vessels may displace the tumor-tissue, and varicosities are common. The walls may undergo a peculiar thickening and hyalinosis which may terminate in occlusion and formation of lamellated homogeneous, or partly calcified masses. Proliferation of the cells in the walls of vessels may be considerable, but a true neoplastic character I have not observed in this process. The capacity of the blood-vessels is very great and the blood content is subject to wide 
variations. Hence the course of glioma is marked by intermittent symptoms dependent on variations in the blood content, and hemorrhage with apoplectic symptoms is frequent. Several observers have noted an accumulation of glia fibrils about the blood-vessels, a feature which I have seen, especially in neuro-epithelioma. Small vessels may form the nodal points of neuroepithelial rosettes and the entire tumor may consist of thick perivascular cell sheaths, an arrangement determined by the demands for nutrition.

The growth of gliomas is one of the best examples of tumors of the infiltrative type. The edge of the tumor often merges insensibly into the surrounding tissue and usually fails of demarcation by vascular reaction or cellular exudate or signs of compression. Yet the hard gliomas and the neuro-epitheliomas may be sharply circumscribed from the surrounding tissue by virtue of expansive growth. Pressure symptoms are slowly established with all varieties of glioma, infiltrative or expansive, but these symptoms are partly dependent on the blood content and may therefore be intermittent. Flattening of convolutions often occurs and demonstrates the existence of a permanent mechanical element in the pressure symptoms. On the edges of infiltrating tumors one finds a zone of round cells with few or no fibrils, and

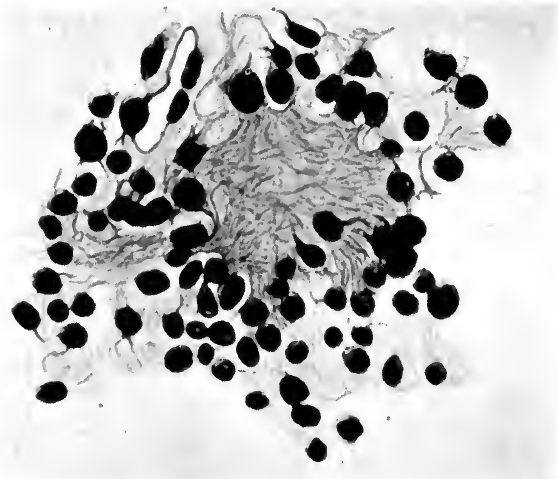

FIG. 167.-Detail of fibril formation in a neurocytoma. (After Landau.)

Ribbert points out that it is only such cells withqut fibrils which are capable of explaining the infiltration. Just within the growing edge the cells develop fibrils which permanently fix their location. Hence the growth of typical glioma is slow. Cell-division by mitosis, symmetrical or multipolar, and by amitosis has repeatedly been observed.

The fate of the invaded tissue is gradual atrophy and absorption, but ganglion-cells may persist for some time on the edges of the tumor, and Stroebe finds in all cerebral gliomas traces of medullated or naked nervefibers derived from the original tissue. Large vacuolated phagocytic cells containing fatty detritus and pigment may appear on the edges of the tumor or throughout its substance.

The possibility that the normal glia-cells are partly transformed into tumor cells while actively opposed by Ribbert, is considered as uncertain by Stroebe and others. It is not yet clear what relation is borne by certain structural anomalies observed in the central nervous system and the occurrence of very diffuse forms of glioma and the uncertain criteria which separate glioma from certain glioses render somewhat hazardous a definite opinion on this point. In a peculiar form of diffuse cerebral glioma I find that the 
ganglion-cells seem to become hypertrophied, they acquire multiple pyknotic nuclei, and become merged with the numerous glia-cells of the tumor.

Resistant structures offer a rather firm barrier to the advance of glioma even to the most cellular varieties. The pia mater terminates the extension of most cortical gliomas, but is itself often split up by strands of cells which fill the natural spaces of this membrane, leading to adhesions, but not to metastases. The retinal tumors, however, infiltrate the perivascular sheaths. Spinal tumors also may extensively infiltrate the pia and subdural space.

The differential diagnosis of glioma from sarcoma may offer difficulties, especially with the small round- or spindle-cell tumors. According to Stroebe the small glia-cells show very scanty cell body, but some fine fibrils are always attached to the cells. The margin of the glioma shows infiltration by tumor cells, but lacks the sharp demarcation and reactive inflammation often observed with sarcoma. The glia-cells are of uniform character throughout the tumor and fragments of medullated nerve-fibers or myelin droplets are always present. Gliomas do not infiltrate the vessel sheaths and they either stop at the pia or, if infiltrating its layers, do not destroy this structure and cause its fusion with the tumor. Yet Pels-Leusden saw very

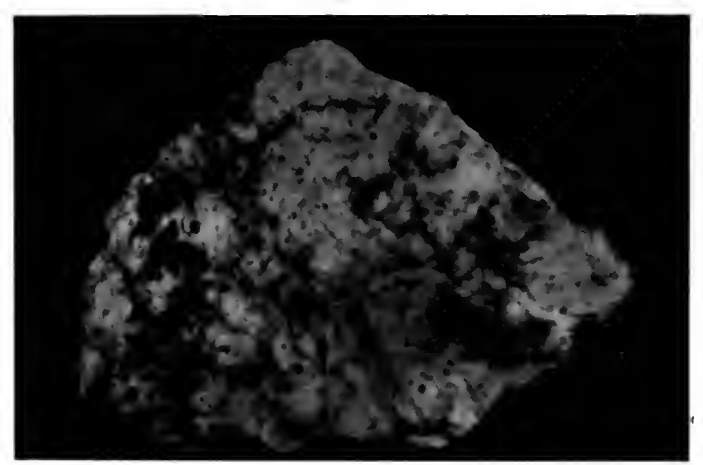

FIG. I68.-A solid papillary pedunculated neuroblastoma of the fourth ventricle. $\times 3$ (Case of Dr. John A. Hartwell.)

extensive infiltration of the pia up to the medulla from a lumbar glioma. A fibrin network is said to closely simulate glia fibrils, but fibrin fibrils radiate from nodal points and are not connected with cells, but exhibit numerous anastomoses.

Sarcoma-cells may possess fibrils, but they are less numerous and more granular than glia-fibers. Glioma without fibrils probably occurs in rare cases, but cellular tumors of this character almost always prove to be sarcomas. The existence of glioma with sarcoma of the vessels of the central nervous system has not been satisfactorily demonstrated. In some cases the diagnosis may remain in doubt. Borst studied two sarcomas of the brain which showed all the gliomatous characters except abundant fibrils, and Stroebe claims to have observed a primary infiltrating round-cell sarcoma of the supramarginal gyrus with fatal hemorrhage. Such tumors suggest the existence of glioma without fibrils. In a tumor of the lumbar cord Thiele saw cellular glioma in the upper portion changing to spindle-cell sarcoma below. Henneberg also found purely sarcomatous areas without fibrils in a glioma of the brain. It is therefore highly probable that anaplastic gliomas occur in which no trace of fibrils may be demonstrable. Gowers and Bruns 
both concluded that glioma-cells frequently resemble sarcoma-cells and may exhibit no signs of specific fibrils, but Borst is inclined to exclude uncertain cases from the category of glioma. It is probable that the careful use of modern staining methods will greatly limit the number of doubtful cases.

Etiology of Glioma.-Although occurring at any age, the predominance of gliomas in early life, their frequency in childhood, and the occurrence of bilateral congenital gliomas of the retina indicate that the tumor usually results from an embryogenic disturbance in the structure of the nervous system. v. Rindfleisch assumed the existence of superfluous embryonal tissue distributed in the glia structures as furnishing the cells of origin of gliomas. Lenhossek conceived that these embryonal cells must take the

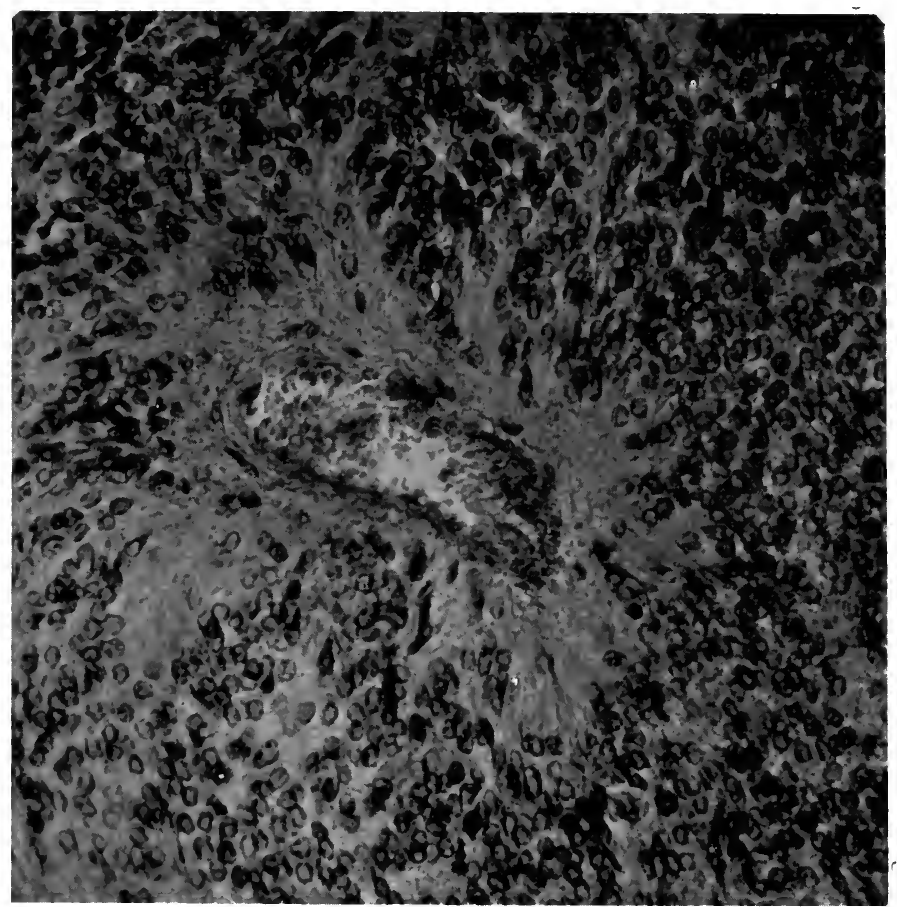

FIG. I69.-Neuroblastoma of fourth ventricle. Same case as Fig. I68.

form of indifferent round cells without fibers, but mitotic figures have been observed in adult glia-cells in forms of gliosis, and multiplication of gliacells and especially of fibers occurs in chronic sclerosis. It is quite clear that these highly differentiated and firmly bound cells are by no means devoid of regenerative power.

The association of glioma with various gross deformities is further evidence of the embryonal origin. Schule observed multiple glioma of the cord with fissure of the medulla. Schmidt interpreted a glioma of the nasal sinus as a form of encephalocele and referred to several other cases of the same nature. Hildebrand collected a series of cases of glioma associated with gross abnormalities in the central nervous system.

Heterotopia or local malformations of the cortex have been observed 
which suggest a probable origin for some cerebral gliomas. Ernst described misplacement of circumscribed foci of cerebellar tissue which exhibited some of the characters of incipient glioma. Baumann observed multiple thickened areas of cerebral gyri in which there were groups of hypertrophic ganglion cells. Hartdegen found a dozen very hard areas in several convolutions in a newborn infant with spina bifida. These areas contained numerous groups of large polygonal cells resembling large ganglion-cells. In the ventricles projected several nodular swellings of the same structure.

Schultze observed reduplication of the central canal with glioma of cerebellum and gliosis of the cord. In cases of congenital hydrocephalus there may appear multiple nodular outgrowths in the cortex which resemble

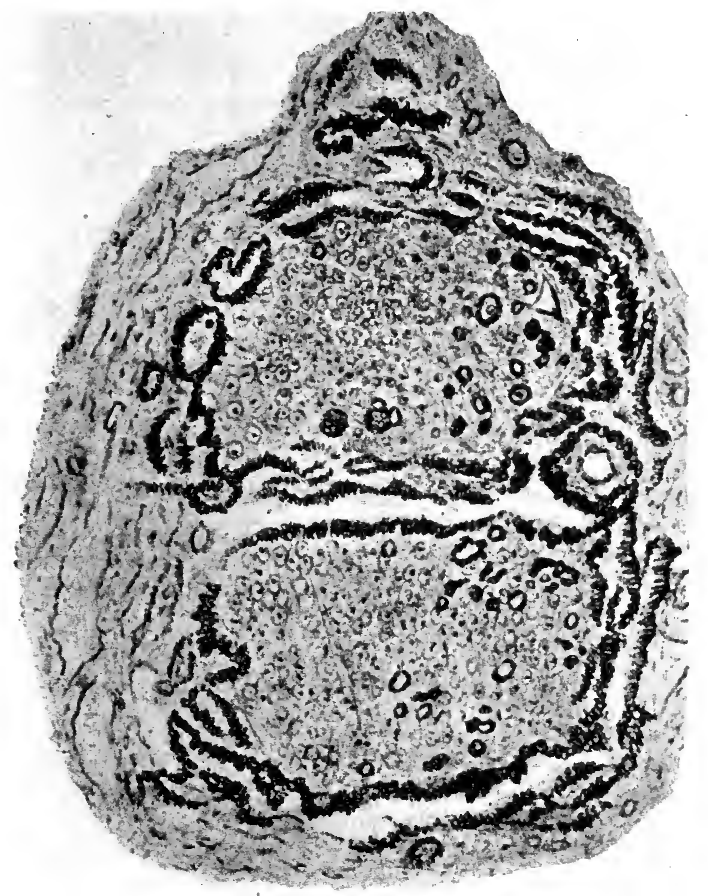

FIG. I70.-Early multiple extramedullary cylindrical cell tumors of ependyma and spinal arachnoid. Section of cauda equina. (Pfeiffer's case, $Z . N, 5$.)

tumors. They consist of glia-tissue rich in fibers and of ganglion-cells and they are associated with focal sclerosis and hypertrophies (Lubarsch, Borst). Small gliomas probably of this class are described by Simon and Meschede.

The embryonal character and eccentric position of the neuro-epitheliomas indicate their derivation from embryonal and misplaced cells, and this origin becomes certain in the numerous group of gliomatous teratomas in which the structure is usually that of neuro-epithelioma.

On the other hand, the embryogenic disturbance becomes less prominent and less essential with the slowly growing and diffuse gliomas of the brain which may exhibit a very wide distribution. Such cases have suggested to 
Rindfleisch and many others that here the glioma is not a true tumor, but a chronic proliferation of glia-tissue resulting from a disturbance of nutrition. Stroebe was much impressed with this view and even went so far as to describe protozoön-like structures in the cells of old gliomas. In the cord the chronic gliosis associated with syringomyelia passes through many gradations up to true glioma with central softening. (Saxer, Schlesinger), and Miura finds it difficult to distinguish between central gliosis and glioma. Meyer and Beyer describe focal lesions in the brain, the inflammatory or neoplastic nature of which they find it difficult to determine. Hererotopia of ependymal epithelium seems to favor both the gliosis of syringomyelia and ependymal glioma, and Schultze and Hoffmann expressed the view that both processes arise in superfluous tissue about the ventricles and central canal.

In a study of 7 cases of ependymal gliomatosis Margulis found the lesion distributed over the ventricles, especially the lateral, and affecting also the subcortical tissues. It was often associated with other proliferative processes affecting the glia-tissue, but inflammatory changes in the vessels were missing. Many stages of the progress terminating in sclerosis may usually be observed in the same case. He interprets the condition as a local or general response of the glia-tissue to infectious or toxic irritation. The symptomatology was variable and not specific.

Pfeifer found the entire ventricular system covered with projecting nodules from which extended a diffuse infiltration of the surrounding brain tissue. The nodules consisted of small polymorphous cells with fine, short processes, but true astrocytes were scanty. Roccavilla demonstrated diffuse gliomatosis of brain and medulla in a child with spina bifida and reduplication of the central canal of the cord. He regarded the gliosis as the result of proliferation of congenitally superfluous glia-cells.

All of these observations form a rather broad basis for the conception of an anatomical predisposition as an essential factor in the etiology of glioma. The formative influence of misplaced epithelium may be a factor of importance in such cases, and the etiological inquiry leads back to the influences which cause the misplacement. Here structural abnormalities, chronic inflammation, and possibly trauma all claim attention (Henneberg).

Thus the etiology of glioma reveals in this group of tumors many grades of neoplastic growth, from processes which are difficult to distinguish from replacement or slightly formative glioses, up to highly embryonal tumors.

The relation to trauma has already been discussed.

Clinical Types of Gliomas.- (a) Cerebral gliomas have been chiefly considered in the foregoing general discussion. These tumors are usually soft and hemorrhagic, ill-defined or rarely firm and nodular, and their position is subpial, intermediate, or central. Multiple flat or nodular growths of variable structure project into the ventricle. The cerebellum, basal ganglia and medulla, and pons furnish the majority of cerebral cases. The centrum ovale is a favorite seat. In structure the great majority of cerebral gliomas fall in the class of astrocytomas with small cells richly provided with fibrils, but gliomas with large ganglionic cells are not infrequently observed, and there are many other variations in structure.

The histological classification includes the following varieties of intracranial gliomas:

I. Embryonal heterotopias of small segments of gray and white matter, with slight hypertrophy and hyperplasia of cells and fibers.

2. Hard gliomas approaching in structure simple gliosis.

3. Typical astrocytomas with or without canals lined with low or high epithelium. 
4. Astrocytomas with many large ganglionic glia-cells.

5. Neuro-epithelioma.

6. Diffuse atypical glioma, with many imperfect astrocytes and formation of many large multinucleated cells and phagocytic cells distended with myelin.

7. Gliosarcomas, round- or spindle-cell tumors with partial or complete loss of glia fibrils.

(b) Nasal Glioma.-A peculiar form of cerebral glioma appears in children as a swelling at the base or tip of the nose or in the nasal sinus (Schmidt). They arise as a form of encephalocele and Reid and Muhr traced the origin through the foramen cecum, Meyer through the cribriform plate. In 2 cases of Clark's large ganglionic glia-cells were well represented, but other cases have shown only glia-tissue with scanty evidence of progressive growth.

(c) Glioma of the spinal cord appears in several widely different forms dependent upon the origin, location, and varying grade of the neoplastic character of the process.

r. Solid central glioma is a common form of the spinal tumor of which Schlesinger has collected i 8 cases. The growth forms a cylindrical mass inclosing or obliterating the central canal and surrounded by a sheath of the distended white matter which in places may be entirely destroyed. It extends over several segments in any portion of the cord or involves almost the entire organ (Daxenberger, Miura). A tumor limited to the filum terminale was observed by Lachmann, and an irregularly distributed growth yielding Brown-Séquard's syndrome was studied by Volkmann. The tumor seldom reaches the pia, but this structure may become involved, and in a case described by Pels-Leusden the arachnoid was diffusely infiltrated from cauda to medulla. Grund collected several similar cases.

The structure of the tumors varies considerably. Not a few belong in the group of neuro-epithelioma with the predominance of polyhedral or spindle-cells with spaces lined by high epithelium and comparatively few astrocytes and glia-fibers. Such tumors exhibit expansive growth, but Ribbert has observed both types of growth in the same tumor. In others the structure is that of the usual type of cellular glioma. Astrocytes and glia fibrils are abundant. Large glia-cells are also present, but the adult characters are prominent, the tumors grow slowly and they infiltrate the surrounding tissue. Canals and spaces lined by ependyma-like cells and compact groups of such cells are present. The majority of spinal gliomas fall in this latter class.

2. In a group of cases a definite gliomatous process is associated with syringomyelia at one or both poles of the tumor, which itself may exhibit elongated canals lined by cubical cells. A typical case is that of Rosenthal in which the tumor was composed largely of a series of canals lined by embryonal ependymal cells lying in a cellular glioma with many astrocytes. Other cases of this class have been collected by Miura. The associated syringomyelia has been referred by some to disturbance in circulation caused by the tumor, while others regard the tumor as an advanced stage of a process dependent on developmental anomalies which lead also to the syringomyelia. There can be little doubt that the chief factor in the softening which produces the canal is the presence of fluid under pressure. Saxer describes a central glioma with syringomyelia extending far beyond the ends of the tumor. In the central portions of the tumor the canal was lined by neoplastic epithelium, extensions of which formed a part of the growth. Elsewhere in the tumor the canal was walled by softened tumor tissue. Above and below the tumor the canal lay in an area of simple gliosis which Saxer refers to disturbances in circulation caused by the tumor. In a case studied by Strauss in this 
laboratory the canal extended from the cauda to the pons. It was surrounded by a well-circumscribed zone of dense gliosis, beyond which the cord was compressed or soft, edematous, and contained many granule-cells. The proportion of glia fibrils and cells varied at different levels, fibrils greatly predominating, but ganglionic glia-cells were occasionally found. Ependymal epithelium was entirely missing and all stages of central softening of the tumor could be seen. Here was a low-grade but definite tumor process leading to syringomyelia by central softening. This case supports Saxer's view that no embryonal anomaly is needed for the development of central glioma

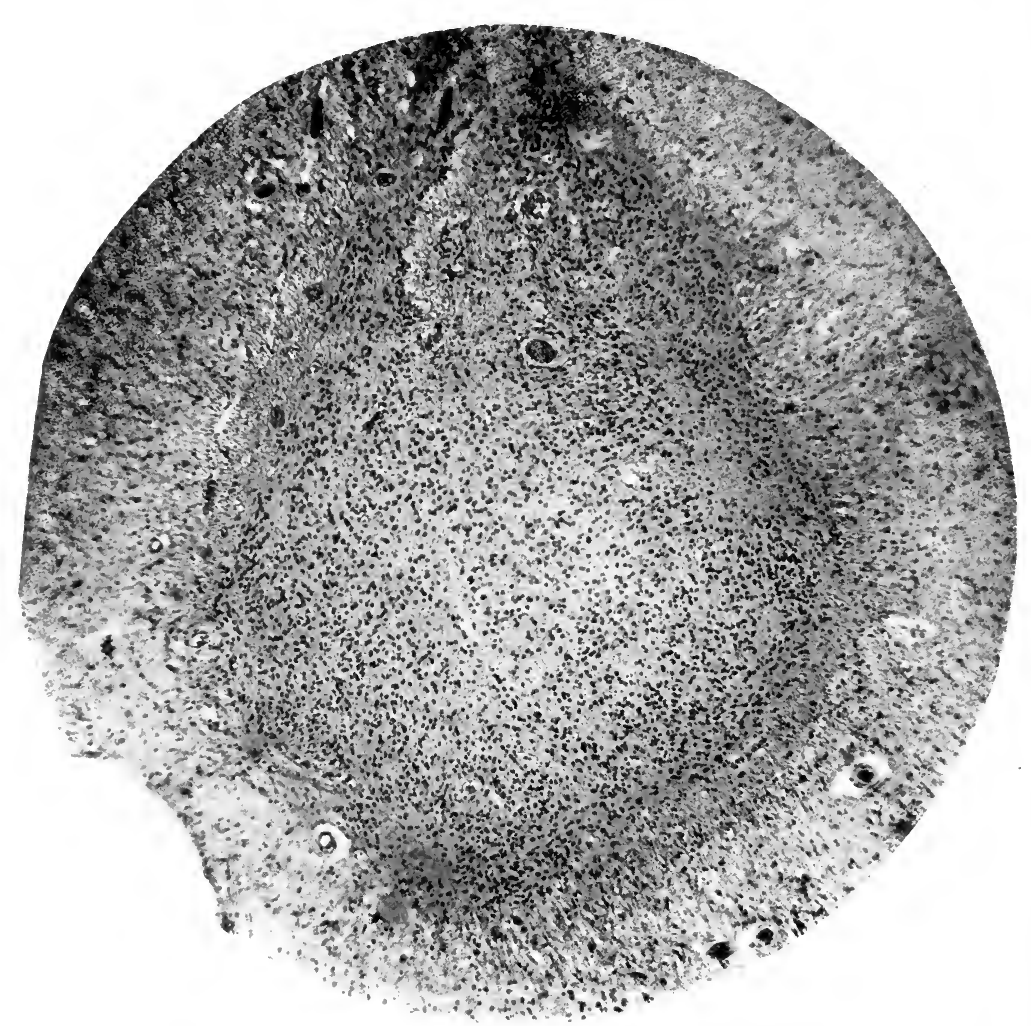

FIG. I7I.-Miliary glioma of spinal gray matter. (From a specimen of Dr. I. Strauss.)

with syringomyelia, and that there is no essential connection between these cases of glioma and the central canal or its epithelium.

3. In an important group of cases a pronounced central gliosis is associated with extensive syringomyelia. The new glia-tissue is comparatively acellular, but its dimensions are considerable and the tracts are displaced and the cord deformed. Definite histological features of neoplastic growth are lacking, but not more so than in some slowly growing diffuse gliomas of the brain. The central space is wide and an epithelial lining may be wanting, in which case the canal seems to be formed by progressive softening of the new tissue. Or the canal may be lined by epithelium which may show numerous invaginations extending into the new tissue. In some cases the canal 
may be compressed by a bulky overgrowth of rather cellular glia tissue (Hoffmann).

The significance of these cases of syringomyelia with overgrowth of glia tissue has been the subject of much discussion by observers whose views are summarized by Saxer. Some regard the process as a true neoplasm, while others consider it as a form of inflammatory gliosis. In favor of the neoplastic theory is the congenital character of some cases in which the syringomyelia follows hydromyelia with extensive gliosis and secondary degeneration (Saxer, Schlesinger). Lateral invaginations of epithelium occur with hydromyelia and may favor the gliosis. In certain cases without hydromyelia the presence of outlying groups of epithelial cells about the central canal suggests the deposit of superfluous material in this locality. Hence Hoffmann, who finds considerable proliferation of cells in the new tissue, regards the process as neoplastic. Storck, having observed angiomatous glioma in the upper cervical cord combined with syringomyelia below, in-

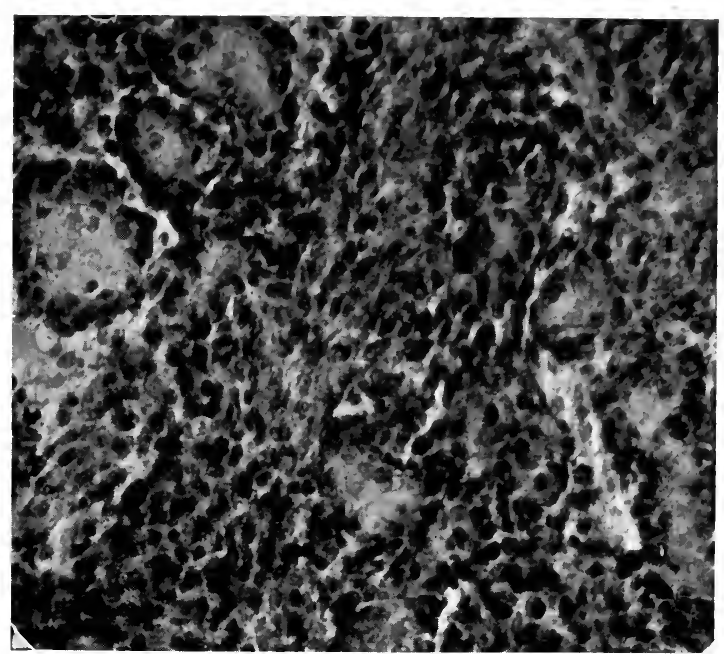

FIG. 172.-Neurocytoma. (From a section of Symmers' case.)

clines to the same view. There is no doubt also that pronounced glioma is occasionally associated with extensive cavity formation, as in the cases of Pels-Leusden, Saxer, Strauss, etc. On the other hand, it is clear that syringomyelia may result from many processes not connected with tumor growth, as in myelitis, meningomyelitis, hematomyelia, simple regressive tissue atrophy (Chiari), lymph or blood stasis (Langhans, Storck) and various chronic inflammatory processes (Saxer). The softening and solution of tissue about the central canal may be referable to changes in the blood-vessels to which this region is prone, and the outgrowth of epithelium is probably often a natural extension of the lining cells of the canal in the attempt to cover defects thus created (Müller, Meder, Miura). Finally, the symptoms of genuine tumors of the cord are pronounced and progressive, while those of the present group of syringomyelia are suppressed or mild, or of very slow progress (Baumler, Simon).

It seems necessary therefore to exclude most of the present group of cases 
from the class of true glioma, and to regard them as representing that common type of process which falls into an intermediate position between neoplasms and inflammatory or simple tissue hyperplasia. Syringomyelia occurs in the course of central glioma, but unless the structure of the process is clearly gliomatous the case should not be classed in that category.

(d) Pial glioma occurs as a secondary invasion of this structure from central tumors (Pels-Leusden, Grund), or as a primary tumor arising from misplaced glia tissue. Inglis reports a pial glioma composed of scanty astrocytes and many fibrils, originating from the root of the seventh dorsal nerve and forming a small elongated intradural tumor. In the numerous cases of extradural tumors collected by Leyden, Goldscheider, Grund, and others it does not appear that any uniform effort was made to recognize glioma, so that

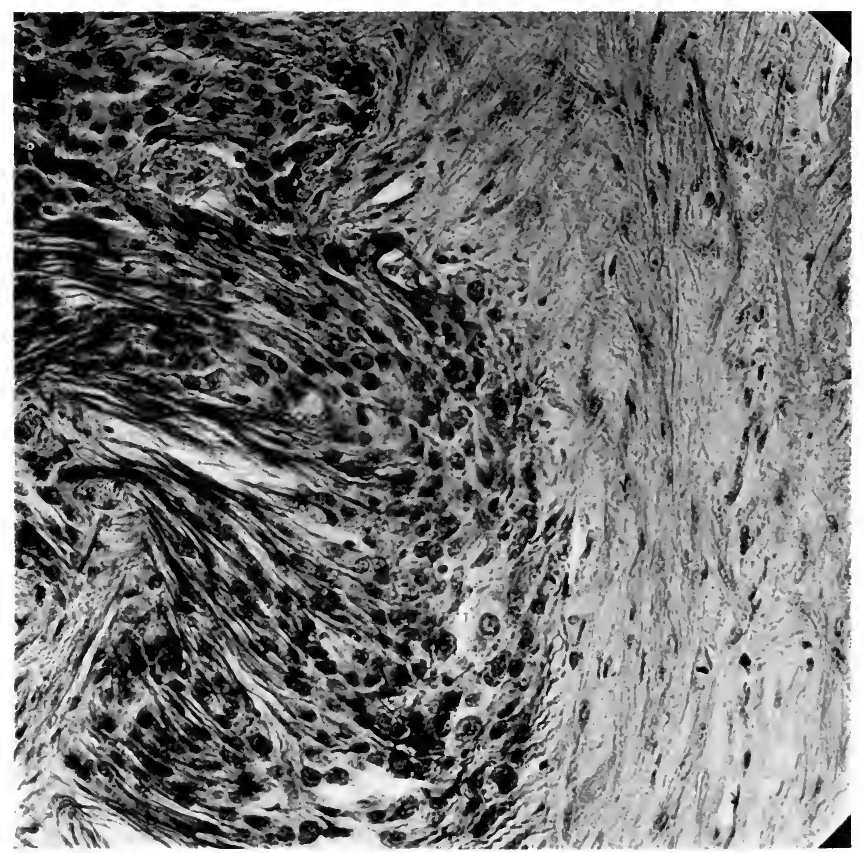

FIG. 173.- Sacral neurocytoma, with rich development of fibrils. (Mallory's specimen and stain.)

the frequency of extradural glioma cannot at present be stated. Thus in 2 cases recorded by Spiller as sarcoma, the author later states his impression that they were gliomatous. That many supposed multiple gliomas of the cord are artefacts was shown by Van Giesen.

(e) Neurocytoma.-Under this term Wright has collected a series of tumors, chiefly of the adrenal with metastases in the liver, but also occurring in the cranium and elsewhere. They have the structure of embryonal neuro-epithelioma. In some the cells exhibit abundant fibrils, in others cells greatly predominate over fibrils and assume the rosette arrangement. Wright believes that certain tumors reported as sarcoma, lymphoma, and lymphosarcoma are in reality highly embryonal forms of neuro-epithelioma, as in the cases of Pepper, Tileston and Wolbach, and Hutchinson. For the so-called con- 
genital sarcomas of the adrenal and liver Kretz has expressed the view that they arise from the formative cells of the sympathetic.

(f) Teratomatous Glioma.-Nervous tissue occurs in a considerable proportion of teratomas. In teratoma testis, besides portions of brain, retina, ganglia, and ependyma, neuro-epithelial cells frequently occur and may occasionally form a considerable portion of the tumor (Ohkubo, Lit.). In ovarian teratoma nervous structures are rare.

From the sacral region Mallory has described a large neuro-epithelioma presenting the structure of carcinoma, but exhibiting an extensive development of neurofibrils. The origin of the tumor he referred to remnants of the neural canal (Fig. I73).

\section{TUMORS OF THE CHOROID PLEXUS AND EPENDYMA}

A considerable variety of pathological processes occur in the choroid plexus and ependyma, many of which have a relation to tumors of these structures.

Pigmentation, and calcific, fatty, and amyloid degeneration are frequently observed in the choroid plexus and have been described in detail by $\mathrm{E}$. Hackel. Common structural changes include varicosity and overgrowth of blood-vessels, thickening, induration, and mucoid degeneration of the stroma. Calcific changes in the vessel walls may lead to the formation of angioliths, which are commonly observed in subjects over 50 years. A diffuse overgrowth of stroma and lining cells with well-marked squamous metaplasia may result from chronic inflammation (Delamarre, Merle). Cysts arising from mucoid degeneration of the stroma occurred in I 5 of 23 cases studied by Loeper. Rarely such cysts reach a large size, and in the case of an infant observed by Cayley and Brown there was protrusion of the parietal bone by a very large cyst. In the infant the choroid plexus is very highly developed and the abundance of lipoids, pigment, and glycogen have led to the assumption that it possesses a secretory function. Petit and Girard class this structure with the hypophysis and other glands of internal secretion.

The ependymal epithelium is also capable of marked proliferation with changes in form and size in acute and chronic simple and specific inflammations (ependymitis granularis). In several conditions, but chiefly in tumors, close relations are revealed between ependymal and glia-cells, the one readily becoming transformed into the other. Thus, in inflamed and edematous brain tissue, polyhedral cells often inclosing lumina appear in multiple foci, by a type of embryonal reversion of glia-cells. In many gliomas alveoli, spaces, and cysts form with a lining of cubical or cylindrical cells of ependymal form (Saxer, Muthmann, Sauerbeck, Lit.). In certain multiple ependymal gliomas the ependymal and the modified glia-cells are richly commingled and one type of cell passes into the other (Linck). With both ependymal and plexus epithelium nutritional and inflammatory changes seem to pass through many gradations into tumor processes.

The excessive pigmentation observed by Virchow in the pia-arachnoid, with tumor-like proliferation of cells, appears in exaggerated form in the melanomas arising from these cells (Pick, Esser, Thorel). Extensive calcification of the plexus may apparently lead to the rare osteoma of the ventricle (Bonnet). The common angioliths appear frequently in cellular tumors of the plexus. Myxomas and angiomas recall the mucoid stroma and varicosities of the chronically inflamed plexus. Hypertrophy of the plexus may lead to extensive overgrowth of this structure, and Bruchanow interpreted his large papillary tumor as a simple hypertrophy and hyperplasia. Ependy- 
mitis granularis involves both epithelium and subepithelial glia tissue, and seems to present a frequent preliminary to multiple ependymal gliomas of the ventricles (Jeremias, Saltykow).

General Etiology.- Tumors of this entire group are comparatively rare. Boudet and Clunet found 32 cases on which to base their study, but their list does not include many recent cases. The majority of subjects are children or young adults. A history of typhoid fever, or coincident tuberculosis, has frequently been noted. In several cases trauma had recently preceded the outbreak of symptoms (Muthmann, Sauer-

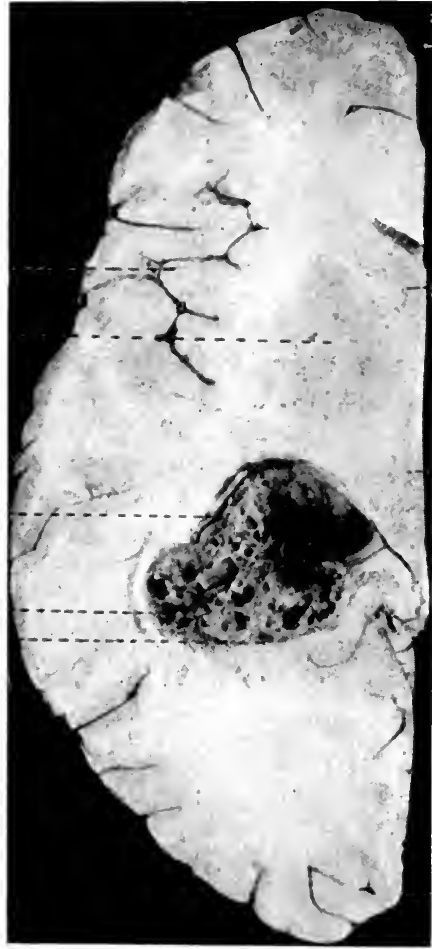

FIG. I 74.--Papillary carcinoma of choroid plexus distending lateral ventricle. (After Boudet and Clunet.)

is a unique observation.

Gross Appearance.-The tumor is usually single except in the case of the ependymal glioma, which is regularly multiple. While several small growths have given rise to perplexing cerebral symptoms, the tumors frequently reach the size of an egg or an orange and produce definite focal symptoms. While usually circumscribed, they may invade the brain tissue. The ventricles are frequently distended. The papillary conformation predominates, but some growths are spongy or polycystic, a few resemble thyroid gland tissue (Mackay, Bruce), and nearly all are highly vascular.

Structure.- Tumors of the ependymal epithelium vary from the perivascular neuro-epithelioma toward the true glioma (astrocytoma) with spidercells and glia fibrils, while tumors of the plexus retain a papillary adeno- 
carcinomatous type and may show squamous metaplasia. Von Willer points out that the ependymal epithelium is ciliated at birth and long retains the

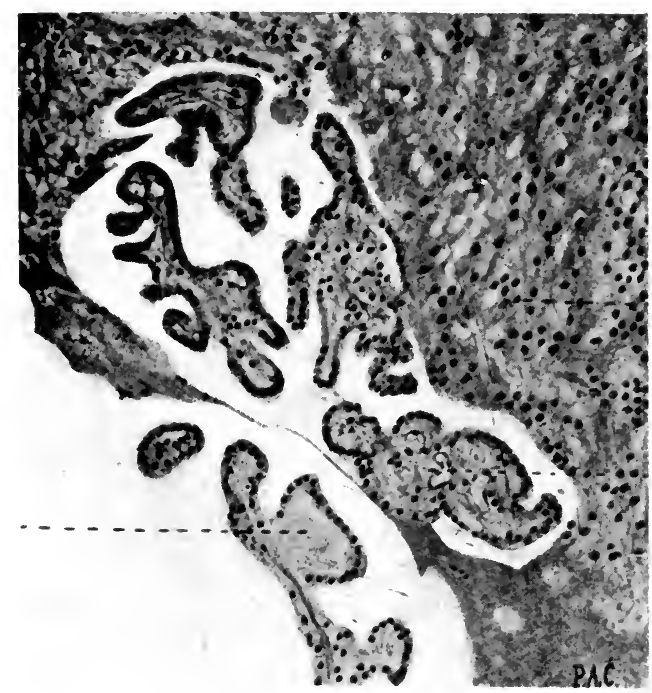

FIG. I75.- Structure of papillary carcinoma of choroid plexus in lateral ventricle, (After Boudet and Clunet.)

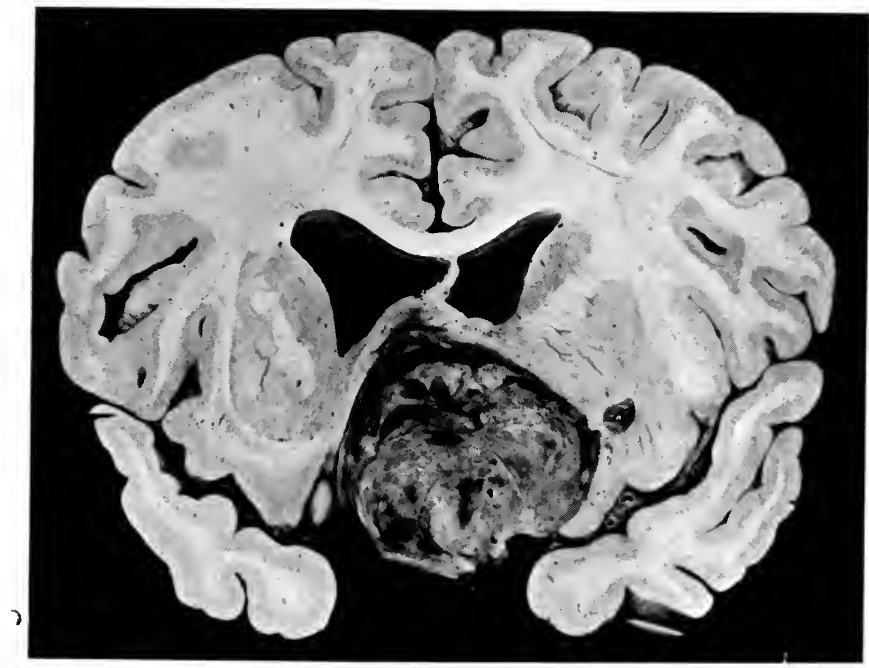

FIG. I76.-Papillary adenocarcinoma of choroid plexus in third ventricle.

condensed border and intracellular fibrils of ciliated cells, whereas the plexus epithelium is granular, reticulated, and secretory in type. These original characters may assist in the separation of the two types of tumors. 
Three main structural types are observed: (I) Papillary adenocarcinoma, (2) squamous-cell carcinoma, and (3) ependymal glioma.

I. Papillary adenocarcinoma is the usual form of tumor of the choroid plexus. The papillary axis contains a well-developed blood-vessel supported by scanty connective tissue which often undergoes mucoid degeneration. Rare fibromyxomas probably represent a predominance of this structure. The blood-vessels are thin walled, often varicose, and subject to hemorrhage. Calcification of the walls often leads to complete occlusion by angioliths. The lining cells present one or more rows, the first usually cylindrical, the others cubical. Numerous capillary blood-vessels surrounded by a single layer of cubical cells are a common picture. In cellular growths adjoining papillæ fuse, the epithelium may grow diffusely, and an angiosarcomatous

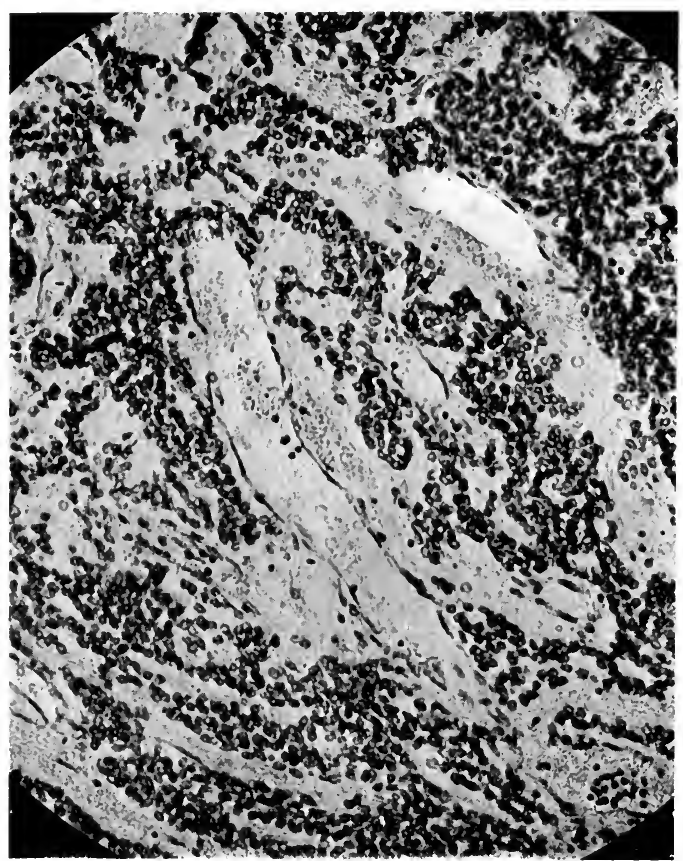

FIG. 177.- Structure of a papillary carcinoma of choroid plexus in the third ventricle.

structure may result. In one case I found all the cells very small, cubical, consisting chiefly of nuclei, and suggesting a gliomatous tendency (cf. Boudet). Cysts form by mucous degeneration of the stroma and they are usually bounded by palisade cells.

Malignant tumors yielding multiple metastases in the pia or brain tissue have been observed. Spat found a primary tumor of the plexus or ependyma of the lateral ventricle with small multiple metastases in the brain substance. Bielschowsky and Unger describe a large vascular papillary growth arising from the plexus of the lateral ventricle. A second tumor developed in the lateral horn of the fourth ventricle and I4 metastatic nodules appeared in the pia over both hemispheres. The structure presented capillary vessels surrounded by radiating high cylindrical cells. E. Meyer found a tumor in the 
third ventricle composed of numerous hyaline blood-vessels surrounded by small closely packed round cells, while two small nodules of similar structure appeared in the fourth ventricle. Although this tumor was described as a sarcoma, a relation to the small-cell epithelial tumors of ependyma or plexus is suggested. Kaufmann also pictures round-cell sarcoma of the pia with hyaline blood-vessels.

2. Squamous-cell carcinoma is the type presented by certain tumors of the choroid plexus. Occurring in the third ventricle they may be difficult to distinguish from acanthoma of the hypophysea! duct, but similar growths are observed in the fourth and lateral ventricles and without relation to the hypophysis (Cornil, Boudet, Clunet).

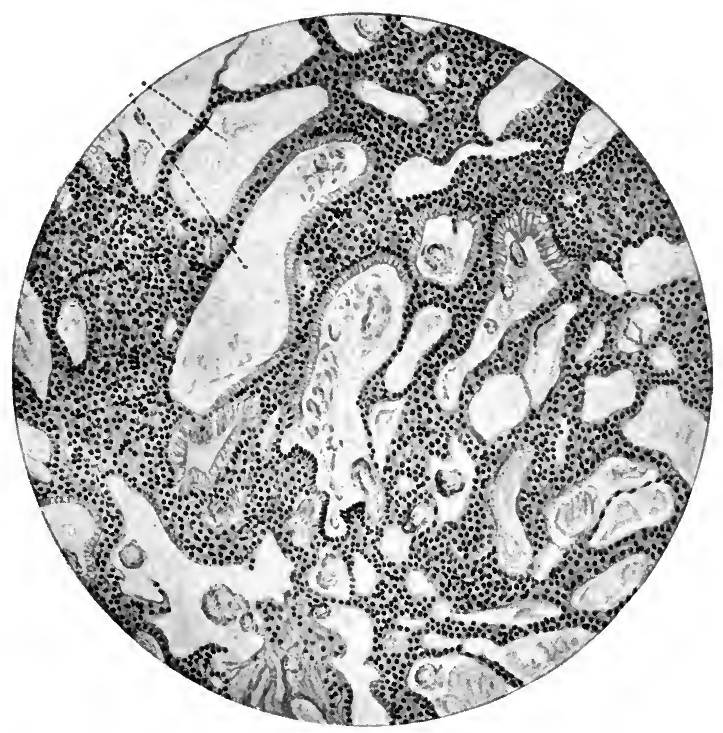

FIG. I78.-Papillary adenocarcinoma of choroid plexus. A specific tumor of ependymal cells. (From Saxer.)

The structure is essentially a duplicate of the adenocarcinoma. The squamous cells appear in scanty groups or diffusely and they form pearls or diffuse sheets usually surrounded by cylindrical cells.

Boudet and Clunet collected 7 cases in which keratinization was present, but the origin of some of their cases is doubtful. Mott and Barrat in a tumor attached to the choroid plexus observed intercellular bridges between flat cells. E. Wagner described a villous papillary tumor near the chiasm without involvement of the hypophysis. It was covered externally with many long papillæ lined in places by cylindrical cells, in other places by flat stratified cells. Selke's case has been variously interpreted. It was a large tumor of the third ventricle passing by the foramen of Monro into the lateral ventricles. The hypophysis was intact. The ependyma about the base of the papillary tumor was represented by layers of squamous cells lying on connective tissue and the entire tumor contained flat stratified cells. Selke regarded the growth as originating from the ependyma of the third ventricle, while Erdheim considers it a cystic tumor of the hypophyseal duct. Saxer has described a papillary tumor of the choroid plexus of the third ventricle, a compact papillary adenocarcinoma of the fourth ventricle, and a papillary squamous cell growth from the hypophyseal duct, all of which form in structure comparative types of these tumors. 
3. Ependymal glioma is a rare, but well-defined tumor. Linck collected 6 fully described cases and to these may be added those of Saxer (Wunscheim) and Mallory. Cimbal refers to many fragmentary reports. Its chief seat is in the fourth ventricle, where it commonly produces a bulky, solid, rather firm but vascular tumor which produces marked pressure symptoms. Cimbal's tumor showed extensive central necrosis and Henneberg described a gelatinous growth as large as a hen's egg. The ependyma of the ventricle is usually the seat of multiple or diffuse, granular or papillary outgrowths, and from such lesions the tumors probably originate. The structure varies chiefly in the relative proportions of ependymal epithelium and astrocytes with their fibers. At one extreme the tumors approach the ependymal adenocarcinomas (Saxer's case), and at the other they may be chiefly gliomatous (Linck's case). Alveolar groups of neural epithelium are regularly present in the form of cubical or cylindrical cells inclosing ill-defined lumina or surrounding capillary blood-vessels. In some cases the cells are very long and cylindrical. By suitable stains (Mallory) neuroglia fibrils derived from the cells may be

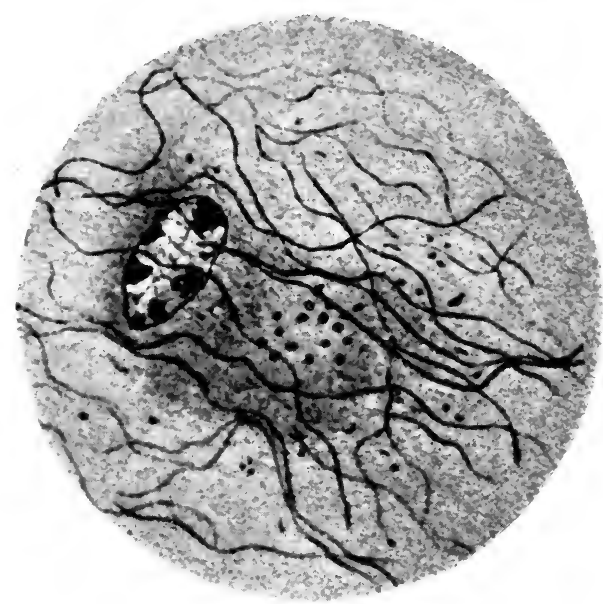

FIG. I79.-Characteristic fibrils and granules of ependymal cells. (After Mallory.)

demonstrated. In very cellular tumors the cells become more numerous, of small size, round or spindle, the fibrils diminish, and a picture resembling roundcell sarcoma may result. That gliosarcoma in the vicinity of the ventricles may develop in this way may safely be assumed. The prominence of bloodvessels may suggest an angiosarcoma, or very cellular sheaths about bloodvessels may yield a peritheliomatous structure.

The origin of these tumors has been traced satisfactorily to the ependymal epithelium and the underlying glia tissue. In ependymitis granularis the erosion of segments of ependymal cells permits minute extrusions of the underlying glia layer, forming the characteristic granules of ependymitis (Jeremias). Proliferation of the glia tissue and the neighboring ependymal cells follows and the tumor develops with varying proportions of these elements (Linck). A transformation of ependymal cells into astrocytes probably occurs in some tumors, but mitotic figures are limited to the rounded or cubical epithelium (Saxer).

Tumors of the Infundibular Ependyma.-The hypophyseal region and the third ventricle are the seat of a group of papillary cystic tumors which 
strongly suggest an origin from ependymal epithelium. These tumors lie outside of the brain tissue, but within the dura. In advanced cases it may be difficult to determine their original relations. They are composed of a loose edematous meshwork of connective tissue covered by one or more layers of small cubical epithelium which may be gathered into broad sheets or thrown up into papillary projections. The great vascularity and the perivascular arrangement of the cells often recalls the structure of the choroid plexus. Saxer, Hart, and others have attributed such tumors to the ependymal epithelium and their occurrence largely beneath the base of the brain and in close relation to the pedicle of the hypophysis indicates that they may arise from the ependymal prolongation of the infundibulum. I have studied such a tumor in a young girl with a long history of cerebral compression. The growth was cystic, $4 \mathrm{~cm}$. in diameter, lay above the hypophyseal lobes, had destroyed the pedicle, was separated by a thin partition from the distended ventricle, and the structure resembled Saxer's (Case I) ependymal carcinoma. A somewhat similar case is described by Mullaly.

\section{GLIOMA OF RETINA}

Characteristic clinical behavior and peculiar structure render glioma retine one of the most striking examples of a specific tumor process. Many of the old cases of fungous hematodes described by Wardrop were probably of this nature. The first histological descriptions were given by Robin in I 854, since which time the interpretation of the tumor has been the subject of very extensive study and speculation, which have not yet reached a final conclusion (Parsons, Lit.).

These tumors are soft and bluish in early stages, but soon become milky or gray from fatty degeneration and focal calcification. They are very subject to necrosis, and are often hemorrhagic; 23 per cent. are bilateral, and of these 94 per cent. are under 4 years of age. The involvement of both eyes is usually noted at the first examination, when it appears that the disease is less advanced in one eye. Occasionally the involvement of the other eye is noted sometime later; and in a few cases a long interval, 3, 4, or even I 3 years, has elapsed between the enucleation of the first and the affection of the second eye (A. Knapp). The initial growth may be very slow.

The tumors arise chiefly from the ciliary portion or from the posterior segment of the retina. According to Wintersteiner the origin is often multiple and chiefly from the posterior pole. The origin has been traced in different cases with considerable certainty to the internal granular laver (Knapp, Hirschberg), from the inner side of external granular layer (Delafield), from the external granular layer (Schweiger, Flexner), from the fibrillar layer (Iwanoff). The early anatomic and ophthalmoscopic appearances are tabulated by Eisenlohr. The tumors grow expansively, splitting the retina and stripping it from the choroid or covering it with a mass of tissue which encircles the vitreous or penetrates it in dendritic masses. Invading the choroid at the papilla it may form a discoid mass in this structure, and traveling within the optic nerve it reaches the base of the brain (Knapp). In the eyeball it later replaces the vitreous, invades all portions of the bulb, perforates the sclerotic or cornea by way of the vessels, invades the orbit, from which in advanced cases it protrudes as a vascular necrosing tumor of large dimensions. Metastases are late or absent, but recurrence is common, and secondary growths appear in brain, skull, spinal cord, regional lymph-nodes, and internal organs. Most cases have proved fatal (66 per cent., Wintersteiner), but Collins reports four recoveries (?) from bilateral tumors after double enucleation. Regressive 
changes rather frequently occur in eyes that retain vision, especially in the second eye, and in a few instances the regression appears to have rendered the tumor quescent, as in Meller's case. Renewed growth may begin after considerable periods. Regression of the tumor may follow rupture of the eyeball, with phthisis bulbi, necrosis of the tumor, and calcific or bony deposits.

The ophthalmoscopic appearances of regressing glioma consists in irregular atrophic areas in the choroid and about the macula and optic nerve, with detachment of the retina by whitish masses.

Glioma retinæ is considerably influenced by applications of heavily filtered radium over the eyeball, but the regression produced is usually temporary.

The structure of glioma retinæ was recognized by Virchow as recalling that of many cerebral gliomas. The tumor is composed of small round cells appearing often as naked compact nuclei, but exhibiting a scanty cytoplasm from which, as a rule, no definite fibrils can be traced. The cells lie usually in perivascular groups between which there may be diffuse cellular tissue or more

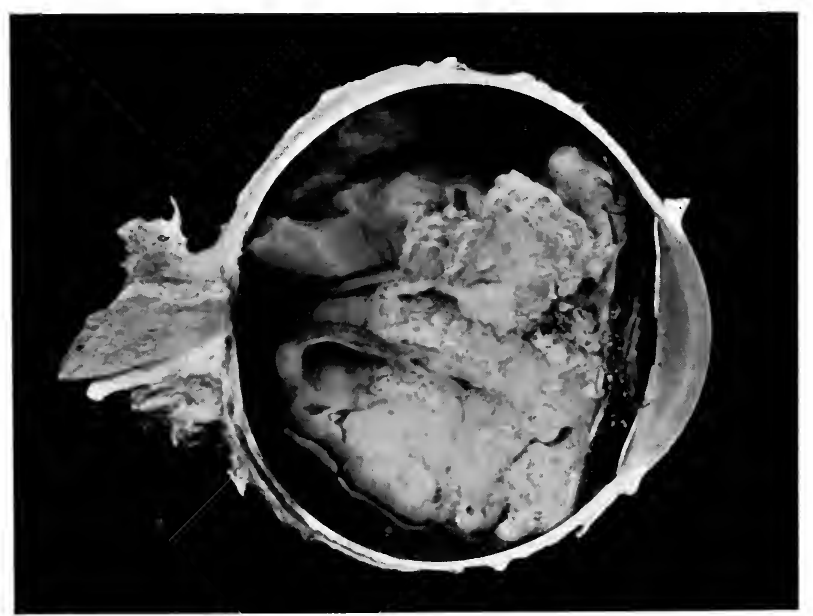

FIG. I80.-Glioma of retina.

often necrotic detritus. Besides the perivascular groups two other features are revealed on close inspection.

(I) The cells may assume a low cuboidal form and be arranged in small alveoli or rosettes after the manner of neuro-epithelial rosettes (Flexner, Becker). Such structures were observed in II of 32 cases by Wintersteiner, and in several cases I have found them the predominating feature. By means of serial sections Steinhaus showed that these rosettes are spheroidal and not tubular bodies. On account of this structure Flexner applied the term "neuro-epithelioma retinæ." Eisenlohr also described occasional very minute canals lined by a thin membrane, but not bounded by cylindrical cells.

The inner border of the typical rosettes is lined by a thin membrane which has been interpreted as a reproduction of the membrana limitans externa, while the cells of the rosettes are believed to be derivatives of the rods and cones. Similar structures have been found in malformed eyes and in inflammatory conditions (Parsons, Lit.).

2. Even when the rosettes are not prominent the small cells are not diffusely distributed, but are arranged in rows or indistinct circles which indicate 
traces of the same tendency which produces the complete rosettes. This appearance is observed even in the perivascular cell mantles, but in old tumors it is gradually obliterated (Steinhaus). In the local extensions of the tumor through the choroid, in the orbit or into the brain, all traces of structure are commonly missing and the tumor is composed of small round- or spindle-cells which tend to travel along the perivascular lymph-sheaths. Yet Greef found that in extrabulbar extensions the tumor cells might show more of the characters of ordinary glioma-cells.

The demonstration of glia fibrils in retinal glioma requires special technic. Ordinary staining methods usually fail to reveal any trace of fibrils and Scaffidi and others have been unsuccessful with Weigert's and Mallory's stains. Greef, however, using the Golgi-Cajal silver impregnation, found many cells with scanty fibrils, resembling the embryonal spider-cells of the optic nerve, and in some areas these fibrils were very numerous. Borst pictures the fibers as short and rudimentary and he finds them only on the cylndrical cells forming rosettes. Greef also demonstrated large ganglionic cells with many branching processes and more numerous medium-sized tripolar cells. These observations have been verified by Hertel, de Gama Pinto, and others. Yet Scaffidi believes that these large cells are persistent remnants of the original retina. It may be urged that the silver method is not a reliable means of distinguishing ganglion-cells from large multipolar glia-cells.

From the descriptions of many authors it is evident that the structure varies considerably. In one group of cases the perivascular arrangement is most prominent, in another the rosettes are the most striking feature, and in still others the tumors are diffuse and large and small glia-cells are demonstrable. These variations do not warrant the assumption that the tumors may be of different nature, but they have formed the basis of much difference of interpretation.

Etiology.-Glioma of the retina appears almost exclusively in infants and two-thirds of the cases occur before the fourth year. The congenital character is most remarkable. Newton reports a family of 16 children, ro of whom died of retinal glioma, in 7 of which the disease was bilateral. Wilson saw 8 children in one family affected and several other similar reports have been collected by Williams. An hereditary element may possibly be traced in the cases of Steinhaus and of Feinstein in which several first cousins were victims of the disease.

The tumor has several times been associated with ocular deformities, as microphthalmos (Helfreich, Ginzberg, Salzmann), or persistent embryonal vessels in the lens (Eisenlohr). The embryonal structure accords with the congenital origin. Although there has been much discussion as to the nature of the growth, the general resemblance to other gliomas in which fibrils are demonstrated with difficulty and the demonstration of spindle-cells by Greef and others, are satisfactory evidence of the embryonal gliomatous nature. The exact nature of the embryogenic disturbance can only be surmised, while its cause is entirely obscure. The tumor bears a strong resemblance to the outer granule layer of the retina to which its early stages have been traced, so that one may assume the existence in this locality of superfluous or misplaced tissue material. Wintersteiner concludes that the tumor arises from misplaced elements of the outer retinal layers which have been found as rosettes in a series of malformed inflamed eyes. He assumes that the disturbance must occur late in the development of the retina, but the highly embryonal structure of the growth and its association with gross abnormalities favor an earlier origin (Greef, Axenfeld, Emanuel). Trauma has been held responsible for the outbreak of many cases, but usually on uncertain evidence. 


\section{SARCOMA OF BRAIN AND MENINGES}

The only well-defined form of sarcoma of the brain and meninges is derived from the walls of blood-vessels, and since these tumors are often quite vascular they may be regarded as essentially angiosarcomas. Very cellular tumors composed of round cells arise from the glia-tissue and are best designated as gliosarcomas or malignant gliomas. Cellular endotheliomas also present many features of spindle-cell sarcoma, but in all but rare instances they may be identified on minute structural features, such as the presence of areas of flat cells and a concentric arrangement around small canals. Owing to the general similarity of structure it is probable that the sarcomas recorded in the literature include tumors of each of the three above groups. There has been much controversy over the use of the term "gliosarcoma." Strictly speaking, this word signifies a mixed tumor of glia-cells and some mesodermal cell. It is not clear that any such mixed tumor exists, although Stroebe describes a rare growth with commingling of glia and mesodermal cells, and Merzbacher saw reactive proliferation of glia about invading pial sarcoma. Wohlwill also describes as true gliosarcoma a tumor composed of glia-cells in one portion and mesoblastic cells in another. Entirely separate glioma and sarcoma in the same brain are recorded by Hanel.

Gross Anatomy.--(I) Sarcoma arises chiefly from the dura and pia and probably some deeply placed tumors develop from the blood-vessels of the brain. Their position is, therefore, extra- or intracerebral, and about equally frequent in all parts of the brain. They produce firm or soft opaque cellular well-circumscribed tumors which may attain large dimensions. Putnam describes one case in which the tumor replaced nearly one-half of the cerebrum. It was successfully removed, but recurred after some months. Henschen's series includes a large cystic sarcoma replacing a parietal lobe, one involving vertex, dura, and base, one arising from the occipital portion of the falx, and one involving both hemispheres. Stern saw the entire corpus callosum destroyed, while a very large tumor of chronic course occupied most of the frontal region. Many of the large tumors are soft, cystic, or hemorrhagic. Arising from the membranes they at first displace but eventually tend to invade the brain tissue. At all times they are rather sharply defined from the surrounding brain tissue, occasionally by a capsule.

Perforating dural sarcomas are rarely observed and most of these tumors probably arise from the bone. In certain instances an intradural tumor has forced its way through the dura, eroded the skull, and appeared beneath or even on the scalp (Bruns).

Arising from the outer side of the dura they may perforate the bone and appear externally, or most of the growth may remain in the skull at the expense of the brain tissue. Weisewange has collected a series of perforating tumors, a few with the structure of alveolar sarcoma, which he traces to dura, diploë, or periosteum. The typical osteosarcomas of bone also perforate the skull in either direction.

Osteochrondrosarcoma arising within the cerebellum and extending into the spinal canal is described by Jacobson and Jamane.

2. Diffuse Sarcoma of Cerebral Meninges.-While local invasion of the meninges by malignant tumors is not uncommon, a/wide-spread extension over meninges, ependyma, nerve roots, and down the spinal cord is observed in a limited number of characteristic cases which include glioma, sarcoma, endothelioma, melanoma, and one tumor of the pituitary gland (Schlesinger's). Verdun has collected 28 cases of this group of sarcomas, to which may be added several others from the reports of Nonne and M. Rindfleisch, as well as 
recent cases of Markus and Almecrona. The same condition usually involves both brain and spinal cord, but in many cases only one of the regions was examined. Occasionally the brain alone or the cord alone is affected.

The anatomical conditions vary. In some cases there is a large primary tumor from which the diffuse invasion spreads through the arachnoid space (Weaver). Or small multiple tumors may appear at several points connected by diffuse invasion of the pia. This condition is observed chiefly with subependymal gliomas of the ventricles (Martens, Seiffer, Verdun).

No definite tumor mass may be present anywhere, but the meninges exhibit a moderate diffuse opacity. To this form Orith's term, pachymeningitis interna sarcomatosa, best applies. In such a case Marcus found the changes to consist of proliferation of large hyperchromatic cells in the walls of blood-vessels occluding the lumen. Almecrona described a very similar case as endothelioma. It was complicated by invasion of the brain tissue and metastases in the liver.

In other cases of this group the structure has been described as large round-cell sarcoma (Weaver), spindle-cell sarcoma (Carnot-Bougle), gliosarcoma (Fischer), ependymal glioma (Verdun).

While it is thus evident that diffusion in the pia is not peculiar to any one form of sarcoma, yet the cases without any localized tumor stand out from the others as a disease sui generis for which a special etiology must probably be found. Many of the patients are young. The clinical course is that of chronic meningitis.

Structure.-The structure of the typical angiosarcoma of the brain is often an exact duplicate of that observed for this tumor in other regions. There are many blood-vessels lined by endothelium and supported by a thin layer of connective tissue, beyond which lies a broad sheath of polyhedral, rounded, or spindle-cells. The unit of the vessel and its cell sheath is very prominent. The vessels may be widened, the walls imperfect, and the tissue infiltrated with blood. Areas of necrosis and cyst formation may greatly alter the appearance. Oppenheim describes pigmentation of these and of less vascular sarcomas. In fact, not a few diffuse melanomas of the pia closely resemble the non-pigmented sarcomas, differing chiefly in the presence of pigment. A possible relation between these processes may well be considered. Berblinger found perithelial sarcoma associated with diffuse melanoma, but he regarded the two processes as distinct.

Very cellular forms of this tumor probably give rise to diffuse large roundcell or giant-cell sarcoma. Coste and Levy describe both these structures in the rapidly growing recurrence of an original typical perithelioma which was possibly of traumatic origin. In another group of cases the abundant sheaths of perithelial cells are not prominent, the vessels are more numerous and smaller, and the tumor cells are arranged concentrically about the vessel, forming a thickened wall lined by tumor cells or a single layer of endothelium. These tumors resemble very cellular granulation tissue. With increasing proliferation of tumor cells and loss of vessels they pass into spindlecell sarcoma. Such tumors develop both in the pia and in the substance of the brain. The great difference in the structure of these two forms of sarcoma suggests a difference in origin. One is embryonal or anaplastic in type, the other more adult. In neither case has the origin been traced.

Myxomatous changes occur in vascular sarcomas as well as in glioma and endothelioma, and the exact origin of the growths which are largely myxomatous is not clear. Oppenheim described a calcified chondromyxoangioma as large as the fist developing in the frontal region of a boy of 16 years, 8 years after trauma. 
The malignancy of most brain sarcomas is considerable and some show extremely active growth, especially after operative interference. The perithelioma of Coste and Levy recurred promptly and reached a very large size with a change to a diffuse structure. Putnam's large tumor recurred only after an interval of several months. Boettiger examined the site of a sarcoma ro years after its removal and found only a cyst and a scar, but he failed to describe the original growth. Not a few of the successful operative results appear to be with angiosarcoma or perithelioma. The prolonged course (Stern) as well as the successful removal of some of these tumors indicate that the prognosis may not be entirely unfavorable. Yet their natural tendency is to grow rapidly and steadily until within a few months there is extensive destruction of brain tissue, and a fatal outcome is assured. General metastases are not observed. 


\section{CHAPTER XXIII}

\section{TUMORS OF NERVE TRUNKS}

Neuroma is a tumor composed of nerve-cells and nerve-fibers, in which form it is usually designated as neuroma ganglionare or ganglionic neuroma. Extensive overgrowth of nerve-fibers, medullated and non-medullated, occurs without participation of ganglion-cells, and this process is termed neuroma myelinicum or amyelinicum.

Although ganglion-cells and their process stand as the most highly differentiated and immutable of the soft tissues, yet both cells and processes occasionally reveal surprising powers of growth, the cells yielding genuine neoplasms, and the fibers multiplying or sprouting to an extent which closely approaches the nature of a neoplasm.

Intermediate grades of proliferation of nerve-fibers which do not constitute genuine tumors are rather numerous.

Traumatic Neuroma.-In amputation stumps and after severe injuries to peripheral nerves the fibers may exhibit protracted and excessive proliferation, giving local thickenings which resemble tumors. The connective tissue of the severed nerve bundles first proliferates and forms a mass of cellular tissue into which the nerve-fibers push their way. The axis-cylinders elongate, divide, and sprout into the surrounding tissue in the form of twisted bundles in which medullary sheaths are present or absent. The nuclei of the medullary sheaths multiply and provide new sheaths for the axis-cylinders. The result is a marked thickening of the ends of the nerves and when several masses join with each other and with the thickened fasciæ and scar tissue a nodular tumor of considerable size is produced. Since very similar changes occur in the simple regeneration of nerve-trunks (Bunger) the amputation neuroma must be regarded as a form of regenerative overgrowth comparable to proud flesh (Thoma). Yet Ziegler holds that the process may exhibit certain neoplastic characters.

Plexiform Neuroma.-While in most cases of neurofibromatosis the nerve-fibers are passive and gradually atrophy, there are rare forms of overgrowth of nerve-trunks and ganglia in which the nervous elements take part in the hyperplasia. In certain plexiform neuromas of neck, back, eyelid, and face the elongated, nodular, and contorted nerve-trunks show preservation of nerve-fibers, and Ziegler, Birch-Hirschfeld, and Klebs believe there may be an active new growth of nerve-fibers (Bruns, Lit.).

In the cirsoid neuroma, which consists of a congeries of elongated and irregularly thickened nerve-trunks, Ziegler points out that the bulk of nervetissue is greatly in excess of that normally provided for the affected part. These processes seem to stand in an intermediate position between genuine neuroma and neurofibroma. Thoma describes neuroma of the Pacinian bodies of the skin, producing small painful tumors which are chiefly fibrous, but may show overgrowth of nerve-fibers.

True Fibroneuroma.-Virchow divided tumors of nerve-trunks into true and false neuromas. The false neuromas arise by proliferation of connective tissue and endothelium of the nerve-trunks with passive atrophy of nervefibers, and this group is numerously represented in the multiple neurofibroma of Recklinghausen. Since his work true neuroma of peripheral nerve-trunks has nearly disappeared from the literature. Yet Virchow gave a fullde- 
scription of tumors of outlying tissues in which he recognized new growth of nerve-fibers with, and more often without, myelin sheaths. The tumor is composed of masses of long, straight running, branching, and anastomosing nerve-fibers in which lie many long oval nuclei. These fibers he identified as naked nerve-fibers, and in some tumors he traced them into fibers possessing myelin-sheaths. The amyelinic tumors he regarded as an undeveloped form of the myelinic. Virchow collected a series of cases of this class in all of which there seemed to be a new growth of atypical nerve-fibers. In recent literature this group of tumors seems to have been divided among the neuro-

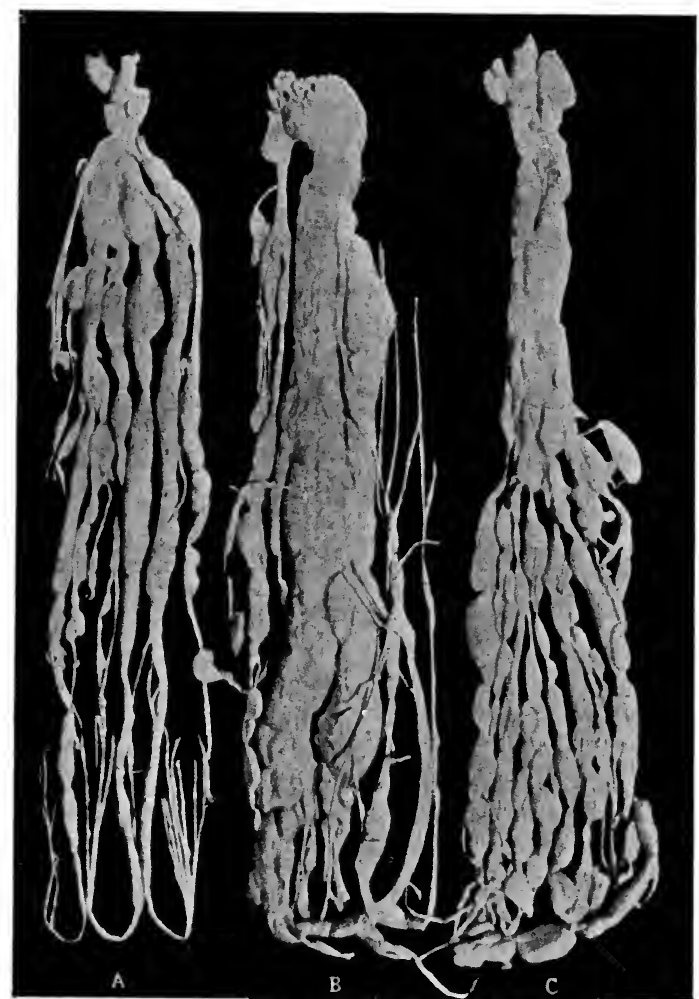

FIG. I8I.-Multiple neuromata of the peripheral nerves: $A$, Nerves of the right arm; $B$, the left sciatic with its branches; $C$, the left anterior crural with its branches. (Pruden's case.)

fibromas on the one hand and the ganglionic neuromas on the other. The existence of true fibroneuroma has been emphasized anew by Knauss, but his tumor was a ganglionic neuroma.

Borst and Ribbert are quite convinced that a neoplastic growth of nervefibers apart from ganglion-cells does not exist, but this conclusion does not dispose of the possibility of marked overgrowth of peripheral nerve-fibers without corresponding multiplication of ganglion-cells. I have seen several tumors of this type of small size and occurring beneath the skin.

Bruce and Dawson, in an elaborate study of a remarkable case of multiple miliary myelinic neuromas of medulla, cord, and pia, have fully disposed of 


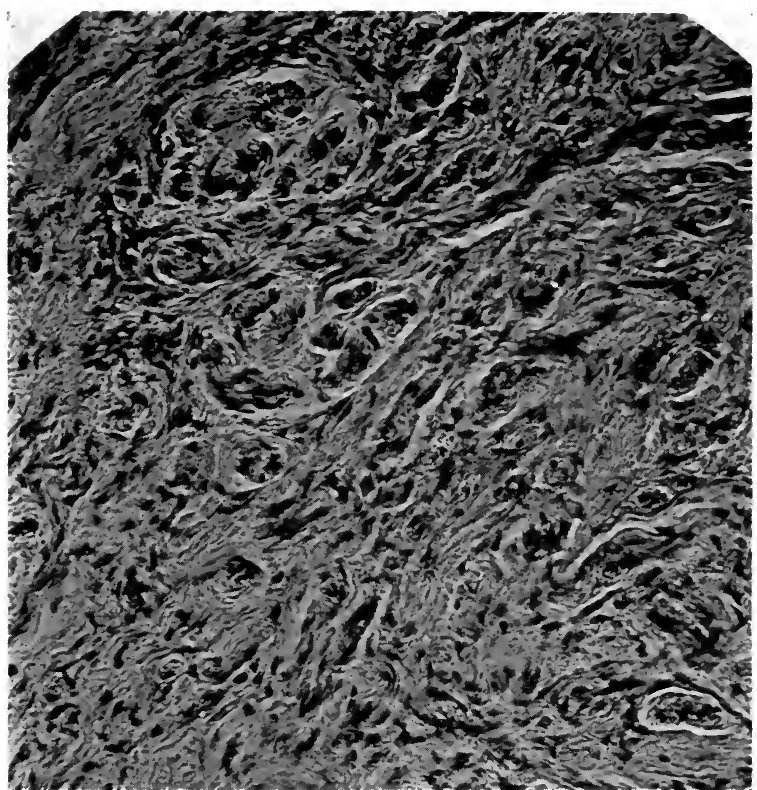

FIG. I82.- Structure of a true neuroma with well-formed medullated nerve-fibers.

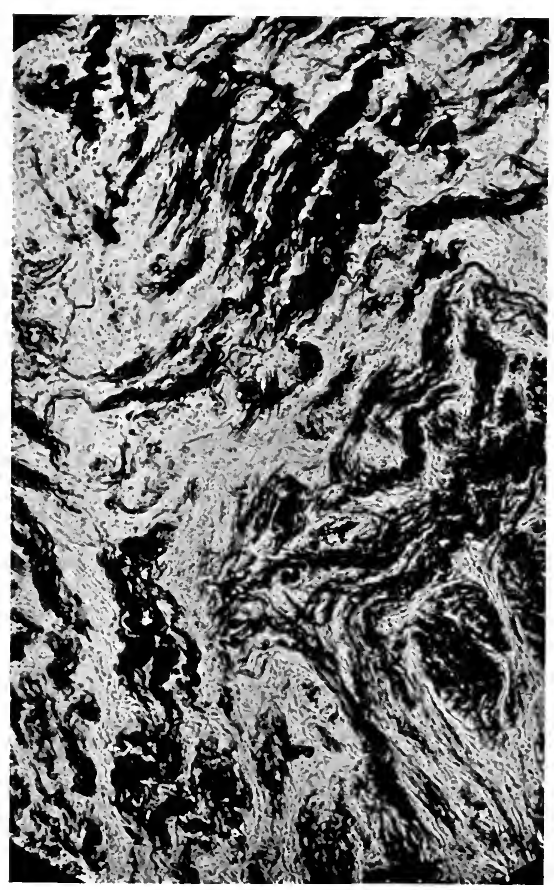

FIG. I83.-Nerve fibrils in a true neuroma. (Stained by Weigert's method.) 
the theory that medullated nerve-fibers cannot develop without the trophic or functional influence of ganglion-cells. In this case the very numerous neuromas developed from indifferent widely scattered cells which the authors regarded as embryonic residue. They clearly traced the origin of medullated nerve-fibers in chains of peripheral cells of the above type. It is interesting to note that these cells and the resulting fibers in the tumors showed a notable tendency to develop in the adventitia of blood-vessels. These observations may have an important bearing on the histogenesis of various more cellular tumors of the nervous system, in which myelinization does not occur, especially the sarcomas of nerve-trunks and pia mater.

Ganglionic Neuroma.-Tumors composed of ganglion-cells and nervefibers, while rare outside of the central nervous system, have occurred in a small group of cases and present rather notable characteristics. They form small, nodular, and multiple growths, or bulky, single tumors located beneath

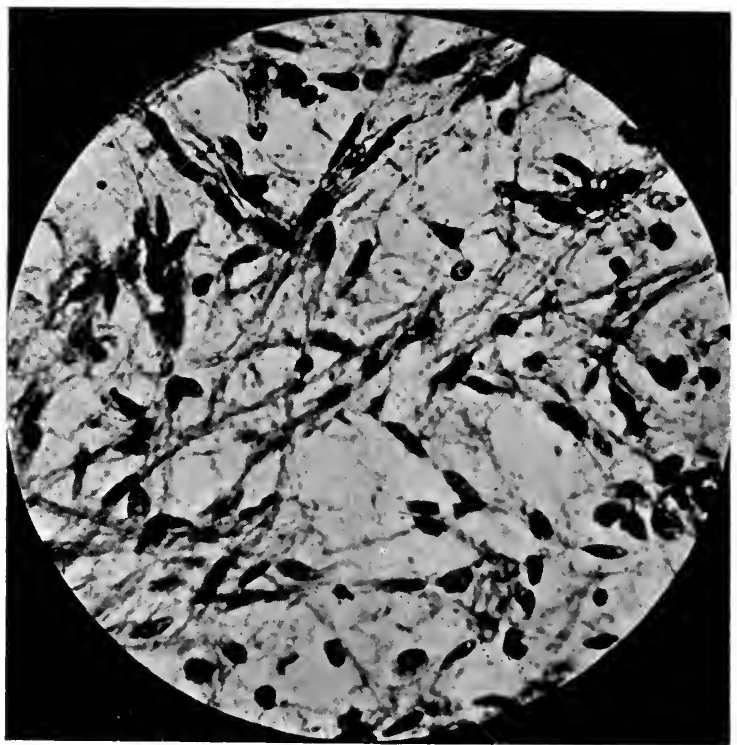

FIG. 184.-Structure of myelinic neuroma. (Bruce and Dawson.)

the skin or more often in the course of large nerve-trunks or plexuses, in the adrenal, gastro-intestinal tract, retroperitoneal region, skin, and about the cerebral and spinal dura. They seem to be chiefly or exclusively derived from the sympathetic system (Knauss, Falk, Lit.), arising usually from the thoracic and abdominal sympathetic trunks and ganglia and from the peripheral circumvascular sympathetic plexuses. These tumors may be regarded as the counterpart of the ganglionic gliomas of the central nervous system.

In the gross the tumors resemble soft fibromas, with radiating markings which correspond to the bundles of nerve-fibers. Their position may suggest their relation to the sympathetic. The microscopical structure shows bundles of nerve-fibers coursing among ganglion-cells and supported by connective tissue and blood-vessels. Fat-cells may also be present. The fibers may be chiefly medullated or wholly non-medullated. The ganglion-cells are of ordinary characters or hypertrophied to giant size, but deficient in 
chromatic substance, or they may be very numerous and approach the form of large round cells. Falk believes that the connective tissue participates in the growth and that the neoplasms are mixed tumors. They differ in composition from the neuro-epithelioma or neurocytoma which Wright described as arising from the sympathetic. The true ganglionic neuroma may be difficult to distinguish from fibroma of ganglionic nerve-trunks, in which both cells and fibers may long persist. In the multiple neurofibroma of stomach and intestine the tumors are probably derived from sympathetic nerves and ganglia and ganglion-cells may long persist (Askanazy). The ganglion may become greatly distended with myelin-like material. Beneke describes mitoses, and many forms of degeneration and atypical cell growth.

The adrenal is the chief seat of ganglionic neuroma, where tumors from the size of a cherry to that of a man's fist have been observed by Weichselbaum, Bruchanow, and Ribbert. In a case of Schmidt's the tumor lay be-

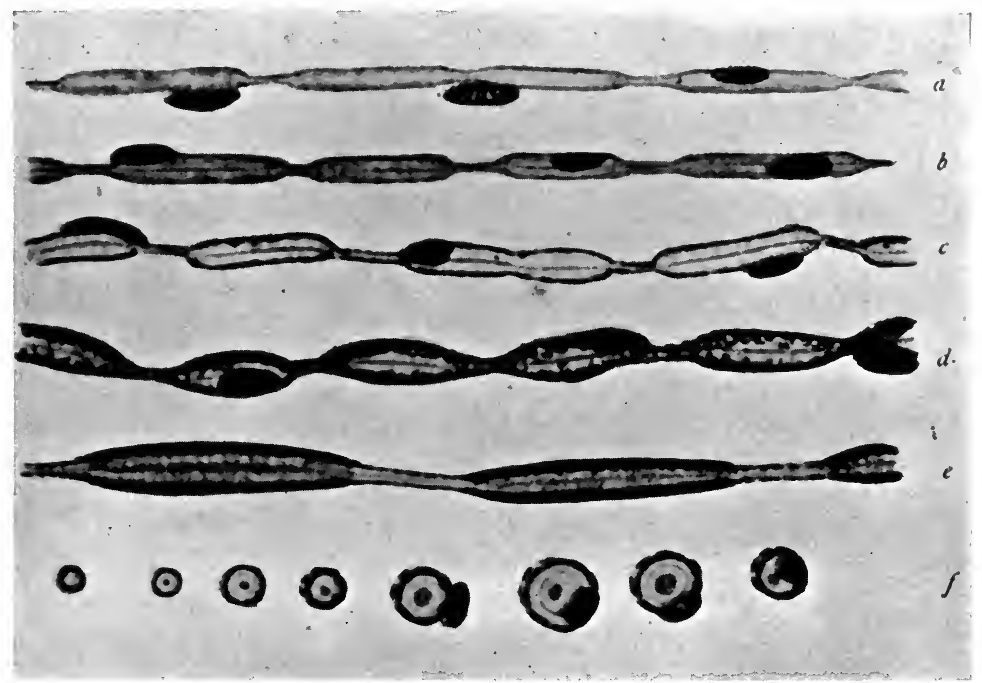

FIG. I 85.-Structure of medullated nerve-fibers in myelinic neuroma. (Bruce and Dawson.)

tween kidney and adrenal, yet the suprarenal plexus was normal. A combination of suprarenal tumor with multiple growths on the surface of the cerebellum, cerebrum, spinal cord, in the cauda equina, spinal ganglia, auditory nerve roots, and axillary plexus, and sciatic nerve occurred in a remarkable case described by Soyka. In the thorax Loretz and Borst have described subpleural tumors lying near the spinal column and doubtless derived from the thoracic plexus. Multiple subcutaneous tumors probably originating from perivascular plexuses, recurrent or associated with internal tumors, are recorded by Soyka and by Knauss. In Kredel's case scores of small and large tumors were scattered over the body of a 5-year-old child. Chiari, Busse, and Beneke found unusually large tumors arising from the abdominal or pelvic plexuses.

Malignant neuroma occurred in a remarkable case of Beneke's.

In a child of 10 years a tumor the size of a man's head, of seven years' duration, originating in the region of the celiac axis, was found to consist largely of typical ganglionic 
neuroma, but in some wide areas the ganglionic cells could be traced into smaller rounded cells without processes and arranged in the form of alveolar sarcoma, which structure also appeared in much enlarged lymph-nodes. A much less cellular tumor with secondary growths in neighboring lymph-nodes is described by Miller. These cases form a transition group between ganglionic neuroma and malignant neuroblastoma, which will be described under the tumors of the adrenal, from which organ they chiefly arise. The entire group has been systematically discussed by Wahl and by Dunn.

In the etiology of ganglionic neuroma one must assume an embryonal disturbance of the structure of the sympathetic nervous system with the presence of superfluous undifferentiated tissue in localities giving origin to the tumors. This view is favored by the congenital or early appearance of the tumors, by the undeveloped character of their component structures, by their wide and multiple distribution. As to the nature and cause of this

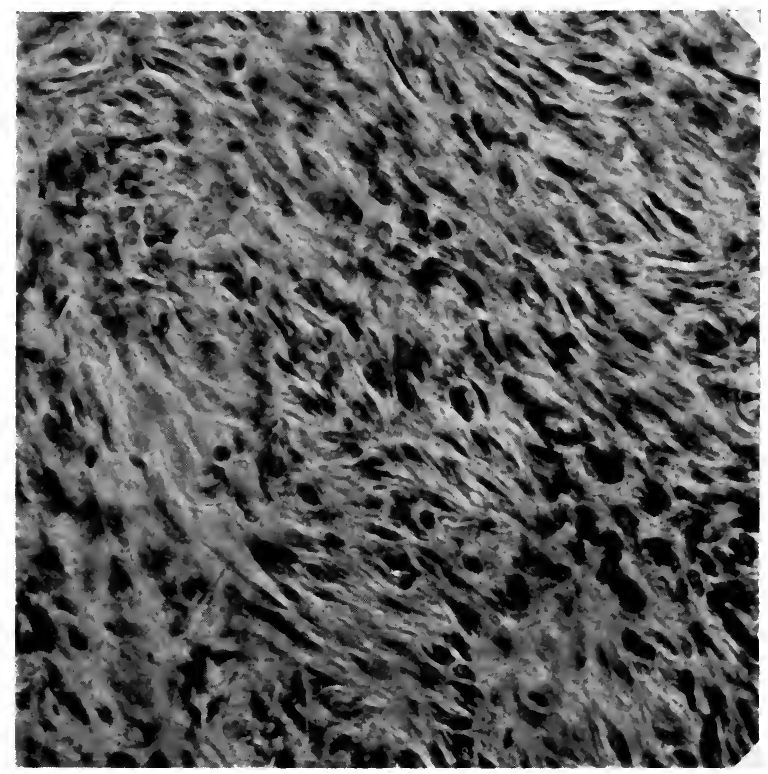

FIG. I 86.-Advancing edge of infiltrating neurofibrosarcoma.

disturbance nothing is known, but congenital variations in the size of various nerve-trunks and ganglia have frequently been observed (Virchow).

Acoustic Neurofibroma.-Intracranial tumors of the nerve-trunks affect chiefly the acoustic, rarely the trigeminus nerves, and form a relatively frequent and characteristic group of brain tumors (Henneburg and Koch; Fraenkel and Hunt, Lit.). They are usually single, rarely bilateral, and may form a part of general neurofibromatosis. Neuromas of the other cranial nerves are regularly extracranial. The condition was reported by Forster in 1862 and fully described by Monakow in 1900.

The tumors occur usually before the 5 oth year, and often in young persons. They may be associated with other abnormalities of the brain, as hyperplasia and hypertrophy of the glia-cells of the cortex (Henneberg: Koch), endothelioma and psammoma of the dura, sarcoma of brain, multiple hernias of brain tissue through the dura (Fraenkel, Hunt). These abnor- 
malities suggest an underlying anatomical predisposition to tumor growth. In one of Henneberg's cases there were neuroma of the acoustic, fibroma of the falx, fibrosarcoma in right ventricle, 3 small tumors in the medulla, and multiple psammomas of pia and dura. The comparative frequency with which isolated brain tumors, fibroma, sarcoma, and glioma are observed with peripheral neurofibroma has an obvious bearing on the origin of many solitary intracranial neoplasms.

The tumors begin in a portion or involve the whole of the nerve-trunk, producing a fusiform or round swelling which has, been encountered when as small as a cherry or as large as a hen's egg. They are firm, lobulated, and encapsulated, but often adherent. They compress the cerebellum, medulla, and adjoining nerves. The diffuse swelling of the nerve may penetrate the internal auditory meatus.

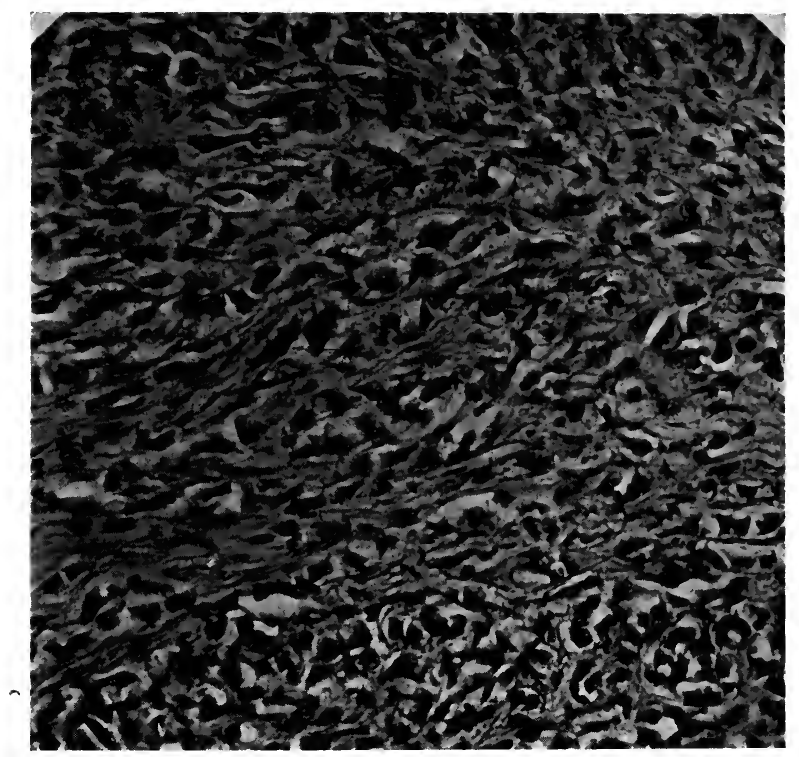

FIG. 187.- Structure of a malignant neurosarcoma.

The structure is usually that of simple neurofibroma, but some very cellular growths approach the type of neurosarcoma. Glia-tissue has several times been demonstrated, and from this element a sarcomatous process may be derived. Regressive changes include fibrosis and cyst formation.

Sternberg points out that the trigeminus, acoustic, facial, glossopharyngeal, and vagus arise from the neural ridge which appears at an early date when the tissues are comparatively undifferentiated, while the other cranial nerves develop from the cerebral vesicles. Hence the former nerves may readily include embryonal cell groups which explain the complex structure of their tumors.

The clinical course is of a slowly growing tumor of the posterior fossa, preceded by symptoms referable to the fifth or eighth nerves. The slow course and encapsulation place them among the operable cases, but the inaccessibility, adhesions, destruction of neighboring vital tissues, and occasional malignancy yield an unfavorable prognosis. 
Neuromyxoma of the Optic Nerve.-The optic nerve is the seat of a characteristic neuromyxoma which has been described by Collins, Marshall, Reese, and others, and which presents quite uniform anatomical and clinical features. Through the kindness of Drs. Knapp, Krug, and Reese I have had the opportunity of studying several cases. The tumors occur in children and young persons, they grow slowly until the whole nerve is destroyed, but they do not invade the eyeball or orbital tissues until very late. They form soft elastic fusiform swellings of the entire nerve. The central portions at first reveal the nerve trunk surrounded by tumor tissue, but eventually the tissue softens and the nerve-fibers are destroyed. The structure presents numerous small groups of cells of the type of glia-cells without fibrils lying in compact foci or scattered in a mucinous matrix. Remnants of nerve-fibers in various stages of degeneration may be found in the softened matrix. Small blood-vessels may be abundant. The location of the tumor in early stages, surrounding the nerve trunk, suggests that it arises from the peripheral portions of the nerve or from the endothelial sheath, but this peculiarity may

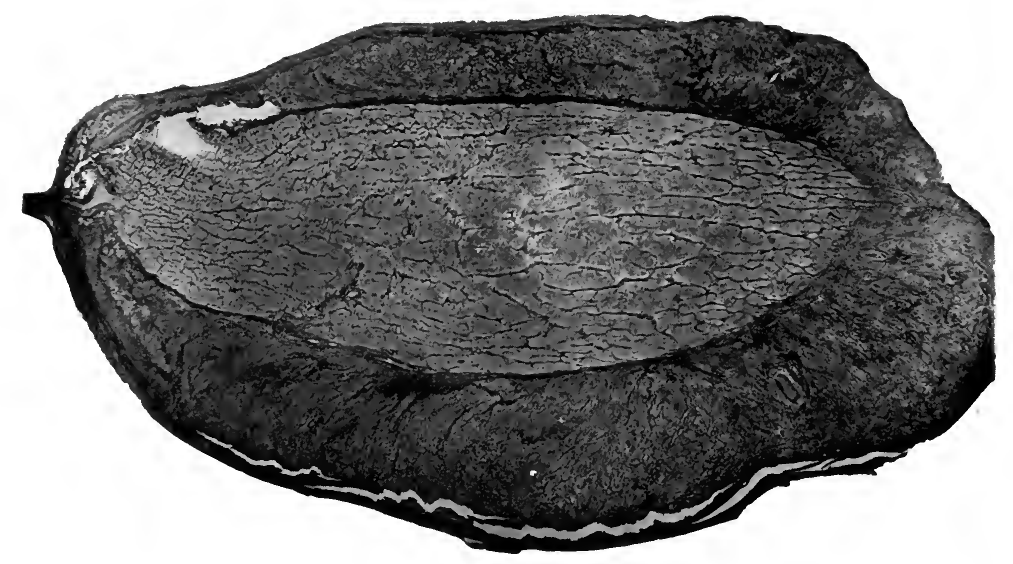

FIG. I 88.-Benign myxoglioma of optic nerve ensheating the nerve, but arising from another segment and extending along the sheath. (Case of Dr. R. G. Reese.)

also be referred to an origin from one end of the nerve, the tumor growing forward about the trunk. The nerve trunk shows definite neoplastic changes, as in Reese's case. Some of the tumors are very cellular, in some the mucinous stroma is prominent, and others show excess of blood-vessels. They are usually removed successfully without disturbing the eyeball, and recurrence of encapsulated growths is not to be anticipated.

Tumors of the Gasserian ganglion are of considerable surgical interest, and form a definite pathological basis in one type of trigeminal neuralgia, which is their chief clinical symptoms. Sachs collected $2 \mathrm{I}$ cases, and the conditions have been reviewed in detail by Spiller and by Giana. The pain in these cases usually becomes continuous, the results of surgical treatment have been very unfavorable, and the disease terminates fatally in I to 3 years. The tumors involve and destroy the ganglion or arise in the capsule and compress the ganglion. They reach the size of a marble or pigeon egg. Several cases appear to have had an inflammatory origin and were associated with tuberculosis (Giana), or extensive plasma-cell overgrowth (Hoffmeister, 
Meyer). The structure has varied from fibrous to very cellular growths, the latter presenting alveoli surrounded by several layers of polyhedral cells. In some the structure resembles perivascular endothelioma. In others glio-

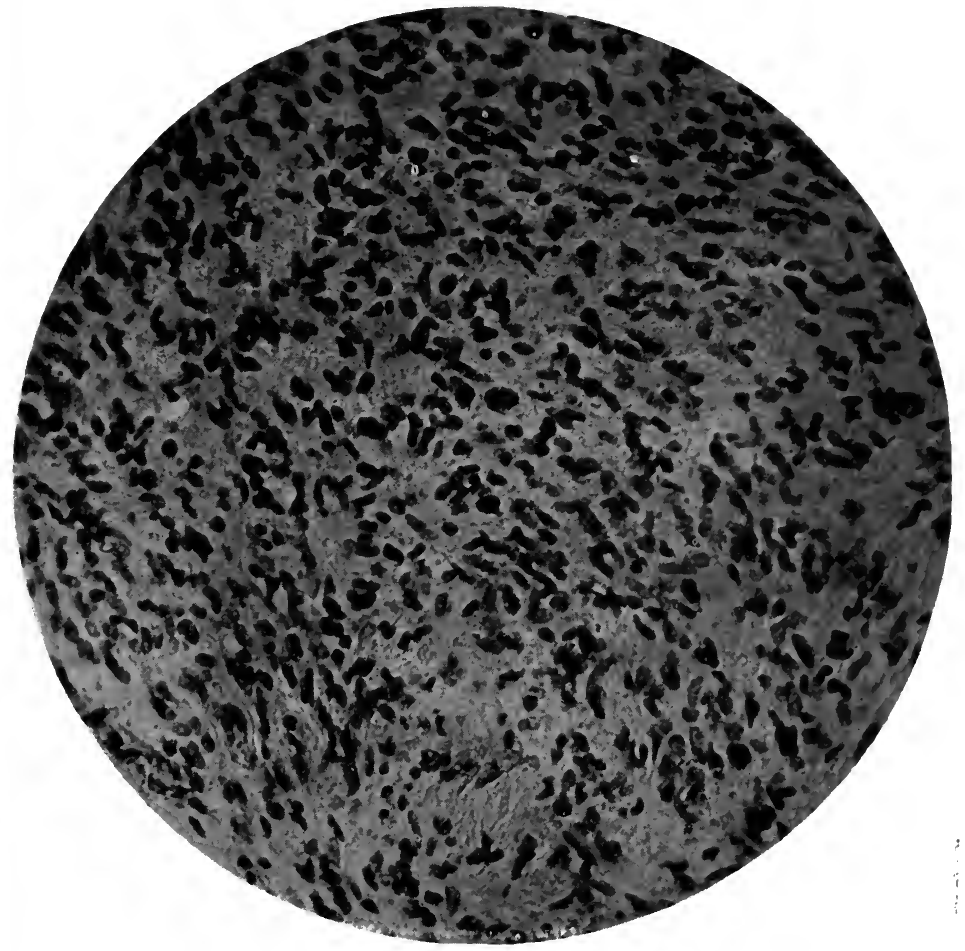

FIG. 189.- Structure of a benign myxoglioma of optic nerve.

matous features were noted. Marchand believed that they represented various types of neurocytoma. Others have derived their tumors from the pericellular lymph-sheaths. 


\section{CHAPTER XXIV}

\section{TUMORS OF SPINAL CORD AND MEMBRANES}

General Etiology.-All tumors of the spinal cord or membranes that compress the cord fall in a single clinical group which can be differentiated only after complete study. They include gummas, tubercle, and parasitic cysts, as well as neoplasms.

Statistical studies of Schlesinger give I5I such tumors among 35,000 autopsies at Vienna ( 0.43 per cent.). Of 400 cases collected by this author, 302 were intradural and 88 extradural. The sarcomas head the list with I07 cases; followed by tubercle, 64; echinococcus, 44; neurofibroma, 37; gumma, 28; glioma and gliosarcoma, 27; psammoma, ı8; myxoma and lipoma, each, II ; endothelioma, 6. Excluding I44 cases of tubercle, gumma, and parasitic cysts, the proportion of sarcomas becomes even more prominent.

Practically, the spinal tumors are chiefly sarcomas, of which a considerable proportion are extradural, 3 I of ro7 cases; glioma and gliosarcoma, which are always intradural, 27 cases; neurofibroma, 37 cases; and psammoma with its congener endothelioma, 24 cases. Lipoma and myxoma are distinctly rare, and some of the latter are probably degenerating forms of growths originating in glia-tissue or nerve-trunks.

It is of interest to note that tumors of the spinal column in the Vienna series of 35,000 autopsies were far more frequent than in the meninges and cord ( 107 cases to 44); while brain-tumors of all types were six times as common (994 cases) as the spinal cord growths (I 5 I cases). Primary tumors of the spinal meninges were twice as common as the secondary metastatic growths. During this same period 427 cases of tuberculous spondylitis were observed.

Trauma.-Less frequently than in the brain, severe trauma has appeared to be the direct exciting cause of spinal cord tumors, usually of the gliomatous type. Severe injury capable of reaching the meninges and followed by symptoms continuing until the existence of a tumor at the injured point was established, occurred in the gliomas of Silberkuhl, Wichmann, Schuppel, Schlapp, and others.

Sarcomas appeared to develop on a traumatic basis in reports of Traube, Forster, Ollivier, Hunt and Woolsey, and myxomas in the cases of Gowers, Bruce, and Mott. Very similar histories have appeared in cases of fibroma (Marshall) and psammoma (Oustaniol). Gaupp's angiofibroma appeared many years after a severe spinal injury.

In many instances symptoms proved to result from tubercle, gumma, or echinococcus cyst have followed immediately or soon after trauma (Schlesinger, Lit.).

It thus appears that trauma may be the direct exciting factor, or may accelerate the growth, or reveal or intensify the symptoms of spinal tumors, or may have no connection whatever with an accidentally associated lesion.

Hereditary influences have been suggested in some interesting cases. Two sisters of Huppel's patient with multiple sarcoma suffered from the 
same symptoms. A brother of Hunt's case of traumatic sarcoma died of the same lesion, also of traumatic onset.

AgE INCIDENCE OF 187 SPINAL CORd TUmors

\begin{tabular}{|c|c|c|c|c|c|c|}
\hline \multicolumn{4}{|c|}{ Sarcoma. } & \multirow{2}{*}{ Glioma. } & \multirow{2}{*}{ Psammoma. } & \multirow{2}{*}{ Fibroma. } \\
\hline Years. & Intradural. & Extradural. & Diffuse. & & & \\
\hline \multirow{8}{*}{$\begin{array}{c}1-9 \\
10-19 \\
20-29 \\
30-39 \\
40-49 \\
50-59 \\
60-69\end{array}$} & I & I & 7 & 3 & o & I \\
\hline & o & 3 & 4 & 6 & O & 3 \\
\hline & I 6 & 0 & 5 & I 3 & o & 3 \\
\hline & I 3 & 7 & 2 & IO & 2 & 3 \\
\hline & 13 & IO & I & 6 & 7 & $t$ \\
\hline & 6 & 9 & $\mathbf{I}$ & 5 & 4 & 3 \\
\hline & I & I & o & 2 & 8 & 3 \\
\hline & $5^{\circ}$ & $3 I$ & 20 & 45 & $2 \mathrm{I}$ & 20 \\
\hline
\end{tabular}

The above table includes $8 \mathrm{I}$ cases tabulated by Schlesinger in $\mathrm{I} 898$, many of the cases collected by Collins, and some recent reports up to I9I 5 .

The main conclusions from these data are as follows: Solitary sarcoma occurs chiefly in young adults between 20 and 40 years. Diffuse sarcomas are practically limited to children or young adults under 30 years of age. Gliomas are well distributed in the age periods. Psammoma is not reported under 30 years. Among extremes are Anderseck's sarcoma of the cord in a newborn infant and Duseberg's angiosarcoma in a man of 79 years.

The great majority of lipomas occur in children under 5 years. Neurofibromas occur at all ages.

In children under ro years a spinal cord tumor is in order of frequency a solitary tubercle, a diffuse or unfavorable form of sarcoma, or rarely a lipoma. After 40 years the benign tumors and favorable forms of circumscribed sarcomas become more frequent. Tubercle is distributed over all periods, but gummas are rare before 30 years.

While customary subdivisions of spinal tumors are numerous it is probable that they arise from three main structures: (I) glia-cells and neural epithelium, (2) blood- and lymph-vessels and their endothelial lining, and (3) the nervetrunks. From this histogenetic standpoint they may be classified as follows:

Glia-tissue - Glioma, gliosarcoma.

Neural epithelium-Neuro-epithelioma. Some so-called angiosarcomas.

Blood-vessels-Angioma, angiosarcoma, sarcoma.

Lymphatics-Lymphangioma, endothelioma, psammoma, sarcoma.

Nerve-trunks - Neurofibroma, fibroma, myxoma.

Spinal fat tissue-Lipoma.

Only the spinal sarcomas will here be considered in detail, the others being considered elsewhere.

Sarcoma of Cord and Meninges.-Sarcoma of the cord or meninges occurs in several anatomical forms:

I. A solitary tumor.

2. Multiple, large, small, or miliary tumors.

This form merges into

3. A diffuse infiltration which results from: (a) Extension from a single large primary meningeal growth, or $(b)$ as a diffuse infiltration without localized primary tumor, and $(c)$ as a secondary invasion from extramedullary growths.

Extradural tumors of a variety of types arising from the bone, connective 
tissue, fat tissue, and nerve trunks are commonly included in this clinical group. Of $6 \mathrm{r}$ sarcomas in the vertebral canal Schlesinger found $\mathrm{I} 8$ extradural. Of 42 primary intradural sarcomas $I 6$ arose from the dura, 14 from pia, 6 from arachnoid, 4 from nerve roots, and 2 from the ligamentum denticulatum.

I. The solitary sarcoma is a relatively frequent form of cerebral and spinal tumor. It arises from the dura, pia, or nerve roots, and extends in elongated form along the cord with local compression if firm and encapsulated or adapting itself to the spinal canal if soft. The hard tumors remain permanently encapsulated or become pedunculated and do not invade the cord. They are composed of closely packed spindle-cells, with few vessels which escape the calcification of psammoma. They are probably related in origin to the neurofibromas and neurosarcomas, but lack the specific structure of these tumors.

Myxosarcoma is a rare but characteristic form of spinal tumor of which Oustaniol collected 9 cases. The cauda is a favorite site. They are occasionally extradural and here the frequent admixture with fat indicates an origin from misplaced mesodermal tissue. Berthelot and Merklen described multiple myxomas of the cord.

Round-cell sarcomas form soft, vascular growths which are prone to hemorrhage and cystic degeneration and while long remaining encapsulated they may eventually invade the cord or produce metastases. Their sources have not been traced. The great abundance of blood-vessels in certain cases suggests a perivascular origin (Schlesinger, 30). As a rule the cellular tumors are difficult to distinguish from endotheliomas, and when the cells are quite round and very numerous the separation from gliosarcoma is required.

2, 3. Multiple and Diffuse Sarcomatosis of Meninges.-This remarkable and highly specific neoplasm appears in the form of multiple nodules or plates or a diffuse infiltration of the pia-arachnoid. It may extend over many segments of the cord, or over the entire cord from medulla to cauda, or may cover the cerebral pia over the base and even extend into the ventricles (Nonne, Schroder). The nervous tissue usually escapes infiltration, but may be involved in the later stages. A localized primary tumor of the meninges is occasionally present and rarely the tumor appears to originate in the nervous tissue or its sulci.

The alteration in the exposed cord may be so slight as to escape the naked eye (Nonne), but usually there is a nodular or diffuse thickening or opacity from grayish-red tumor tissue which appears first on the posterior aspect of the cord, encircles it more or less completely, and passes along the nerve roots.

In R. Schulz's case the entire cord was surrounded by a layer of new tissue $\frac{1}{2} \mathrm{~cm}$. in thickness, and in the cases of Schataloff and Nikoforoff the investing layer was I $\mathrm{cm}$. deep. Bausch found a uniform tumor sheath extending from cauda to brain, irregularly over the cerebral pia, and into the ventricles along the choroid plexus. When a localized primary tumor appears as the source of the diffuse infiltration it usually lies in the cerebellum or base (Hippel, Nonne), or in the substance of the cord (Westphal). Of 18 cases collected by Schlesinger, I4 were associated with large tumors, I 2 of the cerebellum, and 3 of the medulla. The occurrence of miliary nodules over the peripheral areas indicates that the tumor advances by transfer of cell groups along the arachnoid, but in many cases the advancing edge merges gradually into healthy tissue. Cerebral involvement often leads to hydrocephalus.

The structure of the diffuse sarcoma is somewhat uniform, but owing to 
the activity of growth the exact origin of the tumor has never been satisfactorily determined and, in fact, the exact nature of the process in some cases is obscure.

In most cases the tumors contain many fine blood-vessels sheathed by round or polyhedral or spindle-cells. When the vessels are very numerous the tumor has been called angiosarcoma or perithelioma (Nonne, Busch). Hyaline changes in the vessel walls led Kramer to designate his tumor as cylindroma. In 2 cases $\mathrm{W}$. Rindfleisch found the vessels overgrown or obliterated by a diffuse growth of large and small round cells. Lenz analyzes the process as an angioblastic sarcoma to which is added a marked proliferation of the pial endothelium, yielding many large cells in alveolar arrangement. In his case a cerebral extension was purely sarcomatous and failed to show the secondary endothelial overgrowth. Other cases of this type are the original observation of Ollivier, 2 cases of Coupland, and that of Westphal. Cramer and Lowenfeld described multiple nodules of the same structure which probably represent an early stage of the process. Marked regressive changes were observed by Cramer and Ganguillet.

The main histological features in this group point to an origin from the adventitial cells of the blood-vessels, so that the recognition of a specific form of diffuse angioblastic meningeal sarcoma seems warranted.

In a second group of cases endothelial characters are more prominent and the tumor appears as an alveolar sarcoma. Schulz's bulky growth contained large endothelial cells in alveolar arrangement.

Diffuse melanoma of the meninges has been observed in a considerable series of cases. In many of these the perivascular arrangement of the cells was prominent (Schopper, Lua).

The clinical course of diffuse meningeal sarcoma presents certain features of special interest. Most cases occur in children or young adults, but Rind-

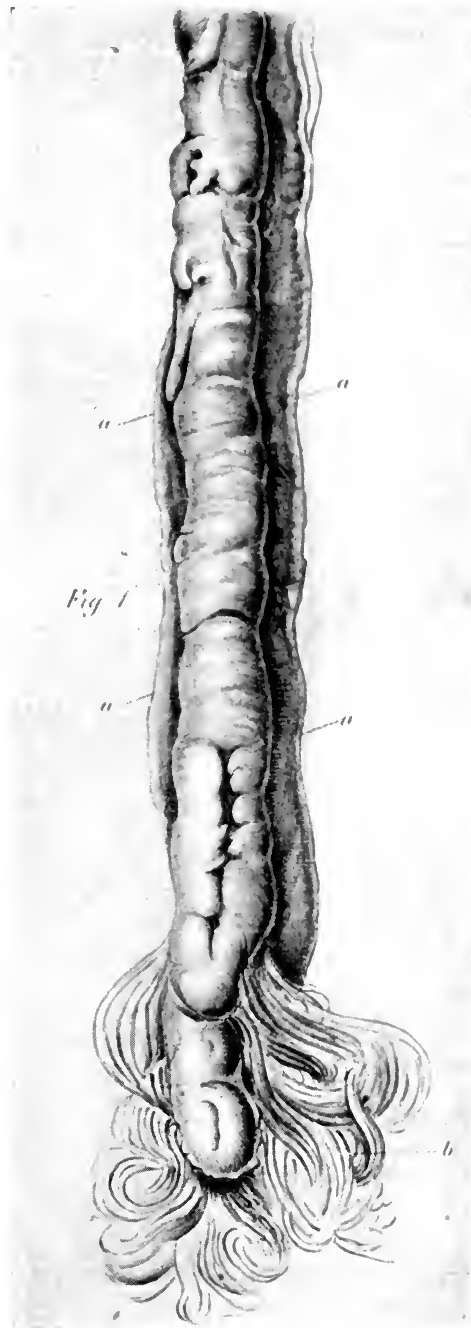

FIG. 190.-Diffuse sarcoma of spinal pia mater. (After Schulz. A.P., I6.)

fleisch reports the condition at 49 years. When there is a large primary tumor the symptoms are usually dominated by local pressure phenomena, while the infiltration reveals its course by increasing spinal disturbance. Rindfleisch established a diagnosis during life by the presence of many large 
cells in the spinal fluid. Many cases are very acute and resemble tuberculous or syphilitic meningitis. In fact, it is not always possible to distinguish the disease from cellular meningitis. Thus, Grund found rather typical gross lesions of tuberculous meningitis with miliary tubercles in liver and kidney in a child of I I years, with a duration of 4 months. Under such conditions his diagnosis of multiple miliary gliosarcoma suggests at least that a tuberculous element was concerned in the meningeal process.

Central spinal sarcoma has occurred in a series of cases of which Schlesinger has collected I3. Gliomas and gliosarcomas are much more frequent in this region. The upper portions of the cord as chiefly involved and in 3 cases the entire cord was affected (Forster, Glaser, Schultze). The tumor mass forms a narrow cylinder or an irregular mass $\frac{1}{2}$ to $\mathrm{I} \mathrm{cm}$. in thickness. Schiff found a minute spindle-cell sarcoma in the anterior horn. Elongated cavities filled with serum or bloody fluid may form within the

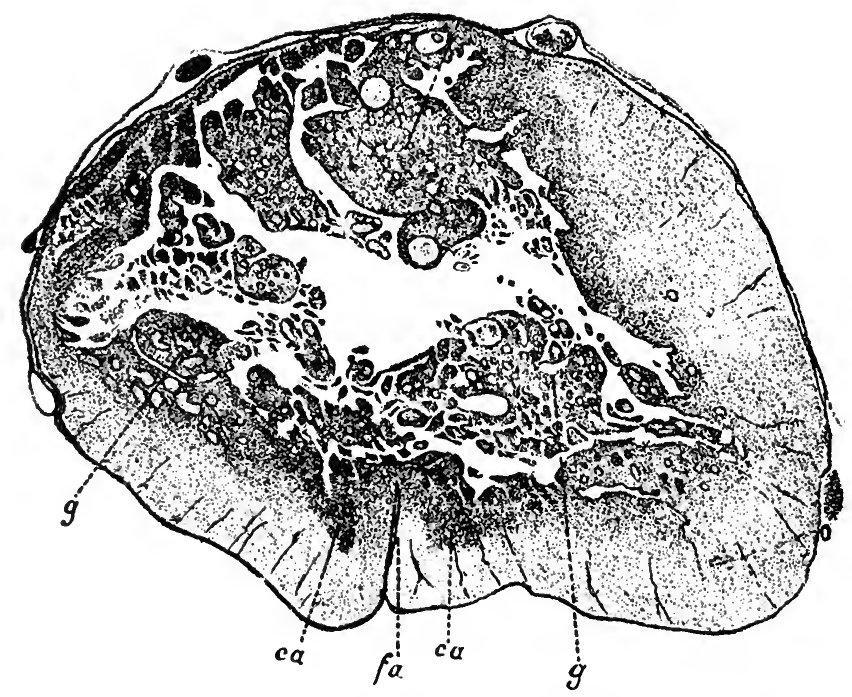

FIG. 191.-Central glioma of spinal cord with syringomyelia: $f a$, Anterior fissure; $c a$, anterior horn; $g$, tumor. (After Saxer.)

tumor (Glaser, Seebohm). The central canal is usually obliterated, but in some cases beyond or within the tumor mass the canal may be dilated and the wall softened and eroded. Sharp encapsulation from the compressed nerve tissue is usually noted (Fenger, Adamkiewicz). The gliosarcomas, on the contrary, begin with central gliosis and gradually invade the cord with central softening. The structure of the more specific forms of these tumors is that of round- or spindle-cell angiosarcoma. They are not always clearly distinguishable from glioma or neurofibroma, which are often highly vascular. Nonne noted the resemblance of his round-cell tumors to gliomyxosarcomas, from which he separates them chiefly on gross characters. In Glazer's case the structure resembled neuro-epithelioma. The true sarcomas appear to originate exclusively from the vessels of the gray matter.

Psammoma arises from the spinal dura or arachnoid and usually produces a single, firm, well-encapsulated growth compressing the cord. Its 
progress is slow and several years are commonly required to reach rather limited dimensions which seldom exceed $2 \times 4 \mathrm{~cm}$. Pressure on the cord begins early, motor and sensory symptoms are prominent, and extensive secondary degenerations are commonly established. In Steudener's case the cord was completely severed in the course of 2 years' growth. The tumors usually lie on the dorsal and lateral aspects of the cord, and chiefly in the lower half. The structure presents either a cellular and vascular form of sarcoma or a less cellular sclerosed tumor with much calcification. In the cellular forms there are many small canals surrounded by concentric layers of spindle- or polygonal cells. Calcification of the walls of these small vessels may appear early when the tumors are cellular, yielding the sarcome angiolithique of Cornil and Ranvier. Very cellular forms of this tumor differ from the common spindle-cell sarcoma, chiefly in the presence of these calcific vessels. In late forms the growth may be largely composed of calcific granules and hyaline tissue.

Multiple neurofibroma of the spinal cord is relatively frequent. Schlesinger collected I 5 cases, of which Io were intradural, while 7 sprang from the membranes and 3 from the nerve roots. All portions of the cord are about equally affected, but the cauda is a somewhat favorite seat. They are frequently a part of general neurofibromatosis (Hunt), and in Vast's case both cerebral and spinal nerve-trunks were involved. Schlesinger observed 3 large tumors compressing the medulla and very numerous small nodules throughout cerebral and spinal meninges and in the cauda.

The solitary fibroma is probably in most cases a hard neurofibroma. While usually appearing as small, hard masses attached to dura or pia, they are occasionally cystic, and at times rather cellular and vascular. Merzbacher found a trilobed cervical intradural fibroma II $\mathrm{cm}$. in length and of I 8 years' duration in a man of 55 years. Heurtaux traced to an intraspinal origin a very large congenital fibroma projecting from the neck.

Endothelioma of the spinal meninges appears in the form of a large solitary tumor or as multiple nodules.

Spinal lipoma occurs in solitary, multiple, and diffuse forms. It is usually extradural, and extravertebral tumors may grow between the vertebræ into the canal (Holmes, Temoin); or an extradural growth may perforate the dura (Johnson); while others originate in the arachnoid (Obre). The extravertebral forms may be connected with spina bifida. Braubach's intradural tumor in a child of 5 years extended from the fourth cervical to fourth dorsal segment. Multiple lipomas extending over much of the lower dura and the posterior nerve sheaths are described by K. Hoffmann. Multiple angiolipoma is described by Berenbuch, and multiple malignant myxolipoma by Virchow. Wolbach reports diffuse lipomatosis of the cord in an infant. Gowers saw striated muscle-fibers in caudal lipoma.

Spinal angiomas present a benign illustration of the proliferative capacity of the meningeal blood- and lymph-vessels. They show many gradations up to angioblastic sarcoma and endothelioma. Gaupp pictures a cavernous angioma of the pia, and a cellular capillary angioma, occurring in the same cord with central glioma and 2 fibromas. Barenbruch found $12 \mathrm{~cm}$. of the cord partly replaced by a coarse angiomatous structure. Angiomatous tumors of the cauda have been described as cylindroma, owing to the hyaline degeneration of the vessel walls (Ganguillet). Cobb collected several cases of cavernous angioma of spinal meninges and cord, and points out the association with cutaneous nevi in the same regions.

Zanda describes multiple osteomas arising from the calcified walls of blood-vessels. Cavernous and cystic lymphangiomas are reported by Laquer 
and by Taube. A dermoid cyst of the dorsal cord is reported by $\mathrm{H}$. White. Trachtenberg has described a remarkable case of multiple dermoids of cerebral and spinal meninges.

Metastatic sarcoma may extend over considerable segments of the cerebral or spinal meninges. Such cases are reported with renal sarcoma by L. Muller and after ovarian sarcoma by Hensen. The entire spinal meninges

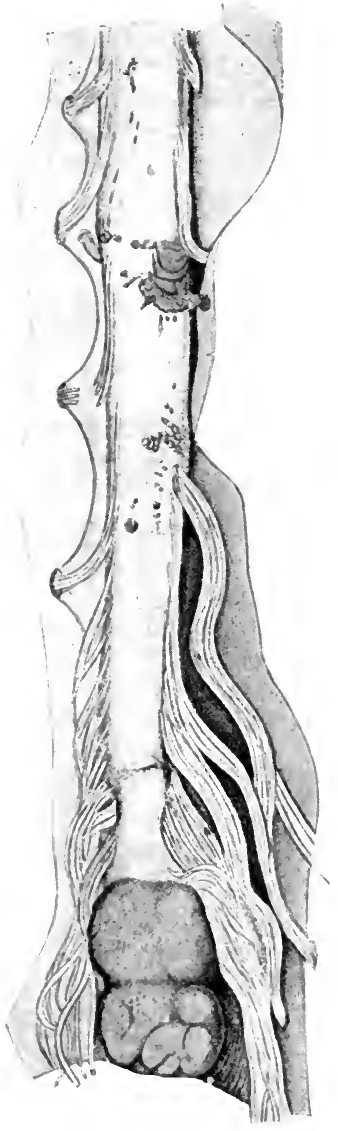

FIG. I92.--Multiple dermoids of spinal meninges. (Trachtenberg's case. V. A., 154.)

(154.) was found microscopically to be diffusely infiltrated from a primary intestinal lymphosarcoma, in a case described by Stursberg. Schlesinger describes a glioma and endothelioma of the pituitary gland with diffuse infiltration of the spinal meninges.

Secondary changes in the cord, meninges, and canal, following tumor growth, are of much practical importance.

Simple compression without destruction of nerve-tissue may greatly narrow the cord directly opposite the tumor. About the compressed area the cord is swollen and hyperemic and there may be much accumulation of fluid in the central canal, in the arachnoid, or in a cystic compartment which often forms about the tumor. Upon removal of the tumor all the changes may rapidly disappear, even during the operation (Kummel). Functional recovery may be almost immediate. That long-standing compression is not necessarily accompanied by irreparable damage is shown by the recovery from complete paralysis after such duration as 8 months (Lichtheim), I 3 months (Schultze), I8 months (Macewen). Eventually there may be complete atrophy of nerve tissue and fusion of the envelopes in a fibrous cord to which the tumor may adhere. Acute degenerative changes may supervene in the course of the compression and seem to be referable not merely to pressure but also to an inflammatory process accompanying the growth. These changes may appear suddenly in the course of benign, slowly growing tumors, as psammoma, and are especially frequent when the pia is invaded by locally aggressive tumors. The nervous tissue is then found infiltrated with blood, softened, and diffluent. The hemorrhage may extend far from the tumor along the tracts or canal and the myelin sheaths of the fibers are swollen and degenerated. Above and below the main lesion Schlesinger describes focal areas of swollen axis-cylinders and myelin sheaths which he refers to a local inflammatory process. Following the early lesions the usual forms of secondary degeneration of the tracts become established, but in very variable degree.

The spinal column itself may suffer from local absorption of vertebral bodies and at the weakened point scoliosis and sharp kyphosis may develop.

The changes in the lumbar spinal fluid have been extensively pursued. The presence of increased albumin in a fluid of distinctly yellowish tinge (xanthochromia) is frequently observed in spinal cord tumors, but is by no 
means pathognomonic (Froin, Nonné). The yellowish color is probably derived from altered blood-pigment. The onset of meningitis is indicated by admixture of fibrin and many round cells. Rindfleisch found many tumor cells in the aspirated fluid.

Meningeal Pseudoneoplasms.-Aside from parasitic cysts, meningeal neoplasms may be simulated, in both clinical and anatomical features, by tuberculous, syphilitic, and traumatic meningitis.

Spinal tuberculosis occurs in several forms which resemble extra- or intradural tumors with or without bone involvement. It produces grayish, vascular, or partly necrotic plates or masses extending over several segments on either side of dura or in the pia-arachnoid. The structure is that of hyperplastic granulation tissue in which the overgrowth of endothelial cells in and about the vessels is very marked. Giant-cells may be absent and lymphocytes abundant, so that the tissue may resemble round-cell sarcoma. Fungous masses of tuberculous granulation tissue arising from carious bone foci may penetrate the subdural space in much the same manner as sarcoma (Schlesinger). The nerve roots in the lumbar and cauda region have been found agglutinated by tuberculous tissue in a tumor-like mass (Schamaschin, Fischer, Schlesinger).

Meningitis (arachnitis) serosa circumscripta in both brain and cord may give rise to cystic accumulations of serous or milky fluid which cause symptoms of tumors (Adler, Spiller, Lit.). The cysts form in the thickened arachnoid, extend over a segment $\frac{1}{2} \mathrm{~cm}$., incompletely encircling the cord, distending the dura and effectively compressing the cord of convolutions. The adjoining meninges may be normal or adhesions may extend over a wide area. The hydrops may involve also the central canal, and in Schwartz's case there were cavities in the anterior horns, and considerable myelomalacia. Secondary degenerations emanating from the central lesion may be extensive. The disease occurs at all ages, chiefly in middle life, and many of the subjects are luetic or tuberculous. Trauma, infectious diseases, and vertebral caries have been charged with other cases. It is relatively frequent, having occurred in 6 of $2 \mathrm{I}$ laminectomies by Oppenheim and Krause for supposed spinal tumor. Quincke has described cerebral cases. The symptoms are those of a mildly progressive tumor (Horsley).

Hodgkin's granuloma may produce well-circumscribed tumors in or about the meninges. A supposed dural sarcoma recently submitted to me proved to be of this nature and mediastinal and cervical tumors developed later. In Welch's case of Hodgkin's disease terminating in sarcoma a paraplegia of many months' duration was found at autopsy to have resulted from a large intradural mass. Nonne also mentions a case of spinal granuloma malignum.

Pachymeningitis cervicalis hypertrophica, often of luetic origin, produces a diffuse thickening of the spinal envelope which may approach the character of an angiofibroma, but apart from the abundance of vessels the structure is quite different from any tumor (Charcot, Koeppen, Wieting). While ordinary gummas are readily distinguished from tumors, the scope of structures in tertiary lesions of the meninges is as yet imperfectly defined.

Trauma following spinal fracture in I case and sharp scoliosis in 2 cases led to diffuse fibromatoid growths, as reported by MacEwen.

Symptomatology of Spinal Cord Tumors.-Elaborate analysis of the symptoms of spinal cord tumors has been required as a guide to the surgical treatment of these growths (Bruns, Starr, Malaise, Nonne). Its main features may be briefly outlined.

Level of the Tumor.-Bruns formulated the law that root pains should 
be referred to the segment from which the root arises and not to pressure on the root in its intraspinal course. They may persist for years before medullary symptoms appear or may fail entirely, especially in ventral growths. They are usually very severe and persistent, continue for a long period, especially in extradural growths, but may eventually disappear when the nerve-trunks are destroyed. Widely disseminated pains point to a medullary lesion. A zone of hyperesthesia extending somewhat above the tumor is often present in both meningeal and medullary growths. Vertebral symptoms, pain and tenderness, are more characteristic of vertebral than of cord tumors, but may be present in both. Sharp kyphosis has once followed bone absorption by dural growths. Girdle sensations have little localizing value, and Hunt finds the root symptoms more reliable than local tenderness. Motor paresis or paralysis appear later than the pains, and a more extensive lesion involving two or three roots is required before paralysis occurs. Painful cramps may denote the onset of anterior root or horn lesions. BrownSéquard's paralysis, usually continuing for a limited period only, may result from unilateral pressure and is rather more frequent in extramedullary tumors, especially in the dorsal region, yet it occurs also with intramedullary growths. The location of the tumor is generally found to be two to four inches above the level of the anesthesia. This rule is based on the fact that with small tumors the pains, anesthesia, and pareses may be due not to root pressure, but to compression of cord well below the roots adjacent to the tumor.

Relation to Cord, Membranes, and Vertebra.-A spinal tumor may be intramedullary, intradural, extradural, or vertebral, and a somewhat different prognosis attaches to each location.

Intramedullary tumors, usually gliomas, present as cardinal symptoms, loss of pain and temperature sense with preservation of tactile sense due to destruction of the intermediate gray matter and the commissure. Yet this symptom-complex occurs usually in unilateral and transitory form in compression of the cord from without (Schlesinger). Invasion of the posterior horns eventually destroys the tactile sense. An initial neuralgic period is usually wanting, or brief and mild, while bilateral motor paresis appears early. Irregularities in course, remissions, and eventually a wide extent of the symptoms accord with the cellular nature of gliomas. Yet meningitis may give pronounced root pains, and Kurtz observed continuous neuralgia for 3 years with multiple gliomas of the cord. Vasomotor and atrophic disturbances frequently occur, and spinal deformity without pain or tenderness is sometimes observed. In many cases the symptoms are those of transverse myelitis.

Extramedullary tumors almost always begin with neuralgic pains. Malaise found them absent in only 5 per cent. of the cases. Absence of pain may be due to a location away from the roots, to the resistance of the roots to pressure from soft sarcomas or to compression of cord above the involved roots (Schultze). The posterior roots have been found incased in tumor, but without pain (Quensel, Sailer). Neuralgia was the only symptom for 8 years in a case of Schultze, and for I 8 months in Sach's caudal sarcoma. Following the neuralgic period come progressive motor disturbances.

An extradural position is indicated especially by long-continued BrownSéquard's paralysis with unilateral root symptoms, followed by unilateral medullary symptoms (Malaise). Because of the elongated form of many extradural tumors muscular cramps and paralysis are relatively commoner than with intradural growths, but owing to the protection of the dura, medullary symptoms may be long delayed. 
Vertebral tumors are suggested by diffuse or local spinal pain and tenderness and by sharp kyphosis or marked degrees of vertebral disturbance which may be determined by the radiograph.

The duration of spinal tumors varies widely. Malignant vertebral tumors are the most rapid (average 8.5 months); 20 extradural sarcomas gave an average duration of $\mathbf{1} 2.7$ months; Io intramedullary sarcomas, I6.8 months; I8 intramedullary gliomas, 23 months; and 20 intradural sarcomas, 24.2 months (Schlesinger). The extremes for tumors diagnosed as sarcoma vary from I month to 25 years. Of prognostic import is the rule that a tumor of more than 3 years' duration is usually intradural. The slowest courses are those of the benign fibroma ( 3 I to 38 months) and psammoma (.30 to 36 months). Yet the duration of symptoms in psammoma has varied from a few weeks to 14 years. The diffuse sarcomas may clinically resemble meningitis. Tubercle and gummas progress slowly (4 to 6 years). 


\section{CHAPTER XXV \\ GENERAL PATHOLOGY OF EPITHELIAL TUMORS \\ PAPILLOMA; ADENOMA; CARCINOMA \\ PAPILLOMA}

From cutaneous, mucous, and serous surfaces local outgrowths of tissue composed of lining cells and subjacent connective tissue occur in many portions of the body, and in a wide variety of forms, to which the general term papilloma is applied.

A papillary structure is often observed in tumors of glandular organs which pursue the course of adenoma or carcinoma of these organs and are

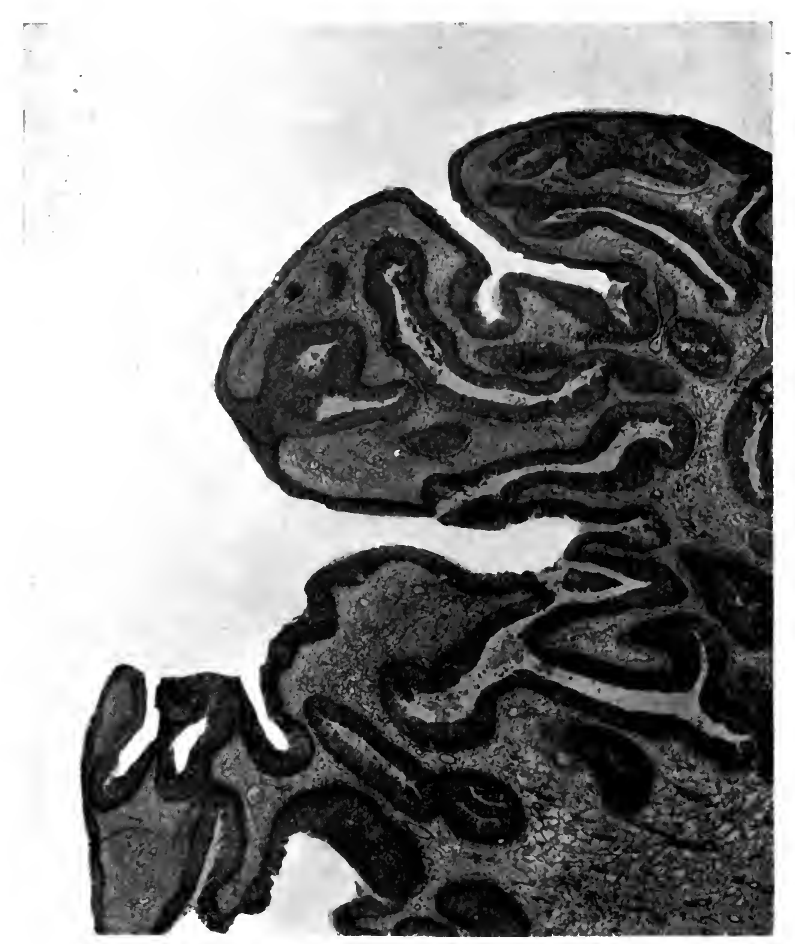

FIG. 193.-Topography of a fibro-epithelial papilloma of nares.

therefore properly designated as papillary adenoma or carcinoma. They usually develop in cysts of these organs where the conditions nearly duplicate those obtaining on free surfaces. Pronounced papillary tendencies appear in papillary adenoma of the ovary and in papillary fibroma of the breast, etc., which, however, are conveniently discussed with other adenomas and fibromas of these organs. 
In certain cases a somewhat specific structure, a characteristic clinical course and well-defined etiological factors, combine to render these processes more or less distinctive diseases and demand for them special consideration. Otherwise the term "papilloma" might well be abandoned or replaced by the adjective "papillary" attached to the particular inflammatory or tumor process concerned.

The form of the papilloma is chiefly determined by the local conditions surrounding the tumor. Outward growth of hyperplastic cells is in the line of least mechanical resistance. Irritation leading to overgrowth of surface epithelium, hyperemia, dilatation, and elongation of blood-vessels, and cellular or fluid exudate, give rise to a large series of inflammatory polyps, especially in mucous membranes, and these lesions pass not infrequently into true tumors.

With the papillomas of true neoplastic type it has long been questioned whether the initial process affects epithelium or connective tissue. No

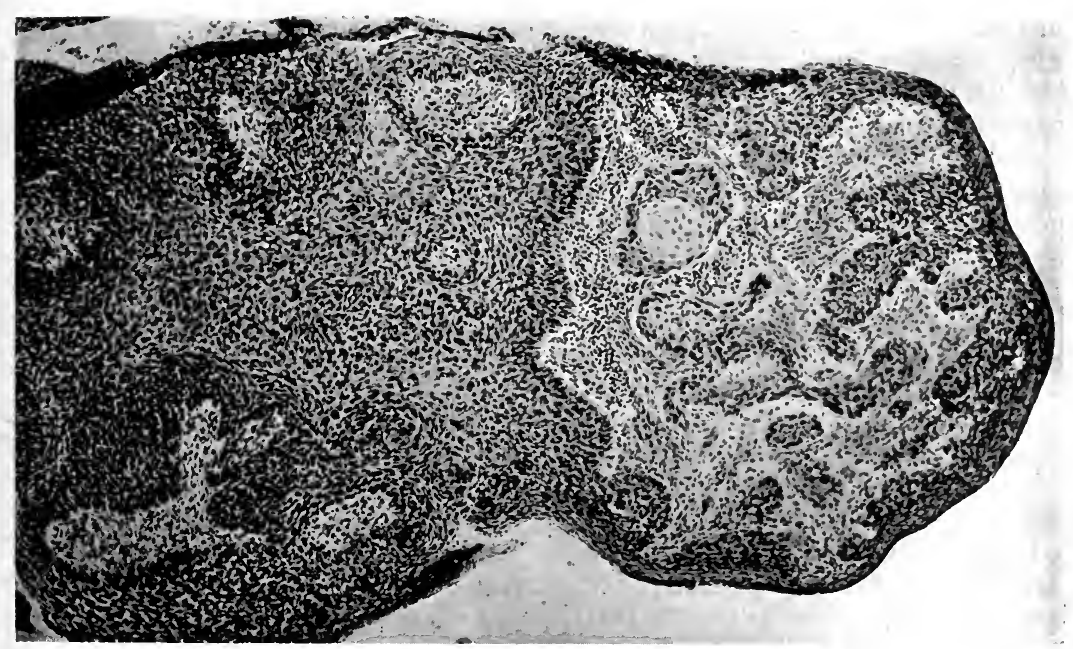

FIG. 194.-Malignant papillary epidermoid carcinoma of larynx.

single factor covers all cases. Growing epithelium reacts on the underlying connective tissue; projecting masses of stroma demand increase of epithelium for their covering; mechanical torsion gradually elongates an originally flat wart; and inflammatory changes modify the contained blood- and lymphvessels. Each of these elements becomes prominent in different papillomas, and several of them are so often combined that the bulk of a papilloma is usually far greater than would ordinarily result from any neoplastic element that may reside in it. In most neoplastic papillomas both epithelium and connective tissue exhibit the tumor tendency.

While the majority of specific papillomas affect cutaneous and mucous surfaces the same processes appear in the ducts and parenchyma of glands, and here the same caution must be exercised in judging of their nature.

The clinical course of papilloma is usually benign, and in most instances the growth capacities are limited. Exceptions to this rule result from persistence of exciting factors, progressive development of new tumors, and malignant changes. The natural history varies with each locality. In the 
skin the common wart has a transitory life, papillomas of the bladder and intestine are usually multiple, and in the larynx the papilloma is often malignant from its inception.

The development of clinical signs of malignancy is probably much more frequent than a corresponding change in the growth capacities of the tumor. Such a change may be simulated when an original carcinoma is associated with secondary simple papillomas on the surface or in the vicinity which mask the malignant character, or an originally malignant process may for a time present a simple papillomatous appearance. The first two conditions are exhibited in papillomatosis of the intestine and the last in some malignant papillomas of the larynx. Nevertheless the gradual transformation of a benign papilloma into a malignant tumor has been fully demonstrated in the cervix uteri, bladder, larynx, and other locations, and has usually followed the trauma of incomplete operation, or prolonged chronic inflammation.

In structure the papillary tumor may fall in one of four groups: (I) An inflammatory hyperplasia of epithelium, connective tissue, vessels, and tissue cells, which belongs in the field of chronic productive inflammation (nasal and uterine polyps, condyloma latum); (2) a simple overgrowth of histologically normal tissues, for which a congenital origin is probable; (3) overgrowth of atypical tissues of the type of adenoma (polypoid adenoma of mucous membranes); (4) distinctly atypical and malignant epithelial overgrowth with supporting stroma which is normal or neoplastic (papillary carcinoma of bladder, malignant papilloma of larynx).

All papillomas do not fall readily into these classes. It may be extremely difficult to determine whether a mucous polyp is purely inflammatory or partly neoplastic. Vesical papilloma often appears to be dependent upon chronic irritation, as of uric-acid crystals or of excretory products in anilin workers, but the resulting polyp is usually distinctly neoplastic. Hence the study of etiology may or may not assist in the classification. The bulk of a condyloma latum surpasses that of many papillary epidermoid carcinomas, and the hyperplastic epithelium of cervical polyps may exhibit every gradation from normal to adenomatous or carcinomatous cell types. In many instances the transformation of clinically benign into malignant growth is attended with few demonstrable changes except a softening of the underlying tissue and a downward heterotopia of exactly the same type of cell which formed the polyp. Abundant mitoses and a change in the type of the misplaced cells are to be regarded as suspicious features. Induration and fixation of the base are early signs of the desmoplastic process that accompanies beginning carcinoma.

Cutaneous Warts.-Verruca vulgaris, the common wart of the skin, is to be distinguished from papillary fibroma of the skin and from the papillary nevus. It is a specific form of epithelial overgrowth, is probably of inflammatory origin, and any neoplastic element it may possess reveals itself in ways peculiar to this process.

It occurs on exposed regions of the skin, especially on fingers, hands, and face. The duration is very variable, some disappearing after a few weeks, others persisting for years. A tendency to recurrence is often noted. The characteristic single discrete wart may become multiple, conglomerate, and occasionally bulky. Rarely, elongated forms develop with numerous thin papillæ. In young subjects very numerous small flat warts may appear on the backs of both hands (verruca plana juvenilis).

The structure exhibits an overgrowth of the entire Malpighian layer with folds and projections into which extend thin strands of stroma. In 
some cases there is considerable increase of blood-vessels approaching the type of the papillary nevus, and for these a congenital origin is probable.

There are numerous clinical observations suggesting the contagiousness of warts, but on analysis they can be dismissed as examples of the transfer

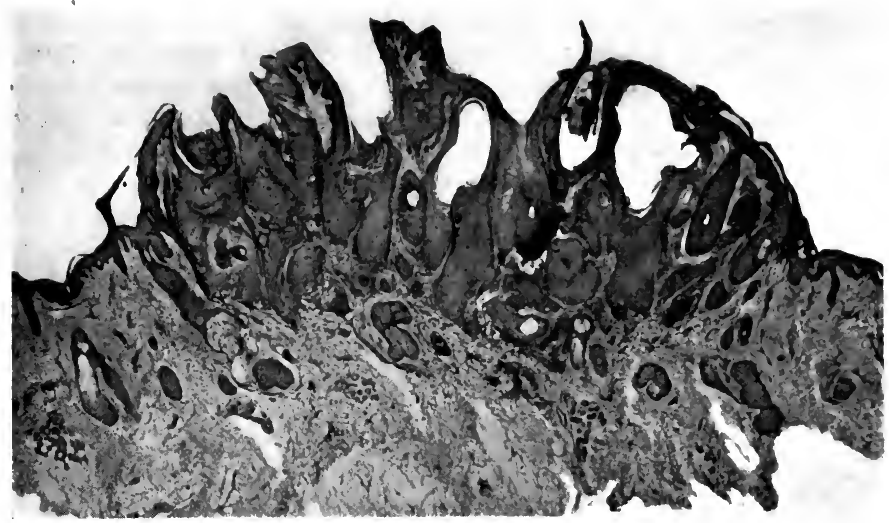

FIG. 195.-Topography of an epithelial papilloma with beginning epidermoid carcinoma

of peculiar chemical irritants, or exposure to the same irritant, or the actual transfer of proliferating epithelial cells. Payne saw three warts develop under the nail of his thumb which he had used to separate a wart from its

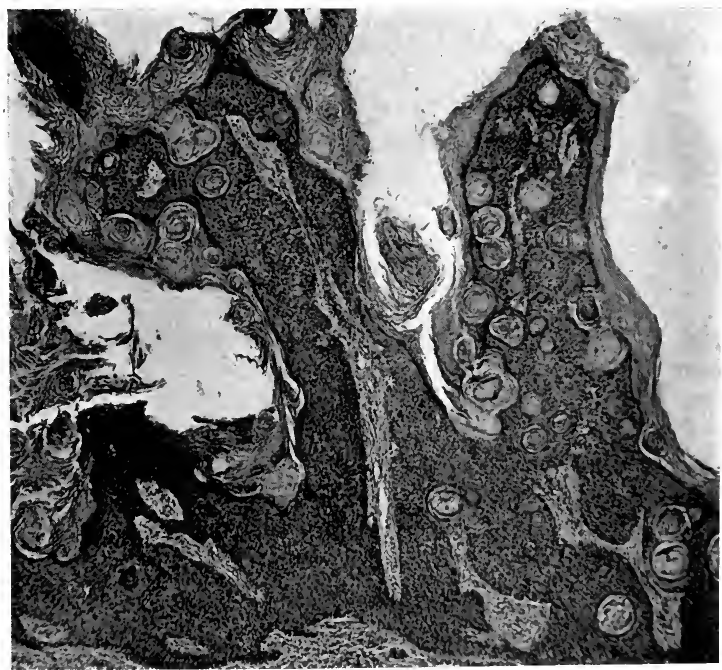

FIG. 196.- Structure of a malignant wart. The lesion was covered with many long papillæ and exfoliated many scales.

base. In many instances warts have developed on the skin of opposed surfaces. Lanz implanted a series of warts on the back of his gardener's hand by inserting the comminuted fragments under the epidermis. From 74 in- 
oculations from warts in 4 adults Judassohn secured in 2 to 6 months 33 warts. Leale successfully employed sections of warts to promote healing of ulcerated surfaces. The etiological importance of chemical irnitants appears in the cases where warts develop after contact with irritating substances. Many of the observations suggesting the contagiousness of cutaneous warts have been duplicated with gonorrheal condyloma and papilloma of the larynx.

Occasionally warts become soft, scaly, and inflamed, they develop atypical cell changes, numerous fine pearls form, invasion of the derma follows, and the process proves malignant.

Cornu cutaneum is a peculiar form of cutaneous wart marked by excessive and progressive keratosis. The cutaneous horn occurs chiefly on the scalp, but also on the face, eyelids, buttock, penis, and scrotum. Lebert collected I04 cases of general distribution. Wilson analyzed 90 cases. Pick reported Io cases of horns on the penis. Bötge observed 6 horns on the face of one patient. Multiple horny growths approach the character of the diffuse ichthyoses. They may reach a length of several inches. Rodriquez reported a horn of the scalp I4 inches in circumference.

The growth is slow but variable, and the course when uninterrupted continues for many years. Lebert found that carcinoma developed in I2 per cent. of his cases.

The growth begins as a wart on the normal skin or in scars. Some horns take origin in the epithelium of sebaceous cysts, a fact which suggests that the originating cells have a peculiar functional tendency to hornification. The structure presents elongated papillæ of the derma covered by lamellæ composed chiefly of hornified epithelium. The persistence of blood-vessels in very fine papillæ coursing through the horny material accounts for the extensive development of the greatly elongated forms. The chief oncological interest centers in the excessive development and persistence of the function of keratosis. The process may be interpreted as a tumor of horny substance (Dubreuilh).

Condyloma Acuminatum. - The pointed moist venereal wart occurs chiefly about the genitals, but also on the thighs and abdomen, while similar growths are occasionally seen in the axillæ and on the face. They are single, multiple or conglomerate, and while usually elongated, some multiple forms are small and low. They are composed of few or many villous projections or tufts which commonly spring from a single pedicle. They are usually moist and bathed in rancid, serous or mucinous fluid, the irritation of which is believed to be the cause of the overgrowth. Yet similar warts occur in positions which are free from moisture. While commonly associated with gonorrhea, they occur in children and the aged. In the irritating fluid many bacteria are found, among which are spirochetes sometimes in enormous numbers. The growth may be very rapid, especially in pregnancy, but they persist for long periods, and may reach a large size or cover a widening area. Hyde mentions a compound venereal wart of the penis in a negro which was as large as an orange. Contagiousness is often observed and must be referred to the transfer of peculiar irritating secretions and of the microörganisms which find in such fluids a suitable habitat.

The structure presents a central core which is more vascular than in other warts, and is covered by a variable thickness of epithelium in which hornification is imperfect or absent. Kuhneman finds the chief peculiarity in a thickening of the granular layer and an imperfect hornification which leaves the epithelial nuclei still visible in the superficial cells. Through this loose epithelial covering there is a slight constant exudation of serum. In the core of acuminate condylomas Herxheimer found varicose nerve-fibers. 
Condyloma latum is the broad, flat wart of syphilis, and occurs about the genitals and especially about the anus. This orifice may be surrounded and partially occluded by a luxuriant fungating mass several inches in diameter and markedly protuberant. The lesion may advance within the external sphincter and over a considerable portion of the buttock. It is subject to fissuring, infection, and ulceration, and in some cases a destructive form of carcinoma develops from it.

The structure shows an extensive overgrowth of the entire Malpighian layer which becomes greatly thickened and much convoluted. The cells are hypertrophic, but otherwise typical. Hornification may be defective. The connective-tissue papillæ are thickened, moderately elongated and bifurcated, and present the characteristic changes of secondary syphilis.

Molluscum contagiosum is a peculiar, contagious, and possibly infectious disease of the skin which produces multiple, wart-like, projecting nodules

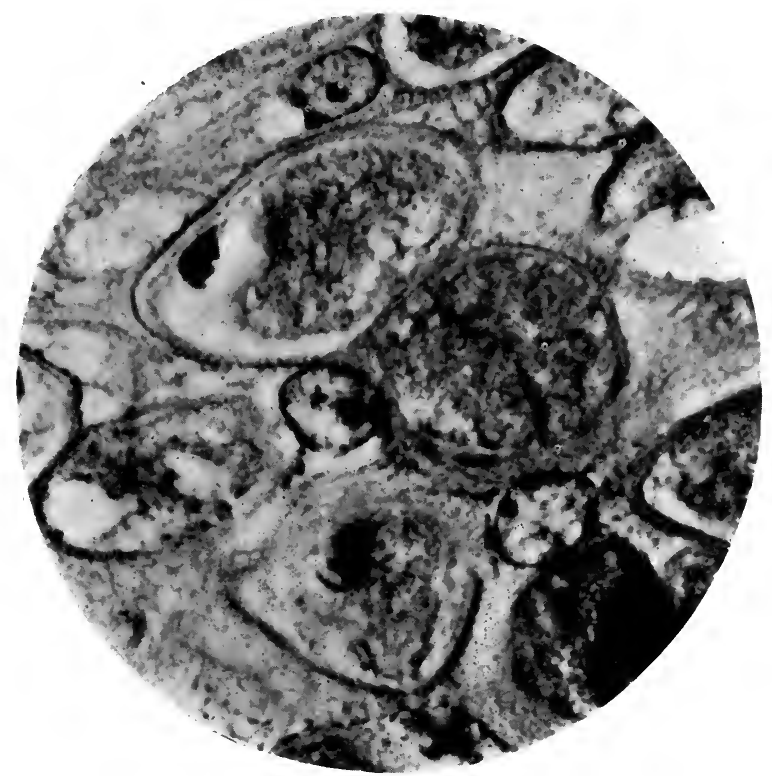

FIG. 197.-Intracellular bodies of molluscum contagiosum.

distributed over the hands, face, neck, or over much of the body. The disease appears chiefly in children, continues for months or years, but eventually subsides. A somewhat similar condition is observed in chickens.

The nodules are single or conglomerate, wax-like, or almost transparent, as large as a pea or bean, and usually present a central depression. On section they exhibit a series of swollen rete pegs composed of a moderately increased number of markedly hypertrophic swollen epithelial cells which produce convolution and projection of the Malpighian layer. Many of the lesions develop in the hair or sebaceous follicles of the skin (Kaposi). Between opposed layers there is considerable exfoliation of epithelium. The structure of the cells is quite peculiar. The cytoreticulum becomes thickened and coarse at the expense of material from the nucleus, and a large portion of the cytoplasm thus becomes converted into a well-defined reticulated struc- 
ture imperfectly separated from nucleus and cell-membrane and appearing as a foreign body. The nucleus eventually shrinks to one side of the cell. The variations in the minute structure of these molluscum bodies are quite numerous. They appear to form in much the same manner as the vaccine bodies of variola, or as the psorosperms of Darier, and similar structures appear in ordinary hyperkeratosis (Petersen). Lubarsch finds them in warts, secondary papillomas, and in various forms of epithelial hypertrophy. Many have assumed that the bodies are parasitic, but their minute analysis has failed to support this view (Kromayer). Juliusberg reports that the virus of human molluscum is filterable.

Papilloma of Tongue.-The lingual and buccal mucosa is frequently the seat of papillomas, which present peculiarities in structure, origin, and clinical course.

The papillomas occur on any portion of the tongue, affecting the lateral borders, under surface, and dorsum. The median raphe of the dorsum is a frequent site. Many are of congenital origin. Butlin mentions multiple papillomas of the fungiform papillæ, and bulky, warty outgrowths of the dorsum of tongue in infants. Riga's disease refers to multiple papillomas on either side of the frenum caused by irritation of coughing in pertussis. In adults papilloma arises in the middle line of the dorsum from a congenital basis, from leukoplakia and other forms of syphilis, from simple glossitis due to tobacco, and irritation of bad teeth. The etiology, therefore, almost exactly duplicates that of lingual carcinoma. The form is either acuminate or flat, and while some remain single and small, others become bulky or multiple. They may be painful and bleed, but ulceration points to a malignant process. In some cases a large portion of the tongue and floor of mouth may be covered by papillomatous growths and from various points multiple carcinomas may develop (diffuse buccal papillomatosis).

Lymphatic varicosity affecting the papillæ may produce a bulky, papillomatous growth of tongue or buccal mucosa (Robin-Leredde, Kaufmann, Rehn).

While some papillomas long remain benign and enjoy a limited growth there is a strong tendency toward malignant change which is indicated by increasing growth, induration, and fixation of the base, and ulceration. A large proportion of lingual carcinomas exhibits a brief preliminary papillomatous stage, and the more pronounced this character as a rule, the slower the progress of the disease.

The chief interest in lingual and buccal papilloma lies in the differential diagnosis from carcinoma. The former readily responds to radium or to simple excision of the lesion, and many successful operations for lingual carcinoma refer to simple papilloma or papilloma with precancerous or miniature carcinomatous changes. A careful microscopical study is therefore indicated before operation.

\section{ADENOMA}

Adenoma is an organoid tumor which reproduces the structure of a gland. The attempt to further define this neoplasm meets at once with limitations. A complete and typical adenoma reproduces epithelial alveoli, gland ducts, membrana propria, stroma and vessels, in a somewhat orderly and functionating organ, as in mamma and thyroid. More atypical adenomas are devoid of definite function, of differentiated ducts, of specific stroma, and of membrana propria, these features being lost in the order named.

Langhans and Lubarsch find a definite membrana propria in most adenomas, but not in all. Dreyfuss could find it only in traces in mammary 
adenoma, but he describes a proliferation of the cells of the membrana in certain cases, in which it is difficult to distinguish between sustentacular epithelium and stroma-cells. Hyaline thickening of the membrana is also observed.

In adenoma malignum the hyperplastic and hypertrophic cells maintain their polarity so that orderly but giant alveoli and glands constitute the tumor unit. Such a tumor is still an adenoma, preserving the gland type, and. the common suggestion to abandon the term "malignant adenoma" seems ill-advised.

In gross appearance the adenomas are usually well circumscribed or encapsulated tumors which do not infiltrate the surrounding tissue as do carcinomas. They are well nourished by blood-vessels running in a sufficient stroma and are therefore not prone to necrosis. On superficial surfaces they often produce polypoid tumors, and in the organs they frequently assume a

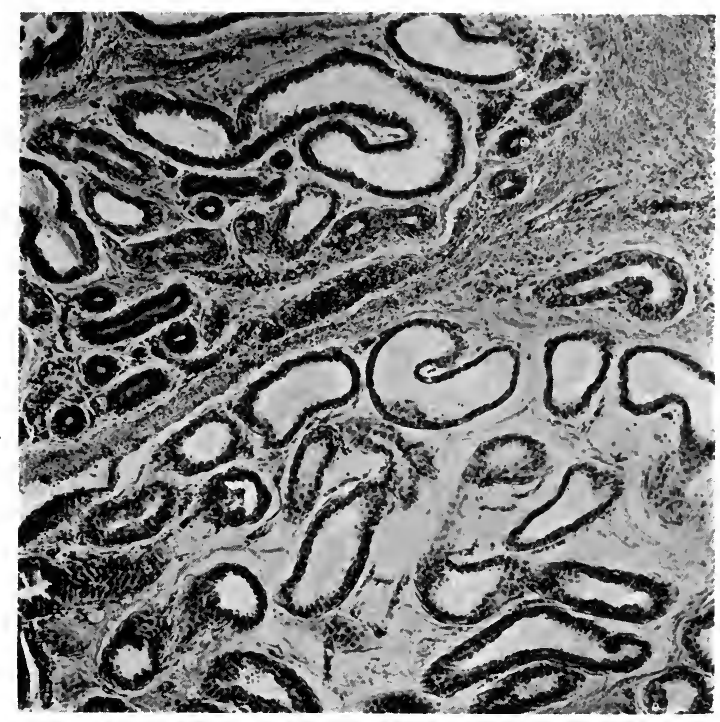

FIG. 198.-Adenoma of axillary sweat glands.

cystic or papillary character. While some are of miliary size and multiple, others are single and large, while a few by virtue of good nutrition or cyst formation reach voluminous dimensions.

Clinically true adenomas are usually slow of development and of limited growth capacity. Their disturbance of the organism is chiefly mechanical. The occurrence of metastases would lead many to separate the tumor from the group of adenomas, while others would classify such growths as a form of malignant adenoma. Most metastasizing simple adenomas exhibit areas of adenocarcinoma in some portions of the tumor.

Even in the benign metastasizing thyroid struma, which is the best example of simple adenoma yielding secondary tumors, many have claimed that the morphology is that of adenocarcinoma. The peculiar relations of thyroid and adrenal alveoli to the blood-vessels are exceptionally favorable for cell emboli from these growths. Encapsulation is a strong barrier against 
malignant change so that adenomas of embryonal origin long retain their type, while those arising on a basis of diffuse chronic inflammation or on mucous or serous surfaces more readily become malignant.

In judging of the probable course of an adenoma it is important to consider which organ is affected. Simple adenomas of the thyroid and stomach are frequently malignant, those of the rectum rarely.

While the study of single sections of an adenoma may fail to yield a correct estimate of malignancy, the finer histological details are usually of distinct prognostic significance. Uniformly atypical cell forms, nuclear hyperchromatism, abundance of mitoses, and reduplication of cell layers are commonly associated with dangerous types of adenomas, but the significance of these signs must be learned for each organ.

Functional capacity is retained by many adenomas; occasionally it is much increased, and probably in all cases the product is abnormal. Fatty material

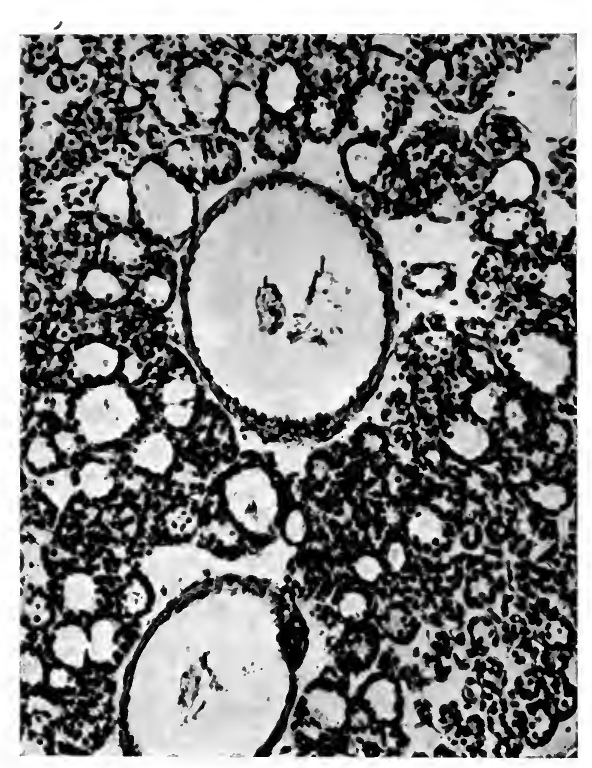

FIG. I99.- Structure of a thyroid tumor of frontal bone. Subject, male, 65 years. Thyroid gland apparently normal.

appears in adenoma sebaceum (Barlow) and in tumors of the breast. I have found rudimentary hairs in adenoma of the hair follicles. Overproduction of mucus is excessive in some ovarian tumors, but Schmidt regards this as a pathological activity not shared by the cells of origin, which do not secrete mucus. In gelatinous adenoma malignum of the colon the production of mucus may be enormous, even destroying portions of the tumor. Bile is often seen in adenoma of the liver (Schmidt, Lit.), and Marckwald found the secretion excessive. Thyroid adenomas occasionally contain colloid both in the main tumor and in heterotopic and metastatic growths (Wolfler). Schmidt points out that the structure of adenoma or adenocarcinoma is regularly assumed in functionating malignant tumors of thyroid and liver. Early adrenal adenomas contain much lipoid material, but the formation of adrenalin in such growths has not, I believe, been demonstrated. Yet some adrenal 
adenomas are extensively pigmented, and in a well-known group remarkable hirsuties and sexual precocity point to disturbed or excessive functional activity.

Contrasted with this group of functionating adenomas stands another, chiefly of embryonal origin, in which there is a sharp suppression of function. Hence it appears that function in adenomas represents an acquired character of the cells of origin and that it is not an essential feature of this tumor group.

Mode of Growth. - In adenoma the neoplastic process resides either in the gland-cells alone or in both cells and stroma. In the former case highly cellular tumors develop, in the latter fibro-adenomas. The original impulse is probably always located in the epithelium which exerts a formative influence over the connective-tissue structures. Yet in some adenomas the growth appears to begin in both elements simultaneously, and occasionally the stroma overgrows the glands. The vessels may also become extremely prominent. The key to the morphology of most adenomas is to be sought in the course of the normal embryonal development of the gland, which is repeated in the tumor (Ribbert). Yet many secondary factors, as mechanical pressure, retention of secretion, and overgrowth of stroma, modify or dominate the structure.

Tubular adenoma results chiefly from elongation of the affected gland, as in the colon, but the same process may give rise to an alveolar appearance in cross-sections of folded and twisted glands. White cut serial sections of a lobule of mammary adenoma, finding that all the alveoli represented a single tubule entering the lobule from the main tumor. Saccular or follicular adenoma results from the formation of lateral sacculi from original glands. This process occurs in many organs, but is best seen in some adenomas of the breast and thyroid. In serial sections of a rectal polypoid adenoma White found lateral sacculi, communications between adjoining acini, and multiplication of surface openings.

Papillary cystic adenoma is a form assumed by many adenomas in which with or without retained secretion the original alveoli are distended by fluid or by papillary outgrowths of epithelium and connective tissue. There has been much discussion of the mechanism which causes the distention in cystic adenoma (Dreyfuss, Lit.). Both retention of secretion and primary overgrowth of epithelium and connective tissue with secondary enlargement of the alveolus are concerned in the result. Fusion of neighboring alveoli is also a factor, while serous and bloody exudates and extravasations may cause rapid enlargements of cystic tumors. Paget divided cystic tumors according to the prominence of these processes. Rokitansky regarded the true cystic adenoma as a structure tending essentially to grow in cystic form, and Dreyfuss emphasizes the importance of proliferation of the membrana cells as leading to a widened lumen. He found the ducts patent in some cystic adenomas of the breast and the smaller alveoli empty. It has often been urged that pressure alone causes atrophy rather than overgrowth of lining cells.

Structure.-Adenoma malignum maintains the alveolar structure and the polarity of the lining cells, but early loses ducts and specific membrana propria, while the stroma is deficient or absent. The alveolar structure may be rigidly maintained even in metastases, but the cells are usually hypertrophic and atypical, and the nuclei hyperchromatic. In this form the tumor has infiltrative capacity and produces metastases. Adenoma malignum often passes into adenocarcinoma.

Morphological distinctions between benign adenoma and adenoma malignum vary in different organs. Adenoma of the thyroid may yield metastases 
of quite typical form. In an interscapular metastasis of gastric adenoma I found regular alveoli lined by a single row of normal-sized cuboidal cells with slight nuclear hyperchromatism. In a splenic metastasis from adenoma of the corpus uteri the alveoli were more typical than in the uterus, but the cells were twice as large as the columnar cervical epithelium. Hence the statement is correct, that the alveoli of adenoma malignum may not differ from those of ordinary adenoma or of glandular hyperplasia. As a rule, however, the alveoli of adenoma malignum are atypical, the cells larger, the nuclei more hyperchromatic, the cell layers are multiplied, secondary alveoli form, quite atypical cells begin to appear, and there is an entire absence of membrana propria. For each organ a special standard is needed in estimating the grade of anaplasia and malignancy. The relation of glands and stroma is also of much importance. Borst emphasizes the promiscuous distribution of glands and stroma in malignant adenoma.

Adenocarcinoma is a tumor of gland-cells resembling adenoma, but failing to completely preserve any of the normal features of the gland. The orderly alveolus is the essential element of an adenoma, but in adenocarcinoma even the alveolus is imperfectly formed and the epithelial cells begin to lose their polarity. Markedly atypical cell morphology and extensive overgrowth are also characters of adenocarcinoma, and this tumor may lose all adenomatous characters and pass into carcinoma. Yet many infiltrating and highly malignant tumors exhibit numerous traces of alveolar formation.

The attempt to classify adenomas on purely morphological features is quite unsatisfactory. In many cases tumors which show simple adenomatous structures in one portion exhibit adenocarcinomatous areas in another, and pure carcinomatous infiltration in still other areas. This instability of the adenomatous structure is more notable in some organs than in others, but is a rather general characteristic of many adenomas.

While the tendency is usually toward greater anaplasia with continued growth, occasionally the metastases of an adenocarcinoma are more distinctly adenomatous than the original tumor. Apolant was able to transform an adenocarcinoma of the mouse into an adenoma by transplantation in partially immune animals. The study of many adenocarcinomas indicates that the pure adenomatous structure may represent an intermediate or transitory stage in the evolution of the tumor process which is rapidly traversed and soon passes into a more atypical carcinomatous phase.

There may be considerable or pronounced variation in the structural type of the adenoma in the same tumor. Thus in an adenocarcinoma of the prostate I have found hypertrophied alveoli recalling adenoma malignum; many small alveoli resembling simple tubular adenoma; distended tubules filled with neoplastic papillary ingrowths; as well as fibrocarcinoma. Clinically this tumor pursued the course of carcinoma.

Accordingly, if the term "adenoma" is to enjoy any great pathological significance, its application must be made with due reference to the gross anatomical and the clinical features of the disease. From this standpoint there are still many tumors which tend to maintain the morphology of adenoma, with some persistence of the function of the originating gland, and pursue a clinical course which is less malignant and destructive than that of carcinomas of the same organ.

Examples of this true adenoma in the general biological sense are those of the cutaneous glands, fibro-adenoma of the breast, follicular adenoma of thyroid, adrenal adenoma, the adenoma of the islands of Langerhans, Kaufmann's adenoma of the testis, and some adenomas, chiefly papillary, of different organs. All of these tumors arise under somewhat peculiar conditions, 
exhibit little morphological variation, and run a uniform and commonly benign clinical course.

Physiological Conception.-When one considers the great variety of conditions in which an adenomatous morphology is presented and analyzes their physiological and clinical significance, the conclusion must be reached that adenoma is rarely a distinct morphological entity, but is rather to be regarded as a series of structures which tissues assume in response to various forms of irritation, functional activity, and overgrowth. It is chiefly, if not exclusively, the adenomas of embryonal origin which fail to show this complex significance. It were better, therefore, in most instances to avoid the rigid morphological conception of adenoma and to regard the diseases marked by adenomatous hyperplasia as specific clinical and pathological entities. The justification of this view will appear in dealing with some of the many forms of adenomatoid and adenomatous hyperplasia.

Most authors distinguish sharply between true adenoma and adenomatous hyperplasia of organs and tissues. It is highly desirable that the distinction should be made wherever possible, but it cannot always be accomplished on morphological data alone. It is usually necessary to consider the conditions under which the abnormal process develops and its clinical course. In the extensive group of adenomatoid hyperplasias it is often impossible to distinguish the morphology from that of adenoma of other types, but reference to the clinical data reveals that the process is dependent on chronic inflammation and tends to run a self-limiting course. In the thyroid and hypophysis adenomatoid changes result from functional hyperplasia. In many situations adenomatoid hyperplasia becomes progressive and passes into a neoplastic process and when this stage is reached the conditions exhibit the same potentialities as other adenomas. In intestinal polyposis different portions of the intestine exhibit peculiar inflammatory overgrowth, adenomatoid hyperplasia, adenoma, and even carcinoma.

The adenoma arising under such conditions may differ in some respects from other adenomas, but still deserves recognition as a neoplasm. All of these considerations, which apply equally to adenoma and carcinoma, urge the necessity of regarding these tumors from the biological or clinical standpoint rather than from the morphological side alone.

On physiological analysis adenomas are found to represent several different processes, and from this standpoint the interpretation of the mode of origin, structure, and significance is most satisfactory.

Thus some adenomas are traceable to a localized inflammatory overgrowth. Others represent chiefly an excessive response to functional stimulus. Some appear to owe their existence to minor anatomical disturbances in the blood-supply or configuration of the organ. Many true adenomas represent pronounced neoplastic growth of definitely misplaced and embryonal portions of gland tissue. Probably more than one of these factors may be combined in origin of the same adenoma.

Inflammatory adenomatoid hyperplasia is of wide-spread occurrence.

In the mucous membranes of the gastro-intestinal tract, uterus, and nares it produces diffuse overgrowth or single or multiple polypoid excrescences which constitute definite clinical entities, and it is a feature of the late stages of chronic catarrhal inflammation of these tissues. The process affects both glands and stroma. The glands become hypertrophic or cystic and the lining cells are increased in size and number. There is usually increased and abnormal secretion. The stroma shows new and tortuous blood- and lymph-vessels, multiplication of stroma-cells, atrophy of lymph-follicles, and perivascular infiltration by lymphocytes. The process tends first to produce a diffuse 
thickening, but focal overgrowth of the tissue, edema, venous stasis, and mechanical traction lead to papillary and polypoid outgrowths which may become numerous and bulky. In this condition there is a tendency to further overgrowth of the predominating element, glands, stroma, or vessels. In the nares the mucous polyps show wide variations in structure, some being glandular, others myxomatoid, other angiomatous, but the tendency to true tumor growth is slight.

In gastritis polyposa the development of polypoid outgrowths may reach an astonishing grade, covering most of the mucosa and leading to dilatation of the organ.

In colitis polyposa the entire colon from cecum to anus may be the seat of myriads of small or pedunculated outgrowths, the pedicle may be elongated and ruptured and the polyp discharged through the anus. The ileum or jejunum is rarely affected, but Kaufmann and Hauser saw involvement of the entire mucosa from cardia and pylorus to anus, and lesions in duodenum, jejunum, and ileum are reported by Lubarsch, Niemarck, and Petrow. Peculiar catarrhal symptoms mark the onset of the disease, and hemorrhage, anemia, and emaciation, intussusception, and prolapse occur in advanced cases (Virchow, Luschka, Hauser, Schwab, Smoler). The condition affects both old and young subjects and exhibits an hereditary, individual, and local predisposition (Cripps, Smith, Bickerstedt, Port, Verse). In the early stages I find one of the earliest changes is a pronounced hypertrophy of the epithelial cells, a tendency which Verse regarded as the sole element in the local predisposition. Although many cases maintain their inflammatory character throughout there is a striking tendency toward the development of malignant adenoma and carcinoma. According to Quénu and Landel about half the cases are associated with carcinoma, while Hauser found this combination as the rule, and Doering, who analyzed 50 cases, found 37 fatalities, 3I from carcinoma. There is probably no other tissue which illustrates so clearly the transition of an inflammatory overgrowth into a malignant tumor.

Hypertrophic Endometritis.-Fungous or glandular endometritis.

In a somewhat limited group of cases associated with myoma, carcinoma, misplacement of the uterus, or disease of the adnexa, the uterine mucosa becomes much thickened $(0.5$ to $\mathrm{I} \mathrm{cm}$.), very vascular, prone to hemorrhage, cystic, and covered with papillary or polypoid projections. There is extensive overgrowth of large, irregular, compact, or dilated glands. Infoldings of the cell lining may yield one or two epithelial rings within the lumina on section. The cells are considerably enlarged, nuclei hypertrophic and often in mitosis, and the layers may be multiple. In a series of cases every gradation may be observed from normal glands to those of adenoma malignum, and one often finds single glands outstripping the others in this transition. New growth of interstitial tissue, with large flat decidua-like cells, lymphocytes, and plasma-cells may appear, especially in the polypoid projections (endometritis interstitialis). Hitschman and Adler have shown that many cases of so-called glandular endometritis represent merely the cyclic changes of menstruation and they deny the existence of any but an interstitial endometritis. Yet the occasional occurrence of definite conditions of the type described is fully attested and the origin must be attributed to the usual factors connected with chronic productive inflammation of mucous membranes, to which is here added the influence of tumors and inflammations in the uterus and adjoining tissues (Weishaupt-Schickele).

That chronic glandular endometritis may pass into adenoma is strongly indicated by its frequent occurrence with myoma which nearly always shows glandular hypertrophy and often adenoma, and by the histological evidence 
of the transition. The malignant change seems to occur suddenly and affects first single glands, or larger areas. It is marked by striking increase in the size of the epithelial cells, pronounced hyperchromatism, atypical mitoses, multiplication of cell layers, and reduction of stroma. These changes are pronounced and unequivocal when the diagnosis of adenoma is justified, and it is only in rare instances that the decision remains doubtful. When the adenoma is sufficiently established to reach the surgeon all traces of the original hyperplasia may, as Heurlin found, be obliterated.

Prostatic Hypertrophy.-In the prostate inflammatory overgrowth is responsible for the great majority of cases of chronic prostatic enlargement $(q . v$.$) . Here the results are peculiar, depending on the structure and func-$ tions of the organ and the changes must be referred not exclusively to the inflammatory factors but also to secondary disturbances of nutrition and function. In fact, a primary disturbance in the function of the prostate may possibly prove to be an essential factor in the causation of hypertrophy, since most cases of active prostatitis tend toward atrophy of the organ. The inflammatory process begins about the ducts, retains a periductal character throughout, and causes more or less new growth of fibromuscular stroma about the systems of alveoli. The nearest parallel to this condition is seen in chronic mastitis. The overgrowth of epithelium is complicated by stasis of secretion which may produce small cysts with relatively atrophic epithelium. Or the epithelium is more active and lateral sacculations and papillary projections form. Ciechanowski emphasizes the absence of any new glands in prostatic hypertrophy, but in many cases focal adenomatous areas appear and the process passes into adenoma or carcinoma. In other cases the natural tendency is toward fibrosis and atrophy.

Many examples of functional or congenital overgrowth of gland tissue should be separated from true adenoma, although no sharp line divides these conditions from true tumors, and in many instances the one passes into the other.

(I) Functional Adenomatoid Hyperplasia.-In the breast, hypertrophy, which is chiefly of functional significance, produces extensive enlargement of both organs, at first of slight degree, but later, often at puberty, increasing rapidly until both organs become very bulky (30 kg., Kaufman). The condition may remain stationary in this stage or may increase during gestation and enormous amounts of very fatty milk are secreted. The structure shows uniform increase of cellular connective tissue and of hypertrophied gland lobules, but there are many variations in the proportions of these elements. In some cases the structure strongly resembles a true fibro-adenoma. The series of cases analyzed by Voges and by Kirchheim illustrate many peculiarities of the process, including an hereditary element, involvement of one breast or both, or of one or two supernumerary organs, its appearance before puberty or in the eighth gestation, after amenorrhea and with other ovarian disturbances, regression partial or complete after pregnancy, marked variations in structure, and frequent occurrence of pain and emaciation. The condition is best interpreted as an excessive response to physiological, functional, and formative stimulus in congenitally predisposed organs.

(2) Congenital hypertrophy of thyroid is a rare condition observed in infants. The organ is uniformly enlarged to twice or three times its normal dimensions. The structure shows increase in number of acini and hypertrophy of lining cells, with absence of colloid. It may cause early death by pressure on the trachea and larynx (Kamann, Fabre-Therenot).

The adult thyroid in Graves' disease presents the most striking example of adenomatoid functional hyperplasia. The gland becomes diffusely enlarged, many new alveoli form, colloid disappears, cysts with papillary pro- 
jections develop, and atypical alveoli and giant-cells may form. Yet in man this condition rarely leads to a true neoplasm. At the acme of the process the cellular overgrowth reaches a limit and becomes stationary, or is overtaken by atrophy and by fibrosis of stroma or interstitial thyroiditis (Ewing, Lit.). In the fish this form of hyperplasia sometimes results in infiltrative growth and a true benign or malignant tumor (Gaylord).

Simple goiter presents less pronounced features of adenomatoid hyperplasia. In the so-called parenchymatous struma the cellular overgrowth is extensive, but orderly and typical. New follicles form by budding from the old, following the normal course of growth of the thyroid (Wölfler). A somewhat similar picture may be produced experimentally in dogs by administration of iodin, while a restoration to the normal structure may be secured by adjusting the diet. This instability of structure and the ready

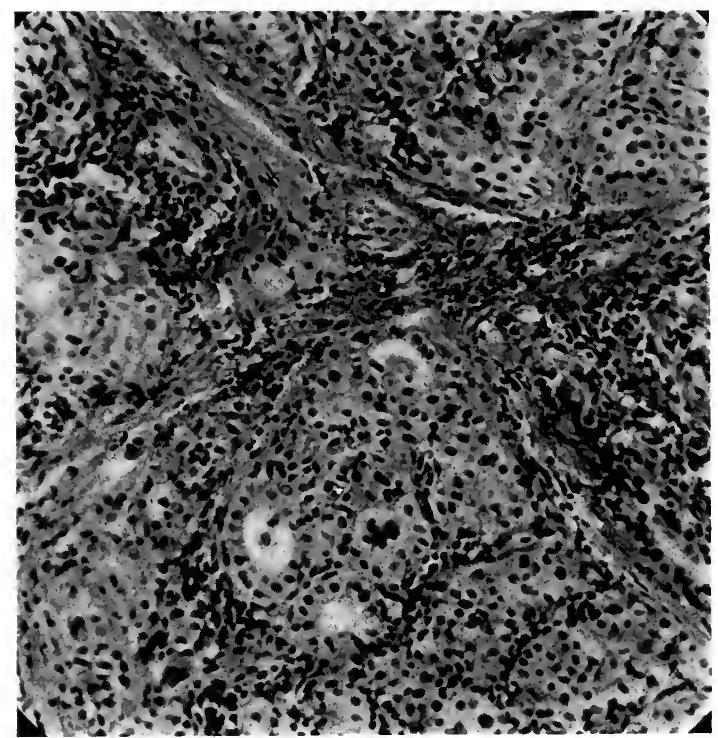

FIG. 200.--Adenomatoid hyperplasia of thyroid gland in Graves' disease.

response to diet and drugs assures that the hyperplasia is in no sense neoplastic (Kocher, Marine).

In colloid struma there is abundance or excess of colloid in and about alveoli and the thyroid may become very large from its accumulation. Colloid cysts form from the fusion of distended alveoli and hemorrhage may occur in these cysts. Overdevelopment of blood-vessels is frequently seen. In the late stages there is fibrosis, softening of cyst contents, atrophy of alveoli, arteriosclerosis, and calcification. All of these changes may occur in an isolated portion of the thyroid producing a tumor-like enlargement. These cases suggest an imperfect alignment of a portion of tissue in the gland with abnormality of blood-supply and possibly retention of embryonal characters.

Nodular hyperplasias of more definite neoplastic character occur in both simple and Graves' goiter, and it is probable that both conditions favor the development of true adenoma and carcinoma. 
Struma Hypophysis.-Nodular or diffuse hyperplasia of the hypophysis is frequently observed in subjects over 35 years of age (Lowenstein, Lit.). Whether these conditions, commonly called adenoma, as well as some of the more pronounced overgrowths associated with acromegaly, etc., are true tumors or functional hyperplasias still remains to be determined. It is probable that many definite adenomas and carcinomas arise from such hypertrophies.

Struma Adrenalis.-Nodular and diffuse hyperplasia of the adrenal is frequently observed. Either cortex or medulla or both may be affected. Between the nodular hypertrophies and true adenomas there appears to be every transition.

Adenoma Sebaceum.- In the sebaceous glands there is a form of overgrowth attended by oversecretion and marked hypertrophy and multiplication of glands which may approach a neoplastic grade (Balzer, Menetrier). The disease occurs chiefly in epileptics and the mentally defective (Crocker) and is usually limited to the skin of the face. In some cases the long duration, diffuse distribution, the orderly character of the cell growth, and the self-limitation indicate a form of functional overgrowth (Caspary's case). Here there is extensive multiplication of large sebaceous glands and proliferation of the cells of the hair-follicles. The epidermis may be thickened and the small vessels dilated. In other cases there is extensive hyperplasia of gland-cells and the lesion resembles a reticulated epithelioma, or adenoma of sebaceous and hair-follicles (Barlow). While usually self-limiting, these lesions are doubtless the source of some multiple epitheliomas of the skin.

The liver and kidney are especially susceptible to focal adenomatoid hyperplasia as a result of regenerative overgrowth. The process may be localized or general. It occurs at all ages, in organs which apparently were normal, in those which were recently the seat of acute degenerative processes, and in many which are the seat of advanced cirrhosis. The hyperplastic nodules are multiple and miliary or solitary and bulky. In young subjects nearly the whole liver may be replaced by adenomatoid nodules. While in many instances the conditions never pass beyond a physiological stage and are found at death from other causes, a variety of somewhat specific tumors take origin in the hyperplastic foci. Their relation to tumors will be considered in detail in connection with the tumors of these organs.

\section{CARCINOMA}

Carcinoma is a tumor process characterized by atypical and destructive proliferation of epithelium.

By emphasizing the atypical morphology this definition separates carcinoma from some destructive adenomatous processes, while recognition of the destructive element sufficiently denotes important physiological and clinical features. From the structural standpoint emphasis may also be laid on the equilibrium between epithelium and connective tissue which in papilloma and adenoma remains more or less intact, while in carcinoma it is lost. Better than any rigid definition is the conception that carcinoma represents more lawless overgrowth than adenoma and leads to a great variety of neoplastic structures which show variable but pronounced grades of anaplasia.

Carcinoma shares with sarcoma the full contrast over against inflammation. This contrast justifies Virchow's general division of tissue changes into three groups, normal growth and physiological changes, inflammation, and neoplasia. Malignant tumors reveal this contrast in the fullest light, but since carcinoma may result from excess of growth energy or from inflam- 
matory hyperplasia there is no wide gap between these processes. This admission does not allow that the processes are essentially the same. With every addition in quantity there is a change in quality. The rate, vigor, and extent of epithelial overgrowth may steadily increase over the normal or over inflammatory hyperplasia, until finally the process expresses itself as carcinoma. There is no necessary change in the nature of the epithelial cell. Hence Ribbert states that there is no such thing as a malignant cell endowed with new biological properties. It is only the process as a whole and its intensity which are new.

It is necessary to recognize two great groups of carcinomas: (I) those arising from misplaced portions of embryonal tissue and (2) those arising from previously normal and adult cells through the influence of chronic irritation.

The latter tumors exhibit many points of similarity with chronic inflammation, in that the etiological factors are very diverse, the structural types

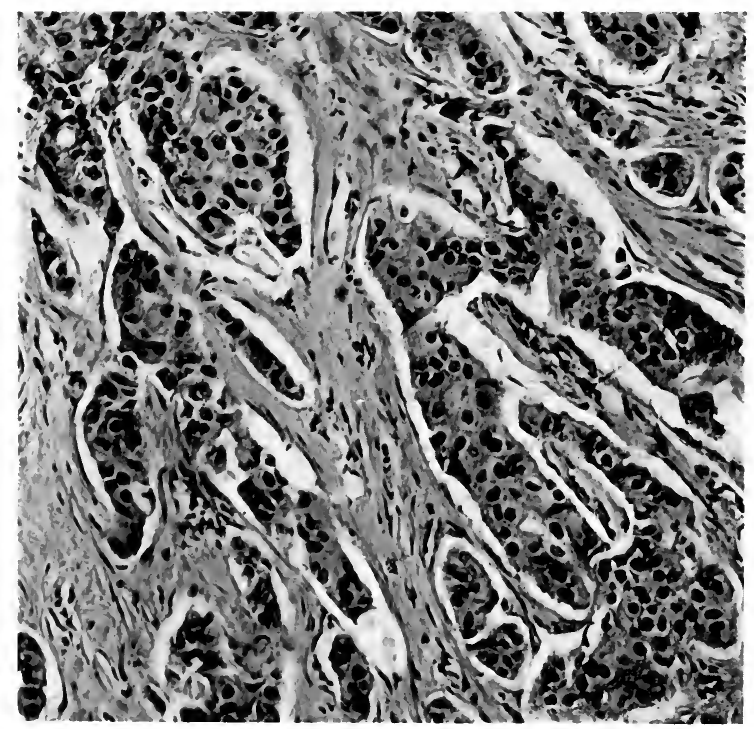

FIG. 20I.-Mammary cancer. Carcinoma simplex. Fully developed carcinoma. Infiltrative growth of atypical cells, with desmoplastic properties.

very numerous and the clinical courses very different, so that they constitute so many different diseases which have in common only the fact that the underlying tissue change is a malignant neoplasm.

For very few general characters of great significance may be stated of the cancer process. Chief among these is its progressive and fatal course, to which there are exceptions. It is much more significant to know the peculiar conditions under which a carcinoma arises, the particular features in its gross and microscopical anatomy, its distinguishing clinical signs, and natural duration. Only in this way can the knowledge of carcinomas become theoretically sound and practically efficient. Hence one may conceive of carcinoma as a vast group of clinical and pathological entities connected only as malignant epithelial neoplasms of extremely varied morphology. 
Gross Anatomy.- - The gross appearance of carcinoma is largely dependent on the tissue of origin and to a considerable extent on its structure, while wide variations are observed in the same and in different organs.

The classical appearance of advanced mammary carcinoma with its central firm mass and many lateral extensions suggested to Galen the resemblance to a crab and led to the designation as cancer.

Induration of the affected tissue is probably the most constant and significant single feature of large and even of miniature carcinomas, and is referable to the desmoplastic property of carcinoma. It is often of very great diagnostic significance, surpassing at times the indications drawn from microscopic structure. The induration of carcinoma is a peculiar physical quality owing to its extreme density, and its presence in suspicious lesions must stand as a strong indication of carcinoma. It is especially to be distinguished from the less resistant and more diffuse induration of chronic productive inflammation.

Associated with certain degenerative changes in the carcinoma cells, as exposed by section through the tumor, induration and fatty degeneration become quite specific of carcinoma. In carcinomatous nodules of the breast, in early carcinoma of lip, tongue, and other mucous membranes, in stenosing carcinomas of stomach and rectum, a translucent matrix of new connective tissue streaked with silvery points and lines of fatty epithelium are absolutely specific of carcinoma. The silvery points lack the deep yellow color of included fat.

Cross-section also reveals the cicatricial character of many carcinomas. The surrounding tissue appears to be drawn toward the carcinomatous center, which may be sharply demarcated from the surrounding tissue, but not encapsulated.

The nodule or mass is often fringed with fine extensions, or from it pass many coarse cicatricial bands through which the tumor-cells are advancing. Induration also marks the beginning of malignant change in the bases of polypoid tumors which thus become fixed to the supporting tissues. It marks the beginning of deep infiltration from superficial and ulcerating carcinomas of mucous membranes.

Induration is a common but alone is not a safe criterion in judging of the nature of enlargements of lymph-nodes. The encapsulation of these small organs permits very marked density to be displayed by inflammatory changes within the nodes. Here fixation is a most important sign of carcinoma. In scirrhous carcinoma induration begins early and continues prominent throughout the process, producing tumor masses of uniform and extreme density. The lesion is usually cicatricial in character, deforming the affected organs, as in the breast, or causing extreme stenosis, as in pylorus or intestine. Yet in some cases a tendency to hyaline swelling of the fibrous tissue limits the contraction or even increases the bulk of the tumor. In cancer en cuirasse a progressive fibrosis accompanies the wide extension of mammary carcinoma over chest, back, neck, and even abdomen, and surrounds the body in a rigid encasement which occludes lymph- and blood-vessels and greatly hampers respiration.

Marked induration usually indicates a slow progress of the local lesion, but very often such quiescent fibrocarcinomas become the source of actively growing and cellular tumors in the neighborhood or at a distance.

Very cellular carcinomas fail to exhibit marked induration, but appear as opaque, whitish nodules or masses presenting a rounded or convoluted border, a peripheral ring of congestion or hemorrhage marking the growing edge, a broad intermediate zone of tumor tissue, and often a central depressed cicatricial area. Secondary cellular carcinoma of this type may be difficult 
to distinguish from massive tuberculosis or syphilis, but the latter lesions usually exhibit more necrosis and less fibrosis than carcinoma. The terms "encephaloid" or "medullary cancer" are often applied to the soft cellular tumors, from a fancied resemblance to brain tissue.

Papillary and adenoid carcinomas usually appear as bulky circumscribed masses which are fragile, crumbly, and of wholly different appearance and consistence as compared with the involved organ. Minute inspection frequently reveals specific markings which give a clue to the microscopical structure. The entire group of papillary carcinomas may usually be recognized by the circumscribed character of the conglomerate nodules and the branching opaque cords of cells. Papillary and adenoid carcinomas are usually vascular and subject to interstitial hemorrhages and necrosis which often lend characteristic gross features.

Many cystic tumors are of carcinomatous structure. The tumor may originate by papillary growth into an enlarging gland-duct or alveolus, and while the tumor remains encapsulated the carcinomatous process remains confined and comparatively harmless. Or the cysts form in previously solid tumors by accumulation of secretion, edema, hemorrhage, or liquefaction necrosis.

Specific degenerative changes are sometimes highly characteristic of certain glandular carcinomas. Chief among these is the excessive mucus production of gelatinous carcinoma of the gastro-intestinal tract, peritoneum, ovary, breast, and other organs, which tends to cause rapid increase in the bulk of the tumor while actually retarding the proliferation of tumor-cells. Yet such tumors may prove highly malignant, since the distention of tissue spaces by the mucus facilitates the dissemination of the surviving cells. The entire abdominal cavity may be distended with mucinous material in colloid cancer of the intestine, or the entire stomach wall may be thickened and honeycombed by such growth. Psendomyxoma peritonei is a form of diffuse implantation tumor arising from mucinous ovarian cancer, in which an originally carcinomatous process becomes greatly altered by a peculiar inflammatory process about masses of mucus.

Calcific changes are relatively uncommon and belong chiefly to the regressive processes. There may be extensive calcification of the pearls of epidermoid carcinoma, and of atrophic portions of rodent ulcer, or in old necrosed areas of glandular carcinoma. In psammocarcinoma of the ovary, parotid, or kidney, actively growing portions of the tumor may present an enormous number of dense sand grains derived from epithelial concretions which seem not to inhibit the progress of the growth. In a form of cylindroma of the skin, growth terminates in diffuse calcification. In many cases the walls of blood-ressels and areas of stroma undergo hyaline transformation followed by calcification, and leading to extensive atrophy of the tumor parenchyma.

Extensive calcific deposits in the organs form a remarkable feature of the metabolic disturbance in rare cases of carcinoma. Thus in a case of mammary cancer Elser found the walls of the pulmonary air-vesicles calcified over wide areas.

Miliary carcinosis is a gross anatomical form of the disease presented by highly malignant and widely disseminated processes. Here the miliary tumor nodules appear widely scattered over serous membranes, peritoneum, pleura or meninges, or extremely numerous foci appear in skin, muscles, or organs, having traveled through the lymphatics or blood-vessels.

Ulcerative lesions commonly result when carcinoma affects cutaneous or mucous surfaces. The characters of the carcinomatous ulcer vary with 
the location and form of the disease. When primary they are usually single. The position is determined by the mucocutaneous junctions or by exposure to irritation. The base is indurated and eventually fixed to the tissues. The secretion is scanty, purulent, bloody, subject to increase from infection, and complicated by hemorrhages from eroded vessels. The edges are raised, hard, usually not undermined, but rigid and opaque. The vicinity shows no essential change, but may be secondarily invaded. The regional lymphnodes are enlarged first by inflammatory changes, later by carcinomatous invasion.

Rodent ulcer rarely invades the lymph-nodes. Carcinomatous ulcers. of the buccal or uterine mucosa early reach the first barrier of lymph-nodes, but rarely pass the second chain, while the ulcer will eventually extend to the invaded nodes and thence laterally until the death of the patient from infec-

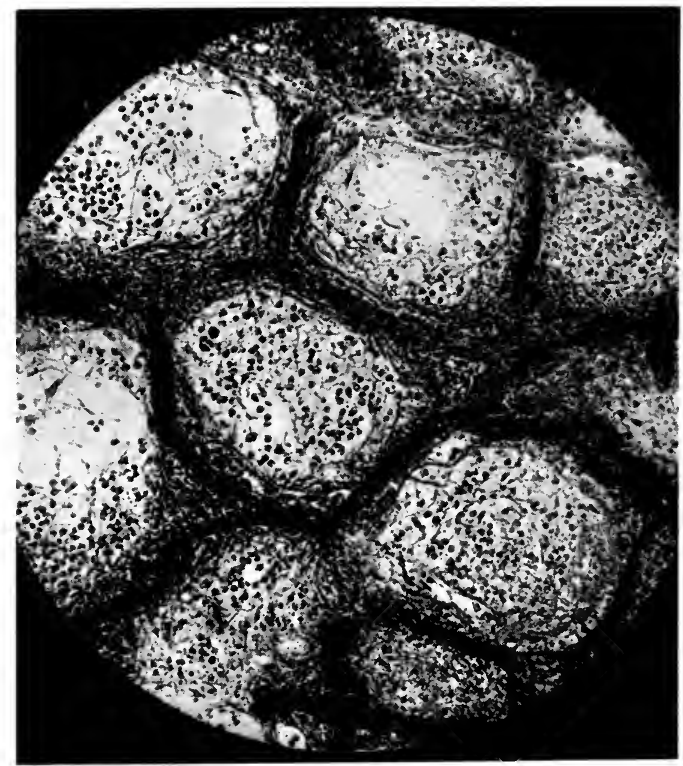

FIG. 202.-Calcification of walls of pulmonary alveoli in a case of advanced mammary carcinoma.

tion and hemorrhage. Ulcerating glandular carcinoma, as of the stomach or uterus, may produce wide-spread metastases.

Microscopic Structure.-No single microscopic feature and no combination of occasional criteria can be claimed as invariably pathognomonic of the cancer process. The history of the microscopic study of cancer presents numerous instances of failure to establish this essential characteristic. The microscopical structure varies so extensively in different organs and at successive stages as to eliminate the possibility of such rigid criteria. It is sometimes impossible to determine whether a given process should be classed as precancerous, as miniature carcinoma, as adenoma or adenocarcinoma, or as carcinoma. Often several of these phases of the evolution of the process are present in the same lesion, and more frequently several structural types of true carcinoma are combined in the same tumor. Hence carcinoma must 
be regarded not as a uniform structural alteration, but as a progressive process which has a diverse origin, a variable course, and an uncertain termination, each phase presenting its own morphology.

Moreover, carcinomas of the various organs and different carcinomas of the same organs may present their own structural peculiarities. It is therefore necessary to learn the specific characters of this disease as it occurs under a great number of conditions. This extremely varied morphology of carcinoma is the source of much confusion of nomenclature, of much conflict of opinion as to what constitutes carcinoma, of great uncertainty regarding histogenesis, and it has especially obscured the distinctions between carcinoma and sarcoma.

Primary Versus Secondary Structure of Carcinoma.-It is highly important to distinguish between the primary structure of a tumor, which usually reflects its original tendencies, and secondary changes in structure which result from inflammation, hemorrhage, adaptation to mechanical environment, and interference by surgical procedures. The primary structure often gives a definite clue to or clearly reveals the origin of the tumor, as in the various forms of epidermoid carcinoma, adenocarcinoma of uterus, embryonal carcinoma of testis, etc. When altered by any of the above influences all carcinomas tend to assume an indifferent, diffuse or perivascular structure in which most of the original features are lost, and from which it is usually hazardous to attempt to reach any conclusions regarding histogenesis. Thus epidermoid carcinoma may appear as a round- or spindle-cell growth, adenocarcinoma of uterus may recur after curetage as perithelioma, and melanoma may recur as a round-cell, diffuse or perivascular, pigment-free tumor.

Structure.-A detailed analysis of the structure of carcinomas reveals at least the following features, any one or all of which may be present:

(I) Cellular overgrowth passing beyond that observed in other processes affecting the same tissue.

Excessive overgrowth indicating abnormal powers of proliferation is a very notable feature of many carcinomas. Its degree may at once reveal that the process differs in kind from functional or inflammatory hyperplasia. It is usually associated with atypical qualities in the cells, but in the thyroid gland, pancreas, and adrenal the cell type may not differ markedly from that observed in other forms of hyperplasia. On the other hand, excessive overgrowth is not an impressive characteristic of some epidermoid carcinomas; the bulk of cells in some scirrhous mammary cancers is probably less than that of the normal breast; while many relatively benign tumors of the gastrointestinal tract are more massive than the highly malignant growths in these tissues.

(2) Atypical Qualities of the Cells.-Although there is no characteristic morphology of the cancer-cell, yet a change in appearance from that of the originating cell is a nearly constant element in the cancer process. This change consists chiefly in an increase in the chromatin content of the nucleus, and signifies increased nutrition and, as the behavior of the cell ordinarily shows, increased powers of growth. The nuclear change may be the only discernible alteration, for such cells may be either larger or smaller than normal and otherwise unaltered.

In some early multiple carcinomas of the skin described by Janeway, the lesions consisted exclusively of a slightly increased number of epithelial cells throughout the Malpighian layer, with very marked hyperchromatism. Usually such cells show some hypertrophy.

In early flat carcinomas of the larynx the lesion may consist of a thickening of the epithelial layer due to hypertrophy of cells with hyperchromatic 
nuclei. Distinct hyperplasia and heterotopia are for a time absent. More frequently still the altered cells exhibit hyperplasia and heterotopia.

In a large group of carcinomas the altered cells are smaller than normal, as in some very malignant tumors of prostate, pancreas, liver, adrenal, and other organs. Indeed, the small cell carcinomas as a class may be regarded as particularly malignant.

The whole series of nuclear changes in cancer-cells forms an extensive chapter in the morphology of cancer which is discussed elsewhere. An abundance of mitoses is observed in many rapidly growing tumors, but is notably absent in others. The atypical quality of the mitoses may or may not be prominent and is not essential. An extensive list of degenerative nuclear and cytoplasmic changes also belongs to the occasional regressive processes in cancer tissue.

The changes in the type of the cancer-cell are often designated as metaplasia, but since this term is more clearly applicable to inflammatory and functional alterations of cells which lack malignant attributes its use in connection with the cancer process is not wholly desirable. Since these changes go with destructive proliferation, and signify loss of organization and restraints to growth, Hansemann's term "anaplasia" appears the more acceptable.

(3) Heterotopia.-The advance of proliferating epithelium beyond its normal limits is nearly identical, as a feature of carcinoma, with distinct invasive properties, but there are instances in which heterotopia is not invasive or destructive. Within giand ducts and alveoli malignant proliferation of epithelium may force the cells into abnormal positions within the confined space. There is heterotopia without invasion of tissues. In many papillary epidermoid carcinomas islands of epithelium may become displaced by inflammatory processes or by elongation and twisting of rete pegs before there is definite invasion of the connective tissue. More pronounced downward growth of surface epithelium is usually preceded by round-cell infiltration and weakening of the connective tissue. The equilibrium between epithelium and connective tissue being thus disturbed, the epithelium begins to pass beyond its normal limits and true cancerous heterotopia is soon established.

(4) Local Invasive and Destructive Properties. - Infiltration of tissue spaces, lymphatics, and blood-vessels is the criterion of fully developed carcinoma. The invasion may be by single cells or cell groups, or by alveoli of adenocarcinoma. Analysis of the process furnishes ground for the belief that several factors are concerned. Chief among these is probably the mechanical pressure of proliferating epithelium. In certain comedocarcinomas of the breast the appearances point most directly to the mechanical forcing of cells through hernias and tears in the distended basement membranes out into the connective tissue, and in many other cases the shape and position of the cells is best explained as the result of pressure. The ameboid properties demonstrated in certain cancer-cells may facilitate their progress through the invaded tissues, but the evidence in favor of this view is scanty.

Chemotaxis exerted by agents toward which proliferating cells travel appears to have been demonstrated in Fischer's experiments with sudan III, but in a process which proves not to be carcinoma.

There appears to be no support for the suggestion that tumor-cells secrete heterolytic ferments which loosen or dissolve the invaded tissues, but an incompetent barrier of round cells, congested and proliferating vessels, and edema often render the tissues less resistant. In some mucous carcinomas the wide diffusion of fluid mucus evidently determines the peculiar manner in which such tumors advance. 
Highly malignant carcinomas penetrate the preformed lymphatics and blood-vessels often like an infection, but when there is much local inflammation and fibrosis the invasion is more localized, slower, and largely through new formed spaces created by the tumor-cells. There is abundant support of the general rule that carcinoma travels by preference through the lymphatics.

The extent of the lymphatic invasion may be extremely wide. In mammary carcinoma the skin of nearly the whole trunk may be invaded, and Handley traced continuous permeation through lymphatics from breast to humerus and femur. Depending apparently on the activity of the process and the resistance of the organism, lymphatic invasion is attended by perilymphangitis with fibrosis and atrophy of tumor-cells, or by no reaction whatever.

The invasion of blood-vessels occurs chiefly in organs in which the vascular supply is unusually favorable to this event, as in thyroid, liver, adrenal, and kidney. It is also relatively frequent in young adults suffering from highly malignant tumors.

Serous cavities are traversed by permeation through the abundant subserous lymphatics or by mechanical dissemination of cells discharged into the cavity. In the peritoneum and especially in the meninges the invasion may be extremely rapid, simulating an inflammatory process.

The destruction of tissues invaded by carcinoma is a common event. Pressure atrophy of supporting structures, especially the blood-vessels, is the chief factor at work, and its influence is most evident in metastatic carcinomas of internal organs. There is much variation in the resistance of different tissues to advancing carcinoma. Arteries and tendons are least affected, but bone and cartilage are readily absorbed and destroyed.

On exposed surfaces mechanical trauma is added to the pressure atrophy, so that on mucous and cutaneous surfaces carcinoma early tends toward necrosis and ulceration. In the stomach the gastric juice promptly attacks the feebly nourished tissues of many early carcinomas, converting them into ulcerating lesions. In Verse's series several early gastric carcinomas were found with superficial erosion of epithelium which permitted access of infection with purulent inflammation. In most epidermoid carcinomas infection becomes established sooner or later, contributes to the destructive process, and may even dominate the clinical course and anatomical picture of the disease.

The mechanical pressure of contracting muscle-fibers seems to account for the form and mode of dissemination of many malignant tumors. The constant contractions of the pylorus probably explain the wide dissemination of gastric cancer along the coats of this organ. The contractions of the bladder serve to maintain the polypoid form of most carcinomas of the urinary bladder. Likewise the activity of the smooth muscle-fibers of the prostate may facilitate the extensions of prostatic cancer. Embolic cells travel long distances down the lymphatics of the esophagus. The movements of the tongue may hasten the dislodgment of cells from invading lingual tumors.

(5) Desmoplastic Properties.-Among the most significant features of the cancer process is its power of exciting the growth of new cellular connective tissue. This tissue reaction may be a very prominent and very early element in carcinoma, so that it deserves important consideration as a diagnostic sign of malignant disease. In some carcinomas of the prostate it may antedate characteristic changes in the epithelium, and in carcinoma of the breast it is often prominent in very early and minute lesions. In true fibrocarcinoma of breast, or stomach, in linitis plastica, and in peritoneal metastatic nodules, the bulk of new connective tissue may far exceed that of the tumor-cells, which may eventually disappear. 
That this product has the same significance as the connective tissue of productive inflammation is shown by the fact that it is often replaced or preceded by or associated with lymphoid infiltration and commonly terminates in sclerosis. For the same reasons it must be interpreted as a reaction of immunity on the part of the tissue. In this respect carcinoma exhibits a parallel with chronic inflammatory processes. According to the activity and age of the reactive process the new tissue is composed of lymphocytes and plasma-cells, capillary blood-vessels and endothelium, proliferating fibroblasts with soft mucinous stroma, or acellular fibrous tissue. Polynuclear leukocytes take no part in the uncomplicated reaction to invading carcinoma. In linitis plastica there is marked proliferation of endothelium and fibroblasts in the gastric submucosa, and these cells mingled with scanty tumor-cells present a highly peculiar picture of a process which is essentially desmoplastic.

(6) Loss of Polarity.-Disturbance in the relation of epithelial cells to one another belongs chiefly to the advanced phases of carcinoma. Gland cells normally stand upon a basement membrane and adhere in orderly fashion to their neighbors, and this arrangement may be assumed as essential to normal growth and function. Complete loss of these relations is observed in diffusely growing carcinomas arising from such glands and signifies advanced anaplasia. Traces of normal polarity may long persist in small alveolar carcinomas in which a definite lumen appears in the centers of cell groups. Persistent retention of polarity is a peculiar feature of adenoma malignum and adenocarcinoma, appearing in the secondary alveoli forming in distended gland spaces, and reappearing, often in striking distinctness, in some distant metastases from such tumors. The significance of loss of polarity as a measure of anaplasia appears in the series of comedo carcinomas of the breast, some of which produce definite secondary alveoli within the ducts, while in others the cells grow diffusely. The former long spare the lymph-nodes, while the latter rupture the basement membrane and invade the breast and axillary nodes. Much the same variations may be traced among the epidermoid and papillary carcinomas.

(7) Metastases.-Apart from the continuous permeation of lymphatics and tissue spaces it is a highly significant property of carcinoma to give rise to cell emboli which pass through the blood- or lymph-vessels and originate distant secondary tumors. While this feature belongs especially to the actively growing tumors it is also observed in very early stages of the evolution of carcinoma. In the benign metastasizing thyroid struma extremely abundant secondary tumors may appear in lungs or bone-marrow in which the structure closely approaches that of the normal gland. Early adrenal adenoma may nearly duplicate this phenomenon. In the liver adenoma may pass easily into the numerous venules of this organ, but its cells do not readily survive in other organs. In vascular tissues, as the tongue, upper lip, stomach, testicle, very small carcinomas may be found to have yielded metastases. On the other hand, some true carcinomas with wide local extensions, as rodent ulcer, seldom or never invade lymph-nodes. The property of producing metastases cannot therefore be demanded of all carcinomas and cannot be regarded as a reliable measure of the degree of anaplasia. In so far as the embolic cells are able to survive transplantation and show even accelerated powers of growth, metastases reveal a property entirely foreign to normal cells.

A survey of the different forms of cancer occurring in various organs demonstrates that the above microscopical features may be combined in very different degrees. Many highly malignant and fully developed carci- 
nomas exhibit all of them from a very early period in their evolution. In a large group of tumors the characteristic features are much more pronounced in one portion of the growth than in another. It is usually observed that the recurrence of a carcinoma is more atypical and malignant than the original tumor. In a series of four recurrences of an epidermoid carcinoma derived from the enamel organ the tumor exhibited increasing grades of anaplasia and finally grew as a diffuse round-cell carcinoma. In the group of adenocarcinomas some of the characters of fully developed carcinoma are regularly lacking. Finally, in the beginnings of carcinoma in many organs, in lesions designated as precancerous, it is sometimes possible to trace the gradual addition of one after another of the microscopical features of the fully developed disease.

From these observations several important conclusions follow:

(I) Carcinoma does not necessarily spring full-fledged into being, but is to be regarded as a process which often exhibits stages of evolution which gather momentum as they progress.

(2) It is not possible nor advantageous to distinguish sharply between processes which show more or less of the microscopic characters of carcinoma. It is necessary to learn by clinical experience the natural history of microscopical structurai changes and to class with cancer all those which tend to progress to the fully developed forms of the disease.

(3) The doctrine of precancerous lesions finds abundant support in the progressive evolution of processes which from the first have commonly been classified as carcinoma.

Structural Classification of Carcinoma.-While the structure of carcinomas is extremely varied, it is possible to recognize several well-defined anatomical types.

Adenocarcinoma.-As previously indicated, adenocarcinoma is a type in which the growth reproduces more or less completely the original gland alveoli from which the tumor springs. It is a partially developed form of carcinoma in which the arrangement of cells in alveoli with central lumina, the polarity of the cells, and frequently some trace of their function, are partially preserved. In some cases the relation of cells to stroma is maintained. The alveoli usually differ, sometimes very notably, from the original type, but occasionally it may be difficult to distinguish the neoplastic from the normal alveolus. The reproduction of alveoli does not identify the tumor with adenoma of the particular organ, for adenocarcinoma lacks the restrained growth, the orderly proliferation, and more typical reproduction of alveoli, of adenoma. Adenocarcinoma commonly exhibits a strong tendency to assume the structure of typical carcinoma in certain portions of the tumor, so that it is ordinarily invasive and destructive and clinically malignant. Adenoma malignum is also invasive, but tends to maintain throughout an orderly glandular structure. It also may exhibit the transformation into true carcinoma, in which case it is practically identical with adenocarcinoma.

The tendency to form acini with central lumina is persistently retained in many malignant tumors of the organs, and it becomes extremely difficult at times to determine whether the tumor is an adenocarcinoma or a diffusely infiltrating growth in which the cells appear in small groups.

Papillary carcinoma is a form of adenocarcinoma arising from glands, mucous surfaces, and occasionally from cutaneous surfaces. Its relation to adenocarcinoma is revealed in the partial preservation of cell polarity and of the supporting stroma. It produces tufts and finger-like branching projections of exuberant epithelium which may be supported by vascular strands of connective tissue. The epithelial layer is multiple and the over- 
growth, far outstripping the connective tissue, may form thick masses, or convoluted layers which fuse with one another. In this manner the original polarity of the cells is completely lost and the papillary structure becomes diffuse. Papillary carcinomas also exhibit malignancy by epithelial invasion of the bases or cores of the papillæ. A large proportion of cystic tumors are papillary adenocarcinomas.

Cystic Carcinoma.-Many glandular carcinomas develop cysts, within gland alveoli or ducts which become greatly distended by a growth which long remains confined by the thickened wall of the resulting cyst. The distention is favored by internal pressure from the epithelial mass, from retained secretion, and from hemorrhage and exudate, while the cyst wall may exhibit intrinsic powers of growth and participate in the process.

The epithelial growth within the cyst may be papillary or glandular and is usually of the order of adenocarcinoma. The papillary structures may be very numerous and low or very long and complex, and present all the features of other papillary carcinomas. They are especially prone to perforate the cyst wall and continue their growth in papillary or diffuse form in the invaded tissues or in adjacent cavities.

Glandular cystadenocarcinoma presents extensive multiplication of glandular alveoli of the ordinary type of adenocarcinoma.

Certain organs, as ovary and breast, are particularly prone to develop cystic carcinomas, and the form of the tumor seems dependent upon the particular conditions under which it develops. In the breast cystic carcinoma arises chiefly from the malignant transformation of a previous cyst or cystadenoma. The tumor perforates the capsule, which lacks muscle tissue, before reaching large dimensions. In the ovary carcinoma appears in very small cysts which by fusion form relatively large tumors. The capacity of the fibromuscular stroma of the organ to resist pressure as well as invasion may partly account for the great bulk attained by some malignant ovarian tumors. Yet perforation of the capsule frequently results at an early stage.

Secondary cysts form in many carcinomas by liquefaction necrosis, edema, lymphangiectasis, and hemorrhage.

Fully developed carcinoma appears in a great variety of structural forms for which there is a wide choice of terms.

Small alveolar carcinoma is a specific term which may properly designate a carcinomatous structure in which the cells appear in small groups supported by a moderate amount of connective tissue. The cell groups do not as a rule surround a central lumen, but lie compactly. Yet in many cases the restoration of a small lumen very readily appears in occasional cell groups and should not release the tumor from consignment to the anaplastic class of true carcinoma. Large alveolar carcinoma equally well designates a similar structure in which the cell groups are larger, but still well defined. As these structures are probably the most frequent histological types of the disease, they are often described as carcinoma simplex.

Tubular carcinoma refers to the appearance of elongated cords of tumorcells, of the type of carcinoma simplex, which may or may not inclose a lumen. When small lateral cell groups branch off from such central tumor cords the term acinar carcinoma is sometimes applied.

In different organs many special peculiarities in the structure of carcinoma appear and some have received special designation as indicated in the descriptions of these tumors.

The attempt to designate a given carcinomatous tumor by a specific term meets the difficulty that several structural types may be presented in different portions of the growth. Not only the most advanced form, but the simplest, 
and the most prominent are of importance in determining the nomenclature, and in this dilemma the structure is often passed as carcinoma varia.

Diffuse Carcinoma.- In the most malignant and highly anaplastic forms of carcinoma the cells lose their specific features, all trace of polarity and capacity to excite connective-tissue reaction, and they grow diffusely, presenting no trace of alveolar formation. Such structures may be designated as diffuse carcinoma. They are observed in very active or fulminant tumors of many organs, especially in young subjects, in the breast during gestation, in certain tumors arising from embryonal structures, and in the accelerated growth of simpler tumors recurring after operation. In the most pronounced stages of such growth the structure may be difficult to distinguish from lymphosarcoma, but the perfectly fixed cells usually remain polyhedral. Many diffuse carcinomas have figured in the literature as sarcomas, especially of the testis and adrenal.

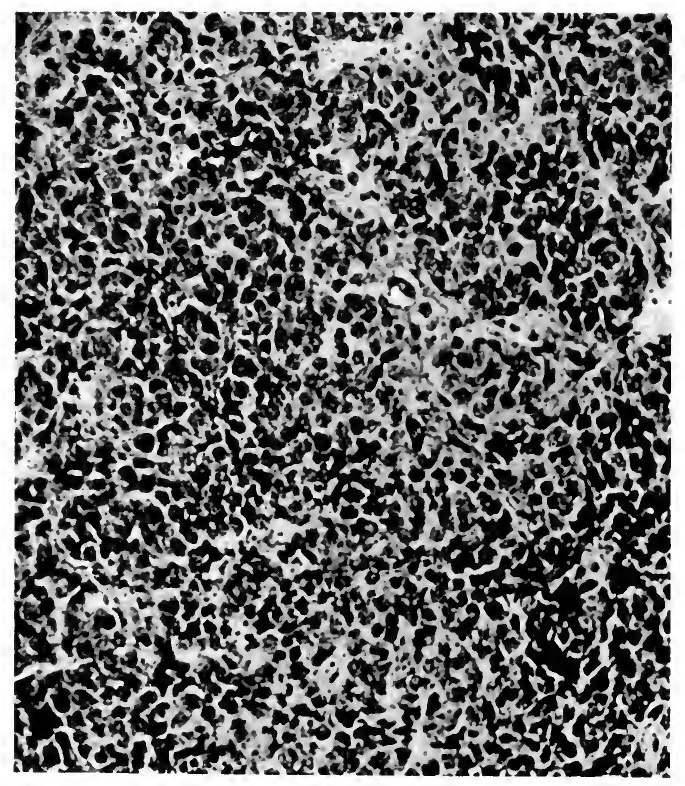

FIG. 203.-Atypical diffuse carcinoma of nares.

Embryonal Carcinoma.-Certain tumors present a structure which resembles that of the embryonal type of the organ from which they are derived. They may be adenomatous, adenocarcinomatous, or carcinomatous. Some fall in the structural class of diffuse carcinoma. A typical example is the epithelial type of Wilms' tumor of the kidney which, even when composed almost entirely of epithelium, at once declares its embryonal nature. At the other extreme stands the hornifying epidermoid carcinoma, which is distinctly adult in type. In nearly all glandular organs are occasionally encountered cellular carcinomas recalling the embryonal structure of the organ, and many of these, as in the thyroid, are composed of small cells consisting chiefly of. nuclei.

The embryonal nature of a tumor should not be confused with an anaplastic character. While many embryonal carcinomas are also highly ana- 
plastic, others are relatively slow in growth and vary little from the cells of origin. The failure to distinguish between embryonal and anaplastic characters gives rise to much misapprehension regarding the prognosis of cellular carcinomas, since embryonal carcinomas are very prone to sudden variations in growth, they often respond to $x$-ray treatment, and their course is distinctly less malignant than that of anaplastic carcinoma of corresponding structure.

Embryonal carcinomas must be conceived as arising from embryonal structures, often misplaced, and since the cells. of origin are imperfectly developed the tumor reflects this character throughout. The embryonal character does not signify that there has been any reversion of the originating cells of the tumor from an adult to an embryonal type. It is possible to grade the carcinomas of many organs according to this principle, as adult or

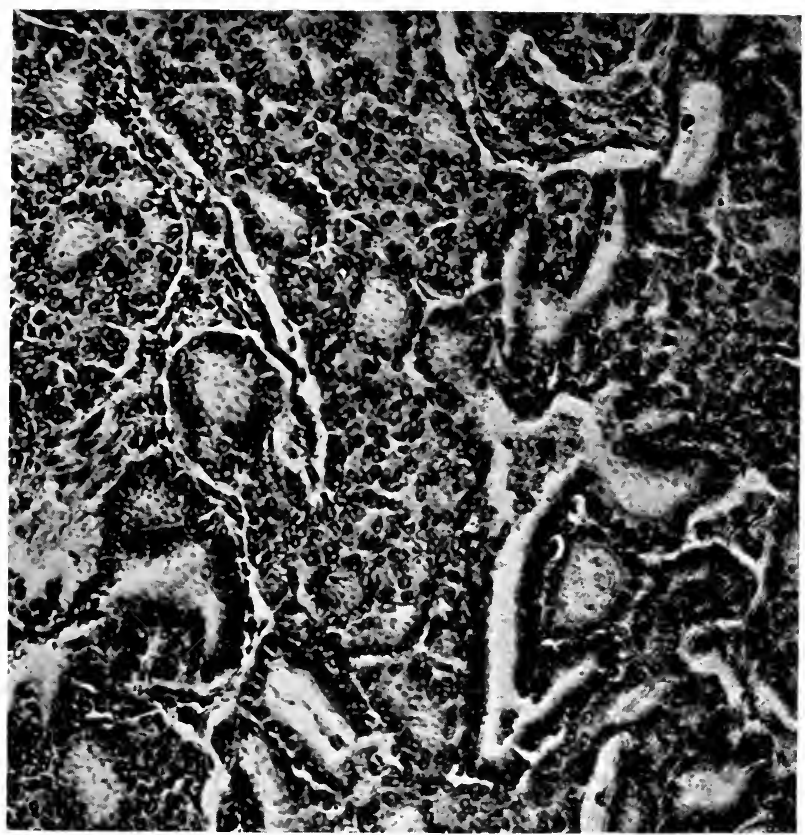

FIG. 204.-Mammary cancer. Embryonal adenocarcinoma producing a bulky, rapidly growing malignant tumor.

embryonal, and in the kidney especially it appears that the classification corresponds with the stage of development which the originating cells have reached at the time the tumor develops from them.

Fibrocarcinoma.-While most carcinomas excite some connective-tissue reaction, in some the connective tissue is excessive in amount, it is subject to hyaline changes, and it compresses the cells so that their growth is impeded. Fibrocarcinomas are usually of slow growth, of dense consistence, and of cicatricial appearance. They are most frequently seen in elderly and resistant subjects. The peculiar structure may appear from the beginning, as in mammary scirrhus, or it may develop later in regressing areas. Eventually the tumor-cells may entirely disappear. Typical examples are observed in sclerosing fibrocarcinoma of the pylorus, in metastatic peritoneal nodules, and especially in cancer en cuirasse. 
In most cases the development of fibrocarcinoma appears to depend chiefly on local conditions, since a localized fibrocarcinoma may develop very cellular secondary tumors at a distance or even in the same organ.

Epidermoid Carcinoma.-From surfaces lined by squamous cells, and by metaplasia of other epithelial cells, develop tumors presenting the characters of squamous epithelium. The general term "epithelioma" is commonly applied to this entire group, but should doubtless be displaced in favor of the designation "epidermoid carcinoma."

There are two main groups of epidermoid carcinoma, those presenting flat squamous cells and sometimes called acanthoma, and those presenting smaller cells of the basal type, and called basal-cell carcinoma.

These terms may give a false inference that the one tumor is derived from the squamous cells of the Malpighian layer, the other from the basal cells. Yet acanthoma arises from downgrowth of both basal and overlying cells,

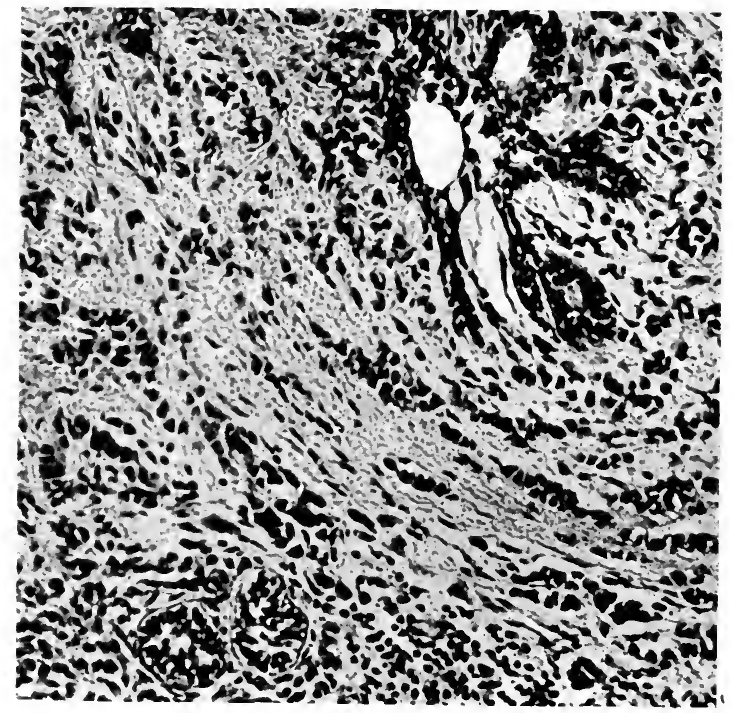

FIG. 205.- Mammary cancer. Primary scirrhus or fibrocarcinoma in a young subject.

which immediately exhibit some of the normal changes into flat cells, often with intercellular spines. Basal-cell carcinoma arises perhaps exclusively from basal cells, often from misplaced and embryonal groups of such cells, but the normal transformation into squamous cells entirely fails.

Many subvarieties of epidermoid carcinoma are observed. Acanthoma presents adult flat epithelium, with hornification, concentric groups of cells flattened by pressure (epithelial pearls), and often intercellular fibrils. Keratohyaline granules may usually be demonstrated by appropriate stains. The younger cells of such tumors tend to lose their adult characters and infiltrate the tissues, producing cords of very opaque polyhedral cells (tubular epidermoid carcinoma).

The squamous cells may become more and more atypical, producing cellular carcinomas of many types, eventually growing diffusely, and even appearing as round-cell or giant-cell carcinoma. Many epidermoid carcinomas. as in the cervix uteri, reproduce the entire epithelial layer of the originating 
structure which becomes convoluted, invaginated into the underlying submucosa, and appears as a structure which may be designated as plexiform epithelioma. Others break up early into small groups of cells which rapidly infiltrate the tissues. They are more malignant than the plexiform type. An extensive group maintains a papillary structure. Or an original glandular tendency may appear pronounced in the tumor, as when it arises from the ducts of cutaneous glands or the enamel organ, and produces a glandular epidermoid carcinoma. So great is the variety of structures in epidermoid carcinoma that they must be dealt with separately in the organs in which they occur.

Squamous metaplasia, affecting the cells before the development of the tumor, or appearing in the course of glandular carcinoma, or as an element in teratomas, accounts for the presence of epidermoid carcinoma in unusual situations.

Precancerous Lesions. - The beginnings of carcinoma have been sought in most organs without wholly satisfactory results. As a rule the cells of the smallest established carcinoma appear to be entirely separate from the normal tissues and to differ essentially from those phases of atypical hypertrophy or inflammatory overgrowth which accompany many carcinomas. These well-established observations have led Ribbert to assert that the cells of carcinoma are isolated throughout their entire course and hence the cells of origin must be isolated. This author has affirmed that no one has ever seen the beginnings of carcinoma of the breast, all true carcinomas of this organ being so well differentiated from the normal gland that the attempt to trace the neoplastic from the normal cells invariably fails.

This tenet conveys an important principle in the histogenesis of carcinoma, namely, that the initial proliferation of cancer-cells proceeds rapidly, usually obliterates any traces of the transformation of normal into tumor-cells and renders extremely difficult the decision just what form the progenitors of cancer-cells presented. It is therefore necessary to regard with caution the interpretation of many forms of atypical cell growth as preliminary to the development of true cancer.

Moreover, the comprehensive lists of tissue abnormalities as catalogued by R. Williams, R. Meyer, and many others, has established on a very broad basis the theory of Cohnheim that tumors develop from misplaced and embryonal cell groups which have never enjoyed a normal structure. This theory must also include structural abnormalities and tissue predispositions which are not revealed by obvious microscopical changes, but which certainly contribute to the origin of tumors. In all this group of cases the origin of cancer is from congenitally misplaced or abnormal cells and not from transformed normal cells.

In another and very extensive group the evidence points to the origin of carcinoma from previously normal adult cells which pass through a series of changes induced by chronic irritation and terminating in carcinoma. This class of tumors has been called the "irritation group," and the preliminary cell changes have been called "precancerous lesions."

The evidence in support of this view is both clinical and morphological.

Clinical observation has long indicated that the majority of important tumors are not dependent on congenital abnormalities in tissue structure, but arise from once normal but previously altered tissues, and that various forms of chronic inflammation are observed to precede the appearance of most tumors.

In 1872 Hutchinson termed various manifestations of buccal syphilis precancerous. Billroth observed that cancer almost never arises in a normal 
breast and later studies have shown that chronic mastitis frequently precedes mammary cancer. V. Bergmann stated that primary cancer of the skin without previous cutaneous lesions does not exist. In the various organs it is universally recognized that certain pathological conditions are followed in a variable but high proportion of cases by carcinoma. For these conditions the term "precancerous diseases" has been employed by Orth, but it should be emphasized that these diseases possess in themselves no essential element of the cancer process, and are merely observed to precede and favor the development of cancer.

The so-called precancerous conditions fall into several distinct classes, but the majority of them represent specific forms of chronic productive inflammation.

I. Chronic Inflammation.-In the skin well-known forms of cancer arise in the cicatrices of burns, tuberculous and syphilitic lesions, and amputation wounds. A long period usually elapses before cancer appears in scars and the tumor is often multicentric or diffuse in origin. Lupus carcinoma may arise in the active tuberculous lesions, but in 30 per cent. of the cases it occurs in the scar tissue (Steinhauser). It develops usually toward the thirtieth year of the disease and never before the fifth. Ashihara reports several carcinomas in syphilitic scars. $X$-ray dermatitis is a remarkable instance of a destructive and inflammatory process tending to develop cancer. A period of 3 to II years precedes the malignant process, and is occupied by necrosis of tissue cells, occlusion of vessels, fibrosis, and epithelial overgrowth (Porter, Wolbach).

In glands and mucous membranes chronic inflammation is frequently followed by cancer. In the gall-bladder cancer was preceded by gall-stones in Ioo per cent. of Janowski's cases and the proportion of cases of cholelithiasis developing carcinoma was placed by Slade at 18 per cent. The irritation of cholesterin seems to have a peculiarly effective influence in inducing atypical epithelial proliferation.

In the uterus chronic catarrhal endocervicitis precedes cancer in the majority of cases (34 of 48 , Polese). Cervical erosions, leukoplakia of portio and canal have frequently been traced into carcinoma. For corpus carcinoma, the hyperemia and endometritis accompanying myoma may be held responsible for the development of carcinoma. It usually develops opposite the point of contact of the myoma and the eroded endometrium. Acanthoma and adenoacanthoma follow leukoplakia of the endometrium (Benckizer).

In routine material I find transition stages from hypertrophic endometritis to carcinoma rather frequently, but clinical observations are still inadequate to prove the close dependence of the malignant upon the inflammatory process. In 44 cases of corpus carcinoma Heurlin failed to find any transition stages, but 29 of his cases were diffuse.

In the urinary bladder most cancers may be traced to previous or coexistent cystitis (Stoerk, Cohen). Specific vesical irritants, as in anilin workers, produce many tumors, chiefly at the ureteral orifices, 50 per cent. of which are malignant (Rehn, Seyberth). In Bilharzia disease the development of carcinoma on venous and lymph stasis, with cystitis from irritation by ova and lithiasis, has often been traced in detail. Various forms of balanitis precede carcinoma of the penis. Demarquay found phimosis in 85 per cent. of his cases, and Kaufmann observed warty vegetations in 29 of 33 cases.

Buccal and lingual cancer is almost always referable to one or all of three factors, syphilis, tobacco, or decayed teeth. The syphilitic process usually takes the form of leukoplakia, of which 30 per cent., according to Fournier, develop carcinoma. In these cases there is hypertrophy and hyperplasia of 
epithelium, hyperkeratosis, lymphocytic infiltration of submucosa, papillomatous overgrowth, and finally destructive invasion of deep tissues. Darier finds that the complication of an ulcer or fissure is usually necessary before leukoplakia becomes cancer. Syphilitic warts, fissures, gummas, and atrophic glossitis also precede cancer.

Excessive use of tobacco produces the well-known smoker's tongue and throat, in which there is hyperemia, edema and lymphocytic infiltration of submucosa, erosion, and then downward growth of epithelium. Ragged teeth produce superficial erosions, thickening of regenerating epithelial layer, lymphocytic infiltration, heterotopia, and then downward growth of atypical cells.

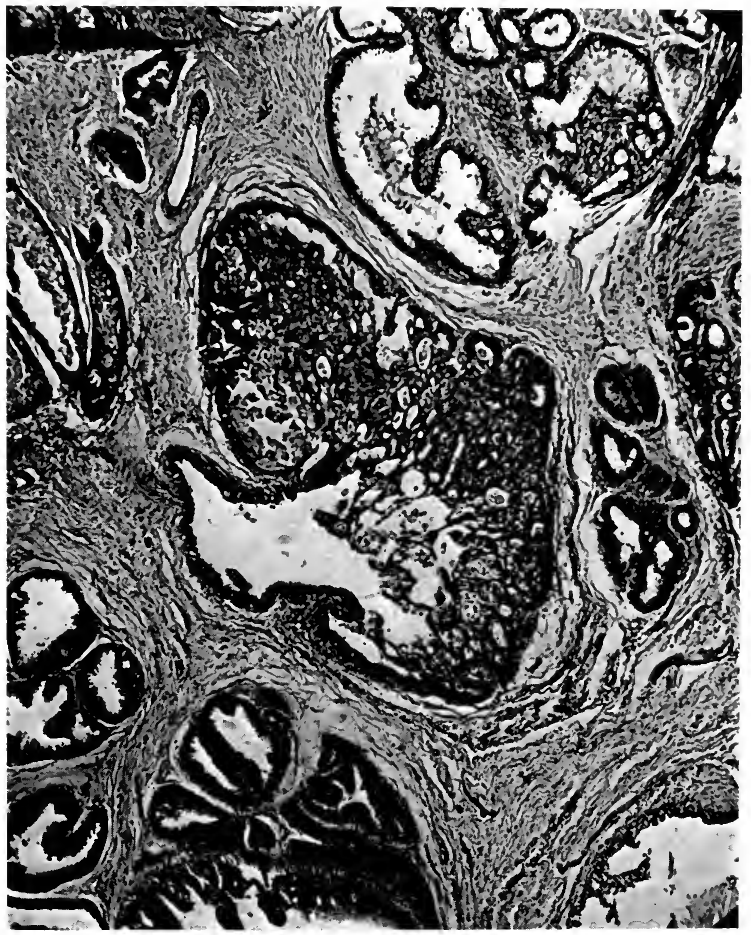

FIG. 206.-Atypical adenomatous proliferation in ducts in chronic mastitis.

In all this group of cases it is evident that the carcinoma is only an indirect result of the irritation, and the long period which precedes the malignant process, as well as the infrequency of the malignant change, give opportunity for other local or constitutional factors to come into play. Nevertheless it is clear, as in Kangri-oven and betel nut cancer of Indian natives, that local and general predisposition may be ignored, since the tumor develops wherever the irritation is effectively applied.

2. Physiological involution of organs and complicating mechanical, nutritional, and inflammatory processes precede the development of cancer chiefly in the breast and prostate. Definite neoplastic tendencies in the "maladie de Reclus" were pointed out by Brissaud and Schimmelbusch. Reclus, 
Saar, and many others have declared that the step from chronic mastitis to carcinoma is short. It is especially in that form of chronic mastitis in which cysts and epithelial proliferation are prominent from the first that cancer is observed to develop, but no anatomical form of the disease is free from the danger of malignant change. Bloodgood prefers to regard the underlying condition as pure senile involution. The proportion of cases of chronic mastitis developing cancer has been variously estimated from io to ${ }_{5} 5$ per cent. (Tietze, Speese).

In the prostate the chief condition predisposing to cancer is chronic prostatitis usually with hypertrophy. Whatever the nature of prostatic hypertrophy may be, it is clear that the influence of physiological involution is usually concerned. The principal age of incidence of cancer and hypertrophy is the seventh decade, in which 68 per cent. of the carcinomas occur. Notable examples of the development of carcinoma on hypertrophy have been recorded by many observers, the clinical evidence being a long history of hypertrophy terminating in cancer. Probably ro per cent. of prostatic carcinomas give such a history. The proportion of enlarged prostates after 50 years which prove malignant has been estimated at 13.3 to $\mathrm{r} 6.5$ per cent. (Freyer, Walker).

Senile degeneration of the skin as observed in seaman's skin and other disorders often leads to multiple carcinoma. It affects regions exposed to sunlight, heat, and cold, and often shows an hereditary element. The changes begin with hyaline degeneration of the derma, sclerosis of vessels and atrophy of Malpighian layer, followed by keratosis, scaling, papillary outgrowths, and elongation of deep papillæ. Finally the invasive features of carcinoma are added.

3. Regenerative hypertrophy may be regarded as the predominant underlying influence in the development of certain forms of cancer observed in the liver and in the glands of internal secretion.

In fish, functional overactivity and hypertrophy of the thyroid, observed in crowded ponds where the animals are fed on meat, leads in a small proportion of cases to a peculiar form of cancer. This condition has been produced experimentally by Gaylord. Few cases of thyroid carcinoma in man develop in subjects with entirely normal thyroid history, but many follow goiter, interstitial thyroiditis, and Graves' disease. Probably many tumors of the adrenal and hypophysis are of the same nature.

In the liver an important group of primary carcinomas or hepatomas represents malignant overgrowth of regenerating lobules following injury with degeneration or cirrhosis (Menetrier).

4. The transformation of benign into malignant tumors is a frequent source of carcinoma occurring in many organs and has important bearing on the origin of cancer. Some of these benign tumors appear to originate from inflammatory processes and the organs may present every gradation between simple inflammatory overgrowth, adenoma, and carcinoma. The most striking example is colitis polyposa which exhibits a peculiar overgrowth of epithelium with polypoid adenomas, leading in about half the cases to carcinoma. In the gastric, intestinal, uterine, and vesical mucosa the transformation of benign polyps into carcinoma has repeatedly been observed. Menetrier has shown that gastric ulcer usually leads to cancer through the preliminary development of polypoid adenomas on the edge of the ulcer. In the solid glandular organs histological rather than clinical evidence indicates that the passage from benign to malignant tumors may be readily accomplished, although as a rule the type of benign adenoma usually remains constant.

Thus clinical observation reveals several groups of pathological processes 
which in a small but notable proportion of cases tend to become malignant. Such clinical data do not, however, show that there is any essential connection between the preliminary process and the subsequent cancer. It may be urged that when carcinoma follows chronic mastitis a new disease has been grafted upon an entirely different condition. Histological evidence, however, has fully demonstrated that an extensive series of structural changes takes place in the precancerous diseases leading up to the fully developed cancer, the one passing slowly or rapidly, but often by insensible gradations into the other. The details of these changes will be presented under the various forms of carcinoma of the organs. It may here be pointed out that precancerous changes differ in each organ as do also the true carcinomas of those organs.

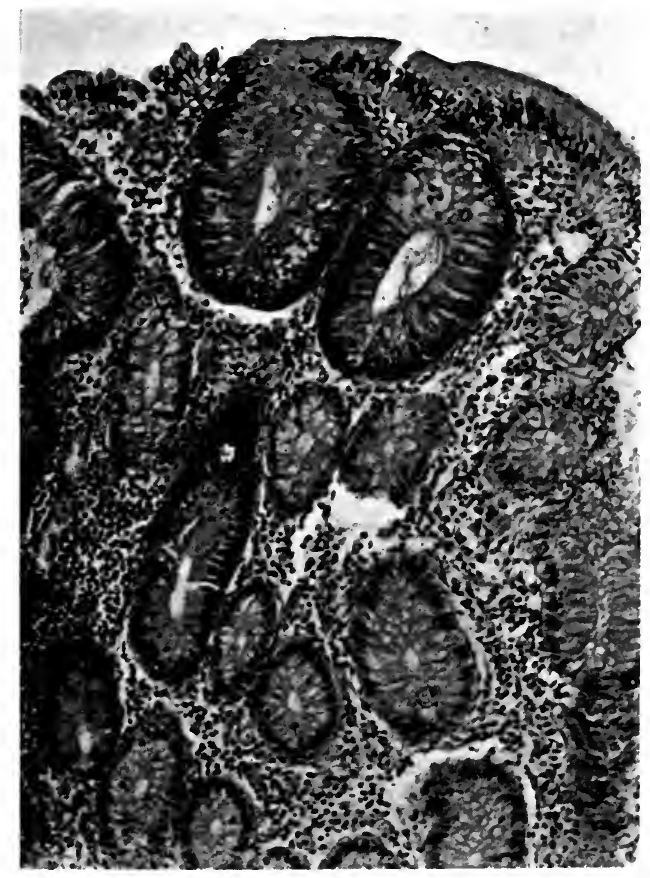

FIG. 207.-Diffuse hypertrophy of glands in colitis polyposa.

As a rule considerable time is required in the transformation as is indicated by the long duration of the preceding disease. Yet in many cases it would appear that the liberation of the restraints to growth is experienced rapidly, so that the resulting cancer although small is found fully developed, and soon overgrows and obliterates any successive stages which may have occurred.

It is not to be assumed that all carcinomas develop after a preliminary series of changes in previously normal tissue such as is now under discussion. In the entire group of tumors arising from misplaced and embryonal cells, while certain phases of abnormal growth probably precede the appearance of the true carcinoma, these changes are of a different nature and morphology from those occurring in the irritation group of tumors arising from normal cells. Such changes may be traced in the development of melanoma from pigmented moles, and with carcinoma from adrenal rests. They indicate 
that even here the carcinoma does not spring full-fledged from the cells of origin, but evolves gradually with increasing freedom from the restraints to growth. Of the factors which incite the malignant growth of embryonal cells little is known, but one may reasonably search for them among the causes of local hyperemia. Melanoma often develops after mechanical trauma.

Against the theory of precancerous lesions stands the common observation that many very small cancers cannot be shown to follow such preliminary changes. Verse describes very early gastric carcinomas in which a limited segment of mucosa exhibited all the criteria of malignant adenoma. Very small but fully developed mammary carcinomas are observed in chronic mastitis which cannot be traced to the suspicious forms of atypical cell growth so common in mastitis. Clinical studies show that the majority of cases of chronic mastitis pursue a prolonged but benign course. These considerations suggest caution in assuming that any given precancerous lesion will develop cancer, and show that many cancers may be preceded by little or possibly by none of the precancerous stages, but they seem not to affect the general validity of the theory that carcinoma as a rule develops by definite stages both from adult and from embryonal cells.

Mode of Extension of Carcinoma.-The events which follow the establishment of a carcinomatous focus in an organ vary greatly with the mode of origin of the tumors, the rapidity of growth, and the character of the affected tissue.

Influence of the Mode of Origin.-Carcinomas arising from misplaced and embryonal cells are isolated from their inception, probably remain so throughout their history, and illustrate in the purest form the principle that tumors grow from their own resources and not by the progressive transformation of normal into tumor-cells. Equally important with this principle, so strongly urged by Ribbert, is the fact that many carcinomas, during the period of their inception and for some portion of their early growth, involve increasing areas of glands or mucous surfaces in their points of origin and thus extend in part by gradual transformation of previously normal cells. Thus one may observe epidermoid carcinoma of the glans penis or prepuce covering a wide area, in different portions of which are presented every gradation from fully developed infiltrating carcinoma in the central areas, to markedly hypertrophic, hyperplastic, and atypical epithelium in the peripheral zones. In very extensive papillary adenocarcinoma of the rectum involving several inches of the bowel a gradual extension of an originally small lesion must be assumed. Hauser and Beneke have traced this transformation in intestinal carcinoma. In carcinomas of multicentric origin the separate foci may eventually fuse, forming one continuous area of origin. In carcinoma following chronic mastitis the malignant process may originate in several foci, even producing different types of carcinoma, and soon involving the entire breast. It is, therefore, not to be assumed that the observed mass of carcinoma is always the result of local invasion from a single minute focus, but, on the contrary, this mass may depend chiefly on the mode of origin of the tumor.

The rapidity of growth of a carcinoma determines in many cases its mode of extension. Rapidly growing tumors soon define their field of origin, no subsequent additions are made to the originating cells, and the tumor rapidly extends through preformed and artificial paths, growing from its own resources. Many but not all such carcinomas arise from isolated or embryonal cells.

The character of the affected tissue influences the mode of extension in many cases. On mucous membranes ulceration and infection early convert many 
carcinomas into ulcerating lesions, in which the inflammatory process seems at times to retard the lateral extensions of the disease, at other times to facilitate them. The wide extension of some gastric carcinomas is probably referable to the contractile movements of the stomach and to a comparatively resistant muscular coat. Growing into ducts as in the breast the extension may long be confined chiefly to these canals as in comedocarcinoma, while in the ovary cystic carcinomas may long be confined by the fibromuscular cyst wall. The whole course of gelatinous carcinoma of the peritoneum may depend upon the early rupture of a small colloid tumor into the free peritoneal cavity.

Collateral Hyperplasia.-About the edges of many carcinomas, especially of the mucous membranes, the neighboring glands commonly exhibit hypertrophy, hyperplasia, and a somewhat atypical form of the lining cells, which may approach the appearance of carcinoma. In adenoma malignum of the rectum the surrounding zone of mucosa exhibits greatly elongated glands lined by very large cells which strongly suggest a gradual transformation of hypertrophied into neoplastic glands. These changes are only imperfectly reproduced in pure inflammatory lesions, for the collateral hyperplasia of tumors is rather specific. In some cases it is extremely difficult to determine where the hyperplastic glands cease and the neoplastic begin. Yet the most careful histological studies indicate that such hyperplastic glands do not become transformed into neoplastic unless it be in the early stage of the definition of the tumor focus. Careful study usually discloses that tumor epithelium grows into the enlarged glands from many sides, gradually replaces the lining cells, and occupies the gland compartments. At the most critical points the transition between hyperplastic and neoplastic epithelium is usually sharp, but in some cases it cannot, I think, be denied that it is impossible to say whether or not a transformation of hypertrophied into tumorcells takes place.

Collateral hyperplasia also occurs in many glands invaded by carcinomatous foci and has led to the unproved assumption that the presence of foreign tumor-cells may excite a tumor process in an invaded organ. For example, in the adrenal invaded by carcinoma from the kidney I have seen adrenal acini assuming a form which strongly suggested neoplastic qualities. In hepatoma the remaining liver tissue may exhibit extensive hypertrophy of liver-cells which is not duplicated in simple inflammatory or regenerative changes of this organ.

Collateral hyperplasia seems to be referable chiefly to overnutrition of cells in the vicinity of the actively growing tumor. Inflammatory factors may also contribute a share in the process. Yet the peculiar morphology suggests that other influences are at work, and Beneke assumes that the proximity of tumor-cells exerts a peculiar trophic action of the neighboring tissues in addition to ordinary inflammatory, nutritive, and regenerative effects.

Local Invasion.-The advance of carcinoma from its focus of origin occurs through the continuous extension of tumor-cells into normal tissues. The paths pursued are usually those of least resistance and include the natural channels in glandular organs, lymph-vessels and spaces, blood-vessels, and nerve-trunks. The ducts of glands become filled with tumor-cells which replace the original lining cells over wide areas. In the breast duct carcinoma may thus invade the entire organ before reaching the lymph-nodes or penetrating the fascia of the gland. Carcinoma of the lung may pass along the air passages, filling air vesicles, replacing the desquamated alveolar epithelium, and consolidating one lobe or a whole lung. In the liver, the bile-ducts, and in the kidney the glomeruli and tubules often conduct the proliferating 
cells. Along mucous surfaces, as of stomach or uterine cervix, carcinoma may spread laterally by replacing the lining cells of the glands. In the peritoneum the flat endothelium may be widely replaced by cylindrical cells derived from glandular carcinoma of the intestine.

The lymphatics form the main channel of local extension. The cells may very early penetrate lymph spaces and lymph-vessels and form new spaces in the connective tissue. It is especially the perivascular lymph-channels which are invaded and from these the route to larger lymph-vessels and nodes is most direct. The lymphatic endothelium remains passive or suffers atrophy. Proliferation of these cells cannot, however, be excluded in the

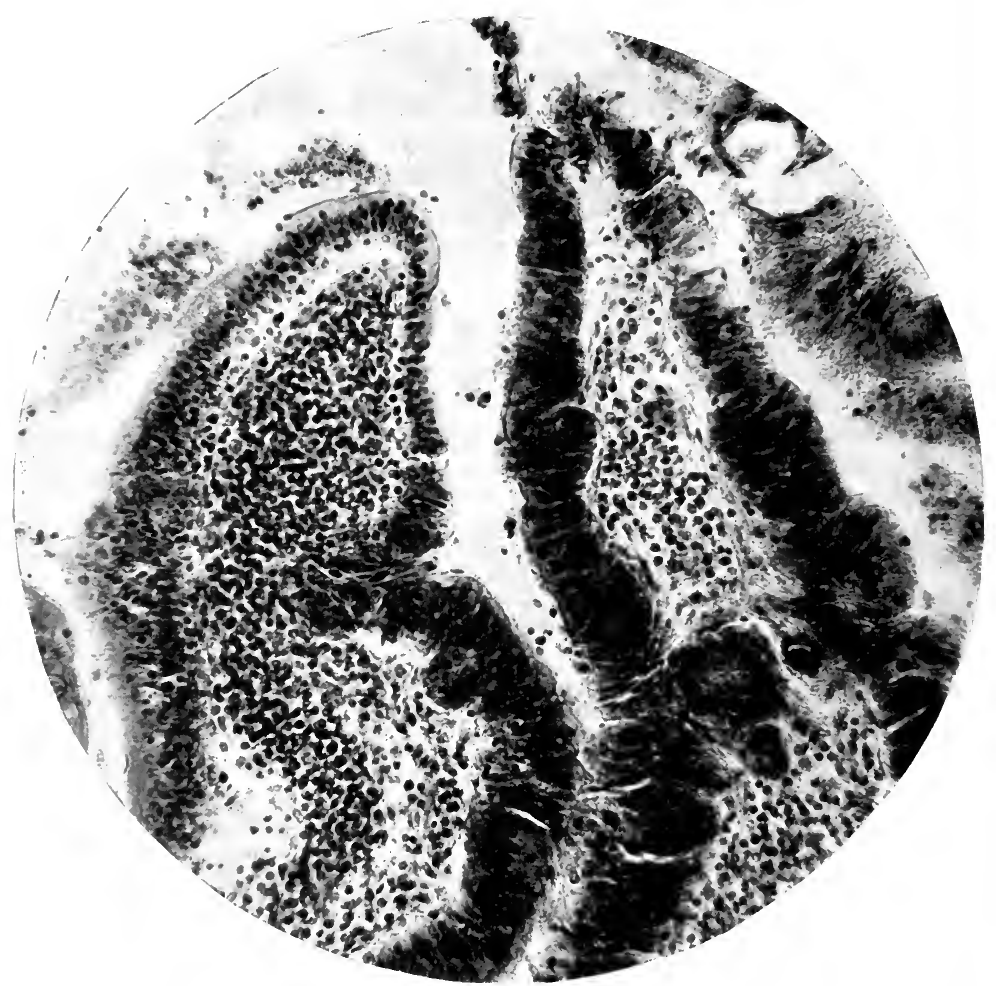

FIG. 208.-Edge of advancing adenoma malignum of rectum. Note sudden transition of normal to neoplastic epithelium.

formation of the new connective tissue that often accompanies local invasion. While the growth in neighboring lymphatics is usually continuous, local metastases may occur and surround the main tumor with many small secondary nodules. Yet the fine strands of tumor-cells demonstrable in the lymphatics connecting the nodules of rodent ulcer suggest that a continuous permeating cord of cells may be broken by fibrous perilymphangitis. The invasion of lymphatics is greatly retarded or suppressed in favorable cases by extensive collections of lymphocytes which gather in the vessels and may even surround the tumor-cells with a rich lymphoid ring. Occasionally the entire tumor is infiltrated with lymphocytes, and in such cases the occurrence of 
many degenerating tumor-cells indicates that the lymphoid infiltration is a phenomenon of immunity.

The lymphatics of nerve-trunks are readily penetrated by many tumorcells, especially in epidermoid carcinoma, and through these minute channels the process may travel over wide areas. It is probable that the spinal ganglia and meninges are occasionally reached by this method. The nerve-fibers suffer atrophy. Much of the pain of carcinoma as well as pareses, paresthesias, and the rare attacks of zoster, must be referred to invasion of nervetrunks and ganglia.

The paths of access to the local blood-vessels vary much in the different organs. In the liver, kidney, adrenal, and thyroid the small vessels are early penetrated by tumor-cells of otherwise moderate infiltrative capacity. Hence tumors of these organs may early present bulky intravenous masses from which vascular emboli are distributed or which completely fill the vena cava up to and into the right auricle. The intravascular position of the fetal villi accounts for the extension of chorioma through the uterine vessels.

In many carcinomas the local veins are penetrated by the cells filling perivascular lymphatics. Goldmann's injections of the veins of carcinoma show that these vessels are often occluded by tumor-cells and that the local advance of the growth may be largely through these vessels. Primary carcinoma of the liver may be rapidly disseminated over the entire organ, exclusively through the blood-vessels. The formation of granulation tissue about ulcerating carcinomas often facilitates the local extension, probably by providing many new capillary channels for invading cells.

The invaded vessel is usually filled by tumor-cells and the lumen occluded, but in many cases one may observe flat or papillary mural implantations, or the endothelium may be replaced by a flat layer of tumor-cells while the lumen remains pervious. Interference with the circulation accounts for much of the edema in tumors, for the tendency toward central necrosis, for bulky areas of necrosis from infarction, for hemorrhage, and for the partial suppression of growth in the centers of carcinomatous areas.

Through these various channels the cells of an originally small carcinoma gradually involve successive portions of the organ or even pass continuously from one organ to neighboring structures. The wider dissemination of the tumor-cells, especially by loosened cell groups, constitutes the process of metastasis, the complex details of which have been separately considered. 


\section{CHAPTER XXVI}

\section{EPITHELIAL AND OTHER TUMORS OF THE BREAST}

Hypertrophy of Breasts.-Mammary hypertrophy occurs as a rare congenital condition in infancy or childhood; it arises most frequently at puberty, and is seen in adults (Deaver, McFarland, R. Williams, Lit.).

I. Infantile Hypertrophy.-Congenital enlargement is recorded by Horritt, Hahn, and Wilson. Infantile hypertrophy is usually associated with precocious sexual development, and is more common in the tropics. It is possibly connected with pituitary disorders, and some of the cases first observed in infancy seem to have begun at birth (Mallett, Sagra).

2. Diffuse Hypertrophy of Adults.-Most cases arise at puberty, others occur late in life, and a considerable number is connected with pregnancy. An hereditary element has been noted in a few cases (Rousseau, Kirchheim). Delayed onset of menstruation, or suppression of menses frequently precede the overgrowth. The disease may best be interpreted as an excessive response to functional and formative stimuli in congenitally predisposed organs.

Either one breast or both, or one or more supernumerary organs, are involved. A partial hypertrophy has been described by Richet and Billroth. The degree of enlargement may be moderate or very great, Kaufmann reporting a weight of $30 \mathrm{~kg}$. for both breasts. The structure varies considerably. Lipomatosis and glandular hypertrophy were the chief elements in the cases of Robert and Warren. Connective- and fat-tissue overgrowth, with moderate glandular hypertrophy, retention of secretion, and marked vascularity are observed in a considerable group. Porter described multiple intracanalicular fibromatous growths with fibrosis, and in many cases the structure resembles diffuse fibro-adenoma. A low grade of adenomatoid hypertrophy is frequently seen.

In a well-marked case, age 24 , I found much excess of fat tissue surrounding a greatly hypertrophied breast. The gland presented a structure resembling the lactating organ. The alveoli were greatly increased in number and while some were compact with narrow lumen others were dilated. Many ducts were widely dilated. There was a uniform overgrowth of fine cellular connective tissue between the acini.

Billroth describes very active overgrowth of glands resembling in places adenocarcinoma. Diffuse carcinoma appears to have followed diffuse hypertrophy (Billroth, Aitken).

The course is usually active during the first few months, and after reaching certain dimensions a quiescent period is maintained for some years with exacerbations during pregnancy. The tumors often become so large as to interfere with general health. Inflammation, suppuration, and gangrene have occurred, or death may result from general exhaustion. Very active cases are recorded by Billroth and Huston in which the breasts reached a large size in a few months, while Durston's case was fatal from gangrene in 4 months. The tumor may regress after pregnancy and operative removal is usually successful.

Gynecomastism, hypertrophy of the male breast, with and without changes in the testicles and in the secondary sexual characters, has occurred in a series of cases collected by R. Williams. 
Chronic Productive Mastitis.-From the extensive literature recording the history of the knowledge of chronic productive inflammation of the breast it is becoming more and more apparent that the various forms of this condition are essentially one and the same disease. Some of the variations depend chiefly on the state of the breast at the time the inflammatory process occurs, others represent exaggerations of single features of chronic inflammation, and still others may be explained as different stages of the same process. Much of the long debate over the inflammatory as against the neoplastic nature of the processes could have been avoided had it been recognized that chronic productive inflammation may consist in much overgrowth of connective tissue and glandular epithelium and that inflammatory passes insensibly into neoplastic hyperplasia. The disease begins as an inflammation

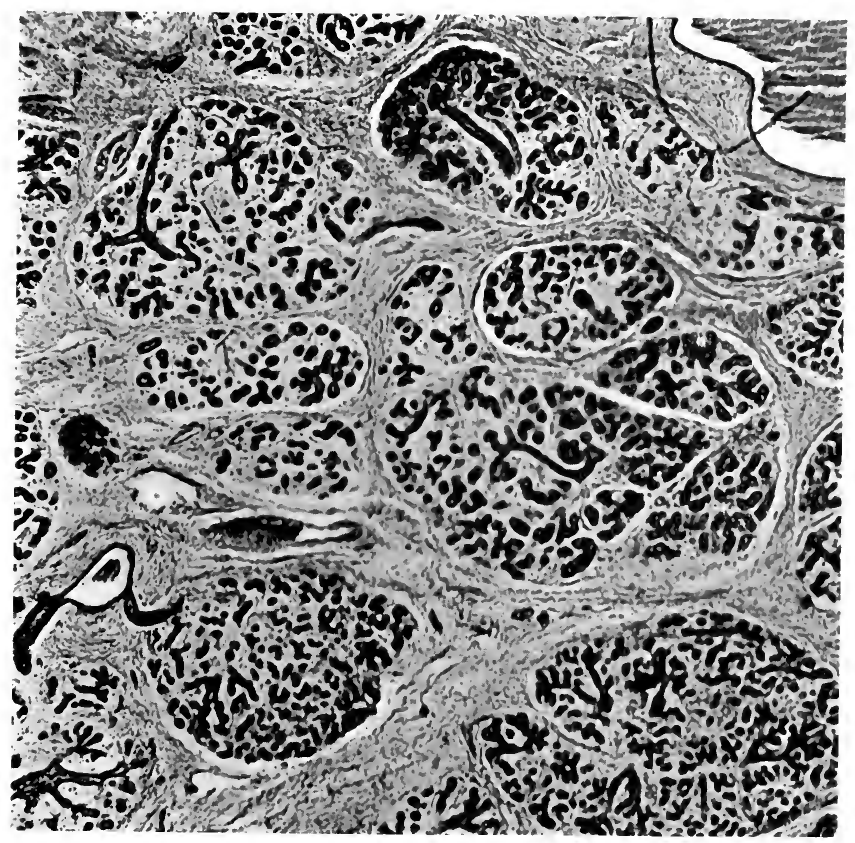

FIG. 209.-Chronic adenomatoid mastitis. A diffuse overgrowth of well-formed lobules.

and often ends as a neoplasm. Nevertheless extreme examples of the different forms of the disease are widely separated.

The importance of the condition is found not only in its frequency, but especially in its relation to the development of tumors of the breast.

(1) Astley Cooper, 1837, first gave a lucid description of cystic mastitis under the term "hydatid disease." He described a slow painless increase in the size of the breast affecting the whole or a portion of the organ, resulting in the appearance of many cysts as large as a pea or bean, some of which might open externally, giving rise to sinuses. The breast was not as hard as scirrhous cancer, the axillary nodes were not affected, and the general health remained good. In $18+6$ Brodie accurately described the same condition in one or both breasts. In 1883 Reclus gave a full description under the term "maladie kystique." He emphasized the bilateral distribution and the large number of cysts throughout all portions of the gland, especially at the periphery.

Brissaud, 1883 , found that the formation of cysts was preceded by signs of abnormal 
activity of the gland, the structure resembling the fetal breast. Both acini and ducts became dilated. Upon these appearances, suggesting a neoplastic tendency, he designated the process as "epitheliome kystique intra-acineux." Phocas, I886, emphasized the occurrence of many shotty nodules throughout the organ and employed the term "maladie noueuse." In Germany König (I875) described the condition in some detail, and later (1893) he followed the successive changes of prolonged cases, pointing out many distinctions from a tumor process. Schimmelbusch, however, reached the same conclusions as Brissaud, that marked activity of the gland preceded the growth of connective tissue and persisted with the development of cysts, and that the process was essentially neoplastic. This view has been maintained by many other writers, Cornil, Lecene, Lenormant. The disease is often referred to as Schimmelbusch's multiple cyst adenoma. This interpretation has been actively contested by König in Germany and Delbet in France, who maintain that the process is essentially inflammatory. Theile finds it impossible to distinguish between adenofibroma, a tumor, and "maladie kystique," an inflammatory process in the breast, and Delbet admits that the inflammatory process passes into the adenomatous. La Roy places the condition among the pathological hyperplasias which should be separated from both inflammatory and neoplastic processes.

(2) A somewhat different form of mastitis was first described by Werner as cirrhosis mammæ, by Velpeau as "induration chronique en masse," and by Virchow as "elephantiasis dura." It is much less frequent than the common cystic disease. Billroth was long uncertain of its existence, but describes a very advanced case with extensive cicatricial contraction and deformity. Labbe and Coyne described a case in which there was diffuse growth of very cellular connective tissue infiltrated with round cells, while a small collection of pus was found in the axillary border of the gland. König recognized a rare form of diffuse chronic mastitis with a painful onset and a marked tendency to atrophy. R. Williams collected several cases in both sexes. Many cases of apparently suppurative mastitis with cicatrization and even calcification (Bryk, Houdoupe) he classifies as circumscribed chronic mastitis. They should be separated from the nonsuppurative diffuse inflammations. Delbet recognizes the peculiar features of this form of mastitis, but on account of the transitional cases he merges it with the common cystic disease.

(3) Finally, it has long been known that senile involution or precocious atrophy of the breast is often accompanied by irregular overgrowth of stroma and glands with the formation of cysts, and various authors have assumed that chronic mastitis is essentially an exaggeration or sequel of the processes concerned in senile involution. R. Williams states that the new tissue in senile involution is usually free from any signs of inflammation, although it may be abundant, as in cystic disease. Altmann observed much epithelial proliferation in 34 cases of senile and precocious atrophy. Tietze found marked similarity between the epithelial changes in about 25 per cent. of senile organs and those of cystic disease.

Bloodgood finds a close relation between cystic mastitis and senile involution. He designates the former as senile parenchymatous hypertrophy and traces accurately the stages of hyperplasia, ectasia, and cystic dilatation. He recognizes two types of the disease, one with small cysts and moderate epithelial hyperplasia, and another with larger cysts and pronounced epithelial overgrowth.

An essential relation between cystic disease and senile involution must, however, be questioned. Involution is chiefly an atrophic process with slow replacement fibrosis and often fails to exhibit the cystic and productive changes. When these are added they are best interpreted as an additional process which may become established in an involuting breast.

From the above brief review of the literature it is evident that two welldefined forms of chronic mastitis have been under discussion which may be designated anatomically as interstitial and glandular, while a third less prominent group of cases includes those in which normal involution atrophy is prominent. While accepting the essential identity of all forms of primary productive inflammation of the breast, as maintained recently by Delbet and Baumgartner, yet many clinical and anatomical peculiarities seem to me to warrant the subdivision of the disease into at least three types-interstitial, glandular, and senile.

Etiology.-The various forms of chronic mastitis are comparatively common, but satisfactory statistical data are unavailable. The increasing attention to the early stages of cancer is bringing under observation a much larger number of cases of chronic mastitis. Many cases pass as fibro-adenoma, 
cystadenoma, and carcinoma. When these conditions develop on chronic mastitis the condition of the whole breast is commonly overlooked.

The majority of cases are first observed at the menopause, but many are encountered much earlier. Of 67 cysts of the breast Bryant found 4 under 30 years, I I between 30 and 40 years, 33 between 40 and 50 years, I 6 from 50 to 60 years, and 3 after 60 years. Delbet believes that most cases begin some years before recognition. Phocas observed a nodular mastitis at is years and Baumgartner at 19 years. Rodman finds the condition more frequent in married women and suggests that a rapid sequence of pregnancies lead to nutritional and inflammatory changes.

Delbet considers the disease a bacterial infection, in which the Staphylococcus albus is the most common but not the sole agent. He compares the infection to the infectious mastitis of cattle described by Nocard and Mollereau and assumes that the microörganism enters through the lacteal ducts and penetrates throughout the lobules. The structure in the active diffuse lesions strongly indicates the presence of an irritant passing out from the acini, but for the more chronic forms this explanation is less satisfactory.

The course of the disease and the structure of the gland vary considerably in the different anatomical forms.

Senile Involution.- The natural history of the mamma includes a terminal stage of atrophy. This change is regularly initiated at the menopause, continues at varying rates and degrees throughout life, and is associated with decline in the nutrition and function of other sexual organs.

Precocious atrophy is observed with sterility and disuse (Williams, Reynolds, De Sinety). An atrophic condition of the breasts of Bavarian women has been attributed by Altmann to a local custom against nursing. Ovarian disease or extirpation appears to notably affect the breast only when occurring before the development of secondary sexual characters (Battey). Yet some observers note pronounced diminution in the size of the breasts following ovariotomy (Keppler), while others report hypertrophy and increased secretion (Baumgartner).

The scope of the changes which may be attributed to simple atrophy is defined with difficulty, since the process is often complicated by other disturbances in structure. In many cases the fat, connective and gland tissue suffer alike and the organ is greatly reduced in size, the cellular connective tissue becomes fibrous and hyaline, and the gland lobules are reduced to a trace, all without reactive processes in any element. The contracted ducts persist, usually with some desquamation of lining cells and a notable increase or prominence of smooth muscle-fibers about the walls. That extreme atrophy may occur without other notable alterations or inflammatory changes is shown in many of the 34 cases studied by Altmann, and it therefore seems clear that many structural variations often ascribed to senile involution are not essentially connected with this condition.

In other cases, especially in obese subjects, the atrophy takes the form of fat invasion, scanty atrophic lobules and ducts being widely scattered in much adipose tissue.

In many cases the process is marked by proliferation and desquamation of lining cells of ducts, the low epithelium may form short lateral outgrowths or papillary projections, small retention cysts may form, connective tissue invades the lobules and disturbs their outlines, and a low grade of chronic productive inflammation is established. These changes do not properly belong to normal involution, but they are so frequently observed in breasts, many of which have suffered from the effects of repeated lactations or previous disturbances of structure, that their presence in moderate degree must be 
included in the usual picture of involution. A pronounced degree of these changes should be classed with chronic productive mastitis.

The advanced atrophy of simple involution distinctly favors forms of atypical proliferation of the lining cells of persistent ducts.

Chronic Interstitial Mastitis.-The characteristic type of this condition is a diffuse interstitial productive inflammation, with a new growth of connective tissue about ducts, lobules, and acini.

Usually one breast only is involved, and the lesion may be more pronounced about the larger ducts. When affecting a circumscribed portion of the breast, the condition resembles a true adenofibroma, but without en-

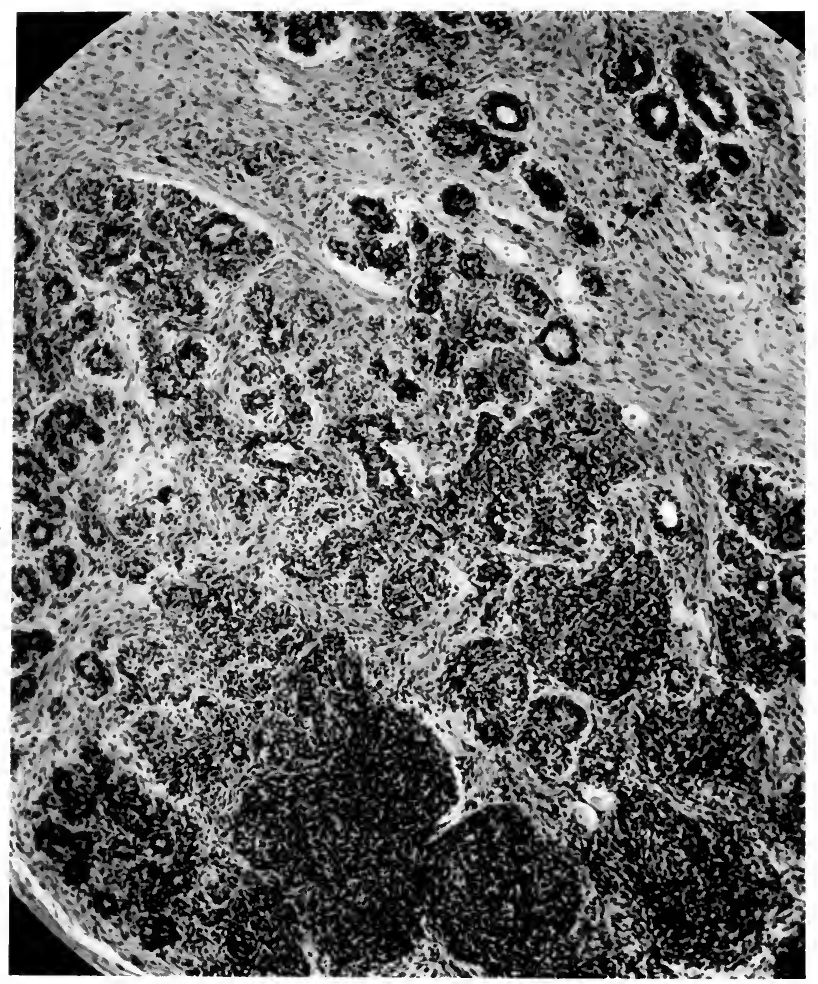

FIG. 210.-Chronic mastitis. Atypical proliferation of acinar cells.

capsulation. It begins with diffuse and somewhat painful enlargement of the breast, which progresses slowly until the organ is moderately increased in size. Exacerbations occur chiefly at menstruation, when the breast may become painful and the axillary nodes enlarge, after which the process remits, leaving new nodules or indurated areas. After months or years contraction and sclerosis begin, the skin becomes roughened (Labbe and Coyne, peau d'orange), adherent and contracted, and the breast may be reduced to a deformed area of hard cicatricial tissue (Billroth). Usually the whole organ remains permanently enlarged, diffusely fibrosed, and with or without small cysts. The whole original area of gland distribution may become converted into a bulky solid mass of elastic connective tissue. Cysts, isolated fibromas, 
and foci of carcinoma frequently interrupt the smooth texture. The gross appearance of these organs is quite specific.

In the acute stage the lesion consists in a diffuse growth of cellular connective tissue which surrounds ducts and lobules and penetrates between the acini. This tissue may be richly infiltrated with lymphocytes. The acini are compressed and show only moderate hyperplasia, but the ducts may be filled with desquamated epithelium. Cyst formation is not prominent. In the later stages the new tissue may contract with atrophy of the parenchyma. In some cases, especially in circursscribed areas, the process resembles adenofibroma, but on careful analysis the structure is found to consist of a limited growth of fibroblasts chiefly about ducts and acini, proliferation of capillaries, and infiltration by lymphocytes and plasma-cells. The very abundant overgrowth of fibroblasts and the encapsulation observed in true adenofibroma are missing. That true adenofibromas may develop on such lesions has been maintained by many authors (Cornil, Theile, Lecene and Lenormand, Delbet, Borst). My own observations lead to the same conclusion. Yet the natural tendency of the process is progressive fibrosis. Epithelial tumors develop in the course of diffuse interstitial mastitis, but rather less frequently than in the glandular cystic mastitis of Reclus and Schimmelbusch.

Chronic Glandular Mastitis.-Cystic Mastitis.-The common form of productive mastitis is marked by the production of many small cysts, by considerable epithelial proliferation, and by diffuse growth of firm fibrous tissue. On account of the prominence of epithelial changes the process may be designated as glandular mastitis, and the presence of many cysts has led to the use of the term "cystic mastitis." It is often called "maladie de Reclus," or "Schimmelbusch's disease," and when many small nodules are present it has been called by Phoca "maladie noueuse." Typical cases differ notably from the diffuse interstitial mastitis of Werner and Billroth, but there are transitional cases which connect the two forms, and it is probable that some cases of diffuse interstitial mastitis later become cystic. Senile atrophic breasts also develop cysts with epithelial proliferation.

The disease usually develops first in one breast and often later in the other. The onset is slow and usually painless and the condition may not be recognized until a cyst of some size attracts attention. There may be a serous discharge from the nipple. A bloody discharge usually but not always indicates a cystadenoma or carcinoma (Sasse, Shield). On palpation the whole organ is firm, of normal or increased size, and many hard, movable nodules as large as a pea or bean may be detected. No part of the breast escapes. There is no adherence to the muscle, nor as a rule to the skin, but the nipple may be retracted (Renon).

The process continues slowly progressive with exacerbations for years. It may subside spontaneously, but cases lasting I5, 30, and 33 years are reported by Monod, Trelat, and Reclus. In the late stages the cysts may open and leave sinuses through the skin. Many cases are complicated with cystadenoma, fibro-adenoma, and carcinoma.

On horizontal section of the breast the organ is found to consist of an irregular mass of fibrous tissue broken by fat lobules, cysts of various sizes, and hard opaque nodules. The cysts may be small and extremely numerous, or larger and infrequent. The contents are serous, fatty or bloody, and thin or inspissated and yellowish. Larger cysts may show all stages of papillary ingrowths, and some (butter cysts) contain much caseous fatty material.

The structure presents a great variety of abnormalities. The cellular or fibrous connective tissue surrounds ducts, lobules, and acini. It is usually 
acellular and cicatricial in type, but at various points it may show local overgrowth of embryonal type. About small ducts there may be very pronounced nodular thickenings. Between the acini the tissue is more cellular, and lymphocytes and plasma-cells are often present.

The epithelial changes take many forms, but proliferation predominates over atrophy. Most acini show a compact lining of two rows of well-nourished cells. The lumen is collapsed or contains globules of acidophile material, or various stages of ectasia are visible. More pronounced overgrowth produces more abundant cells of larger size, with hyperchromatic nuclei. All the acini and ducts of a lobule may show much overgrowth, while adjoining lobules are normal or atrophic. Suspicious or definitely precancerous changes appear when dilated acini or ducts become filled with

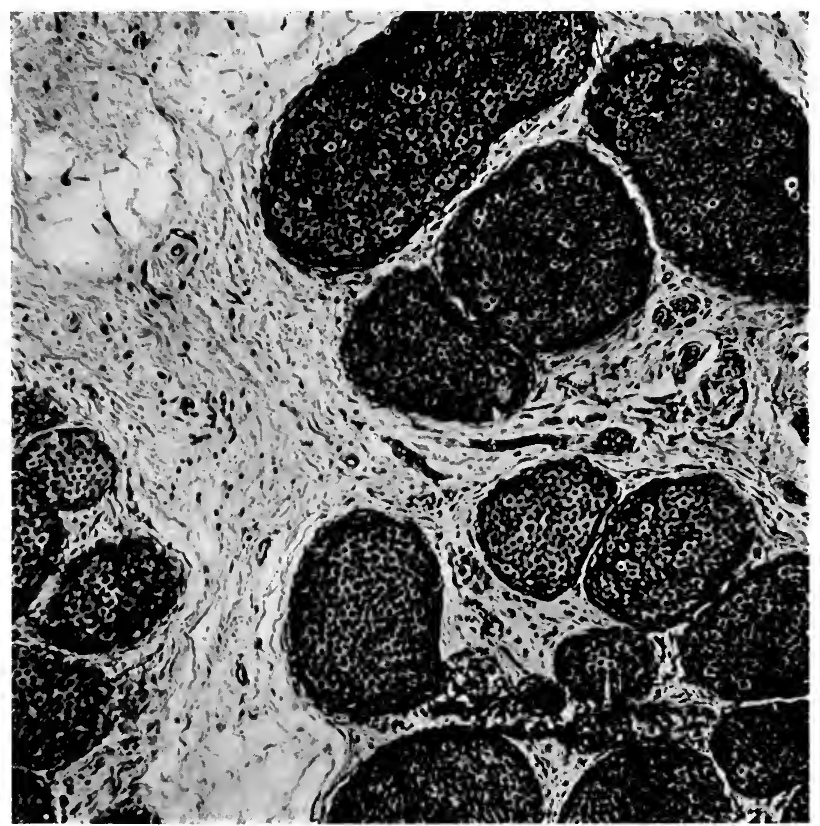

FIG. 21 I. - Precancerous changes in breast. Filling of small ducts and acini with atypical cells. No infiltration.

overnourished cells in compact masses or forming secondary alveoli. Such foci may be scanty and require many sections in different portions of the breast for their detection. In the fresh tissue they appear in the form of shot-like solid nodules. Another precancerous change consists in a multiplication of small alveoli with increase in the size and nuclear chromatin of the cells. According to Dreyfuss and Sasse it is the inner row of secreting cells which multiply in precancerous foci, while McCarthy locates the hyperplasia in the sustentacular cells. I have been unable to recognize these distinctions.

Cysts form from dilatation of alveoli or ducts and are lined by clear polygonal epithelium. Many stages of the proliferation of these cells are observed, including formation of low papillæ, dendritic outgrowths accom- 
panied by stroma, up to pronounced papillary adenoma. From the simple adenomas arise malignant adenoma and adenocarcinoma. Hemorrhage and exudation in these cysts may be associated with malignant changes in the wall of the cyst. Degenerating butter cysts often show such changes in their walls. Another type of cyst forms in the interlobular ducts which may be lined by excessive layers of large cells which form papillary projections or fill the lumen diffusely. Such lesions may pass rapidly into a form of large alveolar or adenocarcinoma of the breast (tubular or duct cancer, Labbe and Coyne, R. Williams). Sasse finds that fibrosis about larger ducts gives rise to one or more large and several smaller cysts in which there is a notable tendency to develop adenoma and carcinoma.

A third form of cyst develops from the sweat-glands incorporated in the breast (Moullin, Dreyfuss, Krompecher). These are easily recognized by the lining of columnar, strongly acidophile cells which are often thrown into papilary projections. Beneath the epithelium there may be overgrowth of muscle spindles. All stages of the development of papillary projections, papillary adenoma, adenocarcinoma, and carcinoma have been traced by Creighton, Borst, Kuru, Krompecher, and others.

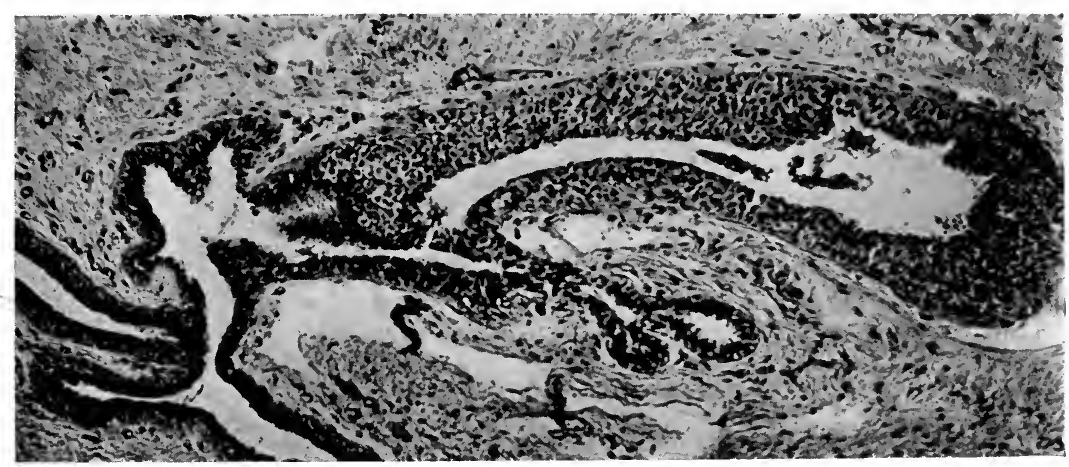

FIG. 21 2.-Precancerous changes in the breast. Atypical proliferation in a segment of a duct.

The frequency of the development of benign or malignant tumor processes in cystic mastitis is a question of prime importance. Delbet represents a large body of surgical opinion in stating that the affection is too benign to justify extirpation, and while he admits that there is no other satisfactory treatment, he recommends pressure, iodid of potash, and injections of carbolized glycerin into the cysts. This conclusion is based on the rather frequent observation that characteristic cases pursue a very chronic course without any neoplastic complication, benign or malignant. In support of this view may also be cited the fact that the epithelial overgrowth is at first inflammatory and that the suspicious carcinomatous areas cannot in any one case be traced into fully developed carcinoma and may never pass beyond the stages actually seen in the simple cystic breast. It is also assumed that the great majority of carcinomas are not associated with chronic mastitis, or if so, that the carcinoma is a wholly distinct process. Baumgartner concludes that the essential sequence of inflammation-adenoma, carcinoma-is not based on irrefutable proof nor disproved by established facts. This conclusion is not helpful. 
Against this view stand the interpretations of Brodie, Brissaud, Sourice, Schimmelbusch, and Saar that there are neoplastic qualities in the process and the step to carcinoma is short. The very chronic cases which remain free from complications are clinically impressive, but when cancer develops little attention is commonly paid to the condition of the remaining portions of the organ. I find that a very large proportion of mammary cancers occur in breasts which are the seat of chronic mastitis. Billroth long ago stated that small cysts are very often seen about carcinoma of the breast. Tietze in 43 cases of cystic disease found cancerous areas in 3, and computes that about ro per cent. of cases of cystic mastitis develop cancer. He describes

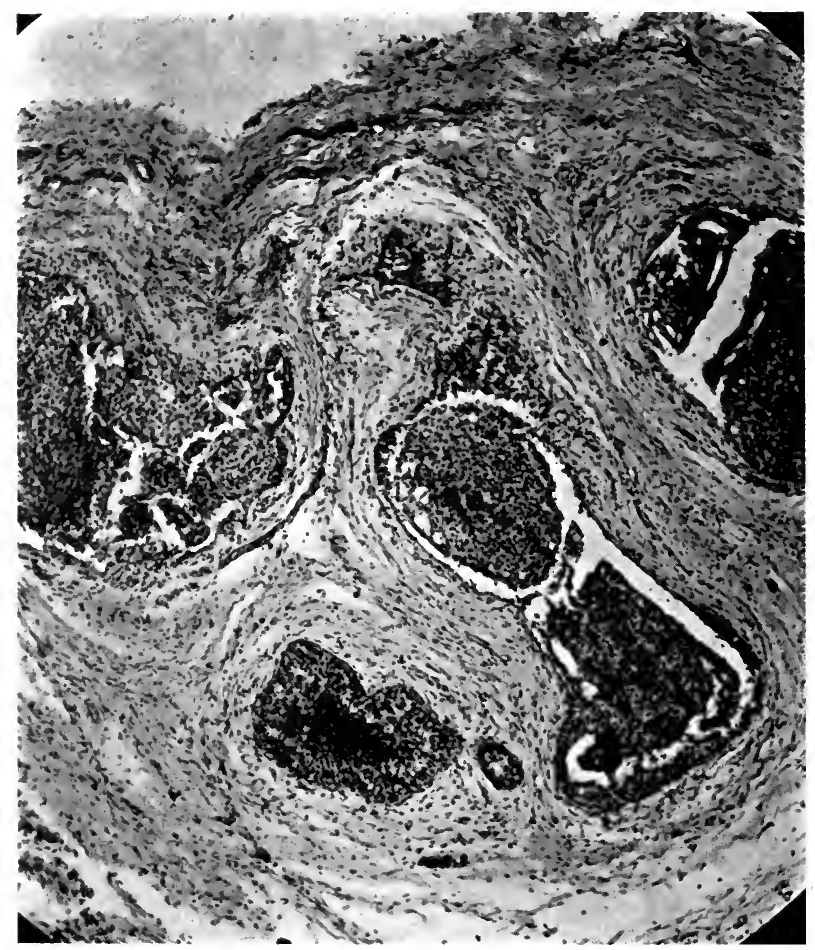

FIG. 2I3.-Diffuse proliferation of atypical cells in ducts in chronic mastitis. A precancerous lesion.

5 cases of cancer with cystic disease in the outlying breast tissue. In 5 of I 8 breasts classed as senile involution he found suspicious areas suggesting cancer, especially in the peripheral portions. Of 295 cases of various types of chronic mastitis collected by Speese, I 5 per cent. were reported as showing carcinomatous changes. In 28 cases of cancer of breast Verga found that in 5 the organ was the seat of cystic mastitis with pronounced carcinomatous metaplasia. In my own material about jo per cent. of the breasts excised for cystic disease show pronounced precancerous changes or miniature carcinomas. Very few cancerous breasts fail to show phases of chronic mastitis in the outlying portions of the parenchyma.

It is therefore clear that chronic mastitis is a very important predisposing 
condition to mammary cancer. It appears also from the histological evidence that many cancers arising in chronic mastitis do not represent wholly new processes, but, on the contrary, are the natural result of steadily increasing epithelial overgrowth which is originally inflammatory and affects not embryonal or misplaced tissues, but the normal and adult glandular epithelium.

When carcinoma develops in chronic mastitis it usually arises early or progresses actively so that the malignant tumor overshadows the inflammatory changes. When the cystic disease passes a critical period without the development of a tumor it tends to maintain a benign course over many years. Hence the clinical impression of the benign character of the process is strongly emphasized by a limited group of cases. Yet I find precancerous

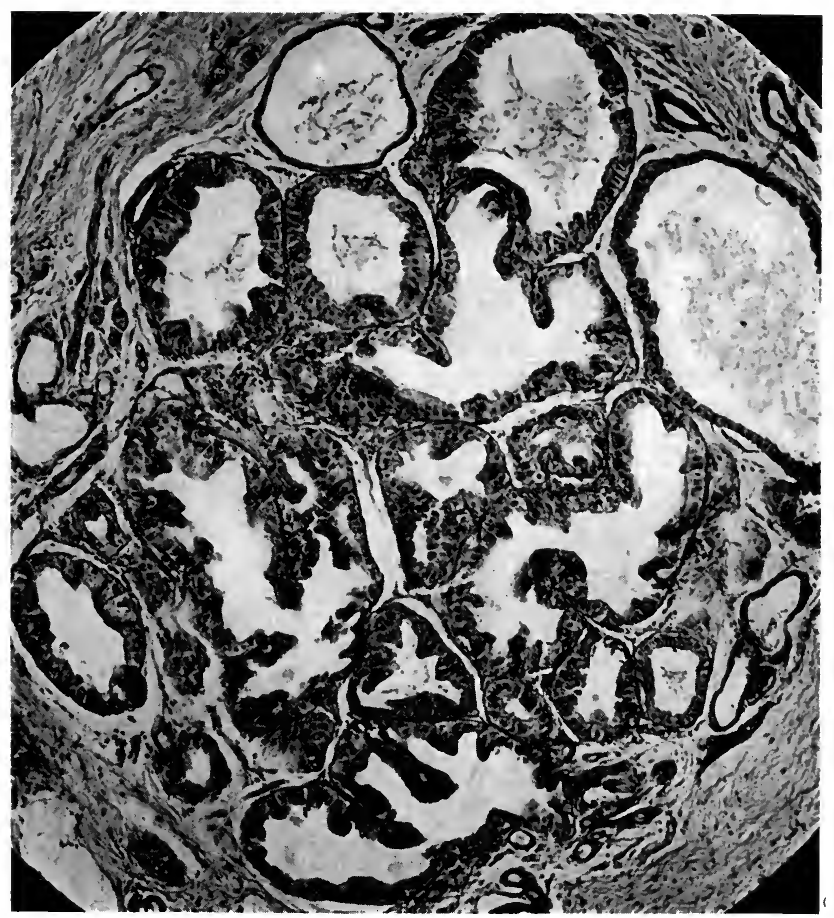

FIg. 2I4.-Adenoma of axillary sweat-glands. An occasional source of mammary carcinoma.

changes in the outlying atrophic and cystic breast tissue in cases of cancer developing after 70 years of age.

The treatment of chronic mastitis must be determined after consideration of all the factors in the case. To remove all such breasts would certainly entail much useless surgery. It seems to be a safe rule to remove the whole breast or the whole affected segment when an incision is required for diagnosis. The presence of carcinoma can nearly always be made by the gross examination of the amputated breast, and the correct surgical procedure can then be determined with certainty. The decision to leave untouched a definite grade of cystic mastitis should be made only with the knowledge that carcinoma frequently develops in such organs. On the other hand, 
simple cysts often disappear spontaneously and do not constitute a definite indication for surgical interference. Yet the course of the established disease is generally progressive and most cases eventually terminate in carcinoma or surgical removal. Physical agents are worthy of trial.

\section{MIXED TUMORS OF BREAST}

Of mixed tumors of the breast in which a tumor process affects more than one element there are two main groups and several varieties.

In one group the structure is comparatively simple and presents adult connective tissue and glandular epithelium, as in adenofibroma and its malignant derivative adenosarcoma. In a second group the structure is more complex and often embryonal, as illustrated in growths which contain connective-tissue, cartilage, bone, or mucous tissue, and epithelium of both glandular and squamous types. Such tumors are usually malignant.

There is a tendency for one element of the tumor to predominate. Thus some pure fibromas, of which $\mathrm{R}$. Williams has collected several cases, probable belong with the adenofibromas in origin, and the pure osteomas, chondromas, and sarcomas are probably often one-sided developments of the complex tumors. Yet the majority maintain a uniform growth of more than one tissue and must be classed with mixed tumors. Many writers have interpreted these growths as simple tumors, chiefly epithelial. Beneke has shown that the development of the early gland acini exerts a strong stimulus on the supporting connective tissue. At puberty and in pregnancy there is an active new growth of acini which may incite proliferation of the supporting stroma. Hence a tumor process originating in the epithelium may be assumed to affect secondarily the connective tissue. Many French authors hold that the majority of benign mammary tumors are primarily adenomas (Delbet). On the other hand, many German authorities locate the primary influence in the connective tissue (Virchow). The attempt to interpret the growths as simple thus meets with difficulties. Since the tumors are usually well encapsulated and both epithelium and connective tissue are well represented it seems most probable that they arise from superfluous or misplaced portions of breast tissue containing both elements. In the more complex and embryonal tumors the mixed composition is unequivocal.

An inflammatory origin for many of the simpler fibro-adenomas and especially of the cystadenomas has been maintained by many authors. Theile, studying I9 tumors, concludes that it is impossible to establish a sharp limitation between the cystic changes of chronic mastitis and fibroadenomas. Cystadenoma is often multiple and associated with chronic mastitis. Occasionally one finds several stages of the growth of diffuse or intracystic fibro-adenoma in the same case of chronic mastitis. I find as a rule that the tumor-like areas in chronic mastitis are not well circumscribed and show many inflammatory changes, such as the growth of fine capillaries and infiltration with round cells, while the pronounced tumors are free from inflammatory signs. It seems probable that an inflammatory process is a frequent inciting factor especially with the cystadenomas, but that a true tumor arises only when there is a tissue predisposition in the form of superfluous or misplaced material. A constitutional element may also be assumed.

The earliest stages of fibro-adenoma have been observed by Borst in the form of small groups of acini which are not sharply circumscribed but exhibit neoplastic characters in the epithelium. Ribbert pictures a very 
small, sharply circumscribed, typical fibro-adenoma. Very early and circumscribed cystadenomas are frequently observed. The isolation of the blood-supply, which is usually from one set of vessels without free lateral anastomoses, points to the origin from misplaced tissue, as do also the congenital and aberrant adenomas.

Adenofibroma; Fibro-adenoma.-Two gross forms of fibro-epithelial tumors occur in the breast: (I) Massive and (2) papillary or intracystic fibroadenoma.

(I) Diffuse massive fibro-adenoma is best illustrated in some cases of diffuse hypertrophy of the whole breast which exhibit neoplastic characters. More frequently the tumor forms a circumscribed mass of considerable

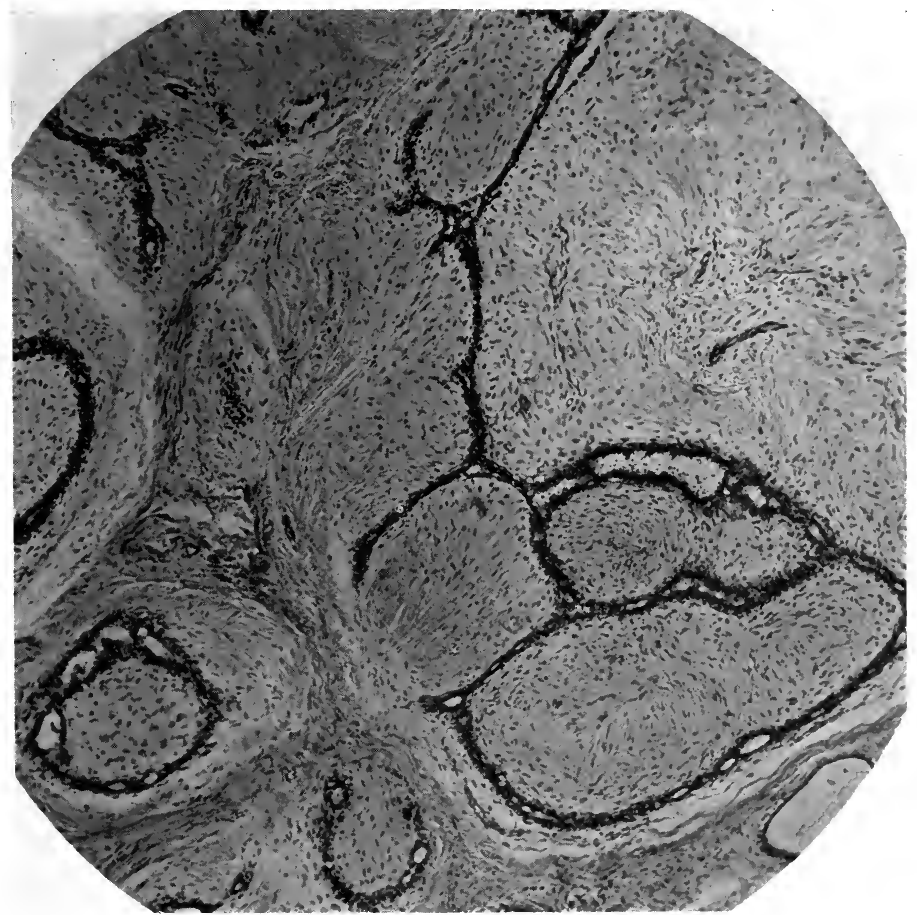

FIG. 21 5--Intracanalicular fibro-adenoma of breast.

dimensions which is firm and fibrous or more often soft and vascular. A very common tumor occurring especially in young persons is the solid encapsulated fibroma which forms a firm, translucent, lobulated, and freely movable growth. In cystic fibro-adenoma the acini are often dilated, forming cysts which are single or multiple, large or small, while the contents are serous, mucous, bloody, or semifluid fatty material containing cholesterin crystals.

The structure of these tumors presents varying proportions of stroma and epithelium. In diffuse adenomatous hypertrophy both glands and stroma are well represented and active, but the adenomatous element usually predominates. In the large soft circumscribed adenomas the alveoli are abundant, large, and lined with several rows of overnourished cells, while the stroma is abundant, very cellular, and sometimes quite vascular. The 
firm solid encapsulated tumors are chiefly fibromatous. The connective tissue is cellular and either surrounds the acini in broad bands (pericanalicular), or grows diffusely, or forms complex papillary projections into the acini (intracanalicular fibroma). In these growths the epithelium is quite subordinate and merely covers the enlarging connective-tissue papillæ. In the typical cystic fibromas the cysts are lined by one or several rows of cubical epithelium which may be thrown into low papillary projections. In one case Buday found ciliated epithelium.

(2) Papillary Intracystic Fibro-adenoma, Cystadenoma.-Cystadenoma is a comparatively common tumor. It appears usually after the menopause (average 50 years), but occasionally in young or very old subjects. The usual features are of a single or multiple rounded tumor, movable beneath the nipple, which may be retracted, of slow growth, and often accompanied by a

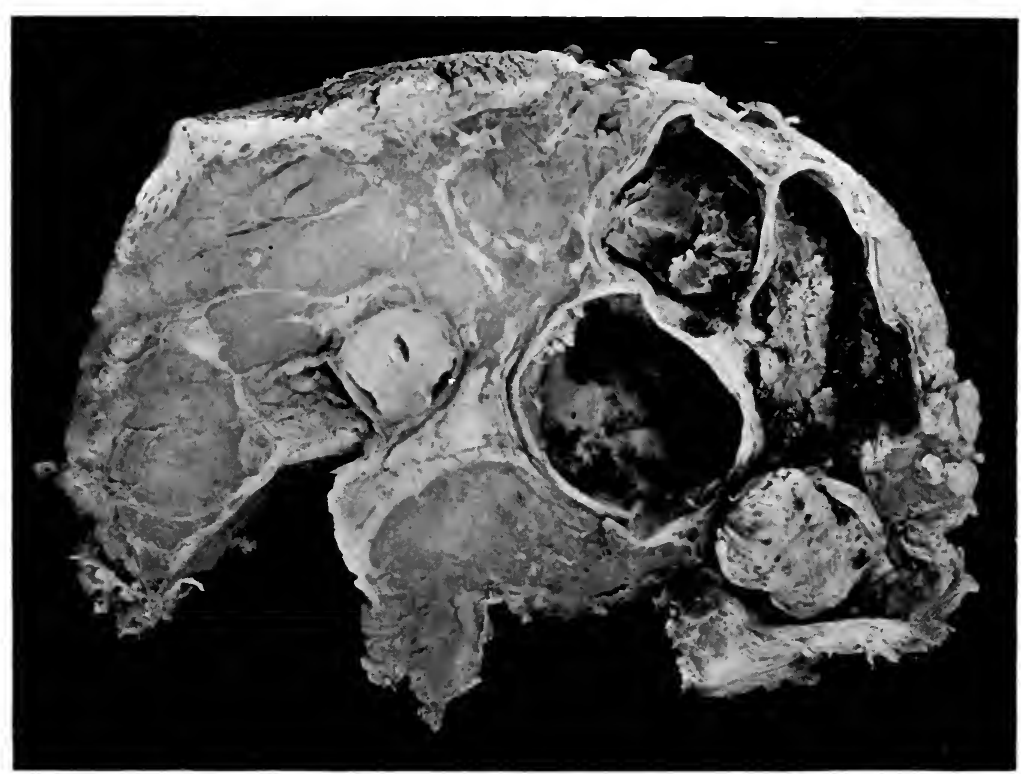

FIG. 2I6.-Multiple cysts of breast, in some of which are papillary adenomas.

serous or bloody discharge from the nipple. The typical cystadenoma is inclosed in a cyst wall. The tumor may appear as a papillary growth attached to one segment of the wall and incompletely filling the cyst, or it may occupy the entire cavity distending the wall, or numerous points of fusion unite the tumor with the wall. These tumors develop by papillary growths into the larger ducts. They are usually encountered when they have reached the size of a hen's egg, but may grow considerably larger. They are usually single, but occasionally multiple and rarely bilateral. The position in the central portion of the breast is characteristic, but not invariable. Cornil describes cases exhibiting rapid growth which is usually due to accumulation of fluid or myxomatous degeneration. Rarely they distend or adhere to the skin or open externally. Johada found an adenoma floating free in the pus of an infected cyst. Delbet and Mintz consider a bloody discharge as a sign of probable malignant degeneration. During pregnancy and lacta- 
tion these tumors may rapidly enlarge and secrete milk, so that they suggest a malignant process or galactocele (Deaver, McFarland).

The structure shows a papillary or complex dendritic growth of connective tissue covered by epithelium. In some tumors the connective tissue predominates and forms coarse branching masses which are fibrous, mucoid, vascular or cellular, or infiltrated with lymphocytes. In "cystosarcoma

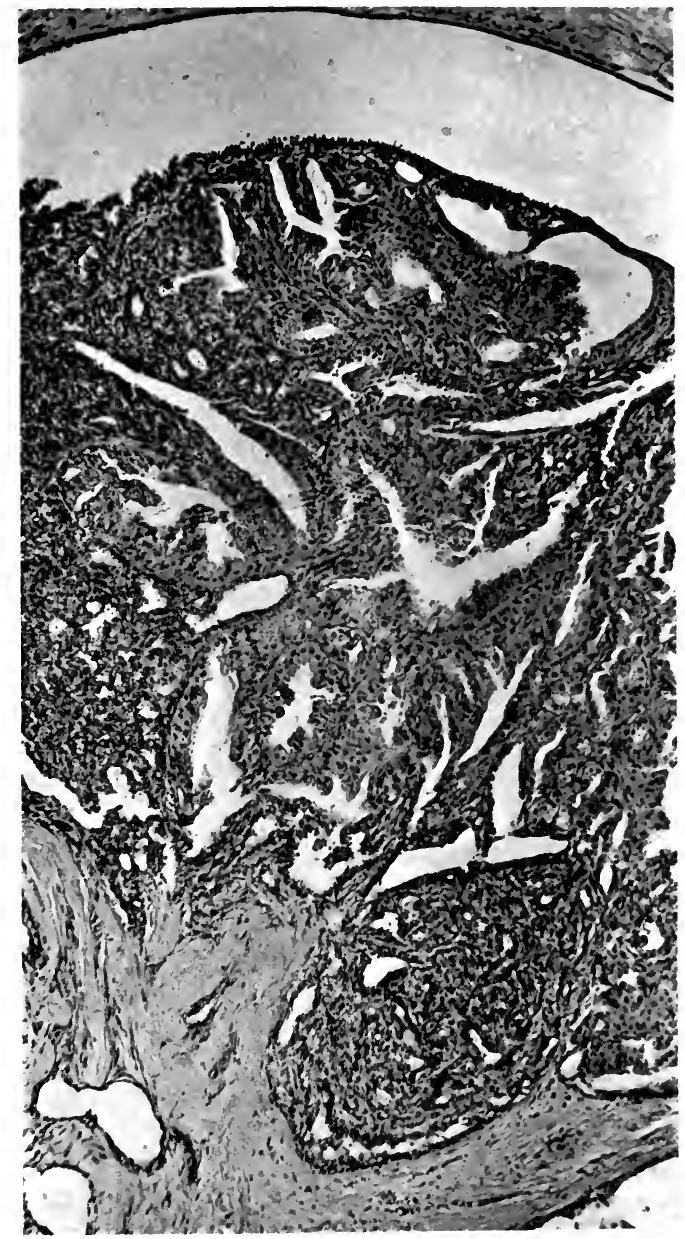

FIG. 217 .- Segment of benign fibro-adenoma growing into a large cy'st in chronic mastitis.

phyllodes" the papillæ are largely of connective tissue which is very cellular, and when this tumor perforates the skin a false appearance of malignancy is presented. This term was first employed by J. Muller to designate the cauliflower-like polypoid masses of connective tissue which fill a cystic cavity in the breast. The same tumor Virchow called intracanalicular myxoma. It is evident that these observers encountered much later stages of these growths than are now commonly seen. 
In most cystadenomas the epithelium predominates and the growth may resemble the ovarian cystadenomas. The lining cells are cubical or cylindrical epithelium in one or several rows. Malignant forms of intracystic fibro-adenoma are relatively common, but whether they represent malignant transformations of a benign tumor or are malignant from the first is difficult to determine. The malignant forms are described by many writers as duct or villous cancer. Delbet holds that the transformation is so common as to demand radical treatment of all intracystic fibro-adenomas. Malignant changes have been recorded by Guinard, Speese, and others. Hansen extirpated a carcinoma which appeared at the base of a fibro-adenoma excised I6 years previously. In 20 cases of cystadenoma Greenough and Simmons found adenocarcinomatous areas in.3. The structure of the malignant tumors is characteristic and follows the type of adenoma destruens, malignant

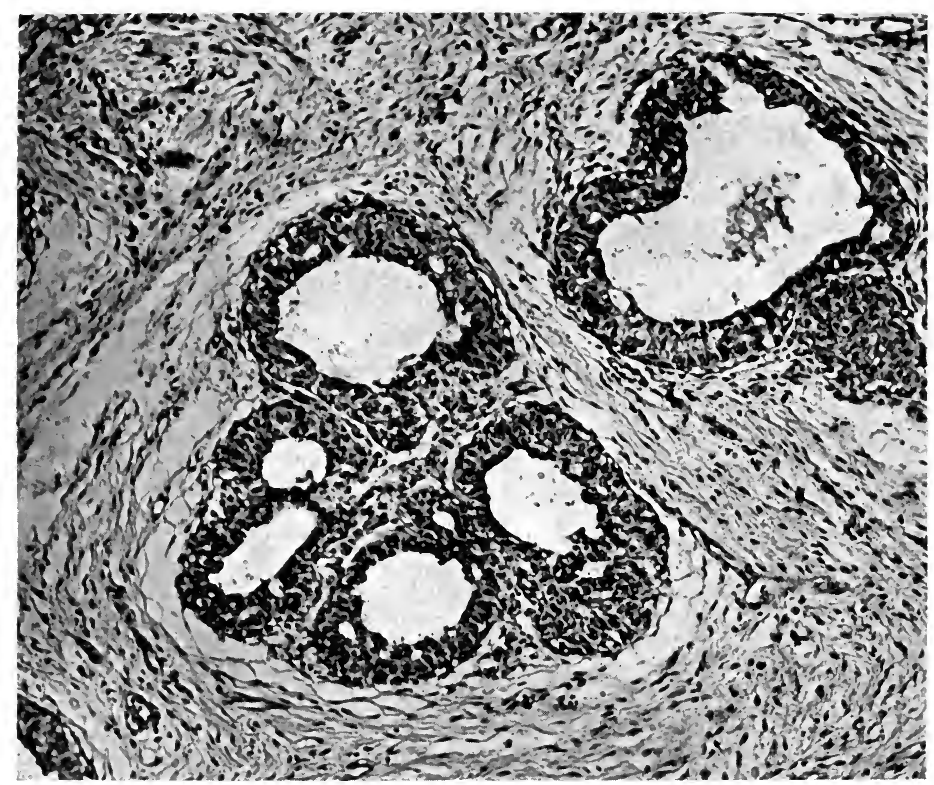

FIG. 218.-Fetal fibro-adenoma of breast in a girl of Io years. Marked overgrowth of epithelium.

adenoma (Tietze), or adenocarcinoma. This structure is often preserved in the metastases which appear late. Fully developed carcinomas are not infrequently seen which give every appearance of having developed in a segment of a duct. These tumors are solid and sharply encapsulated. An outlying cyst or cystadenoma may accompany the solid tumor. The structure usually preserves traces of a papillary or adenocarcinomatous type. Long immunity of the lymph-nodes may also characterize this group of mammary carcinomas. When mucous degeneration attacks the stroma very bulky tumors may result. I have examined such a tumor as large as a child's head of ro years' duration, without involvement of lymph-nodes.

The prognosis of cystadenoma varies widely, corresponding to the structural type. The simple adenomas are benign and an extirpation of the whole breast or even of the tumor alone is all that is called for (Warren). 
Adenoma destruens is long confined by the cyst wall and when well encapsulated may safely be removed with the breast. When the capsule is traversed these tumors are fully malignant and the radical operation is indicated, especially the dissection of the axilla. A definite tendency to invade the muscles is not common.

(3) Fetal Fibro-adenoma.-A peculiar form of fibro-adenoma presents several structural features of the fetal breast, including very cellular connective tissue, alveoli without membrana propria, and imperfect differentiation between ducts and acini. In a characteristic case the tumor was soft, circumscribed, and as large as a goose egg. The structure showed a moderate number of alveoli lined by several layers of cubical cells without membrana propria or the peculiar sustentacular "basket" cells of the adult organ. The cellular overgrowth was pronounced and suggested malignant tendencies. These tumors occur usually between the ages of $I_{5}$ and 25 years. They grow rapidly, producing soft, vascular, well circumscribed, lobulated tumors, which reach much larger dimensions than the fibromas, and are often regarded as sarcoma. Yet they do not recur.

Pure adenoma of the breast is a very rare tumor, but has been described by several authors (Kuru, Lit.). Ziegler describes tubular and acinous varieties. In most of these tumors the growth of connective tissue is considerable and approaches that seen in fibro-adenoma with which they are connected by transitional forms. The gross appearance and course are very similar to those of fibro-adenoma.

Pure fibroma also is rarely observed and bears a close relation to fibroadenoma. Williams has collected several cases from the older literature.

Teratoid Mixed Tumors.-Mixed tumors in which mesodermal or ectodermal tissues differentiate toward the adult type are not infrequent in the human breast. In the dog this type of complex mixed tumor is very frequently observed and the tissue changes are presented far more extensively and clearly than in man (Virchow, Cornil and Petit, Cushing).

Two main groups of teratoid tumors of the breast may be recognized, which are chiefly (I) mesodermal, or (2) ectodermal (Baumgartner).

(I) Mesodermal tumors appear to be closely related to adenosarcoma both in origin and structure. Thus Bowlby described a cystic adenosarcoma throughout which were many nodules of hyaline cartilage. Pilliet found myeloid giant-cells about the bony trabeculæ in an adenosarcoma. In the dog breast I find that most adenosarcomas yield islands of bone or cartilage. Diffuse calcification of a large sarcoma of 3 years' duration was observed by $B$. Clark, and in an axillary tumor Dubar found much diffuse osteoid tissue.

Frequently true bone tissue is found replacing islands of cartilage in normal development, as occurred in cases reported by Stilling, Battle, Cornil, and Souligoux.

Nearly pure chondroma of the breast is not extremely rare (Lange, Cambria, I.eser, Davidsohn). Happel's tumor was large and cystic. Soulier observed extension to the superior maxilla. Not a few are cellular and embryonal in type and malignant (Desoil). Myxomatous degeneration is often present and tends to increase the size of the growth. Chevrier and Debral found a large tumor occupying the whole breast and containing fibroma, myxoma, chondroma, osteoma, and gland alveoli. Combinations of chondroma with carcinoma are recorded by Wagner, Coen, and others. Kaufmann has described a large cystic chondrocarcinosarcoma. Warren saw 3 cases combined with scirrhous cancer. S. W. Gross described a bony stroma inclosing islands of lymphoid bone-marrow with giant-cells and sur- 
rounded by carcinoma simplex. Coen analyzed a slowly growing tumor with vascular well-formed bone, which he attributed to metaplasia. Hacker's case contained bone, cartilage, carcinoma, and fibrocystadenoma, and he regarded it as a terminal product of an original cystadenoma. I have examined a very large solid and cystic tumor containing chiefly masses of poorly developed cartilage, myxomatous tissue, and areas of diffuse embryonal carcinoma. Metaplasia could not have accounted for the extensive development of cartilage.

Chondroma and osteoma of the breast are usually associated with sarcoma or carcinoma, but in the series of reported cases there is a gradual reduction in the malignant elements, leaving eventually pure chondroma or osteoma. Some of these growths are therefore probably one-sided developments of mixed tumors.

(2) Ectodermal mixed tumors take the form of multiple cholesteatoma or squamous-cell carcinoma, often combined with sarcoma. The multiple sebaceous cysts of the breast were early noted by Astley Cooper and by Virchow and have later been studied by Grohe, Wilms, and others. The true tumors resemble cystic fibro-adenomas with characteristic sebaceous material and epithelial detritus in the cysts, which are lined by squamous epithelium. This lining may show differentiation into strata, both horny and Malpighian, with eleidin granules. Stoerk and Erdheim point out that

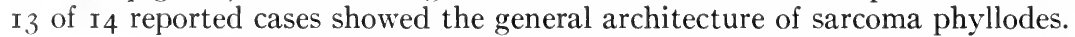
The gland alveoli may show adenomatous growth and the stroma is often sarcomatous. In Konjetzney's case the squamous epithelium had suffered malignant transformation.

In another group of cases squamous epithelium appears in isolated foci associated with fibro-adenoma or glandular carcinoma without cholesteatomatous features. Beginning with the case of Stoerk and Erdheim, in which scanty islands of squamous-cells appeared in a fibro-adenoma, one finds a series of cases in which squamous epithelium becomes more and more abundant. I have studied one case in which glandular and squamous epithelium were about equally divided, while the transformation of one type into the other seemed obvious. In Duval's tumor the cells were squamous, cuboidal, or giant. Walther described a large tumor the periphery of which was pericanalicular fibroma, in the center cystic adenoma, while a single cyst was lined by well-developed skin. Nadal found many squamous-cell groups scattered in a sarcomatous matrix.

The course and prognosis of the teratoid mixed tumors vary with their structure, but as a rule they are slowly growing and comparatively benign. Yet carcinomatous and sarcomatous metastases are observed reproducing the original structure. Nadal found in the spleen a nodule of chondro-adenoma resembling the original tumor.

The origin of the teratoid tumors of the breast has been attributed to complex embryonal inclusions containing mesodermal skeletogenous material and fetal ectoderm. This view has been maintained by Wilms, Lecene, Nadal, Menetrier, and others. The encapsulation of the early growths and the wide diversity and fully adult character of the tissues produced favor this view. Some rapidly growing complex chondrosarcomas strongly suggest an origin from cells of undiminished and embryonal growth capacity. On the other hand, it has been clearly shown that this series of tumors includes many transition stages from fibro-adenoma up to the most complex forms. Close histological study appears to show every stage of metaplasia from the ordinary gland- and stroma-cells of the breast into cartilage, bone, and squamous epithelium. In the dog tumors this histological evidence is unequivocal. 
Hence it appears necessary to accept the theory of autochthonous or local development of mammary mixed tumors as maintained by Grohe, Cornil, and Herrenschmidt.

In the breast one sees the most remarkable forms of metaplastic tumor growth, and while this view does not exclude the origin of the tumors from embryonal and misplaced cell groups it renders this assumption no more urgent for the teratoid growths than for the simpler fibro-adenomas.

Adenosarcoma.-Adenosarcomas represent the malignant form of adenofibroma, but their many striking clinical and anatomical features render them a rather specific form of mixed tumor of the breast. It is quite probable, as Delbet suggests, that most pure sarcomas are identical in origin with adenosarcoma. This view must be accepted at least for the true spindle-cell sarcomas. Not only is their structure identical with that of adenosarcoma, but recurrence of adenosarcoma may take the form of spindle-cell sarcoma (Finsterer). These tumors form from 7 to ro per cent. of mammary neoplasms (Gross, Schmidt, Schouler). While the chief age of incidence is between 30 and 50 years (average 40 ) the condition has been observed by Gross at 9 years and at 75 years. Labbe and Coyne early pointed out that nearly half the cases represent a malignant transformation of a long-standing fibro-adenoma, and that gestation, lactation, and trauma appear to be exciting causes of the change. Ropke and Gebele emphasize the relation to trauma, but identical histories are observed without trauma. Tumors of very long duration eventually becoming malignant are satisfactorily recorded also by Gross (40 years), Schouler (39 years), Mornard, Masson (24 years), Borchmeyer (6 years).

The form of the tumor is usually rounded and lobulated. Cornil describes solid and papillary cystic types, the former developing from solid adenofibromas, the latter from cystadenofibromas. The same breast may contain adenosarcoma and benign fibromas. The cystic forms produce the largest tumors seen in the breast, Velpeau's tumor weighing $20 \mathrm{~kg}$. Labbe and Coyne evacuated a liter of fluid. In the early stages the tumors are circumscribed or encapsulated, but active growth leads to dissemination through the organ, and fungating masses perforate nipple and skin with ulceration. Edema, hemorrhage, necrosis, and suppuration complicate the advanced stages. In some cases myxomatous changes and overgrowth of blood-ressels are prominent.

The structure presents considerable variations. Most tumors are composed chiefly of spindle-cells, large or small, surrounding gland acini or ducts, or growing within distended channels or spreading diffusely. The glands may participate for a time in the overgrowth, but in advanced cases they usually disappear, or are found in isolated islands. Round-cell growths are less numerous and rather more malignant. Such cells usually show traces of a spindle or polygonal form, and sometimes an alveolar arrangement. Myxomatous changes may appear in large areas of bulky tumors. Giant-cells of myeloid type occur in spindle- and round-cell growths.

It is evident that some European authors have included among adenosarcomas certain rather cellular adenofibromas which have no distinct malignant qualities, but have reached a large size by virtue chiefly of long duration. It also seems highly probable that some cases reported as adenosarcoma are rapidly growing carcinomas in which the anaplastic tumor cells assume a spindle or rounded form and grow about hypertrophied ducts. The true adenosarcoma of the breast is very rare and usually follows a long-standing adenofibroma.

Definite association of sarcoma with carcinoma is reported by Lecene. 
Cornil, and Kettle. In several such cases I have not been satisfied that the spindle-cell areas were not modified epithelium.

The course of the true adenosarcoma when once established is usually rapid and fatal. Yet in early stages remissions in the growth are recorded, without satisfactory explanation. Gross emphasized the capricious character of the growth of both cystic and solid sarcoma, but a rapid growth once established was usually maintained. In the late stages the progress is continuous or fulminating, the skin becomes adherent and ulcerated, dilated veins appear over the surface, and cachexia develops from absorption of degenerating and necrotic material, and from metastases. The relative immunity of the lymph-nodes has frequently been noted. Gross in ${ }^{5} 6$ cases of various types of sarcoma found the nodes invaded in only 3 ,

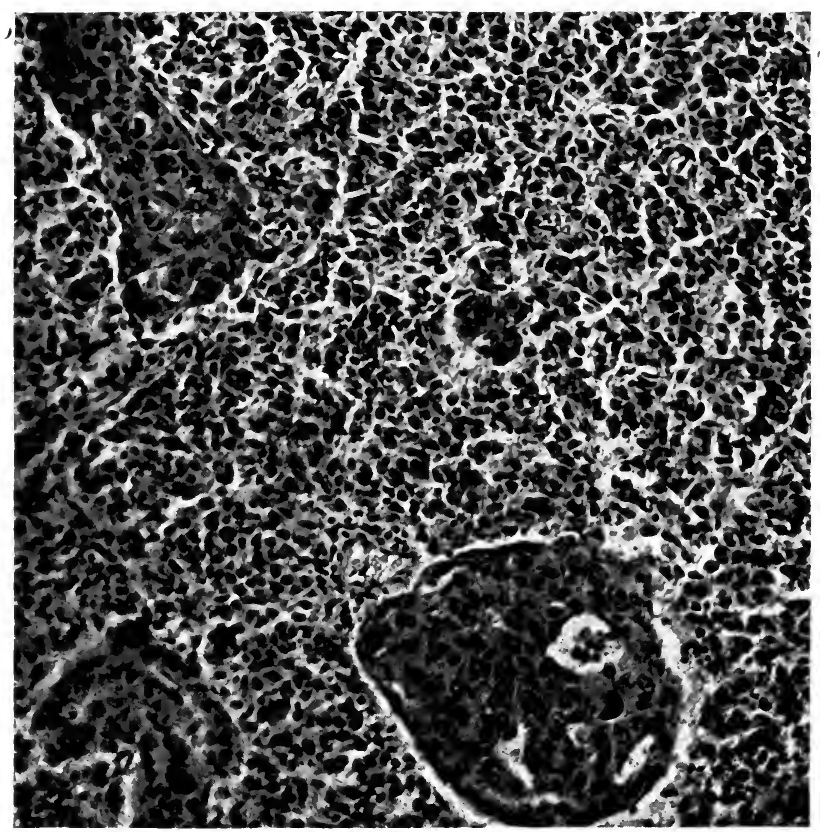

FIG. 219.-Mammary cancer. Alveolar and diffuse carcinoma, simulating carcinosarcoma.

one of which was a melanoma. A much higher proportion of invasion of lymph-nodes is recorded in Finsterer, Gebele, and Rosenstein. Generalization by the blood-vessels with internal metastases is recorded by Schouler in I 2.4 per cent. of the cases. Secondary tumors have been found in lungs, liver, pancreas, bones and brain, and elsewhere. Persistent local recurrence is observed in a high proportion of cases. In Hoffmann's remarkable case proliferating gland acini persisted in the twelfth recurrence, but were missing in metastases in the abdominal wall. Recurrence as round-cell sarcoma and as carcinoma are recorded by Poulsen. Pean reported local return after 25 years. Gross observed recurrence in $5 \mathrm{I}$ per cent. and metastases in I 2 per cent. of cystic sarcomas, with 12 per cent. of cures by operation. Many cases requiring multiple operations are recorded, on one of which by $\mathrm{S}$. D. Gross $2 \mathrm{I}$ excisions during 4 years eventually eradicated the disease. 


\section{PURE SARCOMAS OF THE BREAST}

When one excludes from the group of mammary sarcomas the adenosarcomas, the malignant forms of mixed tumoss containing cartilage, bone, mucoid, or fat tissue, and certain malignant round-, spindle-, and giant-cell alveolar pseudosarcomas which are really atypical carcinomas, there is little remaining of a once formidable group of mammary neoplasms. There are reasons for believing that such a disintegration of the group is justifiable. In fact, the situation today is much as it was in 1894 , when Williams found it impossible to write the history of pure mammary sarcoma because of the absence of requisite data.

Of Gross' collection of ${ }^{6} 6$ cases, 33 per cent. were adenoid, 50 per cent. cystic, and 50 per cent. solid. On clinical grounds Billroth and König concluded that the cystic sarcomas were closely related to adenofibroma, and Schimmelbusch, from thorough anatomical study, reached the same conclusion. Gross mentions that adenoid structures persist chiefly in the spindlecell sarcomas, and that myxomatous changes are almost peculiar to spindle-cell and cystic tumors. The great predominance of the forms thus entangled in the group of malignant fibro-epithelial growths is indicated in Finsterer's recent series-cystosarcoma, I8 cases; spindle-cell, Io; myxosarcoma, 5; round-cell, 6; lymphosarcoma, I.

The pseudosarcomas of epithelial origin probably cover a considerable subgroup of mammary sarcomas. A definitely alveolar structure points at once to an atypical epithelial tumor process. Not a few such tumors are included in the lists of sarcoma. They are difficult to distinguish in the gross from medullary carcinoma. Billroth fully recognized the difficulties of diagnosis and seems to have reached the conclusion that they belong in a group of carcinosarcoma. I have studied several rapidly growing cellular tumols of alveolar or diffuse structure and failed to find any definite evidence of a mesoblastic origin. On the contrary, as Billroth observed, areas can usually be found which are duplicated by portions of undoubted medullary carcinoma. Finsterer saw a supposed alveolar sarcoma recur as frank carcinoma. G. B. Schmidt's angiosarcomas consisted of cells of "distinct epithelial form" in alveolar arrangement. The uncertain significance of perithelial structures in breast tumors is well elucidated in the discussion of Nadal's case of mixed osteosarcoma and perithelioma.

Melanoma also figures among the alveolar and giant-cell sarcomas. Fioriana describes a melanotic lymphangiosarcoma, a form not infrequently assumed by melanoma. The lobular and encapsulated form of some reputed cases of giant-cell sarcoma suggest an origin from cystic adenocarcinoma in which diffuse areas of round and giant-cells are not uncommon.

The round-cell sarcomas of various authors form an ill-defined group. They probably include very atypical tumors of the same nature as the spindlecell sarcomas, malignant and atypical carcinomas, and some pigment-free melanomas. Gross found their chief occurrence at 48 years, the period of beginning physiological decline of the breast, while the spindle-cell tumors appear earlier, 36 to 38 years. Six of Finsterer's 40 cases, 5 of Gebele's 34 cases, and 27 per cent. of the series collected by Gross were round-cell tumors. That the nature of these tumors is often highly uncertain is indicated by the simultaneous occurrence of small round-cell sarcoma in one breast and glandular carcinoma in the other, as in Finsterer's case $2 \mathrm{I}$; or by his case 23, of lymphosarcoma of alleged traumatic origin. That the structure of roundcell sarcoma may be duplicated by carcinoma is demonstrable in certain atypical acinar carcinomas in which considerable areas may fail to show any 
alveolar arrangement. Billroth's case of acute lymphosarcoma of both breasts arising in gestation and fatal in 5 months is very suggestive of the atypical carcinomas of pregnancy. Battle in reporting a large fungating round-cell sarcoma states his belief that the growth was some form of fungating tumor arising within a cyst.

True sarcomas composed of rounded or short spindle-cells probably represent an atypical form of spindle-cell sarcoma. They are highly malignant, 60 per cent. recurring and 25 per cent. becoming generalized (Gross). In one of Finsterer's cases the malignant tumor followed excision of a cystosarcoma, and in most cases a slowly growing tumor has existed for some months.

Pure spindle-cell sarcomas of the breast form a rather well-defined group which closely resemble both clinically and anatomically their more complex homologues, the adenosarcomas. The most characteristic form of this tumor begins as a well-circumscribed growth presenting the lobulated appearance

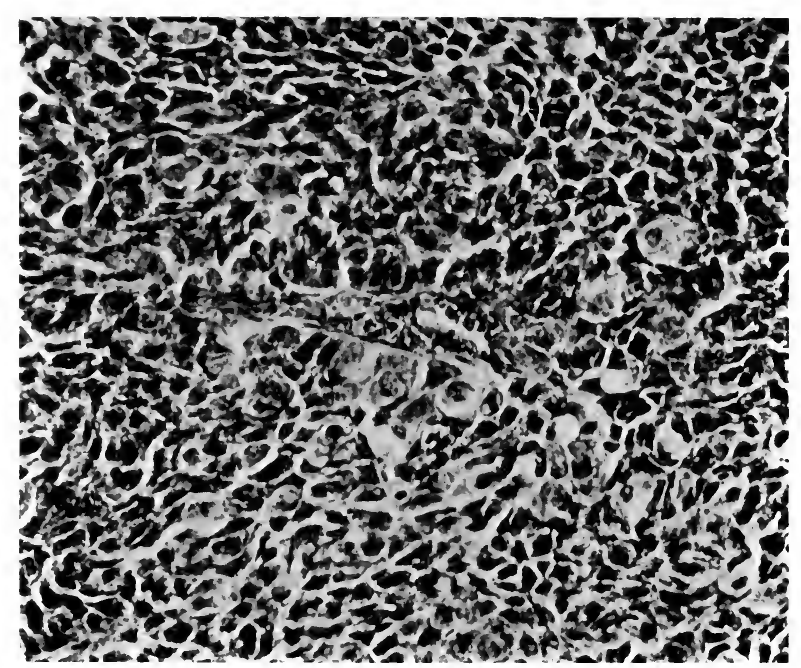

FIG. 220.-Mammary cancer. Diffuse growth of large polyhedral and spindle-cells.

of a soft fibroma. The rate of growth, however, is very rapid, and the tumors reach very large dimensions in a few months or weeks, fungating through the skin, undergoing central liquefaction, and eventually becoming infected with extensive necrosis, fever, and cachexia. Radical removal in an early stage may be successful, but local recurrence is common, and pulmonary metastases frequently develop. They vary greatly in consistence, cell content and form, vascularity, and in the degree of secondary, especially myxomatous, degeneration. Combinations with osteoma, chondroma, and lipoma are occasionally observed (Gross). They formed 68 per cent. of Gross' cases and the great majority of Finsterer's. The prominence of cysts emphasizes their relation to adenosarcoma. In clinical course and gross appearance they are not to be distinguished from the adenosarcomas, but there is a tendency toward occurrence at earlier ages and persistent local recurrence.

Even the presence of an apparently pure spindle-cell structure does not positively assure a mesoblastic origin. Diffuse or atypical carcinomas, especially the malignant forms, may yield broad areas of small spindle-cells, and 
only a thorough study of many areas as well as of the history of the tumor can establish a positive diagnosis.

Osteochondromyxosarcoma is a relatively common variety of complex mammary sarcoma. Its relation to the teratoid growths is clearly brought out in the combinations with dermoid cysts, adenoma, and carcinoma. More often the tumors are purely mesoblastic (St. Arnold, Lit.). The tumors are large, solid or cystic, actively growing, and tend to ulcerate and suppurate. The sarcomatous areas are composed of spindle-, round, or giant-cells (Gross, Manz, Durham). Two tumors of this type in the same breast with areas of spindle- and round-cell sarcoma were observed by Borst.

\section{MISCELLANEOUS TUM ORS OF THE BREAST}

The usual benign tumors of the skin occasionally develop in the mammary region.

Angioma, beginning near the nipple, after trauma, and gradually extending as a pulsating tumor throughout the breast is described by lmage and Hake and by Langenbeck. Subcutaneous angiomas, cavernous or cystic, and erectile, are reported by Williams, and Quenu and Küss. A peculiar diffuse erectile angioma involving the stroma of the gland and the peri- and interacinar connective tissue with atrophy of gland tissue has been studied by Malapert. Lymphangiomas of large dimensions are described by Wegner, Müller, and Pinner, and some large serous cysts in this region probably arise from lymphangiomas. A metastasi zing angioma of breast is described on page 228.

Sebaceous cysts and papillomas occur chiefly about the nipple and may be the source of carcinomas.

Leiomyoma of the nipple or skin has been recorded by Virchow, Sokolow, and Niklas.

Melanoma has been reported by many authors, and presents its usual malignant features. It occurs in both sexes, chiefly about the nipple. Vieregge records a case in an infant. Billroth and Cornil and Ranvier described melanotic carcinoma involving the breast tissue, but there is no evidence that the tumor arose from the gland tissue. In these and other cases an origin from moles of the skin or included epidermal rests, or a secondary pigmentation from hemorrhage must be assumed.

Dermoid cysts of the breast are rare, but several cases have been coilected by Williams and Baumgartner. They are of interest in connection with the mixed tumors and cholesteatomas. A large dermoid described by Reverdin and Mayor was associated with fibro-adenomas. In the middle line, between manubrium and gladiolus, there is sometimes a congenital fossa, and here simple dermoids have been observed (Williams, Lit.).

Lipoma of Breast.-Diffuse lipomatosis is often associated with diffuse hypertrophy. Intraglandular lipomas are very rare, but are described by A. Cooper, Koehler, and Begouin. They show lobulated overgrowth of fat tissue with scattered gland alveoli. Hofer found a small mass of bone in the center of a mammary lipoma.

Retromammary lipomas may reach very large dimensions. Billroth's case measured $43 \mathrm{~cm}$. in its long diameter. Queirel's tumor arose from the tissues of the pectoralis and weighed $800 \mathrm{gm}$. Paget describes a case in a male subject. The subcutaneous lipomas may be single, multiple, or bilateral (Reclus, Baker, and Bowlby). They are subject to central softening and in a group of cases some of which are congenital myomatous areas appear (Moore, Ashby, and Wright). 
Epithelioma of the skin of the breast occurs along thoracic and mammary fold, where it is favored by intertrigo (Richet, Winiwarter), along the axillary border, and in the nipple, whence it may invade the breast and simulate carcinoma. In this last situation it requires differential diagnosis from Paget's disease which is more superficial and wide-spread (Hauser), and from metaplastic squamous changes in duct carcinoma. Cornil traced a squamous epithelioma of the nipple extending some distance into the large ducts. Morestin observed epithelioma of the nipple associated with true carcinoma of the gland. Robinson described a rodent ulcer at the nipple. The tumors may be preceded by a papilloma or a sebaceous cyst (Bryant). They do not differ essentially from epitheliomas in other regions, but in the nipple the course may be unusually active.

\section{CARCINOMA OF BREAST}

General Etiology.-Incidence.-The U. S. Census for I9I4 reports 5423 deaths from cancer of the breast among $5^{2,420}$ total cancer deaths. The mortality rate rose enormously between I900 and I9I2, the average annual death rate from I90I to 1905 being 18.45 per cent., from I906 to I9I0, 30 per cent. There has certainly been no such increase in the incidence of the disease and the recorded increase must be due largely to improved diagnosis and registration. Since a certain proportion of breast cancers are cured by operation the morbidity record must be somewhat higher. R. Williams from 4628 cases of cancer in women in London Hospitals, I868 to I888, calculates that 40.3 per cent. were mammary, 34 per cent. uterine. English mortality statistics, however, placed uterine and ovarian cancer, 34.7 per cent.; mammary cancer, 2 I.2 per cent. In Ireland the ratio was: stomach, 22.4 per cent.; breast, 2 I.5 per cent.; uterus, I4.I per cent. In Frankfurt-am-Main: I860 to I889, uterus, 27.5 per cent.; stomach, I8.3 per cent.; breast, II.3 per cent.

In I9I 2 the mortality from mammary cancer in England and Wales was I9.8 per I00,000 of population. Hoffmann points out that there is great variation in the mortality in different countries. Thus from I906 to I9ro the rates per 100,000 of population were: England and Wales, I7.9; United States, I3.3; Bavaria, 9.I; Italy, 5.8; Japan, I.8.

Of all mammary tumors carcinoma forms 82 to 84 per cent. (Delbet).

Age Incidence.-

U. S. Census, 1914. Age Incidence of Mammary Cancer

\begin{tabular}{c|c|c|c|c|c|c|c|c|c|c|c|c|c|c|c|c|c|c}
\hline \hline $\begin{array}{c}\text { Years } \\
\text { I 5-19 }\end{array}$ & $20-4$ & $25-9$ & $30-4$ & $35-9$ & $40-4$ & $45-9$ & $50-4$ & $55-9$ & $60-4$ & $65-9$ & $70-475-9$ & $80-4$ & $85-9$ & $90-4$ & $95-100$ \\
\hline $\begin{array}{c}\text { Cases } \\
4\end{array}$ & 6 & 44 & I I I & 301 & 460 & 646 & $67 \mathrm{I}$ & 658 & 551 & 529 & 435 & 311 & 207 & 93 & 23 & 6 \\
\hline
\end{tabular}

British mortality statistics, I90I to I903, place the highest mortality between 55 and 65 years. Mortality statistics of Williams, Paget, Nunn, and of most surgeons place the mean age of incidence at 45 to 50 , or about the 48 th year (Schmidt).

The earliest authentic cases recorded appear to be those of Kaufmann, I7 years; Henry, 2 I years, and Williams, 24 years. Brewer reports a fibroadenoma with carcinomatous areas in a negress of 16 years.

Lactation. Of 628 cases of Velpeau, Gross, and Winiwarter, 44I had 
nursed children, I 87 had not. Lehmann offers statistical evidence that nursing is a prophylactic against mammary cancer. Pregnancy is without definite influence.

Hereditary influence has appeared to exist in 5 to ro per cent. of various series of cases studied specifically for this purpose (Delbet). With more rigid criteria Guleke reduces the possible hereditary cases to 2.36 per cent. Williams found cancer in the family in 24.2 per cent. and quotes Sibley's observation of a mother and 5 daughters who died of cancer of left breast.

There is a slight predominance of the disease in the left breast. It appears to originate in both breasts in about I.5 per cent. The frequency of bilateral cancer at the time of observation is, however, much greater. Handley finds 5 per cent. of advanced cases bilateral and Hubbart 9 per cent. Benassey rightly distinguishes between (I) cancer of the second breast developing after amputation of the first, which is not uncommon, and (2) cancer developing in the second breast from an original focus in the first, and (3) primary bilateral cancer, which is rare. The upper axillary segment is the chief site of origin (Delbet). Traumatism has been asserted or suspected in 5 per cent. by Schmidt, I 2.5 per cent. by Schultheis, and 12.7 per cent. by Fink. Its essential relation to mammary cancer is still unproved, but injury not infrequently accelerates growth. In an organ constantly exposed to trauma as is the breast an apparent relation to cancer must be subjected to the most rigid criteria. Thus Williams who obtained a history of antecendent trauma in 25 per cent. of his cases found not a single instance in which the disease had developed out of induration or other obvious lesion thus induced.

Previous exudative inflammation often leaves tissue changes predisposing to cancer. Winckel found such lesions in 17.5 per cent. of a series of cases, including abscesses in $\mathrm{I}_{5}$; lesions of the nipple, 8 ; and inflammation without suppuration in one of 24 cases.

Developmental anomalies are traceable chiefly in the origin of cancer from sequestrated portions of the breast. Of 132 cancers observed by Williams I3 arose from aberrant tissue, in axillary, sternal, or infraclavicular regions. In not a few of these aberrant tumors the structure is nearly pure adenoma with evidences of functional capacity, but the true carcinomas in these regions are often highly malignant and may be atypical. The milk secreting adenomas of Birkett and Power and the tumor-like swellings of aberrant breast tissue described by others are regarded by Williams as hypertrophic rests rather than true neoplasms. Williams calculates that i4 per cent. of fibro-adenomas arise from such rests. Many of the complex mixed tumors are probably of identical origin, but it seems unnecessary always to include skeletogenous material to account for the bone and cartilage found in them. Epidermal inclusions may be held responsible for certain dermoids and melanomas of the breast, but metaplasia is a sufficient explanation for the majority of cholesteatomas and the squamous-cell islands of mixed tumors.

The alveoli of sweat-glands, which are nearly constantly found scattered through the breast, especially in the neighborhood of the larger ducts, give rise to a specific form of mammary carcinoma which corresponds to the duct cancer of many writers.

According to Creighton the duct system of the breast is a derivative of the cutaneous sweat-glands. Superfluous or aberrant portions of this system retain the characters of sweat-glands, persist in the adult organ, and give rise to most of its tumors. The secreting gland-cells he derives from the mesoblastic fat-cells about the ducts, which readily become transformed into the acinar epithelium when the demands for functional activity arise. This 
interesting conception once had considerable authoritative support, but is now almost entirely ignored. There are not a few facts in the physiology and pathology of the organ which are consistent with this hypothesis.

The effective causation of mammary cancer is to be traced in the combination of several predisposing, contributing, and exciting factors. There is ground for assuming that many subjects are predisposed to mammary neoplasms. Some of these subjects appear to develop simple glandular hypertrophy in early life, and this same instability of the organ may under favorable conditions be an important factor in precipitating malignant tumor growth in later life. Developmental anomalies leading to fibro-adenoma and sequestration of breast tissue form a tissue predisposition in a certain group of cases. By far the most important element in the tissue predisposition is chronic productive mastitis. The great majority of cancers develop in organs altered by reactive inflammatory processes. Minute histological analysis of the conditions surrounding the beginnings of cancer in chronic mastitis point to mechanical isolation of cell groups in fibrocarcinoma, and irritation by chemically altered secretion and exudate in adenocarcinoma, as the immediate precursors of atypical overgrowth. This irritation may very well come from various microörganisms and animal parasites, such as the Demodex folliculorum described by Borrel, none of which can be regarded as specific exciting factors. In fact, there appears to be no need of introducing the conception of a specific exciting factor in mammary cancer, since the disease is satisfactorily accounted for as the liberation of growth tendencies of overnourished and proliferating cells, under peculiar anatomical relations and often but not necessarily in predisposed subjects.

Pathological Anatomy:- While there are several well-defined anatomical forms of mammary cancer the specific features characterize chiefly the early stages of the disease. Arising under markedly different conditions of a wide variety the anatomical distinctions are often obliterated when the disease is fully established and the local lymphatics are invaded.

On chiefly anatomical features the disease may be considered under the following forms:

I. Adenocarcinoma arising chiefly in cysts of ducts or sweat-glands.

2. Duct carcinoma arising from the lining cells of ducts.

3. Acinar carcinoma arising from the epithelium of the acini.

Of these main groups there are several subdivisions, such as gelatinous or mucous carcinoma, fibrocarcinoma, and carcinosarcoma, while striking clinical features stamp certain cases of the disease as highly specific.

Although no other form of carcinoma absorbs more attention from surgeons and pathologists, the morphology of mammary tumors still requires more searching analysis than it has yet received. So variable and complex are the forms and structure of mammary cancer that the attempt to employ a histogenetic classification may be premature. The present effort must be acknowledged as only tentative. Yet the rough grouping of these tumors in the current fashion, according to cell content, as carcinoma solidum, medullare, and scirrhus, while involving no risk, is wholly lacking in any illumination of the subject. Not until the anatomical structure and histogenesis of these tumors is more fully understood can any significant knowledge be obtained of their etiology, prognosis, and the value of treatment. The separation of the sweat-gland tumors of the breast may be accomplished with considerable certainty on morphological grounds, but specific clinical features of this group remain to be defined.

Ribbert has stated that no one has ever seen a beginning carcinoma of the breast. While few have accepted this dictum, it is not necessary to ob- 
serve the initial stages in order to deduce the origin of many cystadenocarcinomas, duct cancers, or even of certain acinar tumors.

Clinical features seem to be a less satisfactory basis of nomenclature and classification of cancer of the breast than with many other organs. When the lymph-nodes or blood-vessels are once invaded the further course, while extremely varied, may be much the same with tumors of very divergent antecedent history. On the other hand, the early stages of most tumors are closely correlated with their histogenesis, structure, and the conditions under which they arise.

The condition of the breast at the time of the development of the tumor has a prominent influence on the anatomical characters and clinical course of the disease. In elderly subjects various forms of carcinoma develop, but the disease is usually of slow course, and fibrosis and cicatrization are promi-

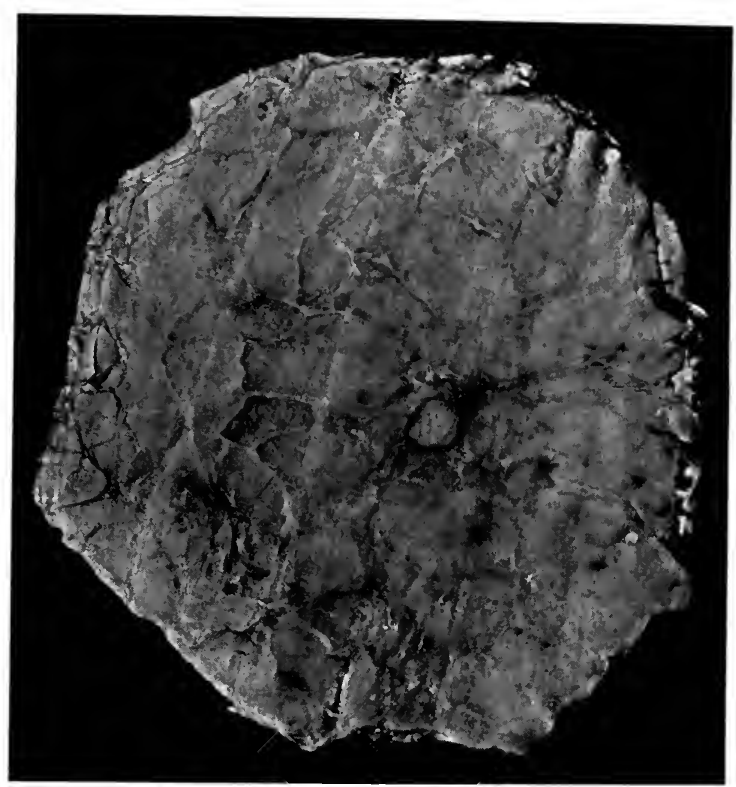

FIG. 221.- Single small nodule of carcinoma in the center of an atrophic and fatty breast.

nent. In small atrophic breasts at any age, glandular tissue being deficient, carcinoma usually takes the form of duct cancer, of the comedo type, and often affects the sweat-glands.

In atrophic but very fat breasts there is often a very small fibrous carcinoma, difficult to detect in the organ, with bulky, extensive, and cellular growths in lymph-nodes.

Very active, anaplastic, and atypical forms of carcinoma develop usually in comparatively young women in the child-bearing period. During pregnancy the disease is usually particularly active, anatomically atypical, and rapidly fatal.

Acute carcinosis with fulminant febrile course occurs usually in women under 35 years of age, with well-developed breasts. The anatomical type varies, but often presents an adenocarcinomatous structure in primary areas, or a diffuse and highly anaplastic structure. 
An origin in cysts, in adenofibromas, in the congenitally hypertrophic breast, or after diffuse fibromatosis, or other pathological conditions, stamps the early stages of the disease with rather definite clinical features.

The more chronic forms of mammary carcinoma, especially those recurring after operation, take a great variety of anatomical types, which compose an extensive chapter in the history of the disease.

Adenocarcinoma is characterized by its origin chiefly in cysts, by the markedly circumscribed character of the growth, the bulky local tumor often produced, the long immunity of the lymph-nodes, the relatively favorable prognosis, and the specific structure. Various terms have been employed to designate types of adenocarcinoma, as carcinome villeux (Cornil), epitheliome iniracanalicu!aire (Labbe, Coyne), villous duct cancer (Williams). A sharp distinction between the benign adenomas and adenocarcinomas has

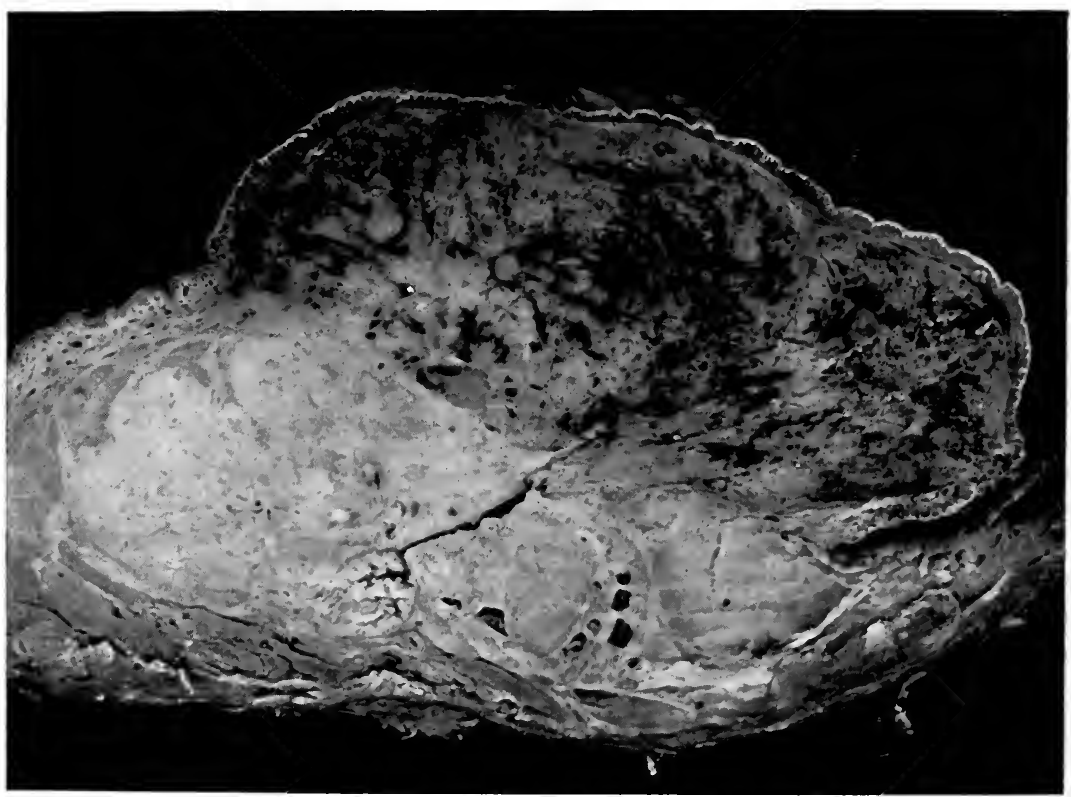

FIG. 222.-An adenocarcinoma of breast. The upper half is slightly pedunculated and the vascular system telangiectatic.

not always been attempted, and the more malignant forms have not been separated from other mammary cancers as clearly as they should be.

The majority of these tumors arise in the cysts of chronic mastitis. They are frequently multiple, and simple cysts, or cysts with papillary adenomas, may accompany the main tumor. In the early stages and often to a late period they form sharply circumscribed masses near the nipple or in outlying regions. They are large, solid, opaque, usually soft, and free from fibrosis. Later they may break through the skin, producing fungating superficial masses which bleed and ulcerate, and they perforate the capsule, invading the breast and the lymphatics. There is much variation in the rapidity of growth and malignancy. Some take the form of papillary or villous adenoma with carcinomatous areas; others are uniformly solid and carcinomatous from the first, but encapsulated. A few are highly atypical and malignant 
and early perforate the capsule and invade the breast and lymph-nodes. In this form they yield some of the tumors commonly called "encephaloid."

Secondary changes are relatively frequent in the advanced tumors. Fatty degeneration is often observed and considerable areas of necrosis may appear in gross section. Overdevelopment of blood-vessels produces a vascular tumor prone to hemorrhage, infarction, and necrosis. I have found the entire tumor infarcted and black, or necrotic without infarction. Repeated hemorrhages may leave much pigment and give the appearance of secondary melanoma.

The structure shows marked variations from a typical benign papillary adenoma to nearly diffuse growth in which only traces of papillary or ade-

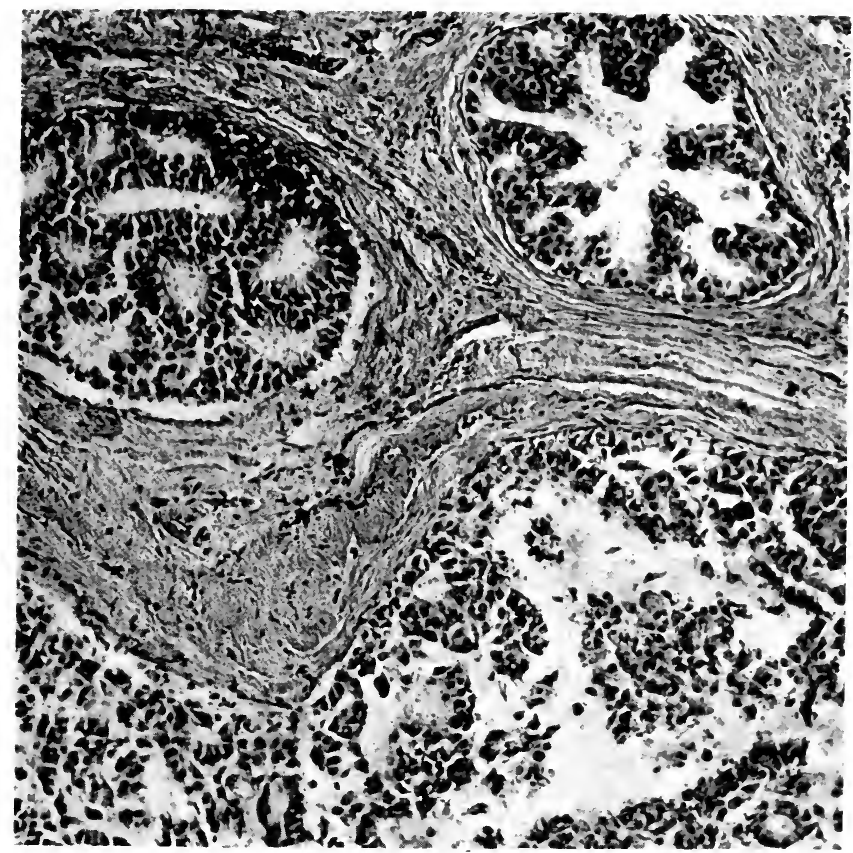

FIG. 223.-Mammary cancer. Adenocarcinoma of ducts.

nomatous structure are retained. Portions of the growth may be quite diffuse. Typical adenoma malignum or destruens is occasionally observed.

I. The simplest structure is a papillary adenoma with carcinomatous foci. This type covers the majority of the cases described by Cornil, Williams, and many of the older authors, who have clearly presented its relatively benign clinical features. The papillæ are low, and freely anastomosing bands of stroma tend to yield an alveolar structure with many secondary sacculations; or the outgrowths are long and villous. The cells are cuboidal or low and cylindrical, granular, opaque, but usually overnourished and atypical. This tumor is of slow growth, long remains encapsulated, is slow to involve lymphnodes, and in this stage a radical operation is not required.

2. A more malignant form shows little evidence of papillary structure in the gross, but is solid and opaque. The structure presents the complex features 
of papillary carcinoma or adenoma malignum. The few strands of connective tissue or the large alveoli are covered or lined by large cuboidal or cylindrical cells with hyperchromatic nuclei. Everywhere epithelium predominates over stroma. These tumors frequently perforate the cyst capsule and grow diffusely in the form of small alveolar carcinoma. In the lymph-nodes, lungs, and especially in the blood-vessels their growth tends to revert to the type of adenoma malignum or papillary carcinoma.

3. The most malignant forms of adenocarcinoma developing in cystadenoma produce bulky tumors invading most of the breast, perforating the skin as fungating masses, and early involving the lymphatics in various directions. The adenomatous structure is visible only in the central portions or in metastases, while the infiltrating portions resemble other forms of mammary can-

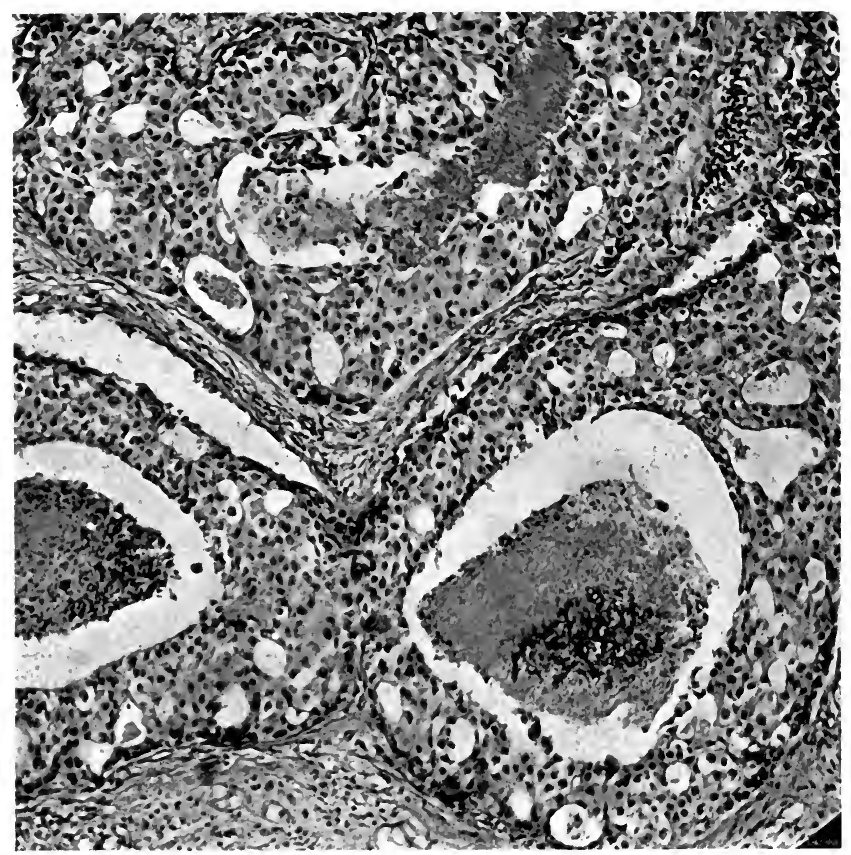

FIg. 224.-Mammary cancer. Adenocarcinoma of ducts, beginning to infiltrate.

cer. These tumors occur at all ages and are among the most malignant in clinical course. A characteristic form presents one or more circumscribed nodules of moderate size with more bulky tumors in the axilla. Or the main tumor is found of large size having long remained localized until a rather sudden access of growth leads to rapid infiltration of vessels and wide dissemination.

Hisiogenesis.-The gross appearance, transitional types, and minute structure point clearly to the origin of these tumors from the papillary ingrowths of mammary cysts. The remaining portions of the breast when available often show the changes of cystic mastitis, but adenocarcinoma develops in breasts in which any preceding inflammatory changes are localized and soon obliterated. Two types of cysts are included in the sites of 
origin: (r) dilated interlobular ducts, and (2) the sweat-glands of Moullin, Creighton, Dreyfuss, and Krompecher. From the study of this group of tumors I have been led to conclude that the lacteal ducts produce most of the low papillary and glandular adenocarcinomas with cuboidal clear cells, while the sweat-glands give rise chiefly to papillary and villous adenocarcinomas with cylindrical cells of acidophile character. This latter group constitutes a well-defined structural group of mammary carcinomas characterized by large, strongly acidophile cells. Many of them take the form of comedo carcinoma in shrunken breasts.

Mucoid carcinoma is usually an adenocarcinoma with mucous degeneration of stroma or cells and owes its slow course both to its comparatively adenomatous structure and to the mucoid changes in its stroma which greatly interfere with nutrition. Gelatinous changes may also occur in the stroma of ordinary duct cancers. The mucoid tumors reach the largest dimensions of any mammary carcinomas and their encapsulation or local confinement

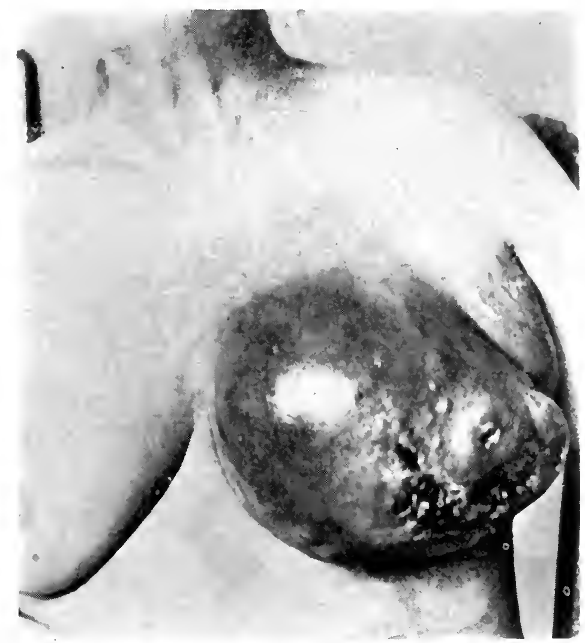

FIG. 225.-Mammary cancer. Bulky fungating adenoma malignum.

may be maintained for several years. They are easily recognized by their gelatinous texture and large bulk. Doutrelepont's case was bilateral. These tumors are comparatively rare. Gaabe collected 49 (I.66 per cent.) among 2944 mammary cancers and analyzed in detail their clinical peculiarities.

The average duration before operation was 31.43 months, as compared with 13.28 months with ordinary carcinoma. Involvement of the lymphnodes occurred at 20 to 36 months, of skin, 36 to 54 months, and of muscle, 28 to 60 months, all about three times as long as the periods fixed by Guleke for ordinary carcinoma. Late metastases constitute a striking feature, the average period being 40 months (ordinary cancer $I_{3}$ months). A limitation of metastases to the thoracic organs is often observed. In 3 cases the undisturbed course of the disease was II, I2, and I4 years (ordinary cancer 20 to 27 months). The duration of life after operation was 5 years, and the recoveries after 3 years 53.85 per cent., as compared with 30.68 months and 28.79 per cent. respectively with ordinary breast cancer.

The structure shows a small alveolar carcinoma or adenocarcinoma with 
extensive mucinous degeneration. The mucinous material is usually found in the stroma, while the epithelium is compressed into small islands of inactive cells. Much discussion has occurred regarding the source of the mucus. Its usual position outside of and surrounding compact groups of well-preserved cells is not in accordance with an epithelial origin and has not been satisfactorily explained by those who maintain this origin. Lange found the source of the material exclusively in the stroma. Most authors trace its origin to the epithelium with subsequent discharge into the stroma and solution of epithelial cells (Cornil, Ziegler, Ribbert, Zimmerman). Kaufmann finds that both elements in different tumors give origin to the mucus. In 4 cases I have not seen mucous degeneration of epithelium, but the compact alveoli lay in broad areas of mucinous stroma. There was strong evidence that the mucinous change had involved and partly originated in the fat tissue

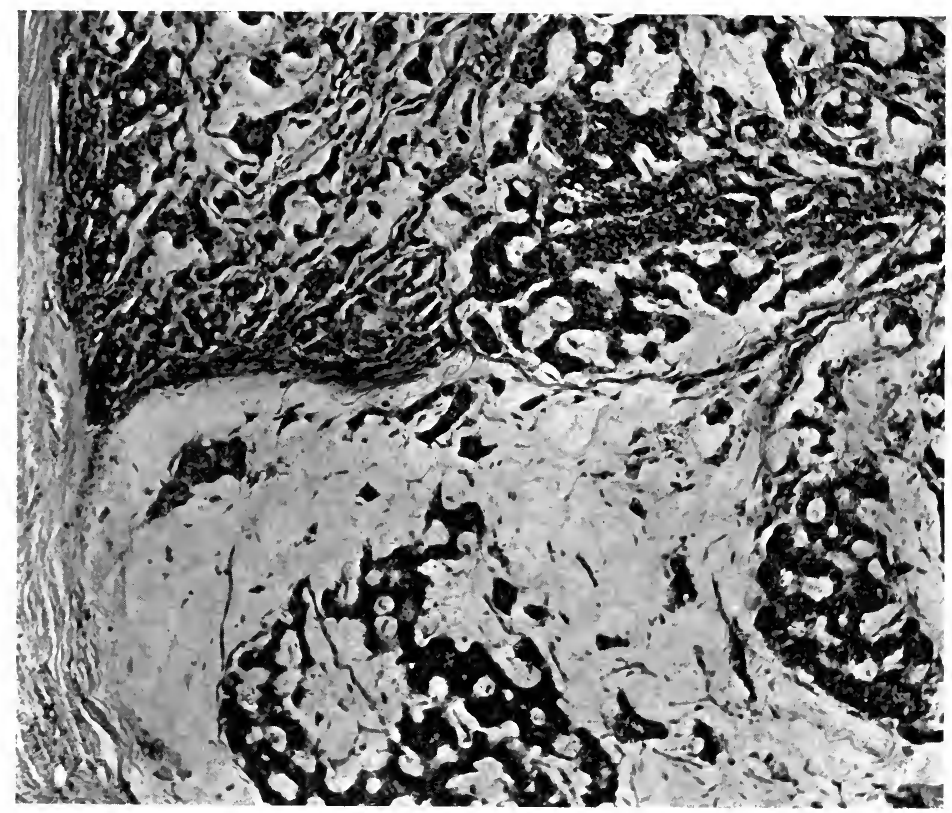

FIG. 226.-Mucoid carcinoma of breast. Mucoid degeneration of stroma and fat tissue.

in the stroma. In another case of adenomatous structure the alveoli were filled with mucus as described by Cornil. There are thus evidently two forms of gelatinous carcinoma of the breast.

The causes of mucous degeneration are obscure and the origin of some of the tumors is uncertain. The long initial quiescent period observed in some cases by Lange suggested that the tumor arose through transformation of an adenoma. Elsasser describes a mucoid stroma in a carcinomatous fibroadenoma. One of my cases showed the usual structure of duct carcinoma with large groups of clear polyhedral cells quite isolated in gelatinous stroma. The metastases are not always gelatinous. In Walther's case they appeared in the cicatrix in a form like sago grains.

Duct Carcinoma.-Carcinoma arising from the lining cells of ducts produces the majority of mammary cancers. The gross anatomical features 
of this group are very variable, and the stages through which any one case passes are numerous, so that these tumors are difficult to classify. Yet two forms of duct carcinoma present in surgical material such striking gross anatomical peculiarities as to deserve special recognition. These are: (I) Cicatrizing comedo carcinoma, and (2) carcinoma associated with diffuse chronic productive mastitis.

(I) The typical cicatrizing comedo carcinoma presents a very characteristic gross appearance. Usually the entire breast is transformed into a dense fibrous tumor mass, smaller than the original organ, involving the nipple and often the overlying skin, which are firmly fixed to the deep fascia over the muscle. On section this mass is marked by prominent yellow streaks or spherical points which are ducts filled with necrotic detritus. Although the points are seldom black, this lesion recalls the pustules of acne.

This process appears to begin in the larger ducts and extends over the entire breast by gradual invasion through the ducts, while progressive fibrosis draws the outlying portions of the organ toward the central area beneath the

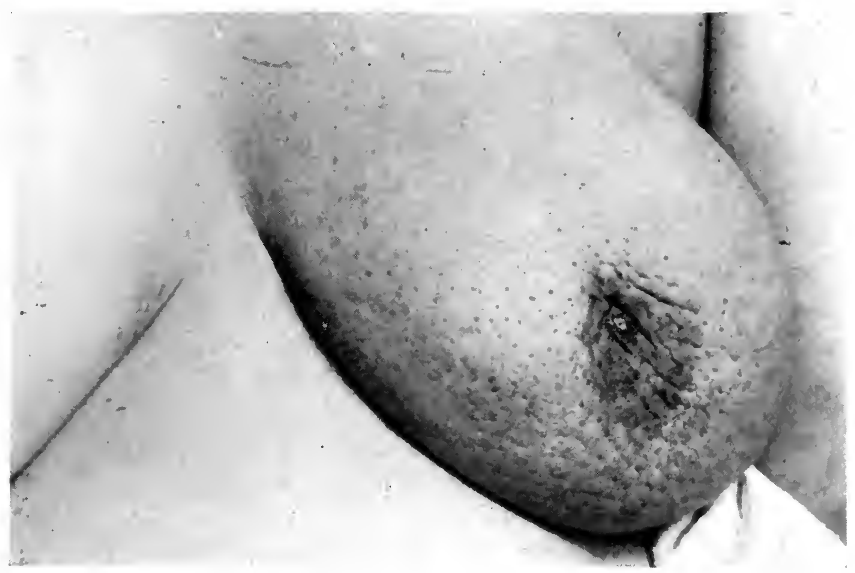

FIG. 227.-Mammary cancer. Advanced carcinoma simplex. Retraction of nipple; dimpling of skin.

nipple. Earlier stages of this process are encountered in which the distended ducts appear over a very wide area, and even in the various prolongations of the breast which have not yet been drawn together by any cicatricial process. Such early forms of comedo carcinoma are difficult to recognize by palpation, since the breast may be diffusely swollen, and no definite resistant mass can be detected. Segmental forms of comedo carcinoma also occur in which only a portion of the organ is involved. The final form of the disease depends much on the original type of breast. Small compact breasts yield single hard tumor masses beneath the nipple, while widely disseminated breasts in fat subjects produce diffuse ill-definied tumors of larger dimensions. In all forms cicatrization is prominent.

Paget's disease of the nipple and skin is sometimes associated with typical comedo carcinoma, but more often with ordinary duct carcinoma.

The structure of comedo carcinoma presents widened ducts lined by large flat or even squamous-like epithelial cells, and filled with acidophile necrotic material. Lateral sacculations of the ducts are frequent and through these 
the tumor cells invade the lymphatics of the cellular connective tissue, disseminate throughout the breast, and early reach the lymph-nodes.

(2) Carcinoma arises in the bulky forms of chronic productive mastitis, usually as a single nodule, but often in multiple foci. This combination produces a very characteristic gross anatomical form of the disease which is not easily recognized by palpation. The breast presents a solid mass of firm elastic connective tissue, of large or occasionally of reduced dimensions, and in it are found one or more areas of dense fibrocarcinoma. The malignant process arises in ducts or sweat-glands which are early broken through by groups of tumor-cells.

The clinical recognition of this group of cases may be based on a long history of chronic mastitis with its evident physical signs, and the discovery of one or more particularly resistant nodules among the more elastic areas of the indurated breast. Since the tumor may arise in any portion of the breast, fixation of nipple, skin, or deep fascia is accidental.

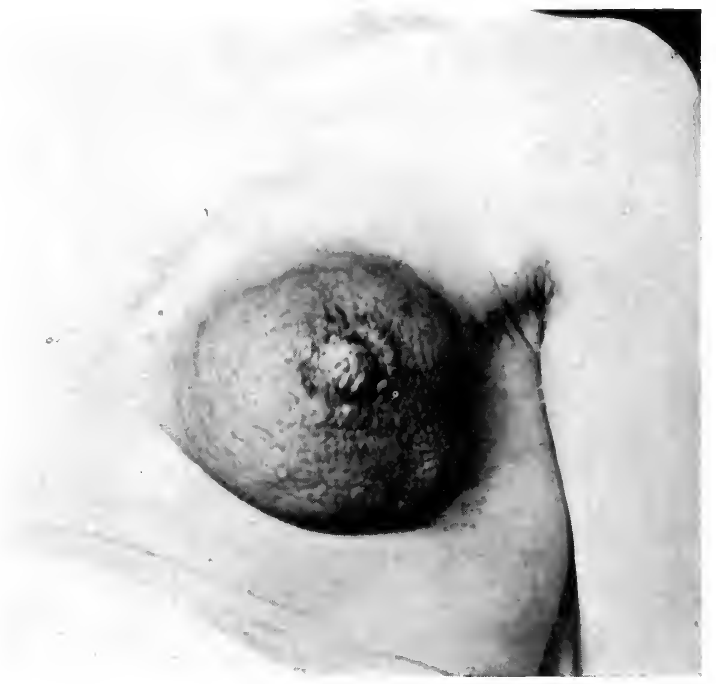

FIG. 228.- Mammary carcinoma. Diffuse fibrocarcinoma with contraction and fixation of breast.

A single very hard nodule in an otherwise normal breast is nearly always carcinoma, but in the present group of cases the hard nodule lies in rather dense new connective tissue, is often overlooked, and many of these cases are detected only after amputation of the breast for chronic mastitis.

(3) Other gross anatomical forms of duct carcinoma, including those arising from sweat-glands, while numerous, are less well defined than the two groups just described Among them may be mentioned:

(a) Single nodules of carcinoma in fat atrophic breasts.

(b) Very cellular types of carcinoma, in which the breast is little altered by mastitis, and in which the duct carcinoma develops rapidly.

(c) Diffuse anaplastic carcinoma developing in young subjects or in gestation, enlarging the breast, and extending widely in skin, muscle, and lymphnodes.

The structure presents marked proliferation of the lining cells, first of the 
larger, then of smaller ducts, or vice versa. The walls of the ducts are thickened, fibrous, or hyaline, and the lumen moderately dilated. Cysts wider than $2 \mathrm{~mm}$. are uncommon.

The form and arrangement of the cells vary with the degree of malignancy, of which three grades may be considered with advantage:

I. Small Cystic Adenocarcinoma.-The cells are cylindrical, only slightly atypical, and they line the dilated ducts in the form of fusing papillæ or in a solid multiple layer in which secondary alveoli appear at regular intervals. Wide areas of such cells with secondary alveoli appearing at regular intervals may form in dilated ducts. The process may be traced from the nipple to the peripheral lobules. This structure constitutes a form of tubular adenocarcinoma.

Tumors of this type are of slow growth, the tumor-cells long remain confined to the ducts and do not break through the basement membrane, the lymph-nodes are usually free from involvement, and extirpation of the breast

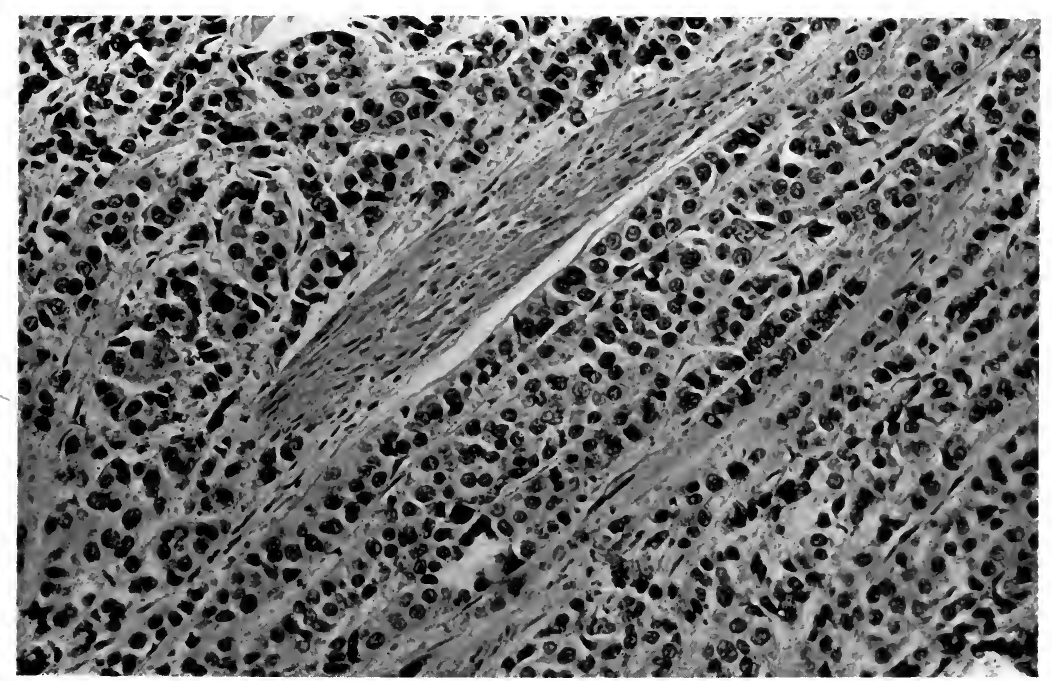

FIG. 229.-Mammary cancer. Carcinoma simplex. Tubular carcinoma.

is frequently successful. Unfortunately, most duct carcinomas are more atypical in the whole or in portions of the growth and the tumor falls in the next subgroup.

2. Small Cystic Duct Carcinoma.-The alveoli are lined by stratified layers of atypical overnourished, large, clear, polyhedral cells resembling squamous epithelium, without secondary alveoli. The ducts are partly or completely filled with these cells and with homogeneous detritus. Many of them are distended and ruptured and the cells, under evident signs of compression, are found in all stages of infiltration of the stroma. Many lateral sacculi form, the process invades the intralobular ducts and even the immature or atrophic acini, and the whole breast may become transformed into a tubular or large alveolar carcinoma in which the prevailing cell is large, clear, and polyhedral or squamous-like. Very varied pictures are produced in the different phases of these tumors. Some are very cellular, plexiform, or show broad sheets or very numerous small groups of clear cells. 
Many are strongly desmoplastic. Some present areas of very large giantcells. Nearly all the structures commonly designated as alveolar, tubular, encephaloid, simplex, solid, scirrhus, and small cystic carcinoma may be produced in the established forms of duct carcinoma. All are locally aggressive, highly invasive of lymph- and blood-vessels, and fully malignant.

3. Fibrocarcinoma arising in chronic mastitis presents a varying structure chiefly of the type of duct carcinoma. The earliest stages include the lesions described under Chronic Mastitis as precancerous changes or miniature carcinomas and consist in single ducts or groups of ducts in and between the lobules, which are filled with atypical overnourished cubical or flat cells. The altered acinar epithelium also frequently participates in these early changes. Soon the distended ducts rupture and groups of large clear cells

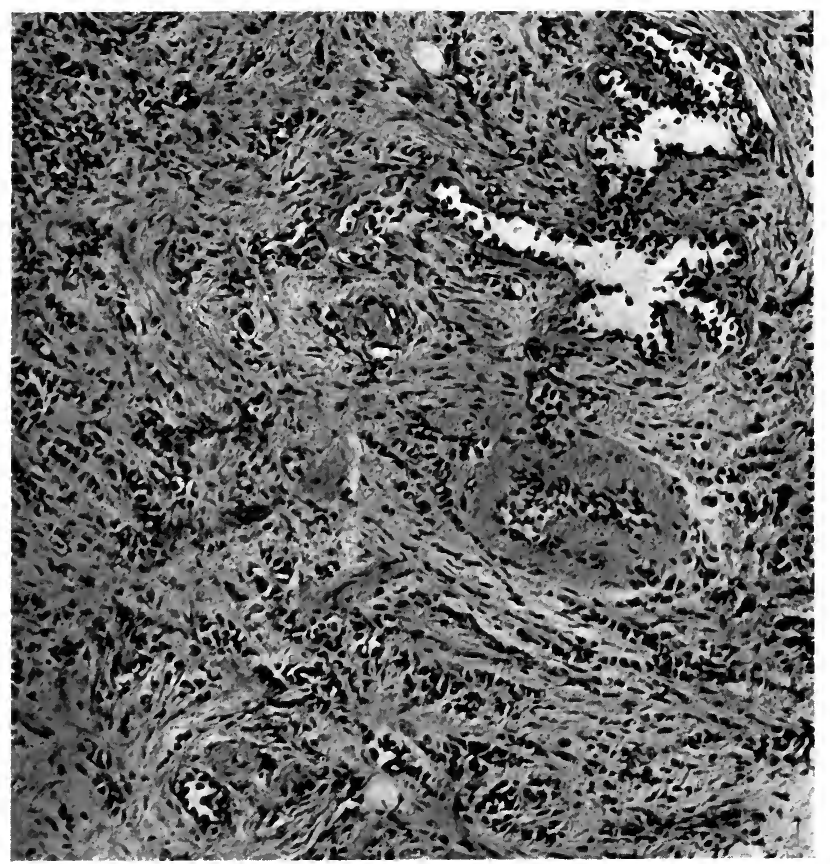

FIG. 230.-Mammary cancer. Primary fibrocarcinoma in a young woman.

infiltrate the lymphatic spaces. Marked increase of connective tissue often richly infiltrated with round cells accompanies the process, and it is this excess of fibrous tissue which alters the gross and microscopical features of ordinary duct carcinoma and stamps the disease with peculiar characters.

Distinctly fibrous carcinomas are, as a rule, less malignant than the cellular forms, but their clinical course varies greatly. Some take the course of scirrhous cancer and in elderly subjects may long remain localized. In the absence of a notable tumor surgical interference is often long delayed so that little advantage is gained from the slower course. In a well-known group of cases there is a relatively small mammary tumor, while the lymphnodes are found involved by a very cellular growth.

Duct carcinoma, originally typical, may develop anomalous forms which are very active, diffuse, sometimes pseudosarcomatous, and highly malig- 
nant. In the case previously mentioned in pregnancy, the main tumor was composed of a diffuse growth of polyhedral round and spindle-cells which soon disseminated through blood- and lymph-vessels. In other cases of diffuse carcinoma with indifferent rounded or spindle-cells I have found areas of more typical plexiform or large alveolar carcinoma with large clear cells, indicating an origin from duct epithelium.

Histogenesis. - The origin of this form of mammary carcinoma has been satisfactorily traced to the lining cells of the interlobular ducts. In central tumors lying beneath the nipple the larger ducts are especially involved

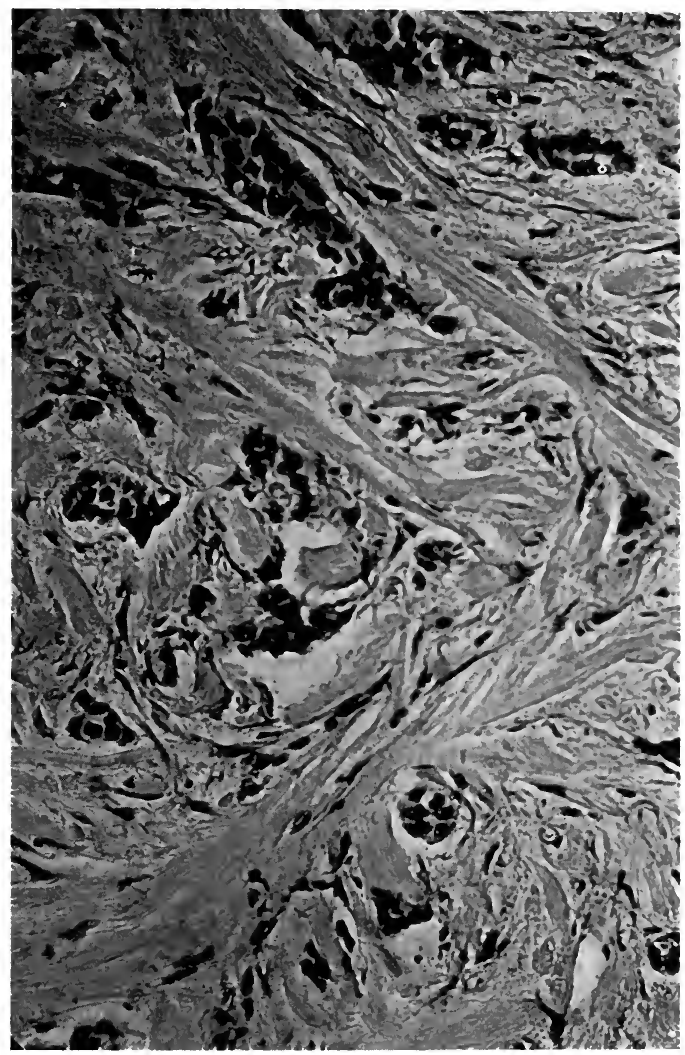

FIG. 23I.-Mammary cancer. Sclerosing fibrocarcinoma. Typical hard scirrhus.

and the lesion may extend to the nipple or even to the skin, as in some cases of Paget's disease. The lesion also extends to the intralobular ducts. That the acini themselves may become involved and contribute to the sources of these tumors is indicated by minute examination of early cases. The present group of duct carcinoma, especially the fibrocarcinomas, does not therefore arise exclusively from duct epithelium. Yet specific acinar epithelium is very scanty in the lobules of many cases of chronic mastitis and may be practically absent in involuting breasts in which duct carcinoma usually develops. The intralobular ducts, on the other hand, persist and 
widen and the scanty acinar epithelium assumes the morphology of duct tumor-cells.

It cannot, I think, be denied that the minute analysis of these lesions indicates a gradual diffusion of the cancer process over adjoining lobules until at least the focus of origin reaches considerable dimensions, after which the tumor progresses from its own resources. Every portion of the organ may be completely invaded without demonstrable increase in size. Nadal

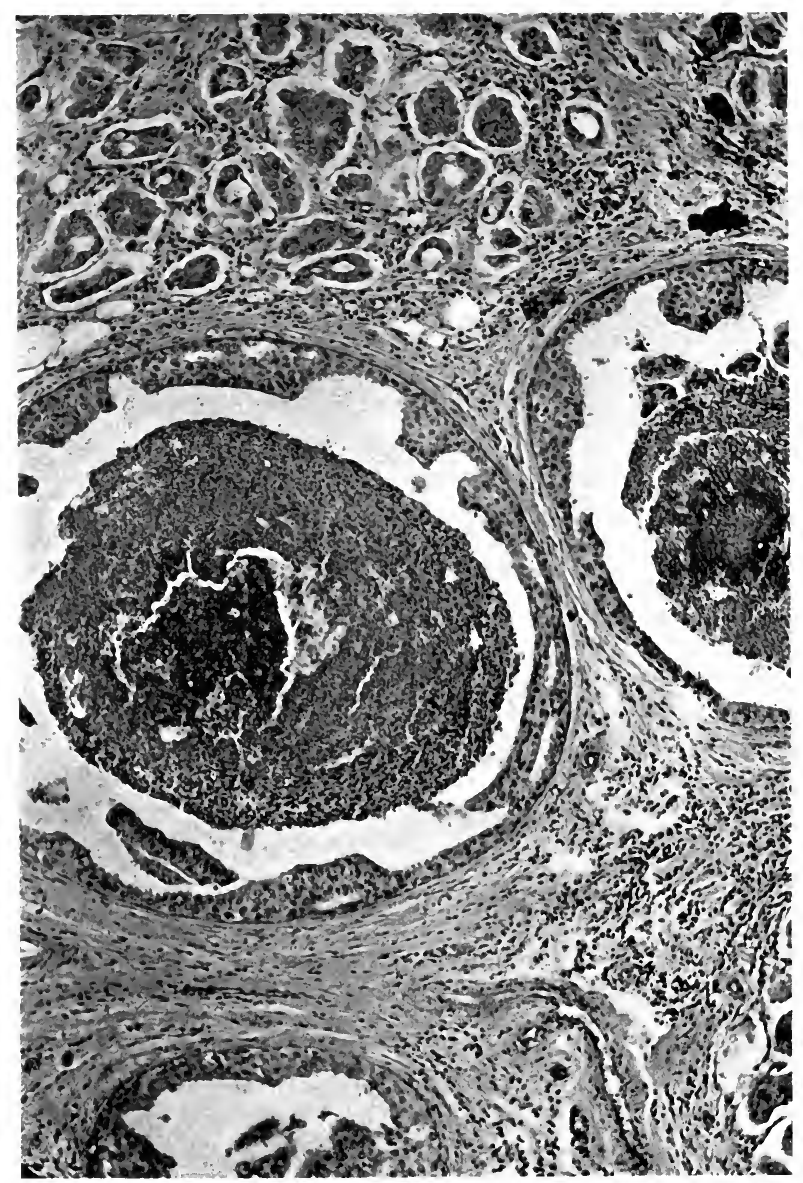

FIG. 232.-Mammary cancer. Large and small alveolar carcinoma. Sweat-gland type.

traces the origin of such diffuse forms of the disease and concludes that the neoplastic process may originate from all portions of the breast.

The aberrant sweat-glands of the breast give rise to tumors which may be difficult to distinguish from true duct carcinoma. The separation is especially difficult between different cases of comedo cancer, many of which arise from the sweat-glands. As a rule, the cells of the duct carcinoma are clear and as they become more atypical the cytoplasm becomes less prominent. The cells of the sweat-gland tumors are large and the cytoplasm is 
opaque and strongly acidophile, while these features tend to persist in extensions and metastases.

The present classification of duct carcinomas somewhat enlarges the scope of this group as recognized by some authors. It includes especially the tubular and some acinar carcinomas of Cornil and others. It is impossible to claim that every tubular carcinoma originates from ducts, yet many such growths are certainly of this origin, while their development from true acinar epithelium is less certain. The cell characters are of much importance in the diagnosis, and wherever the cells are large and clear, of polyhedral form and mosaic arrangement, an origin from ducts is indicated. An exclusive origin from ducts or acini is not always to be assumed. In cellular duct cancers the acinar epithelium may be involved and in many acinar tumors the duct epithelium, as Cornil points out, shows various phases of malignant proliferation. Under these conditions the tumor must be classified according to its predominant features.

Acinar Carcinoma.- Although the earlier observers, as Waldeyer and Langhans, traced certain mammary carcinomas to the acinar epithelium, minute distinctions were not at first attempted between intralobular ducts and acini. In 1865 Cornil described in detail the cancerous transformation of acinar epithelium, and his views were elaborated by Cornil and Ranvier, and lately by Cornil in his work on tumors of the breast. Many of the structures interpreted by Cornil as demonstrating the acinar origin are of somewhat uncertain significance and, as Lecene and Lenormant conclude, may equally well be regarded as the invasion of acini by tumor-cells from other sources. In the previous discussion of the histogenesis of duct carcinoma it has been pointed out that the acini often contribute to the origin of tumors which are chiefly derived from the ducts. Such tumors are here classified with duct carcinoma.

There are, however, mammary carcinomas which are derived chiefly or exclusively from the acinar epithelium, and such growths may be separated from the duct carcinomas and included in the present group. They occur under several different conditions, but their total number is distinctly less than that of the duct carcinomas. Acinar carcinoma in this restricted sense develops in the malignant transformation of fibro-adenoma, it occurs in rare cases of small alveolar carcinoma, and it produces a type of fibrocarcinoma. These tumors are characterized in general by a diffuse growth and lack of encapsulation, by absence of the specific gross features of many duct cancers, by a structure in which small alveoli predominate, while the cells are usually small, and lack the pavement form and clear cytoplasm of the duct epithelium. Rarely they suffer mucoid degeneration of the stroma.

(a) Acinar Carcinoma Following Fibro-adenoma.-While the malignant transformation of intracystic adenoma is frequently observed and forms a well-defined group of mammary adenocarcinomas, the development of a malignant epithelial tumor from fibro-adenoma is rare. Diffuse adenomatous hypertrophy of the breast is said to have terminated in adenocarcinoma in cases described by Billroth and by Aitken.

The common fibro-adenoma appears to have developed carcinoma in a few authentic instances. Birch-Hirschfeld regarded the transformation as not infrequent, but specific, and detailed reports are rare. Langhans described a bilobed tumor, one portion of which was fibro-adenoma and the other scirrhous cancer. Elsasser studied two large fibro-adenomas with small cysts and single well-defined masses of carcinoma. One of the tumors had the structure of small alveolar acinar carcinoma, the other was very vascular, 
pigmented, the stroma mucinous, and both ducts and acini contributed to the growth. J. Müller describes multiple areas of carcinoma in a fibroadenoma. Many new acini filled with atypical cells and diffuse invasion of the connective tissue appeared in these foci. Kuru described a large elastic tumor, $\mathrm{I} 4 \times 9 \mathrm{~cm}$., in one portion of which was a hard area. Here the alveoli were much increased in number and filled with atypical cells, giving the appearance of carcinoma medullare solidum. This observation does not support Borst's view that the carcinoma develops not from the fibro-adenoma, but from another misplaced embryonal tissue mass. I have encountered several moderately malignant mammary carcinomas which appeared to have been derived from fibro-adenomas. They contained considerable cellular connective tissue and many small alveoli, with or without lumen, lined by atypical cuboidal epithelium. In a recent case the tumor had the gross appearance of an encapsulated fibroma, but with fatty points of carcinomatous epithelium. In most of these tumors the structure suggests an acinar origin, and this origin is accepted by the authors, but in some both ducts and acini appear to have been involved. The usual history indicates that a slowly growing fibro-adenoma existed for some years and eventually grew much more rapidly with the onset of malignant changes. The malignancy has been moderate.

(b) Primary Acinar Carcinoma.-Although Cornil and others have traced the development of many common mammary cancers to the acinar epithelium, reasons have been advanced to show that the majority of the tubular and small alveolar tumors are forms of duct cancer or are derived chiefly from the ducts, while the acini are subsequently invaded by the tumor-cells or contribute only secondarily or to a slight degree to the neoplasm. The present group includes tumors of a peculiar and specific histology which is rarely observed.

In one form this tumor is partly circumscribed, rather soft, vascular, and solid. The structure presents a very large number of extremely small cell groups or acini lined by small atypical epithelium.

In another form which Cornil has described there is an extensive multiplication of the acini in rather well-defined lobules. The lumen is long retained, but the cells are small cuboidal and atypical and a disordered growth may extend beyond the lobules. The structure somewhat resembles the lactating breast. No signs of proliferation of duct epithelium are observed. Rarely the cells are extremely small, the acini imperfectly formed, and the structure resembles an embryonal alveolar sarcoma.

All these tumors show limited malignancy, especially in the early stages, but when fully established they invade the lymph-nodes and pursue the usual course of advanced carcinoma.

C. Fibrous Acinar Carcinoma.-In some cases of productive mastitis and possibly in otherwise unaltered breasts the acinar epithelium may give rise to a malignant form of scirrhus or fibrocarcinoma. The structure is highly characteristic. About the unaltered larger and the intralobular ducts the acini become increased in number and break up into many small groups of atypical cells with hyperchromatic nuclei. The connective tissue is much increased and soon becomes fibrous, while the tumor cells are compressed into narrow rows or small groups. Many cases of primary scirrhous cancer develop in this manner. The process is fully malignant.

Gross Anatomical Diagnosis of Mammary Carcinoma.-In no department of surgery is familiarity with gross anatomical diagnosis of greater service than in dealing with mammary tumors. Moreover, the great majority of mammary cancers are rather easily recognizable by inspection and palpation, 
and microscopic examination, especially by frozen section, may usually be dispensed with or reserved for later study.

A single very hard nodule, with restricted mobility, in an otherwise unaltered breast is nearly always carcinoma. Cysts and fibromas may be very firm, but they are elastic and quite mobile in the breast. Trauma from blows or from hypodermic injections may produce cicatricial masses which increase in size, restrict the skin and breast, and are very difficult to distinguish on clinical examination from carcinoma. On gross section they fail to show the specific characters of carcinoma.

Marked induration and restricted mobility characterize nearly all forms of established carcinoma in which there is a well-marked tumor, but there are diffuse forms of carcinoma without tumor, and carcinoma may begin in the walls of cysts. Single or multiple nodules of carcinoma in cases of chronic mastitis cannot often be recognized positively by clinical examination.

With the excised breast or nodule presented for examination, the characters of carcinomatous tissue are usually specific. Chief among these is the cicatricial appearance of the nodule, which may present an opaque welldefined central area surrounded by fine radiating strands of connective tissue. On close inspection, with or without a hand glass, one detects yellowish chalky points and streaks in the translucent connective tissue, which occur only in carcinoma. In many duct carcinomas these chalky streaks are large and prominent. They are produced by fatty degeneration or desquamation of tumor-cells. Even in diffuse carcinoma without tumor formation the chalky points are visible. When dealing with a bulky mass of tissue, such as a breast excised for chronic mastitis, it is obvious that the examiner must rely on gross features to pick out any suspicious areas for section, and that unless one is familiar with the gross appearance of carcinoma small foci may be overlooked even although many sections are made.

Small areas of carcinoma located in the walls of cysts require careful sectioning of all thickened points in the wall, but when present any carcinomatous focus will again present the opaque texture and chalky points.

After trauma of various sorts cicatricial processes develop in the breast, and these often involve much fat tissue which becomes opaque and indurated, or in certain cases, described by Lee and Adair, extensively necrotic. Yet such lesions fail to exhibit the chalky points and other positive features of carcinoma.

The writer has encountered solid adenocarcinoma filling small cysts which did not show a cicatricial character or chalky points, but had to be identified on the opaque solid texture of a cellular tumor.

In certain cases it would appear that the gross diagnosis of carcinoma of the breast is a safer guide to surgical procedure than the discovery by the microscope of questionable minute points of precancerous changes concerning which doubts and differences of opinion are so frequently expressed. When a suspicious focus is so small that it cannot be detected by the trained naked eye it is doubtful if a diagnosis of carcinoma in the clinical sense is justified.

Modes of Extension.- - I. In the Breast.-Extension in the mammary parenchyma must be interpreted in some cases as a local evolution of the tumor; in other cases as a local dissemination. Some acinar and many duct cancers arising at one focus gradually extend over adjoining areas by a gradual transformation of normal duct and acinar epithelium into neoplastic cells. The whole organ may thus be found to be cancerous while only slightly increased in size. Long segments of ducts may show many grades of epithelial proliferation without filling of the lumen by growth from a distance. 
Extension through the ducts also occurs in several forms of the disease, but not to the extent observed in the first-named process (Nadal). Perforation of ducts by cells traveling in lymphatics has been demonstrated by Goldmann. The chief mode of extension is undoubtedly through the lymphatics of the breast, by cells which have broken into lymph-spaces and artificial clefts, and this method is almost exclusively followed by highly malignant tumors, fibrocarcinoma, acinar carcinoma, and adenocarcinoma. The stage of growth at which extension begins as well as the early paths pursued vary widely with the character of the growth, which is therefore the chief index of malignancy. At one extreme stands the encapsulated adenocarcinoma, at the other the locally invasive forms of duct carcinoma.

Rapid local extension may be accompanied by signs of inflammation, as in the mastitis carcinomatosa of pregnancy. On the other hand, the whole organ may be invaded without attracting the patient's attention.

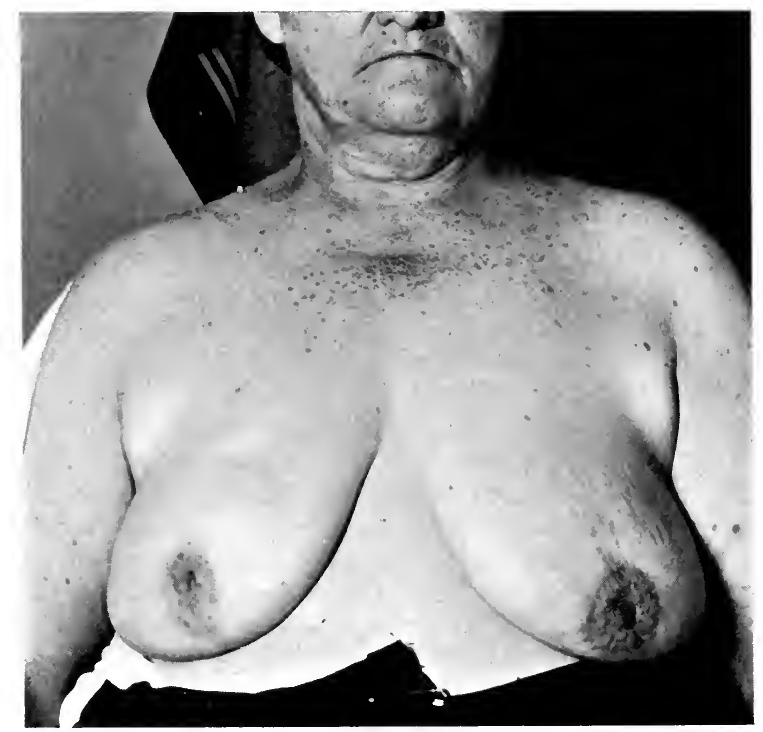

F1G. 233.-Mammary cancer. Fibrocarcinoma. Reduction in size of breast; retraction and elevation of nipple; retraction of skin about nipple; invasion of axillary and supraclavicular nodes.

The effects of local extension are usually cicatricial changes in the affected tissue, which is the most reliable sign of the presence of carcinoma, or increase in the size of the part, which may be local or diffuse. Handley attributes the effects largely to a sclerosing perilymphangitis which follows the permeation through lymphatics, and to this condition he attributes the retraction of the nipple and fixation of the skin. There is also the desmoplastic property of the cancer process which is not connected with lymphangitis and which produces cicatrizing effects beyond the zone of invasion.

The condition of the unaffected portions of the breast varies widely, but is almost never normal. The most distant portions usually show diffuse or cystic mastitis with periacinar infiltration, and overnutrition or proliferation of epithelium (Waldeyer, Leopold). Very small tumors beneath the 
nipple or in any segment may involve all the breast tissue that can be found, suggesting either a congenital hypoplasia or an extreme grade of involution. On the other hand, outlying lobes not previously noticeable may become indurated, as the sternal prolongation of Rieffel, the axillary lobe of Kermisson, and the xiphoid appendix of Zocher and Hennig. Each of these lobes may be the seat of carcinoma. In some cases I have found tumors originating in these lobes atypical in structure and relatively malignant.

2. Invasion of Skin.-The chief mode of extension to the skin is by way of the subpapillary lymphatic plexus from the periductal lymphatics. Hence extensive cutaneous cancer of the breast is observed in central duct cancers which early reach the nipple. In such cases the derma is infiltrated by nodules and masses, discrete or diffuse, encroaching more and more upon the subcutaneous fat tissue. The area of skin thus involved may be much wider than the underlying tumor. Many of the wide-spread cancers "en cuirasse" involving the skin of the entire thorax originate in this way. Very extensive pustular and ulcerating lesions of the skin involving much of the trunk are described by Handley as illustrating an extreme grade of lymphatic permeation, not through the dermal, but by way of the deep aponeurotic plexus.

A second mode of skin invasion is through the lymphatics of Cooper's ligaments which join the subcutaneous lymphatics from the outer surface of the breast. These ligaments connect outlying tooth-like projections of gland tissue with the derma and are markedly developed in many subjects (Stiles). Invasion of this type causes dimpling, retraction, corrugation, and fixation of the skin, without marked thickening.

A third mode of invasion of the skin consists in the destruction of the epidermis by carcinoma. This process may begin at the nipple by a continuation of the lesion in the ducts as in Paget's disease. Or extensions from below may traverse the derma and lead to erosion and ulceration of the epidermis. Early perforation and extensive ulceration of a fungating type belong to the adenocarcinomas.

Active superficial extension through dermal lymphatics may be accompanied by hyperemia and reddening of the skin. Rarely a vesicular eruption accompanies the erythema. It has been attributed to dilatation of the subpapillary lymphatics.

Cancer en cuirasse is a term applied by Velpeau to a remarkably extensive cancerous invasion and thickening of the skin and subcutaneous tissues. He described the condition as involving the thorax, neck, arms, and trunk down to the umbilicus, the skin being greatly thickened, very hard, and over the chest thickly sown with scirrhous ulcers and nodules. The rigidity of the tissue greatly interfered with movement of the limbs and with respiration. Erichsen describes also a brownish discoloration, formation of desquamating crusts resembling the bark of a tree, but without ulceration. Handley applies the term to a condition of elephantiasis best seen in cases of extensive edema of the arm in which there is no definite carcinomatous invasion, and he interprets the process as the result of perilymphatic fibrosis in the deep fascial plexus. In many cases, however, there is diffuse cancerous infiltration from the epidermis to and even through the chest wall. Simple edema or elephantiasis may precede or accompany the cancerous process.

Dissemination.- Lymphatics of the Breast.- The knowledge of the important lymphatics of the breast in relation to cancer is based on anatomical studies of Sappey, Gerota, Grossman, Bartels, and others, and the clinical and pathological observations of Heidenhain, Oelsner, Stiles, Handley, and others. 
The subpapillary plexus is a rich network encircling the dermal papillæ and is particularly well developed at the areola. It is limited at the level of junction of the superficial and middle thirds of the derma. From this plexus vessels run vertically communicating with those of the breast, over the organ, and with the deep fascial plexus elsewhere.

The fascial plexus lies just below the subcutaneous fat and is the main channel of dissemination of cancer. This entire plexus is divisible into three thoracic regions, by the median line, the clavicles, and a line running through the umbilicus, along which the main trunks are interrupted by five branches capable of arresting cell emboli (Handley).

Yet Oelsner found in 2 of 9 cases large trunks in the fascial plexus connecting the mammary region with the opposite axillary nodes. Tumors of the internal segment

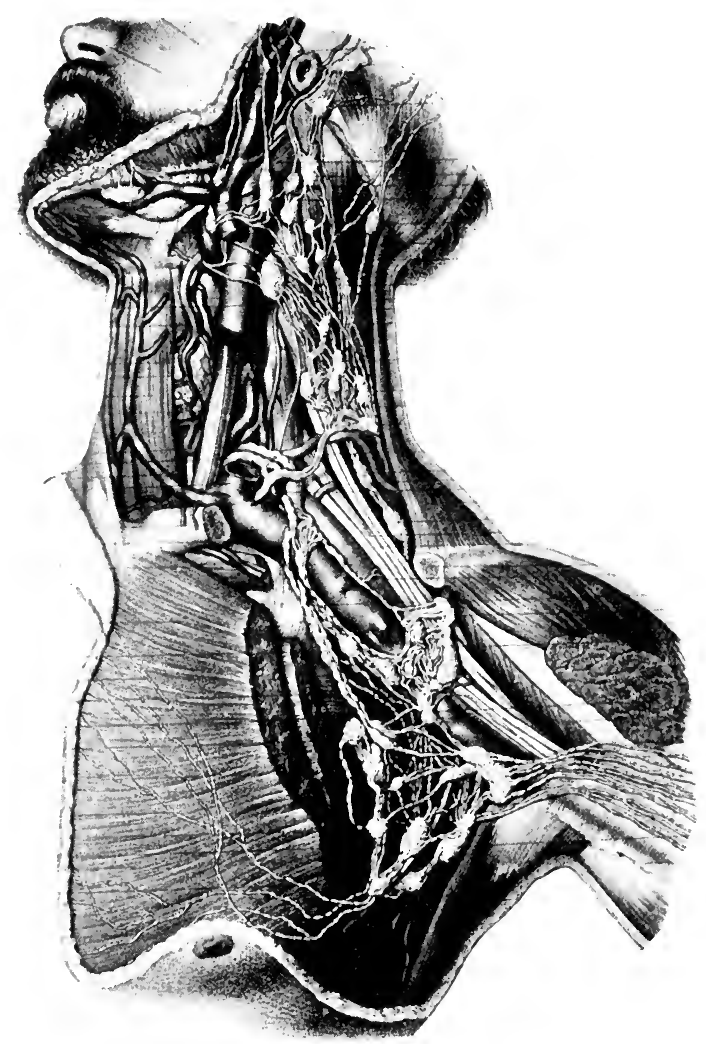

FIG. 234.-General plan of cervical and axillary lymphatic system. (After Bonamy and Beau.)

of the breast are in the most favorable location for extension to the opposite axillary nodes, as in Volkmann's case (Billroth). Riefel found that mercury injected into the skin of this quadrant passed into the opposite axillary nodes. Such crossed metastases are rare in early cases (I per cent.), but more frequent in advanced cases ( 7 per cent.) (Handley), while at autopsy Williams observed them in 10 of 44 cases.

The lymphatics draining the breast belong in this system. Fine lymph-spaces are found in the intralobular connective tissue, but the first definite vessels or sinuses demonstrable by injection encircle the lobules (Waldeyer). They unite to form the main efferent trunks which pass in several directions.

(a) Axillary trunks, usually two, pass from the outer upper and under segments, along the border of the pectoralis major to the axillary nodes. One or two nodes, Sorgius' group, may be interposed at the third digitation of the serratus muscle. A 
paramammary trunk and node may also exist at the edge of the pectoral in the anterior axillary line, at the fourth intercostal space.

The axillary trunks are the chief channel of dissemination. They are especially involved from tumors in the upper outer quadrant of the breast, which is the most frequent location of carcinoma, and they are commonly invaded by all tumors which have extended through the breast tissue.

They are reached not only by the main axillary trunks, but through anastomoses with the subpectoral and infraclavicular groups which drain the intermuscular trunks passing from the under surface of the breast through the pectoral muscle (Hyrtl, Grossmann). By the latter route the nodes at the extreme apex of the axilla may be involved while the lower superficial axillary nodes escape (Stiles). Deep axillary nodes lie along the external mamnary artery and the nerve of the serratus magnus, and these connect freely with the humeral chain accompanying the axillary vein and with the subscapular nodes. These three groups are frequently the seat of recurrences.

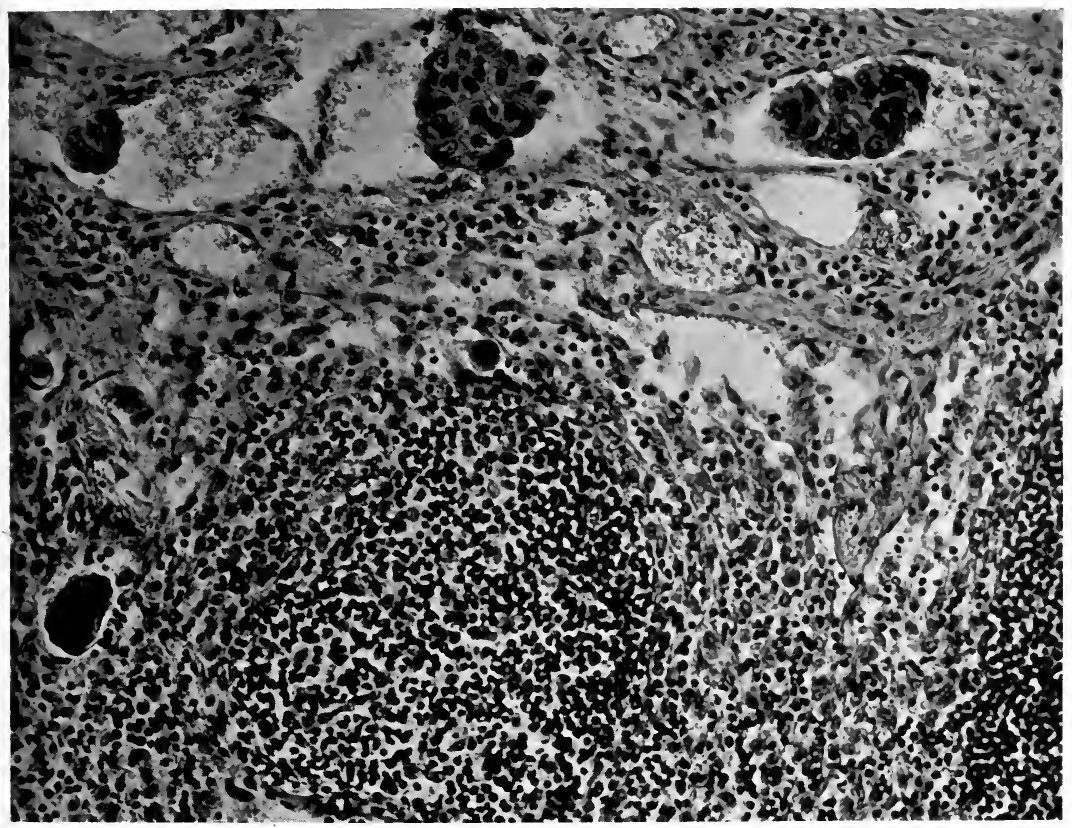

FIG. 235.-Multiple recent tumor-cell emboli in the sinuses of an axillary lymph-node. From a case of mammary cancer which had been roughly manipulated before operation.

The number and position of the axillary nodes are subject to variations. All stages of the development of new nodes in cases of mammary cancer are described by Fage. The number varies also with the physiological state of the breast. On the other hand, fat invasion and atrophy of the nodes is frequently observed and influences the spread of the disease. Those usually present may be grouped as follows: (I) Pectoral group, on inner wall of thorax, in angle between pectoral muscles and serratus magnus, draining breast and front of chest; (2) humeral group, lying on axillary vessels and draining the arm. The highest of these (subclavian) lie in Mohrenheim's space behind the costocoracoid membrane; (3) subscapular group along subscapular vessels on the posterior wall of the axilla.

The close apposition of many of these nodes to veins and nerves favors early invasion of the lumen of veins and the sheaths of nerves. It is chiefly from such secondary foci that the lumen of veins is reached in mammary carcinoma. The significance of this event in the generalization of the disease is not clear, but it does not always lead to immediate wide-spread metastases. The sheaths of nerve-trunks are readily invaded, with pain and degeneration of fibers, and within the lamellar sheaths tumor-cells may permeate long distances (Mousseau). 
(b) Intermuscular trunks pass from the under surface of the breast in the pectoral fascia and over and through the pectoral muscle leading to nodes lying between the pectoralis major and minor or to infraclavicular nodes. Between the axillary and infraclavicular nodes there are limited communications, but between the infra- and supraclavicular nodes the connections are free so that both groups are usually involved together. Exceptions in which the nodes above the clavicle are involved, while those below are free, Grossmann refers to extension from the axillary nodes by way of the brachial lymph-plexus. Since the brachial plexus often communicates with the cervical vessels, the cervical and supraclavicular nodes are often involved together. In these cases a cancerous node of the supraclavicular group is often found at the inner end of the clavicle in close contact with the thoracic duct which is easily ruptured on removal of the node.

(c) Intercostal lymphatics pass from the inner and under segments of the breast through the pectoralis and intercostal muscles to the sternal nodes lying behind the sternum in the parasternal line. Stiles found these vessels invaded by carcinoma, and Oelsner was able to inject them in the first and fourth intercostal spaces. Extensions also pass into the thorax along the intercostal nerves.

The importance of the intermuscular trunks in the dissemination of carcinoma was pointed out by Heidenhain and confirmed by Rotter, Grossmann, and others, and forms the ground for excision of the pectoral muscle. Rotter in 15 of 33 operable cases, several of which were early, and only 5 adherent to the muscle, found invaded lymphnodes beneath the pectoralis major. The remarkably close apposition of the breast to the pectoral muscle, especially in cases of chronic mastitis, facilitates early invasion of these deep trunks. Moreover, Heidenhain found islands of gland tissue within the muscle. Yet actual invasion of the muscle is not frequent. Heidenhain, who found the pectoral fascia involved in 12 of 18 cases, observed growth $1 \mathrm{~mm}$. within the muscle only once. Hence many operators deem it sufficient to remove the pectoral fascia without sacrificing the muscle (Vanverts). Invasion of the pectoral fascia occurs without fixation of the breast, but when the organ is adherent to the muscle the latter is usually invaded by fine nodules or diffuse cicatrizing carcinoma. In advanced cases such invasion of the muscle is often observed. Yet Hardouin found recurrence within the pectoral after operation on an early case, and Cabot reports recurrence in the pectoral alone after removal of a small central carcinoma. Such experience must be rare. Nadal describes miliary carcinosis of the pectoral.

It is obvious that no uniform rule can apply to the treatment of the muscle and that an effort should be made to determine its condition from all the data in the case. Since the subpectoral nodes are involved in some comparatively early cases and the muscle tissue usually escapes even in advanced conditions, the attack should be on the nodes and fascia rather than on the muscle. The question of continuous permeation through the muscle must also be considered, but is rarely demonstrated.

In a recent study of the breasts of 100 infants up to $2 \frac{1}{2}$ years Mornard finds several types of lymphatic distribution:

I. In 25 per cent. of cases the lymphatics carrying off methylene-blue injected into the breast pass along the lower border of pectoralis major, under that muscle to central axillary trunks, meeting nodes on axillary vein which communicate readily with subclavicular nodes.

2. In 12 per cent. of cases a trunk passes from the above to humeral nodes at the insertion of the tendon of the pectoralis.

3. In $5+$ per cent. of cases there is a double set of axillary trunks: $(a)$ Same as above. (b) Superior external trunk passes beneath pectoralis minor near its costal insertion, to subclavicular nodes. They do not penetrate the muscle.

4. The above main trunks may pass between the pectoralis major and minor.

5. Three cases showed a deep trunk passing beneath muscles directly to supraclavicular nodes.

6. In Io per cent. of cases the injection passed from the inner quadrant along the internal mammary artery reaching nodes in second or third interspace.

In no case were any lymphatics found perforating muscle.

Generalization.-After reaching the axillary nodes and the muscular aponeuroses, mammary cancer usually becomes generalized and nearly any tissue in the body may become involved. The location and course of the metastases present some of the most remarkable features of the disease, which have called for very searching analysis.

Gross, from 423 autopsies, placed the organs in the frequency of involvement in the order: pleura, 50.9 per cent.; lungs, 49.9; liver, 48.6; bones, 20.5; 
brain, 9.4; ovary, 8; opposite breast, 7.8; dura mater, 5.9; kidneys, retroperitoneal nodes, and uterus, 5.7 to 5.2 , other organs, less than 5 per cent. Pearce Gould in I 28 autopsies found the nodes involved in II 5 , the liver in 55 , the lungs, excluding pleura, in 28 . Williams, also excluding pleural extensions, finds the liver more frequently invaded than the lungs, but the grounds for such exclusion may be questioned. In 2534 mammary cases collected from Gross, Colwell, Paget, Handley, Gould, and Torok the liver was invaded in 928 , or 36.6 per cent. Torok and Wittelshofer, finding in 366 autopsies I9I free from enlarged nodes, among which 62 per cent. had metastases, concluded that failure of axillary invasion is no guarantee against internal metastases. Yet they did not examine all the nodes microscopically. Handley reports 2 cases of abdominal metastases without axillary invasion.

The pleura is invaded by ( $\mathrm{r}$ ) fine lymphatics which pass from the pectoral fascia straight through to the subpleural lymphatics and (2) from the sternal and anterior or deep mediastinal nodes. The latter route is uncommon, as Torok and Wittelshofer in 366 autopsies found these nodes invaded in only 6.5 per cent. Occasionally cancerous, subclavian, supraclavicular, or bronchial nodes invade the pleura at adjacent points. The first effect is a permeation through the parietal and later the visceral lymphatics which may be outlined with remarkable delicacy. A serous or bloody pleurisy usually follows. Breaking into the pleural cavity the cells gravitate below, cause thick adhesions, and rarely perforate the thickened diaphragm. Handley observed polypoid pleural tumors. He estimates the frequency of pleural invasion at 38 per cent., and reports 3 cases in which an adherent pleura of the side of the growth escaped while the other pleura was non-adherent but cancerous.

The lungs are reached superficially from the pleural lymphatics, while from the bronchial nodes extensions pass along the lymphatics of the bloodvessels. Extension to lungs through the blood-vessels is rare.

The importance of extensions along the muscular aponeuroses has been strongly emphasized by Handley.

By means of vertical gross sections of the skin and muscles hardened in Orth's fluid, dehydrated, and cleared in cedar oil he was able to trace lymphatic invasion with unusual success. He finds that mammary cancer travels by continuous permeation chiefly in the vessels of the muscular aponeuroses, and extends not only over the pectoralis but over the serratus magnus, obliquus, rectus, below the xiphoid, and across the median line, covering an oval area $25 \mathrm{~cm}$. in diameter. Approaching the center of this area and the main tumor the underlying muscles and overlying skin become more and more involved. By the same methods he has concluded that invasion of the peritoneum from the epigastric aponeuroses by way of the parietes or the falciform ligament of the liver is of frequent occurrence (44 per cent.). Thus in 422 autopsies the abdominal organs were involved and the lungs were free in 53 , indicating that the abdominal extensions did not pass through the thorax. In 3 cases by microscopical sections he traced peritoneal invasion through the rectus muscle and into the falciform ligament and liver.

Metastases in humerus and femur he also brings in relation to the growth in the aponeuroses since both humerus and femur appear to be invaded at the insertions of tendons and aponeuroses. All the very wide extensions in the subcutaneous tissue of the trunk, which rarely pass beyond elbow and knee, are most readily traced to the same route.

The conclusions of Handley favor wide excision of the aponeuroses rather than of the skin, and have greatly influenced surgical procedure. Gerota ( 1897 ), in a subject of recurrent mammary carcinoma with invasion of epigastric and inguinal nodes, was able to inject the lymphatics from the breast along the superior and inferior epigastric arteries to the inguinal nodes. He assumed that the recurrent tumor had widened these vessels by disturbing the normal lymph flow. Stiles (1899) points out that the xiphoid is only $2 \frac{1}{2}$ 
$\mathrm{cm}$. from the inferior border of the breast, less than the distance to the mediastinal nodes, and that here only a thin fibrous layer rich in lymphatics separates the mammary fat tissue from the subperitoneal fat tissue and the suspensory ligament of the liver.

Retroperitoneal, adrenal, renal, lumbar, and spinal extensions Handley traces from a preëxisting pleural invasion with growth downward along the diaphragmatic crura. Yet in 6 cases there was retroperitoneal invasion with the thorax free.

The bones most frequently involved are the sternum and ribs, femur, vertebral column, cranium, humerus, and clavicle. The humerus is invaded usually at the junction of the upper and middle thirds, the femur in its superior third, where spontaneous fractures occur. Williams collected 533 cases, of which the cranium was invaded in 36 , the vertebræ in 12 , the femur and humerus each 8 . $x$-Ray examinations are considerably increasing these proportions. Kaufmann in 63 autopsies found 33 (53.3 per cent.) with bone metastases. The osseous invasion is sometimes very wide-spread and about I4 per cent. (Kaufmann) show osteoplastic changes.

Peritoneal invasion occurs chiefly through the epigastric region, through the crura of the diaphragm and rarely by the blood-stream. Epigastric infection alone was demonstrated in at least r 2 per cent. of Handley's cases, combined with thoracic infection in 37 per cent. of early and 46 per cent. of late cases. If it does not occur early it seldom develops at all. The event is often indicated by epigastric pain and tenderness. The first effect is usually extension to the liver, which yields superficial implantations beginning at the falciform ligament, or involvement of the portal nodes, with subsequent central invasion of the liver by retrograde growth. Handley describes efferent lymphatics leading from portal nodes through falciform ligament and diaphragm, thence between pericardium and chest wall, connecting with internal mammary lymphatics.

The hepatic lesions vary greatly, from a single nodule to complete transformation of the organ into cellular or fibrous carcinoma. During the progress of this lesion primary or superficial tumors often regress. The liver may be the only abdominal organ involved, as in 36 cases of Handley's series, but frequently the peritoneum itself, the omentum, intestines, and pelvic organs are invaded. The subserous lymphatics may be injected over a large area, but soon there is rupture into the cavity and transportation of cells to the surfaces of viscera. A low grade of peritonitis may be established, with opacity, thickening and adhesions, and serous or chylous exudates. The omentum may be contracted to a small epigastric mass. Gravitation of cells to the pelvis leads to adhesions of all pelvic organs and invasion of ovary, tubes, uterus, and vaginal vault. The ovaries are a favorable nidus, especially before the menopause, and the lesion here presents the structure of Krunkenberg's tumor, or of fibrocarcinoma.

Diaphrenic invasion of the abdomen occurs only in the late stages of thoracic disease. It passes through the substance of the diaphragm with invasion of the liver, or through the crura, when the further course more often leads to the retroperitoneal nodes and kidneys.

Cerebral metastases occur in about 4 per cent. of autopsies. Any portion of the brain may be chosen, most frequently the cerebellum. Their late appearance indicates an origin through blood-vessels. The dura and skull may be affected when the brain is free, suggesting an access by cranial lymphatics.

In a notable group of cases paraplegia develops early in the course of mammary carcinoma from dural or spinal metastasis. Very remarkable are the cases of diffuse carcinoma of spinal and cerebral meninges, of which 
Peabody's report from this laboratory is illustrative. The tumor-cells in flat strands and groups extend from the cauda to the vertex. Humbert and Alexieff have collected several cases of this type.

The part played by the blood-vessels in the dissemination of mammary carcinoma is distinctly less than that of the lymphatics. Invasion of veins is commonly observed about cancerous lymph-nodes, in the deep fascia, and less often in the breast itself. It becomes a prominent feature in very cellular and rapid growths with which also the metastases are more widespread. The occurrence of metastases sometimes early, in the brain, spinal cord, spleen, bone-marrow, and heart muscle, indicate that intravenous foci often escape the encapsulation by fibrin and organization described by M. B. Schmidt. Cases of general miliary carcinoma also indicate dissemination at least in part by the blood-vessels (Petit).

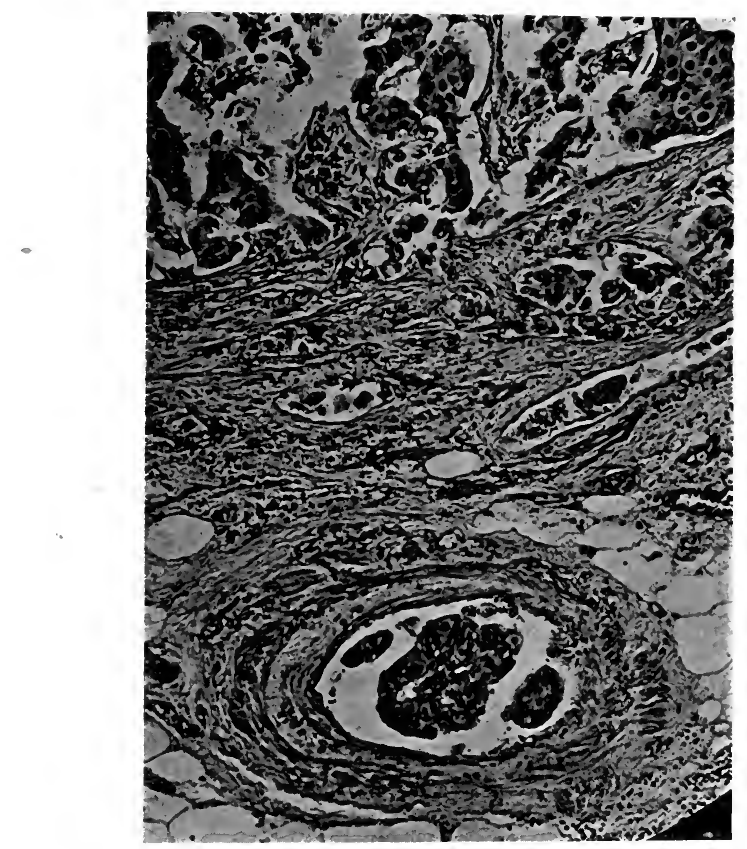

FIG. 236.-Mammary cancer. Alveolar carcinoma invading vein.

Regressive Phenomena.-Regression in mammary carcinoma is often observed in the older lesions. Partial or complete atrophy of tumor-cells, with cicatrization, occurs especially in cutaneous nodules and ulcers when internal metastases are growing actively. It is commonly assumed that the lymph-nodes are capable of destroying not only embolic cells, which may be granted, but even established secondary tumors, an event which must be held excessively rare or even as yet unproved. Handley accounts for the discontinuous character of lymphatic permeation by assuming that perilymphatic fibrosis causes pressure atrophy of tumor-cells in advancing segments of invaded lymphatics, so that the zone of active growth is ringshaped rather than disk shaped. Union of cancerous fractures occurred in several cases in the Middlesex Hospital series. 
A constitutional immunity is the only satisfactory explanation for the resistance which some subjects exhibit to the progress of a disease, whose course varies from a few weeks to 20 years. The slow atrophic scirrhus is the best illustration of a repressed and yet progressive carcinoma. In Hodenpyl's case in which large superficial tumors disappeared, I found the liver almost completely replaced by hyaline connective tissue with comparatively few active foci in the extremely emaciated body. Gould and Mackay have reported cases of apparently complete disappearance of advanced mammary cancer with restoration of health, but histological studies of the tissues of such subjects are still wanting. Osler records shrinkage of a spinal metastasis with relief of paraplegia.

Clinical Varieties. - The clinical course of mammary carcinoma is influenced by the conditions of origin and the structure of the tumor; by the age, susceptibility, and physiological condition of the patient; and by many intercurrent factors. Most prominent is the influence of structure and conditions of origin, and most current clinical classifications show a definite relation to these features. Age has a pronounced influence on most anatomical varieties. The relatively malignant course of most structural types of the disease in young subjects is uniformly recognized. The cellular varieties belong to the early decades, the scirrhous forms to the late periods. Yet there are many exceptions to this general rule. Cystic adenocarcinoma and colloid tumors are of relatively late occurrence. In some old and resistant persons the disease seems to reach a certain limit of growth. I have observed an apparently stationary large adenocarcinoma of ro years' duration in a woman of 80 years who suffered little apparent inconvenience. The very marked aggravations in the disease produced by gestation and lactation are commonly observed. Cancer may apparently arise during gestation and prove fatal before its termination. While there are doubtless marked variations in the susceptibility of patients to the progress of the disease, the nature of this constitutional element is as obscure here as elsewhere.

A possible reflex influence of the ovaries led Beatson to perform ovariotomy in two young subjects. The results were disappointing. Lett in 99 inoperable cases saw notable amelioration in 23 per cent., slight change in I 3 per cent. Rarely, as in the cases of Quinard and of Reynes, distinct inhibition appears to follow castration.

More or less striking clinical peculiarities have led to the recognition of several clinical varieties of mammary cancer.

(1) Cystadenocarcinoma corresponds in part to a specific anatomical type arising in mammary cysts or large ducts. It occurs after the chief age of incidence and in many elderly women who have nursed children (Bowlby, Delbet). A circumscribed tumor appears in the center, less often in the periphery, of the gland. It is preceded by an intermittent, serous or bloody discharge from the nipple, which may temporarily reduce the size of the tumor. A definite tumor may be difficult to demonstrate, especially in fat subjects, or the growths may be multiple. The progress is slow and growth may be inhibited for long periods. Invasion of lymphatics and nodes is delayed until the tumor capsule is perforated, or the breast invaded or the skin eroded. As previously pointed out, there are many grades of malignancy observed in the late stages and atypical forms of this tumor, but many of them are comparatively benign, and they furnish the highest proportion of successful extirpations, as well as the most difficult field for properly adjusted surgica! treatment.

(2) Medullary or encephaloid is the term commonly applied to the average bulky, soft, cellular tumor which pursues the course of very malignant 
mammary cancer. The majority of these tumors are duct carcinomas, but the clinical group includes also cellular acinar carcinomas, malignant adenocarcinomas, and pseudosarcomas. They are characterized by the occurrence at relatively early age, by their considerable or large bulk, fungating form, rapid progress, early invasion of skin, muscle, and lymph-nodes, extensive dissemination, and early cachexia. Ulceration, necrosis, hemorrhage, and gangrene may be prominent. Yet some of them by attracting attention early may be cured by radical operation, so that the formidable appearance is no bar to active measures.

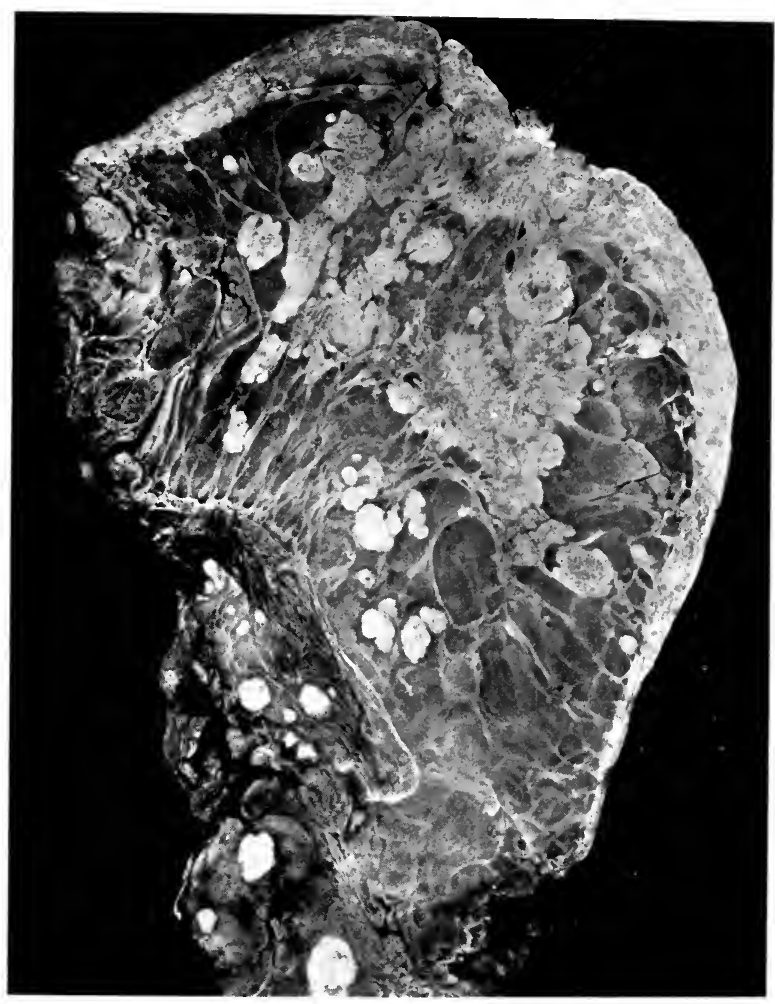

FIG. 237.-Disseminating carcinoma of breast. Extensive invasion of skin reaching beyond line of incision on both sides. Miliary carcinoma of pectoral muscle.

(3) Acute Carcinosis.-Very rapid progress with local inflammatory signs, fever, and early intoxication are observed in a remarkable group of cases occurring at early ages and notably in gestation. The process begins actively and one or both breasts rapidly increase in size without localized tumor formation. There is well-marked hyperemia, local heat, and slight tenderness. The consistence is uniformly firm, but the tissues about the breast or over the chest and back may be edematous. The lymph-nodes are swollen from the first and invaded probably very early. After reaching a certain stage the subsequent course may vary. Some cases are rapidly fatal with generalization or pneumonia, as in Billroth's, 6 weeks; Aitken's, 38 days (?); 
and Schmidt's, 3 months. The shortest duration I have observed was 4 months. Others are subacute, but succumb within a year with very widespread lymphatic and visceral metastases. A patient of Delbet's survived amputation first of one breast then of the other for 3 years. In 2 cases I found this clinical course with large-cell duct carcinoma, in one with a tumor composed of diffuse hyperchromatic round and polyhedral cells.

(4) Scirrhous carcinoma is an ill-defined anatomical variety, but clinically quite characteristic. The majority of mammary cancers are distinctly fibrous in much of their growth, but the typical scirrhus is a small and very hard acellular process which usually fails to enlarge the organ and often results in atrophy and shrinkage. It is a form frequently assumed by local recurrences. Atrophic scirrhus occurred in 7.9 per cent. of Gross' series, and chiefly but not always in elderly subjects. The breast is usually flat, hard, and nodular. The skin is commonly adherent, the nipple retracted,

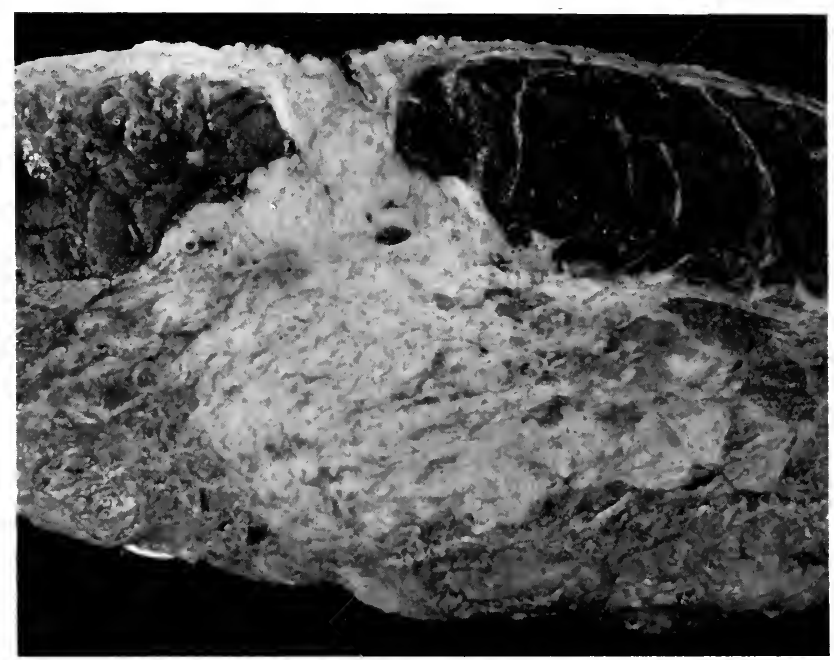

FIG. 238.-Mammary carcinoma arising on chronic mastitis and involving nipple and ducts.

and the breast becomes fixed to the chest. Superficial erosion of the epidermis or ulceration may occur. The progress is very slow and may be prolonged to or even 20 years. Yet the typical small scirrhous carcinoma is also notable for the extensive dissemination in lymph-nodes and distant organs which occurs in some cases. Hence the breast always requires careful examination in cases of visceral carcinoma of uncertain origin.

The skin may become the seat of very numerous secondary nodules or small ulcerating tumors derived from a scirrhous tumor in the breast. In a notable case of Handley's scores of nodular tumors and excavated ulcers covered the skin from chin to navel, illustrating the effects of wide dissemination in the deep fascia with multiple excursions to the derma through the vertical lymphatics. These cases were described by Velpeau as "scirrhus pustuleux." Moderate grades of the cutaneous eruption are relatively common in recurrent cases. The nodules may disappear at one point while developing at another.

Cancer en cuirasse develops in the late stages of cases in which the early 
history is variable, but some cases follow primary scirrhous carcinoma and maintain this character throughout. The pronounced cases show deep) thickening and wooden induration, eroded spots, ulcerated areas, reddish and brownish discoloration of the skin over a wide area, including arm, neck, chest, and even encircling the trunk, with pain, elephantiasis, and embarrassment of respiration. 'The typical condition is rare, and many authors have described imperfect copies of the startling pictures this condition may present.

(5) Fibrocarcinoma following chronic mastitis constitutes one of the most definite of the clinical entities in mammary cancer. After a variable period of chronic induration of one or both breasts, which often escapes the attention of the patient, one or more areas of the organ become very hard,

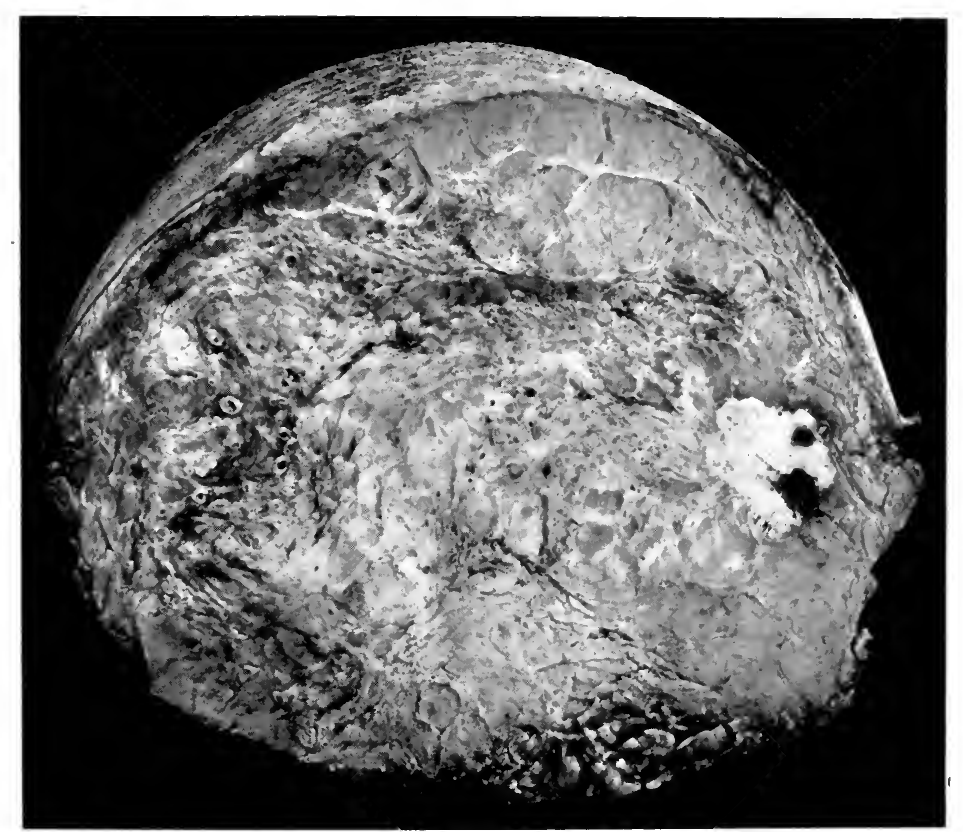

FIG. 239.-Chronic diffuse productive mastitis, with many small cysts, and a single nodule of carcinoma.

the skin becomes dimpled and adherent, the nipple often retracted. A definite tumor being difficult to define, the suspicion of carcinoma is delayed, the growth slowly increases, often pain is added, the breast becomes adherent to the muscle, and the axillary nodes enlarge. The progress may be very slow and usually extends over several months before its serious nature is realized, or it may develop actively while remaining hard and fibrous. The familiar picture of fibrocarcinoma without marked tumor formation is thus unfolded.

It is especially in this group of cases that errors of diagnosis, expectant treatment, and indecision of patient and physician prove costly. Many of these cases recur after early operation, and when they have infiltrated the breast or reached the lymph-nodes they form one of the least favorable for 
successful treatment. Very small fibrocarcinomas occur which cannot be definitely located by palpation, but which early give rise to well-marked tumors in the axillary nodes. Occasionally the dissection of the amputated breast has been pronounced negative, but subsequent recurrence in the lymphnodes proves that a carcinoma has been overlooked.

(6) Mucoid carcinoma is a relatively benign form which has been discussed in the anatomical section.

When the different subvarieties of mammary cancer have been sufficiently distinguished little remains of the so-called banal form of the disease. Such a result is desirable owing to the individual character of the various clinical forms of this protean malady.

The simple diagnosis, "mammary cancer," must be regarded as inadequate.

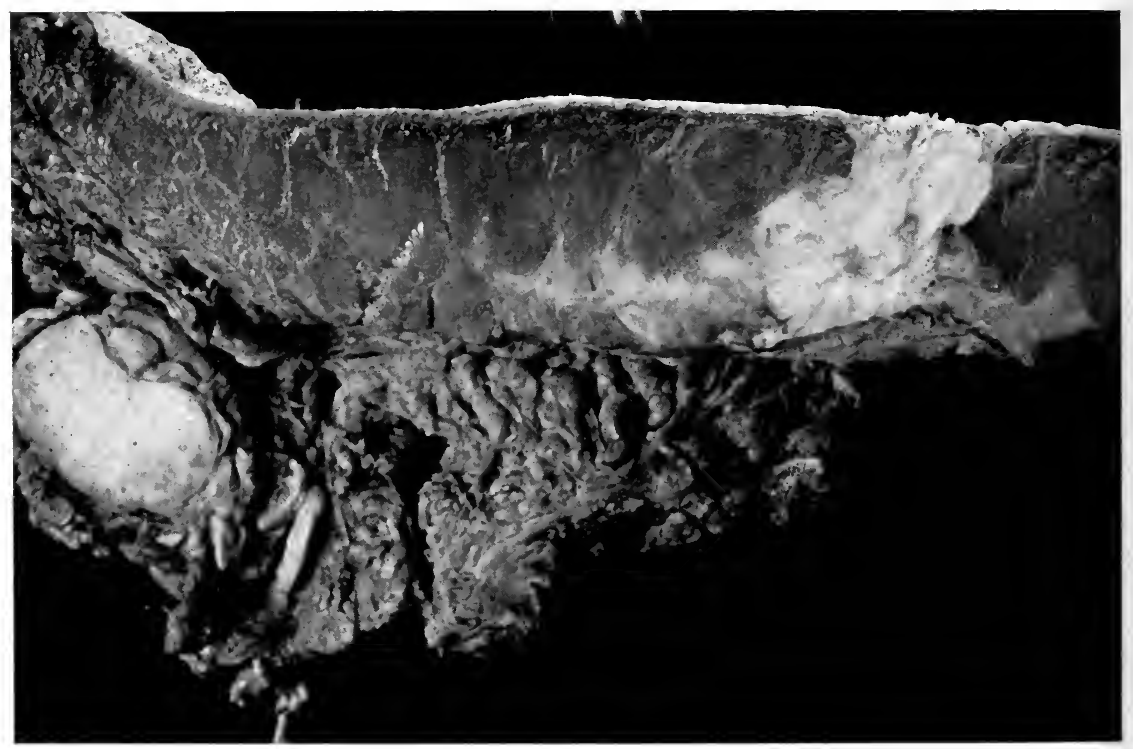

FIG. 240.-Cicatrizing carcinoma in an atrophic breast. Bulky metastasis in axillary lymph-node.

Mammary Carcinoma in Males.-Since the thesis of Hourteloup, I872, carcinoma in the male breast has attracted much attention. The estimates of its frequency have varied from 0.86 per cent. of all mammary cancers (Williams) to 6 per cent. by La Forgue. Of 269 tumors of the male breast, 244 were carcinoma and 3 sarcoma (Schuchardt). Contributing factors observed include repeated trauma, zona, chronic eczema, and attempts at nursing (Baumgartner, Lit.). It occurs at slightly later age than in women, but has been observed at 20 years. Poirier saw affection of both breasts. Unusual development and activity of the breast are predisposing conditions. Owing to the prominence of the large canals, duct cancer is relatively frequent, and the scirrhus type is often followed, but cellular tumors are not uncommon. Williams classified 89 of roo cases as of acinar or alveolar structure. Chronic inflammatory processes may long precede the development of the tumor which is slow and insidious. When fully established the 
process is less malignant than in women, the growth is persistent, many of the features of ordinary mammary cancer are observed, and local or general metastases may develop. Of I6 fatal cases Williams found the average duration 6I months, in I3 unoperated cases, 38 months. Recurrences were notably early, average period 9.7 months.

Development of Surgical Treatment.-Moore introduced the modern operation for mammary cancer in 1867 , when he contended that recurrences were due not to blood infection, but to incomplete extirpation of the tumor. He recommenced a general removal of the breast, skin, fat, axillary nodes, and pectoral muscle. He even undermined the skin-flaps. His views were supported by Lister and Banks. Gross adduced convincing statistics showing the necessity of removing the axillary nodes in every case, and he excised the skin widely, looking for aberrant lobules of the gland, and portions of the pectoral muscle if suspicious. The free excision of underlying muscle followed Heidenhain's demonstration of the invasion of deep intermuscular lymphatics. Stiles limited the excision of skin, enlarged the ablation of mammary fat tissue, and excised all underlying muscle.

Halsted excised the sternal half of the pectoral and divided the clavicular portion and pectoralis minor in order to expose the upper axilla. He had three successful results after removal of supraclavicular nodes. Handley limits the excision of skin, greatly extends that of the muscles and aponeuroses to a circle ro inches in diameter, and clears the epigastric space.

Many other surgeons in England, France, Germany, and America have contributed observations influencing the present accepted form of the operation. Statistical reports of the improving results obtained have shown that the wider the operative field, the better the result. The proportion of cases presenting themselves at the Middlesex Hospital with recurrence fell from 54.5 per cent. in 1858 to 1875 to $\mathrm{I} 3.8$ per cent. (plus operative mortality of I. 5 per cent.) after 1894 . Survival of recurrent cases after operation decreased from 34 to 26 months, partly because of the increased scope of cases judged operable. The same improvement appears in De Page's report of cases well after 3 years in different periods: From I 865 to I875, 9.4 per cent.; I 875 to I885, Io per cent.; I885 to I $895,33.8$ per cent.; I 895 to I $905,46.5$ per cent. Walther's statistics from Paris hospitals gave 52 per cent. of cures after 3 years.

The effects of late recurrences are illustrated in Le Dentu's table showing 47.45 per cent. well after 4 years, but only 6.77 per cent. known to survive after in years. Many others could not be traced.

Surgical statistics may be made more significant by more detailed attention to the gross anatomy and microscopical structure of the tumor. Statistical studies include encapsulated adenocarcinoma, miniature cancers or even precancerous lesions, and early circumscribed duct cancers, all of which may be eradicated by ablation of the breast, along with the highly malignant forms of the disease. It is possible to select cases which will give roo per cent. of recoveries and others which will invariably prove fatal. Too often the radical operation is performed on comparatively innocent growths and the good result attributed to the sacrifice of tissue. No uniform rule can guide in the excision of skin when some cases show cutaneous invasion over a very wide area, others none at all. It would be interesting to know but is impossible to state how much a constitutional resistance influences a favorable result after some very wide excisions. Experimental and clinical data strongly urge great caution against producing mechanical emboli in blood- and lymph-vessels by vigorous preparation of the skin and blunt dissection, especially in those advanced cases subjected to wide excision. 
General Prognosis. - The prognosis in mammary cancer can be estimated only after consideration of the many factors involved, including the age of the patient, the duration and extent of the disease, the structural type and position of the tumor, and the condition of the patient. According to Handley the condition is inoperable when there is extensive ulceration of the skin; with tumors adherent to chest; when axillary nodes are fixed; with edema of arm; when supraclavicular nodes are involved; when there are signs of distant metastases; and in acute carcinosis.

The proportion of cases presenting themselves as inoperable was estimated by Primrose at 32 per cent., after an average duration of the disease of 14 months, and by Deaver at 25 per cent. Before 30 years of age mammary cancer is extremely fatal, so that some surgeons prefer not to operate during this period. Schwartzoff reports ${ }_{5} 5$ such cases all rapidly fatal in spite of early operation, yet Deaver reports three cures in patients under 30 years. In aged women, on the other hand, the disease usually runs a prolonged course, and according to Deaver many of the patients with well-established cancer

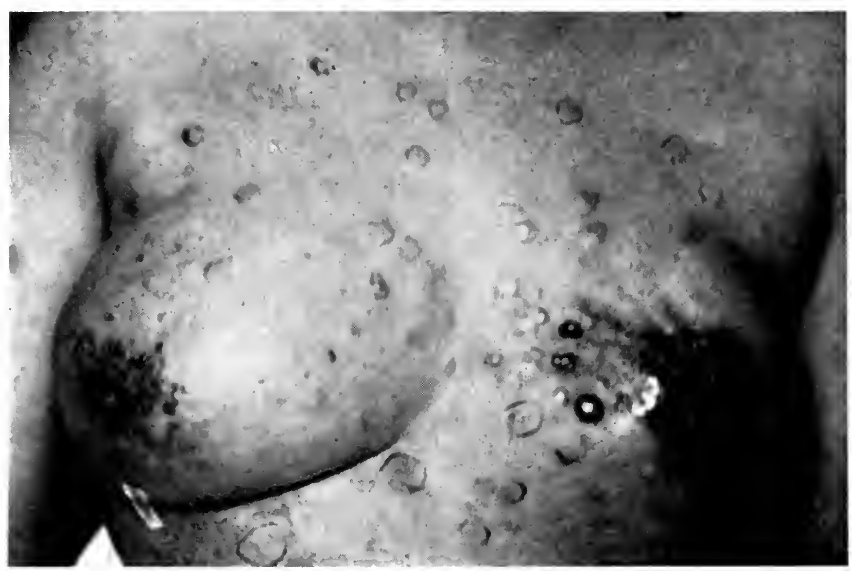

FIG. 24I.-Mammary cancer. Postoperative recurrence.

live longer with palliative instead of operative treatment. In obese subjects with much fat invasion of the breast and atrophy of parenchyma, small tumors very often metastasize early and give disappointing operative results.

The position of the tumor is often of importance. The most favorable position is at the nipple. Aberrant tumors in axillary, sternal, or xiphoid prolongations are frequently atypical and anaplastic, and the initial extensions are relatively inaccessible. Small deep-seated tumor masses may develop on the under surface of the breast, immediately invade the pectoral fascia or muscle, and soon reach the deep nodes or chest wall.

The duration, extent, and structural type of the tumor are the common factors influencing the results of operation. They may be taken together in considering the sources of recurrence.

Recurrences.--Recurrent tumors develop in the skin, in the outlying lymph-nodes, and in distant organs. In each instance they develop from undisturbed tumor foci or loose tumor-cells which have escaped removal. In or under the skin recurrent tumors may arise from aberrant portions of the breast in axillary, sternal, or epigastric region, of which Williams col- 
lected 29 cases. Many incisions supposed to surround the breast really pass through outlying portions of the organ which are not distinctly aberrant. L. Heidenhain long ago, and later Stiles, pointed out that all portions of the breast in carcinoma must be removed and that many recurrences are due to failure to accomplish this object and to the late development of carcinoma in such remnants of the organ. Cancer may develop anew in the opposite breast.

In young subjects recurrences develop earlier (average $2 \frac{1}{2}$ months) than in patients over 65 years ( $8 \frac{1}{2}$ months) (Durand). In the malignant histological forms recurrences are earlier and more frequent than in relatively benign cases, but the more important influence is the extent of the disease. Both these rules are amply apparent in operative statistics. Mucoid carcinoma is notable for the long delay in recurrences. Of 47 recurrences Williams recorded before 3 months, 4; I year, I7; 2 years, Io; 3 years, 6; 4 years, 2; after 4 years, 8. Ransohoff collected a series of very late recurrences. Thirtyseven cases of which 26 were local occurred after 7 years, others at late periods up to 25 years. Recurrence in brain, spine or liver, alone, after Io to I 3 years was observed by Bull.

The striking influence on prognosis exerted by the type of carcinoma is illustrated in Halsted's table.

\begin{tabular}{|c|c|c|c|}
\hline & Cases. & Cures. & Per cent. \\
\hline 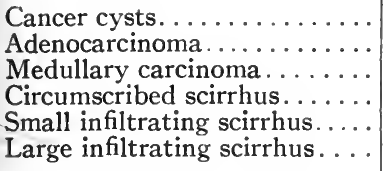 & $\begin{array}{r}6 \\
32 \\
25 \\
28 \\
80 \\
39\end{array}$ & $\begin{array}{r}2 \\
24 \\
12 \\
13 \\
30 \\
8\end{array}$ & $\begin{array}{l}33 \cdot 3 \\
75 \cdot 0 \\
48.0 \\
46.4 \\
35 \cdot 5 \\
20.5\end{array}$ \\
\hline Total.................. & 210 & 89 & 42.4 \\
\hline
\end{tabular}

In this series the neck operation was performed II9 times. Of 44 cases in which both axillary and supraclavicular nodes were found microscopically to be involved, 3 were definitely cured. Pilcher reports 3 cured cases of this type.

The influence of the extent of the disease was even more notable.

\begin{tabular}{l|r|r|c}
\hline \hline & No. & Cured, per cent. & Well 3 years. \\
\hline & & & \\
\hline Axilla and neck negative.... & 60 & 75.0 & 85 \\
Axilla positive, neck negative. & I IO & 24.5 & 3 I \\
Axilla and neck positive..... & 40 & 7.5 & IO \\
\hline
\end{tabular}

At the Massachusetts General Hospital Greenough reports that of 236 cases with palpable axillary nodes only I 2 per cent. were cured by operation, while of 275 similar cases Finsterer reports only (I2) 4.3 per cent. cured. I believe that the last figures represent the average success obtained by surgical treatment, which means that when a woman presents an established mammary cancer with axillary nodes palpably infected she has about one chance in 25 of being cured by operation.

In mucoid carcinoma Gaabe found 53.8 per cent. of 3-year recoveries. 
The influence of improving operative technic is strikingly shown in De Page's report (1907):

Recurrences After Operation (De Page)

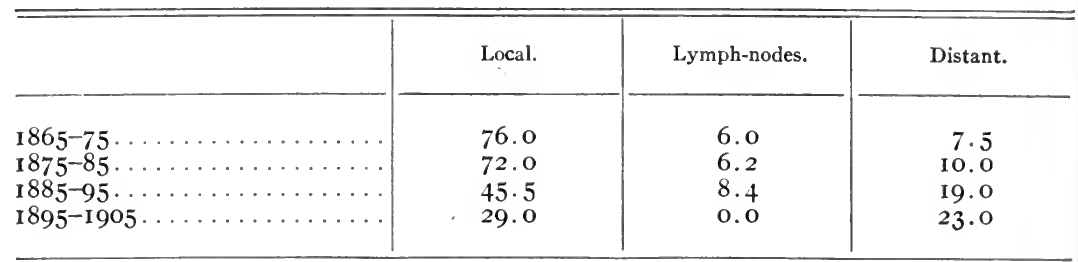

Many authors have stated their belief that the extent of the disease rather than the extent of the operation determines the prognosis. It is regarded as proved that loose tumor-cells may become implanted in the wound and give rise to recurrent nodules. The implantation experiments of Bergmann and Hahn, and the occurrence of tumors at the site of injection of tumor vaccine, favor this view, while the location of nodules exclusively in the line of incision is highly suggestive. An equally or even more important source of cutaneous recurrence is the incomplete removal of infected, cutaneous, and deep lymphatics, while the mechanical displacement of tumorcells from invaded into normal lymphatics is probably rather common. The persistence of cutaneous recurrences in spite of the best modern technic seems to justify the use of various adjuvants to surgery, which may destroy superficial cell groups, and not a few operators claim to secure better results by $x$-ray, radium, fulguration, or escharotics at or soon after operation (Ochsner, Cabot, Deaver).

Recurrence in the lymph-nodes and muscles is largely under the control of the operator, and the conditions governing their appearance have been fully indicated in discussion of the paths of dissemination. It is notable that this form of dissemination has been reduced to a minimum.

The increased proportion of visceral recurrences has been referred chiefly to the increased scope of operability, but it seems quite possible that mechanical dissemination of tumor-cells may account for some of the distant recurrences. It may be suggested also that the removal of a tumor withdraws the natural local barriers against it and may leave the system in a less favorable position to inhibit the growth of vagrant cells. Gaylord (I916) offers experimental evidence to show that prolonged anesthesia and hemorrhage reduce the resistance to cancer. Against such a possibility stands an apparent increased tenure of life of operative cases even after recurrence. The average duration of life in untreated cases was placed by Paget at 4 years, by Odekop at 29 months, by Sprengel at 27 months. After operation Handley's recurrent cases lived an average of 29.6 months and many others were cured. Reports of Sibley, Baker, Gross, and Williams give an average life in unoperated cases of 34 months, of operated cases 52 months.

The operative mortality was estimated by Delbet, I898, to average about 5 per cent., but in many clinics it is now much less. De Page reports 2.8 per cent. (I906). M. Richardson lost 4 patients in 1500 breast operations.

Regarding the significance of these statistics the following comments seem to the writer permissible.

(I) The figures (4 years to 27 months) are too divergent to permit accurate conclusions regarding the natural duration of mammary cancer. The attempt to establish an average duration of this disease should be replaced 
by the systematic classification of cases according to the factors known to influence prognosis. The total duration of life in all forms of this disease is of no clinical significance.

(2) The comparison of operated and unoperated cases is sophistical. The choice of operable cases tends to throw into the untreated class the majority of rapid and unfavorable cases.

(3) Statistics favor the conclusion that operation, on the whole, shortens life in recurrent cases, although sometimes rendering it more tolerable. Handley's recurrent cases lived 29.6 months, while in the above series the duration of life of unoperated cases was $27,29,34$, and 48 months respectively. This conclusion is strengthened by theoretical considerations, as well as by observations on the rapid course of many recurrent cases. It is clearly proved in many instances by the increasing anaplasia exhibited in the structure of recurrent tumors.

(4) The high proportion of clinical cures from the modern operation has resulted largely from the earlier recognition of cancer and the inclusion of a larger number of minute carcinomas or precancerous lesions in the operated class.

(5) The choice of therapeutic measures should not be made under the impression that the duration of the untreated disease is 27 months and that 40 per cent. of operated cases are cured. Since the duration of the disease may vary from 6 weeks to 25 years and the favorable results of operation from o to roo per cent., the first essential in treatment is accurate diagnosis and prognosis of each individual case.

(6) In estimating the economic importance of the surgical treatment of mammary cancer there must be charged up the cost of acquiring surgical skill, and the deplorable conditions following local recurrence. There can be no doubt that operation shortens life and aggravates the terminal suffering in the great majority of recurrent cases. Most of those who deal with the great number of these unfortunate patients would welcome a judicious limitation of the scope of operability in this disease.

From clinical and pathological studies I have drawn the impression that in dealing with mammary cancer surgery meets with more peculiar difficulties and uncertainties than with almost any other form of the disease. The anatomical types of the disease are so numerous, the variations in clinical course so wide, the paths of dissemination so free and diverse, the difficulties of determining the actual conditions so complex, and the sacrifice of tissue so great as to render impossible in a majority of cases a reasonably accurate adjustment of means to ends.

The scope of the operative field having apparently reached a limit, the chief hope for a reduction in the mortality from mammary carcinoma lies in its prevention and earlier diagnosis.

Both these objects point to the excision of many breasts before carcinoma has become established in the clinical sense. The growing tendency to remove the breast for recognized chronic mastitis or suspected carcinoma, while probably sacrificing some organs unnecessarily, has justified itself in the writer's material by securing the early removal of some miniature carcinomas and more precancerous lesions. In this field too much reliance should not be placed on the examination of resected areas and nodules. When the condition of $t$ he organ becomes sufficiently suspicious to demand a partial excision for diagnosis it is usually safer to excise the whole breast, make the diagnosis complete, and remove a source of anxiety or actual danger.

The use of $x$-ray and radium in the treatment of mammary cancer has been 
widely employed in recent years, and with so much success that these agents cannot be disregarded in any competent discussion of this subject.

Local recurrences in the chest wall, opposite breast, axilla and supraclavicular space, and elsewhere very often regress and disappear completely under $x$-ray therapy. Pleural extensions seem to be controlled to some extent. Whether the life of the patient is definitely prolonged in such cases, in most of which the disease is generalized, may be doubted, but the local conditions are very much ameliorated.

Thorough treatment by $x$-ray to the limit of skin tolerance before and shortly after operation has been endorsed by all who have employed it systematically. So great has been the regression of the tumor after such preliminary treatment in many cases as to indicate that the limit of operability may well be reduced in borderline cases in favor of the $x$-ray. Striking histological changes in tumors so treated support the conclusion that such treatment has a powerful effect on the local tumor, but the possibility that metastases may occur from regressing and even quiescent tumors must be considered.

As a rule, radium is more effective with mammary cancer than the $x$-ray, with which it is often advantageously combined. In the hands of several observers the insertion of radium into the tumor and its extensions, followed by external radiation, has caused marked regression and even standstill of the local growths in advanced inoperable cases. The pursuit of this method in operable cases, in which there is some contraindication to operation, offers a very important field of study. 


\section{CHAPTER XXVII}

\section{CANCER OF UTERUS, VULVA, VAGINA}

\section{CANCER OF UTERUS}

General Etiology and Classification.--Statistics may be cited to show that the uterus is first in the list of organs affected by primary cancer. Welch collected (from the literature) over 3r,000 cases of cancer, of which 29.5 per cent. were of the uterus and $2 \mathrm{r} .4$ per cent. of the stomach. Orth states that uterine cancer forms 30 per cent. of all those occurring in women, and BirchHirschfeld places it first in order of frequency. The British statistics of Williams gave 38 per cent., 3 r.4 per cent., and 22.5 per cent. in 1868 , I 888 , I900. The U. S. Census, I9II, shows a very marked predominance of cancer of the stomach and liver over cancer of the female generative organs, viz., 17,365 to 6707 . The uterus is still probably the most frequent seat of the disease in women. Kaufmann, dealing with the material of the Basel and Göttingen institutes, found cancer of the uterus in a smaller proportion, I4.75 per cent. and I 5.59 per cent. of the total in both sexes, and Borst places the uterus fourth in the list of organs. Owing chiefly to better separation of sarcoma, myoma, and cancer of the vagina and ovaries from cancer of the uterus, the cases of the latter disease have tended to diminish in statistical observations (Weinberg).

About ro per cent. of uterine cancers affect the corpus (Koblanck). R. Williams, however, finds the proportion of corpus carcinoma in clinical material much lower, while Bäcker and Blumenfeld found it at autopsy below 3 per cent.

The frequency of uterine cancer must be referred in general partly to its anatomical and physiological characteristics, more especially to its exposure to various forms of trauma and irritation. Cervical carcinoma is strongly influenced by childbirths, which average over 5 in such patients (Gusserow, Koblanck, Williams). While carcinoma seldom develops in scars, yet repeated cervical lacerations disturb the normal structure and functions of this tissue, interfere with its nutrition, and expose its weakened structure to chronic irritation and inflammation. A chronic endocervicitis precedes cancer in the great majority of cases ( 34 out of 48 , Polese), and the routine examination of this tissue reveals abnormalities in the morphology and position of the epithelium which constitute precancerous conditions. The most prominent of these conditions is the cervical erosion, many of which show suspicious hypertrophy and heterotopia of the lining epithelium. Beckmann saw the development of carcinoma in an erosion which he had treated for 5 years.

For corpus carcinoma the chief definite etiological factor is the association with myoma, ro per cent. of which are combined with carcinoma (Olshausen). Taussig, in a personal communication from the Mayo Clinic, learned that of 40 cases of corpus carcinoma there were ro associated with myomas. Local hyperemia, chronic endometritis, and ulceration of the mucosa are frequent concomitants of myoma which favor the development of carcinoma (Winter, Sutton). The carcinomas which arise from adenomyoma, localized or diffuse, present peculiar anatomical and clinical features which usually permit of their identification. Genital tuberculosis has been ob- 
served with carcinoma in several instances (Koblanck), but as, in Cullen's cases, the association may be accidental.

Age.-Uterine cancer chiefly affects child-bearing women in the fifth decade, but occurs from the second to the eighth decades. Cervical cancer appears rather earlier than disease of the corpus. A peculiar case of carcinosarcoma of the cervix in a child of 2 years is reported by Rosenstern, and several cases in very young subjects are collected by Engelhorn. Cervical carcinoma in a child of 7 years is described by Glockner.

Two main histological and clinical varieties of uterine carcinoma are recognized: (I) Squamous-cell carcinoma of the cervix, and (2) glandular carcinoma of the body. The vaginal portion of the cervix is almost exclusively the seat of epidermoid carcinoma; in the cervical canal the two types meet and intermingle, while glandular carcinoma, or malignant adenoma, predominate in the corpus. These relations naturally accord with the structure of the organ, squamous epithelium lining the portio and extending a variable distance up the canal; high cylindrical cells of pavement type line the corrugated surface and gland ducts of the cervical canal; and specific glandular epithelium covers the endometrium and forms its glands.

Anatomy of Cervical Carcinoma.-The earliest stages of the disease are seldom recognized grossly. Very small tumors appear in the form of (I) a hard nodule in the substance of the cervical lip, or (2) a circumscribed indurated ulcer of portio or cervical canal, or (3) more diffuse low papillary outgrowths, covering a portion of the lip or canal.

As usually observed the lesion is more advanced and exhibits (I) an extensive induration and swelling of the cervix; (2) an excavated ulcer, or (3) extensive papillary or cauliflower outgrowths covering much of the canal and portio.

Three stages of the progress of the lesion are emphasized by Cullen: (I) Induration without loss of tissue; (2) disintegration, and (3) excavation.

The established and advanced process varies greatly according to the predominance of certain of these tendencies. In one group of cases there is wide superficial extension of the process, and the whole canal and even portions of the endometrium, the posterior fornix, and much of the vagina are covered by polypoid or cauliflower outgrowths. These may become bulky and obstruct the canal, causing retention of secretions or filling the vagina. Ulcerating carcinoma produces wide excavations of tissue in the cervix and vagina, or completely destroys the cervix, and may perforate into the bladder or rectum, or both.

Infiltrating carcinoma thickens the cervix, extends along the vagina, and up the canal into the endometrium, causing obstruction to the canal with pyometra, firmly fixing the uterus to the parametria, and invading the bladder and rectum. In some cases the entire uterine mucosa and part of the wall is replaced by tumor tissue extending from the cervix, and eventually becoming excavated.

Ruge and Veit distinguish sharply between cancers of the portio vaginalis and those of the cervical canal. Their distinctions are anatomical and clinical. Squamous-cell tumors and adenocarcinoma frequently arise from both regions. Portio cancer produces marked thickening with induration of one lip or the whole lower portion of the cervix, while the canal long remains intact. Cervical tumors involve the canal from the first and may long spare the portio while extensively thickening the whole length of the cervix and ultimately excavating it. The portio carcinoma arises superficially and tends to involve the vaginal mucosa and wall, often by a progressive hypertrophy and cancerous transformation of normal epithelial 
papillæ. Contact metastases are also claimed to occur. On the other hand, portio carcinoma seldom invades the canal and never beyond the os internum, while metastases in the endometrium have not been demonstrated.

Cervical carcinoma may be regarded as a true uterine tumor which does not tend to invade the portio or vagina, although the whole cervix may be excavated. It readily invades the peritoneum. In a series of cases metastases in the endometrium have been observed (Winter, Lit.), for which several modes of origin are under discussion, including multiple primary growths, contact implantation, and extension through the lymphatics.

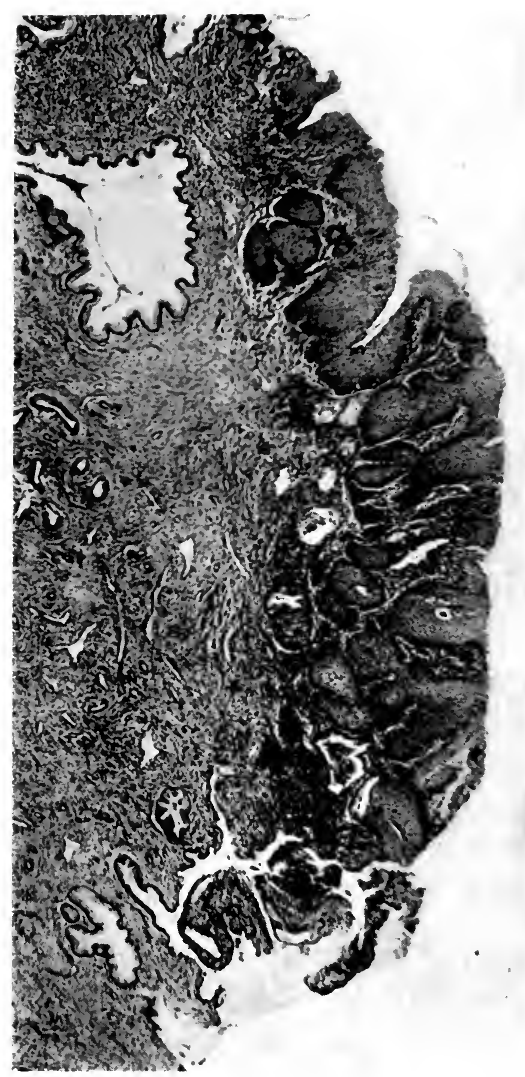

FIG. 242.-Island of beginning carcinoma in a cervical erosion.

Structure and Histogenesis.-In cervical carcinoma two histological types of structure appear, epidermoid carcinoma and adenocarcinoma, but the two types are often combined. The most frequent form presents cords of pavement epithelium in which neither alveoli, pearls, spine-cells, nor hornification are demonstrable. Rarely adult acanthoma is observed with abundance of pearls and much hornification. These structures usually affect the portio and early ulcerate.

The earliest cervical carcinoma that I have seen consisted of localized downward growth of hypertrophied papilla in portions of chronic erosions. 
Since heterotopia of the epithelium was missing these cases might be designated as precancerous. In one case a localized indurated patch, $I \frac{1}{2} \times I \mathrm{~cm}$. in the lower cervical canal, was composed of atypical hypertrophied squamous epithelium with elongated papillæ extending to a depth of $25 \mathrm{~mm}$. In another case a chronic erosion showed early squamous epithelioma of its edges and beginning neoplastic overgrowth and metaplasia of cylindrical cells in the gland ducts. Early stages in the development of carcinoma of the cervix are described by Waldeyer, Ruge and Veit, Cullen, Kermauner, Stone, and others.

The established tumor is usually composed of columns of transitional epithelium with cells of large dimensions, polyhedral or rounded, without a trace of pearl formation or keratosis. Giant-cells are commonly present. The stroma is usually scanty, vascular, and infiltrated by mononuclear or eosinophile leukocytes. It may be completely wanting in certain areas so that neighboring columns of cells cohere. Evidently this structure is

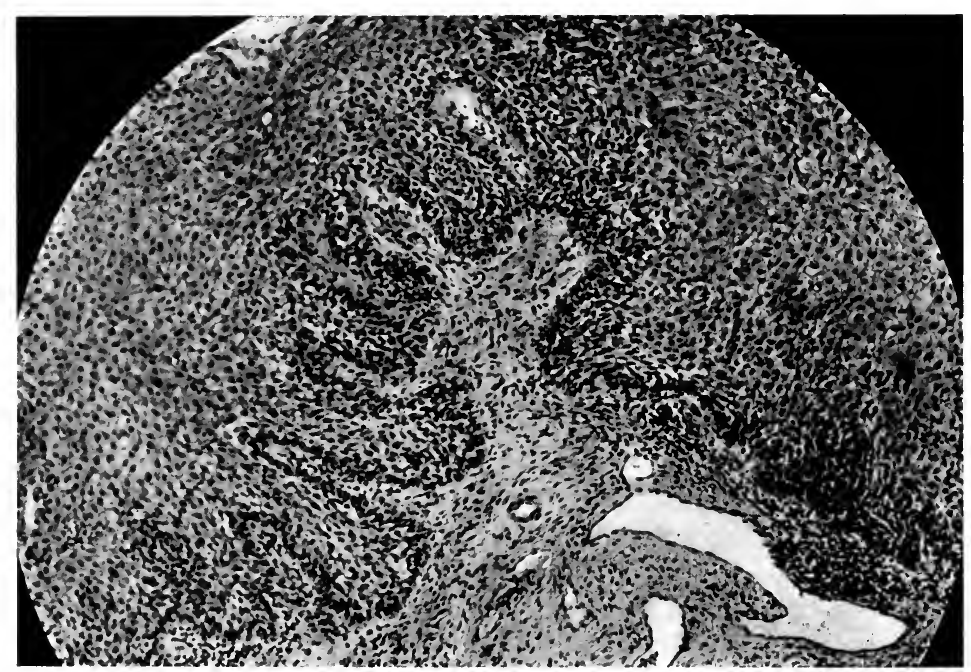

FIG. 243.-Atypical epithelial overgrowth in cervix uteri.

produced by the growth of the epithelial layer as a whole, which, in order to accommodate its enlarged dimensions, becomes variously folded, incurved or everted, thus producing a bulky tumor of essentially papillary type. Lateral outgrowths from the original layer progressively complicate the picture, but the cells do not early split off in small groups as in acanthoma, so that lymphatic involvement is relatively late. The presence of free lying foci of pus surrounded by tumor-cells, and the abundant growth of blood-vessels in elongated papillæ, are structural features resulting from this mode of growth. From the same point of view Ruge has described these tumors as carcinoma evertens and carcinoma invertens, the former growing outward in papillary form, the latter inward, pushing and invading the tissues before it. This structure produces the majority of the papillary or cauliflower, superficial or deep, and widely distributed tumors of the cervix. Their histogenesis has been traced to the stratified epithelium lining the cervical canal and the ducts of the glands. The distribution of this type 
of epithelium is subject to considerable variations, and it is probable that before and during the development of carcinoma the cylindrical epithelium is transformed into stratified cells, or replaced by these cells from a lower level. This metaplasia may extend even to the endometrium and its phases may often be traced in chronic erosions.

Changes in the endometrium occur in many cases of cervical carcinoma. Abel and Landau describe a form of glandular and interstitial hypertrophy, with warty projections of enlarged and dilated glands, overgrowths of spindleand epithelial cells, and exudation of round cells in the stroma. These changes constitute chronic endometritis. A transformation of cylindrical epithelium into stratified flat or polyhedral cells is a form of epithelial metaplasia seen with cervical carcinoma (Kraus) and in chronic endometritis.

Adenocarcinoma, reproducing the alveoli of the cervical glands, occurs in a small proportion of cervical carcinomas. They first appear as submucous nodules in cervix or portio, but in their later stages they reach the surface as papillary, cauliflower, or ulcerating growths which cannot be distinguished

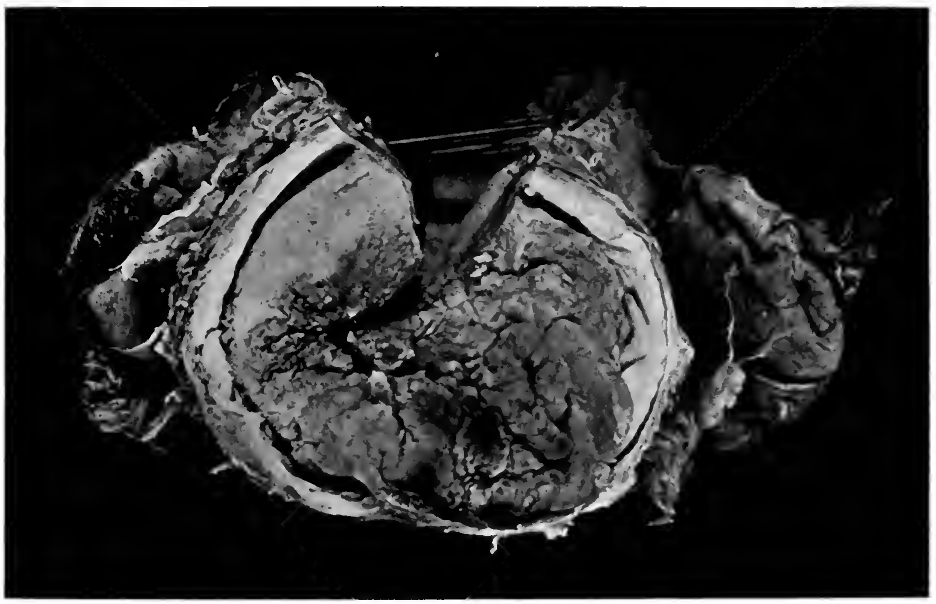

FIG. 244.-Fungating epidermoid carcinoma of the cervix.

from other types of cervical tumors. At the same time, their structure tends to approach that of epidermoid carcinoma. The tumor process begins in the ducts of fundi of one or more cervical glands, where the cells multiply, become stratified, and often assume squamous characters. Cullen pictures a very early but probably secondary focus of origin of carcinoma affecting the superficial cells of the cervical lining. The alveoli have become elongated, dilated, and filled with tumor-cells which have usually lost their cylindrical form. Yet the alveolar arrangement is long retained and reasserts itself in lymphatic metastases. Large alveoli filled with tumor-cells, forming secondary alveoli, is a common structural type of cervical adenocarcinoma. In advanced and in some early cases there is the usual variety of structures of established carcinoma, including alveolar, diffuse, and scirrhous types.

Adeno-acanthoma.- Tumors composed of glandular and squamous elements are often designated as adenocancroids, or adeno-acanthoma. 'They arise in the portio, cervical canal, or in the endometrium, and their histogenesis has formed the topic of much discussion (Fränkel, Wiener, Hitsch- 
mann, Offergeld, Hauser, Buttner). It is now rather apparent that such structures do not, as a rule, signify a multicentric origin from cylindrical and squamous cells, and do not require an original heterotopia of squamous cells, but that metaplasia of cylindrical tumor-cells into squamous is a frequent characteristic of uterine growths as of some other processes in the endometrium. Hitschmann has shown that the metaplasia is complete, producing spine-cells and keratohyalin. On the other hand, Hauser reports a case of adenocarcinoma of the cervix with separate acanthoma of the portio, showing that with multicentric origin the tumors may remain separate. Finally, in several cases the histological study strongly suggested that the adenocancroid developed from a double origin, and not through metaplasia (Hofmeier, Buttner, Sitzenfrey). In a chronic erosion I have observed rarely

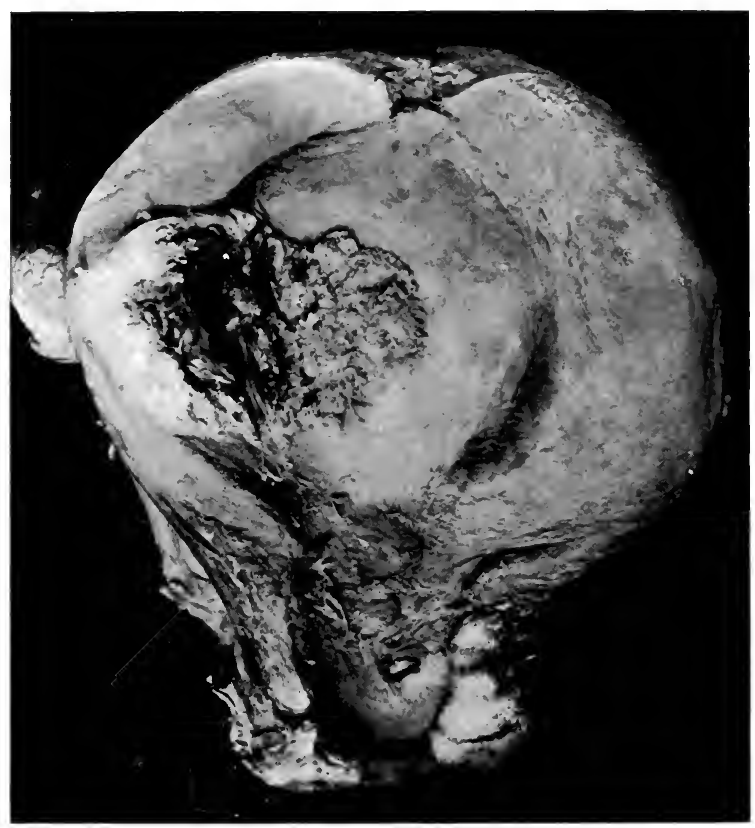

FIG. 245.-Adenocarcinoma of fundus uteri developing over point of greatest pressure from a myoma.

acanthoma arising from the squamous lining and precancerous changes in a neighborhing group of cervical glands. It thus appears that a variable histogenesis must be assumed for the cervical adeno-acanthomas.

The Cervical Erosion.- The facility with which cylindrical mucous cells and squamous cells replace each other in the cervix appears in the study of erosions and pseudoerosions. Ruge and Veit concluded that the mucous cells lining pseudo-erosions were the transformed basal cells of the squamous layer. Meyer and Adair fail to find evidences of this transformation, and Meyer states that true mucous lining cells always develop from the glands of the pseudo-erosion. He finds that erosions always result from inflammation and are attencled by extrusion of the glands into cervical tissue and a replacement of squamous by cylindrical gland epithelium. Schottländer holds that the proliferating glands may pierce the squamous lining of the cervix. In healing, the squamous epithelium from edges or persistent islands grows beneath the mucous cells even into the glands, causing exfoliation of gland-cells and eventually complete replace' 
ment by squamous cells, if the lesion heals completely. The attendant overyrowth readily gives rise to papillomas and adenomas, and these and other secondary changes determine the different features of chronic erosions, as simple, papillary, cystic, and glandular. Fischel holds that the pseudo-erosion of the adult is usually in part a congenital condition. The indifferent epithelium of the fetal cervix differentiates in the third month into glandular and squamous types, the latter covering much of the cervical canal. Later the glands and mucous cells encroach upon the squamous lining and often extend over segments of the portio, producing the congenital pseudo-erosion of infants. At birth the squamous cells begin to regain their position in the canal, undermining the mucous layer, so that both types of epithelium are often seen in contiguity. Meyer recognizes persistent congenital erosions. Most atuthors accept the direct transformation of mucous into squamous lining cells, and, in this respect Mever's view, which sharply separates the two cells types, is probably too rigid. The influence of simple ectropion is also emphasized by many, especially for multipare. All these observations bear directly on the histogenesis and variable structure of cervical carcinoma.

Mucous adenocarcinoma of the cervix is rare. Miller collected 5 cases in which the alveoli were filled or distenderl with mucus, while the stroma was

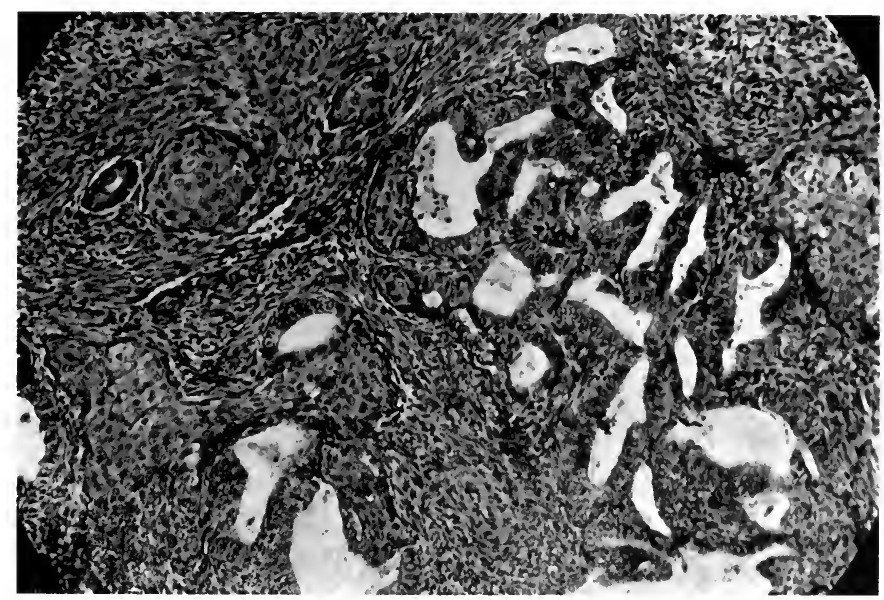

FIG. 246.-Adeno-acanthoma of endometrium.

unaffected. In Albrecht's case there was extensive diffuse infiltration of the cervix, corpus, parametrium, and lymph-nodes. In Cullen's case the tumor involved both cervix and corpus. For these cases an origin from an aberrant group of intestinal or ovarian glands is suggested by Williams.

Atypical Forms of Cerical Carcinoma.-(a) Scirrhous carcinoma of the cervix has been described by several observers who had to deal with overgrowths of cellular or fibrous stroma and corresponding suppression of eipthelial cells, which here may assume a small spindle form (Wagner, Gebhard, Cullen). This structure usually occurs in late cases, and signifies a local inhibition of tumor growth. As in other localities, the outlying areas of the tumor may exhibit undiminished vitality.

(b) Simple Adenoma or Cystadenoma of the Cervix.-The cervix is occasionally the seat of a characteristic adenoma composed of regular alveoli lined by a single layer of cylindrical cells, reproducing the structure of the normal cervical glands (Ruge, Veit). While many of these tumors are benign, some recur locally, and have produced metastases in the vagina. The microscopic structure has also revealed adenocarcinomatous and carcinomatous changes 
(Ruge, Krukenberg). Winter interprets these tumors as a slowly developing adenoma of cervical glands with carcinomatous tendencies. Stone describes precancerous changes in these glands.

(c) Endothelioma.-Unusually small tumor-cells growing in long narrow columns or in small groups, or diffusely, may give the appearance of endothe. lioma, and hence have led several authors to assume an endothelial origin for certain cervical tumors (Kroemer, Graefe, R. Meyer, Poworecké). Since the gross appearance of these tumors is identical with that of certain epitheliomas, and since they are usually quite malignant, it seems most probable that they represent atypical forms of epithelioma (see Endothelioma of Uterus).

(d) Perithelioma.- - In certain rapidly growing and atypical epitheliomas of the cervix the cells become arranged about blood-vessels, giving a peritheliomatous structure. Hansen describes an intravascular hemangioendothelioma. The existence in the uterus of a tumor of vasoformative cells is uncertain.

(e) Sarcomatoid structures are sometimes seen in portions of early or advanced cervical carcinoma, but they are more frequently observed in the corpus (Forssner).

The cells have no definite squamous or glandular character, they grow diffusely with little stroma, and giant-cells may be numerous. The gross appearance of such tumors is nevertheless rather characteristic of carcinoma, and thorough microscopical study may reveal epithelial and alveolar structures. In all such cases the gross appearance of the Ilesion should weigh strongly in the diagnosis. Tumors of this type are described by Cullen and many others, and they are sometimes wrongly called carcinosarcoma (see Sarcoma of Uterus).

Precancerous Changes in the Cervix.-The alterations in structure which precede the development of definite carcinoma of the cervix have been variously interpreted. Schauenstein, Sitzenfrey, and Schottländer describe as characteristic of beginning carcinoma the following changes:

(I) The appearance of groups of irregular, hypertrophied epithelial cells with hyperchromatic nuclei, with irregularity and indistinctness of cell borders.

(2) Loss of regular stratification of cell layers, especially of the proliferating basal cells. Schottländer also emphasizes the importance of marked nuclear granulation.

The significance of these changes is contested. Heurlin and Pick interpret them as atypical hypertrophy and regeneration. Yet when to them is added the features of (3) downward growth of epithelial papillæ and definite heterotopia, there is little doubt that one has to deal with the early stages of carcinoma. In a uterus partly lined by squamous cells, Sitzenfrey describes early downward growth of squamous-cell groups and cylindrical cell alveoli into a tissue infiltrated with round cells, a condition which must be accepted as beginning carcinoma. Rubin pictures extremely early but quite definite proliferation of atypical, cylindrical, and squamous cells on the surface and in the glands of old erosions, and the routine study of such erosions reveals many phases of such precancerous lesions. Schauenstein's cases illustrate extensive epidermization of the canal and corpus, and downward growth of atypical papillæ at a few points. In my own material the changes consist chiefly of epidermization of ducts and fundi of glands, with atypical proliferation of both squamous and cylindrical cells. Pronai would not insist on heterotopia and downward growth of epithelium, but describes as early carcinoma a flat extension over cervix of foci of atypical epithelium. Yet 
there is a condition of epidermization of the canal which seems to have no definite tendency to develop into carcinoma. It need not be assumed that every case presenting the above changes will necessarily develop cancer, focal epidermization often remaining stationary, but it is highly important to recognize that the majority of cervical cancers develop from such altered cells. When atypical hypertrophic and hyperchromatic cells are growing downward from the epidermis or fill enlarged gland alveoli the diagnosis of beginning carcinoma is justified.

Stone has shown that several different anatomical forms of cervical carcinoma develop from equally specific precancerous lesions, such as leukoplakic patches, areas of epidermization in erosions, atypical cervical polyps, and adenomatoid overgrowth of cervical glands.

Anatomy of Corpus Carcinoma.- The rather numerous anatomical forms of this group of tumors may be divided into (I) circumscribed and (2) diffuse growths (Ruge and Veit). The latter usually represent the advanced stages

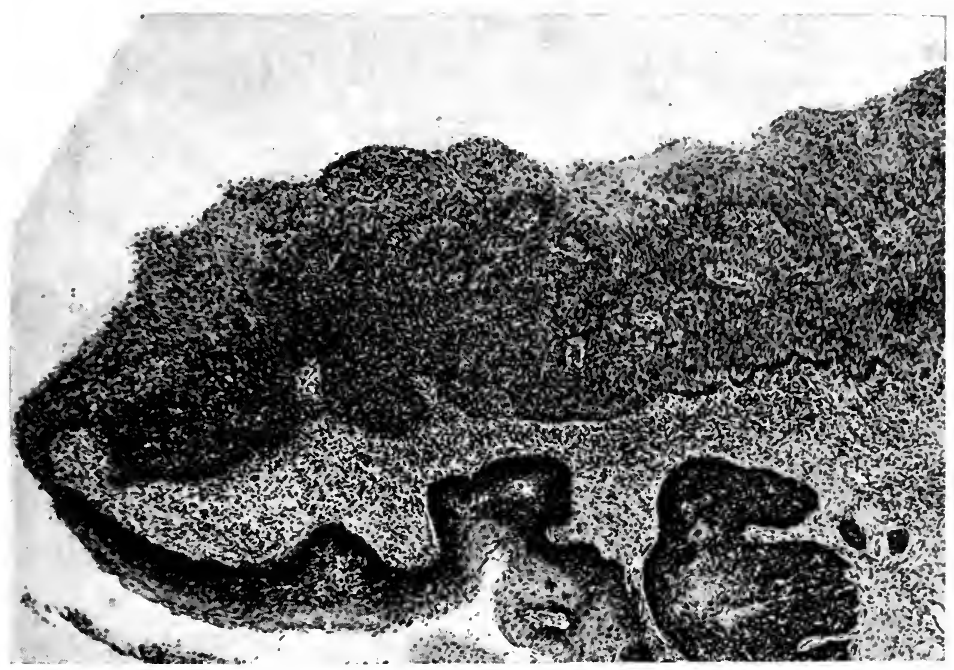

FIG. 247.-Beginning epidermoid carcinoma of uterus.

of the former, but it is probable that certain diffuse tumors originate over a very wide area, and present specific features from their inception.

The earliest stages of the circumscribed growths appear as low thickenings of a segment of the mucosa, usually in the fundus, or at one horn, or near the os internum. Very soon there is a definite outgrowth reaching into the cavum, with papillary or polypoid projections. Some tumors long remain distinctly papillary and are designated as papillary adenoma, or adenocarcinoma (Lindquist, Kaufmann). The circumscribed tumors are usually well deniarcated from the adjoining mucosa, sometimes very sharply, but others, especially early forms, grade off insensibly. In advanced stages they produce bulky growths which destroy the cavum, and invade the wall.

The diffuse tumors either originate from a wide area or early extend laterally in all directions. They are more frequent than the circumscribed forms, Huerlin finding in his series 29 diffuse tumors, 15 circumscribed and 2 papillary, and of these 9 covered the entire mucosa, i 8 others at least one-half. Other authors classify as diffuse only those tumors which involve 
the entire endometrium. The character of the advancing edge should here be considered. The cervical canal is very rarely involved in corpus carcinoma, although a short cervix in an atrophic uterus may suggest a cervical lesion, the cervical mucosa may be partly undermined, and rarely it may show implantation metastasis.

A marked general enlargement of the uterus results from the diffuse thickening of the mucosa, for these tumors produce considerable bulk of tissue before invading the wall. Or the enlargement may be due to myomas which are often associated with malignant adenoma, less frequently with carcinoma. Rarely there is hypertrophy of the muscular wall (Theilhaber, Hollinger). When fully established both localized and diffuse growths may be sharply defined from the muscular wall which gradually becomes thin while still free from invasion.

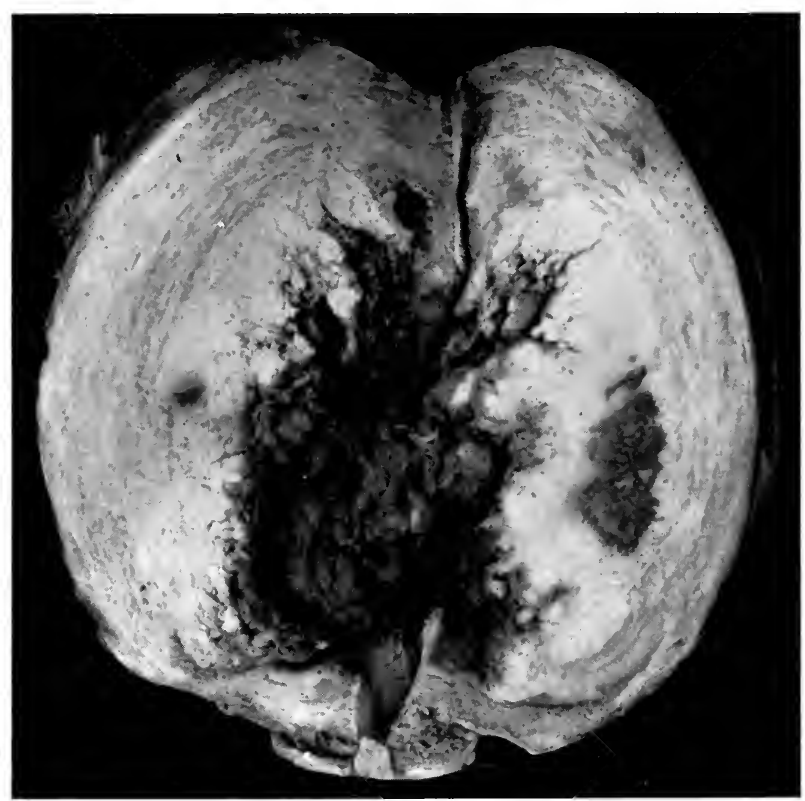

FIG. 248.-Adenocarcinoma involving the entire endometrium.

The integrity of the myometrium is the chief factor in the favorable prognosis of many uterine cancers. In early stages the growth may be strictly limited to the mucosa, and Vassmer has reported the successful eradication of the disease by the curet. Eventually the thin wall is perforated, subperitoneal nodules appear, and the peritoneal cavity, or bladder, or rectum may be invaded. In more malignant cases there is an early tendency toward invasion of the myometrium by groups of tumor alveoli, and the whole wall may thus be infiltrated and thickened. Rarely a deep invasion of adenocarcinoma occurs early at one point; and in such areas I have seen the subserous tissue reached by infiltration along vessels from a flat tumor $2 \mathrm{~cm}$. in diameter. Gravity figures in the early extension of corpus carcinoma so that the vagina from the introitus to the fornix may be the seat of secondary ulcerating growths through blood- and lymph-ressels. 
Secondary changes in the tumor mark the advanced stages, and include excessive polypoid formation, ulceration, necrosis, and calcific deposits. These degenerative changes may greatly alter the gross and microscopical appearance. Pyometra occurs rarely. Loose calcified myomas were found by Cullen and Thorn, in cases of carcinoma.

In the late stages the growth may extend into the parametrium, ovaries, tubes, and lymph-nodes, beyond which its progress is very similar to that of cervical adenocarcinoma.

Structure of Corpus Carcinoma.-Corpus carcinoma appears in several distinct histological varieties.

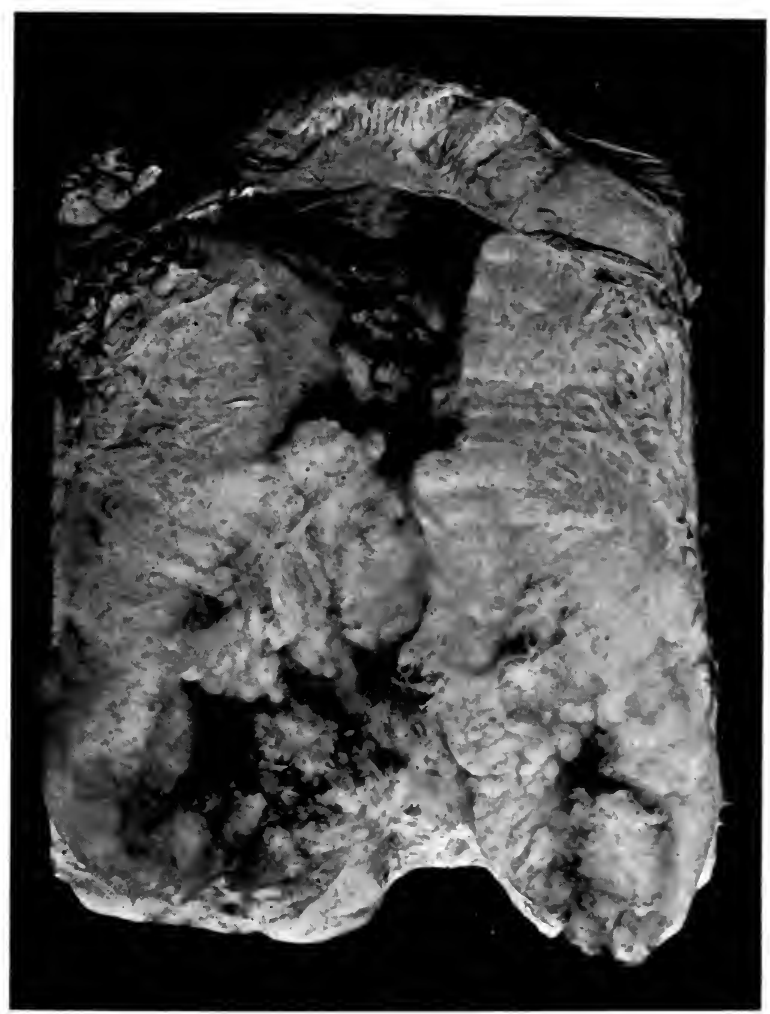

FIG. 249.-Adenocarcinoma involving cervix and lower half of fundus uteri.

(I) Malignant adenoma, the most frequent type, presents greatly enlarged and elongated alveoli, giant reproductions of the uterine glands, lined by several compact layers of cuboidal and cylindrical cells. The cell bodies are usually paler than the normal lining cells, but the large hyperchromatic nuclei give a dark staining character to the gland linings. There is much variation in the number and depth of the cells in the tumor alveoli. Some tumors rigidly maintain the pattern of the enlarged uterine glands and in the lymph-nodes, liver, and spleen the same orderly but malignant structure persists. Secondary alveoli form by convolutions of the lining within the distended alveolus and yield a structure characteristic of a large group. In 
all these cases the stroma is greatly reduced and adjacent alveoli become contiguous, and may fuse.

(2) Papillary adenocarcinoma is a form assumed by certain tumors which probably arise from superficial cells or adenoid forms. They may closely resemble in structure and gross appearance the coarser papillomas of the bladder, but are usually more diffuse and compact (Cullen). Many adenocarcinomas exhibit a certain papillary tendency in their superficial portions. Various stages of malignant transformation of benign polyps are observed. Special interest attaches to one group of polypoid carcinomas because, while the curetings show carcinoma, the extirpated uterus retains no trace of the disease. This situation may arise when a polypoid tumor attached by a narrow pedicle is completely removed by the curet (Gessner, Ladinski, Lit.). Of Ladinski's cases I found one to be an atypical embryonal adenocarcinoma, another was malignant adenomyoma.

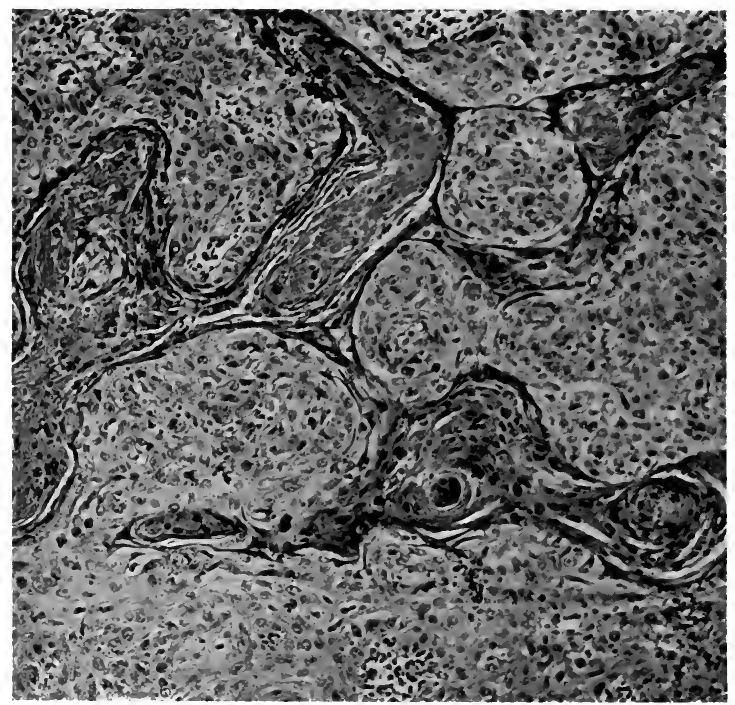

FIG. 250.-Acanthoma of endometrium with extensive hornification.

(3) Alveolar carcinoma is rare in the uterus, but some tumors early show solid masses of cells forming alveoli and smaller cell groups infiltrating spaces and vessels. Extremely small, numerous, and indistinct alveoli may form, giving a structure that is almost or quite diffuse. These structures are more aggressive than the malignant adenoma, and they are subject to degenerative changes which may lead to the diagnosis of giant-cell or round-cell sarcoma.

(4) Squamous cells may form a prominent element in adenocarcinoma of the corpus, and in rare cases they predominate over the glandular structure and produce a true adeno-acanthoma. While in many cases neither spinecells nor keratohyaline granules are demonstrable, in others both these criteria of true squamous epithelium are present, and pearl formation is added. The squamous cells usually appear in foci in the alveolar lining where the sharp transformation of cylindrical into flat cells may be followed. Such cell foci usually fail to show hornification. Or the tumor may be composed exclusively of large and small groups of squamous cells without hornification. 
Rarely all the characters of adult acanthoma are observed. At least three primary acanthomas of the corpus have been described, all in elderly women (Gebhard, Kaufmann, Flaischlen). As secondary changes one may find hyaline degeneration of masses of squamous cells or calcification of pearls (C. psammosum, Hitschmann). In elderly subjects in which the uterus is probably the seat of an old leukoplakia, squamous-cell acanthoma may develop in many superficial foci or diffusely, giving an opaque, warty appearance in the gross and leading to infiltration of the mucosa. Some of these cases probably represent an extension of a cervical acanthoma (Ruge, Benckiser). A combination of this superficial acanthoma with adenocarcinoma is described by $\mathrm{E}$. Kaufmann, who holds that one has to deal with a double origin from previously altered superficial lining cells and from gland-cells.

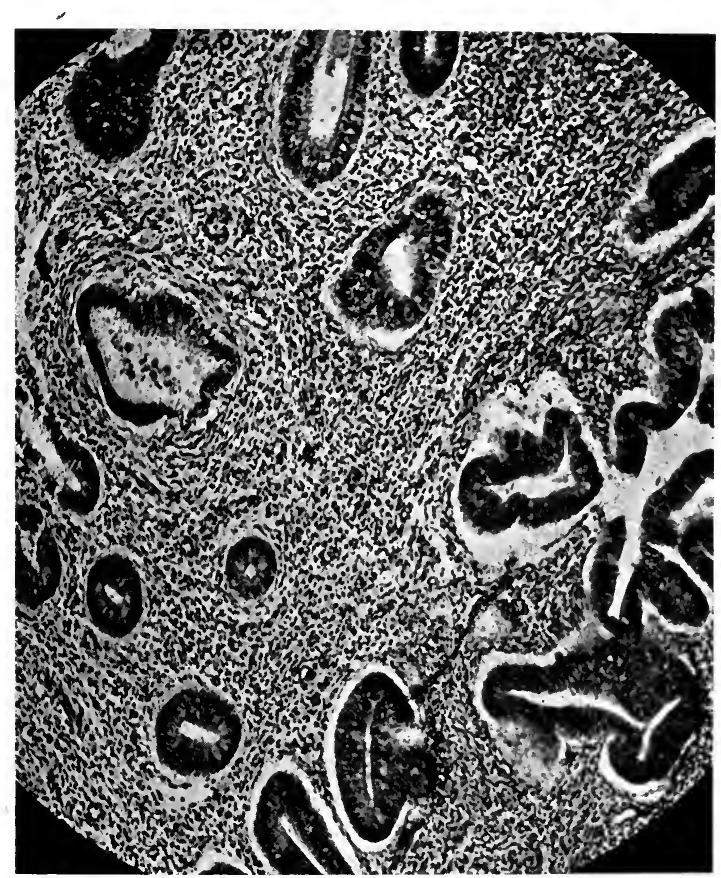

FIG. 251.-Atypical proliferation of uterine glands in a case of myoma uteri.

The histogenesis of corpus carcinoma presents many difficulties, and in spite of much labor the exact origin of the different histological types remains uncertain. Although Gebhard claimed that malignant adenomas arise from hypertrophied glands in chronic endometritis, this simple history has not received verification. Most observers fail to find the transformation from simple hypertrophied gland-cells to the neoplastic. On the contrary, most early adenomas show a sharp demarcation from the surrounding glands, and Heurlin, in 44 cases, failed to find any transitions or any signs of glandular hypertrophy in the endometrium. Such observations indicate that adenoma uteri arises in a circumscribed group of glands and extends laterally by displacing the normal glands even over the whole endometrium. The frequent association of adenocarcinoma with myoma suggests that the originating 
glands may be in some way connected with a myomatous area or other developmental anomaly. But aside from the rare and peculiar carcinomas derived from adenomyomas, there are no facts to support this hypothesis. Hence one is forced to the conclusion that adenoma arises from the normal glands of the endometrium under conditions which cannot be accurately defined, but which are probably associated with a peculiar local irritation. Doca describes early multiple adenocarcinomatous foci in the hypertrophic mucosa in a case of myoma uteri. Here the hyperemia and local irritation of myoma appear to have caused the neoplastic overgrowth of normal glands. I have seen 3 cases of carcinoma arising in the hypertrophied glands overlying the most prominent points of submucous myomas. One of these myomas was calcified.

The papillary adenocarcinomas have been traced with considerable certainty to proliferating superficial cells (Cullen), but here again no morphological signs of a tissue predisposition are usually observed. An exception to this rule occurs in the forms of adenocarcinoma arising from polyps. The transformation of benign polpyi into malignant tumors of the corpus by proliferation and metaplasia of lining and gland-cells has repeatedly been observed. Heurlin describes the implantation of carcinoma from such a malignant polyp upon the opposite side of the uterus, and somewhat similar implantations are reported by Noble and Flaischlen.

The mode of extension of the common adenoma and adenocarcinoma is still under discussion. While most of these tumors present well demarcated edges, the gradual inclusion of normal glands in the tumor area may often be observed in the uterus as in the stomach and colon. It is difficult to account for certain very diffuse tumors on any other basis. Lancereaux observed a diffuse tumor covering the entire endometrium and the mucosa of both tubes (Cit. by Williams). Heurlin's view in this matter is certainly too extreme. Likewise for the adenocarcinomas which arise after epidermization of the endometrium, an origin from multiple foci of altered superficial or gland-cells and a gradual extension of the process must be accepted. In the vicinity of all these tumors there are often observed signs of collateral hyperplasia, low papillary projections of vessels from the stroma capped by epithelium, and groups of squamous cells.

The most definite origin of any corpus carcinoma is that of the rare tumors derived from circumscribed or diffuse adenomyoma. Arising in circumscribed adenomyomas, the carcinoma produces a submucous or intramural tumor which tends to infiltrate deeply and extend to neighboring tissues, often with extensive metastases (E. Kaufmann, Rolly, Dillmann, Cullen).

Diffuse adenomyomatosis gives origin to characteristic forms of uterine cancer. The uterus is uniformly enlarged and most of the myometrium is the seat of a diffuse myomatous process in which are many gland alveoli exhibiting more or less neoplastic character. Often they present the structure of an orderly adenoma, with large alveoli lined by one or more layers of cylindrical cells. In this form they are capable of producing metastases, which I have observed in the ovary. Islands of squamous cells may also be present. Or the epithelial elements may yield various forms of adenocarcinoma or carcinoma (Winter, Schwab, Heurlin). Myomas are frequently the seat of metastatic carcinoma from ovarian sources.

Precancerous Changes in the Corpus.-From the processes which tend distinctly to develop into cancer of the endometrium must be excluded a simple increase in the cell layers lining the surface or glands. In chronic glandular endometritis multiplication of regular cell layers lining the glands has repeatedly been observed without any indication of a cancerous tendency 
(Borst, Cullen, Heurlin). Likewise a simple overgrowth of surface epithelium with partial or complete epidermization cannot be regarded as a definite approach toward a neoplastic process (Heurlin, Lit.). Yet the distinction between such benign overgrowth and a progressive malignant process is extremely difficult, and opinions differ as to the essential morphology of this early form of corpus carcinoma.

Leukoplakia uteri is a term often applied to this epidermization of the endometrium (Gebhard). It occurs under a variety of conditions. After curettage, especially when the uterus has been treated with escharotics and styptics, the regeneration of surface epithelium may yield groups or a continuous lining of stratified flat cells (Werth, Sitzenfrey). In certain cases, especially in atrophic uteri, this epidermization may progress extensively with atrophy of the mucosa, and scaly material with cholesterin may gather in quantities in the cavum, producing the condition observed by E. Kaufmann, as cholesteatoma uteri.

Rarely diffuse benign epidermization occurs as a form of endometritis (Zeller, Borst). It has often been observed in connection with chronic pyometra. In epdermoid carcinoma of the cervix much of the lower endometrium may be found covered with stratified cells, which may be regarded either as an extension of the tumor process or as an independent process affecting the endometrium. In many cases of adenocarcinoma of the corpus, especially when arising from polypi, extensive epidermization of the endometrium has been observed (Hofmann, Hitschmann). Here the process may affect not only the adenocarcinoma, but other portions of the endometrium. Finally, since islands of squamous epithelium may occur in the normal fetal endometrium, it may be assumed that its occurrence in the adult is sometimes only a result of such early malformation (Ribbert, R. Meyer).

The decision regarding the malignancy of uterine epidermization must in each instance be based on its finer peculiarities. In definitely malignant processes there are irregularity in cell form and arrangement, atypical mitoses, hypertrophy and hyperchromatism, and often pearl formation. One may very well insist, with Heurlin, on heterotopia as an essential sign of malignant qualities, of which Oeri's cases furnish examples. Such conditions are true early carcinomas. On the other hand, in the absence of such definite signs of early carcinoma, extensive epidermization must still be regarded with suspicion and classed among precancerous changes.

Course of Uterine Cancer.-From the nature of the initial symptoms of carcinoma it is obvious that the disease must exist for some time before it attracts attention, and since the rate of progress must vary considerably, observations on the duration of the disease when first recognized must have a somewhat uncertain value. Under these circumstances the early recognition of uterine cancer is a very difficult clinical problem. Winter claims to have observed I 3 per cent. of his cases in the first month of symptoms, 30 per cent. in the second to third months, 27 per cent. in the fourth to sixth months, 8 per cent. after one year. Hammer recognized 50 per cent. of cervical cases in the first 3 months, but only 19 per cent. of corpus carcinoma. Taylor found that in 50 per cent. of the cases the symptoms had lasted less than 3 months, in 40 per cent. between 3 and 6 months, and in only ro per cent. for more than 6 months. The rapid progress of the disease is illustrated by Mackenrodt's observations, who found the disease beyond the uterus in half of 18 cases examined within 4 weeks after the earliest definite symptoms. After the second month 20 per cent. of his cases were inoperable, after 6 months 40 per cent. Waldstein points out that cases are very apt to fall into advanced 
inoperable and localized operable tumors if observed during the first year. Early age, pregnancy, special susceptibility, a malignant structure, and a. location in the cervical canal are more important factors than the duration of the disease. Cervical carcinoma yields symptoms earlier, but the established forms of corpus carcinoma give more urgent manifestations.

A serous or mucous discharge is the earliest symptom, long preceding the tumors arising from erosions, or many of the corpus carcinomas, and being established early in deep cervical tumors. It results from the preëxisting inflammation, from excessive mucous secretion of affected glands, and from exudates from eroded surfaces. Only when fetid and mixed with blood, in the more advanced stages, can it be regarded as highly significant.

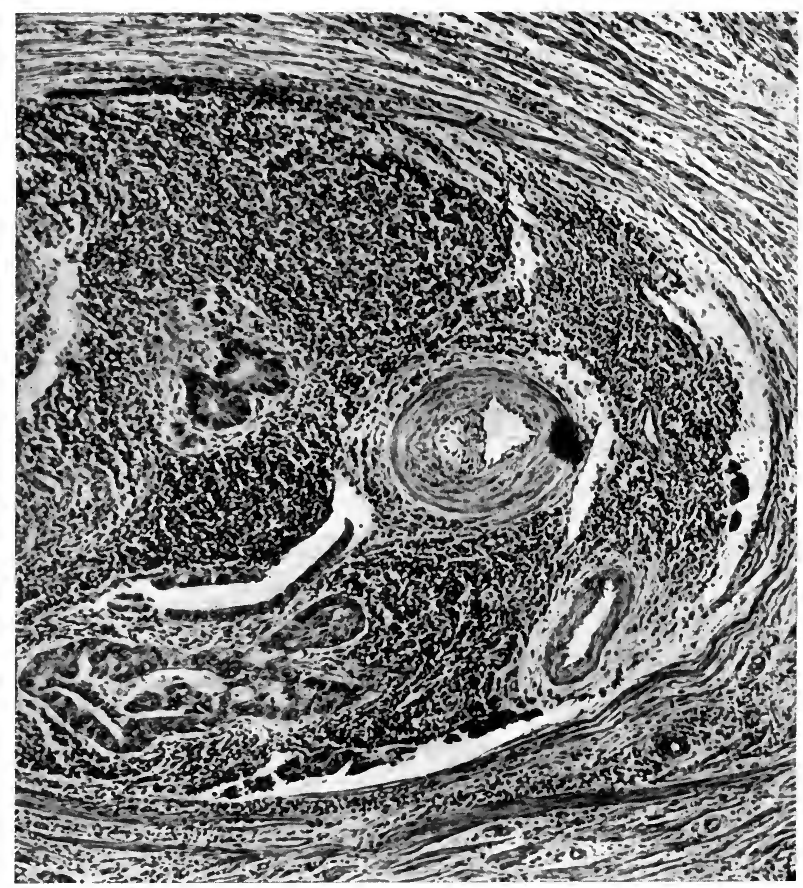

FIG. 252.- Uterine adenocarcinoma penetrating the wall of the uterus by way of the perivascular lymphatics.

Bleeding, irregular, at the climacteric, after slight trauma, or spontaneous, or as prolonged metrorrhagia, is also an early symptom in the majority of cases.

Aside from the uterine colic of early corpus carcinoma, pain is a late symptom, denoting ulceration, or invasion of sensitive structures, usually beyond cervix or endometrium.

A definite stage of the established disease is reached by the invasion of the parametrium, which usually occurs by continuous extension on one or both sides, through lymph-spaces, lymph-vessels, nerve-trunks, and occasionally by the veins. Isolated carcinomatous foci also develop in the lymph-nodes, about the vessels and in the looser tissues of the parametrium, but the parametrium may be thickened while still free from carcinoma (Scheib, Samson). 
In such tissue one finds thickening and sclerosis of vessels, perivascular lymphocytic infiltration, development of many new lymph-nodules, and edema. Bacterial infection of the parametrium by staphylococcus or streptococcus has often been demonstrated (Veit, Bumm, Fromme).

The period of lymphatic invasion varies greatly with the location and type of the disease, but is relatively early in cervical carcinoma. It has been present in more than half ( 43 to 73 per cent.) of patients coming to operation (Scheib). In later stages the parametrium is perforated and peritoneal nodules form, chiefly in the sac of Douglas. Diffuse carcinoma of the peritoneum, peritonitis, and ascites are occasionally observed.

Lymphatic Invasion.-The course of the lymphatics has been carefully studied by many observers with reference to the extensions of carcinoma. Two main groups of lymphatics from (I) the body and (2) the cervix are demonstrable, between which, however, there are rather free and variable anastomoses.

The main cervical vessels follow the uterine artery through the parametrium, meeting small nodes in the broad ligament and at the region where the artery crosses the ureter, and further on the iliac nodes in front of the external iliac artery and about the obturator foramen. From this plexus other vessels pass along the ureter to the hypogastric vessels and nodes. A posterior plexus passes forward and backward along the sacro-uterine ligament, around the rectum, and to nodes at the promontory of the sacrum (Sappey, Kroemer, Poirier, Lucas-Championniére).

The lymphatics of the corpus begin in the mucosa, pass upward and outward and leave the uterus in 4 to 5 trunks just beneath the tubes, pass through the broad ligament, anastomosing with the ovarian plexus and about the ovarian artery in the pelvic ovarian ligament, to end in lumbar nodes above the bifurcation of the aorta. From the middle of the corpus other vessels mingle with those from the cervix, reach the iliac nodes, and some pass along the round ligament to the inguinal nodes (Sappey, Bruhns). These six groups of nodes, parametrial, iliac, hypogastric, sacral, lumbar, and inguinal, form the regional nodes of the uterus. Beyond them there are rich communications with each other and with the vessels of the bladder, rectum, kidney, and abdomen (Baisch).

Uterine carcinoma as a whole cannot be classed among the tumors which early invade the lymph-nodes. Postmortem observations often suggest the striking tendency of the disease to remain localized, either to the uterus, or to this organ and its immediate vicinity, including the regional nodes and the tissues actually destroyed by the tumor. The autopsy records collected by Kroemer showed the lymph-nodes free in 66 per cent. of the fatal cases. There is, however, much variation in the course of the disease. As a rule, tumors of the fundus long remain confined by the muscular wall, while cervical carcinoma early infiltrates the cervix and invades the parametrium. Baisch found the regional nodes involved in only 4 of 24 cases of corpus carcinoma, and Cullen regularly found the nodes free. The duration and the type of the disease are doubtless the controlling factors in the rapidity of extension, but their influence does not appear seriously to alter the above rule, so that permanent operative cures of corpus carcinoma are often observed (Döderlein, Fritsch, Landau, Cullen). In cervical carcinoma the early involvement of the parametrium and its nodes was fully demonstrated by Kundrat, who in 160 cases found this structure involved in 55 per cent., while Lamaris and Kermauner saw such extension in 57.5 per cent., Baisch in 36 per cent., Schauta in 64 per cent. From a large series of reports of various types of cases Baisch concludes that the regional nodes are involved in 33 per cent. of operable cases of cervical carcinoma; when the parametrium is macroscopically free the nodes are affected in 5 to 16 per cent., and with invaded parametrium the nodes are affected in 50 per cent. of the cases. Rarely the regional nodes are said to escape, while more distant ones are involved, as in Schauta's postmortem material. V. Herff also reports such a 
case, but these isolated instances do not alter the rule that uterine carcinoma extends progressively. The invasion may be by continuous permeation, or sound tissue may separate the nodes from the cervix (Kundrat). Rarely extensive lesions may fail to involve the nodes (Cullen), but in many cases they were already involved with cervical lesions which had the appearance of simple erosions, or consisted of a small circumscribed nodule (Baisch, Lit.). It has repeatedly been shown that only microscopical examination is a safe guide to the conditions of both swollen and small lymph-nodes. Distinct metastatic nodules in the uterine wall may develop from cervical cancer (Blau, Winter).

Among Sampson's 27 cases he found only io in which the parametrium was free. The direct extension of the disease through fine lymphatics is the most frequent manner in which this tissue is invaded by cancer, occurring in $\mathrm{I}_{4}$ of $\mathrm{I} 7$ cases in which parametrial extension was found. Metastases in the lymph-nodes of the parametrium were also present in 6 of these cases. Of much clinical importance is the fact that the parametrium may be cancerous while the pelvic nodes are free, demonstrating that the parametrium may be able to check the disease for a time. Hence if one can remove the entire local growth it may be possible to cure a certain percentage of such cases. Induration of the parametrium without cancerous invasion occurred in I case, while in 3 cases the parametrium was invaded, but felt normal on palpation. Of I9 lymph-nodes examined, 9 were found invaded. Gellhorn, from a full review of the literature and from his own experience, concludes that cervical carcinoma involves the pelvic nodes in less that onethird of all cases. R. Peterson examined the lymph-nodes removed in 29 of a series of $5 \mathrm{I}$ cases and found invasion in only 5. At autopsy on cervical carcinoma the striking feature is the usual limitation of the disease to the pelvis or even to the uterus and immediately contiguous tissues. In these respects cervical carcinoma follows the usual rule of acanthoma, viz., to destroy the originating tissue, extend with induration and ulceration to the first barrier of lymph-nodes, and then cease its progress. There are, however, highly anaplastic epidermoid carcinomas and glandular carcinomas of the cervix which extend widely.

Many efforts have been made to establish a relation between prognosis and the structure of the tumor, from which it appears that the transitional cell cervical carcinomas are distinctly more malignant than the squamous epitheliomas of the portio. Thus when the parametrium is free Baisch finds invaded lymph-nodes in 30 per cent. of cervical carcinomas, but in only ro per cent. of portio acanthomas.

Pregnancy has an unfavorable influence on the course of many uterine carcinomas, but this influence is not always apparent. The age of the patient is also of some importance. Between 20 and 30 years the course of the disease is often unusually rapid. Kroemer finds the everted cauliflower growths of the cervix and portio are of slight malignancy; the deep unicentric nodules are more so; while the diffuse forms are highly malignant. Cullen and Lubarsch attribute special infiltrative powers to the tumors composed of small cells, a view which accords with the small size of the cells in many highly anaplastic growths.

The microscopical study of pelvic and other lymph-nodes has revealed the occasional presence in normal nodes of structures resembling isolated carcinomatous alveoli. These structures appear as single or multiple wellformed alveoli lined by cylindrical or ciliated cells. They have been interpreted as inclusions of the Wolffian duct (Borst), or as groups of altered lymphatic endothelium (R. Meyer). They are usually located in the tra- 
beculæ where embolic cancer-cells are not found, but when situated in the parenchyma they may be difficult to distinguish from carcinomatous cell groups (Sheib, Lit.). Carcinomatous invasion of nodes is usually preceded by parenchymatous hypertrophy, and often by catarrhal or exudative lymphadenitis. Streptococcus infection of such nodes appears to be rather prominent in cases of uterine carcinoma, and may give rise to suppuration and peritonitis after operation (Ruhle).

The clinical course of the advanced disease is dominated by the secondary invasion of neighboring organs. The bladder is invaded by direct extension from the cervix, and the wall is infiltrated with the formation of metastatic nodules in the submucosa, or more often with ulceration, necrosis, and fistulæ. Invasion of the bladder is relatively common, Williams finding it in 56 of 75 autopsies, while in 29 vesicovaginal fistulæ had formed.

The natural termination of most cases of uterine cancer is through uremia from occlusion of the ureters. This termination very frequently occurs while the disease remains well localized in the pelvis. These important structures are commonly invaded from without through the parametrium, and while the wall long resists destruction, partial or complete occlusion is readily established by cancerous infiltration or by inflammatory processes. The ureter may also be invaded by extension through the bladder wall, and it may be occluded by inflammation extending upward from the bladder. Dilatation of the ureter, hydronephrosis, and chronic nephritis with gradual suppression of the renal function regularly follow. Suppurative nephritis may arise in cases complicated by cystitis.

The rectum is often compressed by infiltration of the wall and rectovaginal fistulæ form in not a few advanced cases (6 per cent., Beckmann).

With invasions of bladder or rectum the pelvic connective tissue is usually involved, with compression of vessels and edema of vulva and lower limbs.

Visceral metastases, while occurring in many cases, are often conspicuous by their absence. Yet the disease may become widely generalized, and secondary tumors have been observed in many organs. Since visceral deposits occur chiefly in the terminal stages of the disease, they are not distinctly influenced by the location or type of the original disease, but the more adult acanthomas rarely extend beyond the pelvis. In rare instances there is early and very wide extension through the lymphatics, with continuous permeation through the abdominal and thoracic nodes to the supraclavicular and cervical regions.

The liver has been invaded through the portal vessels and by retrograde lymph flow in 5 to 15 per cent. of autopsies.

The lungs have been invaded probably through the blood-vessels in 5 to 7 per cent. Pleural metastases extend from bronchial nodes in rare instances. Offergeld collected 56 cases of peritoneal metastases located in a great variety of positions, and he found records of 7 secondary tumors in the thyroid, II in the adrenals, $3 \mathrm{I}(2.39$ per cent.) in the kidney, 5 in the pancreas. The brain is rarely affected (Williamsky 0.2 per cent.). A few cases of carcinomatous meningitis have occurred from uterine cancer. The bones are involved in many cases.

Characteristic cachexia in uterine cancer develops in the terminal stages of the generalized disease, but when the lesion is localized in the pelvis cachexia is missing, the nutrition may remain surprisingly good, and, if infection can be avoided, death follows from uremia. Local and general infection, usually by streptococcus, may be a prominent feature. Fever is almost always due to infection and local necrosis of tissue. 
The mortality from uterine cancer is very high. Jacobson, from an elaborate analysis, shows that of the women who enjoy the services of the best American operators 35 per cent. are inoperable, the operative mortality is 15.17 per cent., after 5 years 8.39 per cent. are well, and I per cent. of the total are permanently cured. Cullen states that of cervical cases 50 per cent., and of corpus cases 16 per cent., are inoperable when first seen.

In an effort to extend the field of operability Ries and J. G. Clark proposed the more radical operation which was developed especially by Mackenrodt and Wertheim, but the results, at first encouraging, have proved unsatisfactory. Mackenrodt's original 72 per cent. of successes fell first to 42 per cent., then after 8 years to 22 per cent., and after io years to 12 per cent. Wertheim's statistics have suffered a similar revision. The widest discrepancies exist between the claims made of permanent cures, as Mackenrodt, 58 per cent.; Rosthorn, 2.65 per cent. Operable carcinoma of corpus offers a better prognosis, prominent clinics reporting 53 per cent. of permanent cures of this form of the disease (Koblanck). In a series of ro3 cases, Cullen reports 16 per cent. of cures of adenocarcinoma of cervix, 21 per cent. for acanthoma of cervix, and 66 per cent. for corpus carcinoma. Yet in the latter group fall many comparatively benign tumors.

In America the Wertheim operation has never been distinctly popular because of the feeling that the additional cases saved by this method do not justify the higher mortality. Some support of the extraradical operation in advanced cases has come from a few paradoxical cures. Thus Scheib collected I4 cases which remained well for several years although the regional nodes had been involved. From the economic standpoint it must be considered that a competent Wertheim operation is beyond the reach of the great majority of the population and that all the phases of the cost of acquiring technical skill should be counted in estimating its utility. Because of these considerations a marked change has occurred during the last few years in the aspects of surgical treatment of uterine cancer, most surgeons preferring to reduce the scope of operability and others abandoning all operations for uterine cancer, in favor of radium and $x$-ray treatment. Earlier diagnosis has doubtless contributed to improving surgical statistics and is equally important for radium therapy. In these directions lies the hope of real advance in the therapeutics of uterine cancer.

\section{EPIDERMOID CARCINOMA AND EPITHELIAL TUMORS OF VULVA}

These tumors are peculiar because of the special conditions under which they originate, their relatively great malignancy, and the late period of life at which they commonly arise.

Carcinoma of the vulva is not a rare disease, forming ro per cent., according to Gurlt, of all cancers in women. The majority of the tumors are epidermoid carcinomas arising from the mucous membrane of the vulva, but the clitoris is also a frequent source of epithelioma, and glandular cancers develop from the sweat-glands and from the gland of Bartholin. It is most frequent in the sixth decade (Dittrich), but has been observed in a girl of 20 years (Arnot).

The disease first appears as (I) a thickening of the epidermis which soon exhibits cracks and fissures, or (2) a localized papule, vesicle, or wart, or (3) deeper tumors of sweat-glands or of Bartholin's gland precede the appearance of superficial lesions. The initial lesions may be multiple (Fromme). The chief seat of the disease is in the follicles, in the sulci between the labia, and about the urethral orifice. Sitzenfrey traced the origin of one case to the duct 
of Bartholin's gland. Two notable conditions, pruritus and leukoplakia, often antedate the malignant process.

Pruritus is a very common precursor of carcinoma of the vulva, but its source is not always clear. Dittrich reports a case of pruritus lasting I2 years due to a benign wart, which terminated in epidermoid carcinoma. Weir observed a long period of pruritus in a young girl, associated with kraurosis and ending in epithelioma. Frequently the pruritus exists for several months before the carcinoma is discovered. The parakeratosis and round-cell infiltration of the corium in pruritus are distinctly favorable to the development of epithelioma.

The relation of kraurosis and leukoplakia of the vulva to epithelioma was first pointed out by Weir and later emphasized by Butlin, Jung, Franque, and many others. A syphilitic origin is not always demonstrable, but some form of local infection or irritation is usually present. It produces a pearly white, warty condition of the mucosa which appears at many discrete points or affects nearly the whole orifice. The condition begins as a chronic inflammation of the mucous membrane with thickening, edema, and cellular infiltration of the corium, and hypertrophy and keratosis of epithelium, and it results later in extensive sclerosis and atrophy of the corium with contractures (Peter). From one or more points or from a broad area of this lesion epithelioma develops.

Other local conditions related to the disease are psoriasis, syphilitic scars, elephantiasis, abscess, and various forms of trauma.

When fully developed the carcinoma appears as: (I) a prominent warty or lobulated tumor; (2) a deep ulcer; or (3) a diffuse infiltration. In the ulcerating cases a severe infection is often added which greatly complicates the local condition, causes enlargement of inguinal nodes on one or both sides, and hastens the progress of the disease. Involvement of lymph-nodes has been observed as early as $3^{\frac{1}{2}}$ months from the beginning of the disease and has been delayed for more than 3 years. Schwarz found that the swollen inguinal nodes were invaded by cancer in only about one-half of his cases. Dittrich finds that involvement of lymph-nodes occurs in about 50 per cent. of the cases during the second 6 months of the disease. Extensions occur along the deep tissues to pubic bones, to the pelvis, and through inguinal and pelvic lymph-nodes of one or both sides. Fritsch saw extensive growth about the urethra. The vaginal mucosa usually escapes involvement, but the vaginal wall may be invaded and even the cervix uteri. Local metastases occur about the anus, in the abdominal wall, and in the thighs. General metastases have been observed in abdominal nodes, lungs, heart, liver, spleen, and kidneys (Kustner).

Implantation by contact on the opposite labium has been reported by several observers, but the probability of an extension through the lymphatics is not eliminated (Dittrich, Lit.).

The course of vulvar carcinoma is usually rapid and terminates, if without operation, as a rule within 2 years after the discovery of the lesion. Deschamps observed a fatal case terminating in 4 months. The explanation of this rapid course is probably to be found in the abundant vascular and lymphatic supply of the tissues, in the late discovery of the disease, and in complicating infections. Dittrich found no recorded case in which the patient remained free from recurrence for over 6 years. Several cases showed recurrence after 5 years. H. Schultze in II 4 cases found 14 free from recurrence after 5 years. Yet it is probable that not a few cases recognized early have been cured by operation. It may also be noted that in many cases the early stages of the disease may be slow and that several years may elapse 
before the lymph-nodes become involved and the growth becomes accelerated. Kaufmann, Teller, and Rupprecht report cases of long duration. Rupprecht gives a comparatively favorable prognosis for superficial lesions, and reports cures after invasion of the nodes.

Structure.-Epithelioma of the vulva usually presents a structure of narrow cords and columns of relatively undifferentiated squamous cells. Hornification and pearl formation are not prominent. The malignant clinical character of the process is often apparent in the histological structure. In the advanced stages the proportions of cells and stroma varies, and some authors speak of scirrhous and encephaloid types. Pick and H. Ruge state that adenoma of the sweat-glands may become malignant and pursue the course of epithelioma of vulva.' The structure of the benign tumor recalls that of the sweat-glands (tubular adenoma), and the malignant form occurs as an adenocarcinoma. Bartholin's gland is the source of rare adenomas and adenocarcinomas (Veit, Lit.). These tumors usually reach larger dimensions than epithelioma and present an adenoid structure (Chaboux). Many cases of melanoma of the vulva are recorded (P. Meyer, Hinselmann, Lit.).

\section{EPIDERMOID CARCINOMA OF VAGINA}

Primary epidermoid carcinoma of the vagina is a comparatively rare condition, forming 0.24 per cent. (Schwarz) or 0.I9 per cent. (Gurlt) of all carcinomas, or about 0.43 per cent. of all carcinomas in women ( $R$. Williams). Secondary carcinoma, however, is rather common, the vagina being invaded in most cases of cervical cancer, and from the bladder, rectum, ovaries, and other more distant organs. The chief age of incidence is between 30 and 40 years, but Winckel saw 7 cases between 20 and 30 years and many occur late in life. Cases reported in infants are of doubtful nature.

Exciting factors include the irritation of pessaries which have been directly connected with vaginal carcinoma in several reported cases (Wille, Lit.); the traumatism of repeated childbirth, a factor emphasized by West and Bernard; and leukoplakia, which has been observed to precede carcinoma in rare cases discussed by Reclus, Bex, and v. Franque.

In form and location vaginal carcinoma appears as: (I) a papillary or (2) infiltrating growth arising $(a)$ chiefly on the posterior wall of the upper segment, $(b)$ on the anterior wall and about the urethra, $(c)$ on lateral wall, $(d)$ in the fornix, whence it invades both cervix and vagina, producing the "epitheliome luminaire" of Pozzi, $(e)$ in the lower segment and invading the vulva, and $(f)$ as an annular or diffuse infiltration of much of the vaginal wall (Olshausen).

The initial stages of the lesion are rarely observed, but appear as single or multiple warts; as an elastic nodule in the epithelial tissue; or as a flat infiltration. In advanced stages cauliflower, villous, highly vascular, and more frequently ulcerated tumors form. Schwartz described a multiple fungoid epithelioma of upper vaginal segment and cervix.

The extensions of vaginal carcinoma are determined by the location of the lesion. Ulceration often occurs early, the muscular wall offering little resistance. With or without ulceration extension through the lymphatics is established early in the disease. From the upper segment the lymphatics enter the iliopelvic chain and join with those of the cervix uteri; from the middle segment the vessels enter the lowest nodes of the iliopelvic and hypogastric groups; while from the lower portion drainage is toward the inguinal nodes (Poirier, Bruhns). Along these channels the nodes become enlarged, vessels are constricted with edema, and the urethra may become obstructed with cystitis and hydronephrosis. Ulceration produces fistulous tracts to 
rectum or bladder. Extensive suppuration and peritonitis may develop. The broad ligaments, uterine wall, ureters, and ovaries may be infiltrated. Metastatic deposits have been observed in the tubes, ovaries, kidneys, liver, lungs, and bones.

The prognosis is very unfavorable. R. Williams calculated the average duration of life at 16.5 months, longest 26.25 months, shortest 8 months. Longer immunity from recurrence has followed extensive operations in early cases, but no definite cure of the established disease seems to have been recorded (Lohnberg). The early cases respond well to radium.

Structure and Histogenesis. - Vaginal carcinoma usually arises from the squamous epithelial lining and takes the form of acanthoma or tubular epithelioma. Pearl formation and hornification are not prominent. The upper cervical segment contains cysts and follicles lined by cylindrical cells from which atypical adenoid epithelioma may develop. The variable structure and origin of these cysts have been discussed by Cullen. Davidsohn regards the vaginal glands as heterotopic cervical or (below) vulvar structures. R. Meyer traces the ampulla of Gartner's duct into the lateral vaginal wall, where its structure is often to be seen in infants. He also traces the formation of follicles by the dipping down of the basal layer of epithelium and reports several forms of congenital abnormalities in the epithelial lining of the fetal vagina, as well as adenocarcinoma arising from such abnormalities. In the adult he finds small cysts and aberrant groups of squamous epithelium in the submucosa. In a remarkable case the entire vagina was the seat of a cystadenofibromatous degeneration. To what extent these abnormalities are concerned in the genesis of carcinoma in the adult remains to be shown, but the occurrence of such conditions must be considered in all atypical forms of carcinoma of cervix, vagina, and vulva.

Cylindrical cell carcinoma of the vagina is reported by Pintor and by Polosson and Violet, and adenocarcinoma by Hoehne and Hirsch. 


\section{CHAPTER XXVIII}

\section{CHORIOMA (CHORIONEPITHELIOMA)}

Historical.-The characteristic anatomical and clinical features of destructive placental polyp were clearly described and depicted as early as $\mathrm{I} 867$ by R. Volkmann and later by many others (Sanger, Lit.), but the nature and origin of other highly malignant tumors connected with gestation proved to be a much more difficult problem, chiefly because the absence of a core of the villus in these tumors removed the chief basis for connecting them with fetal structures. The idea gained currency that the chorion produced only the destructive polyp, while the more malignant tumors arose from decidual cells, and this view was maintained notably by Sanger, I 888 to I89I, who employed the term "deciduoma malignum" or "sarcoma deciduocellulare."

In this group, I judge from the literature of the period, were included the more malignant chorionic tumors, certain sarcomas of the endometrium, and possibly rare tumors of the placenta. The large fusiform and polyhedral cells of the decidua were supposed to give origin to the tumors, but Pestalozza thought the uterine muscle also contributed to the tumor-cells. A combination of deciduoma with destructive hydatid or placental moles was recognized, especially by Gottschalk and Schmorl, but such cases were regarded as of complex origin.

The fetal origin of the tumors was maintained by a number of authors, especially by Gottschalk, who, however, believed that the connective tissue of the villi suffered sarcomatous transformation. In this situation Marchand, by a minute comparison of the tumor-cells with the fetal villi, concluded that the tumors arose from the chorionic epithelium, reproducing both syncytium and Langhans' cells. At the same time J. W. Williams interpreted his case as of exclusive syncytial (and maternal) origin, and thus was led to retain the term "deciduoma."

Marchand's view of the exclusive chorionic origin of the tumors has proved entirely correct, and has served to connect the various grades of proliferation of chorionic epithelium in one general class, while the clinical relations of the different forms of the disease have been greatly elucidated, especially the connection with gestation and hydatid mole. Marchand divided the tumors into two main classes, typical and atypical, the former covering the actively growing and metastasizing tumors, the latter designating certain less active or degenerating forms composed chiefly of large giant-cells. In I910 it seemed to the writer that the anatomical and clinical distinctions long recognized to exist between destructive moles, typical chorionic carcinoma, and atypical degenerating tumors deserved definite emphasis, and he proposed for them the terms "chorioadenoma," "choriocarcinoma," and "syncytial endometritis." Subsequent experience has confirmed this conclusion and interpretation.

In recent years many authors have contributed numerous details of our knowledge of the origin, course, and treatment of the disease, and numerous monographic studies have appeared (Pestalozza, Veit, Teacher, Risel, Frank. Schmauch). 
Etiology.-The frequency of malignant proliferation of chorionic epithelium cannot at present be determined. The number of reported cases has increased notably in recent years, so that Pollasson and Violet (rgr4) were able to collect 455 . Findley thought that about 16 per cent. of hydatid moles were malignant. Senarclens found that of 49 hydatid moles, 3 (I3 per cent.) developed chorioma, while 4 others died of other complications. In the cases collected by Pollosson and Violet the disease occurred in the following relations to pregnancy: after moles, 203 (44 per cent.); abortion, I35 (30 per cent.); labor near term, 99 (22 per cent.); ectopic, 12; doubtful, 6. Multiple gestations seem to increase the liability to the disease, since Ollivier Bauregard in 178 cases found that 66 had borne more than 5 children, while 22 were nulliparæ. Curtis and Oui noted an average of 7 confinements, and a minimum of 3 , in women between 40 and 53 years suffering from destructive placental mole. The average periods of occurrence have been estimated as 8 weeks after hydatid mole, 7 weeks after abortion, and 5 weeks after labor at term.

Destructive mole usually yields symptoms between 4 and 5 months after the last menstruation. Briquet found that in $\mathrm{I} 4$ of 30 abortions followed by chorioma and in I 7 of 35 hydatid moles, the age of the ovum was 3 to 4 months. The age of the patients has been from I 7 to 58 years. At either extreme of age the probability of a teratomatous origin must be considered.

Latent chorioma has occurred in some remarkable cases in which the disease appeared several years after the last gestation. This interval was 3 to 4 years in several cases, 5 years in Caturani's case and ro years in Polano's (Outerbridge, Lit.). The capacity of fetal villi to maintain their existence in the uterus for a long period is indicated by Ries' observation of intact villi in a superficial uterine sinus 18 years after the last pregnancy. It is, of course, possible to doubt the accuracy of the history in this case. Yet the villi had become fused with and were nourished by the walls of the veins.

Gross Anatomy and Structure.-Three main types of the disease may be distinguished by gross and microscopical structure, while between these there are transition cases.

(I) Chorio-adenoma destruens. Destructive placental mole.

(2) Choriocarcinoma.

(3) Syncytioma and syncytial endometritis.

(I) Chorio-adenoma Destruens. - The destructive recurring and perforating placental mole yields a characteristic gross appearance. The uterus is usually much enlarged, reaching at times a length of $\mathrm{I}_{3}$ to $\mathrm{I} 7 \mathrm{~cm}$. (Huguenin). The wall is thick and honeycombed, or a definite cavity appears in the musculature, or a transverse or longitudinal septum separates tumor cavity from uterine canal (Volkmann). In advanced cases the serous surface may present nodular elevations due to subserous veins distended by tumor masses, and similar extensions may be traced into parametrium, tube, cervix, and vagina. Perforation of the wall has been recorded in 7 cases (Curtis, Lit.). In Waldo's case hemorrhage appeared to be controlled by a fringe of omentum, and the patient recovered. All the others died. In Engstrom's cases separated vesicles were found on the peritoneal surface.

The contents of the uterus vary with many circumstances. When the mole remains in situ it presents the usual bulky tumor adherent over the implantation site and altered by hemorrhage or suppuration. When the mole has been expelled coarse shreds of necrotic tumor or larger lobulated masses resembling blood-clot adhere to a portion of the wall, and are continuous with the distended sinuses. Usually after curetage the tumor is represented by a ragged cavity in the wall. 
The endometrium may be normal in early cases or the seat of implanted vesicles (Moller) or extensively eroded.

The gross diagnosis of malignant mole may sometimes be approached from inspection of the discharged material. The occurrence of great variations in the size of the vesicles, with many small opaque coherent nodules,

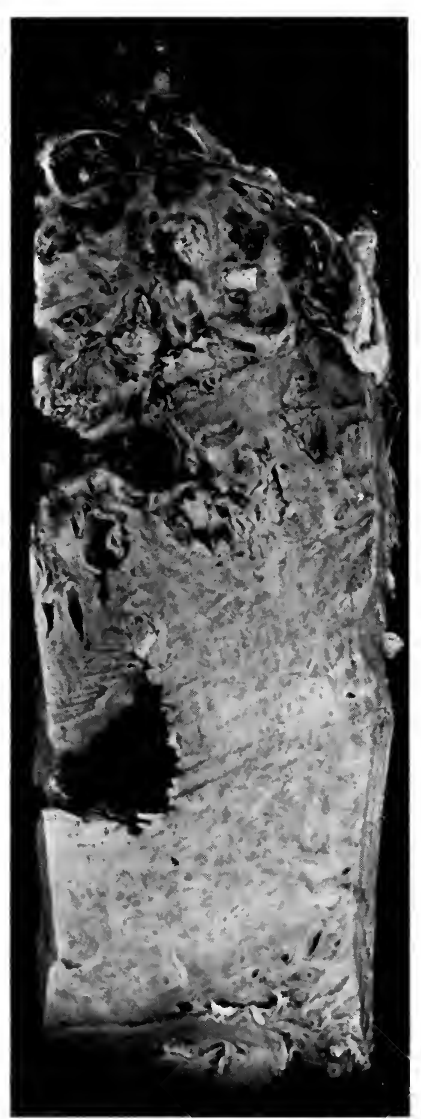

FIG. 253.-Chorio-adenoma. Inin material which comes away in fragments with difficulty and in rather reduced amount, are suggestive signs.

The structure of chorio-adenoma is quite specific. It presents an overgrowth of all the elements of the chorionic villi, connective-tissue core, Langhans' cells, and syncytium. The connective tissue is compact cellular, but not very vascular. It may be greatly increased in thickness and swollen by edema, but mucinous changes are absent or slight. An unusual overgrowth of fibroblasts has occasionally suggested a sarcomatous tendency. Neumann described rows of large round or elongated cells in the stroma, which he derived from the syncytium and regarded as a sign of malignancy. They are by no means constant.

The Langhans cells appear in greatly increased number, forming multiple layers at the bases of villi and long broad sheets not directly connected with the villi. Their size varies little from the normal, but the nuclei are hyperchromatic. They are sharply separated from the overlying syncytium. When denuded of syncytium they are usually covered by fibrin, which suggests that they yield a coagulating ferment (Curtis). These cells present a clear cytoplasm, contain glycogen, and rarely suffer necrosis.

The syncytium appears in the early stages in the form of well-nourished actively sprouting buds of strongly acidophile cytoplasm with abundant compact hyperchromatic nuclei. The syncytial buds may be very large and abundant and constitute the chief element of the neoplasm (Solowij, Gottschalk). Some early observers were thus led to believe that the tumor process resided exclusively in the syncytium. A pure syncytioma, however, has not been demonstrated.

In more fully developed cases the syncytium grows not only in buds, but in elongated sheets covering the increasing masses of Langhans' cells.

"Syncytial wandering cells" is a term applied to derivatives of the syncytium, which are large or giant polyhedral cells occurring singly or in groups, lying in the folds of villi, or loose in the blood-spaces, or infiltrating the walls of veins or the musculature. They are present in normal chorions and in benign hydatid moles, and are distinctly increased when a malignant mole is altered by hemorrhage or inflammation. They are, therefore, less vigorous than either of the other cell types, if not distinctly degenes tive. 
The differential diagnosis between a benign and a malignant hydatid mole usually presents no difficulty. The distance separating the ordinary epithelial proliferation of the hydatid mole from a typical chorio-adenoma is very great and may be recognized at a glance, as may be seen from the accompanying figures. There is, however, an intermediate group of cases in which an unusual overgrowth especially of syncytial buds, less markedly of Langhans' cells, suggests a malignant capacity and leaves the observer in doubt. After encountering several of these cases and learning of their wholly favorable course, I have had to extend my ideas of the limits of epithelial hyperplasia in benign moles. There may be very considerable overgrowth of epithelium in moles which pursue a favorable course or in which an unfavorable outcome results from hemorrhage or infection. It is possible, however, that the same structure may not always signify the same degree of penetration of the uterine sinuses. In Waldo's case a perforating mole

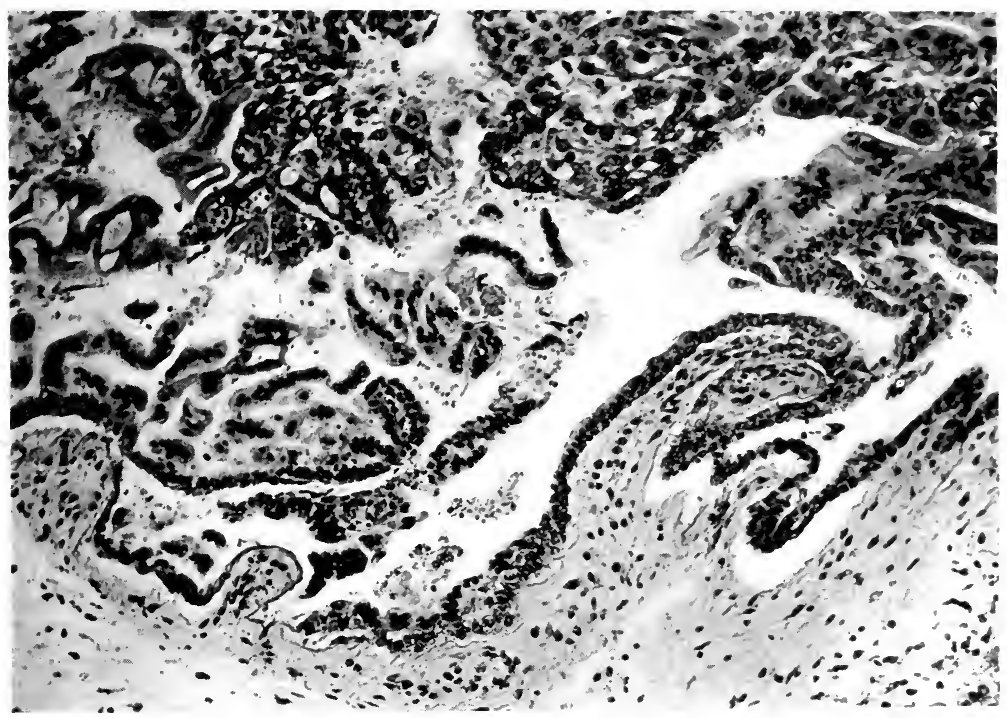

FIG. 254.-Chorio-adenoma. Preservation of villi, orderly syncytium, and Langhans' cells.

was regarded as of benign structure. It must be admitted that serious damage to the uterus may result from hydatid moles which are not distinctly neoplastic, but such cases are extremely rare, and do not belong in the present category. In Solowij's case, with deported villi in the lung, it was stated that the epithelium of the villi in the extensively invaded uterus showed comparatively little proliferation.

Metastases in chorio-adenoma are comparatively rare and their significance is peculiar. The intravascular position of the infiltrating villi favors the separation of portions of the epithelium and even of the core, which become lodged in various capillary systems, including parametrium, vagina, and lungs. As a rule, the metastases of chorio-adenoma are limited to the pelvic region, but occasionally they occur in the lung. In the vaginal wall they form small circumscribed bluish projecting tumors. Several of these have been removed after the expulsion or extirpation of the original tumor 
and the patient has recovered (Neumann, Pick, Engstrom, Kaufmann). The metastases consisted chiefly of syncytium.

Similar vaginal tumors have occurred also with benign hydatid mole. In such a case Pick found in the vaginal tumor segments of villi with hyaline stroma and syncytium largely necrotic. In 2 cases of abortion and 3 of hydatid mole Dunger found mild proliferative changes in embolic syncytium. It is evident, therefore, that vaginal emboli do not necessarily signify the presence of a malignant mole in the uterus. The structure of the vaginal tumor is of first importance in the prognosis. When actively growing Langhans' cells are present the case may safely be regarded as malignant. I have once found only feebly proliferating syncytium in a vaginal tumor in a case which progressed unfavorably. When the cores of villi are present the

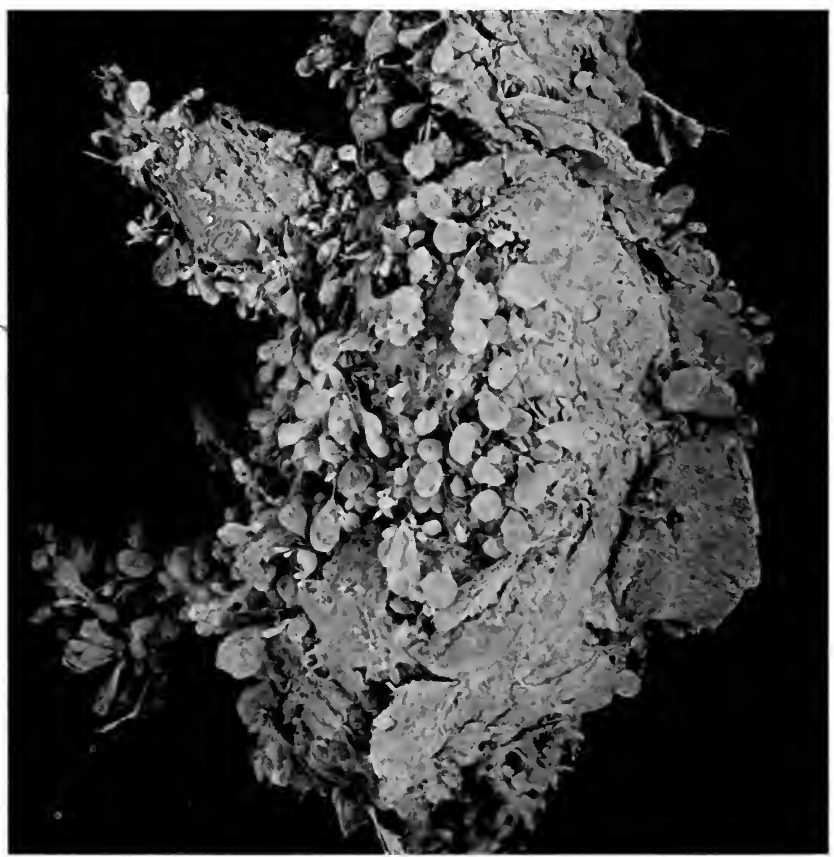

FIG. 255.-Portion of a benign hydatid mole.

case is at least relatively benign, but in Lindfors' case, which was of this type, pulmonary metastases followed. It should be remembered that normal villi may be deported and lodged in small veins. The structure of the original tumor has important bearing on the prognosis of vaginal metastases. In 15 cases with vaginal metastases which recovered I find that the original tumor contained villi in 9, the structure was atypical (syncytioma) in 3, and indefinitely described in 2. In Schlagenhaufer's case the structure was possibly that of choriocarcinoma with excess of vacuolated syncytium.

Pulmonary metastases, as indicated by the symptoms of cough and hemoptysis, occurred in 7 recovering cases of chorioma collected by Fleischmann.

(2) Choriocarcinoma.- The majority of cases of chorioma pursue a fatal course, are marked by a characteristic structure in which villi are absent, and 
the epithelial growth is anaplastic and disorderly. 'They produce wide-spread metastases, and are best designated as "chorionic carcinoma."

In choriocarcinoma the uterus is only moderately enlarged and may appear normal in size, but is usually the seat of a relatively small compact, opaque, infiltrating tumor, commonly located at the placental site. In a few cases no tumor has been demonstrable in the uterus, it having been removed with the placenta or by the curet, or the growth has arisen in tube or ovary. Schmorl reported a case of malignant chorioma in the organs,

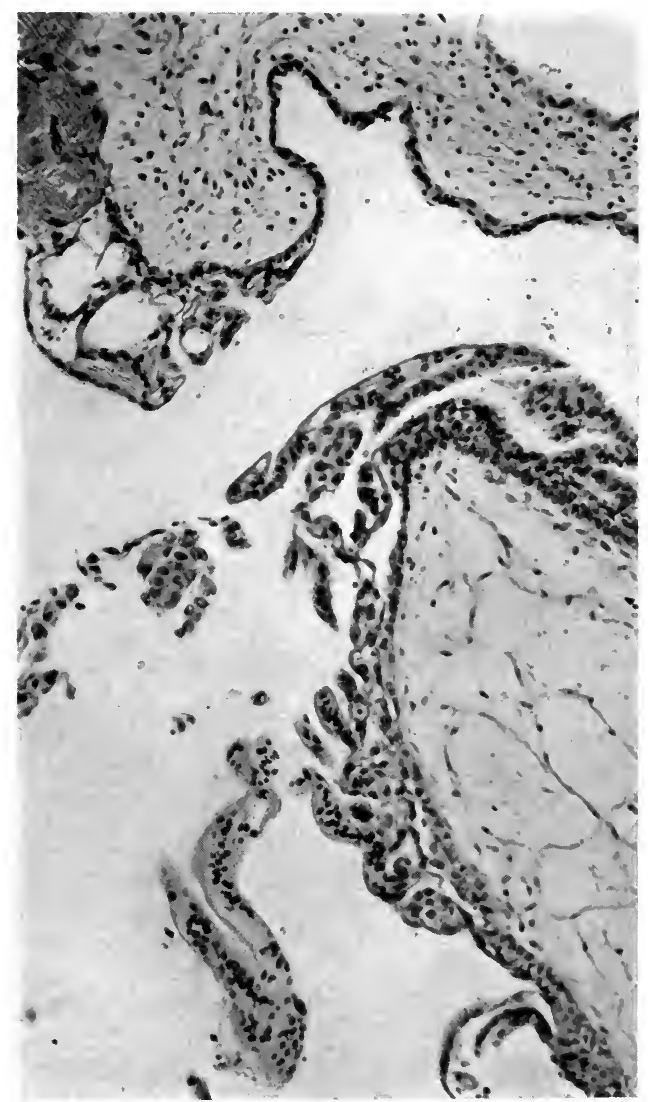

FIG. 256.-Benign hydatid mole. Slight epithelial proliferation.

appearing first in the vagina, I8 weeks after normal labor with a normal uterus.

The chorionic carcinoma is locally aggressive. It invades the sinuses without distending them as does the dissecting mole, it splits up the musculature, and it rapidly traverses or perforates the wall, traveling mainly by the sinuses. While often infiltrated with blood, the uterine tumor is usually cellular and compact, but friable. It is not capable of complete removal by the curet, except possibly in very rare cases.

The structure is rather uniform and highly characteristic. The tumor 


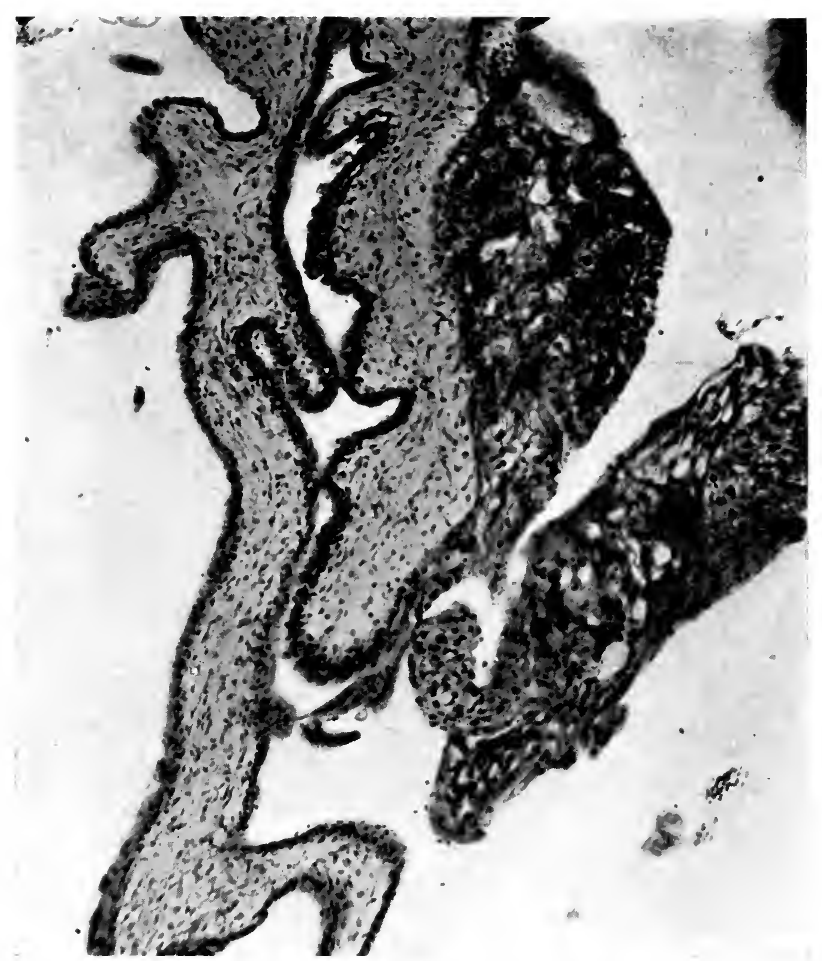

FIG. 257.-Benign hydatid mole. Moderate proliferation of syncytium.

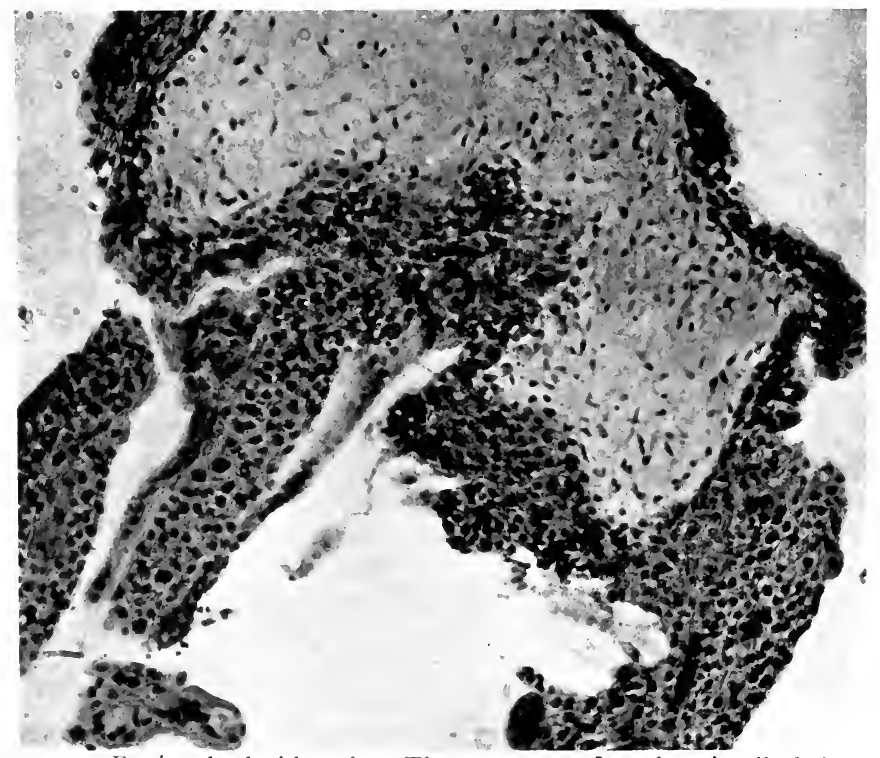

Fiti. 25\%.- Benign hydatid mole. The numerous Langhans' cells belong to the early periods of gestation. 


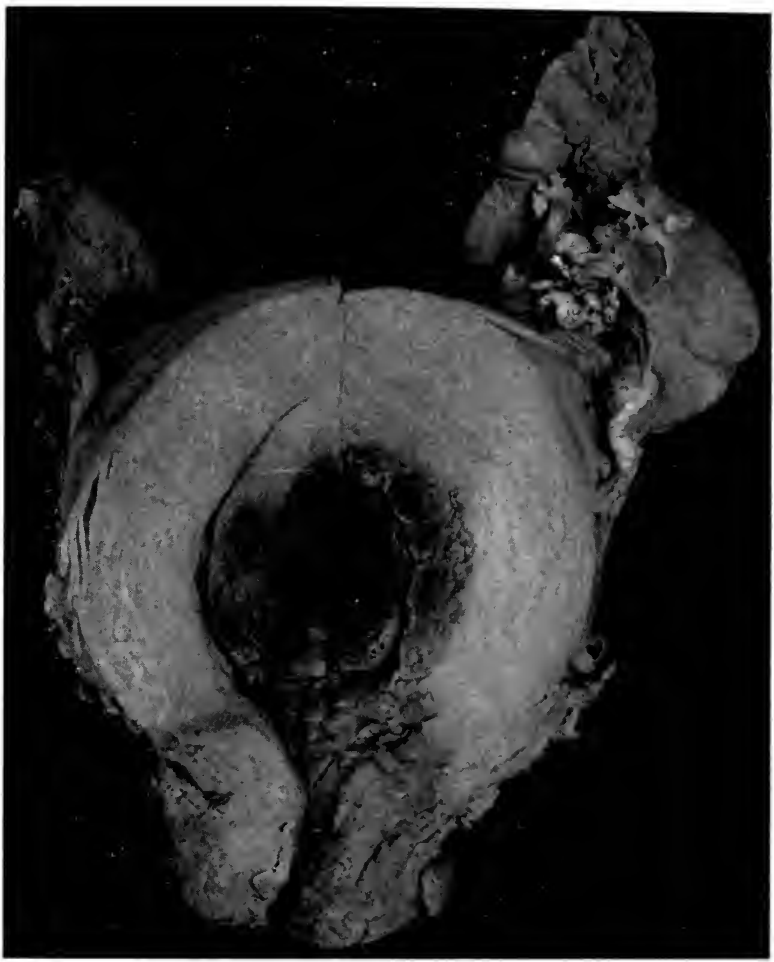

FIG. 259.-Cross-section through a uterus the seat of choriocarcinoma.

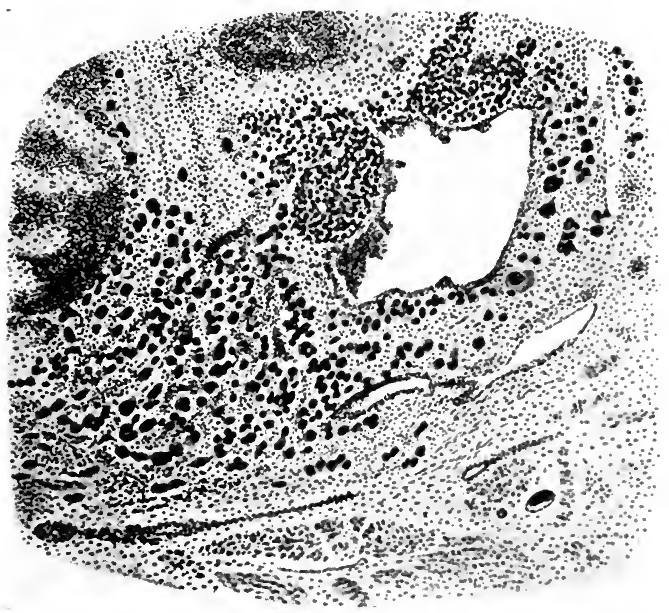

Fig. 260.-Structure of vaginal nodule in Polano's case of latent choriocarcinoma. 
consists of bands and masses of acidophile syncytium commingled in disorderly relations with islands of actively growing Langhans' cells. The stroma of villi is absent, showing that the cells, as in other carcinomas, are capable of growing apart from their ordinary sources of nutrition. The proportions of the two cell types vary, but both are always represented. The syncytium may appear in abundant isolated, elongated, and coherent buds resembling the malignant mole, or it may form diffuse sheets with large vesicular nuclei and resemble giant squamous epithelium. A preponderance

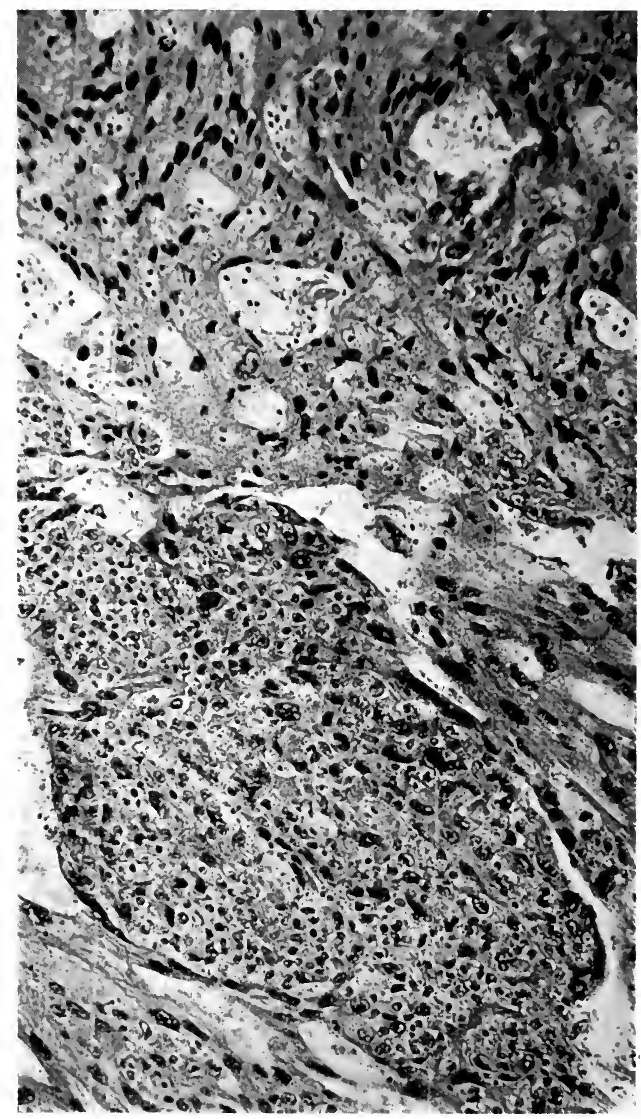

FIG. 26I.-Choriocarcinoma. Above, masses of syncytial cells resembling squamous epithelium. Below, group of Langhans' cells.

of orderly syncytium belongs to the less anaplastic growths. Some authors, as L. Fraenkel, have surmised that variations in the type of syncytium in chorioma signify a varying origin from villi, or endothelium, or uterine epithelium. Lebret and others describe pseudochoriomas arising from the uterine epithelium. Yet there is no satisfactory evidence that the syncytium of choriocarcinoma has more than one source, which is from villi.

The Langhans cells may appear in compact masses sheathed by syncytium and differing little from the normal type, or in the more anaplastic 
tumors these cells are hypertrophied, nuclei hyperchromatic, and the separation from syncytium is imperfect. Mitoses are limited to the Langhans cells.

In the sinuses the tumor masses may lie loosely or they become adherent to the walls and infiltrate through the endothelium. Fibrin masses are often mingled with the tumor-cells, especially in the sinuses, and here many of the syncytial wandering cells appear. In all forms of chorioma infiltration of the uterine wall by syncytial wandering cells is more marked than in normal gestation or hydatid mole.

Reactive inflammation occurs about necrosing portions of choriocarcinoma and has been interpreted as a sign of local regression.

Metastases in choriocarcinoma are of early occurrence and in advanced cases are invariably present. Among 455 cases of all types of chorioma collected by Pollosson and Violet vaginal metastases occurred in 93, pulmonary in $\mathrm{r} 33$, cerebral in 40 .

They may appear first in the vaginal veins and prove to be the initial symptom. On excision these tumors present the usual malignant structure.

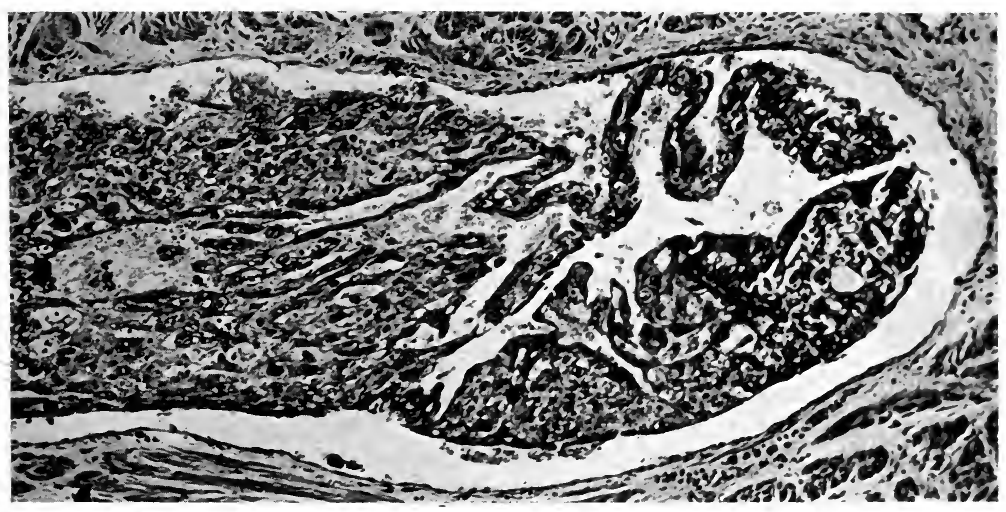

FIG. 262.-Choriocarcinoma invading uterine sinus.

In the lungs they form multiple protuberant hemorrhagic nodules, well circumscribed by the walls of the veins in which they lie. Here the structure often varies from that of the uterine growth, syncytium is scanty or absent, Langhans' cells small and abundant, and the structure may be difficult to separate from other cellular carcinomas. In liver, brain, spleen, and kidney the tumors resemble those in the lungs.

(3) Syncytioma. Atypical Chorioma of Marchand. Syncytial Endometritis.-In a limited group of cases the uterus fails to present a well-defined tumor process of the type of chorio-adenoma or choriocarcinoma, but a portion or the whole of the wall of the cavum is infiltrated by many large or giant acidophile cells of the general type of syncytial wandering-cells. To this process the term "syncytioma" is applied. In Schmauch's list about 5 per cent. of the fatal cases were of this type. The uterus is usually much enlarged and may contain a bulky mass composed of uterine stroma infiltrated with the above cells mingled with much fibrin, and necrotic detritus, and swollen by exudate and hemorrhage. The writer's case 9 illustrates this condition. Or the process may be limited to a small area of mucosa (v. Velits). Or the entire wall may be thickened, as in Cullen's case, but a definite intra- 
uterine tumor is wanting. Rarely the process extends through the wall into the broad ligament.

The walls of vessels are swollen and infiltrated by large cells. The stroma contains lymphocytes, plasma-cells, leukocytes, blood, and fibrin. A reactive growth of endothelial cells and fibroblasts may appear. The musclecells develop multiple nuclei and split up into acidophile fragments which resemble syncytial wandering-cells. In 3 cases $I$ have been unable to trace any participation of uterine epithelium. The decidual cells probably participate in the process or form the main element in certain cases in which the lesion is diffuse. Such a condition is that described by Cullen in which the uterus, as large as a 5 months' pregnancy, was lined by a thick, irregular, largely necrotic tissue covering the entire endometrium. Here many areas of degenerating decidual cells were described. In many cases it is difficult to

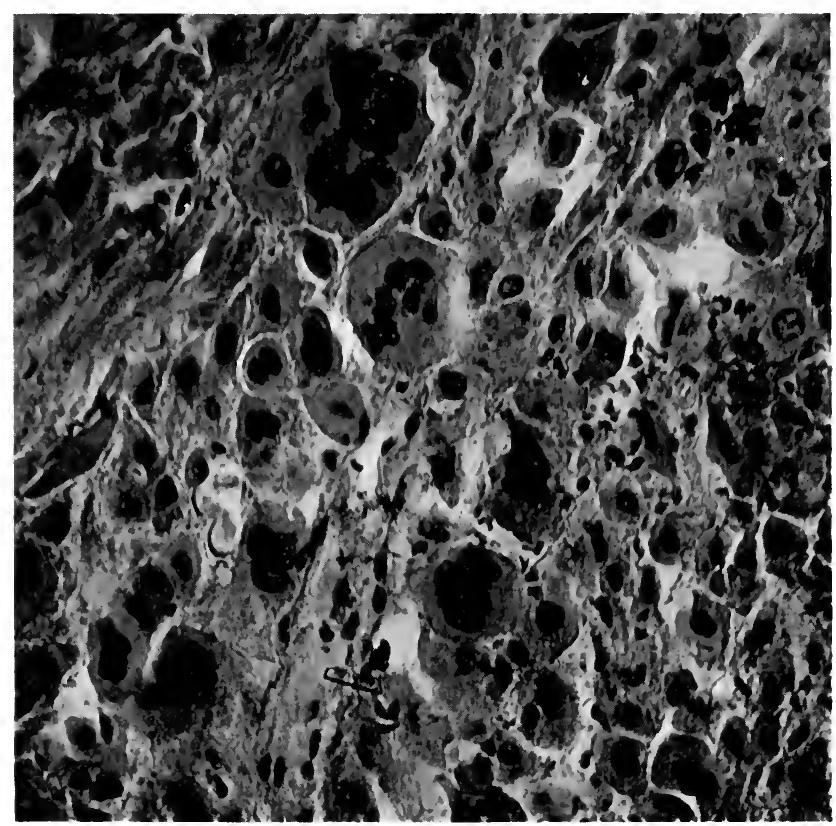

FIG. 263.-Giant-cells in syncytioma.

distinguish between decidual cells and wandering syncytial cells, and this difficulty is increased when the tissue is degenerating and infiltrated by leukocytes.

Progressive metastases are not observed, but Fleischmann described a vaginal tumor presenting the structure of atypical chorioma, which may have arisen from deported villi.

The conditions included in the category of syncytioma probably arise from several antecedent processes, and chiefly from abortion, hydatid mole, and chorio-adenoma. It is unlikely that fully developed choriocarcinoma ever suffers such extensive regression as to produce this anatomical picture. Excessive invasion of the uterine muscle by syncytial wandering cells is observed with retained chorion, hydatid mole, and especially with chorioadenoma. After spontaneous expulsion or imperfect curetage of a malignant 
mole proliferation of wandering-cells may continue, and when hemorrhage and infection follow, the condition called syncytioma may apparently develop. When the process has perforated the wall it must have resulted from a dissecting malignant mole which has undergone the spontaneous regression which many observations show may overtake this process.

Whatever may be the original conditions which result in excessive infiltration by syncytial wandering-cells, it is clear that the disease is not a progressive neoplasm, but a regressive and inflammatory process appearing in various stages and after variable antecedents. I have elsewhere considered at some length the observations supporting this view (S. G. O., I9Io).

When the syncytial wandering-cells are abundant, well nourished, and form more or less compact sheets, a partial neoplastic quality is suggested and the term syncytioma may be employed. When the lesion is more diffuse and complicated by exudative and productive inflammation it appears to be well designated as syncytial endometritis.

The remarkable extent to which the syncytial wandering-cells may invade the uterine wall in normal gestation has been emphasized by many observers and especially by $\mathrm{R}$. Meyer. He shows that these cells may be extremely abundant in the superficial portions of the musculature, often approaching the form of decidual cells, invading the walls and even the lumen of veins, and extending to the serosa. While normally disappearing shortly after gestation, in various pathological conditions, as carcinoma, myoma, retroversion, or mole, they persist and multiply by mitotic division, yielding a picture which may readily be mistaken for sarcoma. Their behavior indicates active ameboid properties. An imperfect development of decidua favors deep invasion of villi and syncytial cells (Bauereisen).

Clinical Course of Chorioma.-The different forms of chorioma vary considerably in their clinical features.

Chorio-adenoma regularly follows hydatid mole, and occurs usually in multiparæ over 40 years of age. Of 23 cases collected by Curtis and Oui the mole was found in situ in I3, and had been expelled in ro. After expulsion of the mole there may be a period of a few days or a few months in which the patient is free from symptoms. Hemorrhage, interpreted as profuse menstruation or metrorrhagia, or more alarming, is the first symptom. In Pick's doubtful case a vaginal nodule was first noticed. The bleeding begins uniformly in the third month of a supposed pregnancy or at any period after expulsion of a mole. The bleedings become more severe and if unchecked the patient may succumb in 2 to 8 months from exsanguination. In about I3 per cent. of the cases infection occurs with fatal septicemia. Rupture of the uterus, spontaneous or from curetage, is an occasional fatal complication.

The treatment commonly employed is hysterectomy. It was followed by recovery in II of I4 cases, with 3 deaths (Curtis). Manual extraction of the mole and curetage were successful in 2 cases reported by Herz and one patient subsequently gave birth to a healthy child.

Yet the anatomy of the disease does not encourage the belief that many of these cases can be completely freed of the infiltrating villi by the curet. Radium may prove of value in this condition, but the danger of hemorrhage and infection complicates its use.

Veit emphasizes the necessity of minute examination of all portions of the placenta extracted after abortion, mole, or normal gestation, as the best means of prevention of chorioma. He would even extirpate the uterus when the wall is found to be very thin over the implantation site of a retained placental remnant, but here microscopical examination of the tissue seems to be a better guide. 
Choriocarcinoma gives less definite clinical manifestations of its onset than do the other forms of the disease. It follows normal labor promptly or after a long interval, ectopic pregnancy, retained placenta, hydatid mole, and abortion. Hemorrhage is again the chief symptom, but it is not as severe as with malignant polyps. The uterus is often only slightly enlarged. Many cases are first recognized by examination of curetings for hemorrhage. All pelvic symptoms may be wanting and the illness begins with hemoptysis, hemiplegia, peritonitis, severe vomiting, or the appearance of a vaginal tumor. Occasionally the disease masks a puerperal fever (Curtis).

If not interrupted by hysterectomy the disease progresses with hemorrhages, anemia, appearance of metastases with their special symptoms, or septicemia. Death results from cachexia, hemorrhage, pulmonary embolism, peritonitis, or cerebral invasion.

With or without hysterectomy the disease usually lasts from 6 to I 8 months. Much more rapid courses are occasionally observed in which death results from infection or hemorrhage. Schlagenhaufer recorded a fatal issue 34 days after normal birth, with an infected uterine tumor and metastases in vagina, lungs, kidney, and spleen. More prolonged duration with an initial latent period probably accounts for the late appearance of symptoms in some of those cases developing years after the last gestation. In advanced stages the symptoms are dominated by the metastases, cough, hemoptysis, and dyspnea with pulmonary lesions, and paraplegia, delirium, and convulsions with cerebral extensions.

Hysterectomy was performed in I8I cases of chorioma of all types collected by Curtis and Oui, I903 to I9I2, with I7 operative deaths. I have been unable to find any record of operative cure of choriocarcinoma. Schmauch emphasizes the necessarily fatal character of the process and advises against operation as hastening the fatal issue. When metastases have occurred operation is certainly contraindicated, but it seems impossible to affirm that the removal of a uterine focus and even of accessible metastases may not in rare instances affect a cure. On the other hand, it seems almost impossible to avoid displacing intravascular fragments during hysterectomy, and thus accelerating dissemination.

Syncytioma.-The early symptoms of syncytioma vary widely with the different antecedent conditions. The disease has followed abortion, hydatid mole, and placental polyp, but it is notable that a definite history of such relation is often lacking. Until such clinical data are more fully supplied the exact nature of many of these cases must remain in doubt.

The first symptom is usually irregular and profuse metrorrhagia. The uterus is then found enlarged, often to considerable dimensions. Infection of the uterus is readily established and many cases die of sepsis or peritonitis after a comparatively brief course. Curetage yields masses of necrosing material, or a tumor tissue which is of uncertain significance and is often regarded as sarcoma. Many cases, however, recover after curetage, which Menge recommended as the operation of choice. When the uterus is extensively damaged, the wall thin, and infection active, hysterectomy has usually justified itself. Yet the disease has a high mortality. The mortality from chorioma as a whole is high but decreasing. In Teacher's statistics of I88 cases there were 65 recoveries, but some were doubtless reported prematurely.

Recovery from Chorioma After Curettage or Partial Removal.-O Of 8 cases of chorioma collected by v. Velits which recovered after curettage, in 5 I find that the tumors were composed chiefly of wandering syncytial cells and belonged in the group of syncytioma or syncytial endometritis, while 2 were proliferating hydatid moles (Risel, Blumreich), and I was inade- 
quately described. Even in this group of cases curettage, although followed promptly by hysterectomy, has a high mortality.

In 8 cases recovery followed partial removal of the tumor, metastases or extensions remaining (Ewing, Lit.). In 5 of these the tumors were wholly or chiefly syncytial. Some of these cases point to a possibility that an isolated metastasis in the milder forms of chorioma may regress after removal of the main tumor. For typical choriocarcinoma this possibility cannot be admitted.

Deportation of Normal Chorionic Elements.-That syncytium frequently lodges in the pulmonary capillaries has been shown by Schmorl, who found them in 80 per cent. of ${ }_{1} 8$ cases. Dunger has stated that portions of villi

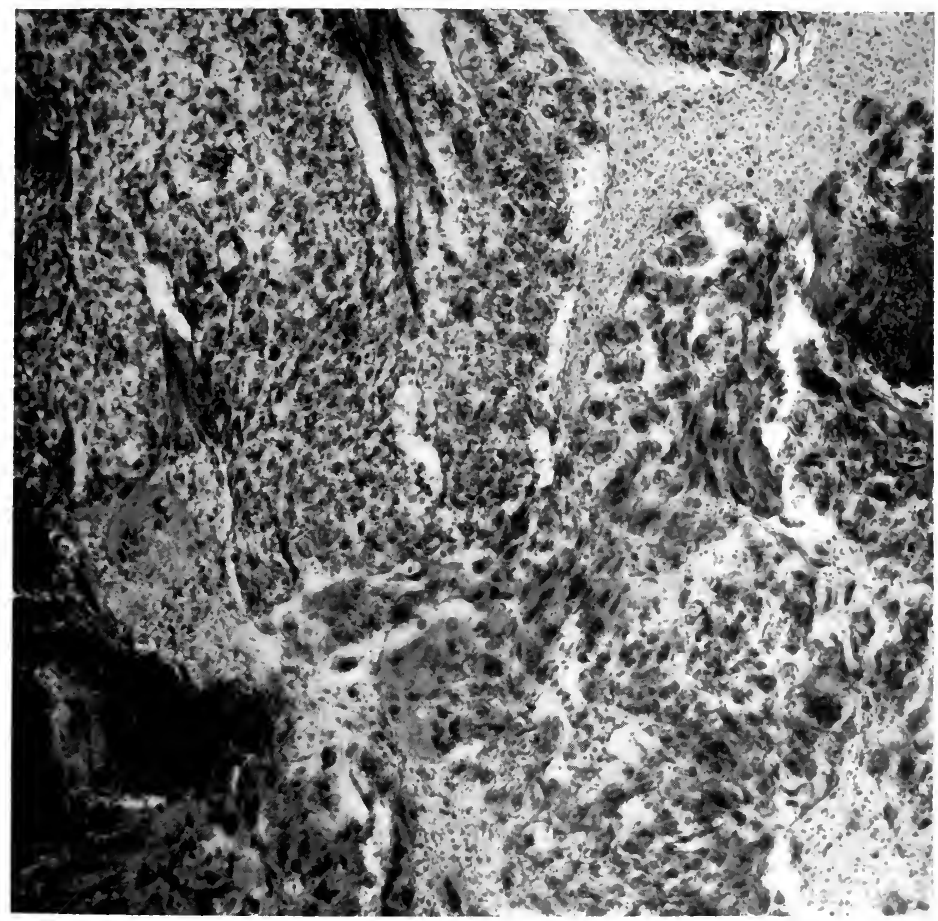

FIG. 264.-Curettings in a case of choriocarcinoma.

even including stroma may be lodged in pulmonary and pelvic vessels, especially after violent labor, and he claims that such deported cells may exhibit transitory proliferation. It must, therefore, be assumed that benign moles may give rise to similar metastases, and Pick has described such a vaginal metastasis containing several segments of villi with stroma and syncytium largely necrotic. The occurrence of villi or other chorionic elements in distant veins should not be regarded necessarily as a sign of malignancy. Veit states that metastases can be regarded as certainly malignant only when they occur beyond the usual pelvic depots for deported villi. Pick claims that the histology of deported villi in cases which recover may not differ from that in cases which develop malignant tumors. It may readily be sup- 
posed that all emboli from uterine tumors do not succeed in producing metastatic tumors. Some may regress because of local factors, while others go on growing. I have seen very extensive regression, hyaline change, and fibrin formation in a vaginal metastasis in a case which succumbed to cerebral invasion. Yet this vaginal tumor contained scattered small islands of wellnourished Langhans' cells. Moreover, the disease in this patient developed 3 years after the last gestation. As a rule the harmless emboli are found embedded in fibrin and degenerating, as is sometimes seen in the veins of the broad ligament in tubal gestation.

The further question arises whether a malignant tumor may arise from deported normal chorionic cells. Schmorl has reported a case of choriocarcinoma in the organs, appearing first in the vagina, I 8 weeks after normal labor, with a normal uterus. Chorioma has frequently arisen after normal labor at term, and since it is unlikely that a malignant change in the placenta can escape detection, the suspicion has arisen, and has been entertained by Marchand, that a malignant tumor may arise from deported normal placental elements. Strongly against this possibility stand the frequency of partial hydatid degeneration especially after abortion (8o per cent., Stoerk), the frequent failure to examine the placenta, and the occurrence of ectopic or teratomatous choriomà.

After abortion (Schlagenhaufer, Schmidt) and after hydatid mole (Pick, Schmidt) the uterus was found normal, but vaginal tumors containing syncytium and Langhans' cells were excised and the patients recovered. In these cases it must be held that the vaginal tumors were deported normal villi or fragments of moles, with, at most, transient proliferation. In 3 of them there were marked signs of degeneration in the vaginal tumors, but in Schmidt's case, which was reported rather soon, the tumor was depicted as an active chorio-adenoma.

On the other hand, after normal labor (Schmorl) and during gestation at term (Walthard) vaginal tumors of the type of choriocarcinoma and the uteri which proved to be normal, were extirpated, but the patients died with metastases. In Lindfors' case the uterus was found normal at autopsy. These and other cases collected by Risel are very difficult to explain, and they suggest that deported normal villi or their derivatives may develop into choriocarcinoma. It should be noted that in some of the cases the placenta was not examined, but this objection does not apply to Walthard's observation.

Ovarian Changes in Chorioma.-In I 895 Marchand pointed out the notable frequency of ovarian cysts in chorioma, and later (I905) Patellani found that in 68 fully reported cases at that date 62, or 9I per cent., presented bilateral cystic changes. This proportion is probably too high unless one includes marked grades of the small cystic cavities of gestation, but it is quite evident that pronounced cystic changes in both ovaries are so frequent in chorioma as to constitute a specific anatomical feature of the disease, and one which invites explanation. In several cases the cysts have receded after successful extirpation of the tumor or the mole, but in fatal chorioma they persist.

The cysts may be very numerous and small, or the ovaries may be ballooned out by roomy multilocular chambers to the size of an orange. The contents are thin, yellowish, serous fluid containing albumins, lipoids, and a little mucin. The walls of the large cysts are lined by a deep layer of large overnourished granular polyhedral cells exactly resembling lutein cells of the early corpus luteum.

The origin of the cysts has not been traced with entire certainty. Most of them probably arise by distention and overgrowth of corpora lutea of various ages (Jaffe, 
Orthmann). The small multiple cysts of gestation are not so clearly traced. Seitz regards them as arising from the atresic follicles of the ovary, which begin to hypertrophy in the sixth week of gestation up to term, and he adheres to the view that these follicles have a different significance from the corpora lutea. Stockel finds that during gestation the theca cells of the follicles wander out into the ovarian strona forming lutein cell groups from which the small cysts arise, and Schaller and Pforinger found such cell groups so abundant as to suggest a tumor process. Whatever the origin, the structure and behavior of the cysts indicate that they have an identical significance in representing a disorder of lutein secretion.

L. Fraenkel concluded that interference with the lutein function resulted in death of the ovum and chorioma, since excision of the corpus luteum prevented implantation and growth of the ovum. L. Pick interpreted the cysts as evidence of hypersecretion leading to excessive growth of the chorion.

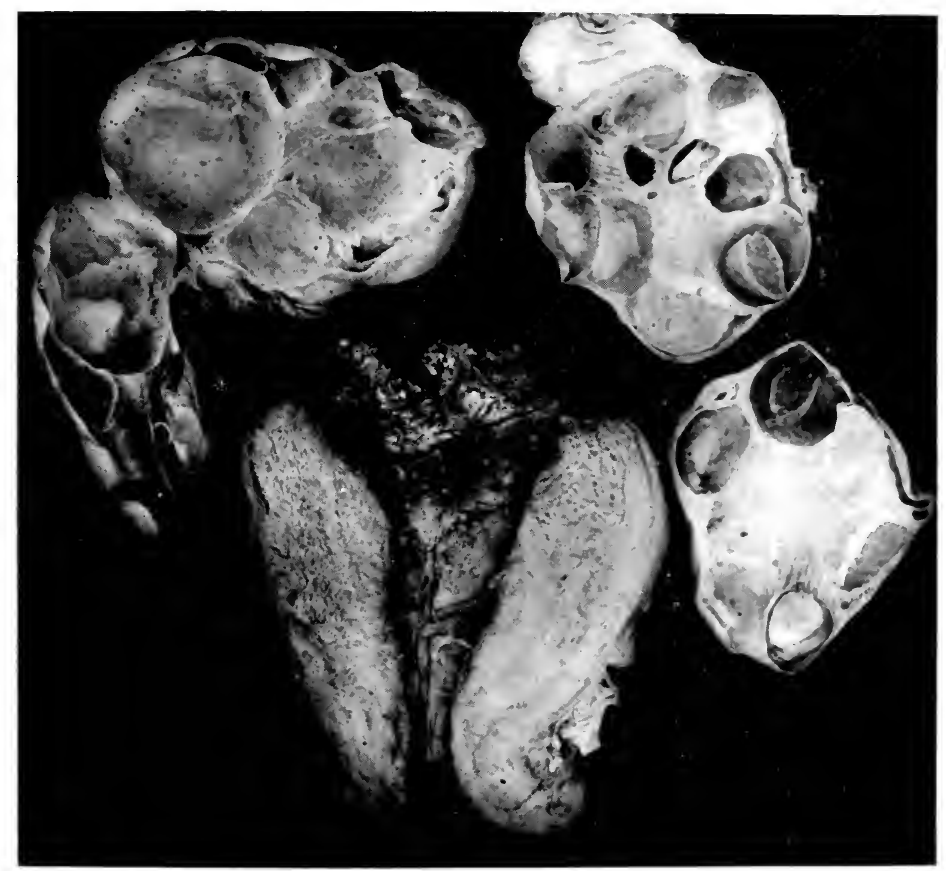

FIG. 265.-Choriocarcinoma of uterus. Cystic ovaries.

The suggestions that the cysts result from venous congestion (Seitz), that they are equally characteristic of normal pregnancy (IVallert), and that they belong to the ordinary nutritional changes of pregnancy (Dunger), seem quite inadequate explanations.

Veit considers the possibility that a primary ovarian disease yields a diseased ovum which degenerates and entails abnormal proliferation in the chorion. Yet normal labor may be followed by chorioma.

Chorioma after tubal pregnancy occurred in 12 cases collected by Risel, to which may be added later reports by Garkisch and Bazy. None was recognized clinically, all were malignant forms, and all but one fatal. Ovarian pregnancy seems to have been the origin of a fatal choriocarcinoma described by Kleinhaus.

Teratomatous chorioma in the female has been described by Lubarsch, Pick, and Bock. 


\section{CHAPTER XXIX}

\section{CYSTS AND EPITHELIAL TUMORS OF THE OVARY}

Ovarian Cysts.-Simple cysts form in the ovary under several conditions. They are often small and bilateral, giving rise to a characteristic appearance designated as small cystic ovaritis or degeneration. Larger single or multiple cysts of inflammatory or mechanical origin pass under the term "hydrops follicularis." The first appearance of mild neoplastic processes in dilated follicles deserves recognition by the term "simple cystoma." Both benign and malignant epithelial tumors of the ovary are usually cystic and thus form a large and important group of varied and uncertain origin. The corpus luteum is a frequent source of cysts of peculiar type. A group of combination cysts includes tubo-ovarian cystoma, dermatocystoma, and combinations of corpus luteum cysts with other forms. The simple dermoid regularly assumes a cystic form, and many teratomas become cystic.

Small multiple cysts (cystic degeneration) represent several different anatomical processes:

(a) Chronic ovaritis with thickening and fibrosis of the cortex may be associated with dilatation of many follicles which appear as small translucent elevations of the surface (Hegar, Bulius). It occurs chiefly in early sexual life, and is interpreted by Nagel as a premature maturation of follicles. Yet it is observed also in children and newborn infants. I have observed it with general edema of the ovary due to torsion. A moderate and variable degree of development of small cysts must be regarded as physiological and occurs especially in infants and in gestation (Felix).

(b) In elderly subjects the deeper portions of the cortex may be the seat of multiple small follicular cysts lined by overnourished but undifferentiated epithelium. v. Kahlden regarded these structures as multiple adenomas and Walthard derived them from superfluous groups of germ epithelium which have failed to develop into follicles.

(c) Remnants of the primitive nephros have been identified in the ovary and Babo traces certain multiple small cysts to these structures. v. Franque describes such cases, the cysts being lined by flat epithelium.

(d) Multiple small or minute translucent cysts may be found over the surface of ovary and tubes and their ligaments, and are lined by flat, or ciliated, or goblet-cells. Their origin is probably not uniform. Some are referred by Walthard to superfluous undifferentiated germ epithelium. Others give evidence of a proliferation of peritoneal epithelium with metaplasia of the inclosed cells. Pick regards them as miniature tumors which reveal the great proliferative capacity of germinal and peritoneal epithelium, and believes that they may go on to produce cystadenomas. They have been extensively discussed and variously interpreted by Schickele, v. Franque, R. Meyer, and others.

Simple follicular cysts (hydrops follicularis) arise from distention of Graafian follicles by an inflammatory serous exudate. These cysts are of small size, seldom as large as the fist, and when several develop in the same ovary they may be mutilocular. Occasionally they fuse with lutein or dermoid cysts. In some cases the condition must be interpreted as an exaggeration of the small cystic chronic ovaritis. The contents are serous albuminous 
fluid, without pseudomucin, but blood and fatty detritus may be added. The wall is composed of the distinctly ovarian tissue in a state of atrophy and fibrosis. Little ovarian stroma may be recognizable and the epithelial alveoli of cystadenomas are missing. The follicular epithelium is usually destroyed. Low, warty, fibrous excrescences may appear in the inner wall. The fairly constant occurrence of inflammation of the pelvic organs points to the inflammatory origin of these cysts. Single or multiple cysts are observed in the newborn (Virchow, Klob, Waldeyer).

Simple Serous Cystoma.-This specific form of ovarian cyst is closely related in structure and identical in origin with the simple follicular cyst, but differs from it in the greater size, preservation and overgrowth of lining epithelium, and in the greater vitality of the connective tissue. These features justify the view that the process is essentially neoplastic. Although there

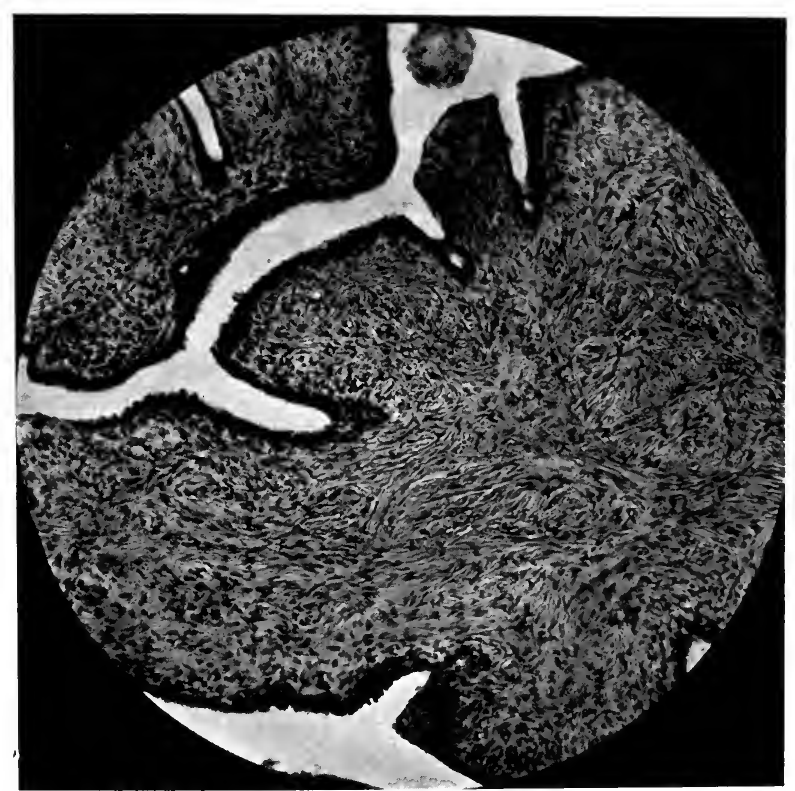

FIG. 266.-Structure of wall of simple cystoma of ovary.

are transitional cases between the simple follicular cyst and the serous cystoma there is practical value in distinguishing between them. The former loses its epithelial lining, its walls are acellular, and it remains of comparatively small size. The cystoma grows to bulky dimensions, its walls are cellular and actively growing, and the lining cells multiply, even forming low papillary projections. In size the cystoma is usually as large as a child's head and may reach the dimensions of the bulky cystadenoma. Rarely it is bilateral (Olshausen, Bulius, Orth). The cavity is usually single. The contents are serous fluid, free from mucin, but often mingled with blood or with fatty detritus or crystals. The lining is of cylindrical epithelium, single cells or syncytium, and these may form low warty projections. The wall is composed of cellular, vascular connective tissue without epithelial structures, and it may form nodular or papillary projections with the structure of an 
active fibroma. The simple cystoma probably arises from ovarian follicles, but from which form of these structures is not known. Fully formed Graafian follicles may develop papillary ingrowths (Williams), and it is probable that some cystomas are of such origin. Hence many authors class these cysts with follicular hydrops. The occurrence of transition forms leading to cystadenoma indicates that some are derived in the same manner as cystadenoma, from misplaced and embryonal cell masses.

Tuho-ovarian Cysts.- The simple ovarian cyst may communicate with a fusiform dilatation of the tube forming a composite cyst resembling in form a glass retort. Such cysts may reach considerable dimensions. The walls are thin and contents usually serous. At the ostium of the tube there may be one or more folds on the inner surface from which radiate the elongated fimbriæ embedded in the inner wall. The tubal portion may present several folds or partial contractions. The lining of the ovarian compartment is of flat cells, that of the tubal part is of cylindrical and sometimes ciliated epithelium. Corpus luteum cysts may fuse with the tube, in which case the wall presents the usual feature of such cysts. Shreds of smooth muscle persist in the wall of the tubal portion, but the ovarian section is thin and fibrous. The origin of the tubo-ovarian cyst is probably to be referred to the union of an already formed ovarian cyst with the lumen of a tube which has become adherent to the cyst wall through chronic productive inflammation. Evidences of this inflammatory process are regularly found in the form of adhesions of the cyst, peritoneal thickenings, salpingitis, and peri-ovaritis (Veit). The communication is established by pressure atrophy. The occurrence of slight and early dilatation of the tube accords with this view. Finding the ovarian remnant limited to a segment of the wall in $\mathrm{I} 2$ of $\mathrm{I} 8$ cases, Zahn concluded that the composite cyst arises chiefly in the tube with subsequent involvement of the ovary, but the eccentric position of the ovarian remnant is common to other ovarian cysts. Moreover, the ovarian portion is usually much the larger (Martin).

Corpus Luteum Cysts. - The corpus luteum is frequently the seat of dilatation with overgrowth of lutein cells, and gathering of serous fluid, which produces cysts. In size the cysts are first small and easily recognized by the yellow layer of lutein tissue. Later they are found as large as an orange or a child's head, but the larger ones are often complicated by hemorrhage or suppuration. While usually single and unilocular, they appear as bilateral multilocular cysts with hydatid mole. The wall is composed of atrophic ovarian tissue and is lined by a corrugated layer of yellow lutein tissue which is often incomplete, but seldom absent. The contents are clear serous fluid, but blood is often extravasated by capillary hemorrhages, bulky spontaneous bleeding, or by strangulation. Rupture of the cyst may lead to retro-uterine hematocele simulating extra-uterine gestation (Lunzer, Lit.). Suppuration, chiefly from gonococcus infection, frequently occurs, replacing the lutein tissue with granulation tissue, and distending the cyst with thick mucopus. A definite group of ovarian abscesses are of this origin. They are lined by hypertrophic granulation tissue in which are remnants of lutein tissue, eosinophile leukocytes, plasma-cells, and often demonstrable gonococci (Langer, E. Fraenkel). Garkisch observed tuberculous infection. The lutein-cell layer is early subject to local infarction and necrosis. The structure reveals notable variations, indicating several modes of origin of these cysts. All present an outer coat of a cellular connective tissue derived from the external layer of the follicle or the distended ovary.

(I) Many cysts show an internal lining of hyperplastic lutein-cells covered by fibrin. This structure is best explained as a dilatation of the cor- 
pus luteum beginning before any regressive changes have set in, and is observed in cysts of all sizes. It is also observed in the multiple cysts arising from atresic follicles in hydatid moles.

(2) The lining may be composed of hyaline and lamellated tissue beneath which is a layer of lutein-cells. Such cysts probably arise after pronounced regressive changes have occurred in the corpus luteum. Many such cysts contain chiefly blood in various stages of absorption and organization, and should be interpreted as hematomas in corpora lutea (Fraenkel, Orthmann).

(3) An epithelial lining is occasionally observed, and is composed of a single layer of cylindrical or flat cells, which lie either directly upon luteincells as in type (I), or upon the hyaline connective tissue as in type (2). The origin of this lining is still undetermined. If the cyst arises from an atresic follicle the epithelium could be derived from the cells of the membrana granulosa. Ciliated epithelium was found by E. and L. Fraenkel, who remained in doubt as to its origin, but Pick and Pfannenstiel hold that such cells must have been derived from the germinal epithelium entering the cyst at the time of rupture. Connective tissue may appear in the centers of lutein cysts and the flat character of the lining cells is consistent with an endothelial origin.

(4) Bilateral multilocular lutein cysts commonly appear with hydatid mole and chorioma. Kroemer finds them in 59 per cent. of moles and Patellani in 90 per cent. Since the condition is probably an excessive grade of the small cystic changes of gestation the estimated frequency will vary according to the size of the cysts included. I find them by no means constant in chorioma.

In pronounced cases the cysts reach large dimensions, the walls are thin, and neighboring cysts fuse. The inner lining is of remarkable hypertrophic and hyperplastic lutein-cells, which may slightly infiltrate the walls. In small cysts remnants of the granulosa cells may persist (Stoeckel). Lymphcysts may appear in the walls.

The natural course of the cysts is regression after removal of the mole. Albert saw complete disappearance of large bilateral tumors 2 months after expulsion of the mole. Yet Pfannenstiel reports 2 cases of rapid increase after curetage.

That a true neoplastic process exists in any form of lutein cyst appears doubtful, although the morphology of some early cysts is suggestive of unrestrained overgrowth. In the early stages of some cystic corpora lutea I find overnourished alveolar groups of small lutein-cells with hyperchromatic nuclei and layers of such cells splitting up the ovarian tissue. The appearance is not distinctive of a true neoplasm. The late course of the lutein cysts fails to develop the essential features of a neoplasm, as progressive growth, infiltration, or metastases. The most advanced cases retain as a rule the cystic form and the lutein-cells tend toward atrophy. The process must be interpreted as a form of overgrowth due to overnutrition, or excessive function, aided by inflammatory factors. The existence of a true tumor of luteincells is not thereby excluded, but the reported cases of lutein-cell tumors are not entirely satisfactory.

Lutein-cell Tumors.-The suggestion that the corpus luteum might give rise to characteristic tumors of the ovary was first made by Rokitansky in I859 and later endorsed by Klob and by Klebs. Since that time several authors with more or less confidence have attributed the origin of certain tumors to the lutein-cells. Some of these tumors have been described as sarcoma, others as carcinoma (Kroemer, Lit.). Voight's case was a large solid tumor, grayish yellow on section, and showing a perithelial or diffuse arrangement of large cells resembling lutein-cells. The tumor obstructed 
labor. Sante also reported a large tumor appearing in the third month of pregnancy. It was soft, reddish yellow, and infiltrated with blood, and the section showed the structure of alveolar sarcoma with septa inclosing areas of cells many of which had all the characteristics of lutein-cells. Six months later the patient succumbed with nodular tumors of omentum. Grousdew found in the walls of a large cystic tumor numerous groups of polyhedral cells which he interpreted as lutein-cells. He concluded that the tumor arose from the cells of one of the corpora albicantia. The patient died with peritoneal recurrence in the form of spindle-cell sarcoma, at the age of 60 . Michelazzi referred to the corpus luteum a large yellowish tumor in an elderly subject. Wendeler speaks of an ovarian tumor resembling a giant corpus luteum the structure of which confirmed the diagnosis of corpus luteum sarcoma.

I have observed two forms of tumor-like overgrowth of lutein-cells. In one the cells retain their original characters and the convoluted layer of the corpus luteum. In the other the cells are smaller, very compact, and a diffuse growth obliterates the convoluted layer. I have examined a tumor, with Dr. L. W. Strong, which strongly suggests a lutein-cell origin. It measured $2 \frac{1}{2} \times 3 \frac{1}{2} \mathrm{~cm}$., was solid, yellowish, encapsulated in the ovary, and resembled an enlarged corpus luteum. The structure was that of alveolar sarcoma, the septa and cells merging insensibly. The cells were large and small, polyhedral, with clear or slightly granular cytoplasm and dense borders. Many contained hyaline acidophile globules and scanty pigment particles.

While, as Seitz concludes, the positive proof of the lutein-cell origin has not been furnished, yet the gross appearance of these rounded yellow tumors and their peculiar structure render such an origin probable.

Considerable proliferation of lutein-cells often occurs in the cystic ovaries of hydatid mole, and Schaller and Pforringer have described a "carcinomatous" proliferation in such a case.

Adenoma.-Adenomas of the ovary are usually cystic, but there are rare forms of solid and of superficial papillary tumors. Two main classes of cystic adenoma are distinguished chiefly on the character of the cyst contents and on the epithelial structure. These are the serous and the pseudomucinous adenomas. Both these types may show a glandular (pseudopapillary) or a papillary structure, and some authors, as Olshausen and Kaufmann, prefer to classify the tumors upon this structural peculiarity rather than upon the character of the cyst contents. Either plan requires the recognition of transitional cases and the histogenesis is not sufficiently clear to form a basis of classification. In each class also there are pure benign adenomas, adenocarcinomas, and carcinomas, the structure varying in different cases and in different parts of phases of the same case.

Serous Cystadenoma.-The serous cystadenomas form about one-third of the cystic tumors of the ovary. In their earliest stages they are probably identical with the relatively small multiple cysts of V. Kahlden and some of them probably begin as simple follicular cysts. When fully developed they reach large dimensions, but never the very great size of the pseudomucinous tumors. Most of them are pedunculated growths lying in the peritoneal cavity, but the papillary type especially may be largely intraligamentary, displacing the pelvic organs in its growth. Most of these tumors are unilateral, but the more actively growing papillary forms frequently ( 60 per cent.) affect both ovaries either as primary tumors or through implantation. The wall, at first lobulated, becomes smooth and tense as the tumor increases. The simple adenomas may be translucent, the more active papillary growths 
are opaque, and papillary nodules appear on the surface and vicinity, while malignant processes are indicated by numerous adhesions and implantations.

In some tumors simple cystic compartments may project in isolated form, and an exaggeration of this process gives rise to peculiar hydatidiform cystic adenomas composed of clusters of cysts. The walls of these cysts are thin, the lining is of ciliated epithelium, and contents are serous (Olshausen). Of 9 cases collected by Jayle and Bender, 6 sprang from the ovary and 3 from the posterior surface of the broad ligament. The extra-ovarian growths probably arise from supernumerary ovaries. Somewhat similar tumors lined externally by epithelium occasionally form by myxomatous degenera-

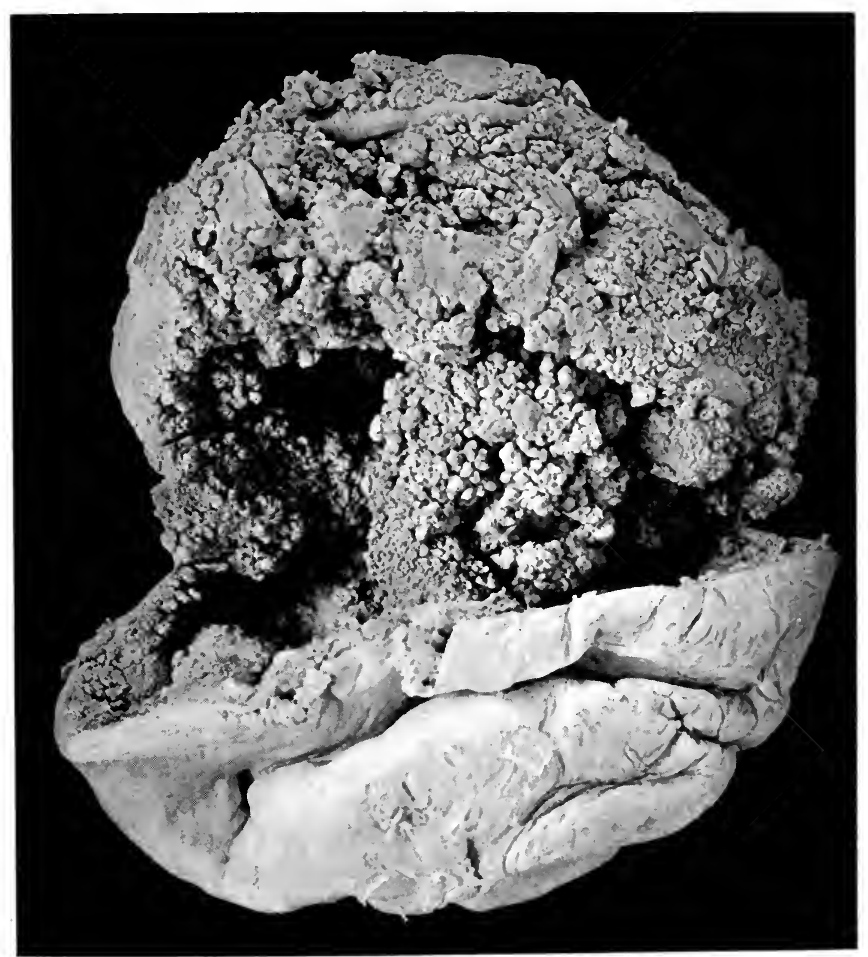

FIG. 267.-A large papillary cystadenoma of ovary.

tion of the stroma of superficial papillary adenomas (Odebrecht). As might be expected from the position of the epithelium some of these tumors prove malignant (Hoffmeier). Rarely the superficial papillary growths are extremely abundant and the intracystic growth much reduced.

On section one large central cavity usually represents the fusion of original multiple chambers some of which often persist until the tumor reaches a large size. The contents are originally yellowish alkaline serous fluid rich in albumin, but free from pseudomucin, but later it may contain increasing traces of pseudomucin, epithelial detritus, fatty substances, and often much blood from rupture of the delicate papillæ. Sand grains of calcific deposit in the wall and papillæ may become extremely abundant. When papillæ 
appear on the surface of the tumor they may give rise to implantation metastases over the adjoining peritoneum. This type of dissemination occurs in many of these tumors, and its extent and severity accords with the histological signs of malignancy. Ascites regularly accompanies these implantations. In general, the serous cystadenomas differ from the pseudomucinous, in their smaller size, the greater frequency of bilateral tumors, the tendency to produce metastases, the predominance of ciliated epithelium, and the usual absence of pseudomucin in the contents.

Structure.-The lining cells are cylindrical or cuboidal epithelium which has a more granular character than the clear cells of the pseudomucinous adenoma. In the simpler tumors the cell layers are single, lying upon a rich stroma. In the more active growths the cells increase in number, their form becomes irregular, secondary papillæ develop, the wall is honeycombed, and sections of the compact papillæ may present a glandular appearance. A papillary structure predominates over the glandular. In more malignant areas the growth of cells may be more diffuse and less dependent upon the stroma. In most cases ciliated epithelium may be found and sometimes it is uniformly present. Calcific granules may appear within or between the epithelium or stroma-cells (psammoma). They occur at all stages and have little relation to the vitality of the growth. The stroma forms low, thick buds or longer cellular and vascular branching twigs. Occasionally it is finely villous. Very abundant and cellular stroma, suggesting a sarcomatous property, is limited chiefly to distinctly adenocarcinomatous tumors.

The course of the serous cystadenoma is slow and relatively benign. The simple tumors are uniformly benign, unilateral, and do not produce local metastases or recur after extirpation. They may cause pressure symptoms, but local peritonitis, adhesions, and ascites are rare. The more active tumors with rich development of papillæ are clinically more serious. While slowly growing, and requiring 3 to I 5 years to reach bulky dimensions, the tumor capsule may at any time be perforated and ascites and implantation metastases follow. Likewise their frequent intraligamentary position and bilateral occurrence give rise to more severe pressure symptoms. Recurrent ascites marks the course of many cases and may require paracentesis over a period of many years, during which there is progressive anemia and emaciation. Pyr-Smith records 229 tappings in 9 years, and Peaslee 665 in $\mathrm{I} 3$ years. The peritoneum becomes thickened and opaque, adhesions form, and implantation metastases occur in I3 per cent. (Pfannenstiel). In Peaslee's case they were absent. Rupture of the cyst and discharge of the serous contents and tumor fragments are rarely observed. It may be followed by a slight fever, or by prolonged chronic peritonitis (Werder). As a rule the implantations develop from papillæ on the surface or growing through the wall without rupture.

Implantation metastases occur early or late and remain localized or extend over a large portion of the peritoneum. Their course varies with the general malignancy of the tumor, but their fate cannot always be predicted from the structure of the original tumor, since some adenomatous tumors have given rise to carcinomatous implantations. In a well-known group the metastases disappear after extirpation of the tumor. This favorable outcome occurs almost exclusively with structures which are comparatively non-malignant and in which the lining cells of the papilla are but slightly atypical and appear in single rows. This spontaneous retrogression has been observed by many writers and is described with anatomical details by Froschel, Bumm, H. Freund, E. Fraenkel, Pfannenstiel, and others. It seems probable that regression of tumor fragments disseminated by operation occurs very fre- 
quently (Olshausen). Even without the removal of the primary tumor the implanted nodules may regress (E. Fraenkel, Werder). Several factors seem to combine in this result. Chief among them is the low vitality of the transported cells which tend to atrophy. A chronic inflammatory process usually incloses the nodule in fibrin, granulation tissue, or multiplying endothelium, which cuts off the nutrition of the tumor-cells. The nodule becomes flat and fibrous, and finally disappears, leaving a slight scar. A constitutional element is probably at work, but the mechanism of the destruction is largely cellular.

In another group the implanted nodules maintain a low grade of nutrition or gradually extend over much of the peritoneum, causing adhesions and ascites and persisting until the death of the patient from cachexia or intercurrent disease. These cases survive in poor health until the peritoneum becomes so badly altered that the intestinal functions are gravely disturbed. The ascites appears to be a less important factor in the cachexia.

In a third group the tumor recurs after extirpation in the form of papillary nodules at the stump, in the wound, or on the peritoneum. Pfannenstiel finds that 77 per cent. of the papillary serous adenomas can be pronounced cured by operation after 5 years, most of the recurrences following intraligamentary growths. An unusually long period of observation is required, since recurrences have been observed at the site of the tumor or in the peritoneum after I3, I5, 20, and 2r years (Malcolm, Opitz, Pozzi, Holzapfel), and in the line of incision after 2I years (Olshausen). Even after recurrence the patient may survive many years in fair health (Schroeder).

Finally, the implantations may rapidly or gradually change their type and become malignant, spreading over the peritoneum, producing more bulky infiltrating local tumors and causing increasing cachexia. Definite metastases of papillary cystic type in diaphragmatic and retroperitoneal nodes, and in subcutaneous tissue are recorded.

The serous cystadenomas show with other cystic ovarian tumors a series of anatomical peculiarities and accidental complications. The pedicle of these tumors may become elongated or divided, or it may remain short and thick. The movable tumors even when benign often form adhesions to omentum, intestine, appendix, opposite ovary or bladder, and much of their nutrition may eventually come from such channels. Intraligamentary tumors, on the other hand, extend through the pelvic fascias about uterus, bladder, and rectum, which are displaced, compressed, or elongated. The Fallopian tube is usually stretched over the tumor, but in some instances the tube is quite movable. Elevation out of the pelvis is regularly accompanied by rotation on the pedicle, and in the further wandering of tumors with long pedicles various grades of torsion occur. Martin estimates that 6 per cent. of all ovarian tumors exhibit this condition. It occurs chiefly in relaxed abdomens and is especially frequent in pregnancy. The results are edema, hemorrhage into the tumor, peritoneum or uterus, sometimes fatal, or complete infarction leading to peritonitis or rupture. The symptoms vary with the intensity of the process, but in slow cases they take the form of cachexia. Suppuration in ovarian cysts is not infrequent. The microörganisms recovered include Bacillus coli, streptococcus, Bacillus typhosus, and the tubercle bacillus.

Papillary Serous Adenocarcinoma. - The clinical malignancy of many simple papillary adenomas becomes more pronounced in those tumors in which the structure reveals the histological signs of malignancy and which must, therefore, be classed as adenocarcinoma. A considerable proportion (50 per cent., Pfannenstiel) of papillary cystic tumors reveal adenocarcino- 
matous structure in at least some portions of the growth, but most of such tumors being encapsulated, are successfully removed. The typical malignant tumor is adenocarcinomatous throughout and its variations extend toward alveolar and diffuse carcinoma. There is little doubt that many of the former group represent malignant transformations of more typical adenomas. A similar transformation has repeatedly been observed in the recurrences of serous adenomas and in their peritoneal implantations. Yet the majority of adenomas long retain their benign type and there is some ground for the view that the malignant tumors are usually carcinomatous from the first. Gebhard takes exactly the opposite view, interpreting every papillary adenocarcinoma as a degenerated adenoma. He holds that it is never possible to determine even approximately their duration. Yet unless these tumors are exceptions to the rule, the transformation often occurs so early as to constitute a primary adenocarcinoma. The tendency of papillary adenomas in other regions to become carcinomatous, the occasional occurrence of adenoma in malignant form, the frequent mingling of benign and malignant areas in the same tumor, and the association of adenocarcinoma in one ovary with adenoma in the other, all indicate that ovarian adenomas are to some extent specially prone to malignant change.

The papillary adenocarcinoma is usually cystic, but the cysts are smaller and less numerous than in adenoma, the inverted tumor masses are larger, and much of the tumor is solid. The finely honeycombed texture of pseudomucinous adenocarcinoma is missing. Some malignant tumors are pedunculated and movable, like the adenomas, but a larger porportion are intraligamentary or bound by adhesions. The cyst contents are seldom clear, since degenerating epithelium is thrown off in large numbers, and small hemorrhages discolor the fluid brownish. The capsule is commonly perforated and papillary growths cover portions of the surface and invade the peritoneum. The tumors are frequently bilateral (66 per cent.), and the malignant structure may be more advanced on one side. Only a small proportion of these bilateral tumors can be referred to implantation.

In advanced stages metastases appear in the pelvic region and extend over the peritoneum and even through the diaphragm to the pleura, accompanied by serous effusions. These secondary carcinomatous growths are more a. gressive than the adenomatous, they do not regress, but invade the serosa, intestinal wall, diaphragm and liver, and produce firm adhesions or fistulous tracts. From the surface the peritoneal lymphatics may be extensively invaded. The intestinal functions are severely disturbed.

Intraligamentary growths less frequently produce ascites, but they invade the ligaments, pelvic tissues, uterus, vagina, rectum, and retroperitoneal and inguinal nodes, but distant metastases in the organs rarely occur. Virchow's observation of the excessive rarity of distant organ metastases in ovarian carcinoma applies especially to the adenocarcinomas.

The structure varies with the malignancy, but regularly presents a pronounced papillary form or at least its traces. A uniform alveolar, medullary, or tubular structure has not been traced to this origin, although in some solid portions the growth may be nearly diffuse. Multiplication of cell layers, cohesion of adjoining papillæ, filling of intermediate spaces and of pseudoalveoli, are the usual features of malignancy, but the adenocarcinomatous type is commonly preserved. Pfannenstiel, however, finds mixed forms in which there is solid medullary carcinoma without evidence of glandular structure. The cells may vary little from those of adenoma, but tend to increase in size, and to become irregular in form, while nuclear hyperchromatism is pronounced. Giant-cells are prominent in some tumors. Calcific 
granules forming about capillaries in the stroma or in epithelial masses may be a very prominent feature (psammocarcinoma). The formation of these granules is preceded by colloid degeneration.

The stroma forms branching vascular papillary strands; it may be invaded and split up by tumor alveoli, and in solid alveolar areas it may be reduced to a trace. Very cellular types of stroma occur, suggesting a neoplastic process in the connective tissue. These tumors grow with unusual rapidity, but the stroma always appears to be in a certain relation to the epithelium; it does not follow the metastases and its designation as sarcoma is hardly justified. Loeb, however, secured a pure sarcomatous tumor by successive transplants of such a growth in the rat. Ovarian remnants are rarely preserved in the stroma, but in one case I found the abundant septa containing many groups of large polyhedral cells resembling lutein-cells.

The course of ovarian papillary carcinoma, while slower than that of other varieties, is unfavorable, because of local recurrence after apparently

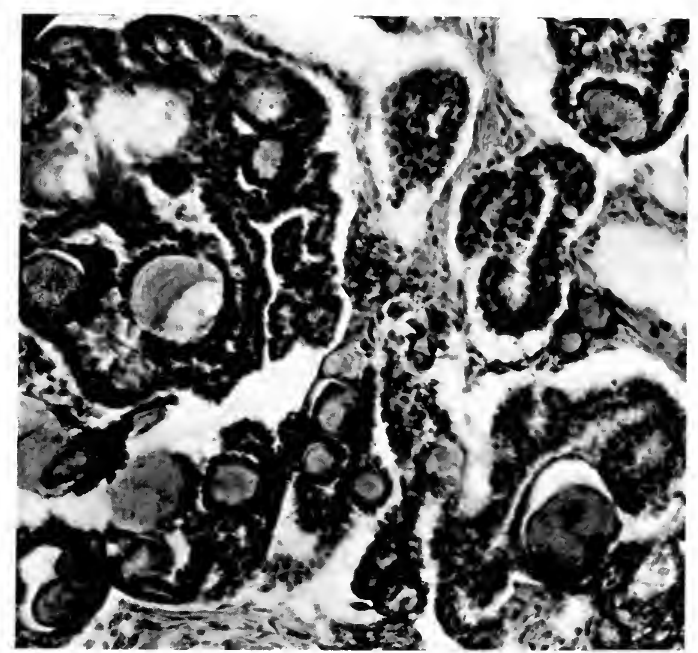

Fig. 268.-Adenocarcinoma of ovary with abundant calcific concretions. Psammoma.

complete extirpation, intraligamentary position, early peritoneal implantations, and later appearance of new tumors in the opposite ovary. Pfannenstiel estimates the recurrence of papillary carcinoma at 83.3 per cent., of other types of carcinoma at 66 per cent. These data are contrasted with those of papillary adenoma of which I6 per cent. recur. The glandular type recurs later, but more frequently than the papillary (95 per cent.). Observations on bilateral tumors vary greatly. Glockner's cases all recurred, while Hoffmeier found only 50 per cent. of recurrences; and there are not a few bilateral carcinomas of miscellaneous types, which permanently recovered (Pfannenstiel, Lit.). The removal of both ovaries is probably indicated, but permanent recovery without this more radical procedure is recorded by Hoffmeier, Glockner, and Tauffer.

Pseudomucinous Cystadenoma.-Typical pseudomucinous cystadenomas are distinctly different in gross appearance from the serous tumors. They are usually of larger dimensions, the walls are thin, a multilocular structure is pronounced and persistent, they are more frequently unilateral and pedun- 
culated and the contents are firm or soft gelatinous material or highly mucinous fluid. They form the majority of ovarian cystadenomas. Bilateral growths occur in 17.7 per cent., and intraligamentary in 9.65 per cent. (Pfannenstiel). The size of some of these tumors has been remarkable. Zacharias reports a case in which the tumor contents weighed $\mathrm{r}_{32} \mathrm{~kg}$. He has collected a series of these cases, some of which occurred in young subjects and most of which were fatal.

On section the cyst presents several roomy compartments with broad, thin partitions. As the cyst enlarges the partitions rupture, atrophic fragments and strands fall into the fluid, and the cyst is occupied by one large central and several peripheral cavities. The walls are usually thin, but certain areas may be rather compact with many small cysts, or solid portions may appear. Scanty low papillary nodules may usually be found on the

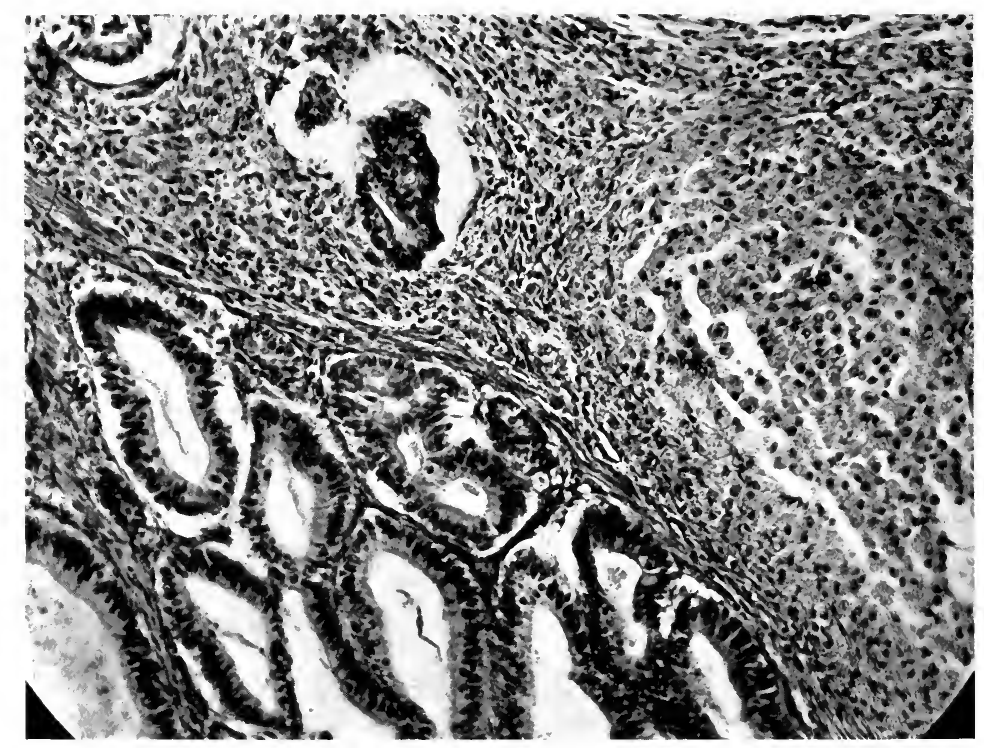

FIG. 269.-Adenocarcinoma of ovary with hyperplasia of interstitial cells.

inner surface, and in one group of cases these papillary projections are highly developed (papillary pseudomucinous cystadenoma). In most tumors the papillary tendency is not highly developed and the growths belong in the group of glandular cystadenoma. The pseudomucinous adenoma may show a very large number of small cysts, honeycombed areas, and exactly resemble stroma ovarii. In fact, Bauer holds that all cases of struma ovarii are pseudomucinous adenomas derived from superficial ovarian epithelium. Yet some are associated with other teratoid elements, and the colloid contains iodin (Pick, R. Meyer). The contents are first clear, thin gelatinous material, which may become condensed and firmer, or thinner and fluid. The addition of much cell detritus, fatty matter, cholesterin, serum, or blood alters the color.

The main ingredient is pseudomucin (Hammersten). This substance is soluble in water, precipitable by alcohol but not by acetic acid, and when treated with dilute 
mineral acid it yields a substance which reduces copper sulphate in alkaline solution. It is, therefore, a glycoproteid and on chemical analysis yields small proportions of hydrogen, nitrogen, and sulphur. Iodin is absent (Kretschmar, Bauer). The mucinous substance does not always occur in the form of typical pseudomucin. In certain cases, sometimes called pseudomyxoma ovarii, the material is much firmer, strongly alkaline, insoluble in water, but dissolved by strong alkali. This substance, Pfannenstiel's pseudomucin B, has a very low nitrogen content. A thin mucin, very soluble in water and with high nitrogen content, is designated by Pfannenstiel as pseudomucin G. Paramucin is a substance obtained from ovarian cysts by Mitjukoff, which reduces copper without boiling in acid. The pure pseudomucin occurs only in small cysts containing clear gelatinous material. It is usually mixed with albumin, producing the paralbumin which Eichardt wrongly supposed to be a single substance. In the demonstration of pseudomucin in albuminous mixture the fluid may be precipitated and washed in alcohol, treated on a water-bath for half an hour with Io per cent. $\mathrm{HCl}$, and after cooling, the albumin precipitated by phosphotungstic acid. The filtrate gives Fehling's reaction. True mucin may be separated by chemical means, but does not occur in ovarian tumors. To separate pseudomucin, a part of the material is extracted in water and another part in I per cent. $\mathrm{NaOH}$ for 2 days. The filtrates are then treated with increasing amounts of acetic acid. If no precipitate falls, mucin is absent. If present, the precipitated mucin is filtered off, and filtrate, heated with acid, is tested for pseudomucin by Fehling's test. Mucin appears not to occur in ovarian tumors. Pseudomucin, on the other hand, is not limited to ovarian cysts, but occurs under many other conditions (E. Herter, Lit.).

The structure presents narrow and slightly vascular septa or partitions, lined by one layer of high cylindrical cells, in which all stages of secretion are observed. The nuclei lie at the bases of the cells, the cell bodies are low, granular and opaque in the resting stage, high and clear in active secretion. Goblet cells may be abundant and occasionally the cells are ciliated. Pressure of firm secretion may cause flattening or complete atrophy of cells and septa. The production of mucus is a secretory function and does not usually entail the degeneration of the cell. In well-preserved areas of typical adenomas there is little desquamation of cells, but in more actively growing tumors the secretion is less abundant, many cells are exfoliated, the cells become atypical and granular, and the nuclei hyperchromatic. In such areas the cysts are small and epithelial papillæ become very numerous. By exaggeration of this process adenocarcinoma develops. A false appearance of malignancy may be produced by compression of many thin septa with low papillary outgrowths, and this appearance may be exaggerated by the collapse of any cystic tumor after aspiration.

The two main structural types, glandular and papillary, are designated by Gebhard as "cystadenoma evertens" and "cystadenoma invertens." No satisfactory explanation of their different growth tendencies is available. In the papillary type Waldeyer assumed a primary proliferation of the connective tissue. Gebhard and Pfannenstiel consider that the primary factor always resides in the epithelium. Gebhard thinks that the presence of secretion prevents the growth of papillæ in tensely filled cysts, while in other portions of the same tumor a papillary growth may be observed. It is possible that the conditions under which the tumor originates determine the structures. Thus in the alveolar strictly glandular tumor, described by Bauer, the tumor originated by downward growth of surface epithelium which early formed alveoli. The papillary tumors appear to develop by growth into preëxisting cysts or cavities.

The course of the pseudomucinous tumors is slow, and most of them being unilateral and pedunculated are successfully removed by operation ( 98 per cent., Pfannenstiel). When a papillary structure is pronounced the tumors are most often bilateral, more difficult to eradicate, implantation metastases may develop, and recovery follows extirpation in a smaller proportion, estimated by Frommel at 85 per cent. Strassmann, however, estimated the 
recoveries as low as 44 per cent. Fatalities result from recurrence of incompletely extirpated tumors, from later development of a tumor in the remaining ovary, from implantations in the peritoneum or abdominal incision, and from progressive pseudomyxomatous changes in the peritoneum. Ascites is relatively infrequent. The elongated pedicle of many of these tumors renders them especially susceptible to torsion, which occurred in $\mathbf{1 4 . 5}$ per cent. of Martin's cases. The recurrence usually reproduces the structure and follows the slow course of the original tumor. A period of many years ( $14, \mathrm{r}$ 7, Olshausen) may intervene before the recurrent tumor appears. Rarely

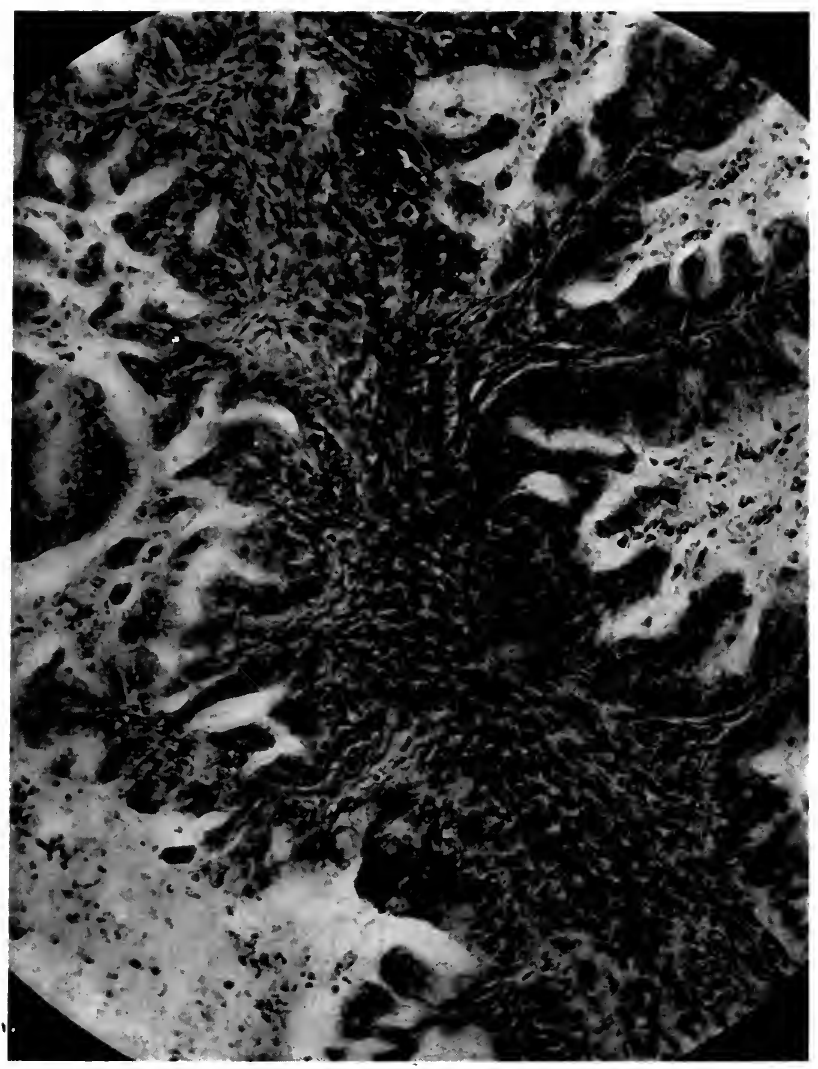

FIG. 270.-A type of malignant cystic adenocarcinoma of ovary.

the implantation or recurrence assumes a malignant carcinomatous form (Frank, Olshausen, Pfannenstiel). Recurrence in the opposite ovary several years after extirpation of the first tumor has been observed by Martin, Hofmeier, and others, and must be interpreted as a new primary tumor.

Owing to the thin walls of the cysts spontaneous or traumatic rupture not infrequently occurs, and the contents and cells are discharged into the peritoneum. When this event occurs at operation and the material is removed no ill effects follow. Spontaneous rupture is followed by prostration, fever, and a marked swelling of the abdomen. The further results depend 
much on the character of the tumor and its contents, but a condition regularly follows known as pseudomyxoma peritonei. The first effect is the passage of the pseudomucinous material into the peritoneal lymphatics. These spaces become distended and ruptured, the material infiltrates the meshes of the peritoneum, round-cell infiltration, growth of granulation tissue and proliferation of endothelium result, and the peritoneum is everywhere thickened by a painless form of chronic peritonitis (Werth, Gunzberger, Westphalen). - When many fragments from papillary tumors are carried along these may become implanted and produce small papillary outgrowths or small subserous cysts, but as a rule the organs are found embedded in gelatinous material without any sign of cellular invasion. The large amount of material produced in some cases has led to the assumption that a true myxomatous process becomes established in the peritoneum (Mennig, Wendeler), but this conception is negated by the complete recovery which sometimes follows. When the condition progresses steadily and spontaneous rupture has not occurred the chief factor must be found in a dissemination of tumor-cells throughout the peritoneum and this process has been fully described, especially with papillary tumors, by Olshausen, Ackermann, and Pfannenstiel. The passage of mucus apparently without tumor-cells in the diaphragm, along the portal canals, into the liver and into the appendix has been reported by Werth and Polano. The prognosis in this condition is unfavorable. Of 40 cases collected by Gunzberger, I7 were said to have recovered. Early cleansing of the peritoneum greatly favors recovery and the final absorption of mucus and tumor-cells. When firm adhesions form the material cannot be removed and a slowly fatal course is the rule. The peritoneum becomes thickened, adhesions and fistulous tracts form, and intestinal paralysis eventually follows. Occasionally a rapid course is observed with all the signs of a carcinomatous process.

Pseudomucinous Adenocarcinoma.-Malignant changes in previously benign pseudomucinous cystadenoma occur, as previously mentioned, under several conditions. (a) Implantations from a benign tumor develop in the stump, peritoneum, abdominal wall, or navel, and pursue a malignant course. (b) Pseudomyxoma peritonei may develop malignant neoplastic tendencies and structure. (c) In portions of benign cystadenomas the structure is malignant, but being encapsulated the tumor is completely extirpated. All of these events are comparatively rare.

In addition, there is a group $(d)$ of ovarian adenocarcinomas which represent malignant forms of the cystadenoma. Some of these evidently represent a malignant change in an originally benign cystadenoma. The history reveals the long existence of an ovarian tumor which on examination proves to be in whole or in part carcinomatous. Gebhard points out that the long duration of these tumors eliminates the probability that they were malignant from the first. Others develop more rapidly, present a uniform adenocarcinomatous structure, and are probably malignant from their inception.

The adenocarcinomas of these types still contain cysts, but large portions of the tumor are solid and present the opaque cellular texture of carcinoma. The cysts represent natural cavities produced in the growth of the tumor and spaces of more ragged outline formed by degeneration and necrosis of central areas. Small hemorrhages frequently occur. The mucus is greatly reduced in amount, but still persists in the small alveoli and spaces of the tumor. It is seldom clear, but more often discolored by cell detritus and blood. The capsule is often perforated, adhesions form, implantations develop and metastases occur in pelvic, retroperitoneal, or inguinal nodes.

The structure is usually of the glandular type with large and small alveoli 
lined by convoluted multiple rows of cells and filled with mucus or occupied by numerous secondary alveoli. A diffuse growth of cells is unusual, the most active areas retaining the structure of malignant adenoma. The cells vary widely from the clear columnar type of simple adenoma. They increase in number and height, lose most of the features of mucus secretion, and become granular and opaque, while the nuclei are very large and hyperchromatic.

Solid Ovarian Adenoma.-Many cystic pseudomucinous adenomas present solid areas and when such areas predominate over the cysts the commonest form of solid adenoma is produced. Stratz estimates that 3 per cent. of all epithelial ovarian tumors are solid adenomas. Usually the solid tumor

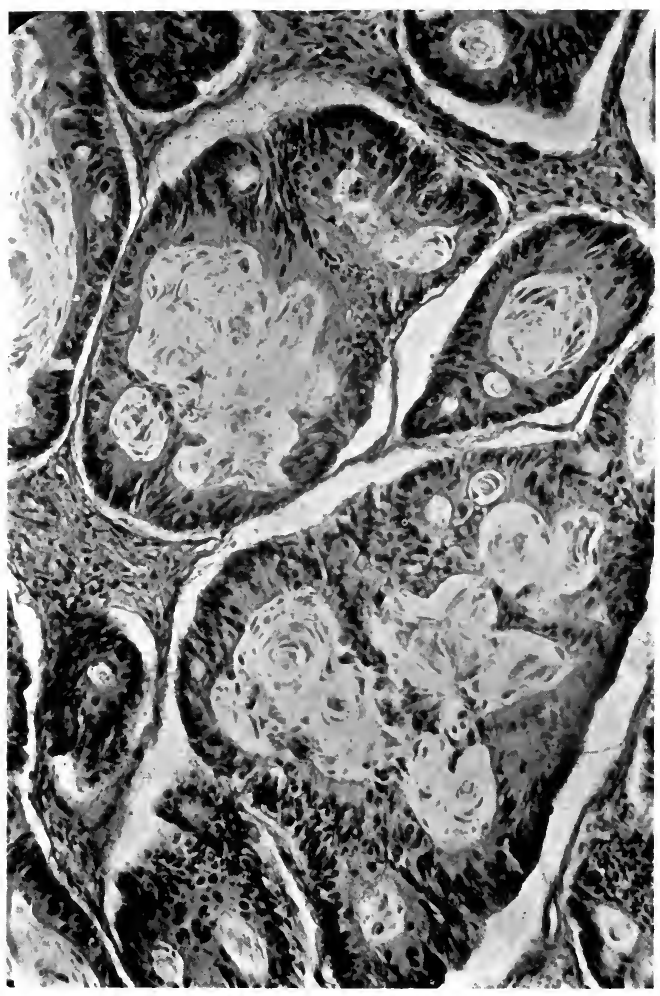

Fig. 271.-A type of artenocarcinoma of ovary.

presents on close inspection a finely honeycombed texture and this tumor may be designated as the parvilocular ovarian adenoma. It is a pseudomucinous tumor, the small spaces being filled with firm mucus or with mucus and cell detritus. In accordance with its solid character it is far more cellular than the cystic growth and the epithelium is usually atypical, granular, and overnourished. Many transitional cases connect this adenoma with solid and with cystic adenocarcinoma. Yet the typical solid adenoma reaches a large size without exhibiting malignant characters. Ascites is common. Pfannenstiel described 3 cases in which large partly cystic tumors of the above type were successfully removed and the patient remained well. 
In a case studied in this laboratory the structure approached adenocarcinoma. Glockner describes a large, solid pure adenoma without cystic tendency, the convoluted alveoli being lined by cylindrical epithelium and containing little mucus.

The superficial or everted papillary adenoma forms a distinct variety of solid tumor. Including the small and early forms this tumor is relatively frequent. Of the more advanced and bulky growths J. W. Williams has collected $\mathrm{I}_{5}$ cases. One or both ovaries present numerous bulky compact papillary outgrowths which spring from a central core, the altered or disintegrated ovary. The appearance in the advanced stages is extremely characteristic. Ascites is common and implantation metastases occur early, but the essentially benign character is attested by the successful extirpation recorded in most cases. An origin from superficial ovarian epithelium is clearly indicated. Gebhard suggests that an early rupture of a papillary cystic tumor permits the entire growth to become everted, but some very early papillomas are superficial from the first. Local inflammatory processes have apparently contributed to the inception of the tumor process in many cases.

Adenofibroma.-Epithelial alveoli are occasionally observed in ovarian fibromas and rarely these epithelial structures increase to such an extent as to constitute an adenofibroma. Orthmann describes carcinomatous changes in such a tumor. Cystic dilatation of the alveoli may occur, and Orthmann describes carcinomatous changes in such cysts.

Adenofibroma colloides designates a rare form of this tumor in which gelatinous changes are prominent. Amann described a bulky tumor covered with warty excrescences and containing some small cysts and many alveoli lined by high cylindrical cells and filled with mucus. Orthmann found a small tubal metastasis from a bilateral tumor of this same structure. Another form of superficial fibroma with carcinomatous infiltration by cubical or flat cells is interpreted by Orthmann as an invasion from the superficial ovarian epithelium. In all these tumors the stroma greatly exceeds the cellular portions.

Carcinoma.-Waldeyer classified ovarian carcinomas according to the proportions of cells and stroma as simple (alveolar), medullary, and scirrhous. Gebhard found it more serviceable to distinguish between the malignant forms of cystadenoma and genuine solid carcinomas of other types, thus introducing the histogenetic principle. Due consideration of the histology, histogenesis, and specific anatomical and clinical characters requires the recognition of several distinct groups of these tumors as follows: Cystic adenocarcinoma; solid carcinoma; carcinoma mucocellulare, Krukenberg; carcinoma folliculoides; ovulogenic carcinoma.

There are also rare forms of carcinoma which cannot be grouped with any of the above classes, as squamous-cell carcinoma, malignant forms of adenofibroma and melanoma. There is also considerable support for Rokitansky's belief that certain carcinomas develop from lutein cells.

I. Cystic adenocarcinoma has been discussed with the cystic adenomas with which it is identical in origin and very similar in course.

2. Solid Carcinoma.-In this class may be conveniently grouped all the tumors of large or small alveolar or medullary structure which are not connected with the cystic adenocarcinomas, and which do not exhibit the specific structure of Krukenberg's tumor nor of carcinoma folliculoides. That some of the small alveolar carcinomas are atypical examples of cystic adenocarcinoma is possible, since the alveoli may contain cylindrical cells, as in Glockner's cases. Nevertheless their gross appearance and microscopical structure 
are very different from malignant cystic adenocarcinoma, of which even the solid forms regularly maintain an adenocarcinomatous structure. The medullary carcinomas with ova-like cells present a somewhat distinctive subvariety, but until their histogenesis is much clearer than can now be claimed a separate classification seems hardly warranted. Until a more thorough understanding of the many forms of ovarian carcinoma is attained no classification can hope to escape revision.

The alveolar and medullary carcinomas produce as a rule solid tumors maintaining the form of the ovary in the early stages, but eventually becoming

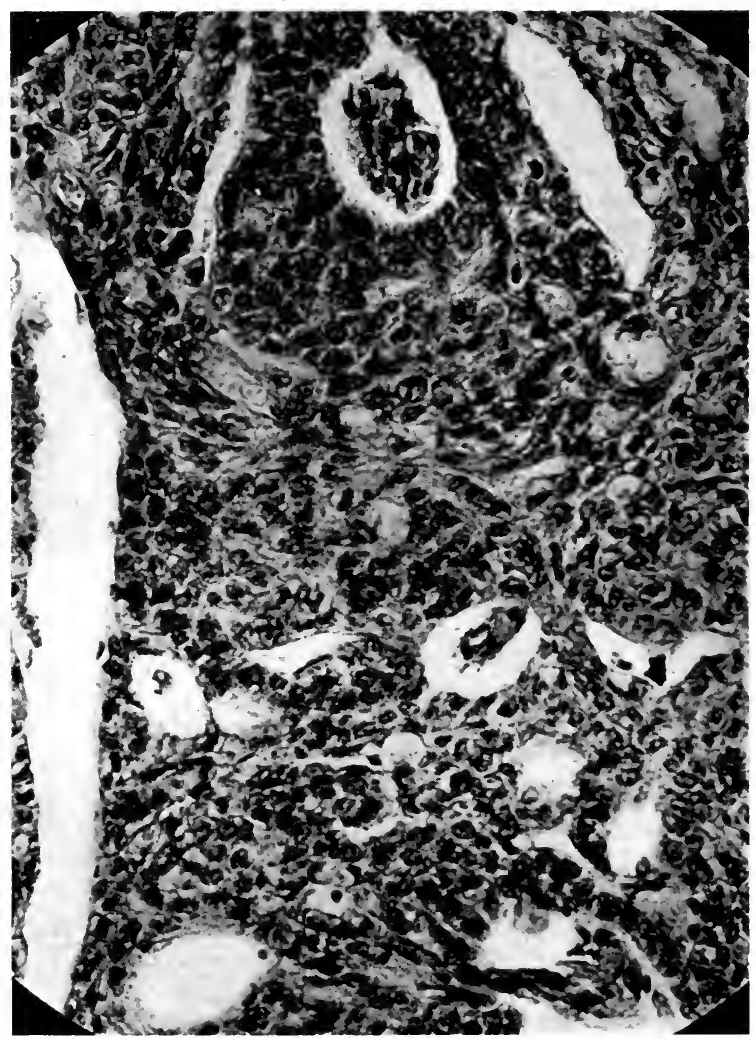

FIG. 272.- -Solid adenocarcinoma of ovary.

irregular and lobulated. On section many are cystic, but the cysts are chiefly the result of necrosis and liquefaction and not of dilatation of alveoli. The tumors reach considerable and even large dimensions, but rarely beyond I 5 to $20 \mathrm{~cm}$. in diameter. They are at first usually unilateral, but metastasis and recurrence in the other ovary are very frequent. If pedunculated they become bound to the pelvic tissues by adhesions and are held by a broad short pedicle composed of the ovarian and broad ligaments. Many occupy the pelvis in a retroligamentary position (Winter). The consistence is firm or soft in accordance with the cellular character. On section they exhibit 
an opaque uniform texture broken by many lobules, small cysts, areas of necrosis and hemorrhage, and foci of gelatinous degeneration.

The structure presents a confusion variety of types. Small alveolar carcinoma presents many small groups of granular polyhedral cells without

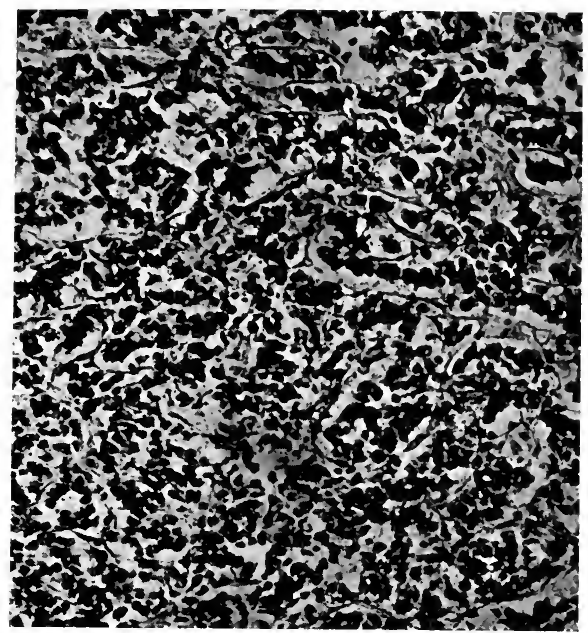

FIG. 273. - Structure of a widely metastasizing small alveolar bilateral carcinoma of ovary.

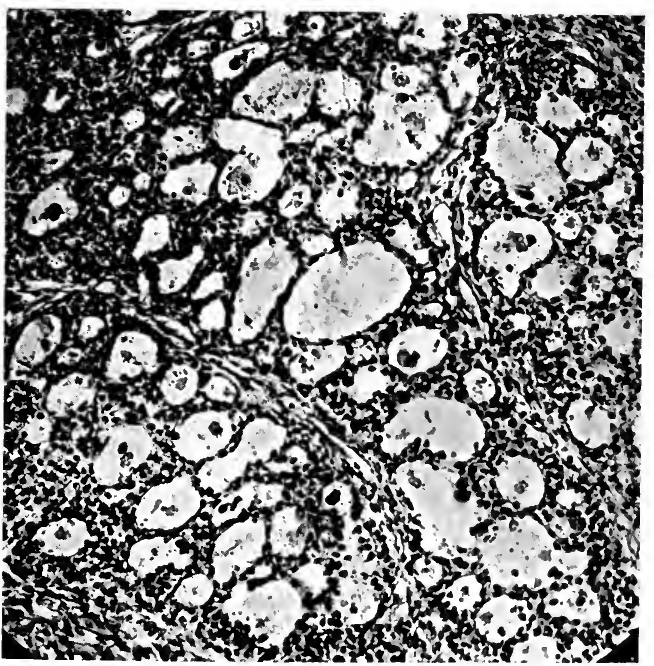

FIG. 274.-Carcinoma of ovary with ova-like cells.

definite lumen, supported by scanty cellular stroma. It is characteristic of this group of tumors that the outlines of alveoli may be lost and the cells not only grow diffusely, but become more difficult to distinguish from stromacells, suggesting myxoma, sarcoma, or a mixed tumor. This tendency toward 
diffuse growth and mucoid degeneration of cells and stroma is observed in many ovarian carcinomas and appears to reach its height in Krukenberg's tumor. Careful study of such growths usually reveals definite alveolar areas and supports Heinrich's conclusion that no true mixed carcinoma and sarcoma of the ovary exists outside the ovulogenic group. Glockner, however, describes a rapidly growing mixed tumor in a girl of I9 years, which resembled the parotid cylindroma and for which he suggested an origin from the Wolffian body. I have studied tumors of this general type and concluded that some belong with the medullary carcinomas with ova-like cells. Others may very well be true mixed tumors originating from the complex alien tissue masses described by Walthard.

The typical small alveolar carcinoma presents well-defined alveoli. The early stages are probably represented by v. Kahlden's case, and characteristic examples are described by Glockner, Orthmann, Heinrichs, and others.

In the medullary type the cell columns are large, broad, and freely anastomosing. The cells are large or small, polyhedral or rounded, opaque, and granular. Scattered at notably regular intervals may appear large clear ova-like cells, the significance of which has been much discussed. These cells are large, spheroidal, with clear cytoplasm, well-marked membrane, and one or more vesicular nuclei. They may be surrounded by a radiating circle of tumor-cells (Schroeder). They may resemble ova in every detail (Gebhard) and they have been so interpreted by Emanuel, Kworostansky, Schroeder, and others. Acconci found them in a cystadenoma. Somewhat similar structures may be produced by cross-sections of strands of mucinous stroma, but the cells in question are spheroidal. Very similar cells may undoubtedly result from mucous degeneration and this origin of all of them is accepted by the majority of observers. Gebhard, however, will not deny the possibility that they represent primordial ova, but the more recent embryological data do not favor such a possibility (cf. Ingier). Ova-like cells appear in the epithelial layer of primordial follicles, although these cells are not derived from germ epithelium (W. Nagel).

In a very cellular tumor which probably belongs in this group I found numerous small pearls composed of flattened cells. Since most of the tumor was composed of granular polyhedral cells, the squamous characters were metaplastic. Hansemann has described a squamous epithelial tumor which he referred to metaplasia of ovarian epithelium.

In a well-defined group the cells are usually small, and grow in indistinct alveoli or groups which vary greatly in size. Such tumors may present areas of diffuse growth and when the cells become intimately mingled with the stroma or appear in rows between stroma fibrils or arranged about bloodvessels they suggest an endothelial origin. Not a few so-called endotheliomas of the ovary are probably of this nature (cf. Pfannenstiel, Figs. I6I, I63).

In another group the cell masses are large, circular on section, and composed of large polyhedral, clear, or slightly granular cells. These tumors have often been described as endothelioma. Yet this structure is occasionally seen in distinctly alveolar and epithelial tumors and there is no necessity of assuming an endothelial origin. Polano divides the pseudo-endotheliomas among (I) alveolar carcinoma, (2) adenocarcinoma, and (3) sarcoma. To these may be added the peritheliomatous structure which is often assumed by epithelial tumors.

In young subjects there occurs a form of solid ovarian carcinoma which is bilateral, of rapid growth, producing wide-spread local invasions, and numerous bulky metastases, while presenting a structure of diffuse growth of small rounded cells resembling lymphosarcoma. Stone has described such a case 
in which a large metastasis in the breast first attracted attention, and the ovarian tumors were overlooked. In the ovary the supposed sarcomatous

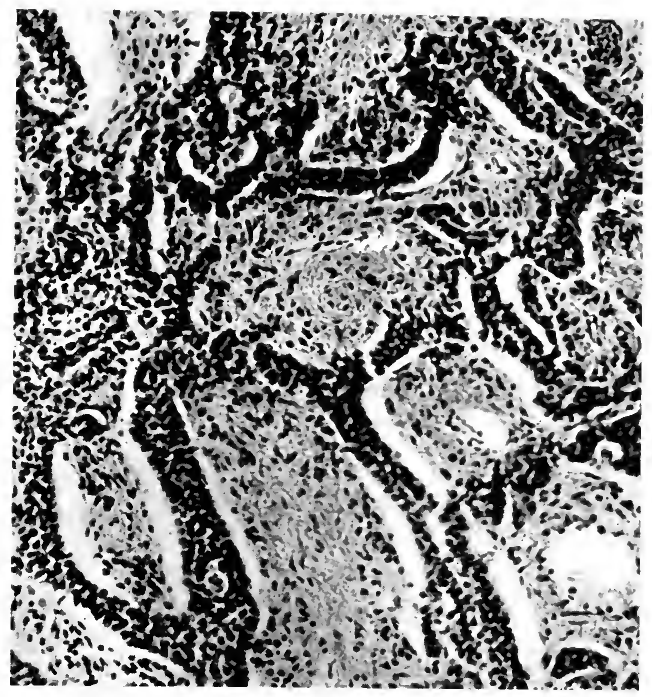

FIG. 275.-Structure of a peculiar carcinoma of ovary, presenting anastomosing cords of small polyhedral cells.

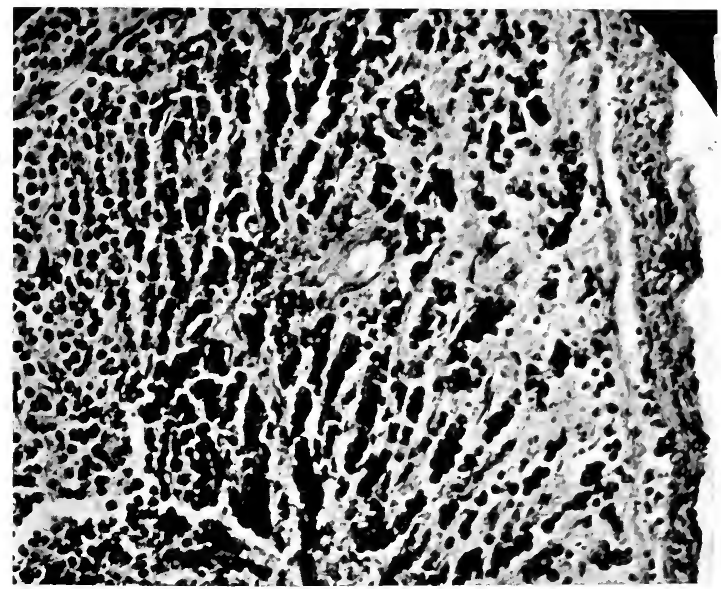

FIG. 276.- Structure of a highly malignant small cell carcinoma of both ovaries in a young subject. There were metastases in breast and other organs, in which the structure suggested lymphosarcoma.

growth revealed its primary alveolar structure. I have seen other very similar cases.

A distinctly scirrhous carcinoma of the ovary is rare and is observed chiefly in elderly subjects. 
Carcinoma develops in fibromas either from the downward growth of surface epithelium (Orthmann), or from the proliferation of glands in a fibroadenoma. In either case the connective-tissue areas are predominant and the tumor usually presents the gross appearance of the original fibroma.

Folliculoma malignum ovarii is a term employed by S. Gottschalk in describing a peculiar small alveolar carcinoma of the ovary. Several apparently related but structurally somewhat different tumors have been grouped under this term, and while their origin and significance are not clear, the tumors are peculiar in many respects and deserve separate consideration. Their chief characters are a comparatively small size, honeycombed texture, small alveolar structure, embryonal cell character, and slight malignancy. Metastases are absent. In Ingier's cases the tumors were bilateral. Ascites may occur. In Gottschalk's case the tumor, Io $\times 8 \times 3^{\frac{1}{2}} \mathrm{~cm}$., maintained the form of the ovary, presented numerous small and minute cysts, interstitial hemorrhages, and a tumor process affecting chiefly the ovarian cortex. The structure resembled struma ovarii and presented many small alveoli

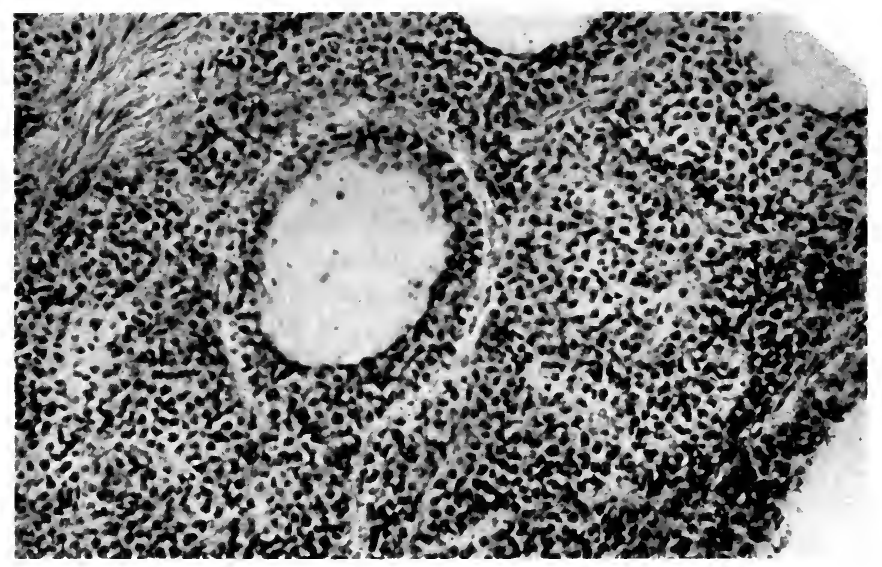

FIG. 277.- Structure of granulosa cell carcinoma of ovary. (After Werdt, Z. B., 59.)

lined by small cubical epithelium with prominent nuclei and surrounded by many small groups of cells. The small cysts contained mucinous material. Very similar tumors have been described by M. Voight, Lonnberg, and Ingier. Other cases sometimes included in this group seem to belong rather with the medullary carcinomas with ova-like cells (Mengershausen, Schroeder). The presence of ova-like cells led some to assume an origin from primary follicles. Voight derived his tumor from downgrowth of superficial ovarian epithelium. L. Pick regarded Gottschalk's case as struma ovarii. Ingier attacks the whole group because the follicular origin is not demonstrable. Yet Bauer has shown that a form of struma ovarii may develop from the superficial ovarial epithelium, and since the early stages of his tumor were practically identical with Gottschalk's, one is led to believe that folliculoma malignum is a form of ovarian tumor in which the alveoli resemble thyroid structures, while the secretion of mucus is scanty. These tumors must, therefore, be distinguished from very similar growths of teratoid origin. In the latter group other teratoid elements are usually demonstrable and iodin may be present in traces. Among the tumors which produce multiple small cysts 
in an enlarged ovary are the granulosa cell carcinoma of Werdt, and the oöphoroma folliculare of Brenner.

The term folliculoma malignum seems applicable also to a group of tumors resembling in many respects those described by Voight and Ingier, of which I have studied four examples. In the gross these tumors are of moderate size, preserve the form of the ovary, and present a finely honeycomb or solid texture. In structure they are glandular adenocarcinomas and carcinomas. The alveoli are small and compact, or larger with a free lumen. The cells are unusually large and polygonal. The cytoplasm is clear and the borders very distinct. The stroma is abundant and consists of hyperplastic ovarian tissue. An origin from undeveloped follicles is indicated by a diffuse neoplastic overgrowth of the lining cells of the small cortical follicles.

Carcinomatous tumors of this class present an alveolar, diffuse, or scirrhous structure. The cells are very large, giant-cells are abundant, and the cyto-

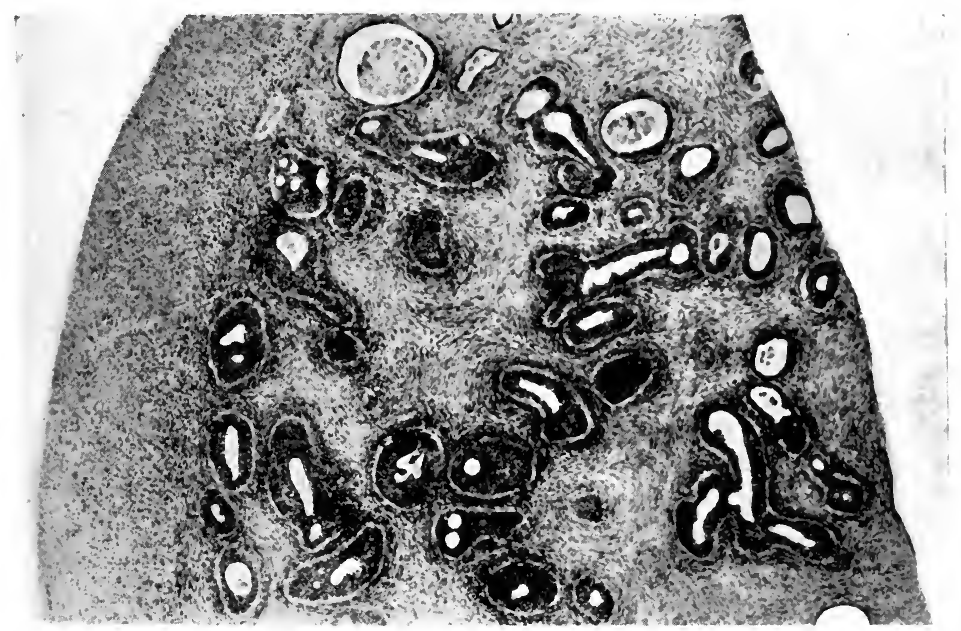

FIG. 278.-Oöphoroma folliculare. A rare ovarian tumor arising from multiple Graaffian follicles. (Brenner.)

plasm is clear or granular. The structure differs in these particulars from other forms of ovarian carcinoma.

Fibrosarcoma Mucocellulare Carcinomatodes (Krunkenberg).--Under this term Krunkenberg in 1896 described a peculiar malignant tumor of the ovary, usually bilateral, of considerable dimensions, maintaining the form of the ovary, of myxomatous appearance, occurring in young or old subjects, growing slowly, usually with ascites, sometimes chylous, and eventually fatal by extension and recurrence. The structure presented small groups or a diffuse growth of large polyhedral or rounded cells with mucoid contents compressing the nucleus into a signet ring form. In the diffuse areas the cells were intimately mingled with the stroma so as to suggest an origin from stroma cells or endothelium. Krunkenberg's first case presented a large cyst with carcinomatous wall in one side, a solid tumor on the other, while there was extensive invasion of lymphatics of abdomen, stomach, pleura, and bronchial and axillary nodes, a condition not commonly observed in other ovarian carcinomas. Krunkenberg failed to point out the close resem- 
blance of his tumors to secondary carcinoma of the ovary and he was unable to determine from which ovarian element the tumors arose. These deficiencies were soon supplied by Wagner, Romer, Schlagenhaufer, and Glockner, with the demonstration that this peculiar tumor may be exactly reproduced by secondary carcinoma from stomach and other abdominal organs, and that the great majority of such growths are secondary to tumors in other organs.

It is still generally admitted that there are primary tumors of the ovary of the Krunkenberg type, and cases reported by Glockner, Schenk, and Rosthorn are regarded as examples. It is obvious that cases without autopsy, as that of Outerbridge, are unavailable in the discussion. In Glockner's case both ovaries were much enlarged and extensions from one had eroded the spinal column. The entire tumor had a myxomatous texture and there were liquefaction cysts. One portion presented diffuse myxomatous tissue, while in another the structure was distinctly alveolar. Schenk described

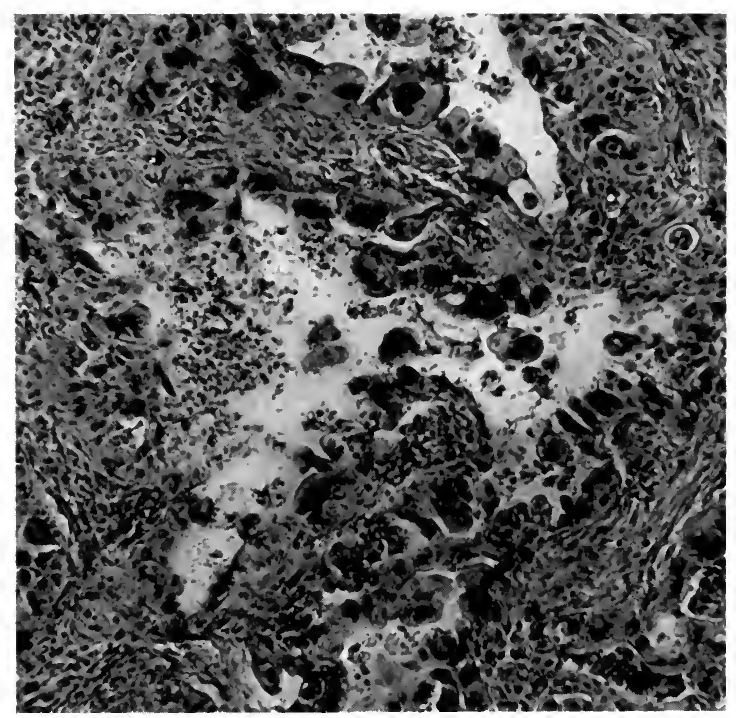

FIG. 279.-Follicular carcinoma of ovary.

bilateral tumors in a subject 27 years of age, one weighing $2300 \mathrm{gm}$. and presenting the typical structure.

Although Marchand and Chiari were unable to find any primary tumors in the cases of Krunkenberg and Schenk, and doubtless no such tumors were demonstrable, it still seems uncertain that the typical structure of secondary mucoid carcinomas can be exactly duplicated by primary carcinoma of the ovary. It is not always possible to demonstrate the primary focus in cases of wide-spread carcinoma of the abdomen with involvement of the ovary, although the structure is not always that of Krunkenberg's tumor.

Glockner states that the typical structure may be seen in portions of alveolar carcinoma of the ovary, but the typical cases show little or no alveolar structure. I have studied 5 secondary ovarian tumors of this type and I primary carcinoma with myxomatoid areas. The structures while similar were far from identical, the primary tumor containing distinctly alveolar areas. None of these tumors gave evidence that the stroma reaction was 
neoplastic or sarcomatous, or that the large cells were derived from the stroma. I am inclined to believe, therefore, that the pure Krunkenberg tumor is always secondary, and that primary carcinomas presenting this structure regularly yield areas of a different type.

The clinical course of ovarian carcinoma varies greatly. The clinical features of the adenocarcinomas have been considered under the cystic tumors. The alveolar and medullary carcinomas are uniformly malignant, but statistical reports of this group are wanting. The majority of these tumors are originally bilateral or soon become so. When pedunculated they may remain movable for some time, even after general metastases have occurred. After extirpation local recurrence in the stump or in the opposite ovary is common. Peritoneal metastases are less common than with cystic adenocarcinoma, but through the lymph- and blood-vessels there may be general dissemina-

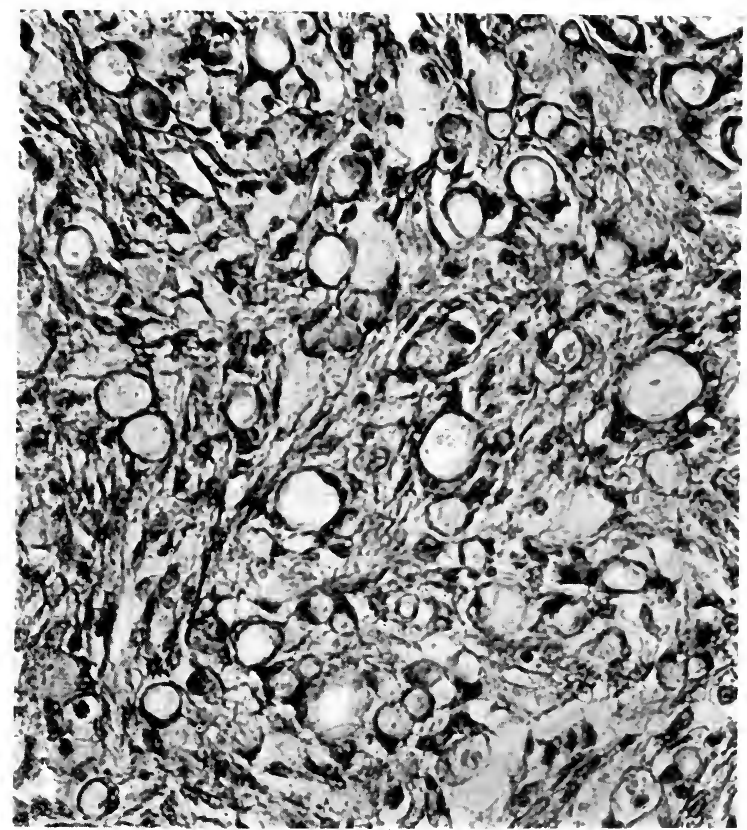

FIG. 280.-Structure of a Krunkenberg tumor of ovary, secondary to gastric carcinoma.

tion in the early stages of growth. In such cases the general condition of the patient may long remain remarkably good, but cachexia eventually supervenes. That the prognosis in this group of tumors is not wholly unfavorable is shown by Glockner's report of 5 survivals out of $\mathrm{I} S$ cases after 3 years. Yet some of his tumors were adenocarcinomas.

In Martin's table of $4 \mathrm{I}$ cases of various types only one solid carcinoma failed to prove fatal.

The Krunkenberg tumors, both primary and secondary, are uniformly fatal. Tumors of the type of carcinoma folliculoides are of slow growth and offer a better prognosis, a fact which commends the retention of this tumor as a special form of ovarian carcinoma. The so-called endotheliomas of the 
ovary are not distinguished from carcinomas by any clinical peculiarities, but are usually malignant (Apelt, Pfannenstiel).

General Etiology.- In view of the remarkable embryological history, the complex structure, and the series of functional changes which the organ suffers, tumors of the ovary are not notably frequent. Dippert refers to 58,787 female clinic patients of all types among whom were 94I ovarian tumors, I.6 per cent. Gurlt collected 947 ovarian tumors, 8.5 per cent., among I I, I 40 women with tumors of all types.

Hereditary influence is suggested only in isolated instances such as are mentioned by J. Simpson, Lohlein, and others. Trauma is a negligible factor, owing to the protected position of the organs. Inflammatory changes are held to influence the development only of the fibromas and simple cysts (Pfannenstiel).

Single women, furnishing 18 per cent. of ovarian tumors, are relatively more susceptible than the married, especially for dermoids ( 32 per cent.) and parovarian cysts (26.19 per cent.) (Olshausen, J. Williams, Lippert, Wedekind). Childbirth seemed to entail less liability in Martin's 1005 cases, of which 554 were nulliparæ. Yet Lippert found that 73.6 per cent. of his 638 cases had borne children.

Age.-All ages are susceptible, from birth to senility, but the period of sexual activity, 25 to 35 years, is most prominent. A fetal cystadenoma and sarcoma are described by Doran, and a bilateral sarcoma by Lonnberg. Wiel collected 24 cases under 5 years and 60 under ro years of age, and Hubert I 75 cases in children. The predominance of cysts, dermoids and sarcomas, and of malignant tumors in early life is notable. Kelly emphasizes the high operative mortality in children in a collection of 55 cystic, 47 dermoid, and 24 solid tumors. Many cases, chiefly cystic, occur also after 70 years. Most of the carcinomas occur between 40 and 50 years (Martin).

The relative frequency of the different forms of tumors I have calculated from the reports of Martin and Libbert, over 200 cases, as follows: Cystadenoma, 55 per cent.; carcinoma, 22 per cent.; embryoma, 9.2 per cent.; parovarial, 8.4 per cent.; sarcoma, 2.9 per cent.; fibroma, 2.5 per cent. Pick emphasizes the relative frequency of ovarian tumors in pseudohermaphrodites.

From the above data it appears that the effective causes of ovarian tumors are quite as obscure as with other organs. Neither predisposing nor exciting factors can be accurately defined, but it may be assumed that chronic hyperemia exaggerating the natural regenerative processes in the organ figures in most cases. The studies of histogenesis, on the other hand, indicate that developmental anomalies are essential conditions in the growth of the great majority of ovarian tumors.

Histogenesis.- Virchow and the older pathologists held that the Graafian iollicle produces only simple cysts and that some other source must be sought for the cystic tumors. Waldeyer (Eierstock u., Ei, I870) traced the origin of the tumors to Pflüger's tubes, which he held were either fetal remnants or newly formed in later life by the ingrowth of superficial ovarial epithelium. Olshausen contrasted the papillary serous tumors with the pseudomucinous and chiefly because of the prominence of ciliated cells in the former he derived them from the Wolffian medullary cords of Kolliker. This view he later abandoned, but it long received support from Fischel, Coblentz, Doran, and others. Marchand (I878) studied an ovary the cortex of which was filled with minute cysts containing papillæ of ciliated epithelium, and he thus showed that the presence of ciliated cells did not require an origin from medullary Wolffian tubes. He was inclined to refer them to included portions of tubal epithelium or to Graafian follicles. 
In a similar cystic ovary Malassez and Sinety traced the origin of these small cysts to the superficial ovarian epithelium, finding the cysts often connected by cords of cells with the surface. Ciliated columnar epithelium they found on the surface in the closed tubes (Pflüger's) and in the cysts. Flaischlen also traced the downward growth of surface epithelium forming tubes which developed into cysts with ciliated cells, in the intact portions of a cystomatous ovary.

Elaborate confirmation of these observations has been furnished by Walthard who in serial sections of many ovaries of all ages demonstrated the downward growth of surface epithelium forming cords, tubes, and cysts lined by cubical, columnar, ciliated, and even flat epithelium. He describes the formation of granulosa cells of the primitive follicles by downgrowths of surface epithelium, chiefly in the fetus and during the first year of life. A somewhat similar downgrowth occurs up to old age, but without relation to follicles, and these cell groups may produce small cysts. Several varieties of ciliated cell groups are found in the cortex and connected with surface epithelium. Most of these undergo atrophy, but others, in which there is a row of supporting cells and the stroma is very cellular, go on to develop cystomas of various types. Larger areas of this type with cellular stroma he regarded as misplaced tubal mucosa. All the tumor-bearing areas give the impression of alien tissue. Mesonephric (Wolffian) rests are commonly found in the hilus and penetrate the zona vasculosa, rarely the zona parenchymatosa (v. Franque), in the form of compact groups of cubical cylindrical or ciliated cells surrounded by connective tissue and smooth muscle. They appear not to develop beyond small cysts or minute cystic adenomas.

Many of the structures observed by Walthard had been described by Pflüger and Kolliker, both of whom demonstrated a postembryonal and periodically recurring formation of ovarial tubes. It appears from Walthard's study that the probable sources of tumors are not to be identified with Pflüger's tubes, which are connected with the formation of granulosa cells, but with congenitally misplaced groups of goblet, ciliated, or squamous cells with which a portion of cellular stroma is associated. It should be noted that while these alien tissue masses exhibit many of the elements regarded as essential to tumor growth, the actual development of the tumors from these sources, although highly probable, is still unproved.

The superficial ovarian epithelium has been clearly shown to give origin to the superficial papillomas of the ovary (Malassez, de Sinety, Flaischlen, Pick). Recently Bauer has shown that pseudostruma ovarii is derived by downward growth of surface epithelium. Since the papillary serous cystoma is identical in structure with the papilloma, and since downward growth of this epithelium is commonly seen at various ages there is considerable ground for assuming that this form of cystoma is derived from embryonal or adult surface epithelium. When one considers that these same cells may secrete much mucus and assume the goblet form (Walthard), the same origin is indicated for the pseudomucinous tumors. The direct transformation of these cells into alveolar or solid carcinoma is described by Glockner, Linnell, and Bauer.

The follicular origin of ovarian cystomas has long been a popular theory, but still lacks definite proof. Wendeler erroneously assumes the embryonic origin of the granulosa cells from the connective tissue of the Wolffian body, which might eliminate them as sources of epithelial tumors. That the superficial ovarian epithelium as well as that of the follicles may be ciliated has been shown by v. Velits, who also found an ill-defined ovum in such a follicle. Lateral sprouts from primitive follicles have also been observed by Steffeck, 
Hoffmeier, and v. Franque, but these may represent the granulosa cords of Walthard, which tend to atrophy. Low and even branching papillary outgrowths into follicles have been described by W. Williams, but it is not clear that these slight grades of proliferation go on to produce definite tumors. They are seen in simple follicular cysts. v. Müller and Pozzi traced ovarian carcinomas to altered follicles in which they identified ova. Yet it is doubtful if the identity of these structures was established.

The presence of ova-like cells in early cystoma does not prove their follicular origin, since these cells are probably not true ova and the granulosa cells are not sex cells or capable of producing such. Pfannenstiel holds the frequent association of cystoma with dermoids as an argument in favor of the follicular origin of the cystoma.

It is commonly assumed that ovarian tumors arise from a narrowly defined tissue segment or single follicle. Yet the early stages of many tumors

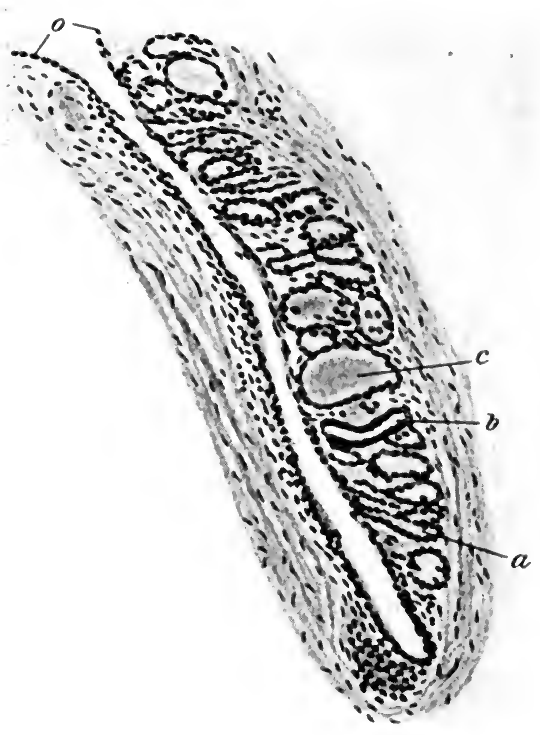

FIG. 28I.-Development of pseudostruma ovarii from superficial epithelium. (After Bauer, Z.G.G., 75.) suggest that considerable areas of superficial epithelium or many follicles are successively involved in the origin of the growth. This mode of origin has been proved, for the pseudostruma of Bauer is strongly indicated by those tumors which preserve the form of the ovary, and by those which present multiple cysts throughout the cortex, and is suggested by the hyperplasia in remaining follicles observed in many cases of cystadenoma.

The Wolffian remnants in hilus and body have been urged as the source of pseudomucinous and serous tumors by Rokitansky, Kossmann, Nagel, and others. The intraligamentary position accords with this origin, and Walthard found one small adenoma in a mesonephric tubule.

The occasional presence of smooth muscle-cells, the association with struma ovarii or dermoids, and the striking resemblance of the columnar and mucous cells to intestinal epithelium led Ribbert to propose a teratoid origin for pseudomucinous cystoma. Misplaced portions of tubal mucosa or of accessory Fallopian tubes have been held by Marchard and by Kossmann as sources of papillary tumors with ciliated cells. Walthard identified some of his alien tissue masses as tubal remnants.

It thus appears that moderate proliferation of epithelium has been observed in primitive and adult follicles, in the superficial epithelium and its various invaginations in embryonal and adult life, and in the Wolffian tubules of the hilus, but that only the surface epithelium has been fully traced into tumor growth. That the proliferating cells are not the normal surface epithelium, but are misplaced and embryonal is strongly suggested by many features of ovarian tumors. Wendeler would combine the views of March- 
and, Walthard, Kossmann, and others by assuming that the originating cells are misplaced Mullerian epithelium, which in its upper segments goes to form the Fallopian tube. This theory would seem consistent with most of the observed facts and may for the present be accepted. That ovarian tumors may also arise from other structures is not thereby excluded. It is highly probable that tumors of adult type are derived from adult epithelium. The ovulogenic origin of certain atypical adult and embryonal carcinomas, sarcomas, and mixed tumors should be entertained for the ovary as for the testis. The search for elements demonstrating the tridermal composition has not been pursued as actively in ovarian tumors as in testicular growths, but there appears to be no reason why the one-sided development of teratomas should be less frequent in one organ than in the other. It is especially in tumors of bizarre and varying structure, which do not fall distinctly in the recognized classes of intrinsic ovarian neoplasms, that the ovulogenic origin should be considered.

Tumors of the Parovarium (Epoöphoron).- The parovarium is the remnant of the sexual portion of the Wolffian body. It lies in the membrane connecting ovary and tube and consists of a main channel running close to the tube, and of Io to I 8 lateral branches arranged like the teeth of a comb. The main channel ends in a blind pouch which may be dilated into a small cyst, the hydatid of Morgagni. Portions of these tubules may be found in the wall of the tube, where they may be confused with secondary Fallopian canals, and in the hilus of the ovary. The tubules are small, lined by low cylindrical cells, and walled by circular and longitudinal muscle-fibers. Slight dilatation of the tubules is common and has been interpreted as an evidence of functional activity (Bucura).

Cysts of the parovarium are observed in about i I per cent. of ovariotomies (Olshausen). They commonly reach the size of the fist, but very large cysts weighing 42 pounds, or containing 33 liters of fluid, are recorded by Kummel and Nagel. Kossmann holds that only the small multiple or multilocular cysts rise from the parovarium. The large cysts he refers to dilatation of accessory Fallopian tubes, hydroparasalpinx. Favoring this view are the presence of muscle-tissue in the wall, the preservation of parovarian tissue noted in some cases, the unilocular character, the difficulty of deriving a single large cyst from the tubules of the parovarium, and the close contact of the cyst with the tube wall. Orth states that the cysts which show parovarian tubules in their walls are derived from the par-epoöphoron.

Parovarian cysts are characterized by their elliptical form, thin walls, close contact with an elongated tube, a movable serous covering, and the presence of an intact ovary. The parovarian cysts are subserous, so that the peritoneal coat is movable over the tumor. The tube is intimately adherent and stretches over the cyst wall in greatly elongated form, measuring in Payer's case $76 \mathrm{~cm}$., the fimbriæ I I $\mathrm{cm}$. The ovary may be stretched to a flat plate. The chamber is usually single. While commonly pedunculated and subject to torsion, these cysts may be extraperitoneal and displace the elongated uterus out of the pelvis. The thin wall easily ruptures. The cyst wall is composed of connective tissue and blood-vessels, and muscle-fibers are occasionally seen. Elastic fibers may be abundant and to their contraction or that of the muscle-fibers is attributed the numerous foldings of the inner surface. The lining is of low cylindrical and ciliated epithelium which is often degenerated and desquamated. The contents when clear are of thin, opalescent alkaline fluid of low gravity, I005. Mucin and pseudomucin are absent or present in traces only, and albumin is scanty. Urea may occur in considerable traces, and a reducing substance is usually demon- 
strable. The salts are chiefly chlorids and sulphates. Hemorrhage and fatty degeneration often alter the character of the fluid. Papillary fibroepithelial growths from the cyst wall occur in a considerable proportion of cases and Pfannenstiel refers to an extensive cauliflower growth with invasion of the wall.

A variety of other forms of tumors has also been referred to the parovarium on account of their structure and location. These include adenomyomas (Pick), multilocular hydatidiform cystoma, adenosarcoma (Werth), fibro-adenoma, fibrosarcoma, and carcinoma (Pick, R. Meyer).

Mesonephric Tumors.-While most observers find that the Wolffian tubules (epoöphoron) often fourid in the hilus of the ovary show little tendency to develop tumor growth, a small group of cystic tumors have been referred to this origin. Usually they produce harmless cysts of small size lying in the hilus or medulla (v. Franque, Walthard). Other tumors are solid and composed of tubules presenting the comb-like arrangement of the epoöphoron (Schickele, Lit.). The cells are high and cylindrical and they may show moderate proliferation.

A peculiar malignant tumor of mixed structure, containing myxosarcomatous and gland-like structures, somewhat resembling the embryonal adenomyosarcoma of the kidney, is described by Glockner and referred to a mesonephric origin.

Adenomyoma of Ovary.-A rare form of ovarian tumor reproduces exactly the structure of Recklinghausen's adenomyoma. They are small, multicystic, and benign. They present tubules with dichotomous branching and ampullary sacs lying in a very cellular stroma. Such cases have been described by Babo, Aschoff, Neumann, and Vassmer, and referred to a mesonephric origin, but since they are usually associated with adenomyoma of uterus or tubes it is probable that Russell's interpretation of his case as of Müllerian origin is correct. The mesonephric tubules are free from a very cellular stroma (Schickele, Lit.).

Adrenal tumors of the ovary occur in typical form and present the usual variations in structure of adenoma, adenocarcinoma, and perithelioma. They commonly reach a large size, are of yellowish color, and prove malignant. They arise from the broad ligament, hilus, or body of the ovary, where adrenal tissue is often present. Typical tumors of the hilus or broad ligament are described by Weiss, L. Pick, and Peham, and in the body of the ovary by Peham, Sternberg, and Pfannenstiel. It would seem difficult to distinguish between such tumors in the body of the ovary and tumors of the corpus luteum.

Testicular Adenoma of Ovary.-L. Pick has described a peculiar adenoma of the ovary and brought forward satisfactory evidence to show that it is derived from a testicular element in the ovary of the hermaphrodite subject. The tumor is a well circumscribed, yellowish, solid growth of moderate dimensions and lobular conformation. The structure presents regular elongated tubules about $35 \mathrm{~m}$. in diameter, lined and filled by uniform cuboidal epithelium. The stroma is slightly developed and may contain cells resembling the interstitial cells of the testis. The tubules resemble those of the sweatglands and are exactly similar to the tubular adenomas of undescended testes.

\section{CARCINOMA OF THE FALLOPIAN TUBE}

Since Orthmann's first description of primary carcinoma of the tube (i886) about I 20 cases have been reported (Doran, Fromme, Heynemann. Lit.). It occurs chiefly between 43 to 50 years, but cases have been observed 
as early as the 27 th year (Norris). In its etiology the influence of chronic inflammation is of first importance. Many cases begin as multiple papillary outgrowths of the mucosa such as arise on inflamed mucous membranes. Sterility, which may also be referred to inflammation, was noted by Kerhrer in 14 and a single birth in 20 , of 52 cases. Association with tubo-ovarian cysts and uterine myoma is often observed (Orthmann), and various abnormalities in the position and structure of the tube may serve as predisposing factors to tumor growth.

In gross appearance the tumors are solid and nodular, or more diffuse and papillary. They arise from the middle and outer portions of the tube, the uterine end usually being free. Very early tumors have been found in the form of small superficial excrescences, while the advanced carcinomas may reach the size of a child's head. Distention of the tube with fluid may add to the bulk and lead to perforation with peritonitis (Kiwisch).

The tumors were bilateral in about one-third of the cases. Stolz collected ro such cases which had the structure of malignant adenoma and I have recorded one other. In a recent case studied by L'Esperance in this laboratory the mucosa of uterus and both tubes was the seat of diffuse adenocarcinoma and co-extensive tuberculosis.

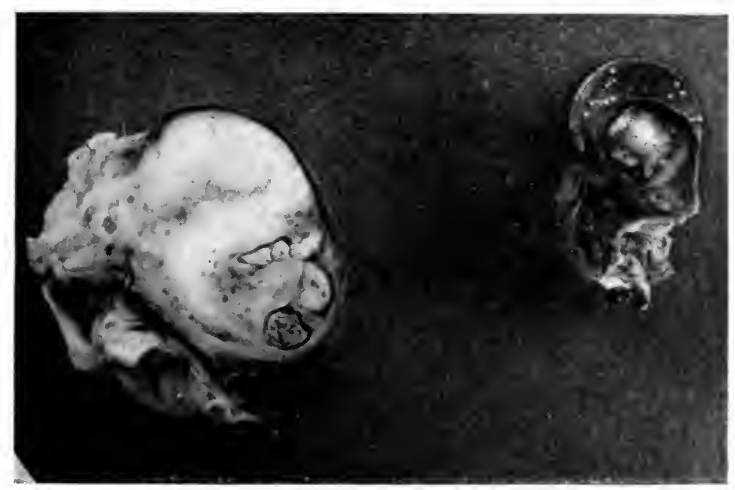

FIG. 282.-Double primary adenocarcinoma of Fallopian tubes.

The papillary forms of the tumor long remain benign, but others invade the wall, cause adhesions, perforate the peritoneum, and cause local and distant metastases. Extensions have often been observed in the ovary, uterus, opposite tube, and pelvic nodes (Stolz), in omentum, stomach and, intestine (Amann), rectum (Cullen), and inguinal nodes (Rosthorn). Tuboovarian carcinoma is difficult to trace to its exact origin on account of the resemblance of the epithelial tumors of these organs. In 3 reported cases this condition was bilateral (Dittrich, Edebohls, Sanger). Ascites and ovarian abscess are occasional complications of tubal carcinoma.

Structure.-The earliest cases observed have appeared as numerous low papillary outgrowths of the tubal folds, covered with a single layer of cuboidal cells, and resembling ovarian papilloma. Some tumors retain this distinctly papillary structure with increase in the cell layers. Others exhibit an alveolar structure, probably from fusion of adjacent papillæ, and resemble uterine adenocarcinoma. Sanger and Barth believe that the latter develop from the former by a gradual change in structure and malignancy.

Orthmann describes a diffuse acanthoma of the tube with hornification 
and pearl formation, and the transformation of cylindrical cells into squamous.

For some tumors apparently originating in the wall an origin from an accessory tube or from Gartner's duct has been suggested (Friedenheim, Doran).

Secondary carcinoma of the tube is relatively frequent, especially from the uterus and ovary (Sanger). Direct extension of corpus carcinoma into both tubes was described by Iancereaux and 3 cases of tubal metastases from corpus carcinoma were collected by Kundrat. Primary carcinomas of both tube and uterus are recorded by Hofbauer and by Novy. The prognosis of tubal carcinoma is no better than that of cervical carcinoma and after infiltration of the wall it is distinctly unfavorable (Doran, Stotz).

Miscellaneous Tumors of the Fallopian Tube.-Extensive proliferation of peritoneal and vascular endothelium of inflammatory origin is described by Kworostansky. A peculiar tumor composed of carcinoma arising from the mucosa, sarcoma of the wall, and endothelioma of the blood-vessels is described by v. Franque. A teratoid chondrosarcocarcinoma is reported by Amann.

Primary sarcomas and myxosarcomas are described by Janvrin, Jacobs, Sanger, and v. Kahlden. They were of variable and uncertain structure and origin. 


\section{CHAPTER XXX}

\section{THE OVARIAN TERATOMA}

The commonly recognized varieties of this tumor, the cystic dermoid and the solid teratoma, represent adult and embryonal types of the same growth. Although varying greatly in gross anatomy and clinical course they are probably identical in origin and many intermediate forms connect the extremes of either type in one nearly continuous series. The chief distinction is the clinical malignancy of the solid teratoma, the simple dermoid being relatively benign.

Clinical Course.-Dermoids constitute about ro per cent. of all ovarian tumors, while solid teratomas are much less frequent in occurrence (Kroemer, Lit.). They appear at all ages, often in childhood, most frequently between 30 and 40 years, the malignant growths occurring rather earlier than the dermoids. The early growth of both varieties is rapid, but after attaining a certain development the dermoid reaches its limit and may remain stationary for many years, its later course being determined by accumulating secretion in the accompanying cystoma, or by malignant changes, or other complications. The dermoid may migrate from the ovary and become implanted elsewhere or perforate the hollow viscera. Extensive calcification may lead to complete arrest of growth. Rupture of the cyst and discharge of its contents if sterile lead to a low grade of peritonitis, with encapsulation of discharged detritus or to multiple implantation cysts. Purulent peritonitis results if the cyst is infected. Malignant processes develop in about 3 per cent. of dermoids and commonly lead to invasion of the peritoneum. These secondary processes take the form of pseudomyxoma peritonei, or numerous cellular nodules, or more diffuse pelvic growths of sarcomatous type.

The solid embryonal tumors develop rapidly and are malignant from the first. They soon reach considerable dimensions, become adherent to surrounding structures, perforate the capsule and become implanted in the pelvis, on opposite ovary, abdominal wall, or over the diaphragm and omentum, with ascites and peritonitis. Later, distant metastases occur in retroperitoneal nodes, lungs, and liver.

The prognosis of simple ovarian dermoids is favorable, as the tumors are well circumscribed, often pedunculated, and readily extirpated. The various complications alter this prognosis and with malignant changes the operative mortality is considerable and recurrence is the rule. With bilateral dermoids malignant processes appear to be comparatively frequent (Hohne).

The solid malignant tumors are usually fatal, directly, or from operation, or recurrence. Yet when the tumor is circumscribed or pedunculated and early, it may be successfully removed. Neuhauser estimates the cured cases at 27 per cent. Struma ovarii is usually benign, but many give recurrence and metastases. Chorioma ovarii is uniformly fatal.

Gross Anatomy.-The ovarian dermoid is a cystic tumor of globular outline and smooth surface and varies in size from a small nodule to a bulky cyst. It arises within the ovary, destroying the organ, or leaving a small nodule lying upon the cyst. It may be attached to the ovary by a pedicle which may break from torsion, leaving the tumor attached to surrounding tissues. The polypoid form has been referred to a superficial position of the 
follicle supposed to give origin to the dermoid. They may develop in the broad ligament or in the retroperitoneal space, and both ovaries may be normal when the tumor arises from a supernumerary organ, traces of which are found in these extra-ovarian growths. The solid embryonal and malignant teratomas exhibit the same variations in position, they often contain many small cysts and they may reach very large dimensions, Falk's tumor weighing $25 \mathrm{~kg}$.

Both dermoids and solid teratomas are frequently (I4 per cent. Arnsperger) combined with ovarian cystomas, either serous or pseudomucinous or

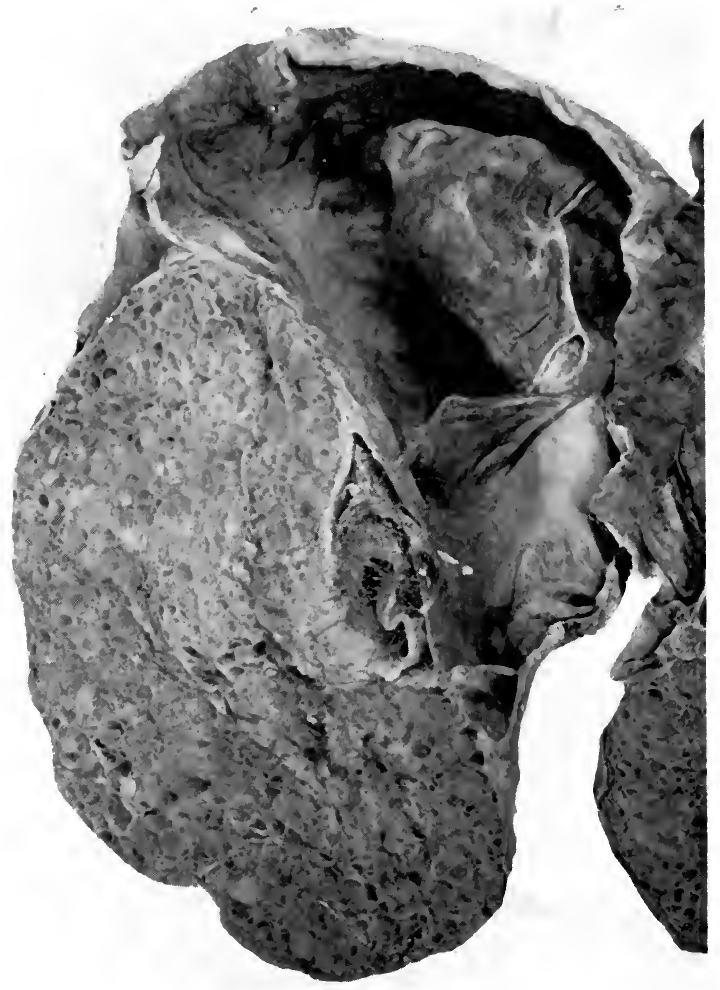

FIG. 283.-Dermatocystoma of ovary. Simple cystoma above. Dermoid with hair in center. Thyroid-like tissue below.

lined by ciliated epithelium. A corpus luteum cyst may also be associated with the dermoid, and Schottlander believes such cysts are often overlooked. In these cases the dermoid portion is limited to a segment of the wall of a large cyst, or the dermoid projects as a finger-like protrusion at one point of the cyst (Rokitansky's protuberance), or two partly or completely separate cysts, dermoid and adenoid, are in apposition or show various stages of fusion. The dermoid portion may be represented also by an embryonal teratoma. In not a few cases the dermoid is found in one ovary, a cystadenoma in the other. 
Dermoid cysts usually contain hair and teeth. The hair is very long like that of the head, or short and fine as lanugo, or curled and stiff as of the pubes, and it is often silvery in tumors in young subjects. Sebaceous or oily material is mingled with the hair. It may be condensed in the form of firm globules, or in a large mass consisting of fatty crystals forming about hair, or in epidermal concretions. The occurrence of these masses has been referred to albuminous bloody exudate following torsion, to mixture with secretion from sweat-glands, or to a sort of butter formation. Bonney counted 4000 small globules in one case and Opitz reports their appearance in the stool through an intestinal fistula.

In the combined cystomas the mucinous material mingles with the fatty, and when blood is added, the dermoid element may be obscured and require careful identification on the cleansed interior. A small tuft of hair or a pigmented spot, or a bony plate, often mark the dermoid area. Calcification transformed the entire mass into a stone in Pfannenstiel's case. Teeth occur in about half the dermoids and in many solid tumors. They lie loosely on the surface or firmly fixed in a rudimentary maxilla, or deeply embedded in dental follicles. The teeth are well formed and may be identified as incisors or molars, etc. The embryonal tumors usually contain only small milk teeth, while those of the dermoid are more adult. Rokitansky observed the eruption of permanent after the discharge of milk teeth which fall into the cyst contents and become absorbed. Saxer's dermoid consisted of a single well-formed tooth, while Schnabel and Plouquet counted from roo to 300 or more.

The maxillæ have resembled the lower jaw with coronoid process or the upper with antrum Highmori. Other bony masses have been interpreted as sternum (Wilms), skull (Grechen), ribs (Schramm), or pelvis (Kuster). Portions of long bones connected by joints are described by Kappeler. Wellformed extremities have occasionally been observed, as a finger with three phalanges and nail (Amor), several fingers (Reverdin), short extremity with long nails (Thornton). Axel Key identified two lower limbs with toes, skull, brain, and jaw. Even more complete rudimentary fetuses are described by Repin, Askanazy, Shattock, and Goffe, who identified such structures as skull, cystic brain vesicles, pigmented optic cups, complete extremities, pelvis with hairy pubes, vulva, clitoris, spinal cord, and blind coil of intestine. The development of these structures, however, falls considerably below that of the epignathi. In the embryonal tumors the complex structure usually renders the gross identification of organ rudiments impossible.

Glandular organs may occasionally be detected. Areas of thyroid tissue are rather frequently present, as small colloid foci, or large masses of typical gland tissue, or the entire tumor may be composed of thyroid tissue, constituting the well-known "struma ovarii." Salivary glands lying near a maxillary bone are described by Axel Key and Flaischlen.

Brain tissue appears in the form of convolutions of soft gray tissue (Baumgarten). It is often the seat of hemorrhage. A portion of cerebellum was observed by Askanazy. Pigmented optic cups have been found embedded within or lying near the brain tissue. Baumgarten described double rudimentary eyes, one of which exhibited a cornea-like structure. Kordi recognized a lens and eyelid, and Graves found eyelid with lashes.

The embryonal teratomas may usually be distinguished from the more adult dermoids by the absence of any large cystic cavities with hair or sebaceous material, or definite limbs, or organs. The solid tumors usually contain many small cysts and close inspection may reveal islands of bone or cartilage, rudimentary teeth, minute dermoids with fine hair, pigmented 
retinal structures, and in general the cellular structure of an embryonal tumor. Transitional cases between the main types occur, and overgrowth of one element, as in struma ovarii, gives rise to peculiar forms requiring special consideration.

Microscopical study reveals the extensive details of the structure of ovarian teratomas, strengthens the interpretation of the various forms of fetal mimicry, and reveals the essential tridermal nature of these growths.

The hairy dermal papilla is covered with a thin epidermis and a corium of normal structure which contains many sebaceous, sweat, and hair glands and presents an almost exact duplicate of the scalp. Retention in the glands often produces comedones and small sebaceous cysts. The epidermis may be traced into the mucous membrane which covers the maxillæ and the tissues in which teeth are found. This point may be indicated by a shallow depression or deep pocket suggesting a buccal cavity. All stages of the

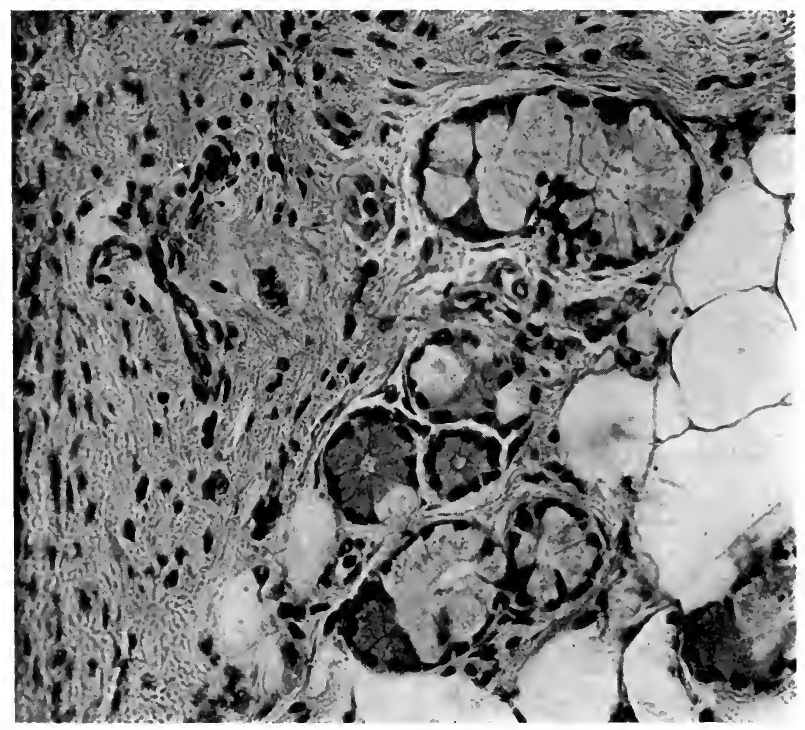

FIG. 284.--Salivary gland alveoli in a small ovarian dermoid.

development of teeth have been traced, from a simple enamel organ to a perfectly formed adult tooth. Here have been found the rudiments of buccal and salivary glands. The subcutaneous tissue contains embryonal fat, connective tissue, and blood-vessels. Beneath this layer one commonly finds plates of bone or cartilage which may be interpreted as fragments of skull, since brain tissue, medullary canal, spinal cord, and ganglia may sometimes be identified in this region (Kroemer). Here also small cysts may present an ependymal lining. Cerebral elements may be reduced to pigment streaks, ependymal tufts, or concretions (brain sand). Askanazy points out that the nerve tissues of ovarian dermoids include the central, peripheral, and sympathetic, as well as special sense organs, as of eye and ear. The dominant system is the sympathetic. The adult forms of these tissues are represented by brain cortex, ganglia, and nerve trunks, the embryonal by neuro-epithelial cell groups. The chromatophores of the central nervous system are often 
abundant. The respiratory tract is represented by canals lined by columnar or ciliated epithelium in a cellular lymphoid mucosa walled by cartilaginous rings and strands of smooth muscle. Mucous glands, islands of thyroid tissue, and occasionally striated muscle bundles complete the resemblance to the fetal trachea. Kroemer interprets certain sharp infoldings of this mucosa, with change to squamous lining, as vocal cords, and he identifies the nares by ciliated epithelium lying on curved cartilaginous plates. Wilms describes a rudiment of lung connected with trachea by a bronchus. Mammary gland tissue is described by Sutton and Reverdin. Gastro-intestinal tract appears in segments which resemble stomach, ileum, or colon. A single layer of low columnar epithelium, lining a flat mucosa with many glands without lymphoid tissue or valvulæ, but backed by layers of smooth muscle, recalls more or less definitely the stomach. In the portions of small intestine valvulæ, villi, crypts, and simple or agminated lymphoid follicles are found, the lymphoid tissue being frequently present in abundance. Segments of

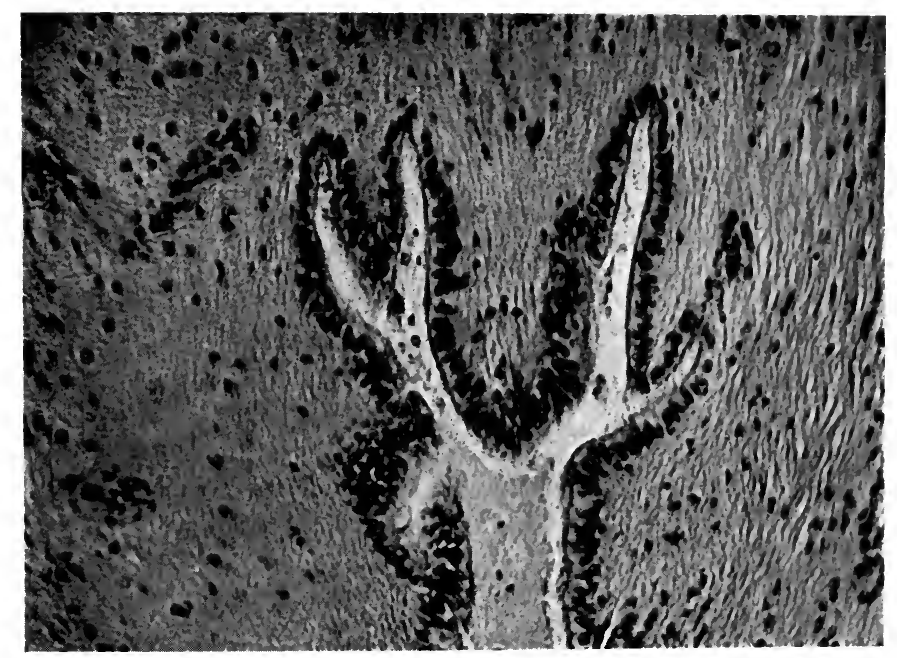

FIG. 285.-Ependymal structure in brain tissue in a small ovarian dermoid.

colon are often observed. Pommer described cecum and appendix. Colon with meconium has been described by Perls, Repin, and others. Arnsperger identified a portion of esophagus, but Askanazy finds that the esophageal remnant is commonly missing, and that the intestinal tract opens directly into the respiratory, constituting a condition which is characteristic of teratomas. Kroemer identified a Müllerian duct with cervical and uterine glands, myometrium, and vaginal mucosa. In the embryonal tumors the respiratory and intestinal structures are reduced to short canals or small cysts in which one type of epithelium often changes sharply into another.

The ocular structures vary from rudimentary eyes with retina, choroid, lens, and cornea, which have appeared separately, although not all in the same case, down to isolated groups of pigmented choroid or retinal cells. The most frequent structure is a layer of pigmented cells lying upon a vascular membrane which incloses an optic vesicle. The pigmented cells resemble those of the choroid, or they are hexagonal plates reproducing the mosaic 
of the retinal epithelium. These structures may be bilateral and lie near or within areas of brain tissue with which no definite connection by an optic nerve-trunk has yet been observed.

Mesodermal elements, besides those recognized in the gross, appear in many forms. Islands of hyaline or elastic cartilage occur in both adult and embryonal tumors, and in the latter they may grade into myxomatous or sarcomatous tissue. Spongy bone masses may contain lymphoid marrow, but without giant-cells (Coe, Kroemer). Muscle-fibers are usually smooth, but scanty striated cells have been observed, as well as heart-muscle cells (Wilms, Askanazy, Katsurada).

The outer wall of a dermoid cyst varies greatly in structure and probably passes through certain transformations. Any ovarian tissue remaining is confined to a small, often cystic, mass of tissue located at one point or distributed along the wall. When the original dermoid sac is preserved the lining is of cuboidal or columnar epithelium and is derived by many from the cells of the Graafian follicle. This membrane with low epithelial lining has been interpreted as a form of amnion. An extension of squamous epithelium from the dermoid papilla over much of this sac is regarded by Kroemer as a late and secondary change. Erosion of this lining epithelium is accompanied by formation of giant-cells lying on granulation tissue. Fat crystals and fragments of hair may be embedded in this tissue and considerable calcification of the wall may occur, especially after separation. The gradual absorption of the oily contents of the cyst may result in honeycombing of the wall, the fat passing into dilated lymphatics lined by proliferating endothelium.

Schottlander traces the large serous cysts accompanying some dermoids to dilated lymphatics. The gradual absorption of the thin wall of the original dermoid chamber leads to communication with surrounding cavities which include corpora lutea, serous cysts, and adenomatous cysts. As these communications widen, the wall of the cyst changes and its lining varies in different portions and at different periods.

Metastases develop from dermoids in which malignant changes have become established and more frequently from the solid and embryonal teratomas.

Local dissemination of the tumor elements over the peritoneum follows rupture of the cyst wall or tumor capsule. These implantation metastases are of very variable structure. Lazarus found nodules on diaphragm and in Douglas' pouch, the latter containing cartilage and cysts with cylindrical cells. In several cases multiple epidermoid and dermoid cysts have resulted. Neuhauser described multiple miliary nodules of glia-tissue along the vessels of a portion of omentum removed at operation on a patient who recovered. Falk demonstrated at autopsy complete regression of peritoneal metastases 3 years after laparotomy in an inoperable tumor.

Through the lymph-vessels the retroperitoneal nodes are involved by metastases which are of simple or complex type. Many of these are of ectodermal and neuro-epithelial nature. Ewald found ectodermal and entodermal cysts and cartilage in a large recurrent retroperitoneal tumor. Pick demonstrated a long bone with marrow cavity, lying in a retroperitoneal node. Falk described a complex metastasis in the abdominal wall containing brain tissue, and intestinal canal with musculature. All of the complex secondary tumors are probably the result of direct extensions or implantations.

Through the blood-vessels metastases have appeared in lungs, liver, and kidney, and are usually of sarcomatous or indifferent embryonal type. It is evident from these observations that several types of differentiated cells 
may be detached from ovarian teratomas and give rise to metastatic tumors. That undifferentiated cells capable of developing tridermal structures may be dislodged from the ovarian tumors is as yet unproved. All the tridermal secondary growths appear to be local recurrences or implantations in or about the peritoneum. The various cells of the original tumor appear to become separated by the embolic process and to develop only their particular kind in metastatic tumors. Yet it must be admitted that the embolic cells or cell groups are capable of developing organoid structures, such as brain tissue and dermoid cysts (cf. Saxer).

Atypical Forms of Ovarian Dermoids.- Not all ovarian dermoids exhibit the tridermal and complex structures of the fully developed tumor. Suppression of one germ layer, overgrowth of single elements, and secondary changes give rise to several well-defined subvarieties.

(I) Bidermal Atrophic Teratomas.-Simple dermoid cysts without demonstrable elements of a third germ layer constitute about 5 per cent. of all ovarian teratomas (Wilms). The ectodermal lining presents the usual features of a typical dermoid and the wall contains dermal glands, teeth, connective, fat, smooth muscle and bone or cartilage, but entodermal structures are missing. While only a very thorough study can determine the absence of entoderm, there is little doubt that this layer is the least vigorous of the three in this as in other teratomas. Askanazy saw complete loss of entodermal structures in a petrified dermoid containing teeth. Kroemer observed partial separation and beginning atrophy of the entire dermoidal area, which hung by a narrow pedicle to the cyst wall. It seems possible that the entire dermoid might be destroyed in this way, especially when associated with a large adenocystoma. Kroemer pictures a very large multilocular cystoma in which the dermoid was reduced to a diminutive hairy nodule. These observations, as well as the occurrence of a dermoid in one ovary and a separate adenocystoma in the same or the other ovary, led Hanan, Ribbert, Landau, Pick, and others to conclude that the pseudomucinous cystadenoma is a one-sided entodermal development of a teratoma. Askanazy includes among typical dermoids some which seem to consist exclusively of epidermis. Saxer's dermoid consisting of a single tooth must have resulted from early suppression of other elements. Portions of the main dermoid, including epidermis and hair or even bone (Wilms), may be transplanted into adjoining cysts, giving a false impression of multiplicity of dermoids. After rupture of ovarian dermoids multiple implantation dermoids may appear over the peritoneum.

(2) Multiple dermoids in one or both ovaries have repeatedly been observed (Novak, Lit.). Schroeder reported seven isolated dermoids in one tumor and Pfannenstiel saw five, all of uniform size. Wulkow found two dermoids in one cystoma and four in a similar tumor of the other ovary. Some of these may have been the result of transplantation, but Heinsius described five complete dermoids in four separate compartments. Hoffmeier found four in one ovary and seven in the other. The largest number observed was by Novak, who found ten in one ovary and eleven in the other. Keitler observed in one ovary a dermoid cyst with hair and teeth, in the other two dermoids and a cyst enclosing a solid teratoma. The genesis of the true multiple dermoids may be referred either to the simultaneous growth of separate ova (oöcytes), or to the separation of blastomeres of one original ovum.

Retroperitoneal and intraligamentary dermoids containing portions of ovarian tissue have been reported by Coen, Dunnwald, and others. Rarely this location may be referred to the separation from the ovary and secondary 
implantation elsewhere (Reinprecht). More often a supernumerary ovary is the probable source (Hermann, Wilms).

An elongated pedicle may permit unusual migration and attachments of the dermoid. I have examined a complex dermoid, showing epithelioma of the skin, hanging in the cecum. Perforations of bladder, rectum, or ileum are recorded.

(3) Secondary changes may greatly alter the appearance and structure of dermoids. Torsion leads to hemorrhagic infiltration and necrosis, and this may be followed by extensive calcification or by suppuration. All traces of the original dermoid structure may thus be lost and the tumor becomes a solid calculus (Busse, Askanazy).

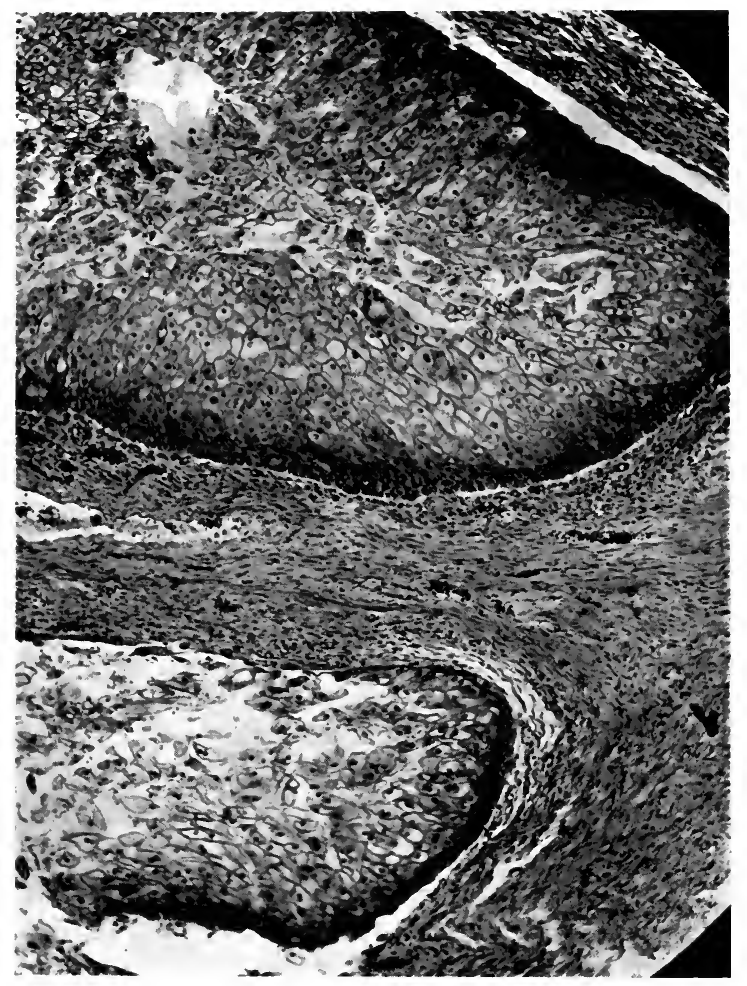

FIG. 286.-Atypical epidermal growth in an ovarian dermoid.

(4) Malignant characters in the dermoid itself or in the associated cystoma are important features of many cases.

The epidermis may develop epithelioma invading the other tissues of the dermoid, of which Bab has collected 37 cases. A mammary carcinoma in a dermoid is reported by Yamagiva, and adenoma of the sweat-glands by Friedlander. Seeger observed bilateral carcinomatous dermoids. Sarcomatous changes occur in dermoids, while in many teratomas much of the tissue may be of embryonal and sarcomatous character (Iudwig, Lit.). Pigmented sarcoma arising in the cyst wall is recorded by Amann and by Lorrain. Roundcell sarcoma in the wall and in hepatic metastases is recorded by Litten. 
Bierman described a large spindle-cell sarcoma and Neumann a perithelial alveolar tumor, arising from the corium of dermoids. The wall of the associated cystoma appeared to be the source of spindle-cell sarcomas in the cases of Flaischlen and Ludwig. A typical perivascular and alveolar sarcoma is described and pictured by Schwertassek. Kroemer traced an alveolar sarcoma of the cyst wall to the vascular endothelium, and the endothelial origin of many of these tumors is also maintained by Mirabeau and Schottlander. The ovarian tissue is believed to furnish some of the dermoid sarcomas, as in the cases of Geyer and Thornton. It would appear that the tumor process is incited in some way by the dermoid, according to Kroemer in some cases by the absorption of oily material.

The malignant processes extend beyond the dermoid with rupture of the cyst and produce metastases in peritoneum and liver. The rupture of the cyst may also be followed by multiple dermoidal implantations or by encapsulation of fatty material which lodges on the peritoneum.

(5) Struma Orarii.- - The occurrence of thyroid tissue in ovarian tumors has long been recognized and variously interpreted (Bell). It is relatively frequent in ovarian, while very rare in testicular, teratomas. An origin from metastatic thyroid cells or from mucous degeneration of a carcinoma (Kretschmar, Voight) has now fully yielded to the view that these tumors represent one-sided growths of a teratoma. This view was strongly supported by Walthard and others, who found in several such growths islands of cartilage, bone, epidermis, and acinous glands. Moreover, Pick in $2 \mathrm{I}$ dermoids found islands of thyroid tissue. Both these observations are frequently verified (Trapl, Lit.).

The tumors occur in both benign and malignant forms. The benign tumors may reproduce almost exactly the structure of the normal adult thyroid gland. Meyer demonstrated iodin in the tissues. They produce rather bulky tumors resembling a colloid goiter in the gross and they are subject to hemorrhage, cyst formation, and regressive changes. Trapl brings evidence to show that the ovarian struma may functionate vicariously for the thyroid gland.

The malignant tumors are more cellular, invade the surrounding tissues, and produce metastases (Polano, Katsurada). To what extent the malignant cellular tumors may lose their structural resemblance to thyroid tissue is not yet clear, but it appears not unlikely that some solid carcinomas of the ovary are derivatives of teratoid thyroid tissue.

That the typical structure of struma ovarii may be produced by a tumor originating from downgrowth of superficial epithelium is clearly shown by Bauer, but his claim that all ovarian strumas originate in this manner cannot be entertained.

(6) Chorioma Ovarii.-While chorioma testis is comparatively frequent, the ovary has furnished only rare or uncertain examples of this type of atypical teratomas.

A notable example is described by L. Pick in which both microscopical and gross features were complete reproductions of the primary uterine chorioma. Small polyhedral Langhans' cells rich in glycogen and with many mitoses were combined with large vacuolated syncytial masses. The syncytium appeared to spring from neuro-epithelial cell groups, invaded the vessels, and added a hemorrhagic character to the tumor. In addition, there was a sarcomatous framework holding epidermis and glands lined by mucous cells.

The occurrence of choriomatous tumors of the pelvis and liver without demonstrated involvement of the ovary leaves a well-founded suspicion 
that an ovarian teratoma may have been suppressed or overlooked. Kroemer described a pure chorioma of the ovary in a woman of $5^{\mathrm{I}}$ years, without other teratoid elements. A typical malignant chorioma ovarii was also described by Glinski and Rosner. Lubarsch examined a large tumor mass in the pelvis of a I3-year virgin, which consisted of polyhedral vacuolated cells and syncytium. He concluded that this structure might be duplicated by tumors arising from various epithelial structures of the genito-urinary tract.

In several instances it has been shown that epithelial tumors of ordinary type may in metastases yield a structure much resembling chorioma. Hence Risel discards many of the reported choriomas of tubes, ovary, and other organs as spurious imitations derived from other than teratomatous sources or gestation products. Monckberg considers many of these growths to be derived from endothelium. Not a few of the so-called choriomas of the liver appear to me to be derived from primary carcinomas of the liver-cells. L'Esperance, in this laboratory, has described such a spurious hepatic chorioma and pointed out its notable distinctions from true chorioma.

Nevertheless the gross and microscopical features of a group of heterotopic choriomas in both sexes exactly reproduce those of uterine chorioma. On the other hand, the secondary chorioma-like structure occurring with carcinoma and other tumors fails to yield such complete reproductions of the true chorioma. Hence I am disposed to think that many of the above tumors are true teratomas derived originally from ovary or testis and that the original tumor is suppressed or overlooked, or that such tumors arise from deported chorionic structures from uterine gestation. For the diagnosis of such true heterotopic choriomas it is necessary to insist on complete identity in gross characters including pulmonary metastases and not to rely merely on occasional microscopical resemblances.

(7) The Relation of Simple Ovarian Tumors to Teratoma.-The demonstration that one element of an ovarian teratoma may suppress the others raises the question how far the apparently simple tumors of the ovary may prove to be of teratomatous origin.

Under the term "epithelioma chorio-ectodermale" I. Pick would include certain embryonal ovarian tumors resembling adenoma, carcinoma, and endothelioma. He describes 5 cases of cystic or solid growths in which the cells were rich in glycogen, and were arranged in cystic adenomatous or solid carcinomatous structure. The main tumor-cell he identifies as the Langhans' cell, while syncytium-like masses were often present.

Pick's interpretations of these tumors has not been supported. Kroemer did not find a convincing resemblance of the tumor-cells to chorionic epithelium, and Risel notes the absence of a vasodestructive property in the tumorcells. It seems probable that the tumors described by Pick were embryonal growths in which the syncytial masses were developed from the main tumorcells of ordinary ectodermal or entodermal origin and that they were not of chorionic nature. This conclusion, however, does not dispose of the possible teratomatous origin of many apparently simple embryonal tumors of the ovary. Especially for the extensive group of sarcomas of the ovary, roundand spindle-cell, perivascular, alveolar, and "endothelial," the observations by many authors of identical structures arising from dermoids and teratomas, strongly suggest a teratomatous relation. This question must, however, await further observations.

Chondroma of the ovary is described by Reiss and Jung as a mesodermal teratoma, and Meyer accepts this interpretation. The chondrosarcomas being embryonal tumors should from this point of view be regarded as 
teratomatous. Ovarian melanoma may also be interpreted as of teratomatous origin.

In all of these cases further observations are necessary before a teratomatous element can be accepted. While the observations on tumors of the testis point by analogy to the simple origin of many ovarian tumors, the conditions in the ovary are much more complex than in the testis.

Etiology.-The origin of ovarian dermoids and teratomas is now referred by the great majority of observers to the sex cell or ovum. The essentially tridermal character of these tumors requires that the originating material be totipotent. Only two possible sources of such material have been seriously considered, the isolated blastomere of Marchand-Bonnet, and the primitive unfertilized ovum. Bandler constructed an elaborate argument for the Wolffian origin of ovarian dermoids, but this view has not met with favor. Several considerations speak against the theory of an isolated blastomere. Chief among these is the frequency of such tumors in the sex glands, both ovary and testis. While it is not necessary to regard all tridermal tumors from the same viewpoint, those of the sex glands find a direct and obvious explanation in the autonomous growth of a totipotent cell which is constantly present in these organs in considerable numbers. On the other hand, it is almost impossible to conceive how isolated blastomeres can gather in sufficient numbers to explain the incidence of dermoids. The occurrence of as many as seven dermoids in one ovary and eleven in both organs practically eliminates the blastomere or polar body as a possible source. The field of the isolated blastomere lies with the parasitic implantations, and its extension to the teratomas of the sex glands is unwarranted.

The direct evidence in favor of the origin from sex cells is considerable. Ovarian dermoids occur during the period of functional activity of the ovary. They are excessively rare in the fetus and they are practically unknown as a tumor arising after the climacteric. The multiplicity is readily explained by the abundance of ova in the organ. The spontaneous growth of the unfertilized ovum is a reasonable hypothesis, in view of the very numerous factors which are known to incite the growth of ova in lower animals. It is a matter of little consequence whether this growth be designated as parthenogenetic or otherwise. No one has tracer the dermoid to an ovum, but the frequent association of cystoma with dermoids suggests, as Kroemer holds, that the cystoma arises from the epithelium of the Graafian follicle and the dermoid from the contained ovum. The occasional combination with a corpus luteum cyst finds a parallel explanation. Malformation of the genitals has been observed with ovarian dermoids, but not with sufficient frequency to indicate a definite relation of such gross anomalies with the tendency to teratomatous growths (Freund). These anomalies have included pseudohermaphroditism, infantilism, supernumerary ovaries, elongated cervix with cysts of Wolffian duct (Amann), and myomas in many cases.

That the dermoid and the teratoma have an identical origin is indicated by the occurrence of intermediate types of tumors, and by the association of both embryonal and adult tissues in the same tumor. Yet the difference in the structure of the dermoid and the teratoma has not been satisfactorily explained. Askanazy assumes that the adult tissues of the dermoid are of equal age with those of the host and the tumors are congenital, while the teratoma arises later in life and yields embryonal tissues. Yet congenital dermoids are excessively rare, and since their initial stages are never seen the early growth must be rather rapid. After attaining a certain size they may remain stationary, so that the age of a dermoid extirpated after the climacteric is difficult to determine. Hence it is probable that both dermoids and 
teratomas arise chiefly in young adults, especially about the 2oth year (Kroemer). It is possible that the varying structure depends upon the degree of differentiation of the originating sex cell. Back of the mature ovum are the öocytes, the oögonia, and the primary sex blastomere, each of which may be assumed to give origin to tumors. From this point of view the two theories of origin, from sex cell and from isolated blastomere, approximate each other. The embryonal tumors may thus be assumed to develop from early members of the sex cell series, the dermoids from late members. Yet the localization in the ovary requires that all the tumors must arise from cells included within this organ, a condition which the isolated blastomere does not readily meet. It seems probable, therefore, that the varying structure is determined by local factors, still undefined, acting upon cells of. a uniform character. 


\section{CHAPTER XXXI}

\section{CARCINOMA OF STOMACH}

Statistical.-The stomach is probably the most frequent seat of cancer in males as is the uterus in females. The exact proportions of these two forms of cancer are matters awaiting expert statistical study. Welch (I885), from an analysis of $3 \mathrm{I}, 482$ cases of cancer in cities, found the proportions: stomach, 2 I.4 per cent.; uterus, 29.5 per cent. These figures include Gurlt's Vienna statistics, which are much too low for the stomach, Io per cent. in 4I 3 I cases. I should be inclined to balance Gurlt's data with Haberlin's figures for Switzerland, which are much too high for most localities, $4 \mathrm{I} .5$ per cent. in 27,5 II cancers. Intermediate but high ratios are recorded by Reiche (Hamburg), 35.5 per cent.; Virchow (Wurzburg, I 855), 34.9 per cent.; Martin (compiled from 70,000 cases), 33 per cent. The U. S. Census, I9I 2 , reports 46,534 deaths from cancer, I8,5I 7 of stomach and liver, 39.75 per cent., 7089 of female genital organs, $443 \mathrm{I}$ of breast, indicating a distinct predominance of gastric cancer. This census also shows a more rapid increase of reported gastric cases than of uterus or breast cancer during the past decade. Marked variations in different countries and cities and slight relative immunity of negroes and in the tropics, appear in various studies.

Males are slightly more often affected than females ( 5 to 4 Welch, 3 to 2 Haberlin, 65 to 35 Kaufmann, 588 to $4 \mathrm{I} 2$ Friedenwald). Of deaths from all causes gastric cancer gives I per cent. (Brinton); $\mathrm{I} \frac{1}{2}$ per cent. (Gussenbauer, v. Winniwarter, Vienna); $3 \frac{1}{2}$ per cent. (Welch, Prague); 2 per cent. (U. S. Census, I9II, stomach and liver).

Etiology.-Age.-The average age of incidence is 6r.2 years (U.S. Census, I9II). The distribution is indicated in the following table:

\begin{tabular}{|c|c|c|c|c|c|c|c|c|c|c|}
\hline & Decade & $\begin{array}{r}0 \\
10\end{array}$ & $\begin{array}{l}10 \\
20\end{array}$ & $\begin{array}{l}20 \\
30\end{array}$ & $\begin{array}{l}30 \\
40\end{array}$ & $\begin{array}{l}40 \\
50\end{array}$ & $\begin{array}{l}50 \\
60\end{array}$ & $\begin{array}{l}60 \\
70\end{array}$ & $\begin{array}{l}70 \\
80\end{array}$ & $\begin{array}{r}80 \\
100\end{array}$ \\
\hline Welch, $1885 \ldots \ldots \ldots \ldots \ldots \ldots$ & $\begin{array}{ll}10 \text { otal } \\
2,038\end{array}$ & & $\mathrm{I}$ & $2 \cdot 7$ & I3.3 & $24 \cdot 5$ & 30.4 & $2 \mathrm{I}$ & 6.85 & I. 15 \\
\hline Reiche, $1900 \ldots \ldots \ldots \ldots \ldots \ldots$ & - 2,000 & & $\mathrm{O} 4$ & .87 & 5.85 & 14.15 & 25.9 & 32.1 & I 8.1 & 2.76 \\
\hline Martin, $1908 \ldots \ldots \ldots \ldots \ldots \ldots$ & 7,000 & & .08 & I. 5 & 8.8 & I 8.0 & 28 & 28 & 14 & 2.0 \\
\hline $\begin{array}{l}\text { U. S. Census, I9I I : } \\
\text { Stomach and liver... }\end{array}$ & 17,365 & & .26 & I. 15 & 4 & 13.2 & $25 \cdot 5$ & 30 & 20.8 & $5 \cdot 5$ \\
\hline $\begin{array}{l}\text { U. S. Census, I9I4: } \\
\text { Stomach alone... }\end{array}$ & I I, 733 & 12 & 6 & 92 & 457 & 1345 & 2857 & $|3587|$ & $|2647|$ & 725 \\
\hline
\end{tabular}

The average age for carcinoma of liver is 47.2 years (Rolleston).

While the disease is distinctly one of advanced age its rather frequent occurrence between 30 and 40 years, and even between 20 and 30 , is noteworthy. A high proportion of the early cases appear to be adenocarcinomas. A congenital adenoma of the pylorus in an infant 5 weeks old was reported 
by Cullingworth. Of several other cases under io years, collected by $\mathrm{R}$. Williams, none appeared to be true carcinoma of the stomach. In the second decade Osler and McCrae collected I 3 cases, illustrating ulcerating and fungating carcinomas of cardia and pylorus (Moore, Dock, Glynn, Wilde), scirrhus of the pylorus (Koster, Dittrick, Landouzy), and encephaloid cancer (Scheffer). Carcinoma developing on an ulcer in a girl of i 8 years is described by Bernoulli. In the third decade the usual forms of the disease are not infrequently represented, and they tend to run a rapid course (Osler, McCrae, Matthieu).

Heredity.-From a study of I 744 gastric cases from various authors Welch found that in about 14 per cent. other members of the family suffered from cancer. Napoleon I, his father, brother, and two sisters died of gastric cancer. Twenty-three families known to Manishon included 69 cancerous members, of which in 57 the disease was in the stomach. In these and other cases collected by $\mathrm{R}$. Williams it is possible to assume a tissue predisposition. The rarity of these observations emphasizes the usual absence of any element of heredity in gastric cancer. Yet this group of cases seems to be a particularly favorable field for the clinical study of heredity in cancer.

Trauma.-Many observers have endeavored to establish a definite relation between trauma and gastric cancer. Menne cites a series of cases designed to illustrate a direct or indirect relation to trauma, and concludes that the trauma may be the effective etiological factor, or may excite malignant growth in a latent and quiescent focus, or may accelerate the growth of an existing carcinoma. He also describes various traumatic lesions of the stomach of benign course which might serve as sources of carcinoma. The legal verdict in these cases strongly favored the victim, on the ground that the evidence created a probability approaching certainty that the carcinoma resulted from the trauma. Although the violence was sufficient to injure the stomach and gastric symptoms began within a reasonable time, there was in the history and anatomical findings in these cases insufficient data from the pathological standpoint to prove the traumatic origin. Boas reported a traumatic history in six gastric carcinomas, but holds that trauma never produces carcinoma in a healthy stomach. Most observers fail to find any traumatic history in their experience with gastric cancer. With the exception of rare cases following ulcer, the known beginning of the disease is inconsistent with a traumatic origin.

Idiosyncrasy.- R. Schmidt describes two types of subjects who develop gastric carcinoma, the dynamic and the adynamic. The former are wellnourished individuals whose parents were long lived and whose digestion has always been so good that they may be designated as "gastric athletes." They develop the disease in adult or later life. The latter are poorly nourished, present a phthisical habit, a low pilosity, and suffer from epistaxis, gastric ulcer, or achylia. They develop the disease early.

Alcoholism was noted in the majority of cases of gastric carcinoma in adults by Martin and R. Schmidt, but Friedenwald found it in only I5 per cent.

Location.-Three-fifths of all gastric cancers originate in the pyloric region, another 20 per cent. arise along the lesser curvature and cardia, and the remainder from the walls of the greater curvature. The fundus is rarely involved. Welch's table from I 300 cases exhibits this distributionpyloric region, 60.8 per cent.; lesser curvature, II.4 per cent.; cardia, 8 per cent.; posterior wall, 5.2 per cent.; diffuse, 4.7 per cent.; multiple tumors, 3.5 per cent.; greater curvature, 2.6 per cent.; anterior wall, 2.3 per cent.; fundus, I.5 per cent. 
By more minute distinction between the pylorus proper and the vicinity of the pylorus, Boas in 125 cases located 27.2 per cent. in the pylorus, and 26.4 per cent. in the lesser curvature, and in 40 later cases he found 25 of the lesser curvature and 6 in the pylorus. Mathieu in 234 cases locates 56 per cent. at pylorus, I 2 per cent. at cardia, 4 per cent. on lesser curvature, I.2 per cent. on greater curvature, 23.5 per cent. on the lateral walls.

Tabora connects the occurrence of cancer at the pylorus and on the lesser curvature with varying physiological conditions in the stomach. Cancer of the lesser curvature is usually associated with achylia, pyloric cancer with hypersecretion. In achylia the pyloric region is relaxed, the stomach is promptly emptied in $\frac{1}{2}$ to $\frac{3}{4}$ hour after a test meal, and motor insufficiency develops late. Hence the pyloric region escapes irritation, while the fixed and vascular lesser curvature is chiefly exposed. With hypersecretion the lesser curvature is equally exposed, but the long closure of the pyloric ring (Pawlow) exposes unduly the prepyloric mucosa where primary cancer chiefly develops.

Gross Anatomy.-Gastric carcinoma appears in several characteristic anatomical forms. A classification of these forms may be reached on strictly histological data as done by Kaufmann. Yet the location and gross form of the tumor determine very marked differences in the symptoms and course of the disease, which it seems desirable to emphasize by a classification according to etiology, gross anatomy, and structure. From this point of view one may recognize the following types:

(I) Bulky adenocarcinoma.

(2) Gelatinous carcinoma.

(3) Carcinoma following peptic ulcer:- Ulcerocancer.

(4) Carcinoma simplex. Diffuse carcinoma.

(5) Diffuse scirrhous carcinoma.

(6) Sclerosing fibrocarcinoma. Linitis plastica:

Of I22I cases reviewed by Welch, 79 I (64.8 per cent.) were medullary, 399 (32.7 per cent.) scirrhus; 3I (2.5 per cent.) colloid. Adenocarcinoma is the most frequent variety in my material.

(I) Bulky Adenocarcinoma.- The distinguishing features of this group depend upon a relatively benign adenocarcinomatous structure, which presents a circumscribed polypoid or fungating and eventually ulcerating growth. This tumor is the gastric counterpart of adenoma destruens of the colon and uterus. As in other situations it may reach large dimensions before invading the lymph-nodes, but it is rather more malignant in the stomach than elsewhere.

The location is chiefly near the pylorus, less frequently at the cardia, in both of which situations it soon ulcerates. Or it may develop from the lesser or greater curvatures where it tends to reach large dimensions. This type of carcinoma may be multiple in the stomach and similar tumors may appear in the colon (Hauser). In the early stages, as described by Verse, it appears as a flat, warty thickening of the mucosa, which may develop into a polypoid outgrowth, but more frequently, especially at the pylorus, it early ulcerates, infiltrates the wall, and runs the course of an ulcerating carcinoma. These cases are sometimes difficult to distinguish from secondary carcinoma developing on a peptic ulcer. Their broad base, markedly overhanging edges, and histological structure usually serve to separate them from the secondary carcinomas arising from ulcers.

A second mode of origin is through the carcinomatous transformation of an adenomatous polyp, all stagés of which are described by Hauser and Verse. It appears probable that most of the bulky, polypoid, and fungating 
adenocarcinomas develop from such preliminary lesions. It is characteristic of these bulky tumors that they grow to large size without prominent gastric symptoms or invasion of lymph-nodes. Ulceration becoming established rapidly transforms the clinical picture, adding hemorrhage, pain, vomiting, and cachexia. The excavation of such tumors may be extensive and deep and lead to fatal hemorrhage or perforation.

All of these growths are comparatively solid on section, resembling medullary carcinoma, but some show accumulation of mucus, and areas of fatty degeneration, hemorrhage, and necrosis. In a small subgroup the gross structure is distinctly villous, resembling the coarser tumors of the bladder.

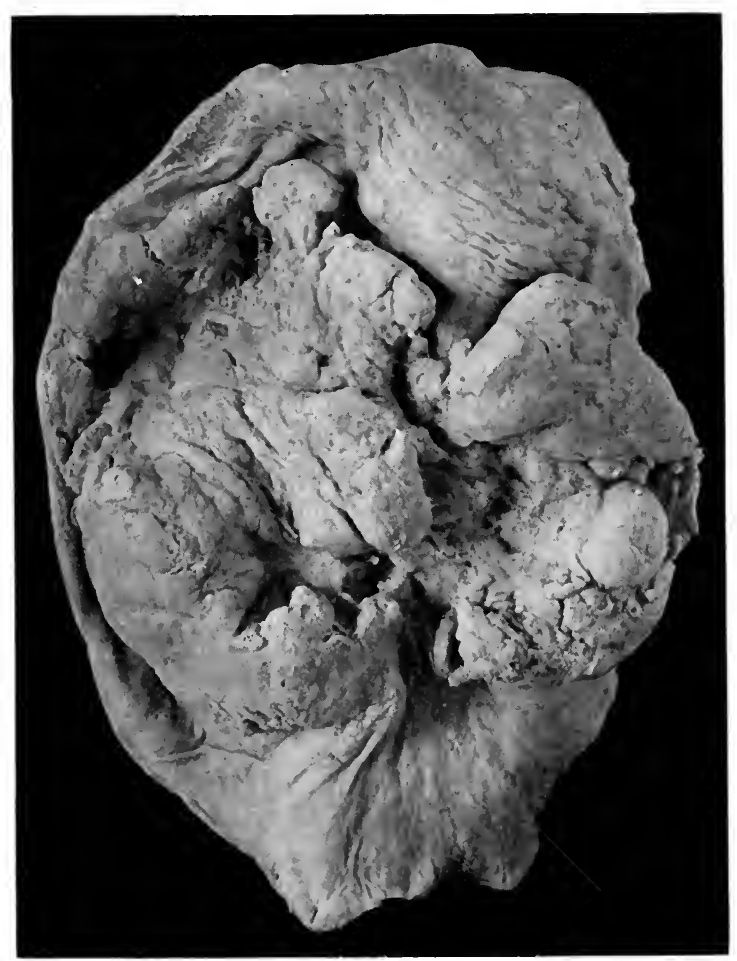

FIG. 287.-Bulky ulcerating adenocarcinoma of stomach.

Their structure shows well-developed branching, vascular papillæ covered with multiple layers of atypical cylindrical cells which may infiltrate the wall and have been known to produce hepatic metastases (Matsuoka, Matti). In a case of empyema Verse found six carcinomas in the stomach, some of which were of elongated papillary or villous type.

The adenocarcinomas are regularly embedded in the submucosa which may be widely infiltrated and in the muscularis which is usually perforated, but extensions to the lymph-nodes may be long delayed. Yet the nodes, liver, and other organs are eventually invaded by growths which reach considerable dimensions, and precocious metastases in distant localities have been observed. Finlay found multiple subcutaneous metastases, the struc- 
ture of which led to the diagnosis of a latent cancer of stomach, verified at autopsy. I have seen a similar case in which a single nodule excised from the midscapular region presented the typical structure of malignant adenoma of the stomach, which was later found at autopsy. Roseler's multiple ulcerating carcinomas of the skin, although not examined microscopically, were probably of this type. A metastatic growth in the humerus was the first sign of gastric cancer in a case of Tilling's.

The structure of gastric adenocarcinoma presents somewhat orderly imitations of the gastric tubule. The originating tubes become much elongated, bifurcated and sacculated, and the tumors are composed of such alveoli compactly grouped with little stroma. The cells are cylindrical or cubical, the cytoplasm clear, and the nuclei hyperchromatic. It is notable that in many gastric tumors the infiltrating and metastasizing alveoli are lined by single rows of cells, and that multiple layers of very large cells are less common than in tumors of the colon. Hence the structure often suggests a benign adenoma. The lumina of the alveoli are usually small, or they may be distended with mucus and exudate, or secondary alveoli may form within the acinus.

Carcinoma cylindrocellulare microscysticum is a type described by Hauser and presenting many large or cystic alveoli distended with thin mucus and serum.

The gastric mucosa is often the seat of glandular and polypoid overgrowths and in the neighborhood of the tumor the hyperplastic glands may gradually pass into carcinomatous structures (Hauser, Verse). Kulbs described an infiltrating adenocarcinoma with ciliated cells in original tumor and in metastases in adrenals and lung.

Adenocarcinomas of the pylorus, with scanty groups of squamous cells more abundant in metastases, are described by Herxheimer and Lubarsch. The squamous cells were probably of metaplastic origin. Primary squamous-cell carcinoma of the cardiac region, probably derived from esophageal epithelium, is described by Kaufmann, Ziegler, and Borst.

Simple adcnoma of the stomach occurs in single or multiple polypoid form. Rarely this tumor is submucous. The single tumors usually remain of small size and give no symptoms unless located near the orifices. Others become the seat of adenocarcinoma. Rarely they reach large dimensions while retaining an adenomatous structure. Chaput described a pedunculated tumor of the fundus as large as a child's head, which was free from ulceration and presented a pure adenomatous structure. Multiple adenomas usually form a part of the lesions of gastritis polyposa.

(2) Gelatinous Carcinoma.-Mucous changes are a frequent microscopic feature of most forms of gastric cancer, but when this change becomes very pronounced it greatly alters the gross appearance and course of the tumor, which in structure is usually an adenocarcinoma.

The characteristic gelatinous carcinoma usually begins in the pyloric region and infiltrates all coats of the stomach wall, which may measure 2 to $3 \mathrm{~cm}$. in thickness. The organ may be reduced in size, but not so markedly as with true scirrhus. The gelatinous material infiltrates and largely replaces the tissues of mucosa, submucosa, and muscularis, and appears in translucent nodules on the serosa. Advanced cases of this type are described by Storer, Amidon, Verse, and others. In Amidon's case the stomach measured 7 inches in length, the wall $\frac{7}{8}$ inch in thickness. The pylorus was but slightly thickened. The mucosa was everywhere replaced by nodules of gelatinous carcinoma, and the peritoneum was extensively invaded. Verse's tumor (64) was a bulky mass encircling the pylorus and infiltrating the vicinity. In Hauser's case $(32)$ the secondary hepatic tumors were pure adenocarcinoma 
without colloid. Adhesions to neighboring organs are frequent with this type of growth. Invasion of lymph-nodes while frequent is usually late and colloid visceral metastases are rare.

In an important group of cases the gastric wall is penetrated early and while the original tumor may remain comparatively small there are numerous and bulky extensions over a portion or the whole of the peritoneum, with ascites. The stomach outranks the colon and gall-bladder as a source of colloid carcinoma of the peritoneum (Kaufmann).

The structure of gelatinous carcinoma in the stomach is very similar to that of simple adenocarcinoma, with the addition of extensive formation of thin mucus. This material fills all but the youngest alveoli to distention and after rupture it infiltrates the supporting tissues and invades lymphatics and the sheaths of vessels and nerves. The lining cells of the alveoli present all stages of its formation and many cells are desquamated and dissolved in the mucus. Complete disappearance of all traces of epithelium may occur in large areas of such tumors, which thereby reach a standstill while growth proceeds in other areas. A partial absorption of this mucus may follow.

In infiltrating portions of mucous carcinoma the diffusely growing cells become ballooned with mucus and the nuclei are compressed into signet-ring forms. These cells are commonly present both in this and other anatomical forms of gastric cancer, either actively growing or regressing, and their presence is an important aid in diagnosis between chronic inflammation and cancer of the stomach.

(3) Carcinoma Following Peptic Ulcer.-The relation of gastric ulcer to carcinoma has long been an actively debated question. Rokitansky, I840, recognized that cancer might develop from ulcer. Dittrich, I848, among I60 cancers found 6 developing near ulcers, and 2 in which the carcinoma was limited to portions of ulcers. Lebert, 1878 , calculated that 9 per cent. of cancers arise from ulcers, while Zenker, 1882 , went so far as to conclude that all gastric cancers are secondary to some form of ulceration. He noted the persistence of free $\mathrm{HCl}$ with cancers following peptic ulcer. In I890 Hauser described several cases of cancer of characteristic anatomical form following ulcer. G. Futterer, I902, concluded that ulcers in the pyloric region frequently give rise to cancer, especially in those portions of the edges most exposed to irritation. The onset of cancer may be marked by increasing pain, anemia, and emaciation. The free $\mathrm{HCl}$ steadily diminishes (Riegel). Mayo Robson finds a history suggestive of ulcer in 59.3 per cent. of his cases of gastric cancer, but reports only one specific case with micioscopic evidence. Recently the Mayo Clinic reports that $7 \mathrm{I}$ per cent. of their gastric cancers were associated with ulcer and 68 per cent. of ulcers were complicated with carcinoma.

In determining the relation of gastric ulcer to cancer, statistical, clinical, gross anatomical, and microscopic data are available (Ewing, Lit.). Statistics show that ulcer is much more common and cancer much less common in women than in men, and this conclusion is not seriously altered by distinguishing between simple and chronic ulcer. The clinical history of precedent ulcer and of persistent free $\mathrm{HCl}$, while suggestive and important as corroborative evidence, cannot demonstrate a direct connection between ulcer and cancer.

The gross anatomy, however, furnishes very strong evidence. In characteristic cases, as described by Hauser, there is a deep crater-like ulcer with thick and very dense base free from cancer, indurated and undermined or everted edges, exactly reproducing the chronic peptic ulcer. In addition 
there are marked contracture of the wall, swelling and induration of lymphnodes and occasionally visceral metastases. Secondary ulceration of primary carcinoma may, but does not often, produce such a lesion and these cases must be regarded as probably carcinoma following ulcer. In most cases of this type microscopical evidence is required and when it appears in unequivocal form there can be no doubt that a carcinoma has been engrafted upon a peptic ulcer.

The uncertain territory in this field includes (I) certain superficial ulcerative processes which the microscope shows are slearly carcinomatous and (2) certain characteristic chronic peptic ulcers which have been classed as carcinomatous on less definite microscopical structure.

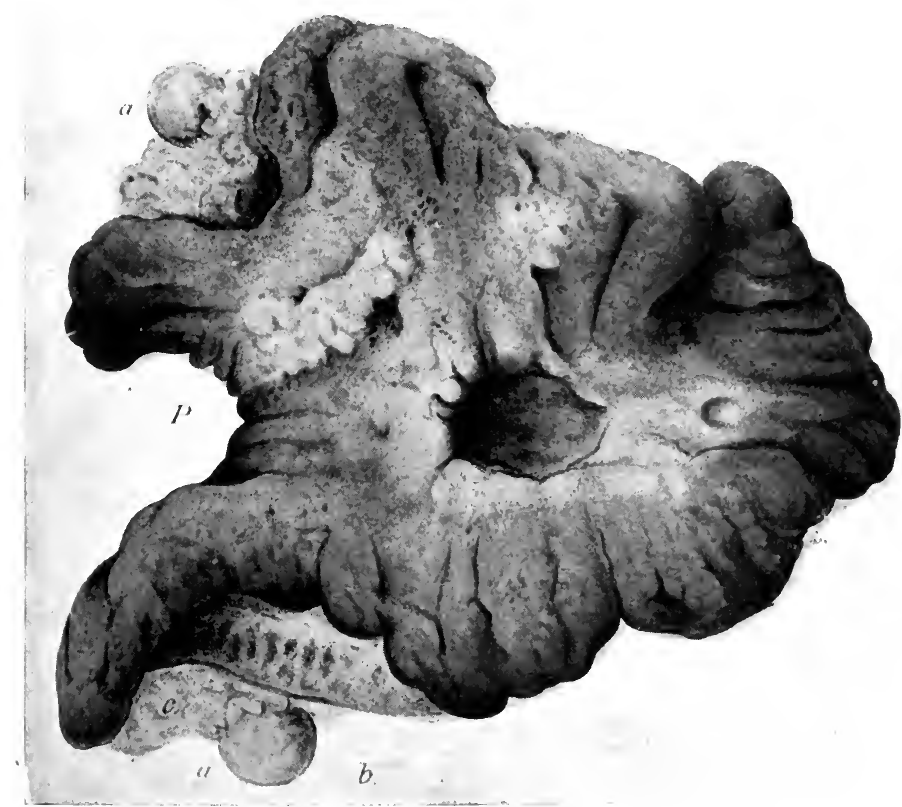

FIG. 288.- - Hauser's figure of peptic ulcer with secondary carcinoma of stomach. Note isolated polypoid outgrowths on edge of ulcer and elsewhere.

(I) That localized superficial carcinomas of the stomach may early become ulcerated has long been known and is most clearly demonstrated by Verse's descriptions of such early cases. These lesions are chiefly located in the pyloric region and lesser curvature, they are comparatively superficial and usually lack the deep funnel form and extensively indurated base and borders of the carcinomatous peptic ulcer. They may eventually closely simulate the latter.

Microscopically they present pronounced adenocarcinoma, or alveolar gelatinous carcinoma in both base and edges and the surrounding mucosa is hyperplastic. Verse finds that the glands on the edges of such ulcers may show a gradual transformation into carcinomatous structures.

(2) Peptic ulcers exhibit many transitional stages suggesting carcinomatous change. Some, and in the experience of most observers the majority, fail to 
show any evidence whatever of carcinoma. In Vienna, 2 per cent., in Copenhagen, 20 per cent. of the subjects coming to autopsy show scars of old healed ulcers (Hirschfeld). Others show suspicious or pronounced precancerous changes in a portion or the whole of the edges. The proportion of ulcers classed as carcinomatous depends on the criteria employed by the observer. Hirschfeld places the proportion of ulcers which become cancerous at 5 to 6 per cent. and he summarizes clinical and statistical data indicating that the proportion is small. Futterer, however, places the proportion very much higher and holds that the tendency toward carcinomatous change greatly influences the prognosis of gastric ulcer. Hauser emphasizes the inflammatory overgrowth of glands in the edges of ulcers, but interprets as carcinoma only very advanced stages of the process. Recently Wilson and MacCarty in the Mayo Clinic report that 68 per cent. of their gastric ulcers were associated with carcinoma. They secure this high proportion by inter-

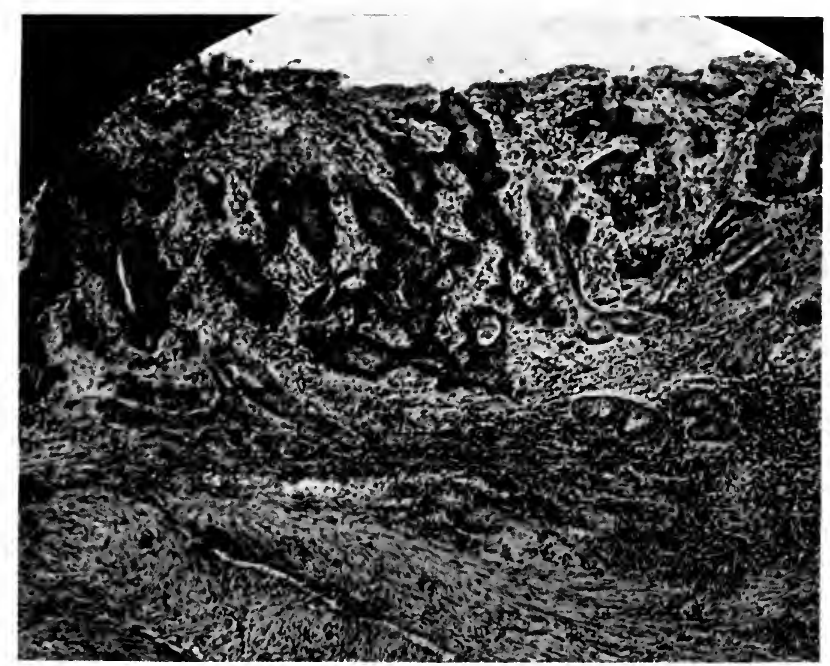

FIG. 289.-Atypical proliferation of gastric glands on edge of peptic ulcer. A precancerous lesion, not to be classed as cancer.

preting as carcinomatous many appearances which it is difficult to separate from chronic inflammatory overgrowth and heterotopia of gland tissue. They recognize many cases in which it is impossible to determine whether the ulcer or the carcinoma was the primary lesion. Boekelmann collects a series of estimates varying from 3 to 50 per cent. In France opinions are widely at variance. Moutier in 35 cases from operation and autopsy found I9 simple ulcers, I5 distinctly cancerous, and one cancerous duodenal ulcer.

I believe that the higher estimates (above 2 or 3 per cent.) indicate too high an average for the carcinomatous transformation of ulcers. Where definite carcinomatous alveoli are present throughout the base of an ulcer, especially if early, the condition is probably better regarded as primary carcinoma. MacCarty states that where irregular epithelial structures occur in the submucosa one is surely dealing with carcinoma. Yet heterotopia of epithelium may be of inflammatory origin and it would seem better to designate many of these uncertain lesions as at most precancerous. In my own 
material I find it very difficult to trace the development of cancer in the edges of peptic ulcers. The cases seem to fall distinctly into the group of ulcerating adenocarcinomas or among simple ulcers without any trace of cancer. The structure of the malignant cases varies greatly, indicating that there are several histological types of early ulcerating cancers. The mucosa surrounding simple ulcers is sometimes extensively altered, the glands hypertrophied, the cells enlarged, nuclei hyperchromatic, and alveoli occasionally misplaced, but definite precancerous changes in cases of undoubted simple ulcer I have rarely found. It has appeared, however, that the digestive fluids may completely excavate a superficial carcinoma leaving the base

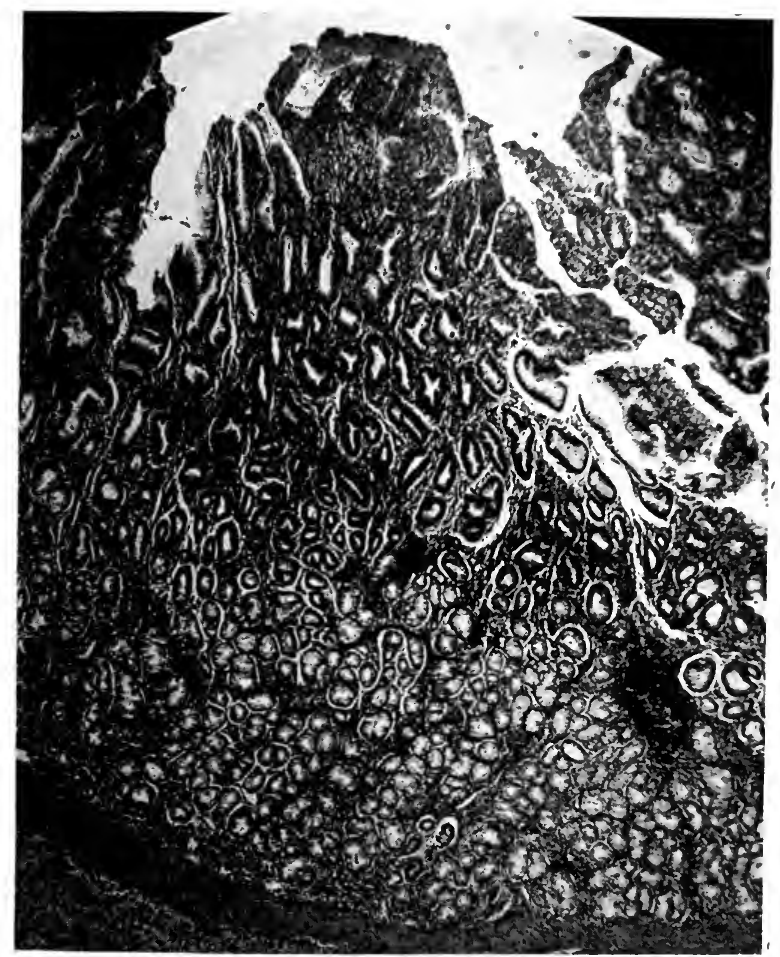

FIG. 290.-Hypertrophic gastritis in a stomach the seat of peptic ulcer.

free, but a submucous ring of cancer encircling the ulcer. More often islands of carcinoma remain throughout the base. The complete destruction of all traces of carcinoma has been demonstrated by Thalheimer. Therefore, the presence of small areas of carcinoma in the edges of an ulcer does not necessarily mean a carcinomatous change in a simple ulcer.

It is at least evident that quite specific criteria must be employed in the diagnosis of cancer following ulcer, since the conditions surrounding this process in the stomach are peculiar.

Against a very great frequency of carcinomatous change of peptic ulcer stand several general considerations emphasized by R. Williams. Sixty per cent. of gastric cancers, he finds, are located at the pylorus, while only 
I 2 per cent. of ulcers occur in this situation. The commonest seat of ulcer is the lesser curvature (36.3 per cent., Welch), whereas only I 2.2 per cent. of cancers occur in this situation. Ulcer of the duodenum very rarely gives rise to cancer. In other regions there are very few ulcers which are known to be followed by cancer. One of these is lupus, in which the average interval is 30 years. A few varicose ulcers of the leg develop epithelioma, but nearly always after many years. The age incidence of the two diseases is very different. Yet I cannot agree with Williams that such evidence is conclusive. It is much too general and uncertain. Thus Boas found 27.2 per cent. of his cancers at the pylorus, but a later series of 40 cases gave only ${ }_{5} 5$ per cent. at

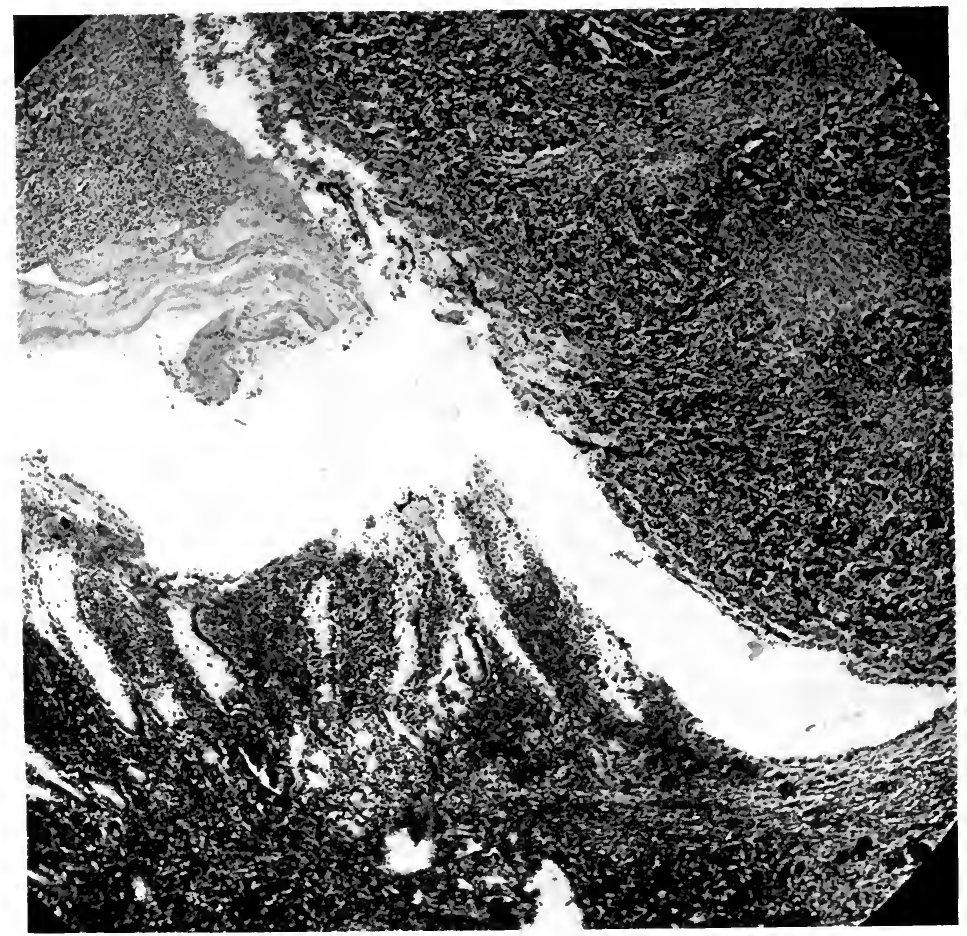

FIG. 29I.-Edge of ulcerating carcinoma of stomach. The carcinoma was limited to a narrow ring encircling the ulcer.

this location. Bamberger reports that after ro25 gastro-enterostomies for ulcer observed for some time cancer developed in 22 (2.I per cent.). Among 152 cases in which ulcer was treated by excision cancer developed in r.9 per cent. These results are quite incompatible with a frequent origin of cancer from peptic ulcer.

Experimental production of adenocarcinoma by irritation of artificial ulcers in animals is reported by Hemmeter, Futterer and Maniscalio, but the supposed malignant process although interesting in itself, lacked the progressive character of cancer. On the other hand, Haga's experimental adenoma of the stomach, which closely approaches a genuine tumor, always showed glandular overgrowth first, and only later, if at all, ulceration. 
Gross Anatomy. - The type of carcinoma which is said to follow peptic ulcer presents in its early stages the same appearance as the uncomplicated peptic ulcer and it may be impossible to detect the malignant process except by microscopical study. The carcinoma is said to begin on the pyloric side of the ulcer and causes increased swelling and density of the borders and fixation of the ulcer to the muscularis and serosa. No great change in the density of the base can be expected since this portion of ulcers is always very firm and carcinoma can invade the base only from below. The shortening of the curvature, thickening of serosa, and cicatricial contraction drawing up the lymph-nodes, seen in old ulcers, are increased in carcinoma, and the swollen lymph-nodes become more dense. These changes observed in surgical cases are usually somewhat aggravated in subjects coming to autopsy. The vicinity of the ulcer then shows more extensive thickening from invasion of all coats of the organ, the lymph-nodes are larger, and there may be extensions to liver, gall-bladder, and peritoneum, but extensive metastases are rare and in many fatal cases the disease is localized to stomach and adjacent nodes. Although pyloric stenosis is frequently established, the tendency to dilatation of the stomach is usually counteracted by cicatricial contraction of the lesser curvature. Perforation of the ulcer occasionally terminates the progress.

The structural type of carcinoma following ulcer is usually a small alveolar or adenocarcinoma, and thís type was exclusively represented in Hauser's cases. From the hyperplastic glands on the edges of the ulcer there is a downward growth of atypical glands which soon break up into irregular alveoli lined by atypical, cylindrical, or cubical cells. From

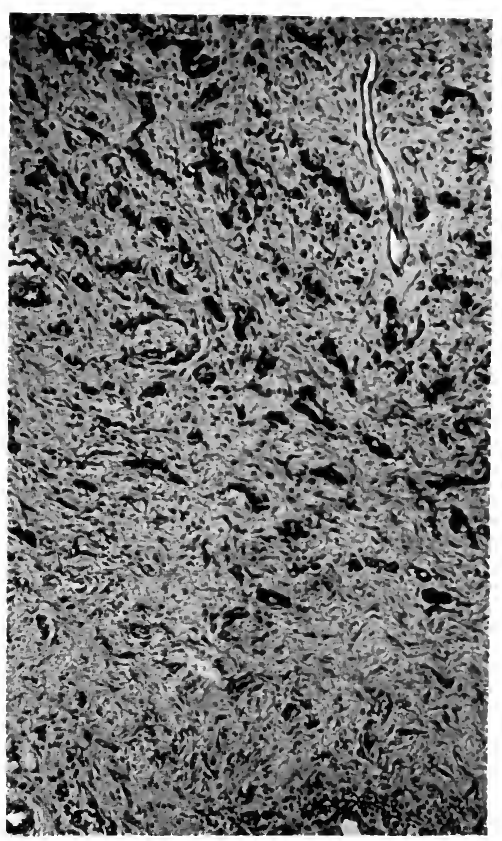

FIG. 292.-Ulcerating gastric cancer. Groups of tumor cells and cellular connective tissue forming base of ulcer. these structures solid cell groups commonly develop and extend laterally in the wall and through the lymphatics into the lymph-nodes.

(4) Carcinoma Simplex. Medullary and Diffuse Carcinoma.-The more malignant types of gastric carcinoma are composed of more atypical cells arranged in alveoli or growing diffusely. These tumors produce two well-defined gross anatomical forms.

(I) Soft, often ulcerating, rounded, more or less circumscribed tumors of considerable extent occur chiefly in the cardia and fundus. They are often vascular (carcinoma telangiectatica) and subject to severe hemorrhages. Fatty degeneration and necrosis lead to ulceration of wide extent, to erosion of large arteries and to perforation. The neighboring organs, liver, pancreas, colon, duodenum, may be involved by continuity of growth. The lymphnodes are early invaded and metastases are very frequently seen in liver, 
lungs, peritoneum, and distant organs. This is the most aggressive and rapidly progressive form of gastric carcinoma.

(2) Diffuse carcinoma may arise from any portion of the mucosa, usually near the pylorus, and maintains an infiltrating tendency from the first. The original tumor may then be difficult to locate and a large segment of the wall may show diffuse thickening of all coats with little ulceration or only superficial erosion of the mucosa. Early invasion of lymph-nodes and widespread metastases occur as in the preceding form. Occasionally there is deep ulceration at the pylorus and diffuse infiltration over a wide area. Most of the perforations in gastric cancer occur in this type. Among 507 cases of cancer Brinton found perforation into the peritoneum in 17 , into colon, II.

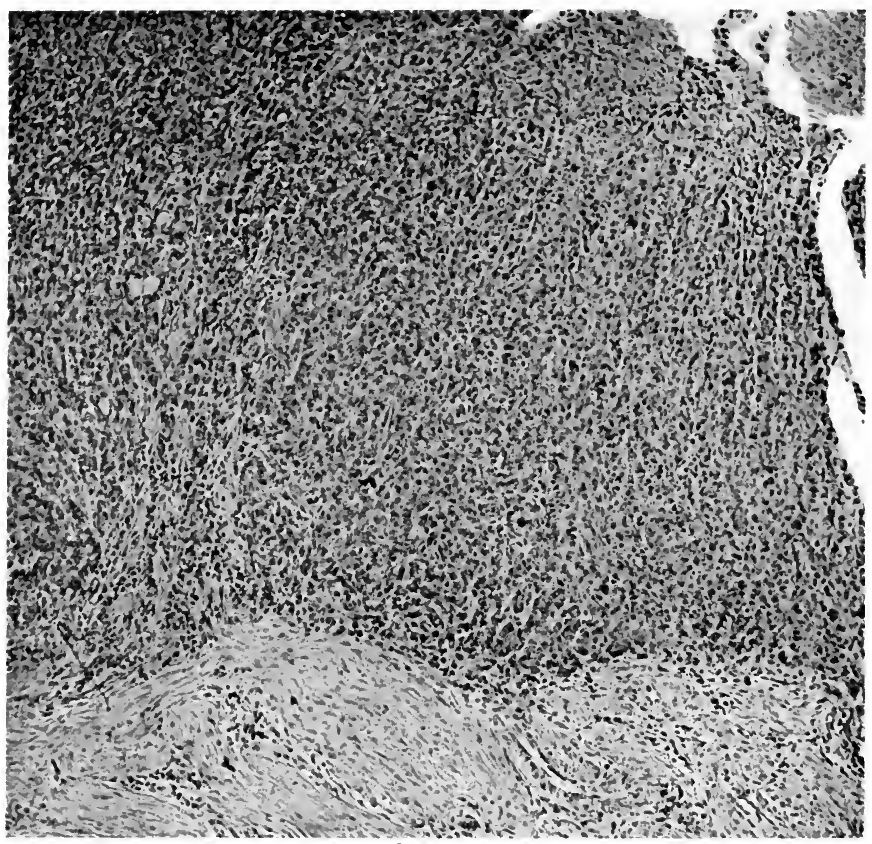

FIG. 293.- Section of mucosa in diffuse superficial carcinoma of stomach.

Mislowitzer collected $\mathrm{I} 7$ cases of perforation of the abdominal wall. Lang in $2 \mathrm{IO}$ cases reports peritoneal perforation in $\mathrm{I} 2$.

The structure presents small or large alveoli or a diffuse growth of atypical cells, which are usually small, polyhedral or round, and with relatively large hyperchromatic nuclei. A desmoplastic quality may be entirely lacking, and some of these carcinomas are readily mistaken for lymphosarcoma. The cell form may vary considerably and in different portions of the same tumor one may find large alveoli, small alveoli, or diffuse growth. Giantcells may occur in considerable numbers and when they appear with roundcells of various sizes in a tumor lacking alveolar structure the resemblance to sarcoma may be pronounced (cf. Kaufmann). The invasion of muscular wall, nerve-trunks, and lymph-vessels is remarkably free. In less active foci mucous globules appear in many cells. In metastases an alveolar struc- 
ture is apt to reappear or the structure may be even more atypical than the original.

(5) Diffuse scirrhous carcinoma is a slowly progressive type of the disease which differs from carcinoma simplex in its slow course, desmoplastic properties, absence of ulceration, prominence of pyloric stenosis, and in the peculiarities of its metastases. It differs from sclerosing pyloric fibrocarcinoma in its wider extent, more cellular character, and frequent metastases.

The gross appearance is characteristic. A considerable portion of the wall beginning at the pylorus shows smooth thickening of all coats, especially

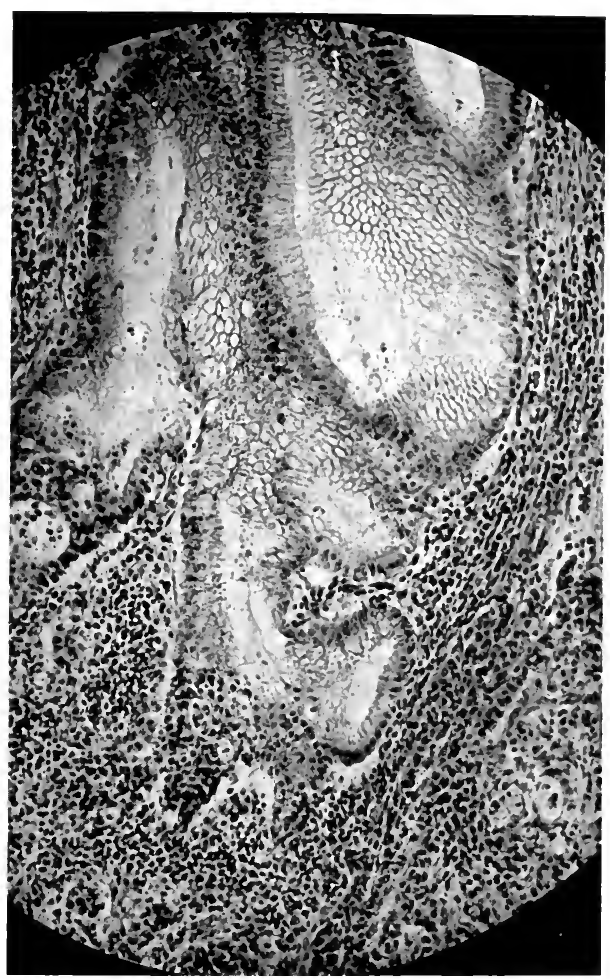

FIG. 294.-Diffuse superficial carcinoma of stomach. The ducts of the mucous glands are intact, the bases are breaking up into small carcinomatous alveoli.

of the submucosa and muscularis. The entire organ may be involved and greatly reduced in size by cicatricial contraction. The pyloric and other areas of mucosa may be eroded over a wide extent, but there is seldom destructive ulceration. The serosa is opaque, fibrous, and covered with small nodules from invaded lymphatics. There may be a remarkable freedom from adhesions, but usually the pylorus is fixed to neighboring tissues and much contracted.

The lymph-nodes are regularly involved and in these structures and in the liver and other organs bulky tumors, often much larger than the original, develop from a freer growth of tumor-cells. The peritoneal lymphatics may 
be extensively involved with constriction and adhesions of the intestine. The omentum may be drawn up into a small cancerous mass attached to the lower border of the stomach.

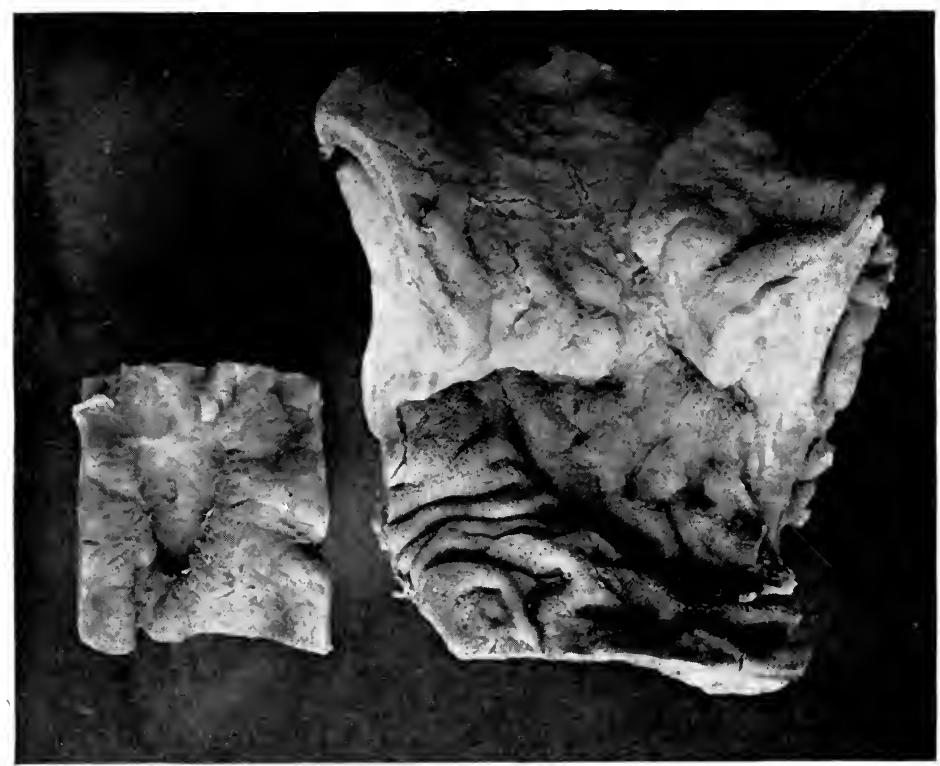

FIG. 295.-Diffuse carcinoma of pylorus with three secondary peptic ulcers, one at pyloric ring, one $3 \mathrm{~cm}$. and another $5 \mathrm{~cm}$. from ring.

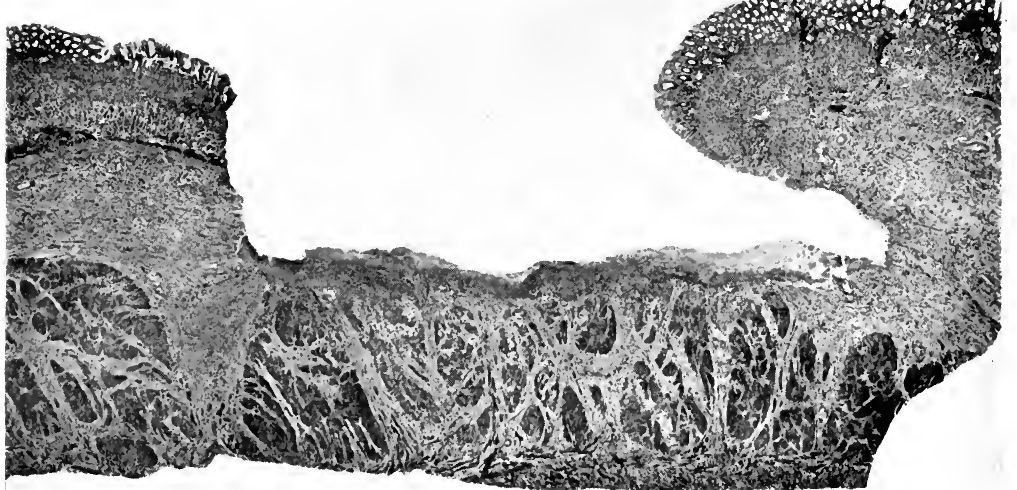

FIG. 296.-Cross-section of secondary peptic ulcer arising on diffuse carcinoma of pylorus.

In a well-defined group of cases the original tumor is small and poorly defined, but bulky metastatic tumors develop, especially in the liver and ovary, and the disease runs its course as a primary tumor of these organs; 
24 per cent. of the secondary carcinomas of the liver are derived from the stomach (Rolleston) and not a few develop from relatively small and usually fibrous cancers of the stomach. In the ovary the metastases are often bilateral. A scirrhous tendency may be associated with adenocarcinoma, and in the pyloric region this tendency is frequent and pronounced, so much so that Hauser describes a special variety of scirrhous adenocarcinoma.

The great majority of scirrhous tumors present small or large solid groups of polyhedral cells which are easily recognized as carcinomatous. As the fibrosis increases the tumor-cells diminish in number, and in wide areas they may be reduced to small groups of degenerating cells with mucous globules,

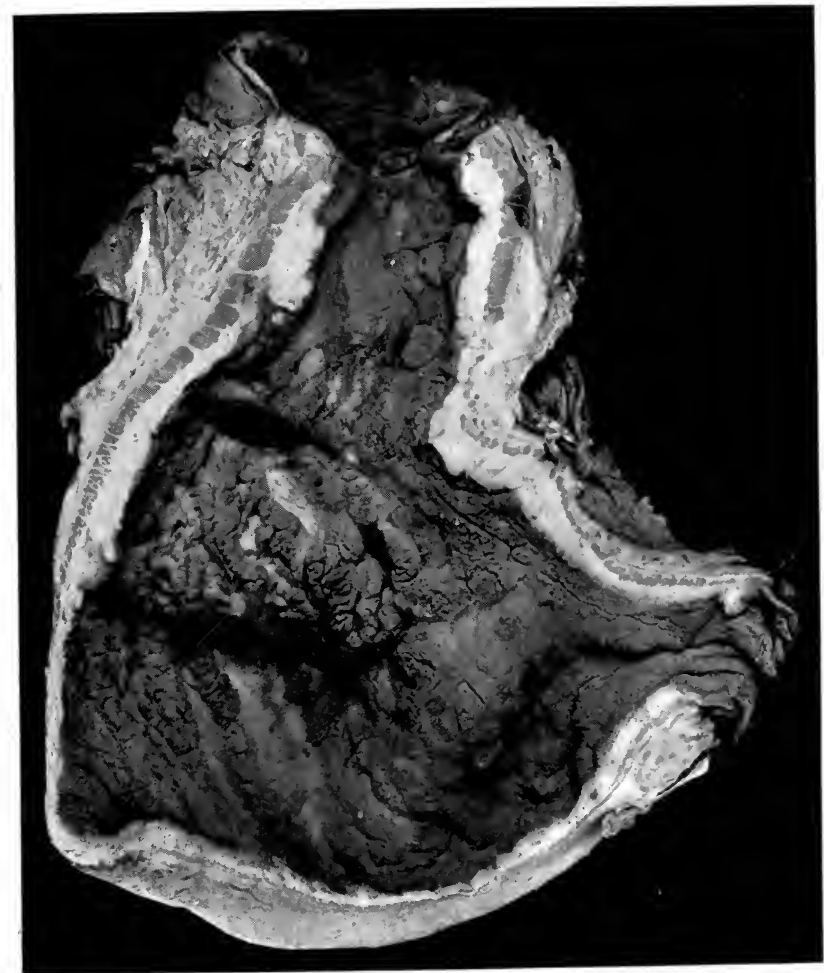

FIG. 297.-Diffuse carcinoma of entire stomach. Gastro-enterostomy.

or to small granular opaque cells which resemble plasma-cells. In the nervetrunks, lymphatics, and capillaries about such tumors much larger and more typical cells are usually found, while in the lymph-nodes and peritoneal nodules the growth may be quite typical.

(6) Sclerosing Pyloric and Diffuse Fibrocarcinoma. Linitis Plastica.The usual form of scirrhous carcinoma of the stomach is easily recognized by the diffuse thickening of all the coats, the contraction of the organ often to a rigid tube, the frequent presence of metastases, and especially by the microscopic evidence of round-cell, or alveolar or gelatinous carcinoma. It has long been known and is specifically mentioned by Rokitansky and by 
Kaufmann that the epithelial celis in scirrhous cancer of the stomach may be reduced to a minimum or may largely disappear, so that a very careful microscopical examination is required to detect the traces of carcinoma. Indeed, the last traces of epithelial structures may completely disappear over considerable areas of thickened submucosa and muscularis, so that no suspicion of malignant process past or present may be suggested.

Neglect of this fact has led to the accumu-

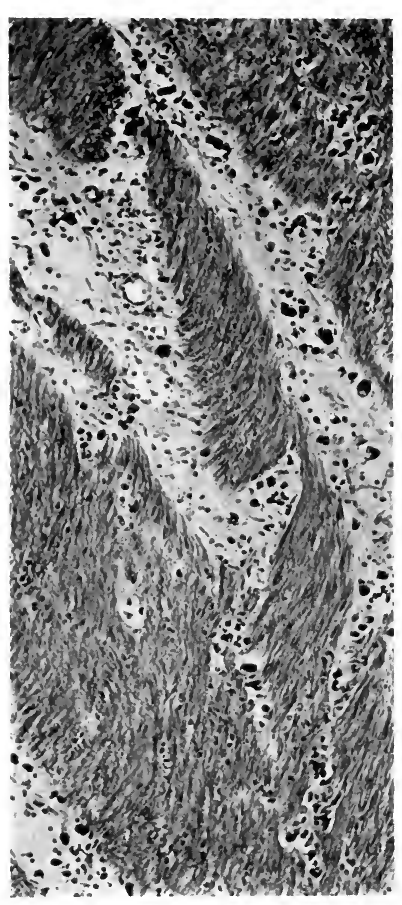

FIG. 298.-Characteristic atypical structure of certain carcinomas of pylorus. Single tumor cells with hyperchromatic nuclei infiltrating muscularis. resembled scirrhous carcinoma. (Schmidt, Viti, Rosenheim, Deguy, Marcy, and Griffith). In Sheldon's case gastric symptoms antedated operation I5 years and the patient was well $3 \frac{1}{2}$ years later. v. Eiselberg's patient was well 5 years after gastro-enterostomy.

In several cases there were signs of old superficial ulcers (Formad, Allbutt, Turner, Meinel). Nothnagel's case ran the course of pernicious anemia. Many cases would suggest classification with polyserositis and showed multiple thickenings throughout the peritoneum, intestine, colon, and rectum (Lebert, Marignac, Wilks, Henrot, v. Kahlden, Gabbi). Yet very similar cases are reported by Bret and Paviot, who point out that the intestina! lesion is chiefly subserous and that extensions readily pass from the stomach to omentum and colon.

Krompecher, under the term "gastro-intestinal sclerostenosis," emphasized a group of benign cases which he attributed to cardiac disease, chronic con- 
gestion, edema, and fibrosis, but he later abandoned the idea that these cases were not probably carcinomatous. Leith describes a case of this type as non-cancerous cirrhosis. The lesion involved stomach, ileum, and colon, but the microscopic report is somewhat lacking in details, although the author positively excludes carcinoma.

In dealing with such an extensive collection of anomalous conditions it is obviously impossible to urge that any uniform process is represented. It appears quite possible that rare cases of chronic gastritis, the scarring of ulcess, late syphilis, peculiar traumas, and atrophying fibrous processes may simulate fibrocarcinoma, but it may be claimed that no well-defined group of cases exists apart from fibrocarcinoma. As Krompecher points out, the

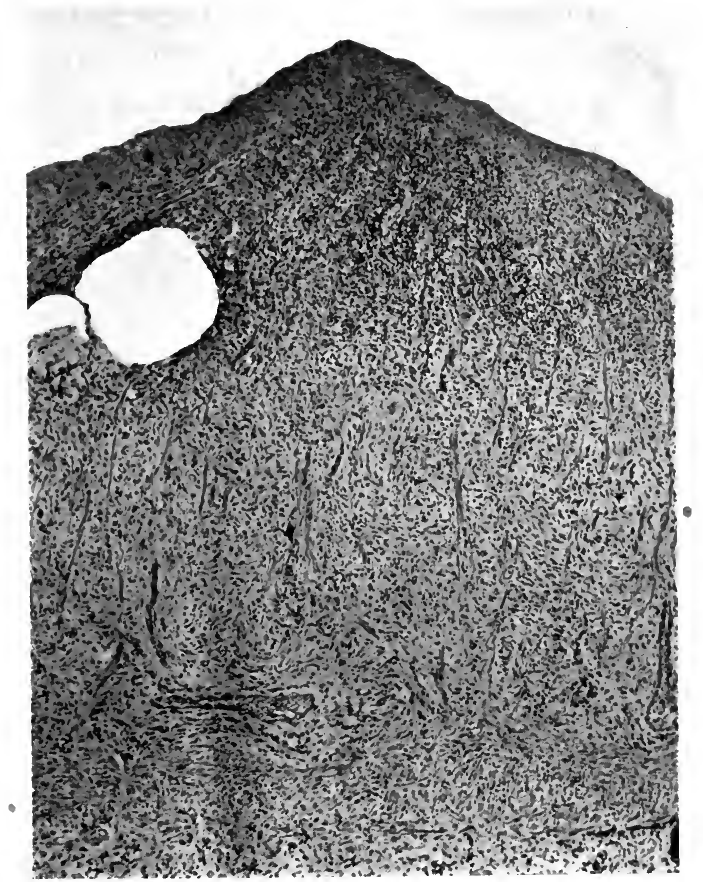

FIG. 299.-Sclerosing fibrocarcinoma of pylorus. Linitis plastica. Erosion of mucosa. Small groups of tumor cells with mucous contents limited to serosa.

microscopic study of older cases and even of the latest reports is open to serious criticism, since the histology of regressing fibrocarcinoma of the stomach is seldom fully recognized. Thus Tilger wrote an elaborate description, completely excluding traces of carcinoma, in a case which on subsequent study Meinel found to be carcinomatous.

In a series of 17 cases of linitis plastica Krompecher and Makai found 9 which they recognized as disseminated fibrocarcinoma, by the discovery of islands of atypical epithelium, or scanty acini of adenocarcinoma, or groups of cells with mucous degeneration and typical signet-ring nuclei. In other portions of these same tissues they find only cellular granulation tissue or hyaline areas and cells of undetermined origin, all of which made up the bulk 
of the sclerotic areas in the remaining 8 cases in which no definite signs of carcinoma were present. In the peritoneal plaques and thickenings of intestinal wall and peritoneum similar structures were present. They therefore conclude that all their cases are of identical nature and belong in a group of atypical disseminated sclerosing carcinoma. They also incline to agree with Meinel that a benign acquired cirrhosis of pylorus and stomach of the type of linitis plastica probably does not exist. The interpretation of these cases as endothelioma, offered by Jungmann, Szobolew, v. Hansemann, Fick, Cignozzi, Aldegarmann, and Donath, they also discard. Yet of the cases reported as endothelioma, only those of Fick and Cignozzi bore any gross resemblance to linitis plastica.

My own study of a group of these cases has led to the same conclusions, modified as above stated, because of the very miscellaneous nature of the reported cases.

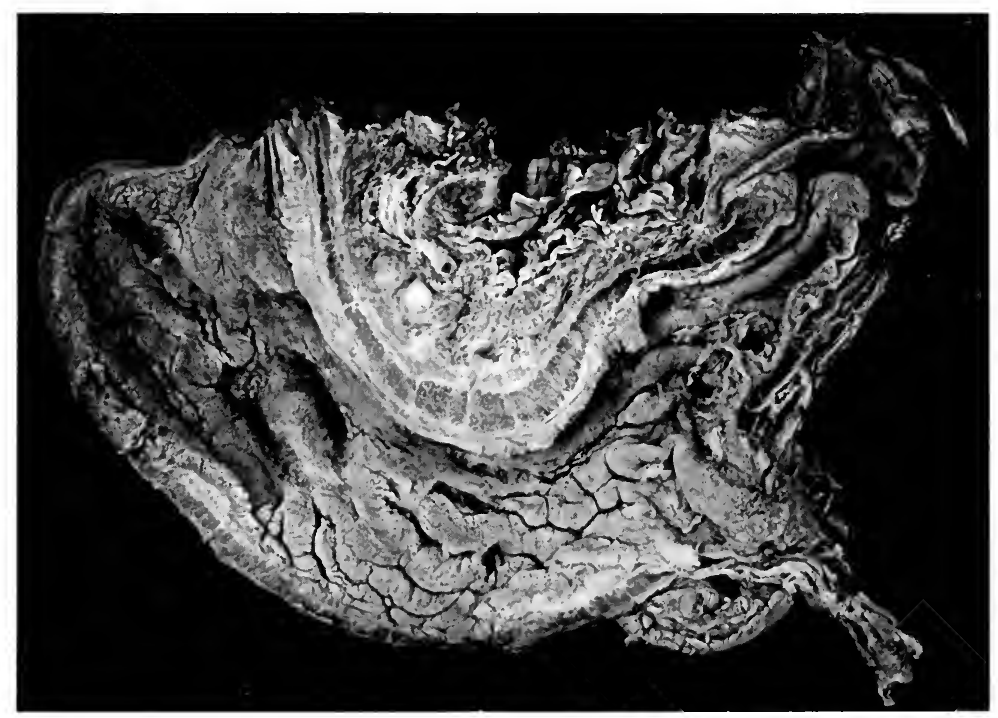

FIG. 300.-Diffuse sclerosing fibrocarcinoma of stomach. Linitis plastica. Leatherbottle stomach.

I find every gradation from small-cell, diffuse, and scirrhous carcinoma, with pronounced signs of malignancy, down to overcellular granulation tissue containing isolated cells with mucous globules, to vascular tissue with overgrowing capillaries suggesting endothelioma, or hyaline connective tissue with scanty cells of undetermined origin, and even to connective tissue without a trace of atypical cells of any type. As a rule extensive search reveals some foci of characteristic mucous cells in submucosa or muscularis. The nervetrunks sometimes show infiltration by polyhedral cells with hyperchromatic nuclei, when the surrounding tissue is free. The last traces of epithelial cell groups may appear as foci of mucus lying in fibrous tissue. Single rows of cells in fibrous tissue may resemble plasma-cells. In 4 cases regarded as benign pyloric stenosis, and narrowly limited to this orifice, I found none of the usual signs of carcinoma, but only such atypical regressing cell groups. It therefore seems necessary to conclude that all cases of hypertrophic pyloric 
stenosis and diffuse cirrhosis of the stomach in adults are atypical fibrocarcinoma. Miscellaneous cases of apparently benign character must be regarded with suspicion.

From this point of view the origin and significance of this type of carcinoma becomes of great interest. The atypical cells may safely be traced to the disordered glands in the mucosa. This structure commonly shows advanced chronic inflammation with hypertrophy or erosion, rarely with atrophy and replacement by granulation tissue covered by flat epithelium. 'The presence of scars of old superficial ulcers in many cases suggests that the cells may be derived from the heterotopic glands in the edges of these lesions. Meinel argues for the derivation of the cells from the endothelium or lymph spaces and uses the term "lymph-epithelioma." I find it often difficult to distinguish atypical epithelium from the hyperplastic endothelium in these tissues, but have not observed any definite indications that the atypical cells are of endo-

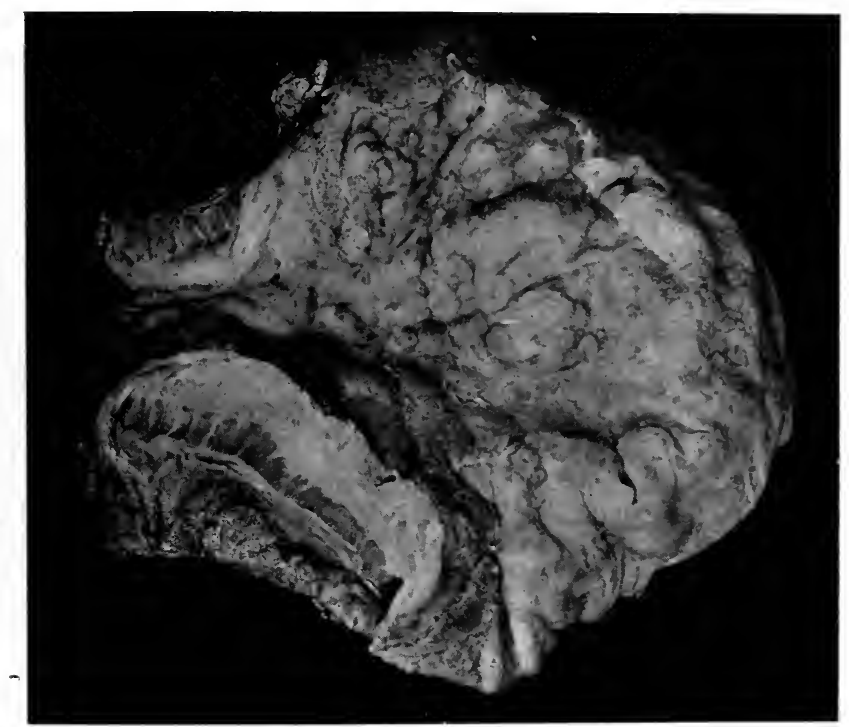

FIG. 301.-Diffuse pyloric carcinoma with erosion.

thelial origin. Most of the cases described as endothelioma of the stomach are quite different in gross appearance from linitis plastica. G. Hayem derives the tumor from the granular acidophile peptic cells. In a case of linitis plastica extending from the cardia to the prepyloric region he found an intact muscularis mucosa, but a diffuse infiltration with large, irregular, granular, acidophile, vacuolated cells with large nuclei, which he would trace to a diffuse breaking down of the peptic glands.

The movements of the stomach provide unusual facilities for the mechanical transfer of proliferating epithelial cells which might otherwise be quite unable to disseminate themselves over such wide areas. It seems necessary to assume that the growth capacity of these cells is much less than that of frank carcinoma. Hence the course of the disease is slow, fibrosis and mucous degeneration overtake most of the cell colonies, and in many areas the carcinomatous element disappears entirely. The wide dissemination over duodenum, peritoneum, and colon suggests that the process was at one time more 
active, but eventually regressed with fibrosis. A final standstill of the entire process may thus be assumed in the cases which appear to recover after gastroenterostomy.

In linitis plastica there appears to exist a peculiar form of gastric carcinoma probably occurring in resistant subjects and originating from cells of limited growth capacity, which pursues a chronic course and tends to spontaneous regression of the tumor process, but to the death of the patient. The occurrence only in adults, 20 to 80 years, clearly separates the condition from congenital stenosis. Yet congenital cancer of the stomach is reported by Mathieu. Danosky described in a Io weeks' old infant a diffuse and very dense thickening of the submucosa with pyloric stenosis. Torkel attributes the benign congenital stenosis of the pylorus to a congenital malformation with inclusion of islands of Brunner's glands in the pyloric muscle. It is possible that some later cases of pyloric stenosis may develop from this same anomaly and that a malignant process may originate in these aberrant glands.

In the typical condition of linitis plastica the stomach is much thickened and contracted (leather-bottle stomach). The lesion begins in the pylorus and may be confined to this region or to the curvatures, from which it extends over a part or the whole of the stomach and occasionally to the duodenum. The pyloric orifice projects into the duodenum as the cervix uteri into the vagina. The stomach wall is four to eight times the normal thickness. The new tissue is chiefly in the submucosa, while the muscularis is also much thickened and traversed by opaque bands of fibrous tissue. The tissue is very dense, resisting incision almost like cartilage. The mucosa is usually thrown into folds or nodular elevations, or it is thin, eroded, or ulcerated. The pylorus may be movable when the disease is limited to the stomach, but is often fixed by adhesions. The pyloric lymph-nodes are usually moderately enlarged. Extensions to the peritoneum give rise to fibrous thickenings constricting the intestine and causing marked cicatricial contraction of the omentum. Fibrous areas may be seen over the liver and spleen. Hanot and Gombault found the portal vein converted into a rigid tube. The microscopical structure is indicated in the preceding discussion.

The Gastric Mucosa in Cancer of the Stomach.-The condition of the gastric mucosa varies widely, depending on the type and progress of the tumor and the previous condition of the mucous membrane. There is a constant tendency toward diminished functional capacity, associated with atrophy of peptic cells and a metaplastic transformation of gastric into an intestinal type of cell lining, with marked increase of goblet cells (J. E. Schmidt, Lit.). An increased secretion of alkaline mucus tends to neutralize acid. This change begins in the pyloric region where it may become very marked and simulate heterotopia of duodenal glands. Observing it in marked degrees Gosset and Masson were led to believe that all pyloric cancers might be derived from heterotopic duodenal glands.

Throughout the altered mucosa many large eosinophile cells and hyaline globules often appear, about the nature of which there has been much discussion. They appear also in the stroma of cancers. They have been regarded as capillary red-cell thrombi (Saltykow), degenerated fibroblasts (Marchand, Lubarsch), or epithelium (Thorel), altered plasma-cells (Schridde, Fabian), or englobed secretion (Verse). Very marked polypoid outgrowths are observed in cases developing on gastritis polyposa. A relatively intact mucosa often persists in cancer following ulcer. Thus in 15 cases examined by Rosenheim, Hammerschlag, Lenek, and Boekelmann the mucosa was intact in $\mathrm{I} 2$ and showed slight increase of peptic cells in 3. In some cases an adenocarcinoma is associated with general hypertrophic gastritis. Chronic productive 
interstitial gastritis with atrophy is the most frequent condition, as was early. shown by Fenwick. In all of I4 cases Rosenheim found catarrhal, interstitial, and atrophic gastritis, most marked near the tumor, but appearing in foci throughout the fundus. Similar results were obtainerl by Mathieu and Cohnheim. In 17 cases Boekelmann found interstitial gastritis in all, focal lesions in 7 , diffuse advanced changes in 8, and early complete loss of glands in 2. In diffuse carcinoma the mucosa has been found atrophic, with or without extensive erosion. In diffuse colloid cancer the gelatinous nodules may be widely distributed over the mucosa. The various changes in the production of $\mathrm{HCl}$ and ferments depend closely upon the state of the mucosa, but

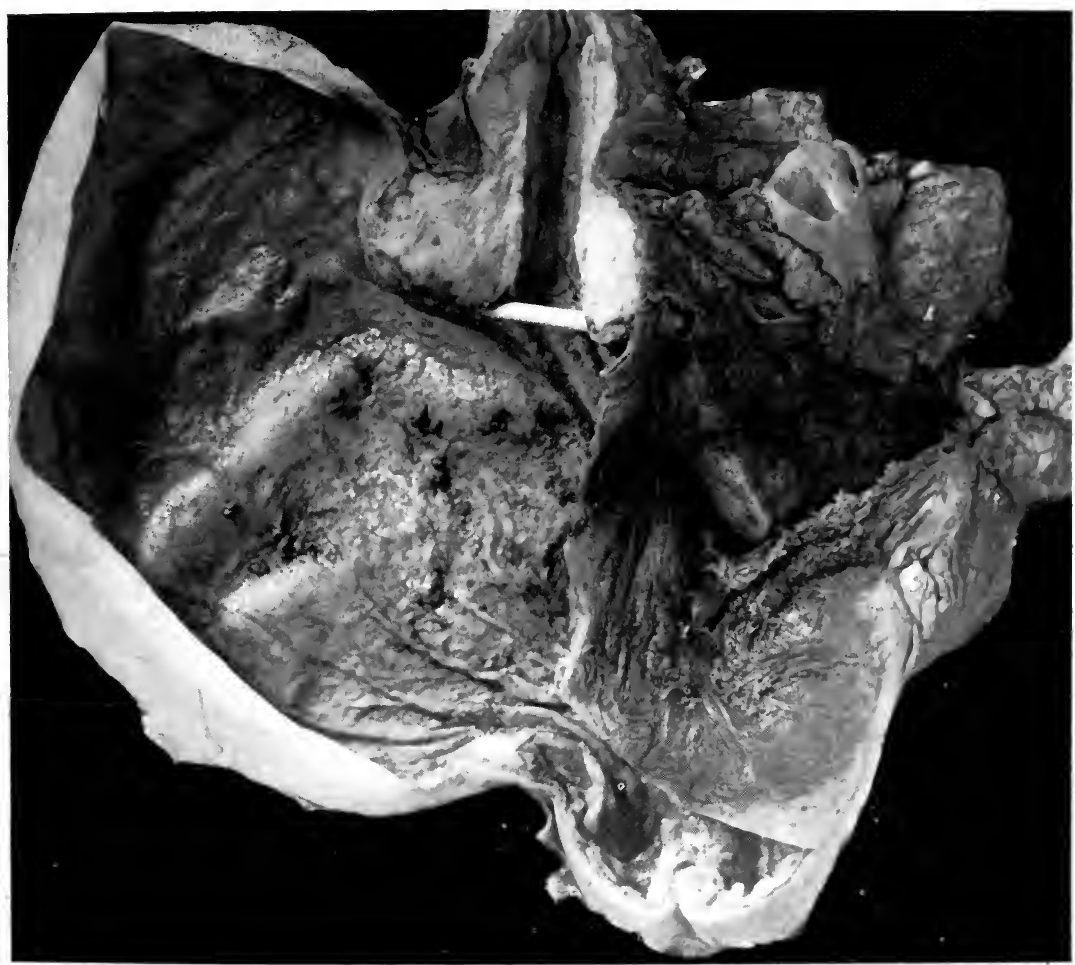

FIG. 302.-Gastric cancer at cardiac orifice,

portions of the fundus may be nearly intact in cases with loss of $\mathrm{HCl}$ (A. Schmidt, Kokubo). Hence function may be restored after partial gastrectomy. The relation of the gastritis to the carcinomatous process varies. Fenwick pointed out that atrophy is most marked with scirhous cancer which occurs in subjects with general fibroid changes. Here the atrophy niust be more or less independent of the carcinoma. There is little ground for the assumption that gastric atrophy itself leads to carcinoma, although the disease may rarely arise in atrophic mucosæ (Riegel). Gastric airophy occurs with carcinoma of other organs, as observed by Fenwick in r 6 of 50 cases. Schneider also found an acidity in 22 of 29 cases of extragastric cancer. It is, therefore, necessary to assume that carcinoma affects the gastric mucosa 
chiefly through a deleterious influence on the organism as a whole. To a considerable extent the two conditions must be regarded as coördinate.

Extensions of Gastric Cancer.- The carcinomatous focus having become established, the mode and rate of extension are determined chiefly by the growth energies of the cells. Hence there are very wide variations in the behavior of different tumors.

Adenocarcinomas first remain localized in the mucosa, but early perforate the muscularis mucosæ, invade the lymphatics of submucosa and muscularis, and thus reach the lymph-nodes. They also penetrate the blood-vessels of the stomach wall, as is indicated by the distant subcutaneous metastases observed in some early cases. A wide superficial extension in the mucosa is less common than in the colon, but occasionally one finds a large portion. of the fundus covered by a superficial adenocarcinomatous ulcer.

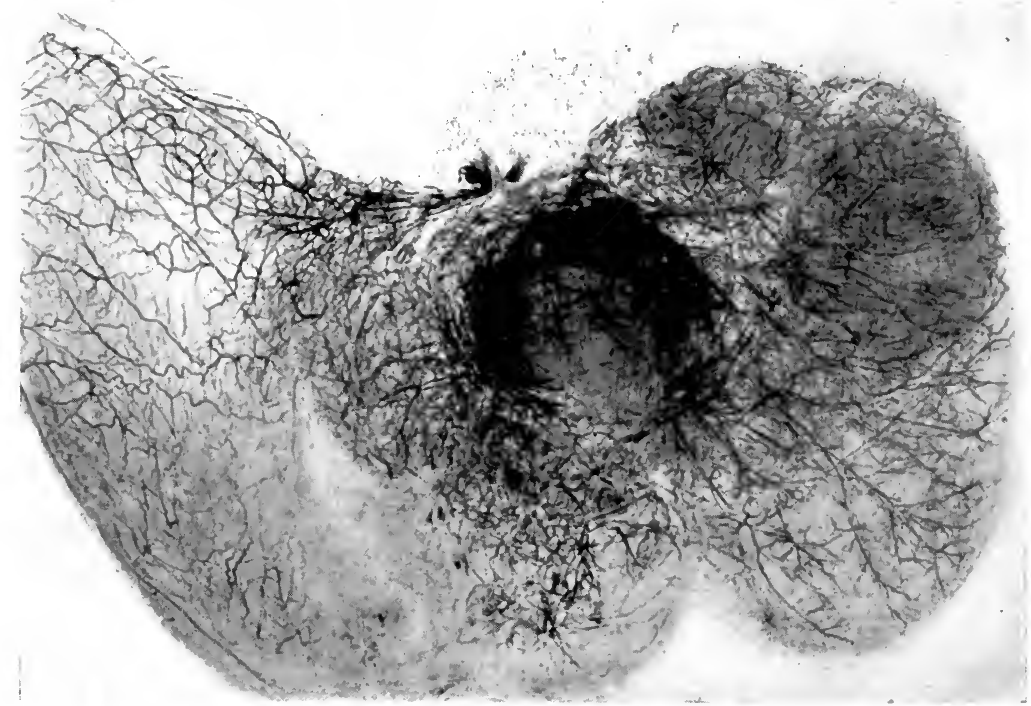

FIG. 303.-Carcinoma of stomach.

$x$-Ray after injection of vessels with bismuth. (Schmidt.)

The diffuse and scirrhous tumors not only penetrate the lymph-vessels early, but extend widely through all coats of the stomach. The entire pyloric region may be the seat of a diffuse infiltration of the mucosa and submucosa with superficial erosion, giving a characteristic form of pyloric carcinoma with stenosis, but without ulceration or early metastases. Many cases of linitis plastica arise in this way. All forms of diffuse and scirrhous cancer invade the submucosa by preference and extend widely through the large and small lymphatics. Submucous invasion is said to extend usually I to $2 \mathrm{~cm}$. beyond the superficial lesion, so that this amount of tissue requires excision (Carle, Fontino, Terrier, Hartman). Yet no general rule can apply to all tumors. Through this coat the pylorus may be passed and the duodenum invaded for $\mathrm{I}$ or $2 \mathrm{~cm}$. Carle and Fontino had three pyloric cancers which extended I to $3 \mathrm{~cm}$. beneath Brunner's glands, while Brinton in 210 cases found 15 invasions of the duodenum. More distant submucous metastases in ileum and colon mentioned by Welch (Lit.), Kaufmann, and others 
suggest that embolic cells may be propelled long distances before finding lodgment. Rectal metastases described by Bensaude probably travel outside the intestinal wall. Passage through the ventral lymphatics of the muscularis is readily accomplished, usually with thickening and fibrosis of this tissue. In the subserous lymphatics permeation becomes much freer. As a rule the serosa backing a carcinoma is thickened, contracted, and adherent to neighboring tissues, with approximation of the contiguous lymph-nodes, and it is not unusual to find the lymphatics leading from such a focus clearly outlined by infiltrating growth over a considerable area or even over much

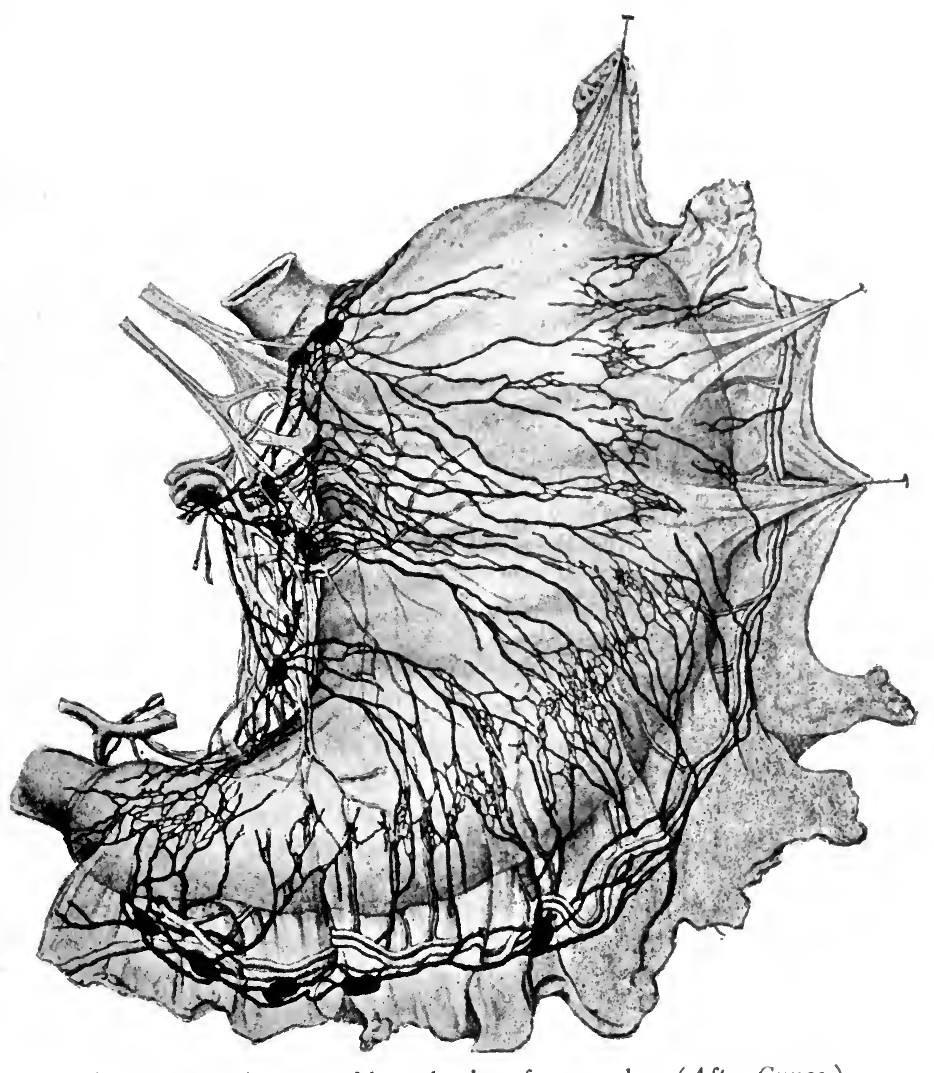

FIG. 304.-Diagram of lymphatics of stomach. (After Cuneo.)

of the serous surface. This process has much influence over the form and motility of the stomach, tends to limit dilatation of the organ, and contributes to the hour-glass contractions. Extension to the peritoneum occurs directly from the serosa or from secondary foci in other organs. It is facilitated especially by mucous changes in the tumor.

The Lymphatics of the Stomach.- Cunee describes three main groups of perigastric lymph-nodes: (I) Nodes of the cardia and lesser curvature. The coronary nodes are two to six in number and are closely applied to the coronary artery. The nodes of the lesser curvature form two groups. One group 
lies in the lesser omentum in front and behind the vertical portion of the cardiac orifice. These nodes may be absent. The other group is constant and lies closely applied to the stomach wall in the course of the coronary artery and contiguous to the vagi. The upper surface of the pyloric region is free of nodes. When invaded by carcinoma this group of nodes is often contracted and bound to the stomach wall.

(2) The hepaic (pyloric) chain drains the lower two-thirds of the greater curvature and the pyloric region. The nodes form a subpyloric and a retropyloric group. The former lie between the vessels and the stomach, but not in direct contact with the organ. Some may be distinctly aberrant and lie 5 to $6 \mathrm{~cm}$. from the stomach in the gastrocolic ligament. They receive the lymph-flow from most of the greater curvature and unite with the retropyloric group or directly with nodes along the hepatic artery.

The retropyloric nodes lie with the gastroduodenal artery and are in relation with the posterior surface of the pylorus and behind with the pancreas. They receive lymph from the posterior surface and upper border of the pylorus and from the first portion of the duodenum and unite with the main hepatic chain.

(3) The splenic group lies along the splenic artery from stomach to hilus of spleen, in the pancreatic-splenic omental fold. They drain the fundus of the stomach.

The lymphatics of the gastric mucosa are very rich but fine. Passage of lymph to the submucosa is somewhat restricted by the muscularis mucosa, through which small vessels pass to larger sinuses in the submucosa. Thence narrow vessels pass between the muscle bundles to larger subserous spaces. The submucous and subserous plexuses communicate freely with those of the esophagus, but only the submucous plexus joins freely with that of the duodenum. The subserous vessels encircle the pylorus, but do not freely join with those of the duodenum. While the above descriptions indicate the general course of lymphatic flow, yet there are notable variations in the lymphatic vessels and still further anomalies in the distribution of lymphatic metastases (Lengeman, Mayo).

Lymphatic invasion usually begins as an embolic process with secondary growth which may fill the lymphatics in all directions (Lengemann, Renner). Yet in slowly growing carcinoma Borrmann's view of continuous permeation may be correct.

General statements regarding the frequency of lymph-nodes invasion have little value, since this event varies so greatly in different forms of tumors. In postmortem material it is rare to find the lymph-nodes free and this condition may be expected only in slow scirrhous cancer and linitis plastica. In none of Verse's I 2 early carcinomas, accidentally found at autopsy, were the nodes invaded, although several were of considerable dimensions and ulcerated and one was recognized during life. There is, therefore, a considerable period when the therapeutic problem is purely local.

Operative material includes chiefly tumors of pylorus or lesser curvature. When a sufficient number of nodes are carefully searched few cases will be found free from some invasion. Cuneo in 8 autopsies found lymphatic invasion in all, but in I3 gastrectomies the nodes were invaded in only 2 . Lengemann, however, made a very thorough study of gastrectomies, finding all nodes free in only one, an extensive adenocarcinoma of pylorus with areas of carcinoma solidum. In 20 cases I IO of 189 nodes examined ( 58 per cent.) were free. The retropyloric nodes and those of the lesser curvature were more often involved than the subpyloric. The invasions were rarely continuous and hence must have occurred by embolism. The size of the nodes gives 
uncertain indications of their condition. Kausch believes that lymphatic invasion is practically constant in operative cases. In resected ulcerating carcinomas I find invasion of accompanying nodes practically constant, but careful search of the nodes may be required to detect small groups of embolic cells in the sinuses. Practically it must be assumed that all accessible nodes should be removed in gastric resections for carcinoma.

Observations on the occurrence of extragastric metastases concern autopsy material. In such cases Cuneo found invasion of perigastric, diaphragmatic, or retroperitoneal nodes in 85 per cent. Extensions to the thoracic nodes are not uncommon. The subhepatic and pancreatic nodes are among the first involved. Mesenteric nodes were invaded in 4I cases and the lumbar and aortic in ro6 of 603 cases collected by Gussenbauer and Winiwarter. From these trunks the inguinal nodes may be affected (Belin). After pleural invasion the axillary nodes may suffer.

The importance of enlargement of supraclavicular nodes in the diagnosis of abdominal cancer was first emphasized by Troisier, and of these cases gastric cancer furnishes the largest proportion (Tarchetti). An inflammatory swelling of these nodes is quite as frequent as carcinomatous invasion. Thus Hechler found swelling in 25 per cent. of 70 abdominal cancers, but the clinical observations were not followed by microscopical. The left nodes are usually the ones affected, but either or both sides may be involved according to the anastomoses of the thoracic duct. The course of the invasion is through the thoracic duct, which in several cases has been found completely infiltrated (Hosch, Lit.). Chylous ascites may accompany the thrombosis (Leydecker, Hektoen). From the supraclavicular nodes the breast may be involved (Moutier, March). Extensions to the thoracic duct are discussed at length by Piat. In rare cases there are wide-spread metastases strictly limited to the lymph-nodes after the manner of lymphosarcoma, and in these cases the cells may be small and round (Israel, Hosch). Multiple cutaneous metastases may be the chief or even the sole form of secondary tumors. The liver is invaded in about 33 per cent. of autopsy cases (Kaufmann) either by direct extension across the adherent peritoneum, or backward from the subhepatic nodes, or by embolism from perforated portal vein (Spaeth, Acker). Very large tumors may then form in the liver and the disease may simulate hepatic carcinoma.

Along the splenic lymphatics or through the splenic vein the spleen may be involved and drawn toward the stomach.

The pleura and lungs were involved in 7.3 per cent., the pancreas in 7.6 per cent., and the peritoneum and intestines in 27.6 per cent. of 2156 cases collected by Martin.

Ovarian metastases occur in 2.3 per cent. of the cases of gastric carcinoma (Borrmann) and form an interesting feature of the disease. Characteristic cases are described by Schlagenhaufer, Glockner, and others. The original tumor is usually scirrhus, less frequently a small, diffuse, or gelatinous carcinoma. The ovarian growths are generally bilateral and of such size that they have often been regarded as primary ovarian growths. Kaufmann found two ovarian tumors weighing i6 pounds. Their structure varies greatly and may reproduce an alveolar or diffuse carcinoma or adenocarcinoma with mucous changes. In one variety the cells are large, contain mucous globules, and are distributed in small groups or diffusely in the hyperplastic ovarian'stroma. This structure is nearly identical with that described by Krukenberg as fibrosarcoma mucocellulare. It has been shown by Schlagenhaufer and others that many of the Krukenberg tumors are secondary carcinomas from mammary, gastric, intestinal, and uterine growths. That 
some of the so-called endotheliomas of the ovary are secondary growths is claimed by Polano and by Papaioannou. Bilateral ovarian tumors and even unilateral growths of this type are so frequently secondary as to call for a careful search for a primary growth and the removal of both ovaries if one is to be resected. The mode of extension to the ovaries is by peritoneal implantation, permeation throughi abdominal lymphatics, and probably also through the blood-stream. When the peritoneum is extensively invaded implantation on the ovary readily follows. Kraus argues that this is the most frequent method, and Sitzenfrey found cancer-cells implanted between the germinal epithelium. In several of the scirrhous cases the abdominal nodes have been extensively involved to the exclusion of other organs and the permeation to the pelvis and ovary has been traced. In some cases the original tumor is small and metastases are practically limited to the ovary. Here it has been assumed that tumor-cells have passed the pulmonary bloodvessels and found a peculiarly favorable soil in the ovary, but the possibility that the ovarian growth is primary must be considered (Stickel, Pfannenstiel).

Of the miscellaneous locations of metastatic growths may be mentioned brain, bones, kidney and adrenal, and spinal cord or membranes.

Direct extensions may also pass far beyond the adjacent organs, penetrating the diaphragm, the spinal column, and abdominal wall. In 48 cases of umbilical cancer collected by Longuet and Quénu, I4 originated in the stomach and passed along the umbilical lymphatics.

Histogenesis. - The origin of gastric cancer has been traced satisfactorily to altered, but previously normal, gastric tubules. Only in certain rare cases is there ground for assuming an origin from congenitally misplaced or embryonal cell groups. Waldeyer described in detail the hypertrophy, cellular overgrowth, and downward extension of small groups of gastric tubules with the development of carcinomatous alveoli and invasion of lymphatics. Hauser describes early adenocarcinomatous changes in small areas of the gastric mucosa. These changes consist in elongation of a group of glands which become very narrow or occasionally dilated and joined to one another by numerous lateral outgrowths, so that they form a network of communicating acini in the thickened mucosa. The epithelial cells become more opaque and the nuclei hyperchromatic, but they long remain confined to a single layer. The stroma is usually thinned and, while rich in nuclei of connective-tissue cells, is poorer in lymphocytes than the normal mucosa.

These changes affect a small area, I to $3 \mathrm{~cm}$. of the mucosa, which on microscopic examination reveals multiple foci of altered glands separated by normal mucosa. From these early foci develop flat adenomatous thickenings and papillomatous elevations which form a relatively brief transitional stage to adenocarcinoma.

Verse describes 12 cases of beginning carcinoma of the stomach (l.c., $3 \mathrm{I}$ to 42 ). The lesions consisted of flat or papillary or polypoid thickenings of the mucosa, from I to $5 \mathrm{~cm}$. in diameter. They occurred in subjects succumbing to other diseases, and in several there were general hyperplastic or atrophic gastritis and a history of alcoholism. The structure presented elongated, branched, and anastomosing tubules, lined with one or more rows of hypertrophic cylindrical or cubical epithelium with hyperchromatic nuclei. Many of these tubules had perforated the muscularis mucosæ. At the edges the gradual transformation of normal into neoplastic tubules could often be followed. In some areas the line of separation was sharp, or single tubules of neoplastic type lay among normal tubules. These small lesions were often the seat of purulent inflammation and superficial ulceration sometimes resulted. While the stroma was at times overcellular, Verse points 
out that the initial change was in the glands and often existed without any proliferation of stroma.

From these observations it seems thoroughly well attested that carcinoma of the stomach arises from the previously normal glands through a process beginning as localized overgrowth and that the process is located primarily in the epithelial cells which usually show preliminary disturbances of overnutrition and excessive function. There is, however, a considerable gap between the ordinary condition of inflammatory overgrowth and definite adenocarcinoma in the stomach, and this gap appears to be bridged, if at all, by a rather sharp morphological change which is established rapidly. Thus single glands and minute groups of glands exhibit a pronounced alteration while surrounded by normal or slightly altered tubules. The original cancerous area may be single or composed of multiple foci which later fuse. From this area of origin the tumor tends to grow from its own elements. Yet during the formation of the original focus and to a variable extent

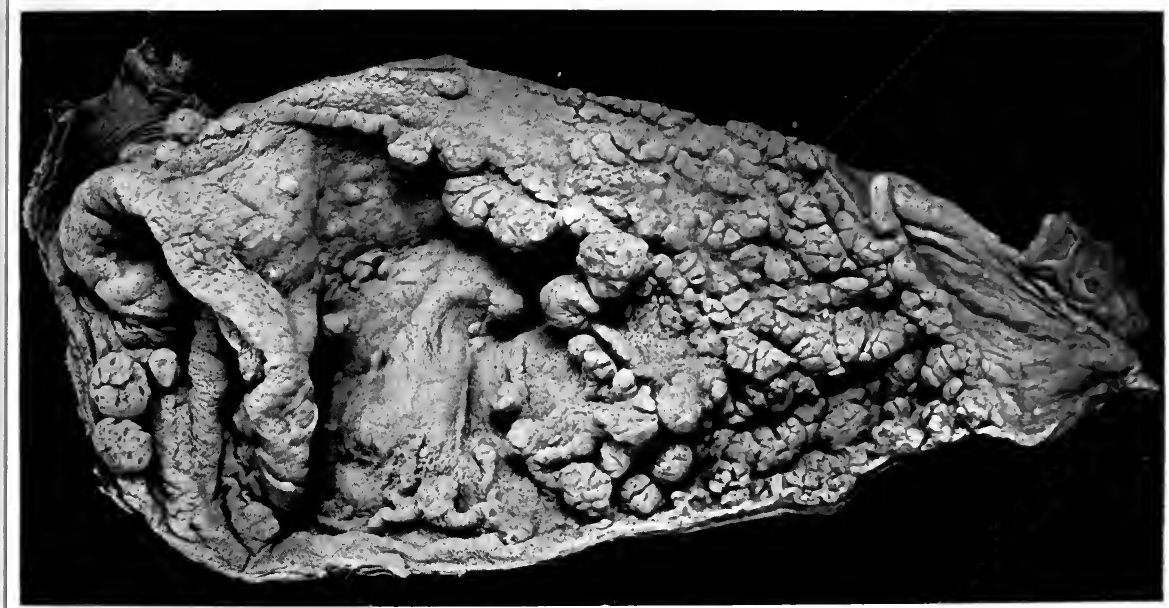

FIG. 305.-Gastritis polyposa.

thereafter normal glands continue to be transformed into neoplastic structures. This lateral extension is more pronounced in the colon than in the stomach.

G. Hayem describes the development of a diffuse scirrhous cancer from a diffuse parenchymatous gastritis in which the peptic glands seemed to break down and the granular acidophile peptic cells spread through the stroma giving origin to the tumor. I have seen one early ulcerating tumor which recalls this description.

While the majority of gastric carcinomas probably arise from such focal lesions as are described by Verse, another group originates from more advanced polypoid or adenomatous structures. The progress of carcinomatous changes in gastric polyps has been followed in detail by Verse. In gastritis polyposa a large portion of all of the mucosa may be the seat of multiple outgrowths and from one or several of these carcinoma may develop. As shown by Hause and by Doering the natural termination of this condition is death from carcinoma. 
In the development of carcinoma from peptic ulcer the usual preliminary changes may be traversed, including hypertrophy and hyperplasia of lining cells, enlargement of alveoli, stratification of cell lavers, and elongation and lateral sacculation of tubules. Or the cells of slightly hyperplastic tubules may at once break up into small groups of atypical cells which from the first grow more or less diffusely in the mucosa (cf. Hayem). The former method yields adenocarcinomas which later become more atypical, the latter produces small alveolar and diffuse carcinoma.

The importance of developmental anomalies in the genesis of gastric carcinoma appears to be slight. Torkel describes aberrant Brunner's glands in the pyloric mucosa and finds a connection between them and congenital stenosis, but they do not appear to give origin to malignant tumors. Islands of intestinal mucosa are frequeritly found in the pyloric region and it is possible that they give origin to certain carcinomas, especially those of the cylindrical cell type. Such islands are difficult to distinguish from the local or general metaplasia with assumption of intestinal characters occurring in chronic gastritis, ulcer, and cancer (Sachs, Lubarsch, A. Schmidt, Kukobo, J. E. Schmidt).

Symptomatology.-For a more detailed discussion of this subject consult Welch, Martin, Riegel, and Mathieu.

Onset.-The initial symptoms of gastric cancer may be as vague and variable as their early recognition is important. The previous digestive or gastric history is almost wholly unknown. Two groups of incipient cases present themselves for diagnosis, one in which there has been a definite history of gastric disturbance, and another in which the disease appears to arise in a perfectly healthy subject. The spontaneous development of the disease in the "athletic stomach" without demonstrable exciting factors must be doubted, yet many patients date their illness from a specific dietetic error or a trauma, or some acute disease (Hammerschlag). Long-continued abuse of the stomach is commonly overlooked until the disease reaches a certain stage, when it produces symptoms which when once established are usually persistent.

Oppression after eating is a very early sign. Its persistence after an acute onset distinguishes it from simple functional disturbance. Pain is a very important early symptom which is influenced by diet, appears one or two hours after eating, or when the stomach is empty, often worse at night, and aggravated by exertion. Chronic gastritis does not, as a rule, give persistent pain. Loss of flesh is an early significant symptom in many cases, especially with disturbed motility, but is often long delayed. Regurgitation appears early and may soon be succeeded by vomiting. When pain and indigestion persist, a systematic search for definite signs of carcinoma is indicated. The first of these definite signs is usually the presence of occult blood in stomach washings or stools. Repeated radiographs of the stomach may be of much value in early cases, especially of pyloric tumors (Cole). Gastroscopy may locate very early lesions of the cardia and curvature, and a portion of a suspicious ulcer may be successfully removed for examination (Janeway). Early loss of $\mathrm{HCl}$ may be expected only in cases preceded by chronic gastritis. Gluzinski's test of relative deficiency of free $\mathrm{HCl}$ is highly recommended by Tabora. Detection of tumor fragments in vomitus or in wash water and especially in blood-clots, is always worth attempting. Reineboth found such fragments in 5 of 8 cases. Zoeppitz points out the nearly constant occurrence of ulceration or erosion in early lesions and estimates the frequency of early symptoms as follows: occult blood, 94.5 per cent.; emaciation, 89.9 per cent.; anacidity, 88.9 per cent.; lactic acid, 67.3 per cent.; long bacilli, 64.4 per cent.; palpable tumor, 63.4 per cent. 
Complete loss of free $\mathrm{HCl}$ and pepsin, presence of lactic acid and BoasOppler bacilli, marked vomiting, hematemesis, and a palpable tumor belong to the established disease, but may appear as early symptoms. In all cases accurate determination of the secretory and motor powers of the stomach should form a basis for the interpretation of symptoms.

General Pathogenesis.- The relation of the symptoms and course to the anatomical character of the tumor is not always distinct, so that clinical conceptions of the disease are not constructed from the anatomical standpoint. While it is desirable to emphasize such relations wherever they exist, clinical records are not usually available for such analysis.

- Several factors widen the discrepancy between clinical records and anatomical type. Chief of these is the necessary period which is clinically

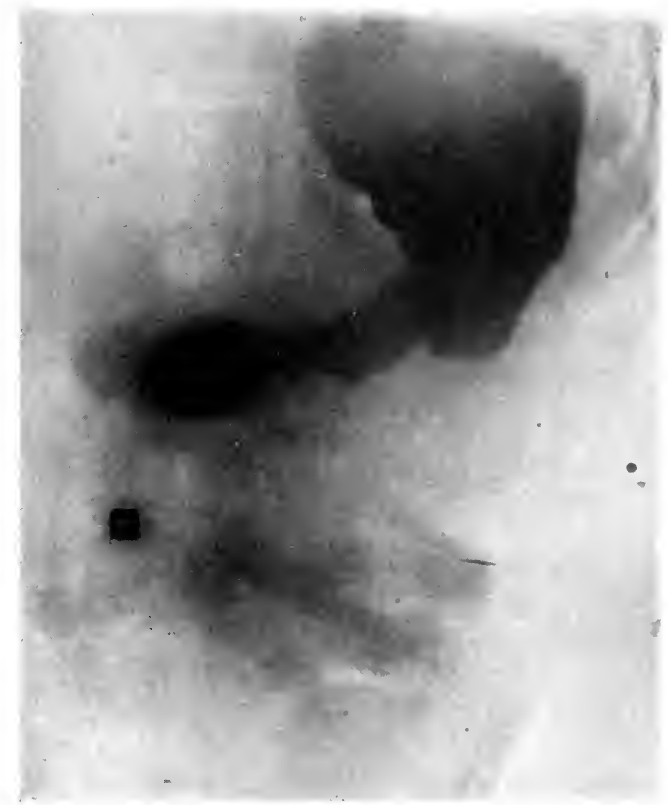

FIG. 306.- $x$-Ray photograph of an extensive gastric carcinoma, involving whole of pyloric region, without stenosis.

unobserved. It is extremely difficult to accurately determine the duration of gastric carcinoma. Thus Osler and MacCrea classify as clinically acute carcinoma lesions which gave observed symptoms for one month, but which must have existed very much longer. It seems probable that the use of the $x$-ray will eliminate many uncertainties regarding the duration of gastric cancer. The studies of Rieder, Fraenkel, Holzknecht, Jonas, Haudek, v. Schmieden, Cole, and others show that it is possible to locate and even to outline the lesion in gastric carcinoma and to follow certain physiological peculiarities of the cancerous stomach, so that when other data confirm the diagnosis, attention may be directed to the anatomical character of the tumor, its operability and prognosis. By serial radiographs Cole has been able to recognize 90 per cent. of gastric carcinomas. The increasing attention of 
surgeons to the operative possibilities on these cases also bids fair to throw much light on the early stages of carcinoma. Hence there are some indications that it will soon be possible to construct a clinical classification of gastric carcinoma with close reference to its anatomical types.

The location of the tumor determines to a large extent its course and symptoms. Pyloric cancer is rather sharply distinguishable from lesions of the lesser curvature. Ulcero-cancer of the pyloric region is usually associated with hypersecretion which tends to cause spasmodic contraction of the pylorus and severe pain. Motor insufficiency develops early and profuse hemorrhages are more frequent with this type of carcinoma than with any other. Primary carcinoma of the pylorus is not associated with hyper-

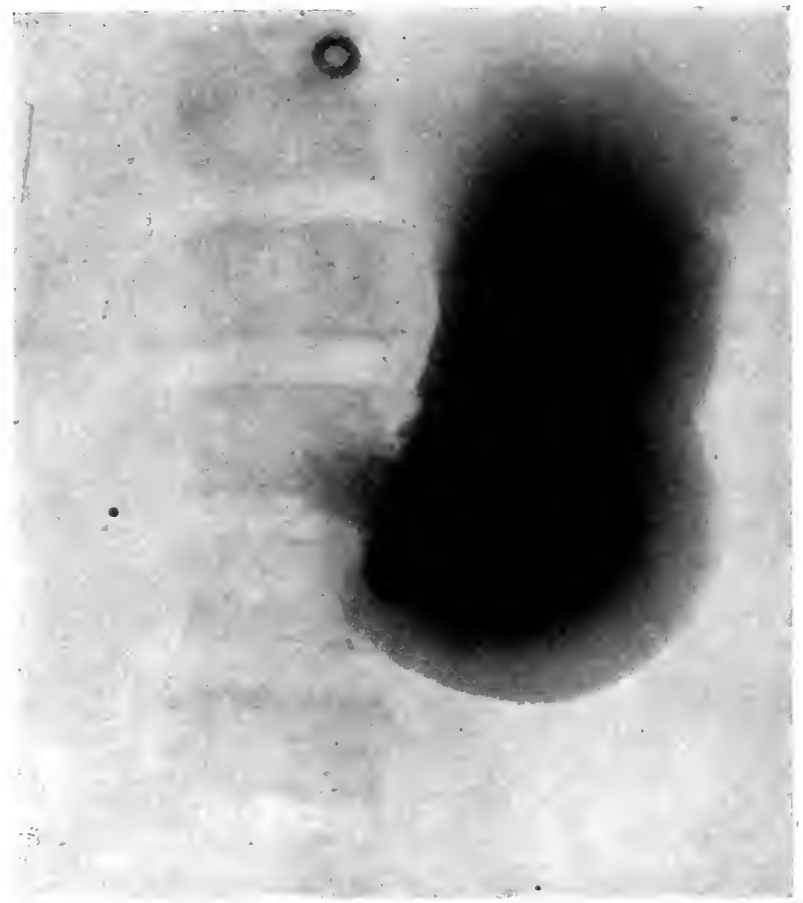

FIG. 307.- $x$-Ray photograph of localized carcinoma causing complete obstruction of pylorus and dilatation of stomach.

secretion, but even in its early stages there is relative or pronounced acid insufficiency (Gluzinski, Tabora). Pain is less severe, motor insufficiency occurs early, and small bleedings are the rule.

Carcinoma of the lesser curvature usually arises in cases with established achylia. The pylorus being open, insufficiency is delayed. Pain, hemorrhage, and cachexia are not prominent, and the disease may run the course of chronic gastritis for one or more years. Periods of marked improvement with gain in weight are not infrequently observed.

Cachexia, in gastric carcinoma, is a complex condition in which two main factors are usually combined. (I) General dyscrasia referable to infection, ulceration, hemorrhage, anemia, pain, and loss of digestive capacity, and 
(2) the more specific terminal cachexia of carcinoma. The latter is rarely observed, while the former group of factors dominate the clinical history throughout. In the absence of such complicating conditions cancer of the stomach often produces no effect upon health. In fact, a specific cachexia of cancer may be practically eliminated from the symptomatology of the gastric disease. Even the toxic destruction of protein reported by $\mathrm{F}$. Müller and Klemperer may be separated with difficulty from non-specific influences. A similar phenomenon occurs in starvation. Hence some patients remain in fair health until a late period and even the digestion may be little affected (Boas). For the same reason marked temporary improvement with gain in weight and relief of gastric symptoms may occur (Friedenwald).

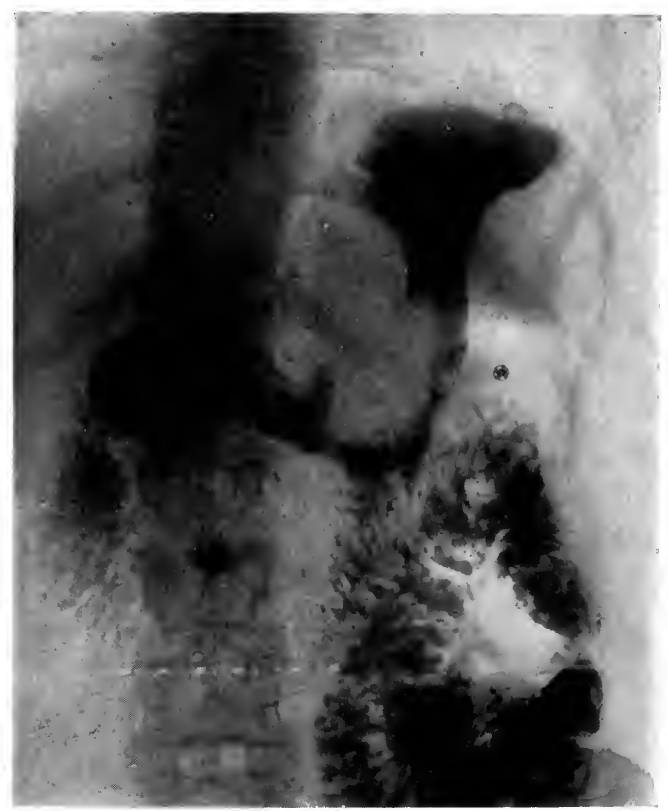

FIG. 308.- $x$-Ray photograph of a bulky adenocarcinoma arising from lesser curvature, occupying much of the fundus, without pyloric stenosis or dilatation.

From the hematological standpoint the cachexia is marantic or anemic. In the marantic cases the blood is concentrated often to a remarkable degree by vomiting and defective absorption, so that the patient may succumb with over Ioo per cent. of $\mathrm{Hb}$ and polycythemia.

Anemia in cancer of the stomach is usually secondary and of the chlorotic type, but it often becomes so severe as to suggest a progressive pernicious type. Yet a low $\mathrm{Hb}$ index and leukocytosis are commonly present. In these cases malnutrition and repeated losses of blood from ulcerating tumors are the chief factors. Progressive pernicious anemia is occasionally associated with gastric carcinoma. In one group of cases the anemia develops as a sequel of the gastric disease and is preceded by hemorrhages and malnutrition and occasionally by metastases in the bone-marrow (Frese). The tumor is then extensive and ulcerating, but in Israel's case it was small but bleeding. I have seen 3 cases, all ulcerating. In another group the gastric tumor is small 
and ulceration is missing. Ehrlich and Lazarus refer to 3 cases of this type, one a ring-shaped growth of the pylorus. In a third group the carcinoma arises in an atrophic mucosa and the anemia may be referred chiefly to the gastric atrophy. All these relations illustrate the peculiar susceptibility of the bonemarrow in predisposed subjects to functional disturbances of the stomach.

Acidemic coma was first observed in gastric cancer by v. Jaksch and later by Senator, Riess, Klemperer, Osler, and others. The usual symptoms are present and the urine contains acetone bodies in moderate amount. Klemperer noted a reduction in the total urinary nitrogen in cases previously showing an increase, and concluded that the coma was referable to toxic derivatives of protein destruction. The tumors are usually large and ulcerating, but the stomach is not, as a rule, dilated. In Osler's case there was a small ulcerating pyloric growth. Tetany is occasionally observed with dilatation of the stomach from carcinoma (Leube). Progressive degenerative neuritis

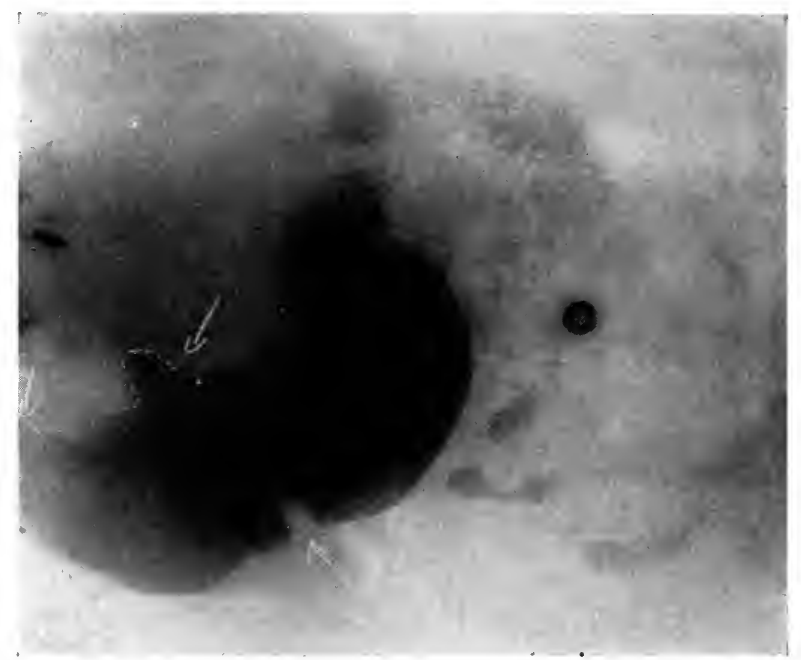

FIG. 309.- - x-Ray photograph of chronic indurated ulcer of lesser curvature of stomach. Note the deep pocket outlined by dotted line, leading into ulcer. On greater curvature is a normal peristaltic constriction.

has been described by Miura and Klippel. Sudden blindness from toxic amaurosis is reported by Deutschmann.

Relation to Anatomical Varieties.-(I) Adenocarcinoma occurs at relatively early ages. It is the chief tumor observed in the second decade, becomes less prominent in the third decade, while one group of cases follows gastritis polyposa, and miscellaneous cases occur at any period. Its course is chiefly determined by the stage at which ulceration is established, and this complication again depends much on the location. It is the usual type of tumor occurring at the cardia, in which situation it produces unusually severe pain, dysphagia, and obstruction to the esophageal bougie, while a palpable tumor is absent.

(a) At the pyloric or cardiac orifice it ulcerates early and this destructive process yields most of the pronounced gastric symptoms, as pain, hemorrhage, vomiting, and a local tumor, followed by progressive cachexia. When obstructing tumors at the pylorus break down the event is often indicated 
by hemorrhage, some relief of vomiting, increased pain, and diarrhea replacing constipation. Clinical observations indicate that bulky tumors may be excavated, and broad superficial adenocarcinomas may be widely eroded in a few days, during which the vomitus, previously free from blood, becomes bloody or chocolate colored. The onset of symptoms, which may be abrupt, is doubtless preceded by a latent period, but the active course is usually severe and relatively rapid, but surgically considered this class of patients is more favorable than the general condition might indicate.

Gastric carcinoma occurring at early ages is usually of rapid course and since adenocarcinoma is the usual type in young subjects the age of incidence rather than any peculiarity in the growth seems to determine its active progress. Yet in adults adenocarcinoma is occasionally very rapidly fatal. Rohrer's adult patient lived only 3 months with a rapidly enlarging epigastric tumor and fracture of femur from metastatic adenocarcinoma.

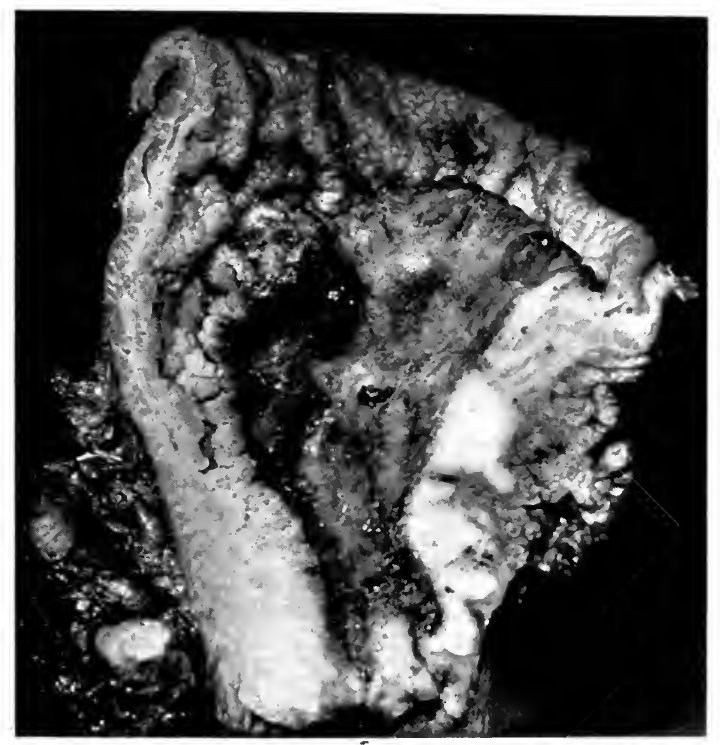

FIG. 3I0.-Carcinoma of pylorus, with pyloric stenosis and invasion of subpyloric nodes.

In a similar case in a young man of 27 years the first definite symptoms observed were abdominal pain and ascites, which led to laparotomy, disclosing multiple metastases over the peritoneum from a relatively small adenocarcinoma of the pylorus.

(b) When ulceration is long delayed these tumors may reach large dimensions which are readily palpable and when located away from the orifices or even near them, they may long remain latent. Ulceration becoming established leads to rapid excavation and at once transforms the clinical picture. Martin describes such a case with duration of symptoms of 5 weeks, with a bulky tumor at the pylorus and extensive metastases in the liver. They form a well-defined group of cases seen at autopsy and the clinical history can usually be reconstructed from the gross appearance of the tumor. There are, of course, many variations due chiefly to factors which control the progress of ulceration, infection, and metastases. Occasionally after a severe initial 
period the course becomes chronic. Pronounced anemia is common and may take a pernicious type.

(2) Carcinoma following ulcer is the best defined clinical group, but the certainty with which this type of tumor may be distinguished from ulcerating primary carcinoma is often overestimated. Mathieu considers the condition so distinctive that he describes it as a separate clinical entity under the term "ulcerocancer." Characteristic cases are preceded by a variable period, months or years, of symptoms of ulcer. The development of carcinoma is marked by gradual diminution of free $\mathrm{HCl}$, which, however, may persist to the end; by increasing anemia; an advancing cachexia; and the development of a tumor which is usually small and fixed. As a rule, the pain becomes more severe and constant, and the vomiting may be aggravated. Many authors mention a selective anorexia against meats. The later course of the disease varies extensively and is determined chiefly by the location. Apart from the previous history it is not, as a rule, to be distinguished from other forms of advanced ulcerating carcinoma.

The total duration of the disease including the period of simple ulcer is much longer than that of ulcerating primary carcinoma. Boekelmann observed cases for $\mathrm{I}_{5}, 20$, and 29 years. Yet, as a rule, after the establishment of cancer the course is steadily progressive and rather rapid (Mathieu).

(3) Gelatinous carcinoma is one of the more chronic types of the disease, but it presents few distinctive clinical features. Certain adenocarcinomas undergo mucoid degeneration and the history of these cases is similar to that of the original tumor. The diffuse infiltrating types of gelatinous carcinoma usually cause contraction of the stomach and great thickening of the walls which may be demonstrated by $x$-ray examination. Ulceration and hemorrhage are less pronounced than with many other types of the disease. Many cases pursue a "latent" course. Storer's case was remarkable for the slight disturbance of digestion, and in Amdion's case gastric symptoms appear to have attracted little attention. In a case reported by Osler and McCrae no marked gastric symptoms were noted, the patients presenting chiefly nervous and mental symptoms. In another case the symptoms began with bloody ascites and the only gastric symptom was occasional vomiting of mucus. Extension to the peritoneum with ascites is relatively common, but occurs also with carcinoma simplex and scirrhus.

(4) Carcinoma simplex is characterized clinically by its steadily progressive course and relatively short duration. The localized form of this tumor yields severe gastric symptoms, among which hemorrhage and pain are prominent. Deep ulceration may lead to fatal hemorrhage as an early symptom. In the diffuse form of carcinoma simplex the gastric symptoms are less marked. The metastases which develop in peritoneum, liver, lungs, ovary, nervous system, and elsewhere produce appropriate symptoms which may divert attention from the stomach.

A characteristic case originating in the cardial region is that of Hosch: age 62 years, initial symptoms: vomiting, dysphagia, loss of flesh for 4 weeks, then ascites, supraclavicular nodes enlarged in sixth week, epigastric tumor in fourteenth week. Autopsy revealed a contracted stomach diffusely infiltrated from cardia nearly to pylorus, with extensive metastases limited to lymphatic system.

(5) Scirrhous carcinoma is regularly of slow development, but the early stages are often latent and the period of observed symptoms may be brief. The clinical characters vary greatly and depend on the location of the growth.

Pyloric scirrhus is one of the more characteristic clinical types of the disease. It frequently, but not always, produces pyloric stenosis and dilata- 
tion of the stomach with characteristic symptoms of this condition. Defective motility, constant presence of lactic acid, and absence of free $\mathrm{HCl}$ are prominent, while hemorrhage is slight or absent. Since stenosis may be produced by any type of tumor of the pyloric region these symptoms have only limited diagnostic value.

Diffuse scirrhous carcinoma tends almost invariably to contract the stomach, so that its capacity is small. Ulceration is absent or superficial and hemorrhages are reduced to a minimum. When the stomach is very small it may refuse to retain even small quantities of fluid.

(6) Sclerosing Fibrocarcinoma. Linitis Plastica.-The clinical features of this condition resemble the scirrhous carcinoma to which it is closely related anatomically. The course of the disease is always prolonged, but the observed symptoms may be brief. It usually begins with a period of months or years marked by dyspepsia. Later there is pronounced anorexia, pain, tenderness, and frequent vomiting. A transverse elongated tumor may be demonstrated. Owing to the integrity of the mucosa, hemorrhage is usually absent. Free $\mathrm{HCl}$ is diminished or absent. The $x$-ray reveals a contracted organ, especially at the pylorus (Jonas). In the later stages peritoneal extensions fix the pylorus, increase the gastric symptoms, and cause ascites. Pernicious anemia may dominate the picture. The terminal stages are marked by persistent vomiting, emaciation, and cachexia. Yet in several instances the gastric condition was masked by other diseases (Viti, v. Sury).

The duration in 43 benign cases was found by Lyle to average 49 months, the shortest course being 3 months, the longest 20 years. In 37 definitely malignant cases the average duration was 23.9 months.

The lethal tendencies of the disease have long been noted. The apparent recoveries 3 and 5 years after gastro-enterostomy do not affect the conceptions of the nature of the process, since long survival after this operation has occurred with ordinary scirrhous cancer (Le Count).

Surgical Treatment.-Operability.-When the disease is recognized early, is confined to the stomach wall, inflammatory adhesions limited, and lymphnodes not invaded, the prognosis from resection may be regarded as good. In such cases much depends on the type of growth and the probability of discontinuous metastases. Most cases are encountered late, with infected adhesions, and involved lymph-nodes, and require extensive resection of gastric tissue and all accessible nodes. Even here results are encouraging. Extensive adhesions and invasion of nodes and surrounding tissue preclude resection, but Petersen calculates that average life is prolonged 5 months by gastro-enterostomy. Le Count's case indicates that the prolongation may be much greater in slowly advancing tumors which may be induced to undergo fibrosis. Rohdenburg reports a case which survived gastro-enterostomy for Io years. At death from pneumonia, cellular carcinoma was still found in stomach wall and liver, but it had produced no notable symptoms (N. Y. Path. Soc., I920).

The operative mortality has steadily diminished. Matti (I905) collected rates from German clinics from 68 to 17 per cent. Petersen at Heidelberg saw the mortality from resection fall from 35 to 18 per cent., and from gastroenterostomy from 35 to $\mathrm{I} 7$ per cent. From I 366 resections Martin derived a mortality of 25 per cent. Kocher's mortality was 17.7 per cent.; Mayo's, I3 per cent.

Curability.-Leriche collected 89 resections for cancer well after 3 years. H. J. Paterson calculated that about 8 per cent. of resections for carcinoma were surgically cured. Of IOI resections by Kocher up to I905, Matti reported 7 I leaving the hospital, of whom 72 per cent. died in one month to 
6 years, average life 18 months, while 20 patients remained well. Of $25^{2}$ pyloric resections by the Mayos, the condition of r9I was known, of whom 39 (20 per cent.) were well after 3 years. Other similar statistics are available, indicating that of selected operable cases about 20 per cent. may be surgically cured. Many of the cured cases appear to have been very early and possibly doubtful ulcerocarcinomas. Even with recurrence Petersen ventured to calculate that resection prolongs life an average of 7 months. Against this result it appears necessary to charge up the natural duration of the disease, the operative mortality, and certain accelerations of course in unfortunately chosen cases. On the other hand, many cases, eventually fatal, enjoy freedom from gastric symptoms and the stomach is found free from the disease (Matti). Recurrences are chiefly in liver, peritoneum, and retroperitoneal nodes.

Over against this encouraging outlook based upon carefully selected material it would appear from Peck's study that the average case of gastric cancer has little to hope from surgery. This author collected 527 cases of gastric cancer in the New York hospitals, which gave 480 operations, I 43 exploratory, $\mathrm{I} 67$ gastro-enterostomies, 98 radical extirpations with an operative mortality of 28 per cent., 33 patients traced, 2.3 recoveries for a variable period, and 8 known to be alive after 3 to 4 years. Friedenwald reports from rooo cases 266 operations, I38 exploratory, 9 gastrectomies, I living over I 8 months, but all dying of the disease.

The study of individual cases is more significant than combined statistics. Rapidly growing diffuse carcinoma carries a fatal prognosis even with early resection. Not a few cases surviving 3 years eventually succumb. The permanent cures observed up to i6 years at Kocher's clinic appear to have been adenocarcinomas, sometimes bulky and ulcerating, but confined to the wall, and ulcerocarcinomas.

The surgical results support the view that certain gastric carcinomas, as a rule, are not among the very malignant tumors. A more careful analysis of operative results with reference to anatomical condition is highly desirable. 


\section{CHAPTER XXXII}

\section{CARCINOMA OF INTESTINE}

Statistics collected by Stengel from German sources include 26,340 cases of carcinoma, 2255 intestinal, 8.56 per cent. The U. S. census (I9I4) shows among 52,420 deaths from cancer, 6234 (II.8 per cent.) of the peritoneum, intestine, and rectum; 2690 in males, 3544 in females, 4 in infants under I year, I9 under 5 years, and the highest incidence between 60 and 65 years. The cecum and appendix follow the rectum as the chief location. The increasing recognition of carcinoma of appendix will probably raise the proportion above previous records. Of 3563 cases of malignant tumors of the intestine Brill found only 89 (2.5 per cent.) in the small intestine.

Carcinoma of Duodenum.- From Geiser's statistics it appears that about 4 per cent. of intestinal carcinomas occur in the duodenum. The age of incidence is much later than with other intestinal carcinomas, being placed at 54 years by v. Heurlin, or 56 years by Geiser.

Three distinct forms of this disease may be recognized: (I) Carcinoma following ulcer: (2) carcinoma at the papilla of Vater, and (3) carcinoma of the third portion of the duodenum. This classification corresponds in general to the topographical division given by A. Pic, viz., parapyloric, peri-ampullary, and prejejunal. Of 40 cases collected by Rolleston, 8 were in the first portion, 24 in the second, and 3 in the third portion, while the distribution of Geiser's 7 I cases was in the same order, II, 5I, and 9.

(I) Carcinoma following duodena! ulcer has been recorded in ro cases by Letulle, Perry and Shaw, and Nattan-Larier. Its location, chiefly near the pylorus, accords with the distribution of duodenal ulcer, which, in Collin's 262 cases, occurred 242 times in the first portion of the duodenum, I4 times in the second portion.

The gross anatomy and the symptoms are very similar to those of pyloric ulcerocarcinoma. Stenosis and adhesions are usually present and metastases are frequent and wide-spread. The stomach is usually much dilated. Letulle's case showed colloid changes and gave secondary tumors. In Eichorst's case the ulcer followed a burn. Ewald found a carcinomatous area in the scar of a healed ulcer. In one case I found it difficult to determine the exact significance of displaced and hyperplastic gland tissue in a scarred duodenum. Butz reports carcinoma arising in an actinomycotic ulcer. I have seen one carcinoma of pylorus extending over the first $2 \mathrm{~cm}$. of the duodenal mucosa, ulcerating into gall-bladder, and causing intense jaundice.

(2) Carcinoma in the second portion of the duodenum usually arises in or about the biliary papilla. Jaundice is, therefore, an early and very constant symptom occurring in 23 of 25 cases quoted by Mathieu. The jaundice is less severe and persistent than with carcinoma of the ampulla. Even in advanced cases the occlusion of the duct may be incomplete, jaundice may be absent, and gastric symptoms prominent (Descos, Beriel). Dilatation of biliary and pancreatic ducts commonly occurs and fat necrosis has been observed by Krause, Gerster, and Geiser.

The early tumors are usually papillary growths surrounding the papilla, and in this stage they may prove fatal by biliary obstruction and severe 
infection. In a case recently studied in this laboratory a small duodenal papillary carcinoma surrounding the papilla proved fatal from a single continuous hemorrhage. More extensive growths cause much dilatation of biliary and pancreatic ducts, they form protuberant ulcers surrounding the common duct, or extend laterally over a considerable area. Geiser describes an ulcerating carcinoma which secondarily invaded the papilla. Many of these tumors are difficult to distinguish from carcinoma of the ampulla. Nothnagel points out that the former growths are sharply circumscribed about the papilla, while the true duodena! cancer tends to encircle or extend along the intestine.

The structure is commonly that of cylindrical-ce!l adenocarcinoma derived from the intestinal mucosa. An origin from Brunner's glands has been suggested by Orth, and from aberrant pancreatic tissue by several writers. Many of the cases are difficult to separate from primary cancer of the pancreas.

(3) Carcinoma of the third portion of the duodenum, prejejunal, is rare. Geiser has collected 9 cases. Most of these took the form of a broad, flat ulceration with stenosis. In Fenwick's case the ulceration extended over 8 inches of the mucosa, and the duodenum was 1.3 inches in circumference. Above the stricture there is dilatation of duodenum and stomach, and gastric symptoms with vomiting of considerable quantities of bile are prominent. Icterus is absent. In Syme's case the ulceration was slight and the vomitus was free from bile. In 2 of Geiser's cases there were more bulky tumors with ulceration and perforation of the wall, but without much stenosis. Local extensions or local or general metastases were usually present. The structure presented cylindrical-cell adenocarcinoma or alveolar carcinoma.

Carcinoma of Ileum and Jejunum.-Carcinoma of the small intestine is comparatively rare, forming about 3 per cent. of all intestinal cancers. It occurs at a comparatively early age, average $46 \frac{1}{2}$ years, relatively often before 40 years, and Duncan records a scirrhous cancer in a child of $3 \frac{1}{2}$ years. Schleips in I908 analyzed 43 cases, 28 in males. In location the tumors increase in frequency as one approaches the stomach above and the colon below.

The disease occurs in three somewhat distinct forms as (I) a part of a local or general intestinal polyposis, (2) a late development of single or multiple carcinoid tumors, and (3) as a localized adenocarcinoma with carcinomatous variations in structure.

In all of these forms certain general features appear. The tumors may produce polypoid outgrowths obstructing the lumen, or the lesions may ulcerate early, or the main result may be stenosis from invasion of muscular and subserous coats. Often there is dilatation of the bowel above the tumor, and when the tumor lies in the upper jejunum the disease may simulate pyloric stenosis (Riegel, Routier).

(I) Carcinoma following intestinal polyposis is rare in the small intestine, the great majority of such cases affecting the colon.

Niemack reported papillary adenoma in the lower ileum in a child of 12 years in whose colon were many small polyps. Petrow found many polyps in stomach, duodenum, and jejunum, with adenocarcinomatous structure in the jejunum. In Kukula's case there was a cylindrical dilatation extending over $8 \mathrm{~cm}$. of the jejunum, with polypoid outgrowths in the vicinity and carcinomatous metastases in the mesentery. Thorel found a large adenocarcinoma of the lower ileum, 20 polyps in the ileum and one in the colon. Kuttner considers that some multiple carcinomas of the ileum with stenosis arise from intestinal polyposis, as in Hahn's case in which there were four strictures in the lower ileum, but it seems more probable that the multiple stenosing carcinomas arise from the carcinoid tumors of Lubarsch. Yet in 
one of Kuttner's cases there were 18 strictures in the small and 4 in the large intestine.

(2) Multiple benign embryonal carcinoid tumors of the intestine constitute a peculiar group of tumors of the small intestine first described by Lubarsch. They are found in ileum, jejunum and mesentery, and probably in the appendix, in the form of single or multiple, firm, opaque nodules as large as a pea or bean. They lie in the mucosa or submucosa and the muscularis mucosa may be lost in the stroma of the tumor. In structure they form three groups, resembling $(a)$ pancreatic island tissue, $(b)$ heterotopic intestinal mucosa, or (c) Brunner's glands. Most of the tumors have been referred to heterotopic

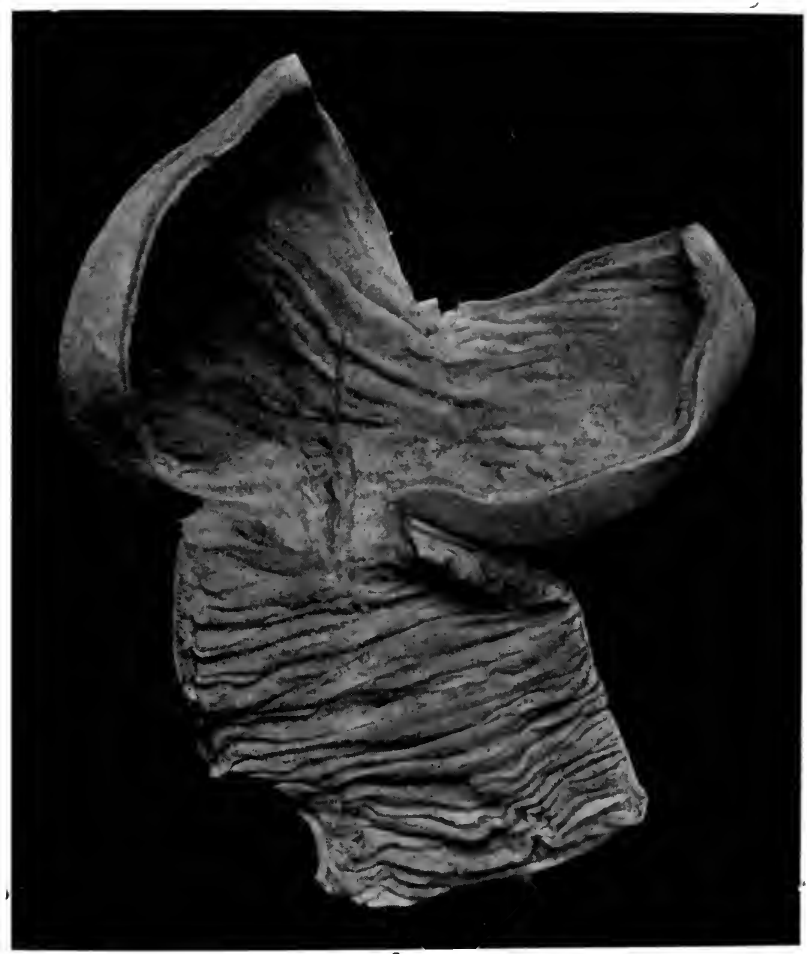

FIG. 3Ir.-Small carcinoma of ileum causing marked stenosis, with dilatation above.

intestinal glands, and Notthaft found a direct connection with the normal follicles. The cells then form compact densely staining groups with traces of alveolar arrangement or with mucous foci producing the appearance of cylindroma. The endothelium of the lymph spaces may be hyperplastic, but does not participate in the tumor process (Lubarsch). 'The resemblance to basalcell carcinoma has been noted by Bunting and Oberndorfer. Smooth muscletissue may be found in the stroma and such growths have been described by Trappe and others as adenomyoma. The prominence of muscle-tissue and the more typical adenomatous structure serve to distinguish the adenomyomas from the carcinoid tumors. A congenital misplacement of tissue must be assumed for the adenomyomas, while the carcinoid tumor may be 
satisfactorily derived from acquired inflammatory heterotopia and overgrowth.

In another group the structure resembles the parenchyma or islands of Langerhans of the pancreas. In fact, Saltykow considers that all the tumors represent heterotopic pancreatic nodules. The cells are cylindrical, cubical or rounded, and they surround pervious or closed capillaries, sometimes in rosette form, or exactly reproducing islands of Langerhans. Heterotopic Brunner's glands were identified in portions of a carcinoma described by Schapper.

The significance of these small tumors has been much discussed. In their simplest form they probably represent anomalies of development and misplacement of gland tissue and are not true tumors (Toennissen, Saltykow).

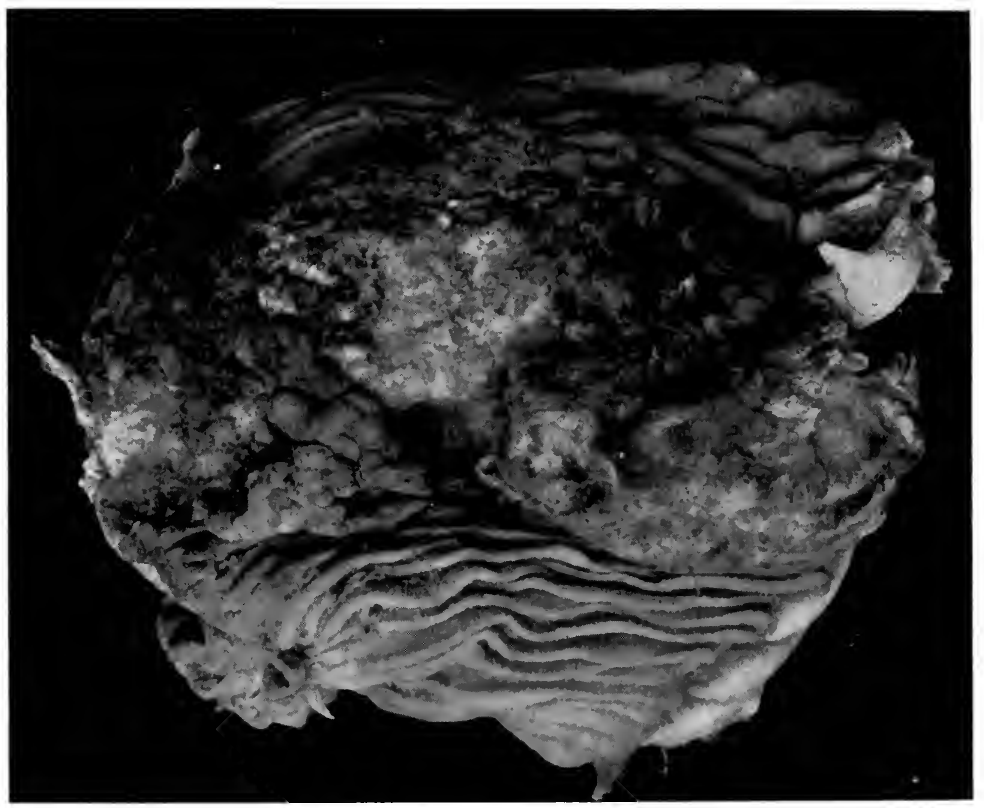

FIG. 3I2.-Gelatinous carcinoma of duodenojejunal flexure.

Yet others show various grades of cellular overgrowth and invade the submucosa and muscularis and are true scirrhous neoplasms (Verse). In this form they long remain localized, although sometimes reaching sufficient size to obstruct the lumen. Others again form the source of malignant carcinomas and produce metastases. 'Thus Ransom found extensive secondary tumors in the liver. Verse found metastases in the lymph-nodes with a small intestinal growth and malignant cases are described by Saltykow and Schapper. It is probable that many small simple primary carcinomas of the intestine and appendix with metastases originate in this way.

(3) The third group of intestinal carcinomas includes lesions which arise under various conditions. Some develop from single intestinal polyps and tend to maintain an adenocarcinomatous structure. The majority of intestinal carcinomas are of this type. When ulceration is delayer! the tumors may reach a size sufficient to obstruct the lumen (Riedel). Ulceration and 
stricture more commonly result and the structure becomes more atypical and scirrhus. Metastases in mesentery, liver, lungs, peritoneum, or elsewhere were present in 16 of 42 cases collected by Schleips and adhesions were much more frequent. That carcinoma may result from catarrhal, typhoidal, or other forms of intestinal ulcers seems possible, but definite observations are wanting. It is difficult to separate tumors of this group from those arising from carcinoid growths, but the former are usually adenocarcinomatous, bulky, and more malignant; the latter atypical, scirrhous, and comparatively benign. Gelatinous adenocarcinoma is described by Marckwald and by Kanzler.

Carcinoma of Appendix.-The occurrence of carcinoma of the appendix has long been recognized, but its importance was first suitably emphasized in 1903 by Elting and by Moschkowitz. It is interesting to note how the recorded cases of this form of carcinoma have increased in proportion to the attention devoted to it.

Of intestinal carcinomas the appendix furnished about 0.39 per cent. in Batzdorff's collection of 2336 cases, but this proportion is probably much too low. Of 13,083 appendices removed at operation and included in the lists of Batzdorff, McCarthy, and the Bender Laboratory, there were 60 cases of carcinoma, 0.46 per cent. Batzdorff refers to I 86 recorded cases. Over 50 per cent. are found with chronic appendicitis (Zaaijer), or 85 per cent., according to Milner. The age of incidence has been from 5 to 80 years, and chiefly in the third decade, while other intestinal carcinomas occur chiefly in the sixth decade.

Gross Anatomy.-The tumors are located chiefly at the tip of the appendix (59 per cent.), which is moderately swollen and stenosed or contains a small tumor nodule which is readily overlooked and requires microscopic identification. Over half the tumors were recognized only after laying open the appendix (McWilliams). In more advanced stages there is a definite tumor and the appendix is inflamed and bound by adhesions. In several cases the whole organ was diffusely enlarged (Kudo). Cystic forms of the disease occur, especially when there is mucous degeneration of the tumor and the mass may be composed of a fibrous wall and gelatinous contents (Rokitansky). Whether the disease may produce the typical condition known as mucocele remains uncertain, but is not improbable. Many mucoceles, however, are free from any tumor element. An irregular cicatrix deforming the organ and obliterating the lumen has been described in several instances. Rarely the lesion takes the form of an ulcer (Baldauf). At the ileocecal valve the tumor may project into or constrict the cecum and be difficult to distinguish from a tumor of the cecum (Draper).

Extension of the tumor process beyond the appendix is relatively uncommon, occurring in about 6 per cent. of the cases (Batzdorf). The mesenteric and retroperitoneal nodes are first involved. Extensive peritoneal carcinosis was observed by Elting, Konjetzny, Kelly, and Neugebauer. In Whipham's case the patient died with secondary growths in nodes, liver, and ovary. Ruyter's tumor was found in the stump of an amputated appendix. As a rule the cases with metastases have been of the columnar cell or gelatinous type.

The clinical course is usually merged with that of chronic appendicitis (McWilliams, Rolleston, Jones, Lit.).

Structure.-Two main varieties of the tumor appear: (I) columnar cell or gelatinous adenocarcinoma, and (2) small polygonal, spheroidal cell, alveolar carcinoma. The former type presents the same age incidence $(52$ years) and general malignancy as other similar intestinal carcinomas, while 
the former occur at any early age and are almost invariably benign (Rolleston, Jones). Transitional forms of intermediate age incidence are observed.

The columnar cell tumors show the usual structure of small or large alveolar adenocarcinoma. In the gelatinous tumors mucous degeneration may be extensive and destroy most of the tumor-cells. In the small alveolar type the cells are composed chiefly of nuclei which are large and hyperchromatic. They are compactly grouped in small or large masses and foci of mucous degeneration may give the structure of cylindroma. The appearance often resembles that of basal-cell carcinoma. The stroma may be scanty or so abundant as to give a scirrhous structure. Both of these tumors are probably derived from the gland crypts. The small-cell tumors seem to be homol-

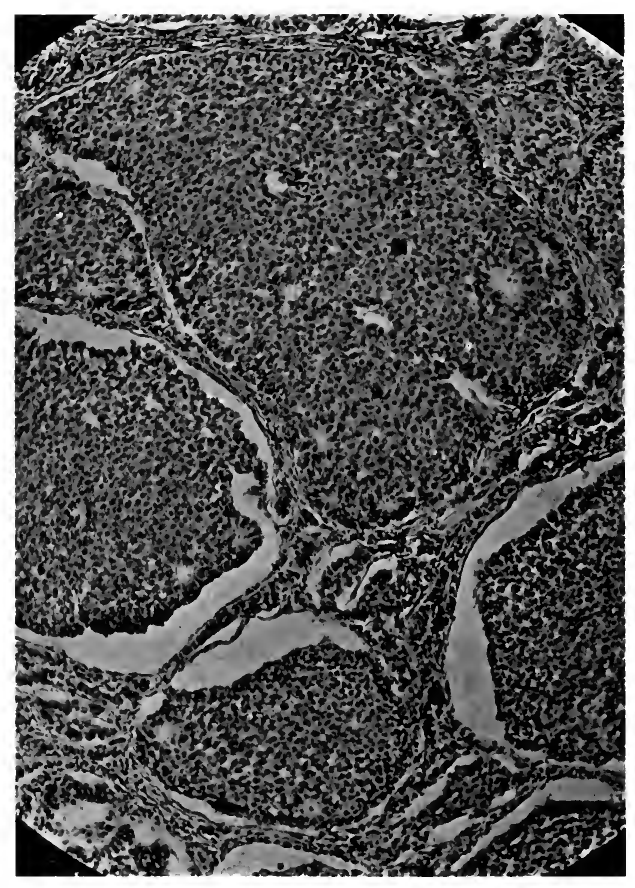

FIG. 3I3.-Carcinoma of appendix.

ogous with the small multiple tumors of the ileum described by Lubarsch, Bunting, and others.

In some cases the epithelial cells possess little growth capacity and become more intimately incorporated with the increasing stroma so that the structure resembles that of endothelioma arising from the lymph spaces of inflammatory connective tissue. These cases have apparently been interpreted as endothelioma by Glazebrook, A. O. J. Kelly, and others. Sudsuki and Milner regard this structure as indicating an inflammatory origin and hold that the great majority of appendix carcinomas are spurious. Yet the tendency of fibrosing basal-cell carcinoma to suggest an endothelial origin is well known.

The occurrence of a specific type of carcinoma in an atrophying organ which is often the seat of chronic inflammatory changes is of much theo- 
retical interest, and its peculiar structure and benign course serve to emphasize the principle that each organ has its own forms of carcinoma.

Carcinoma of Large Intestine.-Statistical.-At the Pathologic Institute at Vienna, between 1870 and 1893,343 intestinal carcinomas came to autopsy, of which 164 were in the colon and 162 in rectum, while only 7 were found in the duodenum and I I in the ileum (Nothnagel). Schleips collected 542 intestinal carcinomas, of which 259 were in the colon, 257 in rectum, 20 in duodenum, 16 in ileum. In Brill's statistics covering 3563 intestinal tumors 97.5 per cent. were in the colon, appendix, or rectum. The tumors increase in frequency from the cecum toward the rectum. Of I 23 intestinal carcinomas Kaufmann found 36 in the colon, 28 in sigmoid, and 5 in rectum. He also states that over 60 per cent. of intestinal carcinomas and 5.25 per cent. of all carcinomas occur in the rectum, which is, therefore, fifth in the list of primary seats of cancer. Of 297 colon carcinomas reported by Korte, Petermann, and Anschutz, 47 were in cecum, 22 in ascending colon, i9 at hepatic flexure, 44 in transverse colon, 31 at splenic flexure, Io in descending colon, I 24 in sigmoid.

Lonart collected $6 \mathrm{r}$ cases of carcinoma of large intestine between the ages of 20 and 30 years. The disease is rather frequently observed at much earlier periods, in the cecum at I 2 years (Nothnagel), in the rectum at is to I 7 years (Czerny, Stern, Schoning), in the sigmoid at I 2 years (Garrard), in the colon at 3 years (Clar).

General Etiology.-A tissue predisposition appears definitely only in those cases arising on multiple polyposis. The carcinomatous transformation of single polyps is described by Hauser, Kraske, Verse, Allen, and many others. In the origin of these polyps a previous abnormality of structure is to be assumed.

Although carcinoma often develops in subjects of hemorrhoids, and this relation has been emphasized by Volkmann, there is no satisfactory evidence that cancer develops in tissues altered by hemorrhoids, fistulæ, or cicatrices (Kraske). The slow development of rectal carcinoma extending over 2 or 3 years suggests rather that the early symptoms commonly referred to hemorrhoids are due to the beginning carcinoma. On the other hand, the sudden development of carcinoma is a common result and an early symptom of rectal carcinoma. Zinner reports that 38 per cent. of his cases of carcinoma of pars perinealis complained of hemorrhoids. It is somewhat remarkable that carcinoma very rarely develops in chronic ulcerative proctitis or colitis.

The traumatic origin of rectal carcinoma has occasionally been asserted. Illustrative cases have been reported by Goeckel, Korte, and Anschutz. The age of incidence is later than with other intestinal carcinomas. Cripps in 380 rectal cases found between 20 and 30 years 3 cases; 30 and 40 years, $2 \mathrm{I} ; 40$ and 50 years, $78 ; 50$ and 60 years, $93 ; 60$ and 70 years, II $9 ; 70$ and 80 years, 53 ; over 80 years, 4 . The incidence of colon carcinoma is much earlier.

Some general and many special factors favor the development of carcinoma in this region. Anatomical relations influence the development of tumors at the ileocecal valve, at the junction of the appendix, at the rectal folds, and at the anus, where there are more or less definite interruptions of the structure of the intestinal wall. The considerable number of cases occurring in children indicates that developmental anomalies at these points serve as sources of carcinoma. While various gross abnormalities, as megacolon or megasigmoideum, atresia recti, and diverticula sufficiently indicate the frequency of disturbed development, none of these conditions seems to be directly connected with tumors (Mya, Lowenstein, Keibel, Stieda). Kraske 
could find no grounds for assuming that misplaced embryonal tissue is concerned in the development of rectal carcinoma. He reported 2 cases of adenocarcinoma, probably derived from embryonic rests, but the tumors, which were of considerable size, lay behind the rectum, were attached to the bones, and secondarily involved the rectal mucosa. Similar cases are reported by R. Meyer and Cohn. The chief factors leading to carcinoma are irritations acting most effectively at relatively fixed points, as cecum, the flexures, and the rectal valves. More than half the rectal cancers arise between 2 and 4 inches above the anus ( $\mathrm{R}$. Williams). Kraske, however, finds most carcinomas in the upper rectum, so that excision requires opening the peritoneum. Zinner explains the high proportion of rectal carcinomas on the anterior wall ( 75 per cent.) as the result of pressure against the prostate.

The gross and microscopical anatomy presents several distinct forms which give a satisfactory basis for clinical and pathological classification. These include: (I) Adenoma destruens; forming a broad superficial ulcerating tumor with obstruction, (2) stenosing fibrocarcinoma; (3) gelatinous adenocarcinoma; (4) bulky polypoid or papillary carcinoma derived from polyps; (5) multiple carcinoma following polyposis; (6) acanthoma; (7) melanoma. The frequency is in the same order.

In nearly all cases secondary infection plays a prominent part in the progress of the lesion, causing local or spreading suppuration, peritonitis, fistulous tracts, and communications between adjoining coils of colon, or with stomach, small intestine, and bladder. Above the tumor the intestine is usually dilated, hypertrophied, chronically inflamed or thinned, and even subject to perforation. These secondary conditions are almost wholly responsible for the cachexia which develops and which may be duplicated by similar inflammatory processes without carcinoma. Hence few subjects of this disease succumb because of the extent of the tumor.

It is a rule to which there are notable exceptions that carcinoma of the colon and rectum is only moderately malignant. The course is comparatively slow, extensions beyond the intestinal wall occur late, and metastases are less, frequent than with similar growths in other regions. Hauser points out that gelatinous tumors tend to perforate the wall and produce metastases in peritoneum and adjoining nodes, and rarely in the bone-marrow, medullary carcinoma limits its metastases to lymph-nodes, while scirrhous tumors often produce growths in the liver. Zinner supports these conclusions.

Lymphatics.-The lymphatics of the colon follow the blood-vessels of the mesenteric system. In the rectum the lymph system is more complex. Gerota describes four rectal groups: (I) The anal vessels form 4 to 5 branches which traverse the skin of the perineum and thigh and reach inguinal nodes in Scarpa's triangle. Other deeper branches join with those of the zona intermedia and reach the anorectal nodes. (2) Branches from the zona intermedia pass backward and follow the superior hemorrhoidal veins, to the anorectal nodes, but occasional branches pass to a node at the foramen ischiadicum (Quénu's node). (3) Branches from the zona columnaris follow the same course. (4) The pars pelvina is drained by ressels which pass below to the anorectal nodes and above to the mesenteric nodes of the colon. Lymphnodes are missing in the wall of the anal portion, but in the fat tissue lying between the muscular wall of pelvic and intermediate segments and the rectal fascia lymph-nodes (anorectal) are abundant. Metastases in adjacent lymph-nodes, while common in autopsy material, are less frequent in operative cases. In 59 postmortems collected by Kraske and Iverson metastases were present in 32 in nodes, liver, peritoneum, lungs, and brain. They usually reproduce the original structure. From these data and from the long dura- 
tion of most cases it is clear that carcinoma of the intestine is a favorable field for aggressive surgery.

The surgical treatment of rectal carcinoma is in a very unsatisfactory state, chiefly because of the failure of early diagnosis. Although this disease gives symptoms in its early stages, it is a general experience to find that 60 to 70 per cent. of these cases are treated for weeks or months for piles, or catarrh, or other vague benign conditions, and that a competent rectal examination is delayed or omitted. Moreover, true adenoma destruens early penetrates the muscularis, transforming a comparatively simple into a very difficult therapeutic problem.

The proportion of cases deemed operable was found by Kuttner to be 32 per cent.; by Cripps, 25 per cent.; by Tuttle and Lynch, 31 per cent. The operative mortality runs rather uniformly about 15 per cent., but varies in German clinics from 6.4 to 32 per cent. Pennington collected 966 records of operations which gave 218 3-year survivals. Of 753 cases applying at the Mayo Clinic, 430 were operated upon; 364 recovered from the operation: of these, excluding cases operated upon less than 3 years before, 33 per cent. lived 3 years or more, 28.5 per cent. 5 years or more. Of Kuttner's IO2I cases, 32 per cent. (3I6) were operable, of whom 32.5 per cent. (II 7 ) survived more than 3 years, 2 I.6 per cent. (76) over 5 years, I6.4 per cent. (58) over 8 years, I 2.8 per cent. (45) over Io years, while 42 died from recurrence between the third and tenth years.

Histogenesis. - The histogenesis of carcinoma of the large intestine has been fully traced by Hauser, Verse, and others. It has been shown that the disease arises usually in a circumscribed area of mucosa in which the glands become enlarged, the lining cells hypertrophied and multiplied, the production of mucous cells increased, and the lumina elongated and bifurcated. The neoplastic alveoli soon break through the muscularis mucosæ and extend along the submucosa, often reaching the surface at lateral points, and thus extending the lesion or penetrating the muscularis along lymph and blood paths. In the early stages of most cases and in some throughout the disease there is a gradual extension of the area of origin by the progressive transformation of normal into neoplastic alveoli. This process is more pronounced in the colon than in the stomach and is often indicated by a peripheral zone of papillary outgrowths of mucosa about the ulcerated tumor, as well as by microscopical evidence of such transition. Similar transitions have been traced in originally benign polyps.

The extent to which the lateral growth proceeds by this method is difficult to determine. In some cases there is a very wide superficial tumor involving Io to $15 \mathrm{~cm}$. of the mucosa, a form strongly suggesting a gradual inclusion of normal areas of mucosa. The structure is then typical malignant adenoma.

In more atypical and rapidly growing tumors the lateral extension is slight and exclusively $b_{y}$ invasion and replacement of normal glands by tumor-cells.

In intestinal polyposis the transition of hypertrophic mucosa and papillæ into multiple carcinoma has been clearly traced (Bardenhauer, Wulf, Verse, Doering). In certain cases of chronic colitis in young subjects preceding definite polyposis the entire mucosa is lined by markedly hypertrophic epithelium closely resembling adenoma destruens, but lacking the heterotopia of a neoplasm.

Anatomical Varieties.-(I) Adenoma destruens produces at first circumscribed elevations in the mucosa which gradually extend deeply and laterally, obstructing the bowel and soon ulcerating. At the ileocecal valve it pro- 
duces a fungating tumor inclosing the orifice without marked stenosis (Kaufmann). In the cecum the tumors are usually bulky and ulcerating, with obstruction of the lumen, fistulæ, and anastomoses. Particularly wide superficial extension is often observed in the colon proper, as in Hauser's cases. I have seen $\mathrm{I}_{4}$ inches of the transverse colon involved in a cylindrical tumor with few adhesions. In the sigmoid and rectum adenoma destruens is the common tumor, and here early ulceration, obstruction, and local extensions are relatively early.

The structure presents the most typical form of malignant adenoma, and its usual variations. In some cases the structure is more atypical and car-

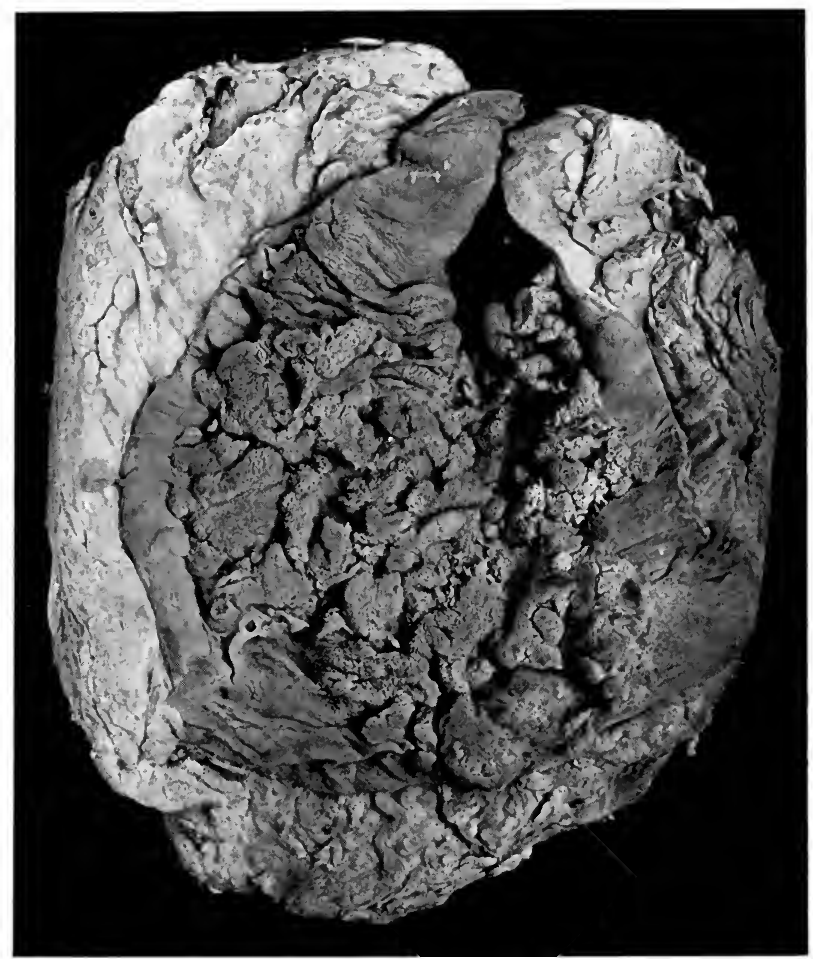

FIG. 3I 4.-An early circumscribed malignant adenoma of sigmoid.

cinomatous and occasionally the growth is highly atypical, diffuse, and resembles lymphosarcoma.

(2) Stenosing fibrocarcinoma produces first a superficial ulcer with pronounced induration due to fibrosis. The lesion may be circumscribed while involving all the coats, but there is a tendency to encircle the lumen and produce a tight annular structure, with its various complications. In advanced stages of sclerosing and other carcinomas the length as well as the lumen of the affected segment is reduced at times to a remarkable degree. This result is due to cicatricial contraction and, as Schuchardt explains, to loss of tissue by ulceration. Small annular strictures of the intestines may occur at any point. Korte reports three of the transverse colon, six of the 
splenic flexure, and several of sigmoid. These annular tumors may be multiple (Brosch).

The structure of this tumor usually presents diffusely growing small groups of atypical cells widely infiltrating the coats of the intestine and the abundant new connective tissue. Transition stages from adenoma destruens and adenocarcinoma are often found and suggest that the process begins as adenoma destruens and that early ulceration in a relatively resistant tissue leads to early fibrosis and cicatrization. Yet this tumor infiltrates neighboring tissues and nodes rather early and offers an unfavorable surgical prognosis, unless excision occurs before the intestine becomes fixed.

(3) Gelatinous adenocarcinoma of the large intestine is rather frequent and occurs both in the colon and in the rectum. Kanthack and Furnival described such a tumor of the ascending colon in a boy of 17 years. Israel

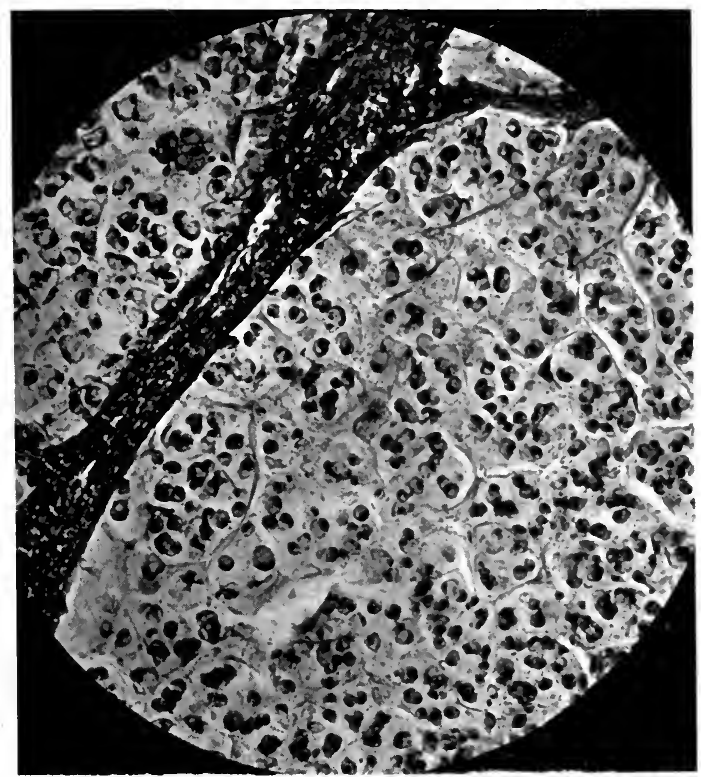

FIG. 315.-Structure of a bulky gelatinous carcinoma of colon.

reports a case in the splenic flexure in a boy of 13 years. Among 5 I colon tumors Korte had six of the gelatinous type, while of Zinner's I 23 cases of rectal tumors $4 \mathrm{I}$ per cent. were gelatinous. It is characterized by a tendency to spread widely over a considerable length of intestine, by the production of a bulky tumor mass in which the original tissues are extensively replaced by gelatinous material. The usual tendency to stenosis may be missing, but the tumor ulcerates early and excavates the lumen in a thick rigid tube. Extension to the peritoneum produces miliary nodules or bulky masses of gelatinous tissuc. I have found the pelvis completely filled by gelatinous material containing little tumor tissue derived from a localized ulcerating tumor of the rectum. Lymphatic invasion is not prominent. Kaufmann describes a case of colloid rectal cancer with a single metastasis in the liver invading the hepatic duct with fatal icterus.

(4) Carcinoma Developing from Solitary Polyps.-The most common seat 
of these polypoid adenomas is the posterior raphe of the lower third of the rectum, where Rotter finds them often the source of carcinoma. They often develop in childhood and become carcinomatous in later years.

Of the benign polyps there are several varieties:

(a) The broad flat adenoma is usually found in the rectum just above the squamous junction. It is of simple cylindrical cell adenomatous structure and its position renders it specially liable to malignant change.

(b) The common pedunculated adenoma occurs throughout the intestinal tract, chiefly in rectum and colon. It is single or multiple, small or as large as the fist, and intussusception, obstruction, or prolapse may result. An original structural anomaly or local tissue predisposition is probably present,

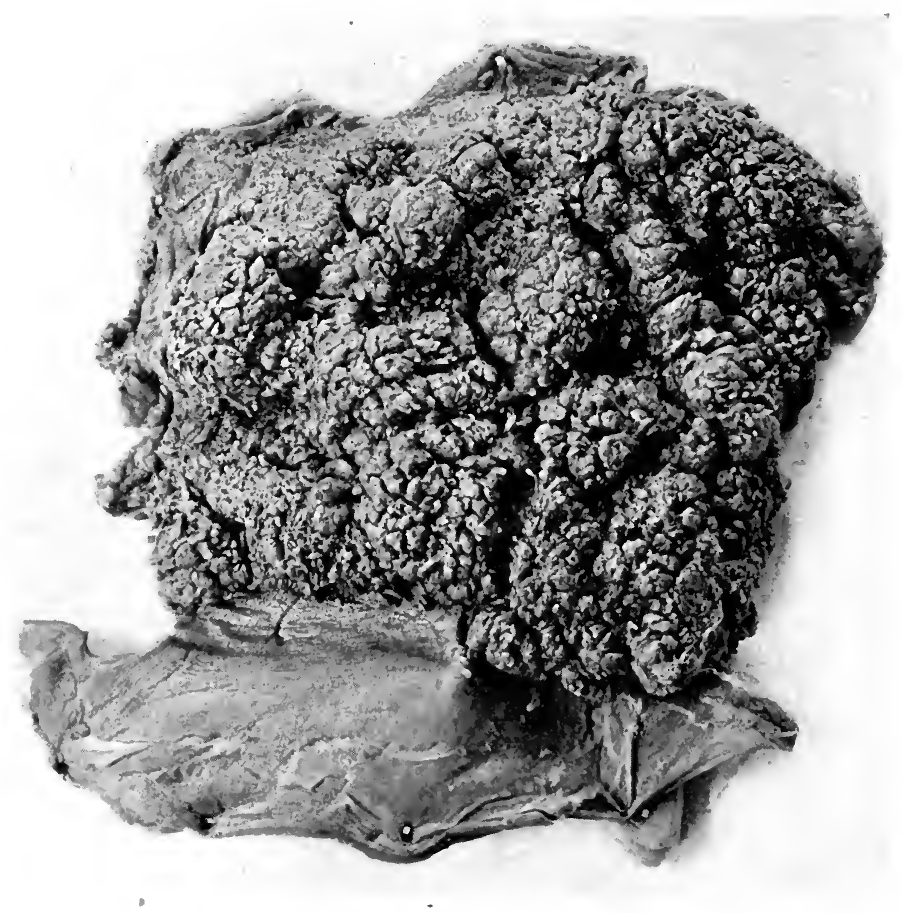

FIG. 316.-A diffuse papillary adenoma of rectum.

but mechanical and inflammatory factors have much influence on the progressive growth. The multiple polyps are probably related to intestinal polyposis. The structure presents large and small alveoli lined by high cylindrical epithelium and often filled or distended with mucus. All grades of atypical cell growth are observed and some localized pedunculated tumors have the typical structure of adenoma destruens with slight invasion of submucosa. When the stroma predominates the tumor may be designated "fibro-adenoma." The carcinomatous transformation of these polyps has been observed by Hauser, Verse, and many others. The change may begin at the base or tip or throughout the tumor. Albu saw the recurrence as carcinoma of a polyp showing some years before suspicious signs of adeno- 
carcinoma. Yeomans describes several conditions in which malignant transformation seemed to occur.

(c) Villous papilloma is a well-defined variety characterized by a finely papillary appearance, great vascularity, hemorrhages, and a pronounced tendency toward recurrence and malignant changes (Esmarch). It occurs at a later age than other intestinal polyps, the youngest of Quénu's I4 patients being 28 years old. The structure shows single or multiple layers of cylindrical cells supported by delicate vascular adenoid tissue. They require thorough extirpation of the base. I have observed a remarkable tumor of this type which covered an area of $13 \times 9 \mathrm{~cm}$. in the sigmoid. It appeared to be composed of a congeries of papillomas which had become fused. The papillæ, $\frac{1}{2}$ to $\mathrm{I} \frac{1}{2} \mathrm{~cm}$. in height, were closely packed, coherent, and flattened at the tips. The muscularis was intact.

(5) Intestinal Polyposis. Colitis Polyposa.-The chief seat of intestinal polyposis is the rectum and the great majority of carcinomas following this condition develop in the rectum or colon. The ileum or jejunum is rarely affected, but Hauser and Kaufmann saw the involvement of the entire mucosa from pylorus or cardia to anus, and lesions in duodenum, jejunum, and ileum are reported by Lubarsch, Niemack, Petrow, and others. Albu found the lesion in isolated segments of sigmoid and rectum. Of 34 cases collected by Thorbecke, 23 were in rectum, 5 in colon and rectum, and 6 in colon alone.

The disease affects both old and young subjects and exhibits an hereditary, individual, and local predisposition (Cripps, Smith, Bickerstedt, Port). In the early stages I find a diffuse and pronounced hypertrophy of the entire epithelial lining, a condition which supports Verse's view that the sole element in the predisposition is the excessive reaction of the epithelium to irritants. Peculiar catarrhal symptoms mark the onset of the disease, and hemorrhage, anemia, emaciation, intussusception, and prolapse occur in advanced cases. Some of the polyps may be discharged per anum.

Although many cases maintain their inflammatory character throughout, there is a striking tendency toward the development of malignant adenoma and carcinoma. According to Quénu and Landel about half the cases are associated with carcinoma, while Hauser found this combination as the rule, and Doering analyzed 50 cases in which of 37 fatalities 31 were from carcinoma. Spontaneous regression has been observed after sidetracking the affected region and after partial excision (Rotter). Some respond to the $x$-ray.

The anatomical form of the carcinoma varies extremely. The polyps may be very small, very numerous, widely distributed, and the entire mucosa hyperplastic, or they may be larger and less numerous. The carcinomatous process usually begins in a single polyp, involving others later. Steinthal found a larger single carcinomatous polyp in rectum and many small benign polyps in the vicinity. Annular carcinoma with many benign polyps scattered over a wide area are reported by Wechselmann and Brentano. Diffuse polyposis of entire colon and adenocarcinoma of submucosa throughout is described by Babler. As a rule one or more of the polyps are malignant, the others benign. Thorbecke comments on the high mortality of the disease, which is referable to metastases from the carcinomatous polyps, to the progressive malignant changes in previously benign areas, and to the functional disturbance of the organ. Oseki found metastases in many retroperitoneal nodes, liver, lung, adrenal, and ovary.

In structure the tumors show various types of malignant adenoma, adenocarcinoma, and alveolar carcinoma. In Babler's case there was diffuse adenocarcinoma and alveolar carcinoma in the submucosa without metastases. 
Wechselmann describes carcinomatous invasion of the base of a polyp, the tip of which was also carcinomatous.

(6) Squamous-cell carcinoma arises from the anal epidermis or by metaplasia from the lower rectal mucosa. The frequency of this tumor is estimated at 3 to 4 per cent. (Funke) or 20 per cent. (Quénu) of all rectal carcinomas. It presents the usual features of this tumor in its more malignant form. Kraske describes 4 cases, 3 of which produced ulcerating nodules destroying the sphincter, while I extended laterally over a very wide ulcerating area. I have seen a very extensive and highly destructive tumor following condyloma latum.

(7) Melanoma of the rectum is rare, but nearly all writers in this field describe one or more cases (Kraske, Cripps, Ball, Heaton, Strasberger, De Buck, Vanderlinden). Paneth collected 8 cases, and Wiener refers to several others. Bulky, firm, relatively benign melanomas of the rectum are frequent in horses (Eiselt).

The tumors are usually of considerable size, and form circumscribed or pedunculated growths which obstruct the lumen and later suffer ulceration and necrosis. In structure they have been described as carcinoma or sarcoma. Kraske's carcinoma was a medullary growth composed of large polyhedral cells with little stroma. The pigment was limited to the epithelium and adjacent lymph-nodes. Two polypoid melanotic tumors were composed of pigmented spindle-cells. The source and significance of the rectal tumors present the same obscurities as surround other heterotopic melanomas. 


\section{CHAPTER XXXIII}

\section{EPITHELIAL HYPERPLASIA AND TUMORS OF LIVER}

Classification.-The difficulties in presenting an orderly arrangement of primary epithelial tumors of the liver, so obvious in the older literature, are still apparent in many recent studies.

There is first the occurrence of a wide variety of very rare tumors which requires the admission of small groups of cases distinguished by their peculiar etiology, gross anatomy, and histogenesis. Thus Simmonds finds that local hyperplasia in the liver occurs as: (I) A solitary focus of congenital origin in a normal liver; (2) multiple nodular hyperplasia; (3) multiple adenoma; (4) solitary adenoma. All of these conditions occur with and without cirrhosis and many of the simple hyperplastic processes lead through benign tumors into malignant neoplasms, thus complicating the task of separation into groups. Secondly, the remarkable regenerative capacity of the livercells leads to the frequent occurrence of partially neoplastic processes which have been variously graded and named by different authors. Third, while the typical structure of liver-cell tumors cannot readily be confused with that of typical bile-duct adenomas and carcinomas, there remain many structures of uncertain significance, resulting probably from a growth derived from both these elements. Hence there will always be a fluctuating group of mixed epithelial tumors of the liver. Moreover, not a few cases of homogeneous type and evidently single origin are so far removed from the usual morphology that some doubt must remain regarding their true origin. Finally there are highly atypical tumors of the liver which have been named and classified on rather superficial resemblance to various forms of carcinoma or sarcoma, which still require more careful analysis before their exact nature can be established.

Two main classes of primary epithelial tumors of the liver are universally recognized as derived from (I) liver-cells, and (2) intrahepatic bileducts. A third or subgroup includes (3) mixed tumors, in which both livercells and bile-ducts contribute. The simplest terms designating these tumors are liver-cell, biliary, and mixed hepatic carcinoma, or adenoma. The term "hepatoma" has come into very general use and has the great advantage of avoiding implications with adenoma and carcinoma, while it sufficiently designates any tumor of liver-cells. Cholangioma is a parallel but less satisfactory term designating tumors of bile-ducts. Rigid terminology calls for such phrases as "hepatoma adenoides," etc., or "adenoma hepatocellulare, vel cholangiocellulare." According to their gross anatomy the tumors are solitary or massive, multiple or nodular, and rarely diffuse.

\section{SCHEME OF Forms OF EPITHELIAL HyPERPLASIA AND TUMORS OF THE LIVER}

(I) Simple hypertrophy and hyperplasia:
(a) Regeneration.
(b) Congenital solitary hyperplasia.
(c) Nodular hyperplasia in stasis, or cirrhosis.
(d) Diffuse hyperplasia (diabetes). 
(2) Neoplastic hyperplasia:

(a) Hepatoma:

(I) Adenoma.

(2) Adenocarcinoma.

(3) Carcinoma, solitary, multiple, atypical.

(b) Cholangioma:

(I) Adenoma, solid, cystic.

(2) Adenocarcinoma.

(3) Carcinoma.

(c) Mixed tumors.

Hepatic Regeneration.-The regenerative capacity of the liver as exhibited in destructive and inflammatory conditions furnishes necessary standards in estimating neoplastic processes in this organ.

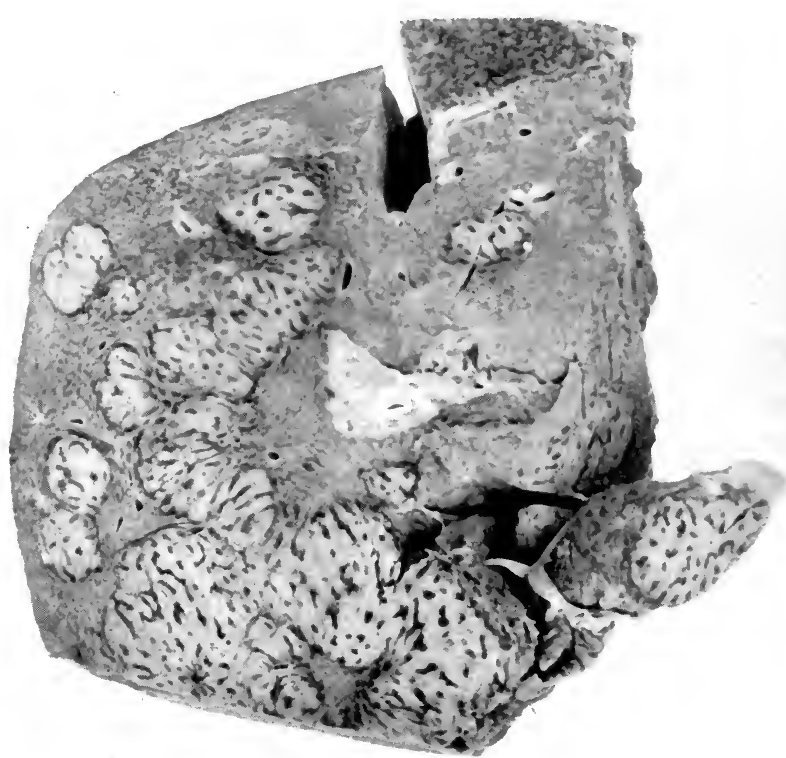

FIG. 317.-Adenomatoid hyperplasia of liver following acute yellow atrophy. Specimen of Dr. Elser's.

Experimental excision of large portions of the liver in lower animals reveals rather astonishing capacity for restitution (Ponfick, Podwyssoski, Jr.). V. Meister extirpated up to four-fifths of the organ in rabbits, rats, and dogs. The remaining lobes exhibited hyperemia, hypertrophy, mitoses and multiplication of peripheral cells of lobules, great increase in the size of the lobules, and eventual restoration of much of the lost tissue. This type of regeneration has been called morphallaxis (Morgan). Functional hypertrophy of the whole organ is observed in diabetes.

Extensive regeneration of necrotic areas is observed after focal lesions produced by hemolytic agents (Jackson, Pearce), and especially in acute yellow atrophy. Prolonged cases of this disease may show many islands of new liver tissue springing from the surviving liver-cells on the peripherics 
of lobules and producing irregular masses of deformed lobules which may present a very bizarre and almost adenomatous appearance (Stroebe). In one of Barbacci's cases a hyperplastic mass reached the size of an apple and the structure approached that of adenoma. In these cases Meder traces rather clearly the origin of new liver-cells from proliferating bile-ducts, but most observers have failed to determine this origin with certainty and believe that the regeneration begins in islands of liver-cells. In Laennec's cirrhosis the incarcerated lobules are often markedly hypertrophic, and in Hanot's cirrhosis in younger subjects the hypertrophy is diffuse and associated with hyperplasia.

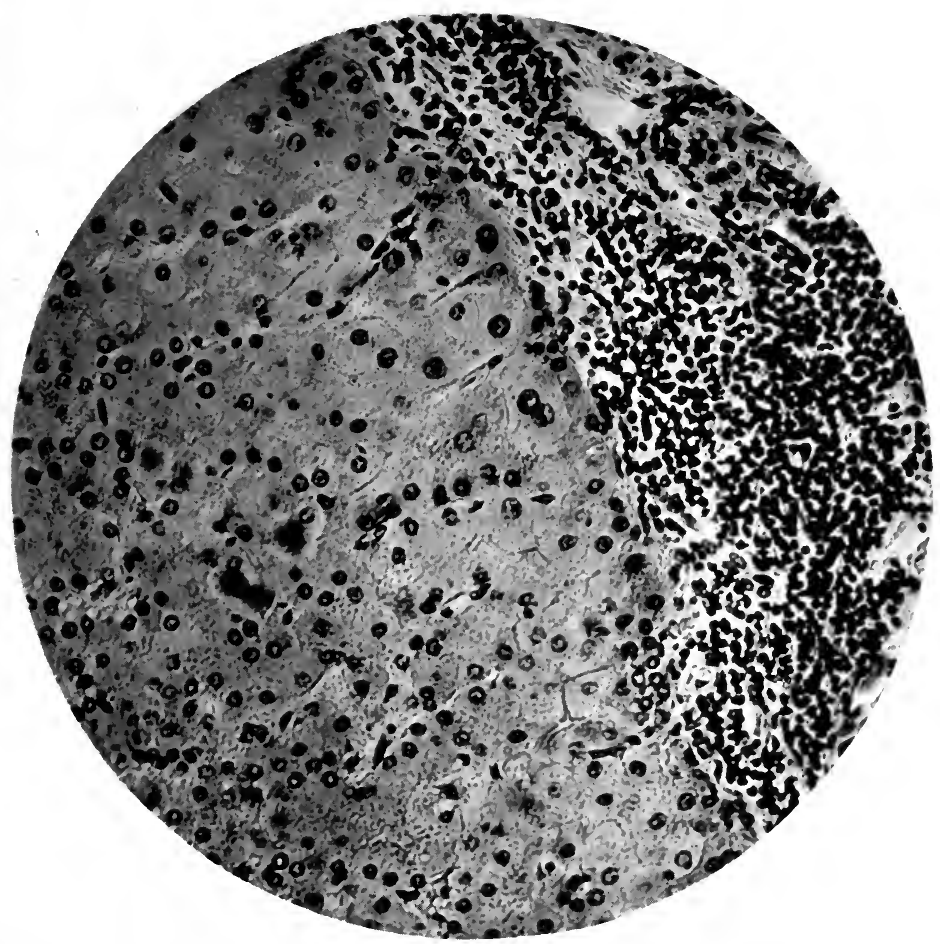

FIG. 3I 8.-Adenomatoid hyperplasia of liver following acute yellow atrophy.

Multiple nodular hyperplasia is a term applied to pronounced focal overgrowth of liver-cells in cirrhosis and other conditions. In cirrhotic livers these nodules may be very numerous, firm, opaque, and yellowish, or softened, fatty, or hemorrhagic. In malarial cirrhosis, Kelsch and Kiener described adenomatoid nodules arising in areas of compressed liver tissue, composed of regular cords of large liver-cells often undergoing fatty degeneration, or caseation, with hemorrhage and liquefaction. In both of these conditions loose masses of hypertrophic liver-cells may be found in the portal and hepatic veins and should not be confused with the solid intravenous growths of malignant tumors (Delepine).

In acute yellow atrophy the regenerating tissue may yield a pronounced picture of multiple nodular hyperplasia without genuine neoplastic features 
(Meder, Marchand). It is difficult to separate the reported cases of nodular hyperplasia from those of the more advanced cancerous cirrhosis and adenoma. Sabourin's observations refer to areas of simple hyperplasia which he finds about proliferating branches of the portal biliary connective tissue.

A very pure form of nodular hyperplasia without cirrhosis or previous destructive lesion was observed by $\mathrm{A}$. Jacobi and the writer in a child suffering from hepatic stasis from enlarged retrohepatic lymph-nodes. Here the otherwise normal liver was studded with myriads of miliary nodules resembling tubercles, and composed of hypertrophic cords of liver-cells regularly incorporated in the liver lobules. Very similar changes, but not in normal livers, are described by Simmonds.

That all of these forms of hyperplasia may pass into single or multiple progressive adenoma is fully attested by the difficulty of separating them from destructive adenomas and cancerous cirrhosis. Nevertheless the condition actually observed in many cases at autopsy is quite different from that of true adenoma.

\section{PRIMARY EPITHELIAL TUMORS OF LIVER}

General Etiology.-Orth found primary carcinoma of the liver four times among $25^{8}$ cases of hepatic cancer and $7 \mathrm{I}_{3}$ cancers of all types ( 0.28 per cent.). The statistics of Orth, Hansemann, and Rindfleisch give 0.5 per cent. of all cancers as primary in the liver. Yet Goldzieher and Bokay saw i 8 cases, I.3 per cent., among 6000 general autopsies, in 5 years.

The chief age of incidence is between 40 and 60 years, especially 50 to 60 , average 43 to 53 . Biliary carcinoma occurs later than hepatoma, rarely before 40 years, and more frequently in women. Aside from the congenital local hyperplasia of Simmonds, congenital malignant adenoma has been described by Ribbert, Prescott, Miller, Cleland, Karsner, and Milne, and multiple hemorrhagic hepatoma by Mair. Pepere concludes that all solitary adenomas are congenital. Of 29 reported cases in children Philipp accepts I 2 as genuine. Rolleston adds several others. Reports of sarcoma show much the same age incidence (Vecchi, Guerrini). Rolleston collected 32 cases under ro years of age.

Congenital maladjustment of groups of liver-cells predisposing them to tumor growth seems to be a very rare factor in adults, while possibly of more importance in infants and with solitary tumors. Among 20 cases of solitary adenoma Caminiti found only 4 with cirrhosis. All liver-cells appear to be capable of neoplastic overgrowth. A previous fatty and atrophic degenerative process is described by Oertel as a preliminary to further neoplastic degeneration in islands of cells separated by connective tissue, but this preliminary change is not constant.

Yamagiwa describes several cases of hepatoma in children, in two of which he found islands of bone and mucoid tissue. The tumors lay deep, were partly encapsulated, cells small, embryonal and rich in glycogen, and the vessels dilated. These features he interprets as those of an autochthonous teratoma, with metaplasia of superfluous mesenchymal material. Islands of metaplastic cartilage and squamous epithelium occurred in cases of v. Hippel and Philipp.

Relation to Cirrhosis.-Cirrhosis is the chief predisposing factor, occurring in about 85 per cent. of hepatomas, and 50 per cent. of biliary tumors (Eggel). The cirrhosis is usually portal and biliary in type and often of alcoholic or syphilitic origin. Carcinoma was associated with hemochromatosis in the cases of Lohlein and Rindfleisch. Echinococcus cysts were ob- 
served in the cases of Dibbelt, Lohlein and Necker, and Yamagiwa reports cases with distomiasis and schistosomiasis. There is little doubt that cirrhosis and the factors that lead to it cause degeneration followed by regenerative overgrowth which may become excessive and neoplastic. This excessive hyperplasia signifies failing compensation and is favored by fatty degeneration and thrombosis of veins (Rolleston). Hence the cirrhotic process must be regarded as both contributing to and coincident with the tumor growth. There seems little point in the effort to disconnect these interrelations. A secondary fibrosis may develop about old nodules of liver-cell tumors, while biliary carcinoma is desmoplastic from the first.

Hepatic stasis frequently antedates the cirrhosis and the tumor process, is very effective in stimulating overgrowth in the form of nodular hyperplasia, is often observed without cirrhosis. and is a prominent predisposing factor in hepatic tumors. Local interference with the blood supply appears responsible for some solitary tumors.

Histogenesis.- The growth of hepatoma from the hypertrophic liver cords was first clearly demonstrated by v. Heukelom, has been traced in greater detail by Goldzieher and Bokay, and these observations have been verified by many others. It has also been shown that there is a uniform gradation between nodular hyperplasia, multiple adenoma, and multiple carcinoma. These gradations may even be observed in the same liver, so that, as Muir has stated, there is no essential distinction between the comparatively benign and the atypical malignant forms of the tumor. Ribbert and Heussi contest the multiple origin of many hepatomas, finding very sharp separation between hypertrophic and invading tumor-cells without great disorder of the cords. Yet such a sudden transformation of hyperplastic into neoplastic cells is characteristic of many tumor processes. During the transition the cells may retain the granular character of liver-cells while staining more intensely with basic dyes, or they may lose granules and pigment and assume the transparent supposed embryonal character described by Adler. Bile secretion diminishes with increasing anaplasia, but may be entirely missing in massive adenocarcinomas. Nuclear hypertrophy and hyperchromatism is very constant, multinucleated and giant-cells appear, and mitosis and amitosis are frequent. Notable changes in the nuclei are often seen in neighboring liver tissue, forming a feature of collateral hyperplasia. The first generations of tumor-cells are usually large, later they become smaller and atypical. The preservation and new growth of capillaries is a remarkable feature, may accompany the intravascular growths (Parcelier), and reaches its highest activity in the so-called angioplastic pseudosarcomas. Renon, Geraudel, and Monier-Vinard particularly emphasize the presence of a neoplastic process residing in the endothelium of the capillaries and they conceive the hepatoma as a new formation of complete embryonal liver tissue from the adult. It seems possible to overestimate the importance of the endothelium in these cases. Yet Dominici and Merle trace a sarcomatous process to the excessive growth of endothelium in an atypical hepatoma.

Biliary carcinomas have also been traced satisfactorily to the proliferating bile-ducts in cirrhotic and other livers, and the usual series of graded cases from adenoma to carcinoma is abundantly supplied in the literature ( $\mathrm{r}$. Hippel, Fischer, Herxheimer). Precancerous changes are supplied by Milne and Yamagiwa in the form of angiocholitis proliferans. The multiple nodules must be referred to the universally distributed small ducts, while the solitary and cystic growths show some predilection for the hilus and subcapsular 
areas. Yamagiwa finds that tumors of the larger bile-ducts yield tubular and papillary adenocarcinomas, while the small ducts produce chiefly carcinoma simplex.

For the atypical and highly malignant growths from both sources there are no observations to indicate their exact origin. They probably originate in few or single foci, as Ribbert urges, and are rapidly disseminated throughout the liver.

The histogenesis of the tumors of mixed type is not clear. Most of these cases arise in extensively cirrhotic organs in which the formation of pseudobile-ducts from incarcerated liver-cells and the elongation of tortuous bileducts render the picture very complex. The uniform mingling of two tumor processes affecting liver-cells and bile-ducts must be arcepted with caution, and Muir and Rolleston emphasize the fact that typical hepatomas may produce canals resembling bile-ducts.

Metastases.-Both single and multiple hepatomas very early invade the capiliaries, even when devoid of other malignant properties. Hence loose intravascular thrombi may appear in relatively benign tumors which are incapable of surviving a dislocation from the liver. They are, however, capable of forming secondary nodules in the liver, and by this means of dissemination malignant tumors may be rapidly distributed over most of the organ. Ribbert and Heussi, and many others, believe that this process, rather than a multicentric origin, explains the distribution of tumor nodules in many cases. Yet in not a few instances bulky tumor masses are found in the portal and hepatic veins without very many secondary nodules. Thrombosis of the hepatic or portal veins may occur without extensive tumor invasion, by a passive discharge of softened tumor tissue. Occasionally the tumor thrombus extends into the vena cava and even up to the heart. While no point of perforation of the large veins can usually be found, yet Engelhardt describes the penetration of the wall of a large vein by infiltrating tumor-cells.

The frequency of metastases is not great. Of 163 cases tabulated by Eggel 46 were free from definite extensions, 50 showed growths limited to the portal or hepatic branches, 30 gave metastases in thorax or lungs, I 8 in regional lymph-nodes only, and 9 secondary tumors were distributed in colon, pancreas, ovary, kidney, omentum, thyroid, and cranium. Giachetti's massive carcinoma gave very numerous metastases only in the brain.

Extrahepatic metastases are much earlier and more frequent in biliary cancer than with hepatoma. In many instances the distant metastases of hepatoma have shown secretion of bile (Schmidt, Hanot, Gilbert, Clan, Pryn, Wegelin, Mair). Secondary hepatoma is often distinctly organoid in structure, exhibiting liver cords, Kupfer's cells (Mirolubow), capillaries, and bile secretion.

Clinical Course.- The history of carcinoma of the liver varies greatly with the type of the disease. The presence of a malignant tumor is usually indicated by anemia and emaciation, but Rolleston refers to cases which gained 19 and 7 pounds in weight during rapid terminal growth of the tumor, the liver weighing I6 pounds. After the appearance of a malignant tumor life is seldom prolonged over 4 months (Hale White). Fever is observed with rapidly growing tumors and from infections. Eggel found fever in I4 per cent. of his cases, jaundice in $6 \mathrm{I}$ per cent., ascites in $5 \delta .5$ per cent. In the urine no specific changes have been demonstrated. Several well-defined clinical groups are observed:

(I) No symptoms are detected and the patient dies suddenly from hemorrhage, or after an illness of a few days, as noted by Karsner. 
(2) Latent carcinoma is found in patients succumbing to cirrhosis or other diseases.

(3) The usual history of cirrhosis terminates rapidly with hepatic tumor, jaundice, ascites, and cachexia.

(4) The usual history of a malignant tumor pointing from the first to the liver, develops in previously healthy subjects.

Recovery from the disease is very rare, but Keen and Yeomans report successful excisions, some of which appear to have recovered permanently. Yeomans states that a patient from whom he excised a portion of a large tumor mass in the liver remains well after 5 years. A section of this tumor I found to show typical trabecular and perithelial adenocarcinoma.

\section{ANATOMICAL AND Clinical Varieties}

(I) Hepatoma.-(a) Solitary Adenoma. Solitary Hepatoma.-Solitary liver-cell adenomas are rarely encountered, but typical cases are described

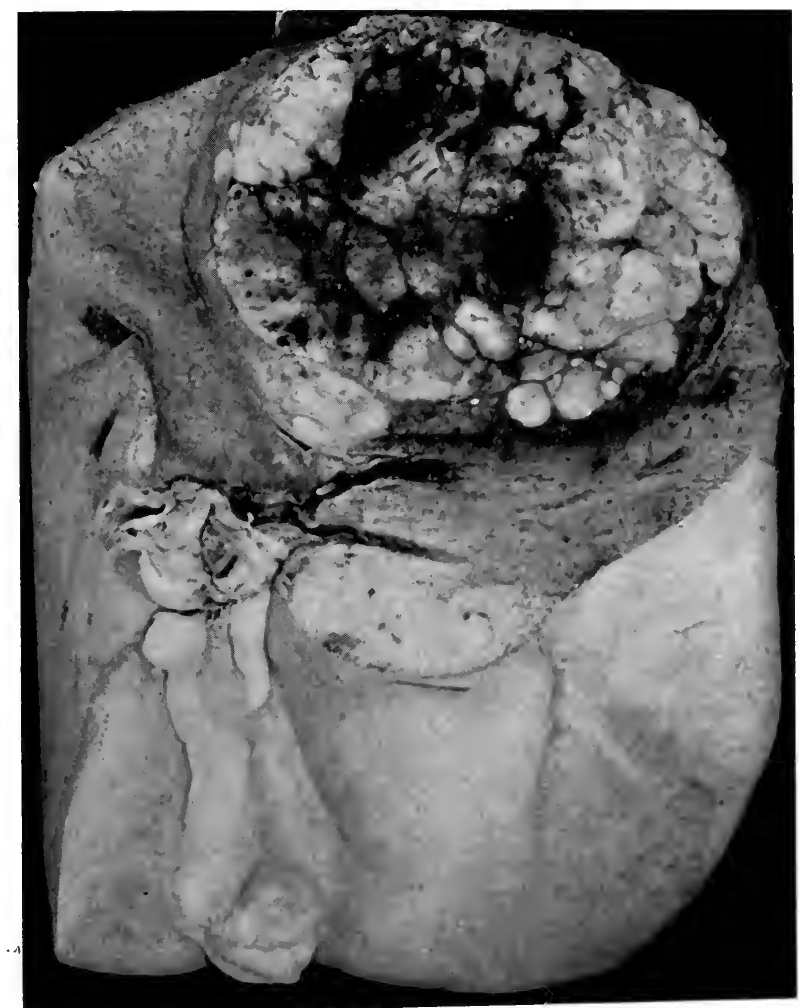

FIci. 319.- - Solitary adenoma of liver.

by Rokitansky, Salter, Engelhardt, Wegelin, Rolleston, and others. 'Two or more massive tumors in one liver are properly included in this group. Caminiti collected 20 cases, several of which occurred in cirrhotic livers. Many appear in early life from 3 months and later (Milne). Hence they are 
often regarded as of congenital origin, but it is probable that acquired lesions may give rise to neoplastic overgrowth of isolated portions of the organ. Christiani describes congenitally displaced lobules of liver tissue in Glisson's capsule, and Pepere found such lobules in liver and scattered over the peritoneum. In Engelhardt's case the quadrate lobe was missing.

The solitary adenoma produces a gray, yellow, or bile-stained, projecting and encapsulated tumor, varying in size from a small nodule to a mass 8 inches in diameter.

The tumor is composed of cords, tubes, and alveoli closely resembling the structure of the liver. In a case of the writer's all three structures were illustrated in different areas. The cells are granular and acidophile, or very fatty. Bile stasis is usually absent, but many phases of its secretion are often observed (Ciechanowski). The stroma is composed chiefly of capillaries.

The veins are not invaded in the typical solitary adenoma, but the benign character is due chiefly to encapsulation and transitional forms to adeno-

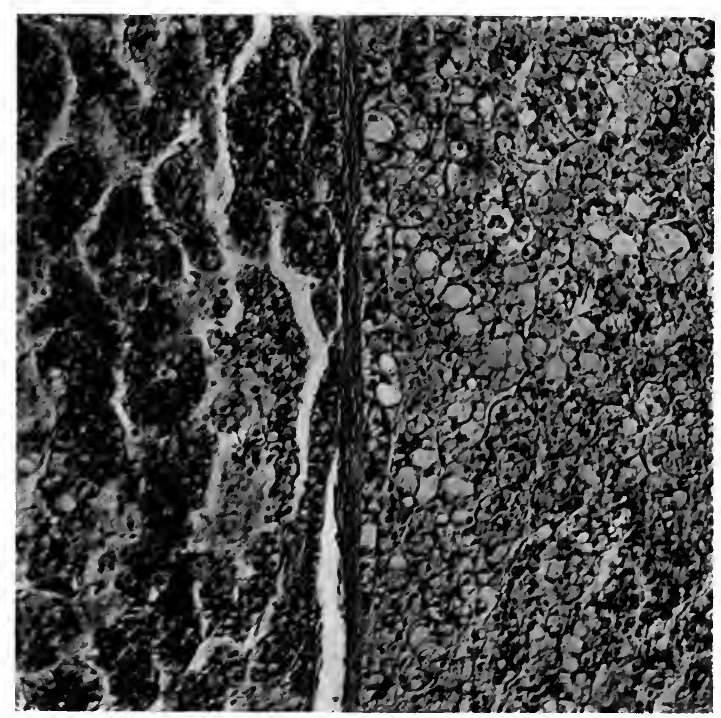

FIG. 320.--Varying structure in adjoining lobules of malignant adenoma of liver cells.

carcinoma occur in which there is invasion of veins and multiplication of tumors. B. Fischer describes a solitary adenocarcinoma with metastases in lymph-nodes.

Adrenal rests in the liver have been described by Schmorl and others, and yellow tumors possibly derived from these structures and closely resembling adrenal tumors in other situations are described by Schmorl, Pepere, de Vecchi, et al. Glynn, however, properiy doubts the adrenal origin of these tumors. Hirschler emphasizes the structure and very fatty character of his tumor, but the lipoids were chemically unlike those of the adrenal.

(b) Primary Massive Liver-cell Carcinoma.-This form of primary carcinoma of the liver is highly characteristic. It occurs in young adults, but more often in elderly subjects, and apart from the cirrhosis which frequently accompanies the tumor it yields few symptoms until a brief terminal period is 


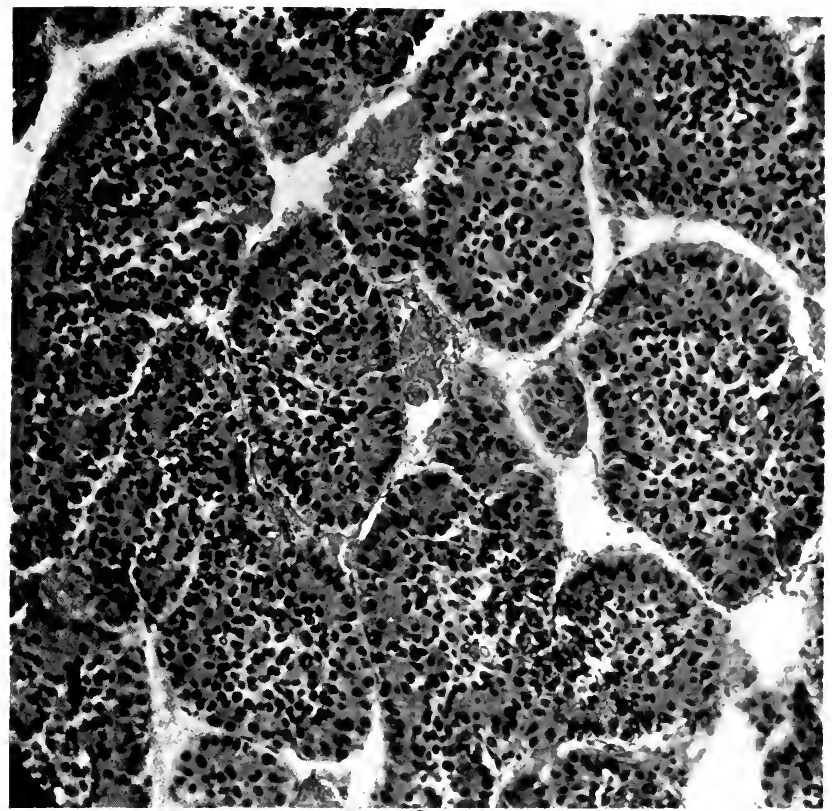

FIG. 321.-Adenocarcinomatous structure in a primary tumor of liver-cells.

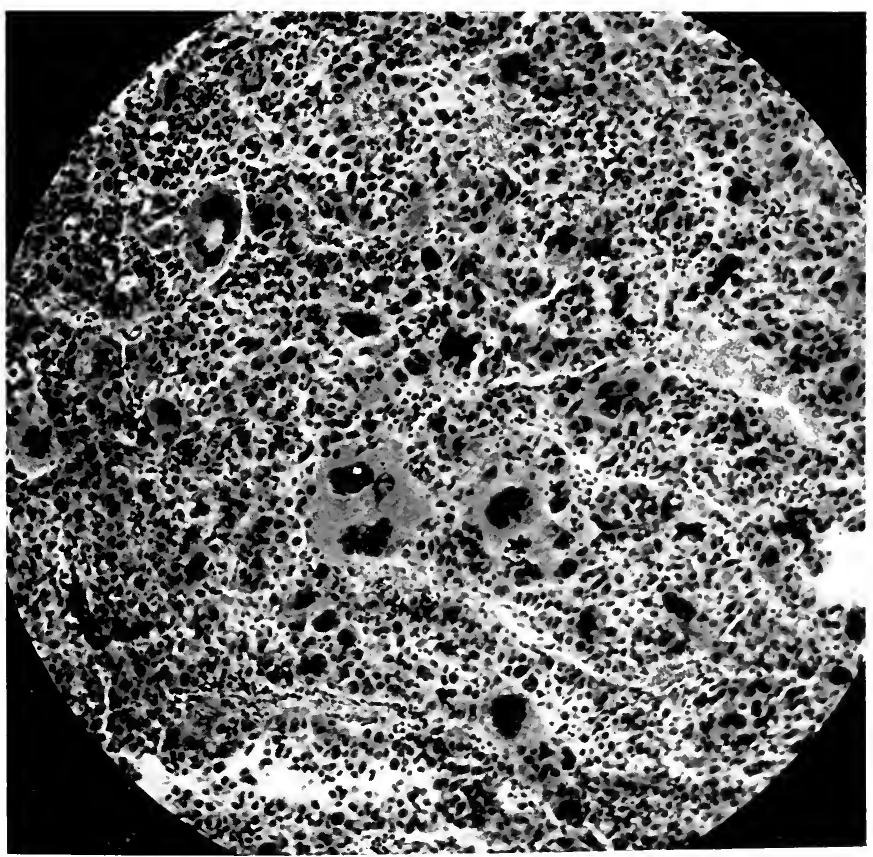

FIg. 322.- Variations in cell types in malignant hepatomia. 
reached. Eggel collected I4 cases at ages from $2 \mathrm{I}$ to 67 years, duration 2 weeks to 6 months. I have reported a case in an old man who died suddenly and unexpectedly from abdominal hemorrhage.

The tumor appears as a large single yellowish friable mass, as large as a child's head or occupying the whole right or left lobe. Small secondary tumors in the liver may be present. The consistence is usually soft, and extensive liquefaction necrosis and hemorrhage may produce a cystic appearance, or rupture into the peritoneum. The large veins are usually invaded and thrombosed and metastases, while occasionally absent, may be wide-spread and bulky, especially in the lungs.

The structure presents wide variations, but always reveals at some points a definite resemblance to liver-cells. In the writer's case four separate structures were presented in different portions: (I) Trabecular adenocarcinoma, composed of cords of very large granular acidophile cells resembling large liver-cells, and separated by capillaries. Giant-cells of very large dimensions were abundant in these areas. These cells have been described by

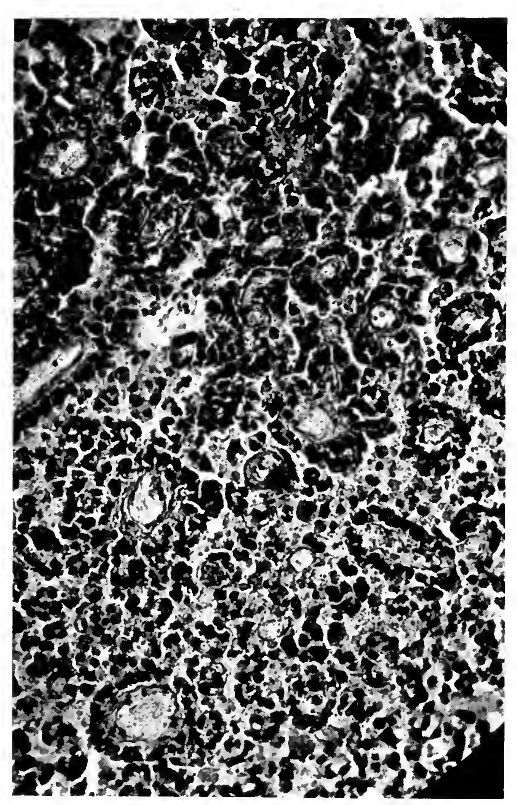

FIG. 323.-Pseudoperitheliomatous structure in multiple hemorrhagic hepatoma. (L'Esperance.) Hanot and Gilbert and by C. P. White as a characteristic feature. (2) Alveolar adenoma. These areas presented compact groups of smaller granular epithelium sharply bounded by very numerous wide capillaries. (3) Peritheliomatous areas composed of capillaries surrounded by one or more rows of cubical granular cells appeared in certain portions. This structure has been noted by Goldzieher and Bokay, Yamagiwa, and others. (4) Diffuse carcinoma. In many softened or necrosing areas all traces of the orderly arrangement of cells were lost, and the growth was composed of diffuse round, polyhedral, or spindle-cells with strongly hyperchromatic nuclei.

These structural types cover most of those observed by other authors. It is evident that this tumor represents a more rapidly growing atypical and malignant form of the solitary adenoma.

(c) Multiple Liver-cell Carcinoma or Hepatoma.--In this group are included the highly malignant rapidly growing tumors occurring in livers in which cirrhosis is either absent or so slight as to be of secondary importance. The peculiar clinical course, gross appearance of the liver, and the microscopical structure which is often atypical, justify the separate consideration of these cases. There is, however, no sharp division between this group and solitary massive carcinoma, on the one hand, and multiple carcinoma following cirrhosis on the other.

While the highly characteristic cases are somewhat rare, the group as a whole is rather numerous. Eggel collected many examples, most of which were associated with advanced cirrhosis. Hanot and Gilbert describe several characteristic cases. There is reason to believe that many of the so-called 
angiosarcomas of Arnold, v. Kahlden, Steinhaus, and Marx are atypical forms of multiple hepatoma, and it is probable that other cases described as alveolar or lymphosarcoma are of the same nature (Dominici, Merle).

The clinical course is chiefly notable for its rapidity, many cases lasting only a few weeks, few continuing more than 3 or 4 months, and some dying suddenly without very definite previous disturbance. Jaundice is uncommon, but serous or bloody peritoneal effusions are often observed. Rapid cachexia with enlargement of the liver and ascites are the usual clinical signs. These tumors occur chiefly after middle life, but De Haan, Mair, and others report cases in infants and children.

The liver is usually enlarged, at times to very considerable dimensions, and is the seat of multiple nodules and tumor masses which are grayish or bile stained or hemorrhagic and necrotic. A resemblance to multiple secondary chorioma has been noted by Marx, Teacher, and L'Esperance. Although the

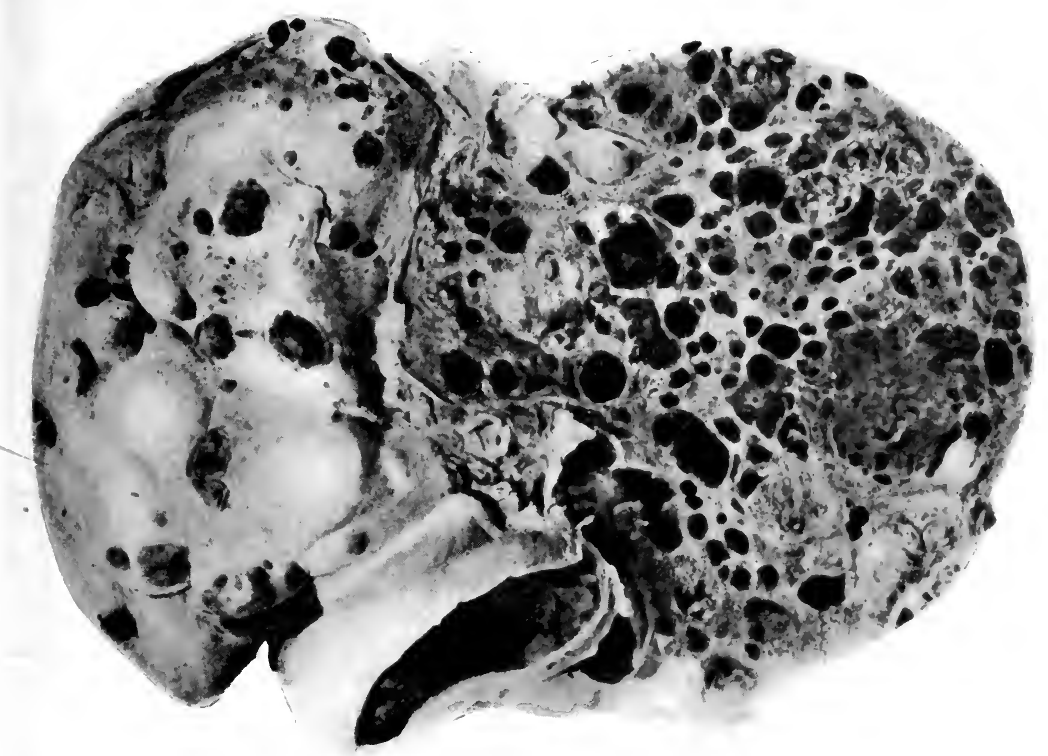

FIG. 324.-Multiple hemorrhagic hepatoma. (After L'Esperance.)

size of the tumor masses varies, it may be impossible to choose any one as a single primary focus. Many small nodules are doubtless secondary. In other cases, usually of slower development, there are one or more larger masses with more numerous and probably secondary tumors in the liver.

Metastases are usually missing, but local extensions to the portal nodes, diaphragm, and gall-bladder are observed, and in the more prolonged cases they may be found in the lungs and other organs. Most tumors vielding extensive metastases are associated with cirrhosis. The structure varies greatly even in different portions of the same tumor and reproduces the more malignant structures of solitary carcinoma. Thus one finds trabecular carcinoma with giant-cells, small alveolar carcinoma with numerous capillaries, perithelioma, and diffuse carcinoma. A definite encapsulation of tumor masses does not occur, but Mirobolew points out that distended renules may inclose tumor nodules. 
L'Esperance has described an atypical form of primary multiple hemorrhagic hepatoma, which probably includes many cases of pseudosarcoma. In portions of these tumors the original structure appears chiefly as a perithelioma, with large or small cubical granular cells surrounding capillaries. In hemorrhagic and necrotic areas this structure is lost and the tumor detritus appears as a large spindle-cell or alveolar sarcoma. The occurrence of such pseudosarcomatous areas in combination with typical hepatoma, as occurred in one of my cases, reveals the true nature of the atypical forms of hepatoma. The structure may somewhat resemble chorioma of the liver, as noted by Cruikshank and Teacher and, Marx, but secondary chorioma from uterus or testis regularly reveals its specific features and there is no reason to believe that chorioma is ever primary in the liver.

(d) Carcinomatous Cirrhosis. Multiple Adenoma, Carcinoma, or Hepatoma with Cirrhosis.-A more advanced stage of hyperplasia with atypical morphology, local aggressive properties, invasion of veins, and occasionally with metastases is described by many authors as carcinomatous cirrhosis

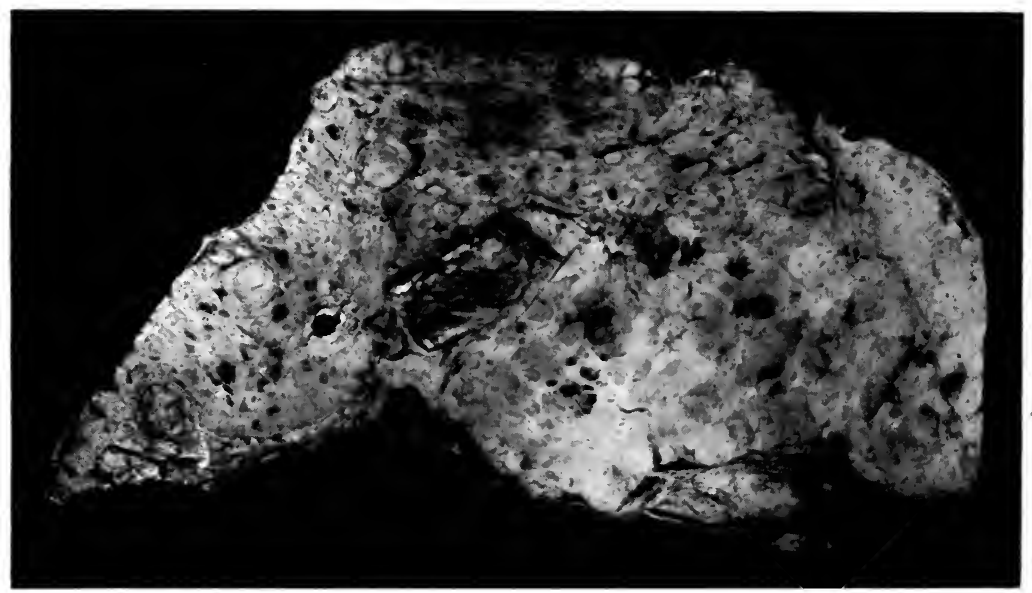

FIG. 325.-Gross appearance of universal cancerous cirrhosis of liver.

(Griesinger, Hanot and Gilbert, v. Heukelom, Thorel, Rolleston). Others designate the condition as multiple adenoma or carcinoma (Marckwald, Lit., Lancereaux).

No sharp division exists between multiple adenoma without cirrhosis, multiple adenoma with cirrhosis, and carcinoma. Each of these conditions exhibits progressive, invasive, and malignant tendencies (Muir).

The distinguishing feature of the present group of cases is the relation to cirrhosis. The tumor process appears to be the direct sequel of, or essentially connected with, the cirrhosis. Hence the symptomatology is much influenced by the history of cirrhosis, and both the clinical picture and pathological anatomy are somewhat distinctive.

Cancerous cirrhosis develops in elderly subjects of 40 years or more, and often in children, previously in good health, who develop symptoms of portal stasis with ascites and slight jaundice. A history of cirrhosis regularly precedes the development of the tumor, but is not always pronounced. With the onset of the tumor process there are added increasing anemia, 
cachexia, diarrhea and hemorrhages, and the disease progresses steadily to a fatal issue. After the appearance of ascites the duration is seldom more than a few months and often only a few weeks.

The liver is usually contracted, but in some cases is normal or greatly increased in size. The surface presents multiple, projecting, yellowish, or bile-stained nodules which on section may be found to represent a large part. of the parenchyma. The nodules may be numerous, small, and almost confluent, or larger, discrete, and encapsulated. One portion of the organ may be quite free, but frequently the lesion is nearly universal. A portal cirrhosis is usually present and many of the nodules are surrounded by connective tissue. In many cases the gross appearance of the organ resembles that of Hanot's hypertrophic cirrhosis. The spleen is diffusely enlarged. Borst found in one case much the same process in both liver and pancreas.

Many authors describe a diffuse form of hepatic carcinoma (Eggel, Rolleston). Yet these cases commonly represent a very extensive development of nodular carcinoma in a cirrhotic liver and not a diffuse growth of tumorcells. Regressive changes in the tumor and overgrowth of hyaline connective tissue may also disturb the original structure and produce areas of diffuse carcinoma.

The microscopical structure shows extensive replacement of parenchyma by adenomatous and carcinomatous nodules. The process begins with the hypertrophy and hyperplasia of cell groups within the acinus and apparently at any point in the lobule. These cell groups enlarge, forming nodules which rapidly encroach upon the remaining parenchyma with atrophy. The tumorcells are of large size, forming thickened liver cords (trabecular adenoma), or the nuclei multiply actively and numerous smaller cells result. Peculiar forms of nuclear division, chiefly of the amitotic type, are observed. Giant and syncytial masses often appear. Fatty degeneration may be prominent and liquefaction may be added. The cells may contain globules of bile and many hyaline cytoplasmic bodies. The structure may vary in the same or different nodules. The cells may be arranged in small regular alveoli with a fine lumen, or the lumen may be wide and the lining cells low cuboidal. Many transitions from adenoma to adenocarcinoma and diffuse carcinoma are observed. v. Heukelom was able to trace the origin from the liver-cells and to follow the growth of early nodules into the central veins and portal and hepatic radicles. By this route the tumor grows into the larger veins which may be occluded by tumor masses, although their walls are intact and no point of penetration may be found. These tumor thrombi may fill the hepatic or portal veins and enter the vena cava. Yet metastatic growths are often wanting, but in about 20 per cent. secondary deposits are found in lungs or peritoneal nodes (Kelsch and Kiener, Brissaud, Hanot and Gilbert, Frohmann, Goldzieher, and Bokay).

The part played by the bile-ducts varies. In most cases they are considerably increased in number in the cirrhotic areas, but do not contribute to the tumor process. In other cases the proliferation of bile-ducts is more active and they appear to become transformed into tumor-cells (BirchHirschfeld, Thorel, Marckwald). The structure is then apt to present many small groups of tumor-cells lying chiefly in connective tissue, as well as the typical hepatic adenoma. In such cases Marckwald, Theodorow, and Parcelier and Fromaget conclude that two separate tumor processes exist, one affecting the liver-cells, the other the bile-duct cells. Pure adenoma or carcinoma of the bile-ducts presents a rather specific structure and will be separately considered.

The relation of the tumor process to the associated cirrhosis is not always 
identical. In many cases the rapid progress of the disease and the moderate grade of cirrhosis indicate that the latter is secondary to the former process (Lancereaux, Marckwald). Moreover, extensive nodular hyperplasia, adenoma, and primary carcinoma occur without cirrhosis. Since focal hypertrophy is often observed in portal cirrhosis and the grade of cirrhosis is often extreme, the liver being contracted and deformed, it is highly probable that the condition in such cases represents a sequel of the cirrhotic process (Simmonds, Orth, Schmieden, Travis, Peabody). In most cases the two processes are probably coördinate (Watzoldt).

The many gradations presented by different cases between simple nodular hyperplasia, multiple adenoma and adenocarcinoma, the multiple origin, the peculiar localization of the disease to the liver, and the frequent absence of local aggressive properties, have raised doubts regarding the genuine carcinomatous nature of the disease. Borst, while admitting its morphological identity with carcinoma, urges a distinction between secondary epithelial proliferation after injury of liver tissue with hypertrophy, hyperplasia of adenomatoid or carcinomatoid structure, and true primary autonomous neoplasms of the liver. This point of view may be emphasized by the occurrence of congenital solitary hyperplastic areas, and solitary adenoma and carcinoma of this organ. Yet all the features of the malignant primary tumors are observed in multiple adenoma or carcinomatous cirrhosis. Different cases present every gradation from nodular hyperplasia to malignant carcinoma, so that the distinction between multiple adenoma with cirrhosis and true carcinoma without cirrhosis appears to be the condition of origin and intensity of the process rather than in the essential nature.

Renon and Geraudel contrast the biological features of all the liver-cell tumors (hepatoma) with (other) true cancers. They point out the multiple origin, the lack of complete emancipation of the cells which do not survive well outside the environment of the liver or frequently produce metastases, the preservation of functional capacity in secreting bile, and hence they discard the term "carcinoma" in favor of "hepatoma." I can only endorse the employment of a specific term for such a peculiar process, but must still regard the process as essentially carcinomatous with certain features which are duplicated by some carcinomas in other organs. Geraudel contends that the hepatic parenchyma is of mesoblastic origin. Yet the liver-cells are derived from exactly the same endodermal bud as the bile-ducts, and the only part of the parenchyma which is of mesodermal origin is the system of blood-vessels.

\section{CYSTS AND TUMORS OF BILE-DUCTS. CHOLANGIOMA}

Cysts of the Bile-ducts.-Biliary obstruction is the cause of a variety of hepatic cysts.

(I) In cirrhosis obstructed bile-ducts may dilate, giving rise to very numerous small or microscopic cysts containing fluid bile. Rolleston describes a case with many subserous cysts containing inspissated bile. In cirrhosis with nodular hyperplasia or adenoma the hypertrophic nodules may break down and yield small cysts in the parenchyma.

(2) Large single or multiple cysts from retention of bile are occasionally observed. Bayer found a cyst containing $13^{\frac{1}{2}}$ pints of fluid, Aldous 12 pints, and North 5 pints, mostly clear or bloody fluid from which the bile pigment had been absorbed. Doran's cyst contained $2 \frac{1}{2}$ pints of colored bile and was evidently more recent. In all these cases the wall is of fibrous tissue in which are embedded numerous bile-ducts. The lining may be of cubical, 
cylindrical, or flat cells, but the epithelium is much desquamated. Zahn collected I4 cases of cysts lined by ciliated epithelium. These suggest an origin from congenitally misplaced aberrant bile-ducts (Moschkowitz).

(3) Congenital multiple cystic disease of the liver is a not infrequent condition which is nearly always associated with similar changes in the kidneys (Bristowe, Still, Moschkowitz). Cystic kidneys, however, are usually found without cystic liver (73 per cent., Lejars; 94 per cent., Luzatto). The pancreas and spleen may also be involved.

The condition is rather common, although not always pronounced, in malformed infants, especially females, and in various ways leads to early death. Another group of cases is observed in adults who escape the initial dangers or in whom the condition develops late. The chief symptoms are uremic.

The liver may be of normal size or greatly enlarged. MacDonald records a cystic liver weighing 14 pounds. When the cysts are very small the organ may appear fibrosed, otherwise a portion or the whole of the liver may be the seat of a large number of cysts from $\frac{1}{10} \mathrm{~mm}$. to several centimeters in diameter. The parenchyma may be reduced to a trace and cirrhotic. The kidneys are usually more altered than the liver. The fluid contents are clear, albuminous, mucoid, bloody or inspissated, and contain urea, salts, and cholesterin, but no bile. The earliest stages of the process show a multiplication of slightly dilated but otherwise normal bile-ducts in widened portal canals. By progressive dilatation and fusion of smaller cysts the larger develop. In adults the lining cells are cylindrical or cubical, occasionally ciliated (Lehars) and often flattened or absent.

The structure does not favor the old theory of a fetal cholangitis, nor the origin from hepatic degeneration with widening of new formed bile-ducts. A neoplastic element was discerned by Rindfleisch and designated as cystic fibrosarcoma. Any neoplastic element present is more probably adenomatous, as appeared in unusual distinctness in cases of Siegmund, v. Kahlden, and Workman, and especially in the kidneys in the cases of Nauwerck and Hufschmidt. A congenital malformation is undoubtedly the original factor. Shattuck suggests that the tubules represent remains of mesonephros blended with the metanephros, and for the liver Still traces the tubules to irregular diverticula from the duodenal invagination which develops along with the normal bile-ducts. Moschkowitz finds aberrant atypical ducts along with normal bile-ducts in the portal canals of cystic livers, but Rolleston doubts this interpretation of these structures. I do not find them in my cases, in which all the bile-ducts are alike. On the basis of this congenital anomaly progressive dilatation may occur or adenomatous processes may be established. Some single and multiple cystic adenomas of the liver probably develop in this way, and in the kidney I have seen highly cystic Wilms' tumors which suggested a transition from the congenital cystic kidney to congenital sarcoma.

Tumors of the Bile-ducts. - The epithelium of the bile-ducts gives origin to several varieties of true tumors. While the congenital cystic liver rarely or never gives rise to a progressive neoplasm, single and multiple cystic adenomas arise from the bile-ducts in cirrhosis; multiple solid adenomas of the bile-ducts are rather frequently observed (Watzold); and a specific form of multiple carcinoma develops from these structures.

Cystic Adenoma of Bile-ducts.-Definite tumors of this origin are rare and the neoplastic nature of some of the recorded cases, of which Leppman collected 9 , is doubtful. Thus Shattuck removed a large cyst containing a gallon of clear fluid, in the wall of which were many ducts and minute cysts interpreted as adenomatous. A true multilocular cystadenoma was suc- 
cessfully removed by Keen. It weighed I 3 gm. and contained many cavities lined by cylindrical cells and supported by fibromuscular tissue. In Siegmund's case a large part of the liver formed an isolated cystic mass in which were many areas suggesting active neoplastic growth of cubical cells.

A remarkable development of multiple cystic adenomas involving the whole of a cirrhotic liver is described by Dmochowski and Janowski. The organ weighed Io,850 gr., contained very numerous cysts, minute and as large as a child's head, filled with clear fluid. There was extensive epithelial proliferation filling some of the smaller cysts.

Multiple Adenoma of the Bile-ducts.-Usually observed as a secondary condition after death from other causes and of itself produces no symptoms (Watzold, Hippel). The liver is usually the seat of stasis or cirrhosis. In Brigidi's cases the liver weighed 6 pounds, the adenomas were small and numerous, and an extensive cirrhosis was present. V. Hippel found very little cirrhosis. The tumors lie beneath the capsule or deeper in the parenchyma and appear as grayish-white nodules 0.5 to 2 or $3 \mathrm{~cm}$. in diameter. The gross appearance may resemble that of thyroid tissue when the alveoli are filled with secretion (v. Hippel, Dreschfeld). Occasionally there is bile stasis in the alveoli and the tumors are discolored. The nodules are usually incased in connective tissue and are free from bile deposits.

The structure presents many small alveoli with definite lumina lined by one or more layers of small cubical or higher cylindrical cells in which the nuclei are very prominent, and the cytoplasm clear. These alveoli resemble the proliferating bile-ducts in cirrhotic livers. The adenomatous alveoli are usually confined to the widened portal canals, while the hepatic lobules are pushed aside, but in some cases there is more active proliferation and the alveoli grow between or within the lobules. The lumina of the alveoli are usually empty or contain granular or hyaline detritus, but dilatation and lateral outgrowths may occur, polypoid projections may develop, and there are many transitions to cystadenoma and adenocarcinoma. Thus Watzold found 30 to 40 nodules under the capsule of the liver and showing many stages of cystic dilatation. Kika describes these cases as proliferating cystadenoma. They develop chiefly from the larger ducts.

Multiple Carcinoma of the Intrahepatic Bile-ducts.-Incidence.-Malignant tumors of this origin occur under much the same conditions as multiple liver-cell carcinoma, but less frequently associated with cirrhosis. Regarding their frequency opinions are widely at variance. Basing the diagnosis on a structure presenting alveoli lined by cylindrical cells, Eggel found that 32 per cent. of primary carcinomas of the liver were of this origin. Pepere, on much the same data, reduced the proportion to 14 per cent. B. Fischer, on the other hand, encountered 2 typical cases and hastily undertook to eliminate the entire group of liver-cell tumors, assuming that practically all primary carcinomas of the liver arise from the bile-ducts. In the literature definite reports of bile-duct carcinoma are much less numerous than cases of livercell carcinoma. Of the 53 cases collected by Herxheimer some are of uncertain origin.

Bile-duct carcinoma appears chiefly in adult or later life, in subjects of cirrhosis. A history of severe disturbance in the evacuation of bile, as in Fischer's case, is relatively common.

Gross Anatomy.-The liver is usually enlarged sometimes to very notable dimensions, intensely jaundiced, and often the seat of advanced cirrhosis, chiefly of the biliary type. The dilated larger bile-ducts may be visible in the gross. The tumor process affects most or all of the organ, and produces very numerous, usually small, firm nodules which may become confluent. 
Fischer found the most extensive tumor growth in the region of the hilus where it probably arose from the larger bile-ducts. In most cases it is impossible to locate any single primary focus and the origin is multicentrie. Massive forms of adenocarcinoma of bile-ducts with transitions to carcinoma in multiple secondary nodules are described by Greenfield and Thorel.

The extensive hemorrhages, necrosis, the bulky soft tumor masses, and prominent invasion of large veins which characterize hepatoma are missing. A tendency toward cicatricial fibrosis of the tumors has often been noted in the gross.

The structural features usually form a sharp contrast with those of livercell carcinoma. The prevailing structure is that of adenocarcinoma or alveolar carcinoma. The cells are cylindrical, high or low, or cubical, resembling those of bile-ducts, and the cytoplasm is clear, lacking the granular acidophile character of liver-cells. The nuclei are small and vesicular and mitoses may be very numerous. Giant-cells are rare. Ciliated epithelium is described by Sokalow and Cagnetto in otherwise typical cases. In more atypical malignant tumors the resemblance to duct-cells may be lost (Waldeyer). Many observers describe the transformation of bile-duct-cells into liver-cells in tumors originating from the former (Fischer, Herxheimer). Others reverse the order and trace bile-ducts forming from liver-cells (Rindfleisch, Muir, Rolleston). In many of these cases it is quite possible that the tumor has a dual origin, as held by Wiegert, Witwicky, Beneke, Marckwald, and others, or that the tumor arises from cells which had previously assumed the characters of pseudobile-ducts.

The stroma is abundant and reveals the desmoplastic property of true carcinoma, while in hepatoma the stroma is composed only of capillaries. Elastic fibers derived from the thickened portal canals and formed anew are demonstrable, and there is a strong tendency toward cicatricial contraction of the tumor nodules. Most of the nodules are inclosed in capsules of connective tissue and the gradation into hepatic parenchyma seen in hepatoma is usually missing. Yet malignant tumors may widely infiltrate the parenchyma. With overgrowth of fibrous tissue a scirrhous structure may develop which is not observed with hepatoma.

The outlying portal canals commonly exhibit marked proliferation of bile-ducts as in biliary cirrhosis, and these ducts may be dilated, lined by one or more layers of proliferating cells or filled with papillary projections of epithelium. From such precancerous overgrowth every stage up to true adenocarcinoma has been traced (Kika).

The arrangement of the cells in small alveoli is characteristic and at once distinguishes most of the tumors from the coarse trabecular tumors of livercells. In the slower growths the alveoli are regular and adenomatous, in more malignant cases they become smaller, more numerous and irregular, and in very active processes they break up into compact small groups of cells which grow diffusely, as in mammary carcinoma. The lumina are empty or filled with hyaline material or inspissated bile. When bile is present in these tumors it must be referred to retained secretion derived from neighboring parenchyma (Lohlein).

When small cubical or indifferent cells form compact anastomosing groups, Goldzieher and Bokay liken the structure to basal-cell carcinoma. They recall the basal-cell carcinoma of the ductus choledochus described by Burkhardt and suggest that these hepatic tumors may arise from the larger bile-ducts. The liver tissue undergoes passive atrophy or degeneration, but in rare cases hypertrophy of cords and cells is observed.

Although the small veins may often be invaded by carcinoma of the bile- 
ducts, metastases are not commonly observed except with the very active tumors. The portal and mediastinal lymph-nodes may be involved. In Dreschfeld's and Lohlein's cases, probably derived from the bile-ducts, the metastases were general. The structure of the secondary tumors may reproduce the original or appear much more atypical. Bile formation is absent. Bonnet describes the production of mucoid material in secondary tumors, and Baschko saw a cystic growth with watery secretion reproduced in metastatic tumors of lymph-nodes and rib.

\section{SARCOMA OF LIVER}

The possible.sources of true sarcoma of the liver and the probability of their occurrence are suggested from the embryology of the organ. The hepatic parenchyma, liver cords and bile-ducts, and gall-bladder develop from a single endodermal bud, from which an early subdivision goes to form the gall-bladder: The remainder branches out into a mesodermal matrix which supplies blood-vessels to the growing parenchyma and connective tissue in the portal canals. The primitive liver is a hemopoietic organ, with marked activity of the endothelial cells, but later this function is replaced by metabolic and secretory activities.

The presence of superfluous mesodermal material in the liver is indicated in the cases of hepatoma in which mucous tissue and cartilage of metaplastic origin have been found. Great proliferative capacity of hepatic endothelium is exhibited in hepatoma, in which the growth of capillaries keeps pace with that of the livel-cells, or in some cases of perivascular type seems almost to outstrip the epithelial growth. Dominici has described a true sarcomatous proliferation of the endothelial cells in a case of multiple hepatoma, and one nodule seemed to be composed exclusively of endothelial overgrowth.

There is, therefore, somewhat scanty but direct evidence that both the stroma-cells and the endothelium of the capillaries are susceptible of tumor growth. In the early embryonal liver there are collections of blood-forming cells which might be drawn into possible sources of round-cell sarcoma, but in the normal liver at birth there is no structure which might reasonably be expected to give rise to round-cell or lymphosarcoma. Arnold's lymphnodules are islands of normoblasts.

The supposed origin of sarcomas suggested by the observers who have reported cases of this disease is extremely varied. Arnold thought of the connective tissue about blood-vessels or acini. Delepine argued in favor of the cellular elements of the vessels. Byrom suggested the perilymphatic connective tissue. Demel derived the tumors from the normal connective tissue. De Vecchi and Guerrini concluded that the new connective tissue in cirrhosis was the source of the polymorphous and angiosarcomas. Marx derived his angiosarcoma from a cavernoma. Rolleston and Trevor called their case malignant hemangio-endothelioma. It is obvious that the source of these tumors has never been actually traced.

Considering the structures accepted as indicating a mesoblastic origin there is again direct conflict of opinion. Arnold included in his table of 26 cases of sarcoma several reported as carcinoma. De Vecchi and Guerrini discard 20 and accept $2 \mathrm{I}$ reported cases. Marx questions the majority of cases on various grounds, as incomplete autopsies and the presence of other and possibly primary tumors. Several reported cases were probably leukemic. Rolleston warns against mistaking syphilitic lesions for sarcoma in infants.

The clinical history of sarcoma fails to show any distinguishing features 
from carcinoma (Rolleston). The disease occurs as a congenital or very early tumor in infants, in children, and also in old age. Its course is usually very rapid and the symptoms are those of malignant carcinoma. The gross appearance of the organ recalls various forms, chiefly of hepatoma, as massive, multiple, hemorrhagic, and necrotic tumors, usually in much enlarged organs. Rolleston and Trevor collected 7 cases in cirrhotic livers.

According to histological structures the sarcomas may be grouped as angiosarcoma, alveolar sarcoma, spindle- and round-cell sarcoma.

Angiosarcoma is much the most frequent variety (Arnold, v. Kahlden, De Vecchi, Marx, Nazari). Yet in the descriptions of the cases one finds the prominence of blood-vessels, the polymorphous granular cells, the giantcells, as well as the necrosis and hemorrhage so prominent in atypical carcinoma. The structure in Marx' case is exactly duplicated by areas of L'Esperance's hepatoma. I do not believe, therefore, that the existence of a true angiosarcoma arising from blood-vessels of the liver has been satisfactorily demonstrated. The sole direct observation pointing to such an origin is that of Dominici in a mixed spindle-cell sarcoma and embryonal hepatoma.

The alveolar sarcomas also fail to commend themselves as true mesoblastic tumors. Typical cases are those of Theodorow, Holm, and de Haan. Rolleston and Trevor mention an alveolar arrangement of polymorphic, round, polygonal and giant-cells, with perithelial structures resembling the angioepithelioma of Renon.

Some of the spindle-cell sarcomas seem more probably of mesoblastic origin, but a pure spindle-cell tumor with uniform structure has yet to be reported. Ford's tumor in a cirrhotic liver was largely necrotic, but a metastatic tumor was composed exclusively of spindle-cells. Bramwell and Leith mention areas of spindle-cells with long oval nuclei, gelatinous foci, and multinuclear giant-cells in a large cystic hemorrhagic tumor replacing the right lobe. Steinhaus describes eight large hemorrhagic and necrotic tumors in a much enlarged liver, the edges of which contained large spindle- and giant-cells. The condition recalls atypical hepatoma. Some of the polypoid and spongy spindle-cell tumors of Pepere's collection were probably mesoblastic. Myxosarcomas in infants are reported by Meissenbach and Bernhinz.

Primary melanoma of the liver is a very difficult diagnosis to establish. Rolleston in referring to 9 reported cases points out that no case can be accepted unless the uveal tract has been examined after death. This precaution has usually been omitted. In 2 cases very small uveal melanomas were discovered only at autopsy. Marx refers to other reports and accepts the occurrence of primary melanoma on somewhat uncertain grounds.

\section{CARCINOMA OF GALL-BLADDER}

On account of its clinical interest carcinoma of the gall-bladder has been very fully studied: by Frerichs (I86r); Musser, in roo cases, I889; Courvoissier, I03 cases, I890; and Futterer, 268 cases, I901. A very full analysis of the disease is given by Rolleston (Lit.).

It forms 5 to 6 per cent. of all carcinomas (Kaufmann), affects women in the ratio of 4 or 5 to one man, and occurs almost always after 40 years, the average in both sexes being 58 years. Maxon reported a villous carcinoma in a child of 4 years, while Thomas and Noica observed the disease at 90 years. A remarkable etiological relation with cholelithiasis is one of the most interesting features of this disease. Gall-stones were present in 69 per cent. of Musser's cases, 70 per cent. with Futterer, $8_{5}$ per cent. Zenker, 9I 
per cent. Courvoisier, 95 per cent. Siegert, and roo per cent. Janowski. In some cases gall-stones may have been passed earlier or they may have been small and overlooked, or the catarrhal inflammation which commonly leads to gall-stones excites a cellular overgrowth without the mechanical irritation of the formed stone. The proportion of cases of cholelithiasis which develop cancer is estimated from 4 to I8 per cent. (Rolleston, Slade). Candler, finding only 2 cases of carcinoma in 315 cases of gall-stones among the insane, argues that hospital statistics show an unduly high proportion. Yet Slade found microscopical cancer in Io of I 7 (59 per cent.) gall-bladders with calculi. Since secondary cancers rarely produce gall-stones these calculi cannot be regarded as the result of the tumor (Siegert).

Mechanical irritation of calculi, the relation to a peculiar form of lipoid metabolism (cholesterin), and the irritative and digestive action of bile

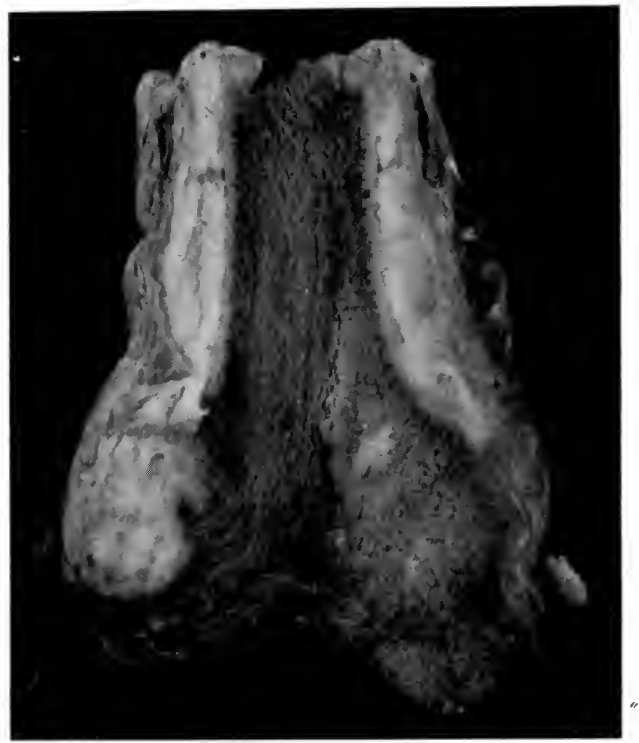

FIG. 326.-Adenocarcinoma with columnar cells diffused through the wall of the gallbladder.

seem to combine in producing the remarkable susceptibility of this mucous membrane to cancer.

Gross Anatomy.- The chronic inflammation associated with gall-stones produces either a papillomatous outgrowth or an original downward growth with metaplasia. These initial tendencies or precancerous conditions determine the later course of the tumor, yielding three main forms: (I) Villous, papillomatous or fungating, (2) gelatinous, and (3) diffuse, flat, infiltrating carcinoma.

The disease first appears as a papilloma or as a flat induration, or as an eroded ulcer. The location is usually at the fundus, or the neck, or at the cystic duct.

(I) The papillary form grows out into the bladder as a coarse villous or solid fungating mass which eventually distends and obliterates the bladder and forms a bulky, well-circumscribed tumor (Michaux). Early papillary 
tumors are rarely seen, but may appear as fragile, villous, or warty growths in a distended cavity. These may grow along the cystic duct into the bileducts or the common duct (Devic, Gallavardin).

Secondary incrustations with bile-salts and tissue detritus may form. Villous tumors gave rise to hemorrhage in 7 of Musser's cases. Most or all of the bulky tumors appear to originate as papillary adenocarcinomas which reach considerable size before infiltrating the liver. The thickened and distended wall of the bladder may become adherent to adjoining tissues. The gall-stones may be found embedded in the mass. In advanced stages the infiltration and adhesions render it difficult to determine the origin and early type of the tumor.

(2) The gelatinous carcinomas form bulky tumors which infiltrate the wall and fill the cavity of the bladder and early extend to liver, lymph-nodes, and peritoneum. After the stomach the gall-bladder is the most frequent source of gelatinous carcinoma of the peritoneum (Kaufmann). Yet reports of such cases distinctly traced to the gall-bladder are not numerous. Musser found 6 .

(3) The infiltrating tumors begin as a submucous growth or as a localized thickening or ulceration in a mucosa which has been the seat of chronic inflammation. It early infiltrates the wall of the bladder which becomes universally thickened and contracted. Extensions to the liver and adjoining lymph-nodes are soon formed and the disease may run its course chiefly as a secondary hepatic carcinoma. This event is particularly common when the tumor originates on the hepatic aspect of the bladder wall.

The majority of infiltrating tumors follow the scirrhous type, converting the bladder into a hard contracted mass without increase in bulk, or constricting it in hour-glass form (Rolleston), and fusing the organ to adjacent viscera.

Extensions of the tumor to neighboring tissues are observed in all but early cases, and metastases are frequent. The tumor is usually adherent to the liver, stomach, duodenum, or colon, and bound down by adhesions which inclose tumor-cells and along which further extensions are wont to travel (Courvoisier). Extension along the walls of the bile-ducts into the liver has several times been traced (Willigk). In my cases the sheaths of nerve-trunks have formed a prominent channel of dissemination. Peritoneal extensions may constrict the cystic or common ducts, the colon, duodenum, or pylorus. Metastases are usually located in liver, abdominal nodes, and peritoneum. Musser in Ioo cases found 55 with secondary tumors, besides cases with direct extensions to colon, duodenum, and stomach. The liver was involved in 54 , abdominal nodes in 16 , lungs or pleura 10 , and pancreas and adrenals occasionally. Mediastinal and supraclavicular nodes may be extensively enlarged (Beadles, West). Tumors have also been found in ribs, navel, rectum, uterus, ovary, and kidney. Adrenal metastases may give rise to cutaneous pigmentation suggesting Addison's disease (Warthin). Fistulous tracts form in much the same manner as with migrating gall-stones and are especially common when the carcinoma begins in the fundus. The fistulous openings lead chiefly to the colon, duodenum, and stomach, rarely into the peritoneum or externally (Riedel, Moutier).

Suppuration, lateral sacculation, thrombosis of the portal veins, angiocholitis, and peritonitis are frequent complications.

Structure.-Precancerous changes are often pronounced in cases of cholelithiasis. Janowski mentions the frequency of papillary outgrowths and disorder of glands, without attributing to them any special significance. Zenker describes elongation of glands, overgrowth of epithelium, fibrosis of submucosa, and round-cell infiltration as preliminaries of cancer. Slade 
found a high proportion of microscopical carcinomas in cases of cholelithiasis, but the details of these lesions are not given. McCarthy in a considerable series found only 3 cases of chronic catarrhal cystitis with carcinomatous changes.

It is probable that the markedly scirrhous character of many cases of early carcinoma of the gall-bladder results from the chronic productive inflammation preceding the new growth. In some cases there are no precancerous changes in the mucosa. In an early carcinoma at the neck I found a submucous nodule as large as a pea lying beneath practically unaltered mucosa and suggesting an origin from aberrant gland tissue. Other probable modes of origin are suggested by the rare cases of cholesterin cysts of the mucosa and of cystic adenomas which may project internally or externally and may contain cholesterin concretions (Rolleston, Kaufmann).

In a case of cholelithiasis of long standing the enlarged and thickened gall-bladder I found to present over its eroded mucosa very numerous flat or nodular opaque plaques, 2 to $5 \mathrm{~mm}$. in diameter. On section these proved to be foci of cellular connective tissue surrounding groups of atypical glandular epithelium showing numerous mitoses. Here was evidently the beginning of a carcinoma arising from multiple foci and destined to produce a diffuse carcinoma of the organ.

The natural course of papilloma appears to be that of the benign growth or of the rare villous carcinoma. Sand and Mayer and Chappet describe advanced cases without loss of villous type. M. Dominici describes diffuse papilloma of the entire mucosa without a trace of atypical growth. PelsLeusden, however, finds atypical downward as well as outward growth of epithelium in early papillomas.

Luschka's glands consist of epithelial invaginations of the mucosa which extend into the muscular layer and often to the serous surface of the gallbladder. Aschoff and others find very marked proliferation of these canals in cholelithiasis, and Pels-Leusden believes that the irritation of cholesterin granules and bacteria in these crypts is an important source of carcinoma following gall-stones.

Aside from minor variations the established disease presents two main structural types: (I) Adenocarcinoma and (2) alveolar carcinoma.

Adenocarcinoma is the most frequent form, and it produces papillary, gelatinous, and scirrhous tumors. The growths present elongated vascular papillæ of connective tissue covered by cylindrical cells and infiltrated with secondary alveoli lined by cuboidal cells. Mucous oversecretion and degeneration is a very common feature of all the adenocarcinomas. It may appear in isolated foci in the papillary and scirrhous growths and become universal in certain cases which run the course of bulky and wide-spread gelatinous carcinoma. They early tend to perforate the wall and extend to the peritoneum.

In the scirrhous type extensive new growth of fibrous tissue surrounds the isolated adenomatous alveoli in the bladder, but in the lymph-nodes and liver the cells grow more rapidly and fibrosis is wanting. The extent of the fibrosis is often remarkable, and only pyloric cancers show such desmoplastic quality in ordinary adenomatous alveoli.

Alveolar carcinoma presents in the gall-bladder the usual features of this neoplastic process. The most malignant growths are composed largely of pseudo-alveoli of small cuboidal or rounded cells. Others show many traces of adenocarcinomatous structure, and the frequent transition of one type into the other indicates that both arise from the same glandular structures in the mucosa.

Squamous-cell carcinomas, pure or associated with cylindrical cell carci- 
noma, are not infrequently observed (Rolleston, Lit.). Deetz reports several cases in some of which the squamous characters with pearls and spine-cells were highly developed. In Monckberg's case the two-cell types were intimately mingled in the original tumor, but grew in greater isolation in the lymph-nodes. These tumors probably arise in lesions which have previously developed a squamous metaplasia. Thus Lubarsch found a resical papilloma the size of a bean resembling an acuminate condyloma. Or they signify a progressive tendency, illustrated in Monckberg's case, toward squamous metaplasia of the cylindrical cells of an adenocarcinoma.

Hamdi. referred to (I) the preëxisting cholelithiasis, (2) the local effects of the tumor, and to (3) extensions and metastases (Rolleston).

Symptoms of gall-stones are absent in the majority of cases (Kehr), but have preceded the tumor for 25 years (Jourdan). Initial symptoms may be local pain, dyspepsia, or (50 per cent.) a local tumor. Jaundice suddenly established and persistent occurs in most cases. Many cases simulate hepatic carcinoma. Ascites is usually added from thrombosis of large veins or peritoneal extensions, and the effusion may be chylous. The cachetic stages are marked by fever from infections, cholemia, hemorrhages, and emaciation. The total duration is extremely variable, but after the appearance of jaundice few cases last more than 6 months.

\section{SARCOMA OF GALL-BLADDER}

Considerable uncertainty as well as theoretical interest surround the interpretation of certain tumors recorded as primary sarcoma of the gallbladder (Bayer, Rolleston, Lit.). All of these cases appear to have occurred in adults who long suffered from gall-stones and usually from suppurative cholecystitis. The organ was much enlarged, once to the size of a man's head (Bayer), and the cystic central cavity contained fluid. One or many gallstones appeared in the cavity or wall. The course of the disease was prolonged. It is evident that a long period of chronic inflammation had preceded any neoplastic process which may have developed by the time the tumor came under observation. Therefore in the interpretation of these cases a wide range of productive inflammatory changes must be taken into account. Iwasaki's very large tumor appears to have been composed largely of inflammatory tissue, and in the case of Carson and Smith, a localized large round-cell growth $8 \mathrm{~cm}$. in diameter, the bladder wall was the seat of a pronounced productive and exudative inflammation.

It appears, however, that two forms of sarcoma arise on such an inflammatory basis, one derived from an overgrowth of smooth muscle-cells, the other from the inflammatory new growth of connective tissue and blood-vessels.

Myosarcoma with the above general characters was described by Landsteiner. The wall of the much enlarged bladder was $3 \mathrm{~cm}$. in thickness. There was beginning infiltration of the liver. The tissue was composed of areas of spindle-cells arranged in parallel bands, and presenting the characters of smooth muscle. Other areas presented larger rounded atypical cells apparently derived from the spindle-cells. The liver nodules contained spindle-cells. Other spindle-cell sarcomas are recorded by Griffon and Segall and Rolleston, and in the latter's case there were metastases in aortic and inguinal glands. In connection with these cases Sutherland's adenomyoma is of interest. 
Sarcomas of fibroblastic type are of somewhat less definite nature. Parlavecchio evacuated 2 liters of purulent fluid from a thickened gall-bladder the wall of which contained sarcomatous tissue with polymorphous cells.

Bayer describes two very large gall-bladders containing many stones, one $8 \mathrm{~cm}$. in diameter, with fluid detritus. The wall was composed of sar-

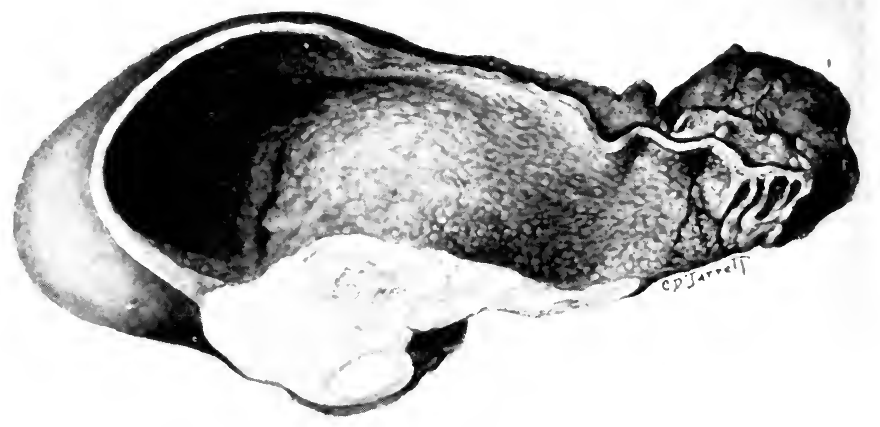

FIG. 327.-Sarcoma of gall-bladder. (After Carson and Smith, A. S., 62.)

comatous tissue containing vascular connective tissue and areas of spindle-, rounded and giant-cells, and epithelial remnants. One of the tumors had invaded the liver and gave peritoneal nodules. In the other, areas of cartilage and bone were found. An angiosarcomatous structure was described by Klingel, the neoplastic nature of which is doubted by Landsteiner. Seibert

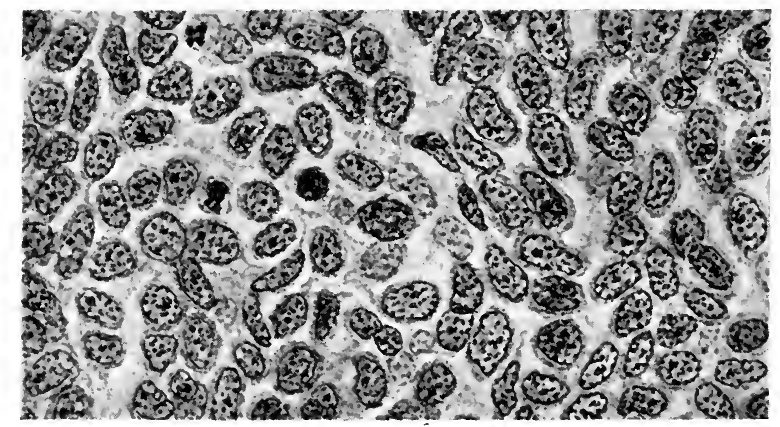

FIG. 328.-Structure of tumor in Fig. 327. Sarcoma of gall-bladder.

described a very large cystic and necrotic tumor as a degenerating lymphosarcoma. Becker's endothelioma seems of uncertain nature.

\section{CARCINOMA OF THE LARGER BILE-DUCTS}

Epithelial tumors of the large bile-ducts present much the same etiological, gross anatomical, and microscopical features as carcinoma of the gallbladder, but their location favors early mechanical obstruction of important channels, which promptly declares itself in the form of severe jaundice and a rapidly fatal course of the disease. The fatal effects of such comparatively 
miniature and relatively benign tumors render these growths of much surgical interest.

The chief locations of these tumors are: (I) Ductus choledochus, (2) ductus cysticus, (3) ductus hepaticus. A common seat of carcinoma is the point of junction of the three ducts, of which Donati collected 33 cases. Rolleston finds the following distribution in 90 cases: Common bile-duct, 44; junction of three ducts, 27; hepatic duct, 22; cystic duct, 7. Lapointe and Raymond collected 32 cases in the hepatic duct and 37 at the junction. Miodowski analyzed $4 \mathrm{I}$ cases.

Etiology.-The relative infrequency of gall-stones (22 per cent. in 264 cases) and the predominance of the disease in males (5o to 35 ) contrast carcinoma of the larger bile passages with that of the gall-bladder. Possibly the trauma of migrating calculi may be a factor.

Gross Anatomy.-Chiefly because of the early appearance of mechanical obstruction carcinoma of the bile-ducts is encountered when the tumor is small. These early tumors are villous, nodular, or diffuse:

(I) Villous growths may be single or multiple, and may fill and distend the duct. Extensions may form while the original tumor remains small (Lamble, Schmidt).

(2) Nodular masses appear in the submucosa and muscular wall and the very small size of some of these tumors is notable. They tend early to encircle and constrict the passage, and may appear merely as a cicatricial stenosis, the cancerous nature of which can be determined only by the microscope (Devic, Gallavardin, Lit.).

(3) A more diffuse growth may extend along the duct converting it into a smooth rigid tube from the inner surface of which project minute carcinomatous vegetations (Jenner, Chapper). Bulky tumors rarely develop from the bile-ducts.

The extensions are usually limited to the walls of the ducts, but in later stages adjoining viscera are involved by various channels. In I 2 cases of carcinoma of the hepatic ducts Lecene and Pagniez found the tumor limited to the ducts. Beginning at one point the growth infiltrates the wall until it involves a large portion of several ducts or the gall-bladder. Extensions of papillary tumors through the hepatic ducts may invade the liver. More frequently the liver is invaded through the lymphatics of the duct. In either case the condition may simulate hepatic carcinoma. Extending to the pancreas the head of this organ may be infiltrated, simulating pancreatic carcinoma. Carcinoma of the cystic duct is rarely demonstrated, but in not a few cases the tumor involves both cystic duct and bladder so that its original source cannot be determined. The gastrohepatic and other nodes are frequently involved.

The condition of the gall-bladder varies with the location of the tumor. Carcinoma of the hepatic ducts leads to contraction of the bladder or permits its moderate distention by mucus or calculi. The intrahepatic ducts may then become the seat of catarrhal, suppurative, or gangrenous cholangitis, and the liver parenchyma may undergo focal icteric necrosis (Futterer). Similar results follow when the tumor is lower down in the common duct. Tumors involving the junction usually lead to distention of the bladder, but when the cystic duct is occluded the bladder remains small (Lapointe, Raymond). Tumors of the common duct regularly permit filling and distention of the gall-bladder.

Courvoisier stated the principle that the gall-bladder is usually distended with cancer and contracted with calculi of the ductus choledochus. Very moderate compression is capable of bringing about bile-stasis, so that the 
stenosed duct may readily admit a probe or become pervious on manual compression of the bladder.

The structure of carcinoma of the bile-ducts presents the same types as in the gall-bladder, differing chiefly in the greater tendency to fibrosis. In some cases it is remarkable to find such extensive areas of fibrous tissue about the hilus of the liver or head of the pancreas with so little epithelial tissue, so that small carcinomas in these regions frequently require microscopical detection.

The usual structure is that of adenocarcinoma with cylindrical cells and mucus production. Occasionally the alveoli are small, the lining cells cuboidal or spherical. Leith describes a colloid carcinoma. Squamous metaplasia is occasionally observed.

According to their chief clinical features Devic and Gallavardin divide the cases as follows: (I) Enlargement of the liver with distended gallbladder. This form is regularly associated with tumors of the lower segments (supraduodenal), rarely with tumors of the upper subhepatic segments of the ducts. (2) The liver varies in size and the gall-bladder is not enlarged, with most tumors limited to the hepatic ducts. (3) Hypertrophic biliary cirrhosis may be simulated by tumors in various locations. Persistent jaundice and local pain are nearly constant. The duration has varied from 3 to I9 months.

Carcinoma of the Ampulla of Vater.-The duct formed by the junction of the common bile-duct and the pancreatic duct (Wirsung) and opening into the duodenum at the papilla is called the ampulla of Vater. Carcinoma arises in the corrugated mucosa of this structure and at the duodenal papilla. Cases have been collected by Busson (I) Georges (20), and Rolleston (I9) (Lit.). Except in very early cases it is difficult to determine the exact origin of these tumors. Some arise at the termination of the common bile-duct, others in the mucosa of the pancreatic duct, still others in the ampulla proper or at the papilla. The etiological factors are similar to those of carcinoma of the bile-ducts.

Gross Anatomy.--The tumors are small villous or papillary growths or diffuse infiltrations of the wall. McCarthy found the papilla excavated by a cancerous ulcer. They early cause stenosis of the duct with cholemia. Aynoud found that 20 per cent. of the cases progress far enough to produce secondary extensions and metastases, chiefly in the adjacent lymph-nodes. The structurally benign growths are almost equally effective with the malignant in producing obstruction, and the anatomical relations are so intimate that the mechanical effects are very similar in all the various locations. The gall-bladder and the bile-ducts from the point of the tumor to the intrahepatic radicles are usually dilated. Jaundice, at first intermittent, later persistent, is a nearly constant symptom, but is often absent with tumors of the papilla. Suppurative cholangitis with fever may result especially with tumors of the papilla. The pancreatic duct becomes widely dilated, usually without secondary changes in the pancreas (Dieulafoy).

Structure.-Columnar-cell adenocarcinoma is the type almost always observed. The architecture is villous, fungoid, or infiltrating, without much variation in structure. Alveoli lined by smaller cubical or rounded cells appear in tumors of Wirsung's duct (Letulle), and have been described in a growth traced to the duodenal mucosa or Brunner's glands by Klotz. In a small villous growth at the termination of Wirsung's duct Carnot and Harvier found all transitions from finely villous papilloma with cylindrical cells to infiltrating alveolar carcinoma. Tumors of the duodenal papilla arise chiefly from the duct mucosa and contain columnar cells (Schuller). A highly pig- 
mented but otherwise typical villous adenocarcinoma of the ampulla was described by Duval, an observation which speaks strongly in favor of the local origin of certain melanotic tumors.

The results of surgical treatment of carcinoma of the gall-bladder and ducts is highly unsatisfactory. In 93 cholecystectomies collected by Quénu the operative mortality, chiefly from hemorrhage, was i 8 per cent., and only I4 of $5^{2}$ cases were known to be living after one year. Quénu mentions 9 excisions of tumors of the bile-ducts, and Mayo 2. The final results were unfavorable. For carcinoma of the ampulla Quénu in 9 cases found 6 deaths, and Oehler the same proportion. Borelius reports several fatal operations from Sweden. The early stage of growth at which these tumors declare themselves encourages surgical interference. L'Esperance, in this laboratory, has observed just within the papilla a papillary carcinoma $5 \mathrm{~mm}$. in diameter which had caused a single fatal hemorrhage. 


\section{CHAPTER XXXIV}

\section{TUMORS OF PANCREAS}

Adenoma of Pancreas.-Adenomas are very rarely observed in the pancreas. Borst states that he has seen nodular hyperplasia of the pancreas associated with a similar condition in the liver.

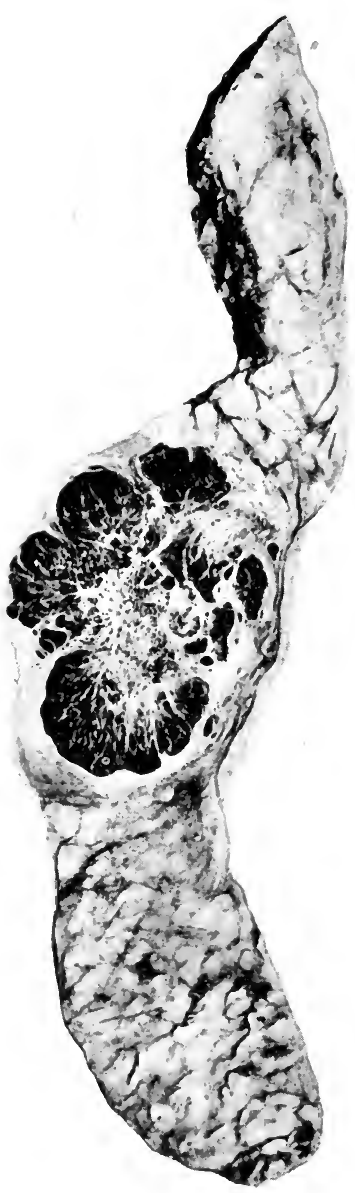

FIG. 329.-Cystadenoma of pancreas. (After Roman.)

Cystadenomas may be separated from retention cysts by the abundant growth of epithelial papillæ. Lazarus describes many stages in the development of cystadenomas from dilated ducts. Edling collected I 3 cases from the literature, which illustrate single and multiple cystadenomas, lined by single layers of cubical or cylindrical cells, or partly or completely filled by epithelial papillæ. Atypical overgrowth and signs of malignancy appear in some cases. Kaufmann saw malignant changes in a portion of a large cystadenoma of the cauda, and Sotti's tumor gave papillary metastases.

Solitary adenomas appear as small solid tumors well circumscribed from the parenchyma. Neve's scirrhous adenoma measured $2 \frac{1}{2} \times 2$ inches and constricted the duodenum. Thierfelder shelled out of the pancreas a small tumor composed of alveoli of cylindrical cells. Other cases are reported by Biondi and Chauffard. All of them appeared to arise from the ducts. Cesaris-Demel describes an adenoma which suggested an origin from the glandular acini.

Adenoma of island tissue has been described by Nicholls, Helmholtz, and Cecil. They were single small yellowish nodules as large as a pea and presented a structure strongly resembling that of island tissue. Cecil, in describing such a structure, traces the transition from the hypertrophic islands of various conditions and concludes that the small tumors are more properly to be regarded as markedly hypertrophic islands rather than neoplasms.

Carcinoma of Pancreas. - Cancer of the pancreas has long been a subject of much clinical interest. It was extensively discussed by Classen in I842 
(older Lit.), by Ancelet, who analyzed 200 cases in 1860 , and by Miraillie in 1893 .

Bard and Pic (1888) emphasized certain features of the disease as constituting a specific syndrome, viz.: distention of the gall-bladder, absence of hepatic enlargement, jaundice, and rapid cachexia.

General Etiology.-Cancer of the pancreas formed 1.76 per cent. of Kaufmann's autopsies on malignant tumors. Of 2943 autopsies on cancer referred to by Korte there were 59 pancreatic cases, 2 per cent. Bashford collected 1000 cases of primary malignant tumors of the pancreas among 84,000 cancers. Owing to the difficulty of accurate diagnosis the value of miscellaneous statistics may be doubted. Ollivier has pointed out the necessity of very careful histological study in order to avoid confusion with cancer of the duodenum and bile-ducts. The disease occurs between 30 and 50 years, but often in younger subjects. A. Kuhn describes a cylindrical-cell carcinoma in a cirrhotic pancreas with pulmonary metastases in a child of 2 years and refers to other cases at an early age. I have observed one case associated with a large pancreatic cyst.

Many cases occur in cirrhotic organs and a parallel may here be drawn between the liver and the pancreas since it appears that carcinoma of the ducts follows periductal fibrosis, carcinoma of the parenchyma appearing after interstitial fibrosis (Hulst). Only rarely are there preliminary symptoms, as in Leriche's case of transient jaundice, pointing to a cholelithiasis or pancreatic calculus. Lazarus mentions 5 cases of adenocystoma with pancreatic calculi. The gall-bladder is usually free of calculi and contains thick bile and mucus. Reasoning from analogy with the liver, carcinoma of the pancreatic parenchyma may be regarded as beginning in a functional hyperplasia following cirrhosis, and carcinoma of the ducts from chronic irritation and stasis in these canals.

The occurrence of aberrant pancreatic tissue in the duodenal mucosa and about the head of the pancreas suggests that certain tumors, especially those involving both duodenal wall and pancreas, may arise from such aberrant tissue (Heinrich, Lit.). In some of these accessory glands there is extensive multiplication of ducts from which a cylindrical-cell tumor might arise.

Gross Anatomy.-The location of the tumor is usually in the head or extending diffusely over most of the organ. Tumors limited to the body are much less common and the tail is rarely affected. Of 386 cases collected from the literature, ${ }_{5} 8$ were diffuse, $I_{5} 6$ limited to the head, 28 in body, I 2 in tail. The organ is regularly enlarged, but in scirrhous tumors the gland may be small but very hard.

While small tumors may be limited to the pancreas ( 12 of $\mathrm{I} 27$ cases, Segrè), more advanced growths early infiltrate the surrounding tissues and cause compression. The common duct is occluded with bile-stasis, dilatation of the bladder, and varicosities of the bile-ducts up to and into the liver. The pancreatic duct may be distended and tortuous and filled with mucinous fluid. Lachmann reported rupture of the gall-bladder. Muller observed displacement of the stomach with hour-glass contraction. The duodenum was stenosed in 8 scirrhous cases collected by Hagenbach. Stenosis of the aorta is reported by Boldt. Portal thrombosis has been recorded in 5 cases (Korte). The soft tumors may necrose and perforate the stomach or the large veins with fatal hemorrhage.

Metastases are very commonly observed and appear first in adjacent lymph-nodes and liver. Bard and Pic emphasize the occurrence of very numerous minute nodules in the liver without enlargement of the organ, whereas secondary gastric carcinoma causes bulky growths in the liver. Very 
numerous nodules may appear over the peritoneum and in the peripancreatic fat tissue. Extensive invasion of the pancreas and adjoining tissues may be complicated by hemorrhages and fat necrosis. General metastases are not common, probably because the disease is rapidly fatal.

Structure.-Two main types of pancreatic carcinoma are observed: (I) Cylindrical-cell adenocarcinoma, arising from the ducts, and (2) carcinoma simplex arising from the parenchyma. The former may rarely show extensive gelatinous changes, and most cases of the latter are scirrhous (Kaufmann, Miraillie).

(I) Carcinoma of the ducts may be associated with proliferation of lining cells in the outlying regions, which Hulst observed in a case of periductal fibrosis and interpreted as precancerous. Ollivier also traced his case of cylindrical-cell tumor from such preliminary changes. Muckenbeck, how-

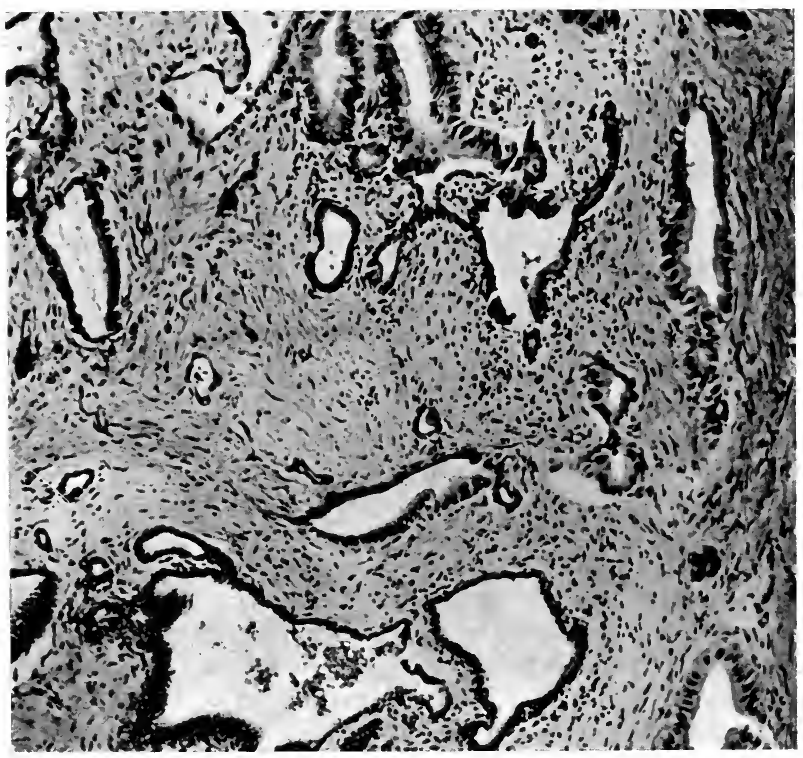

FIG. 330.- Structure of an infiltrating and fibrosing adenocarcinoma of the head of the pancreas.

ever, found reason to believe that cylindrical-cell tumors might develop from the parenchyma.

The tumors are regularly located in the head or corpus and form somewhat massive growths which are imperfectly separated from the parenchyma. They are firm from fibrosis or contain many small cysts, or the central mass is broken down, suggesting an origin from cystadenomas.

The structure is composed of papillary outgrowths and alveoli lined by cylindrical or cuboidal cells. In atypical areas the cells may lose their cylindrical form and grow more diffusely. Yet Kulst found the cylindrical type preserved in secondary tumors of lymph-nodes and liver, and Satti reports a cystadenoma papilliferum with typical metastases in lymph-nodes, lungs, and peritoneum.

The local extensions of the tumor have been traced by Ollivier through 
ducts, lymphatics, and nerve-trunks. Kaufmann found the large and small veins invaded, but without metastases.

(2) Carcinoma of the parenchyma produces a more diffuse, rapidly growing tumor which is firm or soft according to the proportion of fibrous tissue. The cells are small or large, granular, hydropic, or fatty, resembling those of the pancreatic alveoli. The cell borders are often indistinct, and the vesicular nuclei occupy much of the cell. Nucleoli are poorly developed. The relatively large size of the nuclei is often a notable feature. The cells are arranged in small or large alveoli separated by a fine stroma and in some cases the appearance suggests a simple cirrhosis in an otherwise unaltered pancreas (Bard, Pic). In the larger alveoli the outer cells may be cubical. In some cases there is a definite lumen in the cell groups, filled with coagulum. These spaces may then be lined by high cuboidal or low cylindrical cells. Large mononuclear giant-cells may appear in numbers and persist in metastases.

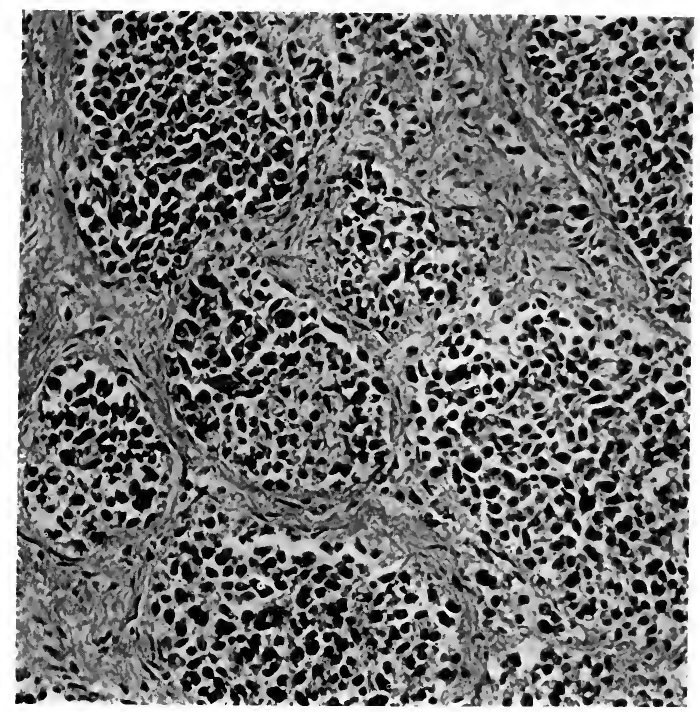

FIG. 331.-Structure of a carcinoma of pancreas.

In more atypical and malignant tumors the cells are smaller and embryonal in type and the arrangement may be irregular and diffuse. Such cases may resemble lymphosarcoma with a faint alveolar tendency. In one of my cases the original tumor recalied lymphosarcoma, while the pulmonary metastases contained small cuboidal or spindle-cells.

The origin of this tumor has been traced by Ollivier, in an early case, to the pancreatic alveoli and the transformation of gland-cells into tumor-cells he has sketched in detail. Usually all early stages of such origin are lost.

The desmoplastic properties of pancreatic carcinoma are somewhat unusual and probably account for the large proportion of scirrhous cases. A preëxisting cirrhosis is also a factor. Extensions throughout the pancreas occur early and travel by ducts, lymphatics, blood-vessels, nerve-trunks, and alveolar spaces. In one of my cases the organ was diffusely infiltrated and the seat of very numerous small cysts, dilated ducts containing minute calculi.

The parenchyma shows interesting changes. There may be a collateral 
or preëxisting hyperplasia of the gland tissue which is difficult to distinguish from invading carcinoma. The islands of Langerhans are usually hypertrophied and may be much increased in number, showing many transitions from secreting to island tissue. The islands may also exhibit a peculiar hypertrophy approaching the structure of the tumor. It was apparently this appearance which led Fabozzi to conclude that all parenchymatous cancers arise from the islands. This interpretation has been discussed by Reitmann and Helmholtz. Another feature which might suggest such an origin consists in focal adenocarcinomatous areas which somewhat resemble hypertrophic islands. In an invaded lymph-node I found such structures exclusively represented. These various peculiarities seem to justify the claim of Bard and Pic that the histology to pancreatic cancer is specific.

The symptoms frequently present the characteristic syndrome of Bard and Pic, but only when the tumor is located in the head. Progressive jaundice without remissions, great distention of the ball-bladder, absence of hepatic enlargement, subnormal temperature, and rapid emaciation and cachexia, form a rather specific group of clinical features observed in the majority of cases. A palpable tumor is often missing, but was detected in about one-fourth of Miraillie's II 3 cases. The liver may be considerably enlarged (Miraillie, Eloesser), and the metastases may be bulky (Oser). When the growth involves only body and cauda, the ampulla of Vater may remain pervious, the gall-bladder is not distended, and jaundice is absent. Such clinically atypical cases are described by Pic and Tolot. An unusual predisposition to hemorrhage is emphasized by Eloesser. Pain is very constant. The loss of pancreatic fluid and bile leads to acholic and fatty stools. This condition, however, is by no means constant, and occurred in only 20 cases collected by Miraillie, Oser, and Fitz. Imperfectly digested bulky stools are described by Oser. Glycosuria is rare. In 30 cases of carcinoma of pancreas ( 3 primary). Pearce found intermittent glycosuria in one, diabetes in another. Persistent glycosuria occurred in $2 \mathrm{I}$ cases collected by Miraillie and Oser.

Sarcoma of Pancreas.-Very few satisfactory reports of pancreatic sarcoma are available. Schilling collected 24 cases and Kakels 21, most of which are of uncertain nature, but from these data it appears probable that spindlecell sarcoma and lymphosarcoma arise in the pancreas.

K. Ehrlich described two large cystic tumors the walls of which were composed, one of spindle-cells, the other of alveoli of rounded, spindle-, and giantcells which he regarded as of endothelial origin. Each tumor gave local or visceral metastases. The author concluded that they arose in the walls of primary cysts. Weil also found spindle- and giant-ceils in the wall of a pancreatic cyst. Kakels found a large solid tumor of the tail, composed of spindle-, round, and giant-cells. Some very vascular tumors are reported as angiosarcomas (Kronlein). Michaelson's case of carcinosarcoma may signify a participation of the stroma of the gland or metaplastic changes in a carcinoma.

Of lymphosarcomas, L'Huillier found a small tumor in the head of the pancreas of a newborn infant. It was composed of lymphocytes and giantcells. Litten's case occurred in a child of 4 years and gave intestinal metastases. Schirokogoroff describes a diffuse tumor growth in the pancreas of a man of 54 years, with extensive visceral metastases. The cells were embryonal in type and of quite uncertain origin. Some of the difficulties in the diagnosis of pancreatic sarcoma are illustrated in Piccoli's cases, which differ from most of the reports in being so fully described that several critics have recognized them as carcinomas (Borrmann, v. Kahlden). 
CHAPTER XXXV

\section{MAXILLARY TUMORS OF DENTAL ORIGIN}

The dental origin of certain cystic and dentigerous tumors of the maxillæ has been recognized for at least a century, and the structural details and probable histogenesis were rather clearly conceived by several writers since about $185^{\circ}$ (Barnes, Guzack, Delpech, Dupuytren, Forget, Nélaton). At this time it was generally supposed that all the cystic tumors arôse from dilated dental follicles, and in 1872 Magitot classified the series upon this basis, excluding certain cysts which he thought to develop between the periosteum and the tooth. In several solid tumors also some authors detected epithelial structures which they interpreted as derivatives of the enamel organ (Robin, Wedl). The odontomas were systematically classified by Broca in I 868 . The entire subject was greatly simplified in 1885 by the classic study of Malassez, who referred most of the tumors to embryonal remnants of the enamel organ.

Débris epitheliaux paradentaires.--Under this term Malassex (I885) described numerous cell groups which he found in the fetus scattered along the borders of the teeth from apex to crown. They are divisible into three groups: (I) Superficial cells lying just beneath the gin-

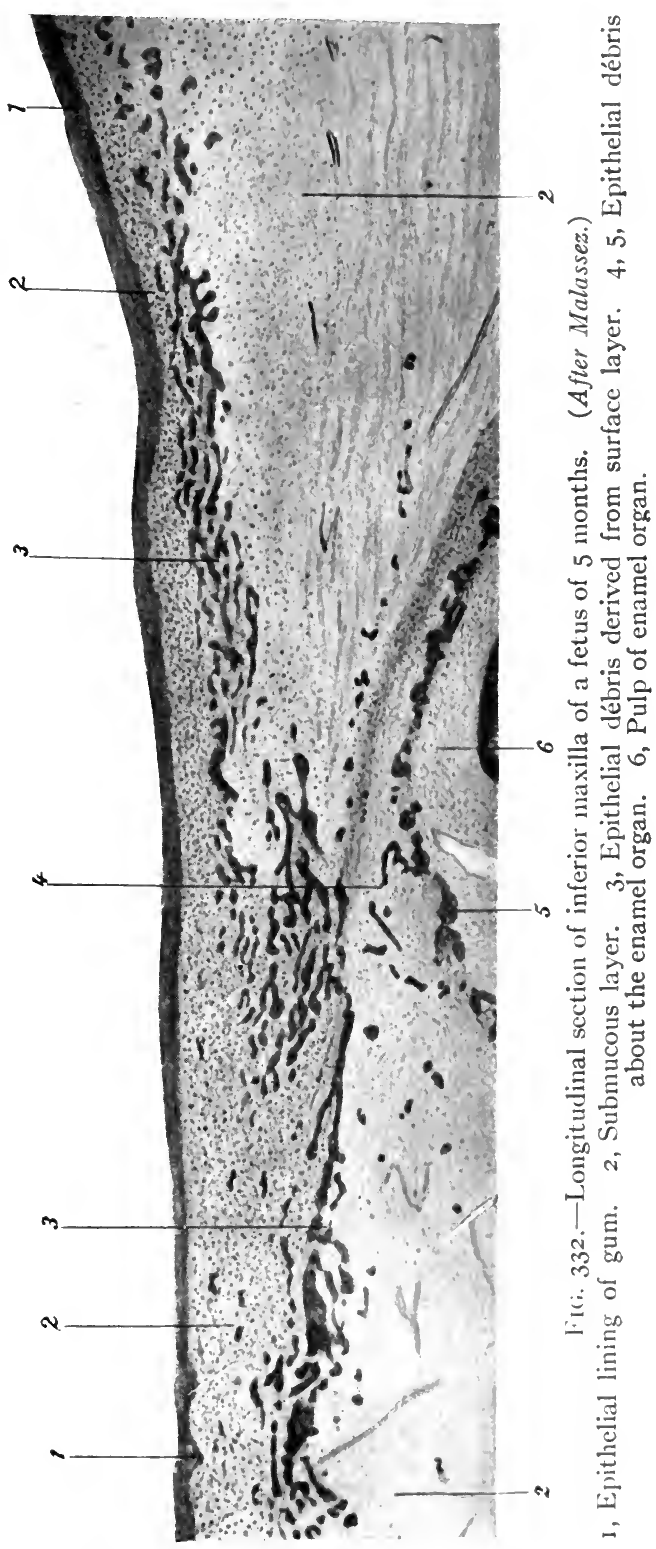


gival epithelium; (2) intermediate, lying along the sides of the tooth; and (3) deep, connected with the enamel organ. The structure of the cells varies considerably. Some are round and of indifferent character and are found in all three situations. The superficial groups also include round cells encircled by cylindrical, and canals lined by one layer of cylindrical cells. About the enamel organ lie groups of cells, which show transitions between cylindrical enameloblasts, round cells, and stellate cells. Many of these groups he was able to show persist in the adult. Malassez interpreted the cell groups, not as accidental offshoots or misplacements of the enamel organ, but as analogues of the very rich dental apparatus of some lower vertebrates and as giving rise to the supernumerary teeth of the so-called third dentitions. They are derived from the invaginations of gingival epithelium which go to form the enamel organs.

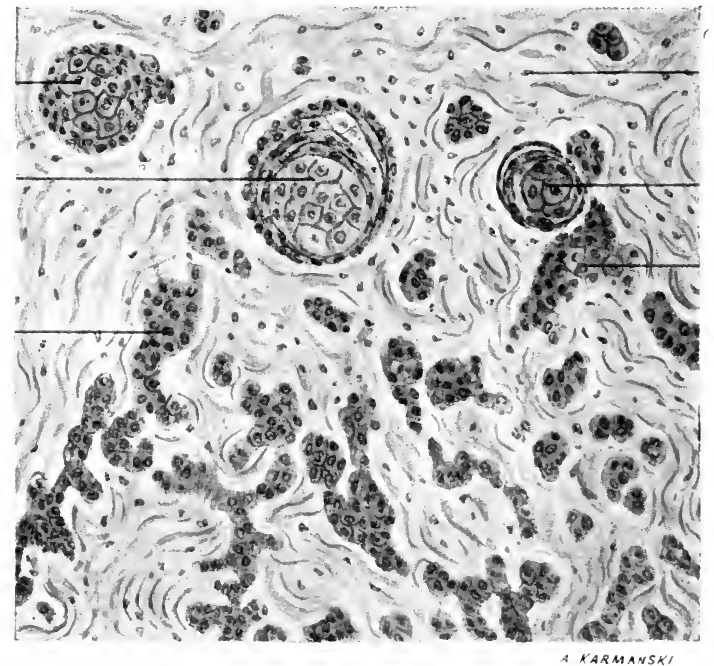

FIG. 333.- Section of inferior maxilla of fetus of 5 months, showing epithelial cell groups beneath gum.

(After Malassez.)

These observations of Malassez have formed the basis of our knowledge of many inflammatory and neoplastic processes connected with the teeth. About inflamed and carious teeth the cells multiply, hypertrophy, wander out into the tissues, become infiltrated with leukocytes, and participate in the expulsion of the teeth in pyorrhea alveolaris. They are concerned with various fibrous or myxomatoid outgrowths attached to the borders of the teeth (fungosites radiculo-dentaires). Dilatation of the lumina of these vestigial alveoli gives rise to various simple cysts found in contact with or in the neighborhood of the roots. In many epulides atrophic or proliferating groups of epithelial cells are found which are derived from the original paradental structures. The numerous cysts of the maxillæ with single or multiple chambers and containing walls or cavities and imperfect or well-formed teeth appear to be derived from these paradental structures and from the specialized mesodermal elements over which the enamel organ exerts a formative control. In the various forms of odontoma enameloblasts and enamel are usually more or less prominent, and these elements Malassez derives 
from the paradental structures. Finally, in the entire group of cystic and solid, malignant, epithelial growths arising in the maxillæ the sole source is to be found in the paradental epithelium.

The views of Malasse $z$ regarding the importance of the paradental structures have received general acceptance. Galippe has recently reviewed the entire subject, tracing in greater detail the natural history of the paradental structures and illustrating by selected cases their pathological relations.

It still remains uncertain to what extent the dental follicle itself is concerned in the formation of dentiferous and dentigerous cysts. Many believe that the complex odontomas and dentiferous cysts arise from original or supernumerary dental follicles and Malassez and Galippe admit such an origin for certain cases. It is reasonable to suppose that all transitions exist between simple groups of paradental epithelium and true supernumerary enamel organs and that the structure and contents of maxillary cysts will vary accordingly. In the very complex processes located in the maxillæ in connection with the formation of two sets of teeth abundant opportunity is presented for abnormal overgrowths of several structures in various stages of evolution. It is, therefore, unlikely that any single embryonal event such as gives rise to the ordinary paradental débris is responsible for all the very numerous clinical forms which these abnormalities present.

Adamantinoma.- Tumors of this group arise from the paradental epithelial débris. They are solid or cystic growths located within the alveolar borders, excavating a cavity within the maxilla, distending the tissues until they become surrounded by a thin parchment-like bony capsule which crepitates on pressure. Eventually the capsule may rupture and the disease extends further by continuity into antrum, orbit, or other tissues and cavities. The cystic tumors may reach a large size, Bryck's tumor weighing $\mathrm{I} .5 \mathrm{~kg}$. I found at autopsy a solid tumor of the upper jaw as large as a child's head, projecting externally into the nasopharynx, with dysphagia, and into the orbit with, extreme

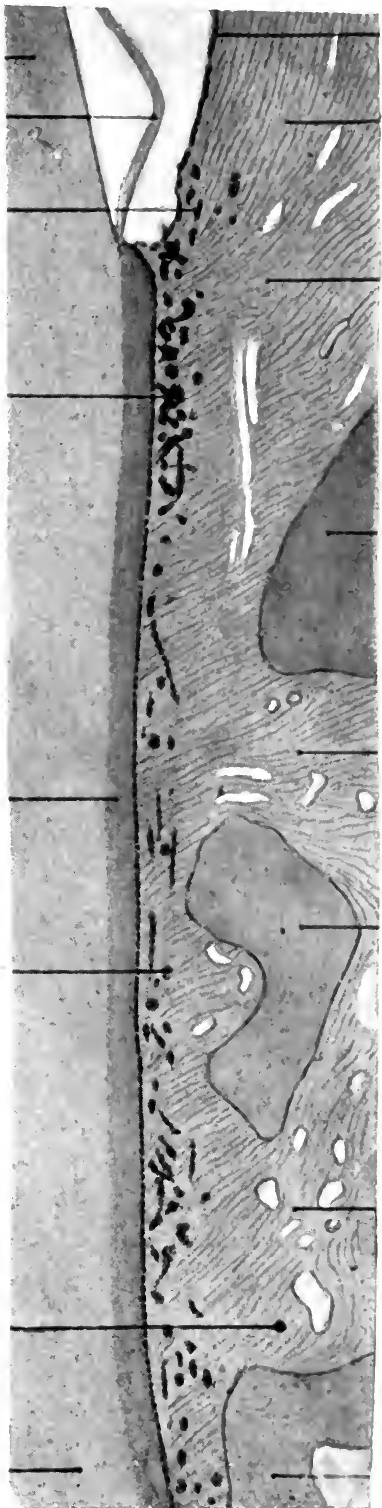

FIG. 334-Ciroups of superfluous epithelial cells found in five successive sections of the alveolar dental ligament of an adult man. (After Malassez.) exophthalmos. 


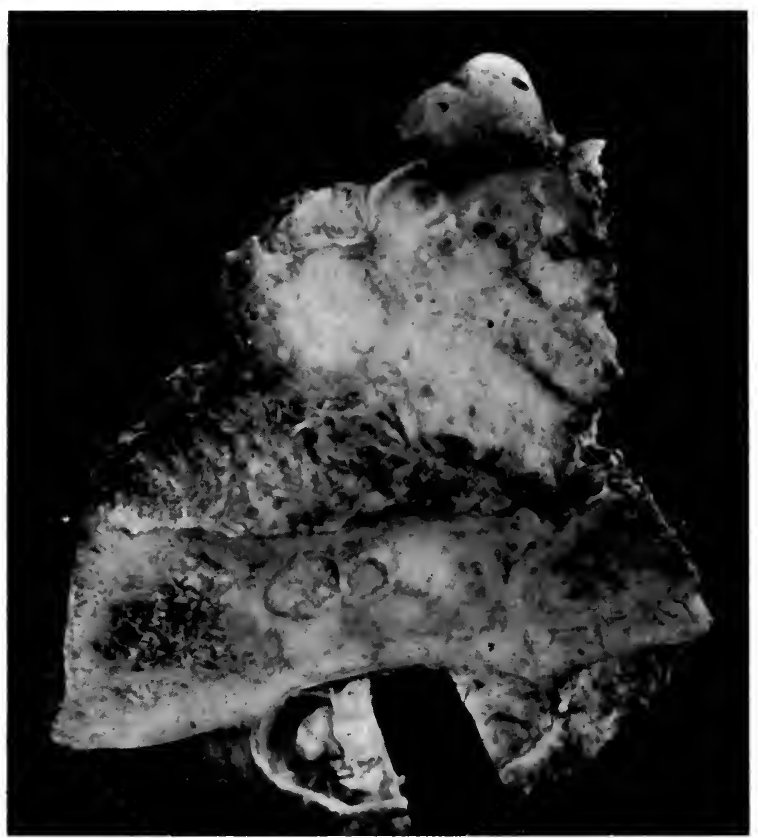

FIG. 335.-Adamantinoma of inferior maxilla.

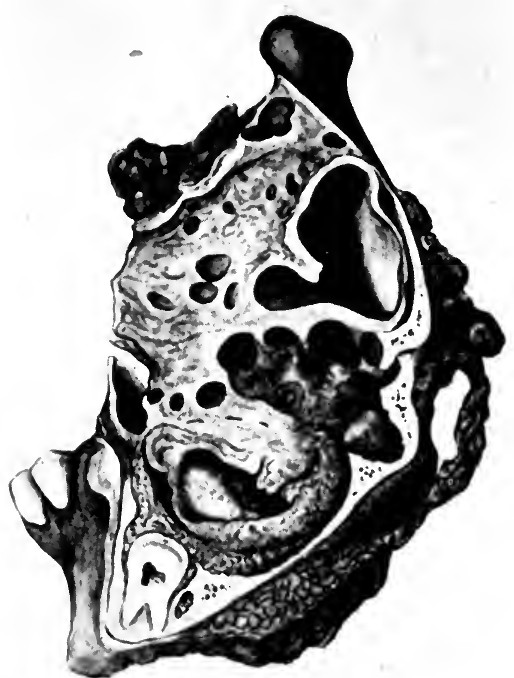

FIG. 336.-Relations of a cystic adamantinoma of inferior maxilla. (After F*itsch.) 
The cystic tumors are unilocular or multilocular and the small cystic ramifications may penetrate extensively into the cancellous tissue (Beneke). The cysts contain serous fluid or mucous or inspissated fatty and scaly material. Calcific particles, probably remnants of imperfect enamel, may be mingled with the fluid. The cavity may be smooth walled or lined by papillary projections of epithelium. In the cyst wall one finds cellular or fibrous connective tissue, calcified areas, or masses of bone or cementum. Cellular overgrowth may give the structure of spindle-ceil or myxosarcoma (Albarran), and it is probable that by exaggeration of this process apparently pure sarcomas may arise. Bilateral cystic tumors were observed by Flaubert.

The tumors composed of small cysts are often traversed by fibrous trabecula which constitute the bulk of the tumor. In such a case Chibret found

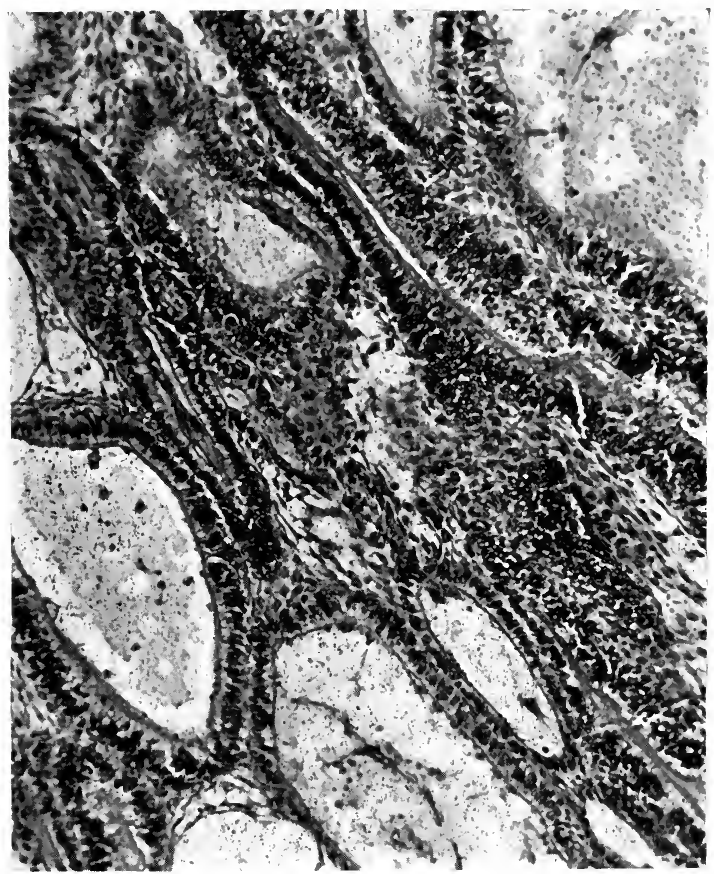

FIG. 337.-Glandular adamantinoma. Many small cysts lined by columnar enameloblasts.

areas of well-formed enamel lying on shallow beds of dentine. Smaller deposits of enamel were observed by Bernays and by others.

The solid tumors present every gradation from the small cystic variety. As a rule, they are more cellular and malignant. On section they usually present a papillary appearance and many fine cysts may be detected. Or by overgrowth of the stroma they may appear firm, solid, and sarcomatous. Some of the solid tumors from their early stages project from the alveolar border as a form of epulis, and when the vessels are overabundant their gross appearance may closely resemble that of a vascular epulis of sarcomatous type. The ordinary epulis, however, fails to distend the ramus of the jaw.

That all these tumors are essentially of one nature is indicated by the 
numerous intermediate forms described in the literature and by the occasional transformation of unilocular into multilocular, cystic, or solid tumors in recurring cases (Guibout, d'Amiens, Malassez).

Structure.-The epithelial cells of adamantinoma exhibit all variations in form between stratified squamous epithelium and specialized adult enameloblasts, thus recalling the changes traversed in the normal development of the enamel organ.

Superficial adamantionomas of the alveolar border present many of the characters of acanthoma, but the central cells in the epithelial columns usually exhibit the peculiar reticulated structure of the central portion of the enamel organ. In some cases there is a direct connection between the gingival epithelium and the deeper groups of characteristic reticulated enameloblasts, so that Buchteman and Eve assumed that such tumors arise from the gingival lining. Malassez is probably correct in referring these appearances to secondary union by upward growth of the tumor, or to supernumerary invag-

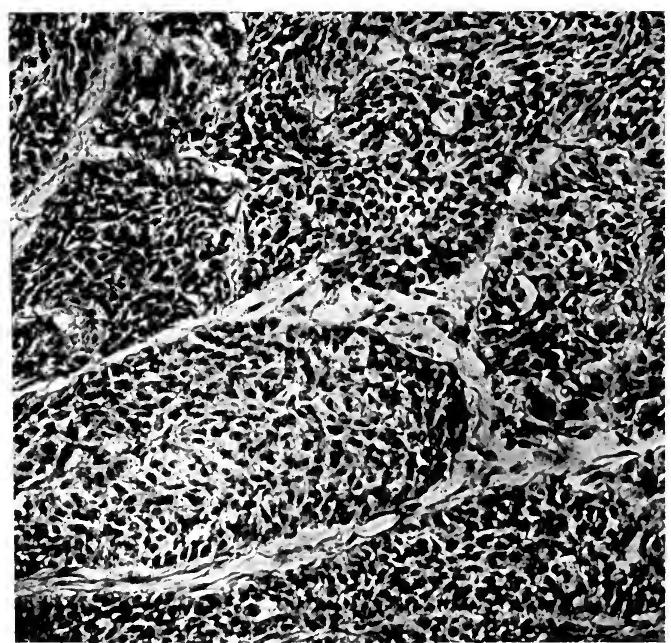

FIG. 338.- Structure of adamantinoma resembling epidermoid carcinoma.

inations of epithelium, but it is sometimes difficult to distinguish between primary acanthoma of the gum and squamous-cell adamantinoma. Galippe holds that the adult gingival epithelium has lost all capacity to differentiate toward enameloblasts and that the squamous cells of superficial tumors arise from the superficial débris paradentaire, which may be regarded as the atrophic duct of the enamel gland.

The epulis of adamantinoma presents a fibrous or mucoid or giant-cell, sarcomatous stroma, supporting cords and groups of epithelium of the enamel type. The cells vary as usual from squamous-cell to columnar and vesicular forms and the origin of the squamous cells presents the same problems as in the case of other superficial tumors of the gums. In various cases described by Malassez, Heath, and Eve, the features of adamantinoma were prominent. Haasler finds that the common epulis is usually connected with a granuloma of the dental root.

The solid intramaxillary or projecting tumors present a variable structure, the cells exhibiting many gradations from enameloblasts to squamous epithe- 
lium. Three main types of structure may be recognized: (I) Acanthoma; (2) plexiform epithelioma; (3) glandular adamantinoma (L'Esperance). Malassez's classification included squamous, cylindrical, and carcinomatous cell types. All the growths are locally aggressive and fall in the general class of carcinoma. The glandular tumors are adenocarcinomas. Some tumors present chiefly one structure, others show sharp and extreme variations in the cell type, and the arrangement of the cells is very diverse. Acanthoma presents anastomosing cords of squamous epithelium in which are spine cells and many pearls, but the characters of the enamel organ appear in isolated foci of large vesicular cells.

Plexiform epithelioma presents broad convoluted columns of epithelium without marked squamous character, lying in dense or cellular connective tissue. Many small cystic tumors present this structure.

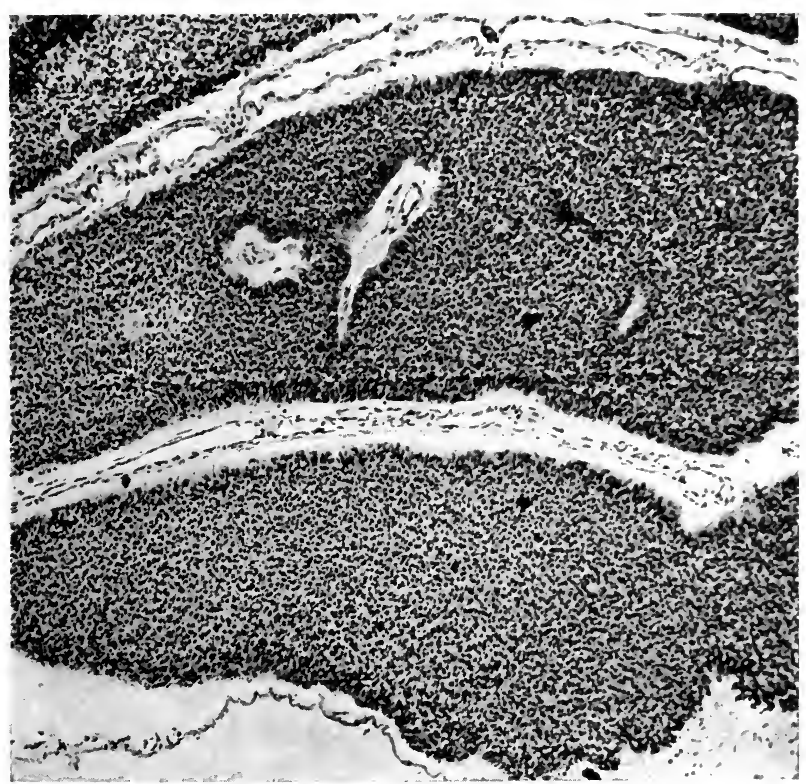

FIG. 339.-Adamantinoma. Large cell groups composed of very small epithelial cells surrounded by compact columnar enameloblasts.

Glandular adamantinoma (adenocarcinoma) covers a variety of structures in which the columnar enameloblasts predominate. The tumors may be composed exclusively of such cells arranged in long columns or alveoli. Or the columnar cells may inclose islands of reticulated cells. Or numerous pearls may be surrounded by reticulated cells with a border of columnar enameloblasts. Considerable areas of spindle-cells may simulate sarcoma. Such spindle-cells may be extremely small, suggesting round-cell sarcoma, but a limiting border of columnar cells usually indicates the embryonal epithelial character of such areas. Extensive metaplasia and extreme anaplasia may be observed in recurring tumors. During a period of 4 years in five recurrences of an original plexiform and squamous epithelioma I observed a continuous loss of specific characters, the tumor eventually spreading widely as a large round-cell perivascular growth without a trace of its epithelial origin. 
Secretory products appear in the form of mucous droplets in the cells or as small cysts with mucous, fatty, or calcific elements. Deposits of wellformed enamel lying on beds of dentine have been observed.

The stroma may be very dense and hyaline as in the gubernaculum dentis, or cellular, myxomatous, or sarcomatous. In the stroma of some adamantinomas one may find an exact reproduction of the structure of the giantcell epulis. Portions of cementum or bone may appear in the capsule. The blood-vessels may be overdeveloped. In one large tumor projecting from the alveolar border the vessels became thrombosed and the stroma was everywhere infiltrated with blood.

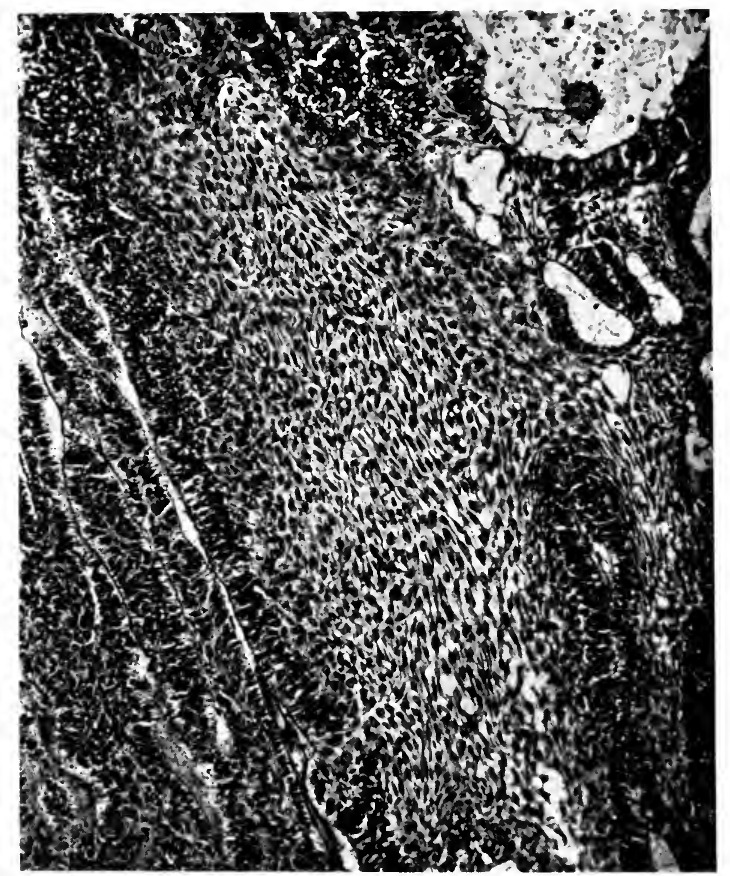

FIG. 340.-Glandular adamantinoma. Sheets of spindle-shaped epithelial cells surrounded by columnar enameloblasts.

Clinical Features. - The tumors occur chiefly in adults, but have been observed as early as the sixth year (Coate), and many occur from 60 to 70 years (Börnig). The duration of the process is usually prolonged, I 8 years in Kruse's case, 23 years in one of Malassez's, 26 years in Bryk's, and $3^{\delta}$ years in Albarran's. A history of extraction of a carious tooth usually precedes by some years the appearance of the tumor. Hence the age of onset is earlier than would appear in many reports and probably begins shortly after the full development of the teeth. Women are more of ten affected than men. The lower jaw is the chief seat of cystic tumors (Pincus), while the upper yields most of the solid and more malignant growths.

The course of adamantinoma varies extremely with the type and location of the tumor. All the crsts and tumors are of slow development and their natural history is prolonged. The superficial growths and small cysts may be 
readily extirpated, but the deep-lying multilocular cysts recur persistently and often require the sacrifice of the maxilla. Tumors of the upper jaw are much the more serious, and the solid adamantinomas of all types recur locally and invade antrum, orbit, and nasopharynx. In spite of their relatively benign structure the prognosis in this group is unfavorable. Yet the lymphnodes are almost never involved, except by malignant recurrences. In one very extensive fibro-epithelial tumor I found metastases in one cervical node and a small nodule in the lung. In another very malignant case the fourth recurrence was in the cervical nodes and loose tissues of the neck and probably in the lungs.

Adamantinoma of Tibia.-B. Fischer describes a tumor involving the outer portions of the shaft of the tibia, with mucoid stroma and many cell groups presenting the typical structures of adamantinoma. In the origin of this tumor he assumes a downward growth of embryonal ectoderm reaching the bone and differentiating as enamel organ, just as the gingival epithelium penetrates the maxilla.

Radiculodental Cysts.-The roots of carious or normal teeth frequently exhibit small excrescences composed of cellular connective tissue in which lie strands and groups of epithelial cells. Malassez showed that these structures are derived from the deeper débris paradentaire, portions of which he finds closely applied to the apex of the root and even extending into the pulp canal.

Small radiculodental cysts frequently develop by proliferation of these epithelial structures and dilatation of the lumina which they surround. Suppuration may destroy or alter the epithelial lining of such cysts and the lesion appears as a small abscess. Larger radiculodental cysts of this same origin have been described by several authors (Sirantoine). They inclose a portion or the whole of the root and may reach the size of a robin's egg, or hen's egg, with considerable absorption of bone. Galippe shows how similar cysts may

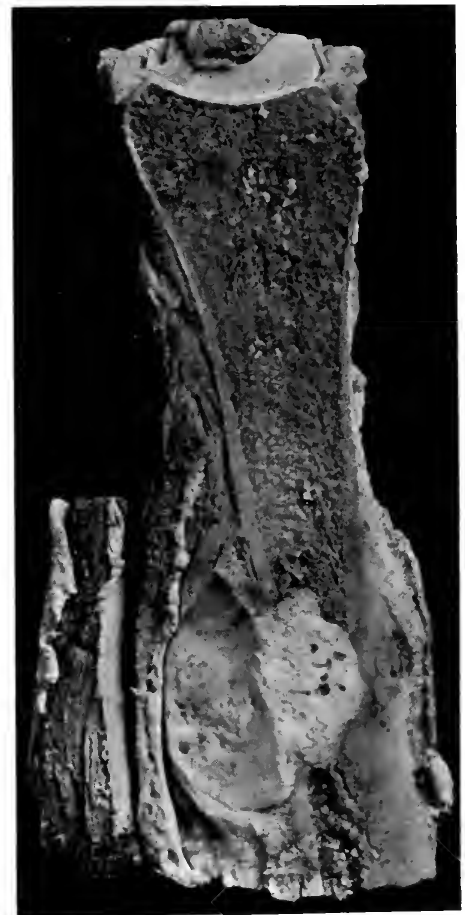

FIG. 34I.-Adamantinoma of tibia. develop without directly exposing the root. The lining of these cysts is of squamous epithelium, so that they have sometimes been regarded as of dermoid nature. Yet occasionally the lining is partly or wholly of columnar cells which indicates their paradental origin. Witzel holds that these cysts arise from dilatation of v. Brunn's sheath, which is the continuation of the enamel epithelium over the root of the tooth. Malassez includes this atrophic sheath with the "débris paradentaire." The walls of the cysts may be very cellular or sarcomatous and the contents are serous, mucous, fatty, or caseous and inspissated (Galippe, Mickulicz).

Multiple cysts of this character were found by Wilks, five separate carities surrounding the roots of as many teeth in a child of 12 years. Or a single 
cyst may surround several roots (Gosselin). The cyst may lie at some distance from the root (Vitalis), or project through the absorbed ramus of the jaw externally (Ancelot, Sourier), or into the maxillary sinuses (Berger). The radiculodental cysts are much more frequent in the upper than in the lower jaw ( 76 to 29 , Witzel).

Coronodental Cysts. Dentigerous Cysts.-All the dental anomalies thus far considered relate to already erupted teeth. The remaining cysts and tumors affect the tooth before its eruption and usually prevent its full development.

Coronodental cysts present small intra-alveolar cavities into which project one or more imperfectly formed teeth. The wall is fibrous and the lining is composed of squamous or cylindrical epithelium or both types. The tooth is usually misplaced, lying horizontally, a position which prevents its eruption.

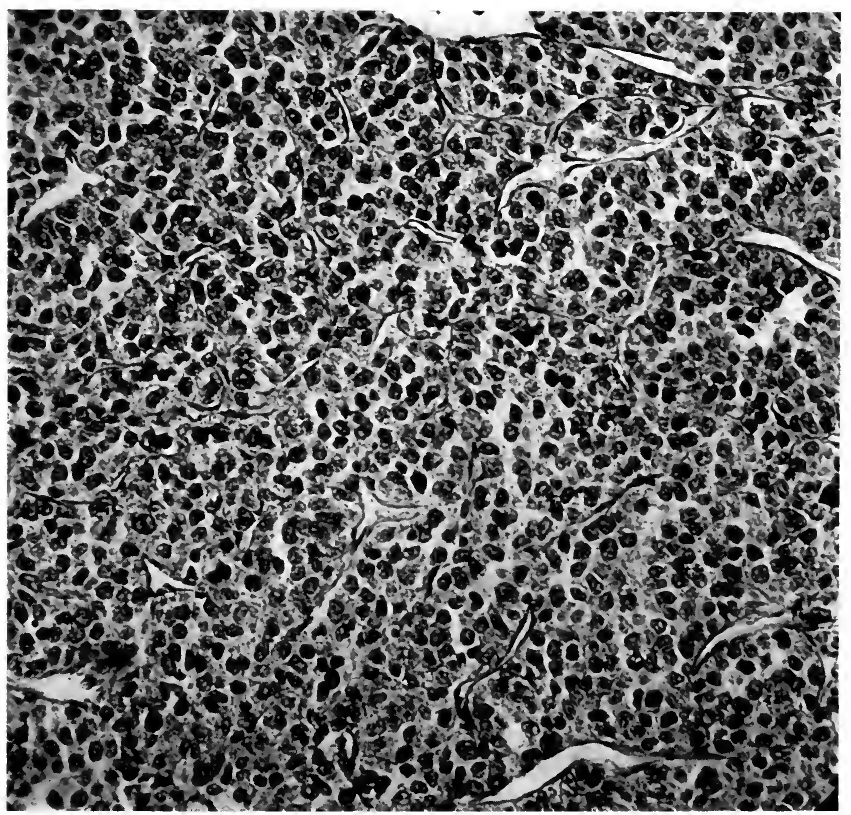

FIG. 342.-Atypical structure of adamantinoma in late recurrence.

The root is imperfectly formed owing to fibrosis of the pulp, the cementum may be hypertrophied, and the dentine may be eroded (Albarran). Heath observed a cyst in each maxillary sinus, one containing a canine, the other a molar, while in another case the tooth lay loose in the cyst cavity. Legouest found two teeth in the same cyst, and Broca saw three. Bayer identified a milk canine and a permanent canine, indicating the fusion of two original cysts. Nélaton found one well-formed tooth projecting into the cavity and numerous rudimentary teeth in the wall. Grosse saw eight well-formed teeth in the same cyst in the middle of the upper jaw. These 2 cases indicate that the hypertrophic dilated enamel organ is still capable of inciting the formation of nearly complete teeth. In several cases the tooth was completely inclosed in the cyst wall, or in a secondary cyst in the wall (Nélaton, RemyDuret). Haasler describes cases in which separate cysts seem to have formed 
about crown and root. Finally Allgayer found a small solid fibrous tumor involving four teeth and containing groups of epithelium. This tumor probably originated in the gubernaculum of one or more teeth, but without the usual formation of cysts.

The origin of these coronodental cysts is referred by Galippe to the stimulus exerted upon the débris paradentaires of the gubernaculum by an imperfectly formed tooth which becomes arrested in the course of its eruption. Fibrosis of the gubernaculum, opposing the eruption of the tooth, with dilatation of the paradental alveoli extending to the original enamel organ, would also account for most of the relations observed. Others regard them as true follicular cysts arising by hypertrophy and dilatation of the enamel organ (M. B. Schmidt).

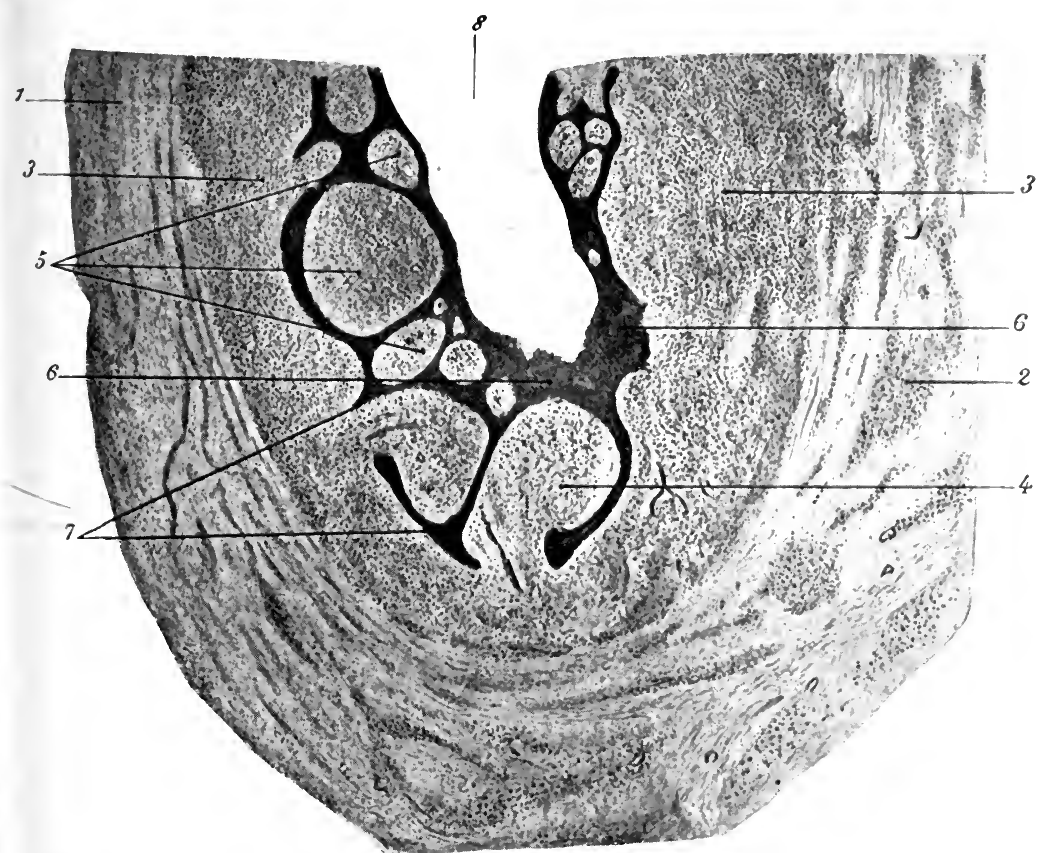

FIG. 343.-Topography of a radiculodental cyst. (After Malassez.) I, 2, Connective tissue of wall. $3,4,5$, Cellular connective tissue of inner layer of cyst, infiltrated with lymphocytes. 6, 7, Epithelial lining of cyst. 8, Cyst cavity.

Dentigerous Cysts and Odontoma.--In this group of anomalies are included cysts and solid tumors caused by an increase in number of more or less well-formed teeth. The most striking illustration of dental overgrowth is seen in the rare case of third dentition described by F. J. Hunter.

The extensive hypertrophy of a single tooth is described by Virchow, Wedl, and Billroth.

A unique case of remarkable multiplication of teeth is described by Hildebrandt. In a boy of 9 years whose erupted teeth were normal in number but misplaced, both upper and lower jaws were swollen and contained $5_{50}$ to 200 teeth lying singly or variously fused, most of them being well formed. After two thorough extirpations the condition recurred and again about the same number of new teeth were removed, leaving a thin shell of bone. In 
this case it is clear that there had been an enormous overproduction of dental follicles and that the dentigerous cells remaining after the first two operations were capable of reproducing well-formed teeth. In the curetted tissues Hildebrand found many rudimentary teeth, enamel organs, and tubules resembling the débris paradentaires. Somewhat similar cases are recorded by Coleman, Bland Sutton, and Matthias.

Dentigerous cysts appear in several forms. Supernumerary teeth are sometimes inclosed in a cyst formed probably from the dilated gubernaculum or follicle, containing one or more well-formed teeth, and serous or mucinous fluid. They lie in the alveolar ramus or at considerable distances, especially below the orbit.

In many cases the tooth is very imperfectly formed or appears in rudiments only, but these rudiments are multiple. Thus Annandale found a large cyst with bony and fibrous wall in which were areas of enamel. Broca described a cyst containing three irregular masses chiefly composed of dentine, and fused in a mass of tissue containing islands of dentine, imperfect enamel, and pulp tissues. Broca cites a very similar case in which a large central mass of dentine, with several roots and crowns, was covered with enamel.

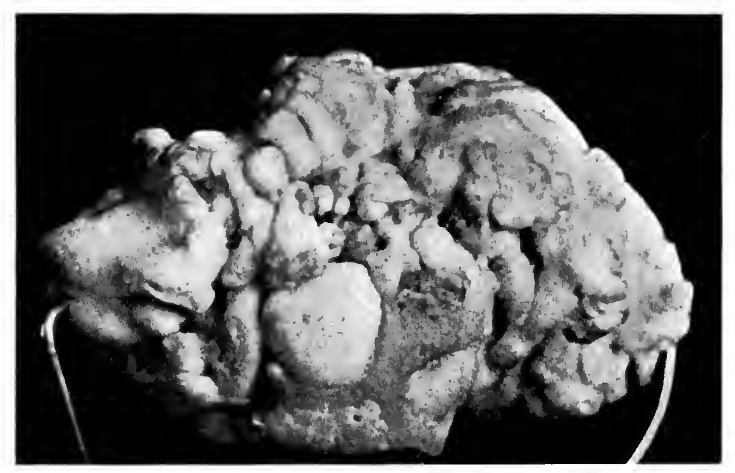

FIG. 344.-Composite odontoma. (After Gilmer, J. A. M. A., 56.)

Odontoma.- The typical odontoma is composed of a congeries of more or less perfectly formed teeth usually surrounded by an epithelial membrane and fibrous capsule. Since there are many grades of development of the teeth and these may incompletely fill the inclosing cysts, there is no sharp dividing line between dentigerous cysts and odontomas. The coronodental cysts are also closely related to odontoma, since they present one or more imperfect tooth crowns in a roomy cavity. In Chibret's case the dentigerous cyst contained irregular masses of dentine and enamel. When the enamel and dentine exhibit an orderly arrangement and pulp tissue and canals appear, the tumor becomes a true odontoma.

Localized hypertrophies of portions of the teeth are also conditions related to odontoma. Enamel pearls are small nodules of enamel which form along the borders of the teeth as a result of overactivity of a group of enameloblasts. The verucous crowns of Satler consist of overgrowth of enamel which may at times reach considerable dimensions. These overgrowths may consist of both dentine and enamel and may even contain a diverticulum of the pulp canal, producing a form radicular odontoma.

The typical odontoma occurs chiefly in young subjects and almost invariably in the lower jaw. Lloyd's mixed odontoma occurred in the upper 
jaw. It forms a solid tumor in the maxilla and is sharply separated from the adjacent bone by an inclosing cyst wall. There is usually an absence of one or more teeth, usually wisdom teeth, whose eruption is obstructed or whose structure is included in the tumor. Incision may evacuate serous fluid or pus from the cavity in which the tumor lies. Looking into this cavity may often be found the crown of an obstructed tooth which, after removal of the tumor, may assume its normal position in the alveolar border (Krogius). On gross section the tumor is composed of fused teeth in which enamel, dentine, and pulp tissue reproduce the form of the teeth which lie in disorderly arrangement. The surface is nodular from the projecting ends of the teeth. Well-formed teeth may not be discernible and the tumor may be composed of irregular masses of dentin, enamel, and pulp canals, all covered by a layer of enamel (Forget). Enamel may be missing and the tumor be composed of masses of dentin and bone (Uskoff). Or enamel is reduced to traces and the tumor composed of dentin and cementum (Krogius). Such tumors may be designated as amorphous odontomas.

A complex cystic odontoma is described by Robin. It contained three well-formed molars in adjoining cysts, two rudimentary teeth rooted in bone, and projecting into small cysts, and three small pedunculated fibromas.

Tapie describes a cyst in the angle of lower jaw, of 5 years' standing. A thick fetid sebaceous discharge drained from two sinuses. The wisdom tooth on that side was missing. A molar crown projected into the cyst, its root embedded in the wall, which presented also a large number of small conical teeth and calcareous points. Free in the cyst lay a composite odontoma with several teeth fused by cementum. The cyst was lined by stratified epithelium. Tapie interpreted the case as an invagination of dentigerous gingival mucosa. Galippe thinks the cyst arose from paradental débris and that the odontoma arising outside penetrated the wall.

Soft Odontomas.-In some cases the odontoma is composed chiefly of connective tissue in which lie many rudimentary teeth (Perthes, Partich). In Schlossmann's case, in a boy of ro years, the last molar and wisdom teeth were missing from the lower jaw which presented a tumor 3.5 to $3.2 \mathrm{~cm}$., composed of firm connective tissue in which lay many small hard structures as large as a pea or bean. The structure presented numerous well-formed enamel organs about which were layers of dentin in various stages of development. Enamel was found only where dentin came in contact with the enamel organs. There were no traces of dental papillæ. Regressive changes affected the older teeth in the center of the tumor, where vessels were invading the enamel organ at the expense of the active cells. The authors concluded that the tumor arose from the follicles of both missing teeth, which became displaced and disturbed in growth by neighboring teeth in the confined region of the angle of the jaw.

General Etiology.-Odontoma almost always appears before the age of 25 years, or before the full development of the teeth has been reached. In this period also most dentigerous cysts are observed, while adamantinoma, as a rule, appears in later life. Odontoma affects almost exclusively the lower jaw, adamantinoma frequently the upper. This contrast suggests a somewhat different mode of origin for the two groups. The second or the first molar is frequently missing with odontoma, indicating that the tumor arises from the structures which normally give origin to the tooth. The factors which initiate the abnormal growth are at present undetermined. Trauma has been only a rare antecedent. Schlossmann traces the origin to misplacement of a dental follicle during the early period of growth, which is especially favored in the confined quarters of the angle of the lower jaw. The exten- 
sive variations in the composition and relations of odontoma, as illustrated in the preceding cases, renders quite difficult the task of tracing its origin.

Broca recognized four periods in the development of the tooth: (a) embryoplastic, before the appearance of definite dental structures; $(b)$ odontoplastic; $(c)$ coronal, and $(d)$ radicular, and he divided odontomas accordingly. He assumed that all the tumors arose from dental follicles, the formative process residing chiefly in the pulp tissue. By multiplication of pulps several teeth might form as in the composite odontoma. But the structure of our tumors does not always correspond to this direct plan, which is not fully in accord with the development of the teeth (Wedl). A purely anatomical classification was used by Sutton, who recognized cementomas, dentomas, osteodentomas, and mixed dentomas. Yet cementum is probably only a secondary product added to stationary odontomas (Schlossman). Malassez and Galippe refer the majority of odontomas to proliferation of the "débris paradentaire," but it is difficult to adjust all the types of these tumors, as well as of adamantinoma, to this single source.

Several modes of origin seem to be involved in the different forms of odontoma and of the closely related dentigerous cysts. The absence of a normal tooth indicates that the odontoma arises in the follicle of this missing organ and the growth of several well-formed teeth in its place indicates that all the structures, especially the pulp, are concerned in the tumor process. Many of these tumors lie in a cyst or are surrounded by a wall in which are proliferating islands of enameloblasts, showing that this structure also participates in the process. The varying proportions of enamel, dentin, and pulp tissue point directly to overgrowth of particular elements, but not to distinctly different modes of origin.

Where all the normal teeth are present a supernumerary follicle first presents itself as the most probable source of the tumor.

A congeries of imperfectly developed teeth, as in Schlossmann's case, suggests an extensive multiplication of enamel organs and dental pulps, which are as readily derived from a single original follicle as from multiple foci of paradental débris. Hildebrand's case shows that practically all the follicles of both jaws may be incited to this form of organ multiplication. It is difficult to conceive of such orderly but active growth from embryonal paradental débris.

Cystic dilatation of the original enamel organ in the follicle adequately explains the dentigerous cyst with a single tooth, and multiplication of pulps may be assumed where multiple teeth are observed. Since the enamel organ almost completely surrounds the developing tooth (v. Brunn), the inclosure of many odontomas in an epithelial cyst is readily understood.

Cementum is added to many odontomas after the limit of growth has been reached. The extensive overgrowth of bone, sometimes observed, must be referred to the periosteum, and it seems probable that some nearly pure maxillary osteomas may arise under these conditions.

This conception would, therefore, limit the domain of the débris paradentaire to the adamantinomas and the cystic tumors lined by epithelium and containing only irregular rudiments of dental structures, while the cysts with multiple well-formed teeth and the various odontomas may be referred to the enamel organ and the pulp tissue.

Tumors of Maxillary and Nasal Sinuses (Antrum of Highmore).-The nasal passages and their accessory sinuses are the seat of a series of tumors, benign and malignant, which form one of the most important departments of rhinology. The complex embryology of this region gives abundant oppor- 
tunity for the origin of tumors from developmental anomalies, while the peculiar anatomy of the part gives unusual chances for the growth of tumors as a result of inflammatory processes. The frequency of these tumors is considerable. At the Memorial Hospital during the years I9I6 to 1917, out of I 892 cases of cancer of all types admitted, 48 (2.53 per cent.) were in this region, and of these, 35 involved the maxillary sinus.

From the diagnostic standpoint it will be serviceable to review the tumors occurring in this region:

Nares.

Polyps. Mucous, myxomatous, fibromatous, angiomatous.

Mucosa. Choanal angioma, angiomyxoma. Myxosarcoma. Schneiderian adenoma, carcinoma. $\begin{array}{lll} & \text { Lymphosarcoma. Melanoma. } \\ \text { Antrum. Mucosa. Cysts. (Empyema.) Mucoid. Cystic myxoma. Fibroma. } & \text { Epithelial papilloma. Carcinoma. }\end{array}$

Bones.

Bones. Osteoma. Fibro-osteoma. Osteosarcoma. Chondroma.

Ethmoidal sinuses. Dental cysts. Odontoma. Adamantinoma.

Sphenoidal sinus.

Frontal sinuses.

Pharyngeal vault.

Fibroma. Osteoma. Carcinoma.

Polypoid myxoma.

Osteoma. Carcinoma.

Hypophyseal duct.

Angiosarcoma. Chondroma. Chondrosarcoma.

Epidermoid carcinoma. Adamantinoma. Dermoids.

Epignathi. Teratoma.

Any one of this formidable array of neoplasms may present itself as a mass obstructing the nares, with or without purulent or bloody discharge, and requiring for its successful treatment an accurate identification. Of the above list the benign tumors have been briefly discussed elsewhere, but the epithelial tumors of the nasal mucosa and maxillary sinus present many special features which may be considered at this point.

Cysts of Antrum.-Chronic empyema of the antrum may become confined by closure of the orifices, the inner wall may yield to pressure, giving a protruding tumor of the nasal passage, while the contents may become inspissated and cheesy. The bony wall may undergo absorption or necrosis, while the lining may be transformed into squamous epithelium. Chronic inflammation and edema of the mucosa may lead to filling of the cavity with myxoma-like tissue which has often led to difficulty in diagnosis from the material excavated from the antrum. In many cases, but by no means in all, a chronic empyema has preceded carcinoma or papilloma of the antrum. Certain osteomas of the antrum have been described as containing one or more cysts. They were probably of dental origin. Polypoid fibroma or myxoma frequently develops from the orifice or the fundus of the antrum. Those arising from the fundus fill and may considerably distend the antrum, but many protrude through the orifice by elongated pedicles and appear as ordinary nasal polyps. They are usually firmly attached to the periosteum and may require opening the antrum for complete eradication. In fact, some rhinologists recommend opening the antrum for all antral polyps (Moore).

Epithelial papilloma occasionally arises in the antrum. It has considerable growth capacity, recurring after operation and until the base is removed. I have observed a case in which the papilloma recurred after many operations over a period of Io years and eventually became carcinomatous.

Carcinoma of Antrum.-There are two separate sources of malignant epithelial tumors of the antrum: (I) from the mucosa, and (2) from epithelial dental structures, tumors of which may first appear in the antral cavity. In each of these classes there is a considerable variety of structure and many peculiarities in course. 
From the mucosa arise:

(I) Papillary carcinomas, some of which represent malignant transformations of papillomas, as above described. These are originally of slow growth, but eventually become fully malignant.

(2) Carcinomas of the basal-cell type arise within the antrum and also from the nasal mucosa. They are composed of anastomosing cords of small cells consisting chiefly of nuclei. The stroma is scanty and undergoes hyaline or mucoid degeneration, so that the structure resembles thyroid gland, or the mucous masses are more abundant and the tumor is reported as adenoid cystic epithelioma, or cylindroma. Endothelioma is also a favorite designation of these tumors. They are of relatively slow growth, but recur persistently

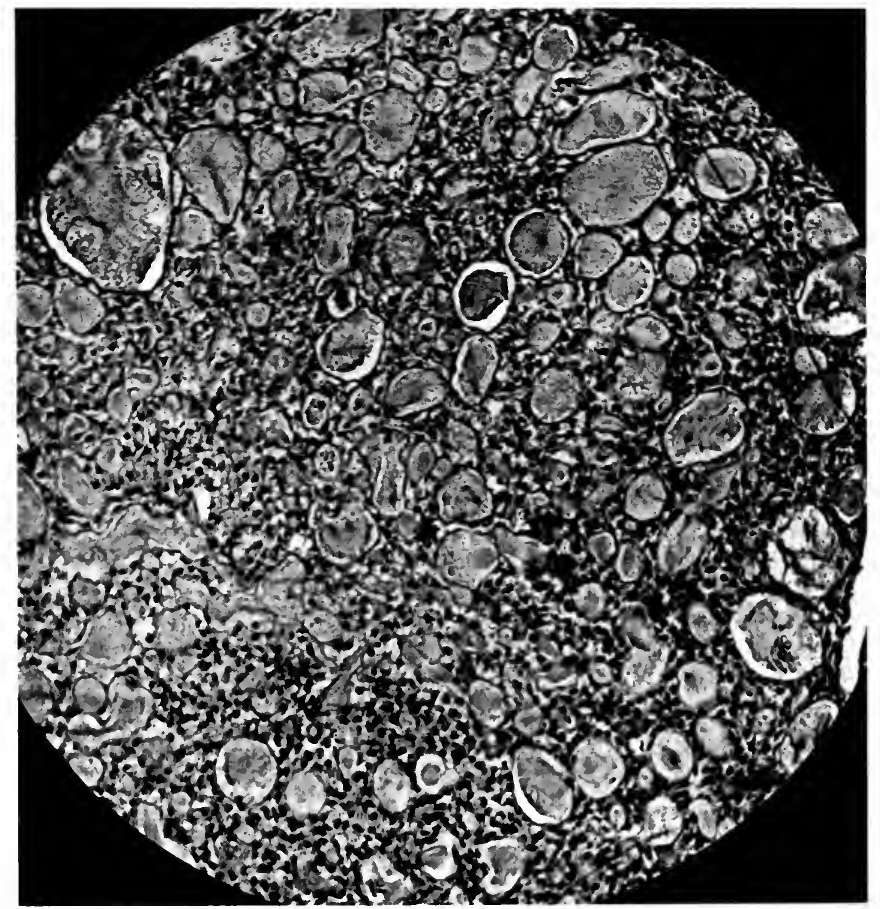

FIG. 345.-Carcinoma of antrum, probably arising from the mucosa, and showing many globules of mucus.

after incomplete removal, and they may invade the bone and eventually the lymph-nodes.

(3) Squamous-cell carcinoma is rarely seen in the antrum except as a secondary invasion, but either from metaplasia or through origin from previously altered lining epithelium this type of growth may arise. It pursues the usual course of acanthoma.

(4) Cylindrical-cell carcinoma is a common form of antrum cancer. It forms a bulky rapidly growing tumor which distends the cavity, protrudes into the nares, erodes bone, ulcerates and bleeds, and usually extends widely with recurrence after operation. The structure is adenocarcinomatous, or the cells grow in large sheets and in polyhedral form. This same structure is 
seen in tumors arising from the maxillary sinus, the ethmoid cells, the frontal sinuses, and the Schneiderian membrane. Wisotski has collected 3 cases of frontal sinus carcinoma and I acanthoma.

(5) Round-cell carcinomas of atypical structure composed chiefly of rounded or polyhedral cells consisting chiefly of nuclei arise in the nasal passages and accessory sinuses. They probably form the majority of the so-called sarcomas of this region. On account of the absence of alveolar structure and definite epithelial characters the true origin is easily overlooked, while many reporters of cases have assumed that the highly malignant course indicates a sarcomatous nature. The diffuse growth of undifferentiated cells is facilitated by the recurrence after incomplete operation to which most of these tumors are subjected, but if the primary structure of the original tumor is secured or the tissue in recurrences is carefully prepared and examined, the epithelial character is sufficiently revealed. The conditions of origin and the course of these tumors are those of carcinoma, and the existence of a mesoblastic tumor of this type has not been demonstrated. Lymphosarcoma occurs in the nares as a part of a more general disease, but I have seen one case of lymphosarcoma in which the main lesions requiring attention were nasal and laryngeal.

Melanoma of the nares is a very rare tumor, of which I have studied one example, reported by Coley, in which the pigmentation was prominent and the structure carcinomatous.

(6) Dental tumors not infrequently develop in the antrum. They include radiculodental cysts, dentigerous cysts, and squamous and glandular types of adamantinoma $(q . \vartheta)$. They can usually be recognized on their peculiar structure and also by the occurrence of disorder in the adjacent teeth early in the course of the disease. Yet the antral growth may be well advanced before the teeth are disturbed, so that this group of dental tumors is of relatively unfavorable prognosis.

(7) True sarcomas of the nares and antrum are chiefly angiosarcomas and myxosarcomas arising from the mucosa. Many authors describe fibrosarcomas without reference to their exact nature (Richou). Osteogenic sarcomas of this region are comparatively rare and are easily recognized by their structure. From the choana and vault of pharynx arises the chondromyxosarcoma of infants and children.

Clinical Course of Malignant Tumors of Antrum.--Since the prognosis of established malignant tumors of this region is extremely unfavorable almost the only real service the physician can render in these cases is the early recognition of the disease. At the Memorial Hospital it is found that most of these patients have passed the period of hopeful therapeutics unnoticed, or with nasal douches, or extraction of teeth.

Richou separates the initial symptoms into two groups, in one of which the disease is preceded or accompanied by purulent or bloody discharge from the nares, while in the other no such signs are observed, but the tumor is discovered only after it has reached such a size as to produce pressure symptoms. It is important to note that many cases are long regarded as pyorrhea, toothache, neuralgia, or simple sinusitis, only to reveal themselves later as malignant tumors. Occasionally the histological report on a benign process removed from the outskirts of a malignant tumor has obscured the true condition (Richou). Thus the thorough exploration of every suspicious case seems to be indicated as soon as possible. The condition of the antrum should be determined by an $x$-ray photograph.

Among the conditions simulating the early stages of malignant tumors Jacques and Gaudier mention disorders in the development of the teeth, 
misplaced molars and wisdom teeth with cysts arising therefrom, simple osteitis and periostitis, mucocele of the antrum, empyema of antrum, and forms of syphilis and tuberculosis.

Three stages in the course of malignant tumors are emphasized by Jacques and Gaudier. In the stage of latency the presence of a tumor may be suspected from aching or neuralgic pain in the jaw, purulent discharge, epistaxis, polypoid growths about the orifice of the sinus, and in the case of dental tumors loosening of the teeth. In the stage of deformation of the maxilla there is a swelling of the walls of the sinus, projecting externally, or into the nares, or into the orbit, together with more definite symptoms of the earlier stage. In the stage of invasion there is destruction of the walls and adjacent structures, hemorrhage, ulceration, and extension to skin, pharynx, orbit, skull, or lymph-nodes. The general duration of the disease is from 4 months to .2 years, and is closely related to the degree of histological differentiation of the tumor-cells. Recovery from the squamous-cell or true carcinomas is very rare.

The lymph-nodes were invaded in 19 of 64 cases reported by Windmuller, and in 19 of $5^{\mathrm{r}}$ cases of Winiwarter. On the whole, this invasion occurs rather late and infrequently, considering the activity of the process. The nodes chiefly affected are those behind the angle of the jaw and above the expansion of the aponeurosis of the sternomastoid (Sebileau). The lymphatics of the superior maxillary region tend to pass posteriorly, join with those from the nasal mucosa and vault of pharynx, and descend along the deep cervical chain. I have observed bulky pulmonary and hepatic metastases in 2 cases of antral and Schneiderian carcinoma in which no invasion of lymph-nodes could be discovered.

The best results in the treatment of carcinoma in this region are secured by early operation combined with or preceded by radium. The English rhinologists especially seem to have developed considerable reliance on this latter agent. 


\section{CHAPTER XXXVI}

\section{EPITHELIAL TUMORS OF THE SALIVARY GLANDS}

Three groups of epithelial tumors are observed in the salivary glands:

(I) Benign adenoma,

(2) Malignant adenocarcinoma or carcinoma, and

(3) Autochthonous mixed tumors.

The parotid is by far the most frequently involved, but all the above growths occur in the submaxillary, the sublingual is occasionally affected, while mixed tumors of the salivary gland type are also found in the lip, cheek, palate, nares, and pharynx. The lacrimal gland may also be included with the salivary organs in the study of its tumors.

Adenoma of the salivary glands is rare. In the parotid, Nasse describes 4 cases and others are reported by Lecene, Lexer, Ribbert, and Wood. In the submaxillary, Chevassu analyzed five reported adenomas, concluding that two were cases of chronic inflammation (Duplay, Poncet), one a cystic dilatation of the ducts (Bougle), one a carcinoma (Wolfler), and one a papillary cystic adenoma (Talazac).

Sublingual adenomas are described by Duplay, Zeisel, Ferreri, and Kuttner. Of $\mathrm{I}_{32}$ tumors of the lacrimal glands, Warthin found $\mathrm{I}_{3}$ described as adenomas. In these cases the tumors have exhibited very slow growth, the duration in Nasse's cases being from 8 to I 2 years. They are encapsulated, cystic or solid, and well incorporated in the gland or attached to it. In the gross the tumors are lobulated by traversing septa, or composed of solid gland tissue, or of many minute cysts or of one large cyst. The structure is alveolar, reproducing the acini of the glands, or cystic and papillary. The stroma is of moderate development and all traces of mucoid or cartilaginous deposits are missing. In the cysts there may be much mucous or serous secretion. Transitions to malignant adenocarcinoma are observed (Chevassu), and Nasse describes in upper lip and nose combinations of adenoma with basalcell carcinoma. Several authors have described transitions to myxochondrocarcinoma.

Carcinoma.-Pure malignant epithelial tumors of the salivary glands are by no means rare. Most of the parotid carcinomas are reported in studies of the mixed tumors, and appear in the series of Paris theses (Pailler, Lit.), and in the collections of Volkmann, Ehrich, Chevassu, and others. Pure carcinomas without any trace of the features of mixed tumors are described in the parotid by Pailler, Ehrich, and Nasse, in the submaxillary by Prengrueber, Waldeyer, Volkmann, Kuttner, Lowenbach, Nasse, and Chevassu. Warthin collected six carcinomas of the lacrimal gland. Most of the malignant tumors of this organ are of the basal-cell type. 'I have studied three pure carcinomas of the parotid. It is evident that many of the pure endothelial tumors described by Volkmann and others are of glandular epithelial origin, but being chiefly of the basal-cell type they are included with the mixed tumors.

Whatever may be their relation to the mixed tumors, pure carcinomas of the salivary glands are highly characteristic both clinically and histologically.

The carcinomas usually develop rapidly, and while at first they may be encapsulated, they soon invade the whole gland, the capsule, and regional nodes. The more adenomatous tumors may contain cysts, while the more 
malignant growths are solid, firm, and opaque and in many cases lobulated. After extirpation prompt recurrence is usually observed, even when the structure suggests an adenomatous nature (Chevassu). In advanced cases there are local and general metastases, and in Nasse's case many bones were involved. The clinical malignancy of these tumors is, therefore, pronounced. In Prengrueber's case the growth was accompanied by continuous salivation. In some cases there is a history of a quiescent growth of long standing which eventually became active, indicating a change from a benign to a malignant structure. I have observed carcinoma developing on a granuloma of 3 years' duration in the submaxillary gland.

Structurally there are two main varieties, adenocarcinoma and alveolar carcinoma. To these must probably be added a third group of diffusely growing tumors of spindle- or rounded cells which are atypical carcinomas (Cherassu).

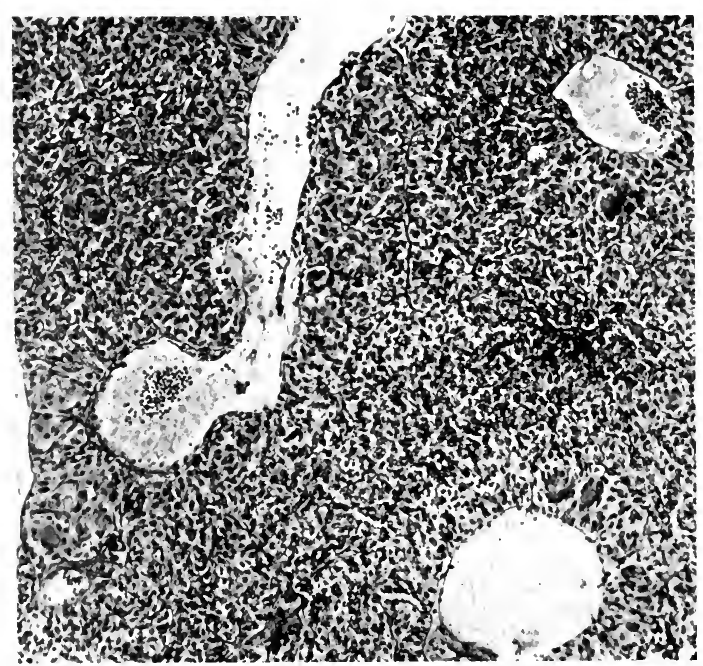

FIG. 346.- Structure of an adenocarcinoma of submaxillary gland.

The adenocarcinomas are alveolar or papillary. The former develop from the acini and the latter from the ducts. The alveolar growths reproduce more or less exactly the acini of the gland. The lumina and the cells may contain hyaline, mucous, or serous secretion. Chevassu describes a dendritic adenocarcinoma arising in the ducts, in which very numerous papillæ were lined by multiple layers of cubical or cylindrical cells. This tumor promptly recurred after operation and grew rapidly.

Under the term cylindroma has been described a rather frequent form of adenocarcinoma of the salivary glands (Lowenbach, Lit.). These tumors are composed of anastomosing cords or broad masses of epithelial cells, inclosing many spaces filled with mucus. There are coarse trabeculæ of connective tissue which may undergo hyaline or mucoid degeneration. The structure suggests a relation to basal-cell carcinoma of the type of adenoid cystic epithelioma, but Lowenbach was able to trace the origin in one case to the ducts and in another to the acini of the gland.

The fully developed carcinomas of the salivary glands usually exhibit traces of the acinar structure of the adenocarcinomas, but the most anaplastic 
tumors grow in broad, cellular masses or diffusely, while some highly atypical growths take the form of so-called round-cell sarcoma. Three structural peculiarities suggesting an origin from the salivary glands are often present, in three structural varieties of these tumors.

(a) Marked squamous metaplasia, affecting very many small groups of cells, appears in carcinomas composed of anastomosing columns of opaque granular, cubical, or cylindrical epithelium. These features suggest an origin from the ducts in which squamous epithelium may result from inflammatory changes.

(b) Peculiar large mucoid globules may distend many cells in small alveolar carcinomas, yielding a structure which is quite specific.

(c) Collections of mucus, such as occur in cylindroma, may persist in more cellular and malignant tumors.

Round-cell Carcinoma.-Malignant round-cell tumors of the salivary glands have been described as round-cell sarcomas by Kuster, Nasse, Koch, Degen, and Schridde (Lit.). Kuttner collected 6 cases of round-cell sarcoma of the submaxillary. Some of the tumors presented a distinctly alveolar structure, while most others exhibited nests of polyhedral cells strongly resembling epithelium. Schafer found well-marked epithelial characters in portions of a tumor chiefly "sarcomatous." Schridde describes among the round cells remnants of gland tissue in markedly hypertrophic condition, which he interprets as collateral hyperplasia. As the tumors were large and rapidly growing, none of the authors was able to trace the origin of the growth. In some cases there was pronounced perithelial arrangement such as is often seen in the mixed tumors (Dubreuil, Volkmann). All of these features are much more satisfactorily explained as belonging to atypical rapidly growing carcinomas. Chevassu argues strongly in favor of this view. Lymphosarcoma originating in the reticulum cells of the lymph-follicles is not infrequently seen in the salivary glands, as in Mikulicz's disease, and may be difficult to distinguish from round-cell carcinoma, but apart from these lymphoid tumors sarcoma of the salivary glands has not been satisfactorily demonstrated.

Mixed Tumors of Salivary Glands, etc.-In the salivary glands, buccal mucosa, palate, lips, neck, orbit, and lacrimal gland and face occurs a group of tumors about the origin of which has long been maintained an active controversy. These tumors are of complex structure, usually presenting epithelial elements in the form of cell strands, alveoli, or diffuse masses, and mesoblastic tissues chiefly cartilage, mucous tissue, and cellular connective. Any one of these elements may predominate, giving nearly pure chondromas, sarcomas, or carcinomas, but usually all the cell types are represented.

These tumors were originally regarded as carcinomas, and while Virchow derived the cartilage from the connective tissue by metaplasia, Cohnheim regarded it as a derivative of remnants of the branchial arches. The epithelial nature of the tumors was undisputed until C. Kaufmann, on the strength of peritheliomatous and sarcomatous features, placed them among the sarcomas.

In 1879 Wartmann maintained that the polyhedral cells were derived from the lymphatic endothelium, and from this time the endothelial theory gained general acceptance in Germany, especially through the study of Volkmann. Although the main conclusion of this author has been proved untenable, his study was of much value in revealing the extent to which embryonal epithelial cells may simulate endothelium, and his view that the mesoblastic structures in the tumors are derived by metaplasia from embryonal connective tissue still remains a matter of discussion.

In France the endothelial theory failed to receive much attention, and Collet in 1896 , Pitance (1900), and many later writers adhered consistently to the epithelial interpretation.

The first definite attack on Volkmann's theory was made by Hinsberg (1899) with 
Ribbert's support. He strongly maintained the cancerous nature of the tumors, demonstrating that the pearls observed in many cases by himself and previously by Nasse, Volkmann, Mauclaire, Cavazzani, and others exhibited spines, and disposing of many histological arguments which had been used in favor of the endothelial theory. By embryological studies he endeavored to show that the tumors are derived from very early invaginations of buccal epithelium and inclusion of mesoblastic tissue connected with the mandible arches. He found islands of salivary gland tissue included within the periosteum of the maxilla, and concluded that the mixed tumors arise from the isolation of a mass of tissue including both salivary gland and periosteal tissue. His conclusions have been accepted with certain reservations by Wilms and Landsteiner, and represent one of the prevailing opinions at the present day. In America they have been endorsed especially by Wood and Verhoef. Yet Borst and Kaufmann will not abandon the possibility that some of the tumors are of endothelial origin, although they do not specify their distinctive features. Recently Steinhaus and Martini espouse the endothelial theory, but with arguments which appear to the writer inadequate.

The arguments favoring the endothelial theory relate chiefly to the morphology of the cells and their relation to surrounding structures. The cells are small, often polyhedral, and under pressure readily assume a flat or spindle form. They are often very intimately adherent to the supporting connective tissue and may give the appearance of merging with that tissue in a manner rarely observed in carcinoma. It has repeatedly been pointed out that the cells may be continuous with those lining lymph-spaces and capillaries. In certain cases diffuse masses of these cells reproduce a structure closely resembling spindle-cell sarcoma, and in the various forms of cylindroma and perithelioma structures appear which have commonly been recognized as sarcomatous.

Especially the relation of the tumor-cells to the growing masses of hyaline, mucinous, or cartilaginous material suggests their mesoblastic nature. The early tumors may be sharply separated from the gland structures, and many observers have been unable to find any signs of derivation of the tumor-cells from gland-cells. Finally, in no other situation do any tumors of known epithelial origin exhibit such peculiar relations to mesoblastic tumor elements, whereas in known forms of endothelioma, sprouting of capillary endothelium, persistence of lumina, pearl formation, phagocytosis of bloodpigment, intimate relation or fusion with the stroma, and ultimate transformation into hyaline connective tissue, are observed.

While the above arguments appeared at one time as conclusive, they must today be regarded as inadequate or even superficial. The cells show no minute characters which permit their identification as endothelium. Instead of a clear cytoplasm, condensed cell border, and small nuclei with indistinct nucleoli, their cytoplasm is granular, the borders ill-defined, and the nucleoli often prominent. The long strands of cells resembling sprouting vessels are reproduced in carcinoma, as is also the intimate contact of cells with supporting stroma. In the pearls many have found spines and fibrils connecting the cells, thus furnishing the strongest evidence of their squamous epithelial nature. The structures identified as lymph-spaces may equally well represent lumina of gland alveoli. In fact, this latter origin has been clearly demonstrated in some cases, whereas the existence of many dilated lymph-spaces in these tumors has not been satisfactorily proved. The alveoli often contain secretion similar to that found in the atrophic salivary gland alveolus. Many of the tumors are encapsulated and do not involve the normal lobules of the gland, so that the opportunity to trace the fate of gland-cells when included in the tumor is rarely given. Yet this encapsulation is often incomplete. Krompecher, Pailler, Ehrich, and others have clearly traced the derivation of the flat cells lining narrow spaces from the glandular epithelium. I have studied cases in which normal gland alveoli were being inclosed in hyaline material on the edge of the advancing process. Here the epithelial cells became transformed into flat cells exactly resembling those lining supposed lymph-vessels, and giving the appearance of sprouting capillaries. The structure of cylindroma or perithelioma does not indicate an endothelial tumor, since this structure is often produced by carcinoma. The mixed epidermal and mesodermal origin is rendered acceptable by embryological studies, and it is in accordance with this view that nearly pure chondromas or carcinomas may occur when one element becomes predominant. It is only in the intermediate group of cases where both elements are commingled that the question of endothelioma arises.

Hence it seems necessary to abandon the theory of the endothelial origin of the common tumors of the salivary glands. If, as Borst, Kaufmann, and others assume, true endotheliomas occur in these organs their specific characters remain to be pointed out.

With the demonstration of the epithelial nature of the tumors the subject of their histogenesis is by no means completed. 
In a series of studies Krompecher reaches the conclusion that the mixed tumors of the salivary glands and many other regions belong in the class of basal-cell carcinoma, and especially in the subgroup of adenoid cystic epithelioma. In the soft pliable stroma of these growths he finds that the epithelial cells readily split off, fibrillate, and assume the spindle or star shape of mucoid or embryonal connective-tissue cells. Under these conditions he finds that true connective tissues, and by further metaplasia cartilage, mav be derived from basal epithelium. Hence, Krompecher interprets the salivary growths as of purely epithelial origin, with peculiar mucoid and cartilaginous metaplasia. Concerning the exact source of the originating epithelium he does not specifically state whether the cells are embryonal and misplaced, or located in the salivary ducts. For many of the tumors of salivary

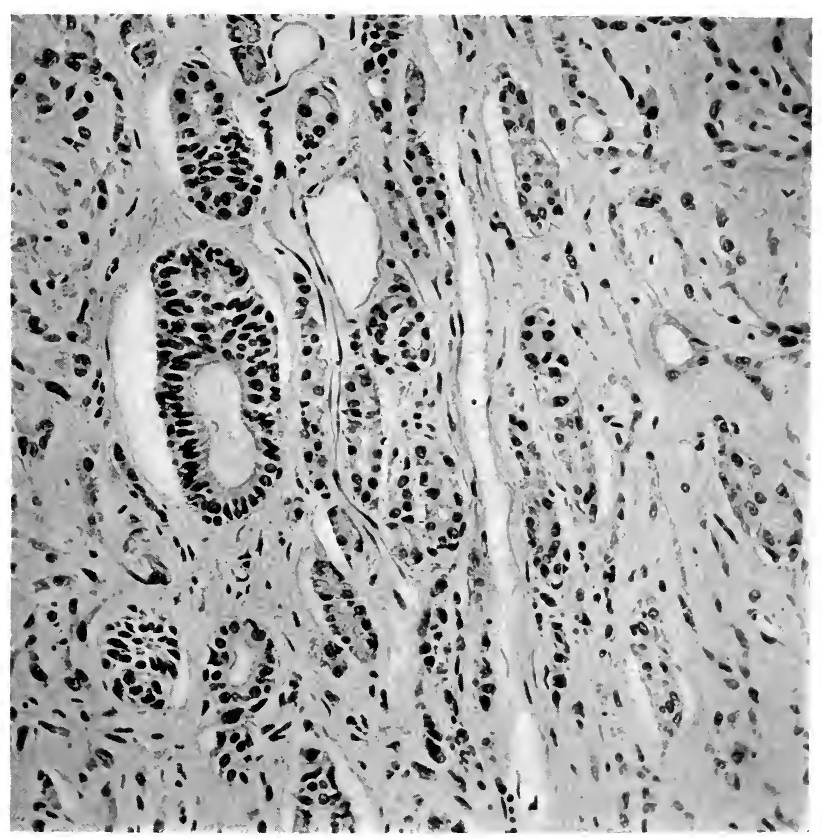

FIG. 347.-Mixed tumor of parotid gland. Showing transformation of epithelium into spindle-cells.

glands and mucous membranes Krompecher's interpretation must, I think, be accepted. Especially the encapsulated growths consisting of anastomosing cords of small cubical or spindle-cells with hyaline stroma and cartilaginous areas, this mode of development appears to be followed. Both the structure and the clinical course of these tumors are identical with those of adenoid cystic epithelioma of the skin. Misplaced portions of buccal or branchial epithelium or superfluous duct-cells may well serve as origins of such tumors.

Not all of the tumors, however, may be disposed of in this manner. A large portion of them, covering a wide variety of structures, are probably derived from the ducts and acini of the salivary and mucous glands. The evidence in favor of this view is well presented by Ehrich, and is duplicated 
in much of my own material. He finds that many tumors are not encapsulated, but uniformly fuse with the gland and reproduce hypertrophic lobules of the gland bounded by regular septa. In several cases the tumors have been multiple, indicating that the process originated in several lobules of the gland (Ehrich, Nasse, Krieg).

In these tumor lobules neoplastic ducts and acini multiply and break up, and the cells become disseminated in the soft mucoid stroma. Cartilage appears chiefly in the centers of such lobules where the metaplastic process is oldest and most advanced. The cartilage cells are derivatives of the epithelium. The part played by the original stroma is subordinate, although in fibrous areas elastic fibers may be derived from the stroma and blood-vessels. Yet genuine collagen and elastic fibrils develop in the mucoid material under the influence of the modified epithelium. The mucoid material is a secretion from the epithelial cells and its diffusion prepares the way for the dissemina-

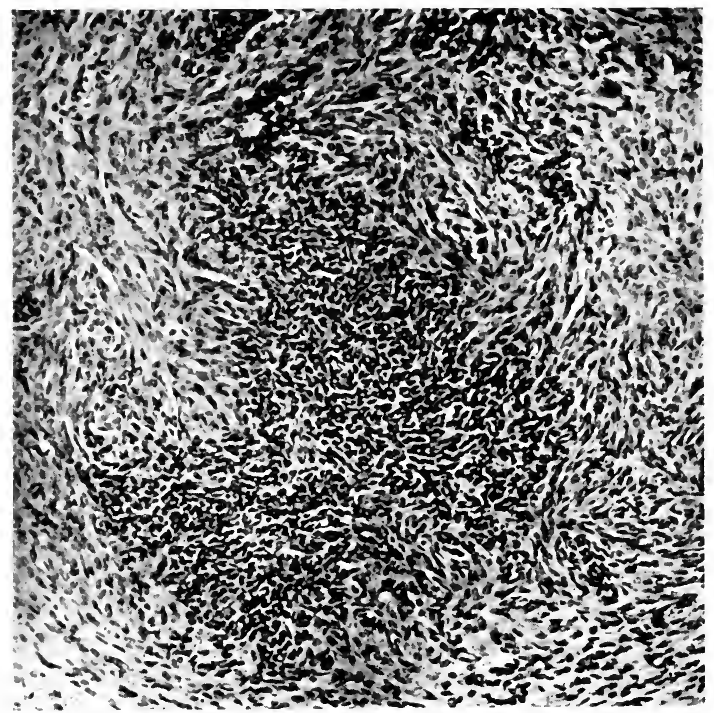

FIG. 348.-Structure of a portion of a mixed tumor of parotid gland, showing transition of cell types.

tion of modified glandular and pavement epithelium. Squamous metaplasia occurs readily in the proliferating cells of both ducts and acini, as in pure carcinomas.

According to this view many of the salivary tumors are pure adenocarcinomas with peculiar and exceptional metaplasia, and not requiring any periosteal or other mesoblastic derivative in their cells of origin. However violently this interpretation may conflict with long-established views regarding tissue growth, it appears to be necessary to accept the facts so well attested by the close study of these tumors. To a considerable extent Krompecher's studies have rendered the exclusive epithelial origin more acceptable. It is not necessary to assume that similar violations of the ordinary laws of histogenesis occur in other tumors, since the salivary gland tumors, under the influence of mucus production and ptyalin secretion, appear to form an exceptional class. Yet a very similar inclusion of epithelial cells in cartilage may some- 
times be traced in chondrocarcinoma of the dog's breast. 'That the ductcells possess a notable capacity for multiplication and squamous metaplasia is shown in the results of ligation of the ducts or partial extirpation reported by Ribbert, Broussis, and Carraro. Lowenstein describes notable hyperplasia of acini occurring in areas infiltrated by lymphocytes in chronic inflammation of the salivary glands and he finds in this altered groundwork the cause of the proliferation which leads to tumor growth.

While the above theory satisfactorily explains the mode of growth of the established tumor it is less convincing in regard to the exact cells of origin of the beginning growth. Some very small encapsulated tumors do not exhibit any relations to the ducts or acini of the gland. The basal-cell type of growth

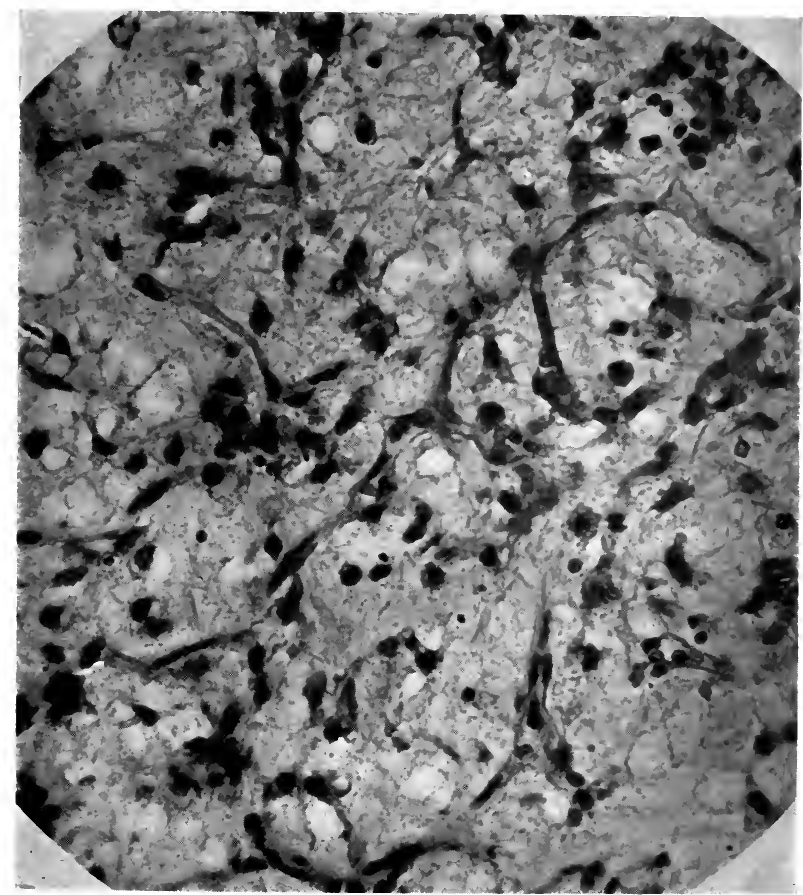

FIG. 349.-Dissemination of epithelial cells in mucinous stroma of a mixed tumor of parotid.

is also less clearly connected with the normal gland structures than is the adenomatous. The theory of branchial origin seems to cover this deficiency and deserves careful consideration. The grounds of this theory have been stated by Hinsberg, Cuneo and Veau, Chevassu, and others, and attacked by Forgue, Vialleton, and Massabuau. Chevassu has shown that branchial cysts may contain pharyngeal epithelium and salivary glands, which furnish a satisfactory origin for some of the salivary tumors. An inclusion of fragments of the aural cartilages is strongly suggested for certain tumors which early involve the ear. Weisshaupt thinks that the tumors which are sharply separated from the gland tissue probably arise from an embryonal diverticulum of the parotid duct, which regularly exists in man.

The position of some tumors, attached to rather than lying within the 
gland, is readily explained by the branchial theory. The occurrence of much striated muscle tissue in a mixed parotid tumor, described by Prudden, shows that an extensive local malformation was

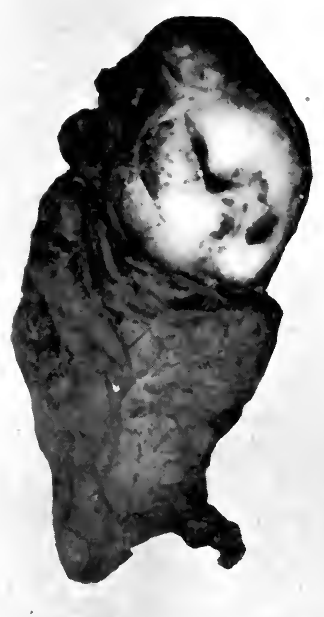

FIG. 350.-Early encapsulated mixed tumor of parotid.

concerned in its origin. The epithelial structures in this tumor were, however, quite peculiar. Of similar significance is a congenital tumor of the usual type associated with an accessory tragus and auricular fistula, described by Wood. On the other hand, the wide distribution of the tumors in lips, cheek, and palate is not easily adjusted to an exclusive branchial origin, but suggests rather that the conditions of their origin are fully provided in salivary and mucous glands.

The view expressed by Forgue and Massabuau that the tumors are true embryomas is without adequate foundation. Teratomas of the neck are reported by Weyl, Poult (Lit.), McGregor and Workman, Ficheaux, and others, but these tumors were quite different from the common mixed growths.

The present status of our knowledge of the origin of mixed tumors of the salivary glands may be summarized as follows:

(I) The endothelial origin has been disproved.

(2) No single source of the mixed tumors meets all the requirements. Some are distinctly adenomatous and probably arise from the acini and ducts of the gland in which they are well incorporated. Others are encapsulated

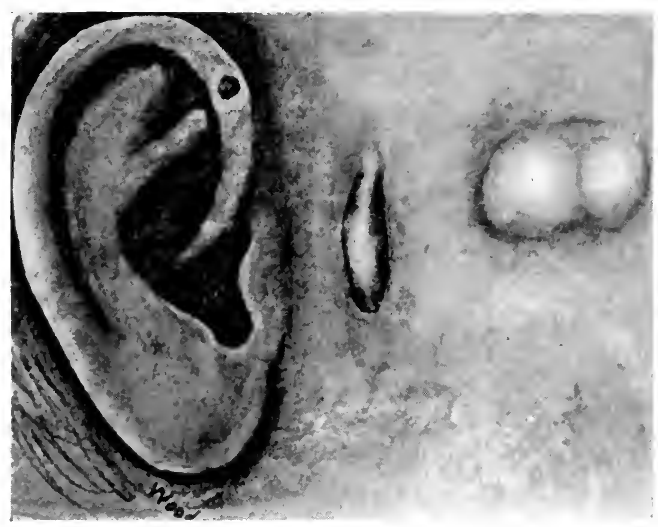

FIG. 351.-Parotid tumor with congenital malformations of ear and face (accessory tragus and auricular fissure). (After Wood.)

or extraglandular and take the form of basal-cell or adenoid cystic epithelioma. These probably arise from misplaced and occasionally embryonal 
portions of gland tissue. Branchial remnants may possibly be connected with this group.

(3) The derivation of mucous tissue and cartilage from gland epithelium has been satisfactorily proved and there is no necessity of including in the originating tissue any cartilaginous structures.

General Etiology.-Epithelial mixed tumors of the salivary glands are comparatively common, and when all the subvarieties occurring about the pharynx and face are included they become quite numerous. The literature contains many reports, each covering from 30 to 60 cases.

They occur at all ages, Pailler observing one case in a girl at I I months, and another at 73 years. Wood reports a rapidly growing mixed parotid tumor at 7 months and Wagner a mixed sublingual growth in an infant of I2 weeks. While thus frequently observed in younger subjects, the majority occur between 20 and 40 years. Of 50 submaxillary tumors Chevassu found I4 occurring between Io and I9 years and I4 between 20 and 29 years. In several cases a quiescent or slowly growing tumor had existed from birth. Both sides and both sexes are about equally affected. According to Bohme and Kuttner the parotid furnishes about 90 per cent. of the tumors, the submaxillary ro per cent., and the sublingual I per cent., but this proportion of parotid growths is probably too high. Most tumors in the floor of the mouth are probably derived from aberrant portions of the submaxillary (Ehrich). In the lacrimal gland Warthin collected $\mathrm{r}_{3}$ cases reported as adenoma and 5 as carcinoma. Verhoef records five mixed tumors of the lacrimal gland. If one includes all the deep orbital tumors of basal-cell and mixed types, this group at once becomes numerous.

In the cheek and lips characteristic basal-cell or mixed tumors are described by Wood, Sempnooff, Krompecher, Lenormant, and Collet; in the buccal mucosa and vault of palate by Laraberie and Bergem; in the antrum of Highmore by Marchand and Gutekunst; in the nares by Dembrowski; in the tonsil and alveolar border by Krompecher. Few of these miscellaneous tumors duplicate the complex structure of the salivary growths. Most of them are more simple and fall in the group of adenoid cystic epithelioma or cylindroma.

Clinical Course.- - In the great majority of cases a quiescent tumor has long preceded the development of an active growth. Very often the quiescent nodule was observed for 8 to ro years, Pailler reporting an inactive period of 37 years and Wood 53 years. Trauma rarely appears as a possible factor. Once established, active growth proceeds slowly, but varies with the histological type. The average duration of operative cases is about 8 years. A limitless growth is by no means a necessary attribute. Thus in Martland's case, after 2 years' active growth, the tumor remained stationary for I3 years, the patient dying from phthisis. This fact has a bearing on the therapeutic results of radium, which is of much value in this group of tumors, as well as on the wisdom of surgical removal, which is often followed by recurrences of increasing malignancy. On the other hand, some old tumors becoming active soon infiltrate the capsule and gland and even invade the lymphnodes in a few months (Wood, Case IV). There are some indications that the addition of malignant properties may come from some secondary neoplastic process established in the adjoining gland, or by perforation of the tumor capsule. There are thus very wide variations in the clinical behavior of the tumors. Occasionally the growth is steadily progressive from the first. After surgical interference encapsulated growth do not, as a rule, recur, but others are very prone to return at once or after an average interval of $2 \frac{1}{2}$ years (Ehrich) or as late as 9 years. In Wood's 37 cases there is a record 
of $\mathrm{I} 7$ recurrences, 45 per cent.; while I 2 cases were checked by a secondary operation, and 20 (55 per cent.) were permanently cured. Successive recurrences with many operations have extended over a period of 20 years (Wood) and 23 years (Billroth). In the recurrent tumors the structure may remain constant, but more often it becomes increasingly cellular, atypical, and malignant.

Undisturbed tumors rarely invade the lymph-nodes, but after unsuccessful operation, while the recurrences are usually local, the cervical nodes may be progressively involved. In the case of Barozzi and Lesne both cervical and tracheal nodes were invaded by an epithelioma with mucous stroma. I have observed soft cartilage in the supraclavicular nodes. In the case of Griffini and Trombetta a chondrocarcinoma invaded cervical and bronchial nodes, lung, and pleura, with carcinoma in the nodes and cartilage in lung

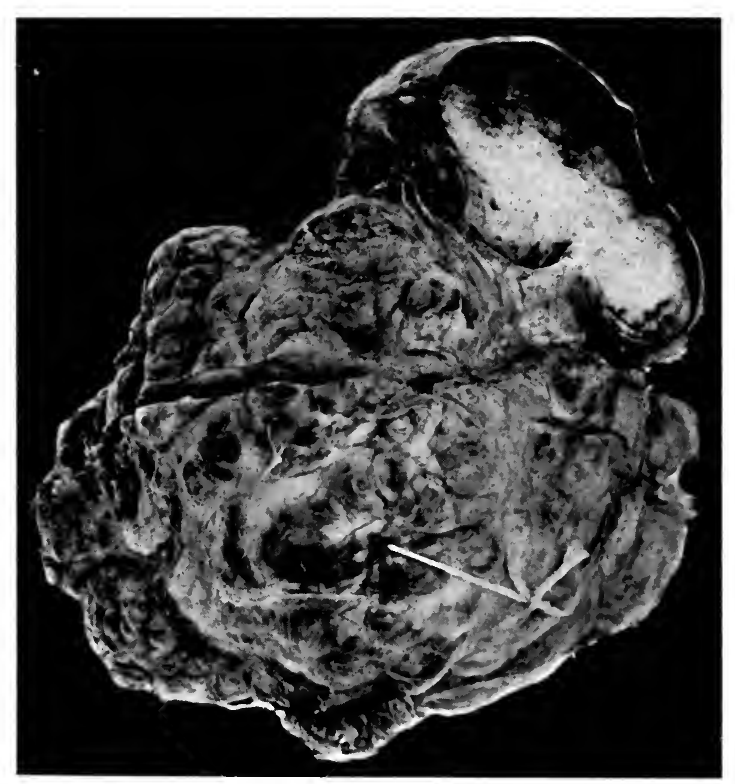

F1G. 352.-Mixed tumor of parotid apparently involving the entire gland.

and pleura. Very cellular growths tending toward the carcinomatous or so-called sarcomatous types may generalize. Wide local extensions and displacement of surrounding structures marked the natural history of a prolonged case of adenomyxoma of parotid, which I studied at autopsy. The growth occluded the pharynx, eroded the superior maxilla and orbit, caused extreme exophthalmos, and displaced the inferior maxilla after ro years' duration in a girl of 20 years.

Gross Anatomy.-Beginning as a small firm nodule in or upon the salivary gland, the tumor usually grows within a capsule until it reaches considerable size. Fusion with the septa of the gland is commonly observed and in lobulated tumors the dividing trabeculæ seem to be continuous with the interlobular septa. While small tumors may be completely separable, larger growths show increasing fusion and some are fully incorporated with the gland from the first. It is usually impossible to excise the tumor without 
including fragments of the gland. Nasse, Ehrich, and Krieg found multiple early tumors in the same gland. The location of the tumors is not always in obvious connection with any gland. They may first appear in the retropharyngeal region or in any part of the lateral wall of the pharynx or floor of the mouth. Some develop along Steno's duct, others appear below or behind the ear and seem to involve the aural cartilages, while a few are found well below the submaxillary. The consistence is usually very firm or hard, but cellular and mucoid growths may be soft and elastic. Small cysts are frequently seen and rarely the tumor consists of one large cyst. Cross-section reveals opaque cellular areas which may be vascular, softened gelatinous masses exuding mucus or serum, hard fibrous or hyaline zones, or areas of cartilage and rarely of bone. Few of the tumors are free from the evidences of mucous secretion. Predominance of one element may give nearly pure myxoma, chondroma, fibroma, carcinoma, or sarcoma.

Structure.-While the majority of the tumors are of rather uniform type, the variety of structures to be included in this group is very great. Although the entire series is connected by transitional cases a serviceable classification may be given as follows:

(I) Myxochondrocarcinoma.

(2) Basal-cell carcinoma with hyaline stroma.

(3) Adenoid cystic epithelioma.

(I) Myxochondrocarcinoma.-The majority of the tumors present broad areas or narrow zones of epithelial cells which surround and fade into masses of mucoid, connective, or cartilaginous tissue. The epithelium shows all gradations from adenomatous alveoli derived from the glandular acini and duct, to solid masses or narrow strands of small flattened compact epithelium of basal type. The transformation of gland structures into tumor tissue can at times be fully traced and has been described in great detail by Pailler, Ehrich, and others. As previously stated these appearances represent the mode of extension rather than the necessary mode of origin of the tumor. Small alveoli lined by cubical cells and filled with dense acidophile secretion are frequently seen, and these tubules often become flattened so as to resemble lymph-spaces lined by endothelium. It is conceivable that such flattened tubules may actually fuse with capillary endothelium, as claimed by Hansemann. The mucoid tissue is in variable quantity and usually most abundant in the central portions of lobules walled by epithelium. Its source is chiefly from the dissemination of epithelium into the swollen interacinar and interlobular stroma which has been infiltrated by mucous secretion of the epithelium. Into the composition of this tissue all preëxisting ibroblasts and endothelium are entitled to enter, but appearances indicate hat the epithelium plays the predominant part. The fully formed cartilage ells must be regarded as derivatives of the epithelium. In the fibrous or nucinous matrix many collagen and elastic fibers may form, but it is difficult o trace their origin. Many foci of lymphocytes or areas of diffuse lymphoid issue may be found in the stroma. Collections of fat-cells are rather freluently included in the tumor, but fail to show neoplastic growth.

Squamous cells appear in many tumors, and exhibit fibrillation, interellular bridges, pearl formation, and keratohyaline granules. They were ound in I4 of Ehrich's 26 cases. Glandular acini may predominate and everal authors have found chondro-adenoma in one portion, with pure denoma in other parts of the same tumor. In slowly growing tumors the pithelium may almost entirely disappear, leaving nearly pure myxoma or hondroma. Peritheliomatous structures may be found in the original tumor $r$ develop in recurrences, and it is probable that many angiosarcomas of 
the salivary glands develop in this way, since some myxocarcinomas are vascular.

Yet $\mathrm{v}$. Haberer has described a large hemangioma simplex of the parotid, and more cellular or slightly sarcomatous growths of this type are reported by Hartmann, Herxheimer, and others. In true angiosarcomas of the salivary glands there is an obvious circulation of blood through the tumor units. Lymphangiomas of the parotid or submaxillary, usually congenital and once symmetrical, have been described by Lannelongue and Achard, Robinson, Fuhr, and Hagenbach, but they suggest no relation to the atypical mixed tumors.

In recurrences the original structure may be retained, but the secondary growth is often more cellular and malignant. Thus spindle-cell, round-cell, and perithelial sarcomas have been observed after extirpation of chondro-

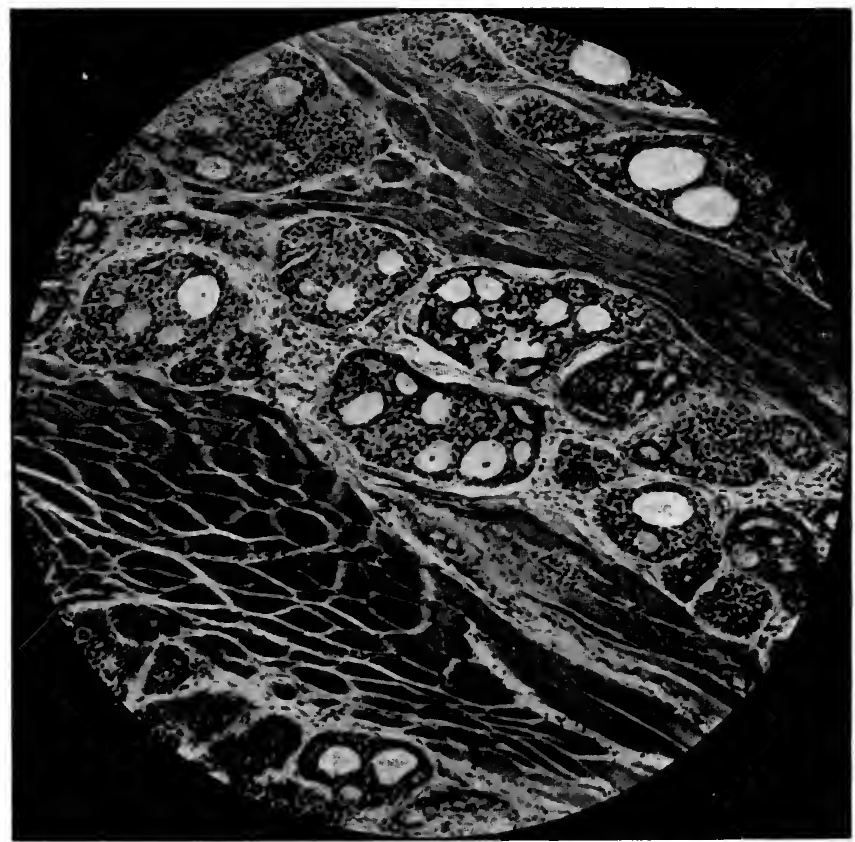

FIG. 353.-Adenocarcinoma of parotid infiltrating sternomastoid muscle.

carcinoma, and it seems probable that most of the so-called sarcomas of the salivary glands are atypical epithelial growths (Menetrier, Chevassu, Wood). Rarely these large cell sarcomas are pigmented, but they lack the malignancy of true melanoma. The more cellular structures of the original tumor may exclusively appear in the recurrence. Forster reports a tumor containing mucoid tissue and cartilage in one area, and sarcomatous tissue in another, the latter alone appearing in the recurrence. In several cases only carcinomatous portions of the original tumor appeared in the recurrence (Landsteiner, Volkmann, Case XVII). In some cases the recurrence probably represents the development of a new tumor in the remaining portions of the gland.

(2) Basal-cell carcinoma with hyaline stroma is the type observed in many small early tumors, it forms the bulk of a minority of the larger growths 
and it appears frequently in tumors outside the salivary glands. This structure may be uniform throughout, or associated with chondrocarcinoma or adenoid cystic structures, but squamous changes are rarely added.

The cells are small, cubical or spindle, darkly staining, with hyperchromatic nuclei, and arranged in narrow anastomosing cords, or thin flattened strands. They are often intimately fused with the hyaline stroma and appear to inclose spaces like the endothelium of lymph-spaces (Krompecher, l. c., Figs. 12, I3, I6). The hyaline stroma appears in narrow strands or large cords or rings inclosing the cells, and is quite variable in bulk.

(3) Adenoid cystic epithelioma observes the same distribution as the other basal-cell types. It usually presents a specific structure which has long been passed as cylindroma. Throughout broad coherent masses of darkly staining cubical or polygonal cells appear small droplets or globules or larger rounded masses of mucus which are sharply outlined against the surrounding epithelium. This material may contain much or little mucin. Its sources are two, first, a secretion of the epithelial cells, when it appears in broad rounded cellular areas inclosed in dense connective; and, second, from mucous degeneration of fine strands of stroma originally supporting the epithelial cords. When these tumors are entirely free from myxomatous and chondromatous areas they are properly classed with the pure adenocarcinomas $(q . v$.$) .$

In the submaxillary gland simple benign epithelial tumors are extremely rare.

Malignant dendritic adenocarcinoma arises from the ducts and produces a firm circumscribed tumor which, in the case of Prengrueber, involved only a portion of the gland. In Chevassu's case the tumor, although encapsulated, recurred promptly after two operations.

Infiltrating carcinomas of tubular or alveolar structure arising from the acini, and proving quite malignant, are described by Waldeyer, Volkmann, and Chevassu. 


\section{CHAPTER XXXVII}

\section{TUMORS OF KIDNEY}

\section{EPITHELIAL TUMORS OF KIDNEY}

While indications of the structure of epithelial tumors of the kidney appear in the early descriptions of J. König, Rayer, and Cruveilhier, the first definite success in tracing the epithelial origin of these tumors was accomplished by Robin in I855, who showed that the tubular epithelium proliferated, destroyed the membrana propria, and produced cancerous nodules. Waldeyer in I867 also traced the beginnings of renal cancer to proliferating cells of the renal tubules. The validity of these observations, which today may be questioned, was accepted by Klebs, Recklinghausen, Lancereaux, and by writers generally, who classified the tumors as circumscribed benign adenoma and malignant infiltrating carcinoma. Ebstein as late as 1877 evidently identified the sarcomas of infants with the carcinomas of adults. More specific details of the origin of renal tumors were obtained by Sturm, I875, who distinguished between solitary adenoma of normal kidneys and multiple adenoma of sclerotic organs, and recognized the slow transformation of adenoma into carcinoma. Sabourin described two forms of multiple adenoma, with lining of cubical or cylindrical cells, and compared these small tumors with multiple adenomas of the liver. In I 883 Weichselbaum and Greenish designated the two forms of adenomas as papillary and alveolar.

Up to this time the epithelial origin of the small tumors was regarded as proved and their transformation into the common malignant tumors appears to have been accepted, while the existence of a separate group of yellowish tumors of the cortex, of fatty nature, was recognized. In 1883 Grawitz contested the lipomatous nature of the small yellowish tumors, showed that their structure was often identical with that of the cortical hyperplasia of the adrenal (Virchow's "struma suprarenalis"), proposed for the renal tumors the term "strumæ suprarenales aberratæ renalis," established the occurrence of adrenal rests in the capsule and cortex of the kidney, and traced the origin of an important group of malignant renal tumors to these rests. Grawitz's interpretation was supported and elaborated by many later authorities.

Beneke traced the origin of certain sarcomas of the kidney to adrenal cells. In 1892 Birch-Hirschfeld introduced the term "hypernephroma" for the adrenal tumor of the kidney. By some the scope of this group has been regarded as including the majority of renal tumors and structures of various types, and even the papillary growths (Horn). Sudeck in I893 contested the adrenal theory, holding that the papillary and alveolar tumors are essentially the same and that the majority of the hypernephromas are renal adenomas. Stoerk has adduced considerable evidence to show that the identification of adrenal rests from fatty renal epithelium may be difficult and has been too hastily undertaken; that renal epithelium may undergo marked fatty changes, especially in the adenomas of sclerotic kidneys; that papillary structures and lumina so common in hypernephroma are rare in tumors of the adrenal itself; and that many of the features regarded as typical of hypernephroma are reproduced in renal adenocarcinomas. 
The discussion of the adrenal theory has also been carried through phases of chemistry, comparative pathology, and embryology without reaching a final solution, but tending, on the whole, to restrict the Grawitz tumors to a relatively small group in which the structure is exactly duplicated by tumors of the adrenal gland itself.

Epithelial tumors in which the cells are grouped about blood-ressels have been erroneously interpreted by Paoli as angiosarcoma, and by Driessen, Hildebrandt, Hansemann, and others as endothelioma. Manasse, in a comprehensive study, recognized many varieties of renal growths, including renal adenomas and carcinomas, adrenal tumors of various types, endotheliomas from both blood- and lymph-vessels, and various sarcomas of mesoblastic origin. Birch-Hirschfeld's group of adeno-angiosarcoma, derived from Wolffian remnants, and the lipomyosarcomas remain, however, the only well-defined varieties of renal sarcoma which have been fully divorced from a probable epithelial origin.

Classification.-Four groups of epithelial tumors of the kidney require recognition:

I. Adenoma, single and multiple, essentially benign, and arising from renal tubules.

2. Adenocarcinoma and carcinoma, arising from renal tubules, and often from adenomas.

3. Papilloma and papillary carcinoma of the pelvis.

4. Adrenal tumors. Hypernephroma.

\section{ADENOMA}

Renal adenomas appear first as single or multiple tumors as large as a pea or bean in the cortex or capsule of the kidney, where they are easily detected as gray, white, or yellowish nodules. The multiple tumors are usually seen in sclerotic kidneys in which they may be very numerous, while in normal kidneys the nodules are usually single. The great majority never pass beyond a small size, but tumors as large as an egg and of cystic character are occasionally observed, and some becoming malignant progressively increase in size. Nobiling refers to a case in which one kidney was the seat of miliary cystadenomas, while the other presented a Grawitz tumor. The frequency of these tumors is variously estimated. Zehbe collected 250 small tumors, of which 40.5 per cent. were solid, 59.5 per cent. papillary or alveolar. They are more frequent in elderly subjects and Weichselbaum and Greenish found them in ro per cent. of all persons over 70 years of age.

The structure of small adenomas presents several distinct varieties, some of which appear to be very similar to adrenal rests.

A papillary structure, occurring in a small cyst and exhibiting papillary characters from the first, has been generally accepted as indicating a renal origin. The papillæ are composed of thick branching strands of connective tissue lined by one layer of cubical or cylindrical epithelium. Or the connective-tissue strands are thin and vascular and the epithelium more abundant. Sabourin pictures papillary adenomas consisting chiefly of convoluted layers of granular epithelium with very little stroma.

This group of tumors represents the renal form of cystadenoma arising in organs which are the seat of chronic inflammation with the production of cysts. Along with the tumors cysts without papillary ingrowths are often seen. The small growths usually present a single cavity, while the larger ones may be multilocular, as in Edmund's case. The tumors may reach the size of a child's head while retaining a benign papillary structure (Scudder). 
There is every reason to believe that the cells of origin are the adult epithelium of the affected tubules. While the lining cells are often granular and opaque, it is important to note that very early papillary tumors may contain large clear cells containing fat and doubly refractive lipoid material (Stoerk, Zehbe). Progressive growth and malignant transformation of these papillary cystadenomas has repeatedly been observed, and it is probable that some papillary adenocarcinomas of the kidney arise from such benign lesions (Sturm, Beneke).

An alveolar structure in early renal adenomas has been described by Klebs, Sabourin, Weichselbaum and Greenish, Sturm, Stoerk, Zehbe, and others, and there is little doubt that true renal adenomas may follow this type. The cells are cubical or cylindrical and of large size. They are often very fatty and fail to stain with eosin. Yet in a group of solid nodules, described by Zehbe, the cells are granular and free from fat. When the cells

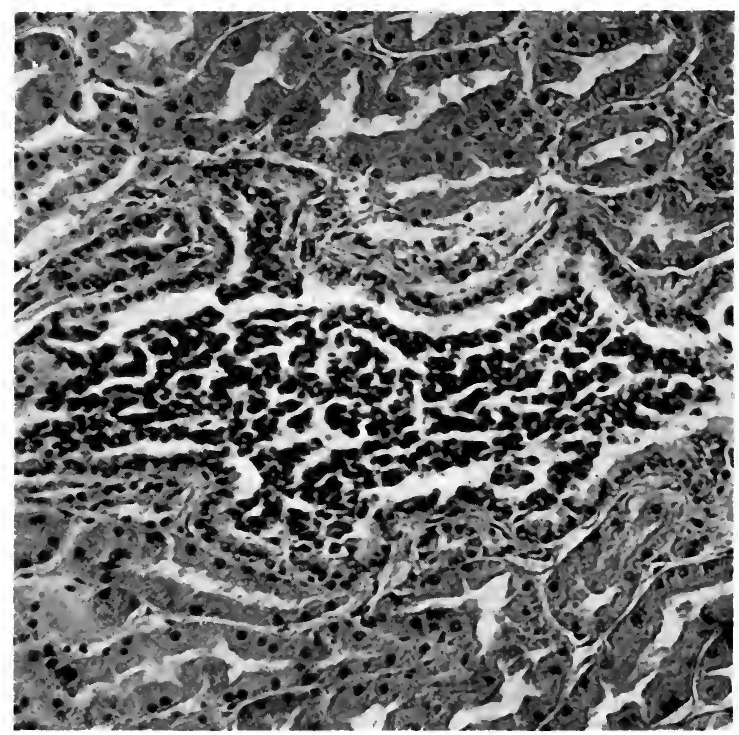

FIG. 354.-Group of isolated embryonal tubules in cortex of a normal kidney. A probable source of renal carcinoma.

contain much fat it becomes very difficult to distinguish such small tumors from adrenal rests, so that there has been much conflict of opinion regarding the nature of this tumor. Stoerk traces the development of the alveoli from convoluted tubules with coincident transformation of opaque granular epithelium into swollen fatty cells. Since the typical adrenal rest fails to show lumina surrounded by cells, the uniform presence of such spaces in the cell groups of small renal tumors must stand strongly in favor of their renal origin.

The fate of the alveolar adenomas has not been satisfactorily determined. Many long remain of small size. The development of a progressive and malignant process would produce a tumor of adenocarcinomatous and alveolar or papillary type with clear cells, resembling certain hypernephromas. This relation is claimed by many, but still remains to be definitely proved.

Some of the large congenital adenomas of children described by Weigert, 
Targett, Kelynack, and others presented a purely alveolar arrangement, suggesting a relation to the small alveolar adenomas of the adult.

Tubular adenoma is a form of renal tumor described by Ricker, and composed of elongated and irregular canals lined by small cells free from fat and consisting chiefly of nuclei. Similar structures appear in some of Sabourin's cases. The stroma is abundant and vascular. The peripheral alveoli may pass gradually into the surrounding renal tubules, while in the central portions the tubules are wider and may become cystic.

In the histogenesis of renal adenoma two conditions appear to be chiefly concerned. In the group of cystadenomas in sclerotic kidneys the tumors arise in cysts resulting from the sclerotic process. The character of the cell lining may be assumed to vary with the location of the cysts, which affect tubules of all types, including especially the convoluted and collecting tubules. Single tumors in otherwise normal organs may be attributed to localized cicatrices.

Congenital anomalies in the structure of the organ are probably connected with the origin of certain adenomas, especially those of embryonal type with small cells, some cystic tumors, and the tubular adenomas. The occurrence of a variety of structural defects in the kidney is fully attested, but few of them have actually been traced into tumor development. Displacement and imperfect development of renal tubules has been described by Albarran in the renal cortex and capsule. Luzzato and Antona have traced the development of adenoma and adenocarcinoma from such pararenal rests. In Antona's case the tumor lay outside the kidney. Beneke and Ulrich identified renal tubules in the adrenal, and Ricker found such islands of renal tubules in adrenal connected with the kidney by strands of connective tissue. Since the renal tubule

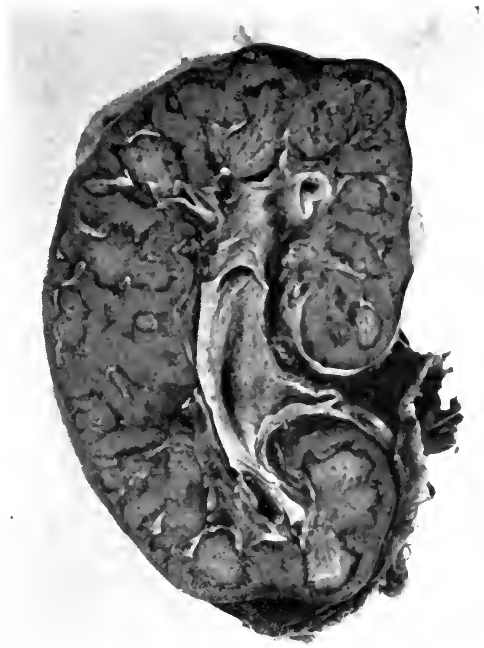

FIG. 355--Congenital malformation of kidney of newborn infant. The organ is enlarged and the cortex everywhere the seat of superfluous undifferentiated tubules.

is formed of two originally separate portions, secreting and discharging, which later fuse, there is abundant opportunity for the miscarriage of this process, resulting not only in the congenital cystic kidney, but in isolated cysts which give rise to cystadenomas. Nauwerck and Hufschmidt and v. Kahlden have pointed out the probable relation between the cystic kidney and some cystadenomas. The case of Keyes in which the entire kidney was the seat of very numerous solid adenomatous nodules as large as $i \frac{1}{2}$ $\mathrm{cm}$. recalls the distribution of multiple congenital cysts. Microscopic islands of renal tubules lined by small densely staining cells are not infrequently encountered in normal kidneys.

Under the term "spotted kidneys" Guillebeau and Vaerst have described in the cow, and Schmey in the horse, characteristic wedge-shaped areas in the enal cortex which they attribute to imperfect development of the cortex. They resemble adenomas, but are properly interpreted as hamartomas, in he sense of Albrecht. They are not associated with nephritis. E. Meyer 
describes a spotted kidney in a child, the areas involving both cortical and medullary tissue, with defect in the convoluted tubules. R. L. Thompson found in a 2-year child a hyperplastic pyramid adjacent to a normal cortical area. In the intermediate zone the structures were greatly disarranged. In a well-developed but stillborn infant with large cyst of umbilical cord I found both kidneys much enlarged. The renculi were of unequal size and irregularly placed. The cortical tubules were deficient and many glomeruli immature. Many tubules, probably the collecting, were of embryonal type lined by very large cells with hyperchromatic nuclei, and appeared in numerous foci throughout the organ, resembling miliary fetal adenomas (Fig. 355).

\section{CARCINOMA}

Malignant tumors of renal epithelium appear in two main forms: Papillary adenocarcinoma, and (2) alveolar carcinoma.

The structural subvarieties of these main groups are rather numerous and constitute a field still awaiting investigation from the histogenetic and clinical standpoint.

In general it may be stated that the two forms of carcinoma accord with the two chief groups of adenoma from which many of them arise. The cystadenomas form a continuous series from the simple benign growths up to highly cellular and malignant tumors. This group includes many vascular growths with clear cells, which are often confused with hypernephroma. The relation of the true alveolar carcinoma to alveolar adenoma is less distinct. These tumors are less frequent than the papillary type and many of them are of embryonal origin.

Papillary Adenocarcinoma.--These tumors are much the most frequent of renal growths. They occur chiefly in adults, but occasionally in children. Most of them are recorded in the literature as hypernephroma, so that statistical data regarding them are scanty and unreliable. For general clinical information the reader may refer to the elaborate monograph of Albarran and Imbert. The group includes two well-defined varieties and one small subgroup of minor importance: (a) Papillary adenoma and carcinoma with clear or glassy cells; (b) Papillary adenocarcinoma or carcinoma with granular cells; $(c)$ Malignant tumors arising from simple cystadenoma.

Of these tumors the first (a) produces large single, less often multiple, yellowish circumscribed vascular or hemorrhagic tumors, composed of villous or thin papillary strands of connective tissue, lined by one layer of cubical or cylindrical clear fatty epithelium. These tumors are often confused with hypernephroma, but their pronounced papillary structure and the occasional presence of areas containing characteristic renal epithelium free from fat, usually permit their recognition. Characteristic cases are described by Kelynack under the term "malignant cystadenoma." The necessity of distinguishing this renal tumor from others and of separating it especially from hypernephroma is recognized by most recent writers (Albarran, Stoerk, Ipsen, Willson, and Willis).

The second form $(b)$ appears as multiple, solid whitish and cellular growths, less distinctly encapsulated, commonly free from hemorrhage, and composed of numerously branching strands of connective tissue, lined by one or several layers of opaque, granular epithelium, free from fat.

The third group $(c)$ includes cystadenomas which have become malignant.

It must be admitted that the character of the cytoplasm of the tumorcells is an uncertain basis for classification. Stoerk has shown that fatty changes occur readily in foci of small renal adenomas in which the cells are 
mainly granular. Zehbe finds that several types of renal adenomas present, indifferently, clear or granular cells. In a tumor composed chiefly of small alveoli lined by low opaque cells I have found sharp transitions to papillary adenoma with clear cells. It is probably impossible to establish a markedly different histogenesis for the two types. Nevertheless the above cases are exceptions to the rule that tumors with clear cells and those with opaque granular cells arise under different conditions and yield peculiar gross as well as microscopical appearances.

(a) Papillary Carcinoma with Clear Cells.-The gross appearance of the papillary carcinoma with clear cells is usually characteristic. The tumor

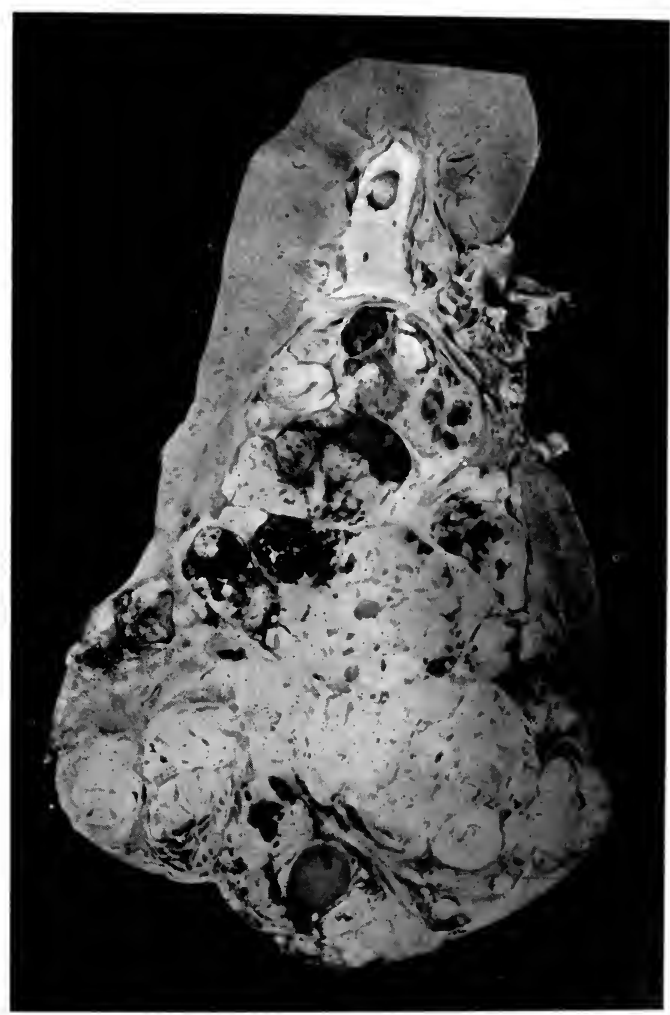

FIG. 356.-Circumscribed papillary and cystic adenocarcinoma of kidney.

begins as a small circumscribed cortical or medullary solid nodule of light yellow color and considerable vascularity. The growth commonly reaches considerable dimensions and when encountered in surgical material it is as large as a hen's egg or the fist and lies within the renal capsule which is distended. On section portions of unaltered or thinned renal tissue appear at one or several segments of the mass. Of I94 renal tumors chiefly of this type, Kuster located 80 in the middle segment, 60 in the lower pole, and 54 in the upper pole. Ipsen, who recognized the renal origin of his tumors, found I 3 in the upper, ro in the middle, and 12 in the lower lobe. The gross structure is divided into many small cysts containing clear or brownish or bloody 
or gelatinous material. Areas of necrosis and hemorrhage may be frequent and these may fuse, yielding large cystic cavities. There may be considerable calcific deposits. As a rule, these tumors are not encapsulated.

From the presence of numerous small cysts with gelatinous contents, gross evidences of a papillary architecture, and a greater tendency to invade surrounding renal tissue, these tumors can usually be distinguished by the naked eye from tumors of adrenal rests.

In advanced and fatal cases the tumors become very large, destroying most of the kidney and undergoing further necrosis, hemorrhage, and cystic softening. They extend by continuity to the kidney, renal pelvis with hydronephrosis, adrenal, lymph-nodes, and abdominal wall, and they invade the renal and other veins often very early. In the renal vein they produce tumor masses reaching through vena cava to the right heart (Coyne, Troisier, Wyler, Gardner, and Coats). Yet invasion of the veins is not always followed at once by metastases, and Albrecht records recovery after extirpation of such a tumor. Stoerk noted that the histology is not always a safe guide to the

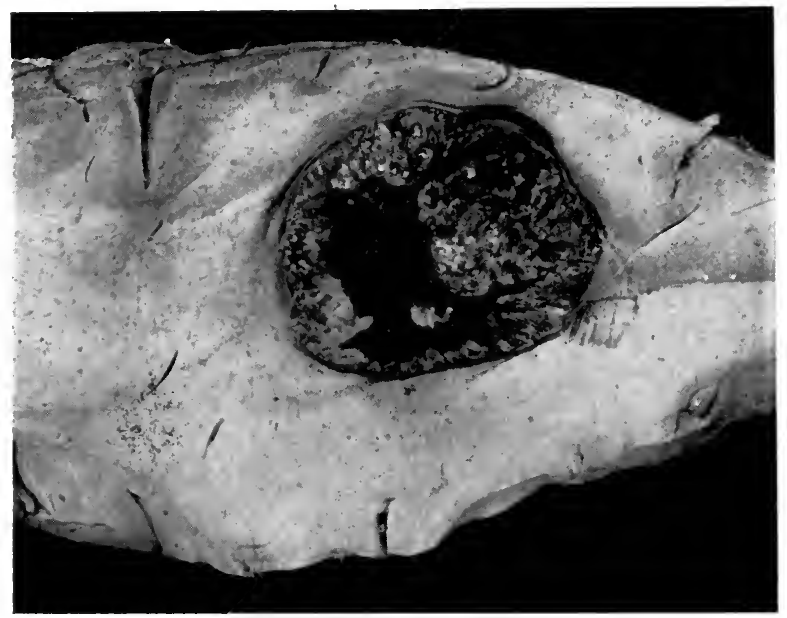

FIG. 357.-Adenocarcinoma of kidney metastatic in brain.

viability of the cells, some apparently simple structures proving exquisitely malignant.

Israel, in I8 cases of "hypernephroma," observed characteristic fever curves, remittent and intermittent, initial, intercurrent, and terminal. $\mathrm{He}$ states that fever occurs in all malignant tumors of the kidney without relation to histological structure, size, rapidity of growth, or invasion of vicinity. Cabot records high leukocytosis in several cases.

Metastases are very frequent. Albrecht found the lungs involved in all his advanced cases. Albarran, in 249 collected cases of renal carcinoma, found the lungs involved in 75 , liver 71 , lymph-nodes 60 , renal vein and cava 23 , pleura 14 .

The frequency of bone metastases has long been recognized. In most reports it has been assumed that the tumors arose from adrenal rests, but it is probable that most of these cases were renal carcinoma with clear cells. $\mathrm{P}$. Albrecht found bone metastases in 8 of I4 fully studied cases of hypernephroma. They occur chiefly in ribs, spine, skull, scapula, and long bones. 
They are sometimes mistaken for primary tumors or other diseases of the bones, the renal tumor being discovered only late in the disease or at autopsy. In a well-known group of cases a solitary bone-tumor arises from a comparatively small renal growth. Such tumors have been successfully extirpated, the patient succumbing later to the renal disease.

Late bone metastases, occurring 5, 8, 9, and even Io years after removal of the primary tumor, are also recorded (Clairmont, Albrecht). The slow development of the tumor-cells may be referred to the early invasion of veins with accompanying phlebitis which for a time blocks their further transport. The structure of the bone-tumors often reproduces exactly that of perivascular or papillary adenocarcinoma, but giant-cells have been found in very large numbers on the advancing edge of the tumor, while occasionally the secondary growth is much more atypical and malignant than the primary. Osteoplastic tendencies were noted by Albrecht.

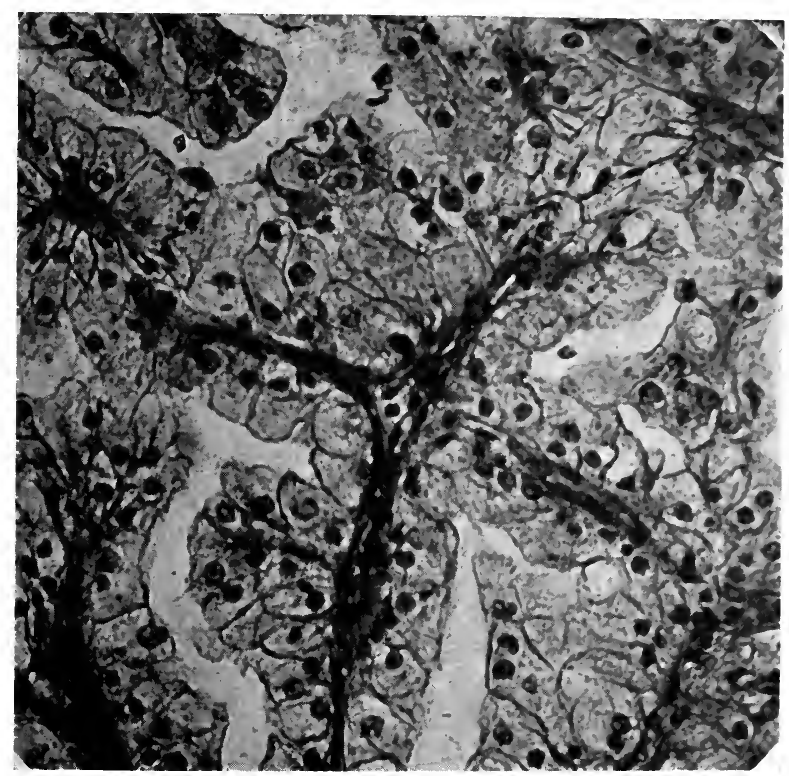

FIG. 358.-Papillary carcinoma of kidney with clear cells.

The structure of the primary tumor is usually papillary. In some cases there are cross-sections of very numerous small blood-vessels surrounded by one layer of larger glassy epithelial cells. In other cases the trabeculæ are long and thin, covered by one layer of high cubical or cylindrical epithelium resembling those of the collecting tubules. Adjoining trabeculæ may seem to inclose long sections of tubules. Rarely an alveolar appearance is produced. In a third group the lining cells appear in several layers, the cells are extremely large and elongated and the layers of adjoining trabeculæ fuse. Yet a diffuse carcinoma of this type probably does not occur. Even in metastases the papillary character and the glassy appearance of the cells are retained. Albarran has described this tumor under the term adenoma alveolare a cellules claires. Sabourin groups it with the papillary growths. Sudeck reported several cases, but failed to distinguish them clearly from 
adrenal growths. Ipsen describes papillary, alveolar, and solid structures and concludes that all the tumors are essentially papillary. The structure often resembles some cases of hypernephroma of perithelial type, but careful study of different sections reveals a predominant papillary and alveolar type. In hypernephroma the cells form thick anastomosing sheets or columns between which lie many dilated blood-vessels. The presence of alveoli filled with mucous secretion, or with basic staining concentrically striated globules, also belongs to renal adenoma.

In very cellular growths nutrition is poor, the cells may become widely distended with fat and watery fluids, and giant-cells may form. Appropriate stains reveal the presence of much glycogen, fat, and lipoid material, but in very variable amounts.

The study of the chemistry of these tumors has been pursued elaborately by Lubarsch, Sisson, Stoerk, Ipsen, and others, but it does not appear that a differential diagnosis can be established on the character of the lipoids. The glassy cells may be almost entirely free from fat, or very large deposits of material reacting to osmic acid or sudan III may be present. Glycogen is usually abundant, but sometimes missing.

Ipsen found that the renal tumors yield about 10.7 per cent. of fat and 0.2 per cent. of phosphorus, while the horse's adrenal contains 30.8 per cent. of fat and I per cent. of phosphorus. The lipoids are cholesterin esters.

Histogenesis.-Papillary carcinoma with clear cells has been subject to several different interpretations. The older authors uniformly regarded it as of renal origin, and this view is maintained perhaps by the majority of recent authors. With the advent of Grawitz's theory these tumors were frequently classed as hypernephroma because of the clear cytoplasm of the cells. The pronounced papillary character appears to the writer as incompatible with an adrenal origin, although contrary to Stoerk, it must be admitted that adrenal tumors occasionally present a certain papillary tendency. Strong evidence of the renal origin of the growths is the occasional presence of tubules reproducing the exact features of hypertrophic renal tubules, which are not observed in tumors of proved adrenal origin. The adrenal tumors which simulate papillary carcinoma most closely present a pseudopapillary structure and are composed of thin but usually solid anastomosing columns of clear cells supported by thin vascular septa.

Papillary carcinoma with clear cells has also figured as endothelioma and as angiosarcoma in the reports of Driessen, Hildebrandt, Manasse, and Hansemann. Yet it is now recognized that the close approximation of large clear celis about the walls of capillaries, or the presence of extravasated blood in the lumina of vascular adenocarcinomas, does not indicate an angioblastic tumor. Many so-called peritheliomas are true adrenal tumors. It is possible also that the intravascular proliferation of endothelium may give rise to new tumors of the kidney, as described by Hansemann under the term "adenoma endotheliale," but there is no satisfactory evidence that these growths may reach the size or follow the course of renal adenocarcinoma.

(b) Papillary adenocarcinoma wilh gramular cells, resembiing those of the renal tubules, occurs under several conditions.

(I) Multiple adenocarcinoma in sclerotic kidneys is one of the best defined varieties of renal carcinoma. It was early recognized and fully described by Sabourin, Klebs, Weichselbaum and Greenish, and Sturm, who encountered chiefly simple adenomas. One or both kidneys may be involved by very numerous small circumscribed light colored tumor nodules which may become so abundant as to leave little intact renal tissue. The organ retains its form and may be only slightly or not at all enlarged. In the early stages 
the tumors may be small and miliary, later they become as large as peas or beans, but bulky tumors are rarely if ever produced.

The structure follows a papillary adlenomatous type with thin strands of vascular connective covered with one layer of cubical or polyhedral, slightly granular cells. Fatty degeneration is not entirely missing, but the abundant large clear cells of other adenomas are not found. In some cases the cell layers are multiplied and malignant features appear. The course of these tumors is slow and they seldom display malignant clinical qualities. In a case of Keyes, Sr., the patient, 49 years of age, complained chiefly of hematuria. The kidney was removed and the disease did not recur. Manasse's 2 cases were found at autopsy.

The multiple tumors have been regarded as the sequel of inflammatory and regenerative hyperplasia. From this point of view the true neoplastic

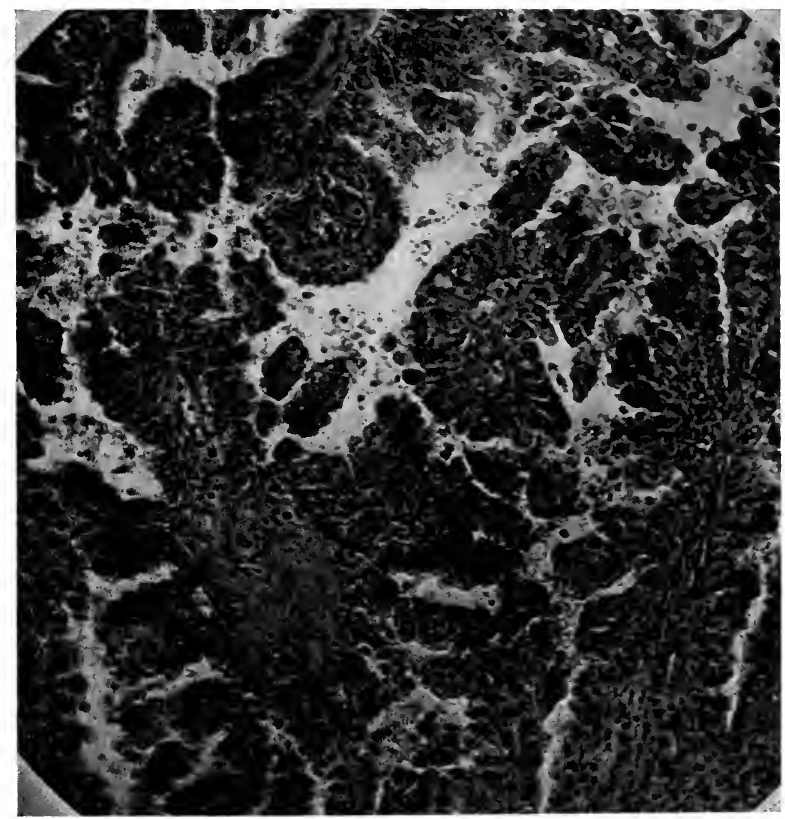

FIG. 359.--Papillary adenocarcinoma of kidney.

nature of the simple forms may be doubted, as done by Sudeck and Hansemann. Yet these growths pass insensibly into true and sometimes active adenomas or adenocarcinomas.

(2) Papillary adenomas and adenocarcinomas with granular cells occur not only in sclerotic kidneys, but frequently in otherwise normal organs. The tumors are single or more often multiple, and appear as solid white masses in cortex or medulla. The solitary tumors may reach considerable dimensions, but the very large growths composed of clear cells are not duplicated in this variety. Fatty changes and hemorrhage are usually wanting, but many of the tumors are locally aggressive and invade the kidney diffuse!y, perforate the pelvis and capsule, and extend into the lymphatics. Invasion of the veins is less prominent than with other renal growths.

On gross anatomical features this group of tumors has often been divided 
into nodular and infiltrating forms, the former being adenomatous, the latter adenocarcinomatous. Yet the transitions are frequent and occur in the same tumors. With increasing malignancy the encapsulation, which is seldom distinct, becomes obliterated. The whole organ may thus be much enlarged while retaining its form.

The structure presents several variations of the papillary type. Usually the connective tissue appears in thin strands, frequently branching, covered by one or more layers of opaque granular cells. The vascularity is much less than in most other renal growths, so that the gross appearance has been described as snow white. The cells somewhat resemble those of the convoluted tubules, but the resemblance is seldom sufficiently distinct to impress such an origin. Nevertheless not a few observers claim to have traced the growth from renal tubules (Waldeyer, Manasse, Stoerk).

In some cases the cells are extremely small, the papillæ minute, and the resulting structure in section resembles in part an alveolar growth and suggests an embryonal origin. Sudeck argued that all the tumors are essentially alveolar. Wiecheselbaum and Greenish found two distinct types with transitions. I have seen rare tumors with both types of structure in adjoining areas, but find that the alveolar growths, as a rule, differ markedly from the papillary, suggesting different conditions of origin.

In the malignant growths the cell layers multiply, adjoining papillæ fuse, and the renal tissue is invaded as papillary, alveolar, or diffuse carcinoma.

(3) The malignant transformation of cystadenoma is described by Klebs, Ziegler, Ricker, and others. These tumors are single or multiple, well encapsulated, and sometimes of large dimensions. The papillary and cystic character is evident on gross inspection, and the cyst may contain considerable fluid with blood and fatty detritus. The capsule may be invaded and the parenchyma may be infiltrated by secondary nodules, or the malignancy may be demonstrated by an unexpected recurrence. The structure presents coarse trabeculæ, or vascular connective tissue, covered by cubical or cylindrical granular or clear epithelium which is usually in a single layer, but in many areas becomes multiple, atypical in form, and produces pseudoalveoli. Characteristic tumors of this type are rare.

Alveolar Adenocarcinoma.-Although the early writers uniformly recognized an alveolar adenoma of renal origin, the advent of Grawitz's theory led many to refer the majority of alveolar growths to an adrenal source. Only a superficial acquaintance with the morphology of renal tumors could favor such an interpretation, since alveolar adenomas form a highly characteristic and important group of renal growths which are wholly different from the adrenal derivatives. In this group also the derivation from preformed renal tubules has been traced by Waldeyer, Manasse, Stoerk, and others.

The structure may almost exactly reproduce that of the renal cortex. Stoerk has shown that regenerating tubules may multiply and ramify, producing localized tumor-like nodules resembling adrenal rests. Most observers have been unable to trace alveolar adenomas to preformed tubules and believe that these growths are chiefly of embryonal origin. In accordance with this view is the fact that the renal carcinomas in children are chiefly of alveolar type. Stoerk found foci of spindle-cells in alveolar adenoma, suggesting a relation to adenosarcoma. Weigert, in a congenital case, observed abortive glomeruli associated with alveolar adenoma. In adults both embryonal and adult types of alveolar adenoma are observed, and the resemblance to adult tubules is occasionally so striking as to indicate that an embryonal origin is not constant. Finally, the islands of displaced renal cortex in the capsule or cortex of the kidney, described by Albarran, were of alveolar type 
and are believed to have given rise to alveolar tumors. Several forms of small adenomas previously described also present themselves as possible sources of larger adenocarcinomas.

The varieties of alveolar adenomas which present sufficiently distinct clinical and structural features to warrant recognition are somewhat numerous. They may be conveniently described in the following classes:

(a) Adenocarcinoma of infants.

(b) Embryonal adenocarcinoma of adults.

(c) Tubular adenocarcinoma reproducing renal tubules.

(a) Adenocarcinoma of Infants.-Compared with the mixed tumors pure epithelial growths are rare in children. Of I 70 cases, Albarran found four adenomas, seven carcinomas, two adenocarcinomas, and four adrenal tumors. In the literature attention is seldom given to the recognition of carcinoma

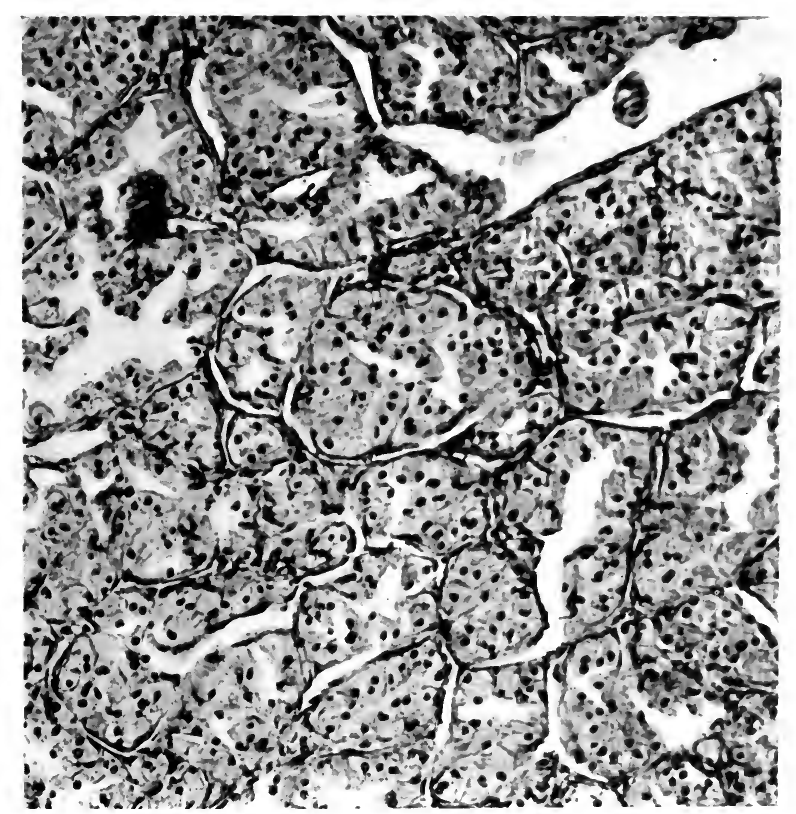

FIG. 360.-Adenocarcinoma of adult kidney, reproducing convoluted tubules.

in infants and many cases have doubtless been included in the literature of congenital sarcoma. It is quite possible that some of the infantile adenocarcinomas are related in origin to the congenital mixed tumors, as Weigert's cases, in which there were abortive glomeruli, but others appear to be of more adult type and probably arise from portions of well-differentiated renal blastema. A relation to the congenital cystic kidney is also probable. Von Kahlden has shown that marked epithelial overgrowth may occur in this condition. The scope of the structure is illustrated in the following cases:

Weigert described small bilateral renal tumors in a stillborn fetus which projected slightly beneath the capsule. They were composed of well-formed atveoli resembling renal tubules, and of vascular foci in which loose polyhedral cells surrounding capillaries recalled the structure of imperfect glomeruli. A large adenocarcinoma, interpreted as a later stage of Weigert's tumors, is described by Barth. It was as large as a child's head, 
replaced most of the kidney in a child of 5 years, had caused hydronephrosis, and was composed of alveoli, cords and diffuse growth of polyhedral cells. Perthes describes three large tumors in children, of adenomatous and carcinomatous structure. The resemblance of the acini to renal tubules was noted. In Malcolm's case the tubules were separated by a delicate stroma and the tumor, a malignant adenoma, was lacking in embryonal characters. In Manasse's alveolar carcinoma (Case VIII) the resemblance to renal epithelium was also noted. The most malignant of the growths show central softening.

Small cysts may appear in the adenomatous growths, as in the cases of Iliot described by Kelynack, in which the structure resembled thyroid gland, and in Edmund's case. Some of the tumors reach a very large size, especially the malignant adenoma involving the whole kidney.

Metastases are comparatively rare. Even the actively growing very cellular carcinomas may be free from secondary growths (Bokai).

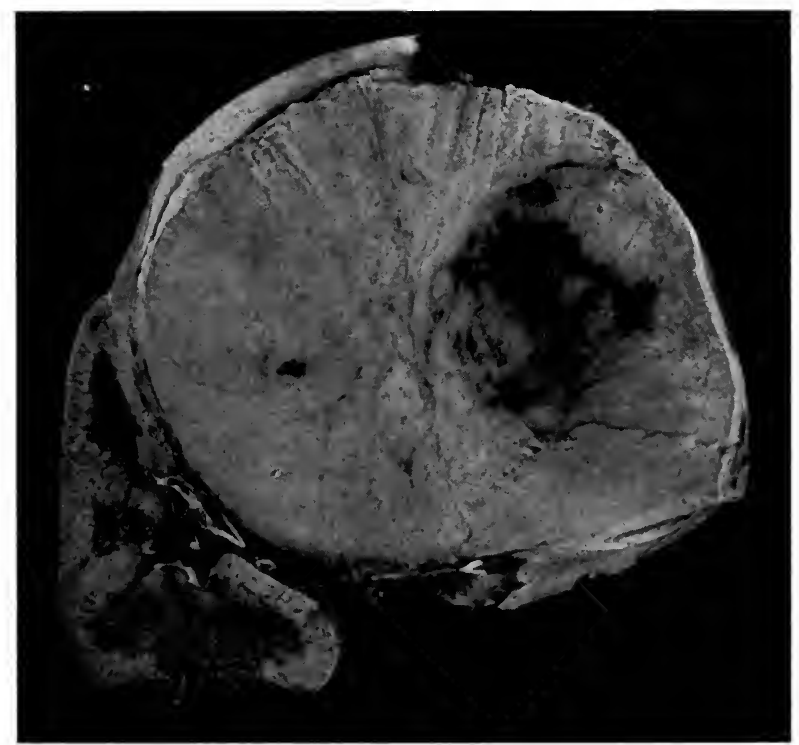

FIG. 361.-Embryonal tumor of renal blastema in the kidney of a child.

(b) Embryonal adenocarcinoma of adults is a rare but characteristic tumor. It is probably of the same origin as some of the malignant adenomas of children, which it resembles in structure.

In a case in the New York Hospital the patient, a woman of 35 years, complained of pain for I year and hematuria for 3 months before operation. The tumor, $24 \times$ i $4 \times 8$ $\mathrm{cm}$., was well circumscribed laterally, but fused gradually with the remnant of kidney. It was solid, lobulated, cream colored, and moderately soft. There were no metastases. Section showed a uniform structure of large alveoli lined by large granular, cylindrical, or cubical cells with darkly staining large nuclei. Many of the alveoli contained homogeneous acidophile material. In some foci the alveoli were indistinct. The embryonal character of the cells and structures was pronounced.

(c) Tubular Adenocarcinoma and Carcinoma of Adults.--In this group are included the majority of malignant alveolar tumors of the renal epithelium. They are clearly separable from the carcinoma with clear cells, from papillary carcinoma, and from adrenal growths. The structure is uniformly alveolar 
or tubular, adult in type, and resembles the renal parenchyma. These tumors probably originate from well-differentiated renal blastema or from adult cortical tubules. Waldeyer, Manasse, and others claim to have traced the development of such tumors from the renal tubule cells, but most observers have not succeeded in this task. Sharkey believed he could trace the origin of his tumor to the glomerular epithelium. Beneke also has observed tumor-like hyperplasia of glomerular epithelium. It seems more probable that the growths develop from misplaced renal tissue or from the well-known types of tubular or alveolar adenoma, but Albarran's early case (I66) was as well diffused as a tuberculous lesion, and the outlines of the cortex were unaltered.

While there are many records of tumors of this group they are distinctly less frequent than other forms of renal carcinoma. They arise from any

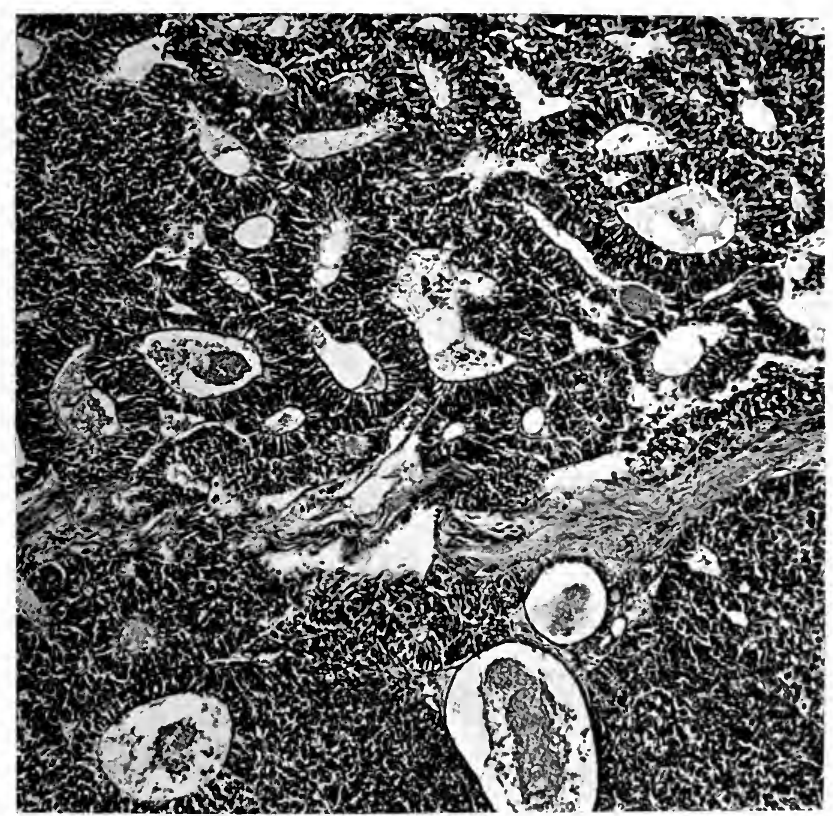

FIG. 362.-Embryonal adenocarcinoma of renal blastema.

portion of the kidney and may be located chiefly in the cortex or pelvis, which are widely distended, or beneath the capsule, or they may be extrarenal. Very early circumscribed but non-encapsulated tumors are described by Albarran. In Brault's case a very small tumor was associated with metastases. Most of the tumors reach considerable and sometimes large dimensions. The adenomatous tumor mav long remain encapsulated, but infiltrating growths may greatly enlarge the kidney while preserving its form. Fatty changes, hemorrhage, and necrosis are not prominent, but the solid, lobulated, opaque texture serves to distinguish most of them from adrenal growths and papillary carcinoma. Small cysts containing gelatinous material are not infrequent, and in a group of cases the abundance of such material has led to the designation as colloid carcinoma (Newman, Dickinson, Schueppel). The highly malignant tumors infiltrate the lidney diffusely, perforate 
pelvis and capsule, invade both veins and lymphatics, and produce metastases in many organs.

The structure presents many variations, but usually follows that of certain segments of the renal tubules. That they are actually derived from such portions of the tubules, while possible, cannot at present be claimed.

The convoluted tubules were reproduced to a remarkable degree in a case of A. Frazer's, kindly placed at my disposal. The tumor was a large solid circumscribed growth presenting mainly large alveoli and tubules lined by high cubical granular cells exactly duplicating those of the convoluted tubule of the adult organ. Numerous variations from this structure appeared in more actively growing hemorrhagic and degenerating portions of the growth, and in some foci the growth was diffuse.

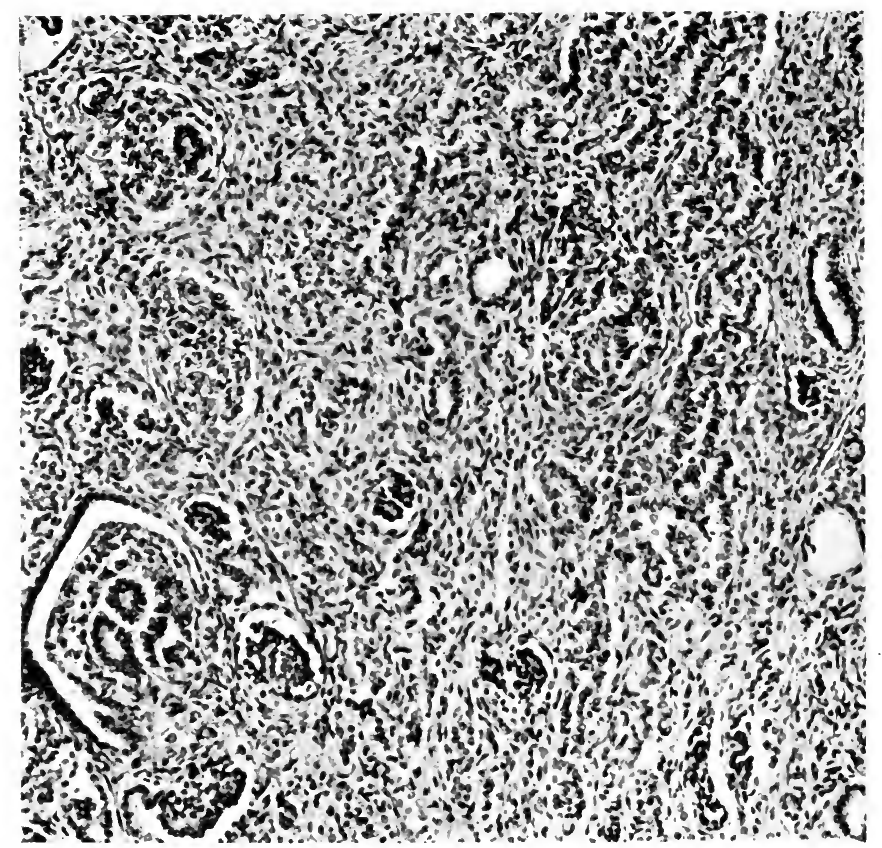

FIG. 363.-Embryonal tumor of renal blastema, with production of abortive tubules and glomeruli.

The smaller renal tubules are simulated by many small alveolar adenocarcinomas and carcinomas, but the size of the alveoli seems to depend on the tissue of origin (adenomas), the rate of growth and associated fibrosis, rather than on any inherent capacity to reproduce normal renal structures. In some tumors the alveoli are quite small, and lined by small flat cubical epithelium. Or the cells are larger and form small compact groups with no definite lumen. Invasion and replacement of preformed tubules may determine the structure of infiltrating growths. Tumors composed of small alveoli are relatively frequent and are depicted by Albarran, Stoerk, and many others. Diffuse growth derived from such structures may simulate various types of sarcoma. Markedly elongated tubules lined by high cylindrical cells of opaque granular type are also encountered. Striking variations in 
structure are frequently observed in the same tumors, including especially low papillary projections into distended alveoli, collections of secretion, giantcell formation, and peculiar cell inclusions.

\section{EMBRYONAL ADENOMYOSARCOMA}

Whatever its origin and its interrelations with other renal tumors may prove to be, the embryonal sarcoma of infants is one of the most characteristic neoplasms of the kidney. Its rather frequent occurrence in infants, the complex embryonal structure, rapid growth to very large size, fatal course, and obscure origin, render it a specific clinical disease and a topic of much theoretical interest. Hedren has extracted many of the case reports up to I907, but includes many simple embryonal tumors which do not certainly belong in this group.

The great majority of cases are observed in the first 3 years of life, it is rarely found after the roth year, but Hedren reports a bilateral tumor at 54 years, while Schaffer describes an early stage of the disease in the single kidney of a deformed fetus. This kidney was twice the normal size, presented abortive renal structures, islands of cartilage, and areas of smooth and striated muscle. Several reports are of bilateral tumors.

Gross Anatomy.-The tumors lie within a distended renal capsule, their position may suggest an origin from any part of the kidney, mid-cortex, upper pole, papillæ (Manasse), or pelvis, and while usually sharply separated from the remnant of the organ, they may fuse insensibly with it (Busse), or a thin rim of renal tissue may inclose a large segment of tumor. The growth may be entirely extrarenal (Broch, Brandt, Borst).

The large size of these tumors is a notable feature, since at any given age they are usually the largest of the renal growths, and this volume is attained rapidly. In several cases the largest diameter has reached 35 to $37 \mathrm{~cm}$. (Hoisholt, Jenckel), while in a subject of 34 years Muus found a tumor of $40 \mathrm{~cm}$. Heineke's tumor weighed $3580 \mathrm{gr}$.

The growth is often solid, opaque, and variously subdivided into lobules or areas by preponderance of glandular, fibrous, muscular, or very cellular tissue. The larger tumors are usually cystic and some approach the appearance of the congenital cystic kidney. Hemorrhage and necrosis are late complications. Extensions and metastases are exceptional, but in Merckel's case a tumor thrombus extended in the vena cava to the heart. The liver is the most frequent seat of metastases. Pulmonary metastases have been observed in several cases, in Brandt's 5 months after extirpation of the tumor. Ribbert's tumor extended along the spine.

The embryonal structure is the most distinguishing feature and presents a great variety of tissues usually suggesting abortive renal elements. The usual composition is of isolated tubules of high cylindrical or cubical cells with indistinct lumina, surrounded by broad zones of indifferent spindlecells, on which is based the designation of adenosarcoma. Either tubules or spindle-cells may be in excess, the tumor approaching embryonal adenocarcinoma or sarcoma.

Occasionally a tuft of spindle-cells projects into an invaginated tubule, like an abortive glomerulus. Both spindle- and cubical cells may be very small and numerous, producing a structure of round-cell or alveolar sarcoma. Multilobed giant-cells may be prominent. Cysts are usually lined by cubical epithelium. Squamous epithelial nests with keratohyalin were found by Muus, and Jenckel saw cysts lined by squamous cells. Most of the cells are rich in glycogen. 
Smooth or striated muscle-cells are usually present in some portions of the tumors and either may make up the bulk of the growth. Some have derived the latter from the former, but Hedren maintains that the two types are separate, the striated cells first appearing in the usual tubular form of the embryo. The cellular connective may be edematous or myxomatous, and often contains elastic fibers. Fat tissue appears in single cells or definite lobules. Cartilage appears in the form of small isolated islands, rather more frequently in older subjects. In one case islands of bone were observed (Hedren). The metastases are usually of round cells, but Blau reports striated muscle-cells in the lung.

Histogenesis.- The interpretation of the origin of renal mixed tumors has passed through several phases and covered all apparent possibilities.

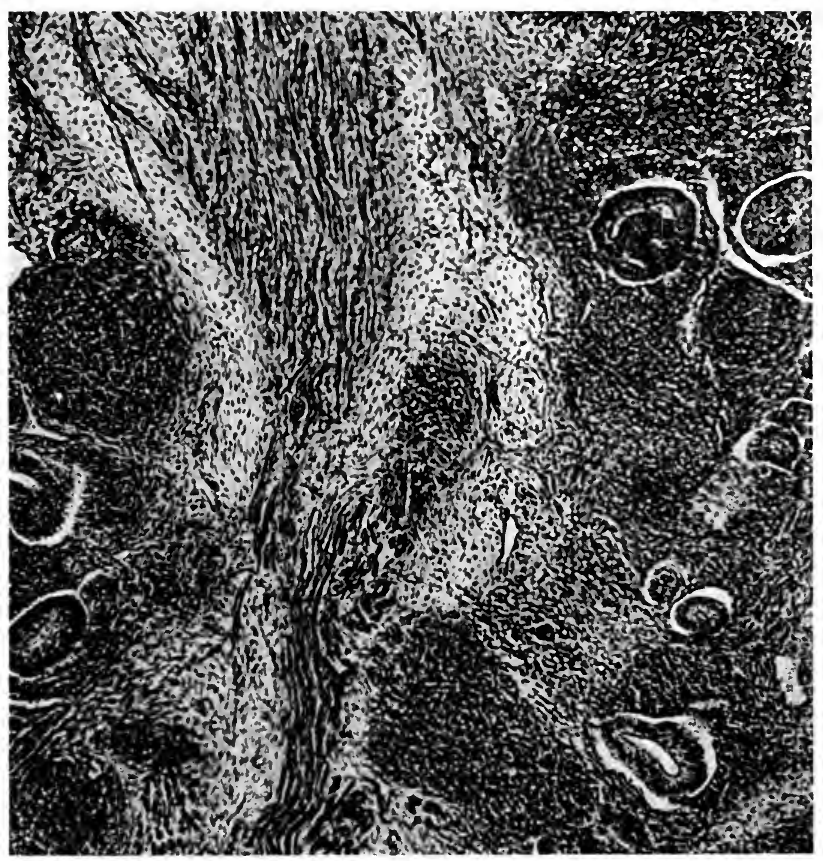

FIG. 364.-Topography of Wilms' embryonal tumor of kidney. Epithelial tubules lying in masses of spindle and polyhedral cells. Striated muscle-cells in center.

An origin from an aberrant sex cell has been deemed necessary by Ribbert, who interpreted some of the epithelial rosettes as neuro-epithelium and who emphasizes the presence of all three germ layers.

Wilms places the period of the embryogenic disturbance later, but considers that the originating tissue must be defined early, must involve ectoderm and mesoderm, and include nephrotome, sclerotome, and myotome.

The earliest conception was that of Cohnheim and Birch-Hirschfeld who assumed an origin from the Wolffian body. Busse points out that no Wolffian remnant has ever been found in the kidney, so that this interpretation has been generally abandoned. Birch-Hirschfeld's service was chiefly in emphasizing the existence of a definite group of embryonal mixed tumors. 
Busse and Muus place the origin at a still later teratogenic termination period and see no reason why all the structures observed may not be derived from the renal blastema. This view attributes a prominent part to metaplasia, thus accounting for adult squamous epithelium,. striated muscle, cartilage, and bone. In this respect it is in accord with modern tendencies, which are not in favor of the rigid specificity of germ layers. It also serves to explain why some of the tumors approach the type of simple embryonal adenocarcinomas of the kidney, of which I have seen one striking example. It accords further with the gradual fusion of some tumors with renal parenchyma, and permits considerable latitude in the exact period in the differentiation and growth of the renal blastema at which the tumor may arise. On the whole, this view is the most acceptable, as well as the simplest of the proposed hypotheses. Hedren was much impressed by the presence of bone in his unique case, which he could not accept as of metaplastic origin, and concludes that embryological data and casuistic studies are inadequate to establish any single origin of the renal mixed tumors.

In addition to the typical embryonal sarcomas and their recognizable atypical forms, there are other renal tumors whose structure suggests a relation with the main group.

Quiescent masses of fat and cartilage are not infrequently observed in the kidney (Hansemann, Borst). Fibromyomas, single or multiple, of cortex or capsule (Busse, Larkin), fibromyo-osteosarcoma (Hildebrand), fibrolipomyoma (Busse), and myxoliposarcoma are recorded. In all of these renal epithelial elements are missing. Apparently pure carcinomas of the kidney occur in infancy (Birch-Hirschfeld). All of these observations suggest that the embryonal kidney is subject to a variety of developmental disturbances resulting in tumor growth and that no single source of these anomalies should be assumed to exist.

\section{RENAL TUMORS OF ADRENAL TISSUE. HYPERNEPHROMA}

In $1883 \mathrm{P}$. Grawitz formulated the conception that certain tumors of the kidney were derived from adrenal rests. In this group he included the so-called lipomas of the cortex, angioma cavernosum, various myomas, as well as the adenomas and adenocarcinomas of Klebs, Sturm, Sabourin, and (I884) of Weichselbaum and Greenish. The argument favoring this view was quite ably prepared. The chief evidence consisted in: (I) The location of the tumors under the capsule where adrenal rests are found. (2) The character of the cells, which differed markedly from renal epithelium in their high content of fat without signs of degeneration. (3) The sharp encapsulation separating the tumors from the renal parenchyma. (4) The resemblance of the structure to that of the adrenal. (5) The presence in the small tumors of a fibrous core and a more cellular glandular cortex. Even when not encapsulated the tumor was sharply separated from the renal parenchyma.

Grawitz theory was soon endorsed by Chiari, Horn, Lubarsch, Beneke, Ambrosius, Marchand, and many others, and came to be generally accepted. Sudeck in 1893 first offered objections, and in recent years other contrary views have been frequently expressed by Stoerk, Zehbe, Wilson and Willis, Ipsen, Glynn, and others. It is now evident that the scope of Grawitz's tumors was much too widely extended, and it has become necessary to determine anew whether adrenal tumors of the kidney exist and how they can be identified.

On these questions it may be said that the presence of adrenal rests in the kidney is fully attested, although they are probably less frequent than 
many have supposed. It also appears that certain tumors arise from these rests, although clear descriptions of their structure have not been fully given. Finally, recent studies have demonstrated that a large proportion of the reported hypernephromas are renal adenocarcinomas.

Occurrence of Adrenal Rests (Pick, Goupel, Lit.).-The adrenal cortex is developed from the mesothelium of the Wolffian ridge, as are also the ovary and testis, while the medulla is of neuro-ectodermal origin and belongs to the sympathetic system, although mingled with the neuro-epithelium are derivatives of the cortical cells. According to Aichel the interrenal bodies of lower vertebrates and the adrenals of higher vertebrates arise from the Wolffian body funnels, which are of mesothelial origin. Marchand's

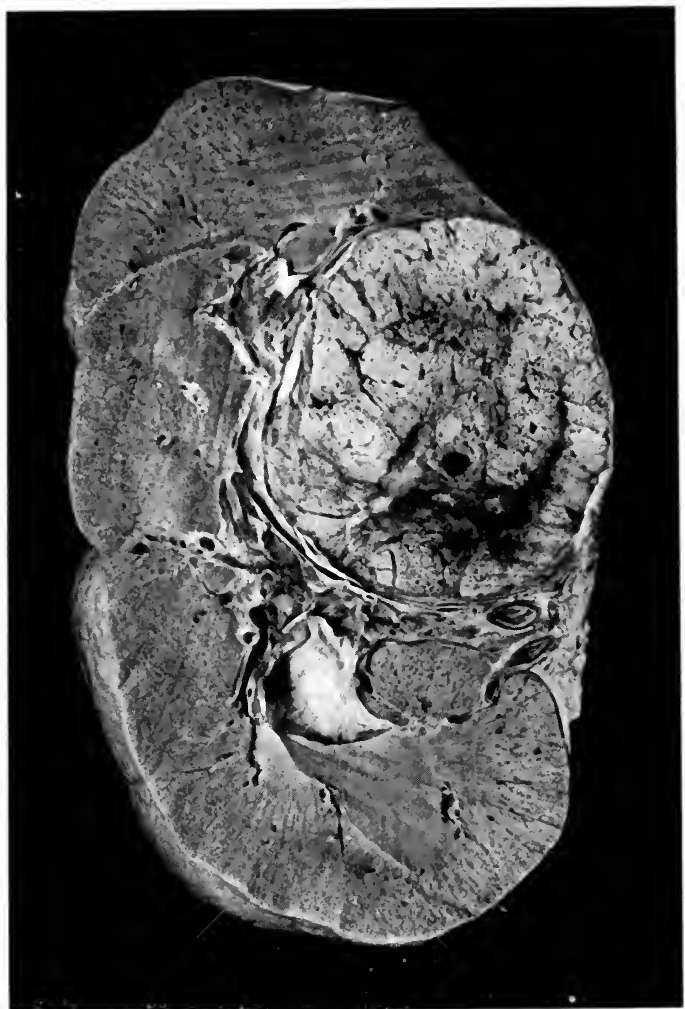

FIG. 365.-An adrenal tumor of the kidney.

adrenals, in the broad ligament of the female and along the spermatic cord of the male, arise from segmental tubules of the IVolffian body and correspond to the suprarenals of lower vertebrates.

The renal cortex, on the other hand, is formed from the renal blastema, a mass of indifferent mesothelium which lies some distance below the adrenal and is separated from it by the Wolffian body.

The adrenal is at first larger than the kidney because of the hypertrophy of the fetal cortex, which atrophies and is replaced after birth by the adult cortex derived from a rim of small cells (Elliott, Armour). The position of the early adrenal is not at first favorable to the inclusion of its tissue in the kidney, as shown by Wilson. Yet after the second month it is closely applied to the kidney and surrounds a large portion of the renal body (Peter). Inclusions in the kidney may therefore reasonably be assumed to result. The occurrence of diffuse adrenal tissue without encapsulation is described by 
R. Meyer. That the adult peritoneal epithelium is capable of developing foci of adrenal tissue is suggested by Pick and Poll. Total subcapsular location of both adrenals, portions remaining on the kidney after decapsulation, are recorded by Klebs, Grawitz, Weiler, and Ulrich. Fusion of adrenal in the renal capsule and intralobular inclusion of rests surrounded by renal parenchyma are described by Grawitz and Ulrich. Interlobular rests connected with the adrenal by a strand of fibrous tissue are more frequent conditions. Most of the adrenal rests contain only cortical tissue, but medullary tissue also was found by N. Pitt, Grawitz, and Manasse.

Yet it appears clearly that adrenal rests in the kidney are much less frequent than the supposed adrenal tumors. Glynn could find only about 17 in the literature and obtained none in 1500 kidneys. Yet Lubarsch in 300 autopsies found eight adrenal rests in the kidney. On the other hand, such rests are relatively frequent in other situations (Marchand); in the neighborhood of the epididymis, in 76.5 per cent. of newborn children (Wiesel); on the under surface of the liver, in 92 per cent. of cadavers (Neusser); in 90 per cent. of all bodies (Holmes); on the broad ligament and spermatic veins of 24 females (Aichel). In adults they are much less common, and many observers find them in only a small proportion of cases, as Schmorl in 4 of 510 livers; Hanau, about spermatic veins, in 5.9 per cent. of children under 5 years. They are occasionally found in the testis (Dagonet, Michael, Meyer, Kirkbride); pancreas (Ribbert); transverse colon (Nicholson). The facility with which different observers are willing to identify adrenal rests doubtless explains the varying results.

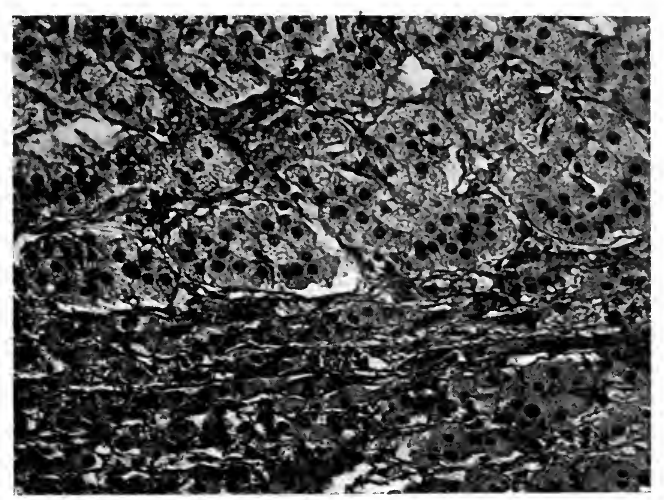

FIG. 366.-Adrenal adenoma in kidney.

It must be admitted also that tumors of misplaced adrenal tissue outside of the kidney are distinctly rare, although the diagnosis of such an origin is frequently made on insufficient grounds. The location of adrenal rests in the kidney is chiefly in the upper pole, but not exclusively between the renculi, as is sometimes,claimed. On the other hand, the tumors commonly attributed to these rests occur with about equal frequency in all segments of the organ.

Notwithstanding these discrepancies it is clear that adrenal rests occur with moderate frequency in the kidney, where they may give rise to tumors.

Gross Appearance of Adrenal Rest Tumors. - The anatomical features commonly attributed to Grawitz's tumors apply to a large group of renal growths. They are large, well-circumscribed, yellowish, fatty, and vascular tumors, prone to hemorrhage, necrosis, and cyst formation. A segment of intact kidney is usually found connected with the tumor. Grawitz emphasized especially the presence of a central fibrous area surrounded by a more cellular cortical portion, but in most descriptions of hypernephroma these features have been absent or disregarded. Yet the true adrenal tumor of the kidney, at least in its early stages, regularly presents such distinguishing landmarks (Fig. 365). It also, as a rule, lacks the markedly lobulated sub- 
divisions, numerous small cysts filled with gelatinous or bloody material, and gradual merging with the renal parenchyma, which belong to renal adenocarcinoma with clear cells. The adrenal tumor is usually more solid, although cysts form from necrosis and hemorrhage. The malignant growths invade the perirenal tissues, peritoneum, and lymph-nodes more readily than renal carcinoma. Both invade the renal pelvis and the large veins, but metastases are earlier and more wide-spread with adrenal growths.

Many so-called renal hypernephromas actually arise in the adrenal gland and, invading the kidney, fuse the two organs in a diffuse mass. When the adrenal cannot be found the renal origin of the tumor at once becomes doubtful.

Structure.-The scope of the structure attributed to adrenal hyper-nephroma should be controlled by the known structure of tumors of the adrenal itself, as seen in the human subject. Observations on tumors of the adrenal in lower animals cannot safely be used as criteria.

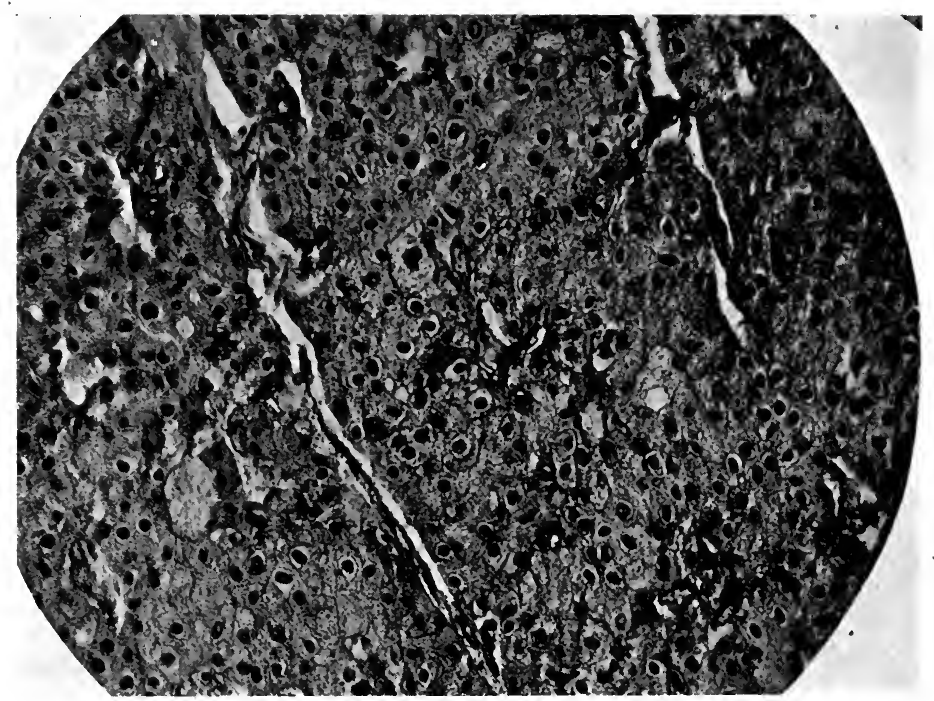

FIG. 367.-Adrenal tumor of kidney. Note the broad sheets of cells without alveolar formation.

From this standpoint it is necessary to eliminate all tumors with distinct lumina and especially those of papillary structure. There remains a considerable number of trabecular, or solid, or diffusely growing or sarcomatoid tumors which differ essentially from renal adenocarcinoma.

The simplest structure reproduces one or more zones of the adrenal cortex. Some resemble the hyperplastic nodules of the adrenal,itself (struma suprarenalis). The cells are arranged in small circular groups separated by fine strands of connective tissue (zona glomerulosa). This structure may be uniform in tumors several centimeters in diameter. Or the elongated cords of the zona fasciculata may predominate. The characteristic cytoplasm is not clear, but slightly granular, with numerous lipoid globules and glycogenic granules, yielding a foamy texture. Hydropic degeneration may considerably enlarge the cell borders. The attachment of the cells to the stroma is loose and the palisade arrangement of rigid cylindrical cells of renal car- 
cinoma is absent. Tumors of this type may be designated as adrenal adenoma of the kidney.

A peritheliomatous structure is presented by certain adrenal tumors, but this type is extremely difficult to distinguish from similar structures in renal carcinoma. Section of many portions of the tumor and attention to the gross relations are then of much value. Peritheliomatous structures are more atypical and malignant than the adenomatous. The cells are loosely attached, lacking the clear mosaic appearance of renal carcinoma, and presenting a granular and vacuolated texture. Large granular giant-cells may form, and some tumors are composed almost exclusively of giant-cells, both in the original tumor and in metastases.

A trabecular arrangement is followed in some adrenal tumors of the kidney in which the granular and fatty cells appear in broad anastomosing sheets

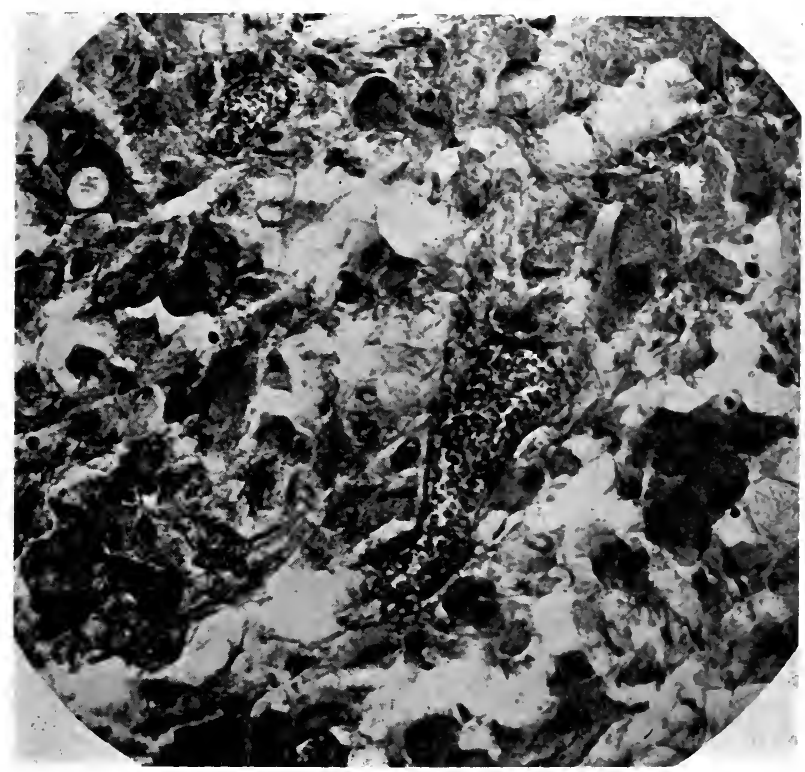

FIG. 368.-Adrenal medullary tumor of kidney.

supported by vascular stroma. These tumors also approach the type of some renal carcinomas, but the absence of lumina or papillæ, and the smaller size and granular characters of the cells strongly indicate their adrenal nature.

The most malignant forms of adrenal tumors of the kidney present a carcinomatous or sarcomatous structure. The cells appear in small groups (alveolar sarcoma) or grow diffusely. In well-nourished areas the tissue may resemble overcellular liver tissue. Giant-cells may be very numerous. The form is polyhedral or round or elongated. In some cases the mesoblastic tendencies, emphasized by Adami, yield areas of large spindle-cells and the appearance is distinctly sarcomatous. The cell borders are indistinct and the cytoplasm is still more granular and opaque and less fatty. Areas of pigmented cells, doubtless representing the chromaffin substance of the medulla, may be present, as in cases described by Weichselbaum and Greenish, Ambrosius, and Manasse, and in one case of the writer's. Lassagna describes 
a bilateral carcinosarcoma presenting both ganglion-cells and chromaffin pigment.

All the above structures are duplicated by tumors of the adrenal gland. Whether other observed types of malignant adrenal tumors contribute any portion of the so-called round-cell or alveolar sarcomas of the kidney remains to be shown.

Differentiation Between Adrenal and Renal Tumors.-The debate over the structure of adrenal tumors of the kidney has been continuous since r893, when the question was opened by Sudeck, and was accelerated by Stoerk's publication in I908. It concerned chiefly the significance of lumina, the evidence of a papillary structure, and the chemistry of the cells.

The presence of lumina in adrenal tumors has been maintained by Grawitz, Marchand, Askanazy, Dobbertin, and Manasse. In the normal adrenal lumina are described by Kolliker, Stilling, and Eberth. In the adrenal of the horse and cow lumina appear to be constant. Yet in the human adrenal, adrenal rests, and adrenal tumors lumina are conspicuous chiefly by their absence, while their occasional presence is hardly sufficient ground for assuming that tumors of the kidney, in which lumina are the predominant feature, are derived from adrenal tissue. Pseudolumina may form by central degeneration of tumor cords, but this origin cannot explain the well-defined structure described by Manasse. Dobbertin's tumor was an embryonal, alveolar, and papillary tumor of uncertain origin in a child. Winkler often speaks of lumina in his adrenal tumors, but depicts only pseudolumina caused by degeneration. It is also possible that certain adrenal tumors are derived from renal rests. Beneke and Ulrich describe renal tubules in the adrenal cortex. Ricker found groups of renal tubules in the adrenal joined to the kidney by cords of connective tissue, and concludes that hypernephroma may have a mixed adrenal and renal origin. The presence of lumina therefore is a somewhat uncertain criterion on which to separate adrenal from renal growths, but it cannot be questioned that a predominance of this feature tells strongly against an adrenal origin. Yet small adrenal adenomas may show an irregular alveolar structure.

The same considerations apply to the papillary structures of supposed adrenal rest tumors. The markedly papillary character of renal carcinoma is not approached in any authentic case of human adrenal growth. Nevertheless a certain papillary structure which is less pronounced than in renal carcinoma, may appear in adrenal tumors which are chiefly of perivascular type. Winkler describes such a structure in the pulmonary metastases of a bilateral adrenal growth. A low papillary growth may be present also in small adrenal adenomas. In and about cystic cavities which form in adrenal carcinoma from hemorrhage and softening localized papillary growth may occur.

The characters of the cells in adrenal growths differ distinctly from those of most renal tumors. In the former the cytoplasm is foamy, granular, and interspersed with lipoid granules. In most tumors the granular character increases and the fatty appearance diminishes with the increase in malignancy. In the renal tumors the cells are remarkably clear throughout, resemble vegetable cells, and contain large globules or crystals of fat with hydropic fluid, and little or no granular material. Lubarsch thought the abundance of glycogen a strong diagnostic point in adrenal growths, but it may be equally abundant in renal tumors. Glycogen is scanty in the normal adrenal. Hydropic degeneration is usually missing in adrenal growths (Zehbe), but very marked in Grawitz's tumors.

Fat is present in both renal and adrenal tumors, and in both the lipoids 
consist chiefly of cholesterin esters (Panzer) and are doubly refractive (Lohlein, Stoerk). The fat content in Ipsen's cases ran from 4 to 10.7 per cent., while that of the horse's adrenal was 30.8 per cent. Sisson finds the fat very unevenly distributed in Grawitz's tumors, but quite uniformly in adrenal growths.

The loose relations of the cells to one another and to the stroma in many adrenal growths contrasts with the rigid cell borders and coherence of the cells in renal papillary carcinoma. Areas of diffuse growth, of indiflerent spheroidal cells, and of spindle-cells are practically unknown in the renal tumors, but are frequent in adrenal growths. Giant-cells form in both varieties of tumors, perhaps to a greater extent in those from the adrenal. Pigmented cells belong to adrenal tumors. Fraser points out that the primary structure differs markedly in adrenal from that of renal tumors, and that while secondary structures due to malignant change or degeneration may cause the renal to resemble the adrenal growths, the primary structures of both types are distinct. Hence the diagnosis must be based only on the primary structure and not on non-specific secondary changes. Many sections from various portions of the tumor may be required to detect the primary structure.

Among miscellaneous data may be mentioned the fact, pointed out by Stoerk, that Grawitz's tumors while very frequent in the kidney are very rare in the adrenal. Ellis collected $\mathrm{r}_{57}$ in the kidney, 3 in adrenal, 2 in liver, and $I$ in uterus. Garceau mentions 176 in the kidney, while Woolley could find only 22 in the adrenal. Glynn points out that abnormal sex characters are commonly present with adrenal growths, but invariably absent with Grawitz's tumors. Wells failed to find adrenalin in a typical Grawitz tumor.

Croftan observed that a watery extract of the adrenal caused rapid decolorization of starch blue from action of iodin, and transforms starch or glycogen into maltose or dextrose, while injection of this extract into dogs and rabbits produces glycosuria. He employed this test for the recognition of adrenal tumors of the kidney. Results of the test in the hands of Korber and Winkler were not uniform. Ellis found the reaction in a variety of tissues.

Conclusions.- - Sufficient evidence has been adduced to prove, in the writer's opinion, that the group of adrenal tumors of the kidney differs, as a whole, quite distinctly from the renal adenocarcinoma with clear cells. The main distinctive features of the adrenal growths are the central fibrous core and exact reproduction of the adrenal by benign growths, and the general mesoblastic tendencies of the malignant forms. Chromaffin cells are not infrequently present. For an alveolar or papillary structure it is extremely difficult to establish an adrenal origin in man. Yet the opponents of Grawitz's theory seem to have gone too far in practically eliminating the adrenal rest as a source of renal tumors. In atypical cases of both varieties it may be impossible to establish a positive differentiation.

Tumors of Extrarenal Adrenal Rests.-The distribution of adrenal structures outside the kidney corresponds to the location of groups of tumors which present a structure suggesting an origin from adrenal tissue. Three main divisions of aberrant adrenal tissue are found:

(a) Along the suprarenal vein, solar plexus, and inferior surface of the liver;

(b) Along the internal spermatic vein, in the broad ligament and about the uterus, ovary, and tube in the female;

(c) In the spermatic cord and corpus Highmori in the male.

These adrenal rests are usually of comparatively simple structure, presenting only cortical tissue, but in several instances pigmented medullary tissue is present. The absence of pigmented cells is not a certain indication of entire lack of medullary elements, since pigment is late in appearance even in the normal adrenal. From the usual construction of the rests only 
cortical tissue can be expected in the tumors. In the rare cases of hyperplasia of aberrant adienal tissue described by Marchand, Gunkel, and Pick only cortical tissue was apparent. Yet Chiari,'s and Schmorl's tumors contained brownish or yèllow pigmented cells.

The tumors attributed to these rests have often shown such striking resemblance to adrenal adenoma as to leave little doubt of their true nature. In others the structure has been less specific, often peritheliomatous or carcinomatous, and the derivation from adrenal tissue must remain doubtful. In some reported cases, especially in the liver, ovary, and testis, the adrenal origin is highly questionable. Thus, Pepere's hypernephroma of the liver is clearly an hepatic adenoma, while some of the supposed hypernephromas of the ovary and testis can equally well or better be interpreted as atypical forms of well-known carcinomas of these organs.

In general, the tumors are well encapsulated, of large size, cystic or solid, of light yellow color, with a tendency toward necrosis and hemorrhage. They possess considerable malignancy and local extensions and general metastases are frequent.

Hepatic Adrenal Tumors.-Schmorl, who reported finding four examples of adrenal rests in the right lobe of the liver, also described a small yellowish tumor in this region presenting the structure of adrenal cortical adenoma. Larger malignant tumors probably of the same nature are recorded by Vecchi and Noyes.

Peritheliomatous structures in hepatic tumors have often been regarded as of possible adrenal origin. An angiosarcoma of the pancreas was so interpreted by Ribbert and Kronlein.

Lateral Retroperitoneal Tumors of Adrenal Rests.- The retroperitoneal tissues above and below the kidney and extending into the pelvis are the seat of a wide variety of tumors of sarcomatous and carcinomatous types. Goebel has tabulated Ior cases, illustrating the wide scope of this group of tumors. They include benign connective-tissue growths, lipoma, fibroma, fibromyomas, and ovarian cysts, various types of sarcomas, rare carcinomas, teratomas and cyst, and four adrenal rest tumors.

Chiari's case, the first reported definite extrarenal hypernephroma, was a large tumor lying below the kidney on the quadratus lumborum, reaching the true pelvis, and extending into the mesocolon and along the crural vessels. The gross and microscopical structure was rather typical of adrenal adenocarcinoma. Weiss described a very similar case. Goebel's tumors both grew through the mesocolon in the peritoneum or abdominal wall.

Adrenal Tumors of the Broad Ligament, Ovary, Tube, and Uterus.Peham has described two large cystic and solid yellowish tumors of the ovary which presented a structure recalling that of hypernephroma. Scudder's case of bilateral ovarian tumor, of very similar structure, does not strengthen the probability of an adrenal origin. The rare tumors of the corpus luteum offer a strong resemblance to hypernephroma, and certain fatty and hydropic ovarian carcinomas closely resemble adrenal growths.

In the broad ligament Weiss and L. Pick have each described large yellowish, hemorrhagic, and necrotic tumors, displacing uterus and ovary, and exhibiting many microscopical features of hypernephroma. In a large tumor attached to the fundus uteri Eastwood describes rather typical reproduction of the zona glomerulosa of the adrenal, as well as alveoli filled with colloid, as in the thyroid gland.

Adrenal Tumors of Spermatic Cord and Testis.-Adrenal tumors of the testis are described by Chevassu and Debarnardi, but their exact nature appears rather uncertain. 


\section{EPITHelial Tumors of ReNal Pelvis aNd URETER}

The renal pelvis and the ureters are the seat of tumors very similar to those of the bladder. They take the form of:

(I) Papilloma.

(2) Papillary epithelioma (epidermoid carcinoma).

(3) Alveolar carcinoma.

Of 54 such tumors collected by Albarran and Imbert, 22 were benign papillomas, I6 epitheliomas, and I6 carcinomas. Savory and Nash have tabulated 49 cases. The tumor occurs chiefly between the ages of 35 and 65 years, much more frequently in males, and in 8 of 53 cases there were calculi.

(I) Papilloma is a benign growth which affects any portion of the renal pelvis or ureter. In 7 cases both were involved, while in 6 the ureter was the primary seat, especially the upper or lower orifices. Pelvis and ureter throughout were affected in the cases of Lancereaux, Fenwick, and Hebb.

The tumors are usually multiple and may be extremely numerous, covering the entire mucosa with fine vegetations. Often there is one large composite tumor and several small polyps scattered over pelvis and calices (Kohlhardt). Albarran holds that multiple tumors are often the result of implantation, but it seems more probable that in cases of very extensive distribution the tumors develop all along the genito-urinary tract as the result of factors affecting the entire mucous membrane. In the cases of Murchison and of Neelson the bladder was involved with pelvis or ureter, and Murchison's case was bilateral. The tumors are villous or wart-like, attached by a narrow pedicle and expanding in fan shape. Very fine branching growths collapse on removal and require floating in water for restitution of the original form. The great vascularity is a source of hemorrhage, which is the chief symptom. Incrustation with salts and association with calculi is frequently observed, and the intervening mucosa may be the seat of chronic pyelitis sometimes cystic (Stoerk). Various grades of hydronephrosis often result from partial occlusion, chiefly by ureteral tumors. The structure presents elongated branching blood-vessels sometimes accompanied by smooth muscle-cells, covered by multiple layers of transitional epithelium. The tumor-cells are cubical, cylindrical or much elongated and spindle-shaped, but the form and arrangement are orderly and typical. At the bases of the tumors there is round-cell infiltration.

While the course of these tumors is usually benign, not a few of them have recurred in malignant form after curettage or incomplete removal. They are distinctly capable of developing recurrences from implantation in the wound. In Tikhoff's case the recurrence developed locally as alveolar carcinoma, ro years after operation. Very suggestive is the case of Drewvillous papilloma of pelvis invading the kidney, simple papillary excrescences throughout the ureter, and a large papilloma at the vesical orifice.

(2) Papillary epithelioma produces more numerous and more compact tumors than the benign papilloma. They appear as closely set, opaque, warty outgrowths which are usually found to cover a considerable area. Both pelvis and ureter may be involved, as in the cases of Rayer, Drew, Israel, and Poll, or a recurrence of a pelvic tumor may later appear in the ureter (Israel, Fenwick). Pelvis, ureter, and bladder may be simultaneously involved (Rayer, Drew, Israel). Even in early stages of the growth there s a tendency toward encroachment on the submucous tissues and renal parenchyma. In a case pictured by Kuster the entire visceral layer of mucosa was involved by low warty growths and the renal cortex was perforated. It is sometimes difficult to determine in the gross whether the original growth 
is renal or pelvic (Graupner). In advanced cases the pelvis may be distended and obliterated and fused with surrounding tissues, and the kidney may either be invaded and deformed, or multiple cysts may distend the cortex in hydronephrosis.

Finally, there is a form of diffuse infiltrating carcinoma of the kidney, the organ being much enlarged but not greatly deformed, which probably originates as papillary carcinoma of the pelvis (Graupner, Hildebrandt, Beneke, Milne). A case of this type, which first suggested a diagnosis of endothelioma, is described by Beneke and Namba and attributed to a trauma. Yet the neighboring lymph-nodes were tuberculous.

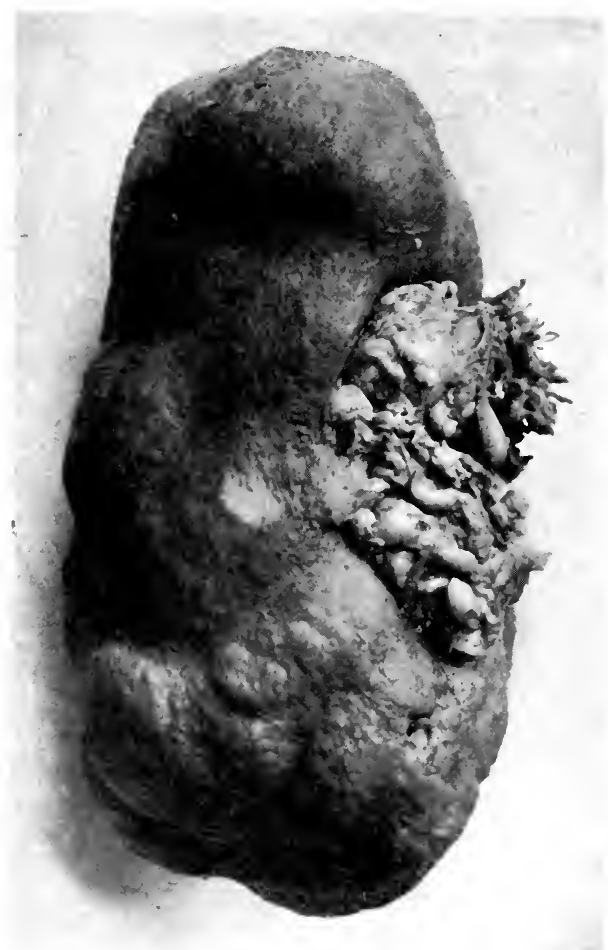

FIG. 369.-Papillary epidermoid carcinoma of renal pelvis.

Metastases are often observed in adjoining nodes, adrenal, peritoneum, liver, lung, and in Kischensky's case, in the femur. Mesenteric, aortic, and perirenal nodes may be invaded (Rayer, Drew, Volcker). Along the genitourinary tract extension occurs by $(a)$ lateral encroachment of the tumor through the lumen, submucosa, or mucosa of the ureter; $(b)$ by the development of new tumors over increasing segments of the mucosa, and $(c)$ according to Albarran, by implantation.

The structure presents two distinct types: (I) Simple papillary epithelioma, suggesting a relation to benign papilloma, and (2) squamous-cell carcinoma.

(I) Papillary epithelioma shows overgrowth of the cell layers of benign 
papilloma, atypical cell forms, and infiltrating qualities. In infiltrating tumors the papillary structure is soon lost and the growth is alveolar or diffuse or scirrhous.

The transformation of benign into malignant papilloma has been made clear by Albarran. In Battle's case simple papilloma curetted from the pelvis soon recurred with malignant structure. Pantaloni observed a recurrence in malignant form in the scar after nephrectomy for a uniformly benign papilloma. In portions of chiefly benign papillomas, especially at the base, atypical overgrowth is sometimes observed. The long duration of symptoms preceding the discovery of a malignant papilloma suggests the development of a slowly growing benign tumor followed by malignant transformation. Yet benign papillomas appear to have existed for 6, I2, and

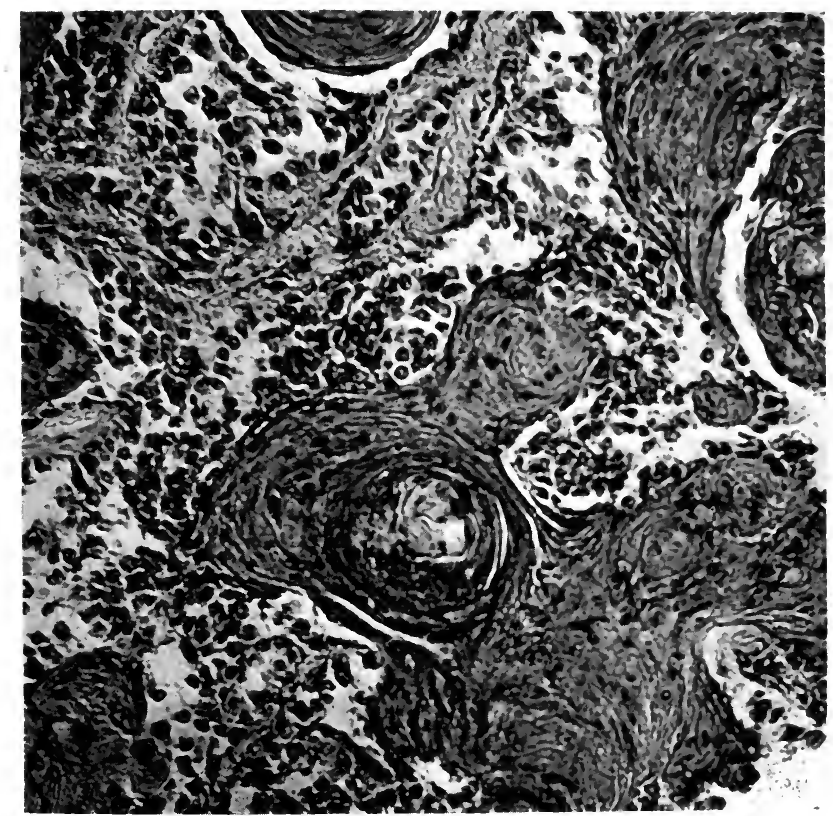

FIG. 370.-Epidermoid carcinoma distending the renal pelvis. Alveolar growth of small polyhedral cells with abrupt and extensive pearl formation.

27 years (Battle, Albarran, Pantaloni) and the malignant properties have developed only after incomplete extirpation.

(2) Squamous-cell carcinoma of the pelvis was described by Kundrat in I89I and later cases have been reported by Rundle, Graupner, Kischensky, Beisenbruch, and Scheel. These remarkable tumors are usually of large size when discovered, but Battle observed squamous changes in a small villous tumor of the pelvis and Rundle found the upper ureter invaded by a squamous carcinoma of moderate dimensions. In other cases the pelvis has been distended and the wall infiltrated by a bulky growth. The kidney is either transformed into large cysts by hydronephrosis or infiltrated and destroyed.

The squamous changes are very pronounced and much of the growth 
may be composed of epithelial pearls many of which are hornified or calcified. In Schmorl's case distant metastases showed the same structure.

That the pelvic and ureteral epithelium is capable of extensive epidermization is well attested by the reports of Wendel of numerous cases of leukoplakia, usually associated with calculi. Rokitansky described a very advanced condition, with much scaly desquamation, as "cholesteatoma." Ziegler found marked epidermization in pyelitis calculosa, and Beselin reported an advanced case with tuberculous pyelitis. Yet all cases are not associated with calculi or leukoplakia, so that the excessive hornification must be regarded as a tendency inherent in the growth.

In a case studied by the writer in a woman of 58 years, the kidney and tumor measured $14 \times 19 \mathrm{~cm}$. The tumor involved chiefly the extrapelvic fat, but had occluded the lower half of the pelvis, along which it invaded the kidney. The renal cortex was thinned out over several large smooth-walled cysts. The ureter was destroyed down to the bladder. There were metastases in aortic and mesenteric nodes and in the uterus. The main bulk of this large tumor was composed of hornified and slightly calcified epithelial pearls surrounded by a moderate number of spindle or cubical granular epithelium. The transition from one to the other type of cell was very sharp.

(3) Alveolar carcinoma of the renal pelvis is not clearly distinguished from papillary epithelioma, which in advanced and malignant cases may lose its papillary character and present the structure of alveolar or diffuse or scirrhous carcinoma. Hildebrandt describes a large tumor of pelvis and kidney, the pelvic portions of which were papillary, the renal being scirrhous. Yet Albarran describes papillary adenoma with cylindrical cells in pelvis and ureter, and Giordano found chiefly cylindrical cells in a pelvic tumor, while several others have appeared to show no trace of papillary but exclusively glandular and alveolar carcinoma (Hedenius, Wirsing, Hektoen, Hartmann, Israel). In Grohe's case the structure resembled thyroid gland and he attributed its origin to the tubular glands of the pelvic mucosa.

The early pelvic and ureteral tumors are described as flat and infiltrating rather than papillary. The advanced tumors are large, infiltrating and highly malignant.

\section{TUMORS OF THE RENAL HIIUS}

The tissue of the renal hilus gives origin to several forms of simple and of complex tumors, which may be distinguished from tumors of renal parenchyma, or pelvis, or adrenals, and for which special embryological errors appear to be responsible. Tumors of the hilus develop about the renal vessels and the ureter, which are soon compressed, and the kidney is often found stretching over the tumor mass as a shell incloses a nut. The tumors are often bilateral. Most of them are observed in children, but not a few appear in adults.

Lipoma of the hilus is not infrequent. It may be difficult to separate from hypertrophy of the fat tissue which follows atrophic conditions of the kidney. The hilus fat tissue is also the seat of a chronic productive inflammation in which the fat is infiltrated with lymphocytes and plasma-cells, and thickened by new connective tissue, so that it is much increased in bulk. Hollen described a well-circumscribed lipoma.

Angiolipoma surrounding the vessels and pelves of both kidneys is described by Salomon.

Myxoma and myxosarcoma of the hilus produce bulky masses which infiltrate the surrounding tissues and cause displacement, stretching, and atrophy of the kidney. In four large tumors of this type $I$ have found considerable admixture of actively growing fat tisste. 
Schluter reported a large myoma which had caused nearly complete atrophy of the kidney.

Diffuse Sarcoma of Hilus and Renal Pelvis.-A peculiar form of sarcoma of the hilus and pelvic tissue has been described by de Vecchi and by Salomon. The tumors were bilateral and occurred in infants under 2 years of age. The tumor tissue encircled the ureter, great vessels, pelvis, and papillæ, but was well demarcated from the renal parenchyma. The structure was complex, presenting areas of round, spindle, and giant-cells, which invaded and destroyed the fat and muscle tissues. de Vecchi found areas of chromaffin cells which he regarded as a portion of the neoplasm derived from misplaced chromaffin tissue, but Salomon interpreted these cells as remnants of the normal paraganglia or adrenal structures of this region which had resisted destruction by the tumor. Both authors were inclined to regard the tumors as derived from remannts of the nephrotome or sclerotome and therefore related to the Wilms' tumors of the kidney, but the grounds for this interpretation are not clear. 


\section{CHAPTER XXXVIII}

\section{TUMORS OF THE ADRENAL}

The embryology of the gland and the very variable histology of its tumors render it difficult to choose a satisfactory terminology of adrenal growths. Since the adrenal cortex is of mesothelial origin, neoplasms of this tissue mav be designated as mesotheliomas. The tendency of many of these tumors to reveal mesoblastic or sarcomatous properties also supports the suggestion of Adami and Woolley that recognition of this fact be granted by employing the term "mesothelioma." Yet the acquired epithelial characters of the glandcells predominate in most of the growths, which behave as adenomas or carcinomas. In others the structure of epithelial alveoli is mingled with areas of spindle-cells as in carcinoma-sarcoma. In a well-known group the cells are large, round, and atypical and the structure presents itself as roundcell sarcoma. Yet there is very good reason to believe, as pointed out by $\mathrm{J}$. $\mathrm{H}$. Wright, that all the round-cell sarcomas of children are derived from the nervous elements of the sympathetic. Hence they may properly be called neurocytoma. Of these some show definite ganglion-cells and nerve-fibers, and have been called neuroma, or glioma ganglionare. Finally, there are rare tumors composed of chromaffin cells, and although these cells are originally derivatives of the neural elements, their acquired characters dominate the neoplastic process and demand for them a separate classification.

The entire group falls into two divisions, (I) cortical and (2) medullary, in which the following special forms are observed.

\section{ADRENAL TUMORS}

Cortical: (a) Hyperplasia, nodular or diffuse.

(b) Adenoma.

(c) Carcinoma.

Medullary: (a) Focal hyperplasia of

I. Glia-tissue or

2. Chromaffin cells.

(b) Neuroma ganglionare.

(c) Neurocytoma, "sarcoma."

(d) Suprarenal chromatophoroma.

Hyperplasia.-Various grades of focal hyperplasia of cortical tissue are well known but rather rare conditions in the adrenal. All gradations exist between simple focal hyperplasia and true adenoma, and the distinctions between them are not always emphasized. Letulle observed both conditions in the same adrenal. Virchow described the group as struma suprarenalis.

The nodules appear as pinhead or pea-sized masses of light yellow or brownish color, sharply circumscribed, but not encapsulated. Larger masses of apparently the same significance may reach the size of a cherry and form a well-defined projecting tumor.

Diffuse hyperplasia of the adrenal is also observed. It affects the cortes and is usually associated with feminine pseudohermaphroditism (Glynn. Lit.). In these cases the adrenal may be as large as the kidney (Marchand. Febiger). 
The structure reproduces that of the cortical zones. The cells and cell groups are often larger than normal and may be irregularly arranged. The tumors are usually very fatty and have been mistaken for lipomas, but usually they present the structure of the zona glomerulosa or reticularis.

Letulle has shown that nodular hyperplasia of the adrenal may be associated with a similar condition in the liver and occurs in a variety of diseases. Sex abnormalities, as overgrowth of hair and genital organs, are observed occasionally with nodular hyperplasia, but especially with definite tumors of the adrenal.

Adenoma of the adrenal is distinguished from nodular hyperplasia by its atypical and neoplastic structure. From the presence of adenomatous areas in portions of malignant adrenal growths it is evident that many of the latter arise from the former. Adrenal adenoma is comparatively rare, Kelynack finding 3 cases in 1500 autopsies. In some of the cases of pseudohermaphroditism the process in the hyperplastic adrenals approaches adenoma.

The tumors are usually single, but may be multiple and bilateral. They form projecting masses of yellowish or reddish color, well circumscribed from the cortex of the gland, but often deforming or destroying the organ. Hemorrhage and central softening may occur. They may reach a considerable size, weighing 2 to 4 ounces while retaining the adenomatous structure. A fibrous stroma is nearly constant and may be abundant, rendering the tumors quite firm and fibrous (fibro-adenoma). More often the stroma is fine and vascular. Rarely the tumors are surrounded by a definite capsule. The structure may reproduce exactly that of the adrenal cortex, with increase in the size of the cells, hyperchromatism of nuclei, and often with marked fatty changes. Or the cells may be smaller, more numerous, granular, and free from fat. Irregular alveolar structures with lumina and low papillary growths are described by Kelynack in benign tumors. Lumina surrounded by cylindrical cells are also described in Manasse in adrenal adenoma, and this structure appears to be very frequent in horses.

Rolleston described a brownish fibro-adenoma as large as a walnut. Extensive fatty changes may produce a structure resembling lipoma. Auvray and Pfeiffel described a large tumor, in a case of pseudohermaphroditism, as adeno-angiolipoma. In a few reported cases the cells were markedly pigmented, but these tumors were probably medullary.

The limits of growth of adrenal adenoma are restricted. In pure form they do not reach large dimensions or produce metastases and most of them are encountered at autopsy. Malignant transformation into adenocarcinoma or still more atypical growth appear to be of relatively common occurrence.

Carcinoma.- Malignant tumors presenting epithelial characters are much the most frequent of adrenal growths. Winkler observed Io such cases to three sarcomas, and suggests that even the sarcomas arose from cortical epithelium. Rolleston and Marks, however, interpreted only 9 of 26 collected cases as carcinoma or malignant adenoma, but they assume that sarcoma may arise from the cortical epithelium. The average age of incidence in 9 cases was 44 years. Winkler observed 2 cases, at I9 years and at 66. Several of his cases gave a history of trauma.

The tumors are usually found at autopsy, of large size, involving the whole of the adrenal, adherent to or fused with the kidney and bound to neighboring structures by many extensions.

The earlier tumors are smaller and embedded in the enlarged adrenal, of which the outline may be partly retained. At all stages they are soft, yellowish, and prone to hemorrhage and necrosis. Central softening may yield cysts of large size, but the very cellular tumors are usually rather solid. 
Early and wide-spread extensions and metastases are a prominent characteristic of adrenal carcinoma. Although there are some reports of a stationary tumor persisting for months or years before active growth occurred, most of the adrenal carcinomas progress rapidly from the first symptom.

The local extensions invade the kidney and perirenal tissue so that it may be difficult to separate the tumor from the kidney. The adrenal is often obliterated. The suprarenal and renal veins are early invaded as in hypernephroma. The renal pelvis may be distended by tumor masses (Winkler). The peritoneum is often the seat of numerous implantations. The opposite adrenal is frequently involved, and there are extensions to the spleen, liver, and abdominal organs. The lymphatics are invaded with enlargements of retroperitoneal, mesenteric, and thoracic nodes. The intravenous growths may reach in a solid column to the right heart (Gerber), but usually they break up and give origin to very numerous distant metastases. Hardly any organ of the body may escape secondary deposits, but they are most frequent in liver, lungs, and brain. The frequency of bone metastases in tumors of supposed adrenal origin has long been emphasized, but in most of the reported cases the tumor arose in the kidney and its exact nature was not fully determined. In cases of fully verified adrenal carcinoma bone metastases are infrequent. They occurred in 3 of Winkler's Io cases. In 48 cases of various malignant adrenal tumors collected by Hartmann and Lecene, bone metastases were demonstrated in only $\mathrm{I}$, while the liver was involved in 23; lungs, 9; preaortic nodes in 8 ; peritoneum, 5; stomach, pancreas, heart, and brain, occasionally.

The structure of atypical adrenal carcinoma is essentially specific, but there are atypical forms which are difficult to separate from sarcoma.

(a) Adenocarcinoma or adenoma.malignum is a type which resembles adenoma, but shows atypical areas of malignant character and yields metastases. Yet the arrangement of cells is orderly and reproduces the alveoli of the zona glomerulosa or the anastomosing sheets of enlarged columns from the zona fasciculata. The diffuse growth of atypical cells as in true carcinoma is missing. There is usually considerable vascular stroma. Lumen formation is rare. The cells closely resemble the fatty granular cells of the normal gland, but are large and the nuclei are hyperchromatic. Various secondary changes result from necrosis and hemorrhage, including especially the formation of giant-cells, cysts, and pigmentation.

As interpreted by Rolleston and Marks, the group of malignant adrenal adenoma includes a large proportion of the malignant tumors, and transitional stages to carcinoma are so frequently found in these growths that a sharp separation from other types is impossible. Clinically they are fully malignant, although Winkler described a very large tumor of this type without extensions.

(b) Fully developed carcinoma also presents several peculiarities of structure. A prominent type, often encountered, shows chiefly large granular and fatty cells in perivascular arrangement. This structure is but slightly removed from the vascular adenocarcinomas, but is usually more active, atypical, and malignant. By softening of central lines of cells the structure may assume a pseudopapillary type.

Giant-cells of large size may become very numerous in tumors which are chiefly perivascular carcinomas. Almost the entire tumor and its metastases may be composed of giant-cells. Such structures have been interpreted as sarcoma, as in Affleck and Leith's case. They are fully paralleled by giant-cell types of hepatoma. Carcinosarcoma is a complex structure seen in not a few adrenal carcinomas (Adami, Woolley). Certain areas of 
these growths may present any one of several carcinomatous types, as alveolar or perivascular, while in other large areas or sheets the cells are elongated, spindle shaped, compact, and granular. It appears possible that the spindle form represents a recrudescence of mesoblastic tendencies, but in many cases such cells occur owing to purely local factors.

Diffuse carcinoma appears in the most malignant forms of adrenal carcinoma. The cells lose all resemblance to adrenal parenchyma, and appear as. rounded or polyhedral granular cells notably free from fats and glycogen. They grow diffusely or in small groups, rapidly penetrate surrounding tissues, and produce bulky metastases. That some of the so-called sarcomas of the adrenal are highly atypical growths derived from the renal parenchyma appears quite possible.

The course of malignant adrenal tumors is usually rapid and fatal. In several cases the usual course has been interrupted by a successful extirpation (Hartmann, Lecene, Lit.). Beginning with signs pointing to a growth in the region of the kidney, there usually follow pain, palpable tumors, asthenia, and cachexia. Pigmentation of the skin was stated by Fox to be very rare, but this symptom and others of the Addison complex appear more frequently in recent reports. Winkler reports 2 cases of well-developed Addison's disease associated with cortical tumors of the adrenal. In a series of cases various disturbances of the sexual characters, hirsuties, pseudohermaphroditism, precocious development of the genitals, and obesity have been associated with adrenal tumors. It has been clearly shown by Glynn that these sexual and nutritional disturbances are observed only with cortical tumors, hyperplasia, adenoma, or carcinoma.

\section{MedUllary AdRenal Tumors}

Tumors of the adrenal medulla include three well-defined types:

(I) Neuroma ganglionare.

(2) Neurocytoma. Neuroblastoma.

(3) Chromaffin cell-tumors. Paraganglioma.

Virchow refers to the occurrence of multiple nodules in the adrenal which he assumed to be gliomatous.

While pure examples of each of these tumors are observed there are many transitions between them, and in Wahl's case all three were represented in portions of the same tumor. The more adult neoplasms occur chiefly in adult or elderly subjects, the malignant growths are practically limited to children, while the chromaffin tumors are often associated with some phase of neurofibromatosis. All these features accord with the origin of the tumors, which arise from the sympathetic anlage which goes to form the adrenal medulla, and the varying structure of the tumors indicates an origin at successive stages of the differentiation of the sympathetic neuroblasts. Similar tumors have been reported, and will doubtless be recognized more frequently, throughout the entire distribution of the sympathetic system and its chromaffin glands (Herxheimer, Wahl, Lit., Dunn, Lit.). The great majority occur in the adrenal medulla and in the abdominal sympathetic.

(I) Neuroma ganglionare is a rare tumor in the adrenal and belongs in the group of ganglionic neuromas which arise throughout the sympathetic system.

A small glioma composed of medullated nerve-fibers and proliferating ganglion-cells in the adrenal was described by Weichselbaum in $188 \mathrm{I}$, and similar cases have been reported by Dagonet in a subject of Addison's disease by Schmidt and by Bruchanow. Schmidt's tumor arose from the 
suprarenal plexus and only partly involved the gland. These tumors may reach considerable dimensions, but they are not locally aggressive and metastases are absent. They probably arise from well-differentiated sympathetic nerve tissue. The structure shows a preponderance of medullated and nonmedullated nerve-fibers, among which lie groups of more or less well-formed proliferating ganglion-cells. Many of these are of relatively small size and approach the form of indifferent polyhedral cells. Others are large and multipolar and may contain lipoid pigment. Dagonet's tumor contained much

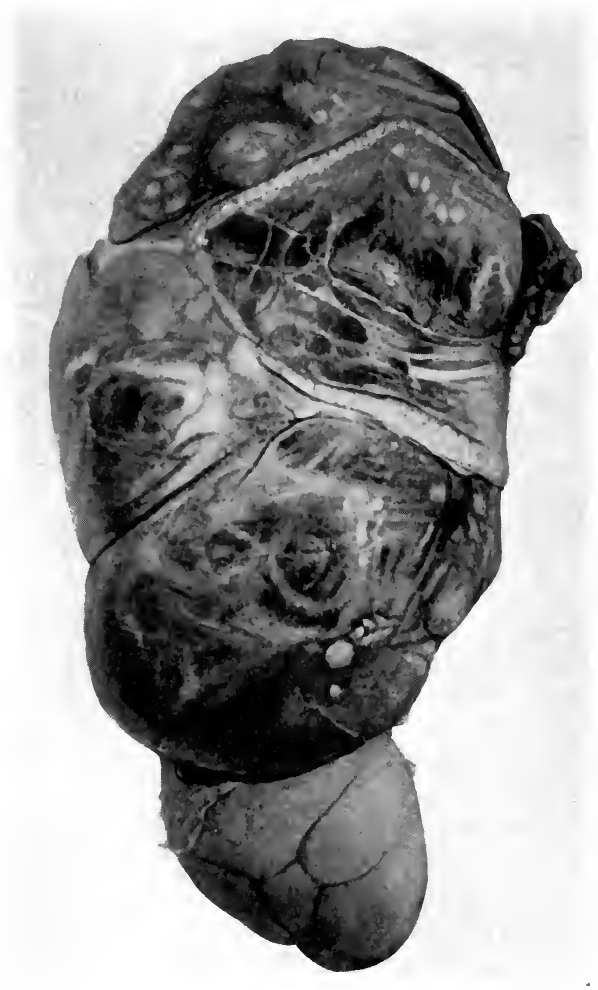

FIG. 371.-Drawing of neuroblastoma of right suprarenal, viewed from anterior aspect. The outline of the flattened residue of the suprarenal body is distinguishable on the front of the upper part of the tumor. The hemorrhagic character of the tumor is visible through the thin capsule. The kidney is seen below and behind the lower pole. (After Dunn, J. P. B., I8.)

fibrous tissue and some smooth muscle-cells. Ohse's tumor appears to have developed largely within the adrenal in a child of 5 years and was more cellular than others of this group.

(2) Neurocytoma.-Marchand, I89I, described a cellular tumor of the adrenal in an infant of 9 months and interpreted the structure as a reproduction of the anlage of the sympathetic nerve tissue of the organ. A fuller description of the structure and origin of these tumors was furnished by Kuster in I905. In a child of I4 weeks the right adrenal was transformed into a tumor as large as a watch, the medulla of the other adrenal showed early 
stages of the same growth, while the liver was extensively invaded. In a second case, in an adult, the tumor was as large as the fist, but metastases were absent. The tumors were sharply circumscribed from the cortex, gray, with dark vascular areas. The structure presented very numerous cell nuclei without definite cytoplasm, very often arranged in the form of rosettes. Some of the cells were large, better defined, and resembled the polyhedral cells of the medulla. The matrix was chiefly granular, but in the adult case fine fibrils were demonstrable. The stroma supported fine blood-vessels. Kuster interpreted the tumors as of neuro-epithelial origin, and Wiesel supported this general view, pointing out that in the development of the adrenal just such rosettes of cell nuclei appear in the sympathetic anlage. From them are developed both ganglion-cells and chromaffin cells. Lapointe and Lecene in

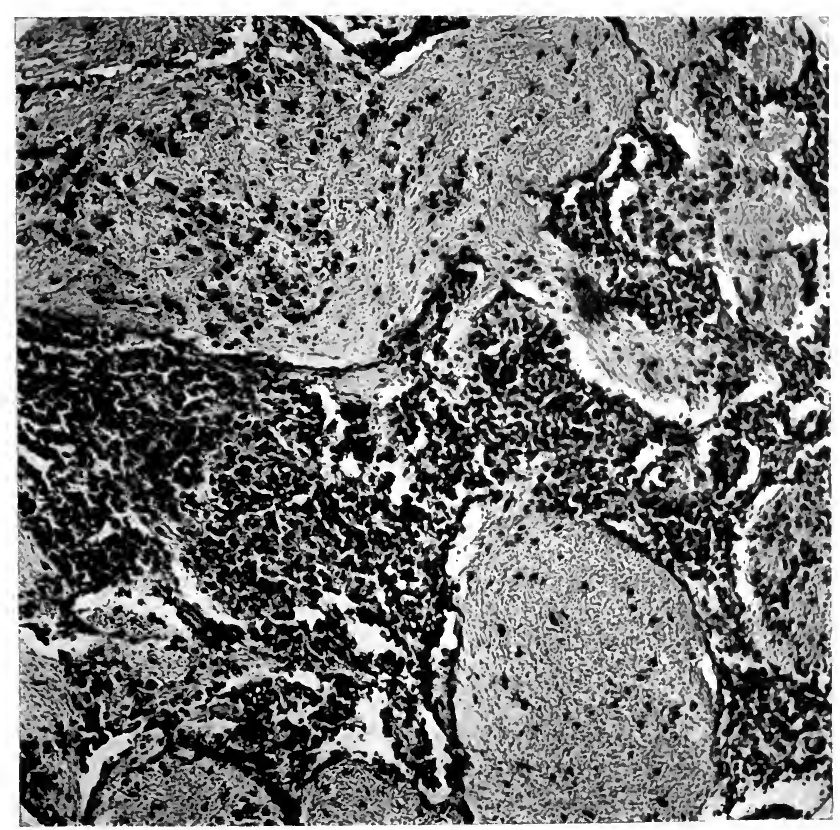

FIG. 372.-Neurocytoma of adrenal in an infant. Note bulky masses of glia fibrils.

describing a typical case demonstrated glia fibrils in the matrix and concluded that the tumors arise in misplaced portions of adrenal nerve tissue, but not in the normal anlage that forms the sympathetic elements, which yield tumors with medullated fibrils and ganglion-cells. Yet it seems that there are transition forms between the two groups, indicating a common origin.

The resemblance between the cellular "gliomas" and the so-called congenital sarcomas and lymphosarcomas of the adrenal immediately became obvious, and Kretz in 190.3 suggested that a series of lymphosarcomatous tumors of the liver were derived from the sympathetic formative cells. In Igro J. H. Wright pointed out these resemblances in detail, concluded that the adrenal and hepatic round-cell sarcomas of infants and many other territories, were probably of neurogenic origin, and he proposed for them the term "neurocytoma." 
This conclusion has been fully justified. The grounds for assuming the existence of true mesoblastic sarcomas of the adrenal have never been adequately established. There is no evidence that the stroma or blood-vessels contribute to these growths. Of traces of fetal blood formation the adrenal preserves none. The existence of lymph-follicles in the normal adrenal is not proved. Dagonet described groups of lymphocytes in the cortical zones, but derives the parenchyma from these cells. The lymphocytes, lymph-follicles, and plasma-cells occasionally seen in the adrenal are probably the result of circulatory and nutritional disturbances (Oberndorfer). The diagnosis of sarcoma has been based on the presence of indifferent round cells, the origin of which has not, as a rule, been considered. Yet in many cases the supposed sarcomas have presented the typical rosettes of neuro-epithelium, while the clinical and anatomical characters are so uniform as to indicate a single origin. Hence the work of many clinical observers who have shown that adrenal "sarcoma" appears in certain characteristic clinical types is not reduced in value by the demonstration of the true origin of these tumors. Not a few of them appear to be of bilateral origin (F. Holst, Sabrazes, Husnot).

In recent years the retroperitoneal malignant neurocytomas of infants and similar tumors in many other regions have frequently been recognized and the structure has repeatedly been analyzed in detail (Landau, Anitschow, Symmers, Wahl, Dunn). These histological studies have extended the scope of the tumor so that it is necessary to include in this group many if not most of the retroperitoneal round-cell sarcomas of infants, and many embryonal and peculiar growths of cervical, thoracic, and peripheral nervous system, while a few cases have been found in the brain. The structure varies in the degree with which nerve elements are developed, but in the most immature types are imperfect rosettes, very numerous but ill-defined fibrils giving an abundance of hyaline stroma, or many hyaline globules staining with eosin. The hyaline globules resolve themselves into bundles of imperfect nerve fibrillæ connecting groups of neurocytes. Various other structures may be interpreted as imperfect ganglion-cells, axis-cylinder processes, or glia fibrils. Falk and others attribute the excess of fibrillar material to the activity of sheath cells which differentiate from the tumor-cells and lay down nervefibers. The fibrils do not stain well by Weigert's neuroglia stain, nor by Mallory's method, yet they may be detected in formalin sections stained by eosin and hematoxylon. Landau secured excellent results with iron-hematoxylon and Van Gieson's stain. Pick and Bielschowsky stained the fibrils by the latter's method, which is not always successful. Dunn, by means of Bielschowsky's and Levaditi's stains, was able to show that the great bulk of the fibrillar material is composed of naked axis-cylinders. The fibrillæ forming the rosettes may resist all the specific stains. The metastases are usually of round cells only, with or without fibrillar material, but in the cases of Miller and of Jacobsthal the secondary tumors contained ganglion-cells.

In a recent case of the writer's, in a child of 2 years, the tumor first appeared on the inner side of the left thigh below Poupart's ligament and grew rapidly to enormous dimensions. It destroyed all the muscles of the thigh from knee upward, invaded and filled the pelvis, destroying the muscles and invading kidney and adrenal. The retroperitoneal nodes were extensively involved, and by way of the lymphatics the lungs were largely replaced by very numerous masses originating from the walls of bronchi and vessels. The liver escaped. The tumor appeared to originate from the femoral sympathetic plexus. The structure of the main tumor was that of round-cell sarcoma with indistinct alveolar arrangement and occasional excess of hyaline stroma. In the lungs there were many rosettes surrounding hyaline globules and great excess of hyaline stroma in which many fine fibrils were discernible. In many areas there were occasional large isolated multipolar cells suggesting abortive ganglion-cells. The fibers in material fixed in formalin failed to take Bielschowsky's stain, but were well defined by picroacid-fuchsin.

Clinical Types of Adrenal Neurocytoma.-(a) Adrenal Sarcoma in Infanis with Cranial Metastases (Hutchinson's Type).-Hutchinson described a 
peculiar clinical syndrome in $\mathrm{I} 3$ cases of adrenal sarcoma occurring in children from 3 months to 9 years of age. The disease begins spontaneously or after trauma, with ecchymosis of one or both eyelids, soon followed by exophthalmos, a tumor of the orbit and temporal region, with extensions to the auricular and submaxillary nodes. The orbital tumor reaches large dimensions, while an abdominal growth may be discovered only at autopsy. The adrenal growth may be as large as a walnut or a child's head, but shows little tendency toward local extension. Secondary growths appear also in ribs, spine, and long bones, as well as in liver and other organs.

The structure of the tumors is usually described as round-cell sarcoma, but in 2 cases the diagnosis was hypernephroma (Aisenstein, McCarty), and in I, melanotic carcinoma (Reimann, Chiari).

In the case of Tileston and Wolbach the tumor contained many rosettes very similar to those observed in retinal and other gliomas. These authors accept the adrenal origin of Hutchinson's tumors because the structure was identical in all, while in 6 cases only adrenal, lymph-nodes, and bones were involved.

There is thus no doubt that certain adrenal tumors in children follow Hutchinson's type, but it appears also that other tumors, hypernephroma, etc., may produce the same syndrome. Moreover, all round-cell tumors of the adrenal do not produce orbital metastases.

(b) Congenital Sarcoma of Adrenal and Liver in Infants (Pepper's Type).The prominence of the hepatic metastases gives a somewhat striking clinical character to a group of cases described by Pepper. The symptoms are those of a rapidly enlarging abdominal tumor caused by diffuse and nodular growths in the liver and adrenal. The other organs were not involved. One or both adrenals may be affected and the tumors may reach large dimensions with little tendency toward local invasion except to the liver. The duration varied from ro days to r6 weeks.

Structurally the tumors were described as round-cell or lymphosarcoma. Winkler describes a case in an infant of 2 years in which the adrenal and kidney were destroyed and there were bulky metastases in liver, lungs, skull, musculature, and many lymph-nodes. Hence the presence of hepatic metastases does not exclude them in other organs.

Much light has been thrown on the various anatomical and clinical characters of these tumors by the study of P. S. Frew. He points out that the medullary origin is indicated by the frequent persistence of a portion of adrenal cortex along one segment of the tumor. It is quite evident, as emphasized by Glynn, that the structure differs entirely from that of the cortical growths. Frew regards the entire group as a type of medullary carcinoma which extends along the lymphatics and yields secondary growths in different territories, depending upon which adrenal is primarily involved. The lymphatics of the left adrenal pass out with the vein at the lower pole of the gland and join with the renal vein and its lymphatics, which have rich connections with the lumbar and preaortic nodes. Tumors of the left adrenal accordingly invade the regional nodes extensively, passing $(a)$ downward along the iliacs to the pelvis and groin, $(b)$ across to the mesenteric nodes, $(c)$ upward to the hilus of the liver and thence into the liver by the portal spaces, $(d)$ through the posterior mediastinum, $(e)$ along the intercostal lymphatics, and $(f)$ through the deep cervical chain along the carotid artery to the base of the skull. Hence with left-sided tumors deposits occur in the above locations, especially in the ribs and cranium (Hutchinson's type). Secondary growths occurred in the substance of the liver in 4 of 22 cases and only in very young subjects 
succumbing to very rapid growths (Pepper's type). The lungs were never involved with tumors of the left side.

From the right adrenal the vein and lymphatics pass from the upper pole and join the vena cava and main lymphatic trunk. This portion of the adrenal is uncovered by peritoneum and is in contact with the bare area of the liver. Hence local lymphatics are less involved, while the extensions are to the surface of the liver ( 20 to 29 cases); the pleura and lung (9 to I6 cases in which the disease extended beyond the diaphragm); and to the right cervical nodes with right-sided exophthalmos. The tumors of the right gland were

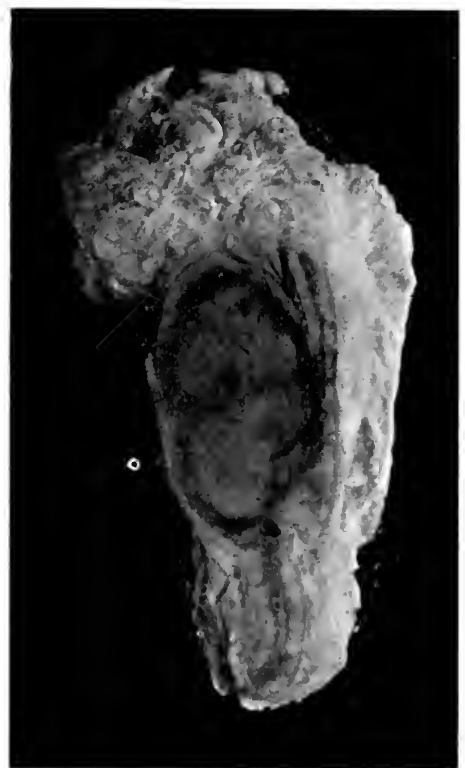

FIG. 373.-Medullary tumor of adrenal. also more confined to the abdomen ( 12 cases), reached a larger size, and tended to involve the kidneys by direct extension into the pelvis (i I cases). These rules were followed by the neurocytomas of Kuster and Lapointe and Lecene.

(c) Medullary Tumors with Adrenalin Content and Nephritic Symptoms.-In a male subject of 47 years, suffering from active nephritis with glycosuria, albuminuria, cardiac hypertrophy and hypertension, and showing finely granular dark red kidneys, Orth found a medullary tumor of the right adrenal, $7 \times 4 \frac{1}{2} \mathrm{~cm}$. A watery filtrate of the tumor gave a green color when treated with ferric chlorid, and contracted the living frog's pupil, showing the presence of adrenalin, while an extract from a secondary carcinoma in the adrenal failed to give these reactions. Orth concludes that the cardiac hypertrophy was referable to overproduction of adrenalin by the tumor.

The structure of this growth was identical with many of the round-cell sarcomas occurring in children. The small round cells he regarded as forerunners of the chromaffin cells, while others resembled the larger polyhedral cells of the normal medulla. Vaquez and many others have noted high arterial tension with adrenal tumors (Ellis, Lit.).

(3) Medullary Chromaffin Cell Tumors.-Nodular hyperplasia of the pigmented cells is not infrequently observed in the adrenal, and appears in the form of small brownish tumors as large as a pinhead or bean (Schmorl). Such nodules are more common in the cow (Stilling) and a pigmented tumor in the cow's adrenal is described by Zanfroginni.

Definite pigmented tumors of the adrenal medulla have been described by Berdez, Manasse, Susuki, Kawashima, and Laignel-Iavastin. Observations on tumor growth of chromaffin cells extend, however, over most of the territory known to contain elements of the chromaffin system.

Weichselbaum found chromaffin cells in a renal alveolar adenoma which may have been derived from the adrenal. This observation is not infrequently repeated. In a tumor of moderate size occupying the whole of the medulla of the adrenal I found very large polyhedral non-pigmented cells in alveolar arrangement. The tumor was evidently derived from the chromaffin cells of the medulla. A similar peculiar structure appears in certain 
renal tumors. Two small pigmented tumors penetrating the upper pole of the renal cortex are mentioned by Wiesel and Stoerk. Stangl found a hard brownish tumor at the bifurcation of the aorta, which he derived from the pigmented cells of Zuckerkandl's organ. Monckberg describes a tumor at the angle of the jaw, and another in the left hyoid region, containing scanty pigmented cells yielding chromaffin reaction, which he derived from the carotid gland. For this entire group of tumors Alezeis and Peyron propose the name "paraganglioma."

The tumors in the adrenal have been of relatively small size, usually as large as a walnut, but Susuki's tumor measured $10 \mathrm{~cm}$. in diameter. 'They

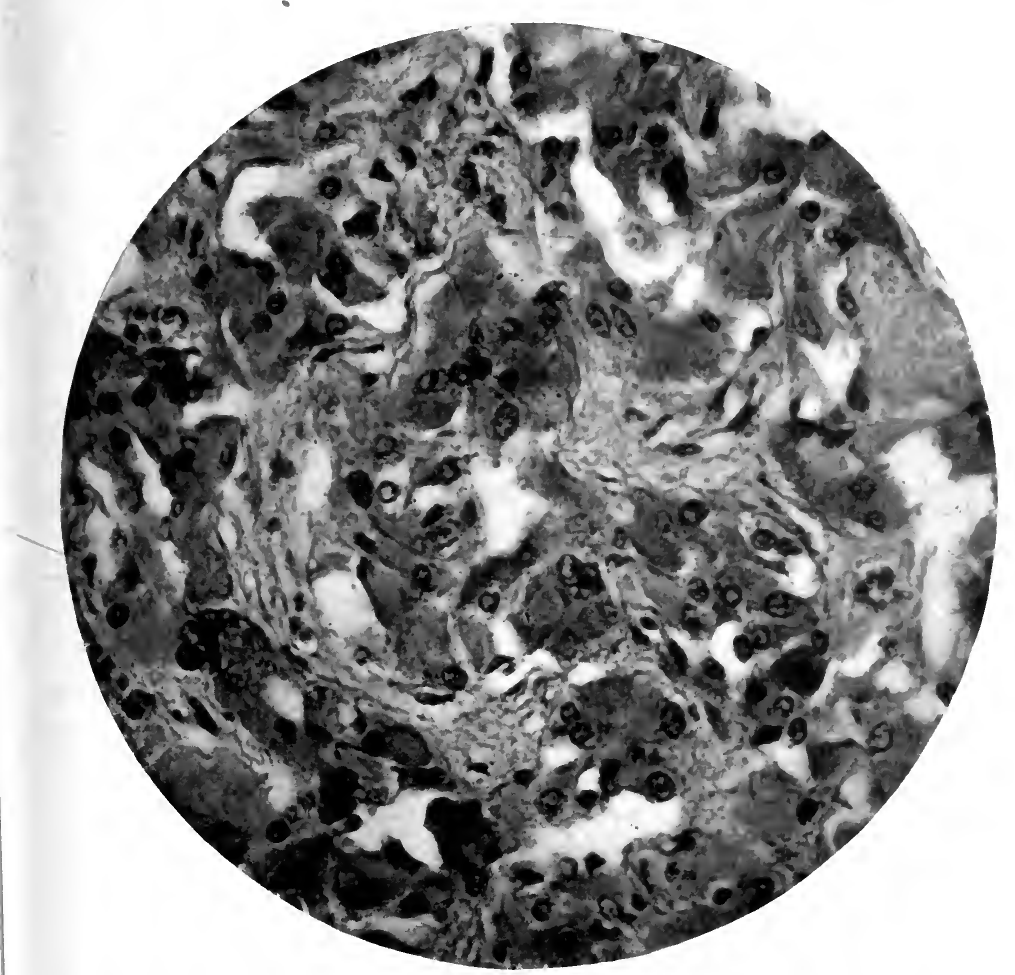

FIG. 374.- Structure of tumor of chromaffin cells of medulla of adrenal.

are sharply demarcated from the cortex, of which a portion usually remains at one segment. The substance is soft, cellular, and vascular, with areas of hemorrhage and necrosis. Manasse found the large venous sinuses invaded, but without metastases.

The structure presents small groups or a diffuse growth of round, oval, spindle-, or polygonal cells most of which are small. Giant-cells with single or multiple nuclei also occur. The pigment is irregular in distribution, but gives the morphology and the many special staining reactions devised for chromaffin substance. Homogeneous acidophile cell inclusions are usually present. In Susuki's case some of the larger cells approached the type of sympathetic ganglion-cells. 'The stroma is composed chiefly of capillaries. 
An interesting physiological relation of this tumor appears in several cases which occurred in subjects of cutaneous neurofibromatosis and pigmentation of the skin (Susuki, Kawashima, Herxheimer).

Adrenal Melanoma.-Small brownish benign cortical tumors of the adrenal are described by Lucksch. They occurred in adults, were composed of cortical cells containing pigment resembling melanin, and they seem to form a probable source of the malignant pigmented tumors of the adrenal.

Primary bilateral melanotic malignant tumors of the adrenal are described by Davidsohn, Goldzieher, Tuczek, and Maclachlan. The tumors occurred in adults, were of moderate size, and sometimes produced extensive metastases, but primary sources in the skin or choroid were missing. The origin of these tumors it is difficult to determine. Not a few of the typical adrenal carcinomas contain areas of pigmented cells of the adrenal type. Davidsohn thought he recognized the structure of the zona fasciculata in a mesenteric metastasis, claimed to have demonstrated adrenalin in this tissue, and derived the tumor from pigmented adrenal cortical cells. Tuczek found the pigment a true melanin and not a lipochrome as is found in the adrenal cortex. He derived his tumor from the pigmented ganglion-cells of the medulla, which contain melanin. Maclachlan concluded that the adrenal melanoma arises from the wandering chromatophores which are said to exist in the loose tissue about the adrenal. Yet the tumors seem to involve the whole organ, which long preserves its form. 


\section{CHAPTER XXXIX}

\section{TUMORS OF PROSTATE}

Hypertrophy of Prostate.--In about 33 per cent. of men over 60 years of age, and occasionally much earlier, but never over 70 years, the prostate becomes notably enlarged (Moullin). Greene and Brooks believe that the condition may begin in youth, becoming noticeable only in later life. The process probably begins in middle life (Gross, White), but very early cases, collected by Belfield, appear to be of uncertain nature and probably neoplastic.

The growth affects one or both lateral lobes, or the anatomical median lobe, or the glandular tissue surrounding the orifice of the urethra, producing growths of variable configuration. Obstruction to the urethra is caused especially by enlargement of the tissue about the urethra which, as Home (I8Ir) showed, produces a valvular, polypoid or ring-shaped tumor at this point (Home's lobe). Lesions originating at this point may spread to the trigone, producing low or bulky intravesical growths. The dimensions are often considerable, Gross reporting a prostate weighing $288 \mathrm{gm}$. Tortuosity of urethra, retention of urine, and hypertrophy and sacculation of bladder are frequently observed, and dilatation of ureter and renal pelvis may result from torsion of the vas deferens where it crosses the ureter (Tandler, Zuckerkandl). After reaching a certain size most cases remain stationary, and in these fibrosis may occur. Or a tendency toward fibrosis may be prominent from the beginning.

The enlarged gland is hard or soft, depending on the prominence of connective tissue and fibrosis or of gland tissue. On section there is diffuse smooth translucent tissue, or nodular masses some of which may shell out of their capsule. Many dilated alveoli may yield a spongy or cystic appearance.

The structure shows two main types: (I) diffuse growth of connective tissue with islands or strands of smooth muscle and (2) glandular overgrowth. Brooks found fibrous overgrowth the predominant process in $4 \mathrm{I}$ of $5^{8}$ cases. The connective tissue is cellular, edematous, infiltrated with lymphocytes and large hydropic cells, or firmer, fibrous, or hyaline. The latter is often a late stage of the former. Atrophic glands may be included in the new tissue. Rarely there is a definite increase of smooth muscle-cells, usually in foci resembling small myomas. More often the smooth muscle is atrophic. The true prostatic myoma of Virchow should be separated from prostatic hypertrophy. The cases of hypertrophy in which there is pure overgrowth of smooth muscle are extremely rare (Halle, Albarran, Ciechanowski).

The glandular overgrowth yields normal prostatic alveoli, or the cells may be more numerous, appear in two or three layers, or in papillary projections into enlarged lumina. Many areas are composed of cystic alveoli lined by flat cells or partly filled by low papillary projections. Desquamation of lining cells, retained secretion, concretions, and round-cell infiltration of stroma are nearly constant, and while the structure varies in different areas, the whole picture indicates a chronic catarrhal and productive inflammation with unusual epithelial overgrowth. 
Occasionally the multiplication of small alveoli is extensive, secretion is missing, the stroma scanty, the cells hypertrophic, and the condition assumes an adenomatous character.

Rarely the alveoli are filled with atypical large or small hyperchromatic cells, lateral sacculations form, and the process passes into carcinoma. The frequency of this change to carcinoma is at present uncertain. It has been observed by Klebs, Socin, Kaufmann, and others, and Greene and Brooks find that it occurs in 5 to Io per cent. of cases of prostatic hypertrophy. Halle and Albarran found suspicious changes which they designated as adenocarcinoma in I4 of Ioo cases, and they note the frequency with which elderly men, long suffering from prostatic hypertrophy, die with symptoms of cancer. I have repeatedly seen miniature carcinomas, as judged by structure, in portions of enlarged prostates. The carcinomatous change probably begins rather early and sharply, if it is to occur at all.

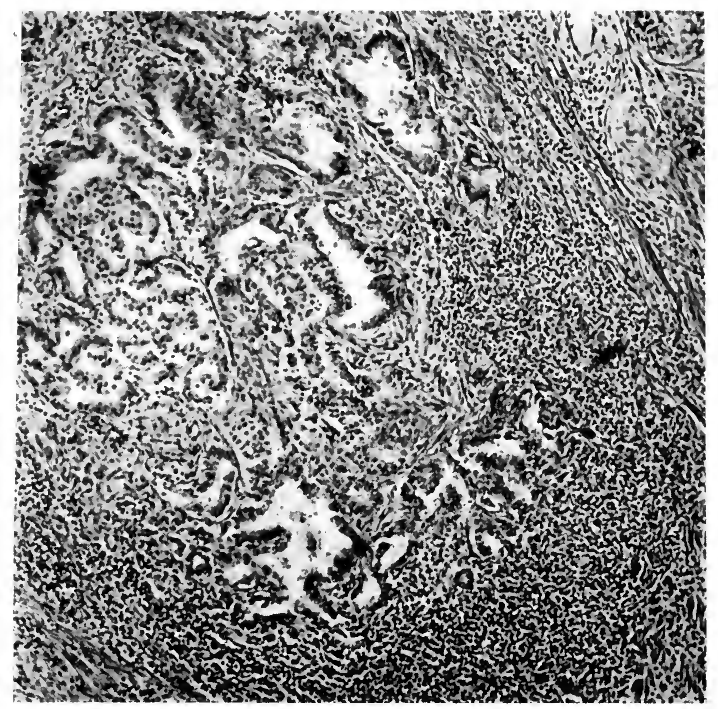

FIG. 375.-Focus of atypical proliferating alveoli in chronic prostatitis. Carcinoma in other portions of prostate.

Etiology.-The older authors looked for predisposing and exciting factors, in gout, syphilis, horse-back riding, alcoholism, sedentary habits, constipation, gonorrhea, strictures, and stone.

Later histological studies led many to conclude that prostatic enlargement is a tumor process, and chiefly a myoma, occasionally adenomyoma, or adenoma. Velpeau likened the tumor to myoma uteri. Yet the prostate is not the homologue of the uterus and the histology and history are not those of myoma. Although Casper found I9 myomatous cases in 24, later interpretations have shown that true myomatous prostates are very rare. More or less isolated or pedunculated myomas arise in the prostate, but the structure of the new fibromuscular tissue in ordinary hypertrophy is that of a simple overgrowth and quite different from that of uterine or other myomas. Several features stand against the neoplastic theory for the glandular hypertrophy. This process begins diffusely or in multiple foci, 
it is associated with periglandular fibrosis, and the process is self-limited. The structure is not that of a neoplasm, and when true adenoma of the prostate arises in the course of hypertrophy it presents a very different structure. In my material adenoma is rare in enlarged prostates.

An inflammatory origin has been maintained by most recent observers, but is firmly opposed by Thompson, Frische, and many experienced clinicians. Ciechanowski attributes the condition to chronic posterior urethritis chiefly of gonorrheal origin: and he emphasizes the constant presence of inflammatory lesions. The inflammation begins in the ducts, and causes first partial or complete occlusion. The retained secretion leads to enlargement of alveoli, new growth of epithelium, perialveolar fibrosis, and hypertrophy of musclefibers. Exudative changes may be limited to portions of the enlarged gland, while other areas show chiefly cellular overgrowth. This distribution is not inconsistent, as some assume, with the inflammatory hypothesis. A centrally located inflammatory process leads to hypertrophy of glands, while a peripheral localization tends toward glandular atrophy and fibrosis (Ciechanowski, Rothschild). Greene and Brooks and Motz also find the ducts occluded, and trace the progress of overgrowth to infection and retained excessive secretion. My own material all indicates the presence of an inflammatory element, but it is not clear that the inflammation is always primary. In this respect the inflammatory theory is distinctly in need of further support. That all cases are not gonorrheal is shown by Keyes, who in 433 cases of hypertrophy found only 18 giving a history of previous prostatitis. Yet he places the proportion of males who have had gonorrhea as high as 80 per cent. While the histology of the process may perhaps be satisfactorily explained as a result of productive inflammation in a peculiar organ yet the clinical data cannot be so readily dismissed.

Launois and Guyon find that all prostates of elderly men show periacinar fibrosis, hypertrophy and diminution in number of muscle-fibers, disorder of gland tissue, and arteriosclerosis. Hypertrophy or atrophy may follow. In line with this theory stand the speculations regarding a functional disturbance in the gland. Furbringer suggests that the marked overgrowth is a compensatory process following senile insufficiency, and Rovsing assumes that there may be increased rather than senile failure of functional capacity. There appears to be a relation to testicular function, but its details are not clear. White, Moullin, and others find that prostatic hypertrophy recedes after castration, but this event has been too exceptional to establish this method of surgical treatment (Keyes). There does not appear to be any complete parallel inflammatory condition in other glands, but in the thyroid a very similar process results from functional disturbance, and involution of the breast approaches in some cases the type of prostatic hypertrophy. Considerable importance attaches to these various hypotheses, since somewhere in this field must be found the key to the unusual glandular overgrowth which often exceeds the limits of productive inflammation. It may become possible to divide the cases into inflammatory and true hypertrophic forms, as suggested by Young and Geraghty, but at present it seems necessary to assume that the condition results from a combination of both factors.

Carcinoma of Prostate.- Until recently carcinoma of the prostate was held to be a rare disease, forming 0.27 per cent. of the carcinomas in men, according to Gurlt, or 0.4 I 8 per cent. in Heimann's series. Probably through more areful examination of apparently simple hypertrophies of the gland and more accurate diagnosis, carcinoma of the prostate now appears more frequently. Engelbach, I888, collected II4 reported cases of malignant tumors. E. Kaufmann personally observed 22, Wolff collected reports of 89 carcinomas 
and 22 sarcomas. Since there is some uncertainty in the diagnosis of both these tumors, present statistical data are still open to revision.

Etiology.-The chief condition predisposing to prostatic cancer is chronic hypertrophy. Notable examples of the transformation of simple hypertrophy into carcinoma are recorded by many observers. The evidence in these cases usually consists in a long history of hypertrophy in cases presenting as carcinoma, and about ro per cent. of prostatic cancers give such a history (Wolff, Kaufmann). Likewise the careful study of enlarged prostates reveals early cancerous changes in about I 9 per cent. (Albarran, Halle, Greene, Brooks). From these data one may safely conclude that early or suspicious cancerous changes in the prostate are much more frequent than is the established disease.

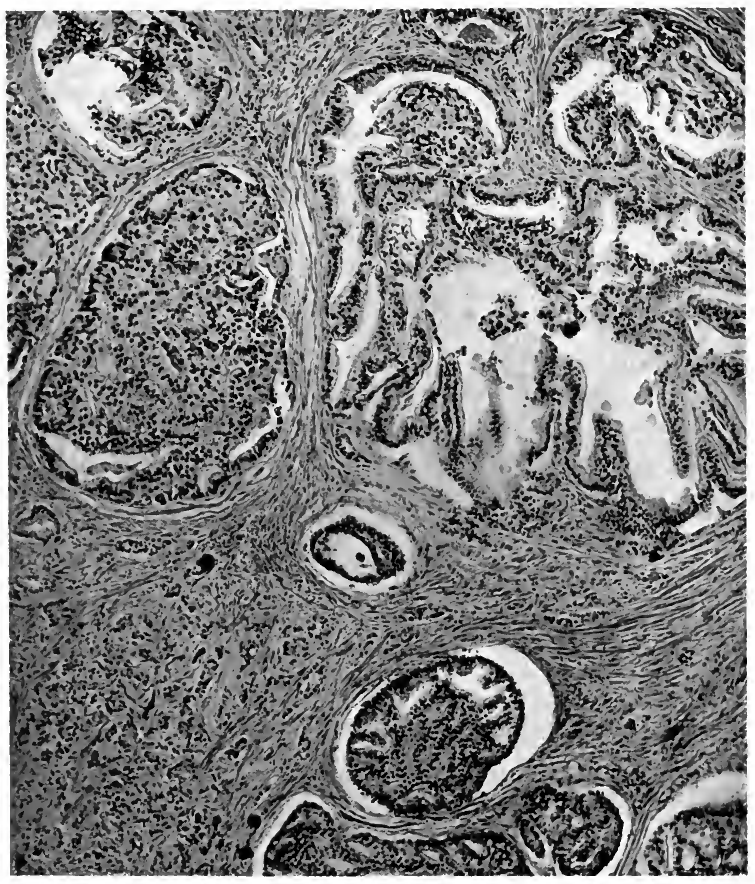

FIG. 376.-Adenocarcinoma of prostate.

A satisfactory elimination of previous hypertrophy has not commonly been attempted, so that the relation of the two processes still remains to be determined.

The chief age of incidence, like that of hypertrophy, is the seventh decade, when 68 per cent. of the cases occur, but the disease has been observed between 40 and 50 years. Wolff collected 6 cases under 40 years, at least one of which (Billroth's) was probably true glandular carcinoma in a subject of $3 \mathrm{c}$ years. A rapid carcinoma with regional metastases in a youth of 17 years is reported by Gardiner and Cummins.

An influence of heredity is not apparent, and the antecedent persona history is usually declared negative. Wolff saw carcinoma develop after incomplete removal of an enlarged prostate. The susceptibility of workers 
in anilin products to bladder tumors does not appear to extend to the prostate, but Kaufmann's case of "lymphosarcoma" occurred in an anilin worker.

Gross Anatomy.-In the early stages carcinoma may appear in simple or multiple foci of firm opaque texture, in a hypertrophied organ. Usually the whole organ is enlarged, hard, and fixed, but one lobe is frequently the chief or sole seat of the disease, while the pars intermedia only was affected in the cases of Billroth and Tyson. The disease may also arise in the glands of the prostatic urethra. To what extent the seminal vesicles are the primary seat of carcinoma involving the prostate is uncertain. Berger, Fenwick, and Walter describe carcinomas which appeared to originate in the vesicles, but the exact source of these tumors is questionable. Guelliot's case of vesicle cancer in a man of 50 years failed to involve the prostate or bladder. Kaufmann's tumor in a man of 87 years diffusely invaded the prostate, but a larger mass involved both vesicles. Arising in an atrophic gland the prostate may remain small while metastases become general (Jolly, v. Recklinghausen, Hebb). A markedly indurated prostate in a man over 50 years of age producing urinary retention is highly suspicious of carcinoma, especially if it is very hard and not very large. When with the indurated prostate there is induration along the seminal vesicles with pain and hematuria the presence of carcinoma is practically certain (Young).

The capsule resists invasion, but is commonly penetrated first along the seminal vesicles. Per rectum the enlarged gland usually presents a hard nodular surface, and extensions to neighboring structures and lymph-nodes with fixation of the organ may be detected. In advanced cases a bulky tumor fills the pelvis, fuses the organ with neighboring tissues, or invades bladder, rectum, and lymph-nodes.

The bladder is invaded in 57 per cent. of cases (Kaufmann). Frequently the invasion passes through the lymphatics, producing multiple or confluent submucous nodules or diffuse infiltration in the fundus ( 32 per cent.). Direct invasion through the trigone, simulating primary vesical cancer, is also common. Belfield has described a bulky tumor filling the bladder. In late stages the secondary vesical tumors may ulcerate with hemorrhage, or perforate the wall, producing peritonitis (Tyson, Engelhardt) or a rectovesical fistula. The bladder may become hypertrophied and sacculated. The ureters are invaded from the vesical wall as in bladder carcinoma, or occluded by nodules at the orifices, or compressed by enlarged lymph-nodes.

The seminal vesicles are early invaded on one or both sides and may be obliterated. Rarely the urethra is involved by submucous infiltration usually by way of the lymphatic connection with the seminal vesicles, and the growth may extend well along the urethra and into the corpora cavernosa (Tailhelfer, Kaufmann).

Diffuse prostatopelvic carcinoma (Guyon) results from diffuse invasion which binds prostate, seminal vesicles, bladder, and rectum in a firm mass :o the pelvic bones. Here the chief symptom may be obstipation. Frisch bserved perforation of the obturator foramen and a large perineal tumor.

Lymphatic Extensions.-According to Sappey, the prostate is very richly upplied with lymphatics. They arise in the glandular lobules and pass hiefly backward and anastomose with the rectal vessels. Two main trunks on each side drain the peripheral plexus, the inferior trunk passing outward o a lymph-node lying on the lateral wall of the true pelvis. The superior essels pass upward along the wall of the bladder, communicating with lymphatics in the muscle tissue and leading to a chain of nodes on the external liac vessels. There are rich connections between the vessels of prostate nd seminal vesicles. The vesical mucosa is very poor in lymphatics, but 
in the trigone small vessels may be infected from the peri-urethral tissues which, in turn, communicate with those of the seminal vesicles. From these relations it appears that urethral invasion must be by retrograde transport, that invasion of seminal vesicles is early, that secondary growths readily form in the pelvis, while metastases in the fundus of the bladder do not early reach the mucosa or ulcerate.

The extent of lymphatic invasion in prostatic carcinoma varies extremely, but is less than with many carcinomas. As a rule only the regional nodes are involved, especially those of the true pelvis in close relation to the seminal vesicles. The frequency of invasion of the inguinal nodes (I 6 per cent.) must be referred to lymphatic connections with the seminal vesicles, pelvis, and urethra, and retrograde transport seems to occur in these cases (Courvoisier). From the true pelvis extensions are along the great vessels, iliac, lumbar, vena cava, to mesenteric and retroperitoneal nodes, liver, and kidneys. Further extensions to the thoracic and cervical nodes are not infrequently observed. The supraclavicular nodes may be involved (6 per cent.) with or without invasion of other cervical chains, and with freedom of the thoracic duct (Carlier). Very general lymphatic invasion is described by Baumgarten, and extensive visceral metastases by Kaufmann. The organs involved in 22 cases were: liver Io, kidneys 3 , adrenals 3 , pancreas 3 , peritoneum 4, Jungs 9 , pleura I 2, dura 5, brain, heart, thyroid, spleen I in each.

Skeletal metastases of markedly osteoplastic character occur in a notable group of prostatic carcinomas (Silcock, Thompson, Recklinghausen, Courvoisier). Similar osteoplastic processes are observed, especially with mammary carcinoma, and with tumors of stomach, gall-bladder, thyroid, etc., but the early and frequent occurrence and extensive distribution belong chiefly to the prostatic disease. Kaufmann calculated that about 70 per cent. of prostatic carcinomas cause skeletal metastases, as compared with 37 per cent. for thyroid carcinoma (Limacher) and I4 per cent. for mammary cancer. Of I 6 cases with skeletal metastases, Kaufmann found more or less osteoplastic tendency in I4, and from the literature he collected 20 cases with extensive growth of bone.

In these cases the prostate may be relatively small and fibrous, the local extension moderate, the organs free, while the bones are the seat of widespread osteoplastic carcinoma. Under these circumstances the prostatic tumor may be overlooked. More often the pelvis contains a bulky tumor involving prostate, vesicles, and bladder, and the viscera are also invaded. The spinal column, pelvis, long bones, skull, ribs and sternum, scapula, and clavicle have been affected with extensive thickenings, deformities, excavations, and fractures. The new bone may be diffuse, obliterating the shafts and marrow cavity, or perisoteal and stalactite, and the structure is spongy or eburnated. The osteoplastic process predominates over the osteoclastic; but both may be equally prominent. Severe anemia may result from destruction of bone-marrow (Schmorl, Braun).

The histological process is described by Recklinghausen as carcinomatous osteitis. The tumor-cells lodge in the small venous sinuses, cause stasis and hemorrhage, which is followed by reactive growth of fibrillated, osteoid, and finally osseous tissue. The tumor-cells become inclosed in the bone, assuming the function of osteoblasts, and bony tissue may be most abundant in very cellular areas of tumor-cells where connective tissue is wanting (Wolff Braun, Sasse). Participation of connective-tissue osteoblasts is also observed (Erbsloh). Extensive resorption of the new bone by osteoclasts may follow the plastic process. Schmorl refers to a similar lesion in the lungs as metastatic osteosarcoma. 
Axhausen attributes the osteoplastic process entirely to the chemical influence of the carcinoma cells. The transformation of epithelial tumorcells into bone-cells, accepted by Wolff and Kaufmann, he did not observe. There is little actual new growth, but chiefly an absorption and redeposit of the old. Cyanotic hyperemia (Recklinghausen), inflammatory changes in the marrow (Lenziger, Kaufmann), and primary bone necrosis (Askanazy) he finds of secondary importance. Lacunar resorption by osteoclasts furnished by the connective tissue is the chief factor in the absorption process, and the new bone forms in the connective tissue both by a metaplastic and a neoplastic process.

The course of prostatic carcinoma varies from that of rapid cases occurring chiefly in middle life and terminating within a few months, to the more prolonged cases, which extend over 3 years or more. Wolff in 46 cases found

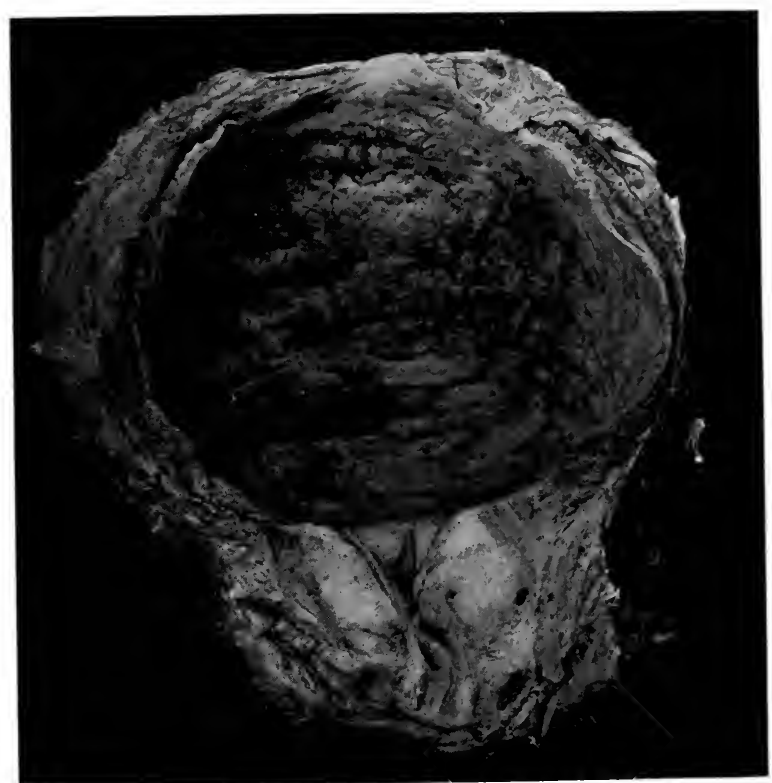

FIG. 377.-Carcinoma of prostate, involving one-half of the prostate, without much enlargement, but giving distant metastases.

39 per cent. lasting less than I year, 28 per cent. I to 2 years, 8.7 per cent. $2 \frac{1}{2}$ years, 24 per cent. 3 years or more.

Sarcoma is much more rapid and has usually proved fatal within 6 months, but firm spindle-cell sarcomas may continue as long as 2 years. Iymphosarcoma is usually of very short duration.

The clinical course of prostatic carcinoma is very notably dependent on the structural type of the tumor, so that different forms appear as radically different diseases.

(I) Adenoma or adenocarcinoma arising on chronic prostatitis may give the symptoms of chronic hypertrophy and the carcinoma be discovered only in the extirpated gland.

(2) Adenocarcinoma, usually arising on hypertrophy, may give a large, hard, nodular prostate, which exists for many months before involving lymph- 
nodes. Eventually it produces local extensions, and finally it generalizes. It is often extirpated, and regularly recurs.

(.3) Fully developed carcinoma, alveolar or diffuse, may fail to cause much enlargement of the gland, prostatic symptoms are moderate, and the disease is overlooked, or the metastatic tumors are regarded as primary, or the condition is discovered at autopsy.

Structure.-Malignant epithelial tumors of the prostate appear in three structural types-adenoma, adenocarcinoma, and carcinoma.

(I) Adenoma.-A pure adenoma of the prostate is rare. Diffuse circumscribed areas of adenomatoid overgrowth occur in many cases of chronically enlarged prostate, and at times this process becomes more pronounced

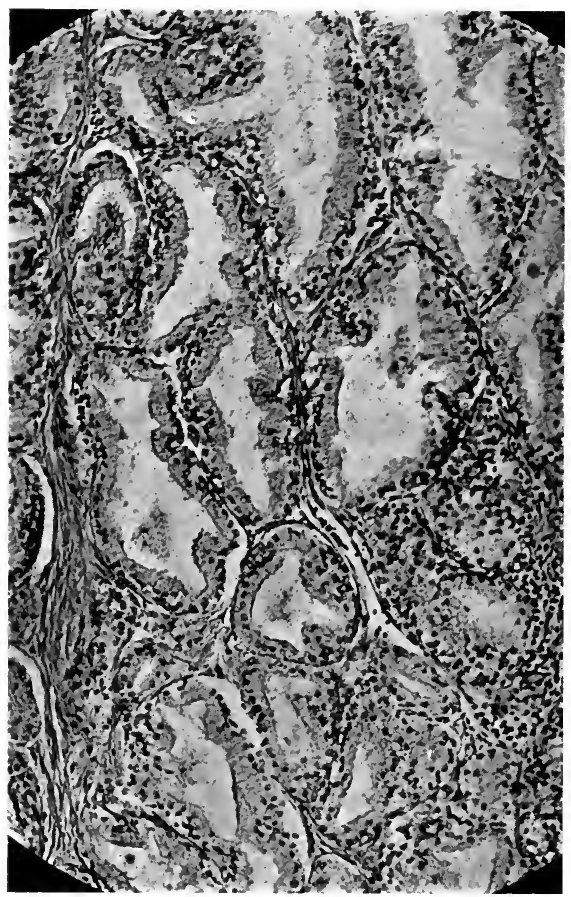

FIG. 378.- True adenoma in an enlarged prostate.

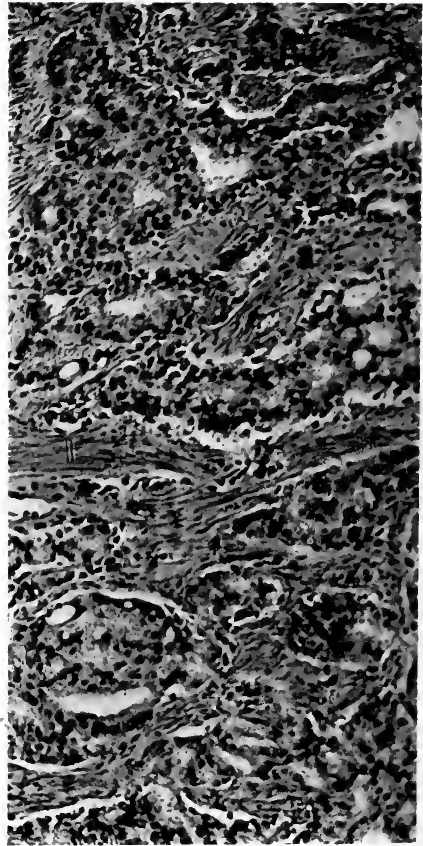

FIG. 379.--Malignant adenocarcinoma of prostate.

and assumes the characters of adenoma. Yet such areas usually form only a small portion of the enlarged prostate and grade off into inflammatory overgrowth. When a true adenoma develops in the prostate it appears always to be locally aggressive, and in this respect is malignant. More often it passes freely into adenocarcinoma or carcinoma.

I have examined I case in which the tumor exhibited throughout a rather uniform structure of malignant adenoma. It formed a partly circumscribed mass, $3 \mathrm{~cm}$. in diameter, lying in a prostate successfully removed for chronic hypertrophy. The lymph-nodes were free. The structure presented compact groups of small alveoli lined by high cuboidal clear epithelium with hyperchromatic nuclei. The stroma was reduced to a trace. In some foci the outlines of the alveoli were indistinct, the cells more atypical, and the 
growth almost diffuse. The tumor capsule was infiltrated with small groups of cells without definite lumen. The remaining portions of the prostate showed the changes of interstitial and glandular hypertrophy. A somewhat similar case is described by Ciechanowski as adenoma malignum, and this term seems properly applicable to the condition.

(2) Adenocarcinoma is the structure assumed by many malignant tumors, but it rarely occurs in pure form, passing readily into alveolar carcinoma. Kaufmann found no exception to this rule and my own material is of the same character. Not infrequently one finds large alveoli of typical adenocarcinoma scattered in wide fields of diffuse or small alveolar carcinoma and

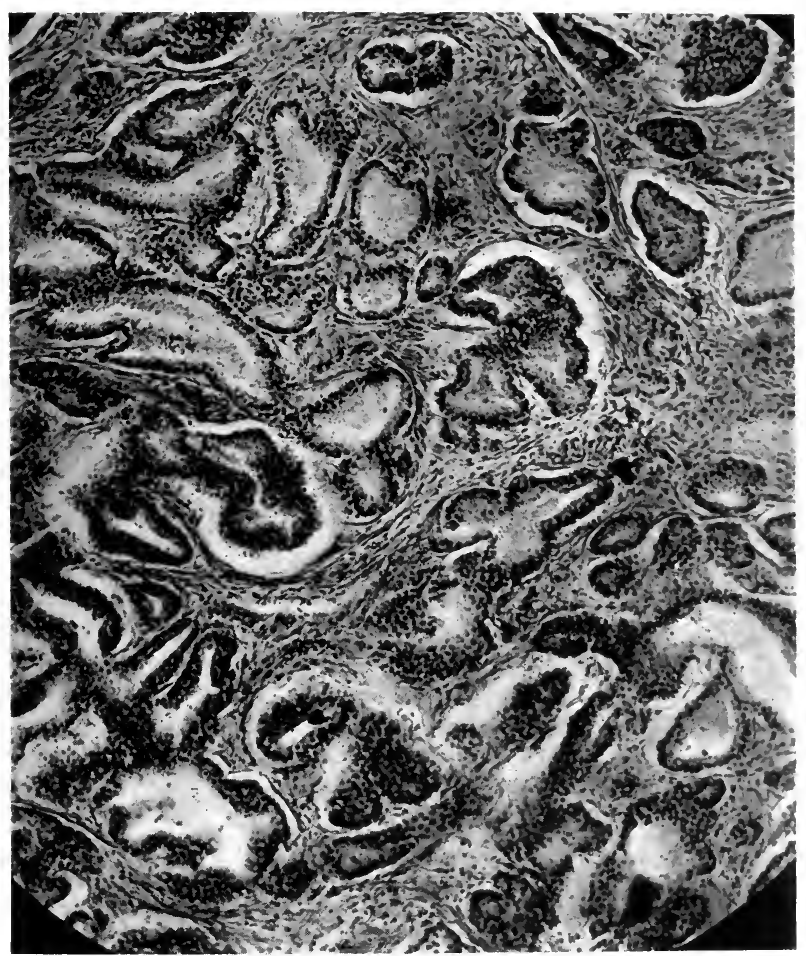

FIG. 380.-An area of adenocarcinoma in a chronically enlarged prostate.

it is evident that the latter represents a more rapidly growing derivative of the former structure, of which transitions may often be clearly traced.

The typical adenocarcinoma presents large spaces filled with masses of somewhat atypical cells forming numerous secondary alveoli. The size of these cells varies from large, clear prostatic epithelium, to small granular acidophile cells, and the carcinomas derived from them present corresponding variations. The simplest form of adenocarcinoma is the structure usually observed in the suspicious or precancerous areas of chronic hypertrophy, but compact groups of small alveoli, as in malignant adenoma, also occur in these cases, and occasionally multiple layers of atypical cells completely fill the alveolus and the structure passes directly into solid carcinoma. Con- 
siderable difficulty may be met in interpreting single fields presenting these various structures, but as a rule the usual signs of malignancy, as atypical cell form and excessive multiplication of alveoli, leave no doubt as to the nature and tendencies of the process.

Pure adenocarcinoma is doubtless not so active and aggressive as alveolar carcinoma and its presence probably signifies that a preliminary period of relatively slow growth has preceded the more malignant carcinoma with which it is usually associated. The change to carcinoma is so nearly constant that the later course of the disease is practically identical in the two conditions. Adenocarcinomatous structures are rarely observed in extensions and metastases.

(3) Carcinoma of the prostate presents many variations in structure. A pseudo-alveolar type is assumed when very numerous small groups of cells, inclosing a definite lumen, are closely packed together and infiltrate stroma, gland, capsule, and nodes. This is really a more malignant form of adenocarcinoma. Usually the cells grow loosely, forming small groups or large masses, or diffuse infiltrations, in which case the growth is indistinguishable from carcinomas from other sources. When the cells are small and cytoplasm scanty the structure closely resembles round-cell sarcoma and it is probable that some reported cases of prostatic sarcoma are of epithelial origin. In McKenzie's case, observed in this laboratory, the general structure was identical with that of lymphosarcoma, and it was only after repeated and very careful search in various sections that the clear evidence of its carcinomatous nature was obtained.

Hydropic swelling, formation of giant-cells, collections of colloid material, inclusion of fatty crystals, and round-cell infiltration of stroma are commonly observed, but it is noteworthy that extensive necrosis of prostatic carcinoma is very rare. Schlagenhaufer finds much doubly refractive lipoid material (cholesterin esters) in the cells of prostatic carcinoma, which he interprets as a sign of exaggerated functional activity of these cells.

Scirrhous carcinoma is observed in rare cases in which the prostate remains small and hard, as well as in foci of cellular tumors (Thompson, Barton, Matthias, Berger, Kaufmann). Boyd designated a case as colloid scirrhus.

Squamous Epithelioma.-In the upper anterior portions of the fetal prostate the ducts are lined by squamous epithelium up to the third month of life or later (Aschoff, Schlachta). In chronic supprative prostatitis Schmidt finds extensive squamous epithelial metaplasia and in a prostate from a man of 53 years he described beginning acanthoma. Klebs and Marchand describe acanthoma of the prostatic urethra, and acanthoma of the prostate is reported by Beyer and Buchal.

Sarcoma of the Prostate.-Sarcoma rarely occurs in the prostate and many of the reported cases are of uncertain nature. Kaufmann collected only 24 authentic cases, but discards many others for lack of microscopical report. Three occurred in infants under I year, seven others in the first decade, and only seven from 30 to 73 years.

The only well-defined variety of prostatic sarcoma is the rhabdomyosarcoma, which is also a probable source for any genuine mesoblastic roundcell tumor that may exist. Few of the older reports of sarcoma are of any value for critical analysis. While true spindle- and round-cell tumors probably arise from mesoblastic elements in children, it is highly probable that most of the cases reported as such, especially in adults, are diffuse carcinomas.

Round-cell sarcoma is the most frequent and the least definite form and no uniform description will apply to it. Socin describes a bulky tumor between bladder and rectum in an infant of 8 months, which was soft, vas- 
cular, cystic, and composed of diffuse small round and polyhedral cells without uniform stroma.

Kaufmann's case was a large myxomatous round-cell sarcoma in a child of $\mathrm{I} \frac{1}{2}$ years. In adults, I8, 2I, and 35 years, round-cell sarcomas are reported by Oliva, West, and Hughes. These tumors are difficult to distinguish from lymphosarcoma and from rapid atypical carcinoma. Thus Graetzer and Dupraz found an alveolar structure closely resembling carcinoma. Dupraz's tumor occurred in a man of 73 years, and gave osteoplastic metastases. Kapsammer reports as lymphosarcoma a very rapidly growing tumor arising from an old stricture of the bulbous urethra and invading the urethra. He refers to 2 other cases of round-cell prostatic tumors, in which an origin from Cowper's glands was suggested.

Lymphosarcoma in adults is described by Coupland, Kaufmann, and others. In Kaufmann's case in an anilin worker the prostate was much enlarged, fused with seminal vesicles, tumor folds projected into bladder, regional nodes with one exception free, while visceral metastases were wide-spread, and the cervical lymph-nodes were involved.

The existence of a true lymphosarcoma of the prostate is not satisfactorily proved. The structure of the gland does not favor the occurrence of such tumors. One of the cases most fully described is Kaufmann's, but there was nothing in the gross anatomy of this case to distinguish it from ordinary diffuse carcinoma and the metastases were exactly as one would expect in carcinoma, the regional nodes being practically free. The structural details were inconclusive. In Lefmann's case the cutaneous metastases suggest sarcoma, but the lymph-nodes were only slightly involved by very numerous metastases. Since studying McKenzie's case of pseudolymphosarcoma I have come to doubt the existence of lymphosarcoma of the prostate.

Rhabdomyosarcoma occurred in 3 cases described by Kaufmann, 2 in infants, $I$ in an adult of 26 years. The tumor grew rapidly to large dimensions. In the adult there were invasion of prostatic veins and widespread metastases, especially in the bones, with pernicious anemia.

The tissue was chiefly composed of spindle-cells and resembled ordinary cellular spindle-cell sarcoma with myxomatous tendencies. Round-cells also were abundant. The specific elements consisted in fusiform, round, and tubular structures, presenting various stages of differentiation of striated muscle-fibers from which all the tumor elements were derived. It is obvious that the distinction between simple spindle-cell sarcoma and myosarcoma may be difficult. Veil's spindle-cell tumor, with large polymorphous cells and growth into gland tubules, strongly recalls the myosarcomas. Of the nature of the embryogenic disturbance which gave origin to these tumors there are no indications.

Spindle-cell sarcoma occurs chiefly in children and produces a bulky tumor destroying the prostate and compressing adjoining organs. Tordens described a tumor as large as the child's head, arising from the left lobe, reaching the navel, of myxomatous character and partly cystic. Other soft sarcomas were also myxomatous, while the firm tumors are free from this element (Isambert, Marsh, Wind). An angiosarcomatous structure occurred in Matthias' case. A rapidly growing tumor in a child of 2 years, composed of adenoma and sarcoma, is mentioned by Birch-Hirschfeld. That the spindlecell sarcomas arise from the prostatic stroma is strongly indicated by their structure.

The seminal vesicles were the primary seat of a round- and spindle-cell sarcoma, with metastases in mesentery, kidney, and heart muscle, described by Zahn. 


\section{CHAPTER XL}

\section{TUMORS OF TESTIS}

In the testis more notably than in any other organ it is possible to maintain a single embryogenic origin of the great majority of tumors. It appears that all the common and nearly all the rarer tumors of this organ arise from totipotent sex-cells, and that the monodermal forms of these growths represent one-sided developments of tridermal teratomas. Very rarely the stroma, duct-cells, interstitial cells, and adult seminiferous tubules give origin to characteristic growths. Whether any of the malignant epithelial tumors may originate from fully differentiated cells of rete, epididymis, or testis it remains for future observations to determine. It is, therefore, of advantage to discuss testicular tumors from the point of view of their teratomatous origin and to trace their derivatives as far as may be possible. Later it may be necessary to reduce the scope of these teratomatous derivatives.

\section{TERATOMA TESTIS AND ITS DERIVATIVES}

Historical.- - In I 696 Saint Donat identified a rudimentary skull and pigmented optic cups in a testicular tumor and thus established the fetal character of some of these growths. In 1803 Prochaska found fetal limbs, and in 1833 Andre de Perrone observed hair, teeth, and bones in testicular tumors. The tridermal composition was first pointed out by Johnson, who distinguished ectodermal cysts lined by squamous cells, entoderm with columnar ciliated cells and mesodermal tissues. Numerous later observations have furnished a nearly complete series of fetal organ rudiments which may be encountered (Ohkubo, Lit.). Astley Cooper (I 845) described the cystic tumors under the term "hydatid disease," and Curling divided the growths into benign cystic and malignant solid types. With the aid of the microscope he detected that cancer regularly begins in the rete. Langhans' work with Kocher marked a distinct epoch, since with the aid of microscopical sections he was able to classify the tumors on structure. He classed many of the apparently simple tumors with teratoma, recognized the carcinomatous nature of the "alveolar sarcomas," and suspected that a large proportion of all tumors of the testis were of teratoid origin. Wilms advanced this work, showing that all the complex tumors are tridermal, and dividing them into adult cystic embryomas and more solid embryoid teratomas. Later Wilms, Ribbert, Pick, Ohkubo, and Chevassu showed that one element of the teratoma may overgrow the others, producing simple growths, and that many tumors called adenoma, sarcoma, carcinoma, chondroma, myxoma, etc., contain scanty traces of all three germ layers and are teratomas. The writer's study led to the conclusion that all the common tumors of the testis are of teratomatous nature, while pure adenoma, fibroma, myoma, and sarcoma are rare.

Varieties of Teratoma Testis.-Three main varieties of the tumor occur:

(I) Adult embryomas or teratomas.

(2) Embryoid, teratoid, or mixed tumors.

(3) Embryonal malignant tumors.

Numerous intermediate cases connect these types; the same tumor may present several structures; and some peculiar forms call for special description. Indeed, the variations in structure and course of these neoplasms are so numerous and definite as to require their description as distinct clinical and pathological conditions, connected only by a single etiological factor.

(I) Adult and complex embryomas constitute a relatively small group, characterized by the presence of definite rudimentary organs which may be so arranged as to resemble a parasitic fetus. 
The typical testicular dermoid is a sharply circumscribed tumor lying within the tunica, separated from the testicular tissue by a capsule and fused with the rete. On section the growth presents a large cyst containing hair and sebaceous material and lined by epidermis. A protuberance containing the main body of the embryo projects into or fills the cyst, and itself exhibits numerous small cysts and the various organ rudiments. The tumor is thus encysted by a continuous layer of epidermis which covers the embryo as well as the inner surface of the capsule.

In other cases the embryo is fused with the capsule and the dermal tissues appear in the form of solid portions, while multiple cysts occur at different points of the tumor.

Microscopical study reveals an extensive series of organ rudiments belonging chiefly to the cephalic extremity. The cutaneous covering presents hair, sebaceous and sweat-glands, and fat tissue, with islands of smooth muscle

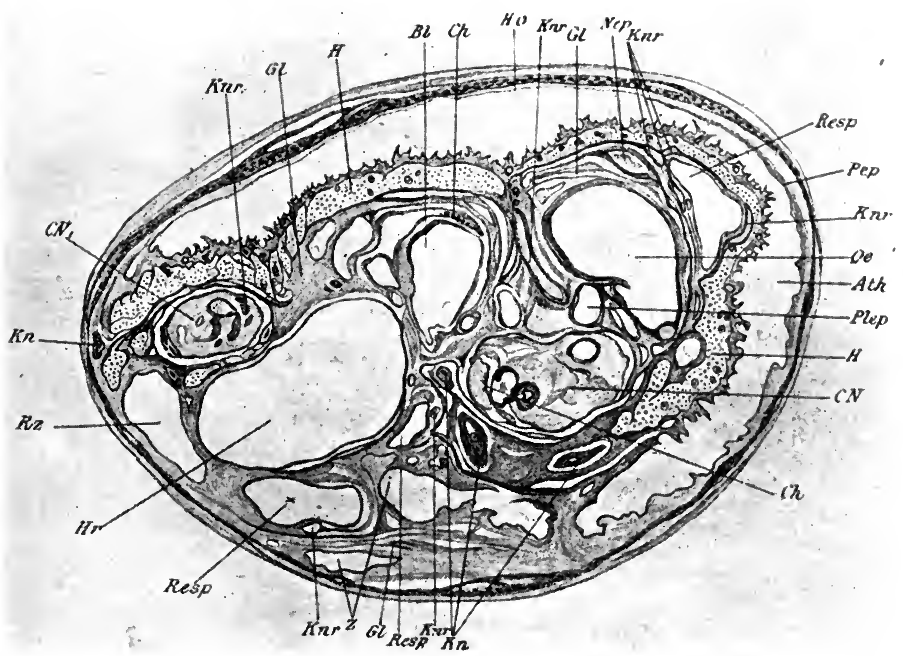

Fig. 381.-Structure of an encysted embryoma of testis. (After A. Muller.) Ho, Testicular tissue in capsule. $H$, Skin and appendages. $P e p$, Squamous epithelium. $A$ th, Cystic cavity containing sebaceous matter. CN, Central nervous system. $G l$, Glia tissue. Nep, Neuro-epithelial cell groups. Resp, Respiratory tract. Krn, Hyaline cartilage. Oe, Esophagus. $C h$, Choroid plexus.

tissue. Beneath lies brain tissue in which cellular and fibrillar layers may be discerned. Choroid plexus, dura, and pia have been traced in connection with cerebral tissue, and wide canals lined by flat cells have been interpreted as ventricles or central canal. Amyloid concretions, or brain sand, may be present. Bony plates are often found about the brain rudiment and in Geinitz's case these plates consisted of two lamellæ inclosing diploë and marrow arranged as a skull. Cavazanni found nucleated red cells in the marrow. Adult ganglion-cells in the course of nerve-trunks represent the sympathetic system. Isolated groups of ganglion-cells, neuro-epithelium, and glia tissue may also be found.

Many other organ rudiments have occasionally been observed. Optic vessels with choroidal and retinal pigment cells have frequently been seen. Mammary gland tissue, normal or fibro-adenomatous, is described by Chevassu, Durr, and many others. Striated muscle bundles were first recognized 
by Senftleben, and Koslowski found cardiac musle arranged about four chambers as in the heart.

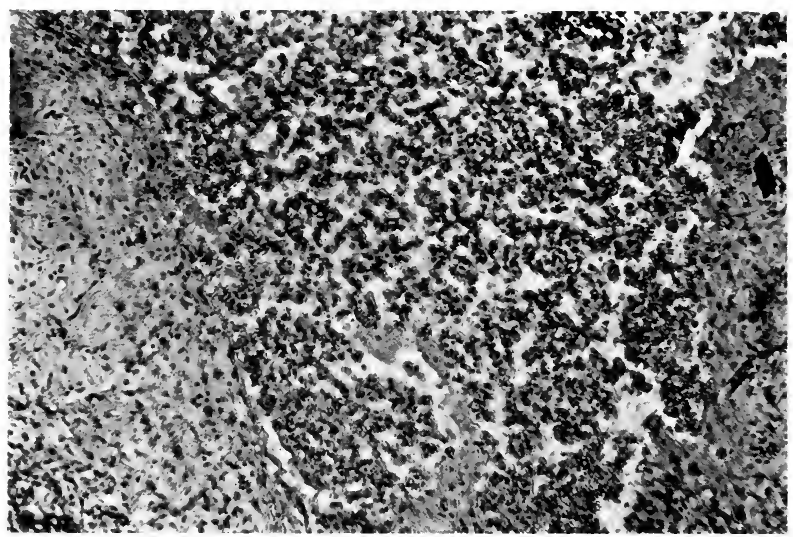

FIG. 382.-Teratoma testis. Tissue resembling liver cords.

Chevassu and Picque thought they could indentify liver-cells and cords. Rudiments of lung have been interpreted by Schlagenhaufer, Pepere, and

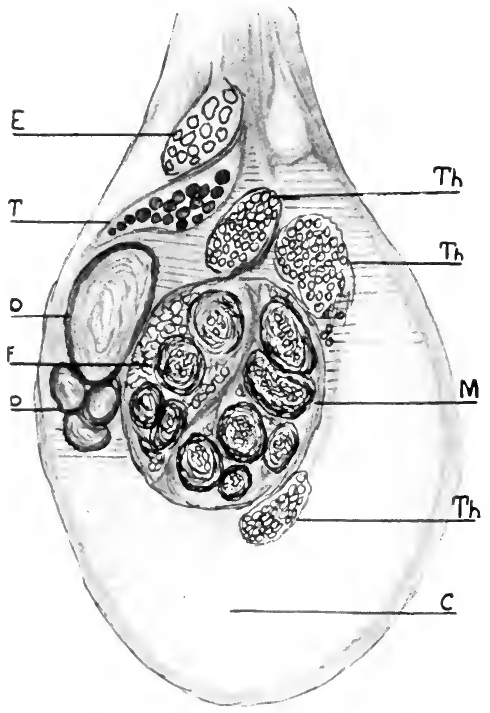

FIG. 383.-Construction of a teratoma testis: $C$, Carcinoma with lymphoid stroma. $D$, Epidermoid cysts. $E$, Epididymis. $F$, Fat tissue. $M$, Fibromuscular organ. $T$, Remains of testis. Th, Thyroid tissue.
Gessner. Chevassu pictures characteristic renal tubules and glomeruli. Gessner described ovarian tissue. The writer found a fibromusclar organ with $\mathrm{mu}-$ cous lining, which resembled stomach or uterus.

A buccal cavity is located by a superficial area of mucous membrane and here have been found in rare cases bicuspid and molar teeth embedded in a bony mass (Wilms, Heinen). Chevassu and Ohkubo identified salivary glands. The respiratory tract is represented by canals lined by columnar or cilited cells and supported by islands of cartilage.

The intestinal tract appears in the form of canals lined by cuboidal mucous cells forming crypts. The cellular lymphoid mucosa may be thrown up into villi. A double layer of smooth muscle surrounds these canals.

Ohkubo detected crystalline lens, spleen, and adrenal. Thyroid tissue is extremely rare, and Szulcewski interprets tissue of this type as a form of mucous degeneration, but I have described four lobules of adult thyroid resembling the normal organ in the minutest details.

The stroma supporting these organ rudiments may be of adult type or 
are reduced to irregular canals or cysts lined by various types of epithelium, to neuro-epithelial cell groups, and to different glandular structures.

The clinical course of the adult teratoma is usually slow. Many of the tumors are present at birth and slowly enlarge until they are removed between the tenth to fortieth years (Tilanus, Kalning, Macewen, Boeckel). In these cases no malignant changes are present.

Rarely adult teratomas terminate with the development of malignant neoplastic processes, which lead to local recurrence after operation and to general metastases. In a tumor described by the writer, containing adult

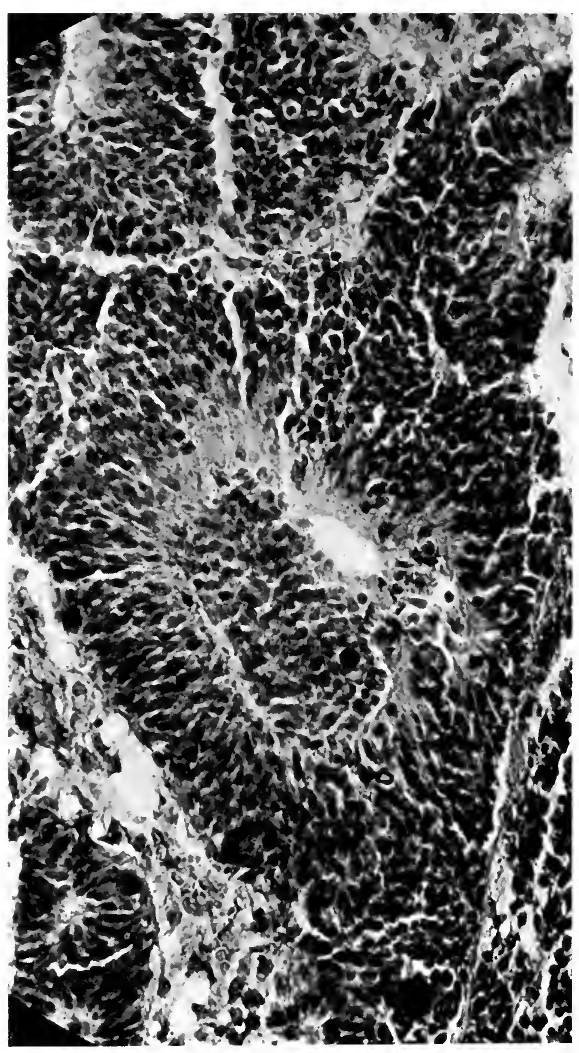

FIG. 386.-Teratoma testis. Adenocarcinoma. Neuro-epithelial cell groups.

dermoid cysts, uterus, and thyroid, much of the tumor was surrounded and infiltrated with embryonal carcinoma with lymphoid stroma. The patient died of internal metastases. The source of the carcinoma is undetermined.

(2) Teratoid, Embryoid, or Mixed Tumors.-A large proportion of testicular tumors are composed of more or less embryonal structures, derived from all three germ layers, but arranged in such confusion as to eliminate any resemblance to an embryo. These tumors have been described under many terms, but Wilms showed that they belong in a single group of tridermal growths which he designated as embryoid or teratoid.

In gross appearance the tumors are of moderate or large size and solid or 
riddled with many small cysts. They develop from the rete and are well encapsulated, the testicular tissue being stretched over them in a thin laver included in the capsule. Occasionally they arise in the tunica. The section is very varied, depending on the proportions of cysts, cartilage, cellular areas, hemorrhage, and necrosis.

In structure the cysts are lined by squamous or cylindrical cells and there may be many islands of squamous cells, while the solid portions contain masses of embryonal cartilage, osteoid tissue, cellular connective and smooth muscle. While these tissues may be comparatively adult in type and the tumor quite benign, there is a prominent tendency for the derivatives of one germ layer to overgrow the others and to exhibit true neoplastic and malig-

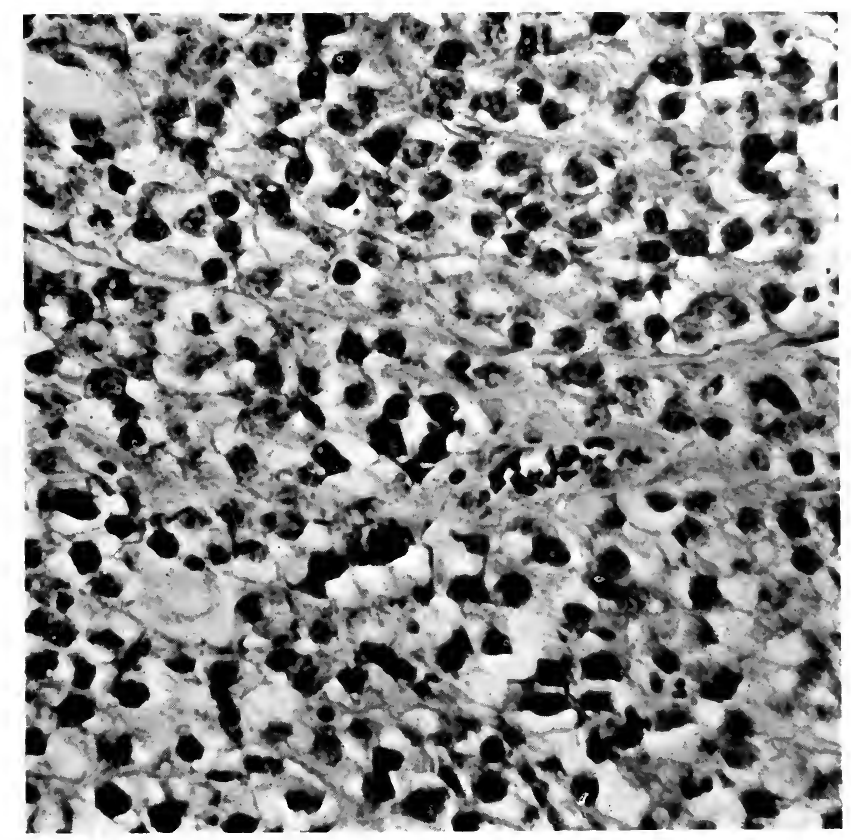

FIG. 387.-Embryonal carcinoma of testis. ("Seminome" of Chevassu.)

nant qualities. Thus the solid areas often show cellular foci composed of embryonal connective and myxomatous tissue, or very cellular masses of smooth muscle-cells, either of which may compose the bulk of the tumor and give origin to metastases.

Neuro-epithelial cell groups may be very abundant and carcinomatous areas of this origin may develop. Entodermal derivatives may be prominent, in the form of small cysts or alveoli lined by cylindrical cells. In many of these cysts the type of epithelium may suddenly change, as from squamous to cylindrical. Adenocarcinomatous foci derived from the cyst lining are frequently seen and these areas grade into various structural forms of carcinoma.

(3) Embryonal Tumors of the Testis. (1) Sarcoma Testis. Seminome (Chevassu).-Embryonal carcinoma with lymphoid stroma.

The most frequent tumor of the testis is composed of large round or poly- 
hedral cells of embryonal type, lying diffusely or in large alveoli. The stroma is often richly infiltrated with lymphocytes. Through the demonstration by Wilms, Ohkubo, and others that many of these tumors contain teratoid elements, the suspicion first stated by Langhans, that the tumors are teratoid, has been confirmed. Yet Chevassu, Debarnardi, and others derive this tumor from the spermatoblasts or their embryonal equivalents and separate this group from the teratomas with which they are so often associated.

Several considerations forced the writer to conclude that this common tumor of the testis is always a one-sided development of a teratoma and is not derived from the adult spermatoblasts.

(I) The characteristic structure is often seen in teratomas, adult or embryonal. (2) In a very early embryonal carcinoma I found minute traces of cartilage, entodermal alveoli, and squamous epithelial cell groups. (3) The rapid growth of the malignant embryonal element gives unusually favorable opportunity for the overgrowth and suppression of other elements. (4) The only known tumor of the adult spermatoblasts is very different from

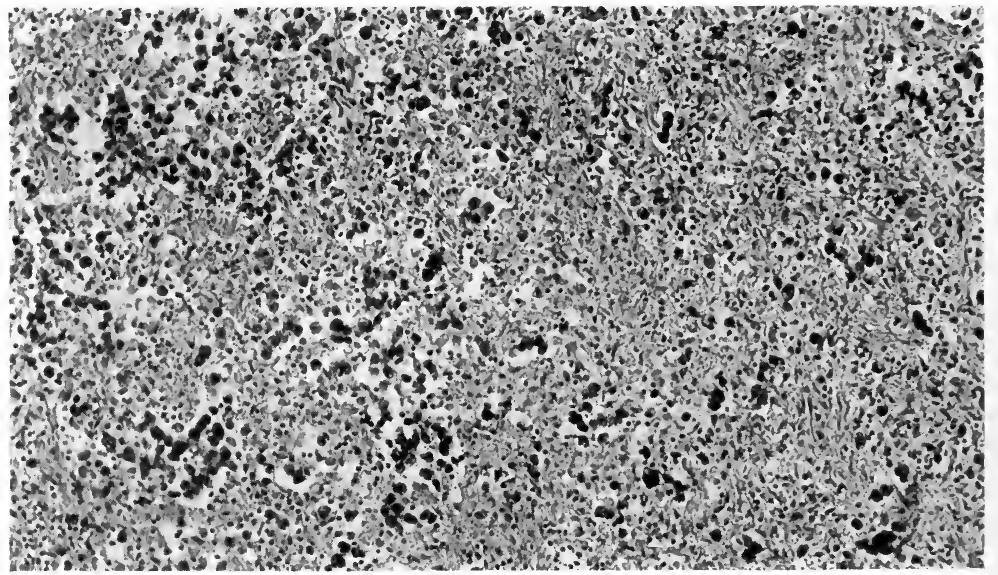

FIG. 388.-Teratoma testis. Embryonal carcinoma. Spontaneous regression.

the embryonal carcinoma. Barring the possibility of unrecognized forms of metaplasia, the teratomatous relations of our tumor seem to be demonstrated. It remains possible that the embryonal carcinoma may arise from spermatoblasts incited to growth by the presence of a teratoma. The occurrence of extratesticular carcinoma speaks against this possibility (Koslowski, the writer).

The embryonal carcinoma is a rapidly growing, soft, opaque, often necrotic tumor arising in the rete and invading and destroying the testis. A portion of the organ may persist as a thin layer in the capsule, where it is found atrophic. The tumors reach a considerable size and then undergo central necrosis, often complicated by hemorrhage, ulceration, or suppuration.

Excision in the early stage may permanently eradicate the disease, but usually there is local recurrence, metastases in the upper abdominal nodes, lungs, brain, and other organs. Chevassu reported 22 recoveries after one year from castration in 59 cases of embryonal carcinoma, and ${ }_{3} 3$ after later periods. No case died after 3 years' immunity. Of 55 mixed tumors only 6 remained well for 2 years, 4 others showing no recurrence after 6 months. 
The rapid course of recurrences is notable. Definite chorioma appears to be uniformly fatal.

Structure.- The structure is usually characteristic, but shows several variations. As a rule one finds large round or polyhedral cells with vesicular hyperchromatic nuclei lying in wide sheets or diffusely. The cells are rich in glycogen. The stroma may be very scanty, or strands of lymphoid tissue may be prominent, or more fibrous bands may produce alveoli. Definite lymph-nodules may form in the stroma. Necrosis is common. A form of regression may be observed with atrophy of tumor-cells and formation of granular or cellular interstitial tissue.

Embryonal adenocarcinoma is a somewhat distinctive type of testicular tumor. It grows rapidly, is very vascular, and is often the seat of extensive hemorrhage. Encapsulation is usually as marked as in adult teratoma, and

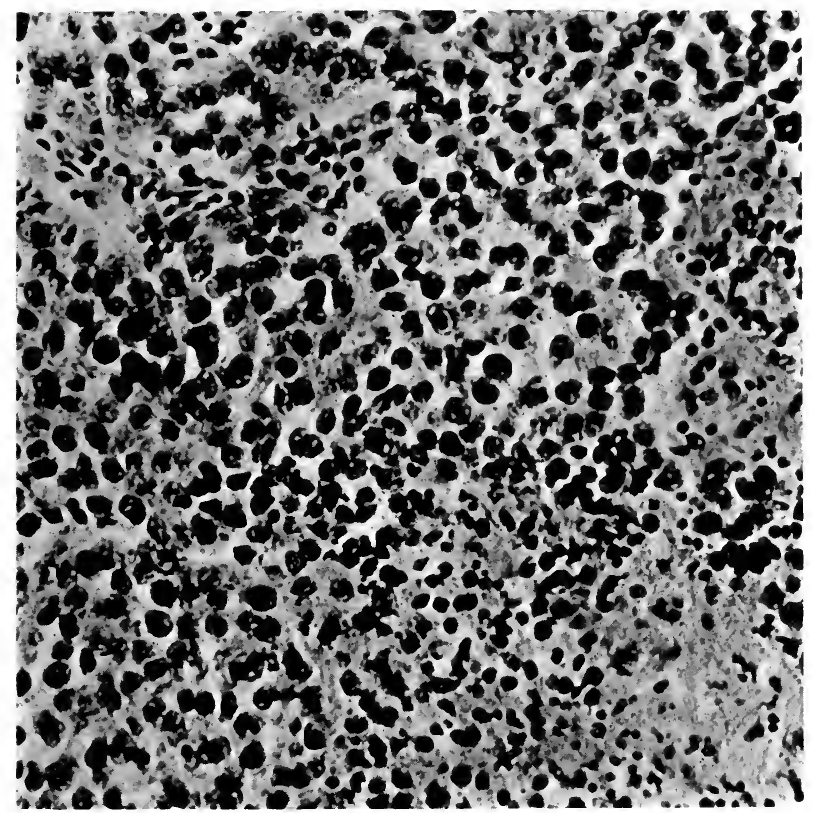

FIG. 389.-Embryonal carcinoma of testis, with lymphoid stroma.

while traces of teratomatous elements are not infrequently found, this structure may be nearly pure carcinoma. It is sometimes associated with chorioma. The structure presents irregular alveoli lined and traversed by rows of large cuboidal cells with large hyperchromatic nuclei. Or the alveoli may be indistinct, the cells smaller and more undifferentiated, and the structure may even resemble lymphosarcoma.

Chorioma Testis. - This remarkable form of teratoma has passed through several stages of study and interpretation. In I 868 Waldeyer observed polypoid tumor masses, extending from a testicular teratoma into the pelvic veins, which he compared in appearance with hydatid mole. A typical case was described by Breus, 1878 , in which the polypoid masses extended into the heart. In the French literature a series of these cases was reported under the term "sarcome angioplastique" by Malassez and Monod, Carnot and 
Marie, and others, who also noted the resemblance to hydatid mole. MacCallum likewise interpreted his case as lymphendothelioma. In I902 Schlagenhaufer, Wlassow, and Steinert all pointed out the exact resemblance of this tumor to chorioma, traced the origin of the syncytial masses from epithelium of the testicular growth, identified glycogen-holding Langhans' cells, noted the hemorrhagic character of the metastases, and showed that chorioma testis reproduces exactly the essential features of chorioma uteri. Warthin and Gabarini have reported cases in which there was hypertrophy of breasts with secretion of colostrum, thus adding physiological features of pseudogestation in the male. While Risel denies the origin from a true chorionic element in the teratoma and points out that syncytial masses may form from any epithelial structure, there is no doubt that the general significance of chrioma testis has been correctly interpreted by Schlagenhaufer. The course and structure of chorioma testis is an exact duplicate in all details of that of the uterine

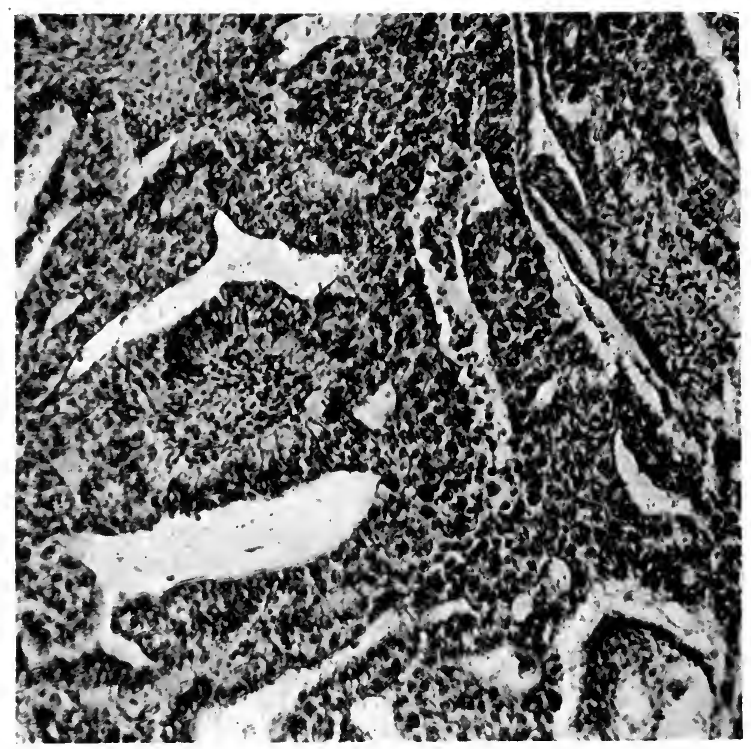

FIG. 390.--Teratoma testis. Tumor composed of adenocarcinoma with cylindrical cells.

tumor, which is not the case with the pseudochoriomatous growths of miscellaneous epithelial origin to which Risel's criticism applies.

The testicular tumors giving origin to chorioma are usually small and may be overlooked during life. They are described as soft, succulent, cystic, or hemorrhagic. In Kanthack's case the tumor was composed of many small cysts of various types. A large cystic tumor chiefly of adult type was found by Steinert. In one case (Blatteis) I found the small original tumor largely destroyed by hemorrhage and presenting the structure of embryonal adenocarcinoma with several areas of syncytium. In another case the testis was very slightly enlarged, but the rete and cord presented a continuous series of pea-sized dermoid cysts. On section a small focus of proliferating syncytium was discovered in the rete. In the gross this area appeared as a hemorrhagic spot. There were bulky metastases of complex structure in the mediastinal and cervical nodes, dermoid cysts and chorioma in the lungs, and a 
single large tumor resembling a placenta in the liver from which fatal hemorrhage occurred. Steinert's case also gave complex metastases in mediastinum, lungs, and liver, among which chorioma was prominent.

The exact origin of the syncytial cells is not clear. Pick has traced them to the neuro-epithelial cell groups. In 2 cases I have found the minute foci of chorioma in a very limited field and lying in or near adenocarcinomatous

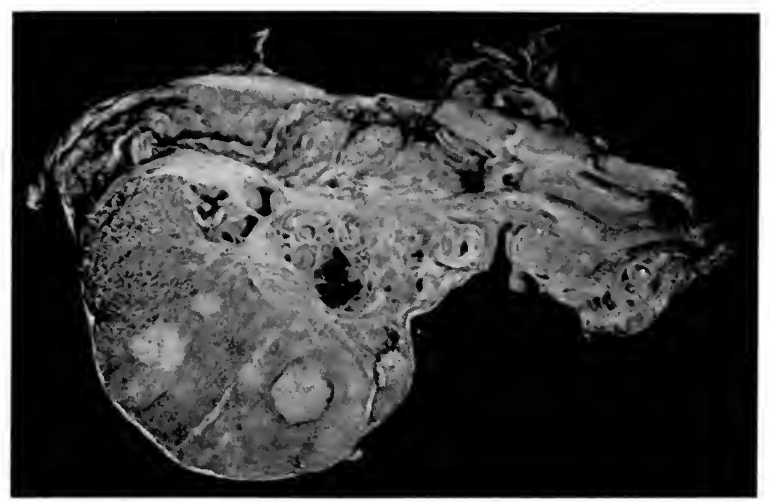

FIG. 39I.-Teratoma testis, composed of epidermoid structures and a dark central rodule of chorioma. In the body of the testis are nodules of large cell embryonal carci1oma. Same case as Figs. 392 and 393 .

ureas of which the cells showed none of the features of neuro-epithelium. Nor was there any special relation to blood-vessels. No gross chorion-like tructures have been identified in the testis. Moreover, similar cells may ppear in tumors which have not yielded choriomatous metastases. Hence $t$ would appear hazardous to attempt the diagnosis of chorioma testis in the bsence of pulmonary or hepatic metastases.

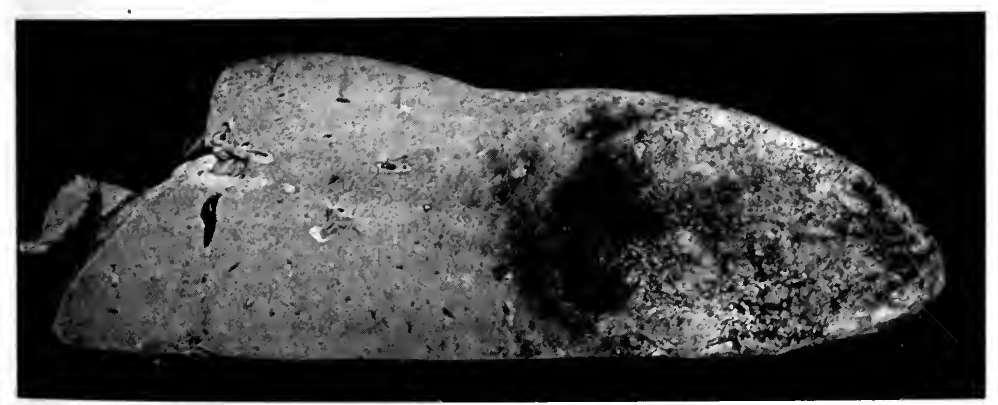

G. 392.- Teratoma testis. Same case as Fig. 393. Choriomatous metastasis in liver resembling a chorion and giving rise to fatal hemorrhage.

Certain apparently primary tumors of the liver in males have been deribed as chorioma for which no satisfactory source has been determined. seems probable that these are either primary carcinomas of the liver-cells nulating chorioma, or metastatic chorioma of the testis, the primary tumor ving been overlooked. 
Metastases of Teratoma Testis.-Nearly all varieties of the embryonal and mixed tumors may prove malignant and give rise to metastases which are often of complex types. The course and character of these metastases present many interesting features.

The retroperitoneal nodes at the celiac axis are often involved through the lymph-channels of the spermatic veins, and an epigastric tumor is often the first sign of recurrence. This form of recurrence is especially common with the highly malignant embryonal carcinomas.

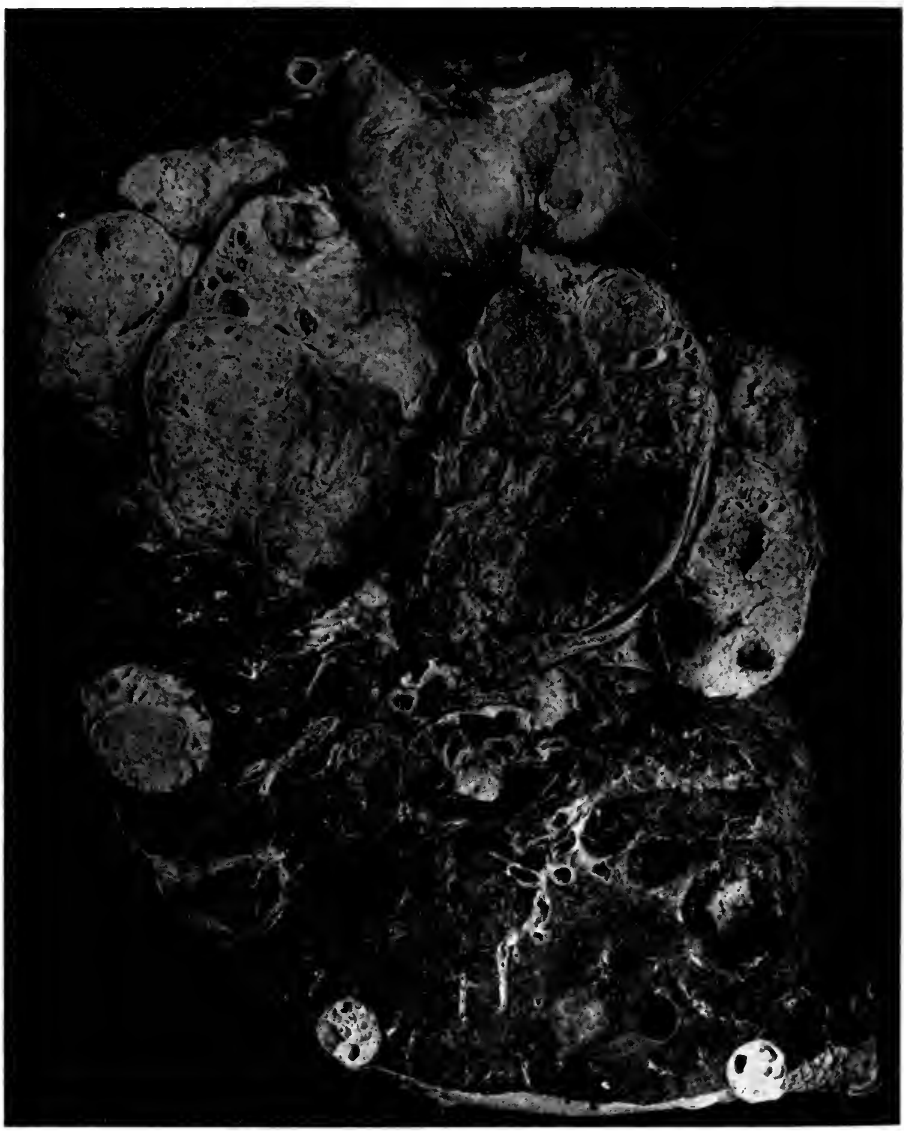

FIG. 393.- Metastases in lung and cervical lymph-nodes from a small teratoma testis shown in Fig. 392. The lung shows whitish epidermoid cysts and dark nodules $c$ choriocarcinoma.

From this point there may be rapid progress upward, so that mediastina and even cervical tumors may divert attention and become the largest tumor in the body. Invasion of the spermatic and iliac veins, with continuou tumor growth extending as far as the heart, has been observed both wit chondrosarcoma (Paget) and chorioma. Discontinuous metastasis by wa of the veins is most frequent, and gives rise to tumors of lungs, liver, braii kidney, and stomach. 
The structure of the metastases may reproduce several features of the original growth, or only one. As a rule the individual secondary tumors represent a single germ layer in either adult or embryonal form. Well-formed adult ectodermal cysts may appear in the lungs and chondromatous nodules may exhibit adult characters.

In the retroperitoneal and thoracic nodes one may find many adult and embryonal structures reproducing in a remarkable degree the original tumor. Yet the derivatives of the germ layers are usually distinct, showing that each embolic cell group has produced only its own kind. In a case of chorioma testis I found three entirely separate groups of metastases in the lungs, one composed of chorioma, another of epidermoid cysts, and a third of myxosarcomatous tissue. The chorioma appeared also in the liver, while the two latter tissues made up the bulk of the mediastinal tumors.

In a case of embryonal adenocarcinoma there was local recurrence, tumors of abdominal and thoracic nodes, very extensive diffuse invasion of both lungs, large tumors of the brain, and seven globular tumors of the gastric mucosa, from the central excavations of which fatal hemorrhage occurred. In Westenhofer's case the hepatic metastases had the structure of lymphosarcoma. Steinert describes combined tridermal metastases in the liver, which contained encapsulated nodules composed of cysts lined by cylindrical cells backed by a muscularis, and associated with epidermis, cartilage, and bone. The presence of such structures in one compact metastatic tumor it is difficult to refer to any other source than to emboli of undifferentiated cells capable of producing all three germ layers. Further observations are needed to elucidate the questions here involved.

4. Miscellaneous Tumors of the Testis, Tunica and Cord, and Their Relation to Teratoma.-The study of teratoma testis has greatly narrowed the scope of pure simple tumors of this organ, and reduced their numbers to a comparatively few cases whose real nature often remains uncertain. In no other organ has the principle of the overgrowth of one element of a teratoma been proved to be so predominant, and while it is possible to carry this principle too far, the data seem to demonstrate its great importance in the interpretation of tumors of this region. Hence tumors which are apparently of other origins must be scrutinized carefully.

Fibroma of the testis is very rare, but apparently pure cases are described by Curling and Kocher. These were large, firm, cystic, or calcified tumors of very slow growth. Fibromas of the tunica albuginea are reported by Fergusson and Pean.

A large fibroma, $\mathrm{I}_{4} \times 9 \mathrm{~cm}$., replacing the testis, is described by Chevassu. It contained pseudocysts and sarcomatous areas and the author was unwilling to eliminate a teratomatous origin.

Plexiform fibroma of the cord is described by Ehrendorfer. Fibrolipoma of the spermatic cord is a well-recognized tumor which Kolisko regards as of teratomatous origin.

Chondroma.- Cartilage is nearly constantly present in the testicular teratoid tumors and often forms the bulk of the growth. It occurs in the form of adult or embryonal hyaline masses of small size or large dimensions. Neumann found bone in one case. Myxoma may arise from imperfect formation of cartilage.

The tendency of cartilage to overgrow other elements of the tumors has long been recognized. Kocher accepted as pure chondroma only 5 of the 28 cases described by Dauvè, adding 3 of his own, but later writers (Ribbert, Ohkubo) question the existence of pure chondroma testis apart from a teratomatous origin. Paget's case of malignant chondroma is now gener- 
ally regarded as a teratoma (Wilms, Ohkubo). Cartilaginous metastases in lungs or lymph-nodes occurred in his and Kocher's cases. Since the origin of these tumors appears to be in the rete, since many are cystic and occur in infants, and since it is practically impossible to eliminate teratomatous elements by microscopical study, it seems necessary to assume that all these tumors are of teratomatous origin.

Myxomatous areas are frequently present in mixed tumors of the testis and may form the bulk of the tumor. Most reported myxomas have been associated with chondroma, sarcoma, adenoma, or cystoma, and clearly belong in the teratoid group. Kocher and Wilms could find no cases of pure myxoma.

Myoma.-Smooth muscle-tissue is nearly constant in the mixed tumors, but seldom forms a prominent part of the tumor, nor does it commonly present blastomatous changes. Reports of leiomyoma testis are likewise rare. Nepveu's case contained both smooth and striated muscle and was probably of teratoid nature. Leiomyoma of epididymis or vas is described by Trelat, following trauma, and by Hericourt. It is assumed that these tumors arise from the cremaster internus muscle.

Striated muscle is rarely seen in testicular tumors, but in a few cases it has been present in abundance, usually with cartilage and epithelial canals, thus demonstrating its teratomatous relations. Mixed tumors of this type are described by Arnold, Kocher, Ribbert, Chevassu, Wood, and others. Apparently pure rhabdomyoma attached to the lower pole of the tunica albuginea and probably derived from the gubernaculum of Hunter are described by Rokitansky and Neumann.

The scope of myoma and myosarcoma of the testis is not yet determined. Neumann and Ribbert found many spindle- and round-cells which were not clearly separable from the muscle-cells, and Neumann suggested that some spindle-cell sarcomas may be of myogenous origin. Benenati describes striated muscle, large spindle-cells lacking striation, and round cells in a testicular tumor with metastases containing only the round-cell tissue.

Sarcoma.-Spindle-cell sarcoma of the testis has rarely been reported and it is difficult to determine the true nature of these cases. Chevassu, out of I 26 testicular tumors, found only one which appeared to be purely sarcomatous. Yet the cells were of mixed type, spindle and round, and since there were also alveoli lined by epithelioid cells, the opinion is expressed that the tumor might be of teratoid origin. The probability that teratoid elements have been overlooked in these cases is shown by Wilms, who, on re-examining an old tumor called plexiform spindle-cell sarcoma, found also cysts lined by cylindrical or squamous cells and an island of cartilage undergoing mucoid degeneration.

In view of these observations considerable doubt surrounds the interpretation of other so-called spindle-cell sarcomas. Some of these tumors contained myxomatous areas and cell groups of uncertain nature (Ehrendorfer). Others contain many giant-cells suggesting a myomatous element (Kocher, Krompecher). Or both these structures are combined with spindle-cells (Debarnardi). There is no doubt that tumors composed chiefly of spindlecells occur in the testis, but their true nature and the possible relation to myoma and teratoma remain uncertain.

Adenoma of the testis has been described in 2 cases by Chevassu and Pick. They were found in undescended organs, one of them in an hermaphrodite. The tumors appeared as pinhead or bean-sized yellowish nodules in the body of the atrophic testis. Some small cysts resulted from dilation of alveoli. They were composed of regular tubules lined by cylindrical cclls about I $_{5}$ micra in height, with finely granular cytoplasm and compact nuclei 
and arranged in palisade fashion. The transition from normal tubules was followed step by step. Chevassu likens the tubules to those of a sweat-gland.

While adenoma of the testis appears in many older reports it is now evident that the authors were dealing with teratomas.

Interstitial Cells.-Tumors or tumor-like hyperplasias of the interstitital cells of the testis are rare conditions observed in undescended and atrophic testes, in which the atrophy of tubules is associated with moderate hyperplasia of the large cells of the stroma. These tumors are usually limited to very moderate dimensions, but in Kaufmann's case it measured $9 \times 6 \mathrm{~cm}$. They are well encapsulated and of rusty brown color due to the lipoid pigment in the cells. The structure exhibits alveolar groups of large polygonal cells

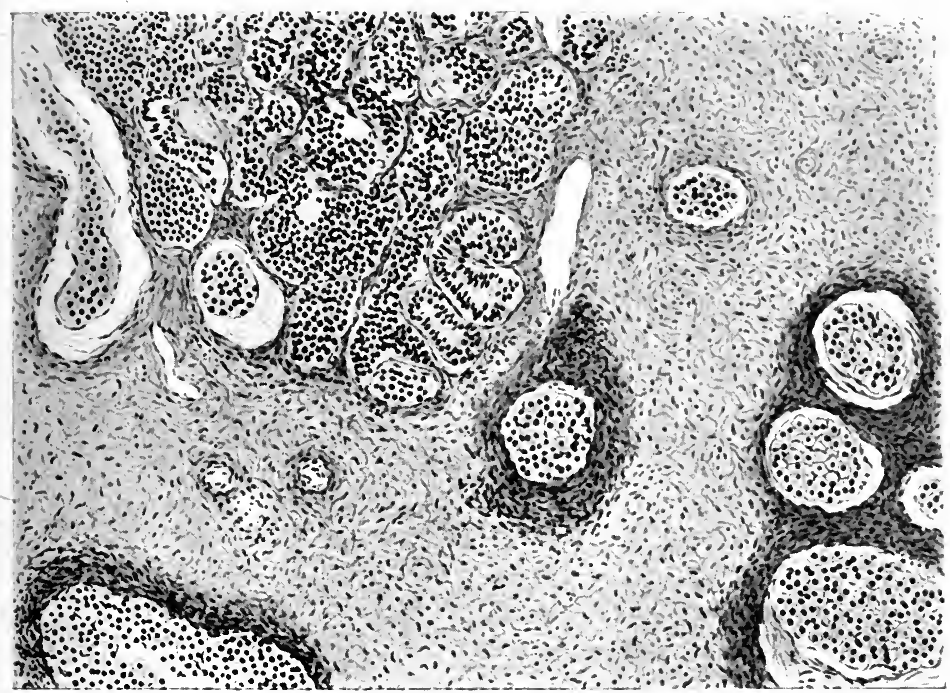

FIG. 394.-Adenoma of testicular tubules. (After Chevassu.)

closely resembling the interstitial cells. Neoplastic characters may be distinct (Durck). Cases of this characteristic type are described by Chevassu, Pick, and Kaufmann.

Waldeyer ( 1872$)$ derived certain plexiform angiosarcomas from the interstitial cells, and Hansemann (I895) attributed certain alveolar sarcomas to the same origin.

Adrenal Tumors of the Testis.-Rarely a solid carcinoma of the testis presents a structure recalling that of adrenal carcinoma or perithelioma. Debarnardi has described such a tumer and attributed its origin to an adrenal rest.' It occurred in a child of ro monchs and involved the upper pole of the testis and extended up the cord. A $:$ the testis and rete were intact it was not strictly a tumor of the testis, but rather of the epididymis and cord. The structure was complex, presenting sarcomatous and adenocarcinomatous areas.

The occurrence of adrenal islands in and about the testis has been fully demonstrated by Marchand, Wiesel, and Kirkbride, and such cell groups may well give origin to tumors.

In Debarnardi's case, and in others of my collection, in which the question of adrenal origin arose, the structure presented alveoli of large polyhedral 
cells which lacked the embryonal character of the ordinary carcinoma and resembled adrenal carcinoma. A perithelial structure was not prominent, nor were the gross features strongly indicative of adrenal nature. In one case the tumor proved to be secondary to a tumor in the adrenal. The structure of perithelioma appeared also in a recurrence of embryonal carcinoma. Further observations would appear necessary to establish the existence of a testicular carcinoma of adrenal origin.

Lymphosarcoma Testis.-An apparently distinct group of lymphosarcoma of the testis was described by Malassez in I 877 and later by Talavera and other French observers. More recently Chevassu has shown that the majority of these tumors are embryonal carcinomas, but his further claim that true lymphosarcoma in the testis is always secondary must be questioned. Curling observed the disease in I866 and Ehrendorfer described a typical case with cutaneous metastases, and recent cases are reported by Debarnardi and by the writer. The disease is distinguished by its development in most cases, in both testicles, by its occurrence chiefly in children, by

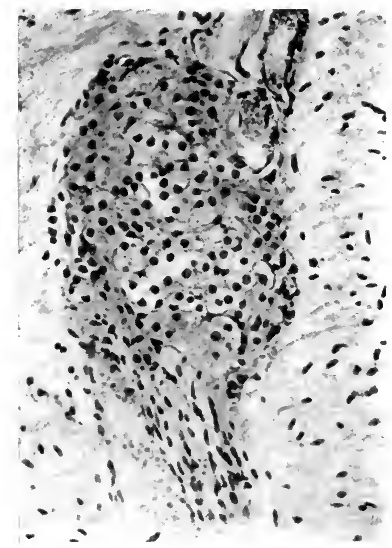

FIG. 395.-Island of adrenal tissue in testis of newborn infant. (After Kirkbride.)

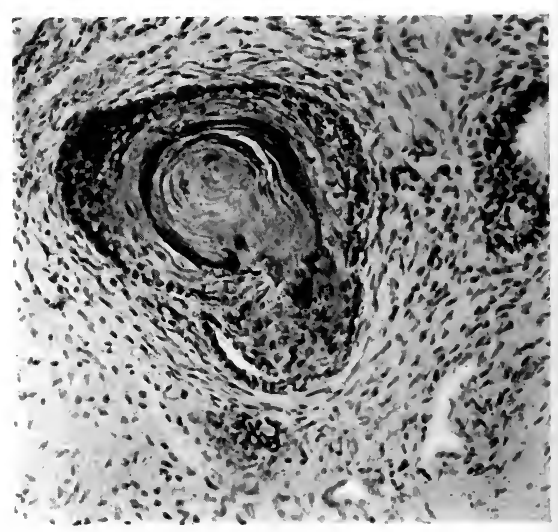

FIG. 396.-Epidermoid in rete testis of newborn infant. (After Kirkbride.)

its rapid course, and by multiple metastases in the skin. The structure is that of a diffuse tumor composed of embryonal large round cells resembling large lymphocytes, lying in a reticular stroma.

The origin of lymphosarcoma of the testis would naturally be referred to lymphoid tissue of the normal organ, but no such structures are commonly present in this organ. It seems possible that the tumor may be derived from some elements of a teratoma of which the rapidly growing cells lose all differentiation. Areas of such embryonal round cells may occasionally be seen in teratomas. Yet there are no definite observations which may be used in support of the hypothesis that lymphosarcoma of the testis is of teratoid nature and its origin remains quite undetermined.

Etiology.-The study of the formal genesis of teratoma testis, quite as much as any other teratoma, has contributed important data in the field of teratology. The questions involved almost exactly duplicate those of teratoma ovarii, but the testicular group is distinguished by the wide variety of simple tumors derived from the parent teratoma. 
Virchow held that the testicular mixed tumors arise from the normal elements of the organ, in which theory he attributed to metaplasia a very wide scope. While modern views greatly restrict the field of metaplasia, it may still be well not to ignore entirely the possible influence of this factor in the structure of mixed tumors. The idea that metaplasia alone can account for the organic structures of these growths cannot, however, be entertained, and since the entire series is connected by abundant intermediate types which demonstrate their single origin, the theory of metaplasia has been abandoned. The testicular embryomas, for the same and other reasons, must be excluded from the group of fetal inclusions. The results of the various forms of double embryos are seen in the sacral and cephalic teratomas, where the conditions

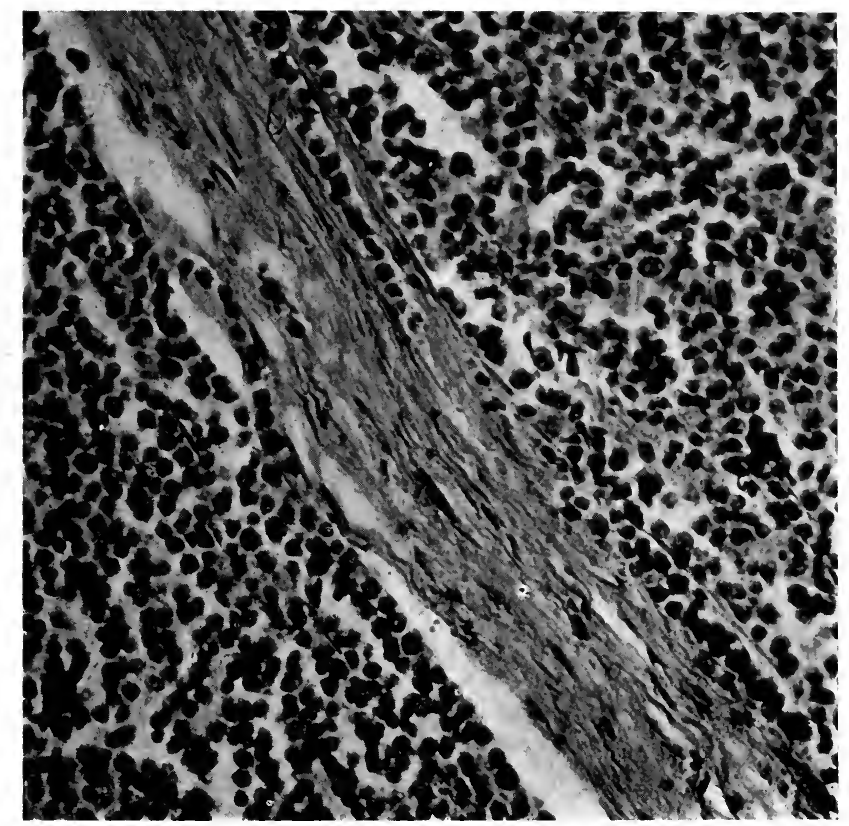

FIG. 397.-Embryonal carcinoma of testis. So-called alveolar sarcoma.

are favorable for the separation or attachment of multiple embryos, but these conditions are not provided in the testis.

The Marchand-Bonnet theory of origin, from polar bodies or isolated blastomeres, is less satisfactory for the testicular growths and their peculiar derivatives than for any of the fields to which it may conceivably apply.

An origin from some member or members of the sex-cell series is now generally accepted as applicable to the testicular tumors. This theory fully accounts for the predilection of teratomas for the sex glands and for the peculiarities of these growths as compared with extragenital teratomas. The exact form of this originating sexual cell cannot well be determined, but the conceptions held have included every stage from an original sex blastomere down to the adult spermatoblast. Unless one chooses to distinguish essentially between adult embryomas and embryonal mixed tumors, for which there are no adequate reasons, the entire group must be derived from a cell commonly present in 
the testis. Hence a nearly mature spermatoblast or its near antecedents are most probably the source of the tumors.

Cavazanni distinguishes between the benign congenital embryomas of early life and the malignant tumors of young adults following trauma. Both arise, he thinks, in cell groups of much more limited potencies than an ovum, probably from remnants of the Wolffian body. Yet his bilobed teratoma exhibited, in one lobe, tumor structures which he interprets as essentially different in origin from those of the other lobe. A misplacement of spermatoblasts with incomplete development would strengthen the sex-cell theory, and these conditions are provided at the junction of tubules and rete where the tumors regularly arise and where Kirkbride finds much disorder in the structure of the embryonal and fetal testis. Teratoma testis is also particularly prone to develop in atrophic undescended organs.

Why one teratoma grows slowly and produces adult tissues, while another develops rapidly and remains embryonal in type, cannot readily be explained and this variation may possibly call for a wide difference in the stage of development of the originating cell. A more obvious factor is probably found in the influence of the blastomatous process which is usually added to the embryonic growth. Such a process at once interferes with the orderly progress of simple embryonal growth and converts the embryo into a true tumor. Beginning early, the blastoma may completely suppress the embryo, or if late the embryo may reach the limit of its growth before the blastoma assumes serious proportions. The reported cases illustrate both of these relations.

The incitement to growth seems to have been furnished in many instances by trauma, and while it is difficult to establish an essential connection, the traumatic theory is in accord with experimental data showing that trauma may readily excite spontaneous growth of ova.

Undescended testes are unusually prone to develop malignant tumors, of which Bulkeley has collected 59 cases. In three the tumors were bilateral (O. C. Smith). 


\section{CHAPTER XLI}

\section{TUMORS OF LUNG}

\section{CARCINOMA OF LUNG}

Primary tumors of the lungs have long attracted much clinical and pathological interest. Bayle in r8ro vaguely described pulmonary cancer, and Stokes, 1842 , recognized several varieties of the disease. Ebermann as early as 1857 collected 72 cases, one occurring before the age of 9 years. Jaccoud appears first to have sharply distinguished the disease from phthisis. Behier pointed out the prominence of pressure symptoms and the frequent invasion of supraclavicular nodes. Bennett's Lumleian Lectures in 1872 dealt fully with

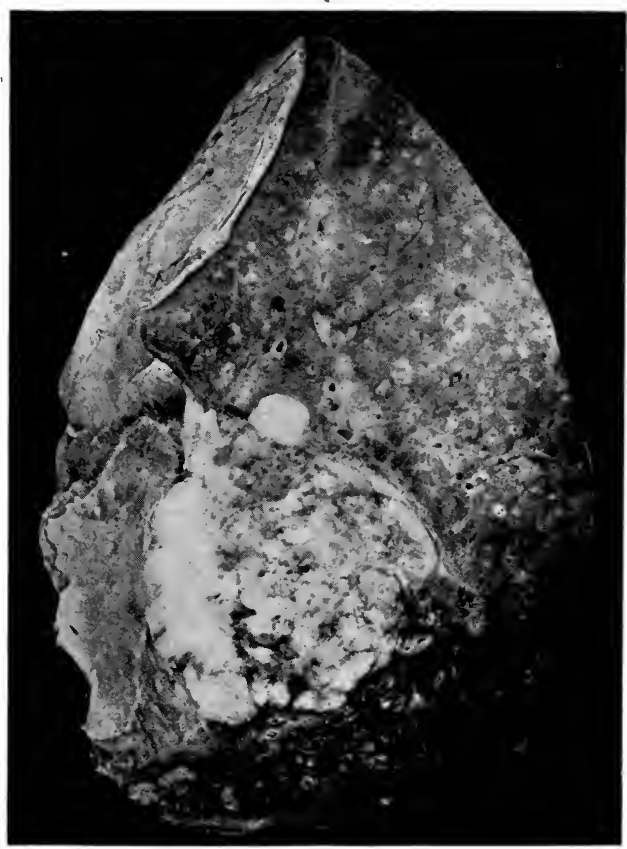

FIG. 398.-Carcinoma of lung.

the clinical aspects, from an analysis of 39 cases. Rokitansky recognized several gross varieties of the pulmonary lesions, but the earliest microscopical studies were those of Langhans, Marchiafava, and Malassez, I87 I to I876.

The more detailed interest and knowledge date chiefly from the later studies of Wolf, r895, and Passler, r896. Since that time the literature has grown to very considerable proportions, so that Adler in IgII was able to tabulate 374 cases of carcinoma and 90 of sarcoma. Since 1922 Scott and Forman find reports of I 20 new cases. 
Etiology.-Primary malignant tumors of the lung form about I per cent. of all cancers. Among I6,578 cancer autopsies from various Continental sources I68 pulmonary cancers were recorded (compiled from Karrenstein and Adler). Kaufman places the proportion much higher, at I.83 per cent. It is evident that increased attention has greatly augmented the list of observed cases. Knierim (I g09) reported that at Leipsic, during 9 years, 66 cases of bronchial carcinoma, 7 acanthomas, and 2 alveolar tumors had been observed.

Males are much more frequently affected than females, 71.9 per cent. to 24.8 per cent. Alder gives the following analysis of age incidence:

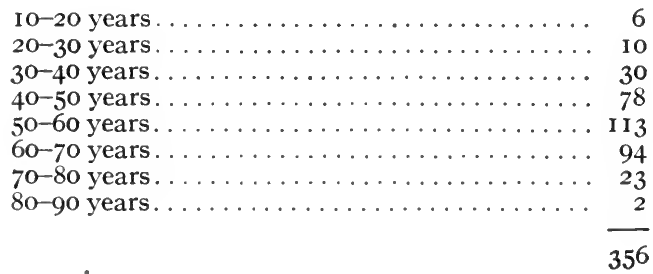

Horn observed an adenocarcinoma in a girl of I8 years. A congenital multiple tumor appears to have been encountered by McAldowie in an infant of $5 \frac{1}{2}$ months, while Nuscheler reported a case in a girl of 7 years, and Werner described a small cell carcinoma in a female of ro years. Sarcoma occurs at somewhat earlier ages, chiefly between 30 and 40 years, and 6 cases have been reported in the first decade. The right lung is more frequently affected than the left. Of ro6 cases Perrutz located 35 per cent. on the left side, 54 per cent. on the right, while ro per cent. were bilateral.

The chief etiological factor is tuberculosis. Of Wolf's 3 I cases, I3 were associated with tuberculosis. Squamous-cell carcinoma developing in the wall of a tuberculous cavity is described by Schwalbe, Friedlander, and Perrone. Wolf found tuberculous lesions throughout diffuse carcinoma, or surrounding tumor masses. In the old scleroses, atelectases, and reparative lesions of tuberculosis may be found many alterations of bronchial and pulmonary epithelium, marked by considerable cellular overgrowth. Oertel points out that the association may be accidental, at other times the two processes exist in symbiosis, while rarely tuberculosis appears to inhibit the carcinoma. Several cases in which there was active miliary tuberculosis with carcinoma suggest that the malignant process may light up latent tuberculous foci (Wolf).

Rupture of anthracotic nodes into the bronchi is held by Wolf as responsible for some cases of bronchial carcinoma. A history of chronic bronchitis has often preceded bronchial carcinoma, and the lesions of chronic bronchitis often present very marked overgrowth of bronchial epithelium.

In chronic interstitial pneumonia also the overgrowth and metaplasia of alveolar epithelium is sometimes excessive. Menetrier offers evidence to show that primary sclerotic lesions in the lung may lead to cancer, and this view is supported by Ribbert. Yet many alveolar carcinomas appear to have developed in previously normal parenchyma.

Trauma has figured prominently in the history in many series of cases and sometimes the relation between the two has been rather striking (Aufrecht).

In many carcinomatous lungs anthracosis is extremely marked and in the much quoted cancer of the Schneeberg miners this condition has been held chiefly responsible for the disease. While some of these cases have proved to be tuberculosis or other infectious granuloma, a few must be accepted 
as primary carcinoma. In a recent case Arnstein found chiefly squamouscell carcinoma of bronchial origin, while portions of the tumor contained spindle-cells which also appeared in the hepatic metastases.

Classification.-Tumors of the upper third of the trachea are relatively common, but they closely resemble laryngeal growths with which they are usually grouped. The lower portion of the trachea is very rarely the seat of tumors.

Among pulmonary tumors are considered the growths arising from the bronchi and pulmonary parenchyma.

According to histogenesis three groups of pulmonary carcinoma must be recognized, as rising from $(a)$ bronchial epithelium, $(b)$ bronchial mucous glands, $(c)$ alveolar epithelium.

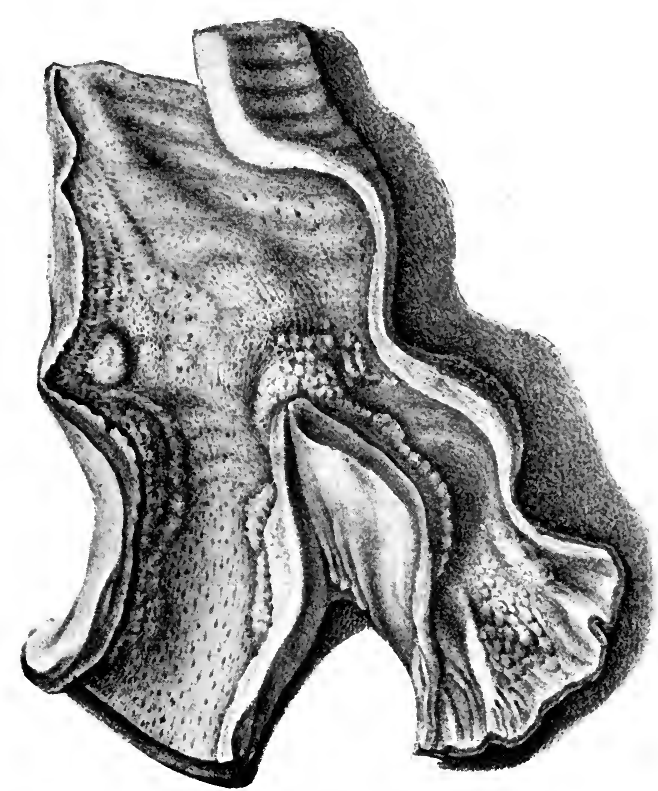

FIG. 399.-Beginning carcinoma of bronchial mucosa. (After Langhans, V. A., 53.)

To a considerable extent these tumors are distinguished by gross anatomical features and probably also by certain clinical characters. It is, however, not possible to separate all advanced cases, especially of the more malignant types, and the specific features belong chiefly to the earlier stages of the tumors.

(a) Bronchial Carcinoma of Lining Epithelium.-Carcinoma of the bronchial lining epithelium produces extensive changes in the bronchial mucosa, advancing along the walls of the bronchi, septa, and pleura, but seldom producing a diffuse tumor of the lung. The process usually begins at or below the bifurcation and extends toward the trachea above and the smaller bronchi below. The bronchi may be filled with papillary projections over a wide area, while the walls are thickened and the parenchyma invaded. Wolf describes a case in which the lower half of the trachea and both main bronchi were filled with papillary masses and encased in dense connective tissue infiltrated with tumor-cells. 
Domeny found the main bronchus of the left lower lobe occluded by a protruding tumor mass, while the mucosa was the seat of large carcinomatous ulcers. Packward describes a large tumor involving the two lower tracheal rings and both bronchi and extending through the lung to the pleura. There were numerous bronchiectases. A distinctly polypoid tumor of the right bronchus with pulmonary metastases is recorded by Merklen and Girard.

Excavations of the superficial tumor tissue and bronchiectasis are frequently established and lead to the formation of one or several cavities filled with mucopus and walled by disintegrating tumor tissue. In tuberculous bronchiectatic cavities one or many polypoid tumors may project into or fill the cavity, while the walls are infiltrated by tumor tissue. Localized stenosing and ulcerating tumors may surround bronchi of the first or second order and on account of their limitation to the hilus may readily be overlooked (Kaufmann, Harbitz).

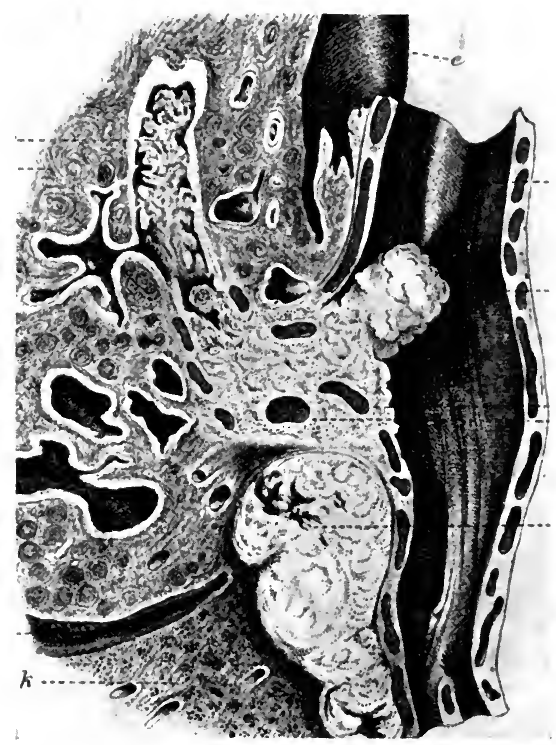

FIG. 400.-Relations of a squamous-cell carcinoma of bronchus. (After Ernst.)

In advanced stages a main tumor mass surrounds the hilus and large bronchi, while ulcerating and usually dilated bronchi form cavities in the centers of tumor masses in the affected lobes. Extension to the pleura is frequently observed in the form of wide-spread lymphatic injection or of very marked thickening. The ribs and chest wall may be eroded.

Secondary complications greatly alter the picture. The remaining lung tissue becomes emphysematous, atelectatic, and sclerosed, or pneumonic areas appear in the terminal stages. Putrefaction of retained mucus, pus, and tumor detritus often excites fatal gangrene. Large vessels are eroded with fatal hemorrhage. The tumor process extends into mediastinum, pericardium, auricles, and great veins. Serous or bloody pleural effusion is very frequent.

The structure of this type of bronchial carcinoma is that of squamous-cell or cylindrical cell alveolar carcinoma. While the squamous-cell tumors usually 
maintain a uniform structure throughout, the cylindrical cell growths often vary extensively both in the type and in the arrangement of the cells. Thus many histological varieties of carcinoma are represented in these bronchial tumors. Watsuji calculates that 32 per cent. of all pulmonary carcinomas are squamous.

Wolf found 8 acanthomas and 7 cylindrical cell tumors among $\mathrm{r}_{5}$ of bronchial origin. The cylindrical cells are arranged in small or large groups or they grow diffusely. Adler describes a very cellular growth, medullary carcinoma, which it was difficult to separate from round-cell sarcoma. Harbitz describes a bilateral gelatinous tumor with bronchiectatic cavities, of which the structure was adenocarcinomatous. He also found high cylindrical cells, with mucoid changes, proliferating in small bronchi which formed the centers of multiple tumor nodules.

Horn's tumor in a young subject was also adenocarcinomatous and grew from a broad pedicle into a bronchiectatic cavity penetrating large and small bronchi. Chiari described a cylindrical cell tumor of distinctly papillary structure. Ernst's papillary tumor contained hornifying squamous epithelium.

Fibrocarcinomatous areas are often observed in old lesions and in the pleura, while in some contracted organs the lesion is chiefly of this type. Spindle-cell areas are occasionally observed, and in Hodenpyl's and Ärnstein's cases this form persisted in metastases. It is highly probable that certain so-called spindle-cell sarcomas of the lung are atypical carcinomas.

(b) Bronchial Carcinoma Arising from Mucous Glands.-In a considerable series of cases tumors of this origin have yielded somewhat characteristic gross and microscopical features, which are: limitation chiefly to the walls and especially to the submucosa of the bronchi; relatively intact condition of the bronchial lining; stenosis rather than dilatation of the bronchi; often considerable infiltration of the parenchyma; and a structure of glandular carcinoma recalling that of the bronchial mucous glands. Typical cases of this group are described by Langhans, Beck, Fuchs, Ebstein, Ehrlich, Rondeau, Decreton, Kretschmar, Wolf, and others. Hansemann considers that most bronchial cancers arise from the mucous glands.

A very early stage is described by Langhans. The lower $4 \mathrm{~cm}$. of the trachea and $2 \frac{1}{2} \mathrm{~cm}$. of the bronchi presented very numerous nodular elevations of the mucosa chiefly on the posterior aspect, while submucosa and adventitia were diffusely thickened. The mucous lining itself was intact. The changes were most advanced at the bifurcation of the trachea, where the nodules projected $7 \mathrm{~mm}$. into the lumen and caused a ring-like constriction. There were no metastases. The nodules proved to be markedly hyperplastic and carcinomatous mucous glands. The solid alveoli were composed of small polyhedral or cylindrical cells and extended through the layers of mucosa and submucosa down to the cartilage.

A tumor of the size of a cherry of glandular carcinomatous structure and with numerous metastases is described by Lehmkuhl. An advanced but characteristic condition is reported by Domeny. The tumor surrounded and compressed the larger bronchi with sclerosis, atelectasis, atrophy, and contraction of the lung. The pleura was much thickened and the hilus nodes were invaded. There was marked thickening of the bronchial mucosa with stenosis, but, while the mucosa was thrown into folds, its ciliated lining was every where intact. In another case the chief lesion was bronchial stenosis. Rondeau describes a markedly contracted and sclerosed right lung in which the bronchi were almost completely occluded, while the pleura was 4 to $6 \mathrm{~cm}$. in thickness. The indurated tissues and pulmonary lymphatics were infiltrated with adenocarcinoma in which the alveoli contained colloid masses. A sharp limita- 
tion of the peribronchial tumor from the parenchyma is mentioned by Beck, Meunier, and Ducreton. Gelatinous changes were prominent in several cases, and the presence of much mucoid material led Willert to designate his tumor as carcinoma myxomatodes. Others have referred mucoid carcinomas to the bronchial lining epithelium and to the alveolar epithelium (Harbitz, Le Sourd).

The structure presents an adenocarcinoma or alveolar carcinoma recalling the mucous glands of the trachea and bronchi. The alveoli may be regular, lined by two or more layers of cubical cells, and dilated or compactly filled. The cells are usually of small size and cubical or polymorphous, while high cylindrical and squamous cells are missing. Highly malignant tumors may yield alveolar or diffuse and atypical carcinoma. The secretion of mucus is a prominent feature and, while not wholly limited to mucous gland cancers, is strongly indicative of this origin. In many cases the mucous secretion is limited to single cells, but it may fill or distend alveoli and lead to pronounced gelatinous or even cystic changes (Coats). From this typical structure there are many variations, corresponding to the duration and malignancy of the process and its secondary changes.

(c) Carcinoma arising from the pulmonary alveoli is either (I) diffuse or (2) multiple and nodular. In the diffuse form one whole lobe or the whole lung is uniformly consolidated, while cavities may form from necrosis. The lesion may resemble organizing pneumonia or croupous pneumonia in gray hepatization (Levene, Pepere), and there are indications that the condition may really follow an atypical acute pneumonic process (Loser, Cahen). The pleura is usually involved, and local and general metastases are frequent.

In the nodular form one or both lungs are the seat of many poorly defined tumor foci from miliary to marble size or larger. Even the small nodules may show necrotic areas, and the lesions are not always readily distinguished from tubercles (Marchiafava). The larger tumors may be solid or cystic.

The structure of the diffuse tumors presents partial or complete filling of the air vesicles with cuboidal, cylindrical, or flat cells. The walls of the vesicles may be preserved or obliterated by diffuse growth or by inflammatory processes. Domeny and others describe diffuse carcinomas of the parenchyma largely composed of squamous cells. Extensive inflammatory changes may reduce the proportion of cells and give excess of new connective tissue. Kretschmar in a diffuse tumor described a papillary structure with cylindrical cells. Extension through the lung occurs by way of the air passages, septa, lymphatics, and blood-vessels, which may be found infiltrated by tumor-cells (Edlavitch).

The structure of the nodular tumors was clearly pictured by Malassez, and presents groups of slightly dilated air vesicles filled with papillary projections of cylindrical cells. The central spaces may be occupied by desquamated cells, mucus, or necrotic material (La Sourd). Multiple tumors composed of high, cylindrical or goblet-cells of adenomatous type and without metastases are reported by Kelly. A combination of high cylindrical cells in papillary arrangement and solid masses of polygonal cells was observed by Knierim and attributed to a dual origin from alveolar and bronchiolar epithelium. Bjornsten found many cystic tumors of both lungs in a child of 9 years, of which the structure was that of papillary cystadenoma proliferum.

The metastases of pulmonary carcinoma are usually very numerous and wide-spread, but vary greatly with the grade of malignancy. The vascularity of the organ and the frequent invasion of blood-vessels facilitate dissemination, while the abundant lymphatic channels lead to infection of the bronchial nodes in a high proportion of cases and to early extension throughout lung and pleura. The tumors extend also along the lumen of the bronchi 
replacing the mucosa, but Stilling's conclusion that aspirated tumor particles produce secondary growths may be doubted. In 374 cases Adler reports invasion of bronchial nodes in II 7 , mediastinal 45 , tracheal 26 , cervical 23, retroperitoneal 23, and in many other regions less frequently. The liver was invaded in 103 cases, pleura $5^{2}$, pericardium 39 , heart 30 , kidney $5^{8}$, adrenal 38 , spleen 18 , brain 53 . The bones were involved 57 times and often extensively, the ribs, spine, skull, and sternum suffering in the order named. In a case reported by Turnbull and Worthington the bones were the sole seat of metastases. Metastases were absent in 33 cases.

Few organs fail to appear as possible sites of secondary growths, which have been noted in skin, finger-tip, nasal septum, eye, bladder, tubes, ovary, uterus, pancreas, thyroid, spinal cord, and hypophysis. Deposits in the voluntary muscles were observed in 9 cases and in $I$ case they were found only in this tissue.

Histogenesis.-The exact sources of pulmonary carcinoma have been traced in detail by many observers who have shown that these tumors arise from the bronchial mucosa, the bronchial mucous glands, and the alveolar epithelium. The impression of Schottelius that the pulmonary endothelium gives rise to carcinomatoid tumors has not been verified.

Comparatively few cases have furnished any opportunity to trace the beginnings of pulmonary carcinoma. On the other hand, when the evidence of histological structure, gross anatomy, and clinical history is combined, the conclusions regarding histogenesis may be regarded as reasonably certain and acceptable.

An origin from bronchial lining epithelium is indicated by extensive involvement of the bronchial mucosa and by invasion chiefly along the bronchial tree. Papillary projections from atypical prolifeation of lining epithelium were observed by Reinhard and Chiari and speak strongly in favor of an origin from this epithelium. A high papillary structure, as in Chiari's case, has a similar significance. While cylindrical cells may be present in all forms of pulmonary carcinoma, the presence of very high cylindrical cells and their persistence in metastases accords best with an origin from the bronchial mucosa (Chiari, Tillmann, Peck). In a case of alveolar carcinoma with high cylindrical cells Ravenna traced the rather sharp transition of the bronchial lining into the tumor tissue.

Bronchial papillomas are probably a source of malignant tumors. Such growths are much less frequent in the bronchi than in the trachea. Siegert described such a tumor at the bifurcation and assumed that it arose from a misplaced fetal remnant. Werner described a bronchial papilloma with ciliated cells. The great majority of squamous-cell tumors arise from bronchial epithelium. Squamous metaplasia of the bronchial mucosa has been shown to be of frequent occurrence in tuberculosis and other pulmonary lesions, and this change probably precedes the development of carcinoma in many instances (Griffini, Kitamura, Haythorn).

E. Froelich found extensive bronchial pachydermia with squamous-cell cancer. It is especially in and about tuberculous cavities that squamous alterations are observed, and in these situations squamous-cell cancer is most frequent.

In many cases squamous epithelium is mingled with cuboidal and cylindrical cells, as in Kretschmar's case, indicating that the metaplasia may take place in the established tumor. In a bulky tumor of bronchial origin Henrici found non-keratinizing squamous mingled with cylindrical cells, while in the main branches there were papillary projections and foci of squamous metaplasia. 
Bronchial malformations appear to be connected with the origin of certain tumors which are characterized by a cystic or papillary structure and by abundant secretion of mucus. Stoerk describes cases of cystic malformation of the lung in infants which he refers to two types of embryogenic disturbance: (I) overgrowth of misplaced and fetal portions of the bronchial tree; (2) overgrowth of areas of fetal bronchiectasis resulting from inflammation and leading to occlusion of bronchus and retention of mucus. He reports a spongy fetal cystic adenoma replacing the middle lobe of the right lung, and presenting many cavities lined by bronchial epithelium. The septa were deficient in elastic fibers and contained many glandlike alveoli. Some tumors of children and adults seem to belong in this series. Aschenborn found the right lung in a boy of I 2 years replaced by a cystocarcinoma containing several large cysts and eroding the ribs. Linser found a large cystadenoma with cellular stroma reproducing fetal bronchi in a boy of 13 years. Couvelaire described a large cystadenoma in a newborn infant with many adenomatous alveoli branching off from abortive bronchi. Dionisi's tumor recalled the structure of thyroid gland. Lohlein describes a sharply circumscribed tumor of the right upper lobe exuding much mucus from cystic cavities which were dilated interlobular bronchioles. The structure was of papillary projections of cylindrical epithelium into spaces filled with mucus. This tumor was interpreted as originating from an area of fetal atelectasis. Weichselbaum's small coarsely papillary adenosarcoma in the lung of a woman of 67 years may represent a late development in a malformed bronchiole.

The bronchial mucous glands have been shown to give origin to a welldefined group of pulmonary growths. In a multiple nodular submucous carcinoma of trachea and bronchi Langhans traced an unbroken series of hyperplastic glands, showing extensive overgrowth of saccules, their dislocation between the layers of connective tissue, the loss of membrana propria, and finally breaking up into infiltrating cords of tumor-cells. Beck and Rondeau also were able to trace the transition from hypertrophic to neoplastic glands in more advanced tumors. In most cases the diagnosis of bronchial gland origin has been based on gross anatomy and microscopical structure. The tumors are often distinctly glandular, but many other histological varieties probably arise from this source (Ehrich, Fuchs, Passler). A large scirrhous tumor with small round or cubical cells is referred by Wolf to the bronchial glands.

In the pulmonary parenchyma it is difficult to distinguish the parts played by vesicular and by bronchiolar epithelium, and in many instances tumors appear to arise from both sources. In a multiple nodular tumor Malassez described groups of slightly widened alveoli lined by papillæ of low cylindrical epithelium, but he was unable to determine whether the cells were alveolar or bronchiolar. Since alveolar epithelium readily changes to cuboidal in atelectatic foci, it is clear that tumors with such cells may originate in the alveoli. By exaggeration of the papillary structure and fusion of adjoining foci some more diffuse tumors may perhaps be safely traced. Thus Knierim describes a diffuse tumor of the midlobe exuding much mucus and resembling a confluent lobular pneumonia. There was mucus in outlying alveoli. The tumor presented alveoli and spaces lined by cylindrical cells in papillary outgrowths and solid masses of polygonal cells. The papillary structure persisted in lymphnodes. The author suggested an origin from both bronchioles and alveoli.

The exact mode of origin of the solid diffuse carcinomas is somewhat obscure. In many cases the air vesicles are filled with solid masses of cubical or cylindrical cells suggesting an origin from altered alveolar epithelium or from the bronchioles. Ramon and Boidin, among others, found all transitions 
from flat to cubical to neoplastic epithelium. The secondary changes of atelectasis seem to precede the advance of the tumor.

In other cases the history and the minute structure indicate that the neoplasm has arisen as a sequel or incident of croupous, productive, or tuberculous pneumonia. In a case of supposed croupous pneumonia Loser was unable to decide whether he had an inflammatory proliferation of endothelial or epithelial cells, or a true carcinoma. Yet there were subpleural nodules of alveolar carcinoma.

Pepere's small contracted lung resembled interstitial pneumonia, but was the seat of alveolar carcinoma. Cahen found a structure which he likened to desquamative pneumonia. Diffuse or miliary tuberculous lesions in or about the tumor have suggested that the neoplasm may arise from exaggerated proliferation connected with this infection (Wolf, Domeny). Many of the diffuse carcinomas arising from the alveolar epithelium contain pavement or distinctly squamous cells (Fuchs, Grunewald, Edlavitch, Domeny). Menetrier could not demonstrate eleidin granules in these flat cells, while pearls and spine-cells appear to be limited to the bronchial tumors. A bulky colloid acanthoma in the lung of a dog was referred by Rievel to the alveolar epithelium.

Symptomatology.-The symptoms and course of pulmonary carcinoma vary extremely according to the type of the tumor and the conditions which have led to its development.

A large number of cases have been regarded clinically as acute or chronic phthisis, and since tuberculosis is often present with bacilli in the sputum a differential diagnosis may be impossible. Other cases appear as sequels of prolonged pneumonia. An apparently sudden onset is often due to acute changes in a long existant tumor. A long history of bronchitis with or without hemoptysis has preceded the discovery of bronchial tumors. The uncomplicated bronchial tumor usually gives physical signs of a progressive intrathoracic growth, with cough, hemoptysis, pain, recurring pleural effusion, ' serous or bloody, dyspnea, and cyanosis. According to Herrmann about half the cases are of the pleuritic type. Extensive infiltration of the pleura with adhesions may prevent effusion. Fever must be referred to complications. The diagnosis of a neoplasm has repeatedly been made from tumor fragments in sputum or pleural effusion (Betschart, Rondeau), by $x$-ray photography (Muser), and by the bronchoscope (Karrenstein). The duration has varied from 4 years (Adler) to ro days (Jaccoud). A fatal hemoptysis may be the only symptom (Beveridge, Degen).

Cachexia is slight in uncomplicated cases, but terminal stages are often marked by suppuration, pneumonia, and gangrene. Immediate causes of death also include rupture of large veins, thrombosis, pulmonary edema, and asphyxia.

In not a few cases the effects of metastatic growths have attracted chief attention, and have centered chiefly in the central nervous system (Coats, Domeny, Wolf). Finally, pulmonary tumors have occasionally appeared as wholly unsuspected conditions at autopsy.

Operative interference with pulmonary tumors has been attempted by Lenhartz, and in I case the excision of the affected lobe was survived for is months.

\section{SARCOMA OF LUNG}

From both clinical and pathological standpoints sarcoma of the lung is a very ill-defined group of processes of varied origin and course. While Adler was able to select 90 cases from the literature, only a minority of the 
reports present sufficient evidence to justify their separation from diffuse carcinoma.

In not a few cases the sarcoma has been associated with extensive chronic exudative necrotic or productive inflammation, with abscesses, gangrene, empyema, or fistulous tracts, which in a reactive tissue like the lung may readily lead to overgrowth of reparative tissue simulating round- and spindlecell sarcoma. In another group active tuberculous lesions have been present and so intimately associated with the whole process as to render a distinction between tumor and inflammatory product somewhat hazardous. Thus Pater and Rivet found tubercle bacilli in multiple nodules of large round-cell sarcoma at both bases and in the liver, while Schnick describes as spindle-cell sarcoma a large excavated tumor mass, partly calcified, in an actively tuberculous lung. Sangalli's round-cell fibrosarcoma, irregularly distributed throughout an old tuberculous lung, does not accord with the usual behavior of sarcoma.

Old syphilis is strongly suggested in a curious cystic tumor filled with mucus and pus described by Milian and Mante. The precocious development of pulmonary tumors secondary to growths in other organs, especially in the bones, stands in the way of accepting certain bulky spindle- and giantcell tumors as primary in the lung. Before sarcoma or carcinoma can be regarded as primary in the lung a very thorough search for primary tumors in other organs must be made. There are possibly on record less thoroughly studied cases than that of Lesieur and Rome, who found a massive apparently primary carcinoma of the lung, but in the rectum a carcinomatous ulcer. Of the lymphosarcomas very few, if any, can be regarded as primary in the lung, while in many instances it is clear from the anatomical descriptions that the tumor arose in the bronchial or mediastinal nodes or the thymus. It also appears that many authors describe as lymphosarcoma any tumor in which the cells are small and round. Nearly all the primary large and small round-cell sarcomas of the lung, as of many other organs, are under suspicion because of the fact that atypical carcinoma may yield exactly similar pictures in rapidly growing areas. Adler among others has pointed out the necessity of extensive study of all portions of such tumors and their metastases before the mesoblastic origin can be assumed. I can support Fuchs' statement that areas of spindle-cells may be found in pulmonary carcinoma. Hodenpyl found spindle-cells in the hepatic and lymphatic metastases of a carcinoma. The carcinosarcomas of the lung are probably thus explained.

Finally, no one has succeeded in tracing the origin of any of the malignant sarcomas of the lung, and the general field of pathological changes in this organ does not point clearly to specific conditions under which mesoblastic tumors may arise. The very marked overgrowth of endothelium in some organizing pneumonias may perhaps have some significance.

Nevertheless these uncertainties hardly warrant the conclusion, once drawn by Hertz, that primary sarcoma of the lung does not exist, for the literature offers not a few reports indicating that mesoblastic tumors occasionally arise in this organ.

A provisional classification of pulmonary sarcoma based on anatomical features may be employed as follows:

(I) Diffuse spindle-cell sarcoma.

(2) Peribronchial sarcoma.

(3) Large round-cell sarcoma.

(4) Lymphosarcoma.

(I) Diffuse spindle-cell sarcoma produces a characteristic gross and microscopical picture and is the best defined type of pulmonary sarcoma. 
The tumors are usually bulky, exceeding in size the largest carcinomas. Bock's tumor in a child filled the entire left thorax, obliterating all pulmonary structures. The ribs may be eroded (Elkan) and chest wall perforated (Koblynski). Cavities may form from central necrosis and hemorrhage (Ranglaret), or the whole tumor may be very soft and hemorrhagic (Rolleston, Trevor). In several cases the tumor, while large, has been well encapsulated (Fuchs).

Smaller firm tumors are usually fibrous. In Chiari's case there was extensive calcification. Blumental describes a large myxosarcoma. Metastases are usually absent, but there are extensions through lung and pleura and to the neighboring nodes.

The structure presents large and small spindle-cells, or occasionally giantcells, with varying proportions of stroma. This structure suggests an origin from the pulmonary connective tissue, as mentioned by Mironescu. The bronchi may be normal (Rutimeyer).

(2) Peribronchial sarcoma presents a specific anatomical distribution which indicates an origin from the bronchial wall, with extension along the bronchus. The infiltration follows the bronchi from the root of the lung or trachea throughout the parenchyma and into the septa (Peritz, Pollak). The bronchial walls may be ulcerated and weakened with ectasiæ (Anderson, Levitt) or compressed.

Nearly all the peribronchial tumors are composed of small round-cells and some are often designated as lymphosarcoma. Their origin is obscure. Since the bronchial nodes are constantly involved, the condition may represent a pulmonary invasion from these nodes. In Hildebrand's case the tumor arose in a phthisical lung and tubercle bacilli were numerous. Small cell carcinoma of the bronchi may be difficult to distinguish from the round-cell tumors of this class.

(3) Large Round-cell Sarcoma.-This highly indefinite term may be applied to a group of tumors which do not appear to belong to lymphosarcomas and which cannot be positively identified with small cell diffuse carcinomas. They differ from lymphosarcoma by their limitation chiefly to the lung. Several cases have been reported in children or young subjects, Bjornsten observing the disease at 2 years, Lehndorff at 3 years, Box at 5 years, Davies at I8 years, and Poore and Pitot at 20 years. They are usually bulky, involving the whole of one lung with pleura, in a solid opaque tumor which obliterates most pulmonary structures. In some cases the tumor is smaller and encapsulated (Lehndorf, Roth). Areas of necrosis and large cavities may form. Metastases are local or absent, but there may be extensions to hilus, nodes, ribs, and vertebræ. In the case of Millian and Bernard, of 4 months' duration, the left lung was represented by a large cavity with sarcomatous walls.

The structure presents small or large round cells in diffuse, perivascular, or alveolar arrangement. The alveolar structure described by Roth, Meyer, Adami, and McDonnell strongly suggests a carcinomatous nature.

(4) Lymphosarcoma.-Although a large proportion of the round-cell tumors of the lung have been described as lymphosarcoma, there is no satisfactory evidence that true lymphosarcoma arises in the pulmonary parenchyma. It seems much more probable that the lymphosarcomas arise in the bronchial or mediastinal nodes or elsewhere and invade the lung along the bronchi. Such tumors present bulky masses about the hilus and more or less extensive invasion of the parenchyma. Cases of this type are described by Powell, Cohen and Kirkbride, Coats, and others. 


\section{CHAPTER XLII}

\section{EPIDERMOID CARCINOMA}

\section{EPIDERMOID CARCINOMA OF SKIN}

Carcinoma of the skin is remarkable for diversity of origin and course and variety of histological structure.

Two main histological types of the tumor occur: (I) Hornifying cancroid, acanthoma, (2) basal-cell carcinoma.

Of each of these there are several subvarieties which are distinguished by structural, etiological, or clinical features.

(I) Acanthoma.-This tumor is distinguished by the presence of adult squamous cells, hornification, and pearl formation. The tumors are single or multiple. The multiple tumors may be very numerous and widely distributed over face, trunk and limbs, and their early stages may be marked by erythema, seborrhea, eczema, or pruritus.

The early lesion of acanthoma of the skin appears in two main types which it is highly important to distinguish.

(a) Many acanthomas pass through a preliminary papillomatous stage, in which they appear as definite elevated warty outgrowths movable on the superficial fascia. Histologically these lesions are malignant, but they long remain localized and tend to spread laterally. In this phase they offer a good prognosis, which is lost when the lesion becomes fixed, ulcerated, and depressed.

(b) Other acanthomas are flat, depressed, indurated, and infiltrating from a very early period. They are less impressive externally, but early ulcerate and invade the deeper tissues and lymph-nodes, usually with a tendency to assume the structure of tubular carcinoma, in which squamous characters are lost.

In the more advanced stages of both types there is erosion and ulceration, and the lesion gradually extends in the form of a broad ulcer with raised nodular indurated edges and granulating base. Acanthoma of the skin differs from rodent ulcer chiefly in its early papillary appearance, more rapid lateral extension, and more destructive course. Yet both types of carcinoma may occur in the same lesion. Occurring in tissues previously altered by syphilis, tuberculosis, etc., its progress is facilitated by this association. The local aggressive tendencies of acanthoma tend to produce deep ulceration with extensions along blood-vessels and nerves with pain and hemorrhage, and invasion of lymphatics with metastases in lymph-nodes and occasionally in internal organs. Bulky internal metastases, however, are rare. The largest I have observed were in the inguinal nodes and liver, from epithelioma of prepuce. Secondary infection by streptococcus is nearly constant with deep ulcers, may accompany the metastases, greatly influences the course and termination, or may even dominate the clinical picture.

The seats of single acanthoma of the skin are chiefly the mucocutaneous junctions, the face, scalp, chest, breast, back of hands, shins, and toes. The location varies with the numerous peculiar etiological factors. Thus chim- 
ney-sweep's and paraffin cancers occur on scrotum and thighs; Kangri cancer on the abdominal wall, while the scars of burns, chronic ulcers, $x$-ray dermatitis, and various forms of trauma are followed by tumors in many regions. Acanthoma occurs chiefly in advanced life, but has been observed as early as the I4th year (Borrmann). Selberg has collected a series of cases occurring in infants and children.

Structure.-Acanthoma of the skin presents the most typical form of adult epithelioma and tends to maintain this structure rather rigidly. Hence flat squamous epithelial cells, intercellular fibrils, and pearl formation are often observed both in the primary tumor and in local or distant metastases. Thus one finds in metastatic nodules in liver or bone-marrow extensive hornification, spine-cells, and small cysts filled with exfoliated scales, and these

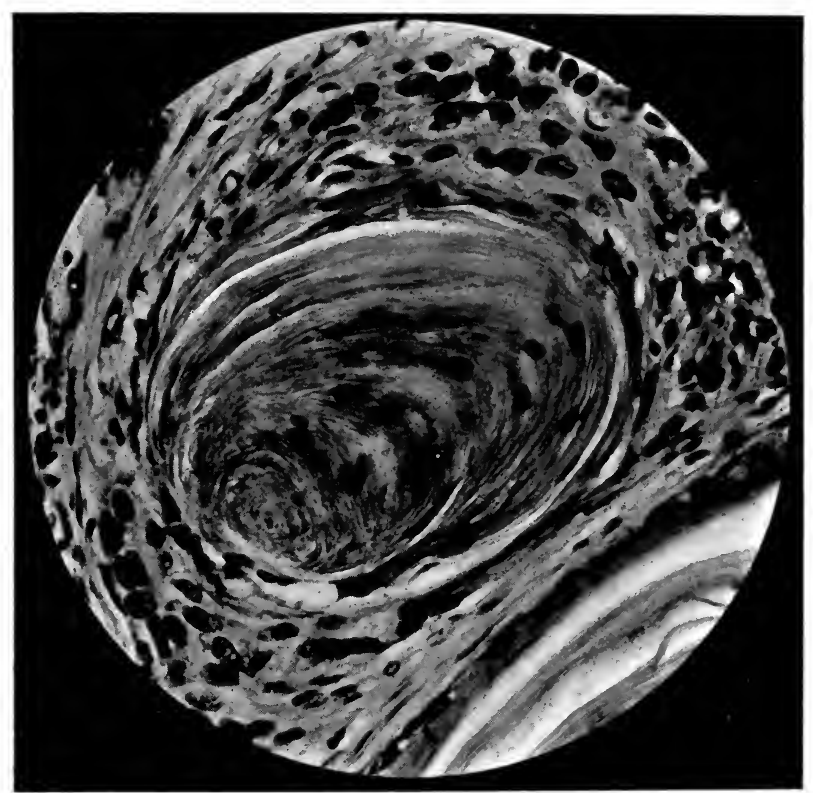

Fig. 40I.-A hornified pearl in acanthoma. (Photo. by B. H. Buxton.)

adult features may be more pronounced than in the primary tumor. On the other hand, many acanthomas lose their adult characters in the growing edges of the primary tumor, where the cells appear in narrow cords of opaque granular cells with hyperchromatic nuclei. This structure may be called "tubular acanthoma." It is usually associated with a high grade of malignancy. In certain acanthomas the coherent masses of epithelial cells are replaced by a diffuse growth of rounded cells each of pavement character. Giant-cells may be very numerous in such tumors and may even predominate over other forms. The cause of such marked variations in structure is not clear, but edema seems to play a part. Many peculiarities of cell structure appear in acanthoma, which Unna has made an effort to classify. These include many of the bodies once regarded as parasites and elaborately pictured by Pianese. Unna has called attention to a peculiar $\mathrm{X}$-cell seen in condyloma and acan- 
thoma. Its cytoplasm is homogeneous and basophilic, and its microchemical reactions have been studied by Pansini.

In the metastases there may be great anaplasia, and after repeated recurrences there may be entire loss of adult epithelial characters and the tumor may appear as an indifferent round- or spindle-cell growth. Unless the history of such changes has been followed an erroneous diagnosis may be made.

Secondary changes in acanthoma are numerous and frequent. Polynuclear leukocytic infiltration follows from infection. Round cells, lymphocytes, and plasma-cells may gather in large numbers about early lesions, but diminish as the tissue resistance fails and the rate of growth increases. Extensive calcification appears in some tumors without greatly influencing the course. Giant-cells form from fusion of tumor-cells or from phagocytic tissue cells which gather about the tumor masses. Fibrosis is prominent in most cases,

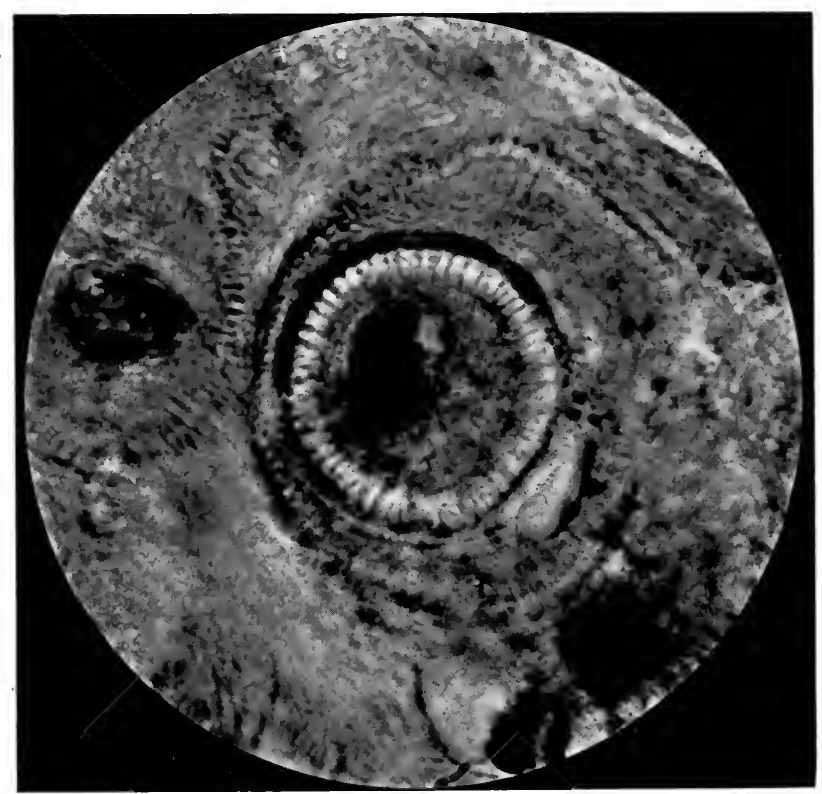

FIG. 402.-Prickle-cell in acanthoma. (Photo. by B. H. Buxton.)

but seems to have little effect in limiting growth. Extensive hornification and calcification of tumor-cells with overgrowth of hyaline connective tissue Orth regards as evidence of spontaneous healing.

In an interesting group of cases fatty degeneration, necrosis, and calcification may become very extensive, so as to destroy many or all of the epithelial cells. Growth may then be very slow, but in time the tumor may reach a large size. In Landau's case it weighed $250 \mathrm{gm}$. Walkohff reports a case of 50 years' duration. Strassberg observed extensive bone formation. The exact nature of these "petrified epitheliomas" has remained in doubt. Barlow regards them as forms of adenoma sebaceum, but Ribbert and most authors consider that all of them are acanthomas.

Etiology.-Acanthoma of the skin is almost exclusively the result of chronic traumatism, but the forms of the irritation are extremely varied, and the 
relation to the tumor is indirect. Yet from the practical standpoint irritation is the important factor, and all other influences, such as heredity, or local predisposition, are secondary. This tumor arises from previously normal epithelium after a period of overnutrition and overgrowth, during which the subepithelial tissues become altered and less resistant. Lymphocytic infiltration, swelling with mucoid or other forms of degeneration, followed by atrophy of elastic tissue, and chronic edema or fibrosis, usually but not always precede the downward growth of epithelium. Yet the controlling influence must, I believe, be regarded as inherent in the epithelial cell. All cutaneous epithelium must be considered capable of developing acanthoma, for which it is only necessary to supply the proper conditions. These conclusions seem to be justified especially by study of the conditions under which acan-

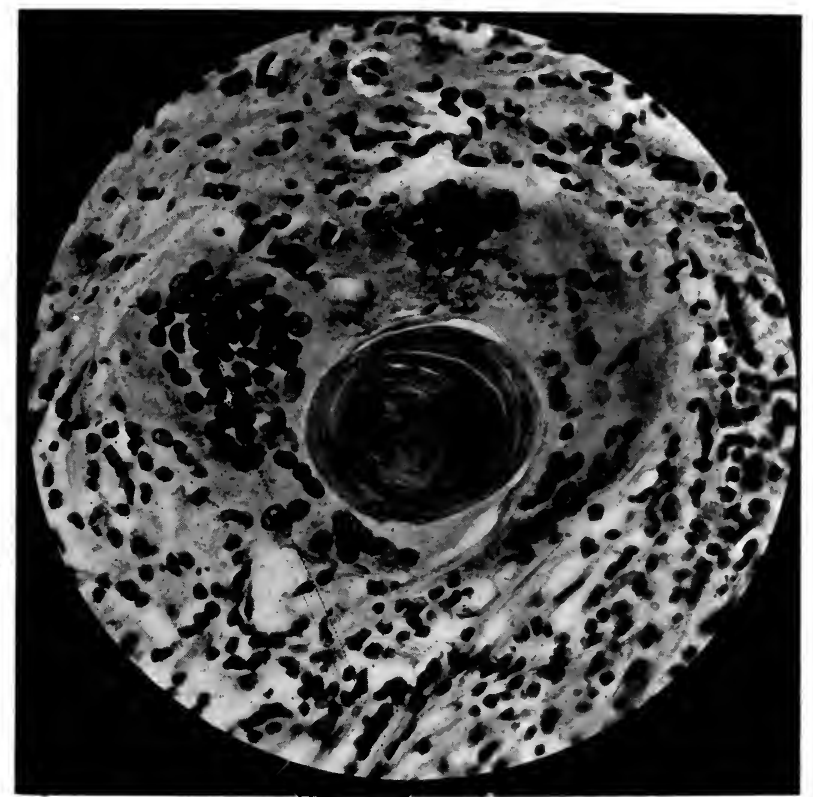

FIG. 403.-Giant-cell forming about an epithelial pearl in acanthoma. (Photo. by B. H. Buxton.)

thoma occurs. Thus the peculiar irritation to which chimney sweepers and paraffin workers are sometimes exposed determines a peculiar localization of the disease. The observations on Kangri cancer of Indian natives, which develops on the abdominal wall at the point of irritation of a hot earthen oven, is a very direct demonstration of the rule. $x$-Ray dermatitis appears to occur indifferently in any region where the rays may have been applied. Yet there is some evidence of a personal predisposition to $x$-ray dermatitis and its sequelæ. Chronic ulceration, eczema, lupus, and the scars of burns present somewhat more complex but essentially the same conditions in which acanthoma develops. In the localization about mucocutaneous junctions there is an added factor in the anatomy of these regions, as well as increased exposure to trauma. Cheatle believes that cutaneous cancer develops mainly at the points where nerve-trunks reach the skin, and tend to spread chiefly 
in such areas of nerve distribution. Warts, both simple and venereal, may be the starting-points of acanthoma.

Some very malignant acanthomas have developed after the bites of animals, especially the horse, and of insects.

In a series of cases of acanthoma, chiefly of the face, Borrel has found a parasitic insect, Demodex folliculorum, which he regards as the direct exciting cause of the tumor. He finds that about 50 per cent. of all subjects show an invasion of the skin by this insect. I have observed the structures he depicts in acanthoma, but find it difficult to draw any conclusions concerning their significance.

B. Fischer, by injections of sudan III in olive oil, produced hyperplasia of epithelium and downward growth resembling epithelioma, but the process ceases when the oil is extruded. Specific "attraxines" of this and other types may well exist, including derivatives of insect bodies, but the scope of their activity in human acanthoma remains to be determined.

Certain deep acanthomas arising from particular conditions require mention. The sebaceous cyst sometimes develops a somewhat malignant

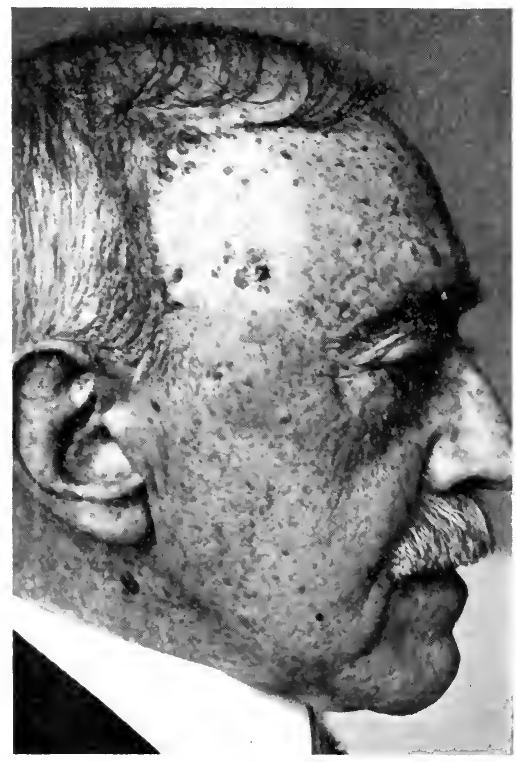

FIG. 404.-Xeroderma pigmentosum with multiple epidermoid carcinomas. deep epithelioma, in which the characters of the duct-cells are prominent. The true congenital epidermoid cyst of the skin is another source of deep epithelioma. The branchiogenic cyst is a frequent source of deep and often very malignant acanthoma of the neck. This tumor may appear anywhere from the mastoid to the clavicle and often reaches considerable development before its true nature is recognized.

Many other congenital dermoid cysts have given rise to deep epithelioma. These occur chiefly in the neck, head, buttock, navel, and wherever embryonal fissures form (Krische, Mertens, Linser). In the breast Kaufmann found a deep dermoid with epitheliomatous changes, associated with carcinoma of the breast. The frontal dermoid appears beneath the skin of the forehead, or within the tables of the skull, or within the cranial cavity, where it may present the changes of acanthoma, usually with bulky lamellated concretions of horny scales (Hartley). Occasionally it shows an opening through the scalp. Embryomas of ovary or testis may be the seat of epitheliomatous change of the skin. I have studied an ovarian dermoid which ruptured into the colon, and presented a papillary acanthoma at one point invading the rest of the complex tumor.

Xeroderma pigmentosum is a condition regarded by many as an aggravated form and sequel of the common lentigines (freckles). It is marked by overgrowth of pigmented epithelium, scaling, even ulceration, and eventually by atrophy of the affected skin (Crocker). Several tumor-like processes in the derma have been described as secondary to the disease, as angioma, my- 
oma, pigmented sarcoma, and especially epithelioma. The epitheliomas may be of the type of acanthoma or of rodent ulcer (Kreibich, Pernet, Halle). They are often pigmented, as in sailor's carcinoma (Unna). Hutchinson described as lentigo maligna juvenilis, v. senilis, cases of progressive freckles of cheek and eyelid which become the seat of epithelioma. It occurs chiefly between the seventh and ninth years (juvenile) or in old age. It appeared in 7 of 8 boys in one family in which 5 girls were unaffected (Ruder). The exciting cause is exposure to sunlight, acting on the skin of constitutionally and congenitally predisposed subjects (Councilman, Magrath). According to Dalous and Constantin the most marked feature of xeroderma is atrophy and scarring of the derma, with secondary carcinoma, while Unna's pigmented epithelioma is a primary miniature carcinoma.

That the prolonged use of arsenic has an influence upon the development of epithelioma of the skin has been urged especially by Hutchinson, who reported 2 cases. The first effects are a hardening and pigmentation, the appearance of palmar or plantar psoriasis, followed later by epithelioma. Darier observed multiple carcinoma following xeroderma pigmentosum which resulted from excessive use of arsenic. Fordyce described characteristic cell changes in the epidermis.

Lupus Carcinoma.--Epithelioma develops in a considerable proportion of cases of lupus vulgaris and much less frequently with 1. erythematodes (Ashihara, Lit.). It arises both in the active regions of the tuberculous process in skin or mucous membranes and in about 30 per cent. of cases in the lupus scar tissue (Steinhauser), and as early as the fourth or as late as the fifty-fifth year of the original disease, the average period being about the thirtieth year. In many cases but not in all the course is relatively rapid for cutaneous carcinoma, with invasion of lymph-nodes and internal growths. The prognosis is very grave, only 9 cases of Ashihara's list of I 22 recovering.

The initial stages of lupus carcinoma may be somewhat difficult to separate from the usual hyperplasia and displacement of epithelium in the lupus process. As a rule the tumor appears in multiple foci with the usual local signs and increased destruction of tissue. The structure is that of acanthoma, becoming tubular and atypical in advanced stages and metastatic areas. Lupus erythematodes has been followed by carcinoma in 7 reported cases collected by Ashihara. Hartzell reports I case after tuberculosis cutis verrucosa, and Blaschko observed a case with leprosy. Several cases developing in leutic scars are mentioned by Ashihara, and Rabaioye reported a case following blastomycosis. Senger reports a case of malignant recurring sarcoma following lupus.

$x$-Ray Dermatitis and Carcinoma.-A single severe exposure to $x$-ray, or repeated slight exposures, lead to a peculiar form of dermatitis which in many instances has been followed by multiple acanthoma of the skin, and in not a few cases by fatal extension of the tumor process to internal organs. The growth of knowledge in this field has been coincident with the development of the lesions in the unfortunate early workers with $x$-ray, who watched the appearance first of the acute dermatitis, then of the thickened skin, alopecia, telangiectases, ribbed nails, and keratosis, and later of the warty outgrowths, ulceration, and finally malignant carcinoma. Since the first formal report of these lesions (Marcuse, I896) observations have multiplied rapidly. In I900 Kienbock gave a very full description of the dermatitis and its immediate sequelæ and pointed out methods of avoiding the "soft" or burning rays. The development of fatal carcinoma began to be reported in 1904 (Porter), and at the time the urgent interest in the subject brought 
out the careful histological study of Unna and the experimental work of Gassmann and of Linser.

Clinical Course. $-x$-Ray dermatitis appears in the form of an erythema which follows a few hours after the application and varies in intensity and character with that of the energy employed. The immediate effects have been compared with heat burns of various degrees. Many of these lesions soon run into ulceration which long remains painful and indolent.

After repeated slight exposures a period of incubation is commonly observed, lasting from 3 to II years and marked by thickening of the skin, dryness, scaling, fissures, keratosis, alopecia, and cyanosis. At any time in this period local warty areas of keratosis may form or painful ulceration develops. The deeper tissues being involved, whole phalanges or limbs may be destroyed by a dry gangrenous process. Carcinoma develops either on a severe acute burn after prolonged ulceration (Porter) or after the more chronic changes which have not been preceded by any severe acute dermatitis, and it has frequently arisen as a combined result of a lupus or other lesion treated by $x$-ray. Da Costa found that I8 per cent. of lupus cases develop carcinoma after

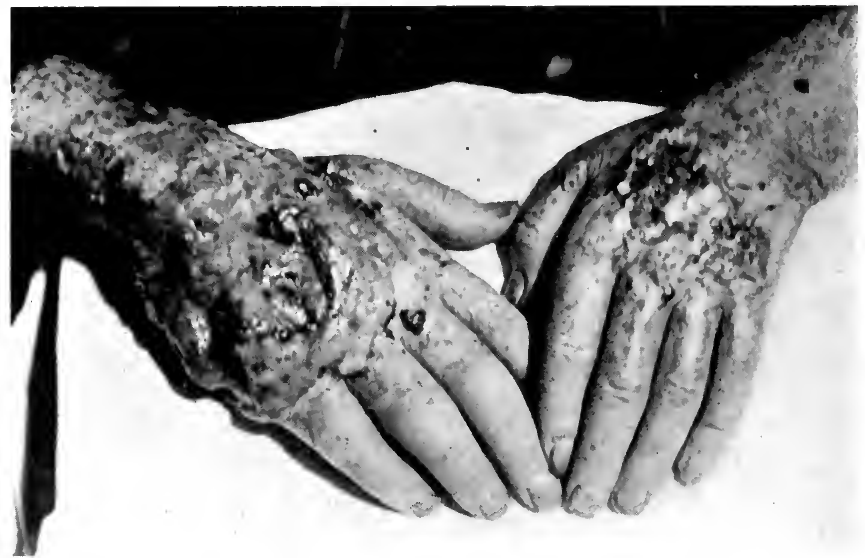

FIG. 405.-Epidermoid carcinoma following $x$-ray dermatitis.

$x$-ray treatment, but carcinoma is by no means uncommon in untreated lupus. The carcinoma occurs in the bases and edges of ulcers, or beneath multiple foci of keratosis, and both because of its initial malignancy and from the progressive development of new foci it is extremely difficult to combat. Involvement of lymph-nodes and internal metastases follow, with death, as recorded in at least 9 cases (Porter).

Structure.-The early lesions are those of a subacute exudative inflammation in which vascular lesions are prominent. Linser, who excised pieces of skin at intervals during treatment for lupus, found thrombi in the vessels in 4 days, which with swelling and desquamation of endothelium reached an acme in 8 eight days, and was followed by obliterating endarteritis. Unna emphasizes the telangiectasis, which he refers to deep obliterating endophlebitis. In a severe burn of 30 days' standing I find hyaline necrosis of endothelium and entire wall of venules, wide subepithelial telangiectases, extensive edema, general infiltration with lymphocytes, swelling of collagen fibrils, hydrops of the epithelium, and beginning keratosis. 
In more chronic lesions fibrosis and atrophy are predominant, so that the condition resembles premature senescence (Lindhorn). Wolbach, who finds foci of necrosis in the corium after many months, interprets the lesions as the result of progressive degeneration from vascular changes and imperfect efforts at repair. The lymphatics are obliterated by endothelial proliferation (Gassmann). The muscle-tissue, at first swollen and vacuolated, atrophies and disappears. The connective-tissue cells are swollen and multiplied and their nuclei may be hyperchromatic. Exaggeration of this feature has led

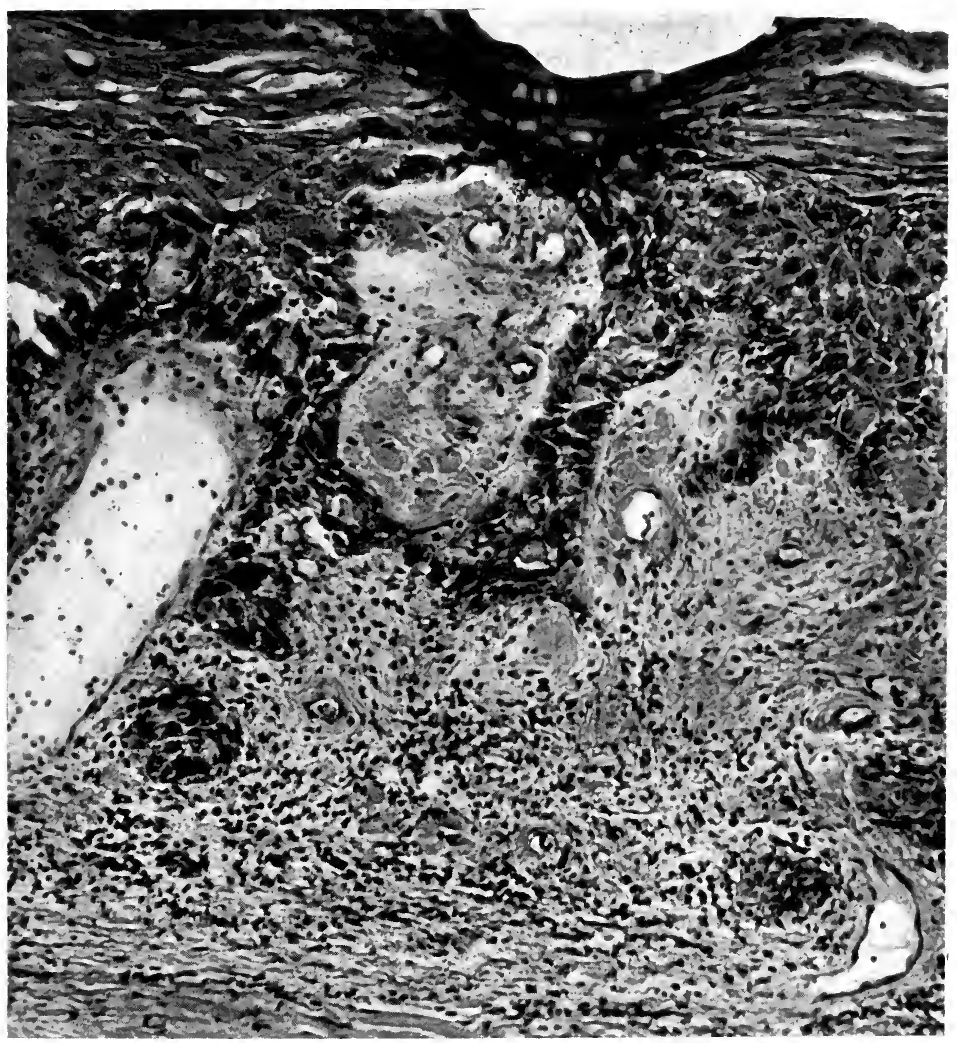

FIG. 406.-Chronic $x$-ray dermatitis. Precancerous stage. Showing keratosis, telangiectasis, destruction of collagen fibrils, lymphocytic infiltration, hypertrophy and hyperchromatism of epithelial cells, and beginning heterotopia of epithelium.

to conditions interpreted by some as sarcoma (Schumann, Lit.). I have seen one case of this type, but the conditions were too obscure for positive diagnosis. The structure resembled that of Clunet's experimental $x$-ray sarcoma in a rat. The epithelium is altered from the first, showing hydropic degeneration, keratosis or scaling, and erosion and overnutrition of the deeper layers of cells. Proliferation of these cells, usually under a thick covering of keratin, gradually leads to downward growth into the edematous corium, or into dilated thrombosed vessels (Wolbach). In this stage the structure is usually that of acanthoma or tubular epithelioma. 
Much use has been made of this experimental cancer, especially by Wyss, for its bearing on the genesis of epithelioma, but it does not appear that the factors concerned in $x$-ray cancer are simpler than in other conditions. A specific change in the blood rendering it injurious to vessels and cells (Unna), repeated efforts at regeneration (Wolbach), disturbed relations of tissue tension from dermal fibrosis (Wyss), and acceleration of autolytic processes, all deserve consideration. Those who assume that the dense fibrosis of the deeper corium is the essential factor have perhaps underestimated the importance of the changes in the epithelium which are constantly present.

Paget's disease is a specific chronic progressive disease of the epithelium of the mammary nipple and adjoining skin which is closely related to and almost invariably followed by carcinoma. It is probably to be interpreted as

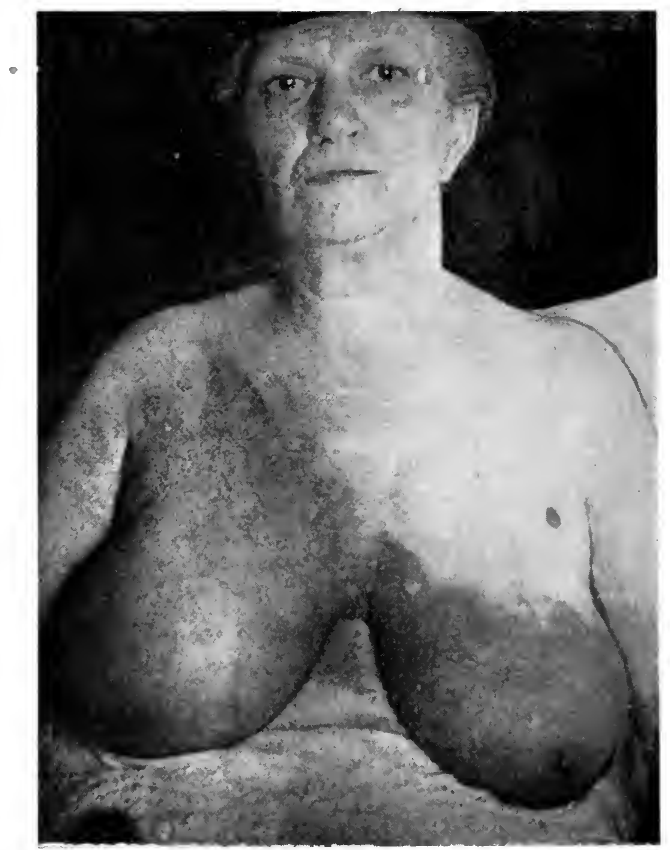

FIG. 407.-Diffuse carcinoma of breast (Paget's disease).

a precancerous affection at first limited to the epidermis and the gland ducts, but later becomes true carcinoma. Commonly. included under this term are also cases of diffuse carcinoma of the breast which invade the skin at the nipple and extend widely over one or both breasts, often with marked hyperemia.

It first appears as a keratosis of the nipple, soon followed by an itching eczematoid condition of the nipple and surrounding skin which extends in continuous concentric areas over most of the breast, producing a moist, slightly indurated, glazed, partly eroded or scaling surface that shows no tendency to heal. Extensions over the axilla and much of the thorax have been observed (Kaufmann, Vignoli-Lutati), and the disease may occur in the skin of penis, scrotum, vulva, navel, abdominal wall, or axilla (Zieler, Lit.). 
It begins usually between the ages of 40 and 60 years and commonly runs a very chronic course of 10 or even 20 years before death from the associated carcinoma or from intercurrent diseases. The nipple is usually retracted and a definite carcinoma of this portion of the breast may be present from the first, or develop later. These tumors may run a rapidly fatal course (Krogius), but more often their progress is relatively slow.

Structure.-The earliest changes have been located in the squamous epithelium of the nipple, or in the milk-ducts below the nipple (De Page), or in the parenchyma of the gland (Kryle, Elbogen), but it is doubtful if the very earliest stages of the process have been observed. Usually both epidermis and milk-ducts are found to be affected, and the fact that the lesion is not always continuous from one to the other is referred by Jacobeus to a sphincter action

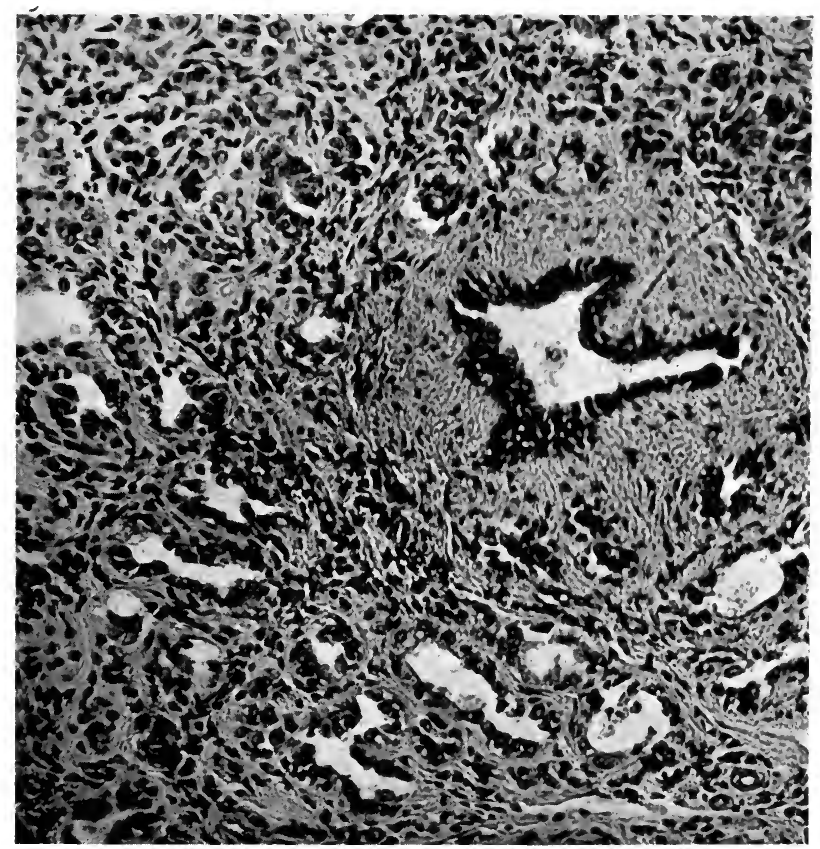

FIG. 408.-Mammary cancer. Carcinoma simplex. Duct carcinoma associated with Paget's disease.

of the tissues at the mouths of ducts. In the epidermis characteristic "Paget's cells" appear as swollen, rounded, clear staining hydropic cells, single or in small groups, with hyperchromatic nuclei often in mitosis. Many observers have traced their derivation from the normal prickle cells, by a process of hydropic degeneration with loss of fibrils (cellules dyskeratosiques, Darier). Sekiguchi regards them as wandering carcinoma cells. The marked isolation of some of these cells, especially when small, rounded, and pigmented, led Darier to regard them as psorosperms. The Malpighian layer containing these cells is usually thickened and the papillæ elongated. The corium is usually rich in plasma-cells. In advanced stages much of the epidermis is replaced by Paget's cells, which appear in alveolar groups or sheets or extend along the basal portions and into sweat-glands. The basal layer alone may exhibit the 
characteristic changes (Zieler). In the milk-ducts a similar process is usually present, but Unna and Karg both found lesions limited to the epidermis, with milk-ducts intact. A notable feature is the long integrity of the corium, but eventually there may be invasion of the connective tissue from the enlarged rete pegs. Many have noted the long delay in the appearance of the usual structure of carcinoma, which takes the form of an adenoid growth. Pearl formation was observed by Darier. In Krogius' case the secondary carcinoma was thought to be derived from the sweat-glands.

The exact nature and origin of Paget's disease remain a subject of active discussion and it seems probable that the disease in its various stages is not always one and the same. The long duration of the disease, the very wide

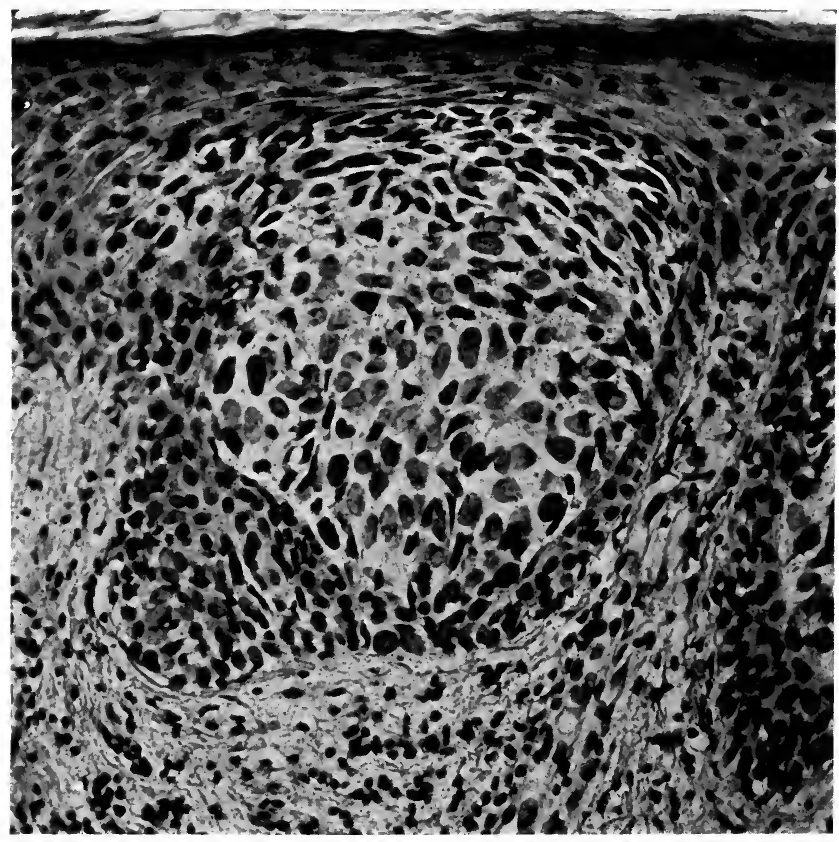

FIG. 409.-Paget's disease of the buttock, showing edema and vacuolization of cells. (From collection of Dr. J. A. Fordyce.)

and superficial extent of the lesion, the occurrence in several regions besides the breast, and the long absence of any definite tumor render it unlikely that the condition can be dismissed as a peculiar form of skin invasion by duct carcinoma. There are many cases of epidermal invasion by mammary and other cancers, but they rarely take the form of Paget's disease. Hence Darier, Unna, and many others regard the condition as a disease sui generis, and a type of precancerous condition. Zieler draws an apt parallel between Paget's disease and xeroderma pigmentosum, both of which represent primary disturbances of the epidermis which tend to result in cancer. For many very extensive lesions without definite localized carcinoma this interpretation is probably correct. Such a case is that of Fox and McLeod, who were unable to find any definite primary tumor associated with Paget's disease of the 
navel of II years' duration. Others, finding a definite tumor of the breast or nipple regard the disease as nothing more than an early and extensive intraepidermal growth of a primary cancer of the mamma (Jacobaeus, Ribbert, Schambacher, Hirschel, Sekiguchi). My own observations indicate that Paget's disease occurs in two quite different forms: (I) The characteristic changes in the epithelium affect the epidermis about the nipple, but do not extend deeply into the milk-ducts. There is no tumor of the breast. The progress is slow, and the prognosis favorable. (2) A rapidly progressive carcinoma of the breast, probably arising from the ducts, extends through the nipple to the skin and spreads widely over the breast. There is often no definable tumor of the breast, but rather a diffuse invasion of much of

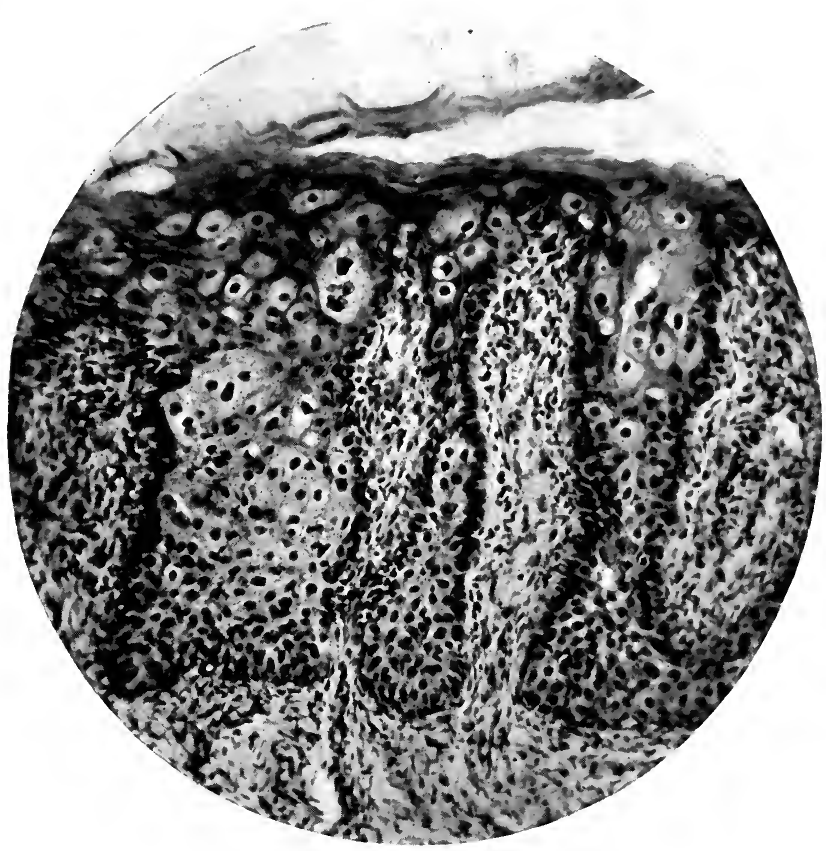

FIG. 4I0.-Paget's disease. Lesion of the buttock showing epidermal hyperplasia, edema, vacuolation, loss of prickles, and multinuclear cells. (Photo. from collection of Dr. J. A. Fordyce.)

the organ, with general swelling and early metastases. The course of the disease is rapid and the prognosis highly unfavorable.

Hannemuller and Landois, while accepting the origin from a primary carcinoma of the breast, interpret the Paget cells as swollen overactive edematous epidermal cells. Others again, and among them some of the earlier observers, regard the process as a peculiar form of primary cancer of the skin (Thin, Duhring and Wile, Ehrhardt). Thus the three main theories cover a precancerous condition, a definite carcinoma of the skin, and a growth, sometimes early, more often terminal, of the breast, so that the composite picture of Paget's disease presents prolonged stages of the evolution of carcinoma.

Whatever the histogenesis may be, remarkable clinical and anatomical characters are quite sufficient ground for recognizing the condition as a specific 
malady. According to Fabry and Trautmann there is also a specific etiological factor in the presence of an yeast which they were able to cultivate, but which is probably a secondary invader.

(2) Basal-cell Epithelioma.-Two somewhat distinct but related histological types of tumors develop chiefly or exclusively from the basal cells of the Malpighian layer of the skin, viz., (a) Reticulated epithelioma, and (b) adenoid epithelioma.

(a) Reticulated Epithelioma. Rodent Ulcer.-The characteristic clinical history of this disease is associated with a specific histological structure. It occurs chiefly on the face, neck, and scalp, at mucocutaneous junctions, especially about eye, nares, or ear, and is notably limited to adult or advanced ages. Williams finds the average age 42 to 44 years, but records a case in a girl at i 4 years fatal at 36 years.

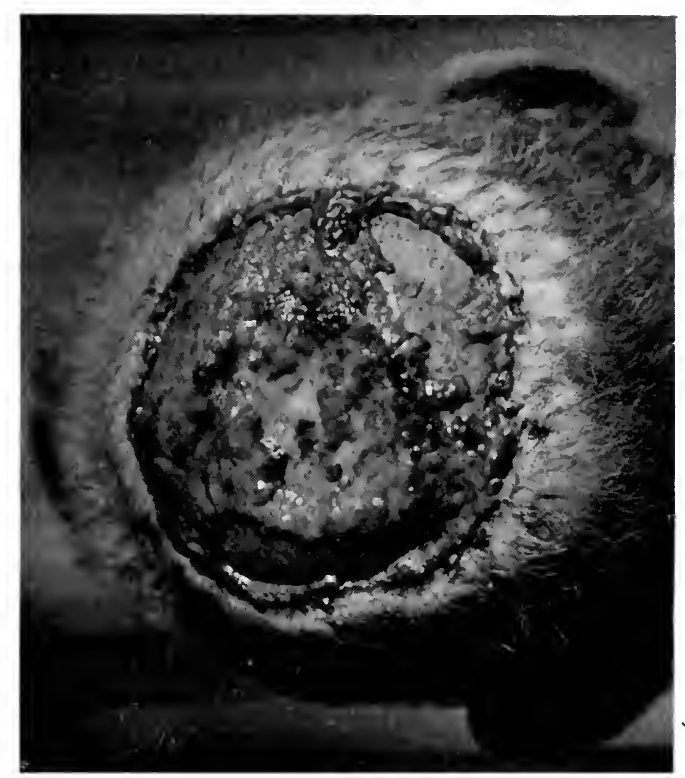

FIG. 4II.-Basal-cell carcinoma of scalp.

It first appears as a flat papule or smooth wart which long remains without marked change, or what is mistaken for a pimple maintains a persistent ulceration which resists efforts at cure. The early lesions may appear as broader flat elevations or as multiple small thickenings which coalesce. Occasionally in cases of multiple tumors there is a history of local erythema, eczema, or seborrhea (Janeway). After a period which may cover several years and often marked by inadequate therapeutic procedures and other forms of trauma the lesion ulcerates. The characteristic rodent ulcer shows a remarkable tendency to remain superficial. It destroys the skin down to superficial fascia, where its progress is long restrained. Later it strips the tissues down to bone or muscle, where again it makes slow progress. Thus the base varies in depth, but is usually covered by granulation tissue and warty islands of tumor. Extensive fibrosis of the base may limit the downward growth and at 
the same time obstruct any natural or artificial efforts at cure. The secretion is first thin and serous, later purulent, and with frequent small bleedings. The edges are raised, nodular, indurated, pearly, and constantly hyperemic. The vicinity may be glazed and hyperemic over a wide margin or the indurated border may be sharply marked. Extension being through the superficial lymphatics, new areas when involved first show subepithelial nodules which later break through the epidermis. As the ulcer widens there may be a distinct tendency toward healing in the older areas.

In advanced stages the dimensions of the rodent ulcer become very wide, involving much of the face, neck, or scalp. 'The destruction of tissue becomes

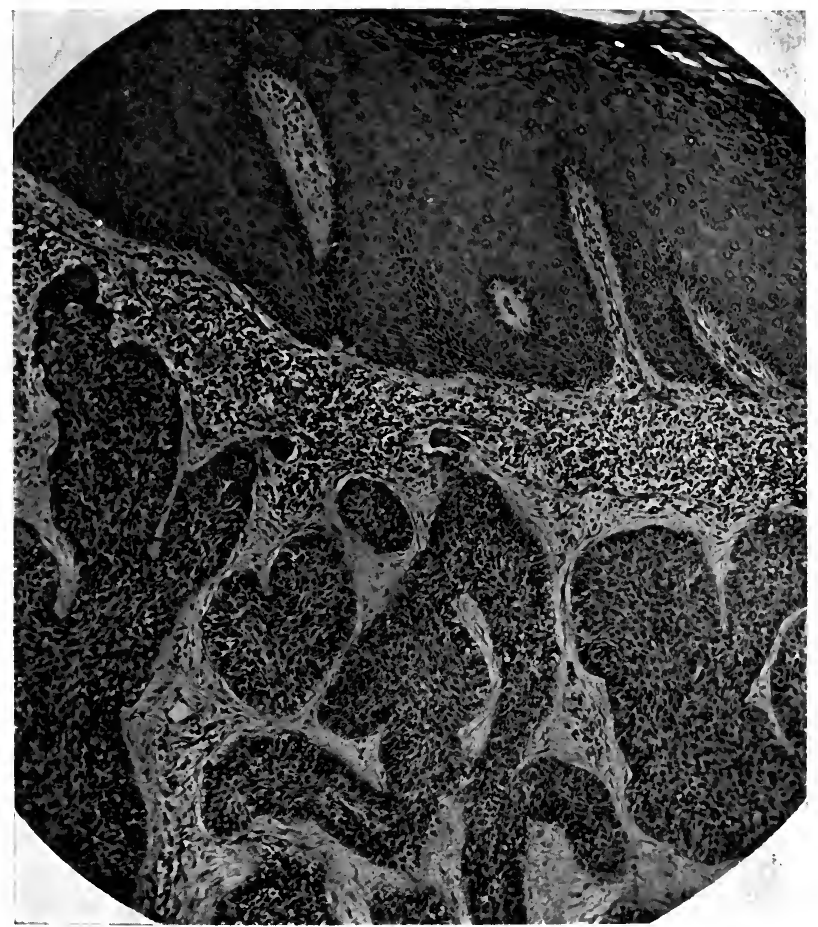

IG. 412.- Structure of a basal-cell carcinoma of skin. Widely infiltrating under normal epidermis.

eeper, especially with the aid of incomplete excision. The eye, ear, and ose are often completely destroyed. The periosteum is invaded with deruction of bone, with penetration of bony sinuses, cranium and middle ear, bone-marrow. Erosion of large vessels may lead to fatal hemorrhage. achexia results chiefly from chronic suppuration, hemorrhages, and mental pression.

Rodent ulcer seldom involves the lymph-nodes even after many years growth, but in a few cases the regional nodes become invaded and the ogress is more rapid with certain changes in structure (Dubreuilh, Auche, prdyce).

Crateriform ulcer is a special variety of rodent ulcer which appears on 
the face in the form of a conical induration which resembles a furuncle. In a few months it ulcerates deeply, involves lymph-nodes, and proves fatal in I to 2 years, but early excision is usually successful (Hutchinson).

Many of the clinical features of rodent ulcer may be simulated by a less malignant low papillary acanthoma of wide extent.

The structure of rodent ulcer is usually characteristic and uniform. Scction shows relatively bulky compact masses of darkly staining cells connected by narrow strands of similar cells, all lying in dilated lymph-channels or artificial spaces from which they readily shrink on hardening (reticulated epithelioma). Or the cells appear in narrower columns separated by hyaline connective tissue after the manner of cylindroma. Or large cell masses may exhibit numerous small clear areas resulting from mucous degeneration, and these spaces may reach the size of small cysts. In cases with a certain adenoid character the cystic structure may approach that of adenoid cystic epithelioma.

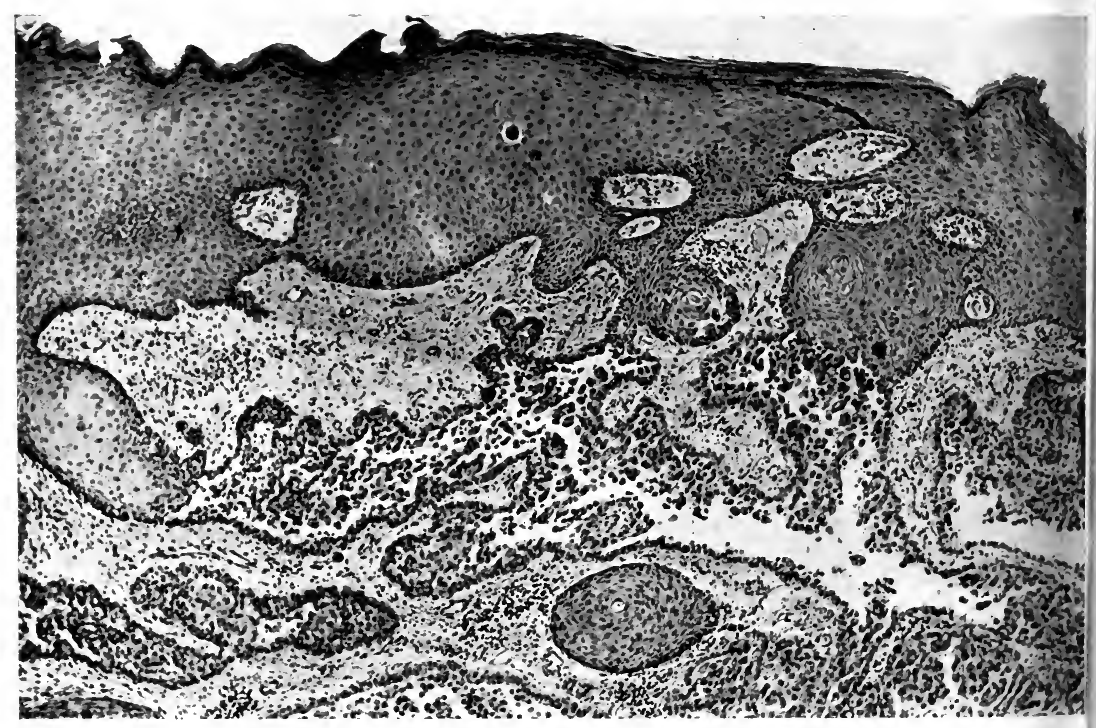

FIG. 4I3.-A basal-cell carcinoma of skin, showing origin from rete pegs.

The whole tumor mass usually appears well circumscribed and separated from the overlying epithelium, and in all ordinary forms of the disease a derivation from the overlying epithelium cannot be demonstrated. This rule is, however, not invariable and is quite inapplicable to the earliest stages. In advancing lesions long slender strands of tumor-cells may be traced for some distance through the lymphatics and eventually connecting with what may appear to be multiple foci of origin. This histological feature explains the persistent recurrence of these tumors after apparently thorough extirpation.

The tumor-cells resemble the basal cells of the epidermis and are small, polyhedral or spindle, with relatively large vesicular nuclei, minute nucleoli, and scanty cytoplasm. Flat pavement characters, spines, hornification, and pearl formation are regularly absent, but in rare cases may appear in traces. Definite combinations of acanthoma and reticulated epithelioma have been observed. In certain cases which clinically and anatomically 
resemble rodent ulcer the structure presents convoluted or lenticular rows of columnar cells which suggest an approach to the type of adenoid epithelioma. In a few cases all the cells are of spindle form and closely resemble spindle-cell sarcoma. Thus the cells of rodent ulcer tend to maintain their original and partly embryonal character throughout, but the varying origin of these tumors gives opportunity for the exhibition of many cell forms.

The histogenesis of reticulated epithelioma and rodent ulcer has been the subject of much careful study. Krompecher, I900, first adequately emphasized the specific structure of the group of epidermal tumors which maintain the characters of basal cells and introduced the term "basal-cell

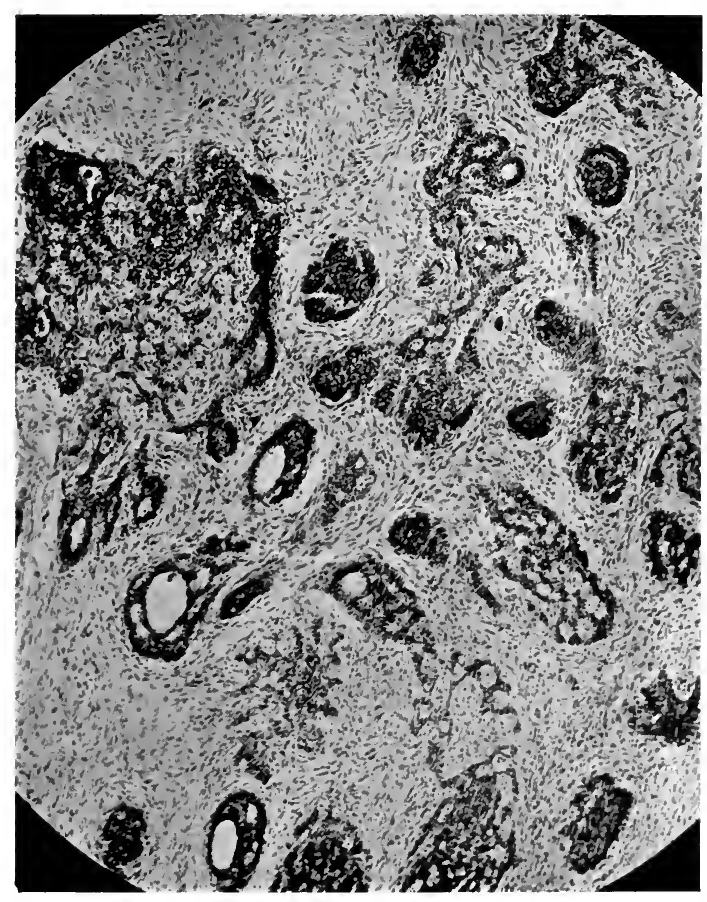

FIG. 4I4.-Epidermoid carcinoma of skin arising from hair-follicles and reproducing small hair shafts.

carcinoma." He traced the origin to the basal cells of the Malpighian layer and of the ducts of sweat, hair, and sebaceous follicles. He described in detail the clinical and histological characters of many tumors of this class occurring in various situations. Many such tumors had previously been regarded as endothelioma of skin, mucous membranes, and salivary glands (Coenen, Lit.). Hansemann argues against the use of the term "basal cell." urging that all epidermal tumors arise from basal cells. Yet this view is probably not entirely correct even for rodent ulcer, and Krompecher's choice of terms may be justified on the ground that the tumors maintain the characters of the basal cells from which, also, they probably arise.

Bormann, in an elaborate study of epidermal carcinoma, also derives 
the reticulated epithelioma from basal epithelium. He concludes that the origin is not from the normal epidermis, but from misplaced superfluous and embryonal cell groups derived from the skin and appendages (corium carcinoma). The apparently complete isolation from the epidermis of many early tumors, their occurrence chiefly along embryonal fissures, on the face, and in salivary glands, as well as their partly embryonal character, are considerations which favor this view. Some basal-cell tumors with compact groups of small elongated cells, or occasionally with adenoid characters, arise from the hair follicles. Mallory finds that these tumors differ from the others in producing long fibrils recalling the formation of the hair shaft. Janeway has clearly shown that epithelioma of basal-cell type may arise either from isolated epithelial structures in the corium or from areas of basal cells which are normally incorporated in the epidermis. The two origins impress somewhat different characters on the resulting tumors. Preceding the neoplastic change in the basal cells he observed transient erythema and a dilatation of subepithelial lymphatics, about which the epithelial cells first showed hypertrophy and hyperchromatism and into which the first downward growth occurs. Ribbert accepts the origin from multiple foci of normal basal cells and emphasizes the changes in the connective tissue which frequently precede the downward growth of epithelium. Whether these changes are constant it is difficult to determine. Bonney, who analyzed the connective-tissue changes in detail, found them of very constant occurrence. Wyss, in a series of early carcinomas of skin and other regions, found extensive infiltration of the connective tissue in 77 per cent. of 36 cases, and some form of alteration in 9r per cent. In some cases the changes are so slight as to escape demonstration by the usual methods of detection. They appear to be more constant with acanthoma than with reticulated epithelioma.

(b) Adenoid Epithelioma of Skin.-Many epithelial tumors of the skin show a tendency more or less pronounced to reproduce the dermal glands and are, therefore, designated as adenoid. They differ from the rarer true adenomas of the dermal glands in the predominance of squamous or basal epithelial cell characters. Several varieties of these tumors are observed.

(I) Adenoid Cystic Epithelioma (Brooke).-This tumor was long grouped with the adenomas of sebaceous glands and confused with many other conditions, from which it was separated by the careful clinical and histological studies of Brooke, Fordyce, Unna, Walters, and others. It differs from adenoma sebaceum in its occurrence in much smaller numbers and in the absence of the definite histological characters of sebaceous gland tumors and prominence of squamous cells.

It occurs in the form of a limited number of small, firm, nodular, painless, circumscribed tumors in the skin of forehead, eyelids, nose, cheek or scalp, notably about the age of puberty. They are also seen on back, chest, and limbs, but not below the pelvis (Brooke). Fordyce observed them in mother and daughter, and Brooke, in three members of a family. The early lesions may resemble milium and they slowly reach but do not exceed the size of a pea or bean, continuing to enlarge slightly for some years. While no serious grade of malignancy has been reported, there is no tendency toward regression. The color differs little from that of the skin, with a tendency to become darker, yellowish, shiny, and occasionally translucent.

The structure varies, but is dominated by the presence of embryonal epithelial cells arranged in cords, masses, and alveoli, and of groups of concentric hornifying epithelium inclosing homogeneous material. Brooke found more of the adenomatous structure than is usual. Unna emphasizes the squamous-cell elements in his designation, "acanthoma adenoides cysticum," 
and he regards the tumor as a pure embryonal acanthoma with marked tendency to cyst formation.

The adenoid structures appear as finger-like projections from the basal epithelium or ducts of glands, and they commonly contain minute or large cystic areas of hyaline, colloid, or mucoid material. The cells inclosing these foci are often cylindrical. The squamous cells in concentric form undergo extensive hornification or calcification, and these structures may compose the bulk of the tumor (Schapper). Or the structure of reticulated epithelioma, resembling rodent ulcer with many thin cell strands, may be prominent (Czillag, Walters).

The origin of the tumor is referred by Brooke to the normal cells or to embryonal cell groups derived from the hair follicles (lanugo) and from the basal cells of the epidermis. While this view is supported by most recent observers, Torok and Darier derived their tumors from the sweat-glands and W. Pick from the sebaceous glands. The identity of these cases with Brooke's tumor is doubtful, but it seems probable that in different cases the origin may in-

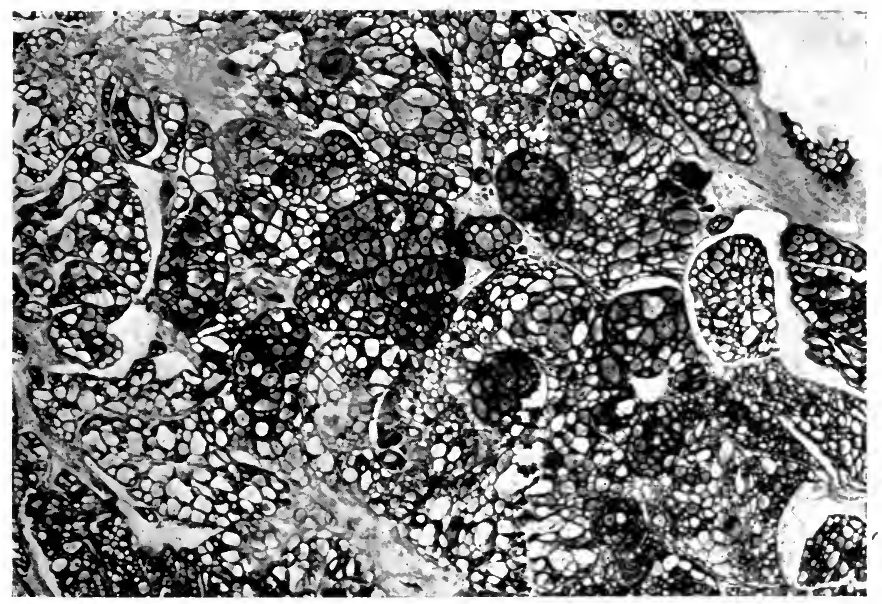

FIG. 415.-Low magnification of epithelioma adenoides cysticum.

volve different elements with corresponding variations in structure. Thus Dorst and Delbanco observed multiple tumors of the scalp in the same patient, some of which had the structure of adenoma sebaceum, others that of adenoid cystic epithelioma.

In addition to the relatively small and multiple tumors of the above type, the structure of adenoid and cystic epithelioma occurs in larger and even bulky single tumors of the skin and subcutaneous tissue about the face and neck. Such a tumor has been described by Buxton as arising at the orifice of Steno's duct. It ran a slow course for 20 years, producing a growth as large as a hen's egg, and finally involved the submaxillary lymph-nodes. The structure presented many cystic cavities lined by convoluted layers of basal cells, among which, in turn, were many small spaces filled with mucoid material.

(2) Simple Adenoid Epithelioma.-At the usual seats of rodent ulcer larger and usually more active tumors often arise in which there are large alveoli lined and filled with cylindrical or cuboidal epithelial cells of a type intermediate between squamous epithelium and the duct cells of dermal glands. 
These tumors are not cystic, they are usually sharply circumscribed and relatively large, and their course is progressive, with occasional involvement of lymph-nodes or ulceration.

The structure often suggests a large alveolar carcinoma, but certain areas may usually be found containing flat squamous epithelium without spines of hornification, and in many cases the derivation from the rete pegs and especially from the basal cells is revealed with striking clearness. In one group of cases clinically resembling rodent ulcer I found alveoli composed of low cylindrical epithelium which was probably derived from hair follicles. In another, large alveoli are lined by low columnar cells and filled with cuboidal granular epithelium and occasional squamous cells. In a third type all the rete pegs over a large area, I to $\mathrm{I} \frac{1}{2} \mathrm{~cm}$., are hypertrophied and continuous, with broad cellular columns of cuboidal cells which compose a rather bulky tumor of the derma.

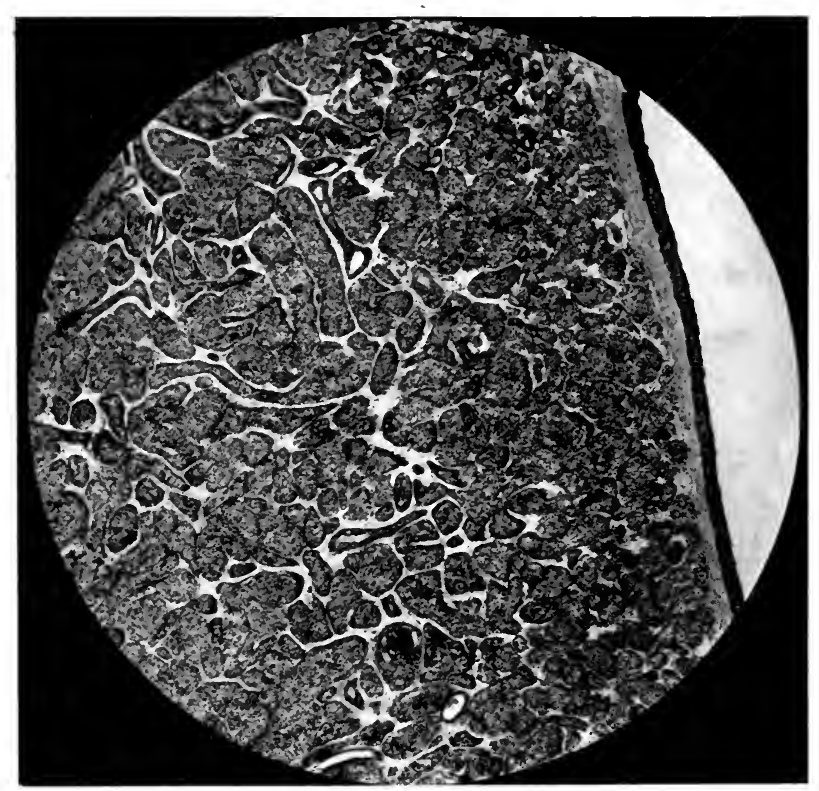

FIG. 416.- Topography of sweat-gland tumor of skin. (Photo. by B. H. Buxton.)

Tumors of Sweat-glands.-The sweat-glands have been assigned as the source of a variety of tumors which have figured extensively in dermatological literature under many different terms (Petersen, Lit.). The tumors vary in structure and form according to their exact point of origin and the conditions under which they arise. From the alveoli arise the somewhat specific spiradenomas, with many well-formed alveoli surrounded by membrana propria and filled with small cuboidal cells. They appear as pearly white tumors beneath the epidermis, and they may be small and multiple. Carcinomas of this type are occasionally encountered. Dilated alveoli of spiradenoma probably constituted the tumor described by Jarisch as lymphangioma tuberosum multiplex. 'The ducts of the glands may be dilated, forming cysts, and into these papillary outgrowths occur, which have been described as papillary adenomas (Elliott). Neither rodent ulcer nor epithelioma adenoides cysticum 
have been positively traced to the ducts of sweat-glands, but it appears probable that the ducts give origin to a certain proportion of the adenoid epithe-

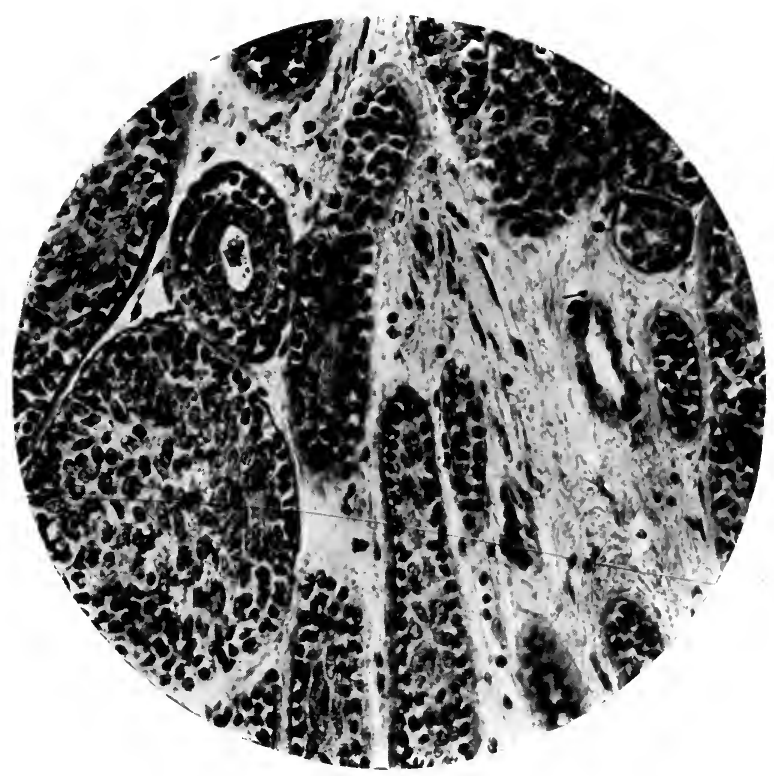

FIG. 417.-Adenocarcinoma of sweat-glands.

liomas of the skin. The adenomas are regularly benign, while the adenocarcinomas are relatively slow in invading lymph-nodes.

\section{EPITHELIOMA OF THE LIP}

Epithelioma of the lip is chiefly a disease of elderly men, 50 to 70 years but occurs in women and in young adults. Among 73 cases collected by Warren, 4 were in women, 3 of whom were smokers. Of 1338 cases Fricke found 9 I per cent. in men, 4 of which were multiple. A definite group of cases follows seborrheic keratosis (Montgomery, Sutton). Many cases are clearly traceable to the irritation of pipe or cigar. Hence it appears usually on the side of the lower lip, rarely on the upper. Dugue believes that tobacco causes a leukoplakia of the lip, as on the tongue, which is followed by epithelioma. Many forms of trauma have preceded the disease, as simple scratches, bee itings, erosion by teeth. One patient, syphilitic in youth, a smoker until 45 vears, developed a minute chronic fissure of the lip at 50 , which remained ndolent and indurated for three years, when ulceration supervened and radually extended over $\mathbf{I} \frac{1}{2} \mathrm{~cm}$. for $\mathrm{I}$ year longer before it was recognized as pithelioma. Contact infection has been held responsible for the appearance ff epithelioma on the upper, opposite a lesion on the lower lip, but lymphatic onnections offer an alternative explanation, since both blood- and lymphessels encircle the lips.

Two main forms of the lesion occur, (I) papillary and (2) ulcerative inltrating types. The papillary form appears as a wart-like thickening which ong remains elevated, at times with much elongated papilla. It extends lowly in all diameters, as a flat thickening in the epidermis, is slow to invade 
the deeper tissues or lymph-nodes, and the underlying tissues remain soft. Later it may ulcerate and follow the usual course of infiltrating epithelioma.

This type of epithelioma is of long

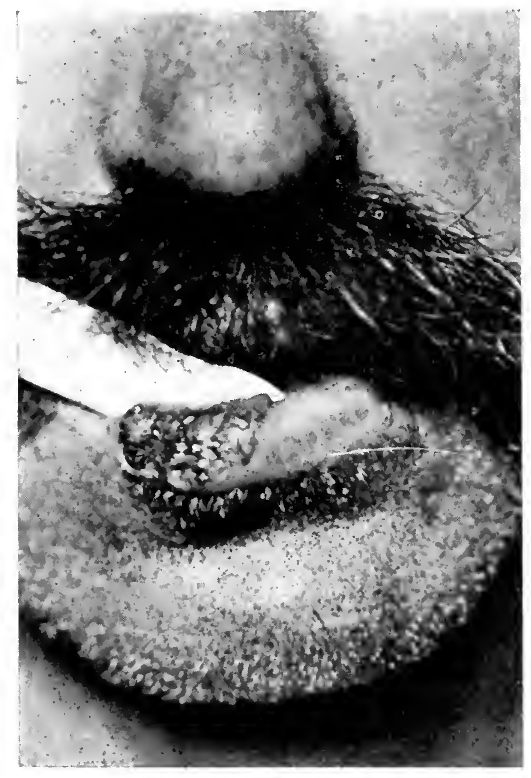

FIG. 4I8.-Epidermoid carcinoma of lip. duration and of somewhat characteristic gross appearance, and since it yields to conservative operation it should be carefully distinguished from the more malignant variety.

The early infiltrating process may appear as a flat thickening of the epithelium, or as a nodular growth invading from the first the submucosa, and lying beneath rather than' in the epithelial layer. Early ulceration produces a broad deep ulcer with pearly indurated edges, or a more bulky excavated tumor which may become fungating. With these lesions there is often much inflammatory reaction, extension along the floor of the mouth, involvement of periosteum, and early invasion of submental, lingual, and maxillary nodes.

The structure is usually that of typical acanthoma with many variations in details. A preliminary softening and cellular infiltration of the submucosa usually precedes the overgrowth of epithelium. Ribbert insists

that downward growth of epithelium never occurs into normal connective tissue, but only after infiltration by round cells. In the papillary form the

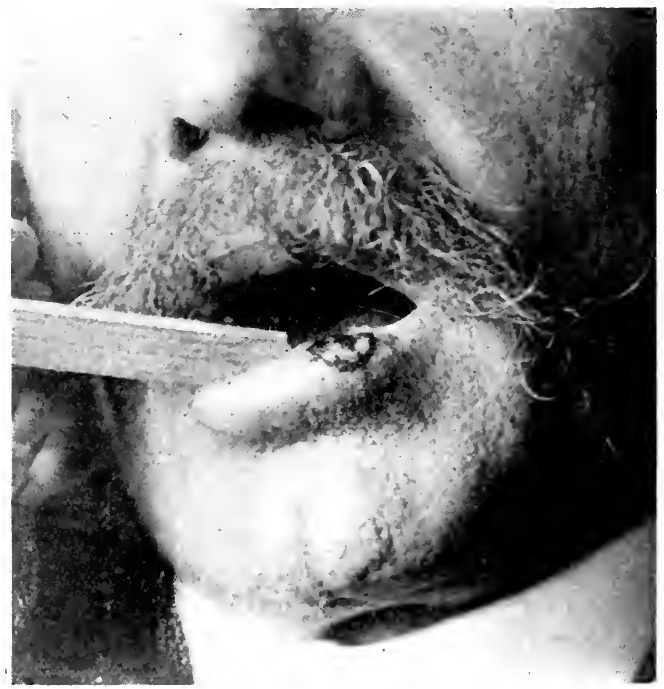

FIG. +19.-Early carcinoma of lip. 
outgrowth produces long papillæ with or without hyperkeratosis. Some downward growth of altered papillæ is nearly constant, but may long be held in check by round-cell exudate and fibrosis of deeper tissues. In the ulcerating tumors the invasion of tissues is preceded by edema and perivascular infiltration by leukocytes. After the tumor has become established there is no evidence of lateral extension over normal epithelium, but the papillæ over a wider area may be much hypertrophied.

The tumor-cells may retain the type of adult squamous epithelium (acanthoma) or they may lose these characters and appear in anastomosing columns of opaque granular polyhedral cells. Infiltration of perivascular sheaths; nerve-trunks, and areolar tissue is attended by much growth of firm con-

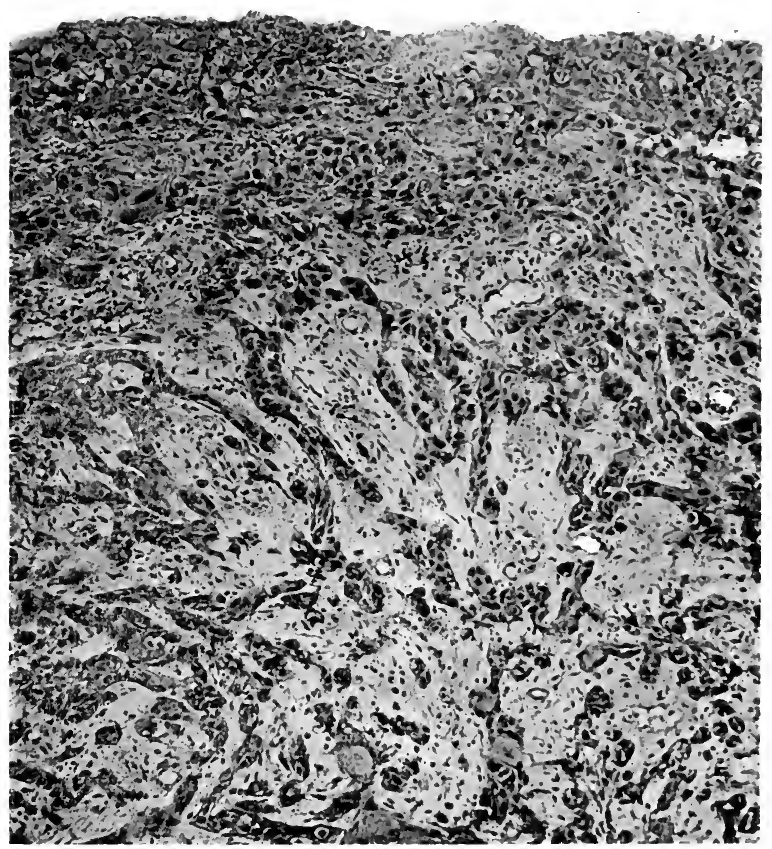

FIG. 420.--Infiltrating epidermoid carcinoma of lip.

nective tissue. This infiltration tends to follow preëxisting lymph paths, but is by no means limited thereto.

While the lymph-nodes may be involved early, they seldom reach a large size, and visceral metastases are occasionally observed. Yet I have seen the right submental nodes as large as an orange, while the left were uninvaded from a left-sided tubular epithelioma of lip. Secondary infection usually by Streptococcus pyogenes plays a prominent part in the progress of the disease, and leads to extensive local suppuration, erysipelas, septicemia, thrombosis of vessels, edema of glottis, and pneumonia.

The operative treatment of carcinoma of the lip is comparatively favorable. Fricke placed the cures at 60 per cent. and Steiner (I go8) reported 35 definite cures after a 5-year limit out of 43 cases ( 70 per cent.). The decisive influence of the character and extent of the disease appears in the fact that three- 
fourths of the fatal results occurred within a year after operation. Surgical experience emphasizes the necessity of removing the lymph-nodes of both sides, even with comparatively early lesions. Of 32 cases in which only the tumor but not the lymph-nodes were removed Bloodgood found only 2 cured. After invasion of the maxillary bone he could find no record of recovery. The more favorable statistics probably include many comparatively benign

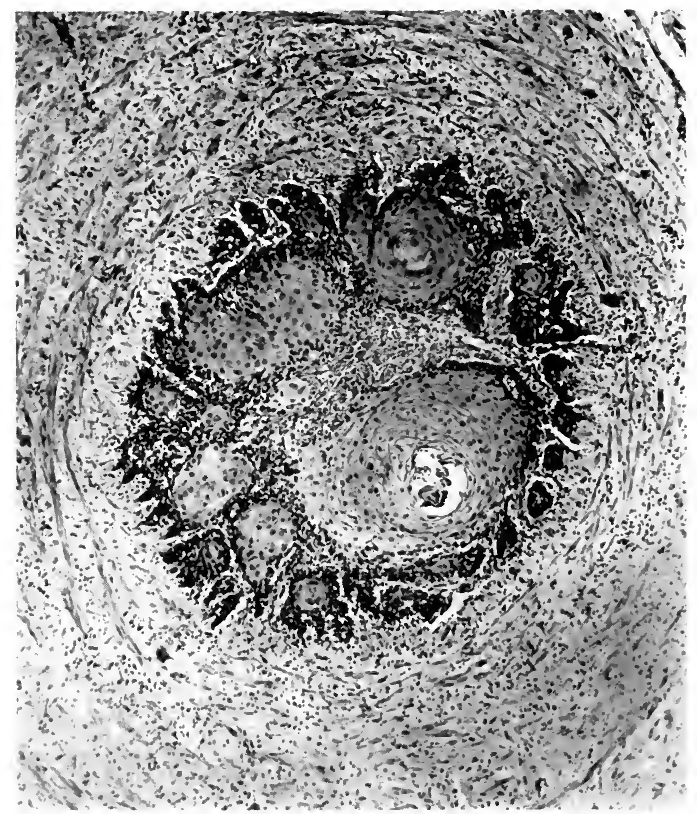

FIG. 42I.-Invasion of labial artery by recurrent epidermoid carcinoma of lip. Note the evidences of growth restraint.

lesions. Fully developed carcinoma of the lip is a very fatal disease, and only when the lesion is early and limited can a favorable result be assured by operation. Such lesions also respond well to radium.

\section{EPIDERMOID CARCINOMA OF TONGUE AND MOUTH}

Several general peculiarities distinguish this form of cancer. In frequency it is placed by Jessett second only to uterine cancer, by Jacobsen third, and by Winiwarter fourth. While among the most accessible of cancers and readily recognizable in its early stages, it is yet one of the most fatal of malignant diseases, with a mortality of 75 to 90 per cent. (Warren, Butlin, Meller). Although its microscopical anatomy is rather uniform, the gross features and clinical course vary extensively, so that a very detailed acquaintance with the disease is demanded of one who hopes to deal with it successfully. The failure to recognize the significance of early or precancerous conditions, temporizing with small but fully developed lesions, and extravagant sacrifice of tissue in mild cases, are common errors committed in the case of these patients, and are largely responsible for the ultimate mortality and for that dread of the disease which leads many to conceal its existence as long as possible. 
Etiology.-The disease occurs chiefly in the fourth to sixth decades. Sigel observed a case in a boy of 6 years. Piquantin estimated that 17 per cent. of the cases occur in women.

The important factors in the causation of buccal cancer are syphilis, tobacco, and defects of the teeth. In a large group of cases all three factors are combined, while one or more are nearly constantly present.

The frequency of syphilis is variously estimated, Meller finding signs of lues in only 7 of 207 cases of buccal cancer, while Fournier found the disease in 155 of 184 cases. The action of syphilis is indirect and the epithelioma is always preceded by a definite and usually prolonged luetic lesion. The pearly white thickening of the epithelium in leukoplakia is a characteristic picture which must always be regarded with suspicion as furnishing the beginning of cancer. According to Fournier leukoplakia is followed by cancer in 30 per cent. of the cases, but many authors hold all such lesions under suspicion, and there is no doubt that the disease has a definite tendency to become malignant and should be treated in every case as a specific precancerous condition. Of $x_{59}$ cases of cancer of tongue, v. Bergmann found leuko-

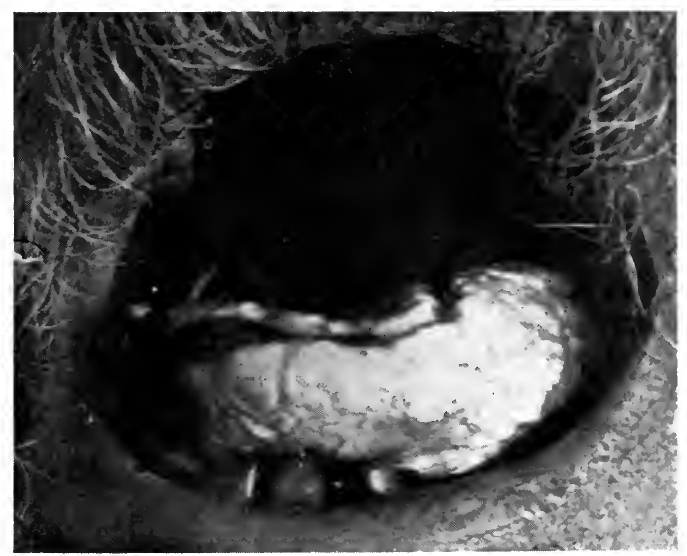

FIG. 422.-Leukoplakia of tongue.

plakia in 34.6 per cent. Other syphilitic lesions which lead to cancer are ocalized wart-like epithelial hypertrophies, fissures, gummas with or without alceration, and chronic glossitis with atrophy of papillæ (Jacobson). The pithelial overgrowth, submucous edema, lymphocytic infiltration, and evenual scarring with loss of elastic tissue are microscopical features explaining he remarkable relation between lues and cancer of this organ. It is a notable act that the syphilitic process may subside or even completely heal under reatment, while a coexistent cancer may continue or develop later in the car, or appear in the lymph-nodes under a smooth epidermis (Landau, Sachs, Narren, Lang, Lydston).

Tobacco has a predominant influence in the development of cancer of he buccal mucosa. Its action is not fully understood, but the irritant effects timulate the epithelium, produce chronic hyperemia, local erosions, lymphoytic infiltration, edema, and ulceration. The effects of heat must also be onsidered, while mechanical irritation appears in the cancers of the tip of nngue in pipe smokers. Tobacco chewers often develop cancer at the point here the quid is held. Many of the tobacco cancers arise at the base of the 
tongue, on palate, tonsil or pharynx, while the relation to laryngeal cancer is well known.

An imperfect condition of the teeth is directly responsible for many buccal cancers. The most obvious cases are those in which a projecting or ragged tooth continually abrades the edge of the organ, along the sides, or at the frenum. It is often difficult to detect the exact source of the effective dental irritation and it is often overlooked. An imperfect alignment of the teeth, a sharp although smooth edge, or chronic caries especially of the roots, pyorrhea, chronic alveolar abscesses, or the traumatism of extraction, may be sufficient to incite a cancerous growth, especially in luetic smokers.

Many other forms of irritation resulting in chronic inflammatory changes have been observed to precede buccal cancer, and they were early designated

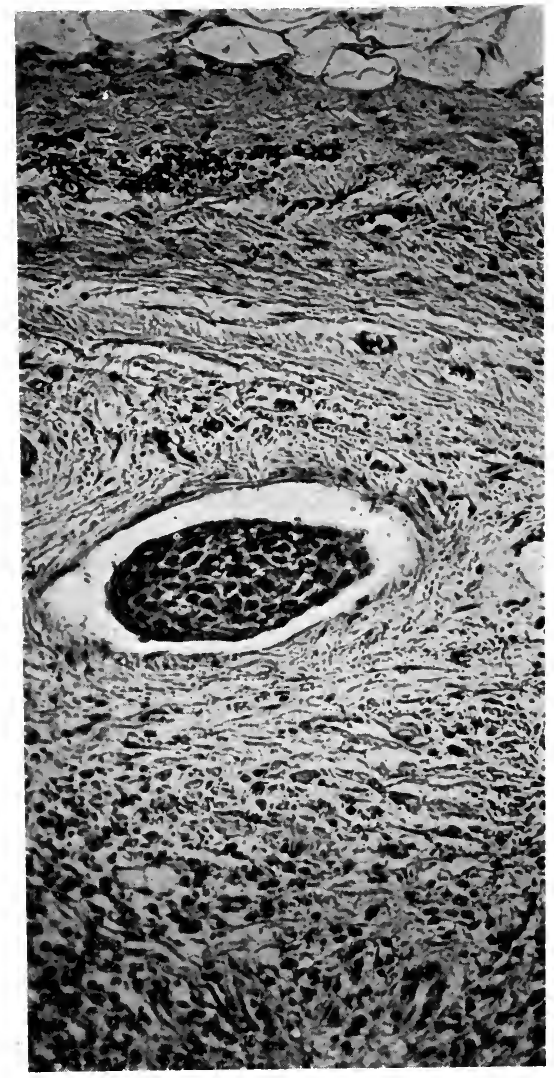

FIG. 423.- Invasion of capsular vessel of lymph node by epidermoid carcinoma.

by Hutchinson as precancerous states. They are thus enumerated by Jacobson: (I) Chronic glossitis with hypertrophy and fissuring, (2) leukoplakia, especially when warts appear, (3) ichthyosis, or late stage of leukoplakia, (4) chronic atrophic glossitis, "bald tongue," (5) papillomas of various types, (6) fissures, (7) ulcers of any type.

Structural abnormalities or other forms of tissue predisposition are not commonly considered in the genesis of buccal cancer, and probably have little relation to the superficial acanthomatous forms, but for many tumors located in the floor of mouth, tonsil, or pharynx, which exhibit the structure of adenoid or embryonal epithelioma, such factors cannot be ignored and, indeed, demand more attention than they have received. This entire region is beset with mucous glands and their ducts, and with tortuous ducts of large salivary glands, while the remnants of branchial clefts and other embryonal fissures must also be considered as contributing to certain forms of epithelioma, especially the deeper lying tumors. The thyroglossal duct is a source of cysts at the base of the tongue, and it seems probable that remnants of the endodermal portions of the upper branchial clefts may also be included in the sources of buccal cancer. A characteristic lingual cancer develops in the middle line of the organ, often appearing first as a slowly growing papilloma.

Gross Anatomy. - The usual form of buccal epithelioma is an indurated ulcer, which extends with considerable activity and much pain and discharge, over a widening area. The indurated base and edges are characteristic. The 
lesion arises from fissures, abrasions and erosions, and is ulcerated and superficial from the first.

Beginning as a deeper submucous nodule another type of the lesion remains for a time as a solid tumor of moderate dimensions, but eventually breaks down, producing a deep ragged excavation.

After leukoplakia epithelioma appears as a broad, flat, pearly white induration which may long escape necrosis, but infiltrates the tissues over a wide area and finally ulcerates. Multiple points of origin may exist in this type, and after excision the disease may recur in new areas.

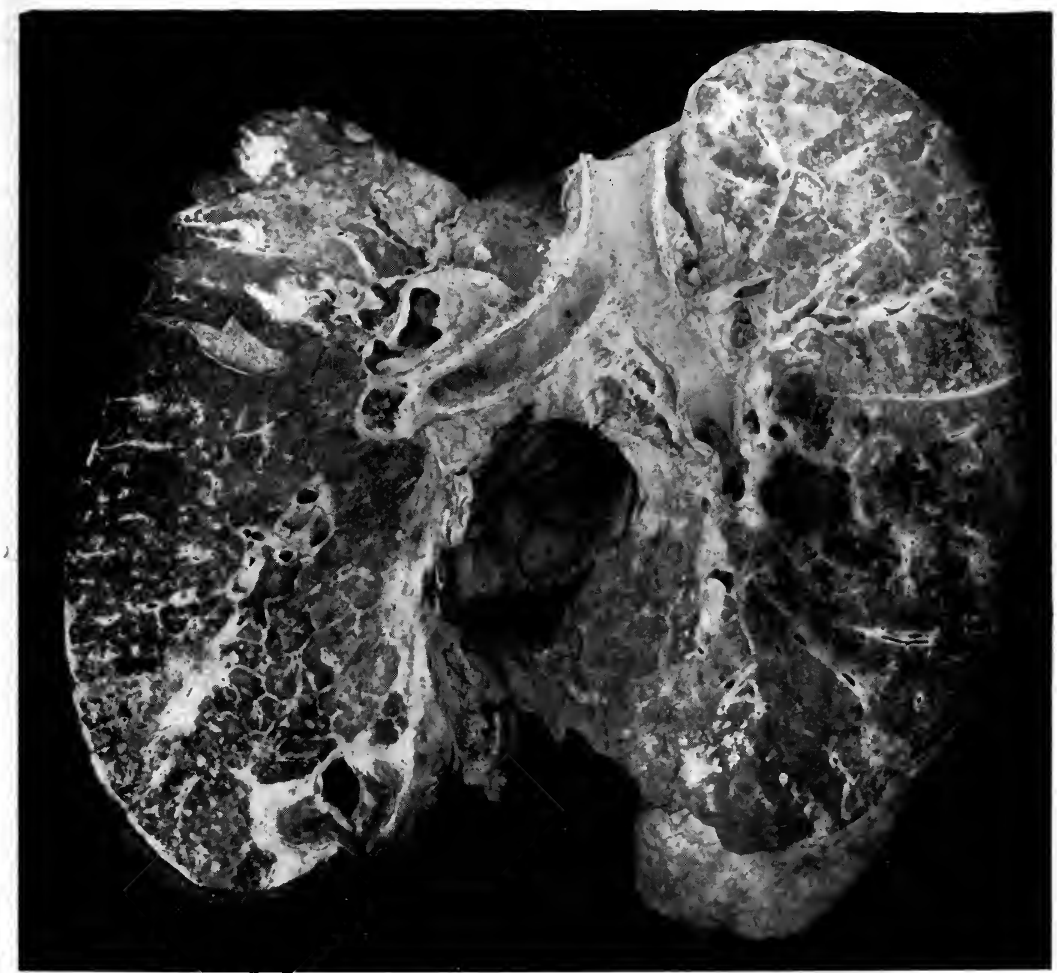

FIG. 424.-Diffuse permeation of pulmonary lymphatics from squamous carcinoma of tongue.

A diffuse infiltration of one or both sides of the tongue occurs in rare cases and leads to extensive increase in the size of the entire organ, interfering with deglutition and preventing extrusion. Simple papilloma of the tongue may become malignant, the enlarged papillæ invading the submucosa. Steiner observed malignant changes in a lingual wart of 6 years' duration. The change is indicated by an increase in the dimensions of the lesion, but ulceration may long be delayed. Sachs describes an extensive papillary epithelioma of the tongue, with very slight structural indications of malignancy, but with invasion of lymph-nodes.

Diffuse papillomatosis of any part of the lingual or buccal mucosa presents a most noteworthy lesion, from one or many points of which carcinoma, usually of moderate malignancy, may develop. 
I have seen cases of very extensive lingual cancer without invasion of lymph-nodes, in which the lesion consisted of plexiform infolding of the entire epithelial layer without invasion by loose cell groups.

The location of the lesion may be in any part of the lingual or buccal mucosa. The edges of the tongue, the under surface near frenum, the tip or base, are most frequently affected. The dorsum is relatively immune. Equally frequent is a primary location in the floor of the mouth. Tonsillar lesions constitute about to per cent. of the cases, while a still smaller proportion occur in pharyngeal wall, gums, and palate (Meller, Warren).

Local extensions of buccal cancer are usually early and wide. In the tongue the arrangement of blood-vessels and lymphatics may somewhat restrict the growth to one lateral half or to the base, but advanced lesions invade both sides, extend from the base in all directions, and from the tip and edges to the floor of the mouth. Lesions of the cheek remain relatively localized; those of the tonsil invade pharyngeal wall, larynx, nares and soft and hard palate, and base

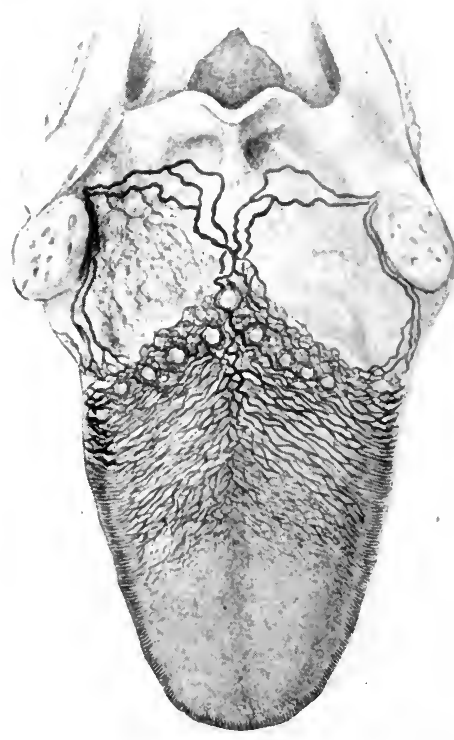

FIG. 425,-Vertical view of lymphatics of tongue in relation to tonsil. (After Kuttner.) of tongue; those of the buccal floor extend actively to tongue, gums, lips, and deeper tissues.

Lymph-node involvement occurs early in the course of buccal cancer and may be the first symptom to attract notice, but there is great variation in the period of its occurrence, depending on the very variable structure. Meller describes extensive carcinoma of tongue, tonsil, and buccal floor without invasion of nodes, while Sachs' superficial papilloma of indistinct cancerous structure had already invaded the nodes. Most observers urge that it is impossible to establish any rule regarding the occurrence of metastases. Yet I venture to think that this impression is due largely to lack of acquaintance with the anatomical varieties of the disease. Papillary, plexiform, superficially diffuse, and leukoplakic lesions are of relatively slow course.

Metastases are unquestionably favored by a malignant atypical structure, by an origin from deep rather than superficial structures, by deep ulceration, by long duration of the disease, and by the presence of inflammatory complications. Reclus mentions the later occurrence of metastases in cases following leukoplakia. Tumors of tonsil, and especially of antrum, are often slow in involving lymph-nodes.

The affected nodes are usually those directly draining the diseased focus, but in many cases the extension seems to violate anatomical rules. Thus both sides of the neck may early be involved, and in a notable group of cases there is extensive involvement of one or both sides from a primary lesion which it is difficult to locate even at autopsy.

The mode of extension of buccal and lingual cancer to the lymph-nodes, I have been led to conclude, is chiefly by embolism. This conclusion is based 
on the very early invasion of nodes in some cases, the appearance of infected nodes at anomalous points while intervening nodes escape, and the failure to find permeated lymphatics in sections of tissue leading from the original lesion. The great vascularity of the tissues and the active muscular movements of the parts seem to be a sufficient explanation of the events observed.

Metastases in the organs are rare, but have been observed in liver, heart, adrenal, and mesentery. Extensions to lungs and pleura are even rarer. The total number of cases with internal metastases is small (I per cent., Crile) and bulky internal growths are rare. In $\mathrm{I}_{4} 8$ autopsies at the Middlesex Hospital there were metastases in the liver (8), lungs (7), pleura (4), adrenal (3), heart (2).

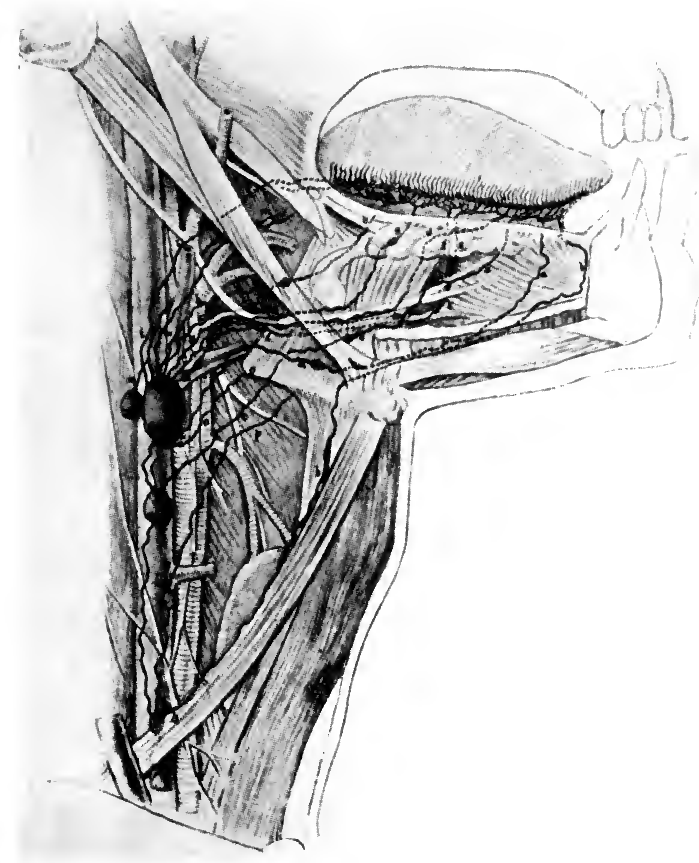

1G. 426.-Diagram of lymphatics of tongue. (After Kuttner.) Note chain leading directly from tip of tongue to supraclavicular node.

The distribution of metastases is determined by the course of the cervical imphatics, which has formed the subject of careful study by many observers Poirier, Kuttner, Crile). The main groups are defined by Warren. The ibmaxillary group drains the border of the tongue as far back as the fauces, 1e middle section of the anterior half of tongue, and under surface of tip and oor of mouth. The superior deep cervical group, lying on internal jugular in and carotid, drains all parts of mouth, tongue, fauces, and upper part of harynx. Nodes at the lower end of the parotid drain the anterior surface palate. The inferior deep cervical nodes, lying on the jugular vein and stending down behind the clavicle, receive branches directly from apex and ise of tongue, and anterior portion of floor of mouth. The submental nodes ain the lips and anterior portions of the buccal floor. The submaxillary and, often extirpated, is rarely invaded. 
Structure.-The great majority of buccal epitheliomas are of the simple acanthomatous type. Their early stages illustrate many phases of the development of epidermoid carcinoma from chronically inflamed, hypertrophied papillæ. Lymphocytic infiltration and edema of submucosa and hyperkeratosis nearly always precede the downward growth of epithelium. The adult characters of the epithelial cells may long be preserved, or they may assume the type of indifferent polyhedral cells as in tubular epithelioma. An original papillary structure may persist, even after metastases have occurred. Rarely the structure is that of basal-cell epithelioma. Berkeley saw both types in the same tumor. An alveolar arrangement of polygonal cells devoid of squamous characters suggests an origin of some tumors from ducts of mucous glands (Steiner, Hulsmever, Kaufmann).

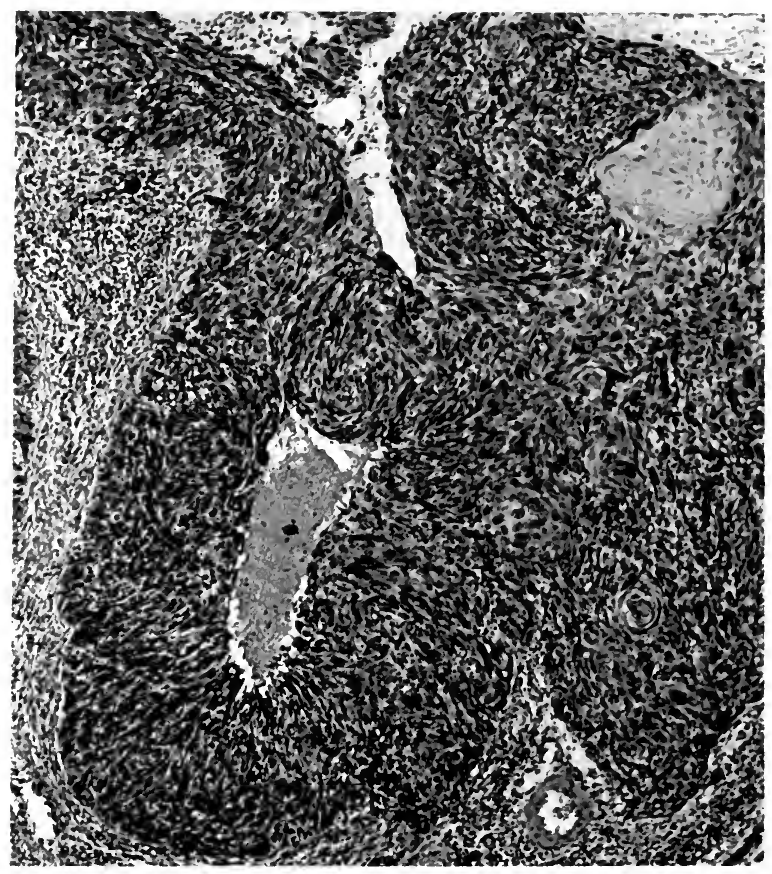

FIG. 427.- Spindle-cell epidermoid carcinoma of tonsil.

Embryonal forms of epithelioma arise in the base of tongue, tonsil, and pharynx, but their exact source is undetermined. The cells lack squamous characters and they grow in columns or indistinct alveoli.

In all types of structure the cells infiltrate surrounding tissues, following chiefly the lymph-vessels and nerve-trunks. Goldmann observed the invasion of a vein, but the blood-vessels are usually occluded by the extensive new connective tissue which follows the process. Secondary infection by the streptococcus complicates or dominates the later stages and gives rise tc suppuration and necrosis.

The clinical course of buccal cancer is usually progressive and terminate: fatally in an average period of 2 years. More rapid courses are commonly observed in lingual cases ( 6 to 12 months) and in tonsillar (6 to Io months) 
and pharyngeal tumors ( 4 to ro months) (Meller). In a large series in all locations Meller found that operation prolonged life on the average 13.4 months and cured I 4.6 per cent., while the operative mortality was 13 per cent. While the duration and extent of the disease chiefly determined the result, the cured list includes several with large ulcers, extensions to lymphnodes, and some with local recurrences.

The chief causes of death are pneumonia, septicemia, edema of glottis, and hemorrhage.

It seems probable that the prognosis of buccal cancer will be influenced in the future by the introduction of radium. This agent, either alone or in combination with surgery, has produced encouraging results, especially in localized cases of lingual, tonsillar, and pharyngeal cancers in inaccessible positions and in advanced stages.

\section{TUMORS OF THE LARYNX}

Papilloma of Larynx.- Of benign tumors of the larynx epithelial papilloma constitutes 39 per cent. (Juracz), but the proportion varies with different histological criteria from 59 to 8 per cent. (Janeway, Schech). The chief location is the anterior and middle portion of the under surface of the true vocal cords, but the epiglottis and various mucous folds may be involved. All ages are found to develop papillomas, especially in the fourth decade and in males far more than in females. An hereditary influence is rare, but more papillomas appear in infants and children, indicating a congenital predisposition (Casnit, Gerhardt). Overuse of the voice, irritant inhalations, the sequelæ of exanthems, alcohol, and tobacco, have been recognized as etiological factors, and all probably act by producing some form of chronic laryngitis. Yet some cases develop without a demonstrable trace of previous inflammation. L. Browne attributes many laryngeal polyps to the influence of nasal and pharyngeal adenoids.

The tumors appear as (I) a single smooth red to gray, firm, pea-sized nodule, or as (2) a group of elongated papillæ resembling a cock's-comb, or (3) a more bulky cauliflower tumor. The base may include a small circumscribed area, or much of the mucosa may be the seat of papillary outgrowth and lead to suffocation, or stenosis after extirpation. Laryngologists generally agree that while most benign papillomas may be recognized through the laryngoscope, yet in some cases a full microscopical study is necessary.

The early stages have been traced by Werner to a condition first of hyperemia and round-cell infiltration of submucosa, which is followed by nodular overgrowth of epithelium. The area affected by these changes may be considerable and a basis is thus furnished for the appearance of multiple tumors at once or at intervals. In the condition called "singer's nodes" the epithelial hypertrophies become chronic, more or less keratinized, associated with dilatations of blood-vessels, and sometimes give origin to papilloma.

The structure shows an active overgrowth of normal or slightly altered epithelium, in many projecting folds and with some downward elongation of papillæ, but with an intact basement membrane. The stroma consists of branching strands of vascular connective tissue which may be very scanty or so abundant as to resemble papillary fibroma.

The epithelium is of squamous type with much or little hornification. small concentric cell groups and mitotic figures may be found in benign growths, but when these features are prominent they should be regarded with uspicion, especially when combined with irregularity in the form and arrangenent of the cells. Papillomas removed shortly after cauterization or imperect curetage may show great disorder of the epithelium. 
In the diagnosis of malignancy in early papillary tumors of the larynx chief attention should be given to atypical cells in the epithelial layer. I have seen a fatal course in so many apparently simple laryngeal papillomas in which the sole sign of malignancy consisted in atypical hypertrophic hyperchromatic cells in a uniformly thickened layer, that $I$ am compelled to regard this appearance as of grave significance. Reliance on this criterion leads to the conclusion that most epithelial papillomas of the larynx tend to become malignant, especially in adults, and this conclusion is strengthened by the frequent occurrence of long-existing bulky papillary growths which have eventually invaded the lymph-nodes.

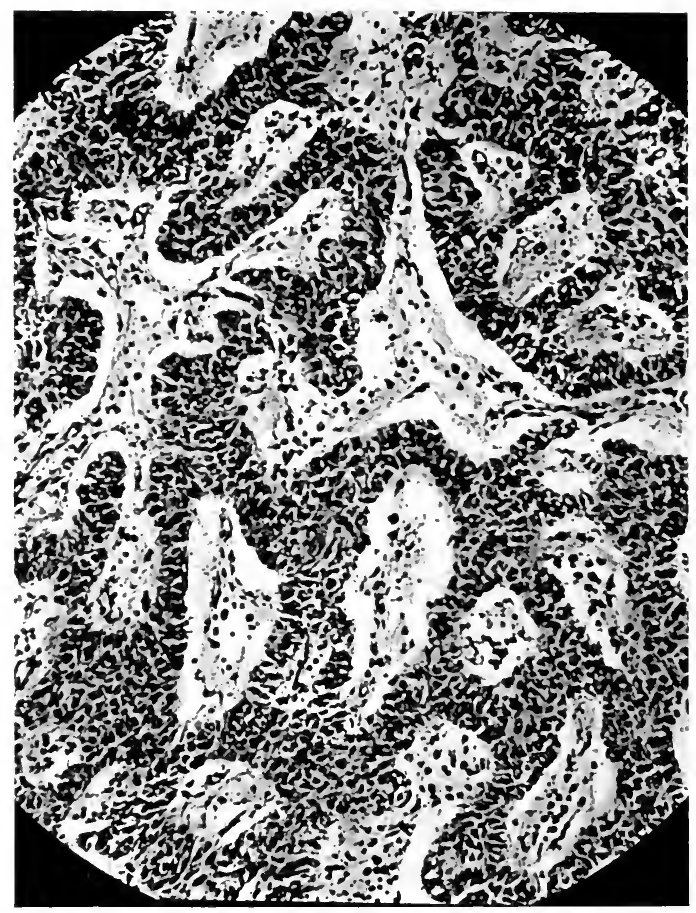

FIG. 428.- Atypical epidermoid carcinoma of tonsil.

While most papillomas are completely eradicable, not a few recur locally, either immediately or after an interval which may be as long as 9 (Curtis) or even 22 years (Lincoln). Partial extirpation of a broad base is responsible for some recurrences which appear immediately with apparent acceleration of growth. Or a new tumor may slowly develop in a predisposed mucosa. There are no sufficient grounds for assuming a local infectiousness of these tumors.

Rapid and extensive recurrence in histologically benign form is observed especially in children, and illustrates the remarkable regenerative powers of the laryngeal epithelium. Laryngotomy and excision or cauterization has been performed three, four, and even seven times in a relatively short period before the disease could be arrested (Wilkinson, Lendon, Clubbe). In this group of cases radium treatment has given excellent results.

The question of the transformation of a benign papillomatous into a 
malignant process has been extensively discussed. Semon could find only 45 definite reports of this event, I 2 spontaneous, 33 after operation, and of these he discards all but $\mathbf{2}$. It is difficult for the laryngologist to secure satisfactory material for histological study, which must include a considerable portion and especially the deeper parts of the growth. Tissue from the edges of a carcinoma may show the structure of simple papilloma. Even with these sources of error histological data have often proved more reliable than laryngoscopic appearance. When the microscopical study can be made of satisfactory material its evidence must be regarded as final, and on such evidence it seems probable that laryngeal disease not infrequently appears first as simple papil-

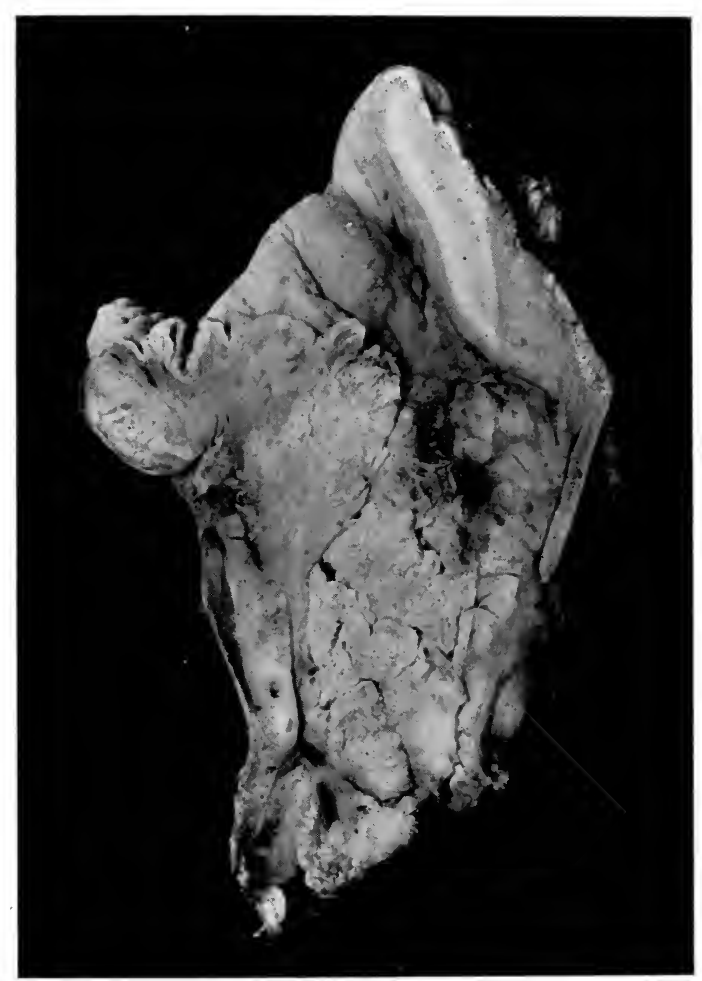

FIG. 429.-Bulky papillary epidermoid carcinoma filling larynx. No invasion of lymphnodes.

loma, later as carcinoma. I have examined the whole of an excised papilloma in a case which developed carcinoma 3 years later, possibly at another point.

In cases in which such a transformation appears to occur there are several possible explanations: ( $\mathrm{I}$ ) The original disease is a simple papilloma which really changes its clinical character and develops infiltrative growth. (2) The original disease is carcinoma, but the examination of tissue is incompetent or inadequate. (3) An original papilloma is removed, but the disease recurs elsewhere as carcinoma. Such events are observed in other mucous membranes. (4) An original papilloma is imperfectly removed and the remnant is stimulated to atypical growth. 
Miscellaneous Benign Tumors of the Larynx.-Fibroma is a frequent, with some authors the most frequent, tumor of the larynx. It appears on the true or false cords, near the anterior commissure, or on the epiglottis, rarely elsewhere. Fibromas are usually single, small, and pedunculated, but may be multiple, and present a broad base and reach a large size. Solis-Cohen observed six in one case. The structure varies with the consistence. Hard tumors are composed of adult connective tissue, while the soft growths are edematous or mucoid or contain an excess of vessels. The surface lining of squamous epithelium may be quite smooth or convoluted. Very small fibromas constitute a portion of the so-called singer's nodes (A. Chiari). In some cases the structure shows hornification of epithelium, dilated lymph-spaces, dilated gland ducts, and collections of lymphocytes, all of which indicate an inflammatory origin. When the mucoid element is prominent the tumors have been called myxomas (Fauvel, Bruns).

Chondroma.-Bruns collected i4 reports of chondroma of the larynx. The largest reached the size of a walnut. The chief location was in the cricoid cartilage, and the structure showed hyaline cartilage often with calcification.

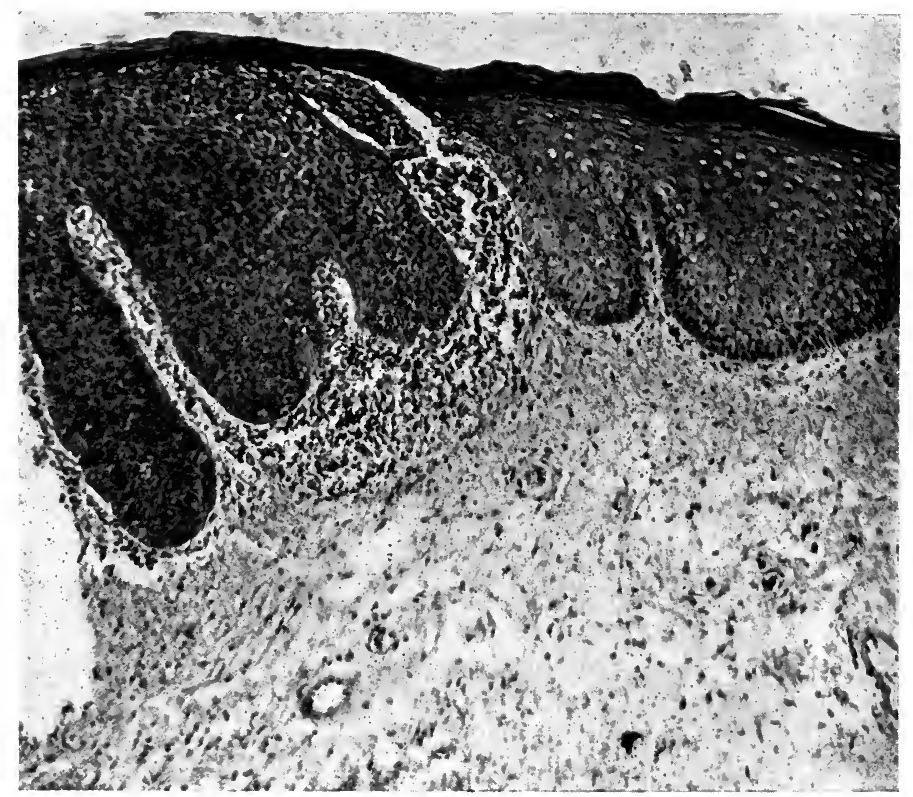

FIG. 430.-Pachydermia laryngis. A form of beginning carcinoma of the larynx.

Lipoma has been reported in and about the larynx in 9 cases (Jurasz). They were usually small and single, but Seyfert's tumor was lobulated and of considerable bulk.

Bruns described a rather large adenoma arising from the vocal cord and consisting of alveoli lined by cylindrical cells. A few other cases are mentioned by Jurasz.

Angioma has been observed by many authors. Phillips and Ruh collected 26 cases from the literature and report I case in a child following measles, in which there was extensive dilatation of vessels below the cords and evidences of chronic laryngitis. The structure indicates a close relation to fibroma, both in histogenesis and in etiology. Many of these tumors consist of simple varices, capillary or cavernous, and are not neoplastic. Others reach considerable size, and show a definite overgrowth of new vessels. In Loomis' case there were also angiomas of tongue, pharynx, and neck.

A large cavernous lymphangioma is described by Koschier.

A primary lymphoma of the sinus pyriformis is recorded by J. Mackenzie, and lymphosarcoma of pharynx and larynx by Stoerk. Generalized lymphosarcoma may occlude the larynx. Aberrant thyroid tissue in the wall of the larynx may be the source of submucous tumors (Ziemssen, Heise). 
Carcinoma of Larynx.-Carcinoma and sarcoma of the larynx form I to 5 per cent. of all malignant tumors and about $x 6$ per cent. of all laryngeal tumors (Jurasz).

The chief age of incidence is the fifth decade, but many cases occur between 5o and 90 years, and several cases have been observed in children of 3 to 6 years (Rehn, Steiner). The evidence suggesting an hereditary influence in cancer of the larynx is probably not more definite than with other forms of cancer in the adult (Semon, Lit.). Syphilis and tuberculosis seem to have no definite relation to the disease, although a few cases have arisen in tuberculous or syphilitic lesions (Schmidt, Wolfenden, Crone). Baumgarten has observed tubercle diffused throughout a laryngeal carcinoma. Males are much more often affected than females ( 400 to 68 , Sendziak). The special

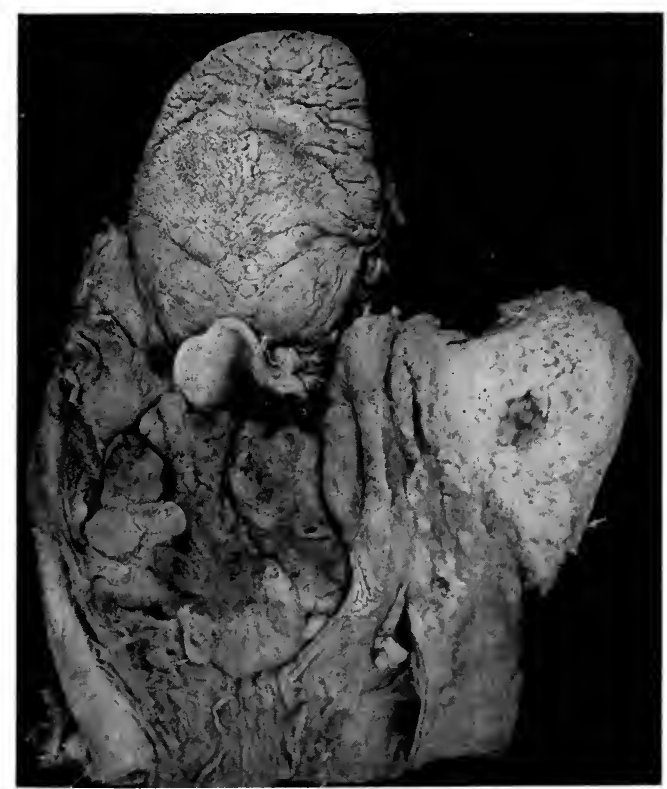

FIG. 43I.-Bulky hornifying papillary carcinoma of larynx, with invasion of cervical lymph-nodes.

factors concerned in the etiology of carcinoma are very similar to those of papilloma. Chronic laryngitis and the misuse of alcohol and tobacco probably determine the great predominance of the disease in men.

Molinie emphasizes the combined influence of syphilis, alcoholism, and tobacco.

Pachydermia laryngis or leukoplakia is a condition of epithelial hypertrophy and hyperkeratosis affecting the posterior portions of the vocal cords. The mucosa appears smooth or warty and scaly. The papillæ are elongated and the submucosa is infiltrated with round cells, while the glands are hyperplastic (Virchow, Fraenkel). It is not known to precede cancer in the larynx, but its relation to cancer in other tissues renders it suspicious in all situations. With C. Jackson I have seen one characteristic case in which the downward growth of papillæ and atypical qualities of the cells were very prominent. 
Anatomy.-The earliest stages of carcinoma present to the laryngoscope (a) a broad but circumscribed thickening of the mucosa, or (b) a projecting whitish nodule, $(c)$ a broad warty excrescence. Only 4 cases of carcinoma with a definite pedicle have been reported (Schmidt). These lesions are commonly associated with signs of laryngitis which precedes or accompanies the growth. A characteristic light opaque color may or may not be prominent. The early carcinomas may not differ in any gross feature from benign papilloma, but usually they are broader, more dense and opaque, and more firmly incorporated in the submucosa.

The anterior portion and commissure of vocal cords and vicinity are the chief seat of carcinoma, but it may develop in any part of the organ and its various folds and sinuses. Immobility of the vocal cord occurs with tumors

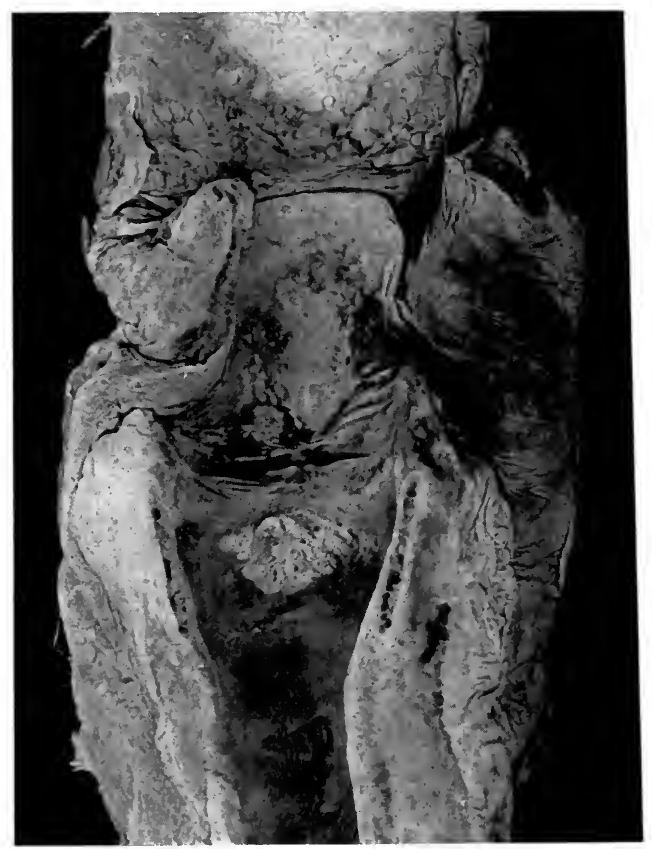

FIG. 432.-A flat papillary subglottic carcinoma which gave origin to bulky metastases in cervical lymph-nodes.

of the posterior segment. In frequency the various locations stand in the following order: true vocal cords, false cords, posterior surface of cricoid, epiglottis, and the lateral plicæ and sinuses. 'These locations suggest the division of laryngeal carcinoma into intrinsic and extrinsic forms (Krishaber). The former are much the more numerous except in females (Molinie).

The established disease takes the form of a circumscribed papillary growth or a more diffuse infiltration. The cordal tumors long remain localized to one side, but diffuse growths spread backward and to the opposite side (circular carcinoma), and many extrinsic tumors early involve both sides. Ulceration supervenes in the second year in nearly all cases, greatly altering the gross appearance and giving rise to many complications.

Arising in the submucous tissues of the ventricle of Morgagni the tumor 
reaches a moderate size, displacing the false cord and causing a protrusion of the overlying mucosa. Later it perforates the mucosa and reveals itself unexpectedly as an extensive ulcerating carcinoma (Sendziak).

Larval Carcinoma.-M. Schmidt described a form of deep-seated carcinoma of the larynx associated with superficial benign papillomas. It may cause considerable destruction of the cartilages before it appears on the surface and discloses its malignant nature.

The advanced disease varies considerably in its form and mode of extension. Many reach a large bulk while retaining a papillary form. Others infiltrate early and cause only minor laryngeal symptoms. Intrinsic cancer long remains confined to the larynx and is slow to infiltrate the lymph-nodes,

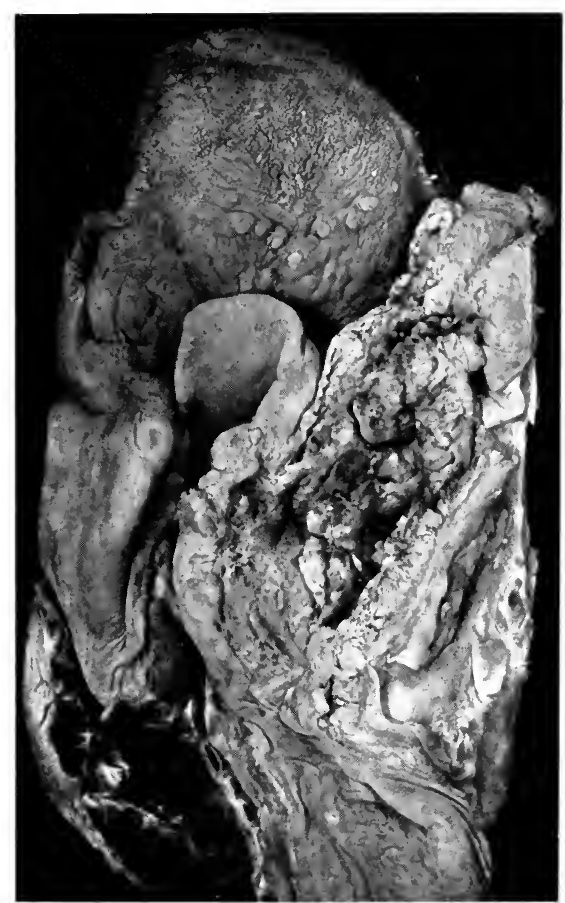

FIG. 433.-Epidermoid carcinoma involving tonsil and pyriform sinus of larynx.

but the outlying or extrinsic tumors extend to esophagus, tongue, pharynx and treachea, and to the neighboring lymph-nodes. Quite bulky tumors may develop in the late stages, and the extensions may be remarkably wide. Thiersch observed invasion of the cervical vertebræ, Moure saw extension to the submaxillary tissues, and Gougenheim and Gaston reported a large secondary thyroid tumor.

Contact implantation from one vocal cord to the other has several times been observed (Butlin, Newmann, Semon, Jurasz). With the invasion of the soft tissues above the glottis there is soon involvement of the submaxillary and cervical nodes which may reach large dimensions even with small laryngeal growths. In some cases the cervical tumors long precede definite laryngeal symptoms. Intralaryngeal tumors may first affect the prelaryngeal nodes. 


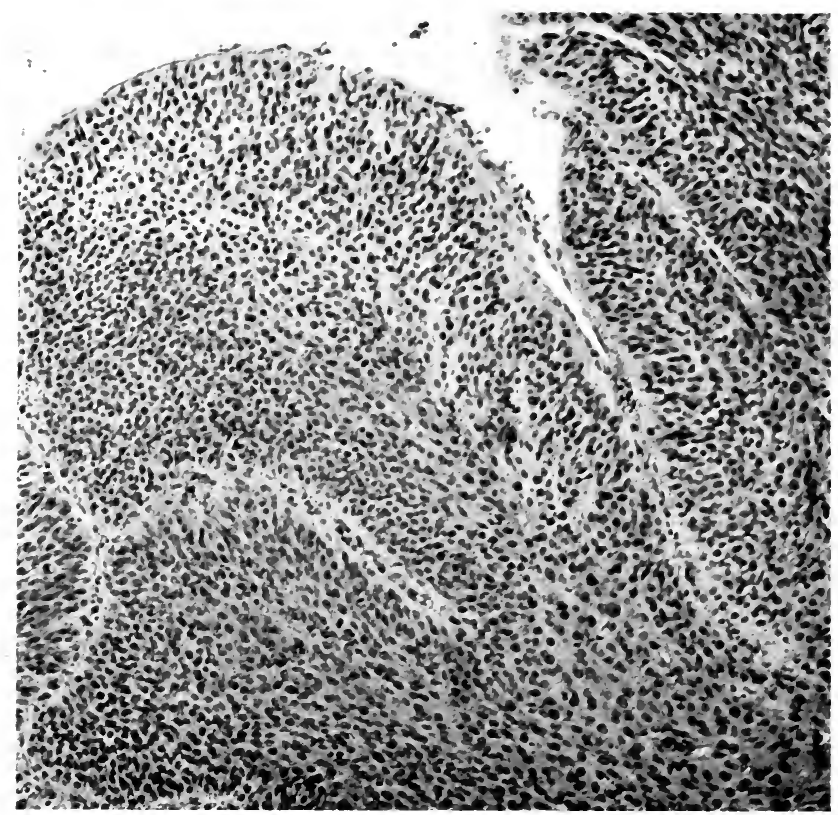

FIG. 434.-Malignant papillary carcinoma of larynx.

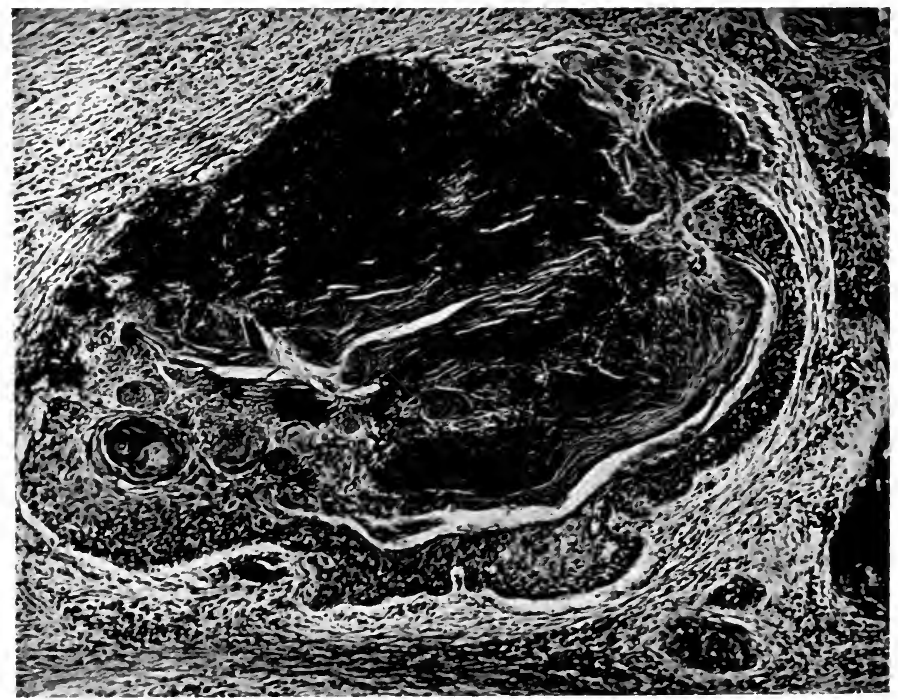

FIG. 435.-Epidermoid carcinoma of larynx. Extensive hornification and calcification in cervical lymph-node.

The structure is usually that of adult acanthoma with abundant keratosis and pearl formation. These tumors are locally destructive but of slow 
growth. Many laryngeal cancers exhibit transitional epithelial characters with cylindrical or cuboidal cells, free from keratosis. They arise from the folds and sinuses of the larynx and are the source of extensive tumors in the lymph-nodes (Kosinski, Maydl). Glandular carcinoma arising from the alveoli and ducts of mucous glands is rare (Schmidt, Schmiegelow, B. Fraenkel, Krieg). Its chief location is in the ventricle of Morgagni.

In some tumors, probably arising from the stratified cells lining the crypts, the cells rapidly become changed to an indifferent rounded or polyhedral form and grow in very small groups or diffusely. Such a structure may readily be mistaken for sarcoma.

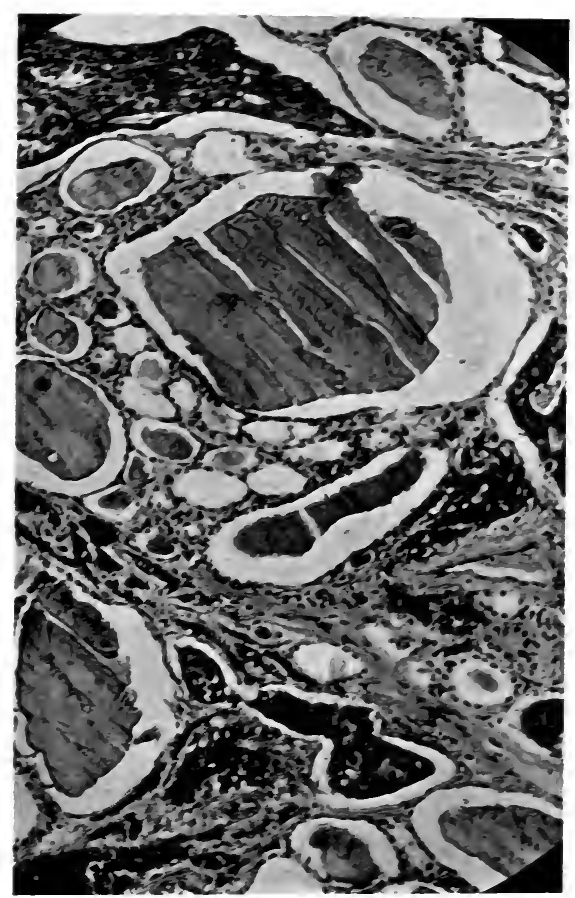

FIG. 436.-Epidermoid carcinoma of larynx invading the lymphatics of the thyroid.

Lymphatic Extension.-Based on Cuneo's studies of the laryngeal lymphatics, Molinie shows that: (I) Supraglottic cancer early invades the superior nodes of the external jugular chain; (2) cancer limited to the vocal cords rarely invades any nodes; (3) subglottic cancer invades the deep nodes about larynx and trachea, subcricoid, and peritracheal, as well as inaccessible posterior groups along the recurrent nerves and behind the esophagus.

The course of laryngeal carcinoma is much influenced by the type and location of the tumor. Acanthoma of the vocal cords is of relatively slow development, long remains confined to the larynx, but in the ulcerative stage extends widely and involves neighboring tissues and nodes. Periodic remissions are often observed and 2 or 3 years may elapse before a small carcinoma begins to grow rapidly. Extensive perichondritis may become established, in which the carcinomatous process combined with secondary infection per- 
forates and sequestrates the cartilage. Diffuse carcinoma of the cords may cause asphyxia. The usual termination is by cachexia, from dysphagia, suppuration, and hemorrhage with terminal pneumonia. The extrinsic carcinomas usually progress more rapidly, early invade lymph-nodes, and are very subject to ulceration and extensive suppuration. Many of these are composed of cylindrical or cuboidal cells without hornification. Glandular carcinoma is usually less malignant. In advanced stages secondary deposits may occasionally appear in distant organs, as the lungs, liver, kidney, humerus, dura mater (Shappers, Desnos, Sands, Virchow).

Secondary carcinoma of the stomach, probably from extension through the lymph-vessels, is recorded by Gougenheim.

Sarcoma of Larynx. - Sarcomas form in per cent. of malignant laryngeal growths (Molinie). The series of I I 7 cases analyzed by Bergeat, 50 by Sendziak, and 23 by Butlin show that sarcoma arises in much the same situations and in its early stages closely resembles the epithelial tumor. Thus they are extrinsic or intrinsic in location and polypoid or diffuse in form. Some develop a long pedicle (McWhinnie).

Many histological varieties are described, most frequently spindle-cell, mucoid, round-cell, and giant-cell types. Lymphosarcoma is described by Koschier and Stoerk. Spindle-cell sarcoma is practically limited to the vocal cords and epiglottis, while the so-called alveolar types (carcinoma ?) appear in the folds and sinuses (Sendziak). The surface of the early sarcoma is usually smooth. In late stages ulceration occurs, but less frequently than with carcinoma, the lymph-nodes may be invaded (I5 per cent.), and the conditions resemble the advanced carcinomas. It is probable that many cases recorded as sarcoma are of epithelial origin. 


\section{CHAPTER XLIII}

\section{EPIDERIMOID CARCINOMA (Continued)}

\section{CARCINOMA OF ESOPHAGUS}

Carcinoma of the esophagus is characterized by the variety and often by the obscurity of its symptoms, the peculiarities of its structure, and by its high mortality.

Etiology.-Males are affected in 75 per cent. of the cases (Kraus), and while the disease occurs chiefly about the fiftieth year, Kaufmann saw a small lesion in a woman of 90 and Heiman reports a case in a girl of 19 years.

Alcohol, irritating hot foods, tobacco, and wounds of various kinds stand as contributing factors. Leukoplakia and tuberculous ulcers have also furnished the basis of the disease.

The anatomy of the organ has a prominent bearing on the location and incidence of esophageal cancer. The three points of predilection, (I) upper, (2) middle, and (3) lower thirds, are also points of normal constriction, the narrowest being at the level of the cricoid. Mehnert holds that there are I3 natural constrictions at the vertebræ. Others enumerate narrowings and points of election, as at cricoid, aorta, tracheal bifurcation, left bronchus, and cardia. At these points also diverticula form and these abnormalities either on their edges or in the depth may be the seat of carcinoma (Heller-Arndt, Ritter).

Anomalies of structure of the esophageal mucosa seem to be an important predisposing factor in carcinoma, but their exact importance has not been determined. The great majority of subjects exhibit interruptions in the squamous lining of the mucosa by islands of mucous glands resembling those of the stomach (Schaffer). The most notable of these islands appear at the level of the cricoid to fifth tracheal cartilage, as bilateral spots 2 to ro $\mathrm{mm}$. in diameter, resembling gastric mucosa, or they are much smaller, irregularly distributed, and require microscopic identification. The cardiac end of the esophagus is also regularly beset with similar glands. The glands are of branched tubular type, lined by cylindrical cells, containing mucus and often becoming cystic. Mucous and parietal cells are often to be distinguished. Much has been written on the origin and significance of these glands and their relation to carcinoma (Hewlett, Lit.). They doubtless give origin to the mucoid and adenoid varieties of carcinoma, and the sudden transition of epithelial types which they present offers a predisposing factor for epithelioma. They are probably not to be regarded as misplaced islands of gastric mucosa, but as a type of normal development of the embryonal esophageal epithelium (Eberth, Neumann). The general incidence of carcinoma does not seem definitely related to these structures, Kraus finding $15^{8}$ cases in the upper hird to 699 in the middle and lower thirds.

Another series of abnormalities of importance in the origin of esophageal ancer is described by Glinski, Ciechanowski and Mosher, and consists' of I) canals lined by cubical or squamous cells lying in the submucosa, ori (2) leeper canals perforating the muscular coat, and (3) of complete tracheo- 
esophageal fistulæ. Many of these canals open on the mucous surface of the esophagus and always on the anterior surface in the middle line. Stoeber, Kern, and others describe cysts of this origin. Kathe found in a case of esophageal cancer an opening in the esophagus in the above position, $18 \mathrm{~cm}$. below the laryngeal orifice, leading into a canal which passed in the submucosa downward $5.5 \mathrm{~cm}$., where it again opened in the middle line. All these structures may be referred to incomplete separation of trachea and esophagus at an early embryonal period. That epidermoid carcinoma may arise from such structures is indicated by Grabowski's case, in which a tumor protruded into esophagus and also to a less extent into the trachea. An origin from such deep structures may also explain the common failure to find early superficial lesions of the lining epithelium, the early onset of deep ulcerations, the wide vertical extensions of the disease, and prob-

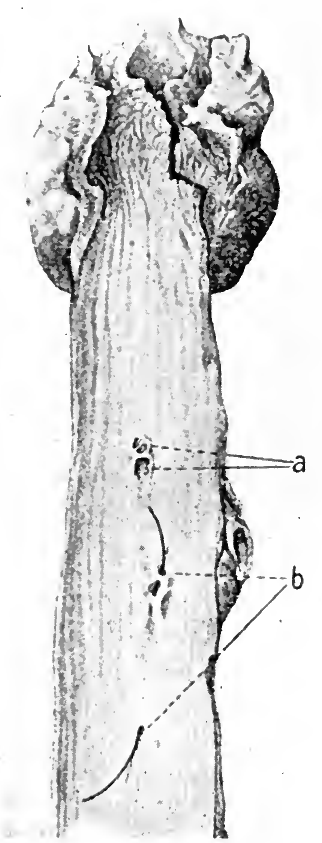

FIG. 437.-Congenital fistulous tracts in esophagus. Probable sources of carcinoma. (After Glinski, V. A., I99.) ably, also, the embryonal character of certain very malignant cases.

Gross Anatomy.-The tumor appears as (I) a flat infiltrating ulcer, or (2) a more bulky polypoid or globular mass, or (3) a diffuse infiltration.

(I) The ulcer may encircle the tube, causing constriction and invading surrounding tissues. Above, it invades larynx; below, gastric wall, and externally it permeates lymph-nodes and ruptures into trachea, bronchus, lung, aorta (Stadelmann, Kaufmann), and mediastinum. Fatal hemorrhage results also from erosion of pulmonary vessels, or carotid and thyroid arteries. Infection and suppuration constantly supervene. In many cases of this type the initial lesion is small and resembles a peptic ulcer, while the invasion of nodes and tissues is extensive. Thus there may be bulky tumors in the neck, or extending down the mediastinum, or involving pleura and pericardium, and these tumors may cause secondary stenosis or perforations. A second ulcer of the mucosa may appear, probably formed in this manner (Seelig).

Pressure on the recurrent laryngeal nerve is a frequent and early symptom recalling aneurysm. It occurred in 18 of 236 cases (Sakata). Ehret once noted hoarseness as the initial symptom.

(2) Globular or polypoid tumors are usually of the adenocarcinomatous type (Coplin, Lit.). They form circumscribed growths within lumen, wall, or lying externally.

(3) A diffuse infiltration of much of the esophagus occurs, of which Cæsar describes a typical case. The organ from larynx to cardia, as well as a portion of lesser gastric curvature, was uniformly $\frac{3}{4} \mathrm{~cm}$. in thickness, stenosed, but free from ulceration, and the mediastinal nodes were much enlarged.

The early and extensive metastases of esophageal carcinoma are favored by the abundant blood and lymphatic supply and by the movements of the organ. The influence of the latter element is only less prominent than in the stomach. Of the lymphatics Sakata describes a rich plexus in the mucosa which is chiefly longitudinal and a plexus of much finer vessels in muscularis 
and outer coats. Hence growth along the tube is readily accomplished and this mode of extension probably accounts for the multiple growths and supposed implantations in lower esophagus and stomach (Borrmann). Once beyond the fibrous coat extension through lymph-vessels occurs with unusual rapidity, giving secondary tumors in neck, thyroid, pleura, lung, and liver. The blood-vessels appear to be penetrated in rare cases. Dür found a large metastasis in the muscles over the pubic bone. Francke sectioned a case supposed to be a brain tumor, finding an adenocarcinoma of esophagus with metastases in lymph-nodes, pericardium, pleura, spleen, ovaries, pancreas, and brain.

The structure is usually that of acanthoma, but pearls, hornification, and prickle cells are often missing and the tumor has an embryonal type. The adenocarcinomatous varieties resemble similar tumors of the stomach and may exhibit an alveolar or a more diffuse structure. Mucous production may be abundant. The structure may vary in different portions of the same tumor and Kaufmann mentions mucous production in the secondary deposits from an acanthoma.

Among atypical varieties may be mentioned a highly embryonal growth described by Norris. In this case, with an ulcer of the usual gross appearance in the upper esophagus, there were enormous tumors in the liver. In both the cells were of indifferent large polyhedral, or spindle, or round type.

Extensive involvement of cervical lymph-nodes with comparatively small tumors of the esophagus occurs in an important group of cases. Large tumors of one or both sides of the neck may form when esophageal symptoms are indefinite or absent. The structure of the tumors in the nodes may be typical acanthoma or more embryonal and atypical.

Carcinosarcoma of the esophagus is an atypical growth of uncertain sig-

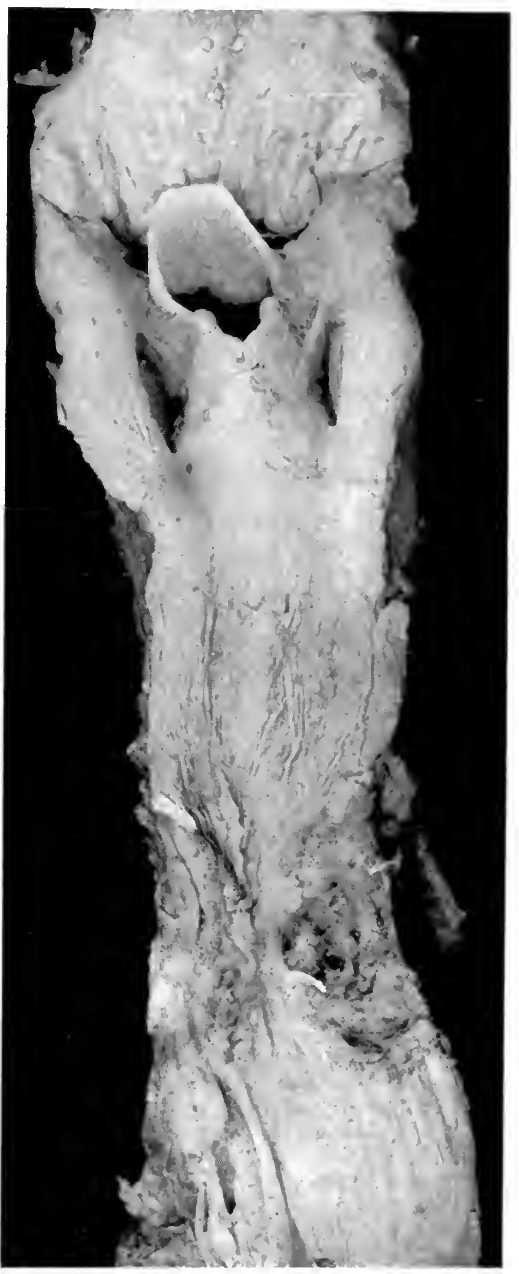

FIG. 438.-Carcinoma of esophagus. nificance, of which several cases have been reported. In some of them there was adult acanthoma in sarcomatous stroma (Herxheimer, Lit., Donath).

The very early stages of esophageal carcinoma are rarely encountered. Janeway examined in this laboratory an early epithelioma $\mathrm{I} \mathrm{cm}$. in diameter. It had not yet broken the line of basal cells, but the cells were hypertrophic, nuclei very hyperchromatic, the focus was rather sharply demarcated, and 
the whole area resembled a very early basal-cell carcinoma. Probably many tumors arise from embryonal submucous or the deeper structures previously described.

\section{EPITHELIAL TUMORS OF BLADDER}

The natural history of epithelial tumors of the bladder is determined by the structure of its mucosa, and the physical conditions to which it is

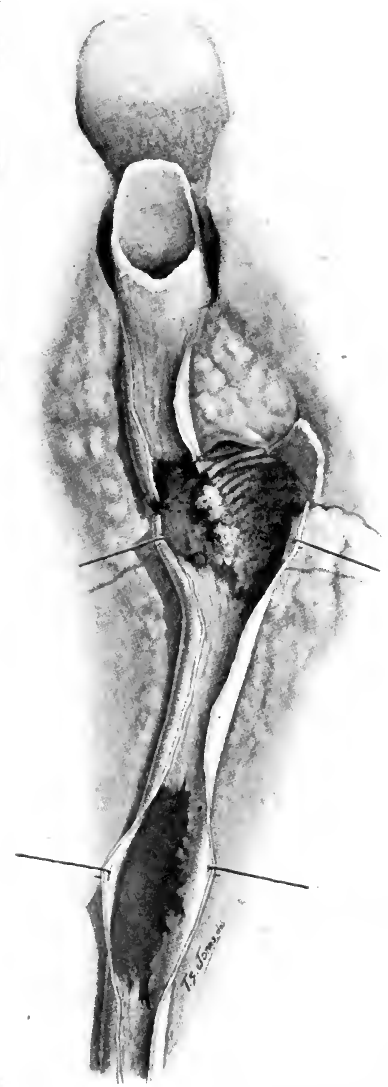

FIG. 439.-Double ulcerating epitheliomas of esophagus. (After Seelig, A. S., 46.) exposed. The transitional character of the vesical epithelium, which exhibits both pavement and glandular properties, accounts for the histological varieties of tumors arising from it. Thus the superficial cells are chiefly of the squamous type, while the deeper cells rapidly become polygonal and in many cases deep lying crypts, often called Brunn's follicles, are present and exhibit a lining of polygonal or cylindrical cells. Muscular activity and a constant bathing in fluid explain the villous form of the tumors. The villous form probably determines the long confinement of the tumors to the site of origin, which was once wrongly attributed to absence of lymphatics. Multiplicity may be referred to ready diffusion of the irritants which cause inflammatory and then neoplastic overgrowth.

There are two common varieties of these tumors: (I) Papilloma, which is fibrous, benign, or malignant; and (2) Carcinoma, which may be of adenoid or diffuse type. Adenoma is very rare. Several subvarieties are mentioned by various authors, as Albarran and Fenwick. The bladder furnishes between 0.96 per cent. of all carcinomas (Hadda) and 0.25 per cent. (Albarran). Rauenbusch finds the disease three times as common in men as in women. Yet Stein observed I6 simple papillomas in women to I 4 in men. Sarcomas are much more frequent in women and children.

Papilloma.-This common tumor occurs usually as a coarse villous growth with narrow pedicle, or the papillæ may be feathery or fleecy, or the growth may be rather flat and lobulated, or the tumor has a broad flat base and is sessile. Rarely long cylindrical papillomas are observed (Albarran). The surface of some papillomas may be of low warty character or nearly smooth, and it may vary in different portions of the same tumor. Half the tumors are multiple; as many as twenty different growths may be scattered over a wide area; and in some cases much of the bladder wall is the seat of very numerous villous outgrowths. Hence the discovery of one papilloma indicates a search for others at once or later. The multiple villous tumors must be distinguished from a form of diffuse low papillary inflammatory hypertrophy of epithelium, which has been known to cover almost the entire mucosa, producing a velvety surface. This condition is a curious exhibition of the regenerative tendencies 
of the irritated mucosa, is seen in exstrophy of the bladder, with calculus, and about other tumors, and may be arrested by curetage (Keyes, Fenwick).

The size of the papillomas varies extremely. Some long remain small, not exceeding $2 \mathrm{~cm}$. in length. Others, while pedunculated, attain the size of a small orange. The more solid and malignant tumors may also attain considerable bulk. A very large pedunculated myo-epithelioma is described by Albarran. The chief location is about the uretera! orifices and along the edges of the trigone. Fenwick notes a relative immunity of the trigone itself, where the mucosa is less mobile.

The course of the tumors is generally prolonged. Hadda refers to cases lasting 18 years. Guyon observed a case of 30 years' duration, and Fenwick

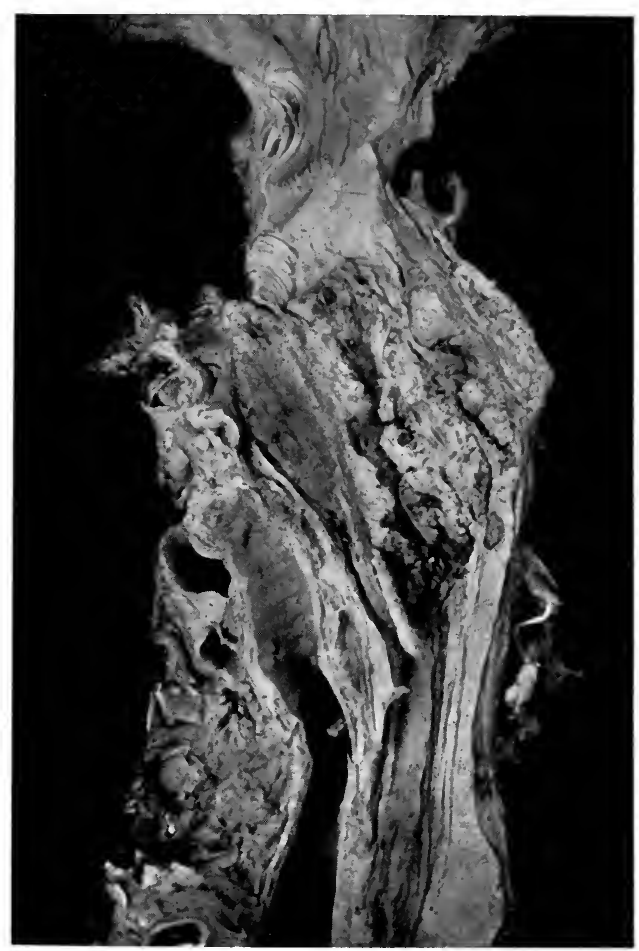

FIG. 440.-Advanced carcinoma of esophagus with rupture into bronchus.

attributed to I case a duration of 60 years. They arise at any period of ife from $2 \frac{1}{2}$ to 79 years (Albarran, Frisch), but are most common after middle ife, especially in the sixth decade. The life of most papillomas remains onfined chiefly to the bladder. Some remain benign for many years, and arely spontaneous expulsion occurs. Others (25 per cent.) become maligant after a variable period. Some are malignant from the first (Albarran). lmost 75 per cent. reach an urgent condition or are fatal in 3 years. Albarran ound 68 malignant cases to $\mathrm{I} 3$ benign papillomas. There is to be considered, $\mathrm{n}$ addition, the tendency of the benign discase to become malignant. Most uthors are agreed that the macroscopic appearance is no safe criterion of linical course, while the microscopic structure usually indicates considerable 
growth capacity which in other situations would always prove malignant. The clinical malignancy is also influenced by the tendency of new tumors to progressively develop in a predisposed mucosa.

In many cases the removal of the original tumor is followed by recurrence at another site. It is probable that the recurrence usually represents a development of a new tumor from preëxisting lesions, but not a recrudescence or implantation of the old growth. Frankly malignant cases, however, doubtless exhibit a growth of retained cell groups in the submucosa. Of 103 simple papillomas Frisch, after suprapubic cystotomy, saw 2I recurrences in 53 cases which he could follow. Of 49 papillomas which gave microscopical signs of

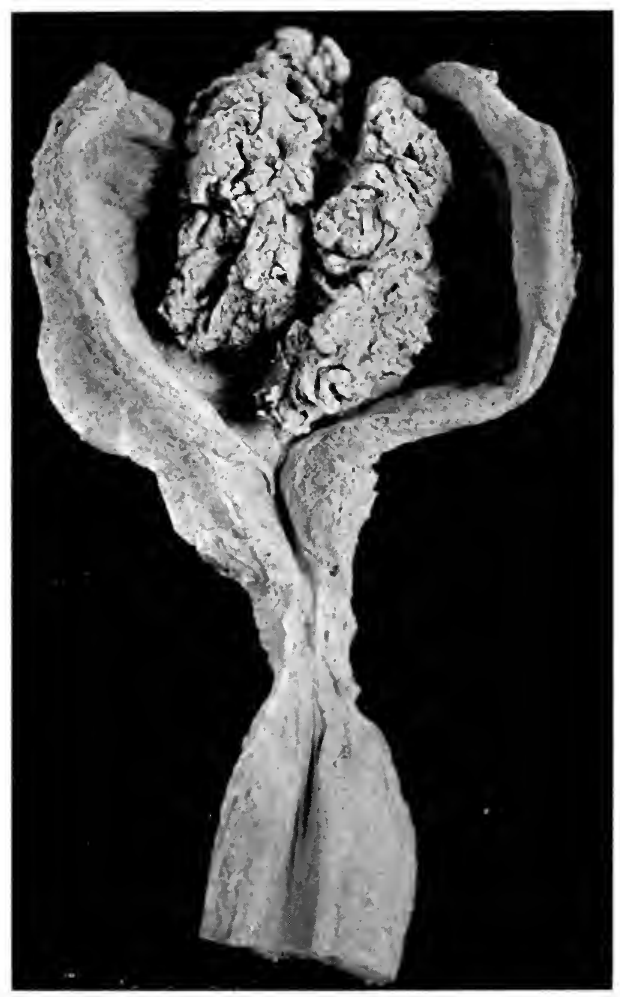

FIG. 44I.-Bulky papillary carcinoma of bladder.

malignancy, 29 recurred, 3 in the form of flat carcinomas. Of 95 definite cancers, 62 recurred and the others were not traced. In 3 cases a simple papilloma recurred as single or multiple carcinoma.

Extensions occur through the wall of the bladder to the pelvic tissues, up the ureters toward the kidneys, along the pelvic lymphatics to the prevertebral lymph-nodes. Rarely there is a superficial extension of a papillary cancer of the urethra into the bladder (Fluss, Lit.), or vice versa (Adenot). Metastases are not common, but have been observed in liver, lung, pleura, kidney, and inguinal and axillary lymph-nodes.

Albarran saw a pulmonary metastasis 2 years after removal of a solitary 
papilloma, the bladder remaining free. He accepts frequent propagation by contact, but does not insist on the actual implantation of tumor-cells. The bulky wide-spread metastases of prostatic cancer are regularly wanting.

The complications of malignant papillomas and of other cancers are hemorrhage with anemia, infection with cystitis and pericystitis, hydronephrosis, renal suppuration, and septicemia. Extension occurs late and true cancerous cachexia from generalization is rare.

The structure of the vesical papilloma is rather simple and uniform. It consists of a series of branching tufts of stroma covered by multiple layers of epitheluim resembling that of the bladder. The variable element is the stroma, which may consist of a single delicate capillary sometimes in immediate contact with the epithelium, more often supported by delicate strands of connective tissue, occasionally accompanied by smooth muscle-fibers. The muscular stroma may be quite abundant and in Albarran's myo-epithelioma it

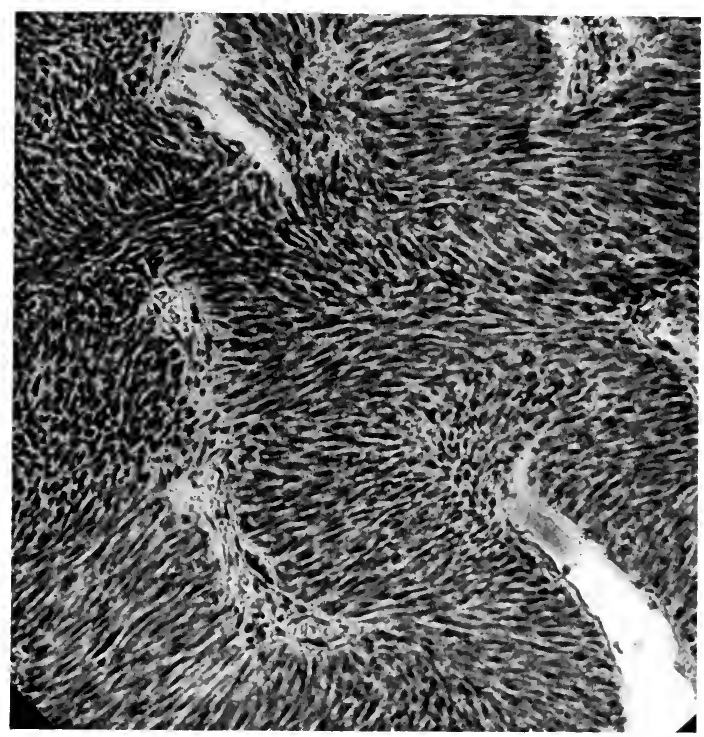

FIG. 442.-Spindle-cell epidermoid carcinoma of bladder.

mposed the bulk of a large malignant tumor. Fibrous polyps are described, nsisting chiefly of localized overgrowth of submucous tissue. They are of flammatory nature. Stein found mucous polyps almost as common as pilloma. True fibromyxomas are rare, but may reach large dimensions tein, Albarran). The epithelium is usually overnourished, with hyperromatic nuclei, but mitoses are rare. The cell form may exactly reproduce at of the bladder, or the cells may be elongated, or rounded, or extensively cuolated. Albarran describes as the "allantoid type" a simple papilloma of nich the villi are capped with cylindrical cells as in the intestine. Venulet scribed a structure resembling chorioma. The papillæ are closely packed pether in the more solid tumors, so that the section may resemble alveolar cinoma.

Signs of malignancy in papillomas include much variation in the size and the of the cells and extensive and irregular overgrowth, but the most im- 
portant indications may be found only in the pedicle which is seldom accessible in operative material. Downward growth of the convoluted epithelium invading the pedicle and subajcent tissue is the chief factor in local recurrence and malignancy. There is reason to believe that chronic inflammation with edema and round-cell infiltration facilitates this downward growth and renders it possible without any great change in the growth capacities or morphology of the cells. Yet in some papillomas the invasive tendency is present from the first. That a slowly growing and originally benign papilloma may become malignant is abundantly attested (Albarran, Fenwick, Frisch). Frankly, malignant papillomas exhibit throughout the atypical and lawless growth of carcinoma.

Buerger places the proportion of carcinomas preceded by papilloma at 30 to 36 per cent. While it is impossible to determine from the structure which papillomas will become malignant, the actual presence of malignant characters, he finds, may be determined from examination of portions accessible to the cystoscope. The changes, as usual, consist in atypical qualities of the cells and nuclear hyperchromatism.

Degenerative changes in papillomas include fragmentation, infection and ulceration, edema, necrosis, cellular infiltration, and calcific incrustation

Adenoma.- The literature contains isolated reports of adenoma and ade nocarcinoma of the bladder (Albarran, Fenwick). These tumors are usually small, single or multiple, located on trigone or other portion of wall, anc they may be flat or pedunculated. They are composed of large alveoli linec by multiple layers of cells some of which are usually cylindrical. They shor a tendency to mucous degeneration, as in Kaltenbach's case. Some of thi large glandular cysts of the bladder are probably related to adenoma.

The occurrence of cylindrical cell and mucoid adenoma and carcinomi in the bladder suggests an origin from mucous glands, which are not alway: attributed to the structure of this organ. Yet short tubular glands are reg ularly found in the bladder mucosa, especially in the trigone and about th urethral orifice (Albarran, Aschoff). They have been variously interpretec as aberrant prostatic glands (Virchow), or urethral glands (Aschoff), or as th result of snaring off of papillæ of epithelium by thickened connective-tissu septa (v. Brunn's follicles). In cystitis cystica many of the invaginate epithelial tubules are lined by cylindrical cells and the modified epitheliun develops secretory tendencies. Albarran describes in the trigone tubula glands in the submucosa lined by cylindrical and cuboidal cells and simple tubular crypts scattered over the bladder wall. No true secretory processe have been established in these glands, but the lumina are filled with des quamated cells. It is probable that such glandular structures increase i: number with increasing age and in cases of chronic cystitis (Prezwosky).

Glandular Carcinoma.-A rare type of carcinoma of the bladder take the form of a diffuse infiltration of the wall, with extensive mucoid degenert tion. Rauenbusch has collected ro cases of this type and offers a descriptio which indicates that such tumors may result from an extensive malignar growth of the same type as the mucoid adenomas. Yet the well-known diff culty of distinguishing secondary and primary carcinoma of the bladder, an the occurrence of somewhat similar conditions from secondary invasion $b$ intestinal cancer, leaves a reasonable doubt as to the exact origin of some the colloid carcinomas. Montfort, for example, was able to find only primary glandular cancers of the bladder in 83 cases fully examined. The of servations of many authors indicate the importance of this point of view.

Secondary invasion of the bladder by carcinoma may closely simula primary tumors. Carcinoma of the prostate frequently invades the bladd 
at the trigone, and Klebs once thought that all carcinomas of the bladder arose in the prostate. The differential diagnosis is often difficult and at times impossible. Many observers have found that only a small proportion of true carcinomas involving the bladder are primary in that organ. Besides the prostate, the uterus and rectum furnish a considerable proportion of the invading tumors (Fere). The invasion of the trigone from the prostate may take the form of a fungating mass or a flat ulceration; or the urethral orifice, trigone, and prostatic urethra may be the seat of hard projecting nodules; or the growth originating in the anterior portion of the prostate may invade and thicken the anterior wall of the bladder (Fenwick). Sarcomas of the prostate or vagina, especially in children, may grow extensively in the bladder. From the seminal vesicle a carcinoma, described by Fenwick, invaded prostate and bladder wall, forming a hard cup-shaped ulcer involving one-half of the trigone and ureteral opening.

Primary ureteral carcinoma has occurred in rare cases (Voelcker, Rundle), and downward growth of this tumor may yield a tumor mass at the urethral orifice (Drew). Yet it is difficult to exclude a primary growth at the ureteral orifice, as in Fenwick's case (l. c., p. 18). The cases in which the renal pelvic mucosa, ureter, and ureteral orifice are involved by nodular or villous carcinoma probably signify the development of multiple primary tumors over a wide area. The possible extent of this predisposed territory is indicated by various cases of pyelitis, ureteritis, and cystitis cystica (Stow, Lit.). A similar explanation probably applies to the alleged cases of implantation of renal carcinoma along ureter and in bladder. This view is strengthened by the occurrence of benign papillomas in the bladder in cases of malignant renal or ureteral growths (Fenwick, Lit.). Several observers have noted that the invasion of the bladder by malignant tumors of other organs may excite the growth of villous papillomas in the adjacent mucosa.

Etiology.-The great majority of bladder cancers can be traced to a previous or coexistent cystitis. In one group the cystitis long antedates the tumor and may be forgotten. Vesical calculus is associated with carcinoma in a rather small proportion of cases, varying greatly with different authors. It is especially common when stones form in Bilharzia cystitis (Goebel).

Stoerk has traced a definite relation between cystitis cystica and its forerunners with multiple papilloma, and he and Cohen report cases of cystitis cystica developing into carcinoma. I have observed one case in which a large portion of the bladder was the seat of low, nodular, and cystic outgrowths, from many points of which carcinoma had developed. The multiple tendency of villous carcinoma and the heterotopic recurrences indicate the existence of wide-spread lesions in the mucosa from which tumors may develop.

In Bilharzia disease there is a series of changes, including venous and lymph stasis, catarrhal cystitis, irritation by ova, and lithiasis, which have been shown by Goebel and others to lead in many instances to epithelial and other tumors. The frequency of chronic cystitis, papilloma, carcinoma, and even sarcoma among anilin workers has been pointed out by Rehn, Wendel, Leichtenstern, and lately by Nassauer. Here the cystitis and the tumors are often located at the ureteral orifices. A large proportion (50 per cent., Seyberth) of these tumors are malignant.

Miscellaneous Tumors of Bladder-Among the rare tumors sarcoma of the bladder is of chief interest. Albarran collected $5^{\mathrm{I}}$ cases. They occur hiefly in young subjects. They produce a large globular or polypoid inravesical growth or diffusely invade the wall. The structure varies, round, pindle, and mixed cells predominating. Vascular myxosarcomas of large 
size are reported by Kaufmann. Osteochondrosarcoma is reported by Beneke and W. Fischer. Secondary invasion of the bladder by the myxosarcoma of the vagina in children is a well-recognized condition.

Rhabdomyoma has been described in a few cases (Monckberg). Leiomyomas appear as polypoid tumors, sometimes of large dimensions (Terrier,

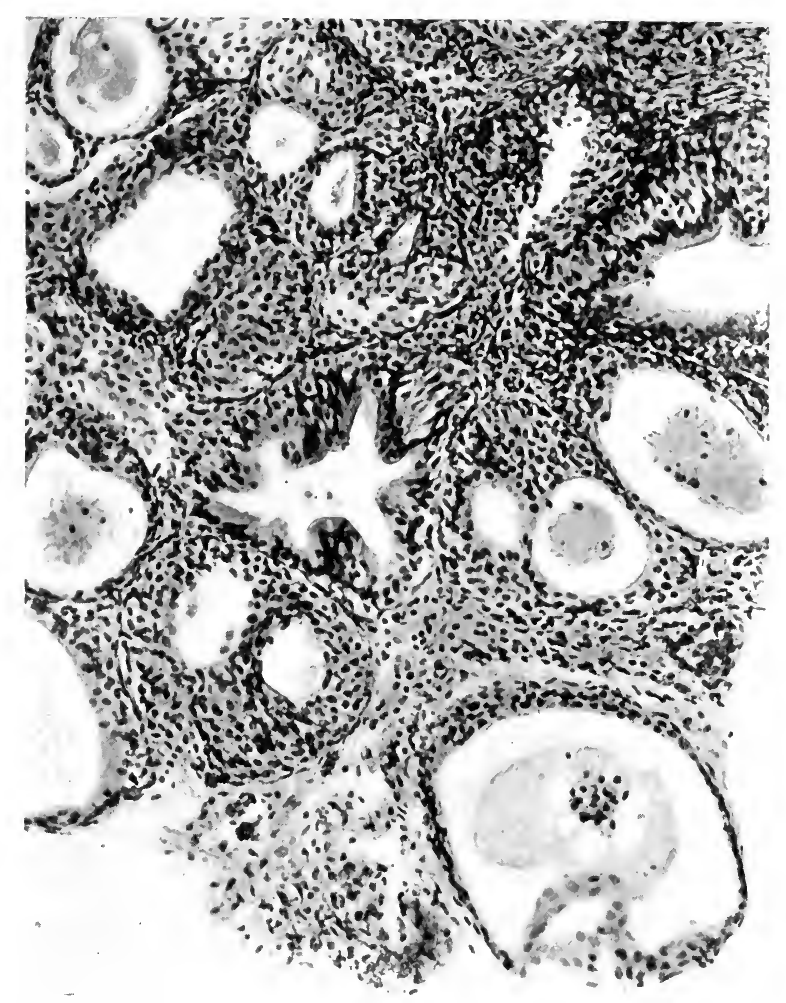

FIG. 443.-Cystitis cystica. A lesion often followed by carcinoma of bladder.

Hartmann). Various mixed tumors of the bladder occurring chiefly in children are described by Husler and R. F. Müller. Probable sources of these tumors and their interpretations are discussed under Teratology.

\section{TUMORS OF PENIS}

Epithelioma of the penis forms from $\mathrm{I}$ to 3 per cent. of all cancers in males (Paget, Billroth) and occurs most frequently in the sixth, fifth, and seventh decades of life (C. Kaufmann). Curtis refers to a case of epithelioma at 18 years, and Demarquay saw 9 cases between 20 and 30 years. Creite describes an atypical embryonal carcinoma of the corpora cavernosa of undetermined origin in a child of 2 years.

The disease arises chiefly on the glans (69 per cent., Barney) and on the dorsal aspect nera the corona (Thomson). Kaufmann found it more frequently on the prepuce. Cancer arises from the normal or chronically inflamed urethral mucosa. Hottinger collected 20 cases of this type, which 
took the form of epithelioma or carcinoma with mucoid degeneration and occasionally with alveoli lined by cylindrical cells. Cowper's glands were the source of carcinoma in 3 cases described by Kaufmann. Contributing factors are chiefly phimosis and syphilis. Demarquay and Barney noted phimosis in 80 to 85 per cent. of their cases, and Kaufmann found a chronic balanitis with warty vegetations, the result of irritating secretions, in 29 of 33 cases. Hebrews are practically free from the disease. Thomson describes as a precancerous condition of the penis a catarrhal balanitis with desquamation or, later, epithelial hypertrophy with overgrowth of subepithelial connective tissue. Schuchardt calls this condition "psoriasis preputialis."

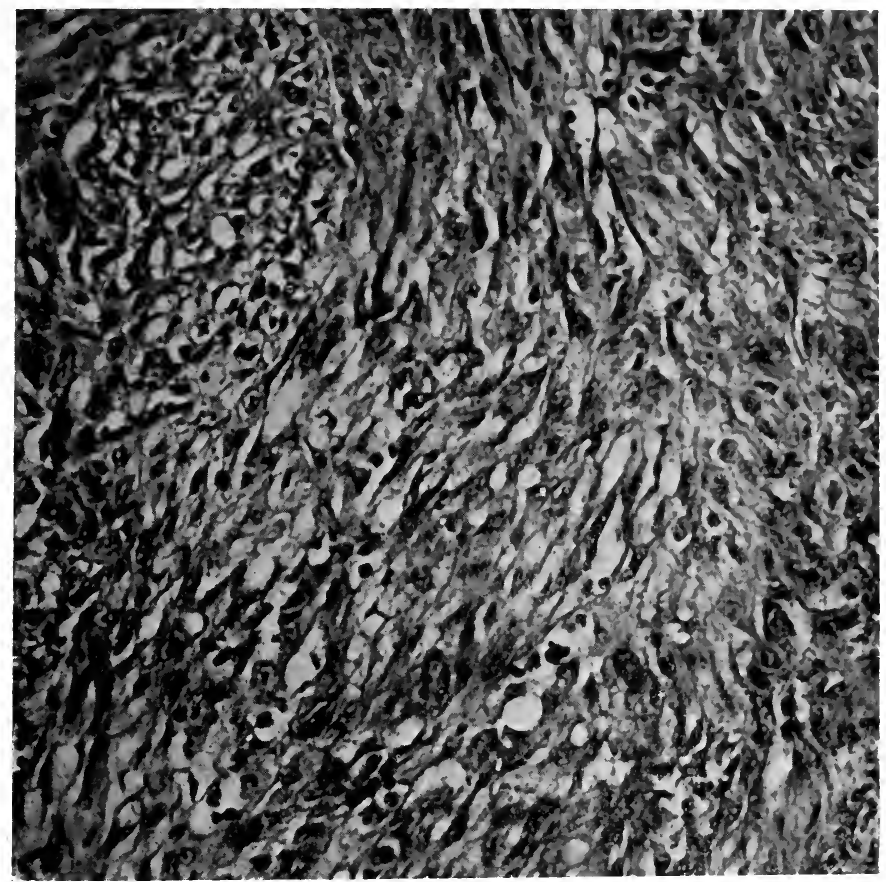

FIG. 444.- -Spindle-cell epidermoid carcinoma of penis.

Syphilitic scars and venereal warts are not infrequently the starting-points of epithelioma, and the trauma of circumcision is occasionally mentioned. Buday reports a urethral carcinoma following elephantiasis of the penis.

Implantation by contact with a cancerous cervix has been maintained as a source of penile carcinoma by Demarquay, Martin, and others, but without satisfactory data.

The initial lesion is usually regarded as a simple wart, or it appears as an eroded papule, more dense and pearly than a chancre, or as a smooth lump or thickening of the epithelium, or finally, as a definite ulcer. Many cases are not recognized until an irritating purulent discharge calls for the relief of phimosis and discloses an established lesion, and most cases have progressed for months or years before recognition.

The established disease regularly takes the form of a papillary epithelioma 
and may long remain a relatively warty tumor of considerable dimensions, but ulceration commonly supervenes, excavates the central portions, and leads to infection and suppuration. Early ulceration and infiltration of the base may obscure the papillary character from the first and lead to a more rapid and malignant course. C. Kaufmann speaks of bulky non-papillary lobulated tumors arising from the glans. Papillary tumors of the glans are nearly always malignant.

Local extensions of the disease occur late, but eventually the glans is destroyed, the corpora cavernosa are invaded, and the whole penis may be infiltrated, especially in the ulcerative form. In most cases, however, the corpora cavernosa long resist invasion and the corpus spongiosum remains free, so that urination through the urethra or a fistulous tract is maintained.

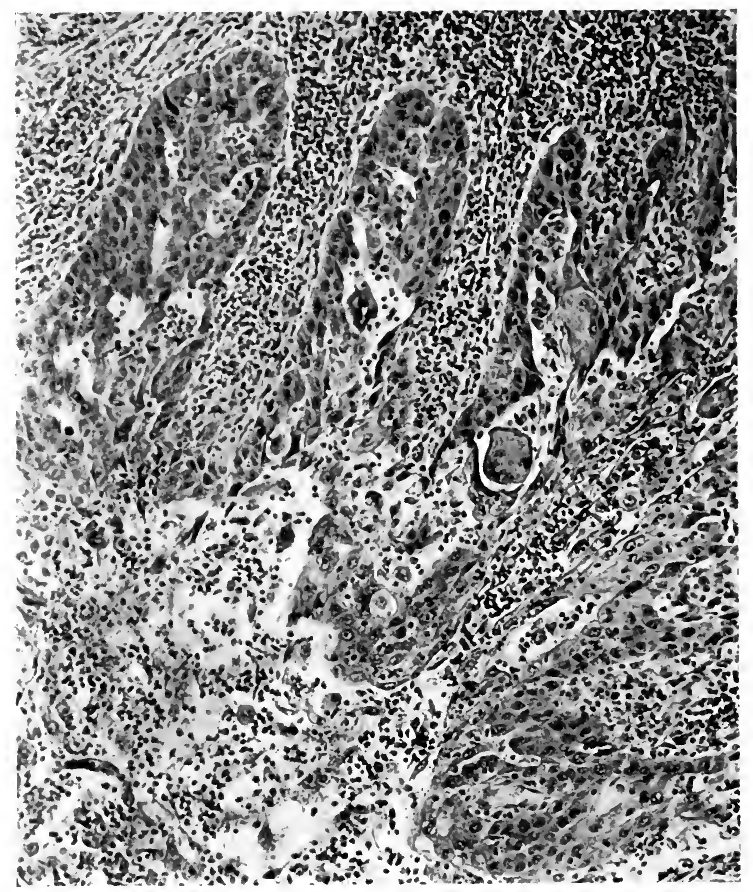

FIG. 445.-Carcinoma of penis. Suppurating carcinomatous inguinal lymph-node.

Lymphatic extension occurs through the superficial vessels to the inguinal nodes and through the deep lymphatics of the urethra and along the dorsal vein to the pelvic nodes. The inguinal nodes are commonly involved and often on both sides. Kaufmann found the inguinal nodes free in only 8 of 48 cases. Martin found 30 out of 40 invasions bilateral. Kuttner proved that many swollen nodes were free from cancer. He records invasion of pelvic nodes and in prevesical region in 2 cases without the affection of inguinal nodes, and emphasizes the connections of the deep urethral and dorsal vein lymphatics with the iliac vessels. A notable case is that of Taylor who amputated the penis without removing the nodes in the sixth year of the disease, the patient remaining well for Io years. Permeation along the dorsal 
lymphatics is the rule, and continuous cords have been traced up to groin, pubes, and even to umbilicus. Localized nodules may develop in the course of the lymphatics and break through the skin. Highly infected cancerous ulcers develop in this way and hasten cachexia. In advanced cases there may be wide-spread ulceration and extensions with metastases in pelvic and abdominal nodes and internal organs. The liver is a favorite seat of metastases from carcinoma of penis, which may occur early in the disease. The lungs, heart, stomach, and central nervous system have also been invaded. Rarely the disease appears to become implanted by contact from glans to prepuce or scrotum.

The course of penile carcinoma is relatively slow, especially in cases which remain papillary and escape ulceration. Barney shows that most cases run

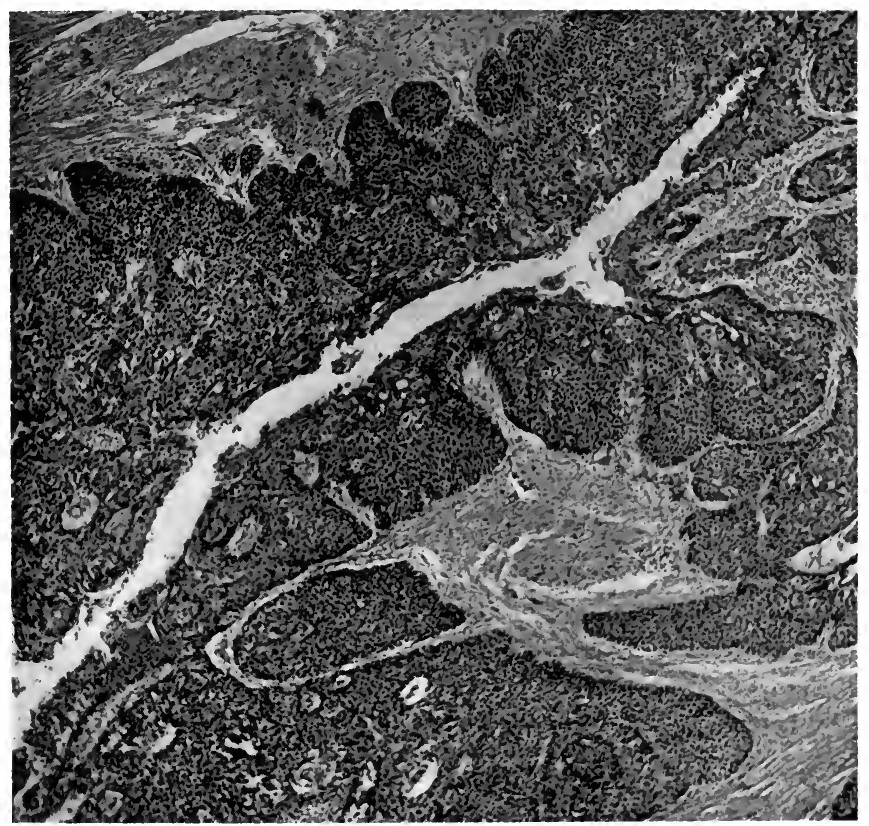

FIG. 446.-Beginning epidermoid carcinoma of female urethra involving a superficial area of 2 sq. $\mathrm{cm}$.

to 3 years before reaching a critical condition, but he reports i c cases of over ; years' duration and I case living I I years without operation. The gross nortality was 32 per cent., while I 5 per cent. developed visceral metastases, he others succumbed to local and pelvic lesions, and of all types of cases 38 er cent. were cured. Recurrences are usually observed in the first year, ut often develop after 5 years. Ten of Barney's 26 recurrent cases died of ancer after an average duration from the beginning of the disease of about years.

The structure of epithelioma of the penis is uniformly that of adult acanhoma of papillary type and with abundant development of pearls and hornication. This structure is usually maintained in the secondary growths and ray be very prominent even in hepatic metastases. 
Urethral carcinoma presents the structure of epithelioma of the bladder (Hall, Lit.). It is quite possible that the endothelioma intravasculare of the corpora cavernosa is a urethral carcinoma (cf. Borrmann). The malignant tumors arising from Cowper's glands show the structure of glandular carcinoma (Beck).

Melanoma of the glans penis is described by Payr (Lit.).

Scrotal cancer is interesting, especially because of the variety of specific exciting factors to which it has been attributed. Chimney-sweeps' cancer figured frequently in the literature from I820 to 1870 , but has now largely disappeared from observation. It was attributed to the irritation of carbon and oils derived from the soot. It was first recognized by Pott and fully described by Curling. It first appears as a "soot-wart" which may long remain innocent and may long grow as a harmless process, or with ulceration may develop malignant features. The lesion may develop in the cutaneous glands and produce a subcutaneous nodule. Creighton states that the epitheliomatous nature of chimney-sweeps' cancer, although always assumed, is far from clear in the recorded cases. He notes that the lesions appear first as subcutaneous nodules, that there are visceral metastases in fatal cases, that the structure is not that of adult acanthoma, and he traces the origin to the sweat-glands of the skin. Many parts of the body have been affected. Curling relates several histories indicating that the disease may develop long after exposure. It has been observed chiefly in England.

Paraffin cancer of the scrotum has occupied a somewhat less definite position. V. Volkmann was chiefly responsible for the view that irritation by products occurring in the manufacture of paraffin gives rise to scrotal cancer. He described the chronic irritation of the skin of the genitals in paraffin workers and stated that the lesions had a notable tendency ( 90 per cent.) to run into epithelioma.

J. K. Mitchell and Lewin studied the cutaneous eruptions among workers in petroleum, but found no cases of carcinoma. Lewin describes a form of chronic acne which in advanced cases leads to diffuse chronic inflammation with crusting. It was observed especially among the handlers of the byproducts of petroleum refining.

That cancer of the scrotum often develops in subjects that are free from exposure to bituminous products is generally recognized. Exposure to trauma and irritating secretions are probably important factors in its genesis. While many of the cases are of slow progress, very active and malignant forms occurring at an early age ( 8 to 15 years) are reported by Curling and Heath. 


\section{CHAPTER XLIV}

\section{MELANOMA}

Melanoma is a pigmentiferous tumor arising from a specific mesoblastic cell, the chromatophore, and possibly also from epithelial cells which have been modified by pigment production. It arises chiefly in the skin and the choroid coat of the eye, less frequently in the meninges, rectum, and other organs. In the skin its origin is connected with a congenital abnormality consisting of overgrowth and pigmentation of epidermis and often of the cutaneous glands, occasionally with angiomatous processes, and especially by the presence of many peculiar abnormal cells in the derma, forming a lesion known as the congenital nevus. In many cases the process terminates in wide-spread extension of the proliferating cells, giving a highly malignant tumor process. The peculiar and obscure conditions of its origin, the remarkable physiological properties of the chromatophores in the animal kingdom, the eccentricities of its clinical course, and its interesting history as a field of debate render this tumor one of the most notable topics in oncology.

History.-The congenital character, the origin from various forms of pigmented moles, and the numerous clinical peculiarities were fully recognized by the early writers before Virchow (1864, Lit.), while their views concerning the exact position of the process were about equally divided. Virchow recognized both a sarcomatous and a carcinomatous melanoma, the former exhibiting a diffuse structure of spindle-cells, the latter an alveolar structure, and he employed the term "melanoma" for the entire group. Demieville in I880 traced the origin of nevus cells to the perithelium and endothelium of blood-vessels, and in $\mathrm{I} 882$ Recklinghausen discussed the origin of melanoma in connection with multiple fibroma of the skin. He accepted by exclusion the endothelial origin, but could not trace any transitions of capillary endothelium into nevus cell masses. He dealt especially with the older fibrous nevi which may resemble fibroma.

In I893 Unna presented in substantial form the evidence in favor of the cpithelial origin of nevus cells, and while at first his views received little support (Delbanco, Hodara, Scheuber, Gilchrist), or were actively combated (Bauer, Jadassohn, Lubarsch, Hansemann, Borst), they have been actively supported by many later writers up to the present time (Favera, Lit.).

According to Unna "All pigmented and non-pigmented flat or wart-like nevi of newborn infants and children exhibit a direct connection between the cutaneous epithelium or hair follicles or ducts of sweat-glands and the cell cords of the nevus. In these structures there is a progressive transformation of prickle-cells into clumps of pliable ameboid cells without spines or fibrils, whose $€$ pithelial origin and nature is still attested by their clear oval vesicular nuclei and their immediate contact with each other and with the neighboring epithelium without the intervention of intercellular substance. This metaplastic process shows a constant tendency toward the complete isolation of groups of altered epithelial cells which are completely surrounded by the connective tissue of the derma."

Definite limitations to this explicit doctrine were soon $\epsilon$ countered, concerning especially the relation of the nevus cells to the abundant connective 
tissue in older nevi, and their behavior toward the lymph- and blood-vessels of the cutis.

The rather definite indications that the nevus cells actively participate in the formation of the cellular and fibrous tissue which surrounds them have been interpreted by Kromayer as evidence of the desmoplastic property of epithelium and this phase of the subject has been pursued by Kromayer in a series of studies 1898 to 1905 and by others, with interesting results. Judalewitsch especially has confirmed Kromayer's views and depicts the gradual loss of fibrils, pigmentation followed by depigmentation, and isolation of epithelial cells and their transformation into fusiform cells with fibrillar prolongations. Yet there is a strong probability favoring the conclusion of Favera and others that this fibrous tissue is a product derived from the preëxisting tissue of the derma. Riecke and Pini, however, consider the nevus cells to be modified fibroblasts. The relation of the nevus cells to the endothelium of vessels has remained a difficult question and a few more recent observers have clung to theory of endothelial origin of certain nevi, while admitting that many are derived from epithelium (Lowenbach, Walsch, Herxheimer, Borrmann, Moller, Fox, Johnston). The particular form which this compromise takes, and the grounds for it, vary considerably with the authors, and specific features distinguishing the two types of tumors have not been demonstrated. According to Soldan the endothelium of the nerve sheaths gives origin to nevus cells.

The presence of many large polygonal or multipolar heavily pigmented cells in nevi and melanomas and certain considerations bearing on the question of pigment production in general, as well as observations on melanoma of the uveal tract, have led Ehrmann and Ribbert to maintain the origin from the highly specialized mesodermal cell, the melanoblast or chromatophore, and to attribute the nevus and its tumors to aberrations of this cell (Ribbert's chromatophoroma).

The evidence accumulating in recent years from the comparative study of the physiology of the color function in the animal kingdom is a very formidable argument in favor of the specific mesoblastic nature of the chromatophore. It is much less cogent evidence that all melanomas are derived from mesoblastic chromatophores. Melanoma undoubtedly arises from nevus cells and the histological signs point very strongly toward the origin of nevus cells from the epidermis. The relation of chromatophores to the nevomelanoma is not yet clear. The theoretical considerations favor the origin of all melanomas from the mesoblastic chromatophore, while the histology of human tumors favors the origin from epithelial cells which have taken on pigmentary functions. The established tumors exhibit either carcinomatous or sarcomatous structure, or both.

Under these circumstances the writer confesses his inability to reach a conclusion regarding the nature of melanomas, and he will therefore present the data regarding them as impartially as possible, awaiting further elucidation of the many subjects involved.

Although the chromatophore is a mesoblastic cell, it arises very early in the formation of this germ layer and exhibits many specific properties some of which resemble those of epithelium, so that the identification of this tumor with carcinoma or sarcoma seems undesirable. The term "melanoma" should be employed to emphasize the specific character of the tumor and its cells of origin. The possible origin from epidermal cells may be indicated by the term "melanocarcinoma," and the sarcomatous character of other tumors is indicated as "melanosarcoma."

- Clinical Characters. - Modes of Origin.-The pigmented mole or nevus 
which is the source of cutaneous melanomas occurs chiefly on face, neck, and back, but may be found on any portion of the body. It may even appear on the sole of the foot, and in this and other exposed positions is more liable to malignant change. A relation to nerve-trunks has sometimes been noted, as well as a unilateral or symmetrical distribution. It appears as a minute pigmented spot (n. spilus), or soft, flat elevation, and often grows to a warty excrescence (n. verrucosus), or papillary tumor (n. papillaris). Many exhibit a growth of soft or coarse hairs, pointing to the implication of hair follicles (n. pilosus), and larger tumors may contain much loose connective tissue and fat tissue suggesting a complex disturbance of dermal structure (n. lipomatodes).

Remarkably wide extension over much of the body occurs in rare cases of "bathing trunk" type, of which Fox has collected 25 cases. The color varies from a light brown to black, but not a few notable cases of pigmentfree moles have been described (Moullin). The consistence is usually soft, in older fibrous lesions firm, and the vascularity slight. Eventually they may become hard and pedunculated (molluscoid degeneration). The clinical distinction from capillary nevi, port-wine stains, and lentigines (freckles) is usually obvious, but other pigmented tumors and processes in the skin may require microscopical study.

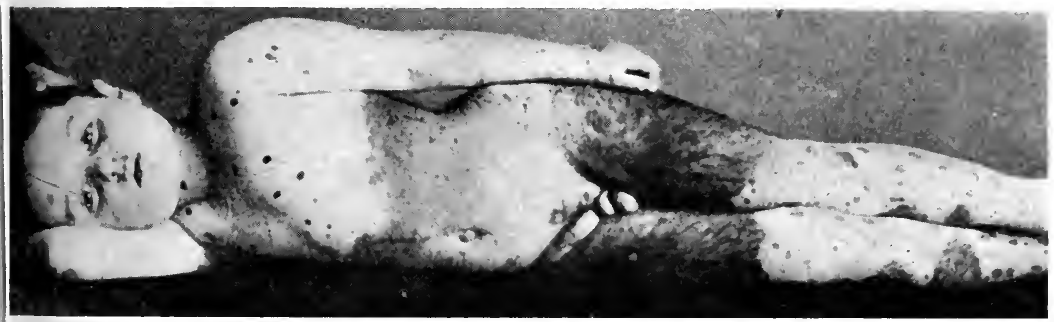

FIg. 447.-Extensive hairy nevus. Bathing trunk type. (After Fox, J. A. M. A., 58.)

Pigmented moles are sometimes associated with vascular nevi and with nolluscum fibrosum. These various lesions may be wholly distinct in the ame region, or so intimately combined as to greatly complicate the question ff their essential relations.

Melanotic whitlow (Hutchinson) is a heavily pigmented malignant epihelioma which begins as a swelling of the lateral nail fold of finger or toe. Che lesion soon ulcerates and early involves the regional lymph-nodes. The lisease has all the essential features of melanoma (Faguet, Plantier, Galloway). he process may take origin in the flat pigmented lesions called lentigo, which nay or may not show irritation or ulceration (Bayet).

Finally, in rare cases no local point of origin of the disease can be demontrated clinically or at autopsy.

In the lower animals moles and melanomas are relatively frequent. Their ccurrence in white horses was noted by Virchow as evidence of a constituonal dyscrasia.

Course.-The natural history of the vast majority of pigmented nevi cludes a long period of slow growth, a stage of inertia, followed by a process regression. A congenital disturbance of the structure of the derma ust be assumed for all cases, and some moles are visible at birth. Many ore appear in early life, and according to Unna these always show 
evidences of progressive growth. Having reached the limit of growth, which is commonly restricted to small innocuous tumors, they tend to undergo fibrosis and atrophy. Some become hard, flat, and circumscribed (fibromatous degeneration), others become pedunculated, fissured, or extruded (molluscoid degeneration). Repeated trauma often causes a limited overgrowth.

Malignant melanoma develops in a small proportion of cases, usually after trauma or incomplete extirpation. Familiarity with these apparently insignificant lesions constantly invites meddlesome interference by patient, surgeon, dermatologist, and other specialists. A slight enlargement or increase of pigmentation of the tumor may be the first signs of malignant change, which may be checked by wide extirpation. Following operation or without, there may be rapid local growth and extension, but some cases remain localized, with repeated excisions, for many years. In another group of cases there is immediately or after years wide-spread dissemination with invasion of regional nodes, or more often of the blood-stream. The skin is the chief seat of the first metastatic tumors, which may be extremely numerous. Painless firm nodules or larger hemorrhagic masses appear in many regions, grow larger for a time, and then may tend to become circumscribed, hard, or even pedunculated. Successive series of superficial tumors may develop before visceral metastases occur in lungs, liver, kidney, brain, bone-marrow, serous membranes and other tissues, with melanemia, melanuria, and cachexia. The duration of the generalized disease is about 3 years, but ro years may intervene between the extirpation of a primary tumor and the appearance of secondary lesions (Dobbertin). Fisher and Box observed a large melanoma of liver 14 years after removal of a primary intra-ocular tumor, and Albert reported a local recurrence 24 years after excision of a primary tumor.

The melanemia may be an early, pronounced, and persistent symptom, and the serous membranes, meninges, and intima of blood-vessels may become diffusely pigmented.

A very acute course is observed in rare cases in which localized tumors do not appear in the skin, but the disease proves fatal in a few weeks with melanemia, melanuria, and cachexia.

The melanuria has been attributed to the excretion of pigment grains or the passage of tumor-cells through the glomeruli (Eberth). Johnston found the cortex sparsely dotted with small metastases and light pigmentation of endothelium, Bowman's capsule and epithelium of Henle's loop, but no evidences of emigration of pigment cells. In the early stages of dissemination the urine is usually of deep brownish color, probably from dissolved melanogen. Later it may become nearly black (Eiselt).

Histology.- The structure of pigmented moles varies in different stages of their natural history.

(I) In the congenital mole of infants and young children one finds chiefly the early stages of development and a process limited chiefly or exclusively to the epidermis and the immediately adjacent derma. The first change is a clearing of the cytoplasm, swelling of the cell body, and nuclear hyperchromatism in foci of cells in the deeper layers of the epidermis. Sharply isolated groups of such cells thus appear in the epidermis and similar groups in the adjacent derma represent cross-sections of columns of cells that seem to have pushed their way downward and appear beneath the basal epithelium (Fig. 448). These same changes may be more diffuse and affect mostly the basal layer, from which single cells appear to break off and penetrate the derma. The epithelium of hair follicles and sweat-glands often participates 
in the process. Pigmentation usually accompanies these early changes, but may be absent. It appears uniformly in all affected cells, or single elongated, polyhedral or multipolar epithelial cells may usurp the chief part of this function. This mode of origin of pigmented cells can readily be followed. As these proliferating cells become more and more displaced they may lose their polygonal form, spines, and fibrils, and become converted into rounded coherent clear staining cells with dense nuclei, lying in alveolar groups or elongated columns. Depigmentation proceeds with the isolation of the cells and usually is complete when the cells become quiescent in a matrix of fibrous tissue. Pigment may then be found in this fibrous tissue, in the surrounding loose tissue cells, and in walls of capillaries. It must be noted, however, that all these interpretations of the epithelial origin of nevus cells are regarded by Ribbert and others as erroneous. These authors regard all the peculiar cells as inwandering chromatophores.

In the congenital mole the blood- and lymph-vessels play a passive part. The endothelium is usually normal, sometimes hypertrophic, and the lumen patent. About the vessels one often finds pigmented cells and the endothelium may be laden with pigment. Neither the lumen nor the

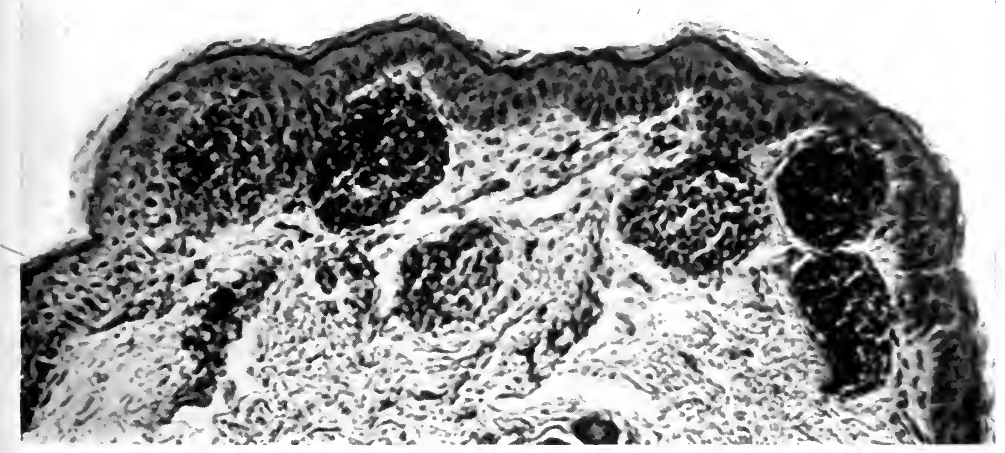

FIG. 448.--Structure of quiescent pigmented nevus in an infant. Notice apparent continuity of one nest of nevus cells with rete peg.

perivascular spaces seem to serve as channels for the advancing epithelium. I have been unable to trace any connection between the endothelium and the nevus cells of congenital moles. Capillary nevi and capillary angioma or endothelioma may be associated with pigmented mole. In these cases the two processes remain distinct and the separation of endothelium from epithelium is unusually sharp.

(2) In adults the structure of the pigmented mole varies with the stage of development. Many such moles become elevated, warty or papillary, and in these one finds groups or elongated vertical cords of clear rounded cells lying in acellular fibrous tissue. Scanty blood- and lymph-vessels of normal structure traverse these areas. I have not been able to find any connection between endothelium and adult nevus cells. All connections of the cell cords with the epidermis are missing. The deeper cells become spindle shaped and are intimately incorporated with the fibrous tissue and fine fibrils usually pass between the cells (Favera).

In not a few cases the pigmented mole of adults continues to exhibit appearances suggesting a focal or diffuse process of swelling and splitting off of basal epithelium. These moles are usually of large size and of slow 
but progressive growth. The structure then fails to show the clear cells of the quiescent nevus, but presents small groups or alveoli or a diffuse growth of larger opaque polygonal cells with hyperchromatic vesicular

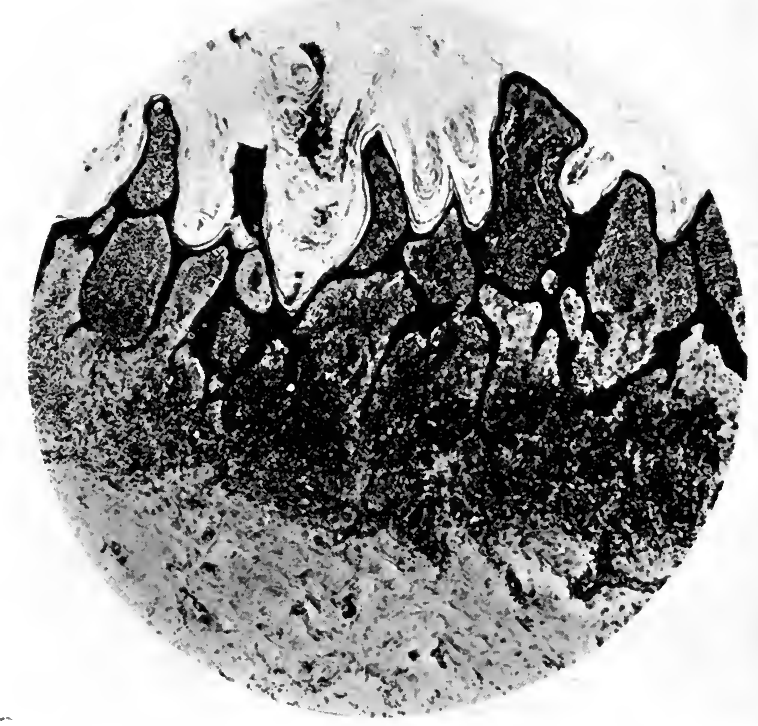

FIG. 449.-A quiescent papillary pigmented nevus. (After Johnston.)

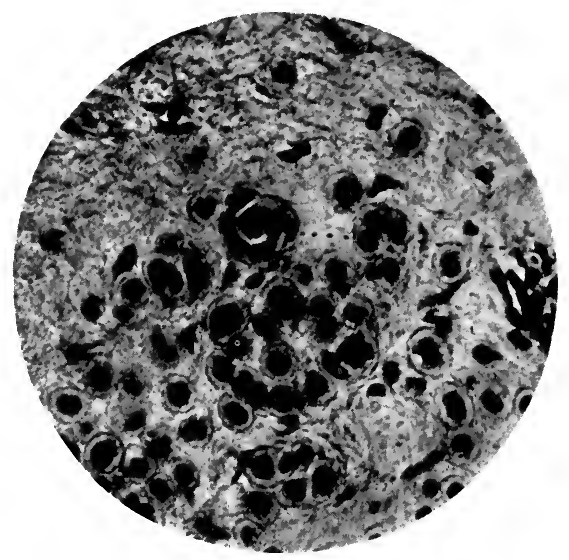

FIG. 450.-Cells in a resting congenital nevus.

nuclei. Pigmented cells are scanty in the quiescent adult mole and appear chiefly in the outskirts of the cell masses. The basal cells of the epidermis may, however, be deeply pigmented and swollen.

In rare cases the mole may be entirely free from pigment. 
(3) Fibromatous and molluscoid regression overtakes many moles in their late history. Much new fibrous tissue replaces the cellular areas and surrounds sweat, sebaceous, and hair glands, and this tissue may be cellular and mucinous. The nevus cells become atrophic, compressed, and disappear. Pigmentation is scanty or absent. The included fat lobules may be enlarged. The mole may be much elongated, pedunculated, or extruded.

(4) Malignant changes supervene spontaneously or after trauma or incomplete extirpation. The apparently innocent histological character of certain moles which have given rise to secondary tumors is one of the mysterious features of this disease, but, as a rule, the dangerous lesions give gross and microscopical evidence of active growth and especially of increased vascularity. The vigorous preparation of the skin is perhaps responsible for some unsuccessful operations on these vascular growths. Deep pigmentation is commonly observed to belong to dangerous moles.

The histological signs of malignant tendencies are readily detected and consist of increased vascularity, hypertrophy of cells, with nuclear hyper-

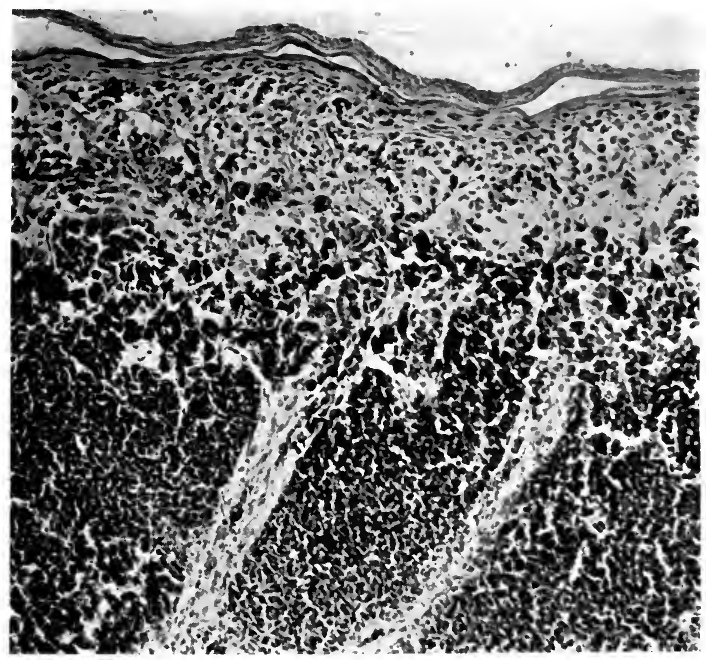

IG. 451.-Section of a flat heavily pigmented mole on skin of forearm, which had given rise to metastases in axillary lymph-nodes.

bromatism and occasional mitotic figures. An alveolar grouping of polyedral cells with prominence of epithelioid characters are observed in many roles which prove malignant. Fibrous lesions containing only cords of ear cells are seldom dangerous. Pigmentation usually increases with the dvent of new growth, but this rule has shown exceptions. The local growth nevi is usually the result of multiplication of the cells originally isolated. some cases, however, there is evidence of a progressive involvement previously normal rete pegs. The histological appearances indicate that is secondary process begins in the basal epithelium, is often accompanied pigmentation of these cells, but is not associated with the presence of ecific chromatophores.

The extensions of melanoma pass through either lymph- or blood-vessels. the former case the regional nodes are involved, the generalization is atively slow, and repeated local extirpation may accomplish a cure or 
long delay the fatal termination. Preceding the invasion of the nodes there may be pronounced hyperplastic lymphadenitis. Through the blood-vessels dissemination is usually general. There is little histological indication of the factors which determine the mode of extension, but the accumulation of tumor-cells about blood-vessels often reveals an imminent danger of rupture into the vessels. Numerous dilated blood sinuses are occasionally present and offer a ready access to the general blood-stream. In some malignant moles I have found great variations in the size and grade of anaplasia of the cells, some reassuming their epithelioid characters, others multiplying in the form of small indifferent round cells.

The structure of metastatic tumors presents the same wide variations. The tumor may appear as an alveolar or diffuse carcinoma, as a large spindlecell sarcoma, often as perithelioma, and even as lymphosarcoma. Well

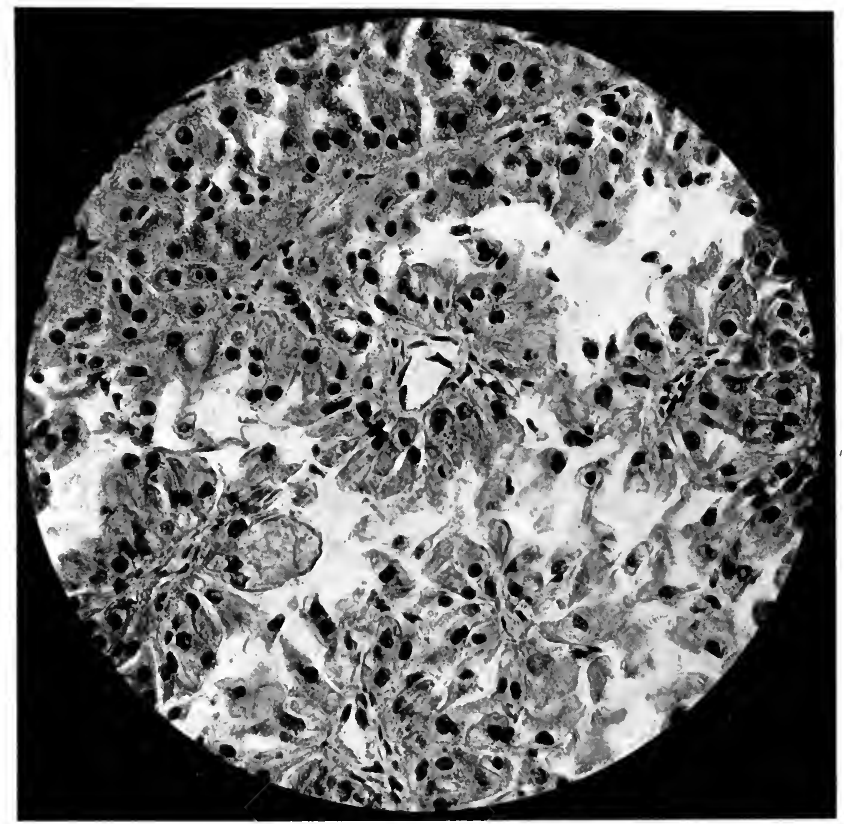

FIG. 452.-Melanoma of pseudoperitheliomatous type.

circumscribed groups of characteristic large round or polyhedral clear cells are often seen in cutaneous metastases, pigmented or not, and at once suggest their true origin. The appearance of such tumors may be the first indication of the existence of an offending mole. Metastatic nodules may at first be surrounded by hemorrhage from dilated blood-vessels and the absorption of the blood may be followed by cicatrization and marked regression of the tumor. In some cases there are very numerous pigmented points and spots in skin and mucous and serous membranes. These lesions may contain so much pigment and so few traces of tumor-cells as to suggest that pigment emboli have formed. The assumption that such pigment may incite tumor growth of normal cells is without definite support, but there is no satisfactory explanation of the great number of small metastatic tumors arising from some very small primary tumors. 
In the organs the metastatic tumor may contain the common large polyhedral cells, or smaller round cells of highly anaplastic type. In a large hepatic tumor I found the cells all of spindle form resembling sarcoma. In another case all the numerous visceral metastases contained small polyhedral cells whose desmoplastic properties yielded the structure of alveolar carcinoma. In 2 cases I have found adenomatoid hypertrophy and hyperplasia of polyhedral non-pigmented cells in the medulla of the adrenal.

The pigmentation of metastatic tumors varies greatly, being excessive in some tumors or parts of tumors, entirely absent or scanty in others. When excessive it may lead to destruction of tissue and the formation of cysts containing black fluid. There is no constant relation between the degree of pigmentation of primary and metastatic tumors. Heavily pigmented primary tumors may give origin to pigment-free metastases. The absence of pigment is observed in some of the most rapid and malig-

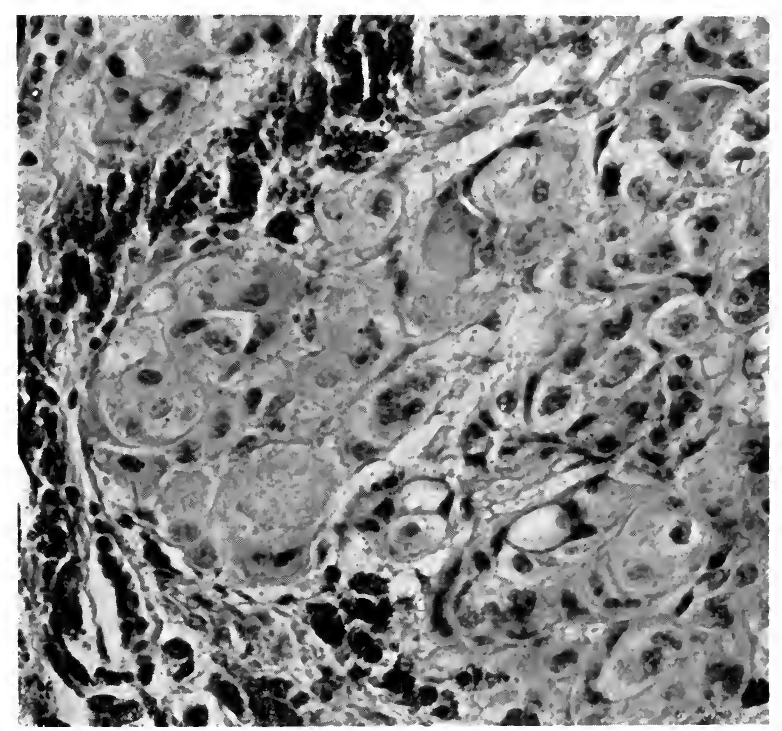

IG. 453. - Structure of metastatic melanoma in lymph-node. Note the limitation of pigment to invaded stroma cells.

ant cases. Not infrequently the pigment is limited to the large fusiform ells in the connective tissue about tumor nodules. In large areas of nonpigmented cells small foci may contain swollen, apparently hydropic, and reavily pigmented cells. The conditions of pigment formation appear to e very delicately balanced.

The Pigmentation of Melanoma.-The origin and nature of the pigment a melanoma have been the subjects of long and elaborate investigation urth (Lit.). Special interest attaches to this study since variations in this ubstance offer a very delicate indicator of the functional activity of the ells and a unique opportunity to trace the relation between a functional ctivity and the growth capacity of tumor-cells. In fact, it is not unlikely hat a complete elucidation of the pigment problems may reveal at the same me the secret of the excessive nutrition and overgrowth of this particular umor-cell. 
Morphology.-The earliest indications of pigment deposit in nevi appear in the form of fine yellowish granules in the swollen basal cells of the mole, and these appearances are nearly identical with those of normal pigmentation. As the epithelial proliferation progresses the foci of new cells continue to exhibit increasing pigmentation and single cells in the groups may develop excessive deposits of pigment, increase in size, and become elongated or multipolar. Multipolar chromatophores are usually present in pigmented moles, lying beneath the epidermis, passing between the basal cells and occasionally lying within the Malpighian layer. When absent it is permissible to assume that they have yielded their pigment to the epithelium and then disappeared.

With the further progress of the cell growth the pigment may appear in very large quantities, and in these cases evidences of intranuclear formation of pigment appear and it becomes scattered widely in neighboring cells and tissues. The intranuclear formation has been described by several authors (Ritter, Mertsching, Kromayer, Jarisch), and is frequently observed. A parallel formation of pigment from the chromidia of protozoa has been described by Hertwig. The nuclear origin led Bohn to assume that the pigment granule is a biological entity with nuclear properties. Rossle noted an excess of nucleolar material in pigmented tumor-cells and traced its escape into the cytoplasm in the form of pigment grains. He concluded that the production of pigment reduced the regenerative capacity of the cell. This relation of the nucleoli to pigment production has been elaborated by Staffel and by Meirowsky.

The dissemination of pigment to neighboring cells may be very extensive and in metastatic nodules it may be almost exclusively present in the stroma of invaded tissues while scanty or absent in the tumor-cells. This condition may be attributed to the phagocytosis of free pigment, to the assumption of pigment production by normal cells (Fuchs), or to absorption of extravasated blood. Pigment streaks leading from cutaneous tumors, patches of pigment in serous membranes, extensive deposits in lymphnodes and bone-marrow, and englobed granules in the vascular endothelium of many organs, melanemia, and melanuria mark the advanced stages of the process. Invaded organs may show much diffuse pigmentation. Katsurada observed diffuse pigmentation of cerebral capillaries without cerebral tumor. The extensive pigmentation of lymph-nodes led Gierke to conclude that the tissue cells must be induced to elaborate pigment by some agent derived from the tumor-cells. Berdez and Nencki have calculated that as much as $500 \mathrm{gr}$. of pigment may be produced in one case.

That the pigment of melanoma is the product of a special metabolic function of the cell is the conclusion of the great majority of observers. Virchow regarded the local deposits as the expression of a general dyscrasia. Many find a close parallel between the physiological formation of pigments, that occurring in Addison's and other metabolic diseases, and that of melanoma. Lubarsch concludes that melanomas, contrary to the rule with many tumors, contain no glycogen and that from a specific disturbance of metabolism they convert protein material into pigment. Nevertheless many facts have been interpreted in favor of the direct hematogenous origin of the pigment. Langhans has described the englobement of red cells by tumor-cells, but this event must be rare. The perivascular position of many chromatophores may be explained as an adaptation in the interests of nutrition and appears in a wide variety of non-pigmented tumors. The view that ameboid chromatophores carry the pigment to the nevus cells is in conflict with some definite histological appearances in early nevi. Their movement is probably in the opposite direction. 
The evidence of microchemical reactions favors the view that the pigment is essentially of metabolic origin, but that secondary pigmentation from the absorption of extravasated blood is of frequent occurrence. In the early mole pigment almost constantly fails to give Perls' reaction for free iron, but in more advanced cases this reaction may often be obtained (Vossius, Hamburger, Walter), although not always (Perls, Rindfleisch, Lubarsch, Ravenna). Melanin stains with some basic dyes, as polychrome methylene-blue, after which careful treatment by tannic acid leaves melanin colorless, hemosiderin greenish blue (Unna). Barlow finds that the pigment granules blacken with osmic acid. The comparative study of pigment production in lower animals points to its metabolic nature (Furth, Lit.).

Chemical studies have thrown much light on the nature and origin of tumor pigments, while leaving many questions still unsettled. The black pigment of the hair, choroid, melanotic tumors, and sepia pigment, known as melanin, usually contains $\mathrm{C}, \mathrm{H}, \mathrm{N}$ while $\mathrm{S}$ is by no means constant. The elements $\mathrm{C}, \mathrm{H}, \mathrm{N}$ occur in the general proportions of $\mathrm{I}, 5,5$ (Hoffmeister). The presence of $\mathrm{Fe}$ also appears to be variable and inconstant (Berdez, Nencki), so that one may conclude that melanin is essentially free from $\mathrm{S}$ and $\mathrm{Fe}$, but possesses a strong tendency to unite with these elements. It may be dissolved slowly by strong alkalies, and bleached by $\mathrm{H}_{2} \mathrm{O}_{2}$, chlorin, and by Whitefield's method, which includes laying sections some hours in potassium permanganate Io per cent., followed by dilute sulphurous acid.

Heated with strong alkali melanin from tumors yields indol, skatol, volatile fatty acids, and an ether-soluble acid which gives a dark blue color with ferric chlorid. The chief product is the melanic acid of Nencki. Under dry heat melanin gives off pyrrhol.

Thormahlen found that the urine of advanced cases of melanoma gives a characteristic reaction with sodium nitroprusside. When this reagent is added with $\mathrm{KOH}$ a deep reddish-violet color appears, which on acidification becomes blue. The reaction occurs in other diseases and is given by indol, and probably depends on the presence of the pyrrhol ring in the reacting substance (Eppinger). Melanin appears in the urine of advanced cases either constantly or periodically. Stiller observed it only during febrile periods, and Ganghofner noted an increase during regression of cutaneous tumors. The urine may be very dark on passage, but usually darkens only on standing. Hence the pigment is chiefly in the form of dissolved melanogen which oxidizes to melanin on exposure to the air. Various oxidizing agents hasten the reaction.

Many investigators have attempted to determine the construction of melanin and its relation to the protein molecule. Nencki concluded that the protein molecule contains a heterocyclic chromogenic radicle which is the source of both melanin and hemoglobin, and may be split off by pancreatic digestion. Hopkins and Cole isolated from pancreatic digestive products a crystalline tryptophan which they regarded as the mother substance of the chromogen. The demonstration that the ferment tyrosinase is concerned in the remarkable pigments of many insects and in the cuttlefish (Furth) led to the search for this ferment in melanoma. Gessard first demonstrated the presence of tyrosin and tyrosinase in melanoma of the horse, and he concluded that the pigment of tumors and that of the cuttlefish are produced in the same way by the action of tyrosinase on tyrosin and other aromatic compounds. Eppinger isolated from the urine of a case of melanoma of the liver a crystalline melanogen which he regards as a derivative of tryptophan. On dry distillation it yielded pyrrhol, treated with strong alkali it gave indol and skatol, blackened with oxidizing agents, and gave a blue color 
with sodium nitroprusside (Thormahlen's reaction). He concluded that the disturbance of metabolism in melanoma consists essentially in the inability to split the pyrrhol ring and thus destroy the excessive pigment produced by the tumor-cells. He would regard the excess of pigment and its products, especially indol and skatol, as the cause of the overgrowth of the cells. Stoeber and Wacker observed that indol and skatol cause marked proliferation of epithelial cells. In a melanoma, regarded by Orth as originating in the adrenal, Neuberg demonstrated a ferment which failed to act on tyrosin, but split adrenalin. Alsberg in a melanoma of liver found a ferment which acted on pyrocatechin and hydrochinon, but not on tyrosin. In all of these fields, as Furth remarks, the various hypotheses require further substantiation.

$\mathrm{O}$. Adler by treating tryptophan or tyrosin with strong $\mathrm{H}_{2} \mathrm{SO}_{4}$ secures black pigments which he regards as very similar to those occurring in melanoma. He devised a color test which seemed to be specific of melanoma.

That the pigment or its derivatives exert a toxic action in the disease, while probable, is without definite proof. Rosenfeld increased the excretion of pigment by administering iodid of potash, without causing any toxic symptoms. Most of the pigments artificially produced or extracted from tumors or urine are toxic for animals, but it is difficult to trace these effects in the cachexia of the disease. The antigenic properties of choroid pigments have been studied by Elschnig.

Orbital Melanoma.-Pigmented nevi and melanomas occur in the conjunctiva, iris, choroid, and in the sheath of the optic nerve and surrounding tissue. In each of these situations they present characteristic features of origin and course, and variations in the aspect of the problem of histogenesis, all of which have been very fully studied by ophthalmopathologists (Wintersteiner, Lit.).

In the eye there occur processes which correspond to the cutaneous nevus and to the malignant tumors derived from them, as well as pigment-free tumors which are difficult to separate from the pigmented forms.

In the conjunctiva pigment spots appear at birth or later and may remain unchanged throughout life or become the source of malignant tumors. Parsons describes them as of epithelial origin reproducing the picture of cutaneous pigmented moles. There is epithelial hyperplasia, shallow extensions into the deeper tissues, and appearance of chromatophores. The lesions may gradually extend over conjunctiva and eyelid and when incompletely removed may be followed by the usual wide-spread dissemination through blood-vessels. I have followed one congenital case for to years which gradually extended, was twice excised, and eventually became disseminated.

In the iris Fuchs and Parsons describe brownish nodules which appear on the pupillary margin, in the horse nearly constantly, and are said to be derived from the pigmented epithelium. They also describe brownish tumors of the iris which grow into the anterior chamber, and are derived from the chromatophores of the iris. Both begin as slow benign tumors which may become malignant.

The ciliary body is a frequent seat of melanomas, of which Groenouw has collected 50 cases. The tumor first appears as a protuberance on the ciliary body and extends in various characteristic forms as a flat tumor involving a segment of the ciliary body and contiguous choroid, as a projecting mass displacing the lens, or as a diffuse growth of entire ciliary body, the so-called ring sarcoma of Evetsky. It may infiltrate the iris in various ways (Verhoeff), extend over the choroid throughout most of the eyeball, or appear as discontinuous multiple foci in the choroid (Meyerhoff), 
or perforate the cornea along the ciliary vessels. The variety of histological structure is considerable, spindle-, rounded, and polyhedral cells appearing diffusely or in alveoli, or in perithelial form, while pigment may be scanty or absent (Rogman). An alveolar structure with pronounced epithelial character is described by Groenouw, and appears in one of my cases. In a very early case Derby claims to have traced the origin to the pigmented cells of the intermuscular connective tissue.

The choroid is the most frequent site of ocular melanoma, yet the disease is relatively rare, occurring in 0.58 per cent. of ophthalmic patients (Wintersteiner). About one-third of all melanomas originate in the choroid (Johnston). The earliest stages of the growth have seldom been observed. Fuchs encountered several isolated elongated pigmented spots near the macula. On section these consisted of groups of proliferating cells between the large vessels of the choroid and apparently from cells lining the elastic lamella.

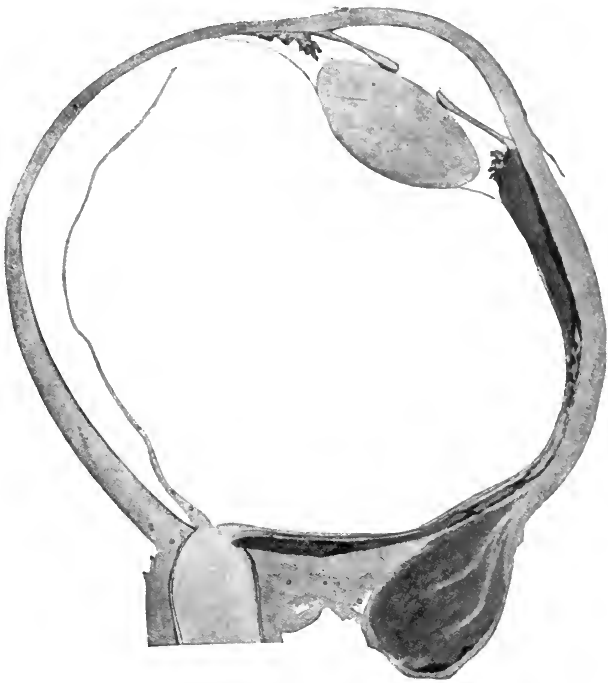

IG. 454.-Flat infiltrating melanoma of choroid, with epibulbar nodule. (A. Knapp.)

he pigmented epithelium of retina and the choriocapillaris were normal. ery similar tumors are described by Wintersteiner and by Purtscher.

In more developed stages the tumor takes various forms. Usually a lobular mass forms in the choroid, which displaces the basal lamella (Bauch) ad retina into the vitreous. Perforation of the basal lamella is followed by stension along the retina. A flat growth long confined by the basal memane, on perforating this structure, grows more rapidly, producing a globular Imor connected by a narrow pedicle with the flat base. Rarely the flat owth in choroid is maintained until almost the entire eyeball is encircled by diffuse tumor (Evetsky). About the entrance of the optic nerve the tumor ay encircle the nerve, in which position early extension along the nerve must feared (Fehr). The intrabulbar extensions are usually by continuity. he retina is detached, infiltrated, split up by layers of tumor-cells, and deroyed. Reaching the anterior chamber free cells or pigment may become ssened and deposited on the iris or cornea or choke the canal of Schlemm amocki, Michel). The ciliary body and iris are invaded and the ciliary 
vessels may be penetrated by tumor-cells (Gutman), thus opening the way to intravascular dissemination. The sclerotic may be perforated by the same channels or along the optic nerve, producing a retrobulbar tumor which extends even to the chiasm. Focal or extensive necrosis of the tumor may result from occlusion of blood-vessels, or complete phthisis bulbi may result. In such cases extensive arteritis has usually been found, which Evetsky refers to toxic products derived from the disintegrating tumor. The complicating sympathetic ophthalmia has been referred to the same factor.

Fuchs divides the natural history of the tumor into four stages, including the periods before and after the onset of glaucoma, the stage of extra-ocular growth, and that of general dissemination. Excision in the first stage has

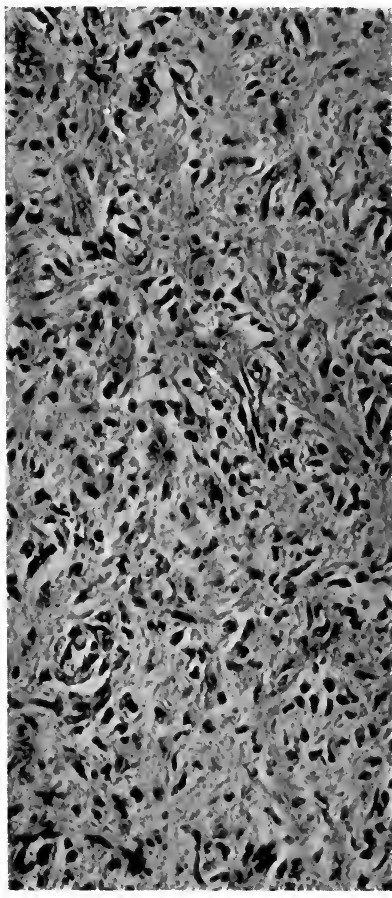

FIG. 455.-Metastatic melanoma of choroid. Spindle tumorcells growing a mong atrophic liver cords. pigment, not yielding the hemoside reaction and with cells chiefly of spindle form, belongs to the primary pigmentation. Yet since hematogenous pigment may be diffuse and may lose the reaction for free iron, and since hemorrhage may occur in true melanoma, these distinctions cannot be applied rigidly. They may perhaps serve to eliminate certain pigmented tumors which have no relation to melanoma.

The arrangement of the tumor-cells varies. The majority of the tumors in early stages appear superficially as large spindle-cell sarcomas, but on careful study an alveolar arrangement of large polyhedral cells may often be detected in small tumors. A concentric arrangement about vessels has 
led many to employ the term "perithelioma," and an extensive growth of bloodvessels has received the designation "angiosarcoma." Each of these structures has been called by some endothelioma. None of them offers any definite indication of histogenesis. After penetrating the retina and after passing the eyeball the tumors grow more rapidly, the cells enlarge, pigment is less abundant, and the structure soon duplicates that of melanoma from other sources.

In the etiology of ocular melanoma hereditary influence rarely appears, but Silcock observed the disease in three generations. A frequent traumatic origin has been claimed by Leber and Krahnstover, but on uncertain data. Sex is without influence. The age incidence is chiefly between 40 to 60 years. but a few cases have occurred in infants (Wintersteiner).

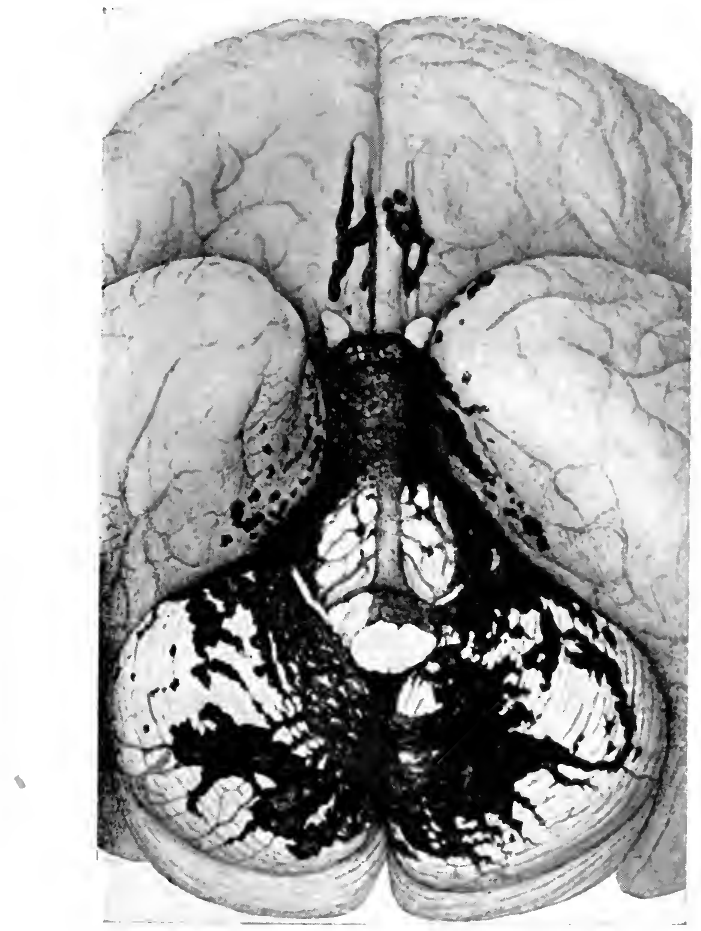

Fig. 456.-Primary melanomatosis of cerebral pia. (After Schopper, Frankf., Z. P., I3.)

Melanoma of Brain and Cord.- In a series of cases which now number at east 9, primary melanoma of brain or cord has been observed (Boit, Lit.). Beginning with the normal chrornatophores which are sometimes found along he vessels of pia and even in the brain tissue, a considerable increase of these -ells, producing definite pigment spots and streaks, has repeatedly been bserved (Obertsteiner, Rokitansky). In a notable case of Grahl's an extenive skin nevus of the bathing trunk type was associated with profuse pignent spots of pia mater, and Oberndorfer saw the same extensive cutaneous evus with symmetrical pigment spots and tumors of cerebral pia. Thorel bserved diffuse pigmentation of the pia of brain and upper cord, which 
merged into a tumor process only at the cauda. Some of the tumors have been multiple (Virchow, Stoerk), others single (Minelli). Boit's tumor arose from the outer side of the spinal dura, Hirschberg's developed in the substance of the lumbar cord, and Minelli's in the centrum ovale.

Although intracranial metastases of cutaneous or orbital melanoma are frequent, in several of the above cases a careful autopsy eliminated the possibility of a primary tumor elsewhere. There is, therefore, no doubt that primary melanoma of the central nervous system occurs and that it arises from the pigmented cells often found along the cerebral vessels.

The origin of the cerebral chromatophores appears more difficult to establish than in any other situation. The structure of the simple pigment spots and areas has suggested to many that these cells are specialized perithelial cells of mesodermal origin, and in this field Ribbert's views find their best support. The structure of the tumors is that of a diffuse growth of elongated or polyhedral heavily pigmented cells without intervening fibrils, often sheathing blood-vessels as in perithelioma.

In a remarkable case described by Berblinger, in an infant of 9 months, multiple melanomas of the skin and neurofibromatosis were associated with a melanotic tumor of the gyrus hippocampus, multiple pigmented spots in various portions of the brain, glioma of pons, diffuse perithelial sarcoma of cerebrospinal pia with secondary deposits of pigment and a true accessory adrenal. The author's interpretation of the diffuse sarcoma as an independent process may be questioned, since many observers have regarded such perithelial growths as derived from perivascular chromatophores. The chromatophores were very widely distributed throughout the brain, sheathing the larger vessels, lining the endothelium of capillaries, and forming rosettes resembling those of neuro-epithelium. The case presents a universal disease of the fetal pigment-producing function, and involving disturbances in skin, central nervous system, and even the adrenal gland.

Melanoma of Intestine.-Diffuse melanosis of the colon is not an uncommon condition in elderly subjects and is generally referred to the elaboration of blood extravasated in the mucosa. I have observed it in pernicious anemia as well as in many other conditions. Its extent recalls the diffuse pigmentation by chromatophores in the intestinal tract of lower vertebrates. Pigmented tumors of the rectum are relatively common in horses (Eiselt).

Melanoma of the intestine, occurring almost exclusively in the rectum, was recognized by Virchow, has been described in isolated reports by various observers, as Paneth, Dietrich, Tuffier, Wiener, while recently Chalier and Bonnet have collected and fully analyzed 64 cases. They form about 2 to 3 per cent. of all melanomas.

The tumors occur chiefly in adults, but I have examined a cecal melanoma from a child of 8 years. They arise from the anal orifice or from the rectal ampulla, and produce circumscribed nodules or bulky masses as large as a child's head. A polypoid form is very common, but Meurier found the rectum encased in a cylindrical mass. I found the ileocecal orifice surrounded by a lobulated tumor. The growth originates and expands within the submucosa so that the mass is movable and only later do ulceration and fixation supervene. Metastases in pelvic nodes, liver, and other organs are extremely common. Only 2 of 64 cases were reported free after operation.

The structure presents the usual appearance of melanoma. Chalier and Bonnet emphasize the resemblance to epidermoid carcinoma and they derived their tumor from the anal epidermis. Yet most tumors occupy the ampulla and some lie 5 to $6 \mathrm{~cm}$. above the anus, necessitating an origin, if epidermal, from a misplaced tissue rest. Most authors have been unable to 
trace the origin, so that the observations on intestinal melanoma do not strengthen the epithelial theory of the nature of chromatophoromas.

Melanoma of Uncertain Origin.-In a series of fatal melanomas careful search at autopsy has failed to demonstrate the origin of the tumor. In some of these the presence of a large tumor of the adrenal has suggested a primary melanoma of this organ. In Orth's case the structure of some metastases resembled the adrenal cortex, and Neuberg, finding in them a ferment acting on adrenalin with the production of a black pigment, suggested that disturbance of adrenal function might be concerned in the pigmentation of melanoma. In Davidsohn's case there were many tumors of brain and colon and a large growth in the adrenal. Reiman found a large growth in adrenal with small tumors elsewhere. Wieting and Hamdi described a supposed primary melanoma of gall-bladder. In Johnston's case Elser was quite unable to fix any point of origin for a massive hepatic tumor. I have studied a large melanoma of the spleen in which the structure presented very large polyhedral cells in perithelial and alveolar arrangement. There were no other tumors in the body.

Histogenesis of Melanoma.-The extant theories of the origin of melanoma are as follows:

(I) Exclusively from mesoblastic chromatophores (Ribbert).

(2) Exclusively from epithelial cells and epithelial chromatophores (Post, Wieting and Hamdi, Favera).

(3) From epithelial nevus cells in the skin, and mesoblastic chromatophores in choroid and meninges.

(4) From endothelial cells of blood- and lymph-vessels, or of nerve-trunks.

It is of first importance to determine the origin and function of chromatophores before considering their probable relation to tumors.

Origin and Function of Chromatophores.-A competent opinion regarding the nature of chromatophores must be based on a knowledge of the color function in the whole animal kingdom. This comprehensive point of view will inevitably influence the interpretation of phenomena observed in man. The comparative physiology and morphology of the color function, as presented by R. Fuchs, strongly supports the view that pigment production belongs essentially to a specific mesoblastic cell, the chromatophore. Only in the highly pigmented vertebrates, as the frogs and reptiles, in which the ova are pigmented and pigment is required in unusual quantities, is there any reasonable doubt of the mesoblastic origin of chromatophores and of the exclusive relation of this cell to pigment production and transport. In these animals, however, there is some evidence to show that pigment production may overflow to epithelial cells. Yet the conditions here are not favorable for a ready solution of the problem of pigment production by epithelium. Even in the frog Ehrmann concluded that pigmentation is controlled exclusively by the wandering chromatophores. In the higher vertebrates the evidence swings distinctly in favor of the mesoblastic chromatophore.

In fundulous embryos, 48 hours old without a circulation, Stockard has watched with the binocular microscope the first appearance of pigment in large mesoblastic wandering cells of such huge size that they can be traced with great certainty. These cells have a notable tendency to gather along blood-vessels and they can be readily followed in the act of depositing pigment in the ectoderm. In cephalopods the migratory movements and changes in form of the chromatophores are facilitated by muscular processes. Many of them are provided with rich connection with sympathetic nerve fibers (Hoffmann). In some species the chromatophores exhibit many properties of protozoa (Chun). In Amphioxus chromatophores exist only in the 
central nervous system, and in crustaceans intestinal chromatophores are the rule. In man the prominence of the chromatophore was demonstrated by Kolliker, and has been maintained by an unbroken line of subsequent observers. These observations establish the mesoblastic origin of the primitive pigment cells, and they are especially significant in emphasizing certain characters which reappear in human melanoma, viz., extreme motility, peculiar relations to blood-vessels, and distribution throughout central nervous system, choroid, and intestinal tract.

This extensive chapter of natural history must unquestionably incline the student of tumors to regard all manifestations of melanoma in terms of the chromatophore. It forms a strong theoretical basis for Ribbert's view of the origin of melanoma exclusively from chromatophores.

On the other hand, there has always been a considerable number of observers, occupied chiefly with the direct study of pigment processes in man, who have maintained the epithelial origin of the pigment. Post attributed pigment production in mammals almost exclusively to the epithelium. Pigmented connective-tissue cells he regarded as carrying off excess pigment from the epithelium. Caspary and Kaposi maintained the epithelial origin of pigments in skin and hair. It is generally agreed that chromatophores cannot be traced in the hair follicles. The great majority of close histological studies of nevi and early melanoma have led to the conclusion that epidermal pigment arises in the epithelial cells (Abesser, Wieting and Hamdi, Favera). It must be admitted that the course of pigment production in man is not as clearly defined as in lower mammals and that the doctrine of specific chromatophores may not be rigidly applicable to man.

Results of the Studies of Nevi.-The minute studies of nevi by many observers have told strongly in favor of the epithelial origin of nevus cells and their pigmented derivatives. Unna's derivation of the resting nevus cell from the rete pegs has been verified in detail by Judalewitsch, Favera, and many others, who have traced every stage of the passage of cell groups from the Malpighian layer into the derma, where they lose their fibrils and become converted into the glassy rounded nevus cells. Kromayer has further traced these cells into fusiform cells with intercellular fibrils, a process which he interprets as the transformation of basal epithelium into fibroblastic elements. These intercellular fibrils Ribbert regards as an indication of a mesoblastic origin of nevus cells which he identifies with depigmented chromatophores. The sharp circumscription of the pigmented cells in the Malpighian layer he interprets as the result of inwandering of superfluous chromatophores.

I believe that the histological data do not permit a final decision between these contending hypotheses. The histological data appear to me strongly but not decisively in favor of the epithelial origin, while the theoretical considerations are all against the epithelial theory.

Subordinating the evidence drawn from comparative morphology, the following conception of the origin of moles and cutaneous melanoma might be suggested: The deposit of excessive pigment by mesoblastic chromatophores in certain cells of the fetal epidermis excites these cells to proliferation and causes them to assume the function of pigment production, thus producing the early congenital nevus. This process is favored by excess of blood-vessels in the derma, and possibly by some abnormality in the innervation of the part. Chromatophores are attracted by blood-vessels and probably somewhat controlled by the nervous system, and some nevi are associated with capillary angioma, and some appear to be in relation to peripheral nerve-fibers.

The congenital nevus tends to regress or remain quiescent, the cells de- 
scending into the derma and losing their pigment as in the adult nevus. Through irritation of simple traumatic, or nervous, or more complex origin the quiescent nevus cells may be incited to overgrowth and renewed pigment production, developing a malignant localized tumor. The overlying epidermis soon participates in this tumor process, developing pigment, the presence of which still further stimulates growth and often the extension of the tumor process along the basal cells of previously normal rete pegs. From both these sources actively proliferating epithelial cells, bearing pigment and devoid of fibrils, become disseminated by lymph- or blood-vessels. In this scheme the part assigned to chromatophores, while initial and essential, is subordinate. Histological appearances do not indicate that these cells are more than camp followers during the advance of the tumor process. On the other hand, assuming that the nevus cell is a mesoblastic chromatophore, the tumor process becomes simply a neoplastic overgrowth of a specific cell, while the appearances suggesting participation of rete pegs and hair follicles must signify invasion of these structures by tumor-cells. In this dilemma one may turn to evidence presented by melanoma in other situations.

In the eye much the same obscure histological pictures as in the skin present themselves for interpretation. Although Wieting and Hamdi claim that embryological studies indicate the epithelial origin of all ocular pigments, yet the mesoblastic origin of the choroidal chromatophores must be conceded. The origin of the tumors from these cells is somewhat less certain. Leber found the retinal epithelium in active proliferation and from them he derived the uveal melanoma. Very early melanomas have, however, been traced by Fuchs, Wintersteiner, and Purtscher, to the pigmented cells of the choroid, while the retinal epithelium was intact. In the iris and ciliary body exactly the same relations between pigment cells and epithelium are encountered as in the skin, and here many authors have derived their tumors from the epithelium (La Grange, Bard, Griffith, Mitrolsky, Weinbaum, Kerschbaumer). In very early artificially depigmented ciliary melanomas I find the usual indications of the splitting off of heavily pigmented basal epithelial cells.

In primary ocular melanoma spindle-cells are more prominent than with cutaneous tumors, but in metastatic growths the structure of each type shows identical variations. The occurrence of melanoma in the sheath of the optic nerve is difficult to reconcile with an epithelial origin, but is quite consistent with the distribution of chromatophores.

The melanomas of the central nervous system arise from the wandering chromatophores which are scattered over the meninges, about the vessels, along nerve sheaths, and in the subependymal tissue. That these cells are homologues of the mesoblastic chromatophores appearing abundantly in the meninges of lower animals there can be no reasonable doubt. The association of these tumors with extensive cutaneous nevi strongly indicates that in both situations the process is identical in nature. While the melanomas of the nervous system resemble sarcoma or perithelioma in structure, these features are frequently seen in cutaneous tumors and their metastases.

The series of intestinal melanomas may safely be referred to groups of chromatophores located in the submucous tissue. Their presence recalls the great abundance of intestinal chromatophores in crustaceans.

For the rare cases of melanoma occurring in the internal organs as in the spleen, an origin from aberrant chromatophores is the most reasonable assumption. Pigmented adrenal tumors belong in a special group which need not be considered in this connection.

Thus the review of the different forms of melanoma reveals a direct con- 
flict between the histological data derived from cutaneous melanomas on the one hand, and on the other the occurrence of melanoma in several situations where an epithelial origin is a strained hypothesis, as well as the formidable body of evidence presented by comparative morphology. If the epithelial derivation of nevus cells were not so strongly supported by observation and argument there would no longer be any reason for delay in accepting Ribbert's theory, to which the author inclines. Until more light is thrown on the nature of the supposed epithelial changes in congenital nevi, a final decision may be withheld.

The endothelial origin of the nevus has received little new support in recent years. The general behavior of malignant tumors arising from nevi is strongly against their endothelial nature. No other known endothelioma approaches melanoma in its cell forms, peculiar course, and malignancy. The endothelial theory can best be pursued in old quiescent nevi, where it is quite impossible to reach any conclusion regarding the origin of the cells which have lost their original form, while their relations are disturbed by fibrosis and atrophy. From the study of sections in which others have claimed to find lymph- and blood-vessels terminating in nests of nevus cells, I have been impressed with the unsuitable nature of the material for such analysis. The cell columns of epithelial tumors have been found canalized and filled with blood. Where angioma is associated with nevi I find the two processes invariably distinct.

Adrenal Melanoma.- Small brownish benign cortical tumors of the adrenal are described by Lucksch. They occur in adults and are composed of cortical cells containing pigment resembling melanin, and they seem to form a probable source of the malignant pigmented tumors of the adrenal. Not a few of the typical adrenal carcinomas contain areas of pigmented cells of the adrenal type.

Primary bilateral melanotic malignant tumors of the adrenal are described by Davidsohn, Goldzieher, Tuczek, and MacLachlan. The tumors occurred in adults, were of moderate size, and sometimes produced extensive metastases, but primary sources in skin or choroid were missing.

The origin of the tumors it is difficult to determine. Davidsohn thought he recognized the zona fasciculata in a mesenteric metastasis, claims to have demonstrated adrenalin in this tissue, and derives the tumor from pigmented adrenal cortex cells. Tuczek found the pigment a true melanin and not a lipochrome, as is found in the adrenal cortex. He derived the tumor from the pigmented ganglion cells of the medulla, which contain melanin. MacLachlan concluded that the adrenal melanoma arises from the wandering chromatophores which are said to exist in the loose tissue about the adrenal. Yet the tumors seem to involve the whole organ, which long preserves its form. 


\section{CHAPTER XLV}

\section{TUMORS OF THE THYROID}

Epithelial overgrowth in the thyroid occurs in three main conditions:

(I) Simple goiter, and the benign tumors or tumor-like processes associated with it.

(2) Graves' disease.

(3) True tumors.

These conditions are not sharply separated from each other, simple goiter may assume the characters of the exophthalmic type, and true tumors may develop in the course of either form.

The correct interpretation of hyperplastic processes in the thyroid demands special standards dependent upon the development, structure, and functions of this gland, and not applicable elsewhere.

In the development of the organ there is progressive centrifugal growth of separate new vesicles from central embryonal foci which produce independent lobules. Hence the central portions of lobules contain the older vesicles, a relation which is specially apparent in certain fetal adenomas. The independence of the vesicles and lobules is most clearly shown in the wide dissemination of thyroid acini in the fish (Gudernatsch), and of accessory thyroids in man. More striking evidence of unusual capacity of independent growth is seen in the cases of structurally benign but metastasizing goiter. Dissociated thyroid alveoli tend to grow rather than to regress, a fact demonstrated more recently by the survival of thyroid tissue in transplants and cultures.

A second important principle governing pathological processes in the thyroid is the remarkable response to functional stimulus, and the wide variations in the functional activity and structure of the gland in different physiological states. Marked cellular hyperplasia may readily be induced by changes in diet and by drugs, and correspondingly reduced. The excessive hyperplasia of severe Graves' disease, which is probably the result of functional stimulus, equals that of some malignant tumors, but may promptly regress under suitable treatment. No other gland, and only the lymphoid tissues, exhibit such adaptations.

A third dominating factor is found in the structure of the gland. The vascularity is enormous and subject to wide variations, owing to the great capacity of the venous sinuses. Capillaries encircle the alveoli and the bloodcurrent is separated from the epithelium only by an endothelial barrier. A basement membrane is absent in the thyroid gland, and the mobility of the epithelium is thereby increased. The colloid secretion of the organ varies rapidly in quantity and in composition and exerts a marked influence on the structure of the diseased organ.

Simple Goiter.-Under this term are included self-limiting but often bulky enlargements of the thyroid of congenital or spontaneous origin and usually unattended by other than pressure symptoms. Two chief forms are observed: (I) colloid, and (2) parenchymatous.

(I) Colloid goiter is a very common disease of sporadic, endemic, and even epidemic occurrence, and is commonly referred to some influence residing in 
drinking-water. Reference to the extensive discussion of its etiology may here be omitted (Bircher, Lit.).

The enlarged gland reaches considerable, sometimes enormous, dimensions, the growth affecting single nodules, or one lobe, or the isthmus, or the

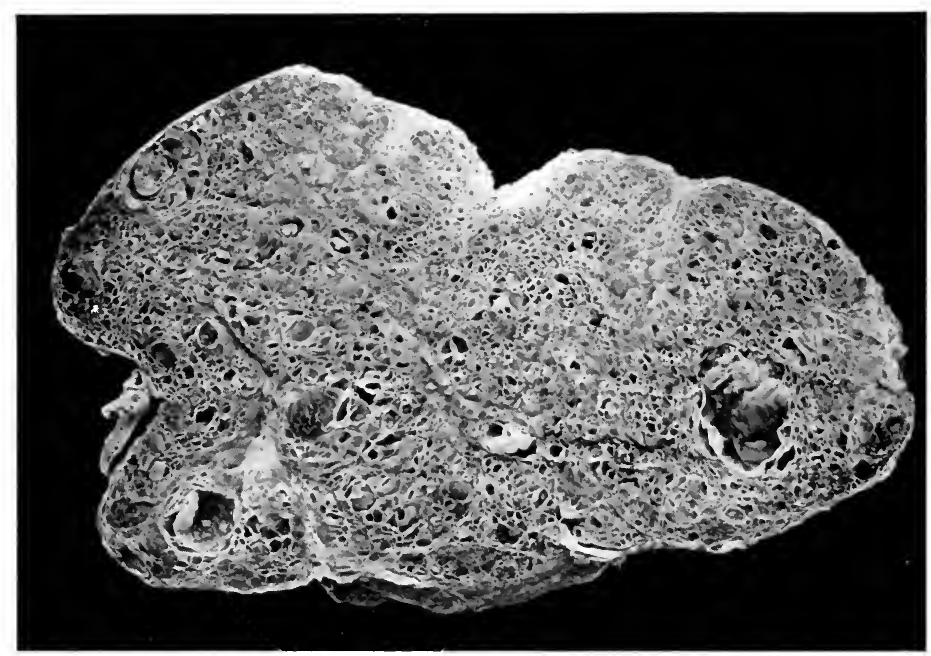

FIG. 457.-Cross-section through a simple goiter.

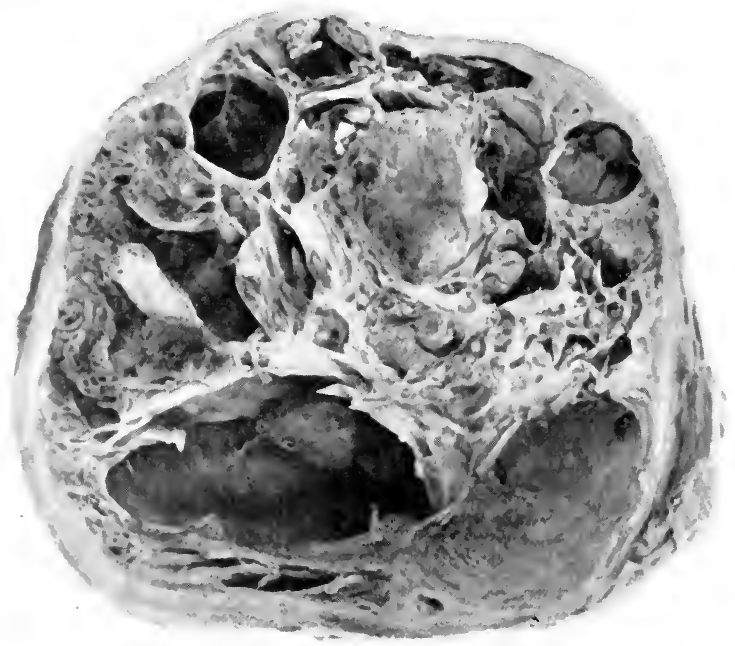

FIr. 458.-Cystic adenoma of thyroid. A form of encapsulated cystic goiter.

entire gland, or accessory glands in many situations. The chief accessory goiters are substernal, intratracheal, retropharyngeal and esophageal, and lingual (Payr, Martina, Lit., Meerwein). 
On section the gland presents distended spaces filled with colloid to which the enlargement is due, and which yields a translucent, shiny, brownish, honeycomb appearance. Cysts of all sizes may form by fusion of adjoining spaces, liquefaction, and hemorrhage (cystic goiter). The appearance is greatly altered by hemorrhage, pigmentation, fatty degeneration, and by late fibrosis and calcification. Smooth shiny areas may be produced by hyaline changes in the stroma. Amyloid deposits in stroma and bloodvessels may be very prominent (v. Eiselberg). Bone deposits are often observed in late stages (Sehrt, Heinziger, Pfister). The blood-vessels may be overdeveloped, at times so much that the gland pulsates.

The structure of colloid goiter presents chiefly normal alveoli distended with colloid. The lining cells are flattened, the walls of adjoining alveoli

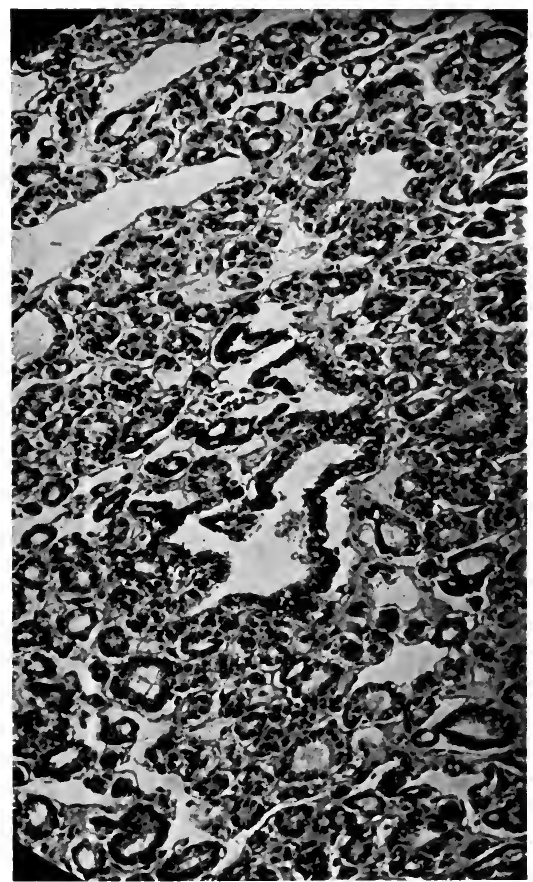

FIG. 459.-Benign cystic adenoma of thyroid. Tissue from wall of cyst.

melt away and large spaces and cysts form. New alveoli are commonly observed in small foci in the stroma and abundantly beneath the capsule. Wolfler finds an increase of alveoli in all cases of colloid goiter and hence speaks of the process as adenoma gelatinosum.

The colloid is of normal appearance, or more often soft or fluid. It may infiltrate the stroma, collecting in lymphatics, vessels, and tissue spaces, often in great abundance. Small capillary hemorrhages or bulky extravasations are common, producing cysts and leaving pigment. Into these hemorrhagic areas new alveoli sprout, and gradually fill with colloid as the blood is absorbed. Fatty and hyaline degeneration, calcification, and fibrosis regularly mark the advanced stages. 
Notwithstanding the great size of colloid goiter the process lacks any definite neoplastic character, and consists in a functional hyperplasia, retention of colloid, simple regenerative growth, and regressive and inflammatory changes. Yet true tumors may arise in its course, as papillary adenomas growing out into cysts, and the low-grade interacinous adenomas. Malignant tumors if they arise in colloid goiter usually develop early, but the great majority of colloid strumas maintain their individual character for many years. A few cases develop Graves' symptoms and structure.

Interacinous Adenoma.- In many cases of colloid goiter the overgrowth of epithelium reaches considerable proportions, many alveoli are lined by clusters of small round or cubical cells, many new alveoli form between the older acini and the condition suggests a low grade of neoplastic process. Such areas may occur in circumscribed portions of the gland and recur after extirpation. In all cases they are of slow growth and after reaching variable

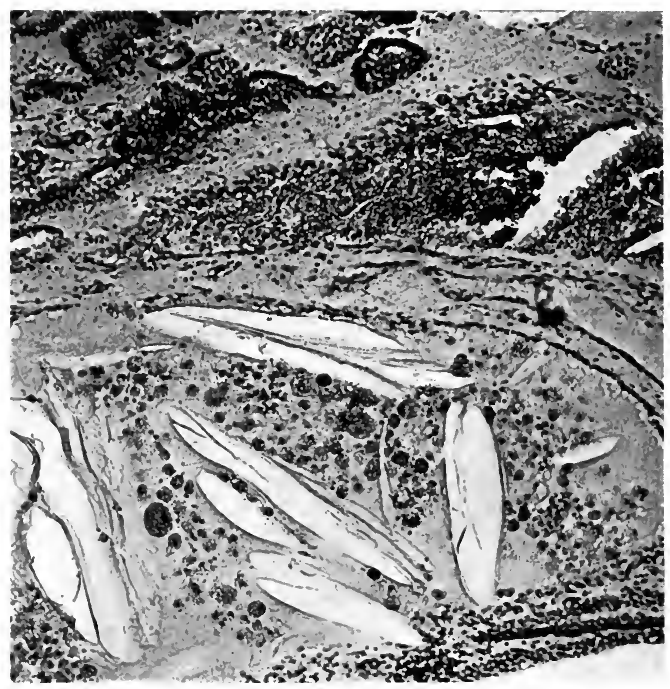

FIG. 460.- - Wall of a cystic adenoma of thyroid. Active growth of alveoli with very small cells, and formation of cholesterin crystals in cyst contents.

dimensions they remain stationary. The new alveoli thus formed tend to develop into normal colloid-holding vesicles. Wolfler derives these focal and diffuse overgrowths from the same superfluous cell groups which give origin to the fetal adenoma. Interacinous adenoma is distinguished from fetal adenoma chiefly by the more adult character of the cells, and by its occurrence in colloid goiters.

(2) Parenchymatous goiter is less frequent, but of wider significance than the colloid form. It is congenital or acquired.

(a) Congenital Goiter.- In the newborn infant the thyroid may be much enlarged, pressing upon the trachea and causing death by asphyxia. In many instances there has been a history of chronic goiter or idiocy in the parents (Demme). It is rather common in goitrous regions (Escherich). The infants are often stillborn or survive only a few hours, but occasionally there is a gradual regression and the goiter disappears after some years (Kamann). 
Wolfler divides the congenital strumas into parenchymatous, angiomatous, cystic, and adenomatous. The anatomical condition is usually a diffuse parenchymatous hypertrophy, with overgrowth of well-formed alveoli lined with cells somewhat larger than in the normal fetus, and without any collection of colloid (Demme, Fabre, Therenot). In a case of the writer's this condition was remarkably uniform. Hewetson reports a very large goiter in which parenchymatous hypertrophy with desquamation of cells was combined with extensive development of blood-vessels. Cysts and nodular hypertrophy are usually wanting. Simple distention of the blood-vessels in face presentation may give a marked swelling of the fetal thyroid which disappears in a few hours or days. The existence of a true angiomatous condition is doubtful. Berry describes congenital circumscribed adenoma and sarcoma, and Zahn a case of congenital osteochondrosarcoma. In a few cases the congenital parenchymatous goiter has given origin to a very early carcinoma (O. Ehrhardt, Lit.).

(b) Acquired parenchymatous goiter is less frequent than the colloid form, from which it is distinguished by the prominence of new alveoli and the rela-

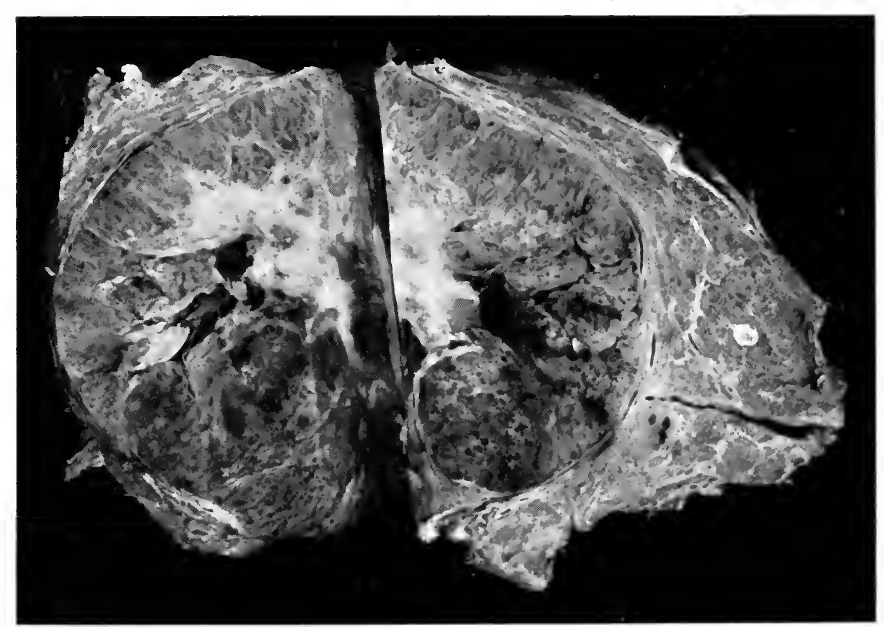

FIG. 461.-Circumscribed follicular adenoma of thyroid.

tive diminution of colloid. The new growth of alveoli is diffuse or nodular. The diffuse type gives a uniform or focal distribution of alveoli containing little or no colloid, intermingled with alveoli filled or distended with colloid. The structure, therefore, may approach that of colloid goiter. The nodular masses usually exhibit the structure of Wolfler's fetal adenoma.

Fetal Adenoma.-This condition appears in the form of small or large opaque nodules in one or both lobes. They develop slowly, reach considerable dimensions, form nodular projections of the capsule, but eventually become stationary. The larger tumors may reach the size of the fist, replacing nearly the entire lobe. They are often the seat of hemorrhage which alters the gross appearance and structure. The structure presents numerous small alveoli lined by low cubical epithelium, and without colloid. Or the cells are small and round with densely staining nuclei, while a definite lumen is wanting. The growth is centrifugal, so that the center of the nodules often show alveoli with colloid and abundance of stroma, while on the periphery 
there are many small closely packed cell groups in scanty stroma. The embryonal character of the cells is quite prominent, and when they become closely packed the structure resembles sarcoma. When interstitial hemorrhage occurs the structure is disordered. The alveoli sprout with renewed energy into the blood mass, which undergoes hyaline transformation and absorption, or it becomes mucoid and gradually replaced by new growth of fetal alveoli (secondary adenoma). Small cysts may appear, into which the epithelium grows in low papillary form (papillary fetal adenoma). The circulation in the fetal adenoma is of the lacunar type with many pouched blood sinuses, a structure which favors hemorrhage on moderate congestion.

Wolfler traces the fetal adenoma to somewhat ill-defined groups of superfluous embryonal thyroid epithelium which he finds throughout the

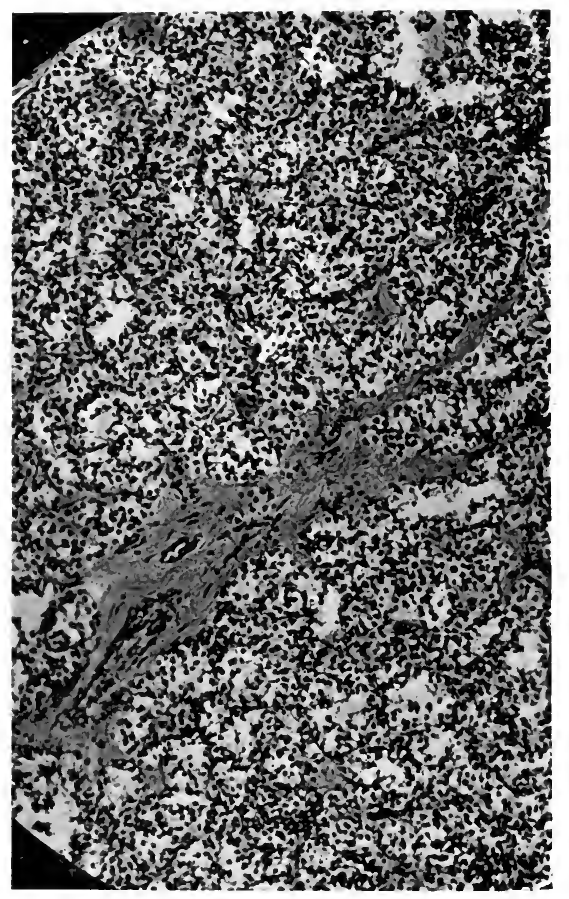

FIG. 462.-Follicular adenoma of thyroid, with very small cells. Benign course.

organ. The existence of these cell groups is not easily demonstrated. The diffuse colloid goiter he refers to the same sources, more diffusely distributed, and not to proliferation of adult follicles. In this latter view he is not confirmed by the majority of observers. The fetal adenoma may give origin to adenocarcinoma, carcinoma and, as I believe, to many so-called sarcomas. It arises in early life, and appears as a definite tumor usually before the twentieth year. Both colloid and parenchymatous goiter may develop Graves' symptoms, in which case the structure assumes the peculiarities of exophthalmic goiter.

(c) Exophthalmic Goiter.-The relation of the symptoms of Graves' disease to the structure of the thyroid gland is variable, complex, and in many respects obscure. These symptoms in mild form are rather frequently observed in 
the course of simple colloid goiter, and less frequently a colloid goiter precedes the development of progressive Graves' disease (Moebius). In the course of relatively benign, as well as with certain malignant tumors, Graves' symptoms may also appear (Koeber, Ehrhardt).

In the great majority of cases Graves' disease is associated with a series of structural changes in the thyroid which signifies functional overgrowth. While in the early stages these changes may occasionally be duplicated in other conditions, yet in nearly all stages the changes are peculiar and, taken as a whole, the series is distinctly characteristic of the disease (Greenfield, Ewing).

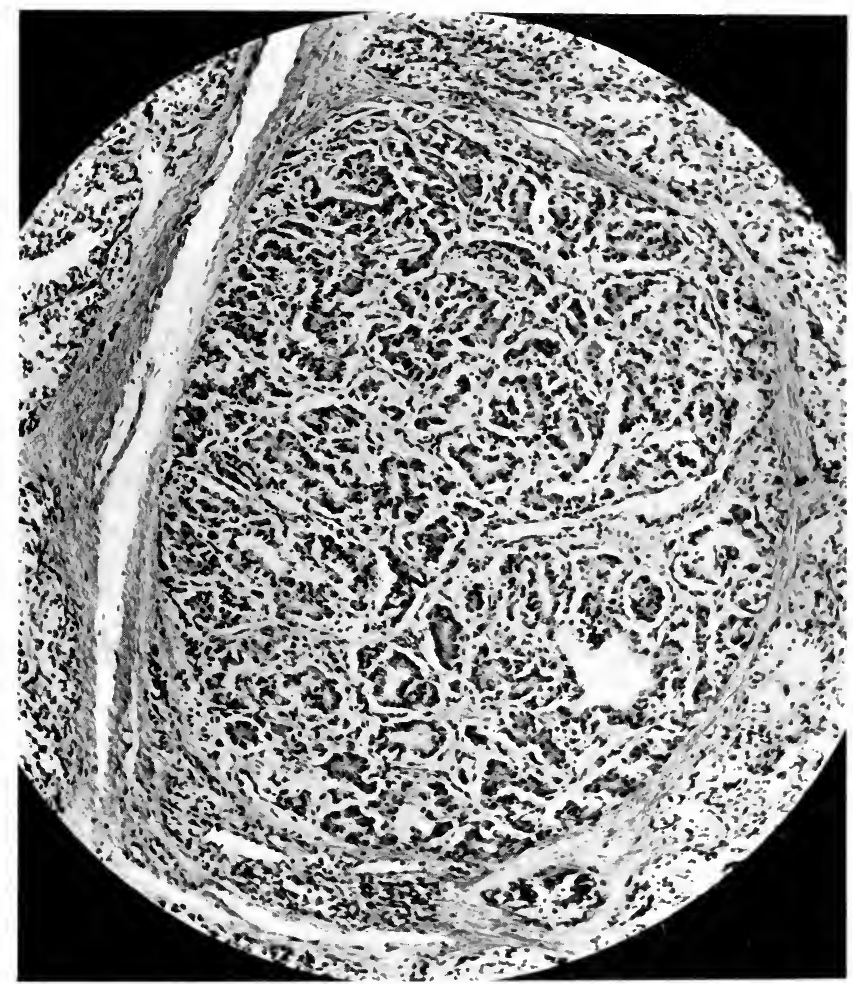

FIG. 463.-Miliary adenoma of thyroid in exophthalmic goiter.

Several phases of the natural history of the exophthalmic goiter may be traced. Not all cases pass through every stage of the disease. Some are mild and early arrested; others are fatal at the height of cellular activity; not a few pass a critical period and become chronic: while the remainder pursue a very chronic course, ending in glandular atrophy and myxedema. The determining factor seems to be the intensity of the process.

Four somewhat distinct stages may be recognized in the exophthalmic goiter.

(I) Hyperemia with increased secretion of colloid showing diminished staining reaction with eosin. In these cases the disease is early and the symptoms are mild. 
(2) Increased vascularity, abundance of abnormal colloid, and beginning cellular hyperplasia. These changes are observed in established cases, often of considerable duration and intensity. Some of them are long preceded by simple goiter and the lesions differ from those of simple colloid goiter in the greater vascularity, reduction of colloid, and greater cellular activity.

(3) Varicosities of large vessels with diminished capillary circulation, comparative or complete absence of colloid, extensive cellular hyperplasia, and interstitial thyroiditis or fibrosis may be present.

(4) In chronic cases of long duration cellular hyperplasia is succeeded by atrophy, arteriosclerosis, cyst formation, and calcification, and the structure approaches that of myxedema. These cases are of long duration, with urgent symptoms, and often fatal.

There is much variation in the structure of the gland in the advanced disease, but the lesions regularly present the most pronounced types of parenchymatous goiter. The follicles proliferate, forming many new alveoli without colloid and almost diffuse cellular areas. The cells are hypertrophied, nuclei are hyperchromatic, and giant-cells may form. Or the outlines of follicles are better preserved, but the lining cells are enlarged and appear in multiple layers often filling the lumen. Dilated spaces may appear in which there are papillary projections of epithelium lying on thickened septa. In a bulky thyroid of this type the cellular hyperplasia far exceeds that of some true tumors, but the Graves process is not neoplastic. The chief histological distinction is the preservation of a more or less typical cell form and orderly arrangement. The capsule, septa, lobules, and alveoli remain intact, the course of the disease is prolonged, and the natural tendency of the lesion is toward regression, atrophy, and fibrosis.

True tumors rarely develop in the Graves thyroid. When miliary adenomas appear their structure is at once distinguished from that of the hyperplastic gland. When malignant tumors are associated with Graves' symptoms it is possible that the initial hyperplasia belonging to the general disease passes rapidly into a malignant overgrowth, which thereafter dominates the clinical picture. In several cases marked Graves' symptoms continued throughout the course of thyroid carcinoma (Ehrhardt, Lit.). I have observed Graves' symptoms with a fetal adenoma of the thyroid during a period in which the thyroid tumor was invaded by mammary carcinoma.

Adenocarcinoma. Malignant Adenoma.-This tumor appears in the form of a rather large, solid, well-encapsulated growth presenting a nodular or lobulated texture. The lobules show a soft obaque periphery and a firmer or fibrous central area. The red color indicates an abundant or excessive blood-supply, and hemorrhage is frequent. Yellowish opaque or pigmented areas represent fatty and cellular portions and the sites of hemorrhage. On section the tumor may yield a mucinous exudate, and colloid portions may resemble the normal thyroid. Papillary cystadenocarcinoma is a welldefinied subvariety, in which small cysts and a papillary structure may be detected in the gross examination.

The appearance of the tumor is usually preceded by a period of slow enlargement of the gland which may extend over several years.

The rate of growth is active, but not often rapid, 2 to 3 years being required for the appearance of serious pressure symptoms. Local recurrence is frequently observed, as a single tumor arising from remnants of the old, or in the form of primary multiple nodules. Metastases are observed in some cases, but less frequently than with carcinoma. 
The structure is much the same in each of the constituent lobules, which present a peripheral cellular zone of small alveoli without definite lumina, or cellular cords of secondary alveoli, and a central zone in which alveoli are more adult and may contain colloid, while the stroma is more abundant. This structure has often been interpreted as representing a centrifugal growth from an original focus of embryonal thyroid tissue, passing through the natural course of development of the thyroid lobule. Hence the peripheral alveoli are the youngest. There is much variation in the size of the cells, the distinctness of alveoli, and the presence of colloid, but there are always signs of an alveolar tendency. The cells are sufficiently atypical to indicate the presence of a true neoplasm. They are regularly larger and usually more adult in type than the fetal adenoma, from which, however, this tumor may arise.

The stroma is usually very vascular, the vessels showing the characters of sinusoids, a definite adventitia is often lacking, and in the usual absence of membrana propria the tumor-cells lie on the vascular endothelium. Or convoluted columns of tumor-cells surround thick-walled veins. A perithe-

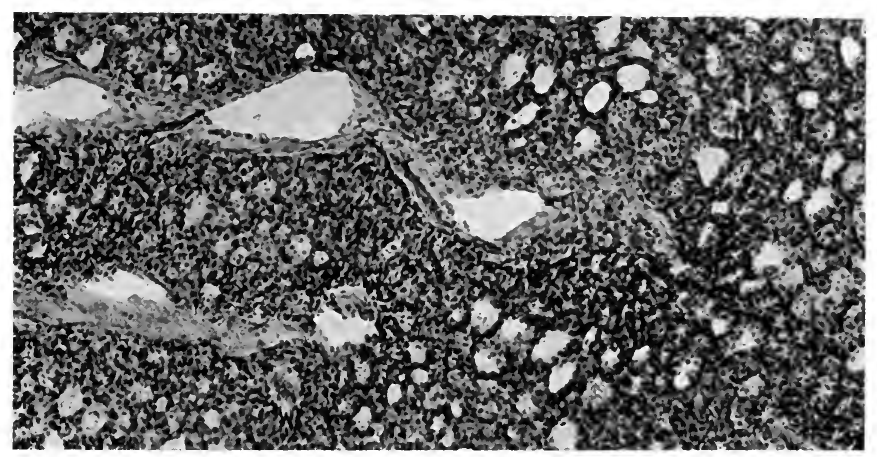

FIG. 464.-Embryonal follicular adenocarcinoma of thyroid.

lial arrangement may be assumed and has led some observers to seek a specific origin for these particular forms of adenocarcinoma.

In some of these very vascular tumors the alveolar structure is reduced to a minimum or entirely lost and the tumor is composed of a network of columns of round or spindle-cells surrounding the blood spaces and resembling angiosarcoma. Many of these growths pulsate.

In different portions of the same tumor the structure of simple goiter, of adenocarcinoma, and of solid carcinomatous cell masses may appear. In the metastases the structure is usually of the more adult type and many alveoli contain colloid. Hence this type of growth contributes some of the so-called benign metastasizing strumas.

In rare cases the original tumors contain alveoli lined by cylindrical cells. Some authors regard such growths as belonging in a separate class and the very peculiar structures sometimes observed, as by Wolfler, suggests a specific origin. Yet the thyroid cells often assume a cylindrical form, no specific origin has been demonstrated for these growths, and it seems necessary to assume that they arise from the thyroid alveoli by metaplasia.

Papillary epithelial tumors of the thyroid form a well-defined subgroup which usually takes the form of adenocarcinoma. Yet some are relatively simple and benign (adenoma), while others are atypical, malignant, and 
carcinomatous. They arise as single or multiple nodular tumors which fuse in a single lobulated mass usually of moderate dimensions. They are soft in the early stages, but later there is fibrosis, thickening of stroma, and calcific deposits. Small cysts ( $\mathrm{I}$ to $2 \mathrm{~mm}$.) and a papillary structure are visible in the gross and occasionally the cysts are of larger dimensions. In Rose's case the cyst contained $65^{\circ}$ c.c. of fluid. Hinterstoisser makes a separate group of large cystic papillary cystadenomas. The papillary tumors are usually preceded by chronic enlargement of the thyroid and growth is relatively slow, but the malignancy and rate of progress vary with the structure. Local recurrence is common and probably results from the development of new independent tumors. Barker observed five recurrences in one case.

The adenomas are slow to infiltrate capsule or nodes, while the adenocarcinomas recur locally, infiltrate the capsule, and generalize. A common history is that the original tumor recurs after operation, becoming more and

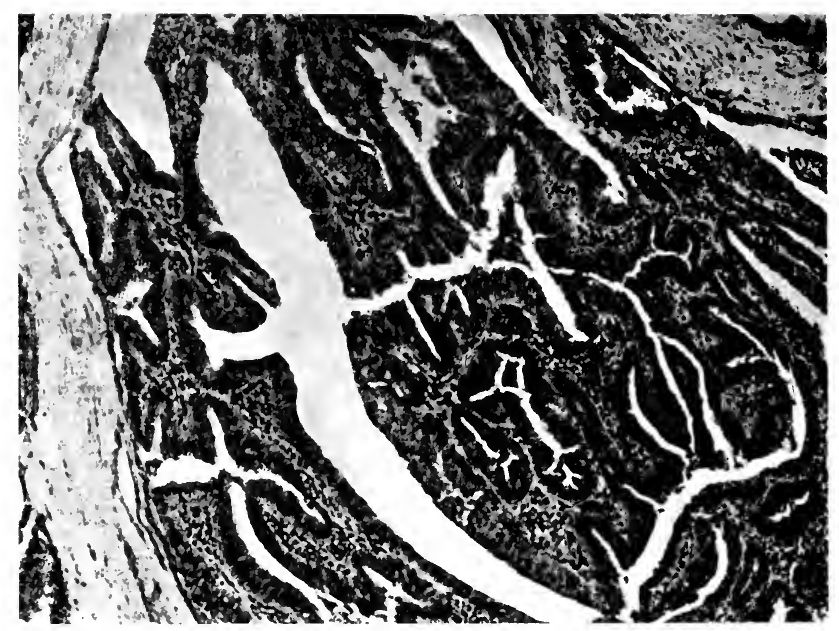

FIG. 465.-Papillary adenocarcinoma of thyroid.

more malignant, and eventually generalizing. Graves' symptoms are associated with many papillary adenomas.

The structure presents a complex network of vascular stroma enclosing alveoli and small cystic cavities into which there are many papillary projections. The papillæ are low and composed of epithelial buds, or higher, with connective-tissue strands, inclosing groups of epithelium and lined by one or more layers of epithelial cells. These cells are cubical, cylindrical or syncytial, and the cytoplasm may be clear or acidophile. The cylindrical character is prominent and uniform in a group of cases which many authors are inclined to separate from the others (Langhans, Kocher, Ehrhardt). The connective tissue of the papillæ may become very thick and hyaline, while the lining cells desquamate. Colloid is scanty or absent. Calcific granules may appear in stroma or alveoli. In 3 of 5 cases Langhans found numerous lymph-follicles in the surrounding gland tissue.

Papillary adenoma in one of the thyroid tumors that may readily be traced to an origin from the adult gland tissue, and the structure is throughout that of an adult, though often atypical and malignant neoplasm. In one early 
case I find very numerous foci where the normal alveoli throughout a large portion of the lobe are becoming transformed by a pronounced neoplastic overgrowth of epithelium. In this case also there were transitions from regular adenomatous to infiltrating carcinomatous growth. In some cases the tumor process becomes established on the hyperplastic gland of Graves' disease, the symptoms of which long precede the appearance of a neoplasm. A certain papillary tendency may also be observed in tumors of embryonal type. In a series of cases papillary cystic tumors of the neck have been traced to accessory thyroids (Madelung, Plauth, Kapsamer).

Struma Postbranchialis (Getzowa). Small Alveolar, Large Cell Adenocarcinoma (Langhans).- In a group of thyroid tumors, which in other respects do not differ from malignant adenocarcinoma, the highly specific structure suggests a peculiar origin. These tumors are composed of relatively small well-formed alveoli lined by one or more layers of very irregular cells. Some are clear, cuboidal or cylindrical, or irregularly polyhedral. Others are large, sometimes of giant size, finely granular, eosinophile and opaque, and resemble granular adrenal or liver-cells. The nuclei are small, vesicular, with visible nucleoli. In some alveoli the cells are twisted about one another with concave and convex borders, or separated by clefts, and occasionally syncytial masses appear. Rather numerous globules of colloid are usually present, and the original structure reappears in metastases. The cells contain neither fat nor glycogen.

Langhans considers the possibility of the origin of these tumors from parathyroid or carotid gland, but concludes that they arise from certain cell groups in the thyroid, identified by Getzowa as remnants of the lateral thyroid anlage (postbranchial body). I have examined two tumors answering this description and suggesting that the acidophile cells might represent hypertrophic Hurthle's cells of the thyroid alveoli.

Carcinoma.-The separation between adenocarcinoma and carcinoma of the thyroid is very imperfect and many transitional tumors are observed. Langhans finds that the great majority of tumors which he would class as carcinoma still show a tendency to produce alveoli which sometimes contain colloid. The same tendency appears in many of Wolfler's carcinomas. Aside from these pure carcinomatous growths which produce solid groups of atypical infiltrating cells, the class of thyroid carcinoma must take account of the gross anatomical characters, such as infiltration of stroma, capsule, and blood-vessels, invasion of neighboring tissues and lymph-nodes, as well as the formation of local and distant metastases.

Thyroid carcinoma is a disease of advanced life, $5^{\circ}$ to 60 years, but has been observed between Io and 90 years. Berry described a round-cell sarcoma in a boy of 3 years and Demme a carcinoma in a boy of 5 years. This tumor is slightly more frequent in females than in males ( 85 to 65 , Ehrhardt). Three cases were imputed to trauma (Braun, Cornil, Suskind). Not a few are attributed to or aggravated by gestation. Most cases arise in goiterous glands, Ehrhardt finding this history in 104 of 200 cases, the goiter dating from I to 50 years.

The average duration is about 2 years. Very acute and usually febrile courses are occasionally seen (Braun, Hochstetter, Karst). I have observed 2 such cases in young women. The structure was alveolar or diffuse, with local and general metastases. Adenocarcinoma progresses more slowly, v. Eiselsberg recording a duration of 8 years. Papillary and cystic adenocarcinomas are even more prolonged, as in Wolfler's case (I8 years) and Smoler's (27 years).

In the gross thyroid carcinoma produces a single, firm, opaque, encap- 
sulated tumor mass which grows steadily until it invades capsule, gland, and surrounding tissues. A multicentric origin in five separate nodules is described by Langhans and is probably not infrequent. Or the growth may begin as a diffuse infiltration, of which Hinterstoisser has collected I7 cases. Friedland describes carcinoma without enlargement of the gland. Most carcinomas grow to considerable size and yield symptoms from pressure, local invasion, and metastasis. Occasionally the original tumor remains small while metástases develop, and in a few cases the thyroid tumor has been overlooked and a metastatic tumor in bone or lungs has first attracted attention. The trachea is displaced, constricted, or flattened. Billroth's scirrhous cancer encircled this organ like a band. The wall becomes infiltrated or perforated, and polypoid masses may appear in the lumen or fill a bronchus (Herb, Semon). A diffuse carcinomatous lymphangitis of the tracheal mucosa is described by Mermet and Lecour. Beneath the obstructing growth the bronchi may be dilated, and some have assumed that pulmonary metastasis may arise from inspired particles. The esophagus suffers in much the same manner as the trachea. The blood-vessels are early invaded by malignant as well as by some histologically benign tumors. The thyroid veins may

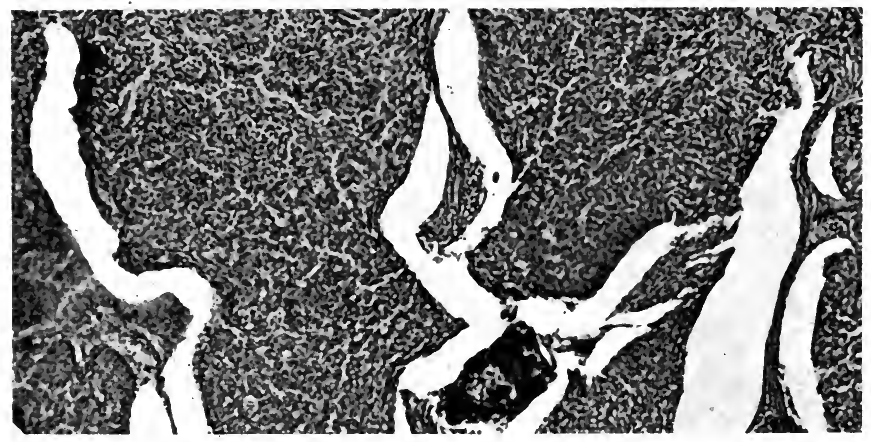

FIG. 466.- Solid carcinoma of thyroid.

contain tumor thrombi, and in Ehrhardt's case the thrombus extended to the jugular and the innominate.

Invasion along the vessel sheaths extends further, to the base of the skull, to the axilla, and to the heart. The recurrent laryngeal nerve is frequently compressed or infiltrated, the vagus and sympathetic less often. Thus of 34 malignant tumors, Meyer noted laryngeal paralysis in 20. Direct invasion of muscles of neck, cervical spine, mediastinum, and lungs are commonly observed.

Metastases pass chiefly through the blood-vessels (47 cases), by lymphpaths (I6), or by both channels. Metastases were absent in $\mathrm{I} 4$ of 94 cases. The organs in 238 cases of carcinoma and sarcoma were affected as follows: lungs, I 29 cases; bones, 66; liver, 36; kidneys, 20; pleura, I6; brain, I2; other organs, I3 (Ehirhardt).

The main lymphatics of the thyroid are: (I) the deep jugular plexus, which drains the organ laterally and continues downward in the inferior cervical nodes into mediastinum; (2) posterior trunks which run between trachea and esophagus; (3) superior vessels leading to submaxillary and lingual nodes; and (4) inferior vessels leading directly to the mediastinal nodes. The blood-vessels first invaded are the main thyroid veins. 
The pulmonary tumors are large or small and disseminated. The bronchi may be surrounded and perforated by polypoid masses. Hemorrhage is early excited in the pulmonary parenchyma and hemoptysis is a frequent result.

The bones are involved only less frequently than with mammary and prostatic cancer, and in the following order: skull, sternum, spine, ribs, humerus, femur, and pelvis. The metastatic growths appear at epiphyses or along sutures, and produce large encapsulateci tumors or diffuse central or periosteal infiltrations. The process may be osteoplastic and the fractures occurring may heal (v. Eiselsberg). The claim that bone metastases may appear at points of hemorrhage following trauma, must be received with caution (Ehrhardt, Lit.). Pulsating bone tumors have in several instances been identified as metastatic thyroid growths (Coats, Feurer, Middledropf, Morris). Swelling of such a tumor was interpreted by v. Eiselsberg as a menstrual phenomenon. Retrosternal pulsating tumors were regarded as aneurysms and treated by appropriate arterial ligatures, by Cramer, Langenbeck, and Sheen. Solitary benign bone metastases have been observed by v. Eiselsberg and others. Here a thyroid adenocarcinoma produces a single benign metastasis (sternum), which may be successfully extirpated, although the patient dies with later metastases in other organs. Ehrhardt shows that many reputed cases of this type exhibit multiple bone metastases. In either case the surgical procedure is of little avail.

Comparing the structure of primary and secondary thyroid tumors Jaeger finds the following relations: (I) both are benign; (2) primary tumor malignant, secondary benign; (3) primary tumor benign, secondary malignant; (4) both malignant.

That many metastatic thyroid tumors functionate is indicated by the presence of colloid and has been proved experimentally by v. Eiselsberg. His patient suffered from tetany after extirpation of a large carcinomatous thyroid, but was gradually relieved upon the appearance of an adenomatous tumor in the sternum, after the removal of which the tetany reappeared. The influence of the parathyroids seems to have been ignored in the numerous citations of this case. The presence of iodin in metastatic adenocarcinoma, demonstrated by K. Ewald, is a further indication of functional activity, and since the original tumor was free from iodin the result indicates a partial restoration of function in the metastases.

Metastasizing Colloid Goiter.-That a simple colloid goiter, presenting a structure that usually remains harmless for years, may give rise to metastatic tumors of benign or malignant type is one of the striking anomalies of the pathological thyroid. Such a case was first described by Cohnheim, who found the structure of colloid goiter in both primary and secondary tumors. The lobes of the thyroid were uniformly enlarged and presented the appearance of simple goiter, while in the left lobe were three large nodules of ordinary gelatinous adenoma, one of which had penetrated the lumen of the inferior thyroid artery. Extensive metastatic tumors were found in bronchial nodes, lungs, femur, and lumbar spine. All of these tumors gave the typical alveolar structure of thyroid tissue, and only in a very few cell groups was a lumen with colloid absent. Other somewhat similar cases are reported by Runge, Coats, Middledorpf, Feurer, Langhans, Honsell, Deraun, A. Meyer.

Langhans found several opaque gelatinous nodules in the thyroids, but no trace of invasion of veins. Hence he considers that the extension occurred through the lymphatics. While none of the observers admits the existence of suspicious actively proliferating areas in the original tumor, they usually describe areas containing a few solid cell groups. It is evident that the growth 
capacity of the cells in colloid goiter is greater than its clinical course indicates. Possibly an accidental access to the blood- or lymph-stream may release the cells from mechanical restraint and permit them to exhibit malignant qualities in more favorable situations. In Litten's case the metastases showed both colloid struma and carcinoma, and Hofmann's metastases were adenocarcinomatous. In a case of Jeffries' I found in the dural growth the normal regularity of alveoli with colloid, but the cells were large and nuclei hyperchromatic. In Middledorpf's case the original "adenoma" had perforated

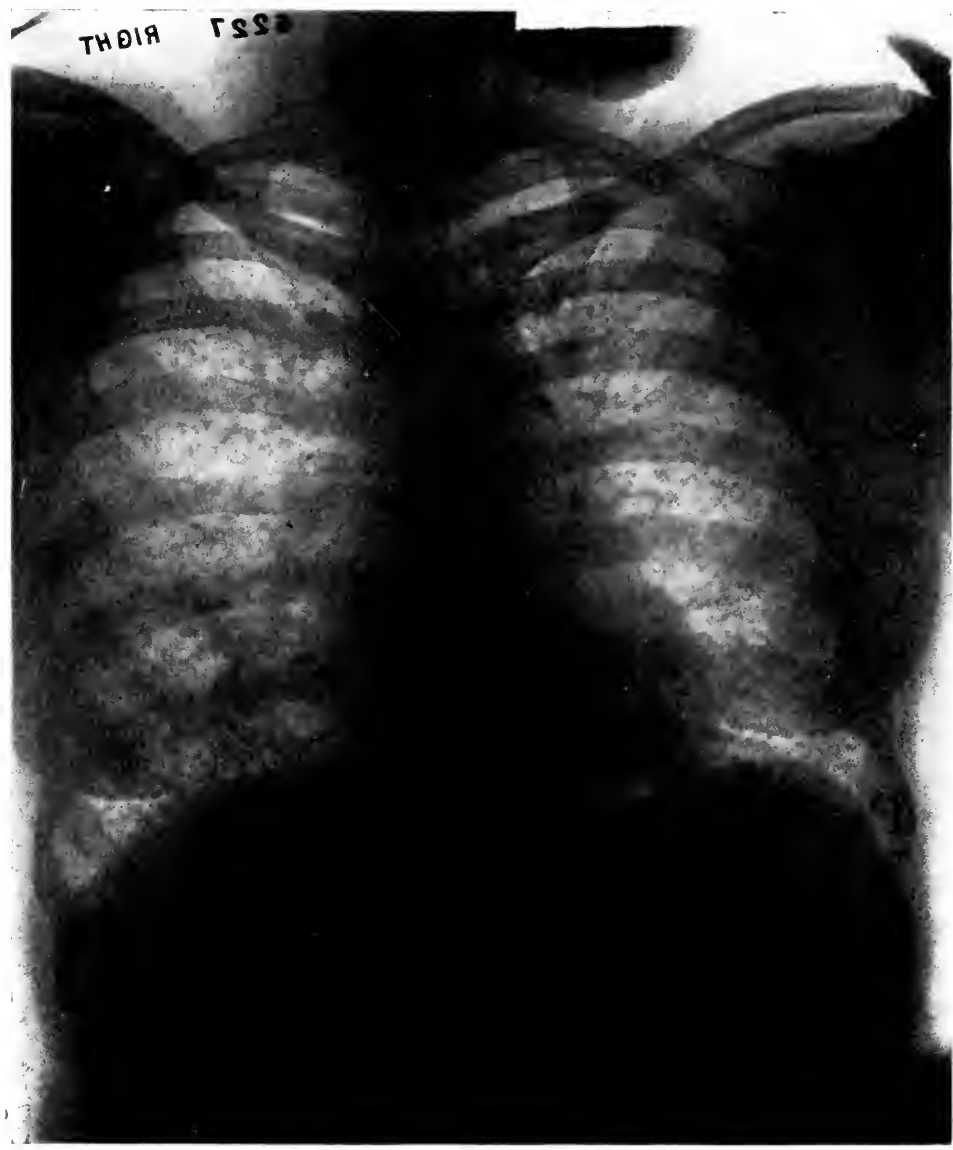

FIG. 467 . - $x$-Ray photograph of lungs showing multiple metastases in benign metastasizing thyroid struma (adenocarcinoma).

a partly calcified capsule and the wall of a vein. The natural tendency of the metastatic thyroid cells to develop into normal thyroid tissue may progressively alter the structure of a secondary growth, so that an original carcinomatous area may eventually appear adenomatous. This principle, first stated by Eberth, is occasionally illustrated.

Metastases from the Normal Thyroid.-In a few instances tumors of thyroid tissue have developed under circumstances which suggested an origin 
from aberrant cells from the normal thyroid. Riedel removed a tumor of the inferior maxilla composed of normal thyroid tissue, which recurred locally after ro years. The thyroid gland is said to have remained normal throughout this period of observation.

Oderfeld and Steinhaus excised a perforating tumor of the skull, composed of normal thyroid tissue. There was no enlargement of the thyroid or of any accessory gland. Jeffries reported a parietal subdural tumor composed of somewhat embryonal thyroid tissue, in a subject whose thyroid appeared to be entirely normal.

In explanation of these cases it seems necessary to assume an origin either from aberrant thyroid tissue or from dislodged cells from the normal thyroid. Yet in none of the cases has the supposed normal thyroid been submitted to microscopical examination. In Becker's case a supraclavicular tumor probably represented an aberrant thyroid.

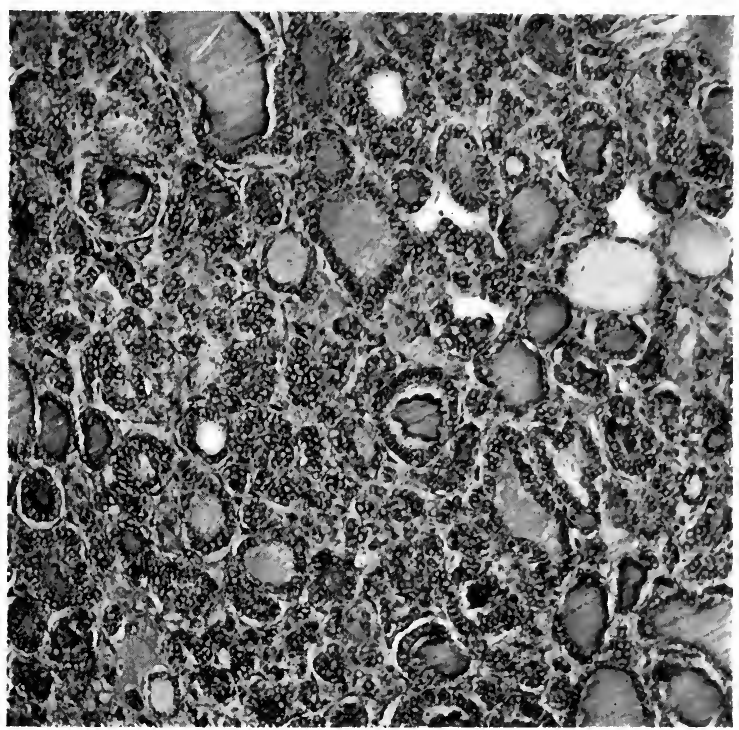

FIG. 468.-Tumor of aberrant thyroid tissue in cerebral dura. So-called benign metastasizing struma. (Specimen of Dr. Jeffries.)

The structure of thyroid carcinoma presents an alveolar type with large or small groups of atypical cells and nearly constant absence of colloid.

In some cases the structure approaches that of adenocarcinoma, but the alveoli are closely packed with hyperchromatic cells, and invasive tendencies are pronounced. Langhans (his Fig. 20) classes as carcinoma a tumor containing many colloid deposits in irregular masses of atypical cells. Wolfler (his Fig. 48) describes as carcinoma a tumor composed chiefly of very small groups, apparently alveoli, of large atypical cells. Such tumors seem to belong among adenocarcinomas. In more typical cases the cell groups are compact without a trace of colloid, and little or no evidence of formation of alveoli. Considerable areas of diffuse cell growth may appear and here the cells may be small with very hyperchromatic nuclei, or fusiform, resembling sarcoma. 
Scirrhous carcinoma, first described by Billroth as a ring-shaped growth surrounding the trachea, has been reported by later observers, and is not infrequently found in portions of slowly growing and very hard tumors.

In the typical scirrhus the gland may not be enlarged, but is extremely hard, resembling cicatricial tissue. The groups of tumor-cells are small, scanty, and limited chiefly to the peripheral portions. The exact nature of some of the cases described as scirrhus appears uncertain (Ehrhardt, Lit.). Other regressive changes are also observed, as fatty degeneration, Icyst formation, necrosis, and calcification. Glycogen is scanty or absent in most carcinomas, but abundant in parathyroid struma. It is probable also that bone may form in the central areas of old carcinomas, and when the epithelial cells are incorporated in fibrous tissue osteosarcoma may be simulated.

Histogenesis. - The question of the histogenesis of thyroid carcinoma is involved with that of all other thyroid growths. Wolfler and Langhans assume that all the ordinary epithelial tumors arise not from the adult vesicles, but from cell groups which remain embryonal through disorders of development and later give origin to fetal adenoma, gelatinous adenoma or colloid goiter, adenocarcinoma, and carcinoma. Yet neither the existence of these embryonal cells nor their participation in many tumor processes have been studied in sufficient detail to extend their significance over such a broad field. Only the fetal adenoma can be certainly referred to embryonal cell groups.

On the other hand, Virchow, Ehrhardt, and others have found satisfactory evidence that many adenomas, adenocarcinomas, and carcinomas arise from the adult follicles. Ehrhart pictures very early focal hyperplasia of follicular cells in carcinoma. I have seen such focal areas especially in papillary adenocarcinoma. This view accords with the usual clinical history, which indicates that a long period of benign overgrowth precedes the malignant tumor.

There are many indications that tumors of the thyroid, as in many other organs, fall into two groups, embryonal and adult, and that each variety includes forms of adenoma and carcinoma. Some atypical, usually rapidly growing tumors, described as sarcoma, appear to find their true explanation in an embryonal epithelial origin.

Squamous-cell epithelioma in the thyroid has been observed in a series of cases and pursues the usual course of destructive acanthoma. It arises not from branchial remnants, but from the thyroglossal duct and its pyramidal process (Langhans). Secondary acanthoma from esophagus, larynx, and branchiogenic cysts is frequently observed.

Sarcoma of Thyroid.- Tumors interpreted as sarcoma are almost as frequent in the thyroid as carcinoma. Thus Ehrhardt collected 150 carcinomas with 99 sarcomas. Ewald states that the proportions are about 3 or 4 carcinomas to I sarcoma. In goiterous regions they are said to be very common. Kocher states that the thyroid is one of the chief seats of "acute" sarcoma.

The general etiology is identical with that of carcinoma. Sarcoma occurs chiefly after 40 years, about equally in both sexes. An hereditary tendency and a history of previous goiter is very common. The rapid course of thyroid sarcoma is its chief distinguishing feature. Morf found the shortest duration 4 weeks, the longest, I7 months.

The gross anatomy fails to show any definite variations from carcinoma. The tumors are large, circumscribed or diffuse, locally aggressive, invading lymph-nodes and veins, and producing distant metastases, with or without affection of the nodes. 
The histological varieties are numerous. Of 40 cases analyzed by Morf, the distribution was: round cell ro, spindle- 6 , mixed 7 , alveolar 5 , fibrous 3 , osteoid 2, etc. Giant-cells are prominent in some tumors. Lymphosarcomas are described by Braun and Ehrhardt.

Notwithstanding the wide acceptance of numerous reported cases as sarcoma of the thyroid, there is a strong reason for believing that the great majority of these tumors are of epithelial origin. It is clear that the diagnosis of sarcoma has usually been accepted on the most superficial resemblance to a mesoblastic tumor, and without any effort to critically interpret the structure and origin. Hence many authors have recognized the uncertain standing of thyroid sarcoma, but dismissed the matter by stating that there is no sharp dividing line between carcinoma and sarcoma. The close resemblance of the general etiology, clinical course, and gross anatomy of the two conditions fully bears out this conclusion. There remain only histological distinctions, but when these are examined in detail they fail to establish the frequent occurrence of malignant mesoblastic tumors of the thyroid.

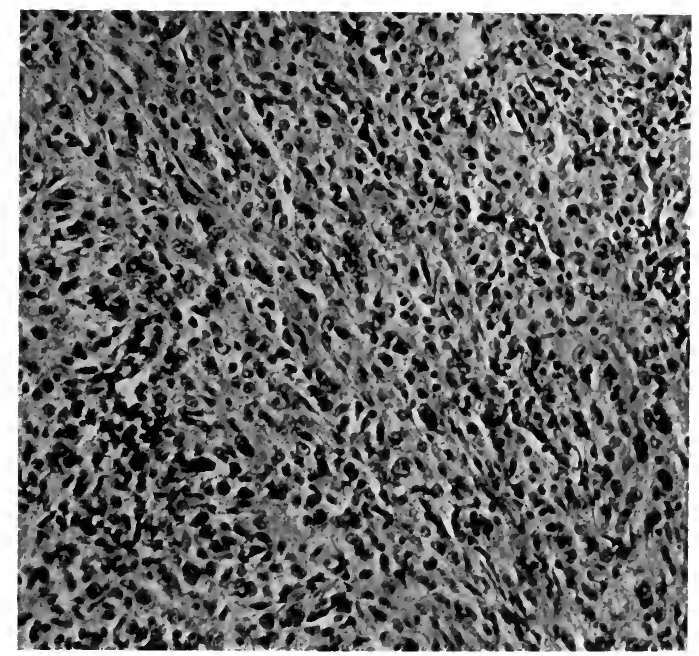

FIG. 469.- Spindle-cell area in thyroid carcinoma.

Of the reported cases many showed an alveolar structure which is strongly indicative of an epithelial origin (Forster, Newmann, Braun). Wolfler's alveolar sarcomas (his Figs. 62,63) are indistinguishable from small alveolar or highly vascular embryonal carcinoma. C. Kaufmann based his diagnosis of a round-cell sarcoma on a minute histological study, but his tumor was alveolar, a striking resemblance to carcinoma was noted in some cases, his drawing shows a typical adenocarcinoma with the common infiltration of stroma by large polyhedral cells, and the intimate relation of blood-ressels to tumor-cells, on which he lays chief stress, is characteristic of thyroid carcinoma. He also describes carcinoma and spindle-cell sarcoma in adjoining portions of the same tumor. Limacher traces in great detail the origin of a typical fibrocarcinoma to the endothelial cells of blood-ressels. Ehrhardt mentions the great difficulty of distinguishing many reported cases of fibrosarcoma from scirrhus. Some osteosarcomas appear to represent bone formation in old fibrous strumas. Pick's osteosarcoma was probably second- 
ary to a growth in the maxilla, and Ehrhardt concluded that his case was secondary to a tumor in the femur. Fraenkel's melanosarcoma showed cutaneous moles. Braun's lymphosarcoma presents none of the distinguishing features of this disease.

My own material, which includes Lartigau's case, illustrates so fully the transformation of thyroid epithelium into spindle-, round, and giant-cells, producing a structure resembling sarcoma, as to lead to the conclusion that the mesoblastic origin of most of the sarcomas reported in the literature is highly improbable, and that the occurrence of true sarcoma in man still requires demonstration.

Benign Granuloma of the Thyroid. Riedel's Struma.--In I 896 Riedel described a form of chronic inflammation of the thyroid gland which transformed the organ into a bulky tumor composed of dense fibrous and sclerotic tissue. The entire gland was involved and converted into a mass of almost iron-hard consistence, and so generally adherent that extirpation was difficult or impossible. Although the disease was variously diagnosed as syphilis,

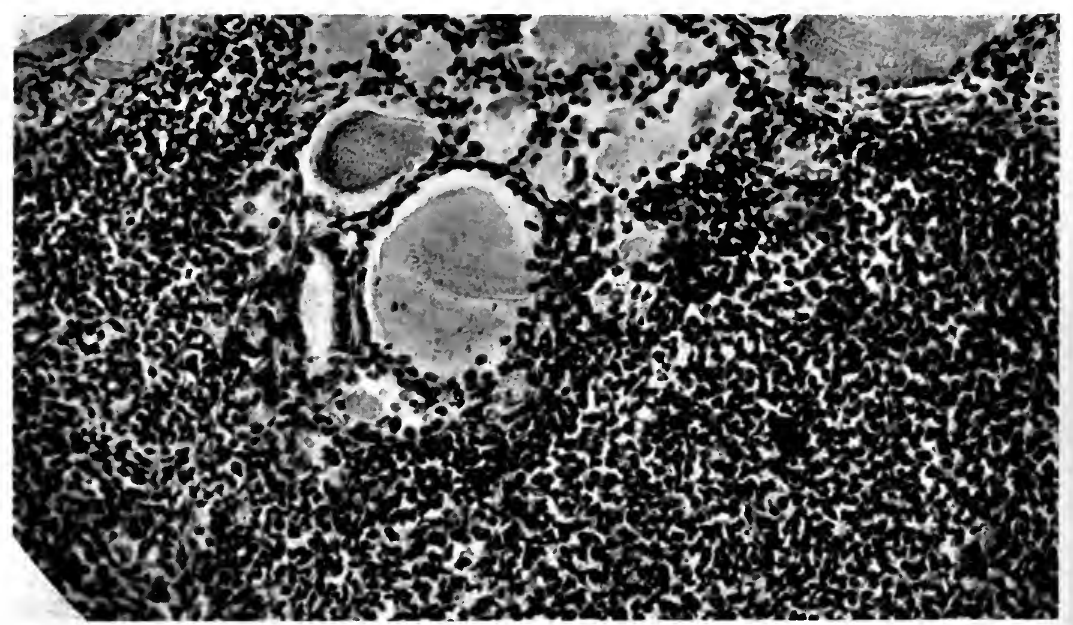

FIG. 469a.-Structure of early stage of benign granuloma of thyroid gland.

tuberculosis, or cancer, potassium iodid was without effect, and cases observed for years did not become cachectic, the neighboring nodes were not invaded, and metastases did not develop. Selitschek observed spontaneous recovery of a moderately advanced case. Occasionally after a partial resection the remainder of the tumor receded. Riedel and others made no mention of extensive infiltration of the gland with lymphocytes or lymph-follicles.

In I9 2 Hashimoto described 3 clinically similar cases in which the acini of the gland were largely replaced by very numerous lymph-follicles with prominent germ centers. In places these follicles became diffuse. He concluded that he had to deal with a new disease which was not to be identified with Riedel's iron-hard struma.

I have studied 4 cases which illustrate both the above conditions, and in 2 of them a very extensive and peculiar sclerosis had overtaken and largely replaced the lymphoid tissue. It appears, therefore, that Hashimoto and Riedel have described the early and the late stages of the same pathological process. 
These cases occurred in adults and produced tumors of considerable size involving the whole gland. The growth was rather active for a few months, after which the process became slower or stationary. The regional nodes were unaffected. There were no symptoms of hypothyroidism. One very large tumor produced severe dyspnea from pressure, but receded rapidly under radium. The form of the gland was retained.

In the early stages the gland is thickly beset with very numerous lymphfollicles with active germ centers, the reticulum cells of which become so numerous as to suggest lymphosarcoma. In one instance the condition had been diagnosed as carcinoma. The persisting reticulum cells incarcerated between the hyaline strands of fibrous tissue yield a picture resembling small cell alveolar carcinoma. All stages of atrophy of the alveolar epithelium can be traced. Fibrosis and hyaline transformation begin early and eventually, as in Riedel's cases, the enlarged gland becomes very firm and dense. The specific features of Hodgkin's granuloma are missing.

Tumors of the Parathyroid.--Hyperplasia of the parathyroids has been found in a considerable number of cases of osteomalacia, osteoporosis, and

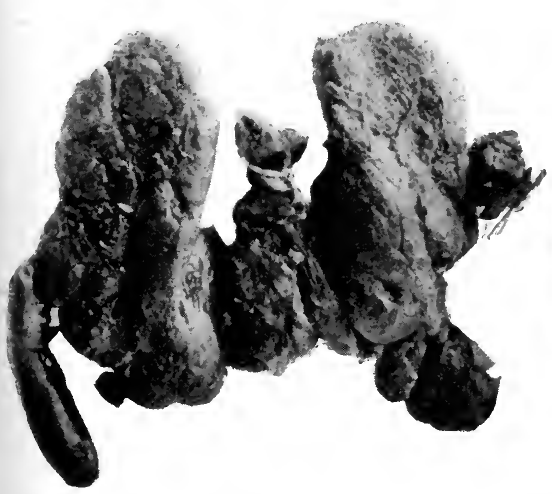

FIG. 470.- Symmetrical tumors of parathyroid glands. (After Harbitz.)

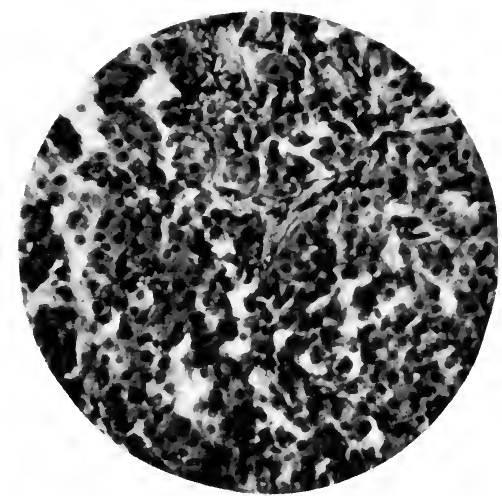

FIG. 47 I.-Adenoma of parathyroid gland in osteomalacia. (After Harbitz.)

rickets, and has been interpreted as an indication of hyperfunction with disturbance in calcium metabolism (Harbitz, Lit.). In several cases the hyperplasia has been regarded as adenomatous.

The recognition of extrathyroid tumors of parathyroid origin may be accomplished with sufficient certainty, but in the case of intrathyroid growths it is difficult to establish a parathyroid origin unless the tumor presents very definite structural peculiarities. There is thus ground for the doubt expressed by Harbitz regarding the nature of certain intrathyroid tumors referred by various authors to the parathyroid.

The peculiar structure and rich glycogen content of certain thyroid tumors was interpreted by Kocher, Jr., in I 899 as evidence of a parathyroid origin. Very similar but smaller extrathyroid growths were later recognized as of parathyroid origin by Benjamins, Erdheim, and MacCallum. The occurrence of aberrant parathyroid tissue in the thyroid (Getzowa, Erdheim) accounts for the presence of these growths within the confines of the organ.

The parathyroid struma produces an encapsulated tumor involving a part or the whole of one side, or appearing as a separate mass at the lower 
pole of the thyroid. The extrathyroid tumors are small and adenomatous (Erdheim, MacCallum) or large and malignant (Benjamins). Within the thyroid they produce firm, nodular, adherent, rapidly growing and malignant tumors, with pain, dyspnea, invasion of nodes, and metastases (Kocher, Sr.). Small cysts may form from dilated ducts.

The structure recalls that of the parathyroid and presents chiefly compact columns of opaque epithelium often arranged in palisade form. There is much variation in the size and appearance of these columns. In one of my cases they vary from very thin strands to large alveoli, bordered by palisade cells and filled with large cuboidal cells. These areas are very vascular, especially when the cell columns are small, and the vessels are very thinwalled sinuses. Areas of very large clear cells with dense cell borders and cytoplasm rich in glycogen, recalling the structure of hypernephroma, are a second characteristic feature. Occasionally there are canals lined with cylindrical epithelium, which Langhans derives from the embryonal tubules traced by Kursteiner from the parathyroid to the thymus. Groups of strongly acidophile cells may also be present. The cell columns are not always intact, but there is a strong tendency in rapidly growing tumors toward

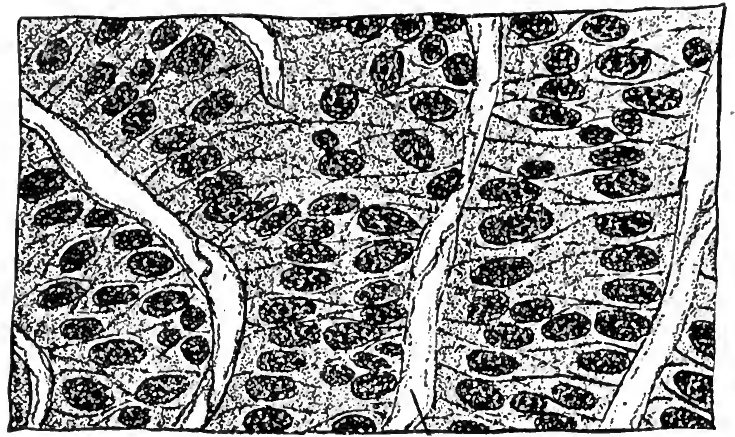

FIG. 472.-Detail of adenoma of parathyroid. (After Erdheim.)

a diffuse growth of small round or spindle- or giant-cells, producing a pseudosarcoma. Such a structure is described by Langhans, and appears in one of my cases. In the smaller tumors the structure is more regular and adenomatous, and in MacCallum's case the question arose whether the process was a simple hypertrophy or a neoplasm. Hulst's small tumor lay in a calcified capsule. In several cases there were present droplets of colloid, or definite alveoli filled with colloid or mucin, and these tumors may be separated with difficulty from thyroid growths.

Cysts of Thyroid and Parathyroid.-The middle lobe of the thyroid is developed from an invagination of the buccal epithelium, just behind the tuberculum impar, which marks the junction of the second and third branchial clefts and goes to form the anterior portion of the tongue. Behind this invagination later rises a fold formed by the second and third branchial arches, which goes to form the base of the tongue. The thyroid invagination (thyroglossal duct) passes down in front of the hyoid bone and its lower portion gives origin to the middle lobe of the thyroid. The upper end may remain patent, opens at the foramen cecum, and is lined by pavement cells. The middle portion usually atrophies, but may persist as a canal lined by ciliated epithelium. Bochdalek found many salivary gland acini opening by lateral ducts into this canal (Bochdalek's duct or gland). Secretion in the upper 
portion of this canal (lingual duct) is readily discharged through the foramen cecum, but in the deeper portions (thyroid duct) secretion is retained and gives rise to median cysts lined by squamous or cylindrical cells and often containing thyroid follicles in the wall (König). Cysts lined by ciliated epithelium, lying in the middle line between the base of the epiglottis and the upper border of the hyoid bone, have been traced to dilatation of the ducts of Bochdalek's gland opening into the lingual duct (Schmidt). Some of these cysts lie at or below the hyoid bone and are single or multilocular, with a lining of squamous or ciliated cells, and mucinous contents (Streckeisen). Others may appear in the floor of the mouth as forms of ranula (Neumann).

Accessory thyroid gland tissue may be found along the entire length of the thyroglossal duct, and according to their position have been termed supra-, pre-, and intrahyoid. The uppermost glands are very prone to hypertrophy at puberty and produce tumors presenting at the foramen cecum. In 2 cases extirpation of these tumors was followed by myxedema, showing that the lingual thyroid was the only portion of the gland which developed (Seldowitsch, Chamisso).

In cases of thyroid aplasia in cretins, or of unilateral aplasia of adults, small multilocular cystic tumors regularly appear in the region of the lingual duct. The cysts are lined by squamous or ciliated cells and remnants of the aplastic thyroid are found in the walls (Erdheim, Lit.).

As with the median lobe, so the lateral thyroid lobes develop from invaginations of entodermal epithelium. These divide into a dorsal portion, giving origin to the parathyroids and a ventral or thyroid portion. Either of these ducts may persist and yield cysts in thyroid or parathyroid, or separate ducts from third and fourth buccal pouches lead to the lower and upper parathyroids, as well as to various accessory parathyroids, of which Erdheim has found as many as eight. In cases of unilateral thyroid aplasia Erdheim finds normal parathyroids and a cyst with tubular glands representing the atrophic lateral thyroid. 


\section{CHAPTER XLVI}

\section{THE THYMUS AND ITS TUMORS}

No group of tumors has more successfully resisted attempts at interpretation and classification than those of the thymus. The problems involved include those which have complicated the embryological and histological study of the gland, while added difficulties arise from the comparative rarity and considerable diversity of the tumors, and from the somewhat imperfect knowledge of the general pathology of the thymus.

A short résumé of the knowledge of the origin and structure of the gland will facilitate the interpretation of its tumors (Hammar, Lit.).

Anatomy.-The thymus is a paired organ arising from evaginations of the third branchial clefts (Thymus III). Its anlage is contiguous with that

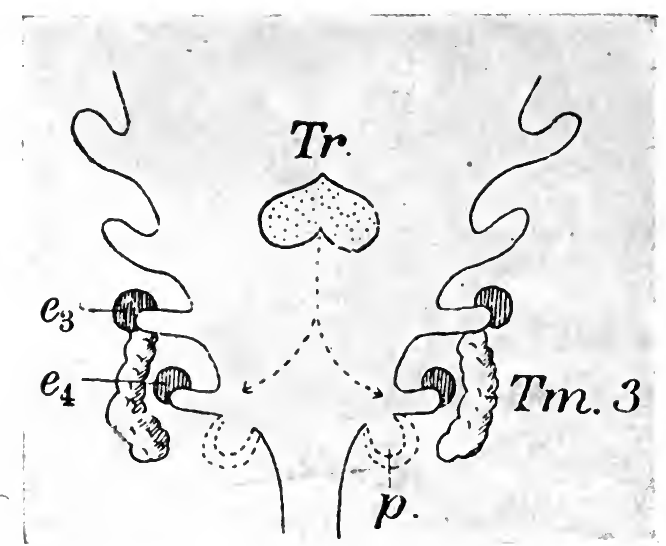

FIG. 473.--Scheme of derivatives of branchial clefts in the human embryo. (After Sobotta.) Tr, thyroid; $e$, parathyroids; $T m$, thymus; $p$, postbranchial body.

of the parathyroids, arising from the same clefts, a relation which explains the occasional presence of parathyroid alveoli in the thymus, as well as the rare association of thymus tissue with parathyroid in the thyroid gland. A second portion of the thymus arises from the fourth cleft, where it holds the same relation to the parathyroids developed from this cleft (Thymus IV). From these four sources the growing entodermal epithelium coalesces to form a four-lobed fetal organ which, with the descent of the heart, becomes drawn out in an elongated double-pear shape. In Hammar's early fetal models the stems stretch from the lateral lobes of the thyroid down below the sternal notch where the main mass of the gland develops, the stems disappearing. This mode of origin only partly explains the occurrence of accessory thymus lobes beside and below the thyroid (Erdheim), and laterally in the neck. Sharp describes a large accessory thymus extending from the anterior border of the trapezius behind sternomastoid and clavicle.

The fully developed organ consists of a stroma and reticulum, parenchyma, and capsule. The supporting stroma is chiefly found in the net- 
work of arterioles, capillaries, and venules, to which is confined practically all of the connective tissue within the organ.

The finer stroma is a derivative of the original epithelium of the gland, which becomes elongated into a fine reticulum in the meshes of which lie the parenchyma cells. This reticulum has nodal thickenings or syncytia, or may be stretched into fine fibrils. By accumulation of its cells in the medulla are formed concentric groups of flat cells (Hassall's corpuscles), with which the reticulum is directly continuous, while on the periphery in many fetal glands the epithelium appears in groups and cords in cubical or cylindrical form. Hassall's corpuscles are, therefore, not remnants of embryonal epithelium, but collections of adult reticulum cells. In the medulla the reticulum is far more abundant than in the cortex.

The parenchyma cells have the appearance of very small lymphocytes, from which they have not been successfully distinguished by many studies

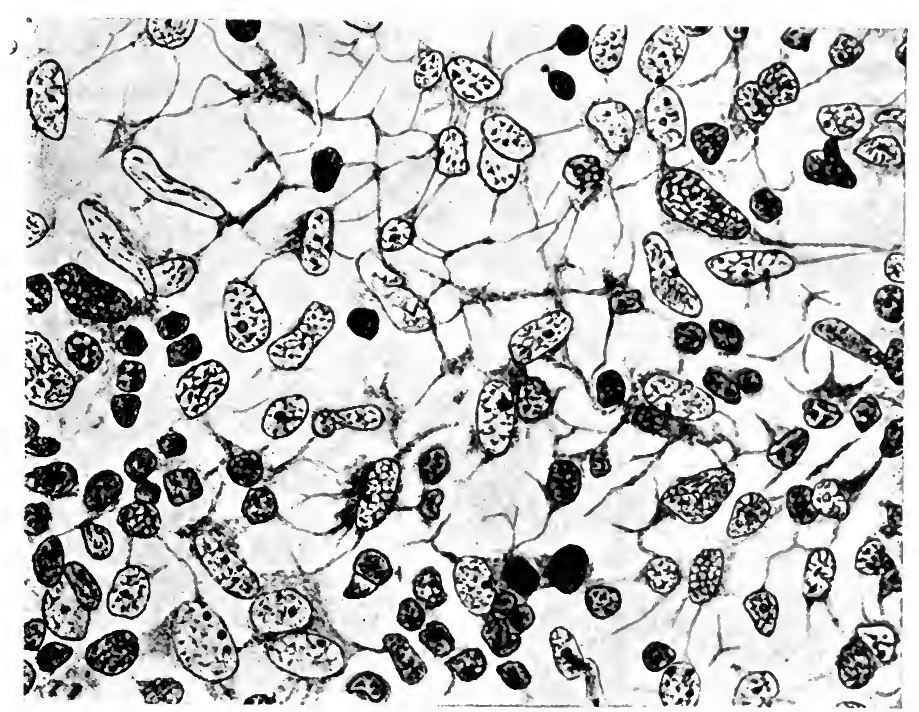

FIG. 474.-Structure of thymus of human embryo of $70 \mathrm{~mm}$. Coarse epithelial reticulum, beginning infiltration by lymphocytes. (Hammar.)

directed to their morphology, microchemical reactions, serological relations, or behavior in most pathological conditions.

Their origin is as yet undetermined. In favor of the view that they are epithelial derivatives, as claimed by Beard, Stohr, and others, the following facts may be cited: (I) The occurrence of transition forms between the reticulum cells and the lymphocytes (Prenant, Bell). Yet Maximow is able to distinguish between the small darkly staining epithelium and the lymphocytes. (2) The very early appearance of lymphocytes in the thymus. (3) The apparent absence of any signs of infiltration by lymphocytes from without. (4) The apparent transformation of small thymus cells into epithelium. Against the epithelial origin stand chiefly the evident identity of the cells with other lymphocytes, the development of other lymphoid organs by the inwandering of lymphocytes, and the behavior of these cells in pathological conditions. Most observers, including Maximow, Hammar, and Wiesel, 
consider the thymus an organ with peculiar reticulum of epithelial origin infiltrated by lymphocytes. Yet the organ never assumes, either in structure or functions, the position of a simple lymphoid organ. In many conditions it fails to participate with other lymphoid organs in systemic diseases (Hart).

In addition to the lymphocytes other cells are often seen in the thymus. Shaeffer observed many plasma-cells derived from the lymphocytes in the involuting gland, and with these may occur eosinophile cells and mastcells (Maximow). Watney describes giant phagocytes. Myoid cells with cross striation are scanty, but very constant derivatives of the epithelium. Wasutoschkin, however, considers that they are derived from the muscle-cells in the capsule. The formation of red cells appears to be limited to lower animals, but evidences of the formation of leukocytes are frequently observed in man. The efforts to establish the thymus as a gland of internal secretion and a unit in the chromaffin system are reviewed by Wiesel.

General Pathology.-A horn of thymus tissue reaches upward to the thyroid gland in 20 per cent. of young subjects (Rieffel), and strands of thymus tissue may encircle the great vessels of the neck and the vagus nerve at the level of the hyoid bone in the infant (Harman, Bien). Accessory lobes appear occasionally in the thyroid and in connection with the parathyroids.

The weight of the thymus at birth varies greatly from 2 to $14 \mathrm{gm}$., but averages $7.7 \mathrm{gm}$. (Bovaird and Nicoll). It increases in size at least to the ${ }_{5} 5_{\text {th }}$ year, when the average weight is from 20 to $28 \mathrm{gm}$. (v. Sury, Ronconi). It then steadily diminishes, but persists in most subjects throughout life. Its proportion to the body weight is highest at birth, 0.42 per cent., but after 25 years the permanent ratio of 0.3 per cent. is reached. Owing to numerous accidental variations it is extremely difficult to establish the existence of hypertrophy unless of very marked degree. Involution takes the form chiefly of fat invasion of the parenchyma, with persistence of many Hassall's corpuscles.

Simple hypertrophy of the thymus occurs in infants, in which the enlarged gland exerts at least in part a mechanical effect in fatal thymic asthma by compression of the trachea. The structure of these glands is usually normal. In status lymphaticus the thymus usually exceeds the normal weight for the age and weight of the patient, and at times the excess is very marked. In Graves' disease thymus hypertrophy is nearly constant and often marked. In all of these conditions the hypertrophy is due to lymphocytic hyperplasia, and does not reach the grade of a neoplasm. In aberrant thymus tissue the hyperplasia has at times been very active, as in Sharp's lymphadenoma of a cervical thymus. Hyperplasias interpreted as lymphadenomas are described by Rolleston, Edmunds and McKenzie, Pepper and Stengel, and Hektoen. In these cases the organ was several times the normal size, the capsule was intact, the medulla largely obliterated, and Hassall's corpuscles widely scattered. Tarozzi describes as simple hyperplasia a very large encapsulated tumor occupying all the anterior mediastinum in a boy of 18 years.

Proliferation or, more correctly, increase in number of Hassall's corpuscles occurs in Graves' disease (Soupault) and in hemophilia (Acland). Exfoliation of very numerous large reticulum cells in the involuting thymus has been described by Lochte in gangrenous gingivitis and in leukemia. Hahn and Thomas collected several cases of thymic tuberculosis. It is a notable fact that in most cases of leukemia and pseudoleukemia the thymus fails to participate in the process. In a series of such cases Schridde found no thymic enlargement. Yet a leukemic blood-picture has been observed in a series of large sarcomatous tumors of the thymus (Fabian). In a case of Coenen's, 
with leukemic blood, it was difficult to determine whether the thymic lesion was leukemic or granulomatous. Lit.).

Cysts form in the thymus from several sources (Pigache, Beclere, Heuter,

(I) The epithelial canals of the embryonal thymus may persist and form one or several small or large cysts in or along the horns of the gland. They are especially frequent in syphilitic infants. Each lobe of the organ may be converted into a large cyst (Bednar). Pollosson and Piery describe a congenital multilocular cyst of a cervical thymus, extending from behind the sternum to the midcarotid region. It was lined by flat pavement cells. In a sclerosed and luetic thymus in a man of 25 years Hueter found general cystic alterations. The cysts were lined by flattened cells, filled with mucoid and lipoid material, and into many of them grew polypoid masses of thymus

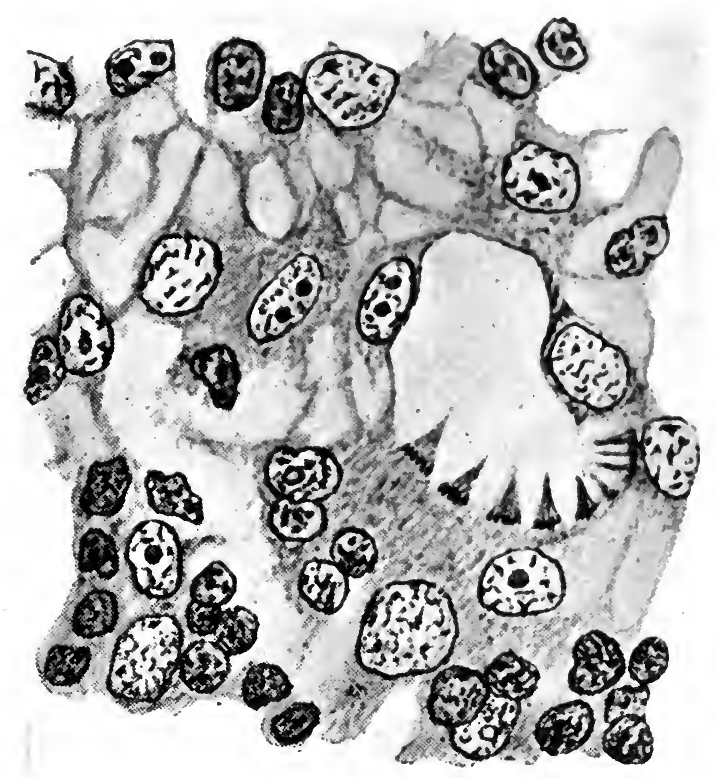

FIG. 475.-Ciliated epithelial cyst in thymus of human embryo of $70 \mathrm{~mm}$. (Hammar.)

tissue. The origin was attributed to persistent epithelial cell groups. Westernak recognized a mediastinal cyst, lined by ciliated epithelium, by means of thymus tissue in the wall, and he attributed its origin to the thymus duct. The demonstration of lymphoid tissue and Hassall's corpuscles in the wall aided Funke in the recognition of a thymus cyst in the thyroid.

In Graves' disease Soupault describes multiple cysts lined by columnar epithelium. In a man of 60 years he found a thymus I $_{5} \mathrm{~cm}$. in length, hyperplastic above, but in the lower half diffuse overgrowth of epithelial cells, and small cysts filled with mucus.

(2) Dermoid cysts, of which Hare has collected 9 cases, may arise from portions of the ventral ectoderm or from the branchial clefts. Rolleston describes a compound cyst with adenomatous structures resembling Lieberkühn's follicles and areas of cartilage and sarcoma.

(3) Invasion and distention of Hassall's corpuscles by lymphocytes is 
of common occurrence, and many small cysts may form throughout the gland by degeneration of these wandering cells. Chiari showed that Dubois' abscesses consist of distended corpuscles filled with lymphocytes. The lining of these corpuscle cysts is of cubical or flat epithelium. All these cysts are regarded by Ribbert as derived from persistent embryonal tubules.

(4) Cystic lymphangioma is described by Seidel in an infant of 2 years. The entire organ was the seat of many small cysts filled with bloody fluid and lined by flat endothelial cells.

\section{PRIMARY TUMORS OF THE THYMUS}

Primary tumors of the thymus are probably not as rare as the scanty reports would indicate. Rubaschow collected 69 cases, but questioned the thymic origin of many. The age of incidence of 33 sarcomas was: before

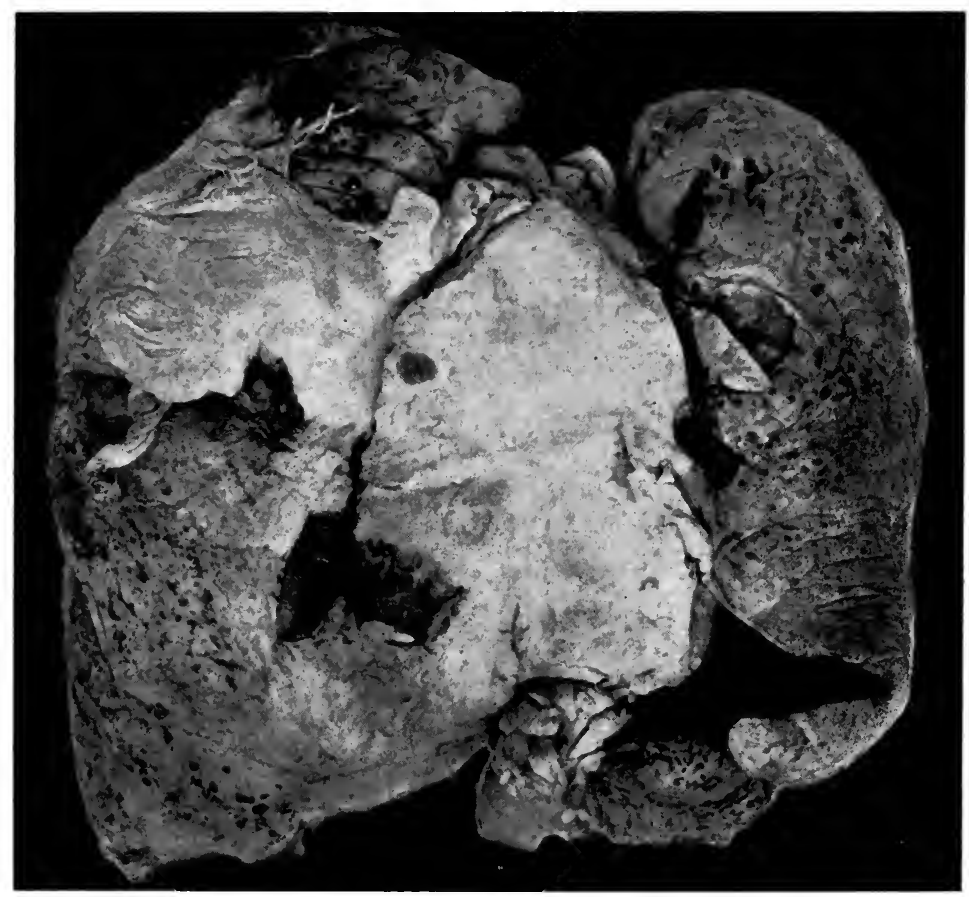

Fici. 476.-Anterior view of a large thymoma. The tumor covers the pericardium, sur. rounds the bronchi and great vessels, and extends slightly to pleura and lung.

2.5 years, I 8 cases; from 25 to 40 years, 8 cases; over 40 years, 7 cases. Carcinomas occur in later years and usually after 50. Steudener found a large lymphosarcoma in an infant of one year. While Virchow believed that thymic hypertrophy led to tumor growth, Bartel's statistics do not show that cases of status lymphaticus are especially prone to develop thymic tumors. Eisenstadt's case gave a history of trauma. The age of incidence and the usual course of involution strongly suggest that thymic carcinoma is affected by disturbances in the natural process of involution. The origin of the sarcomas seems closely connected with that of lymphosarcoma in general, and not 
a few of these cases show marked resemblances to, or a practical identity with, granuloma malignum. Analogy suggests that the peculiar reticulum cells of the thymus may at times respond to infection by inflammatory and eventually neoplastic overgrowth.

Classification.-Thymic tumors fall into two main groups:

(I) Lymphosarcoma or thymoma, composed of a diffuse growth of round, polyhedral, and giant-cells. The chief source of this tumor is probably the reticulum cell, but lymphocytes are often present in abundance.

(2) Carcinoma arising from the reticulum cells.

To these may be added very rare and somewhat questionable cases of tumors attributed to the stroma and called (3) Spindle-cell or myxosarcoma.

Owing to the uncertainty which still surrounds the nature of the thymic round cells, the term "thymoma" has been suggested by Thiroloix and

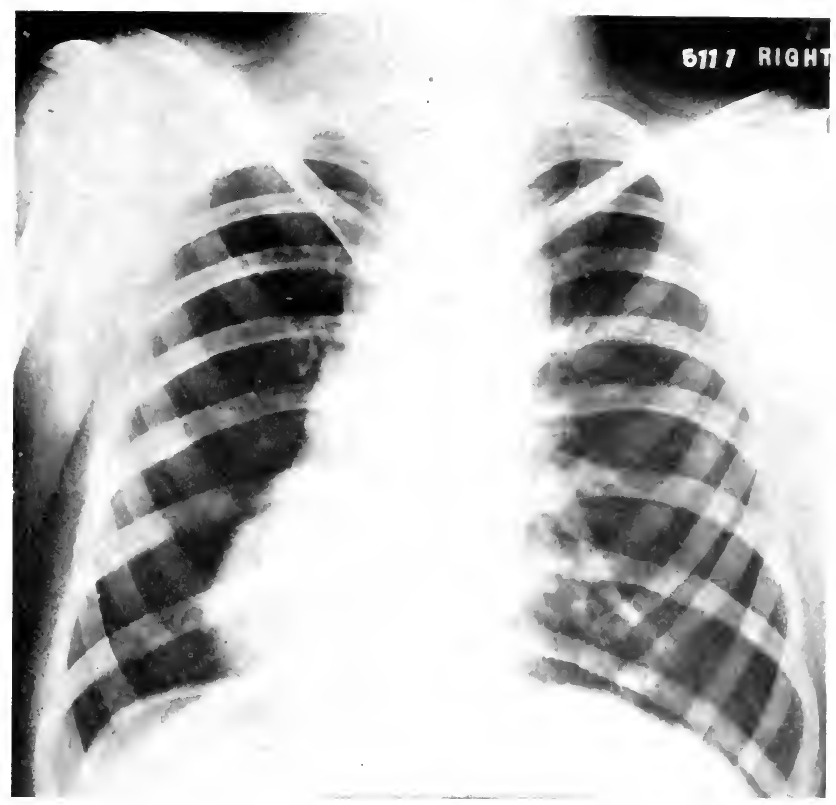

FIG. 477.- $x$-Ray photograph of a thymic tumor. The growth forms a pyramid capping the pericardium.

Debert, Simmonds, and others, for tumors of this origin, while Schridde employs the phrase "malignant thymus tumors."

The exact origin of the so-called lymphosarcomas of the thymus remains undetermined. My own study of several cases has led to the conclusion that the thymic round-cell tumors differ from round-cell tumors of lymphnodes, that the reticulum cell is here the chief or sole source of the tumor, the lymphocytes being largely passive, that many of these tumors seem to fall in the class of granuloma malignum. The term "thymoma" may perhaps deserve recognition as a parallel to lymphoma.

(I) Lymphosarcoma or thymoma is the most frequent form of thymus tumor. The tumors occupy the anterior mediastinum in the position of the thymus, and usually extend from the sternal notch, or as high as the thyroid, 
down to the diaphragm. Many authors have questioned the diagnosis of thymic origin based on the location of the tumor, but the objections seem to apply chiefly to clinical diagnosis. There is little difficulty in distinguishing thymic tumors at autopsy from tumors of mediastinal lymph-nodes, lung, or sternum. They usually surround and compress the trachea, bronchi, pericardium, and great vessels. Both by compression and less often by invasion of vessels and air passages they cause death by asphyxia and venous obstruction, which may increase gradually or supervene suddenly. The more rapidly growing tumors are soft, but as a rule they are found to be remarkably dense from diffuse fibrosis. The soft tumors may be vascular and hemorrhagic, while the firm growths exhibit a characteristic lobulation from dense fibrous septa. Rarely areas of softening and cyst formation are observed. In many cases the tumors exhibit a characteristic creamy yellow or lemon color.

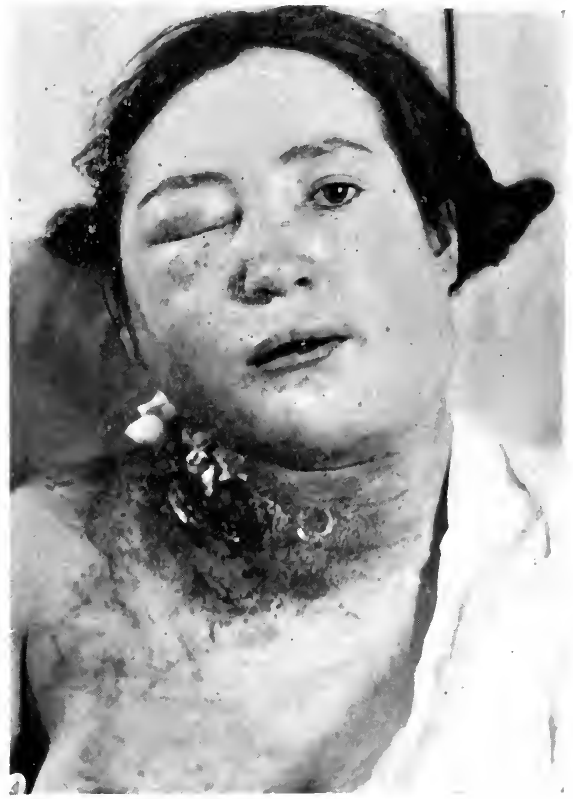

FIG. 478.-Malignant thymoma; extensions to axillæ, neck, pharynx, and orbit.

A strict encapsulation within the mediastinum has been a notable feature in some histologically malignant growths, but the more malignant forms regularly become adherent to surrounding organs, and invade pleura, lung, pericardium, walls or lumina of vessels, and trachea. The bronchial and cervical nodes are frequently invaded. The axillary nodes may early be enlarged and in Gabcke's case the invasion of nodes was very wide-spread. Tumors of the axillary nodes without known origin should call for the investigation of the thymus by the $x$-ray, especially before any operation is attempted on the axilla. Occasionally there are metastases in the organs, spleen, liver, adrenal, pancreas, and kidney (Zniniewicz). Perforation of the chest wall has occurred in several cases and was the first localizing symptom in a case I have recently observed (Seebohm, Zniniewicz, Le Tulle). Fracture 
of the humerus from bone-marrow metastases is recorded by Zniniewicz, and infiltration of the orbits, brain, and other organs by Meigs and de Schweinitz.

The structure of these tumors varies greatly. Exactly the same diffculties are encountered in their histological classification as one meets with tumors of lymph-nodes. In one group the structure resembles that of an infectious granuloma of the type of Hodgkin's disease. The tissue presents lymphocytes, plasma-cells, and larger polyhedral cells irregularly distributed. Several cases of this type have been recorded, with emphasis on the presence of many large polyhedral or giant-cells (Ertmann, Weigert, and Laquer). These large cells must be derived from the reticulum. When they become very numerous the lymphocytes largely disappear and the tumor may be classed as a carcinoma, as has commonly been done by French writers (Le Tulle). In another group the reticulum cells are said to be missing and the

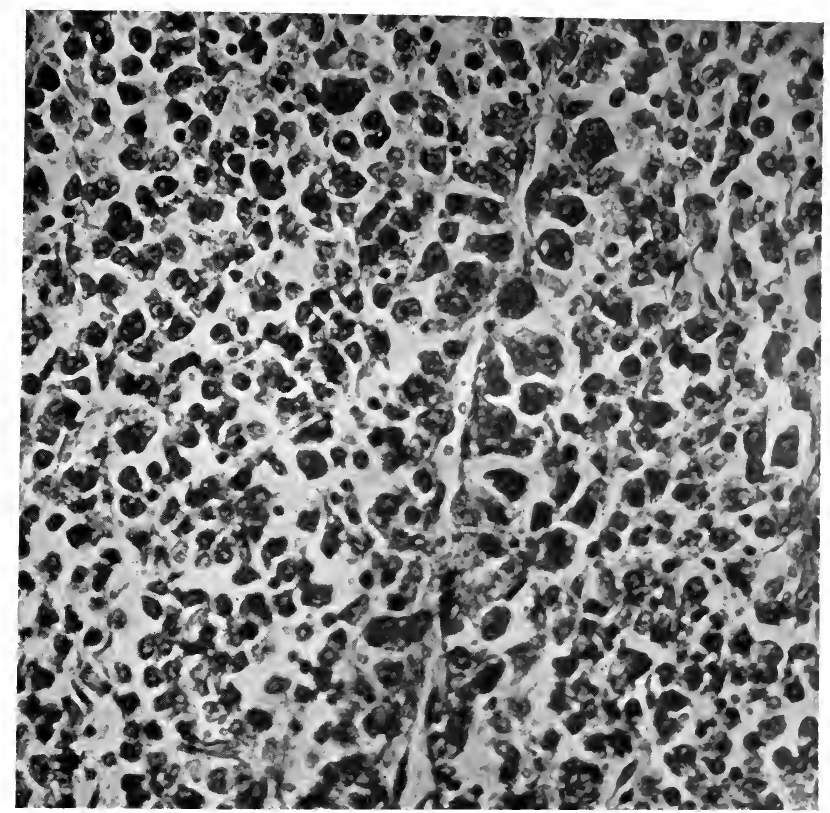

FIG. 479.-Malignant thymic tumor composed of large round polyhedral and columnar cells.

tumor is composed of a diffuse growth of small round cells (Le Tulle, Stockart). These tumors have not been distinguished from other lymphosarcomas, but it does not appear that any definite effort has been made to do so. The existence of a pure lymphocytoma of the thymus apart from leukemia does not appear to have been established.

The blood-vessels may be very numerous and in some cases cells of medium size may form sheaths about the ressels. When the perivascular arrangement becomes very marked and lymphocytes are scanty the diagnosis of endothelioma or perithelioma may be suggested, as in the cases of Hahn and Thomas and Mandelbaum and Celler.

It seems highly probable that these cells arise from the reticulum, producing an analogue of perivascular endothelioma of the lymph-nodes. In 
the same manner may be explained the mixed tumors described by Gabcke and Schneider, who found round and spindle- and many giant-cells in their tumors, all of which may readily be derived from the reticulum.

On close analysis the round-cell tumors of the thymus are found to differ in structure from the round-cell tumors of lymph-nodes. The lymphocytes are scanty. The chief cell showing mitosis is often polyhedral, with acidophile cytoplasm, vesicular nucleus, and well-developed nucleoli. They often cling to the walls of numerous small capillaries where they assume a cubical or even cylindrical form. They may produce abortive Hassall's corpuscles. The giant-cells are of two main types: (I) pale staining reticulum cells with irregular outlines, distended with vacuoles and red cell detritus, and (2)

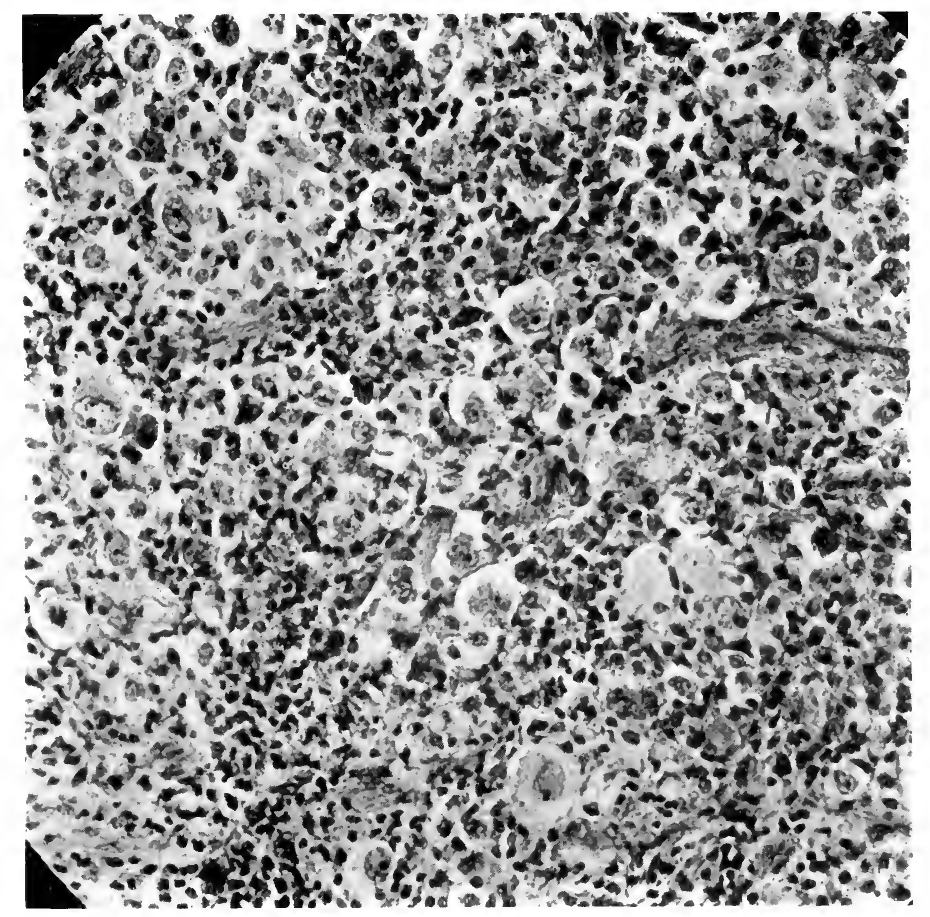

FIG. 480.-Portion of thymoma perforating sternum. Note the many peculiar giant-cells.

myeloid giant-cells with opaque acidophile cytoplasm and many vesicular nuclei. These giant-cells differ from the smaller giant-cells of lymphatic Hodgkin's disease. The marked fibrosis suggests the desmoplastic property of carcinoma. In a report of 3 cases I have described these and other structural features in detail.

(2) Thymic Carcinoma.-In many cases the main tumor-cell appears in the form of pavement, cubical, or rarely cylindrical epithelium and the growth must be classed as carcinoma.

The gross anatomy of thymic carcinoma is identical with that of the hard thymomas of round-cell type. Although metastases may occur, it is notable that the invasion of surrounding organs is less active than is usual with a distinctly carcinomatous tumor. An aberrant thymic carcinoma, containing 
lymphoid tissue and numerous bodies resembling Hassall's corpuscles, was observed in the thyroid by Achard and Paisseau.

The structure in typical cases presents coherent sheets, cords, and columns of large flat or polyhedral cells lying in dense connective tissue. Hornification is absent, but concentric layers of flat cells may form structures resembling Hassall's corpuscles (Thiroloix, Debret, Paviot-Gerest). In other cases the pavement characters are less evident and the cells are chiefly cubical and form alveoli. Le Tulle and Ambrosini found accumulation of mucus in the spaces of an alveolar carcinoma. In many cases both round cells and epithelium participate in the tumor process, and the authors speak of the growth as carcinosarcoma or adenosarcoma. Thus in Rubaschow's case

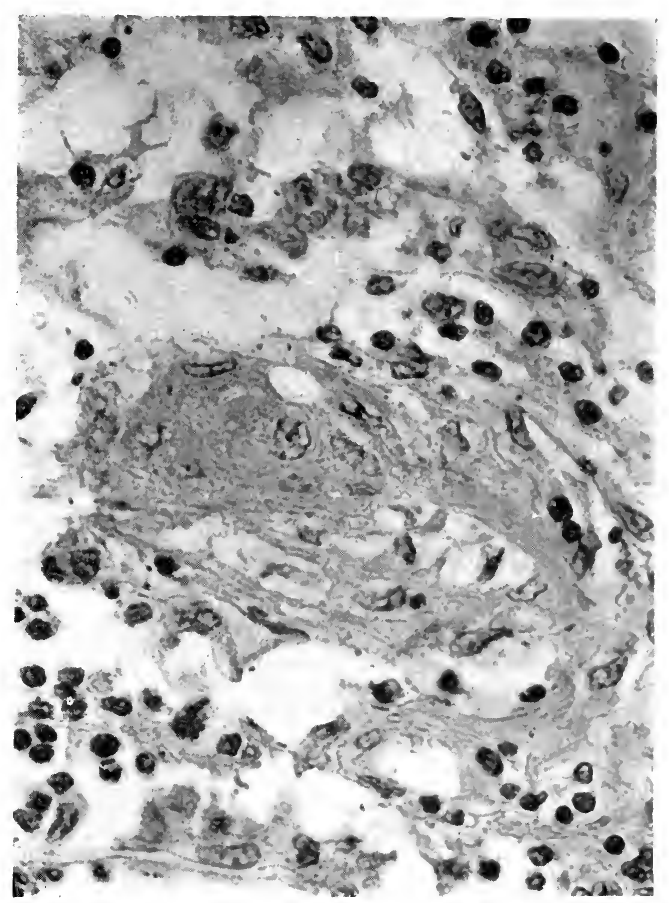

FIG. 481.-Thymoma. Duration 3 years. Structure resembling Hassall's corpuscles.

the main mass was composed of round cells, in which lay many foci of flat epithelium forming pearls or surrounding blood-vessels. Giant-cells of a variety of forms are frequently present.

(3) Thymic Sarcoma.-Although it has been commonly assumed that various spindle-cell or alveolar or perivascular tumors arise from the connective-tissue stroma of the thymus, this origin has never been fully traced and there are strong grounds for concluding that the so-called spindle-cell sarcomas and endotheliomas are varieties of thymoma. Congenital myxoma, ro $\times 8 \mathrm{~cm}$. in dimensions, weighing $182 \mathrm{gm}$., containing lymphocytes and Hassall's corpuscles in the tumor tissue, was observed by Caso in an infant of $2 \frac{1}{2}$ months, and a similar case is described by Winogradoff. 
Interpretation of Thymomas.-The foregoing review of the structure of thymus tumors reveals extreme confusion in the nomenclature employed by different authors, great difficulty in establishing sharply defined varieties and the existence of transitional forms connecting the two types. The great polymorphism of the cells noted by Ambrosini has been emphasized by later writers as the chief characteristic of thymic neoplasms and has led to the use of the term "thymoma."

It is significant that the carcinomas have been recorded almost entirely by French observers, while practically all the German reports are of sarcoma. Yet Le Tulle and Ambrosini describe as carcinoma tumors which have many of the features which Ertmann and Zniniewicz have designated as sarcoma. It is also clear, as in Dansac's, Hauser's, and Rubaschow's cases, that many tumors present an overgrowth of both reticulum and parenchyma cells. A

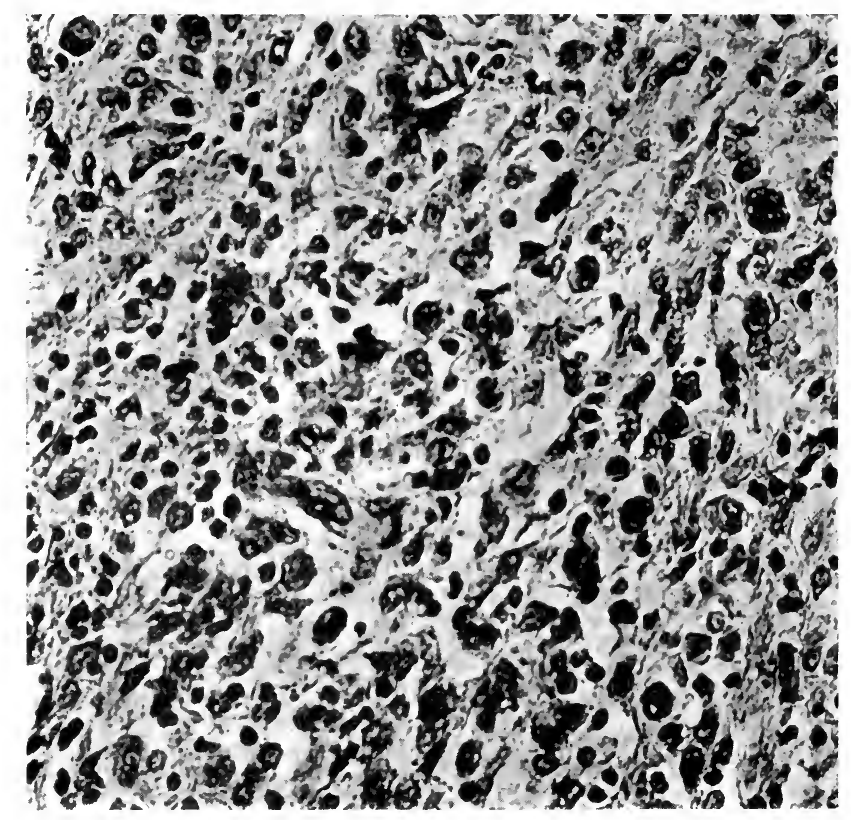

FIG. 482.-Thymoma of granulomatous type. Proliferation of large reticulum cells, scanty lymphocytes.

full survey of the structural variations reveals at one extreme a mixed process involving lymphocytes and reticulum cells, with giant, plasma, and eosinophile cells, producing a structure nearly identical with Hodgkin's granuloma. At the other extreme are nearly pure tumors of rounded or polyhedral reticulum cells, $i$. e., lymphosarcoma and carcinoma. Exactly similar relations exist between tumors of lymph-nodes, including Hodgkin's granuloma and the "sarcomas" arising from it, pseudoleukemia, and pure endothelioma. Hence the conclusion is reached that the great majority of thymus tumors and especially the mixed growths, represent infectious granulomas or particular forms of cell overgrowth arising on the basis of an infectious granuloma. Detailed evidence supporting this conclusion is presented in the writcr's study of endothelioma of lymph-nodes. This etiological point of view offers a 
simple explanation of the great variety of structural forms which thymus tumors present.

In a series of cases of Hodgkin's granuloma the granulomatous process has shown malignant properties both in local aggressiveness and in the production of metastases. Such cases are recorded by Yamasaki, Chiari, J. E. Welch, Symmers, Karsner, and Beitzke. In most of these cases it is stated that the main tumor was mediastinal and occupied the region of the thymus, while the structure presented a diffuse growth of cells larger than lymphocytes and many giant-cells of myeloid type. In the report of Symmer's case, studied in this laboratory, a thymic origin was suggested. I have reexamined various portions of this tumor and find in it all the features of thymoma, including Hassall's corpuscles, polyhedral reticulum cells, and myeloid giant-cells. In the light of this and other cases it seems highly probable that the mediastinal Hodgkin's disease of the above writers is a thymic tumor which should be separated from other forms of Hodgkin's disease and owes its malignancy to its origin from the peculiar reticulum cells of the thymus.

Clinical Course.-Many thymus tumors are highly malignant and prove rapidly fatal from asphyxia, but the actual duration is difficult to determine. Ambrosini's 5 cases were all fatal in from 2 to 9 months. The very rapidly. growing tumors are usually very cellular and vascular. In I of Zniniewicz's cases, lasting ro weeks, there were general metastases, while in Ambrosini's case of 2 months' duration the extensions were local. Ertmann's tumor of 2 months' course was vascular, contained very large polyhedral cells, areas of perivascular growth and collections of colloid, but metastases were limited to the pleura. The tumor described by Hahn and Thomas reached dimensions of $26 \times 18 \times 9 \mathrm{~cm}$. in I year.

Constitutional symptoms suggesting a sympathetic disturbance of the chromaffin system are not observed, but Gabcke records for his case that the adrenals were very large and the skin pigmented.

In a notable group of cases thymic tumor has been associated with myasthenia gravis (Oppenheim, Weigert and Laquer, Buzzard). Of 45 cases of myasthenia gravis, Mandelbaum and Celler found thymus lesions recorded in II. The thymic tumor has usually shown the structure of lymphosarcoma mingled with epithelioid cells. In Hun's case the epithelioid cells were abundant, but showed no tendency to form Hassall's corpuscles, while plasma-cells, eosinophile cells, and focal hemorrhages are often present. Mandelbaum and Celler found a tumor composed of small concentric groups of polyhedral cells, while the numerous vessels were sheathed with lymphocytes and surrounded by tumor-cells. The tumors are usually of moderate size, and in several cases the lesion was regarded as simple hyperplasia (Link, Burr, Buzzard).

Throughout the skeletal muscles and often in the organs are found foci of lymphocytes with varying numbers of polynuclear leukocytes, and eosinophile, plasma, or epithelioid cells. Weigert and Laquer regarded these lesions as metastatic foci, while others consider them as of local inflammatory origin. Their occurrence favors the view that many thymic tumors are manifestations of an infectious granuloma. 


\section{CHAPTER XLVII}

\section{TUMORS OF THE HYPOPHYSIS}

Because of the remarkable clinical phenomena with which they are associated, uncertainty regarding the origin and nature of the processes concerned, and the peculiar problems involved in their diagnosis and treatment, tumors of the hypophysis form one of the most interesting of all groups of neoplasms.

The history of our knowledge of pituitary enlargements begins with isolated anatomical reports dating from 1700 (Bonnetus) and 1705 (Vieussens) and continuing until I8S6, during which period tumors discovered at autopsy were known to have caused chiefly cerebral pressure symptoms. In I856 Marie initiated a second period of more minute clinical study, by pointing out the essential connection between pituitary tumors and acromegaly, while, later, gigantism was included among the clinical phenomena by Cunningham, Tamburini, Brissaud and Meige, and Launois and Roy.

Although the association of obesity with hypophyseal tumors was noted as early as 1840 by Mohr, and hypoplasia of the genital organs as well as obesity were described by Pechkranz, the considerable frequency of this association was first emphasized by Froelich, I901, and by Bartels and Cushing, I906. The modern period of elaboration of the clinical features may be said to date from these observations which have led directly to more accurate diagnostic methods especially by the $x$-ray and to the development of physical and surgical treatment. Cushing especially has demonstrated that the natural history of primary hypophyseal disease passes through an initial period of overactivity of the gland with acromegaly, into a later status of underactivity with adiposogenital dystrophy.

The use of the $x$-ray introduced by Beclere (IgO2) greatly facilitated the diagnosis and clinical study of the tumors, and this agent was successfully employed in treatment by Gramegna and Beclere in I909. Hypophysectomy was first performed by Horsley; Schloffer, and v. Eiselsberg in I90\%, and successful results were soon reported by Hochenegg, Hirsch, Cushing, Lecene, and others. Minute histological studies were contributed by Benda (Igoo to Iç04), Erdheim (I904 to I9I0), Lowenstein, I907, Cushing, Lewis, and many others, and considerable success has followed the attempt to attach the various syndromes to different types and locations of the tumors. The discovery of accessory pharyngeal pituitary tissue by Haberfeld served to elucidate some problems, while complicating others. The obscurity surrounding the interrelations of the ductless glands includes the functions of the hypophysis and its influence on glycosuria, polyuria, and many other disturbances of internal secretions, and most of these questions still await final answer. This phase of the subject is at present being rather actively pursued.

General Etiology.-Tumors conveniently regarded as hypophyseal are relatively uncommon and many of the recorded cases must be interpreted as simple hyperplasia. Nevertheless the series of cases now available for statistical study is numerous. Collarit in 1905 collected 52 cases without acromegaly or glycosuria. Creuzfeld, I908, in 60 autopsies on cases of acromegaly, reported 47 hypophyseal tumors and collected 55 tumors without acromegaly. Eleven tumors were associated with gigantism. FranklHochwart, I009, collected $\delta_{5}$ cases of various types of tumors. Strada, I9II, 
collected 33 cases of tumor without acromegaly, but with obesity and genital hypoplasia.

Among 3620 autopsies at Prague 4 hypophyseal tumors were found by Klebs, while Boyce and Beadles collected 6 tumors in 3000 autopsies.

Owing to the uncertainties of histological diagnosis the relative frequency of the different types of tumors cannot be accurately estimated. Epithelial neoplasms of the anterior lobe, with much excess of eosinophile or chromophobe cells and associated with acromegaly, are the most common. Of 47 such cases Creuzfeld found i 5 described as sarcoma, to which he added one glioma of the posterior lobe. On the other hand, this author collected 55 cases without acromegaly, of which I $_{5}$ were designated as sarcoma, is as glandular, r9 as squamous-cell growths, one teratoma, and one lipoma of the posterior lobe. Frankl-Hochwart's series contained I 2 carcinomas (7 squamous); 22 glandular ( 13 adenomas); 27 sarcomas; I 5 cysts, 3 gliomas, and 2 teratomas. Of Strada's 3 I cases with obesity and genital hypoplasia 2 were described as simple struma, 5 as adenoma, 5 as carcinoma, Io as acanthoma of infundibulum, and 9 as sarcoma. It is probable that all the sarcomas were really epithelial tumors. Cushing reports about 29 hypophyseal tumors examined microscopically with analyses of symptoms and refers to I 48 cases of hypophyseal disease observed clinically.

An hereditary element appears distinctly in certain cases of gigantism which has affected several members of the same family (Cushing, Case 3 I, de Neuville). Cerebral trauma occurred in 6 of Cushing's cases of hypopituitarism, in 5 of which there was an interpeduncular tumor. The marked functional hyperplasia of pregnancy appears to have led to progressive changes with acromegaly and secondary hypopituitarism. Lowenstein and Erdheim found several definite adenomas in the glands following pregnancy. Ascenzi observed acromegaly develop after ten rather rapidly succeeding gestations, the hypophysis containing a large cystadenoma. Occasionally acromegaly has followed attacks of pulmonary tuberculosis, typhoid fever, or pneumonia (Massedaglia, Cushing).

Acromegaly has been referred to severe fright by Ingermann and Poindecker and has followed ovariotomy in reports of Strumpell and Goldstein.

Anatomy of the Hypophysis. - The normal anatomy of the hypophysis is complex and several embryological and histological features are of prime importance in tracing the origin of tumors of this organ. The weight of the hypophysis from the third to seventh decade averages $61.2 \mathrm{gm}$., and its size $21.5 \times 14.4 \times 5.5 \mathrm{~mm}$. Variations in weight from 30 to $75 \mathrm{gm}$. are probably normal (Erdheim).

The organ consists of (1) an anterior glandular lobe which is developed by an upward evagination of oral ectoderm, (2) a posterior nervous portion which is an elongated portion of nervous tissue derived from the tuber cinereum at the floor of the third ventricle, and (3) a stalk or pedicle which passes through the arachnoid and connects the organ with the tuber cinereum.

(1) The anterior lobe or pituitary gland proper, is partly subdivided into two lateral portions by constriction of the encircling posterior lobe. A long lingual process of this lobe encircles the stalk as a glove over a finger, covering the anterior surface below and completely surrounding it above and almost to the optic chiasm. A cross-section of the stalk therefore presents a central cavity, the prolongation of the third ventricle (infundibulum), surrounded by a zone of nervous tissue which is sheathed in turn by the lingual process of the anterior lobe. This lingual process is often designated as pars intermedia. The lingual process often contains small cysts, and Erdheim showed that it very frequently contains groups of squamous epithelium, remains of the oral ectoderm, which give rise to tumors. The presence of such epithelial débris had previously' been described by Luschka, Saxer, and Launois, who also suggested its probable relation to tumors of the brain.

The anterior lobe is separated from the posterior by a connective-tissue septum which usually contains a multilocular cleft, the remains of Rathke's ectodermal pouch. About this cleft Erdheim describes the remains of salivary gland alveoli. 
(2) The posterior lobe (pars nervosa) is a club-shaped mass of glia-tissue which descends from the stalk and partly surrounds and constricts the anterior lobe. It is largely surrounded by a thin sheathing of neutrophile epithelium merging with a similar sheathing of the stalk, and continuous with the epithelium of the anterior lobe from which it is derived. The glia-cells contain variable amounts of insoluble pigment which is probably derived from the wandering cells of the anterior lobe or pars intermedia (Kohn, Vogel).

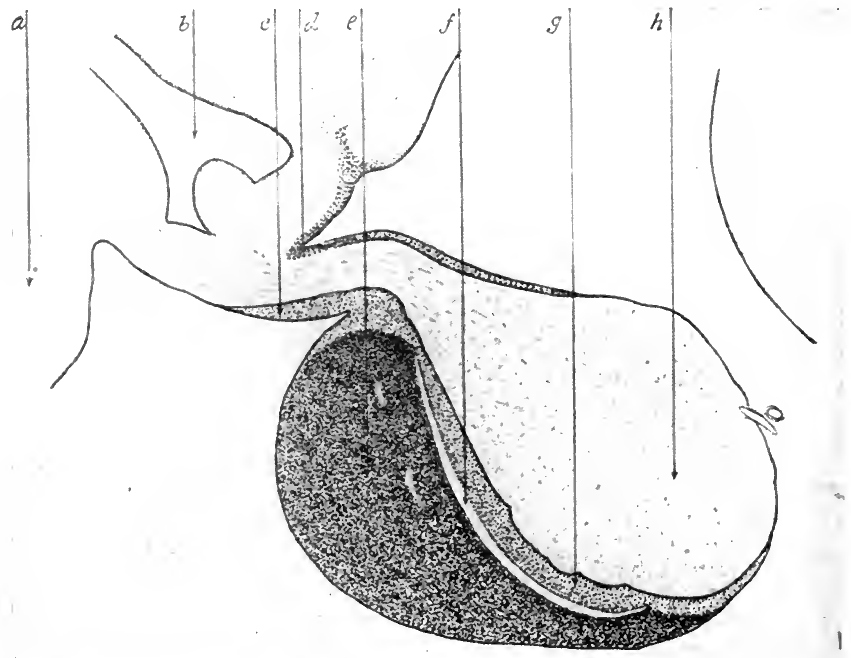

FIG. 483.-Median sagittal section through pituitary of monkey; semidiagrammatic. (Herring.) $a$, Optic chiasma; $b$, third ventricle; $c, g$, pars intermedia; $d$, epithelium of pars intermedia extending round neck of pars nervosa; $e$, pars glandularis seu epithelialis; $f$, intraglandular cleft, lying between pars glandularis $(e)$ and pars intermedia $(g) ; h$, pars nervosa.

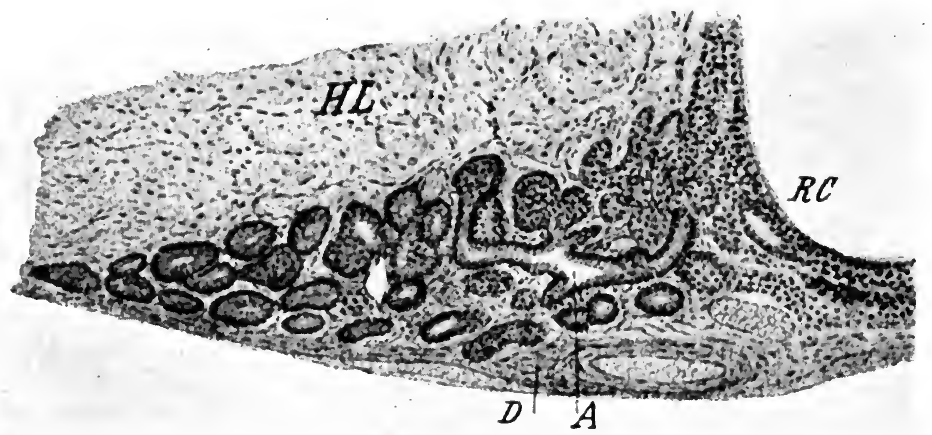

FIG. 484.- Salivary gland alveoli in the neighborhood of Rathke's cyst between anterior and posterior lobes of hypophysis. They give rise to cysts of the hypophysis. (After Erdheim.)

The pars intermedia is all that sheath of epithelium which surrounds the posterior lobe and stalk, including the lingual process. Its peculiar relations to the inclosed nervous tissue render it of much physiological interest. Its epithelial cells exhibit evidences of secretion, in the form of many colloid globules which collect in round masses between the cells and have been traced apparently passing through the pars nervosa into the iniundibulum and thence to the third ventricle and cerebrospinal fluid (Herring, Cushing, Goetsch). 
(3) The stalk or pedicle connects the organ with the tuber cinereum, passing through the dural septum and arachnoid. It contains a central cavity, infundibulum, which is the downward prolongation of the third ventricle, surrounded by a mass of nerve tissue which enlarges below to form the pars nervosa, while externally it is sheathed by the lingual process of the anterior lobe.

The whole organ is enclosed in a dural capsule which has an upper diaphragm stretching from the four clinoid processes and is perforated by the pedicle. Pressure of an enlarging gland is therefore slightly limited above and laterally, while below there is bony resistance.

The blood-supply of the posterior lobe is derived from one of three slender vessels entering below from the internal carotid, while the vessels of the anterior lobe pass down the stalk. Sympathetic nerve-fibers also pass down the stalk (Dandy).

Accessory Pituitary Glandules.-Rathke's pouch, which is the remnant of the oral ectodermal evagination, does not always undergo complete involution, but regularly in some lower animals, and occasionally in man, it persists and gives rise to accessory gland-

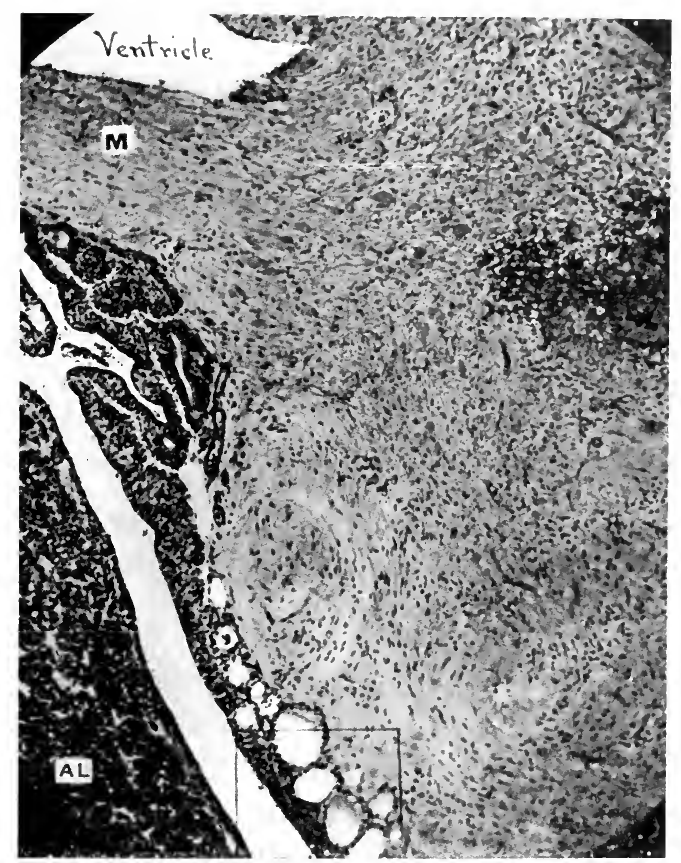

FIG. 485.-Topography of dog's hypophysis. $A L$, Anterior lobe. Note hyaline material in epithelial investment of pars nervosa. (After Cushing.)

ules. These were first observed by Erdheim'in the pharyngeal mucosa and later by Arai and Civalleri. Haberfeld in 51 human subjects of various ages regularly found a thin glandular strand, 3 to $9 \mathrm{~mm}$. in length, 5 to $5 \frac{1}{2} \mathrm{~mm}$. in breadth, in the pharyngeal submucosa just behind the alæ of the vomer, and extending inward to the base of the skull. These glandules are composed chiefly of chromophobe cells, but acidophile cells are commonly present and flat epithelium may be observed at the lower pole. Dandy and Goetsch found similar glandules in a central pit in the sellar floor of dogs. Erdheim has described an intra-osseous tumor of acidophile cells, with acromegaly, which probably arose from such an accessory glandule, and he derives certain squamous-cell tumors from this same source. Distinct heterotopia of the entire pituitary body below the sella has been reported (Haberfeld, Lit.).

Histology. - The structure of the pituitary body is subject to wide variations which are of much significance in the interpretation of functional changes and neoplasmis of this organ.

The normal gland contains two main classes of cells, chromophile and chromophobe 
(Flesch). A third cell type is chromophobic or neutrophile. The chromophile cells are either $(a)$ acidophile or $(b)$ basophile, by virtue of the microchemical reactions of their abundant granules (Schoneman). The acidophile cells are usually more numerous along the central sinuses, while the basophiles occupy the periphery and anterior portion of the lobe. It is not yet clear whether the two types are distinct or represent different functional phases of the same cell order. Benda and others accept the former view, while Erdheim supports the latter. The acidophile granules after fixation in Müller-formol stain deeply with eosin, black with Heidenhain's hematoxylon, deep red by Mallory's stain, and usually decolorize in Gram's stain. These cells are normally the most abundant of the three types. The basophilic cells are less numerous, but larger than the acidophiles, and the large granules stain deep blue by Mallory or Gram, violet with hematoxylon, and are decolorized by complete differentiation in iron hematoxylon. The nuclei are large and pale.

The chromophobe cells are the least numerous of the cell types, and the smallest. The cytoplasm is scanty and the nuclei are relatively compact. They often appear in groups resembling acini, and surrounded by chromophile cells.

In the pars intermedia the cells lie in small groups surrounded by connective tissue. Often they form alveoli of cubical or cylindrical cells surrounding colloid globules, and

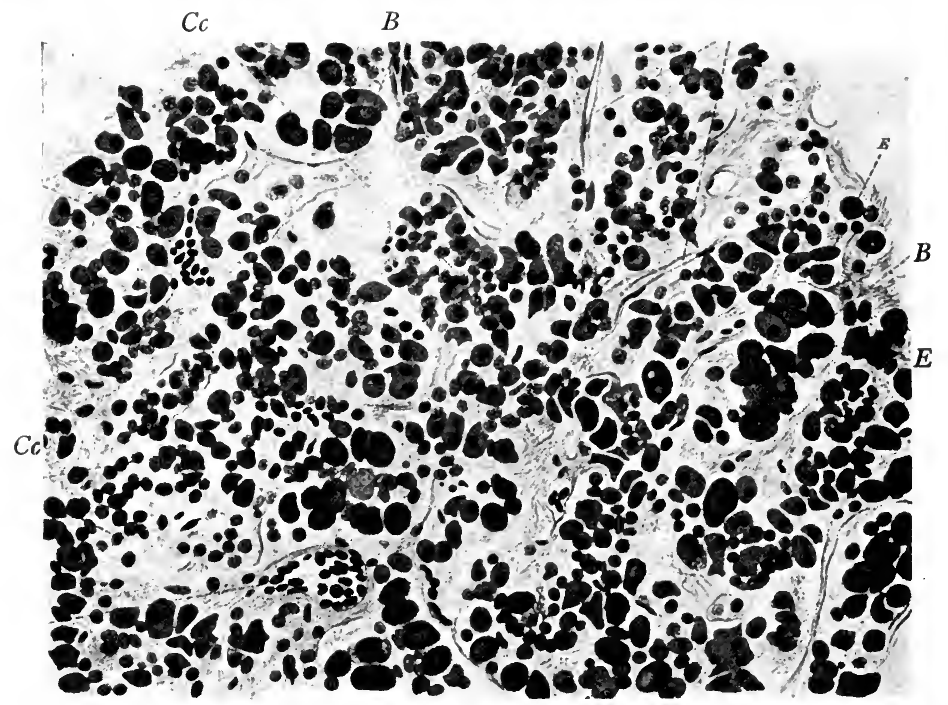

FIG. 486.-Normal hypophysis of a subject of 37 years. Stain, hematoxylon and eosin. $E$, Eosinophile cells, large and small; $B$, basophile cells, large and granular; $C c_{\text {, }}$ chief cells, small with little cytoplasm. (After Erdheim and Stumme.)

they have a close relation to the blood-vessels. The cytoplasm is clear, chromophobic, and lacking in specific granules (Lewis). Numerous special methods for the demonstration of cell granules have been recommended, but none appears to have an essential advantage over fixation in Müller-formol and staining by hematoxylon and eosin. For demonstration of basophilic granules Erdheim prefers long staining in kresofuchsin, preceded by lithium carmine as a nuclear stain. Many prefer Zenker fixation.

Functional changes in the pituitary gland are frequent and so pronounced as often to simulate neoplasms. The range of alterations associated with disturbed function is even greater than occurs in the thyroid gland, and it is probable that true tumors develop on the bases of functional hyperplasia. Probably the most marked series of changes occurs in gestation, in which extensive and specific hyperplasia has been described by Comte, Launois and Mulon, Erdheim, and others. According to Erdheim, hyperplasia begins early in the first gestation, reaches its height at term, persists several weeks or months after parturition, and is more pronounced in succeeding pregnancies. 
The size of the normal hypophysis averages $6 \mathrm{r} .2 \mathrm{gm}$. in adults, but reaches an average size of $84.7 \mathrm{gm}$. in primiparæ, and $106 \mathrm{gm}$. in multiparæ. The hyperplasia affects only the chief or chromophobe cells which greatly increase in size and number, becoming more abundant than the chromophiles. The cytoplasm contains many fine granules staining slightly with Heidenhain's hematoxylon. These cells multiply by mitosis, form large solid groups, and displace the chromophile elements. In so per cent. of the cases the specific cells of pregnancy form single or multiple focal adenomas I to $4 \mathrm{~mm}$. in diameter, free from fat globules, but without marked variation from the normal type and without encapsulation. They probably regress after the puerperium and are not true tumors. Transitions to atypical adenoma of malignant appearance may be observed, in which the cords are narrow or very broad or present a peritheliomatous arrangement, but the clinical course of such adenomas cannot be predicted from their structure. In 4 cases of gestation-hypertrophy Erdheim saw adenomas of chromophile cells. Pressure

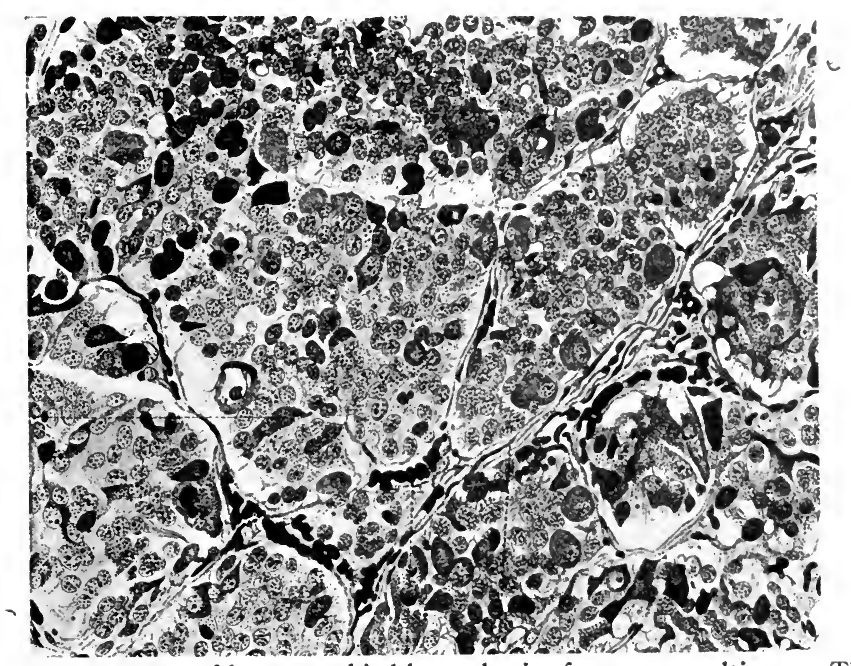

FIG. 487.- Structure of hypertrophied hypophysis of a recent multipara. The cords are composed chiefly of the hyperplastic cells of gestation, among which are many large basophile cells. The eosinophiles are black. Stained by Heidenhain's hematoxylon. (After Erdheim and Stumme.)

symptoms, as hemianopsia, may apparently result from the hyperplasia of gestation (Reuss).

Varying grades of pituitary hyperplasia have been observed in infectious diseases. Experimental studies of the effects of extirpation or ingestion of the substance of other glands of internal secretion have supported the view that there is a close interdependence of all these glands, but the exact types of pituitary hyperplasia produced by these methods still remain to be accurately defined.

Gross Anatomy of Hypophyseal Tumors.-The several portions of the hypophysis each give origin to tumors which may be classified accordingly as:

(I) Tumors of the pituitary body;

(2) Tumors of the hypophyseal duct and its derivatives;

(3) Tumors of the pars intermedia;

(4) Tumors of the pars nervosa. 
I. Tumors of the pituitary proper include:

(a) Diffuse hyperplasia and focal adenoma;

(b) Adenocarcinoma;

(c) Malignant atypical carcinoma (sarcoma).

(a) Diffuse hyperplasia arising from functional overgrowth occurs in most advanced form after multiple pregnancies when the gland may reach a weight of $165 \mathrm{gm}$. (Erdheim). The growth distends the dural diaphragm, spreads laterally, may produce hernia of the capsule and in cases with some anatomical predisposition may press upon the optic chiasm with hemianopsia. Considerable widening of the sella may exist with all the simple hyperplasias. On section the overgrowth may be diffuse, or in about ro per cent. of the cases focal adenomas may appear as opaque spots 3 to $7 \mathrm{~mm}$. in diameter.

The natural course of these hyperplasias is slow regression, but some probably pass into malignant and progressive adenocarcinoma. The structure presents compact groups or narrow or wide cords of chromophobic cells derived from the chief cells of the gland.

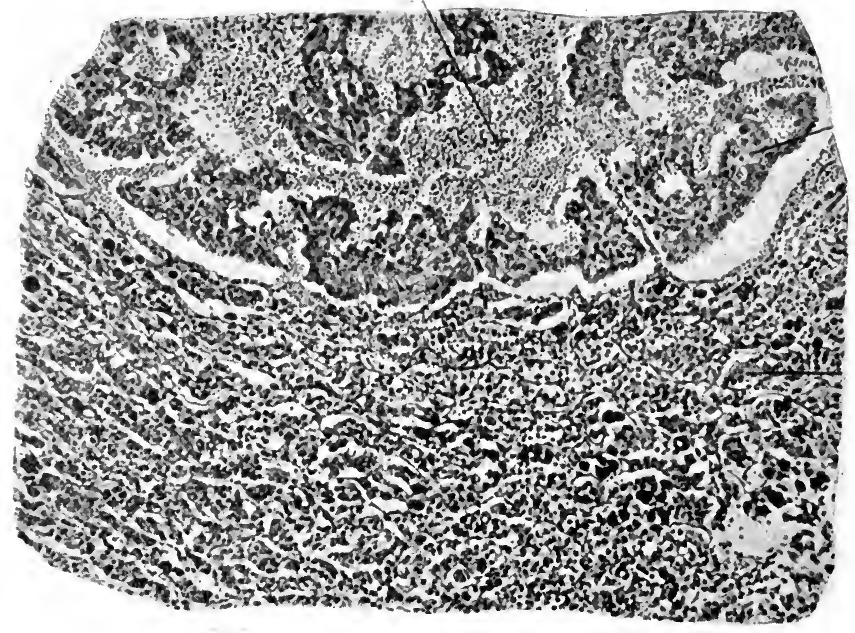

FIG. 488.- Hypophyseal adenoma of gestation. (After Erdheim.)

In many cases of acromegaly the glandular lesion takes the form of a diffuse hyperplasia often with adenomas. In 56 cases of acromegaly Creuzfeld found I 2 glands with simple hyperplasia, 8 designated as struma, I 2 as adenoma, I5 as sarcoma, and 5 without demonstrated hypophyseal lesions. There is thus a wide variety in the extent of the glandular lesion associated with acromegaly, and it appears that this disease may result from very moderate hyperplasia or from very small adenomas. As a rule the changes in acromegaly pass far beyond those observed in gestation, and yet in many instances the hyperplasia is succeeded by regression and the signs of overactivity are followed by glandular insufficiency.

Many of the glandular lesions in acromegaly are therefore not true tumors, but self-limiting functional hyperplasias. The structure of these enlarged glands often presents an overgrowth of eosinophile cells which are increased in size and number, form compact masses or adenomatoid accumulations and displace the other cell types. The arrangement of the cells is orderly 
and mitoses are rare. While the gland usually remains solid and firm, small cysts filled with colloid may develop and hemorrhages may occur.

The areas of eosinophile cells accord with the view that the overactivity of the gland resides in these cells. Yet many of the moderately enlarged glands of acromegaly contain not acidophile but chromophobic cells (Cushing). Eosinophile adenomas are observed also in gestation.

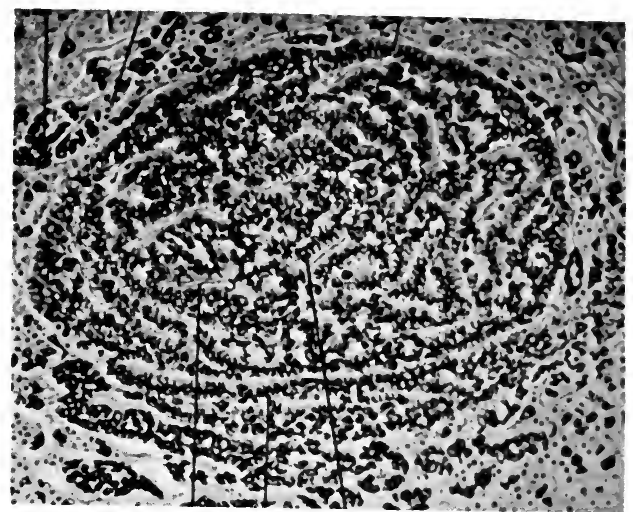

FIG. 489.-Miliary basophile adenoma of hypophysis. (After Erdheim.)

Roussy and Clunet describe a form of pathological hyperplasia occurring in alcoholism, nephritis, Parkinson's disease, and arteriosclerosis, and signifying a reaction to the failure of thyroid function. Hyperplasia of the basophilic cells with the production of small adenomas is described by Erdheim, who found such a nodule $I \frac{1}{2} \mathrm{~mm}$. in diameter in the anterior lobe. In a case of acromegaly he found both eosinophile and basophile adenomas of small size.

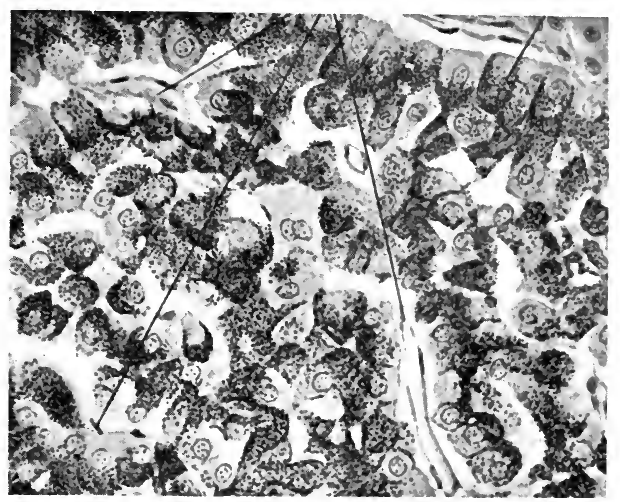

FIG. 490.-Detail of miliary basophile adenoma of hypophysis. Stain, kresofuchsin. (After Erdheim.)

Nothdurft describes a small basophilic adenoma in the posterior lobe. In none of these cases does it appear that the adenoma was responsible for any local symptoms. In the giant acromegalic subject of Huchard and Launois the tumor appeared to be composed chiefly of basophilic cells. A malignant tumor of such cells has not yet been demonstrated, although Nagaeli assumed 
that a large peritheliomatous growth composed of non-granular cells had such an origin. Pick describes an extensively calcified tumor in a case of adiposogenital dystrophy which he designated as basophile adenoma or adenocarcinoma.

(b) Adenocarcinoma.-True adenoma of the pituitary may be difficult to recognize by its size and structure, but it is probable that true neoplasms of this gland are always atypical in structure and malignant. In a considerable group of cases the gland exceeds in size the limits of simple hyperplasia, the structure is distinctly atypical, the capsule is ruptured, extension beyond the limits of the sella is observed, pressure symptoms are marked, and the process must be classed as malignant adenoma or adenocarcinoma.

The size of these tumors varies greatly, but they regularly distend or rupture the dural diaphragm, they widen and absorb the sella so that the $x$-ray shows extensive excavation, the clinoid processes are often destroyed and the basilar portion of the sphenoid may be extensively eroded. Growing beyond the sella the tumor compresses the optic chiasm, extends along the

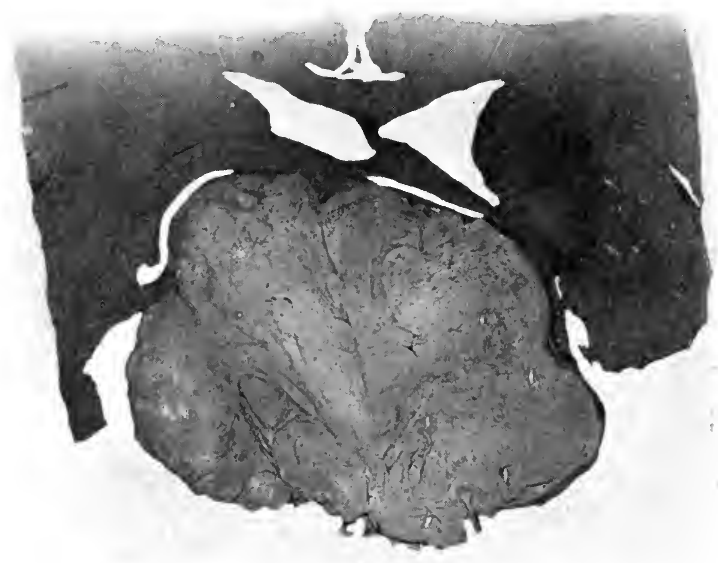

FIG. 49I.-Cross-section through a large hypophyseal adenoma.

frontal lobes, and penetrates the third and even the lateral ventricles. With reference to their bony relation the growths may be described as intrasellar, with uniform widening of the bony wall, or as extrasellar when the clinoid processes are absorbed, and as voluminous when tumors absorb much of the bone and extend into the brain. Usually the tumors long remain solid, but cysts form from colloid secretion, hemorrhage, and necrosis. In one group the tumor tends to become cystic from the first and many areas filled with soft colloid material are traversed by thin strands of epithelium, or wellmarked papillary outgrowths develop. These tumors are difficult to distinguish from papillary epithelioma of the ventricles, but their location emanating from the sella is usually decisive. While the majority of growths long remain encapsulated and preserve the general form of the organ, extension into the brain is often accompanied by diffuse invasion of this tissue.

In advanced cases the tumor has perforated the cavernous sinus, invaded frontal and temporal lobes, compressed many of the cranial nerves, and extended to the peduncles, medulla, and even to the cerebellum. Pechkranz's tumor penetrated the orbit, and Kruger's large round-cell "sarcoma" 
eroded the atlas at the base of the skull. In several cases the growth has communicated with the superior nares and produced a watery or bloody discharge (S. Ingermann, O'Malley, Sommer). Açcess to the ventricles may be accompanied by hydrocephalus.

Metastases occasionally occur. With comparatively typical glandular tumors Smoler found a cerebellar metastasis; Stolpe reports a large secondary growth in the occipital lobe; Bruns saw a nodule in the tongue; Cagnetto describes subpial nodules along the spinal cord from a colloid adenocarcinoma in a remarkable case of acromegaly.

The structure of the pituitary adenocarcinomas varies extensively. Some resemble the adenomatoid hyperplasia with solid cords or compact groups of atypical polygonal epithelium. These tumors are of moderate malignancy,

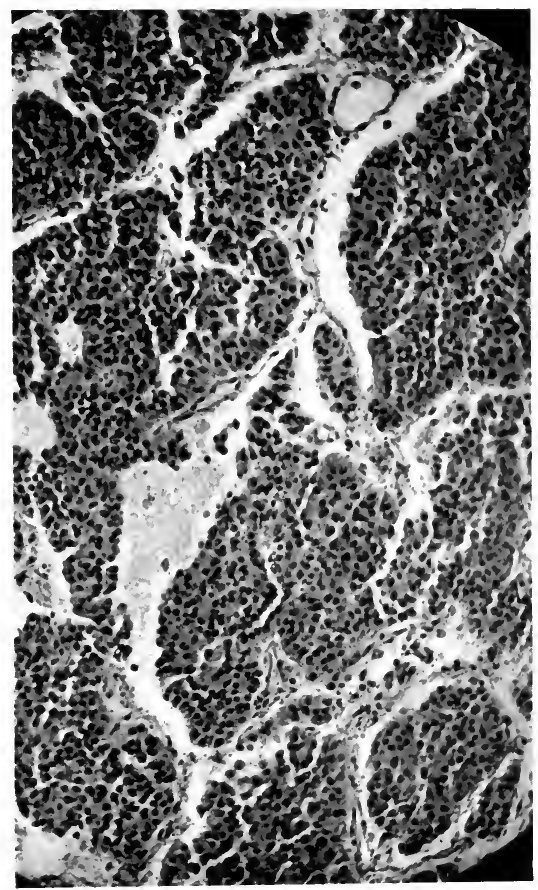

FIG. 492.- Hypophyseal adenoma, with large imperfectly walled blood sinuses.

and while most of the cells are distinctly chromophobic, traces of the chromophile differentiation may at times be detected. Chiefly acidophile cells occurred in the cases of Benda, Tamburini, Zak, Alquier, Presbeanu, Marinesco, Cagnetto, and Erdheim (1903 to 1904, I909, r910). Cushing describes most of his cases as chromophobe struma, as do Carbone, Lecene, Strada, Krumbhaar, and others. In general the latter group is more distinctly neoplastic.

Other tumors are more atypical, composed strictly of chromophobic cells, arranged in cords or groups, or often forming distinct acini in which colloid secretion may accumulate. Excess of colloid or fluid may yield soft cystic growths in which a papillary structure may appear. A perivascular arrangement of cubical or cylindrical cells is common (Moskalew), and the 
numerous blood-vessels with their epithelial sheaths may simulate a papillary adenoma or angiosarcoma. Many cellular growths fail to show any subdivision of the cells into groups or acini and the structure is diffuse. In different portions of the same tumor the structure may vary widely. Thus Launois and Roy and Strada describe an alveolar structure in intrasellar portions, and a diffuse growth in extrasellar areas of their tumors. Meyer describes new growth of nerve-cells in the pars nervosa of a case of acromegaly and diabetes with hypophyseal tumor.

(c) Malignant atypical carcinoma has usually been reported as sarcoma, angiosarcoma, myxosarcoma, perithelioma or endothelioma, and a considerable proportion of pituitary tumors falls in this class. Of 243 tumors collected by Marie, Creuzfeld, Frankl-Hochwart, and Strada, 64, or 26 per cent., were entered as sarcoma. Since the demonstration by Benda of specific epithelial cell granules in the cells of supposed sarcomas and the recognition

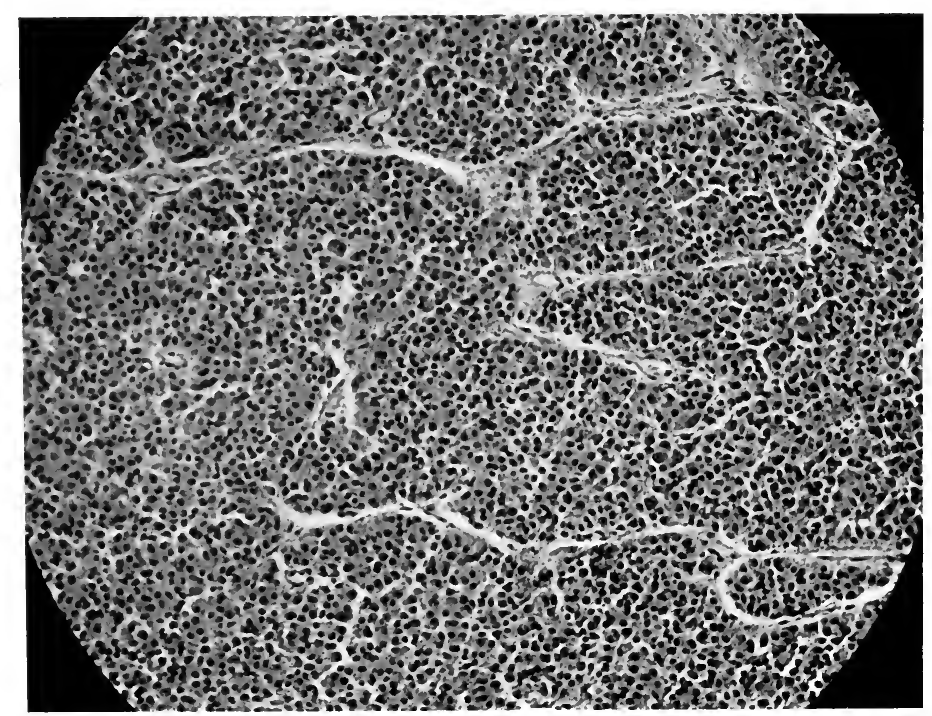

FIG. 493.--Section of large hypophyseal adenocarcinoma.

that epithelial growths of the pituitary very readily assume various atypical structures, the diagnosis of sarcoma has practically disappeared from the literature. With very rare possible exceptions all these tumors must be classed by atypical carcinomas. Only in their more atypical structure do they differ from malignant adenocarcinoma.

Angiosarcomas are described by Pechkranz, Jakubowsky, Kon, and others, and perithelioma by Kon, but the descriptions strongly recall the structure of vascular pituitary adenocarcinomas. Roth, Beck, and others described spindle- and round-cell sarcomas, but the data are inadequate to exclude an epithelial origin. In many cases the epithelium of the gland merged with the sarcoma, so that the author was in doubt as to the nature of the growth, and employed the term "adenosarcoma" (Hippel, O'Malley). The presence of colloid in round-cell tumors sometimes warns against the diagnosis of sarcoma (v. Bonin). "Sarcomatous struma" was the term applied by Hansemann to a tumor of the pituitary composed of large ovoid cells. Benda, whose minute studies threw many sarcomas into the epithelial class, accepts the occurrence of true sarcoma of the hypophysis and Ingerman's case in particular. This was an actively growing, circumscribed, soft, gelatinous tumor $7 \times 6 \frac{1}{2} \times 3 \mathrm{~cm}$., producing pressure symptoms and obesity in a 
woman of 35 years. It was composed of closely packed cells slightly larger than lymphocytes, while some cells were polygonal or spindle. Intercellular substance was lacking. The structure resembled overvascular splenic pulp and a perithelial arrangement was prominent. This description varies little from that of many atypical gland-cell tumors. Caussade and Laubry describe a nodular growth, $3 \frac{1}{2} \times 3 \mathrm{~cm}$., involving the anterior lobe. There were many spindle-cells arranged in whorls, groups of polygonal cells, and some acidophile cells. This tumor resembled certain dural endotheliomas, but its exact nature appears uncertain. Wolf designates as sarcoma cylindromatodes a structure with hyaline changes in the vessels.

Agostini in a prolonged case of dyspituitarism with mental symptoms described a spindle-cell melanotic fibrosarcoma with ossification of the capsule.

Degenerative changes occur in the advanced simple hyperplasias and frequently in the true tumors. Colloid secretion may be very abundant,

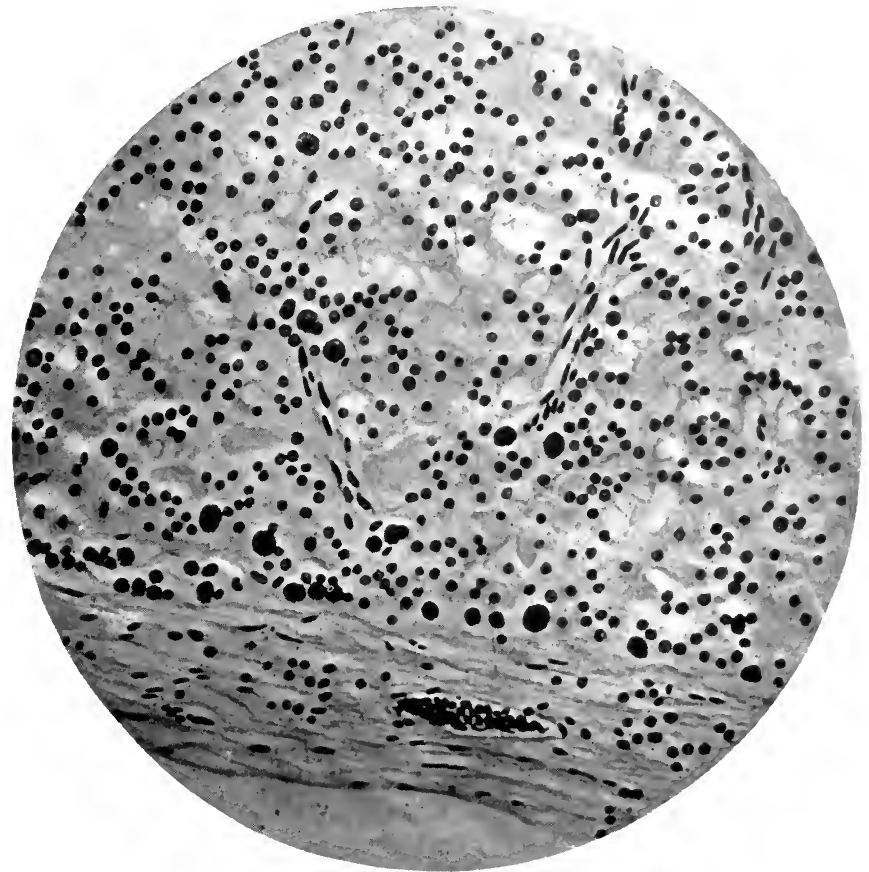

FIG. 494--Hypophyseal adenoma. (After Martius.)

diffused through the stroma, and lead to myxomatoid softening. From this source as well as from central necrosis and hemorrhage large tumors may be converted into cysts with mucus or chocolate colored fluid. Calcification appears in granules in and between cells, in the blood-ressels, and diffusely (Pick). In Konjetzny's cases the entire tumor was calcific.

2. Tumors of the Hypophyseal Duct and its Derivatives.- Owing chiefly to the studies of Erdheim, a well-defined group of hypophyseal tumors has been traced to the remnants of the hypophyseal duct. These growths are usually of squamous epithelial type, but many present the features of basalcell carcinoma, and it is probable that certain mixed tumors, or teratoids of the salivary gland type, arise from epithelium of the duct or from salivary gland acini carried along with the oral evagination. The distribution of these epithelial derivatives has been discussed with the anatomy of the hypophysis. 
The hypophyseal duct tumors arise from any point along the course of this embryonal structure, from the squamous epithelial nests in the lingual lobe from the chiasm downward, from Rathke's cyst between the anterior and posterior lobes, beneath the hypophysis in the floor of the sella or the sphenoidal spaces, and in the wall of the pharynx at the entrance of the duct.

The gross form of these tumors presents a number of variations.

(a) Simple cysts develop from distentions of Rathke's pouch. They arise between the anterior and posterior lobes, compress both portions, and contain serous or gelatinous or chocolate-colored material. According to Erdheim they are relatively common in infants. In adults they may reach a considerable size, chiefly by distention with fluid derived from the salivary acini in their walls. Occasionally they may rupture or the walls become so richly infiltrated with lymphocytes as to resemble a lymph-node. It is probable that some of the cysts lined by ciliated epithelium are derived from this source (Weichselbaum).

Cysts are believed to arise from separated portions of the infundibular extension of the ventricular cavity into the nervous portion of the stalk.

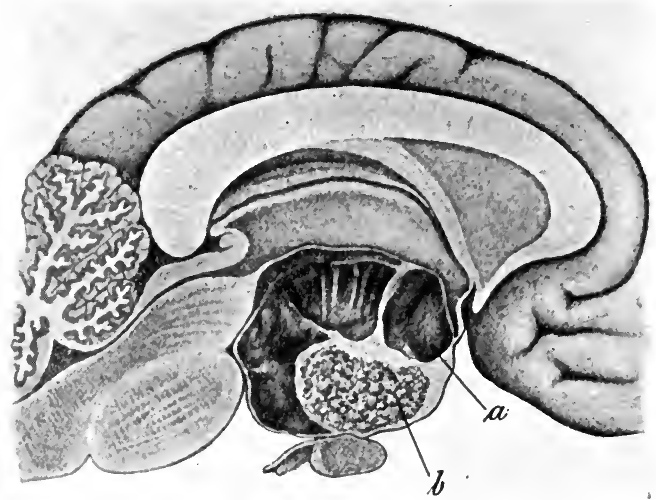

FIG. 495.- Relations of a cystic papillary epidermoid carcinoma of hypophyseal duct. (After Strada.)

They develop above the hypophysis, are lined by ependymal, rarely ciliated, epithelium, and contain serous fluid. From them may possibly develop some of the papillary tumors of ependymal epithelium (Saxer, Hart, Langer).

Cystic tumors also develop from distention of dermoids and other tumors derived from the hypophyseal duct, as well as from liquefaction of pituitary adenomas. Some cystic tumors of Boyce and Beadles appear to be of this type. In one of these the wall was partly ossified and the contents inspissated.

(b) Epidermoid carcinoma is the most frequent type of tumor derived from the hypophyseal duct. It arises from the remnants of oral epithelium found by Erdheim in the lingual process along the pedicle, or from similar remnants lying between the lobes (Launois). That the ependymal cells of the infundibulum may give rise to squamous epidermoid carcinoma remains unproved and appears improbable, although many papillary tumors of cylindrical covered by flat cells have been referred to the ependyma or choroid plexus (Fahr, Selke, Saxer, Boudet, Clunet). It appears impossible to determine where many recorded cases really belong, and quite likely that too many have been referred to misplaced remnants of the hypophyseal duct. The simple squamous-cell tumors of the duct are to be separated from more 
complex dermoids and teratoid growths, and probably from cholesteatomas, which Bostroem and Erdheim trace to invaginations of cranial epidermis carried into the brain vesicles in the closure of the neural canal. This latter group of tumors contains true skin and appendages, hair and sebaceous follicles, the tumors are scattered widely over the base of the brain, and Erdheim pointed out that they rarely lie in the median line. The occurrence of chorda-like tissue in Kon's case also indicates an origin apart from the duct.

Yet it is not easy to sharply distinguish all tumors of these classes. The absence of keratohyalin granules emphasized by Erdheim is not invariable, for both granules and keratin have been demonstrated in duct tumors by

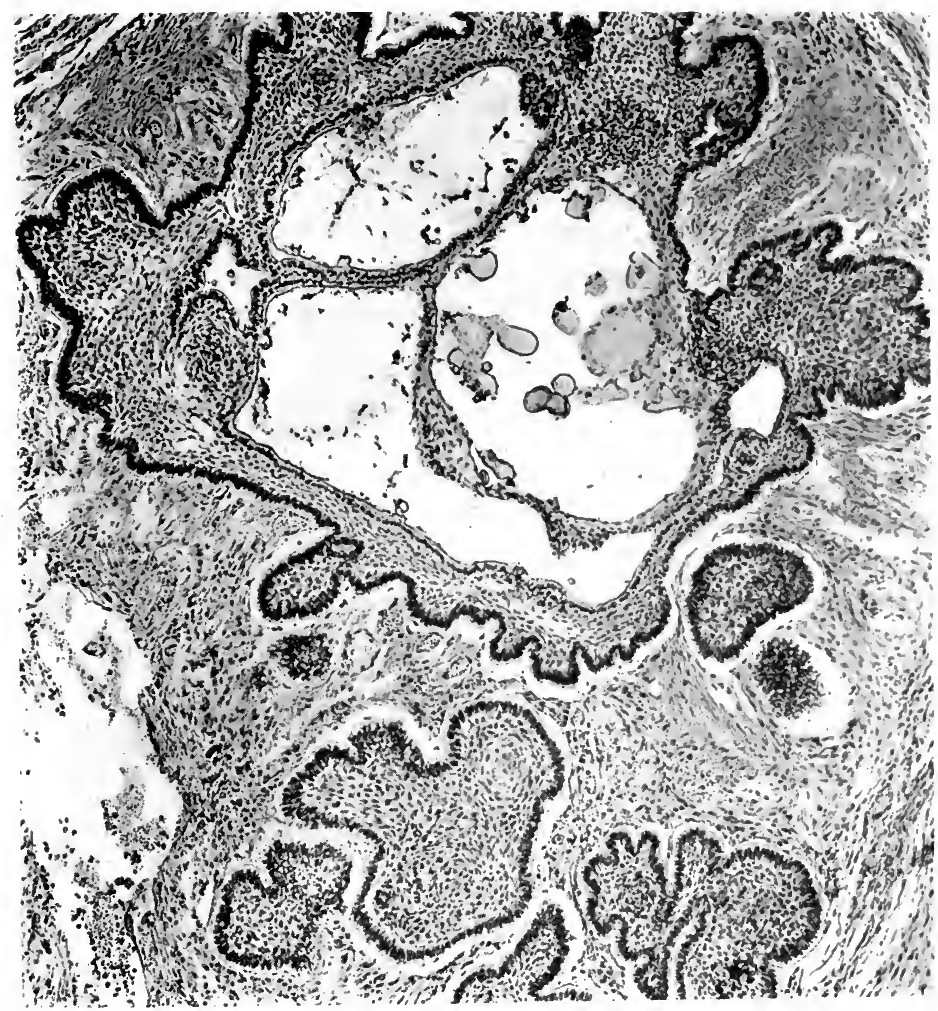

FIG. 496.-Epidermoid carcinoma of hypophyseal duct. Adamantinoma.

Allgayer, Bartels, and Strada. Intercellular spines occur in both groups. In some of the duct tumors extensive scaling occurs, recalling cholesteatoma, and metaplastic changes in the stroma may yield bone and cartilage (Bregmann and Steinhaus). The presence of hair and much sebaceous material points clearly to a dermal origin.

The distinction between epithelial tumors of the hypophyseal duct and papilloma of the choroid plexus is also difficult and must be based chiefly on the location and attachments of the tumor. The structure in both groups may be very similar. Most tumors of the choroid plexus lie well within the ventricles and only when they involve the third ventricle do they suggest a 
hypophyseal origin. For the demonstration of a hypophyseal origin one may demand a close relation to the pituitary gland, destruction of the stalk alone or both stalk and gland, and an intact choroid plexus. Many of these tumors are separated by a membrane from the cavity of the ventricle. The structure of the hypophyseal growths is more solid, or cystic with inverted papillæ, and the squamous characters are prominent. Tumors of the plexus are very vascular, even angiomatous, the papillæ are everted, mucoid degeneration of the stroma is common, and the cells are usually small and delicate, while squamous changes are less prominent or absent.

Epidermoid carcinoma as a rule develops from the hypophyseal stalk, lies above the hypophysis, widens the chiasm and circle of Willis, protrudes toward the ventricle, and compresses the hypophysis below. Arising within the gland the tumor may destroy the organ. The growth is solid, spongy, or cystic. Erdheim describes a unilocular cyst $6 \mathrm{~cm}$. in diameter, lined by squamous' epithelium. From the wall many low warty projections may form, and it is probable that the epithelial lining may be lost, giving rise to cysts of uncertain origin containing chocolate fluid, such as are described by Benda. Saxer's markedly papillary tumor was composed of very vascular connective tissue covered by squamous cells. Other tumors are comparatively solid or contain only few and small cysts. While commonly well encapsulated there may be invasion of surrounding soft tissues and bone. The main structural type is that of adenoid cystic epithelioma. In acellular connectivetissue stroma lie anastomosing masses of epithelium the outer layers of which are cylindrical, while the inner layers become flattened and vacuolated. There is often a distinct tendency to reproduce the reticulated structure of adamantinoma, and many small cysts form in the liquefied central areas, as well as in the edematous stroma. Or squamous metaplasia is pronounced, pearls and intercellular bridges form, and keratohyalin granules appear. In some cases squamous cells are wanting and the tumor is composed of many closely packed papillæ lined chiefly by cylindrical cells (Lewis). Either in the epithelial masses or in the walls of vessels calcific concretions may form.

The stroma may present a variety of features. The cellular connective tissue may be infiltrated with blood and contain cysts with chocolate fluid. Portions of the nervous tissue of the posterior lobe or the gland tissue of the anterior lobe may be scattered through the tumor. By metaplasia areas of cartilage or bone may form as in the salivary gland tumors. Walker described his tumor as osteoma. Benda's teratoma in a dwarf contained epidermoid cysts with cholesterin and bony plates in the wall. All the so-called teratomas (teratoids) of the hypophysis may safely be included in this group. While there is thus a wide variety of structure attributable to this origin and illustrated in many reported cases, the great majority of these tumors are comparatively simple and resemble adamantinomas.

Duffy, in a careful analysis, recognizes three groups of hypophyseal duct tumors: (a) Intracystic papillomas, which are relatively benign; (b) solid or cystic basal cell carcinomas of the type of adamantinoma, which may infiltrate locally, but do not metastasize; $(c)$ squamous carcinoma which may yield metastases to the cervical nodes, as in Fahr's case. In the second group calcific deposits are common and yield a characteristic $x$-ray picture.

3. Tumors of the Pars Intermedia. - In 3 cases the relations of a hypophyseal tumor have strongly suggested an origin from the pars intermedia.

Boyce and Beadles describe a firm tumor as large as a pea attached to the pedicle of the hypophysis above the sella. It was composed of large polygonal cells with single or multiple nuclei, lying in slightly acinar arrangement in a vascular stroma. Erdheim mentions a similar growth composed 
of large polygonal cells with densely staining cytoplasm. Cushing studied a large adenomatous tumor which compressed the anterior lobe in such a way as to indicate an origin from the pars intermedia alone. The structure resembled that of the thyroid gland.

4. Glioma of the posterior lobe is briefly reported by Bury. A soft cellular growth with colloid deposit and composed of small round and spindlecells merging with the normal tissue of the posterior lobe was regarded by Roxburg and Collis as a glioma or sarcoma.

Two small lipomas were found replacing the posterior lobe by Weichselbaum. They might arise from fat tissue occasionally seen about Rathke's cyst. W. Hutchinson describes a fibrosed gland in a dwarf.

Miscellaneous Tumors of the Hypophysis. - Cholesteatomas and true dermoids containing hair and sebaceous material occasionally appear in or near the sella turcica, but they seldom originate within the hypophysis. Bostroem has shown that they take origin from ectodermal rests carried in with the closure of the brain vesicles. Erdheim has collected a series of $\mathrm{I}_{5}$ tumors of this group in various locations, none of them strictly hypophyseal. Complex teratomas are described by Beck, Hecht, Cushing, and others. They are probably autochthonous teratoids developed by metaplasia from hypophyseal duct remnants. Margulis records an adult tridermal teratoma in the rabbit's hypophysis. Fetal implantations apparently attached to the hypophysis were recorded by Wegelin and Rippmann.

Symptomatology.- The combined clinical, anatomical, and experimental studies of tumors of the hypophysis have not only thrown much light on the nature and behavior of these growths, but rather unexpectedly have opened up fields of research which have a broad relation to many problems in the physiology and pathology of the glands of internal secretion. The outline of a brief presentation of this complex subject, which is an essential part of the study of hypophyseal tumors, includes, first, a clinical classification of the tumors, and second, a discussion of the pathogenesis of symptoms.

In general, hypophyseal tumors are associated with two main groups of symptoms. Some produce almost exclusively cerebral pressure symptoms, and thereby greatly lose in interest. Others not only yield pressure symptoms, but are accompanied by peculiar forms of general dystrophy usually involving other glands of internal secretion and thus adding greatly to their interest as well as their clinical complexity. Rarely the tumors are found at autopsy, having run a latent course. These groups may be termed (I) cephalic, (2) dystrophic.

(I) Cephalic Type.-The usual symptoms of headache, vertigo, and vomiting are typical signs of onset which are common to many cerebral tumors. Rising beyond the sella, ocular symptoms first appear and take a variety of forms which may permit very accurate localization of the point of pressure. Dimness of vision often appears very early on one or both sides, and results in blindness of one eye in 33 per cent. of the cases and of both eyes in 16 per cent. In Henneberg's case ocular disturbance appeared I3 years before death. Bitemporal hemianopsia, with loss of pupillary reaction to light, is a characteristic symptom resulting from pressure on the chiasm with atrophy of the nasal half of the retina. Later, homonymous hemianopsia occurs from pressure on the whole optic tract. The directness of the pressure on the optic tract renders stasis of the retinal papilla relatively rare ( 5 per cent.). Bilateral non-pulsating exophthalmos occurs in ro per cent. of acromegalics and without acromegaly (Scalincie).

Oculomotor paralysis occurred in ro of I74 cases of acromegaly (Hertel) and in 29 per cent. of tumors without acromegaly (Lapersonne, Cantonnet). 
Any of the cranial nerves may be affected. When the tumor reaches the peduncle hemiplegia or more extensive paralyses result. Diffuse cerebral symptoms characterize many cases and are exclusively present in some. Narcolepsy, brief or prolonged, occurs in 25 per cent. of the cases without acromegaly (Fr. Hochwart), but is not observed in acromegaly itself (L'Hermitte). In 775 cases of cerebral tumors with psychoses, mania, hypochondria, hallucinations, or dementia, Schuster found 6I tumors of the hypophysis, I 2 of which were associated with acromegaly. Glycosuria and diabetes, at times very severe, is comparatively frequent in acromegaly. Launois and Roy collected 16 cases. Without acromegaly glycosuria is rare, but simple polyuria is more frequent.

Radiographic examination is constantly indicated in hypophyseal tumors, but the interpretation of enlargements of the sellar outlines is surrounded by many uncertainties. Individual variations in the size of the sella, physiological swelling of the hypophysis, widening of the sella by increased intracranial pressure from other cerebral tumors, and tuberculous or syphilitic lesions of the bones are possible sources of diagnostic error. Actual erosion of bone beginning with the posterior clinoid processes is far more reliable evidence than simple enlargement of the sella.

(2) Dystrophic Type.- Three forms of general dystrophy occur with hypophyseal tumors; giantism or dwarfism, acromegaly, and obesity with genital hypoplasia.

Giantism is the result of precocious activity of the hypophysis in infancy, while dwarfism follows loss of pituitary function in early life (Brissaud, Meige, Launnois, Roy). Acromegaly results from overactivity of the hypophysis in the adolescent or adult. The adiposogenital syndrome is the expression of functional underactivity of the gland, chiefly of the posterior lobe. There are giant acromegalics in whom the overactivity established in infancy persists after the limit of height has been reached and many cases of giantism reveal traces of the adiposogenital dystrophy when the glandular function is exhausted. The passage of acromegaly into the adiposogenital dystrophy is easily conceivable, and this tendency has been emphasized especially by Cushing in cases showing symptoms of both classes. While the relation of these dystrophies to the functional state of the gland appears with sufficient clearness to constitute a general principle, yet numerous apparent violations of the principles are observed, and other complicating factors are found in associated disturbances of other glands of internal secretion and in combinations of individual features of the various dystrophies. Hence it has become increasingly evident that each case of dystrophy connected with hypophyseal disease is best analyzed as a form of polyglandular disturbance. The necessity and the difficulties of this method are well illustrated in Cushing's studies.

(a) Giantism.-Pure cases of giantism fully studied from the polyglandular standpoint are rare. In Cushing's case (32), age 37 , the body measured $251.5 \mathrm{~cm}$. in length, the brain weighed $1884 \mathrm{gm}$., spleen $1000 \mathrm{gm}$., thymus atrophic, thyroid not enlarged, adrenals extremely small, testes atrophic, pancreas fibrosed. There were marked signs of pituitary insufficiency with impotence, hypotrichosis, and obesity. The sella measured $2.2 \times 2.7 \mathrm{~cm}$. and was occupied by a large cystic tumor in the walls of which were irregular alveoli of chromophobe cells. This case represents a preadolescent hyperpituitarism with terminal insufficiency.

In Bassoe's case (Winkler) there were leontiasis of the skull and a large osteosarcoma compressing hypophysis and brain. The enlarged hypophysis contained focal eosinophilic adenomas, areas of small, round, densely staining cells, alveoli filled with colloid, hyaline vessels, hemorrhages and pigmenta- 
tion. There was a large colloid goiter, thymus and testes were atrophic, adrenals and pancreas normal.

Giantism with acromegaly is a relatively numerous class. Sternberg states that 40 per cent. of all giants are acromegalic, but the literature contains few fully reported cases. The increase in height begins in early life and ceases after a period of years, but the acromegalic changes continue, and evidences of dyspituitarism are often added.

In Dana's acromegalic giant there was extensive enlargement of the pituitary which led to central hemorrhage and softening, probably a pure adenoma (cf. Hutchinson).

In the case of Lady Aama Hutchinson found the hypophysis as large as a pigeon's egg, but no microscopical study could be made.

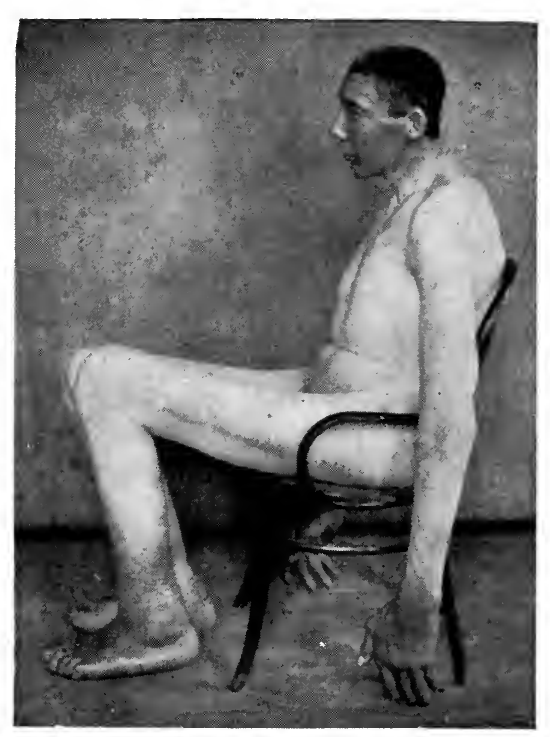

FIG. 497.

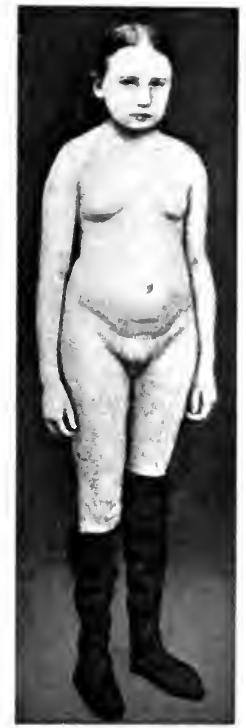

FIG. 498.

FIG. 497.- Preadolescent hyperpituitarism with giant overgrowth. Enlarged sella, containing a cystic struma pituitaria. No neighborhood symptoms. Terminal glandular insufficiency (hypopituitarism). Note extreme length of arms and thighs in relation to usual 18-inch chair. (Cushing, "The Pituitary Body and Its Disorders.")

FIG. 498.-Pituitary dystrophy, Lorain type. Pituitary tumor, small adult body, retarded sexual development, no adiposity. (After Stewart.)

(b) Infantilism.-Various types of imperfect development of the skeleton and especially of the genital organs are associated with hypophyseal disease. In general, pituitary infantilism results from pre-adolescent hypopituitarism, but the influence of disorders of the thyroid, thymus, and adrenal are often added. Considering the very numerous clinical forms and complex etiology of the conditions designated as infantilism it is evident that pituitary lesions have a very restricted importance in this field (cf. Strauch). Dwarfism in pronounced form was associated with a diffuse fibroma or fibrosis of the pituitary in Hutchinson's case.

A combination of gigantism with infantilism is described by Lemos. 
There is excessive growth of long bones and some facial hypertrophy, but the general bodily conformation is infantile and there is genital hypoplasia. The mentality is defective. It is referred to a congenital hypertrophy of the pituitary which manifests itself early in life.

Lorain described a form of infantilism characterized by a general smallness and delicacy of body with correspondingly small genitals, but without obesity. Growth is arrested, $\mathrm{b}_{\mathrm{u}} \mathrm{t}$ adult proportions of the body are maintained. While

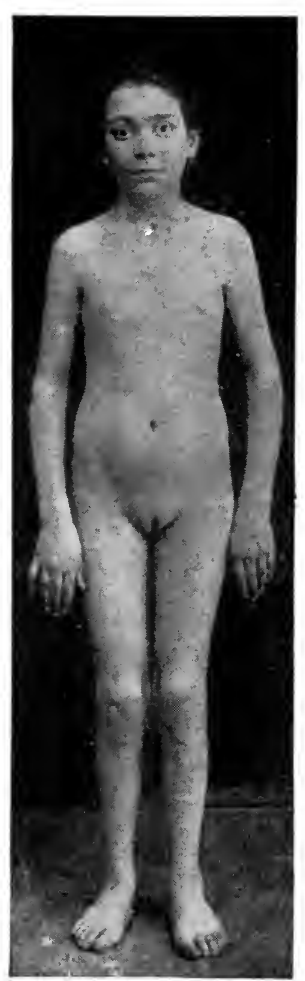

FIG. 499.-A typical case of infantilism of type Lorain, with an enlarged $2 \mathrm{~cm}$. sella. Patient aged 20 years, 6 months; height $4 \mathrm{ft}$., 5 in. $(133 \mathrm{~cm}$.$) . (Ettore$ Levi.) by microscopical examination (Dallemagne, Mitchell, LeCount, Huchard, Launois, Lewis, Widal, Roy, Froin, and Petren).

B. Fischer, however, rejects all such cases, and affirms that there is no recorded instance of acromegaly in which the absence of specific changes in the pituitary has been proved. This position may be too radical, but it is obvious that changes once present may disappear. Thus Huchard's and one of Cushing's cases showed a small sclerosed pituitary in an enlarged sella. The primary pituitary origin of acromegaly is strongly indicated by the fact that the great majority of cases show pronounced hyperplasia or 
tumors of this organ. The hypophysis, however, is not the only organ involved in acromegaly. The disease is of complex etiology and pathogenesis and several clinical varieties depend upon associated lesions.

Many cases of pituitary enlargement fail to give acromegaly even when, as in Cagnetto's and Zak's cases, the eosinophile cells are greatly in excess. The disease is observed with pineal tumors, and the thyroid and sex glands are commonly involved. Hypophyseal disease is therefore an essential, but not a sufficient cause of acromegaly (Parisot). Acromegalic changes are also observed with hydrocephalus from cerebellar cyst (Cushing), in neurofibromatosis (Nichols and Favre), and in syringomyelia (Petren).

An hereditary element is observed, especially in negroes. Infantile acromegaly has been observed in a series of 20 cases collected by Hutinel, and Babonneix and Paisseau. Matassari has shown that this condition may be prevented by precocious ossification of epiphyseal cartilages (Claude, Franchini, Giglio). There may be, however, very marked overgrowth of skull, face, hands, and feet. Many cases show acromegalic overgrowth with adiposogenital dystrophy.

Acromegalic conditions of moderate degree combined with myxedema and associated with hypophyseal tumors are of not infrequent occurrence (Grahaud, Lit.). In such a case Calderara found an atrophic thyroid, while the pituitary was doubled in size by a chromophobe struma. Boyce and Beadles in a myxedematous obese subject found the hypophysis much enlarged, while in a similar case Dalton reports an atypical "sarcoma" destroying pituitary and posterior lobe. Other reports worthy of mention include a "sarcoma" (Pechkranz), endothelioma compressing the hypophysis (Stewart), carcinoma extending into the face (Zollner), and a cystic tumor as large as an orange (Sainton, Rathery).

Colloid goiters are frequently observed in acromegaly, but hyperthyroidism rarely. Exophthalmos may result from the pituitary tumor. Thyroid hyperplasia occurred after partial hypophysectomy in 2 of Hochenegg's cases (Exner).

Acromegalics, especially in the late stages of dyspituitarism, may give evidence of adrenal insufficiency in the form of pigmentation of the skin, extreme asthenia, low blood-pressure, and hypoglycemia. While the adrenals are usually reported as normal, yet in a giant acromegalic with cystic pituitary struma (32) Cushing found atrophic adrenals. Hyperactivity of the adrenal is sometimes indicated in acromegaly, and Renon and De Lille observed adrenal hypertrophy after injections of hypophyseal extracts. In rare cases of acromegaly there is marked hypertrophy of the adrenals. Schultze and Fischer report cases in which hyperplasia of the Z. fasciculata reached an almost adenomatous grade.

Glycosuria and diabetes are often associated with acromegaly. The pancreas is usually reported as normal, but Hansemann found interstitial pancreatitis and a colloid goiter, and pancreatic lesions are reported by Dallemagne and Pineles. In one case with malignant hypophyseal adenoma I found interstitial pancreatitis and giant-cell formation in hypertrophied island's. Although polyuria is induced by injections of extracts of the pars nervosa its occurrence with hypophyseal tumors is probably to be classed with the polyuria of other brain tumors.

Grahaud has collected II cases of young acromegalics with marked or extreme obesity. Such a condition is referred by Fischer to a tumor of the anterior compressing the posterior lobe. It may also be explained as a combination of acromegaly with primary testicular hypoplasia. Analysis of the case reports shows that both these explanations may apply. Fischer's case 
was a perivascular round-cell tumor with an epithelial colloid cyst compressing the remainder of the pituitary and pars nervosa.

Nearly all cases of acromegaly are accompanied by genital dystrophy, a fact which accords with the view that a slight long-continued pressure on the pars nervosa affects the genital glands (L. Pick, Fischer).

In the adult evidence of hypophyseal failure appears in the tendency of most acromegalics to lay on fat. This feature is pronounced in Dercum's disease, adiposis dolorosa, marked by local or general adiposity, pains and tenderness, asthenia, and psychoses. Hypophyseal lesions observed in this condition include: pituitary glioma (Burr); adenocarcinoma (McCarthy); alveolar carcinoma (Guillain, Alquier); processes suggesting carcinoma, 2 cases (Price); epithelial tumor (Launois, Clunet); aneurysm compressing hypophysis (Lyon). Dammann, however, reports a normal hypophysis and emphasizes the frequency of various phases of chronic thyroid disturbance in this disease.

Status lymphaticus with persistent thymus and lymphoid hyperplasia is occasionally observed in acromegaly, but belongs more clearly with adiposogenital dystrophy. In 2 such cases Cushing reports a chromophobe adenoma and a cerebellar cyst. Epileptic seizures are also common in this group of cases. Amenorrhea has long been recognized as an early symptom in many acromegalics, and most male subjects of the disease are sexually deficient (Marie, Cushing). The ovaries are usually cystic or atrophic, and in the testes the interstitial cells are deficient or absent (Cushing). In a peculiar syndrome of precocious sexual development, hypertrichosis, obesity, asthenia, and blindness Cushing observed some hyperplasia of the interstitial cells.

Finally, in a group of cases, many organs and tissues are affected by developmental anomalies and acquired disorders, revealing a disturbance of growth and function which is nearly universal. Thus in Harbitz's case there was a large chromophobic adenoma with apparent destruction of pars nervosa, associated with acromegaly, hemiatrophia facialis, overgrowth of lung, trachea, liver, spleen, kidney, heart, larynx, thyroid, pancreas, and adrenals. In Amsler's case an adenoma, chiefly eosinophile, was associated with enlargement of tongue, spleen, liver, kidneys, thyroid and heart, and atrophy of testis.

(d) Dystrophia adiposogenitalis is the most characteristic form of hypophyseal dyscrasia. Its numerous subvarieties suggest the implication of other glands of internal secretion. Both clinical and experimental studies indicate that the glandular insufficiency affects chiefly the posterior lobe. The secretion of this lobe raises blood-pressure and exerts a peculiar influence on the metabolism of fats and carbohydrates. The subjects exhibit hypoglycemia and high sugar tolerance, 300 to $400 \mathrm{gm}$. of glucose by mouth failing to produce glycosuria. Apparently carbohydrates are converted into fats and deposited in the depot regions, subcutaneous and subserous tissues, and the liver. The associated genital hypoplasia doubtless favors fat deposit by some other mechanism. While other glands may be secondarily concerned the predominance of the hypophysis is indicated by the remarkable improvement observed after operation. V. Eiselsberg, Cushing, and others have reported improvement in pressure symptoms, restoration of sexual function, growth of hair, etc., after extirpation of various tumors. Moreover, Cushing has shown that partial extirpation of the anterior lobe leads to obesity and genital hypoplasia. In subsequent experiments, however, he attributed this result to injury to the pars nervosa.

The cases differ chiefly in the feature of overgrowth and in the condition 
of the genitals. In the type described by Froelich a hypophyseal tumor is associated with diminished growth, genital hypoplasia, and adiposity. In childhood the condition produces many of the characteristic excessively fat children with genital hypoplasia (Neurath). The fully developed condition presents many or all of the following features: fat deposits in the skin of hips, abdomen, and genitals; atrophy and loss of function of the genital organs, amenorrhea, anaphrodisia, hypotrichosis; limitation of skeletal growth and traces of infantilism, malformation of genitals, cryptorchismus, hypospadias, absence of one testicle, transformation of sexual characters, hypertrophy of breasts, and widening of pelvis in males. In many details these cases may present the features of status lymphaticus, but the hypoplasia of the aorta and hyperplasia of the lymphatic structures are usually not noted.

Genital hyperplasia and skeletal overgrowth with obesity are observed without hypophyseal tumor in cases of hydrocephalus (Marburg). Or hydrocephalus without hypophyseal tumor may yield skeletal overgrowth, obesity, and genital hypoplasia (Cushing, Case 24).

In adolescence the male develops feminine outlines, genital hypoplasia, hypotrichosis, and adiposity, and when lymphoid hyperplasia and persistent thymus are added, the condition merges with status lymphaticus (Cushing, 5, 8, 10), Mixter and Quackenboss, Strada, Souques, and Chauvet.

In general, the tumors associated with adiposogenital dystrophy are characterized by a wide diversity of origin and structure, by a frequent failure to involve the pituitary, by a tendency to grow into the third ventricle and to destroy the infundibulum, and by their comparative inaccessibility to surgical attack. Of 34 cases collected by Strada, Ottenberg, and Pick, 6 left the hypophysis

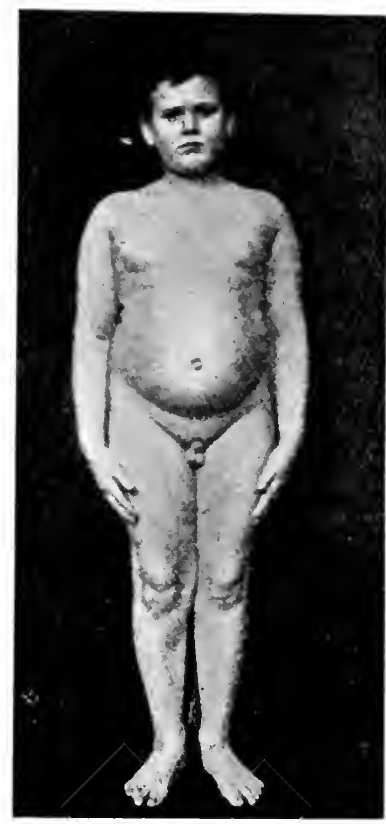

FIG. 500.-Hypopituitarism. Adiposogenital dystrophy, type Fröhlich. No sign of tumor. (After Batten.) normal, 8 were derived from the hypophyseal duct, 23 represented nearly all the histological varieties of pituitary neoplasms, and some arose from the choroid plexus or ependyma, or from the pial endothelium. Pick emphasizes the bipartite character of many tumors, which present a small intrasellar and a larger extrasellar or intraventricular portion connected by a pedicle.

Relation Between the Type of Tumor and the Symptom Complex.-Throughout the above clinical types of hypophyseal disturbance a certain relation may be traced between the type and location of the tumor and the symptoms. Skeletal and tissue overgrowth and the various form of acromegaly and giantism, at least during their active stages, are associated with hyperplasia of the eosinophile cells of the pituitary. Although the gland may be considerably enlarged by this process, it is doubtful if any of the eosinophile hyperplasias can be regarded as true neoplasms. Even in actively progressive and early acromegaly it cannot be claimed that eosinophile cells are exclusively concerned, since acromegaly develops from the hyperplasia of gestation which 
affects the specific cells of Erdheim. Moreover, the great majority of glands examined microscopically from advanced cases of acromegaly show not eosinophile overgrowth, but adenomas of chromophobic or quite atypical small or large round cells. Such cases usually show evidences of dyspituitarism. Hence the pituitary lesion beginning with hyperplasia of specific functionating cells, if it assumes the character of a neoplasm, does so at the expense of form and function of the secreting cells. It is clear that the same type of atypical adenoma may occur in both

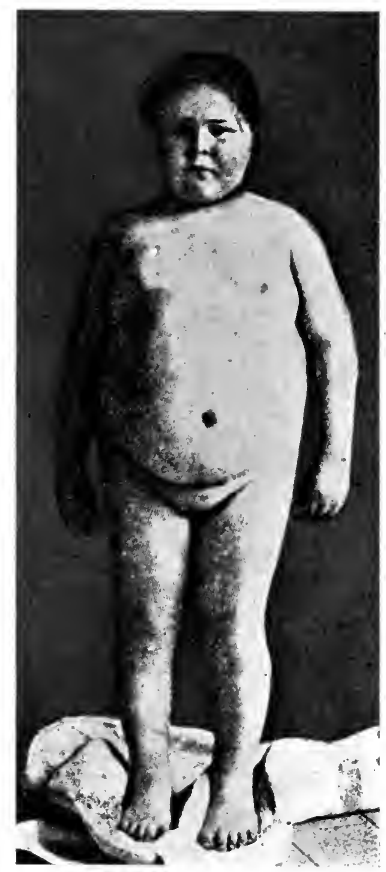

FIG. 50I.-Adiposogenital dystrophy, from bullet wound of hypophysis. (Madelung.) acromegaly and in adiposogenital dystrophy (Wurmbrand).

The natural course of these hyperplasias varies considerably. Some appear to be long maintained as simple functional hyperplasia and are found in this condition at operation or autopsy. Others reaching a large size tend to suffer central softening and terminate in cysts with gelatinous or blood-stained contents. Gradual atrophy with calcification, which may be complete (Pick, Konjetzny), may supervene. In many cases the functional hyperplasia passes rapidly or slowly into a neoplastic process and distinctly malignant features may appear. In these relations the hypophysis presents a close parallel with the thyroid. Yet it is frequently noted that malignant histological structures are associated with slow clinical course, and in several instances the symptoms have regressed after partial removal of the tumors (Pick). Nearly all the extreme forms of pituitary overgrowth are associated with functional insufficiency, while the period of functional excess is early and transient. In this stage there appears to be little relation between the various histological peculiarities of the tumor and the clinical symptoms. The former represent local phases of tumor growth, while the latter reveal an increasing participation of other glands of internal secretion, and the complicating pressure symptoms.

While cases of pure acromegaly are comparatively rare, signs of glandular insufficiency overtake most cases of acromegaly, and various forms of primary hypophyseal atrophy are relatively common. The lists of pronounced adiposogenital dystrophy contain a large proportion of atypical overgrowths and true tumors (Strada, Ottenberg). Extrapituitary tumors and those derived from the hypophyseal duct almost invariably lead to primary dystrophy without acromegaly. Wurmbrand and others have noted a prompt response of the thyroid and genital organs after operations on hypophyseal adenoma.

Three theories have been advanced in explanation of the adiposogenital dystrophy: (a) deficiency of the internal secretion of the hypophysis (Frohlich), (b) pressure from any source on a supposed cerebral center (Erdheim), and (c) a lesion of the posterior lobe or the infundibulum. In most cases the anatomical condition is consistent with any one of the three theories. In considering the evidence bearing on these theories it is necessary to distinguish between the secondary adiposogenital dystrophy which fol- 
lows acromegaly and the primary form which develops without a preliminary period of hyperpituitarism.

(1) The chief evidence in favor of the theory of pituitary deficiency is the fact that dystrophy overtakes most acromegalics in later stages and is associated with destructive tumors of the hypophysis and in general with chromophobic strumas. This condition has also arisen from a bullet wound of the pituitary (Madelung), and from solitary tubercle of the anterior lobe (Cushing). An old hemorrhage destroying two-thirds of the pituitary was the sole lesion found in a case of Maranon's. On the other hand, there have been many destructive lesions of the pituitary, including tumors, tubercle, and gummas, which have failed to produce dystrophy (L. Pick, Lit.). Atrophy of the pituitary leads to dwarfism rather than to adiposogenital dystrophy. Many considerations favoring the theory of pituitary insufficiency are collected by Marburg, Melchoir, and Strada.

(2) While a certain grade of cerebral adiposity has appeared with miscellaneous brain tumors and hydrocephalus, yet the existence of such a center as Erdheim assumes is quite hypothetical, and in many cases of dystrophy the element of cerebral pressure has been absent.

(3) Many observations favor the importance of disturbed function of the pars nervosa in adiposogenital dystrophy. In many cases there is a tumor of the hypophyseal duct which compresses the infundibulum and the pars nervosa while leaving the pituitary intact. It appears to be the location rather than the type of tumor which determines the dystrophy. Tumors involving the third ventricle frequently give rise to dystrophy, apparently by occluding the infundibulum (Erdheim, Selke, Bartels, Zak). Fischer describes a tumor of the third ventricle which he details as meeting these conditions exactly. Extensive lesions of the anterior lobe without involvement of the posterior, as by tubercle or gummas (Pick), have repeatedly failed to yield the characteristic dystrophy (Stroebe, Schmidt). There is a theoretical difficulty in assuming that hyperfunction with acromegaly can coexist in the same gland with hypofunction. Finally, Cushing has shown that experimental lesions of the pars nervosa alone lead to adiposogenital dystrophy, and in not a few cases marked improvement has followed operative removal of cystic tumors compressing chiefly the posterior lobe and infundibulum. The weight of evidence is thus distinctly in favor of the mechanical theory of pressure on the pars nervosa. L. Pick, also, after a critical review of much of the literature, is inclined to favor the mechanical theory. 


\section{CHAPTER XLVIII}

\section{THE PINEAL GLAND AND ITS TUMORS}

Histology. - The pineal gland at full development measures 10 to $12 \times 5$ to $8 \mathrm{~mm}$., but suffers gradual atrophy beginning at the seventh year. Its normal weight in children is 20 to 25 centigrams. It is surrounded by a thin capsule of connective tissue which closely invests the gland, joins with many septa supporting groups of parenchymal cells, and is connected with a loose vascular tunic derived from the choroid plexus. The stroma steadily increases in amount with age, fine acellular strands appear subdividing the cell groups and fusing with them, while hyaline or calcific granules appear in increasing numbers. Blood-ressels are scanty.

The parenchyma is composed of groups of polyhedral cells irregular from mutual pressure. The cells are finely granular and often contain small vacuoles. The nuclei are somewhat peculiar in their reticular network, small nucleoli, and large size, in comparison with the cytoplasm. They stain more deeply in young subjects. At this age both nuclei and cytoplasm may contain hyaline globules which Dimitrowa regards as secretion. The lipoid mitochondria demonstrated by Ruggeri are much more definite indications of a secretory function. Krabbe in 100 human glands always found basophilic granules in and about the nucleus which he regarded as secretory. The cells are distinctly epithelioid in infancy and form well-defined groups without lumina, but in adults they are less regular and the groups are less compact. By Weigert's or Heidenhain's stains glia fibrils may be demonstrated arising from the cells, passing between cells, and surrounding cell groups. Compact areas of glia fibers may appear in the adult. Marburg demonstrated medullated fibers. This structure suggests that the gland is composed essentially of modified glia-cells which first resemble neuro-epithelium with secretory functions and later assume the form of glia-cells.

About the vessels may sometimes be seen small cells with compact nuclei and acidophile cytoplasm (Galasescu, Urechia). According to Marburg the gland always contains ependymal cells derived from the ependymal recess in which it lies.

Striated muscle-fibers, common in the beef, have occasionally appeared in the stroma of the human gland. Pappenheimer regards them as analogues of the myoid cells of the thymus and as derivatives of epithelium.

General Pathology.-Hypertrophy of the epiphysis is recorded by Virchow in an infant and in an aged woman with dural psammoma; by Heurot in myxedema; and by Marburg in a case of general cerebral hypertrophy. In a polyglandular syndrome with hypophyseal adenoma Bartlett describes hyperplasia of the pineal gland. Atrophy of the gland of quite unusual degree has been observed by Laignel and Lavastine.

Calcific deposits are constantly present after the seventh year. While usually limited to coarse granules the process reached an extreme degree in the case of Drelincourt in which the gland was a stony mass as large as a pigeon egg. Schnepf in an idiot found the gland replaced by two small concretions. Extensive hematomas are described by Ziegler and Simon.

Cysts are frequently observed at all ages. Many small cysts without definite lining may form during involution by softening of the increasing areas of gliosis. Ependymal cysts lined by cubical or high epithelium arise from distention of alveoli of included ependymal cells (Marburg). Either variety may be found without symptoms and some have produced hydrocephalus and fatal tumor syndromes (Bouchut, Joukowsky, Nieden, Neumann, Campbell, et al.).

\section{TUMORS OF THE PINEAL GLAND}

Of the very rare tumors those of the pineal gland are of much interest because of the peculiarities of their structure and origin and because of the remarkable disturbance of growth with which they are often associated (Seigneur, Lit.).

Occurrence.- Sixty cases were collected by Bailey and Jelliffe in I9II, while Seigneur, г9г2, found reports of 65 . To these may be added the cases of Wood, Goldzieher, and Rorschach. Other reports, as those of Pellizzi, are of clinical diagnoses. 
Classification.-Pineal tumors form three well-defined groups:

(I) Cysts.

(2) Teratomas.

(3) Ependymal gliomas.

Cysts of various types constituted I $_{2}$ of the 65 tumors collected by Seigneur. Most of them were simple cysts without tumor growth, but their general and local symptoms did not appear to be influenced by that fact.

The simple cysts vary in size from that of a pea (Garrod) to a tumor containing 20 c.c. of fluid (Joukowsky). They project into or fill the third ventricle and compress the corpora quadrigemina or the optic tracts. By pressure on the veins of Galen and occlusion of the ventricle they almost invariably lead to hydrocephalus. They have occurred in the newborn and at the age of 59 years (Joukowsky, Friedreich). They produce the usual symptoms of pineal tumors, but simple cysts have not as yet been observed with genital overgrowth.

The simple hydrops cysticus produces multiple small cysts filled with clear fluid and lined by flattened, cubical, or cylindrical epithelium. Very similar multiple cysts from softened areas of gliosis fail to show a definite cell lining. In 5 cases the cyst formed in a true neoplasm, usually of the type of gliosarcoma (Ogle, Verger, Neumann). The wall in Falkson's tumor contained cartilage, and in Friedrich's tumor the solid tissue was psammomatous.

Teratoma.-The pineal teratoma is exclusively observed in young males from 4 to 16 years of age, and is regularly associated with precocious sexual development, hirsuties, and sometimes with adiposity and general overgrowth. About I 2 cases have been recorded, but the distinctly teratomatous nature of some of them is doubtful. Thus Oestreich and Slawyk describe as cystic psammosarcoma a cystic growth as large as an apple, which Askanazy regarded as a teratoma on account of the presence of islets of tissue resembling cartilage.

The complex teratomas are of moderate size, solid or cystic, circumscribed, and produce the usual local changes from pressure. They may be largely dermoidal, containing hair, sebaceous material, epidermoid cysts, and cartilage (Bailey and Jelliffe). Weigert's tumor was a complex growth containing cysts lined by squamous or cylindrical cells, hair, sebaceous glands, sebum, calcific grains, cartilage, fat tissue, and non-medullated nerve-fibers. Gutzeit's tumor presented skin, sebaceous glands, fat, and smooth muscle.

A layer of normal or adenomatous pineal tissue may be found alongside of the tumor (Hochwart, Gutzeit). Less complex growths classed as teratomas have yielded the structure of adenochondrosarcoma with smooth muscle (Coats). Somewhat uncertain data are available in the cases of Gauderer, Ogle, and P. Neumann.

In a hemorrhagic growth Askanazy found the typical structure of chorioma with well-defined syncytium and glycogen-holding Langhans' cells.

The significance of the teratomas has been variously interpreted. The development of the tumor anteriorly from the gland and the presence of nerve-fibers seemed to Marburg to support the theory that the tumor represents an abortive third eye. Ogle ventured to liken the high epithelial lining of a cyst to the retina of a third eye. Askanazy applies the theory of origin from a totipotent sex cell and traces in the condition a form of pseudogestation. It may be noted that the breasts were enlarged in Oestreich's case. This change as well as the genital overgrowth he would refer to the presence of fetal tissue in the body. Yet the same genital precocity occurs in simple tumors of the epiphysis.

Were it not for the presence of hair, skin, and sebaceous glands it would 
be possible to regard all these simple tumors as autochthonous teratoids. A partial choriomatous structure is produced in testicular tumors by alterations in the cylindrical cell lining of blood spaces. While the structure of Askanazy's tumor closely approaches that of chorioma, Goldzieher's very similar tumor was traced to the cells lining numerous blood spaces. The presence of cartilage may be satisfactorily explained by metaplasia. Smooth and striated muscle is an occasional element of the normal epiphysis. Ependymal epithelium may become high and cylindrical or flat, and squamous and sharp transitions from one to the other type are observed in the so-called teratomas (Weigert). Thus all the elements except dermal structures may be derived from the pineal gland itself. The dermal structures, as hair and sebaceous glands, if they have been correctly interpreted, seem to require an ectodermal tissue, which may possibly reach the pineal gland by the same developmental disturbance that yields dermal cholesteatomas at the

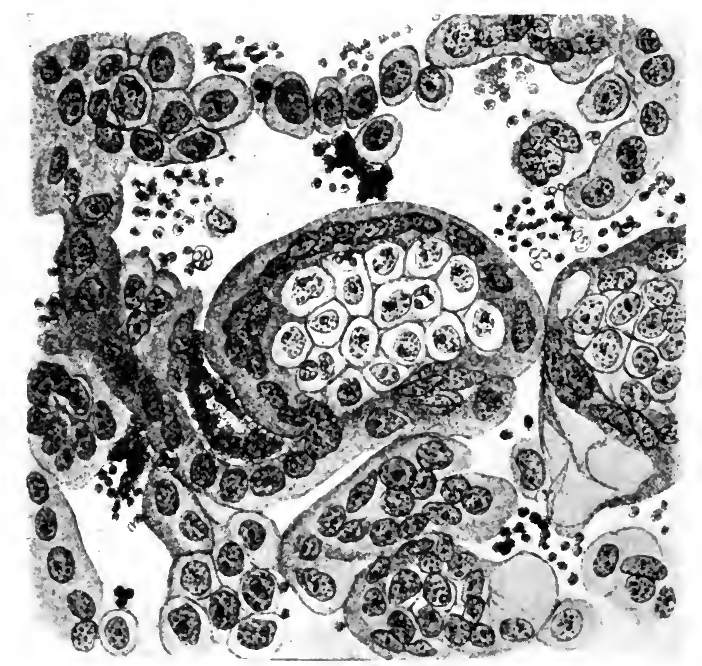

FIG. 502.-Chorioma of pineal gland. (After Askanazy.)

cerebral base. The pure pineal lipoma of Hirtz indicates that misplaced tissue may rest in this gland as well as in the hypophysis.

The few recorded cases, especially those of Falkson and Hochwart and Coats, seem already to furnish many transitions from autochthonous mixed tumors, as adenochondrosarcoma, to teratomas, so that the pineal teratomas are not so sharply separated from teratoids as to warrant their final acceptance as genuine tridermal neoplasms.

Ependymal Glioma.-The majority of pineal tumors present a structure best interpreted as formal variations of the modified ependymal cells from which the human epiphysis is immediately derived.

They occur at later ages than the other tumors, raising the average age of incidence of all pineal growths to 20 years for males and, for the 14 cases in females, to $2 \mathrm{I}$ years. The usual duration is from $\mathrm{I}$ to $\mathrm{I} 8 \mathrm{months}$ (Falkson, Oestreich). A hard tumor of Blane's, not examined microscopically, and Hertz's lipoma, progressed for 3 and 4 years. Hempel's patient died 3 years after trauma. As a rule they grow more rapidly and reach a larger size than 
other pineal tumors, although many are soon fatal. Turner's mixed tumor reached the size of a kidney and Hempel's solid carcinoma was as large as a hen's egg. The larger growths may distend the third, lateral, and fourth ventricles, with hydrocephalus, which in children may widen the cranial sutures. Finkelburg's sarcoma involved the corpora quadrigemina, hypophysis, floor of third ventricle, chiasm, and posterior commissure. Howell's first case was quite as extensive. Forster's carcinoma with general metastases is out of line with all later reports. The exact origin of some other large tumors of this region must also be accepted with reserve.

The structure of the mixed pineal tumors is extremely varied and these growths have been reported under many terms which served to designate the amin structural type. Thus there appear 3 pure gliomas, 4 gliosarcomas, 4 angiosarcomas, 4 psammosarcomas, 2 chondrosarcomas, and 4 round-cell or spindle-cell sarcomas, while one tumor was called adenoma, and 3 carcinoma (Seigneur). There is no good reason to doubt that all these types represent variations in the growth tendencies of the fully differentiated cells of the gland.

An adenomatous structure is approached by tumors which reproduce the features of the normal gland and in which many well-defined groups of polyhedral cells appear in a scanty fibrous stroma. The polyhedral cells are, however, fused with the stroma and lumina are absent or ill-defined, so that the resemblance to a glandular adenoma is seldom pronounced.

Ependymal neuroglioma is the term employed by Pappenheimer to designate a cellular mixed tumor, and may well be extended to cover the entire group, to the exclusion of such terms as adenoma, carcinoma, and sarcoma. The cells are usually abundant and are arranged in groups surrounded by stroma, or they grow diffusely between fibrous strands, or sheath numerous blood-vessels. They are polyhedral, with clear cytoplasm and sharp borders resembling ependymal epithelium, and such cells may inclose fine lumina. Wide lumina lined by high cylindrical cells appear in teratomas and also in mixed tumors without other teratomatous characters. Many cells are rounded, loosely distributed, and exhibit many fibrils. Lymphocytes may be mingled with the tumor-cells. Diffusely growing tumors of this type resemble round-cell sarcoma, but are properly interpreted as gliomas or gliosarcomas. Excessive development of blood-vessels may produce the appearance of perithelioma or angiosarcoma. In these very vascular tumors syncytial masses may form, probably from ependymal epithelium. In Wood's tumor flattened or spindle-cells surrounded many small blood-vessels. Heidenhain's or Mallory's stains may demonstrate neuroglia fibrils running from and between the cells. Best's stain demonstrates glycogen in cell vacuoles.

In the stroma calcific deposits are very common and chondromatous metaplasia may yield the masses of cartilage occasionally observed. Marburg traced the origin of the tumor-cells from the pineal parenchyma, finding an external zone of gland tissue, an intermediate layer of proliferating cells of ependymal type, and a central area chiefly gliomatous. In Howell's cases the central area was composed of diffuse large round cells without intercellular substance, while the periphery yielded alveoli somewhat larger than those of the normal gland.

Symptomatology.-The chief interest in pineal tumors lies in their symptomatology, the study of which is susceptible of the same elaboration as has been given to hypophyseal growths.

The clinical features present two phases: (I) Pressure symptoms, general and specific, and (2) disturbances in the physiology of growth.

(I) Pressure symptoms common to this and other brain tumors, head- 
ache, vomiting, paresthesias, and vertigo, mark the onset of most cases. The usual absence of brachycardia is referred by M. Neumann to early closure of the aqueduct of Sylvius which spares the vagus in the fourth ventricle from pressure. More specific of the location of the tumors are the staggering gait, cerebellar ataxia from pressure on the vermis, and peculiar ocular disturbances, including nystagmus, ophthalmoplegia, and loss of pupillary reactions. Seigneur found paralysis of the common oculomotor nerve in I 8 cases, of the pathetic in 7 , and of the externus in 9. The oculomotor is affected by destruction of its nucleus and the pathetic and externus by pressure on the trunks. Auditory disturbance may be referred to pressure on the auditory nerve or corpora quadrigemina. Unilateral facial palsy may occur. Failure of mentality is marked by dulness, delirium, or prolonged somnolence in which hydrocephalus probably plays a part. Epileptic convulsions frequently occur. Polyphagia, polyuria, and glycosuria have been observed.

(2) The disturbance of growth affects chiefly the genitals, but is often associated with adiposity and occasionally with general and symmetrical overgrowth. Hypertrophy of the penis and testes, growth of pubic hair, and precocious sexual instinct have been observed with most tumors classed as teratomas, as well as with simple, benign, and malignant tumors. In the testes there is marked development of the interstitial cells (Goldzieher). In Oestreich's case the enlarged breasts in a boy of 4 secreted colostrum and the testes were much enlarged. In the case of Raymond and Claude the genital organs were small, but there was a growth of fine hair on the lip and cheeks. Hirsuties has been much more frequent than hypertrophy of the genitals. Hochwart mentions a deepening of the voice. In Schmidt's case conception occurred at 15 years and menstruation ceased at 22 years. General overgrowth of the body is described by Oestreich, Raymond, Hochwart, and Bailey and Jelliffe. Daly's patient, aged 23, with a large carcinoma, had polyphagia and gained 30 kilos in 6 months.

Adiposis has been observed in all varieties of tumors and is apparently not to be distinguished clinically from hypophyseal obesity. Marburg stated expressly that the hypophysis was normal in his case and yet the usual obesity was present.

The pathogenesis of the metabolic symptoms remains obscure. The combination of genital overgrowth with obesity is contrasted with the genital hypoplasia and adiposity of hypophyseal tumors. It may be assumed that the pineal tumor excites genital overgrowth by a sympathetic influence on the interstitial cells of the testis, which in some cases have been found hyperplastic. The obesity may then be attributed to pressure on the hypophysis, pars nervosa, or distention of the infundibulum. This interpretation rests on the theories of hyperpinealism and hypophyseal insufficiency. It is not entirely consistent with Raymond's case of large glioma, hydrocephalus, obesity, and testicular atrophy. Yet most observations indicate that pineal overgrowth stimulates growth of the testes.

Adrenal tumors are often associated with overgrowth, obesity, and genital precocity. With hypophyseal tumors the adrenals were found normal by Marburg and Goldzieher, but were hyperplastic in Raymond's case. In each of these cases there was genital overgrowth. A direct influence of the adrenal may therefore be eliminated.

Signs of status lymphaticus with persistence of thymus were noted by M. Neuman and Marburg.

Experimental studies are already not without suggestive results (Dana, Berkeley, Lit.). Exner and Boese destroyed the pineal in 95 rabbits without observing any change in 22 surviving animals. Foa, however, observed 
increase of weight and genital overgrowth in 3 roosters deprived of the pineal gland in the fifth week of life. Biach and Hulles produced marked atrophy of the pineal gland by castrating kittens. Berkeley produced acceleration of growth in young animals by injections of pineal nucleoproteid, and he with Cornell and Goddard observed considerable mental improvement in backward children fed on gland substance. McCord also observed considerable acceleration of growth and sexual precocity in chicks and guinea-pigs fed on the substance of calves' pineal.

The foregoing clinical, pathological, and experimental data seem to point rather strongly to the conclusion that normal activity of the pineal gland facilitates normal growth in general and sexual development in particular. Acceleration of these functions occurring in the course of pineal tumors may therefore be interpreted as hyperpinealism. In the absence of further data the obesity and hypertrichosis may be regarded as a part of the general and sexual overgrowth, but the hypophyseal failure must be considered as a possible factor in the adiposity. The existence of hypopinealism is less clearly established. There is evidently a close relationship between pineal and testicular functions which is probably not of an antagonistic nature, but the observations are as yet inadequate to define the exact position of the pineal with reference to other glands of internal secretion. 


\section{CHAPTER XLIX}

\section{TERATOLOGY}

Only a very limited portion of the extensive subject of teratology is of interest to the oncologist, and that portion is probably of least importance to teratology. Hence in the great scope of Ballantyne's "Antenatal Pathology" one finds an impressive catalogue of the multitudinous types of human deformities and monsters, but little regarding human teratomas. Marchand in Eulenberg's Realencyclopedia (I9II) presents an orderly and very exhaustive list of forms and ably discusses the subject of causal genesis of double monsters, without entering exactly into the details of many other questions. Schwalbe's elaborate treatise presents the sum total of knowledge up to rgo6, the scope of which necessarily renders specific details somewhat inaccessible. He emphasizes the existence of an unbroken series from identical twins, through double monsters, teratomas, and mixed tumors down to some simple tumors. In tracing the origin of these structures he introduces the term "teratogenic termination period" to indicate the latest period of embryonal development at which a given structure could have originated. A recent compilation is that of Hubner.

It must be admitted, I think, that while teratological studies have narrowed down the range of possibilities in the origin of identical twins and have defined the probable origin of most double monsters, yet the divergent views regarding the nature of fetal inclusions and adult teratomas are almost as numerous as ever.

Experimental teratology, as presented by Fischl, illuminates the principles of embryogenesis, but points out how teratomas may develop rather than demonstrating how they actually arise.

Under these conditions it seems most serviceable for the present purpose to indicate the present position of the various forms of abnormal growths which belong to oncology, their general relation to one another in a series of diminishing complexity, and to briefly review the factors probably present in their origin. For the more complete discussion of all these subjects the reader should refer to the above-mentioned works.

There are, however, two important tendencies discernible in recent embryological research which seem to be insufficiently considered in most text-books on teratology. These are the growing recognition of frequent impurities in specific germ layers and the increasing scope now being given to the process of budding in embryogenesis.

It must be admitted that the doctrine of the rigid specificity of the germ layers has suffered some limitation in recent years. The possibility that in the formation of these layers the separation may be incomplete and that portions of one layer may be carried over into another has been entertained with increasing freedom. The further possibility that the formative capacities of anatomically pure germ layers may not always be restrained within the rigid limits formerly set must also be considered. Especially in the field of teratogenesis these infractions of the set laws of growth become of first importance, for the practical inviolability of these laws has always been 
assumed as a necessary basis in the construction of theories of origin of teratomas. To what extent recent studies in this direction will remodel the conceptions of teratomas remains for the future to determine.

Secondly, the importance of budding of originally simple embryonal tissues as a source of complex teratomas has probably been underestimated by many observers who have been inclined to attribute all such complex growths to originally complex sources, as isolated blastomeres, totipotent sex cells, or embryonic inclusions. This principle, long since recognized and amplified by R. Williams, applies especially to the parasitic implantations and complex mixed tumors, although it may be of less importance than that author seems to imply in the origin of the irritation group of malignant tumors. In many respects this conception appears in the references to the "genius loci" and the development of secondary growth centers, which appear in many discussions of the origin of teratomas.

Oncology is usually assumed to deal with the following list of developmental anomalies and their sequelæ:

(I) Asymmetrical double monsters, parasitic fetuses, or fetal inclusions.

(2) Teratomas.

(3) Complex dermoids or teratoid tumors.

(4) Mixed tumors.

(5) Simple dermoids and epidermoids.

\section{DOUBLE MONSTERS}

Double monsters are (I) symmetrical or (2) asymmetrical.

(I) Symmetrical double monsters consist of two equal or nearly equal fetuses which are joined together at the cephalic ends (cephalopagi); or at
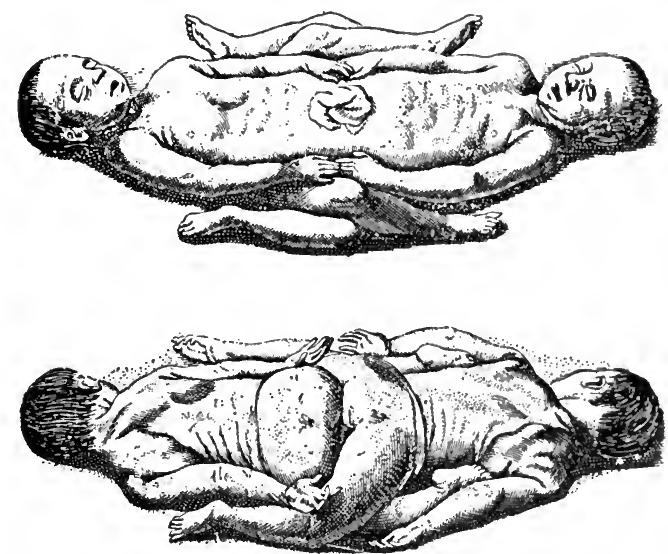

FIG. 503.-Case of ischiopagnus duplicatus, born at Hanau, Germany, Mar. II, 1643. (After Wilder.)

the thorax (thoracopagi); or at the pelvis (ischiopagi). Such conditions may result from the partial fusion of two originally separate embryos derived from one ovum, or from the incomplete splitting of one original embryo. Which of these two events actually occurs cannot at present be determined. According to Marchand, all symmetrical double monsters have a single chorion, as do monochorial twins, and nearly all have a single placenta. 
In the causation of symmetrical double monsters there are several possibilities. In the case of the asymmetrical double monster the parasitic fetus is rudimentary, and this fact is the chief basis of the view that it originates from a structure of much reduced embryonic value. Marchand holds that the parasitic fetus is derived either from a polar body or from an early isolated blastomere. In the first instance the isolated structure is derived from an unfertilized, in the latter from a fertilized, ovum. Yet the possibility of fertilization of polar bodies even in the human subject may perhaps be entertained, since in the flat worms this event is of regular occurrence and has a definite function in the early embryo. The sacral parasites and epignathi, from their structure, position, and mode of attachment, may be regarded as developing within the amnion, while the abdominal parasites may arise

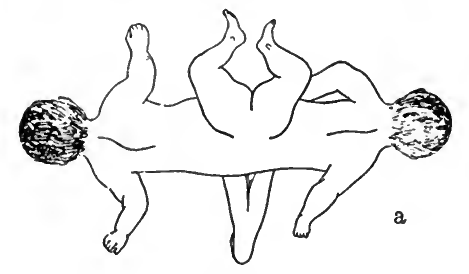
on the belly stalk outside the amnion and become included during the closure of the abdominal wall. A development from two separate embryos is inconsistent with the position of the parasite, which would have to lie free on the yolk sac of the autosite without connection with the chorion, as held by Ahlfeld and Taruffi. Yet umbilical cords and even rudimentary

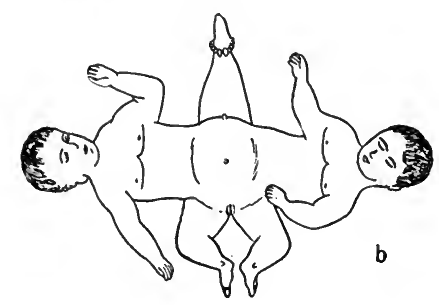
placentæ have been observed with epignathi (Rathke) and abdominal inclusions. Bonnet has accepted and elaborated this view of the origin of parasitic inclusions and many teratomas.

Schwalbe concludes that epignathi and other teratomas arise from isolated blastomeres, but regards the development of polar bodies as improbable. Fischel argues at length against the origin from polar bodies, and reasons that all fetal inclusions arise (I) from a second fertilized ovum attached to the first, or (2) from isolation and dislocation of portions of the germ layers. This point of view renders most intelligible the apparently unbroken series of complex to simpler structures occurring at the caudal and cephalic extremities, but, as Marchand says, it probably overestimates the potencies of fragments of differentiated germ layers. The ovum may be abnormal and contain multiple germ disks, which have often been observed in human ovaries, or there may be an abnormal distribution of germ material. The entrance of more than one spermatozoön FIG. 504. $-a$ and $b$, Case of imshowing on one side a double bilateral limb, composed of halves belonging to each component, born in Cadiz, May 30, 1818; $c$, Monstrum Anglicum, born in Salisbury, Eng., Oct. 26, 1664. (Licetus.) (After Wilder.) into an ovum occurs in reptiles, but in man it probably entails an abnormality
of the ovum which tends to result in its death. Yet this outcome cannot of the ovum which tends to result in its death. Yet this outcome cannot
be assumed as certain. The occurrence of double headed and other abnormal spermatozoa has been emphasized especially by Bromann and by Hofer and considered as a possible source of double monsters. The formation of the double germinal areas necessary for the origin of double monsters must occur after the union of male and female pronuclei, since twinning is hereditary. 
Experimental studies have demonstrated some of the conditions which may exist in the origin of double monsters. Roux produced one-sided embryos some of which were able to develop almost perfect embryos of reduced size. This process he called "post-generation." The bilateral char-

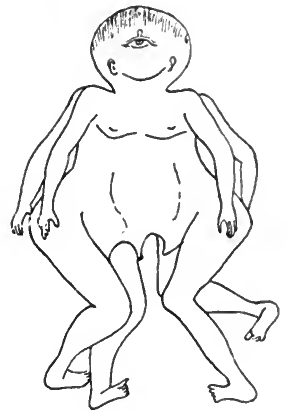

a

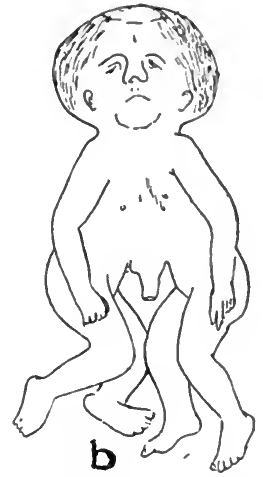

b

FIG. 505.- Lateral view of two cephalothoracopagi (Janus monsters), showing different degrees of separation. The face which is toward the observer is duplicated by another on the opposite side. Each face is contributed by the two components. (After Wilder.)

acter of the human embryo may favor complete splitting, but there is no evidence that double monsters result from such factors. Mechanically, inverting the ova to r8o degrees (Schultze, Wetzel) and the distorting effects of

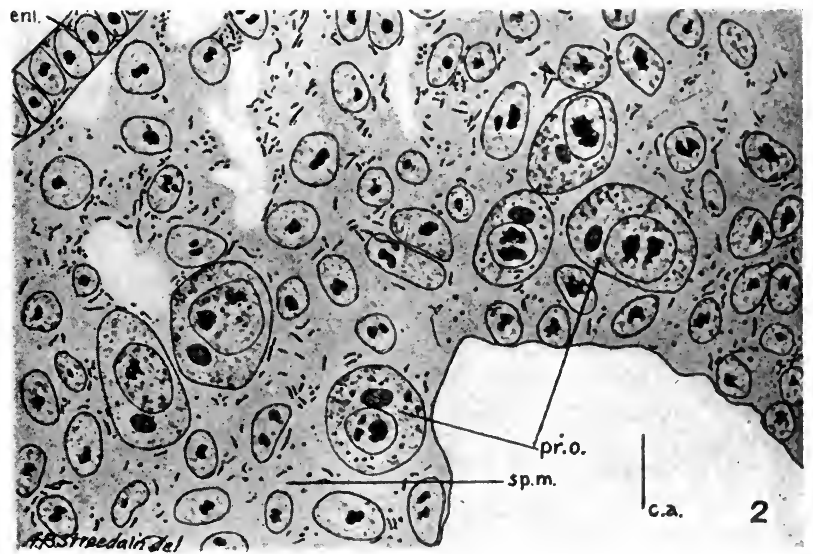

FIG. 506.-Position, form, and grouping of primordial germ cells in 33 somite chick embryos: pr.o., Germ cells; sp.m., splanchnic plate of mesoderm; c.a., cœlomic angle; ent, entoderm. (After Swift.)

distilled water (J. Loeb) have produced double embryos, but this method is probably not followed in nature. Two primitive streaks and embryonal areas have often been observed in ova, and double monsters of this origin have been traced in the chicken egg by Dareste. Marchand concludes that all 
cases of symmetrical double monsters arise from two originally separate embryonal areas which imperfectly fuse by ectoderm or entoderm and mesoderm, and that this process occurs during gastrulation. In symmetrical monsters either member of the pair may be considerably dwarfed through defects of the ovum or disturbances in placenta, cord, or amnion.

The importance of aberrant sex cells as sources of fetal implantations has been emphasized by the observations of Nussbaum, Allen, and Swift, who have found groups of such cells marooned in considerable numbers all along the embryonal entoderm from which they are derived. In some cases only a portion of these cells reach their normal haven in the sex glands. That the teratomas of the sex glands are derived from primitive sex cells is now generally accepted, and it is highly probable that many extragenital teratomas have the same origin. Since most observers agree with Schwalbe and Marchand that no essential morphological distinctions separate the genital and extragenital teratomas and the fetal implantations, it seems not impossible that the entire group may be referred to a single origin. At present the collateral evidence hardly justifies the extension of this theory to the epignathi and sacral parasites, but the sex-cell theory has one great advantage in that aberrant sex cells have been observed in numbers and their common occurrence in man is established, whereas isolated blastomeres and developing polar bodies in the human subject are hypothetical structures.

It thus appears that while the possible modes of morphogenesis of fetal inclusions have been fully covered, the real source or sources of these structures remain undetermined. It can only be stated that they arise from nearly or quite totipotent material. The field of divided single or fused double embryonal areas appears to be exhausted in the fused twins, thoracopagi, etc. The weight of opinion seems to favor the view that the fetal implantation arises from material of much less growth energy than is possessed by any form of double embryo and must be referred to isolated blastomeres, to marooned sex cells, possibly in some instances to polar bodies, and very probably in many instances to the process of budding.

Of the causal genesis of double monsters and fetal implantations nothing is definitely known. Clinical observations have given no hint of the underlying conditions. Signs of syphilis are rarely present, and no case of repeated births of double monsters has been recorded. The extensive field of experimental teratology has, in the opinion of Schwalbe, added nothing to our knowledge of causal genesis. Hubner's review consists of a repetition of the data regarding multinucleated ova, multiple germ disks and embryonal areas, abnormal or supernumerary spermatozoa, excessive germ material, and other conditions which form the basis of the discussions of formal genesis.

\section{THE PARASITIC FETUS}

This form of parasitic growth appears chiefly in the sacral region, or protruding from the pharynx (epignathi), or in the thoracic or abdominal cavities. Its structure presents the organs of a fetus, but always in imperfectly developed or rudimentary form, and often in disorderly arrangement. An umbilical cord is often present and fetal membranes and even a placenta or its homologous structure may be found. Schwalbe and Nakayama are unable to separate sharply between such complete fetuses and much less complex growths occurring in the same situations.

The origin of the fetal implantation may in certain cases be attributed to a second embryo which in some way becomes attached to the tissues of 
its host, the autosite. This mode of origin is, however, probably the rarest of events, for the evidence of comparative embryology points to the process of budding as the most probable source of parasitic implantations, especially when the parasites are multiple and their development markedly imperfect.

Therefore in the origin of the parasitic fetus a wholly different process is probably concerned from that which give rise to true twin fetuses. True twins may result from the fertilization of two separate ova derived from one Graafian follicle, or from simultaneous ovulation from both ovaries as shown by the presence of bilateral corpora lutea, or by rupture of more than one follicle in the same ovary, an event which is common in lower animals. In these cases the sex and form of the twins may differ and there are two separate placentas and fetal membranes.

Identical twins develop from one ovum and are of the same sex and characteristics, but the conformation of the placenta and membranes varies. Several theories of the origin of identical twins are maintained, including multinucleated ova, formation of two germinal vesicles, or complete separation in equal parts of an originally single fertilized ovum, fusion of two embryos, post-generation, separation of the two initial blastomeres. Of these Wilder favors the last.

\section{TERATOMAS}

The term "teratoma" has a restricted application to a group of tumors composed of recognizable tissues and complex organs derived from more than one germ layer. While in the parasitic fetus the organs are arranged in normal or nearly normal relations, producing a rudimentary fetus, in the teratoma there is a notable lack of orderly arrangement, producing merely a veritable potpourri of fetal tissues. The less complex tumors of this class may be designated as teratoids. The term "embryoma" is applied by Wilms and others to various members of this group. It is best limited to teratomas closely resembling embryos. The tissues of teratomas may be of adult or embryonal type, and on this ground some authors refer to the former as co-etaneous, the latter as embryonal. Since both types of tissue occur in the same teratoma the analysis furnishes no indications of the origin of the growths, and it must be assumed that secondary factors determine the rate of differentiation of the cells of origin.

While the parasitic fetus does not give rise to secondary blastomatous processes, the teratoma frequently originates true tumors, benign from the adult elements, malignant from the embryonal structures.

An important principle of the growth of teratomas is the tendency of one element, either adult or embryonal, to overgrow and suppress the others. The obverse of this principle, viz., the progressive degeneration of germ layers, is probably of equal importance. Thus bidermal teratomas are frequently observed, but such cases seem to diminish with the increased persistence of the search for a third germ layer. Saxer observed a single tooth as the sole element of an ovarian teratoma, and all chondromas of the testis are of teratoid nature (Ribbert). The studies of Wilms on ovarian dermoids, by showing the uniform tridermal nature of these tumors, furnished the basis of this principle, which has been elaborated by L. Pick and many others. The application of the rule is open to error when the various germ-layer derivatives are not rigidly scrutinized. Borst and others warn against the unscrupulous identification of embryonal derivatives in teratomas. Yet the tridermal nature of certain groups of teratomas is well established and the warning may apply chiefly against fantastic interpretations of 
organ rudiments. Another source of error lies in the possibility that metaplasia or abnormal self-differentiation may occasionally break down the barriers formerly erected between the germ layers. It is assumed that the separation of germ-layers of the normal embryo holds also for the teratoma, but if the abnormal environment of the teratoma may serve to disturb the normal laws of differentiation the views of the origin of teratomas may have to be altered. The "genius loci" has already been invoked to support the local origin of certain apparently heterochthonous sacral teratomas. The overgrowth of one element in an embryonal teratoma produces a malignant tumor of nearly uniform type, since it has been shown that many malignant tumors of the sex glands originate in this way. The same principle probably applies to many embryonal tumors of other regions, but to what extent remains for future observations to determine.

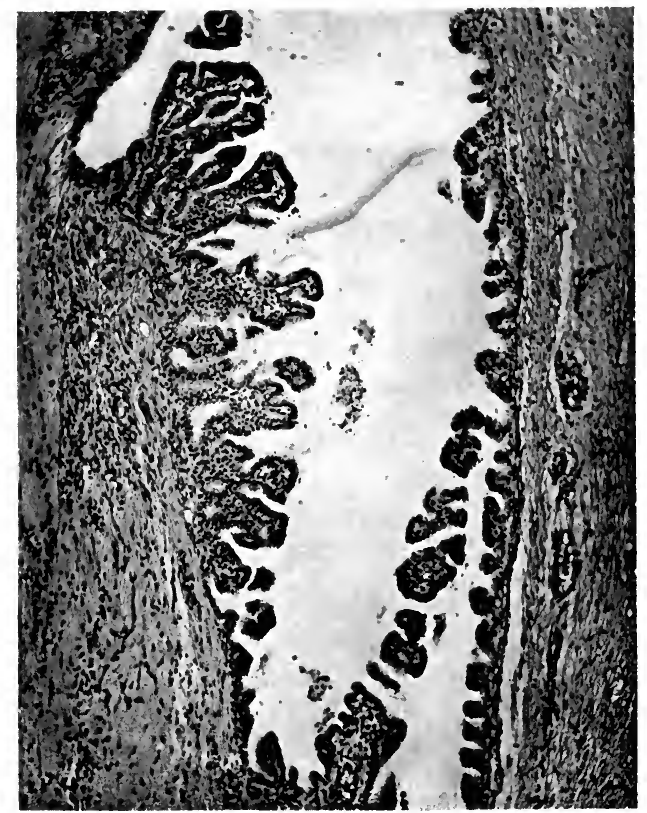

FIG. 507.-Ependymal structures surrounded by brain tissue in a small teratoma of ovary.

In discussing the morphogenesis of teratomas it must be admitted that no sharp dividing line can be drawn between these disorderly embryomas and fetal implantations, on the one hand, and the complex teratoids and mixed tumors on the other. Yet this fact does not lessen the probability that special conditions determine the growth of the large group of typical teratomas and that a particular material, not connected with fetal implantations or simple mixed tumors, gives origin to teratomas. The clinical evidence in the field lies in favor of this view, but is somewhat neglected. The age incidence, location, and course of many typical teratomas strongly suggest a peculiar formal and causal genesis.

The chief source of teratomas is probably the aberrant sex cell. This origin alone adequately accounts for the predilection of teratomas for the sex 
glands. The observation of numerous sex cells displaced along the entire length of the embryonal entoderm offers abundant source for most abdominal and thoracic teratomas. Some reported abdominal and thoracic teratomas may possibly represent metastatic growths from minute teratomas of ovary or testis. I have recorded a mediastinal teratoma secondary to a small testicular growth which escaped several examinations during life.

For the pharyngeal and sacral teratomas no single mode of origin can be adduced. The most highly developed of the sacral tumors are fetal implantations. Another larger group, of which Nakayama traces many transitions from comparatively simple up to very complex growths, are true teratomas. Others probably represent forms of partial reduplication or are of strictly local origin. Exactly similar variations are observed in the structure and complexity of the epignathi (cf. Arnold). The complex developmental proccesses and the definite growth centers located at the cephalic and caudal extremities offer unusual opportunities for the appearance of complex teratoid structures derived by budding from purely local sources. An extreme variety of structural defects in neighboring tissues is observed with this class of growths, including cranial fissures, spina bifida, rhachischisis, etc., and the discovery of such defects is of much value in establishing the nature of the growth.

For the majority of extragenital teratomas an origin from isolated blastomeres is maintained by Marchand and accepted in some form by many authors. The occurrence of these growths in median positions and in much the same localities with fetal implantations, the difficulty of separating them morphologically from the parasitic fetus, and their obvious origin from totipotent or nearly totipotent material, strongly suggest an origin very similar to that of the asymmetrical double monster. The isolation of an early blastomere would theoretically account for such structures, but this theory has little if any advantage over the origin from sex cells. The process of budding, however, presents strong claims for consideration in this field.

Thus, three main groups may be recognized of tumors containing adult or embryonal organs in disorderly arrangement: (I) Teratomas derived from aberrant sex cells and found chiefly in the sex glands. (2) Extragenital teratomas, many of which approach the development of the parasitic fetus and which may be derived from isolated nearly totipotent blastomeres, or from early budding of the blastoderm. (3) Teratomas (or teratoids) derived from multipotent material of distinct regional stamp and reproducing the organs of these regions. These tumors are usually associated with a defect in the formation of the parts from which they spring.

Regarding the causal genesis of teratomas there are few data. The isolation of blastomeres is not known to occur-much less the causes of such isolation. For the teratomas of sex glands there is much clinical evidence that trauma figures in many cases and excites the parthenogenetic development of the aberrant sex cell. Harvey has tabulated many methods for the artificial production of parthenogenesis, some of which, including trauma, are successful in frogs. Teratoma testis arises at the junction of rete and spermatic tubules, and at this junction Kirkbride has shown that there may be very marked disorder in the structure of the fetal testis favoring the displacement of cells. Teratoma testis is also relatively frequent in the rudimentary undescended testis.

\section{COMPLEX DERMOIDS}

The great majority of complex dermoids are imperfectly developed teratomas and in the sex glands it is very probable that all dermoids are of this 
nature. Since the bulk of the early embryo is composed of ectoderm, the overgrowth of this germ layer in the development of teratomas is a natural result and favors the theory of origin from sex cells. The comparatively frequent chorioma testis is the embryonal equivalent of the testicular dermoid. Chevassu's series contains intermediate stages between the embryonal and the adult ectodermal tumor.

A local origin by budding off of multipotent material appears to be responsible for certain complex dermoids of the cephalic and caudal extremities. They are usually associated with defects in neighboring structures. Mediastinal and retroperitoneal dermoids may be of comparatively simple structure, but it is unusually difficult to refer such growths to a local origin.

\section{MIXED TUMORS}

The scope of this term has been greatly restricted, especially by the studies of Wilms, who reviewed the once numerous group and demonstrated the tridermal and teratomatous nature of many of the tumors included therein. Through his work and that of many later observers the mixed tumor has largely become the modified teratoma, and the idea of a predominant metaplasia in the growth of many tissues has been replaced by the conception of a natural unfolding of embryonal cell potencies. Hence the term "mixed tumors" is now confined to comparatively simple, chiefly embryonal growths, of purely local origin, resulting from overgrowth of embryonal structures with or without misplacement.

Most of the accepted forms of mixed tumors do not contain derivatives of three germ layers, but are bidermal or monodermal. Yet tridermal tumors of local origin are observed, especially at the caudal and cephalic extremities. Since it is not always possible to determine whether a tumor is a poorly developed teratoma of heterochthonous origin or a complex neoplasm of local origin, a rigid classification of such cases cannot be accomplished. In general one may follow the rule of designating as mixed tumors growths in which embryonal and blastomatous features are prominent, and grouping as teratomas those growths in which the structure is more complex and rudimentary organs are present. It is obvious that the mixed tumors are closely related in origin to the extensive series of simple embryonal tumors occurring in many regions.

The restricted group of mixed tumors is designated by Adami as teratoblastomas. Yet many true teratomas are blastomatous, and it seems desirable to distinguish as far as possible between teratomas and mixed tumors.

In the true mixed tumor the blastomatous process affects both germinal derivatives, producing adenosarcoma or carcinosarcoma, etc., and the tendency of one element to overgrow the other appears to be less marked than with teratomas.

The mixed tumor may therefore be defined as a complex embryonal tumor of local origin which reproduces the normal development of the tissues and organs of the affected part. They may be classified according to the region in which they occur, as renal, genito-urinary, salivary and facial, and mammary.

The most typical examples of the restricted class of mixed tumors are found in a series of complex embryonal and congenital tumors of the kidney. These are mesodermal growths derived from the primitive kidney and neighboring tissues and reproduce the structure of the nephrotome and sclerotome. They are called adenomyosarcoma. Muus found also epidermal 
inclusions. Certain mixed tumors of the male bladder and gubernaculum testis are probably of the same origin.

The congenital sarcoma of the vagina contains spindle-cells, striated muscle-fibers, and myxomatous tissue, and is derived from mesoderm misplaced during the development and degeneration of the Wolffian duct. More complex sarcomas of the adult cervix uteri contain muscle and cartilage. The adenomyoma uteri and its malignant derivatives constitute a welldefined variety of mixed tumor derived from the mesonephros or paroöphoron.

The salivary and lacrimal glands are the seat of mixed tumors containing mesodermal derivatives and basal-cell carcinoma, and are probably derived from buccal ectoderm with a possible admixture from the maxillary periosteum or bronchial cartilages.

The mixed tumors of the breast probably originate from aberrant and superfluous material derived from the fetal ectoderm and mesoderm during the early development of the breast. They contain glandular tissue and carcinoma and various mesodermal derivatives. It is probable that metaplasia is responsible for some of these mesodermal structures (Beneke).

Carcinoma sarcomatodes constitutes a miscellaneous group of tumors of variable and uncertain origin (Lippmann, Herxheimer, Lit.). Most of these tumors have occurred in the uterus, but out of a considerable number Herxheimer found only 5 which could withstand critical analysis. Others have been observed in the ovary, and in Lippmann's case there were both carcinomatous and sarcomatous metastases.

In the thyroid several cases of supposed carcinosarcomas are reported. Especially difficult is the interpretation of Schmorl's case, of thyroid adenoma recurring as carcinoma with sarcomatous stroma and with sarcomatous metastases. Tumors of the esophagus containing epithelioma and sarcoma are described by Hansemann and Herxheimer, and the former has described similar tumors of pharynx and gall-bladder. Landsteiner reports a tumor of the gall-bladder in which acanthoma and myosarcoma encroached upon each other. Of 2 cases occurring in the stomach, that of Lindemann gave numerous metastases in which both elements were intimately mixed. In the liver Lubarsch cautiously interpreted a large tumor as an adenocarcinosarcoma. In many respects it recalls the complex pictures of primary carcinoma of liver-cells. Michelsohn believed he could trace the origin of a carcinosarcoma from the gland-cells and stroma of the pancreas. Arising in the nasopharynx and invading the orbit Klein found a vascular round-cell sarcoma containing islands of acanthoma.

In the lower animals tumors of mixed structure are not infrequently observed, especially in the mammæ of dogs and in the thyroid in rats (Wilms, Loeb).

The transformation of carcinoma into carcinosarcoma, followed by elimination of the epithelial element in the course of transplantation of tumors of mice and rats, observed by Loeb, Ehrlich and Apolant, Bashford, and others, is an interesting experimental contribution in this field. Loeb saw the sarcomatous element in the first transplant, but it usually occurs much later, either gradually or suddenly, and with or without persistence of the carcinoma. That the original tumors contained mixed tumor elements is improbable. That the spindle-cells are modified epithelium is rejected, although transitions of cell forms have occasionally been observed. It is generally assumed that the carcinoma cells exert a stimulating influence on the stroma-cells, which in the course of the transfers yields a tumor of fibroblastic origin.

The correct interpretation of the carcinosarcomatous structures in man and lower animals is a matter of much difficulty. No single explanation will probably apply to all cases. The chief source of these structures is, I believe, the transformation of epithelial cells into spindle-cells. This change is of wide-spread occurrence in epithelial tumors, but only rarely is it interpreted as carcinosarcoma. It is frequently observed in the 
course of recurrences and metastases of melanoma, basal-cell epithelioma, adamantinoma, adenocarcinoma of thyroid or ovary, primary carcinoma of liver, and in embryonal epithelial tumors. The change is facilitated by rapid growth, relief of pressure, and inflammatory exudate. Krompecher's studies of the metaplasia of squamous epithelium have an important bearing on this subject. The change may often be traced in the same tumor where polygonal cells merge into spindle forms. The most striking instances are furnished in the recurrences and metastases of epithelial tumors. Especially in thyroid tumors the form of the epithelial cells is subject to wide variations, a fact which renders Schmorl's interpretation of his carcinosarcoma very questionable. I have seen pure spindle-cell metastases in epithelioma of lip, melanoma of skin, adamantinoma, and other epithelial tumors. In experimental tumors of mice I have twice seen convincing indications of change of epithelial cells into more actively multiplying spindle-cells in cases now figuring as carcinosarcoma. Thus in several departments there is reason to think that transformation of polygonal into spindle-cells contributes a share of carcinosarcomas.

A second group of cases may be referred to the intermingling of contiguous primary tumors of the same region, and on this ground Herxheimer rightly discards certain reported cases of carcinosarcoma.

That certain tumors originate through neoplastic growth of both stroma and epithelial cells of a tissue complex, the epithelial cells at first outstripping the fibroblasts, must be conceded, but it is difficult to determine which if any of the so-called carcinosarcomas originate in this manner. Known examples of this type, as the adenomyoma uteri and its malignant forms, are rather well defined. It is possible that some of the uterine and ovarian adenosarcomas originate thus and constitute a special variety of mixed tumor.

In the lower animals the ready response of fibroblasts to various stimuli renders more acceptable the belief that transplanted stroma may occasionally assume neoplastic properties during a series of transfers, but does not guarantee the existence of such a process in the course of tumor growth in man.

\section{SIMPLE DERMOIDS AND EPIDERMOIDS}

The simple dermoid consists of epidermis, derma, and dermal glands, and usually appears in the form of a cyst. In the epidermoid definite dermal structures are wanting.

The simple dermoid commonly originates by the inclusion of a portion of ectoderm during the closure of embryonal fissures, or at the point of union of ectodermal with other structures, along the course of ectodermal invaginations (hypophyseal duct), or from persistent embryonal ectodermal structures. In the sex glands, especially the ovary, a simple dermoid may be the sole remnant of a true teratoma. A traumatic origin of both dermoids and epidermoids has been demonstrated in many regions. Epidermoids are usually of traumatic origin, but certain well-defined forms are of embryonic derivation and produce characteristic tumors, as cholesteatoma. A defect of the overlying skin or other abnormality is sometimes present with congenital dermoids (Heschl). The dermoid cyst is single or multilocular, and the contents are sebaceous material and hair. The character of the hair corresponds to that of the region affected. Torok concluded that very early separation of ectodermal material is followed by the development of dermal structures, late separation giving origin to the epidermoid.

Secondary changes in dermoids are frequent and include suppuration, rupture, papillary ingrowths, overgrowths of dermal structures, and neoplastic changes.

The experimental production of dermoids has been successful in the hands of many authors, the result being a variable period of growth of the implanted skin, a long period of quiescence, followed in every instance by slow atrophy. 

SPECIAL FORMS OF EPITHELIAL CYSTS, DERMOIDS, MIXED TUMORS,
TERATOMAS, AND FETAL IMPLANTATIONS

A regional classification of this extensive group of tumors is the most serviceable, and is rendered almost imperative on account of the difficulty of separating the different types on the basis of formal genesis.

Epithelial Cysts of Skin.- The variety of cystic structures lined by squamous epithelium in skin and subcutaneous tissues is considerable.

(I) Atheromatous cysts arising from retention in dermal glands, chiefly sebaceous, are of frequent occurrence. They are usually connected with the epidermis. Chiari, however, from the study of a notable case, showed

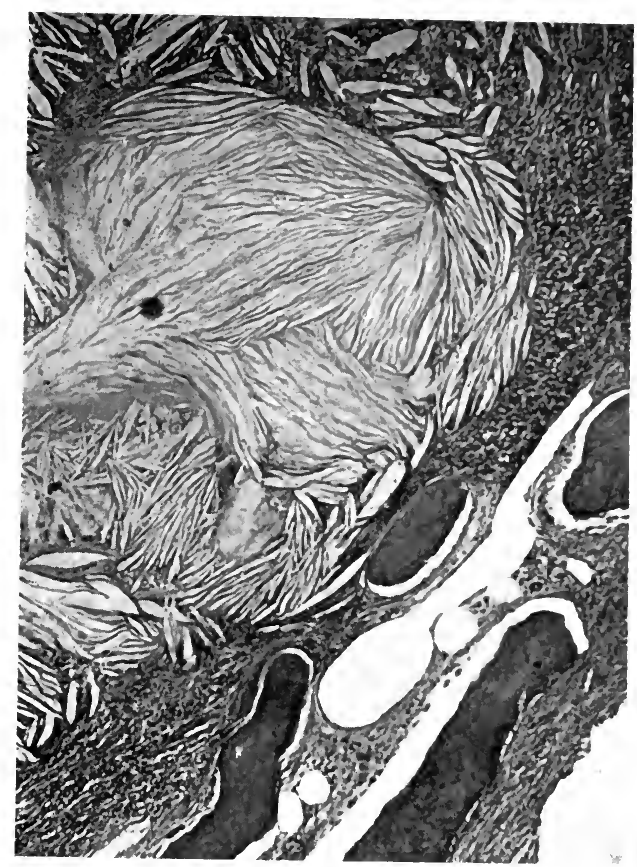

FIG. 508.-Fat crystals in a dermoid of skin.

that the common multiple cysts of the derma may arise, as does the smaller comedo or milium, by retention in hair, sebaceous and sweat-glands, and that these retention cysts may grow deeper as they enlarge and may eventually lose all connection with the epidermis. The lining is of simple stratified epithelium which is compressed and atrophic.

(2) Congenital Epidermoids.-The origin of true dermoids of the skin from ectodermal cysts was established by Remak. The majority of small or multiple epithelial cysts of the skin was also shown by Chiari, Heschl, and Franke to be the result of congenital misplacements of epithelium. The lining of these cysts is of well-formed epidermis, often with papillæ, but without other structure of the derma. On account of the simple epidermal lining Heschl called them epidermoid cysts. It is probable that deep epitheliomas of the skin arise from such cysts, including certain reticulated epitheliomas. 
(3) Implantation Dermoids (Sutton). Cystes Epidermiques (Reverdin).Traumatic misplacement of fragments of epidermis produces simple cysts which are of wide-spread distribution and may reach considerable dimensions. The importance of this origin has been emphasized especially by Reverdin, Jonnesco, and others, who have pointed out their occurrence in exposed portions of the body, as the palms and soles, their main clinical features, and the varying character of the lining. Schwenninger, Kaufmann, and Dooremale showed that such cysts may be produced experimentally by implanting portions of epidermis in deep tissues, or in the interior chamber of the eye. The character of the resulting cyst depends much upon the conformation of the displaced fragment. A complete fragment of skin with epidermis and derma yields cysts with papillæ, while the epidermis alone produces smooth-walled cysts or pearly nodules (Garre, Le Fort). Yet Hoesch and Aschoff concluded that definite growth of displaced epithelium and cyst formation required that some connective tissue must accompany the epithelium. Fetal epithelium is more active than adult (Nicholls). The extent of the misplacement is often surprising, and the original injury may excite little notice. Although usually found on exposed surfaces in working people, traumatic epidermoids occur in the cornea and iris (Franke, Graefe). Yet the origin of these intra-ocular cysts is not fully determined (Collins, Greef). A traumatic frontal intracranial dermoid resembling cholesteatoma is described by Hartley. The growth capacity of the traumatic dermoid is moderate and malignant changes are not observed.

(4) True Dermoids.- The true or congenital dermoid is, as a rule, a larger, more complex, and more deeply situated structure than the retention cysts and traumatic dermoids. It is situated in locations which connect it with the embryonal disturbances of development to which it owes its crigin (fissural dermoids). The structure presents all the elements of the skin, including epidermis, derma, and dermal glands, which may become atrophic, but usually exhibit overgrowth or at times malignant changes. Accessory structures are sometimes added, as lymphoid tissues, fat tissue, muscle, bone, cartilage, and normal or cystic blood- and lymph-vessels.

Dermoid cysts present particular clinical, pathological, and embryological problems in the several situations in which they occur. It is especially difficult to distinguish between certain complex dermoids and teratoid growths with predominance of ectoderm. The congenital dermoids occur in the scalp, neck, back, along median line of chest and abdomen, sacral region and buttocks, where they are readily connected with embryonal fissures, clefts, and junctures. Teratomas are usually found in deeper organs and along the vertebræ from neck to coccyx. Some observers give a very wide scope to the congenital dermoid at the expense of the traumatic forms and would include not only those which occur at points of recognized predisposition (clefts, fissures), but those in many other regions, especially when a traumatic history is missing. Thus Labougle found a traumatic history in only I6 of $4^{2}$ dermoids of the vola manus; the others he would refer to congenital separation of epidermis at interdigital folds.

Cervical Dermoids. - An extensive variety of congenital anomalies in the neck results from irregularities in the closure of the branchial clefts, which give rise to reduplication, partial or complete, of organs; fistulous tracts; epidermoids; dermoids; blood- and lymph-cysts, and true tumors. Various combinations of these conditions may be observed, as multilocular proliferating cystoma with dermoid cyst, or multilocular cysts of various types with cystic lymphangioma (Samter). The location of the different clefts and of the abnormalities referable to them may be seen from the accompanying 
sketch from Cusset. This subject was extensively investigated by many early observers, more recently by Kostaniecki and Mielecki, who conclude that the great majority of cervical dermoids arise from the second cleft. Yet many fissures, cysts, dermoids, and tumors in particular locations are connected with each of the other clefts (Virchow, König).

(I) The first branchial cleft locates a series of abnormalities which are chiefly found in the aural and submaxillary regions. These consist of supernumerary ears (Sutton), aural fistulas, chondromas from the aural cartilages, and many outgrowths of the skin which often contain cartilage (Virchow, Hennes). It is probable, however, that most of the superficial anomalies in and about the ear result from secondary disturbances in the formation of this organ and are not connected with the branchial clefts. Congenital epithelial cysts of the external ear result from irregular closure of the fistula auris congenita (Steinbrugge).

Dermoid cysts from the first cleft appear to be relatively rare. Hildebrand out of 20 epithelial cysts of neck observed one which he referred to the first cleft. It lay in the suprahyoid triangle away from the middle line. König reported a cyst and fistulous tract probably derived from the first cleft, which ran under the angle of the jaw along the facial nerve and terminated between tragus and antitragus. Another group is found along the floor of the mouth, constituting the sublingual dermoids (Kostanecki, Lit.). These are lined by epidermis and contain sebaceous material, and the walls are free from lymphatic tissue. They originate from the ectoderm of the first branchial

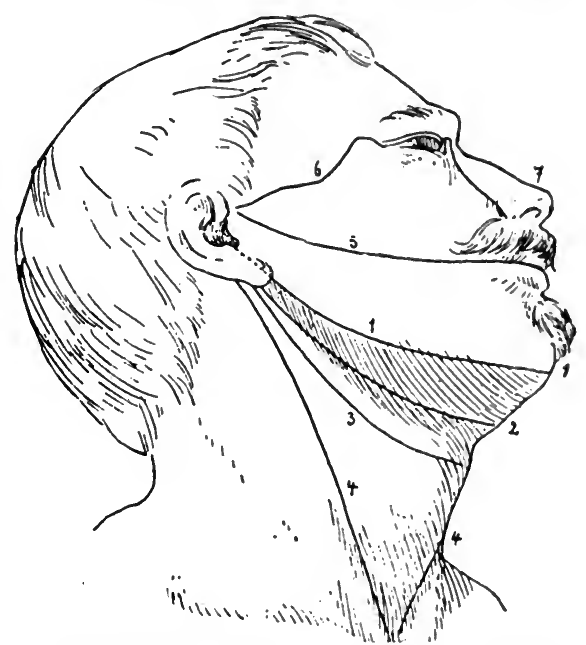

FIG. 509.--Situations of congenital fissures in neck and face: $1,2,3,4$, First, second, third, and fourth clefts. 5, Intermaxillary cleft. 6, Frontomaxillary cleft. 7 , Nasomaxillary cleft. (Cusset.) arch which goes to form the lateral portion of the base of tongue. Incomplete union of the two opposite portions of the first branchial arches may leave a triangular defect beneath the skin in the middle line in the submental region (mesobranchial space of His). Thus, portions of ectoderm may become adherent to entoderm and form mesobranchial dermoids which lie at base of tongue in middle line, or may appear anteriorly in suprahyoid region.

(2) The course of the second branchial cleft follows the stylohyoid ligament from the styloid process to the lesser cornu of the hyoid bone. It thus runs from beneath the ear externally and from the tonsillar fossa internally, along the posterior belly of the digastric muscle. According to Kostanecki and more recent observers, the great majority of branchial fistulæ and cysts originate from this cleft. They lie anterior to the sternomastoid muscle and may develop at any point from the external ear to the sphenoid bone, or they may extend along the whole length of this region. König suggests that the position may be influenced by the course of the facial nerve or artery. Portions of the cyst may lie within the parotid gland and numerous lateral sacculations may develop. 
Structure.-The lining reproduces the structure of ectoderm or of entoderm. Both embryonal layers may be represented when the membranous septum is ruptured and both inner and outer layers contribute to this lining. Some cysts present a lining of epidermis with dermal glands. Their contents are usually sebaceous and they may contain hair. Others show squamous epithelium of a mucous membrane with basal layer of columnar cells and their contents are mucoid. A third group shows ciliated epithelium as well as squamous. These variations depend on the character of the embryonal structures involved, the mucous membrane and ciliated cells being derived from the entoderm which originally possesses a ciliated lining. Foreign body giant-cells often replace much of the epithelial lining, especially when the contents are fatty or oily. Groups of thyroid alveoli may be present in the wall. Lymphoid tissue is abundant in the embryonal entoderm and reappears in cysts derived from this layer, but is absent in the others. In the wall may be found striated muscle, mucous glands, and islands of cartilage, derived from the mesodermal tissues of the branchial arches.

Secondary changes in cervical dermoids are of much importance. Extravasation of blood into the cyst not infrequently occurs from the small vessels in the wall. Rarely a communication is formed with the jugular vein, producing a blood-cyst (Thomas). The dermal glands in the wall may become cystic or may develop cystadenomas (Zahn, Samter).

Branchiogenic Carcinoma.-That many cervical carcinomas and primary epitheliomas of the neck arise from branchiogenic cysts and epithelial rests was first shown by Volkmann. These tumors occur usually after the fortieth year, producing globular, cystic or solid, often externally ramifying growths which are extremely difficult to extirpate. Pharyngitis, difficulty in swallowing, inflammatory edema, and lymphadenitis often mark their onset and course. Invasion of lymph-nodes is early or late, depending on the integrity of the cyst capsule. The structure is commonly that of adult acanthoma. To what extent other carcinomas of the neck of obscure origin are referable to branchial remnants is at present undetermined. Many of the multilocular blood-cysts of the neck probably arise in this manner. The lymph-vessels may be highly developed and it is probable that these vessels may be the source of hygroma colli (Lucke, Zahn). Virchow designated as fissural angioma certain vascular tumors of this region. The fragments of cartilage in the wall are held to be the source of certain branchiogenic chondromas.

The diagnosis of branchiogenic carcinoma, after it has advanced to the cervical lymph-nodes, may be difficult. It should be emphasized that the usual form of the tumor is a cystic growth in and about which are carcinomatous areas, and that most cases are encountered when the cysts are recognizable, and when a squamous-cell cover is present or predominant. In the same region are observed carcinoma of the lymph-nodes secondary to lesions of adjoining mucosæ, buccal, nasal, ethmoidal; carcinoma from aberrant thyroid follicles; tumors of the carotid gland; and primary endothelioma of lymph-nodes. In all the secondary carcinomas it should be possible to demonstrate the primary growth. The structure of carotid gland tumors is specific and that of endothelioma is partially so. There remains a group of apparently primary carcinomas of the cervical lymph-nodes whose origin is obscure, and some of these may be derived from the entodermal epithelium of branchial cysts. Definite observations in this field are lacking, but in Samter's case of complex branchiogenic cyst there were adenomatous proliferation in several of the compartments and infiltrating carcinoma with metastases in lungs and abdomen. 
Cervical Teratoids and Teratomas.-Complex teratoid tumors of the neck are comparatively rare and are found chiefly in connection with the branchial clefts and the thyroid gland. Partial reduplication of the lower jaw has given rise to large tumors of the neck containing bone, cartilage, teeth, muscle, connective tissues, and fat (Wilms). More complex tumors containing bone, teeth, muscle, ectodermal and entodermal cysts, portions of respiratory tract, and brain tissue, are described by Pupovac and Wetzel, and interpreted as of bigerminal origin. Several cervical teratomas have been connected with the thyroid gland (Poult, Lit.). In Henle's case a large congenital tumor contained nerve tissue and choroid plexus, cysts lined by cuboidal and ciliated epithelium, thyroid follicles, and connective tissue. Some cystic sarcomas, as in the thyroid, are of complex structure (Zahn). In Pupovac's case the lymph-nodes contained metastases of ganglionic nerve tissue.

A headless but otherwise well-formed fetus was found embedded in the cervical tissues by Rosenstiel, and Grass observed a complete fetus of the size of the finger lying in the neck of a woman. Several reports of fetal implantation in the branchial clefts have been collected from the older literature by Heusinger.

Cysts of Thyroglossal Duct.-The thyroglossal duct is a rare source of dermoid cysts.

When the base of the tongue is formed by the union of two lateral arches an epithelial-lined canal persists, leading from the foramen cecum to the middle lobe of the thyroid The upper portion of the duct may remain patent (lingual duct), while the lower region from thyroid isthmus to hyoid bone forms the thyroid duct. Bochdalek in several cases found segments of this canal, 2 to $3 \mathrm{~cm}$. in length, patent and with lateral sacculations lined by ciliated epithelium. Streckeisen found small mucous cysts lined by ciliated cells along this tract, and Hildebrand has collected other similar cases in some of which the wall contained thyroid tissue (Berard, Chalier, Erdheim, Lit.). Dermoid cysts in the middle line of the tongue are probably derived from portions of this duct. They may reach a large size, appearing in the floor of the mouth and displacing the tongue upward (Bryck, Dirmstrey). Rarely cysts are found behind the right lobe of thyroid. They are lined by ciliated epithelium, and are probably derived from the esophagus, the upper part of which in the early embryo is covered by ciliated epithelium.

Mediastinal Dermoids.- These tumors are located below the sternal notch in the anterior mediastinum or between the heart and root of lung or in the lung, or they occupy the pleural cavity (Christian, Lit.; Lambert and Knox, Lit.). They form single or multilocular cystic growths which may reach large dimensions. Most of them appear in young adults, and run a slow course of I to I6 years. Symptoms may be wanting, but most cases show the results of pressure on the bronchi and trachea, with cough, hemoptysis, and in 8 cases with expectoration of hair, as well as sebaceous material.

According to structure they fall into two classes: (I) Simple dermoids.

(2) Teratoid tumors.

The simple dermoids are single or multilocular cysts with smooth or polypoid epidermal lining containing dermal glands and with contents of hair and sebaceous material. Teeth are frequently present. 'The epidermal lining may be lost, and plates of bone or cartilage may be present. Some have been connected with portions of thymus (Marchand). The complex tumors are tridermal and contain besides epidermis, bone, cartilage, nervous tissue, intestinal tract, respiratory ciliated epithelium (Loewenmeyer), and thyroid (Mandelbaum) (Dangschaat, Lit.). The older cases have been col- 
lected by Riegel. Malignant changes were observed in 3 cases, all of which were probably teratoid. Pinders described lymphosarcoma probably from the thymus, Jores spindle-cell sarcoma extending into lung, and Virchow carcinoma and sarcoma with nodules in liver.

A single origin through one-sided developments of teratomas cannot be excluded for the entire group. Ekehorn points out that the great majority of mediastinal dermoids prove to be tridermal teratomas. Yet most authors regard the simple tumors as derived from the third branchial arch which produces the deep sinus cervicalis and the thymus. The intimate relations of ectodermal and entodermal layers of the third and fourth arches may explain the variety of epithelium and the connection with the thymus and thyroid, while the descent of the heart may carry these structures deep into the thorax.

Dermoids of the lower mediastinum may result from imperfect closure of the anterior chest wall, which gives origin to presternal dermoids (Andoly). Bergmann observed a dermoid divided into two compartments by the sternum, but joined by a canal through the bone.

Congenital dermoids occur along the orbitonasal fissıres: (I) At the outer angle of the orbit next to pericranium covering external process of frontal bone. (2) At inner angle of orbit. (3) In upper eyelid between the frontonasal plate and the cutaneous fold from which the eyelid is formed.

At the root of nose, over the fontanels, at the occipital protuberance, and elsewhere in the scalp congenital dermoids are also found (Hartley). The origin of these dermoids is explained by the imperfect separation of dura and skin during the intramembranous formation of the cranial bones. As the bone forms between the dura and skin portions of the latter may adhere to the dura, become enclosed in bone and form dermoids which lie within the skull and may be connected with the skin by a pedicle. With the formation of intra-orbital dermoids the invagination of ectoderm in the development of the lens is prominently concerned.

Orbital Teratomas.- Teratomas occur in the orbit, usually as bulky growths present at birth (Hippel, Lit.). Their analysis reveals, as a rule, derivatives from three germ layers, but only two layers may be identifiable. The development of rudimentary organs is not pronounced, but segments of intestine, ocular cups, and portions of central nervous system have been identified. Hippel suggests that certain orbital dermoids are of teratomatous nature, since ganglia and gland alveoli lined by mucous cells have been found in the wall of the dermoid. Monogerminal mixed tumors of the orbit are illustrated by the case of Weigert, Broer, which contained fat, bone, cartilage, and cysts lined by cylindrical or ciliated or squamous epithelium.

Pharyngeal Dermoids, Teratomas, and Epignathi.-A somewhat numerous group of complex tumors and developmental anomalies appears in the form of polypoid growths of the nasopharynx, which may be subdivided into three main classes: (I) Dermoids, (2) teratoid tumors, and (3) epignathi (Arnold, Lit.). All these conditions are congenital and are usually encountered in deformed stillborn fetuses, but the simpler pharyngeal polyps may first be recognized as late as the twenty-second year.

(I) The dermoids are usually complex. They appear at birth or after some years, producing dysphagia, or dyspnea, or are described as cases of hairy tongue. They are attached to the hard or soft palate, or wall or vault of pharynx, in or near the middle line, and fill the nares or buccal cavity. Some may show an intracranial portion connected through a perforation in the skull with the pharyngeal tumor.

The tumors are covered by skin, often hairy, and containing dermal glands. 
The main mass is composed largely of fat tissue, with occasional fragments of striated muscle, cartilage, or bone. They are, therefore, bidermal.

(2) The teratoid tumors differ from the dermoids chiefly in the greater complexity of structure, larger size and earlier development, and they are frequently associated with extensive deformities of the skull, as anencephalus (Otto), hemicrania (Wollmann), and palatal fissures (Sonnenberg). The presence of these local deformities suggests that the overgrowth of tissue constituting the teratoid results entirely from local factors and that they are not true embryomas. These tumors are all tridermal, and Schwalbe further subdivides them into two groups, in one of which the three germ layers produce definite organs, $i$. e., into teratoids and teratomas.

The structures which form the basis of the classification as teratoids consist of tridermal elements, fat, muscle, cartilage, bone, acinous glands,

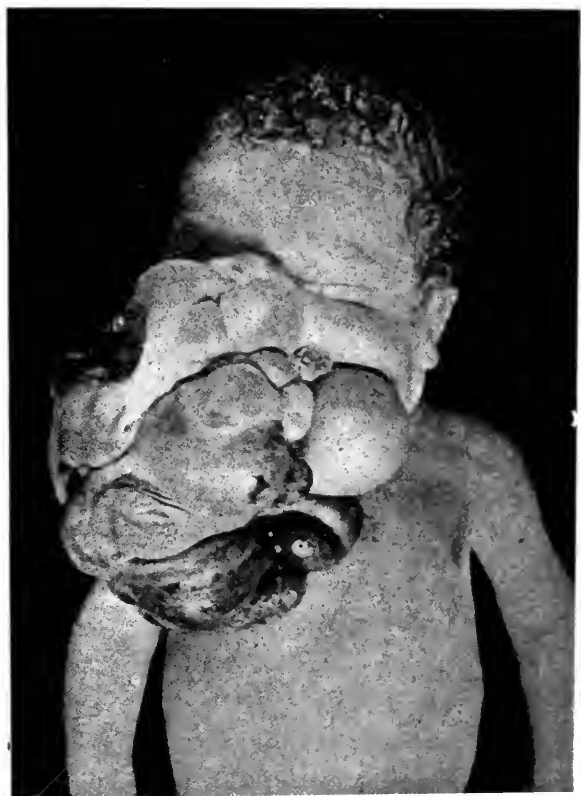

Fig. 510.-Epignathus. (Low, Studies in Pathology, Univ. of Aberdeen, 1906.)

teeth, cysts lined by various forms of epithelium, and nervous tissue. Cases in which the bony masses imitate a rudimentary skull form a transition to the epignathi. In Koch's case the growth was composed chiefly of brain and glia-tissue. In several cases the structure has suggested an involvement of the branchial arch, as when there are deformities of tongue, palate, jaw, and ear, and the tumor contains rudiments of the ear (Wallmann).

(3) Epignaihi.-These remarkable anomalies differ from other pharyngeal teratomas in presenting well-formed organs and limbs of a parasitic fetus. There has been much variation in the criteria demanded for identification of this group, but since the studies of Arnold and Schwalbe the term "epignathus" has been limited to the growths that present unmistakable parts of a fetus. Even thus the observed cases are rather numerous. The extent to which the fetal organs are represented varies greatly. In some 
the main tumor consists of a rudimentary head with or without brain tissue (Ahlfeld). In many cases well-formed extremities with fingers, toes, and nails are present. Otto observed a cystic growth containing portions of intestine and a rudimentary testis. Vrolich found intestinal canal with appendix, long bones, and a placenta-like mass. Wegelin identified sacrum, foot with five toes, intestine, crystalline lens, choroid, and brain. Rindfleisch described a large tumor at the base of the brain attached at the sella turcica, and containing fetal extremities with fingers and toes, normal liver, brain, and navel, while passing through a fissure in the base of the skull and projecting from the mouth was a bulky polypoid tumor containing chiefly mesodermal structures. He assumed an origin from the hypophysis and characterized this organ as a veritable mine of undifferentiated tissues.

The case of Baart de la Faille is thus reported by Schwalbe. In a case of quadruple pregnancy the first fetus was well developed; the second fetus formed a large epignathus hanging from the mouth of the first; while the

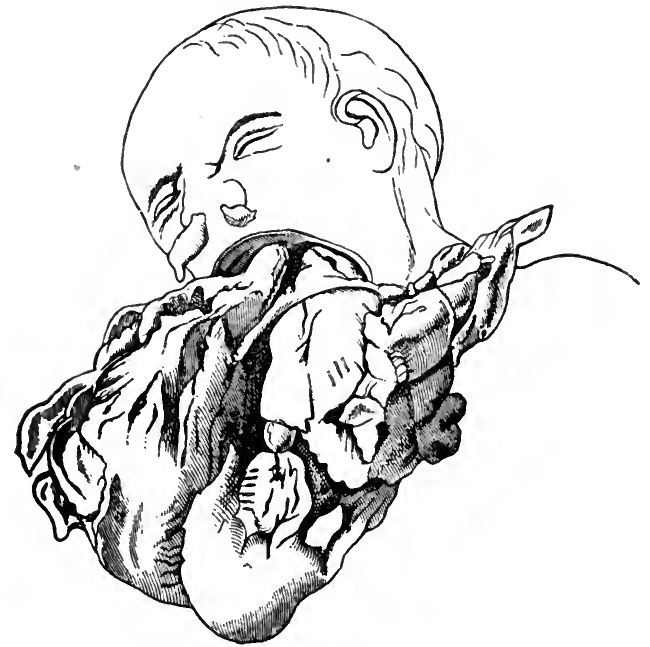

Fig. 5II.-Complex epignathus. (A case of Baart de la Faille.)

third and fourth were joined by a forked umbilical cord to the palate of the first, where its vessels joined with the sphenopalatine branches.

In the genesis of pharyngeal dermoids and teratomas various theories have contended for recognition. In the extensive discussions of this subject it has been recognized not only that the general principles of teratogenesis are involved, but that special local factors in development must be considered.

Arnold maintained that the simpler bidermal and the complex tridermal growths must be referred to local disorders of development, while the true teratomas, which according to his definition must present definite rudimentary organs, were true fetal implantations. Here as elsewhere an effort has been made to distinguish between autochthonous teratomas (developed from local tissues) and heterochthonous (developed from sources alien to the locality). From this point of view two entirely different embryonic disturbances must be held responsible for the present group of conditions: (1) Local disturbances of development involving the buccal cavity, the hypophyseal duct, and the first branchial arch; (2) the aberrant growth of a totipotent blastomere, polar body, sex cell, or tissue bud.

The hairy polyps and the simpler tridermal teratoids have been regarded by nearly all authors as adequately explained by the first mentioned factors. The invagination of ectoderm in the formation of the buccal cavity and the fusion of this cavity with the 
fore-gut through the primitive pharyngeal membrane give opportunity for the separation of ectodermal and mesodermal rests which may explain some of the growths attached to the palate. Rather more definite sources are provided in the course of development of the hypophysis and its duct, for some of the pharyngeal growths are clearly connected with these structures and many of them are attached at the sella turcica or perforate the skull at this point. The hypophysis may be missing. That the first branchial arch may also be concerned in some cases is indicated by the occurrence of other anomalies in the course of this arch and by the complex teratoids and epignathi located in the course of other arches. Finally, the theory of local origin is favored by analogy with parallel conditions at the caudal extremity and by the series of simpler embryonal tumors of the nasopharynx. The definite teratomas and epignathi Arnold, Askanazy, and others interpret as forms of fetal implantation without designating their particular history.

Somewhat definite discrepancies, however, exist between the above theory and the observed facts. The sharp separation attempted between complex autochthonous and only slightly more complex heterocthonous growths proves extremely difficult, for no sharp distinction in morphology exists. In fact, in the not overnumerous series every gradation seems to be represented, from the simple bidermal polyps up to the wellformed epignathi, so that a single origin for the entire group is strongly indicated. Hence, Schwalbe concludes that all the complex pharyngeal growths are derived from isolated blastomeres, and that the varying structure of the growths depends upon the stage of development of the blastomeres at the time of their isolation. It is more probable that they result from early budding. He employs the term "teragogenic termination-point" to indicate the stage of differentiation which the separated blastomere or originating cell group must possess in order to produce just the particular form of teratoid, teratoma, or parasitic fetus observed. When, however, Schwalbe includes with isolated blastomeres more complex and differentiated cell complexes, his theory closely: approaches the standpoint of Arnold.

Adami explains epignathi and sacral monstrosities as the result of overgrowth of cells at the two "growing points" of the embryo, after the rudiments of the local organs have been laid down. After the brain vesicles are formed any further growth of the superior growing point, which lies in the region of the sella turcica, would produce superfluous tissue involving three germ layers.

In the origin of the nasopharyngeal dermoids three possible sources must be considered: (1) Incomplete absorption of the membrane separating buccal cavity (stomadeum) and fore-gut. (2) The ectodermal pouch, hypophysis. (3) The first branchial cleft in its relations especially to the Eustachian tube, which, however, is wholly endodermal in origin. The relation of the hairy pharyngeal dermoids to the complex teratoid tumors of this region remains undetermined, but since their structure is comparatively simple and they contain only such elements as may arise from normal tissues in that neighborhood, they probably originate from a local disturbance in the first branchial cleft and not from a totipotent cell or cell group (Askanazy).

Hypophyseal Dermoids.-Closely related to the pharyngeal growths is a group of more complex intracranial dermoids lying at the base of the skull, in the sella turcica (Beck), near the olfactory lobes (Bonorden), or at optic chiasm (Rokitansky). The neighboring pineal gland may be hyperplastic. These tumors are lined by epidermis and filled with granular or lamellated detritus as in cholesteatoma and the wall may contain bone, cartilage, muscletissue, and fat. Bonorden found I4 teeth, cysts lined by ciliated epithelium, and fragments of thyroid tissue, in a case which suggests a teratoid origin. These tumors are commonly referred to buccal ectoderm misplaced in the development of the hypophysis (Wilms, Lit.). While there are some grounds for assuming a teratoid origin of the entire group, the structural scope is somewhat limited, and represents chiefly the local organs from which different elements might well be contributed by some early embryona! disturbance, such as budding at the cephalic extremity. The alternative hylothesis would explain these growths as abortive epignathi.

A few cases of intracranial teratoid tumors have been referred to the hypophysis. Beck found in the sella turcica a small tumor containing cartilage, bone, myxoma, cysts with ciliated epithelium, and I4 teeth.

Intracranial Dermoids. - The relations of the medullary groove to the 
ectoderm, the complex steps in the formation of the brain and ventricles, and the formation and union of the cranial bones give abundant sources for the development of epidermal growths in the skull. The interpretation of these tumors is further complicated by certain properties of endothelial growths to copy the structure of cholesteatoma, and finally traumatic implantation of portions of ectoderm accounts for a small proportion of intracranial processes. Several types of epidermal tumors are included in this group.

(I) Cholesteatoma is a tumor composed of lamellated waxy or scaly material enclosed in a wall of stratified squamous cells. It occurs in all parts of the brain, ventricles, and basal regions, usually near the midline, and is regularly connected with the meninges. Bostroem, in an elaborate study, concludes that all cholesteatomas arise from embryonal epidermal inclusions. Beneke and Bonorden support this view, and refer a basal cholesteatoma to an inclusion from the pharyngeal wall connected with the hypophysis. Erdheim reports 13 cholesteatomas at the base of brain, none of which seemed to be connected with the hypophysis and few were in the median line. He finds only one case of cholesteatoma of the infundibulum (Beneke's). Blasius, in a tumor of the convexity, found epithelium resembling that of the skin. A traumatic origin must be accepted for Hartley's frontal cholesteatoma. An endothelial origin for the deep tumors is assumed by Glaeser, $\mathrm{H}$. Frank, and others, and Borst distinguishes intracranial dermoids, epidermoids, and endothelial cholesteatomas. Finally, Benda holds the ependymal epithelium responsible for some deep cholesteatomas. In several cases I have found no evidence of any other than an epithelial origin.

(2) The intracranial dermoids are walled by epidermis and derma with sebaceous and hair glands, and contain sebaceous material and hair. They lie (I) between the olfactory lobes and corpora mammillaria, or (2) between medulla and pons, and their positions indicate that the embryogenic disturbance occurred at the formation of the second brain vesicle. Bostroem collected i8 cases, all of which were connected with dura or pia.

(3) Dural dermoids lie close to the skull, but grow deeply, pushing the pia before them. They usually lie in the median line in tentorium cerebelli, at internal occipital protuberances or torcular herophili, or they may be epicranial. They are often connected with the skin by an epithelial canal or a fibrous strand, and there may be a defect in the skull or an absence of hair in the overlying scalp (Heschl). They must arise late after the skin is formed, from adhesions between skin and dura, and they are observed at birth or in infancy (Bostroem, Ziegler). Some of the tumors of the hypophyseal duct described by Erdheim contained cysts lined by derma in which were bony trabeculæ. Benda has also described a hypophyseal dermoid containing bone in its wall.

Multiple complex dermoids scattered over pia of cord and brain and in ventricles, appearing as yellowish nodules of connective tissue, fat, nervecells and fibers, smooth muscle, and flat epithelium, occurred in a remarkable case recorded by Trachtenberg. The large complex intracranial dermoids are probably to be interpreted as teratomas (Askanazy).

Intracranial teratoids and teratomas have been observed in a few cases, the position of which it has often been difficult to determine. Arnold described a frontal tumor partly extracranial, but extending into the ventricles, which contained fat, cartilage, and bone, and which he interpreted as a fissural mixed tumor. Eberth observed an intracranial tumor connected by a pedicle with the dura, and composed of fat, muscle, lymphoid tissue, and nerves. 
In the ventricle Strassmann and Streker described in a boy of 3 years a tumor of the right choroid plexus, composed of connective tissue, fat, bone, cartilage, muscle, nerve-cells, glands, and cysts lined by epithelium. In the third ventricle of an infant Saxer found a complex teratoid containing cartilage, bone, muscle, chorda tissue, gland alveoli, cysts lined by cylindrical or ciliated or squamous epithelium, pigmented retinal epithelium, choroid plexus, and fetal brain tissue and ganglia. Both of these tumors may be referred to complex portions of the medullary plate.

Certain intracranial teratoids and teratomas arise in connection with the hypophysis and the pineal gland $(q . v$.$) .$

\section{Teratoma of Pineal Gland.-(See Pineal Gland.)}

Peritoneal, Mesenteric, and Omental Cysts, Dermoids, and Teratomas.-A comprehensive résumé of this subject was first contributed by Hahn in 1887. A more minute analysis and history were given by Braquehaye (I892), who recognized especially the dermoids and cysts derived from neighboring organs. Dowd (r900) pointed out the probable identity and embryonal origin of many glandular, serous, and blood-cysts. A complete résumé of the entire subject with reference to 184 cases is presented by Niosi.

Four main varieties of cystic tumors appear in the peritoneum: Lymphatic or chylous cysts. (2) Enteric cysts. (3) Urogenital cysts. Dermoids and teratoids. Hydatid cysts and cyst of complicate the clinical diagnosis.

(r) Chylous cysts form very large, single, usually multilocular tumors, or very numerous small swellings of mesentery, omentum, intestinal wall, and retroperitoneal regions (Klemm, Lit.). The contents are clear fluid or chyle, or more inspissated fatty material, and blood is often added. The walls are of fibrous tissue in which are many round cells or lymph-follicles, and often dilated lymph spaces. Bundles of smooth muscle tissue may be present, as in enteric cysts. The lining of recognizable endothelium, which may be hyperplastic (Tilger). Many giant-cells may form about fatty detritus, or the cellular lining may be lost.

Klemm and Rittner interpret all mesenteric chylous cysts as cystic lymphangiomas. That some represent merely dilated lymphatics is shown by Kostlivy's study and by the cases of lymph-cysts arising after occlusion of local lymphatics in cancer. In some cases the cyst appears to involve chiefly certain lymph-nodes (Lion, Spaeth). Since enteric cysts may contain chylous fluid a sharp distinction from chylous lymph-cysts is not always possible.

The so-called serous cysts probably represent rare forms of other varieties of this group. Tuffier found clear fluid in one compartment and chylous fluid in two other portions of a lymph-cyst. The presence of muscle-fibers in the walls of serous cysts suggests an enteric origin.

Blood-cysts usually arise from extravasation. Wagener has described a true cystic hemangioma of the mesentery.

(2) Enteric cysts constitute a rather well-defined group of intraperitoneal cysts. They form single or multiple large or small cysts lying usually along the lower end of the ileum, in the wall of the intestine, at the point of Meckel's diverticulum (Roth), in the mesentery, or near the navel (Wyss). When originating within the muscular wall of the intestine they usually remain connected with this organ and are enclosed by a muscular wall. Arising in the subserosa they remain attached to the convex side or project into the mesentery. There may be definite malformation of the intestine (Nasse). The cavity is usually single and the contents are mucinous, colorless, yellowish, or brownish fluid. The wall resembles that of the intestine, and may contain smooth muscle, mucosa, crypts, lymphoid tissue, and a lining of cylindrical 
or cuboidal or stratified epithelium (Colmers). The epithelium may show papillary proliferation. Secondary changes in the cyst may destroy much or possibly all the epithelial lining, and many stages of the atrophy of muscle tissue have been observed (Niosi). Both cylindrical and squamous cells may be found in the same cyst (Gfeller).

There are probably several modes of origin of enteric cysts. Many originate from Meckel's diverticulum and are located at the lower ileum where they may communicate with the bowel (Ruge, Roth). At the navel they may be referred to the omphalomesenteric duct (Wyss). The term "entodermoid" has been employed by Beneke to designate enteric cysts which arise from definitely misplaced portions of intestine. Many of these lie in the mesentery, and are multiple. Roth and Hennig found a large cyst in mesentery and one in posterior mediastinum, both in infants. Sanger and Klopp, in a newborn infant, found 5 cysts, in the walls of 2 of which were portions of liver tissues. Honl has reported 2 remarkable cases of multiple enteric cysts in which there were 37 and 89 small cysts lined by intestinal mucosa.

(3) Intraperitoneal cysts of nephrogenic origin form a less definite and less numerous group, of which Niosi collected 5 cases. Many other cases in which the structure was obscure are held by Dowd and Niosi to belong in this group. These cysts are of large size, single or multilocular, involving mesentery and adjacent regions or extending into the pelvis, and occur chiefly in adult women. The contents are brownish serous fluid containing pseudomucin. The wall is composed of fibrous tissue and the lining is of high cylindrical or cuboidal glandular epithelium, which may be deficient in some areas. In Niosi's case the structure was complex and the wall contained definite portions of adrenal cortex, and structures resembling the embryonal kidney.

The origin of these cysts presents a difficult problem, but it seems probable that they are derived from aberrant remnants of the Wolffian body and that the embryogenic disturbance occurs at various periods in the history of this structure. Dowd regarded his case as arising from an ovarian rest, while Niosi's case must have included more complex elements.

(4) Dermoid cysts of the mesentery and peritoneum are rare (Niosi, Lit.). They are located in any portion of the mesentery from celiac axis to pelvis and their dimensions may be considerable. In Fraenkel's case the cyst was attached to the diaphragm. The epithelial lining may be lost (Marie). They are usually lined by squamous epithelium and contain hair and sebaceous material. Ruysch and Martel observed in the omentum large cysts containing hair. Others are very complex and contain hair and teeth. In a large cyst extending in mesentery from ribs to pelvis of an infant of 2 years Dickinson found connective tissue, fat, bone, and cartilage.

A few peritoneal teratoid tumors have been reported. Bonfigli describes a cyst adherent to liver and stomach containing I9 loose teeth and 2 embedded in well-formed bone. The wall was lined by hairy skin. A very similar case is that of Schutzer.

Various sources appear to contribute to these tumors. Many simple intraperitoneal dermoids are referred by Wilms to imperfect closure of the abdominal plates. Others are commonly regarded as derived from the ovary, by separation of an original ovarian tumor, or by development from a supernumerary ovary, or by implantation from a ruptured ovarian dermoid.

The position of the complex dermoids strongly suggests that many of them belong in the group of abdominal teratomas and that more complete examination would have revealed a tridermal structure. 
Abdominal Fetal Implantations and Teratomas. - Seventeen cases of definite fetal implantation in the abdomen have been collected by Lexer. They occurred in newborn infants or in subjects between the ages of a few months and 6r years. Their location in the transverse mesocolon or behind liver in bursa epiploica indicates that they were originally celomic implantations. The vascular supply is from the aorta or the vessels of the colon. Rudimentary limbs and organs and well-formed membranes and umbilical cords have been recognized.

Several cases of tridermal teratomas of the peritoneum have been collected by Lexer and by Askanazy, and to these may be added other bidermal growths which are probably of similar origin. Their position has been behind the liver and extending downward behind the kidney.

A sharp separation between peritoneal and retroperitoneal teratomas cannot be made. The tumors occur in young or adult subjects and reach large dimensions. The scope of structure includes solid portions composed of bones with marrow cavity, cartilage, fat tissue, muscle, and connective, and cystic portions with cavities containing hair or mucus, and lined by epidermis or cylindrical or ciliated epithelium. Hosmer found a portion of intestine with mesentery. Marchand observed intestine with calculi, prostate gland, and rudimentary brain. Ahren's case consisted chiefly of a segment of gastro-intestinal canal, with lining of intestinal mucosa, or squamous or ciliated cells. There was ulceration, and pepsin and acid were demonstrated in the contents of the cyst. Whether this case was a teratoma reduced to gastro-intestinal canal, or a derivative of misplaced entoderm, it is difficult to determine. Yet Englander's case, containing intestine, cartilage, and fat, furnishes a transition to genuine tridermal teratoma. Pilliet describes sarcomatous areas, and in Montgomery's case the original sarcomatous and carcinomatous areas recurred. In Goebell's case adenosarcoma derived from the entoderm produced numerous metastases. The peritoneum is occasionally the seat of malignant embryonal tumors of carcinomatous or sarcomatous structure whose position suggests a relation to the malignant forms of peritoneal teratoma. Fleischmann found pure neuroepithelioma in the metastases of a complex malignant tumor of the omentum.

Primary abdominal chorioma in the male, located in the omentum, is described by Bonney, and other abdominal tumors have been interpreted as chorioma by Bostroem and Djewitsky.

Retroperitoneal Dermoids and Teratoids.-Three varieties of epidermal tumors occur in retroperitoneal regions:

(I) Tridermal teratomas. These may be unattached teratomas, as in Brouha's or Schonholzer's cases, or derived from the ovary, normal, displaced, or supernumerary (Borst, Bolzano). Funke would derive all retroperitoneal dermoids from germ epithelium, or from ovary, or from supernumerary testes, of which Merkel has collected several cases.

(2) Epidermal rests derived from the Wolffian duct are probably the source of certain dermoids of kidney (Schlegtendal, Wedeman), ligamentum latum, spermatic cord, and epididymis (Wilms, Lexer, R. Meyer). They contain no dermal glands.

(3) True dermoids are derived from imperfect closure of abdominal folds. Ruge describes a large cyst with two compartments, one lined by skin with dermal glands and containing hair, the other reproducing intestinal mucosa, which he would derive from a remnant of the vitelline duct. Of the abdominal fetal implantations, Lexer finds 2 cases (Buhl, Phillips) in which the location was retroperitoneal. The tumors lay on the aorta behind pancreas, and extended from diaphragm downward and behind kidney. 
Dermoid cysts of the pelvic connective tissues are rare but well-recognized conditions giving rise to pelvic symptoms (Germain, Lit.). They appear chiefly in women in the childbearing period, and are commonly situated behind the rectum and above the levator ani muscle, and reach the size of a hen's egg, or child's head, or larger. Six cases have occurred in male subjects. Sanger collected II cases in which the tumors were found in various positions, chiefly in contact with and behind the rectum. The presence of hair in the stools has been the first symptom observed, or the bladder may be reached and hair appear in the urine (Le Gendre). The occasional presence of muscle tissue and renal structures suggest a teratoid origin, but it is more probable that they result from misplacement of portions of ectoderm and mesoderm during the formation of the anus, rectum, and urinary passages (proctodeal membrane and neurenteric canal). As a group they correspond to the pharyngeal and cervical dermoids of the cephalic extremity. They differ in location, origin, and symptoms from the more superficial dermoids which project externally and are usually present at birth. Some very extensive pelvic and retroperitoneal dermoids probably belong in this group. Zweifel removed a large cyst lined by hairy skin, which extended from diaphragm behind kidney and into pelvis, and Bardenhauer's cystic tumor was even more extensive in the same regions.

Ciliated Epithelial Cysts. Enterocystomas.-In many regions of the body occur small or large cysts lined by ciliated epithelium, the origin of which has given rise to much speculation. One group of these cysts is relatively numerous along the gastro-intestinal canal, and since they are usually lined by mucous membrane resembling that of the intestine they are commonly named enteric cysts. Others, while of much the same structure, are widely distributed in organs where an enteric origin is improbable, as in brain, thorax, pharynx, lung, and genital organs, and in each situation they are probably referable to local disorders of development. They seem to represent an endodermal parallel of the dermoid cysts, but the presence of ciliated cells does not necessarily indicate an endodermal origin. In general, their structure may be explained by the fact that many embryonal canals are originally lined by ciliated epithelium, as the neural, enteric, respiratory, and genital, and that an early misplacement of cell groups from these structures would naturally have the lining cells in an embryonal state. In fact, all undifferentiated epithelium is generally ciliated. In the brain the cysts occur in the region of the ventricles, with which they may be connected by cell strands. Their origin is probably from the epithelium of the neural canal (Eberth). In the regions of the branchial clefts, nasopharynx, mouth, neck, tongue, and mediastinum many cysts of this type are observed. Their origin may be traced to the same conditions that lead to branchiogenic dermoids, with which they are often combined. In the skin Hess has described a number of cysts with ciliated lining, for which no explanation is apparent.

Along the esophagus in the fibrous or submucous coats the cysts have frequently been observed and referred to misplacements of the embryonal epithelial lining which is originally ciliated (Wyss, Zahn, Trespe). In the pleura, hilus of lung, arch of aorta (Stilling), and in mediastinum between diaphragm and lung, simple or complex cysts occur, often associated with mucous glands, muscle, or cartilage. They have been referred to misplaced portions of the respiratory tract (Virchow, Zahn). In the liver the cysts have appeared on the anterior surface, near the suspensory ligament, and along the inferior border. They lie between the peritoneum and capsule of the organ (Zahn), and their origin is not readily explained.

About the female generative organs, internal and external, the cysts are 
particularly frequent, and may be referred to the several embryonal structures connected with these organs.

Sacrococcygeal Dermoids, Mixed Tumors, and Teratomas.-The complex embryonal processes occurring in the caudal extremity form the basis of a series of fistulæ, cysts, and tumors in this region. Very similar conditions exist at the cephalic extremity with parallel results.

In the sacrococcygeal region the abnormalities resulting from embryonal disorders include: (I) Simple dermoids, (2) complex dermoids, (3) teratoid tumors, (4) teratomas, (5) fetal implantations. In many cases these conditions are associated with various defects of the spinal cord and its membranes and the spinal column (spina bifida).

While some of these conditions are simple and readily referable to particular embryonal structures, others are complex, difficult of interpretation, and seem to involve more than one embryonal structure. In dealing with

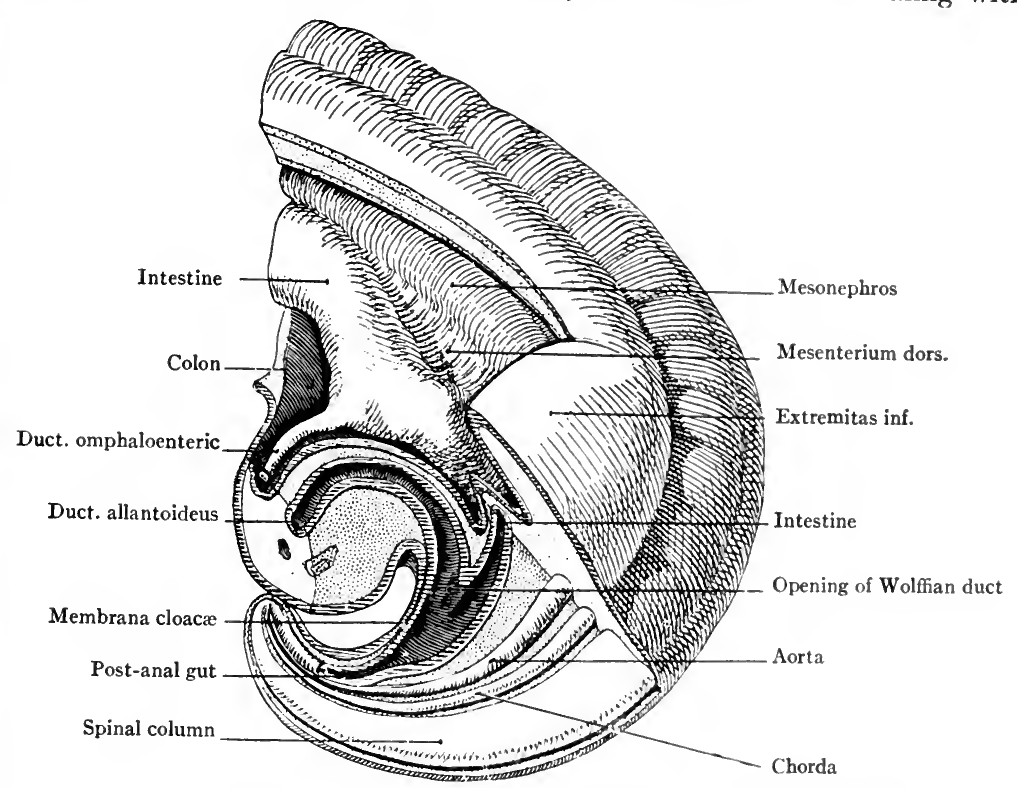

Fig. 512.-Model of caudal extremity of embryo. (After Kollmann.)

the more complex growths one encounters teratoid tumors which seem to have a purely local origin and transitional cases which seem to belong to the true bigeminal teratomas, among which are also pronounced forms of fetal implantation. Thus the entire group is not only numerous but complex, and a rigid classification is at present impossible (Borst, Lit.).

The embryonal structures which give rise to these growths are chiefly: (I) The fovea coccygea and the coccygeal vestiges of the neural canal, (2) the neurenteric canal, (3) the postanal gut, (4) the proctodeal membrane. The location of these structures and the general conformation of the caudal extremity is partially indicated in the accompanying sketch.

Fovea Coccygea. Coccygeal Vestiges of Neural Canal.-Up to the third month the spinal cord reaches to the third coccygeal vertebra, beyond which it is continued to the tip of coccyx and to the overlying skin as a fibrous cord containing groups of epithelial cells. Up to the fifth month or later there 
is further growth of the cells of this structure, producing irregular spaces lined by polyhedral or pavement cells. The later development of the soft parts about the anus and the gradual atrophy of the remannt of cord often produces a superficial depression over the coccyx - the fovea coccygea (Tourneux, Hermann, Mallory). Fistulas lined by pavement or polyhedral cells, dermoids, and more complex tumors may develop from these structures. Tourneux has found remnants of gland tissue along with these coccygeal vestiges. Perman describes a large tumor resembling a meningomyelocele which was not connected with the spinal canal, but contained much neuroglia tissue and cysts lined by cubical or stratified squamous epithelium. Nasse describes a simple fistula lined with epidermis and many sweat-glands, and Wendelstadt reports a case in which the tract contained skin and hair. Wette observed a dermoid in the coccygeal canal, with a fistula leading to the skin. Schmidt found a sacral dermoid with several fistulous openings, associated with a complex mesodermal tumor in which were tubules lined by cylindrical cells. Mallory has collected a series of cases of fistulous tracts, cysts, dermoids, and a glioma in this region, and demonstrated in several fetuses the embryonal structures from which they spring. Bergmann pointed out that the dermoid tumors in this locality show no free communication with the spinal canal, which is regularly present with meningocele. Yet some spinal dermoids are connected with the canal by a narrow pedicle.

Neurenteric Canal.-In the lower vertebrates there is constantly present a short narrow canal connecting the lower end of the medullary groove with the hind-gut. This neurenteric canal has been observed by Graf Spee in a human embryo with still widely open medullary groove, and Eternod has repeated this observation. Although the canal is a very minute structure and there is no evidence that it exists in any form in the adult (Keibel and Mall), it has been drawn into the possible sources of certain tumors of the ventral side of the coccyx, especially those which combine cysts lined by intestinal wall with portions of nervous tissue.

Of these tumors Hildebrand's case may serve as an example. A female infant of 6 months presented a tumor as large as its head, lying between rectum and sacrum and protruding externally. It was composed of many cysts lined by cuboidal or cylindrical or ciliated or hornifying squamous epithelium. In some cysts the lining closely resembled the intestinal mucosa, and into these cysts projected two papillary masses of neuroglia tissue. Other complex cases which seem to belong in this group are reported by Jantreboff and Ritchl. Bergmann described sarcomatous changes, and cases in which the course is rapid and the tumor exhibits several types of sarcoma, but without metastases (cystosarcoma).

Postanal Gut.-The proctodeal portion of the anal canal, formed by an invagination of the ectoderm, does not meet the lower end of the intestine, but joins the intestinal wall at some distance anterior to its extremity. The terminal portion of the intestine, lying on the ventral side of the coccyx, becomes closed and atrophic, and this embryonal remnant appears to be connected with many tumors lying between coccyx and rectum. It was first employed by Middledorpf in explanation of a cystic tumor containing a convoluted portion of intestine surrounded by much fat tissue.

These tumors are observed at birth. They form papillary external tumors between anus and coccyx, displacing the genitals, and they extend upward behind the rectum, and occasionally outward over the coccyx.

Simple tumors of this group are composed of segments and convoluted folds of intestinal wall and mucosa. Middledorpf's tumor had an external fistula and was adherent to rectum. Ganz found a segment of intestine 7 
cm. long, and other similar cases are described by Freyer and Nasse. Widened sacral vessels coursing over the tumor may yield a bruit (Jordan). The well-developed muscular walls may yield spontaneous or electrically excited peristaltic movements (Preuss, Bergmann, Stolper). Striated muscle-fibers from the sacral region may be added to the capsules and contribute to the movements (Ahlfeld).

Cloacal Membrane.-The various steps in the union of anus with gut, the absorption of the cloacal membrane, and the formation of bladder and external genitals, are concerned with tumors of these organs and may lead to formation of dermoids. Some of these appear in the pelvic connective tissue (Germain, Lit.). Small nodules along the raphé of penis and scrotum composed of epithelial pearls are referred to imperfect closure of the dermal cleft at this point (Epstein).

A variety of cysts lined by epidermis or by cylindrical or mucous cells, and probably derived from cloacal remnants, have been collected by Mermet and Marchadier.

A dermoid of the bladder with atresia ani et urethræ is described by Martini.

There are thus four somewhat definite groups of tumors which may be referred with considerable certainty to single embryonal structures in the sacrococcygeal regions. Yet the majority of congenital tumors of this area are most complex and seem to involve more than one of the embryonal remnants or some additional anomalies of development.

Sacrococcygeal teratoid tumors constitute a somewhat heterogeneous group in this field (Braune, Stolper, Lit.). These are solid and cystic large nodular congenital tumors lying at the lower end of the spinal column in front of sacrum and coccyx. They project below, displacing the anus forward, and may extend over the dorsal surface of coccyx and sacrum. A definite connection with the bones is occasionally seen (Kummel), and the sacrum may be rudimentary (Pannwitz). The spinal dura is, as a rule, not involved. In these features they differ from the tumors on the dorsal surface of the sacrum. The hypertrophied sacral arteries and the branches of the nerves of the sacrococcygeal plexus course over the tumors. One-third of the subjects are born dead, and 90 per cent. of the others die in the first few days.

The structure is very varied and complex, a histological potpourri which is yet sufficiently restricted for a local origin, although three germ layers are represented.

The solid portions contain cellular connective tissue, fat, smooth and striated muscle, bone, and cartilage. Chorda rests were identified by Hennig. Portions of central nervous system are often observed in the form of masses of glia tissue or ganglia. The cysts are of numerous types, being lined by cubical, cylindrical, ciliated epithelium, or with pavement cells, or they resemble true dermoids with hornification, epithelial papillæ, sebaceous material, hair, and sweat-glands (Nasse). The cyst walls are composed of fibrous tissue or contain cartilage, bone, adenoid tissue with lymph-follicles or nervous tissue. Areas of glia tissue may contain small cysts lined by neuroepithelium (Perman, Stolper). Very large cysts lined by cuboidal cells probably represent a distended neural canal simulating a cerebral ventricle. Borst describes a cystic tumor which he interpreted as a rudimentary caudal brain. Pigmentation of various tissues is frequent and certain pigmented epithelial structures have been interpreted as rudimentary retinal vesicles (Spondly, Kummel). Well-defined retinal structures are described by Hennig, and fully developed or rudimentary teeth by Port, Kronlein, and Danzel. Fetal bones have also been identified by Kiderlen, Borst, and Menzel. 
Sacral Teratomas.-The presence of definite organs or their rudiments which cannot well be referred to neighboring structures constitute the basis of classification in this group of tumors. This principle was first employed by Nasse and its validity will be considered later.

The general relations of these growths are very similar to those of the teratoid tumors. They are bulky masses, present at birth, lying on the dorsal surface of the sacrum and coccyx and adherent to or enclosed within the periosteum or connected to the bone by a pedicle. Others lie anterior to the sacrum and connected with this bone (Kauffmann), or with the rectum (Feldmann), and projecting into the pelvis. The structure presents cystic and solid portions similar to those of the teratoid tumors, including cysts lined

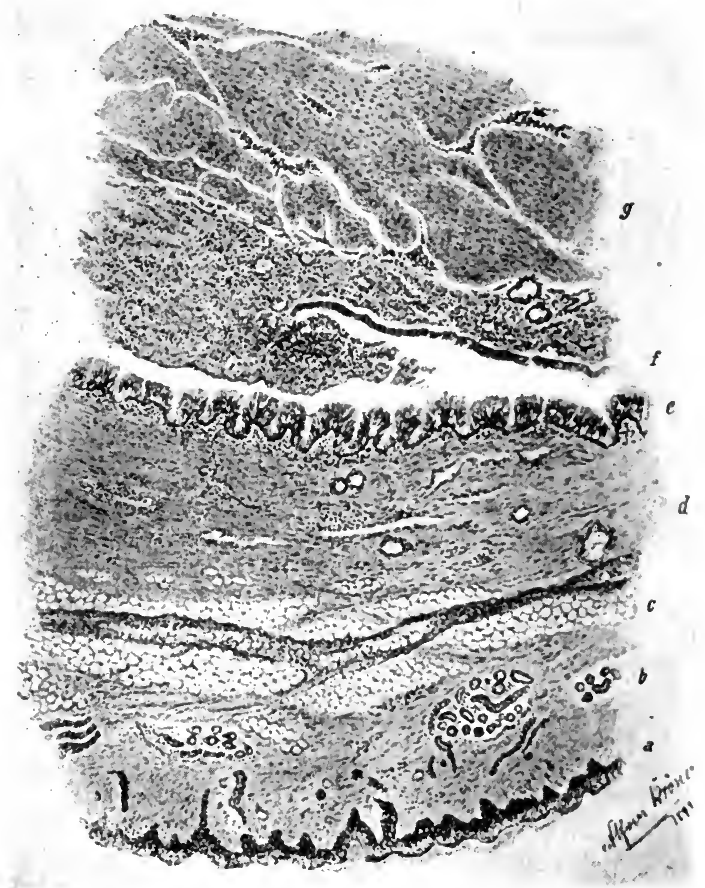

FIG. 5I3.-Structure of a congenital sacral teratoma: $a, b, c$, Dermal layers; $d$, smooth muscle; $e$, intestinal epithelium; $g$, glia tissue. (After Stolper.)

by various types of epithelium, dermoids, segments of intestinal mucosa, gland structures, fat, muscle, cartilage, and bone, and, finally, portions of nervous and glia tissue.

In addition, a great variety of rudimentary organs are obselved. These include segments of intestine with mesentery, rudimentary esophagus, stomach, and buccal cavity with salivary glands (Linser, Kleinwachter); pulmonary parenchyma, bronchi with cartilaginous rings (Piper, Linser); thyroid (Hagenthorn), pancreas, spleen, adrenal (Stroh), kidney, brain with ventricles and choroid plexus (Sperling, Stroh). The bones may reproduce well-formed extremities, as forearm and hand (Böhm), tibia, femur, and joint (Kaufmann), pelvis and extremities (Kleinwachter), toes (Feldmann), 
eyes (Frank) (Dépaul). In fact, Askanazy regards the sacral teratomas as the most prolific in the production of rudimentary organs. An abortive fetus enclosed in amniotic membrane lay under the skin in a case of Jordan's. Thus many transition stages up to definite fetal implantations are provided.

Secondary changes of importance occur in sacral teratomas, giving rise to simple malignant tumors. Overgrowth of muscle-cells led Virchow to speak of myosarcoma in these tumors. Bergmann described a variety of sarcomatous changes. Nakayama observed infiltrating endothelioma and myxo-angiosarcoma, and a metastatic growth consisting of fetal brain tissue. In a sacral tumor reported by Buzzi as angiosarcoma Perrier afterward found teratoid structures. A recurring sarcomatous teratoma described by Hinterstoisser gave extensive metastatic tumors, "alveolar sarcoma," in lungs, liver, and lymph-nodes. Askanazy suggests that more thorough

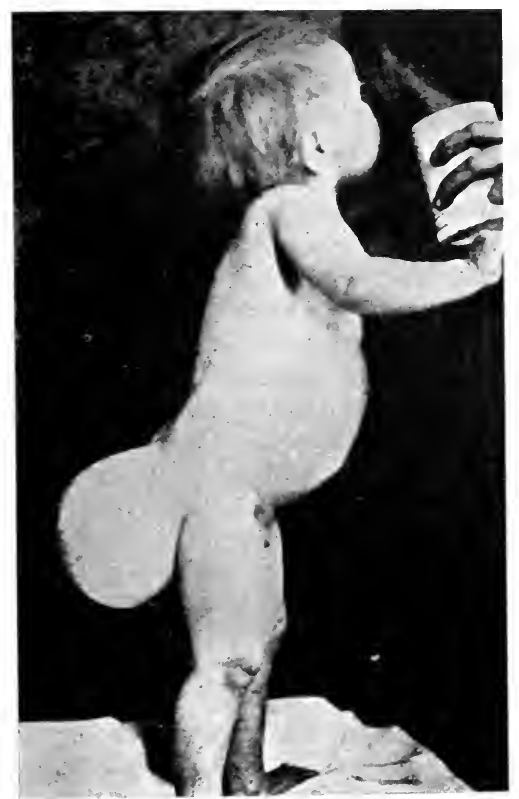

FIG. 5I4.-A case of spina bifida, with meningocele.

examination of other simple tumors of the sacral region might disclose teratoid elements. The sacral and ischiorectal region is a frequent seat of angiosarcomas, whose origin may thus be explained. The sacral neurogliomas constitute a definite subdivision (Mallory, Keller, Scheuermann).

Tumors Associated with Spina Bifida.-While the common meningocele, myelocele, myelocystocele, etc., have no neoplastic character, spina bifida is often associated with tumor processes. The association with spina bifida throws some light on the pathogenesis of the entire group of sacral teratomas, since spina bifida signifies a lack of coherence of embryonal parts, and a failure of growth momentum, which is favorable to spasmodic and aberrant growth of the type of budding.

Sacral hygroma or cystic lymphangioma is the simplest tumor of this class and arises from extensive cystic dilatation of the spinal subarachnoid 
spaces. Such a cystic tumor may show extensive connection with the spinal canal (Marchand, Kroner), or these connections may be lost and the tumor appear as an isolated lymphangioma lying on the dorsal or ventral side of the spine. Marchand describes hernial protrusions of spinal meninges between vertebræ, and Borst believes that many cervical and sacral hygromas arise in this manner, referring to cases of Lotzbeck and Brauner, and to one of his own observation in which the spinal connection was reduced to a narrow canal.

Dilatation and overgrowth of lymph- and blood-vessels in the walls of such cysts may lead to the growth of angioma and lymphangioma associated with spina bifida (Hildebrand), and overgrowths of fibrous or fat tissue may produce fibroma or lipoma. Such lipomas may contain cysts or a canal leading to the spine.

Spina bifida occulta is a condition in which the original fissure in the spinal column has become closed by a fibrous or bony membrane, while the hernial sac and its accompanying tissues persist. This condition has been observed with a variety of malformations, sacral hypertrichosis, club-foot, congenital luxation of hip, with atrophy of limbs, elephantiasis, syndactyly, disturbances of innervation, mal perforant, and in a series of cases it has been associated with sacral tumors (Recklinghausen). Most of these tumors consist of fibrolipoma with areas of nerve-fibers and striated muscle, and are connected by a fibrous or fatty tissue pedicle with the sacral fissure. The so-called "false tail" is a pendulous lipoma which is often connected by a strand of fibrous or muscular tissue with the sacral dura (Arnold, Bartels, Hagenbach). The spinal cord may be extensively deformed (Bohnstadt). The nerve plexus in the tumor may show myxomatous changes (Borst). Muscatello described cases with extensive defects in sacral and dorsal spine and cord, with a dermoid in the dorsal region and an epidermoid at the sacrum.

On the convexity, at the base of the skull, and in the dorsal region hairy lipomas are described which probably have the same origin (Arnold, Virchow, Bartels). That portions of brain and cord may be displaced with the membranes in spina bifida is indicated in the reports of glioma with meningocele (Hildebrand). Much more complex tumors and deformities arising from spina bifida and imperfect development of the spinal cord are described by Arnold and Borst.

Origin of Congenital Sacral Mixed Tumors.-The recent tendency has been to regard the congenital mixed tumors of the sacral region as forms of true teratoma, a view which is favored by many considerations. It has long been recognized that many gradations exist between the comparatively simple mixed tumors, teratoids, teratomas, and fetal implantations in this region, and that there are no definite anatomical features on which they can readily be separated. The resemblances to teratomas of the sex glands have often been pointed out. Nakayama has recently reviewed the subject, with the conclusion that all or nearly all sacral mixed tumors are of tridermal structure and arise from totipotent material, and Askanazy accepts this conclusion.

Against the sweeping application of this view there stand, however, the well-known embryogenic disturbances peculiar to the caudal and cephalic extremities. It seems quite clear that certain complex growths must be referred to the coccygeal vestiges of the neural canal, the postanal gut, probably also to the neurenteric canal, while the complex forms of spinal rachischisis doubtless account for other complex tumors. Many other special factors pertaining to the mechanics of development of the caudal extremity have been adduced in support of the local origin of sacral tumors (Borst, Lit.). 
Therefore, in view of the several important possible sources of complex sacral tumors, it seems necessary to continue the attempt to analyze each case and place it in a proper category and not merge the group into one class of teratomas. As previously pointed out, there are special reasons for attributing many of the sacral growths as well as the cervical to budding processes. In this aspect the sacral tumors differ from those of the sex glands, which probably always arise from totipotent sex cells.

The difficulty of accomplishing this separation is illustrated in a case described by Borst. A large congenital antesacral tumor, composed of solid and cystic portions, was connected by a pedicle with the spinal column. It appeared to represent an immature brain with gyri, bilateral ventricles, choroid plexus, and pigmented ganglia. Small cysts were lined by cubodial or flat epithelium. The solid parts contained much cellular connective, fat tissue, and glandular alveoli, nerve-trunks, and ganglia. Borst refers this tumor exclusively to derivatives of the medullary groove and its mesenchymal investments. 



\section{BIBLIOGRAPHY}

Note.-The bold-face figures refer to page numbers in this book.

\section{KEY to ABBREVIATIONS OF JOURNALS Cited}

The common names of journals, rather than their official titles, are usually employed.

Articles are not entered more than once in the bibliography of one chapter, although they may be cited twice in the text of that chapter. Accordingly, if a reference appears to be missing, it may often be found by looking backward in the lists.

A. m. A. Archiv. f. microscopische Anatomie.

A. D. Archiv. f. Dermatologie.

A. G. Archiv f. Gynæcologie.

A. K. Archiv. f. Kinderheilkunde.

A. P. Archiv. f. Psychiatrie.

A. J. M. S. Amer. Jour. of Med. Sciences.

A. S. Annals of Surgery.

Arch. gen. Archives generale de Medecine.

B. M. J. British Medical Journal.

B. B. Bruns' Beitrage z. kl. Chirurgie.

B. W. Berliner kl. Wochenschrift.

C. B. Centralblatt f. Bacteriologie.

C. C. Centralblatt f. Chirurgie.

C. G. Centralblatt f. Gynæcologie.

C. P. Centralblatt. f. allg. Pathologie.

D. A. Deutsches Arch. f. kl. Med.

D. P. G. Deutsche patholog. Gesellschaft.

D. W. Deutsche med. Wochenschrift.

Erg. Path. Ergenbnisse d. allg. Pathol. Lubarsch, Ostertag.

J. A. M. A. Journal of Amer. Med. Association.

J. C. D. Journal of Cutaneous and Genito-urinary Diseases.

J. H. Bull. Bulletin of Johns Hopkins Hospital.

J. P. B. Journal of Pathology and Bacteriology.

L. A. Lagenbeck's Archiv. f. Chirurgie.

M. G. Mittheilungen a. d. Grenzgebieten d. Med. u. Chir.

M. G. G. Monatshefte f. Geburtshilfe u. Gynecologie.

M. W. Münchener med. Wochenschrift.

S: G. O. Surgery, Gynecology, and Obstetrics.

Soc. Anat. Bulletin d. Soc. Anat., Paris.

Soc. Chir. Bulletin d. Soc. Chir., Paris.

V. A. Virchow's Archiv.

W. Wochenschrift.

Z. B. Ziegler's Beitrage $z$. path. Anat.

Z. G. G. Zeitschrift f. Geburtshilfe u. Gynecologie.

Z. K. Zeitschrift f. Krebsforschung.

Z. M. Zeitschrift f. kl. Medezin.

Z. N. Deutsche Zeitschrift f. Nervenheilkunde.

Z. C. Deutsche Zeitschrift f. Chirurgie.

\section{HISTORICAL •}

The author has made no research in the older literature of cancer. Most of the older titles cited in this section are taken from Gurlt, Geschichte d. Chir., Berlin, 1898; from 
Wolff, Die Lehre v. d. Krebskrank., Berlin, I907; and from Garrison, History of Medcine, Philadelphia, I9I4.

17 Herodotus, Thalia, Lib. III, Cap. 133. Celsus, De Medecina. Cato, De re rustica. Pliny, sec., Historia naturalis. Wolff, Die Lehre v. d. Krebskrankheit., 1907 (Lit.). Albert, Das Carcinom im histor. exper. u. path. Beziehung, Jena, I887. 18 Leonides, cit. by Wolff from Aetius. Paulus Aeginetæ, Totius rei., Med. Lib. VII. Avicenna, Canon medecinæ. Avenzoar, Liber Theisir., Cap. I8. Walsh, Popes and Science. Andreas Vesal, Med. Opera. Fabricius, Med. et. Chir. Opera. Severinus, De recondita obscessuum natura. Sennert, Opera, cit. by Wolff. Lusitanus, cit. by Wolff. Paracelsus, cit. by Wolff. Louis, Observ. sur les effets du virus carc., Paris, 1749. LeDran, Mem. Acad. Roy. de Chir., Paris, I757, T. IV. Astruc, Abhandl. v. Geschwulsten., I76I, Bd. II. 19 Morgagni, De Tumoribus, Opera, V. Peyrilhe, Diss. acad. de Cancro., Paris, I776. Stahl, Theoria med. vera., cit. by Wolff. Hoffmann, Opera omnia, Geneva, I740, cit. by Wolff. London Cancer Soc., Edinb., M. S. J., I 806, 2, 382. 'Hunter, Invest. on Blood and Inflam., etc. Hey, Pract. Observ. on Surg., 1814. Wardrop, Obs. on Fungus hematodes., Edinb., 1809. Abernethy, An attempt to form a Classification of Tumors, London, I804. Maunoir, Fungus medullaris u. hematodes. Pott, Surgical Works, 1783, Vol. 3, p. 225. 20 Home, A Short Treatise on the Formation of Tumors, London, I830. Dupuytren, Consid. gen. s. 1. cancer, Paris, I807. Laennec, Dict. d. sci. med., Paris, I8I 5, T. 2, T. I2. Bichat, Anat. generale, I801. Bayle, Cayal, Dict. d. sci. med., Paris, I812, T. 3. Broussais, Exam. d. Doct. Med. d. Syst. de Nosologie, Paris, I 82 I. Lobstein, Traitè Anat. path., Paris, 1829. Recamier, Recherch. s. 1. Traitement de Cancer, Paris, I829. Andral, Precis. d. Anat. path., Paris, I829. Cruveilhier, Essai s. 1. Anat. path., Paris, I8I6. Velpeau, Arch. gen. de Med., I 825. Richter, Anfangsgrunde d. Wundarzneykinst, Gottingen, 1782. Walther, Jour. der Chir. u. Angenheilk., Berlin, I820, I, 55. Langstaff, Med. Chir. Transac., I81 7, 8, 286. Carswell, Cyclop. Practice of Med., London, 1834. 21 Raspail, Bull. de Sci. nat., Paris, I 826, Io, 25I. Collard, Compt.-rend. Soc. Anat., I826. Schwann, Froriep's Notizen., I 838. Müller, J., U. d. fein. Bau. u. d. Formen d. krankhaften, Geschwulsten, Berlin, I838. Lebert, Physiol. path., Paris, I845, 2, 254. Hannover, Das Epithelioma, Leipzig, I 852. Müller's Arch., I 844. Bruch, Diagnose d. bosart. geschwulsten, Mainz, I847. Velpeau, Traite des mal. du. sein, Paris, I854. Virchow, V. A., I85I, 3, I97. Vogel, Path. Anat., I, 258. 22 Rokitansky, Handb. Path. Anat., $I, 342,424,552$. Führer, Deutsche Klinik., I 852, 222. Langenbeck, Schmidt's Jahrb., I840, 99. Follin, Traitè de path. ext., I861, I, 303. Meckel, Sömmering, Path. Anat., II, 30I, 34I. Virchow, V. A., I852, 4, 375. Remak, Entewickelung d. Huhnchens im Ei., I 843; Deutsche Klinik., I854, 7, I7o. His, Arch. f. micr. Anat., I. Thiersch, Der Epithelialkrebs, Leipsig, I865. 23 Waldeyer, V. A., I867, 4I, 470. Popper, Wiener Zeitsch., I865, 22, 57. Volkmann, V. A., I 870, 50, 543. Weber, V. A., I 866, 35, 522. Gussenbauer, L. A., I 872, 14, 561. Classen, V. A., I870, 50, 56. Rollett, U. Elementarteile u. gewebe. Graz., I871. Recklinghausen, Graefe's Arch. f. Opth., I 864, I2, 70. Koster, V. A., I 867, 40, 468; Die Entw. d. Carc., Wurzburg, I869. Carmalt, V. A., I $872,55,487$.

\section{DEFINITION. CLASSIFICATION. GENERAL PATHOLOGY}

25 Virchow, Dir krankh. Geschwulste, I 863. Ziegler, Path. Anat. D. W., I894, I 898. Birch-Hirschfeld, Path. Anat., 1896. Ribbert, Geschwulste: Das path. Wachstum, Bonn, I 896. Lubarsch, Erg. Path. Anat., I 895. Borst, Die Lehre v. d. Gesch., 1902. Adami, Text-book of Path., I914. Prudden, Text-book of Path. (Delafield \& Prudden), I9I I. von Heukelom, C. P., I908. Bonney, Lancet, I908, I, I389. Karg, Z. C., 34, I33. Daniels, Z. K., 3, 540. Tolot, Rev. d. Med., I904, 948. 35 Janeway, Z. K., 8. I36. 36 Oertel, V. A., 180, 499. Woolley, Am. J. M. S., 125, 33. Hauser, V. A., I38, 482 ; I4I, 485. Hauser, Das cylinder-epithel. d. Magans u. d. Darms, Jena, I8go. 
Petersen, V. A., 164, 570. Marchwald, V. A., I4I, I28. 37 Symmers, Rep. Path. Dept. N. Y. City Hosp., 1906. Hansemann, Z. K., I, I83. Redlich, Z. K., 5, 26 I. Walter, L. A., 53, I. Woolley, Boston M. S. J., 148, I. Hauser, D. A., 55, 429. Abrikossof, V. A., I73, 335. 38 Wells, A. J. M. S., 128, 837. Symmers, Arch. Int. Med., 4, 2 I8. Marine, Arch. Int. Med., 1908, 1909. Stroebe, Z. B., II. V. Muller, V. A., 130, 512. Cornil, Arch. d. Phys., 18, 310. Arnold, V. A., 78, 279; 93, 501. Hansemann, D. Specif. d. Zellen, etc. Pianese, Z. B., Suppl. I. 39 Galeotti, Z. B., I4, 249 ; 20, 192. Pfitzner, V. A., I03, 275. Hertwig, Erg. Path. (Benda), 2, 54I. Farmer, Moore, Walker, Proc. Roy. Soc., 1903. 72, 104; B. M. J., 1903, 2, 1664. Nedjelsky, Z. B., 27, 43I. 40 Trambusti, Z. B., 22, 88. Howard, Fest, f. R. Hertwig. Klebs, Allg. Path., I887-9, II, 399. Recklinghausen, Die Adenomyome d. Uterus, 1896. Auerbach, Sitz. d. pr. Akad. Wissen, I891, 713. Bashford, Scientific Reports, 1904, I, 16. Schleich, D. W., I891, 83. Watson-Cheyne, B. M. J., I908, I, I174. Czerny, Z. K., 1909, 7, 295; B. B., 25. 42 Brosch, V. A., 162, 32. Fabian, I. D., Rostock, 1901. 43 Kaiserling, Orgler, V. A., I67, 296. White, J. P. B., I3, 3. 44 Virchow, V. A., 3, 197. Best, Z. B., 23, 2 I3. Brault, Arch. gen. d. Med., 1899. v. Muller, V. A., 130. Gierke, Z. B., 37, 502, Lit. Best, Z. B., 33, 585. Brault, Le Prognostic des tumeurs base sur las recherche du glycogene, Paris, I899. Lubarsch, V. A., I83, I88. Muller, I. D., Zurich, 1899. 45 Wagner, A. f. Phys. Heilkunde, 1857. Billroth, Beitr. z. path. Histol., I 858. Burow, L. A., I8, 228. Langhans, V. A., 38, 497. Siegert, V. A., I29, 413. Aoyama, V. A., 106, 575. Hildebrand, V. A., 140, 249. 46 Weber, Blendinger, J. P. B., II, 59. Schuller, C. B., I904, 37, 547. 47 Ewing, J. Med. Res., 12, 509. Meser, V. A., I63, III. LeCount, J. Med. Res., 7, 383. Borrel, Annal. Pasteur, 15, 49. Heidenham, A. micr. Anat., I8. 48 Hansemann, Diag. bos. Ges. Benda, Deut. Gesell. f. Chir., I902, 73. Greenough, J., med. Res., 13, 137. Nosske, Z. C., 64, 352. Honda, V. A., I74, 96. Klimenko, C. P., 13, 837. Plimmer, Practitioner, I 899, 62, 430. Apolant-Embden, Z. Hyg., 42, 353. Virchow, V. A., I. Foa, C. B., I2, 185. Thoma, Fort. d. Med., 189, 413. Soudakewitch, Annal. Pasteur, 1892. Leyden, Z. K., I, 293. Ruffer, Walker, B. M. J., I892, 2, II3. Feinberg, D. W., 1902, 1051. 50 Klebs, Arch. exp. Path., 3, 154, Waldeyer, V. A., 4I. Blumenthal, Erg. d. Exp. Path. Ther., 1907, I, 65. 51 Fuginami, V. A., I6I, II5. Orth. Z. K., I, 399. 53 v. Hansemann, Z. B., I, I9I; B. W., I 890, 901. Heller, Verh. Ges. deut. Naturf., I 895, I I, 2, I0. Perls, V. A., 56. v. Eiselsberg, L. A., 48, 489. Ewald, Nothnagel's Handb., 2, 1896, 22. Wells, J. Med. Res., I7, 461. 54 Woolley, J. H. Bull., I4, 21. Williams, J. H. Reports, 1900, 9, 293. 55 Langhans, Deut. Chir. L., 50, 414. Clowes, Beslack, Med. News, 1905, 87, 968. Gaylord, Clowes. S. G. O., I906, 2, 633. 56 v. Dungern, Coca, Z. f. Immunit., 2, 39 I. Da Fano, Zeit. f. Immunit., 5, I. Boll, Das princip. des. Wachstum, Berlin, 1876. 57 Eberth, V. A., 49, 51. Belapolsky, Entwickelungs ges. d. Krebsig. Entartung, Móskow, 1881. Von der Kolk, Observ. Anat. Path., I828. Goldmann, B. B., I8. 59 Marchand, W., I894, I. Risel, Arb. path. Inst. Leipsig, 1903. 60 Martland, N. Y. P. S., 1909, 9, 123. Starck, cit. by Ribbert, p. 51. Nasse, Volkmann's Vort., 125. Randolph, Phila. Path. Soc., 7. Watson, Lancet, 1902, I, 300. Kaposi, B. B., 30. Tripier, Lyon Med., 1876. Ewing, S. G. O., 1910, 366. Rotter, L. A., 48, 357. 61 MacKay, Brit. M. J., 1907, 2, 138. Hodenpyl, N. Y. Med. Record, 77, 359. Bryant, Dis. of Breast, 1887, 142. Gould, Chir. Soc. Trans., London, 1897, 30, 205. Broca, Traite d. Tumeurs, 1866, I, 240. Hutchinson, Arch. of Surgery, 1891, 2, 354. Williams, I. c., p. 484, 488. Petersen, B. B., 32, 605; 34, 683. Teacher, J. P. B., I908, 88.

\section{MALIGNANCY AND ITS EFFECT ON THE ORGANISM}

65 Piquand, Les degen. d. fibromyomes de l'ut., Paris, 1905. Klob, Path. d. weibl. Geschlechtsorgane, 1864, 163. Rolly, V. A., 150, 555. Schafter, V. A., I29, 61. Bender, Bull. Soc. Anat., Paris, 1904. 66 v. Noorden, Path. d. Stoffwechsel, 456. Cramer, III Report Imp. Cancer Com., 1908. Stockard, Jour. Zoöl., 1909, 6, 433. F. Müller, 
Z.! M., I 889, 16, 496. Wilks, Guy's Hosp. Rep., I868, 4. Klemperer, Char. An., I891, 16, 138. Widal, Arch. Verdauungsk, 1899, 5, 540. Setti, Maly's Jahrb., 1899, 741. Braunstein, Z. K., 1904, I, I99. Clowes, Biochem, Centr., 1905. Lewin, D. W., 1905, 218. Schapp, D. W., I893, II 55. Moraczewski, Z. M., I897, 33, 385. Benedict, Gorslin, Z. K., I912, II, I40. 67 Petry, Zeit. phys. Chem., I899, 27, 398; Hoffmesiter's Beitr., 1902, 2, 94. Wolff, Z. K., 1905, 3. Beebe, Amer. J. Phys., 1905, 13, I43. Shaffer, Amer. J. Phys., 1905, 14, 231. Buxton, J. Med. Res., I903, 9, 356. Neuberg, B. W., 1904, 1080; Z. K., 1904, 1905. Jacoby, Hoffmeister's Beitr., 1903, 3, 446. Blumenthal, Wolff, Erg. Exper. Path. u. Ther., 1907, I, 65. Ewing, Arch. Int. Med., 1908, I, 175. Kepinow, Z. K., 1909, 7, 517. Ed. Müller, D. A. kl. Med., 1907, 91; 1908, 92, 199. Aberhalden, Z. K., I9I0, 9, 266. Osterspey, B. W., I892, 27I, 308. Cabot, Clin. Path. of Blood, N. Y., I902. Laache, Die Anemie, I883. Leichtenstern, Unters. u. d. Hb. gehalt., etc., Leipsig, I878. Patrigeon, Thèse de Paris, 1877. Ewing, Clin. Path. of the Blood, 1903. Hampeln, cit. by Neubert. Neubert, I. D., Dorpat, I889; St. Petersb. med. Woch., I 889. 68 Maragliano, B. W., I892, 765. Polk, J. Med. Res., 1904, 12, 263. Elsberg, Amer. J. M. S., 1910, 138, 264. Kullman, Z. M., 1904, 53, 293. Bard, Sem. med., I901, No. 25. Dieballa, D. A., 57, 302. Peiper, Cent. inn. Med., I891, 217. Grawitz, Klin. Path. d. Blutes, I896, 316. Wendelstadt, Bleibtreu, Z. M., 1894, 25, 204. H. Rieder, Beitr. z. Kennt. d. Leucocytose, 1892. 69 Alexander, Thèse, Paris, I887. Price-Jones, Arch. Middlesex Hosp., I902, I. Kurpjuweit, D. A., I903, 77. Martin, Matthewson, B. M. J., I896, Dec. 5. Loeper, Loeste, Sem. med., I904, 24, 36. Janeway, Blood Pressure, 1904, 237. Bierfreund, L. A., 4I, I. 70 Schmidlechner, Z. K., 1905, 3, 247. del Conte, Inst. mic. anat., Univ. Neapel, 1904. Klemperer, Charite An., I890. Peiper, V. A., II6, 337. Rumpf, Cent. in. Med., I89I, 44I. Herter, N. Y. Path. Soc., I900. H. Strauss, Z. M., 1896, 30, 317 . Moore, Wilson, Biochem. Jour., Liverpool, 1906, I, 297, 398. Watson, J. P. B., I909, 13, 729. Gamble, Ibid., 1906, II, 124. Royle, Lancet, 1910, 2, 450. Sturroch, B. M. J., 1913, 2, 780. Freund, Wien. med. Bl., I885. Trinkler, Cent. med. Wissen., I890, 486. Matrai, Pest. med-chir. Presse, I885. Lewis, Benedict, Soc. exp. Biol., N. Y., I914. Theis, Stone. J. Cancer Research, I919, 4, 349. Van der Velden, D. A., 1879, 23, 369. Richter, Arch. Verd., 1900, 5, 379. Schneider, V. A., I48, 243. Rosenheim, Z. M., I890, I7, I 6. Riegel, Nothnagel's Syst. 71 Reissner, Z. M., 44, 71. Stahelin, cit. by Emerson, D. A., 72, 4I 5. Rosenberger, Ibid. Salomon, D. W., I903, 546. Moore, Palmer, B. M. J., I906, I, 274, 297. Copeman, Hake, Lancet, 1906, II, I276. Oppler, Arch. Verdauungsk, I896, 2, 40. Glassner, B. W., I902, 675. Emerson, D. A., I902, 72, 415. Rosenberg, Z. M., 1905, 56, 449. Fischer, D. A., 1908, 93, 98, 456. Neubauer, Fischer, D. A., 1909, 97, 495. 72 Strauss, Z. M., 1894, 26, 514. B. Wagner, Arch. Verd., 1905, 11, I. Seelig, B. W., 1895, 100. Sick, D. A., 86, 370. Wasbutski, A. Exp. P. P., 1889, 26, I33. Cario, U. d. Einfl. d. Fiebers u. d. Inanition, I888. 73 Brandenburg, B. W., I896, I37. Blumenthal, Char. An., I896, 21, I44. Setti, Maly's Jahrb., I899, 74I. Salomon, Saxl, Med. Klin., 1910. Salkowski, B. W., 1906, I581 ; 1910, 533. M. Weiss, Biochem. Z., I910, 27, I75. Salomon, Saxl, Wien. k. Woch., I91 I, 449. Lehmann, D. A., I913, 112, 376. Saxl, Biochem. Z., 1913, 55, 224. Beneke, Path. d. Stoffwech., 1874, 61. Müller, Z. M., I889, I6, 496. Braunstein, Z. K., I904, I, I99. Schopp, D. W., I893, II 55. Laudenheimer, Z. M., I892, 2, 513. Royle, Med. Chronicle, I909. 74 Gerhardt, Z. M., I897, 32, 303. Hoppe-Seyler, V. A., 1891, 124. Blumenthal Path. d. Harns., 1903, 356. Brieger, Z. M., I88I, 3, 465. Haberlin, D. A., I890, 45, 339. Hennige, D. A., 1879, 23, 27I. Lewin, Fest. f. Salkowski, 1904, 225; D. W., 1905, 218. Waldvogel, Die Acetonkerper. Hirschfeld, Z. M., I895, 28, 176. v. Jaksch, Z. M., I 886, 10, 362. Klemperer, B. W., I889, 869. Riess, Z. M., I884, 7, 34. Senator, Z. M., I 884, 7, 325. 75 Von Noorden, Stoff wechsel, I 893, 464. Campbell, B. M. J., I895, 2, 776. Ury, Lilienthal, Ar. Verd., I905, II, 72. Maixner, Z. M., 8, 234. Pechanowski, Z. M., 9, 429. 


\section{METASTASIS}

76 Handley, Cancer of Breast, London, I9ı. 77 Borrmann, Z. B., 48, 576. Gussenbauer, Zeit. f. Heilk., I88I, 2, 17. Kuster, Deutsch. Ges. Chir., 12, 288. 78 Moore, Lancet, I889, 2, 4r8. Cuneo, De l'envahis. d. syst. lymph. dans le cancer de l'estomac, Paris, 1900. Colwell, Arch. Middlesex Hosp., 1906, 7, 151. Renner, Mitt. Grenzgeb., 13. Butlin, Sarcoma and Carcinoma, 1882. Ewing, S. G. O., I9I I, 12, 232. 79 Rousseau, These, Paris, 1895. Raw, Brit. M. J., 1905, $x$, I380. Poncet, Lyon Med., 1893. Hurlemont, These, Lille, I 896. Williams, London, P. S., I890, 4r, 302. Leydbecker, V. A., 134, I I8. Winkler, V. A. Suppl., 151, 195. 81 Gross, A. J. M. S., 1879. 82 Fuhrmann, I. D., Marburg, I889. Risel, Arb. path. Instit., Leipsiz., I903. M. B. Schmidt, Die Verbreitungswege d. Carc., Jena, I903. 83 Goldmann, Z. B., 28, 595. Gay, Boston M. S. J., 1909, 161, 207. Freund, Caminer, Wien. kl. W., 1910, 1221; Biochem. Zeit., I910, 26,313. Ewing, Z. K., II, I47. Tyzzer, J. Med. Res., 28, 309. 84 Arnold, V. A., 124, 385. Heller, D. A., 7. Bonome, Arch. Med. Exper., 13. Ernst, V. A., 151, 69. Recklinghausen, V. A., I00, 503. Poncet, Lyon Med., 1893, 25, 53. Most, V. A., 154, 138. Vogel, V. A., 125, 495. Zahn, V. A., 115, 71; Z. C., 1885, 22, 22. 85 Ernst, B. B., I900, 28, 255. Langerhans, Berl. W., I893, 338. Beetson, Festschr. f. Orth., 1903. Schlagenhaufer, Wien. kl. W., I902, 523. Moser, D. W., 1903, I33. Borrmann, Mitt. Grenzg., 6. Demarquay, cit. by Pianese, Z. B., Suppl. I. Gueillot, Union med. du Nord-Est, 1891, 15, 33, 106. Hartmann, Lecene, Annal. de. Gyn., etc., 1907, 4, 65. Semon, London, P. S., 39, 38. Williams, B. M. J., I887, 2, 1369. C. Kaufmann, V. A., 75, 317. Bucher, Z. B., 14, 71. Walter, L. A., 53. Thorn, C. G., 1894. Butlin, B. M. J., 1907, 2, 225. 87 Heurteaux, Arch. Prov. de Chir., 1899, No. 2. Verneuil, Sem. med., I 888, I 12 . English, Lancet, 1904, 2, 596. Guinard, Bull. soc. chir. Paris, I903. Fischer, Box, N. M. J., I900, 1, 639. Pomard, La Gynecologie, 1899. Gross, Amer. Syst. Gyn., 2, 247. 88 Wells, Ovarian Tumors. Kratzenstein, Z. G., 36, 6r. Neugebauer, C. G., r890. Williams, Nat. Hist. of Cancer, 464. Velits, Z. G., 22.

\section{CHEMISTRY; SEROLOGY}

89 Wolff, Z. K., 3, 95. Petry, Hoffmeister's Beitr., I902, 2, 94. Beebe, Am. J. Physiol., 1905, 13, 341. Bergell, Dorpinghaus, D. W., 1905, 1426. Bang, Hoffm. Beitr., 4, 362. Neuberg, Fest. f. Orth., 1906, 593. Beebe, Am. J. Phys., 12, 167. Clowes, Ibid., 14, I73. Beebe, Shaffer, Am. J. Phys., 15, 23I. Neuberg, B. kl. W., 1905, I 8. Fulci, Gazz. internat. d. med., 1910. B. Wolter, Biochem. Z., 55, 260. 90 Fasal, Biochem. Z., 55, 88. Bossart, I. D., Basel, 1902. Wells, Long, Z. K., 1912, I2. Long, J. Exper. Med., I8, 512. Michaelis, Z. K., 4, I. Beebe, N. Y. M. J., 92, 1058. Buxton, J. M. Res., 9, 356. Buxton, Shaffer, Ibid., 13, 543. Aberhalden, Z. phys. Chem., 1909, 60,$411 ; 62,145$. Weil, J. A. M. A., 1910, 55, 1532. Aberhalden, Medigreceanu, Z. Phys. Chem., I910, 66, 265. Yoshimoto, Biochem. Z., 1909, 22, 299. 91 Blumenthal, Wolf, Med. Klinik., I905, I, 364; Erg. d. exper. Path., 1907, 1, 65. Neuberg, Chemie d. Neubild. Oppenheim, Handb. d. Biochemie, 1909, 2 (Lit.); Biochem. Z., 26, 344. Baer, Ettinger, cit. by Blumenthal. Kepinow, Z. K., 7, 517 (Lit.). Hess, Saxl, Wien. kl. W., 1908, 248. Joachim, Pfluger Arch., 1903, 93, 558. Wolff, Hoff m. Beitr., 5, 208; Z. K., 3, 95. R. Weil, J. Med. Res., 23, 85. Umber, Z. kl. Med., 48, 364. Eppinger, Z. Heilk., 25, 378. K. Wiener, Biochem. Z., 4I, r49. J. W. Vaughan, J. A. M. A., 59, I 764. Cann, M. W., I9II, 73I. A Foerster, Lancet, I9II, I, I695. Noguchi, Serum Diagnosis of Syph. (Philada.). F. J. Fox, Med. Rec., 84, 283 (Lit.). T. Cohn, Neur. Cent., I910, 688. W. Barrett, Z. K., $I I$, 245. 92 De Marichis, Lo Sper., I910, 63, 969. v. Dungern, Munch. m. W., I912, 65; Z. Immun., 1912, Ref. 525. Edzard, B. kl. W., I912, 2488, Coca, J. Can. Res., 2, 61. Shenk, Wien. kl. W., 1913, 529. Rosenberg, D. W., I912. 1225. Yamanouchi, Lytchkowsky, Z. Immunitatsf., 20, 374. Brieger, Trebing, B, W., 1908, 1349, 2260. Bergman, Meyer, B. W., I908, 1673. Herzfeld, B. W., 1908. 
2182. Roche, Arch. Int. Med., 3, 249. Wiens, Schlecht, D. A. kl. Med., 96, 44. Weil, Arch. Int. Med., 5, I09. S. M. Lewin, Z. Immun. (Ref.), I913, 7 I6. Ascoli, Munch. W., 1910, 62. Ascoli, Izar, Munch. W., 1910, 403. 93 Burmeister, J. Inf. Dis., I2, 459. N. Blumenthal, Z. Immun., 24, 42. Izar, Wien. kl. W., 1913, 698. Michaeli, Cottoretti, Munch. W., 1910, II22. Kohler, Luger, Wien. kl. W., I912, III4; I913, 295. Izar, Z. Immun., 20, 303. Weichardt, Munch. W., I911, 662. Jozca, Tokioka, D. W., 1914, 590. E. Rosenthal, Z. Immun., 15, 37. Stammler, D. Gesell. Chir., I9I I. Ransohoff, J. A. M. A., 6I, 8. F. Green, Z. Immun., 23, $55^{8}$.

\section{THEORIES OF THE NATURE OF CANCER}

94 Lobstein, cit. by Wolff. Recamier, cit. by Wolff. Rokitansky, K. Acad. d. Wissen. Wien., 1852, 9, 350. Houel, Traitè d. Anat. path. gen. (Cruveilhier), I864, 5, 327. Remak, Deutsche Klinik, 1854, I60. Paget, Surgical Pathology, I85.3, 2, 490. Durante, Arch. di polasciano, 1874, cit. by Pianese, Z. B., I896, Sup., I. 95 Lebert, Jahrb. f. Kinderheil., 1884, 21, 276. Reiman, Prag. Woch., 1902, 297. Philip, Z. K., 5, 395. Ahfeld, A. G., I890, 16, 135. Cullingworth, B. M. J., 1877, 2, 253. Bohn, Jahrb. f. Kinderheil., I885, I43. Braun, L. A., I893, 45, I86. Selberg, V. A., 145, I76. Kron. lein, Cor. Schweizer. Aertze, 1894. Muus, V. A., 176, r80. Defosses, Jour. d. Anat. et de Physiol., I88I, 364. Ricker, V. A., 142, 193. Kraske, Volkmann's Samml. N. F., I897, No. 183-4. Chiari, Centr. f. wissen. Heilk., I884. Askanazy, Z. B., I4, 633. Lubarsch, V. A., 135. Langenbeck, L. A., I86I, I, I. Volkman, C. C., I882. 96 Sazarin, These, Paris, 1895. Ehrmann, I. D., Heidelberg, 1889. Grosch, Z. C., 26, 307. Kottnitz, Z. C., 38, 75. Brigidi, Marcacci, A. D., 1882. Lubarsch, Erg. Path., 1901, 884. Borrman, Erg. Path., 1900, 7, 833. R. Meyer, Z. G. G., 49, 464; Erg. Path., 1903, 2, 518. Wiessel, Z. f. H., 24; V. A., I76. R. Williams, Nat. Hist. of Cancer, 1908. 97 Aschoff, Erg. Path., I 898. Ribbert, Das. path. Wachsthum. Bonn., I896; V. A., I4I; Bibliot. med., I897, C., 9, 71; Beitr. z. Entsteh. d. Ges. Bonn, 1906, 1907. 98 Weigert, V. A., 88, 3c8. Roux, V. A., 64, I13. Lubarsch, Arb. path. Inst., Posen, I90I, 205. 99 Adami, B. M. J., 1901, 1, 621. Benecke, Z. B., 9, 440. 100 Oertel, V. A., I80, 499. Rulf, Z. K., 4, 4I7. Morgan, Regeneration, N. Y., 1909. Weigert, V. A., 70, 72, Verh. d. gesell. d. Naturf., 1896. Wilson, Jour. Morph., 1893, 8, 579. Zoja, Arch. f. Entwick,. 1895, I, 578; 2, I. Hansemann, Studien u. Zellen, Berlin, I893. 102 Farmer, Moore, Walker, B. M. J., 1903, 2, I664. Klebs, Allg. Path., I887, 2, 399. Waldeyer, D. W., I 887 , 925. Recklinghausen, Die Adenomyome d. Uterus, Berlin, 1896. Auerbach, Sitz. d. k. Pr. Akad., 1891, 7I3. Bashford, Imp. Cancer Res., 1904, 16. Schleich, D. W., 1891, 83. L. Pick, C. G., 1903, 1033. Marchand, Z. G. G., 32, 405. H. Janeway, Z. K., 1910, 8, 403. Ribbert, Das Kar. d. Mensch., 1911. 103 Israel, V. A., I67, 533. 104 Borst, 1. c., 95. Beneke, Constitution u. const. Kranksein., Marburg, I887. Wells, Ovarian Tumors. Kratzenstein, Z. G. G., 36, 6r. Neugebauer, C. G., I890, I. Sticker, L. A., 1902, 65. 105 Adams, Trans. Path. Soc., London, 1905, 66, 189. Guthrie, B. M. J., 1907, 2, 747. Beard, Med. Press \& Circular, 1905, 66I. Warren, Observ. on Tumors, Boston, 1837. Broca, Traitè d. Tumeurs, I866, I, I5I. I. Levin, Z. K., I912, II, 547. Warthin, Arch. Int. Med., 1913, I2, 546. Sibley, Trans. Chir. Soc., London, 1859, 42, r ro. Korteweg, Verof. d. Com. f. Krebsf., 1902, Jena. Paget, Med. Times and Gazette, 1857. Newton, Austral. Med. Gaz., 1902, 236. Wilson, Trans. Path. Soc., Dublin, 1871-74, 108. 106 Baker, Med. Chir. Trans., 1862; St. Bart's. Hosp. Rep., I864. Velpeau, Traitè d. mal. d. sein, I858, 685. Leichtenstein, Ziemssen's Handb. Campiche, Lazarus-Barlow, Arch. Middlesex Hosp., 1905, 83. Butlin, cit. by Williams. Nunn, Cancer of Breast, London, I882. Leaf, Clin. Causes of Cancer, London, 1904. Lebert, Traite. pract. de. mal. cancer, Paris, 1851. Sibley, Trans. Med. Chir. Soc., I859, 12. Winiwarter, Beitr. z. Statis. d. Carcinome, 1878. Billroth, Deutsche Chir., I880, Lief., 41. Tillmann, L. A., 1895. Ziel. U. d. Einfl. d. hered. Anlage a. d. Ensteh. d. Carcin., Erlangen, 1892. Cripps, St. 
Bart's. Hosp. Rep., I878. Hillier, Tritsch, Arch. Middlesex Hosp., I904, 2, I04. Pearson, Ibid., 127. Bashford, Proc. Royal Soc., 1909, 2, No. 3, 63. Weinberg, Z. K., 1904, 2, 195. Guillot, Rev. de Med., 1908, 28, Suppl., I I2. 107 Manichon, These, Paris, 1896. Le Tulle, Arch. de Med. exper., 1907, 658. Le Doux-Le Bard, Rev. de med., 1908, 28, Suppl., 92. Bashford, Murray, Imper. Can. Res. Reports, 1911, 114. Tyzzer, J. Med. Res., I7, 199; 21, 519. Slye, Z. K., 13, 500; J. Med. Res., 30, 281; 32, I 59; 33, 171. J. Can. Res., I, 2, 4, 5 .

\section{THE SPECIAL ETIOLOGY OF TUMORS; TRAUMA}

111 P. Ziegler, Munch. W., 1895, 621. Lowenthal, L. A., 1895, 49, 1. Jordan, Munch. W., I901, 1741. Segond, Assoc. Franc. de Chir., 1907, 745. Berard, II Intern. Cancer Conference, 1910, 356. 112 Ribbert, Aertz. Sach. Zeit., I898, 389. Kottnitz, Z. C., 38. Werner, Rowe, Diss. Kiel, 1899. Kempf, Diss. Gottingen, 1900. Adler, Arch. f. Unfalheilk., I 897, 2, I89. Lowenthal, L. A., I895, 49, I. Schimmelbusch, Erg. Path., I 895, 2, Abt., 527.

\section{THE PARASITIC THEORY}

113 Arnaudet, L'Union med., 1889. Guelliot, Gaz. d. Hop., I 892. Webb, Birmingham Med. Rev., 1892, 32, 342. Fiessinger, Rev. de Med., I 893, 13, I3. D'Arcy Power, B. M. J., I894, I, I240, I302. Bosc, Le Cancer, Paris, 1898. Behla, C. B., 24. Haviland, Geog. Dist. of Disease in Gt. Brit., 1892. Park, Practitioner, 1902; B. M. J., I899, I, 812. Mason, B. M. J., I902, I, I39. Nason, B. M. J., I898, I, 679. LloydJones, B. M. J., I899, I, 813. Poppelman, Z. K., 4, 39. Noel, Rev. de Mal. Canc., Paris, I896, 2, I37. Symons, Public Health, London, I898. Williams, Nat. Hist. of Cancer, 50. Sticker, Z. K., 5, 21 5. Prinzing, Z. K., 5, 224. Loeb, Medecine, Detroit, 1900, 6, 286; C. B., 37, 235. Gaylord, J. A. M. A., 48, I 5. Borrel, C. R. Soc. Biol., 1905, 770. Loeb, Univ. of Penn. Bull., 1907. 114 Bashford, Lancet, I907, I, 802. Pick, B. W., 1905, I435. Plehne, Z. K., 4, 525. Gaylord, J. A. M. A., 54, 227. Gudernatsch., J. Morphol., I9II, 21, 709. Marine, Lenhardt, J. Exper. Med., 12, 3II. Hoffman, Mortality from Cancer, Newark, 1915. Riechelman, B. W., I902, 728. Willcox, Jour. Cancer Research, 1917; Proc. Royal Soc., 1917. Boinet, C. R. Soc. Biol., I894, Io, II. Dagonet, Arch. de Med. exper., 1904, 16, 345. Juergens, Verh. d. deutsch. Gesell. Chir., Berlin, I896, I897. Werner, v. Dungern; Das Wesen d. bos. Geschw., I907, 146. Lusitanus, cit. by Wolff, 522. Tulpius, cit. by Wolff, 522. Lebert, Traitè d' Anat. Path., Paris, 1855. Friedreich, V. A., 36, 465. Langenbeck, Schmidt's Jahrb., 1840, 25, 99. Follin, Traitè de Path. ext., Paris, I861. Velpeau, Traitè de mal. du Sein., Paris, I854, 544. Budd, Lancet, I 887, 2, 727-1 145. Guelliot, Gaz. d. Hop., 1892, 1209. Demarquay, Mal. chir. du Penis, r876. Bossi, Gaz. d. Osped., 1902. Alibert, Mal. de la Peau., I 806, I 18, cit. by Pianese. Wickham. Senn, J. A. M. A., 46, 1255. 115 Rappin, C. R. Soc. Biol., I887, 4, 756. Schill, D. W., I887, 1034. Francke, Munch. W., r 887, 57. Lampiasi, La Rif. med., 1888, 4, 20. Scheurlen, D. W., 1887, 1033. Baumgarten, C. B., 3, 397. Koubassoff, C. B., 7, 317. Doyen, Etiol. et Trait. du Cancer, Paris, 1904. Shattock, Ballance, Trans. Path. Soc., London, 1887-1888. Verneuil, Rev. de Chir., 1889, 9, 793. Zahn, V. A., 1889, 117, 209. Richet, C. R. Acad. Sci., 1895, 2. Maragliano, Gaz. d. ospedali, 1901. Darier, Anal. derm. et syph., I899, Io, 597. Sudakiewitsch, Annal. Pasteur., I 892, 6, I45. Adamkiewicz, Unters, u. d. Krebs. Wien, I893. Clarke, J., Protozoa and Disease, I908. Korontoneff, Sporozoen als Krankheitser., Berlin, I893. Eisen, N. Y. Med. Record, 58, 6. Podwyssoski, C. B., II, 491 ; C. B., 27, 97. Sawtschenko, C. B., 12, I7. Ruffer, Walker, J. P. B., 1892, I, 198. Kahane, C. B., I5, 413. Schaudinn, Berl. Acad. d. Wissen., I896, 39, 951. 116 Foa, C. B., I2, I85. Feinberg, D. W., I902, I85. Hertwig, D. IV., I902, 221. L. Pfeiffer, Die Protozoen als Krankheitser., Jena., I891. Behla, Zeit. f. Hyg., 1899, 32, 123. Podwyssoski, La Presse Med., 1900, 77. Gaylord, IV Report Buffalo 
Lab., 1902, 20. Robertson, Wade, Lancet, 1904, 2, 469. Sjöbring, Fort. d. Med., I890; C. B., 27, I29. Schüler, Die Parasiten im Krebs., Jena., I901; C. B., 27, 5 I I. v. Tubeuf, Verb. d. Com. d. Krebsf., Berlin, 1902, 74. E. F. Smith, J. Cancer Res., 1916. Volcker, D. W., I901, 494. Borrel, C. R. Soc. Biol., 1905, 59, 770. Wenyon, J. Hyg. Cambr., 1906, 6, 580. Gaylord, J. Infect. Dis., I907, 4, I55. Calkins, Ibid., I71. Tyzzer, Soc. Exp. Biol., I907, 4, 85. 117 Mulzer, B. W., 1905, 880. Lowenthal, Ibid., 1906, 283. Russell, B. M. J., I890, II, I356. Fox, Med. Chir. Trans., London, I 858, 4I, 361. Klein, Z. B., II, I25. Lubarsch, Verh. d. Naturf. Gessel. Rostock, I 892. San Felice, Zeit. f. Hyg., I 896-98, 21; 32, 394; 23, I71; 26, 298; 20, 463; Z. K., 7, 564 . Plimmer, Practitioner, 62, 43I. Roncali, C. B., I8, 533. Corselli, Frisco, Ibid. Curtis, Annal. Pasteur, IO, 448. Monsarrat, Proc. Royal Soc., 1900, 66, 58. Leopold, A. G., 6I, 77. Wlaeff, C. R. Soc. Biol., 52, 1030; 53, I06, 285; Rev. de Obstet., 1904, I64. Foulerton, J. P. B., 5, 57; 6, I54. Richardson, J. Med. Res., 5, 312. Klein, London, P. S., 52, 270. Meser, V. A., I63, II I. Mafucci, Sirleo, Zeit. f. Hyg., I 898, 27, I. Rabinowitsch, Zeit. f. Hyg., I 896, 21 , II. Peterson, Exner, B. B., 1899, 15, 769. Nichols, J. Med. Res., 7, 312. Sternberg, Z. B., 25, 554;32, I. Schmidt, O., C. B., 47, 342; 52 , I I. 118 Baisch, Deut. W., 1908, 278. Schuberg, Munch. W., I909, No. 16, cit. by Schmidt. Fischer, Munch. W., 1906, 2041. Jores, Munch. W., 1907, 879. Stahr., Munch. W., 1907, I178. Stoeber, Munch. W., I910, 739. Stoeber, Wäcker, Munch. W., 1910, 947. Galeotti, Pentimalli, C. B., 56, 312. Langenbeck, Schmidt's Jahrb., I 840, 25, 99. Boinet, C. R Soc. Biol., I 894, I0, I I. Juergens, B. W., I895, 331, 447, 465. Dagonet, Arch. de med. exper., I904, I6, 345. Goujon, Gaz. d. Hop., I867, 339. Lanz, D. W., 1899, I46. Bosc, Vedel, Sem. med., I898, I66. Roux, Metchnikoff, Bull. Acad. de Med., I903, 3. 119 Firket, Sem. med., I893, 8. Gaylord, A. J. M. S., I901, I2I, 503. Lewin, L. K., I906, 4, 55. Shattock, Ballance, Proc. Royal Soc., I890, 48. Sticker, Z. K., 2, 4I3. Hemmeter, A. J. M. S., 125, 666. Herzog, J. Med. Res., 8, 74. Tyzzer, J. Med. Res., 2I, 479. Lubarsch, Path. Anat. u. Krebsf., Weisbaden, 1902. Rous, Jour. Exper. Med., 13, 397. 120 Mayet, Gaz. hebdom., 1902, 64. Hemmeter, A. J. M. S., 125, 666. Francotte, deRechter, Bull. R. Acad. de med. Belg., I892, 999. 121 Dubreuilh, Annal. de Dermat., I910. Hutchinson, Lancet, I9I I, I, 976. 122 Wilms, Die Mischgeschw., Leipsig, 1900. Berent, C. B., 1902, 13, 406. Cramer, IV Report Imp. Cancer Res. Com. Brumpt, Precis. de Parasit., 1910. Lowenstein, B. B., I9I0, 69; I9II, 76. Haaland, IV Report Imp. Cancer Res. Wasielewski, C. B., 54. Askanazy, D. W., 1904. 123 Katsurada, Z. B., 1900, 28. Langenbeck, Deut. Klinik., 1863. Babes, C. B., 42. Fibiger, Z. K., 13, 217 . Borrel, Annal. Pasteur., 1909, 23, 97. Bridre, Rev. de Med., I910, 29, Suppl., 318. Saul, C. B., 47, 440; 55, 15. 124 Smith, J. Cancer Res., I916. Levin, Levine, J. Cancer Res., 5, 243.

\section{EXPERIMENTAL CANCER RESEARCH}

126 Lengemann (Lubarsch), Zur Lehre v. d. Gesch., Wiesbaden, I899. Zahn, Cong. internat. sci. med., Geneve, 1877. Leopold, V. A., 85, 283. Kaufmann, V. A., 97, 236. Nichols, J. med. Res., 8, I45. Lack, J. P. B., I900, 6, I54. Boycott, Proc. Roy. Soc., 1911, 4, Path., 225. Fraenkel, C. P., I4, 666. Stilling, D. P. G., 1903, 6, I22. Lubarsch, Erg. d. Path., I899, 6, 958. 127 Eiselsberg, Wien. kl. W., 1882, I890, I892. Krauer, C. G., I 896. Ribbert, Arch. f. Entwickel., 6, 7. Loeb, J. A. M. A., 1903, 40, 974. Tiesenhausen, V. A., 195, I 54. Birch-Hirschfeld, Garten, Z. B., 26, I32. Askanzy, D. P. G., 1907, II, 82. Jentzer, Rev. med. Suisse rom., 1908, 28, 329. Shattock, Proc. Roy. Soc., I910, 3, Path., I32. Rous, J. Exper. Med., I91 I, 13, 248. Wilms, D. P. G., 1904, 8, 79. Fichera, Arch. med. exper., I909, 21, 617. Freund, Z. B., 51, 490. v. Hippel, D. P. G., 1906, 10, 56. Rous, J. Exper. Med., I91 I, 13, 239. Traina, C. P., 13. 128 Lewin, Z. K., 4, 55. Stieve, Z. B., 54, 415. Reinke, D. med. Zeitung, 1907, 28, 579. Askanzy, Wien. m. Woch., 1909, 2518. Freund, C. P., 1909, 1039. Fischer, D. P. G., 1906, 10, 20. McConnell, J. A. M. A., 1907, 49, 1498. Jores, M. W., 1907, 879. 
Stohr, M. W., 1907, I178. Meyer, Z. B., 46, 437. Stoeber, Wäcker, M. W., 1910, 947. White, J. P. B., 14, 450. Benthin, Z. K., I0, 227. 129 I. I.evin, J. Exper. Med., 10, 815. Wacker, Schmincke, M. W., I9II, r607. Bullock, Rohdenburg, J. Med. Res., 33, 53; J. Cancer Res., 1918, 3, 227. Haga, Z. K., 12, 525. Clowes, Internat. Cong. Med., London, 1913. 131 Brosch, V. A., 162, 32. Yamagiwa, Ichikawa, J. Cancer Res., I918, 3, I. 132 Cazin, Des Origins et modes de transmis. de Cancer, Paris, 1894. 133 Marie, Clunet, Raulot-Lapointe, Assoc. franc. p. l'etude du cancer, I912, 239. Febiger, Z. K., 13, 21 7; 14, 295. 134 M. B. Stark, J. Cancer Res., 3, 279. Roux, Metchnikoff, Bull. acad. d. med., 1903, 50, 10r. Jobling, cit. by Flexner, Med. Record, 75, 783. 135 Cornil, Sem. med., I891, II, 259. Milner, I.. A., 74, 669. Hahn, Berl. kl. W., I887, 892. Coca, Z. Immunitatsf., 1912, 13, 549. Sticker, Z. K., I, 413; 4, 227; L. A., 78, 773. Funk, J. Exper. Med., 1915, 21, 571. Murphy, J. Exper. Med., 1915, 22. Strauch, Z. K., 12, 577. Happe, Z. Augenheilk., 1913, 30, 72. Novinsky, Med. Vestnik., St. Petersb., I876, 16, 289. Hanau, L. A., 39, 678. Wehr., L. A., 39, 226. v. Eiselsberg, Wien. kl. W., I890, 927. Moreau, Arch. d. med. exper., I 894, 6, 677. 136 Firket, Bull. acad. Roy. de med., I892, 6, I I47. Velic. Wien. med. Blät., I898, 2I, 7 II. Loeb, J. Med. Res., 6; 1902, 8. Jensen, C. B., 34, 28. Borrel, Annal. Pasteur, 1903, I12. Woglom, Crocker Reports, I913, I (Lit.). Bashford, Murray, Cramer, Imper. Cancer Res. Rep., 1905. Apolant, Arb. Inst. exper. Therap., I906, 1, 60. 137 Bashford, Murray, Imper. Cancer Res. Rep., 3. Haaland, Annal. Pasteur, 19, I88. Ehrlich, Arb. Inst. Exper. Ther., 1906, I, 65. Tyzzer, J. Med. Res., 2I, 459, 479. Stohr, C. P., I4, I. Herzog, J. Med. Res., 1909, 3, 74. Flexner, Jobling, J. A. M. A., 1907, 48, 420. Lewin, Michaelis, D. m. W., 1907, 657. Wernicke, Z. K., xo, 168 (Lit.). Beebe, Ewing, J. Med. Res., 10, 209. Ehrenreich, Z. K., 4, 586. Pick, Berl. W., 1903, 669. Fujinami, Inamoto, Verh. Japan path. Gesell., I91 I, I14. Rous, J. Exper. Med., 1910, 12, 696; J. A. M. A., 55, 56, 58. 139 v. Dungern, Coca, Z. Immun., 2, 391. Ehrlich, Z. K., 5, 70. Gierke, Z. B., 43, 340. Bridrè, Ann. Pasteur, 21, 762. Stohr, C. P., 20, 874. 140 Moore, Walker, Lancet, I908, I, 226. Gaylord, J. Infec. Dis., 5, 443. Haaland, Berl. kl. W., 1906, 40. Bashford, Murray, Cramer, Imper. Cancer Res. Rep., 1906, No. 2, Part 2, 48; 1908, 287, 326. 141 Goldmann, Arb. Instit. Exper. Ther., 1906, 1, 79. Uhlenhuth, Arb. Kaiserl. Ges., I9I1, 36, 477. Beebe, Van Alstyne, J. Med. Res., 29, 217 . Sweet, Carson, Saxon, J. Biol. Chem., 15, I81. Benedict, Proc. Soc. Exper. Biol., I913. Moreschi, Z. Immun., I909, 2, 651. Rous, Soc. Exper. Biol., I9I I, 8, 128. Woglom, J. Exper. Med., 1915, 22. Centanni, Tumori, I9I3, 466. Oser, Z. B. exper. Path., I913, I2. Loeb, J. Med. Res., I901, I, 34. Clowes, J. Hopk. Bull., 16, 130. Baeslack, Med. News, 87, 968. Robertson, Burnett, Jour. Exper. Med., I913, 17, 344; J. Cancer Res., 1918, 3, 75. Sweet, Corson-White, Saxon, J. Biol. Chem., I91 5, 21, 309. Luden, J. Biol. Chem., 1916, 27, 273. Gaylord, Clowes, S. G. O., 1906, 2, 633. Bashford, B. M. J., I906, 2, 209. 142 Bashford et al., Imper. Cancer Res. Rep., 1908, 302, 340; 1911, 131. Apolant, M. W., 1907, 1720. Murray, Berl. W., 1907, I520. Ehrlich, Apolant, Berl. W., 1905, 873; Z. K., 5, 62. Loeb, Univ. Penn. Bull., I906, 19, I13. Lewin, Z. K., 6, 273. Bashford, Murray, Haaland, Berl. W., I907, I238. Russell, J. Path. and Bact., I910, I4, 344. 143 Orth, Z. K., 6, 43 I. Ewing, J. Cancer Res., I916. Bashford, Imper. Can. Res. Rep., 1908, 322. Thorel, D. P. G., I908, 12, 60. Clunet, Rech. exp. s. 1. tum. mal., Paris, I910. 144 Haaland, Berl. kl. W., 1907, 718. Albrecht, Hecht, C. P., 1909, 1039. Stohr, C. P., 1907, 628. Cuenot, Mercier, C. R. Acad. Sci., I908, 167, I003. Slye, J. Cancer Res., I920, 5, 25. Gaylord, Clowes, Baeslack, Med. News, 1905, 86, 91; S. G. O., 1906, 2, 633. Michaelis, D. W., 1907, 827; Z. H., 5, I92. Ehrlich, Z. K., 5, 73. Clowes, B. M. J., 1906, 2, 1550. Haaland, Lancet, 1910, I, 787. Bashford, B. M. J., 1906, 2, 209. Schone, M. W., 1906, 2517. Borrel, Bull. Pasteur, 1907, 5, 605. Higuchi, Sei-I-Kwai M. J., I911, 30, 91. Moreschi, Z. Immun., 2, 675. Russell, Imper. Can. Res. Rep., 1908, 3+5; 1912, 29. Woglom, J. Exper. Med., 16, 629; 12, 29. 145 Haaland, J. Path. and Bact., I4, 407. Coca, Z. Immunit., I3, 524. Michaelis, Med. Klinik, 1905, I, 205. Ehrlich, Z. K., 5, 
75. Haaland, Berl. kl. W., 1907, 717. Vidal, Internat. Cancer Cong., 1910. Beebe, Berkley, N. Y. Med. Rec., I912, 81, 513. Ehrlich, Arb. Inst. exp. Ther., 1906, 83; Z. K., 1907, 5, 76; D. P. G., 1908, 12, I4. Uhlenhuth et al., Z. Immun., 6, 657. Borrel, Bull. Pasteur, 1907, 5, 594. Hertwig, Poll, K. Preus. Acad. Wissen., 1907, 31. Bashford, Imper. Can. Res. Rep., 1908, 386. Beebe, Crile, J. Med. Res., 18, 385. Lambert, Hanes, J. Exper. Med., I4, I29. 147 Burgess, J. Med. Res., 21, 575. Apolant, Z. Immun., 17, 219. Baeslack, Z. Immun., 20, 421. Murphy, J. Exper. Med., 1914, 19, 513; I9I3, I7, 482. 148 Dungern, Munch. W., I 899, I228. Metalnikoff, Ann. Pasteur, I900, 577. Halpern, Z. f. Immun., II, 609. Adler, Z. f. Immun., 3. v. Dungern, Hirschfeld, Z. f. Immun., 8, 332. Schenk, Munch. W., I9I0, 903. Dunbar, Z. f. Immun., 4, 740; 7, 454. Lambert, Hanes, J. Exper. Med., 13, 505; 14, 453. v. Dungern, Coca, Z. Immun., 2, 391. R. Weil, Soc. Exper. Biol., N. Y., I9II, 9, 32.

\section{FIBROMA}

150 Mallory, Jour. Exp. Med., I90 I, 5, I 5. 151 Kosinski, C. C., I 874, 241. Chiari, Prag. Woch., I889. Arnold, Z. B., 8. Ribbert, 1. c., I07. Williams, 1. c., I66. 152 Hitchcock, Amer. J. M. S., I 862, 220. Murray, Lancet, I873, I, 4 IO. Atkinson, N. Y. M. J., I875, II, 60I. Balzer, C. R. Soc. Biol., I879. Winiwarter, V. A., I876. Recklinghausen, U. d. Mult. Fibrome d. Haut., Berlin, I882. 154 Bruns, Die Geschwulste d. Nervensystems, Berlin, I 897. Sarazanes, These, Paris, 1904. Verocay, Z. B., 48. Genersich, V. A., 49, 33. Herxheimer, Roth, Z. B., 58 (Lit.). Jordan, Z. B., 8, 7I. 155 Delore, Poncet, Soc. med. de Lyon, 1896; Annal. de dermat. et syph., I898, 238. Vallas, Provence med., I 899. Mouchet, Gaz. Hebdom., I900. Robin, Mem. soc. de. Biol., 1854. Lotzbeck, cit. by Recklinghausen. Czerny, L. A., I874, 17, 357. 157 Pomorski, V. A., III. 158 Adrian, Cent. f. Grenz., 1903. Simon, Hoche, C. R. Soc. Biol., I905, II, 487. Suzuki, B. W., I910, 1623. Hoffmann, These, Geneve, 1910. Kawashima, V. A., 203. Vignolo-Lutati, Mon. f. Derm., 52, 126. Harbitz, Arch. Int. Med., I909, 3, 32. Leisrink, D. Klinik, I869. 159 Naegeli, cit. by Recklinghausen. Morgan, London P. S., I 875, 26, 2. P. Bruns, V. A., I870, 50, 80. V. Mott, Med. Chir. Trans. I 854,37 , 155 . Wernher, Z. C., 5. Carter, London P. S., I862, I3, I4. Gerhardt, Jahrb. d. Kinderheilk., I87I, 4. Barensprung, Charite Annalen, I863, II. Unna, Histopath. d. Hautkrankh., I894, II63. Simon, U. Nervennevi. A. D., I872, o. Philipson, Monatsh. f. Derm., I890, II. Pott. Jahrb. f. Knderheilk., N. F., 28. Schönberg, D. W., I895, $35^{\circ}$ (Lit.). Haegele, I. D., Wurzburg, I 886 (Lit.). Beigel, V. A., I 869, 47, 367. Neumann, Oester, Jahrb. f. Ped., I 877, 2. 160 Elliot, J. Cut. Dis., I893. Marie, Revue Neurol., I900, 919. Chauffard, Soc. d. Hop., I 896, I I I9. Bernard, Soc. med. de. Lyon, I897, Gaz. d. Hop., I896, 203. S. Brickner, Amer. J. Obst., 53, I91. Gumbel, V. A., I7I. Wilms, B. B., 23. Goldmann, B. B., $3 I$ (Lit.). Ribbert, Diss. Zurich, I899 (Lit.). 162 Porter, Ann. Surg., 50. Wilson, cit. from Waldeyer. Smith, Arch. d. Derm., 1870. Poensgen, V. A., I02, 2. Korach, D. A., I 882, 32. Kobner, A. D., 1888, 393. Ehrmann, Diss. Heidelberg, I 889. Chambard, Arch. de. Physiol., 1879, 690 (Lit.). Johnston, J. Cut. D., I900, I8, 387. Torok, Annal. de. Derm., I893, I 109, I261. Virchow, V. A., I871, 52, 504. 163 Stoerk, Sitz. d. Kais. Acad. Wien., 11 5, 96, Abt. 3, 31. Pinkus, Pick, D. W., 1908, 1426. Touton, Viertelj. f. Derm. u. Syph., I 885. Hallopeau, Annal. de. D., I903, 4, 595. Waldeyer, V. A., I $87 \mathrm{I}, 52,308$. Hebra, Atlas d. Hautk. Geyer, A. D., II. Knauss, I. D., Wurzburg, I888. Pollitzer, J. Cut. Dis., I897, 15, 367. Blauel, B. B., 37. 164 Ledderhose, Deutsche Chir., Lief, 52. Bodenstein, M. W., I892, 4. Kramer, L. A., 52, 34 (Lit.). Greig, Edin. M. J., I915, I5. Bouffleur, A. S., 30, 587. Genewein, Z. f. Heilk., I905, 26. 165 Tillmann, Hygeia, I891, 277. Claus, Deut. Ges. f. Chir., I 885. Wilks, London P. S., I890, 20, 224. Busse, V. A., 157, 346 (Lit.). Reinach. Jahrb. f. Kinderheilk., 58 (Lit.). Hess, Z. B., Suppl., 7. Lartigau, Larkin, J. Med. Res., I90I, 6, 25. Langhans, Deutsche Chir., I 887, 50, 4I4. Ewing, S. G. O., I9II, 320. 
Chevassu, Tumeurs. d. Test., Paris, 1906. 166 Adler, C. G., 1906. Brothers, Amer. J. Obst., 4I, 50. Rokitansky, Allg. Wien. med. Z., I 859 . Bosworth, The Nose and Throat, N. Y., I892. Heymann, Handb. d. Rhin. Wien., I 899. Zarniko, Krankh. d. Nase., Berlin, I9Io. 167 Kalischer, Arch. f. Laryng., 1895, 2. Stork, Nothnagel's Handb., I895, I3, I. Zaufal, Prag. Woch., 1893. Zarniko, V. A., I892, 128. Herxheimer, V. A., I74. Syme, Jour. of Laryng. Rhin., I916, 3I, 515. 1. Moore, Proc. Royal Soc., 1917, No. 5, Lar., 71. 168 Bensch, I. D., Breslau, 1878; Voltolini, Die Krankh. d.Nase, I888. Ballo, Zeit. f. Ohrenheilk., 1908, 55, 3 10. Grünwald, M. W., I 890, 353. König, Lehrb. d. Spec. Chir., I898. Bruns, B. B., I894, II, 568. Naab, B. B., $1898,22$.

\section{MYXOMA}

172 Malherbe, Rech. s. 1. sarcome, 1904, I90. 173 Barling, Ann. Surg., 26, 504. Wilms, cit. by Virchow. Sattler, Fetschr. f. Billroth, 1 892. Parsons, Text-book of Ophthal. Salzmann, Arch. f. Oph., 39. 174 A. Knapp, Arch. f. Oph., 1915. Martino, Z. C., 83. Bloodgood, A. S., 72, 712. 175 E. Kaufmann, V. A., I2I, 513. Brenner, Frankf. Z. P., 1907, 492 (Lit.). 176 Czapek, Prag. m. W., I 891. Marchand, B. W., I894. Thorel, Ergeb. Path., 1907. Jacobsthal, V. A., 159. Oppenheimer, N. Y. P. S., 1912, 214. Curtis, Arch. de. Physiol., I871. Binder, Frankf. Z. P., 1914, 15, I9I4 (Lit.). Winogradow, C. P., 9. Robertson, J. Med. Res., 35. 177 Sanarelli, C. B., 23, 865. Splendore, 48,300 .

\section{LIPOMA}

180 Müller, V. A., 145 (Lit.). Beriel, Delachanal, Arch. d. med. exper., 24. Dreschfeld, cit. by Adami. Murchison, Edin. M. J., 1857, 1091. Voeckler, Z. C., 99, I 49. Broca, quoted by Warthin, Ref. Handb. Med. Sci. Wells, Arch. Int. Med., IO, 297. 181 Blaschko, V. A., I24, I75. Meerbeck, I. D. Wurzburg, 1887. Petren, V. A., I47, 560. Payr, Wien. kl. W., I 895, 733, 756, 776. Alsterg, Diss., Berlin, I 892 . Goebel, C. P., I895, 6, 4 (Lit.). Virchow, V. A., II, 28I. Askanazy, V. A., 158, 407 (Lit.). Curling, Med. chir. Trans., 33. Madelung, L. A., 37, I06. Kottnitz, Z. C., 38, 75. Kolliker, Toldt, Anat. Anzeig., 1888. Volcker, B. B., 21. Merkel, Z. B., 29. Sazarin, These, Paris, I 895 (Lit.). Ehrendorfer, L. A., 27, 352. Porges, Wien. kl. W., I 896, 649. Selter, V. A., I34. Lubarsch., V. A., I35. Manasse, V. A., 143. Bostroem, C. P., 8. 182 Grosch, Z. C., 26, 307. Shattock, Proc. Roy. Soc., I909; Path. Sec., 225. Dercum, Amer. J. M. S., I04, 52 I. König, Lehrb. d. chir. Filter, I. D. Greifswald, I890. Bruns, Pract. Chir. Abt. II, B. A. I, I46, I I34. Ransohoff, A. S., 65. Gant, Lancet, I856, 2. 620. 178 Wallmann, V. A., I4, 385. Hackel, V. A., I6, 272. Taubner, V. A., IIO, 95. Bernhard, Hirngeschw., Berlin, 1881. P. Ernst, Z. B., Suppl., 7. Würth, A. P., 36, Benjamin, V. A., I4, 552. Chiari, Wien. m. W., 1879, 515. Shouppe, Arch. de. Physiol., I873, 209. Coats, B. M. J., I $874, I I, 75.183$ v. Sury, Frankf. Z. P., I, 484 (Lit.). Nippe, Ibid., II, 466. Weil, Prag. m. W., I 890. 184 Lacrampe-Loustan, These, Paris. Rayer, Traitè d. mal. d. reins, Paris, I84I. Epstein, Ziemssen's Handb., I 875, 9, 88. Warthin, J. P. B., I 897, 404. Lauwers, Annal. soc. Belg. de. chir., I895, 313. Windle, J. Anat. and Physiol., I 884, I8, I50. Dewis, Boston M.S. J., 1906, 155, 427. Ehrlich, B. B., 71 (Lit.). Adami, Montreal M. J., I 897, 25, 529. Rokitansky, Path. Anat., 1861, 3, 39. Czerny, Wien. med. W., I875, I66. Gussenbauer, I.. A., 43, 323. Cruveilhier, Traitè d' anat. path., 1856, 3, 3 ro. Conner, N. Y. Path. Soc., I $897,43$. Ewing, Assoc. Amer. Phys., 1905, 20, 66. Fitz, Assoc. Amer. Phys., 1905, 20, 57 (Lit.). Carless, Proc. Roy. Soc., 1908, I, Clin. S., 233. Wehrsig, C. P., 1910, 21, 243. Orth, Lehrb. d. Path. Dittrich, C. P., 20, 108r. Spalty, Diss. Zurich, 1901 (Lit.). Hagedorn, C. P., 19, 825. Merkel, Z. B., 29, 507. Gebhardt, Z. f. G. G., 1902. Pollack, Wien. kl. W., 1903, 68. 


\section{CHONDROMA}

185 Weber, Die Knochengeschwulste, Bonn, I856; V. A., 35. Recklinghausen, D. P. G., I (Lit.). Mischaikoff, Diss., Zurich, I888. v. Recklinghausen, V. A., II8, 4. 186 Siegert, V. A., I29, 419. 187 Ranvier, Soc. d'Anat., I865, 40. 188 Foerster, Wien. med. W., 8. Biesiadecki, Sitz. Wien. Akad. Wissen, 57. 189 Ernst, Z. B., 28. 190 Nasse, Volkmann's Vort., I895, N. F., I24 (Lit.). Hagen, L. A., 4I, 420. Honeij, Arch. Int. Med., I920, 25, 584. Carman, Fisher, A. S., 61. Ashhurst, A. S., 63. Lenormant, Revue d'Orthop., I6, I93. Deganello, V. A., 168 (Lit.). Pfeiffer, Dis. Erlangen, I890. Clark, Atwood, N. Y. M. J., I907, 86, 97. Bidder, V. A., 120, 194. Recklinghausen, D. W., I892, 339. 191 Zahn, V. A., I15. Deichert, V. A., I4I. Spisharny, D. W., I 892, 853. Boeckel, cit. by Spisharny. 192 Kramer, V. A., 156, I 88 . Williams, Diseases of Breast, London, 1894. Williams, Uterine Tumors, London, I90I. 193 R. Meyer, V. A., 167, 8I. Ewing, S. G. O., I91 I, 230 (Lit.). Paget, Med. chir. Trans., 1 855, 38, 247. 'Ohkubo, Arch. f. Entwick, 1908, 26, 509 (Lit.). Kolliker, Gewebelehre, I889, I, 301, 317. H. Müller, Zeit. f. rat. Med., I 858, 202. Ribbert, Geschwulstlehre, 149; C. P., 5, 457 (Lit.). 194 Grahl, I. D. Gottingen, 1903, cit. by Fischer. Klebs, Allg. Path., I889, 2, 693. Hassner, V. A., 210, 385. Nebelthau, I. D. Marburg, I897. Fischer, Z. B., 40, 109. 195 Linck, Z. B., 46, 573. Feldmann, Z. B., 48. C. Vecchi, L. A., 99, 575. Albert, S. G. O., 21, 766.

\section{OSTEOMA}

197 Sancerotte, cit. by Virchow, 1. c., II, 24. Heymann, V. A., 104, 145. Reinecke, B. B., I891, 7. 198 E. Müller, Z. B., 57, 232 (Lit.). Hartmann, L. A., 35 (Lit.). Mischaikoff, I. D., Zurich, 1894. Heymann, V. A., I16, 329 (Lit.). Kammerer, Annal. Surg., 10. Nakel, Z. C., 33, 308. Zimmerman, Z. C., 57 (Lit.). 199 La Grange, Tumeurs de l'Oeil, 2, 346. Panas, Mal. des Yeux, 1894. Lesser, Allg. Chir., 1905, 2, 288. A. Knapp, Arch. of Ophth., 25, 353. 200 Bornhaupt, L. A., 26, 589 (Lit.). Tillmanns, Ibid, 32, 677. 201 Busse, Z. C., 1904, 73. Berndt, L. A., 1906, 79. Rapke, L. A., I907, 82. Luschka, V. A., 10, 500. Wagner, Arch. f. p. Heilk., I 859. Heschl, Zeit. f. p. Heilk., 8. Forster, V. A., 13, 105. Bostrom, Sitz. Phys-med. Soc. Erlangen, I875, I 58 . Picchini, Giorn. intern. d. Sci. med. Napoli, I885. Triboulet, Bull. soc. anatom., Paris, 1892, 340. Arnsperger, Z. B., 21 (Lit.). Port, cit. by Virchow, Geschwulste, II, 102. Cohn, V. A., IOI, I56. Rullier, Arch. gen., I824. V. Browning, London Path. Soc., I861, 13, 25. Le Diberder, L'Union med., 1867, 57. 202 Krauss, cit. by Arnsperger. Nusser, Zeit. f. p. Heilk., 1855. Gross, Amer. J. Med. Sci., 1879. Lenhossek, V. A., 60, r. Konig, Lehrb. d. Chir., 2, 551. Zanda, Z. B., 5 (Lit.). Meschede, V. A., 55. Ebstein, V. A., 49. Bidder, V. A., 88. De Vecchi, Lo Sperimentale, 37. C. Cohn, V. A., 106. O. Klotz, Arteriosclerosis, I9II. Lexer, L. A., 50. Schultz, Schuler, B. B., 33. Munchmayer, Z. nat. Med., 1869, 24, 9. Elliott, J. A. M. A., I9I I, 57, 873 (Lit.). De Witt, Amer. J. M. S., I20, 295 (Lit.). Mays, V. A., 74. Kaufmann, Lehrb. path. Anat., I909, Ir91. Kroemer, Veit's Handb. Gyn., 1908, 4.

\section{MYOMA}

205 Reich, Arb. path. Instit. Gottingen, 1893. Gottschalk, A. G., 43. 206 Krische, I. D. Gottingen, 1869. Langerhans, Berl. W., 1893, 338. Beetson, Festschr. f. Orth, 1903. Schlagenhaufer, Wien. kl. W., I902, 523. Ulesko-Stroganow, Mon. f. Geb., I8, 357. Evans, S. G. O., 1920, 30, 225. Hannsemann, Naturf. Vers., 95. Moser, D. W., 1903, 133. Eising, J. P. B., 1903, 233. Borrmann, M. G., 6. Williams, Zeit. f. Heilk., 1894, 15. Basso, M. G. G., 28. Hansemann, Diag. bos. Geschwulste. Evelt, M. W., I903, I414. 207 Winter, Z. G. G., 57. Hofmeier, Z. G. G., 5, 96. Flatau, Munch. W., I90I, 558. Cullen, J. A. M. A., I903, 4I, I013. Kelly, Cullen, Myomata of Uterus, 
Phila., 1909. Ricker, V. A., 142. v. Franque, Z. G. G., 40. Hansen, Nord. med. Arch., 1903. 208 Cruveilhier, Anat. path. I, II, 5. Stone, Amer. J. Obst., I899, 519. Fehling, Lehrb. d. Frauenk. Cullen, J. H. B., I896, I898; Fest, Orth., 1903 (Lit.). Aschoff, M. G. G., 9, 25. Turner, Edin. M. J., I861, 698. Simpson, Dis. of Women., Edin., 1872. Leyden, Z. G. G., 26, 434. Kustner, Ibid., 33, 338. Kelly, Operative Gyn. Hunter, cit. by Gebhard. 209 Severanu, cit. by Williams, 1. c., 67. Martin, C. G., 1888, 90. Tillaux, Gaz. des Hop., 1867. Bertelsmann, A. G., 50, 178. 210 Lennander, C. G., I 895, I59. Wyder, A. G., 13, 35. Fabricius, A. G., 50. Bulius, Z. G., 23, 358. Kuster, Beitr. z. Geb. u. Gyn., 1, 7. Kotschau, C. G., 1887, 757. Hedren, A. G., 83. Martin, A. G., 32, 470. Menge, C. G., 1895, 453. 211 Duncan, C. G., 1894, 222. Gusserow, Deutsche Chir., I885. Stratz, Z. G. G., I7. Henocque, Arch. de Phys., 1873. Everett, Amer. J. Obstet., 12. Payr, Z. C., 81. Wylie, N. Y. J. Obstet and Gyn., I 894. Liebmann, V. A., II7. Schafer, V. A., I20. Rolly, V. A., I50, 555 (Lit.). Gebhard, Veit's Handb., I 897, 2, 409. Rosger, Z. G. G., 18. Hertz, V. A., 36, 235. Freund, Beitr. z. G. u. G., 3, I50. Bennet, cit. by Williams, 1. c. Kworostansky, Z. B., 32. Amos, Z. G. G., 54. 212 Klob, Path. anat. d. weib. Sexualorgane, I864. Bayle, Dict. d. sci. med., I, VIII. Tillaux, Ann. de. Gyn., 26. Williams, Uterine Tumors, 1901. 214 Kleinwachter, Z. G. G., 9, 68. Landau, Berl. W., I901, 205. Pick, A. G., 60; M. G. G., 1897. Meyer, Z. G. G., 37, 38, 49. Pick, A. G., 52, 54, 60; V. A., 156. Schroeder, Handb. Krank. weib. Geschl., I881. Ruge, Z. G. G., I7. v. Franque, C. G., 1900, 660. Babes, Wien. allg. med. Z., I882. 215 Recklinghausen, Die Adenomyome, d. Ut., I896. Schroeder-Hofmeier, Handb. Krank. w. Geschl., 1892. Pick, A. G., 54 (Lit.). Schickele, C. P., 1904. Ernst, A. G., 85 (Lit.). Meyer, Z. G. G., 49 (Lit.). Grossmann, A. G., 54. v. Franque, Z. G. G., 47. Lockstadt, M. G. G., 7 (Lit.). Cohen, V. A., 158. Aschoff, M. G. G., 1899. Borst, Geschwulste, I, 217. Klein, V. A., 154 (Lit.). Breus, Cystenbild. in Uterusmyomen, Wien, I 894. Herpf, Deut. gyn. Gesell., 1897. Pfannenstiel, Deut. gyn. Gesell., I897. R. Meyer, A. G., 54. 216 Pichler, Prag. W., 1897. Illig, I. D. Giessen, 1894. Miodowski, V. A., I73. Laboulbene, Traitè anat. path., 1879, 120. Nazzari, Gas. med. ital., 1902. Steiner, B. B., 22 (Lit.). Mercer, N. Y. Med. Rec., 36, 67. Trappe, Frankf. z. P., I, I09. Boetticher, V. A., I04. Lode, Wien. kl. W., I894, 381. Babes, Ziemsenn's Bandb., I4, 499. Besnier, Annal. de. Dermat., I 885. Forster, Wien. med. W., I858. Challard, Bull. Soc. Anat., I87 I. Axel, Key, V. H. Jahresb., I877, I, 27I. Czerny, L. A., 17. Chambard, Gouilloud, Annal de Dermat., 1883. S. Pollitzer, J. Cut. Dis., I894, 15, 367. Hess, V. A., I20. Marc, V. A., I25, 543 (Lit.). Judassohn, V. A., I21. Walters, A. D., 25. Sobotka, A. D., 89, 323 (Lit.). Stein, Tumors of Bladder, N. Y., I881. Terrier, Hartmann, Rev. de Chir., 1895 (Lit.). Volkmann, L. A., 18. Buttner, Z. G. G., 28. 217 Becker, V. A., I63. H. Müller, V. A., I45 (Lit.). Abramow, C. P., I2. Jacobaeus, Nord. m. Arch., 1903, 3. Larkin, J. Med. Res., 6.

\section{RHABDOMYOMA}

Wolfensberger, Z. B., 15. Fujinami, V. A., I60, 203. Billroth, V. A., 9. Zenker, V. A., 120. Ribbert, V. A., I06, I30. Eberth, V. A., 55. Benenati, V. A., I7I, 418 (Lit.). 218 Marchand, V. A., Ioo. Kaschewarowa, V. A., 54, 63. Arnold, Z. B., 8. Girode, C. R. Soc. Biol., I892. Nehrkorn, V. A., I5 I. 219 Himwich, Jour. Cancer Research, 1920. Hauser, V. A., 88. 220 Cattani, Arch. sci. med., 19. Vincenzi, Riv. clin., Bologna, I887. Stumpf, Z. B., 50. Pfannenstiel, V. A., I27 (Lit.). Gow, St. Bart's Hosp. Rep., 27, 97. Pick, A. G., 46 (Lit.). Demme, Ber. Jenner Kinderhosp., 19. Franque, M. W., 1898, I301. Kalustow, A. G., 40. Rosthorn, Wien. kl. W., I889. Kolisko, Wien. kl. W., I 889. Rein, A. G., 15. Pernici, V. A., I13. Ribbert, V. A., I30, 249. 221 Neumann, V. A., I03, 497. Wood, N. Y. Path. Soc., 1902. Ewing, S. G. O., 12, 230 (Lit.). Stoerk, Z. f. Heilk., 22. Ponfick, D. P. G., 1901, 4, 226. Cesaris-Demel, Arch. sci. med., I9, I39. 222 Wolbach, J. Med. Res., I6, 495 (Lit.). Seiffert, Z. B., 
27. Kolisko, Wien. m. Jahrb., I 887. Knox, Shorer, Arch. Ped., N. Y., 1906. Bonome, Atti Instit. Veneto d. sci., 63. Glinski, V. A., 167. Brodowski, V. A., 67. Pende, Z. f. Heilk., 18. Prudden, Am. J. M. S., 85. Billroth, V. A., 18. Kaufmann, Med. Gesell., Basel, 1902. Helbing, C. P., 9. Buhl, Z. f. Biol., I, 263. Erdmann, V. A., 43. Kuttner, Deutsche Chir., I9I3, I, 25a. H. R. Müller, J. Cancer Res., I917.

\section{ANGIOMA}

225 Seifert, A. f. D., 59, 197 (Lit.). Fox, N. Y. Derm. Soc., I 895 . Jackson, J Cut. Dis., I 895. Unna, Histopath. d. Hautk., I 194. Muscatello, V. A., 135 (Lit.). Pupovac, L. A., 54, 555. 226 Sutter, Z. C., 76, 368 (Lit.). Nauwerck, V. A., III, 21 I. Lowenthal, L. A., 49. Borrmann, Z. B., 40, 372. 227 Gascoyen, London Path. Soc., I860, II, 267. Cruveilhier, Atlas d' Anat. Path., LXXX, pl. 5. 228 Falkowski, Z. B., 57, 385. Hildebrand, Z. C., 30 (Lit.). Esmarch, V. A., 6, 34. Schuh, Wien. med. Woch., I861, 763. Shennan, J. Path. and Bact., I9 (Lit.). 230 Recklinghausen, V. A., II8, 4. Albrecht, D. P. G., I904, 7, I53. 231 Reiche, Rust's Mag., I836, 46. Wegner, L. A., 20, 641. Delbau, Union med., I857, 478. Morton, Amer. J. M. S., I865, 378. di Ricci, Dublin Quart. Jour., I 865, 338. Quackenboss, Annal. Ophth. (St. Louis), 1908, 17. 232 Schoene, Z. B., Sup., 7, 1905 (Lit.). Gerhardt, Charite Annal., 20. Muthmann, V. A., I72, 324 (Lit.). Veeder, Austin, Amer. J. M. S., I43, I02. Image, Hake, Med. Chir. Trans., I847, 30, 109. Dowd, Ann. Surg., 62. H. Albrecht, Z. f. Heilk., 1902. Ribbert, V. A., I5I. Schmieden, V. A., I6I. Orth, Spec. Path. Anat., Bd. II, 570. La Villette, These, Paris, I906. Albert, Arch. f. Gyn., 56. Williams, S. G. O., 32, 523. 233 Luschka, V. A., 6, 458. Lafora, N. Y. M. J., 1912, 66 , 1007. Enders, M. m. W., I908, I646. Wergman, I.ancet, I914, I, I746. Orbison, J. A. M. A., I915, 64, I575. Deetz, V. A., I68, 34I (Lit.). Schuck, I. D., Berlin, I885. Heine, Prag. Vierteljahrs., r869. Lableé, Gaz. d. Hop., I872. Kretschmann, I. D., Halle, r881. Emanuel, D. Z. f. Nervenheil., I4. Ritschl, B. B., 15. Langhans, V. A., 75. 234 Wegner, B. B., I 894. 235 Nasse, L. A., 44, 233. Sutter, Z. C., 77, 368. Novack, L. A., 86, 873 (Lit.). Kuttner, B. B., 18. Ribbert, V. A., I5I. 236 Haug, cit. by Borst. T. and C. Fox, London P. S., 1879, 30, 470. Hutchinson, Ibid., I880, 31, 342. Noyes, Torok, Brit. J. Derm., I89r. Freudweiler, A. D., 4I. Waelsch, Arch. D., 5I. Kaposi, Hebra, Hautkrankh., 3, 387. Beneke, V. A., I23 (Lit.). 237 Crocker, Dis. of Skin, I893, 654 (Lit.). Perry, Brit. J. Derm., I892. Kromayer, V. A., 139, 282. Steudener, V. A., 59, 413. Bull. Amer. J. Ophth., I878. Wintersteiner, A. f. Ophth., 45 (Lit.). Sachs, Z. B., 5, I0I (Lit.). 238 Zeyneck, Z. phys. Chem., 20. Suckstorff, B. B., 27 (Lit.). Krauss, V. A., 125. Schmidt, D. P. G., I898. Ritter, Zeit. f. Heilk., I900, 21. Blatteis, N. Y. Path. Soc., 1910, 10, 102. Thalheimer, Ibid., 1913, 13, 5. Michel, Graefe-Saemisch, 4. Bryck, L. A., 24, 273. Klein, A. G., 46. Nasse, L. A., 38, 614. Otto, cit. by Borst. Frobenius, Z. B., 6. Takano, Z. B., 53 (Lit.). Sick, V. A., 170, 9. Smoler, B. B., 32. Schwarzenburg, B. B., II. Lion, V. A., 167. Hedinger, V. A., I64. Ranvier, C. R. Acad. Sci., 1896, 122, 578. Reimers, L. A., 23, 632. Tilger, V. A., 139. 239 Maresch, Z. f. Heilk., 24. Kroemer, Veit's Handb., 1908, 4.

\section{SARCOMA}

240 Malherbe, Rech. s. 1. sarcome., Paris, I904. 243 Wakabayashi, V. A., 205, 54. Howard, Fetsch. f. R. Hertwig. Virchow, Geschwulste, 2, 214.244 Favre, Regaud, C. R. Soc. Biol., 74, 608, 688. Brault, Arch. gen. de. Med., I899. 245 W. C. White, J. H. Bull., I900, 209. Seelig, S. G. O., 4, 319. 246 Ricker, L. A., 50, 573. Müller, I. D., Zurich, I894. Ewing, Jour. Med. Res., 1913, 28, I. v. Hansemann, Diag. bos Geschw. 247 Berndt, L. A., 65. Kolisko, Wien. kl. Woch., 1906, 1429. Heukelom, V. A., 107. Ackermann, Volkmann's Vort., 233, 234. 


\section{SPINDLE-CELL SARCOMA; FIBROBLASTIC SARCOMA}

249 Walter, I. D., Wurzburg, 1896. 250 Goldmann, B. B., I8. Hedinger, V. A., 164. Heukelom, V. A., I07. Lubarsch, Erg. Path., 1894. Stroebe, Z. B., 11. 251 Babes, Cent. m. Wis., I883. Ackermann, Volk. Vort., 233. Paltauf, Mracek's Handb. Boeck, A. D., 73. Spiegler, A. D., 27. Joseph, A. D., 46. 252 Pollard, A. D., 104. Fano, A. D., 73. Kaposi, A. D., 1872. Mariani, A. D., 98. De Amicis, Mon. D., 25. Phillipsohn, V. A., 167. 253 W. Pick, A. D., 87. Justus, A. D., 99, 446. 254 Linser, B. B., 26. Moldovan, Prag. W., 1905, 403. Perret, London P. S., 1902, 360. Johnston, J. Cut. D., I901, 1903. 256 Lowenstein, B. B., 48. Gross, Soc. de Chir., 1878, 284. Paquet, Ibid., 705. Reverdin, Rev. Med. Suisse. Rom., I885, 67r. Heurteaux, Arch. Gen., I 891, 40. Spiess, Frankf. Z. P., I3, I. 258 Fleissig, Z. C., I22. Chambe, Thèse, Paris, 1895. Pasteau, Soc. Anat., 1895. Bodenstein, M. W., 1892, 4. Sokalow, V. A., 57, 321. Guitton, Thèse, Paris, 1894. Peters, Med. News, 42, 279. W. Schaffer, I. D., Heidelberg, I 887. Hacker, Mitt. Grenzg., I9 (Lit.). 259 Howard, J. A. M. A., 38, 392 (Lit.). Herxheimer, Z. B., 1908, 44, 150. Stephan, Jahrb. Kinderh., 30, 354. Euchen, Z. C., 65. Shaw, London P. S., 42. Stark, V. A., I62. Rolleston, London P. S., 44. Targett, Ibid., 40. Ogle, Ibid., 47. Livingood, J. H. Bull., I898. Norris, N. Y. Path. S., I9I I. Wolfensberger, Z. B., I5. Glinsky, V. A., I67. Lippmann, Z. K., 3. Baur, I. D., Tubingen, 1905. Ziesche, Davidsohn, Mitt. Grenzg., 20. Brodowski, V. A., 67. Cantwell, An. Surg., 1899. Baldy, J. A. M. A., 30, 523. Manges, Med. News, 87. Schlesinger, Z. kl. M., 32, Suppl. Ewald, Klin. Verdauungsk., I 893. Hosch, Z. C., 90. Moser, D. A., 1903, 133. Steudel, B. B., 23. Alsleben, V. A., 173, 137. Finlay'son, B. M. J., I 899, 2, I 535. 260 Dreyer, I. D., Gottingen, I894. Leube, Spec. Diag. Cammidge, B. M. J., I90 I, I, I316. Wilson, Ibid. Kehr., L. A., 58. Baltzer, L. A., 44, 717. Rademacher, I. D., Jena, 1908. Key, Nord. med. Arch., 1905. Lehmann, I. D., Wurzburg, I888. C. Stern, B. W., I894, 802. Sandner, I. D., Erlangen, I904. Treves, Intestinal Obstruction, I899, 268. Carwardine, B. M. J., 1907, 2, 1771. Warren, Boston M. S. J., 138, I77. 262 Kroemer, Veit's Handb. Gyn., 1908, 4. Basso, A. G., 74. Gangolphe, La Gyn., 1898. Pinto, La ginecol. Riv. Firenze, 1904, 368. Walker, J. Obs. Brit. Empr., 1903. Segalowitz, I. D., Konigsberg, 1903. Glockner, A. G., 72. Krukenberg, A. G., 50. Reis, I. D., Berlin, I882, cit. Kroemer. W. Gibb, Glasgow M. J., 1903, 60, 33. Donati, Arch. p. 1. sci. med., Torino, 28. 263 Kehrer, Hegar's Beitr., 4. Kiwisch, cit. by Kroemer. Buet, L. Union med., igoo. Jung, Z. G., 52. Callender, London P. Soc., 9. Gusserow, A. G., I. Terillon, Gaz. d. Hop., 63. Gessner, Handb. d. Gyn. (Veit), I Aufl. Piquard, Thèse, Paris, 1905 (Lit.). Meyer, Handb. d. Gyn. (Veit), 2 Aufl. (Lit.). v. Kahlden, Z. B., 14. Busse, D. W., 1904, 373. 264 Lubmann, I. D., Kiel, 1902. Moraller, Mon. G. G., I3. v. Franquè, C. G., 1893, Z. G. G., 40. Heinreich, I. D., Leipsig, 1903. 266 Mastny, Z. Heilk., 22, I90I. v. Winckel, A. G., 3. Amann, U. Neubild. d. Cervical portion d. Uterus, Munchen, 1892. Griffith, cit. by Meyer. Simpson, Edin. M. J., 1876. Bechman, Z. G. G., $4 I$. W. Williams, Z. Heilk., 15, I894. 267 C. Ruge, Winter's Handb., Leipsig, I907. Spiegelberg, A. G., I4. Wolgren, Mon. G. G., 10. Fellander, A. G., 83. Wilischamin, A. G., I4. Wagner, Verh. Ges. Naturf. u. Arzte., 1902. Gow, Trans. London Obstet. Soc., I 890. Herxheimer, Z. B., 44. H. Albrecht, Frankf. Z. P., 2. Niebergall, A. G., 50. 268 Schaller, D. W., I906, 959. Nebesky, A. G., 73. Gebhard, Path. Hist. d. weib. Sex. org., Leipsig, 1899. Ballin, I. D., Leipsig, 1903. Lindemann, Z. K., 6. Klein, M. W., I890, I70. Riederer, I. D., Zurich, I893.

\section{SARCOMAS OF BONE AND BONE-MARROW}

269 A. Cooper, Surg. Essays, London, 1818. Boyer, cit. by Poncett. Lebert, Phys. Path., I845. Paget, Surg. Path., I853. 270 Robin, C. R. Soc. Biol., I849. Gray, Med. Chir. Tran., I856, 21, I43. Nélaton, Tumeurs a myeloplaxes, Paris, I860. Gross, S. W., Amer. J. M.S., I 879, 78 (Lit.). Rindfleisch, Path. Hist. Wyss, V. A., 35. Lieg- 
ler, V. A., 73. Wegner, V. A., 56. Malassez, Monod, Arch. de Physiol., 1878. Fehr, L. A., I7. 272 Marullaz, Z. B., 40. Muir, Glasgow M. J., 1899, 438. 274 Gaylord, A. S., 37. Nakayama, B. B., 64. Bloodgood, A. S., 52. 275 Rydygier, Z. C., 82, 211. Garre, B. B., 7. Marsh, Lancet, I898, 2, I330. Weir, N. Y. M. J., I886. Dowd, A. S., 56. Moser, Z. C., 98 . Coley, A. S., 45, 52. Goebel, L. A., 87. Jackson, London P. S., I8, $215 . \quad$ Holmes, Syst. Surg., 2d ed., 3, 823. 276 Le Dentu, L'Union med., I 877. Zahn, Rev. med. Suisse rom., I 886, 580. Poncet, Traitè de Chir. (Duplay, Reclus), 1897, 2, 930. Le Count, J. H. Bull., 1909. Pitts, London P. S., I886, 374. Cocks, Wilks, Med. Times Gazette, I859. Kuster, L. A., 12, 630. Allin, Med. Record, I3, I 16. Meakin, London P. S., 36, 33. Hektoen, Med. News, 63, 571. West, London P. S., I8, 215. Jenckel, Z. C., 64. Feistmantel, Wien. m. Woch., 1904, 2014. McAuliffe, Chicago P. S., I9II, 8, 21 I. 277 Bristowe, London P. S., 7, 254. 280 Stimson, Med. Record, I877, I1, 524. 283 Mallory, J. Med. Research, 24. Malassez, Arch. de Physiol., 1878. 285 Kramer, L. A., 66, 792. Kocher, B. B., 50. Reinhardt, Z. C., 47. 286 Jeanbreau, Riche, Rev. de Chi., 32, 153. McCosh, A. S., 40. Nasse, L. A., 39. Butlin, Treat. of Malig. Dis., 1900. 287 Haberer, Z. f. Heilk., 1906, 27 (Lit.). Hinds, B. M. J., I898, I, 555. Recklinghausen, Fest. f. Virchow, I891. 292 Tietze, B. A., 52. Konig, D. Gesell. Chir., I906. Monckberg, D. P. G., 1904. Heincke, B. B., 40. Rehn, L. A., 97, 35. Haberer, L. A., 82, 873 (Lit.). Gaugele, L. A., 83, 953. Knaggs, Gruner. Royal Soc., 1908, I, P. S., 26. Kolisko, Wien. kl. W., 1906, I429. Martland, N. Y. P. S., 1915. McIntyre, Med. Chir. Trans., 1850. Marchand, B. W., I896, 486. Zahn, Z. C., 22. Buch, I. D., Halle, I873. 293 Rusticky, Z. C., 3. Kahler, Wien. Med. Presse, I889. Wright, J. Med. Res., I900, 4, 195. Hoffmann Z. B., 35 (Lit.). Winkler, V. A., I61. Abrikassoff, V. A., 173 (Lit.). Seegelken, D. A., I897, 58. Grawitz, V. A., 94. Saltykow, V. A., 173. Aschoff, M. W., 1906, 337. 294 Magnus Levy, Cong. inn. Med., I900. O. Williams et al., Lancet, I910, 2, 1403. 295 Weber, J. P. B., I898, 6. Scheele, Herxheimer, Z. M., 54. Ewald, Wien. kl. W., I897, I69. Hueter, Z. B., 49, IoI. Christian, J. Exper. Med., 1907, 325. Wieland, V. A., 166. 296 Norris, N. Y. P. S., 1906, 6, 128. Jochmann, Schumm, Z. kl. M., 46, 445. 297 Hirschfeld, Folia Hem., I9I0, 9, I. Marchwald, V. A., I4I. Sternberg, Z. Heilk., 1904, 25. Lubarsch, V. A., 184, 213. Charles, Sanguinetti, B. M. J., I907, I, 1916. Menne, V. A., 183 (Lit.). Herz, Wien. med. W., 1908, Nos. 23, 24. Kaufmann, Spec. path. Anat., 1909, 732. 298 Hammer, V. A., 137. Baumgarten, Arb. path. Instit. Tubingen, I894-99. Rubinstein, Z. M., 6r. Stokvis, Zeit. f. Biol., 1883. Ribbert, C. P., 1904, 337. Jellinek, V. A., I77. Vignard, Gallavardin, Rev. d. Chir., 1903.

\section{ENDOTHELIOMA}

299 Huxley, Anat. of Invert. Animals. Lancaster, Quart. J. Med. Sci., 1877, I7. Balfour, Ibid., I880, 20. Hertwigs, Die Coelomtheorie, Jena, I88I. His, Die erste Entwickel. d. Hunchens, I868. Ziegler, A. m. A., 32. Uskow, Mem. acad. d. Sci., St. Petersb., I 887, 35. 300 Ruckert, Biol. Centralbl., I888, 8. Councilman, Harvey Lectures, I906-7. Schmidt, Z. B., II. Remak, Canstatt's Jahresber., I84I. Stockard, Amer. J. Anat., 1915, 18. Graser, L. A., 50; Z. C., 27. Roloff, I. D., Tubingen, I894. 301 Lubarsch, V. A., 135. Marchand, Z. B., 4. 302 Ribbert, Z. B., 6. Renngli, I. D., Zurich, I 894. R. Meyer, I. D., Zurich, 1895. Golgi, V. A., 51, 3 II. Kaufmann, L. A., 26. Wartmann, Diss., Strassburg, I879. Billroth, L. A., II, 244. Tillmann, Arch. Heilk., I4, 530. Jaffe, L. A., I7, 91. 303 Pagenstecher, V. A., 45, 490. Knapp, Arch. f. A. u. O., 4, 209. Schweninger, Aertz. Intelligensbl., I876. Massey, Gaz. d. Hop., I867. Hubl, Wein. med. Woch., I879. Kaposi, Lehrb. d. Hautk., II, 282. Jarisch, A. D., 28, I63. Marchand, I. D., Halle, 1879. Leopold, A. G., 6. Wagner, Arch. f. Heilk., 1870, II, 509. Neelson, D. A., 31, I882. Amann, U. Neubild. d. Cervical Portion, 1897. Kirschgessner, Z. G. G., 49. Sperber, I. D., Leipsig, 1904. Hannsemann, D. W., I896, 52. Billroth, Unters. u. d. Entsteh. d. Blutgefasse, Berlin, I856. v. Ohlen, Z. B., I3, 450. 
304 Hildebrandt, Z. C., 3I, 263. Borrmann, V. A., 157, 297. Barth, Z. B., 19. Hippel, Z. B., I4. Malassez, Arch. de Physiol., 1883. Berget, Rev. de Chir., 1897. Collet, Thèse de Paris, 1895. Sternberg, C. P., I6. 305 Ackermann, Volkmann's Vort., 233, 234. Langhans, V. A., 75. Limacher, V. A., 151, Suppl. Perthes, B. B., 12, 589. Franke, V. A., I2I, 465. 306 Rosthorn, A G., 4I, 328. Best, Z. B., 23. Neelsen, D. A., 31. Schottelius, I. D., Wurzburg, I874. Schweninger, Annal. d. allg. Krankenh., Munchen, 1878. Lubarsch, V. A., III, I22. Schulz, Arch. Heilk., I7. Wichern, I. D., Tubingen, I902. Martland, N. Y. P. S., 1909, 123. Mulert, L. A., 54, 658. Weichselbaum, V. A., 85. Zahn, Z. C., 22, I. 308 Marchwald, V. A., I4I, 128. Braun, L. A., 43, 196. Nauwerck, V. A., III. Narath, L. A., 50. 310 Glockner, Z. B., 26 (Lit.). Brosch, V. A., 144. Rindfleisch, Harris, V. A., I03. Ritter, Z. C., 50, 349. Best, Z. B., 23 (Lit.). Lubarsch, Z. Lehre, v. d. Geschwulsten., I 899, 282. Schmidt, Z. B., 8. 313 Eberth, Spude, V. A., 153, 71. Schmidt, V. A., 170. 315 Anitschkow, Z. B., 57. Lutz, Z. B., 58. Schlagenhaufer, V. A., 187. Risel, Z. B., 46. Henschen, Z. B., 49. Eppinger, Z. B., 49. Hippel, Z. N., 2. Schlesinger, Ruckenmarkstumoren, Jena, 1898. Engert, V A., 160. 316 Lindner, Z. f. Heilk., 23. Albrecht, Munch. Woch., 1902, I135. 317 Ernst, Z. B., 11. Schlapp, Jour. Nerv. and Mental Dis., 191 I. 318 Neumann, Arch. f. Heilk., I872, 305. Bizzozero, Bozzolo, Wien. Jahrb., 1874, 284. Zenoni, Reale Ac. di med. Torino, 1898, 61. Lenz, Z. B., 19, 663. Dufour, Soc. Anat., 10, 1896. Taylor, A. J. M. S., 127. Lunz, D. W., 1902, 225. Tailor, Annal. di Ottalmol., 1894. Braunschweig, Graefe's Arch., 39. Fester, B. W., I 878, 97. Billroth, Clin. Surg., 1881. 319 Parsons, Pathology of Eye, II. Verhoef, J. Med. Res., 1905, 13. Spiller, J. A. M. A., 34, 1026. M. Frank, N. Y. M. Rec., 65, 50. Gutmann, D. A., 75, 337. Scagliosi, D. W., 1904, 1715. Benda, D. W., 1897, 324. 310 Eberth, V. A., 49, 60. Bassoe, Chicago P. S., 6. I. Adler, J. M. Res., I901, 6,1 75. Pollmann, Z. B., 26. Malassez, Arch. d. Phys., I 876, 351. Borst, 1. c., I, 320. Bostroem, I. D., Erlangen, I870. 320 Rossier, Z. B., 13. Perls, V. A., 56, 438, Neelsen, D. As., 3I, 375, old lit. Glockner, Z. f. Heilk., I8 (Lit.). Brosch, V. A., 144, 289. Fraenkel, B. W., I892, 497. Kaufmann, 1. c., 317. Wichern, I. D., Tubingen, 1902. Hibler, Jahrb. f. Heilk., 59. Waldeyer, V. A., I867, 4I, 470. Miller, Wynn, J. P. B., 1908, 12, 267. Korner, I. D., Wurzburg, 1901. 321 Nager, Z. B., 36. Henke, C. P., IO, II. 322 Hueter, Z. B., 4I. Monckberg, Erg. P., 1906, 789. Recklinghausen, V. A., 36, 465. Erbsloh, V. A., I63. Albrecht, L. A., 77. Grosheintz, Z. f. Urol., 1907. Gierke, V. A., 170. Pick, A. G., 64, 746. Stilling, Rev. de Med., I888, 859. Ritter, Z. G., 50. 323 Schlagenhaufer, D. P. G., 13, 222. Rustisky, Z. C., 1875. Kahler, Wien. med. Presse, I889. Wieland, I. D., Basel, I893. 325 Howard, Crile, Annals Surg., 42, 358. Coletti, Rif. med., 1904, No. 35. 329 Jacquet, Darier, Annal de. Derm., I887, 317. Fick, Monatsh. Derm., 48, 199 (Lit.). Stockman, A. D., 1908, 92, 145 (Lit.). Krompecher, Z. B., 28, I. Spiegler, A. D., 50, 163. Borrmann, Z. K., 2. Juliusberger, A. D., 89, 77. 330 Coenen, L. A., 76, 1 100. Haslund, A. D., 82, 247. 331 Recklinghausen, Fibrome d. Haut., Berlin, I882. Demieville, V. A., 81. Lowenbach, V. A., 157. Johnston, J. Cut. Gen. U. Dis., I905. Schutz, A. D., 63. Unna, Histopath. d. Hautk. Winkler, V. A., 178 (Lit.). Wise, A. J. M. Sci., 1919, 137, 236. Linser, B. B., 26. Volkmann, Z. C., 41. Maurer, I. D., Halle, I883. Alexander, Dunham, J. Cut. G. U. Dis., 1897. Hildebrandt, Z. C., 48, 209. Colmers, Z. B., 34. Borrmann, Erg. Path., 1900, 7. Creite, Z. C., 79. Leopold, A. G., 6. Marchand, Habil-Schrift., Halle, 1879. 332 Flaischlen, Z. G. G., 7. Olshausen, Deutsche Chir., I886, 58. Eickardt, Z. G. G., I6. Pomorski, Z. G. G., 18. Velits, Z. G. G., I8. Rosthorn, A. G., 4I. Pick, B. W., I894, I017. Krunkenberg, A. G., 50. Rosinski, Z. G. G., 35. Linck, I. D., Konigsberg, 1900. Krunkenberg, Z. G. G., 41. Schlagenhaufer, M. G. G., 15. Glockner, A. G., 75. Polano, Z. G. G., 5I. Papaioannou, M. G. G., 20. W. S. Stone, S. G. O., I9I6. R. Meyer, Z. G. G., 63. 333 Vogel, I. D., Munchen, I895. Ribbert, D. Carc. d. Mensch., I9II. Jamagiva, cit. by Ribbert. Monckberg, V. A., rgo. Franque, Z. G. G., 48. Gebhard, Z. G. G., 47. Apelt, Hegar's Beitr., 5. Kubo, A. G., 87. Pfannenstiel, Veit's Handb., 1908, IV, 1, 372. 334 Schurmann, Z. G. G., 50. Lange, C. G.. 1903. Monckberg, Erg. 
Path., I906, 801. Kworostansky, A. G., 85. Eymer, A. G., 88. Rosthorn, A. G., 4 I. Amann, A. G., 46. Pfannenstiel, Verh. d. Gesel. Gyn., 99. R. Meyer, Veit's Handb. III, I, 504 (Lit.). 335 Pick, A. G., 49. Hansen, V. A., 17I. Silberberg, A. G., 67. Braetz, A. G., 62. Pohorecky, A. G., 60. Kroemer, A. G., 65. Kirschgessner, A. G., 49. Hurdon, Johns H. Bull., I 898. Robb, Amer. J. Obstet., 38. Graefe, A. G., 72. Lyle, Ann. Surg., 54 (Lit.). Fick, Z. C., 48. Tilger, V. A., 132 (Lit.). Cignozzi, Rif. med., 1905, 17. Aldegarmann, I. D., Wurzburg, 1899. Szobelow, V. A., I6I. Krompecher, Z. B., 49. Chambard, Rec. mens. de med., I880, I0; Prag. med. W., I 889. Hoffmann, Schottelius, Mit. Path. Instit., Marburg, I88I, 23. Recklinghausen, Wien. kl. Woch., 1897, 350. 336 Parlavecchio, L. A., 86, 738 (Lit.). Ewing, J. Med. Res., I913, 28, I. 337 Zahn, Arch. f. Heilk., I874, 14, I43. Ravenna, Arch. med. Exper., 1905, 17, 325. 338 Gallina, V. A., I72. Da Gradi, de Amicis, V. A., 207, 323 (Lit.). 339 Saxer, Anat. Hefte, I 890, 6, 349. Gulland, J. P. B., I 894, 2, 447. 342 Waldeyer, V. A., 55. Paltauf, Z. B., II. Eberth, V. A., 49. Arnold, V. A., 38. Sertoli, V. A., 42. Luschka, J. de 1. Anat. et Phys., I 868. Kolaczek, Z. C., 9 , I3. Hanke, V. A., I48. Roussy, Sem. med., I9II, 3I, 385. 344 Eisenmenger, Z. C., 39. Arndt, V. A., 51, 72.345 Wells, Amer. J. M. S., 122. Kocher, V. A., 44. Lucke, V. A., 35. Howard, Crile, An. Surg., 42 (Lit.). 346 Schmidt, L. A., 36. Amann, A. G., 46. Paoli, Z. B., 8. Arnold, Z. B., 8. L'Esperance, J. Med. Res., I9I 5, 32, 225. Marchand, Intern. Beitr. wissen. Med., I891. Kohn, A. micr. A., 56, 62. Stilling, Revue de Med., I891. Monckberg, Z. B., 38. 347 Keen, Funke, J. A. M. A., 47, 469, Illus. Leithoff, I. D., Wurzburg, 1904. Reclus, Chevassu, Rev. de Chir., 28. Heinleth, C. P., 1900, 599. Kauffmann, Ruppaner, Z. C., 80 (Lit.). Kretschmar, I. D., Giessen, 1893. Kopfstein, Wien. kl. Runds., 1895. Licini, Z. C., 96. Oberndorfer, C. P., I905, 225. Reid, J. H. Bull., 1920.

\section{LYMPHOMA AND LYMPHOSARCOMA}

349 Flemming, A. m. Anat., I885. Gulland, J. Path. Bact., I 894, 2, 447. Ewing, J . Med. Res., I9I3, 28, I. 350 Le Count, J. Exper. Med., I 899, 4, 559 (Lit.). 351 Wagner, Hewitt, Chicago Path. Soc., I912, 8, 273. 352 Paltauf, Erg. d. Path., I896, I, 652. Bartels, Wien. kl. W., 1905, 881. 354 Schmorl, Munch. W., 1904, 537. Pappenheim, Hirschfeld, Fol. Hem., I908, 347. Meyer, Heinicke, D. A., 88. Walz, C. P., 12, 967. Kelly, U. Penna. Med. Bull., I903. Rosenfeld, Z. M., 42. 355 Oertel, J. Exper. Med., I 899, 4. Nekam, Mon. f. Derm., I 899, Erganzheft. Pincus, A. f. Derm., 50. Nicolau, Annal. d. Derm., I 904. Hazen, J. Cut. Dis., 29, 521. Arndt, J. A. M. A., 63, 1268. 356 Dominici, Arch. med. exper., 12, 733. Sternberg, Erg. Path., 1903, 9, 435. 359 Schwartz, Z. f. Heilk., 22, Path. Abt. Michaelis, Cong. in. Med., I901, 573. Ribbert, D. W., I907, 329. 360 Gulland, Goodall, The Blood, London, 1912. Leube, Munch. W., I900, I I21. 361 Nagaeli, D. W., I900, 287. Preis, Z. M., 57. Neuwerck, Moritz, D. A., 85. Weber, V. A., 174. Pappenheim, Z. M., 52. Seelig, D. A., 54, 537. v. d. Wey, D. A., 57, 287. Wilkinson, Lancet, 1903, I, I 739. Mellard, Lancet, I905, I, 497. Wolf, Michaelis, D. W., I90I, 65I. Turk, Wien. kl. Woch., I903, 1073. Hirschveld, Fol. Hem., I905, 2, 743. Wolff, Berl. kl. Therap. Woch., I904. Lehndorff, Zak. Fol. Hem., 1907. Banti, C. P., 1904. Askanazy, cit. by Sternberg. Fabian, C. P., 19. Heubner, Berl. W., 1904, 1128. 362 Moritz, Fol. Hem., 1907, 627. Warthin, Trans. A. A. Phys., 1904. Grawitz, V. A., 76. Lazarus, I. D., Berlin, 1890. Lucke, V. A., 35. Martin, Matthewson, Brit. M. J., 1896, 2, I634. Wolff, Z. M., 45. Babes, C. P., 13. King, Jour. Med. Sci., Edin., I853. Aran, Arch. gen., 1854, 4, 385. Lebert, Traitè d. Anat. Path., I857, I, 323. Recklinghausen, Tagebl. Naturforsch., Strassburg, 1885. Huber, Arch. d. Heilk., I878, 19, I29. Dock, Amer. J. M. S., I893, 106 (Lit.). Dock, Warthin, Tr. Assoc. Amer. Phys., I904, Med. News, 85, 1025. 363 Klein, Steinhaus, C. P., I904. Sternberg, Z. B., 37. Fabian, Z. B., 43 (Lit.). 364 Stevens, Glasgow M. J., 1903. Hitschmann, Wien. kl. Woch., I903, I470. Bramwell, B. M. J., I902, I, III, 453. Trevithick, Lancet, 1903, 2, 530. Risel, D. A., 72. Lang, Arch. gen. de Med., I893, 
4. Lubarsch, Erg. d. Path., I895, 585. Waldstein, V. A., 91. Weinberger, Wien. kl. Woch., 1903, 461. Horing, Arb. path. Instit., Tubingen, I. Schmidt, I. D., Gottingen, I 895. Hoffmann, Z. B., 35. Gluzinski, Reichenstein, Wien. kl. W., I905, 336. Schridde (Aschoff), Munch. W., 1906, 160. 366 Cohnheim, V. A., 33, 451. Pel, Berl. W., 1887, 644. Ebstein, D. A., 44, 389. Renvers, Deut. m. W., I 888, 753. Combemale, Rev. de Med., I892, 540. Delafield, N. Y. Med. Rec., I887, 3I, 425. Waetzoldt, C. inn. Med., 189o. Brentano, Tangl, D. W., r891, 588. 367 Sabrazes, Gaz. hebdom., Bordeaux, 1907. Sternberg, Z. Heilk., I 898, 19, 2 I. Christian, Tr. Assoc., Amer. Phys., 1907, 397. Warthin, Osler's System, IV, 829. H. Ziegler, Die Hodgk. Krank., Jena, I9I I (Lit.). 368 Rolleston, Brit. M. J., I909, 2, 852. Bramwell, Hodgkin's Disease, Clin. Studies, Edin., 1909, 7, I3I. Bunting, Yates, J. H. Bull., I91 I, 22, 368. Hippel, Munch. Woch., 1910, 364. Reed, J. Hopk. Rep., 1902. Fabian, Sammelreferat., C. P., I91 I. Benda, D. P. G., I904. Hirschfeld, Isaak, Med. Klinik, 1907, I580. Beitzke, D. P. G., Gesell, I909, 224. Symmers, Arch. Int. Med., I909, 4, 2 I 8 . Kummel, cit. by Ziegler. Donhauser, Bull. Ayer Clin. Lab., 1908. 369 Nowak, Berl. kl. W., I905, 704. Stoerk, Wien. k1. W., I904, 91. 370 Coupland, London Path. S., I878. La Roy, Arch. intern. de Chir., 1907. Palma, D. Woch., I892, 784. Lorrain, Bull. Soc. Anat., I905, 275. Schottelius, V. A., 185, 226. Jacquet, V. A., 185, 251. Brigidi, Piccoli, Z. B., I6, 388. Weber, Ledingam, D. A., 96. Coenen, L. A., 73, 443. Longcope, Bull. Ayer Clin. Lab., 1903. Gutig, Berl. W., I905, 1067. 371 Brun, B. B., 45. Fleischer, Monatsh. f. Augenheilk., Io, 289. Haeckel, L. A., 69, 191. Jacobaeus, Fol. hem., 10, 152. Grocz, Z. B., 39. Arndt, V. A., 209, 432. Jessup, N. Y. Path. Soc., I912, 3. 374 Yamasaki, Z. Heilk., 1904, 25, 269. Karsner, Arch. Int. Med., 6, I75. Welch, N. Y. Path. Soc., I910, 10, 161. Ewing, S. G. O., 1916. 375 Longcope, Bull. Ayer Clin. Lab., 1907, I8. Fraenkel, Much. Z. H., 67. O. Meyer, Frankf. Z. P., 8. 376 Arrigo, C. B., 28. Sticker, C. B., 55. Pick, D. W., 1909, 2033. Mosler, Ziemssen's Handb., I 875, 8, cit. Ardnt. Bunting, Yates, Arch. Int. Med., 1913, 12, 236. Torrey, Jour. Med. Res., 1916, 34, 65. Bloomfield, Arch. Int. Med., 1915, 16, 197. Cohnheim, V. A., 33, 451. Wunderlich, Arch. f. ph. Heilk., 1858, I23. 377 Baumgarten, Arb. a. d. path. Instit., Tubingen, I899. Pincus, Nothnagel's Spec. Path., VIII, Bd. II. Moritz, Fol. hem., 1907, 4, 627. Pappenheim, L. A., 7I. Runeberg, D. A., I 883, 33, 629. Litten, Berl. kl. W., I877, 748. 378 Rubinstein, Z. kl. M., 61. Senator, Z. kl. M., 54 (Lit.). Domarus, Munch. W., I909, I173. Nothnagel, Fests. f. Virchow, I901. Strauss, Rohnstein, D. Blutzus. bei Anemien, Berlin, I90I. Bloch, Hirschfeld, Berl. kl. Woch., I90I, I014. Hirschfeld, Fol. hem., I910, 9, 33. Briquet, Cruveilhier's Atlas, 1835, 2, 34. 379 Wells, Maver, Amer. J. Med. Sci., 128, 837. Glinski, V. A., I67. Flexner, J. H. H. Rep., I 893. Sternberg, Deut. path. Ges., I904, 8, 141. 380 Turk, Wien. kl. W., I899, 985. Klein, C. inn. Med., I903. 381 Kundrat, Wien. kl. W., I893. Paltauf, Erg. path. Anat., I 896, I, 652. Sadler, Fort. d. Med., I892, 38. Reinbach, L. A., 46, 486. Warthin, Tr. Assoc. Amer. Phys., 1904, 421. Birch-Hirschfeld, Ziemsen's Hb., N. Y., I877, 16, 837. Longcope, Tr. Amer. Assoc. Cancer Res., 1911. 382 Ruff, Wien. kl. W., I906, 531. Koschier, Wien. kl. W., 1910, 618. 383 Kanter, C. P., 5, 299. 384 Harbitz, C. P., 14, 759. Wieland, C. P., 13, 94. 385 Freudweiler, D. A., 64. Schlagenhaufer, V. A, I64. Martin, J. Med. Res., 4, 249. Goppert, V. A., I44, Suppl. (Lit.). Schridde, Handb. Pathol. (Aschoff). Chiari, Wien. kl. W., I895, 39. Eisenmenger, Wien. kl. W., I893, 936. 386 Haberer, Mitt. a. d. Grenzg., I6, 371. Pitt, London P. S., 40. Cayley, London P. S., I869, 20. Hammerschlag, Arch. f. Verd., I896, 2. Manges, Med. News, 87, 206. Thursfield, Lancet, 1900, 2, 1652. Kaufmann, Lehrb. spec. Path. Stanelin, Arch. Verdauk, I4. Howard, J. A. M. A., 1902, 38, 392. Maas, D. W. v. b., I 895,6 . 387 Ziesche, Davidsohn, Mitt. a. d. Grenzg., 20. Fleiner, Lehrb. d. Verdauungsk. Baltzer, L. A., 44. Key, Nord. Med. Arch., 1905. Glinski, V. A., I67 (Lit.). Libman, Am. J. M. S., 120. Jopson, White, Ibid., 122. Munk, B. B., 60. Sedziak, Monatschr. f. Ohrenh., I892. Romberg, D. W., I 892, 419. Kutzner, I. D., Greifswald, 1889. Arning, D. W., I 891, 1372. Packard, Univ. Penna. Med. Mag., I891, 387. Schleinzer, Z. C., IOO (Lit.). Fripp, Iwan, Guys. Hosp. Rep., 1902. Foote, Amer. 
J. M. S., 142; Tr. N. Y. Path. Soc., 1912, I2, 49. 388 Chevassu, Les. tum. d. Testicule. Malassez, Soc. anat. Paris, $1877,52,176$. Ehrendorfer, L. A., 27, 352. Debarnardi, Z. B., 40, 534. Ewing, S. G. O., I9I I, 12, 230. Martland, communicated. Turner, London P. S., 29. Dowd, An. Surg., 1912. Kusunoki, Frank, V. A., 212, 391. 389 Ricker, L. A., 50. Muller, I. D., Zurich, I894. Brandt, Munch. W., 1908, 735. 390 Freudweiler, D. A., 64. Weichselbaum, V. A., 85, 562. Jepson, Albert, An. Surg., 40. Foix, Roemmele, Arch. med. exp., 24 (Lit.). Heinricius, Cent. Chir., I898. Langhans, V. A., 75. Theile, V. A., I78. Jores, C. P., I9, 662. 391 Bunting, Univ. Pa. Bull., I6, 180. Hacker, D. Ges. Chir., 13, 30. Clarke, Brit. M. J., I883, I, 418. Grohe, V. A., I50, 324. Herczel, Orvosi Hetilap, I895, 59, 607. Menetrier, Traitè path. gen. (Brouardel). Simon, B. B., 35. Asch, A. G., 33. Janvrin, Amer. J. Obst., 4I, 85.

\section{TUMORS OF BRAIN AND NERVE TRUNKS}

393 Hirschi, D. W., 1912, 2243. Bruns, Geschw. d. Nervensystems, Berlin, 1908. Tooth, Proc. Roy. Soc., 1912, 6, Neurol., I. Seydel, Deut. Gesell. f. Chir., I892. Beck, B. B., I894, 12. Starr, Med. News, I889, 54, 29. Lowenthal, L. A., 49. Bailey, Accident and Injury, N. Y., 1905. 394 Hitzig, Berl. kl. W., I892, 713. Eppinger, Aerzt. Sachv. Zeit., 1909. Keen, Amer. J. M. S., I 888, 96,329 . Annandale, Edinburg M. J., 1894. Carara, Viertel. f. ger. med., 1896. Adler, Arch. f. Unfallheilk., 1897, 2, I89. P. Knapp, Rep. Boston City Hosp., 1889. Osler, Amer. J. M. S., I885, 89, 31. 395 Hanel, A. P., 3I. Kuttner, J. A. M. A., I914, 63, 1530. Henschen, Path. d. Gehirns, Upsala, I9II. v. Eiselsberg, Ranzi, L. A., 102, 309. Eichelberg, Z. N., 51, 288. Duret, Les tum. de l'Encephale, I905 Cramer, Hippel, D. m. W., 1912, 2243. 396 Alexander, Unger, Berl. kl. W., I914, I408. Oppenheim, A. P., 21, 560. F. Krause, Chir. Gehirn. u. Ruckenm., Berlin, 1908, r9r1. 397 Wernicke, Lehrb. d. Gehirnk. J. Collier, Brain, 27, 490. Merzbacher, M. W., 1909, 2051. 398 Hawthorne, Prevention of Heart Dis., etc., London, 1902. Elschnig, Neur. Cent., 1894. Batten, Collier, Brain, 22, 473. Verdun, Thèse, Paris, I9I I-I2. 399 Scaffidi, V. A., I73 (Lit.). 400 Linck, Z. B., 33. Bielschowski, Z. N., 22. Pfeiffer, Ibid., 5. Miura, Z. B., II (Lit.). Saxer, C. P., 9 (Lit.) Schlesinger, Die Syringomyelie, Wien, 1895. 401 Stroebe, Z. B., I8 (Lit.). 402 Renaut, Gaz. med., 1884, 614. Storch, V. A., 157. 404 Mallory, J. Med. Res., 8. Weigert, C. P., I890, 736; Anat. Anz., I890. E. J. Kraus, V. A., 217, I21. Golgi, U. d. Gliome d. Geh., Jena, 1884. Aschoff, Erg. Path. Anat., 1898, 5. Buchholz, A. P., 22. Arnold, Z. B., II. 406 Thiele, I. D., Erlangen, 1902. 407 Henneberg, A. P., 30. Gowers, Dis. of Nerv. Syst. Bruns, Encyclop. Jahrb., 5, 1893. 408 Schule, D. A., 20. Schmidt, V. A., 162. Hildebrand, Z. C., 36. 409 Ernst, Z. B., 25. Baumann, Z. B., 2, 500. Hartdegen, A. P., II. Schultze, V. A., 87. Simon, V. A., 6I. Borst, 1. c., 254. Meschede, Allg. Z. Psych., 21. 410 Meyer, Beyer, A. P., 12. Hoffmann, Z. N., 3. Margulis, A. P., 51, 788 (Lit.). Pfeifer, Z. N., 5, 405. Roccavilla, Riv. pat. nerv. e ment., 1914, I9, 208. Gerhardt, cit. by Borst. Lowenthal, D. A., 49. Adler, Arch. Unfallh., 2. Flesch, Wien. m. Woch., 1905. Eppinger, Aerzt. Sachv. Z., 1909. 411 Schmidt, V. A., 162 (Lit.). Muhr, A. P., S. Meyer, V. A., I20. Clark, Amer. J. M. S., I29. Schlesinger, Ruckenmarkstumoren, Jena, I 898. Daxenberger, J. D., I890. Lachmann, A. P., 13. Volkmann, D. A., 42. Grund, Z. N., 3 I (Lit.). Rosenthal, Z. B., 23. Saxer, Z. B., 32. 413 Hoffmann, Z. N., 3. Chiari, cit. by Saxer. Langhans, V. A., 85. Müller, Meder, Z. M., 28. Baumler, D. A., 40. Simon, A. P., 5. 414 Inglis, N. Y. M. J., 92, I006. Spiller, Amer. J. M. S., I26;Z. N., 32. I. Strauss, N. Y. M. J., 88, I079. Wright, J. Exper. Med., I2, 556. Pepper, Amer. J. M. S., 121, 287. Wolbach, Tileston, Amer. J. M. S., 135, 871. Hutchinson, Quar. J. M. S., I, 33. 415 Kretz, Erg. Path., 1902, 8, II, 532. Ohkubo, Arch. f. Ent., 26. Mallory, J. Med. Res., 13, I13. E. Hackl, V. A., I6, 267. Delmarre, Merle, Arch. med. exper., 21, 458. Loeper, Ibid., 16, 473. Cayley, Brown, London P. S., 1876. Muthmann, Sauerbeck, Z. B., 34. Petit, Girard, Arch. d'anat. micr., 1902. Linck, Z. B., 33. Pick, Berl. W., 1906, 476. Esser, Z. C., 32. Thorel, Munch, W., 1907, 725. 
Bonnet, Soc. anat., I86I. Bruchanow, Prag. m. W., I898. 416 Jeremias, Arb. path. Instit., Posen, 1901. Saltykow, Z. B., 42. Cornil, Soc. Anat., 1901. Marchand, Ibid., 1904, 71 5. Saxer, Z. B., 20. Weisenburg, Brain, 33. Bassoe, J. A. M. A., 1916, 67, 1423. Fauret, Thèse, Paris, I910-I I. J. R. Hunt, A. J. M. S., 127, 504. Mackay, Bruce, Rev. of Neurology, etc., 1909. 417 Vonwiller, V. A., 204, 230. 418 Spat, Aertzl. Intellegenzbl., I 883, 305. Bielschowsky, Unger, L. A., 81. E. Meyer, A. P., 32, 320. 419 Boudet, Clunet, Arch. med. exper., 22. Mott, Barratt, Arch. of Neurology, 1899, cit. by Boudet. Selke, I. D., Konigsberg, I891. Saxer, D. Path. Ges., 1902. 420 Cimbal, V. A., I66. Rosenblath, Z. N., 31. Hart, A. P., 47. 421 Mullaly, Arch. of Int. Med., 1913, II, 523. Robin, Gaz. med., Paris, 1854. Parsons, Pathol. of Eye, I905, II, 626. A. Knapp. Arch. of Ophthal., 1920. Wintersteiner, Wien. kl. Woch., I894, 493; Das Neuroepitheliom retinæ, Wien, 1897. H. Knapp, Intraocular Tumors, 1869; Arch. of Ophth., 2. Hirschberg, Arch. f. Ophth.,I6. Delafield, Arch. f. Ophth., N. Y., I 871, 2, 58. Schweigger, Arch. f. Ophth., 6. 422 Flexner, Johns Hops. Bull., I 89I. I wanoff, Arch. f. Ophth., 45. Eisenlohr, V. A., 123, 429. Becker, Arch. f. Ophth., 39. 423 Steinhaus, C. P., II, 257. Greef, Deut. m. W., I 896. Hertel, Monatsbl. f. Augenh., I 897 da Gama Pinto, Unters. u. intraoc. Tumoren, Wiesbaden, i 886 . Newton, Austral. med. Gaz., 1902, 236. Wilson, Dublin Path. Soc., I871-74, 108. Williams, Nat. Hist. of Cancer, 362. Feinstein, Rev. sci. med., I 896, 30I. Helfreich, Arch. f. Ophth., 21. Ginsberg, Ibid., 48. Salzmann, Ibid., 39. Axenfeld, Erg. d. Path., I896, III, 2. Emanuel, V. A., I6I. 424 Wohlwill, Mitt. Hamburg Statsan., II, 19. Putnam, Boston M. S. J., I899, I40, I29. Weiswange, I. D., Tubingen, I897. Jacobson, Jamare, A. P., 29. 425 Almecrona, V. A., 217, I61. Marcus, A. P., 51,322 . Weaver, J. Exper. Med., 3, 669. Nonne, Z. N., 2I. W. Rindfleisch, Z. N., 26 (Lit.). Mertens, Seiffert, Berl. kl. Woch., 1908, I477. Carnot, Bougle, Soc. anat., I9I I, 535. Fischer, Arch. f. Heilk., I90I, 22, II, 344. Oppenheim, A. P., 21, 560. Coste, Levy, L. A., 96, I049. Berblinger, V. A., 219, 328. 426 Boettiger, D. m. W., 1913, 244. 427 Bungner, Z. B., Io. Bruns, L. A., 42. Thoma, Text-book of Pathology, 463. Virchow, 1. c., III, 283. 428 Knauss, V. A., 153 (Lit.). Bruce, Dawson, Review of Neur. and Psych., I913!'(Lit.)., Illus. 430 Falk, Z. B., 40 (Lit.). Askanazy, Fest. f. Neumann, 2. Wiechselbaum, V. A., 85, 554. Bruchanow, Z. f. Heilk., 20. Schmidt, V. A., 155, 557. Soyka, Prag. Viertelj., I35. Loretz, V. A., 49. Knedel, Z. C., 67 (Lit.). Chiari, Verh. Naturf., Dusseldorf, I898. Busse, V. A., I5I, Suppl. Beneke, Z. B., 30; Z. C., 67, 239. 432 Miller, V. A., I9I. Wahl, J. M. Res., 30, 205. Dunn, J. Path. and Bact., 19, 456. Virchow, 1. c., III, 265. Foerster, Wurz. m. Zeit., 1862. Monakow, Berl. k. W., I900, 721. Henneberg, Koch, A. P., 36. Fraenkel, Hunt, N. Y. M. Rec., 1903, 64, 1001. 433 K. Sternberg, Z. f. Heilk., 1900, 2I, 163. 434 Collins, Marshall, Ophth. Soc. Unit. Kingd., 1900, 156. A Knapp, Arch. of Ophth., 1916. E. Sachs, A. S., 66. Spiller, J. A. M. A., 34, 1026; A. J. M. S., I36. Giani, Mitt. G. G., 19, 452. Marchand, Festschr. f. Rindfleisch, 1907.

\section{TUMORS OF SPINAL CORD AND MEMBRANES}

436 Schlesinger, Ruckenmarkstum., Jena, I 898 (Lit.). Silberkuhl, I. D., Griefswald, 1892. Wichmann, I. D., Tubingen, I887. Schueppel, Arch. f. Heilk., 8, II3. Schlapp, J. Nerv. and Ment. Dis., I9I I, 38, I29, 221 . Traube, Ges. Abhand., I871, 2, 1005. Forster, cit. by Schlesinger. Gowers, Med. Chir. Trans., 1888. Hunt, Woolsey, Annals Surg., 52. Bruce, Mott, Brain, 1887. Marshall, cit. by Gowers. Oustaniol, Thèse, Paris, 1892. Gaupp, Z. B., 2, 5 I0. 437 Collins, N. Y. Med. Rec., 1902, 62, 882. Andersech, cit. by Schlesinger. Duseberg, I. D., Giessen, I893. 438 Berthelot, Philada. P. S., I874-75. Merklen, Prag. med., I883, 306. Nonne, Z. N., 21, 396. R. Schulz, A. P., I6, 592. 439 Bausch, Z. N., 9. Lens, Z. B., 19, 603. Ollivier, Tr. de mal. moelle epin., Paris, 1837. Coupland, London P. S., 1887, 38, 26. Westphal, A. P., 26, 770. Cramer, I. D., Marburg, I888. Lowenfeld, Wien. m. Presse, 1873. Ganguillet, I. D., Bern, 1878. S. Cobb, An. Surg., 62. Schopper, Frankf. Z. P., 13, 77. Lua, A. P., 53, 
895 (Lit.). W. Rindfleisch, Z. N., 26, I35. 440 Schiff, C. P., I 895, 84.441 Holmes, Med. News, I885, 2, I7. Temoin, Arch. prov. de Chir., I892, 179. Obre, London P. S., I85I, 3, 248. Johnson, Ibid., I856, I6. Braubach, A. P., 15. K. Hoffmann, Z. nat. med., 34. Berenbruch, I. D., Tubingen, I890. Wolbach, Boston M. J., I913, I48, $68 \mathrm{r}$. Zanda, Z. B., 5. Laquer, Neur. Cent., 1980, 193. Taube, Ibid., I887. H. White, Clin. Soc., London, I900, 33, I40. 442 Trachtenberg, V. A., I54. L. Müller, D. A., 54, 472. Hensen, Z. N., 21, 235. Sturzberg, Ibid., 33, 66. Lichtheim, D. m. W., 189I, I 386. Schultze, Z. N., I6. 443 Spiller, Univ. Penna. Med. Bull., I903, 16, 27. S. Adler, Berl. W., 1908, I596. Schwartz, Wien, kl. Woch., I897, 177. Quincke, Volk. Vort., I893; Z. N., 9. Oppenheim, Beitr. Diag. d. Ges. im Centraln., I907. Krause, D. Gesell. Chir., 1907. Horsley, B. M. J., 1909, I, 513. Charcot, Gaz. med., I879. Koeppen, A. P., 27, 918. Wieting, 1. D., Marburg, I 893. MacEwen, Med. News, I888, 2, I69. Bruns, Gesch. cl. Nervensystems, Berlin, I908. Malaise, D. A., 50. Nonne, Z. N., 47, 48. 444 Kuntz, cit. by Malaise. Quensel, Neur. Cent., I 898. Sailer, Philada. P. S., I898-99. B. Sachs, N. Y. Med. Rec., I900, 57, 7.

\section{GENERAL PATHOLOGY OF EPITHELIAL TUMORS}

449 Payne, Brit. J. Dermat., I89I, I86. Lanz, D. W., I899, 313. 450 Leale, N. Y. Med. Rec., I878, I4, I 88. Judassohn, Verh. II Cong. Dermatol., I896, 497. Lebert, Ueber Keratosis, etc., Breslau, I 864. Wilson, Med. Chir. Transac., 1844. Pick, Viertelj. f. Dermat. u. Syph., I875, 315. Botge, Z. C., I875. Rodriguez, cit. by Crocker, Dis. of Skin. Dubreuilh, Annal. de dermat., 1896. Kuhnemann, B. M. J., I889, I, 328.452 Petersen, C. B., I4. Lubarsch, Erg. Path., I 894-95. M. Juliusberg, D. W., I905, I 598. Kromayer, V. A., I32. Robin, Leredde, Arch. med. exper., 1896, 459. Rehn, L. A., 88, 1053. Langhans, V. A., 58; Deutsch. Chir., 1887. Lubarsch, V. A., III. Dreyfuss, V. A., I13, 535. 454 Barlow, D. A., 55, 61. Schmidt, V. A., I48. Marckwald, V. A., 144. Wolfler, L. A., 29. 455 P. White, J. P. B., 1901. Paget, Surg. Path., I853. Selberg, V. A., I60. 458 Luschka, V. A., 20; D. A., 55. Hauser, Das Cylinderepithelcarc. d. Magens, Jena, I890. Schwab, B. B., I8. Cripps, London P. S., I882. Smith, St. Bart's H. R., I887. Bickerstedt, Ibid., I890. Port, Z. C., 42. Verse, Arb. a. d. P. Inst., Leipsig, 1908 (Lit.). Smoler, B. B., 36. Lubarsch, V. A., III. Niemack, Ann. Surg., 1902. Petrow, C. C., I 896, 542. Quenu, Landel, Rev. d. Chir., I 899. Doering, L. A., 83 (Lit.). Hitschman, Adler, Z. G., 60; A. G., 10o. Weishaupt, Z. G., 62. Schickele, B. Geb. Gyn., I3, I913. 459 Heurlin, A. G., 94. Voges, Wien. kl. W., I902, 1255. Kirchheim, L. A., 68. Kamann, W. k1. Runds, 1903. Fabre-Therenot, Le goitre chez les nouv. ne., Lyons, 1907. 460 Ewing, N. Y. M. J., 84, 106I. A. Kocher, Mitt. G. g., I902; T. Kocher, L. A., 92, 1166. Marine, J. Exper. Med., I910, 12, 511 . 461 Lowenstein, V. A., I88. Balzer, Menetrier, Arch. d. Phys., 1885. Caspary, A. D., 23. Barlow, D. A., 55 (Lit.). Crocker, Dis. of Skin. 466 Janeway, Z. K., 8, 403.476 Orth, Z. K., IO. Steinhauser, B. B., I2. Ashihara, A. D., 57. Porter, Wolbach, J. Med. Res., 21. Janowski, Z. B., Io. Slade, Lancet, I905, I, 1059. Polese, C. G., I906, 284. Alshausen, Veit's Handb., 1908. Benckizer, Z. G., 22. Huerlin, A. G., 94. Rehn, L. A., 50. Seyberth, Munch. m. W., I907, 1573. Demarquay, Mal. chir. d. penis, Paris, 1877. Kaufmann, Deut. Chir. L., 53. Stoerk, Z. B., 26. Cohen, V. A., I13. 478 Brissaud, Arch. de Physiol., 1884. Schimmelbusch, L. A., 44. Saar, L. A., 84. Bloodgood, S. G. O., 1903, 721. Tietze, Z. C., 75, I17. Speese, Annal. Surg., 51, 212. Freyer, Arch. internat. d. chir., I9I4, 6. Walker, Lancet, I909, I, I054. Menetrier, Assoc. franc. d. cancer, 1908. 481 Beneke, Biblioth. d. med. Wissen, 1900. 483 Goldmann, B. B., 18, 595; Z. K., 5, 39.

\section{EPITHELIAL AND OTHER TUMORS OF THE BREAST}

484 Deaver, McFarland, The Breast, Phila., I917. Roger Williams, Dis. of Breast, London, I 894. Howitt, Lancet, I837, I, 5.37. Hahn, Schmidt's Jahrb., 5, I38. Wilson, 
Gas. d. Hop., 1854, 315. Mallett, Gaz. med., 1832, 620. Sagra, C. R. Acad. Sci., 61, 570. Rousseau, Riv. de Chir., 1856, 596. Kirchheim, L. A., 68, 582. Richet, Gaz. d. hop., I881, 322. Billroth, Deut. Chir., 4I. Robert, Amussat. L'Union med., 1851. Warren, Surg. Obs. on Tumors, 1837, 228. Porter, Boston M. S. J., 1892. Aitken, Med. Times and Gaz., I 858, I1, 608. Huston, A. J. M. S., 1834, 374. Durston, cit. by Williams. 485 Brodie, Lect. on Path. and Surg., London, 1846. Reclus, Rev. de Chir., 1883, 76r. 486 Brisasud, Arch. de Physiol., 1884. Phocas, Thèse, Paris, I886. Konnig, Lehrb. d. Chir.; Cent. f. Chir., I 893. Schimmelbusch, i.. A., 44. Delbet, Traitè de chir. Duplay, Reclus, I898, 5. Theile, L. A., 88, 261. Cornil, Tum. du Sein., Paris, 1908. Lecene, Lenormant, Prec. de path. chir., I9II, 3. La Roy, Arch. intern. de Chir., I912. Werner, Zeit. f. rat. Med., I 85I, I 854. Velpeau, Mal. du sein., I854. Virchow, Geschw., 1, 325. Labbe, Coyne, Tum. benign. du sein., I876, 243. Bryk, L. A., 25. Houdoupe, Gaz. d. Hop., I887, 841. Tietze, Z. C., 56, 512; 75, 117 . Bloodgood, S. G. O., 1903, 721 . Baumgartner, Nouv. Tr. de Chir., 1913, 23. 487 T. Bryant, Lancet, I900, I, I201. Rodman, Dis. of Breast, 1908. Nocard, Mollereau, Annal. de l'Instit., Pasteur, I887. Reynolds, Lancet, I884, I, 782. De Sinety, Traitè de Gyn., 918. Altmann, V. A., III. R. Batty, Amer. Syst. Gyn., I 888, 2, 849. Keppler, Ann. de Gyn., I 890.489 Sasse, L. A., 54. Schield, B. M. J., I901, I, II37. Renon, Gaz. d. Hop., 1904. Monod, cit. by Baumgartner. 490 Dreyfuss, V. A., II3. 491 Creighton, Tumors of Breast, London, 1902. Moullin, J. Anat. and Physiol., 188I. Krompecher, D. P. G., I9I3 (Lit.). Borst, D. P. G., 1904. Kuru, Z. C., 98 . 492 Sourice, Thèse, Paris, 1 887. Saar, L. A., 84 (Lit.). Speese, Am. Surg., 5I, 212 . Verga, Soc. med. Chir., Paris, 1907; C. P., 1908, ref. Grohe, Z. C., 55. Stoerk, Erdheim, Wien. kl. W., 1904, $35^{8}$ (Lit.). 496 Buday, V. A., 156. Johada, Wien. m. W., I891, I869. Mintz, Wratch, 1912. 497 Deaver, McFarland, The Breast, 1917. 498 Guinard, Rev. de chir., 1910, 657. Greenough, Simmons, An. Surg., 45. Hansen, cit. by Baumgartner. 499 Warren, J. A. M. A., 1905, 45, 149. Cushing, J. H. Bull., I905, 179. Bowlby, London P. S., I882, 33, 306. Pilliet, Soc. anat., I 890, 552. B. Clark, Lancet, I 890, I, I I 79. Dubar, Thèse, Paris, 1888. Wagner, Arch. f. Heilk., 2, 275. Doen, Boll. d. sci. med., Bologna, I89I. Chevrier, Delval, Soc. anat., 1910. Stilling, Z. C., 15, 247. Battle, London P. S., 1886, 37, 473. Cornil, Souligoux, Soc. anat., 1907. Lange, N. Y. M. Rec., 1881, 20, 161. Cambria, Riv. clin. sci. med., 1887. Leser, Z. B., 2, 379. Davidsohn, Cent. Gyn., I909, 1357. Soulier, Dauphinè med., I891. Happel, B. B., 14, 3. Desoil, Thèse, Lille, I 895. S. W. Gross, Med. News, I883, 42. Coen, cit. by St. Arnold. Hacker, L. A., 27, 614. 500 Wilms, Mischgeschw., I902, III. Konjetzny, B. B., 78, 504. Duval, Soc. anat., 1909. Walther, Soc. chir., I910. Nadal, Soc. anat., I910; Rev. de chir., 1912, 45, 630. Lecene, Rev. de Chir., 1906, 33, 434. Menetrier, Assoc. franc. etude cancer, I910. Herrenschmidt, Ibid. 501 Gross, A. J. M. S., 94, 17. Schmidt, B. B., 4, 40. Schouler, Corresp. schw. Aertze, 1890, 283. Mornard, Masson, Rev. de gyn., 1909. Borchmeyer, I. D., Wurzburg., I 889. Rapke, L. A., 78. Gebele, B. B., 29. Lecene, Soc. anat., 1905. Kettle, Lancet, 1912, 2, 750. 502 A. Hoffmann, L. A., 48. Poulsen, L. A., 42. Pean, Lec. clin. chir., I 892. S. D. Gross, Syst. Surg., 6 ed., 2, 974. Rosenstein, L. A., 63. 503 Finsterer, Z. C., 86 (Lit.). Fioriani, La clin. chir., 1902. G. B. Schmidt. L. A., 36, 421. Nadal, Assoc. Franc. p. cancer, 1910, 476. Battle, Lancet, 1901, I, 177. 505 St. Arnold, V. A., I48. Manz, B. B., 13. Durham, B. M. J., I883, 2, IoI9. Image, Hake, Med.-Chir. Trans., 30. Quenu, Kuss, Soc. anat., 1908. Malapert, Rev. de Chir., 29, 200. Sokolow, V. A., 58. Niklas, Leiomyoma mammæ, Wurzburg, I889. Vieregge, Nor. Western. Lancet (St. Paul), ı 891. Wegner, L. A., 20. Müller, Cent. Chir., 1885. Pinner, Ibid., I880. Reverdin, Mayer, Rev. med. Suisse rom., I887, 96. Koehler, Charite Annal., I888. Begouin, J. de med., Bordeaux, I891. Hofer, Holmes' Syst. Surg., I 883, Vol. 3. Queirel, Cong. franc. de chir., I889. Paget, Lancet, I894, I, I173. Reclus, Clin. chir., 1888. Baker, Bowlby, Med.-Chir. Trans., 69. Moore, Dublin J. Med. Sci., 63. Ashby, Wright, Dis. of Children. 506 Richet, Gaz. d. hop., I883. Winiwater, Beitr. z. stat. d. Canc., Stuttgart, 1878. Hauser, I. D., Heidelberg, 1 886. Morestin, cit. by Baumgart- 
ner. Robinson, London P. S., I893. Bryant, Dis. of Breast, London, I887. W. R. Williams, Ann. Surg., 1891. 507 Lehmann, I. D., Munchen, I903. Guleke, L. A., 42. Paget, Lect. Surg. Path., I853. Nunn, Cancer of Breast, I 882. F. L. Hoffman, The Mortality from Cancer, Newark, 1915. Henry, cit. by R. Williams. Gross, Internat. J. Med. Sci., I888, 347. Handley, Cancer of Breast, I910. Hubbart, Boston M. S. J., 1912. Benassey, Thèse, Paris, I913. Schultheiss, B. B., 4. Fink, Zeit. f. Heilk., I888, 9, 453. Winchel, Path. u. Ther. d. Wochenbet., I878. 508 Borrel, Ann. Pasteur, 1909. 513 Doutrelepont, L. A., I2. Gaabe, B. B., 60 (Lit.). 514 Lange, B. B., I6. Zimmermann, I. D., Strassburg, I902. Elsasser, V. A., 82. 520 Nadal, Assoc. Franc. p. cancer, I911. 521 Langhans, V. A., 58; A. G., 8, I875. Cornil, Jour. de l'Anat., I865. J. Müller, I. D., Zurich, 1907. 523 Lee, Adair, Ann. Surg., 72, I88. 524 E. Goldmann, B. B., 1897. Waldeyer, V. A., 4. Leopold, A. G., 5. 525 Rieffel, Thèse, Paris, 1890. Kirmisson, Soc. anat., 1882. Zocher, I. D., Liepsig, 1869. Hennig, A. G., 2. Stiles, B. M. J., I899, I, 1452. Erichsen, Science and Art of Surgery. Sappey, Anat. descript., Paris; I 888. Gerota, L. A., 54. Grossmann, I. D., Berlin, I896. P. Bartels, Anat. d. Mensch., Bardelben, I909, III, 4. 526 I. Heidenhain, L. A., 39. Oelsner, L. A., 64. Fage, Thèse, Paris, 1909. Mousseaux, Thèse, Paris, 1902. 528 Rotter, L. A., 58. Van Verts, Assoc. franc. cancer, 1909, 1910. Hardouin, Soc. anat., 1910. A. T. Cabot, Ann. Surg., 46. Mornard, Rev. de Chir., 5I. 529 Colwell, Arch. Middlesex Hosp., 1905. Paget, Lancet, I889, I, 57 I. Toroi, Wittelshofer, L. A., 25. Handley, Lancet, 1905, I, 1048. 531 Peabody, Trans. Assoc. Amer. Phys., 1907, 17. Humbert, Alexieff, Rev. de Med., 1913. Petit, Thèse, Paris, 1895. 532 Gould, Clinical Journal, 1900. Mackay, B. M. J., I907, 2, I38. Osler, B. M. J., I906, I, I. Beatson, Lancet, I896, 2, 104. Lett, Edinburg Med. Chir. Soc., 1905. Guinard, Soc. chir., 1904. Reynes, Cong. franc. de Chir., 1907. Bowlby, St. Barts. Hosp. Rep., 25. Klotz, I. D., Halle, I869. Volkmann, Beitr. z. Chir., Leipsig., I875. 533 Aitken, Med. Times and Gazette, I857. 534 M. B. Schmidt, Verhut. d. Carc., I903. 536 La Forgue, Thèse, Toulouse, I 895. Poirier, Thèse, Paris, 1863. Schuchardt, L. A., 31. Moore, Tr. Roy. Med. Chir. Soc., I867, $r$, 245. 537 Banks, B. M. J., I882, 2, I I38. Gross, Surg. Cong., London, I88I. Depage, Cong. intern. chir., Bruxelles, 1908. Walther, Assoc. franc. p. cancer, I910. Le Dentu, Cong. intern. chir., 1908, 223. Durand, Gaz. hebdom., I895. 539 Halsted, Johns Hop. Rep., I894; Ann. Surg., 46. Willy Meyer, Amer. J. Surg., I909, 77. Ransohoff, Ann. Surg., 46. Pilcher, Ann. Surg., 46. Greenough, Ann. Surg., 46, 1907. Finsterer, Z. C., 89, I43. $540 \mathrm{Hahn,} \mathrm{Berl.} \mathrm{W.,} \mathrm{I888,} \mathrm{413.} \mathrm{Ochsner,} \mathrm{Ann.} \mathrm{Surg.,}$ 46. Cabot, Ibid. Odekop, L. A., 24. Sprengel, L. A., 27. Sibley, Med. Chir. Trans., 42. M. Baker, Ibid., 45. Richardson, J. A. M. A., I91 I, 56, 315.

\section{CANCER OF UTERUS}

543 Welch, Pepper's System Med., I885, II, 533. R. Williams, Natural History of Cancer, 54, 55. Weinberg, Z. K., 4. Koblanck, Veit's Handb., III, 2, 672. Taussig, Amer. Gyn. Soc., 37, 327. R. Williams, Uterine Tumors, 1896. Gusserow, Volkmann's Vortr., No. I8. Bäcker, A. G., 53, 47. Blumenfeld, M. W., I899, 409. Polese, C. G., 1906, 284. Beckmann, Z. G. G., 45, 492. Sutton, Giles, Diseases of Women, 1904, 255. 544 Cullen, Cancer of Uterus, Phila., I90. Engelhorn, Beitr. G. G., 13, 278. Glockner, Z. G. G., 63, I82. 546 Waldeyer, V. A., 4I, 470; 55, 67. Ruge, C., Z. G. G., 31,47 I. 547 Abel, Landau, A. G., 35, 214 . Krauss, Z. G. G., 54, 383. 548 Frankel, Beitr. G. G., 2, 351. Hitschmann, A. G., 69, 629. Offergeld, A. G., 78, 289. Buttner, A. G., 94, 794 . Hauser, A. G., 99, 339. Sitzenfrey, Z. G. G., 59, 385. Hoffmeier, Z. G. G., 32, 17 I. Ruge, Veit, Z. G. G., 2, 4I 5; 5, 232; 8, 405. Meyer, Z. G. G., 65, 218; A. G., $91,579$. Adair, S. G. O., 10, 337. Schottlander, Mon. G. G., 26, I. 549 Fischel, A. G., 15, 76. Miller, A. G., 89, 76. Albrect, Mon. G. G., 23, 285. Wagner, Gebarmutterkrebs, Leipsig, 1858. Gebhard, Path. Anat. d. weibl. sex. organe, 1899. Ruge, Veit. Z. G. G., 7, 231. 550 Krukenberg, Mon. G. G., 5, 138. Pohorecky, A. G., 60, 252. Meyer, Veit's 
Handb., 1908, 3. Graefe, A. G., 72, 373. Kroemer, A. G., 65, 626. Forsner, A. G., 87, 445. Hansen, V. A., I7I, I8. Schauenstein, A. G., 85, 576. Schottlander, XII, Cong. Gyn., 1907. Schottlander, Kermauner, Uterus carcinom, Berlin, 1912. Heurlin, Pick, A. G., 94, 402. Ribbert, Das carcinome d. Menschens, I9I I. Pronai, A. G., 89, 596. 551 Stone, S. G. O., 23, 248. Lindquist, Uterus Papillom, Upsala, 1909. 552 Theilhaber, Hollinger, A. G., 73, I. Vassmer, A. G., 75, 668. 553 Thorn, Z. G. G., 28, 75. 554 Gessner, Z. G. G., 34, 387. Ladinski, S. G. O., 191 5, 20, 325. 555 Gebhard. Z. G. G., 24, I. Flaischlen, Z. G. G., 32, 347. Ruge, C., Z. G. G., 31, 471 . Renckiser, Z. G. G., 22, 337. 556 Doca, Z. G. G., 58 , I Noble, Amer. Jour. Obstet., 1904, 49, 306. Flaischlen, Z. G. G., 58, 343. Rolly, V. A., 155, 555. Dillmann, Z. K., 2, 333. Schwab, Hegar's Beitr., I2, I02. 557 Gebhard, Z. G. G., 24, I. Werth, A. G., 49, 369. Kaufmann, C. G., I906, I07I. Zeller, Z. G. G., II, 56. Borst, D. P. G., 7, 1907. Meyer, A. G., $9 I$, 579. Heurlin, C. G., I 898, 38. Oeri, Z. G. G., 57, 384. Winter, Die Bekampfung des Uteruskrebses, Stuttgart, I904. Taylor, H. C., Amer. Gyn. Soc., 37, 313. Mackenrodt, Z. G. G., 54, 5I 4. Waldstein, A. G., 6I, 52. 559 Scheib, A. G., 87, I. Veit, B. W., I889, 701. Bumm, Z. G. G., 55, I73. Fromme, A. G., 79, 197. Sappey, Traitè d'Anat., I88. Kroemer, A. G., 73, 57. Poirier, Le Progress Med., I889, 2, 491; I890, 1, 4I. Lucas-Championniere, Thèse, Paris, 1875. Bruhns, Arch. f. Anat. u. Phys., Anat. Abt., I898, 57. Baisch, A. G., 75, 273. Kroemer, A. G., 73, 57. Veit, A. G., I3, 470. Doederlein, Hegar's Beitr., 9, i73. Fritsch, A. G., 29, 359. Landau, A. G., 44, 567. Kundrat, A. G., 60, 355. Kermauner, Lameris, Hegar's Beitr., 5, 87. Schauta, Mon. G. G., 19, 475. v. Herf, Corresp. Schweitz. Arzte, 1904. 560 Blau, I. D., Berlin, 1870. Lubarsch, Erg. d. allg. Path., 2. Meyer, Z. G. G., 49, 54. 561 Ruhle, Z. G. G., 74, 321. Williams, Brit. Gyn. J., 2, 529. Beckmann, Z. G. G., 45, 492. Offergeld, A. G., 87, 298; Mon. G. G., 22, 5I4. Willinsky, I. D., Berlin, I904. J. H. Jacobson, Trans. A. M. A., Section Obst. Gyn., I910, I88; cf. J. A. M. A., 67, I219. Ries, S., Am. Jour. Obst., 44, 29. Clark, J. G., Johns Hopkins Hosp. Bull., July-August, 1895. 562 Mackenrodt, C. G., I902, 808. Peterson, B. B., 34. J. W. Miller, A. G., 89, 76. Aldergott, I. D., Leipzig, 1905. Sampson, Amer. Gyn. Soc., 31, 387. Gellhorn, Ibid, 30, I 37. R. Peterson, Ibid, 37, 295.

\section{VULVA}

Gurlt, L. A., 25, 446. Dittrich, Amer. J. M. S., 1905, I30 (Lit.). Arnot, London P. S., 1873, 24. Fromme, Beitr. G. G., 9, 382. Sitzenfrey, Z. G. G., 58, 363. 563 Weir, Amer. J. M. S., I 875, 21, 240. Butlin, B. M. J., I90I, 2, 61. P. Jung. Mon. G. G., I7, 985. v. Franque, Z. G. G., 60, 237. Peter, Mon. G. G., 3. Schwarz, I. D., Berlin, I893. Fritsch, cit. by Veit. Kustner, Z. G. G., 7, 70. H. Schulze, I. D., Leipsig, I903. Teller, Z. G. G., 6I. Rupprecht, Z. G. G., 72. 564 L. Pick, V. A., 175. H. Ruge, Z. G. G., 56. Veit, Handb. d. Gyn., I910, IV, 2, 724, 74I. P. Meyer, A. G., 85. Hinselmann, Z. G. G., 62. Chaboux, Les tum. mal. prim. d. glande Barth., Lyone, I906.

\section{VAGINA}

Schwarz, U. d. Erfolg. d. Oper. Vul. u. Vag., Berlin, 1893. Wille, I. D., Erlangen, 1903. West, Lessons on the Dis. of Women, London, 1870. Reclus, Gaz. hebdom., I 887. Bex, Thèse, Paris, 1887. Bernard, Thèse, Paris, I 895 (Lit.). v. Franque, Z. G. G., 60. Pozzi, Traitè de Gyn. Schwarz, Deut. Cong. Gyn., I886. Olshausen, Cent. Gyn., I895, I. Poirier, Prag. Med. W., I889, 491. Bruhns, Arch. A. u. P., A. Abt., I 898. 565 Lohnberg, Z. G. G., 73, 755. R. Williams, N. Y. M. Rec., I901, 60, 84 I. R. Meyer, Erg. P., 9, 518 (Lit.); Z. G. G., 7I. Davidsohn, A. G., 61. Cullen, Johrs Hopk. Hosp. Bull., I6. Pintor, Ac. d. Med. Torino, 1900. Pollasson, Violet, Ann. de Gyn., 32, 675. Hoehne, Z. G. G., 67. Hirsch, Z. G. G., 67. 


\section{CHORIOMA (CHORIONEPITHELIOMA)}

566 R. Volkmann, V. A., 6I. Sanger, A. G., 44. Pestalozza, Il Morgagni, I89I, 33. Schmorl, C. G., I 893, I69. Gottschalk, A. G., 46. Marchand, Mon. G. G., I895. J. W. Williams, Gyn. Trans., I 895. Ewing, S. G. O., I9II, II, 366. Pestalozza, Soc. Ital. Obstet., I9I3. Veit, Handb. Gyn., I908, 3, Th. 2. Teacher, Obstet. Trans., London, 45. Risel, Arb. path. Instit., Leipsig, 1903. Frank, N. Y. M. J., 83. Schmauch, S. G. O., 5, 259. 567 Pollasson, Violet, Ann. de Gyn., I9I3, 70. Findley, J. A. M. A., 43, I35I. Ladinsky, Amer. J. Obstet., 45. Olliveir Bauregard, Thèse, Paris, 1904. Curtis, Oui, Ann. de Gyn., I913, 70. Senarclens, Thèse, Lausanne, 1902. Polano, Z. G. G., 75. Outerbridge, Amer. J. Obs., 72. Ries, Ibid., 57. Caturani, Ibid., 63. Waldo, Ibid., 62. Huguenin, An. de Gyn., I905, 2, 649. Engstrom, Mitt. gyn. klin. Engstrom, Io. 568 Moller, Stud. u. Blasenmolen, Wiesbaden, I9I2. Solowij, Mon. G. G., I2. 570 Pick, Berl. W., I897, 1069. Kaufmann, Z. G. G., 60. Schlagenhaufer, Wien. kl. W., I 899, 486. Fleischman, Mon. G. G., I903, I7. 574 Lebret, Thèse, Paris, I9I I. Fraenkel, D. W., I899, I77. 575 v. Velits, Z. G. G., 52, 56. 577 R. Meyer, Z. G. G., 58. Bauereisen, Z. G. G., 53. 580 H. Schmidt, C. G., I900; Wien, kl. W., I90I, I077. Lindfors, Upsala Lak. For., 6, I77. Walthard, Z. G. G., 59. Patellani, C. G., I905, 388. 581 Stockel, Fests. f. Fritsch, 1902. Schaller, Pforringer, Hegar's Beitr., I899. Jaffe, A. G., 70. Orthmann, D. Ges. Gyn., I 897, 35I. Seitz, A. G., 75. Fraenkel, Anat. Anz., 20; A. G., 68. Pick, Berl. W., 1902, I189. Wallert, Z. G. G., 53. Dunger, Z. B., 37. Risel, Z. G. G., 56. Garkisch, Z. G. G., 60, II5. Basy, Ann. de Gyn., I9I3, Io. Kleinhaus, C. G., 1902. Lubarsch, Arbeit, path. Inst., Posen, 1901. Bock, Soc. Belge de Gyn., I 899, II3.

\section{CYSTS AND EPITHELIAL TUMORS OF THE OVARY}

582 Felix, Embryology (Keibel \& Mall). Bulius, Hegar's Festschr., I889. Nagel, Handb. d. Anat. v. Bardeleben, I896. v. Kahlden, Z. B., 30, 31. Walthard, Z. G. G., 49, 233. Babo, V. A., I6I, 3 I I. v. Franque, Z. G. G., 39, 326. Pick, Berl. W., 1900, 219. Schickele, V. A., I69 (Lit.). v. Franque, Z. G. G., 43. R. Meyer, V. A., I7I. 583 Bulius, Z. G. G., 15. Klob, Path. Anat. weibl. Sexorg. Wien., I864. Waldeyer, A. G., I. Olshausen, Krankh. d. Ovar., I886. Rokitansky, Allg. Wien. m. Zeit., I859, 253. 584 Zahn, V. A., I5I (Lit.). Martin, Krankh. weibl. Adnexorg., I899. Garkisch, Z. G. G., 63. Lunzer, Z. f. Heilk., 28. Langer, A. G., 49, 87. E. Fraenkel, A. G., 48, 57, 5I I. 585 Orthmann, Verh. Gyn. Gesell., I 897. L. Fraenkel, A. G., 56, 355. L. Pick, Cent. Gyn., 1903. Pfannenstiel, Handb. d. Gyn. (Veit), IV. Kroemer, D. W., 1907, 1246. Patellani, Cent. Gyn., 1905, 388. Stoeckel, Fest. f. Fritsch, 1902, I36. Albert, Cent. Gyn., 1900, I328. Voight, A. G., 49. 586 Grousdew, A. G., 70. Michelazzi, La Riforma med. Roma, 3. Santi, Annali di Ost. e. Gin., Milano, 27. Seitz, A. G., 75. Schaller, Pforringer, Beitr. G. G., 2. Wendeler, Martin's Handb., I899, 2, 416. Seitz, A. G., 77. 587 Jayle, Bender, Rev. de Gyn., 7, 755. Nebesky, Cent. Gyn., I 895, I052. Odebrecht, Z. G. G., 54, I60. Hoffmeier, Frauenkrankheiten, 1908. 588 PyeSmith, cit. by Pfannenstiel. Peaslee, Ovarian tumors, N. Y., I872. Troschel, Arb. a. d. Mackenrodt's Klin., H. 3. 589 E. Fraenkel, Mon. G. G., 2I, 67. Werder, Amer. J. Obst., 38, 668. Olshausen, D. m. W., I902, 750. H. Freund, Z. G. G., I7. Malcolm, London Obst. Soc., I899, 226. Opitz, Z. G. G., 42. Pozzi, Rev. de Gyn., I904, 407. Holzapfel, D. Gyn. Ges., 1905, 358. Schroeder, Z. G., 54, 19. Marchand, Beitr. z. Kennt. Ovarialtum, I879, Halle. v. Velits, Z. G. G., I7. Wagner, A. F. Heilk., 5. H. Freund, Saml. kl. Vort., 301, 362. 590 Virchow, V. A., 75, 348. Flaischlen, Z. G. G., 7, 434. 591 Glockner, A. G., 83. Tauffer, D. Gyn. Gesell., 1905, 374. 592 Zacharias, M. IV., I904, I386 (Lit.). Bauer, Z. G. G., 75, 617. L. Pick, A. G., 64, 670 (Lit.). R. Meyer, V. A., I72. 593 Pfannenstiel, A. G., 38, 407 (Lit.). Mitjukoff, A. G., 49, 278. Kretschmar, Mon. G. G., 19, 389. E. Herter, Martin's Handb., I899, 2, 61 5. Gebhard, Path. Anat. weibl. Geschl., I 899. Waldeyer, A. G., I, 252. 594 Olshausen, Z. G. G., 4I, 278. Fromme, I. D., Tubingen, 1902. Frank, Prag. m. Woch., I891, 266. Martin, 
Krank. d. Eierstock. R. Strassmann, Z. G. G., 22, 308. 595 Werth, A. G., 24, 103. Gunzberger, A. G., 59, I. Westphalen, A. G., 59, 632. Mennig, I. D., Kiel, I880. Wendeler, Mon. G. G., 3, I86. Netzel, Nord. med. Archiv., 1885. Polano, Mon. G. G., 13, 734. 596 Stratz, Die Geschw. f. Eierstocks, Berlin, I 894. 597 Glockner, A. G., 75. J. W. Williams, J. H. Rep., I892, 20. Orthmann, Mon. G. G., 9, 780. Amann, Mon. G. G., I 897, 5, 224. Orthmann, Handb. K. weibl. Adnexorg. 600 Heinrichs, Martin's Handb., I 899, 2. v. Kahlden, Cent. Path., 6, 257. Acconci, Cent. Path., I890, 739. Emanuel, Z. G. G., 27, 62. Kworostansky, A. G., 57, I. Schroder, A. G., 64, 193. W. Nagel, Anat. d. Mensch. (Bardeleben), 1896, 72, 1. Hansemann, D. path. Ges., 1904, 85. Polano, Z. G. G., 51, I. Stone, S. G. O., 22. 602 S. Gottschalk, A. G., 59, 676. Ingier, A. G., 83 (Lit.). M. Voight, A. G., 70, 87. Lonnberg, Nord. m. Arch., 190 I. Mengershausen, I. D., Freiberg, 1894. L. Pick, Berl. W., 1902, 618. 603 Werdt., Z. B., 59. Krukenberg, A. G., 50. 604 Wagner, Wien. kl. W., 1902, 519. Romer, A. G., 66. Schlagenhaufer, M. G. G., I5 (Lit.) Glockner, A. G., 72. Schenk., Z. G. G., 5 I. Rosthorn, Mon. G. G., 24. Outerbridge, Am. J. Obstet., 64 (Lit.) . M. E. Hall, N. Y. Path. Soc., 1912, 57. 605 Apelt, Beitr. G. G., 5, 360. 606 Lippert, A. G., 74. J. Simpson, Lancet, 1905, I, 799. Lohlein, Mon. G. G., 3. John Williams, West London M. J., 1897. Wedekind, I. D., Giessen, 1907. Doran, London P. S., I889. Lonneberg, C. f. Gyn., I899, 953. Wiel, J. H. R., I905, I02. Hubert, I. D. Giesson, I90I. Kelly, Dis. Child. Keating, Suppl., 1901. Virchow, Geburtsh. Verh., Berlin, I848, 3, 220. Olshausen, Krank. d. Ovar., I 877. Fischel, A. G., 15. Coblentz, V. A., 84. Malassez, de Sinety, Arch. de. Physiol., 1878. 607 Flaischlen, Z. G. G., 6, 7. Walthard, Z. G. G., 49. Pfluger, D. Eierstock d. Saugetier, Leipsig, 1863. L. Pick, Berl. kl. W., I900, 219. E. Bauer, Z. G. G., 75. Glockner, A. G., 75. Limnell, A. G., 63. Wendeler, Krank. weibl. Adnexorg. (Martin), 4I I. Steffeck, Z. G. G., 19, 28. Hoffmeier, Schroeder's Handb., I898. v. Franque, Z. G. G., 39. 608 J. W. Williams, J. Hop. Bull., ı 89ı, I 49. v. Müller, A. G., 42. Pozzi, Beaussenat, Rev. de Gyn., I897. 260. Kossmann, Mon. G. G., I. Nagel, Veit's Handb., 1. Kossmann, Martin's Handb., 2, 919. 609 Bucura, Z. f. Heilk., 28. Bennecke, I. D., Gottingen, 1902. Payer, Mon. G. G., 14. 610 L. Pick, V. A., 156. Werth, Munch. W., I 895, 765. R. Meyer, Erg. P., 9, II, 534. v. Franque, Z. G. G., 39. Schickele, Cent. Path., 1904. Aschoff, Mon. G. G., 9. Babo, A. G., 6r. Neumann, A. G., 58. Vassmer, A. G., 64. W. N. Russell, J. H. Bull., I 899. Schickele, Cent. Path., 1904. Weiss, Z. B., 24. L. Pick, A. G., 64. Peham, Mon. G. G., Io. Sternberg, Cent. Gyn., 1906, 732. L. Pick, A. G., 76. Schickele, Beitr. G. G., 6; Cent. Path., 1904, 261. Pick, Arch. micr. Anat., 84. Orthmann, Z. G. G., 15, 21 2; 58, 376; 63. Doran, J. Obst. Brit. Emp., I910. Fromme, Heynemann, Veit's Handb., 1908, V. Norris, S. G. O., 1909, 272. 611 Kiwisch, cit. by Fromme. Stolz, A. G., 66, 365 (Lit.). Rosthorn, Prag. Z. f., Heilk., I7. Sanger, Barth (Martin), Krank. d. Eileiter. 612 Friedenheim, B. kl.W., I 899, 542. Kundrat, A. G., 80. Hofbauer, A. G., 55. Novy, Mon. G. G., I1. Kworostansky, A. G., 85. v. Franque, Z. G. G., 47. Amann, Cent. Gyn., 1909, 1684. Janvrin, N. Y. M. J., 49, 609. Jacobs, Le Prog. med. Belge., 1905. Sanger, Cent. Gyn., I 886, 601. v. Kahlden, Z. G. G., $2 x$.

\section{THE OVARIAN TERATOMA}

613 Kroemer, Veit's Handb., Gyn., I908, 4 (Lit.); A. G., 57, 322. Höhne, D. Ges. f. Gyn., II, 360. Neuhauser, A. G., 79, 696. 614 Falk, Mon. f. Gyn., 12, 351. Arnsperger, V. A., 156. Schottlander, A. G., 78, I 37. 615 Bonney, Obstet. Soc., London, 4, 354. Opitz, Z. G. G., 42, 188; 47, I1 8. Pfannenstiel, Veit's Handb. Gyn., 1908, 4. Wilms, D. A., 55, 289 (Lit.). Saxer, Z. B., 31. Grechen, cit. by Kroemer. Schramm, Mon.f. Gyn., 13, 845. Kuster, Berl. kl. W., I 887, 24, 517. Kappeler, I. D., Zurich, 1896. Reverdin, Nouv. Arch. d. Obstet. et de Gyn., 2, 52. Thornton, London Obstet. Soc., 1882. Repin, C. P., 3, 98I. Askanazy, Dermoidcysten d. Eierstocks, Stuttgart, 1904. Shattock, Lancet, 1904, 2, 1284. Goffe, Amer. J. Obstet., 49, 675. Axel-Key, cit. by Wilms, Hygeia, 6, 300. Flaischlen, Z. G. G., 6. Baumgarten, V. A., 107. 617 Sutton, 
London P. S., I888. Pommer, C. P., ז890, 260. Perls, D. A., 17, 443. 618 Katsurada, Z. B., 30. Lazarus, I. D., Giessen, I 888. Neuhauser, A. G., 79, 696. Ewald, Wien. kl. Woch., 1897. L. Pick, Berl. kl. Woch., 1902, 442, I I89; 1904, 158, 195; Cent. Gyn., 1905, 545, 82 I. 619 Novak, Z. B., 45 (Lit.). Landau B. kl. W., I904, 162. Schroeder, Krank. weibl. Geschl., I 893. Wulkow, I. D., Marburg, I901. Heinsius, Z. G. G., 56, 259. Hoffmeier, D. ges. Gyn., II. Keitler, Z. f. Heilk., I900, 21. Coen, La Gyn., 1903, 478. Dunnwald, I. D., Munchen, I901. 620 Reinprecht., Wien. Med. W., 1902, 33. Herrmann, Z. G. G., 44, 217. Bab, Char. An., 30. Yamagiwa, V. A., 147. Friedlander, V. A., 56. Seeger, U. sol. Tum. d. Ovar., Munchen, 1888. Amann, D. Ges. Gyn., 8. Lorrain, Soc. Anat., Paris, 1905. 621 Litten, V. A., 75. Ludwig, W. kl. W., 1905, 7 I 5 (Lit.). Schwertassek, A. G., 47 (Lit.) Flaischlen, Z. G. G., 7. Geyer, I. D., Greifswald, 1895. Biermann, Prag. m. W., I885. Mirabeau, Monatsch. f. Gyn., Io, 462. Bell, J. Obst. Brit. Emp., 1905. Kretschmar, D. Ges. Gyn., 9, 459. Walthard, Z. G. G., 50, 567. Meyer, Z. G. G., 54, 370; V. A., 173. Katsurada, Z. B., 30. Trapl., Z. G. G., 70 (Lit.). Polano, Munch. W., I904, 45. Bauer, Z. G. G., 75. 622 Lubarsch, Arb. Inst., Posen, I901, 230. Monckberg, V. A., rgo. Risel, Arb. p. Inst., Leipsig, I903. L'Esperance, J. Med. Res., 32, 225. Reiss, I. D., Berlin, I882. Jung, Z. G. G., 52. 623 Bandler, A. G., 6I. Amann, C. P., II, 793.

\section{CARCINOMA OF STOMACH}

625 Welch, Pepper's System, I885, II, 530. Gurlt, L A., 25. Haberlin, D. A., 44, Friedenwald, A. J. M. S., I48. Reiche, D. m. W., I900, I20. Virchow, Verh. m. Ges.. Wurzburg, 1860. Martin, Osler's System, 1908, 5, 220. Brinton, Brit. For. Med.-Chir. Rev., 1857. Gussenbauer, Winiwarter, L. A., 19, 372. Cullingworth, B. M. J., I877, 2, 253. R. Williams, Lancet, I897, I, I I94. 626 Koster, C. C., 1888, 372. Dittrich. Prag. Viert., I 845, I I6. Landouzy, Soc. anat., I872, 27. Scheffer, Jahrb. d. Kinderheilk., I880, 15, 425. Bernoulli, Arch. Verdk., 13. Osler, McCrae, Cancer of Stomach, Phila., 1900. Moore, London P. S., 1885, 36, 195. Dock, A. J. M. S., I897, I13, 665. Glynn, Lancet, I896, 2, I232. Wilde, I. D., Kiel, I 892. Mathieu, Sem. med., I895, 225. Menne, Z. C., 81; Arch. f. Orthrop. Mechanot., etc., I905. Boas, D. W., I897, 707. R. Schmidt, Bos. Neubild. d. Bauchorg. Wien., I9II. 627 Tabora, D. W., 1905, 570. 628 Matti, Z. C., 77. Matsuoka, B. B., 46. Finlay, London P. S., 34, 102. 629 Roseler, V. A., 77, 372. Tilling, cit. by Martin. Kulbs, W. kl. W., 1901, 762. Herxheimer, Z. B., $4 I$ (Lit.). Lubarsch, D. path. G., 1906, 200. E. Kaufmann, 1. c., 433. Chaput, Soc. anat., 1905. Storer, Boston M. S. J., 1872, 93. Amidon, N. Y. Path. Soc., 1879, 3, 38. Verse, Arb. p. Inst., Leipsig, 1908. 630 Ewing, An. Surg., 67. Rokitansky, Schmidt's Jahrb., 25, 40. Dittrich, Prag. Viert., I 848 . Lebert, Krank. d. Magens, Tubingen, 1878. Zenker, cit. by Martin. Hauser, Das Cylinderepithel. Carc., Jena, 1890. Futterer, U. d. Etiol. d. Carcinoma, Wiesbaden, I90I. Riegel, Nothnagel's System, 1909. MayoRobson, Cancer of Stomach, N. Y., 1907. 631 Hirschfeld, Cong. inn. Med., 1902, 279. 632 Wilson, MacCarty, A. J. M. S., 138, 846. MacCarty, Mayo Clinic, 1910. Moutier (Matlieu et al.), Mal de l'estom., I9I3. Thalheimer, A. S., 67, 215. 634 Bamberger, Behand. d. chron. magenges., Berlin, I909. Hemmeter, A. J. M. S., 1903, 126, 33. Maniscalio, Rif. med., I905. 636 Brinton, Brit. and For. Med.-Chir. Rev., 1857. Mislowitzer, I. D., Berlin, I889. Lange, I. D., Berlin, I877. 639 Rokitansky, Anat. Path., 3, 2. 640 Cruveilhier, Anat. path., I 835, 3, 25. Lyle, Ann. Surg., 54 (Lit.). Brinton, Dis. of Stomach, I864. Krompecher, Z. B., 49. Krompecher, Makai, Z. K., II. Meinel, Z. B., 3 I. Bret, Paviot, Rev. de Med., I 894,384 . Schoch, I. D., Zurich, I857. Nauwerck, D. A., 21. Schmidt, Rev. med. de l'Est., I88I. Viti, Rev. des sci. med., 1887, 31, 543. Rosenheim, Zeit. kl. med., 17, I16. Deguy, Soc. anat., I896, 314. Marcy, Griffith, A. J. M. S., 88, 1 82. Sheldon, Ann. Surg., 39, 341; 44, 666. v. Eiselberg, S. G. O., 7, 253. Formad, J. A. M. A., I 887, 4, 599. Albutt, cit. by Leith, Allbutt's System, I900, 3, 440. Turner, London P. S., I887. Nothnagel, D. A., 24, 352. Mariganac, 
Soc. Anat., I 877, 519. Wilks, London P. S., I86r, 13, 83. Henrot, Soc. d. hop., 1878, 975. v. Kahlden, C. kl. M., I897, 281. Gabbi, Rif. med., I893. 642 Tilger, V. A., 132. Jungmann, I. D., Wurzburg, 1892. Szobolew, V. A., I6I, 56. Hansemann, Verh. D. Naturf., Lubeck, I892. Fick, Z. C., 1898. Cignozzi, Rif. med., 1905, 21, 449. Aldegarmann, I. D., Wurzburg, 1899. Donath, V. A., 195, 341. 643 G. Hayem, Bull. soc. anat., 1905, 649. 644 Mathieu, Thèse, Lyon, 1884, cit. by Bret, Paviot. Danosky, Arch. gen. de. med., 1843. Torkel, V. A., 180, 316. Hanot, Gombault, Arch. de Phys., 1882. J. E. Schmidt, Mitt. G. G., 22 (Lit.). Gosset, Masson, Presse med., 1912, I, 225. Saltykow, V. A., 153. Marchand, Eulenberg's Encyc. III, Auf. 5, 52. Lubarsch, Erg. P., I895, 2; Abt., 2, 180. Thorel, V. A., 151. Schridde, A. D., 73. Fabian, C. P., 18, 689. Rosenheim, Berl. W., I888, I02 I. Hammerschlag, A. Vdk., I896, 2, I. Lenk, Z. kl. M., 37, 296. Boekelmann, Z. kl. M., 44, I28. Mathieu, Arch. gen. de Med., I889, 402. P. Cohnheim, Arch. Vdk., I 896, I, 278. Fenwick, Lancet, I 877, 2, I. 645 Kokubo, Festschrift f. Orth., 1903. A. Schmidt, V. A., 143. Schneider, V. A., I48, 266. Carle, Fontino, L. A., 56. Terrier, Hartman, Chir. de. l'estomac. Bensaude, Soc. hop., r9io, 29, 382. 646 Cuneo, Thèse, Paris, 19ı. Most, L. A., 59. 647 W. J. Mayo, St. Mary's Clin., 1910. Renner, Mitt. G. G., 13. Borrmann, Mitt. G. G., I, Suppl. Colwell, Arch. Middlesex Hosp., 1906, 7, I5I (Statistical). 648 Kausch, Handb. d. Chir. Bergman-Bruns, r907, 3. Belin, Thèse, Paris, 1888. Tarchetti, D. A., 67. Troisier, Gaz. heb., 1886, 23, 683. Hechler, I. D., Berlin, I 897. Hosch, Mitt. G. G., I8 (Lit.). Leydecker, V. A., 134. Hektoen, V. A., 135. Piat, Thèse, Paris, I9I1. Moutier, Maure, Arch. med. Exper., 1910, 22, 433. Israel, Berl. W., I 897, 68. Spaeth, V. A., 35, 432. Acker, D. A., II, I73. 649 Borrmann, Deut. p. Ges., I904, 80. Schlagenhaufer, M. G. G., 15, 485. Glockner, A. G., 72. Stickel, A. G., 79. Krukenberg, A. G., 50. Pfannenstiel, Veit's Handb., 1908, 4, 187. Polano, Z. G., 51. Papaioannou, M. G. G., 20. Kraus, M. G. G., I4. Sitzenfrey, Mitt. Grenz., I9. Lonquet, Quenu. Rev. de Chir., 1906, 97. 651 G. Hayem, Bull. soc. anat., 1905, 649. Doering, L. A., 83 (Lit.). 652 Sachs, Arch. exp. P. P., 24. Lubarsch, Achylia gastrica, Martius, Leipsig, 1887. A Schmidt, V. A., I43. Hammerschlag, Arch. Verdk., 2. Reinboth, D. A., 58. Gluzinski, M. G. G., Io. Zoeppitz, M. G. G., 24, 538. Janeway, J. A. M. A., 1913, 61, I339. 653 Rieder, M. W., I910, 2508. Holzknecht, Jonas, Rad. Diagnostik. Wien., 1908. Haudek, M. W., 1911, 399. v. Schmieden, L. A., 96, 253; Mitt. G. G., 27. Cole, N. Y. M. J., I914, 99, 305. 654 F. Müller, Z. k1. M., I6. Frese, D. A., 68. 656 Ehrlich, Lazarus, Nothnagel's Syst. v. Jaksch, II Cong. inn. Med. Senator, Z. kl. M., 7. Riess, Ibid., Suppl. Klemperer, Berl. k. W., I889, 869. Osler, J. H. B., 1902. Miura, B. kl. W., I891, 905. Deutschmann, Beitr. z. Augenheilk., I, 34. Klippel, Thèse, Paris, 1889. Rohrer, M. W., I897, 1826. 658 Hosch, Mitt. G. G., 18. 659 Jonas, Wien. m. W., 1909, 260. v. Sury, Arch. Verdk., 1907, 13. Le Count, Amer. Assoc. Cancer Research, r912. 660 Matti, Z. C., 77. Petersen, D. Chir. Cong., 1903. Leriche, Rev. de Chir., I906, 34, I 10. H. J. Patersen, Lancet, 1906, I, 574. Peck, S. G. O., 24.

\section{CARCINOMA OF INTESTINE}

660 Stengel, Mod. Med., Osler, 5. Brill, A. J. M. S., 128, 824. v. Heurlin, Thèse, Paris, 1897. A. Pic, Rev. de Med., I894, I 895. Rolleston, Lancet, I90I, I, I1 2 I. Geiser, Z. C., 86 (Lit.). Letulle, Soc. anat., 1897, 72 I. Perry, Shaw, Guy's Hosp. Rep., 1893, 274. Nattan, Larier, Gaz. hop., I 897 ; Soc. anat., 1900, 732. Collin, Thèse, Paris, 1894. Eichorst, Hand. spec. P. u. Ther., I890, 2, 234. Ewald, B. W., I 886, 527. Butz, I. D., Greifswald, 1900. Descos, Beriel, Rev. de Med., I899, 631. Krause, I. D., Kiel, 1901. Gerster, N. Y. P. S., 1905, 5, 139. 662 Orth, Lehrb. spec. Path., I887, I, 850. S. Fenwick, Edinb. M. J., IO, 316 . J. A. Syme, Lancet, 1904, I, I48. Duncan, Edinb. M. J., 1 886. Schleips, B. B., 58 (Lit.). Riegel, D. W., I 890, 861. Routier, Soc. de Chir., Paris, 1899. Niemack, Ann. Surg., 36. Petrow, Cent. Chir., 1896, 542. Kukula, L. A., 6o. Kuttner, B. B., 23. Hahn, D. W., I 897, 674. Thorel, M. IV., 1905, 2062. 663 
Lubarsch, V. A., III, 280. Notthaft, D. A., 54, 555. Bunting, J. H. Bull., I904, 389. Oberndorfer, Z. B., 29; Frankf. Z. P., I, 426. Trappe, Frankf. Z. P., I, I09. 664 Verse, D. P. G., I2, 95. Ransom, Lancet, I890, 2, 1020. Saltykow, Z. B., 54 (Lit.). Schapper, D. P. G., I6, 387. 665 Riedel, D. Chir. Gesel, I896. Marckwald, M. W., I905, 1033. Kanzler, B. B., 48. Elting, A. S., 37. Moschkowitz, A. S., 37. Batzdorff, L. A., 98 (Lit.). McCarthy, cit. by Batzdorff. Bender, Lab. Reports, 1909. Zaaijer, B. B., 54. Milner, D. W., I910, I190; Z. C., I02. McWilliams, A. J. M. S., 135, 822. Baldauf, Albany Med. An., 1905. Kudo, Z. K., 6. Rokitansky, W. med. Presse, I866, 675. Draper, Boston M. S. J., I884, 131. Konjetzny, Z. C., 103, 365. A. O. J. Kelly, A. J. M. S., 135. Neugebauer, B. B., 67, 328. Whipham, Lancet, 1901, 1, 320. Rolleston, Jones, A. J. M. S., I38, 95I. 666 Lubarsch, V. A., III, 280. Glazebrook, Virginia M. M., 22, 22 I. Sudsuki, Mitt. G. G., 7, 5I6. Vassmer, Z. C., 9I. 667 Nothnagel, Erk. d. Darms, Wien., 1898. Lonant, Thèse, Paris, 1900. Garrard, Quart. J. Med., I897. Nothnagel, Syst. Med., 1907, 412. Czerny, B. W., I880. Stern, Nothnagel's Syst. Schoning, Ibid. Clar. Ibid. Goeckel, Arch. Vdk., 2. Anschutz, Mitt. G. G., 3 (Lit.). Zinner, L. A., 9o. Mya, Lo Sperimentale, I 894. Lowenstein, C. P., 1907, 429 (Lit.). Keibel, A. D., I 896-97. Stieda, L. A., 70. Kraske, Kl. Vort., I83, I84. R. Meyer, V. A., 195. Cohn, L. A., 94. 668 Rotter, Band. d. f. Chir., r913, 3; L. A., 98 . Petermann, L. A., 80, 86. Kuttner, Munch. Woch., 1920, 799. Pennington, J. A. M. A., 7I, I892. Mayo, A. S., 64, 304. Bardenhauer, L. A., 4I. Wulf, I. D., Kiel, I892. Doering, L. A., 83 (Lit.). Rotter, L. A., 58, 357. 670 Schuchardt, L. A., 61, 340. Korte, L. A., 61, 403. Brosch, D. A., 57. Kanthack, Furnival, London P. S., 68, 99. Israel, cit. by Korte. $\mathbf{6 7 2}$ Albu, B. W., I912, I 847. F. C. Yeomans, N. Y. M. R., 90,537 . Quenu, Mal. du rectum, I 899. 673 Petrow, C. C., I896, 542. Cripps, London P. S., I882. Smith, St. Bart's Hosp. Rep., 1887. Bickerstedt, Ibid., I 890 . Port, Z. C., 42. Thorebecke, Z. C., I26. Quenu, Landel, Rev. de Chir., I 899. Wechselmann, B. B., 7o. Babler, J. A. M. A., 52, 1235. Brentano, C. C., 1909. Oseki, Z. C., 118. Ball. Dis. of Rectum, 2d ed., 341. Heaton, London P. S., 45. Strasberger, I. D., Bonn, I894. 674 De Buck, Vanderlinden, Belg. med., 1899. Paneth, L. A., 28. Wiener, Z. B., 25 (Lit.). Eiselt, Prag. Viertelj., I862.

\section{EPITHELIAL HYPERPLASIA AND TUMORS OF LIVER}

674 Simmonds, D. A., 34, 388. 675 Ponfick, V. A., II8, II9, I38; Suppl. Fest., Virchow, I89I. Podwyssoski, Jr., Z. B., I. v. Meister, Z. B., 15. Morgan, Regeneration, N. Y., 1907. Jackson, Pearce, J. Exp. Med., 1907, 9, 577. 677 Stroebe, Z. B., 21. Barbacci, Z. B., 30. Meder, Marchand, Z. B., 17. Friedreich, V. A., 33. Kelsch, Kiener, Arch. d. Physiol., I876. Sabourin, Rev. de med., 1884, I, 321. Delepine, London P. S., I890, 41, 362. 678 A. Jacobi, N. Y. M. Rec., I 897, 52, 109. Hansemann, B. W., I890, 552. Ribbert, D. W., I910, I43. Prescott, Boston City Hosp. Rep., I895, 6, 245. Miller, Cleland, Arch. Path. Lond. Hosp., I906, I, 5. Milne, J. P. B., I909, 348. Karsner, Arch. Int. Med., I9I I, 8, 238. Mair, J. P. B., I9II, 6, 389. Phillipp, Z. K., 5, 326. Rolleston, Dis. Liver, 469. Vecchi, Guerrini, Med. News, 79, 816. Oertel, V. A., I80. Caminiti, L. A., 49, 630. Lohlein, Z. B., 42. Rindfleisch, N. W., I901, 283. Dibbelt, I. D., Greifswald, 1903. Necker, Z. Heilk., 1905. Adler, Z. B., 35. 680 Giachetti, Riv. nerv. e. ment., 12, I49. Schmidt, V. A., 148. Clan, Prag. m. W., I901. Prym, Frankf. Z. P., 2, I7o. Hale White, Guy's Hosp. Rep., I89o, 47, 59. 681 Keen, Ann. Surg., 30. Yeomans, J. A. M. A., 52, 1741. Rokitansky, Allg. Wien. m. Z., I859. Salter, London P. S., I 869. Engelhardt, D. A., 6o. Wegelin, V. A., I79. Heussi, I. D., Zurich, I898. B. Fischer, V. A., 174. Christiani, J. d'anat. et Phys., I891, 27, 27 I. 682 Ciechanowski, cit. by Caminiti. Pepere, Arch. p. 1. sc. med., 1902, 26, 117 . Schmorl, Z. B., 9. De Vecchi, V. A., 177. Glynn, Quart. J. Med., I91 1, 5, 157 . Hirschler, Frankf. Z. P., g. (Lt.). Eggel, Z. B., 30 (Lit.). Ewing, N. Y. P. S., 1906, 6, 168. 684 C. P. White, B. M. J., 1899, 2, 1347. Goldzieher, Bokay, V. A., 203. Arnold, Z. B., 8. v. Kahlden, Z. B., 21. Steinhaus, C. P., 1900, 87 I. Marx, Z. B., 36. 685 De Haan, Z. B., 
34. Dominici, Merle, Arch. med. exper., 21. 686 Cruikshank, Teacher, J. P. B., I9ro, 282. Griesinger, Arch. f. Heilk., I864. Hanot, Gilbert, Etude s. 1. mal. d. Foie., Paris, I 888. v. Heukelom, Z. B., I6. Thorel, Z. B., 18. Marckwald, V. A., 144. I.ancereaux, Gaz. med., Paris, I 868. 687 Borst, 1. c., II, 567. Brissaud, Arch. gen. de Med., 1885, 2, 129. Birch-Hirschfeld, Lehrb. d. Kinderk. (Gerhardt), 827. Frohmann, I. D., Koningsberg, 1894. Theodorow, V. A., 193. Parcelier, Fromaget, Arch. med. exper., 24. 688 Orth. Path. Anat., I887, 955. Schmieden, V. A, 159, 290. Watzoldt, Z. B., 39. Travis, J. H. B., 1902. Peabody, Tr. Assoc. Am. Phys., 19. Renon, Geraudel, MonierVinard, Arch. med. exper., 22. Geraudel, J. d'Anat. et d. physiol., 1907. Yamagiwa, V. A., 206. Rolleston, Lond. P. S., I 898, 49, I 33. Bayer, Prag. W., I 892, 637. Aldaus, B. M. J., I9I I, 2, 688. North, N. Y. M. R., I882, 344. Doran, Med. Chir. Tr., I904, 87, I. 689 Zahn, V. A., 143. Moschkowitz, A. J. M. S., I3r, 674 (Lit.). Bristowe, Lond. P. S., I 856, 6, 229. Still, Lond. P. S., 49, I 55. Lejars, Thèse, Paris, I 888. Luzatto, cit. by Boinet; Rev. de Med., 23. MacDonald, N. Y. State Jour. Med., 1908, 185. Siegmund, V. A., I15. v. Kahlden, Z. B., 13. Workmann, Glasgow Hosp. Rep., 1900, 2, 363. Nauwerck, Hufschmidt, Z. B., 12. Shattock, Lond. P. S., 37, 287. Leppmann, Z. C., 54. Shattuck, Boston M. S. J., 143, 427. 690 Keen, Ibid., 126. Siegmund, V. A., I15. Dmochowski, Janowski, Z. B., 16. Brigidi, Lo. Sper., I881, 337. v. Hippel, V. A., I23, 473. Dreschfeld, J. Anat. and Phys., 1880. Herxheimer, C. P., I902. Kika, cit. by Yamagiwa, V. A., 206. Pepere, I. tum. mal. prim. d. fegato, I902, I7I. 691 Greenfield, London P. S., 25, 166. Thorel, Z. B., 18. Waldeyer, V. A., 55. Sokalow, V. A., 162. Cagnetto, Arch. p. 1. Sci. Med., I910, 34, 495. Muir, J. P. B., I908, 299. Beneke, Z. B., 9. Witwicky, Z. kl. M., I899, 36, 474. Rindfleisch, Arch. f. Heilk., 1864, 5, 394. Weigert, V. A., 67. Burkhardt, Frank, Z. P., 1909, 593. Lohlein, Z. B., 42. 692 Bonnet, I. D., Kiel, 1902. Bascho, Frank, Z. P., 1909, 242. Arnold, Z. B., 8. Delepine, London P. S., 1891, 43, 161. Byrom, cit. by de Vecchi. Cesaris-Demel, Arch. p. 1. Sci. Med., Torino, 1900. de Vecchi, Guerrini, Rif. med., 1901, 16, 365. Marx, C. P., 1904; Z. B., 36. 693 Rolleston, Trevor, J. P. B., 15, 247. v. Kahlden, Z. B., 21. Nazari, Il Policlin., 1905, 12. Dominici, Merle, Arch. de med. exper., 21, I36. Holm, Arb. path. Insti., Tubingen, 5, 1904. Theodorow, C. P., 1908, 507. de Haan, Z. B., 34. Ford, A. J. M. S., 120, 4I3. Bramwell, Leith, Lancet, 1897, I, I70. Meissenbach, St. Louis Med. Rec., I884, 9. Bernhinz, La clin. med., Milano, 1900. Steinhaus, C. P., 82 I. Frerichs, Dis. of Liver, Syd. Soc. Musser, Boston M. S. J., I 889, I21, 525; Tr. Assoc. Am. Phys., I 889. Courvoissier, Path. u. Chir. d. Gallenwege, Leipsig, r890. Futterer, U. d. Atiol. d. Carc., Wiesbaden, I901. Maxon, London P. S., 1867, 140. Thomas, Noica, Soc. Anat., I896, 71, 471. Zenker, D. A., 44. Janowski, Z. B., Io. Siegert, V. A., 132. Candler, Proc. Roy. Soc. Med., I9II, 4; P. S., 87. Slade, Lancet, 1905, I, 1059. 694 Michaux, Soc. de Chir., 1907, 33, I 182. Devic, Gallavardin, Rev. d. Med., 21, 569. Beadles, Lond. P. S., I 897,48 , I 19. West, Ibid., I886, 37, I44. Warthin, Phila. M. J., I900, 6, 38. Willigk, V. A., 48, 524. Riedel, M. W., I9I I, I337. Moutier, Arch. gen. de Med., I905, 164, 2001. 696 McCarthy, Mayo Clin., I910, I5I. M. Dominici, L. A., 96, 387. Sand. Mayer, Arch. med. exper., 23, 523. Chappet, Lyon med., 1894, 76, 146. Pels-Leusden, L. A., 80, I28 (Lit.). Aschoff, D. P. G., 1906, 9, 4I. Lubarsch, Arb. Path. Inst., Posen, 1901. 697 Deetz, V. A., 164, 381. Monckberg, V. A., 169,353 . Wieting, Hamdi, Z. B., 42. Kehr, Diagnosis of Gall-stone Dis., I901. Jourdan, Soc. anat., I891, 66, 323. Bayer, Z. B., 46. Landsteiner, W. kl. W., 1904, I62. 698 Griffon, Segal, Soc. anat., I 897, 72, 586. Iwasaki, L. A., 104. Carson, Smith, An. Surg., 62. Paulavecchio, L. A., 87, 365. Sutherland, Glasgow M. J., I898, 216. Bayer, Z. B., 46. Klingel, B. B., 5, 1889. Seibert, N. Y. M. R., 1882, 21, 299. Becker, J. A. M. A., 1903, 40, 903. 699 Donati, Arch. p. 1. sc. med., 1904, 28. Miodwoski, V. A., 169. Lambl, V. A., I 855, I33. Schmidt, I. D., Giesen, I892. K. Jenner, I. D., Breslau, I 892. Chapper, Lyon med., I 894. Lecene, Pagniez, Arch. gen. d. med., 1901, 187, 176. Futterer, Chicago Med. Rec., I897, 12, 325. Lapointe, Raymond, Arch. gen. de Chir., I908, 2, 375. 700 Leith, Tr. Med. Chir. Soc. Edin., I 896, 15, 59. Busson, Thèse, Paris, I890, 
Georges, Thèse, Paris, 1896. Aynaud, Gaz. d. hop., 1907, 80, 807. Dieulafoy, Presse med., 1907, 657. Letulle, Soc. d. hop., 1905, 1063. Klotz, Montreal M. J., I904, 33, 497. Carnot, Harvier, Soc. d. hop., 1906, 296. 701 Schuller, B. B., 31. Duval, J. Exper. med., IO, 465. Quenu, Rev. de Chir., I909, 39, 245. Mayo, W. J., Ann. Surg., 42, 93. Oehler, B. B., 69, 726. Borelius, B. B., 6I.

\section{TUMORS OF PANCREAS}

702 Lazarus, Z. kl. M., 5I, 52. Edling, V. A., I82. Sotti, Arch. p. sci. med., 1906. Neve, Lancet, I891, 2, 659. Fhierfelder, cit. by Korte. Biondi, Clinica Chirurg., I896, 4, I31. Chauffard, Gaz. d. hop., I896, I385. Cesaris-Demel, Arch. p. 1. sci. med., I895, 225. Nicholls, J. Med. Res., I902, 8, 385. Cecil, J. Exper. Med., I909, II, 266. Classen, Krank. d. Bauchspeicheldrusen. Koln, I 842. 703 Ancelet, Etude s. 1. mal. d. Pancreas, Paris, I 866; Gaz. d. hop., I 860. Bard, Pic. Rep. de Med., I888. Miraillie, Gaz. d. hop., 1893. Fitz, Boston M. S. J., 120. Korte, Deut. Chir., 45, d. A. Kuhn, B. kl. W., I887, 494. Hulst, V. A., I80, 288. Leriche, L. A., 92. Heinrich, V. A., I99, 392 (Lit.). Lazarus, Z. f. Heilk. Chir. Abt., 22. Lachmann, I. D., Griefswald, I889. M. Müller, N. Y. M. R., I 895, 48, 301. Hagenbach, Z. C., 27, I Io. Boldt, I. D., Berlin, I882. Segrè, An. univ. d. med. e. chir., I888, 283, 3. 704 Ollivier, Z. B., 15, 351. Muckenbeck, I. D., Marburg, I 890. Pearce, Albany Med. Ann., 1904, 329. 706 Fabozzi, Z. B., 34. Reitmann, Z. f. Heilk., 1905, 26, I. Helmholtz, J. H. Hosp. Bull., 1907, 185. Pic, Tolot, Prov. Med., I900. Eloesser, Mitt. G. G., I8. Oser, Die Erkrank. d. Panc. Wien, I 898. Schilling, Fort. d. Med., I906. Kakels, A. J. M. S., I23, 471. K. Ehrlich, M. W., I903, 368. E. Weil, Prag. m. W., 1905. Kronlein, B. B., 14. Piccoli, Z. B., 22. Borrmann, E. P., 1900, 824. v. Kahlden, C. P., 9, 33. L'Huillier, V. A., 507. Schirikogoroff, V. A., 193 (Lit.).

\section{MAXILLARY TUMORS OF DENTAL ORIGIN}

707 Magitot, Arch. gen. de Med., I872-73. Barnes, Med. Trans., London, 1813, 316. Guzack, Dublin Hosp. Rep., 1826, 29. Delpech, Chir. clin., 1828. Dupuyten, Chir. clin., I 833. Forget, Thèse, Paris, I840. Nèlaton, Soc. anat., I856. Robin, C. R. S. B., 1862. Wedl, Path. d. Zahne, I870. Broca, Gaz. hebdom., r868, 70. Traitè d. Tum., 1886. Malassez, Arch. de Physiol., 1885. 709 Galippe, Le debris epit. parad., Paris, I910 (Lit.). Bryck, L. A., 25. 711 Benneke, Z. C., 42. Albarran, Rev. de Chir., I 888. Kruse, V. A., 124. Flaubert, Thèse, Paris, 1857. Chibret, Arch. med. exper., I894. Bernays, N. Y. M. Rec., I885, 28, I. 712 Guibout, Un. Med., 1847. d'Amiens, Bull. soc. chir., I 878. Buchteman, L. A., 26. Eve, B. M. J., I883, I, I. Heath, Inf. and Dis. of Jaws, London, I872; B. M. J., I887, 1, 777. Haasler, L. A., 53, 749. 713 L'Esperance, N. Y. Path. Soc., 1912. 714 Coate, Lancet, I857, 2, 363. Bornig, V. A., 190, 421. Kruse, V. A., 124. Albarran, C. R., Soc. Biol., 1887. Pincus, L. A., 72. B. Fischer, Frank. Z. Path., 12, 422. 715 Sirantoine, Thèse, Nancy, 1903. Witzel, Mon. f. Zahnh., I896, 305. Mickulicz, Wien. m. W., 1876, 952. 716 Gosselin, Bull. soc. Chir., I 860, I85. Vitalis, Bull. soc. anat., I858, 326. Ancelot, Gaz. d. hop., I 869. Sourier, Ibid., I34. Berger, Bull. soc. chir., I88I, 422. Albarran, Soc. anat., I887, 497. Legouest, Soc. chir., I862, 345. Broca, Rev. de mal. de l'Enfance, 1906, 521 . Bayer, Rev. de Stomatol., 1904, 414. Nelaton, Soc. anat., 1856, 149. Grosse, L. A., 5I, 436. Remy-Duret, Soc. anat., 1873, 40I; 1874, 686. 717 Allgayer, B. B., 440. M. B. Schmidt, Erg. Path., 7, 332. F. J. Hunter, cit. by Hildebrandt, Z. C., 31 . Billroth, V. A., 8, 426. 718 Hildebrandt, Z. C., 31, 35. Coleman, Tr. Odon. Soc. Gt. Brit., I 862. Matthias, Ibid., I 863. Bland Sutton, Ibid., I887. Annandale, Edin. M. J., I 875, 599. Broca, C. R. Soc. Biol., I 862, 301; Tumeurs, 2, 346. Lloyd, Lancet, 1888, I, 64. 719 Forget, Des. anom. dent., I859, 5. Uskoff, V. A., 85, 537. Krogius, L. A., 50, 275. Robin, C. R. Soc. Biol., I802, I99. Tapie, Gaz. heb., I890, 55. Perthes, Deut. Chir., 1907, 33a. Schloessmann, Z. B., 44. Partsch, Mon. f. Zahnh., I892, 223. 720 Sut- 
ton, Introd. to Gen. Path. v. Brunn, Arch. m. anat., 29. Tumors of nares and accessory sinuses. Moore, Proc. Royal Soc., I917, Io Lar., 6o. 723 Wisotski, Z. C., I24, 605. Coley, A. S. Richou, Thèse, Paris, I905-06. Jacques, Gaudier, Tum. mal. du sin. max., Paris, 1907. Windmuller, I. D., Gottingen, I890. Sebileau, Ann. mal de l'oreille, I90r.

\section{EPITHELIAL TUMORS OF THE SALIVARY GLANDS}

725 Nasse, L. A., 44, 233. Lecene, Rev. de Chir., I908, 37, 1. Lexer, Path. Chir. Chevassu, Rev. de. Chir., I9I0, 4I, 145. Duplay, Arch. gen. de Med., I875, I, 601. Poncet, Gaz. d. hop., I888, 862. Bougle, Soc. anat., 1900, 71 5. Wolfler, L. A., 29, 8I. Zeisel, Oester, med. Jahrb., i881. Ferreri, Bull. soc. osp., Roma, i888. Kuttner, B. B., I6. Warthin, Arch. of Ophth., I90I, 30 (Lit.). Prengrueber, Cour. med., I 884, 435. Waldeyer, V.A., 55, 127. Volkmann, Z. C., 41, 107. Pailler, Thèse, Paris, 1903. Ehrich, B. B., 51. Lowenbach, V. A., 150, 73. 727 Kuster, L. A., I2. W. Koch, I. D., Freiburg, 1897. Degen, I. D., Freiburg, I900. A. Schafer, I. D., Erlangen, I 896. Dubreuil, Gaz. hebd., Montpellier, I89I, 205. C. Kaufmann, L. A., 26. Wartmann, Diss. Straasburg, I879. Volkmann, Z. C., 41 (Lit.). Collet, C. P., I 896. Pitance, Thèse, Paris, 1897. 727 Hinsberg, Z. C., 5 I. Mauclaire, Soc. anat., I897. Cavazanni, Riv. Veneta. di. sci. med., I897, 26, 405. Wilms, Die Mischgesch., I902. Landsteiner, Zeit. f. Heilk., I901, I. Wood, A. S., 39. Verhoef, J. Med. Res., I3. Steinhaus, V. A., I68. Martini, V. A., 189. Krompecher, Z. B., 28, 37, 44. $729 \mathrm{Krieg,} \mathrm{I.} \mathrm{D.,} \mathrm{Tubingen,} \mathrm{1874,} \mathrm{cit.} \mathrm{by}$ Ehrich. 731 Broussis, I. D., Marburg, 1903. Carraro, Frankf. Z. P., 1909, 3, 26 (Lit.). Lowenstein, Ibid., I910, 4, 87. Cuneo, Veau, Cong. intern. de Med. Chir., 1900, 278. Chevassu, Rev. de Chir., 37, I I I. Vialleton, Arch. d. anat. mic., I908, I. Estor, Massbuau, Rev. de Chir., I908, 38, 341. Forgue, Massabuau, Prov. Med., 1908. Weisshaupt, L. A., IOI, 542. 732 Prudden, A. J. M. S., 85. Weyl, I. D., Munich, 1900. Poult, V. A., I8I (Lit.). McGregor, Workman, Lancet, 1906, I, 433. Ficheaux, Thèse, Lille, 1908. 733 E. Wagner, Arch. f. Heilk., 1861, 283. Böhme, I. D., Berlin, I892. Semponoff, I. D., Zurich, 1904 (Lit.). Lenornant, Duval, Cottard, Rev. Chir., I908, 37. Collet, Thèse, Paris, I 895. Laraberie, Arch. gen. de med., I890, 25, 26. Bergen, Rev. de Chir., I 897, I7, 361. Marchand, Z. B., 13. Gutekunst, Arb. Inst., Tubingen, 1904, 5. Dembrowski, Z. C., 32. Martland, N. Y. Path. Soc., 1909, 9, 123. 734 Barozzi, Lesne, Soc. anat., I 897, 266. Griffini, Trombetta, Atti. r. ac., Turin, I883. 735 v. Hansemann, Z. K., 1910, 9, 379. 736 Hartmann, Rev. de Med., 25, 206. Herxheimer, C. P., 1908, 709 (Lit.). Forster, Wien. med. W., 1858, 481. v. Haberer, L. A., 93. Lannelongue, Achard, Traitè d. cyst. congen., Paris, I886. Robinson, London P. S., I895, 47, 255. Fuhr, I. D., Wurzburg, I908. Hagenbach, Z. C., 93. Landsteiner, Z. f. Heilk., I901, 22. Talazac, Thèse, Paris, I869. Duplay, Arch. gen. de med., I875, I, 601 .

\section{TUMORS OF THE KIDNEY AND ADRENAL}

738 Ebstein, Ziemssen Encyc., I 877, Vol. 15. G. König, I826, cit. by Kuster; Deutsche. Chir., 52. Rayer, Traitè d. mal. d. rein, I 841. Robin, Soc. biol., I853. Waldeyer, V. A., 54, 1867. Lancereaux, Gaz. d. hop., I889. Sturm, Arch. f. Heilk., I 875. Sabourin, Arch. de Physiol., I882; Rev. de med., I884. Weichselbaum, Greenish, Wien med. Jahrb., 1883. P. Grawitz, V. A., 93; L. A., 30. Horn, V. A., I26. Lubarsch, V. A., 135, I37, 148. Marchand, V. A., 73; B. W., I 895; V. A., 92. Ambrosius, I. D., Marburg, 1891. A. O. J. Kelly, Z. B., 23. Askanazy, Z. B., 14. Beneke, Z. B., 9. Stoerk, Z. B., 43. 740 Paoli, Z. B., 8. Driessen, Z. B., 12. Hildebrandt, Z. C., I 894; L. A., 47. Hansemann, Diag. bos. Gesch. Manasse, V. A., 142, I43, 145 (Lit.). Nobiling, Z. K., 10. Zehbe, V. A., 20I. Scudder, Amer. J. M. S., IIo. Edmunds, London P. S., 37, 287. 741 Weigert, V. A., 67, 492. Targett, Lancet, 1894, 2, ro95. Eurich, J. P. B., I896, 504. Kelynack, Renal Growths, I5I. Ulrich, Z. B., I8. Albarran, Imbert, Tumeurs du 
rein., 1903 (Lit.). Luzzato, Riv. Veneta de. sc. med., I90ı. Antona, Tumori prim. d. rene., Pisa, I900. v. Kahlden, Z. B., 15. Nauwerck, Hufschmidt, Z. B., 12. Keyes, Sr., Amer. J. M. S., I00. Guillebeau, Vaerst, Anat. Anz., I901, 340. Shmey, V. A., 202. E. Meyer, V. A., I73, 209. 742 R. L. Thompson, V. A., I88, 55 I. 743 Kuster, Cent. f. Harn u. sexorg., I 897, 583. 744 P. Albrecht, L. A., 77. Coyne, Troisier, Soc. anat., 1871, 239. Gardner, Coats, Glasgow M. J., I 870, 3, 22 I. 745 Clairmont, D. G. Chir., 1903, I96. Israel, D. W., I9I1, 57. Albarran, Ann. gen.-ur., I897. 746 Ipsen, Z. B., 54. Hansemann, Z. kl. M., 44. Sudeck, V. A., 133. 748 Waldeyer, V. A., 41, 55. 749 Barth, D. W., I 892, 53I. Malcolm, B. M. J., I894, 1 , 242. Bokai, ref. Cent. Chir., I883, 503. 751 Sharkey, London P. S., I881, 33, 195. Newman, Glasgow M. J., I896, 45, 179. W. H. Dickinson, Renal and Urinary Affections, London, I885. Schueppel, Arch. f. Heilk., I868. Brault, Sem. med., I 891, 249. 752 A. Frazer, N. Y. P. S., 1914, 14, 22. 753 Hedren, Z. B., 40. Weigert, V. A., 67, 492. Paul, London P. S., 37, 292. Schaffer, A. G., 53. Eve, London P. S., 33, 312. Merkel, Z. B., 24. Manasse, V. A., 145. Busse, V. A., 157, 346, 175, 442. Brandt, cit. by Hedren. Hoishalt, V. A., 104, 1 I 8 . Jenckel, Z. C., 60, 500. Muus, V. A., 155, 40I. Heineke, I. D., Erlangen, I897. Broch, V. A., 140. Ribbert, V. A., I06, 282. 754 Blau, I. D., Konigsberg, I898. Wilms, Die Mischgeschwulste, I899. Cohnheim, V. A., 65, 64. Birch-Hirschfeld, Z. B., 24. 755 Chiari, Z. f. Heilk., I 884, 5, 449. Marchand, Festsch. f. Virchow, I891, 569. Larkin, J. Med. Res., 6. Hildebrand, L. A., 48. 756 Goupel, Thèse, Paris, 1907. L. Pick, A. G., 64. Huber, Amer. J. Anat., 1905. Elliott, Armour, J. P. B., 19II. Peter Unters. u. d. Bau. d. Niere, Jena, 1909. 757 Pick, A. G., 64. Poll, Arch. m. Anat., 56. Weiler, I. D., Kiel, 1895. Dagonet, Zeit. f. Heilk., I885, 6. Michael, L. A., 43; I 20. Marchand, D. p. Ges., I 898. R. Meyer, Z. G. G., $7 I$ (Lit.). N. Pitt, London P. S., 1894, 45, 141. Neusser, Wiesel, Erkrank. d. Nebennieren, Wien., 1910. Holmes, J. A. M. A., 2, 405. Wiesel, Wien. kl. W., I 898, 443, V. A., I76. Aichel, Arch. u. Anat., 56. Schmorl, Z. B., 9, 523. Hanau, I. D., Zurich, I 895. Nicholson, cit. by Glynn. Glynn, Quart. J. Med., I912, 5, 157. 759 Adami, Syst. Pathology, I910. 760 Lassagna, V. A., 201. Dobbertin, Z. B., 28. Winkler, Die Gewachsl. d. Nebennieren, Jena, 1909. 761 Panzer, Z. phys. Chem., 48, 519. Ellis, Keen's Surg., 1908, IV, 245. Garceau, Renal Tumors, N. Y., 1909. Woolley, Am. J. M. S., 1903. Fraser, S. G. O., I915. Croftan, J. A. M. A., 1903, 1, 91. Ellis, Amer. Med., 1904, 8, 1039. 762 Gunkel, I. D., Marburg, 1887. Vecchi, V. A., 177. Noyes, N. Y. P. S., I 899-1900. Ribbert-Kronlein, B. B., I895, 677. Goebel, Z. C., 6I (Lit.). Weiss, Z. B., 24. Peham, Mon. G. G., 10, 485. 763 Eastwood, Lancet, I902, I, 90. Debarnardi, Z. B., 40. Kohlhardt, V. A., I48. Lancereaux, Dict. Dechambre, II, 247. Fenwick, Med.-Chir. Trans., I897, 238. Hebb, cit. by Fenwick. Savory, Nash, Lancet, 1904, 2, 1699. Murchison, London P.S., 21, 241. Neelson, Z. B., 3. Stoerk, Z. B., 26. Tikhoff, Arch, provincial de chir., I901, 145. 764 Kuster, Deut. Chir., 52, b. Graupner, Z. B., 24, 399. Rayer, Traitè d. mal. d. reins., I 841, III, 699. Volcker, London P. S., 46, I35. Beneke, Nambe, V. A., 203. Milne, J. med. Res., 25. 765 Battle, London P. S., I895, 235. Pantaloni, Arch. provincial d. chir., I 899, 8, I. Drew, London P. S., I 897, I30. Kundrat, cit. by Albarran. Rundle, London P. S., I897, 47, 128. Kischensky, Z. B., 30. Beisenbruch, I. D., Kiel, 1907. Scheel, V. A., 201. 766 Wendle, Mitt. G. G., 6. Beselin, V. A., 99. Hildebrandt, L. A., 48. Giordano, Ann. mal. gen.-ur., I892, 585. Hedenius, Waldenstrom, Upsula Lak., I3. Wirsing, Blix, Hygeia, I878. Hektoen, Medicine, I 896. Israel, V. A., I 886. Hartmann, Prag. med., I 886. Grohe, Z. C., 6o. Hollen, I. D., Griefswald, I 890.767 Schluter, I. D., Greifswald, I 890. De Vecchi, V. A., I82. Salomon, Z. K., I906, 4, 648. 768 Adami, Woolley, Assoc. Amer. Phys., 17, 627. Marchand, Eulenberg's Realencyc. Missbild. Febiger, V. A., I81. 769 Letulle, Gaz. hebdom., I 892, 29, 306; Arch. d. sc. Med., Bucharest, I896, I, 80. Manasse, V. A., I45, Case 25. Rolleston, London P. S., I895, 46, I50. Awray, Pfeffel, Soc. anat., I9I I. Rolleston, Marks, A. J. M. S., I898, $116,3^{8} 3.770$ Gerber, Wien. m. W., 1904. Affleck, Leith, Edin. Hop. Rep., 1896, 4, 278. Hartmann, Lecene, Trav. de. Chir., I904, II. 771 Fox, Londion P. S., I885. Virchow, Krankh. Ges., 2, I 49. 
Weichselbaum, V. A., 85. Herxheimer, Z. B., 57. Dagonet, Z. f. Heilk., I 885, 6. 772 Schmidt, V. A., I55, 557. Bruckanow, Z. f. Heilk., I899, 20. Ohse, B. B., 50 (Lit.). Kuster, V. A., I80, I17. 773 Wiesel, V. A., 180, 553. Lapointe, Lecene, Arch. med. exper., I907, 19, 69. Kretz, Erg. P., I902, 8; Abt. II, 532. J. H. Wright, J. exper. Med., 1910, 12, 556. 774 Landau, Frankf. Z. P., II. Anitschow, V. A., 214. Symmers, J. A. M. A., 60, 337. Wahl, J. Med. Res., 30, 205. Dunn, J. Path. \& Bact., 19, 456. Falk, Z. B., 40. Pick, Bielschowsky, Z. ges. Neur. u. Psych., 6, 391. Oberndorfer, D. P. G., 1909, 273. F. Holst, I. D., Leipsig, 1904. Sabrazes, Husnot, Arch. med. exper., I9. 775 Hutchinson, Quart. J. Med., 1907, I, 33. Aisenstein, I. D., Zurich, 1905. McCarty, W. C., Berl. kl. W., 1905, I15. Reimann-Chiari, Prag. m. Woch., 1902, 297. Tileston, Wolbach, A. J. M. S., I35 (Lit.). Pepper, A. J. M. S., I901, I21, 287. R. S. Frew, Quart. J. M., I9I I, 4, I23. 776 Orth, K. Preus. Kad. Wissen., 1914. Vaquez, Soc. d. Hop., I904. Ellis, Amer. Med., 1904, 8, 1039. Stilling, cit. by Berdez. Zanfrognini, Ref. Barbacci, C. P., 1904. Schmorl, D. P. G., 1909, 13, 295. Berdez, Arch. med. exper., I892, 412. Manasse, V. A., 133. Susuki, B. W., 1909, 1644. Kawashima, V. A., 203. Poll, Hertwig's Handb., 1906. 777 Stangl, D. P. G., 1902. Wiesel, D. P. G., 1902. Stoerk, Ibid. Laignel-Lavastin, Aubertin, Arch. med. exper., 1908. Monckberg, Z. B., 38. Alezeis, Peyron, C. R. soc. biol., 1908. 778 Davidsohn, D. P. G., 13, 287. Lucksch, Z. B., 53, 324. Goldzieher, D. P. G., 1913, 213. Tuczek, Z. B., 58, 250. Maclachlan, J. Med. Res., 1915, 28, 93.

\section{TUMORS OF PROSTATE}

779 Home, Prac. Obs. on Prostate Gland, London, 1811. Moullin, Enlarg. of Prostate, London, 1894 (Lit.). J. W. White, Ann. Surg., I8. Belfield, Am. J. M. S., Ioo. Greene, Brooks, Dis. G. U. Organs, N. Y., 1908; J. A. M. A., 1902, 38, 1051. S. D. Gross, Dis. Urin. Org., Phila., I876. Tandler, Zuckerkandl, Berl. W., 1908, 2093. Halle, Albarran, Ann. mal. org. urin., 1900, 18. Ciechanowski, Mit. Grenzgeb., 1900. Young, Geraghty, Cancer of Prostate, J. Hop. Hos. Rep., 1906, 14. 780 Velpeau, Lec. or. de clin. chir., Tome, 3. Casper, cit. by Frisch. 781 Thompson, Dis. of Prostate. Frische, Krank. d. Prost., Wien., 1910. Motz, Thèse de Paris, I897; Ann. mal. org. gen. ur., 1897. Keyes, Jr., J. A. M. A., 43, 187. Rothschild, V. A., 173. Launois, Thèse, Paris, 1885. Guyon, Ann. d. mal. org. g. u., I 885, I887, I 895. Rovsing, cit. by Moullin. Furbringer, Nothnagel's spec. path., I9. Keyes, N. Y. M. Rec., I900, 58, 81. Gurlt, L. A., 25. Heimann, L. A., 37. Engelbach, Thèse, Paris, 1888. Wolff, Z. C., 53. E. Kaufmann, Deut. Chir. L., 53 (Lit.). 782 Billroth, L. A., 10, 548. Gardiner, Cummins, J. A. M. A., 1912, 58 , 1282. 783 Berger, Soc. Anat., 1871, 46, 222. Fenwick, London P. S., I887, 38, 199. Walter, I. D., Greifswald, I891. Guelliot, Thèse, Paris, 1882. Billroth, Chir, klin. Wien., 1871-76. Tyson, Am. J. M. S., 1869. Jolly, Arch. gen. d. Med., I869, I, 577; 2, 61. Recklinghausen, Fest. f. Virchow, I891. Hebb, London P. S., I896, I54. Young, J. H. B., 1905, 315. Belfield, J. A. M. A., I888, 10, 120. Engelhardt, V. A., 158. Tailhelfer, Gaz. hebd., I897, 2, 805. Guyon, Bulletin med., I887. Sappey, Anat. descript., I 889, 4, 538. 784 W. Courvoisier, I. D., Basel, r9oı. Carlier, An. mal. org. g. u., I896, 14, I050. Baumgarten, Arb. path. Ins., Tubingen, 1907. Silcock, London P. S., I883, 244. Thompson, London P. S., I 854, 204. L. Braun, Wien. m. W., I896, 481. Schmorl, D. P. G., 13. Wolff, Z. C., 5.3. Sasse, L. A., 48, 593. Erbsloh, V. A., I63. Schmorl, D. P. G., I3; D. m. W., 1907, 207. 785 Axhausen, V. A., 195, 358 (Lit.). Lenziger, I. D., Zurich, 1886. Askanazy, Fest. f. M., Jaffe. 788 Schlagenhaufer, D. P. G., I3. Aschoff, V. A., 138. Schlachta, Arch. m. Anat., 64. Barton, Dublin J. M. S., I88I, 553. Matthias, I. D., Munchen, 1889. Berger, Soc. Anat., I871, 222. Beyer, I. D., Greifswald, I896. Boyd, London P. S., I881, 33, 200. Schmidt, Z. B., 40. Marchand, A. L., 22. Socin, Pitha. Billroth's Handb., 1875. 789 Oliva, Cent. Chir., 1884, 513. West, London P. S., 34, I45. Hughes, Phila. P. S., I884, I89. Kapsammer, Wien, kl. W., 1903, 282. Dupraz, Rev. m. Suisse Rom., 1896, 465. Coupland, London P. S., I877, I79. Lefmann, Munch. W., 1907, 442; 1906, I591. Veil, Berl. W., 1908, 872 . Tordens, 
J. d. med. chir., Brux, 1890, 90, 405. Birch-Hirschfeld, Spec. Path., I895, 1008. Isambert, Soc. anat., I 853,57 . Marsh, Lancet, 1897,1 , I092. Wind, I. D., Munchen, 1888. Matthias, I. D., Munchen, I889. Zahn, Z. C., 22.

\section{TUMORS OF TESTIS}

790 Saint-Donat, cit. by Verneuil, Bull. soc. chir., Paris, 1867. Prochaska, cit. by Verneuil, Ibid. Andre de Perrone, cit. by Verneuil, Ibid. Johnson, London P. S., I 856. Ohkubo, Arch. f. Entw., 1908, 26. Astley Cooper, Diseases of Testis, Philada., I845. Curling, Med.-Chir. Trans., 1854. Langhans, Deut. Chir. Lief., 50, B., I887. Kocher, Ibid. Wilms, Z. C., 49; Z. B., 19. Ribbert, V. A., 130. Pick, Berl. W., 1902, 1189. Chevassu, Tum. d. test., Paris, 1906. Ewing, S. G. O., 191 I, 12, 230 (Lit.). 791 Geinitz, Deut. Klinik., I862, 216. Cavazanni, Z. B., 4I. 792 Dürr, I. D., Freiburg, I894. Senftleben, V. A., 15. Koslowski, V. A., I48. Chevassu, Picque, Bull. soc. Chir., Paris, I898. Schlagenhaufer, Wien. kl. W., I902, 57 I. Pepere, La clin. med., 1903, 9. Gessner, Z. C., 60. Heinen, I. D., Bonn, I893. Szulcewski, I. D., Wurzburg, I904. 794 Tilanus, Schmidt's Jahdb., I 858, I00, I7 I. Kalning, I. D., Dorpat, 1876, cit. by Wilms. Macewen, Glasgow M. J., I878, Io. Boeckel, Bull. soc. chir., Paris, I888. 796 Debarnardi, Z. B., 40. 797 Waldeyer, V. A., 65. Breus, Wien. m. W., I878, 767. Malassez, Monod, Arch. de physiol., 1878, 375. Carnot, Marie, Bull. soc. anat., Paris, 1898, 73, 82. 798 MacCallum, Johns Hop. Rep., 1900, 9, 497. Wlassow, V. A., 160. Warthin, communicated. Garbarini, Il Morgagni, I899, 4I, I37. Risel, Arb. a. d. p. Instit., Leipsig, 1903. Blatteis, communicated. Ewing, N. Y. Path. Soc., I913, 28. 799 Pick, V. A., I80. 800 Paget, Med. Chir. Trans., I855, 38, 247. 801 Westenhofer, D. p. G., I904, 7, 107. Fergusson, cit. by Kocher. Pean, Lec. clin. d. chir., Paris, 1888. Ehrendorfer, L. A., 27, 352. Neumann, Arch. f. Heilk., I875, 16, 92. Dauve, Soc. d. chir., Paris, I 868, 6, 29I. 802 Nepveu, Tum. d. Test., Paris, I 875. Hericourt, Rev. de Med., I 885, 5, 54. Arnold, Z. B., 8. Wood, N. Y. Path. Soc., I902, 52. Benenati, V. A., I7I, 418. Krompecher, V. A., Suppl., 15I. Pick, A. G., 76. 803 Durck, D. P. G., II. Kaufmann, D. p. G., II. Hansemann, V. A., I42, 538. Marchand, Fest. f. Virchow. Wiesel, Wien. kl. Woch., I 898, 443. Kirkbride, Arch. f. Entwick., I91 I, 32, 717. 804 Malassez, Soc, anat., Paris, I877, 52, 176. Talavera, Thèse, Paris, I879. 805 Bulkeley, S. G. O., I7. O. C. Smith, Boston M. S. J., I914, I70, 839.

\section{TUMORS OF THE LUNG}

807 Boyle, Rech. s. 1. phthisie pulmon., Paris, I81o. Stokes, Dublin J. Med. Sci., 1842. Jaccoud, Clin. med. d. Charite, Paris, I867. Behier, Gaz. d. hop., 1867. Langhans, V. A., 53, 470. Perls, V. A., 56, 437. Marchiafava, Riv. clin. de Bologna, 1873, I 50. Malassez, Arch. de Physiol., I876, 353. K. Wolf, Fort. d. Med., I895. Passler, V. A., 145, 191. Adler, Prim. malig. Growths of Lungs, N. Y., 1912. Scott, Forman, N. Y. M. R., 90, 452. Ebermann, De cancer pulmonum, Petropoli, 1857. R. Bennett, Cancerous and Other Intrathoracic Growths, London, I872. 808 McAldowie, Lancet, I 876, 2, 570. Nuscheler, Cor. Sdhweiz. Aertze, I875. Karrenstein, Charite An., 32. Horn, V. A., I89, 414. Werner, I. D., Freiburg, I897. Schwalbe, V. A., I49. Oertel, J. Med. Res., 25. Friedlander, Fort. d. Med., I885, 307. Perrone, Fests. f. Orth., 1906. Perrutz, I. D., Munchen, I897. Aufrecht, Nothnagel's System. Menetrier, Le Prog. med., I886, 436. Ribbert, D. W., I896, 471. 809 Arnstein, D. p. Ges., I913, 16, 332. 810 Domeny, Z. f. Heilk., 1902. M. Packward, Med. News, 1905, 86, 303. Merklen, Girard, Soc. d. hop., 1901, 760. Harbitz, cit. Z. K., 1904, I54; Norsk. Mag. f. Laegevid., 1903, 71 5. 811 Watsuji, Z. K., I, 445. Chiari, Prag. m. W., I883, 497. Ernst, Z. B., 20. Beck, Z. f. Heilk., I884, 459. Fuchs, I. D., Munchen, I886. Ebstein, D. m. W., I89o. 921. Ehrich, I. D., Marburg, I891. Rondeau, Thèse, Paris, 1903. Decreton, Thèse, Paris, 1910. Kretschmar, I. D., Leipsig, 1904. Hansemann, V. A., I6I. Lehmkuhl, 
I. D., Kiel, I893. 812 Meunier, Arch. gen. de Med., 1895, 351. Willert, I. D., Wurzburg, 1905. Le Sourd, Soc. anat., 1899, 587. Coats, London P. S., 1886, 326. Levene, Thèse, Montpellier, 1901. P3pere, C. P., 1904, 948. Loser, Ver. phys. med. Ges., Wurzburg, 1899, 10. Marchiafava, Rev. clin. Bologna, 1873, 150. Cahen, Beitr. z. Histol. d. Lungencar. Edlavitch, J. A. M. A., 1912, 59, 181. Knierim, D. p. G., 1909, 407. Kelly, Z. f. Heilk., 1907, 28, 105. Bjornsten, C. P., 15, 513. 813 Turnbull, Worthington, London Hosp. Arch., 1908, 163. Stilling, V. A., 83. Schottelius, I. D., Wurzburg, 1874. Reinhard, Arch. f. Heilk., 1878, 19, 369. Tillmann, I. D., Halle, 1889. Peck, Z. f. Heilk., 1884. Ravenna, Arch. med. exper., 21, 87. Griffini, Arch. p. 1. sci. med., I884. Kitamura, V. A., 190. Haythorn, J. Med. Res., 26, 523. E. Froelich, I. D., Berlin, 1899. 814 Henrici, J. Med. Res., 26, 395. Stoerk, Wien. kl. W., 1897, 25. Aschenborn, L. A., 25, 140. Lohlein, D. p. Ges., I908, I2, Ir1. Linser, V. A., I57, 281. Couvelaire, Annal. gyn. obstet., 1903. Dionisi, Arch. di biol. Firenze, 1903, 716 . Wiechselbaum, V. A., 85, 559. 815 Loser, Phys. med. Ges., Wurzburg, r899, ro. Rievel, D. tierarztl. Woch., 1906. Herrmann, D. A., 63. Betschart, V. A., I42. Beveridge, Med. Press and Circ., 1869. Degen, I. D., Zurich, 1897. 816 Pater, Rivet, Arch. de med. exper., 1906, 85. Schnick, I. D., Greifswald, 1899. Sangalli, Gaz. med., Lombardi, 1897, 226. Milian, Mante, Soc. Anat., I901, 76, 82. Lesieur, Rome, Lyon Med., 1909, I13, 74. Hodenpyl, N. Y. P. S., 1895, 19. Hertz, Ziemssen's Handb., r874. 817 Bock, St. Louis Med. Rev., 1889, 19, 512. Elkan, I. D., Munchen, 1903. Koblynski, I. D., Greifswald, 1904. Ranglaret, Soc. anat., 1893, 7, 591. Rolleston, Trevor, B. M. J., 1903, I, 361. Fuchs, I. D., Munchen, r886. Blumenthal, I. D., Berlin, 1881. Mironescu, Baroncea, Rev. mens., 1894, 12, 82. Rutimeyer, Cor. Schweiz, Aert., 1886, I6, 169. Peritz, I. D., Berlin, 1896. Pollak, I. D., Wurzburg, 1897. M. Anderson, Glasgow M. J., I893, 39, 243. Levitt, I. D., Erlangen, 1901. Hildebrand, I. D., Berlin, 1887. Lehrdorff, Wien. m. W., 1909. Davies, London P. S., I889, 46. Box, St. Thomas H. R., I896, 260. Poore, Lancet, 1895, I, 170. Pitot, Arch. de med. mil., I 899, 306. Roth, I. D., Munchen, I904. Millian, Bernard, Soc. anat., 1898, 336. Meyer, I. D., Munchen, 1900. Adami, Montreal M. J., r895, 5 ro. 818 Powell, B. M. J., 1879, r, 115. Cohen, Kirkbride, Phil. P. S., 1900, 200. Coats, Glasgow M. J., 1874, 274.

\section{EPIDERMOID CARCINOMA OF SKIN, LIP, TONGUE, AND LARYNX}

Selberg, V. A., 145. Krompecher, Z. B., 28; Die Basalzellenkrebs, Jena, 1903; Z. K., 3; B. W., 1907, 940. 819 Unna, Lehrbuch, 732; Mon. f. D., 38. Pianese, Z. B., Suppl., I. Pansini, Mon. f. D., 39. 820 Orth, Z. K., I, 399. Landau, Z. K., 12, 506 (Lit.). Walkhoff, Festchr. f. Recklinghausen, 1907. Strassberg, V. A., 203. Barlow, D. A. kl. Med., 55. 821 Chaetle, B. M. J., 1903, 05, 07, 08. Ashihara, A. D., 56. Hartzell, J. C. D., 1903, 393. Spitzer, Z. Heilk., 1902, 227. 822 Borrel, An. Instit. Pasteur, 1909, 23, 97. Rabaioye, Lyon Med., 1904, 1107. Krische, B. B., 31, Suppl. Mertens, Ibid. Linser, Ibid. Kaufmann, 1. c., I060. Councilman, Magrath, J. Med. Res., 21, 331. Crocker, Dis. of Skin, 1893. Kreibich, A. D., 57. Halle, W. kl. W., 1901, 765. Hutchinson, D. m. W., 1904, 1378. 823 Dalous, Constantin, Annal. de Derm., 1904, 961. Darier, An. de D., I892, I121. Fordyce, J. A. M. A., 55, 1624. Ashihara, A. D., 57, 193 (Lit.). Steinhauser, B. B., 12, 501. Hartzell, J. C. D., I903, 393. Blaschko, Mon. f. D., 25, 82. Marcuse, D. W., 1896, 481. Kienbock, Wien. kl. Woch., 1900, I153. Senger, B. W., I911, 662. Gassmann, A. D., 70, 97. Porter, An. Surg., 46, 649; J. Med. Res., 2⿰. Linser, Fort. a. d. Geb. d. Rontgens., 1904-05. Unna, Ibid. 824 Da Costa, Rev. prat. d. mal. cut., 1905, 224. Lindhorn, B. B., 59. 825 Schumann, L. A., 84, 855. Wolbach, J. Med. Res., 2I. Clunet, Bull. Assoc. Franc. f. Cancer, 1910, 3, 404. Wyss, Z. C., 93. 826 Paget, St. Bart's. H. R., 1874. Vignolo-Lutati, Mon. f. D., 42, 253. Zieler, V. A., 177. 827 Depage, Ann. d. 1. Soc. belge. d. Chir., 1894. Sekiguchi, An. Surg., 65. Kyrle, A. D., 83. Elbogen, Fests. f. Chiari, Wien., 1908. Darier, La Prat. derm., 3, 1902. Karg, Z. C., 34. 828 Fox, McLeod, B. J. Derm., 1904, 16. Jaco- 
beus, V. A., 178. Ribbert, D. med. IV., 1905, I218. Schambacher, Z. C., 80. Hirschel, Z. B., 1905, Suppl., 7. Schulten, L. A., 48, 913, photo of extensive case. Hannemuller, Landois, B. B., 60. Krogius, Z. C., 73. 829 Thin, B. M. J., I88I, I, 433. Duhring, Wile, A. J. M. S., I884, 88, I4I. Ehrhardt, Z. C., 54. 830 Fabry, Trautmann, A. D., 69. Williams, B. M. J., 1900, 2, 895. 831 Dubreuilh, Auche, An. d. D., I901, 1902. Fordyce, J. C. D., 1902, 20. Hutchinson, D. m. W., 1904, I378. 833 Coenen, B. W., 1907, 662. Borrmann, Z. K., I. Janeway, Z. K., 8. 834 Bonney, Lancet, 1908, I, I389. Wyzz, Z. C., 93. Brooke, Monatsh. f. p. D., I 892, 15, 589. Fordyce, J. C. D., I892, 10, 589. Walters, A. D., 56, 89. Schapper, A. D., 98. Czillag, A. D., 80. Walters, A. D., 56. Torok, Mon. D., 8. Darier, Annal. de D., I887. W. Pick, A. D., 58. 835 Buxton, Cornell Path. Stud., I, I901. Dorst, Delbanco, Mon. f. D., 33, 317 . Petersen, A. D., 25, 44I. Elliot, J. Cut. Dis., II, I68. 837 Fricke, Z. C., 50, 95. Warren, Internat. Surg. Assoc., 1898. Dugue, Thèse, Paris, r901. Montgomery, J. Cut. Dis., 1913, 82. Sutton, J. A. M. A., 60, I774. 838 Ribbert, D. Carc. d. Menschen, 1912. 839 Steiner, Z. C., 97, 243. 840 Bloodgood, J. A. M. A., 55, 1615. Jessett, Cancer of Mouth, London, I886. Jacobson, Guy's Hosp. Rep., I889. Winiwarter, Beitr. z. Statis. d. Carc., Stuttgart, 1878. Warren, An. Surg., 48. Butlin, Brit. M. J., 1903, I, 353. 841 Meller, Z. C., 84. Sigel, I. D., Tubingen, I864. Piquantin, Thèse, Paris, 1905. Fournier, Wien. kl. Rund., 1900. Landau, I. D., Gottingen, I885. Sachs, L. A., 45, 774. Lang, cit. by Sachs. Lydston, N. Y. Med. Rec., 36, 456. Hutchinson, Brit. M. J., I872. 845 Poirier, The Lymphatics, Chicago, 1904. Kuttner, N. B., 21. Crile, Surg., Gyn., Obstet., 1907, 5, 91. 846 Berkeley, N. Y. Path. Soc., 1905, 5, I69. Steiner, B. B., 3. Hulsmeyer, I. D., Wurzberg, I888. Goldmann, B. B., I8. 847 Juracz, Handb. Laryng. (Heymann), 1898. Schech, Krank. d. Kehlk., Wien., 1897. Casnit, Gaz. d. Hop., I866. Gerhardt, Jena, Z. f. Med., I867. L. Browne, The Throat and Nose, London, r89o. Werner, I. D., Heidelberg, I894. 848 Curtis, Intern. J. Surg., I892. Lincoln, N. Y. M. J., I893, 58, 785. Wilkinson, Austral. M. Gaz., 1895. London, Cent. f. Laryng., I892, IO, 222. Clubbe, cit. by Juracz. Simon, Cent. f. Laryng., 1888-89; Lancet, 1904, 2, 1263. J. SolisCohen, N. Y. M. R., I 869, 4, 265. 850 Fauvel, Traitè d. mal. d. Larynx, Paris, I 876. O. Chiari, Arch. f. Lar., I 895 . Bruns, B. B., 3. Seyfert, Wurzb. phys. med. Gesell., I 894. Phillips, Ruh, Amer. Text-book, Dis. Child., I913, 5, I23 (Lit.). Loomis, N. Y. Path. Soc., I 890. Koschier, Wien. m. Blat., I 895. J. Mackenzie, J. A. M. A., I889, I3, 801. Ziemssen, Handb. Spec. Path. Heise, I. D., Tubingen, 1887. Stoerk, Krank. d. Kehlk., I88o. Rehn, V. A., 43. Steiner, Jahrb. d. Kind., I. 851 Baumgarten, Arb. a. d. Inst., Tubingen, I 894. Crone, I. D., Tubingen, I 895. Semon, Cent. f. Laryng., 5, 6. M. Schmidt, Krank. d. ob., Luftwege, I909, 315. Wolfenden, Cent. Laryng., I 893, 555. Sendziak, Bos. Gesch. d. Kehlk., Wiesbaden, I 897. Virchow, Berl. kl. W., I887, 585 . 852 Molinie, Tum. mal. d. Lar., Paris, 1907. B. Fraenkel, Arch. f. Laryng., I 895, 2. Krishaber, Annal. d. mal. d. l'or, etc., 1879, 5. 853 Butlin, B. M. J., I884, I, 457. Neuman, Glasgow M. J., I888. Semon, B. M. J., I888, I, 746. Thiersch, Z. C., I6, I56. Moure, Mal. de Lar., 596. Gougenheim, Gaston, An. mal. or., I888, 654. Kosinski, Cent. f. Chir., 1877. Maydl, Wien. m. Presse, 1884. Krieg, Arch. Laryng., I 893. Schmiegelow, Mon. f. Ohrenh., 1901. 855 Cuneo, Gaz. d'hop., 1902, I4I. 856 Shappers, Rev. mens. d. Laryng., I883. Desnos, Prag. med. W., I879. Sands N. Y. M. J., I 865, I10. Virchow, D. W., I894; V. B., 52. Bergeat, Mon. f. Ohrenh., I895, 320. McWhinnie, N. Y. M. J., 1912, 96, 838. Koschier, Wien. kl. W., I843, 38. Stoerk, Krank. d. Kehlk., I880. Butlin, Malig. Dis. Lar., 1883.

\section{CARCINOMA OF ESOPHAGUS}

857 Kraus, Nothnagel's Handb., 1902, 16. Heimann, L. A., 57. Mehnert. Heller, Arndt, I. D., Kiel, I90 I. Ritter, D. A., 55. Schaffer, V. A., I77. Hewlett, J. Exper. Med., 5 (Lit.). Eberth, Fort. d. Med., 1897, 251. Neumann, Ibid., 366. Glinski, Ciechanowski, V. A., 199, 420. Mosher, S. G. O., 1917, 25, I75. 858 Kern, V. A., 20I, 
135. Stoeber, Z. B., 52, 512. Kathe, V. A., 190, 80. Grabowski, Z. B., 56, 266. Seelig, An. Surg., 46. Stadelmann, C. P., 7, 215. Sakata, Mitt. G., 1903, 77. Ehret, D. W., 1901, V. B., 240. Coplin, Phila. P. S., 1904. Cæsar, M. W., 1904, 944. 859 Borrmann, Z. B., 48, 576. Dürr, C. P., 3, 38. Francke, V. A., 174. Norris, N. Y. P. S., I912. Herxheimer, Z. B., 44 (Lit.). Donath, V. A., 194.

\section{EPITHELIAL TUMORS OF BLADDER}

860 Albarran, Les tumeurs de la vessie, Paris, 1891. Fenwick, Tumors of Bladder, London, 1897. Hadda, L. A., 88. Rauenbusch, V. A., 182. 861 Keyes, Jour. Cut. Dis., I 887, 242. Guyon, Ann. d. mal. d. org. gen. ur., 1884. Adenot, Ibid., I 895.863 Fluss, W. kl. W., 1907, 1227. Stein, Tumors of Bladder, N. Y., 1881. 864 Venulet, V. A., 196. Buerger, S. G. O., 1915, 179. Kaltenbach, L. A., 30. Virchow, V. A., 5. Aschoff, V. A., 138. Lubarsch, Arch. micr. Anat., 41. v. Brunn, Ibid. Prezowsky, V. A., 116. 865 Montfort, Thèse, Paris, 1902. Kuster, L. A., 42, 864. Voelcker, London P. S., 46, 133. . Drew, London P. S., 1 897. Fenwick, Tumors of Bladder, London, 1897. Rundle, London P. S., I 895. Fere, Thèse, Paris, I 881. Stow, An. Surg., 46, 233. Goebel, Z. K., 3. Stoerk, Z. B., 26 (Lit.). Cohen, V. A., I13. Rehn, L. A., 50. 866 Wendel, M. G., 6. Leichtenstern, D. W., I898, 709. Nassauer. Frankf. Z. f. Path., 1920, 22, 353. Seyberth, M. W., 1907, 1573. Beneke, V. A., 16r. W. Fischer, Arb. path. Instit., Tubingen, 1908. Monckberg, V. A., 187. Terrier, Hartmann, Rev. d. Chir., I 895. Husler, Jahrb. f. K., 1905, 62. R. F. Müller, I. D., Leipsig, I904.

\section{TUMORS OF PENIS}

867 Creite, Z. C., 79. C. Kaufmann, Deut. Chir., 50a., I63. Barney, Mass. Gen. Hosp. Rep., 1908, 2, 275. Thomson, B. M. J., 1897, 2, 1841. Hottinger, Cor. Schweiz. Aertze., 1897. Demarquay, Mal. Chir. d. Penis, Paris, I877. Martin, J. Cut. Dis., 1895, 13. Buday, L. A., 49. Schuchardt, S. kl. V., 257. Taylor, J. Cut. Dis., I889, 7. Kuttner, L. A., 59; B. B., 26. 870 Borrmann, Erg. Path., 7, 822. Hall, Annal. Surg., 39. Beck, Internat. Clinics, 1892, 2. Payr, Z. C., 53, 221. P. Pott, Surg. Observations, London, 1775. Curling, Dis. of Testis, London, I878. R. Volkmann, S. kl. V., 3345 , 1889. Mitchell, Med. News, $1888,53,152$. Lewin, V. A., I12. Heath, B. M. J., I883, 2, 327. Bell, Edin. M. J., 1876, 22, 135. Cameron, Glasgow M. J., 12, 40.

\section{MELANOMA}

871 Demieville, V. A., 82. Recklinghausen, Fibrome d. Haut, I882. Unna, Berl. k. Woch., I893, I4; V. A., I43; Histopath. d. Hautk., I894. Delbanco, Monatsh. f. p. Derm., 22, 105. Hodara, Ibid., 25, 205. Scheuber, A. D., 44, 175. Gilchrist, J. Cut. Dis., I 899, 17. Bauer, V. A., 142. Judassohn, A. D., 33. Lubarsch, Erg. P., I 895, 588. Hannsemann, Bosartig. Gesch., 13. Borst, Geschwulste, I, I16, 447. Favera, Z. B., 43 (Lit.). 872 Kromayer, Z. B., 22; Monatsh. f. p. Derm., 4I. Lowenbach, V. A., 157. Walsch, A. D., 39. Riecke, Ibid., 65. Pini, Ibid., 61. Judalewitsch, A. D., 58. Herxheimer, Erg. P., 1897, 1907. Moller, A. D., 62. Fox, Brit. J. Derm., 1906. Johnston, Jour. Cut. Dis., 1905, 23. H. Fox, J. A. M. A., 1912, 58, 1190. Ehrmann, Bibliotheca med., I896, Abt. D., II. Ribbert, Z. B., 21. Soldau, L. A., 59. 873 Moullin, B. M. J., I891, I, 224. Hutchinson, B. M. J., I 886, $I$, 49I. Faguet, Arch. clin. Bordeaux, I 894, 448. Plantier, Thèse, Bordeaux, I 894. Galloway, B. M. J., I 897, 2, 873. Bayet, Annal. de Dermat., I 895, 495. Just, I. D., Strassburg, I888. 874 Dobbertin, Z. B., 28. Eberth, V. A., 58. Fisher, Box, B. M. J., 1900, I, 639. Albert, C. C., 1906. Eiselt, Prag. Viertelj., I 858, 190. 879 Furth, C. P., I904 (Lit.). 880 Ritter, Arch. f. Oph., I864, 10. Mertsching, V. A., II6. Kromayer, Arch. micr. Anat., 42. Jarisch, A. D., 24. Hertwig, Festschr. f. Haeckel, 1904. Bohn, Z. K., 2, 291. Staffel, D. P. G., 1907, 11, 136. Meir- 
owsky, Monatsch. f. Derm., 42, 44. Fuchs, Das Sarcoma d. Uvealt. Wien., I 882 ; Textbook, Ophthal. Baumgarten, Arch. f. Heilk., I875, 16. Lubarsch, Erg. P., 1895, 374. Katsurada, Z. B., 32. Gierke, Z. B., 7, Suppl. Berdez, Nencki, Arch. f. exp. Path., 1886. 881 Vossius, Graefe's Arch., I 885, 3I. Hamburger, V. A., II7. Walter, Monatsch. f. Augenh., I893, 357. Perls, V. A., 39. Rindfleisch, V. A., I03. Barlow, Bibliot. med., I 895; Derm. Abt. D., II, b, 6. Ravenna, V. A., I7I. Whitfield, Brit. J. Derm., 1900, 12. Thormahlen, V. A., 108. Stiller, D. A. kl. M., I875, I6, 414. Ganghofner, Prag. Viertelj., 1876, 77. Eppinger, Biochem. Z., 1910, 28. Hopkins, Cole, cit. by Furth. Gessard, C. R. S. Biol., I902, 54, I304, I398; C. R. A. Sci., I903, I38, 586, 774. 882 Stoeber, Wacker, M. W., I910, 739, 947. Adler, Z. K., 1912, II, I. Rosenfeld, Arch. f. exper. Path., 45. Elschnig, Graefe's Arch., 75, 76, 78. Neuberg, V. A., 192, 514. Alsberg, J. Med. Res., I907, I6, I17. Wintersteiner, Erg. P., I907, I0, I044 (Lit.). Parsons, Path. of Eye; Oph. Hosp. Rep., 1903, 15, 286, 375. Verhoeff, Arch. of Oph., 1902, 30. Meyerhoff, K. Monatsb. f. Augenh., I901, 913. 883 Rogman, Zeit. f. Augenh., I902, 7, 253. Groenouw, Arch. f. Oph., I898, 47. Purtscher, Arch. f. Oph., 1900, 50. Evetsky, A. f. O., 42, 170; 45, 563. Fehr, Cent. f. Augenh., 1903, 129. Michel, Festschr. d. phys. med. Gesell., Wurzburg, I899. 884 Gutman, A. f. Augenh., 31, 158; Zeit. f. Augenh., 1900, 3 , 32. Stock, Ophthal. Klin., 1899. Hirschberg, B. W., 1904, 77. Kerschbaumer, Das Sarcom. d. Auges., Wiesbaden, I900. 885 Silcock, B. M. J., I892, I, I079. Boit, Frankf. Z. f, P., I. Rokitansky, Allg. Wien. m. Zeit., I86I. Grohl, Z. B., 39. Oberndorfer, Erg. Path., 1908. Thorel, Munch. m. W., 1907, 725. 886 Stoerk, V. A., 183. Virchow, V. A., I6. Hirschberg, V. A., 186. Berblinger, V. A., 219, 328. Eiselt, Prag. Vietelj., 1862. Paneth, L. A., 28, I78. Dietrich, L. A., 53. Tuffier, Arch. gen. d. Med., 21, 28. Wiener, Z. B., 25, 322. Chalier, Bonnet, Rev. de Chir., 46, 47 (Lit.). 887 Orth, B. W., 1906, 1084. Davidson, D. W., 1906, I802. Reiman, Prag. m. W., 1902. R. Fuchs, Handb. vergl. Physiol. (Winterstein), I9I3, 3. Stockard, Amer. J. Anat., 1915, I8. Hoffmann, Arch. m. Anat., 70. Chun, Verh. d. Zoöl. Gesell., 1902. Post, V. A., 135, 47.9. 888 Caspary, A. D., I891. Kaposi, A. D., I891. Abesser, V. A., 166, 40. Wieting, Handi, Z. B., 43. 889 La Grange, Les tum. d. Yeux. Mitrolsky, Arch. d. Augenh., 1894, 28. Bard, Man. d. Anat. Path. Weinbaum, Arch. d. Oph., I891. Griffith, Trans. Oph. Soc,, London, I894, I4, I60. 890 Lucksch, Z. B., 53, 324. Goldzieher, D. p. G., I913, 213. Tuczek, Z. B., 58, 250. Maclachlan, J. Med. Res., 1915, 28, 92 (Lit.).

\section{TUMORS OF THE THYROID}

891 Wolfler, L. A., 29. Gudernatsch., Am. J. Anat. 892 Bircher, Med. Klinik., Beiheft. 6, 1908. Payr, Martina, Z. C,, 85. Meerwein, Z. C., 9I (Intratracheal). 893 Eiselsberg, L. A., 72. Sehrt, Cent. Chir., 13. Heinziger, Pfister, Z. C., 82. 894 Kamann, Wien. kl. R., I903, 280. Hewetson, B. M. J., 1903, I, 657. Demme, Handb. d. Kinderk. (Gerhardt), III, I, 392. 895 Fabre, Therenot, Arch. de mal. des Enf., 1907, 403. Escherich, Jahrb. f. Kinderh., I8, 87. J. Berry, Dis. of Thyroid Gland, London, I901. Zahn, Z. C., 1885. 897 O. Ehrhardt, B. B., 35, 343. Moebius, Nothnagel's System, 22. Greenfield, B. M. J., 1893, 2, 1260. Ewing, N. Y. M. J., 1906, 84, 1061. Eiselsberg, L. A., 46, 430, and 48; Deut. Chir., I901. 900 Langhans, V. A., I89. Rose, L. A., 23. Barker, London P. S., I896, 47. 901 Madelung, L. A., 24. Plauth, B. B., 19. Kapsamer, W. kl. W., I899, 46I (Lit.). Getzowa, V. A., I88. Braun, L. A., 28. Cornil, Arch. de Physiol., I 875 . Suskind, I. D., Tubingen, I877. Hochstetter, Wien. m. W., I 888. Karst, I. D., Wurzburg, I858. 902 Friedland, Prag. m. W., I 896. Hinterstoisser, Fest. f. Billroth., Stuttgart, I 892. Herb, I. D., Munchen, 1892. Semon, B. M. J., I893, I, 1267. Mermet, Lecour, Soc. anat., I896. Meyer, Arch. Laryng., 5. 903 Coats, London P. S., I887. Feuer, Fest. f. Kocher, Weisbaden, I891. Middledorpf, L. A., 48. Morris, London P. S., I880. Cramer, L. A., 36. Sheen, B. M. J., I 899, 2, I 102. Jaeger, B. B., 19. K. Ewald, W. kl. W., I896, I86. Cohnheim, V. A., 68, 547. Runge, V. A., 55, 254. Feurer, I. D., Kiel, I889. Honsell, B. B., 24. 904 Litten, B. kl. W., I889, I094. Hof- 
mann, W. kl. W., I897, 1004. Middledorpf, L. A., 48. 905 Riedel, Berlin Chir.-Congresber., 1893. Oderfeld, Steinhaus, C. P., 1902, 209. Becker, Cent. f. Chir., I900. Jeffries, cit. by Ewing, Arch. Int. Med., 1908. Billroth, W. m. W., I888. 906 Ewald, Nothnagel's Spec. Path. Morf, J. A. M. A., 32, 9 II. 907 Braun, L. A., 24, 28. Forster, Wurzb. m. Zeit., I860. Neumann, L. A., 23. C. Kaufmann, Z. C., II. Limacher, V. A., 151, Suppl. Pick, Z. f. Heilk., I3. 908 Lartigau, Amer. J. M. S., I901, I56. Riedel, D. Ges. Chir., r 896-7. Silatschek, B. B., 67, 590. Hashimoto, L. A., 97, 219. 909 Harbitz, J. Med. Res., 32 (Lit.). 910 Kocher, Jr., V. A., 155. Benjamin, Z. B., 31. Erdheim, Z. B., 33, 35. MacCallum, J. H. Bull., 1905. Kursteiner, Anat. Hefte, I898. Hulst, cit. by Verebely, V. A., 187. König, L, A., 51, 578. 911 M. B. Schmidt, Fest. f. B. Schmidt, Jena, 1896. Streckeisen, V. A., I03. Neumann, L. A., 20. Seldowitsch, Cent. Chir., 1897, 499. Chamisso, B. B., 19. Erdheim, Z. B., 35.

\section{THE THYMUS AND ITS TUMORS}

912 Hammar, Erg. Anat., 19; Anat. Hefte, 43. Erdheim, Z. B., 35. Sharp, Lancet, 1906, I, 436. 913 Stohr, Anat. Hefte, 31. Prenant, La Cellule, Io. Bell, Amer. J. Anat., 5. Beard, Anat. Anz., 9. Hart, V. A., 214. Maximow, Arch. m. Anat., 74. Watney, Proc. Royal Soc., 27. Schaeffer, Cen. f. Physiol., 22. Wasutatchkin, C. P., 1913, 61 7. Wiesel, Erg. Path. Anat., 1911. Rieffel, C. rend. acad. sci., 1909, 148. 914 Harman, J. anat. and Physiol., I901, 36. Bien, Anat. Anz., I906, 20. Bovaird, Nicoll, Arch. of Pediat., 1906. v. Sury, Viertelj. f. ger. Med., 1908, 36. Ronconi, Pathologica, 1909. Rolleston, Clin. Jour., I 898, 13. Edmunds, McKenzie, London P. S., 48, 192. Pepper, Stengel, Internat. Med. Mag., 4, 739. Hektoen, Ibid. Tarozzi, C. P., 1908, 154. Soupault, Soc. Anat., 1897, 592. Acland, London P. S., 36, 491. Lochte, C. P., I899, I. Hahn, Thomas, Arch. gen. de med., 1879, 1, 523. Fabian, C. P., 19. 915 Coenen, L. A., 73, 443. Pigache, Beclere, Soc. Anat., I9I I, 86, I (Lit.). Hueter, Z. B., 55. Soupault, Soc. Anat., 1897, 590. Pollosson, Pierry, Prov. med., 1901, 151. Funke, Philadel. P. S., 1908, II, 60. Chiari, Z. f. Heilk., I894, 15, 403. Rolleston, J. P. B., 4. Hare, cit. by Rolleston. Bednar, cit. by Chiari. 916 Seidel, I. D., Leipsig, 1902. Ribbert, Frankf. Z. P., II, 208. Rubaschow, V. A., 206. Bartels, Jahrb. d. Kinderh., I906, 64, 289. Eisenstadt, I. D., Greifswald, 1902. Steudener, V. A., 59, 413. 917 Thiroloix, Debret, Arch. med. exper., 19, 668. Simmonds, Z. K., 12, 280. Schridde, Path. Anat. (Aschoff), C. P., 22, 902. 918 Gabcke, I. D., Kiel, I896. Zniniewicz, I. D., Greifswald, I9I I. Seebohm Hamburg. Statsanst., 1890. Meigs, de Schweinitz, A. J. M. S., 108, 193. 919 Ertmann, I. D., Griefswald, I898. Weigert, Laquer, Neur. Cent., 1901, 594. Le Tulle, Arch. gen. de med., I890, 2, 641. Stockart, I. D., Heidelberg, 1905. Mandelbaum, Celler, Jour. Exper. Med., I908, IO, 308. Schneider, I. D., Griefswald, I892. 921 Achard, Paisseau, Arch. med. exper., 20, 78. Paviot, Gerest, Arch. med. exper., 1896, 8, 699. 922 Ambrosini, Thèse, Paris, I 894. Caso, Gaz. d. osped., Milano, 1897, I8, 401. Winugradoff, Arch. Russi. di. Pat., I 897, 3, 4I. 923 Danzac, Soc., anat. 1 893, 48, I99. Hauser, Soc. anat., I901, 76, I69. Yamasaki, Zeit. Heilk., 1904, 5, 269. Chiari, C. P., 22. Beitzke, D. P. G., 1909, 13, 264. J. E. Welch, N. Y. Path. Soc., 10, 161. Karsner, Arch. Int. Med., 1910, 6, 175. Symmers, N. Y. M. J., 1911, 93, 971. Oppenheim, D. myasthen. Paralyze, Berlin, 1901. Buzzard, Brain, 1905, 28, 438. Hun, Albany Med. An., 1904, 25, 28. Link, D. Zeit. Nervenh., 1902, 23, I I 4. Burr, McCarthy, A. J. M. S., $1901,121,46$.

\section{TUMORS OF THE HYPOPHYSIS}

924 Marie, Rev. de med., I886; Brain, I889. Courtellemont, Des Tum. d. Corps Pituitaire, Paris, 191 I (Lit.). D. W. Cunningham, Trans. Roy. Irish Acad., I891. Tamburini, Int. Med. Cong., Bruxelles, I 894. Brissaud, Meige, Jour. de med. et chir. prat., 1895, 49. Meige, Arch. gen. de med., 1902, 2, 407. Launois, Roy. Rev. neurol., 1902, 1054. Mohr, cit. Strada, Caspar's Wochen., 1840, 565. Pechkranz, Neurol. Cent., I 899, 
203. Froelich, Wien. kl. Rundschau, r901, 883. Bartels, Zeit. f. Augenh., I906, 15, 407; Verh. Deut. Naturf., Strassburg, 1907. Cushing, Jour. Nerv. and Mental Dis., 1906. Beclere, Soc. des Hop., 1902, 1060; 1909, 274. Gramegna, Rev. neurol., 1909, I, 15. Horsley, B. M. J., 1906, I, 323; 2, 4II. Schloffer, B. B., 1909, 50, 767. v. Eiselsberg, Wien. kl. W., I909, 287. Hochenegg, Therap. d. Gegenw., 1908. Hirsch, Wien. kl. W., 1909, 473. Cushing, Annal. Surg., 50, 1002. Lecene, Rev. neurol., 1909, 815. Benda, B. W., 1900, I205; Hand. d. path. Anat. d. Nervens., Flatau, 1904. Erdheim, Kais. Ac. d. Wissen. Wien., 1904; Z. B., 33, 46; Frankf. Z. P., 1910. Lowenstein, V. A., 188. Lewis, J. H. Bull., 1905, 16, 157; J. A. M. A., 1910, 55, 1002. Collarit, Z. N., 1905, 28. Creuzfeld, Jahrb. Hamburg. Staatsk., 1909, 13, 351. 925 Strada, V. A., 203. Cushing, Pituitary Body, etc., I91 I J J. A. M., I914, 63, 1515. de Neuville, Revue d. Revues, I 898. Massedaglia, cit. by Cushing. Ascenzi, Riv. pat. nerv. e. ment., 1910. Poindecker, W. kl. W., 1913, 745. Strumpell, Z. N., II. Goldstein, Munch. W., 1913, 757. Tilney, Mem. Wistar Instit., Philada., 191 I. Rogowitsch, Z. B., 4. Luschka, Die Hirnanh. d. Mensch., I860. Saxer, Z. B., 32. Launois, C. R. Soc. Biol., I903, I578. 926 Kohn, Arch. m. Anat., 75. M. Vogel, Frankf. Z. P., II. Herring, Quart. J. Exper. Phys., I908, I, I21. Cushing, Goetsch, Amer. J. Physiol., 1910, 27, 60. 927 Dandy, Goetsch, Am. J. Anat., I9I I, 12, I37. Arai, Anat. Hefte, 1907, 33, 4 Ir. Civalleri, C. R. assoc. d. anat., 1908, 10 , I28. Haberfeld, Z. B., 46. Flesch, Naturf. Samml., Strassburg, 1885. 928 Schoneman, V. A., I29. Comte, Z. B., 23. Launois, Mulon, C. R. Soc. Biol., I903, I, 448. 929 Reuss, Wien. kl. W., 1908, I I 6.930 Erdheim, Frank, Z. P., 4. 931 Nothdurft, Ibid., 10. Roussy, Clunet, Rev. Neurol., I91 I, 22, 313. Nagaeli, I. D., 1909. Huchard, Launois, Soc. d. hop., I903, I44. 933 Smoler, Wien. kl. W., 1909, 1488. Stolpe, D. W., I904, 689. Bruns, cit. by Courtellemont. Cagnetto, V. A., 176. Kruger, I. D., Greifswald, 1895. Sommer, Zeit. f. Laryng., 1909, 2, 355. Zak, Wien. k. Rund., 1904, 165. Alquier, Schmiergeld, L'Encephale, 1907, 536. Presbeanu, Thèse, Paris, I899. Marinesco, Rev. neurol., I9II, 398. Carbone, Gaz. med., Torino, I902, 17 I. Lecene, Roussy, Rev. neurol., 1909, 815. Krumbhaar, Phila. P. S., 1909, 158. 934 Launois, Roy, Rev. neurol., 1903, 93. A. Meyer, Amer. J. Insan., 1913, 59, 653. v. Bonin, Quart. J. Med., I913, 6, I45. Wolf, Z. B., 13, 629. Hippel V. A., I26. O'Malley, N. Y. M. J., I911, 92, I219. Hansemann, Berl. W., 1897, 417. Boyd, Lancet, 1910, 2, I I29. Ingermann, I. D., Bern., I889. 935 Caussade, Laubry, Arch. med. exp., 21. Agostini, Riv. di pat. nerv. e mentale., I 899, 169. 936 Hart, Zeit. f. Psych., 47. Langer, Zeit. f. Heilk., I892, 13. Bostroem, C. P., I897, 8, 1. Fahr, Munch. m. W., 1903, 1987. Boudet, Clunet, Arch. med. exper., 22. 937 Allgayer, B. B., 2. Bartels, Wien. kl. W., 1908, 273. Bregmann, Steinhaus, V. A., 188. 938 Walker, cit. by Erdheim. Selke, I. D., Königsberg. Boyce, Beadles, Jour. Path. Bact., I893, I, 359. Cushing, A. J. M. S., 1910, 1.39, 473. Duffy, A. S., 72, 537. 939 Bury, B. M. J., I 891, I, I I 79. Roxburg, Collis, B. M. J., I 896, 2, 63. Weichselbaum, V. A., 75, 444. W. Hutchinson, N. Y. M. J., 1900, 72, I34. Hecht, J. A. M. A., I909, 53, I00I. Beck, Zeit. f. Heilk., 1883, 4, 393. Margulis, Neur. Cent., I901, ro26. Wegelin, cit. by Erdheim. Rippmann, Ibid. Scalincie, Il Tommasi, 1906. Hertel, cit. by Courtellemont. Lapersonne, Cantonnet, Arch. d'Ophth., 1910, 65. 940 L'Hermitte, Congres neurol., Brussels, 1910. Schuster, Psych. Storungen. b. Hirntumoren, Stuggart, 1992. Brissaud, Meige, Nouv. Icon. Salpetr., 1904, 165. Sternberg, Nothnagel, Spec. Path. 941 Strauch, A. J. M. S., 148. Lemos, N. Icon. Salpetr., I9I I. 942 Gandy, Soc. d. hop., 1906, 1226; 1907, 478. Brissaud, N. Icon. Salpetr., 1907, I. Sainton, Rathery, Soc. d. hop., 1908, 647. Claude, Gougerot, Soc. biol., 1907, 2, 785. Gilford, Disorders of Growth, etc., London, I91 I. Petren, V. A., 190 (Lit.). Dallemagne, Arch. med. exp., I 895, 589. Mitchell, Le Count, N. Y. M. J., 69, 5 I 7. Huchard, Launois, Soc. d. hop., 1903, I444. Widal, Roy, Froin, Rev. de Med., 1906, 313. B. Fischer, Frankf. 7. P., II, 130. 943 Nichols, Favre, Lyon Med., I910, I 14, 786. Parisot, Rev. Neurol., 1910. Hutinel, La Clinique, 1910, 193. Babonnieux, Paisseau, Gaz. d. hop., I910, I431. Matassari, Rev. Neurol., I91 I, 198. Bertolotti, N. Icon. Salpet., 1910. Claude, L'Encephale, 1907, 295. Franchini, Giglioli, N. Icon. 
Salpet., 1908, 324. Calderara, Arch. Ital. de Biol., 1908, 50, 190. W. Dalton, Lancet, 1892, 2, I 190. Stewart, Boston M. S. J., 1899, I40, 501. Zollner, Arch. f. Psych., 44. 815. Sainton, Rathery, Soc. d. hop., 1908, 647. Exner, W. kl. W., 1909, 108. Renon, De Lille, Soc. biol., 64, 65. Hansemann, B. W., I897, 41 7. Pineles, Ver. f. Psych. Wien., 1899. Schultze, Fischer, Mit. Grenzg., 24. Grahaud, Thèse, Paris, 1910. 944 L. Pick, D. m. W., I91 I, 1930. McCarthy, A. J. M. S., 124, 994. Guillain, Alquier, Arch. med. Exper., 1906. Price, A. J. M. S., 137, 705. Launois, Cleret, Gaz. d. hop., 1910, 83, 57. I. Lyon, Arch. Int. Med., I910, 6, 28. Dammann, Frankf. Z. P., I9I 3, 12, 337. Harbitz, C. P., I9I I, 801. Amsler, B. W., I912, I600. v. Eiselsberg, Ann. Surg., 52. 945 Neurath, Wien. kl. W., 19I 1, 43. Marburg, D. Z. Nervenh., 36, II4. Mixter, Quacken. boss, Ann. Surg., 52. Souques, Chauvet, N. Icon. Salpet., 1913, 67. Ottenberg, N. Y. M. J., 1910, 92, 1222. 946 Wurmbrand, Z. B., 47. 947 Madelung, Deut. Ges. Chir., 1904, 33, 164. Maranon, Bol. Soc. Espan. d. Biol., I9I1. Marburg, Z. N., 1909, 36, i 14. Melchoir, B. W., I9I I, I453. Stroebe, Z. B., 37. Schmidt, Erg. Path., I898, 918.

\section{THE PINEAL GLAND AND ITS TUMOŔ'}

948 Dimitrowa, Thèse, Nancy, I900-or ; Le Neuraxe, 2. Ruggeri, Riv. pat. nerv., 1914, 19, 649. Galasescu, Urechia, C. R. S. Biol., 1910, 1, 623. Krabbe, N. Icon. Salp., I9I I, 257. Pappenheimer, V. A., 200. Joukowsky, Rev. mens. mal. de en Fance, I901, 197. Nieden, Cent. f. Nerven., 1879, 169. M. Neumann, Mon. Psych., 1901, 9, 337. A. W. Campbell, London P. S., I 898, 50, I5. Virchow, Krankh. Ges., II, I48. Heurot, Union med., Rheims, I882. Marburg, Arb. neurol. Instit. Wien., I906; Wien. m. W., 1908, 2617 ; Z. N., 36. Laignel-Lavastine, cit. by Seigneur. Drelincourt, cit. by Seigneur. Scheupf., C. R. Soc. Biol., I850, 2, I67. Simon, Soc. anat., I859, 34, 306. Bouchut, Gaz. hop., I 872, 353. Seigneur, Thèse, Paris, I912. 949 F. C. Wood (Dana), N. Y. M. Rec., 83, 835. Goldzieher, V. A., 213, 353. Rorschach, B. B., 83. Pellizzi, Riv. ital. neuropath., 1910. Garrod, London P. S., 1898, 50, 14. Friedreich, V. A., 33, 165. Ogle, London P. S., I 898, 50. Verger, J. med. de Bordeaux, 1907, 37, 216. Falkson, V. A., 75, 550. Oestreich, Slawyk, V. A., 157, 475. Askanazy, D. P. G., 1906, 58. Gutzeit, I. D., Königsberg, I 896. Bailey, Jelliffe, Arch. Int. Med., I9I1, 8, 85I. Frankl-Hochwart, Wien. M. Woch., 1909, 2328; Z. N., 37, 455. Coats, London P. S., 1886, 38, 44. P. Neumann, I. D., Königsberg. Gauderer, I. D., Giessen, 1889. Weigert, V. A., 65, 212. 950 Hirtz, Soc. anat., I 875, 254. 951 Blane, cit. by Bailey. Hempel, I. D., Leipsig, 1901. Finkelburg (Z. N. 21, Forster), V. A., 13, 27 I. Turner, London P. S., 1884, 36, 27. Howell, Proc. Royal Soc., 1910, 3; Neurol., 65. Schmidt, Med. Zeit., I 837, 6, 32. 952 Raymond, Claude, Bull. Acad. Med., Paris, 1910, 63, 265. Daly, Brain, I888, Io, 234. 953 Dana, Berkeley, N. Y. M. Rec., I9I 3, 83, 835. Exner, Boese, Z. C., I910, 107, 182. Foa, Arch. Ital. de Biol., I912, 57, 233. Biach, Hulles, Wien. kl. W., 1912, 373. McCord, J. A. M. A., 1914, 63, 232.

\section{TERATOLOGY}

954 Schwalbe, D. Missbild. d. Mensch., Jena, 1906. Hubner, Erg. P., I9I I, I, 650 (Lit.). Fischl, D. path. Gesell., 1903. 955 Wilder, Amer. J. Anat., 3. 956 Ahlfeld, Missbild. d. Mensch., Leipzig, I880. Taruff, Stor. d. Teratol, Bologna, I88I-1 895 . Rathke, Müller's Archiv., I838. Bonnet, Lehrb. d. Entwickel., M. G. G., I3, I49. 957 W. Roux, Gesam. Abhand., Leipzig, I895. Brown, Anat. Hefte, I8. Hofer, A. micr. Anat., 74. O. Schultze, C. P., 10, 393; A. f. Entwick., I, 297. Wetzel, A. micr. Anat., 46, 654. J. Loeb, Pfluger's Arch., 55, 525. 958 Nussbaum, Anat. Verhand., I90I. Allen, Amer. J. Anat., 1904, 3, I89. C. H. Swift, Ibid., I914, 15, 484 (Lit.). 961 Harvey, Biol. Bull., I910, 18, 269 (Lit.). Kirkbride, A. f. Entw., 32, 717. 962 Chevassu, Les. tum. d. testicule, Paris, I9II. 963 Herxheimer, Z. B., 44. Lippmann, Z. K., 3. Schmorl, cit. by Herxheimer. Thyroid case. Hansemann, Bos. Ges., 71. Landsteiner, Z. kl. 
M., 62, 427. Lindemann, Z. K., 6. Lubarsch, Z..Lehre. v. d. Ges., I 899, 279. Michelsohn, I. O., Wurzburg, I894. Klein, M. W., 1890, I70. Wells, J. P. B., I901. J. Loeb, Am. J. M. S., 125. Ehrlich, Apolant. Arch, Instit., Frankfort, 1906. Bashford, B. W., 1907, I238. 964 Krompecher, Z. B., 44. Remak, D. Klinik, 1854, 170. 965 Heschl, Z. f. Heilk., I860, 68. Chiari, Z. f. Heilk., 1891, I2. Franke, L. A., 34. Sutton, Jour. of Anat., I 886, I 887 ; Lancet, I 888, $I, 308,357$. Reverdin, Rev. med. Suisse. Rom., I 887 , 7, I2 I. 966 Jonnesco, Bull. Soc. d'Anat., I 888, 240. Nichols, J. Med. Res., 13, I878. Schwenninger, Char. An., I886, 642. Kaufmann, V. A., 97. v. Dooremale, A. f. Oph., I 873, 354. Garre, B. B., II. Le Fort, Rev. de Chir., I894, I898. Hoesch, V. A., 99, 449. : Aschoff, Erg. P., I 895 (Lit.). Collins, Royal Oph. Hosp. Rep., I 89o.. Greef, Arch. f. Augenh., I 892, 28, 395. Hartley, An. Surg., 23, 573. Labougle, Thèse de Bordeaux, I889. Samter, V. A., II2. Kostanecki, Mielecki, V. A., I2O, I2I (Lit,). 967 Virchow, V. A., 30. Hennes, Arch. f. Kinderh., g. Cusset, Cong. français d.. Chir., I 886. Steinbrugge, Opth. Spec. Path. Hildebrand, L. A., 49, I67. F. König, L. A., 48, 164. Goldman, Z. B., 7. 968 Thomas, Bull. soc. Chir., I887, I4I. Zahn, Z. C., 1885, 23. Volkmann, C. C., I882. Lucke, L. A., I. 969 Wilms, D. A., 55. Pupovac, L. A., 53. Wetzel, I. D., Giessen, 1895. Poult, V. A., 18I. Herb, Amer. J. M. S., 131. Rosenstiel, cit. by Wetzel. Maas, cit. by Wetzel. Heusinger, V. A., 33. Bochdallek, Ost. Z. f. p. Heilk،, I866. Streckeisen, V. A., I03. Erdheim, L. A:, 85. Berard, Chalier, A. gen. d. Med., 1908. Bryck, Wien. m. Woch., I4. Dirmstrey, D. W., I895, 573. Christian, J. Med. Res., 7, 54. Lambert, Knox. Trans. Assoc. Amer. Phys., 1920. Marchand, Cysts. Realencyclop., I885. Riegel, V. A., 49. Lowenmeyer, B. W., I888, I35. Mandelbaum, A. J. M. S., 120, 64. Dangschaat, B. B., 38. Pinders, I. D., Bonn, 1887. 970 Jores, V. A., I33. Virchow, V. A., 53, 444. Andoly, Cont. d. Kysten. derm., etc., Paris, I 898. Bergmann, Prag. W., I898, 109. Ekehorn, L. A., 56. Hippel, Arch. f. Ophth., 63. Weigert, Broer, V. A., 67. 971 Otto, A. G., 13. Wallmann, Verh. ph.-m. Ges., Wurzburg, 9. Sonnenberg, Z. C., 5. Koch, I. D., Erlangen, I899. Ahlfeld, A. G., 7 (Lit., Illus.). 972 Rindfleisch, V. A., 30. Adami, Text-book of Pathology. Arnold, V. A., IIr, I 76 (Lit.). 973 Askanazy, D. p. Ges., II, 39. Beck, Z. f. Heilk., I883, 393. Bonorden, Z. B., II. 974 Erdheim, S. d. K. Acad. d. Wissen. Wien., I904; C. P., 1906. Steinhaus, V. A., 188. Bostroem, C. P., 8, 1897 (Lit.). Beneke, V. A., 142, 149. Blasius, V. A., 165. Glaeser, V. A., I22, 389. H. Frank, Allg. Z. f. Psych., I890, 46. Benda, B. W., I897, 167. Benda, B.W., 1900, 1205. Heschl. Trachtenberg,V. A., 154. Arnold, V. A., 50. Eberth, V. A., 153. 975 Strassmann, Streker, V. A., 108. Saxer, Z. B., 20. Falkson, V. A., 75. Weigert, V. A., 65. Ogle, London P. S., I899, 50, 6. Hahn, Berl. W., I 887, 408. Braquehaye, Arch. gen. d. Med., I892, 2. Dowd, An. Surg., 32. Niosi, V. A., 190. Klemm, V. A., I8I, 54I. Rittner, Z. p. Heilk., I910. Tilger, V. A., I39. Kostlivy, Z. C., 9I, 35I. Rokitansky, Path. Anat., II, 388. Virchow, V. A., 7, I30. Lion, V. A., I44. Spaeth, Munch. W., I898, 1083. Tuffier, Bull. Soc. Chir., 1904. Wagener, cit. by Kostlivy. Roth, V. A., 86. Wyss, V. A., 5 I. Nasse, L. A., 45. 976 Colmers, L. A., 79 (Lit.). Gfeller, Z. C., 65. Ruge, Z. G. G., I. Beneke, cit. by Niosi. Hennig, C. G., I880. Sanger, Klopp, A. G., I6. Honl, cit. by Kostlivy. Fraenkel, Wien. m. W., 1883. Marie, Bull. soc. Anat., I 899, 267. Ruysch, cit. by Wilms, D. A., 55. Mantel, Ibid. Dickinson, London P. S., I87 I, 296. Bonfigli, Schmidt's Jahrb., 1876, I80. Schutzer, cit. by Wilms. 977 Marchand, Breslauer. arztl. Zeit., I88I, 25I. Lexer, L. A., 6I (Lit.). Hosmer, Boston M. S. J., I890, 6I. Pilliet, Bull. soc. anat., I 888, 875. Montgomery, J. Exper. Med., I 898. Ahrens, L. A., 64. Englander, C. P., 1902. Goebell, Z. C., 6I. Fleischmann, Z. G. G., 56. Bonney, London P. S., 58. Djewitsky, V. A., I78. Schonholzer, Z. B., 40. Kolaczek, V. A., 75. Frankel, Wien. m. W., I 883, 865. Brouha, Rev. de Gyn., 1902. Bolzano, I. D., Wurzburg, 1901. Funke, Beitr. G. u. G., 3. Merkel, Z. B., 33. Schlegtendal, L. A., 36. Wedeman, I. D., Jena, 1902. R. Meyer, V. A., I68. Ruge, Z. B., 34. Germain, Ann. Surg., 40, 928 (Lit.). 978 Sanger, A. G., 37. Zweifel, C. G., I 868. Bardenhauer, cit. by Wilms. Le Gendre, C. P., 9, 762. Eberth, V. A., 35. Zahn, V. A., I43; Z. C., 22. Trespe, Arch. path. Inst., Posen, I901. Stilling, V. A., II4. Vir- 
chow, V. A., 53. Hess, Z. B., 8. Roth, V. A., 86. Colmers, L. A., 79 (Lit.). 979 Borst, C. P., I 898 (Lit.). 980 Tourneux, Hermann. J. d'anat. et de physiol., I887, 23, 598. Wette, I.. A., 47. Wendelstadt, I. D., Bonn, I885. Mallory, A. J. M. S., I03; J. Med. Res., 8. Perman, L. A., 49. Schmidt, V. A., I12. v. Bergmann, B. W., I884, 76r. Eternod, An. Anz., 1899, 16. Keibel, Mall. Embryology, 1912. Jantreboff, V. A., 49. Ritchl, B. B., 8. Middledorpf, V. A., IOI. Ganz, Prag. W., 1894. Freyer, V. A., 58. Jordan, I. D., Leipzig, I895. 981 Preuss, A. f. Anat. u. Phys., I 868. Stolper, Z. C., 50 (Lit.). Epstein, Z. f. Heilk., I, 589. Mermet, Rev. de Chir., I895, 15. Marchadier, Thèse de Paris, 1893-94. Martini, L. A., 17. Braune, Doppelbildungen, Leipzig, I 862. Kummel,V. A., I18. Pannwitz, I. D., Berlin, I884. Hennig,V. A., 115;Z. B., 28; Digests of I. D. Perman, L. A., 49. Spondly, I. D., Zurich, I894. Port, London P. S., 32. Danzel, L. A., 17. Kiderler, Z. C., 52. Kronlein, L. A., 21, Suppl. Menzel, L. A., 22. 982 Linser, B. B., 29. Kleinwachter, Z. G., 9. Piper, I. D., Wurzburg, 1895. Stroh, I. D., Giessen, I895. Hagenthorn, L. A., 60. Sperling, I. D., Wurzburg, I891. Böhm, B. W., I 872 . Feldmann, I. D., Berlin, I895. Frank, Prag. W., I894. Dépaul, Gas. med. d. Hop., 1869. 983 Hinterstoisser, L. A., 87. Nakayama, Arch. f. Entw., 19, 475. Keller, A. G., 85. Scheuermann, L. A., 88. Marchand, Kroner, A. G., I88I, 17. 984 Recklinghausen, V. A., I05. Hagenbach, L. A., 66. Bartels, Z. C., 20; B. W., I892, 833. Bohnstadt, V. A., I40. Hildebrand, L. A., 46; Z. C., 36. Muscatello, L. A., 47. Arnold, Z. B., I6; V. A., III. Borst, 1. c., 906, 924 . 



\section{N D E X}

AbDominal fetal implantations, 977

teratomas, 977

Hodgkin's granuloma, 370

Acanthoma, 474

adenoides cysticum, 834

of skin, Demodex folliculorum in, 822

etiology, 820

secondary changes, 820

structure, 8I9

types, 818

of vocal cords, 855

tubular, 8I9

Acidemic coma in cancer of stomach, 656

Acidosis of cancer, 74

Acinar carcinoma, $47 \mathrm{I}$

of breast, 508, $52 \mathrm{I}$

fibrous, 522

following fibro-aden-

oma, $52 \mathrm{I}$

primary, 522

Acoustic neurofibroma, 432

Acromegaly, 942

diabetes with, 943

glycosuria with, 943

Adamantinoma, 49, 709

clinical features, 7 I 4

glandular, 7 I3

of tibia, 7 I 5

structure, 712

Adeno-acanthoma of uterus, 547

Adenocarcinoma, 456, 470

of adrenal gland, 770

of breast, 508, 510

forms, 512

histogenesis, 512

small cystic, 5 I 7

structure, 5I I

of cervix uteri, 547

mucous, 549

of kidneys, alveolar, 748

embryonal, in adults, 749

in infants, 749

multiple, in sclerosis, 746

papillary, 742

histogenesis, 746

structure, 748

with granular cells, 746

tubular, 750

of large intestine, gelatinous, 671
Adenocarcinoma of ovary, cystic, 597

papillary serous, 589

pseudomucinous, 595

of pituitary gland, 932

metastases, 933

structure, 933

of prostate, 787

of stomach, 627

occurrence, 656

of thyroid gland, 898

small alveolar, large cell, 9or

papillary, of choroid plexus, 4 I 8

Adenofibroma, I 49

edematodes, I66, I67

of breast, 495

of ovary, 597

Adenoid carcinomas, 464

epithelioma of skin, 834 cystic, 834 simple, 835

Adenoma, 28, 446, $45^{2}$ alveolare a cellules claires, 745

clinical characteristics, 453

destruens of large intestine, 669

endotheliale, 746

functional capacity, 454

gelatinosum, 893

gross appearance, 453

in colitis polyposa, $45^{8}$

in gastritis polyposa, $45^{8}$

malignum, 453,455

of adrenal gland, 770

mode of growth, 455

of adrenal gland, 769

of bile-ducts, cystic, 689

multiple, 690

of bladder, 864

of cervix uteri, simple, 549

of kidney, 739

alveolar, $74^{\circ}$

histogenesis, $74 \mathrm{I}$

papillary, 739

tubular, $74 \mathrm{I}$

of larynx, 850

of liver, multiple, 686 solitary, 68 I

of ovary, 586

everted papillary, 597

solid, 596

superficial, 597

testicular, 610

1033
Adenoma of pancreas, 702 of prostate, 786

of salivary glands, 725

of stomach, simple, 629

of testis, 802

of thyroid gland, fetal, 895 interacinous, 894 malignant, 898

papillary cystic, 455

physiological conception, 457

sebaceum, 46I

structure, 45.5

tubular, 455

Adenomatoid hyperplasia, functional, 459

inflammatory, 457

Adenomyoma, 2 I 2

mesonephric, 2 I 4

of ovary, 610

psammopapillare, 2 I 5

Adenomyomatosis of corpus uteri, diffuse, $\mathbf{5 5 6}$

Adenomyosarcoma of kidney, 220

embryonal, 753

anatomy, 753

histogenesis, 754

structure, 753

Adenosarcoma of breast, $50 \mathrm{I}$ of pituitary gland, 934

Adiposogenital dystrophy, theories, 946, 947

Adrenal gland, adenocarcinoma of, $77^{\circ}$

adenoma of, 769 malignum, 770

cancer of, 769

fully developed, 770

hyperplasia of, 768

melanoma of, 778

neurocytoma of, 772

clinical types, 774

sarcoma of, in infants, congenital, (Pepper type), 775

with craniai metastases (Hutchinson type), 774

tumors of, 768

classification, 768

medullary, $77 \mathrm{I}$

chromaffin cell, 776 with adrenalin content and nephritic symptons, 776 
Adrenal melanoma, 890 rests, extrarenal, tumors of, $76 \mathrm{I}$

tumors of, 755

carcinomatous, 759 gross appearance, $75^{8}$ lateral retroperitoneal, 762

occurrence, 756

peritheliomatous, 759 renal tumors and, differentiation, 760 sarcomatous, 759 structure, $75^{8}$ trabecular, 759

tumors, hepatic, 672

of female genital organs, 752

of ovary, 6Io

of spermatic cord and testis, 762

of testis, 803

Adult tumors, 26, 48 "

Albuminuria in cancer, 75

Aleukemic systemic lymphomatosis, 376

Alimentary tract, myoma of, 2 I 6

Alkalescence of blood in cancer, 69

Allantoid type of papilloma of bladder, 863

Altruism of cells, 100

Alveolar adenocarcinoma of kidneys, 748

adenoma of kidney, 740

cancer, large, 47 I small, $47 \mathrm{I}$

endothelioma, 305

sarcoma, 983

tumor, 346

Alveoli, pulmonary, cancer arising from, 8I2

Amitosis in tumors, 39

Amphipyrenin, 46

in tumor-cells, 46

Ampulla of Vater, cancer of, 700

Amyloid tumors, I 67

Anaplasia of cancer-cells, 467

of tumor-cells, IOI

Anemia from Hodgkin's granuloma, 368

in cancer of stomach, 655 infantum, 379

Anemic type of cancer, 67

Angiocholitis proliferans, 679

Angiochondroma, I 86

Angio - endothelioma bones, telangiectatic, 323 of skin, 33 I

Angiofibroma, I49 blood-vessels in, 151

Angioma, 27, 223 arteriale racemosum, 233
Angioma, cavernous, 227 clinical features, 23 I course, 229 cutaneous, 23 I histogenesis, 230 multiplicity of, 228 neoplastic nature, 231 of liver, 232

of ovary, 232

of tongue, $23 \mathrm{I}$

structure, 230

submucous, 23 I

fissural, 225

histogenesis, 226

of bone, $23 \mathrm{I}$

of brain, 232

of breast, 505

of glands, 232

of larynx, $85^{\circ}$

of muscles, 225

of orbit, 231

of spinal cord, 44I

plexiform, 224

Angioplastic endothelioma, 334

Angiosarcoma, 303, 304, 342 of pituitary gland, 934 orbital, 885

Antigen, autogenous tumor, complement deviation with, 9I

Antitryptic power of blood, 92

Antra, osteoma of, 199

Antrum, maxillary. Maxillary antrum.

Aplastic leukemia, 36I

Aponeuroses, giant-cell sarcoma of, 256

Appendix, cancer of, 665 anatomy, 665 structure, 665

Astrocytes, 40I, 403

Astrocytoma, 4I 6

Astromas, $40 \mathrm{I}$

Ateliosis, 942

Atheromatous cysts of skin, 965

Athletic stomach, 652

Atypical leukemia, 360

Auricular chondroma, I9I

Autogenous tumor antigen, complement deviation with, 9I

BACTERIA in cancer, II 5

Bartholin's gland, tumors of, 564

Basal-cell carcinoma, 474, 833

epithelioma of skin, 830

Bathing trunk type of nevus, 873

Benign giant-cell sarcoma of bone, 280

Bile-ducts, adenoma of, cystic, 689
Bile-ducts, ademoma of, multiple, 690

cysts of, 688

intrahepatic, cancer of, multiple, 690

larger, cancer of, 698

anatomy, 699

etiology, 699

location, 699

structure, 700

tumors of, 689

Bird's-eye inclusion in cancer, I I 5

Bladder, adenoma of, 864 cancer of, etiology, 865 glandular, 864

leiomyoma of, 866

myoma of, 216

osteochondrosarcoma of, 866

papilloma of, 860

allantoid type, 863

structure, 863

rhabdomyoma of, 866

sarcoma of, 865

tumors of, epithelial, 860 miscellaneous, 865

Blastomycetes in cancer, I 7

Blood, alkalescence of, in cancer, 69

antitryptic power of, 92

changes in, in leukemia, 359

in cancer, 67

hyperglycemia in, 70

in chloroma, 363

in stools in cancer, 72

in true pseudoleukemia, 377

regeneration of, in cancer, 69

total quantity of, in tumors, 69

Blood-vessels in dissemination of cancer, $8 \mathrm{I}$ of breast, 531

in gliomas, 405

relation of, to tumors, 56

Bochdalek's gland, 9 Io

Bones, angioma of, 231

endothelioma of, 308, 322

classification of, 323

multiple, 324

solitary bulky cystic, 323

diffuse, 326

fibroma of, 163

formation, heteroplastic, 201

myeloma of, 292

myxoma of, I74, I 75

perithelioma of, 345

sarcoma of, 244,269 . See also Sarcoma of bone.

telangiectatic angio-endothelioma of, 323 
Bone-marrow, lesions in Hodgkin's disease, $37 \mathrm{I}$ multiple myeloma of, 292 sarcoma of, 269 primary multiple, 293

Bone-sarcoma, telangiectatic, 273

Brain, angioma of, 232 cholesteatoma of, 974 melanoma of, 885

sarcoma of, 424 diffuse, 424 gross anatomy, 424 malignancy of, 426 structure, 425

tumors of, 393. See also Tumors of brain.

Branchiogenic cancer, 968 chondromas, 190

Breast, acinar carcinoma of, $52 \mathrm{I}$

fibrous, 522

following fibro-adenoma, 521

primary, 522

adenocarcinoma of, 508 , 510

small cystic, $5 \mathrm{I} 7$

adenofibroma of, 495

adenosarcoma of, 501

angioma of, 505

cancer of, 506. See also Cancer of breast.

carcinosis of, acute, 533

chondroma of, 192

cystadenocarcinoma 532

cystadenoma of, 496

cysts of, dermoid, 505 sebaceous, 505

epithelial tumors of, 484

epithelioma of, 506

fibro-adenoma of, 495 fetal, 499

papillary

496
fibrocarcinoma of, 518 , 535

fibroma of, I 65

hypertrophy of, 484 diffuse, of adults, 484

infantile, 484

of male, 484

leiomyoma of, 505

lipoma of, 505

melanoma of, 505

osteochondromyxosarcoma of, 505

papilloma of. 505

perithelioma of, 345

sarcoma of, pure, 503 spindle-cell, 504

round-cell, 503

senile involution of, 487

tumors of, ectodermal mixed, 500

mesodermal, 499

miscellaneous, 505
Breast, tumors of, mixed, Cancer, Cohnheim's theory 494.

teratoid mixed, 499

Bronchial cancer arising

from mucous glands, $8 \mathrm{I}$ I

structure, 8I2

of lining epithelium, 809

structure, 8 Io

papilloma, 8I 3

Buccal papillomatosis, diffuse, $45^{2}$

Byzantine period, cancer in, I 8

Cachexia in cancer, demineralization in, 75

of stomach, 654

in uterine cancer, $56 \mathrm{I}$

Cancer, 28, 446, 46I

à deux, 85 , I I 4

acidosis of, 74

acinar, $47 \mathrm{I}$

adenoid, 464

albuminuria in, 75

alkalescence of blood in, 69

alveolar, large, 47 I

small, 47 I

anemic type, 67

arising from pulmonary alveoli, 8 I 2

atypical qualities of cells, 466

bacteria in, I 15

basal-cell, 474,833

bird's-eye inclusion in, I 5

blastomycetes in, 1 I 7

blood in, 67 hyperglycemia in, 70 in stools in, 72

blood-vessels in dissemination of, $8 \mathrm{I}$

branchiogenic, 968

bronchial, arising from $\mathrm{m} \mathrm{u} \mathrm{c}$ o $\mathrm{u} \mathrm{s}$ glands, 8I I structure, 812

of lining epithelium, 809 structure, 810

cachexia of, demineralization in, 75

cell autonomy, theory of, 97

cellular overgrowth in, 466

changes in urine in, 72

character of affected tissue, 480

chemical agents in production of, I 9

chimney-sweeps', 870

chorionic, $57 \mathrm{I}$

cicatricial character, 463

classification, 470

coccidia in, I I 5 of, 94

limitations of, 97

collateral hyperplasia in, $48 \mathrm{I}$

coma, 74

congenital, 95

contact infection in, 85

contagiousness of, II 4

cystic, $47 \mathrm{I}$

desmoplastic properties, 468

diffuse, 472

distribution of nutriment in, 99

embryonal, 472

theory of, 94

limitations of, 97

en cuirasse, $463,473,525$, 534

epidermoid, $28,474,857$

glandular, 475

tubular, 474

epiphanin reaction in, 93

families, I05

following intestinal polyposis, 662

from chronic inflammation, 476

from physiological invo-

lution of organs, 477

from regenerative hyper-

from transformation of tumors, 478

fully developed, 47 I

genius loci in, 85

gross anatomy, 463

groups of, 462

hemorrhage in, 67

heredity in, 105

heterotopia in, 467

histological period, 2 I

historic study of, 17

in ancient times, 17

incidence of, I 13

induration in, 463

inflammatory reaction in, 50

influence of intestinal digestion on, 72

of mechanical pressure in, 98

of mode of origin, 480

of organization on, 100

of specialized functions on, 99

on gastric digestion, 70 invasion of serous cavities by, 80

of thoracic duct by, 79

leukocytosis in, 68

local invasion in, 481

invasive and destruc-

tive properties, 467

loss of polarity in, 469

marantic type, 67 trophy, 478 benign into malignant 
Cancer, mechanical pressure of contracting muscle-fibers in, 468

separation of epithelial cells in, $\mathrm{IO}_{2}$

meiostagmin reaction in, 92

metastases in, 469

microörganisms in, I I 5

microscopic structure, 465

mode of extension of, 480

mycetozoa in, I 16

of adrenal gland, 769

fully developed, 770

of ampulla of Vater, 700

of appendix, 665

anatomy, 665

struct ure, 665

of bladder, etiology, 865

glandular, 864

of breast, 506

acinar, 508

fibrous, 522

following fibro-adenoma, 521

primary, 522

age incidence, 506

blood-vessels in dissemination, $53 \mathrm{I}$

cerebral metastases in, $530^{\circ}$

clinical varieties, $\mathbf{5 3 2}$

development of surgical treatment, 537

developmental anomalies in, 507

dissemination, 525

duct, 508, 5I 4 gross anatomy, 5I4

histogenesis, 519

small cystic, 5 I 7

structure, 5 I 6

effective causation, 508

encephaloid, 532

etiology, 506

extension to lungs in, 529

general prognosis, 538

generalization, 528

gross anatomical diagnosis of, 522

heredity in, 507

in males, 536

incidence, 506

incidence, 506

invasion of skin in, 525

involvement of pleura in, 529

lymphatic invasion in, 525

medullary, 532

modes of extension, 523

mucoid, 5 I 3

pathological anatomy, 508

position of, 538

peritoneal invasion in, 530
Cancer of breast, previous exudative inflammation in, 507

radium in, 54 I

recurrences, 538

regressive phenomena in, $53 \mathrm{I}$

scirrhous, 534

$x$-ray in, 54 I

of cervix uteri, 544

anatomy, 544

atypical forms, 549

erosion in cause, 548

sarcomatoid struc-

tures in, $55^{\circ}$

scirrhous, 549

of corpus uteri, $55 \mathrm{I}$

anatomy, $55 \mathrm{I}$

histogenesis, $\mathbf{5 5 5}$

precancerous changes in, 556

structure, 553

of duodenum, 661

of esophagus, 857

anatomy, 858

etiology, 857

structure, 859

of Fallopian tubes, 610

etiology, 6I I

structure, 6x I

of gall-bladder, 693

anatomy, 694

clinical features, 697

squamous-cell, 696

structure, 695

surgical results in, 701

of hypophyseal duct, epi-

dermoid, 936

of ileum, 662

of intestine, 66I

of intrahepatic bile-ducts, multiple, 690

anatomy, 690

incidence, 690

structure, 691

of jejunum, 662

of kidney, 742

metastases in, 744

papillary, with clear cells, 743

tubular structure, $75^{2}$

of large intestine, 667

anatomical varieties, 669

developing from solitary polyps, 67 I

etiology, 667

histogenesis, 669

squamous-cell, 674

statistical, 667

of larger bile-ducts, 698

anatomy, 699

etiology, 699

location, 699

structure, 700

of larynx, 85I

anatomy, 852

course, 855
Cancer of larynx, larval, 853

lymphatic extension, 855

structure, 854

of lining epithelium, bronchial, 809

structure, 8Io

of lip, 838

of liver-cells, multiple, 684 primary massive, 682

of lung, 807

arising from pulmonary

alveoli, 8 I 2

classification, 809

etiology, 808

histogenesis, 813

metastases, 812

symptomatology, 8I 5

of maxillary antrum, 721

clinical course, 723

deformation stage, 724

invasion stage, 724

latent stage, 724

of ovary, 597

clinical course, 605

solid, 597

structure, 599

of pancreas, 702

anatomy, 703

etiology, 703

origin, 705

structure, 704

symptoms, 706

of pituitary gland, atypical, 934

of prostate, 78I

age of incidence, 782

clinical course, 785

course, 785

etiology, 782

gross anatomy, 783

heredity in, 782

lymphatic extensions in, 783

scirrhous, 788

skeletal osteoplastic metastases in, 784

structure, 786

variations in structure, 788

of salivary glands, 725 round-cell, 727

of scrotum, 870

paraffin, 870

of skin, epidermoid, 818

of small intestine, 662

of stomach, 625

acidemic coma in, 656

age in etiology, 625

alcoholism in, 626

anatomy, 627

anemia in, 655

cachexia in, 654

curability, 659

cylindrocellulare microcysticum, 629

diffuse, 635 scirrhous, 637 
Cancer of stomach, etiology, 625

experimental, in rat, I 33

extensions of, 646

following peptic ulcer, 630 anatomy, 635 clinical features, 658

gastric mucosa in, 644 gelatinous, 629

clinical features, 658 structure of, 630

heredity in etiology, 626

histogenesis, $65^{\circ}$

idiosyncrasy in etiology, 626

location, 654

in etiology, 626

lymphatic invasion in, 648

lymphatics involved in, 668

medullary, 635

metastases in ovary in, 649

onset, $65^{2}$

operability, 659

operative mortality, 659

pathogenesis, 653

relation to anatomic varieties, 656

scirrhous, clinical features, 658

simplex, 635

clinical features, 658

statistics, 625

structure, 629

surgical treatment, 659

symptomatology, 652

telangiectatica, 635

trauma in etiology, 626

of testis, embryonal, with

lymphoid stroma, 795

of thymus gland, 920

of thyroid gland, 90I

histogenesis, 906

metastases, 902, 904

structure, 905

of tongue and mouth, epidermoid, $84^{\circ}$

clinical course, 846

embryonal forms, 846

etiology, 84 I

gross a natomy, 842

lymph-node involvement in, 844

metastases in, 844

prognosis, 847

structure, 846

syphilis in etiology, 84 I

tobacco in etiology, 8+ I
Cancer of ureter, primary, 865

of urethra, 870

of uterus, 267

age in, 544

cachexia in, 56I

classification, 543

clinical course, $56 \mathrm{I}$

course, 557

effect of pregnancy on, 560

etiology, 543

evertens, 546

frequency, 543

histogenesis, 545

invertens, 546

lymphatic invasion in, 559

mortality, 562

structure, 545

symptoms, $55^{8}$

termination, $56 \mathrm{I}$

visceral metastases in, 56 I

of vagina, epidermoid, 564 histogenesis, 565

prognosis, 565

structure, 565

of vulva, course, 563

epidermoid, 562

pruritus in, 563

structure, 564

papillary, 464,470

paraffin, of scrotum, 870

parasitic theory of, I I 3

theoretic objections to, 120

percentage of urea in, 72 precancerous lesions in, 475

protozoa in, I I 5

Ransohoff's test in, 93

rapidity of growth, 480

regeneration of blood in, 69

research, experimental, 126

sarcomatodes, 963

scope of relation of parasites to, I 22

simplex, 47 I

specific degenerative changes in, 464

spirochetæ in, 116

squamous-cell, of choroid plexus, 419

Stammler's reaction in, 93 structural classification, 470

structure, 466

primary versus secondary, 466

theories of nature of, 94 tissue tension theory of, 98

tubular, $47 \mathrm{I}$

ulcerative lesions in, 464 uric acid increase in, 72
Cancer, urobilin in, $7+$

varia, 472

Wassermann reaction in, 9I

$x$-ray, 102

dermatitis and, 823

clinical course, 824

structure, 824

Capsular lipoma, I8

sarcoma, 275

Carcinoma. See Cancer.

Carcinomatous cirrhosis of liver, 686

structure in cystic tumors, 464

Carcinome villeux, 5 Io

Carcinosarcoma, 963

of esophagus, 859

Carcinosis, acute, of breast, 533

miliary, +64

Cardiac lipoma, I 84

Carotid gland, perithelioma of, 346

Cavernous angioma, 227

clinical features, 231

course, 229

cutaneous, 23 I

histogenesis, 230

multiplicity of, 228

neoplastic nature, 23 I

of liver, 232

of ovary, 232

of tongue, $23 \mathrm{I}$

structure, 230

submucous, 23 I

Cell autonomy, theory of cancer, 97

division in tumors, 38

Cells, altruism of, roo

endothelial, origin and functions, 299

pathology of, 300

physiology, 300

Paget's, 827

syncytial wandering, 568

Cellular nevus, 33 I

Cellules dyskeratosiques, 827

Central spinal sarcoma, $44^{\circ}$

Cephalic tumors of pituitary gland, 939

Cephalopagi, 955

Cerebral gliomas, 4 I 0

meninges, sarcoma of, diffuse, 424

metastases in cancer of breast, $53^{\circ}$

Cervical dermoids, 966 teratoids, 969

teratomas, 969

Cervix uteri, adenocarcinoma of, $5+7$

mucous, 549

adenoma of, simple, 549 cancer of, 54t. See also

Cancer of cervix uteri. cystadenoma of, 549 
Cervix uteri, endothelioma of, $55^{\circ}$ perithelioma of, $55^{\circ}$ precancerous changes in, $55^{\circ}$

Chemical agents in production of cancer, I 19

Chemistry of tumors, 89

Chimney-sweeps' cancer, 870

Chloroma, 362

blood in, 363

form, 363

gross anatomy, 363

occurrence, 362,363

structure, 364

symptoms, 363

Choanal polyp, I67

Choked disk in tumors of brain, 398

Cholangioma, 688

Cholesteatoma, 766

of brain, 974

Chondroma, 27,185

auricular, I9I

branchiogenic, 190

clinical types of, I90

cystic papillary, 187

etiology of, 189

heredity in, 189

matrix, 187

multiple character of, 186

of breast, 192

of larynx, $85^{\circ}$

of respiratory tract, 192

of salivary glands, 193

of scapula, 190

of testis, 8 or

of uterus, 192

ossification of, $\mathrm{I} 87$

osteoid, I90

Ranvier's classification, I 87

rickets in etiology, 189

skeletal, I90

location of, I 86

structure of, 187

subperiosteal, capsule of, I 86

teratoid, 193

trauma in etiology, 190

Chondromyxoma, I76

Chondrosarcoma, I87, 188

of ovary, 262

Chordoma, 27, 193

coccygeal, 195

identification of, 195

malignant, I 94

Chorio-adenoma, 566

destruens, 567

clinical course, 577

differential diagnosis, 569

metastases in, 569

structure, 568

uterine contents in, 568

Choriocarcinoma, 566, 570 clinical course, 578 \begin{tabular}{|} 
Choriocarcinoma, metas- \\
tases in, 575 \\
structure, 57I \\
Chorioma, 566 \\
clinical course, 577 \\
deportation of normal \\
chorionic elements in, \\
579 \\
etiology, 567 \\
gross anatomy, 567 \\
historical note, 566 \\
of Marchand, atypical, \\
575
\end{tabular}

ovarian changes in, 580

ovarii, 621

recovery after curetage or partial removal, 578

structure, 567

testis, 797

Chorionepithelioma, 566

Chorionic carcinoma, 57 I

Choroid plexus, papillary adenocarcinoma of, 418

squamous-cell carcinoma of, 419

tumors of, 4 I 5

Chromatophores, origin and function, 887

Chromatophoroma, Ribbert's, 872

Chylangioma of mesentery, 238

Chylous cysts, 975

Ciliated epithelial cysts, 978

Cirrhosis mammæ, 486

of liver, cancer with, 686 carcinomatous, 686 relation of primary epithelial tumors to, 678

Cirsoid neurofibroma, I 56

Coccidia in cancer, I 5

Coccygeal chordoma, 195

Cohnheim's theory of cancer, 94

limitations of, 97

Colitis polyposa, 673 adenoma in, $45^{8}$ cancer following, 662

Collateral hyperplasia in cancer, 48I

hypertrophy in tumors, 37

Colloid degeneration of tumors, 45

goiter, 891

metastasizing, 903

struma, 460

Collonema, I 80

Coma, acidemic, in cancer of stomach, 656

cancer, 74

Complement deviation with autogenous tumor antigen, 9I

Complex dermoids, 961

Condyloma acuminatum, $45^{\circ}$
Condyloma latum, 45I

Congenital hypertrophy of thyroid, 459

myxoma of navel, 175

nevus, $87 \mathrm{I}$

rhabdomyoma of heart, 222

tumors, 26

Connective-tissue tumors, 27

Contact infection in cancer, 85

Cornu cutaneum, $45^{\circ}$

Coronodental cysts, 7 I 6

Corpus adiposum malæ, I 82 cavernosum penis, endothelioma of, 33 I

luteum cysts, 584

uteri, adenomyomatosis of, diffuse, 556

cancer of, 55I. See also Cancer of corpus uteri.

Corpuscles, Hassall's, 913

Cranial lipoma, I 82

Crateriform rodent ulcer, $83 I$

Cutaneous lymphosarcoma, 387

neurofibroma, I 53

warts, 448

Cylindroma, 305

Cystadenocarcinoma of breast, 532

Cystadenoma, malignant, of kidney, 742

of breast, 496

prognosis, 498

structure, 497

of cervix uteri, 549

of $\mathrm{k}$ id ney, malignant

transformation in, 748

of ovary, pseudomucinous, 59 I

course, 593

structure, 593

serous, 586

course, 588

implantation metastases in, 588

structure, 588

Cystes epidermiques, 966

Cystic adenocarcinoma of breast, small, 517

of ovary, 597

carcinoma, 47 I

duct carcinoma of breast, small, 5 I 7

goiter, 893

lymphangioma, 983

mastitis, 489

tumors, 464

of carcinomatous structure, 464

Cystoma of ovary, simple serous, 583

simple, 582

Cystosarcoma phyllodes, 497 
Cysts, chylous, 975 coronodental, 7 I 6 corpus luteum, 584 dentigerous, 716,717 dermoid. See Dermoid cysts.

enteric, 975

entodermoid, 976

epidermoid, 965

epithelial, ciliated, 978 special forms, 965

implantation dermoid, 966

in lipoma, 180

in tumors, $3 \mathbf{I}$

intraperitoneal, of nephrogenic origin, 976

mesenteric, 975

of bile-ducts, 688

of hypophyseal duct, 936

of maxillary antrum, 72 I

of ovary, 582

dermoid, atypical forms, 619

small multiple, 582

simple follicular, 582

of parathyroid gland, 9 ro

of pelvic connective tissue, dermoid, 978

of peritoneum, 975 dermoid, 975

of pineal gland, 949

of skin, atheromatous, 965 epithelial, 965

of thymus gland, 9I5

of thyroglossal duct, 969

of thyroid gland, 9ro

omental, 975

radiculodental, 7 I 5

sebaceous, of breast, 505

serous, 975

tubo-ovarian, 584

DEAD osteoma, 200

Débris epitheliaux paradentaires, 707

Deciduoma, 566

malignum, 566

Degeneration, fatty, in tumors, 43

Demineralization in cachexia of cancer, 75

Demodex folliculorum in acanthoma of skin, 822

Dentigerous cysts, 716, 7 I 7

Dermatomyoma, 216

Dermoid cysts, cervical, 966 structure, 968

complex, 96I

fissural, 966

from first branchial cleft, 967

hypophyseal, 973

implantation, 966

intracranial, 973

mediastinal, 969

mesobranchial, 967
Dermoid cysts of breast, Elephantiasis, acquired, I59 505

of mesentery, 976

of omentum, 975

of orbitonasal fissures, 970

of ovary, atypical forms, 6 I 9

etiology, 623

malignant characters in, 620

multiple, 6 I 9

secondary changes in, 620

with cystoma, 6 I 4

of pelvic connective tissue, 978

of peritoneum, 975

pharyngeal, 970

presternal, 970

retroperitoneal, 977

sacrococcygeal, 979

simple, 964

special forms, 965

sublingual, 967

true, 966

tumors, I 64

Diabetes with acromegaly, 943

Diffuse buccal papillomatosis, $45^{2}$

carcinoma, 472

endothelioma, 339

hypertrophy of breasts in adults, 484

melanoma of meninges, 439

sarcomatosis of meninges, 438

spindle-cell sarcoma of lung, 8 I 6

Digestion, influence of cancer on, 70

Double monsters, 955 asymmetrical, 956 symmetrical, 955

Duct carcinoma of breast, 508

thyroglossal, cysts of, 969

Duodenum, cancer of, 66I

Dystrophia adiposogenitalis, 944

Dystrophic tumors of pituitary gland, $94^{\circ}$

Dystrophy, adiposogenital, theories, 946, 947

ECCHONDROSES, I 85

Ecchondrosis physalifora, I 94

spheno-occipitalis, I 93

Ectodermal mixed tumors of breast, 500

Edema in tumors of brain, 397

Elephantiasis, 158 angiomatosa, I 59

dura, 486

durum, 158

fibrosarcomatosa, 267

hemangiomatosa, 225

lymphangiectatica, I 58 , 237

molle, I 58

telangiectatica, I 59

Emaciation from tumors, 65

Embolism, pulmonary metastatic, 84

paradoxic, 84

secondary, 84

tertiary, 84

Embryoid tumors of testis, 794

Embryoma, 28, 959

of testis, adult, 790

complex, 790

Embryonal adenomyosarcoma of kidneys, 753

anatomy, 753

histogenesis, 754

structure, 753

cancer of testis with lymphoid stroma, 795

carcinoma, 472

theory of cancer, 94

limitations of, 97

tumors, 26, 49

of testis, 795

Embryos, post - generation of, 957

Encephaloid cancer of breast, 532

tumors, 5 I I

Enchondroma, I 85, I 86

Endometrial sarcoma, structure, 267

Endometritis, hypertrophic, $45^{8}$

interstitialis, $45^{8}$

syncytial, $566,575,577$

Endothelial cells, origin and functions, 299

pathology of, 300

physiology of, 300

myeloma, 323

sarcoma of spleen, $39^{\circ}$

tumors, 27

Endothelioma, 27, 299, 342

alveolar, 305

angioplastic, 334

calcification in, 3 I I

classification, 305

clinical types, 3 I 3

diagnosis, differential, $3 \mathbf{I} 2$

diffuse, 305, 339

etiology, 313

gross anatomy, 308

growth, 305

histological types of, 305

history of, 302 psammoma of skin, 33 I 
Endothelioma, hyaline de- Ependyma, tumors of, in- Epithelioma of skin, adenoid, generation in, 3 IO interfascicular, 305 mucinous degeneration in, 3 II

multiplicity, 309

nomenclature, 305

of bones, 308,322

classification, 323

multiple, 324

solitary bulky cystic, 323

diffuse, 326

of cervix uteri, $55^{\circ}$

of corpus cavernosum penis, $33 \mathrm{I}$

of ganglia, $3 \mathrm{I} 8$

of lymph-nodes, 309, 335, $34 \mathrm{I}$

clinical types, 336

frequency, 336

gross anatomy, 337

histogenesis, 339

localized, 336

metastases in, 337

structure, 338

systemic form, 336

of meninges, 315

course, 318

forms, 315

location, 3 I 5

structure, 3 I 5

of nerve-trunks, 318

of orbit, 318

of ovary, 309, 33 I

appearance, 333

clinical course, 333

diagnosis, 333

structure, 333

of serous membranes, 3 19, 320

of skin, 309, 328

of stomach, 335

of testis, 308

of uterus, 334

origin, 305

papillary, 305

peritoneal, 320

classification, 322

structure, 320

perivascular, 305, 339

pleural, 3 I 9

classification of, 322

clinical course, 320

origin, 319

structure, 319

plexiform, 305, 339

sarcoma and, combina-

tion of, $3 \mathrm{II}$

structure of, 3 IO

Endothelium, pathology, 300

tumor-like hyperplasia of, $3 \mathrm{I} 3$

Enteric cysts, 975

Enterocystomas, 978

Entodermoid cysts, 976

Ependyma, tumors of, 415

fundibular, 4I 5, 420

Ependymal glioma, 420 of pineal gland, $95^{\circ}$

gliomatosis, 4 10

neuroglioma of pineal gland, 95I

Ependymitis granularis, 4I5

Epidermoid cancer, 28, 474, 857

glandular, 475

of hypophyseal duct, 936

of skin, 818

of tongue and mouth, 840

of vagina, 564

of vulva, 562

tubular, 474

cysts, 965

Epidermoids, 964

congenital, 965

Epignathi, 970

Epiphanin reaction in cancer, 93

Epithelial cysts, ciliated, 978

of skin, 965

special forms, 965

hyperplasia of liver, 675

papilloma of maxillary antrum, 72 I

pearls, 474

proliferation, influence of lipoid solvents on, I 28 tumors, 28

general pathology of, 446

of bladder, 860

of breast, 484

of kidney, 738

of liver, primary, 678

of ovary, $5^{82}$

of renal pelvis and ureter, 763

of salivary glands, 7

of thyroid gland, papillary, 899 structure, 900

of vulva, 562

Epithelioma, 21, 28, 474 adenoides cysticum, 329

chorio-ectodermale, 622

of breast, 506

of lip, papillary, 837

ulcerative infiltrating, 837

of penis, 866

course, 869

established disease, 867

extensions, 868

initial lesion, 867

strict ure, 869

of prostate, squamous, 788

of renal pelvis, papillary, 763

of skin, adenoid, 834 cystic, 834

simple, 835

basal-cell, 830

reticulated, $83^{\circ}$

of ureter, papillary, 763

petrified, 820

plexiform, 475

reticulated, histogenesis, 833

Epitheliome intracanaliculaire, 5 Io

kystique intra-acineux, 486

luminaire, 564

Epithelium, lining, bronchial, cancer of, 809

structure, 8 io

Epoöphoron, tumors of, 609

Erosion as cause of cancer

of cervix uteri, 548

Esophagus, cancer of, 857 anatomy, $85^{8}$ etiology, 857

structure, 859

carcinosarcoma of, 859

leiomyoma of, 2 I 6

sarcoma of, ${ }^{25} 8$

Everted papillary adenoma of ovary, 597

Exophthalmic goiter, 896 stages, 897

Exostoses, 197

Exostosis bursata, 198

cartilaginea, 198

clavata, 197

clinical groups, 198

de croissance, 198

eburnea, I97

medullaris, 197

multiple, 198

of trachea, 198

spongiosa, 197

traumatic, 198

Extradural tumors of spinal cord, 444

Extramedullary plasma-cell tumors, 388

tumors of spinal cord, 444

Extrarenal adrenal rests, tumors of, 761

Eye, lymphangioma of, 237 osteoma of, 202

FACraL bones, osteoma of, I99

Fallopian tubes, adrenal tumors of, 762

cancer of, 610

etiology, 6I I

structure, 6I I

tumors of, 612 adrenal, 762

False tail, 984

Family incidence of cancer, I05 
Fasciæ, fibroma of, 163

Fats in tumors 90

Fatty degeneration in tumors, 43

Febiger's experimental gastric cancer in rat, 133

Ferments in tumors, 67, 90

Fetal adenoma of thyroid gland, 895

fibro-adenoma of breast, 499

implantations, abdominal, 977

special forms, 965

teratomas abdominal, 977

Fetus, parasitic, 958

Fibro-adenoma of breast, 495

acinar carcinoma following, 52 I

fetal, 499

papillary intracystic, 496

Fibroblastic sarcoma, 249

Fibrocarcinoma, 473

of breast, 5 I 8,535

of large intestine, stenosing, 670

of stomach, diffuse, 639 sclerosing, clinical features, 659

pyloric, 639

Fibrolipoma of spermatic cord, 801

Fibroma, 27, 149

blood-vessels in, I49, I 5 I

clinical types, I 52

edematodes cysticum, I66, I 67

simplex, I66, I67

etiology, I6o

gross appearance of, I 49

juvenile nasopharyngeal, I68

course, 160

lipomatodes, I62

lymph-vessels in, $15 \mathrm{I}$

matrix, I 50

molluscum, I 49, I 53

clinical course, 155

typical, I54, 155

natural history of, $I_{5} 1$

of bones, 163

of breast, 165

of fasciæ, I63

of glandular organs, 165

of kidney, I65

of larynx, $85^{\circ}$

of nares, 166

of ovary, I 66

of periosteum, I 63

of spermatic cord, plexi-

form, 801

of testis, I65, 801

origin of, $15 \mathrm{I}$

papillary, I49

structure of, 150
Fibromatosis, diffuse, 158 uteri, 267

Fibromyoma, uterine, 207

Fibroneuroma, true, 427

Fibro-osteoma, 200

Fibrosarcoma, I 50, 251 mucocellulare carcinomatodes of ovary, 262, 603

Fibrous acinar carcinoma of breast, $\mathbf{5 2 2}$

Fissural angioma, 225

dermoids, 966

Fistula auris congenita, epithelial cysts of external ear from, 967

Folliculoma malignum ovarii, 602

Frontal sinus, osteoma of, 200

Functional activity in tumors, 53

adenomatoid hyperplasia, 459

Fungus hematodes, I9, 30

GALEN's theory of cancer, I 7

Gall-bladder, cancer of, 693 anatomy, 694

clinical features, 697

squamous-cell, 696

structure, 695

surgical results in, 7or sarcoma of, 697

Gametoid mitosis in tumors, 39

Ganglia, endothelioma of, 3 I 8

Gangliocellulare, 399

Ganglionic neuroma, 427 , 430

etiology, 432

Gasserian ganglion, tumors of, 434

Gastric cancer, 625. See also Cancer of stomach. digestion, influence of cancer on, 70

Gastritis polyposa, adenoma in, $45^{8}$

Gastro-intestinal Hodgkin's granuloma, 369

lipoma, I 84

pseudoleukemia, 378

sclerostenosis, 640

tract, lymphosarcoma of, 385

Gelatinous adenocarcinoma of large intestine, 671

cancer of stomach, 629

Genius loci in cancer, 85

Giant-cell sarcoma, $25^{\circ}$ of aponeuroses, 256 of bone, benign, 280 prognosis, 287 structure, 282 of tendon-sheaths, 256
Giantism, 940

Glandular adamantinoma, 713

cancer of bladder, 864

epidermoid car'cilnoma, 475

mastitis, chronic, 489

organs, fibroma of, I65

tumors, 28

Glans penis, melanoma of, 870

Glioma, 27, 399

blood-vessels in, 405

cerebral, 4 Io

clinical types, 4 Io

differential diagnosis, 407

durunı, 399

ependymal, 410,420

etiology of, 408

ganglionare, 399

gross anatomy, 399

growth of, 406

intracranial, histological classification, 410

molle, 399

nasal, 4 I I

of pineal gland, ependymal, $95^{\circ}$

of posterior lobe of pituitary, 939

of retina, $42 \mathrm{I}$

etiology, 423

structure, 422

of spinal cord, 4 II

pial, 4 I 4

sarcoma and, differentiation, 407

secondary degeneration in, 400

structural forms, 399

structure of, $40 \mathrm{I}$

telangiectaticum, 400

teratomatous, 4I5

Gliosarcoma, 399, 405, 424

Glycogenic degeneration of tumors, 44

Glycosuria with acromegaly, 943

Goiter, colloid, 89I metastasizing, 903

congenital, 894

cystic, 893

exophthalmic, 896 stages of, 897

parenchymatous, 894 acquired, 895

simple, 460,891

Granuloma, benign, of thy roid gland, 908

Hodgkin's, 367, 443 abdominal. 370 anemia from, 368 clinical course, 368 types, 368 etiology of, 375 gastro-intestinal, 369 mediastinal, 370 structure, $37 \mathrm{I}$ 
Granuloma, Hodgkin's, tuberculous lesions in, 375

with chronic generalized lesions, 368

malignum, 38I

Graves' disease, 459,896

Gynecomastism, 484

HAMARTOMA, 23 I

Hassall's corpuscles, 9 I 3

Heart, myxoma of, 175 rhabdomyoma of, congenital, 222

sarcoma of, primary, i 76

Hemangio - endothelioma, 226, 307

Hemangioma, 223

hypertrophicum, 226

simplex, 224, 736

Hematodes, fungus, 30

Hemorrhage in cancer, 67

Hemorrhagic sarcoma, multiple, 252

Hepatic adrenal tumors, 762

Hepatoma, 68I

multiple, 684

solitary, 68 I

with cirrhosis, 686

Hereditary tumors, 26

Heredity in cancer, 105

Heterolytic action of tumor ferments, 67

Heteroplastic bone formation, 201

clinical forms, $20 \mathrm{I}$

deposits in leukemia, 355

Heterotopia in cancer, 467

Highmore, antrum of, tumors of, 720

Hippocrate's theory of cancer, 17

Histoid tumors, 48

Hodgkin's disease, bonemarrow lesions in, 37 I

mediastinal tumors in, 370

skin lesions in, $37 \mathrm{I}$

splenic, 368

granuloma, 367,443 . See also Granuloma, Hodgkin's.

sarcoma, 373

Homologous tumors, $4^{8}$

Homotypic tumors, 48

Hunter's theory of cancer, I9

Hutchinson's type of sarcoma of adrenal gland in infants, with cranial metastases, 774

Hyaline changes in tumors, $+5$

Hydatid disease, 485

Hydrocystoma tuberosum multiplex, 329
Hydropic degeneration tumors, 43

Hydrops follicularis, $5^{82}$

Hygroma cysticum, I59 colli, 238

sacral, 239, 983

Hylomata, 29

Hyperglycemia in blood in cancer, 70

Hypernephroma, 738, 744, 755

Hyperostosis, 197

Hyperplasia, adenomatoid, functional, 459

inflammatory adenomatoid, 457

neoplastic, morphology of, 4I

versus inflammatory hyperplasia, 40

of adrenal gland, 768

of interstitial cells testis, 803

of liver, epithelial, 675

multiple nodular, 677

of pituitary gland, diffuse, 930

tumor-like, of endothelium, $3 \mathrm{I}_{3}$

Hyperplastic lymphadenitis, $35^{\circ}$

Hypertrophic endometritis, $45^{8}$

granulation tissue, 224

Hypertrophy of breast, 484 diffuse, of adults, 484

infantile, 484

of male, 484

of prostate, 459,779 adenomatous, 780 carcinomatous, 780 etiology, 780 productive inflammation in, 779

structure, 779

of thymus gland, simple, 914

of thyroid, congenital, 459

regenerative, cancer from, 478

Hypophyseal dermoid cysts, 973

duct, cancer of, epidermoid, 936

cysts of, 936

teratoids of, 935

tumors of, 935

Hypophysis cerebri. See

Pituitary gland.

ILEUM, cancer of, 662

Implantation dermoid cysts, 966

metastases in serous cystadenoma of ovary, 588

Induration chronique en masse, 486 nfantile hypertrophy

breasts, 484

Infantilism, 94 I

reversif, 942

tardif, 942

Infants, adenocarcinoma of kidneys in, 749

sarcoma of adrenal gland and liver in, congenital (Pepper type), 775

with cranial metastases (Hutchinson type), 774

Infection by contact in cancer, 85

Infiltrative growth in malignancy, 62

Inflammation, chronic, in experimental production of tumors, 13I

Inflammatory adenomatoid hyperplasia, 457

hyperplasia versus neoplastic hyperplasia, 40 reaction in cancer, $5^{\circ}$

Infundibular ependyma, tumors of, 420

Interacinous adenoma of thyroid gland, 894

Interfascicular endothelioma, 305

Intermuscular lipoma, I 82

Interstitial cells of testis, hyperplasia of, 803

mastitis, chronic, 488

Intestinal indigestion, effect of cancer on, 72

polyposis, 673 cancer following, 662

Intestine, cancer of, 66I

large, adenocarcinoma of, gelatinous, 67 I

cancer of, 667. See also Cancer of large intestine.

fibrocarcinoma of, stenosing, 670

leiomyoma of, 2 I 6

lymphomatous tumors of, 260

lymphosarcoma of, 387

melanoma of, 886

sarcoma of, 260

spindle-cell, 260

tumors of, multiple benign embryonal carcinoid, 663

Intoxication, general, in malignancy, 63

Intracranial dermoid cysts, 973

teratoids, 974

teratomas, 974

Intracystic fibro-adenoma of breast, papillary, 496

Intrahepatic bile-ducts, cancer of, multiple, 690 
Intramedullary tumors of LACHRymaL gland, tumors Linit is plastica, leather-botspinal cord, 444 of, 319, 725

Intraperitoneal cysts of Lactic acid in tumors, 89 nephrogenic origin, 976 Langenbeck's theory of can-

Involution of breasts, senile, cer, 22 487

Irritation group, 475

Ischiopagi, 955

Large alveolar carcinoma, $47 \mathrm{I}$

Larval cancer of larynx, 853

Larynx, adenoma of, $85^{\circ}$ angioma of, $85^{\circ}$

JEJUNUM, cancer of, 662

Juvenile nasopharyngeal fibroma, I 68 course, I69

KANGRI cancer, 82 I

Keloid, 160

from trauma, 160

in negro, 160

neoplastic properties, 162 spontaneous, I60

Kidneys, adenocarcinoma of, alveolar, 748

embryonal, in adults, 749

in infants, 749

multiple, in sclerosis, 746

papillary, 742

histogenesis, 746

structure, 748

with granular cells, 746

tubular, 750

adenoma of, 739

alveolar, $74^{\circ}$

histogenesis, $74 \mathrm{I}$

papillary, 739

tubular, $74 \mathrm{I}$

adenomyosarcoma of, 220

embryonal, 753

anatomy, 753

histogenesis, 754

structure, 753

cancer of, $74^{2}$

metastases in, 744

papillary, with clear cells, 743

tubular, structure, $75^{2}$

fibroma of, 165

leukemic infiltrations in, 355

lymphosarcoma of, 388

myoma of, 217

myxoma fibromatosum of, I65

perithelioma of, 346

spotted, $74 \mathrm{I}$

tumors of, 738

adrenal tumors and, differentiation, 760

epithelial, 738

classification, 739

Krunkenberg's fibrosarcoma mucocellulare carcinamatodes of ovary, 262,603

cancer of, 85I

anatomy, 852

course, 855

larval, 853

lymphatic extension, 855

structure, 854

chondroma of, $85^{\circ}$

fibroma of, $85^{\circ}$

leukoplakia of, $85 \mathrm{I}$

lipoma of, $85^{\circ}$

lymphosarcoma of, 856

papilloma of, 847

structure, 847

sarcoma of, 856

tumors of, 847

benign, $85^{\circ}$

Leiomyoma, 27, 203

cavernosum, 203

clinical types, 207

course of, 205

diagnosis, 205

durum, 203

molle, 203

of bladder, 866

of breast, 505

of esophagus, 2 I 6

of intestine, 216

regressive changes in, 205

structure of, 204

Lentigo, 873

maligna juvenilis, 823

Leontiasis ossea, 197

Lepidomata, 29

Leukanemia, 360

Leukemia, aplastic, 36I atypical, 360

changes in blood in, 359

heteroplastic deposits in, 355 infiltrations in kidney in, 355

lymph-nodes in, 354

lymphoid-cell, 362

mixed-cell, $36 \mathrm{I}$

nature of, 364

plasma-cell, 364

splenic lesions in, 354

Leukemic lymphoma, 353

Leukocytosis in cancer, 68

Leukoplakia of larynx, 85 I uteri, 557

Leukosarcomatosis, $36 \mathrm{I}, 362$

Linin, $4^{6}$

in tumor-cells, 46

Linitis plastica, $.35,639$, $64^{\circ}$

clinical features, 659 the stomach in, 644

Lip, cancer of, 838

epithelioma of, papillary, 837

ulcerative infiltrating,

837
Lipoid solvents, influence of, on epithelial proliferation, I 28

Lipoma, 27, 178

annulare colli, 182

arborescens, 182

capsular, I 8 I

cardiac, I 84

cavernosum, I 79

clinical types, 182

combined with myxoma, I 76

connection with bloodvessels, 179

cranial, I 82

cysts in, 180

etiology, i 80

gastro-intestinal, I 84

intermuscular, I 82

mediastinal, I 84

microscopical structure, I 79

molle, i 78

myelogenous, I 84

of breast, 505

of larynx, $85^{\circ}$

of posterior lobe of pitui-

tary, 939

of renal hilus, 766

of spinal cord, $44 \mathrm{I}$

pelvic, I 84

perirenal, I 84

petrificum ossificans, I 80 renal, I 83

secondary changes in, 179

subcutaneous, I 82

synovial, I 82

telangiectaticum, I 79

trauma as cause, I 8 I

uterine, I 84

Lipomatosis, replacement, I 8 I

Liquefaction cysts, 32

Liver, adenoma of, multiple, 686

solitary, 68 I

cavernous angioma of, 232

cirrhosis of, carcinomatous, 686

hyperplasia of, epithelial, 675

multiple nodular, 677

melanoma of, primary, 693

regenerative power of, 676 sarcoma of, 69

in infants, congenital (Pepper type), 775

tumors of, 675 . Sie also Tumors of liver. 
Liver-cells, cancer of, mul- Lymphoid tissue, tumors of, | Malignancy, significance of, tiple, 684 primary massive, 682

Lung, cancer of, 807 . See also Cancer of lung.

extension to, in cancer of breast, 529

lymphosarcoma of, 817 sarcoma of, 815. See also Sarcoma of lung.

tumors of, 807

Lupus cancer, 823

Lusitanus' theory of cancer, I 8

Lutein-cell tumors, 585

Lymphadenia ossium, 378

Lymphadenitis, chronic, 313 hyperplastic, $35^{\circ}$

Lymphadenoma, 35o, 352, 367

Lymphangiofibroma, 149

Lymphangioma, 233

cavernosum, 234, 237

clinical forms, 235

cutis circumscripta, 236

cystic, 983

cysticum, 238

cystoides, 235

development of, 235

histological structure, 234

mesenteric, 238

of eye, 237

of ovary, 239

of salivary glands, 736

origin of, 235

retroperitoneal, 238

simple, 236

simplex, 234

tuberosum, 303

multiplex, 236, 328

Lymphangitis carcinomatosa, 306

Lymphatic extensions in cancer of prostate, 783

invasion by sarcoma, 80 in cancer of uterus, 559 permeation, metastasis from, 76

tuberculosis, 366

Lymphatics involved in cancer of stomach, 668

of stomach, 647

Lymph-epithelioma, 643

Lymph-nodes, endothelioma of, 309,335

clinical types, 336

etiology, 34 I

frequency, 336

gross anatomy, 337

histogenesis, 339

localized, 3.36

metastases in, 337

structure, 338

systemic form, 336

in leukemia, 354

in lymphemia, 354

metastases in, 77

Lymphogranuloma, 367

348

classification, $34^{8}$

Ly mphoid-cell leukemia, 362

Lymphoma, 27, 348

leukemic, 353

malignant, 380

simple, $35^{\circ}$

etiology, 352

Lymphomatosis,

aleukemic, 376

Lymphomatous tumors of intestine, 260

of stomach, 260

Lymphosarcoma, 348,380

aggressive qualities, 383

anatomic features, 381

clinical features, 381

subvarieties, 384

congenital, 390

cutaneous, 387

duration, 382

etiology, 388

extension, 382

histological character, 383

metastases in, 387

of gastro-intestinal tract, 385

of intestine, 387

of kidney, 388

of larynx, 856

of lung, 817

of prostate, 789

of spleen, primary, 39I

of stomach, 386

of testis, 388,804 .

etiology, 804

of thymus gland, 917 structure, 919

of tongue, 387

origin, 382

primary lesions, 383

pharyngeal, 385

regressive changes in, 383

relation of syphilis to, 246

of tuberculosis to, 246

retroperitoneal, 387

round-cell, large, 382

structure, 382

tuberculosis in, 389

types, 382

MACrocheilia, 238

Macroglossia, 238

Macromelia, 238

Mal perforant, 984

Maladie de Reclus, 477,489

kystique, 485,486

noueuse, 486,489

Malignancy, diagnosis of, 63

effects on body, 65

on organism, 62

formation of metastases in, 62

general intoxication in, 63

infiltrative growth in, 62
62

Malignant adenoma, 453, 455

of thyroid gland, 898

cystadenoma, 742

lymphoma, 380

neuroma, 431

tumors. See Cancer.

Marantic type of cancer, 67

Marchand's atypical chorioma, 575

Mastitis, chronic glandular, 489

cyst contents in, 489

development, 489 of benign or malignant process in, 491

predisposition to cancer in, 492

progress, 489

structure, 489

treatment, 493

interstitial, 488

productive, 485

etiology, 486

glandular, 486

interstitial, 486

senile, 486

cystic, 489

Maxillæ, osteoma of, 199

Maxillary antrum, carcinoma of, 721

cysts of, $72 \mathrm{I}$

epithelial papilloma of, 721

tumors of, 720

sinuses, tumors of, 720

tumors of dental origin, 707

Mediastinal dermoid cysts, 969

lipoma, 184

tumors in Hodgkin's disease, $37^{\circ}$

Medullary cancer of breast, 532

tumors of adrenal gland, $77 \mathrm{I}$

chromaffin cell, 776

with adrenalin con-

tent and neph-

ritic symptoms, 776

Medullocelles, 269

Meiostagmin reaction in cancer, 92

Melanocarcinoma, 872

Melanoma, 87 I

clinical characters, 872

course, 873

diffuse, of meninges, 439

histogenesis, 887

histology, 874

history, $87 \mathrm{I}$

malignant changes in, 877

modes of origin, 872 
Melanoma of adrenal gland, | Metastasis, pulmonary em778,890

of brain, 885

of breast, 505

of glans penis, 870

of intestine, 886

of liver, primary, 693

of rectum, 674

of spinal cord, 885

of uncertain origin, 887

orbital, 882

etiology of, 885

structure of, 884

pigmentation of, 879

Melanosarcoma, 872

Melanotic whitlow, 873

Meningeal pseudoneoplasms, 443

Meninges, diffuse melanoma of, 439

endothelioma of, 3 I 5

sarcoma of, 424,437

diffuse, 424

gross anatomy, 424

structure, 425

sarcomatosis of, diffuse, 438

multiple, 438

Meningitis arenosa, 315

serosa circumscripta, 443

Mesenteric cysts, 975

lymphangioma, 238

Mesentery, chylangioma of, 238

Mesobranchial dermoids, 967

Mesodermal elements in teratoma of ovary, 618

tumors of breast, 499

Mesonephric adenomyoma, 214

tumors, 6 ro

Mesothelioma, 768

Metastasis, 76

cerebral, in cancer of breast, $53 \mathrm{I}$

formation of, 62

from lymphatic permeation, 76

implantation, in serous cystadenoma of ovary, 588

in chorio-adenoma, 569

in choriocarcinoma, 575

in primary epithelial tumor of liver, 680

in syncytioma, 576

in teratoma of ovary, 618

lymph-node, 77

of benign tumor, 84

ovarian, in cancer of stomach, 649

preservation of structural type in, 86

pulmonary embolism from, 84 paradoxic, 84 secondary, 84

gross anatomy, 293 bolism from, tertiary, 84 susceptibility of tissues to, 86

visceral, in cancer of uterus, $56 \mathrm{I}$

Microörganisms in cancer, I 15

Mikulicz's disease, 37 I

Miliary carcinosis, 464

Mixed tumors, 962 of testis, 794 special forms, 965

Mixed-cell leukemia, 36 I

Mole, congenital, 874

pigmented, 872 of adults, 875

Mollities ossium, 292

Molluscum contagiosum, $45^{\mathrm{I}}$

Monsters, double, 955 asymmetrical, 956 symmetrical, 955

Mouth, cancer of, epidermoid, $84^{\circ}$

Mucoid carcinoma of breast, 513

Mucous adenocarcinoma of cervix uteri, 549

degeneration in tumors, 44

glands, bronchial cancer arising from, 8II

Muller's tailed corpuscles, 2 I

Multicentric growth of tumors, 36

Multiple adenocarcinoma in sclerotic kidneys, 746

hemorrhagic sarcoma, 252 neurofibroma of spinal cord, 44I

sarcomatosis of meninges, 438

Muscle tissue tumors, 27

Muscles, angioma of, 225

sarcoma of, 258

voluntary, rhabdomyoma of, 222

Mycetozoa in cancer, I16

Myelogenous lipoma, I 84 pseudoleukemia, 292

Myeloid pseudoleukemia, 379

Myeloma, $25^{6}$

endothelial, 323

of bone, 292

albumosuria in, 293

classification, 298

clinical course, 293

erythroblastic origin, 298

etiology, 298

histogenesis, 297

structure, 295
of bone-marrow, multiple, Necrotic tissue, osteoma of, 292 yoma, 27, 203

malignum, 205

of alimentary tract, 216

of bladder, 216

of kidney, 217

of skin, 216

of stomach, 216

of testes, 217,802

of urinary organs, 2 I 6

of uterus, 20

degenerative changes

in, 210

etiology, 212

form, 208

gross appearance, 2 Io

location, 208

microscopical structure, 2 II

recurrent, 265

Myosarcoma, 27, 207

spindle-cell, of stomach, 259

Myositis ossificans, 202

Myxochondrocarcinoma of salivary glands, 735

Myxochondroma, I 88

Myxoglioma, for

Myxoma, 27, I 70

anatomical characters, I 7 I

clinical course, 172

forms, I 73

combined with lipoma, I 76

congenital, of navel, I75

etiology, I 77

fibromatosum of kidney, I 65

location, I 73

matrix, I 7 I

of bones, I74, I75

of heart, 175

of nervous system, I 73

of optic nerve, I 73

of renal hilus, 766

origin, 176

primary, I 70

structure, I 7 I, I 76

Myxosarcoma of navel, I75

of ovary, 262

of renal hilus, 766

NAREs, fibroma of, 166

melanoma of, 723

Nasal glioma, 4 I I

polyp, I 66

sinuses, tumors of, 720

Nasopharyngeal fibroma, juvenile, I 68

course, I69

Nature of cancer, theories of, 94

Navel, congenital myxoma of, 175

202 
Neoplastic hyperplasia, Nevus vinosus, 224 morphology of, 4I Nipple, Paget's disease of, versus inflammatory hyperplasia, 40

Nephrogenic origin, intraperitoneal cysts of, 976

Nerve nevus, I 59 optic, neuromyxoma of, 434

Nerve-trunks, endothelioma of, 318

neurofibroma of, 155

tumors of, 427

Nervous system, myxoma of, 173

osteoma in, 200

tumors of, 27

Neurinoma, 155

Neurocytoma, $4 \mathrm{I} 4$

of adrenal gland, 772 clinical types, 774

Neuro-epithelioma, 27, 399, 405

of retina, 422

Neurofibroma, I 49

acoustic, 432

cirsoid, 156

cutaneous, I 53

multiple, of spinal cord, $44 \mathrm{I}$

of deeper nerve trunks, I 55

plexiform, 156

visceral, I 55

Neurofibromatosis, 152

visceral, I 57

Neurofibrosarcoma, I 55

clinical course, I 56

Neuroglioma of pineal gland, ependymal, $95 \mathrm{I}$

Neuroma, 27, 427

a myelinicum, 427

ganglionare, $27,427,771$

ganglionic, 430

etiology, 432

malignant, $43 \mathrm{I}$

myelinicum, 427

plexiform, 427

traumatic, 427

Neuromyxoma of optic nerve, 434

Neuropathic papilloma, I 59

Nevus, 872

bathing trunk type, 873

cellular, 33I

congenital, $87 \mathrm{I}$

linearis, 159

lipomatodes, 873

nerve, I 59

papillaris, 873

pilosus, 873

results of studies, 888

spilus, 873

unius lateris, I 59

vascular, 224

vasculosus molluscifor-

mis, 225

verrucosus, 873

Nodes, singer's, 847

Nuclear fluid in tumor-cells, 46

Nuclein, 46

in tumor-cells, 46

Nucleus, cystic degeneration of, in tumors, 46

fragmentation of, in tumors, 46

\section{ODONTOMA, 7 I 8}

etiology, 719

modes of origin, 720 soft, 719

Omental cysts, 975

Oncology, general, 17 special, I 49

Oöphoroma folliculare, 603

Optic nerve, myxoma of, I 73 neuromyxoma of, 434

Orbit, angioma of, 23 I angiosarcoma of, 885 endothelioma of, 318 melanoma of, 882 etiology, 885 structure, 884 osteoma of, 199 perithelioma of, 885 teratomas of, $97^{\circ}$

Orbitonasal fissures, dermoid cysts of, 970

Organization, influence of, on cancer, 100

Osteitis fibrosa, 292

Osteochondroma, nultiple, 190

Osteochondromyxosarcoma of breast, 505

Osteochondrosarcoma, I 88 of bladder, 866

Osteodystrophia juvenilis, 292

Osteogenic sarcoma, 270 course, 275

sclerosing, 275,278

site of origin, 27 I

structure of, 276

Osteoid chondroma, I9o

Osteoma, 27, I 96

dead, 200

heteroplastic, 197

in nervous system, 200

hyperplastic, I97

of antra, 199

of eye, 202

of facial bones, 199

of frontal sinus, 200

of maxillæ, 199

of necrotic tissue, 202

of orbit, 199

of penis, 202

of skin, 202

reticulated, 20 I
Osteoma, secondary, 200 terminology, 196

true, I99

Osteomalacia, senile, 292

Osteophyte, 197

Osteosarcoma, 269

Ovarian metastases in cancer of stomach, 649

Ovarium gyratum, I66

Ovary, adenocarcinoma of, cystic, 597

papillary serous, 589

pseudomucinous, 595

adenofibroma of, 597

adenoma of, $5^{86}$

everted papillary, 597

solid, 596

superficial, 597

testicular, 610

adenomyoma of, 610

cancer of, 597. See also

Cancer of ovary.

cavernous angioma of, 232

chondrosarcoma of, 262

cystadenoma of, pseudomucinous, $59 \mathrm{I}$ serous, 586

cystoma of, simple serous, 583

cysts of, 582. See also Cysts of ovary.

dermoids of, 6r3. See also Dermoid cysts of ovary.

endothelioma of, 309

appearance, 333

clinical course, 333

diagnosis, 333

structure, 333

fibroma of, 166

fibrosarcoma mucocellu. lare carcinomatodes of, 262,603

lymphangioma of, 239

myxosarcoma of, 262

perithelioma of, 346

round-cell sarcoma of, 26 I

sarcoma of, 260

spindle-cell sarcoma of, 26 I

teratoma of, 6r3. See also Teratoma.

tumors of, adrenal, 6ro, 762

age in etiology, 606

epithelial, 582

etiology, 606

histogenesis, 606

origin, 608

PACHydermatocele, I 59

Pachydermia, I 59

laryngis, $85 \mathrm{I}$

Pachymeningitis cervicalis hypertrophica, 443

interna sarcomatosa, 425

Paget's cells, 827 
Paget's disease of nipple, | Parosteal sarcoma, 275 826 origin, 828 structure, 827

Pancreas, adenoma of, 702 cancer of, 702. See also Cancer of pancreas.

sarcoma of, 706

tumors of, 702

Papillary adenocarcinoma of chroid plexus, 4I 8 of kidney, 742 with granular cells, 746

adenoma of kidney, 739 of ovary, everted, 597

cancer, $464,47^{\circ}$ of kidney with clear cells, 743

cystic adenoma, 455

endothelioma, 305

epithelial tumors, 899

epithelioma of lip, 837

fibroma, I 49

intracystic fibro-adenoma of breast, 496

pseudomucinous cystadenoma, 592

serous adenocarcinoma of ovary, 589

Papilloma, 28, 446

bronchial, 8 I 3

clinical course, 447

development of malignancy in, 448

neuropathic, 159

of bladder, 860

allantoid type, 863

structure, 863

of breast, 505

of larynx, 847

structure, 847

of maxillary antrum, epithelial, $72 \mathrm{I}$

of renal pelvis and ureter, 763

of tongue, $45^{2}$

of ureter, 763

structure of, 448

Papillomatosis, diffuse buccal, $45^{2}$

Paraffin cancer of scrotum, 870

Paraganglioma, 777

Paranuclein, 46

in tumor-cells, 46

Parasites, scope of relation to cancer, 122

Parasitic fetus, 958 theory of cancer, II 3 theoretic objections to, 120

Parathyroid gland, cysts of, 910

tumors of, 909

Parenchymatous goiter, 894 acquired, 895

Parosteal exostosis, 198
Parovarium, tumors of, 609

Pars intermedia, tumors of, 938

Pavement tumors, 28

Pelvic connective tissue, dermoid cysts of, 978 lipoma, 184

Pelvis spinosa, 185

Penis, epithelioma of, 866 course, 869

established disease, 867 extensions, 868

initial lesion, 867

structure, 869

osteoma of, 202

tumors of, 866

Pepper's type of congenital sarcoma of adrenal gland and liver in infants, 775

Peptic ulcer, cancer following, 630

Peribronchial sarcoma of lung, 8I 7

Peri-endothelioma, 345

Periosteal sarcoma, 270

Periosteum, fibroma of, I 63

Peripheral nervous system, tumors of, 152

Perirenal lipoma, I 84

Perithelioma, 304, 3I 7, 34 I clinical types, 344

gross appearance, 343

of bones, 345

of breast, 345

of carotid gland, 346

of cervix uteri, $55^{\circ}$

of kidney, 346

of ovary, 346

of pia mater, 344

of subcutaneous tissue, 344

orbital, 885

secondary, $34^{2}$

structure, $3+3$

Peritoneal endothelioma, 320

invasion in cancer of breast, 530

teratoid tumors, 976

Peritoneum, cysts of, 975 dermoid, 975

Perivascular endothelioma, 305,339

Petrified epithelioma, 820

Phagocytosis by tumorcells, 47

Pharyngeal dermoid cysts, 970

teratoma, $97^{\circ}$

Pharynx, lymphosarcoma of, primary, 385

Phosphorus in tumors, 90

Physaliden of Virchow, 43, I 87

Pia mater, perithelioma of, 344

Pial glioma, 414
Pigmented mole, 872 of adults, 875

Pineal gland, 948

cysts of, 949

general pathology, 948

glioma of, ependymal, 950

histology, 948

neuroglioma of, epen-

dymal, 95I

teratoma of, 949

tumors of, $94^{8}$

classification, 949

clinical features, $95 \mathrm{I}$

occurrence, 948

structure, 95 I

symptomatology, $95^{1}$

Pituitary gland, adenocarcarcinoma of, 932 metastases, 933

structure, 933

adenosarcoma of, 934

anatomy, 925

angiosarcoma of, 934

cancer of, atypical, 934

functional changes in, 928

histology of, 927

hyperplasia of, diffuse, 930

posterior lobe, glioma of, 939

lipoma of, 939

tumors of, 924

anatomy, 929

cephalic, 939

dystrophic, $94^{\circ}$

etiology, 924

miscellaneous, 939

relation of type with symptom complex, 945

symptomatology, 939 glandules, accessory, anatomy of, 927

Placenta, angioma of, 232 myxoma of, 173

Plasma-cell leukemia, 364 tumors, extramedullary, 388

Plastin, 46

in tumor-cells, 46

Pleura, endothelioma of, 3 I 9 involvement in cancer of breast, 529

Plexiform angioma, 224 endothelioma, 305, 339 epithelioma, 475

fibroma of spermatic cord, 801

neurofibroma, 156

neuroma, 427

Polyp, choanal, I67 nasal, I66

Polyposis, intestinal, 673 cancer following, 662

Porphyry spleen, 368

Port-wine stain, 224 
Post-generation of embryos, | Pylorus, sclerosing fibro- $\mid$ Rhabdomyoma, miscellane957

Postnatal tumors, 26

Precancerous changes cervix uteri, $\mathbf{5 5 0}$ in corpus uteri, $\mathbf{5 5 6}$ diseases, 476

lesions, 475

Pregnancy, effect of, I 44 on cancer of uterus, 560

Presarcomatous lesions, 246

Presternal dermoid cysts, 970

Primary acinar carcinoma of breast, 522

splenomegaly, 313

Prostate, adenocarcinoma of, 787

adenoma of, 786

cancer of, 78I. See also Cancer of prostate.

epithelioma of, squamous, 788

hypertrophy of, 459, 779 adenomatous, 780 carcinomatous, 780 etiology, 780 productive inflammation in, 779

structure, 779

lymphosarcoma of, 789

rhabdomyosarcoma of, 789

sarcoma of, 788

spindle-cell, 789

tumors of, 779

Proteins, tumor, constitution of, 89

febrile destruction of, 66

toxic destruction of, 66

Protozoa in cancer, I I 5

Pruritus in cancer of vulva, 563

Psammocarcinoma, 59 I

Psammoma, 305

endothelial, of skin, 33 I

of spinal cord, 440

Pseudoleukemia, 366, 367

gastro-intestinal, 378

myelogenous, 292

myeloid, 379

true, 376

blood in, 377

clinical features, 377

general features, 376

histology, 376

Pseudomucinous adenocarcinoma of ovary, 595

cystadenoma of ovary,59 I

Pseudomyxoma peritonei, 464,595

Pseudoneoplasms, meningeal, 443

Psoriasis preputialis, 867

Pulmonary alveoli, cancer arising from, 812

Pulp tumors, 29 carcinoma of, 639

RADICULODENTAL cysts, 7 I 5

Rankenneurome, I 56

Ransohoff's test in cancer, 93

Rapidity of growth in malignancy, 62

Rat, experimental cancer of stomach in, 133

Rathke's pouch, 927

Recklinghausen's disease, I 53

Rectum, melanoma of, 674

Recurrent myoma of uterus, 265

Regeneration of blood in cancer, 69

Regenerative hypertrophy, cancer from, 478

Regressive changes in tumor-cells, 43

Renaissance period, cancer in, 18

Renal hilus, lipoma of, 766 myxoma of, 766

myxosarcoma of, 766

sarcoma of, diffuse, 767 tumors of, 766

lipoma, I 83

pelvis, epithelial tumors of, 763

epithelioma of, papillary, 763

papilloma of, 763

sarcoma of, diffuse, 767

Replacement lipomatosis, I 8 I

Respiratory tract, chondroma of, 192

Retention cysts, 32

Reticulated epithelioma, histogenesis, 833 of skin, $83^{\circ}$

osteoma, 20 I

Reticulosplenome nodulaire, 391

Reticulum-cell sarcoma, $34^{\circ}$

Retina, glioma of, 42 I etiology, 423

structure, 422

neuro-epithelioma of, 422

Retrograde transport in dissemination of tumors, 83

Retroperitoneal dermoids, 977

lymphangioma, 238

lymphosarcoma, 387

teratoids, 977

tumors of adrenal rests, lateral, 762

Rhabdomyoma, 27, 203, 217

appearance, 2 I 7

clinical forms, 220

course, 2 I9

form, 217 ous, 222

of bladder, 866

of heart, congenital, 222

of testis, 221

of urinary bladder, 220

of voluntary muscle, 222

structure, 2 I 7

uteri, 220

Rhabdomyosarcoma of prostate, 789

Ribbert's chromatophoroma, 872

Riedel's struma, 908

Rodent ulcer, 830 crateriform, $83 \mathrm{I}$ histogenesis, 833 structure, 832

Round-cell cancer of salivary glands, 727

sarcoma, 243, 244

of breast, $5^{\circ} 3$

of lung, 8I 7

of ovary, 26I

SABER-SHEATH veins in $\mathrm{tu}$ mors, 57

Sacral cystic lymphangioma, 983

hygroma, 239, 983

mixed tumors, congenital, origin of, 984

teratomas, 982

Sacrococcygeal dermoids, 979

mixed tumors, 979

teratoid tumors, 98 I

teratomas, 979

Salivary glands, adenoma of, 725

cancer of, 725

round-cell, 727

chondroma of, I93

lymphangioma of, 736

myxochondrocarcinoma of, 735

tumors of, epithelial, 725

mixed, 727

Sarcoma, 20, 27

alveolar, 983

alveolare epithelioides, 335

benign giant-cell, of bone, 280

capsular, 275

character of growth, 24 I

clinical types, 249

deciduocellulare, 566

endometrial, structure, 267

endothelioma and, cont-

bination of, 3 I I

epithelioides, 303

etiology, 245

fasciculatum, $25^{\circ}$ 
Sarcoma, fibroblastic, 249 general characters, 240 giant-cell, $25^{\circ}$

benign, of bone, 280

of tendon-sheaths and aponeuroses, 256

gross anatomy, 240

Hodgkin's, 373

invasion of lymphatics by, 80

lamellosum, 250

multiple hemorrhagic, 252 histological struc-

ture, 253

of adrenal gland in infants, congenital (Pepper type), 775

with cranial metastases (Hutchinson type), 774

of bladder, 865

of bone, 244, 269

classification, 270

duration, 277

of life in, 277

etiology, 289

giant-cell, benign, 280 structure, 282

prognosis, 287

historical, 269

prognosis, 284

rapidity of growth, 285

spindle-cell, 277

prognosis, 287

telangiectatic, 273,278 prognosis, 287

trauma in, 289

treatment, 288

of bone-marrow, 269

primary multiple, 293

of brain and meninges, 424

diffuse, 424

gross anatomy, 424

structure, 425

of breast, pure, 503

spindle-cell, 504

round-cell, 503

of esophagus, 258

of gall-bladder, 697

of heart, primary, 176

of intestine, 260

of larynx, 856

of liver, 692

and adrenal in infants,

congenital

(Pepper's

type), 775

of lung, 815

classification, 816

diffuse spindle-cell, 816

peribronchial, 817

round-cell, 8I 7

of muscles, 258

of ovary, 260

round-cell, $26 \mathrm{r}$

of pancreas, 706

of prostate, 788
Sarcoma of prostate, spindlecell, 789

of renal hilus, diffuse, 767 pelvis, diffuse, 767

of seminal vesicles, 789

of skin, 252

of spinal cord and meninges, 437

central, $44^{\circ}$

metastatic, $44^{2}$

solitary, 438

of spleen, 390

endothelial, 390

spindle-cell, 390

of stomach, 259

miscellaneous, 260

of testis, 795, 802

of thymus gland, $92 \mathrm{I}$

of thyroid gland, 906

of uterine mucosa, 266

of uterus, 263

classification, 264

etiology, 266

histogenesis, 266

metastases in, 263

mural, 263

structure, 264

osteogenic, 270

course, 275

sclerosing, 278

site of origin, $27 \mathrm{I}$

structure of, 276

parosteal, 275

periosteal, 270

radiare, $25^{\circ}$

reticulum-cell, 340

round-cell, 243,244

sclerosing osteogenic, 275

spindle-cell, 249. See also

Spindle-cell sarcoma.

stroma of, 244

structure, 242

telangiectatic, of bone, 278

trabeculare, 250

Sarcomatoid structures in cancer of cervix uteri, $55^{\circ}$

Sarcomatosis of meninges, diffuse, 438

multiple, 438

Sarcomatous struma, 934

Sarcome angioplastique, 797

Scapula, chondroma of, 190

Schimmelbusch's disease, 489

Scirrhous cancer of breast, 534

of cervix uteri, 549

of prostate, 788

of stomach, clinical features, 659

diffuse, 637

Scirrhus pustuleux, 534

Sclerosing fibrocarcinoma of stomach, clinical features, 659

osteogenic sarcoma, 275, 278 clerosing pyloric fibrocarcinoma of stomach, 639

Sclerosis, multiple adenocarcinoma of kidneys in, 746

Scrotum, cancer of, 870 paraffin, 870

Sebaceous cysts of breast, 505

Secondary osteoma, 200

Seminal vesicles, sarcoma of, 789

Seminome, 795

Senile involution of breasts, 487

osteomalacia, 292

Sennert's theory of cancer, I 8

Serology, 89

Serous adenocarcinoma of ovary, papillary, 589 cavities, invasion of, by cancer, 80

cystadenoma of ovary, $5^{86}$

cysts, 975

membranes, endothelioma of, 319

Simple cystoma, 582

lymphoma, $35^{\circ}$

etiology, $35^{2}$

mixed tumors, 28

Singer's nodes, 847

Skin, acanthoma of, 818. See also Acanthoma of skin.

angio-endothelioma of, $33 \mathrm{I}$

cancer of, epidermoid, 8 I 8 cysts of, atheromatous, 965

epithelial, 965

endothelial psammoma of, 331

endothelioma of, 309,328

epithelioma of, adenoid,

$$
834
$$

cystic, 834

simple, 835

basal-cell, 830

reticulated, 830

invasion in cancer of breast, 525

lesions in Hodgkin's disease, $37 \mathrm{I}$

myoma of, 2 I 6.

osteonia of, 202

sarcoma of, 252

spindle-cell sarcoma of, 255

Skull ${ }^{255}$ changes in brain tumors, 398

Small alveolar carcinoma, 47 I

cystic adenocarcinoma of breast, $5 \mathrm{I} 7$

duct carcinoma of

breast, 517

Solid ovarian adenoma, 596 
Solitary diffuse endotheli- Spontaneous cure in tu-|Sweat-glands, tumors of, oma of bones, 326

Soot-wart, 870

Spermatic cord, fibrolipoma of, 801

fibroma of, plexiform, 801

tumors of, adrenal, 762

Spina bifida occulta, 984

tumors associated with, 983

Spinal cord and meninges, sarcoma of, 437

angioma of, 44I

degeneration of, in brain tumors, 398

glioma of, 4 I I

lipoma of, 44I

melanoma of, 885

neurofibroma of, multiple, 44I

psammoma of, 440

sarcoma of, central, $44^{\circ}$ metastatic, 442

solitary, 438

tumors of, 436 . See also Tumors of spinal cord.

secondary changes in, $+42$

symptomatology, 443

xanthochromia in, $44^{2}$

membranes, relation of

tumors to, 444

Spindle-cell myosarcoma of stomach, 259

sarcoma, 249

blood-vessels of, 250

diagnosis, 250

histogenesis, 25

of bone, 277

of breast, pure, 504

of intestine, 260

of lung, diffuse, 816

of ovary, $26 \mathrm{I}$

of prostate, 789

of skin, 254

etiology, 256

histogenesis, 255

structure, 254

of spleen, 390

rate of growth, 249

secondary changes in, 250

structure, 249

Spirochetæ in cancer, I 6

Spleen, lesions in, in leukemia, 354

lymphosarcoma of, primary, 39I

porphyry, 368

sarcoma of, 390

endothelial, 390

spindle-cell, 390

Splenic Hodgkin's disease, 368

mors, 60

regression in tumors, 40

Spotted kidneys, 741

Squamous epithelioma of prostate, 788

Squamous-cell cancer of choroid plexus, 419

of large intestine, 674

Stain, port-wine, 224

Stammler's reaction in cancer, 93

Stenosing fibrocarcinoma of large intestine, 670

Sterility in etiology of uterine tumor, 212

Sternberg's disease, 367

Stomach, adenocarcinoma of, 627

occurrence, 656

adenoma of, simple, 629

athletic, 652

cancer of, 625. See also Cancer of stomach.

endothelioma of, 335

fibrocarcinoma of, diffuse, 639 sclerosing, clinical features, 659 pyloric, 639

leather-bottle, in linitis plastica, 644

lymphatics of, 647

lymphomatous tumors of, 260

lymphosarcoma of, 386

myoma of, 2 I 6

sarcoma of, 259 miscellaneous, 260

spindle-cell myosarcoma of, 259

tumors of, lymphomatous, 259

ulcerocancer of, 658

Stools, blood in, in cancer, 72

Stroma of tumors, 53 regressive changes in, 55

reactions, experimental analysis of, 55

Struma adrenalis, $46 \mathrm{I}$

colloid, 460

hypophysis, $46 \mathrm{I}$

ovarii, 6I 5, 62 I

postbranchialis, 90I

Riedel's, 908

sarcomatous, 934

suprarenalis, I 83,758

Strumæ suprarenales aberratæ renalis, 738

Subcutaneous lipoma, I 82 tissues, perithelioma of, $3+4$

Sublingual dermoids, 967

Submaxillary gland, epithelial tumors of, 737

Superficial adenoma ovary, 597
836

Syncytial endometritis, 566 ,

$$
575,577
$$

wandering cells, 568

Syncytioma, 575

clinical course, 578

metastases in, 576

Synovial lipoma, I 82

Syphilis, relation of, to lymphosarcoma, 246

to tumors, 123, I24

Syringomyelia associated with gliosis, 4I2

Systemic aleukemic lymphomatosis, 376

TAIL, false, 984

Teeth, tumors of, 707

Telangiectasis, 224

Telangiectatic angio-endothelioma of bones, 323

bone-sarcoma, 273

sarcoma, 224

of bone, 273,278 prognosis, 286

Tendon-sheaths, giant-cell sarcoma of, 256

Teratoblastomas, 962

Teratogenic termination period, 954, 973

Teratoid chondroma, I93

Teratoids, 959 cervical, 969

intracranial, 974

of breast, mixed, 499

of hypophyseal duct, 935

of testis, 794

peritoneal, 976

retroperitoneal, 977

sacrococcygeal, 98I

Teratology, 954

Teratoma, 28, 959, 975

abdominal fetal, 977

causes, 96I

cervical, 969

intracranial, 974

morphogenesis, 960

of orbit, $97^{\circ}$

of ovary, 6r 3

anatomy, 613

atrophic bidermal, 619

clinical course, 613

contents, 615

dermoids and, differentiation, 615

etiology, 623

mesodermal elements in, 618

metastases in, 6I 8

multiple, 619

prognosis, 613

relation to simple tumors, 622

with cystoma, 6I 4

of pineal gland, 949

of testis, 790 
Teratoma of testis, adult, | Thyroglossal duct, cysts of, clinical course, 794 derivatives, 790 historical note, 790 metastases in, 800 relation to miscellaneous tumors, $80 \mathrm{O}$ structure, 797 varieties, 790 pharyngeal, 970 sacral, 982

sacrococcygeal, 979 source, 960 special forms, 965 tridermal, 977

Teratomatous glioma, 4I5

Testicular adenoma of ovary, 6ro

Testis, adenoma of, 802 cancer of, embryonal, with lymphoid stroma, 795

chondroma of, 801

chorioma of, 797

embryoma of, adult, 790 complex, 790

endothelioma of, 308

fibroma of, 165,801

interstitial cells of, hyperplasia of, 803

lymphosarcoma of, 388 , 804

etiology, 804

myoma of, 217,802

rhabdomyoma of, 22 I

sarcoma of, 795, 802

teratoma of, 790 . See also Teratoma of testis. tumors of, adrenal, 762 , 803

embryoid, 794

embryonal, 795

miscellaneous, relation to teratoma, 801

mixed, 794

teratoid, 794

Theory of cell autonomy, 97

Thiersch's theory of cancer, 23

Thoracic duct, invasion of, by cancer, 79

Thoracopagi, 955

Thymoma, 9I7

interpretation, 922

Thymus gland, anatomy, 912

cancer of, 920

cysts of, 915

general pathology, 914 hypertrophy of, simple, 914

lymphosarcoma of, 9I 7 structure, 919

sarcoma of, 92 I

tumors of, 912

classification, 9 I 7

clinical course, 923

primary, 9 I 6 969

Thyroid

gland, adenocarcinoma of, 898 small alveolar, large cell, 901

adenoma of, fetal, 895 interacinous, 894 malignant, 898

cancer of, $90 \mathrm{or}$. See also Cancer of thyroid gland.

cysts of, 910

granuloma of, benign, 908

hypertrophy of, congenital, 459

sarcoma of, 906

tumors of, 89 I

papillary epithelial, 899 structure, 900

Tibia, adamantinoma of, 7 I 5

Tissue tension theory of cancer, 98

Tongue, cancer of, epidermoid, $84^{\circ}$

cavernous, angioma of, $23 \mathrm{I}$

lymphosarcoma of, 387

papilloma of, $45^{2}$

Trachea, exostosis of, 198

Transplantable tumors, adaptation of, 139

fate of, I 39

nature and origin of, I36

problems of, 136

Transplantation of tumors, I34

acquired resistance to, I 44

between animals of different species, I34

between lower animals of same species, I35

between man and man, I 34

between related species of lower animals, I34 hypersusceptibility to, 147

influence of variations in soil, 140

natural resistance, I 43

nature of factors in resistance, 145

passive resistance to, 145

uniformity of technic in; 140

Transplanted tumors, influence of diet on, I 40 resistance to, I 43

spontaneous absorption of, I4 I

structural changes in, I 4 I
Trauma in etiology of $\mathrm{t} t 1-$ mors, 109, I 10

frequency, I 12

mechanism, I I I

medicolegal aspects, I 12

Traumatic exostoses, 198

neuroma, 427

tumors, 26

Tridermal teratomas, 977

True fibroneuroma, 427

osteoma, I99

pseudoleukemia, 376

Tuberculosis cutis verru. cosa, 823

Hodgkin's disease and, 375

in lymphosarcoma, 389

lymphatic, 366

relation of, to lymphosarcoma, 246

to tumors, I 23, 124

Tuberculum impar, 9 I0

Tubo-ovarian cysts, 584

Tubular acanthoma, 819

adenocarcinoma of kidneys, $75^{\circ}$

adenoma, 455

of kidney, $74 \mathrm{I}$

carcinoma, $47 \mathrm{I}$

epidermoid carcinoma, 474

Tumeur à medullocelles, 269

à myeloplacques, 269

Tumor-cells, anaplasia of, IOI

miscellaneous, peculiarities of, 46

phagocytosis by, 47

regressive changes in, 43

Tumor-like hyperplasia of endothelium, 3 I 3

Tumors, adrenal, hepatic, of $^{762}$ female genital organs, 762

of spermatic cord and testis, 762

rest, 755

carcinomatous, 759

gross appearance, 757

lateral retroperitoneal, 762

occurrence, 756

peritheliomatous, 759

renal tumors and, differentiation, 760

sarcomatous, 759

structure, 758

trabecular, 759

adult, 26,48

alveolar, 346

a mitosis in, 39

a myloid, I 67

associated with spina bifida, 983

autogenous antigen of, complement deviation with, 9I 
Tumors, benign, malignant transformation in, 64 metastasis of, 84

blood-vessel lesions in, 57 cell division in, 38 central growth in, 3 I

chemistry of, 89

classification, 26

collateral hypertrophy in, 37.

colloid degeneration of, 45 color of tissue, 29 complex tissue type, 28

congenital, 26

connective-tissue type, 27 consistence, 30

course, 59

cystic, 464

of carcinomatous structure, 464

cysts in, 31

definition, 25

desmoid, I 64

effects on body, 65

emaciation from, 65

embryonal, 26, 49

encephaloid, 5I I

endothelial type, 27

epithelial type, 28

general pathology, 446 etiological classification, 26

etiology of, special, xo9

expansive growth, 25

experimental production of, 126

by $x$-ray, I 32

chronic inflammation in, I $3 \mathrm{I}$

effect of extract of human tumors, 132

study of, I I 8

extensive growth, 25

fats in, 90

fatty degeneration in, 43 febrile destruction of proteins in, 66

ferments in, 67,90

heterolytic action of, 67 functional activity in, 53 gametoid mitosis in, 39 general morphology, 29 glandular, 28 glycogenic degeneration of, 44

growth of, $3 I, 48$

hereditary, 26

histioid, 48

histological classification, 26,27

histopathology, 34

homologous, 48

homotypic, 48

hyaline changes in, 45

hydropic degeneration in, 43

infiltrative growth, 25,
Tumors, inflammatory reaction in, 50

intermittent periods of growth of, 59

lactic acid in, 89

local circumscription of, 29

extension of, $5^{\circ}$

lutein-cell, 585

lymphomatous, of intestine, 260

of stomach, 260

malignancy in, significance of, 62

markings of, 30

maxillary, of dental origin, 707

mesonephric, 6 Io

mixed, 962

sacral congenital, origin, 984

special forms, 965

modes of origin, 36

mucous degeneration in, 44.

multicentric growth of, 36 muscle tissue type, 27

natural limit in course of, 59

nervous system type, 27

nomenclature, 26

of adrenal gland, 768

classification, 768

medullary, 77 I

chromaffin cell, 776

with adrenalin content and nephritic symptoms, 776

of bile-ducts, 689

of bladder, epithelial, 860 miscellaneous, 865

of brain, 393

age incidence, 393

anatomical classification, 398

choked disk in, 398

circulatory disturbances in, 398

destruction of tissue in, 397.

duration, 396

edema in, 397

general considerations, 393

distribution, 395

heredity in, 393

meningeal complications, 398

occurrence, 393

operability, 395

relative frequency, 394

secondary effects of, 396

sex in, 393

skull changes in, 398

spinal cord degeneration in, 398
Tumors of brain, trauma in etiology, 393

of breast, ectodermal mixed, 500

epithelial, 484

mesodermal, 499

miscellaneous, 505

mixed, 494

teratoid mixed, 499

of choroid plexus and ependyma, 4I5

etiology, 4 I 6

gross appearance, 416

structure, 4 I 6

of epoöphoron, 609

of extrarenal adrenal rests, $76 \mathrm{I}$

of Fallopian tubes, 61 2

adrenal, 762

of Gasserian ganglion, 434

of hypophyseal duct, 935

of hypophysis, 924

of infundibular ependy$\mathrm{ma}, 420$

of intestine, multiple benign embryonal carcinoid, 663

of kidney, 738

adrenal tumors and, differentiation, 760 epithelial, 738 classification, 739

of lacrimal gland, 319, 725

of larynx, 847

benign, $85^{\circ}$

of liver, 675

classification, 675

primary epithelial, 678 anatomical varieties, 68I

clinical course, 680 varieties, $68 \mathrm{I}$

etiology, 678

histogenesis, 679

metastases in, 68I relation to cirrhosis, 678

of lung, 807

of lymphoid tissue, $34^{8}$

classification, 348

of maxillary and nasal sinuses, 720

of nerve trunks, 427

of ovary, adrenal, 610, 762

age in etiology, 606

epithelial, $5^{82}$

etiology, 606

histogenesis, 606

origin, 608

simple, relation to teratoma, 622

of pancreas, 702

of parathyroid gland, 909

of parovarium, 609

of pars intermedia, 938 
Tumors of penis, 866 of peripheral nervous system, I52

of pineal gland, 948 classification, 949 clinical features, 95 I occurrence, 948 structure, $95 \mathrm{I}$ symptomatology, 95 I

of pituitary gland, 924

anatomy, 929

cephalic, 939

dystrophic, $94^{\circ}$

etiology, 924

miscellaneous, 939

relation of type with symptom complex, 945

symptomatology, 939

of placenta, 232

of prostate, 779

of renal hilus, 766

pelvis and ureter, epithelial, 763

of salivary gland, epithelial, 725

mixed, anatomy, 734

clinical course, 733

etiology, 733

structure, 735

mixed, 727

of spinal cord and mem-

branes, 436

age incidence in, 437

classification, 437

duration, 445

etiology, 436

trauma in etiology of, 436

extradural, 444

extramedullary, 444

intramedullary, 444

level of, 443

relation of, to cord, membranes, and

vertebræ, 444

secondary changes in, 442

symptomatology, 443

vertebral, 445

xanthochromia in, 442

of stomach, lymphomatous, 260

of submaxillary gland, epithelial, 737

of sweat-glands, 836

of teeth, 707

of testis, 790

adrenal, 803

embryoid, 794

embryonal, 795

mixed, 794

teratoid, 794

Tumors of thymus gland, | Tumors, transplantation of 912

classification, 9 I 7

clinical course, 923

primary, 916

of thyroid gland, 89I epithelial papillary, 899

structure, 900

of uterus, adrenal, $76^{\circ}$

of vulva, epithelial, 562

parasitic theory of, classi-

fication by, 125

pavement, 28

peripheral growth of, 3 I

plasma-cell, extramedullary, 388

post-natal, 26

precancerous stage of growth, 34

progressive growth, 25, 59 proteins, constitution of, 89

pulp, 29

rate of growth, 59

recurrence of, 86

regional classification, 26 relation of blood-vessels to, 56

retrograde transport in dissemination of, 83

saber-sheath veins in, 57 sacrococcygeal, mixed, 979

simple mixed, 28

spontaneous cure in, 60 regression in, 40

stroma of, 53 retrogressive changes in, 55

structure, 48

teratoid. See Teratoids.

texture of, 30

total quantity of blood in, 69

toxic destruction of protein tissues in, 66

transplantable, adaptation of, I 39

fate of, 139

nature and origin of, 136

problems of, I 36

transplantation of, $I 34$

acquired resistance to, I 44

between animals of different species, I 34

between lower animals of same species, I 35

between man and man, 134

between related species of lower animals, I 34 hypersusceptibility to, I 47

influence of variations in soil, I40 natural resistance, 143

nature of factors in resistance, 145

passive resistance to, 145

uniformity of technic in, 140

transplanted, influence of diet on, 140

resistance to, 143

spontaneous absorption of, 141

structural changes in, I 4 I

trauma in etiology of, I09, IIO

frequency, I 12

mechanism, I I I

medicolegal aspects, I 2

traumatic, 26

ulceration in, 33

unicentric growth of, 36

ULCER, peptic, cancer following, $63^{\circ}$

rodent, 830

crateriform, $83 \mathrm{I}$

histogenesis, 833

structure, 832

Ulceration in tumors, 33

Ulcerative infiltrating epithelioma of lip, 837

Ulcerocancer of stomach, 658

Unicentric growth of tumors, 30

Urea, percentage of, in cancer, 72

Ureter, cancer of, primary, 865

epithelial tumors of, 763

epithelioma of, papillary, 763

papilloma of, 763

Urethra, cancer of, 870

Uric acid increase in cancer, 72

Urinary bladder, rhabdomyoma of, 220 organs, myoma of, 2 I 6

Urine, changes in, in cancer, 72

Urobilin in urine in cancer, 74

Uterine fibromyoma, 207 lipoma, I 84

mucosa, sarcoma of, 266

Uterus, adeno-acanthoma of, $54 \pi$

cancer of, 267,543 . See also Cancer of uterus.

chondroma of, 192

endothelioma of, 334

myoma of, 208 
Uterus, myoma of, degenera- | Vertebral tumors of spinal tive changes in, 210 etiology, 212

form, 208

gross appearance, 2 IO location, 208 microscopical structure, 2 I I

recurrent, 265

rhabdomyoma of, 220

sarcoma of, 263

classification, 264

etiology, 266

metastases in, 263

mural, 263

structure, 264

tumors of, adrenal, 762

VAGINA, epidermoid cancer of, 564

Varicosities, chronic, 224

Vascular nevus, 224

Verruca plana juvenilis, 448 vulgaris, 448 cord, 445

Villous duct cancer, 510

Virchow's physaliden, 43 , I 87

theory of cancer, 22

Visceral metastases in cancer of uterus, $56 \mathrm{I}$ neurofibroma, I 55

neurofibromatosis, I 57

Vocal cords, acanthoma of, 855

Vulva, epidermoid cancer of, 562

tumors of, epithelial, 562

WANDERING cells, syncytial, 568

Warts, cutaneous, 448 soot-, 870

Wassermann reaction in cancer, 9I

Whitlow, melanotic, 873
Xanthelasma, I62

Xanthochromia in spinal cord tumors, 442

Xanthoma, I62

diabeticorum, 162

generalized, 162

multiplex, 162

palpebrarum, 162, I63

planum, I62

structure of, 163

tuberosum, I62

vulgare, 162

Xanthomatosis, 163

Xanthosarcoma, 256

Xeroderma pigmentosum, 822

$x$-ray carcinoma, 102

dermatitis, cancer and, 823

clinical course, 824

structure, 824

experimental production of tumors by, I32

ZoNA glomerulosa, $75^{8}$ 




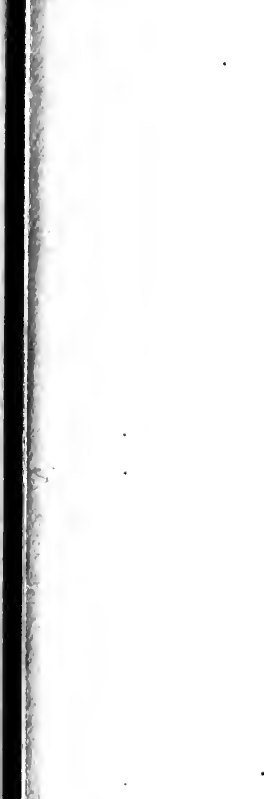





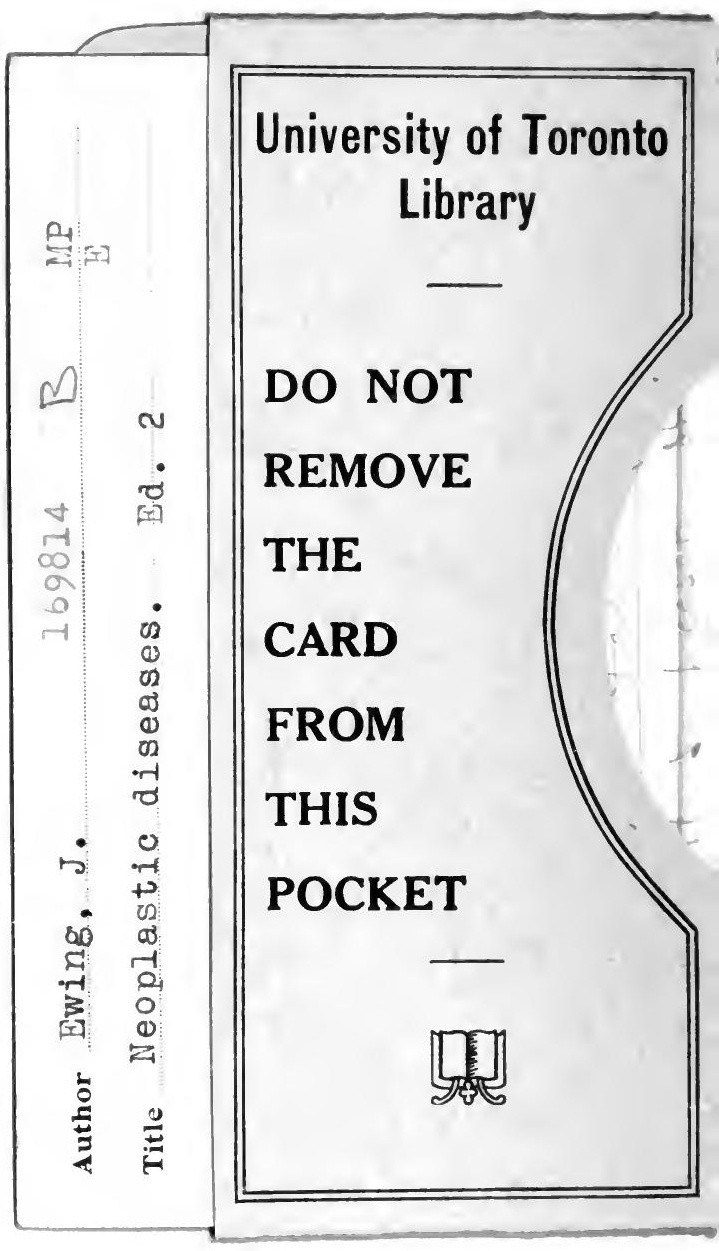


.

,

•

,

,

. 